\title{
Phase Transition Enthalpy Measurements of Organic and Organometallic Compounds. Sublimation, Vaporization and Fusion Enthalpies From 1880 to 2015. Part 1. $\mathrm{C}_{1}-\mathrm{C}_{10}$
}

\author{
William Acree, Jr. \\ Department of Chemistry, University of North Texas, Denton, Texas 76203, USA
}

James S. Chickos

Department of Chemistry and Biochemistry, University of Missouri-St. Louis, One University Boulevard, St. Louis, Missouri 63121, USA

(Received 14 April 2016; accepted 14 April 2016; published online 21 July 2016)

\begin{abstract}
A compendium of phase change enthalpies published in 2010 is updated to include the period 1880-2015. Phase change enthalpies including fusion, vaporization, and sublimation enthalpies are included for organic, organometallic, and a few inorganic compounds. Part 1 of this compendium includes organic compounds from $C_{1}$ to $C_{10}$. Part 2 of this compendium, to be published separately, will include organic and organometallic compounds from $\mathrm{C}_{11}$ to $\mathrm{C}_{192}$. Sufficient data are presently available to permit thermodynamic cycles to be constructed as an independent means of evaluating the reliability of the data. Temperature adjustments of phase change enthalpies from the temperature of measurement to the standard reference temperature, $T=298.15 \mathrm{~K}$, and a protocol for doing so are briefly discussed. (C) 2016 American Institute of Physics for the National Institute of Standards and Technology NIST. [http://dx.doi.org/10.1063/1.4948363]
\end{abstract}

Key words: compendium; fusion enthalpy; sublimation enthalpy; vaporization enthalpy.

\section{CONTENTS}

1. Introduction $\ldots \ldots \ldots \ldots \ldots \ldots \ldots \ldots \ldots \ldots$

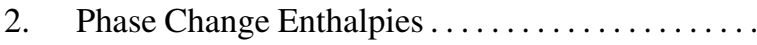

2.1. Estimation of condensed phase heat capacities

2.2. Group values for estimating liquid and solid heat capacities at $T=298.15 \mathrm{~K} \ldots \ldots \ldots \ldots$.

2.3. Statistics in evaluation of group values .....

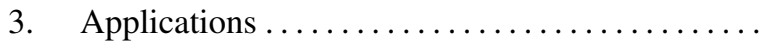

3.1. Estimation of condensed phase heat capacities

3.2. Estimation of gas phase heat capacities .....

4. The phase change enthalpy compendium .......

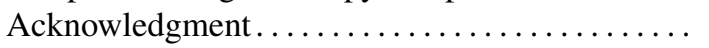

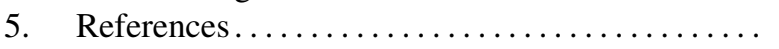

\section{List of Tables}

1. Groups values for estimation of liquid $\Gamma$ (1) and solid $\Gamma$ (s) heat capacities at $T=298.15 \mathrm{~K} \ldots \ldots$.

2. Estimation of L-tryptophan, inosine, 1,3dimethylbarbituric acid and $\beta$-ionone ........

3. List of acronyms and descriptions ...........

4. Phase change enthalpies of $\mathrm{C}_{1}$ organic com-

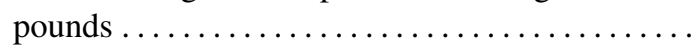

5. Phase change enthalpies of $\mathrm{C}_{2}$ organic com-

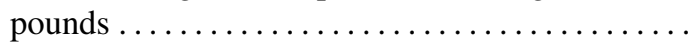

6. Phase change enthalpies of $\mathrm{C}_{3}$ organic compounds
7. Phase change enthalpies of $\mathrm{C}_{4}$ organic com-

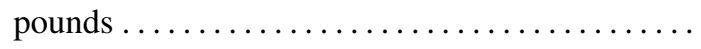

2 8. Phase change enthalpies of $C_{5}$ organic com-

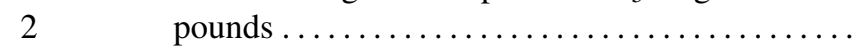

9. Phase change enthalpies of $\mathrm{C}_{6}$ organic com-

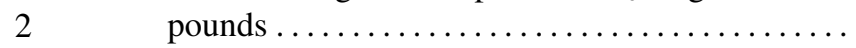

10. Phase change enthalpies of $\mathrm{C}_{7}$ organic com-

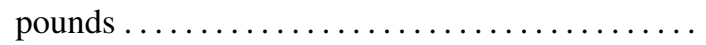

11. Phase change enthalpies of $\mathrm{C}_{8}$ organic com-

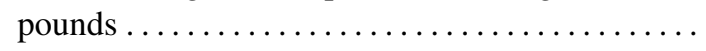

12. Phase change enthalpies of $\mathrm{C}_{9}$ organic compounds $\ldots \ldots \ldots \ldots \ldots \ldots \ldots \ldots \ldots \ldots \ldots \ldots \ldots \ldots \ldots \ldots$

13. Phase change enthalpies of $\mathrm{C}_{10}$ organic compounds $\ldots \ldots \ldots \ldots \ldots \ldots \ldots \ldots \ldots \ldots \ldots \ldots \ldots \ldots$

\section{List of Figures}

1. A comparison of experimental and calculated liquid heat capacities at $T=298.15 \mathrm{~K} \ldots \ldots \ldots$.

32 2. A comparison of experimental and calculated solid heat capacities at $T=298.15 \mathrm{~K} \ldots \ldots \ldots \ldots$.

7 3. Structures of simvastatin $\underline{\mathbf{1}}$, uridine $\underline{\mathbf{2}}$, and risperi-

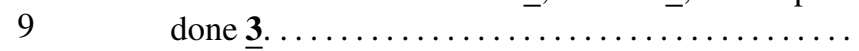

4. Structures of benzoic acid (), 2,2,5,7,810 pentamethylchroman-6-ol () and 6-hydroxy2,5,7,8-tetramethylchroman-2-carboxylic acid (6)...

5. Structures of 2-methylbenzoxazole (ㅁ) and 2,5dimethylbenzoxazole (ㅇ). 


\section{Introduction}

This compendium updates a previous report published in 2010 [2010ACR/CHI] on phase change enthalpies. Part 1 of this compendium is inclusive and includes organic compounds containing from one to ten carbon atoms published over the period of 1880 to 2015 and includes a total of over 6600 entries. Part 2 of this series to be published separately contains over 7400 entries covering both organic compounds from eleven to 192 carbons, organometallic, and organic ionic liquids. Current interest in thermochemical properties of these materials is reflected by the $15 \%$ increase in the number of publications reported during the five year interval since the 2010 publication. While many of these properties are freely available online [http://webbook.nist.gov/ chemistry/, http://trc.nist.gov/thermolit/], each compound in these sources needs to be searched individually. A goal of this publication is to provide to those interested direct access to the entire collection of experimental phase change enthalpies of compounds that have been measured in addition to allowing electronic searching using available software. Since phase change properties are useful to a variety of disciplines they are frequently reported as part of other projects. A goal of this compendium is to provide a single location for identifying and accessing work that may have been published in specialty journals focusing on other topics.

\section{Phase Change Enthalpies}

Sublimation, vaporization, and fusion enthalpies are related to each other by Eq. (1) provided temperature $T$ for all three terms is the same. Unlike fusion enthalpy, sublimation and vaporization enthalpies are frequently measured as a function of temperature, where $T$ frequently refers to the mean temperature of measurement, $T_{\mathrm{m}}$. Fusion enthalpies of pure materials occur on the other hand isothermally at $T_{\text {fus }}$. The complication that arises in applying Eq. (1) is that frequently it is necessary to adjust two or all three measurements to the same temperature, often the reference temperature, $T=298.15 \mathrm{~K}$. In principle, to do so requires information regarding the heat capacity of at least two of the three phases involved, $C p(\mathrm{~g}), C p(\mathrm{l})$, and $C p(\mathrm{cr})$ and is further complicated by their temperature dependence,

$$
\Delta_{\text {sub }} H_{\mathrm{m}}(T)=\Delta_{\mathrm{vap}} H_{\mathrm{m}}(T)+\Delta_{\text {fus }} H_{\mathrm{m}}(T) .
$$

Heat capacities for $C p(\mathrm{l})$ and $C p(\mathrm{cr})$ are available for many compounds and can be measured as a function of temperature. Experimental heat capacities of the gas phase at temperatures when the material is either solid or liquid are more problematic. Gas phase heat capacities for these materials are frequently calculated. Statistical mechanics combined with $a b$ initio calculations are usually employed (see [2010SAN/ROC] for example). An alternative approach that has been developed for adjusting phase change enthalpies with temperature aimed at circumventing the scarcity of experimental gas phase heat capacities is described by Eq. (2) for liquids and Eq. (3) for solids, [1993CHI/HOS]

$$
\begin{aligned}
& \Delta_{\text {vap }} H_{\mathrm{m}}(T / \mathrm{K}) / \mathrm{kJ} \mathrm{mol}^{-1} \\
& =\Delta_{\mathrm{vap}} H_{\mathrm{m}}\left(T_{\mathrm{m}} / K\right)+\Delta_{\mathrm{l}}^{\mathrm{g}} C p_{298 \mathrm{~K}}(\mathrm{l}) \cdot \Delta T, \\
& \text { where : } \Delta_{1}{ }^{\mathrm{g}} \mathrm{Cp}(\mathrm{l}) \cdot \Delta T \\
& =\left(10.58+0.26 C p_{298 \mathrm{~K}}(\mathrm{l})\right) \cdot(\Delta T) / 1000 \text { and } \\
& \Delta_{\mathrm{sub}} H_{\mathrm{m}}(T / \mathrm{K}) / \mathrm{kJ} \mathrm{mol}^{-1} \\
& =\Delta_{\mathrm{sub}} H_{\mathrm{m}}\left(T_{\mathrm{m}} / \mathrm{K}\right)+\Delta_{\mathrm{cr}}{ }^{\mathrm{g}} C p(\mathrm{l}) \cdot \Delta T, \\
& \text { where : } \Delta_{\mathrm{cr}}{ }^{\mathrm{g}} C p(\mathrm{cr}) \cdot \Delta T \\
& =\left(0.75+0.15 C p_{298 \mathrm{~K}}(\mathrm{cr})\right) \cdot(\Delta T) / 1000 .
\end{aligned}
$$

In both Eqs. (2) and (3), $\Delta T$ refers to $T_{\mathrm{m}}-T_{\mathrm{R}}$, where $T_{\mathrm{m}} / \mathrm{K}$ often represents the mean temperature of measurement and $T_{\mathrm{R}}$ is usually the reference temperature, $298.15 \mathrm{~K} ; C p_{298.15 \mathrm{~K}}(\mathrm{l})$ and $C p_{298.15 \mathrm{~K}}(\mathrm{cr})$ refer to the heat capacity of the liquid and solid at $T=298.15$, respectively. Either experimental or estimated heat capacities can be used. Equations (2) and (3) were derived using heat capacities estimated by group additivity. By appropriate manipulation of Eqs. (2) and (3), it is also possible to arrive at a means of adjusting fusion enthalpies with temperature, Eq. (4),

$$
\begin{aligned}
& \Delta_{\mathrm{cr}}{ }^{\mathrm{g}} C p_{298 \mathrm{~K}}(1) \cdot \Delta T-\Delta_{\mathrm{l}}^{\mathrm{g}} C p_{298 \mathrm{~K}}(\mathrm{cr}) \cdot \Delta T \\
& \quad=\Delta_{\mathrm{cr}}{ }^{l} C p_{298 \mathrm{~K}}(\mathrm{cr}) \cdot \Delta T \\
& \begin{aligned}
\Delta_{\mathrm{cr}}{ }^{l} C & p_{298 \mathrm{~K}}(\mathrm{cr}) \cdot \Delta T \\
\quad & {\left[0.15 C p_{298 \mathrm{~K}}(\mathrm{cr})-0.26 C p_{298 \mathrm{~K}}(\mathrm{l})-9.93\right] \cdot \Delta T . }
\end{aligned}
\end{aligned}
$$

The term $\Delta_{\mathrm{cr}}{ }^{\mathrm{g}} C p_{298 \mathrm{~K}}(\mathrm{cr})=\left(0.75+0.15 C p_{298 \mathrm{~K}}(\mathrm{cr})\right)$ has also been used for evaluating values of the gas phase heat capacity of a solid, Eq. (5). [2010MON/ALM] Similarly, gas phase heat capacities of the liquids could be evaluated using a similar approach together with Eq. (2). Although Eq. (2) does not seem to have been used in this manner, Eq. (6) can be derived in the same manner. This is illustrated for both solids and liquids in Sec. 3.2 for several compounds,

$$
\begin{aligned}
\Delta_{\mathrm{cr}}{ }^{\mathrm{g}} C p_{298 \mathrm{~K}}(\mathrm{cr}) & =\left[C p_{298 \mathrm{~K}}(\mathrm{~g})-C p_{298 \mathrm{~K}}(\mathrm{cr})\right] \\
& =\left(0.75+0.15 C p_{298 \mathrm{~K}}(\mathrm{cr})\right) .
\end{aligned}
$$

Rearranging and solving for $C p_{298 \mathrm{~K}}(\mathrm{~g})$,

$$
\begin{aligned}
& C p_{298 \mathrm{~K}}(\mathrm{~g})=0.85 C p_{298 \mathrm{~K}}(\mathrm{cr})-0.75 \\
& C p_{298 \mathrm{~K}}(\mathrm{~g})=0.74 C p_{298 \mathrm{~K}}(\mathrm{l})-10.58
\end{aligned}
$$

\subsection{Estimation of condensed phase heat capacities}

In order to apply Eqs. 2-6, reliable values of the heat capacity of the condensed phase are required. A number of methods have been developed over the years that vary in their 
TABLE 1. Group values for estimation of liquid $\Gamma$ (l) and solid $\Gamma$ (s) heat capacities at $T=298.15 \mathrm{~K}^{\mathrm{a}}$

\begin{tabular}{|c|c|c|c|}
\hline & Group atoms & $\begin{array}{c}\Gamma(1) \\
\mathrm{J} \mathrm{K}^{-1} \mathrm{~mol}^{-1}\end{array}$ & $\begin{array}{c}\Gamma(\mathrm{s}) \\
\mathrm{J} \mathrm{K}^{-1} \mathrm{~mol}^{-1}\end{array}$ \\
\hline \multicolumn{4}{|l|}{ 1. Hydrocarbon groups } \\
\hline Primary $\mathrm{sp}^{3}$ carbon & $\mathrm{CH}_{3}-$ & $34.9(25)$ & $36.6(14)$ \\
\hline Secondary $\mathrm{sp}^{3}$ carbon & $-\mathrm{CH}_{2}-$ & $31.9(24)$ & $26.9(14)$ \\
\hline Tertiary $\mathrm{sp}^{3}$ carbon & $>\mathrm{CH}-$ & $22.4(20)$ & $9.0(11)$ \\
\hline Quaternary $\mathrm{sp}^{3}$ carbon & $>\mathrm{C}<$ & $14.0(17)$ & $-4.9(41)$ \\
\hline Secondary $\mathrm{sp}^{2}$ carbon & $=\mathrm{CH}_{2}$ & $25.8(18)$ & {$[46.0](5)$} \\
\hline Tertiary $\mathrm{sp}^{2}$ carbon & $=\mathrm{CH}-$ & $27.8(22)$ & $21.4(14)$ \\
\hline Quaternary $\mathrm{sp}^{2}$ carbon & $=\mathrm{C}<$ & $21.7(30)$ & $6.9(10)$ \\
\hline Tertiary sp carbon & $\equiv \mathrm{CH}$ & {$[38.8](9)$} & {$[25](1)$} \\
\hline Quaternary sp carbon & $\equiv \mathrm{C}-;=\mathrm{C}=$ & $29.1(15)$ & {$[15.1](3)$} \\
\hline \multicolumn{4}{|l|}{$\begin{array}{l}\text { 2. Aromatic hydrocarbon and nitrogen } \\
\text { groups }\end{array}$} \\
\hline Tertiary aromatic $\mathrm{sp}^{2}$ carbon & $=\mathrm{C}_{\mathrm{a}} \mathrm{H}-$ & $21.8(32)$ & $17.5(27)$ \\
\hline Quaternary aromatic $\mathrm{sp}^{2}$ & $=\mathrm{C}_{\mathrm{a}}<$ & $15.3(32)$ & $8.5(27)$ \\
\hline Internal quaternary aromatic carbon ${ }^{\mathrm{b}}$ & $=\mathrm{C}_{\mathrm{a}}<$ & & {$[8.2]^{\mathrm{b}}(2)$} \\
\hline Aromatic tertiary $\mathrm{sp}^{2}$ nitrogen & $=\mathrm{N}_{\mathrm{a}}-$ & $20.7(12)$ & $13.9(21)$ \\
\hline \multicolumn{4}{|l|}{ 3. Cyclic hydrocarbon groups } \\
\hline Cyclic secondary $\mathrm{sp}^{3}$ carbon & $-\mathrm{C}_{\mathrm{c}} \mathrm{H}_{2}-$ & $25.9(46)$ & $24.6(27)$ \\
\hline Cyclic tertiary $\mathrm{sp}^{3}$ carbon & $>\mathrm{C}_{\mathrm{c}} \mathrm{H}-$ & $20.6(40)$ & $11.7(20)$ \\
\hline Cyclic quaternary $\mathrm{sp}^{3}$ carbon & $>\mathrm{C}_{\mathrm{c}}<$ & $18(13)$ & $6.1(21)$ \\
\hline Cyclic tertiary $\mathrm{sp}^{2}$ carbon & $=\mathrm{C}_{\mathrm{c}} \mathrm{H}-$ & $21.8(17)$ & $18(61)$ \\
\hline Cyclic quaternary $\mathrm{sp}^{2}$ carbon & $=\mathrm{C}_{\mathrm{c}}<$ & $21.2(16)$ & $6.6(60)$ \\
\hline \multicolumn{4}{|l|}{ Functional groups } \\
\hline \multicolumn{4}{|l|}{ A. Monovalent groups } \\
\hline Hydroxyl group (alcohols) & $-\mathrm{OH}$ & $58.7(85)$ & $22.7(42)$ \\
\hline Hydroxyl group (phenols) & $-\mathrm{OH}$ & [74.9] (2) & $23.2(17)$ \\
\hline Fluorine & $-\mathrm{F}$ & $16.9(45)$ & {$[24.0](9)$} \\
\hline Chlorine & $-\mathrm{Cl}$ & $30.8(76)$ & $28.2(32)$ \\
\hline Bromine & $-\mathrm{Br}$ & $34.5(32)$ & $30.9(11)$ \\
\hline Iodine & $-\mathrm{I}$ & {$[39.2](9)$} & {$[27.6](5)$} \\
\hline Nitrile & $-\mathrm{C} \equiv \mathrm{N}$ & $49.9(23)$ & $41.6(12)$ \\
\hline Carboxylic acid & $-(\mathrm{C}=\mathrm{O}) \mathrm{OH}$ & $89.4(16)$ & $51.3(84)$ \\
\hline Aldehyde & $-(\mathrm{C}=\mathrm{O}) \mathrm{H}$ & $57.2(22)$ & {$[36.3](7)$} \\
\hline Primary $\mathrm{sp}^{3}$ nitrogen & $-\mathrm{NH}_{2}$ & $59.4(29)$ & $23.5(48)$ \\
\hline Primary amide & $-(\mathrm{C}=\mathrm{O}) \mathrm{NH}_{2}$ & [41.1] (1) & $49.6(27)$ \\
\hline Nitro group & $-\mathrm{NO}_{2}$ & {$[64.1](7)$} & $54.9(18)$ \\
\hline Monosubstituted urea & $-\mathrm{NH}(\mathrm{C}=\mathrm{O}) \mathrm{NH}_{2}$ & & $86.5(10)$ \\
\hline Isocyanate & $-\mathrm{N}=\mathrm{C}=\mathrm{O}$ & {$[57.3](8)$} & {$[54.5](4)$} \\
\hline Monosubstituted guanidine & $-\mathrm{NH}(\mathrm{C}=\mathrm{NH}) \mathrm{NH}_{2}$ & & {$[69.5](2)$} \\
\hline Thiol & $-\mathrm{SH}$ & $47.2(23)$ & {$[54.5](2)$} \\
\hline \multicolumn{4}{|l|}{ B. Acyclic divalent groups } \\
\hline Ether & $-\mathrm{O}-$ & $28.0(62)$ & $18.5(13)$ \\
\hline Ketone & $-(\mathrm{C}=\mathrm{O})-$ & $49.4(28)$ & [34.5] (9) \\
\hline Ester & $-(\mathrm{C}=\mathrm{O}) \mathrm{O}-$ & $63.9(83)$ & $42.2(19)$ \\
\hline Secondary amine & $-\mathrm{NH}-$ & $47.0(10)$ & {$[9.8](3)$} \\
\hline Secondary amide & $-(\mathrm{C}=\mathrm{O}) \mathrm{NH}-$ & $80.9(10)$ & $46.9(13)$ \\
\hline Tertiary amide & $-(\mathrm{C}=\mathrm{O}) \mathrm{N}<$ & {$[68.8](5)$} & \\
\hline Sulfide & $-\mathrm{S}-$ & $42.3(21)$ & {$[26](4)$} \\
\hline Disulfide & $-\mathrm{S}-\mathrm{S}-$ & [72.7] (3) & {$[40.5](1)$} \\
\hline Sulfoxide & $>(\mathrm{S} \rightarrow \mathrm{O})$ & {$[83.2](1)$} & [47.7] (1) \\
\hline Sulfone & $>(\mathrm{S} \rightarrow \mathrm{O})_{2}$ & & {$[54.8](3)$} \\
\hline Carbamate & $-\mathrm{NH}(\mathrm{C}=\mathrm{O}) \mathrm{O}-$ & & {$[82.1](6)$} \\
\hline Oxime & $=\mathrm{N}-\mathrm{OH}$ & & {$[53.3](2)$} \\
\hline Carbonate & $-\mathrm{O}(\mathrm{C}=\mathrm{O}) \mathrm{O}-$ & {$[77.4](1)$} & {$[68.7](1)$} \\
\hline \multicolumn{4}{|l|}{ C. Acyclic trivalent groups } \\
\hline Tertiary $\mathrm{sp}^{3}$ nitrogen (tert amine) & $-\mathrm{N}<$ & $29.7(14)$ & [37.9] (7) \\
\hline Tertiary $\mathrm{sp}^{2}$ nitrogen & $=\mathrm{N}-$ & [36.6] (3) & {$[18.5](8)$} \\
\hline Tertiary amide & $-(\mathrm{C}=\mathrm{O}) \mathrm{N}<$ & {$[68.8](5)$} & \\
\hline Triphosphate ester & $>\mathrm{O}_{2}(\mathrm{P}=\mathrm{O})-\mathrm{O}-$ & & {$[44.6](2)$} \\
\hline \multicolumn{4}{|l|}{ D. Acyclic quaternary group } \\
\hline Quaternary silicon & $>\mathrm{Si}<$ & $37.6(33)$ & {$[40.2](5)$} \\
\hline
\end{tabular}


TABLE 1. Group values for estimation of liquid $\Gamma$ (l) and solid $\Gamma$ (s) heat capacities at $T=298.15 \mathrm{~K}^{\mathrm{a}}$ - Continued

\begin{tabular}{|c|c|c|c|}
\hline & Group atoms & $\begin{array}{c}\Gamma(1) \\
\mathrm{J} \mathrm{K}^{-1} \mathrm{~mol}^{-1}\end{array}$ & $\begin{array}{c}\Gamma(\mathrm{s}) \\
\mathrm{J} \mathrm{K}^{-1} \mathrm{~mol}^{-1}\end{array}$ \\
\hline \multicolumn{4}{|l|}{ E. Cyclic functional groups } \\
\hline Cyclic anhydride & $-(\mathrm{C}=\mathrm{O}) \mathrm{O}(\mathrm{C}=\mathrm{O})_{\mathrm{c}}-$ & & {$[78.5](4)$} \\
\hline Cyclic carbonate & $-(\mathrm{O}(\mathrm{C}=\mathrm{O}) \mathrm{O})_{\mathrm{c}}-$ & {$[84.5](6)$} & [68.7] (1) \\
\hline Cyclic ether & $-\mathrm{O}_{\mathrm{c}}-$ & $23.2(23)$ & $10.3(30)$ \\
\hline Cyclic ester (lactone) & $-((\mathrm{C}=\mathrm{O}) \mathrm{O})_{\mathrm{c}}-$ & {$[69.4](8)$} & {$[44.6](6)$} \\
\hline Cyclic imide & $-((\mathrm{C}=\mathrm{O}) \mathrm{NH}(\mathrm{C}=\mathrm{O}))_{\mathrm{c}}-$ & & {$[71.4](4)$} \\
\hline$N$-substituted cyclic imide & $-((\mathrm{C}=\mathrm{O}) \mathrm{NR}(\mathrm{C}=\mathrm{O}))_{\mathrm{c}}-$ & & {$[54.5](5)$} \\
\hline Cyclic ketone & $-(\mathrm{C}=\mathrm{O})_{\mathrm{c}}-$ & $48.0(12)$ & {$[31.0](9)$} \\
\hline Cyclic secondary $\mathrm{sp}^{3}$ nitrogen & $-\mathrm{NH}_{\mathrm{c}}-$ & $47.8(14)$ & $17.8(13)$ \\
\hline Cyclic tertiary $\mathrm{sp}^{3}$ nitrogen & $-\mathrm{N}_{\mathrm{c}}<$ & {$[24.4](11)$} & $2.1(12)$ \\
\hline Cyclic tertiary $\mathrm{sp}^{2}$ nitrogen & $=\mathrm{N}_{\mathrm{c}}-$ & $20.7(21)$ & $13.2(21)$ \\
\hline Cyclic secondary amide & $-((\mathrm{C}=\mathrm{O}) \mathrm{NH})_{\mathrm{c}}-$ & [91.3] (1) & $45.9(42)$ \\
\hline Cyclic tertiary amide & $-((\mathrm{C}=\mathrm{O}) \mathrm{N}<)_{\mathrm{c}}$ & [96.3] (1) & $42.4(31)$ \\
\hline Cyclic sulfide & $-\mathrm{S}_{\mathrm{c}}-$ & $33.2(13)$ & $21.7(17)$ \\
\hline Cyclic sulfone & $>(\mathrm{S} \rightarrow \mathrm{O})_{2 \mathrm{c}}$ & & {$[36.5](4)$} \\
\hline Cyclic sulfoxide & $>(\mathrm{S} \rightarrow \mathrm{O})_{\mathrm{c}}$ & & {$[27.5](1)$} \\
\hline Cyclic quaternary silicon & $>\mathrm{Si}_{\mathrm{c}}<$ & [26.9] (8) & [37] (10) \\
\hline Cyclic thiocarbamate & $-(\mathrm{NH}(\mathrm{C}=\mathrm{S}) \mathrm{O})_{\mathrm{c}}-$ & & [64.6] (3) \\
\hline Cyclic urethane & $-(\mathrm{NH}(\mathrm{C}=\mathrm{O}) \mathrm{O})_{\mathrm{c}}-$ & & {$[55.1](6)$} \\
\hline Cyclic urea & $-(\mathrm{NH}(\mathrm{C}=\mathrm{O}) \mathrm{NH})_{\mathrm{c}}-$ & & [61.2] (1) \\
\hline
\end{tabular}

complexity. Some of the earlier methods have been previously described. [1990LYM/REE, 1987CHI, 1966REI/SHE] Most recently, group additivity methods have frequently been used to evaluate values of $C p_{298 \mathrm{~K}}(\mathrm{cr}), C p_{298 \mathrm{~K}}(\mathrm{l})$, and indirectly, $C p_{298 \mathrm{~K}}(\mathrm{~g})$, using Eq. (5). This work updates a previously reported method using group additivity methods to estimate the heat capacities of both liquids and solids. [2010ACR/CHI] The intention of the update is to take advantage of recent data to both bolster the statistics of many of the existing group values and to provide some additional new values. This work has taken advantage of an earlier compendium by Domalski and Hearing, [1996DOM/HEA] the exhaustive two volume compendium reported Zabransky et al. [1996ZAB/RUZ] and two additional supplements published subsequently [2001ZAB/RUZ, 2010ZAB/KOL] together with some recent literature results. Despite the influx of new data, some groups still have tentative values assigned to them. The reader should also be aware of a more sophisticated group additivity method that has also been reported to estimate liquid heat capacities [2004ZAB/RUZ]. These authors compare their results at $T=298.15 \mathrm{~K}$ to those evaluated by this method and note that the percent deviations obtained using their group contributions are generally somewhat less than those obtained by this group method. Their group contributions are represented by second order polynomials and their estimations are combined with some additional structural corrections. The additional complexity of the Zabransky and Ruzicka group method has the specific advantage of providing liquid heat capacities as a function of temperature. The major advantages of the group method described below are its relative simplicity and capability to provide both liquid and solid heat capacities at
$T=298.15 \mathrm{~K}$ within reasonable error limits and the potential of also providing gas phase heat capacities as illustrated below.

\subsection{Group values for estimating liquid and solid heat capacities at $T=298.15 \mathrm{~K}$}

The group additivity method and the group values used to evaluate heat capacities are summarized in Table 1. Groups defined in column 1 of the table are identified on the basis of the substitution pattern and hybridization. Primary, secondary, ... groups are defined by the number of hydrogens on carbon, $3,2, \ldots$. The second column provides a structural basis for the group in question. Group values are assigned in column 3 . Values in brackets refer to tentative assignments while the numbers in parenthesis refer to the number of compounds used in the assignment of the group value. Tentative assignments are characterized by less than ten experimental measurements. A blank entry indicates the lack of any experimental data. In a few cases some group values are based on a single entry. Group values for the hydrocarbon portion of the molecule are for the most part unchanged from previous versions. Exceptions include changes in two liquid and four solid group values due primarily to the inclusion of additional data. Values for the functional groups are changed but most changes are relatively small. For consistency and ease of identification, some group values previously combined have been defined separately. For example, a cyclic tertiary $\mathrm{sp}^{2}$ hybridized nitrogen in imidazoline and also previously used for nitrogen in aromatic heterocycles is identified separately even though the value is the same for both groups. 


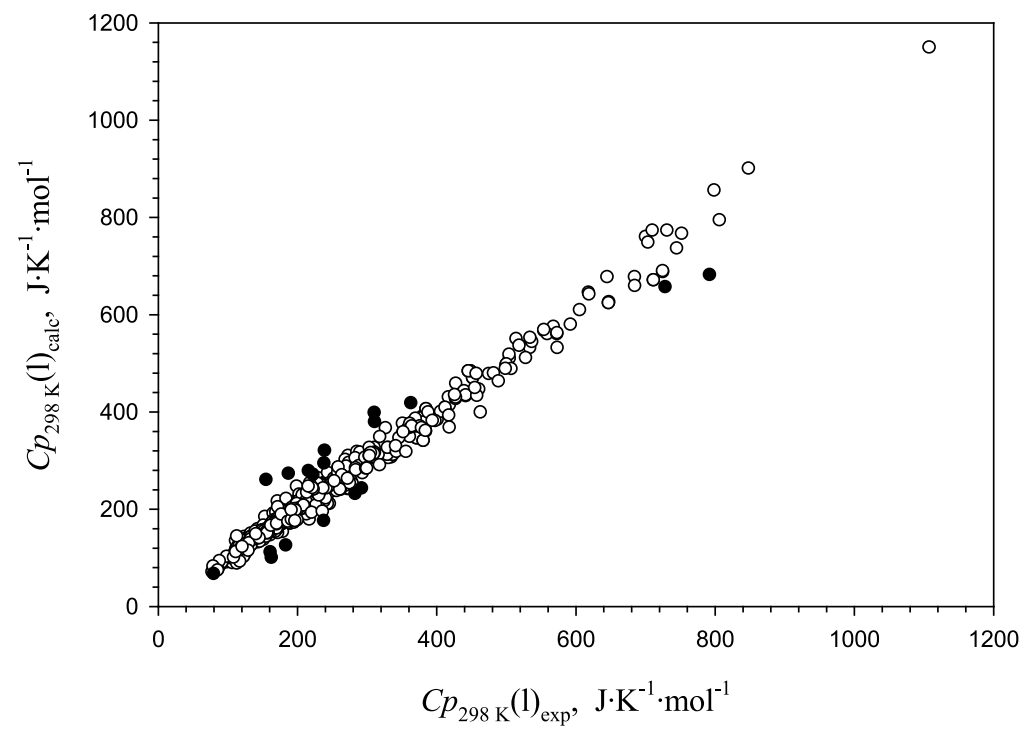

Fig. 1. A comparison of experimental and calculated liquid heat capacities at $T=298.15 \mathrm{~K}$. The solid circles represent data that were not used in evaluating group values nor are they included in the equation of the line which is given by $C p_{298 \mathrm{~K}}(1)_{\mathrm{calc}}=(1.003 \pm 0.004) \cdot C p_{298 \mathrm{~K}}(1)_{\exp }-(4.42 \pm 1.19) ; \mathrm{r}^{2}=0.9878$.

\subsection{Statistics in evaluation of group values}

Values for most hydrocarbon groups were retained from previous work and were not evaluated in this update. A total of 709 liquids and 556 solid heat capacities, mostly substituted hydrocarbons were collected from the compendia and the current literature as noted above. A total of 691 entries were used to evaluate group values for liquids and 520 were used to evaluate the group values for solids reported in Table 1. Not included in this count are both liquid and solid hydrocarbons previously used to evaluate values that have remained unchanged. Heat capacities ranged from (73 to 730 ) and (79 to 1184$) \mathrm{J} \mathrm{K}^{-1} \mathrm{~mol}^{-1}$ for liquids and solids, respectively. Group values were evaluated by minimizing $\mathrm{R}$ in the following function for each functional group:

$$
R=\sum_{1}^{n}\left(\frac{C p_{\exp }-C p_{\text {calc }}}{C p_{\exp }}\right)^{2}
$$

Once a functional group was evaluated it was used as a known in substances containing multiple functionalities. In most cases it was necessary to reiterate the minimization. A total of 19 liquid and 36 solid experimental values were rejected. Compounds rejected were selected on the basis of the difference between estimated and calculated value when the fractional difference either exceeded $25 \%$ of the experimental value or was larger than $50 \mathrm{~J} \mathrm{~K}^{-1} \mathrm{~mol}^{-1}$. The value of $50 \mathrm{~J} \mathrm{~K}^{-1} \mathrm{~mol}^{-1}$ was selected as approximately three standard deviations of the uncertainty associated with previous correlations. The larger number of solids rejected is likely due to the fact that some solids either form plastic crystals, liquid crystals, or are not totally crystalline. These materials frequently exhibit larger heat capacities than conventional solids [2006ACR/CHI], [2013GOB/RAT]. Consistent with this interpretation is the finding that a total of 28 of the 39 experimental values rejected were larger than the calculated value while approximately half of the 18 rejected liquids $(8 / 18)$ exceeded the calculated value. The standard deviation
$( \pm 1 \sigma)$ associated with estimations for 681 liquids was $\pm 14.4 \mathrm{~J} \mathrm{~K}^{-1} \mathrm{~mol}^{-1}$ and $\pm 17.0 \mathrm{~J} \mathrm{~K}^{-1} \mathrm{~mol}^{-1}$ for 524 solids. The absolute average fractional error $\left[\left|\left(C p_{\exp }-C p_{\text {calc }}\right)\right| / C p_{\text {exp }}\right]$ for liquids was 0.045 and 0.053 for solids. Figures 1 and 2 compare experimental and estimated heat capacities for both the liquid and solid compounds included in the estimations. The solid circles in the figures identify values of the compounds not used in evaluating group values. These values were also not included in generating the statistics.

\section{Applications}

Sections 3.1 and 3.2 deal with the application of the group values evaluated to estimate values of $C p_{298 \mathrm{~K}}(\mathrm{cr}), C p_{298 \mathrm{~K}}(\mathrm{l})$, and $C p_{298 \mathrm{~K}}(\mathrm{~g})$.

\subsection{Estimation of condensed phase heat capacities}

Figure 3 illustrates the structure of three compounds in which the groups in Table 1 are used to evaluate their heat capacity; the substances include simvastatin, used to decrease elevated lipid levels in blood, uridine, one of the five standard nucleosides, and risperidone, an antipsychotic medication. Estimations of these three solids are detailed below. Table 2 is included to provide some additional examples of some complex substances that can be addressed by this method. The table includes estimations of L-Tryptophan, 1,3dimethylbarbituric acid, inosine, and $\beta$-ionone. L-Tryptophan is included to illustrate the applicability of using these group values in estimating amino acids despite the fact that they exist as dipolar ions. 1,3-dimethylbarbituric acid and inosine illustrate further examples of estimations in which the available functional groups can be used to evaluate their heat capacity. All but $\beta$-ionone are crystalline materials. The estimation of $\beta$-ionone represents an estimation of a liquid. 


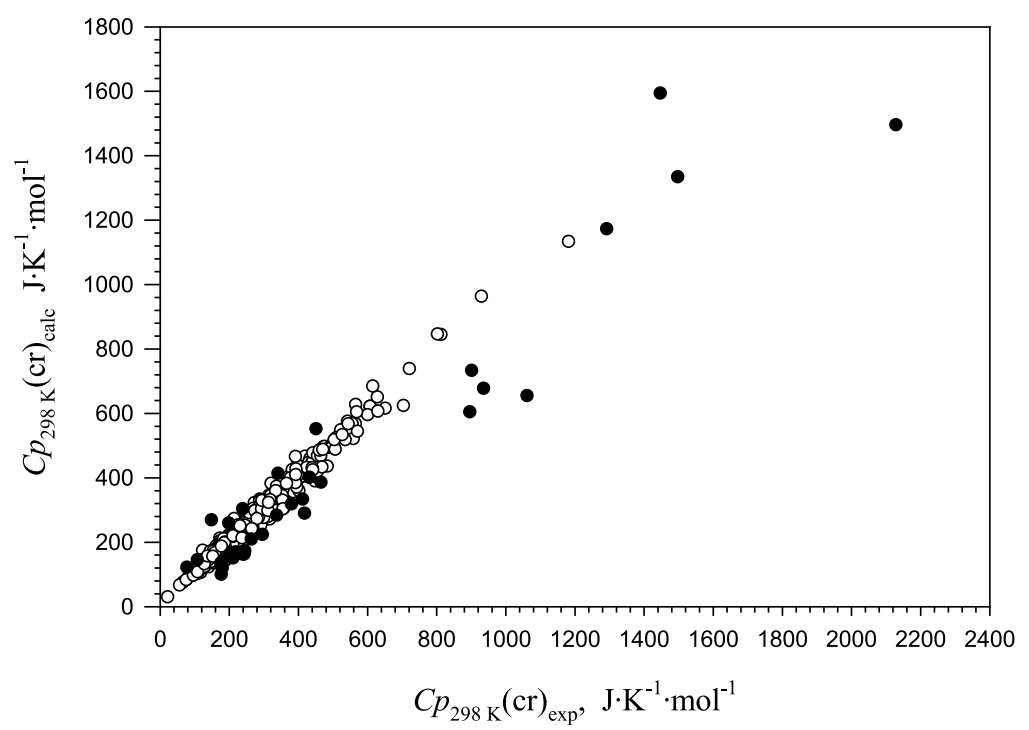

FIG. 2. A comparison of experimental and calculated solid heat capacities at $T=298.15 \mathrm{~K}$. The solid circles represent data that were not used in evaluating group values nor are they included in the equation of the line which is given by $C p_{298 \mathrm{~K}}(\mathrm{cr})_{\mathrm{calc}}=(0.986 \pm 0.006) \cdot C p_{298 \mathrm{~K}}(\mathrm{cr})_{\exp }+(2.09 \pm 1.78) ; \mathrm{r}^{2}=0.9794$.

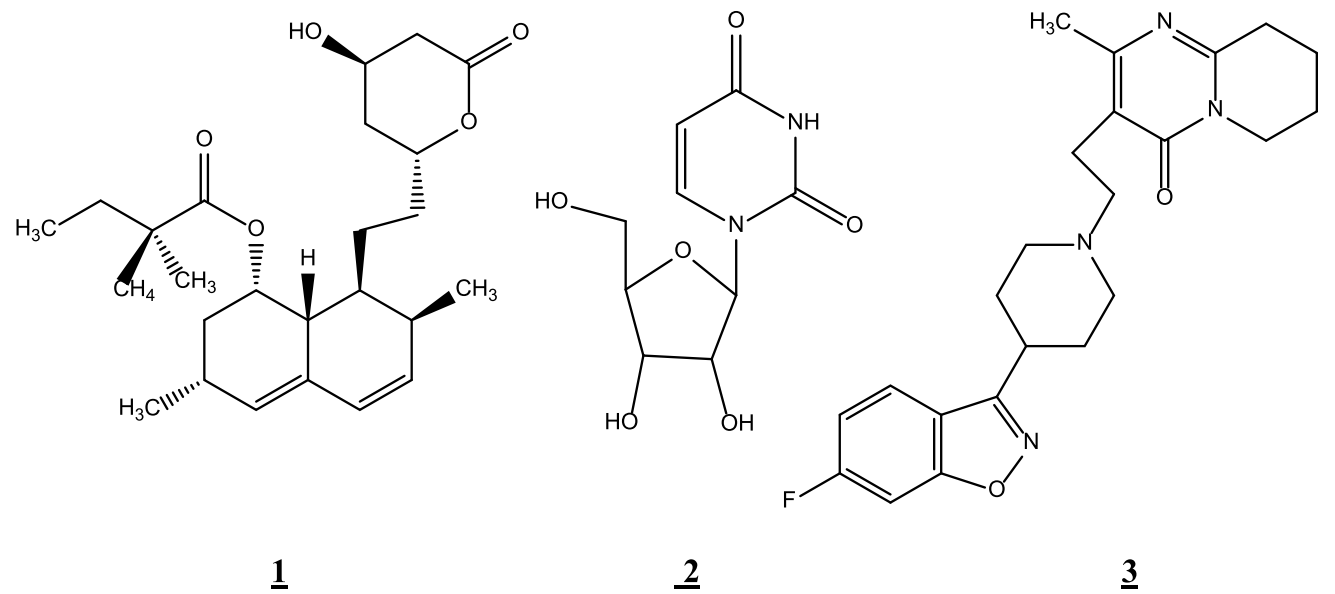

FIG. 3. Structures of simvastatin $\underline{\mathbf{1}}$, uridine $\underline{\mathbf{2}}$, and risperidone $\underline{\mathbf{3}}$.

Following the detailed description of the three compounds in Figure 3, estimation of the compounds in Table 2 should follow.

Simvastatin can be estimated by reducing the structure to the number and types of groups in the molecule. Identifying groups by beginning at the top of Table 1 and working downwards, the carbon component of simvastatin consists of $5\left[\mathrm{CH}_{3}-\right], 3\left[-\mathrm{CH}_{2}-\right], 1[>\mathrm{C}<], 3\left[-\mathrm{C}_{\mathrm{c}} \mathrm{H}_{2}-\right], 7$ $\left[>\mathrm{C}_{\mathrm{c}} \mathrm{H}-\right], 3\left[=\mathrm{C}_{\mathrm{c}} \mathrm{H}-\right], 1\left[=\mathrm{C}_{\mathrm{c}}<\right]$. The three functional groups complete the estimation, $1[-\mathrm{OH}], 1[-(\mathrm{C}=\mathrm{O}) \mathrm{O}-]$, and $1\left[-((\mathrm{C}=\mathrm{O}) \mathrm{O})_{\mathrm{c}}-\right]$, for a total of 26 groups. As a check, adding the carbons, hydrogens, nitrogens, and oxygens reproduces the molecular formula of simvastatin, $\mathrm{C}_{25} \mathrm{H}_{38} \mathrm{O}_{5}$. Adding the values associated with each of these groups results in an estimated value for $C p_{298 \mathrm{~K}}(\mathrm{cr})$ of $(585.2 \pm 17) \mathrm{J} \mathrm{K}^{-1} \mathrm{~mol}^{-1}$ (lit. $576.5 \pm 23.1 \mathrm{~J} \mathrm{~K}^{-1} \mathrm{~mol}^{-1}$. [2013SIM/BER]) Heat capacity differences due to stereochemistry are frequently small. The reader should note that stereochemical considerations and positional isomers on rings are not addressed in this approach.
A similar estimation for uridine, molecular formula $\mathrm{C}_{9} \mathrm{H}_{12} \mathrm{~N}_{2} \mathrm{O}_{6}$, results in the following for $C p_{298 \mathrm{~K}}(\mathrm{cr})$ : $1\left[-\mathrm{CH}_{2}-\right], \quad 4 \quad\left[>\mathrm{C}_{\mathrm{c}} \mathrm{H}-\right], \quad 2 \quad\left[=\mathrm{C}_{\mathrm{c}} \mathrm{H}-\right], \quad 3 \quad[-\mathrm{OH}]$, $1\left[-\mathrm{O}_{\mathrm{c}}-\right],\left[-((\mathrm{C}=\mathrm{O}) \mathrm{NH})_{\mathrm{c}}-\right]$, and $\left[-\left((\mathrm{C}=\mathrm{O}) \mathrm{N}_{\mathrm{c}}\right)<\right]$. Adding the $\mathrm{C}, \mathrm{H}, \mathrm{N}$, and $\mathrm{O}$ of these groups reproduces the correct molecular formula. Adding the values associated with each of these groups results in a value for $C p_{298 \mathrm{~K}}(\mathrm{cr})$ of $(278.9 \pm 17) \mathrm{J} \mathrm{K}^{-1} \mathrm{~mol}^{-1}$ (lit. 292.3 [2008SZT]).

The molecular formula of risperidone is $\mathrm{C}_{23} \mathrm{H}_{27} \mathrm{FN}_{4} \mathrm{O}_{2}$. The groups include $1\left[\mathrm{CH}_{3}-\right], 2\left[-\mathrm{CH}_{2}-\right], 3\left[=\mathrm{C}_{\mathrm{a}} \mathrm{H}-\right], 3$ $\left[=\mathrm{C}_{\mathrm{a}} \mathrm{R}-\right], 8\left[-\mathrm{C}_{\mathrm{c}} \mathrm{H}_{2}-\right], 1\left[>\mathrm{C}_{\mathrm{c}} \mathrm{H}-\right], 4\left[=\mathrm{C}_{\mathrm{c}}<\right], 1[-\mathrm{F}]$, $1\left[-\mathrm{O}_{\mathrm{c}}-\right], 1\left[-\mathrm{N}_{\mathrm{c}}<\right], 2\left[=\mathrm{N}_{\mathrm{c}}-\right], 1\left[-((\mathrm{C}=\mathrm{O}) \mathrm{N})_{\mathrm{c}}<\right]$. Addition of the appropriate atoms reproduces the molecular formula and application of the group values results in a total $C p_{298 ~ K}$ (cr) of $(509.2 \pm 17) \mathrm{J} \mathrm{K}^{-1} \mathrm{~mol}^{-1}$ (lit. $500 \mathrm{~J} \mathrm{~K}^{-1} \mathrm{~mol}^{-1}$ [2014MEA/SVA]).

Simvastatin, risperidone, and inosine were not included in the 550 solid heat capacities used in generating the group values. L-Tryptophan was one of the 48 entries used to evaluate the group value for primary amines, 1,3-dimethylbarbituric 
TABLE 2. Estimation of L-tryptophan, inosine, 1,3-dimethylbarbituric acid and $\beta$-ionone

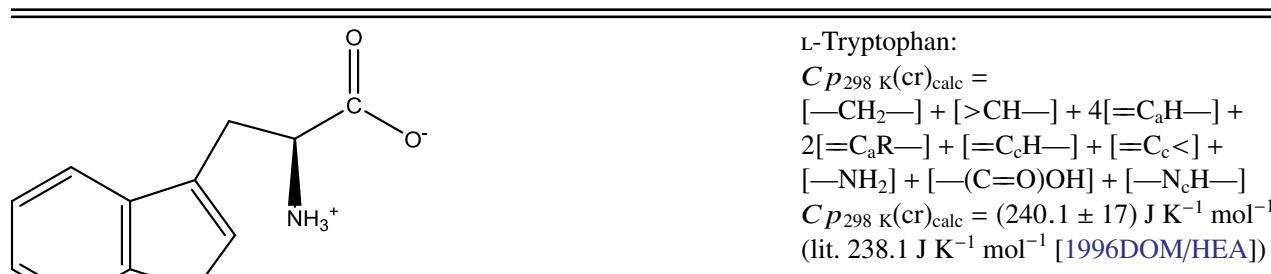<smiles>O=c1[nH]cnc2c1ncn2C1OC2COC1C2O</smiles>

Inosine:

$C p_{298 \mathrm{~K}}(\mathrm{cr})_{\mathrm{calc}}=$

$\left[-\mathrm{CH}_{2}-\right]+4\left[>\mathrm{C}_{\mathrm{c}} \mathrm{H}-\right]+2\left[=\mathrm{C}_{\mathrm{c}} \mathrm{H}-\right]+$

$2\left[=\mathrm{C}_{\mathrm{c}}<\right]+3[-\mathrm{OH}]+\left[-\mathrm{O}_{\mathrm{c}}-\right]+$

$\left[-\mathrm{N}_{\mathrm{c}}<\right]+2\left[=\mathrm{N}_{\mathrm{c}}-\right]+(-(\mathrm{C}=\mathrm{O}) \mathrm{NH}-)_{\mathrm{c}}$

$C p_{298 \mathrm{~K}}(\mathrm{cr})_{\text {calc }}=(277.5 \pm 17) \mathrm{J} \mathrm{K}^{-1} \mathrm{~mol}^{-1}$

(lit. 283.1 J K $\mathrm{Jol}^{-1}$ [2005BOE/HOP])

\section{$\mathrm{C}_{12} \mathrm{H}_{12} \mathrm{~N}_{4} \mathrm{O}_{5}$}<smiles>CN1C(=O)CC(=O)N(C)C1=O</smiles>

1,3-Dimethylbarbituric acid

$C p_{298 \mathrm{~K}}(\mathrm{cr})_{\text {calc }}=2\left[\left[\mathrm{CH}_{3}-\right]+\right.$

$\left[-\mathrm{C}_{\mathrm{c}} \mathrm{H}_{2}-\right]+\left[-((\mathrm{C}=\mathrm{O}) \mathrm{N}<)_{\mathrm{c}}\right]+$

$\left[-((\mathrm{C}-\mathrm{O}) \mathrm{NR}(\mathrm{C}=\mathrm{O}))_{\mathrm{c}}-\right]$

$C p_{298 \mathrm{~K}}(\mathrm{cr})_{\text {calc }}=(194.7 \pm 17) \mathrm{J} \mathrm{K}^{-1} \mathrm{~mol}^{-1}$

(lit. $188.5 \mathrm{~J} \mathrm{~K}^{-1} \mathrm{~mol}^{-1}$ [2011TEM/ROU])

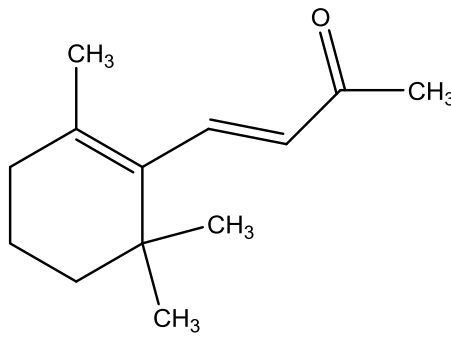

$\beta$-Ionone:

$C p_{298 \mathrm{~K}}(1)_{\text {calc }}=$

$4\left[\mathrm{CH}_{3}-\right]+2[=\mathrm{CH}-]+3\left[-\mathrm{C}_{\mathrm{c}} \mathrm{H}_{2}-\right]+$

$\left[>\mathrm{C}_{\mathrm{c}}<\right]++2\left[=\mathrm{C}_{\mathrm{c}}<\right]+[-(\mathrm{C}=\mathrm{O})-]$

$C p_{298 \mathrm{~K}}(1)_{\text {calc }}=(381.3 \pm 14.4) \mathrm{J} \mathrm{K}^{-1} \mathrm{~mol}^{-1}$

(lit. $377 \mathrm{~J} \mathrm{~K}^{-1} \mathrm{~mol}^{-1}$ [2005BOE/HOP])

$\mathrm{C}_{13} \mathrm{H}_{20} \mathrm{O}$

acid was one of the 38 compounds used to evaluate the group value for cyclic tertiary amides and $\beta$-ionone was one of the twelve entries used to evaluate the group value for liquid cyclic ketones.

\subsection{Estimation of gas phase heat capacities}

As noted above, Eq. (5) has been used to estimate the heat capacity of the corresponding gas phase. Figure 4 illustrates the structures of benzoic acid, 2,2,5,7,8-pentamethylchroman-6-ol, and 6-hydroxy-2,5,7,8tetramethylchroman-2-carboxylic acid. The heat capacity of gaseous benzoic acid has recently been reported [2010SAN/ROC]. A theoretical value of $C p_{298 \mathrm{~K}}(\mathrm{~g})=$
$126 \mathrm{~J} \mathrm{~K}^{-1} \mathrm{~mol}^{-1}$ was calculated and compared to a value of $125.1 \mathrm{~J} \mathrm{~K}^{-1} \mathrm{~mol}^{-1}$ obtained spectroscopically [1996STE/REV]. The heat capacity of crystalline benzoic acid can be estimated as $5\left[=\mathrm{C}_{\mathrm{a}} \mathrm{H}-\right], 1$ $\left[=\mathrm{C}_{\mathrm{a}}<\right]$, and $[-(\mathrm{C}=\mathrm{O}) \mathrm{OH}]$. Addition of the appropriate group values results in a value, $C p_{298 \mathrm{~K}}(\mathrm{cr})=(147.3 \pm$ 17; lit.146.8) $\mathrm{J} \mathrm{K}^{-1} \mathrm{~mol}^{-1}$ [1996DOM/HEA]. Benzoic acid was one of the 84 entries used to evaluate the group value for a carboxylic acid. Applying Eq. (5) results in a gas phase heat capacity of $(124.5 \pm 17) \mathrm{J} \mathrm{K}^{-1} \mathrm{~mol}^{-1}$ in very good agreement with the literature value,

$$
\begin{aligned}
C p_{298 \mathrm{~K}}(\mathrm{~g})(\text { benzoic acid }) & =0.85 \cdot[147.3]-0.75 \\
& =(124.5 \pm 17) \mathrm{J} \mathrm{K}^{-1} \mathrm{~mol}^{-1}
\end{aligned}
$$




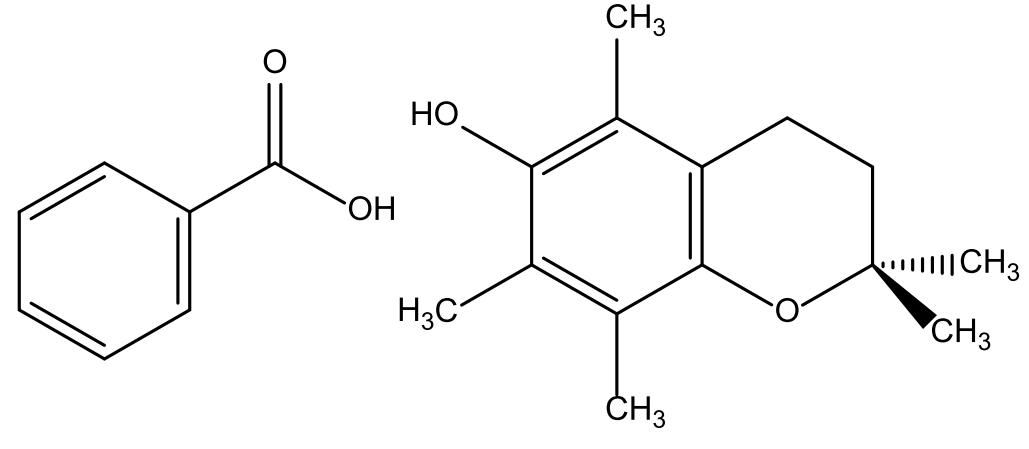

$\underline{4}$

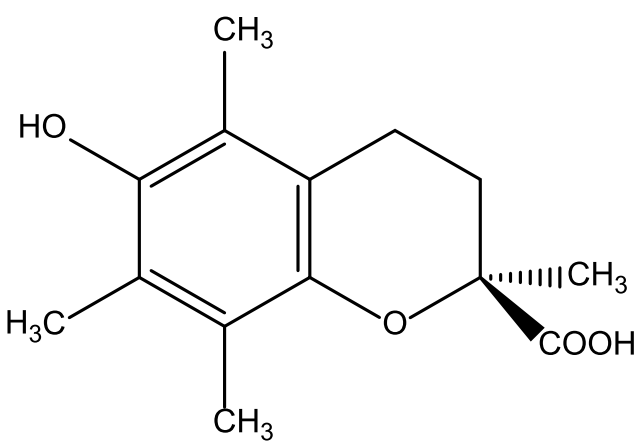

$\underline{6}$

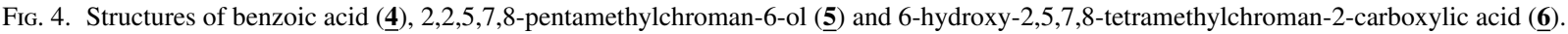

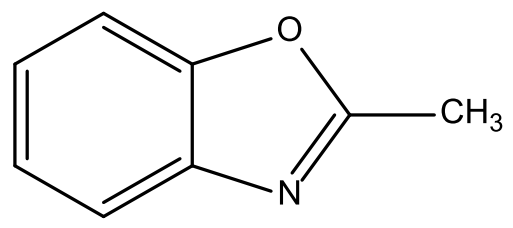

$\underline{7}$

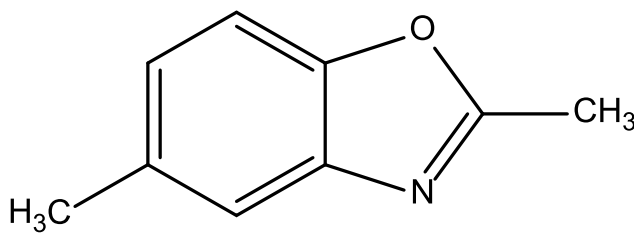

$\underline{8}$

FIG. 5. Structures of 2-methylbenzoxazole (ㅁ) and 2,5-dimethylbenzoxazole (ㅇ) .

Applying the appropriate group values to estimate the heat capacity of 2,2,5,7,8-pentamethylchroman-6-ol $\left(5^{*} 36.6+\right.$ $6 * 8.5+2 * 24.6+6.1+23.2+10.3)$ results in an estimation of $C p_{298 \mathrm{~K}}(\mathrm{cr})=(322.8 \pm 17) \quad \mathrm{J} \mathrm{K}^{-1} \mathrm{~mol}^{-1} \quad[$ lit. $315.3 \pm$ 9.6 J K ${ }^{-1} \mathrm{~mol}^{-1}$ [2014BER/SIM]]. Similarly for 6-hydroxy2,5,7,8-tetramethylchroman-2-carboxylic acid, replacing the value of one methyl group with a carboxylic acid group results in $C p_{298 \mathrm{~K}}(\mathrm{cr})=(337.5 \pm 17) \mathrm{J} \mathrm{K}^{-1} \mathrm{~mol}^{-1}$ [lit. (332 \pm 9.4) $\mathrm{J} \mathrm{K}^{-1} \mathrm{~mol}^{-1}$ [2014BER/SIM]]. Neither of these two materials was included in the database. Applying Eq. (5) to the two estimated $C p_{298 \mathrm{~K}}(\mathrm{cr})$ values results in gas phase heat capacities of $(273.5 \pm 17) \mathrm{J} \mathrm{K}^{-1} \mathrm{~mol}^{-1}$ for the former and $(286.1 \pm 17) \mathrm{J} \mathrm{K}^{-1} \mathrm{~mol}^{-1}$ for the latter compound. These values compare to gas phase values of $(293.9 \pm 20.6) \mathrm{J} \mathrm{K}^{-1} \mathrm{~mol}^{-1}$ and $(311 \pm 21.8) \mathrm{J} \mathrm{K}^{-1} \mathrm{~mol}^{-1}$, respectively, calculated by statistical mechanics based on structural parameters and vibrational frequencies obtained at the B3LYP/6-31+G(d,p) level of theory. The two sets of results appear to agree within their levels of uncertainty.

Figure 5 illustrates the structures of 2-methylbenzoxazole and 2,5-dimethylbenzoxazole. Liquid heat capacities of $(217.8 \pm 14.4) \mathrm{J} \mathrm{K}^{-1} \mathrm{~mol}^{-1}$ and $(244.4 \pm 14.4) \mathrm{J} \mathrm{K}^{-1} \mathrm{~mol}^{-1}$ are estimated for these two materials. Application of Eq. (6) suggests $C p_{298 \mathrm{~K}}(\mathrm{~g})$ values of $(150.6 \pm 14.4) \mathrm{J} \mathrm{K}^{-1} \mathrm{~mol}^{-1}$ and $(171.6 \pm 14.4) \mathrm{J} \mathrm{K}^{-1} \mathrm{~mol}^{-1}$, respectively. Gas phase heat capacities derived from statistical thermodynamics using the vibrational frequencies calculated at the B3LYP/6-31G(d) level of theory predict values of (138.3 and 164.3) $\mathrm{J} \mathrm{K}^{-1} \mathrm{~mol}^{-1}$, respectively, [2013SIL/CIM] in reasonable accord with the estimated values.

\section{The phase change enthalpy compendium}

The phase change enthalpy data reported in this compendium have been reported over the time period of 1880-2015 with a few references from 2016. The data are a combination of the compendium published in 2010 together with results obtained from the literature up to 2016. Phase change enthalpies have been measured for many years and numerous techniques have been developed and used over this period of time. Both direct and indirect methods have been employed. Direct methods include calorimetric measurements and indirect methods usually include a direct or indirect measure of vapor pressure over a range of temperatures. Gas chromatographic techniques have also been developed which rely on established measurements. For many materials, a brief description of the method of measurement is provided by the acronyms defined in Table 3. Some entries may not have an acronym. In these cases either the method of measurement was not clearly evident, the information was not available from the source the information was retrieved (such as an abstract), or the method was not recorded at the time the article was referenced. A significant number of early measurements have been calculated from the Antoine Constants reported in the compilation by Stephenson and Malonowski. [1987STE/MAL] References to the original literature are not available for materials evaluated from this reference. In some cases the original reference has been identified independently by inference. 
TABLE 3. List of acronyms and descriptions

\begin{tabular}{|c|c|}
\hline A & $\begin{array}{l}\text { Calculated from the vapor pressure data reported by the } \\
\text { method of least squares }\end{array}$ \\
\hline $\mathrm{AC}$ & Adiabatic calorimeter \\
\hline $\mathrm{B}$ & $\begin{array}{l}\text { Calculated from the difference of the enthalpies of } \\
\text { sublimation at temperature } \mathrm{T} \text { and fusion at the melting point. }\end{array}$ \\
\hline BG & Bourdon gauge \\
\hline $\mathrm{BP}$ & Boiling point temperature at different pressures \\
\hline $\mathrm{C}$ & Calorimetric determination \\
\hline CATH & Cathetometer \\
\hline $\mathrm{CC}$ & Conduction calorimeter \\
\hline $\mathrm{CCM}$ & Cooling curve method \\
\hline $\mathrm{CE}$ & Critical evaluated value \\
\hline CGC & Correlation-gas chromatography \\
\hline CGC- & Combined correlation gas chromatography-differential \\
\hline DSC & scanning calorimetry \\
\hline CR & Cryoscopy \\
\hline CRT & Chromatographic retention time \\
\hline CVC & Calvet calorimeter \\
\hline DBM & Dibutyl pththalate manometer \\
\hline DM & Diaphram manometer \\
\hline DFM & Differential manometer \\
\hline DP-LPD & Dew point low pressure distillation method \\
\hline DSC & Differential scanning calorimeter \\
\hline DTA & Differential thermal analysis \\
\hline $\mathrm{E}$ & Estimated value \\
\hline EM & Electronic manometer \\
\hline EST & Estimated value \\
\hline $\mathrm{F}$ & Fluorescence \\
\hline FPM & freezing point method \\
\hline FTIR & Fourier transform infrared spectroscopy \\
\hline GC & Gas chromatography \\
\hline GC-RT & Gas chromatography retention time \\
\hline GCC & Gas chromatography-calorimetry \\
\hline GS & Gas saturation, transpiration \\
\hline GSM & Glass spring manometer \\
\hline $\mathrm{HG}$ & Heise gauge \\
\hline HBG & Heise-Bourdon gauge \\
\hline $\mathrm{HFC}$ & Heat flux calorimetry \\
\hline HSA & Head space analysis \\
\hline I & Isoteniscope \\
\hline IPM & Inclined piston manometry \\
\hline IR & Infrared spectroscopy \\
\hline KG & Knudsen gauge \\
\hline $\mathrm{LE}$ & Langmuir evaporation \\
\hline MDSC & Modulated differential scanning calorimetry \\
\hline $\mathrm{ME}$ & Mass effusion-Knudsen effusion \\
\hline MEM & Modified entrainment method \\
\hline MG & McLeod gauge \\
\hline MM & Mercury manometer \\
\hline MS & Mass spectrometry \\
\hline $\mathrm{OM}$ & Oil manometer \\
\hline PG & Pressure gauge \\
\hline QCM & Quartz crystal microbalance \\
\hline QF & Quartz fiber \\
\hline QR & Quartz resonator \\
\hline $\mathrm{RC}$ & Radiation calorimeter \\
\hline RG & Rodebush gauge \\
\hline RS & Recirculating still \\
\hline S-F & Sublimation-fusion \\
\hline $\mathrm{S}-\mathrm{V}$ & Sublimation-vaporization \\
\hline $\mathrm{SC}$ & Solution calorimetry \\
\hline SG & Spoon gauge \\
\hline SRFG & Spinning rotor friction gauge \\
\hline Static & Static method \\
\hline STG & Strain gauge \\
\hline $\mathrm{T}$ & Tensiometer \\
\hline
\end{tabular}

TABLE 3. List of acronyms and descriptions-Continued

\begin{tabular}{ll}
\hline TCC & Tin Calvet calorimeter \\
TCM & Thermal conductivity manometer \\
TE & Torsion effusion \\
TGA & Thermal gravimetric analysis \\
TG-TS & Thermogravimetric transpiration method \\
THBC & Triple heat bridge calorimeter \\
TPD & Temperature programmed desorption \\
TPTD & Temperature programmed thermal desorption \\
TSGC & Temperature scanning gas chromatography \\
U & Unreliable \\
UV & Ultraviolet spectroscopy \\
UV/VIS & Ultraviolet-visible spectroscopy \\
VS & Visible spectroscopy \\
V +F & vaporization + fusion \\
VG & Viscocity gauge \\
VP & Vapor pressure as a function of temperature \\
ZG & Zimmerli gauge \\
\hline
\end{tabular}

Organic compounds provided by the following tables are arranged according to the Hill system. The molecular formula is provided in the first column of the first row of each individual material. This is usually followed by the Chemical Abstracts reference number in the second column and the chemical name. The chemical name is usually the name provided by the authors of the article. Occasionally this may have resulted in redundant entries for some complex materials. The remaining information in subsequent columns includes the phase transition (column 2), the temperature or temperature range if any associated with the transition (column 3), the enthalpy associated with the transition (column 4), the mean temperature associated with the transition if measured over a temperature range (column 5), the acronym associated with the method of measurement (column 6), and reference to the source of information (column 7), usually to the original work. If an entry is not provided in one of these columns, it is likely that the information was not available in the source consulted. For liquids, the vaporization enthalpy $(V)$ in $\mathrm{kJ} \mathrm{mol}^{-1}$ follows the name in subsequent rows. For solids, depending on the nature of the material, the order of entry includes any solid-solid transitions available (TRS), followed in subsequent rows by each available solid to liquid transition (FUS), the sublimation enthalpy or enthalpies available (SUB), and the vaporization enthalpy (ies) if available. Some enthalpy values are cited without an accompanying reference on the same line. In these cases the reference cited directly below should be consulted since multiple enthalpy values may have been taken from the same source, particularly for compounds with multiple solid-solid phase transitions. A brief note summarizing some peculiarity in the data is also provided for some compounds, particularly if the value reported appears to be unreliable, U (e.g., "U $66 \pm 22.1$ ").

The thermochemical data are arranged by carbon number. Table 4 contains thermochemical data for all compounds containing a single carbon atom that has been located. Table 5 continues with all compounds containing two carbons $\left(\mathrm{C}_{2}\right)$, Table 6, with three carbons $\left(\mathrm{C}_{3}\right)$, and so on. Tables 7-13 include compounds containing $\mathrm{C}_{4}$ through $\mathrm{C}_{10}$. 
TABle 4. Phase change enthalpies of $\mathrm{C}_{1}$ organic compounds

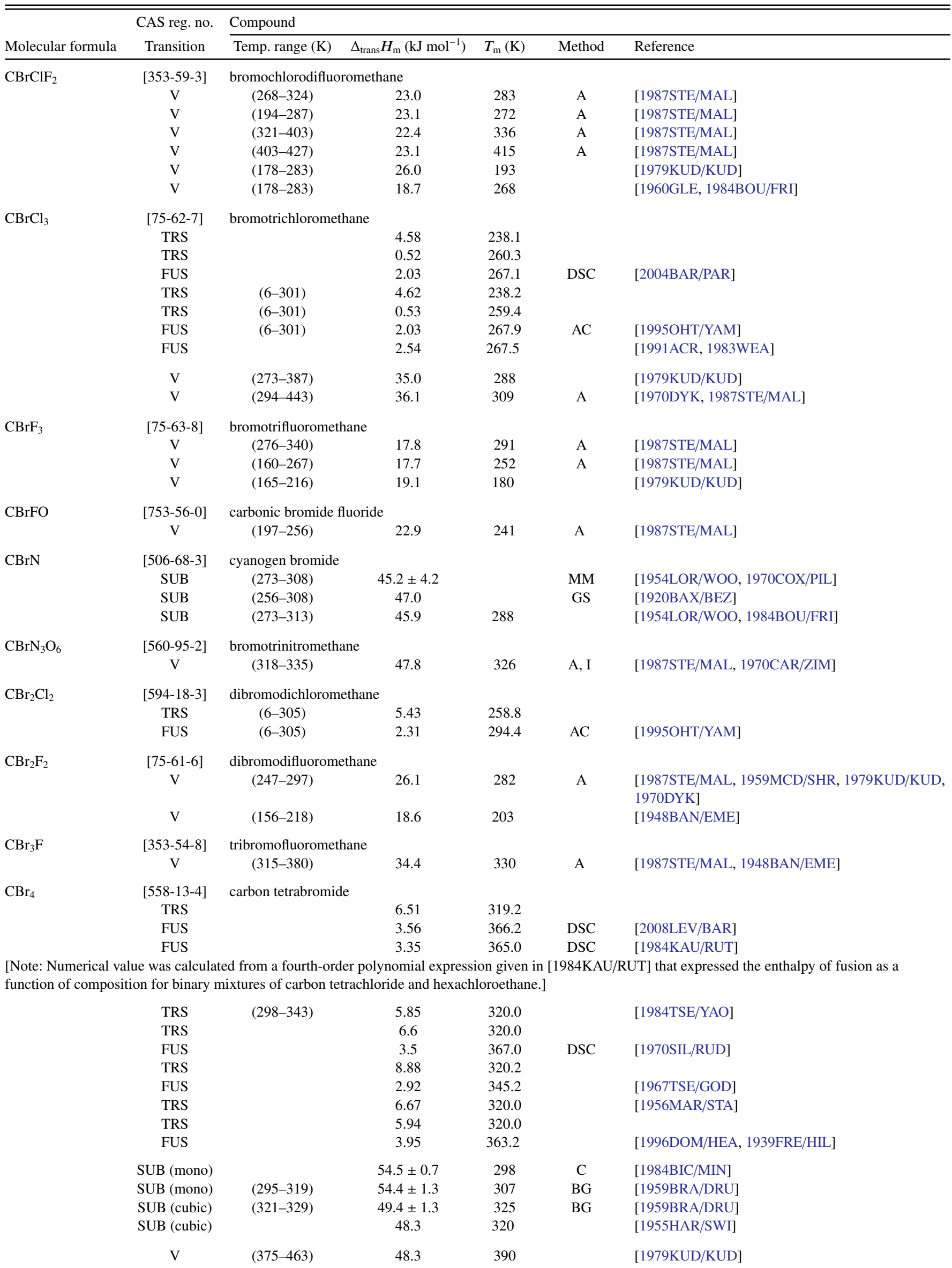


TABLE 4. Phase change enthalpies of $\mathrm{C}_{1}$ organic compounds-Continued

\begin{tabular}{|c|c|c|c|c|c|c|}
\hline \multirow[b]{2}{*}{ Molecular formula } & \multirow{2}{*}{$\begin{array}{c}\text { CAS reg. no. } \\
\text { Transition }\end{array}$} & \multicolumn{5}{|l|}{ Compound } \\
\hline & & Temp. range $(\mathrm{K})$ & $\Delta_{\text {trans }} H_{\mathrm{m}}\left(\mathrm{kJ} \mathrm{mol}^{-1}\right)$ & $T_{\mathrm{m}}(\mathrm{K})$ & Method & Reference \\
\hline & $\mathrm{V}$ & $(369-463)$ & 48.2 & 384 & A & [1987STE/MAL, 1947STU] \\
\hline \multirow[t]{3}{*}{$\mathrm{CClFO}$} & {$[353-49-1]$} & \multicolumn{5}{|c|}{ carbonic chloride fluoride } \\
\hline & $\mathrm{V}$ & $(165-211)$ & 22.7 & 196 & A & [1987STE/MAL, 1964FIS/BUC] \\
\hline & $\mathrm{V}$ & $(157-227)$ & 22.0 & 192 & & [1948EME/WOO] \\
\hline \multirow[t]{2}{*}{$\mathrm{CClF}_{2} \mathrm{NO}$} & [16847-30-6] & \multicolumn{5}{|c|}{ difluorocarbamoyl chloride } \\
\hline & $\mathrm{V}$ & $(189-234)$ & 25.8 & 219 & A & [1987STE/MAL, 1967FRA/SHR] \\
\hline \multirow[t]{9}{*}{$\mathrm{CClF}_{3}$} & {$[75-72-9]$} & \multicolumn{5}{|c|}{ chlorotrifluoromethane } \\
\hline & $\mathrm{V}$ & $(268-302)$ & 16.0 & 283 & A & [1987STE/MAL] \\
\hline & $\mathrm{V}$ & $(133-185)$ & 17.0 & 170 & A & [1987STE/MAL] \\
\hline & $\mathrm{V}$ & $(184-246)$ & 15.7 & 231 & A & [1987STE/MAL] \\
\hline & $\mathrm{V}$ & $(243-271)$ & 15.7 & 257 & A & [1987STE/MAL] \\
\hline & $\mathrm{V}$ & $(145-192)$ & 16.8 & 177 & A & [1987STE/MAL, 1979KUD/KUD] \\
\hline & $\mathrm{V}$ & $(124-191)$ & 17.1 & 177 & A & [1947STU] \\
\hline & $\mathrm{V}$ & $(134-298)$ & NA & & & [1941RIE] \\
\hline & $\mathrm{V}$ & $(161-192)$ & 16.4 & 176 & & [1933THO/BUR] \\
\hline \multirow[t]{3}{*}{$\mathrm{CClF}_{3} \mathrm{O}$} & {$[22082-78-6]$} & \multicolumn{5}{|c|}{ trifluoromethyl hypochlorite } \\
\hline & $\mathrm{V}$ & $(160-226)$ & 21.2 & 211 & A & [1987STE/MAL, 1969SCH/MAY] \\
\hline & $\mathrm{V}$ & $(142-219)$ & 19.6 & 204 & A & [1987STE/MAL, 1969GOU/AND] \\
\hline \multirow[t]{2}{*}{$\mathrm{CClF}_{3} \mathrm{O}_{2}$} & {$[32755-26-3]$} & \multicolumn{5}{|c|}{ peroxyhypochlorous acid, trifluoromethyl ester } \\
\hline & V & $(163-296)$ & 23.4 & 281 & A & [1987STE/MAL, 1971RAT/HAR] \\
\hline \multirow[t]{3}{*}{$\mathrm{CClF}_{3} \mathrm{O}_{3} \mathrm{~S}$} & {$[6069-31-4]$} & \multicolumn{5}{|c|}{ fluorosulfuric acid, chlorodifluoromethyl ester } \\
\hline & $\mathrm{V}$ & (227-309) & 32.1 & 243 & & [1999DYK/SVO] \\
\hline & $\mathrm{V}$ & $(228-310)$ & 34.6 & 243 & A & [1987STE/MAL, 1966DES/CAD] \\
\hline \multirow[t]{2}{*}{$\mathrm{CClF}_{3} \mathrm{~S}$} & {$[421-17-0]$} & \multicolumn{5}{|c|}{ trifluoromethanesulfenyl chloride } \\
\hline & $\mathrm{V}$ & $(247-272)$ & 24.5 & 260 & A & [1987STE/MAL, 1999DYK/SVO, 1953HAS/KID] \\
\hline \multirow[t]{2}{*}{$\mathrm{CClF}_{4} \mathrm{~N}$} & {$[13880-71-2]$} & difluoro(difluoroch & loromethyl)amine & & & \\
\hline & $\mathrm{V}$ & $(209-277)$ & 26.6 & 262 & A & [1987STE/MAL] \\
\hline $\mathrm{CClF}_{4} \mathrm{NO}_{2} \mathrm{~S}$ & [19419-95-5] & chloro(trifluorome & hyl) sulfamoyl fluorid & & & \\
\hline & $\mathrm{V}$ & $(253-288)$ & 28.8 & 273 & A & [1987STE/MAL, 1999DYK/SVO, 1968ROE] \\
\hline $\mathrm{CClF}_{4} \mathrm{NO}_{12} \mathrm{~S}_{4}$ & {$[53684-03-0]$} & fluorosulfuric acid & bis [[(fluorosulfonyl)o & xy]amino] & loromethy & e ester \\
\hline & $\mathrm{V}$ & & 42.6 & 424 & & [1975KIR/LAS] \\
\hline $\mathrm{CClF}_{7} \mathrm{~S}$ & {$[42179-04-4]$} & chlorotetrafluoro ( & rifluoromethyl) sulfur & & & \\
\hline & $\mathrm{V}$ & $(293-353)$ & 25.9 & 323 & & [1999DYK/SVO, 1973ABE/SHR2] \\
\hline $\mathrm{CClF}_{7} \mathrm{O}_{3} \mathrm{~S}_{2}$ & {$[81439-33-0]$} & cis-chlorotetrafluo & o(trifluoromethanesul & ano)sulfur & & \\
\hline & $\mathrm{V}$ & & 39.3 & & & [1982JOH/KAT] \\
\hline $\mathrm{CClN}$ & [506-77-4] & cyanogen chloride & & & & \\
\hline & FUS & & 11.38 & 266.3 & & [1947DOU/WIN] \\
\hline & SUB & $(196-259)$ & 35.7 & 228 & A & [1947STU, 1987STE/MAL] \\
\hline & $\mathrm{V}$ & (196-286) & 32.2 & 271 & & [1947STU] \\
\hline & $\mathrm{V}$ & & 26.8 & & & [1947DOU/WIN] \\
\hline $\mathrm{CCl}_{2} \mathrm{FNO}$ & [32751-02-3] & dichlorocarbamic & uoride & & & \\
\hline & $\mathrm{V}$ & & 40.7 & & & [1972DEM/SHR] \\
\hline $\mathrm{CCl}_{2} \mathrm{~F}_{2}$ & {$[75-71-8]$} & dichlorodifluorom & thane & & & \\
\hline & $\mathrm{V}$ & $(282-345)$ & 20.0 & 297 & A & [1987STE/MAL] \\
\hline & $\mathrm{V}$ & $(173-244)$ & 21.4 & 229 & A & [1987STE/MAL] \\
\hline & $\mathrm{V}$ & $(173-240)$ & 21.6 & 225 & A & [1987STE/MAL] \\
\hline & $\mathrm{V}$ & $(236-285)$ & 20.4 & 270 & A & [1987STE/MAL] \\
\hline & $\mathrm{V}$ & $(341-385)$ & 20.5 & 356 & A & [1987STE/MAL] \\
\hline & $\mathrm{V}$ & $(172-279)$ & 22.9 & 187 & & [1979KUD/KUD] \\
\hline & $\mathrm{V}$ & $(154-243)$ & 21.5 & 228 & & [1947STU] \\
\hline & $\mathrm{V}$ & & 20.1 & 243 & & [1931BUF/FLE] \\
\hline $\mathrm{CCl}_{2} \mathrm{~F}_{3} \mathrm{~N}$ & [24618-60-8] & $N, N$-difluoro-1,1-d & chloro-1-fluoromethy & amine & & \\
\hline & $\mathrm{V}$ & (209-277) & 27.0 & 262 & I & [1970ZAB/SHR] \\
\hline
\end{tabular}


TABLE 4. Phase change enthalpies of $\mathrm{C}_{1}$ organic compounds-Continued

\begin{tabular}{|c|c|c|c|c|c|c|}
\hline \multirow[b]{2}{*}{ Molecular formula } & \multirow{2}{*}{$\begin{array}{c}\text { CAS reg. no. } \\
\text { Transition }\end{array}$} & \multicolumn{5}{|l|}{ Compound } \\
\hline & & Temp. range $(\mathrm{K})$ & $\Delta_{\text {trans }} H_{\mathrm{m}}\left(\mathrm{kJ} \mathrm{mol}^{-1}\right)$ & $T_{\mathrm{m}}(\mathrm{K})$ & Method & Reference \\
\hline \multirow[t]{3}{*}{$\mathrm{CCl}_{2} \mathrm{~F}_{3} \mathrm{~N}$} & {$[13880-73-4]$} & \multicolumn{5}{|c|}{$N, N$-dichloro-1,1,1-trifluoromethylamine } \\
\hline & $\mathrm{V}$ & $(226-291)$ & 25.8 & 276 & A & [1987STE/MAL] \\
\hline & $\mathrm{V}$ & & 25.5 & & & [1967HYN/BIS] \\
\hline \multirow[t]{2}{*}{$\mathrm{CCl}_{2} \mathrm{~F}_{3} \mathrm{~N}$} & {$[33757-11-8]$} & \multicolumn{5}{|c|}{$N, 1$-dichloro- $N, 1,1$-trifluoromethylamine } \\
\hline & $\mathrm{V}$ & $(226-291)$ & 26.4 & 258 & & [1971SWI/ZAB] \\
\hline \multirow[t]{3}{*}{$\mathrm{CCl}_{2} \mathrm{~F}_{3} \mathrm{NS}$} & {$[10564-47-3]$} & \multicolumn{5}{|c|}{ (trifluoromethyl)imidosulfurous dichloride } \\
\hline & $\mathrm{V}$ & $(284-344)$ & 35.4 & 298 & & [1999DYK/SVO, 1966LUS] \\
\hline & $\mathrm{V}$ & $(283-362)$ & 33.7 & 298 & A & [1987STE/MAL] \\
\hline \multirow[t]{2}{*}{$\mathrm{CCl}_{2} \mathrm{~F}_{3} \mathrm{P}$} & [421-58-9] & \multicolumn{5}{|c|}{ (trifluoromethyl)dichlorophosphine } \\
\hline & V & $(208-276)$ & 29.2 & 260 & & [1964PET/BUR] \\
\hline \multirow[t]{2}{*}{$\mathrm{CCl}_{2} \mathrm{~F}_{3} \mathrm{PS}$} & {$[18799-78-5]$} & \multicolumn{5}{|c|}{ dichloro(trifluoromethylthio) phosphine } \\
\hline & V & $(293-363)$ & 31.7 & 308 & A & [1987STE/MAL, 1999DYK/SVO, 1960EME/PUG] \\
\hline \multirow[t]{14}{*}{$\mathrm{CCl}_{2} \mathrm{O}$} & {$[75-44-5]$} & \multicolumn{5}{|l|}{ phosgene } \\
\hline & FUS (I) & & 5.74 & 145.4 & & \\
\hline & FUS (II) & & 5.59 & 142.1 & & \\
\hline & FUS (III) & & 4.73 & 139.2 & & [1960GIA/OTT] \\
\hline & FUS & & 5.73 & 145.3 & & [1948GIA/JON] \\
\hline & $\mathrm{V}$ & $(280-341)$ & 24.5 & 295 & A & [1987STE/MAL] \\
\hline & $\mathrm{V}$ & $(240-281)$ & 25.7 & 266 & A & [1987STE/MAL] \\
\hline & $\mathrm{V}$ & $(338-410)$ & 24.5 & 353 & A & [1987STE/MAL] \\
\hline & $\mathrm{V}$ & $(406-455)$ & 24.4 & 421 & A & [1987STE/MAL] \\
\hline & V & $(253-293)$ & 25.2 & 273 & & [1970FET/EFR] \\
\hline & $\mathrm{V}$ & $(215-248)$ & 27.0 & 233 & & [1948GIA/JON] \\
\hline & $\mathrm{V}$ & & 24.4 & 281 & $\mathrm{C}$ & [1948GIA/JON] \\
\hline & $\mathrm{V}$ & $(180-273)$ & 25.8 & 258 & & [1947STU] \\
\hline & $\mathrm{V}$ & & 23.0 & 277 & & {$[1920 \mathrm{ATK} / \mathrm{HEY}]$} \\
\hline \multirow[t]{11}{*}{$\mathrm{CCl}_{3} \mathrm{~F}$} & {$[75-69-4]$} & \multicolumn{5}{|c|}{ trichlorofluoromethane } \\
\hline & FUS & & 6.9 & 162.7 & & [1996DOM/HEA, 1941OSB/GAR] \\
\hline & $\mathrm{V}$ & $(213-301)$ & 28.5 & 228 & A & [1987STE/MAL] \\
\hline & $\mathrm{V}$ & $(213-249)$ & 28.2 & 234 & A & [1987STE/MAL] \\
\hline & $\mathrm{V}$ & $(295-363)$ & 25.6 & 310 & A & [1987STE/MAL] \\
\hline & $\mathrm{V}$ & $(357-429)$ & 24.7 & 372 & A & [1987STE/MAL] \\
\hline & V & $(424-468)$ & 25.1 & 439 & A & [1987STE/MAL] \\
\hline & V & $(237-293)$ & 27.3 & 251 & & [1979KUD/KUD] \\
\hline & $\mathrm{V}$ & $(237-293)$ & 27.1 & 276 & & [1941OSB/GAR] \\
\hline & $\mathrm{V}$ & & 25.2 & 290 & $\mathrm{C}$ & [1941OSB/GAR] \\
\hline & $\mathrm{V}$ & $(244-334)$ & 26.4 & 259 & & [1940BEN/MCH] \\
\hline \multirow[t]{2}{*}{$\mathrm{CCl}_{3} \mathrm{~F}_{2} \mathrm{~N}$} & {$[24708-52-9]$} & $N, N$-difluoro-1,1,1 & trichloromethylamine & & & \\
\hline & $\mathrm{V}$ & $(252-325)$ & 33.4 & 267 & I & [1987STE/MAL, 1970ZAB/SHR] \\
\hline $\mathrm{CCl}_{3} \mathrm{~F}_{2} \mathrm{~N}$ & {$[33757-10-7]$} & $N, 1,1$-trichloro- $N$ & 1-difluoromethylamine & & & \\
\hline & $\mathrm{V}$ & $(273-319)$ & 27.8 & 296 & & [1971SWI/ZAB] \\
\hline $\mathrm{CCl}_{3} \mathrm{~F}_{2} \mathrm{P}$ & {$[1112-03-4]$} & difluoro(trichlorom & ethyl) phosphine & & & \\
\hline & SUB & $(264-283)$ & 36.8 & 274 & $\mathrm{~A}, \mathrm{~T}$ & [1987STE/MAL, 1964NIX] \\
\hline & $\mathrm{V}$ & $(289-313)$ & 32.5 & 301 & $\mathrm{~A}, \mathrm{~T}$ & [1987STE/MAL, 1964NIX] \\
\hline $\mathrm{CCl}_{3} \mathrm{~F}_{4} \mathrm{P}$ & {$[1184-80-1]$} & trichloromethyl tet & afluorophosphorane & & & \\
\hline & V & $(257-300)$ & 10.4 & & & {$[1965 \mathrm{NIX}]$} \\
\hline $\mathrm{CCl}_{3} \mathrm{NO}$ & {$[3711-49-7]$} & trichloronitrosome & hane & & & \\
\hline & $\mathrm{V}$ & $(253-333)$ & 32.4 & 268 & A & [1987STE/MAL, 1968BRI/PRI] \\
\hline $\mathrm{CCl}_{3} \mathrm{NO}_{2}$ & {$[76-06-2]$} & trichloronitromethe & ne (chloropicrin) & & & \\
\hline & V & $(273-333)$ & 39.3 & 288 & A & [1987STE/MAL, 1948RED/CHA5] \\
\hline & V & $(301-449)$ & 38.5 & 316 & A & [1987STE/MAL, 1970DYK] \\
\hline & $\mathrm{V}$ & $(247-385)$ & 40.0 & 262 & & [1947STU] \\
\hline & $\mathrm{V}$ & $(299-328)$ & 39.3 & & & [1947GOU/HOL] \\
\hline & $\mathrm{V}$ & $(253-303)$ & 39.2 & 283 & & [1920BAX/BEZ] \\
\hline
\end{tabular}

$\mathrm{CCl}_{4}$

[56-23-5] carbon tetrachloride 
TABLE 4. Phase change enthalpies of $\mathrm{C}_{1}$ organic compounds-Continued

\begin{tabular}{|c|c|c|c|c|c|c|}
\hline \multirow[b]{2}{*}{ Molecular formula } & \multirow{2}{*}{$\begin{array}{l}\text { CAS reg. no. } \\
\text { Transition }\end{array}$} & \multicolumn{5}{|l|}{ Compound } \\
\hline & & Temp. range $(\mathrm{K})$ & $\Delta_{\text {trans }} H_{\mathrm{m}}\left(\mathrm{kJ} \mathrm{mol}^{-1}\right)$ & $T_{\mathrm{m}}(\mathrm{K})$ & Method & Reference \\
\hline & TRS & & 4.68 & 225.9 & & \\
\hline & FUS & & 2.52 & 250.3 & DSC & [2004BAR/PAR, 1999PAR/BAR] \\
\hline & TRS & & 4.63 & 225.7 & & \\
\hline & FUS & & 2.56 & 250.3 & $\mathrm{AC}$ & [1996DOM/HEA, 1976MOR/RIC] \\
\hline & TRS & & 4.63 & 225.3 & & \\
\hline & FUS & & 2.54 & 250.3 & & [1974VAN/OON] \\
\hline & FUS & & 2.56 & 250.3 & $\mathrm{AC}$ & [1972ARE/VAN] \\
\hline & TRS & & 4.48 & 226.6 & & \\
\hline & FUS & & 2.49 & 247.8 & DSC & [1970SIL/RUD] \\
\hline & TRS & & 4.59 & 225.8 & & \\
\hline & FUS & & 2.52 & 250.2 & & [1949STA/GUP] \\
\hline & FUS & & 2.46 & 250.3 & $\mathrm{AC}$ & [1947AST/FIN] \\
\hline & TRS & & 4.58 & 225.4 & & \\
\hline & FUS & $(17-300)$ & 2.52 & 250.3 & & [1996DOM/HEA, 1944HIC/HOO] \\
\hline & TRS & & 4.60 & 225.6 & & \\
\hline & FUS & & 2.43 & 250.4 & & [1996DOM/HEA, 1937STU] \\
\hline & TRS & & 4.52 & 225.5 & & \\
\hline & FUS & & 2.41 & 250.3 & $\mathrm{C}$ & [1934JOH/LON] \\
\hline & TRS & & 4.60 & 224.6 & & \\
\hline & FUS & & 2.69 & 249.0 & & [1996DOM/HEA, 1922LAT] \\
\hline & SUB & & 43.3 & 226 & $\mathrm{~B}$ & [1963BON] \\
\hline & SUB & $(209-225)$ & 38.8 & 217 & & [1960JON, 1948NIT/SEK] \\
\hline & SUB & $(227-248)$ & 37.9 & & & [1948NIT/SEK] \\
\hline & $\mathrm{V}$ & $(349-416)$ & 30.4 & 364 & A & [1987STE/MAL] \\
\hline & $\mathrm{V}$ & $(412-497)$ & 29.2 & 427 & A & [1987STE/MAL] \\
\hline & $\mathrm{V}$ & $(494-555)$ & 30.6 & 509 & A & [1987STE/MAL] \\
\hline & $\mathrm{V}$ & & 32.4 & 298 & $\mathrm{C}$ & [1980MAJ/SVA] \\
\hline & $\mathrm{V}$ & & 31.7 & 313 & $\mathrm{C}$ & [1980MAJ/SVA] \\
\hline & $\mathrm{V}$ & & 30.9 & 328 & $\mathrm{C}$ & [1980MAJ/SVA] \\
\hline & $\mathrm{V}$ & & 30.1 & 343 & $\mathrm{C}$ & [1980MAJ/SVA] \\
\hline & $\mathrm{V}$ & & 29.4 & 358 & $\mathrm{C}$ & [1980MAJ/SVA] \\
\hline & $\mathrm{V}$ & & $32.5 \pm 0.1$ & 298 & $\mathrm{C}$ & [1973KON] \\
\hline & $\mathrm{V}$ & $(262-349)$ & 33.7 & 277 & $\mathrm{~A}, \mathrm{~EB}$ & [1987STE/MAL, 1972BOU/AIM] \\
\hline & $\mathrm{V}$ & & $32.4 \pm 0.1$ & 298 & $\mathrm{C}$ & {$[1966 \mathrm{WAD} 2]$} \\
\hline & $\mathrm{V}$ & $(293-351)$ & 32.3 & 308 & & [1959HIL/MCD] \\
\hline & $\mathrm{V}$ & $(313-338)$ & 31.7 & 325 & & [1953BAR/BRO] \\
\hline \multirow[t]{3}{*}{$\mathrm{CCl}_{4} \mathrm{O}_{2} \mathrm{~S}$} & {$[2547-61-7]$} & \multicolumn{5}{|c|}{ trichloromethanesulfonyl chloride } \\
\hline & TRS & & 7.1 & 227.4 & & \\
\hline & FUS & & 7.46 & 418.5 & DSC & [1994DOU/FUE] \\
\hline \multirow[t]{2}{*}{$\mathrm{CFIO}$} & [1495-48-3] & \multicolumn{5}{|c|}{ carbonyl fluoride iodide } \\
\hline & $\mathrm{V}$ & $(230-292)$ & 26.1 & 277 & A & [1987STE/MAL] \\
\hline \multirow[t]{5}{*}{$\mathrm{CFN}$} & {$[1495-50-7]$} & cyanogen fluoride & & & & \\
\hline & SUB & $(147-191)$ & 28.9 & 176 & A, I & [1987STE/MAL, 1964FAW/LIP] \\
\hline & SUB & $(139-192)$ & 24.4 & 166 & & [1947STU] \\
\hline & SUB & $(133-203)$ & 29.3 & 168 & & {$[1931 \mathrm{COS}]$} \\
\hline & $\mathrm{V}$ & $(201-227)$ & 22.4 & 214 & A, I & [1987STE/MAL, 1964FAW/LIP, 1970DYK] \\
\hline \multirow[t]{2}{*}{$\mathrm{CFNO}_{3} \mathrm{~S}$} & {$[1495-51-8]$} & \multicolumn{5}{|c|}{ sulfuryl fluoride isocyanate } \\
\hline & $\mathrm{V}$ & $(294-335)$ & 36.5 & 309 & A & [1987STE/MAL, 1999DYK/SVO] \\
\hline \multirow[t]{2}{*}{$\mathrm{CFNO}_{6} \mathrm{~S}_{2}$} & [27931-74-4] & \multicolumn{5}{|c|}{ pyrosulfuryl fluoride isocyanate } \\
\hline & $\mathrm{V}$ & $(330-405)$ & 40.9 & 345 & A & [1987STE/MAL, 1999DYK/SVO, 1970NOF] \\
\hline \multirow[t]{2}{*}{$\mathrm{CFN}_{3} \mathrm{O}_{6}$} & {$[1840-42-2]$} & \multicolumn{5}{|c|}{ fluorotrinitromethane } \\
\hline & $\mathrm{V}$ & $(274-358)$ & 34.2 & 289 & $\mathrm{~A}, \mathrm{~T}$ & [1987STE/MAL, 1966ZIM/ROB] \\
\hline \multirow[t]{2}{*}{$\mathrm{CF}_{2} \mathrm{~N}_{2}$} & {$[7127-18-6]$} & \multicolumn{5}{|l|}{ difluorocyanamide } \\
\hline & $\mathrm{V}$ & $(179-198)$ & 20.6 & 189 & A & [1987STE/MAL, 1966MEY/FRA] \\
\hline \multirow[t]{2}{*}{$\mathrm{CF}_{2} \mathrm{~N}_{2} \mathrm{OS}$} & {$[19073-57-5]$} & cyanoimidosulfury & fluoride & & & \\
\hline & $\mathrm{V}$ & $(262-354)$ & 37.2 & 277 & A & [1987STE/MAL, 1999DYK/SVO, 1967GLE/BIE3] \\
\hline
\end{tabular}


TABLE 4. Phase change enthalpies of $\mathrm{C}_{1}$ organic compounds-Continued

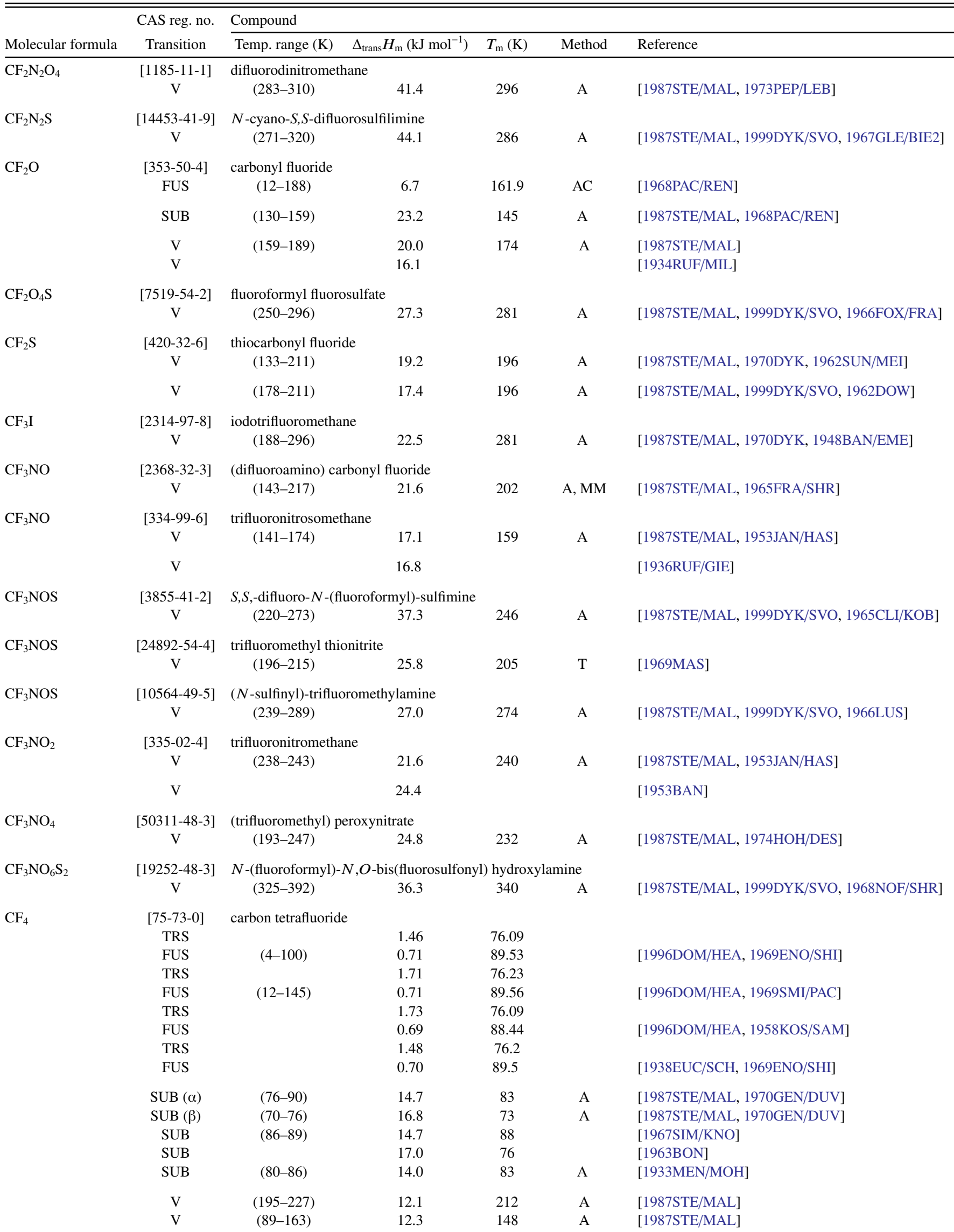


TABLE 4. Phase change enthalpies of $\mathrm{C}_{1}$ organic compounds-Continued

\begin{tabular}{|c|c|c|c|c|c|c|}
\hline \multirow[b]{2}{*}{ Molecular formula } & \multirow{2}{*}{$\begin{array}{l}\text { CAS reg. no. } \\
\text { Transition }\end{array}$} & \multicolumn{5}{|l|}{ Compound } \\
\hline & & Temp. range $(\mathrm{K})$ & $\Delta_{\text {trans }} H_{\mathrm{m}}\left(\mathrm{kJ} \mathrm{mol}^{-1}\right)$ & $T_{\mathrm{m}}(\mathrm{K})$ & Method & Reference \\
\hline & $\mathrm{V}$ & $(160-197)$ & 11.9 & 182 & A & [1987STE/MAL] \\
\hline & $\mathrm{V}$ & $(116-146)$ & 12.4 & 131 & & [1969SMI/PAC] \\
\hline & $\mathrm{V}$ & $(93-146)$ & 12.8 & 131 & & [1933MEN/MOH, 1987STE/MAL] \\
\hline & $\mathrm{V}$ & & 12.3 & & & [1933RUF/BRE] \\
\hline \multirow[t]{2}{*}{$\mathrm{CF}_{4} \mathrm{~N}_{2} \mathrm{O}$} & [815-10-1] & fluoro(trifluoromet & yl) diimidoxide & & & \\
\hline & $\mathrm{V}$ & $(233-267)$ & 27.7 & 252 & A & [1987STE/MAL] \\
\hline \multirow[t]{2}{*}{$\mathrm{CF}_{4} \mathrm{~N}_{2} \mathrm{O}_{3} \mathrm{~S}_{2}$} & {$[25523-80-2]$} & carbonyl bis(imido & ulfuryl fluoride) & & & \\
\hline & $\mathrm{V}$ & $(316-331)$ & 41.3 & 323 & A & $\begin{array}{l}\text { [1987STE/MAL, 1999DYK/SVO, } \\
\text { 1969GLE/MEW2] }\end{array}$ \\
\hline \multirow{2}{*}{$\mathrm{CF}_{4} \mathrm{O}$} & {$[373-91-1]$} & hypofluorous acid & ifluoromethyl ester & & & \\
\hline & $\mathrm{V}$ & $(153-194)$ & 15.5 & 179 & A & [1987STE/MAL, 1948KEL/CAD] \\
\hline \multicolumn{7}{|c|}{$\begin{array}{l}\text { [Note: The table in [1948KEL/CAD] gives the temperatures in }{ }^{\circ} \mathrm{C} \text {; however, all of the equations and graphs in the article suggest that the temperature } \\
\text { should be in Kelvin. We have assumed that the tabulated temperatures are in Kelvin; the results closely correspond to the entry in [1987STE/MAL].] }\end{array}$} \\
\hline \multirow[t]{2}{*}{$\mathrm{CF}_{4} \mathrm{OS}$} & {$[812-12-4]$} & trifluoromethyl sul & nyl fluoride & & & \\
\hline & $\mathrm{V}$ & $(204-271)$ & 22.7 & 256 & $\mathrm{~A}, \mathrm{I}$ & $\begin{array}{l}\text { [1987STE/MAL, 1968RAT/SHR, 1970DYK, } \\
\text { 1999DYK/SVO] }\end{array}$ \\
\hline \multirow[t]{2}{*}{$\mathrm{CF}_{4} \mathrm{O}_{2}$} & [34511-13-2] & hydroperoxyfluoric & acid trifluoromethyl e & ster & & \\
\hline & $\mathrm{V}$ & $(156-203)$ & 18.7 & 188 & A & [1987STE/MAL, 1972DES] \\
\hline \multirow[t]{2}{*}{$\mathrm{CF}_{4} \mathrm{O}_{2} \mathrm{~S}$} & {$[335-05-7]$} & trifluoromethane st & fonyl fluoride & & & \\
\hline & V & $(226-249)$ & 23.4 & 237 & A & [1987STE/MAL, 1999DYK/SVO, 1956GRA/HAS] \\
\hline \multirow[t]{2}{*}{$\mathrm{CF}_{4} \mathrm{O}_{3} \mathrm{~S}$} & {$[926-08-9]$} & trifluoromethyl fluc & rosulfonate & & & \\
\hline & $\mathrm{V}$ & $(194-269)$ & 25.6 & 231 & & [1960VAN/CAD] \\
\hline \multirow[t]{2}{*}{$\mathrm{CF}_{4} \mathrm{O}_{4} \mathrm{~S}$} & {$[13990-10-8]$} & trifluoromethylper & xyfluorosulfonate & & & \\
\hline & $\mathrm{V}$ & $(233-286)$ & 27.7 & 259 & & [1960VAN/CAD] \\
\hline \multirow[t]{2}{*}{$\mathrm{CF}_{4} \mathrm{O}_{5} \mathrm{~S}_{2}$} & {$[21595-44-8]$} & fluorosulfonic acid & trifluoromethane sulf & nic acid anl & dride & \\
\hline & V & $(308-338)$ & 32.9 & 323 & A & [1987STE/MAL, 1999DYK/SVO, 1968NOF] \\
\hline \multirow[t]{2}{*}{$\mathrm{CF}_{4} \mathrm{O}_{6} \mathrm{~S}_{2}$} & {$[6123-47-3]$} & trifluoromethyl fluc & rodisulfate & & & \\
\hline & $\mathrm{V}$ & $(292-351)$ & 34.4 & 321 & & [1960VAN/CAD] \\
\hline \multirow[t]{2}{*}{$\mathrm{CF}_{5} \mathrm{~N}$} & $\begin{array}{l}{[335-01-3]} \\
\text { SUB }\end{array}$ & $\begin{array}{l}\text { pentafluoromethyla } \\
\qquad(128-141)\end{array}$ & mine & 135 & & [1987STE/MAL, 1951COA/HAR] \\
\hline & V & $(151-198)$ & 17.3 & 174 & & [1951COA/HAR] \\
\hline $\mathrm{CF}_{5} \mathrm{NO}$ & $\begin{array}{l}{[4217-93-0]} \\
\mathrm{V}\end{array}$ & $\begin{array}{l}\text { pentafluoromethox } \\
\qquad(167-210)\end{array}$ & 18.5 & 195 & A & [1987STE/MAL, 1965SHR/DUN] \\
\hline $\mathrm{CF}_{5} \mathrm{OPS}$ & $\begin{array}{c}{[52752-66-6]} \\
\mathrm{V} \\
\mathrm{V}\end{array}$ & $\begin{array}{l}\text { phosphorothionic c } \\
\qquad(293-353)\end{array}$ & $\begin{array}{l}\text { fluoride, } S \text {-trifluorom } \\
\qquad \begin{array}{c}23.1 \\
23.0\end{array}\end{array}$ & $\begin{array}{l}\text { ethyl ester } \\
323\end{array}$ & & $\begin{array}{l}\text { [1999DYK/SVO, 1974SPR/SHR] } \\
\text { [1974SPR/SHR] }\end{array}$ \\
\hline $\mathrm{CF}_{5} \mathrm{O}_{3} \mathrm{P}$ & $\begin{array}{c}{[39125-42-3]} \\
\mathrm{V}\end{array}$ & $\begin{array}{l}\text { trifluoromethoxypl } \\
\qquad(225-264)\end{array}$ & $\begin{array}{l}\text { osphoryl difluoride } \\
27.4\end{array}$ & 245 & & [1973BER/DES] \\
\hline \multirow[t]{2}{*}{$\mathrm{CF}_{5} \mathrm{O}_{3} \mathrm{P}$} & {$[39125-42-3]$} & difluoroperoxypho & phoric acid trifluorom & ethyl ester & & \\
\hline & $\mathrm{V}$ & $(241-280)$ & 32.0 & 265 & A & [1987STE/MAL, 1973BER/DES] \\
\hline \multirow[t]{3}{*}{$\mathrm{CF}_{5} \mathrm{PS}$} & {$[52752-65-5]$} & trifluoromethyl thic & difluorophosphine & & & \\
\hline & $\mathrm{V}$ & $(293-353)$ & 22.3 & 323 & & [1999DYK/SVO, 1974SPR/SHR] \\
\hline & $\mathrm{V}$ & & 24.3 & & & [1974SPR/SHR] \\
\hline \multirow[t]{2}{*}{$\mathrm{CF}_{6} \mathrm{~N}_{2} \mathrm{O}_{2} \mathrm{~S}_{2}$} & {$[20094-83-1]$} & $N, N^{\prime}$-(difluorometh & lene) bisimidosulfury & 1 fluoride & & \\
\hline & $\mathrm{V}$ & $(283-308)$ & 36.0 & 295 & & [1968GLE/VON] \\
\hline \multirow[t]{2}{*}{$\mathrm{CF}_{6} \mathrm{~N}_{2} \mathrm{~S}_{2}$} & {$[17686-45-2]$} & difluoromethane bi & $(S, S$-difluorosulfilin & ine) & & \\
\hline & V & $(230-313)$ & 36.0 & 245 & A & [1987STE/MAL, 1999DYK/SVO, 1967GLE/BIE2] \\
\hline \multirow[t]{2}{*}{$\mathrm{CF}_{8} \mathrm{OS}$} & {$[1873-23-0]$} & pentafluoro (trifluo & omethoxy) sulfur & & & \\
\hline & $\mathrm{V}$ & $(217-262)$ & 24.4 & 247 & A & [1987STE/MAL, 1964DUN/CAD] \\
\hline $\mathrm{CF}_{8} \mathrm{O}_{3} \mathrm{~S}_{2}$ & $\begin{array}{c}{[81439-27-2]} \\
\mathrm{V}\end{array}$ & pentafluoro(trifluor & $\begin{array}{l}\text { methylsulfonato)sulf } \\
26.8\end{array}$ & & & [1982JOH/KAT] \\
\hline
\end{tabular}


TABLE 4. Phase change enthalpies of $\mathrm{C}_{1}$ organic compounds-Continued

\begin{tabular}{|c|c|c|c|c|c|c|}
\hline \multirow[b]{2}{*}{ Molecular formula } & \multirow{2}{*}{$\begin{array}{c}\text { CAS reg. no. } \\
\text { Transition }\end{array}$} & \multicolumn{5}{|l|}{ Compound } \\
\hline & & Temp. range $(\mathrm{K})$ & $\Delta_{\text {trans }} H_{\mathrm{m}}\left(\mathrm{kJ} \mathrm{mol}^{-1}\right)$ & $T_{\mathrm{m}}(\mathrm{K})$ & Method & Reference \\
\hline \multirow[t]{3}{*}{$\mathrm{CF}_{8} \mathrm{~S}$} & [373-80-8] & \multicolumn{5}{|c|}{ trifluoro(pentafluorothio)methane } \\
\hline & $\mathrm{V}$ & $(223-252)$ & 20.2 & 253 & I & [2001KUL/DES] \\
\hline & $\mathrm{V}$ & $(205-262)$ & 23.8 & 247 & A & [1987STE/MAL, 1999DYK/SVO, 1950SIL/CAD] \\
\hline \multirow[t]{2}{*}{$\mathrm{CF}_{9} \mathrm{NOS}$} & {$[1840-45-5]$} & \multicolumn{5}{|c|}{ tetrafluoro(difluoroamino)(trifluoromethoxy) sulfur } \\
\hline & $\mathrm{V}$ & $(257-298)$ & 28.7 & 272 & A & [1987STE/MAL, 1964DUN/CAD2] \\
\hline \multirow{2}{*}{$\mathrm{CF}_{10} \mathrm{O}_{5} \mathrm{~S}_{2}$} & {$[60672-59-5]$} & \multicolumn{5}{|c|}{ [ $\mu$-(carbono)diperoxato]decafluorodisulfur } \\
\hline & $\mathrm{V}$ & & 38.1 & & & {$[1976 \mathrm{HOP} / \mathrm{DES}]$} \\
\hline \multirow[t]{6}{*}{$\mathrm{CIN}$} & {$[506-78-5]$} & cyanogen iodide & & & & \\
\hline & SUB & $(337-426)$ & 59.9 & 352 & GSM & [1987STE/MAL, 1943KET/KRU] \\
\hline & SUB & $(298-414)$ & 58.6 & 356 & A & [1947STU] \\
\hline & SUB & $(337-426)$ & $59.8 \pm 0.4$ & & GSM & [1943KET/KRU, 1970COX/PIL] \\
\hline & SUB & $(278-374)$ & 58.3 & 326 & & [1933YOS/STO] \\
\hline & $\mathrm{V}$ & $(419-426)$ & 40.0 & 423 & A & [1987STE/MAL, 1943KET/KRU] \\
\hline \multirow[t]{5}{*}{$\mathrm{CN}_{4} \mathrm{O}_{8}$} & [509-14-8] & tetranitromethane & & & & \\
\hline & SUB & $(255-286)$ & 47.4 & 271 & & [1987STE/MAL, 1941SEK/NIT] \\
\hline & $\mathrm{V}$ & $(286-373)$ & 43.1 & 301 & A & [1987STE/MAL] \\
\hline & $\mathrm{V}$ & $(313-373)$ & 42.9 & 328 & A & [1987STE/MAL, 1984BOU/FRI, 1952EDW] \\
\hline & $\mathrm{V}$ & $(273-313)$ & 46.6 & 288 & & [1987STE/MAL, 1984BOU/FRI, 1949NIC] \\
\hline \multirow[t]{8}{*}{$\mathrm{CO}$} & {$[630-08-0]$} & carbon monoxide & & & & \\
\hline & SUB & $(54-61)$ & 7.6 & 58 & A & [1987STE/MAL] \\
\hline & SUB & $(51-68)$ & 8.1 & 60 & A & [1947STU] \\
\hline & SUB & $(57-68)$ & 7.9 & 62 & A & {$[1931 \mathrm{CRO} / \mathrm{BIJ}]$} \\
\hline & $\mathrm{V}$ & $(68-108)$ & 6.0 & 93 & A & [1987STE/MAL] \\
\hline & $\mathrm{V}$ & $(93-132)$ & 6.2 & 112 & & [1952MIC/WAS] \\
\hline & V & $(69-83)$ & 6.0 & 81 & & [1932CLA/GIA] \\
\hline & $\mathrm{V}$ & & 6.0 & 81 & $\mathrm{C}$ & [1932CLA/GIA] \\
\hline \multirow[t]{6}{*}{ COS } & [463-58-1] & carbonyl sulfide & & & & \\
\hline & $\mathrm{V}$ & $(161-284)$ & 20.4 & 176 & & [1999DYK/SVO] \\
\hline & V & $(284-379)$ & 18.3 & 299 & & [1999DYK/SVO] \\
\hline & $\mathrm{V}$ & $(140-224)$ & 19.5 & 209 & A & [1987STE/MAL] \\
\hline & $\mathrm{V}$ & & $19.0 \pm 0.1$ & 214 & & [1939FRA/CLU] \\
\hline & V & $(162-224)$ & 19.5 & 209 & & [1937KEM/GIA] \\
\hline \multirow[t]{13}{*}{$\mathrm{CO}_{2}$} & [124-38-9] & carbon dioxide & & & & \\
\hline & FUS & & 7.94 & 215.6 & & [1928EUC/HAU] \\
\hline & SUB & $(198-216)$ & 26.1 & 207 & A & [1987STE/MAL] \\
\hline & SUB & (194-216) & 26.1 & 205 & & [1984FER/DEL] \\
\hline & SUB & $(70-102)$ & $27.2 \pm 0.4$ & & LE & [1974BRY/CAZ] \\
\hline & SUB & (179-198) & 25.9 & 188 & & [1956AMB] \\
\hline & SUB & $(139-195)$ & 26.3 & 167 & $\mathrm{~A}$ & [1947STU] \\
\hline & SUB & $(154-196)$ & 25.2 & 195 & & [1937GIA/EGA] \\
\hline & $\mathrm{V}$ & $(273-304)$ & 16.7 & 288 & A & [1987STE/MAL] \\
\hline & $\mathrm{V}$ & $(216-273)$ & 16.4 & 258 & A & [1987STE/MAL] \\
\hline & $\mathrm{V}$ & $(216-243)$ & 16.7 & 231 & & [1984FER/DEL] \\
\hline & $\mathrm{V}$ & $(267-303)$ & 16.5 & 282 & & [1972BOU/AIM] \\
\hline & $\mathrm{V}$ & $(217-276)$ & 16.6 & 247 & & [1950MIC/WAS2] \\
\hline \multirow[t]{10}{*}{$\mathrm{CS}_{2}$} & {$[75-15-0]$} & carbon disulfide & & & & \\
\hline & FUS & & 4.39 & 161.1 & & [1996DOM/HEA, 1937BRO/MAN] \\
\hline & $\mathrm{V}$ & $(255-354)$ & 28.7 & 270 & & [1999DYK/SVO] \\
\hline & $\mathrm{V}$ & $(354-552)$ & 27.1 & 369 & & [1999DYK/SVO] \\
\hline & $\mathrm{V}$ & $(260-353)$ & 28.5 & 275 & A & [1987STE/MAL] \\
\hline & $\mathrm{V}$ & $(338-408)$ & 27.4 & 353 & A & [1987STE/MAL] \\
\hline & $\mathrm{V}$ & $(388-497)$ & 27.0 & 403 & A & [1987STE/MAL] \\
\hline & $\mathrm{V}$ & $(490-533)$ & 28.7 & 505 & A & [1987STE/MAL] \\
\hline & $\mathrm{V}$ & $(255-318)$ & 28.7 & 270 & $\mathrm{~EB}$ & [1972BOU/AIM, 1987STE/MAL] \\
\hline & $\mathrm{V}$ & $(277-353)$ & 28.1 & 292 & $\mathrm{~EB}$ & [1962WAD/SMI] \\
\hline
\end{tabular}


TABLE 4. Phase change enthalpies of $\mathrm{C}_{1}$ organic compounds-Continued

\begin{tabular}{|c|c|c|c|c|c|c|}
\hline \multirow[b]{2}{*}{ Molecular formula } & \multirow{2}{*}{$\begin{array}{l}\text { CAS reg. no. } \\
\text { Transition }\end{array}$} & \multicolumn{5}{|l|}{ Compound } \\
\hline & & Temp. range $(\mathrm{K})$ & $\Delta_{\text {trans }} H_{\mathrm{m}}\left(\mathrm{kJ} \mathrm{mol}^{-1}\right)$ & $T_{\mathrm{m}}(\mathrm{K})$ & Method & Reference \\
\hline & $\mathrm{V}$ & & $28.1 \pm 0.1$ & 282 & $\mathrm{C}$ & [1962WAD/SMI] \\
\hline & $\mathrm{V}$ & & $27.5 \pm 0.1$ & 298 & $\mathrm{C}$ & [1962WAD/SMI] \\
\hline & $\mathrm{V}$ & & $26.7 \pm 0.1$ & 319 & $\mathrm{C}$ & [1962WAD/SMI] \\
\hline & $\mathrm{V}$ & & 27.7 & 298 & & {$[1961 \mathrm{GOO} / \mathrm{LAC}]$} \\
\hline & $\mathrm{V}$ & $(303-358)$ & 27.6 & 318 & & {$[1946 \mathrm{THO}]$} \\
\hline \multirow[t]{3}{*}{$\mathrm{CHBrF}_{2}$} & {$[1511-62-2]$} & bromodifluorometl & tane & & & \\
\hline & $\mathrm{V}$ & $(194-259)$ & 24.0 & 244 & A & [1987STE/MAL] \\
\hline & $\mathrm{V}$ & $(194-288)$ & 24.7 & 209 & & [1979KUD/KUD] \\
\hline \multirow[t]{5}{*}{$\mathrm{CHBr}_{3}$} & {$[75-25-2]$} & tribromomethane & & & & \\
\hline & FUS & & 11.09 & 281.5 & & [1987KAF/DOR] \\
\hline & $\mathrm{V}$ & & $46.1 \pm 0.1$ & 298 & $\mathrm{C}$ & [1972LAY/WAD] \\
\hline & $\mathrm{V}$ & $(320-412)$ & 42.3 & 335 & EB & [1972BOU/AIM, 1979KUD/KUD] \\
\hline & $\mathrm{V}$ & $(303-373)$ & 44.0 & 318 & & [1941KIR/SIT, 1984BOU/FRI] \\
\hline \multirow[t]{10}{*}{$\mathrm{CHClF}_{2}$} & {$[75-45-6]$} & chlorodifluorometl & Iane & & & \\
\hline & TRS & & 0.07 & 59 & & \\
\hline & FUS & & 4.12 & 115.7 & & [1996DOM/HEA, 1957NEI/WHI] \\
\hline & V & $(275-327)$ & 20.0 & 290 & A & [1987STE/MAL] \\
\hline & $\mathrm{V}$ & $(170-233)$ & 21.3 & 218 & A & [1987STE/MAL] \\
\hline & V & $(230-275)$ & 20.4 & 260 & A & [1987STE/MAL] \\
\hline & $\mathrm{V}$ & $(324-366)$ & 20.1 & 339 & A & [1987STE/MAL] \\
\hline & V & (194-310) & 21.8 & 209 & & [1979KUD/KUD] \\
\hline & $\mathrm{V}$ & $(229-236)$ & 21.0 & 232 & & [1964KLE] \\
\hline & $\mathrm{V}$ & & 20.2 & 232 & $\mathrm{C}$ & [1957NEI/WHI] \\
\hline \multirow[t]{8}{*}{$\mathrm{CHCl}_{2} \mathrm{~F}$} & {$[75-43-4]$} & dichlorofluorometl & Iane & & & \\
\hline & V & $(225-282)$ & 26.1 & 267 & A & [1987STE/MAL] \\
\hline & $\mathrm{V}$ & $(279-344)$ & 25.3 & 294 & A & [1987STE/MAL] \\
\hline & V & $(341-399)$ & 24.2 & 356 & A & [1987STE/MAL] \\
\hline & V & $(397-450)$ & 24.1 & 412 & $\mathrm{~A}$ & [1987STE/MAL] \\
\hline & V & $(229-236)$ & U20.9 & 233 & & [1964KLE] \\
\hline & V & $(181-282)$ & 26.2 & 267 & & [1947STU] \\
\hline & V & $(244-317)$ & 36.7 & 259 & & {$[1940 \mathrm{BEN} / \mathrm{MCH}]$} \\
\hline \multirow[t]{2}{*}{$\mathrm{CHCl}_{2} \mathrm{FO}_{3} \mathrm{~S}$} & {$[42016-50-2]$} & fluorosulfuric acid & dichloromethyl ester & & & \\
\hline & V & $(275-293)$ & 36.2 & 284 & A & [1987STE/MAL, 1999DYK/SVO, 1974CAF/SIC] \\
\hline \multirow[t]{18}{*}{$\mathrm{CHCl}_{3}$} & {$[67-66-3]$} & chloroform & & & & \\
\hline & $\begin{array}{l}\text { FUS }(\alpha, \\
\text { microsample) }\end{array}$ & & 9.54 & 209.6 & & \\
\hline & $\begin{array}{c}\text { FUS }(\beta, \\
\text { microsample) }\end{array}$ & & 8.9 & 200.0 & & {$[1979 D U M]$} \\
\hline & FUS & & 8.8 & 209.6 & & [1991ACR, 1972DUB/DEV] \\
\hline & V & $(306-427)$ & 30.8 & 321 & & [1995CHE/WAN] \\
\hline & V & $(227-269)$ & 31.8 & 254 & A & [1987STE/MAL] \\
\hline & $\mathrm{V}$ & $(333-416)$ & 30.4 & 348 & A & [1987STE/MAL] \\
\hline & V & $(410-481)$ & 28.9 & 425 & A & [1987STE/MAL] \\
\hline & $\mathrm{V}$ & $(479-523)$ & 30.1 & 494 & A & [1987STE/MAL] \\
\hline & $\mathrm{V}$ & & 31.1 & 298 & $\mathrm{C}$ & [1980MAJ/SVA] \\
\hline & V & & 30.4 & 313 & $\mathrm{C}$ & [1980MAJ/SVA] \\
\hline & V & & 29.6 & 328 & $\mathrm{C}$ & [1980MAJ/SVA] \\
\hline & $\mathrm{V}$ & & 28.8 & 343 & $\mathrm{C}$ & [1980MAJ/SVA] \\
\hline & $\mathrm{V}$ & $(260-333)$ & 32.5 & 275 & EB & [1972BOU/AIM] \\
\hline & V & $(215-334)$ & 35.0 & 230 & & [1947STU] \\
\hline & $\mathrm{V}$ & $(308-333)$ & 30.9 & 320 & & [1938SCA/RAY] \\
\hline & V & & 29.4 & 334 & & [1926MAT] \\
\hline & $\mathrm{V}$ & & 31.3 & 298 & & [1913FLE/TYR, 2002MAN] \\
\hline \multirow[t]{2}{*}{$\mathrm{CHFI}_{2}$} & {$[1493-01-2]$} & diiodofluorometha & & & & \\
\hline & V & $(299-332)$ & 32.9 & 314 & A & $\begin{array}{l}\text { [1987STE/MAL, 1979KUD/KUD, 1970DYK, } \\
\text { 1936RUF/BRE] }\end{array}$ \\
\hline $\mathrm{CHFN}_{2} \mathrm{O}_{4}$ & $\begin{array}{c}\text { [7182-87-8] } \\
\mathrm{V}\end{array}$ & fluorodinitrometha & ne & 318 & $\Delta$ & [1087STE/MAI 1074PFP/NAT] \\
\hline
\end{tabular}


TABLE 4. Phase change enthalpies of $\mathrm{C}_{1}$ organic compounds-Continued

\begin{tabular}{|c|c|c|c|c|c|c|}
\hline \multirow[b]{2}{*}{ Molecular formula } & \multirow{2}{*}{$\begin{array}{l}\text { CAS reg. no. } \\
\text { Transition }\end{array}$} & \multicolumn{5}{|l|}{ Compound } \\
\hline & & Temp. range $(\mathrm{K})$ & $\Delta_{\text {trans }} H_{\mathrm{m}}\left(\mathrm{kJ} \mathrm{mol}^{-1}\right)$ & $T_{\mathrm{m}}(\mathrm{K})$ & Method & Reference \\
\hline $\mathrm{CHFO}$ & $\begin{array}{c}{[1493-02-3]} \\
\mathrm{V}\end{array}$ & $\begin{array}{l}\text { formyl fluoride } \\
\qquad(178-235)\end{array}$ & 24.4 & 220 & A & [1987STE/MAL, 1964FIS/BUC, 1970DYK] \\
\hline $\mathrm{CHF}_{2} \mathrm{I}$ & $\begin{array}{c}{[1493-03-4]} \\
\mathrm{V}\end{array}$ & $\begin{array}{l}\text { difluoroiodomethan } \\
\quad(227-287)\end{array}$ & 26.0 & 272 & A & $\begin{array}{l}\text { [1987STE/MAL, 1979KUD/KUD, 1970DYK, } \\
\text { 1936RUF/BRE] }\end{array}$ \\
\hline $\mathrm{CHF}_{3}$ & $\begin{array}{l}{[75-46-7]} \\
\text { FUS }\end{array}$ & trifluoromethane (fl & $\begin{array}{r}\text { uoroform) } \\
4.06\end{array}$ & 118 & & [1996DOM/HEA, 1962VAL/BRO] \\
\hline & SUB & $(89-118)$ & 25.6 & 103 & A & [1987STE/MAL] \\
\hline & $\begin{array}{l}\mathrm{V} \\
\mathrm{V} \\
\mathrm{V} \\
\mathrm{V} \\
\mathrm{V}\end{array}$ & $\begin{array}{l}(138-190) \\
(198-298) \\
(146-192)\end{array}$ & $\begin{array}{r}18.1 \\
16.8 \\
18.0 \\
16.7 \\
17.9\end{array}$ & $\begin{array}{l}175 \\
213 \\
177 \\
191\end{array}$ & $\begin{array}{l}\text { A } \\
\text { A } \\
\text { C }\end{array}$ & $\begin{array}{l}\text { [1987STE/MAL] } \\
{[1987 \mathrm{STE} / \mathrm{MAL}]} \\
{[1962 \mathrm{VAL} / \mathrm{BRO}]} \\
{[1962 \mathrm{VAL} / \mathrm{BRO}]} \\
{[1937 \mathrm{HEN}]}\end{array}$ \\
\hline $\mathrm{CHF}_{3} \mathrm{O}_{2}$ & $\begin{array}{c}{[16156-36-8]} \\
\mathrm{V}\end{array}$ & $\begin{array}{l}\text { trifluoromethyl hyd } \\
\qquad(248-285)\end{array}$ & $\begin{array}{r}\text { roperoxide } \\
30.9\end{array}$ & 270 & A & [1987STE/MAL, 1971BER/HOH] \\
\hline $\mathrm{CHF}_{3} \mathrm{O}_{3} \mathrm{~S}$ & $\begin{array}{c}{[1493-13-6]} \\
\mathrm{V}\end{array}$ & $\begin{array}{l}\text { trifluoromethylsulf } \\
\qquad(354-435)\end{array}$ & nic acid 47.7 & 369 & A & [1987STE/MAL, 1999DYK/SVO] \\
\hline $\mathrm{CHF}_{3} \mathrm{~S}$ & $\begin{array}{l}{[1493-15-8]} \\
\text { FUS }\end{array}$ & trifluoromethanethi & 4.93 & 116 & & [1996DOM/HEA, 1960DIN/PAC] \\
\hline & $\begin{array}{l}\mathrm{V} \\
\mathrm{V} \\
\mathrm{V}\end{array}$ & $\begin{array}{l}(167-236) \\
(167-236)\end{array}$ & $\begin{array}{l}21.8 \\
21.0 \\
20.0\end{array}$ & $\begin{array}{l}183 \\
221 \\
235\end{array}$ & A & $\begin{array}{l}\text { [1999DYK/SVO] } \\
\text { [1987STE/MAL, 1999DYK/SVO] } \\
\text { [1960DIN/PAC] }\end{array}$ \\
\hline $\mathrm{CHF}_{3} \mathrm{~S}_{2}$ & $\begin{array}{l}{[55860-39-4]} \\
\mathrm{V}\end{array}$ & $\begin{array}{l}\text { trifluoromethyldisu } \\
\qquad(201-308)\end{array}$ & fane & 254 & & [1975GOM/SEE] \\
\hline $\mathrm{CHF}_{7} \mathrm{~S}$ & $\begin{array}{c}{[420-67-7]} \\
\mathrm{V} \\
\mathrm{V}\end{array}$ & $\begin{array}{l}\text { (difluoromethyl) su } \\
\quad(221-292) \\
(221-293)\end{array}$ & $\begin{array}{l}\text { Ifur pentafluoride } \\
\qquad \begin{array}{c}27.5 \\
25.6\end{array}\end{array}$ & $\begin{array}{l}237 \\
278\end{array}$ & A & $\begin{array}{l}\text { [1999DYK/SVO] } \\
\text { [1987STE/MAL, 1999DYK/SVO, 1950SIL/CAD] }\end{array}$ \\
\hline $\mathrm{CHI}_{3}$ & $\begin{array}{l}{[75-47-8]} \\
\text { SUB }\end{array}$ & $\begin{array}{l}\text { iodoform } \\
\qquad(308-365)\end{array}$ & 69.9 & 323 & & [1943NIT/SEK] \\
\hline $\mathrm{CHN}$ & $\begin{array}{l}{[74-90-8]} \\
\text { FUS }\end{array}$ & hydrogen cyanide & 8.4 & 259.9 & & [1939GIA/RUE] \\
\hline & $\begin{array}{l}\text { SUB } \\
\text { SUB } \\
\text { SUB } \\
\text { SUB } \\
\text { SUB }\end{array}$ & $\begin{array}{l}(236-259) \\
(237-256) \\
(202-254) \\
(236-259) \\
(244-258)\end{array}$ & $\begin{array}{l}35.6 \\
37.7 \\
37.6 \\
35.9 \\
35.7\end{array}$ & $\begin{array}{l}248 \\
247 \\
228 \\
248 \\
251\end{array}$ & A & $\begin{array}{l}\text { [1987STE/MAL] } \\
{[1982 \mathrm{APP} / \mathrm{VAN}]} \\
{[1947 \mathrm{STU}]} \\
{[1934 \mathrm{LEW} / \mathrm{SCH}]} \\
{[1926 \mathrm{PER} / \mathrm{POR}]}\end{array}$ \\
\hline & $\begin{array}{l}\text { V } \\
\text { V } \\
\text { V } \\
\text { V } \\
\text { V } \\
\text { V } \\
\text { V } \\
\text { V }\end{array}$ & $\begin{array}{l}(259-299) \\
(298-457) \\
(257-315) \\
(259-294) \\
(256-319) \\
(256-319) \\
(265-300)\end{array}$ & $\begin{array}{l}28.1 \\
27.8 \\
28.1 \\
25.2 \\
28.0 \\
28.1 \\
27.2 \\
27.8\end{array}$ & $\begin{array}{l}274 \\
313 \\
272 \\
298 \\
277 \\
272 \\
303 \\
282\end{array}$ & $\begin{array}{c}\text { A } \\
\text { A } \\
\\
\text { MM } \\
\text { MM }\end{array}$ & $\begin{array}{l}\text { [1987STE/MAL] } \\
\text { [1987STE/MAL] } \\
\text { [1975IWA/DAT] } \\
\text { [1939GIA/RUE] } \\
{[1934 \mathrm{LEW/SCH}]} \\
{[1926 \mathrm{SIN} / \mathrm{HAR}]} \\
{[1926 \mathrm{SIN} / \mathrm{HAR}]} \\
\text { [1926PER/POR] }\end{array}$ \\
\hline $\mathrm{CDN}$ & $\begin{array}{l}{[3017-23-0]} \\
\text { SUB } \\
\text { SUB }\end{array}$ & $\begin{array}{l}\text { deuterium cyanide } \\
\qquad \begin{array}{l}(227-259) \\
(235-260)\end{array}\end{array}$ & $\begin{array}{l}36.1 \\
36.5\end{array}$ & $\begin{array}{l}243 \\
247\end{array}$ & & $\begin{array}{l}{[1982 \mathrm{APP} / \mathrm{VAN}]} \\
{[1934 \mathrm{LEW} / \mathrm{SCH}]}\end{array}$ \\
\hline & $\begin{array}{l}\mathrm{V} \\
\mathrm{V}\end{array}$ & $\begin{array}{l}(182-282) \\
(265-293)\end{array}$ & $\begin{array}{l}26.2 \\
27.6\end{array}$ & $\begin{array}{l}267 \\
279\end{array}$ & & $\begin{array}{l}{[1947 \mathrm{STU}]} \\
{[1934 \mathrm{LEW} / \mathrm{SCH}]}\end{array}$ \\
\hline $\mathrm{CHNO}$ & $\begin{array}{c}{[420-05-3]} \\
\mathrm{V} \\
\mathrm{V}\end{array}$ & $\begin{array}{l}\text { cyanic acid } \\
\qquad(233-268) \\
(197-267)\end{array}$ & $\begin{array}{l}30.7 \\
\text { NA }\end{array}$ & 253 & A & $\begin{array}{l}{[1987 \mathrm{STE} / \mathrm{MAL}]} \\
{[1938 \mathrm{LIN}]}\end{array}$ \\
\hline CHNS & $\begin{array}{l}{[463-56-9]} \\
\text { FUS }\end{array}$ & thiocyanic acid & 10.87 & 163.2 & & [1940BIR/BUC] \\
\hline
\end{tabular}


TABLE 4. Phase change enthalpies of $\mathrm{C}_{1}$ organic compounds-Continued

\begin{tabular}{|c|c|c|c|c|c|c|}
\hline \multirow[b]{2}{*}{ Molecular formula } & \multirow{2}{*}{$\begin{array}{c}\text { CAS reg. no. } \\
\text { Transition }\end{array}$} & \multicolumn{5}{|l|}{ Compound } \\
\hline & & Temp. range $(\mathrm{K})$ & $\Delta_{\text {trans }} H_{\mathrm{m}}\left(\mathrm{kJ} \mathrm{mol}^{-1}\right)$ & $T_{\mathrm{m}}(\mathrm{K})$ & Method & Reference \\
\hline & $\mathrm{V}$ & $(278-396)$ & 28.0 & 293 & A & [1987STE/MAL] \\
\hline \multirow[t]{5}{*}{$\mathrm{CHN}_{3} \mathrm{O}_{6}$} & [517-25-9] & trinitromethane & & & & \\
\hline & SUB & & $45.2 \pm 2.1$ & 298 & & [1999MIR/VOR] \\
\hline & SUB & & $54.8 \pm 4.2$ & & & [1970BON/CAT] \\
\hline & SUB & & $46.7 \pm 0.4$ & & & [1967MIR/LEB, 1970COX/PIL, 1977PED/RYL] \\
\hline & V & $(290-317)$ & 32.6 & 303 & A & [1987STE/MAL, 1967MIR/LEB] \\
\hline \multirow[t]{3}{*}{$\mathrm{CH}_{2} \mathrm{BrCl}$} & {$[74-97-5]$} & bromochloromethe & & & & \\
\hline & $\mathrm{V}$ & $(226-341)$ & 42.0 & 241 & A & [1987STE/MAL] \\
\hline & $\mathrm{V}$ & $(289-341)$ & 33.5 & 304 & & [1959MCD/SHR, 1979KUD/KUD] \\
\hline \multirow[t]{6}{*}{$\mathrm{CH}_{2} \mathrm{Br}_{2}$} & {$[74-95-3]$} & dibromomethane & & & & \\
\hline & $\mathrm{V}$ & $(273-373)$ & 36.5 & 288 & $\mathrm{C}$ & [1979KUD/KUD] \\
\hline & V & $(293-343)$ & 36.3 & 318 & I & [1973PHI/JAM] \\
\hline & $\mathrm{V}$ & & $37.0 \pm 0.1$ & 298 & $\mathrm{~A}, \mathrm{E}$ & [1972LAY/WAD] \\
\hline & $\mathrm{V}$ & $(290-409)$ & 37.2 & 305 & $\mathrm{~A}, \mathrm{E}$ & [1987STE/MAL, 1956MAN, 1970DYK] \\
\hline & $\mathrm{V}$ & $(238-371)$ & 37.8 & 253 & & [1947STU] \\
\hline \multirow[t]{2}{*}{$\mathrm{CH}_{2} \mathrm{ClF}$} & {$[593-70-4]$} & chlorofluorometha & & & & \\
\hline & $\mathrm{V}$ & $(140-264)$ & 23.3 & 249 & A & [1987STE/MAL, 1970DYK] \\
\hline \multirow[t]{14}{*}{$\mathrm{CH}_{2} \mathrm{Cl}_{2}$} & {$[75-09-2]$} & dichloromethane & & & & \\
\hline & FUS & $(15-301)$ & 6.16 & 178.2 & & [1996DOM/HEA, 1978MOS/RAB] \\
\hline & V & $(286-311)$ & 29.6 & 298 & & [2016VRB/DOH] \\
\hline & $\mathrm{V}$ & & $30.6 \pm 0.1$ & 298 & $\mathrm{C}$ & [1989AN/HU] \\
\hline & $\mathrm{V}$ & $(311-383)$ & 29.0 & 326 & A & [1987STE/MAL] \\
\hline & V & & 28.8 & 298 & $\mathrm{C}$ & [1980MAJ/SVA] \\
\hline & V & & 28.1 & 313 & $\mathrm{C}$ & [1980MAJ/SVA] \\
\hline & $\mathrm{V}$ & & 27.3 & 328 & $\mathrm{C}$ & [1980MAJ/SVA] \\
\hline & V & $(293-308)$ & 29.4 & 300 & I & [1973PHI/JAM] \\
\hline & V & $(264-311)$ & 30.3 & 279 & $\mathrm{~EB}$ & [1972BOU/AIM] \\
\hline & V & $(303-313)$ & 29.2 & 308 & & [1960MUE/IGN] \\
\hline & V & $(233-313)$ & 30.2 & 248 & & [1948GAN/JUN] \\
\hline & V & & NA & & & [1946DZU] \\
\hline & $\mathrm{V}$ & $(186-312)$ & 29.4 & & & [1927PER] \\
\hline \multirow[t]{10}{*}{$\mathrm{CH}_{2} \mathrm{~F}_{2}$} & {$[75-10-5]$} & difluoromethane & & & & \\
\hline & FUS & & 4.36 & 136.4 & $\mathrm{AC}$ & [1996LUE/MAG] \\
\hline & $\mathrm{V}$ & $(208-237)$ & $20 / 8$ & 222 & EB & {$[1993 \mathrm{WEB} / \mathrm{GOO}]$} \\
\hline & V & $(149-245)$ & 20.6 & 230 & & [1987KAN/OI] \\
\hline & V & $(256-321)$ & 19.9 & 271 & A & [1987STE/MAL] \\
\hline & V & $(191-222)$ & 21.2 & 207 & A & [1987STE/MAL, 1968MAL/MEU] \\
\hline & $\mathrm{V}$ & $(191-258)$ & 20.3 & 243 & A & [1987STE/MAL] \\
\hline & $\mathrm{V}$ & $(316-351)$ & 20.3 & 331 & $\mathrm{~A}$ & [1987STE/MAL] \\
\hline & $\mathrm{V}$ & $(191-221)$ & 21.2 & 206 & & [1968MAL/MEU] \\
\hline & V & $(191-242)$ & 20.6 & 227 & & [1968MAL/MEU] \\
\hline \multirow[t]{2}{*}{$\mathrm{CH}_{2} \mathrm{~F}_{3} \mathrm{NS}$} & {$[1512-33-0]$} & 1,1,1-trifluorometh & anesulfenamide & & & \\
\hline & V & $(218-291)$ & 34.1 & 276 & A & [1987STE/MAL, 1999DYK/SVO, 1960EME/NAB] \\
\hline \multirow[t]{6}{*}{$\mathrm{CH}_{2} \mathrm{I}_{2}$} & {$[75-11-6]$} & diiodomethane & & & & \\
\hline & FUS & & 12.05 & 279.2 & & [1987KAF/DOR] \\
\hline & $\mathrm{V}$ & & 45.6 & 298 & GC & [1994CAR/LAY] \\
\hline & $\mathrm{V}$ & & 49.0 & 298 & $\mathrm{C}$ & [1987FUC/CHA] \\
\hline & $\mathrm{V}$ & $(293-455)$ & 48.8 & 307 & & [1979KUD/KUD] \\
\hline & V & $(356-505)$ & 45.4 & 371 & A & [1987STE/MAL, 1970DYK] \\
\hline \multirow[t]{5}{*}{$\mathrm{CH}_{2} \mathrm{~N}_{2}$} & [420-04-2] & cyanamide & & & & \\
\hline & FUS & & 8.76 & 317.2 & & [1991ACR, 1983WEA] \\
\hline & FUS & $(90-330)$ & 7.27 & 318.7 & $\mathrm{AC}$ & [1983DEW/DEK] \\
\hline & SUB & $(227-289)$ & 75.9 & 290 & $\mathrm{TE}, \mathrm{ME}$ & [1983DEW/VAN] \\
\hline & SUB & & 75.2 & 298 & $\mathrm{TE}, \mathrm{ME}$ & [1983DEW/VAN] \\
\hline
\end{tabular}


TABLE 4. Phase change enthalpies of $\mathrm{C}_{1}$ organic compounds-Continued

\begin{tabular}{|c|c|c|c|c|c|c|}
\hline \multirow[b]{2}{*}{ Molecular formula } & \multirow{2}{*}{$\begin{array}{l}\text { CAS reg. no. } \\
\text { Transition }\end{array}$} & \multicolumn{5}{|l|}{ Compound } \\
\hline & & Temp. range $(\mathrm{K})$ & $\Delta_{\text {trans }} H_{\mathrm{m}}\left(\mathrm{kJ} \mathrm{mol}^{-1}\right)$ & $T_{\mathrm{m}}(\mathrm{K})$ & Method & Reference \\
\hline \multirow[t]{2}{*}{$\mathrm{CH}_{2} \mathrm{~N}_{4}$} & [288-94-8] & $2 H$-tetrazole & & & & \\
\hline & FUS & & 18.0 & 430 & THBC & [1993KAB/KOZ] \\
\hline \multicolumn{7}{|c|}{ [Note: Authors of [1993KAB/KOZ] report a small solid state enthalpy of transition of $14 \mathrm{~J} / \mathrm{mole}$ in the range of 230 to $245 \mathrm{~K}$.] } \\
\hline & FUS & & 17.7 & 432.1 & & [1990KOZ/SIM3] \\
\hline & FUS & & 18.4 & 430.7 & $\mathrm{DSC}$ & [1989HIL/MOU] \\
\hline & SUB & & 88.16 & 353 & $\mathrm{C}$ & [1993KAB/KOZ] \\
\hline & SUB & $(333-404)$ & $87.8 \pm 1.4$ & 369 & $\mathrm{ME}$ & [1993KAB/KOZ] \\
\hline & SUB & $(333-404)$ & $88.0 \pm 1.6$ & & ME & [1990KOZ/SIM] \\
\hline & SUB & $(333-363)$ & $97.5 \pm 4.2$ & 348 & $\mathrm{ME}$ & [1951MCE/RIG, 1970COX/PIL] \\
\hline \multirow[t]{4}{*}{$\mathrm{CH}_{2} \mathrm{O}$} & {$[50-00-0]$} & formaldehyde & & & & \\
\hline & FUS & & 7.53 & 155 & & [1998VAS/LEB] \\
\hline & $\mathrm{V}$ & $(184-251)$ & 24.3 & 236 & A & [1987STE/MAL] \\
\hline & $\mathrm{V}$ & $(173-251)$ & 24.2 & 236 & & [1935SPE/WIL, 1987STE/MAL] \\
\hline \multirow[t]{18}{*}{$\mathrm{CH}_{2} \mathrm{O}_{2}$} & {$[64-18-6]$} & formic acid & & & & \\
\hline & SUB & $(268-281)$ & 60.5 & 275 & & [1987STE/MAL] \\
\hline & SUB & $(203-218)$ & $62.1 \pm 1$ & 213 & TE, ME & [1978CAL/CAL] \\
\hline & SUB & $(265-268)$ & 60.7 & 266 & & [1930COO, 1960JON] \\
\hline & SUB & $(253-275)$ & 60.1 & 264 & A & [1947STU] \\
\hline & $\mathrm{V}$ & $(300-392)$ & 35.2 & 315 & EB & [1987AMB/GHI3] \\
\hline & $\mathrm{V}$ & $(283-384)$ & 36.0 & 298 & A & [1987STE/MAL] \\
\hline & $\mathrm{V}$ (monomer) & & $20.1 \pm 0.1$ & 298 & $\mathrm{C}$ & [1970KON/WAD] \\
\hline & $\mathrm{V}$ & & $46.3 \pm 0.5$ & 298 & $\mathrm{C}$ & [1970KON/WAD] \\
\hline & $\mathrm{V}$ & $(310-374)$ & 35.2 & 325 & & [1949DRE/SHR, 1949DRE/MAR] \\
\hline & $\mathrm{V}$ & & 19.9 & 298 & & [1941STO/FIS] \\
\hline & $\mathrm{V}$ & & 29.6 & 303 & & [1934CAM/CAM] \\
\hline & $\mathrm{V}$ & $(273-373)$ & 20.3 & 315 & & {$[1930 \mathrm{COO}]$} \\
\hline & $\mathrm{V}$ & $(273-373)$ & 20.9 & 338 & & {$[1930 \mathrm{COO}]$} \\
\hline & $\mathrm{V}$ & & 20.4 & 315 & $\mathrm{C}$ & [1930COO] \\
\hline & $\mathrm{V}$ & & 21.1 & 338 & $\mathrm{C}$ & [1930COO] \\
\hline & $\mathrm{V}$ & $(273-307)$ & 36.8 & 288 & & [1894KAH] \\
\hline & $\mathrm{V}$ & $(295-374)$ & 47.7 & 374 & & {$[1883 \mathrm{KAH}]$} \\
\hline \multirow[t]{2}{*}{$\left(\mathrm{CH}_{2} \mathrm{O}_{2}\right)_{2}$} & [14523-98-9] & formic acid dimer & & & & \\
\hline & SUB & $(203-218)$ & $64.1 \pm 1$ & 213 & TE, ME & {$[1978 \mathrm{CAL} / \mathrm{CAL}]$} \\
\hline \multirow[t]{2}{*}{$\mathrm{CH}_{2} \mathrm{O}_{3}$} & {$[463-79-6]$} & carbonic acid & & & & \\
\hline & SUB & $(240-255)$ & $71 \pm 9$ & 247 & IR & [2010PEE/HUD] \\
\hline \multirow[t]{7}{*}{$\mathrm{CH}_{3} \mathrm{Br}$} & [74-83-9] & methyl bromide & & & & \\
\hline & TRS & & 0.47 & 173.8 & & \\
\hline & FUS & $(13-276)$ & 5.98 & 179.5 & & [1996DOM/HEA, 1987KAF/DOR, 1938EGA/KEM] \\
\hline & $\mathrm{V}$ & $(223-278)$ & 25.8 & 238 & & [1979KUD/KUD] \\
\hline & $\mathrm{V}$ & $(201-296)$ & 24.6 & 281 & $\mathrm{~A}, \mathrm{E}$ & [1987STE/MAL, 1961LI/ROS] \\
\hline & $\mathrm{V}$ & $(203-277)$ & 25.2 & 262 & & [1947BEE/JUN] \\
\hline & $\mathrm{V}$ & $(203-278)$ & 25.3 & 263 & & [1938EGA/KEM] \\
\hline \multirow[t]{11}{*}{$\mathrm{CH}_{3} \mathrm{Cl}$} & {$[74-87-31]$} & methyl chloride & & & & \\
\hline & FUS & & 6.42 & 174.5 & & [1996DOM/HEA, 1940MES/AST] \\
\hline & SUB & $(130-172)$ & $31.6 \pm 0.1$ & 151 & & [1995BAH/DUP] \\
\hline & SUB & & 28.0 & & $\mathrm{~B}$ & [1940MES/AST] \\
\hline & $\mathrm{V}$ & $(247-310)$ & 22.0 & 262 & A & [1987STE/MAL] \\
\hline & $\mathrm{V}$ & $(368-416)$ & 21.8 & 383 & A & [1987STE/MAL] \\
\hline & $\mathrm{V}$ & $(308-373)$ & 21.0 & 323 & A & [1987STE/MAL] \\
\hline & $\mathrm{V}$ & $(198-278)$ & 22.0 & 263 & & [1948GAN/JUN] \\
\hline & $\mathrm{V}$ & $(183-250)$ & 22.7 & 235 & & [1947BEE/JUN] \\
\hline & $\mathrm{V}$ & $(191-249)$ & 23.5 & 206 & & [1946THO] \\
\hline & $\mathrm{V}$ & $(192-249)$ & 22.6 & 234 & & [1940MES/AST] \\
\hline
\end{tabular}


TABLE 4. Phase change enthalpies of $\mathrm{C}_{1}$ organic compounds-Continued

\begin{tabular}{|c|c|c|c|c|c|c|}
\hline \multirow[b]{2}{*}{ Molecular formula } & \multirow{2}{*}{$\begin{array}{c}\text { CAS reg. no. } \\
\text { Transition }\end{array}$} & \multicolumn{5}{|l|}{ Compound } \\
\hline & & Temp. range $(\mathrm{K})$ & $\Delta_{\text {trans }} H_{\mathrm{m}}\left(\mathrm{kJ} \mathrm{mol}^{-1}\right)$ & $T_{\mathrm{m}}(\mathrm{K})$ & Method & Reference \\
\hline & $\mathrm{V}$ & & 19.7 & 284 & $\mathrm{C}$ & [1932GRI/AWB] \\
\hline & $\mathrm{V}$ & & 20.1 & 293 & $\mathrm{C}$ & [1926YAT] \\
\hline & $\mathrm{V}$ & & 21.6 & 249 & & [1924SHO, 2002MAN] \\
\hline \multirow[t]{2}{*}{$\mathrm{CH}_{3} \mathrm{ClFOP}$} & [753-71-9] & \multicolumn{5}{|c|}{ methylphosphonyl chlorofluoride } \\
\hline & FUS & & 11.85 & 250.7 & $\mathrm{AC}$ & [1964FUR/REI] \\
\hline \multirow[t]{2}{*}{$\mathrm{CH}_{3} \mathrm{Cl}_{2} \mathrm{P}$} & {$[676-83-5]$} & \multicolumn{5}{|c|}{ dichloromethyl phosphine } \\
\hline & $\mathrm{V}$ & $(229-297)$ & 35.5 & 282 & A & [1987STE/MAL, 1963HOL/WAG] \\
\hline \multirow[t]{3}{*}{$\mathrm{CH}_{3} \mathrm{Cl}_{2} \mathrm{OP}$} & {$[676-97-1]$} & \multicolumn{5}{|c|}{ methylphosphonic dichloride } \\
\hline & FUS & & 18.08 & 306.1 & $\mathrm{AC}$ & [1964FUR/REI] \\
\hline & SUB & & 62.3 & & & [1970COX/PIL, 1955NEA/WIL] \\
\hline \multirow[t]{8}{*}{$\mathrm{CH}_{3} \mathrm{~F}$} & {$[593-53-3]$} & methyl fluoride & & & & \\
\hline & $\mathrm{V}$ & $(205-242)$ & 16.9 & 227 & A & [1987STE/MAL] \\
\hline & $\mathrm{V}$ & $(240-288)$ & 16.9 & 273 & A & [1987STE/MAL] \\
\hline & $\mathrm{V}$ & $(133-211)$ & 17.9 & 172 & & [1983OI/SHU] \\
\hline & $\mathrm{V}$ & $(141-208)$ & 17.1 & 193 & $\mathrm{~A}, \mathrm{E}$ & [1987STE/MAL, 1961LI/ROS, 1970DYK] \\
\hline & $\mathrm{V}$ & $(165-217)$ & 16.4 & 202 & & [1987STE/MAL, 1948MIC/WAS, 1984BOU/FRI] \\
\hline & $\mathrm{V}$ & $(170-197)$ & 17.7 & 183 & A & [1987STE/MAL, 1919MOL/BAT, 1984BOU/FRI] \\
\hline & $\mathrm{V}$ & $(268-318)$ & 18.6 & 293 & & [1889COL] \\
\hline \multirow[t]{2}{*}{$\mathrm{CH}_{3} \mathrm{FO}$} & {$[36336-08-0]$} & \multicolumn{5}{|l|}{ methyl hypofluorite } \\
\hline & $\mathrm{V}$ & $(156-225)$ & 23.4 & 190 & & {$[1991 \mathrm{KOL} / \mathrm{ROZ}]$} \\
\hline \multirow[t]{3}{*}{$\mathrm{CH}_{3} \mathrm{~F}_{2} \mathrm{~N}$} & {$[753-58-2]$} & \multicolumn{5}{|c|}{$N, N$-difluoromethylamine } \\
\hline & $\mathrm{V}$ & $(203-257)$ & 23.5 & 242 & A & [1987STE/MAL] \\
\hline & $\mathrm{V}$ & & 22.9 & 257 & & [1960FRA] \\
\hline \multirow[t]{3}{*}{$\mathrm{CH}_{3} \mathrm{~F}_{2} \mathrm{NS}$} & {$[758-20-3]$} & \multicolumn{5}{|c|}{ methylimidosulfurous difluoride } \\
\hline & $\mathrm{V}$ & $(194-258)$ & 28.7 & 226 & & [1999DYK/SVO] \\
\hline & $\mathrm{V}$ & $(194-258)$ & 28.6 & 243 & A & [1987STE/MAL, 1999DYK/SVO, 1966COH/MAC] \\
\hline \multirow[t]{2}{*}{$\mathrm{CH}_{3} \mathrm{~F}_{2} \mathrm{P}$} & [753-59-3] & \multicolumn{5}{|c|}{ difluoromethyl phosphine } \\
\hline & $\mathrm{V}$ & $(174-236)$ & 23.4 & 221 & A & [1987STE/MAL] \\
\hline \multirow[t]{2}{*}{$\mathrm{CH}_{3} \mathrm{~F}_{2} \mathrm{OP}$} & [676-99-3] & methylphosphonyl & difluoride & & & \\
\hline & FUS & $(15-335)$ & 11.88 & 236.3 & $\mathrm{AC}$ & [1964FUR/REI] \\
\hline $\mathrm{CH}_{3} \mathrm{~F}_{2} \mathrm{OPS}$ & {$[25237-37-0]$} & difluorothiophosph & ric, $S$-methyl ester & & & \\
\hline & $\mathrm{V}$ & $(236-298)$ & 31.2 & 251 & A & [1987STE/MAL, 1999DYK/SVO, 1968CHA/CAV] \\
\hline $\mathrm{CH}_{3} \mathrm{~F}_{2} \mathrm{PS}_{2}$ & {$[21348-13-0]$} & difluorodithiophos & horic acid, methyl est & & & \\
\hline & $\mathrm{V}$ & $(253-298)$ & 39.0 & 268 & A & [1987STE/MAL, 1999DYK/SVO, 1968CHA/CAV] \\
\hline $\mathrm{CH}_{3} \mathrm{~F}_{4} \mathrm{NP}_{2} \mathrm{~S}_{2}$ & {$[25741-62-2]$} & $N, N$-bis(difluoroth & ophosphoral) methyla & nine & & \\
\hline & $\mathrm{V}$ & $(273-325)$ & 38.7 & 288 & A & [1987STE/MAL, 1999DYK/SVO, 1970CHA/CAV] \\
\hline $\mathrm{CH}_{3} \mathrm{I}$ & [74-88-4] & methyl iodide & & & & \\
\hline & FUS & & 9.12 & 206.8 & & [1982WRE/VIK] \\
\hline & SUB & $(176-227)$ & $40.2 \pm 0.4$ & 191 & VG & [1982WRE/VIK] \\
\hline & SUB & & U 69.9 & & & [1943NIT/SEK, 1960JON] \\
\hline & $\mathrm{V}$ & $(228-337)$ & 30.4 & 243 & A & [1987STE/MAL] \\
\hline & $\mathrm{V}$ & $(315-502)$ & 26.5 & 330 & A & [1987STE/MAL] \\
\hline & $\mathrm{V}$ & $(208-227)$ & 31.1 & 217 & & [1982WRE/VIK] \\
\hline & $\mathrm{V}$ & $(259-314)$ & 29.2 & 274 & EB & [1972BOU/AIM, 1979KUD/KUD] \\
\hline & $\mathrm{V}$ & $(218-315)$ & 30.4 & 233 & & [1947STU] \\
\hline & $\mathrm{V}$ & $(273-307)$ & 28.2 & 288 & & [1936EWE] \\
\hline $\mathrm{CH}_{3} \mathrm{NO}$ & {$[75-12-7]$} & formamide & & & & \\
\hline & FUS & $(85-290)$ & 8.67 & 275.6 & $\mathrm{AC}$ & [1983DEW/DEK] \\
\hline & FUS & & 7.98 & 275.7 & & [1996DOM/HEA, 1965SOM/COO] \\
\hline & SUB & $(251-273)$ & 72.4 & 264 & TE, ME & [1983DEW/VAN] \\
\hline & SUB & $(251-273)$ & 71.7 & 298 & TE, ME & [1983DEW/VAN] \\
\hline & SUB & & 71.7 & 276 & & [1979DAA/VAN] \\
\hline
\end{tabular}


TABLE 4. Phase change enthalpies of $\mathrm{C}_{1}$ organic compounds-Continued

\begin{tabular}{|c|c|c|c|c|c|c|}
\hline \multirow[b]{2}{*}{ Molecular formula } & \multirow{2}{*}{$\begin{array}{c}\text { CAS reg. no. } \\
\text { Transition }\end{array}$} & \multicolumn{5}{|l|}{ Compound } \\
\hline & & Temp. range $(\mathrm{K})$ & $\Delta_{\text {trans }} H_{\mathrm{m}}\left(\mathrm{kJ} \mathrm{mol}^{-1}\right)$ & $T_{\mathrm{m}}(\mathrm{K})$ & Method & Reference \\
\hline & $\mathrm{V}$ & $(303-376)$ & 60.6 & 340 & GS & [2011EME/VER2] \\
\hline & $\mathrm{V}$ & $(303-376)$ & $62.2 \pm 0.3$ & 298 & GS & [2011EME/VER2] \\
\hline & $\mathrm{V}$ & $(344-484)$ & 69.1 & 298 & $\mathrm{~EB}$ & [1989COX/WAG, 2011EME/VER2] \\
\hline & $\mathrm{V}$ & $(293-377)$ & 70.8 & 308 & A & [1987STE/MAL] \\
\hline & $\mathrm{V}$ & $(415-466)$ & 61.2 & 430 & A & [1987STE/MAL] \\
\hline & $\mathrm{V}$ & & 60.2 & 298 & A & [1985BAR/CAS, 1985MAJ/SVO] \\
\hline & $\mathrm{V}$ & $(391-466)$ & 54.6 & 429 & EB & [1979OLO, 2011EME/VER2] \\
\hline & $\mathrm{V}$ & $(391-466)$ & $59.5 \pm 0.2$ & 298 & EB & [1979OLO, 2011EME/VER2] \\
\hline & $\mathrm{V}$ & $(303-453)$ & 59.3 & 378 & & [1967QUI, 2011EME/VER2] \\
\hline & $\mathrm{V}$ & $(303-453)$ & $62.0 \pm 0.7$ & 298 & & [1967QUI, 2011EME/VER2] \\
\hline & $\mathrm{V}$ & $(251-273)$ & $62.7 \pm 0.7$ & 298 & TE, ME, S-F & [1967QUI/HOF, 2011EME/VER2] \\
\hline & $\mathrm{V}$ & $(298-333)$ & $70.8 \pm 0.3$ & 298 & $\mathrm{ME}$ & [1965SOM/COO, 2011EME/VER2] \\
\hline & $\mathrm{V}$ & $(343-483)$ & 64.0 & 358 & & {$[1947 \mathrm{STU}]$} \\
\hline \multirow[t]{2}{*}{$\mathrm{CH}_{3} \mathrm{NOS}$} & {$[4291-05-8]$} & $N$-sulfinyl methan & mine & & & \\
\hline & $\mathrm{V}$ & $(252-277)$ & 31.8 & 264 & A & [1987STE/MAL, 1999DYK/SVO, 1954BUR/WOO] \\
\hline \multirow[t]{5}{*}{$\mathrm{CH}_{3} \mathrm{NO}_{2}$} & {$[624-91-9]$} & methyl nitrite & & & & \\
\hline & $\mathrm{V}$ & $(218-273)$ & 22.1 & 258 & A & [1987STE/MAL] \\
\hline & $\mathrm{V}$ & $(154-225)$ & 26.2 & 190 & & [1982ROO] \\
\hline & $\mathrm{V}$ & & $22.6 \pm 0.2$ & & & [1958GRA/PRA] \\
\hline & $\mathrm{V}$ & $(241-274)$ & 21.0 & 257 & & [1937THO/DAI] \\
\hline \multirow[t]{17}{*}{$\mathrm{CH}_{3} \mathrm{NO}_{2}$} & {$[75-52-5]$} & nitromethane & & & & \\
\hline & FUS & & 9.7 & 244.8 & & [1996DOM/HEA, 1947JON/GIA] \\
\hline & $\mathrm{V}$ & & $38.5 \pm 0.4$ & 298 & & [1999MIR/VOR2] \\
\hline & $\mathrm{V}$ & $(313-353)$ & 37.2 & 298 & CGC & [1995CHI/HOS] \\
\hline & $\mathrm{V}$ & $(405-476)$ & 35.2 & 420 & A & [1987STE/MAL, 1967BER/WES] \\
\hline & $\mathrm{V}$ & $(328-410)$ & 36.8 & 343 & A & [1987STE/MAL, 1954MCC/SCO] \\
\hline & $\mathrm{V}$ & & $37.2 \pm 0.1$ & 318 & $\mathrm{C}$ & {$[1954 \mathrm{MCC} / \mathrm{SCO}]$} \\
\hline & $\mathrm{V}$ & & $36.3 \pm 0.1$ & 335 & $\mathrm{C}$ & [1954MCC/SCO] \\
\hline & $\mathrm{V}$ & & $35.2 \pm 0.1$ & 353 & $\mathrm{C}$ & [1954MCC/SCO] \\
\hline & $\mathrm{V}$ & & $34.0 \pm 0.1$ & 374 & $\mathrm{C}$ & [1954MCC/SCO] \\
\hline & V & $(283-373)$ & $38.0 \pm 0.4$ & 298 & ZG & [1949HOL/DOR] \\
\hline & $\mathrm{V}$ & & $38.3 \pm 0.1$ & 298 & $\mathrm{C}$ & [1947JON/GIA] \\
\hline & $\mathrm{V}$ & & 34.4 & 374 & $\mathrm{C}$ & [1941PIT/GWI] \\
\hline & $\mathrm{V}$ & $(288-373)$ & 37.2 & 330 & & [1940HOD] \\
\hline & V & & 34.6 & 374 & $\mathrm{C}$ & [1930PHI/WAT] \\
\hline & V & & 33.9 & 373 & $\mathrm{C}$ & [1926MAT] \\
\hline & $\mathrm{V}$ & $(310-374)$ & 34.4 & 342 & & {$[1925 \mathrm{WIL}]$} \\
\hline \multirow[t]{3}{*}{$\mathrm{CH}_{3} \mathrm{NO}_{3}$} & {$[598-58-3]$} & methyl nitrate & & & & \\
\hline & FUS & & 8.24 & 190.2 & & [1996DOM/HEA, 1953GRA/SMI] \\
\hline & $\mathrm{V}$ & $(273-303)$ & 34.8 & 288 & A & [1987STE/MAL, 1952MCK/MOE] \\
\hline \multirow[t]{2}{*}{$\mathrm{CH}_{3} \mathrm{~N}_{5}$} & {$[4418-61-5]$} & 5-aminotetrazole & & & & \\
\hline & SUB & $(383-443)$ & $112.6 \pm 1.2$ & & $\mathrm{ME}$ & {$[1990 \mathrm{KOZ} / \mathrm{SIM}]$} \\
\hline \multirow[t]{14}{*}{$\mathrm{CH}_{4}$} & {$[74-82-8]$} & methane & & & & \\
\hline & FUS & & 0.94 & 90.7 & & $\begin{array}{l}\text { [1929CLU, 1931PAR/HUF, 1961SPE, } \\
\text { 1976VOG/PIT] }\end{array}$ \\
\hline & FUS & & 0.97 & 90.6 & & [1924EUC/KAR] \\
\hline & SUB & $(53-91)$ & 9.7 & 72 & & [1987STE/MAL] \\
\hline & SUB & $(54-90)$ & 9.2 & 72 & & [1963BON, 1955ARM/BRI] \\
\hline & SUB & $(79-89)$ & 10.0 & 84 & & [1960JON] \\
\hline & SUB & $(48-78)$ & 9.7 & 63 & A, MS & [1951TIC/LOS] \\
\hline & SUB & $(67-88)$ & 9.62 & 77 & A & [1947STU] \\
\hline & $\mathrm{V}$ & $(90-120)$ & 8.6 & 105 & A & [1987STE/MAL] \\
\hline & $\mathrm{V}$ & $(115-149)$ & 8.4 & 134 & A & [1987STE/MAL] \\
\hline & V & $(148-189)$ & 8.7 & 174 & A & [1987STE/MAL] \\
\hline & $\mathrm{V}$ & $(91-127)$ & 8.6 & 112 & & [1972PRY/GOO, 1984BOU/FRI] \\
\hline & $\mathrm{V}$ & $(91-190)$ & 8.5 & 175 & & [1972PRY/GOO] \\
\hline & $\mathrm{V}$ & & 8.1 & 137 & & [1971WIL/ZWO] \\
\hline
\end{tabular}


TABLE 4. Phase change enthalpies of $\mathrm{C}_{1}$ organic compounds-Continued

\begin{tabular}{|c|c|c|c|c|c|c|}
\hline \multirow[b]{2}{*}{ Molecular formula } & \multirow{2}{*}{$\begin{array}{c}\text { CAS reg. no. } \\
\text { Transition }\end{array}$} & \multicolumn{5}{|l|}{ Compound } \\
\hline & & Temp. range $(\mathrm{K})$ & $\Delta_{\text {trans }} H_{\mathrm{m}}\left(\mathrm{kJ} \mathrm{mol}^{-1}\right)$ & $T_{\mathrm{m}}(\mathrm{K})$ & Method & Reference \\
\hline & $\mathrm{V}$ & $(100-190)$ & 8.6 & 175 & & [1970AMB/COU] \\
\hline & V & & 8.2 & 112 & $\mathrm{C}$ & [1961HES/WHI] \\
\hline & V & & 7.5 & 130 & $\mathrm{C}$ & {$[1961 \mathrm{HES} / \mathrm{WHI}]$} \\
\hline & V & & 5.9 & 160 & $\mathrm{C}$ & [1961HES/WHI] \\
\hline & $\mathrm{V}$ & & 4.0 & 180 & $\mathrm{C}$ & [1961HES/WHI] \\
\hline & $\mathrm{V}$ & $(109-189)$ & 8.5 & 149 & & [1961HES/WHI] \\
\hline & V & & $8.5 \pm 0.1$ & 99 & & [1939FRA/CLU] \\
\hline & V & $(92-110)$ & 8.6 & 101 & & [1921STO/HEN, 1984BOU/FRI] \\
\hline \multirow[t]{2}{*}{$\mathrm{CH}_{4} \mathrm{~F}_{2} \mathrm{NPS}$} & [31411-30-0] & \multicolumn{5}{|c|}{ difluorothiophosphoric acid, $N$-methylamide } \\
\hline & $\mathrm{V}$ & $(273-325)$ & 39.1 & 288 & A & [1987STE/MAL, 1999DYK/SVO, 1971CAV/CHA] \\
\hline \multirow[t]{2}{*}{$\mathrm{CH}_{4} \mathrm{~N}_{2}$} & {$[12211-52-8]$} & \multicolumn{5}{|l|}{ ammonium cyanide } \\
\hline & V & $(222-305)$ & 47.1 & 237 & & [1947STU] \\
\hline \multirow[t]{3}{*}{$\mathrm{CH}_{4} \mathrm{~N}_{2}$} & [26981-93-1] & \multicolumn{5}{|l|}{ methyl diazene } \\
\hline & V & $(195-236)$ & 27.5 & 221 & A & [1987STE/MAL] \\
\hline & V & $(209-236)$ & 25.7 & 222 & & [1972ACK/HAL] \\
\hline \multirow[t]{2}{*}{$\mathrm{CH}_{3} \mathrm{DN}_{2}$} & [34994-49-5] & \multicolumn{5}{|c|}{$N$-deuteromethyl diazene } \\
\hline & V & & 29.9 & & & [1972ACK/HAL] \\
\hline \multirow[t]{35}{*}{$\mathrm{CH}_{4} \mathrm{~N}_{2} \mathrm{O}$} & {$[57-13-6]$} & \multicolumn{5}{|l|}{ urea } \\
\hline & FUS & & 16.6 & 406.2 & DSC & [2013AGA/MOS] \\
\hline & FUS & & 14.6 & 406.2 & DSC & [2012SHA/SHA] \\
\hline & FUS & & 14.6 & 406.5 & DSC & [2011RAI/MUD] \\
\hline & FUS & & 15.13 & 407.5 & DSC & [2010BAB/KUZ] \\
\hline & FUS & & 13.9 & 405.8 & DSC & [2010ZEN/LI] \\
\hline & FUS & & 14.6 & 407.2 & DSC & [1999RAI/RAI] \\
\hline & FUS & & 14.6 & 406.7 & DSC & [1998RAI/RAI] \\
\hline & FUS & & 13.6 & 405.2 & DSC & [1998JAM/PAL] \\
\hline & FUS & & 15.03 & 407.9 & DSC & [1995FER/DEL] \\
\hline & FUS & & U 11.42 & 408.2 & DSC & [1993RAN/LAK] \\
\hline & FUS & & 14.1 & 406.2 & & [1993STR/ARG] \\
\hline & FUS & & 12.93 & 408.1 & & [1990KAB/MIR2] \\
\hline & FUS & & 14.5 & 406.2 & DSC & [1988GAM/BRO, 1987GAM/GAU] \\
\hline & FUS & & 14.8 & 406.5 & DSC & [1987DEL/FER] \\
\hline & FUS & & 13.9 & 405.8 & & [1986KOZ/DAL] \\
\hline & FUS & & 15.52 & 405.2 & & [1985OZA] \\
\hline & FUS & & 14.41 & 406.2 & & [1983BRO/GAM] \\
\hline & FUS & & 13.61 & 406.0 & & [1980VOG/SCH] \\
\hline & FUS & & 13.47 & 406.3 & DSC & [1972ZOR/HUR] \\
\hline & FUS & & 13.8 & & $\mathrm{CR}$ & [1972ZOR/HUR] \\
\hline & FUS & & 14.52 & 405.9 & & [1934MIL/DIT] \\
\hline & SUB & $(358-402)$ & $95.5 \pm 0.3$ & 298 & GS & [2006EME/KAB] \\
\hline & SUB & $(329-403)$ & $94.6 \pm 2.2$ & 370 & ME & [2003ZAI/KAB] \\
\hline & SUB & $(329-403)$ & $95.1 \pm 2.2$ & 350 & ME & [2003ZAI/KAB] \\
\hline & SUB & & $96.0 \pm 0.6$ & 298 & & [2003ZAI/KAB] \\
\hline & SUB & & $94.6 \pm 0.5$ & 350 & $\mathrm{C}$ & [2003ZAI/KAB] \\
\hline & SUB & & $97.6 \pm 1.0$ & & ME & [1987KRA/KOZ] \\
\hline & SUB & & 90.9 & 381 & & [1987FER/DEL2] \\
\hline & SUB & $(345-368)$ & 87.7 & 357 & A & [1987STE/MAL] \\
\hline & SUB & $(338-362)$ & 96.9 & 351 & TE, ME & [1983DEW/VAN] \\
\hline & SUB & & 98.6 & 298 & & [1983DEW/VAN] \\
\hline & SUB & & 95.4 & 361 & & [1978TRI/VOO] \\
\hline & SUB & $(345-368)$ & $87.9 \pm 2.1$ & 356 & & [1956SUZ/ONI, 1960JON, 1970COX/PIL] \\
\hline & SUB & & 88.2 & 357 & & [1953BRA/CLE2, 1983DEW/VAN] \\
\hline \multirow[t]{2}{*}{$\mathrm{CH}_{4} \mathrm{~N}_{2} \mathrm{O}_{2}$} & [598-57-2] & \multicolumn{5}{|c|}{$N$-nitromethylamine } \\
\hline & FUS & & 11.86 & 324.2 & DSC & [2010FAR/RAJ] \\
\hline \multirow[t]{2}{*}{$\mathrm{CH}_{4} \mathrm{~N}_{2} \mathrm{O}_{2}$} & [1111-78-0] & ammonium carban & & & & \\
\hline & $\mathrm{V}$ & (247-331) & 54.1 & 262 & & [1947STU] \\
\hline $\mathrm{CH}_{4} \mathrm{~N}_{2} \mathrm{~S}$ & {$[62-56-6]$} & thiourea & & & & \\
\hline & FUS & & 15.17 & 450.2 & DSC & [2016WAN/YIN] \\
\hline
\end{tabular}


TABLE 4. Phase change enthalpies of $\mathrm{C}_{1}$ organic compounds-Continued

\begin{tabular}{|c|c|c|c|c|c|c|}
\hline \multirow[b]{2}{*}{ Molecular formula } & \multirow{2}{*}{$\begin{array}{c}\text { CAS reg. no. } \\
\text { Transition }\end{array}$} & \multicolumn{5}{|l|}{ Compound } \\
\hline & & Temp. range $(\mathrm{K})$ & $\Delta_{\text {trans }} H_{\mathrm{m}}\left(\mathrm{kJ} \mathrm{mol}^{-1}\right)$ & $T_{\mathrm{m}}(\mathrm{K})$ & Method & Reference \\
\hline & FUS & & 15.64 & 444.7 & DSC & [2000DEL/JOZ] \\
\hline & FUS & & 12.55 & 452.2 & DSC & [1994KIM/LEE] \\
\hline & TRS & $(140-240)$ & 0.026 & 169 & & \\
\hline & TRS & $(140-240)$ & 0.113 & 200 & $\mathrm{AC}$ & [1993IGA/LOP] \\
\hline & FUS & & 16.96 & 454.2 & DSC & [1993RAN/LAK] \\
\hline & FUS & & 16.0 & & DSC & [1993STR/ARG] \\
\hline & FUS & & 15.0 & 454.4 & DSC & [1993DEM/BUC] \\
\hline & FUS & & 14.42 & 445.5 & DSC & [1990DON/DRE] \\
\hline & FUS & & 15.28 & 455 & DSC & [1988ASH] \\
\hline & FUS & & 13.8 & 453.3 & $\mathrm{DSC}$ & [1972ZOR/HUR] \\
\hline & SUB & & $112.0 \pm 2.0$ & 298 & $\mathrm{ME}$ & [2000DEL/JOZ] \\
\hline & SUB & & $109.0 \pm 2.0$ & 408 & $\mathrm{TE}$ & [1994FER/MAR] \\
\hline & SUB & & $111.0 \pm 3.0$ & 298 & $\mathrm{TE}$ & [1994FER/MAR] \\
\hline & SUB & $(378-396)$ & $103.9 \pm 0.3$ & 387 & $\mathrm{ME}$ & [1994TOR/HER] \\
\hline & SUB & $(348-382)$ & $93.7 \pm 4.7$ & 365 & DTA & [1988ASH] \\
\hline & SUB & $(348-382)$ & $95.2 \pm 4.8$ & 298 & DTA & [1988ASH] \\
\hline & SUB & $(368-395)$ & 106.6 & 384 & $\mathrm{TE}, \mathrm{ME}$ & [1983DEW/VAN] \\
\hline & SUB & & 107.6 & 298 & & [1983DEW/VAN] \\
\hline & SUB & & $112.0 \pm 1.5$ & 298 & $\mathrm{C}$ & [1982TOR/SAB] \\
\hline & SUB & & $93.7 \pm 10$ & & & [1975BAG/AND] \\
\hline \multirow[t]{4}{*}{$\mathrm{CH}_{4} \mathrm{~N}_{4} \mathrm{O}_{2}$} & [556-88-7] & nitroguanidine & & & & \\
\hline & FUS & & 33.46 & 505.2 & DTA & [2014CHE/LI] \\
\hline & SUB & $(463-498)$ & 139 & 480 & DTA & [2014CUD/POD] \\
\hline & SUB & $(402-473)$ & $142.7 \pm 2.0$ & 298 & $\mathrm{ME}$ & [1978CUN/PAL] \\
\hline \multirow[t]{2}{*}{$\mathrm{CH}_{4} \mathrm{~N}_{4} \mathrm{O}_{4}$} & [14168-44-6] & $N, N^{\prime}$-dinitrodiami & omethane & & & \\
\hline & FUS & & 35.85 & 371 & DTA & [1987OYU/BRI] \\
\hline \multirow[t]{33}{*}{$\mathrm{CH}_{4} \mathrm{O}$} & {$[67-56-1]$} & methanol & & & & \\
\hline & TRS & $(78-320)$ & 0.64 & 157.8 & & \\
\hline & FUS & $(78-320)$ & 3.21 & 175.9 & $\mathrm{AC}$ & [2006WAN/NAN] \\
\hline & TRS & $(6-325)$ & 0.64 & 157.3 & & \\
\hline & FUS & $(6-325)$ & 3.22 & 175.6 & $\mathrm{AC}$ & [1996DOM/HEA, 1971CAR/WES] \\
\hline & TRS & & 0.71 & 157.8 & & \\
\hline & FUS & & 3.16 & 175.4 & & [1996DOM/HEA, 1949STA/GUP] \\
\hline & TRS & & 0.65 & 157.4 & & \\
\hline & FUS & & 3.17 & 175.2 & & [1996DOM/HEA, 1929KEL2] \\
\hline & TRS & & 0.59 & 161.1 & & \\
\hline & FUS & & 3.18 & 175.3 & & [1996DOM/HEA, 1925PAR] \\
\hline & $\operatorname{SUB}(\beta)$ & $(157-175)$ & $44.2 \pm 0.5$ & 166 & MS, FTIR & [2005LUC/FER] \\
\hline & $\operatorname{SUB}(\alpha)$ & $(145-157)$ & $46.9 \pm 0.2$ & 151 & MS, FTIR & [2005LUC/FER] \\
\hline & V & $(298-333)$ & 38.0 & 298 & & [2004NAS/ZIM] \\
\hline & $\mathrm{V}$ & & 34.3 & & & [1999FAT] \\
\hline & V & $(175-273)$ & 39.2 & 258 & A & [1987STE/MAL] \\
\hline & V & $(338-487)$ & 36.9 & 353 & A & [1987STE/MAL] \\
\hline & V & $(188-228)$ & 43.7 & 213 & A & [1987STE/MAL] \\
\hline & V & $(224-290)$ & 38.9 & 275 & A & [1987STE/MAL] \\
\hline & V & $(285-345)$ & 38.3 & 300 & A & [1987STE/MAL] \\
\hline & V & $(335-376)$ & 37.0 & 350 & A & [1987STE/MAL] \\
\hline & V & $(373-458)$ & 36.1 & 388 & A & [1987STE/MAL] \\
\hline & $\mathrm{V}$ & $(453-513)$ & 35.1 & 468 & A & [1987STE/MAL] \\
\hline & V & & 32.7 & 373 & $\mathrm{C}$ & [1986YER/WOR] \\
\hline & V & & 28.1 & 423 & $\mathrm{C}$ & [1986YER/WOR] \\
\hline & $\mathrm{V}$ & & 20.6 & 473 & $\mathrm{C}$ & [1986YER/WOR] \\
\hline & V & & 7.4 & 510 & $\mathrm{C}$ & [1986YER/WOR] \\
\hline & V & $(316-336)$ & 37.5 & 331 & EB & [1984CER/BOU] \\
\hline & $\mathrm{V}$ & $(243-303)$ & 37.8 & 298 & & [1983SCH/STR] \\
\hline & V & $(288-337)$ & 38.3 & 303 & & [1974GIB/CRE, 1984BOU/FRI] \\
\hline & $\mathrm{V}$ & $(337-383)$ & 37.0 & 352 & & [1973WIL/ZWO] \\
\hline & $\mathrm{V}$ & & $37.4 \pm 0.1$ & 298 & $\mathrm{C}$ & [1973SVO/VES] \\
\hline & $\mathrm{V}$ & & $36.7 \pm 0.1$ & 313 & $\mathrm{C}$ & [1973SVO/VES] \\
\hline
\end{tabular}


TABLE 4. Phase change enthalpies of $\mathrm{C}_{1}$ organic compounds-Continued

\begin{tabular}{|c|c|c|c|c|c|c|}
\hline \multirow[b]{2}{*}{ Molecular formula } & \multirow{2}{*}{$\begin{array}{c}\text { CAS reg. no. } \\
\text { Transition }\end{array}$} & \multicolumn{5}{|l|}{ Compound } \\
\hline & & Temp. range $(\mathrm{K})$ & $\Delta_{\text {trans }} H_{\mathrm{m}}\left(\mathrm{kJ} \mathrm{mol}^{-1}\right)$ & $T_{\mathrm{m}}(\mathrm{K})$ & Method & Reference \\
\hline & $\mathrm{V}$ & & $36.2 \pm 0.1$ & 323 & $\mathrm{C}$ & [1973SVO/VES] \\
\hline & $\mathrm{V}$ & & $35.6 \pm 0.1$ & 333 & $\mathrm{C}$ & [1973SVO/VES] \\
\hline & $\mathrm{V}$ & & $35.3 \pm 0.1$ & 338 & $\mathrm{C}$ & [1973SVO/VES] \\
\hline & $\mathrm{V}$ & & $34.7 \pm 0.1$ & 343 & $\mathrm{C}$ & [1973SVO/VES] \\
\hline & $\mathrm{V}$ & & $35.2 \pm 0.1$ & 338 & $\mathrm{C}$ & [1973COU/LEE] \\
\hline & $\mathrm{V}$ & & $35.6 \pm 0.1$ & 331 & $\mathrm{C}$ & {$[1973 \mathrm{COU} / \mathrm{LEE}]$} \\
\hline & $\mathrm{V}$ & & $36.2 \pm 0.1$ & 321 & $\mathrm{C}$ & [1973COU/LEE] \\
\hline & V & & $37.0 \pm 0.1$ & 306 & $\mathrm{C}$ & [1973COU/LEE] \\
\hline & $\mathrm{V}$ & $(275-336)$ & 38.7 & 290 & EB & [1972BOU/AIM, 1987STE/MAL] \\
\hline & $\mathrm{V}$ & & $37.43 \pm 0.02$ & 298 & $\mathrm{C}$ & {$[1971 \mathrm{POL} / \mathrm{BEN}]$} \\
\hline & $\mathrm{V}$ & & $37.0 \pm 0.1$ & 308 & $\mathrm{C}$ & [1971POL/BEN] \\
\hline & V & & $36.5 \pm 0.1$ & 328 & $\mathrm{C}$ & [1971POL/BEN] \\
\hline & V & & $35.5 \pm 0.1$ & 328 & $\mathrm{C}$ & [1971POL/BEN] \\
\hline & V & $(288-357)$ & 38.3 & 303 & EB & [1970AMB/SPR] \\
\hline & $\mathrm{V}$ & $(353-483)$ & 36.3 & 368 & & [1967HIR/SUD] \\
\hline & V & & $37.3 \pm 0.1$ & 298 & $\mathrm{C}$ & [1966WAD] \\
\hline & V & & $37.7 \pm 0.1$ & 298 & $\mathrm{C}$ & [1963MCC/LAI] \\
\hline & V & $(278-323)$ & 38.4 & 293 & & [1960KLY/MIS] \\
\hline & V & & 38.5 & 273 & & [1949STA/GUP] \\
\hline \multirow[t]{2}{*}{$\mathrm{CH}_{4} \mathrm{O}_{2}$} & {$[3031-73-0]$} & methyl hydroperox & & & & \\
\hline & $\mathrm{V}$ & $(253-313)$ & 37.7 & 268 & A & [1987STE/MAL, 1951EGE/EMT] \\
\hline \multirow[t]{2}{*}{$\mathrm{CH}_{4} \mathrm{O}_{3} \mathrm{~S}$} & {$[75-75-2]$} & methanesulfonic a & & & & \\
\hline & $\mathrm{V}$ & $(395-440)$ & 73.9 & 410 & A & [1987STE/MAL, 1999DYK/SVO] \\
\hline \multirow[t]{11}{*}{$\mathrm{CH}_{4} \mathrm{~S}$} & [74-93-1] & methyl mercaptan & & & & \\
\hline & TRS & $(15-279)$ & 2.2 & 137.6 & & \\
\hline & FUS & $(15-279)$ & 5.9 & 150.2 & $\mathrm{AC}$ & [1996DOM/HEA, 1942RUS/OSB] \\
\hline & $\mathrm{V}$ & $(208-298)$ & 27.2 & 223 & & [1999DYK/SVO] \\
\hline & $\mathrm{V}$ & $(267-359)$ & 25.2 & 359 & A & [1987STE/MAL] \\
\hline & $\mathrm{V}$ & $(221-283)$ & 25.7 & 268 & A & [1987STE/MAL] \\
\hline & V & $(345-424)$ & 23.7 & 360 & A & [1987STE/MAL] \\
\hline & $\mathrm{V}$ & $(414-470)$ & 24.2 & 429 & A & [1987STE/MAL] \\
\hline & V & & 23.8 & 298 & & [1971WIL/ZWO] \\
\hline & $\mathrm{V}$ & $(222-279)$ & 25.8 & 264 & & [1987STE/MAL, 1942RUS/OSB] \\
\hline & $\mathrm{V}$ & & $24.6 \pm 0.1$ & 279 & $\mathrm{C}$ & [1942RUS/OSB] \\
\hline \multirow[t]{7}{*}{$\mathrm{CH}_{5} \mathrm{~N}$} & [74-89-5] & methylamine & & & & \\
\hline & FUS & & 6.13 & 179.7 & & [1996DOM/HEA, 1937AST/SIL] \\
\hline & V & $(319-381)$ & 24.8 & 334 & A & [1987STE/MAL] \\
\hline & $\mathrm{V}$ & $(373-430)$ & 23.5 & 388 & A & [1987STE/MAL] \\
\hline & $\mathrm{V}$ & $(263-329)$ & 26.1 & 278 & A & [1987STE/MAL] \\
\hline & V & $(223-273)$ & 27.2 & 258 & A & [1987STE/MAL, 1970DYK] \\
\hline & $\mathrm{V}$ & $(190-267)$ & 27.4 & 252 & & [1937AST/SIL, 1984BOU/FRI] \\
\hline \multirow[t]{4}{*}{$\mathrm{CH}_{5} \mathrm{NO}$} & {$[593-77-1]$} & $N$-methylhydroxy & amine & & & \\
\hline & SUB & $(273-308)$ & 56.6 & 288 & & [1987STE/MAL, 1957BIS/PAR] \\
\hline & V & $(293-338)$ & 49.7 & 308 & A & [1987STE/MAL, 1970DYK] \\
\hline & V & $(313-338)$ & 49.3 & 325 & A & [1987STE/MAL, 1957BIS/PAR, 1984BOU/FRI] \\
\hline \multirow[t]{3}{*}{$\mathrm{CH}_{5} \mathrm{NO}$} & {$[67-62-9]$} & $O$-methylhydroxyl & amine & & & \\
\hline & $\mathrm{V}$ & $(228-322)$ & 36.9 & 243 & A & [1987STE/MAL] \\
\hline & V & $(210-321)$ & 38.0 & 225 & & [1957BIS/PAR, 1984BOU/FRI] \\
\hline \multirow[t]{2}{*}{$\mathrm{CH}_{5} \mathrm{~N}_{3} \mathrm{O}$} & [758-19-0] & 1-methyl-1-nitroso & nydrazine & & & \\
\hline & SUB & & $79.5 \pm 0.4$ & 298 & & {$[1998 \mathrm{LEB} / \mathrm{CHI}]$} \\
\hline \multirow[t]{2}{*}{$\mathrm{CH}_{5} \mathrm{~N}_{3} \mathrm{~S}$} & {$[79-19-6]$} & thiosemicarbazide & & & & \\
\hline & SUB & & $125.8 \pm 1.5$ & 298 & $\mathrm{C}$ & {$[1982 \mathrm{TOR} / \mathrm{SAB}]$} \\
\hline \multirow[t]{3}{*}{$\mathrm{CH}_{5} \mathrm{O}_{3} \mathrm{P}$} & [993-13-5] & methylphosphonic & acid & & & \\
\hline & FUS & & 10.41 & 382.1 & DSC & [2015SHA/GE] \\
\hline & SUB & & $48.1 \pm 4.2$ & & & [1955NEA/WIL, 1970COX/PIL] \\
\hline
\end{tabular}


TABLE 4. Phase change enthalpies of $\mathrm{C}_{1}$ organic compounds-Continued

\begin{tabular}{|c|c|c|c|c|c|c|}
\hline \multirow[b]{2}{*}{ Molecular formula } & \multirow{2}{*}{$\begin{array}{c}\text { CAS reg. no. } \\
\text { Transition }\end{array}$} & \multicolumn{5}{|l|}{ Compound } \\
\hline & & Temp. range $(\mathrm{K})$ & $\Delta_{\text {trans }} H_{\mathrm{m}}\left(\mathrm{kJ} \mathrm{mol}^{-1}\right)$ & $T_{\mathrm{m}}(\mathrm{K})$ & Method & Reference \\
\hline \multirow[t]{2}{*}{$\mathrm{CH}_{6} \mathrm{ClN}$} & [593-51-1] & \multicolumn{5}{|c|}{ methylamine hydrochloride } \\
\hline & $\mathrm{V}$ & $(518-593)$ & 114.5 & 533 & A, I & [1987STE/MAL, 1967KIS] \\
\hline \multirow[t]{3}{*}{$\mathrm{CH}_{6} \mathrm{~N}_{2}$} & {$[60-34-4]$} & methylhydrazine & & & & \\
\hline & FUS & & 10.42 & 220.8 & & [1996DOM/HEA, 1951AST/FIN] \\
\hline & $\mathrm{V}$ & $(274-299)$ & 41.8 & 286 & A & [1987STE/MAL, 1951AST/FIN] \\
\hline \multirow[t]{4}{*}{$\mathrm{CH}_{6} \mathrm{~N}_{4} \mathrm{O}_{3}$} & {$[506-93-4]$} & guanidine nitrate & & & & \\
\hline & FUS & & 25.27 & 487.2 & DSC & [2015ZHA/QIA] \\
\hline & TRS & & 1.67 & 296 & & \\
\hline & FUS & & 25.6 & 487 & $\mathrm{DSC}$ & [1993SZA/CZA] \\
\hline \multirow[t]{2}{*}{$\mathrm{CH}_{6} \mathrm{~N}_{4} \mathrm{~S}$} & [2231-57-4] & thiocarbohydrazide & & & & \\
\hline & SUB & & $152.1 \pm 3.0$ & 298 & $\mathrm{C}$ & [1982TOR/SAB] \\
\hline
\end{tabular}


TABLE 5. Phase change enthalpies of $\mathrm{C}_{2}$ organic compounds

\begin{tabular}{|c|c|c|c|c|c|c|}
\hline \multirow[b]{2}{*}{$\begin{array}{l}\text { Molecular } \\
\text { formula }\end{array}$} & \multirow{2}{*}{$\begin{array}{l}\text { CAS reg. no. } \\
\text { Transition }\end{array}$} & \multicolumn{5}{|l|}{ Compound } \\
\hline & & Temp. range $(\mathrm{K})$ & $\begin{array}{c}\Delta_{\text {trans }} H_{\mathrm{m}} \\
\left(\mathrm{kJ} \mathrm{mol}^{-1}\right)\end{array}$ & $T_{\mathrm{m}}(\mathrm{K})$ & Method & References \\
\hline \multirow[t]{2}{*}{$\mathrm{C}_{2} \mathrm{BrCl}_{2} \mathrm{~F}_{3} \mathrm{O}_{4}$} & {$[38217-36-6]$} & \multicolumn{5}{|c|}{ perchloric acid, 1,2,2-trifluoro-1-chloro-2-bromoethyl ester } \\
\hline & $\mathrm{V}$ & $(273-294)$ & 42.5 & 283 & A & [1987STE/MAL, 1973SCH/PIL] \\
\hline \multirow[t]{2}{*}{$\mathrm{C}_{2} \mathrm{BrCl}_{3} \mathrm{O}$} & {$[34069-94-8]$} & \multicolumn{5}{|c|}{ trichloroacetyl bromide } \\
\hline & $\mathrm{V}$ & $(265-416)$ & 42.6 & 280 & A & [1987STE/MAL, 1947STU] \\
\hline \multirow[t]{2}{*}{$\mathrm{C}_{2} \mathrm{BrCl}_{5}$} & {$[79504-02-2]$} & \multicolumn{5}{|c|}{ bromopentachloroethane } \\
\hline & SUB & $(383-433)$ & 44.4 & 398 & A & [1987STE/MAL, 1949HIG/END] \\
\hline \multirow[t]{2}{*}{$\mathrm{C}_{2} \mathrm{BrF}_{3}$} & [598-73-2] & \multicolumn{5}{|c|}{ bromotrifluoroethylene } \\
\hline & V & $(260-340)$ & 25.0 & 275 & A & {$[1987 \mathrm{STE} / \mathrm{MAL}]$} \\
\hline \multirow[t]{2}{*}{$\mathrm{C}_{2} \mathrm{BrF}_{5} \mathrm{O}_{3} \mathrm{~S}$} & [757-02-8] & \multicolumn{5}{|c|}{ 2-bromotetrafluoroethyl fluorosulfate } \\
\hline & $\mathrm{V}$ & $(273-298)$ & 33.2 & 285 & & [1963GIL/CAD] \\
\hline \multirow[t]{2}{*}{$\mathrm{C}_{2} \mathrm{BrF}_{9} \mathrm{~S}$} & {$[63011-81-4]$} & \multicolumn{5}{|c|}{ pentafluoro(1-bromo-1,2,2,2-tetrafluoroethyl) sulfur } \\
\hline & $\mathrm{V}$ & $(294-330)$ & 30.7 & 309 & A & [1987STE/MAL, 1999DYK/SVO, 1977NOF/FOX] \\
\hline \multirow[t]{8}{*}{$\mathrm{C}_{2} \mathrm{Br}_{2} \mathrm{ClF}_{3}$} & [354-51-8] & \multicolumn{5}{|c|}{ 2-chloro-1,2-dibromo-1,1,2-trifluoroethane } \\
\hline & V & $(343-428)$ & 31.4 & 358 & A & [1987STE/MAL] \\
\hline & $\mathrm{V}$ & & $35.0 \pm 0.1$ & 298 & $\mathrm{C}$ & [1981MAJ/SVO] \\
\hline & $\mathrm{V}$ & & $34.2 \pm 0.1$ & 313 & $\mathrm{C}$ & [1981MAJ/SVO] \\
\hline & $\mathrm{V}$ & & $33.5 \pm 0.1$ & 328 & $\mathrm{C}$ & [1981MAJ/SVO] \\
\hline & $\mathrm{V}$ & & $32.6 \pm 0.1$ & 343 & $\mathrm{C}$ & [1981MAJ/SVO] \\
\hline & $\mathrm{V}$ & & $31.6 \pm 0.1$ & 358 & $\mathrm{C}$ & [1981MAJ/SVO] \\
\hline & $\mathrm{V}$ & $(301-365)$ & 33.9 & 333 & & [1981MAJ/SVO] \\
\hline \multirow[t]{3}{*}{$\mathrm{C}_{2} \mathrm{Br}_{2} \mathrm{Cl}_{4}$} & {$[630-25-1]$} & 1,2-dibromotetrach & roethane & & & \\
\hline & SUB & $(383-453)$ & 52.5 & 398 & A & [1987STE/MAL, 1949HIG/END] \\
\hline & SUB & $(323-423)$ & 56.7 & 373 & A & {$[1935 \mathrm{CAR} / \mathrm{DIC}]$} \\
\hline $\mathrm{C}_{2} \mathrm{Br}_{2} \mathrm{~F}_{4}$ & [124-73-2] & 1,2-dibromotetraflu & oethane & & & \\
\hline & FUS & $(8-300)$ & 7.04 & 162.8 & & [1991ACR, 1982KOS/ZHO] \\
\hline & $\mathrm{V}$ & $(283-357)$ & 28.5 & 298 & A & [1987STE/MAL] \\
\hline & $\mathrm{V}$ & (354-443) & 26.9 & 369 & A & [1987STE/MAL] \\
\hline & $\mathrm{V}$ & $(440-488)$ & 27.1 & 455 & A & [1987STE/MAL] \\
\hline & $\mathrm{V}$ & $(298-320)$ & $28.0 \pm 0.5$ & 298 & & [1985PAS/VAR] \\
\hline & $\mathrm{V}$ & & $28.4 \pm 0.1$ & 298 & $\mathrm{C}$ & [1981MAJ/SVO] \\
\hline & $\mathrm{V}$ & & $27.5 \pm 0.1$ & 313 & $\mathrm{C}$ & [1981MAJ/SVO] \\
\hline & $\mathrm{V}$ & & $26.5 \pm 0.1$ & 328 & $\mathrm{C}$ & [1981MAJ/SVO] \\
\hline & $\mathrm{V}$ & $(293-319)$ & 28.8 & 306 & & [1981MAJ/SVO] \\
\hline & $\mathrm{V}$ & $(246-295)$ & 30.0 & 280 & & [1987STE/MAL, 1970DYK] \\
\hline $\mathrm{C}_{2} \mathrm{Br}_{4}$ & [79-28-7] & tetrabromoethylene & & & & \\
\hline & SUB & $(221-310)$ & 44.2 & 236 & A & [1987STE/MAL] \\
\hline $\mathrm{C}_{2} \mathrm{ClFN}_{2}$ & {$[30915-40-3]$} & cis-chloro(fluoroim & o)acetonitrile & & & \\
\hline & $\mathrm{V}$ & $(254-320)$ & 31.7 & 269 & A & [1987STE/MAL, 1971ZAB/SHR] \\
\hline $\mathrm{C}_{2} \mathrm{ClFN}_{2}$ & {$[30915-39-0]$} & trans-chloro(fluoroi & ino)acetonitri & & & \\
\hline & $\mathrm{V}$ & $(257-320)$ & 32.7 & 272 & A & [1987STE/MAL, 1971ZAB/SHR] \\
\hline $\mathrm{C}_{2} \mathrm{ClF}_{2} \mathrm{NO}_{2}$ & [42016-33-1] & chloro(fluorocarbon & )carbamic flu & & & \\
\hline & $\mathrm{V}$ & & 36.8 & 376 & & [1973SPR/WRI] \\
\hline $\mathrm{C}_{2} \mathrm{ClF}_{3}$ & [79-38-9] & chlorotrifluoroethyl & & & & \\
\hline & FUS & $(16-245)$ & 5.55 & 115 & $\mathrm{AC}$ & [1996DOM/HEA, 1951OLI/GRI2] \\
\hline & $\mathrm{V}$ & $(206-262)$ & 21.8 & 247 & A & [1987STE/MAL, 1951OLI/GRI2] \\
\hline & $\mathrm{V}$ & $(298-379)$ & 20.2 & 313 & A & [1987STE/MAL, 1951OLI/GRI2] \\
\hline & $\mathrm{V}$ & $(195-250)$ & 21.9 & 235 & & [1933HEI/MUR] \\
\hline $\mathrm{C}_{2} \mathrm{ClF}_{3} \mathrm{O}_{2}$ & {$[23213-83-4]$} & chloroformic acid, $\mathrm{t}$ & fluoromethyl & & & \\
\hline & $\mathrm{V}$ & $(195-273)$ & 24.1 & 258 & A & [1987STE/MAL, 1969SCH/MAY] \\
\hline & $\mathrm{V}$ & $(195-273)$ & 25.2 & 234 & & [1969SCH/MAY] \\
\hline $\mathrm{C}_{2} \mathrm{ClF}_{3} \mathrm{O}_{4} \mathrm{~S}$ & {$[6069-32-5]$} & difluorochloroacetic & cid, fluorosul & acid anhy & & \\
\hline & V & $(265-352)$ & 39.8 & 280 & A & [1987STE/MAL, 1966DES/CAD, 1999DYK/SVO] \\
\hline $\mathrm{C}_{2} \mathrm{ClF}_{4} \mathrm{NO}$ & [42016-31-9] & chloro(trifluorometl & 1)carbamic flu & & & \\
\hline & $\mathrm{V}$ & & 28.9 & 310 & & [1973SPR/WRI] \\
\hline
\end{tabular}


TABLE 5. Phase change enthalpies of $\mathrm{C}_{2}$ organic compounds-Continued

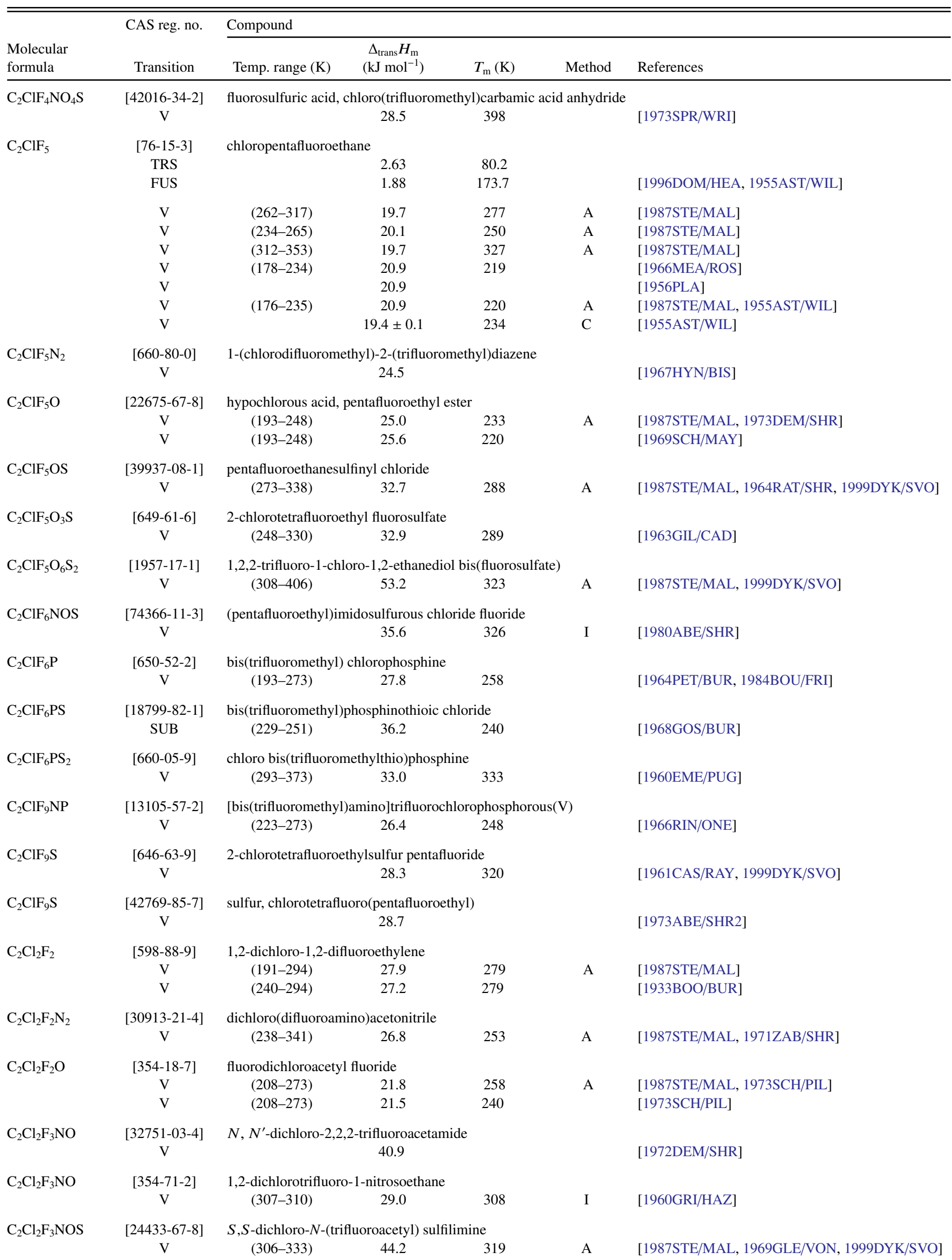


TABle 5. Phase change enthalpies of $\mathrm{C}_{2}$ organic compounds-Continued

\begin{tabular}{|c|c|c|c|c|c|c|}
\hline \multirow[b]{2}{*}{$\begin{array}{l}\text { Molecular } \\
\text { formula }\end{array}$} & \multirow{2}{*}{$\begin{array}{l}\text { CAS reg. no. } \\
\text { Transition }\end{array}$} & \multicolumn{5}{|l|}{ Compound } \\
\hline & & Temp. range $(\mathrm{K})$ & $\begin{array}{c}\Delta_{\text {trans }} H_{\mathrm{m}} \\
\left(\mathrm{kJ} \mathrm{mol}^{-1}\right)\end{array}$ & $T_{\mathrm{m}}(\mathrm{K})$ & Method & References \\
\hline \multirow[t]{2}{*}{$\mathrm{C}_{2} \mathrm{Cl}_{2} \mathrm{~F}_{3} \mathrm{NO}_{2} \mathrm{~S}$} & {$[51587-33-8]$} & \multicolumn{5}{|c|}{ (trifluoromethyl)sulfonyl carbonimidic dichloride } \\
\hline & $\mathrm{V}$ & $(312-405)$ & 44.1 & 327 & A & [1987STE/MAL, 1999DYK/SVO, 1974BEH/HAA] \\
\hline \multirow[t]{6}{*}{$\mathrm{C}_{2} \mathrm{Cl}_{2} \mathrm{~F}_{4}$} & {$[374-07-2]$} & \multicolumn{5}{|c|}{ 1,1-dichloro-1,2,2,2-tetrafluoroethane } \\
\hline & $\mathrm{V}$ & $(231-373)$ & 23.5 & 246 & A & [1987STE/MAL, 1970DYK] \\
\hline & $\mathrm{V}$ & & 23.2 & 233 & BG & {$[1955 \mathrm{MEA} / \mathrm{STA}]$} \\
\hline & $\mathrm{V}$ & & 22.5 & 273 & BG & [1955MEA/STA] \\
\hline & $\mathrm{V}$ & & 20.8 & 313 & BG & [1955MEA/STA] \\
\hline & $\mathrm{V}$ & & 17.7 & 353 & BG & {$[1955 \mathrm{MEA} / \mathrm{STA}]$} \\
\hline \multirow[t]{7}{*}{$\mathrm{C}_{2} \mathrm{Cl}_{2} \mathrm{~F}_{4}$} & {$[76-14-2]$} & \multicolumn{5}{|c|}{ 1,2-dichloro-1,1,2,2-tetrafluoroethane } \\
\hline & TRS & $(8-179)$ & 1.21 & 109.3 & & \\
\hline & TRS & $(8-179)$ & 2.63 & 134.6 & & \\
\hline & FUS & $(8-179)$ & 1.51 & 180.6 & $\mathrm{AC}$ & [1996DOM/HEA, 1981KOL/KOS] \\
\hline & $\mathrm{V}$ & $(277-391)$ & 24.3 & 292 & A & [1987STE/MAL] \\
\hline & $\mathrm{V}$ & $(210-277)$ & 25.1 & 262 & A & [1987STE/MAL] \\
\hline & $\mathrm{V}$ & $(178-277)$ & 25.3 & 261 & & [1947STU] \\
\hline \multirow[t]{2}{*}{$\mathrm{C}_{2} \mathrm{Cl}_{2} \mathrm{~F}_{4} \mathrm{O}_{4}$} & {$[38126-28-2]$} & \multicolumn{5}{|c|}{ perchloric acid, 1,1,2,2-tetrafluoro-2-chloroethyl ester } \\
\hline & $\mathrm{V}$ & $(249-294)$ & 32.6 & 279 & A & [1987STE/MAL, 1973SCH/PIL] \\
\hline \multirow[t]{2}{*}{$\mathrm{C}_{2} \mathrm{Cl}_{2} \mathrm{~F}_{5} \mathrm{NS}$} & {$[10564-48-4]$} & \multicolumn{5}{|c|}{$S, S$-dichloro- $N$-(pentafluoroethyl) sulfilimine } \\
\hline & V & $(297-375)$ & 37.4 & 312 & A & [1987STE/MAL, 1999DYK/SVO, 1966LUS] \\
\hline \multirow[t]{2}{*}{$\mathrm{C}_{2} \mathrm{Cl}_{2} \mathrm{~F}_{8} \mathrm{NP}$} & {$[13105-58-3]$} & \multicolumn{5}{|c|}{ [bis(trifluoromethyl)amino]difluorodichlorophosphorous(V) } \\
\hline & $\mathrm{V}$ & $(262-305)$ & 32.9 & 293 & & [1966EME/ONA] \\
\hline \multirow[t]{7}{*}{$\mathrm{C}_{2} \mathrm{Cl}_{3} \mathrm{~F}_{3}$} & {$[354-58-5]$} & \multicolumn{5}{|c|}{ 1,1,1-trichloro-2,2,2-trifluoroethane } \\
\hline & FUS & & 4.11 & 287.5 & DSC & [1987OTT/WOO] \\
\hline & $\mathrm{V}$ & & $28.1 \pm 0.1$ & 298 & $\mathrm{C}$ & [1980MAJ/SVO] \\
\hline & $\mathrm{V}$ & & $27.2 \pm 0.1$ & 313 & $\mathrm{C}$ & {$[1980 \mathrm{MAJ} / \mathrm{SVO}]$} \\
\hline & $\mathrm{V}$ & & $26.3 \pm 0.1$ & 328 & $\mathrm{C}$ & {$[1980 \mathrm{MAJ} / \mathrm{SVO}]$} \\
\hline & $\mathrm{V}$ & $(297-319)$ & 28.9 & 308 & & [1980MAJ/SVO] \\
\hline & $\mathrm{V}$ & $(286-310)$ & 29.2 & 298 & A & [1987STE/MAL, 1963HIR/HIL] \\
\hline \multirow[t]{14}{*}{$\mathrm{C}_{2} \mathrm{Cl}_{3} \mathrm{~F}_{3}$} & {$[76-13-1]$} & 1,1,2-trichloro-1,2,2 & rifluoroethan & & & \\
\hline & TRS & $(6-299)$ & 0.83 & 82.5 & & \\
\hline & FUS & $(6-299)$ & 2.47 & 236.9 & $\mathrm{AC}$ & [1991ACR, 1981KOL/KOS] \\
\hline & SUB & $(205-233)$ & 32.9 & 219 & A & [1947STU] \\
\hline & $\mathrm{V}$ & $(238-364)$ & 30.9 & 253 & A & [1987STE/MAL] \\
\hline & $\mathrm{V}$ & $(360-473)$ & 26.9 & 375 & A & [1987STE/MAL] \\
\hline & $\mathrm{V}$ & $(297-317)$ & 28.8 & 307 & A & [1987STE/MAL] \\
\hline & $\mathrm{V}$ & & $28.4 \pm 0.1$ & 298 & $\mathrm{C}$ & [1980MAJ/SVO] \\
\hline & $\mathrm{V}$ & & $27.5 \pm 0.1$ & 313 & $\mathrm{C}$ & [1980MAJ/SVO] \\
\hline & $\mathrm{V}$ & & $26.6 \pm 0.1$ & 328 & $\mathrm{C}$ & [1980MAJ/SVO] \\
\hline & $\mathrm{V}$ & & $28.2 \pm 0.4$ & 298 & & [1974VAR/BUL] \\
\hline & $\mathrm{V}$ & $(273-318)$ & 28.2 & 288 & A & [1963HIR/HIL, 1987STE/MAL] \\
\hline & $\mathrm{V}$ & $(248-356)$ & 30.8 & 263 & & [1940BEN/MCH] \\
\hline & $\mathrm{V}$ & $(243-353)$ & NA & & & [1939REI] \\
\hline $\mathrm{C}_{2} \mathrm{Cl}_{3} \mathrm{~F}_{3}$ & & trichlorotrifluoroeth & & & & \\
\hline & $\mathrm{V}$ & $(248-352)$ & 30.5 & 263 & & [1938REI] \\
\hline $\mathrm{C}_{2} \mathrm{Cl}_{3} \mathrm{~F}_{3} \mathrm{O}_{4}$ & [38126-27-1] & perchloric acid, 1,2 , & -trifluoro-1,2- & roethyl e & & \\
\hline & $\mathrm{V}$ & $(273-296)$ & 26.9 & 284 & A & [1987STE/MAL, 1973SCH/PIL] \\
\hline $\mathrm{C}_{2} \mathrm{Cl}_{3} \mathrm{~N}$ & {$[545-06-2]$} & trichloroacetonitrile & & & & \\
\hline & $\mathrm{V}$ & $(289-357)$ & 35.1 & 304 & A & [1987STE/MAL, 1970DYK] \\
\hline & $\mathrm{V}$ & $(289-356)$ & 34.7 & 304 & & [1954DAV/JEN] \\
\hline $\mathrm{C}_{2} \mathrm{Cl}_{4}$ & [127-18-4] & tetrachloroethylene & & & & \\
\hline & TRS & $(6-300)$ & 0.82 & 210 & & \\
\hline & FUS & $(6-300)$ & 10.88 & 250.8 & $\mathrm{AC}$ & [1996DOM/HEA, 1986NOV/RAB] \\
\hline & $\mathrm{V}$ & $(307-393)$ & 38.4 & 322 & & [1995DEJ/BUR] \\
\hline & $\mathrm{V}$ & & 39.7 & 298 & $\mathrm{C}$ & [1980MAJ/SVA] \\
\hline
\end{tabular}


TABle 5. Phase change enthalpies of $\mathrm{C}_{2}$ organic compounds-Continued

\begin{tabular}{|c|c|c|c|c|c|c|}
\hline \multirow[b]{2}{*}{$\begin{array}{l}\text { Molecular } \\
\text { formula }\end{array}$} & \multirow{2}{*}{$\begin{array}{l}\text { CAS reg. no. } \\
\text { Transition }\end{array}$} & \multicolumn{5}{|l|}{ Compound } \\
\hline & & Temp. range $(\mathrm{K})$ & $\begin{array}{c}\Delta_{\text {trans }} H_{\mathrm{m}} \\
\left(\mathrm{kJ} \mathrm{mol}^{-1}\right)\end{array}$ & $T_{\mathrm{m}}(\mathrm{K})$ & Method & References \\
\hline & $\mathrm{V}$ & & 38.9 & 313 & $\mathrm{C}$ & [1980MAJ/SVA] \\
\hline & $\mathrm{V}$ & & 38.2 & 328 & $\mathrm{C}$ & [1980MAJ/SVA] \\
\hline & $\mathrm{V}$ & & 37.4 & 343 & $\mathrm{C}$ & [1980MAJ/SVA] \\
\hline & $\mathrm{V}$ & & 36.6 & 358 & $\mathrm{C}$ & [1980MAJ/SVA] \\
\hline & $\mathrm{V}$ & $(310-393)$ & 38.7 & 325 & A & [1987STE/MAL, 1972BOU/AIM] \\
\hline & $\mathrm{V}$ & $(300-380)$ & 38.9 & 315 & & [1970POL/MUR, 1984BOU/FRI] \\
\hline & $\mathrm{V}$ & $(333-373)$ & 37.6 & 348 & & [1967FRI/GAL] \\
\hline & $\mathrm{V}$ & & $39.6 \pm 0.1$ & 298 & $\mathrm{C}$ & [1960AIH] \\
\hline & $\mathrm{V}$ & & $34.7 \pm 0.1$ & 394 & & [1926MAT] \\
\hline & $\mathrm{V}$ & $(306-391)$ & 35.7 & 397 & & [1913HER/RAT] \\
\hline \multirow{16}{*}{$\mathrm{C}_{2} \mathrm{Cl}_{4} \mathrm{~F}_{2}$} & {$[76-12-0]$} & \multicolumn{5}{|c|}{ 1,2-difluoro-1,1,2,2-tetrachloroethane } \\
\hline & TRS & & 0.79 & 130 & & \\
\hline & FUS & & 3.7 & 297.9 & & [1991ACR, 1978KIS/SUG] \\
\hline & FUS & & 3.7 & 299.7 & & {$[1978 \mathrm{KOS} / \mathrm{KOL}]$} \\
\hline & SUB & $(235-293)$ & 36.4 & 278 & A & [1987STE/MAL, 1947STU] \\
\hline & SUB & $(237-293)$ & 38.2 & 265 & A & {$[1947 \mathrm{STU}]$} \\
\hline & $\mathrm{V}$ & $(313-361)$ & $34.8 \pm 0.4$ & 298 & & [2007VAR/DRU, 1976VAR/BUL] \\
\hline & $\mathrm{V}$ & & $34.6 \pm 0.1$ & 308 & $\mathrm{C}$ & [1992SVO/KUB2] \\
\hline & $\mathrm{V}$ & & $34.1 \pm 0.1$ & 315 & $\mathrm{C}$ & [1992SVO/KUB2] \\
\hline & $\mathrm{V}$ & & $33.6 \pm 0.1$ & 323 & $\mathrm{C}$ & [1992SVO/KUB2] \\
\hline & $\mathrm{V}$ & & $33.1 \pm 0.1$ & 330 & $\mathrm{C}$ & [1992SVO/KUB2] \\
\hline & $\mathrm{V}$ & & $32.6 \pm 0.1$ & 338 & $\mathrm{C}$ & [1992SVO/KUB2] \\
\hline & $\mathrm{V}$ & $(301-365)$ & 36.6 & 316 & A & [1987STE/MAL] \\
\hline & $\mathrm{V}$ & $(235-293)$ & 36.4 & 278 & A & [1987STE/MAL] \\
\hline & $\mathrm{V}$ & $(312-362)$ & 34.0 & 327 & A & [1987STE/MAL, 1976VAR/BUL] \\
\hline & $\mathrm{V}$ & $(283-364)$ & 32.7 & 298 & & {$[1933 \mathrm{HOR} / \mathrm{GEI}]$} \\
\hline \multirow[t]{2}{*}{$\mathrm{C}_{2} \mathrm{Cl}_{4} \mathrm{~F}_{2} \mathrm{O}_{3} \mathrm{~S}$} & {$[665-15-6]$} & \multicolumn{5}{|c|}{ 2-fluorotetrachloroethyl fluorosulfate } \\
\hline & $\mathrm{V}$ & $(311-437)$ & 42.0 & 329 & & [1963GIL/CAD] \\
\hline \multirow[t]{2}{*}{$\mathrm{C}_{2} \mathrm{Cl}_{4} \mathrm{~F}_{2} \mathrm{O}_{4}$} & {$[38126-29-3]$} & \multicolumn{5}{|c|}{ perchloric acid, 1,2-difluoro-1,2,2-trichloroethyl ester } \\
\hline & $\mathrm{V}$ & $(273-294)$ & 30.2 & 283 & A & [1987STE/MAL, 1973SCH/PIL] \\
\hline \multirow[t]{2}{*}{$\mathrm{C}_{2} \mathrm{Cl}_{4} \mathrm{~F}_{4} \mathrm{~N}_{2}$} & {$[35695-53-5]$} & \multicolumn{5}{|c|}{ 1,2-bis(dichloroamino) tetrafluoroethane } \\
\hline & $\mathrm{V}$ & & 43.1 & & & [1972DEM/SHR] \\
\hline \multirow[t]{2}{*}{$\mathrm{C}_{2} \mathrm{Cl}_{4} \mathrm{~F}_{6} \mathrm{OS}$} & {$[762-90-3]$} & \multicolumn{5}{|c|}{ pentafluoro(2-fluoro-1,1,2,2-tetrachloroethoxy) sulfur } \\
\hline & $\mathrm{V}$ & $(314-418)$ & 42.8 & 329 & A & [1987STE/MAL, 1962WIL/CAD] \\
\hline \multirow[t]{2}{*}{$\mathrm{C}_{2} \mathrm{Cl}_{4} \mathrm{O}$} & {$[16650-10-5]$} & \multicolumn{5}{|c|}{ tetrachloroethylene oxide } \\
\hline & $\mathrm{V}$ & $(308-348)$ & 36.9 & 323 & A & [1987STE/MAL, 1957FRA/JOH] \\
\hline \multirow[t]{2}{*}{$\mathrm{C} 2 \mathrm{Cl} 4 \mathrm{O}$} & {$[76-02-8]$} & \multicolumn{5}{|c|}{ trichloroacetyl chloride } \\
\hline & $\mathrm{V}$ & $(305-393)$ & 38.3 & 320 & A & [1987STE/MAL, 1959MCD/SHR, 1970DYK] \\
\hline $\mathrm{C}_{2} \mathrm{Cl}_{6}$ & {$[67-72-1]$} & hexachloroethane & & & & \\
\hline & TRS & & 2.10 & 316.8 & & \\
\hline & TRS & & 6.30 & 345.0 & & [1996DOM/HEA, 1988PET/TSY] \\
\hline & TRS & $(14-360)$ & 2.64 & 317.4 & & \\
\hline & TRS & $(14-360)$ & 6.92 & 344.6 & $\mathrm{AC}$ & [1996DOM/HEA, 1975RAK/GUT] \\
\hline & TRS & & 2.61 & 320.2 & & \\
\hline & TRS & & 6.31 & 344.7 & DSC & [1970MUR/BRE2] \\
\hline & TRS & & 3.12 & 316.2 & & [1967TSE/GOD] \\
\hline & TRS & $(295-351)$ & 2.57 & 318 & & \\
\hline & TRS & $(295-351)$ & 8.22 & 345 & & \\
\hline & FUS & $(295-351)$ & 9.75 & 458 & $\mathrm{CC}$ & [1996DOM/HEA, 1950SEK/MOM] \\
\hline & SUB & $(317-345)$ & 58.9 & 331 & A & [1987STE/MAL] \\
\hline & SUB (m.p. 186.6) & $(306-459)$ & 48.8 & 382 & A & [1947STU] \\
\hline & SUB (triclinic) & $(286-447)$ & $59.1 \pm 0.7$ & 367 & BG & [1947IVI/DAI, 1960JON, 1970COX/PIL] \\
\hline & SUB (cubic) & $(286-447)$ & 51.0 & 367 & $\mathrm{BG}$ & [1947IVI/DAI, 1960JON] \\
\hline & SUB & & NA & & GSM & [1941NIT/SEK] \\
\hline & SUB & $(335-453)$ & 50.5 & & & [1935LEE] \\
\hline & SUB & $(288-333)$ & 59.0 & 310 & GS, A & [1930NEL] \\
\hline
\end{tabular}


TABLE 5. Phase change enthalpies of $\mathrm{C}_{2}$ organic compounds-Continued

\begin{tabular}{|c|c|c|c|c|c|c|}
\hline \multirow[b]{2}{*}{$\begin{array}{l}\text { Molecular } \\
\text { formula }\end{array}$} & \multirow{2}{*}{$\begin{array}{l}\text { CAS reg. no. } \\
\text { Transition }\end{array}$} & \multicolumn{5}{|l|}{ Compound } \\
\hline & & Temp. range $(\mathrm{K})$ & $\begin{array}{c}\Delta_{\text {trans }} H_{\mathrm{m}} \\
\left(\mathrm{kJ} \mathrm{mol}^{-1}\right)\end{array}$ & $T_{\mathrm{m}}(\mathrm{K})$ & Method & References \\
\hline & $\mathrm{V}$ & $(460-513)$ & 40.3 & 475 & A & [1987STE/MAL, 1935LEE] \\
\hline & $\mathrm{V}$ & $(345-460)$ & 51.2 & 360 & A & [1987STE/MAL, 1970DYK] \\
\hline & $\mathrm{V}$ & $(305-458)$ & 53.7 & 320 & & [1947STU] \\
\hline \multirow[t]{2}{*}{$\mathrm{C}_{2} \mathrm{FNO}_{2}$} & [15435-14-0] & \multicolumn{4}{|c|}{ fluorocarbonyl isocyanate } & \\
\hline & $\mathrm{V}$ & $(228-264)$ & 33.5 & 249 & A & [1987STE/MAL, 1967GLE/BIE] \\
\hline \multirow[t]{2}{*}{$\mathrm{C}_{2} \mathrm{~F}_{2} \mathrm{~N}_{2} \mathrm{O}$} & {$[32837-63-1]$} & \multicolumn{4}{|c|}{ difluorocarboncyamidic amide } & \\
\hline & $\mathrm{V}$ & & 29.7 & 383 & & [1973WRI/SHR] \\
\hline \multirow[t]{2}{*}{$\mathrm{C}_{2} \mathrm{~F}_{2} \mathrm{~N}_{2} \mathrm{O}_{2}$} & {$[32837-64-2]$} & \multicolumn{4}{|c|}{ difluorocarbonisocyantidic amide } & \\
\hline & $\mathrm{V}$ & & 33.9 & 327 & & [1973WRI/SHR] \\
\hline \multirow[t]{2}{*}{$\mathrm{C}_{2} \mathrm{~F}_{2} \mathrm{~N}_{4} \mathrm{O}_{8}$} & {$[20165-39-3]$} & \multicolumn{4}{|c|}{ 1,2-difluoro-1,1,2,2-tetranitroethane } & \\
\hline & $\mathrm{V}$ & $(297-323)$ & 62.8 & 310 & A & [1987STE/MAL, 1973PEP/LEB] \\
\hline \multirow[t]{4}{*}{$\mathrm{C}_{2} \mathrm{~F}_{2} \mathrm{O}_{2}$} & {$[359-40-0]$} & \multicolumn{4}{|l|}{ oxalyl fluoride } & \\
\hline & FUS & & 13.4 & 260.7 & & [1996DOM/HEA, 1971HOD] \\
\hline & SUB & $(234-260)$ & 16.7 & 247 & A & [1987STE/MAL] \\
\hline & $\mathrm{V}$ & $(264-272)$ & 29.7 & 268 & A & [1987STE/MAL] \\
\hline \multirow[t]{2}{*}{$\mathrm{C}_{2} \mathrm{~F}_{2} \mathrm{O}_{4}$} & {$[692-74-0]$} & \multicolumn{4}{|c|}{ bis(fluorocarbonyl)peroxide } & \\
\hline & $\mathrm{V}$ & $(226-266)$ & 30.6 & 251 & & [1962VON/AYM, 1984BOU/FRI] \\
\hline \multirow[t]{9}{*}{$\mathrm{C}_{2} \mathrm{~F}_{3} \mathrm{~N}$} & {$[353-85-5]$} & \multicolumn{4}{|l|}{ trifluoroacetonitrile } & \\
\hline & FUS & & 4.97 & 128.7 & & [1996DOM/HEA, 1961PAC/BOB] \\
\hline & $\mathrm{V}$ & $(151-206)$ & 19.3 & 191 & A & [1987STE/MAL] \\
\hline & $\mathrm{V}$ & $(141-203)$ & 19.2 & 188 & A & [1987STE/MAL] \\
\hline & $\mathrm{V}$ & $(197-241)$ & 18.5 & 226 & A & [1987STE/MAL] \\
\hline & $\mathrm{V}$ & $(282-336)$ & 17.4 & 309 & A & [1987STE/MAL] \\
\hline & $\mathrm{V}$ & $(272-311)$ & 17.4 & 287 & A & [1987STE/MAL] \\
\hline & $\mathrm{V}$ & $(142-206)$ & 19.2 & 191 & & [1961PAC/BOB] \\
\hline & $\mathrm{V}$ & & 17.8 & 205 & $\mathrm{C}$ & [1961PAC/BOB] \\
\hline \multirow[t]{2}{*}{$\mathrm{C}_{2} \mathrm{~F}_{3} \mathrm{NO}$} & {$[460-49-1]$} & \multicolumn{4}{|c|}{ trifluoromethyl isocyanate } & \\
\hline & $\mathrm{V}$ & $(195-228)$ & 22.5 & 213 & A & [1987STE/MAL, 1956BAR/HAS2] \\
\hline \multirow[t]{2}{*}{$\mathrm{C}_{2} \mathrm{~F}_{3} \mathrm{NO}$} & {$[2713-04-4]$} & trifluoronitrosoeth & & & & \\
\hline & $\mathrm{V}$ & $(247-250)$ & 25.7 & 248 & $\mathrm{~A}, \mathrm{I}$ & [1987STE/MAL, 1960GRI/HAZ] \\
\hline $\mathrm{C}_{2} \mathrm{~F}_{3} \mathrm{NOS}$ & {$[61951-27-7]$} & trifluoromethanesı & tyl cyanide & & & \\
\hline & $\mathrm{V}$ & & 40.2 & 352 & $\mathrm{I}$ & [1977BUR/SHR] \\
\hline $\mathrm{C}_{2} \mathrm{~F}_{3} \mathrm{NOS}$ & [691-03-2] & trifluoroemethylsu & nyl isocyanat & & & \\
\hline & $\mathrm{V}$ & $(231-293)$ & 27.9 & 278 & A & [1987STE/MAL, 1999DYK/SVO, 1963EME/HAA] \\
\hline $\mathrm{C}_{2} \mathrm{~F}_{3} \mathrm{NO}_{2} \mathrm{~S}$ & [26454-68-2] & 2,2,2-trifluoro- $N$-s & inylacetamide & & & \\
\hline & $\mathrm{V}$ & $(267-302)$ & 36.4 & 282 & A & [1987STE/MAL, 1999DYK/SVO] \\
\hline & V & $(244-268)$ & 35.4 & 256 & & [1970VON/GLE] \\
\hline $\mathrm{C}_{2} \mathrm{~F}_{3} \mathrm{NO}_{2} \mathrm{~S}_{2}$ & {$[51587-30-5]$} & trifluoromethanest & nyl isothiocy & & & \\
\hline & $\mathrm{V}$ & $(297-385)$ & 41.0 & 312 & A & [1987STE/MAL, 1999DYK/SVO, 1974BEH/HAA] \\
\hline $\mathrm{C}_{2} \mathrm{~F}_{3} \mathrm{NO}_{3} \mathrm{~S}$ & [30227-06-6] & trifluoromethanest & nyl isocyanat & & & \\
\hline & V & $(275-345)$ & 36.9 & 290 & A & [1987STE/MAL, 1999DYK/SVO, 1974BEH/HAA] \\
\hline $\mathrm{C}_{2} \mathrm{~F}_{3} \mathrm{NO}_{6}$ & [53340-26-4] & trifluoroacetyl per & nitrate & & & \\
\hline & $\mathrm{V}$ & $(223-293)$ & 33.6 & 258 & & [1997KOP/BEU] \\
\hline $\mathrm{C}_{2} \mathrm{~F}_{3} \mathrm{NO}_{6}$ & [227095-77-4] & trifluoromethoxyc & onyl peroxyn & & & \\
\hline & $\mathrm{V}$ & $(213-263)$ & 38.0 & 238 & & [2005VON/GAR] \\
\hline $\mathrm{C}_{2} \mathrm{~F}_{3} \mathrm{NS}$ & [690-24-4] & thiocyanic acid, tri & oromethyl est & & & \\
\hline & $\mathrm{V}$ & $(226-294)$ & 32.6 & 279 & A & [1987STE/MAL, 1999DYK/SVO, 1963EME/HAA] \\
\hline $\mathrm{C}_{2} \mathrm{~F}_{3} \mathrm{~N}_{3} \mathrm{O}_{6}$ & {$[20165-38-2]$} & 1,1,2-trifluoro-1,2 & rinitroethane & & & \\
\hline & $\mathrm{V}$ & $(313-353)$ & 57.7 & 328 & A & [1987STE/MAL, 1973PEP/LEB] \\
\hline
\end{tabular}


TABLE 5. Phase change enthalpies of $\mathrm{C}_{2}$ organic compounds-Continued

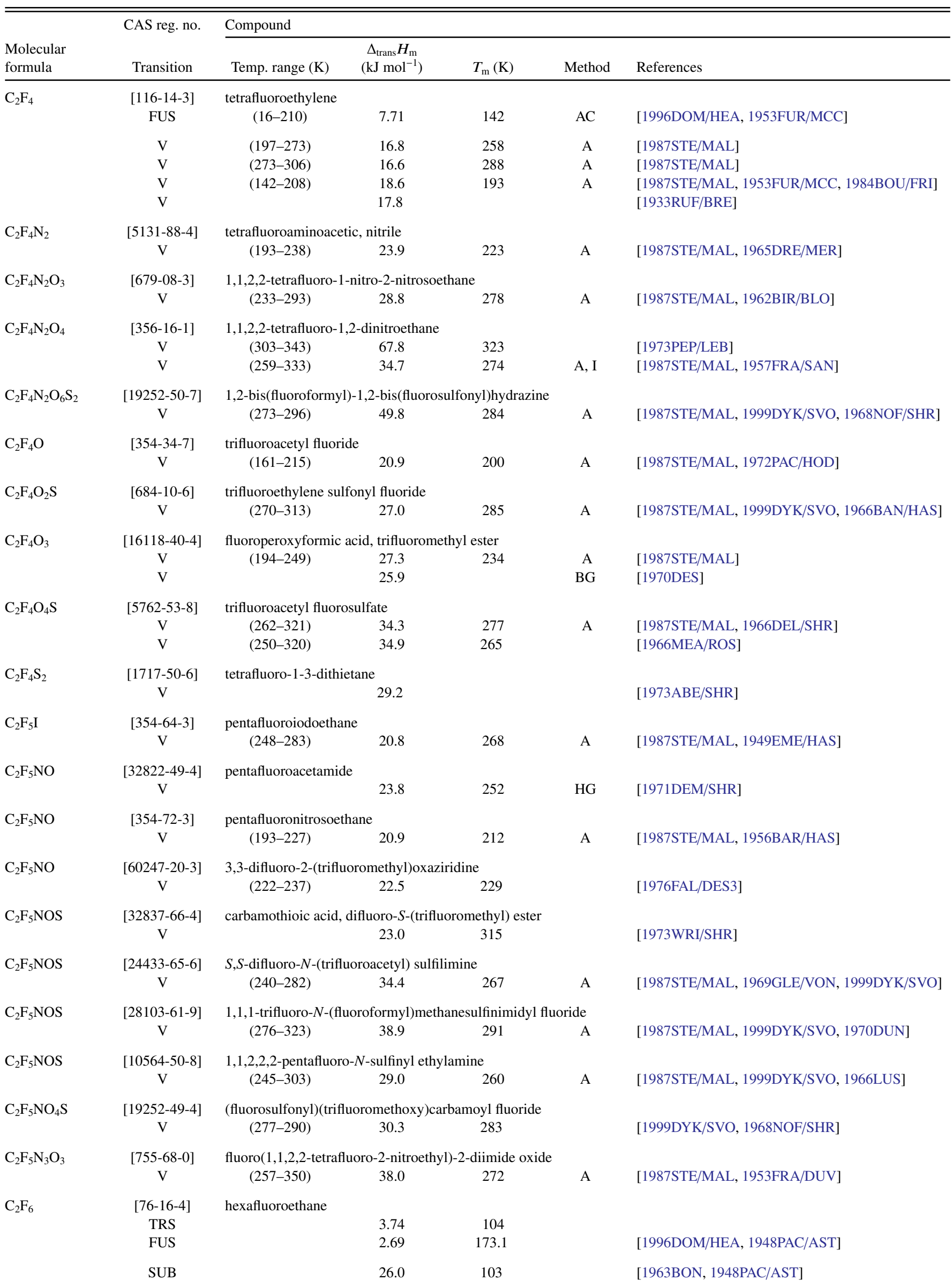


TABLE 5. Phase change enthalpies of $\mathrm{C}_{2}$ organic compounds-Continued

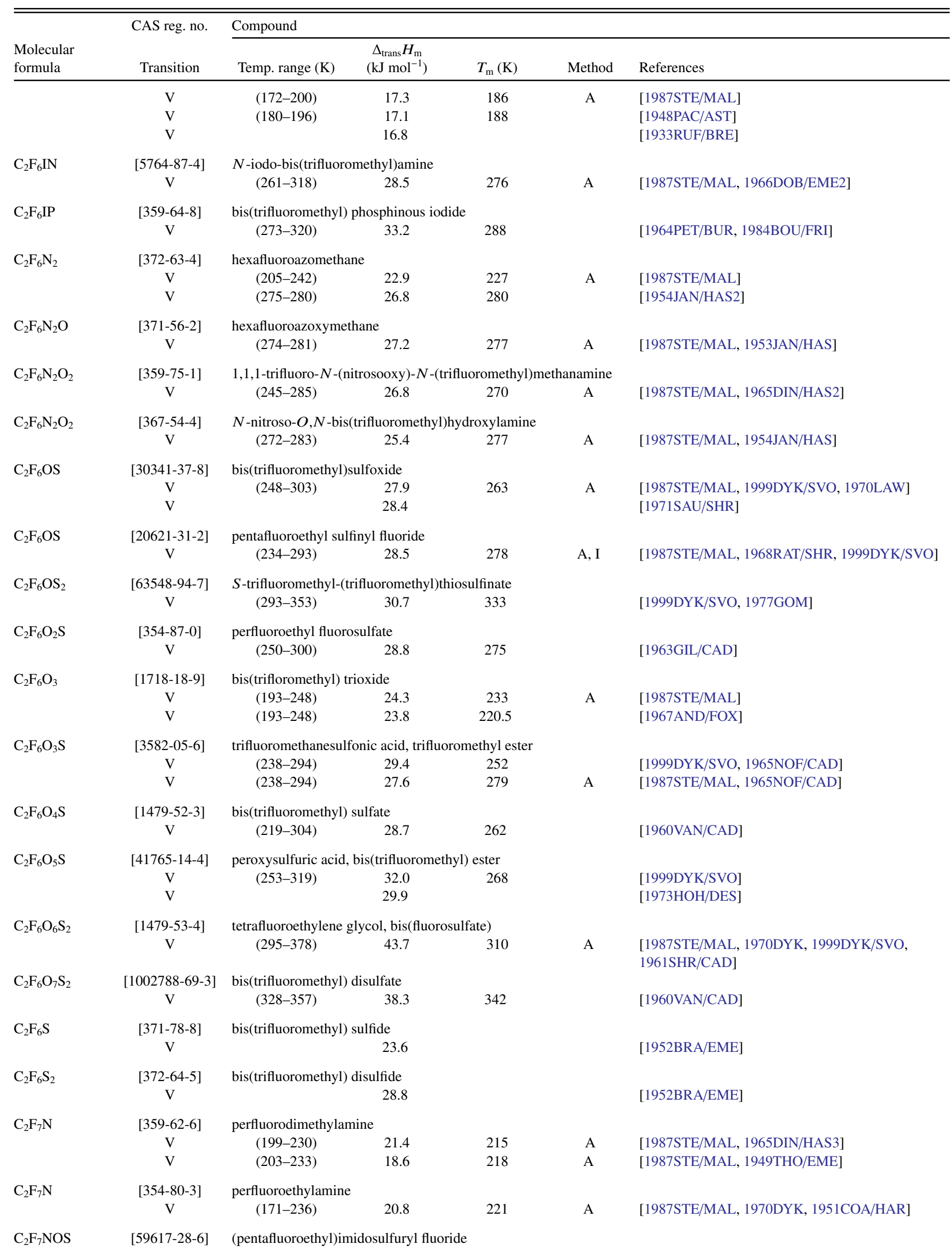


TABLE 5. Phase change enthalpies of $\mathrm{C}_{2}$ organic compounds-Continued

\begin{tabular}{|c|c|c|c|c|c|c|}
\hline \multirow[b]{2}{*}{$\begin{array}{l}\text { Molecular } \\
\text { formula }\end{array}$} & \multirow{2}{*}{$\begin{array}{l}\text { CAS reg. no. } \\
\text { Transition }\end{array}$} & \multicolumn{5}{|l|}{ Compound } \\
\hline & & Temp. range $(\mathrm{K})$ & $\begin{array}{c}\Delta_{\text {trans }} H_{\mathrm{m}} \\
\left(\mathrm{kJ} \mathrm{mol}^{-1}\right)\end{array}$ & $T_{\mathrm{m}}(\mathrm{K})$ & Method & References \\
\hline & $\mathrm{V}$ & & 30.7 & & & [1976STA/MEW] \\
\hline $\mathrm{C}_{2} \mathrm{~F}_{7} \mathrm{NO}_{3} \mathrm{~S}$ & $\begin{array}{c}{[4188-34-5]} \\
\mathrm{V}\end{array}$ & $\begin{array}{l}\text { fluorosulfuric acid } \\
\qquad(262-321)\end{array}$ & $\begin{array}{c}\text { 1,2,2-tetraflu } \\
31.1\end{array}$ & $\begin{array}{l}\text { (difluoro } \\
291\end{array}$ & $\begin{array}{l}\text { ethyl ester } \\
\text { A }\end{array}$ & [1987STE/MAL, 1999DYK/SVO, 1965LUS/RUF] \\
\hline $\mathrm{C}_{2} \mathrm{~F}_{7} \mathrm{NO}_{12} \mathrm{~S}_{4}$ & $\begin{array}{c}{[53684-02-9]} \\
\mathrm{V}\end{array}$ & fluorosulfuric acid & $\begin{array}{c}\text { [bis[(fluoros } \\
43.4\end{array}$ & $\begin{array}{l}\text { 1)oxo]am } \\
418\end{array}$ & 2,2-trifluor & $\begin{array}{l}\text { ethylidene ester } \\
\text { [1975KIR/LAS] }\end{array}$ \\
\hline $\mathrm{C}_{2} \mathrm{~F}_{8} \mathrm{NOP}$ & $\begin{array}{c}{[13105-59-4]} \\
\mathrm{V}\end{array}$ & $\begin{array}{l}\text { [bis(difluoromethy } \\
\quad(233-278)\end{array}$ & $\begin{array}{c}\text { mino] difluo } \\
30.4\end{array}$ & $\begin{array}{l}\text { phine ox } \\
255\end{array}$ & & [1966EME/ONA] \\
\hline $\mathrm{C}_{2} \mathrm{~F}_{8} \mathrm{NOP}$ & $\begin{array}{c}\text { [36544-19-1] } \\
\mathrm{V}\end{array}$ & phosphorous bis(tr & $\begin{array}{c}\text { loromethyl)n } \\
28.0\end{array}$ & $\begin{array}{l}\text { e difluor } \\
288\end{array}$ & & [1973WAN/SHR] \\
\hline $\mathrm{C}_{2} \mathrm{~F}_{8} \mathrm{OS}$ & $\begin{array}{c}{[33716-15-3]} \\
\mathrm{V}\end{array}$ & $\begin{array}{l}\text { difluorooxo-bis-(tr } \\
\quad(239-299)\end{array}$ & $\begin{array}{c}\text { loromethyl)s } \\
22.4\end{array}$ & 254 & A & [1987STE/MAL, 1999DYK/SVO, 1971SAU/SHR3] \\
\hline $\mathrm{C}_{2} \mathrm{~F}_{8} \mathrm{OS}$ & $\begin{array}{c}{[82390-51-0]} \\
\mathrm{V}\end{array}$ & $\begin{array}{l}\text { pentafluoro(trifluo } \\
(162-290)\end{array}$ & $\begin{array}{c}\text { cetyl) sulfur } \\
26.6\end{array}$ & 177 & & [1999DYK/SVO, 1982DEM/FOX] \\
\hline $\mathrm{C}_{2} \mathrm{~F}_{8} \mathrm{O}_{3} \mathrm{~S}$ & $\begin{array}{c}{[60672-61-9]} \\
\mathrm{V}\end{array}$ & pentafluoro (trifluo & $\begin{array}{c}\text { thaneperoxc } \\
28.0\end{array}$ & lfur & & {$[1976 \mathrm{HOP} / \mathrm{DES}]$} \\
\hline $\mathrm{C}_{2} \mathrm{~F}_{8} \mathrm{~S}$ & $\begin{array}{c}{[1186-51-2]} \\
\mathrm{V}\end{array}$ & trifluorovinyl sulfu & $\begin{array}{c}\text { entafluoride } \\
25.1\end{array}$ & 292 & & [1961CAS/RAY] \\
\hline $\mathrm{C}_{2} \mathrm{~F}_{8} \mathrm{~S}$ & $\begin{array}{c}\text { [30341-38-9] } \\
\mathrm{V}\end{array}$ & difluoro bis(trifluo & $\begin{array}{c}\text { nethyl) sulfu } \\
28.8\end{array}$ & & & [1971SAU/SHR] \\
\hline $\mathrm{C}_{2} \mathrm{~F}_{10} \mathrm{OS}$ & $\begin{array}{c}{[1580-07-0]} \\
\mathrm{V}\end{array}$ & $\begin{array}{c}\text { pentafluoro(pentaf } \\
(245-287)\end{array}$ & $\begin{array}{c}\text { roethoxy) su } \\
27.6\end{array}$ & 272 & A & [1987STE/MAL, 1962WIL/CAD] \\
\hline $\mathrm{C}_{2} \mathrm{~F}_{10} \mathrm{O}_{2} \mathrm{~S}$ & $\begin{array}{c}\text { [2004-38-8] } \\
\mathrm{V}\end{array}$ & $\begin{array}{l}\text { tetrafluoro bis(trifl } \\
\quad(246-302)\end{array}$ & $\begin{array}{c}\text { omethoxy) s } \\
29.9\end{array}$ & 261 & A & [1987STE/MAL, 1964DUN/CAD] \\
\hline $\mathrm{C}_{2} \mathrm{~F}_{10} \mathrm{O}_{3} \mathrm{~S}$ & $\begin{array}{c}\text { [41938-43-6] } \\
\text { V } \\
\text { V }\end{array}$ & $\begin{array}{c}\text { (trifluoromethoxy) } \\
\text { (255-317) }\end{array}$ & $\begin{array}{l}\text { ifluoromethy } \\
32.5 \\
30.7\end{array}$ & $\begin{array}{l}\text { y] sulfur } \\
270\end{array}$ & doride & $\begin{array}{l}\text { [1999DYK/SVO] } \\
{[1973 \mathrm{HOH} / \mathrm{DES}]}\end{array}$ \\
\hline $\mathrm{C}_{2} \mathrm{~F}_{10} \mathrm{O}_{3} \mathrm{~S}_{2}$ & $\begin{array}{c}{[68010-32-2]} \\
\mathrm{V}\end{array}$ & pentafluoro[ $[1,2,2,2$ & $\begin{array}{l}\text { trafluoro-1-[ } \\
34.8\end{array}$ & sulfonyl) & hyl] sulfur & [1978DEM/FOX] \\
\hline $\mathrm{C}_{2} \mathrm{~F}_{10} \mathrm{~S}$ & $\begin{array}{c}\text { [42179-02-2] } \\
\mathrm{V}\end{array}$ & $\begin{array}{l}\text { trans-tetrafluoro bi } \\
\quad(233-293)\end{array}$ & $\begin{array}{c}\text { rifluorometh } \\
23.3\end{array}$ & 278 & A & [1987STE/MAL, 1999DYK/SVO] \\
\hline $\mathrm{C}_{2} \mathrm{~F}_{11} \mathrm{NS}$ & $\begin{array}{c}{[13888-13-6]} \\
\mathrm{V}\end{array}$ & $\begin{array}{l}\text { [bis(trifluoromethy } \\
\text { (233-306) }\end{array}$ & $\begin{array}{c}\text { mino] sulfur } \\
29.3\end{array}$ & $\begin{array}{c}\text { Auoride } \\
248\end{array}$ & A & [1987STE/MAL, 1966DOB, 1999DYK/SVO] \\
\hline $\mathrm{C}_{2} \mathrm{~F}_{12} \mathrm{~S}_{2}$ & $\begin{array}{c}{[42060-66-2]} \\
\mathrm{V}\end{array}$ & perfluoro-1,3-dithi & $\begin{array}{c}\text { ne octafluori } \\
35.6\end{array}$ & & & {$[1973 \mathrm{ABE} / \mathrm{SHR}]$} \\
\hline $\mathrm{C}_{2} \mathrm{~N}_{2}$ & $\begin{array}{c}\text { [460-19-5] } \\
\text { FUS }\end{array}$ & cyanogen & 8.11 & 245.3 & $\mathrm{C}$ & [1996DOM/HEA, 1939RUE/GIA] \\
\hline & $\begin{array}{l}\text { SUB } \\
\text { SUB } \\
\text { SUB } \\
\text { SUB } \\
\text { SUB }\end{array}$ & $\begin{array}{l}(202-239) \\
(177-230) \\
(202-245) \\
(198-240)\end{array}$ & $\begin{array}{l}33.0 \\
33.6 \\
34.4 \\
32.4 \\
\text { NA }\end{array}$ & $\begin{array}{l}224 \\
204 \\
224\end{array}$ & $\begin{array}{c}\text { A } \\
\text { A } \\
\text { CATH }\end{array}$ & $\begin{array}{l}\text { [1987STE/MAL] } \\
\text { [1947STU] } \\
\text { [1939RUE/GIA] } \\
\text { [1975GRO, 1925PER/BAR] } \\
\text { [1916TER] }\end{array}$ \\
\hline & $\begin{array}{l}\mathrm{V} \\
\mathrm{V} \\
\mathrm{V} \\
\mathrm{V}\end{array}$ & $\begin{array}{l}(240-253) \\
(246-273) \\
(246-273)\end{array}$ & $\begin{array}{l}24.5 \\
23.9 \\
23.5 \\
\text { NA }\end{array}$ & $\begin{array}{l}246 \\
257 \\
267\end{array}$ & A & $\begin{array}{l}\text { [1987STE/MAL] } \\
{[1925 \mathrm{PER} / \mathrm{BAR}]} \\
{[1925 \mathrm{PER} / \mathrm{BAR}]} \\
{[1916 \mathrm{TER}]}\end{array}$ \\
\hline $\mathrm{C}_{2} \mathrm{~N}_{4} \mathrm{O}_{6}(? ?)$ & SUB & trinitroacrylonitrile & $\begin{array}{l}? ? ?) \\
66.9 \pm 1.3\end{array}$ & & $\mathrm{~V}+\mathrm{F}$ & [2002MAT/LEB] \\
\hline
\end{tabular}

[Note: Authors of [2002MAT/LEB] call the compound trinitroacrylonitrile; however, they give a structural formula of $\left(\mathrm{NO}_{2}\right)_{3} \mathrm{CCN}$ which is not consistent with the name. We have listed the compound under the structural formula and indicated with question marks that there was an inconsistency in how the name and formula were given.] 
TABle 5. Phase change enthalpies of $\mathrm{C}_{2}$ organic compounds-Continued

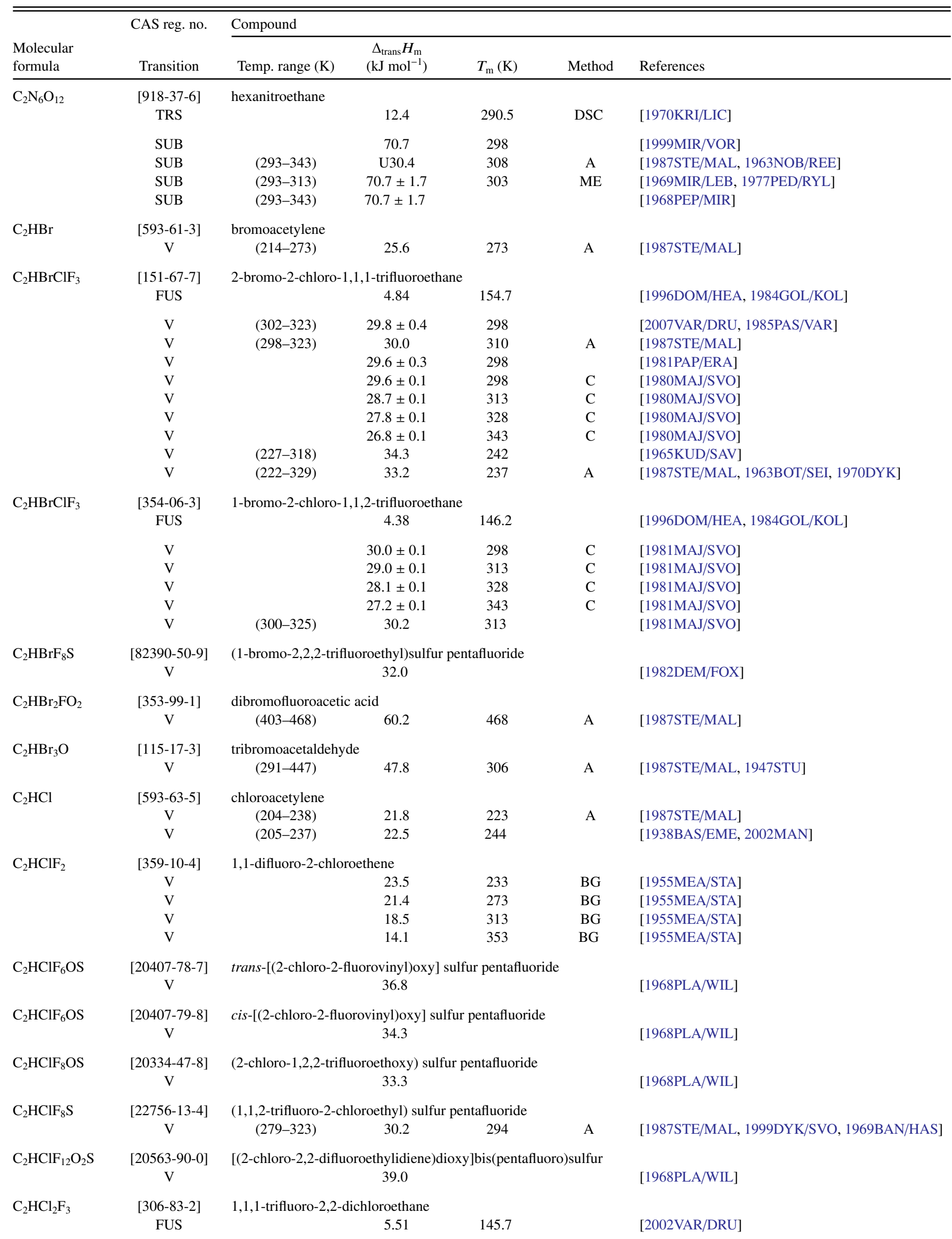


TABle 5. Phase change enthalpies of $\mathrm{C}_{2}$ organic compounds-Continued

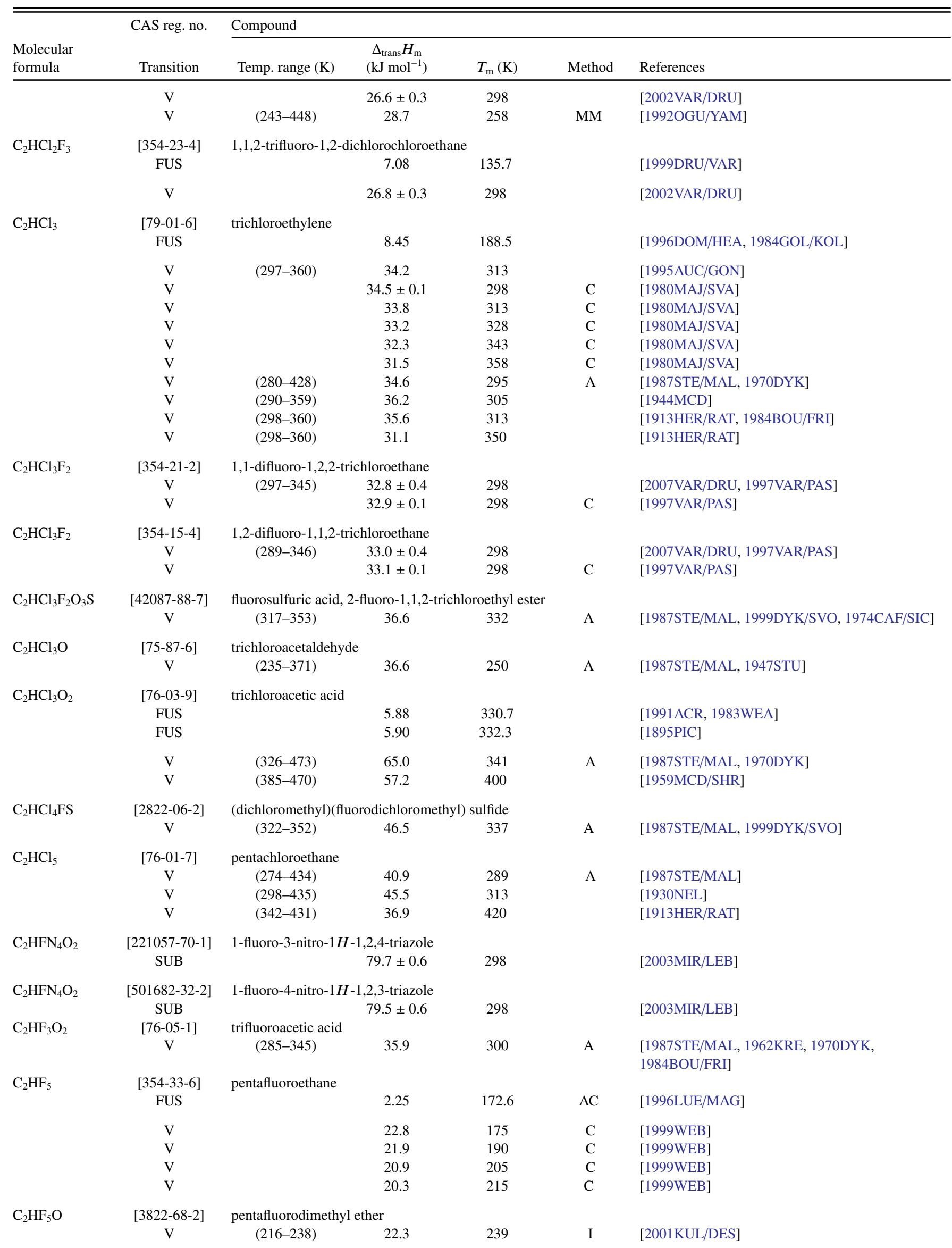


TABLE 5. Phase change enthalpies of $\mathrm{C}_{2}$ organic compounds-Continued

\begin{tabular}{|c|c|c|c|c|c|c|}
\hline \multirow[b]{2}{*}{$\begin{array}{l}\text { Molecular } \\
\text { formula }\end{array}$} & \multirow{2}{*}{$\begin{array}{l}\text { CAS reg. no. } \\
\text { Transition }\end{array}$} & \multicolumn{5}{|l|}{ Compound } \\
\hline & & Temp. range $(\mathrm{K})$ & $\begin{array}{c}\Delta_{\text {trans }} H_{\mathrm{m}} \\
\left(\mathrm{kJ} \mathrm{mol}^{-1}\right)\end{array}$ & $T_{\mathrm{m}}(\mathrm{K})$ & Method & References \\
\hline & $\mathrm{V}$ & $(229-331)$ & 19.3 & 260 & EB & [1996WEB/DEF2] \\
\hline & $\mathrm{V}$ & $(229-331)$ & 17.6 & 280 & $\mathrm{~EB}$ & [1996WEB/DEF2] \\
\hline & $\mathrm{V}$ & $(229-331)$ & 15.6 & 300 & $\mathrm{~EB}$ & [1996WEB/DEF2] \\
\hline & $\mathrm{V}$ & $(240-313)$ & 20.4 & 255 & A & [1992SAL/WAN] \\
\hline \multirow[t]{2}{*}{$\mathrm{C}_{2} \mathrm{HF}_{6} \mathrm{NOS}$} & {$[34556-22-4]$} & \multicolumn{5}{|c|}{$S, S$-bis(trifluoromethyl)sulfoximine } \\
\hline & $\mathrm{V}$ & & 35.1 & 346 & I & [1972SAU/SHR] \\
\hline \multirow[t]{2}{*}{$\mathrm{C}_{2} \mathrm{HF}_{6} \mathrm{NS}_{2}$} & [763-24-6] & \multicolumn{5}{|c|}{ bis(trifluoromethane) sulphenimide } \\
\hline & $\mathrm{V}$ & $(243-293)$ & 36.5 & 268 & & [1960EME/NAB] \\
\hline \multirow[t]{2}{*}{$\mathrm{C}_{2} \mathrm{HF}_{6} \mathrm{OP}$} & [359-65-9] & \multicolumn{5}{|c|}{ phosphinous acid, bis(trifluoromethyl) ester } \\
\hline & SUB & $(233-251)$ & 46.6 & 242 & & [1987STE/MAL, 1962GRI/BUR] \\
\hline \multirow[t]{3}{*}{$\mathrm{C}_{2} \mathrm{HF}_{6} \mathrm{OPS}$} & {$[35814-49-4]$} & \multicolumn{5}{|c|}{ bis(trifluoromethyl) thiophospinic acid } \\
\hline & V & $(283-324)$ & 38.3 & 298 & A & [1987STE/MAL] \\
\hline & $\mathrm{V}$ & $(277-324)$ & 37.4 & 300 & & [1972PIN/CAV] \\
\hline \multirow[t]{3}{*}{$\mathrm{C}_{2} \mathrm{HF}_{6} \mathrm{PS}$} & {$[1486-19-7]$} & \multicolumn{5}{|c|}{ bis(trifluoromethyl)(mercapto)phosphine } \\
\hline & V & $(269-304)$ & 30.7 & 286 & & [1964CAV/EME] \\
\hline & $\mathrm{V}$ & $(217-280)$ & 32.9 & 265 & A & [1987STE/MAL, 1999DYK/SVO, 1965BUR/GOS] \\
\hline \multirow[t]{2}{*}{$\mathrm{C}_{2} \mathrm{HF}_{6} \mathrm{PS}_{2}$} & {$[18799-75-2]$} & \multicolumn{5}{|c|}{ phosphinodithioic acid, bis(trifluoromethyl) ester } \\
\hline & SUB & $(273-287)$ & 41.9 & 280 & A & [1987STE/MAL, 1968GOS/BUR] \\
\hline \multirow[t]{2}{*}{$\mathrm{C}_{2} \mathrm{HF}_{7} \mathrm{~S}$} & {$[58636-78-5]$} & \multicolumn{5}{|c|}{ (2,2-difluoroethenyl) pentafluorosulfur } \\
\hline & $\mathrm{V}$ & \multicolumn{3}{|c|}{27.7} & & {$[1978 \mathrm{DEM} / \mathrm{FOX}]$} \\
\hline \multirow[t]{2}{*}{$\mathrm{C}_{2} \mathrm{HF}_{9} \mathrm{~S}$} & {$[63011-80-3]$} & pentafluoro $(1,2,2$, & etrafluoroeth & & & \\
\hline & V & & 28.0 & & & [1978DEM/FOX] \\
\hline $\mathrm{C}_{2} \mathrm{H}_{2}$ & {$[74-86-2]$} & acetylene & & & & \\
\hline & TRS & & 2.54 & 142.7 & & \\
\hline & FUS & & 3.76 & 192.4 & & [1976MIS/RIE] \\
\hline & SUB & $(98-145)$ & 23.5 & 130 & & [1987STE/MAL] \\
\hline & SUB & $(133-191)$ & 21.8 & 162 & & [1960JON] \\
\hline & SUB & $(151-193)$ & 25.2 & 193 & & [1956AMB] \\
\hline & SUB & $(130-189)$ & 22.7 & 160 & A & [1947STU] \\
\hline & SUB & $(89-169)$ & 22.1 & 129 & A & [1943BUR] \\
\hline & SUB & & 23.0 & & & {$[1907 \mathrm{MCI}]$} \\
\hline & $\mathrm{V}$ & $(258-308)$ & 16.3 & 273 & A & [1987STE/MAL] \\
\hline & $\mathrm{V}$ & $(192-308)$ & 16.7 & 207 & A & [1987STE/MAL] \\
\hline & $\mathrm{V}$ & $(192-225)$ & 16.7 & 210 & A & [1987STE/MAL] \\
\hline & $\mathrm{V}$ & & 17.0 & 214 & & [1971WIL/ZWO] \\
\hline & $\mathrm{V}$ & $(215-308)$ & 16.4 & 230 & & [1964AMB/TOW] \\
\hline & $\mathrm{V}$ & $(193-207)$ & 16.8 & 200 & & [1956AMB, 1984BOU/FRI] \\
\hline $\mathrm{C}_{2} \mathrm{H}_{2} \mathrm{Br}_{2}$ & {$[590-11-4]$} & cis-1,2-dibromoeth & & & & \\
\hline & $\mathrm{V}$ & $(299-351)$ & 40.6 & 314 & A & [1987STE/MAL, 1950NOY/NOY, 1970DYK] \\
\hline $\mathrm{C}_{2} \mathrm{H}_{2} \mathrm{Br}_{2}$ & {$[590-12-5]$} & trans-1,2-dibromo & ylene & & & \\
\hline & $\mathrm{V}$ & $(277-344)$ & 35.2 & 310 & A & $\begin{array}{l}\text { [1987STE/MAL, 1970DYK, 1950NOY/NOY, } \\
\text { 1984BOU/FRI] }\end{array}$ \\
\hline $\mathrm{C}_{2} \mathrm{H}_{2} \mathrm{Br}_{2} \mathrm{Cl}_{2}$ & [75-81-0] & 1,2-dibromo-1,1-di & loroethane & & & \\
\hline & $\mathrm{V}$ & $(354-519)$ & 45.9 & 369 & A & [1987STE/MAL, 1970DYK] \\
\hline $\mathrm{C}_{2} \mathrm{H}_{2} \mathrm{Br}_{2} \mathrm{Cl}_{2}$ & {$[683-68-1]$} & 1,2-dibromo-1,2-di & loroethane & & & \\
\hline & FUS & & 8.3 & 206.3 & & [1991ACR, 1983WEA] \\
\hline & $\mathrm{V}$ & $(320-379)$ & 45.9 & 335 & A & [1987STE/MAL] \\
\hline $\mathrm{C}_{2} \mathrm{H}_{2} \mathrm{Br}_{4}$ & {$[630-16-0]$} & 1,1,1,2-tetrabromo & ane & & & \\
\hline & $\mathrm{V}$ & $(331-473)$ & 61.5 & 346 & A & [1987STE/MAL, 1947STU] \\
\hline $\mathrm{C}_{2} \mathrm{H}_{2} \mathrm{Br}_{4}$ & {$[79-27-6]$} & 1,1,2,2-tetrabromo & ane & & & \\
\hline & $\mathrm{V}$ & $(413-573)$ & 56.9 & 428 & A & [1987STE/MAL, 1970DYK] \\
\hline $\mathrm{C}_{2} \mathrm{H}_{2} \mathrm{ClFO}$ & $\begin{array}{c}{[359-14-8]} \\
\mathrm{V}\end{array}$ & $\begin{array}{l}\text { chloroacetyl fluori } \\
\quad(273-333)\end{array}$ & 38.0 & 288 & $\mathrm{~A}, \mathrm{GS}$ & [1987STE/MAL, 1948RED/CHA4, 1970DYK] \\
\hline
\end{tabular}


TABLE 5. Phase change enthalpies of $\mathrm{C}_{2}$ organic compounds-Continued

\begin{tabular}{|c|c|c|c|c|c|c|}
\hline \multirow[b]{2}{*}{$\begin{array}{l}\text { Molecular } \\
\text { formula }\end{array}$} & \multirow{2}{*}{$\begin{array}{l}\text { CAS reg. no. } \\
\text { Transition }\end{array}$} & \multicolumn{5}{|l|}{ Compound } \\
\hline & & Temp. range $(\mathrm{K})$ & $\begin{array}{c}\Delta_{\text {trans }} H_{\mathrm{m}} \\
\left(\mathrm{kJ} \mathrm{mol}^{-1}\right) \\
\end{array}$ & $T_{\mathrm{m}}(\mathrm{K})$ & Method & References \\
\hline $\mathrm{C}_{2} \mathrm{H}_{2} \mathrm{ClFO}$ & $\begin{array}{c}{[359-06-8]} \\
\mathrm{V}\end{array}$ & $\begin{array}{l}\text { fluoroacetyl chloride } \\
\qquad(273-333)\end{array}$ & 36.7 & 288 & A, GS & [1987STE/MAL, 1948RED/CHA4, 1970DYK] \\
\hline $\mathrm{C}_{2} \mathrm{H}_{2} \mathrm{ClF}_{3} \mathrm{O}_{2} \mathrm{~S}$ & $\begin{array}{c}{[57169-80-9]} \\
\mathrm{V}\end{array}$ & \multicolumn{5}{|c|}{ chlorosulfurous acid, 2,2,2-trifluoroethyl ester } \\
\hline $\mathrm{C}_{2} \mathrm{H}_{2} \mathrm{ClF}_{7} \mathrm{~S}$ & $\begin{array}{c}{[68010-35-5]} \\
\mathrm{V}\end{array}$ & \multicolumn{5}{|c|}{ (2-chloro-2,2-difluoroethyl)pentafluorosulfur } \\
\hline $\mathrm{C}_{2} \mathrm{H}_{2} \mathrm{Cl}_{2}$ & $\begin{array}{l}{[75-35-4]} \\
\text { FUS }\end{array}$ & $\begin{array}{l}\text { 1,1-dichloroethylene } \\
\text { (13-291) }\end{array}$ & 6.51 & 150.59 & $\mathrm{AC}$ & [1996DOM/HEA, 1959HIL/MCD] \\
\hline & $\mathrm{V}$ & $(245-305)$ & 28.4 & 260 & A & [1987STE/MAL, 1959HIL/MCD, 1970DYK] \\
\hline $\mathrm{C}_{2} \mathrm{H}_{2} \mathrm{Cl}_{2}$ & $\begin{array}{c}{[156-59-2]} \\
\mathrm{V} \\
\mathrm{V} \\
\mathrm{V} \\
\mathrm{V}\end{array}$ & $\begin{array}{l}\text { cis-1,2-dichloroethyle } \\
\quad(332-495) \\
(273-334) \\
(292-335) \\
(273-356)\end{array}$ & $\begin{array}{l}\text { ne } \\
\begin{array}{l}29.3 \\
31.5 \\
31.6 \\
31.8\end{array}\end{array}$ & $\begin{array}{l}347 \\
288 \\
307 \\
288\end{array}$ & $\begin{array}{l}\text { A } \\
\mathrm{A}\end{array}$ & $\begin{array}{l}\text { [1987STE/MAL] } \\
\text { [1987STE/MAL, 1970DYK] } \\
\text { [1951FLO/ALP] } \\
\text { [1947KET/VAN] }\end{array}$ \\
\hline $\mathrm{C}_{2} \mathrm{H}_{2} \mathrm{Cl}_{2}$ & $\begin{array}{c}{[156-60-5]} \\
\text { V } \\
\text { V } \\
\text { V } \\
\text { V } \\
\text { V }\end{array}$ & $\begin{array}{l}\text { trans-1,2-dichloroeth } \\
\quad(321-473) \\
(273-319) \\
(263-323) \\
(287-325) \\
(235-358)\end{array}$ & $\begin{array}{l}\text { lene } \\
29.0 \\
30.1 \\
30.4 \\
29.6 \\
31.4\end{array}$ & $\begin{array}{l}336 \\
288 \\
278 \\
306 \\
250\end{array}$ & A & $\begin{array}{l}\text { [1987STE/MAL] } \\
\text { [1983MAC] } \\
\text { [1987STE/MAL, 1970DYK] } \\
\text { [1951FLO/ALP] } \\
\text { [1947KET/VAN] }\end{array}$ \\
\hline $\mathrm{C}_{2} \mathrm{H}_{2} \mathrm{Cl}_{2} \mathrm{~F}_{2}$ & $\begin{array}{c}{[1649-08-7]} \\
\mathrm{V}\end{array}$ & \multicolumn{5}{|c|}{ 1,2-dichloro-1,1-difluoroethane } \\
\hline $\mathrm{C}_{2} \mathrm{H}_{2} \mathrm{Cl}_{2} \mathrm{~F}_{2}$ & $\begin{array}{l}{[1842-05-3]} \\
\text { FUS }\end{array}$ & $\begin{array}{l}\text { 1,2-difluoro-2,2-dichl } \\
\text { (13-313) }\end{array}$ & $\begin{array}{l}\text { oroethane } \\
8.19\end{array}$ & 163.0 & $\mathrm{AC}$ & [1996DOM/HEA, 1992LEB/KUL] \\
\hline $\mathrm{C}_{2} \mathrm{H}_{2} \mathrm{Cl}_{2} \mathrm{~F}_{6} \mathrm{OS}$ & $\begin{array}{c}{[20334-44-5]} \\
V\end{array}$ & \multicolumn{4}{|c|}{ (1,2-dichloro-2-fluoroethoxy)pentafluoro sulfur } & [1968PLA/WIL] \\
\hline $\mathrm{C}_{2} \mathrm{H}_{2} \mathrm{Cl}_{2} \mathrm{~F}_{6} \mathrm{OS}$ & $\begin{array}{c}{[20334-45-6]} \\
V\end{array}$ & (2,2-dichloro-2-fluoro & $\begin{array}{l}\text { ethoxy)pent } \\
38.5\end{array}$ & sulfur & & [1968PLA/WIL] \\
\hline $\mathrm{C}_{2} \mathrm{H}_{2} \mathrm{Cl}_{2} \mathrm{O}$ & $\begin{array}{c}\text { [79-04-9] } \\
\mathrm{V} \\
\mathrm{V} \\
\mathrm{V}\end{array}$ & $\begin{array}{l}\text { chloroacetyl chloride } \\
\qquad \begin{array}{l}(253-379) \\
(301-380) \\
(290-373)\end{array}\end{array}$ & $\begin{array}{l}45.0 \\
40.8 \\
44.1\end{array}$ & $\begin{array}{l}268 \\
316 \\
305\end{array}$ & A & $\begin{array}{l}\text { [1987STE/MAL, 1970DYK] } \\
\text { [1959MCD/SHR] } \\
\text { [1935KIR/POP] }\end{array}$ \\
\hline $\mathrm{C}_{2} \mathrm{H}_{2} \mathrm{Cl}_{2} \mathrm{O}_{2}$ & $\begin{array}{l}\text { [79-43-6] } \\
\text { FUS } \\
\text { FUS }\end{array}$ & dichloroacetic acid & $\begin{array}{l}12.34 \\
\mathrm{U} 7.64\end{array}$ & $\begin{array}{l}286.5 \\
284.0\end{array}$ & & $\begin{array}{l}\text { [1996DOM/HEA, 1961GLA/TIM] } \\
\text { [1996DOM/HEA, 1895PIC] }\end{array}$ \\
\hline & $\mathrm{V}$ & $(317-468)$ & 55.7 & 332 & A & [1987STE/MAL, 1947STU] \\
\hline $\mathrm{C}_{2} \mathrm{H}_{2} \mathrm{Cl}_{4}$ & $\begin{array}{c}{[630-20-6]} \\
\mathrm{V} \\
\mathrm{V} \\
\mathrm{V}\end{array}$ & $\begin{array}{l}\text { 1,1,1,2-tetrachloroeth } \\
(316-447) \\
(332-403)\end{array}$ & $\begin{array}{l}41.1 \pm 0.2 \\
40.1 \\
39.2\end{array}$ & $\begin{array}{l}298 \\
331 \\
347\end{array}$ & A & $\begin{array}{l}\text { [1978GUN/HEA] } \\
\text { [1987STE/MAL, 1970DYK] } \\
\text { [1949DRE/SHR, 1949DRE/MAR] }\end{array}$ \\
\hline $\mathrm{C}_{2} \mathrm{H}_{2} \mathrm{Cl}_{4}$ & $\begin{array}{l}\text { [79-34-5] } \\
\text { FUS } \\
\text { TRS } \\
\text { FUS }\end{array}$ & 1,1,2,2-tetrachloroeth & $\begin{array}{l}\text { ane } \\
10.10 \\
0.54 \\
9.17\end{array}$ & $\begin{array}{l}231.0 \\
207.3 \\
230.8\end{array}$ & $\mathrm{DSC}$ & $\begin{array}{l}\text { [2013NEG/BAR] } \\
\text { [1996DOM/HEA, 1982KOS/KOL] }\end{array}$ \\
\hline & V & $(343-418)$ & 42.5 & 358 & EB & [2006TEO/BAR] \\
\hline & $\begin{array}{l}\mathrm{V} \\
\mathrm{V}\end{array}$ & $\begin{array}{l}(377-419) \\
(371-419)\end{array}$ & $\begin{array}{l}40.4 \\
40.8\end{array}$ & $\begin{array}{l}392 \\
394\end{array}$ & A & $\begin{array}{l}\text { [1987STE/MAL] } \\
\text { [1984CAS/FRA] }\end{array}$ \\
\hline & $\mathrm{V}$ & & 44.7 & 313 & $\mathrm{C}$ & [1980MAJ/SVA] \\
\hline & $\mathrm{V}$ & & 43.8 & 328 & $\mathrm{C}$ & [1980MAJ/SVA] \\
\hline & $\mathrm{V}$ & & 42.8 & 343 & $\mathrm{C}$ & [1980MAJ/SVA] \\
\hline & $\mathrm{V}$ & & 41.8 & 358 & $\mathrm{C}$ & [1980MAJ/SVA] \\
\hline
\end{tabular}


TABLE 5. Phase change enthalpies of $\mathrm{C}_{2}$ organic compounds-Continued

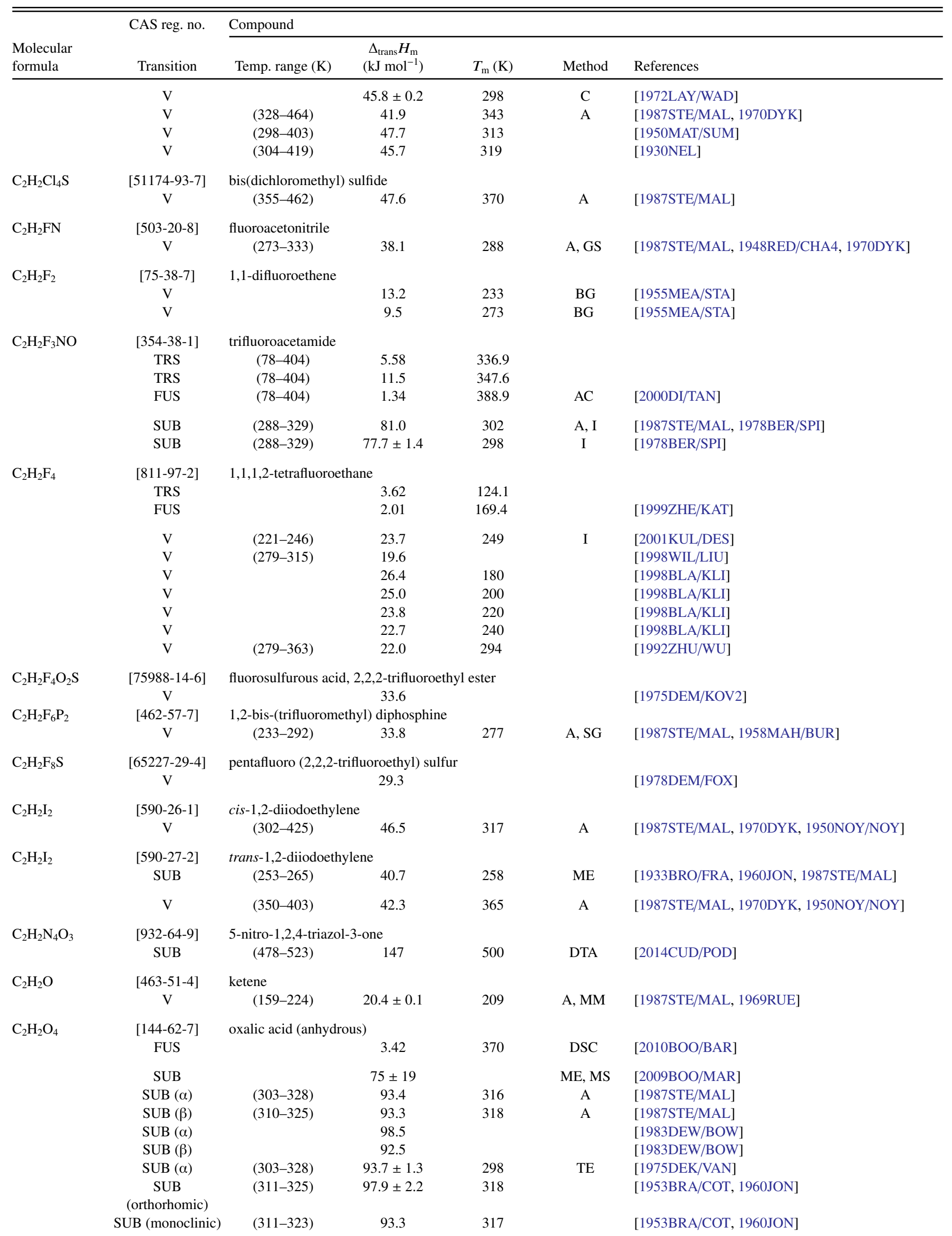


TABle 5. Phase change enthalpies of $\mathrm{C}_{2}$ organic compounds-Continued

\begin{tabular}{|c|c|c|c|c|c|c|}
\hline \multirow[b]{2}{*}{$\begin{array}{l}\text { Molecular } \\
\text { formula }\end{array}$} & \multirow{2}{*}{$\begin{array}{l}\text { CAS reg. no. } \\
\text { Transition }\end{array}$} & \multicolumn{5}{|l|}{ Compound } \\
\hline & & Temp. range $(\mathrm{K})$ & $\begin{array}{r}\Delta_{\text {trans }} H_{\mathrm{m}} \\
\left(\mathrm{kJ} \mathrm{mol}{ }^{-1}\right) \\
\end{array}$ & $T_{\mathrm{m}}(\mathrm{K})$ & Method & References \\
\hline & SUB & $(292-320)$ & 61.8 & 306 & A & [1947GRA] \\
\hline & SUB & $(333-378)$ & 90.6 & & GS & [1926NOY/WEB] \\
\hline \multirow[t]{4}{*}{$\mathrm{C}_{2} \mathrm{H}_{3} \mathrm{Br}$} & {$[593-60-2]$} & vinyl bromide & & & & \\
\hline & $\mathrm{V}$ & (224-319) & 27.3 & 239 & A & [1987STE/MAL, 1970DYK] \\
\hline & $\mathrm{V}$ & $(186-289)$ & 24.8 & 274 & & [1937GUY/SCH, 1984BOU/FRI] \\
\hline & $\mathrm{V}$ & $(207-285)$ & 26.9 & 270 & & [1934MEH, 1984BOU/FRI] \\
\hline \multirow[t]{3}{*}{$\mathrm{C}_{2} \mathrm{H}_{3} \mathrm{BrO}$} & {$[506-96-7]$} & acetyl bromide & & & & \\
\hline & $\mathrm{V}$ & $(289-334)$ & 29.5 & 304 & A & [1987STE/MAL] \\
\hline & $\mathrm{V}$ & $(275-333)$ & 31.4 & 290 & & [1969DEV/ONE] \\
\hline \multirow[t]{5}{*}{$\mathrm{C}_{2} \mathrm{H}_{3} \mathrm{BrO}_{2}$} & {$[79-08-3]$} & bromoacetic acid & & & & \\
\hline & FUS & & 13.9 & 319.2 & DSC & [2001LAG/DIO] \\
\hline & SUB & & $83.5 \pm 3.0$ & 298 & ME & [2001LAG/DIO] \\
\hline & SUB & & $84.0 \pm 3.0$ & 280 & ME & [2001LAG/DIO] \\
\hline & $\mathrm{V}$ & $(327-481)$ & 57.2 & 342 & A & [1987STE/MAL] \\
\hline \multirow[t]{4}{*}{$\mathrm{C}_{2} \mathrm{H}_{3} \mathrm{Br}_{3}$} & {$[78-74-0]$} & 1,1,2-tribromoethane & & & & \\
\hline & FUS & & 9.11 & 244 & & [1991ACR, 1983WEA] \\
\hline & $\mathrm{V}$ & $(368-511)$ & 50.5 & 383 & A & [1987STE/MAL, 1970DYK] \\
\hline & V & $(305-461)$ & 52.9 & 321 & & [1947STU] \\
\hline \multirow[t]{6}{*}{$\mathrm{C}_{2} \mathrm{H}_{3} \mathrm{Cl}$} & {$[75-01-4]$} & vinyl chloride & & & & \\
\hline & FUS & $(60-300)$ & 4.92 & 119.3 & & [1996DOM/HEA, 1967LEB/RAB] \\
\hline & $\mathrm{V}$ & $(243-288)$ & 22.7 & 265 & & [1967DAN/GOL] \\
\hline & $\mathrm{V}$ & $(213-273)$ & 22.9 & 258 & & [1967HAC/MAT] \\
\hline & $\mathrm{V}$ & $(209-260)$ & 23.3 & 245 & A & [1987STE/MAL, 1959MCD/SHR, 1970DYK] \\
\hline & V & $(244-333)$ & 20.1 & 298 & & [1927DAN/BAR, 2002MAN] \\
\hline \multirow[t]{8}{*}{$\mathrm{C}_{2} \mathrm{H}_{3} \mathrm{ClF}_{2}$} & {$[75-68-3]$} & 1-chloro-1,1-difluoroe & thane & & & \\
\hline & FUS & & 2.69 & 142.4 & & [1996DOM/HEA, 1937PER] \\
\hline & $\mathrm{V}$ & $(225-285)$ & 24.2 & 240 & EB & [1993SIL/WEB] \\
\hline & $\mathrm{V}$ & $(248-390)$ & 22.7 & 263 & A & [1987STE/MAL, 1970DYK, 1955MEA/STA] \\
\hline & $\mathrm{V}$ & $(248-390)$ & 24.0 & 233 & BG & [1955MEA/STA] \\
\hline & V & $(248-390)$ & 21.9 & 273 & BG & [1955MEA/STA] \\
\hline & $\mathrm{V}$ & $(248-390)$ & 19.2 & 313 & BG & [1955MEA/STA] \\
\hline & $\mathrm{V}$ & $(248-390)$ & 15.4 & 353 & BG & [1955MEA/STA] \\
\hline \multirow[t]{2}{*}{$\mathrm{C}_{2} \mathrm{H}_{3} \mathrm{ClF}_{3} \mathrm{~N}$} & {$[16276-45-2]$} & $N$-chloro- $N, 1,1$-triflu & loroethanamine & & & \\
\hline & V & $(220-294)$ & 30.8 & 279 & BG & [1987STE/MAL, 1967LUS] \\
\hline \multirow[t]{2}{*}{$\mathrm{C}_{2} \mathrm{H}_{3} \mathrm{ClF}_{3} \mathrm{P}$} & {$[4669-76-5]$} & chloromethyl(trifluorc & omethyl)phosphine & & & \\
\hline & V & $(236-294)$ & 30.9 & 279 & A & [1987STE/MAL, 1966BUR/JOS] \\
\hline \multirow[t]{3}{*}{$\mathrm{C}_{2} \mathrm{H}_{3} \mathrm{ClO}$} & {$[75-36-5]$} & acetyl chloride & & & & \\
\hline & $\mathrm{V}$ & $(273-323)$ & 24.5 & 288 & A & [1987STE/MAL] \\
\hline & V & $(267-324)$ & 31.5 & 282 & A & [1987STE/MAL, 1959MCD/SHR, 1970DYK] \\
\hline \multirow[t]{13}{*}{$\mathrm{C}_{2} \mathrm{H}_{3} \mathrm{ClO}_{2}$} & {$[79-11-8]$} & chloroacetic acid & & & & \\
\hline & FUS & & 16.3 & 334.8 & DSC & [2001LAG/DIO] \\
\hline & FUS & & 12.35 & & DSC & [1992SHA/SHA] \\
\hline & FUS $(\alpha)$ & & 12.28 & 334.4 & & [1983WEA, 1991ACR] \\
\hline & FUS $(\beta)$ & & 13.88 & 329.2 & & [1983WEA, 1991ACR] \\
\hline & FUS $(\alpha)$ & & 16.3 & 334.3 & & [1996DOM/HEA, 1895PIC] \\
\hline & FUS $(\beta)$ & & 13.93 & 329.2 & & [1996DOM/HEA, 1895PIC] \\
\hline & SUB & & $82.2 \pm 0.9$ & 298 & $\mathrm{ME}$ & [2001LAG/DIO] \\
\hline & SUB & & $82.7 \pm 0.9$ & 282 & $\mathrm{ME}$ & [2001LAG/DIO] \\
\hline & SUB & & $75.3 \pm 4.2$ & & & $\begin{array}{l}\text { [1927STE/JOH, 1949DRE/MAR, 1949DRE/SHR, } \\
\text { 1970COX/PIL] }\end{array}$ \\
\hline & V & $(336-463)$ & 61.1 & 351 & A & [1987STE/MAL] \\
\hline & $\mathrm{V}$ & $(377-463)$ & 56.8 & 392 & A & [1987STE/MAL, 1959MCD/SHR, 1970DYK] \\
\hline & V & $(396-460)$ & 55.7 & 411 & & [1949DRE/SHR, 1949DRE/MAR] \\
\hline
\end{tabular}


TABLE 5. Phase change enthalpies of $\mathrm{C}_{2}$ organic compounds-Continued

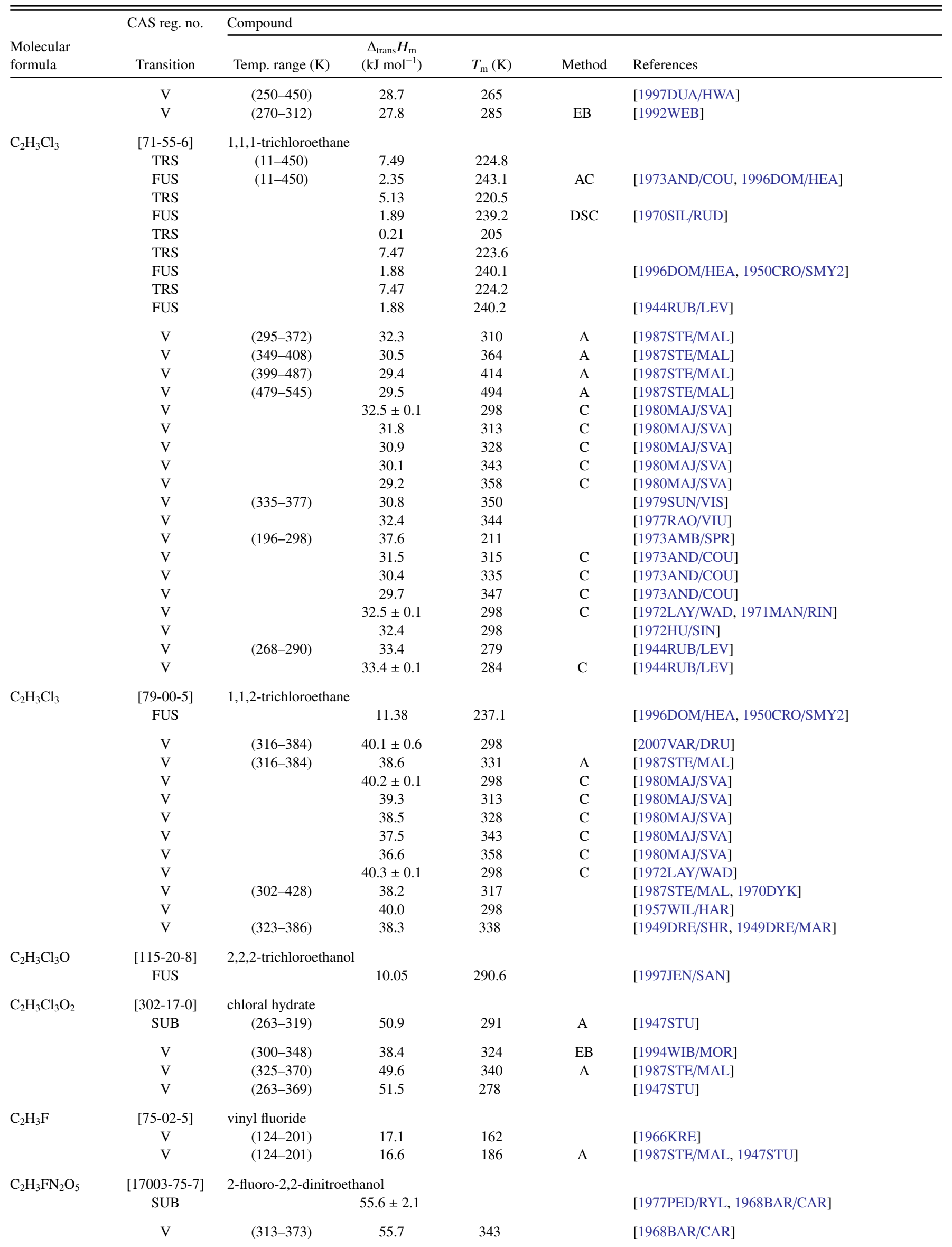


TABle 5. Phase change enthalpies of $\mathrm{C}_{2}$ organic compounds-Continued

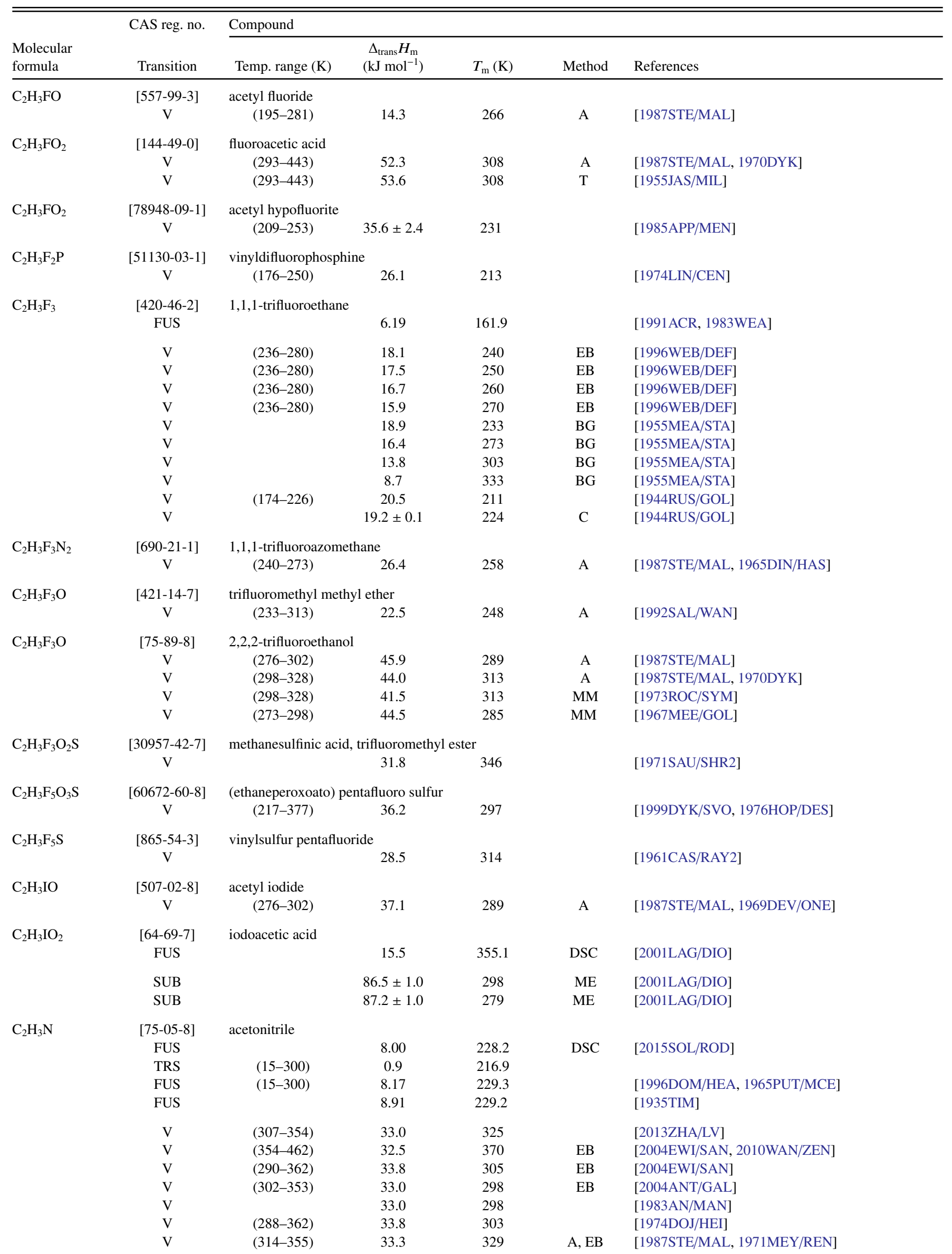


TABLE 5. Phase change enthalpies of $\mathrm{C}_{2}$ organic compounds-Continued

\begin{tabular}{|c|c|c|c|c|c|c|}
\hline \multirow[b]{2}{*}{$\begin{array}{l}\text { Molecular } \\
\text { formula }\end{array}$} & \multirow{2}{*}{$\begin{array}{c}\text { CAS reg. no. } \\
\text { Transition }\end{array}$} & \multicolumn{5}{|l|}{ Compound } \\
\hline & & Temp. range $(\mathrm{K})$ & $\begin{array}{c}\Delta_{\text {trans }} H_{\mathrm{m}} \\
\left(\mathrm{kJ} \mathrm{mol}^{-1}\right)\end{array}$ & $T_{\mathrm{m}}(\mathrm{K})$ & Method & References \\
\hline & $\mathrm{V}$ & $(299-343)$ & 34.8 & 315 & BG & [1971HAL/BAL] \\
\hline & $\mathrm{V}$ & & $32.9 \pm 0.1$ & 298 & $\mathrm{C}$ & [1970HOW/WAD] \\
\hline & $\mathrm{V}$ & $(273-323)$ & 34.2 & 288 & & [1968KUS/MIS] \\
\hline & $\mathrm{V}$ & $(280-300)$ & 33.9 & 290 & & [1965PUT/MCE] \\
\hline & $\mathrm{V}$ & & 33.3 & 298 & $\mathrm{C}$ & [1965PUT/MCE] \\
\hline & $\mathrm{V}$ & & 32.9 & 298 & $\mathrm{C}$ & [1950IWA, 1970HOW/WAD] \\
\hline & $\mathrm{V}$ & & 33.6 & 298 & & [1933HEI, 1970HOW/WAD] \\
\hline & $\mathrm{V}$ & & 31.1 & 387 & $\mathrm{C}$ & [1901KAH2] \\
\hline \multirow[t]{5}{*}{$\mathrm{C}_{2} \mathrm{H}_{3} \mathrm{NO}$} & [624-83-9] & methyl isocyanate & & & & \\
\hline & FUS & & 7.45 & 178.5 & DSC & [2013DAV/KIL] \\
\hline & $\mathrm{V}$ & & $28.7 \pm 0.1$ & 298 & $\mathrm{C}$ & [2013DAV/KIL] \\
\hline & $\mathrm{V}$ & $(265-308)$ & 29.9 & 280 & A & [1987STE/MAL] \\
\hline & $\mathrm{V}$ & $(253-310)$ & 31.7 & 268 & A & [1987STE/MAL] \\
\hline \multirow[t]{3}{*}{$\mathrm{C}_{2} \mathrm{H}_{3} \mathrm{NO}_{3}$} & {$[471-47-6]$} & \multicolumn{5}{|l|}{ oxalic acid, monoamide } \\
\hline & SUB & & $108.9 \pm 2.1$ & 298 & ME & [1988NUN/BAR] \\
\hline & SUB & $(355-363)$ & 107.9 & 359 & $\mathrm{ME}$ & [1953BRA/CLE2, 1960JON, 1987STE/MAL] \\
\hline \multirow[t]{3}{*}{$\mathrm{C}_{2} \mathrm{H}_{3} \mathrm{NO}_{5}$} & {$[2278-22-0]$} & acetyl nitro peroxide & & & & \\
\hline & $\mathrm{V}$ & $(277-330)$ & 34.6 & 292 & A & [1987STE/MAL] \\
\hline & $\mathrm{V}$ & $(277-330)$ & 38.1 & 303 & & {$[1978 \mathrm{KAC} / \mathrm{SOL}]$} \\
\hline \multirow[t]{2}{*}{$\mathrm{C}_{2} \mathrm{H}_{3} \mathrm{NS}$} & {$[556-64-9]$} & methyl thiocyanate & & & & \\
\hline & V & $(259-406)$ & 40.7 & 274 & A & [1987STE/MAL, 1947STU, 1999DYK/SVO] \\
\hline \multirow[t]{4}{*}{$\mathrm{C}_{2} \mathrm{H}_{3} \mathrm{NS}$} & {$[556-61-6]$} & methyl isothiocyanate & & & & \\
\hline & SUB & $(238-293)$ & 31.5 & 266 & A & [1947STU] \\
\hline & $\mathrm{V}$ & $(309-392)$ & 37.4 & 324 & A & [1987STE/MAL, 1999DYK/SVO] \\
\hline & $\mathrm{V}$ & $(283-323)$ & 37.3 & 298 & & [1935BAU/BUR, 1984BOU/FRI] \\
\hline \multirow[t]{7}{*}{$\mathrm{C}_{2} \mathrm{H}_{3} \mathrm{~N}_{3}$} & {$[288-88-0]$} & 1,2,4-triazole & & & & \\
\hline & FUS & & 16.1 & 393.5 & DSC & [1996DOM/HEA, 1989HIL/MOU] \\
\hline & FUS & & 10.76 & 393.3 & & [1999SAB/PER] \\
\hline & SUB & & $80.7 \pm 0.5$ & 298 & $\mathrm{C}$ & [1999SAB/PER] \\
\hline & SUB & & $84.0 \pm 0.7$ & 298 & ME & [1989JIM/ROU] \\
\hline & SUB & & $80.6 \pm 0.5$ & & & [1985SKI/PIL] \\
\hline & SUB & & 84.1 & & $\mathrm{ME}$ & [1961ZIM/GEI] \\
\hline \multirow[t]{4}{*}{$\mathrm{C}_{2} \mathrm{H}_{3} \mathrm{~N}_{3} \mathrm{O}_{6}$} & {$[595-86-8]$} & 1,1,1-trinitroethane & & & & \\
\hline & TRS & & 4.6 & 311.7 & & \\
\hline & FUS & & 11.72 & 329.2 & DSC & [1969ROS, 1969ROS/HOL] \\
\hline & SUB & & $72.0 \pm 8.8$ & 298 & & [1999MIR/VOR] \\
\hline \multirow[t]{3}{*}{$\mathrm{C}_{2} \mathrm{H}_{3} \mathrm{~N}_{3} \mathrm{O}_{7}$} & [918-54-7] & 2,2,2-trinitroethanol & & & & \\
\hline & TRS & & 17.99 & 312.5 & & \\
\hline & FUS & & 2.72 & 344.9 & DSC & [1969ROS/HOL] \\
\hline \multirow[t]{16}{*}{$\mathrm{C}_{2} \mathrm{H}_{4}$} & {$[74-85-1]$} & ethylene & & & & \\
\hline & FUS & & 3.35 & 104 & & [1996DOM/HEA, 1937EGA/KEM] \\
\hline & FUS & & 2.92 & 103.6 & & [1928EUC/HAU] \\
\hline & SUB & $(77-103)$ & 15.0 & & & [1982MEN] \\
\hline & SUB & $(99-104)$ & 19.4 & 99 & & [1977BIG/FUK] \\
\hline & SUB & $(79-104)$ & 18.4 & 91.5 & A, MS & [1987STE/MAL, 1951TIC/LOS] \\
\hline & $\mathrm{V}$ & $(252-282)$ & 14.0 & 267 & A & [1987STE/MAL] \\
\hline & $\mathrm{V}$ & $(170-273)$ & 13.7 & 258 & A & [1987STE/MAL] \\
\hline & $\mathrm{V}$ & $(120-170)$ & 14.4 & 155 & A & [1987STE/MAL] \\
\hline & $\mathrm{V}$ & $(169-211)$ & 13.7 & 196 & A & [1987STE/MAL] \\
\hline & $\mathrm{V}$ & $(209-254)$ & 13.6 & 239 & A & [1987STE/MAL] \\
\hline & $\mathrm{V}$ & $(104-119)$ & 15.7 & 111 & & [1982MEN] \\
\hline & $\mathrm{V}$ & $(120-182)$ & 14.1 & 167 & A & [1987STE/MAL, 1970DYK] \\
\hline & $\mathrm{V}$ & $(150-190)$ & 14.0 & 175 & & [1950MIC/WAS] \\
\hline & $\mathrm{V}$ & $(148-174)$ & 14.3 & 161 & & [1940LAM/ROP] \\
\hline & $\mathrm{V}$ & $(124-171)$ & 14.4 & 156 & & [1937EGA/KEM] \\
\hline
\end{tabular}


TABle 5. Phase change enthalpies of $\mathrm{C}_{2}$ organic compounds-Continued

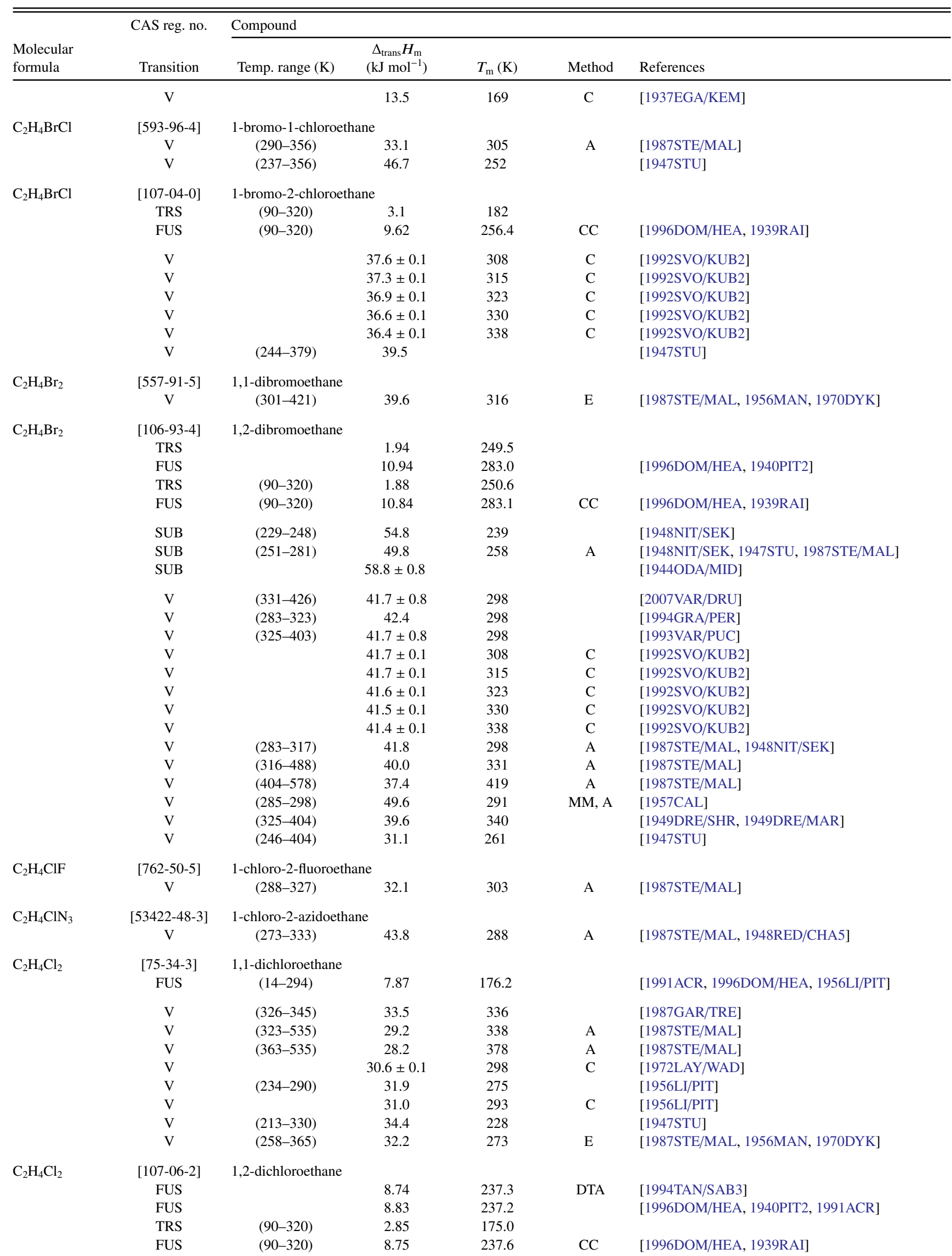


TABLE 5. Phase change enthalpies of $\mathrm{C}_{2}$ organic compounds-Continued

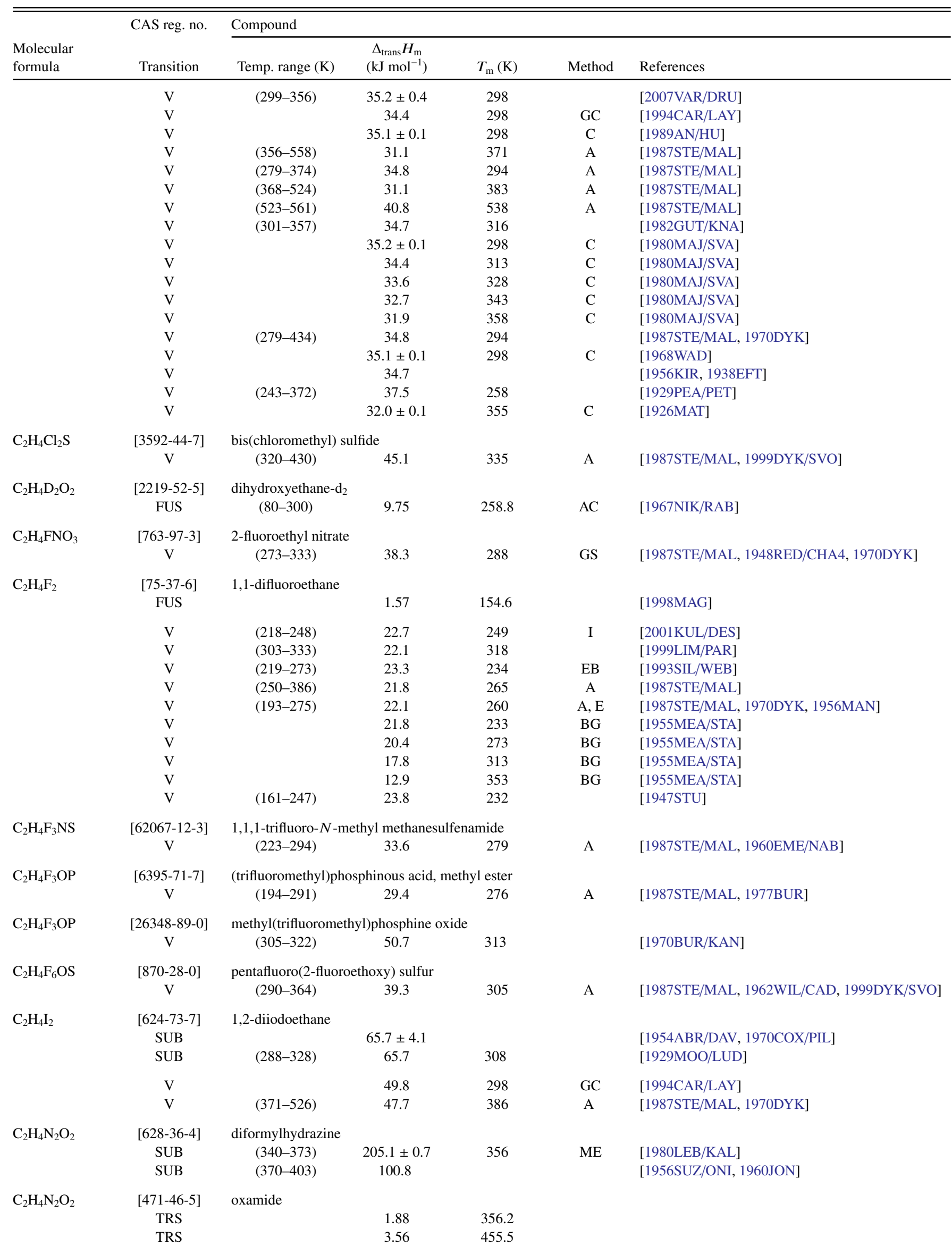


TABle 5. Phase change enthalpies of $\mathrm{C}_{2}$ organic compounds-Continued

\begin{tabular}{|c|c|c|c|c|c|c|}
\hline \multirow[b]{2}{*}{$\begin{array}{l}\text { Molecular } \\
\text { formula }\end{array}$} & \multirow{2}{*}{$\begin{array}{l}\text { CAS reg. no. } \\
\text { Transition }\end{array}$} & \multicolumn{5}{|l|}{ Compound } \\
\hline & & Temp. range $(\mathrm{K})$ & $\begin{array}{c}\Delta_{\text {trans }} H_{\mathrm{m}} \\
\left(\mathrm{kJ} \mathrm{mol}^{-1}\right)\end{array}$ & $T_{\mathrm{m}}(\mathrm{K})$ & Method & References \\
\hline & TRS & & 6.24 & 494.3 & & \\
\hline & FUS & & $\begin{array}{l}\text { Decomposed } \\
\text { prior to melting }\end{array}$ & & DSC & [2006BAD/DEL] \\
\hline & SUB & & $117.3 \pm 1.2$ & 298 & TE, ME & [1988NUN/BAR] \\
\hline & SUB & $(370-398)$ & 115.8 & 387 & TE, ME & [1983DEW/VAN] \\
\hline & SUB & $(353-369)$ & 113 & 361 & $\mathrm{ME}$ & [1953BRA/CLE, 1960JON, 1970COX/PIL] \\
\hline $\mathrm{C}_{2} \mathrm{H}_{4} \mathrm{~N}_{2} \mathrm{O}_{4}$ & $\begin{array}{c}{[600-40-8]} \\
\mathrm{V}\end{array}$ & $\begin{array}{c}\text { 1,1-dinitroethane } \\
\text { (303-363) }\end{array}$ & 51.0 & 318 & A & [1987STE/MAL] \\
\hline $\mathrm{C}_{2} \mathrm{H}_{4} \mathrm{~N}_{2} \mathrm{O}_{6}$ & $\begin{array}{c}{[628-96-6]} \\
\text { V } \\
\text { V } \\
\text { V } \\
\text { V } \\
\text { V } \\
\text { V }\end{array}$ & $\begin{array}{l}\text { ethylene glycol dini } \\
\quad(283-535) \\
(343-465) \\
(240-298) \\
(278-390) \\
(278-390) \\
(293-323)\end{array}$ & $\begin{array}{l}\text { itrate } \\
\qquad \begin{array}{c}70.5 \\
55.3 \\
68.3 \\
62.3 \pm 0.4 \\
61.3 \\
68.6 \pm 0.4\end{array}\end{array}$ & $\begin{array}{l}298 \\
358 \\
255 \\
334\end{array}$ & $\begin{array}{c}\text { A } \\
\text { A } \\
\text { GS }\end{array}$ & $\begin{array}{l}\text { [1987STE/MAL] } \\
\text { [1987STE/MAL] } \\
\text { [1987STE/MAL, 1977PEL] } \\
\text { [1941BEL] } \\
\text { [1940BEL] } \\
\text { [1938BRA] }\end{array}$ \\
\hline $\mathrm{C}_{2} \mathrm{H}_{4} \mathrm{~N}_{2} \mathrm{~S}_{2}$ & $\begin{array}{l}\text { [628-96-6] } \\
\text { SUB } \\
\text { SUB } \\
\text { SUB }\end{array}$ & $\begin{array}{l}\text { dithiooxamide } \\
\qquad \begin{array}{r}(345-372) \\
(360-378)\end{array}\end{array}$ & $\begin{array}{l}103.8 \\
105.1 \\
105.4\end{array}$ & $\begin{array}{l}298 \\
361 \\
369\end{array}$ & $\begin{array}{l}\text { TE, ME } \\
\text { TE, ME } \\
\text { ME }\end{array}$ & $\begin{array}{l}\text { [1988NUN/BAR] } \\
\text { [1983DEW/VAN] } \\
\text { [1953BRA/CLE2, 1960JON, 1987STE/MAL] }\end{array}$ \\
\hline $\mathrm{C}_{2} \mathrm{H}_{4} \mathrm{~N}_{4}$ & $\begin{array}{l}\text { [461-58-5] } \\
\text { FUS } \\
\text { TRS } \\
\text { FUS }\end{array}$ & $\begin{array}{c}\text { dicyandiamide } \\
(330-573) \\
(80-370) \\
(330-500)\end{array}$ & $\begin{array}{c}25.0 \\
2.98 \\
22.96\end{array}$ & $\begin{array}{l}482.7 \\
269.5 \\
487.6\end{array}$ & $\begin{array}{l}\text { DSC } \\
\text { AC } \\
\text { DSC }\end{array}$ & $\begin{array}{l}\text { [2014ZHA/QIA] } \\
\text { [1997ZHA/TAN] }\end{array}$ \\
\hline & SUB & $(420-450)$ & 128.7 & 436 & $\mathrm{TE}, \mathrm{ME}$ & {$[1983 \mathrm{DEW} / \mathrm{VAN}]$} \\
\hline $\mathrm{C}_{2} \mathrm{H}_{4} \mathrm{~N}_{4}$ & $\begin{array}{c}{[16681-77-9]} \\
\text { FUS }\end{array}$ & 1-methyltetrazole & 15.7 & 315 & & [1990KOZ/SIM3] \\
\hline & SUB & $(282-311)$ & $86.7 \pm 1.9$ & & $\mathrm{ME}$ & [1990KOZ/SIM] \\
\hline $\mathrm{C}_{2} \mathrm{H}_{4} \mathrm{~N}_{4}$ & $\begin{array}{l}{[16681-78-0]} \\
\text { FUS }\end{array}$ & 2-methyltetrazole & 12.37 & 286 & & [1990KOZ/SIM3] \\
\hline $\mathrm{C}_{2} \mathrm{H}_{4} \mathrm{~N}_{4}$ & $\begin{array}{c}\text { [4076-36-2] } \\
\text { FUS }\end{array}$ & 5-methyltetrazole & 16.0 & 418 & & [1990KOZ/SIM3] \\
\hline & SUB & $(323-418)$ & $93.8 \pm 0.5$ & & $\mathrm{ME}$ & {$[1990 \mathrm{KOZ} / \mathrm{SIM}]$} \\
\hline $\mathrm{C}_{2} \mathrm{H}_{4} \mathrm{~N}_{4}$ & $\begin{array}{l}{[61-82-5]} \\
\text { FUS }\end{array}$ & 1H-1,2,4-triazol-3- & $\begin{array}{l}\text { amine } \\
21.93\end{array}$ & 428.3 & DSC & [1990DON/DRE] \\
\hline $\mathrm{C}_{2} \mathrm{H}_{4} \mathrm{~N}_{4} \mathrm{O}_{4}$ & $\begin{array}{l}{[145250-81-3]} \\
\text { TRS }\end{array}$ & 1,1-diamino-2,2-dir & $\begin{array}{r}\text { nitroethene } \\
6.31\end{array}$ & 387.2 & DSC & [2006GAO/ZHA] \\
\hline $\mathrm{C}_{2} \mathrm{H}_{4} \mathrm{O}$ & $\begin{array}{c}\text { [75-07-0] } \\
\text { TRS } \\
\text { FUS }\end{array}$ & $\begin{array}{r}\text { acetaldehyde } \\
(13-300) \\
(13-300)\end{array}$ & $\begin{array}{l}2.31 \\
1.72\end{array}$ & $\begin{array}{l}149.8 \\
242.9\end{array}$ & $\mathrm{AC}$ & [1996DOM/HEA, 1988LEB/VAS] \\
\hline & $\begin{array}{l}\text { V } \\
\text { V } \\
\text { V } \\
\text { V } \\
\text { V } \\
\text { V }\end{array}$ & $\begin{array}{l}(293-377) \\
(293-345) \\
(272-294) \\
(293-377) \\
(273-307)\end{array}$ & $\begin{array}{c}26.0 \\
26.3 \\
27.6 \\
26.9 \\
27.0 \\
25.7 \pm 0.1\end{array}$ & $\begin{array}{l}308 \\
308 \\
283 \\
298 \\
307 \\
292\end{array}$ & $\begin{array}{c}\text { A } \\
\text { A } \\
\text { EB }\end{array}$ & $\begin{array}{l}\text { [1987STE/MAL] } \\
\text { [1977KIM/KIM] } \\
\text { [1987STE/MAL, 1970DYK] } \\
\text { [1963BUL/SER, 2003VER/KRA2] } \\
\text { [1950COL/POP] } \\
\text { [1949COL/DEV] }\end{array}$ \\
\hline $\mathrm{C}_{2} \mathrm{H}_{4} \mathrm{O}$ & $\begin{array}{l}\text { [75-21-8] } \\
\text { FUS }\end{array}$ & ethylene oxide (oxi & $\begin{array}{l}\text { rane) } \\
5.17\end{array}$ & 160.7 & & [1996DOM/HEA, 1949GIA/GOR] \\
\hline & $\begin{array}{l}\mathrm{V} \\
\mathrm{V} \\
\mathrm{V} \\
\mathrm{V}\end{array}$ & $\begin{array}{l}(283-385) \\
(239-284) \\
(223-284) \\
(268-313)\end{array}$ & $\begin{array}{l}25.9 \\
26.8 \\
26.8 \\
26.9\end{array}$ & $\begin{array}{l}298 \\
269 \\
269 \\
290\end{array}$ & $\begin{array}{l}\mathrm{A} \\
\mathrm{A} \\
\mathrm{A}\end{array}$ & $\begin{array}{l}\text { [1987STE/MAL] } \\
\text { [1987STE/MAL, 1959MCD/SHR, 1970DYK] } \\
\text { [1987STE/MAL, 1949GIA/GOR] } \\
\text { [1937MOO/KAN] }\end{array}$ \\
\hline $\mathrm{C}_{2} \mathrm{D}_{4} \mathrm{O}$ & $\begin{array}{c}{[6552-57-4]} \\
\mathrm{V}\end{array}$ & $\begin{array}{l}\text { ethylene oxide }-\mathrm{d}_{4} \\
\text { (230-273) }\end{array}$ & $\begin{array}{l}\text { oxide } \\
27.6\end{array}$ & 258 & & [1952LEI/MOR, 1984BOU/FRI] \\
\hline
\end{tabular}


TABLE 5. Phase change enthalpies of $\mathrm{C}_{2}$ organic compounds-Continued

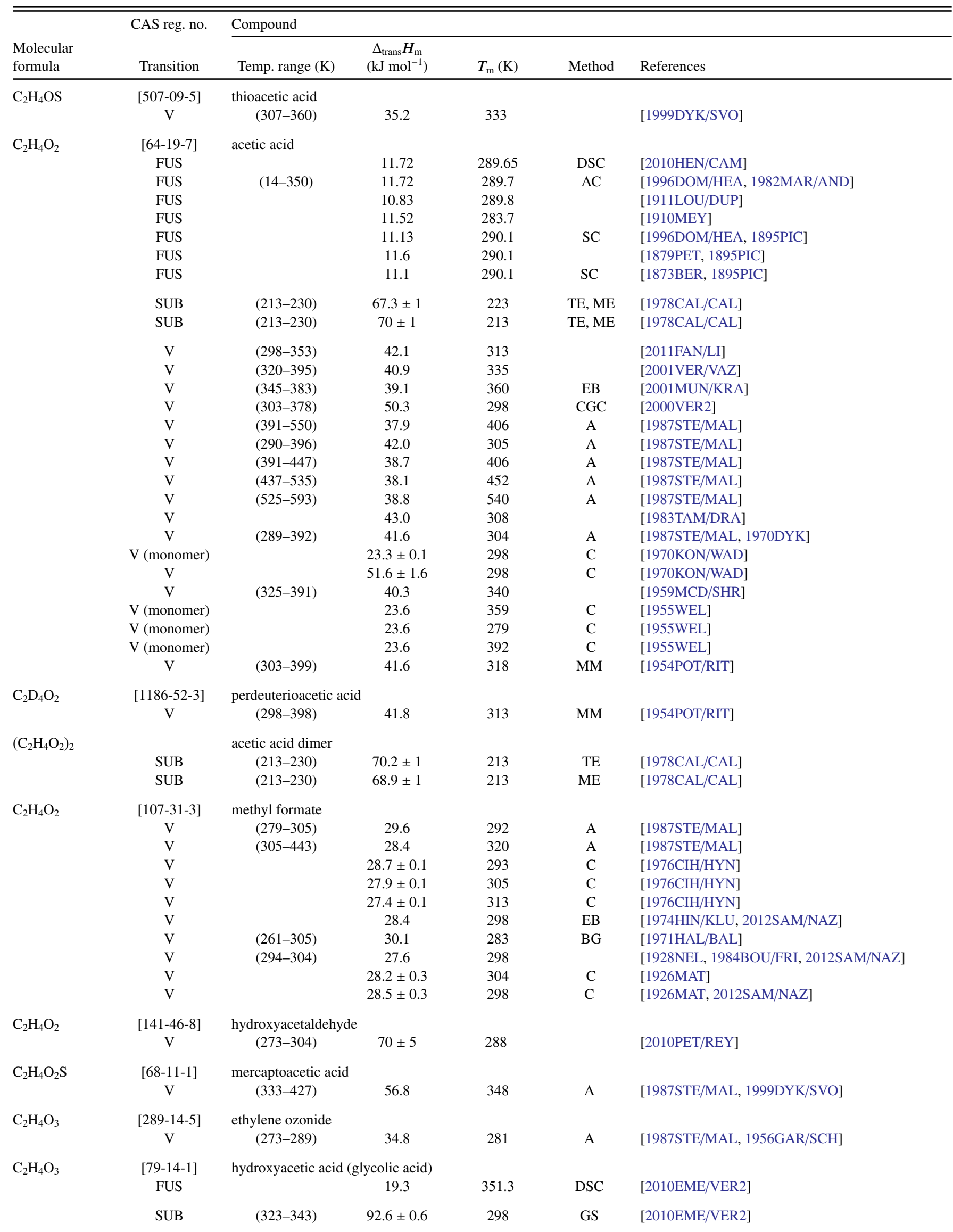


TABle 5. Phase change enthalpies of $\mathrm{C}_{2}$ organic compounds-Continued

\begin{tabular}{|c|c|c|c|c|c|c|}
\hline \multirow[b]{2}{*}{$\begin{array}{l}\text { Molecular } \\
\text { formula }\end{array}$} & \multirow{2}{*}{$\begin{array}{l}\text { CAS reg. no. } \\
\text { Transition }\end{array}$} & \multicolumn{5}{|l|}{ Compound } \\
\hline & & Temp. range $(\mathrm{K})$ & $\begin{array}{c}\Delta_{\text {trans }} H_{\mathrm{m}} \\
\left(\mathrm{kJ} \mathrm{mol}^{-1}\right)\end{array}$ & $T_{\mathrm{m}}(\mathrm{K})$ & Method & References \\
\hline & $\mathrm{V}$ & & $74.6 \pm 0.6$ & 298 & S-F & [2010EME/VER2] \\
\hline & $\mathrm{V}$ & $(350-375)$ & 51.8 & 362 & A & [1987STE/MAL] \\
\hline $\mathrm{C}_{2} \mathrm{H}_{4} \mathrm{O}_{3}$ & $\begin{array}{l}{[79-21-0]} \\
\mathrm{V}\end{array}$ & $\begin{array}{l}\text { peroxyacetic acid } \\
\qquad(273-383)\end{array}$ & 44.2 & 288 & A & [1987STE/MAL, 1970DYK, 1951EGE/EMT] \\
\hline $\mathrm{C}_{2} \mathrm{H}_{4} \mathrm{O}_{3}$ & $\begin{array}{l}{[7456-87-3]} \\
\text { SUB }\end{array}$ & $\begin{array}{l}\text { methyl hydrogen c } \\
\quad(204-237)\end{array}$ & $\begin{array}{l}\text { onate } \\
18.2 \pm 1.6\end{array}$ & 220 & & [1973BEH/GAT] \\
\hline $\mathrm{C}_{2} \mathrm{H}_{4} \mathrm{O}_{4}$ & $\begin{array}{l}{[291-15-6]} \\
\text { SUB }\end{array}$ & $1,2,4,5$-tetroxane & 11.19 & & & {$[2003 \mathrm{ROM} / \mathrm{LEI}]$} \\
\hline $\mathrm{C}_{2} \mathrm{H}_{4} \mathrm{~S}$ & $\begin{array}{c}{[420-12-2]} \\
\mathrm{V} \\
\mathrm{V}\end{array}$ & $\begin{array}{l}\text { ethylene sulfide } \\
\qquad(291-361)\end{array}$ & $\begin{array}{l}30.5 \\
30.3\end{array}$ & $\begin{array}{l}306 \\
298\end{array}$ & A & $\begin{array}{l}\text { [1987STE/MAL, 1952GUT/SCO2, 1999DYK/SVO] } \\
\text { [1971WIL/ZWO] }\end{array}$ \\
\hline $\mathrm{C}_{2} \mathrm{H}_{5} \mathrm{Br}$ & $\begin{array}{c}\text { [74-96-4] } \\
\text { V } \\
\text { V } \\
\text { V } \\
\text { V } \\
\text { V } \\
\text { V } \\
\text { V } \\
\text { V }\end{array}$ & $\begin{array}{r}\text { ethyl bromide } \\
(334-504) \\
(326-454) \\
(452-503) \\
\\
(225-333) \\
(301-348)\end{array}$ & $\begin{array}{c}26.9 \\
26.6 \\
31.0 \\
27.6 \pm 0.1 \\
27.0 \pm 0.1 \\
26.2 \pm 0.1 \\
30.6 \\
27.9\end{array}$ & $\begin{array}{l}349 \\
341 \\
467 \\
305 \\
312 \\
323 \\
240 \\
316\end{array}$ & $\begin{array}{l}\text { A } \\
\text { A } \\
\text { A } \\
\text { C } \\
\text { C } \\
\text { C } \\
\text { E }\end{array}$ & $\begin{array}{l}\text { [1987STE/MAL] } \\
{[1987 \mathrm{STE} / \mathrm{MAL}]} \\
{[1987 \mathrm{STE} / \mathrm{MAL}]} \\
{[1977 \mathrm{SVO} / \mathrm{MAJ}]} \\
{[1977 \mathrm{SVO} / \mathrm{MAJ}]} \\
{[1977 \mathrm{SVO} / \mathrm{MAJ}]} \\
{[1987 \mathrm{STE} / \mathrm{MAL}, 1961 \mathrm{LI} / \mathrm{ROS}, 1970 \mathrm{DYK}]} \\
{[1930 \mathrm{ZMA}, 1984 \mathrm{BOU} / \mathrm{FRI}]}\end{array}$ \\
\hline $\mathrm{C}_{2} \mathrm{H}_{5} \mathrm{BrO}$ & $\begin{array}{c}{[540-51-2]} \\
\mathrm{V}\end{array}$ & 2-bromoethanol & $54.1 \pm 0.4$ & 298 & $\mathrm{C}$ & [2007BER/MIN] \\
\hline $\mathrm{C}_{2} \mathrm{H}_{5} \mathrm{Cl}$ & $\begin{array}{l}\text { [75-00-3] } \\
\text { FUS }\end{array}$ & ethyl chloride & 4.45 & 134.8 & & [1991ACR, 1983WEA] \\
\hline & $\begin{array}{l}\text { V } \\
\text { V } \\
\text { V } \\
\text { V } \\
\text { V } \\
\text { V } \\
\text { V } \\
\text { V } \\
\text { V } \\
\text { V }\end{array}$ & $\begin{array}{l}(285-344) \\
(334-413) \\
(403-460) \\
(207-305) \\
(218-285)\end{array}$ & $\begin{array}{c}25.1 \\
24.4 \\
24.4 \\
27.8 \\
25.9 \\
24.9 \\
24.4 \\
24.8 \pm 0.1 \\
24.2 \\
24.5\end{array}$ & $\begin{array}{l}300 \\
349 \\
418 \\
222 \\
270 \\
288 \\
296 \\
298 \\
294 \\
294\end{array}$ & $\begin{array}{l}\text { A } \\
\text { A } \\
\text { A } \\
\text { A, E } \\
\text { C } \\
\text { C } \\
\text { C }\end{array}$ & $\begin{array}{l}\text { [1987STE/MAL] } \\
\text { [1987STE/MAL] } \\
\text { [1987STE/MAL] } \\
\text { [1987STE/MAL, 1961LI/ROS, 1970DYK] } \\
\text { [1948GOR/GIA] } \\
\text { [1932GRI/AWB] } \\
\text { [1932GRI/AWB] } \\
\text { [1926YAT, 2002MAN] } \\
\text { [1923JEN/SHO, 2002MAN] } \\
\text { [1871REG, 2002MAN] }\end{array}$ \\
\hline $\mathrm{C}_{2} \mathrm{H}_{5} \mathrm{ClFOPS}$ & $\begin{array}{c}{[460-51-5]} \\
\mathrm{V}\end{array}$ & ethyl chlorofluorot & $\begin{array}{l}\text { phosphate } \\
38.0\end{array}$ & & & [1948BOO/MAR] \\
\hline $\mathrm{C}_{2} \mathrm{H}_{5} \mathrm{ClO}$ & $\begin{array}{c}{[107-07-3]} \\
\text { V } \\
\text { V } \\
\text { V } \\
\text { V } \\
\text { V }\end{array}$ & $\begin{array}{l}\text { 2-chloroethanol } \\
\qquad \begin{array}{c}(328-401) \\
(323-363) \\
(363-403) \\
(269-401)\end{array}\end{array}$ & $\begin{array}{c}48.3 \pm 0.4 \\
43.3 \\
46.9 \\
39.1 \\
45.7\end{array}$ & $\begin{array}{l}298 \\
343 \\
338 \\
378 \\
284\end{array}$ & $\begin{array}{l}\mathrm{C} \\
\mathrm{A}\end{array}$ & $\begin{array}{l}{[2007 \mathrm{BER} / \mathrm{MIN}]} \\
{[1987 \mathrm{STE} / \mathrm{MAL}]} \\
{[1973 \mathrm{GOT} / \mathrm{MEN}]} \\
{[1973 \mathrm{GOT} / \mathrm{MEN}]} \\
{[1947 \mathrm{STU}]}\end{array}$ \\
\hline $\mathrm{C}_{2} \mathrm{H}_{5} \mathrm{ClO}$ & $\begin{array}{c}{[107-30-2]} \\
\mathrm{V}\end{array}$ & $\begin{array}{l}\text { methyl(chlorometh } \\
\quad(290-332)\end{array}$ & $\begin{array}{l}\text { ether } \\
32.2\end{array}$ & 305 & A & [1987STE/MAL, 1966MAT/RAS] \\
\hline $\mathrm{C}_{2} \mathrm{H}_{5} \mathrm{ClO}_{2} \mathrm{~S}$ & $\begin{array}{c}{[594-44-5]} \\
\mathrm{V} \\
\mathrm{V}\end{array}$ & $\begin{array}{l}\text { ethane sulfonyl chl } \\
\qquad \begin{array}{l}(349-449) \\
(233-263)\end{array}\end{array}$ & $\begin{array}{l}\text { de } \\
\begin{array}{r}47.7 \\
56.4\end{array}\end{array}$ & $\begin{array}{l}364 \\
248\end{array}$ & A & $\begin{array}{l}\text { [1999DYK/SVO] } \\
\text { [1987STE/MAL, 1999DYK/SVO, 1963QUI/NOW] }\end{array}$ \\
\hline $\mathrm{C}_{2} \mathrm{H}_{5} \mathrm{Cl}_{2} \mathrm{OP}$ & $\begin{array}{c}{[1066-50-8]} \\
\mathrm{V}\end{array}$ & ethylphosphonic a & $\begin{array}{l}\text { dichloride } \\
42.7 \pm 4.2\end{array}$ & & & [1956NEA/WIL, 1982PIL/SKI] \\
\hline $\mathrm{C}_{2} \mathrm{H}_{5} \mathrm{Cl}_{2} \mathrm{OPS}$ & $\begin{array}{c}\text { [1498-64-2] } \\
\mathrm{V}\end{array}$ & ethyl dichlorothiop & $\begin{array}{l}\text { sphate } \\
40.4\end{array}$ & & & [1948BOO/MAR] \\
\hline $\mathrm{C}_{2} \mathrm{H}_{5} \mathrm{Cl}_{2} \mathrm{P}$ & $\begin{array}{c}{[1498-40-4]} \\
\mathrm{V}\end{array}$ & $\begin{array}{c}\text { dichloroethyl phos } \\
\quad(313-385)\end{array}$ & 36.8 & 328 & A & [1987STE/MAL] \\
\hline
\end{tabular}


TABLE 5. Phase change enthalpies of $\mathrm{C}_{2}$ organic compounds-Continued

\begin{tabular}{|c|c|c|c|c|c|c|}
\hline \multirow[b]{2}{*}{$\begin{array}{l}\text { Molecular } \\
\text { formula }\end{array}$} & \multirow{2}{*}{$\begin{array}{l}\text { CAS reg. no. } \\
\text { Transition }\end{array}$} & \multicolumn{5}{|l|}{ Compound } \\
\hline & & Temp. range $(\mathrm{K})$ & $\begin{array}{c}\Delta_{\text {trans }} H_{\mathrm{m}} \\
\left(\mathrm{kJ} \mathrm{mol}^{-1}\right) \\
\end{array}$ & $T_{\mathrm{m}}(\mathrm{K})$ & Method & References \\
\hline \multirow[t]{8}{*}{$\mathrm{C}_{2} \mathrm{H}_{5} \mathrm{~F}$} & {$[353-36-6]$} & ethyl fluoride & & & & \\
\hline & $\mathrm{V}$ & $(200-235)$ & 20.7 & 236 & $\mathrm{I}$ & [2001KUL/DES] \\
\hline & V & $(275-353)$ & 20.2 & 290 & A & [1987STE/MAL] \\
\hline & $\mathrm{V}$ & $(235-280)$ & 20.5 & 265 & A & [1987STE/MAL] \\
\hline & $\mathrm{V}$ & $(343-375)$ & 20.7 & 358 & A & [1987STE/MAL] \\
\hline & $\mathrm{V}$ & $(170-255)$ & $\mathrm{U} 4.2$ & 240 & & [1975IWA/DAT] \\
\hline & $\mathrm{V}$ & $(173-251)$ & 20.8 & 236 & $\mathrm{E}$ & [1987STE/MAL, 1961LI/ROS, 1970DYK] \\
\hline & V & $(156-241)$ & 22.0 & 226 & & {$[1947 \mathrm{STU}]$} \\
\hline \multirow[t]{2}{*}{$\mathrm{C}_{2} \mathrm{H}_{5} \mathrm{FO}$} & {$[371-62-0]$} & 2-fluoroethanol & & & & \\
\hline & V & $(273-333)$ & 44.1 & 288 & GS & [1987STE/MAL, 1948RED/CHA4, 1970DYK] \\
\hline \multirow[t]{2}{*}{$\mathrm{C}_{2} \mathrm{H}_{5} \mathrm{FO}_{3} \mathrm{~S}$} & {$[371-69-7]$} & ethyl fluorosulfonate & & & & \\
\hline & V & $(273-333)$ & 38.5 & 288 & GS & [1987STE/MAL, 1948RED/CHA4, 1970DYK] \\
\hline \multirow[t]{3}{*}{$\mathrm{C}_{2} \mathrm{H}_{5} \mathrm{~F}_{2} \mathrm{~N}$} & {$[758-18-9]$} & $N, N$-difluoroethylam & ine & & & \\
\hline & $\mathrm{V}$ & $(241-259)$ & 27.3 & 250 & A & [1987STE/MAL] \\
\hline & $\mathrm{V}$ & $(241-259)$ & 25.7 & 288 & & {$[1960 \mathrm{FRA}]$} \\
\hline \multirow[t]{2}{*}{$\mathrm{C}_{2} \mathrm{H}_{5} \mathrm{~F}_{2} \mathrm{OPS}$} & {$[460-53-7]$} & \multicolumn{5}{|c|}{ ethyl difluorothiophosphate } \\
\hline & $\mathrm{V}$ & & 32.5 & & & [1948BOO/MAR] \\
\hline \multirow[t]{2}{*}{$\mathrm{C}_{2} \mathrm{H}_{5} \mathrm{~F}_{3} \mathrm{NP}$} & {$[4669-74-3]$} & \multicolumn{5}{|c|}{ methyl(trifluoromethyl) phosphinic acid amide } \\
\hline & $\mathrm{V}$ & $(238-294)$ & 36.8 & 279 & & [1987STE/MAL, 1966BUR/JOS] \\
\hline \multirow[t]{7}{*}{$\mathrm{C}_{2} \mathrm{H}_{5} \mathrm{I}$} & {$[75-03-6]$} & ethyl iodide & & & & \\
\hline & V & $(313-353)$ & 31.7 & 298 & CGC & [1995CHI/HOS] \\
\hline & $\mathrm{V}$ & & $31.9 \pm 0.1$ & 298 & $\mathrm{C}$ & [1968WAD] \\
\hline & $\mathrm{V}$ & $(249-369)$ & 33.6 & 264 & $\mathrm{E}$ & [1987STE/MAL, 1961LI/ROS] \\
\hline & $\mathrm{V}$ & $(219-345)$ & 34.7 & 234 & & [1947STU] \\
\hline & $\mathrm{V}$ & $(254-293)$ & 32 & 278 & & [1944MIL2] \\
\hline & $\mathrm{V}$ & $(303-333)$ & 31.7 & 318 & & [1929SMY/ENG] \\
\hline \multirow[t]{2}{*}{$\mathrm{C}_{2} \mathrm{H}_{5} \mathrm{IO}$} & {$[151-56-4]$} & 2-iodoethanol & & & & \\
\hline & $\mathrm{V}$ & & $57.0 \pm 0.2$ & 288 & $\mathrm{C}$ & [2007BER/MIN] \\
\hline \multirow[t]{2}{*}{$\mathrm{C}_{2} \mathrm{H}_{5} \mathrm{~N}$} & {$[151-56-4]$} & aziridine & & & & \\
\hline & $\mathrm{V}$ & $(274-303)$ & 34.9 & 288 & A & [1987STE/MAL, 1968CAB/CON] \\
\hline \multirow[t]{2}{*}{$\mathrm{C}_{2} \mathrm{H}_{5} \mathrm{NO}$} & {$[107-29-9]$} & acetaldehyde oxime & & & & \\
\hline & V & $(288-388)$ & 48.0 & 303 & A & [1987STE/MAL] \\
\hline \multirow[t]{17}{*}{$\mathrm{C}_{2} \mathrm{H}_{5} \mathrm{NO}$} & {$[60-35-5]$} & acetamide & & & & \\
\hline & FUS & & 15.5 & 355.2 & DSC & [1999SHA/BUD] \\
\hline & FUS & & 15.5 & 354.1 & DSC & [1989NIK/TRI] \\
\hline & FUS & & 15.6 & 353.5 & DSC & [1986EMO/NAU] \\
\hline & FUS & $(90-346)$ & 15.6 & 353.3 & $\mathrm{AC}$ & [1996DOM/HEA, 1983DEW/DEK] \\
\hline & FUS & & 14.86 & & $\mathrm{CCM}$ & [1937HRY/SMO] \\
\hline & FUS & & 16.4 & 355.5 & & [1914MUL, 1989NIK/TRI] \\
\hline & SUB & & $78.5 \pm 0.3$ & & & [1998PRI/HAW] \\
\hline & SUB & $(273-293)$ & 77.8 & 284 & TE, ME & [1983DEW/VAN] \\
\hline & SUB & & 77.2 & 298 & & [1983DEW/VAN] \\
\hline & SUB & & $78.7 \pm 0.3$ & & & [1975BAR/PIL, 1977PED/RYL] \\
\hline & SUB & & $80.3 \pm 1$ & 298 & & [1971MOR2] \\
\hline & SUB & & $80.3 \pm 1.3$ & 298 & $\mathrm{C}$ & [1965WAD] \\
\hline & SUB & $(298-349)$ & $77.4 \pm 0.4$ & 323 & GS & [1959DAV/JON2, 1987STE/MAL] \\
\hline & SUB & $(293-306)$ & U57.2 & 300 & & {$[1952 \mathrm{AIH}]$} \\
\hline & $\mathrm{V}$ & $(381-492)$ & 63.8 & 396 & A & [1987STE/MAL] \\
\hline & $\mathrm{V}$ & $(338-495)$ & 60.9 & 353 & & {$[1947 \mathrm{STU}]$} \\
\hline \multirow[t]{5}{*}{$\mathrm{C}_{2} \mathrm{H}_{5} \mathrm{NO}$} & {$[123-39-7]$} & $N$-methylformamide & & & & \\
\hline & TRS & & 1.23 & 228.1 & & \\
\hline & FUS & & 10.44 & 270.6 & & [1999AHL/LOH] \\
\hline & $\mathrm{V}$ & $(340-440)$ & 53.8 & 355 & Static & [1996USH/SED] \\
\hline & $\mathrm{V}$ & $(340-440)$ & $54.4 \pm 1.3$ & 298 & Static & [1996USH/SED] \\
\hline
\end{tabular}


TABle 5. Phase change enthalpies of $\mathrm{C}_{2}$ organic compounds-Continued

\begin{tabular}{|c|c|c|c|c|c|c|}
\hline \multirow[b]{2}{*}{$\begin{array}{l}\text { Molecular } \\
\text { formula }\end{array}$} & \multirow{2}{*}{$\begin{array}{c}\text { CAS reg. no. } \\
\text { Transition }\end{array}$} & \multicolumn{5}{|l|}{ Compound } \\
\hline & & Temp. range $(\mathrm{K})$ & $\begin{array}{c}\Delta_{\text {trans }} H_{\mathrm{m}} \\
\left(\mathrm{kJ} \mathrm{mol}^{-1}\right)\end{array}$ & $T_{\mathrm{m}}(\mathrm{K})$ & Method & References \\
\hline & $\mathrm{V}$ & $(310-391)$ & 54.5 & 325 & A & {$[1987 \mathrm{STE} / \mathrm{MAL}]$} \\
\hline & $\mathrm{V}$ & & 56.2 & 298 & A & [1985BAR/CAS, 1986VAR] \\
\hline & $\mathrm{V}$ & $(369-472)$ & 53.4 & 384 & A & [1987STE/MAL, 1961HEI/ILA, 1970DYK] \\
\hline \multirow[t]{3}{*}{$\mathrm{C}_{2} \mathrm{H}_{5} \mathrm{NO}_{2}$} & {$[109-95-5]$} & ethyl nitrite & & & & \\
\hline & $\mathrm{V}$ & $(252-276)$ & 25.7 & 264 & A & [1987STE/MAL, 1937THO/DAI] \\
\hline & $\mathrm{V}$ & & 27.8 & & & {$[1934 \mathrm{GOO}]$} \\
\hline \multirow[t]{7}{*}{$\mathrm{C}_{2} \mathrm{H}_{5} \mathrm{NO}_{2}$} & {$[598-55-0]$} & methyl carbamate & & & & \\
\hline & FUS & & 16.46 & 328.8 & AC,DSC & [2010ZEN/LI] \\
\hline & FUS & & 16.7 & 328.6 & & [1996DOM/HEA, 1976BER/BOU] \\
\hline & SUB & $(341-393)$ & 53.0 & 360 & $\mathrm{~EB}$ & {$[2013 X U / L I]$} \\
\hline & SUB & & $73.6 \pm 1.3$ & 298 & $\mathrm{C}$ & [2013SAN/RIB] \\
\hline & SUB & $(287-305)$ & $74.5 \pm 0.8$ & 296 & GS & {$[1959 \mathrm{DAV} / \mathrm{JON}]$} \\
\hline & $\mathrm{V}$ & $(333-388)$ & 45.7 & 348 & A & [1987STE/MAL] \\
\hline \multirow[t]{6}{*}{$\mathrm{C}_{2} \mathrm{H}_{5} \mathrm{NO}_{2}$} & {$[79-24-3]$} & nitroethane & & & & \\
\hline & FUS & $(80-300)$ & 9.85 & 183.7 & $\mathrm{AC}$ & [1996DOM/HEA, 1966LIU/ZIE] \\
\hline & $\mathrm{V}$ & $(324-388)$ & 38.6 & 339 & $\mathrm{~EB}$ & [1987STE/MAL, 1956TOO, 1970DYK] \\
\hline & $\mathrm{V}$ & $(283-383)$ & $41.5 \pm 0.4$ & 289 & $\mathrm{ZG}$ & [1949HOL/DOR] \\
\hline & $\mathrm{V}$ & $(252-387)$ & 41.3 & 267 & & [1947STU] \\
\hline & $\mathrm{V}$ & & 38 & & & {$[1934 \mathrm{GOO}]$} \\
\hline \multirow[t]{4}{*}{$\mathrm{C}_{2} \mathrm{H}_{5} \mathrm{NO}_{2}$} & {$[56-40-6]$} & glycine & & & & \\
\hline & TRS & & 0.09 & 435.5 & $\mathrm{DSC}$ & [2008DIC/SGH] \\
\hline & $\operatorname{TRS}(\gamma$ to $\alpha)$ & & 1.8 & 453.2 & DSC & \\
\hline & $\operatorname{TRS}(\gamma$ to $\alpha)$ & & 1.2 & 438.2 & DSC & [2001PER/HAN] \\
\hline \multicolumn{7}{|c|}{ [Note: Authors of [2001PER/HAN] report that the phase transition was sensitive to the conditions under which the $\gamma$-phase was grown.] } \\
\hline & SUB & $(408-431)$ & $136.5 \pm 2$ & 419 & $\mathrm{TE}, \mathrm{ME}$ & {$[1979 \mathrm{DEK} / \mathrm{VOO}]$} \\
\hline & SUB & $(325-425)$ & $\mathrm{U} 96.2 \pm 4$ & 375 & $\mathrm{LE}$ & [1977GAF/PIE] \\
\hline & SUB & $(413-450)$ & $138.1 \pm 4.6$ & 298 & $\mathrm{C}$ & {$[1977 \mathrm{NGA} / \mathrm{SAB}]$} \\
\hline & SUB & $(453-471)$ & $136.4 \pm 4.0$ & 462 & ME & $\begin{array}{l}\text { [1965SVE/CLY, 1970COX/PIL, 1964CLY/SVE, } \\
\text { 1989CHI/GRO] }\end{array}$ \\
\hline & SUB & $(412-417)$ & $130.5 \pm 2$ & 414 & ME & {$[1959 \mathrm{TAK} / \mathrm{CHI}]$} \\
\hline \multirow[t]{6}{*}{$\mathrm{C}_{2} \mathrm{H}_{5} \mathrm{NO}_{3}$} & {$[625-58-1]$} & ethyl nitrate & & & & \\
\hline & FUS & & 8.53 & 178.6 & & [1996DOM/HEA, 1954GRA/SMI] \\
\hline & $\mathrm{V}$ & $(273-361)$ & 37.0 & 288 & A & [1987STE/MAL, 1970DYK] \\
\hline & $\mathrm{V}$ & $(273-343)$ & 37.3 & 288 & & [1957GRA/PRA] \\
\hline & $\mathrm{V}$ & $(273-333)$ & 37.0 & 288 & & [1956GRA/PRA] \\
\hline & $\mathrm{V}$ & & 38.5 & & & {$[1934 \mathrm{GOO}]$} \\
\hline \multirow[t]{4}{*}{$\mathrm{C}_{2} \mathrm{H}_{5} \mathrm{NS}$} & {$[62-55-5]$} & thioacetamide & & & & \\
\hline & FUS & & 18.36 & 385.7 & DSC & {$[1990 \mathrm{DON} / \mathrm{DRE}]$} \\
\hline & SUB & & $83.3 \pm 0.3$ & 298 & $\mathrm{C}$ & [1982INA/MUR, 1985MUR/SAK] \\
\hline & SUB & & $82.8 \pm 0.3$ & 298 & $\mathrm{C}$ & {$[1982 \mathrm{SAB} / \mathrm{TOR}]$} \\
\hline \multirow[t]{2}{*}{$\mathrm{C}_{2} \mathrm{H}_{5} \mathrm{~N}_{3}$} & $\begin{array}{c}{[871-31-8]} \\
\mathrm{V}\end{array}$ & azidoethane & 315 & 308 & A & [1087STF/MAI 1070DYK] \\
\hline & $\mathrm{V}$ & $(253-298)$ & 28.9 & 268 & A & [1987STE/MAL, 1964GEI/KON, 1984BOU/FRI] \\
\hline \multirow[t]{3}{*}{$\mathrm{C}_{2} \mathrm{H}_{5} \mathrm{~N}_{3} \mathrm{O}$} & {$[1517-05-1]$} & 2-azidoethanol & & & & \\
\hline & $\mathrm{V}$ & $(284-316)$ & $62.2 \pm 0.3$ & 298 & GS & [2011VER/EME2] \\
\hline & V & & $33.9 \pm 1.3$ & & & [1997KOR/API] \\
\hline \multirow[t]{2}{*}{$\mathrm{C}_{2} \mathrm{H}_{5} \mathrm{~N}_{3} \mathrm{O}_{2}$} & & bis(nitrosomethyl) & & & & \\
\hline & $\mathrm{V}$ & $(276-426)$ & 43.5 & 291 & A & [1987STE/MAL, 1947STU] \\
\hline \multirow[t]{2}{*}{$\mathrm{C}_{2} \mathrm{H}_{5} \mathrm{~N}_{5}$} & {$[5422-44-6]$} & 1-methyl-5-aminot & azole & & & \\
\hline & SUB & $(379-438)$ & $116.4 \pm 1.7$ & & ME & [1990KOZ/SIM] \\
\hline \multirow[t]{2}{*}{$\mathrm{C}_{2} \mathrm{H}_{5} \mathrm{~N}_{5}$} & [6154-04-7] & 2-methyl-5-aminot & azole & & & \\
\hline & SUB & $(310-373)$ & $90.6 \pm 1.1$ & & ME & [1990KOZ/SIM] \\
\hline
\end{tabular}


TABLE 5. Phase change enthalpies of $\mathrm{C}_{2}$ organic compounds-Continued

\begin{tabular}{|c|c|c|c|c|c|c|}
\hline \multirow[b]{2}{*}{$\begin{array}{l}\text { Molecular } \\
\text { formula }\end{array}$} & \multirow{2}{*}{$\begin{array}{l}\text { CAS reg. no. } \\
\text { Transition }\end{array}$} & \multicolumn{5}{|l|}{ Compound } \\
\hline & & Temp. range $(\mathrm{K})$ & $\begin{array}{c}\Delta_{\text {trans }} H_{\mathrm{m}} \\
\left(\mathrm{kJ} \mathrm{mol}^{-1}\right)\end{array}$ & $T_{\mathrm{m}}(\mathrm{K})$ & Method & References \\
\hline \multirow[t]{16}{*}{$\mathrm{C}_{2} \mathrm{H}_{6}$} & {$[74-84-0]$} & ethane & & & & \\
\hline & TRS & & 2.28 & 89.8 & & \\
\hline & FUS & & 0.58 & 90.3 & & [1976ATA/CHI] \\
\hline & FUS & & 2.86 & 89.9 & & [1996DOM/HEA, 1937WIT/KEM] \\
\hline & FUS & & 2.79 & 89.5 & & [1996DOM/HEA, 1930WIE/HUB] \\
\hline & SUB & $(80-90)$ & 22.6 & 85 & & [1972REG] \\
\hline & SUB & & 20.5 & 90 & $\mathrm{~B}$ & {$[1963 \mathrm{BON}]$} \\
\hline & $\mathrm{V}$ & $(273-305)$ & 15.3 & 288 & A & [1987STE/MAL] \\
\hline & $\mathrm{V}$ & $(154-185)$ & 15.7 & 170 & A & [1987STE/MAL] \\
\hline & $\mathrm{V}$ & $(95-129)$ & 17.7 & 114 & A & [1987STE/MAL] \\
\hline & $\mathrm{V}$ & $(185-229)$ & 14.9 & 214 & A & [1987STE/MAL] \\
\hline & $\mathrm{V}$ & $(228-274)$ & 14.9 & 259 & A & [1987STE/MAL] \\
\hline & $\mathrm{V}$ & $(91-144)$ & 17.1 & 129 & & [1973CAR/KOB] \\
\hline & $\mathrm{V}$ & & 14.7 & 210 & & [1971WIL/ZWO] \\
\hline & $\mathrm{V}$ & & 14.7 & 184 & & [1937WIT/KEM] \\
\hline & $\mathrm{V}$ & $(136-200)$ & 15.3 & 185 & & {$[1926 \mathrm{LOO} / \mathrm{WAL}]$} \\
\hline \multirow[t]{2}{*}{$\mathrm{C}_{2} \mathrm{H}_{6} \mathrm{BrF}_{4} \mathrm{NS}$} & {$[63324-17-4]$} & \multicolumn{5}{|c|}{ bromotetrafluoro( $N$-methylmethanaminato) sulfur } \\
\hline & $\mathrm{V}$ & & 38.1 & 372 & I & [1977KIT/SHR2] \\
\hline \multirow[t]{2}{*}{$\mathrm{C}_{2} \mathrm{H}_{6} \mathrm{ClF}_{4} \mathrm{NS}$} & {$[63324-16-3]$} & \multicolumn{5}{|c|}{ chlorotetrafluoro( $N$-methylmethanaminato) sulfur } \\
\hline & $\mathrm{V}$ & & 36.0 & 359 & I & [1977KIT/SHR2] \\
\hline \multirow[t]{3}{*}{$\mathrm{C}_{2} \mathrm{H}_{6} \mathrm{ClP}$} & [811-62-1] & \multicolumn{5}{|c|}{ chlorodimethyl phosphine } \\
\hline & SUB & $(233-268)$ & 55.5 & 253 & A & [1987STE/MAL, 1958BUR/SLO] \\
\hline & $\mathrm{V}$ & $(273-306)$ & 32.9 & 288 & A & [1987STE/MAL, 1958BUR/SLO] \\
\hline \multirow[t]{2}{*}{$\mathrm{C}_{2} \mathrm{H}_{6} \mathrm{Cl}_{2} \mathrm{NP}$} & {$[683-85-2]$} & \multicolumn{5}{|c|}{ (dimethylamino)dichlorophosphine } \\
\hline & $\mathrm{V}$ & & $40.8 \pm 0.7$ & 298 & STG & [1995ALM/FIN2] \\
\hline \multirow[t]{2}{*}{$\mathrm{C}_{2} \mathrm{H}_{6} \mathrm{ClO}_{3} \mathrm{P}$} & {$[16672-87-0]$} & \multicolumn{5}{|c|}{ 2-chloroethylphosphonic acid } \\
\hline & FUS & & 14.79 & 347.9 & DSC & [1990DON/DRE] \\
\hline \multirow[t]{2}{*}{$\mathrm{C}_{2} \mathrm{H}_{6} \mathrm{FN}$} & {$[14722-43-1]$} & \multicolumn{5}{|c|}{ fluorodimethylamine } \\
\hline & $\mathrm{V}$ & $(249-273)$ & 29.9 & 261 & A & [1987STE/MAL, 1966WIE/RUF] \\
\hline \multirow[t]{2}{*}{$\mathrm{C}_{2} \mathrm{H}_{6} \mathrm{FO}_{3} \mathrm{P}$} & {$[5954-50-7]$} & \multicolumn{5}{|c|}{ dimethylfluorophosphate } \\
\hline & $\mathrm{V}$ & $(273-333)$ & 44.4 & 288 & A, GS & [1987STE/MAL, 1948RED/CHA4] \\
\hline $\mathrm{C}_{2} \mathrm{H}_{6} \mathrm{~F}_{2} \mathrm{NOP}$ & {$[354-43-8]$} & $N, N$-dimethylpho & horamidic dif & & & \\
\hline & $\mathrm{V}$ & & 50.1 & & & {$[1967 \mathrm{CAV}]$} \\
\hline $\mathrm{C}_{2} \mathrm{H}_{6} \mathrm{~F}_{2} \mathrm{NP}$ & {$[814-97-1]$} & (dimethylamino)di & orophosphine & & & \\
\hline & $\mathrm{V}$ & $(263-313)$ & 29.3 & 288 & I & {$[1964 \mathrm{CAV}]$} \\
\hline $\mathrm{C}_{2} \mathrm{H}_{6} \mathrm{~F}_{3} \mathrm{NOS}$ & {$[22519-52-4]$} & (dimethylaminato) & luorooxo sulf & & & \\
\hline & $\mathrm{V}$ & $(313-357)$ & 44.9 & 335 & & [1968GLE/VON2] \\
\hline $\mathrm{C}_{2} \mathrm{H}_{6} \mathrm{~F}_{3} \mathrm{NS}$ & {$[3880-03-3]$} & (dimethylamino) st & ar trifluoride & & & \\
\hline & $\mathrm{V}$ & $(296-327)$ & 40.5 & 311 & A & [1987STE/MAL, 1999DYK/SVO, 1967DEM/KEN] \\
\hline $\mathrm{C}_{2} \mathrm{H}_{6} \mathrm{~F}_{4} \mathrm{NP}$ & {$[2353-98-2]$} & (dimethylamino) te & fluorophosph & & & \\
\hline & $\mathrm{V}$ & & 37.1 & & & {$[1966 \mathrm{BRO} / \mathrm{FRA}]$} \\
\hline $\mathrm{C}_{2} \mathrm{H}_{6} \mathrm{~N}_{2}$ & {$[503-28-6]$} & azomethane & & & & \\
\hline & $\mathrm{V}$ & $(195-273)$ & 26.4 & 258 & A & [1987STE/MAL] \\
\hline & $\mathrm{V}$ & $(209-236)$ & 25.3 & 222 & A & [1987STE/MAL] \\
\hline $\mathrm{C}_{2} \mathrm{H}_{6} \mathrm{~N}_{2}$ & {$[51283-79-5]$} & methylammonium & anide & & & \\
\hline & $\mathrm{V}$ & $(251-295)$ & 49.1 & 280 & A & [1987STE/MAL, 1973DIE/MAR] \\
\hline $\mathrm{C}_{2} \mathrm{H}_{6} \mathrm{~N}_{2} \mathrm{O}$ & [598-50-5] & $N$-methylurea & & & & \\
\hline & FUS & & 12.5 & 372 & DSC & [2005HAS/TAJ] \\
\hline & FUS & & 16.6 & 375 & DSC & [1995FER/DEL] \\
\hline & FUS & & 14.06 & 378.1 & & [1990KAB/MIR2] \\
\hline & FUS & & 15.75 & 373.8 & DSC & [1987DEL/FER] \\
\hline & FUS & & 13.97 & 375.3 & DSC & [1972ZOR/HUR] \\
\hline & SUB & $(331-365)$ & $95.5 \pm 0.5$ & 298 & GS & [2006EME/KAB] \\
\hline
\end{tabular}


TABLE 5. Phase change enthalpies of $\mathrm{C}_{2}$ organic compounds-Continued

\begin{tabular}{|c|c|c|c|c|c|c|}
\hline \multirow[b]{2}{*}{$\begin{array}{l}\text { Molecular } \\
\text { formula }\end{array}$} & \multirow{2}{*}{$\begin{array}{l}\text { CAS reg. no. } \\
\text { Transition }\end{array}$} & \multicolumn{5}{|l|}{ Compound } \\
\hline & & Temp. range $(\mathrm{K})$ & $\begin{array}{c}\Delta_{\text {trans }} H_{\mathrm{m}} \\
\left(\mathrm{kJ} \mathrm{mol}^{-1}\right)\end{array}$ & $T_{\mathrm{m}}(\mathrm{K})$ & Method & References \\
\hline & SUB & $(322-371)$ & $96.9 \pm 1.2$ & 347 & $\mathrm{ME}$ & [2003ZAI/KAB] \\
\hline & SUB & $(322-371)$ & $96.8 \pm 1.2$ & 350 & $\mathrm{ME}$ & [2003ZAI/KAB] \\
\hline & SUB & & $94.4 \pm 0.8$ & 350 & $\mathrm{C}$ & [2003ZAI/KAB] \\
\hline & SUB & & $96.3 \pm 0.4$ & 298 & & [2003ZAI/KAB] \\
\hline & SUB & & $94.4 \pm 0.8$ & 343 & $\mathrm{C}$ & [1993KOZ/KAB] \\
\hline & SUB & & $97.1 \pm 0.4$ & 298 & & {$[1993 \mathrm{KOZ} / \mathrm{KAB}]$} \\
\hline & SUB & & $94.9 \pm 0.6$ & 337 & $\mathrm{C}$ & [1990KAB/MIR] \\
\hline & SUB & & $93.3 \pm 1.2$ & 355 & TE & [1990PIA/FER, 1987FER/DEL2] \\
\hline & SUB & & 87.3 & 348 & & [1987FER/DEL2] \\
\hline & SUB & & $99.3 \pm 0.7$ & & & [1986KRA/KOZ2] \\
\hline & SUB & & & 78.2 & $\mathrm{E}$ & [1982AIR/CHA] \\
\hline \multirow[t]{3}{*}{$\mathrm{C}_{2} \mathrm{H}_{6} \mathrm{~N}_{2} \mathrm{O}_{2}$} & [4164-28-7] & \multicolumn{5}{|c|}{$N$-nitro- $N$-methylaminomethane } \\
\hline & FUS & & 37.66 & 327 & DTA & [1987OYU/BRI] \\
\hline & SUB & & $67.4 \pm 1.3$ & 298 & $\mathrm{C}$ & [2009MIR/KON] \\
\hline
\end{tabular}

[Note: Authors of [2009MIR/KON] tabulate the experimental value as an enthalpy of vaporization; however, they report that the compound is crystalline and use the value to calculate the standard molar enthalpy of formation. Given the crystaline state and how the value was used, we have tabulated the value as an enthalpy of sublimation.]

SUB

(315-324)

69.9

319

DBM

[1952BRA/COT, 1977PED/RYL]

$\mathrm{C}_{2} \mathrm{H}_{6} \mathrm{~N}_{2} \mathrm{O}_{2}$

[4164-28-7] 2-nitro-2-azapropane FUS

17.26

331.5

DSC

[1997ZEM]

$\mathrm{C}_{2} \mathrm{H}_{6} \mathrm{~N}_{2} \mathrm{~S}$

[598-52-7] N-methylthiourea

FUS

FUS

$19.46 \quad 392.4$

16.3

393

DSC

2000DEL/JOZ

SUB

$112.9 \pm 3$

298

$\mathrm{ME}$

[1993DEM/BUC]

SUB

$111 \pm 3.0$

381

[2000DEL/JOZ]

$\mathrm{C}_{2} \mathrm{H}_{6} \mathrm{~N}_{2} \mathrm{~S} \quad$ [13849-02-0] sulfur diimide, dimethyl

$\mathrm{V} \quad(248-298) \quad 37.2$

$\mathrm{C}_{2} \mathrm{H}_{6} \mathrm{~N}_{4} \mathrm{O}_{4}$

[505-71-5] $\quad N, N^{\prime}$-dinitroethanediamine

FUS

29.5

263

A

[1987STE/MAL, 1999DYK/SVO, 1966COH/MAC]

$\mathrm{C}_{2} \mathrm{H}_{6} \mathrm{O}$

[115-10-6] dimethyl ether FUS

$$
4.94
$$

450

DTA

[1987OYU/BRI]

$\begin{array}{ll}\text { V } & (180-249) \\ V & (293-360) \\ V & (349-400) \\ V & (241-303) \\ V & (171-248) \\ V & (171-248) \\ V & (183-265) \\ V & (195-248) \\ V & \end{array}$

$\mathrm{C}_{2} \mathrm{H}_{6} \mathrm{O}$

[64-17-5] ethanol
FUS

FUS
FUS
TRS
FUS
FUS
FUS
FUS
FUS

$\begin{array}{ll}\mathrm{V} & (317-351) \\ \mathrm{V} & (325-352)\end{array}$

(311-351)

(298-348)

(323-357)

(309-343)

(309-343)

4.94
22.8
21.2
21.1
22.2
18.5
21.4
22.6
22.7
$21.5 \pm 0.1$

131.7

[1996DOM/HEA, 1941KEN/SAG]

234

308

364

256

298

248

250

233

248

[1987STE/MAL]

A [1987STE/MAL]

A [1987STE/MAL]

A [1987STE/MAL]

[1976AMB/ELL]

[1976AMB/ELL]

A [1987STE/MAL, 1969KUD/REE]

[1941KEN/SAG]

C [1941KEN/SAG]

\begin{tabular}{|c|c|c|c|}
\hline 5.08 & 158.6 & DTA & [1994TAN/SAB3] \\
\hline 4.64 & 158.8 & & [1996DOM/HEA, 1977HAI/SUG] \\
\hline 3.14 & 111.4 & & \\
\hline 4.64 & 158.8 & & [1996DOM/HEA, 1967NIK/RAB2] \\
\hline 4.97 & 159.0 & & [1944YOS] \\
\hline 5.02 & 158.5 & & [1929KEL4] \\
\hline 4.96 & 158.7 & & [1925PAR] \\
\hline 4.63 & 156.2 & & [1920GIB/PAR] \\
\hline 41.3 & 332 & & [2011SAP/UUS] \\
\hline $1.2 \pm 0.4$ & & I & [2010GER/PEL] \\
\hline 41.7 & 326 & & [2010MEJ/SEG] \\
\hline 41.7 & 298 & & [2004NAS/ZIM] \\
\hline 38.9 & & & [1999FAT] \\
\hline 39.3 & 338 & & [1999AUC/LOR] \\
\hline 40.7 & 321 & EB & [1995DIO/SAN] \\
\hline 42.4 & 298 & EB & [1995DIO/SAN] \\
\hline
\end{tabular}


TABle 5. Phase change enthalpies of $\mathrm{C}_{2}$ organic compounds-Continued

\begin{tabular}{|c|c|c|c|c|c|c|}
\hline \multirow[b]{2}{*}{$\begin{array}{l}\text { Molecular } \\
\text { formula }\end{array}$} & \multirow{2}{*}{$\begin{array}{l}\text { CAS reg. no. } \\
\text { Transition }\end{array}$} & \multicolumn{5}{|l|}{ Compound } \\
\hline & & Temp. range $(\mathrm{K})$ & $\begin{array}{c}\Delta_{\text {trans }} H_{\mathrm{m}} \\
\left(\mathrm{kJ} \mathrm{mol}^{-1}\right)\end{array}$ & $T_{\mathrm{m}}(\mathrm{K})$ & Method & References \\
\hline & V & $(342-357)$ & 40.5 & 357 & & [1990ORT/SUS] \\
\hline & $\mathrm{V}$ & & 35.2 & 393 & $\mathrm{C}$ & [1989VIN/WOR] \\
\hline & V & & 30.6 & 423 & $\mathrm{C}$ & [1989VIN/WOR] \\
\hline & $\mathrm{V}$ & & 25.7 & 453 & $\mathrm{C}$ & [1989VIN/WOR] \\
\hline & V & & 21.8 & 473 & $\mathrm{C}$ & [1989VIN/WOR] \\
\hline & V & & 17.3 & 493 & $\mathrm{C}$ & [1989VIN/WOR] \\
\hline & $\mathrm{V}$ & & 14.2 & 503 & $\mathrm{C}$ & [1989VIN/WOR] \\
\hline & V & & 40.9 & 320 & $\mathrm{C}$ & [1988DON/LIN] \\
\hline & $\mathrm{V}$ & & 40.4 & 328 & $\mathrm{C}$ & [1988DON/LIN] \\
\hline & $\mathrm{V}$ & & 40.2 & 335 & $\mathrm{C}$ & [1988DON/LIN] \\
\hline & $\mathrm{V}$ & & 39.4 & 344 & $\mathrm{C}$ & [1988DON/LIN] \\
\hline & $\mathrm{V}$ & & 38.8 & 351 & $\mathrm{C}$ & [1988DON/LIN] \\
\hline & V & $(320-359)$ & 41.3 & 335 & A & [1987STE/MAL] \\
\hline & V & $(210-271)$ & 45.6 & 256 & $\mathrm{~A}$ & [1987STE/MAL] \\
\hline & $\mathrm{V}$ & $(193-223)$ & 44.0 & 208 & A & [1987STE/MAL] \\
\hline & V & $(320-359)$ & 41.3 & 335 & A & [1987STE/MAL] \\
\hline & $\mathrm{V}$ & $(349-374)$ & 40.1 & 361 & A & [1987STE/MAL] \\
\hline & $\mathrm{V}$ & $(370-464)$ & 39.1 & 385 & A & [1987STE/MAL] \\
\hline & V & $(459-514)$ & 36.1 & 474 & A & [1987STE/MAL] \\
\hline & $\mathrm{V}$ & $(292-353)$ & 42.5 & 307 & A & [1987STE/MAL] \\
\hline & $\mathrm{V}$ & $(243-303)$ & 42.3 & 298 & & [1983SCH/STR] \\
\hline & V & $(271-373)$ & 42.9 & 286 & & [1973WIL/ZWO] \\
\hline & $\mathrm{V}$ & & $42.26 \pm 0.02$ & 298 & $\mathrm{C}$ & [1971POL/BEN] \\
\hline & $\mathrm{V}$ & & $41.7 \pm 0.1$ & 308 & $\mathrm{C}$ & [1971POL/BEN] \\
\hline & V & & $41.2 \pm 0.1$ & 318 & $\mathrm{C}$ & [1971POL/BEN] \\
\hline & $\mathrm{V}$ & & $40.1 \pm 0.1$ & 328 & $\mathrm{C}$ & [1971POL/BEN] \\
\hline & $\mathrm{V}$ & & $41.0 \pm 0.1$ & 320 & $\mathrm{C}$ & [1970COU/FEN] \\
\hline & V & & $40.0 \pm 0.1$ & 335 & $\mathrm{C}$ & [1970COU/FEN] \\
\hline & $\mathrm{V}$ & & $38.7 \pm 0.1$ & 351 & $\mathrm{C}$ & [1970COU/FEN] \\
\hline & $\mathrm{V}$ & $(293-366)$ & 42.5 & 308 & A, EB & [1987STE/MAL, 1970AMB/SPR] \\
\hline & V & $(288-348)$ & 42.4 & 303 & & [1967VAN/SOC] \\
\hline & $\mathrm{V}$ & & $42.3 \pm 0.1$ & 298 & $\mathrm{C}$ & [1966WAD] \\
\hline & $\mathrm{V}$ & & $42.2 \pm 0.1$ & 298 & $\mathrm{C}$ & [1963MCC/LAI] \\
\hline & $\mathrm{V}$ & $(298-351)$ & 42.2 & 313 & & [1949KRE/WIE] \\
\hline & $\mathrm{V}$ & & 40.0 & 351 & & [1934OGU/ANJ] \\
\hline & $\mathrm{V}$ & $(286-351)$ & 54.1 & 301 & & {$[1883 \mathrm{KAH}]$} \\
\hline \multirow[t]{13}{*}{$\mathrm{C}_{2} \mathrm{H}_{6} \mathrm{OS}$} & {$[67-68-5]$} & dimethyl sulfoxide & & & & \\
\hline & FUS & & 12.34 & 292.2 & DSC & [2015SOL/ROD] \\
\hline & FUS & $(5-350)$ & 14.37 & 291.7 & $\mathrm{AC}$ & [1996DOM/HEA, 1970CLE/WES] \\
\hline & $\mathrm{V}$ & $(402-463)$ & 47.5 & 425 & & [2013ZHA/LV] \\
\hline & $\mathrm{V}$ & & 53.3 & 298 & $\mathrm{CE}$ & [2011FUL/RUZ] \\
\hline & $\mathrm{V}$ & $(377-483)$ & 48.6 & 392 & & [1999DYK/SVO] \\
\hline & $\mathrm{V}$ & $(353-383)$ & 48.1 & 368 & TGA & [1987ALN/ALS] \\
\hline & V & $(305-464)$ & 51.7 & 320 & $\mathrm{~A}$ & [1987STE/MAL] \\
\hline & $\mathrm{V}$ & $(298-318)$ & 52.3 & 308 & & [1974SAS/KON] \\
\hline & $\mathrm{V}$ & $(325-442)$ & 50.6 & 340 & MM & [1972JAK/VAN, 1984BOU/FRI] \\
\hline & $\mathrm{V}$ & $(303-423)$ & 52.1 & 318 & & [1972NIS/HAK] \\
\hline & $\mathrm{V}$ & $(293-323)$ & 52.5 & 308 & & [1969MES] \\
\hline & $\mathrm{V}$ & $(293-323)$ & $52.9 \pm 0.4$ & 298 & RG & [1948DOU] \\
\hline \multirow[t]{2}{*}{$\mathrm{C}_{2} \mathrm{H}_{6} \mathrm{OS}$} & {$[60-24-2]$} & 2-mercaptoethanol & & & & \\
\hline & $\mathrm{V}$ & $(293-440)$ & 54.1 & 308 & A & [1987STE/MAL, 1970DYK, 1999DYK/SVO] \\
\hline \multirow[t]{8}{*}{$\mathrm{C}_{2} \mathrm{H}_{6} \mathrm{O}_{2}$} & {$[107-21-1]$} & ethylene glycol & & & & \\
\hline & FUS & & 11.98 & 261.2 & DSC & [2015SOL/ROD] \\
\hline & FUS & & 11.1 & 261.6 & DSC & [2012ROD/SOL] \\
\hline & FUS & & 9.5 & 256.6 & DTA & [1990KNA/SAB] \\
\hline & FUS & & 9.96 & 260.6 & & [1996DOM/HEA, 1967NIK/RAB] \\
\hline & FUS & & 11.6 & 260.6 & & {$[1925 \mathrm{PAR} / \mathrm{KEL}]$} \\
\hline & FUS & & 11.2 & & & {$[1901 \mathrm{DEF} 2]$} \\
\hline & $\mathrm{V}$ & $(307-384)$ & $62.4 \pm 4.0$ & 345 & & [2010PET/REY] \\
\hline
\end{tabular}


TABLE 5. Phase change enthalpies of $\mathrm{C}_{2}$ organic compounds-Continued

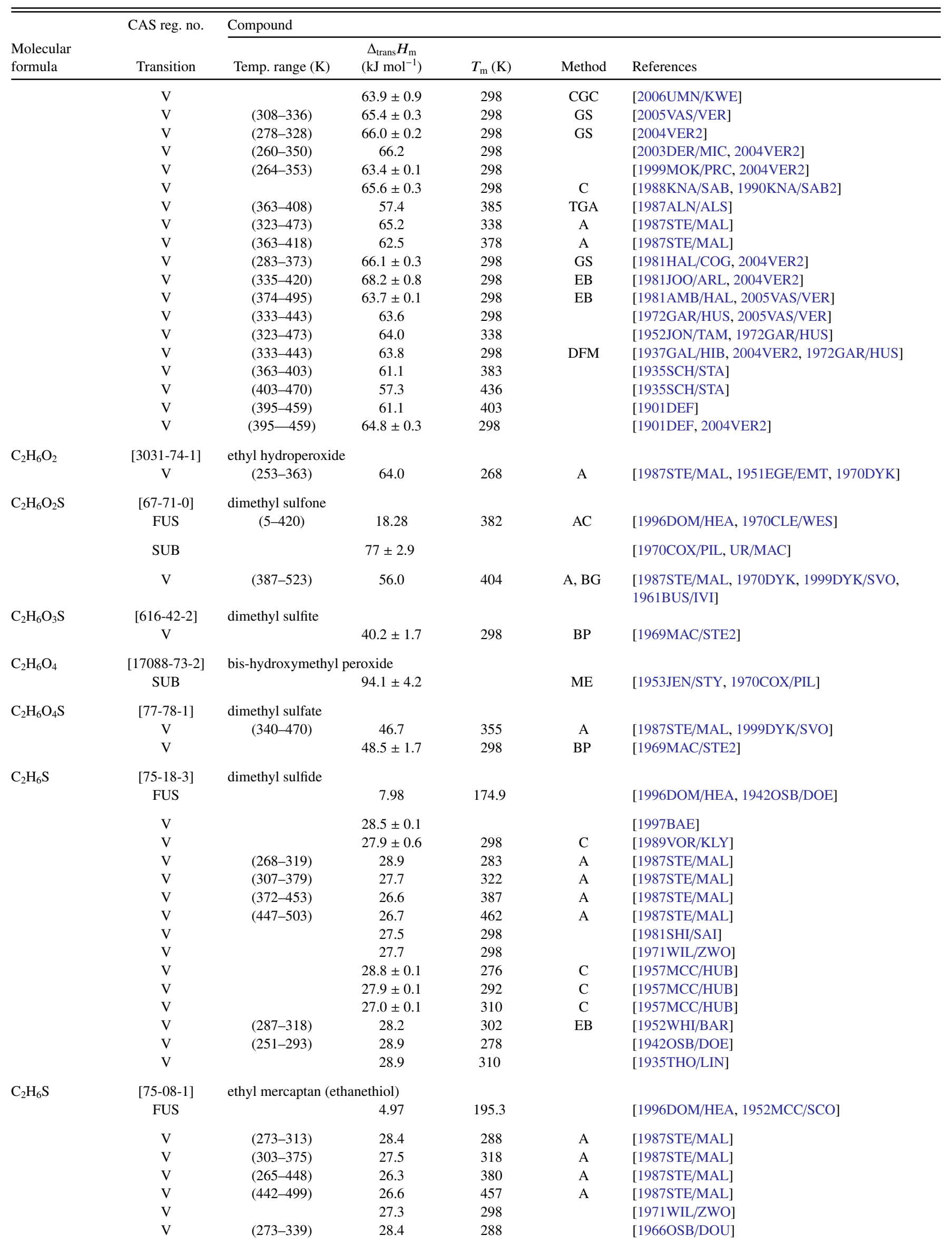


TABLE 5. Phase change enthalpies of $\mathrm{C}_{2}$ organic compounds-Continued

\begin{tabular}{|c|c|c|c|c|c|c|}
\hline \multirow[b]{2}{*}{$\begin{array}{l}\text { Molecular } \\
\text { formula }\end{array}$} & \multirow{2}{*}{$\begin{array}{l}\text { CAS reg. no. } \\
\text { Transition }\end{array}$} & \multicolumn{5}{|l|}{ Compound } \\
\hline & & Temp. range $(\mathrm{K})$ & $\begin{array}{c}\Delta_{\text {trans }} H_{\mathrm{m}} \\
\left(\mathrm{kJ} \mathrm{mol}^{-1}\right)\end{array}$ & $T_{\mathrm{m}}(\mathrm{K})$ & Method & References \\
\hline & $\mathrm{V}$ & $(273-339)$ & 28.4 & 288 & $\mathrm{~A}, \mathrm{~EB}$ & [1987STE/MAL, 1952MCC/SCO, 1966OSB/DOU] \\
\hline & $\mathrm{V}$ & & 28.7 & 306 & & [1935THO/LIN] \\
\hline \multirow[t]{2}{*}{$\mathrm{C}_{2} \mathrm{H}_{6} \mathrm{~S}_{2}$} & {$[540-63-6]$} & 1,2-ethanedithiol & & & & \\
\hline & $\mathrm{V}$ & & $44.7 \pm 0.1$ & 298 & & [1962MAN/SUN] \\
\hline \multirow[t]{13}{*}{$\mathrm{C}_{2} \mathrm{H}_{6} \mathrm{~S}_{2}$} & {$[624-92-0]$} & dimethyl disulfide & & & & \\
\hline & FUS & & 9.19 & 188.4 & & [1996DOM/HEA, 1950SCO/FIN] \\
\hline & $\mathrm{V}$ & & $38.5 \pm 0.6$ & 298 & $\mathrm{C}$ & [1989VOR/KLY] \\
\hline & $\mathrm{V}$ & $(297-402)$ & 37.8 & 312 & A & [1987STE/MAL] \\
\hline & $\mathrm{V}$ & & $37.8 \pm 0.1$ & 298 & $\mathrm{C}$ & [1985KUS] \\
\hline & $\mathrm{V}$ & & 37.8 & 298 & & [1981SHI/SAI] \\
\hline & $\mathrm{V}$ & & 38.4 & 298 & & [1971WIL/ZWO] \\
\hline & $\mathrm{V}$ & & $36.0 \pm 0.1$ & 341 & $\mathrm{C}$ & [1958HUB/DOU] \\
\hline & $\mathrm{V}$ & & $34.9 \pm 0.1$ & 360 & $\mathrm{C}$ & [1958HUB/DOU] \\
\hline & $\mathrm{V}$ & & $33.7 \pm 0.1$ & 383 & $\mathrm{C}$ & [1958HUB/DOU] \\
\hline & $\mathrm{V}$ & $(321-388)$ & 36.7 & 336 & EB & [1952WHI/BAR] \\
\hline & $\mathrm{V}$ & $(334-401)$ & 36.2 & 349 & & [1950SCO/FIN] \\
\hline & V & $(288-333)$ & 38.2 & 303 & & [1950SCO/FIN] \\
\hline \multirow[t]{6}{*}{$\mathrm{C}_{2} \mathrm{H}_{7} \mathrm{~N}$} & {$[124-40-3]$} & dimethylamine & & & & \\
\hline & FUS & & 5.94 & 181 & & [1996DOM/HEA, 1939AST/EID] \\
\hline & $\mathrm{V}$ & $(277-360)$ & 27.0 & 292 & A & [1987STE/MAL] \\
\hline & $\mathrm{V}$ & $(358-438)$ & 23.8 & 373 & A & [1987STE/MAL] \\
\hline & $\mathrm{V}$ & $(202-279)$ & 28.4 & 264 & A & [1987STE/MAL, 1939AST/EID, 1984BOU/FRI] \\
\hline & $\mathrm{V}$ & & 27.2 & 280 & & {$[1935 \mathrm{WIB} / \mathrm{SUT}]$} \\
\hline \multirow[t]{7}{*}{$\mathrm{C}_{2} \mathrm{H}_{7} \mathrm{~N}$} & {$[75-04-7]$} & ethyl amine & & & & \\
\hline & $\mathrm{V}$ & $(213-297)$ & 29.0 & 282 & A & [1987STE/MAL] \\
\hline & $\mathrm{V}$ & $(290-449)$ & 27.2 & 305 & A & [1987STE/MAL] \\
\hline & $\mathrm{V}$ & $(291-387)$ & 27.6 & 306 & A & [1987STE/MAL] \\
\hline & $\mathrm{V}$ & $(377-456)$ & 25.9 & 392 & A & [1987STE/MAL] \\
\hline & $\mathrm{V}$ & $(275-288)$ & 29.1 & 281 & EB & [1962BIT/KAU2] \\
\hline & $\mathrm{V}$ & $(190-290)$ & 28.9 & 275 & & {$[1947 \mathrm{STU}]$} \\
\hline \multirow[t]{2}{*}{$\mathrm{C}_{2} \mathrm{H}_{7} \mathrm{NO}$} & {$[5725-96-2]$} & $N, N$-dimethylhyd & yl amine & & & \\
\hline & $\mathrm{V}$ & $(290-363)$ & 45.7 & 305 & A & [1987STE/MAL, 1957BIS/PAR, 1984BOU/FRI] \\
\hline \multirow[t]{2}{*}{$\mathrm{C}_{2} \mathrm{H}_{7} \mathrm{NO}$} & {$[1117-97-1]$} & $N, O$-dimethylhydr & yl amine & & & \\
\hline & $\mathrm{V}$ & $(228-316)$ & 34.3 & 243 & A & [1987STE/MAL, 1957BIS/PAR, 1984BOU/FRI] \\
\hline \multirow[t]{17}{*}{$\mathrm{C}_{2} \mathrm{H}_{7} \mathrm{NO}$} & [141-43-5] & 2-aminoethanol & & & & \\
\hline & FUS & & 13.32 & 283.2 & DSC & [2015SOL/ROD] \\
\hline & FUS & & 13.8 & 283.9 & DSC & [2014PEN/UUS] \\
\hline & V & $(283-363)$ & 60.6 & 298 & Static & [2009BEL/RAZ] \\
\hline & $\mathrm{V}$ & $(357-435)$ & 55.9 & 372 & EB & [2008KIM/SVE] \\
\hline & $\mathrm{V}$ & $(279-324)$ & $59.6 \pm 0.3$ & 298 & GS & [2005KAP/SLO] \\
\hline & $\mathrm{V}$ & $(358-440)$ & 59.4 & 298 & EB & [1999TOC/AKI, 2005KAP/SLO] \\
\hline & $\mathrm{V}$ & $(352-613)$ & 59.0 & 298 & EB & [1987DAU/JAL, 2005KAP/SLO] \\
\hline & $\mathrm{V}$ & $(310-444)$ & 61.7 & 325 & A & [1987STE/MAL] \\
\hline & $\mathrm{V}$ & $(298-308)$ & 56.1 & 298 & & [1982TOU/OKA, 2005KAP/SLO] \\
\hline & $\mathrm{V}$ & $(293-298)$ & 54.8 & 298 & & [1974GUS/REN, 2005KAP/SLO] \\
\hline & $\mathrm{V}$ & $(325-443)$ & 57.7 & 298 & & [1969DAN/MAT, 2005KAP/SLO] \\
\hline & $\mathrm{V}$ & $(379-443)$ & 54.7 & 394 & & [1959MCD/SHR] \\
\hline & $\mathrm{V}$ & $(273-301)$ & U50.8 & 287 & A, GS & [1957BES/KOC] \\
\hline & $\mathrm{V}$ & $(338-443)$ & 58.9 & 353 & & [1950MAT/SUM, 1984BOU/FRI] \\
\hline & $\mathrm{V}$ & $(303-373)$ & 57.4 & 298 & EB & [1947LEI/SHO, 2005KAP/SLO] \\
\hline & $\mathrm{V}$ & $(341-453)$ & 57.9 & 298 & & [1935WIL, 2005KAP/SLO] \\
\hline \multirow[t]{2}{*}{$\mathrm{C}_{2} \mathrm{H}_{7} \mathrm{NO}_{3} \mathrm{~S}$} & {$[107-35-7]$} & \multicolumn{5}{|c|}{ 2-aminoethanesulfonic acid } \\
\hline & FUS & & 22.20 & 588.45 & DSC & [2012HAN/ZHO] \\
\hline
\end{tabular}


TABle 5. Phase change enthalpies of $\mathrm{C}_{2}$ organic compounds-Continued

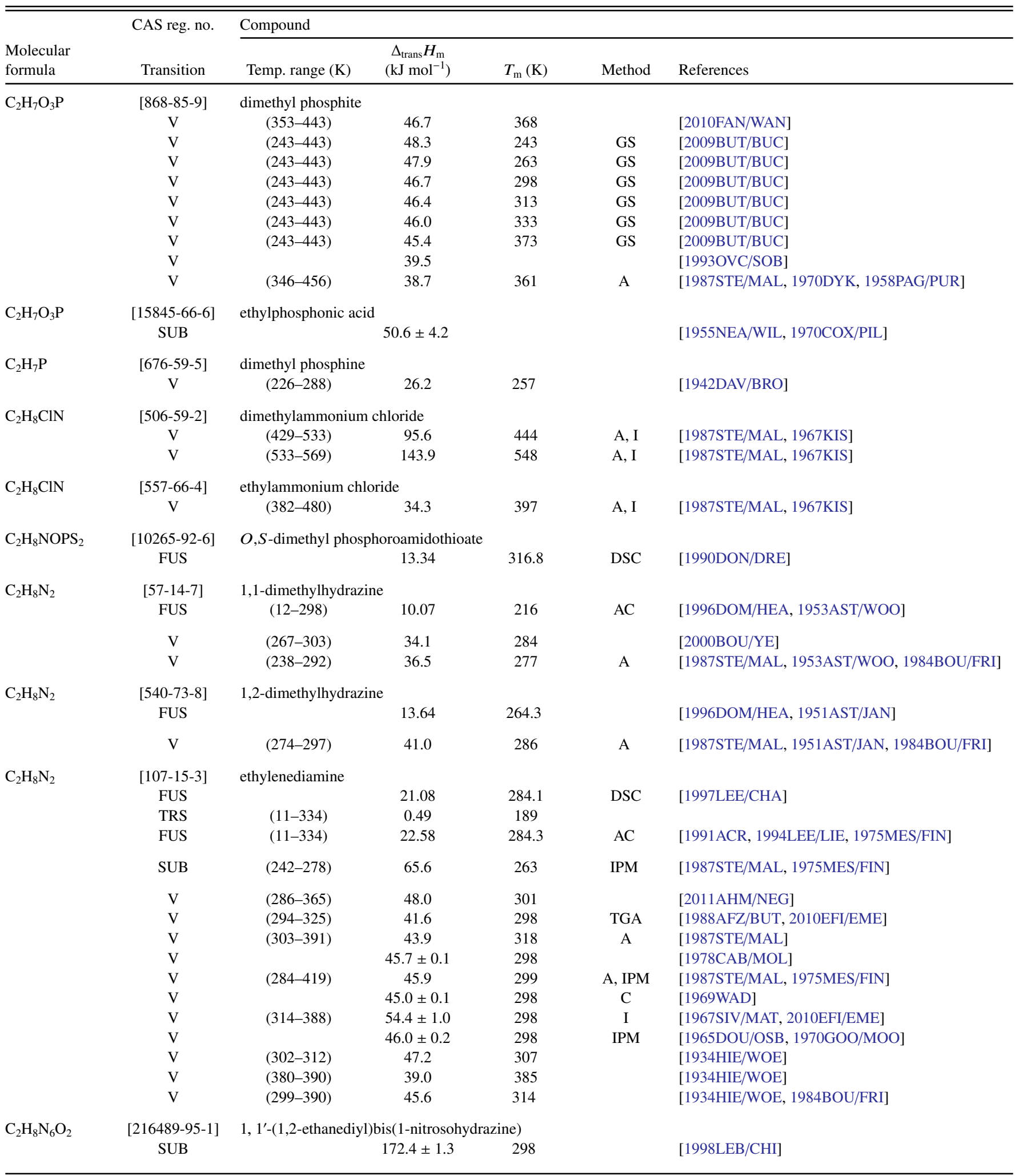


TABLE 6. Phase change enthalpies of $\mathrm{C}_{3}$ organic compounds

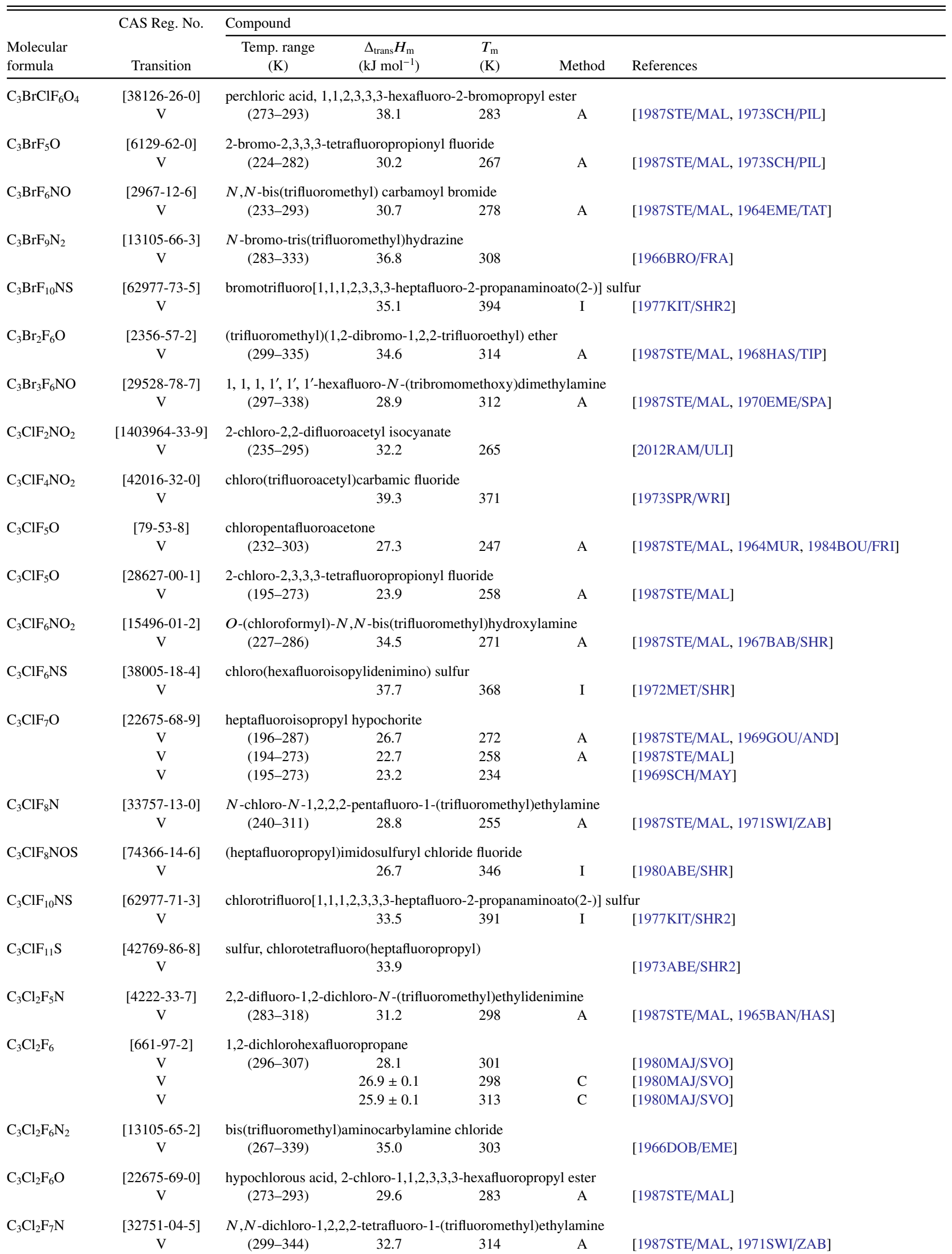


TABLE 6. Phase change enthalpies of $\mathrm{C}_{3}$ organic compounds-Continued

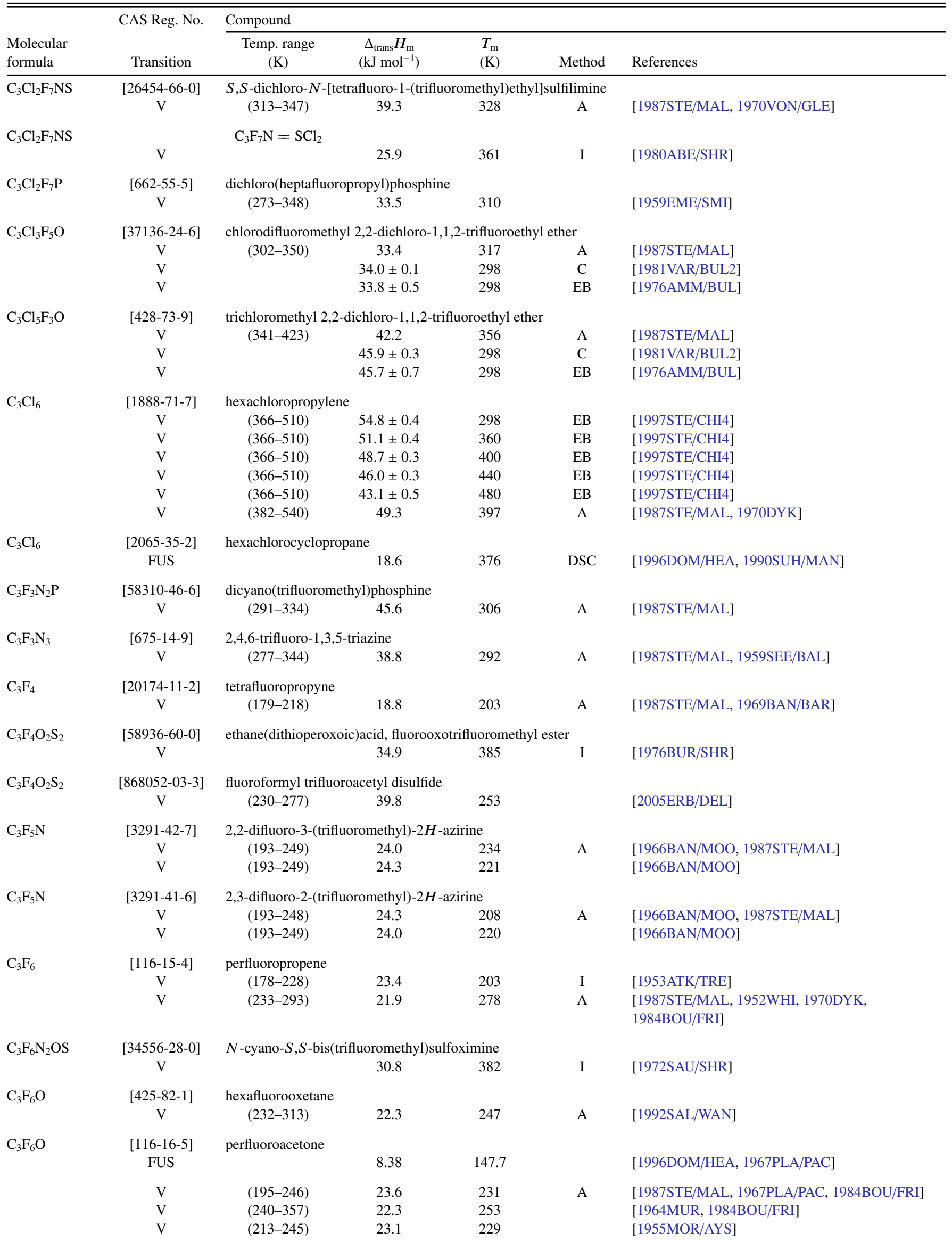


Table 6. Phase change enthalpies of $\mathrm{C}_{3}$ organic compounds-Continued

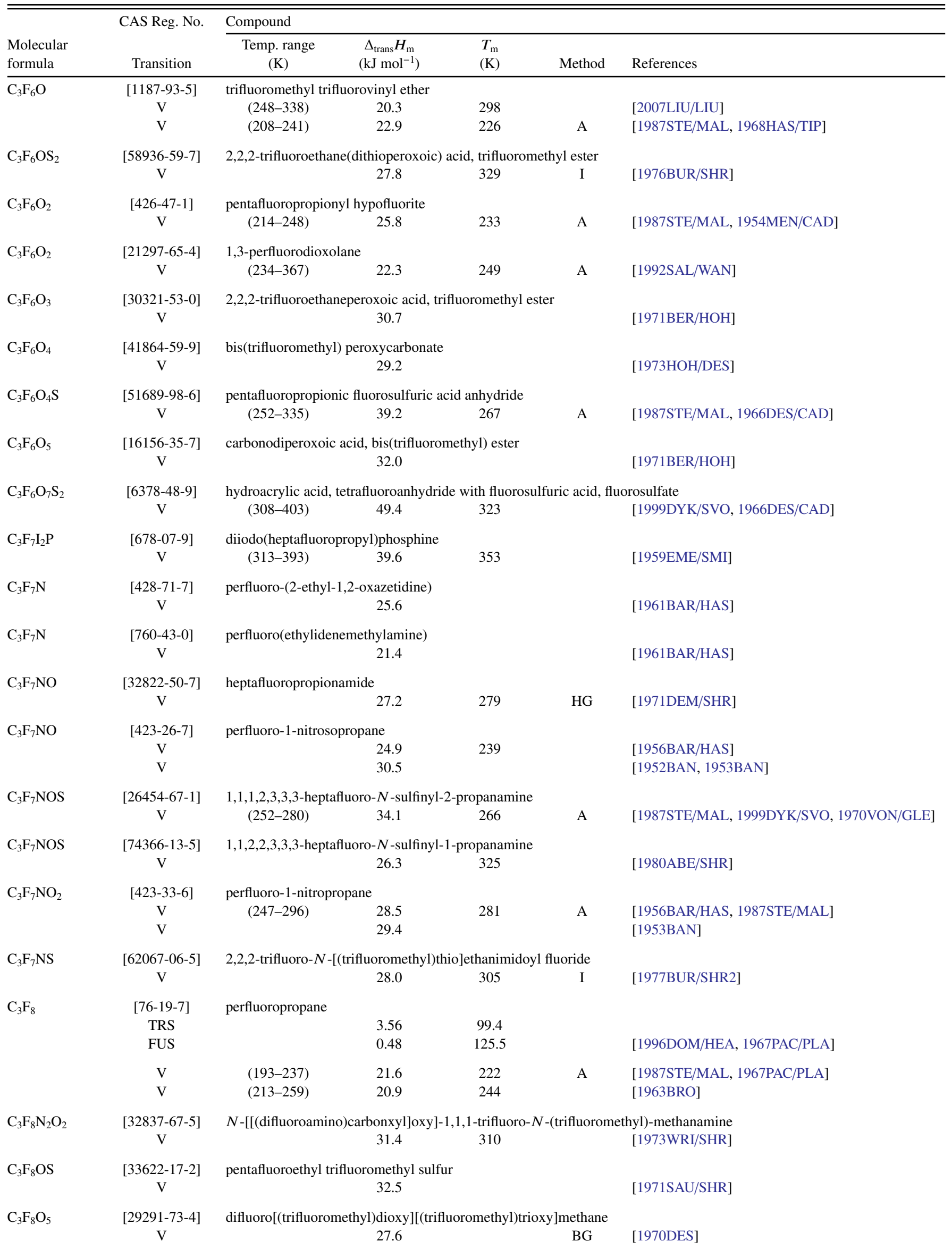


TABLE 6. Phase change enthalpies of $\mathrm{C}_{3}$ organic compounds-Continued

\begin{tabular}{|c|c|c|c|c|c|c|}
\hline \multirow[b]{2}{*}{$\begin{array}{l}\text { Molecular } \\
\text { formula }\end{array}$} & \multirow{2}{*}{$\begin{array}{c}\text { CAS Reg. No. } \\
\text { Transition }\end{array}$} & \multicolumn{5}{|l|}{ Compound } \\
\hline & & $\begin{array}{l}\text { Temp. range } \\
\text { (K) }\end{array}$ & $\begin{array}{c}\Delta_{\text {trans }} H_{\mathrm{m}} \\
\left(\mathrm{kJ} \mathrm{mol}^{-1}\right)\end{array}$ & $\begin{array}{c}T_{\mathrm{m}} \\
(\mathrm{K})\end{array}$ & Method & References \\
\hline $\mathrm{C}_{3} \mathrm{~F}_{8} \mathrm{~S}$ & $\begin{array}{c}{[33547-10-3]} \\
\text { V }\end{array}$ & 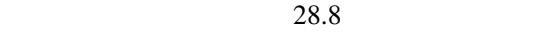 & \multicolumn{3}{|c|}{ pentafluoroethyl trifluoromethyl sulfide } & {$[1971 \mathrm{SAU} / \mathrm{SHR}]$} \\
\hline $\mathrm{C}_{3} \mathrm{~F}_{9} \mathrm{~N}$ & $\begin{array}{c}{[432-03-1]} \\
\mathrm{V}\end{array}$ & \multicolumn{5}{|c|}{$\begin{array}{l}\text { perfluorotrimethylamine } \\
\qquad(193-263)\end{array}$} \\
\hline $\mathrm{C}_{3} \mathrm{~F}_{9} \mathrm{NO}$ & $\begin{array}{c}{[671-63-6]} \\
\mathrm{V}\end{array}$ & \multicolumn{5}{|c|}{ 1,1,1-trifluoro- $N$-(trifluoromethoxy)- $N$-(trifluoromethyl)-methanamine } \\
\hline $\mathrm{C}_{3} \mathrm{~F}_{9} \mathrm{NOS}$ & $\begin{array}{c}{[59617-29-7]} \\
\mathrm{V}\end{array}$ & \multicolumn{4}{|c|}{ [1,2,2,2-tetrafluoro-1-(trifluoromethyl)ethyl] imidosulfuryl fluoride } & [1976STA/MEW] \\
\hline $\mathrm{C}_{3} \mathrm{~F}_{9} \mathrm{NOS}_{2}$ & $\begin{array}{c}{[34556-26-8]} \\
\mathrm{V}\end{array}$ & \multicolumn{5}{|c|}{$S, S$-bis(trifluoromethyl)- $N$-[(trifluoromethyl)thio]sulfoximine } \\
\hline $\mathrm{C}_{3} \mathrm{~F}_{9} \mathrm{NO}_{2} \mathrm{~S}_{2}$ & $\begin{array}{c}{[34556-27-9]} \\
\mathrm{V}\end{array}$ & \multicolumn{5}{|c|}{$S, S$-bis(trifluoromethyl)-N-[(trifluoromethyl)sulfinyl]sulfoximine } \\
\hline $\mathrm{C}_{3} \mathrm{~F}_{9} \mathrm{NO}_{2} \mathrm{~S}_{3}$ & $\begin{array}{c}{[29749-02-8]} \\
\text { V }\end{array}$ & \multicolumn{4}{|c|}{ 1,1,1-trifluoro- $N, N$-bis(trifluoromethyl)thio]methanesulfonamide } & [1987STE/MAL, 1999DYK/SVO] \\
\hline $\mathrm{C}_{3} \mathrm{~F}_{9} \mathrm{~N}_{3} \mathrm{O}$ & $\begin{array}{c}{[10405-30-8]} \\
\mathrm{V} \\
\mathrm{V}\end{array}$ & $\begin{array}{l}\text { nitrosotris(trifluc } \\
\qquad \begin{array}{l}(279-300) \\
(233-294)\end{array}\end{array}$ & $\begin{array}{l}\text { ethyl) hydraz } \\
29.5 \\
33.5\end{array}$ & $\begin{array}{l}289 \\
263\end{array}$ & A & $\begin{array}{l}\text { [1987STE/MAL, 1966HAS/TIP] } \\
\text { [1966DOB/EME] }\end{array}$ \\
\hline $\mathrm{C}_{3} \mathrm{~F}_{9} \mathrm{~N}_{3} \mathrm{O}_{2}$ & $\begin{array}{c}{[10405-31-9]} \\
\mathrm{V}\end{array}$ & \multicolumn{5}{|c|}{ nitrotris(trifluoromethyl) hydrazine } \\
\hline $\mathrm{C}_{3} \mathrm{~F}_{9} \mathrm{P}$ & $\begin{array}{c}{[432-04-2]} \\
\mathrm{V}\end{array}$ & $\begin{array}{l}\text { tris(trifluorometl } \\
\qquad(248-285)\end{array}$ & $\begin{array}{r}\text { hosphine } \\
24.7\end{array}$ & 270 & A & [1987STE/MAL, 1953BEN/EME] \\
\hline $\mathrm{C}_{3} \mathrm{~F}_{9} \mathrm{PS}$ & $\begin{array}{c}{[671-64-7]} \\
\mathrm{V}\end{array}$ & \multicolumn{4}{|c|}{ bis(trifluoromethyl)trifluoromethylthiophosphine } & [1962EME/PAC] \\
\hline $\mathrm{C}_{3} \mathrm{~F}_{9} \mathrm{PS}$ & $\begin{array}{c}{[2025-08-3]} \\
\mathrm{V}\end{array}$ & \multicolumn{5}{|c|}{ tris(trifluoromethyl)phosphine sulfide } \\
\hline $\mathrm{C}_{3} \mathrm{~F}_{9} \mathrm{PS}_{2}$ & $\begin{array}{c}{[36121-49-0]} \\
\mathrm{V}\end{array}$ & \multicolumn{5}{|c|}{ (trifluoromethyl)dithiophosphite acid, bis(trifluoromethyl) ester } \\
\hline $\mathrm{C}_{3} \mathrm{~F}_{9} \mathrm{P}_{3} \mathrm{~S}_{5}$ & $\begin{array}{c}{[26349-17-7]} \\
\text { SUB }\end{array}$ & \multicolumn{5}{|c|}{ 2,4,5-tris(trifluoromethyl)-1,3,2,4,5-dithiatriphospholane-2,4,5-trisulfide } \\
\hline $\mathrm{C}_{3} \mathrm{~F}_{10} \mathrm{OS}$ & $\begin{array}{c}\text { [33564-24-8] } \\
\mathrm{V} \\
\mathrm{V}\end{array}$ & $\begin{array}{l}\text { difluorooxo(trifl } \\
(291-324) \\
(270-324)\end{array}$ & $\begin{array}{c}\text { methyl)(pent } \\
30.6 \\
28.0\end{array}$ & $\begin{array}{l}\text { thyl) } \\
306 \\
297\end{array}$ & A & $\begin{array}{l}\text { [1987STE/MAL, 1999DYK/SVO] } \\
\text { [1971SAU/SHR3] }\end{array}$ \\
\hline $\mathrm{C}_{3} \mathrm{~F}_{10} \mathrm{O}_{3} \mathrm{~S}$ & $\begin{array}{c}{[60672-62-0]} \\
\mathrm{V}\end{array}$ & \multicolumn{4}{|c|}{ pentafluoro (pentafluoropropaneperoxoato)sulfur } & [1976HOP/DES] \\
\hline $\mathrm{C}_{3} \mathrm{~F}_{10} \mathrm{~S}$ & $\begin{array}{c}{[68010-33-3]} \\
\mathrm{V}\end{array}$ & \multicolumn{4}{|c|}{ [2,2-difluoro-(1-trifluoromethyl)ethenyl] pentafluoro sulfur } & {$[1978 \mathrm{DEM} / \mathrm{FOX}]$} \\
\hline $\mathrm{C}_{3} \mathrm{~F}_{10} \mathrm{~S}$ & $\begin{array}{c}{[31222-06-7]} \\
\mathrm{V}\end{array}$ & \multicolumn{5}{|c|}{ difluoro(pentafluoroethyl)(trifluoromethyl) sulfur } \\
\hline $\mathrm{C}_{3} \mathrm{~F}_{11} \mathrm{NO}_{3} \mathrm{~S}_{2}$ & $\begin{array}{c}{[65844-08-8]} \\
\mathrm{V}\end{array}$ & \multicolumn{5}{|c|}{ trifluoro(trifluorosulfato- $O)[1,1,1,2,3,3,3$-heptafluoro-2-propanaminoato(2-)]sulfur } \\
\hline $\mathrm{C}_{3} \mathrm{~F}_{12} \mathrm{O}_{3} \mathrm{~S}_{2}$ & $\begin{array}{c}{[68010-30-0]} \\
\mathrm{V}\end{array}$ & \multicolumn{5}{|c|}{ pentafluoro [2,2,2-trifluoro-1-(fluorosulfonyl)oxo]-1-(trifluoromethyl)ethyl] sulfur } \\
\hline $\mathrm{C}_{3} \mathrm{~N}_{3} \mathrm{P}$ & $\begin{array}{c}\text { [1116-01-4] } \\
\text { SUB } \\
\text { SUB }\end{array}$ & $\begin{array}{l}\text { tricyanophosphi } \\
\quad(293-323)\end{array}$ & $\begin{array}{c}78.3 \\
75.3 \pm 2.9\end{array}$ & $\begin{array}{l}308 \\
298\end{array}$ & ME & $\begin{array}{l}\text { [1987STE/MAL, 1976DAV/FIN] } \\
\text { [1995ALM/FIN, 1976DAV/FIN] }\end{array}$ \\
\hline $\mathrm{C}_{3} \mathrm{HClF}_{6} \mathrm{O}_{2} \mathrm{~S}$ & $\begin{array}{c}{[57169-81-0]} \\
\mathrm{V}\end{array}$ & chlorosulfurous & $\begin{array}{l}\text { 2,2,2-trifluo } \\
36.7\end{array}$ & fluron & thyl ester & [1975KIR/LAS] \\
\hline $\mathrm{C}_{3} \mathrm{HClF}_{10} \mathrm{~S}$ & $\begin{array}{c}{[68010-36-6]} \\
\text { V }\end{array}$ & [1-(chlorodifluo & $\begin{array}{l}\text { hyl) }-2,2,2-\mathrm{tr} \\
31.2\end{array}$ & thyl] 1 & orosulfur & {$[1978 \mathrm{DEM} / \mathrm{FOX}]$} \\
\hline $\mathrm{C}_{3} \mathrm{HCl}_{7}$ & [594-89-8] & $1,1,1,2,2,3,3$-hep & loropropane & & & \\
\hline
\end{tabular}


Table 6. Phase change enthalpies of $\mathrm{C}_{3}$ organic compounds-Continued

\begin{tabular}{|c|c|c|c|c|c|c|}
\hline \multirow[b]{2}{*}{$\begin{array}{l}\text { Molecular } \\
\text { formula }\end{array}$} & \multirow{2}{*}{$\begin{array}{c}\text { CAS Reg. No. } \\
\text { Transition }\end{array}$} & \multicolumn{5}{|l|}{ Compound } \\
\hline & & $\begin{array}{c}\text { Temp. range } \\
\text { (K) }\end{array}$ & $\begin{array}{c}\Delta_{\text {trans }} H_{\mathrm{m}} \\
\left(\mathrm{kJ} \mathrm{mol}^{-1}\right)\end{array}$ & $\begin{array}{l}T_{\mathrm{m}} \\
(\mathrm{K})\end{array}$ & Method & References \\
\hline & $\mathrm{V}$ & $(413-473)$ & 34.8 & 428 & A & [1987STE/MAL, 1949HIG/END, 1970DYK] \\
\hline $\mathrm{C}_{3} \mathrm{HFN}_{4} \mathrm{O}_{4}$ & $\begin{array}{c}{[604739-69-7]} \\
\text { SUB }\end{array}$ & 1-fluoro-2,5-din & $\begin{array}{l}\text {-imidazole } \\
74.1 \pm 0.8\end{array}$ & 298 & & [2003MIR/LEB] \\
\hline $\mathrm{C}_{3} \mathrm{HFN}_{4} \mathrm{O}_{4}$ & $\begin{array}{c}{[604739-70-0]} \\
\text { SUB }\end{array}$ & 1-fluoro-3,4-din & $\begin{array}{c}H \text {-pyrazole } \\
88.3 \pm 1.7\end{array}$ & 298 & & [2003MIR/LEB] \\
\hline $\mathrm{C}_{3} \mathrm{HFN}_{6} \mathrm{O}_{6}$ & $\begin{array}{c}{[604739-71-1]} \\
\text { SUB }\end{array}$ & 1-(trifluorodinit & $\begin{array}{l}\text { hyl)3-nitro-1 } \\
99.6 \pm 0.8\end{array}$ & $\begin{array}{c}\text { 4-triaz } \\
298\end{array}$ & & [2003MIR/LEB] \\
\hline $\mathrm{C}_{3} \mathrm{HF}_{3}$ & $\begin{array}{c}{[661-54-1]} \\
\mathrm{V}\end{array}$ & $\begin{array}{c}\text { 3,3,3-trifluoropr } \\
\text { (138-213) }\end{array}$ & 21.5 & 198 & A & [1987STE/MAL, 1951HAS2] \\
\hline $\mathrm{C}_{3} \mathrm{HF}_{6} \mathrm{~N}$ & $\begin{array}{c}{[3291-64-3]} \\
\mathrm{V}\end{array}$ & $\begin{array}{c}\text { 2,2,3-trifluoro-3 } \\
(268-298)\end{array}$ & $\begin{array}{c}\text { omethylaziri } \\
30.2\end{array}$ & 283 & A & [1987STE/MAL, 1966BAN/MOO] \\
\hline $\mathrm{C}_{3} \mathrm{HF}_{7}$ & $\begin{array}{c}\text { [431-89-0] } \\
\text { V } \\
\text { V } \\
\text { V } \\
\text { V } \\
\text { V } \\
\text { V } \\
\text { V }\end{array}$ & $\begin{array}{r}1,1,1,2,3,3,3-\text { he } \\
(278-308) \\
(293-353) \\
(279-315) \\
(237-370) \\
(237-370) \\
(237-370)\end{array}$ & $\begin{array}{c}\text { oropropane } \\
18.9 \\
22.7 \\
22.6 \\
23.2 \\
22.3 \\
14.5 \\
12.5\end{array}$ & $\begin{array}{l}298 \\
293 \\
308 \\
\\
250 \\
300 \\
325\end{array}$ & & $\begin{array}{l}\text { [2007ECK/HUA] } \\
{[2002 \mathrm{BOB} / \mathrm{ART}]} \\
{[2002 \mathrm{VAL} / \mathrm{COQ}]} \\
{[1998 \mathrm{WIL} / \mathrm{LIU}]} \\
{[1992 \mathrm{SAL} / \mathrm{WAN}]} \\
{[1992 \mathrm{SAL} / \mathrm{WAN}]} \\
{[1992 \mathrm{SAL} / \mathrm{WAN}]}\end{array}$ \\
\hline $\mathrm{C}_{3} \mathrm{HF}_{7} \mathrm{O}$ & $\begin{array}{c}{[2356-61-8]} \\
\mathrm{V}\end{array}$ & $\begin{array}{l}\text { trifluoromethyl } \\
\qquad(232-313)\end{array}$ & $\begin{array}{c}\text { entafluoroetl } \\
27.3\end{array}$ & 247 & A & {$[1992 \mathrm{SAL} / \mathrm{WAN}]$} \\
\hline $\mathrm{C}_{3} \mathrm{HF}_{7} \mathrm{O}_{2} \mathrm{~S}$ & $\begin{array}{c}{[52225-56-6]} \\
\mathrm{V}\end{array}$ & fluorosulfurous & $\begin{array}{l}\text {,2,2-trifluor } \\
33.8\end{array}$ & fluoron & thyl ester & {$[1975 \mathrm{DEM} / \mathrm{KOV} 2]$} \\
\hline $\mathrm{C}_{3} \mathrm{HF}_{8} \mathrm{NOS}$ & $\begin{array}{c}{[34556-23-5]} \\
\mathrm{V}\end{array}$ & $S$-(pentafluoroe & $\begin{array}{c}S \text {-(trifluoron } \\
36.3\end{array}$ & $\begin{array}{l}\text { ulfoxil } \\
358\end{array}$ & I & [1972SAU/SHR] \\
\hline $\mathrm{C}_{3} \mathrm{HF}_{8} \mathrm{NO}_{2}$ & $\begin{array}{c}\text { [60247-19-0] } \\
\mathrm{V}\end{array}$ & $N-[$ difluoro[(tri & $\begin{array}{c}\text { methyl)diox } \\
37.5\end{array}$ & l] 1,1 & oromethan & $\begin{array}{l}\text { mine } \\
\text { [1976FAL/DES3] }\end{array}$ \\
\hline $\mathrm{C}_{3} \mathrm{HF}_{9} \mathrm{~N}_{2}$ & $\begin{array}{c}{[13105-67-4]} \\
\text { V }\end{array}$ & $\begin{array}{l}\text { tris(trifluoromet } \\
\qquad(238-307)\end{array}$ & $\begin{array}{r}\text { ydrazine } \\
29.9\end{array}$ & 273 & & [1966DOB/EME] \\
\hline $\mathrm{C}_{3} \mathrm{HF}_{11} \mathrm{~S}$ & $\begin{array}{c}\text { [68010-34-4] } \\
\mathrm{V}\end{array}$ & pentafluoro $[2,2$ & $\begin{array}{l}\text { luoro-1-(1-tr } \\
30.1\end{array}$ & nethyl & ulfur & {$[1978 \mathrm{DEM} / \mathrm{FOX}]$} \\
\hline $\mathrm{C}_{3} \mathrm{HN}$ & $\begin{array}{c}\text { [68010-34-4] } \\
\text { FUS }\end{array}$ & cyanoacetylene & 14.1 & & $\mathrm{~S}-\mathrm{V}$ & [1963DAN/FLU] \\
\hline & SUB & $(247-279)$ & 42.3 & 264 & A & [1987STE/MAL, 1963DAN/FLU] \\
\hline & $\mathrm{V}$ & $(279-315)$ & 28.1 & 294 & A & [1987STE/MAL, 1963DAN/FLU] \\
\hline $\mathrm{C}_{3} \mathrm{H}_{2} \mathrm{ClF}_{3}$ & $\begin{array}{c}{[2730-43-0]} \\
\mathrm{V}\end{array}$ & $\begin{array}{c}\text { 1-chloro-3,3,3-t } \\
(263-352)\end{array}$ & $\begin{array}{c}\text { ro-1-propene } \\
26.3\end{array}$ & 308 & & [2012HUL/BAS] \\
\hline $\mathrm{C}_{3} \mathrm{H}_{2} \mathrm{ClF}_{3}$ & $\begin{array}{c}{[2730-62-3]} \\
\mathrm{V}\end{array}$ & $\begin{array}{l}\text { 2-chloro-3,3,3-t } \\
\quad(263-373)\end{array}$ & $\begin{array}{c}\text { ro-1-propene } \\
24.3\end{array}$ & 298 & Static & [2014ZHA/WEI, 2013ZHA/YAN] \\
\hline $\mathrm{C}_{3} \mathrm{H}_{2} \mathrm{ClF}_{5}$ & $\begin{array}{l}{[460-92-4]} \\
\text { FUS }\end{array}$ & 3-chloro-1,1,1,3 & $\begin{array}{c}\text { ntafluoropro] } \\
10.47\end{array}$ & 165.4 & & [1996DOM/HEA, 1974VOR/KOL] \\
\hline $\mathrm{C}_{3} \mathrm{H}_{2} \mathrm{ClF}_{5} \mathrm{O}$ & $\begin{array}{c}\text { [13838-16-9] } \\
\text { V } \\
\text { V } \\
\text { V } \\
\text { V } \\
\text { V } \\
\text { V }\end{array}$ & $\begin{array}{c}\text { 1-chloro-1,2,2-t } \\
(274-351) \\
(290-329)\end{array}$ & $\begin{array}{c}\text { ro-2-(difluor } \\
33.8 \\
32.9 \\
32.6 \pm 0.1 \\
31.3 \pm 0.1 \\
30.2 \pm 0.1 \\
29.1 \pm 0.1\end{array}$ & $\begin{array}{l}\text { xy)eth } \\
289 \\
305 \\
298 \\
313 \\
328 \\
343\end{array}$ & $\begin{array}{l}\text { A } \\
\text { C } \\
\text { C } \\
\text { C } \\
\text { C }\end{array}$ & $\begin{array}{l}\text { [1988AMB/GHI2] } \\
\text { [1987STE/MAL, 1978ROD/HIL] } \\
\text { [1984UCH/MAJ] } \\
{[1984 U C H / M A J]} \\
{[1984 U C H / M A J]} \\
{[1984 U C H / M A J]}\end{array}$ \\
\hline $\mathrm{C}_{3} \mathrm{H}_{2} \mathrm{ClF}_{5} \mathrm{O}$ & $\begin{array}{c}{[26675-46-7]} \\
\text { V } \\
\text { V }\end{array}$ & $\begin{array}{l}\text { 2-chloro-1,1,1-t } \\
\quad(280-344) \\
(283-312)\end{array}$ & $\begin{array}{c}\text { ro-2-(difluor } \\
\begin{array}{c}31.7 \\
31.9\end{array}\end{array}$ & $\begin{array}{l}\text { xy)ethe } \\
295 \\
297\end{array}$ & A & $\begin{array}{l}\text { [1988AMB/GHI2] } \\
\text { [1987STE/MAL, 1978ROD/HIL] }\end{array}$ \\
\hline
\end{tabular}


Table 6. Phase change enthalpies of $\mathrm{C}_{3}$ organic compounds-Continued

\begin{tabular}{|c|c|c|c|c|c|c|}
\hline \multirow[b]{2}{*}{$\begin{array}{l}\text { Molecular } \\
\text { formula }\end{array}$} & \multirow{2}{*}{$\begin{array}{c}\text { CAS Reg. No. } \\
\text { Transition }\end{array}$} & \multicolumn{5}{|l|}{ Compound } \\
\hline & & $\begin{array}{c}\text { Temp. range } \\
\text { (K) }\end{array}$ & $\begin{array}{c}\Delta_{\text {trans }} H_{\mathrm{m}} \\
\left(\mathrm{kJ} \mathrm{mol}^{-1}\right)\end{array}$ & $\begin{array}{l}T_{\mathrm{m}} \\
(\mathrm{K})\end{array}$ & Method & References \\
\hline \multirow[t]{2}{*}{$\mathrm{C}_{3} \mathrm{H}_{2} \mathrm{Cl}_{2} \mathrm{~F}_{4}$} & {$[64712-27-2]$} & \multicolumn{5}{|c|}{ 3,3-dichloro-1,1,1,3-tetrafluoropropane } \\
\hline & $\mathrm{V}$ & $(297-333)$ & 31.7 & 312 & A & [1987STE/MAL, 1976VAR/BUL] \\
\hline \multirow[t]{6}{*}{$\mathrm{C}_{3} \mathrm{H}_{2} \mathrm{Cl}_{2} \mathrm{~F}_{4} \mathrm{O}$} & [37031-38-2] & \multicolumn{5}{|c|}{ 2-chloro-1,1,2-trifluoroethyl chlorofluoromethyl ether } \\
\hline & $\mathrm{V}$ & & $37.5 \pm 0.1$ & 298 & $\mathrm{C}$ & [1984MAJ/UCH] \\
\hline & $\mathrm{V}$ & & $36.4 \pm 0.1$ & 313 & $\mathrm{C}$ & {$[1984 \mathrm{MAJ} / \mathrm{UCH}]$} \\
\hline & V & & $35.3 \pm 0.1$ & 328 & $\mathrm{C}$ & [1984MAJ/UCH] \\
\hline & $\mathrm{V}$ & & $34.1 \pm 0.1$ & 343 & $\mathrm{C}$ & [1984MAJ/UCH] \\
\hline & $\mathrm{V}$ & & $32.9 \pm 0.1$ & 353 & $\mathrm{C}$ & [1984MAJ/UCH] \\
\hline \multirow[t]{4}{*}{$\mathrm{C}_{3} \mathrm{H}_{2} \mathrm{Cl}_{3} \mathrm{~F}_{3}$} & {$[7125-84-0]$} & \multicolumn{5}{|c|}{ 1,1,1-trichloro-3,3,3-trifluoropropane } \\
\hline & FUS & $(12-300)$ & 14.07 & 232.7 & $\mathrm{AC}$ & [1991ACR, 1971KOL/VOL] \\
\hline & $\mathrm{V}$ & & $36.8 \pm 0.1$ & 298 & $\mathrm{C}$ & [2007VAR/DRU] \\
\hline & V & $(320-365)$ & 35.2 & 335 & A & [1987STE/MAL] \\
\hline \multirow[t]{3}{*}{$\mathrm{C}_{3} \mathrm{H}_{2} \mathrm{Cl}_{4}$} & {$[60320-18-5]$} & \multicolumn{5}{|c|}{ 1,1,2,3-tetrachloropropylene } \\
\hline & $\mathrm{V}$ & $(347-416)$ & 42.9 & 362 & A & [1987STE/MAL] \\
\hline & $\mathrm{V}$ & $(348-416)$ & 43.4 & 382 & & [1969OTO/BES] \\
\hline \multirow[t]{2}{*}{$\mathrm{C}_{3} \mathrm{H}_{2} \mathrm{Cl}_{2} \mathrm{~F}_{4}$} & {$[64712-27-2]$} & \multicolumn{5}{|c|}{ 1,1,1,3-tetrafluoro-3,3-dichloropropane } \\
\hline & $\mathrm{V}$ & $(297-333)$ & $31.9 \pm 0.5$ & 298 & & [2007VAR/DRU] \\
\hline \multirow[t]{2}{*}{$\mathrm{C}_{3} \mathrm{H}_{2} \mathrm{Cl}_{2} \mathrm{~N}_{2}$} & {$[15965-30-7]$} & \multicolumn{5}{|c|}{ 4,5-dichloroimidazole } \\
\hline & SUB & $(338-395)$ & $94.5 \pm 0.8$ & 298 & Static & [2012ALM/MON] \\
\hline \multirow[t]{2}{*}{$\mathrm{C}_{3} \mathrm{H}_{2} \mathrm{D}_{5} \mathrm{~N}$} & {$[153557-95-0]$} & \multicolumn{5}{|c|}{ cyclopropanamine $\left(\mathrm{D}_{5}\right)$} \\
\hline & V & $(283-330)$ & 31.7 & 298 & & [1993WOL/KIM] \\
\hline \multirow[t]{2}{*}{$\mathrm{C}_{3} \mathrm{H}_{2} \mathrm{FNOS}$} & {$[459-71-2]$} & \multicolumn{5}{|c|}{ fluoracetyl isothiocyanate } \\
\hline & V & $(273-353)$ & 49.3 & 288 & A & [1987STE/MAL, 1970DYK, 1999DYK/SVO] \\
\hline $\mathrm{C}_{3} \mathrm{H}_{2} \mathrm{~F}_{6}$ & {$[690-39-1]$} & 1,1,1,3,3,3-hex & opropane & & & \\
\hline & V & $(283-323)$ & 24.5 & 303 & A & {$[2000 \mathrm{BOB} / \mathrm{CAM}]$} \\
\hline $\mathrm{C}_{3} \mathrm{H}_{2} \mathrm{~F}_{6} \mathrm{~N}_{2} \mathrm{~S}$ & {$[38005-20-8]$} & amino (hexafluc & propylidenim & lfur & & \\
\hline & V & & 37.7 & 388 & $\mathrm{I}$ & [1972MET/SHR] \\
\hline $\mathrm{C}_{3} \mathrm{H}_{2} \mathrm{~F}_{6} \mathrm{~N}_{2} \mathrm{~S}$ & {$[62067-09-8]$} & 2,2,2-trifluoro- 1 & ifluoromethy & ethanim & & \\
\hline & V & $(322-390)$ & 39.8 & 337 & A, I & [1987STE/MAL, 1977BUR/SHR2, 1999DYK/SVO] \\
\hline $\mathrm{C}_{3} \mathrm{H}_{2} \mathrm{~F}_{6} \mathrm{O}$ & {$[57041-67-5]$} & 2-(difluorometh & 1,1,1,2-tetraf & hane & & \\
\hline & $\mathrm{V}$ & $(274-311)$ & 24.9 & 293 & $\mathrm{I}$ & [1996SUS/SMI] \\
\hline $\mathrm{C}_{3} \mathrm{H}_{2} \mathrm{~F}_{6} \mathrm{O}$ & {$[920-66-1]$} & 1,1,1,3,3,3-hex & o-2-propanol & & & \\
\hline & $\mathrm{V}$ & $(294-330)$ & 40.2 & 309 & A, MM & [1987STE/MAL, 1973ROC/SYM] \\
\hline & $\mathrm{V}$ & $(294-330)$ & 41.6 & 298 & MM & [1973ROC/SYM] \\
\hline & V & $(273-296)$ & 47.3 & 284 & & [1967VAN/SOC] \\
\hline $\mathrm{C}_{3} \mathrm{H}_{2} \mathrm{~F}_{6} \mathrm{O}_{2}$ & [30957-44-9] & bis-(difluorome & difluorometl & & & \\
\hline & FUS & & 7.2 & 153 & DSC & [1999MAR/BAS] \\
\hline & $\mathrm{V}$ & $(243-308)$ & $31.5 \pm 0.4$ & & & {$[1999 \mathrm{MAR} / \mathrm{BAS}]$} \\
\hline $\mathrm{C}_{3} \mathrm{H}_{2} \mathrm{~F}_{6} \mathrm{O}_{2} \mathrm{~S}$ & [30957-44-9] & trifluoormethan & nic acid, 2,2, & orometh & & \\
\hline & $\mathrm{V}$ & & 36.8 & 363 & & [1971SAU/SHR2] \\
\hline $\mathrm{C}_{3} \mathrm{H}_{2} \mathrm{~F}_{8} \mathrm{~N}_{2} \mathrm{~S}$ & {$[2433-66-1]$} & $S, S$-difluoro- $N$ & mino-2,2,2-tr & $-1-($ trifli & thyl)ethy & lfilimine \\
\hline & V & $(295-313)$ & 38.7 & 304 & A & [1987STE/MAL, 1969GLE/VON, 1999DYK/SVO] \\
\hline $\mathrm{C}_{3} \mathrm{H}_{2} \mathrm{~N}_{2}$ & {$[109-77-3]$} & malononitrile & & & & \\
\hline & TRS & & 0.43 & 260.9 & & \\
\hline & FUS & & 10.7 & 305 & DSC & [2007BAD/BLA] \\
\hline & FUS & $(6-319)$ & 10.8 & 305 & $\mathrm{AC}$ & [1996DOM/HEA, 1968GIR/WES] \\
\hline & SUB & $(278-299)$ & $78.2 \pm 1.0$ & 298 & & [1990BEC/DOG] \\
\hline & SUB & & $79.1 \pm 8$ & & $\mathrm{ME}$ & [1967BOY/GUH, 1970COX/PIL] \\
\hline $\mathrm{C}_{3} \mathrm{H}_{2} \mathrm{~N}_{2} \mathrm{O}_{3}$ & {$[120-89-8]$} & imidazolidine-2 & trione (paraba & & & \\
\hline & TRS & & 2.1 & 392.3 & & \\
\hline & FUS (decomp) & $(263-473)$ & & & DSC & [2008RIB/RIB2] \\
\hline
\end{tabular}


Table 6. Phase change enthalpies of $\mathrm{C}_{3}$ organic compounds-Continued

\begin{tabular}{|c|c|c|c|c|c|c|}
\hline \multirow[b]{2}{*}{$\begin{array}{l}\text { Molecular } \\
\text { formula }\end{array}$} & \multirow{2}{*}{$\begin{array}{c}\text { CAS Reg. No. } \\
\text { Transition }\end{array}$} & \multicolumn{5}{|l|}{ Compound } \\
\hline & & $\begin{array}{c}\text { Temp. range } \\
\text { (K) }\end{array}$ & $\begin{array}{c}\Delta_{\text {trans }} H_{\mathrm{m}} \\
\left(\mathrm{kJ} \mathrm{mol}^{-1}\right)\end{array}$ & $\begin{array}{c}T_{\mathrm{m}} \\
(\mathrm{K})\end{array}$ & Method & References \\
\hline & SUB & $(381-406)$ & $114.6 \pm 0.6$ & 393.7 & ME & [2008RIB/RIB2] \\
\hline & SUB & $(381-406)$ & $119.4 \pm 0.6$ & 298 & $\mathrm{ME}$ & [2008RIB/RIB2] \\
\hline \multirow[t]{2}{*}{$\mathrm{C}_{3} \mathrm{H}_{2} \mathrm{~N}_{4} \mathrm{O}_{4}$} & {$[5213-49-0]$} & \multicolumn{5}{|l|}{ 2,5-dinitroimidazole } \\
\hline & SUB & & $112.1 \pm 0.8$ & 298 & & [2003MIR/LEB] \\
\hline \multirow[t]{2}{*}{$\mathrm{C}_{3} \mathrm{H}_{2} \mathrm{~N}_{4} \mathrm{O}_{4}$} & [38858-92-3] & \multicolumn{5}{|c|}{ 3,4-dinitro-1 $H$-pyrazole } \\
\hline & SUB & & $112.5 \pm 0.8$ & 298 & & [2003MIR/LEB] \\
\hline \multirow[t]{2}{*}{$\mathrm{C}_{3} \mathrm{H}_{2} \mathrm{OS}_{2}$} & {$[2314-40-1]$} & \multicolumn{5}{|l|}{ 1,3-dithiol-2-one } \\
\hline & SUB & & $73.6 \pm 0.8$ & 298 & & [1973RAU/GEI, 1977PED/RYL] \\
\hline \multirow[t]{2}{*}{$\mathrm{C}_{3} \mathrm{H}_{2} \mathrm{OS}_{3}$} & {$[930-35-8]$} & \multicolumn{5}{|l|}{ 1,3-dithiole-2-thione } \\
\hline & SUB & & $75.4 \pm 0.4$ & 298 & & [1973RAU/GEI, 1977PED/RYL] \\
\hline \multirow[t]{3}{*}{$\mathrm{C}_{3} \mathrm{H}_{2} \mathrm{O}_{3}$} & {$[872-36-6]$} & \multicolumn{5}{|l|}{ vinylene carbonate } \\
\hline & V & $(308-350)$ & 46.9 & 323 & A & [1987STE/MAL] \\
\hline & $\mathrm{V}$ & $(308-400)$ & 41.3 & & MM & {$[1971 \mathrm{CHO} / \mathrm{JON}]$} \\
\hline \multirow[t]{2}{*}{$\mathrm{C}_{3} \mathrm{H}_{3} \mathrm{Cl}$} & {$[7747-84-4]$} & \multicolumn{5}{|l|}{ 1-chloro-1-propyne } \\
\hline & $\mathrm{V}$ & $(200-289)$ & 28.3 & 274 & A & [1987STE/MAL] \\
\hline \multirow[t]{2}{*}{$\mathrm{C}_{3} \mathrm{H}_{3} \mathrm{ClF}_{4}$} & {$[421-73-8]$} & \multicolumn{5}{|c|}{ 2-chloro-1,1,1,2-tetrafluoropropane } \\
\hline & $\mathrm{V}$ & $(268-349)$ & 24.6 & 298 & & [2014YAN/KOU] \\
\hline \multirow[t]{5}{*}{$\mathrm{C}_{3} \mathrm{H}_{3} \mathrm{Cl}_{2} \mathrm{~F}_{3}$} & {$[460-69-5]$} & \multicolumn{5}{|c|}{ 1,1-dichloro-3,3,3-trifluoropropane } \\
\hline & TRS & & 0.2 & 167.7 & & \\
\hline & FUS & & 10.13 & 182.2 & $\mathrm{AC}$ & [1996DOM/HEA, 1972KOL/VOR, 1972VOR/KOL] \\
\hline & $\mathrm{V}$ & & $34.1 \pm 0.1$ & 298 & $\mathrm{C}$ & [2007VAR/DRU] \\
\hline & $\mathrm{V}$ & $(301-342)$ & 33.7 & 316 & A & {$[1987 \mathrm{STE} / \mathrm{MAL}]$} \\
\hline \multirow[t]{6}{*}{$\mathrm{C}_{3} \mathrm{H}_{3} \mathrm{Cl}_{2} \mathrm{~F}_{3} \mathrm{O}$} & {$[428-92-2]$} & 2-chloro-1,1,2-trifluor & roethyl chloro & l ether & & \\
\hline & $\mathrm{V}$ & & $42.4 \pm 0.1$ & 298 & $\mathrm{C}$ & {$[1984 \mathrm{UCH} / \mathrm{MAJ}]$} \\
\hline & $\mathrm{V}$ & & $41.2 \pm 0.1$ & 313 & $\mathrm{C}$ & [1984UCH/MAJ] \\
\hline & $\mathrm{V}$ & & $40.1 \pm 0.1$ & 328 & $\mathrm{C}$ & [1984UCH/MAJ] \\
\hline & $\mathrm{V}$ & & $39.0 \pm 0.1$ & 343 & $\mathrm{C}$ & [1984UCH/MAJ] \\
\hline & V & & $37.8 \pm 0.1$ & 358 & $\mathrm{C}$ & [1984UCH/MAJ] \\
\hline $\mathrm{C}_{3} \mathrm{H}_{3} \mathrm{Cl}_{3} \mathrm{O}_{2}$ & {$[598-99-2]$} & methyl trichloroacetat & & & & \\
\hline & $\mathrm{V}$ & & $48.3 \pm 0.1$ & 298 & $\mathrm{C}$ & [1972LAY/WAD] \\
\hline $\mathrm{C}_{3} \mathrm{H}_{3} \mathrm{Cl}_{5}$ & [16714-68-4] & 1,1,2,2,3-pentachloro & propane & & & \\
\hline & $\mathrm{V}$ & $(365-447)$ & 46.3 & 380 & A & [1987STE/MAL, 1970DYK] \\
\hline & $\mathrm{V}$ & $(365-446)$ & 45.5 & 405 & & [1969OTO/BES] \\
\hline $\mathrm{C}_{3} \mathrm{H}_{3} \mathrm{~F}_{2} \mathrm{P}$ & [37805-59-7] & 1-propnyl difluoropho & ospine & & & \\
\hline & V & $(228-278)$ & 32.7 & 253 & & [1973LIN/CEN] \\
\hline $\mathrm{C}_{3} \mathrm{H}_{3} \mathrm{~F}_{3}$ & {$[677-21-4]$} & 3,3,3-trifluoro-1-prop & ene & & & \\
\hline & V & $(283-363)$ & 22.0 & 298 & A & [1987STE/MAL] \\
\hline $\mathrm{C}_{3} \mathrm{H}_{3} \mathrm{~F}_{3} \mathrm{O}_{3}$ & [33017-08-2] & peroxyacetic acid, trif & fluoromethyl e & & & \\
\hline & V & & 32.5 & & & [1971BER/HOH] \\
\hline $\mathrm{C}_{3} \mathrm{H}_{3} \mathrm{~F}_{4} \mathrm{I}$ & [1737-76-4] & 1,1,1,2-tetrafluoro-3-i & iodopropane & & & \\
\hline & V & $(295-356)$ & 28.4 & 310 & A & [1987STE/MAL, 1970HAS/KEE] \\
\hline $\mathrm{C}_{3} \mathrm{H}_{3} \mathrm{~F}_{4} \mathrm{I}$ & {$[460-74-2]$} & 1,1,1,3-tetrafluoro-3-i & iodopropane & & & \\
\hline & V & $(301-356)$ & 31.2 & 316 & A & [1987STE/MAL, 1970HAS/KEE] \\
\hline $\mathrm{C}_{3} \mathrm{H}_{3} \mathrm{~F}_{4} \mathrm{NO}_{2}$ & {$[72316-36-0]$} & methoxy (trifluoromet & thyl)carbamic & & & \\
\hline & V & & 27.8 & & & [1979SEK/DES] \\
\hline $\mathrm{C}_{3} \mathrm{H}_{3} \mathrm{~F}_{5}$ & {$[679-86-7]$} & 1,1,2,2,3-pentafluorop & propane & & & \\
\hline & V & $(258-353)$ & 30.2 & 273 & A & [2002DIN/PAS] \\
\hline $\mathrm{C}_{3} \mathrm{H}_{3} \mathrm{~F}_{5}$ & [1814-88-6] & 1,1,1,2,2-pentafluorop & propane & & & \\
\hline & $\mathrm{V}$ & $(232-283)$ & 22.9 & 268 & A & [1987STE/MAL, 1970DYK] \\
\hline & V & $(233-379)$ & 23.0 & 248 & & [1967SHA] \\
\hline
\end{tabular}


TABLE 6. Phase change enthalpies of $\mathrm{C}_{3}$ organic compounds-Continued

\begin{tabular}{|c|c|c|c|c|c|c|}
\hline \multirow[b]{2}{*}{$\begin{array}{l}\text { Molecular } \\
\text { formula }\end{array}$} & \multirow{2}{*}{$\begin{array}{c}\text { CAS Reg. No. } \\
\text { Transition }\end{array}$} & \multicolumn{5}{|l|}{ Compound } \\
\hline & & $\begin{array}{c}\text { Temp. range } \\
(\mathrm{K})\end{array}$ & $\begin{array}{c}\Delta_{\text {trans }} H_{\mathrm{m}} \\
\left(\mathrm{kJ} \mathrm{mol}^{-1}\right)\end{array}$ & $\begin{array}{l}T_{\mathrm{m}} \\
(\mathrm{K})\end{array}$ & Method & References \\
\hline & $\mathrm{V}$ & $(273-297)$ & 47.0 & 285 & $\mathrm{~A}, \mathrm{MM}$ & [1987STE/MAL, 1967MEE/GOL, 1984BOU/FRI] \\
\hline & $\mathrm{V}$ & & 44.4 & 298 & MM & [1973ROC/SYM, 1967MEE/GOL] \\
\hline & $\mathrm{V}$ & & 41.3 & 298 & & {$[1967 \mathrm{MUR} / \mathrm{KIV}]$} \\
\hline \multirow[t]{2}{*}{$\mathrm{C}_{3} \mathrm{H}_{3} \mathrm{~F}_{5} \mathrm{O}$} & {$[37031-31-5]$} & \multicolumn{5}{|c|}{ 1,1,2,2-tetrafluoro-1-(fluoromethoxy)ethane } \\
\hline & $\mathrm{V}$ & $(288-317)$ & 33.5 & 303 & I & [2002MUR/YAM] \\
\hline \multirow[t]{2}{*}{$\mathrm{C}_{3} \mathrm{H}_{3} \mathrm{~F}_{6} \mathrm{NOS}$} & {$[34556-25-7]$} & \multicolumn{5}{|c|}{$N$-methyl-S,S-bis(trifluoromethyl)sulfoximine } \\
\hline & $\mathrm{V}$ & & 30.7 & 338 & I & {$[1972 \mathrm{SAU} / \mathrm{SHR}]$} \\
\hline \multirow[t]{2}{*}{$\mathrm{C}_{3} \mathrm{H}_{3} \mathrm{~F}_{6} \mathrm{NS}$} & [13105-12-9] & \multicolumn{5}{|c|}{$N, N$-bis(trifluoromethyl)methanesulfenamide } \\
\hline & V & $(269-309)$ & 31.1 & 284 & $\mathrm{~A}, \mathrm{~T}$ & [1987STE/MAL, 1966EME/TAT] \\
\hline \multirow[t]{2}{*}{$\mathrm{C}_{3} \mathrm{H}_{3} \mathrm{~F}_{6} \mathrm{OPS}$} & {$[71040-58-9]$} & \multicolumn{5}{|c|}{ phosphinothioic acid, bis(trifluoromethyl)-,O-methyl ester } \\
\hline & V & $(263-333)$ & 35.1 & 298 & & {$[1979 \mathrm{CAV} / \mathrm{PIN}]$} \\
\hline \multirow[t]{2}{*}{$\mathrm{C}_{3} \mathrm{H}_{3} \mathrm{~F}_{6} \mathrm{OPS}$} & [71009-87-5] & \multicolumn{5}{|c|}{ phosphinothioic acid, bis(trifluoromethyl)-,S-methyl ester } \\
\hline & V & $(293-353)$ & 33.8 & 318 & & {$[1979 \mathrm{CAV} / \mathrm{PIN}]$} \\
\hline \multirow[t]{2}{*}{$\mathrm{C}_{3} \mathrm{H}_{3} \mathrm{~F}_{6} \mathrm{O}_{2} \mathrm{P}$} & {$[25439-11-6]$} & \multicolumn{5}{|c|}{ bis(trifluromethyl)phosphinic acid, methyl ester } \\
\hline & V & $(258-313)$ & 40.5 & 273 & A & [1987STE/MAL, 1969BUR/MIS] \\
\hline \multirow[t]{2}{*}{$\mathrm{C}_{3} \mathrm{H}_{3} \mathrm{~F}_{6} \mathrm{PS}$} & {$[1486-18-6]$} & \multicolumn{5}{|c|}{ bis(trifluoromethyl) methylthiophosphine } \\
\hline & V & $(273-321)$ & 36.9 & 297 & $\mathrm{~T}$ & {$[1964 \mathrm{CAV} / \mathrm{EME}]$} \\
\hline \multirow[t]{2}{*}{$\mathrm{C}_{3} \mathrm{H}_{3} \mathrm{~F}_{6} \mathrm{PS}_{2}$} & {$[18799-79-6]$} & \multicolumn{5}{|c|}{ bis(trifluromethyl)dithiophosphinic acid, methyl ester } \\
\hline & V & $(273-344)$ & 41.5 & 288 & A & [1987STE/MAL, 1999DYK/SVO, 1968GOS/BUR] \\
\hline \multirow[t]{8}{*}{$\mathrm{C}_{3} \mathrm{H}_{3} \mathrm{~N}$} & {$[107-13-1]$} & acrylonitrile & & & & \\
\hline & TRS & & 1.19 & 162.5 & & \\
\hline & FUS & & 6.23 & 189.6 & & [1996DOM/HEA, 1972FIN/MES] \\
\hline & $\mathrm{V}$ & $(257-352)$ & 33.6 & 272 & A & [1987STE/MAL] \\
\hline & $\mathrm{V}$ & $(283-343)$ & 31.6 & 298 & A & [1987STE/MAL] \\
\hline & $\mathrm{V}$ & $(222-351)$ & 35.5 & 237 & & [1964SEV/SOK] \\
\hline & $\mathrm{V}$ & $(293-343)$ & 32.9 & 308 & & [1964GUB/FER] \\
\hline & V & $(273-353)$ & 32.6 & & & [1945DAV/WIE] \\
\hline $\mathrm{C}_{3} \mathrm{H}_{3} \mathrm{NO}$ & {$[288-42-6]$} & oxazole & & & & \\
\hline & $\mathrm{V}$ & $(293-344)$ & 34.6 & 308 & A, EB & [1987STE/MAL, 1975SOU/BAR] \\
\hline & V & & $32.5 \pm 0.1$ & 298 & $\mathrm{C}$ & [1978MCC/HAM] \\
\hline $\mathrm{C}_{3} \mathrm{H}_{3} \mathrm{NO}$ & {$[288-14-2]$} & isoxazole & & & & \\
\hline & $\mathrm{V}$ & $(314-404)$ & $37.2 \pm 0.2$ & 298 & EB & [1996STE/CHI3] \\
\hline & $\mathrm{V}$ & $(314-404)$ & $37.1 \pm 0.2$ & 300 & EB & [1996STE/CHI3] \\
\hline & V & $(314-404)$ & $35.1 \pm 0.2$ & 340 & EB & [1996STE/CHI3] \\
\hline & V & $(314-404)$ & $33.0 \pm 0.2$ & 380 & EB & [1996STE/CHI3] \\
\hline & $\mathrm{V}$ & & $36.5 \pm 0.1$ & 298 & $\mathrm{C}$ & [1978MCC/HAM] \\
\hline $\mathrm{C}_{3} \mathrm{H}_{3} \mathrm{NO}_{2}$ & {$[17640-15-2]$} & cyanoformic aci & thyl ester & & & \\
\hline & $\mathrm{V}$ & $(273-333)$ & 39.3 & 288 & A & [1987STE/MAL, 1970DYK, 1948RED/CHA5] \\
\hline $\mathrm{C}_{3} \mathrm{H}_{3} \mathrm{NS}$ & [288-47-1] & thiazole & & & & \\
\hline & FUS & & 9.58 & 239.4 & & [1968GOU/WES2, 1966MEY/MET] \\
\hline & $\mathrm{V}$ & $(333-393)$ & 39.7 & 348 & A & {$[1987 \mathrm{STE} / \mathrm{MAL}]$} \\
\hline & $\mathrm{V}$ & $(336-391)$ & 38.9 & 351 & A & [1987STE/MAL, 1969SOU/GOU2] \\
\hline $\mathrm{C}_{3} \mathrm{H}_{3} \mathrm{~N}_{3}$ & [290-87-9] & 1,3,5-triazine & & & & \\
\hline & TRS & $(12-347)$ & 0.07 & 197.7 & & \\
\hline & FUS & $(12-347)$ & 14.56 & 353.4 & $\mathrm{AC}$ & [1991ACR, 1988VAN/VAN] \\
\hline & TRS & $(134-382)$ & 0.07 & 197.6 & & \\
\hline & FUS & $(134-382)$ & 14.58 & 353.9 & $\mathrm{C}$ & [1979BRI/VAN] \\
\hline & TRS & $(100-355)$ & 0.02 & 198.1 & $\mathrm{AC}$ & {$[1978 \mathrm{SMI} / \mathrm{RAE}]$} \\
\hline & SUB & $(212-229)$ & 58.2 & 222 & TE, ME & [1983DEW/VAN] \\
\hline & SUB & & $54.2 \pm 0.2$ & 298 & $\mathrm{C}$ & [1982BYS] \\
\hline
\end{tabular}

[Note: In [1982BYS] the author quotes an unpublished calorimetric value of $S U B 55.2 \pm 0.2 \mathrm{~kJ} / \mathrm{mole}$ determined by K. Arvidsson.]
SUB
(283-313) $\quad 56.5 \pm 2.1$
[1982INI/LOP] 
Table 6. Phase change enthalpies of $\mathrm{C}_{3}$ organic compounds-Continued

\begin{tabular}{|c|c|c|c|c|c|c|}
\hline \multirow[b]{2}{*}{$\begin{array}{l}\text { Molecular } \\
\text { formula }\end{array}$} & \multirow{2}{*}{$\begin{array}{c}\text { CAS Reg. No. } \\
\text { Transition }\end{array}$} & \multicolumn{5}{|l|}{ Compound } \\
\hline & & $\begin{array}{c}\text { Temp. range } \\
(\mathrm{K})\end{array}$ & $\begin{array}{c}\Delta_{\text {trans }} H_{\mathrm{m}} \\
\left(\mathrm{kJ} \mathrm{mol}^{-1}\right)\end{array}$ & $\begin{array}{l}T_{\mathrm{m}} \\
(\mathrm{K})\end{array}$ & Method & References \\
\hline & SUB & $(242-264)$ & $\mathrm{U} 43.1$ & 253 & ME & [1968MAS/RAE] \\
\hline & V & $(342-373)$ & $38.8 \pm 1.9$ & 298 & CGC & [2009LIP/CHI2, 2009LIP/CHI] \\
\hline $\mathrm{C}_{3} \mathrm{H}_{3} \mathrm{~N}_{3} \mathrm{O}_{2}$ & $\begin{array}{l}\text { [461-89-2] } \\
\text { SUB } \\
\text { SUB } \\
\text { SUB } \\
\text { SUB }\end{array}$ & $\begin{array}{c}\text { 6-azauracil } \\
452.6 \\
(377-399) \\
(377-399)\end{array}$ & $\begin{array}{c}118.1 \pm 2.0 \\
119.1 \pm 1.7 \\
120.7 \pm 1.7 \\
141\end{array}$ & $\begin{array}{l}298 \\
388 \\
298\end{array}$ & $\begin{array}{c}\mathrm{C} \\
\mathrm{ME} \\
\mathrm{ME} \\
\mathrm{LE}\end{array}$ & $\begin{array}{l}\text { [2016AMA/SZT] } \\
\text { [2016AMA/SZT] } \\
\text { [2016AMA/SZT] } \\
\text { [1974YAN/VER] }\end{array}$ \\
\hline $\mathrm{C}_{3} \mathrm{H}_{3} \mathrm{~N}_{3} \mathrm{O}_{3}$ & $\begin{array}{c}\text { [108-80-5] } \\
\text { SUB } \\
\text { SUB }\end{array}$ & $\begin{array}{r}\text { cyanuric acid } \\
(440-473)\end{array}$ & $\begin{array}{l}131 \\
133\end{array}$ & $\begin{array}{l}458 \\
298\end{array}$ & ME,TE & $\begin{array}{l}\text { [1983DEW/VAN] } \\
\text { [1983DEW/VAN] }\end{array}$ \\
\hline $\mathrm{C}_{3} \mathrm{H}_{3} \mathrm{~N}_{3} \mathrm{O}_{3}$ & $\begin{array}{c}{[77666-53-6]} \\
\mathrm{V}\end{array}$ & 3-methyl-4-nitr & $\begin{array}{l}\operatorname{zan} \\
51.9 \pm 0.9\end{array}$ & 298 & $\mathrm{C}$ & [2012KON/MAT] \\
\hline $\mathrm{C}_{3} \mathrm{H}_{3} \mathrm{~N}_{5} \mathrm{O}_{10}$ & $\begin{array}{c}\text { [62626-83-9] } \\
\text { SUB }\end{array}$ & 1,1,1,2,2-pentan & $\begin{array}{l}\text { ropane } \\
77.4 \pm 1.3\end{array}$ & 298 & & [1999MIR/VOR] \\
\hline $\mathrm{C}_{3} \mathrm{H}_{4}$ & $\begin{array}{c}\text { [463-49-0] } \\
\text { V } \\
\text { V } \\
\text { V } \\
\text { V } \\
\text { V } \\
\text { V }\end{array}$ & $\begin{array}{l}\text { allene } \\
\qquad \begin{array}{l}(136-274) \\
(193-246) \\
(153-238) \\
(138-189) \\
(203-236) \\
(200-260)\end{array}\end{array}$ & $\begin{array}{l}22.6 \\
19.9 \\
21.3 \\
22.9 \\
20.9 \\
21.5\end{array}$ & $\begin{array}{l}259 \\
231 \\
223 \\
174 \\
220 \\
245\end{array}$ & $\begin{array}{c}\mathrm{A} \\
\mathrm{A} \\
\mathrm{MM}\end{array}$ & $\begin{array}{l}\text { [1987STE/MAL] } \\
\text { [1987STE/MAL] } \\
\text { [1947STU] } \\
\text { [1940LAM/ROP, 1984BOU/FRI] } \\
\text { [1930LIV/HEI] } \\
\text { [1921MAA/WRI, 1984BOU/FRI] }\end{array}$ \\
\hline $\mathrm{C}_{3} \mathrm{H}_{4}$ & $\begin{array}{c}\text { [74-99-7] } \\
\text { V } \\
\text { V } \\
\text { V } \\
\text { V } \\
\text { V } \\
\text { V } \\
\text { V } \\
\text { V } \\
\text { V } \\
\text { V }\end{array}$ & $\begin{aligned} & 1-\text { propyne } \\
&(183-257) \\
&(257-402) \\
&(303-361) \\
&(359-402) \\
&(249-306) \\
& \\
&(162-255) \\
&(323-400) \\
&(194-250) \\
&(200-260)\end{aligned}$ & $\begin{array}{l}23.0 \\
20.8 \\
21.2 \\
21.9 \\
23.2 \\
22.1 \\
23.9 \\
21.6 \\
23.4 \\
21.4\end{array}$ & $\begin{array}{l}242 \\
272 \\
318 \\
374 \\
264 \\
275 \\
240 \\
338 \\
235 \\
230\end{array}$ & $\begin{array}{l}\text { A } \\
\text { A } \\
\text { A } \\
\text { A } \\
\text { A }\end{array}$ & $\begin{array}{l}\text { [1987STE/MAL] } \\
{[1987 \mathrm{STE} / \mathrm{MAL}]} \\
{[1987 \mathrm{STE} / \mathrm{MAL}]} \\
{[1987 \mathrm{STE} / \mathrm{MAL}]} \\
{[1987 \mathrm{STE} / \mathrm{MAL}]} \\
{[1971 \mathrm{WIL} / \mathrm{ZWO}]} \\
{[1967 \mathrm{VAN}]} \\
{[1962 \mathrm{VOH} / \mathrm{KAN}]} \\
{[1933 \mathrm{BOO} / \mathrm{BUR}, 1984 \mathrm{BOU} / \mathrm{FRI}]} \\
{[1921 \mathrm{MAA} / \mathrm{WRI}]}\end{array}$ \\
\hline $\mathrm{C}_{3} \mathrm{H}_{4} \mathrm{Br}_{2}$ & $\begin{array}{c}{[513-31-5]} \\
\mathrm{V}\end{array}$ & $\begin{array}{c}\text { 2,3-dibromopro } \\
(267-415)\end{array}$ & 43.1 & 282 & A & [1987STE/MAL, 1947STU] \\
\hline $\mathrm{C}_{3} \mathrm{H}_{4} \mathrm{Br}_{4}$ & $\begin{array}{c}\text { [54268-02-9] } \\
\mathrm{V}\end{array}$ & $\begin{array}{c}\text { 1,2,2,3-tetrabro } \\
(418-580)\end{array}$ & $\begin{array}{l}\text { opane } \\
57.7\end{array}$ & 433 & A & [1987STE/MAL, 1970DYK] \\
\hline $\mathrm{C}_{3} \mathrm{H}_{4} \mathrm{ClFO}_{3}$ & $\begin{array}{c}{[462-27-1]} \\
\mathrm{V}\end{array}$ & $\begin{array}{c}\text { carbonochloridi } \\
\quad(273-333)\end{array}$ & $\begin{array}{c}\text { d, 2-fluoroeth } \\
46.6\end{array}$ & 288 & GS & [1987STE/MAL, 1948RED/CHA4, 1970DYK] \\
\hline $\mathrm{C}_{3} \mathrm{H}_{4} \mathrm{ClF}_{3}$ & $\begin{array}{c}\text { [460-35-5] } \\
\text { TRS } \\
\text { FUS }\end{array}$ & 1-chloro-3,3,3-t & $\begin{array}{c}\text { ropropane } \\
4.49 \\
5.31\end{array}$ & $\begin{array}{l}169.8 \\
179.4\end{array}$ & & [1996DOM/HEA, 1974KOL/VOR] \\
\hline & $\begin{array}{l}\mathrm{V} \\
\mathrm{V}\end{array}$ & $\begin{array}{l}(297-315) \\
(301-341)\end{array}$ & $\begin{array}{l}29.9 \\
33.7\end{array}$ & $\begin{array}{l}306 \\
316\end{array}$ & A & $\begin{array}{l}\text { [1987STE/MAL] } \\
\text { [1972VAR/DRU] }\end{array}$ \\
\hline $\mathrm{C}_{3} \mathrm{H}_{4} \mathrm{ClF}_{3} \mathrm{O}$ & $\begin{array}{c}{[425-87-6]} \\
\mathrm{V} \\
\mathrm{V} \\
\mathrm{V}\end{array}$ & 2-chloro-1,1,2-t & $\begin{array}{l}\text { roethyl meth } \\
34.4 \pm 0.1 \\
33.4 \pm 0.1 \\
31.1 \pm 0.1\end{array}$ & $\begin{array}{l}298 \\
313 \\
343\end{array}$ & $\begin{array}{l}\mathrm{C} \\
\mathrm{C} \\
\mathrm{C}\end{array}$ & $\begin{array}{l}\text { [1984MAJ/UCH] } \\
{[1984 \mathrm{MAJ} / \mathrm{UCH}]} \\
{[1984 \mathrm{MAJ} / \mathrm{UCH}]}\end{array}$ \\
\hline
\end{tabular}

$\mathrm{C}_{3} \mathrm{H}_{4} \mathrm{ClF}_{3} \mathrm{O}_{2} \mathrm{~S}$ [61915-99-9] trifluoromethanesulfinic acid, 2-chloroethyl ester

$$
\text { (320-403) } \quad 40.5 \quad 335
$$

$\mathrm{C}_{3} \mathrm{H}_{4} \mathrm{Cl}_{2} \mathrm{~F}_{2} \mathrm{O}$ [76-38-0] 2,2-dichloro-1,1-difluoro-1-methoxyethane

$$
\begin{array}{llll}
\mathrm{V} & (279-378) & 40.3 & 294
\end{array}
$$

$\mathrm{C}_{3} \mathrm{H}_{4} \mathrm{Cl}_{2} \mathrm{O} \quad$ [513-88-2] 1,1-dichloroacetone

$\begin{array}{llll}\mathrm{V} & (292-382) & 35.8 & 307\end{array}$

$\mathrm{C}_{3} \mathrm{H}_{4} \mathrm{Cl}_{2} \mathrm{O}$ [534-07-6] 1,3-dichloroacetone 
Table 6. Phase change enthalpies of $\mathrm{C}_{3}$ organic compounds-Continued

\begin{tabular}{|c|c|c|c|c|c|c|}
\hline \multirow[b]{2}{*}{$\begin{array}{l}\text { Molecular } \\
\text { formula }\end{array}$} & \multirow{2}{*}{$\begin{array}{c}\text { CAS Reg. No. } \\
\text { Transition }\end{array}$} & \multicolumn{5}{|l|}{ Compound } \\
\hline & & $\begin{array}{l}\text { Temp. range } \\
\text { (K) }\end{array}$ & $\begin{array}{c}\Delta_{\text {trans }} H_{\mathrm{m}} \\
\left(\mathrm{kJ} \mathrm{mol}^{-1}\right)\end{array}$ & $\begin{array}{l}T_{\mathrm{m}} \\
(\mathrm{K})\end{array}$ & Method & References \\
\hline & $\mathrm{V}$ & $(348-445)$ & 49.6 & 363 & A & [1987STE/MAL, 1970SMI/THO] \\
\hline \multirow[t]{4}{*}{$\mathrm{C}_{3} \mathrm{H}_{4} \mathrm{Cl}_{2} \mathrm{O}_{2}$} & [116-54-1] & \multicolumn{5}{|c|}{ methyl dichloroacetate } \\
\hline & $\mathrm{V}$ & & $47.7 \pm 0.1$ & 298 & $\mathrm{C}$ & [1972LAY/WAD] \\
\hline & $\mathrm{V}$ & $(331-481)$ & 44.9 & 346 & A & [1987STE/MAL, 1970DYK] \\
\hline & V & $(276-416)$ & 47.2 & 291 & & [1947STU] \\
\hline \multirow[t]{2}{*}{$\mathrm{C}_{3} \mathrm{H}_{4} \mathrm{Cl}_{4}$} & {$[812-03-3]$} & \multicolumn{5}{|c|}{ 1,1,1,2-tetrachloropropane } \\
\hline & $\mathrm{V}$ & $(331-469)$ & 42.3 & 346 & A & [1987STE/MAL, 1970DYK] \\
\hline \multirow[t]{4}{*}{$\mathrm{C}_{3} \mathrm{H}_{4} \mathrm{Cl}_{4}$} & [1070-78-6] & \multicolumn{5}{|c|}{ 1,1,1,3-tetrachloropropane } \\
\hline & TRS & & 2.2 & 219.9 & & \\
\hline & FUS & & 10.49 & 237.7 & & [1996DOM/HEA, 1974KOL/VOR] \\
\hline & $\mathrm{V}$ & $(300-377)$ & 57.8 & 315 & A & {$[1987 \mathrm{STE} / \mathrm{MAL}]$} \\
\hline \multirow[t]{3}{*}{$\mathrm{C}_{3} \mathrm{H}_{4} \mathrm{Cl}_{4}$} & [13116-53-5] & \multicolumn{5}{|c|}{ 1,2,2,3-tetrachloropropane } \\
\hline & $\mathrm{V}$ & $(346-415)$ & 42.8 & 361 & A & [1987STE/MAL] \\
\hline & $\mathrm{V}$ & $(335-415)$ & 42.9 & 375 & & [1969OTO/BES] \\
\hline \multirow[t]{2}{*}{$\mathrm{C}_{3} \mathrm{H}_{4} \mathrm{~F}_{2} \mathrm{O}_{2}$} & {$[433-53-4]$} & \multicolumn{5}{|c|}{ methyl difluoroacetate } \\
\hline & $\mathrm{V}$ & $(273-333)$ & 41.9 & 288 & GS & [1987STE/MAL, 1948RED/CHA4, 1970DYK] \\
\hline \multirow[t]{2}{*}{$\mathrm{C}_{3} \mathrm{H}_{4} \mathrm{~F}_{4} \mathrm{O}$} & [32778-16-8] & \multicolumn{5}{|c|}{ 2-difluoromethoxy-1,1-difluoroethane } \\
\hline & $\mathrm{V}$ & $(288-328)$ & 32.9 & 303 & I & [2002MUR/YAM] \\
\hline \multirow[t]{4}{*}{$\mathrm{C}_{3} \mathrm{H}_{4} \mathrm{~F}_{4} \mathrm{O}$} & [76-37-9] & \multicolumn{5}{|c|}{ 2,2,3,3-tetrafluoro-1-propanol } \\
\hline & $\mathrm{V}$ & $(303-380)$ & 47.9 & 318 & $\mathrm{~A}$ & [1987STE/MAL] \\
\hline & V & $(298-333)$ & 50.3 & 313 & MM & [1973ROC/SYM] \\
\hline & $\mathrm{V}$ & $(298-333)$ & 53.6 & 298 & MM & [1973ROC/SYM] \\
\hline \multirow[t]{2}{*}{$\mathrm{C}_{3} \mathrm{H}_{4} \mathrm{~F}_{6} \mathrm{NOP}$} & [31411-29-7] & $N$-methyl- $P, P$ & ifluoromethy & phinic a & & \\
\hline & $\mathrm{V}$ & $(293-342)$ & 40.3 & & & {$[1971 \mathrm{CAV} / \mathrm{CHA}]$} \\
\hline $\mathrm{C}_{3} \mathrm{H}_{4} \mathrm{~N}_{2}$ & [288-32-4] & imidazole & & & & \\
\hline & FUS & & 13.1 & 359.3 & DSC & [2012ALM/MON] \\
\hline & FUS & & 11.18 & 362.3 & DSC & [2002DOM/KOZ2] \\
\hline & FUS & & 12.5 & 363.2 & DSC & [1989HIL/MOU] \\
\hline & FUS & $(90-370)$ & 12.82 & 361.9 & $\mathrm{AC}$ & $\begin{array}{l}\text { [1996DOM/HEA, 1983DEW/DEK, } \\
\text { 1983DEW/OFF] }\end{array}$ \\
\hline & SUB & $(304-358)$ & $81.4 \pm 0.1$ & 360 & & [2012ALM/MON] \\
\hline & SUB & $(304-358)$ & $82.5 \pm 0.1$ & 298 & & [2012ALM/MON] \\
\hline & SUB & $(292-309)$ & $83.1 \pm 0.2$ & 300 & $\mathrm{ME}$ & [1987JIM/ROU] \\
\hline & SUB & & $83.1 \pm 0.2$ & 298 & ME & [1986JIM/ROU] \\
\hline & SUB & $(288-310)$ & 80.8 & 301 & ME,TE & [1983DEW/VAN] \\
\hline & SUB & & $74.5 \pm 0.4$ & 298 & $\mathrm{C}$ & [1980SAB2] \\
\hline & SUB & & 85.3 & 298 & & [1961ZIM/GEI] \\
\hline & $\mathrm{V}$ & $(334-385)$ & $67.3 \pm 0.4$ & 360 & & [2012ALM/MON] \\
\hline & V & $(334-385)$ & $71.3 \pm 0.4$ & 298 & & [2012ALM/MON] \\
\hline $\mathrm{C}_{3} \mathrm{H}_{4} \mathrm{~N}_{2}$ & [288-13-1] & pyrazole & & & & \\
\hline & FUS & & 14.2 & 343.2 & DSC & [1996DOM/HEA, 1989HIL/MOU] \\
\hline & FUS & $(300-385)$ & 13.8 & 333.1 & $\mathrm{DSC}$ & [1983DEW/OFF] \\
\hline & SUB & $(268-287)$ & $74.3 \pm 0.4$ & 275 & ME & [1987JIM/ROU] \\
\hline & SUB & & $74.0 \pm 0.4$ & 298 & & [1987JIM/ROU, 1986JIM/ROU] \\
\hline & SUB & $(253-273)$ & 72.7 & 265 & TE, ME & [1983DEW/VAN] \\
\hline & SUB & & $69.2 \pm 0.3$ & 298 & $\mathrm{C}$ & [1980SAB2] \\
\hline & SUB & & 71.8 & & & [1979DAA/VAN] \\
\hline & SUB & & 67.7 & & & [1961ZIM/GEI] \\
\hline $\mathrm{C}_{3} \mathrm{H}_{4} \mathrm{~N}_{2} \mathrm{O}$ & {$[107-91-5]$} & 2-cyanoacetami & & & & \\
\hline & FUS & & 18.81 & 394.7 & DSC & [2013SIN/PAN] \\
\hline & FUS & & 18.97 & 394.2 & DSC & [2011SIN/RAI] \\
\hline & TRS & $(300-420)$ & 1.2 & 346.5 & & \\
\hline & FUS & $(300-420)$ & 21.7 & 387.3 & DSC & [1996DOM/HEA, 1983DEW/OFF] \\
\hline & SUB & $(325-348)$ & 99.7 & 336 & $\mathrm{TE}, \mathrm{ME}$ & [1983DEW/VAN] \\
\hline
\end{tabular}


TABle 6. Phase change enthalpies of $\mathrm{C}_{3}$ organic compounds-Continued

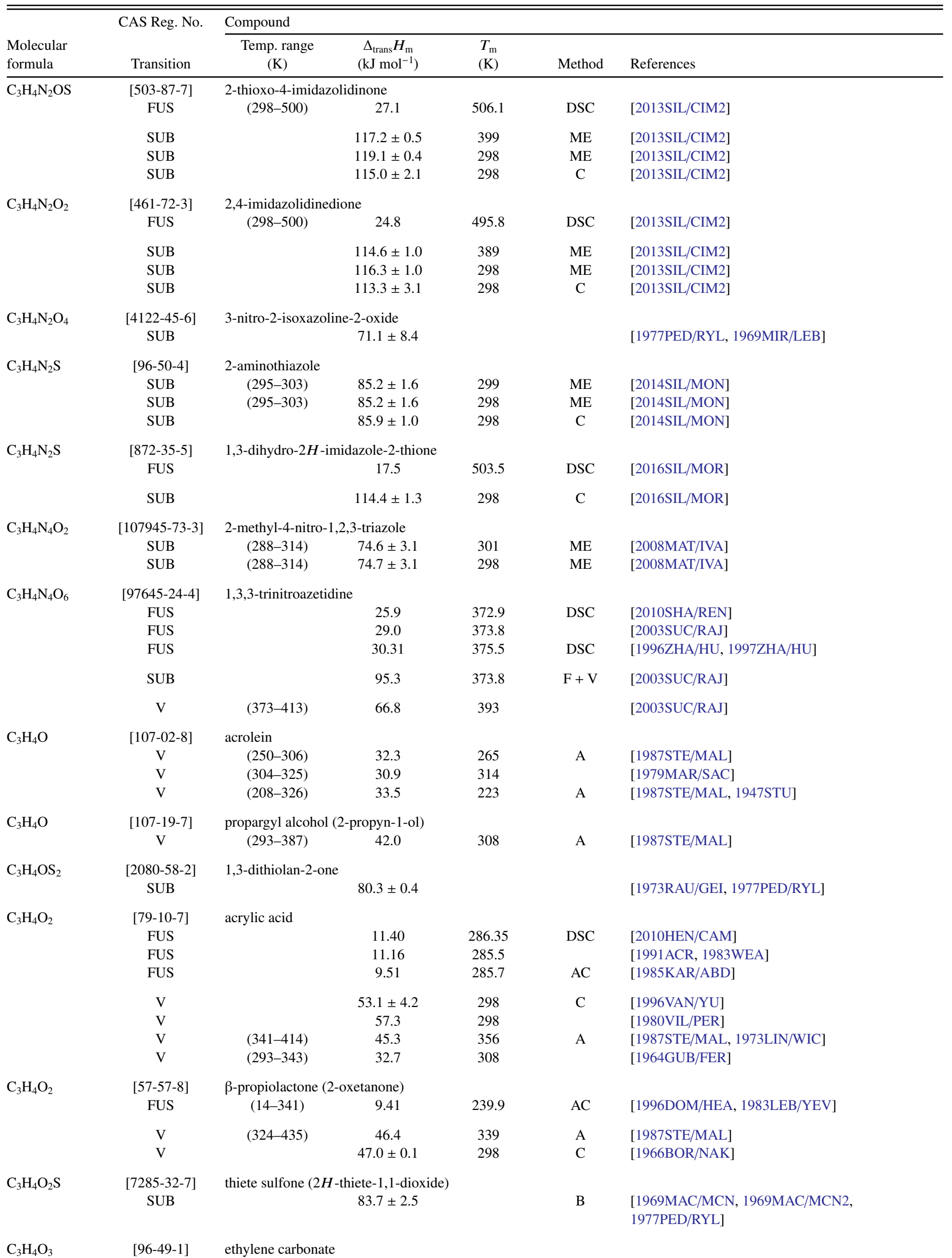


TABLE 6. Phase change enthalpies of $\mathrm{C}_{3}$ organic compounds-Continued

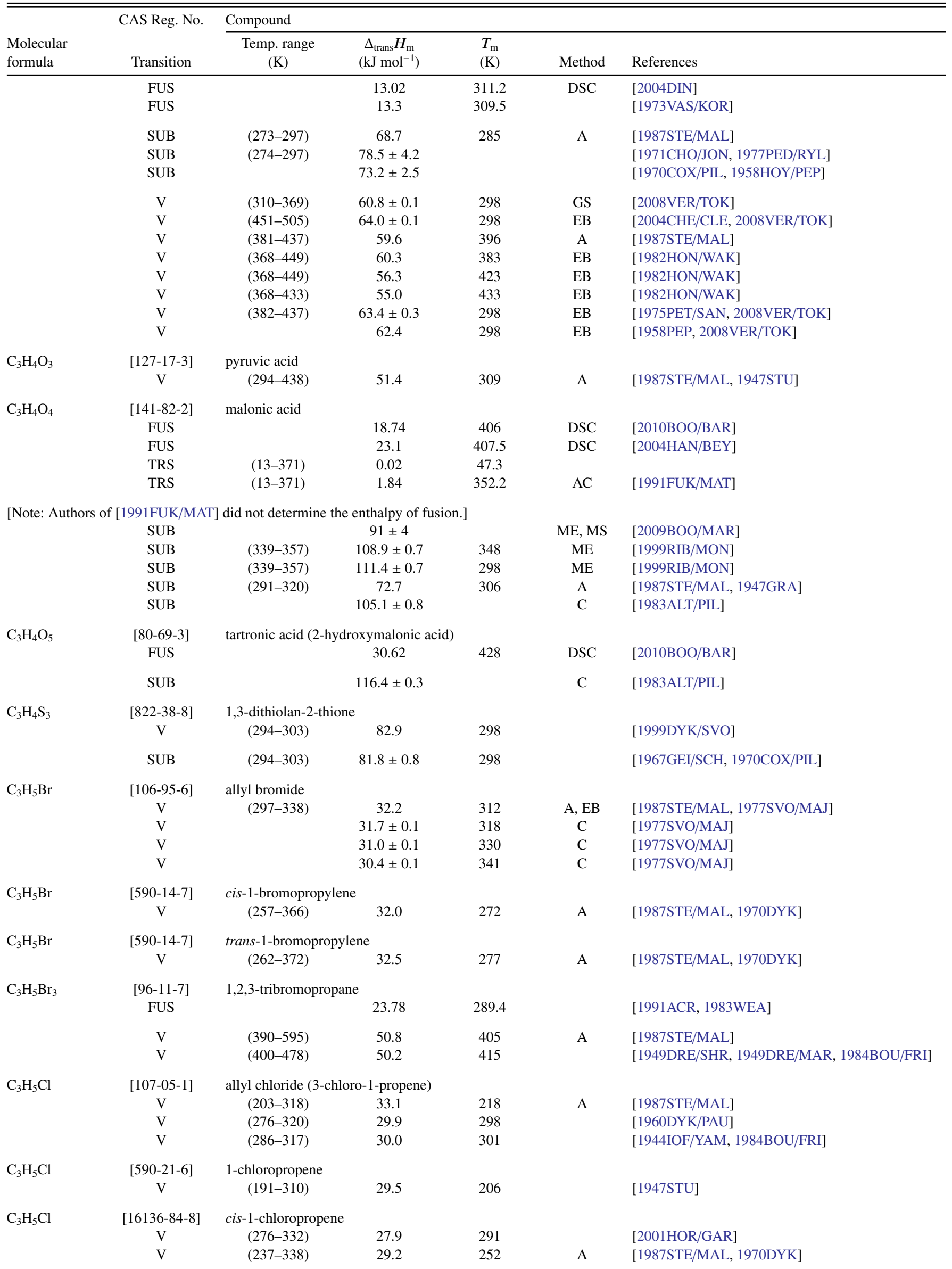


TABLE 6. Phase change enthalpies of $\mathrm{C}_{3}$ organic compounds-Continued

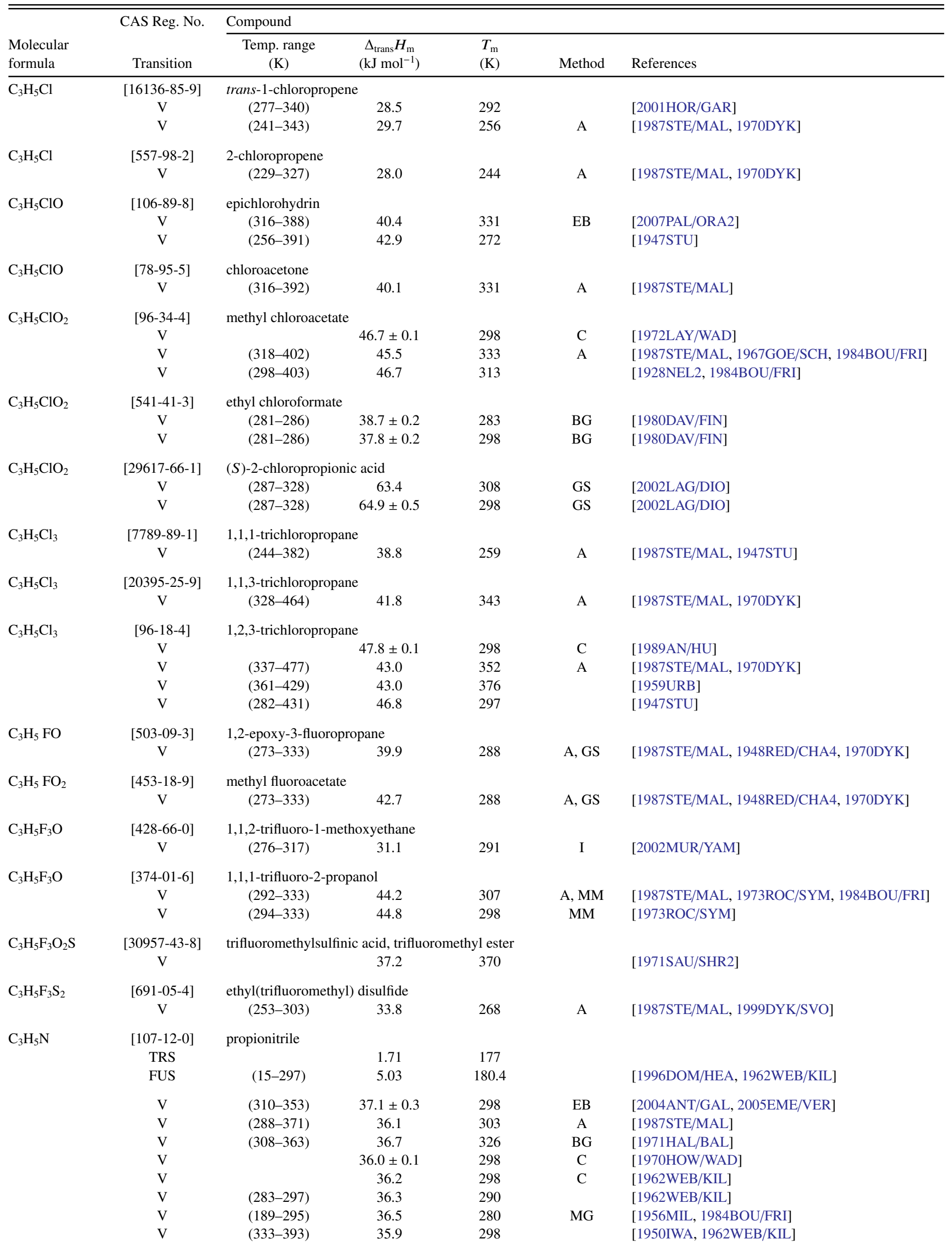


TABLE 6. Phase change enthalpies of $\mathrm{C}_{3}$ organic compounds-Continued

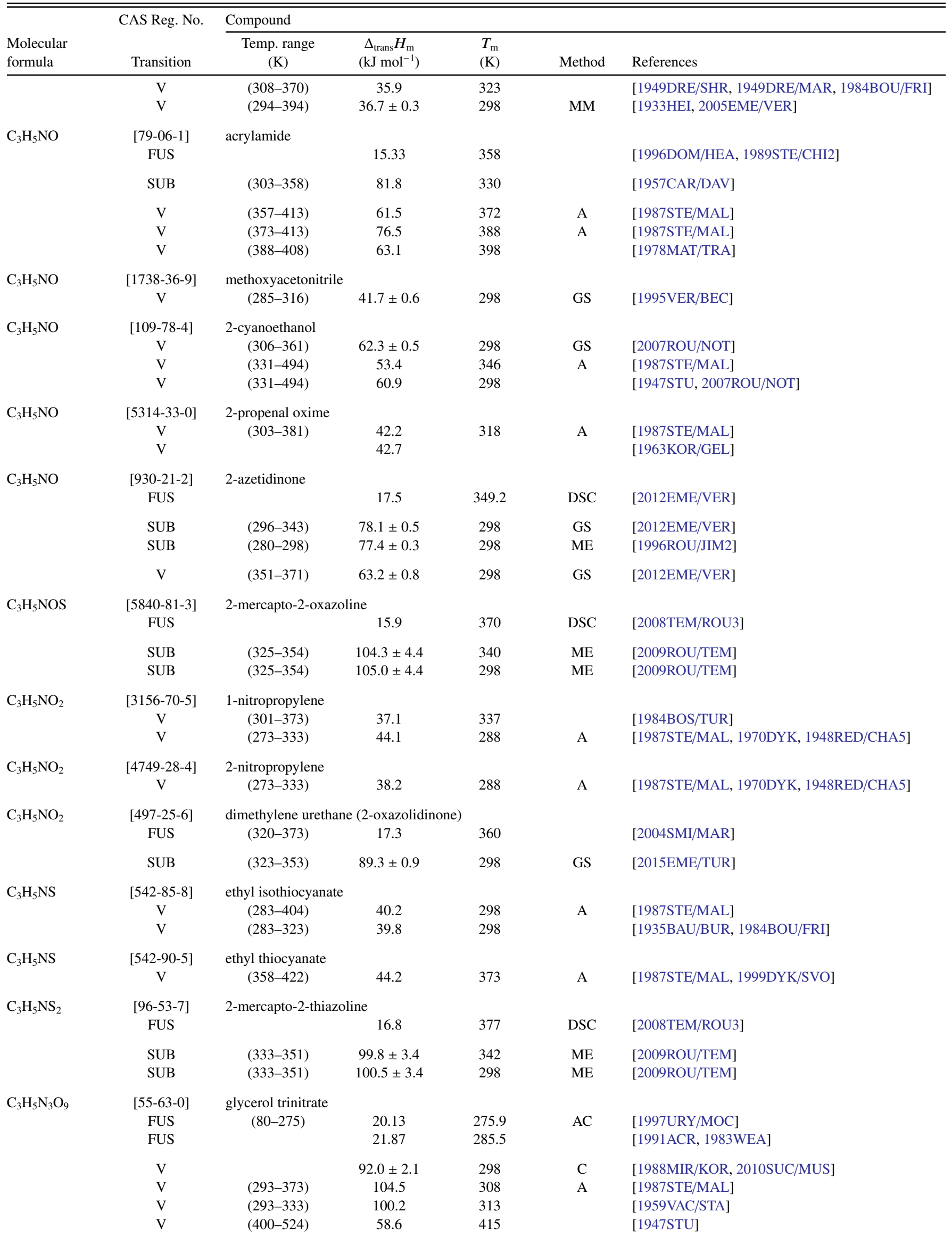

$\mathrm{C}_{3} \mathrm{H}_{5} \mathrm{P} \quad$ [114596-02-0] 2-propynylphosphine 
TABLE 6. Phase change enthalpies of $\mathrm{C}_{3}$ organic compounds-Continued

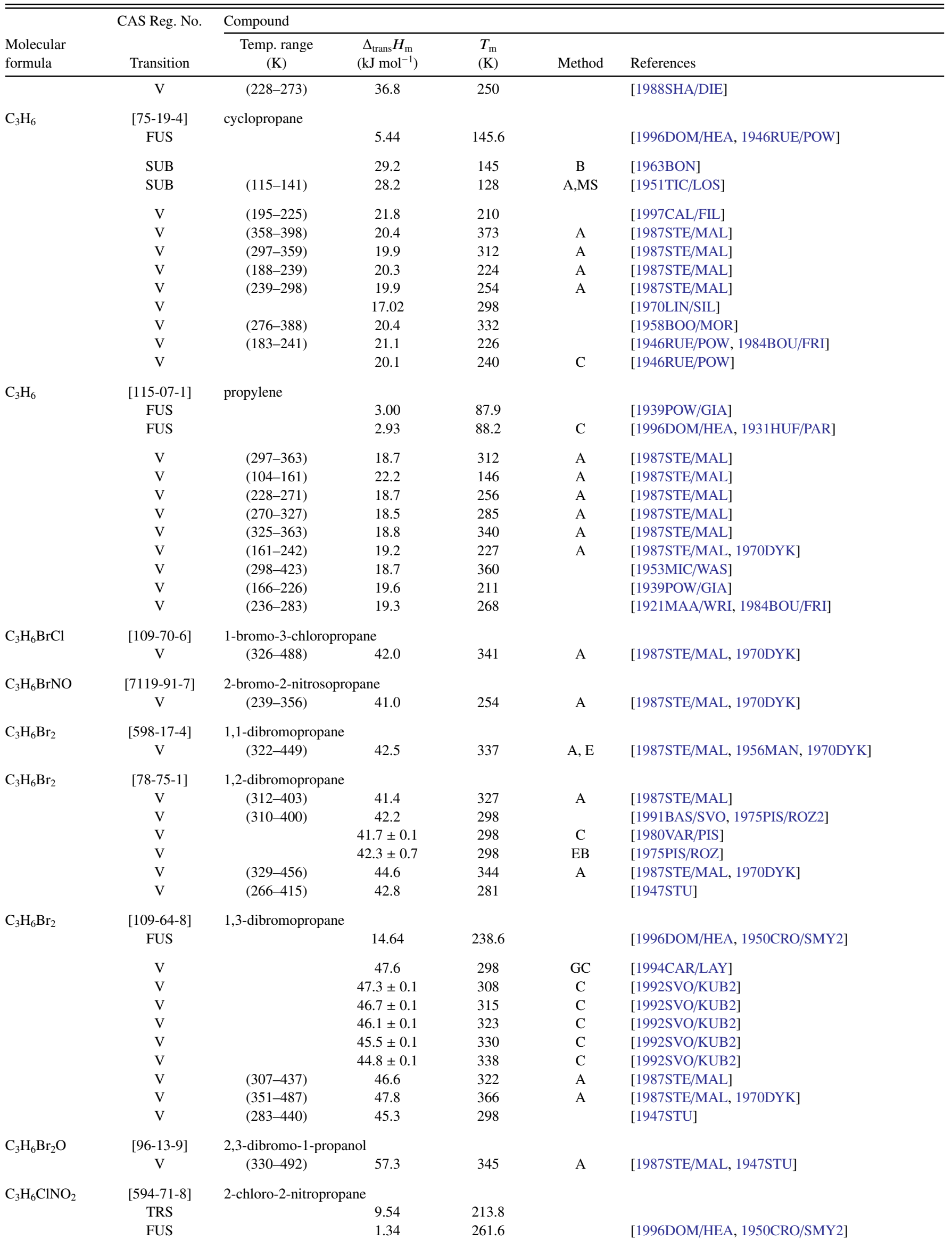


Table 6. Phase change enthalpies of $\mathrm{C}_{3}$ organic compounds-Continued

\begin{tabular}{|c|c|c|c|c|c|c|}
\hline \multirow[b]{2}{*}{$\begin{array}{l}\text { Molecular } \\
\text { formula }\end{array}$} & \multirow{2}{*}{$\begin{array}{c}\text { CAS Reg. No. } \\
\text { Transition }\end{array}$} & \multicolumn{5}{|l|}{ Compound } \\
\hline & & $\begin{array}{c}\text { Temp. range } \\
\text { (K) }\end{array}$ & $\begin{array}{c}\Delta_{\text {trans }} H_{\mathrm{m}} \\
\left(\mathrm{kJ} \mathrm{mol}^{-1}\right)\end{array}$ & $\begin{array}{l}T_{\mathrm{m}} \\
(\mathrm{K})\end{array}$ & Method & References \\
\hline \multirow[t]{4}{*}{$\mathrm{C}_{3} \mathrm{H}_{6} \mathrm{Cl}_{2}$} & {$[78-99-9]$} & 1,1-dichloropropane & & & & \\
\hline & $\mathrm{V}$ & & $35.2 \pm 0.4$ & 298 & $\mathrm{C}$ & [2007VAR/DRU] \\
\hline & $\mathrm{V}$ & $(310-360)$ & 35.2 & 298 & & [1967HAC/MAT, 1991BAS/SVO] \\
\hline & $\mathrm{V}$ & $(282-399)$ & 35.5 & 297 & $\mathrm{~A}, \mathrm{E}$ & [1987STE/MAL, 1956MAN, 1970DYK] \\
\hline \multirow[t]{10}{*}{$\mathrm{C}_{3} \mathrm{H}_{6} \mathrm{Cl}_{2}$} & {$[78-87-5]$} & 1,2-dichloropropane & & & & \\
\hline & FUS & & 6.4 & 172.7 & & [1991ACR, 1983WEA] \\
\hline & V & $(303-368)$ & $36.3 \pm 0.5$ & 298 & & [2007VAR/DRU] \\
\hline & $\mathrm{V}$ & & $36.2 \pm 0.1$ & 298 & $\mathrm{C}$ & [2007VAR/DRU] \\
\hline & $\mathrm{V}$ & $(294-406)$ & $38.4 \pm 0.3$ & 298 & EB & [1997STE/CHI3] \\
\hline & V & $(300-370)$ & 36.3 & 298 & & [1991BAS/SVO] \\
\hline & $\mathrm{V}$ & & $36.1 \pm 0.1$ & 298 & $\mathrm{C}$ & [1989AN/HU] \\
\hline & $\mathrm{V}$ & $(239-373)$ & 39.4 & 254 & A & [1987STE/MAL] \\
\hline & $\mathrm{V}$ & $(321-369)$ & 34.7 & 336 & & [1949DRE/SHR, 1949DRE/MAR] \\
\hline & $\mathrm{V}$ & $(288-373)$ & 34.3 & 303 & & [1933NEL/YOU] \\
\hline \multirow[t]{5}{*}{$\mathrm{C}_{3} \mathrm{H}_{6} \mathrm{Cl}_{2}$} & {$[142-28-9]$} & 1,3-dichloropropane & & & & \\
\hline & $\mathrm{V}$ & & 41.0 & 298 & $\mathrm{GC}$ & [1994CAR/LAY] \\
\hline & $\mathrm{V}$ & $(330-400)$ & 41.0 & 298 & & [1987VAR/LOS, 1991BAS/SVO] \\
\hline & $\mathrm{V}$ & & $40.6 \pm 0.1$ & 298 & $\mathrm{C}$ & [1989AN/HU] \\
\hline & $\mathrm{V}$ & $(307-435)$ & 39.0 & 322 & A & [1987STE/MAL, 1970DYK] \\
\hline \multirow[t]{8}{*}{$\mathrm{C}_{3} \mathrm{H}_{6} \mathrm{Cl}_{2}$} & [594-20-7] & 2,2-dichloropropane & & & & \\
\hline & TRS & $(5-301)$ & 0.01 & 171.6 & & \\
\hline & TRS & $(5-301)$ & 6.08 & 188.2 & & \\
\hline & FUS & $(5-301)$ & 2.39 & 239.6 & $\mathrm{AC}$ & [1999KOB/OGU] \\
\hline & TRS & & 5.98 & 188.0 & & \\
\hline & FUS & & 2.34 & 239.3 & & [1996DOM/HEA, 1972VAN] \\
\hline & $\mathrm{V}$ & $(295-340)$ & 32.1 & 298 & A & [1987VAR/LOS, 1991BAS/SVO] \\
\hline & $\mathrm{V}$ & $(267-378)$ & 33.2 & 282 & A & [1987STE/MAL, 1970DYK] \\
\hline \multirow[t]{2}{*}{$\mathrm{C}_{3} \mathrm{H}_{6} \mathrm{Cl}_{2} \mathrm{O}$} & [616-23-9] & 2,3-dichloro-1-propan & & & & \\
\hline & $\mathrm{V}$ & $(384-419)$ & 48.5 & 399 & A & [1987STE/MAL] \\
\hline \multirow[t]{2}{*}{$\mathrm{C}_{3} \mathrm{H}_{6} \mathrm{Cl}_{2} \mathrm{O}$} & {$[96-23-1]$} & 1,3-dichloro-2-propan & & & & \\
\hline & $\mathrm{V}$ & $(301-448)$ & 50.4 & 316 & A & [1987STE/MAL, 1947STU] \\
\hline \multirow[t]{2}{*}{$\mathrm{C}_{3} \mathrm{H}_{6} \mathrm{~F}_{2}$} & {$[430-61-5]$} & 1,1-difluoropropane & & & & \\
\hline & $\mathrm{V}$ & $(219-311)$ & 27.2 & 234 & $\mathrm{~A}, \mathrm{E}$ & [1987STE/MAL, 1956MAN, 1970DYK] \\
\hline \multirow[t]{2}{*}{$\mathrm{C}_{3} \mathrm{H}_{6} \mathrm{~F}_{2}$} & {$[420-45-1]$} & 2,2-difluoropropane & & & & \\
\hline & V & $(211-302)$ & 25.6 & 226 & A & [1987STE/MAL, 1970DYK] \\
\hline \multirow[t]{2}{*}{$\mathrm{C}_{3} \mathrm{H}_{6} \mathrm{~F}_{2} \mathrm{O}$} & {$[461-57-4]$} & 1,1-difluoro-2-methox & xyethane & & & \\
\hline & $\mathrm{V}$ & $(288-322)$ & 31.8 & 303 & I & [2002MUR/YAM] \\
\hline \multirow[t]{3}{*}{$\mathrm{C}_{3} \mathrm{H}_{6} \mathrm{~F}_{3} \mathrm{NS}$} & {$[62067-13-4]$} & dimethyl(trifluorometl & hylthio)amin & & & \\
\hline & $\mathrm{V}$ & $(273-329)$ & 31.1 & 288 & A & [1987STE/MAL, 1999DYK/SVO] \\
\hline & $\mathrm{V}$ & $(223-295)$ & 30.2 & 259 & & [1960EME/NAB] \\
\hline \multirow[t]{2}{*}{$\mathrm{C}_{3} \mathrm{H}_{6} \mathrm{~F}_{3} \mathrm{OP}$} & {$[26348-84-5]$} & methyl(trifluoromethy & l)phosphinou & , methyl & & \\
\hline & $\mathrm{V}$ & $(232-285)$ & 33.9 & 258 & & [1970BUR/KAN] \\
\hline \multirow[t]{3}{*}{$\mathrm{C}_{3} \mathrm{H}_{6} \mathrm{~F}_{3} \mathrm{OP}$} & {$[26348-91-4]$} & dimethyl(trifluorometl & hyl)phosphin & & & \\
\hline & SUB & $(320-341)$ & 75.4 & 330 & & [1970BUR/KAN] \\
\hline & $\mathrm{V}$ & $(347-360)$ & 52.4 & 358 & & [1970BUR/KAN] \\
\hline \multirow[t]{2}{*}{$\mathrm{C}_{3} \mathrm{H}_{6} \mathrm{~F}_{3} \mathrm{O}_{2} \mathrm{P}$} & {$[684-56-0]$} & (trifluoromethyl)phos! & phonic acid, & yl ester & & \\
\hline & $\mathrm{V}$ & $(237-318)$ & 37.4 & 252 & A & [1987STE/MAL, 1961BUR/GRI] \\
\hline \multirow[t]{2}{*}{$\mathrm{C}_{3} \mathrm{H}_{6} \mathrm{~F}_{3} \mathrm{PS}$} & {$[26348-86-7]$} & methyl(trifluoromethy & l)phosphinot & acid, me & ster & \\
\hline & $\mathrm{V}$ & $(273-313)$ & 38.4 & 293 & & {$[1970 \mathrm{BUR} / \mathrm{KAN}]$} \\
\hline \multirow[t]{3}{*}{$\mathrm{C}_{3} \mathrm{H}_{6} \mathrm{~F}_{3} \mathrm{PS}$} & {$[26348-92-5]$} & \multicolumn{5}{|c|}{ dimethyl(trifluoromethyl)phosphine sulfide } \\
\hline & SUB & $(300-320)$ & 68.0 & 310 & & {$[1970 \mathrm{BUR} / \mathrm{KAN}]$} \\
\hline & $\mathrm{V}$ & $(323-357)$ & 47.2 & 340 & & [1970BUR/KAN] \\
\hline
\end{tabular}


TABle 6. Phase change enthalpies of $\mathrm{C}_{3}$ organic compounds-Continued

\begin{tabular}{|c|c|c|c|c|c|c|}
\hline \multirow[b]{2}{*}{$\begin{array}{l}\text { Molecular } \\
\text { formula }\end{array}$} & \multirow{2}{*}{$\begin{array}{c}\text { CAS Reg. No. } \\
\text { Transition }\end{array}$} & \multicolumn{5}{|l|}{ Compound } \\
\hline & & $\begin{array}{l}\text { Temp. range } \\
\text { (K) }\end{array}$ & $\begin{array}{c}\Delta_{\text {trans }} H_{\mathrm{m}} \\
\left(\mathrm{kJ} \mathrm{mol}{ }^{-1}\right)\end{array}$ & $\begin{array}{l}T_{\mathrm{m}} \\
(\mathrm{K})\end{array}$ & Method & References \\
\hline \multirow[t]{2}{*}{$\mathrm{C}_{3} \mathrm{H}_{6} \mathrm{I}_{2}$} & [627-31-6] & 1,3-diiodopropane & & & & \\
\hline & $\mathrm{V}$ & & 54.1 & 298 & $\mathrm{GC}$ & [1994CAR/LAY] \\
\hline \multirow[t]{9}{*}{$\mathrm{C}_{3} \mathrm{H}_{6} \mathrm{~N}_{2} \mathrm{O}$} & {$[120-93-4]$} & 2-imadazolidinone & & & & \\
\hline & TRS & & 3.6 & 344.6 & & \\
\hline & FUS & & 11.5 & 401.2 & DSC & [2008RIB/RIB] \\
\hline & FUS & & 14.7 & 403 & DSC & [1999DEF/DEO, 1999DEF] \\
\hline & TRS & & 3.27 & 344 & & \\
\hline & FUS & & 5.11 & 397.3 & $\mathrm{DSC}$ & [1984WEI/LEF] \\
\hline & SUB & $(327-349)$ & $94.5 \pm 0.8$ & 338 & $\mathrm{ME}$ & [2008RIB/RIB] \\
\hline & SUB & $(327-349)$ & $96.6 \pm 0.8$ & 298 & $\mathrm{ME}$ & [2008RIB/RIB] \\
\hline & SUB & & $83.7 \pm 1.9$ & 298 & $\mathrm{~V}+\mathrm{F}$ & [1999DEF/DEO, 1999DEF] \\
\hline \multirow[t]{4}{*}{$\mathrm{C}_{3} \mathrm{H}_{6} \mathrm{~N}_{2} \mathrm{O}_{2}$} & {$[591-07-1]$} & acetylurea & & & & \\
\hline & SUB & $(360-407)$ & $102.4 \pm 0.7$ & 383 & $\mathrm{C}$ & [1988IMA/MUR] \\
\hline & SUB & & $103.1 \pm 0.7$ & 298 & $\mathrm{C}$ & [1988IMA/MUR] \\
\hline & SUB & & $103.1 \pm 0.7$ & 298 & $\mathrm{C}$ & [1985MUR/SAK] \\
\hline \multirow[t]{11}{*}{$\mathrm{C}_{3} \mathrm{H}_{6} \mathrm{~N}_{2} \mathrm{O}_{2}$} & {$[108-13-4]$} & malonamide (propar & ediamide) & & & \\
\hline & $\begin{array}{l}\text { FUS } \\
\text { (orthorhombic) }\end{array}$ & & 24.2 & 431.1 & & \\
\hline & $\begin{array}{c}\text { FUS } \\
\text { (monoclinic) }\end{array}$ & & 30.3 & 439.5 & & \\
\hline & FUS (quadratic) & & 28.8 & 444.0 & DSC & [2014COR/GUI] \\
\hline & TRS & & 1.40 & 410.2 & & \\
\hline & FUS & & 23.2 & 445.9 & DSC & [2009CHE/PET] \\
\hline & TRS & & 1.89 & 418.9 & & \\
\hline & FUS & & 29.85 & 444.2 & DSC & [2006BAD/DEL] \\
\hline & TRS & & 1.9 & 393 & & \\
\hline & FUS (quadratic) & & 35.8 & 443 & DSC & [1996DOM/HEA, 1989SAK/IMA, 2014COR/GUI] \\
\hline & SUB & & $126.4 \pm 0.5$ & 298 & $\mathrm{C}$ & [1989IMA/TAK] \\
\hline \multirow[t]{3}{*}{$\mathrm{C}_{3} \mathrm{H}_{6} \mathrm{~N}_{2} \mathrm{O}_{4}$} & {$[601-76-3]$} & 1,1-dinitropropane & & & & \\
\hline & V & $(323-383)$ & 57.9 & 338 & A & {$[1987 \mathrm{STE} / \mathrm{MAL}]$} \\
\hline & $\mathrm{V}$ & $(323-383)$ & $62.5 \pm 0.6$ & 298 & $\mathrm{ZG}$ & {$[1949 H O L / D O R]$} \\
\hline \multirow[t]{8}{*}{$\mathrm{C}_{3} \mathrm{H}_{6} \mathrm{~N}_{2} \mathrm{O}_{4}$} & {$[595-49-3]$} & 2,2-dinitropropane & & & & \\
\hline & TRS & & 1.87 & 259.7 & & \\
\hline & TRS & & 11.28 & 267.7 & & \\
\hline & FUS & & 2.64 & 324.5 & & $\begin{array}{l}\text { [1996DOM/HEA, 1978GOD/RAC, } \\
\text { 1978GOD/RAC2] }\end{array}$ \\
\hline & TRS & & 10.42 & 266.5 & & \\
\hline & FUS & & 2.41 & 326.0 & DSC & [1972ROG/ORT] \\
\hline & TRS & & 12.22 & 268 & & {$[1958 \mathrm{BIL} / \mathrm{NOL}]$} \\
\hline & $\mathrm{V}$ & $(363-553)$ & 46.3 & 378 & A & [1987STE/MAL] \\
\hline \multirow[t]{3}{*}{$\mathrm{C}_{3} \mathrm{H}_{6} \mathrm{~N}_{2} \mathrm{O}_{5}$} & {$[918-52-5]$} & 2,2-dinitro-1-propan & & & & \\
\hline & TRS & & 15.06 & 281.7 & & \\
\hline & FUS & & 2.85 & 366.7 & & [1969ROS/HOL] \\
\hline \multirow[t]{2}{*}{$\mathrm{C}_{3} \mathrm{H}_{6} \mathrm{~N}_{2} \mathrm{O}_{6}$} & {$[6423-43-4]$} & 1,2-propanediol din & rate & & & \\
\hline & V & $(288-328)$ & 63.8 & 303 & A & [1987STE/MAL, 1970DYK] \\
\hline \multirow[t]{2}{*}{$\mathrm{C}_{3} \mathrm{H}_{6} \mathrm{~N}_{2} \mathrm{O}_{6}$} & {$[3457-90-7]$} & 1,3-propanediol din & rate & & & \\
\hline & $\mathrm{V}$ & $(293-313)$ & $74.3 \pm 4.6$ & 303 & A, GS & [1987STE/MAL, 1957KEM/GOL] \\
\hline \multirow[t]{2}{*}{$\mathrm{C}_{3} \mathrm{H}_{6} \mathrm{~N}_{2} \mathrm{O}_{6}$} & {$[2736-80-3]$} & 2,2-dinitro-1,3-prop & nediol & & & \\
\hline & TRS & & 21.34 & 341.2 & & [1969ROS/HOL] \\
\hline \multicolumn{7}{|c|}{ [Note: Decomposes before melting.] } \\
\hline \multirow[t]{3}{*}{$\mathrm{C}_{3} \mathrm{H}_{6} \mathrm{~N}_{4}$} & [5144-11-6] & 1,5-dimethyltetrazol & & & & \\
\hline & FUS & & 14.7 & 349 & & [1990KOZ/SIM3] \\
\hline & SUB & $(303-343)$ & $86.2 \pm 1.0$ & & $\mathrm{ME}$ & [1990KOZ/SIM] \\
\hline $\mathrm{C}_{3} \mathrm{H}_{6} \mathrm{~N}_{4}$ & [4135-93-7] & 2,5-dimethyltetrazol & & & & \\
\hline
\end{tabular}


TABLE 6. Phase change enthalpies of $\mathrm{C}_{3}$ organic compounds-Continued

\begin{tabular}{|c|c|c|c|c|c|c|}
\hline \multirow[b]{2}{*}{$\begin{array}{l}\text { Molecular } \\
\text { formula }\end{array}$} & \multirow{2}{*}{$\begin{array}{c}\text { CAS Reg. No. } \\
\text { Transition }\end{array}$} & \multicolumn{5}{|l|}{ Compound } \\
\hline & & $\begin{array}{c}\text { Temp. range } \\
\text { (K) }\end{array}$ & $\begin{array}{c}\Delta_{\text {trans }} H_{\mathrm{m}} \\
\left(\mathrm{kJ} \mathrm{mol}^{-1}\right)\end{array}$ & $\begin{array}{l}T_{\mathrm{m}} \\
(\mathrm{K})\end{array}$ & Method & References \\
\hline & FUS & & 13.5 & 256.4 & & {$[1990 \mathrm{KOZ} / \mathrm{SIM} 3]$} \\
\hline \multirow[t]{2}{*}{$\mathrm{C}_{3} \mathrm{H}_{6} \mathrm{~N}_{4} \mathrm{O}_{2}$} & {$[5465-96-3]$} & \multicolumn{5}{|c|}{ 4,5-dihydro- $N$-nitro- $1 H$-imidazol-2-amine } \\
\hline & FUS & & 34.24 & 495.1 & DSC & [2011CAI/XIA] \\
\hline \multirow[t]{3}{*}{$\mathrm{C}_{3} \mathrm{H}_{6} \mathrm{~N}_{4} \mathrm{O}_{4}$} & [5754-91-6] & 1,3-dinitro-1,3-c & cyclopentane & & & \\
\hline & FUS & & 25.08 & 410 & & [1996DOM/HEA, 1971HAL] \\
\hline & SUB & & $105.4 \pm 0.8$ & 298 & $\mathrm{C}$ & [2009MIR/KON] \\
\hline
\end{tabular}

[Note: Authors of [2009MIR/KON] tabulate the experimental value as an enthalpy of vaporization; however, they report that the compound is crystalline and use the value to calculate the standard molar enthalpy of formation. Given the crystaline state and how the value was used, we have tabulated the value as an enthalpy of sublimation.]

\begin{tabular}{|c|c|c|c|c|c|c|}
\hline \multirow[t]{3}{*}{$\mathrm{C}_{3} \mathrm{H}_{6} \mathrm{~N}_{6}$} & {$[108-78-1]$} & \multicolumn{5}{|c|}{ 2,4,6-triamino-s-triazine (melamine) } \\
\hline & SUB & $(417-614)$ & $121.3 \pm 4.2$ & 515 & GS & [1960HIR/STE, 1970COX/PIL] \\
\hline & SUB & $(417-447)$ & 123.3 & 432 & A & [1987STE/MAL] \\
\hline \multirow[t]{7}{*}{$\mathrm{C}_{3} \mathrm{H}_{6} \mathrm{~N}_{6} \mathrm{O}_{3}$} & [13980-04-6] & \multicolumn{5}{|c|}{ 1,3,5-trinitroso-1,3,5-triazacyclohexane } \\
\hline & TRS & & 17.78 & 367 & & \\
\hline & FUS & & 3.77 & 376 & DSC & [1996DOM/HEA, 1971HAL] \\
\hline & SUB & $(348-383)$ & 99.5 & & TGA & [2011FEL/RAM] \\
\hline & SUB & $(343-447)$ & $134.3 \pm 0.7$ & 298 & $\mathrm{ME}$ & [1978CUN/PAL] \\
\hline & SUB & $(383-411)$ & 112.1 & & $\mathrm{ME}$ & [1974PEP/MAT] \\
\hline & SUB & & 112.1 & & & [1953EDW, 1960JON] \\
\hline \multirow[t]{2}{*}{$\mathrm{C}_{3} \mathrm{H}_{6} \mathrm{~N}_{6} \mathrm{O}_{5}$} & {$[5755-27-1]$} & \multicolumn{5}{|c|}{ 1,3-dinitro-5-nitroso-1,3,5-triazacyclohexane } \\
\hline & FUS & & 25.97 & 446 & DTA & [1987OYU/BRI] \\
\hline \multirow[t]{11}{*}{$\mathrm{C}_{3} \mathrm{H}_{6} \mathrm{~N}_{6} \mathrm{O}_{6}$} & [121-82-4] & \multicolumn{5}{|c|}{ hexahydro-1,3,5-trinitro-1,3,5-triazine } \\
\hline & FUS $(\beta)$ & & 12.63 & 459.9 & DSC & [2013HUN/SUT] \\
\hline & FUS $(\alpha)$ & & 33.5 & 476.7 & DSC & [2010SHA/REN] \\
\hline & FUS $(\alpha)$ & & 32.98 & 478.5 & & [2003MCK/KRA] \\
\hline & FUS $(\alpha)$ & & 33.60 & 477.4 & DSC & [1997ZEM] \\
\hline & FUS $(\alpha)$ & & 33.43 & 478.3 & DSC & [1995YIN/LIU] \\
\hline & FUS $(\alpha)$ & & 37.66 & 478.2 & & [1996DOM/HEA, 1992MAK] \\
\hline & FUS $(\alpha)$ & & 30.7 & 477.4 & DSC & [1977KIS, 1997ZEM] \\
\hline & FUS $(\alpha)$ & & 35.65 & 478.5 & DSC & [1971HAL, 1997ZEM] \\
\hline & SUB & $(443-473)$ & 147 & 458 & DTA & [2014CUD/POD] \\
\hline & SUB & & $128.0 \pm 1.3$ & 298 & $\mathrm{C}$ & [2009MIR/KON] \\
\hline
\end{tabular}

[Note: Authors of [2009MIR/KON] tabulate the experimental value as an enthalpy of vaporization; however, they report that the compound is crystalline and use the value to calculate the standard molar enthalpy of formation. Given the crystaline state and how the value was used, we have tabulated the value as an enthalpy of sublimation.]

\begin{tabular}{|c|c|c|c|c|c|}
\hline SUB & & 134.3 & 298 & & [1978CUN/PAL] \\
\hline SUB & $(325-360)$ & $112.5 \pm 0.8$ & & $\mathrm{ME}$ & [1974PEP/MAT] \\
\hline SUB & $(329-371)$ & 130.1 & 350 & & [1969ROS/DIC] \\
\hline V & $(503-523)$ & 84.4 & 513 & A & [1987STE/MAL] \\
\hline 07-18-6] & 2-propen-1-ol & & & & \\
\hline V & $(311-355)$ & 46.1 & 298 & EB & [2004LUB/MAL] \\
\hline V & $(323-373)$ & 47.3 & 298 & CGC & [1995CHI/HOS] \\
\hline $03-30-0]$ & oxetane & & & & \\
\hline $\mathrm{V}$ & & 29.8 & 298 & $\mathrm{C}$ & [1981HOS/SCO] \\
\hline 67-64-1] & acetone & & & & \\
\hline TRS & $(78-320)$ & 0.04 & 126.2 & & \\
\hline FUS & $(78-320)$ & 5.77 & 179.0 & $\mathrm{AC}$ & [2006WAN/NAN] \\
\hline FUS & & 5.72 & 176.6 & & [1996DOM/HEA, 1929KEL3] \\
\hline FUS & & 5.69 & 177.6 & & [1928PAR/KEL] \\
\hline FUS & & 4.77 & 178.5 & & [1925MAA/WAL] \\
\hline V & $(310-330)$ & $31.7 \pm 0.4$ & & I & [2010GER/PEL] \\
\hline $\mathrm{V}$ & $(298-318)$ & 32.1 & 308 & & [2008SON/RAM] \\
\hline $\mathrm{V}$ & $(329-488)$ & 29.9 & 344 & A & [1987STE/MAL] \\
\hline
\end{tabular}


TABLE 6. Phase change enthalpies of $\mathrm{C}_{3}$ organic compounds-Continued

\begin{tabular}{|c|c|c|c|c|c|c|}
\hline \multirow[b]{2}{*}{$\begin{array}{l}\text { Molecular } \\
\text { formula }\end{array}$} & \multirow{2}{*}{$\begin{array}{c}\text { CAS Reg. No. } \\
\text { Transition }\end{array}$} & \multicolumn{5}{|l|}{ Compound } \\
\hline & & $\begin{array}{c}\text { Temp. range } \\
\text { (K) }\end{array}$ & $\begin{array}{c}\Delta_{\text {trans }} H_{\mathrm{m}} \\
\left(\mathrm{kJ} \mathrm{mol}^{-1}\right)\end{array}$ & $\begin{array}{l}T_{\mathrm{m}} \\
(\mathrm{K})\end{array}$ & Method & References \\
\hline & V & $(178-243)$ & 32.9 & 228 & $\mathrm{~A}$ & [1987STE/MAL] \\
\hline & V & $(203-269)$ & 33.8 & 254 & A & [1987STE/MAL] \\
\hline & V & $(323-379)$ & 30.6 & 338 & A & [1987STE/MAL] \\
\hline & V & (374-464) & 29.5 & 389 & $\mathrm{~A}$ & [1987STE/MAL] \\
\hline & $\mathrm{V}$ & $(457-508)$ & 29.7 & 472 & $\mathrm{~A}$ & [1987STE/MAL] \\
\hline & V & & 26.1 & 373 & $\mathrm{C}$ & [1986DMI/KAC] \\
\hline & V & & 21.7 & 423 & $\mathrm{C}$ & [1986DMI/KAC] \\
\hline & $\mathrm{V}$ & & 15.3 & 473 & $\mathrm{C}$ & [1986DMI/KAC] \\
\hline & V & & 9.2 & 498 & $\mathrm{C}$ & [1986DMI/KAC] \\
\hline & V & $(285-329)$ & 31.9 & 300 & EB & [1986BAL/GNA] \\
\hline & V & $(305-333)$ & 31.8 & 319 & & [1984CAS/FRA3] \\
\hline & V & & $30.5 \pm 0.6$ & 298 & $\mathrm{C}$ & [1981GAT/STR] \\
\hline & V & $(259-351)$ & 32.8 & 274 & A & [1987STE/MAL, 1974AMB/SPR2, 1975AMB/ELL] \\
\hline & $\mathrm{V}$ & & 31.3 & 298 & & [1975AMB/ELL] \\
\hline & $\mathrm{V}$ & $(261-328)$ & 32.7 & 276 & $\mathrm{~A}, \mathrm{~EB}$ & [1987STE/MAL, 1972BOU/AIM] \\
\hline & $\mathrm{V}$ & $(278-293)$ & 32.6 & 285 & & {$[1963 \mathrm{SOK} / \mathrm{ZHI}]$} \\
\hline & $\mathrm{V}$ & & $30.8 \pm 0.1$ & 300 & $\mathrm{C}$ & {$[1957 \mathrm{PEN} / \mathrm{KOB}]$} \\
\hline & $\mathrm{V}$ & & $29.8 \pm 0.1$ & 318 & $\mathrm{C}$ & {$[1957 \mathrm{PEN} / \mathrm{KOB}]$} \\
\hline & $\mathrm{V}$ & & $29.1 \pm 0.1$ & 329 & $\mathrm{C}$ & {$[1957 \mathrm{PEN} / \mathrm{KOB}]$} \\
\hline & $\mathrm{V}$ & & $28.5 \pm 0.1$ & 338 & $\mathrm{C}$ & [1957PEN/KOB] \\
\hline & $\mathrm{V}$ & & $28.1 \pm 0.1$ & 345 & $\mathrm{C}$ & [1957PEN/KOB] \\
\hline & V & $(310-329)$ & 31.1 & 319 & & [1957BRO/SMI] \\
\hline & $\mathrm{V}$ & (304-329) & 31.3 & 316 & & [1952BAC/SIM] \\
\hline & $\mathrm{V}$ & $(204-339)$ & 35 & 253 & MG & [1926FEL/DUR] \\
\hline & V & (204-339) & 32.1 & 293 & MG & [1926FEL/DUR] \\
\hline & V & $(204-339)$ & 30.7 & 313 & MG & [1926FEL/DUR] \\
\hline \multirow[t]{6}{*}{$\mathrm{C}_{3} \mathrm{H}_{6} \mathrm{O}$} & [107-18-6] & allyl alcohol & & & & \\
\hline & $\mathrm{V}$ & $(310-340)$ & 44.6 & 325 & & [2002LUB/BAN] \\
\hline & $\mathrm{V}$ & $(301-375)$ & 45.1 & 315 & & [1983MAR/SHV] \\
\hline & $\mathrm{V}$ & $(253-370)$ & 46.7 & 268 & $\mathrm{~A}$ & [1987STE/MAL] \\
\hline & $\mathrm{V}$ & & NA & & & [1936EWE] \\
\hline & $\mathrm{V}$ & $(283-313)$ & 44.8 & 298 & & [1935BAU/BUR] \\
\hline \multirow[t]{2}{*}{$\mathrm{C}_{3} \mathrm{H}_{6} \mathrm{O}$} & {$[107-25-5]$} & methyl vinyl ether & & & & \\
\hline & $\mathrm{V}$ & $(278-412)$ & 23.4 & 293 & A & [1987STE/MAL] \\
\hline \multirow[t]{2}{*}{$\mathrm{C}_{3} \mathrm{H}_{6} \mathrm{O}$} & [503-30-0] & trimethylene oxide & & & & \\
\hline & FUS & $(85-270)$ & 6.27 & 173.2 & $\mathrm{C}$ & {$[1985 \mathrm{HAN}]$} \\
\hline \multirow[t]{12}{*}{$\mathrm{C}_{3} \mathrm{H}_{6} \mathrm{O}$} & [123-38-6] & propanal & & & & \\
\hline & FUS & & 8.59 & 171.3 & & [1996DOM/HEA, 1977KOR/VAS] \\
\hline & $\mathrm{V}$ & $(263-373)$ & 31.5 & 278 & & [1977KIM/KIM] \\
\hline & V & $(286-321)$ & NA & 301 & & [1974AMB/SPR] \\
\hline & $\mathrm{V}$ & & 28.3 & 321 & & [1972COU/LEE] \\
\hline & $\mathrm{V}$ & & 29.4 & 303 & & [1972COU/LEE] \\
\hline & $\mathrm{V}$ & & 30.3 & 286 & & [1972COU/LEE] \\
\hline & $\mathrm{V}$ & & 29.6 & 298 & & [1972COU/LEE] \\
\hline & V & $(290-322)$ & 30.3 & 305 & A & [1987STE/MAL, 1970DYK] \\
\hline & $\mathrm{V}$ & & $29.7 \pm 0.4$ & 298 & EB & [1967BUC/COX, 2003VER/KRA2] \\
\hline & $\mathrm{V}$ & & 30.0 & 298 & EB & [1962TJE2, 2003VER/KRA2] \\
\hline & $\mathrm{V}$ & $(250-330)$ & 31.9 & 265 & EB & [1987STE/MAL, 1951SMI/BON] \\
\hline \multirow[t]{8}{*}{$\mathrm{C}_{3} \mathrm{H}_{6} \mathrm{O}$} & {$[75-56-9]$} & propylene oxide & & & & \\
\hline & FUS & $(11-330)$ & 6.53 & 161.3 & $\mathrm{AC}$ & [1996DOM/HEA, 1964OET] \\
\hline & $\mathrm{V}$ & $(225-308)$ & 31.6 & 240 & A & [1987STE/MAL, 1970DYK] \\
\hline & $\mathrm{V}$ & $(292-345)$ & 28.5 & 307 & & [1966BOT/ADL] \\
\hline & $\mathrm{V}$ & & 27.9 & 298 & $\mathrm{C}$ & [1962SIN/HIL] \\
\hline & V & $(249-308)$ & 30.1 & 264 & & [1959MCD/SHR] \\
\hline & $\mathrm{V}$ & $(285-322)$ & 28.2 & 303 & & [1937MOO/KAN] \\
\hline & $\mathrm{V}$ & $(243-306)$ & 32.9 & 273 & & {$[1935 \mathrm{KIR} / \mathrm{POP}]$} \\
\hline \multirow[t]{3}{*}{$\mathrm{C}_{3} \mathrm{H}_{6} \mathrm{O}_{2}$} & {$[646-06-0]$} & 1,3-dioxolane & & & & \\
\hline & TRS & & 2.68 & 142.4 & & \\
\hline & FUS & & 6.57 & 175.9 & & [1996DOM/HEA, 1969CLE/MEL2] \\
\hline
\end{tabular}


TABLE 6. Phase change enthalpies of $\mathrm{C}_{3}$ organic compounds-Continued

\begin{tabular}{|c|c|c|c|c|c|c|}
\hline \multirow[b]{2}{*}{$\begin{array}{l}\text { Molecular } \\
\text { formula }\end{array}$} & \multirow{2}{*}{$\begin{array}{c}\text { CAS Reg. No. } \\
\text { Transition }\end{array}$} & \multicolumn{5}{|l|}{ Compound } \\
\hline & & $\begin{array}{c}\text { Temp. range } \\
\text { (K) }\end{array}$ & $\begin{array}{c}\Delta_{\text {trans }} H_{\mathrm{m}} \\
\left(\mathrm{kJ} \mathrm{mol}^{-1}\right)\end{array}$ & $\begin{array}{l}T_{\mathrm{m}} \\
(\mathrm{K})\end{array}$ & Method & References \\
\hline & $\mathrm{V}$ & $(305-347)$ & 34.6 & 326 & & [1989WU/SAN] \\
\hline & $\mathrm{V}$ & $(280-323)$ & 35.8 & 295 & A & [1987STE/MAL] \\
\hline & $\mathrm{V}$ & $(321-357)$ & 33.7 & 339 & & [1982CAS/FRA] \\
\hline & $\mathrm{V}$ & $(306-346)$ & 33.7 & 326 & & [1980FRA/CAS] \\
\hline & V & $(280-355)$ & 34.1 & 296 & & [1968CHE/TUR, 1984BOU/FRI] \\
\hline & V & & $35.6 \pm 0.4$ & & & [1959FLE/MOR] \\
\hline \multirow{11}{*}{$\mathrm{C}_{3} \mathrm{H}_{6} \mathrm{O}_{2}$} & [109-94-4] & ethyl formate & & & & \\
\hline & $\mathrm{V}$ & $(300-326)$ & 31.4 & 313 & $\mathrm{~EB}$ & [1993FAR/WIC] \\
\hline & V & $(300-326)$ & $32.1 \pm 0.1$ & 298 & EB & [1993FAR/WIC, 2012SAM/NAZ] \\
\hline & V & $(327-498)$ & 29.9 & 342 & A & [1987STE/MAL] \\
\hline & V & & $31.6 \pm 0.1$ & 304 & $\mathrm{C}$ & [1976CIH/HYN] \\
\hline & $\mathrm{V}$ & & $30.9 \pm 0.1$ & 313 & $\mathrm{C}$ & [1976CIH/HYN] \\
\hline & V & & $29.8 \pm 0.1$ & 328 & $\mathrm{C}$ & [1976CIH/HYN] \\
\hline & V & & 32.2 & 298 & EB & [1974HIN/KLU] \\
\hline & $\mathrm{V}$ & $(213-336)$ & 35.8 & 228 & A & [1987STE/MAL, 1970DYK] \\
\hline & $\mathrm{V}$ & & $30.1 \pm 0.2$ & 326 & $\mathrm{C}$ & [1926MAT] \\
\hline & V & & $31.5 \pm 0.2$ & 298 & $\mathrm{C}$ & [1926MAT, 2012SAM/NAZ] \\
\hline \multirow{17}{*}{$\mathrm{C}_{3} \mathrm{H}_{6} \mathrm{O}_{2}$} & {$[79-20-9]$} & methyl acetate & & & & \\
\hline & FUS & $(13-290)$ & 7.49 & 174.9 & $\mathrm{AC}$ & [1992OKA/OGU] \\
\hline & V & $(296-353)$ & 32.6 & 312 & & [1997MON/BUR] \\
\hline & V & $(260-351)$ & 34.1 & 275 & A & [1987STE/MAL] \\
\hline & V & & $31.7 \pm 0.7$ & 298 & $\mathrm{C}$ & [1981GAT/STR] \\
\hline & $\mathrm{V}$ & & $32.3 \pm 0.1$ & 298 & $\mathrm{C}$ & [1980SVO/UCH] \\
\hline & $\mathrm{V}$ & & $29.5 \pm 0.1$ & 343 & $\mathrm{C}$ & [1980SVO/UCH] \\
\hline & V & $(308-338)$ & 31.8 & 323 & DTA & [1980MEY/AWE] \\
\hline & V & & $32.6 \pm 0.1$ & 298 & $\mathrm{C}$ & [1979SUN/SVE2] \\
\hline & V & & $32.2 \pm 0.1$ & 304 & $\mathrm{C}$ & [1977SVO/VES] \\
\hline & $\mathrm{V}$ & & $31.6 \pm 0.1$ & 313 & $\mathrm{C}$ & [1977SVO/VES] \\
\hline & V & & $30.5 \pm 0.1$ & 328 & $\mathrm{C}$ & [1977SVO/VES] \\
\hline & V & & $30.3 \pm 0.1$ & 331 & $\mathrm{C}$ & [1977SVO/VES] \\
\hline & $\mathrm{V}$ & & 32.5 & 295 & & [1976CON/COU] \\
\hline & V & & 30.2 & 330 & & [1976CON/COU] \\
\hline & $\mathrm{V}$ & $(273-318)$ & 34.5 & 296 & BG & [1971HAL/BAL] \\
\hline & V & $(274-329)$ & 33.4 & 289 & A & [1987STE/MAL, 1965MER/POL, 1970DYK] \\
\hline \multirow[t]{15}{*}{$\mathrm{C}_{3} \mathrm{H}_{6} \mathrm{O}_{2}$} & {$[79-09-4]$} & propionic acid & & & & \\
\hline & FUS & & 11.25 & 252.5 & DSC & [2010HEN/CAM] \\
\hline & FUS & $(15-353)$ & 10.66 & 252.7 & $\mathrm{AC}$ & [1996DOM/HEA, 1982MAR/AND] \\
\hline & FUS & & $\mathrm{U} 7.24$ & 253.4 & & [1909MAS/FAU] \\
\hline & SUB & $(225-238)$ & $74.1 \pm 1$ & 233 & $\mathrm{TE}$ & [1978CAL/CAL] \\
\hline & SUB & & $73.2 \pm 1$ & 233 & $\mathrm{ME}$ & [1978CAL/CAL] \\
\hline & V & $(303-378)$ & 54.4 & 298 & CGC & [2000VER] \\
\hline & $\mathrm{V}$ & $(353-393)$ & 54.9 & 298 & $\mathrm{CGC}$ & {$[1995 \mathrm{CHI} / \mathrm{HOS}]$} \\
\hline & $\mathrm{V}$ & $(343-419)$ & 47.0 & 358 & A & [1987STE/MAL] \\
\hline & V & $(414-511)$ & 60.6 & 429 & A & [1987STE/MAL] \\
\hline & V & $(345-401)$ & 46.4 & 360 & A & [1987STE/MAL] \\
\hline & V & & 56.0 & 303 & & [1983TAM/DRA] \\
\hline & $\mathrm{V}$ (monomer) & & $31.1 \pm 0.1$ & 298 & $\mathrm{C}$ & [1970KON/WAD] \\
\hline & $\mathrm{V}$ & & $55 \pm 2$ & 298 & $\mathrm{C}$ & [1970KON/WAD] \\
\hline & $\mathrm{V}$ & $(328-437)$ & 48.3 & 343 & & [1981AMB/ELL] \\
\hline \multirow[t]{3}{*}{$\left(\mathrm{C}_{3} \mathrm{H}_{6} \mathrm{O}_{2}\right)_{2}$} & [32574-16-6] & propionic acid dimer & & & & \\
\hline & SUB & $(225-238)$ & $81.3 \pm 1$ & 233 & TE & [1978CAL/CAL] \\
\hline & SUB & & $79.4 \pm 1.0$ & 233 & $\mathrm{ME}$ & [1978CAL/CAL] \\
\hline \multirow[t]{2}{*}{$\mathrm{C}_{3} \mathrm{H}_{6} \mathrm{O}_{2}$} & [116-09-6] & hydroxyacetone & & & & \\
\hline & $\mathrm{V}$ & $(296-356)$ & $42 \pm 3$ & 326 & & [2010PET/REY] \\
\hline \multirow[t]{2}{*}{$\mathrm{C}_{3} \mathrm{H}_{6} \mathrm{O}_{2} \mathrm{~S}$} & [107-96-0] & $\beta$-thiolactic acid & & & & \\
\hline & FUS & & 16.97 & 291.9 & & [1996DOM/HEA, 1935HUF/ELL] \\
\hline $\mathrm{C}_{3} \mathrm{H}_{6} \mathrm{O}_{3}$ & {$[625-45-6]$} & 2-methoxyacetic acid & & & & \\
\hline
\end{tabular}


TABLE 6. Phase change enthalpies of $\mathrm{C}_{3}$ organic compounds-Continued

\begin{tabular}{|c|c|c|c|c|c|c|}
\hline \multirow[b]{2}{*}{$\begin{array}{l}\text { Molecular } \\
\text { formula }\end{array}$} & \multirow{2}{*}{$\begin{array}{c}\text { CAS Reg. No. } \\
\text { Transition }\end{array}$} & \multicolumn{5}{|l|}{ Compound } \\
\hline & & $\begin{array}{c}\text { Temp. range } \\
\text { (K) }\end{array}$ & $\begin{array}{c}\Delta_{\text {trans }} H_{\mathrm{m}} \\
\left(\mathrm{kJ} \mathrm{mol}^{-1}\right)\end{array}$ & $\begin{array}{l}T_{\mathrm{m}} \\
(\mathrm{K})\end{array}$ & Method & References \\
\hline & V & $(325-477)$ & 54.5 & 340 & A & [1987STE/MAL, 1947STU] \\
\hline \multirow[t]{6}{*}{$\mathrm{C}_{3} \mathrm{H}_{6} \mathrm{O}_{3}$} & {$[96-35-5]$} & methyl glycolate & & & & \\
\hline & FUS & & 11.4 & 272.8 & DSC & [2000JAR/MAR] \\
\hline & $\mathrm{V}$ & $(326-381)$ & $52.5 \pm 6.3$ & 298 & EB & [1996STE/CHI2] \\
\hline & $\mathrm{V}$ & $(326-381)$ & $50.2 \pm 5.2$ & 320 & EB & [1996STE/CHI2] \\
\hline & $\mathrm{V}$ & $(326-381)$ & $47.0 \pm 4.2$ & 360 & EB & [1996STE/CHI2] \\
\hline & $\mathrm{V}$ & $(282-425)$ & 47.4 & 297 & A & [1987STE/MAL, 1947KET/VAN] \\
\hline \multirow[t]{9}{*}{$\mathrm{C}_{3} \mathrm{H}_{6} \mathrm{O}_{3}$} & [616-38-6] & dimethylcarbonate & & & & \\
\hline & TRS & & Not reported & 220.1 & & \\
\hline & FUS & & 11.58 & 278.2 & DSC & [2004DIN] \\
\hline & $\mathrm{V}$ & $(274-304)$ & $38.0 \pm 0.2$ & 298 & GS & [2008KOZ/EME] \\
\hline & $\mathrm{V}$ & $(326-411)$ & 36.4 & 341 & & [2002ROD/CAN] \\
\hline & $\mathrm{V}$ & $(311-397)$ & $37.7 \pm 0.2$ & 298 & EB & [1997STE/CHI4, 1997STE/CHI2] \\
\hline & $\mathrm{V}$ & $(311-397)$ & $36.4 \pm 0.2$ & 320 & EB & [1997STE/CHI4, 1997STE/CHI2] \\
\hline & $\mathrm{V}$ & $(311-397)$ & $33.8 \pm 0.3$ & 360 & EB & [1997STE/CHI4, 1997STE/CHI2] \\
\hline & $\mathrm{V}$ & $(311-397)$ & $30.9 \pm 0.5$ & 400 & EB & [1997STE/CHI4, 1997STE/CHI2] \\
\hline \multirow[t]{2}{*}{$\mathrm{C}_{3} \mathrm{H}_{6} \mathrm{O}_{3}$} & {$[4212-43-5]$} & peroxypropionic acid & & & & \\
\hline & $\mathrm{V}$ & $(273-393)$ & 43.2 & 288 & A & [1987STE/MAL, 1951EGE/EMT, 1970DYK] \\
\hline \multirow{2}{*}{$\mathrm{C}_{3} \mathrm{H}_{6} \mathrm{O}_{3}$} & [38787-96-1] & propylene ozonide & & & & \\
\hline & $\mathrm{V}$ & $(261-296)$ & 36.9 & 281 & A & [1987STE/MAL, 1956GAR/SCH] \\
\hline \multirow[t]{7}{*}{$\mathrm{C}_{3} \mathrm{H}_{6} \mathrm{O}_{3}$} & [110-88-3] & 1,3,5-trioxane & & & & \\
\hline & FUS & $(7-346)$ & 15.1 & 333.4 & $\mathrm{AC}$ & [1996DOM/HEA, 1991ACR, 1988VAN/VAN] \\
\hline & SUB & $(212-231)$ & 57.9 & 223 & TE, ME & [1983DEW/VAN] \\
\hline & SUB & & 55.6 & 298 & & [1983DEW/VAN] \\
\hline & SUB & & 56.5 & 298 & $\mathrm{C}$ & [1975BOG/BER] \\
\hline & SUB & & $56.2 \pm 0.2$ & 298 & $\mathrm{C}$ & [1969MAN/MOR, 1977PED/RYL] \\
\hline & $\mathrm{V}$ & $(329-386)$ & 40.0 & 344 & A & [1987STE/MAL, 1965SER/BYK] \\
\hline \multirow[t]{2}{*}{$\mathrm{C}_{3} \mathrm{H}_{6} \mathrm{O}_{3}$} & {$[50-21-5]$} & $(d l)$-lactic acid & & & & \\
\hline & FUS & & 11.34 & 289.9 & & [1996DOM/HEA, 1944YOS] \\
\hline \multirow[t]{2}{*}{$\mathrm{C}_{3} \mathrm{H}_{6} \mathrm{O}_{3}$} & {$[79-33-4]$} & L-(+)-lactic acid $(S-1$ & actic acid) & & & \\
\hline & $\mathrm{V}$ & & $69.1 \pm 1.0$ & 298 & GS & [2010EME/VER3] \\
\hline \multirow[t]{2}{*}{$\mathrm{C}_{3} \mathrm{H}_{6} \mathrm{~S}$} & {$[1072-43-1]$} & 2-methylthiirane & & & & \\
\hline & $\mathrm{V}$ & $(272-423)$ & 34.6 & 287 & A & [1987STE/MAL, 1970DYK, 1999DYK/SVO] \\
\hline \multirow[t]{6}{*}{$\mathrm{C}_{3} \mathrm{H}_{6} \mathrm{~S}$} & [287-27-4] & thiacyclobutane (thie & tane) & & & \\
\hline & TRS & & 0.67 & 176.7 & & \\
\hline & FUS & $(12-321)$ & 8.24 & 199.9 & & [1996DOM/HEA, 1953SCO/FIN] \\
\hline & $\mathrm{V}$ & $(275-393)$ & 36.5 & 290 & & [1999DYK/SVO] \\
\hline & $\mathrm{V}$ & & 35.8 & 298 & & [1971WIL/ZWO] \\
\hline & $\mathrm{V}$ & $(321-404)$ & 34.6 & 336 & $\mathrm{~A}, \mathrm{~EB}$ & [1987STE/MAL, 1953SCO/FIN, 1966OSB/DOU] \\
\hline \multirow[t]{5}{*}{$\mathrm{C}_{3} \mathrm{H}_{6} \mathrm{~S}_{3}$} & [291-21-4] & 1,3,5-trithiane & & & & \\
\hline & FUS & & 32.22 & 488.4 & DSC & [2002VAN/VAN2] \\
\hline & SUB & & $93.2 \pm 0.2$ & 298 & ME & [2001ROU/JIM] \\
\hline & SUB & $(320-339)$ & 91.5 & 331 & TE, ME & [1983DEW/VAN] \\
\hline & SUB & & 93.9 & 298 & & [1983DEW/VAN] \\
\hline \multirow[t]{10}{*}{$\mathrm{C}_{3} \mathrm{H}_{7} \mathrm{Br}$} & [106-94-5] & 1-bromopropane & & & & \\
\hline & $\mathrm{V}$ & $(301-344)$ & 31.8 & 316 & $\mathrm{~A}, \mathrm{~EB}$ & [1987STE/MAL, 1977SVO/MAJ] \\
\hline & $\mathrm{V}$ & & $31.1 \pm 0.1$ & 322 & $\mathrm{C}$ & [1977SVO/MAJ] \\
\hline & $\mathrm{V}$ & & $30.5 \pm 0.1$ & 332 & $\mathrm{C}$ & [1977SVO/MAJ] \\
\hline & $\mathrm{V}$ & & $30.1 \pm 0.1$ & 339 & $\mathrm{C}$ & [1977SVO/MAJ] \\
\hline & $\mathrm{V}$ & & $29.3 \pm 0.1$ & 352 & $\mathrm{C}$ & [1977SVO/MAJ] \\
\hline & $\mathrm{V}$ & & $31.9 \pm 0.1$ & 298 & $\mathrm{C}$ & [1966WAD] \\
\hline & $\mathrm{V}$ & $(250-368)$ & 34.1 & 265 & $\mathrm{~A}, \mathrm{E}$ & [1987STE/MAL, 1961LI/ROS, 1970DYK] \\
\hline & $\mathrm{V}$ & $(220-344)$ & 35.5 & 235 & & [1947STU] \\
\hline & $\mathrm{V}$ & $(273-303)$ & 32.6 & 288 & & [1906REX, 1984BOU/FRI] \\
\hline
\end{tabular}


Table 6. Phase change enthalpies of $\mathrm{C}_{3}$ organic compounds-Continued

\begin{tabular}{|c|c|c|c|c|c|c|}
\hline \multirow[b]{2}{*}{$\begin{array}{l}\text { Molecular } \\
\text { formula }\end{array}$} & \multirow{2}{*}{$\begin{array}{c}\text { CAS Reg. No. } \\
\text { Transition }\end{array}$} & \multicolumn{5}{|l|}{ Compound } \\
\hline & & $\begin{array}{c}\text { Temp. range } \\
(\mathrm{K})\end{array}$ & $\begin{array}{c}\Delta_{\text {trans }} H_{\mathrm{m}} \\
\left(\mathrm{kJ} \mathrm{mol}^{-1}\right)\end{array}$ & $\begin{array}{l}T_{\mathrm{m}} \\
(\mathrm{K})\end{array}$ & Method & References \\
\hline \multirow[t]{13}{*}{$\mathrm{C}_{3} \mathrm{H}_{7} \mathrm{Br}$} & {$[75-26-3]$} & 2-bromopropane & & & & \\
\hline & FUS & & 6.55 & 184.1 & & [1996DOM/HEA, 1950KUS/CRO] \\
\hline & $\mathrm{V}$ & $(323-363)$ & 30.6 & 298 & CGC & [1995CHI/HOS] \\
\hline & $\mathrm{V}$ & $(236-328)$ & 32.1 & 251 & A & [1987STE/MAL] \\
\hline & $\mathrm{V}$ & $(299-332)$ & 30.1 & 314 & EB & [1987STE/MAL, 1977SVO/MAJ] \\
\hline & $\mathrm{V}$ & & $29.8 \pm 0.1$ & 305 & $\mathrm{C}$ & [1977SVO/MAJ] \\
\hline & $\mathrm{V}$ & & $29.2 \pm 0.1$ & 318 & $\mathrm{C}$ & [1977SVO/MAJ] \\
\hline & $\mathrm{V}$ & & $28.5 \pm 0.1$ & 330 & $\mathrm{C}$ & [1977SVO/MAJ] \\
\hline & $\mathrm{V}$ & & $28.0 \pm 0.1$ & 338 & $\mathrm{C}$ & [1977SVO/MAJ] \\
\hline & $\mathrm{V}$ & $(297-333)$ & 30.4 & 315 & & [1974VAN/HOU] \\
\hline & $\mathrm{V}$ & & $30.2 \pm 0.1$ & 298 & $\mathrm{C}$ & [1966WAD] \\
\hline & $\mathrm{V}$ & $(211-333)$ & 33.4 & 226 & & [1947STU] \\
\hline & $\mathrm{V}$ & $(273-303)$ & 30.9 & 288 & & [1906REX, 1984BOU/FRI] \\
\hline \multirow[t]{5}{*}{$\mathrm{C}_{3} \mathrm{H}_{7} \mathrm{Cl}$} & {$[540-54-5]$} & 1-chloropropane & & & & \\
\hline & $\mathrm{V}$ & $(250-320)$ & 29.0 & 298 & & [1984BOU/FRI, 1991BAS/SVO] \\
\hline & $\mathrm{V}$ & & $28.5 \pm 0.2$ & 298 & $\mathrm{C}$ & [1977MAN/SEL] \\
\hline & $\mathrm{V}$ & $(248-320)$ & 31.0 & 263 & A & [1987STE/MAL, 1969KEM/KRE, 1970DYK] \\
\hline & $\mathrm{V}$ & $(205-319)$ & 33.1 & 219 & & [1947STU] \\
\hline \multirow[t]{7}{*}{$\mathrm{C}_{3} \mathrm{H}_{7} \mathrm{Cl}$} & {$[75-29-6]$} & 2-chloropropane & & & & \\
\hline & FUS & & 7.39 & 155.8 & DTA & [1994TAN/SAB3] \\
\hline & FUS & & 7.39 & 156 & & [1991ACR, 1983WEA] \\
\hline & $\mathrm{V}$ & $(239-310)$ & 30.2 & 254 & A & [1987STE/MAL] \\
\hline & $\mathrm{V}$ & $(271-312)$ & 27.6 & 298 & & [1960DYK/PAU] \\
\hline & V & (194-309) & 30.6 & 209 & & [1947STU] \\
\hline & $\mathrm{V}$ & $(273-303)$ & 27.3 & 288 & & [1906REX, 1984BOU/FRI] \\
\hline \multirow[t]{3}{*}{$\mathrm{C}_{3} \mathrm{H}_{7} \mathrm{ClO}$} & {$[127-00-4]$} & 1-chloro-2-propanol & & & & \\
\hline & $\mathrm{V}$ & $(308-399)$ & $45.0 \pm 2.2$ & 340 & EB & [2002STE/CHI] \\
\hline & V & $(308-399)$ & $42.2 \pm 1.9$ & 380 & EB & [2002STE/CHI] \\
\hline \multirow[t]{2}{*}{$\mathrm{C}_{3} \mathrm{H}_{7} \mathrm{ClO}$} & {$[78-89-7]$} & 2-chloro-1-propanol & & & & \\
\hline & $\mathrm{V}$ & $(316-399)$ & 45.0 & 331 & A & [1987STE/MAL] \\
\hline \multirow[t]{2}{*}{$\mathrm{C}_{3} \mathrm{H}_{7} \mathrm{ClO}_{2}$} & {$[96-24-2]$} & 3-chloro-1,2-propane & diol & & & \\
\hline & $\mathrm{V}$ & $(343-409)$ & 66.6 & 358 & & [1996GIL/WIL] \\
\hline \multirow[t]{4}{*}{$\mathrm{C}_{3} \mathrm{H}_{7} \mathrm{ClO}_{2} \mathrm{~S}$} & [10147-36-1] & 1-propanesulfonyl chl & oride & & & \\
\hline & $\mathrm{V}$ & $(273-362)$ & 52.3 & 288 & & [1999DYK/SVO] \\
\hline & V & $(362-464)$ & 49.9 & 377 & & [1999DYK/SVO] \\
\hline & $\mathrm{V}$ & $(243-273)$ & 60.1 & 258 & A & [1987STE/MAL, 1999DYK/SVO, 1963QUI/NOW] \\
\hline \multirow[t]{2}{*}{$\mathrm{C}_{3} \mathrm{H}_{7} \mathrm{ClS}$} & {$[542-81-4]$} & methyl(2-chloroethyl) & sulfide & & & \\
\hline & $\mathrm{V}$ & $(293-333)$ & 42.4 & 308 & A, GS & [1987STE/MAL, 1948RED/CHA, 1970DYK] \\
\hline \multirow[t]{2}{*}{$\mathrm{C}_{3} \mathrm{H}_{7} \mathrm{~F}$} & [460-13-9] & 1-fluoropropane & & & & \\
\hline & V & $(196-289)$ & 24.0 & 274 & $\mathrm{~A}, \mathrm{E}$ & [1987STE/MAL, 1961LI/ROS, 1970DYK] \\
\hline \multirow[t]{2}{*}{$\mathrm{C}_{3} \mathrm{H}_{7} \mathrm{~F}$} & [420-26-8] & 2-fluoropropane & & & & \\
\hline & $\mathrm{V}$ & $(190-264)$ & 23.7 & 249 & A & [1987STE/MAL] \\
\hline \multirow[t]{5}{*}{$\mathrm{C}_{3} \mathrm{H}_{7} \mathrm{I}$} & {$[107-08-4]$} & 1-iodopropane & & & & \\
\hline & $\mathrm{V}$ & $(171-271)$ & 37.8 & 256 & A & [1987STE/MAL] \\
\hline & $\mathrm{V}$ & & $36.3 \pm 0.1$ & 298 & $\mathrm{C}$ & [1968WAD] \\
\hline & $\mathrm{V}$ & $(271-402)$ & 36.8 & 286 & $\mathrm{~A}, \mathrm{E}$ & [1987STE/MAL, 1961LI/ROS, 1970DYK] \\
\hline & $\mathrm{V}$ & $(237-375)$ & 37.0 & 252 & & [1947STU] \\
\hline \multirow[t]{5}{*}{$\mathrm{C}_{3} \mathrm{H}_{7} \mathrm{I}$} & {$[75-30-9]$} & 2-iodopropane & & & & \\
\hline & $\mathrm{V}$ & $(313-353)$ & 34.0 & 298 & CGC & {$[1995 \mathrm{CHI} / \mathrm{HOS}]$} \\
\hline & $\mathrm{V}$ & $(173-262)$ & 36.7 & 247 & A & [1987STE/MAL] \\
\hline & $\mathrm{V}$ & & $34.1 \pm 0.1$ & 298 & $\mathrm{C}$ & [1968WAD] \\
\hline & $\mathrm{V}$ & $(230-363)$ & 36.3 & 244 & & [1947STU] \\
\hline \multirow[t]{3}{*}{$\mathrm{C}_{3} \mathrm{H}_{7} \mathrm{~N}$} & [765-30-0] & cyclopropylamine & & & & \\
\hline & FUS & $(13-319)$ & 13.18 & 237.8 & $\mathrm{AC}$ & [1991ACR, 1981FIN/MES] \\
\hline & V & & $31.3 \pm 0.4$ & 298 & $\mathrm{~EB}$ & [1971GOO/MOO] \\
\hline
\end{tabular}


TABLE 6. Phase change enthalpies of $\mathrm{C}_{3}$ organic compounds-Continued

\begin{tabular}{|c|c|c|c|c|c|c|}
\hline \multirow[b]{2}{*}{$\begin{array}{l}\text { Molecular } \\
\text { formula }\end{array}$} & \multirow{2}{*}{$\begin{array}{c}\text { CAS Reg. No. } \\
\text { Transition }\end{array}$} & \multicolumn{5}{|l|}{ Compound } \\
\hline & & $\begin{array}{c}\text { Temp. range } \\
\text { (K) }\end{array}$ & $\begin{array}{c}\Delta_{\text {trans }} H_{\mathrm{m}} \\
\left(\mathrm{kJ} \mathrm{mol}^{-1}\right)\end{array}$ & $\begin{array}{l}T_{\mathrm{m}} \\
(\mathrm{K})\end{array}$ & Method & References \\
\hline \multirow[t]{2}{*}{$\mathrm{C}_{3} \mathrm{D}_{7} \mathrm{~N}$} & {$[153557-96-1]$} & \multicolumn{5}{|c|}{ perdeuterocyclopropylamine } \\
\hline & $\mathrm{V}$ & $(283-336)$ & 32.0 & 298 & & [1993WOL/KIM] \\
\hline \multirow[t]{3}{*}{$\mathrm{C}_{3} \mathrm{H}_{7} \mathrm{~N}$} & [107-11-9] & allylamine & & & & \\
\hline & $\mathrm{V}$ & $(273-303)$ & 33.0 & 288 & A & [1987STE/MAL] \\
\hline & $\mathrm{V}$ & $(273-324)$ & 32.6 & 288 & A & [1987STE/MAL] \\
\hline \multirow[t]{2}{*}{$\mathrm{C}_{3} \mathrm{H}_{7} \mathrm{~N}$} & [503-29-7] & azetidine & & & & \\
\hline & $\mathrm{V}$ & $(273-303)$ & 34.6 & 288 & A & [1987STE/MAL, 1968CAB/CON] \\
\hline \multirow[t]{3}{*}{$\mathrm{C}_{3} \mathrm{H}_{7} \mathrm{NO}$} & [127-06-0] & acetone oxime & & & & \\
\hline & SUB & $(313-333)$ & 59.6 & 323 & $\mathrm{I}$ & [1987STE/MAL, 1975MES/BAE] \\
\hline & $\mathrm{V}$ & $(338-352)$ & 51.4 & 345 & A & [1987STE/MAL] \\
\hline \multirow[t]{9}{*}{$\mathrm{C}_{3} \mathrm{H}_{7} \mathrm{NO}$} & {$[79-05-0]$} & propionamide & & & & \\
\hline & FUS & & 12.9 & 352.6 & DSC & [2008ABA/BAD, 2000BRU/DEL] \\
\hline & SUB & $(283-343)$ & $75 \pm 4.0$ & 298 & TE & [2000BRU/DEL] \\
\hline & SUB & & $79.2 \pm 0.3$ & & & [1975BAR/PIL, 1977PED/RYL] \\
\hline & SUB & & 73.3 & & & [1960THO] \\
\hline & SUB & $(318-346)$ & $79.1 \pm 0.4$ & & GS & [1959DAV/JON2] \\
\hline & $\mathrm{V}$ & & $68.6 \pm 2.3$ & 298 & $\mathrm{CGC}$ & [2013GUT/RAT] \\
\hline & $\mathrm{V}$ & $(375-476)$ & 63.9 & 390 & EB & [2004HOR/FIS] \\
\hline & $\mathrm{V}$ & $(338-486)$ & 60.3 & 353 & & {$[1947 \mathrm{STU}]$} \\
\hline \multirow[t]{12}{*}{$\mathrm{C}_{3} \mathrm{H}_{7} \mathrm{NO}$} & {$[68-12-2]$} & $N, N$-dimethylf & mide & & & \\
\hline & FUS & & 8.95 & 212.9 & $\mathrm{AC}$ & [2007SMI/TSV] \\
\hline & FUS & & 8.95 & 212.9 & & [1996DOM/HEA, 1978KAR/RAB] \\
\hline & V & $(463-513)$ & $46.7 \pm 0.5$ & 298 & CGC & [2009PAN/ANT] \\
\hline & $\mathrm{V}$ & $(346-425)$ & 43.1 & 361 & & [2005MUN/MON] \\
\hline & $\mathrm{V}$ & $(377-426)$ & 41.8 & 392 & & [1997BLA/BEL] \\
\hline & $\mathrm{V}$ & $(338-425)$ & 43.6 & 353 & & [1995MAR/GAB] \\
\hline & V & $(301-426)$ & 49.2 & 316 & A & [1987STE/MAL] \\
\hline & V & & 46.9 & 298 & A & [1985BAR/CAS, 1985MAJ/SVO] \\
\hline & V & $(318-423)$ & 42.5 & 370 & & [1979BLU/BAE] \\
\hline & $\mathrm{V}$ & $(331-425)$ & 56.7 & 346 & & [1974MYA/SCH, 1984BOU/FRI] \\
\hline & $\mathrm{V}$ & $(303-363)$ & 46.7 & 318 & & [1968GOP/RIZ] \\
\hline \multirow[t]{2}{*}{$\mathrm{C}_{3} \mathrm{H}_{7} \mathrm{NO}$} & [627-45-2] & $N$-ethylformam & & & & \\
\hline & $\mathrm{V}$ & & 58.4 & 298 & A & [1985BAR/CAS, 1985MAJ/SVO] \\
\hline \multirow[t]{7}{*}{$\mathrm{C}_{3} \mathrm{H}_{7} \mathrm{NO}$} & {$[79-16-3]$} & $N$-methylacetar & & & & \\
\hline & FUS & & 10.11 & 303.7 & & [1999AHL/LOH] \\
\hline & FUS & & 9.73 & 303.8 & $\mathrm{C}$ & [1969KRE/WOO] \\
\hline & FUS & & 8.37 & 303.8 & $\mathrm{C}$ & [1964BON/JOR] \\
\hline & SUB & $(266-278)$ & $70.8 \pm 2.0$ & 298 & ME & [1996ROU/JIM2] \\
\hline & SUB & & $69.9 \pm 0.3$ & 298 & $\mathrm{C}$ & [1984STA/WAD] \\
\hline & SUB & $(288-303)$ & 54.0 & & & [1952AIH, 1960JON] \\
\hline
\end{tabular}

[Note: The value of $54.0 \mathrm{~kJ} / \mathrm{mole}$ disagrees with the value of $70.8 \mathrm{~kJ} / \mathrm{mole}$ given in [1996ROU/JIM2]. Given the disagreement and closeness of the value to other reported $\mathrm{V}$ values we believe that the value might be an enthalpy of vaporization, rather than enthalpy of sublimation.]

$\begin{array}{llllll}\mathrm{V} & (363-414) & 55.5 & 378 & & \\ \mathrm{~V} & (353-428) & 62.0 & 368 & & {[1995 \mathrm{SCH} / \mathrm{PUS}]} \\ \mathrm{V} & (333-443) & 59.6 & 348 & \mathrm{~A} & {[1993 \mathrm{AUC} / \mathrm{MON}]} \\ \mathrm{V} & (353-479) & 53.5 & 368 & \mathrm{~A} & {[1987 \mathrm{STE} / \mathrm{MAL}, 1975 \mathrm{MAL}]} \\ \mathrm{V} & (303-363) & 69.0 & & & {[1968 \mathrm{GOP} / \mathrm{RIZ}]}\end{array}$

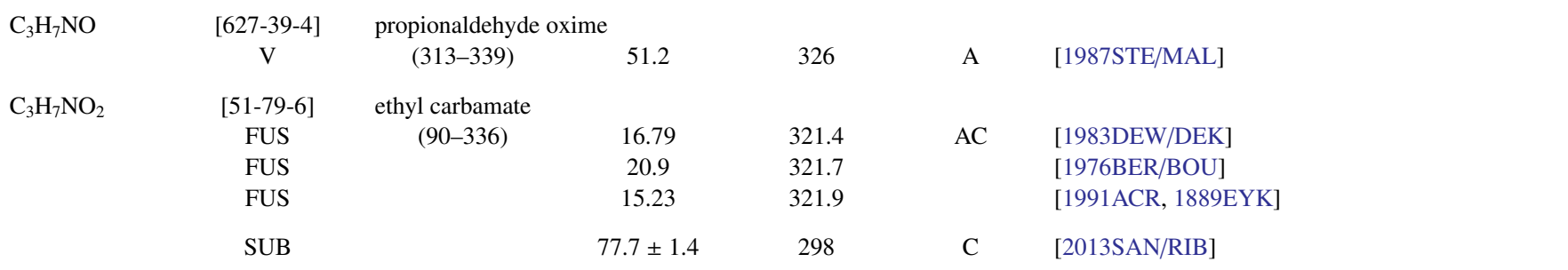


TABLE 6. Phase change enthalpies of $\mathrm{C}_{3}$ organic compounds-Continued

\begin{tabular}{|c|c|c|c|c|c|c|}
\hline \multirow[b]{2}{*}{$\begin{array}{l}\text { Molecular } \\
\text { formula }\end{array}$} & \multirow{2}{*}{$\begin{array}{c}\text { CAS Reg. No. } \\
\text { Transition }\end{array}$} & \multicolumn{5}{|l|}{ Compound } \\
\hline & & $\begin{array}{l}\text { Temp. range } \\
\text { (K) }\end{array}$ & $\begin{array}{c}\Delta_{\text {trans }} H_{\mathrm{m}} \\
\left(\mathrm{kJ} \mathrm{mol}^{-1}\right)\end{array}$ & $\begin{array}{l}T_{\mathrm{m}} \\
(\mathrm{K})\end{array}$ & Method & References \\
\hline & SUB & $(256-273)$ & 77.7 & 265 & $\mathrm{TE}, \mathrm{ME}$ & [1983DEW/VAN] \\
\hline & SUB & & 76.3 & 298 & & [1983DEW/VAN] \\
\hline & SUB & & 71.9 & 322 & & [1976BAR/BOU] \\
\hline & SUB & $(292-307)$ & $89.1 \pm 0.8$ & 299 & GS & [1959DAV/JON] \\
\hline & $\mathrm{V}$ & $(323-373)$ & $\mathrm{U} 25.8$ & 338 & & [2004AHM/GIE] \\
\hline & $\mathrm{V}$ & $(338-457)$ & 56.6 & 353 & A & [1987STE/MAL, 1947STU] \\
\hline \multirow{2}{*}{$\mathrm{C}_{3} \mathrm{H}_{7} \mathrm{NO}_{2}$} & [541-42-4] & isopropyl nitrite & & & & \\
\hline & $\mathrm{V}$ & $(253-268)$ & 26.0 & 260 & A & [1987STE/MAL, 1937THO/DAI] \\
\hline \multirow[t]{5}{*}{$\mathrm{C}_{3} \mathrm{H}_{7} \mathrm{NO}_{2}$} & [108-03-2] & 1-nitropropane & & & & \\
\hline & $\mathrm{V}$ & $(313-353)$ & 43.9 & 298 & CGC & [1995CHI/HOS] \\
\hline & $\mathrm{V}$ & $(293-405)$ & 42.6 & 308 & A, EB & [1987STE/MAL, 1956TOO, 1970DYK] \\
\hline & $\mathrm{V}$ & $(331-404)$ & 40.6 & 346 & & [1949DRE/SHR, 1949DRE/MAR] \\
\hline & $\mathrm{V}$ & $(283-403)$ & $43.4 \pm 0.4$ & 298 & $\mathrm{ZG}$ & [1949HOL/DOR] \\
\hline \multirow[t]{4}{*}{$\mathrm{C}_{3} \mathrm{H}_{7} \mathrm{NO}_{2}$} & {$[79-46-9]$} & 2-nitropropane & & & & \\
\hline & $\mathrm{V}$ & $(313-353)$ & 43.9 & 298 & $\mathrm{CGC}$ & {$[1995 \mathrm{CHI} / \mathrm{HOS}]$} \\
\hline & $\mathrm{V}$ & $(284-394)$ & 40.9 & 299 & $\mathrm{~A}, \mathrm{~EB}$ & [1987STE/MAL, 1947STU, 1956TOO, 1970DYK] \\
\hline & $\mathrm{V}$ & $(283-383)$ & $41.3 \pm 0.4$ & 298 & ZG & [1949HOL/DOR] \\
\hline \multirow[t]{2}{*}{$\mathrm{C}_{3} \mathrm{H}_{7} \mathrm{NO}_{2}$} & [543-67-9] & propyl nitrite & & & & \\
\hline & $\mathrm{V}$ & $(253-268)$ & 28.3 & 260 & A & [1987STE/MAL, 1937THO/DAI] \\
\hline \multirow[t]{4}{*}{$\mathrm{C}_{3} \mathrm{H}_{7} \mathrm{NO}_{2}$} & [56-41-7] & $\mathrm{L}-(d)$-alanine & & & & \\
\hline & SUB & & $132.8 \pm 1.0$ & 414 & TE, ME & [1979DEK/VOO] \\
\hline & SUB & $(413-450)$ & $132.4 \pm 1.3$ & 433 & $\mathrm{C}$ & [1977NGA/SAB] \\
\hline & SUB & & $144.8 \pm 4.2$ & 298 & $\mathrm{C}$ & {$[1977 N G A / S A B]$} \\
\hline \multirow[t]{4}{*}{$\mathrm{C}_{3} \mathrm{H}_{7} \mathrm{NO}_{2}$} & [338-69-2] & $\mathrm{D}-(l)$-alanine & & & & \\
\hline & SUB & $(407-426)$ & 132.8 & 417 & A & [1987STE/MAL] \\
\hline & SUB & $(342-442)$ & $\mathrm{U} 105 \pm 8$ & 392 & $\mathrm{LE}$ & [1977GAF/PIE] \\
\hline & SUB & $(453-469)$ & $138.3 \pm 8$ & 461 & $\mathrm{ME}$ & $\begin{array}{l}\text { [1965SVE/CLY, 1970COX/PIL, 1964CLY/SVE, } \\
\text { 1989CHI/GRO] }\end{array}$ \\
\hline \multirow[t]{3}{*}{$\mathrm{C}_{3} \mathrm{H}_{7} \mathrm{NO}_{2}$} & {$[302-72-7]$} & DL- $(\alpha)$-alanine & & & & \\
\hline & SUB & $(397-419)$ & $135.9 \pm 2.7$ & 408 & ME & [2010RIB/RIB2] \\
\hline & SUB & $(397-419)$ & $138.0 \pm 2.7$ & 298 & ME & [2010RIB/RIB2] \\
\hline \multirow[t]{6}{*}{$\mathrm{C}_{3} \mathrm{H}_{7} \mathrm{NO}_{2}$} & [107-95-9] & $\beta$-alanine & & & & \\
\hline & SUB & $(388-402)$ & $136.2 \pm 1.7$ & 395 & ME & [2010RIB/RIB2] \\
\hline & SUB & $(388-402)$ & $137.9 \pm 1.7$ & 298 & ME & [2010RIB/RIB2] \\
\hline & SUB & $(384-402)$ & $133.1 \pm 0.7$ & 393 & $\mathrm{C}$ & [1983SKO/SAB] \\
\hline & SUB & & $134 \pm 2$ & 298 & $\mathrm{C}$ & [1983SKO/SAB] \\
\hline & SUB & $(318-418)$ & $\mathrm{U} 105 \pm 4$ & 368 & $\mathrm{LE}$ & [1977GAF/PIE] \\
\hline \multirow[t]{5}{*}{$\mathrm{C}_{3} \mathrm{H}_{7} \mathrm{NO}_{2}$} & [107-97-1] & sarcosine $(N-\mathrm{m}$ & glycine) & & & \\
\hline & FUS & & 20.6 & 481.6 & DSC & [2011ROU/NOT2] \\
\hline & SUB & $(381-403)$ & $128.0 \pm 0.7$ & 392 & ME & [2013AMA/SAN] \\
\hline & SUB & $(381-403)$ & $129.2 \pm 0.7$ & 298 & ME & [2013AMA/SAN] \\
\hline & SUB & $(380-413)$ & $146 \pm 1$ & 298 & $\mathrm{C}$ & [1978SAB/LAF] \\
\hline \multirow[t]{4}{*}{$\mathrm{C}_{3} \mathrm{H}_{7} \mathrm{NO}_{2} \mathrm{~S}$} & [52-90-4] & L-cysteine & & & & \\
\hline & SUB & $(410-436)$ & $140.3 \pm 1.4$ & 423 & ME & [2010ROU/FOC] \\
\hline & SUB & $(410-436)$ & $146.4 \pm 1.4$ & 298 & $\mathrm{ME}$ & [2010ROU/FOC] \\
\hline & SUB & $(337-437)$ & $\mathrm{U} 96.2 \pm 4.2$ & 387 & LE & [1977GAF/PIE] \\
\hline \multirow[t]{3}{*}{$\mathrm{C}_{3} \mathrm{H}_{7} \mathrm{NO}_{2} \mathrm{~S}$} & {$[3374-22-9]$} & $(d l)$-cysteine & & & & \\
\hline & TRS & $(6-309)$ & 2.3 & 300 & $\mathrm{AC}$ & [2010PAU/KOV] \\
\hline & TRS & & 1.36 & 283 & DTA & [1979MAN/LAU] \\
\hline \multirow[t]{4}{*}{$\mathrm{C}_{3} \mathrm{H}_{7} \mathrm{NO}_{3}$} & {$[1712-64-7]$} & isopropyl nitrate & & & & \\
\hline & FUS & $(14-300)$ & 10.1 & 190.9 & $\mathrm{AC}$ & [1988LUS/RUB] \\
\hline & $\mathrm{V}$ & & $35.3 \pm 0.6$ & & DSC & [1999JON/FEN] \\
\hline & $\mathrm{V}$ & $(273-343)$ & 39.7 & 288 & A & [1987STE/MAL, 1957GRA/PRA, 1970DYK] \\
\hline
\end{tabular}

$\mathrm{C}_{3} \mathrm{H}_{7} \mathrm{NO}_{3} \quad[627-13-4] \quad$ propyl nitrate 
TABLE 6. Phase change enthalpies of $\mathrm{C}_{3}$ organic compounds-Continued

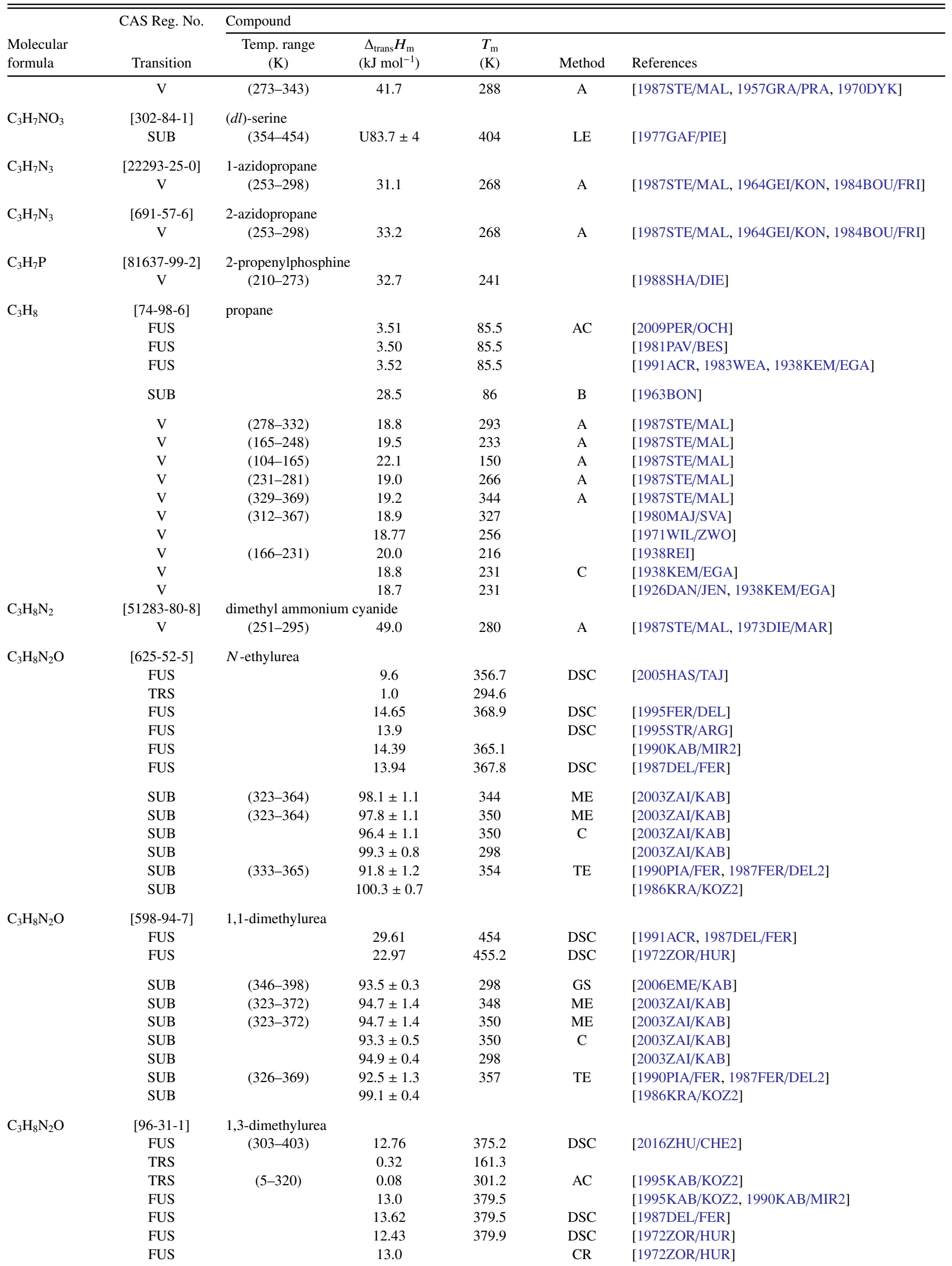


Table 6. Phase change enthalpies of $\mathrm{C}_{3}$ organic compounds-Continued

\begin{tabular}{|c|c|c|c|c|c|c|}
\hline \multirow[b]{2}{*}{$\begin{array}{l}\text { Molecular } \\
\text { formula }\end{array}$} & \multirow{2}{*}{$\begin{array}{c}\text { CAS Reg. No. } \\
\text { Transition }\end{array}$} & \multicolumn{5}{|l|}{ Compound } \\
\hline & & $\begin{array}{c}\text { Temp. range } \\
\text { (K) }\end{array}$ & $\begin{array}{c}\Delta_{\text {trans }} H_{\mathrm{m}} \\
\left(\mathrm{kJ} \mathrm{mol}^{-1}\right)\end{array}$ & $\begin{array}{l}T_{\mathrm{m}} \\
(\mathrm{K})\end{array}$ & Method & References \\
\hline & SUB & $(313-357)$ & $89.3 \pm 0.4$ & 298 & GS & [2006EME/KAB] \\
\hline & SUB & $(317-377)$ & $87.6 \pm 1.0$ & 347 & ME & [2003ZAI/KAB] \\
\hline & SUB & $(317-377)$ & $87.5 \pm 1.0$ & 350 & $\mathrm{ME}$ & [2003ZAI/KAB] \\
\hline & SUB & & $86.6 \pm 0.5$ & 350 & $\mathrm{C}$ & [2003ZAI/KAB] \\
\hline & SUB & & $88.3 \pm 0.4$ & 298 & & [2003ZAI/KAB] \\
\hline & SUB & $(316-373)$ & $87.2 \pm 0.6$ & 353 & $\mathrm{TE}$ & [1990PIA/FER, 1987FER/DEL2] \\
\hline \multirow{2}{*}{$\mathrm{C}_{3} \mathrm{H}_{8} \mathrm{~N}_{2} \mathrm{O}_{2}$} & [4114-31-2] & ethyl carbazate & & & & \\
\hline & FUS & $(78-371)$ & 20.0 & 318.9 & $\mathrm{AC}$ & [2001DI/SUN2] \\
\hline \multirow[t]{8}{*}{$\mathrm{C}_{3} \mathrm{H}_{8} \mathrm{~N}_{2} \mathrm{~S}$} & [534-13-4] & 1,3-dimethylthi & & & & \\
\hline & FUS & & 13.71 & 337 & DSC & [2000DEL/JOZ] \\
\hline & FUS & & 12.7 & 336.9 & & [1994FER/MAR] \\
\hline & FUS & & 11.4 & 339 & $\mathrm{DSC}$ & [1993DEM/BUC] \\
\hline & SUB & & $111.8 \pm 3$ & 298 & $\mathrm{~B}$ & [2000DEL/JOZ] \\
\hline & SUB & & $107.3 \pm 4.0$ & 298 & B & [1994TER/PIA] \\
\hline & SUB & & $108 \pm 3.0$ & 361 & B & [1994FER/MAR] \\
\hline & $\mathrm{V}$ & $(342-375)$ & $93 \pm 4.0$ & 359 & $\mathrm{ME}, \mathrm{TE}$ & [1994TER/PIA] \\
\hline \multirow[t]{5}{*}{$\mathrm{C}_{3} \mathrm{H}_{8} \mathrm{~N}_{2} \mathrm{~S}$} & [625-53-6] & 1-ethylthiourea & & & & \\
\hline & FUS & & 21.34 & 380.8 & $\mathrm{DSC}$ & [2000DEL/JOZ] \\
\hline & SUB & $(360-380)$ & $116.1 \pm 2.0$ & 370 & $\mathrm{ME}, \mathrm{TE}$ & [2007FER/BAD] \\
\hline & SUB & $(360-380)$ & $118.8 \pm 2.1$ & 298 & $\mathrm{ME}, \mathrm{TE}$ & [2007FER/BAD] \\
\hline & SUB & & $118.8 \pm 5$ & 298 & ME & [2000DEL/JOZ] \\
\hline \multirow[t]{2}{*}{$\mathrm{C}_{3} \mathrm{H}_{8} \mathrm{~N}_{4} \mathrm{O}_{2}$} & [39197-62-1] & 1-ethyl-2-nitrog & line & & & \\
\hline & V & & $70.5 \pm 3.3$ & & & [2009AST/DYU] \\
\hline \multirow[t]{5}{*}{$\mathrm{C}_{3} \mathrm{H}_{8} \mathrm{~N}_{4} \mathrm{O}_{4}$} & [13232-00-3] & 2,4-dinitro-2,4- & gentane & & & \\
\hline & FUS & & 16.81 & 327.1 & DSC & [2010FAR/RAJ] \\
\hline & FUS & & 16.79 & 327.6 & DSC & [2003SPI/WAN] \\
\hline & FUS & & 16.36 & 330.2 & DSC & [1997ZEM] \\
\hline & SUB & & $102.5 \pm 0.4$ & 298 & $\mathrm{C}$ & [2009MIR/KON] \\
\hline
\end{tabular}

[Note: Authors of [2009MIR/KON] tabulate the experimental value as an enthalpy of vaporization; however, they report that the compound is crystalline and use the value to calculate the standard molar enthalpy of formation. Given the crystaline state and how the value was used, we have tabulated the value as an enthalpy of sublimation.]

\begin{tabular}{|c|c|c|c|c|c|c|}
\hline \multirow[t]{6}{*}{$\mathrm{C}_{3} \mathrm{H}_{8} \mathrm{O}$} & [540-67-0] & methyl ethyl ether & & & & \\
\hline & $\mathrm{V}$ & $(281-433)$ & 30.1 & 296 & A & [1987STE/MAL] \\
\hline & $\mathrm{V}$ & $(216-299)$ & 37.0 & 231 & A & [1987STE/MAL, 1969KUD/REE] \\
\hline & V & $(281-438)$ & 37.1 & 296 & A & [1987STE/MAL] \\
\hline & V & $(278-281)$ & NA & & & [1967SHA] \\
\hline & $\mathrm{V}$ & $(182-280)$ & 26.3 & 265 & & [1947STU] \\
\hline \multirow[t]{19}{*}{$\mathrm{C}_{3} \mathrm{H}_{8} \mathrm{O}$} & [71-23-8] & 1-propanol & & & & \\
\hline & FUS & $(10-350)$ & 5.4 & 148.7 & & [2004VAN/VAN] \\
\hline & FUS & & 5.37 & 148.8 & & [1968COU/LEE] \\
\hline & $\mathrm{V}$ & $(278-323)$ & 47.4 & 298 & & [2012GIM/MAR] \\
\hline & $\mathrm{V}$ & $(335-370)$ & 44.6 & 350 & & [2011SAP/UUS] \\
\hline & $\mathrm{V}$ & $(298-363)$ & 45.7 & 298 & & [2004NAS/ZIM] \\
\hline & V & $(310-356)$ & 47.8 & 298 & EB & [2004LUB/MAL] \\
\hline & $\mathrm{V}$ & & 41.2 & 371 & & [2000WOR/VIN] \\
\hline & $\mathrm{V}$ & & 35.2 & 423 & & [2000WOR/VIN] \\
\hline & V & & 29.4 & 453 & & [2000WOR/VIN] \\
\hline & $\mathrm{V}$ & & 21.0 & 498 & & [2000WOR/VIN] \\
\hline & $\mathrm{V}$ & & 11.4 & 528 & & [2000WOR/VIN] \\
\hline & V & $(323-373)$ & 49.2 & 298 & $\mathrm{CGC}$ & [1995CHI/HOS] \\
\hline & $\mathrm{V}$ & $(303-370)$ & 47.0 & 318 & & [1995AUC/GON] \\
\hline & V & $(360-377)$ & 42.9 & 375 & & [1990ORT/SUS] \\
\hline & $\mathrm{V}$ & $(200-228)$ & 48.0 & 214 & A & [1987STE/MAL] \\
\hline & $\mathrm{V}$ & $(356-376)$ & 43.5 & 366 & A & [1987STE/MAL] \\
\hline & $\mathrm{V}$ & $(369-407)$ & 42.3 & 384 & A & [1987STE/MAL] \\
\hline & V & $(401-482)$ & 40.1 & 416 & A & [1987STE/MAL] \\
\hline
\end{tabular}


TABLE 6. Phase change enthalpies of $\mathrm{C}_{3}$ organic compounds-Continued

\begin{tabular}{|c|c|c|c|c|c|c|}
\hline \multirow[b]{2}{*}{$\begin{array}{l}\text { Molecular } \\
\text { formula }\end{array}$} & \multirow{2}{*}{$\begin{array}{c}\text { CAS Reg. No. } \\
\text { Transition }\end{array}$} & \multicolumn{5}{|l|}{ Compound } \\
\hline & & $\begin{array}{c}\text { Temp. range } \\
\text { (K) }\end{array}$ & $\begin{array}{c}\Delta_{\text {trans }} H_{\mathrm{m}} \\
\left(\mathrm{kJ} \mathrm{mol}^{-1}\right)\end{array}$ & $\begin{array}{l}T_{\mathrm{m}} \\
(\mathrm{K})\end{array}$ & Method & References \\
\hline & $\mathrm{V}$ & $(478-507)$ & 36.5 & 492 & A & [1987STE/MAL] \\
\hline & V & $(243-303)$ & 46.3 & 298 & & [1983SCH/STR] \\
\hline & $\mathrm{V}$ & $(275-373)$ & 49.3 & 290 & & [1973WIL/ZWO] \\
\hline & $\mathrm{V}$ & & $46.4 \pm 0.1$ & 313 & $\mathrm{C}$ & [1973SVO/VES] \\
\hline & V & & $45.7 \pm 0.1$ & 323 & $\mathrm{C}$ & [1973SVO/VES] \\
\hline & $\mathrm{V}$ & & $44.9 \pm 0.1$ & 333 & $\mathrm{C}$ & [1973SVO/VES] \\
\hline & $\mathrm{V}$ & & $44.0 \pm 0.1$ & 343 & $\mathrm{C}$ & [1973SVO/VES] \\
\hline & V & & $43.2 \pm 0.1$ & 353 & $\mathrm{C}$ & [1973SVO/VES] \\
\hline & V & & $42.4 \pm 0.1$ & 363 & $\mathrm{C}$ & [1973SVO/VES] \\
\hline & $\mathrm{V}$ & & $47.49 \pm 0.02$ & 298 & $\mathrm{C}$ & [1971POL/BEN] \\
\hline & V & & $46.8 \pm 0.1$ & 308 & $\mathrm{C}$ & [1971POL/BEN] \\
\hline & V & & $46.2 \pm 0.1$ & 318 & $\mathrm{C}$ & [1971POL/BEN] \\
\hline & $\mathrm{V}$ & & $45.0 \pm 0.1$ & 328 & $\mathrm{C}$ & [1971POL/BEN] \\
\hline & $\mathrm{V}$ & $(333-377)$ & 44.7 & 348 & EB & [1970AMB/SPR, 1987STE/MAL] \\
\hline & $\mathrm{V}$ & $(292-370)$ & 46.9 & 307 & DTA & [1969KEM/KRE] \\
\hline & $\mathrm{V}$ & $(288-348)$ & 46.7 & 303 & & [1967VAN/SOC] \\
\hline & $\mathrm{V}$ & & $47.3 \pm 0.1$ & 298 & $\mathrm{C}$ & [1966WAD] \\
\hline & $\mathrm{V}$ & $(338-378)$ & 44.3 & 353 & $\mathrm{~EB}$ & [1963BID/COL] \\
\hline & $\mathrm{V}$ & & 46.6 & 298 & $\mathrm{C}$ & [1963MCC/LAI] \\
\hline & $\mathrm{V}$ & $(405-537)$ & 40.7 & 420 & & [1963AMB/TOW] \\
\hline & $\mathrm{V}$ & $(343-385)$ & 44.1 & 358 & & [1961MAT/MCK] \\
\hline & $\mathrm{V}$ & & $43.9 \pm 0.1$ & 343 & $\mathrm{C}$ & [1961MAT/MCK] \\
\hline & $\mathrm{V}$ & & $42.3 \pm 0.1$ & 360 & $\mathrm{C}$ & [1961MAT/MCK] \\
\hline & $\mathrm{V}$ & & $41.2 \pm 0.1$ & 370 & $\mathrm{C}$ & [1961MAT/MCK] \\
\hline & $\mathrm{V}$ & & $40.3 \pm 0.1$ & 378 & $\mathrm{C}$ & [1961MAT/MCK] \\
\hline & $\mathrm{V}$ & & $39.7 \pm 0.1$ & 384 & $\mathrm{C}$ & [1961MAT/MCK] \\
\hline & $\mathrm{V}$ & $(321-367)$ & 45.5 & & & [1959ARO/KAS] \\
\hline & V & & 43.2 & 354 & & [1957WIL/HAR] \\
\hline \multirow[t]{35}{*}{$\mathrm{C}_{3} \mathrm{H}_{8} \mathrm{O}$} & {$[67-63-0]$} & 2-propanol & & & & \\
\hline & FUS & & 5.41 & 185.2 & & [1996DOM/HEA, 1963AND/COU] \\
\hline & FUS & & 5.37 & 184.7 & & [1929KEL3] \\
\hline & FUS & & 5.30 & 184.6 & & [1925PAR/KEL, 1928PAR/KEL] \\
\hline & $\mathrm{V}$ & $(298-353)$ & 44.0 & 298 & & [2004NAS/ZIM] \\
\hline & $\mathrm{V}$ & $(322-355)$ & 43.2 & 337 & & [2002SEG/GAL] \\
\hline & $\mathrm{V}$ & & 39.8 & 355 & & [2000WOR/VIN2] \\
\hline & $\mathrm{V}$ & & 29.7 & 423 & & [2000WOR/VIN2] \\
\hline & $\mathrm{V}$ & & 23.7 & 453 & & [2000WOR/VIN2] \\
\hline & $\mathrm{V}$ & & 16.5 & 483 & & [2000WOR/VIN2] \\
\hline & $\mathrm{V}$ & & 10.5 & 503 & & [2000WOR/VIN2] \\
\hline & $\mathrm{V}$ & & 40.4 & & & [1999FAT] \\
\hline & $\mathrm{V}$ & $(300-355)$ & 44.8 & 315 & & [1995AUC/GON] \\
\hline & $\mathrm{V}$ & $(195-228)$ & 50.3 & 213 & A & [1987STE/MAL] \\
\hline & $\mathrm{V}$ & $(347-368)$ & 42.0 & 355 & A & [1987STE/MAL] \\
\hline & $\mathrm{V}$ & $(350-383)$ & 41.3 & 365 & A & [1987STE/MAL] \\
\hline & $\mathrm{V}$ & $(379-461)$ & 39.2 & 394 & A & [1987STE/MAL] \\
\hline & $\mathrm{V}$ & $(453-508)$ & 35.3 & 468 & A & [1987STE/MAL] \\
\hline & $\mathrm{V}$ & $(273-374)$ & 45.7 & 288 & & [1973WIL/ZWO] \\
\hline & V & $(325-362)$ & 43.1 & 340 & $\mathrm{~A}, \mathrm{~EB}$ & [1987STE/MAL, 1970AMB/SPR] \\
\hline & $\mathrm{V}$ & & $45.34 \pm 0.02$ & 298 & $\mathrm{C}$ & [1971POL/BEN] \\
\hline & $\mathrm{V}$ & & $44.7 \pm 0.1$ & 308 & $\mathrm{C}$ & [1971POL/BEN] \\
\hline & V & & $43.9 \pm 0.1$ & 318 & $\mathrm{C}$ & [1971POL/BEN] \\
\hline & $\mathrm{V}$ & & $42.5 \pm 0.1$ & 328 & $\mathrm{C}$ & [1971POL/BEN] \\
\hline & $\mathrm{V}$ & $(288-348)$ & 45.5 & 303 & & [1967VAN/SOC] \\
\hline & V & & $45.2 \pm 0.1$ & 298 & $\mathrm{C}$ & [1966WAD] \\
\hline & $\mathrm{V}$ & & $42.7 \pm 0.1$ & 330 & $\mathrm{C}$ & [1964BER/LAR] \\
\hline & $\mathrm{V}$ & & $41.0 \pm 0.1$ & 346 & $\mathrm{C}$ & [1964BER/LAR] \\
\hline & V & & $39.8 \pm 0.1$ & 355 & $\mathrm{C}$ & [1964BER/LAR] \\
\hline & $\mathrm{V}$ & & $38.9 \pm 0.1$ & 363 & $\mathrm{C}$ & [1964BER/LAR] \\
\hline & $\mathrm{V}$ & $(329-363)$ & 42.8 & 344 & $\mathrm{~EB}$ & [1963BID/COL] \\
\hline & V & & 44.0 & 298 & $\mathrm{C}$ & [1963MCC/LAI] \\
\hline & $\mathrm{V}$ & $(395-508)$ & 39.1 & 410 & & [1963AMB/TOW] \\
\hline & $\mathrm{V}$ & & 43.2 & 324 & $\mathrm{C}$ & [1963HAL/COX] \\
\hline & V & & 41.7 & 339 & $\mathrm{C}$ & [1963HAL/COX] \\
\hline
\end{tabular}


TABLE 6. Phase change enthalpies of $\mathrm{C}_{3}$ organic compounds-Continued

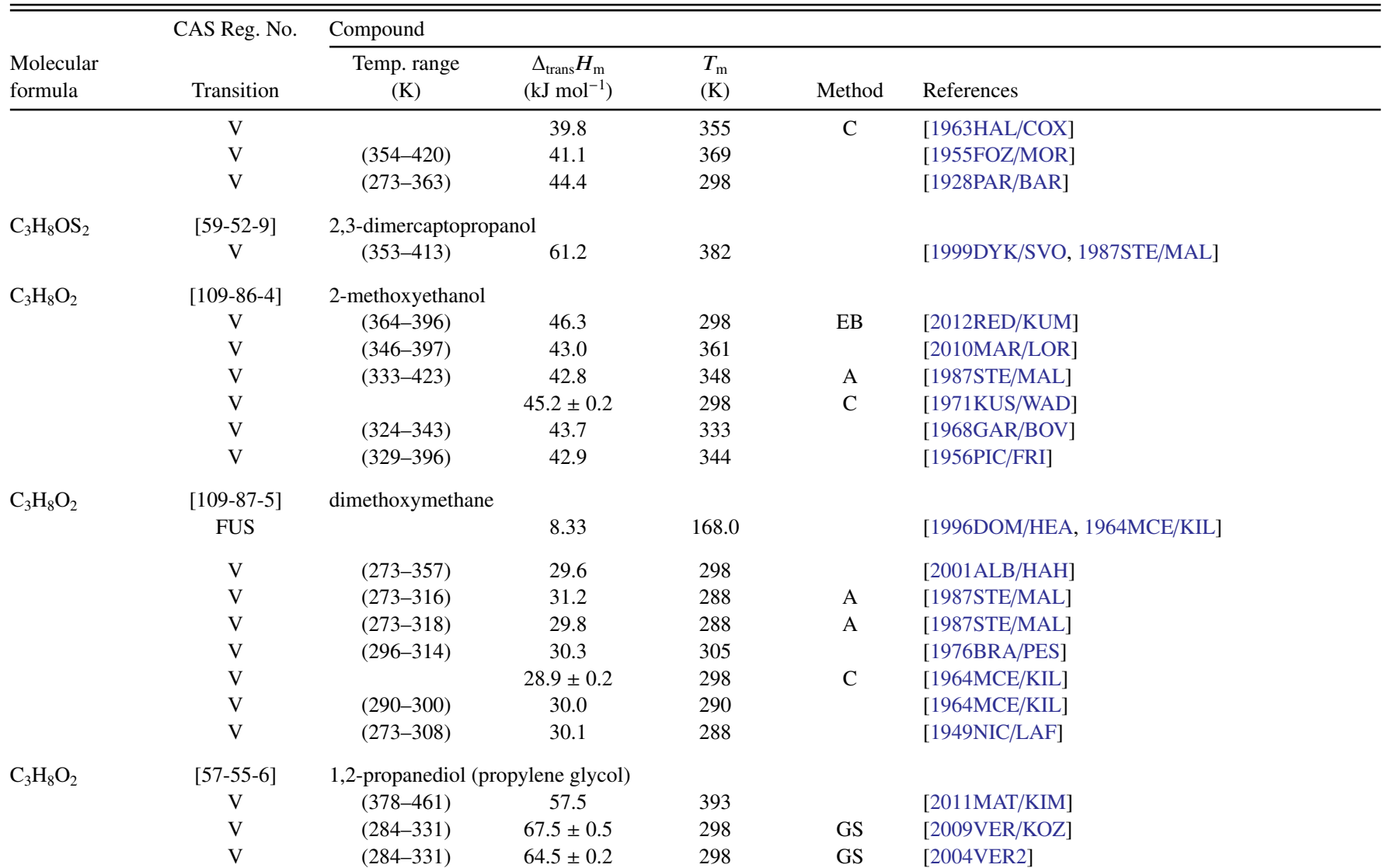

[Note: The author recalculated this earlier value in a later paper [2009VER/KOZ]. The recalculated value was reported to be $68.5 \pm 0.5 \mathrm{~kJ} / \mathrm{mole}$.]

\begin{tabular}{|c|c|c|c|c|c|}
\hline $\mathrm{V}$ & $(293-423)$ & 76 & 298 & $\mathrm{~EB}$ & [2004CHY/FRA2] \\
\hline $\mathrm{V}$ & $(366-396)$ & 62.2 & 298 & TGA & [2002TAT/DOL] \\
\hline V & $(365-496)$ & $60.0 \pm 0.3$ & 380 & EB & [2002STE/CHI3] \\
\hline $\mathrm{V}$ & $(365-496)$ & $56.2 \pm 0.2$ & 420 & EB & [2002STE/CHI3] \\
\hline V & $(365-496)$ & $52.0 \pm 0.3$ & 460 & EB & [2002STE/CHI3] \\
\hline V & $(365-496)$ & $47.5 \pm 0.6$ & 500 & EB & [2002STE/CHI3] \\
\hline $\mathrm{V}$ & $(348-453)$ & $63.6 \pm 0.3$ & 298 & EB & [1991WIL/WIL, 2004VER2] \\
\hline $\mathrm{V}$ & & $\mathrm{U} 71.2 \pm 0.1$ & 298 & $\mathrm{C}$ & [1990KNA/SAB3, 2004VER2] \\
\hline V & $(373-408)$ & 66.5 & 413 & TGA & [1987ALN/ALS] \\
\hline $\mathrm{V}$ & & U51.7 & 298 & I & [1971SUN/EIS] \\
\hline V & $(359-461)$ & 64.7 & 298 & EB & [1966THO/MEA, 2004VER2] \\
\hline $\mathrm{V}$ & $(318-461)$ & 58.6 & 333 & A & [1987STE/MAL, 1947STU] \\
\hline $\mathrm{V}$ & $(353-403)$ & 58.2 & 378 & & {$[1935 \mathrm{SCH} / \mathrm{STA}]$} \\
\hline $\mathrm{V}$ & 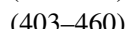 & 56 & & & 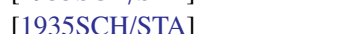 \\
\hline
\end{tabular}

\begin{tabular}{|c|c|c|c|c|c|c|}
\hline \multirow{2}{*}{$\mathrm{C}_{3} \mathrm{H}_{8} \mathrm{O}_{2}$} & [4254-14-2] & \multicolumn{5}{|c|}{$(R)$-1,2-propanediol (propylene glycol) } \\
\hline & FUS & & 8.4 & 240 & DSC & [1995JAB/LET] \\
\hline \multirow[t]{3}{*}{$\mathrm{C}_{3} \mathrm{H}_{8} \mathrm{O}_{2}$} & {$[4254-15-3]$} & \multicolumn{5}{|c|}{ (S)-1,2-propanediol (propylene glycol) } \\
\hline & FUS & & 8.4 & 240 & DSC & [1995JAB/LET] \\
\hline & $\mathrm{V}$ & $(294-338)$ & $69.2 \pm 0.3$ & 298 & GS & [2009VER/KOZ] \\
\hline \multirow{13}{*}{$\mathrm{C}_{3} \mathrm{H}_{8} \mathrm{O}_{2}$} & {$[504-63-2]$} & 1,3-propanediol & & & & \\
\hline & FUS & & 13.47 & 253.2 & DSC & [2015SOL/ROD] \\
\hline & FUS & & 11.4 & 249 & DSC & [1998JAB/LET] \\
\hline & FUS & & 7.1 & 245.5 & DTA & [1990KNA/SAB] \\
\hline & $\mathrm{V}$ & $(293-346)$ & $70.5 \pm 0.3$ & 298 & GS & [2015EME/VER] \\
\hline & $\mathrm{V}$ & $(313-462)$ & $73.6 \pm 0.9$ & 298 & & [2012MOK/SAW] \\
\hline & $\mathrm{V}$ & $(314-460)$ & 66.5 & 298 & EB & [2008RIB/SAN4] \\
\hline & $\mathrm{V}$ & $(353-383)$ & U84.2 & 298 & TGA & [2007BAR/COZ, 2015EME/VER] \\
\hline & $\mathrm{V}$ & $(293-342)$ & $70.5 \pm 0.2$ & 298 & GS & [2007VER] \\
\hline & $\mathrm{V}$ & & $69.8 \pm 1.1$ & 298 & CGC & [2006UMN/KWE] \\
\hline & $\mathrm{V}$ & $(480-716)$ & $69.1 \pm 0.2$ & 298 & & [2002WIL/VON, 2007VER] \\
\hline & $\mathrm{V}$ & $(418-488)$ & $70.1 \pm 1.0$ & 298 & & [1999MUN/LEE, 2015EME/VER] \\
\hline & $\mathrm{V}$ & $(413-458)$ & $70.6 \pm 0.5$ & 298 & EB & [1996OLS, 2007VER] \\
\hline
\end{tabular}


TABLE 6. Phase change enthalpies of $\mathrm{C}_{3}$ organic compounds-Continued

\begin{tabular}{|c|c|c|c|c|c|c|}
\hline \multirow[b]{2}{*}{$\begin{array}{l}\text { Molecular } \\
\text { formula }\end{array}$} & \multirow{2}{*}{$\begin{array}{c}\text { CAS Reg. No. } \\
\text { Transition }\end{array}$} & \multicolumn{5}{|l|}{ Compound } \\
\hline & & $\begin{array}{l}\text { Temp. range } \\
\text { (K) }\end{array}$ & $\begin{array}{c}\Delta_{\text {trans }} H_{\mathrm{m}} \\
\left(\mathrm{kJ} \mathrm{mol}^{-1}\right)\end{array}$ & $\begin{array}{l}T_{\mathrm{m}} \\
(\mathrm{K})\end{array}$ & Method & References \\
\hline & $\mathrm{V}$ & $(402-488)$ & $70.1 \pm 0.3$ & 298 & EB & [1981MAR/SAC, 2007VER] \\
\hline & $\mathrm{V}$ & $(367-489)$ & $71.4 \pm 0.8$ & 298 & EB & [1966THO/MEA, 2007VER, 2015VER/EME] \\
\hline & $\mathrm{V}$ & & $72.4 \pm 0.3$ & 298 & $\mathrm{C}$ & [1988KNA/SAB, 1990KNA/SAB2] \\
\hline & $\mathrm{V}$ & $(332-448)$ & 57.2 & 347 & A & [1987STE/MAL, 1947STU] \\
\hline & $\mathrm{V}$ & $(373-488)$ & 65.2 & 298 & EB & [1937GAL/HIB, 2007VER] \\
\hline & $\mathrm{V}$ & $(383-433)$ & 63.3 & 408 & $\mathrm{~EB}$ & [1935SCH/STA] \\
\hline & $\mathrm{V}$ & $(383-433)$ & $69.5 \pm 1.6$ & 298 & EB & [1935SCH/STA, 2015EME/VER] \\
\hline & $\mathrm{V}$ & $(433-488)$ & 60.4 & 460 & & {$[1935 \mathrm{SCH} / \mathrm{STA}]$} \\
\hline & $\mathrm{V}$ & $(433-488)$ & $69.6 \pm 1.8$ & 298 & & [1935SCH/STA, 2015EME/VER] \\
\hline \multirow[t]{3}{*}{$\mathrm{C}_{3} \mathrm{H}_{8} \mathrm{O}_{2} \mathrm{~S}$} & [594-43-4] & ethyl methyl sulfone & & & & \\
\hline & FUS & & 11.3 & 307.7 & $\mathrm{RC}$ & [1961BUS/IVI] \\
\hline & SUB & & $77.8 \pm 2.9$ & & & [UR/MAC, 1970COX/PIL] \\
\hline \multirow[t]{30}{*}{$\mathrm{C}_{3} \mathrm{H}_{8} \mathrm{O}_{3}$} & {$[56-81-5]$} & glycerol & & & & \\
\hline & TRS & & 0.61 & 229.1 & & \\
\hline & FUS & & 14.7 & 289.7 & DSC & [2011SOU/NIS] \\
\hline & FUS & & 18.28 & 291 & & [1991ACR, 1923GIB/GIA] \\
\hline & $\mathrm{V}$ & $(338-370)$ & $85.5 \pm 0.7$ & 354 & GS & [2015VER/ZAI2] \\
\hline & $\mathrm{V}$ & $(338-370)$ & $90.7 \pm 0.9$ & 298 & GS & [2015VER/ZAI2] \\
\hline & $\mathrm{V}$ & $(304-436)$ & $81.3 \pm 0.1$ & 370 & Static & [2015VER/ZAI2] \\
\hline & $\mathrm{V}$ & $(304-436)$ & $90.5 \pm 0.5$ & 298 & Static & [2015VER/ZAI2] \\
\hline & $\mathrm{V}$ & $(477-521)$ & $75.8 \pm 1.4$ & 499 & $\mathrm{~EB}$ & [2013VEN/BEN, 2015VER/ZAI2] \\
\hline & $\mathrm{V}$ & $(477-521)$ & 94.9 & 298 & EB & [2013VEN/BEN, 2015VER/ZAI2] \\
\hline & $\mathrm{V}$ & $(352-463)$ & $88.2 \pm 0.9$ & 298 & Static & [2012MOK/SAW] \\
\hline & $\mathrm{V}$ & $(497-561)$ & $69.3 \pm 0.1$ & 529 & $\mathrm{~EB}$ & [2010SOU/SAT, 2015VER/ZAI2] \\
\hline & $\mathrm{V}$ & $(497-561)$ & $91.2 \pm 1.7$ & 298 & $\mathrm{~EB}$ & [2010SOU/SAT, 2015VER/ZAI2] \\
\hline & $\mathrm{V}$ & $(463-543)$ & 72.9 & 503 & TGA & [2008YAN/SUP, 2015VER/ZAI2] \\
\hline & $\mathrm{V}$ & $(463-543)$ & $92.3 \pm 2.5$ & 298 & TGA & [2008YAN/SUP, 2015VER/ZAI2] \\
\hline & $\mathrm{V}$ & & $91.7 \pm 0.9$ & 298 & $\mathrm{C}$ & [1988BAS/NIL] \\
\hline & $\mathrm{V}$ & $(469-563)$ & 78.5 & 484 & A & [1987STE/MAL] \\
\hline & $\mathrm{V}$ & $(291-341)$ & 86.8 & 316 & $\mathrm{ME}$ & [1977CAM/SCH] \\
\hline & $\mathrm{V}$ & & 67.5 & 343 & GC & [1977NOV/NOV] \\
\hline & $\mathrm{V}$ & & 66.8 & 353 & $\mathrm{GC}$ & [1977NOV/NOV] \\
\hline & $\mathrm{V}$ & & 66.2 & 363 & GC & [1977NOV/NOV] \\
\hline & $\mathrm{V}$ & & 65.5 & 373 & GC & [1977NOV/NOV] \\
\hline & $\mathrm{V}$ & & 64.8 & 383 & GC & [1977NOV/NOV] \\
\hline & $\mathrm{V}$ & $(278-323)$ & 71.5 & 300 & & [1972MCF/SOM] \\
\hline & $\mathrm{V}$ & $(439-563)$ & $71.4 \pm 0.3$ & 501 & EB & [1972SOK/TSY, 2015VER/ZAI2] \\
\hline & $\mathrm{V}$ & $(439-563)$ & $90.5 \pm 1.5$ & 298 & EB & [1972SOK/TSY, 2015VER/ZAI2] \\
\hline & $\mathrm{V}$ & $(293-343)$ & 85.8 & 308 & $\mathrm{ME}$ & [1987STE/MAL, 1962ROS/HEI, 1970DYK] \\
\hline & $\mathrm{V}$ & $(323-473)$ & $82.8 \pm 0.4$ & 398 & EB & [1928STE, 2015VER/ZAI2] \\
\hline & $\mathrm{V}$ & $(323-473)$ & $91.5 \pm 0.8$ & 298 & EB & [1928STE, 2015VER/ZAI2] \\
\hline & $\mathrm{V}$ & $(456-553)$ & 86 & 471 & $\mathrm{~EB}$ & {$[1886 \mathrm{RIC}]$} \\
\hline \multirow[t]{2}{*}{$\mathrm{C}_{3} \mathrm{H}_{8} \mathrm{O}_{3} \mathrm{~S}$} & {$[10315-59-0]$} & ethyl methyl sulfite & & & & \\
\hline & $\mathrm{V}$ & & $43.5 \pm 1.7$ & 298 & $\mathrm{BP}$ & [1969MAC/STE2] \\
\hline \multirow[t]{11}{*}{$\mathrm{C}_{3} \mathrm{H}_{8} \mathrm{~S}$} & [624-89-5] & ethyl methyl sulfide & & & & \\
\hline & FUS & & 9.76 & 167.2 & & [1996DOM/HEA, 1951SCO/FIN] \\
\hline & $\mathrm{V}$ & $(253-363)$ & 33.7 & 268 & & [1999DYK/SVO] \\
\hline & $\mathrm{V}$ & & 31.5 & 298 & & [1981SHI/SAI] \\
\hline & $\mathrm{V}$ & & 32.3 & 287 & & [1960MAC/MAY] \\
\hline & $\mathrm{V}$ & $(296-373)$ & 31.8 & 311 & $\mathrm{~A}, \mathrm{~EB}$ & [1987STE/MAL, 1951SCO/FIN] \\
\hline & $\mathrm{V}$ & & 31.8 & 298 & & [1971WIL/ZWO, 1966OSB/DOU, 1954HUB/WAD] \\
\hline & $\mathrm{V}$ & & 31.6 & 302 & & [1951SCO/FIN] \\
\hline & $\mathrm{V}$ & & 30.7 & 320 & & [1951SCO/FIN] \\
\hline & $\mathrm{V}$ & & 29.5 & 340 & & [1951SCO/FIN] \\
\hline & $\mathrm{V}$ & & 30.3 & 338 & & {$[1935 \mathrm{THO} / \mathrm{LIN}]$} \\
\hline \multirow[t]{4}{*}{$\mathrm{C}_{3} \mathrm{H}_{8} \mathrm{~S}$} & [107-03-9] & 1-propanethiol & & & & \\
\hline & TRS & $(12-320)$ & 3.97 & 142.1 & & \\
\hline & FUS & $(12-320)$ & 5.48 & 160 & $\mathrm{AC}$ & [1996DOM/HEA, 1956PEN/SCO] \\
\hline & $\mathrm{V}$ & $(254-364)$ & 33.7 & 269 & & [1999DYK/SVO] \\
\hline
\end{tabular}


Table 6. Phase change enthalpies of $\mathrm{C}_{3}$ organic compounds-Continued

\begin{tabular}{|c|c|c|c|c|c|c|}
\hline \multirow[b]{2}{*}{$\begin{array}{l}\text { Molecular } \\
\text { formula }\end{array}$} & \multirow{2}{*}{$\begin{array}{c}\text { CAS Reg. No. } \\
\text { Transition }\end{array}$} & \multicolumn{5}{|l|}{ Compound } \\
\hline & & $\begin{array}{l}\text { Temp. range } \\
\text { (K) }\end{array}$ & $\begin{array}{c}\Delta_{\text {trans }} H_{\mathrm{m}} \\
\left(\mathrm{kJ} \mathrm{mol}^{-1}\right)\end{array}$ & $\begin{array}{l}T_{\mathrm{m}} \\
(\mathrm{K})\end{array}$ & Method & References \\
\hline & $\mathrm{V}$ & & 31.9 & 298 & & [1971WIL/ZWO] \\
\hline & $\mathrm{V}$ & $(297-375)$ & 31.8 & 312 & $\mathrm{~A}, \mathrm{~EB}$ & $\begin{array}{l}\text { [1987STE/MAL, 1956PEN/SCO, 1966OSB/DOU, } \\
\text { 1954HUB/WAD] }\end{array}$ \\
\hline & $\mathrm{V}$ & & $31.6 \pm 0.1$ & 303 & $\mathrm{C}$ & [1956PEN/SCO] \\
\hline & V & & $30.7 \pm 0.1$ & 320 & $\mathrm{C}$ & [1956PEN/SCO] \\
\hline & V & & $29.5 \pm 0.1$ & 341 & $\mathrm{C}$ & [1956PEN/SCO] \\
\hline & $\mathrm{V}$ & $(284-340)$ & 31.5 & 312 & & [1933TAY/LAY] \\
\hline \multirow[t]{6}{*}{$\mathrm{C}_{3} \mathrm{H}_{8} \mathrm{~S}$} & {$[75-33-2]$} & 2-propanethiol & & & & \\
\hline & TRS & & 0.05 & 112.5 & & \\
\hline & FUS & & 5.73 & 142.6 & & [1996DOM/HEA, 1954MCC/FIN2] \\
\hline & $\mathrm{V}$ & $(242-348)$ & 31.9 & 257 & & [1999DYK/SVO] \\
\hline & $\mathrm{V}$ & & 29.5 & 298 & & [1971WIL/ZWO] \\
\hline & $\mathrm{V}$ & $(283-358)$ & 30.1 & 298 & $\mathrm{~A}, \mathrm{~EB}$ & $\begin{array}{l}\text { [1987STE/MAL, 1954MCC/FIN2, 1966OSB/DOU, } \\
\text { 1954HUB/WAD] }\end{array}$ \\
\hline \multirow[t]{4}{*}{$\mathrm{C}_{3} \mathrm{H}_{8} \mathrm{~S}_{2}$} & [109-80-8] & 1,3-propanedithiol & & & & \\
\hline & $\mathrm{V}$ & $(338-446)$ & 50.9 & 353 & & [1999DYK/SVO] \\
\hline & V & $(377-446)$ & 41.6 & 398 & A & [1987STE/MAL] \\
\hline & $\mathrm{V}$ & & $49.7 \pm 0.1$ & 298 & & [1962MAN/SUN] \\
\hline \multirow[t]{5}{*}{$\mathrm{C}_{3} \mathrm{H}_{9} \mathrm{~N}$} & {$[75-31-0]$} & isopropylamine & & & & \\
\hline & FUS & & 7.33 & 178 & & [1991ACR, 1972FIN/MES] \\
\hline & $\mathrm{V}$ & & 28.4 & 298 & & [1979MAJ/SVO2] \\
\hline & $\mathrm{V}$ & & 27.2 & 313 & & [1979MAJ/SVO2] \\
\hline & $\mathrm{V}$ & $(277-334)$ & 29.7 & 292 & $\mathrm{~A}, \mathrm{~EB}, \mathrm{IPM}$ & [1987STE/MAL, 1968OSB/DOU, 1970DYK] \\
\hline \multirow[t]{6}{*}{$\mathrm{C}_{3} \mathrm{H}_{9} \mathrm{~N}$} & [107-10-8] & propylamine & & & & \\
\hline & FUS & & 10.97 & 188.4 & & [1991ACR, 1972FIN/MES] \\
\hline & $\mathrm{V}$ & & 31.3 & 298 & & [1979MAJ/SVO2] \\
\hline & $\mathrm{V}$ & & 30.1 & 313 & & [1979MAJ/SVO2] \\
\hline & $\mathrm{V}$ & & 28.9 & 328 & & [1979MAJ/SVO2] \\
\hline & $\mathrm{V}$ & $(296-350)$ & 31.3 & 311 & $\mathrm{~A}, \mathrm{~EB}, \mathrm{IPM}$ & [1987STE/MAL, 1968OSB/DOU, 1970DYK] \\
\hline \multirow[t]{7}{*}{$\mathrm{C}_{3} \mathrm{H}_{9} \mathrm{~N}$} & {$[75-50-3]$} & trimethylamine & & & & \\
\hline & FUS & & 6.55 & 156.1 & & [1991ACR, 1983WEA, 1944AST/SAG] \\
\hline & $\mathrm{V}$ & $(333-403)$ & 23.0 & 368 & & [1950DAY/FEL] \\
\hline & $\mathrm{V}$ & $(273-313)$ & 24.1 & 288 & & [1945SWI/HOC] \\
\hline & $\mathrm{V}$ & $(193-276)$ & 24.6 & 261 & A & [1987STE/MAL, 1944AST/SAG] \\
\hline & $\mathrm{V}$ & & 24.5 & 250 & $\mathrm{C}$ & [1944AST/SAG] \\
\hline & $\mathrm{V}$ & & 22.2 & 276 & & {$[1935 \mathrm{WIB} / \mathrm{SUT}]$} \\
\hline \multirow[t]{10}{*}{$\mathrm{C}_{3} \mathrm{H}_{9} \mathrm{NO}$} & [109-83-1] & 2-(methylamino)ethar & & & & \\
\hline & $\mathrm{V}$ & $(275-320)$ & $57.8 \pm 0.2$ & 298 & GS & [2005KAP/SLO] \\
\hline & V & $(269-401)$ & 57.9 & 298 & & [1998NOL/VAL, 2005KAP/SLO] \\
\hline & $\mathrm{V}$ & $(340-461)$ & $54.7 \pm 0.5$ & 340 & $\mathrm{~EB}$ & [1997STE/CHI3] \\
\hline & $\mathrm{V}$ & $(340-461)$ & $51.9 \pm 0.4$ & 380 & EB & [1997STE/CHI3] \\
\hline & $\mathrm{V}$ & $(340-461)$ & $50.2 \pm 0.4$ & 400 & $\mathrm{~EB}$ & [1997STE/CHI3] \\
\hline & $\mathrm{V}$ & $(340-461)$ & $46.4 \pm 0.5$ & 440 & $\mathrm{~EB}$ & [1997STE/CHI3] \\
\hline & $\mathrm{V}$ & $(340-461)$ & $57.0 \pm 0.5$ & 298 & EB & [1997STE/CHI3] \\
\hline & $\mathrm{V}$ & $(351-410)$ & 57.6 & 298 & EB & [1987SMI/TER, 2005KAP/SLO] \\
\hline & V & $(298-308)$ & 57.5 & 298 & & [1982TOU/OKA, 2005KAP/SLO] \\
\hline \multirow[t]{2}{*}{$\mathrm{C}_{3} \mathrm{H}_{9} \mathrm{NO}$} & {$[78-96-6]$} & 1-amino-2-propanol & & & & \\
\hline & $\mathrm{V}$ & $(306-431)$ & 51.6 & 321 & A & [1987STE/MAL] \\
\hline \multirow[t]{3}{*}{$\mathrm{C}_{3} \mathrm{H}_{9} \mathrm{NO}$} & {$[156-87-6]$} & 3-amino-1-propanol & & & & \\
\hline & FUS & & 20.42 & 285.2 & DSC & [2015SOL/ROD] \\
\hline & FUS & & 16.9 & 284.1 & DSC & [2003CAC/BAU] \\
\hline \multirow[t]{2}{*}{$\mathrm{C}_{3} \mathrm{H}_{9} \mathrm{NO}$} & [5669-39-6] & $N$-methoxy dimethyl & amine (trim & droxyla & & \\
\hline & $\mathrm{V}$ & $(194-297)$ & 28 & 282 & A & [1987STE/MAL, 1957BIS/PAR] \\
\hline \multirow[t]{3}{*}{$\mathrm{C}_{3} \mathrm{H}_{9} \mathrm{NO}$} & [109-85-3] & 2-methoxyethyl amin & & & & \\
\hline & $\mathrm{V}$ & $(278-318)$ & 38.8 & 293 & A & [1987STE/MAL, 1978CAB/MOL] \\
\hline & $\mathrm{V}$ & $(278-318)$ & $38.3 \pm 0.1$ & 298 & & [1978CAB/MOL] \\
\hline
\end{tabular}


TABle 6. Phase change enthalpies of $\mathrm{C}_{3}$ organic compounds-Continued

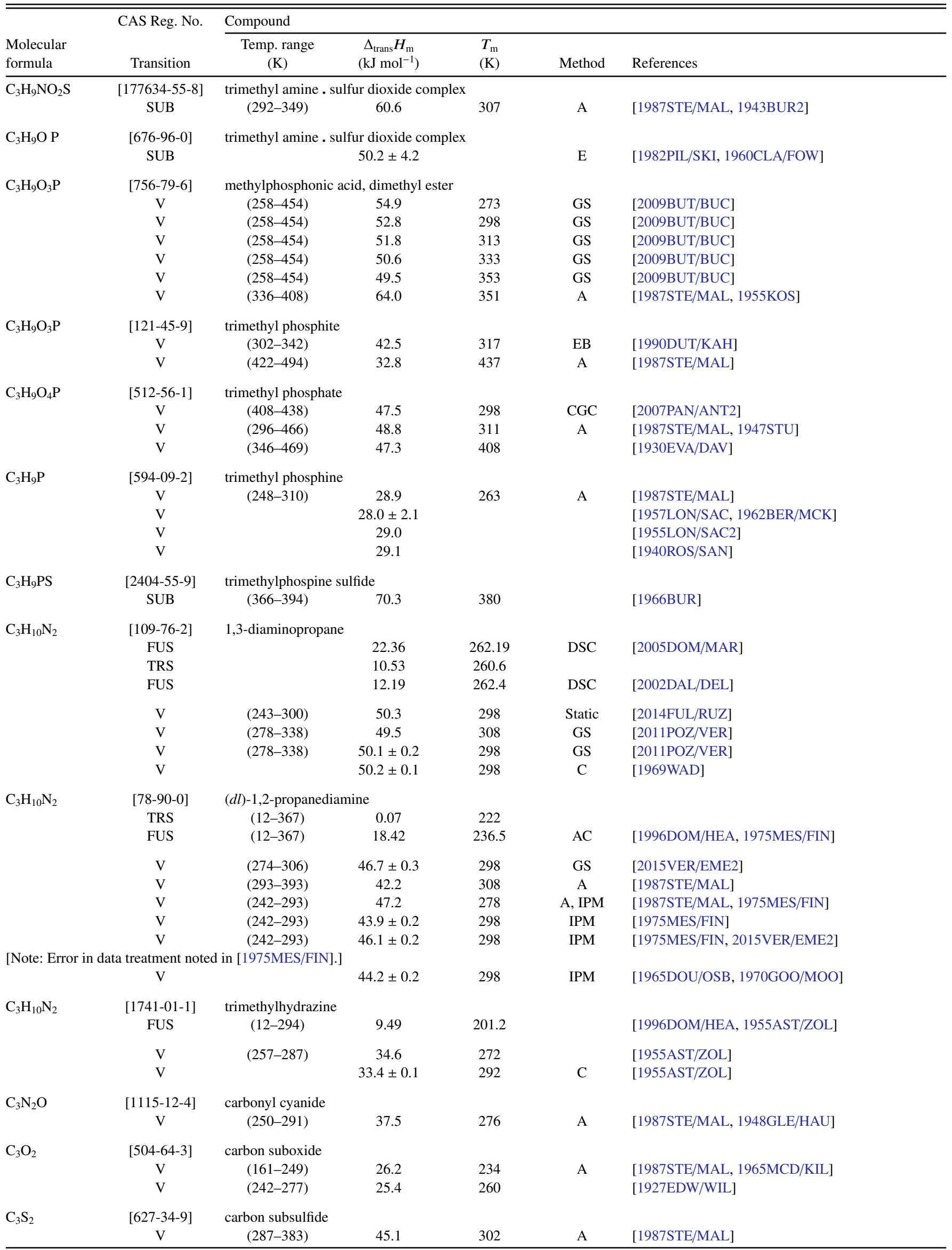


TABLE 7. Phase change enthalpies of $\mathrm{C}_{4}$ organic compounds

\begin{tabular}{|c|c|c|c|c|c|}
\hline \multirow[b]{2}{*}{$\begin{array}{l}\text { Molecular } \\
\text { formula }\end{array}$} & \multirow{2}{*}{$\begin{array}{l}\text { CAS reg. no. } \\
\text { Transition }\end{array}$} & \multicolumn{4}{|l|}{ Compound } \\
\hline & & $\begin{array}{c}\text { Temp. range } \\
\text { (K) }\end{array}$ & $T_{\mathrm{m}}(\mathrm{K})$ & Method & References \\
\hline \multirow[t]{2}{*}{$\mathrm{C}_{4} \mathrm{BrClF}_{9} \mathrm{~N}$} & [4905-97-9] & \multicolumn{4}{|c|}{ 1,1,2-trifluoro-2-chloro-2-bromo- $N, N$-bis(trifluoromethyl)ethylamine } \\
\hline & V & $(329-364) \quad 33.1$ & 344 & A & [1987STE/MAL, 1965HAS/TIP] \\
\hline \multirow[t]{2}{*}{$\mathrm{C}_{4} \mathrm{BrCl}_{2} \mathrm{~F}_{8} \mathrm{~N}$} & {$[4905-98-0]$} & \multicolumn{4}{|c|}{ 2-bromo-1,2-dichloro-1,2-difluoro- $N, N$-bis(trifluoromethyl)ethamine } \\
\hline & $\mathrm{V}$ & $(358-394) \quad 36.5$ & 376 & & {$[1965 \mathrm{HAS} / \mathrm{TIP}]$} \\
\hline \multirow[t]{2}{*}{$\mathrm{C}_{4} \mathrm{BrF}_{6} \mathrm{~N}$} & {$[22130-38-7]$} & \multicolumn{4}{|c|}{ 2-bromo- $N, N$-bis(trifluoromethyl)ethynylamine } \\
\hline & $\mathrm{V}$ & $(311-329) \quad 30.4$ & 320 & A & [1987STE/MAL, 1969FRE/TIP] \\
\hline \multirow[t]{2}{*}{$\mathrm{C}_{4} \mathrm{BrF}_{8} \mathrm{~N}$} & {$[17725-57-4]$} & \multicolumn{4}{|c|}{$N, N$-bis(trifluoromethyl)-2,2-difluoro-1-bromovinylamine } \\
\hline & $\mathrm{V}$ & $(293-320) \quad 31.2$ & 320 & A & [1987STE/MAL, 1968HAS/TIP] \\
\hline \multirow[t]{2}{*}{$\mathrm{C}_{4} \mathrm{BrF}_{8} \mathrm{NO}$} & {$[104550-50-7]$} & \multicolumn{4}{|c|}{ 4-bromo-3-(trifluoromethyl)perfluorooxazolidine } \\
\hline & $\mathrm{V}$ & 30.7 & & & {$[1986 \mathrm{OBR} / \mathrm{LAM}]$} \\
\hline \multirow[t]{2}{*}{$\mathrm{C}_{4} \mathrm{BrF}_{9} \mathrm{O}_{3} \mathrm{~S}$} & {$[14609-27-9]$} & \multicolumn{4}{|c|}{ 2-bromo-3-fluorosulfatooctafluorobutane } \\
\hline & $\mathrm{V}$ & $(313-372)$ & 342 & & [1966EAR/HIL] \\
\hline \multirow[t]{2}{*}{$\mathrm{C}_{4} \mathrm{BrF}_{10} \mathrm{~N}$} & {$[2261-32-7]$} & \multicolumn{4}{|c|}{ 1,1,2,2-tetrafluoro-2-bromo- $N, N$-bis(trifluoromethyl)ethylamine } \\
\hline & $\mathrm{V}$ & $\begin{array}{ll}(289-329) & 30.4\end{array}$ & 304 & A & [1987STE/MAL, 1965HAS/TIP] \\
\hline \multirow[t]{3}{*}{$\mathrm{C}_{4} \mathrm{Br}_{2} \mathrm{Cl}_{2} \mathrm{~F}_{6}$} & {$[375-42-8]$} & \multicolumn{4}{|c|}{ 1,4-dibromo-2,3-dichloro-1,1,2,3,4,4-hexafluorobutane } \\
\hline & $\mathrm{V}$ & $47.7 \pm 0.1$ & 308 & $\mathrm{C}$ & [1992SVO/KUB2] \\
\hline & $\mathrm{V}$ & $46.9 \pm 0.1$ & 315 & $\mathrm{C}$ & [1992SVO/KUB2] \\
\hline \multirow[t]{2}{*}{$\mathrm{C}_{4} \mathrm{Br}_{2} \mathrm{~F}_{9} \mathrm{~N}$} & {$[17725-58-5]$} & \multicolumn{4}{|c|}{ 1,2-dibromo-1,2,2-trifluoro- $N, N$-bis(trifluoromethyl)ethylamine } \\
\hline & $\mathrm{V}$ & $(326-366)$ & 341 & A & [1987STE/MAL, 1968HAS/TIP] \\
\hline $\mathrm{C}_{4} \mathrm{Br}_{4} \mathrm{~S}$ & {$[3958-03-0]$} & tetrabromothiophene & & & \\
\hline & FUS & 21.1 & 391.8 & DSC & [2014YAN/WAN] \\
\hline $\mathrm{C}_{4} \mathrm{ClF}_{8} \mathrm{~N}$ & {$[14003-64-6]$} & 2-chloro-1,2-difluoro- $N, N$-bis(tri & methyl)vi & ine & \\
\hline & $\mathrm{V}$ & $(273-312)$ & 288 & A & [1987STE/MAL, 1968HAS/TIP] \\
\hline $\mathrm{C}_{4} \mathrm{ClF}_{8} \mathrm{NO}$ & {$[82135-35-1]$} & 4-chloro-3-(trifluoromethyl)perfl & xazolidine & & \\
\hline & $\mathrm{V}$ & 27.9 & & & [1986OBR/LAM] \\
\hline $\mathrm{C}_{4} \mathrm{ClF}_{10} \mathrm{~N}$ & {$[54566-79-9]$} & $N$-chloro-1,1,2,2,2-pentafluoro- 1 & tafluoroe & thanamine & \\
\hline & $\mathrm{V}$ & 27.2 & 325 & & [1975PET/SHR2] \\
\hline $\mathrm{C}_{4} \mathrm{ClF}_{10} \mathrm{~N}$ & [53684-04-1] & $N$-chloro-1,1,1,2,3,3,3-heptafluc & -(trifluoro & 1)-2-propa & nine \\
\hline & $\mathrm{V}$ & 28.9 & 325 & & [1975KIR/LAS] \\
\hline $\mathrm{C}_{4} \mathrm{ClF}_{12} \mathrm{NS}$ & {$[62609-69-2]$} & chlorodifluoro[1,1,1,2,3,3,3-hept & -2-propa & $\operatorname{oto}(2-)](\operatorname{tri}$ & oromethyl) sulfur \\
\hline & $\mathrm{V}$ & 37.7 & 402 & & [1977KIT/SHR2] \\
\hline $\mathrm{C}_{4} \mathrm{ClF}_{13} \mathrm{~S}$ & {$[42769-87-9]$} & sulfur, chlorotetrafluoro(nonafluo & l)- & & \\
\hline & V & 37.1 & & & [1973ABE/SHR2] \\
\hline $\mathrm{C}_{4} \mathrm{Cl}_{2} \mathrm{~F}_{6}$ & [20972-44-5] & 1,4-dichloro-hexafluoro-2-butene & & & \\
\hline & V & $(279-330) \quad 34.0$ & 294 & A & [1987STE/MAL] \\
\hline $\mathrm{C}_{4} \mathrm{Cl}_{2} \mathrm{~F}_{6}$ & {$[2418-22-6]$} & cis-2,3-dichloro-hexafluoro-2-but & & & \\
\hline & V & $\begin{array}{ll}(298-341) & 32.5\end{array}$ & 313 & A & [1987STE/MAL, 1958DIC/HIL] \\
\hline $\mathrm{C}_{4} \mathrm{Cl}_{2} \mathrm{~F}_{6}$ & {$[2418-21-5]$} & trans-2,3-dichloro-hexafluoro-2-1 & & & \\
\hline & $\mathrm{V}$ & $\begin{array}{ll}(298-340) & 32.2\end{array}$ & 313 & A & [1987STE/MAL, 1958DIC/HIL] \\
\hline $\mathrm{C}_{4} \mathrm{Cl}_{2} \mathrm{~F}_{7} \mathrm{~N}$ & {$[89033-96-5]$} & 2,3,4,4-tetrafluoro-2,3-dichloro-( & romethyl) & ine & \\
\hline & $\mathrm{V}$ & $(273-333)$ & 288 & A & [1987STE/MAL, 1965BAN/BAR] \\
\hline $\mathrm{C}_{4} \mathrm{Cl}_{2} \mathrm{~F}_{7} \mathrm{~N}$ & {$[4776-86-7]$} & 2,3-dichlorotetrafluoropropyliden & Ioromethy & & \\
\hline & V & $(283-343)$ & 313 & & [1965BAN/BAR] \\
\hline $\mathrm{C}_{4} \mathrm{Cl}_{3} \mathrm{~F}_{7}$ & {$[335-44-4]$} & 2,3,3-trichloroheptafluorobutane & & & \\
\hline & $\mathrm{V}$ & $(350-371)$ & 360 & & [1959YEN/REE] \\
\hline & $\mathrm{V}$ & $(350-371)$ & 298 & & [1959YEN/REE] \\
\hline & $\mathrm{V}$ & $(302-446)$ & 317 & $\mathrm{MM}, \mathrm{A}$ & [1987STE/MAL, 1956CAP/JAC] \\
\hline $\mathrm{C}_{4} \mathrm{Cl}_{4} \mathrm{~F}_{4}$ & {$[357-19-7]$} & 1,2,3,4-tetrachlorotetrafluoro-1-b & & & \\
\hline & $\mathrm{V}$ & $(362-414) \quad 39.4$ & 377 & A & [1987STE/MAL] \\
\hline
\end{tabular}


TABLE 7. Phase change enthalpies of $\mathrm{C}_{4}$ organic compounds-Continued

\begin{tabular}{|c|c|c|c|c|c|c|}
\hline \multirow[b]{2}{*}{$\begin{array}{l}\text { Molecular } \\
\text { formula }\end{array}$} & \multirow{2}{*}{$\begin{array}{l}\text { CAS reg. no. } \\
\text { Transition }\end{array}$} & \multicolumn{5}{|l|}{ Compound } \\
\hline & & $\begin{array}{c}\text { Temp. range } \\
(\mathrm{K})\end{array}$ & $\begin{array}{c}\Delta_{\text {trans }} H_{\mathrm{m}} \\
\left(\mathrm{kJ} \mathrm{mol}^{-1}\right)\end{array}$ & $T_{\mathrm{m}}(\mathrm{K})$ & Method & References \\
\hline \multirow[t]{3}{*}{$\mathrm{C}_{4} \mathrm{Cl}_{4} \mathrm{~F}_{6} \mathrm{O}$} & {$[61136-57-0]$} & \multicolumn{5}{|c|}{ trichloromethyl 2-chloro-1,1,2,3,3,3-hexafluoropropyl ether } \\
\hline & $\mathrm{V}$ & $(325-403)$ & 40.3 & 340 & A & [1987STE/MAL] \\
\hline & V & & $42.8 \pm 0.7$ & 298 & $\mathrm{~EB}$ & [1976AMM/BUL] \\
\hline \multirow[t]{2}{*}{$\mathrm{C}_{4} \mathrm{Cl}_{4} \mathrm{~N}_{2}$} & {$[1780-40-1]$} & 2,4,5,6-tetrachl & rmidine & & & \\
\hline & SUB & & $83.0 \pm 1.0$ & 298 & $\mathrm{C}$ & [2007RIB/AMA] \\
\hline \multirow[t]{3}{*}{$\mathrm{C}_{4} \mathrm{Cl}_{6}$} & {$[87-68-3]$} & perchloro-1,3-b & ene & & & \\
\hline & $\mathrm{V}$ & $(343-484)$ & 58.6 & 358 & A & [1987STE/MAL] \\
\hline & $\mathrm{V}$ & $(343-473)$ & 60.4 & 358 & & [1971GEL/SIM, 1984BOU/FRI] \\
\hline \multirow[t]{2}{*}{$\mathrm{C}_{4} \mathrm{Cl}_{6} \mathrm{O}_{3}$} & {$[4124-31-6]$} & trichloroacetic & dride & & & \\
\hline & $\mathrm{V}$ & $(329-496)$ & 56.0 & 344 & A & [1987STE/MAL, 1947STU] \\
\hline \multirow[t]{2}{*}{$\mathrm{C}_{4} \mathrm{~F}_{2} \mathrm{~N}_{8} \mathrm{O}_{4}$} & {$[604739-72-2]$} & 1,1'-difluoro-3, & nitro-5,5'-bi- 1 & 2,4-triazo & & \\
\hline & SUB & & $105.4 \pm 3.3$ & 298 & & [2003MIR/LEB] \\
\hline \multirow[t]{2}{*}{$\mathrm{C}_{4} \mathrm{~F}_{6}$} & {$[685-63-2]$} & hexafluoro-1,3- & liene & & & \\
\hline & $\mathrm{V}$ & $(273-343)$ & 25.9 & 288 & & [2002BOB/FED] \\
\hline \multirow[t]{5}{*}{$\mathrm{C}_{4} \mathrm{~F}_{6} \mathrm{~N}_{2} \mathrm{~S}$} & {$[71148-78-2]$} & 3,4-bis(trifluorc & yl)-1 $1 \lambda 4-1,2$ & diazole & & \\
\hline & FUS & & 11.5 & 284 & DSC & [2000BRO/DU, 1989HAN] \\
\hline & FUS & & 10.8 & 284 & $\mathrm{~S}-\mathrm{F}$ & [2000BRO/DU] \\
\hline & SUB & $(253-283)$ & $49.0 \pm 1.5$ & 268 & PG & [2000BRO/DU] \\
\hline & $\mathrm{V}$ & & $38.1 \pm 0.5$ & & & [2000BRO/DU] \\
\hline \multirow[t]{2}{*}{$\mathrm{C}_{4} \mathrm{~F}_{6} \mathrm{O}_{3}$} & {$[407-25-0]$} & trifluoroacetic a & Iride & & & \\
\hline & $\mathrm{V}$ & $(271-312)$ & 34.7 & 286 & A & [1987STE/MAL, 1962KRE, 1971DYK] \\
\hline \multirow[t]{2}{*}{$\mathrm{C}_{4} \mathrm{~F}_{7} \mathrm{NO}$} & {$[4222-29-1]$} & \multicolumn{5}{|c|}{ 4,4-difluoro-3-(difluoromethylene)-2-(trifluoromethyl)-1,2-oxazetidine } \\
\hline & V & $(238-283)$ & 31.1 & 268 & A, I & [1987STE/MAL, 1960GRI/HAZ] \\
\hline \multirow[t]{2}{*}{$\mathrm{C}_{4} \mathrm{~F}_{7} \mathrm{NO}$} & {$[4777-13-3]$} & \multicolumn{5}{|c|}{ 3,6-dihydro-2,2,3,3,5,6,6-heptafluoro-2 $\mathrm{H}$-1,4-oxazine } \\
\hline & V & $(249-293)$ & 27.3 & 278 & A & [1987STE/MAL, 1965BAN/BUR] \\
\hline \multirow[t]{2}{*}{$\mathrm{C}_{4} \mathrm{~F}_{7} \mathrm{NO}_{3} \mathrm{~S}$} & {$[26404-53-5]$} & \multicolumn{5}{|c|}{ fluorosulfuric ester 3,3,3-trifluoro-2-(trifluoromethyl)lactonitrile } \\
\hline & V & $(262-320)$ & 31.2 & 277 & A & [1987STE/MAL, 1970LUS] \\
\hline \multirow[t]{4}{*}{$\mathrm{C}_{4} \mathrm{~F}_{8}$} & {$[357-26-6]$} & perfluoro-1-but & & & & \\
\hline & $\mathrm{V}$ & $(203-279)$ & 28.9 & 264 & A, I & [1987STE/MAL, 1971DYK, 1953ATK/TRE] \\
\hline & V & $(250-293)$ & U14.4 & 265 & & [1984BOU/FRI] \\
\hline & V & $(203-279)$ & 28.9 & 241 & I & [1953ATK/TRE] \\
\hline \multirow[t]{9}{*}{$\mathrm{C}_{4} \mathrm{~F}_{8}$} & {$[115-25-3]$} & perfluorocyclob & & & & \\
\hline & FUS & $(17-270)$ & 2.77 & 233.0 & $\mathrm{AC}$ & [1954FUR/MCC] \\
\hline & $\mathrm{V}$ & $(289-348)$ & 23.5 & 304 & A & [1987STE/MAL] \\
\hline & V & $(343-388)$ & 23.2 & 358 & A & [1987STE/MAL] \\
\hline & V & $(233-388)$ & 25 & 248 & & [1967KLE/PET] \\
\hline & $\mathrm{V}$ & $(234-269)$ & 25 & 254 & & [1962MAR, 1984BOU/FRI] \\
\hline & $\mathrm{V}$ & $(233-274)$ & 24.9 & 259 & A & [1987STE/MAL, 1954FUR/MCC] \\
\hline & V & $(235-261)$ & 24.8 & 248 & I & [1953ATK/TRE] \\
\hline & SUB & $(223-233)$ & 30.3 & 228 & I & [1953ATK/TRE] \\
\hline $\mathrm{C}_{4} \mathrm{~F}_{8} \mathrm{~N}_{2} \mathrm{O}_{2} \mathrm{~S}$ & {$[66918-60-3]$} & $N, N^{\prime}$-bis(trifluo & tyl)sulfur dif & liimide & & \\
\hline & V & $(328-383)$ & 43.5 & 355 & & [1978STA/MEW] \\
\hline $\mathrm{C}_{4} \mathrm{~F}_{8} \mathrm{~N}_{2} \mathrm{O}_{3}$ & {$[382-38-7]$} & perfluoro-2-(tet & oro-2-nitroetl & 2-oxazet & & \\
\hline & V & $(273-343)$ & 31.0 & 288 & A & [1987STE/MAL, 1962BIR/BLO] \\
\hline $\mathrm{C}_{4} \mathrm{~F}_{8} \mathrm{OS}$ & [42060-62-8] & perfluorotetram & ene sulfoxid & & & \\
\hline & $\mathrm{V}$ & & 37.1 & & & [1973ABE/SHR] \\
\hline $\mathrm{C}_{4} \mathrm{~F}_{8} \mathrm{O}_{2} \mathrm{~S}$ & [42060-64-0] & perfluorotetram & ene sulfone & & & \\
\hline & $\mathrm{V}$ & & 31.1 & & & [1973ABE/SHR] \\
\hline $\mathrm{C}_{4} \mathrm{~F}_{8} \mathrm{O}_{4} \mathrm{~S}$ & {$[6069-35-8]$} & heptaflurobutyr & id and fluoros & c acid an & & \\
\hline
\end{tabular}


TABLE 7. Phase change enthalpies of $\mathrm{C}_{4}$ organic compounds-Continued

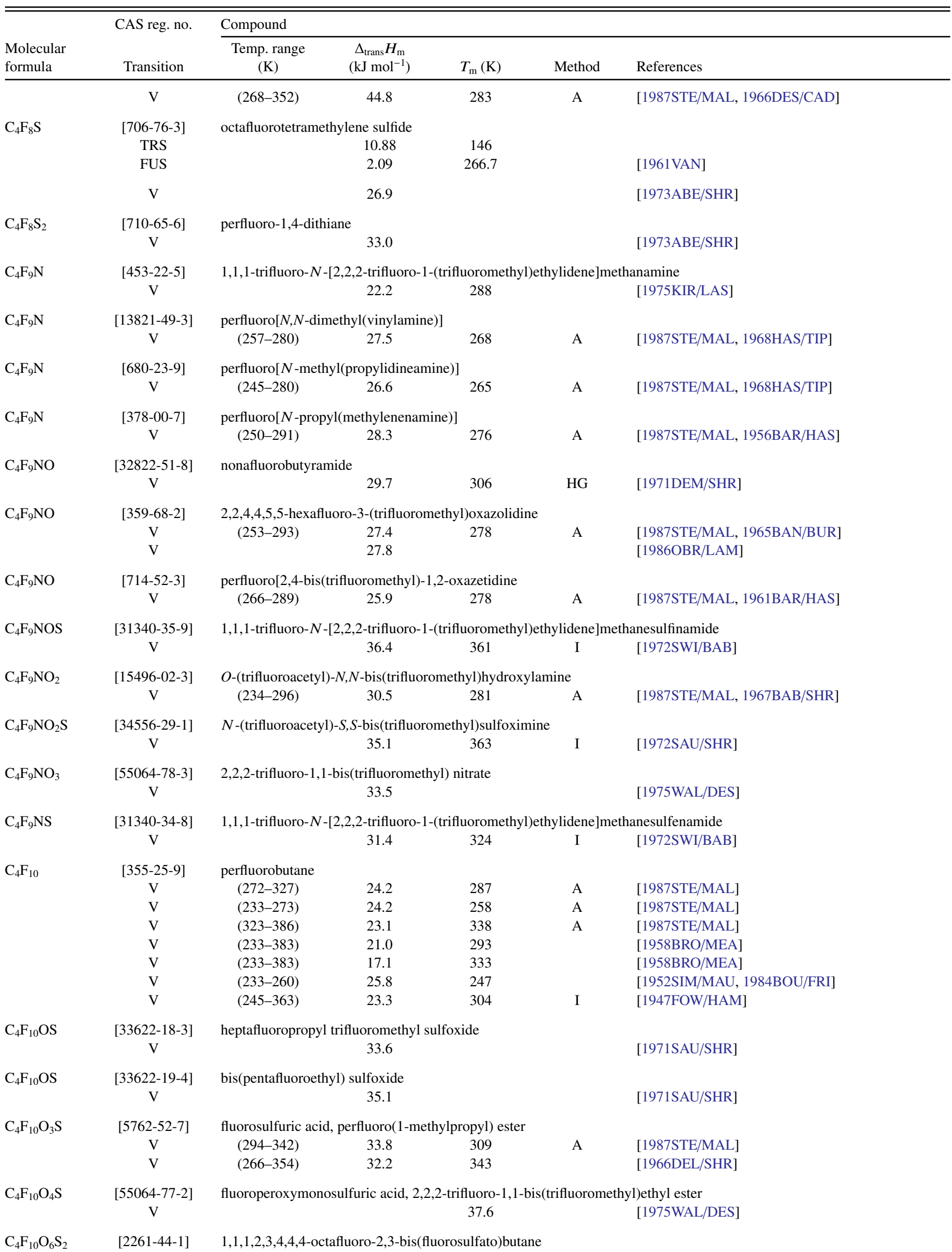


TABLE 7. Phase change enthalpies of $\mathrm{C}_{4}$ organic compounds-Continued

\begin{tabular}{|c|c|c|c|c|c|c|}
\hline \multirow[b]{2}{*}{$\begin{array}{l}\text { Molecular } \\
\text { formula }\end{array}$} & \multirow{2}{*}{$\begin{array}{l}\text { CAS reg. no. } \\
\text { Transition }\end{array}$} & \multicolumn{5}{|l|}{ Compound } \\
\hline & & $\begin{array}{c}\text { Temp. range } \\
\text { (K) }\end{array}$ & $\begin{array}{c}\Delta_{\text {trans }} H_{\mathrm{m}} \\
\left(\mathrm{kJ} \mathrm{mol}^{-1}\right) \\
\end{array}$ & $T_{\mathrm{m}}(\mathrm{K})$ & Method & References \\
\hline & $\mathrm{V}$ & $(316-393)$ & 30.1 & 331 & A & [1987STE/MAL, 1964RAT/SHR] \\
\hline & $\mathrm{V}$ & $(392-411)$ & 27.1 & 401 & A & [1987STE/MAL, 1964RAT/SHR] \\
\hline \multirow[t]{2}{*}{$\mathrm{C}_{4} \mathrm{~F}_{10} \mathrm{~S}$} & {$[42060-60-6]$} & \multicolumn{5}{|c|}{ octafluoroetramethylene sulfur difluoride } \\
\hline & $\mathrm{V}$ & \multicolumn{4}{|c|}{41.5} & {$[1973 \mathrm{ABE} / \mathrm{SHR}]$} \\
\hline \multirow[t]{2}{*}{$\mathrm{C}_{4} \mathrm{~F}_{10} \mathrm{~S}$} & {$[33547-11-4]$} & \multicolumn{5}{|c|}{ heptafluoropropyl trifluoromethyl sulfide } \\
\hline & $\mathrm{V}$ & \multicolumn{3}{|c|}{27.7} & & {$[1971 \mathrm{SAU} / \mathrm{SHR}]$} \\
\hline \multirow[t]{2}{*}{$\mathrm{C}_{4} \mathrm{~F}_{11} \mathrm{NOS}$} & {$[62609-62-5]$} & \multicolumn{5}{|c|}{ difluoro(1,1,1,3,3,3-hexafluoro-2-propaniminato)oxo(trifluoromethyl) sulfur } \\
\hline & $\mathrm{V}$ & & 35.1 & 396 & I & {$[1977 \mathrm{KIT} / \mathrm{SHR}]$} \\
\hline \multirow[t]{2}{*}{$\mathrm{C}_{4} \mathrm{~F}_{11} \mathrm{NS}$} & {$[37826-43-0]$} & \multicolumn{5}{|c|}{ fluoro(trifluoromethyl)[2,2,2,1-tetrafluoro-1-(trifluoromethyl)ethyl]imino sulfur } \\
\hline & V & $(300-333)$ & 32.4 & 315 & A, I & [1987STE/MAL, 1972SWI/SHR] \\
\hline \multirow[t]{2}{*}{$\mathrm{C}_{4} \mathrm{~F}_{12} \mathrm{~N}_{2} \mathrm{O}$} & {$[10405-32-0]$} & \multicolumn{5}{|c|}{ perfluoro(2,3-dimethyl)-4-oxo-diazepentane } \\
\hline & V & $(276-308)$ & 32 & 291 & A & [1987STE/MAL, 1971DYK, 1966HAS/TIP] \\
\hline \multirow[t]{2}{*}{$\mathrm{C}_{4} \mathrm{~F}_{12} \mathrm{~N}_{2} \mathrm{O}$} & {$[6141-72-6]$} & \multicolumn{5}{|c|}{ perfluoro(2,4-dimethyl)-3-oxo-diazepentane } \\
\hline & $\mathrm{V}$ & $(288-318)$ & 30.1 & 303 & A & [1987STE/MAL, 1971DYK, 1966HAS/TIP] \\
\hline \multirow[t]{2}{*}{$\mathrm{C}_{4} \mathrm{~F}_{12} \mathrm{~N}_{2} \mathrm{~S}$} & {$[4101-59-1]$} & \multicolumn{5}{|c|}{ difluoro bis[1,1,2,2,2-pentafluorothananaminato]sulfur } \\
\hline & $\mathrm{V}$ & & 37.0 & & & [1976STA/MEW] \\
\hline \multirow[t]{2}{*}{$\mathrm{C}_{4} \mathrm{~F}_{12} \mathrm{OP}_{2} \mathrm{~S}_{2}$} & {$[35814-50-7]$} & \multicolumn{5}{|c|}{ bis(trifluoromethyl)thiophosphinic anhydride } \\
\hline & $\mathrm{V}$ & $(281-333)$ & 37.1 & 307 & & {$[1972 P I N / C A V]$} \\
\hline $\mathrm{C}_{4} \mathrm{~F}_{12} \mathrm{OS}$ & {$[33564-25-9]$} & difluoro bis $[1,1$ & -pentafluoro & haminato] & & \\
\hline & $\mathrm{V}$ & $(284-341)$ & 33.8 & 299 & & [1999DYK/SVO] \\
\hline & $\mathrm{V}$ & $(284-341)$ & 28.6 & 299 & & [1971SAU/SHR] \\
\hline $\mathrm{C}_{4} \mathrm{~F}_{12} \mathrm{O}_{2} \mathrm{~S}$ & {$[63465-11-2]$} & bis(trifluoromet & is(trifluorom & y) sulfur & & \\
\hline & $\mathrm{V}$ & $(273-325)$ & 29.3 & 288 & $\mathrm{~A}, \mathrm{I}$ & [1987STE/MAL, 1977KIT/SHR3, 1978KIT/SHR] \\
\hline $\mathrm{C}_{4} \mathrm{~F}_{12} \mathrm{O}_{3} \mathrm{~S}$ & {$[66632-46-0]$} & oxo bis(trifluor & hyl)bis(trifluc & thoxy) sul & & \\
\hline & V & $(273-335)$ & 33.4 & 288 & A, I & [1987STE/MAL, 1978KIT/SHR] \\
\hline $\mathrm{C}_{4} \mathrm{~F}_{12} \mathrm{P}_{2} \mathrm{~S}$ & {$[1486-20-0]$} & di[bis(trifluoron & 1)phosphino & & & \\
\hline & V & $(273-335)$ & 42.2 & 304 & $\mathrm{~T}$ & {$[1964 \mathrm{CAV} / \mathrm{EME}]$} \\
\hline $\mathrm{C}_{4} \mathrm{~F}_{12} \mathrm{P}_{4}$ & [393-02-2] & 1,2,3,4-tetrakis & Ioromethyl)te & osphetane & & \\
\hline & SUB & $(292-339)$ & 65.3 & 307 & A & [1987STE/MAL, 1958MAH/BUR] \\
\hline & $\mathrm{V}$ & $(313-375)$ & 43.2 & 328 & $\mathrm{~A}, \mathrm{SG}$ & [1987STE/MAL, 1958MAH/BUR] \\
\hline $\mathrm{C}_{4} \mathrm{~F}_{12} \mathrm{~S}$ & {$[33622-15-0]$} & difluorobis(pent & roethyl) sulf & & & \\
\hline & V & $(284-341)$ & 34.0 & 299 & A & [1987STE/MAL] \\
\hline & $\mathrm{V}$ & & 32.2 & & & [1971SAU/SHR] \\
\hline $\mathrm{C}_{4} \mathrm{~F}_{12} \mathrm{~S}$ & {$[31206-31-2]$} & difluoro(heptafl & propyl) (triflu & ethyl) sul & & \\
\hline & $\mathrm{V}$ & & 32.8 & & & [1971SAU/SHR] \\
\hline $\mathrm{C}_{4} \mathrm{~F}_{13} \mathrm{NOS}$ & {$[65844-09-9]$} & trifluoro $[1,1,1,2$ & 3-heptafluoro & opanamin & -)](trifluor & ethanolato) sulfur \\
\hline & $\mathrm{V}$ & & 33.9 & 389 & I & [1978KIT/SHR] \\
\hline $\mathrm{C}_{4} \mathrm{~F}_{15} \mathrm{~N}_{2} \mathrm{O}_{2} \mathrm{P}$ & {$[36544-20-4]$} & phosphorous bi & (trifluorometl & troxide] d & & \\
\hline & $\mathrm{V}$ & $(303-370)$ & 37.6 & 336 & & [1973WAN/SHR] \\
\hline $\mathrm{C}_{4} \mathrm{~F}_{16} \mathrm{~S}_{2}$ & {$[4556-31-4]$} & hexadecafluoro- & hydro-1,4-dit & & & \\
\hline & $\mathrm{V}$ & $(323-408)$ & 40.5 & 365 & & [1999DYK/SVO, 1973ABE/SHR] \\
\hline $\mathrm{C}_{4} \mathrm{~N}_{2}$ & {$[1071-98-3]$} & dicyanoacetyler & & & & \\
\hline & SUB & (263-273) & 44.3 & 268 & I & [1957SAG, 1975GRO, 1987STE/MAL] \\
\hline & $\mathrm{V}$ & $(295-350)$ & 27.3 & 310 & A & [1987STE/MAL] \\
\hline & $\mathrm{V}$ & & 28.8 & & & [1957SAG] \\
\hline $\mathrm{C}_{4} \mathrm{~N}_{6} \mathrm{O}_{7}$ & {$[152845-81-3]$} & 3,3'-dinitrofuraz & ether & & & \\
\hline & V & (453-713) & 57.4 & 583 & BG & [2016SIN/BUR] \\
\hline $\mathrm{C}_{4} \mathrm{HBrF}_{7} \mathrm{~N}$ & [25273-49-8] & 1-bromo-2-fluo & $N$-bis(triflur & yl)vinylan & & \\
\hline
\end{tabular}


TABLE 7. Phase change enthalpies of $\mathrm{C}_{4}$ organic compounds-Continued

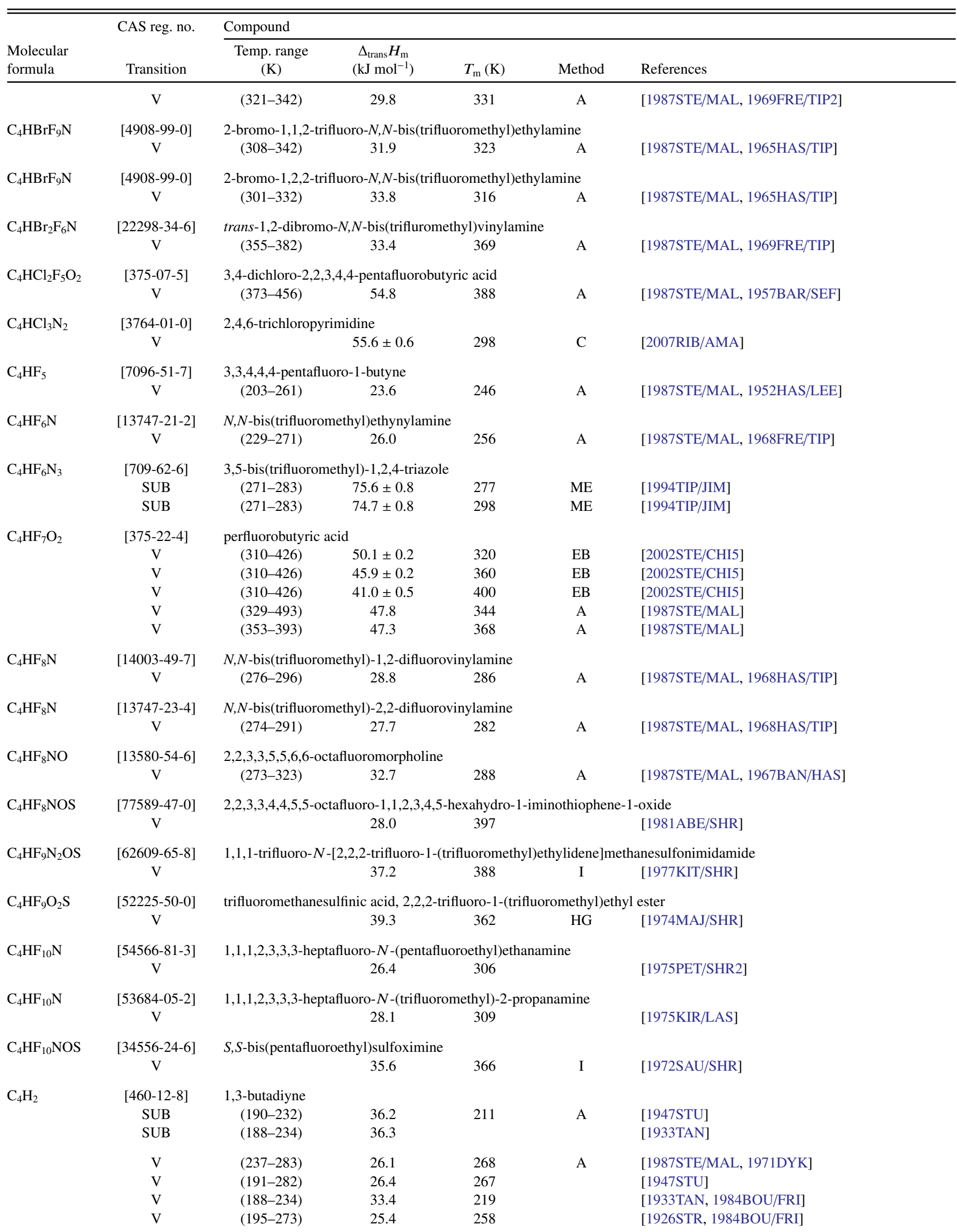


TABLE 7. Phase change enthalpies of $\mathrm{C}_{4}$ organic compounds-Continued

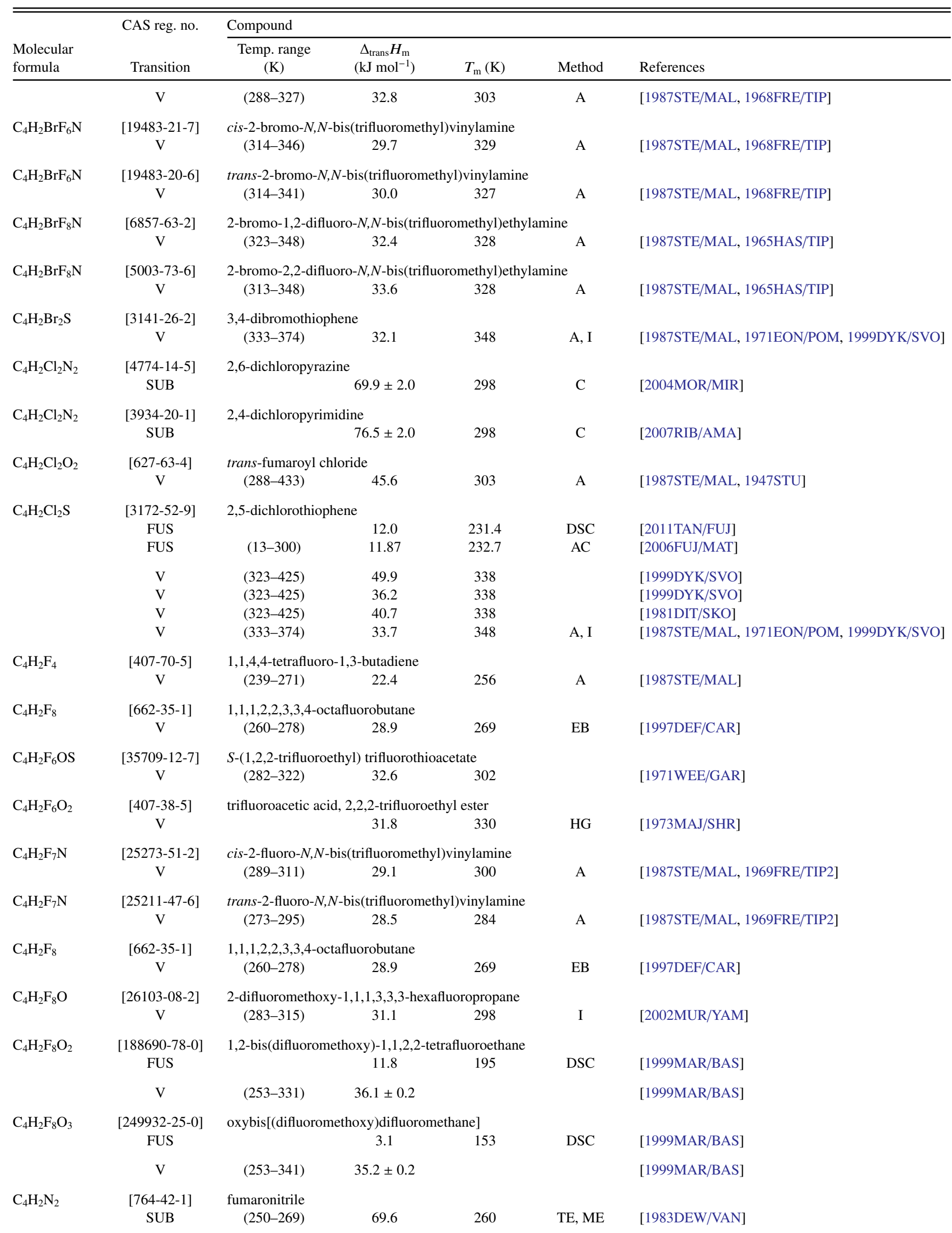


TABLE 7. Phase change enthalpies of $\mathrm{C}_{4}$ organic compounds-Continued

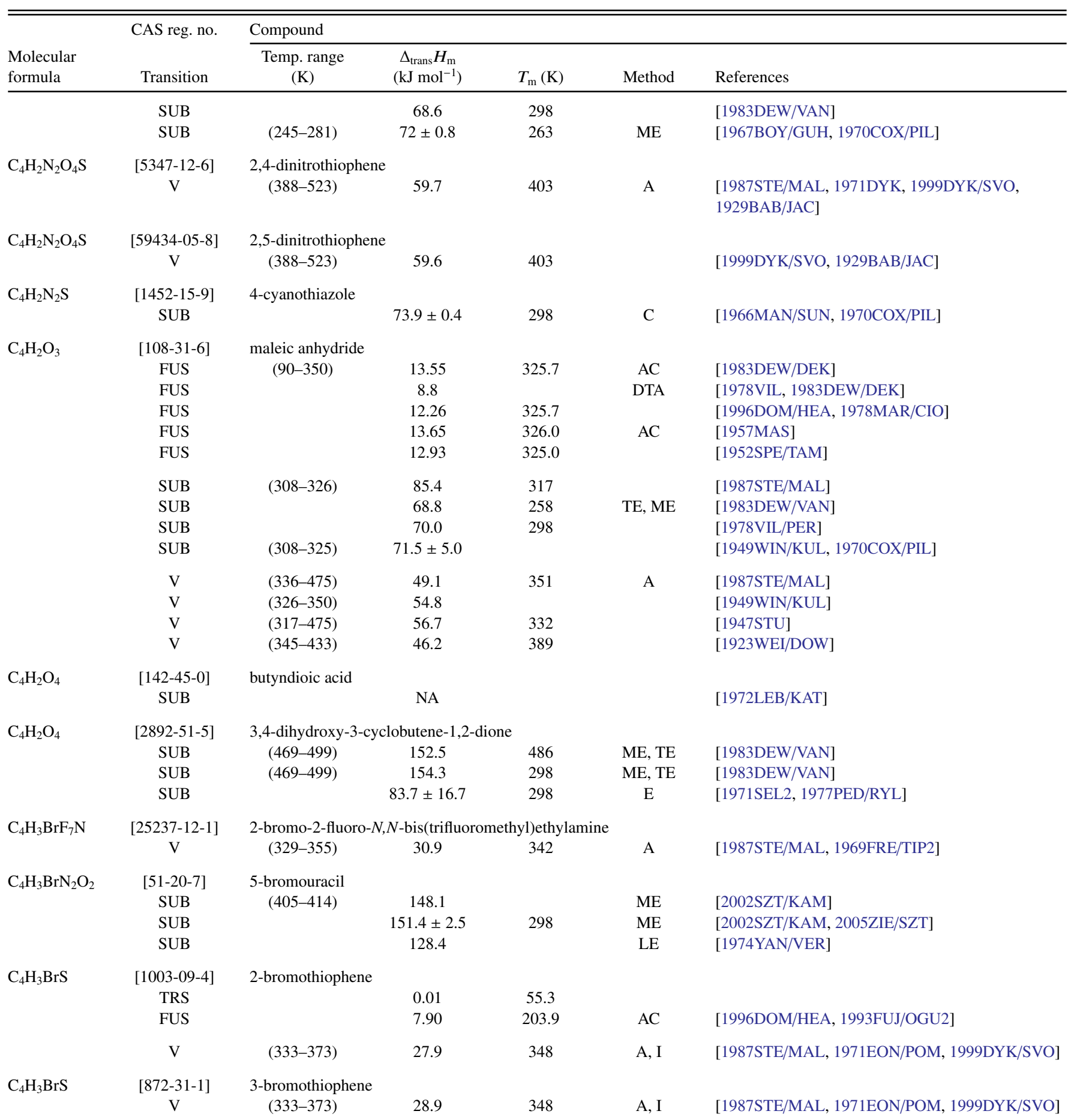

$\mathrm{C}_{4} \mathrm{H}_{3} \mathrm{ClF}_{6} \mathrm{O}_{2} \mathrm{~S}$ [57169-82-1] chlorosulfurous acid, 2,2,2-trifluoro-1-methyl-1-(trifluoromethyl)ethyl ester $\mathrm{V} \quad 39.7$

[1975DEM/KOV2]

\begin{tabular}{|c|c|c|c|c|c|c|}
\hline $\mathrm{C}_{4} \mathrm{H}_{3} \mathrm{ClN}_{2}$ & $\begin{array}{c}{[14508-49-7]} \\
\mathrm{V}\end{array}$ & 2-chloropyrazine & $45.1 \pm 1.5$ & 298 & $\mathrm{C}$ & [2004MOR/MIR] \\
\hline $\mathrm{C}_{4} \mathrm{H}_{3} \mathrm{ClN}_{2}$ & $\begin{array}{l}{[1722-12-9]} \\
\text { SUB }\end{array}$ & 2-chloropyrimidine & $70.1 \pm 1.3$ & 298 & $\mathrm{C}$ & [2007RIB/AMA] \\
\hline $\mathrm{C}_{4} \mathrm{H}_{3} \mathrm{ClN}_{2} \mathrm{O}_{2}$ & $\begin{array}{c}\text { [1820-81-1] } \\
\text { SUB } \\
\text { SUB }\end{array}$ & $\begin{array}{c}\text { 5-chlorouracil } \\
(402-412)\end{array}$ & $\begin{array}{l}145.5 \pm 3.3 \\
148.3 \pm 2.4\end{array}$ & $\begin{array}{l}407 \\
298\end{array}$ & $\begin{array}{l}\mathrm{ME} \\
\mathrm{ME}\end{array}$ & $\begin{array}{l}\text { [2002SZT/KAM] } \\
\text { [2002SZT/KAM, 2005ZIE/SZT] }\end{array}$ \\
\hline $\mathrm{C}_{4} \mathrm{H}_{3} \mathrm{ClN}_{2} \mathrm{O}_{2}$ & $\begin{array}{l}{[4270-27-3]} \\
\text { SUB }\end{array}$ & $\begin{array}{c}\text { 6-chlorouracil } \\
(381-394)\end{array}$ & $134.3 \pm 2.7$ & 387 & $\mathrm{ME}$ & [2002SZT/KAM] \\
\hline
\end{tabular}


TABLE 7. Phase change enthalpies of $\mathrm{C}_{4}$ organic compounds-Continued

\begin{tabular}{|c|c|c|c|c|c|c|}
\hline \multirow[b]{2}{*}{$\begin{array}{l}\text { Molecular } \\
\text { formula }\end{array}$} & \multirow{2}{*}{$\begin{array}{l}\text { CAS reg. no. } \\
\text { Transition }\end{array}$} & \multicolumn{5}{|l|}{ Compound } \\
\hline & & $\begin{array}{c}\text { Temp. range } \\
(\mathrm{K})\end{array}$ & $\begin{array}{c}\Delta_{\text {trans }} H_{\mathrm{m}} \\
\left(\mathrm{kJ} \mathrm{mol}^{-1}\right) \\
\end{array}$ & $T_{\mathrm{m}}(\mathrm{K})$ & Method & References \\
\hline & SUB & & $135.2 \pm 2.0$ & 298 & ME & [2002SZT/KAM, 2005ZIE/SZT] \\
\hline $\mathrm{C}_{4} \mathrm{H}_{3} \mathrm{ClS}$ & $\begin{array}{l}{[96-43-5]} \\
\text { FUS }\end{array}$ & $\begin{array}{l}\text { 2-chlorothiophene } \\
\qquad(13-300)\end{array}$ & 8.97 & 201.3 & $\mathrm{AC}$ & [1993FUJ/OGU] \\
\hline & $\begin{array}{l}\mathrm{V} \\
\mathrm{V} \\
\mathrm{V}\end{array}$ & $\begin{array}{l}(313-401) \\
(320-401) \\
(333-374)\end{array}$ & $\begin{array}{l}34.7 \\
36.9 \\
34.4\end{array}$ & $\begin{array}{l}328 \\
335 \\
348\end{array}$ & $\mathrm{~A}, \mathrm{I}$ & $\begin{array}{l}\text { [1999DYK/SVO] } \\
\text { [1999DYK/SVO, 1981DIT/SKO] } \\
\text { [1987STE/MAL, 1971EON/POM, 1999DYK/SVO] }\end{array}$ \\
\hline $\mathrm{C}_{4} \mathrm{H}_{3} \mathrm{ClS}$ & $\begin{array}{c}\text { [17249-80-8] } \\
\text { FUS }\end{array}$ & $\begin{array}{l}\text { 3-chlorothiophene } \\
\text { (13-300) }\end{array}$ & 9.39 & 214.2 & $\mathrm{AC}$ & [2004FUJ/TOD] \\
\hline $\mathrm{C}_{4} \mathrm{H}_{3} \mathrm{Cl}_{3} \mathrm{OS}$ & V & $\begin{array}{l}\text { 2,3,3-trichloro-2-pr } \\
\quad(383-423)\end{array}$ & $\begin{array}{c}\text { penethioic ac } \\
64.8\end{array}$ & -methyl e: & GC & [1980PIT/KIS] \\
\hline $\mathrm{C}_{4} \mathrm{H}_{3} \mathrm{FN}_{2} \mathrm{O}_{2}$ & $\begin{array}{l}{[51-21-8]} \\
\text { SUB } \\
\text { SUB } \\
\text { SUB } \\
\text { SUB } \\
\text { SUB }\end{array}$ & $\begin{array}{c}\text { 5-fluorouracil } \\
\quad(411-433) \\
(411-433) \\
(394-405) \\
(421-483)\end{array}$ & $\begin{array}{l}130.1 \pm 1.2 \\
132.5 \pm 1.2 \\
129.9 \pm 1.5 \\
133.2 \pm 2.1 \\
150 \pm 2\end{array}$ & $\begin{array}{l}422 \\
298 \\
399 \\
298\end{array}$ & $\begin{array}{l}\mathrm{ME} \\
\mathrm{ME} \\
\mathrm{ME} \\
\mathrm{ME} \\
\mathrm{TE}\end{array}$ & $\begin{array}{l}\text { [2014AMA/SZT] } \\
\text { [2014AMA/SZT] } \\
\text { [2002SZT/KAM] } \\
\text { [2002SZT/KAM, 2005ZIE/SZT] } \\
\text { [2002BRU/POR] }\end{array}$ \\
\hline $\mathrm{C}_{4} \mathrm{H}_{3} \mathrm{~F}_{5} \mathrm{OS}$ & $\begin{array}{c}\text { [35709-11-6] } \\
\text { V }\end{array}$ & $\begin{array}{l}\text { trifluoroacetic acid, } \\
\qquad(282-322)\end{array}$ & $\begin{array}{c}\text { S-(2,2-difluor } \\
39.3\end{array}$ & $\begin{array}{l}\text { 1) ester } \\
297\end{array}$ & A & [1987STE/MAL, 1999DYK/SVO, 1971WEE/GAR] \\
\hline $\mathrm{C}_{4} \mathrm{H}_{3} \mathrm{~F}_{5} \mathrm{O}_{3}$ & $\begin{array}{c}\text { [2195-84-8] } \\
\text { FUS }\end{array}$ & $\alpha-$ (trifluoromethox & $\begin{array}{l}\text { y)- } \alpha, \alpha \text {-difluo } \\
8.51\end{array}$ & $\begin{array}{c}\text { hyl acetat } \\
167.4\end{array}$ & & [1996DOM/HEA, 1984GOL/KOL] \\
\hline $\mathrm{C}_{4} \mathrm{H}_{3} \mathrm{~F}_{6} \mathrm{NO}_{2}$ & $\begin{array}{c}{[22743-78-8]} \\
V\end{array}$ & $\begin{array}{l}N, N \text {-bis(trifluorome } \\
\quad(268-336)\end{array}$ & $\begin{array}{l}\text { thyl)acetamid } \\
40.6\end{array}$ & $\begin{array}{l}\text { oxide } \\
283\end{array}$ & A & [1987STE/MAL, 1968NAS/BAB] \\
\hline $\mathrm{C}_{4} \mathrm{H}_{3} \mathrm{~F}_{6} \mathrm{O}_{2} \mathrm{P}$ & $\begin{array}{c}{[2022-79-9]} \\
V\end{array}$ & $\begin{array}{l}\text { bis(trifluoromethyl) } \\
\quad(273-313)\end{array}$ & $\begin{array}{c}\text { acetoxyphosp } \\
41\end{array}$ & 288 & & [1964PET/BUR, 1984BOU/FRI] \\
\hline $\mathrm{C}_{4} \mathrm{H}_{3} \mathrm{~F}_{7} \mathrm{O}$ & $\begin{array}{c}{[375-01-9]} \\
\mathrm{V}\end{array}$ & $\begin{array}{l}\text { 2,2,3,3,4,4,4-heptaf } \\
\quad(273-298)\end{array}$ & $\begin{array}{l}\text { uoro-1-butanc } \\
43.6\end{array}$ & 286 & A, MM & [1987STE/MAL, 1971DYK, 1967MEE/GOL] \\
\hline $\mathrm{C}_{4} \mathrm{H}_{3} \mathrm{~F}_{7} \mathrm{O}$ & $\begin{array}{c}\text { [171182-95-9] } \\
\mathrm{V}\end{array}$ & $\begin{array}{l}\text { 1-(2,2-difluoroethos } \\
\quad(288-318)\end{array}$ & $\begin{array}{l}\text { y)-1,1,2,2,2-p } \\
\quad 31.5\end{array}$ & $\begin{array}{l}\text { luoroethar } \\
303\end{array}$ & I & [2002MUR/YAM] \\
\hline $\mathrm{C}_{4} \mathrm{H}_{3} \mathrm{~F}_{7} \mathrm{O}$ & $\begin{array}{c}\text { [406-78-0] } \\
\mathrm{V}\end{array}$ & $\begin{array}{l}\text { 1,1,2,2-tetrafluoro- } \\
\quad(283-329)\end{array}$ & $\begin{array}{c}-(2,2,2 \text {-trifluo } \\
34\end{array}$ & $\begin{array}{l}\text { oxy)ethan } \\
298\end{array}$ & I & [2002MUR/YAM] \\
\hline $\mathrm{C}_{4} \mathrm{H}_{3} \mathrm{~F}_{7} \mathrm{O}$ & $\begin{array}{c}{[1683-81-4]} \\
\mathrm{V}\end{array}$ & $\begin{array}{l}\text { 1,1,2,2-tetrafluoro-? } \\
\quad(288-319)\end{array}$ & $\begin{array}{c}\text {-(trifluoromet } \\
31.3\end{array}$ & $\begin{array}{l}\text { propane } \\
303\end{array}$ & I & [2002MUR/YAM] \\
\hline $\mathrm{C}_{4} \mathrm{H}_{3} \mathrm{~F}_{7} \mathrm{O}$ & $\begin{array}{c}{[28523-86-6]} \\
\mathrm{V}\end{array}$ & $\begin{array}{l}\text { 1,1,1,3,3,3-hexafluc } \\
\quad(288-331)\end{array}$ & $\begin{array}{c}\text { ro-2-fluorome } \\
34.1\end{array}$ & $\begin{array}{l}\text { ypropane } \\
303\end{array}$ & I & [2002MUR/YAM] \\
\hline $\mathrm{C}_{4} \mathrm{H}_{3} \mathrm{~F}_{7} \mathrm{O}$ & $\begin{array}{c}{[56860-81-2]} \\
\mathrm{V}\end{array}$ & $\begin{array}{l}\text { 3-(difluoromethoxy } \\
\text { (283-319) }\end{array}$ & $\begin{array}{c}-1,1,1,2,2-\text { per } \\
31.2\end{array}$ & $\begin{array}{c}\text { oropropan } \\
298\end{array}$ & $\mathrm{I}$ & [2002MUR/YAM] \\
\hline $\mathrm{C}_{4} \mathrm{H}_{3} \mathrm{~F}_{7} \mathrm{O}_{2} \mathrm{~S}$ & $\begin{array}{c}{[57169-83-2]} \\
V\end{array}$ & fluorosulfurous acic & $\begin{array}{c}\text { 2,2,2-trifluo } \\
36.4\end{array}$ & nethyl-1-( & romethyl) & $\begin{array}{l}1 \text { ester } \\
\text { [1975DEM/KOV2] }\end{array}$ \\
\hline $\mathrm{C}_{4} \mathrm{H}_{3} \mathrm{IN}_{2} \mathrm{O}_{2}$ & $\begin{array}{l}{[696-07-1]} \\
\text { SUB } \\
\text { SUB }\end{array}$ & $\begin{array}{r}5 \text {-iodouracil } \\
(403-414) \\
(402-413)\end{array}$ & $\begin{array}{l}152.5 \pm 2.9 \\
153.5 \pm 2.5\end{array}$ & $\begin{array}{l}409 \\
407\end{array}$ & $\begin{array}{l}\mathrm{ME} \\
\mathrm{ME}\end{array}$ & $\begin{array}{l}\text { [2002SZT/KAM] } \\
\text { [2002SZT/KAM] }\end{array}$ \\
\hline $\mathrm{C}_{4} \mathrm{H}_{3} \mathrm{IS}$ & $\begin{array}{c}{[3437-95-4]} \\
\mathrm{V}\end{array}$ & $\begin{array}{l}\text { 2-iodothiophene } \\
\quad(333-374)\end{array}$ & 29.0 & 348 & A, I & [1987STE/MAL, 1971EON/POM, 1999DYK/SVO] \\
\hline $\mathrm{C}_{4} \mathrm{H}_{3} \mathrm{NO}_{2} \mathrm{~S}$ & $\begin{array}{c}{[609-40-5]} \\
\mathrm{V} \\
\mathrm{V}\end{array}$ & $\begin{array}{l}\text { 2-nitrothiophene } \\
\quad(378-443) \\
(321-498)\end{array}$ & $\begin{array}{l}48.6 \\
50.4\end{array}$ & $\begin{array}{l}393 \\
336\end{array}$ & A & $\begin{array}{l}\text { [1999DYK/SVO] } \\
\text { [1987STE/MAL] }\end{array}$ \\
\hline $\mathrm{C}_{4} \mathrm{H}_{3} \mathrm{NO}_{3}$ & $\begin{array}{c}\text { [609-39-2] } \\
\text { SUB }\end{array}$ & 2-nitrofuran & $75.3 \pm 2.1$ & & & [1980BAL/LEB, 1986PED/NAY] \\
\hline $\mathrm{C}_{4} \mathrm{H}_{3} \mathrm{~N}_{3} \mathrm{O}_{4}$ & $\begin{array}{l}{[611-08-5]} \\
\text { SUB }\end{array}$ & $\begin{array}{r}\text { 5-nitrouracil } \\
(431-453)\end{array}$ & $159.1 \pm 2.1$ & 442 & ME & [2003ZIE/SZT] \\
\hline
\end{tabular}


TABLE 7. Phase change enthalpies of $\mathrm{C}_{4}$ organic compounds-Continued

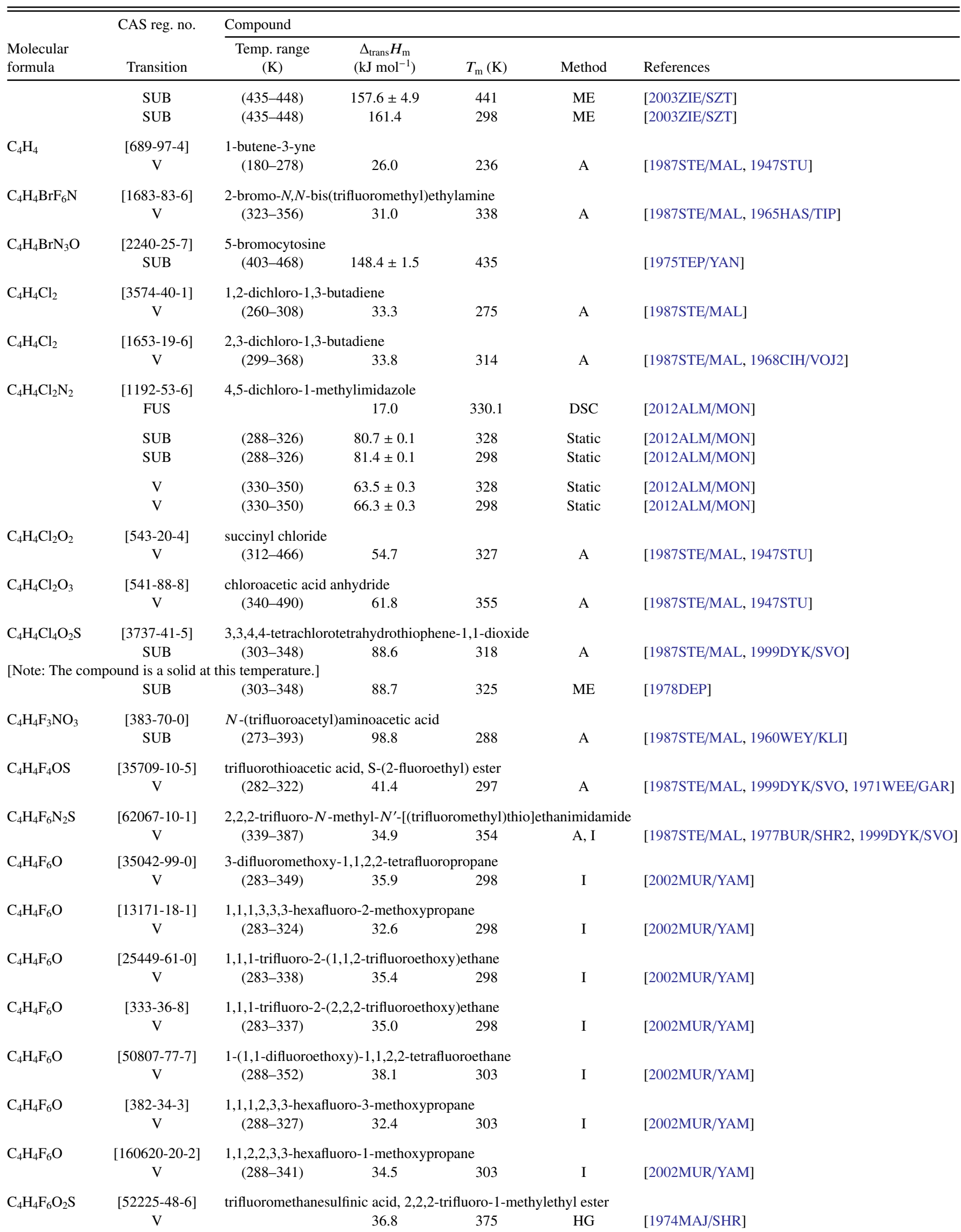


TABLE 7. Phase change enthalpies of $\mathrm{C}_{4}$ organic compounds-Continued

\begin{tabular}{|c|c|c|c|c|c|c|}
\hline \multirow[b]{2}{*}{$\begin{array}{l}\text { Molecular } \\
\text { formula }\end{array}$} & \multirow{2}{*}{$\begin{array}{l}\text { CAS reg. no. } \\
\text { Transition }\end{array}$} & \multicolumn{5}{|l|}{ Compound } \\
\hline & & $\begin{array}{l}\text { Temp. range } \\
\text { (K) }\end{array}$ & $\begin{array}{c}\Delta_{\text {trans }} H_{\mathrm{m}} \\
\left(\mathrm{kJ} \mathrm{mol}^{-1}\right)\end{array}$ & $T_{\mathrm{m}}(\mathrm{K})$ & Method & References \\
\hline \multirow[t]{2}{*}{$\mathrm{C}_{4} \mathrm{H}_{4} \mathrm{IN}_{3} \mathrm{O}$} & [1122-44-7] & \multicolumn{4}{|c|}{ 5-iodocytosine (4-amino-5-iodopyrimidinone) } & \\
\hline & SUB & $(413-463)$ & $144 \pm 1.5$ & 438 & & [1975TEP/YAN] \\
\hline \multirow[t]{16}{*}{$\mathrm{C}_{4} \mathrm{H}_{4} \mathrm{~N}_{2}$} & [290-37-9] & pyrazine & & & & \\
\hline & TRS & & 1.0 & 304.0 & & \\
\hline & FUS & & 15.3 & 326.5 & DSC & [2012VER/EME4] \\
\hline & TRS & $(5-380)$ & 1.09 & 300.5 & & \\
\hline & TRS & $(5-380)$ & 0.05 & 309.8 & & \\
\hline & FUS & $(5-380)$ & 14.78 & 325.4 & $\mathrm{AC}$ & [2003CHI/KNI] \\
\hline & FUS & & 12.95 & 328.2 & & [1978BOU/LEC] \\
\hline & SUB (I) & $(274-300)$ & $57.5 \pm 0.4$ & 298 & GS & [2012VER/EME4] \\
\hline & SUB (II) & $(303-314)$ & $56.4 \pm 0.8$ & 298 & GS & [2012VER/EME4] \\
\hline & SUB & $(288-317)$ & 56.2 & 303 & & [1995SAK/UEO] \\
\hline & SUB & & $56.3 \pm 0.5$ & & $\mathrm{C}$ & [1962TJE, 1970COX/PIL] \\
\hline & $\mathrm{V}$ & $(342-373)$ & $40.5 \pm 1.7$ & 298 & CGC & [2009LIP/CHI2, 2009LIP/CHI] \\
\hline & V & $(354-426)$ & $38.8 \pm 0.1$ & 340 & $\mathrm{~EB}$ & [2002STE/CHI3] \\
\hline & $\mathrm{V}$ & $(354-426)$ & $36.5 \pm 0.2$ & 380 & $\mathrm{~EB}$ & [2002STE/CHI3] \\
\hline & $\mathrm{V}$ & $(354-426)$ & $34.1 \pm 0.4$ & 420 & $\mathrm{~EB}$ & [2002STE/CHI3] \\
\hline & $\mathrm{V}$ & $(332-373)$ & 37.9 & 352 & & [1995SAK/UEO] \\
\hline \multirow[t]{8}{*}{$\mathrm{C}_{4} \mathrm{H}_{4} \mathrm{~N}_{2}$} & [289-95-2] & pyrimidine & & & & \\
\hline & TRS & & 2.6 & 238.4 & & \\
\hline & FUS & & 13.0 & 294.3 & DSC & [2012VER/EME4] \\
\hline & SUB & $(275-292)$ & $58.3 \pm 0.6$ & 298 & GS & [2012VER/EME4] \\
\hline & $\mathrm{V}$ & $(293-328)$ & $44.1 \pm 0.4$ & 298 & GS & [2012VER/EME4] \\
\hline & $\mathrm{V}$ & $(342-373)$ & $41.0 \pm 1.9$ & 298 & CGC & [2009LIP/CHI2, 2009LIP/CHI] \\
\hline & $\mathrm{V}$ & & $49.9 \pm 0.6$ & 298 & $\mathrm{C}$ & {$[1977 \mathrm{NAB} / \mathrm{SAB}]$} \\
\hline & $\mathrm{V}$ & & $49.8 \pm 0.3$ & 298 & $\mathrm{C}$ & [1962TJE] \\
\hline \multirow[t]{4}{*}{$\mathrm{C}_{4} \mathrm{H}_{4} \mathrm{~N}_{2}$} & [289-80-5] & pyridazine & & & & \\
\hline & $\mathrm{V}$ & $(283-343)$ & $54.6 \pm 0.2$ & 298 & GS & [2012VER/EME4] \\
\hline & $\mathrm{V}$ & & $53.1 \pm 2.4$ & 298 & CGC & [2010LIP/PLI] \\
\hline & $\mathrm{V}$ & & $53.5 \pm 0.4$ & 298 & $\mathrm{C}$ & [1962TJE] \\
\hline \multirow[t]{8}{*}{$\mathrm{C}_{4} \mathrm{H}_{4} \mathrm{~N}_{2}$} & [110-61-2] & succinonitrile & & & & \\
\hline & FUS & & 3.7 & 329.7 & DSC & [2009RAI/RED] \\
\hline & TRS & & 6.09 & 233.6 & & \\
\hline & FUS & & 3.75 & 330.3 & DSC & [2007BAD/BLA] \\
\hline & FUS & & 3.72 & 331.2 & DSC & [2004STU/WIT] \\
\hline & TRS & & 6.2 & 233.3 & & \\
\hline & FUS & $(5-350)$ & 3.7 & 331.2 & $\mathrm{AC}$ & [1996DOM/HEA, 1963WUL/WES] \\
\hline & SUB & $(279-298)$ & $70.0 \pm 0.3$ & 289 & & $\begin{array}{l}\text { [1960WOO/MUR, 1977PED/RYL, 1971RAP/WES, } \\
\text { 1969STU/WES] }\end{array}$ \\
\hline \multirow[t]{2}{*}{$\mathrm{C}_{4} \mathrm{H}_{4} \mathrm{~N}_{2} \mathrm{O}$} & {$[4562-27-0]$} & 4(3H)-pyridm & & & & \\
\hline & SUB & & $104.8 \pm 0.8$ & 298 & ME & [2014GAL/ROC] \\
\hline \multirow[t]{4}{*}{$\mathrm{C}_{4} \mathrm{H}_{4} \mathrm{~N}_{2} \mathrm{OS}$} & [141-90-2] & 2-thiouracil & & & & \\
\hline & SUB & (421-443) & $131.8 \pm 1.1$ & 432 & ME & [2013RIB/AMA] \\
\hline & SUB & $(421-443)$ & $138.5 \pm 1.1$ & 298 & ME & [2013RIB/AMA] \\
\hline & SUB & & 129.3 & & LE & [1974YAN/VER] \\
\hline \multirow[t]{2}{*}{$\mathrm{C}_{4} \mathrm{H}_{4} \mathrm{~N}_{2} \mathrm{OS}$} & [591-28-6] & 4-thiouracil & & & & \\
\hline & SUB & & 125.5 & & LE & [1974YAN/VER] \\
\hline \multirow[t]{2}{*}{$\mathrm{C}_{4} \mathrm{H}_{4} \mathrm{~N}_{2} \mathrm{O}_{2}$} & [2423-84-9] & pyrazine $1,4-\mathrm{d}$ & & & & \\
\hline & SUB & & $116.9 \pm 0.8$ & 298 & $\mathrm{C}$ & [1997ACR/POW] \\
\hline \multirow[t]{5}{*}{$\mathrm{C}_{4} \mathrm{H}_{4} \mathrm{~N}_{2} \mathrm{O}_{2}$} & {$[66-22-8]$} & uracil & & & & \\
\hline & SUB & (424-493) & $131.9 \pm 0.5$ & 298 & GS & [2015EME/VER2] \\
\hline & SUB & (424-493) & $131.9 \pm 0.7$ & 298 & GS & [2014ACH/EME] \\
\hline & SUB & $(315-435)$ & $125.3 \pm 0.2$ & 425 & $\mathrm{QR}, \mathrm{ME}$ & [2006DEB/MED] \\
\hline & SUB & $(399-411)$ & 101.3 & & ME & [2002SZT/KAM] \\
\hline
\end{tabular}


TABle 7. Phase change enthalpies of $\mathrm{C}_{4}$ organic compounds-Continued

\begin{tabular}{|c|c|c|c|c|c|c|}
\hline \multirow[b]{2}{*}{$\begin{array}{l}\text { Molecular } \\
\text { formula }\end{array}$} & \multirow{2}{*}{$\begin{array}{c}\text { CAS reg. no. } \\
\text { Transition }\end{array}$} & \multicolumn{5}{|l|}{ Compound } \\
\hline & & $\begin{array}{c}\text { Temp. range } \\
(\mathrm{K})\end{array}$ & $\begin{array}{c}\Delta_{\text {trans }} H_{\mathrm{m}} \\
\left(\mathrm{kJ} \mathrm{mol}^{-1}\right)\end{array}$ & $T_{\mathrm{m}}(\mathrm{K})$ & Method & References \\
\hline & SUB & $(394-494)$ & $127.0 \pm 2.0$ & 439 & TE & [2000BRU/PIA] \\
\hline & SUB & $(452-587)$ & $130.6 \pm 4.0$ & 519 & ME, TE & [1980BAR/BEN] \\
\hline & SUB & $(452-587)$ & $131 \pm 5$ & 298 & TE, GS & [1980BAR/BEN] \\
\hline & SUB & $(378-428)$ & $120.5 \pm 1.3$ & 403 & QR & [1980TEP/YAN] \\
\hline & SUB & & 121.7 & 425 & MS & [1979YAN/TEP] \\
\hline & SUB & $(500-545)$ & $133.9 \pm 8$ & 523 & HSA & {$[1978 \mathrm{NOW} / \mathrm{SZC}]$} \\
\hline & SUB & & 122.9 & 440 & $\mathrm{C}$ & {$[1977 \mathrm{NAB} / \mathrm{SAB}]$} \\
\hline & SUB & & $125.9 \pm 2.5$ & 298 & $\mathrm{C}$ & [1977NAB/SAB, 2015EME/VER2] \\
\hline & SUB & $(393-458)$ & $120.5 \pm 5.2$ & 426 & LE & [1975YAN/TEP, 1974YAN/VER] \\
\hline & SUB & & $115.5 \pm 2.1$ & & ME & [1972ROM/SUK, 2000BRU/PIA] \\
\hline & SUB & & $\mathrm{U} 83.7$ & & & {$[1965 \mathrm{CLA} / \mathrm{PES}]$} \\
\hline \multirow[t]{5}{*}{$\mathrm{C}_{4} \mathrm{H}_{4} \mathrm{~N}_{2} \mathrm{O}_{2} \mathrm{~S}$} & [504-17-6] & thiobarbituric acid & & & & \\
\hline & TRS & $(265-430)$ & 0.7 & 458.9 & & \\
\hline & TRS & $(265-430)$ & 0.2 & 483.8 & & \\
\hline & TRS & $(265-430)$ & 0.3 & 503.1 & & \\
\hline & FUS & $(265-430)$ & 14.8 & 516.4 & DSC & [2012ROU/NOT] \\
\hline
\end{tabular}

[Note: Authors of [2012ROU/NOT] noted that an exothermic decomposition occurred immediately after fusion.]

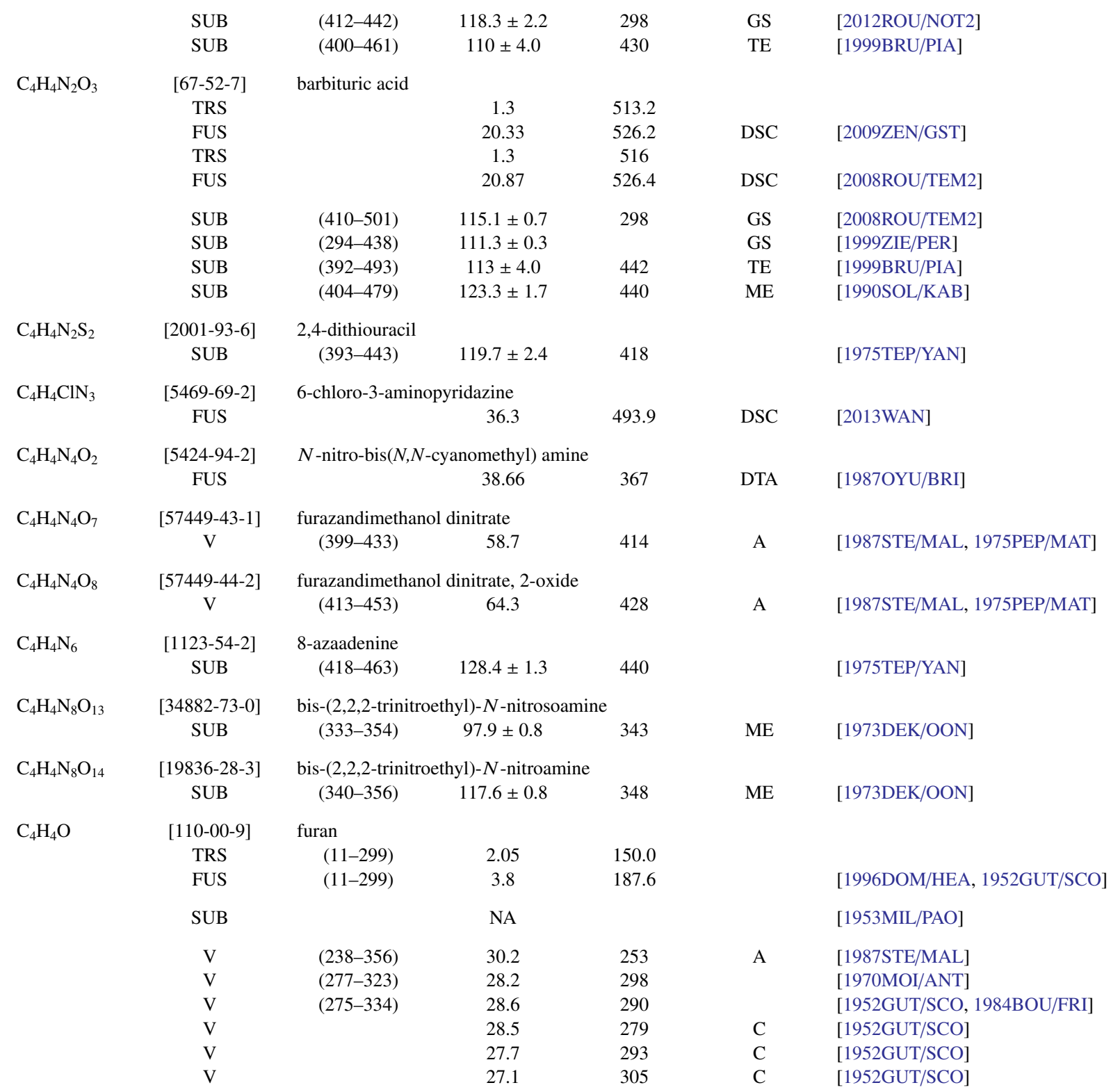


TABLE 7. Phase change enthalpies of $\mathrm{C}_{4}$ organic compounds-Continued

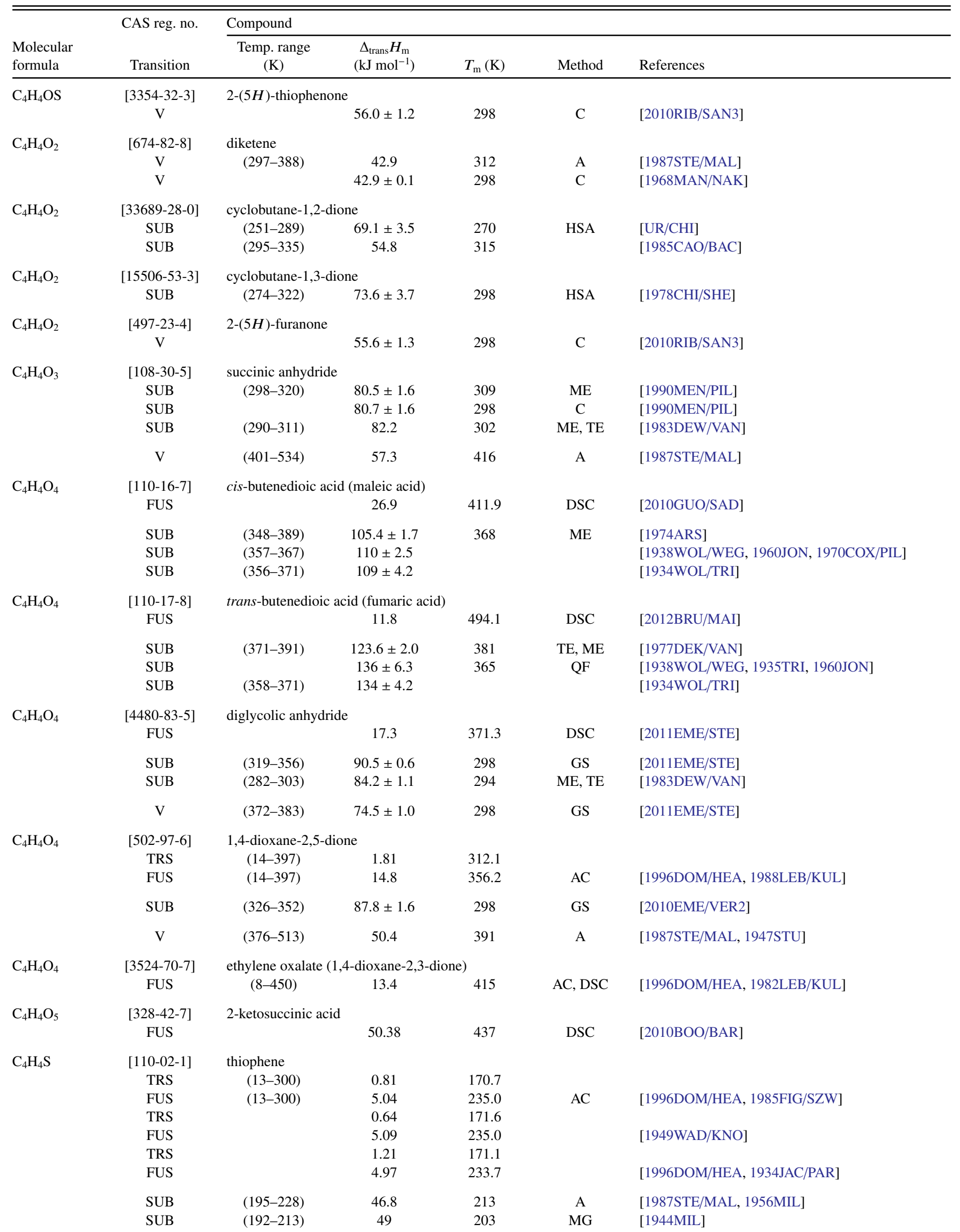


TABLE 7. Phase change enthalpies of $\mathrm{C}_{4}$ organic compounds-Continued

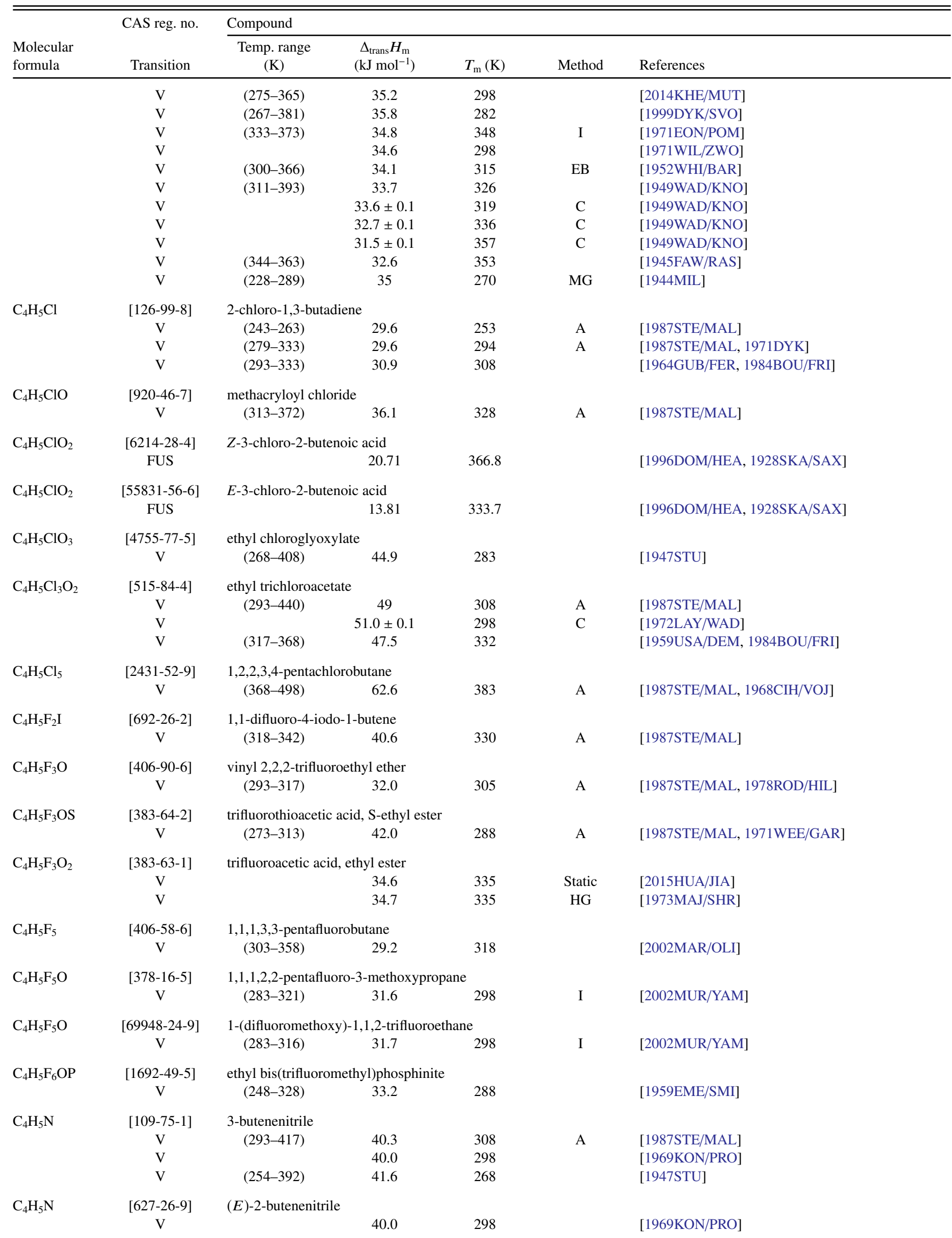


TABLE 7. Phase change enthalpies of $\mathrm{C}_{4}$ organic compounds-Continued

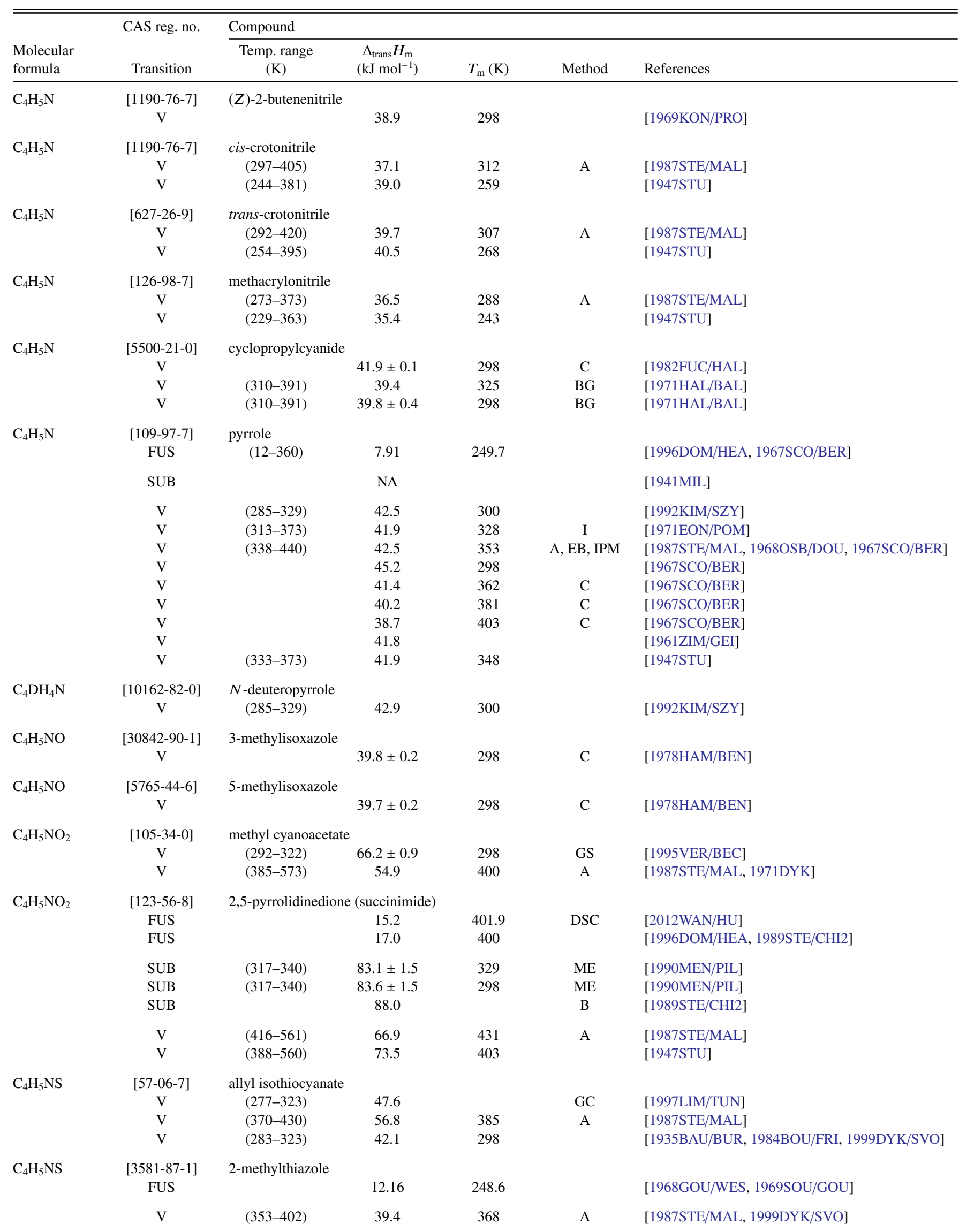


TABle 7. Phase change enthalpies of $\mathrm{C}_{4}$ organic compounds-Continued

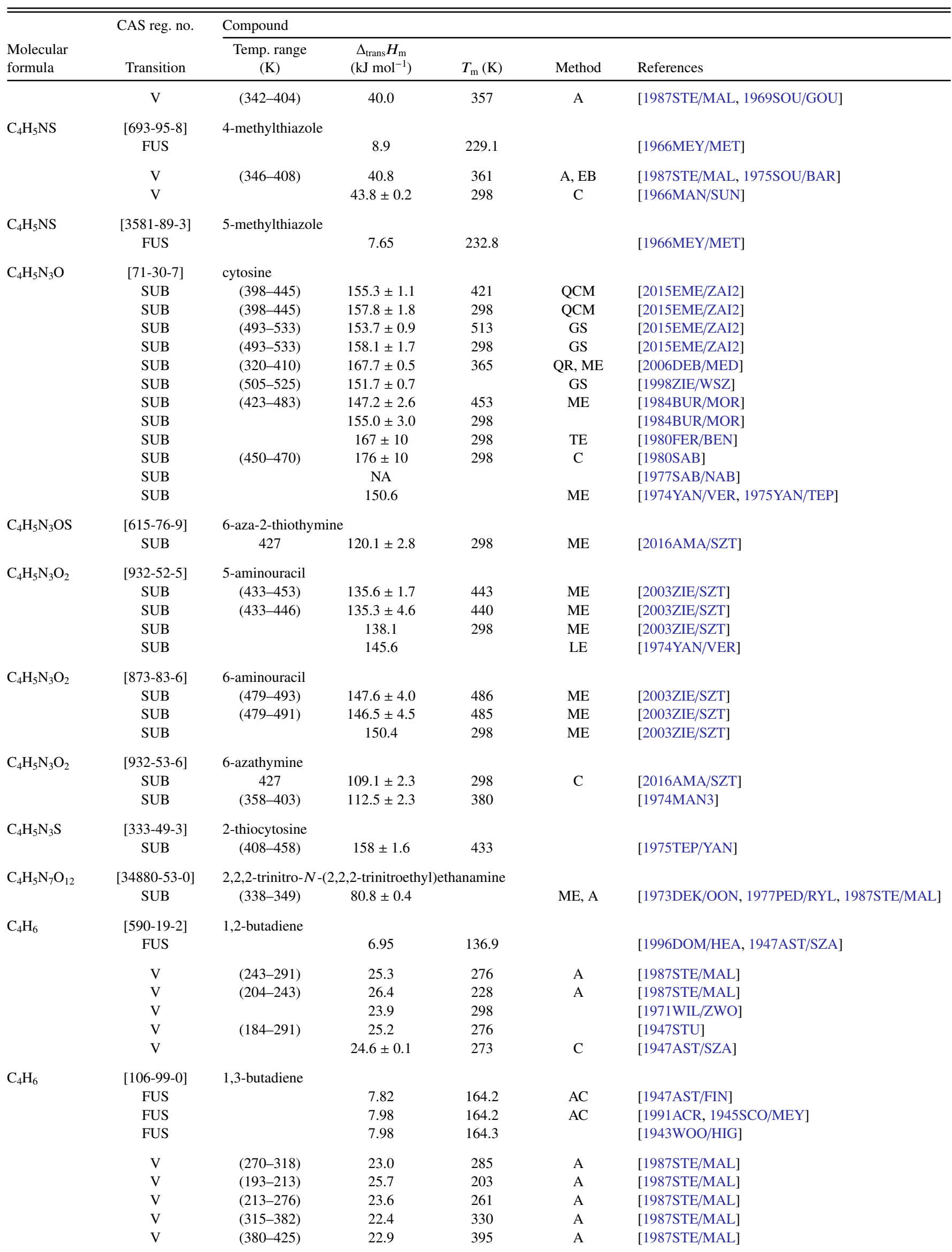


TABLE 7. Phase change enthalpies of $\mathrm{C}_{4}$ organic compounds-Continued

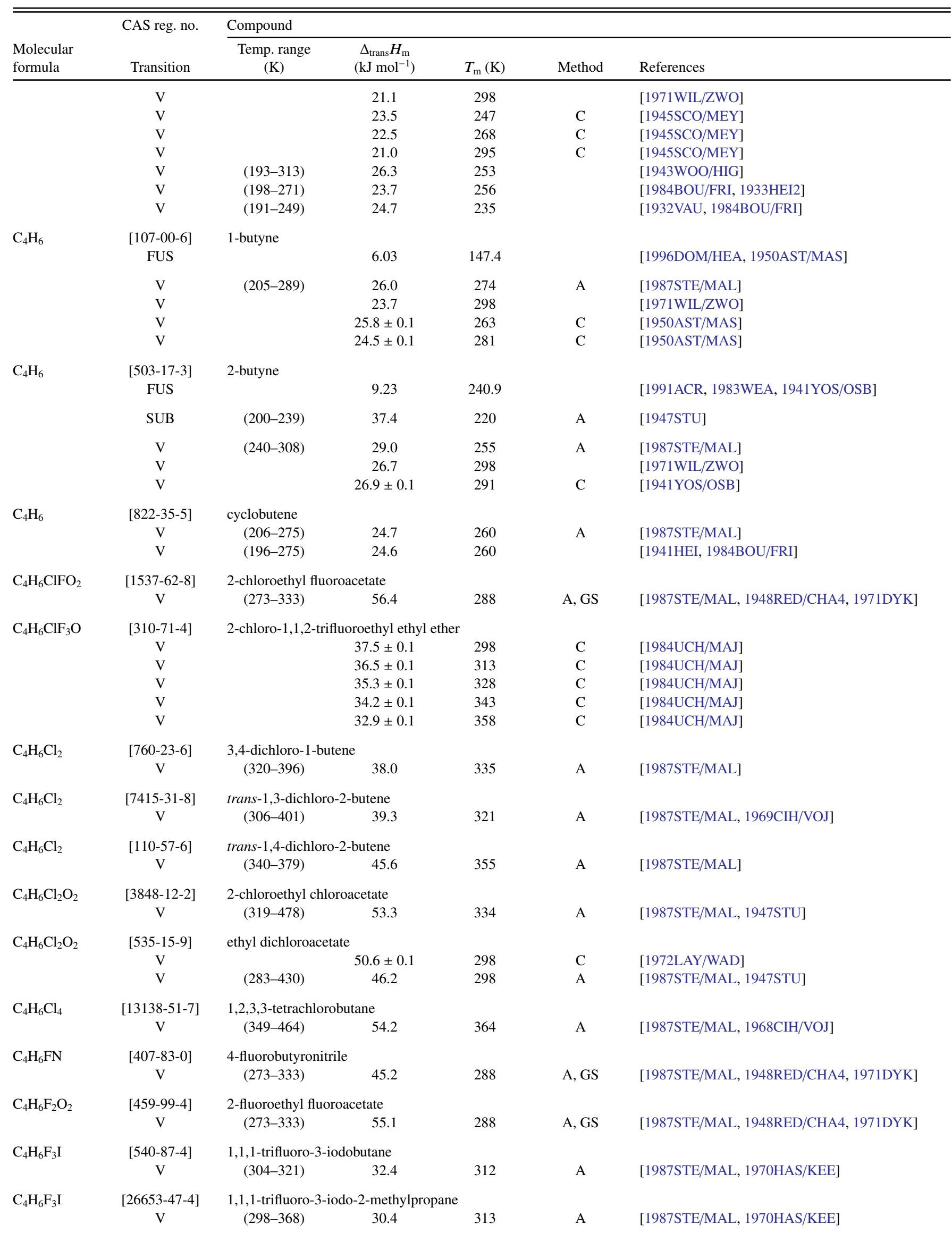


TABLE 7. Phase change enthalpies of $\mathrm{C}_{4}$ organic compounds-Continued

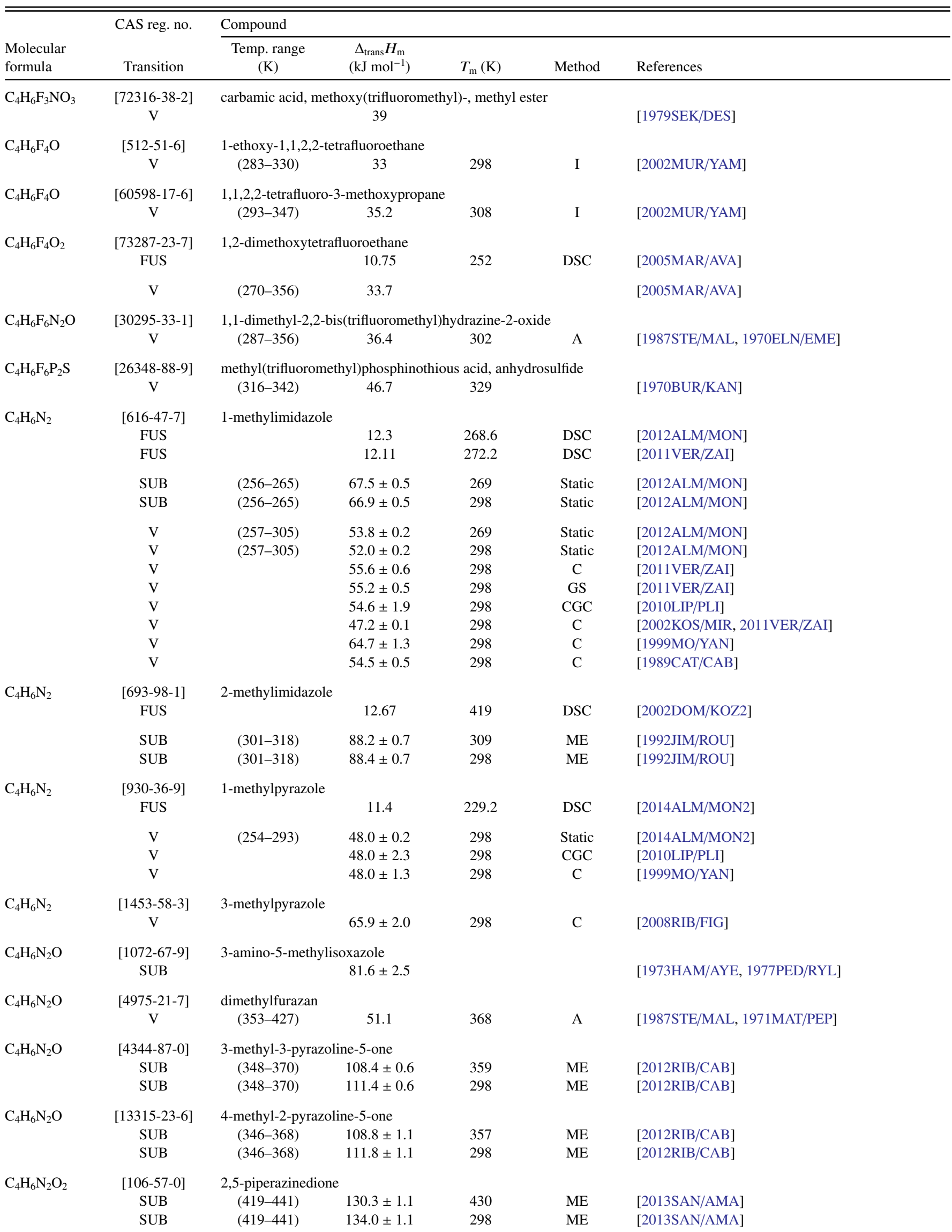


TABLE 7. Phase change enthalpies of $\mathrm{C}_{4}$ organic compounds-Continued

\begin{tabular}{|c|c|c|c|c|c|c|}
\hline \multirow[b]{2}{*}{$\begin{array}{l}\text { Molecular } \\
\text { formula }\end{array}$} & \multirow{2}{*}{$\begin{array}{l}\text { CAS reg. no. } \\
\text { Transition }\end{array}$} & \multicolumn{5}{|l|}{ Compound } \\
\hline & & $\begin{array}{c}\text { Temp. range } \\
(\mathrm{K})\end{array}$ & $\begin{array}{c}\Delta_{\text {trans }} H_{\mathrm{m}} \\
\left(\mathrm{kJ} \mathrm{mol}^{-1}\right)\end{array}$ & $T_{\mathrm{m}}(\mathrm{K})$ & Method & References \\
\hline & SUB & $(413-450)$ & 103.8 & 428 & A & [1987STE/MAL] \\
\hline $\mathrm{C}_{4} \mathrm{H}_{6} \mathrm{~N}_{2} \mathrm{O}_{2}$ & $\begin{array}{c}{[2518-42-5]} \\
\mathrm{V}\end{array}$ & $\begin{array}{c}\text { dimethylfuraza } \\
\text { (353-493) }\end{array}$ & $\begin{array}{l}\text { xide } \\
57.0\end{array}$ & 368 & A & [1987STE/MAL, 1971MAT/PEP] \\
\hline $\mathrm{C}_{4} \mathrm{H}_{6} \mathrm{~N}_{2} \mathrm{O}_{2}$ & $\begin{array}{c}\text { [504-07-4] } \\
\text { SUB } \\
\text { SUB } \\
\text { SUB }\end{array}$ & $\begin{array}{c}\text { 5,6-dihydroura } \\
(377-405) \\
(377-405)\end{array}$ & $\begin{array}{l}114.4 \pm 1.6 \\
114.2 \pm 0.9 \\
116.0 \pm 1.2\end{array}$ & $\begin{array}{l}298 \\
391 \\
298\end{array}$ & $\begin{array}{c}\mathrm{C} \\
\mathrm{ME} \\
\mathrm{ME}\end{array}$ & $\begin{array}{l}\text { [2013GAL/ROC] } \\
\text { [2013GAL/ROC] } \\
\text { [2013GAL/ROC] }\end{array}$ \\
\hline $\mathrm{C}_{4} \mathrm{H}_{6} \mathrm{~N}_{2} \mathrm{O}_{2}$ & $\begin{array}{c}\text { [616-04-6] } \\
\text { FUS }\end{array}$ & 1-methylhydan & 21.5 & 428.9 & DSC & [2014NOG/ILD] \\
\hline $\mathrm{C}_{4} \mathrm{H}_{6} \mathrm{~N}_{4}$ & $\begin{array}{c}\text { [156-81-0] } \\
\text { SUB } \\
\text { SUB }\end{array}$ & 2,4-diaminopyr & $\begin{array}{l}\text { ine } \\
105.4 \pm 0.7 \\
106.3 \pm 0.8\end{array}$ & $\begin{array}{l}347 \\
298\end{array}$ & $\begin{array}{l}\text { ME } \\
\text { ME }\end{array}$ & $\begin{array}{l}\text { [2012RIB/GAL] } \\
\text { [2012RIB/GAL] }\end{array}$ \\
\hline $\mathrm{C}_{4} \mathrm{H}_{6} \mathrm{~N}_{4} \mathrm{O}$ & $\begin{array}{l}{[56-06-4]} \\
\text { SUB }\end{array}$ & $\begin{array}{l}\text { 2,4-diamino-6- } \\
\quad(423-471)\end{array}$ & $\begin{array}{l}\text { oxypyrimidine } \\
147.6 \pm 0.2\end{array}$ & & GS & [1999ZIE/PER] \\
\hline $\mathrm{C}_{4} \mathrm{H}_{6} \mathrm{~N}_{4} \mathrm{O}_{2}$ & $\begin{array}{c}{[107945-67-5]} \\
\text { TRS } \\
\text { FUS }\end{array}$ & 1-ethyl-4-nitro- & $\begin{array}{c}\text { 1,2,3-triazole } \\
2.13 \\
16.40\end{array}$ & $\begin{array}{c}223.6 \text { and } \\
259.7 \\
349.0\end{array}$ & $\mathrm{AC}$ & {$[2013 \mathrm{BLO} / \mathrm{KOH}]$} \\
\hline
\end{tabular}

[Note: Authors of [2013BLO/KOH] report that the peaks for the two phase transitions at $223.6 \mathrm{~K}$ and $259.7 \mathrm{~K}$ overlapped and could not be resolved. The value of $2.13 \mathrm{~kJ} / \mathrm{mole}$ represents the sum of the two transition enthalpies.]

$\begin{array}{lllll}\text { SUB } & (313-343) & 93.5 \pm 0.8 & 328 & \text { ME }\end{array}$

\begin{tabular}{|c|c|c|c|c|c|}
\hline \multirow[t]{3}{*}{$\mathrm{C}_{4} \mathrm{H}_{6} \mathrm{~N}_{4} \mathrm{O}_{3} \mathrm{~S}_{2}$} & {$[59-66-5]$} & \multicolumn{4}{|c|}{ 2-acetamido-1,3,4-thiadiazole-5-sulfonamide } \\
\hline & TRS & 1.2 & 506.2 & & \\
\hline & FUS & 28.6 & 532.2 & DSC & [2009BAR/GAM] \\
\hline \multirow[t]{2}{*}{$\mathrm{C}_{4} \mathrm{H}_{6} \mathrm{~N}_{4} \mathrm{O}_{6}$} & {$[14760-99-7]$} & \multicolumn{4}{|c|}{ 2,5-dinitro-2,5-diazahexane-3,4-dione } \\
\hline & FUS & 23.4 & 397.1 & DSC & {$[1997 Z \mathrm{ZM}]$} \\
\hline \multirow[t]{3}{*}{$\mathrm{C}_{4} \mathrm{H}_{6} \mathrm{~N}_{4} \mathrm{O}_{8}$} & {$[3759-60-2]$} & \multicolumn{4}{|l|}{ 1,1,3,3-tetranitrobutane } \\
\hline & SUB & $97.1 \pm 1.2$ & 298 & $\mathrm{C}$ & [2011MIR/KON] \\
\hline & SUB & $87.9 \pm 0.8$ & 298 & & {$[1999 \mathrm{MIR} / \mathrm{VOR}]$} \\
\hline \multirow[t]{3}{*}{$\mathrm{C}_{4} \mathrm{H}_{6} \mathrm{~N}_{4} \mathrm{O}_{8}$} & {$[20919-97-5]$} & \multicolumn{4}{|l|}{ 2,2,3,3-tetranitrobutane } \\
\hline & SUB & $79.5 \pm 0.8$ & 298 & $\mathrm{C}$ & [2011MIR/KON] \\
\hline & SUB & $78.2 \pm 0.8$ & 298 & & [1999MIR/VOR] \\
\hline \multirow[t]{3}{*}{$\mathrm{C}_{4} \mathrm{H}_{6} \mathrm{~N}_{4} \mathrm{O}_{8}$} & {$[20919-96-4]$} & \multicolumn{4}{|l|}{ 1,1,1,4-tetranitrobutane } \\
\hline & SUB & $102.5 \pm 1.6$ & 298 & $\mathrm{C}$ & [2011MIR/KON] \\
\hline & SUB & 99.6 & 298 & & [1999MIR/VOR] \\
\hline \multirow[t]{3}{*}{$\mathrm{C}_{4} \mathrm{H}_{6} \mathrm{~N}_{4} \mathrm{O}_{8}$} & {$[42216-58-0]$} & \multicolumn{4}{|l|}{ 1,1,1,3-tetranitro-2-methylpropane } \\
\hline & SUB & 91.2 & 298 & & [1999MIR/VOR] \\
\hline & $\mathrm{V}$ & $(304-327)$ & 316 & A & [1987STE/MAL] \\
\hline \multirow[t]{2}{*}{$\mathrm{C}_{4} \mathrm{H}_{6} \mathrm{~N}_{4} \mathrm{O}_{10}$} & {$[99171-29-6]$} & \multicolumn{4}{|l|}{ 4-nitroso-1,2,3-butanetriol trinitrate } \\
\hline & V & $(313-353)$ & 333 & & {$[1959 \mathrm{VAC} / \mathrm{STA}]$} \\
\hline \multirow[t]{2}{*}{$\mathrm{C}_{4} \mathrm{H}_{6} \mathrm{~N}_{4} \mathrm{O}_{11}$} & {$[20820-44-4]$} & \multicolumn{4}{|c|}{ 2-nitro-2-hydroxymethyl-1,3-propanedioltrinitrate } \\
\hline & $\mathrm{V}$ & $(313-353)$ & 328 & A & [1987STE/MAL] \\
\hline \multirow[t]{2}{*}{$\mathrm{C}_{4} \mathrm{H}_{6} \mathrm{~N}_{6} \mathrm{O}_{8}$} & {$[81360-42-1]$} & \multicolumn{4}{|c|}{ 1,3,5,5-tetranitro-1,3-diazacyclohexane } \\
\hline & FUS & 29.37 & 430 & DTA & [1987OYU/BRI, 1995SKA/GOL] \\
\hline \multirow[t]{4}{*}{$\mathrm{C}_{4} \mathrm{H}_{6} \mathrm{O}$} & {$[123-73-9]$} & \multicolumn{4}{|l|}{ trans-crotonaldehyde } \\
\hline & $\mathrm{V}$ & $36.6 \pm 0.1$ & 320 & $\mathrm{~EB}$ & [2002STE/CHI2] \\
\hline & $\mathrm{V}$ & $34.5 \pm 0.2$ & 360 & $\mathrm{~EB}$ & [2002STE/CHI2] \\
\hline & $\mathrm{V}$ & $32.1 \pm 0.5$ & 400 & $\mathrm{~EB}$ & [2002STE/CHI2] \\
\hline \multirow[t]{2}{*}{$\mathrm{C}_{4} \mathrm{H}_{6} \mathrm{O}$} & {$[4170-30-3]$} & crotonaldehyde & & & \\
\hline & $\mathrm{V}$ & $37.3 \pm 0.4$ & 298 & $\mathrm{C}$ & [1996VAN/YU] \\
\hline
\end{tabular}


TABLE 7. Phase change enthalpies of $\mathrm{C}_{4}$ organic compounds-Continued

\begin{tabular}{|c|c|c|c|c|c|c|}
\hline \multirow[b]{2}{*}{$\begin{array}{l}\text { Molecular } \\
\text { formula }\end{array}$} & \multirow{2}{*}{$\begin{array}{l}\text { CAS reg. no. } \\
\text { Transition }\end{array}$} & \multicolumn{5}{|l|}{ Compound } \\
\hline & & $\begin{array}{l}\text { Temp. range } \\
\text { (K) }\end{array}$ & $\begin{array}{c}\Delta_{\text {trans }} H_{\mathrm{m}} \\
\left(\mathrm{kJ} \mathrm{mol}^{-1}\right)\end{array}$ & $T_{\mathrm{m}}(\mathrm{K})$ & Method & References \\
\hline & $\mathrm{V}$ & & 38.8 & 325 & EB & [1994WIB/MOR] \\
\hline & $\mathrm{V}$ & $(288-376)$ & $35.1 \pm 0.5$ & 332 & Static & [1988BAG/GUR] \\
\hline & $\mathrm{V}$ & $(304-377)$ & 37.3 & 319 & & [1979MAR/SAC] \\
\hline & $\mathrm{V}$ & $(306-376)$ & 36.8 & 321 & A & [1987STE/MAL, 1973WAR/SKU, 1984BOU/FRI] \\
\hline \multirow[t]{7}{*}{$\mathrm{C}_{4} \mathrm{H}_{6} \mathrm{O}$} & [1191-95-3] & cyclobutanone & & & & \\
\hline & FUS & & 10.8 & 220.5 & DSC & [1998GON/SZW] \\
\hline & $\mathrm{V}$ & $(301-344)$ & 37.7 & 322 & $\mathrm{~EB}$ & [1994WIB/MOR] \\
\hline & $\mathrm{V}$ & $(283-313)$ & 38.4 & 298 & A & [1987STE/MAL, 1972WOL] \\
\hline & $\mathrm{V}$ & $(317-380)$ & 36.3 & 332 & $\mathrm{~A}, \mathrm{~EB}$ & [1987STE/MAL, 1976MEY/HOT] \\
\hline & $\mathrm{V}$ & $(283-313)$ & $38.2 \pm 0.4$ & 298 & VP & [1972WOL] \\
\hline & $\mathrm{V}$ & $(249-298)$ & 38.5 & 273 & & [1942BEN/KIS, 1984BOU/FRI] \\
\hline \multirow[t]{3}{*}{$\mathrm{C}_{4} \mathrm{H}_{6} \mathrm{O}$} & [109-93-3] & divinyl ether & & & & \\
\hline & $\mathrm{V}$ & $(253-323)$ & 29.2 & 268 & A & [1987STE/MAL] \\
\hline & $\mathrm{V}$ & $(253-323)$ & 26.1 & 301 & I & [1933MIL/MEN] \\
\hline \multirow[t]{3}{*}{$\mathrm{C}_{4} \mathrm{H}_{6} \mathrm{O}$} & [78-94-4] & methyl vinyl ketone & & & & \\
\hline & $\mathrm{V}$ & $(279-355)$ & 32.9 & 294 & A & [1987STE/MAL] \\
\hline & $\mathrm{V}$ & $(300-355)$ & 33.6 & 315 & A & [1987STE/MAL] \\
\hline \multirow[t]{3}{*}{$\mathrm{C}_{4} \mathrm{H}_{6} \mathrm{O}$} & [1191-99-7] & 2,3-dihydrofuran & & & & \\
\hline & $\mathrm{V}$ & $(302-360)$ & $30.8 \pm 0.1$ & 300 & $\mathrm{~EB}$ & [2002STE/CHI5] \\
\hline & $\mathrm{V}$ & $(302-360)$ & $28.6 \pm 0.3$ & 340 & $\mathrm{~EB}$ & [2002STE/CHI5] \\
\hline \multirow[t]{2}{*}{$\mathrm{C}_{4} \mathrm{H}_{6} \mathrm{O}$} & [927-74-2] & 3-butyn-1-ol & & & & \\
\hline & $\mathrm{V}$ & (343-393) & $51.7 \pm 0.9$ & 298 & CGC & [2005VAL/QUI] \\
\hline \multirow[t]{2}{*}{$\mathrm{C}_{4} \mathrm{H}_{6} \mathrm{OS}$} & [1115-15-7] & divinyl sulfoxide & & & & \\
\hline & $\mathrm{V}$ & & $51.2 \pm 0.9$ & 298 & $\mathrm{C}$ & [1989VOR/KLY] \\
\hline \multirow[t]{5}{*}{$\mathrm{C}_{4} \mathrm{H}_{6} \mathrm{O}_{2}$} & [1759-53-1] & cyclopropane carbox & ylic acid & & & \\
\hline & $\mathrm{V}$ & $(357-473)$ & $58.9 \pm 0.3$ & 340 & EB & [2002STE/CHI5] \\
\hline & $\mathrm{V}$ & $(357-473)$ & $55.7 \pm 0.2$ & 380 & EB & [2002STE/CHI5] \\
\hline & $\mathrm{V}$ & $(357-473)$ & $52.4 \pm 0.2$ & 420 & $\mathrm{~EB}$ & [2002STE/CHI5] \\
\hline & $\mathrm{V}$ & $(357-473)$ & $48.8 \pm 0.4$ & 460 & $\mathrm{~EB}$ & [2002STE/CHI5] \\
\hline \multirow[t]{3}{*}{$\mathrm{C}_{4} \mathrm{H}_{6} \mathrm{O}_{2}$} & [431-03-8] & 2,3-butanedione (bia & cetyl) & & & \\
\hline & $\mathrm{V}$ & $(273-348)$ & 38.5 & 288 & A, I & [1987STE/MAL, 1972NEE/HAL] \\
\hline & $\mathrm{V}$ & $(273-293)$ & $39.6 \pm 0.2$ & 283 & & {$[1954 \mathrm{NIC} / \mathrm{SZA}]$} \\
\hline \multirow[t]{2}{*}{$\mathrm{C}_{4} \mathrm{H}_{6} \mathrm{O}_{2}$} & [503-64-0] & cis-2-butenoic acid & & & & \\
\hline & $\mathrm{V}$ & $(306-445)$ & 55.8 & 321 & A & [1987STE/MAL, 1947STU] \\
\hline \multirow[t]{2}{*}{$\mathrm{C}_{4} \mathrm{H}_{6} \mathrm{O}_{2}$} & [107-93-7] & trans-2-butenoic acic & & & & \\
\hline & $\mathrm{V}$ & (353-458) & 56.7 & 368 & A & [1987STE/MAL, 1947STU] \\
\hline \multirow[t]{3}{*}{$\mathrm{C}_{4} \mathrm{H}_{6} \mathrm{O}_{2}$} & [110-65-6] & 2-butyne-1,4-diol & & & & \\
\hline & $\mathrm{V}$ & & $81.5 \pm 0.3$ & 298 & CGC & [2006UMN/KWE] \\
\hline & $\mathrm{V}$ & $(418-520)$ & 69.0 & 433 & A & [1987STE/MAL, 1966GAN/SEM, 1971DYK] \\
\hline \multirow[t]{14}{*}{$\mathrm{C}_{4} \mathrm{H}_{6} \mathrm{O}_{2}$} & [96-48-0] & $\gamma$-butyrolactone & & & & \\
\hline & FUS & $(14-328)$ & 9.57 & 229.8 & $\mathrm{AC}$ & [1991ACR, 1983LEB/YEV] \\
\hline & $\mathrm{V}$ & $(377-477)$ & 48.5 & 392 & & [2011MAT/KIM] \\
\hline & $\mathrm{V}$ & $(293-333)$ & $47.6 \pm 4.0$ & 298 & & [1997KLE, 2008EME/KOZ] \\
\hline & $\mathrm{V}$ & $(378-406)$ & $49.5 \pm 0.1$ & 392 & EB & [1991WIB/WAL] \\
\hline & $\mathrm{V}$ & $(378-406)$ & $55.2 \pm 1.3$ & 298 & EB & [1991WIB/WAL] \\
\hline & $\mathrm{V}$ & $(345-370)$ & $51.8 \pm 0.6$ & 357 & MM & [1991WIB/WAL] \\
\hline & $\mathrm{V}$ & $(345-370)$ & $55.6 \pm 1.4$ & 298 & MM & [1991WIB/WAL] \\
\hline & $\mathrm{V}$ & $(392-555)$ & $48.9 \pm 0.3$ & 298 & & [1990RAM/KUD, 2008EME/KOZ] \\
\hline & $\mathrm{V}$ & & $54.4 \pm 0.4$ & 298 & $\mathrm{C}$ & [1990LEI/PIL2] \\
\hline & $\mathrm{V}$ & $(361-522)$ & $54.9 \pm 0.2$ & 298 & $\mathrm{~EB}$ & [1989STE/CHI2, 2008EME/KOZ] \\
\hline & $\mathrm{V}$ & $(357-435)$ & 51.5 & 298 & $\mathrm{~EB}$ & [1988ISM/GAB, 2008EME/KOZ] \\
\hline & $\mathrm{V}$ & $(392-474)$ & 48.2 & 407 & A & [1987STE/MAL, 1950MCK/COP, 1981YAR/KAL] \\
\hline & $\mathrm{V}$ & $(273-478)$ & $54.6 \pm 0.2$ & 298 & GS & [1980JAR/AFA, 2008EME/KOZ] \\
\hline
\end{tabular}


TABLE 7. Phase change enthalpies of $\mathrm{C}_{4}$ organic compounds-Continued

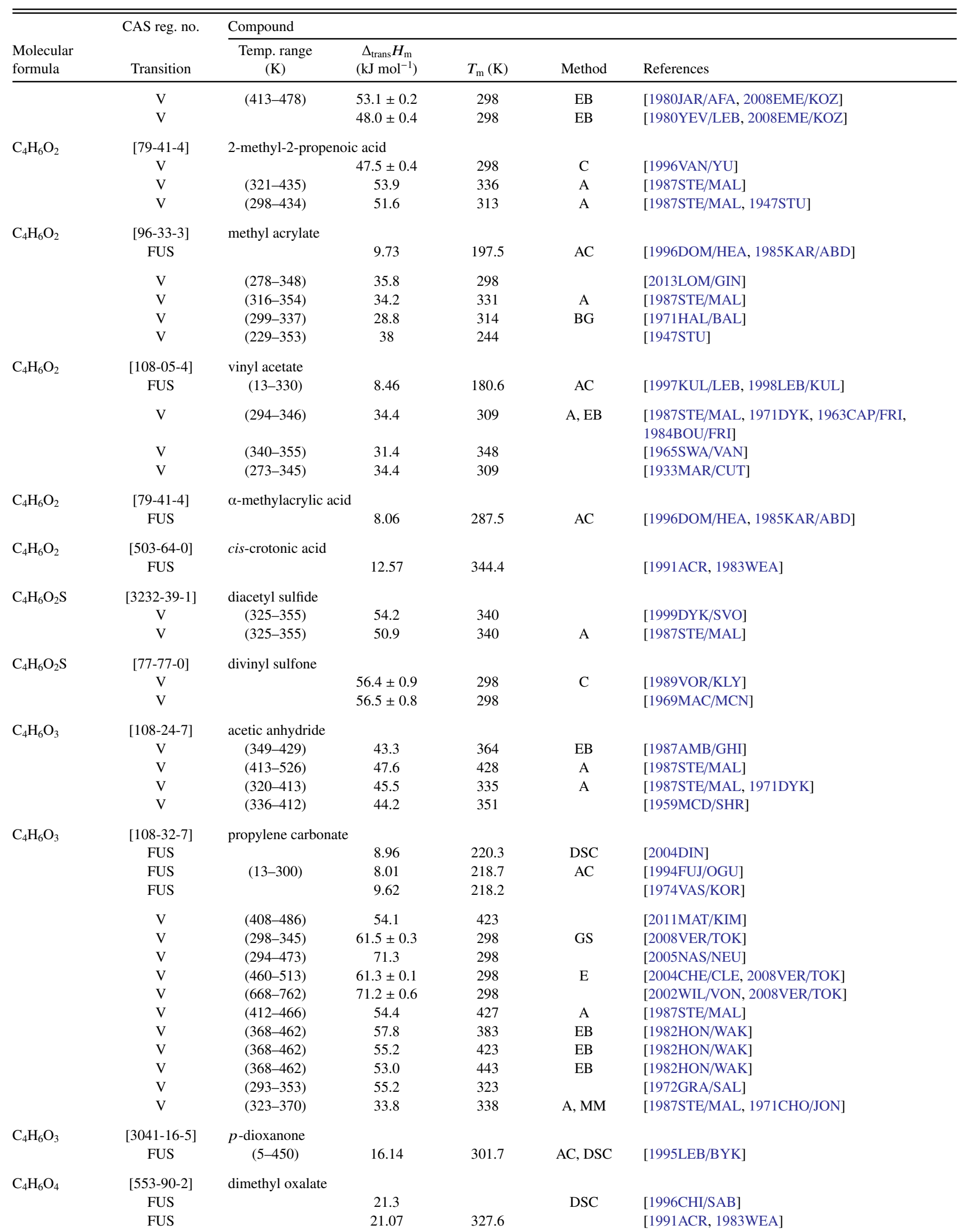


TABLE 7. Phase change enthalpies of $\mathrm{C}_{4}$ organic compounds-Continued

\begin{tabular}{|c|c|c|c|c|c|c|}
\hline \multirow[b]{2}{*}{$\begin{array}{l}\text { Molecular } \\
\text { formula }\end{array}$} & \multirow{2}{*}{$\begin{array}{l}\text { CAS reg. no. } \\
\text { Transition }\end{array}$} & \multicolumn{5}{|l|}{ Compound } \\
\hline & & $\begin{array}{l}\text { Temp. range } \\
(\mathrm{K})\end{array}$ & $\begin{array}{r}\Delta_{\text {trans }} H_{\mathrm{m}} \\
\left(\mathrm{kJ} \mathrm{mol}^{-1}\right)\end{array}$ & $T_{\mathrm{m}}(\mathrm{K})$ & Method & References \\
\hline & SUB & & $74.6 \pm 0.7$ & 298 & $\mathrm{C}$ & [1996CHI/SAB] \\
\hline & SUB & $(268-298)$ & $75.6 \pm 1.6$ & 283 & HSA & [1996CHI/SAB] \\
\hline & SUB & & $75.3 \pm 1.6$ & 298 & & [1996CHI/SAB] \\
\hline & SUB & & $74.9 \pm 0.6$ & & $\mathrm{~B}$ & [1996CHI/SAB] \\
\hline & SUB & $(289-306)$ & $\mathrm{U} 47.4 \pm 0.5$ & 298 & BG & [1976ANT/CAR2, 1975ANT/CAR] \\
\hline & $\mathrm{V}$ & $(330-365)$ & $54.7 \pm 0.3$ & 298 & GS & [2006VER/KOZ] \\
\hline & $\mathrm{V}$ & $(328-443)$ & 52.5 & 298 & & [2004MA/LIU, 2006VER/KOZ] \\
\hline & $\mathrm{V}$ & $(347-485)$ & 44.7 & 416 & $\mathrm{HG}, \mathrm{EB}$ & [1988ASK/DAU] \\
\hline & $\mathrm{V}$ & $(293-437)$ & 48.8 & 308 & A & [1987STE/MAL, 1947STU] \\
\hline \multirow[t]{17}{*}{$\mathrm{C}_{4} \mathrm{H}_{6} \mathrm{O}_{4}$} & [110-15-6] & succinic acid & & & & \\
\hline & FUS & & U15.82 & & DSC & [2014HAS/JIR] \\
\hline & FUS & & 27.4 & 460.1 & DSC & [2013PAL/MCC] \\
\hline & FUS & & 31.26 & 458 & DSC & [2010BOO/BAR] \\
\hline & FUS & & 34.0 & 455.2 & DSC & [2005ROU/TEM] \\
\hline & FUS & & 32.95 & 457 & DSC & [1991ACR, 1974CIN/BER] \\
\hline & SUB & & $93 \pm 6$ & & ME, MS & [2009BOO/MAR] \\
\hline & SUB & $(318-358)$ & $128 \pm 2$ & & TPD & [2007CAP/LOV] \\
\hline & SUB & $(280-302)$ & 119.5 & & TPTD & {$[2005 \mathrm{CHA} / \mathrm{ZIE}]$} \\
\hline & SUB & $(360-375)$ & $123.2 \pm 2.6$ & 298 & ME & [2001RIB/MON2] \\
\hline & SUB & $(356-376)$ & 120.5 & 368 & TE, ME & [1983DEW/VAN] \\
\hline & SUB & & 123.1 & 298 & & [1983DEW/VAN] \\
\hline & SUB & $(372-401)$ & $118.1 \pm 3.3$ & 386 & ME & [1970COX/PIL, 1960DAV/THO] \\
\hline & SUB & & $120.3 \pm 4.4$ & 298 & & [1970COX/PIL, 1960DAV/THO] \\
\hline & SUB & & $121.8 \pm 3.3$ & 298 & & [1960DAV/THO, 1999RIB/MON] \\
\hline & SUB & $(292-320)$ & 73.6 & 306 & A & [1947GRA] \\
\hline & $\mathrm{V}$ & $(424-503)$ & 94.4 & 298 & $\mathrm{CGC}$ & [2005ROU/TEM] \\
\hline \multirow[t]{4}{*}{$\mathrm{C}_{4} \mathrm{H}_{6} \mathrm{O}_{4}$} & {$[516-05-2]$} & \multicolumn{2}{|c|}{ 2-methylmalonic acid } & & & \\
\hline & FUS & & 30.73 & 403 & DSC & [2010BOO/BAR] \\
\hline & SUB & $(341-355)$ & $117.4 \pm 1.9$ & 298 & ME & [2000RIB/MON] \\
\hline & SUB & & $113.2 \pm 0.4$ & & $\mathrm{C}$ & [1983ALT/PIL] \\
\hline \multirow[t]{2}{*}{$\mathrm{C}_{4} \mathrm{H}_{6} \mathrm{O}_{4}$} & [629-15-2] & \multicolumn{3}{|c|}{ ethylene glycol dimethanoate } & & \\
\hline & $\mathrm{V}$ & $(303-325)$ & $50.3 \pm 1.4$ & & GS & [2011MAS/KRA] \\
\hline \multirow[t]{3}{*}{$\mathrm{C}_{4} \mathrm{H}_{6} \mathrm{O}_{4}$} & [931-40-8] & \multicolumn{3}{|c|}{ 4-(hydroxmethyl)-1,3-dioxolan-2-one } & & \\
\hline & $\mathrm{V}$ & $(330-398)$ & $85.4 \pm 0.4$ & 298 & GS & [2008VER/TOK] \\
\hline & $\mathrm{V}$ & $(430-455)$ & $87.8 \pm 0.4$ & 298 & $\mathrm{~EB}$ & [2002WIL/VON, 2008VER/TOK] \\
\hline \multirow{4}{*}{$\mathrm{C}_{4} \mathrm{H}_{6} \mathrm{O}_{5}$} & {$[6915-15-7]$} & $(d l)$-malic acid I & & & & \\
\hline & FUS & & 29.03 & 403 & DSC & [2010BOO/BAR] \\
\hline & FUS (I) & & 33.52 & 402 & & [1996DOM/HEA, 1990CEO/SZW] \\
\hline & FUS (II) & & 30.17 & 396 & & [1996DOM/HEA, 1990CEO/SZW] \\
\hline \multirow[t]{2}{*}{$\mathrm{C}_{4} \mathrm{H}_{6} \mathrm{O}_{5}$} & [636-61-3] & $(d)$-malic acid & & & & \\
\hline & FUS & & 23.01 & 376 & DSC & [1976LEC/COL] \\
\hline \multirow[t]{2}{*}{$\mathrm{C}_{4} \mathrm{H}_{6} \mathrm{O}_{6}$} & [147-73-9] & meso-tartaric acid & & & & \\
\hline & SUB & & 156.9 & & & [1983DEW/BOW] \\
\hline \multirow[t]{3}{*}{$\mathrm{C}_{4} \mathrm{H}_{6} \mathrm{O}_{6}$} & [87-69-4] & L-(+)-tartaric acid & & & & \\
\hline & FUS & & 36.3 & 445.0 & DSC & [2012MEL/PIN] \\
\hline & FUS & & 32.3 & 445.1 & DSC & [1998MUR/BET] \\
\hline \multirow[t]{2}{*}{$\mathrm{C}_{4} \mathrm{H}_{6} \mathrm{~S}$} & {$[5954-75-6]$} & 2-vinylthiirane & & & & \\
\hline & $\mathrm{V}$ & (273-335) & 38.7 & 288 & A & [1987STE/MAL, 1999DYK/SVO] \\
\hline \multirow[t]{2}{*}{$\mathrm{C}_{4} \mathrm{H}_{6} \mathrm{~S}$} & {$[627-51-0]$} & divinyl sulfide & & & & \\
\hline & $\mathrm{V}$ & & $38.3 \pm 0.7$ & 298 & $\mathrm{C}$ & [1989VOR/KLY] \\
\hline \multirow[t]{2}{*}{$\mathrm{C}_{4} \mathrm{H}_{6} \mathrm{~S}$} & {$[1120-59-8]$} & \multirow{2}{*}{\multicolumn{2}{|c|}{$\begin{array}{l}\text { 2,3-dihydrothiophene } \\
\qquad 37.7 \pm 0.4\end{array}$}} & & & \\
\hline & $\mathrm{V}$ & & & 298 & $\mathrm{C}$ & [1962DAV/SUN] \\
\hline
\end{tabular}


TABLE 7. Phase change enthalpies of $\mathrm{C}_{4}$ organic compounds-Continued

\begin{tabular}{|c|c|c|c|c|c|c|}
\hline $\begin{array}{l}\text { Molecular } \\
\text { formula }\end{array}$ & $\begin{array}{l}\text { CAS reg. no. } \\
\text { Transition }\end{array}$ & \multicolumn{5}{|l|}{ Compound } \\
\hline $\mathrm{C}_{4} \mathrm{H}_{6} \mathrm{~S}_{3}$ & $\begin{array}{c}\text { [1748-15-8] } \\
\text { SUB } \\
\text { SUB }\end{array}$ & $\begin{array}{l}\text { 1,3-dithian-2-thione } \\
\quad(321-348)\end{array}$ & $\begin{array}{c}88.6 \\
91.4 \pm 2.5\end{array}$ & $\begin{array}{l}335 \\
298\end{array}$ & & $\begin{array}{l}\text { [1967GEI/SCH] } \\
\text { [1967GEI/SCH, 1970COX/PIL] }\end{array}$ \\
\hline $\mathrm{C}_{4} \mathrm{H}_{7} \mathrm{Br}$ & $\begin{array}{c}\text { [31849-78-2] } \\
\text { V } \\
\text { V }\end{array}$ & $\begin{array}{l}\text { cis-1-bromo-1-butene } \\
\quad(280-397) \\
(229-359)\end{array}$ & 35.1 & $\begin{array}{l}295 \\
244\end{array}$ & A & $\begin{array}{l}\text { [1987STE/MAL, 1971DYK] } \\
\text { [1947STU] }\end{array}$ \\
\hline $\mathrm{C}_{4} \mathrm{H}_{7} \mathrm{Br}$ & $\begin{array}{c}{[32620-08-9]} \\
V\end{array}$ & $\begin{array}{l}\text { trans-1-bromo-1-buten } \\
\quad(234-368)\end{array}$ & 36.1 & 249 & A & [1987STE/MAL, 1947STU] \\
\hline $\mathrm{C}_{4} \mathrm{H}_{7} \mathrm{Br}$ & $\begin{array}{c}{[3017-71-8]} \\
\mathrm{V}\end{array}$ & $\begin{array}{l}\text { cis-2-bromo-2-butene } \\
\quad(234-367)\end{array}$ & 36.5 & 249 & A & [1987STE/MAL, 1947STU] \\
\hline $\mathrm{C}_{4} \mathrm{H}_{7} \mathrm{Br}$ & $\begin{array}{l}{[3017-68-3]} \\
\mathrm{V}\end{array}$ & $\begin{array}{l}\text { trans-2-bromo-2-buten } \\
\quad(228-359)\end{array}$ & 35.7 & 243 & A & [1987STE/MAL, 1947STU] \\
\hline $\mathrm{C}_{4} \mathrm{H}_{7} \mathrm{BrO}$ & $\begin{array}{c}{[816-40-0]} \\
\mathrm{V} \\
\mathrm{V}\end{array}$ & $\begin{array}{l}\text { 1-bromo-2-butanone } \\
\quad(322-428) \\
(279-420)\end{array}$ & $\begin{array}{l}49.9 \\
47.7\end{array}$ & $\begin{array}{l}337 \\
294\end{array}$ & A & $\begin{array}{l}\text { [1987STE/MAL, 1948CAT/ELL] } \\
\text { [1947STU] }\end{array}$ \\
\hline $\mathrm{C}_{4} \mathrm{H}_{7} \mathrm{BrO}$ & $\begin{array}{c}{[814-75-5]} \\
\mathrm{V}\end{array}$ & $\begin{array}{l}\text { 3-bromo-2-butanone } \\
\text { (306-409) }\end{array}$ & 46.4 & 321 & A & [1987STE/MAL, 1948CAT/ELL] \\
\hline $\mathrm{C}_{4} \mathrm{H}_{7} \mathrm{BrO}$ & $\begin{array}{c}{[2736-37-0]} \\
V\end{array}$ & $\begin{array}{l}\text { isobutyryl bromide } \\
\quad(286-436)\end{array}$ & 45.7 & 301 & A & [1987STE/MAL, 1947STU] \\
\hline $\mathrm{C}_{4} \mathrm{H}_{7} \mathrm{Br}_{3}$ & $\begin{array}{c}{[632-05-3]} \\
\mathrm{V} \\
\mathrm{V}\end{array}$ & $\begin{array}{l}\text { 1,2,3-tribromobutane } \\
\quad(394-546) \\
(318-489)\end{array}$ & $\begin{array}{l}54.1 \\
51.3\end{array}$ & $\begin{array}{l}409 \\
333\end{array}$ & A & $\begin{array}{l}\text { [1987STE/MAL, 1971DYK] } \\
\text { [1947STU] }\end{array}$ \\
\hline $\mathrm{C}_{4} \mathrm{H}_{7} \mathrm{Br}_{3}$ & $\begin{array}{c}{[38300-67-3]} \\
V\end{array}$ & $\begin{array}{l}\text { 1,2,4-tribromobutane } \\
\quad(390-541)\end{array}$ & 53.5 & 405 & A & [1987STE/MAL, 1971DYK] \\
\hline $\mathrm{C}_{4} \mathrm{H}_{7} \mathrm{Br}_{3}$ & $\begin{array}{c}{[62127-47-3]} \\
V\end{array}$ & $\begin{array}{l}\text { 2,2,3-tribromobutane } \\
\quad(311-480)\end{array}$ & 51.7 & 326 & A & [1987STE/MAL, 1947STU] \\
\hline $\mathrm{C}_{4} \mathrm{H}_{7} \mathrm{Cl}$ & $\begin{array}{c}{[513-37-1]} \\
\mathrm{V}\end{array}$ & $\begin{array}{l}\text { 1-chloro-2-methyl-1-pr } \\
\text { (285-343) }\end{array}$ & $\begin{array}{r}\text { propene } \\
33.2\end{array}$ & 300 & A & [1987STE/MAL] \\
\hline $\mathrm{C}_{4} \mathrm{H}_{7} \mathrm{Cl}$ & $\begin{array}{c}{[563-47-3]} \\
\mathrm{V}\end{array}$ & $\begin{array}{l}\text { 3-chloro-2-methyl-1-pr } \\
\quad(285-348)\end{array}$ & $\begin{array}{r}\text { propene } \\
33.3\end{array}$ & 300 & A & [1987STE/MAL] \\
\hline $\mathrm{C}_{4} \mathrm{H}_{7} \mathrm{ClO}$ & $\begin{array}{c}{[616-27-3]} \\
\mathrm{V}\end{array}$ & $\begin{array}{l}\text { 1-chloro-2-butanone } \\
\quad(307-411)\end{array}$ & 49.2 & 322 & A & [1987STE/MAL, 1971DYK] \\
\hline $\mathrm{C}_{4} \mathrm{H}_{7} \mathrm{ClO}$ & $\begin{array}{l}{[4091-39-8]} \\
\mathrm{V}\end{array}$ & $\begin{array}{l}\text { 3-chloro-2-butanone } \\
\quad(313-389)\end{array}$ & 38.8 & 328 & A & [1987STE/MAL, 1965PES/NAS] \\
\hline $\mathrm{C}_{4} \mathrm{H}_{7} \mathrm{ClO}$ & $\begin{array}{c}{[40605-42-3]} \\
V\end{array}$ & $\begin{array}{l}\text { 3-chloro-2-butene-1-ol } \\
\quad(345-437)\end{array}$ & 50.0 & 360 & A & [1987STE/MAL] \\
\hline $\mathrm{C}_{4} \mathrm{H}_{7} \mathrm{ClO}_{2}$ & {$[109-61-5]$} & propyl chloroformate & & & & \\
\hline
\end{tabular}


TABle 7. Phase change enthalpies of $\mathrm{C}_{4}$ organic compounds-Continued

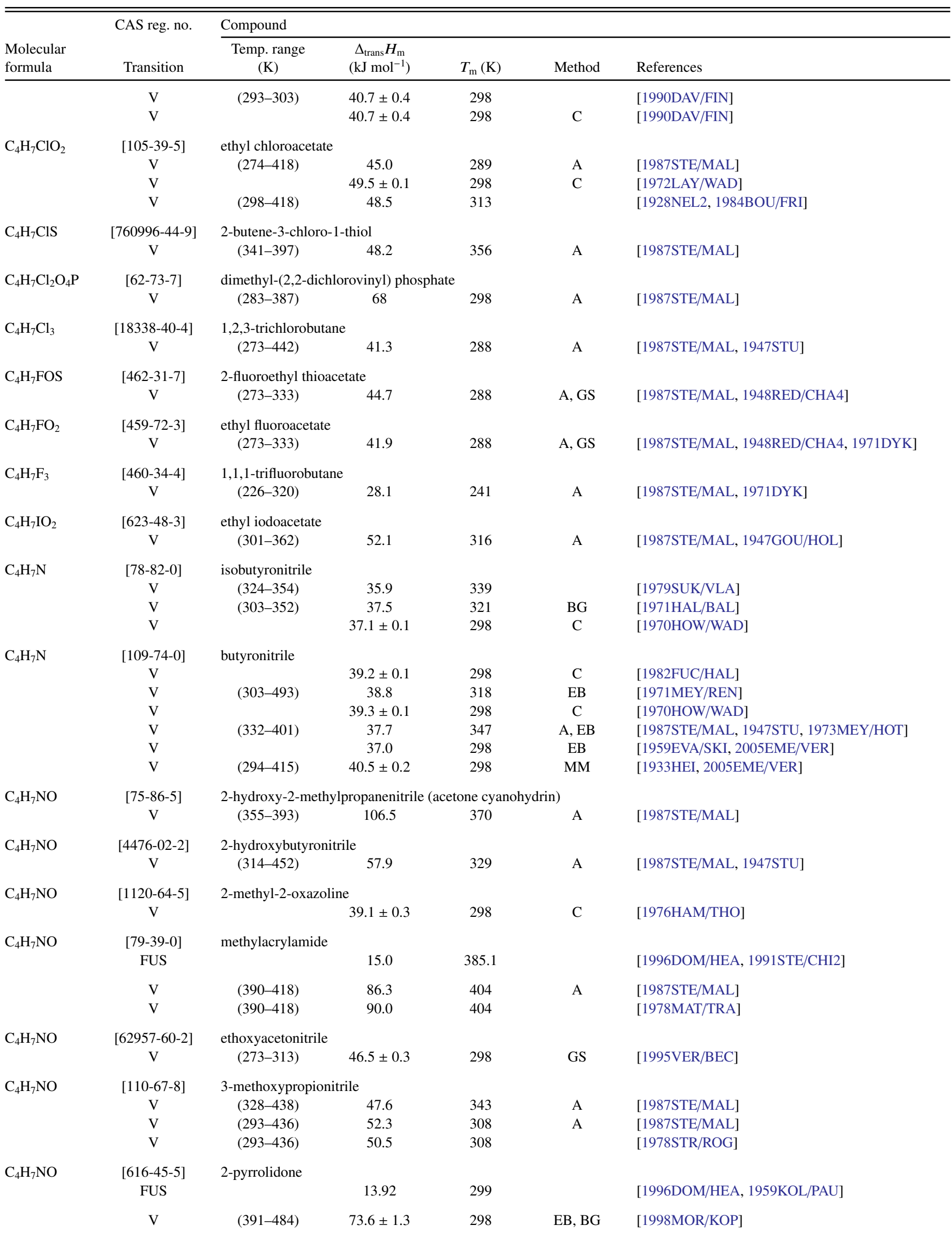


TABLE 7. Phase change enthalpies of $\mathrm{C}_{4}$ organic compounds-Continued

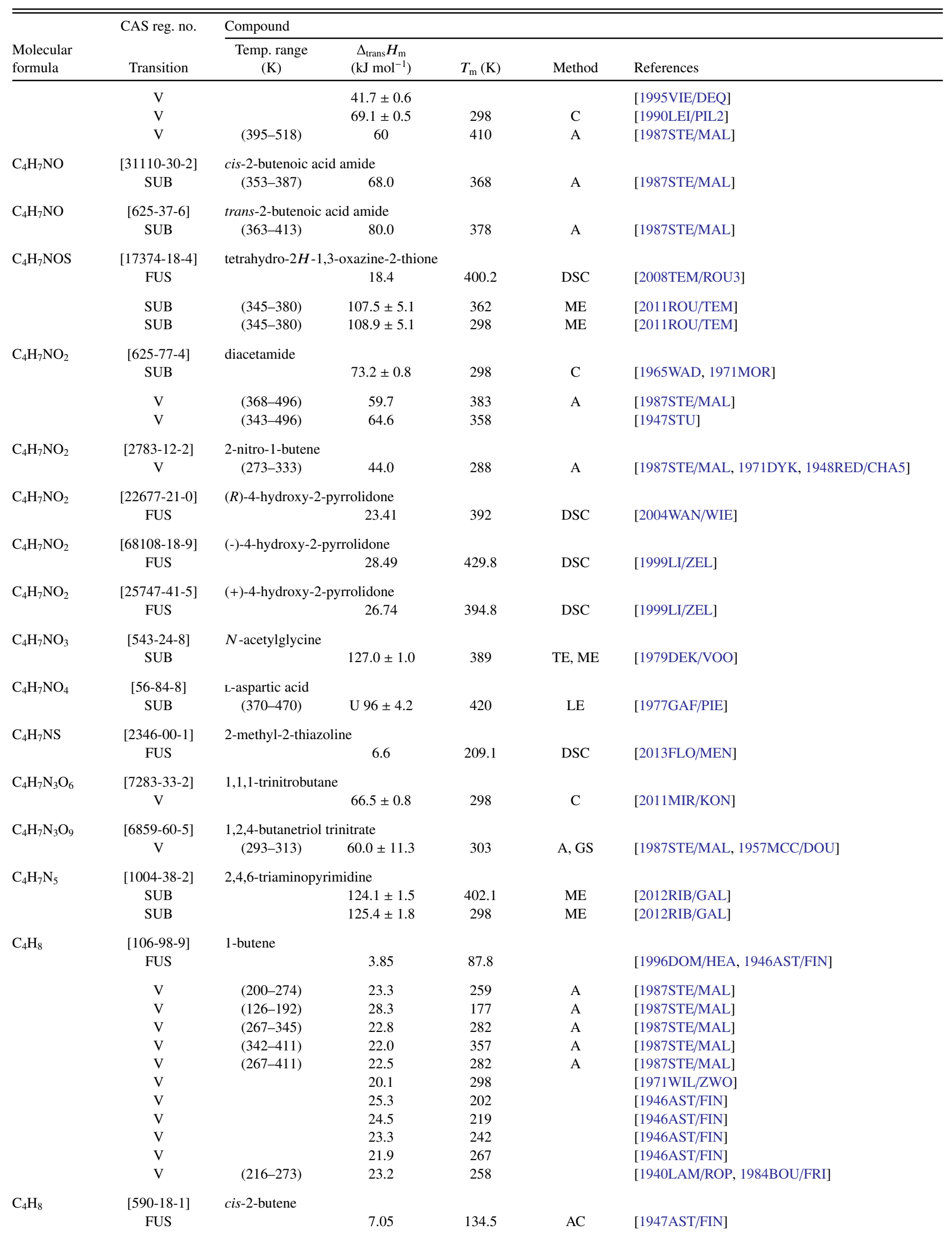


TABLE 7. Phase change enthalpies of $\mathrm{C}_{4}$ organic compounds-Continued

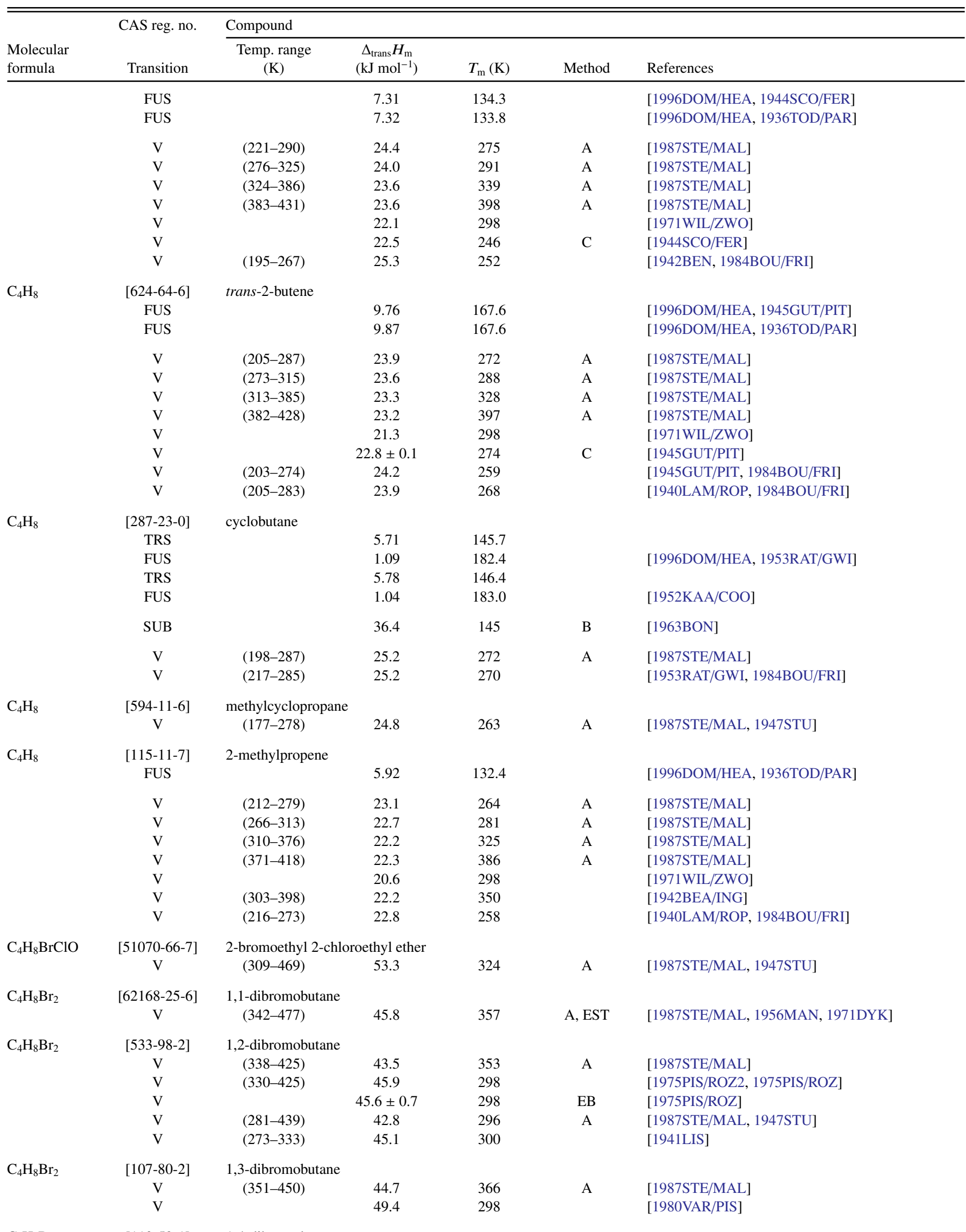

$\mathrm{C}_{4} \mathrm{H}_{8} \mathrm{Br}_{2} \quad$ [110-52-1] 1,4-dibromobutane 
TABLE 7. Phase change enthalpies of $\mathrm{C}_{4}$ organic compounds-Continued

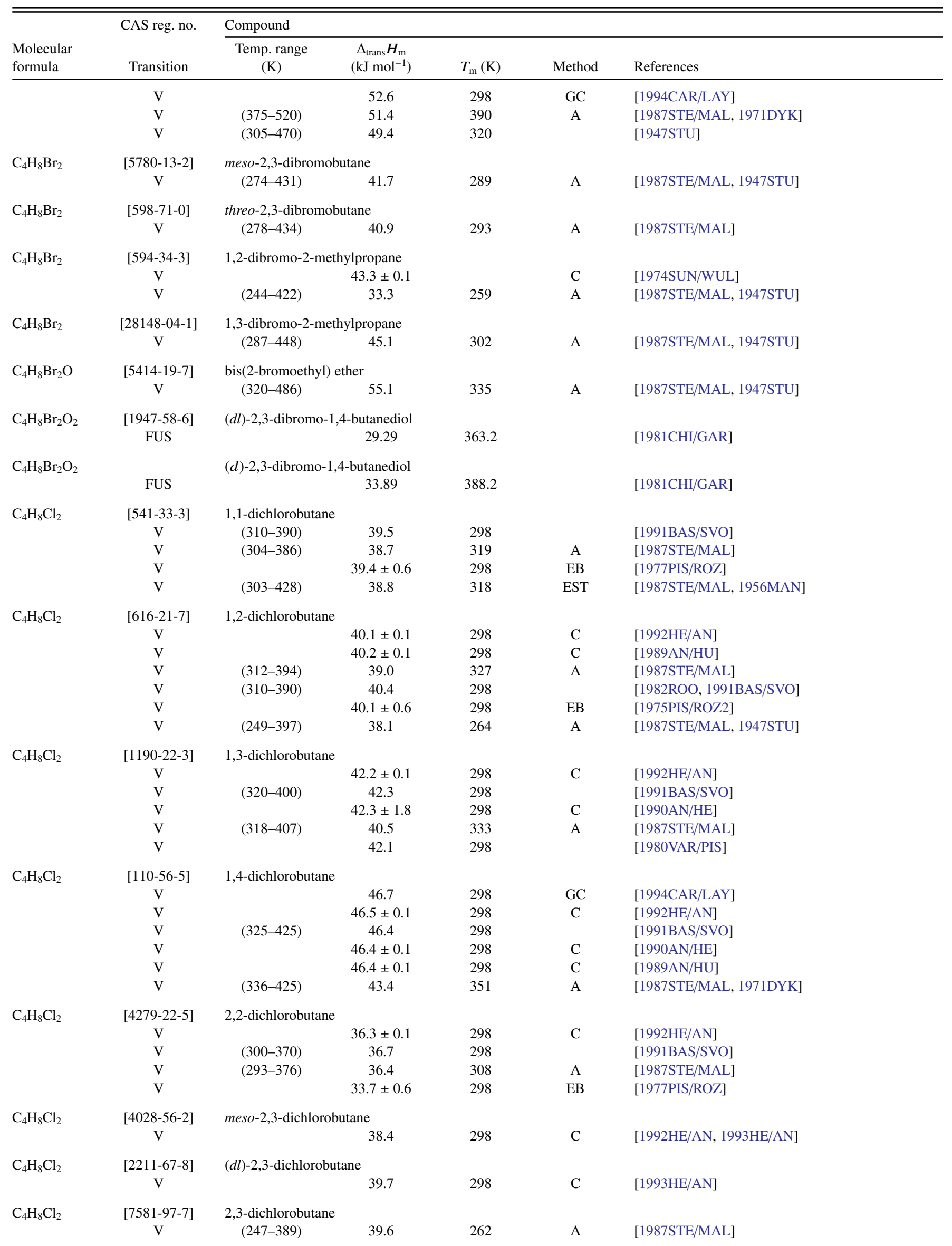


TABLE 7. Phase change enthalpies of $\mathrm{C}_{4}$ organic compounds-Continued

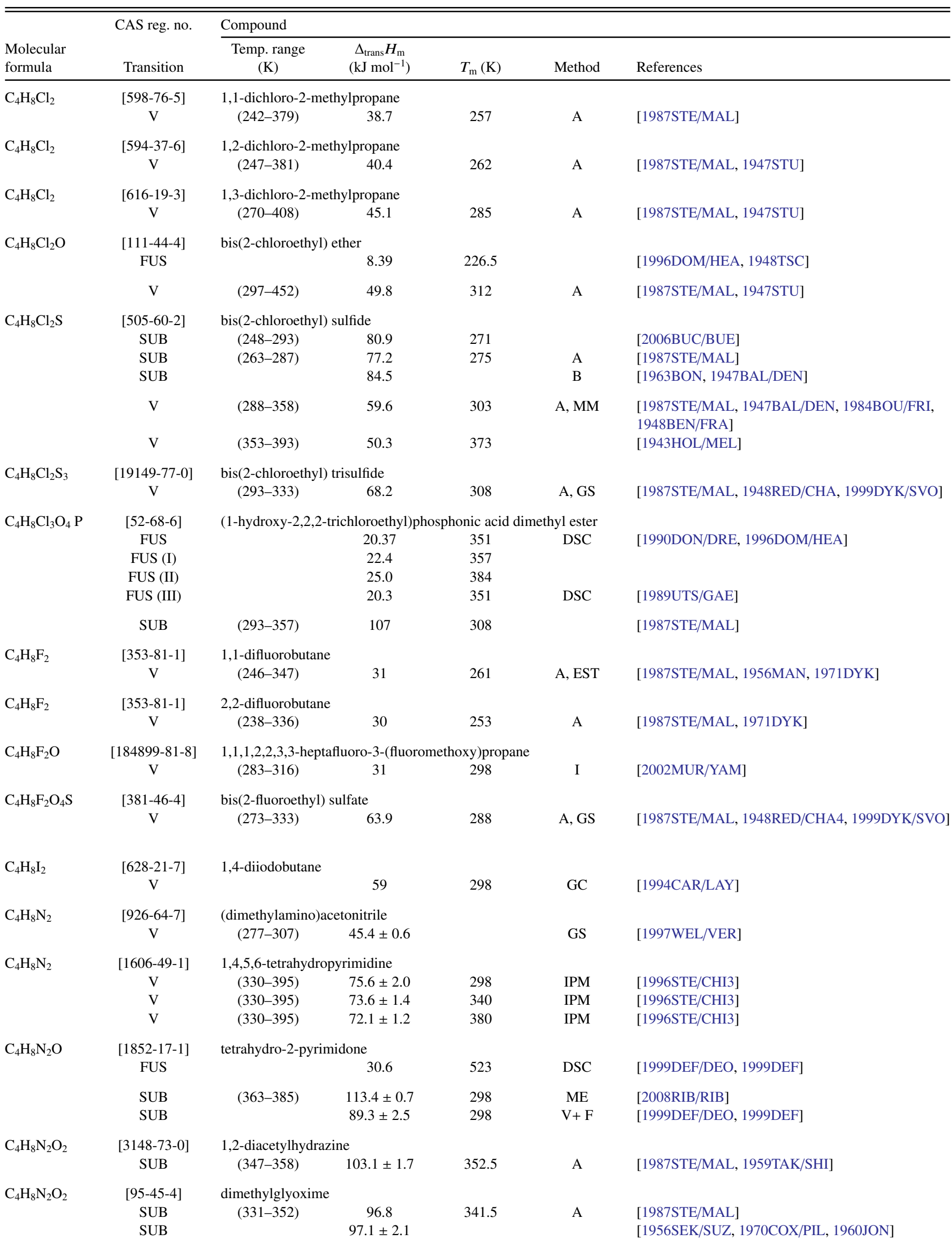


TABLE 7. Phase change enthalpies of $\mathrm{C}_{4}$ organic compounds-Continued

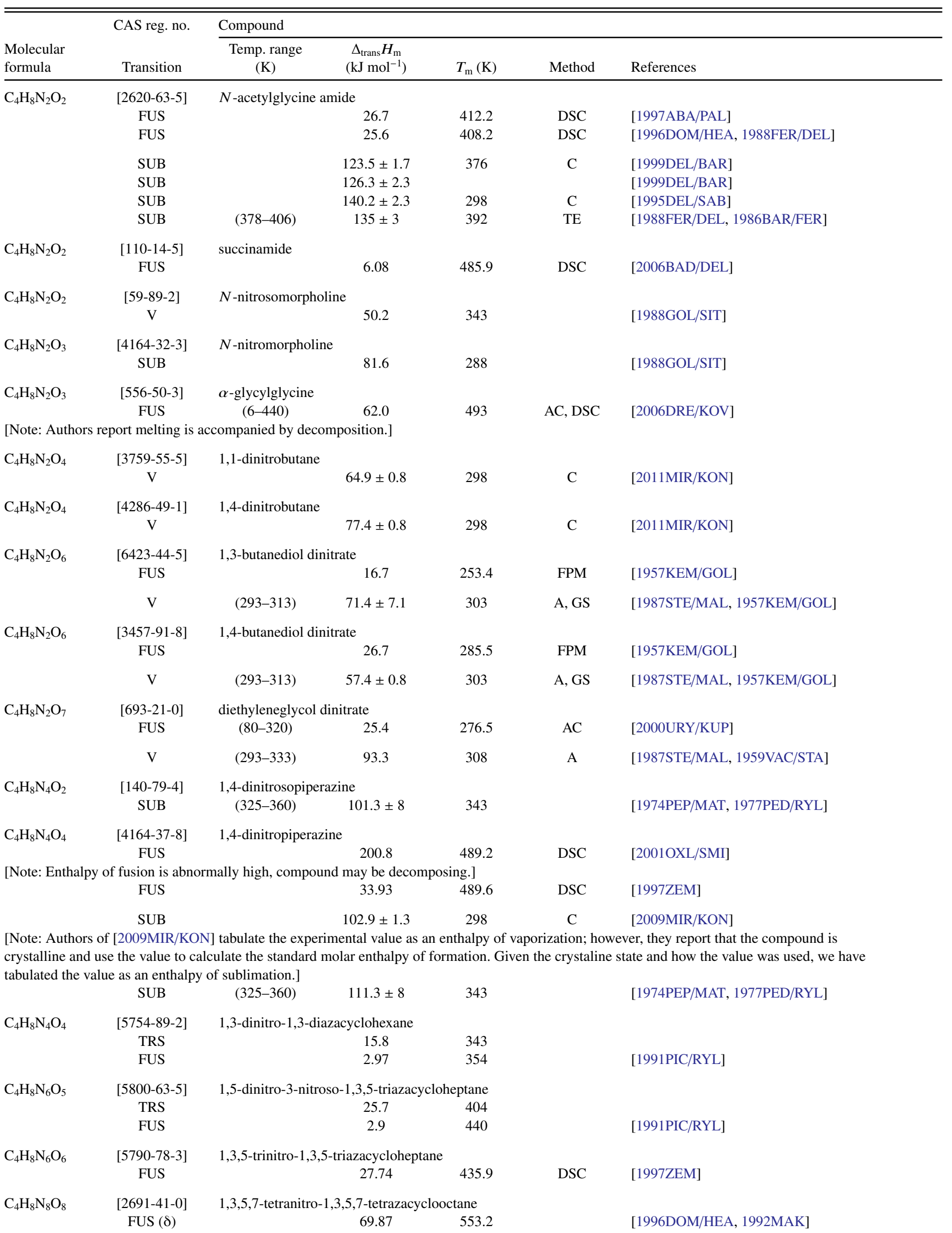


TABLE 7. Phase change enthalpies of $\mathrm{C}_{4}$ organic compounds-Continued

\begin{tabular}{|c|c|c|c|c|c|c|}
\hline \multirow[b]{2}{*}{$\begin{array}{l}\text { Molecular } \\
\text { formula }\end{array}$} & \multirow{2}{*}{$\begin{array}{l}\text { CAS reg. no. } \\
\text { Transition }\end{array}$} & \multicolumn{5}{|l|}{ Compound } \\
\hline & & $\begin{array}{c}\text { Temp. range } \\
(\mathrm{K})\end{array}$ & $\begin{array}{c}\Delta_{\text {trans }} H_{\mathrm{m}} \\
\left(\mathrm{kJ} \mathrm{mol}^{-1}\right)\end{array}$ & $T_{\mathrm{m}}(\mathrm{K})$ & Method & References \\
\hline \multirow{2}{*}{\multicolumn{7}{|c|}{$\begin{array}{l}\text { SUB } \\
\text { [Note: Authors of }[2009 \mathrm{MIR} / \mathrm{KON}] \text { tabulate the experimental value as an enthalpy of vaporization; however, they report that the compound is } \\
\text { crystalline and use the value to calculate the standard molar enthalpy of formation. Given the crystaline state and how the value was used, we have } \\
\text { tabulated the value as an enthalpy of sublimation.] }\end{array}$}} \\
\hline & & & & & & \\
\hline & SUB & $(461-487)$ & 161.9 & 474 & & [1976TAY/CRO] \\
\hline & SUB $(\delta)$ & $(415-479)$ & $161 \pm 0.3$ & 447 & & [1978CUN/PAL] \\
\hline & $\operatorname{SUB}(\beta)$ & $(371-403)$ & 175.2 & 385 & & [1969ROS/DIC] \\
\hline \multirow[t]{2}{*}{$\mathrm{C}_{4} \mathrm{H}_{8} \mathrm{~N}_{12} \mathrm{O}_{6}$} & {$[62209-57-8]$} & \multicolumn{5}{|c|}{ 1,7-diazido-2,4,6-trinitro-2,4,6-triazaheptane } \\
\hline & FUS & & 40.17 & 406 & DTA & [1987OYU/BRI] \\
\hline \multirow[t]{2}{*}{$\mathrm{C}_{4} \mathrm{H}_{8} \mathrm{O}$} & [2919-23-5] & cyclobutanol & & & & \\
\hline & FUS & & 8.53 & 228.4 & & [1982DWO/FUC] \\
\hline \multirow[t]{19}{*}{$\mathrm{C}_{4} \mathrm{H}_{8} \mathrm{O}$} & [78-93-3] & 2-butanone & & & & \\
\hline & FUS & & 8.44 & 186.5 & & [1991ACR, 1972FIN/MES] \\
\hline & $\mathrm{V}$ & $(294-342)$ & 34.6 & 309 & A & [1987STE/MAL] \\
\hline & $\mathrm{V}$ & $(353-403)$ & 32.5 & 368 & A & [1987STE/MAL] \\
\hline & $\mathrm{V}$ & $(397-479)$ & 31.6 & 412 & A & [1987STE/MAL] \\
\hline & $\mathrm{V}$ & $(473-537)$ & 31.1 & 488 & A & [1987STE/MAL] \\
\hline & V & & $34.8 \pm 0.1$ & 298 & $\mathrm{C}$ & [1983UCH/MAJ] \\
\hline & $\mathrm{V}$ & & $35.0 \pm 0.6$ & 298 & $\mathrm{C}$ & [1981GAT/STR] \\
\hline & $\mathrm{V}$ & & $34.5 \pm 0.1$ & 298 & $\mathrm{C}$ & [1979SUN/SVE2] \\
\hline & $\mathrm{V}$ & $(258-362)$ & 35.6 & 273 & & {$[1978 \mathrm{CAV} / \mathrm{CHA}]$} \\
\hline & $\mathrm{V}$ & & 34.7 & & & [1975AMB/ELL] \\
\hline & $\mathrm{V}$ & $(315-363)$ & 33.9 & 330 & $\mathrm{~A}, \mathrm{~EB}, \mathrm{GS}$ & [1987STE/MAL, 1975AMB/ELL, 1965COL/COU] \\
\hline & V & & 33.8 & 315 & $\mathrm{C}$ & [1973GEI/QUI] \\
\hline & V & & $33.8 \pm 0.1$ & 314 & $\mathrm{C}$ & [1961NIC/KOB] \\
\hline & V & & $32.3 \pm 0.1$ & 338 & $\mathrm{C}$ & [1961NIC/KOB] \\
\hline & V & & $31.3 \pm 0.1$ & 352 & $\mathrm{C}$ & [1961NIC/KOB] \\
\hline & $\mathrm{V}$ & & $30.5 \pm 0.1$ & 363 & $\mathrm{C}$ & [1961NIC/KOB] \\
\hline & V & & $30.0 \pm 0.1$ & 370 & $\mathrm{C}$ & [1961NIC/KOB] \\
\hline & $\mathrm{V}$ & $(314-370)$ & 33.9 & 329 & & [1947STU] \\
\hline \multirow[t]{2}{*}{$\mathrm{C}_{4} \mathrm{H}_{8} \mathrm{O}$} & [513-42-8] & 2-methyl-2-propen & $1-\mathrm{ol}$ & & & \\
\hline & $\mathrm{V}$ & (323-373) & 51.9 & 298 & CGC & [1995CHI/HOS] \\
\hline \multirow[t]{5}{*}{$\mathrm{C}_{4} \mathrm{H}_{8} \mathrm{O}$} & [627-27-0] & 3-buten-1-ol & & & & \\
\hline & $\mathrm{V}$ & $(343-393)$ & $50.8 \pm 0.0$ & 298 & CGC & [2005VAL/QUI] \\
\hline & $\mathrm{V}$ & & $50.9 \pm 0.1$ & 313 & $\mathrm{C}$ & [1996ULB/KLU] \\
\hline & V & & $48.8 \pm 0.1$ & 328 & $\mathrm{C}$ & [1996ULB/KLU] \\
\hline & $\mathrm{V}$ & & $46.7 \pm 0.1$ & 343 & $\mathrm{C}$ & [1996ULB/KLU] \\
\hline \multirow[t]{2}{*}{$\mathrm{C}_{4} \mathrm{H}_{8} \mathrm{O}$} & [6118-14-5] & $(d l)$-3-buten-2-ol & & & & \\
\hline & $\mathrm{V}$ & (304-370) & 39.2 & 319 & A & [1987STE/MAL] \\
\hline \multirow[t]{8}{*}{$\mathrm{C}_{4} \mathrm{H}_{8} \mathrm{O}$} & [123-72-8] & butyraldehyde & & & & \\
\hline & FUS & & 11.09 & 176.8 & & [1996DOM/HEA, 1989VAS/LEB] \\
\hline & $\mathrm{V}$ & $(313-353)$ & 33.2 & 298 & CGC & [1995CHI/HOS] \\
\hline & $\mathrm{V}$ & $(293-349)$ & 34.2 & 308 & A & [1987STE/MAL] \\
\hline & V & & $33.7 \pm 0.4$ & 298 & EB & [1967BUC/COX, 2003VER/KRA2] \\
\hline & $\mathrm{V}$ & $(330-348)$ & 32.9 & 339 & $\mathrm{~EB}$ & [1963WOJ] \\
\hline & $\mathrm{V}$ & $(304-347)$ & 33.3 & 319 & & [1959SEP/PAU, 1984BOU/FRI] \\
\hline & V & $(258-353)$ & 33.9 & 306 & & [1938KUC] \\
\hline \multirow[t]{4}{*}{$\mathrm{C}_{4} \mathrm{H}_{8} \mathrm{O}$} & [106-88-7] & $(d l)$-1,2-epoxybuta & & & & \\
\hline & $\mathrm{V}$ & & $33.4 \pm 1.3$ & 298 & EB & [2013MOR/ELL] \\
\hline & $\mathrm{V}$ & $(297-361)$ & 32.1 & 312 & & [1997MON/BUR] \\
\hline & $\mathrm{V}$ & $(254-347)$ & 24.7 & 269 & A & [1987STE/MAL] \\
\hline \multirow[t]{2}{*}{$\mathrm{C}_{4} \mathrm{H}_{8} \mathrm{O}$} & {$[558-30-5]$} & 1,2-epoxy-2-methy & propane $(2,2-$ & hyloxiran & & \\
\hline & $\mathrm{V}$ & $(204-329)$ & 30.6 & 219 & A & [1987STE/MAL, 1947STU] \\
\hline \multirow[t]{2}{*}{$\mathrm{C}_{4} \mathrm{H}_{8} \mathrm{O}$} & [109-92-2] & ethyl vinyl ether & & & & \\
\hline & V & (223-309) & 29.5 & 238 & A & [1987STE/MAL] \\
\hline
\end{tabular}


TABLE 7. Phase change enthalpies of $\mathrm{C}_{4}$ organic compounds-Continued

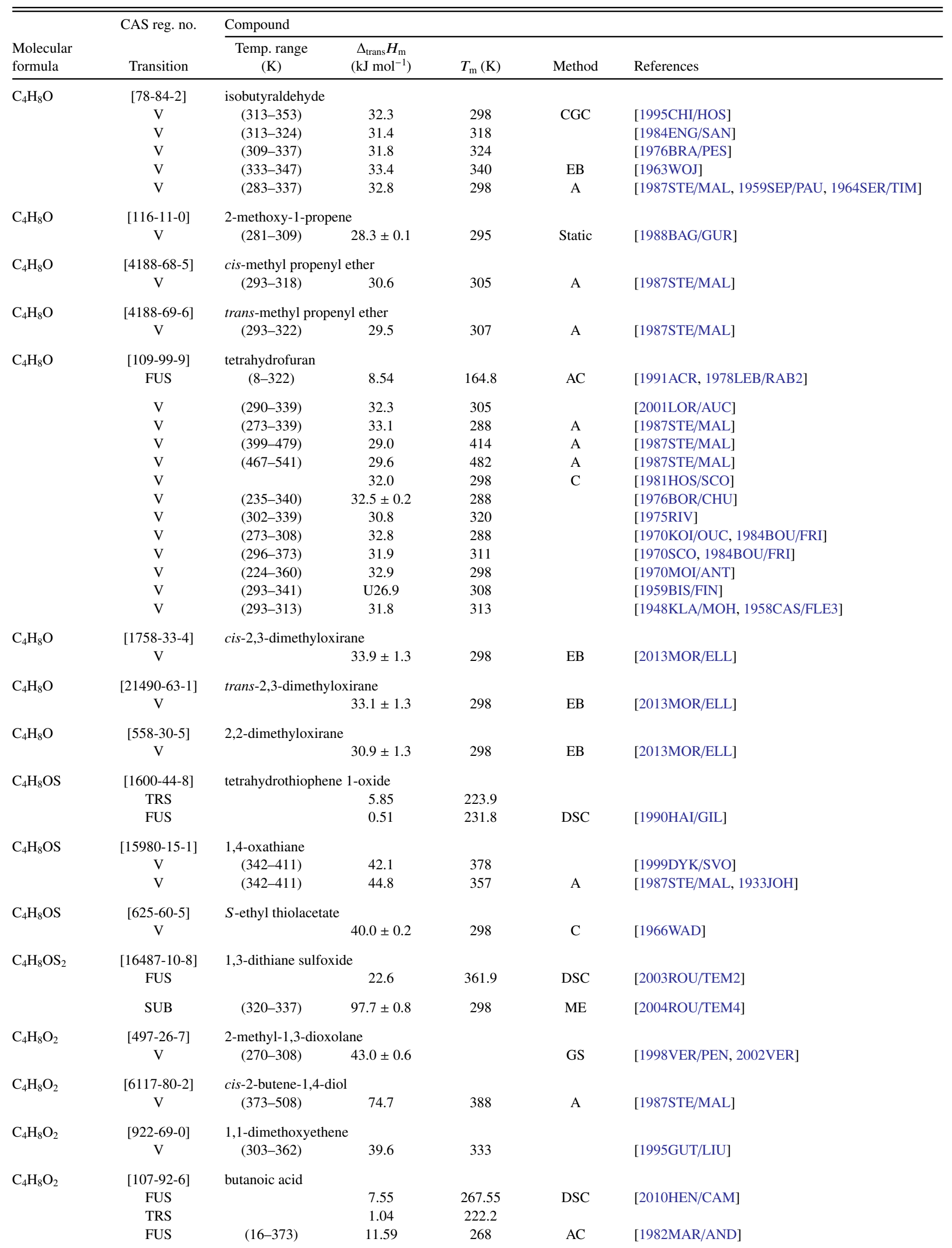


TABLE 7. Phase change enthalpies of $\mathrm{C}_{4}$ organic compounds-Continued

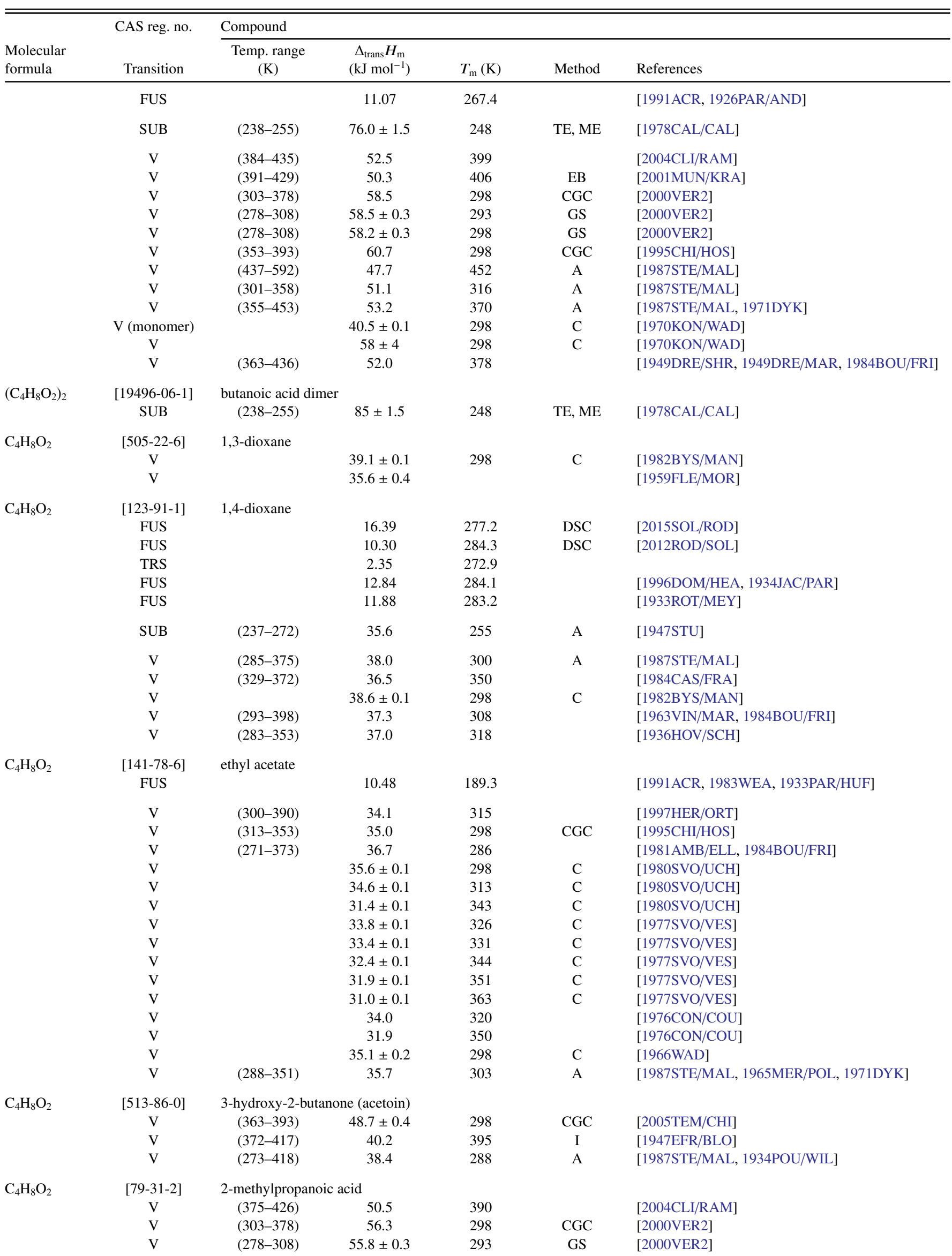


TABLE 7. Phase change enthalpies of $\mathrm{C}_{4}$ organic compounds-Continued

\begin{tabular}{|c|c|c|c|c|c|c|}
\hline \multirow[b]{2}{*}{$\begin{array}{l}\text { Molecular } \\
\text { formula }\end{array}$} & \multirow{2}{*}{$\begin{array}{l}\text { CAS reg. no. } \\
\text { Transition } \\
\end{array}$} & \multicolumn{5}{|l|}{ Compound } \\
\hline & & $\begin{array}{c}\text { Temp. range } \\
(\mathrm{K})\end{array}$ & $\begin{array}{c}\Delta_{\text {trans }} H_{\mathrm{m}} \\
\left(\mathrm{kJ} \mathrm{mol}^{-1}\right)\end{array}$ & $T_{\mathrm{m}}(\mathrm{K})$ & Method & References \\
\hline & $\mathrm{V}$ & $(278-308)$ & $55.5 \pm 0.3$ & 298 & GS & [2000VER2] \\
\hline & $\mathrm{V}$ & $(344-445)$ & 51.6 & 359 & EB & [1987AMB/GHI3] \\
\hline & $\mathrm{V}$ & $(288-428)$ & 50.9 & 303 & A & [1987STE/MAL] \\
\hline & $\mathrm{V}$ & $(428-562)$ & 45.4 & 443 & A & [1987STE/MAL] \\
\hline & $\mathrm{V}$ & $(228-243)$ & $53.4 \pm 3$ & 398 & $\mathrm{TE}$ & [1979DEK/OON] \\
\hline & V (monomer) & & $35.5 \pm 0.1$ & 298 & $\mathrm{C}$ & [1970KON/WAD] \\
\hline & $\mathrm{V}$ & & $53 \pm 4$ & 298 & $\mathrm{C}$ & [1970KON/WAD] \\
\hline $\mathrm{C}_{4} \mathrm{H}_{8} \mathrm{O}_{2}$ & $\begin{array}{c}{[625-55-8]} \\
\mathrm{V}\end{array}$ & $\begin{array}{l}\text { isopropyl formate } \\
\quad(221-342)\end{array}$ & 34.5 & 236 & A & [1987STE/MAL, 1947STU] \\
\hline \multirow[t]{18}{*}{$\mathrm{C}_{4} \mathrm{H}_{8} \mathrm{O}_{2}$} & {$[554-12-1]$} & methyl propanoate & & & & \\
\hline & $\mathrm{V}$ & & $35.7 \pm 1.2$ & 298 & CGC & [2015KOZ/GOB] \\
\hline & $\mathrm{V}$ & $(335-387)$ & 34.1 & 350 & $\mathrm{~EB}$ & [2014RIO/ORT] \\
\hline & $\mathrm{V}$ & $(313-363)$ & 28.9 & 298 & CGC & [1995CHI/HOS] \\
\hline & $\mathrm{V}$ & & $35.6 \pm 0.4$ & 298 & GC & [1987AZA] \\
\hline & $\mathrm{V}$ & $(231-353)$ & 39.1 & 246 & A & [1987STE/MAL] \\
\hline & $\mathrm{V}$ & $(353-486)$ & 32.8 & 368 & A & [1987STE/MAL] \\
\hline & $\mathrm{V}$ & & $36.0 \pm 0.7$ & 298 & $\mathrm{C}$ & [1981GAT/STR] \\
\hline & $\mathrm{V}$ & & $35.9 \pm 0.1$ & 298 & $\mathrm{C}$ & [1980SVO/UCH] \\
\hline & $\mathrm{V}$ & & $34.9 \pm 0.1$ & 313 & $\mathrm{C}$ & [1980SVO/UCH] \\
\hline & $\mathrm{V}$ & & $36.3 \pm 0.3$ & 298 & GCC & [1980FUC/PEA] \\
\hline & $\mathrm{V}$ & & $35.8 \pm 0.1$ & 298 & $\mathrm{C}$ & [1979SUN/SVE2] \\
\hline & $\mathrm{V}$ & & $34.2 \pm 0.1$ & 326 & $\mathrm{C}$ & [1977SVO/VES] \\
\hline & $\mathrm{V}$ & & $33.8 \pm 0.1$ & 331 & $\mathrm{C}$ & [1977SVO/VES] \\
\hline & $\mathrm{V}$ & & $32.8 \pm 0.1$ & 344 & $\mathrm{C}$ & [1977SVO/VES] \\
\hline & $\mathrm{V}$ & & $32.1 \pm 0.1$ & 355 & $\mathrm{C}$ & [1977SVO/VES] \\
\hline & $\mathrm{V}$ & & $31.5 \pm 0.1$ & 363 & $\mathrm{C}$ & [1977SVO/VES] \\
\hline & $\mathrm{V}$ & $(293-353)$ & 35.9 & 308 & A & [1987STE/MAL, 1965MER/POL] \\
\hline \multirow[t]{18}{*}{$\mathrm{C}_{4} \mathrm{H}_{8} \mathrm{O}_{2}$} & {$[110-74-7]$} & propyl formate & & & & \\
\hline & $\mathrm{V}$ & $(328-371)$ & $36.6 \pm 0.1$ & 298 & $\mathrm{~EB}$ & [1999GON/ORT, 2012SAM/NAZ] \\
\hline & $\mathrm{V}$ & $(302-353)$ & 35.3 & 317 & $\mathrm{~EB}$ & [1993FAR/WIC] \\
\hline & $\mathrm{V}$ & $(302-353)$ & $36.4 \pm 0.1$ & 298 & $\mathrm{~EB}$ & [1993FAR/WIC, 2012SAM/NAZ] \\
\hline & $\mathrm{V}$ & $(354-518)$ & 32.7 & 369 & A & [1987STE/MAL] \\
\hline & $\mathrm{V}$ & $(230-355)$ & 36.8 & 245 & A & [1987STE/MAL] \\
\hline & $\mathrm{V}$ & & $37.5 \pm 0.1$ & 298 & $\mathrm{C}$ & [1980SVO/UCH] \\
\hline & $\mathrm{V}$ & & $36.5 \pm 0.1$ & 313 & $\mathrm{C}$ & {$[1980 \mathrm{SVO} / \mathrm{UCH}]$} \\
\hline & $\mathrm{V}$ & & $35.8 \pm 0.1$ & 326 & $\mathrm{C}$ & [1976CIH/HYN] \\
\hline & $\mathrm{V}$ & & $35.4 \pm 0.1$ & 331 & $\mathrm{C}$ & [1976CIH/HYN] \\
\hline & $\mathrm{V}$ & & $34.4 \pm 0.1$ & 344 & $\mathrm{C}$ & [1976CIH/HYN] \\
\hline & $\mathrm{V}$ & & $33.8 \pm 0.1$ & 351 & $\mathrm{C}$ & [1976CIH/HYN] \\
\hline & $\mathrm{V}$ & & $33.5 \pm 0.1$ & 355 & $\mathrm{C}$ & [1976CIH/HYN] \\
\hline & $\mathrm{V}$ & & $32.9 \pm 0.1$ & 363 & $\mathrm{C}$ & [1976CIH/HYN] \\
\hline & $\mathrm{V}$ & $(299-355)$ & 35.6 & 314 & $\mathrm{~EB}$ & [1928NEL, 1984BOU/FRI] \\
\hline & $\mathrm{V}$ & & 36.4 & 298 & $\mathrm{~EB}$ & [1928NEL, 2012SAM/NAZ] \\
\hline & $\mathrm{V}$ & & $32.5 \pm 0.5$ & 353 & $\mathrm{C}$ & [1926MAT] \\
\hline & $\mathrm{V}$ & & $32.5 \pm 0.5$ & 298 & $\mathrm{C}$ & [1926MAT, 2012SAM/NAZ] \\
\hline \multirow[t]{3}{*}{$\mathrm{C}_{4} \mathrm{H}_{8} \mathrm{O}_{2}$} & {$[497-26-7]$} & \multicolumn{5}{|c|}{ 2-methyl-1,3-dioxolane } \\
\hline & V & & 35.6 & & & [2003CHO/DHA] \\
\hline & $\mathrm{V}$ & & 34.9 & & & [1969PIH/HEI, 2003CHO/DHA] \\
\hline \multirow[t]{2}{*}{$\mathrm{C}_{4} \mathrm{H}_{8} \mathrm{O}_{2} \mathrm{~S}$} & {$[16215-14-8]$} & \multicolumn{5}{|l|}{ allyl methyl sulfone } \\
\hline & $\mathrm{V}$ & $(405-450)$ & 68.2 & 420 & $\mathrm{~A}, \mathrm{BG}$ & [1987STE/MAL, 1999DYK/SVO, 1961BUS/IVI] \\
\hline \multirow[t]{8}{*}{$\mathrm{C}_{4} \mathrm{H}_{8} \mathrm{O}_{2} \mathrm{~S}$} & {$[126-33-0]$} & \multicolumn{5}{|c|}{ tetrahydrothiophene-1,1-dioxide (sulfolane) } \\
\hline & TRS & & 7.86 & 288.6 & & \\
\hline & FUS & & 1.37 & 301.7 & & [1999AHL/LOH] \\
\hline & TRS & & 5.35 & 288.6 & & \\
\hline & FUS & & 1.43 & 301.6 & & [1996DOM/MOO] \\
\hline & $\mathrm{V}$ & & $69.1 \pm 1.4$ & 298 & $\mathrm{C}$ & [2004MOR/MAT] \\
\hline & $\mathrm{V}$ & $(423-529)$ & 59.0 & 438 & & [1999DYK/SVO] \\
\hline & $\mathrm{V}$ & $(364-529)$ & 53.7 & 379 & & [1999DYK/SVO] \\
\hline
\end{tabular}


TABLE 7. Phase change enthalpies of $\mathrm{C}_{4}$ organic compounds-Continued

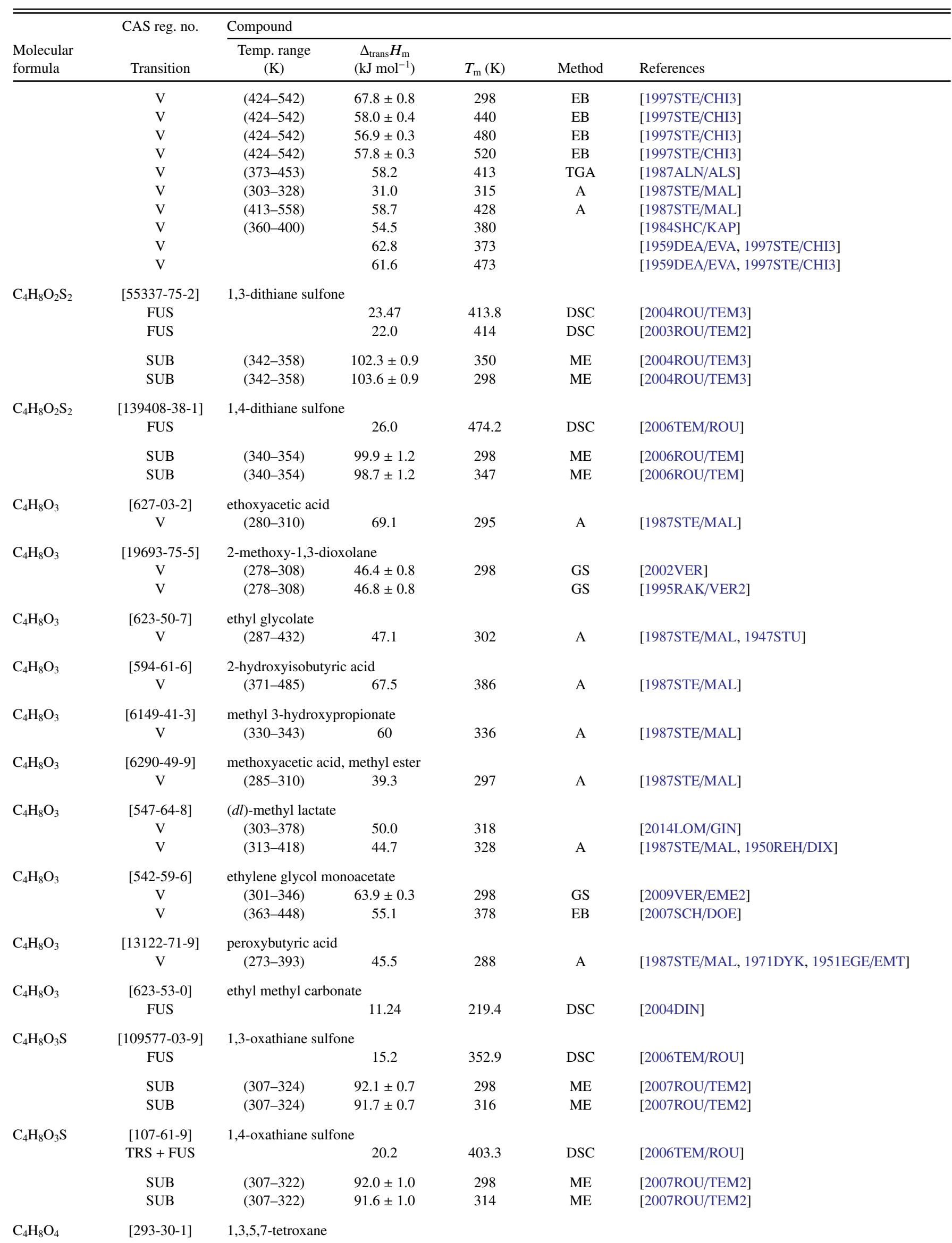


TABLE 7. Phase change enthalpies of $\mathrm{C}_{4}$ organic compounds-Continued

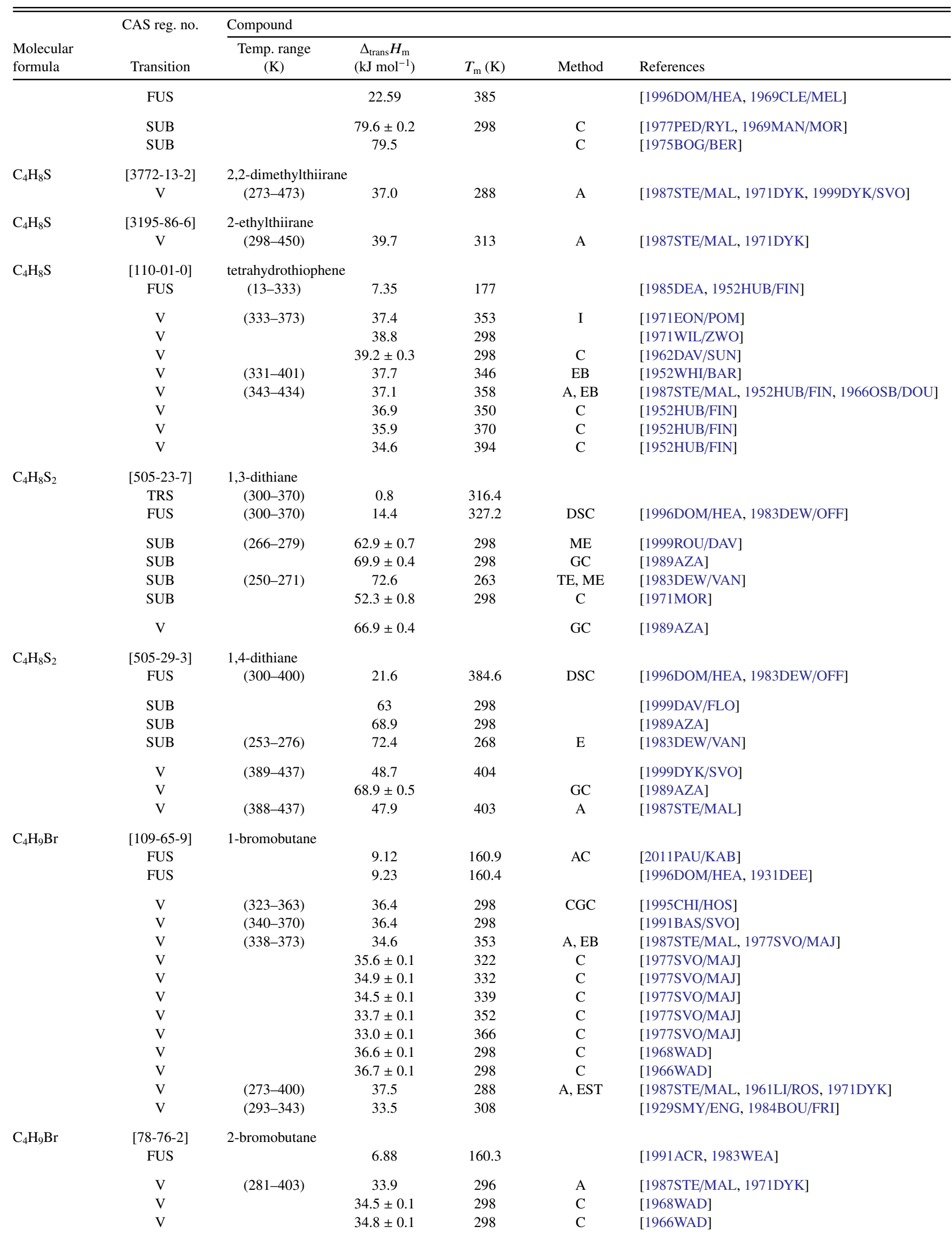


TABLE 7. Phase change enthalpies of $\mathrm{C}_{4}$ organic compounds-Continued

\begin{tabular}{|c|c|c|c|c|c|c|}
\hline \multirow[b]{2}{*}{$\begin{array}{l}\text { Molecular } \\
\text { formula }\end{array}$} & \multirow{2}{*}{$\begin{array}{l}\text { CAS reg. no. } \\
\text { Transition }\end{array}$} & \multicolumn{5}{|l|}{ Compound } \\
\hline & & $\begin{array}{l}\text { Temp. range } \\
\text { (K) }\end{array}$ & $\begin{array}{c}\Delta_{\text {trans }} H_{\mathrm{m}} \\
\left(\mathrm{kJ} \mathrm{mol}^{-1}\right)\end{array}$ & $T_{\mathrm{m}}(\mathrm{K})$ & Method & References \\
\hline \multirow[t]{8}{*}{$\mathrm{C}_{4} \mathrm{H}_{9} \mathrm{Br}$} & {$[78-77-3]$} & \multicolumn{5}{|c|}{ 1-bromo-2-methylpropane } \\
\hline & $\mathrm{V}$ & $(305-363)$ & 34.1 & 320 & $\mathrm{~A}, \mathrm{~EB}$ & [1987STE/MAL, 1977SVO/MAJ] \\
\hline & $\mathrm{V}$ & & $33.1 \pm 0.1$ & 330 & $\mathrm{C}$ & [1977SVO/MAJ] \\
\hline & $\mathrm{V}$ & & $32.6 \pm 0.1$ & 341 & $\mathrm{C}$ & [1977SVO/MAJ] \\
\hline & V & & $32.0 \pm 0.1$ & 353 & $\mathrm{C}$ & [1977SVO/MAJ] \\
\hline & $\mathrm{V}$ & & $31.4 \pm 0.1$ & 366 & $\mathrm{C}$ & [1977SVO/MAJ] \\
\hline & V & $(281-404)$ & 34.0 & 296 & A & [1987STE/MAL, 1971DYK] \\
\hline & $\mathrm{V}$ & & $34.9 \pm 0.1$ & 298 & $\mathrm{C}$ & [1968WAD] \\
\hline \multirow[t]{12}{*}{$\mathrm{C}_{4} \mathrm{H}_{9} \mathrm{Br}$} & [507-19-7] & \multicolumn{5}{|c|}{ 2-bromo-2-methylpropane } \\
\hline & TRS & & 5.85 & 209.3 & & \\
\hline & TRS & & 0.96 & 231.8 & & \\
\hline & FUS & & Not given & & DSC & [1986WEN/SCH] \\
\hline & TRS & & 5.65 & 208.6 & & \\
\hline & TRS & & 1.05 & 231.5 & & \\
\hline & FUS & & 1.97 & 256.1 & $\mathrm{C}$ & [1996DOM/HEA, 1950KUS/CRO] \\
\hline & $\mathrm{V}$ & $(248-346)$ & 31.4 & 263 & A & [1987STE/MAL] \\
\hline & $\mathrm{V}$ & $(270-345)$ & 31.0 & 298 & & [1984BOU/FRI, 1991BAS/SVO] \\
\hline & $\mathrm{V}$ & $(298-323)$ & 31.5 & 313 & & [1969CAL/VAL] \\
\hline & $\mathrm{V}$ & & $31.8 \pm 0.1$ & 298 & $\mathrm{C}$ & [1968WAD] \\
\hline & $\mathrm{V}$ & $(273-346)$ & 31.2 & 288 & & [1951BRY/HOW, 1984BOU/FRI] \\
\hline \multirow[t]{2}{*}{$\mathrm{C}_{4} \mathrm{H}_{9} \mathrm{BrO}$} & [2482-57-7] & \multicolumn{5}{|c|}{ 1-bromo-2-butanol } \\
\hline & $\mathrm{V}$ & $(296-418)$ & 58.4 & 311 & A & [1987STE/MAL, 1947STU] \\
\hline \multirow[t]{13}{*}{$\mathrm{C}_{4} \mathrm{H}_{9} \mathrm{Cl}$} & [109-69-3] & \multicolumn{5}{|l|}{ 1-chlorobutane } \\
\hline & $\mathrm{V}$ & $(260-350)$ & 33.5 & 298 & & [1984BOU/FRI, 1991BAS/SVO] \\
\hline & V & & $33.5 \pm 0.1$ & 298 & $\mathrm{C}$ & [1981TEK/MAJ] \\
\hline & $\mathrm{V}$ & & $32.7 \pm 0.1$ & 313 & $\mathrm{C}$ & [1981TEK/MAJ] \\
\hline & $\mathrm{V}$ & & $31.8 \pm 0.1$ & 328 & $\mathrm{C}$ & [1981TEK/MAJ] \\
\hline & $\mathrm{V}$ & & $30.9 \pm 0.1$ & 343 & $\mathrm{C}$ & [1981TEK/MAJ] \\
\hline & $\mathrm{V}$ & & $30.0 \pm 0.1$ & 358 & $\mathrm{C}$ & [1981TEK/MAJ] \\
\hline & $\mathrm{V}$ & & $29.4 \pm 0.1$ & 358 & $\mathrm{C}$ & [1981TEK/MAJ] \\
\hline & $\mathrm{V}$ & $(256-352)$ & 35.6 & 271 & DTA & [1969KEM/KRE] \\
\hline & $\mathrm{V}$ & & $33.5 \pm 0.1$ & 298 & $\mathrm{C}$ & [1968WAD] \\
\hline & $\mathrm{V}$ & $(257-389)$ & 35.0 & 272 & A, EST & [1987STE/MAL, 1961LI/ROS, 1971DYK] \\
\hline & $\mathrm{V}$ & $(284-350)$ & 33.8 & & & [1933LEN] \\
\hline & $\mathrm{V}$ & $(293-343)$ & 37.2 & 308 & & [1929SMY/ENG, 1984BOU/FRI] \\
\hline \multirow[t]{10}{*}{$\mathrm{C}_{4} \mathrm{H}_{9} \mathrm{Cl}$} & {$[78-86-4]$} & \multicolumn{5}{|l|}{ 2-chlorobutane } \\
\hline & $\mathrm{V}$ & $(315-341)$ & 30.9 & 328 & EB & [1996DAH/WIC] \\
\hline & $\mathrm{V}$ & (266-377) & 33.1 & 281 & A & [1987STE/MAL] \\
\hline & $\mathrm{V}$ & & $31.5 \pm 0.1$ & 298 & $\mathrm{C}$ & [1981TEK/MAJ] \\
\hline & $\mathrm{V}$ & & $30.7 \pm 0.1$ & 313 & $\mathrm{C}$ & [1981TEK/MAJ] \\
\hline & $\mathrm{V}$ & & $29.9 \pm 0.1$ & 328 & $\mathrm{C}$ & [1981TEK/MAJ] \\
\hline & $\mathrm{V}$ & & $29.1 \pm 0.1$ & 343 & $\mathrm{C}$ & [1981TEK/MAJ] \\
\hline & $\mathrm{V}$ & & $28.2 \pm 0.1$ & 358 & $\mathrm{C}$ & [1981TEK/MAJ] \\
\hline & $\mathrm{V}$ & & $31.6 \pm 0.1$ & 298 & $\mathrm{C}$ & [1968WAD] \\
\hline & $\mathrm{V}$ & $(273-312)$ & 31.8 & 288 & & [1928ROL, 1984BOU/FRI] \\
\hline \multirow[t]{3}{*}{$\mathrm{C}_{4} \mathrm{H}_{9} \mathrm{Cl}$} & {$[513-36-0]$} & \multicolumn{5}{|c|}{ 1-chloro-2-methylpropane } \\
\hline & $\mathrm{V}$ & $(219-342)$ & 36.1 & 234 & A & [1987STE/MAL, 1947STU] \\
\hline & $\mathrm{V}$ & & $31.7 \pm 0.1$ & 298 & $\mathrm{C}$ & [1968WAD] \\
\hline \multirow[t]{10}{*}{$\mathrm{C}_{4} \mathrm{H}_{9} \mathrm{Cl}$} & {$[507-20-0]$} & 2-chloro-2-met & opane & & & \\
\hline & TRS & & 2.08 & 183.6 & & \\
\hline & TRS & & 5.75 & 219.8 & & \\
\hline & FUS & & 1.89 & 247.8 & DSC & [2000TAM/LOP] \\
\hline & TRS & $(11-300)$ & 1.84 & 183.3 & & \\
\hline & TRS & $(11-300)$ & 5.61 & 219.7 & & \\
\hline & FUS & $(11-300)$ & 1.92 & 248.6 & $\mathrm{AC}$ & [1999KOB/OGU] \\
\hline & TRS & & 1.87 & 182.9 & & \\
\hline & TRS & & 5.88 & 219.3 & & \\
\hline & FUS & & 1.97 & 248.1 & & [1985DEA] \\
\hline
\end{tabular}


TABLE 7. Phase change enthalpies of $\mathrm{C}_{4}$ organic compounds-Continued

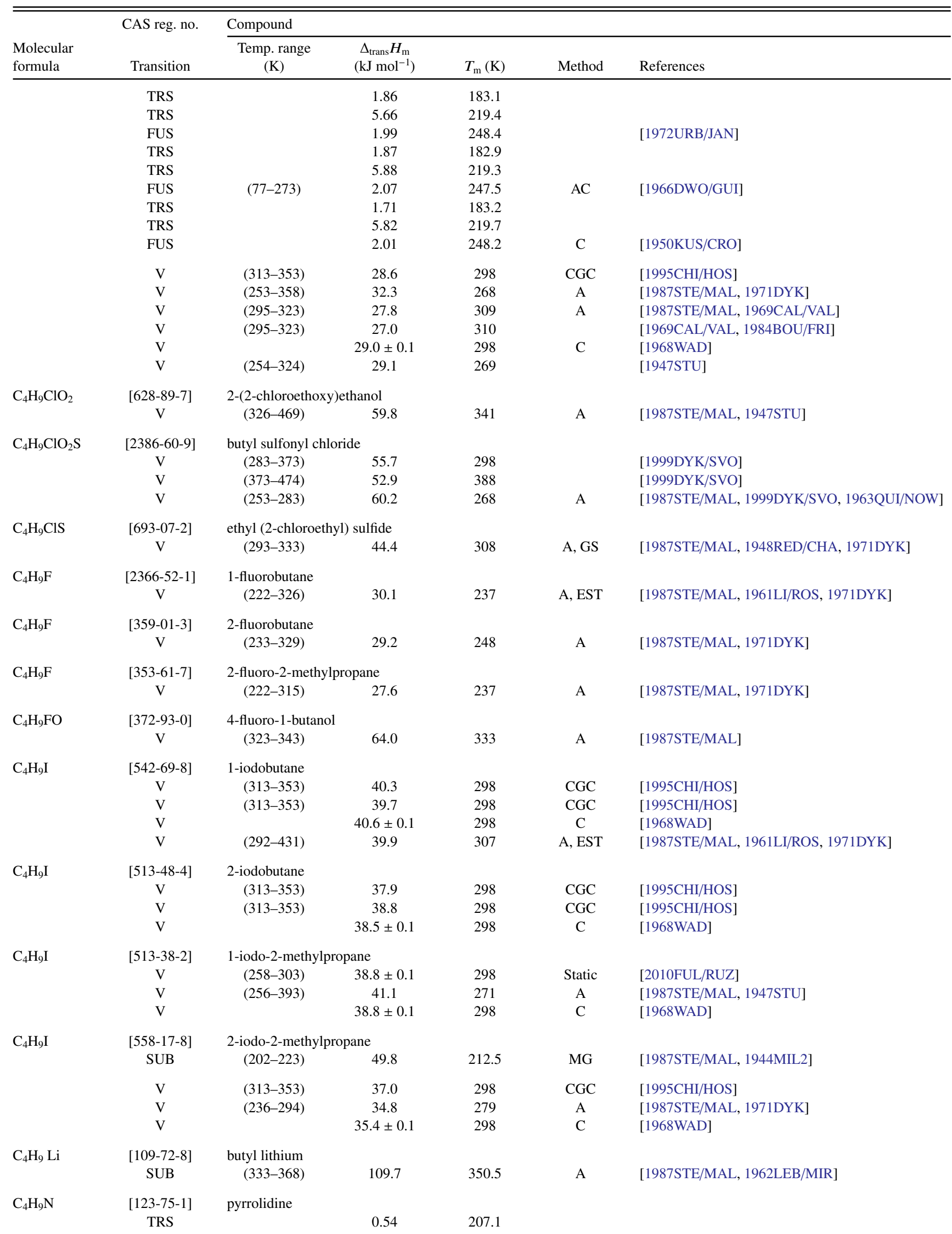


TABLE 7. Phase change enthalpies of $\mathrm{C}_{4}$ organic compounds-Continued

\begin{tabular}{|c|c|c|c|c|c|c|}
\hline \multirow[b]{2}{*}{$\begin{array}{l}\text { Molecular } \\
\text { formula }\end{array}$} & \multirow{2}{*}{$\begin{array}{l}\text { CAS reg. no. } \\
\text { Transition }\end{array}$} & \multicolumn{5}{|l|}{ Compound } \\
\hline & & $\begin{array}{l}\text { Temp. range } \\
(\mathrm{K})\end{array}$ & $\begin{array}{c}\Delta_{\text {trans }} H_{\mathrm{m}} \\
\left(\mathrm{kJ} \mathrm{mol}^{-1}\right)\end{array}$ & $T_{\mathrm{m}}(\mathrm{K})$ & Method & References \\
\hline & FUS & & 8.58 & 215.3 & & [1996DOM/HEA, 1959HIL/SIN] \\
\hline & $\mathrm{V}$ & $(273-313)$ & 38.4 & 288 & A & [1987STE/MAL] \\
\hline & $\mathrm{V}$ & $(273-313)$ & 37.6 & 298 & & {$[1968 \mathrm{CAB} / \mathrm{CON}]$} \\
\hline & $\mathrm{V}$ & $(316-394)$ & 35.8 & 331 & $\mathrm{~EB}, \mathrm{IPM}$ & [1987STE/MAL, 1959MCC/DOU, 1968OSB/DOU] \\
\hline & $\mathrm{V}$ & & $35.8 \pm 0.1$ & 322 & $\mathrm{C}$ & {$[1959 \mathrm{MCC} / \mathrm{DOU}]$} \\
\hline & $\mathrm{V}$ & & $34.5 \pm 0.1$ & 340 & $\mathrm{C}$ & {$[1959 \mathrm{MCC} / \mathrm{DOU}]$} \\
\hline & $\mathrm{V}$ & & $33.0 \pm 0.1$ & 360 & $\mathrm{C}$ & {$[1959 \mathrm{MCC} / \mathrm{DOU}]$} \\
\hline & $\mathrm{V}$ & $(294-360)$ & 37.3 & 309 & & [1959HIL/SIN, 1984BOU/FRI] \\
\hline \multirow[t]{7}{*}{$\mathrm{C}_{4} \mathrm{H}_{9} \mathrm{NO}$} & [96-29-7] & 2-butanone oxime & & & & \\
\hline & $\mathrm{V}$ & $(283-329)$ & $58.6 \pm 0.2$ & 306 & GS & [2009VER/EME4] \\
\hline & $\mathrm{V}$ & $(283-329)$ & $59.1 \pm 0.2$ & 298 & GS & [2009VER/EME4] \\
\hline & $\mathrm{V}$ & $(308-425)$ & 53.7 & 323 & A & [1987STE/MAL] \\
\hline & $\mathrm{V}$ & $(318-343)$ & 55.5 & 330 & A & [1987STE/MAL] \\
\hline & $\mathrm{V}$ & $(313-333)$ & 57.2 & 323 & & [1975MES/BAE, 2009VER/EME4] \\
\hline & $\mathrm{V}$ & $(313-333)$ & 57.7 & 298 & & [1975MES/BAE, 2009VER/EME4] \\
\hline \multirow[t]{2}{*}{$\mathrm{C}_{4} \mathrm{H}_{9} \mathrm{NO}$} & [3376-35-0] & \multicolumn{2}{|c|}{$O$-methyloxime acetone } & & & \\
\hline & $\mathrm{V}$ & (308-344) & 37.0 & 323 & & [2013WAN/LI, 2013YE/DON] \\
\hline \multirow[t]{3}{*}{$\mathrm{C}_{4} \mathrm{H}_{9} \mathrm{NO}$} & {$[625-50-3]$} & \multirow{3}{*}{$\begin{array}{c}N \text {-ethylacetamide } \\
(361-423)\end{array}$} & & & & \\
\hline & $\mathrm{V}$ & & 55.7 & 376 & & [1995SCH/PUS] \\
\hline & $\mathrm{V}$ & & $64.9 \pm 0.2$ & 298 & $\mathrm{C}$ & [1984STA/WAD] \\
\hline \multirow[t]{2}{*}{$\mathrm{C}_{4} \mathrm{H}_{9} \mathrm{NO}$} & [110-69-0] & \multicolumn{2}{|l|}{ buyraldehyde oxime } & & & \\
\hline & $\mathrm{V}$ & $(313-343)$ & 55.8 & 328 & A & [1987STE/MAL] \\
\hline \multirow[t]{12}{*}{$\mathrm{C}_{4} \mathrm{H}_{9} \mathrm{NO}$} & {$[541-35-5]$} & \multirow[t]{2}{*}{ butyramide } & & & & \\
\hline & FUS & & 19.2 & 387.3 & DSC & [2008ABA/BAD, 2000BRU/DEL] \\
\hline & SUB & $(288-354)$ & $82 \pm 4.0$ & 298 & $\mathrm{TE}$ & [2000BRU/DEL] \\
\hline & SUB & $(298-347)$ & $82 \pm 4.0$ & 298 & TE & [2000BRU/DEL] \\
\hline & SUB & & $86.4 \pm 0.4$ & & & [1975BAR/PIL, 1977PED/RYL] \\
\hline & SUB & $(292-304)$ & $85.4 \pm 1.7$ & 298 & ME & [1973LEB/KAT2, 1977PED/RYL] \\
\hline & SUB & $(353-373)$ & 87 & 363 & & [1960JON] \\
\hline & SUB & $(336-382)$ & $86.4 \pm 0.4$ & 359 & GS & [1959DAV/JON2] \\
\hline & SUB & $(298-341)$ & $87.0 \pm 0.8$ & 320 & ME & [1959DAV/JON2] \\
\hline & SUB & & 79.9 & & & [1960THO] \\
\hline & $\mathrm{V}$ & & $72.3 \pm 2.4$ & 298 & CGC & [2013GUT/RAT] \\
\hline & $\mathrm{V}$ & $(397-504)$ & 64 & 412 & A & [1987STE/MAL] \\
\hline \multirow{7}{*}{$\mathrm{C}_{4} \mathrm{H}_{9} \mathrm{NO}$} & [563-83-7] & \multicolumn{2}{|c|}{ 2-methylpropanamide } & & & \\
\hline & FUS & & 19.8 & 401.2 & DSC & [2013GUT/RAT] \\
\hline & FUS & & 19.2 & 400.1 & DSC & [2008ABA/BAD] \\
\hline & SUB & & 82 & & & [2000BRU/DEL] \\
\hline & SUB & $(285-302)$ & $86.1 \pm 0.2$ & 294 & ME & [1989ABB/JIM] \\
\hline & SUB & & $86.0 \pm 0.2$ & 298 & & [1989ABB/JIM] \\
\hline & $\mathrm{V}$ & & $70.7 \pm 2.9$ & 298 & CGC & [2013GUT/RAT] \\
\hline \multirow[t]{12}{*}{$\mathrm{C}_{4} \mathrm{H}_{9} \mathrm{NO}$} & [127-19-5] & \multicolumn{2}{|c|}{$N, N$-dimethylacetamide } & & & \\
\hline & FUS & & 10.2 & 254.2 & & [2007SMI/TSV] \\
\hline & FUS & & 8.2 & 253.2 & DSC & [2000LIS/JAM] \\
\hline & FUS & & 10.42 & 251.4 & & [1999AHL/LOH] \\
\hline & $\mathrm{V}$ & $(463-513)$ & $50.7 \pm 0.7$ & 298 & CGC & [2009PAN/ANT] \\
\hline & V & $(298-423)$ & 45.8 & 298 & & [2005NAS/NEU] \\
\hline & $\mathrm{V}$ & $(371-423)$ & 45.1 & 386 & A & [1987STE/MAL] \\
\hline & $\mathrm{V}$ & & 50.2 & 298 & A & [1985BAR/CAS, 1985MAJ/SVO] \\
\hline & $\mathrm{V}$ & & $45.6 \pm 2.0$ & & & [1978BEA/LEE] \\
\hline & $\mathrm{V}$ & $(297-438)$ & 67.9 & 312 & & [1974MYA/SCH, 1984BOU/FRI] \\
\hline & $\mathrm{V}$ & & 43.7 & 298 & I & [1971SUN/EIS] \\
\hline & $\mathrm{V}$ & $(303-363)$ & 45.2 & 318 & A & [1987STE/MAL, 1968GOP/RIZ] \\
\hline
\end{tabular}

$\mathrm{C}_{4} \mathrm{H}_{9} \mathrm{NO} \quad$ [1187-58-2] $\quad \mathrm{N}$-methylpropionamide 
TABLE 7. Phase change enthalpies of $\mathrm{C}_{4}$ organic compounds-Continued

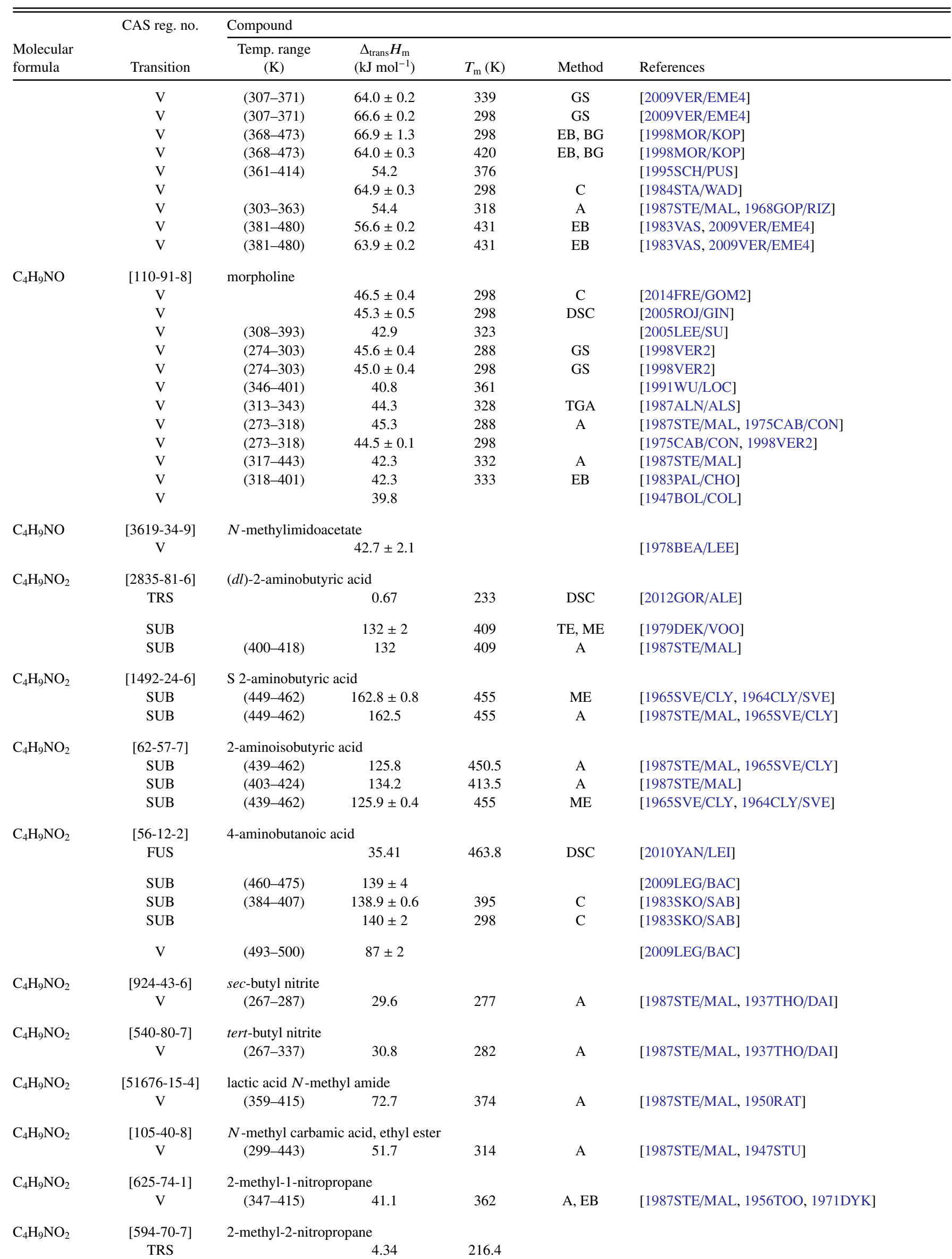


TABLE 7. Phase change enthalpies of $\mathrm{C}_{4}$ organic compounds-Continued

\begin{tabular}{|c|c|c|c|c|c|c|}
\hline \multirow[b]{2}{*}{$\begin{array}{l}\text { Molecular } \\
\text { formula }\end{array}$} & \multirow{2}{*}{$\begin{array}{c}\text { CAS reg. no. } \\
\text { Transition } \\
\end{array}$} & \multicolumn{5}{|l|}{ Compound } \\
\hline & & $\begin{array}{l}\text { Temp. range } \\
\text { (K) }\end{array}$ & $\begin{array}{c}\Delta_{\text {trans }} H_{\mathrm{m}} \\
\left(\mathrm{kJ} \mathrm{mol}^{-1}\right)\end{array}$ & $T_{\mathrm{m}}(\mathrm{K})$ & Method & References \\
\hline & TRS & & 4.64 & 260.0 & & \\
\hline & FUS & & 2.56 & 298.5 & DSC & [1999SAL/LOP] \\
\hline & TRS & & 4.2 & 215.3 & & \\
\hline & TRS & & 4.7 & 260.1 & & \\
\hline & FUS & & 2.6 & 299.2 & & [1997REU/BUS, 1975URB/TOM] \\
\hline & $\mathrm{V}$ & & $42.7 \pm 0.4$ & 298 & $\mathrm{C}$ & [2011MIR/KON] \\
\hline & $\mathrm{V}$ & $(334-401)$ & 39.1 & 349 & EB & [1987STE/MAL, 1956TOO, 1971DYK] \\
\hline \multirow[t]{5}{*}{$\mathrm{C}_{4} \mathrm{H}_{9} \mathrm{NO}_{2}$} & {$[627-05-4]$} & 1-nitrobutane & & & & \\
\hline & $\mathrm{V}$ & & $48.1 \pm 0.4$ & 298 & $\mathrm{C}$ & [2011MIR/KON] \\
\hline & $\mathrm{V}$ & $(313-353)$ & 47.0 & 298 & CGC & [1995CHI/HOS] \\
\hline & $\mathrm{V}$ & $(357-426)$ & 42.7 & 372 & $\mathrm{~A}, \mathrm{~EB}$ & [1987STE/MAL, 1956TOO, 1971DYK] \\
\hline & $\mathrm{V}$ & $(283-423)$ & $48.5 \pm 0.5$ & 298 & $\mathrm{ZG}$ & [1949HOL/DOR] \\
\hline \multirow[t]{4}{*}{$\mathrm{C}_{4} \mathrm{H}_{9} \mathrm{NO}_{2}$} & {$[600-24-8]$} & $(d l)$-2-nitrobutane & & & & \\
\hline & V & & $46.4 \pm 0.8$ & 298 & $\mathrm{C}$ & [2011MIR/KON] \\
\hline & $\mathrm{V}$ & $(345-413)$ & 40.3 & 360 & $\mathrm{~A}, \mathrm{~EB}$ & [1987STE/MAL, 1956TOO, 1971DYK] \\
\hline & $\mathrm{V}$ & $(323-383)$ & $43.8 \pm 0.4$ & 298 & ZG & {$[1949 \mathrm{HOL} / \mathrm{DOR}]$} \\
\hline \multirow[t]{2}{*}{$\mathrm{C}_{4} \mathrm{H}_{9} \mathrm{NO}_{2}$} & [627-12-3] & propyl carbamate & & & & \\
\hline & $\mathrm{V}$ & $(325-468)$ & 61.6 & 340 & A & [1987STE/MAL, 1947STU] \\
\hline \multirow[t]{2}{*}{$\mathrm{C}_{4} \mathrm{H}_{9} \mathrm{NO}_{3}$} & [928-45-0] & butyl nitrate & & & & \\
\hline & $\mathrm{V}$ & $(273-343)$ & 44.1 & 288 & A & [1987STE/MAL, 1971DYK, 1957GRA/PRA] \\
\hline \multirow[t]{2}{*}{$\mathrm{C}_{4} \mathrm{H}_{9} \mathrm{NO}_{3}$} & {$[543-29-3]$} & isobutyl nitrate & & & & \\
\hline & $\mathrm{V}$ & $(273-343)$ & 42.8 & 288 & A & [1987STE/MAL, 1971DYK, 1957GRA/PRA] \\
\hline \multirow{2}{*}{$\mathrm{C}_{4} \mathrm{H}_{9} \mathrm{NO}_{3}$} & {$[80-68-2]$} & $(d l)$-threonine & & & & \\
\hline & SUB & $(341-441)$ & $\mathrm{U} 96 \pm 8$ & 391 & $\mathrm{LE}$ & [1977GAF/PIE] \\
\hline \multirow[t]{11}{*}{$\mathrm{C}_{4} \mathrm{H}_{9} \mathrm{NO}_{3}$} & [76-39-1] & 2-methyl-2-nitro-1 & propanol & & & \\
\hline & TRS & & 14.64 & 311.5 & & \\
\hline & FUS & & 3.17 & 363.9 & DSC & [1999SAL/LOP] \\
\hline & TRS & & 17.2 & 310 & & \\
\hline & FUS & & 3.74 & 361 & DSC & [1996DOM/HEA, 1970MUR/BRE] \\
\hline & TRS & & 15.0 & 312 & & \\
\hline & FUS & & 3.3 & 364 & DSC & [1995BAR/LOP] \\
\hline & SUB (cryst) & $(293-309)$ & $78 \pm 1$ & & & [1995FON/MUN] \\
\hline & SUB (plastic) & $(319-333)$ & $64 \pm 2$ & & & [1995FON/MUN] \\
\hline & SUB (plastic) & & $59.5 \pm 3.0$ & 319 & $\mathrm{C}$ & [1994FON/MUN] \\
\hline & SUB (cryst) & & $73.2 \pm 3.7$ & 311 & $\mathrm{C}$ & [1994FON/MUN] \\
\hline \multirow[t]{7}{*}{$\mathrm{C}_{4} \mathrm{H}_{9} \mathrm{NO}_{4}$} & {$[77-49-6]$} & 2-methyl-2-nitro-1, & 3-propanediol & & & \\
\hline & TRS & & 25.72 & 352 & & \\
\hline & FUS & & 3.84 & 424 & DSC & [1996DOM/HEA, 1970MUR/BRE] \\
\hline & SUB (cryst) & $(330-349)$ & $98 \pm 4$ & & & [1995FON/MUN] \\
\hline & SUB (plastic) & $(361-382)$ & $74 \pm 5$ & & & [1995FON/MUN] \\
\hline & SUB (plastic) & & $79.3 \pm 4.0$ & 368 & $\mathrm{C}$ & [1994FON/MUN] \\
\hline & SUB (cryst) & & $102.0 \pm 5.1$ & 339 & $\mathrm{C}$ & [1994FON/MUN] \\
\hline \multirow[t]{4}{*}{$\mathrm{C}_{4} \mathrm{H}_{9} \mathrm{NO}_{5}$} & [126-11-4] & 2-hydroxymethyl-2 & nitro-1,3-prop & & & \\
\hline & SUB (cryst) & $(332-347)$ & $107 \pm 10$ & & & [1995FON/MUN] \\
\hline & SUB (plastic) & $(354-378)$ & $76 \pm 8$ & & & [1995FON/MUN] \\
\hline & SUB (plastic) & & $77.3 \pm 3.9$ & 368 & $\mathrm{C}$ & [1994FON/MUN] \\
\hline \multirow[t]{2}{*}{$\mathrm{C}_{4} \mathrm{H}_{9} \mathrm{NS}$} & [123-90-0] & thiomorpholine & & & & \\
\hline & $\mathrm{V}$ & & $54.0 \pm 1.5$ & 298 & $\mathrm{C}$ & [2014FRE/GOM2] \\
\hline \multirow[t]{2}{*}{$\mathrm{C}_{4} \mathrm{H}_{9} \mathrm{~N}_{3} \mathrm{O}_{2}$} & & bis(nitrosoethyl)am & & & & \\
\hline & V & $(291-450)$ & 46.4 & 306 & A & [1987STE/MAL] \\
\hline \multirow[t]{2}{*}{$\mathrm{C}_{4} \mathrm{H}_{9} \mathrm{~N}_{3} \mathrm{O}_{2}$} & [216489-98-4] & \multicolumn{4}{|c|}{ 1-[2-(ethenyloxy)ethyl]-1-nitrosohydrazine } & \\
\hline & SUB & & $112.1 \pm 1.9$ & 298 & & [1998LEB/CHI] \\
\hline $\mathrm{C}_{4} \mathrm{H}_{9} \mathrm{ONa}$ & {$[865-48-5]$} & sodium tert-butoxi & & & & \\
\hline
\end{tabular}


TABLE 7. Phase change enthalpies of $\mathrm{C}_{4}$ organic compounds-Continued

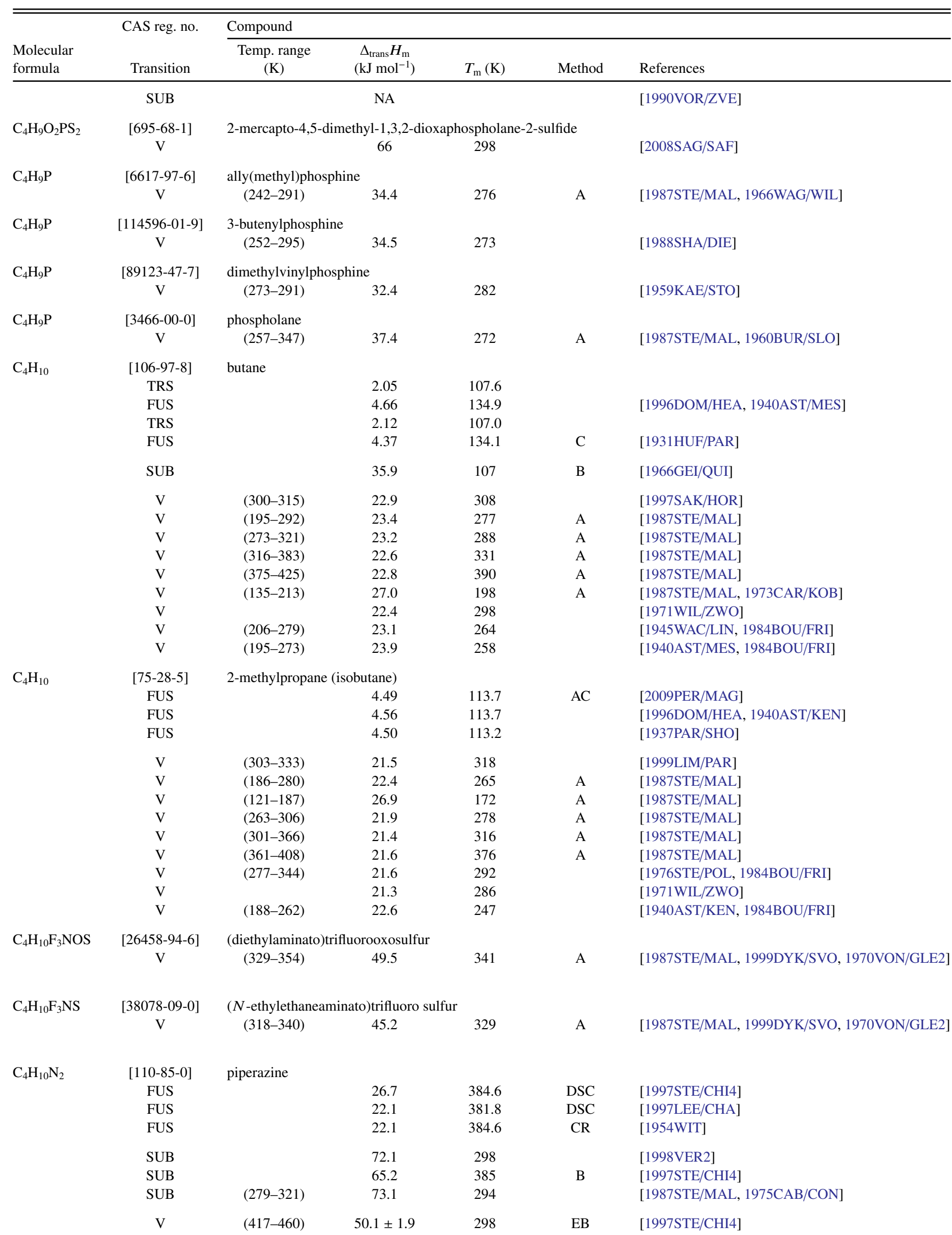


TABLE 7. Phase change enthalpies of $\mathrm{C}_{4}$ organic compounds-Continued

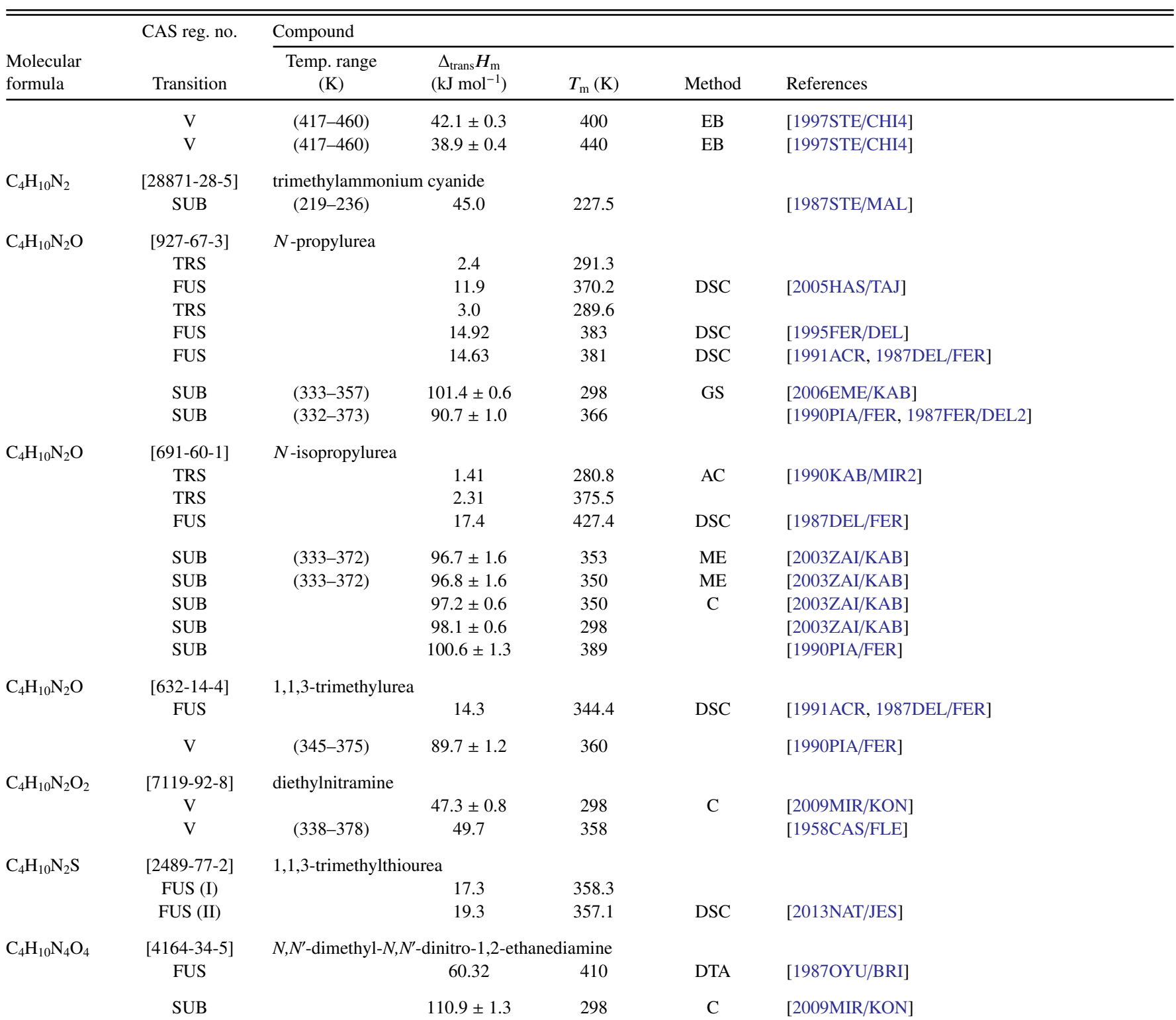

[Note: Authors of [2009MIR/KON] tabulate the experimental value as an enthalpy of vaporization; however, they report that the compound is crystalline and use the value to calculate the standard molar enthalpy of formation. Given the crystaline state and how the value was used, we have tabulated the value as an enthalpy of sublimation.]

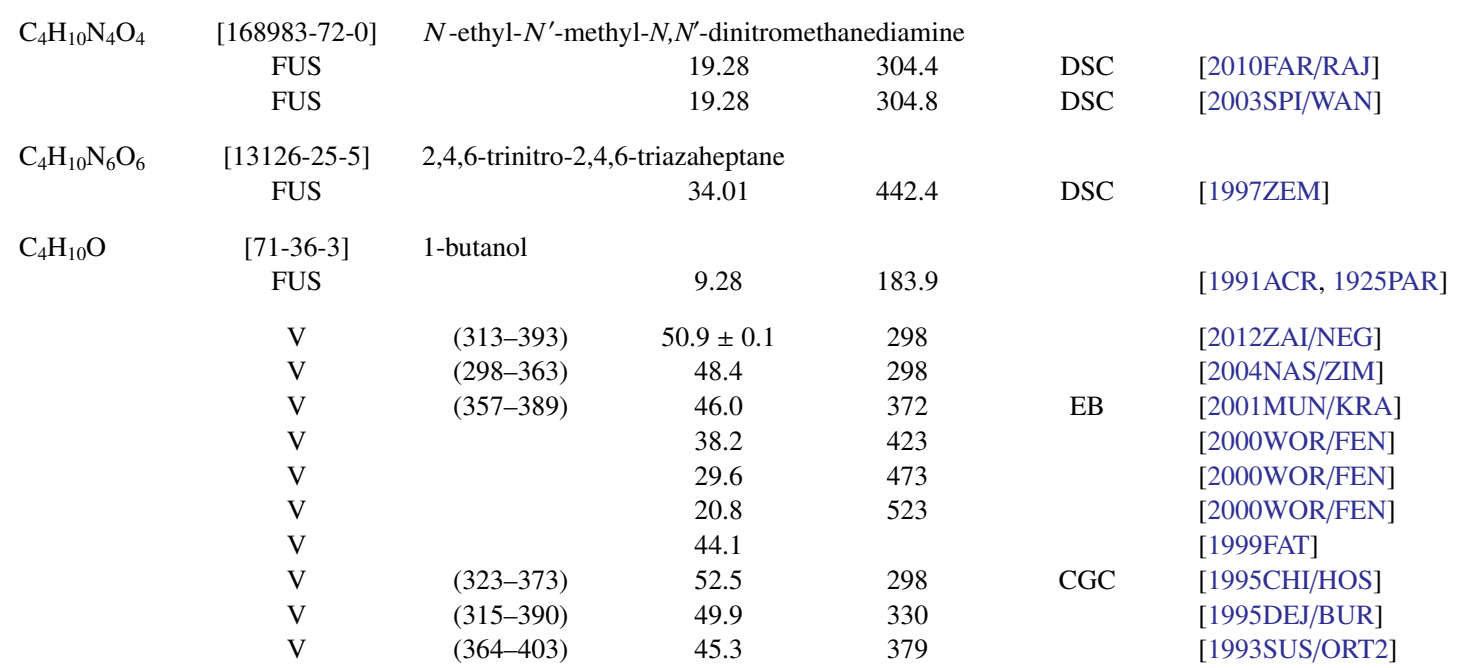


TABle 7. Phase change enthalpies of $\mathrm{C}_{4}$ organic compounds-Continued

\begin{tabular}{|c|c|c|c|c|c|c|}
\hline \multirow[b]{2}{*}{$\begin{array}{l}\text { Molecular } \\
\text { formula }\end{array}$} & \multirow{2}{*}{$\begin{array}{l}\text { CAS reg. no. } \\
\text { Transition }\end{array}$} & \multicolumn{5}{|l|}{ Compound } \\
\hline & & $\begin{array}{c}\text { Temp. range } \\
(\mathrm{K})\end{array}$ & $\begin{array}{c}\Delta_{\text {trans }} H_{\mathrm{m}} \\
\left(\mathrm{kJ} \mathrm{mol}^{-1}\right)\end{array}$ & $T_{\mathrm{m}}(\mathrm{K})$ & Method & References \\
\hline & $\mathrm{V}$ & $(283-323)$ & 55.2 & 298 & & [1992GRA/SAN] \\
\hline & V & $(376-399)$ & 45.3 & 387 & A & [1987STE/MAL] \\
\hline & V & $(323-413)$ & 50.1 & 338 & A & [1987STE/MAL] \\
\hline & $\mathrm{V}$ & $(413-550)$ & 41.9 & 428 & A & [1987STE/MAL] \\
\hline & V & $(209-251)$ & 51.6 & 236 & A & [1987STE/MAL] \\
\hline & $\mathrm{V}$ & $(376-397)$ & 45.4 & 386 & A & [1987STE/MAL] \\
\hline & V & $(391-429)$ & 43.8 & 406 & A & [1987STE/MAL] \\
\hline & V & $(415-501)$ & 41.9 & 430 & A & [1987STE/MAL] \\
\hline & $\mathrm{V}$ & $(497-563)$ & 37.4 & 512 & A & [1987STE/MAL] \\
\hline & $\mathrm{V}$ & $(243-303)$ & 51.7 & 298 & & [1983SCH/STR] \\
\hline & V & $(329-391)$ & 49.0 & 344 & & [1982SAC/PES] \\
\hline & V & & 52.1 & 298 & $\mathrm{C}$ & [1982FUC/PEA] \\
\hline & $\mathrm{V}$ & $(288-404)$ & 55.0 & 303 & & [1973WIL/ZWO] \\
\hline & $\mathrm{V}$ & & $49.5 \pm 0.1$ & 333 & $\mathrm{C}$ & [1973SVO/VES] \\
\hline & V & & $48.6 \pm 0.1$ & 343 & $\mathrm{C}$ & [1973SVO/VES] \\
\hline & V & & $47.5 \pm 0.1$ & 353 & $\mathrm{C}$ & [1973SVO/VES] \\
\hline & V & & $46.4 \pm 0.1$ & 363 & $\mathrm{C}$ & [1973SVO/VES] \\
\hline & V & & $52.34 \pm 0.02$ & 298 & $\mathrm{C}$ & [1971POL/BEN] \\
\hline & $\mathrm{V}$ & & $51.6 \pm 0.1$ & 308 & $\mathrm{C}$ & [1971POL/BEN] \\
\hline & $\mathrm{V}$ & & $51.0 \pm 0.1$ & 318 & $\mathrm{C}$ & [1971POL/BEN] \\
\hline & V & & $49.8 \pm 0.1$ & 328 & $\mathrm{C}$ & [1971POL/BEN] \\
\hline & $\mathrm{V}$ & $(351-397)$ & 47.2 & 366 & EB & [1987STE/MAL, 1970AMB/SPR] \\
\hline & $\mathrm{V}$ & $(295-391)$ & 53.0 & 310 & DTA & [1969KEM/KRE] \\
\hline & $\mathrm{V}$ & & $52.3 \pm 0.1$ & 298 & $\mathrm{C}$ & [1966WAD] \\
\hline & $\mathrm{V}$ & & $47.2 \pm 0.1$ & 356 & $\mathrm{C}$ & [1965COU/HAL] \\
\hline & $\mathrm{V}$ & & $45.4 \pm 0.1$ & 381 & $\mathrm{C}$ & [1965COU/HAL] \\
\hline & $\mathrm{V}$ & & $43.1 \pm 0.1$ & 391 & $\mathrm{C}$ & [1965COU/HAL] \\
\hline & $\mathrm{V}$ & $(419-563)$ & 42.1 & 434 & & [1963AMB/TOW] \\
\hline & $\mathrm{V}$ & $(362-398)$ & 46.6 & 377 & EB & [1963BID/COL] \\
\hline & $\mathrm{V}$ & & $51.0 \pm 0.1$ & 298 & $\mathrm{C}$ & [1963MCC/LAI] \\
\hline & $\mathrm{V}$ & $(337-390)$ & 48.3 & 352 & & [1959BRO/SMI, 1984BOU/FRI] \\
\hline & $\mathrm{V}$ & $(314-390)$ & 48.3 & 352 & & [1898KAH, 1984BOU/FRI] \\
\hline \multirow[t]{29}{*}{$\mathrm{C}_{4} \mathrm{H}_{10} \mathrm{O}$} & {$[78-92-2]$} & 2-butanol & & & & \\
\hline & FUS & & 5.97 & 184.7 & & [1971AND/CON] \\
\hline & $\mathrm{V}$ & $(358-372)$ & 44.2 & 360 & & [2012LAA/ZAI] \\
\hline & $\mathrm{V}$ & $(313-393)$ & $48.1 \pm 0.1$ & 298 & & [2012ZAI/NEG] \\
\hline & $\mathrm{V}$ & $(315-371)$ & 48.8 & 330 & $\mathrm{~EB}$ & [2009GIE/KOS] \\
\hline & $\mathrm{V}$ & $(320-379)$ & 46.2 & 335 & & [2009MAR/LLA] \\
\hline & $\mathrm{V}$ & $(298-563)$ & 46.2 & 298 & & [2004NAS/ZIM] \\
\hline & V & $(306-373)$ & 47.7 & 321 & & [1995DEJ/BUR] \\
\hline & $\mathrm{V}$ & $(303-403)$ & 49.3 & 318 & A & [1987STE/MAL] \\
\hline & V & $(359-381)$ & 43.2 & 370 & A & [1987STE/MAL] \\
\hline & $\mathrm{V}$ & $(372-524)$ & 47.9 & 387 & A & [1987STE/MAL] \\
\hline & $\mathrm{V}$ & $(210-303)$ & 57.5 & 225 & A & [1987STE/MAL] \\
\hline & $\mathrm{V}$ & $(359-380)$ & 43.2 & 369 & A & [1987STE/MAL] \\
\hline & $\mathrm{V}$ & $(368-404)$ & 42.0 & 383 & A & [1987STE/MAL] \\
\hline & $\mathrm{V}$ & $(395-485)$ & 39.6 & 410 & A & [1987STE/MAL] \\
\hline & $\mathrm{V}$ & $(476-536)$ & 35.0 & 491 & A & [1987STE/MAL] \\
\hline & V & $(307-373)$ & 47.8 & 322 & & [1982SAC/PES] \\
\hline & $\mathrm{V}$ & $(293-380)$ & 53.2 & 308 & & [1978CAV/CHA] \\
\hline & $\mathrm{V}$ & $(319-372)$ & 44.1 & 334 & & [1975BRA/AND] \\
\hline & V & $(280-314)$ & 50.2 & 295 & & [1975CAB/CON2] \\
\hline & $\mathrm{V}$ & $(298-393)$ & 48.1 & 313 & & [1973WIL/ZWO] \\
\hline & V & & $49.74 \pm 0.02$ & 298 & $\mathrm{C}$ & [1971POL/BEN] \\
\hline & $\mathrm{V}$ & & $48.8 \pm 0.1$ & 308 & $\mathrm{C}$ & [1971POL/BEN] \\
\hline & $\mathrm{V}$ & & $47.9 \pm 0.1$ & 318 & $\mathrm{C}$ & [1971POL/BEN] \\
\hline & $\mathrm{V}$ & & $46.1 \pm 0.1$ & 328 & $\mathrm{C}$ & [1971POL/BEN] \\
\hline & $\mathrm{V}$ & $(323-373)$ & 46.3 & 338 & & [1969BRO/FOC, 1984BOU/FRI] \\
\hline & $\mathrm{V}$ & & $49.7 \pm 0.1$ & 298 & $\mathrm{C}$ & [1966WAD] \\
\hline & $\mathrm{V}$ & $(422-538)$ & 38.4 & 437 & & [1963AMB/TOW] \\
\hline & $\mathrm{V}$ & $(345-381)$ & 44.1 & 360 & $\mathrm{~EB}$ & [1963BID/COL] \\
\hline
\end{tabular}


TABle 7. Phase change enthalpies of $\mathrm{C}_{4}$ organic compounds-Continued

\begin{tabular}{|c|c|c|c|c|c|c|}
\hline \multirow[b]{2}{*}{$\begin{array}{l}\text { Molecular } \\
\text { formula }\end{array}$} & \multirow{2}{*}{$\begin{array}{l}\text { CAS reg. no. } \\
\text { Transition }\end{array}$} & \multicolumn{5}{|l|}{ Compound } \\
\hline & & $\begin{array}{c}\text { Temp. range } \\
(\mathrm{K})\end{array}$ & $\begin{array}{c}\Delta_{\text {trans }} H_{\mathrm{m}} \\
\left(\mathrm{kJ} \mathrm{mol}^{-1}\right)\end{array}$ & $T_{\mathrm{m}}(\mathrm{K})$ & Method & References \\
\hline & $\mathrm{V}$ & & 48.5 & 298 & $\mathrm{C}$ & {$[1963 \mathrm{MCC} / \mathrm{LAI}]$} \\
\hline & $\mathrm{V}$ & $(340-379)$ & 44.7 & 355 & $\mathrm{~EB}$ & [1987STE/MAL, 1962BER/MCK, 1970AMB/SPR] \\
\hline & $\mathrm{V}$ & & $45.3 \pm 0.1$ & 340 & $\mathrm{C}$ & {$[1962 \mathrm{BER} / \mathrm{MCK}]$} \\
\hline & $\mathrm{V}$ & & $43.3 \pm 0.1$ & 355 & $\mathrm{C}$ & [1962BER/MCK] \\
\hline & $\mathrm{V}$ & & $41.9 \pm 0.1$ & 365 & $\mathrm{C}$ & [1962BER/MCK] \\
\hline & $\mathrm{V}$ & & $40.8 \pm 0.1$ & 372 & $\mathrm{C}$ & {$[1962 \mathrm{BER} / \mathrm{MCK}]$} \\
\hline \multirow[t]{2}{*}{$\mathrm{C}_{4} \mathrm{H}_{10} \mathrm{O}$} & {$[4221-99-2]$} & $(+)$-2-butanol & & & & \\
\hline & FUS & & 6.0 & 177.4 & & {$[1971 \mathrm{AND} / \mathrm{CON}]$} \\
\hline \multirow[t]{30}{*}{$\mathrm{C}_{4} \mathrm{H}_{10} \mathrm{O}$} & {$[78-83-1]$} & 2-methyl-1-pro & & & & \\
\hline & FUS & & 6.32 & 171.2 & & [1968COU/LEE] \\
\hline & $\mathrm{V}$ & $(350-400)$ & 48.8 & 298 & & [1999ORT/HER] \\
\hline & $\mathrm{V}$ & $(350-400)$ & 45.4 & 365 & EB & [1993SUS/ORT] \\
\hline & $\mathrm{V}$ & $(313-411)$ & 49.5 & 328 & A & [1987STE/MAL] \\
\hline & $\mathrm{V}$ & $(381-524)$ & 46.0 & 396 & A & [1987STE/MAL] \\
\hline & $\mathrm{V}$ & $(202-243)$ & 55.0 & 228 & A & [1987STE/MAL] \\
\hline & V & $(369-389)$ & 44.2 & 379 & A & [1987STE/MAL] \\
\hline & $\mathrm{V}$ & $(383-416)$ & 42.6 & 398 & A & [1987STE/MAL] \\
\hline & $\mathrm{V}$ & $(401-493)$ & 41.1 & 416 & A & [1987STE/MAL] \\
\hline & $\mathrm{V}$ & $(483-548)$ & 36.2 & 498 & A & [1987STE/MAL] \\
\hline & $\mathrm{V}$ & & $50.8 \pm 0.1$ & 298 & $\mathrm{C}$ & [1984MAJ/SVO] \\
\hline & $\mathrm{V}$ & & $49.7 \pm 0.1$ & 313 & $\mathrm{C}$ & [1984MAJ/SVO] \\
\hline & V & & $48.3 \pm 0.1$ & 328 & $\mathrm{C}$ & [1984MAJ/SVO] \\
\hline & $\mathrm{V}$ & & $45.0 \pm 0.1$ & 358 & $\mathrm{C}$ & [1984MAJ/SVO] \\
\hline & V & $(320-382)$ & 48.1 & 335 & & [1982SAC/PES] \\
\hline & $\mathrm{V}$ & $(293-388)$ & 52.6 & 308 & & [1973WIL/ZWO] \\
\hline & $\mathrm{V}$ & & $50.79 \pm 0.02$ & 298 & $\mathrm{C}$ & [1971POL/BEN] \\
\hline & V & & $50.1 \pm 0.1$ & 308 & $\mathrm{C}$ & [1971POL/BEN] \\
\hline & $\mathrm{V}$ & & $49.3 \pm 0.1$ & 318 & $\mathrm{C}$ & [1971POL/BEN] \\
\hline & $\mathrm{V}$ & & $47.9 \pm 0.1$ & 328 & $\mathrm{C}$ & [1971POL/BEN] \\
\hline & $\mathrm{V}$ & & $46.2 \pm 0.1$ & 347 & $\mathrm{C}$ & [1970COU/FEN] \\
\hline & $\mathrm{V}$ & & $44.2 \pm 0.1$ & 363 & $\mathrm{C}$ & [1970COU/FEN] \\
\hline & $\mathrm{V}$ & & $41.9 \pm 0.1$ & 381 & $\mathrm{C}$ & [1970COU/FEN] \\
\hline & $\mathrm{V}$ & $(342-389)$ & 46.2 & 357 & $\mathrm{~A}, \mathrm{~EB}$ & [1987STE/MAL, 1970AMB/SPR] \\
\hline & $\mathrm{V}$ & $(333-381)$ & 47.0 & 348 & & [1969BRO/FOC, 1984BOU/FRI] \\
\hline & $\mathrm{V}$ & & $50.8 \pm 0.1$ & 298 & $\mathrm{C}$ & [1966WAD] \\
\hline & V & $(423-548)$ & 40.1 & 438 & & [1963AMB/TOW] \\
\hline & $\mathrm{V}$ & $(353-388)$ & 45.2 & 368 & EB & [1963BID/COL] \\
\hline & $\mathrm{V}$ & & 49.8 & 298 & $\mathrm{C}$ & [1963MCC/LAI] \\
\hline \multirow[t]{21}{*}{$\mathrm{C}_{4} \mathrm{H}_{10} \mathrm{O}$} & {$[75-65-0]$} & 2-methyl-2-pro & & & & \\
\hline & FUS & & 6.42 & 295.1 & DSC & [2011BOG/DAO] \\
\hline & TRS & $(15-330)$ & 0.83 & 286.1 & & \\
\hline & TRS & $(15-330)$ & 0.49 & 294.5 & & \\
\hline & FUS & $(15-330)$ & 6.7 & 299.0 & $\mathrm{AC}$ & [1996DOM/HEA, 1963OET] \\
\hline & FUS & & 6.78 & 298.5 & & [1996DOM/HEA, 1926PAR/AND] \\
\hline & SUB & & 51.5 & & & [2011BOG/DAO] \\
\hline & SUB & $(253-298)$ & 51.3 & 275 & A & [1947STU] \\
\hline & $\mathrm{V}$ & & 45.0 & & & [2011BOG/DAO] \\
\hline & $\mathrm{V}$ & $(306-355)$ & 47.4 & 298 & EB & [2007MAL] \\
\hline & $\mathrm{V}$ & $(323-368)$ & 42.7 & 338 & & [2003ORT/ESP] \\
\hline & $\mathrm{V}$ & $(321-359)$ & 43.4 & 336 & & [1999AUC/LOR] \\
\hline & $\mathrm{V}$ & $(323-373)$ & 45.4 & 298 & CGC & [1995CHI/HOS] \\
\hline & $\mathrm{V}$ & $(299-375)$ & 46.2 & 314 & A & [1987STE/MAL] \\
\hline & $\mathrm{V}$ & $(347-363)$ & 41.4 & 355 & A & [1987STE/MAL] \\
\hline & $\mathrm{V}$ & $(356-480)$ & 43.2 & 371 & A & [1987STE/MAL] \\
\hline & $\mathrm{V}$ & $(347-363)$ & 41.4 & 355 & A & [1987STE/MAL] \\
\hline & $\mathrm{V}$ & $(357-461)$ & 39.8 & 372 & A & [1987STE/MAL] \\
\hline & V & $(453-506)$ & 33.6 & 468 & A & [1987STE/MAL] \\
\hline & $\mathrm{V}$ & & $46.2 \pm 0.1$ & 303 & $\mathrm{C}$ & [1984MAJ/SVO] \\
\hline & $\mathrm{V}$ & & $44.9 \pm 0.1$ & 313 & $\mathrm{C}$ & [1984MAJ/SVO] \\
\hline
\end{tabular}


TABLE 7. Phase change enthalpies of $\mathrm{C}_{4}$ organic compounds-Continued

\begin{tabular}{|c|c|c|c|c|c|c|}
\hline \multirow[b]{2}{*}{$\begin{array}{l}\text { Molecular } \\
\text { formula }\end{array}$} & \multirow{2}{*}{$\begin{array}{l}\text { CAS reg. no. } \\
\text { Transition }\end{array}$} & \multicolumn{5}{|l|}{ Compound } \\
\hline & & $\begin{array}{l}\text { Temp. range } \\
\text { (K) }\end{array}$ & $\begin{array}{c}\Delta_{\text {trans }} H_{\mathrm{m}} \\
\left(\mathrm{kJ} \mathrm{mol}^{-1}\right)\end{array}$ & $T_{\mathrm{m}}(\mathrm{K})$ & Method & References \\
\hline & $\mathrm{V}$ & & $43.0 \pm 0.1$ & 328 & $\mathrm{C}$ & [1984MAJ/SVO] \\
\hline & $\mathrm{V}$ & & $41.0 \pm 0.1$ & 343 & $\mathrm{C}$ & [1984MAJ/SVO] \\
\hline & $\mathrm{V}$ & & $37.2 \pm 0.1$ & 368 & $\mathrm{C}$ & [1984MAJ/SVO] \\
\hline & $\mathrm{V}$ & $(306-357)$ & 44.7 & 321 & & [1982SAC/PES] \\
\hline & $\mathrm{V}$ & $(293-376)$ & 46.5 & 308 & & [1973WIL/ZWO] \\
\hline & $\mathrm{V}$ & & $46.94 \pm 0.02$ & 298 & $\mathrm{C}$ & [1971POL/BEN] \\
\hline & $\mathrm{V}$ & & $45.8 \pm 0.1$ & 308 & $\mathrm{C}$ & [1971POL/BEN] \\
\hline & $\mathrm{V}$ & & $44.6 \pm 0.1$ & 318 & $\mathrm{C}$ & [1971POL/BEN] \\
\hline & $\mathrm{V}$ & & $42.7 \pm 0.1$ & 328 & $\mathrm{C}$ & [1971POL/BEN] \\
\hline & $\mathrm{V}$ & $(313-355)$ & 44.2 & 328 & & [1969BRO/FOC, 1984BOU/FRI] \\
\hline & $\mathrm{V}$ & & $46.6 \pm 0.1$ & 298 & $\mathrm{C}$ & [1966WAD] \\
\hline & $\mathrm{V}$ & $(333-363)$ & 42.1 & 348 & $\mathrm{~EB}$ & [1963BEN/MCK] \\
\hline & $\mathrm{V}$ & & $42.5 \pm 0.1$ & 330 & $\mathrm{C}$ & [1963BEN/MCK] \\
\hline & $\mathrm{V}$ & & $41.3 \pm 0.1$ & 340 & $\mathrm{C}$ & [1963BEN/MCK] \\
\hline & $\mathrm{V}$ & & $40.4 \pm 0.1$ & 346 & $\mathrm{C}$ & [1963BEN/MCK] \\
\hline & $\mathrm{V}$ & & $40.0 \pm 0.1$ & 349 & $\mathrm{C}$ & [1963BEN/MCK] \\
\hline & $\mathrm{V}$ & & $39.0 \pm 0.1$ & 356 & $\mathrm{C}$ & [1963BEN/MCK] \\
\hline & $\mathrm{V}$ & $(329-363)$ & 42.6 & 344 & EB & [1987STE/MAL, 1970AMB/SPR, 1963BEN/MCK] \\
\hline & $\mathrm{V}$ & & 44.9 & 298 & $\mathrm{C}$ & [1963MCC/LAI] \\
\hline & $\mathrm{V}$ & $(373-506)$ & 38.7 & 388 & & [1963AMB/TOW] \\
\hline & $\mathrm{V}$ & $(293-363)$ & 44.7 & 323 & & [1928PAR/BAR] \\
\hline \multirow[t]{13}{*}{$\mathrm{C}_{4} \mathrm{H}_{10} \mathrm{O}$} & {$[60-29-7]$} & diethyl ether & & & & \\
\hline & FUS & & 7.19 & 156.9 & & [1971COU/LEE] \\
\hline & $\mathrm{V}$ & $(298-317)$ & $28.0 \pm 0.4$ & & I & [2010GER/PEL] \\
\hline & $\mathrm{V}$ & $(286-329)$ & 28.1 & 301 & A & [1987STE/MAL] \\
\hline & $\mathrm{V}$ & $(307-457)$ & 26.9 & 322 & A & [1987STE/MAL] \\
\hline & $\mathrm{V}$ & $(305-360)$ & 27.5 & 320 & A & [1987STE/MAL] \\
\hline & $\mathrm{V}$ & $(351-420)$ & 26,6 & 366 & A & [1987STE/MAL] \\
\hline & $\mathrm{V}$ & $(417-467)$ & 26.7 & 432 & A & [1987STE/MAL] \\
\hline & $\mathrm{V}$ & & $27.1 \pm 0.1$ & 298 & $\mathrm{C}$ & [1980MAJ/WAG] \\
\hline & $\mathrm{V}$ & & $26.1 \pm 0.1$ & 313 & $\mathrm{C}$ & [1980MAJ/WAG] \\
\hline & $\mathrm{V}$ & $(250-329)$ & 27.2 & 298 & & [1976AMB/ELL] \\
\hline & $\mathrm{V}$ & $(250-329)$ & 29.5 & 265 & A & [1987STE/MAL, 1972AMB/SPR, 1976AMB/ELL] \\
\hline & $\mathrm{V}$ & $(213-293)$ & 28.4 & 278 & & [1922TAY/SMI] \\
\hline \multirow[t]{7}{*}{$\mathrm{C}_{4} \mathrm{H}_{10} \mathrm{O}$} & {$[598-53-8]$} & \multicolumn{2}{|l|}{ isopropyl methyl ether } & & & \\
\hline & FUS & $(13-311)$ & 5.85 & 127.3 & $\mathrm{AC}$ & [1996DOM/HEA, 1975AND/MAR] \\
\hline & $\mathrm{V}$ & $(250-325)$ & 28.8 & 265 & A & [1987STE/MAL] \\
\hline & $\mathrm{V}$ & & $26.4 \pm 0.1$ & 298 & $\mathrm{C}$ & [1980MAJ/WAG] \\
\hline & $\mathrm{V}$ & & $25.5 \pm 0.1$ & 313 & $\mathrm{C}$ & {$[1980 \mathrm{MAJ} / \mathrm{WAG}]$} \\
\hline & $\mathrm{V}$ & $(260-325)$ & 28.4 & 275 & A & [1987STE/MAL, 1976AMB/ELL] \\
\hline & $\mathrm{V}$ & & 26.4 & 298 & & [1976AMB/ELL] \\
\hline \multirow[t]{12}{*}{$\mathrm{C}_{4} \mathrm{H}_{10} \mathrm{O}$} & {$[557-17-5]$} & \multicolumn{2}{|l|}{ methyl propyl ether } & & & \\
\hline & FUS & $(16-308)$ & 7.67 & 134 & $\mathrm{AC}$ & [1996DOM/HEA, 1975AND/MAR] \\
\hline & $\mathrm{V}$ & $(325-407)$ & 27.2 & 340 & A & [1987STE/MAL] \\
\hline & $\mathrm{V}$ & $(401-476)$ & 26.7 & 416 & A & [1987STE/MAL] \\
\hline & $\mathrm{V}$ & & $27.6 \pm 0.1$ & 298 & $\mathrm{C}$ & [1980MAJ/WAG] \\
\hline & $\mathrm{V}$ & & $26.7 \pm 0.1$ & 313 & $\mathrm{C}$ & [1980MAJ/WAG] \\
\hline & $\mathrm{V}$ & & $25.8 \pm 0.1$ & 328 & $\mathrm{C}$ & [1980MAJ/WAG] \\
\hline & $\mathrm{V}$ & $(253-328)$ & 29.7 & 268 & A & [1987STE/MAL, 1976AMB/ELL] \\
\hline & $\mathrm{V}$ & & 27.5 & 298 & & [1976AMB/ELL] \\
\hline & $\mathrm{V}$ & & $27.9 \pm 0.2$ & 298 & $\mathrm{C}$ & [1975FEN/HAR] \\
\hline & $\mathrm{V}$ & $(273-321)$ & 30.7 & 288 & A & [1987STE/MAL, 1969KUD/REE] \\
\hline & $\mathrm{V}$ & $(273-312)$ & 29.7 & 288 & & [1910BIN, 1984BOU/FRI] \\
\hline \multirow[t]{2}{*}{$\mathrm{C}_{4} \mathrm{H}_{10} \mathrm{OS}$} & {$[70-29-1]$} & \multicolumn{2}{|l|}{ diethyl sulfoxide } & & & \\
\hline & $\mathrm{V}$ & $(298-318)$ & $58.7 \pm 3.3$ & 308 & & [2005MAR/ZAT] \\
\hline $\mathrm{C}_{4} \mathrm{H}_{10} \mathrm{O}_{2}$ & $\begin{array}{c}{[26171-83-5]} \\
\mathrm{V}\end{array}$ & $\begin{array}{l}( \pm)-1,2 \text {-butanediol } \\
\quad(283-332)\end{array}$ & $70.4 \pm 0.3$ & 298 & GS & [2004VER2] \\
\hline
\end{tabular}


TABLE 7. Phase change enthalpies of $\mathrm{C}_{4}$ organic compounds-Continued

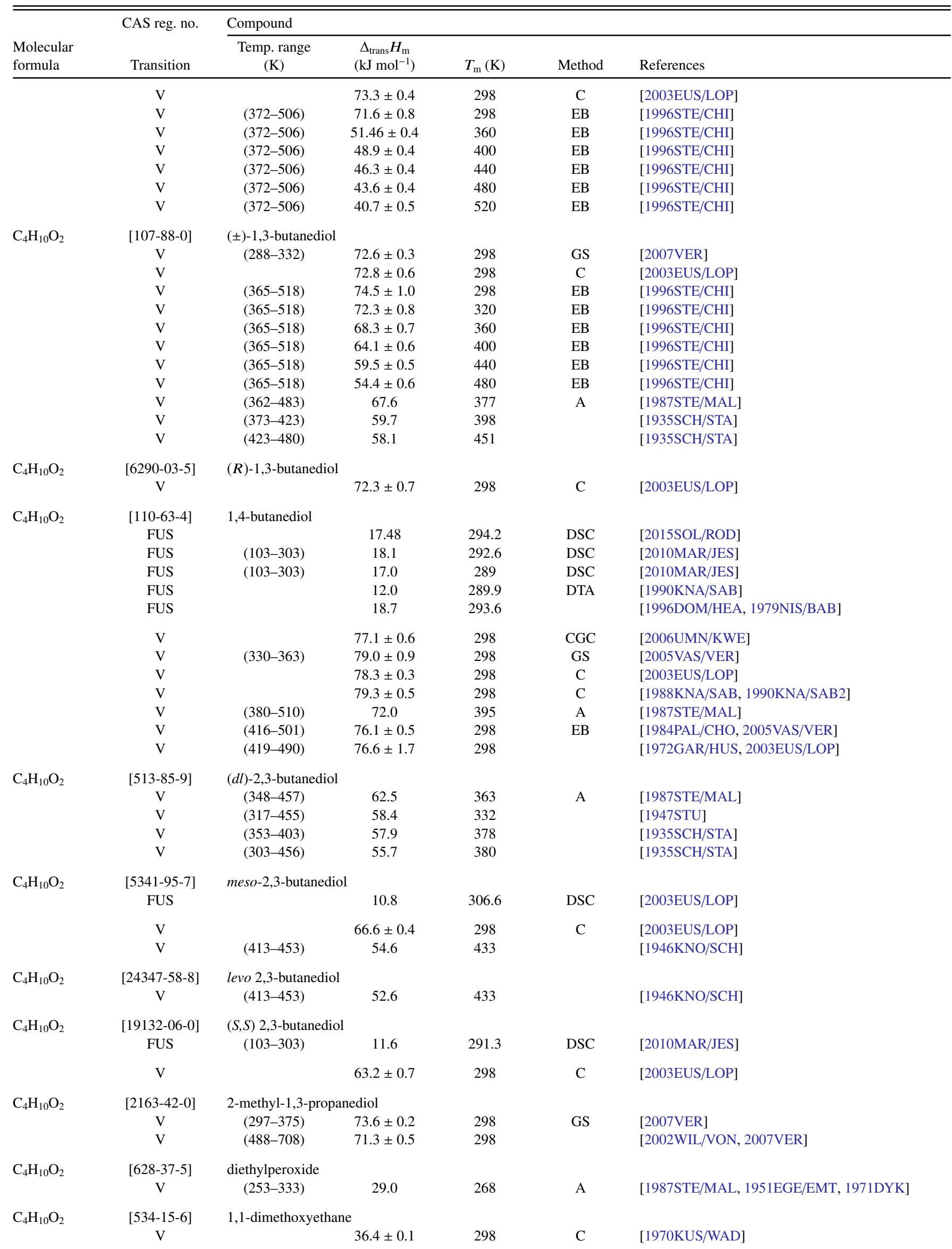


TABLE 7. Phase change enthalpies of $\mathrm{C}_{4}$ organic compounds-Continued

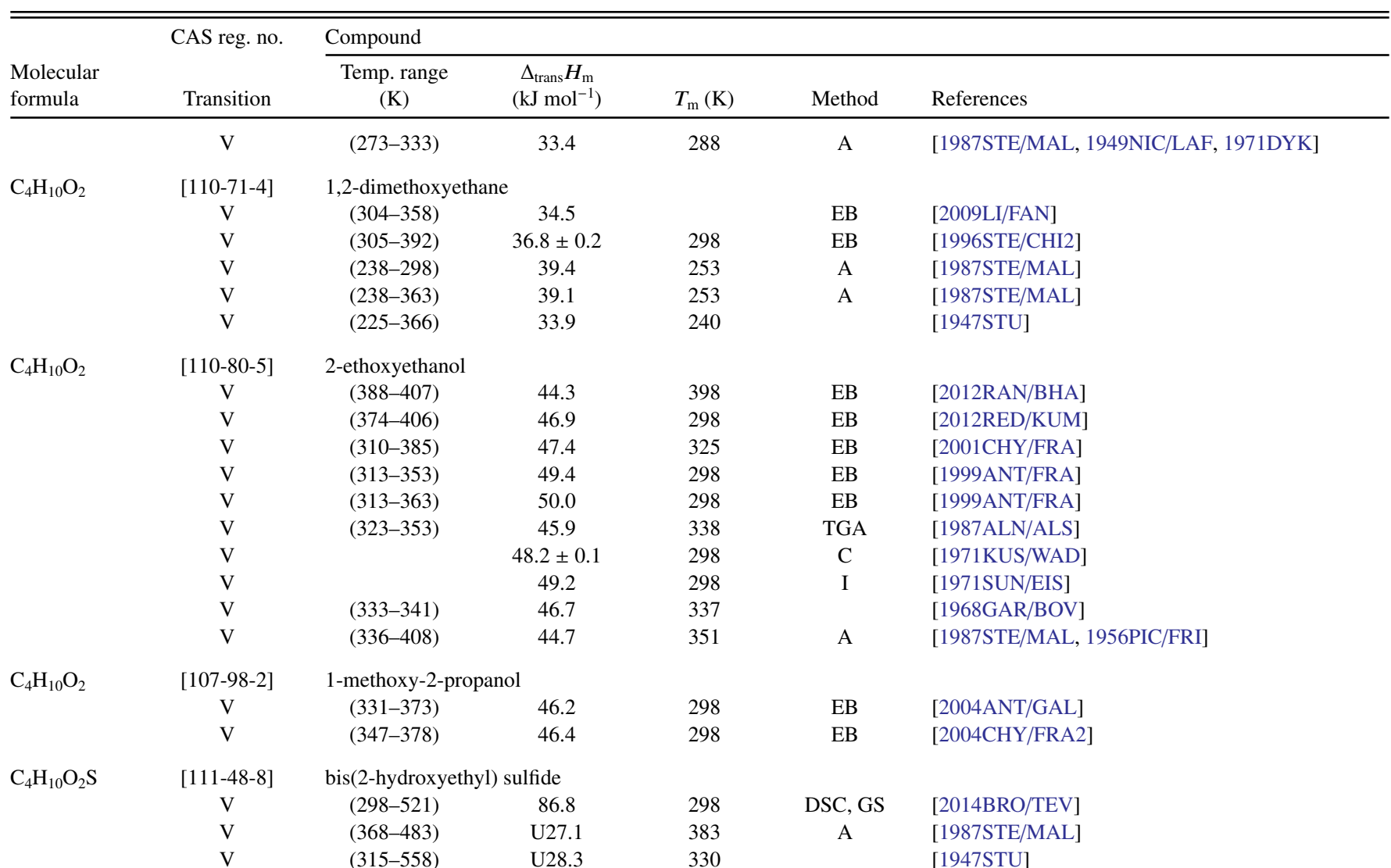

[Note: Authors of [2014BRO/TEV] noted that abnormally low enthalpies of vaporization are calculated from the earlier vapor pressure data.]

\begin{tabular}{|c|c|c|c|c|c|}
\hline $\mathrm{C}_{4} \mathrm{H}_{10} \mathrm{O}_{2} \mathrm{~S}$ & $\begin{array}{l}{[597-35-3]} \\
\text { SUB }\end{array}$ & diethyl sulfone & & & [UR/MAC, 1970C \\
\hline $\mathrm{C}_{4} \mathrm{H}_{10} \mathrm{O}_{2} \mathrm{~S}_{2}$ & $\begin{array}{c}\text { [21884-56-0] } \\
\text { FUS }\end{array}$ & $\begin{array}{c}\text { meso-1,2-bis(methylsufinyl)ethane } \\
34.31\end{array}$ & 446.7 & DSC & [2001CAL/MEL] \\
\hline $\mathrm{C}_{4} \mathrm{H}_{10} \mathrm{O}_{2} \mathrm{~S}_{2}$ & FUS & $\begin{array}{l}\text { racemic 1,2-bis(methylsufinyl)etha } \\
22.18\end{array}$ & 405.4 & DSC & [2001CAL/MEL] \\
\hline
\end{tabular}

[Note: The enthalpy and entropy of fusion values given in the paper are not consistent.]

\begin{tabular}{|c|c|c|c|c|c|c|}
\hline \multirow[t]{8}{*}{$\mathrm{C}_{4} \mathrm{H}_{10} \mathrm{O}_{3}$} & $\begin{array}{c}{[111-46-6]} \\
\mathrm{V}\end{array}$ & \multicolumn{5}{|l|}{ diethylene glycol } \\
\hline & $\mathrm{V}$ & $(410-539)$ & $63.1 \pm 0.3$ & 460 & EB & [2002STE/CHI2] \\
\hline & $\mathrm{V}$ & $(410-539)$ & $59.2 \pm 0.3$ & 500 & EB & [2002STE/CHI2] \\
\hline & $\mathrm{V}$ & $(410-539)$ & $55.1 \pm 0.5$ & 540 & $\mathrm{~EB}$ & [2002STE/CHI2] \\
\hline & $\mathrm{V}$ & $(373-453)$ & 66.5 & 413 & TGA & [1987ALN/ALS] \\
\hline & $\mathrm{V}$ & $(364-518)$ & 59.8 & 379 & A & [1987STE/MAL] \\
\hline & $\mathrm{V}$ & $(412-513)$ & 66.8 & 427 & & [1981AMB/HAL, 1984BOU/FRI] \\
\hline & V & $(403-513)$ & 69.2 & 418 & & [1927RIN, 1984BOU/FRI] \\
\hline $\mathrm{C}_{4} \mathrm{H}_{10} \mathrm{O}_{3}$ & $\begin{array}{c}{[4435-50-1]} \\
\mathrm{V}\end{array}$ & $\begin{array}{l}\text { 1,2,3-butanetriol } \\
(375-537)\end{array}$ & 68.1 & 390 & & [1947STU] \\
\hline $\mathrm{C}_{4} \mathrm{H}_{10} \mathrm{O}_{3}$ & $\begin{array}{c}{[149-73-5]} \\
\text { V } \\
\text { V }\end{array}$ & $\begin{array}{l}\text { orthoformic acid t } \\
\quad(273-358)\end{array}$ & $\begin{array}{l}\text { nethyl ester } \\
39.0 \\
38.1 \pm 0.8\end{array}$ & $\begin{array}{l}288 \\
298\end{array}$ & A & $\begin{array}{l}\text { [1987STE/MAL] } \\
\text { [1971PIH/TUO] }\end{array}$ \\
\hline $\mathrm{C}_{4} \mathrm{H}_{10} \mathrm{O}_{3} \mathrm{~S}$ & $\begin{array}{c}{[623-81-4]} \\
\text { V } \\
\text { V } \\
\text { V }\end{array}$ & diethyl sulfite & $\begin{array}{c}44.7 \\
48.5 \pm 1.7 \\
44.5\end{array}$ & $\begin{array}{l}298 \\
298\end{array}$ & $\begin{array}{c}\mathrm{BP} \\
\mathrm{A}\end{array}$ & $\begin{array}{l}\text { [1975DEM/KOV] } \\
\text { [1969MAC/STE2] } \\
\text { [1987STE/MAL, 1947STU, 1999DYK/SVO, } \\
\text { 1937ISH/TAN] }\end{array}$ \\
\hline $\mathrm{C}_{4} \mathrm{H}_{10} \mathrm{O}_{4}$ & $\begin{array}{l}\text { [149-32-6] } \\
\text { FUS } \\
\text { FUS } \\
\text { FUS }\end{array}$ & meso-erythritol & $\begin{array}{l}42.0 \\
38.9 \\
41.2\end{array}$ & $\begin{array}{l}390.2 \\
391.2 \\
391.8\end{array}$ & $\begin{array}{l}\text { DSC } \\
\text { DSC } \\
\text { DSC }\end{array}$ & $\begin{array}{l}\text { [2008KAI/MAR] } \\
\text { [2005LOP/TOM] } \\
\text { [2004STU/WIT] }\end{array}$ \\
\hline
\end{tabular}


TABle 7. Phase change enthalpies of $\mathrm{C}_{4}$ organic compounds-Continued

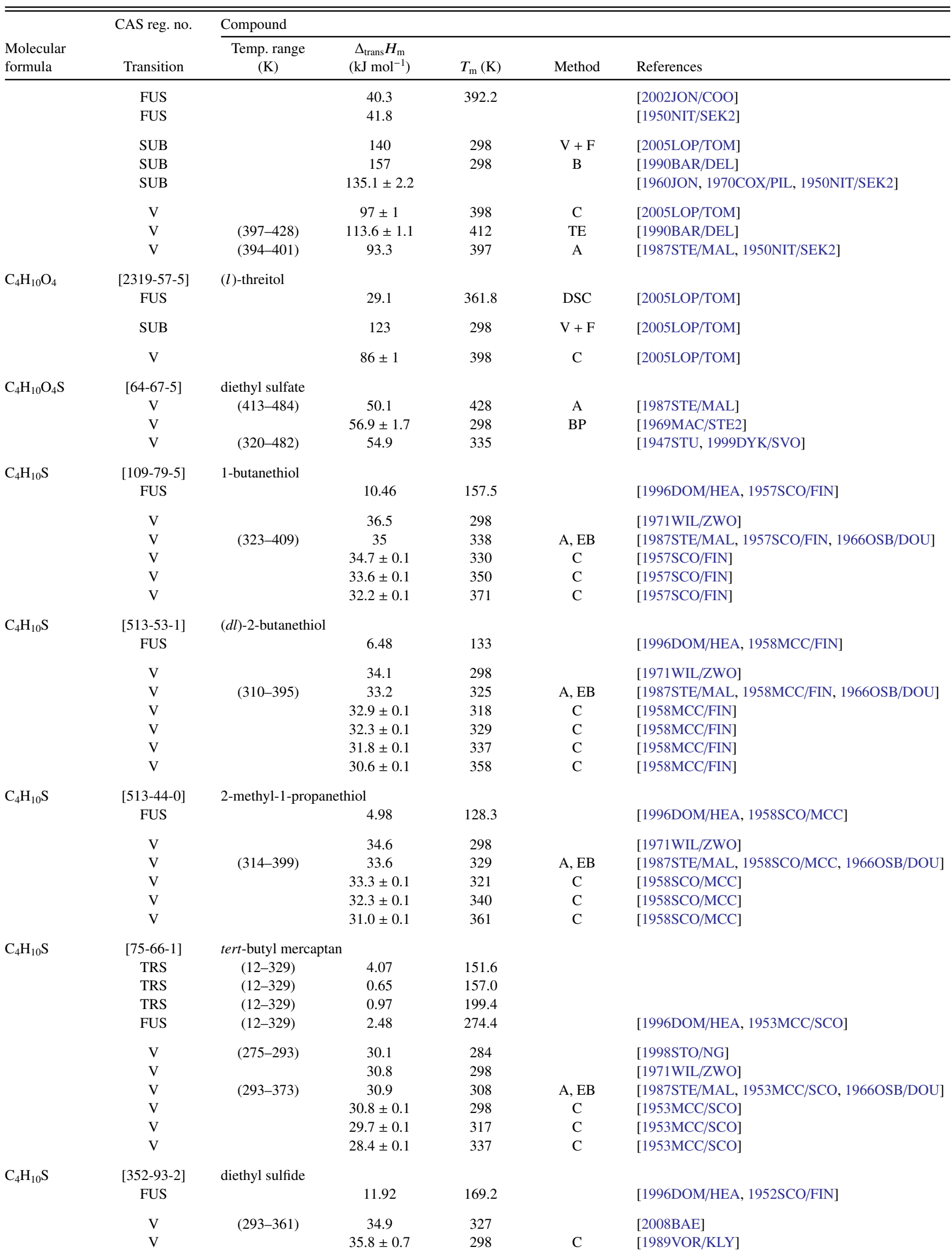


TABLE 7. Phase change enthalpies of $\mathrm{C}_{4}$ organic compounds-Continued

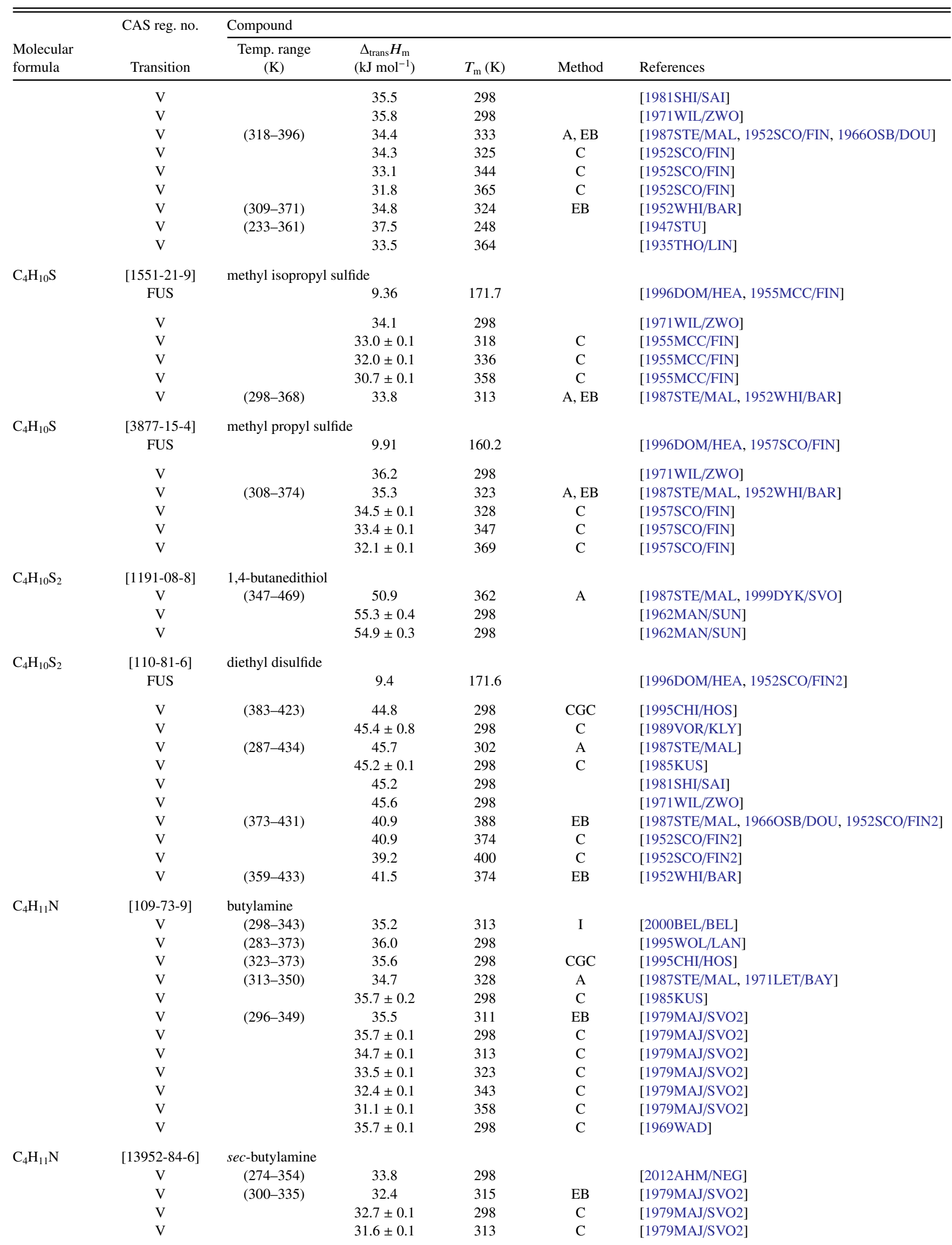


TABLE 7. Phase change enthalpies of $\mathrm{C}_{4}$ organic compounds-Continued

\begin{tabular}{|c|c|c|c|c|c|c|}
\hline \multirow[b]{2}{*}{$\begin{array}{l}\text { Molecular } \\
\text { formula }\end{array}$} & \multirow{2}{*}{$\begin{array}{c}\text { CAS reg. no. } \\
\text { Transition }\end{array}$} & \multicolumn{5}{|l|}{ Compound } \\
\hline & & $\begin{array}{c}\text { Temp. range } \\
(\mathrm{K})\end{array}$ & $\begin{array}{r}\Delta_{\text {trans }} H_{\mathrm{m}} \\
\left(\mathrm{kJ} \mathrm{mol}^{-1}\right)\end{array}$ & $T_{\mathrm{m}}(\mathrm{K})$ & Method & References \\
\hline & $\mathrm{V}$ & & $30.5 \pm 0.1$ & 328 & $\mathrm{C}$ & [1979MAJ/SVO2] \\
\hline & $\mathrm{V}$ & & $29.4 \pm 0.1$ & 343 & $\mathrm{C}$ & [1979MAJ/SVO2] \\
\hline & $\mathrm{V}$ & $(264-371)$ & 34.1 & 279 & A & [1987STE/MAL, 1971DYK] \\
\hline & $\mathrm{V}$ & & $32.6 \pm 0.1$ & 298 & $\mathrm{C}$ & [1969WAD] \\
\hline \multirow[t]{8}{*}{$\mathrm{C}_{4} \mathrm{H}_{11} \mathrm{~N}$} & [78-81-9] & isobutylamine & & & & \\
\hline & $\mathrm{V}$ & $(248-347)$ & 37.6 & 263 & & [1987STE/MAL] \\
\hline & $\mathrm{V}$ & & $33.9 \pm 0.1$ & 298 & $\mathrm{C}$ & [1979MAJ/SVO2] \\
\hline & $\mathrm{V}$ & & $32.7 \pm 0.1$ & 313 & $\mathrm{C}$ & [1979MAJ/SVO2] \\
\hline & $\mathrm{V}$ & & $31.6 \pm 0.1$ & 328 & $\mathrm{C}$ & [1979MAJ/SVO2] \\
\hline & $\mathrm{V}$ & $(297-340)$ & 33.5 & 313 & $\mathrm{~EB}$ & [1979MAJ/SVO2] \\
\hline & $\mathrm{V}$ & & $33.8 \pm 0.1$ & 298 & $\mathrm{C}$ & [1969WAD] \\
\hline & $\mathrm{V}$ & & $33.9 \pm 0.2$ & 298 & IPM & [1965DOU/OSB, 1970GOO/MOO] \\
\hline \multirow{7}{*}{$\mathrm{C}_{4} \mathrm{H}_{11} \mathrm{~N}$} & [75-64-9] & tert-butylamine & & & & \\
\hline & TRS & $(12-332)$ & 0.11 & 91.3 & & \\
\hline & TRS & $(12-332)$ & 6.05 & 202.3 & & \\
\hline & FUS & $(12-332)$ & 0.88 & 206.2 & $\mathrm{AC}$ & [1996DOM/HEA, 1972FIN/MES] \\
\hline & $\mathrm{V}$ & $(283-343)$ & 30.5 & 298 & & [1995WOL/LAN] \\
\hline & $\mathrm{V}$ & & $29.6 \pm 0.1$ & 298 & $\mathrm{C}$ & [1969WAD] \\
\hline & $\mathrm{V}$ & $(292-349)$ & 30.1 & 307 & A, EB, IPM & [1987STE/MAL, 1968OSB/DOU] \\
\hline \multirow[t]{15}{*}{$\mathrm{C}_{4} \mathrm{H}_{11} \mathrm{~N}$} & [109-89-7] & diethylamine & & & & \\
\hline & FUS & & 13.3 & 224.7 & DSC & [2015ROT/VRB] \\
\hline & $\mathrm{V}$ & $(302-328)$ & 31.2 & 315 & A & [1987STE/MAL, 1971LET/BAY] \\
\hline & $\mathrm{V}$ & $(325-437)$ & 30.4 & 340 & A & [1987STE/MAL] \\
\hline & $\mathrm{V}$ & $(431-496)$ & 28.4 & 446 & A & [1987STE/MAL] \\
\hline & $\mathrm{V}$ & & $31.3 \pm 0.1$ & 298 & $\mathrm{C}$ & [1979MAJ/SVO2] \\
\hline & V & & $30.2 \pm 0.1$ & 313 & $\mathrm{C}$ & [1979MAJ/SVO2] \\
\hline & $\mathrm{V}$ & & $29.1 \pm 0.1$ & 328 & $\mathrm{C}$ & [1979MAJ/SVO2] \\
\hline & $\mathrm{V}$ & & $28.0 \pm 0.1$ & 343 & $\mathrm{C}$ & [1979MAJ/SVO2] \\
\hline & $\mathrm{V}$ & & $31.2 \pm 0.1$ & 298 & $\mathrm{C}$ & [1969WAD] \\
\hline & $\mathrm{V}$ & $(273-333)$ & $32.7 \pm 0.2$ & 298 & I & [1969FRA/WAT] \\
\hline & $\mathrm{V}$ & $(292-313)$ & 31.8 & 307 & & [1965KIL/BIT, 1984BOU/FRI] \\
\hline & $\mathrm{V}$ & $(304-323)$ & 31.5 & 319 & & [1962BIT/KAU, 1984BOU/FRI] \\
\hline & $\mathrm{V}$ & $(288-328)$ & 31.5 & 308 & I, EB & {$[1953 \mathrm{COP} / \mathrm{EVE}]$} \\
\hline & $\mathrm{V}$ & & 32.7 & & & [1933MEH] \\
\hline \multirow[t]{5}{*}{$\mathrm{C}_{4} \mathrm{H}_{11} \mathrm{~N}$} & {$[4747-21-1]$} & $N$-methyl isopr & amine & & & \\
\hline & $\mathrm{V}$ & & $30.7 \pm 0.1$ & 298 & $\mathrm{C}$ & [1979PET/MAJ] \\
\hline & $\mathrm{V}$ & & $29.5 \pm 0.1$ & 313 & $\mathrm{C}$ & [1979PET/MAJ] \\
\hline & $\mathrm{V}$ & & $27.1 \pm 0.1$ & 343 & $\mathrm{C}$ & [1979PET/MAJ] \\
\hline & $\mathrm{V}$ & $(293-319)$ & 30.9 & 306 & EB & [1979PET/MAJ] \\
\hline \multirow[t]{7}{*}{$\mathrm{C}_{4} \mathrm{H}_{11} \mathrm{NO}$} & [108-01-0] & 2-(dimethylamir & thanol & & & \\
\hline & $\mathrm{V}$ & $(274-363)$ & 46.7 & 298 & Static & [2013CHI/DER] \\
\hline & $\mathrm{V}$ & $(278-316)$ & $46.5 \pm 0.4$ & 298 & GS & [2005KAP/SLO] \\
\hline & $\mathrm{V}$ & $(350-387)$ & 43.2 & 365 & A & [1987STE/MAL] \\
\hline & $\mathrm{V}$ & $(323-408)$ & 42.7 & 338 & A & [1987STE/MAL] \\
\hline & $\mathrm{V}$ & $(298-308)$ & 47.9 & 298 & & [1982TOU/OKA, 2005KAP/SLO] \\
\hline & $\mathrm{V}$ & $(333-423)$ & 47.6 & 298 & $\mathrm{~EB}$ & [1970QUI/HOF, 2005KAP/SLO] \\
\hline \multirow[t]{3}{*}{$\mathrm{C}_{4} \mathrm{H}_{11} \mathrm{NO}$} & {$[5332-73-0]$} & 3-methoxypropy & & & & \\
\hline & $\mathrm{V}$ & $(278-390)$ & 44.5 & 293 & A & [1987STE/MAL] \\
\hline & $\mathrm{V}$ & & $44.0 \pm 0.1$ & 298 & & [1978CAB/MOL] \\
\hline \multirow[t]{2}{*}{$\mathrm{C}_{4} \mathrm{H}_{11} \mathrm{NO}$} & [124-68-5] & 2-methyl-2-amil & -propanol & & & \\
\hline & $\mathrm{V}$ & $(293-373)$ & 63.3 & 308 & Static & [2010BEL/AHM] \\
\hline \multirow[t]{2}{*}{$\mathrm{C}_{4} \mathrm{H}_{11} \mathrm{NO}$} & [13325-10-5] & 4-amino-1-butar & & & & \\
\hline & FUS & & 21.83 & 292.2 & DSC & [2015SOL/ROD] \\
\hline $\mathrm{C}_{4} \mathrm{H}_{11} \mathrm{NO}_{2}$ & $\begin{array}{c}{[111-42-2]} \\
\mathrm{V}\end{array}$ & $\begin{array}{l}\text { 2,2'-iminodietha } \\
\quad(463-582)\end{array}$ & 69 & 478 & & [1959MCD/SHR] \\
\hline
\end{tabular}


TABLE 7. Phase change enthalpies of $\mathrm{C}_{4}$ organic compounds-Continued

\begin{tabular}{|c|c|c|c|c|c|c|}
\hline \multirow[b]{2}{*}{$\begin{array}{l}\text { Molecular } \\
\text { formula }\end{array}$} & \multirow{2}{*}{$\begin{array}{l}\text { CAS reg. no. } \\
\text { Transition }\end{array}$} & \multicolumn{5}{|l|}{ Compound } \\
\hline & & $\begin{array}{c}\text { Temp. range } \\
(\mathrm{K})\end{array}$ & $\begin{array}{c}\Delta_{\text {trans }} H_{\mathrm{m}} \\
\left(\mathrm{kJ} \mathrm{mol}^{-1}\right)\end{array}$ & $T_{\mathrm{m}}(\mathrm{K})$ & Method & References \\
\hline \multirow[t]{5}{*}{$\mathrm{C}_{4} \mathrm{H}_{11} \mathrm{NO}_{2}$} & {$[111-42-2]$} & diethanolamine & & & & \\
\hline & SUB & & $96.7 \pm 1.2$ & 298 & $\mathrm{C}$ & {$[1982 \mathrm{MIN} / \mathrm{SAB}]$} \\
\hline & $\mathrm{V}$ & $(423-542)$ & 74.4 & 438 & A & [1987STE/MAL] \\
\hline & $\mathrm{V}$ & $(376-454)$ & 77.0 & 391 & & [1969DAN/MAT, 1984BOU/FRI] \\
\hline & $\mathrm{V}$ & $(466-514)$ & 70.6 & 481 & & [1959MCD/SHR, 1984BOU/FRI] \\
\hline \multirow[t]{16}{*}{$\mathrm{C}_{4} \mathrm{H}_{11} \mathrm{NO}_{2}$} & {$[115-69-5]$} & \multicolumn{5}{|c|}{ 2-amino-2-methyl-1,3-propanediol } \\
\hline & TRS & $(78-405)$ & 24.33 & 351.6 & & \\
\hline & FUS & $(78-405)$ & 4.01 & 380.6 & $\mathrm{AC}$ & [2010TON/TAN] \\
\hline & TRS & & 23.55 & 356.7 & & \\
\hline & FUS & & 2.76 & 384.1 & DSC & [2006DIV/CHE] \\
\hline & TRS & & 20.1 & 353.2 & & \\
\hline & FUS & & 2.52 & 383.4 & DSC & [2004STU/WIT] \\
\hline & TRS & & 25.21 & 352 & & \\
\hline & FUS & & 2.99 & 384 & & [1996DOM/HEA, 1994LOP/VAN] \\
\hline & TRS & $(283-393)$ & 5.0 & 352.9 & & \\
\hline & TRS & $(283-393)$ & 18.46 & 353.7 & & \\
\hline & FUS & $(283-393)$ & 2.78 & 384.1 & $\mathrm{AC}$ & [1990ZHA/YAN] \\
\hline & SUB (cryst) & $(330-346)$ & $110 \pm 6$ & & & [1995FON/MUN] \\
\hline & SUB (plastic) & $(354-372)$ & $81 \pm 8$ & & & [1995FON/MUN] \\
\hline & SUB (plastic) & & $86.5 \pm 4.3$ & 368 & $\mathrm{C}$ & [1994FON/MUN] \\
\hline & SUB (cryst) & & $114.5 \pm 5.7$ & 339 & $\mathrm{C}$ & [1994FON/MUN] \\
\hline \multirow[t]{5}{*}{$\mathrm{C}_{4} \mathrm{H}_{11} \mathrm{NO}_{2}$} & {$[929-06-6]$} & \multicolumn{5}{|c|}{ 1-amino-2-(2-hydroxyethoxy)ethane } \\
\hline & $\mathrm{V}$ & $(303-359)$ & 73.4 & 331 & GS & [2011VER/TON] \\
\hline & $\mathrm{V}$ & $(303-359)$ & $75.9 \pm 0.4$ & 298 & GS & [2011 VER/TON] \\
\hline & $\mathrm{V}$ & $(391-516)$ & 59.4 & 454 & $\mathrm{~EB}$ & [1994CUN/JON, 2011VER/TON] \\
\hline & V & $(391-516)$ & $71.2 \pm 0.6$ & 298 & EB & [1994CUN/JON, 2011VER/TON] \\
\hline \multirow[t]{3}{*}{$\mathrm{C}_{4} \mathrm{H}_{11} \mathrm{NO}_{2} \mathrm{~S}$} & {$[6338-68-7]$} & \multicolumn{5}{|c|}{$N, N$-dimethylethanesulfonamide } \\
\hline & $\mathrm{V}$ & $(384-517)$ & 54.3 & 399 & A & [1987STE/MAL] \\
\hline & $\mathrm{V}$ & $(384-517)$ & 54.0 & & & [1978LUK/MAK] \\
\hline \multirow[t]{5}{*}{$\mathrm{C}_{4} \mathrm{H}_{11} \mathrm{NO}_{3}$} & {$[77-86-1]$} & \multicolumn{5}{|c|}{ 2-amino-2-hydroxymethylpropane-1,3-diol } \\
\hline & TRS & & 33.48 & 409.2 & & \\
\hline & FUS & & 3.1 & 444.6 & DSC & [2006DIV/CHE] \\
\hline & TRS & $(294-450)$ & 33.42 & 407.5 & $\mathrm{AC}$ & [1990YIN/LIN, 1994LOP/VAN] \\
\hline & FUS & $(294-450)$ & 2.41 & 443.6 & $\mathrm{AC}$ & [1990YIN/LIN, 1994LOP/VAN] \\
\hline \multirow[t]{5}{*}{$\mathrm{C}_{4} \mathrm{H}_{11} \mathrm{NS}$} & {$[108-02-1]$} & \multicolumn{5}{|c|}{ 2-dimethylaminoethanethiol } \\
\hline & $\mathrm{V}$ & $(275-364)$ & 42.7 & 283 & DSC,GS & [2013WIL/HUL] \\
\hline & $\mathrm{V}$ & $(275-364)$ & 41.1 & 303 & DSC,GS & [2013WIL/HUL] \\
\hline & $\mathrm{V}$ & $(275-364)$ & 39.8 & 323 & DSC,GS & [2013WIL/HUL] \\
\hline & $\mathrm{V}$ & $(275-364)$ & 38.2 & 353 & DSC,GS & [2013WIL/HUL] \\
\hline \multirow[t]{2}{*}{$\mathrm{C}_{4} \mathrm{H}_{11} \mathrm{O}_{2} \mathrm{PS}_{2}$} & [298-06-6] & \multicolumn{5}{|c|}{$O, O$-diethyl phosphorodithioate } \\
\hline & $\mathrm{V}$ & & 67.7 & 298 & & {$[2008 \mathrm{SAG} / \mathrm{SAF}]$} \\
\hline \multirow[t]{3}{*}{$\mathrm{C}_{4} \mathrm{H}_{11} \mathrm{O}_{3} \mathrm{P}$} & {$[762-04-9]$} & \multicolumn{5}{|c|}{ diethylphosphite } \\
\hline & $\mathrm{V}$ & & 49.3 & 298 & & {$[2008 \mathrm{SAG} / \mathrm{SAF}]$} \\
\hline & V & $(338-471)$ & 38.1 & 353 & A & [1987STE/MAL, 1958PAG/PUR] \\
\hline $\mathrm{C}_{4} \mathrm{H}_{11} \mathrm{O}_{3} \mathrm{P}$ & {$[6163-75-3]$} & dimethyl ethylp & phonate & & & \\
\hline & $\mathrm{V}$ & $(333-410)$ & 70.1 & 348 & A & [1987STE/MAL, 1955KOS, 1984BOU/FRI] \\
\hline $\mathrm{C}_{4} \mathrm{H}_{11} \mathrm{P}$ & {$[1605-51-2]$} & ethyldimethylpl & hine & & & \\
\hline & $\mathrm{V}$ & $(273-297)$ & 32.8 & 285 & & {$[1959 \mathrm{KAE} / \mathrm{STO}]$} \\
\hline $\mathrm{C}_{4} \mathrm{H}_{12} \mathrm{ClN}$ & {$[3858-78-4]$} & butylammonium & loride & & & \\
\hline & $\mathrm{V}$ & $(489-508)$ & 62.1 & 498 & $\mathrm{~A}, \mathrm{I}$ & [1987STE/MAL, 1967KIS] \\
\hline $\mathrm{C}_{4} \mathrm{H}_{12} \mathrm{ClN}$ & {$[660-68-4]$} & diethylamine hy & chloride & & & \\
\hline & $\mathrm{V}$ & $(513-558)$ & 177.6 & 528 & $\mathrm{~A}, \mathrm{I}$ & [1987STE/MAL, 1967KIS] \\
\hline $\mathrm{C}_{4} \mathrm{H}_{12} \mathrm{ClN}_{2} \mathrm{P}$ & {$[3348-44-5]$} & bis(dimethylam & chlorophosph & & & \\
\hline & $\mathrm{V}$ & & $45.9 \pm 1.2$ & 298 & STG & [1995ALM/FIN2] \\
\hline
\end{tabular}


TABLE 7. Phase change enthalpies of $\mathrm{C}_{4}$ organic compounds-Continued

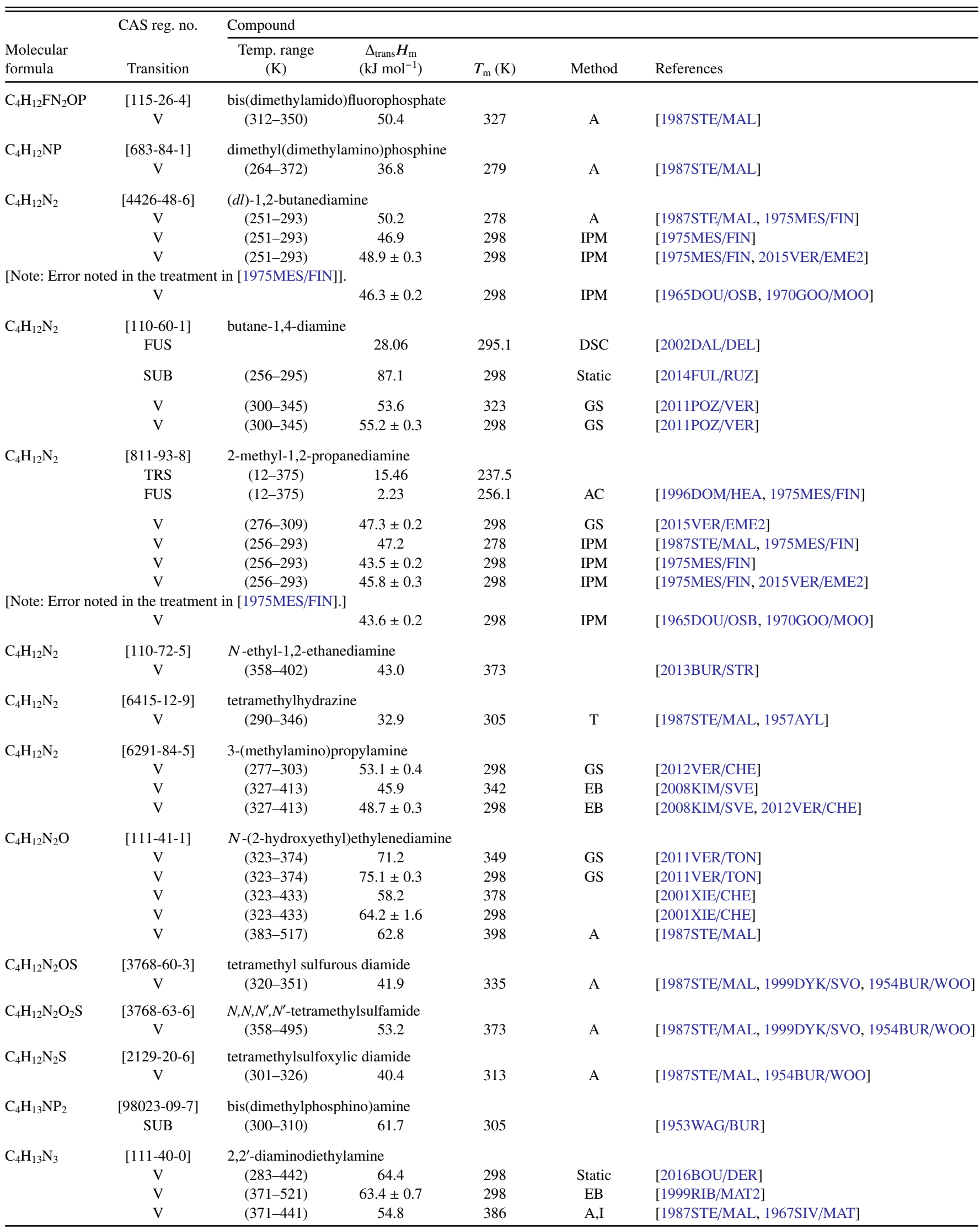


TABLE 8. Phase change enthalpies of $C_{5}$ organic compounds

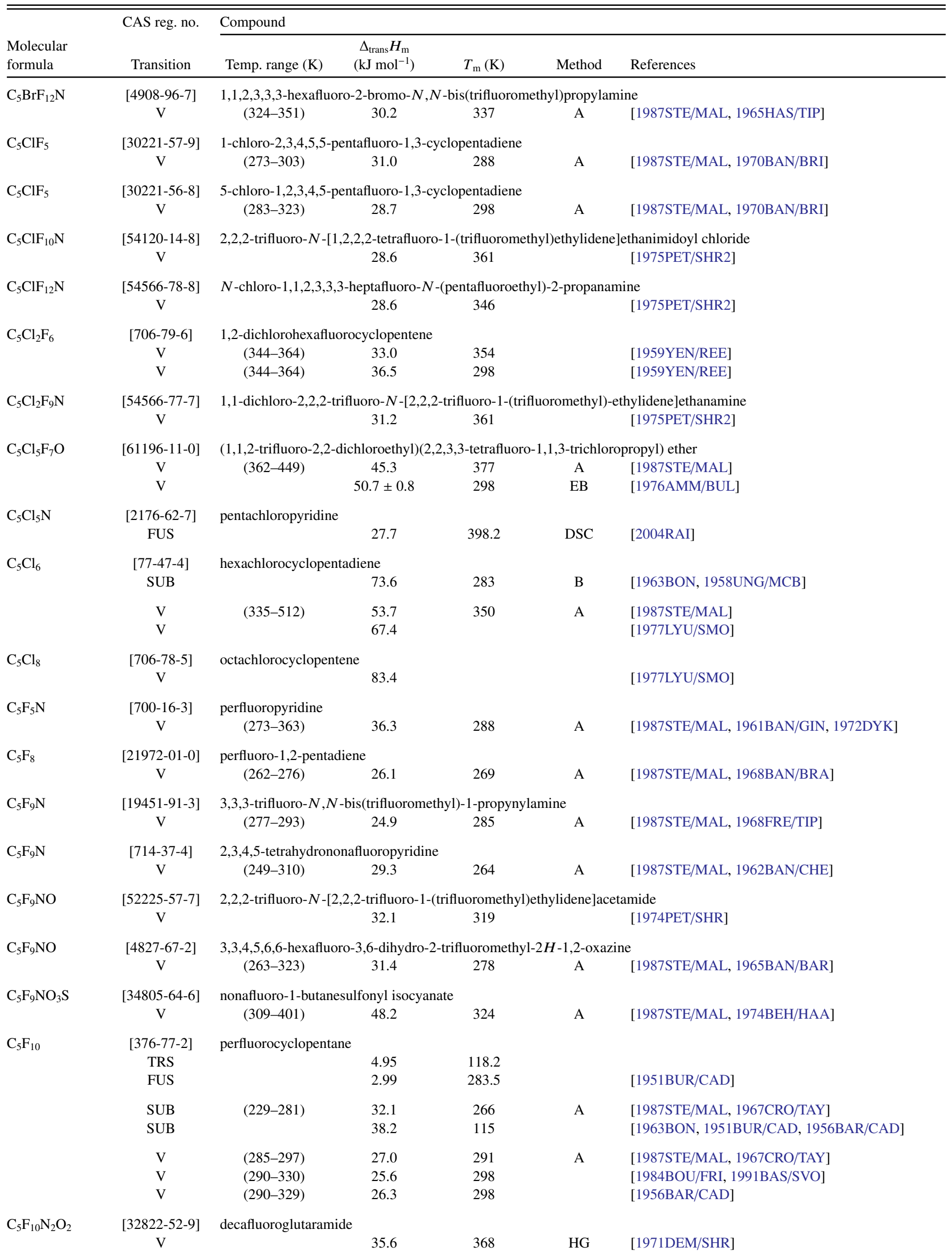


TABLE 8. Phase change enthalpies of $C_{5}$ organic compounds-Continued

\begin{tabular}{|c|c|c|c|c|c|c|}
\hline \multirow[b]{2}{*}{$\begin{array}{l}\text { Molecular } \\
\text { formula }\end{array}$} & \multirow{2}{*}{$\begin{array}{c}\text { CAS reg. no. } \\
\text { Transition }\end{array}$} & \multicolumn{5}{|l|}{ Compound } \\
\hline & & Temp. range $(\mathrm{K})$ & $\begin{array}{c}\Delta_{\text {trans }} H_{\mathrm{m}} \\
\left(\mathrm{kJ} \mathrm{mol}^{-1}\right)\end{array}$ & $T_{\mathrm{m}}(\mathrm{K})$ & Method & References \\
\hline \multirow[t]{2}{*}{$\mathrm{C}_{5} \mathrm{~F}_{10} \mathrm{~N}_{2} \mathrm{O}_{2}$} & [1840-07-9] & \multicolumn{5}{|c|}{ 1-nitrodecafluoropiperadine } \\
\hline & $\mathrm{V}$ & $(283-343)$ & 29.6 & 298 & A & [1987STE/MAL, 1964BAN/CHE] \\
\hline \multirow[t]{2}{*}{$\mathrm{C}_{5} \mathrm{~F}_{10} \mathrm{O}_{2}$} & {$[55064-79-4]$} & \multicolumn{5}{|c|}{ carbonofluoridic acid, 2,2,2-trifluoro-1,1-bis(trifluoromethyl) ethyl ether } \\
\hline & $\mathrm{V}$ & $(275-305)$ & 32.2 & 290 & A & [1987STE/MAL, 1975WAL/DES2] \\
\hline \multirow[t]{2}{*}{$\mathrm{C}_{5} \mathrm{~F}_{10} \mathrm{O}_{3} \mathrm{~S}$} & {$[2993-14-8]$} & \multicolumn{5}{|c|}{ perfluorocyclopentyl fluorosulfate } \\
\hline & $\mathrm{V}$ & $(255-360)$ & 36.6 & 307 & & [1963GIL/CAD] \\
\hline \multirow[t]{2}{*}{$\mathrm{C}_{5} \mathrm{~F}_{10} \mathrm{O}_{6} \mathrm{~S}_{2}$} & {$[741-20-8]$} & \multicolumn{5}{|c|}{ octafluorocyclopentanediol bis(fluorosulfate) } \\
\hline & $\mathrm{V}$ & $(334-423)$ & 49.5 & 349 & & $\begin{array}{l}\text { [1972DYK, 1987STE/MAL, 1999DYK/SVO, } \\
\text { 1961SHR/CAD] }\end{array}$ \\
\hline \multirow[t]{5}{*}{$\mathrm{C}_{5} \mathrm{~F}_{11} \mathrm{~N}$} & {$[836-77-1]$} & \multicolumn{5}{|l|}{ perfluoropiperidine } \\
\hline & TRS & $(12-318)$ & 6.63 & 161 & & \\
\hline & TRS & $(12-318)$ & 1.84 & 171.9 & & \\
\hline & FUS & $(12-318)$ & 2.82 & 274.1 & & [1996DOM/HEA, 1963GOO/TOD] \\
\hline & $\mathrm{V}$ & $(302-355)$ & 30.0 & 317 & $\mathrm{~A}, \mathrm{~EB}$ & [1987STE/MAL, 1963GOO/TOD, 1972DYK] \\
\hline \multirow[t]{2}{*}{$\mathrm{C}_{5} \mathrm{~F}_{11} \mathrm{~N}$} & {$[2344-10-7]$} & \multicolumn{5}{|c|}{ octafluoro-1-(trifluoromethyl)pyrrolidine } \\
\hline & $\mathrm{V}$ & $(249-306)$ & 29.4 & 264 & A & [1987STE/MAL, 1962BAN/CHE] \\
\hline \multirow[t]{2}{*}{$\mathrm{C}_{5} \mathrm{~F}_{11} \mathrm{NO}$} & {$[52225-65-7]$} & \multicolumn{5}{|c|}{$N, 2,2,2$-tetrafluoro- $N$-[1,2,2,2-tetrafluoro-1-(trifluoromethyl)ethyl]acetamide } \\
\hline & $\mathrm{V}$ & & 32.6 & 332 & & [1974PET/SHR] \\
\hline \multirow[t]{5}{*}{$\mathrm{C}_{5} \mathrm{~F}_{12}$} & {$[594-91-2]$} & \multicolumn{5}{|c|}{ perfluoro-2-methylbutane } \\
\hline & V & $(290-340)$ & 26.3 & 298 & & [1984BOU/FRI, 1991BAS/SVO] \\
\hline & $\mathrm{V}$ & $(228-308)$ & 31.0 & 243 & A & [1987STE/MAL, 1967CRO/TAY] \\
\hline & $\mathrm{V}$ & & 26.1 & 303 & & [1967CRO/TAY] \\
\hline & V & $(290-337)$ & 27.4 & 298 & & [1956BAR/CAD] \\
\hline \multirow[t]{7}{*}{$\mathrm{C}_{5} \mathrm{~F}_{12}$} & {$[678-26-2]$} & perfluoropentane & & & & \\
\hline & FUS & & 6.8 & 147.8 & & [1951BUR/CAD] \\
\hline & SUB & & 43.7 & 145 & & [1963BON, 1951BUR/CAD, 1956BAR/CAD] \\
\hline & $\mathrm{V}$ & $(280-340)$ & 26.6 & 298 & & [1984BOU/FRI, 1991BAS/SVO] \\
\hline & $\mathrm{V}$ & $(221-303)$ & 31.1 & 236 & A & [1987STE/MAL, 1967CRO/TAY] \\
\hline & $\mathrm{V}$ & & 26.4 & 302 & & [1967CRO/TAY] \\
\hline & V & $(288-338)$ & 27.5 & 298 & & [1956BAR/CAD] \\
\hline $\mathrm{C}_{5} \mathrm{~F}_{12} \mathrm{~N}_{2}$ & {$[53684-06-3]$} & {$[2,2,2$-trifluoro-1,1 } & is(trifluorome & ethyl](trif & ethyl)diaz & \\
\hline & $\mathrm{V}$ & & 23.7 & 309 & & [1975KIR/LAS] \\
\hline $\mathrm{C}_{5} \mathrm{~F}_{12} \mathrm{O}_{2}$ & {$[20822-11-1]$} & bis(pentafluoroetho & y)difluoromet & & & \\
\hline & V & $(246-299)$ & 32.7 & 261 & A & [1987STE/MAL, 1968HOH/SHR] \\
\hline $\mathrm{C}_{5} \mathrm{~F}_{12} \mathrm{O}_{2} \mathrm{~S}$ & {$[52225-54-4]$} & trifluoromethanesul & nic acid, 2,2, & uoro-1,1- & luorometh & ethyl ether \\
\hline & $\mathrm{V}$ & & 37.7 & 355 & $\mathrm{HG}$ & [1974MAJ/SHR] \\
\hline $\mathrm{C}_{5} \mathrm{~F}_{12} \mathrm{O}_{4} \mathrm{~S}$ & {$[60672-63-1]$} & pentafluoro $(2,2,3,3$ & ,4,5-heptafluc & oxopenta & xoato)sulf & \\
\hline & $\mathrm{V}$ & & 39.2 & & & [1976HOP/DES] \\
\hline $\mathrm{C}_{5} \mathrm{~F}_{13} \mathrm{~N}$ & {$[1481-55-6]$} & $N$-(trifluoromethyl & is(pentafluor & l)amine & & \\
\hline & FUS & & 4.60 & 150.1 & & [1996DOM/HEA, 1984GOL/KOL] \\
\hline & TRS & & 5.53 & 126.0 & & \\
\hline & FUS & $(6-300)$ & 7.16 & 149.7 & & [1996DOM/HEA, 1980ZHO/KOS] \\
\hline & $\mathrm{V}$ & $(298-319)$ & 30.2 & 308 & A & [1987STE/MAL] \\
\hline & V & & $29.4 \pm 0.4$ & 298 & & [1977VAR/AMM2] \\
\hline $\mathrm{C}_{5} \mathrm{~F}_{13} \mathrm{NS}$ & {$[37826-44-1]$} & $N$-[1,2,2,2-tetrafluc & o-1-(trifluoro & l)ethyl]- & (trifluoror & thyl)sulfilimine \\
\hline & V & $(314-360)$ & 31.3 & & A & [1987STE/MAL, 1999DYK/SVO] \\
\hline $\mathrm{C}_{5} \mathrm{~F}_{14} \mathrm{~N}_{2} \mathrm{O}$ & {$[17636-89-4]$} & 1-[difluoro(trifluorc & nethoxy)meth & 2,2-tris $(\mathrm{tr}$ & methyl)hy & razine \\
\hline & $\mathrm{V}$ & $(302-331)$ & 34.7 & & A & [1987STE/MAL, 1967HAS/TIP] \\
\hline $\mathrm{C}_{5} \mathrm{~F}_{14} \mathrm{~N}_{2} \mathrm{O}$ & {$[17636-88-3]$} & 1,1-difluoro- $N$-(tri & loromethoxy) & $N^{\prime}, N^{\prime}$-tri & oromethyl & nethanediamine \\
\hline & $\mathrm{V}$ & $(282-323)$ & 33.7 & 297 & A & [1987STE/MAL, 1967HAS/TIP] \\
\hline $\mathrm{C}_{5} \mathrm{~F}_{14} \mathrm{OS}$ & [736-59-4] & pentafluoro[(nonflu & rocyclopentyl & sulfur & & \\
\hline
\end{tabular}


TABLE 8. Phase change enthalpies of $C_{5}$ organic compounds-Continued

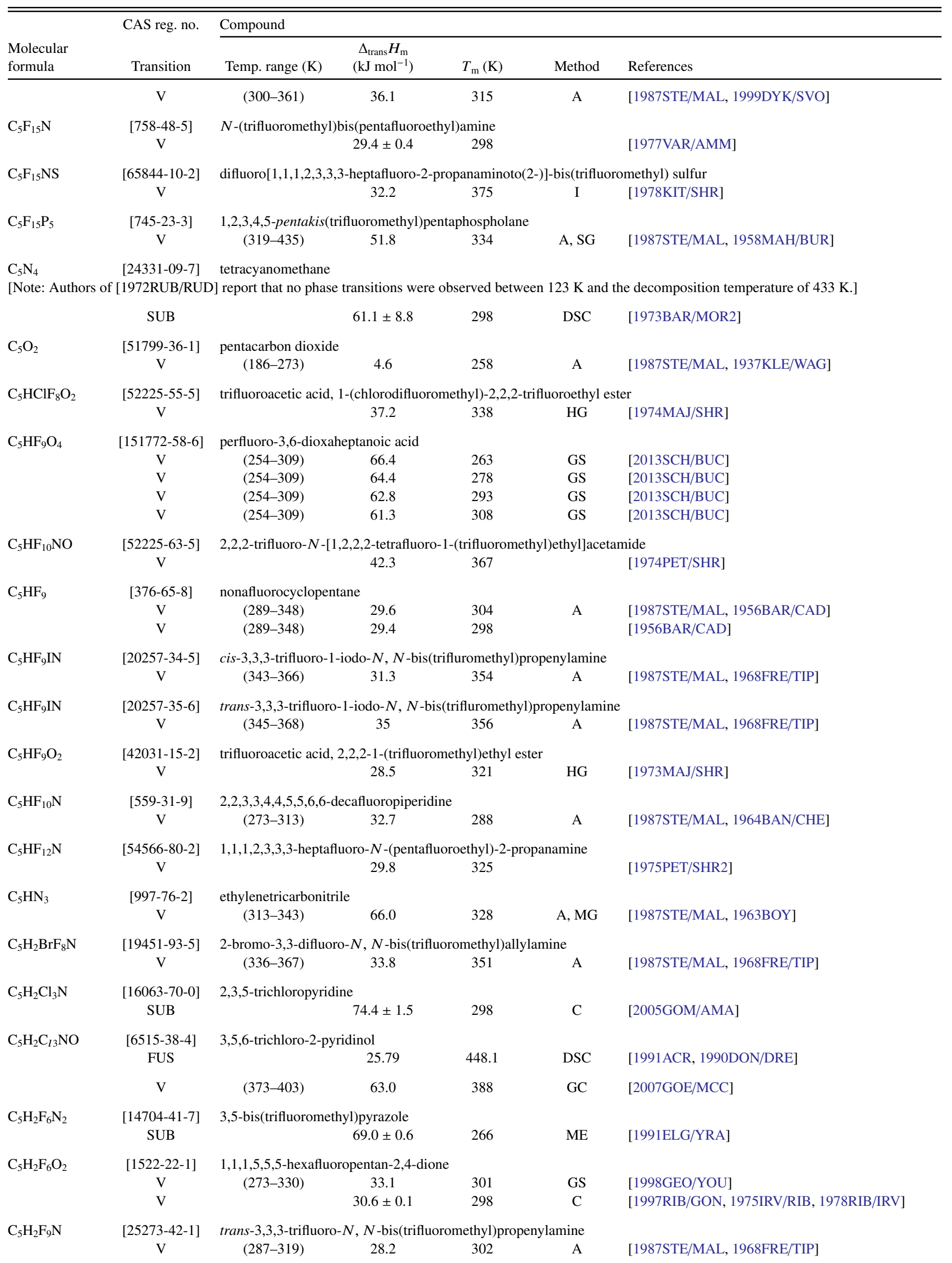


TABLE 8. Phase change enthalpies of $C_{5}$ organic compounds-Continued

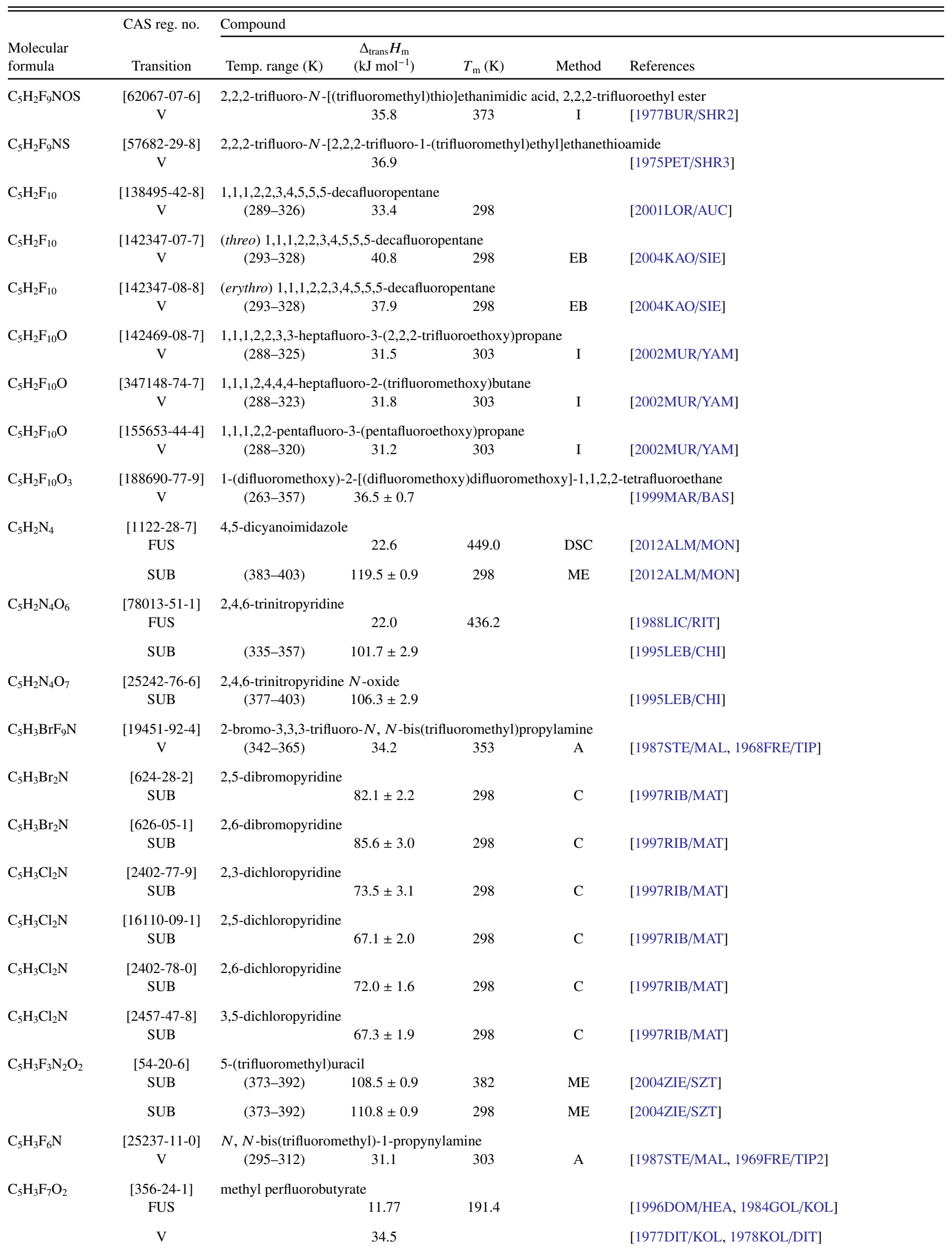


TABLE 8. Phase change enthalpies of $C_{5}$ organic compounds-Continued

\begin{tabular}{|c|c|c|c|c|c|c|}
\hline $\begin{array}{l}\text { Molecular } \\
\text { formula }\end{array}$ & $\begin{array}{l}\text { CAS reg. no. } \\
\text { Transition }\end{array}$ & \multicolumn{5}{|l|}{ Compound } \\
\hline $\mathrm{C}_{5} \mathrm{H}_{3} \mathrm{~F}_{8} \mathrm{NOS}$ & $\mathrm{V}$ & & 33.9 & 330 & & [1981ABE/SHR] \\
\hline \multirow[t]{2}{*}{$\mathrm{C}_{5} \mathrm{H}_{3} \mathrm{~F}_{9} \mathrm{~N}_{2} \mathrm{OS}$} & [62609-63-6] & \multicolumn{5}{|c|}{ 1,1,1-trifluoro- $N^{\prime}$-methyl- $N$-[2,2,2-trifluoro-1-(trifluoromethyl)ethylidene]methanesulfonimidamide } \\
\hline & $\mathrm{V}$ & & 32.6 & 417 & I & [1977KIT/SHR] \\
\hline $\mathrm{C}_{5} \mathrm{H}_{3} \mathrm{~F}_{9} \mathrm{O}$ & $\mathrm{V}$ & $(288-336)$ & 34.0 & 303 & I & [2002MUR/YAM] \\
\hline \multirow[t]{2}{*}{$\mathrm{C}_{5} \mathrm{H}_{3} \mathrm{~F}_{9} \mathrm{O}$} & {$[176310-28-4]$} & \multicolumn{5}{|c|}{ 1-(2,2-difluoroethoxy)-1,1,2,2,3,3,3-heptafluoropropane } \\
\hline & $\mathrm{V}$ & $(288-340)$ & 34.8 & 303 & I & [2002MUR/YAM] \\
\hline \multirow[t]{2}{*}{$\mathrm{C}_{5} \mathrm{H}_{3} \mathrm{~F}_{9} \mathrm{O}$} & {$[50807-74-4]$} & \multicolumn{5}{|c|}{ 1,1,1,2,2-pentafluoro-3-(1,1,2,2-tetrafluoroethoxy)propane } \\
\hline & $\mathrm{V}$ & $(293-343)$ & 35.6 & 308 & I & [2002MUR/YAM] \\
\hline $\mathrm{C}_{5} \mathrm{H}_{3} \mathrm{~F}_{9} \mathrm{O}$ & $\mathrm{V}$ & $(288-326)$ & 31.3 & 303 & I & [2002MUR/YAM] \\
\hline \multirow[t]{2}{*}{$\mathrm{C}_{5} \mathrm{H}_{3} \mathrm{~F}_{9} \mathrm{O}$} & {$[993-95-3]$} & \multicolumn{5}{|c|}{ 1,1,1,2,3,3-hexafluoro-3-(2,2,2-trifluoroethoxy)propane } \\
\hline & $\mathrm{V}$ & $(293-346)$ & 36.1 & 308 & I & [2002MUR/YAM] \\
\hline \multirow[t]{2}{*}{$\mathrm{C}_{5} \mathrm{H}_{3} \mathrm{~F}_{9} \mathrm{O}$} & [69948-43-2] & 1,1,1,2,3,3-hexafluor & o-4-(trifluoron & xy)butane & & \\
\hline & $\mathrm{V}$ & $(288-338)$ & 34.0 & 303 & I & [2002MUR/YAM] \\
\hline $\mathrm{C}_{5} \mathrm{H}_{3} \mathrm{~F}_{9} \mathrm{O}_{2} \mathrm{~S}$ & {$[52225-51-1]$} & trifluoromethanesulfi & nic acid, $2,2,2$ & 1oro-1-me & -(trifluoro & ethyl)ethyl ester \\
\hline & $\mathrm{V}$ & & 34.3 & 385 & HG & [1974MAJ/SHR] \\
\hline $\mathrm{C}_{5} \mathrm{H}_{3} \mathrm{NO}$ & {$[617-90-3]$} & 2-furancarbonitrile & & & & \\
\hline & V & & $44.8 \pm 0.4$ & 298 & $\mathrm{C}$ & [2009RIB/AMA] \\
\hline $\mathrm{C}_{5} \mathrm{H}_{3} \mathrm{NS}$ & [1641-09-4] & 3-thiophenecarbonitr & & & & \\
\hline & $\mathrm{V}$ & & $51.6 \pm 1.9$ & 298 & $\mathrm{C}$ & [2008RIB/SAN] \\
\hline $\mathrm{C}_{5} \mathrm{H}_{3} \mathrm{NS}$ & [10359-20-3] & 2,2-dicyanopropionit & & & & \\
\hline & FUS & & 18.7 & 367.2 & & [1994RAK/VER] \\
\hline & SUB & $(293-333)$ & $73.9 \pm 0.5$ & 313 & $\mathrm{~T}$ & [1994RAK/VER] \\
\hline & $\mathrm{V}$ & $(293-333)$ & 55.2 & & B & [1994RAK/VER] \\
\hline $\mathrm{C}_{5} \mathrm{H}_{3} \mathrm{~N}_{3}$ & [19847-12-2] & pyrazinecarbonitrile & & & & \\
\hline & $\mathrm{V}$ & & $58.7 \pm 1.2$ & 298 & $\mathrm{C}$ & [2005RIB/MIR] \\
\hline $\mathrm{C}_{5} \mathrm{H}_{4} \mathrm{BrF}_{6} \mathrm{~N}$ & {$[25273-47-6]$} & cis-2-bromo- $N, N$-b & is(trifluorome & oropenylar & & \\
\hline & $\mathrm{V}$ & $(346-367)$ & 35.3 & 356 & A & [1987STE/MAL, 1969FRE/TIP2] \\
\hline $\mathrm{C}_{5} \mathrm{H}_{4} \mathrm{BrF}_{6} \mathrm{~N}$ & {$[25273-48-7]$} & trans-2-bromo- $N, N$ & -bis(trifluoron & 1)propeny & & \\
\hline & V & $(336-360)$ & 33.3 & 348 & A & [1987STE/MAL, 1969FRE/TIP2] \\
\hline $\mathrm{C}_{5} \mathrm{H}_{4} \mathrm{BrN}$ & [109-04-6] & 2-bromopyridine & & & & \\
\hline & $\mathrm{V}$ & & $54.4 \pm 1.3$ & 298 & $\mathrm{C}$ & [1997RIB/MAT] \\
\hline & $\mathrm{V}$ & $(233-323)$ & 51.0 & 298 & ME & [1991AUE/WEB] \\
\hline $\mathrm{C}_{5} \mathrm{H}_{4} \mathrm{BrN}$ & {$[626-55-1]$} & 3-bromopyridine & & & & \\
\hline & $\mathrm{V}$ & & $52.1 \pm 1.3$ & 298 & $\mathrm{C}$ & [1997RIB/MAT] \\
\hline & $\mathrm{V}$ & $(233-323)$ & 45.2 & 298 & ME & [1991AUE/WEB] \\
\hline & $\mathrm{V}$ & $(289-447)$ & 47.4 & 304 & $\mathrm{~A}$ & [1987STE/MAL, 1947STU] \\
\hline
\end{tabular}

$\mathrm{C}_{5} \mathrm{H}_{4} \mathrm{ClF}_{7} \mathrm{O}$ [65064-83-7] 3-(2-chloro-1,1,2-trifluoroethoxy)-1,1,2,2-tetrafluoropropane
$\mathrm{V}$
$44.4 \pm 0.2$
298
[1981VAR/BUL2] 
TABLE 8. Phase change enthalpies of $C_{5}$ organic compounds-Continued

\begin{tabular}{|c|c|c|c|c|c|c|}
\hline \multirow[b]{2}{*}{$\begin{array}{l}\text { Molecular } \\
\text { formula }\end{array}$} & \multirow{2}{*}{$\begin{array}{c}\text { CAS reg. no. } \\
\text { Transition }\end{array}$} & \multicolumn{5}{|l|}{ Compound } \\
\hline & & Temp. range $(\mathrm{K})$ & $\begin{array}{c}\Delta_{\text {trans }} H_{\mathrm{m}} \\
\left(\mathrm{kJ} \mathrm{mol}^{-1}\right)\end{array}$ & $T_{\mathrm{m}}(\mathrm{K})$ & Method & References \\
\hline \multirow[t]{4}{*}{$\mathrm{C}_{5} \mathrm{H}_{4} \mathrm{ClN}$} & {$[109-09-1]$} & 2-chloropyridine & & & & \\
\hline & $\mathrm{V}$ & & $51.0 \pm 1.2$ & 298 & $\mathrm{C}$ & [1997RIB/MAT2] \\
\hline & $\mathrm{V}$ & $(283-298)$ & 46.2 & 298 & & [1979ARN/CHA] \\
\hline & $\mathrm{V}$ & $(286-444)$ & 53 & 301 & A & [1987STE/MAL, 1947STU] \\
\hline \multirow[t]{3}{*}{$\mathrm{C}_{5} \mathrm{H}_{4} \mathrm{ClN}$} & {$[626-60-8]$} & 3-chloropyridine & & & & \\
\hline & V & & $47.9 \pm 1.1$ & 298 & $\mathrm{C}$ & [1997RIB/MAT2] \\
\hline & $\mathrm{V}$ & $(283-298)$ & 43.4 & 298 & & [1979ARN/CHA] \\
\hline \multirow[t]{2}{*}{$\mathrm{C}_{5} \mathrm{H}_{4} \mathrm{ClNO}$} & {$[6636-78-8]$} & 2-chloro-3-hydroxy & yridine & & & \\
\hline & SUB & & $89.2 \pm 1.7$ & 298 & $\mathrm{C}$ & [2013MIR/MAT] \\
\hline \multirow[t]{2}{*}{$\mathrm{C}_{5} \mathrm{H}_{4} \mathrm{ClNO}$} & {$[89-64-5]$} & 2-chloro-6-hydroxy & yridine & & & \\
\hline & SUB & & $91.7 \pm 1.2$ & 298 & $\mathrm{C}$ & [2013MIR/MAT] \\
\hline \multirow[t]{2}{*}{$\mathrm{C}_{5} \mathrm{H}_{4} \mathrm{ClNO}$} & {$[610-78-6]$} & 3-chloro-5-hydroxy & yridine & & & \\
\hline & SUB & & $99.5 \pm 2.0$ & 298 & $\mathrm{C}$ & [2013MIR/MAT] \\
\hline \multirow[t]{2}{*}{$\mathrm{C}_{5} \mathrm{H}_{4} \mathrm{~F}_{4} \mathrm{~N}_{4} \mathrm{O}_{10}$} & {$[58715-08-5]$} & \multicolumn{5}{|c|}{ bis(2-fluoro-2,2-dinitroethyl)difluoroformal } \\
\hline & $\mathrm{V}$ & $(323-357)$ & 72.7 & 340 & & {$[1997 \mathrm{MIN} / \mathrm{BEH}]$} \\
\hline \multirow[t]{2}{*}{$\mathrm{C}_{5} \mathrm{H}_{4} \mathrm{FN}$} & {$[372-48-5]$} & 2-fluoropyridine & & & & \\
\hline & $\mathrm{V}$ & $(233-323)$ & 43.5 & 298 & ME & [1991AUE/WEB] \\
\hline \multirow[t]{2}{*}{$\mathrm{C}_{5} \mathrm{H}_{4} \mathrm{FN}$} & {$[372-47-4]$} & 3-fluoropyridine & & & & \\
\hline & $\mathrm{V}$ & $(233-323)$ & 45.2 & 298 & ME & [1991AUE/WEB] \\
\hline \multirow[t]{2}{*}{$\mathrm{C}_{5} \mathrm{H}_{4} \mathrm{~F}_{7} \mathrm{I}$} & {$[1513-88-8]$} & 1,1,1,2,2,3,3-heptaf & oro-5-iodope & & & \\
\hline & $\mathrm{V}$ & $(317-386)$ & 38.7 & 332 & A & [1987STE/MAL] \\
\hline \multirow[t]{2}{*}{$\mathrm{C}_{5} \mathrm{H}_{4} \mathrm{~F}_{8} \mathrm{O}$} & {$[16627-68-2]$} & 1,1,2,2-tetrafluoro-3 & $(1,1,2,2$-tetraf & ethoxy)pr & & \\
\hline & $\mathrm{V}$ & (293-366) & 40.2 & 308 & I & [2002MUR/YAM] \\
\hline \multirow[t]{2}{*}{$\mathrm{C}_{5} \mathrm{H}_{4} \mathrm{~F}_{8} \mathrm{O}$} & [382-26-3] & 1,1,1,3,3-penatafluo & o-3-methoxy- & uorometh & ane & \\
\hline & $\mathrm{V}$ & $(288-343)$ & 34.5 & 303 & I & [2002MUR/YAM] \\
\hline \multirow[t]{2}{*}{$\mathrm{C}_{5} \mathrm{H}_{4} \mathrm{~F}_{9} \mathrm{~N}$} & {$[19451-89-9]$} & 3,3,3-trifluoro- $N, N$ & -bis(trifluoron & propylar & & \\
\hline & $\mathrm{V}$ & $(290-333)$ & 31 & 305 & A & [1987STE/MAL, 1968FRE/TIP] \\
\hline \multirow[t]{2}{*}{$\mathrm{C}_{5} \mathrm{H}_{4} \mathrm{~N}_{2}$} & {$[37580-43-1]$} & cis-2-methyl-2-bute & edinitrile & & & \\
\hline & $\mathrm{V}$ & $(395-467)$ & 58.5 & 410 & A & [1987STE/MAL, 1972DYK] \\
\hline \multirow[t]{2}{*}{$\mathrm{C}_{5} \mathrm{H}_{4} \mathrm{~N}_{2}$} & {$[37580-44-2]$} & trans-2-methyl-2-bu & enedinitrile & & & \\
\hline & V & (339-411) & 47.9 & 354 & A & [1987STE/MAL, 1972DYK] \\
\hline \multirow[t]{2}{*}{$\mathrm{C}_{5} \mathrm{H}_{4} \mathrm{~N}_{2}$} & [4513-94-4] & 2-pyrrolecarbonitril & & & & \\
\hline & V & & $65.3 \pm 1.4$ & 298 & $\mathrm{C}$ & [2012SAN/RIB] \\
\hline \multirow[t]{2}{*}{$\mathrm{C}_{5} \mathrm{H}_{4} \mathrm{~N}_{2} \mathrm{O}_{2}$} & {$[98-97-5]$} & 2-pyrazinecarboxyl & acid & & & \\
\hline & SUB & & $103.6 \pm 2.9$ & 298 & $\mathrm{C}$ & [2005RIB/MIR] \\
\hline $\mathrm{C}_{5} \mathrm{H}_{4} \mathrm{~N}_{2} \mathrm{O}_{3}$ & {$[1124-33-0]$} & 4-nitropyridine- $N$-c & kide & & & \\
\hline & SUB & & $108.9 \pm 0.3$ & 298 & $\mathrm{C}$ & [1995ACR/TUC] \\
\hline & SUB & $(311-335)$ & $89.1 \pm 2.5$ & & & [1995LEB/CHI] \\
\hline $\mathrm{C}_{5} \mathrm{H}_{4} \mathrm{~N}_{4}$ & {$[275-02-5]$} & 1,2,4-triazolo[1,5-a & yrimidine & & & \\
\hline & FUS & & 19.4 & 419.5 & & [1997STE/CHI4] \\
\hline & SUB & & 86.9 & 419 & & [1997STE/CHI4] \\
\hline & $\mathrm{V}$ & & $70.7 \pm 4.5$ & 298 & CGC & [2011LIP/RAT] \\
\hline & $\mathrm{V}$ & & $63.7 \pm 2.7$ & 298 & CGC & [2011LIP/RAT] \\
\hline & $\mathrm{V}$ & $(370-523)$ & $82.5 \pm 13.1$ & 298 & EB & [1997STE/CHI4] \\
\hline & $\mathrm{V}$ & $(370-523)$ & $63.5 \pm 2.2$ & 480 & $\mathrm{~EB}$ & [1997STE/CHI4] \\
\hline & $\mathrm{V}$ & $(370-523)$ & $61.2 \pm 2.0$ & 520 & $\mathrm{~EB}$ & [1997STE/CHI4] \\
\hline $\mathrm{C}_{5} \mathrm{H}_{4} \mathrm{~N}_{4}$ & {$[120-73-0]$} & purine & & & & \\
\hline & SUB & & NA & & & [1974TEP/SUK] \\
\hline $\mathrm{C}_{5} \mathrm{H}_{4} \mathrm{~N}_{4} \mathrm{O}$ & [68-94-0] & hypoxanthine & & & & \\
\hline & SUB & $(423-473)$ & $158.1 \pm 1.6$ & 448 & & [1975TEP/YAN] \\
\hline
\end{tabular}


TABLE 8. Phase change enthalpies of $C_{5}$ organic compounds-Continued

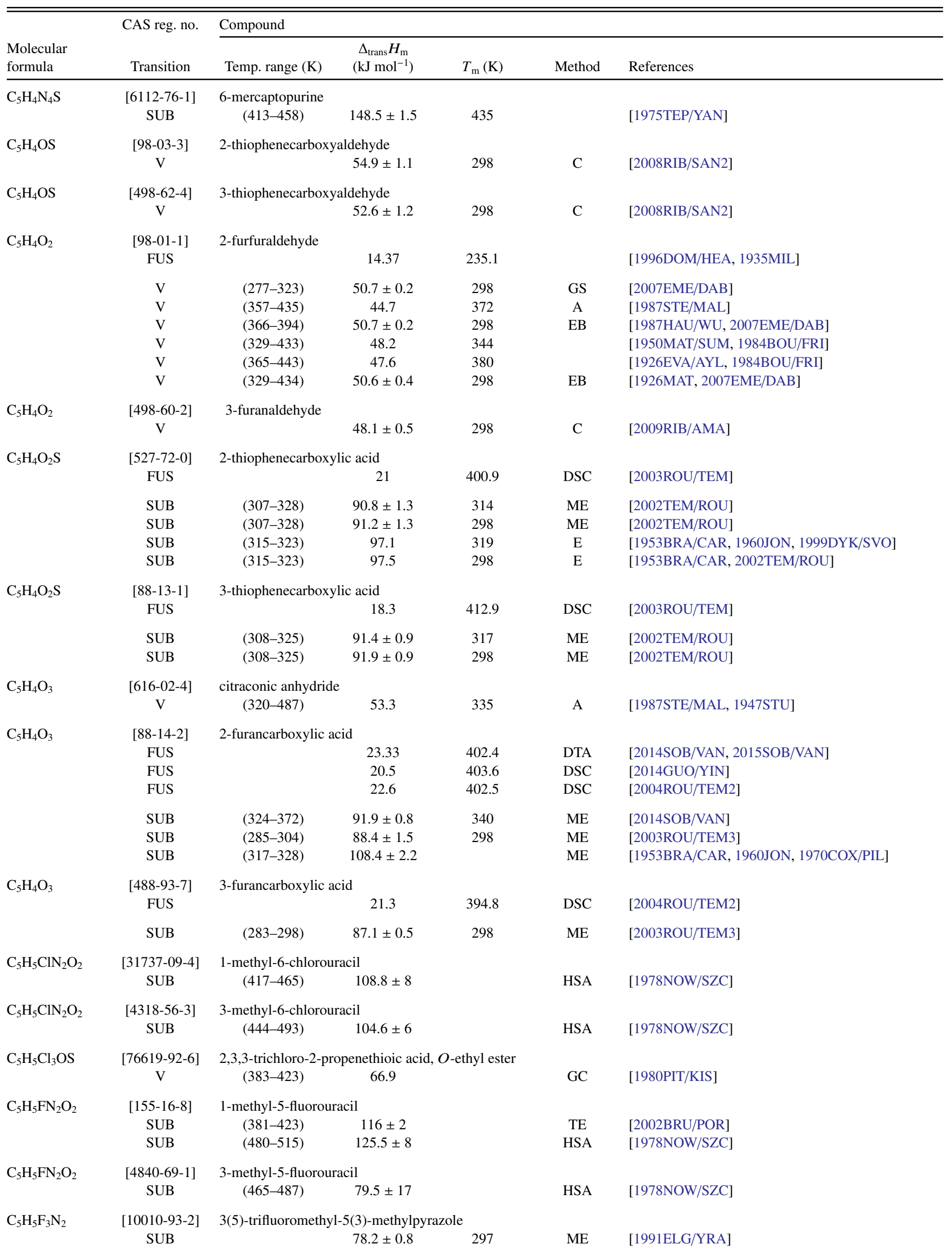


TABLE 8. Phase change enthalpies of $C_{5}$ organic compounds-Continued

\begin{tabular}{|c|c|c|c|c|c|c|}
\hline $\begin{array}{l}\text { Molecular } \\
\text { formula }\end{array}$ & $\begin{array}{l}\text { CAS reg. no. } \\
\text { Transition }\end{array}$ & \multicolumn{5}{|l|}{ Compound } \\
\hline $\mathrm{C}_{5} \mathrm{H}_{5} \mathrm{~F}_{3} \mathrm{O}_{2}$ & $\begin{array}{l}{[7291-30-7]} \\
\text { FUS }\end{array}$ & \multicolumn{5}{|c|}{ trifluoromethyl (2-hydroxy-1-propenyl)ketone } \\
\hline $\mathrm{C}_{5} \mathrm{H}_{5} \mathrm{~F}_{6} \mathrm{NO}$ & $\begin{array}{c}{[22130-39-8]} \\
\mathrm{V}\end{array}$ & \multicolumn{4}{|c|}{ 1-methoxy- $N, N$-bis(trifluoromethyl)vinylamine } & [1987STE/MAL, 1969FRE/TIP] \\
\hline $\mathrm{C}_{5} \mathrm{H}_{5} \mathrm{~F}_{6} \mathrm{NO}$ & $\begin{array}{c}{[22298-35-7]} \\
\text { V }\end{array}$ & \multicolumn{4}{|c|}{ cis-2-methoxy- $N, N$-bis(trifluoromethyl)vinylamine } & [1987STE/MAL, 1969FRE/TIP] \\
\hline $\mathrm{C}_{5} \mathrm{H}_{5} \mathrm{~F}_{7} \mathrm{O}$ & $\begin{array}{c}{[22052-86-4]} \\
\text { V }\end{array}$ & \multicolumn{5}{|c|}{ 1-ethoxy-1,1,2,2,3,3,3-heptafluoropropane } \\
\hline $\mathrm{C}_{5} \mathrm{H}_{5} \mathrm{~F}_{7} \mathrm{O}$ & $\begin{array}{c}{[376-98-7]} \\
V\end{array}$ & \multicolumn{5}{|c|}{ 1,1,1,2,2,3,3-heptafluoro-4-methoxybutane } \\
\hline $\mathrm{C}_{5} \mathrm{H}_{5} \mathrm{~N}$ & $\begin{array}{l}{[2180-69-0]} \\
\text { V } \\
\text { V } \\
\text { V }\end{array}$ & $\begin{array}{c}\text { cis-2,4-pentadienen } \\
(318-383)\end{array}$ & $\begin{array}{l}\text { trile (cis-1-cya } \\
\quad 41.4 \\
40.7 \\
38.3\end{array}$ & $\begin{array}{l}\text { 3-butadiene) } \\
333 \\
348 \\
408\end{array}$ & A & $\begin{array}{l}\text { [1987STE/MAL, 1972DYK] } \\
\text { [1954WIS] } \\
\text { [1954WIS] }\end{array}$ \\
\hline $\mathrm{C}_{5} \mathrm{H}_{5} \mathrm{~N}$ & $\begin{array}{c}{[16955-35-4]} \\
\mathrm{V}\end{array}$ & $\begin{array}{c}\text { bicyclo[1.1.0]butan } \\
(307-349)\end{array}$ & $\begin{array}{l}\text {-1-carbonitrile } \\
48.0\end{array}$ & 319 & BG & [1971HAL/BAL] \\
\hline & V & $(323-373)$ & 40.4 & 298 & CGC & [1995CHI/HOS] \\
\hline & $\begin{array}{l}\text { V } \\
\text { V }\end{array}$ & $\begin{array}{l}(346-362) \\
(295-388)\end{array}$ & $\begin{array}{l}37.6 \\
39.9\end{array}$ & $\begin{array}{l}354 \\
310\end{array}$ & EB & $\begin{array}{l}\text { [1994BLA/BEL] } \\
\text { [1990LEN] }\end{array}$ \\
\hline & $\mathrm{V}$ & $(296-353)$ & 39.7 & 311 & A & [1987STE/MAL] \\
\hline & $\mathrm{V}$ & $(348-434)$ & 37.3 & 363 & A & [1987STE/MAL] \\
\hline & $\mathrm{V}$ & $(431-558)$ & 35.0 & 446 & A & [1987STE/MAL] \\
\hline & $\begin{array}{l}\mathrm{V} \\
\mathrm{V}\end{array}$ & $(552-620)$ & 34.0 & 567 & A & [1987STE/MAL] \\
\hline & $\begin{array}{l}\text { V } \\
\text { V }\end{array}$ & (298-333) & $\begin{array}{l}39.6 \\
40.2\end{array}$ & $\begin{array}{l}313 \\
298\end{array}$ & $\begin{array}{l}\mathrm{C} \\
\mathrm{C}\end{array}$ & $\begin{array}{l}\text { [1986MIC/JOS] } \\
{[1984 \mathrm{MAJ} / \mathrm{SVO} 2]}\end{array}$ \\
\hline & $\mathrm{V}$ & & 39.4 & 313 & $\mathrm{C}$ & [1984MAJ/SVO2] \\
\hline & $\mathrm{V}$ & & 38.5 & 328 & $\mathrm{C}$ & [1984MAJ/SVO2] \\
\hline & $\mathrm{V}$ & & 37.7 & 343 & $\mathrm{C}$ & [1984MAJ/SVO2] \\
\hline & $\mathrm{V}$ & & 36.3 & 368 & $\mathrm{C}$ & [1984MAJ/SVO2] \\
\hline & $\mathrm{V}$ & $(283-298)$ & 40.5 & 298 & & [1979ARN/CHA] \\
\hline & $\mathrm{V}$ & $(340-426)$ & 37.6 & 355 & EB & [1987STE/MAL, 1957MCC/DOU] \\
\hline & $\mathrm{V}$ & & $37.5 \pm 0.1$ & 346 & $\mathrm{C}$ & {$[1957 \mathrm{MCC} / \mathrm{DOU}]$} \\
\hline & $\mathrm{V}$ & & $36.4 \pm 0.1$ & 366 & $\mathrm{C}$ & [1957MCC/DOU] \\
\hline & $\mathrm{V}$ & & $35.1 \pm 0.1$ & 388 & $\mathrm{C}$ & [1957MCC/DOU] \\
\hline & $\mathrm{V}$ & $(320-388)$ & 38.4 & 335 & MG & [1953HER/MAR] \\
\hline & $\mathrm{V}$ & $(290-305)$ & 41.8 & 298 & & [1934HIE/WOE] \\
\hline & $\mathrm{V}$ & $(333-347)$ & 37.7 & 340 & & [1934HIE/WOE] \\
\hline & $\mathrm{V}$ & $(380-388)$ & 36.6 & 384 & & [1934HIE/WOE] \\
\hline
\end{tabular}


TABLE 8. Phase change enthalpies of $C_{5}$ organic compounds-Continued

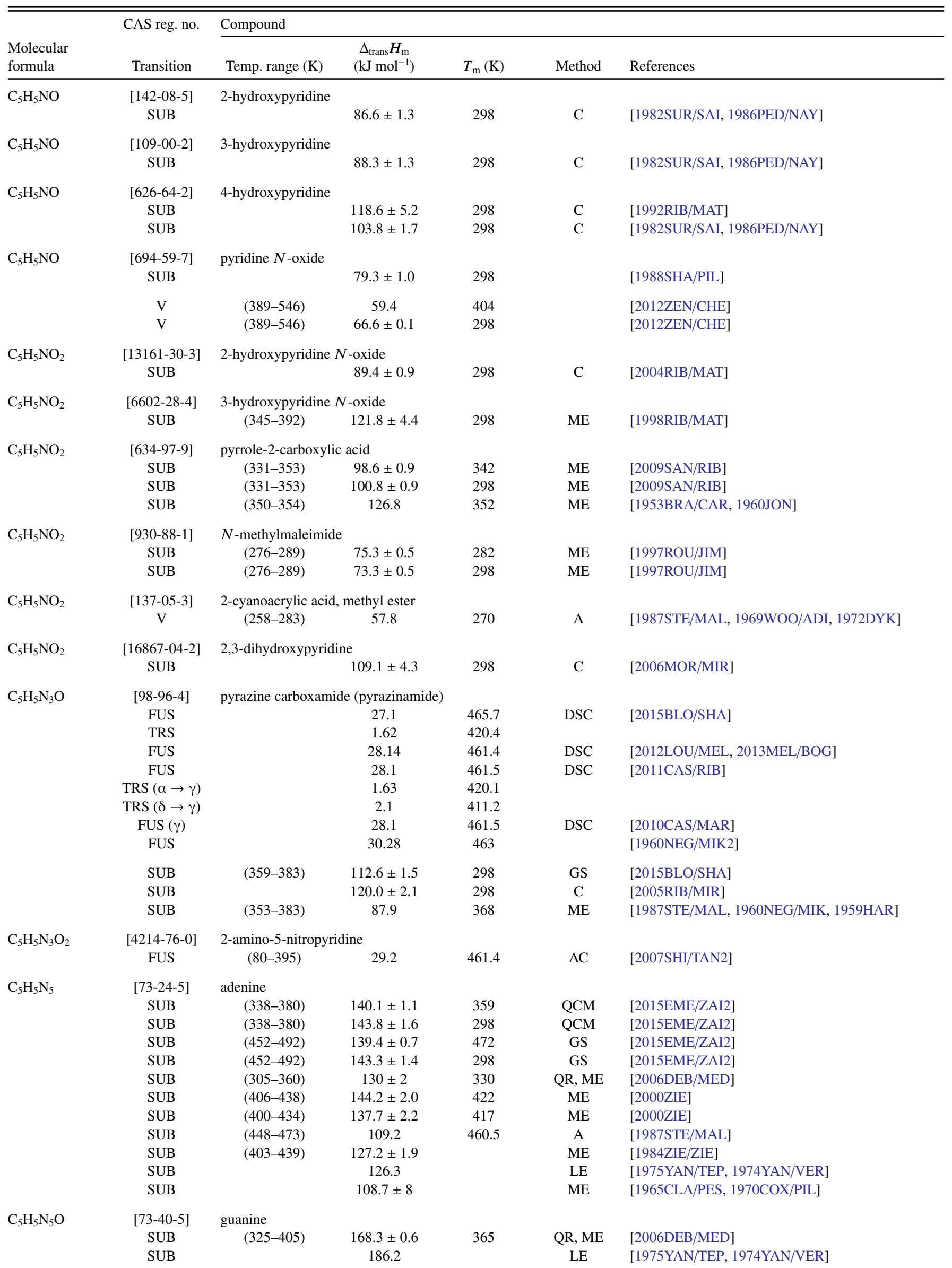


TABLE 8. Phase change enthalpies of $C_{5}$ organic compounds-Continued

\begin{tabular}{|c|c|c|c|c|c|c|}
\hline \multirow[b]{2}{*}{$\begin{array}{l}\text { Molecular } \\
\text { formula }\end{array}$} & \multirow{2}{*}{$\begin{array}{c}\text { CAS reg. no. } \\
\text { Transition }\end{array}$} & \multicolumn{5}{|l|}{ Compound } \\
\hline & & Temp. range $(\mathrm{K})$ & $\begin{array}{c}\Delta_{\text {trans }} H_{\mathrm{m}} \\
\left(\mathrm{kJ} \mathrm{mol}^{-1}\right)\end{array}$ & $T_{\mathrm{m}}(\mathrm{K})$ & Method & References \\
\hline \multirow{3}{*}{$\mathrm{C}_{5} \mathrm{H}_{5} \mathrm{~N}_{7} \mathrm{O}_{14}$} & [20919-99-7] & \multicolumn{5}{|c|}{$1,1,1,3,5,5,5$-heptanitropentane } \\
\hline & SUB & & $114.2 \pm 1.3$ & 298 & $\mathrm{C}$ & [2011MIR/KON] \\
\hline & SUB & & 111.7 & 298 & & [1999MIR/VOR] \\
\hline \multirow[t]{2}{*}{$\mathrm{C}_{5} \mathrm{H}_{5} \mathrm{NO}$} & {$[1003-29-8]$} & \multicolumn{5}{|c|}{ 2-pyrrolecarboxaldehyde } \\
\hline & SUB & & $78.6 \pm 1.3$ & 298 & $\mathrm{C}$ & [2011SAN/RIB3] \\
\hline \multirow{7}{*}{$\mathrm{C}_{5} \mathrm{H}_{6}$} & {$[542-92-7]$} & \multicolumn{5}{|l|}{ 1,3-cyclopentadiene } \\
\hline & FUS & & 8.01 & 176.6 & & [1996DOM/HEA, 1977LEB/LIT] \\
\hline & $\mathrm{V}$ & $(271-314)$ & 28.2 & 286 & & [1967LES/OGO, 1984BOU/FRI] \\
\hline & $\mathrm{V}$ & $(291-314)$ & 28.1 & 302 & $\mathrm{~A}, \mathrm{MM}$ & [1987STE/MAL, 1965HUL/REI] \\
\hline & $\mathrm{V}$ & $(291-314)$ & $28.4 \pm 0.3$ & 298 & MM & [1965HUL/REI] \\
\hline & $\mathrm{V}$ & $(291-314)$ & $27.6 \pm 0.3$ & 312 & MM & [1965HUL/REI] \\
\hline & $\mathrm{V}$ & $(273-287)$ & 29.7 & 298 & & [1965HUL/REI, 1933BAR/BUR] \\
\hline \multirow[t]{2}{*}{$\mathrm{C}_{5} \mathrm{H}_{6}$} & {$[6746-94-7]$} & \multicolumn{5}{|l|}{ ethynylcyclopropane } \\
\hline & $\mathrm{V}$ & $(290-320)$ & 31.1 & 305 & A & [1987STE/MAL] \\
\hline \multirow[t]{2}{*}{$\mathrm{C}_{5} \mathrm{H}_{6}$} & {$[78-80-8]$} & \multicolumn{5}{|l|}{ isopropenylacetylene } \\
\hline & V & & 27.2 & & & [1977LEB/RYA] \\
\hline \multirow[t]{2}{*}{$\mathrm{C}_{5} \mathrm{H}_{6} \mathrm{ClN}$} & {$[32366-08-8]$} & \multicolumn{5}{|c|}{ 4-chloro-3-pentenenitrile } \\
\hline & V & $(349-433)$ & 63.9 & 364 & A & [1987STE/MAL] \\
\hline \multirow[t]{2}{*}{$\mathrm{C}_{5} \mathrm{H}_{6} \mathrm{Cl}_{2} \mathrm{O}_{2}$} & {$[2873-74-7]$} & \multicolumn{5}{|l|}{ glutaryl chloride } \\
\hline & V & $(329-490)$ & 55.9 & 344 & A & [1987STE/MAL, 1947STU] \\
\hline \multirow[t]{2}{*}{$\mathrm{C}_{5} \mathrm{H}_{6} \mathrm{~F}_{2} \mathrm{~N}_{4} \mathrm{O}_{10}$} & {$[17003-79-1]$} & \multicolumn{5}{|c|}{ bis(2-fluoro-2,2-dinitroethyl)formal } \\
\hline & $\mathrm{V}$ & $(323-365)$ & 85.1 & 344 & & [1997MIN/BEH] \\
\hline $\mathrm{C}_{5} \mathrm{H}_{6} \mathrm{~F}_{3} \mathrm{NO}_{3}$ & {$[383-72-2]$} & glycine, $N$-(trifluoroa & acetyl) methy & & & \\
\hline & SUB & $(293-463)$ & 57.3 & 308 & & [1987STE/MAL, 1960WEY/KLI] \\
\hline $\mathrm{C}_{5} \mathrm{H}_{6} \mathrm{~F}_{6} \mathrm{~N}_{2} \mathrm{~S}$ & {$[62067-11-2]$} & 2,2,2-trifluoro- $N, N$ - & -dimethyl- $N^{\prime}$ & uoromet & ]ethanim & mide \\
\hline & V & & 40.4 & 400 & I & [1977BUR/SHR2] \\
\hline $\mathrm{C}_{5} \mathrm{H}_{6} \mathrm{~F}_{6} \mathrm{O}$ & [58705-93-4] & 1,1,1,2,3,3-hexafluoro & o-4-methoxyl & & & \\
\hline & $\mathrm{V}$ & $(293-360)$ & 37.0 & 308 & I & [2002MUR/YAM] \\
\hline $\mathrm{C}_{5} \mathrm{H}_{6} \mathrm{~F}_{6} \mathrm{~N}_{2} \mathrm{~S}$ & [38005-19-5] & dimethylamino(hexafl & luoroisoprop & mino) $\mathrm{su}$ & & \\
\hline & $\mathrm{V}$ & & 39.7 & 383 & I & [1972MET/SHR] \\
\hline $\mathrm{C}_{5} \mathrm{H}_{6} \mathrm{~F}_{6} \mathrm{O}_{2} \mathrm{~S}$ & {$[52225-49-7]$} & trifluoromethanesulfin & nic acid, 2,2, & loro- 1,1 - & ylethyl es & \\
\hline & $\mathrm{V}$ & & 35.6 & 388 & $\mathrm{HG}$ & [1974MAJ/SHR] \\
\hline $\mathrm{C}_{5} \mathrm{H}_{6} \mathrm{~F}_{6} \mathrm{O}_{5} \mathrm{~S}_{2}$ & [61915-97-7] & 3,3-bis[(trifluorometh & yyl)sulfonyl]- & panol & & \\
\hline & V & $(333-418)$ & 32.8 & 348 & $\mathrm{~A}, \mathrm{I}$ & [1987STE/MAL, 1977BUR/SHR, 1999DYK/SVO] \\
\hline $\mathrm{C}_{5} \mathrm{H}_{6} \mathrm{~N}_{2}$ & {$[7321-55-3]$} & dimethylmalononitrile & & & & \\
\hline & TRS & & 9.87 & 302.6 & & \\
\hline & FUS & $(5-350)$ & 4.05 & 307.5 & $\mathrm{AC}$ & [1996DOM/HEA, 1967RIB/WES] \\
\hline & SUB & & $62.0 \pm 0.7$ & 298 & & {$[1990 \mathrm{BEC} / \mathrm{DOG}]$} \\
\hline & $\mathrm{V}$ & $(322-413)$ & 47.5 & 337 & A & [1987STE/MAL, 1967RIB/WES] \\
\hline $\mathrm{C}_{5} \mathrm{H}_{6} \mathrm{~N}_{2}$ & {$[109-08-0]$} & 2-methylpyrazine & & & & \\
\hline & $\mathrm{V}$ & $(342-373)$ & $43.7 \pm 1.9$ & 298 & $\mathrm{CGC}$ & [2009LIP/CHI2] \\
\hline & $\mathrm{V}$ & $(288-392)$ & 42.4 & 340 & & [1995SAK/UEO] \\
\hline $\mathrm{C}_{5} \mathrm{H}_{6} \mathrm{~N}_{2}$ & [1632-76-4] & 3-methylpyridazine & & & & \\
\hline & $\mathrm{V}$ & & $56.6 \pm 2.5$ & 298 & CGC & [2010LIP/PLI] \\
\hline & $\mathrm{V}$ & $(342-373)$ & $49.7 \pm 2.8$ & 298 & CGC & [2009LIP/CHI2] \\
\hline $\mathrm{C}_{5} \mathrm{H}_{6} \mathrm{~N}_{2}$ & [3438-46-8] & 4-methylpyrimidine & & & & \\
\hline & $\mathrm{V}$ & & $43.8 \pm 2.6$ & 298 & CGC & [2010LIP/PLI] \\
\hline & $\mathrm{V}$ & $(342-373)$ & $44.2 \pm 2.4$ & 298 & CGC & [2009LIP/CHI2] \\
\hline $\mathrm{C}_{5} \mathrm{H}_{6} \mathrm{~N}_{2}$ & {$[544-13-8]$} & glutaronitrile & & & & \\
\hline & FUS & & 12.03 & 242 & DSC & [2007BAD/BLA] \\
\hline & FUS & & 12.59 & 244.2 & & [1996DOM/HEA, 1965CLE/WUL] \\
\hline
\end{tabular}


TABLE 8. Phase change enthalpies of $C_{5}$ organic compounds-Continued

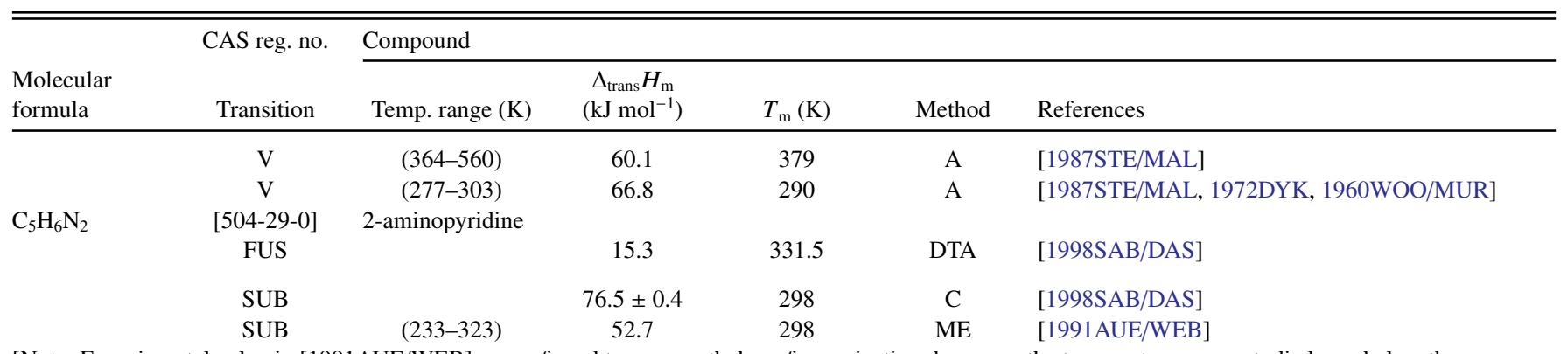

[Note: Experimental value in [1991AUE/WEB] was referred to as an enthalpy of vaporization; however, the temperature range studied was below the melting point temperature.]

\begin{tabular}{|c|c|c|c|c|c|c|}
\hline & SUB & & $38.6 \pm 1.9$ & & DSC & [1985BRO/INI] \\
\hline & SUB & & $78.7 \pm 0.8$ & 298 & $\mathrm{C}$ & [1984BIC/PIL] \\
\hline $\mathrm{C}_{5} \mathrm{H}_{6} \mathrm{~N}_{2}$ & [462-08-8] & 3-aminopyridine & & 3355 & DTA & [1098SAP/DA S \\
\hline & FUS & & 14.4 & 335.5 & $\mathrm{DIA}$ & [1990SAD/DAD] \\
\hline & $\begin{array}{l}\text { SUB } \\
\text { SUB }\end{array}$ & $(233-323)$ & $\begin{array}{c}80.7 \pm 0.3 \\
84.9\end{array}$ & $\begin{array}{l}298 \\
298\end{array}$ & $\begin{array}{c}\mathrm{C} \\
\mathrm{ME}\end{array}$ & $\begin{array}{l}\text { [1998SAB/DAS] } \\
\text { [1991AUE/WEB] }\end{array}$ \\
\hline
\end{tabular}

[Note: Experimental value in [1991AUE/WEB] was referred to as an enthalpy of vaporization; however, the temperature range studied was below the melting point temperature.]

\begin{tabular}{|c|c|c|c|c|c|c|}
\hline & SUB & & $84.0 \pm 1.4$ & 298 & $\mathrm{C}$ & [1984BIC/PIL] \\
\hline \multirow[t]{4}{*}{$\mathrm{C}_{5} \mathrm{H}_{6} \mathrm{~N}_{2}$} & [504-24-5] & 4-aminopyridine & & & & \\
\hline & FUS & & 19.3 & 432.1 & DTA & [1998SAB/DAS] \\
\hline & FUS & & 20.07 & 429.9 & DSC & [1990DON/DRE] \\
\hline & $\begin{array}{l}\text { SUB } \\
\text { SUB }\end{array}$ & $(233-323)$ & $\begin{array}{c}87.1 \pm 0.4 \\
90.0\end{array}$ & $\begin{array}{l}298 \\
298\end{array}$ & $\begin{array}{c}\mathrm{C} \\
\mathrm{ME}\end{array}$ & $\begin{array}{l}\text { [1998SAB/DAS] } \\
\text { [1991AUE/WEB] }\end{array}$ \\
\hline
\end{tabular}

[Note: Experimental value in [1991AUE/WEB] was referred to as an enthalpy of vaporization; however, the temperature range studied was below the melting point temperature.]

SUB

SUB

$53.8 \pm 0.8$

$88.1 \pm 1.1$

$\mathrm{C}_{5} \mathrm{H}_{6} \mathrm{~N}_{2} \mathrm{OS}$

[2361-27-5 SUB SUB

2-th

hiophenecarboxylic acid hydrazide

$$
\begin{array}{ll}
(339-361) & 110.7 \pm 0.5
\end{array}
$$

(339-361) $\quad 113.3 \pm 0.5$

$\mathrm{C}_{5} \mathrm{H}_{6} \mathrm{~N}_{2} \mathrm{OS}$

[636-26-0]

SUB

SUB

5-methyl-2-thiouracil

(413-433)

(413-433)

$131.0 \pm 0.9$

$137.3 \pm 0.9$

[56-04-2]

SUB

SUB

6-methyl-2-thiouracil

(421-447)

(421-447)

$133.6 \pm 0.9$

$140.7 \pm 0.9$

$\mathrm{C}_{5} \mathrm{H}_{6} \mathrm{~N}_{2} \mathrm{O}_{2}$

[615-77-0]

SUB

SUB

SUB

$\mathrm{C}_{5} \mathrm{H}_{6} \mathrm{~N}_{2} \mathrm{O}_{2}$

[608-34-4]

SUB

SUB

1-methyluracil

$$
\text { (343-428) }
$$

(378-418)

(435-480)

$121.7 \pm 4.0$

$112.5 \pm 2.6$

$104.6 \pm 8$

3-methyluracil

(344-419)

(438-498)

$118.8 \pm 3.0$

$75.3 \pm 8$

$\mathrm{C}_{5} \mathrm{H}_{6} \mathrm{~N}_{2} \mathrm{O}_{2}$

[65-71-4]

5-methyluracil (thymine)

FUS

SUB

SUB

SUB

SUB

SUB

SUB

SUB

SUB

SUB

$\mathrm{C}_{5} \mathrm{H}_{6} \mathrm{~N}_{2} \mathrm{O}_{2}$

[626-48-2]

SUB

(426-503)

131

17.51

$$
\begin{aligned}
& (409-472) \\
& (409-428) \\
& (305-355) \\
& (383-438) \\
& (378-428)
\end{aligned}
$$

$126.8 \pm 0.3$

$126.3 \pm 1.2$

$135.8 \pm 0.4$

$125.7 \pm 3.6$

$131.3 \pm 4.0$

$124.4 \pm 1.3$

$138 \pm 10$

$134.1 \pm 4.2$

124.3

[3326-71-4] 2-furancarboxylic acid hydrazide

$\mathrm{C}_{5} \mathrm{H}_{6} \mathrm{~N}_{2} \mathrm{O}_{2}$
$\mathrm{C}_{5} \mathrm{H}_{6} \mathrm{~N}_{2} \mathrm{OS}$

298

$\begin{array}{cc}\text { DSC } & {[1985 \mathrm{BRO} / \mathrm{INI}]} \\ \mathrm{C} & {[1984 \mathrm{BIC} / \mathrm{PIL}]}\end{array}$

350

298

ME

[2008RIB/AMA3]

ME [2008RIB/AMA3]

ME [2013RIB/AMA]

ME [2013RIB/AMA]

298

439

298

ME

ME [2013RIB/AMA]

439

398

457

TE [2000BRU/PIA]

QR [1980TEP/YAN]

HSA [1978NOW/SZC]

382

463

TE

[2000BRU/PIA]

HSA [1978NOW/SZC]

321.3

[1996DOM/HEA, 1889EYK]

298

298

330

411

298

403

298

298

$\begin{array}{cl}\text { GS } & {[2015 \mathrm{EME} / \mathrm{VER} 2]} \\ \mathrm{GS} & {[2014 \mathrm{ACH} / \mathrm{EME}]} \\ \mathrm{QR}, \mathrm{ME} & {[2006 \mathrm{DEB} / \mathrm{MED}]} \\ \mathrm{ME} & {[1984 \mathrm{BUR} / \mathrm{MOR}]} \\ & {[1984 \mathrm{BUR} / \mathrm{MOR}]} \\ \mathrm{QR} & {[1980 \mathrm{TEP} / \mathrm{YAN}]} \\ \mathrm{TE} & {[1980 \mathrm{FER} / \mathrm{BEN}]} \\ \mathrm{C} & {[1977 \mathrm{NAB} / \mathrm{SAB}]} \\ \mathrm{LE} & {[1975 \mathrm{YAN} / \mathrm{TEP}, 1974 \mathrm{YAN} / \mathrm{VER}]}\end{array}$


TABLE 8. Phase change enthalpies of $C_{5}$ organic compounds-Continued

\begin{tabular}{|c|c|c|c|c|c|c|}
\hline \multirow[b]{2}{*}{$\begin{array}{l}\text { Molecular } \\
\text { formula }\end{array}$} & \multirow{2}{*}{$\begin{array}{l}\text { CAS reg. no. } \\
\text { Transition }\end{array}$} & \multicolumn{5}{|l|}{ Compound } \\
\hline & & Temp. range $(\mathrm{K})$ & $\begin{array}{c}\Delta_{\text {trans }} H_{\mathrm{m}} \\
\left(\mathrm{kJ} \mathrm{mol}^{-1}\right)\end{array}$ & $T_{\mathrm{m}}(\mathrm{K})$ & Method & References \\
\hline & SUB & $(309-325)$ & $98.1 \pm 0.7$ & 317 & ME & [2008RIB/AMA3] \\
\hline & SUB & $(309-325)$ & $99.0 \pm 0.7$ & 298 & $\mathrm{ME}$ & [2008RIB/AMA3] \\
\hline \multirow[t]{9}{*}{$\mathrm{C}_{5} \mathrm{H}_{6} \mathrm{O}$} & $\begin{array}{l}\text { [534-22-5] } \\
\text { FUS }\end{array}$ & $\begin{array}{l}\text { 2-methylfuran } \\
(8-307)\end{array}$ & 8.55 & 181.9 & $\mathrm{AC}$ & [1965CAR/WES] \\
\hline & $\mathrm{V}$ & & $33.5 \pm 0.2$ & 298 & $\mathrm{C}$ & [2010RIB/AMA] \\
\hline & $\mathrm{V}$ & $(289-337)$ & 32.4 & 304 & & [2002LOR/AUC] \\
\hline & $\mathrm{V}$ & $(251-338)$ & 34.4 & 266 & A & [1987STE/MAL] \\
\hline & $\mathrm{V}$ & $(309-339)$ & 31.5 & 324 & & [1986KRE/PRA] \\
\hline & $\mathrm{V}$ & $(288-303)$ & 32.5 & 295 & & [1972DYK] \\
\hline & $\mathrm{V}$ & $(333-373)$ & 30.9 & 348 & & [1971EON/POM, 1984BOU/FRI] \\
\hline & $\mathrm{V}$ & $(215-360)$ & 32.2 & 298 & & [1970MOI/ANT] \\
\hline & V & $(213-293)$ & 33.3 & 253 & Static & [1965CAR/WES] \\
\hline $\mathrm{C}_{5} \mathrm{H}_{6} \mathrm{OS}$ & $\begin{array}{l}{[16839-97-7]} \\
\mathrm{V}\end{array}$ & $\begin{array}{l}\text { 2-methoxythiophene } \\
\text { (278-323) }\end{array}$ & $48.3 \pm 0.3$ & 298 & GS & [2014TEM/NOT] \\
\hline $\mathrm{C}_{5} \mathrm{H}_{6} \mathrm{OS}$ & $\begin{array}{c}{[17573-92-1]} \\
\mathrm{V}\end{array}$ & $\begin{array}{l}\text { 3-methoxythiophene } \\
\quad(278-325)\end{array}$ & $48.5 \pm 0.2$ & 298 & GS & [2014TEM/NOT] \\
\hline $\mathrm{C}_{5} \mathrm{H}_{6} \mathrm{O}_{2}$ & $\begin{array}{c}{[591-12-8]} \\
\mathrm{V}\end{array}$ & $\begin{array}{l}\text { 5-methyl-2(3H)-fura } \\
\quad(324-442)\end{array}$ & $\begin{array}{l}\text { none } \\
40.3\end{array}$ & 339 & A & [1987STE/MAL] \\
\hline $\mathrm{C}_{5} \mathrm{H}_{6} \mathrm{O}_{2}$ & $\begin{array}{c}{[591-11-7]} \\
\mathrm{V}\end{array}$ & $(d l)$-5-methyl-2(5H)-furanone & $\begin{array}{c}\text {-furanone } \\
48.2\end{array}$ & 371 & A & [1987STE/MAL] \\
\hline \multirow[t]{2}{*}{$\mathrm{C}_{5} \mathrm{H}_{6} \mathrm{O}_{2}$} & $\begin{array}{l}\text { [98-00-0] } \\
\text { FUS } \\
\text { FUS }\end{array}$ & $\begin{array}{l}\text { furfuryl alcohol } \\
\qquad(78-300)\end{array}$ & $\begin{array}{l}13.1 \\
14.8\end{array}$ & $\begin{array}{l}258.6 \\
253.3\end{array}$ & & $\begin{array}{l}\text { [1996DOM/HEA, 1956PAR/KEN] } \\
\text { [1996DOM/HEA, 1935MIL] }\end{array}$ \\
\hline & $\begin{array}{l}\mathrm{V} \\
\mathrm{V} \\
\mathrm{V}\end{array}$ & $\begin{array}{l}(275-365) \\
(273-412) \\
(304-443)\end{array}$ & $\begin{array}{l}55.7 \\
58.6 \\
53.6\end{array}$ & $\begin{array}{l}298 \\
298 \\
319\end{array}$ & $\begin{array}{l}\text { Static } \\
\text { A }\end{array}$ & $\begin{array}{l}\text { [2014BEN/NEG] } \\
\text { [2014NEG/MOK] } \\
\text { [1987STE/MAL, 1947KET/VAN] }\end{array}$ \\
\hline $\mathrm{C}_{5} \mathrm{H}_{6} \mathrm{O}_{2}$ & $\begin{array}{c}{[15441-65-3]} \\
\mathrm{V}\end{array}$ & $\begin{array}{l}\text { 5-hydroxy-3-pentyn- } \\
\text { (273-333) }\end{array}$ & $\begin{array}{l}\text { 2-one } \\
64.4\end{array}$ & 288 & A & [1987STE/MAL, 1972DYK, 1948RED/CHA5] \\
\hline \multirow[t]{2}{*}{$\mathrm{C}_{5} \mathrm{H}_{6} \mathrm{O}_{3}$} & $\begin{array}{l}{[108-55-4]} \\
\text { SUB } \\
\text { SUB }\end{array}$ & $\begin{array}{l}\text { glutaric anhydride } \\
\qquad(298-320) \\
(298-320)\end{array}$ & $\begin{array}{l}85.9 \pm 1.6 \\
86.1 \pm 1.6\end{array}$ & $\begin{array}{l}309 \\
298\end{array}$ & $\begin{array}{l}\mathrm{ME} \\
\mathrm{ME}\end{array}$ & $\begin{array}{l}\text { [1990MEN/PIL] } \\
{[1990 \mathrm{MEN} / \mathrm{PIL}]}\end{array}$ \\
\hline & V & $(373-560)$ & 60.9 & 388 & A & [1987STE/MAL, 1947STU] \\
\hline $\mathrm{C}_{5} \mathrm{H}_{6} \mathrm{O}_{3}$ & $\begin{array}{c}{[4100-80-5]} \\
\mathrm{V}\end{array}$ & $\begin{array}{l}(d l) \text {-monmethylsuccir } \\
\quad(342-521)\end{array}$ & $\begin{array}{c}\text { nic anhydride } \\
59.3\end{array}$ & 357 & A & [1987STE/MAL, 1947STU] \\
\hline \multirow[t]{2}{*}{$\mathrm{C}_{5} \mathrm{H}_{6} \mathrm{O}_{4}$} & $\begin{array}{c}\text { [598-10-7] } \\
\text { FUS }\end{array}$ & 1,1-cyclopropanedica & $\begin{array}{l}\text { arboxylic acid } \\
17.4\end{array}$ & 413 & DSC & {$[2011 \mathrm{BOO} / \mathrm{MON}]$} \\
\hline & SUB & $(298-318)$ & 126 & 308 & ME & {$[2011 \mathrm{BOO} / \mathrm{MON}]$} \\
\hline \multirow[t]{2}{*}{$\mathrm{C}_{5} \mathrm{H}_{6} \mathrm{O}_{4}$} & {$[498-23-7]$} & \multicolumn{4}{|c|}{ cis-methylbutenedioic acid } & \\
\hline & $\begin{array}{l}\text { FUS (I) } \\
\text { FUS (II) }\end{array}$ & & $\begin{array}{l}17.7 \\
25.3\end{array}$ & $\begin{array}{l}356.4 \\
365.3\end{array}$ & $\mathrm{DSC}$ & {$[2010 \mathrm{LEO} / \mathrm{PIN}]$} \\
\hline \multirow[t]{2}{*}{$\mathrm{C}_{5} \mathrm{H}_{6} \mathrm{O}_{5}$} & $\begin{array}{c}\text { [328-50-7] } \\
\text { FUS }\end{array}$ & $\alpha$-ketoglutaric acid & 28.59 & 388.7 & DSC & [2005CON/CHI] \\
\hline & SUB & $(269-285)$ & 100 & & TPTD & [2005CHA/ZIE] \\
\hline
\end{tabular}

[Note: Values based on TPTD method are not consistent with values determined by other experimental methods.]

$\mathrm{C}_{5} \mathrm{H}_{6} \mathrm{O}_{5}$

[542-05-2] 3-oxopentanedioic acid
SUB
(310-322)
160.2
TPTD [2005CHA/ZIE]

[Note: Values based on TPTD method are not consistent with values determined by other experimental methods.]

$\mathrm{C}_{5} \mathrm{H}_{6} \mathrm{~S}$

[554-14-3] 2-methylthiophene FUS

$\mathrm{V} \quad(333-373)$

$\mathrm{V} \quad(324-391)$
9.47

36.8

38.7

37.2
207.8

348

298

339
[1956PEN/FIN]

I [1971EON/POM, 1984BOU/FRI]

[1971WIL/ZWO]

A, EB [1987STE/MAL, 1952WHI/BAR, 1999DYK/SVO] 
TABLE 8. Phase change enthalpies of $C_{5}$ organic compounds-Continued

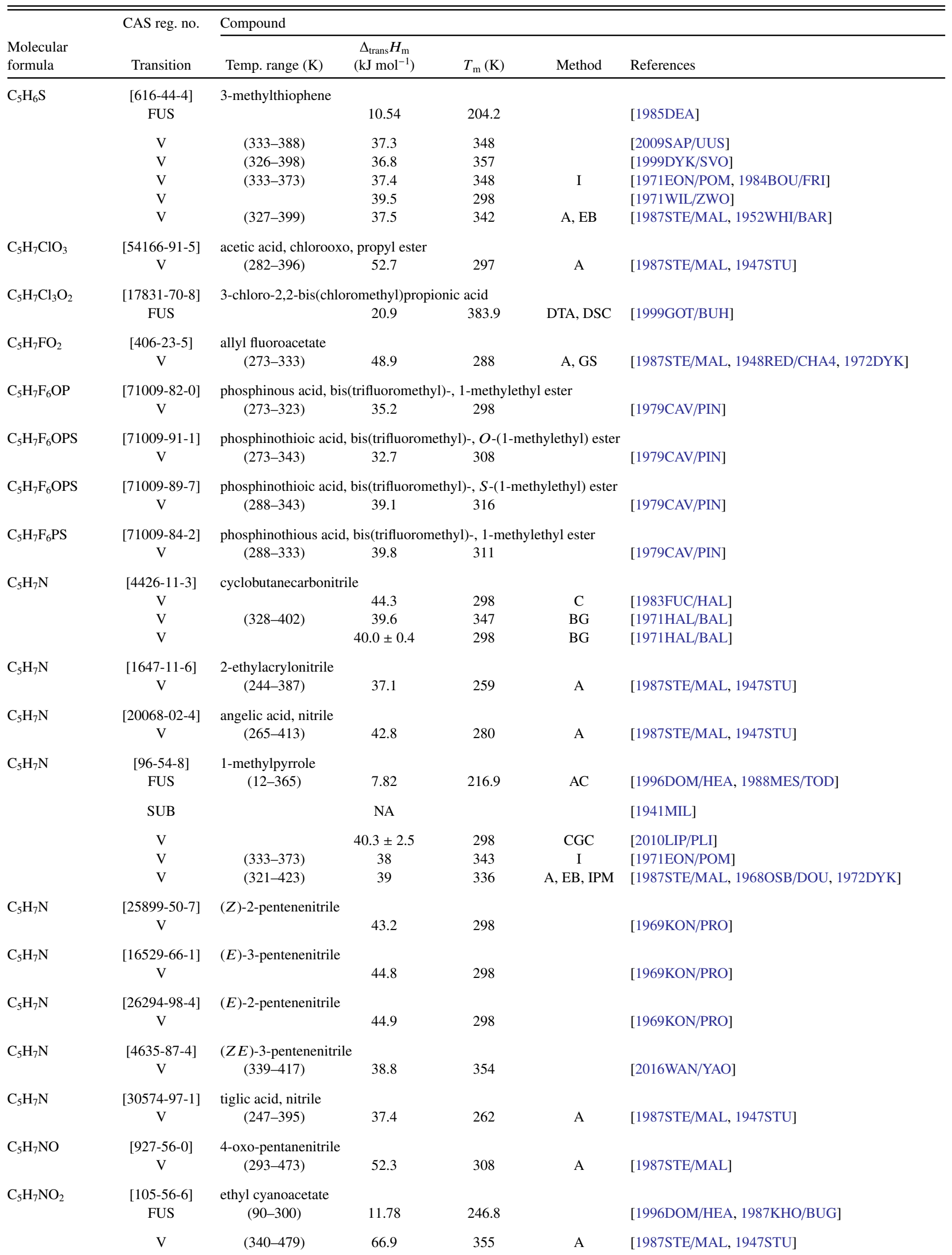


TABLE 8. Phase change enthalpies of $C_{5}$ organic compounds-Continued

\begin{tabular}{|c|c|c|c|c|c|c|}
\hline \multirow[b]{2}{*}{$\begin{array}{l}\text { Molecular } \\
\text { formula }\end{array}$} & \multirow{2}{*}{$\begin{array}{c}\text { CAS reg. no. } \\
\text { Transition }\end{array}$} & \multicolumn{5}{|l|}{ Compound } \\
\hline & & Temp. range $(\mathrm{K})$ & $\begin{array}{c}\Delta_{\text {trans }} H_{\mathrm{m}} \\
\left(\mathrm{kJ} \mathrm{mol}^{-1}\right)\end{array}$ & $T_{\mathrm{m}}(\mathrm{K})$ & Method & References \\
\hline \multirow[t]{3}{*}{$\mathrm{C}_{5} \mathrm{H}_{7} \mathrm{NO}_{2}$} & [1121-89-7] & glutarimide & & & & \\
\hline & SUB & (317-340) & $93.6 \pm 1.6$ & 329 & ME & [1990MEN/PIL] \\
\hline & SUB & $(317-340)$ & $94.1 \pm 1.6$ & 298 & ME & [1990MEN/PIL] \\
\hline \multirow[t]{3}{*}{$\mathrm{C}_{5} \mathrm{H}_{7} \mathrm{NO}_{2}$} & {$[98-79-3]$} & L-pyroglutamic acid & & & & \\
\hline & TRS & & 0.53 & 341.2 & & \\
\hline & FUS & & 16.79 & 435.2 & DSC & [2010WU/REE] \\
\hline
\end{tabular}

[Note: DSC thermogram showed a peak of $1.83 \mathrm{~kJ} / \mathrm{mole}$ at $427.2 \mathrm{~K}$, which the authors attributed to a small amount of $(d l)$-pyroglutamic acid impurity.]

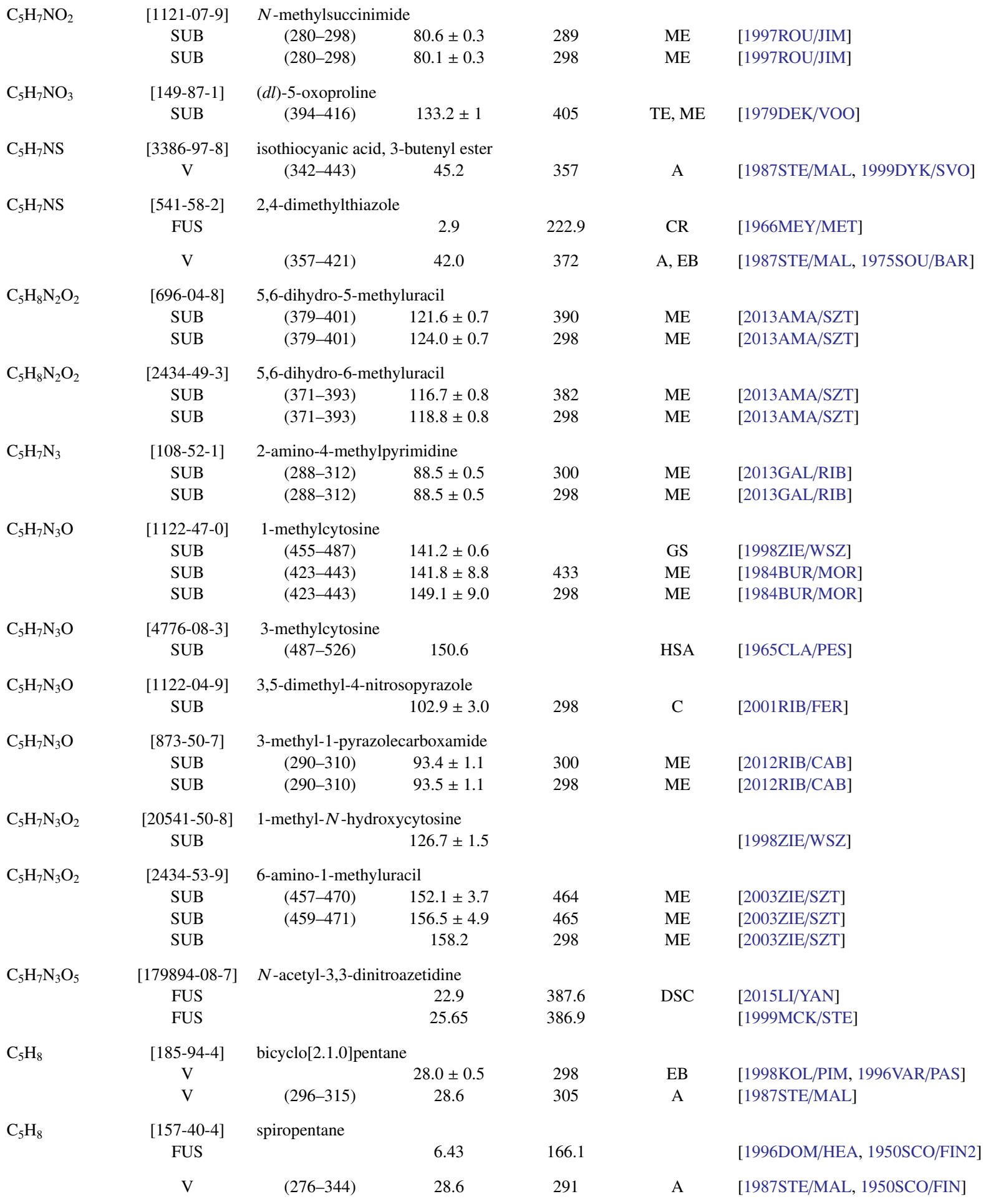


TABLE 8. Phase change enthalpies of $C_{5}$ organic compounds-Continued

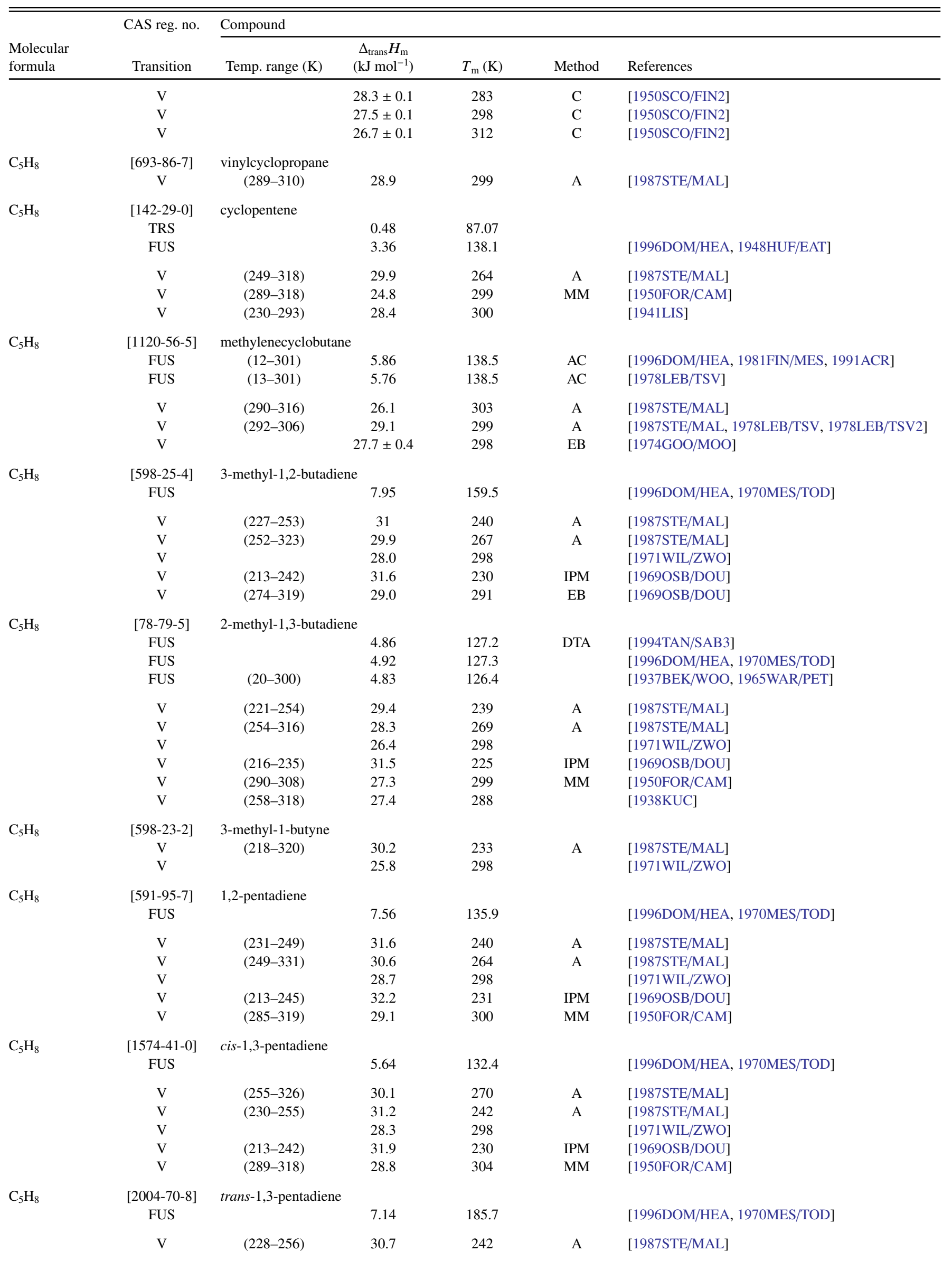


TABLE 8. Phase change enthalpies of $C_{5}$ organic compounds-Continued

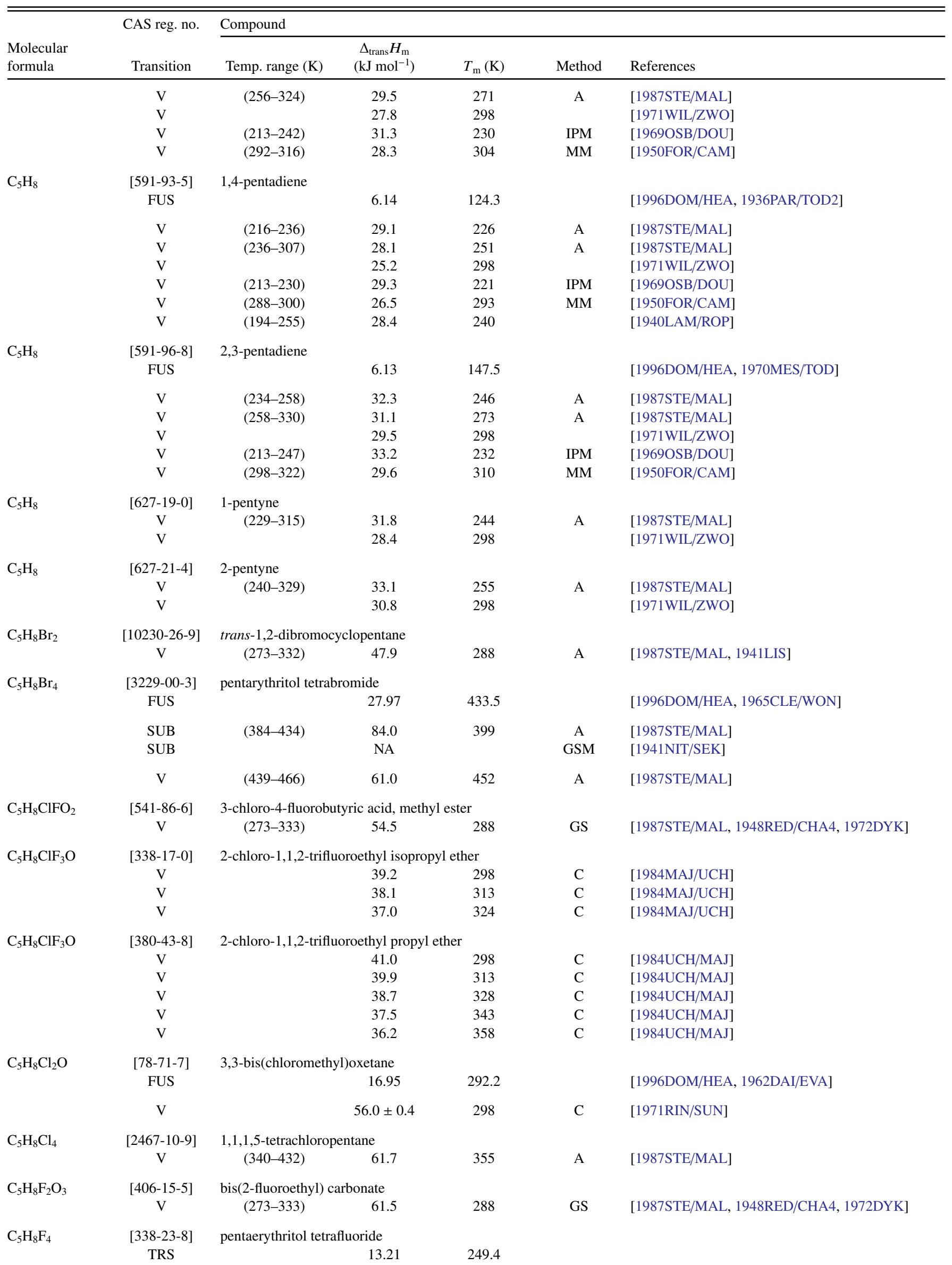


TABLE 8. Phase change enthalpies of $C_{5}$ organic compounds-Continued

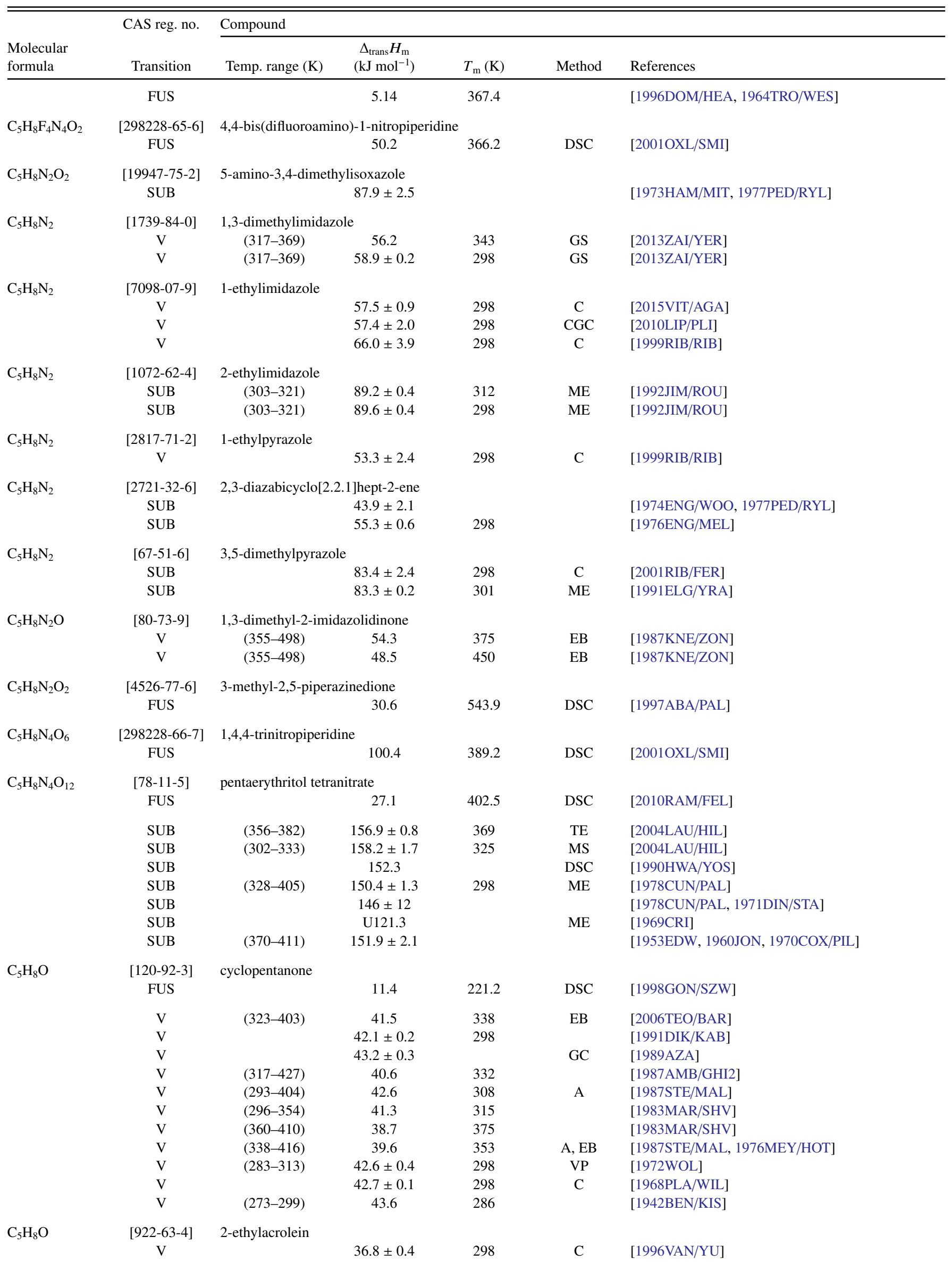


TABLE 8. Phase change enthalpies of $C_{5}$ organic compounds-Continued

\begin{tabular}{|c|c|c|c|c|c|c|}
\hline \multirow[b]{2}{*}{$\begin{array}{l}\text { Molecular } \\
\text { formula }\end{array}$} & \multirow{2}{*}{$\begin{array}{l}\text { CAS reg. no. } \\
\text { Transition }\end{array}$} & \multicolumn{5}{|l|}{ Compound } \\
\hline & & Temp. range $(\mathrm{K})$ & $\begin{array}{c}\Delta_{\text {trans }} H_{\mathrm{m}} \\
\left(\mathrm{kJ} \mathrm{mol}^{-1}\right)\end{array}$ & $T_{\mathrm{m}}(\mathrm{K})$ & Method & References \\
\hline \multirow[t]{5}{*}{$\mathrm{C}_{5} \mathrm{H}_{8} \mathrm{O}$} & {$[765-43-5]$} & \multicolumn{5}{|c|}{ cyclopropyl methyl ketone } \\
\hline & $\mathrm{V}$ & $(361-387)$ & 37.6 & 374 & A & [1987STE/MAL] \\
\hline & $\mathrm{V}$ & & 39.4 & & & [1984KOZ/TIM] \\
\hline & $\mathrm{V}$ & & $39.4 \pm 0.1$ & 298 & $\mathrm{C}$ & [1983FUC/HAL] \\
\hline & $\mathrm{V}$ & & $40.5 \pm 0.5$ & 298 & $\mathrm{C}$ & [1981GAT/STR] \\
\hline \multirow[t]{2}{*}{$\mathrm{C}_{5} \mathrm{H}_{8} \mathrm{O}$} & {$[25512-65-6]$} & \multicolumn{5}{|l|}{ dihydro- $2 \boldsymbol{H}$-pyran } \\
\hline & $\mathrm{V}$ & $(273-288)$ & 32.2 & 280 & A & [1987STE/MAL, 1972DYK, 1958CAS/FLE3] \\
\hline \multirow[t]{2}{*}{$\mathrm{C}_{5} \mathrm{H}_{8} \mathrm{O}$} & {$[497-03-0]$} & \multicolumn{5}{|c|}{ trans-2-methyl-2-butenal } \\
\hline & V & $(248-390)$ & 39.2 & 263 & A & [1987STE/MAL, 1947STU] \\
\hline \multirow[t]{2}{*}{$\mathrm{C}_{5} \mathrm{H}_{8} \mathrm{O}$} & {$[814-78-8]$} & \multicolumn{5}{|c|}{ 3-methyl-3-buten-2-one } \\
\hline & $\mathrm{V}$ & $(313-371)$ & 26.2 & 328 & A & [1987STE/MAL, 1972DYK] \\
\hline \multirow[t]{4}{*}{$\mathrm{C}_{5} \mathrm{H}_{8} \mathrm{O}$} & {$[115-19-5]$} & \multicolumn{5}{|c|}{ 2-methyl-3-butyn-2-ol } \\
\hline & $\mathrm{V}$ & $(333-377)$ & 41.0 & 353 & A & [1999ZAR/CHA] \\
\hline & $\mathrm{V}$ & $(294-380)$ & 43.9 & 337 & A & [1987STE/MAL, 1972DYK] \\
\hline & $\mathrm{V}$ & $(294-380)$ & 49.5 & 309 & & [1984BOU/FRI, 1950CON/ELV] \\
\hline \multirow[t]{2}{*}{$\mathrm{C}_{5} \mathrm{H}_{8} \mathrm{O}$} & {$[1629-58-9]$} & \multicolumn{5}{|l|}{ 1-penten-3-one } \\
\hline & $\mathrm{V}$ & $(303-376)$ & 36.7 & 318 & A & [1987STE/MAL] \\
\hline \multirow[t]{4}{*}{$\mathrm{C}_{5} \mathrm{H}_{8} \mathrm{OS}$} & {$[1072-72-6]$} & \multicolumn{5}{|c|}{ tetrahydro- $4 H$-thiopyran-4-one } \\
\hline & SUB & & $73.4 \pm 1.4$ & 298 & $\mathrm{C}$ & [2010FRE/GOM3] \\
\hline & SUB & & $71.7 \pm 1.7$ & 317 & I & [1972GEI/SAW] \\
\hline & SUB & & $72.6 \pm 1.7$ & 298 & & [1972GEI/SAW, 1977PED/RYL] \\
\hline \multirow[t]{3}{*}{$\mathrm{C}_{5} \mathrm{H}_{8} \mathrm{O}_{2}$} & [111-30-8] & \multicolumn{5}{|l|}{ glutaraldehyde } \\
\hline & V & $(347-382)$ & 51.4 & 362 & & [1998OLS] \\
\hline & $\mathrm{V}$ & $(327-436)$ & 56.2 & 342 & & [1998OLS] \\
\hline $\mathrm{C}_{5} \mathrm{H}_{8} \mathrm{O}_{2}$ & [2868-37-3] & methyl cyclopropan & carboxylate & & & \\
\hline & V & $(273-313)$ & $42.6 \pm 0.4$ & & GS & [1998VER/KUM] \\
\hline & $\mathrm{V}$ & & $41.3 \pm 0.1$ & 298 & $\mathrm{C}$ & [1983FUC/HAL] \\
\hline $\mathrm{C}_{5} \mathrm{H}_{8} \mathrm{O}_{2}$ & [123-54-6] & 2,4-pentanedione (a & etylacetone) & & & \\
\hline & $\mathrm{V}$ (diketone) & & $51.2 \pm 2.2$ & 298 & CGC & [2005TEM/ROU] \\
\hline & V (enol) & & $50.8 \pm 0.6$ & 298 & CGC & [2005TEM/ROU] \\
\hline & V & $(307-414)$ & 39.2 & 322 & $\mathrm{~EB}$ & [1985RAV/RAO] \\
\hline & $\mathrm{V}$ & $(295-313)$ & 40.6 & 304 & & [1981INO/ARA] \\
\hline & $\mathrm{V}$ & $(378-411)$ & 35.2 & 393 & $\mathrm{~A}, \mathrm{I}, \mathrm{EB}$ & [1987STE/MAL, 1972NAK/TOY] \\
\hline & $\mathrm{V}$ & $(288-378)$ & 42.7 & 303 & $\mathrm{~A}, \mathrm{~EB}$ & {$[1987 \mathrm{STE} / \mathrm{MAL}]$} \\
\hline & V (84\% enol) & & $41.8 \pm 0.2$ & 298 & $\mathrm{C}$ & [1970IRV/WAD] \\
\hline & V (100\% enol) & & 43.2 & 298 & $\mathrm{C}$ & [1970IRV/WAD] \\
\hline & $\mathrm{V}$ & $(297-398)$ & 39.4 & 347 & & [1969MEL/MER] \\
\hline $\mathrm{C}_{5} \mathrm{H}_{8} \mathrm{O}_{2}$ & {$[1522-20-9]$} & acetylacetone enol & & & & \\
\hline & FUS & $(80-300)$ & 14.5 & 254.8 & & [1969MEL/MER] \\
\hline $\mathrm{C}_{5} \mathrm{H}_{8} \mathrm{O}_{2}$ & {$[600-14-6]$} & 2,3-pentanedione & & & & \\
\hline & FUS & & 7.84 & 221.2 & DSC & [2006DOM/MOR] \\
\hline $\mathrm{C}_{5} \mathrm{H}_{8} \mathrm{O}_{2}$ & {$[565-63-9]$} & cis-2-methyl-2-bute & oic acid & & & \\
\hline & $\mathrm{V}$ & $(361-458)$ & 61.8 & 376 & A & [1987STE/MAL] \\
\hline $\mathrm{C}_{5} \mathrm{H}_{8} \mathrm{O}_{2}$ & {$[3586-58-1]$} & 2-ethylpropenoic ac & & & & \\
\hline & $\mathrm{V}$ & & $52.1 \pm 0.4$ & 298 & $\mathrm{C}$ & [1996VAN/YU] \\
\hline $\mathrm{C}_{5} \mathrm{H}_{8} \mathrm{O}_{2}$ & {$[3586-58-1]$} & 2-ethylacrylic acid & & & & \\
\hline & $\mathrm{V}$ & $(320-453)$ & 62.2 & 335 & A & [1987STE/MAL, 1947STU] \\
\hline $\mathrm{C}_{5} \mathrm{H}_{8} \mathrm{O}_{2}$ & {$[626-96-0]$} & 4-oxovaleraldehyde & levulinaldehyde) & & & \\
\hline & $\mathrm{V}$ & $(301-460)$ & 48.8 & 316 & A & [1987STE/MAL, 1947STU] \\
\hline $\mathrm{C}_{5} \mathrm{H}_{8} \mathrm{O}_{2}$ & {$[541-47-9]$} & 3-methylcrotonic ac & & & & \\
\hline & $\mathrm{V}$ & $(363-473)$ & 57.7 & 378 & A & [1987STE/MAL] \\
\hline $\mathrm{C}_{5} \mathrm{H}_{8} \mathrm{O}_{2}$ & $\begin{array}{c}{[140-88-5]} \\
\mathrm{V}\end{array}$ & $\begin{array}{c}\text { 2-ethyl acrylate } \\
(278-368)\end{array}$ & 39.5 & 298 & & [2013LOM/GIN] \\
\hline
\end{tabular}


TABLE 8. Phase change enthalpies of $C_{5}$ organic compounds-Continued

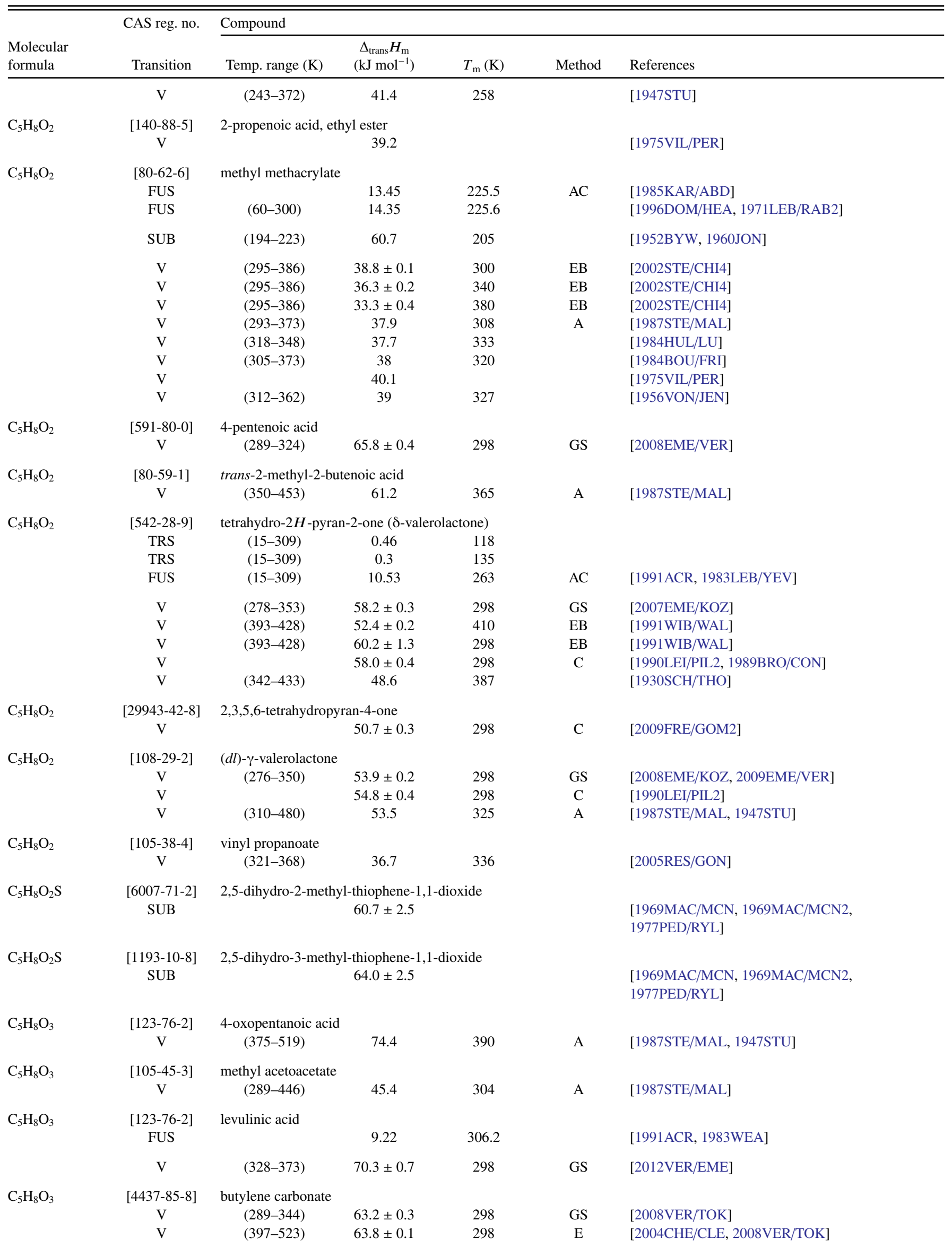

$\mathrm{C}_{5} \mathrm{H}_{8} \mathrm{O}_{4} \quad$ [108-59-8] dimethyl malonate 
TABLE 8. Phase change enthalpies of $C_{5}$ organic compounds-Continued

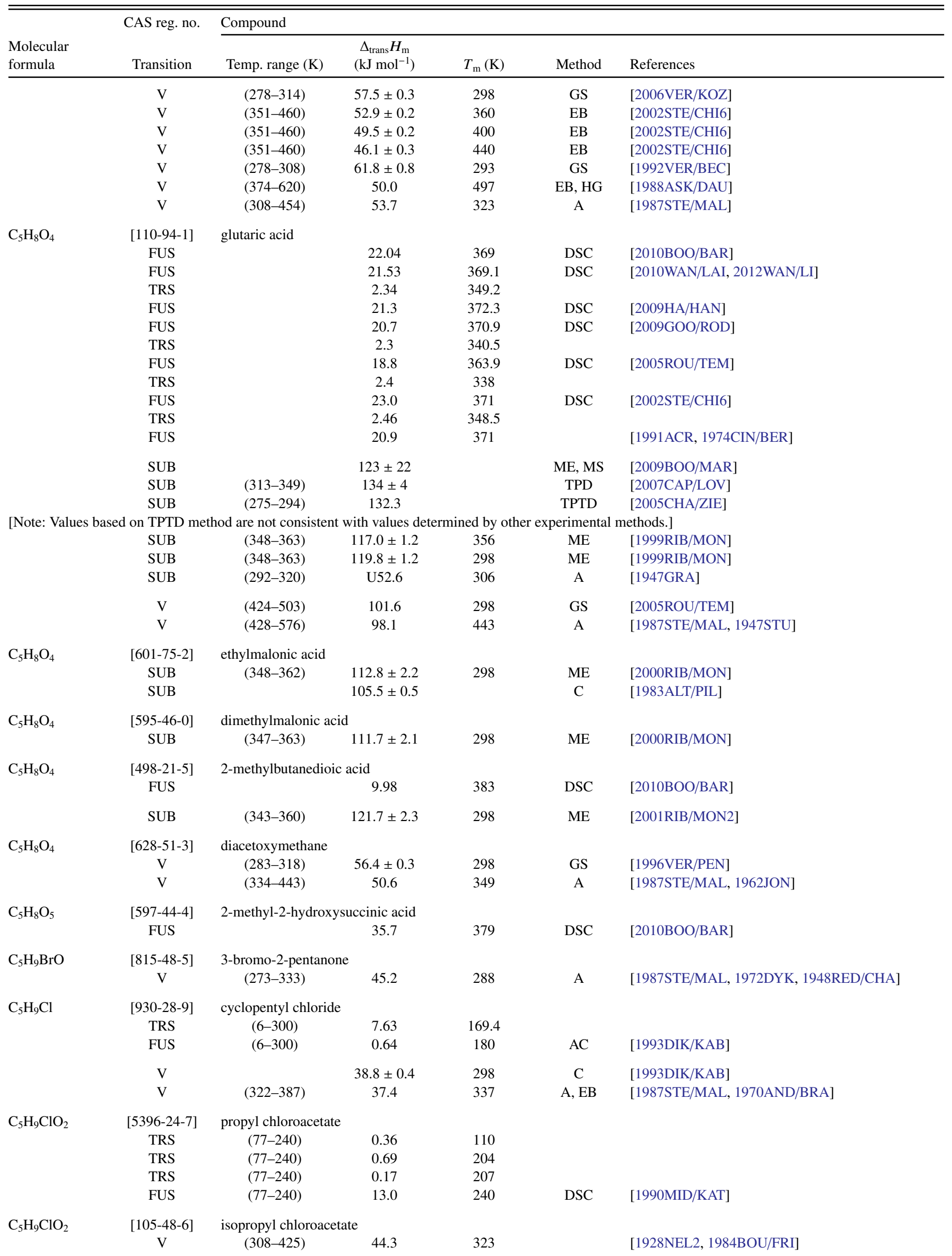


TABLE 8. Phase change enthalpies of $C_{5}$ organic compounds-Continued

\begin{tabular}{|c|c|c|c|c|c|c|}
\hline $\begin{array}{l}\text { Molecular } \\
\text { formula }\end{array}$ & $\begin{array}{l}\text { CAS reg. no. } \\
\text { Transition } \\
\end{array}$ & \multicolumn{5}{|l|}{ Compound } \\
\hline $\mathrm{C}_{5} \mathrm{H}_{9} \mathrm{ClO}_{2}$ & $\begin{array}{c}{[623-71-2]} \\
\mathrm{V}\end{array}$ & $\begin{array}{l}\text { 3-chloropropionic a } \\
\text { (316-358) }\end{array}$ & $\begin{array}{l}\text { id, ethyl ester } \\
56.0\end{array}$ & 331 & A & [1987STE/MAL] \\
\hline $\mathrm{C}_{5} \mathrm{H}_{9} \mathrm{Cl}_{3} \mathrm{O}$ & $\begin{array}{c}\text { [1067-09-0] } \\
\text { TRS } \\
\text { FUS }\end{array}$ & 2-chloromethyl-2-m & $\begin{array}{l}\text { thyl-1,3-dichl } \\
12 \\
2.5\end{array}$ & $\begin{array}{l}\text { opane } \\
246.6 \\
291.3\end{array}$ & DTA, DSC & [1996DOU/FUE] \\
\hline $\mathrm{C}_{5} \mathrm{H}_{9} \mathrm{FO}_{2}$ & $\begin{array}{c}{[406-20-2]} \\
\mathrm{V}\end{array}$ & $\begin{array}{l}\text { 4-fluorobutyric acid } \\
\text { (273-333) }\end{array}$ & $\begin{array}{c}\text { methyl ester } \\
\quad 47.3\end{array}$ & 288 & A, GS & [1987STE/MAL, 1948RED/CHA4, 1972DYK] \\
\hline $\mathrm{C}_{5} \mathrm{H}_{9} \mathrm{FO}_{2}$ & $\begin{array}{c}{[406-06-4]} \\
\mathrm{V}\end{array}$ & $\begin{array}{l}\text { isopropyl fluoroacet } \\
(273-333)\end{array}$ & 44.3 & 288 & A, GS & [1987STE/MAL, 1948RED/CHA4] \\
\hline $\mathrm{C}_{5} \mathrm{H}_{9} \mathrm{FO}_{3}$ & $\begin{array}{c}\text { [25309-12-0] } \\
\mathrm{V}\end{array}$ & $\begin{array}{l}\text { 3-fluoro-2-hydroxyl } \\
\text { (273-333) }\end{array}$ & $\begin{array}{l}\text { tryic acid, me } \\
\quad 62.3\end{array}$ & $\begin{array}{l}\text { ester } \\
288\end{array}$ & GS & [1987STE/MAL, 1948RED/CHA4, 1972DYK] \\
\hline $\mathrm{C}_{5} \mathrm{H}_{9} \mathrm{~N}$ & $\begin{array}{c}\text { [630-18-2] } \\
\text { TRS } \\
\text { TRS } \\
\text { FUS } \\
\text { V } \\
\text { V } \\
\text { V } \\
\text { V }\end{array}$ & $\begin{array}{c}\text { 2,2-dimethylpropan } \\
(5-350) \\
(5-350) \\
(5-350) \\
(299-365) \\
\\
(313-371) \\
(313-371)\end{array}$ & $\begin{array}{l}\text { nitrile (pivalor } \\
\quad 0.23 \\
1.91 \\
9.29 \\
37.0 \\
37.4 \pm 0.1 \\
36.5 \\
37.8\end{array}$ & $\begin{array}{c}213 \\
232.7 \\
292.1 \\
318 \\
298 \\
328 \\
298\end{array}$ & $\begin{array}{l}\mathrm{AC} \\
\mathrm{BG} \\
\mathrm{C} \\
\mathrm{A}, \mathrm{I} \\
\mathrm{I}\end{array}$ & $\begin{array}{l}\text { [1996DOM/HEA, 1967WES/RIB] } \\
\text { [1971HAL/BAL] } \\
\text { [1970HOW/WAD] } \\
\text { [1987STE/MAL, 1967WES/RIB] } \\
\text { [1967WES/RIB] }\end{array}$ \\
\hline $\mathrm{C}_{5} \mathrm{H}_{9} \mathrm{~N}$ & $\begin{array}{l}{[110-59-8]} \\
\text { V } \\
\text { V } \\
\text { V } \\
\text { V } \\
\text { V } \\
\text { V }\end{array}$ & $\begin{array}{l}\text { valeronitrile } \\
\qquad \begin{array}{r}(313-418) \\
(342-414) \\
(394-439)\end{array}\end{array}$ & $\begin{array}{c}42.3 \\
43.6 \pm 0.1 \\
44.3 \\
44.2 \\
44.1 \pm 0.2 \\
33.4\end{array}$ & $\begin{array}{l}328 \\
298 \\
298 \\
\\
298 \\
402\end{array}$ & $\begin{array}{l}\mathrm{A} \\
\mathrm{C} \\
\mathrm{EB} \\
\mathrm{MM} \\
\mathrm{C}\end{array}$ & $\begin{array}{l}\text { [1987STE/MAL] } \\
\text { [1970HOW/WAD] } \\
\text { [1969KON/PRO] } \\
\text { [1949DRE/SHR, 1949DRE/MAR, 2005EME/VER] } \\
\text { [1933HEI, 2005EME/VER] } \\
\text { [1901KAH] }\end{array}$ \\
\hline $\mathrm{C}_{5} \mathrm{H}_{9} \mathrm{NO}$ & $\begin{array}{l}\text { [872-50-4] } \\
\text { FUS } \\
\text { FUS }\end{array}$ & $N$-methyl-2-pyrroli & $\begin{array}{l}\text { one } \\
\begin{array}{c}11.04 \\
18.1\end{array}\end{array}$ & $\begin{array}{c}249 \\
248.5\end{array}$ & $\begin{array}{l}\text { DSC } \\
\text { DSC }\end{array}$ & $\begin{array}{l}{[2005 \mathrm{DOM} / \mathrm{LAC}]} \\
{[2000 \mathrm{LIS} / \mathrm{JAM}]}\end{array}$ \\
\hline & $\begin{array}{l}\mathrm{V} \\
\mathrm{V} \\
\mathrm{V} \\
\mathrm{V} \\
\mathrm{V} \\
\mathrm{V} \\
\mathrm{V} \\
\mathrm{V} \\
\mathrm{V} \\
\mathrm{V}\end{array}$ & $\begin{array}{l}(430-474) \\
(380-475) \\
(352-378) \\
(415-475) \\
(330-373) \\
(340-476) \\
(340-476) \\
(291-299) \\
(333-473) \\
(361-477)\end{array}$ & $\begin{array}{l}47.4 \\
49.5 \\
61.9 \\
49.1 \\
53.1 \\
53.4 \\
47.7 \\
55.3 \\
49.3 \\
49.2\end{array}$ & $\begin{array}{l}445 \\
395 \\
298 \\
430 \\
345 \\
350 \\
425 \\
295 \\
403 \\
376\end{array}$ & $\begin{array}{l}\text { EB } \\
\text { EB } \\
\text { EB } \\
\text { GS } \\
\text { EB } \\
\text { EB } \\
\text { A } \\
\\
\text { A }\end{array}$ & $\begin{array}{l}\text { [2012RED/KUM] } \\
\text { [2007PAL/ORA2] } \\
\text { [2004CHY/FRA2] } \\
\text { [1997BLA/BEL] } \\
{[1996 \mathrm{LIN} / \mathrm{WIC}]} \\
{[1987 \mathrm{KNE} / \mathrm{ZON}]} \\
{[1987 \mathrm{KNE} / \mathrm{ZON}]} \\
{[1987 \mathrm{STE} / \mathrm{MAL}]} \\
{[1979 \mathrm{BLU} / \mathrm{BAE}]} \\
{[1987 \mathrm{STE} / \mathrm{MAL}, 1972 \mathrm{DYK}, 1966 \mathrm{GAN} / \mathrm{SEM}]}\end{array}$ \\
\hline $\mathrm{C}_{5} \mathrm{H}_{9} \mathrm{NO}$ & $\begin{array}{c}{[111-36-4]} \\
\mathrm{V} \\
\mathrm{V}\end{array}$ & $\begin{array}{l}\text { butyl isocyanate } \\
(293-388) \\
(273-389)\end{array}$ & $\begin{array}{l}38.5 \\
46.8\end{array}$ & $\begin{array}{l}308 \\
288\end{array}$ & A & $\begin{array}{l}\text { [2004AHM/GIE] } \\
{[1987 \mathrm{STE} / \mathrm{MAL}, 1974 \mathrm{ZHU} / \mathrm{MON}]}\end{array}$ \\
\hline
\end{tabular}


TABLE 8. Phase change enthalpies of $C_{5}$ organic compounds-Continued

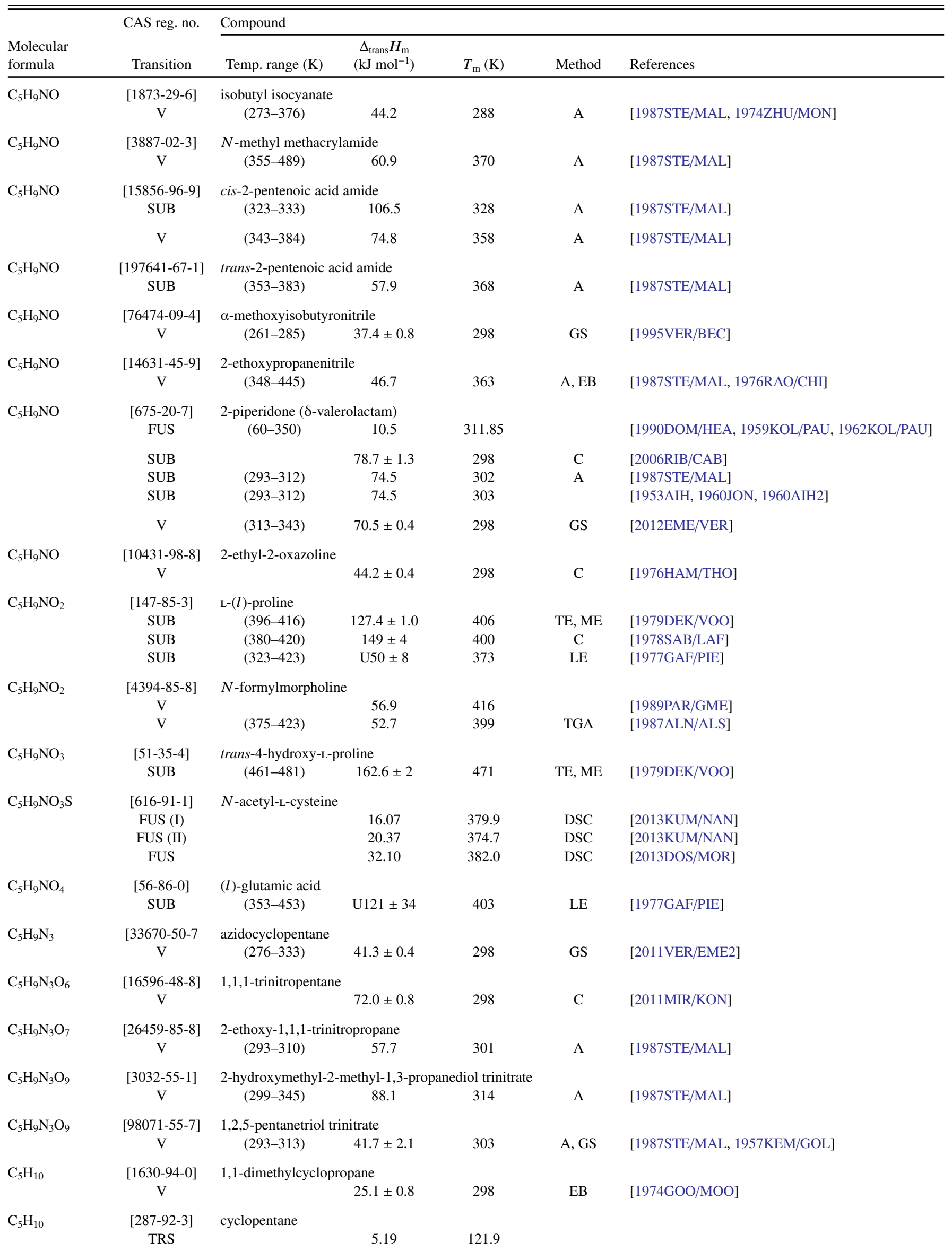


TABLE 8. Phase change enthalpies of $C_{5}$ organic compounds-Continued

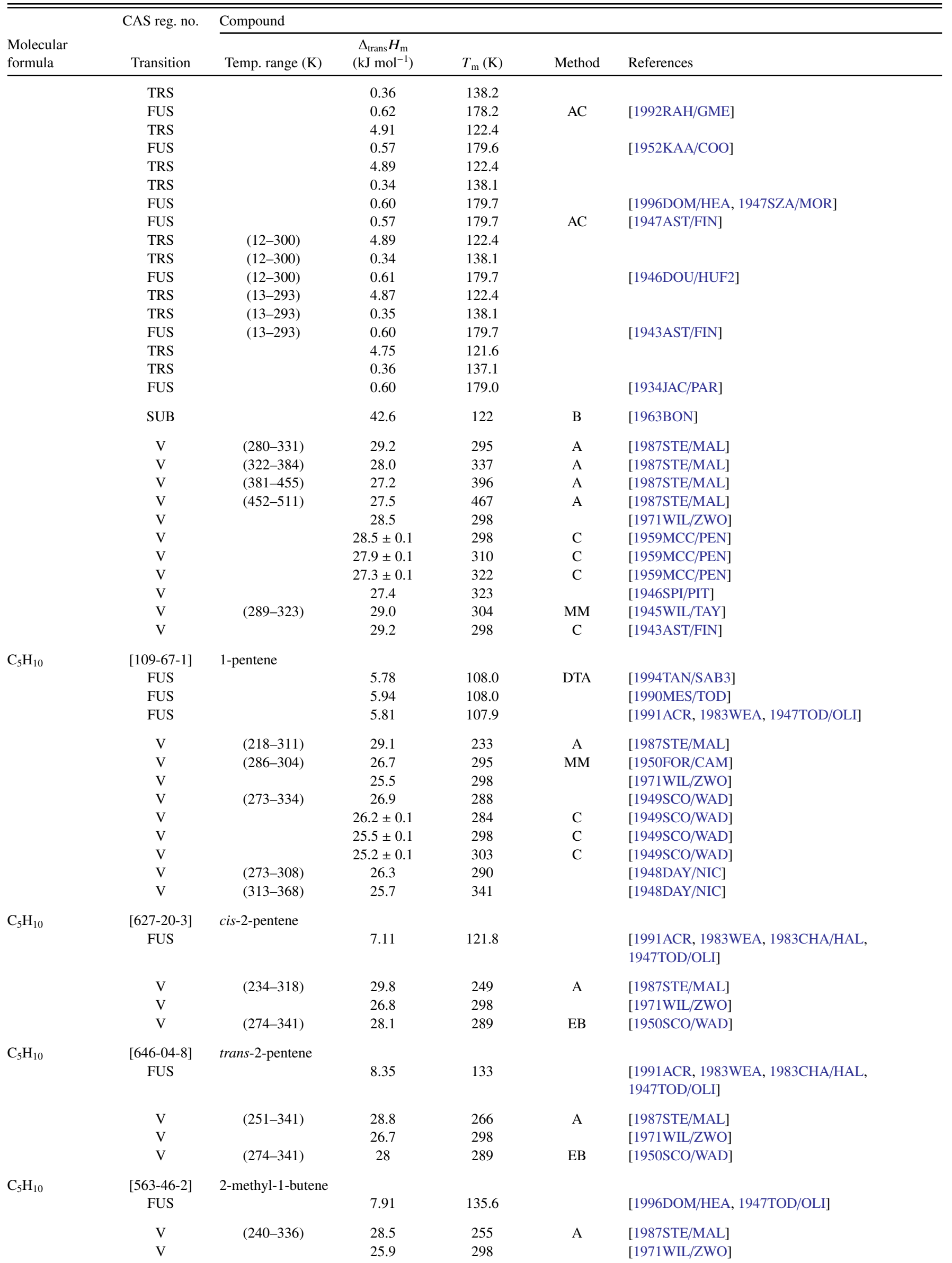


TABLE 8. Phase change enthalpies of $C_{5}$ organic compounds-Continued

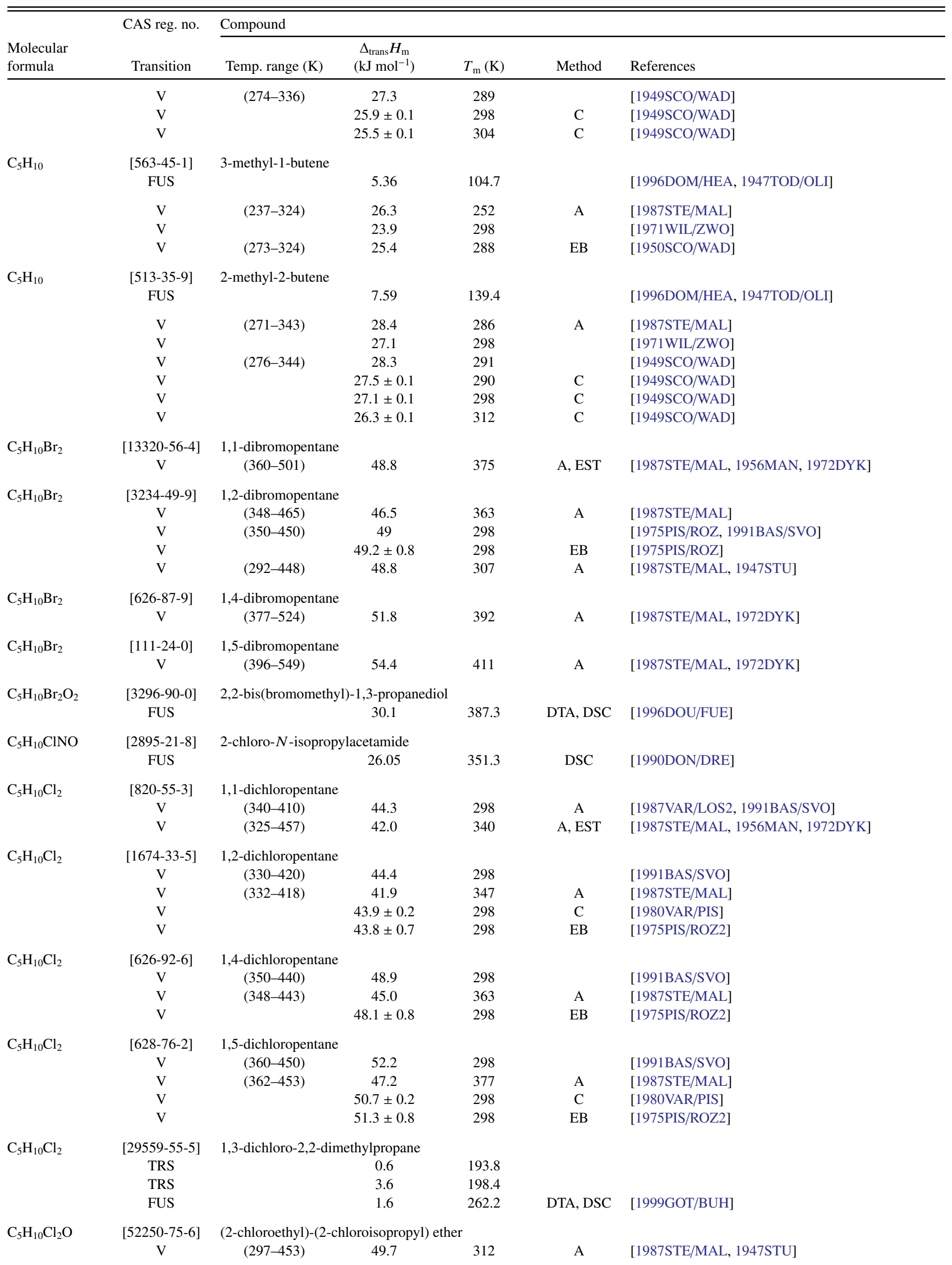


TABLE 8. Phase change enthalpies of $C_{5}$ organic compounds-Continued

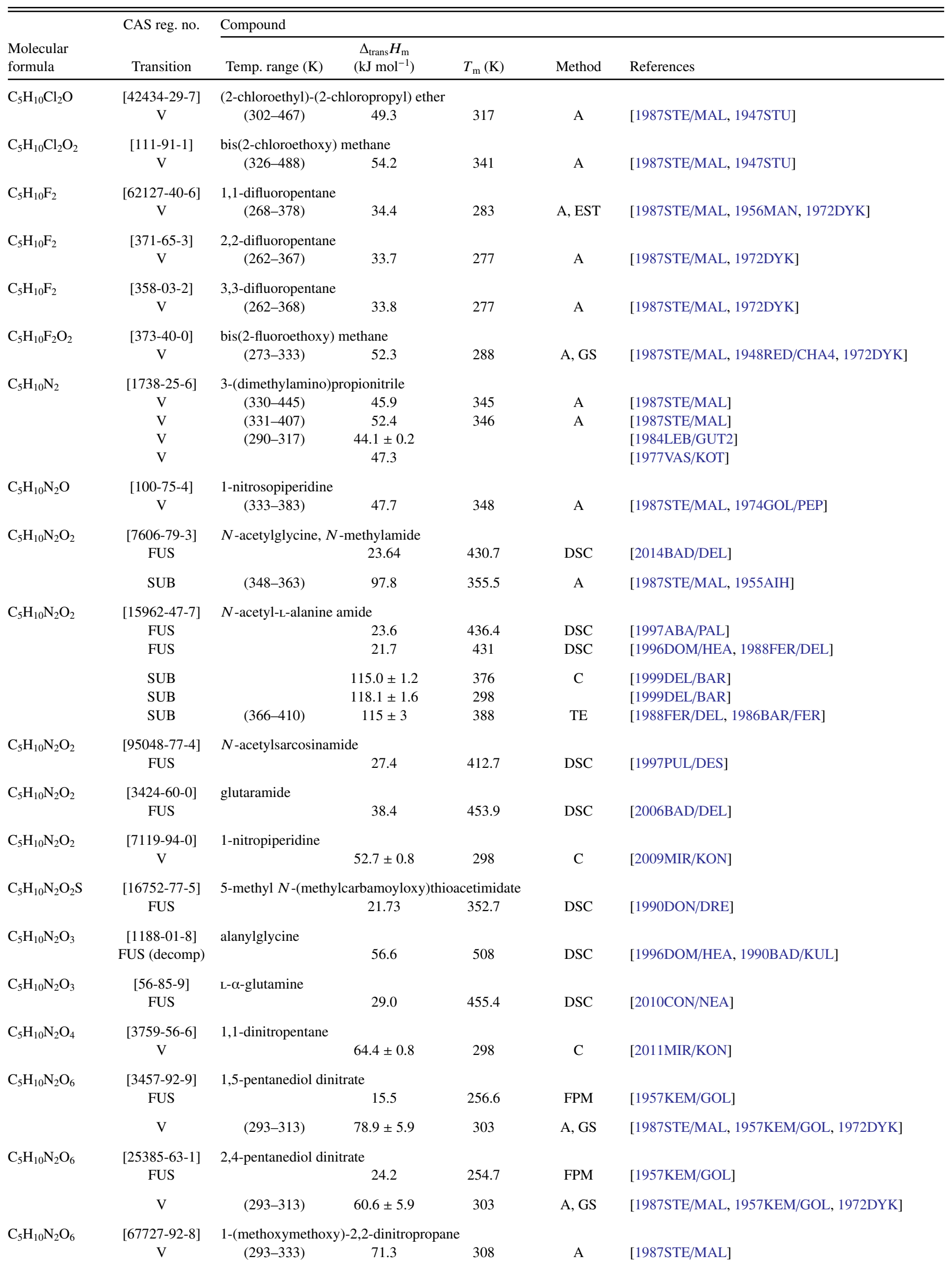


TABLE 8. Phase change enthalpies of $C_{5}$ organic compounds-Continued

\begin{tabular}{|c|c|c|c|c|c|c|}
\hline \multirow[b]{2}{*}{$\begin{array}{l}\text { Molecular } \\
\text { formula }\end{array}$} & \multirow{2}{*}{$\begin{array}{c}\text { CAS reg. no. } \\
\text { Transition }\end{array}$} & \multicolumn{5}{|l|}{ Compound } \\
\hline & & Temp. range $(\mathrm{K})$ & $\begin{array}{c}\Delta_{\text {trans }} H_{\mathrm{m}} \\
\left(\mathrm{kJ} \mathrm{mol}^{-1}\right)\end{array}$ & $T_{\mathrm{m}}(\mathrm{K})$ & Method & References \\
\hline \multirow[t]{3}{*}{$\mathrm{C}_{5} \mathrm{H}_{10} \mathrm{~N}_{4} \mathrm{O}_{4}$} & {$[5754-90-5]$} & \multicolumn{5}{|c|}{ 1,3-dinitro-1,3-diazacycloheptane } \\
\hline & TRS & & 21.8 & 369 & & \\
\hline & FUS & & 2.8 & 374 & & [1991PIC/RYL] \\
\hline \multirow[t]{2}{*}{$\mathrm{C}_{5} \mathrm{H}_{10} \mathrm{O}$} & {$[557-31-3]$} & \multicolumn{5}{|l|}{ allyl ethyl ether } \\
\hline & $\mathrm{V}$ & $(244-401)$ & 34.6 & 259 & A & [1987STE/MAL] \\
\hline \multirow[t]{4}{*}{$\mathrm{C}_{5} \mathrm{H}_{10} \mathrm{O}$} & [616-25-1] & \multicolumn{5}{|l|}{ 1-penten-3-ol } \\
\hline & V & & $49.9 \pm 0.1$ & 313 & $\mathrm{C}$ & [1996ULB/KLU] \\
\hline & $\mathrm{V}$ & & $48.4 \pm 0.1$ & 328 & $\mathrm{C}$ & [1996ULB/KLU] \\
\hline & $\mathrm{V}$ & & $46.8 \pm 0.1$ & 343 & $\mathrm{C}$ & [1996ULB/KLU] \\
\hline \multirow[t]{2}{*}{$\mathrm{C}_{5} \mathrm{H}_{10} \mathrm{O}$} & [556-82-1] & \multicolumn{5}{|c|}{ 3,3-dimethyl-2-propen-1-ol } \\
\hline & $\mathrm{V}$ & $(348-372)$ & 48.7 & 360 & $\mathrm{~EB}$ & [1989WAN/YIN] \\
\hline \multirow[t]{16}{*}{$\mathrm{C}_{5} \mathrm{H}_{10} \mathrm{O}$} & [115-18-4] & \multicolumn{5}{|c|}{ 2-methyl-3-buten-2-ol } \\
\hline & TRS & $(5-370)$ & 0.17 & 88.1 & & \\
\hline & FUS & $(5-370)$ & 5.20 & 245.0 & $\mathrm{AC}$ & [2015ZAI/PAU] \\
\hline & V & $(280-320)$ & $48.5 \pm 0.1$ & 298 & Static & [2015ZAI/PAU] \\
\hline & $\mathrm{V}$ & & $47.6 \pm 1.6$ & 298 & $\mathrm{C}$ & [2015ZAI/PAU] \\
\hline & $\mathrm{V}$ & $(322-370)$ & $42.2 \pm 0.4$ & 350 & EB & [2003LEI/LI, 2015ZAI/PAU] \\
\hline & $\mathrm{V}$ & $(322-370)$ & $47.8 \pm 0.5$ & 298 & $\mathrm{~EB}$ & [2003LEI/LI, 2015ZAI/PAU] \\
\hline & $\mathrm{V}$ & $(333-370)$ & $40.1 \pm 1.4$ & 352 & $\mathrm{~EB}$ & [1999ZAR/CHA2, 2015ZAI/PAU] \\
\hline & $\mathrm{V}$ & $(333-370)$ & $46.0 \pm 1.4$ & 298 & $\mathrm{~EB}$ & [1999ZAR/CHA2, 2015ZAI/PAU] \\
\hline & $\mathrm{V}$ & $(327-364)$ & $42.8 \pm 0.6$ & 346 & $\mathrm{~EB}$ & [1992RAA/BRO, 2015ZAI/PAU] \\
\hline & $\mathrm{V}$ & $(327-364)$ & $48.0 \pm 0.7$ & 298 & $\mathrm{~EB}$ & [1992RAA/BRO, 2015ZAI/PAU] \\
\hline & $\mathrm{V}$ & $(290-372)$ & $43.1 \pm 0.1$ & 331 & & [1988BAG/GUR] \\
\hline & $\mathrm{V}$ & $(345-390)$ & 40.6 & 369 & Static & [1986PAV/PES, 2015ZAI/PAU] \\
\hline & $\mathrm{V}$ & $(345-390)$ & 48.3 & 298 & Static & [1986PAV/PES, 2015ZAI/PAU] \\
\hline & $\mathrm{V}$ & $(358-379)$ & 41.6 & 369 & EB & [1974BLA/OGO, 2015ZAI/PAU] \\
\hline & $\mathrm{V}$ & $(358-379)$ & 49.3 & 298 & $\mathrm{~EB}$ & [1974BLA/OGO, 2015ZAI/PAU] \\
\hline \multirow[t]{2}{*}{$\mathrm{C}_{5} \mathrm{H}_{10} \mathrm{O}$} & {$[763-32-6]$} & \multicolumn{5}{|c|}{ 3-buten-3-methyl-1-ol } \\
\hline & V & $(338-409)$ & 55.6 & 353 & A & [1987STE/MAL] \\
\hline \multirow[t]{2}{*}{$\mathrm{C}_{5} \mathrm{H}_{10} \mathrm{O}$} & & \multicolumn{5}{|c|}{$(d l)$-3-buten-3-methyl-2-ol } \\
\hline & $\mathrm{V}$ & $(358-379)$ & 41.0 & 368 & A & {$[1987 \mathrm{STE} / \mathrm{MAL}]$} \\
\hline $\mathrm{C}_{5} \mathrm{H}_{10} \mathrm{O}$ & {$[96-41-3]$} & cyclopentanol & & & & \\
\hline & TRS & $(78-300)$ & 3.71 & 202.8 & & \\
\hline & FUS & $(78-300)$ & 1.54 & 257.4 & & [1996DOM/HEA, 1956PAR/KEN] \\
\hline & $\mathrm{V}$ & $(323-373)$ & 57.1 & 298 & $\mathrm{CGC}$ & {$[1995 \mathrm{CHI} / \mathrm{HOS}]$} \\
\hline & $\mathrm{V}$ & $(346-437)$ & 52.7 & 361 & $\mathrm{~A}, \mathrm{~EB}$ & [1987AMB/GHI2] \\
\hline & $\mathrm{V}$ & $(283-321)$ & 56.1 & 298 & A & [1987STE/MAL] \\
\hline & $\mathrm{V}$ & $(283-323)$ & 56.4 & 298 & A & [1987STE/MAL] \\
\hline & $\mathrm{V}$ & $(279-314)$ & 57.1 & 294 & & [1975CAB/CON2] \\
\hline & $\mathrm{V}$ & & $57.5 \pm 0.2$ & 298 & $\mathrm{C}$ & [1968PLA/WIL] \\
\hline & $\mathrm{V}$ & & $57.5 \pm 0.3$ & 298 & $\mathrm{C}$ & {$[1966 \mathrm{WAD}]$} \\
\hline $\mathrm{C}_{5} \mathrm{H}_{10} \mathrm{O}$ & {$[142-68-7]$} & tetrahydropyran & & & & \\
\hline & $\mathrm{V}$ & $(286-361)$ & 36 & 301 & & [2006ROD/GIN] \\
\hline & $\mathrm{V}$ & & $38.2 \pm 1.1$ & 298 & DSC & [2005ROJ/GIN] \\
\hline & $\mathrm{V}$ & $(335-412)$ & 33.2 & 350 & & [2000ROD/ART] \\
\hline & $\mathrm{V}$ & $(273-362)$ & 35.0 & 288 & A & [1987STE/MAL] \\
\hline & $\mathrm{V}$ & $(273-288)$ & 35.0 & 281 & & [1972DYK, 1958CAS/FLE] \\
\hline $\mathrm{C}_{5} \mathrm{H}_{10} \mathrm{O}$ & {$[96-47-9]$} & 2-methyltetrahydrc & & & & \\
\hline & $\mathrm{V}$ & $(283-353)$ & 34.0 & 298 & A & [1987STE/MAL] \\
\hline & $\mathrm{V}$ & & 33.7 & 298 & & {$[1970 \mathrm{MOI} / \mathrm{ANT}]$} \\
\hline $\mathrm{C}_{5} \mathrm{H}_{10} \mathrm{O}$ & {$[563-80-4]$} & 3-methyl-2-butano & & & & \\
\hline & FUS & & 9.34 & 180 & & [1996DOM/HEA, 1968AND/COU2] \\
\hline & $\mathrm{V}$ & $(311-369)$ & 35.5 & 326 & A & [1987STE/MAL] \\
\hline & $\mathrm{V}$ & $(363-415)$ & 33.8 & 378 & A & [1987STE/MAL] \\
\hline & $\mathrm{V}$ & $(405-500)$ & 32.6 & 420 & A & [1987STE/MAL] \\
\hline
\end{tabular}


TABLE 8. Phase change enthalpies of $C_{5}$ organic compounds-Continued

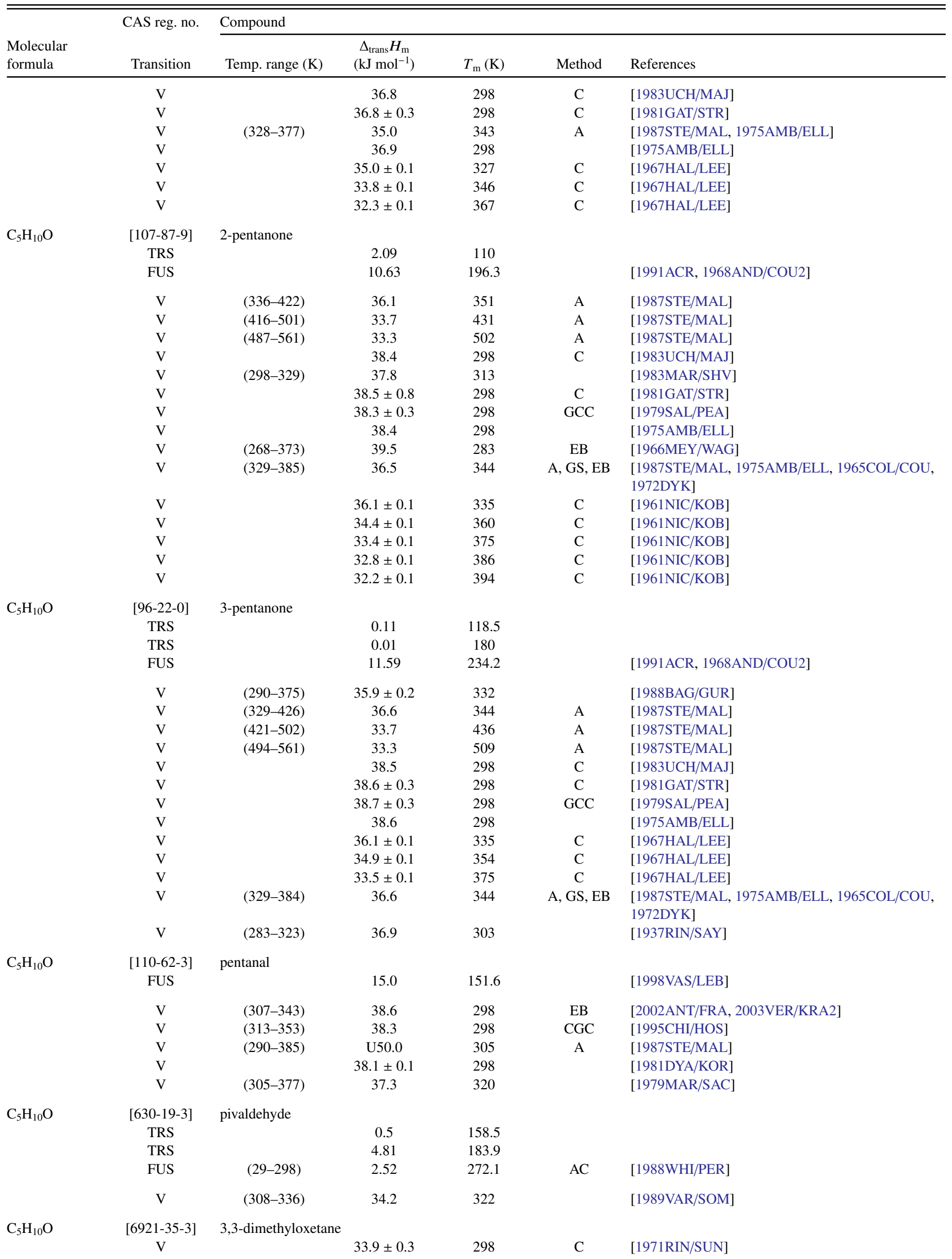


TABLE 8. Phase change enthalpies of $C_{5}$ organic compounds-Continued

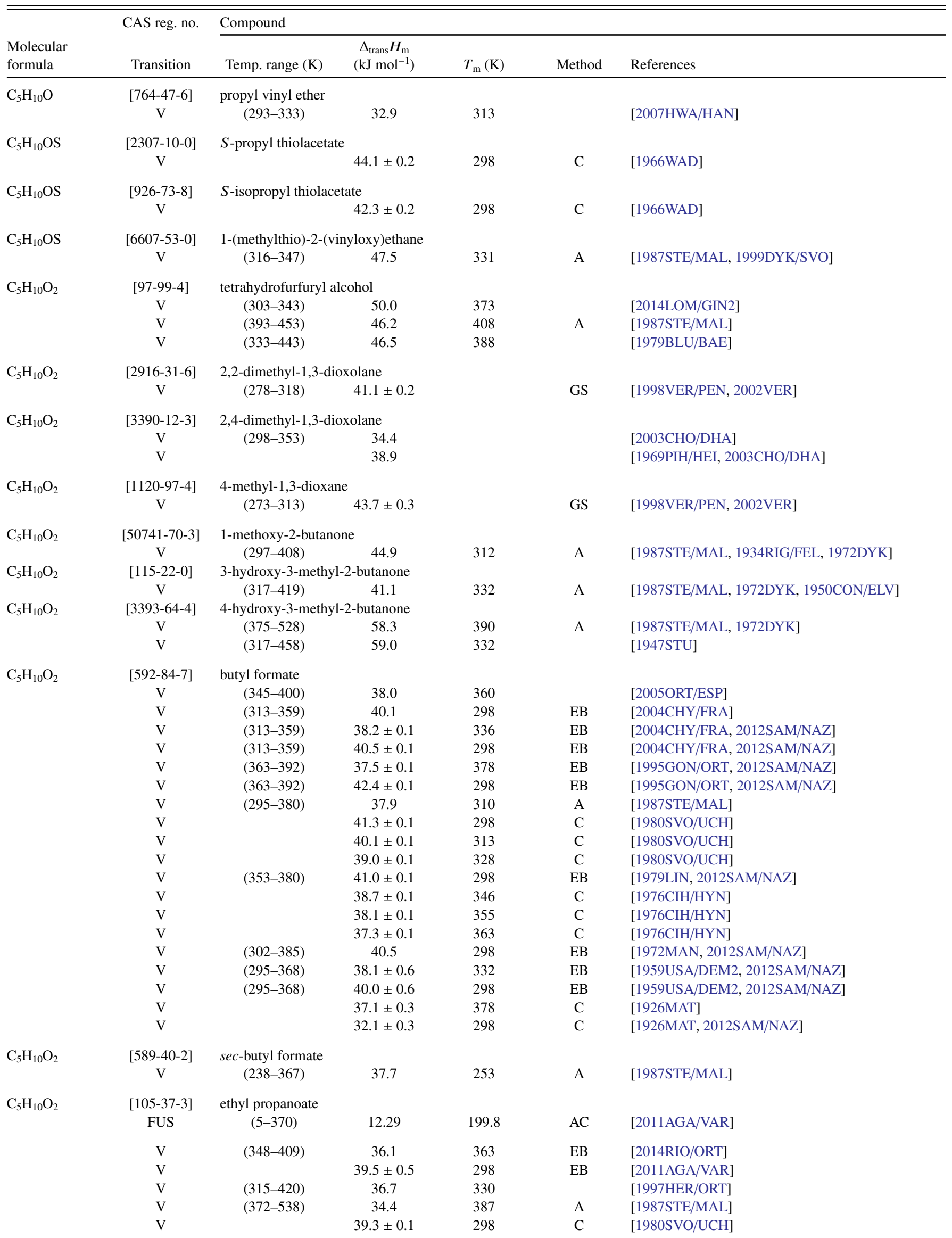


TABLE 8. Phase change enthalpies of $C_{5}$ organic compounds-Continued

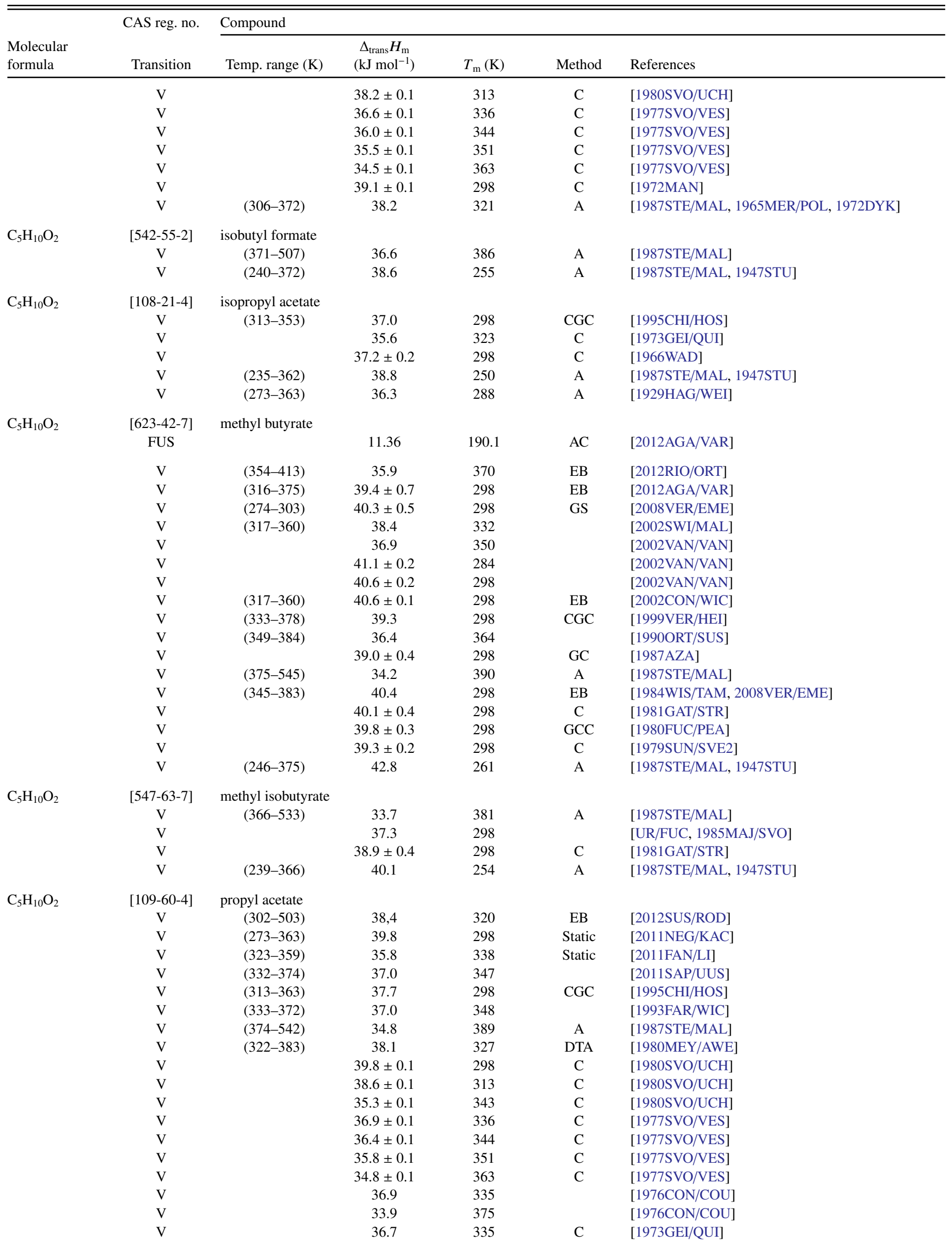


TABLE 8. Phase change enthalpies of $C_{5}$ organic compounds-Continued

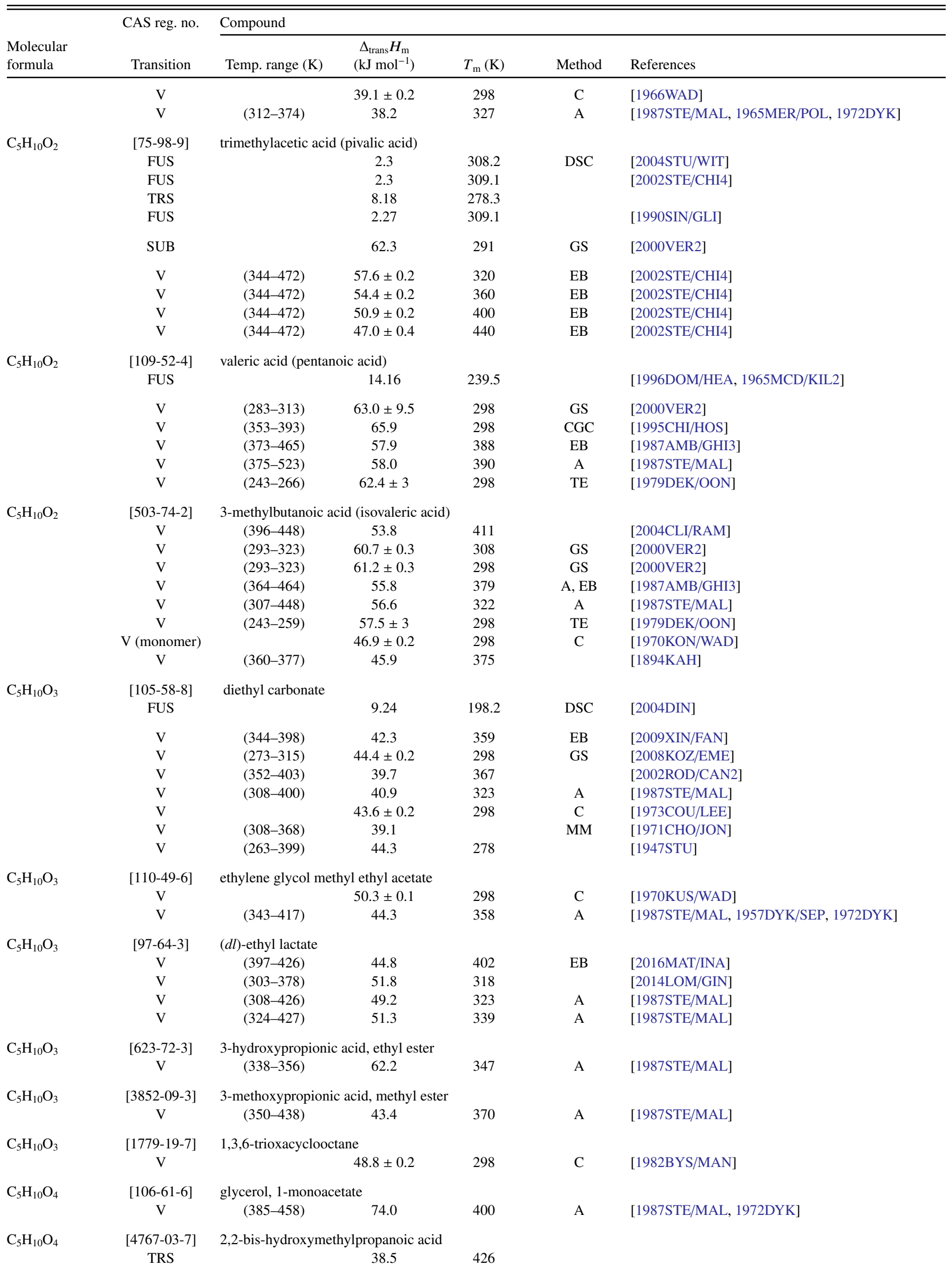


TABLE 8. Phase change enthalpies of $C_{5}$ organic compounds-Continued

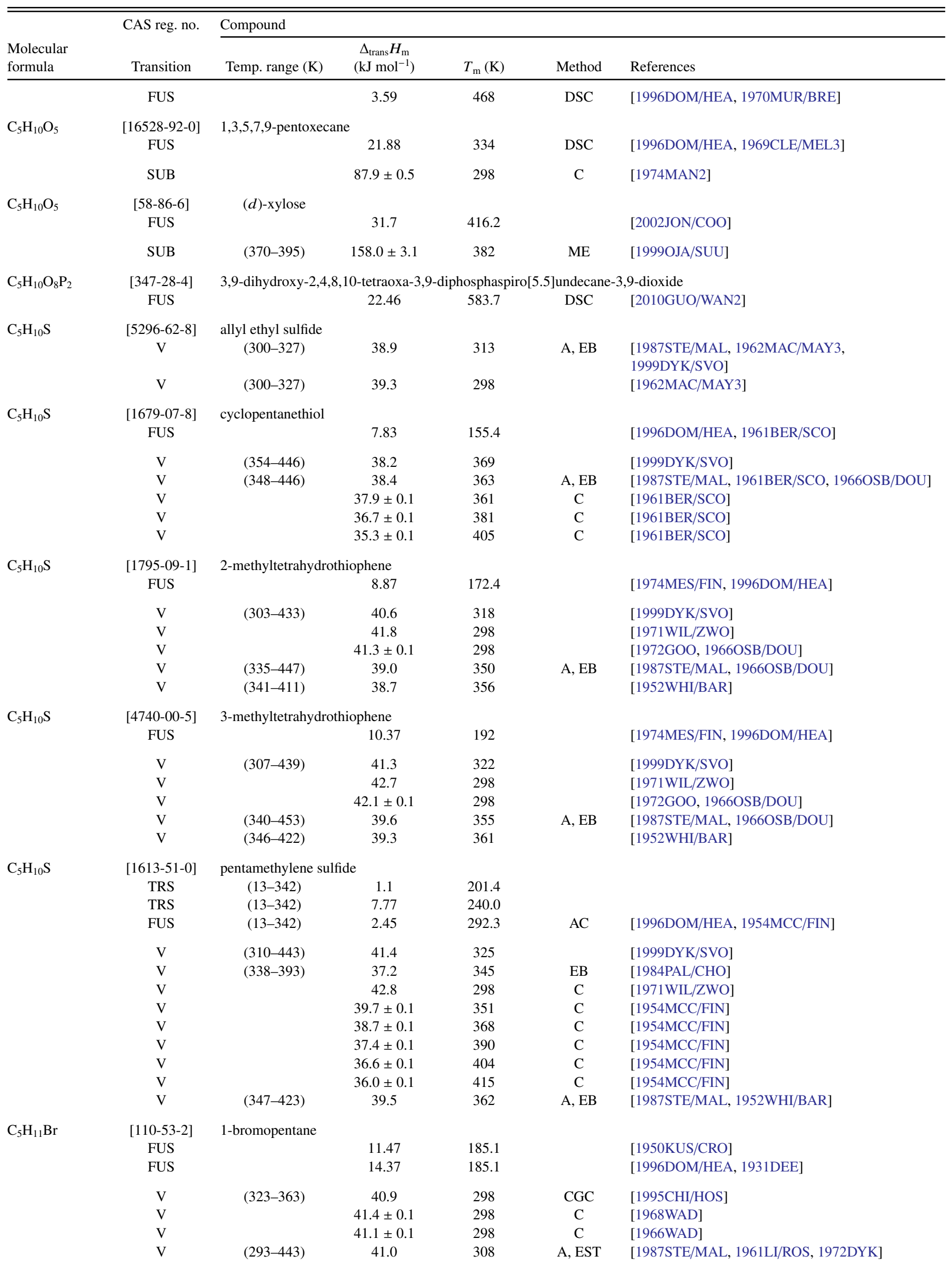


TABLE 8. Phase change enthalpies of $C_{5}$ organic compounds-Continued

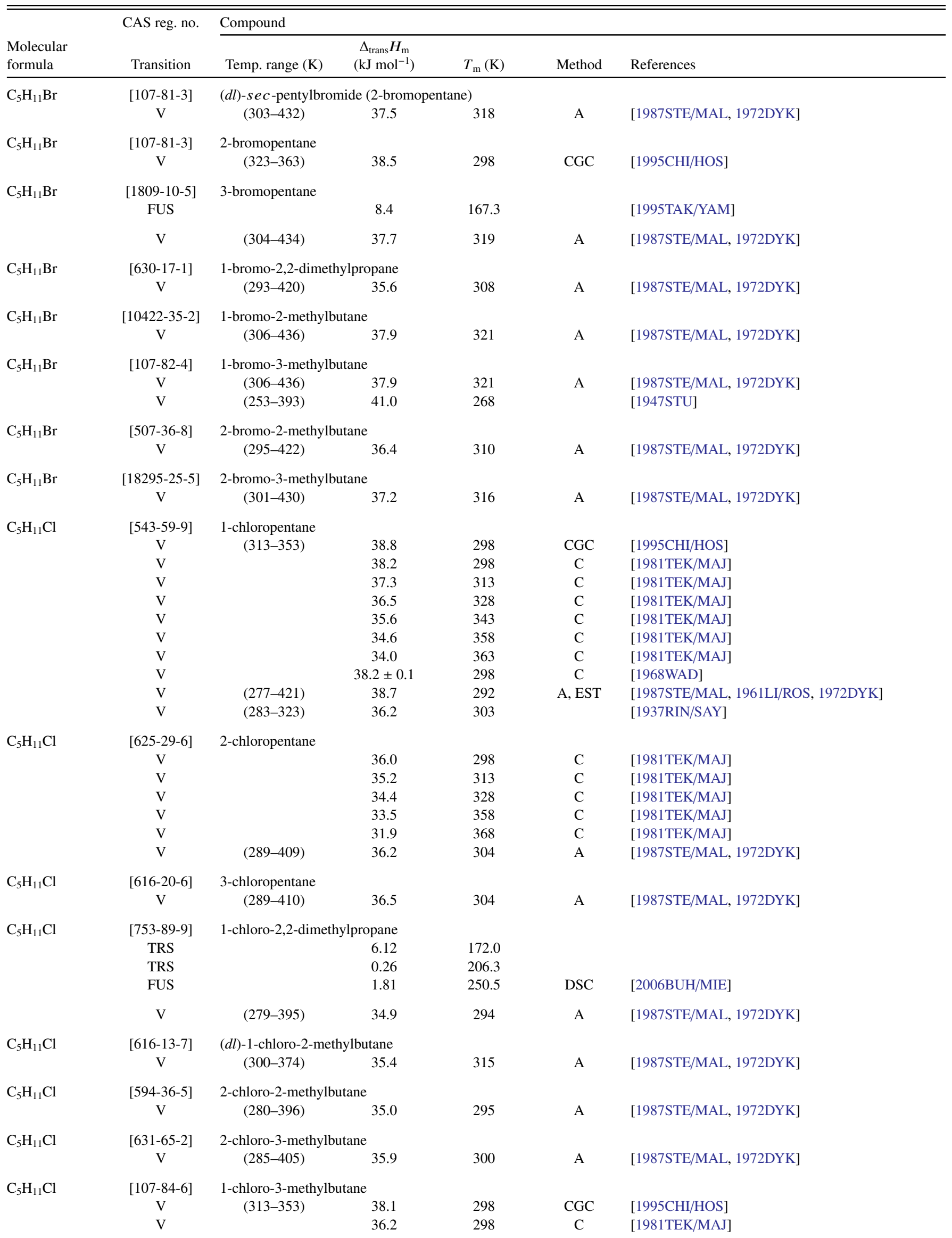


TABLE 8. Phase change enthalpies of $C_{5}$ organic compounds-Continued

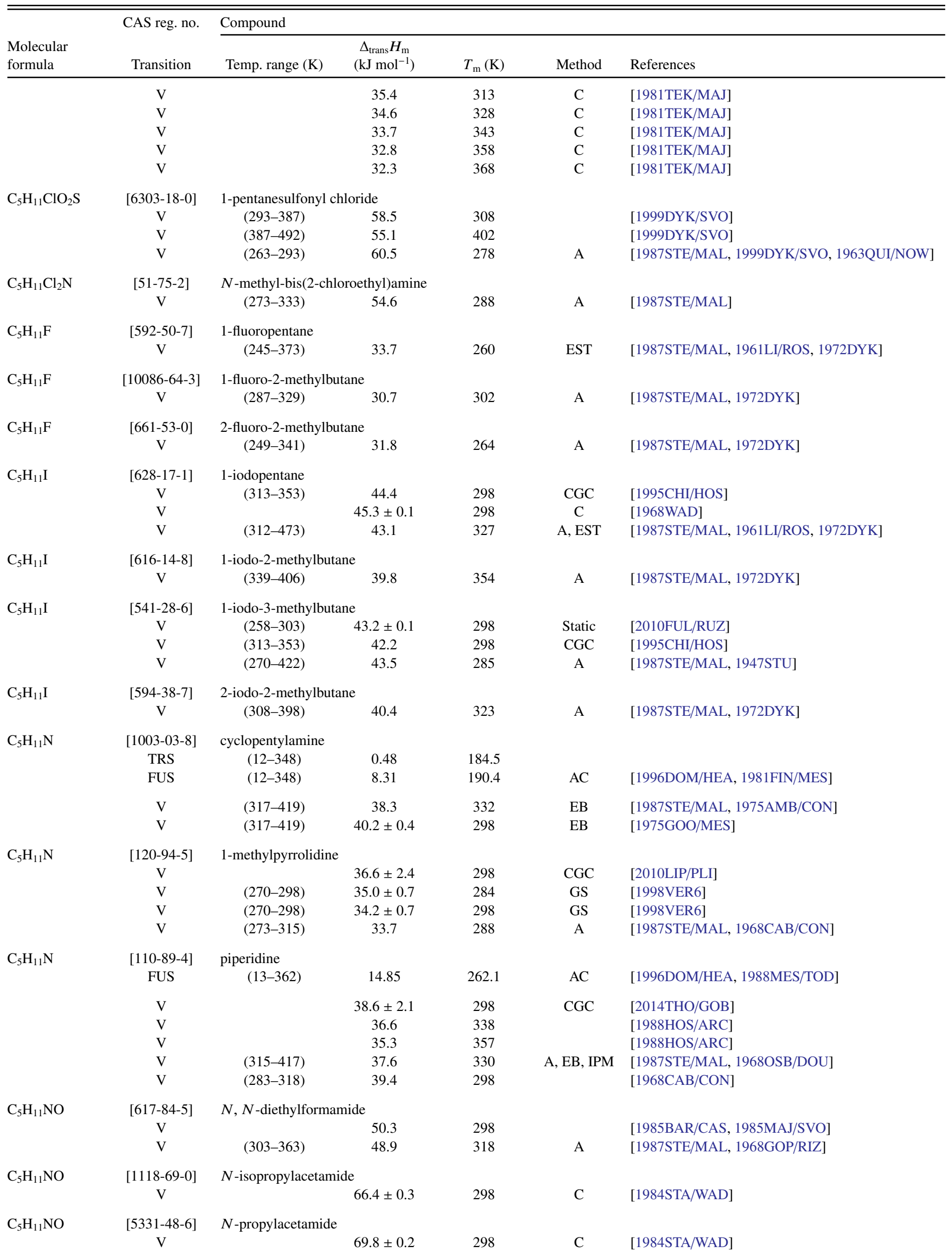


TABLE 8. Phase change enthalpies of $C_{5}$ organic compounds-Continued

\begin{tabular}{|c|c|c|c|c|c|c|}
\hline \multirow[b]{2}{*}{$\begin{array}{l}\text { Molecular } \\
\text { formula }\end{array}$} & \multirow{2}{*}{$\begin{array}{l}\text { CAS reg. no. } \\
\text { Transition }\end{array}$} & \multicolumn{5}{|l|}{ Compound } \\
\hline & & Temp. range $(\mathrm{K})$ & $\begin{array}{c}\Delta_{\text {trans }} H_{\mathrm{m}} \\
\left(\mathrm{kJ} \mathrm{mol}^{-1}\right)\end{array}$ & $T_{\mathrm{m}}(\mathrm{K})$ & Method & References \\
\hline \multirow[t]{7}{*}{$\mathrm{C}_{5} \mathrm{H}_{11} \mathrm{NO}$} & [626-97-1] & pentanamide & & & & \\
\hline & TRS & & 1.9 & 211.8 & & \\
\hline & TRS & & 1.2 & 365 & & \\
\hline & FUS & & 17.9 & 377.2 & DSC & {$[2008 \mathrm{ABA} / \mathrm{BAD}]$} \\
\hline & SUB & $(333-374)$ & $89.3 \pm 0.4$ & & GS & [1959DAV/JON2, 1970COX/PIL] \\
\hline & SUB & $(353-373)$ & 89.1 & & & {$[1960 \mathrm{JON}]$} \\
\hline & $\mathrm{V}$ & & $75.9 \pm 3.2$ & 298 & CGC & [2013GUT/RAT] \\
\hline \multirow[t]{4}{*}{$\mathrm{C}_{5} \mathrm{H}_{11} \mathrm{NO}$} & {$[754-10-9]$} & 2,2-dimethylpropan & mide & & & \\
\hline & FUS & & 24.1 & 425.4 & DSC & {$[2008 \mathrm{ABA} / \mathrm{BAD}]$} \\
\hline & SUB & $(298-359)$ & $89.0 \pm 2.0$ & 298 & $\mathrm{TE}$ & [2000BRU/DEL] \\
\hline & SUB & $(288-306)$ & $86.6 \pm 0.4$ & 298 & & {$[1989 \mathrm{ABB} / \mathrm{JIM}]$} \\
\hline \multirow[t]{3}{*}{$\mathrm{C}_{5} \mathrm{H}_{11} \mathrm{NO}$} & {$[758-96-3]$} & $N, N$-dimethylprop & onamide & & & \\
\hline & V & $(326-424)$ & 53.5 & 341 & A & [1987STE/MAL] \\
\hline & $\mathrm{V}$ & & 52.9 & & & [1977VAS/KOT] \\
\hline \multirow[t]{2}{*}{$\mathrm{C}_{5} \mathrm{H}_{11} \mathrm{NO}$} & {$[2675-88-9]$} & $N$-methyl-2-methyl & ropionamide & & & \\
\hline & $\mathrm{V}$ & & $67.1 \pm 0.2$ & 298 & $\mathrm{C}$ & [1984STA/WAD] \\
\hline \multirow{2}{*}{$\mathrm{C}_{5} \mathrm{H}_{11} \mathrm{NO}$} & [7148-06-3] & methyl 2- $(N, N-\operatorname{din}$ & ethylamino)p & oate & & \\
\hline & $\mathrm{V}$ & $(278-308)$ & $43.9 \pm 0.4$ & 293 & GS & [1992VER/BEC] \\
\hline \multirow[t]{12}{*}{$\mathrm{C}_{5} \mathrm{H}_{11} \mathrm{NO}$} & {$[109-02-4]$} & $N-$ & & & & \\
\hline & & methylmorpholine & & & & \\
\hline & $\mathrm{V}$ & $(273-353)$ & 39.5 & 298 & Static & [2009BEL/RAZ] \\
\hline & $\mathrm{V}$ & $(273-353)$ & 38.9 & 313 & Static & [2009RAZ/HAJ] \\
\hline & $\mathrm{V}$ & $(273-353)$ & 39.8 & 298 & Static & [2009RAZ/HAJ] \\
\hline & $\mathrm{V}$ & & $38.2 \pm 1.1$ & 298 & DSC & [2005ROJ/GIN] \\
\hline & $\mathrm{V}$ & $(274-304)$ & $40.2 \pm 0.3$ & 288 & GS & [1998VER2] \\
\hline & $\mathrm{V}$ & $(274-304)$ & $39.6 \pm 0.3$ & 298 & GS & [1998VER2] \\
\hline & $\mathrm{V}$ & $(323-363)$ & 33.6 & 343 & TGA & [1987ALN/ALS] \\
\hline & $\mathrm{V}$ & $(297-389)$ & 38.4 & 312 & A & [1987STE/MAL] \\
\hline & $\mathrm{V}$ & $(276-319)$ & 40.0 & 291 & $\mathrm{~A}$ & [1987STE/MAL, 1975CAB/CON] \\
\hline & $\mathrm{V}$ & $(276-319)$ & $39.4 \pm 0.1$ & 298 & & {$[1975 \mathrm{CAB} / \mathrm{CON}]$} \\
\hline \multirow[t]{2}{*}{$\mathrm{C}_{5} \mathrm{H}_{11} \mathrm{NO}$} & {$[15364-56-4]$} & 1-(dimethylamino)- & -propanone & & & \\
\hline & V & $(298-338)$ & $43.6 \pm 0.3$ & 298 & GS & [1994WEL/VER] \\
\hline \multirow[t]{2}{*}{$\mathrm{C}_{5} \mathrm{H}_{11} \mathrm{NO}$} & [1188-11-0] & 3-pentanone oxime & & & & \\
\hline & V & $(318-425)$ & 55.8 & 333 & A & [1987STE/MAL] \\
\hline \multirow[t]{3}{*}{$\mathrm{C}_{5} \mathrm{H}_{11} \mathrm{NO}_{2}$} & {$[628-05-7]$} & 1-nitropentane & & & & \\
\hline & $\mathrm{V}$ & & $51.0 \pm 0.4$ & 298 & $\mathrm{C}$ & [2011MIR/KON] \\
\hline & $\mathrm{V}$ & $(278-318)$ & $50.3 \pm 0.2$ & 298 & GS & [1997VER3] \\
\hline \multirow[t]{2}{*}{$\mathrm{C}_{5} \mathrm{H}_{11} \mathrm{NO}_{2}$} & {$[543-28-2]$} & isobutyl carbamate & & & & \\
\hline & V & $(356-479)$ & 58.8 & 371 & A & [1987STE/MAL, 1947STU] \\
\hline \multirow[t]{2}{*}{$\mathrm{C}_{5} \mathrm{H}_{11} \mathrm{NO}_{2}$} & {$[35123-06-9]$} & $N, N$-dimethyl lact & nide & & & \\
\hline & $\mathrm{V}$ & $(351-417)$ & 73.7 & 366 & A & [1987STE/MAL] \\
\hline \multirow[t]{5}{*}{$\mathrm{C}_{5} \mathrm{H}_{11} \mathrm{NO}_{2}$} & {$[760-78-1]$} & $(d l)$-2-aminopentan & c acid (DL-nc & ne) & & \\
\hline & TRS & & 0.36 & 156.3 & & \\
\hline & TRS & & 1.59 & 197.1 & DSC & [2010CHA/PAP] \\
\hline & SUB & $(439-461)$ & 120 & 450 & & [1987STE/MAL, 1965SVE/CLY] \\
\hline & SUB & $(439-461)$ & $121.1 \pm 0.4$ & 455 & $\mathrm{ME}$ & [1965SVE/CLY, 1964CLY/SVE] \\
\hline \multirow[t]{6}{*}{$\mathrm{C}_{5} \mathrm{H}_{11} \mathrm{NO}_{2}$} & [592-35-8] & butyl carbamate & 1715 & 3260 & 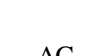 & [20147FN/YAN] \\
\hline & ros & $(299-453)$ & $1 / .45$ & 320.2 & AC & [20142EN/YAN] \\
\hline & SUB & & 92.9 & 298 & $\mathrm{~F}+\mathrm{V}$ & [2014ZEN/YAN] \\
\hline & SUB & $(292-316)$ & $94.1 \pm 8$ & & $\mathrm{ME}$ & [1959DAV/JON] \\
\hline & $\mathrm{V}$ & $(372-409)$ & 64.8 & 390 & & [2014ZEN/YAN] \\
\hline & $\mathrm{V}$ & $(372-409)$ & 76.6 & 298 & & [2014ZEN/YAN] \\
\hline
\end{tabular}


TABLE 8. Phase change enthalpies of $C_{5}$ organic compounds-Continued

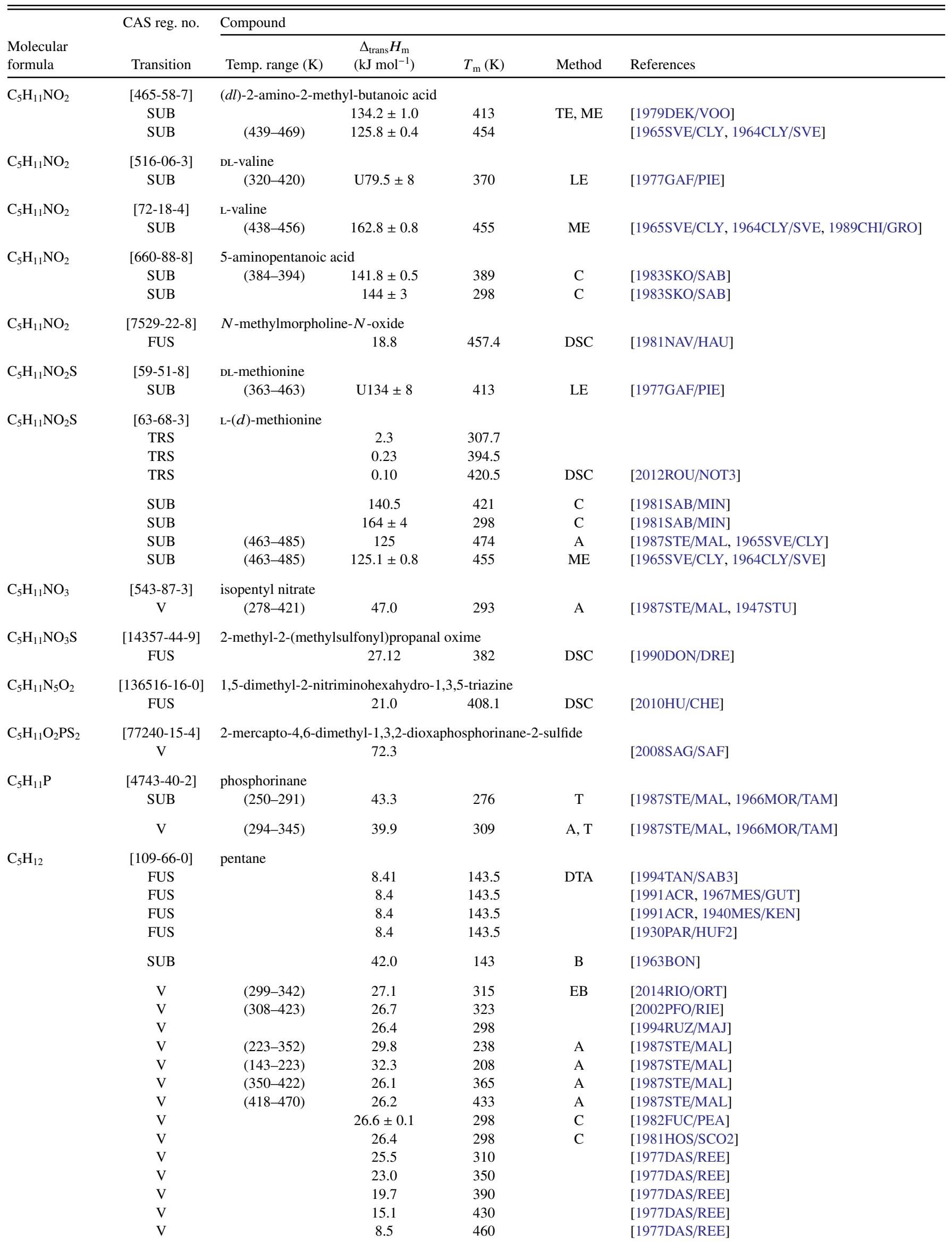


TABLE 8. Phase change enthalpies of $C_{5}$ organic compounds-Continued

\begin{tabular}{|c|c|c|c|c|c|c|}
\hline \multirow[b]{2}{*}{$\begin{array}{l}\text { Molecular } \\
\text { formula }\end{array}$} & \multirow{2}{*}{$\begin{array}{l}\text { CAS reg. no. } \\
\text { Transition }\end{array}$} & \multicolumn{5}{|l|}{ Compound } \\
\hline & & Temp. range $(\mathrm{K})$ & $\begin{array}{c}\Delta_{\text {trans }} H_{\mathrm{m}} \\
\left(\mathrm{kJ} \mathrm{mol}^{-1}\right)\end{array}$ & $T_{\mathrm{m}}(\mathrm{K})$ & Method & References \\
\hline & $\mathrm{V}$ & $(269-341)$ & 27.9 & 284 & EB & [1987STE/MAL, 1974OSB/DOU] \\
\hline & $\mathrm{V}$ & & 26.4 & 298 & & [1971WIL/ZWO] \\
\hline & $\mathrm{V}$ & & 26.4 & 298 & $\mathrm{C}$ & [1947OSB/GIN] \\
\hline \multirow[t]{2}{*}{$\mathrm{C}_{5} \mathrm{DH}_{11}$} & [55620-30-9] & 1-deuteropentane & & & & \\
\hline & $\mathrm{V}$ & $(223-303)$ & 26.2 & 298 & & [1975HOE/HOE] \\
\hline \multirow[t]{2}{*}{$\mathrm{C}_{5} \mathrm{DH}_{11}$} & [55620-31-0] & 3-deuteropentane & & & & \\
\hline & $\mathrm{V}$ & (213-294) & 26.3 & 298 & & [1975HOE/HOE] \\
\hline $\mathrm{C}_{5} \mathrm{D}_{12}$ & [2031-90-5] & pentane- $d_{12}$ & & & & \\
\hline \multirow{12}{*}{$\mathrm{C}_{5} \mathrm{H}_{12}$} & FUS & & 3.10 & 256.8 & & [1970CHA/WES, 1969ENO/SHI] \\
\hline & TRS & & 2.56 & 139.0 & & \\
\hline & FUS & & 3.24 & 253.8 & DSC & [1970SIL/RUD] \\
\hline & TRS & & 2.58 & 140 & & \\
\hline & FUS & & 3.26 & 256.5 & & [1996DOM/HEA, 1936AST/MES] \\
\hline & SUB & $(223-256)$ & 28.2 & 241 & A & [1987STE/MAL] \\
\hline & SUB & & 33.2 & & & [1933WHI/FLE, 1936AST/MES] \\
\hline & SUB & $(171-249)$ & 23.9 & 210 & A & [1947STU] \\
\hline & $\mathrm{V}$ & $(268-313)$ & 24.0 & 283 & A & [1987STE/MAL] \\
\hline & V & $(312-385)$ & 23.1 & 327 & A & [1987STE/MAL] \\
\hline & $\mathrm{V}$ & $(382-433)$ & 23.1 & 397 & A & [1987STE/MAL] \\
\hline & $\mathrm{V}$ & & $22.8 \pm 0.1$ & 283 & & [1936AST/MES] \\
\hline \multirow[t]{17}{*}{$\mathrm{C}_{5} \mathrm{H}_{12}$} & {$[78-78-4]$} & 2-methylbutane (is & entane) & & & \\
\hline & FUS & & 5.13 & 113.4 & & [1996DOM/HEA, 1942SCH/AST] \\
\hline & FUS & & 5.11 & 112.6 & & [1996DOM/HEA, 1930PAR/HUF] \\
\hline & $\mathrm{V}$ & $(255-323)$ & 26.9 & 270 & & [1991EWI/GOO] \\
\hline & $\mathrm{V}$ & $(216-323)$ & 28.5 & 231 & A & [1987STE/MAL] \\
\hline & $\mathrm{V}$ & $(300-460)$ & 25.2 & 315 & A & [1987STE/MAL] \\
\hline & $\mathrm{V}$ & $(320-391)$ & 25.2 & 335 & A & [1987STE/MAL] \\
\hline & $\mathrm{V}$ & $(385-416)$ & 24.8 & 400 & A & [1987STE/MAL] \\
\hline & $\mathrm{V}$ & $(412-460)$ & 25.3 & 427 & A & [1987STE/MAL] \\
\hline & $\mathrm{V}$ & & 24.4 & 310 & & [1977DAS/REE3] \\
\hline & $\mathrm{V}$ & & 21.5 & 350 & & [1977DAS/REE3] \\
\hline & $\mathrm{V}$ & & 18.0 & 390 & & [1977DAS/REE3] \\
\hline & $\mathrm{V}$ & & 12.9 & 430 & & [1977DAS/REE3] \\
\hline & $\mathrm{V}$ & & 24.8 & 298 & & [1971WIL/ZWO] \\
\hline & $\mathrm{V}$ & $(190-300)$ & 30.2 & 205 & & [1947STU] \\
\hline & $\mathrm{V}$ & $(289-301)$ & 26.2 & 295 & MM & [1945WIL/TAY] \\
\hline & $\mathrm{V}$ & & 25.0 & 298 & $\mathrm{C}$ & [1942SCH/AST] \\
\hline \multirow[t]{2}{*}{$\mathrm{C}_{5} \mathrm{H}_{12} \mathrm{ClF}_{3} \mathrm{~N}_{2} \mathrm{OS}$} & [63265-73-6] & chloro bis $(N$-meth & nethanaminto & trifluoror & sulfur & \\
\hline & $\mathrm{V}$ & & 40.2 & 477 & I & [1977KIT/SHR2] \\
\hline \multirow[t]{2}{*}{$\mathrm{C}_{5} \mathrm{H}_{12} \mathrm{ClF}_{3} \mathrm{~N}_{2} \mathrm{~S}$} & [63265-71-4] & chloro bis $(N$-meth & nethanaminat & fluoromet & ulfur & \\
\hline & $\mathrm{V}$ & & 38.1 & 368 & I & [1977KIT/SHR2] \\
\hline
\end{tabular}


TABLE 8. Phase change enthalpies of $C_{5}$ organic compounds-Continued

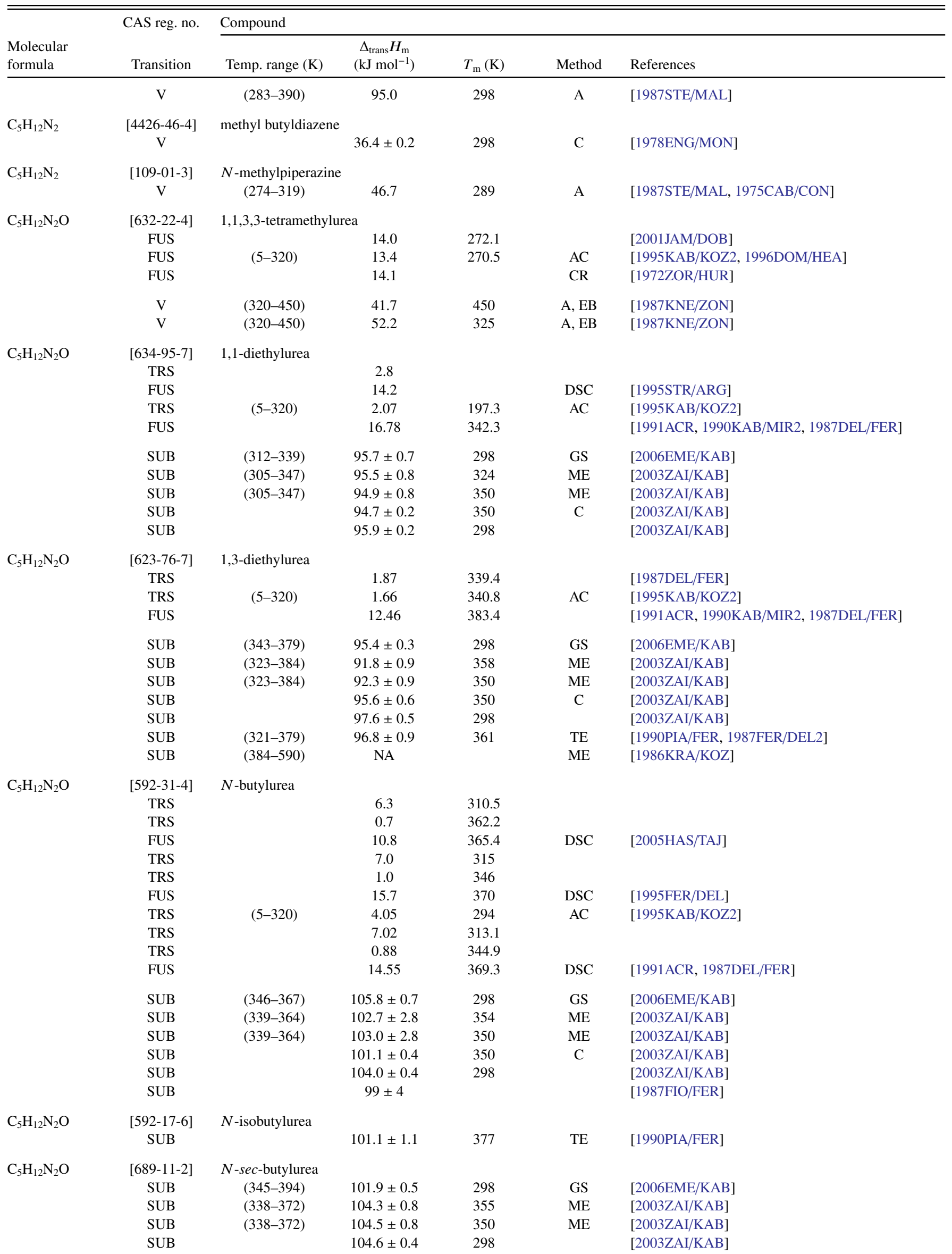


TABLE 8. Phase change enthalpies of $C_{5}$ organic compounds-Continued

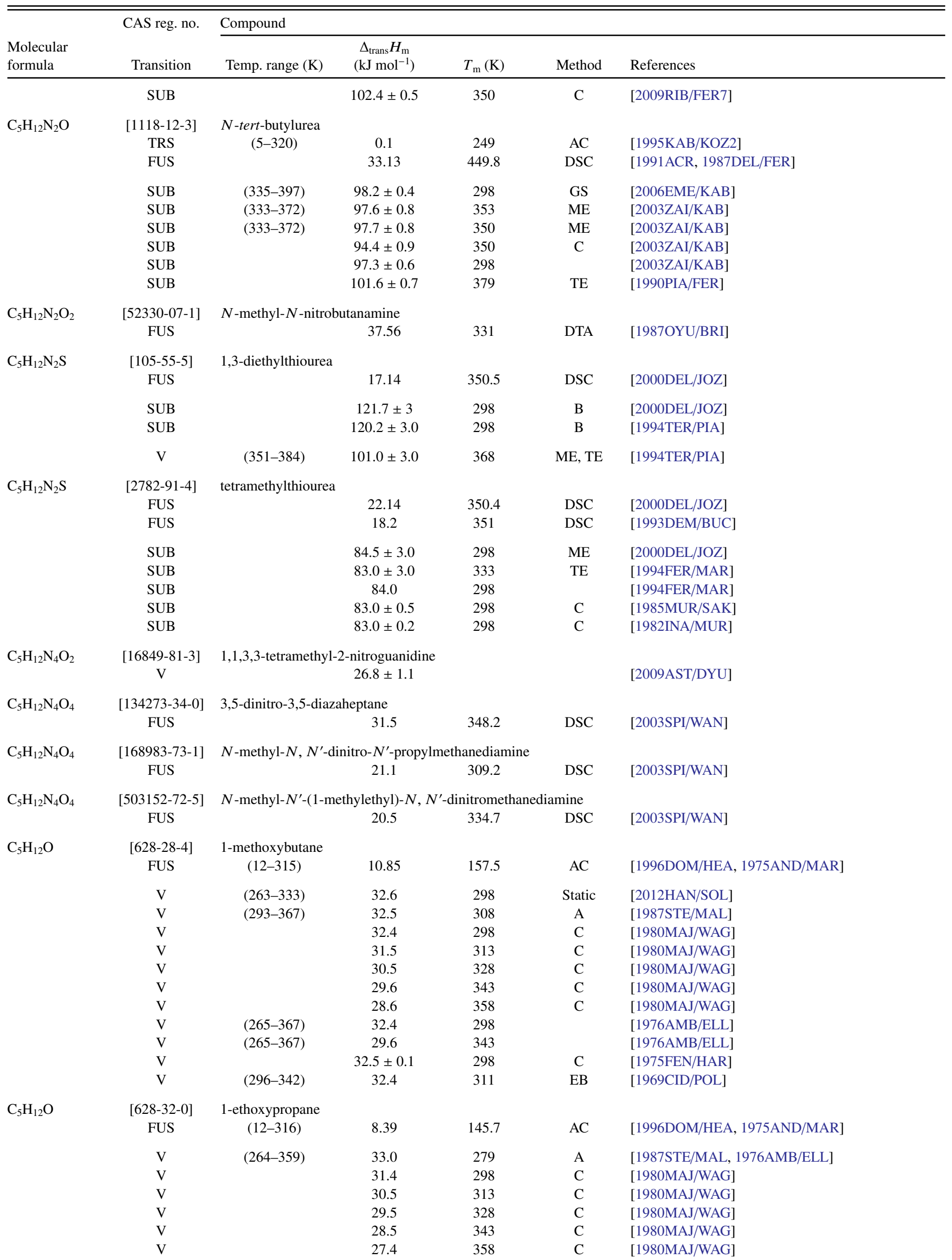


TABLE 8. Phase change enthalpies of $C_{5}$ organic compounds-Continued

\begin{tabular}{|c|c|c|c|c|c|c|}
\hline \multirow[b]{2}{*}{$\begin{array}{l}\text { Molecular } \\
\text { formula }\end{array}$} & \multirow{2}{*}{$\begin{array}{l}\text { CAS reg. no. } \\
\text { Transition }\end{array}$} & \multicolumn{5}{|l|}{ Compound } \\
\hline & & Temp. range $(\mathrm{K})$ & $\begin{array}{c}\Delta_{\text {trans }} H_{\mathrm{m}} \\
\left(\mathrm{kJ} \mathrm{mol}^{-1}\right)\end{array}$ & $T_{\mathrm{m}}(\mathrm{K})$ & Method & References \\
\hline & $\mathrm{V}$ & $(264-359)$ & 29.0 & 336 & & [1976AMB/ELL] \\
\hline & $\mathrm{V}$ & & $31.4 \pm 0.1$ & 298 & $\mathrm{C}$ & [1975FEN/HAR] \\
\hline & $\mathrm{V}$ & $(293-335)$ & 31.6 & 308 & & [1969CID/POL] \\
\hline & $\mathrm{V}$ & & 29.2 & 313 & $\mathrm{C}$ & [1980MAJ/WAG] \\
\hline & $\mathrm{V}$ & & 28.1 & 328 & $\mathrm{C}$ & [1980MAJ/WAG] \\
\hline & $\mathrm{V}$ & & 27.1 & 343 & $\mathrm{C}$ & [1980MAJ/WAG] \\
\hline \multirow[t]{13}{*}{$\mathrm{C}_{5} \mathrm{H}_{12} \mathrm{O}$} & [1634-04-4] & methyl tert-butyl ethe & & & & \\
\hline & FUS & $(12-308)$ & 7.6 & 164.6 & $\mathrm{AC}$ & [1996DOM/HEA, 1975AND/MAR] \\
\hline & $\mathrm{V}$ & $(298-322)$ & 30.0 & 310 & & [1995BEL/AIT] \\
\hline & $\mathrm{V}$ & $(300-411)$ & 31.2 & 315 & EB & [1994KRA/GME] \\
\hline & $\mathrm{V}$ & $(287-326)$ & 30.4 & 302 & & [1991WU/PIV] \\
\hline & $\mathrm{V}$ & & 29.8 & 298 & $\mathrm{C}$ & [1980MAJ/WAG] \\
\hline & $\mathrm{V}$ & & 28.9 & 313 & $\mathrm{C}$ & [1980MAJ/WAG] \\
\hline & $\mathrm{V}$ & & 28.0 & 328 & $\mathrm{C}$ & [1980MAJ/WAG] \\
\hline & $\mathrm{V}$ & & 27.0 & 343 & $\mathrm{C}$ & [1980MAJ/WAG] \\
\hline & $\mathrm{V}$ & $(287-351)$ & 30.2 & 302 & A & [1987STE/MAL, 1976AMB/ELL] \\
\hline & $\mathrm{V}$ & & 29.6 & 298 & & [1976AMB/ELL] \\
\hline & $\mathrm{V}$ & & 27.9 & 328 & & [1976AMB/ELL] \\
\hline & $\mathrm{V}$ & & $30.4 \pm 0.1$ & 298 & $\mathrm{C}$ & [1975FEN/HAR] \\
\hline \multirow{25}{*}{$\mathrm{C}_{5} \mathrm{H}_{12} \mathrm{O}$} & $\mathrm{V}$ & & 31.7 & 498 & & [2000WOR/JAM] \\
\hline & $\mathrm{V}$ & & 26.4 & 523 & & [2000WOR/JAM] \\
\hline & V & & 22.0 & 548 & & [2000WOR/JAM] \\
\hline & $\mathrm{V}$ & & 14.1 & 573 & & [2000WOR/JAM] \\
\hline & $\mathrm{V}$ & & 7.1 & 586 & & [2000WOR/JAM] \\
\hline & $\mathrm{V}$ & & 43.5 & & & [1999FAT] \\
\hline & $\mathrm{V}$ & $(323-373)$ & 57.8 & 298 & CGC & [1995CHI/HOS] \\
\hline & V & $(323-373)$ & 57.4 & 298 & CGC & [1995CHI/HOS] \\
\hline & $\mathrm{V}$ & $(335-410)$ & 51.5 & 350 & & [1994AUC/BUR] \\
\hline & V & $(388-420)$ & 47.2 & 403 & A & [1987STE/MAL] \\
\hline & V & $(326-411)$ & 54.3 & 341 & A & [1987STE/MAL] \\
\hline & $\mathrm{V}$ & $(408-441)$ & 45.4 & 423 & $\mathrm{~A}$ & [1987STE/MAL] \\
\hline & $\mathrm{V}$ & & $55.7 \pm 0.2$ & 313 & $\mathrm{C}$ & [1985MAJ/SVO2] \\
\hline & $\mathrm{V}$ & & $54.4 \pm 0.2$ & 328 & $\mathrm{C}$ & [1985MAJ/SVO2] \\
\hline & $\mathrm{V}$ & & $53.0 \pm 0.2$ & 343 & $\mathrm{C}$ & [1985MAJ/SVO2] \\
\hline & V & & $51.2 \pm 0.2$ & 358 & $\mathrm{C}$ & [1985MAJ/SVO2] \\
\hline & $\mathrm{V}$ & $(343-403)$ & 55.4 & 298 & & [1983SCH/STR] \\
\hline & $\mathrm{V}$ & $(310-411)$ & 55.0 & 325 & & [1973WIL/ZWO] \\
\hline & V & & $50.5 \pm 0.1$ & 362 & $\mathrm{C}$ & [1970COU/FEN] \\
\hline & $\mathrm{V}$ & & $49.2 \pm 0.1$ & 374 & $\mathrm{C}$ & [1970COU/FEN] \\
\hline & $\mathrm{V}$ & & $47.0 \pm 0.1$ & 392 & $\mathrm{C}$ & [1970COU/FEN] \\
\hline & $\mathrm{V}$ & & $44.4 \pm 0.1$ & 411 & $\mathrm{C}$ & [1970COU/FEN] \\
\hline & $\mathrm{V}$ & $(347-429)$ & 51.6 & 362 & EB & [1987STE/MAL, 1970AMB/SPR] \\
\hline & $\mathrm{V}$ & $(307-411)$ & 56.2 & 322 & DTA & [1969KEM/KRE] \\
\hline & $\mathrm{V}$ & & $56.9 \pm 0.2$ & 298 & $\mathrm{C}$ & [1966WAD] \\
\hline
\end{tabular}


TABLE 8. Phase change enthalpies of $C_{5}$ organic compounds-Continued

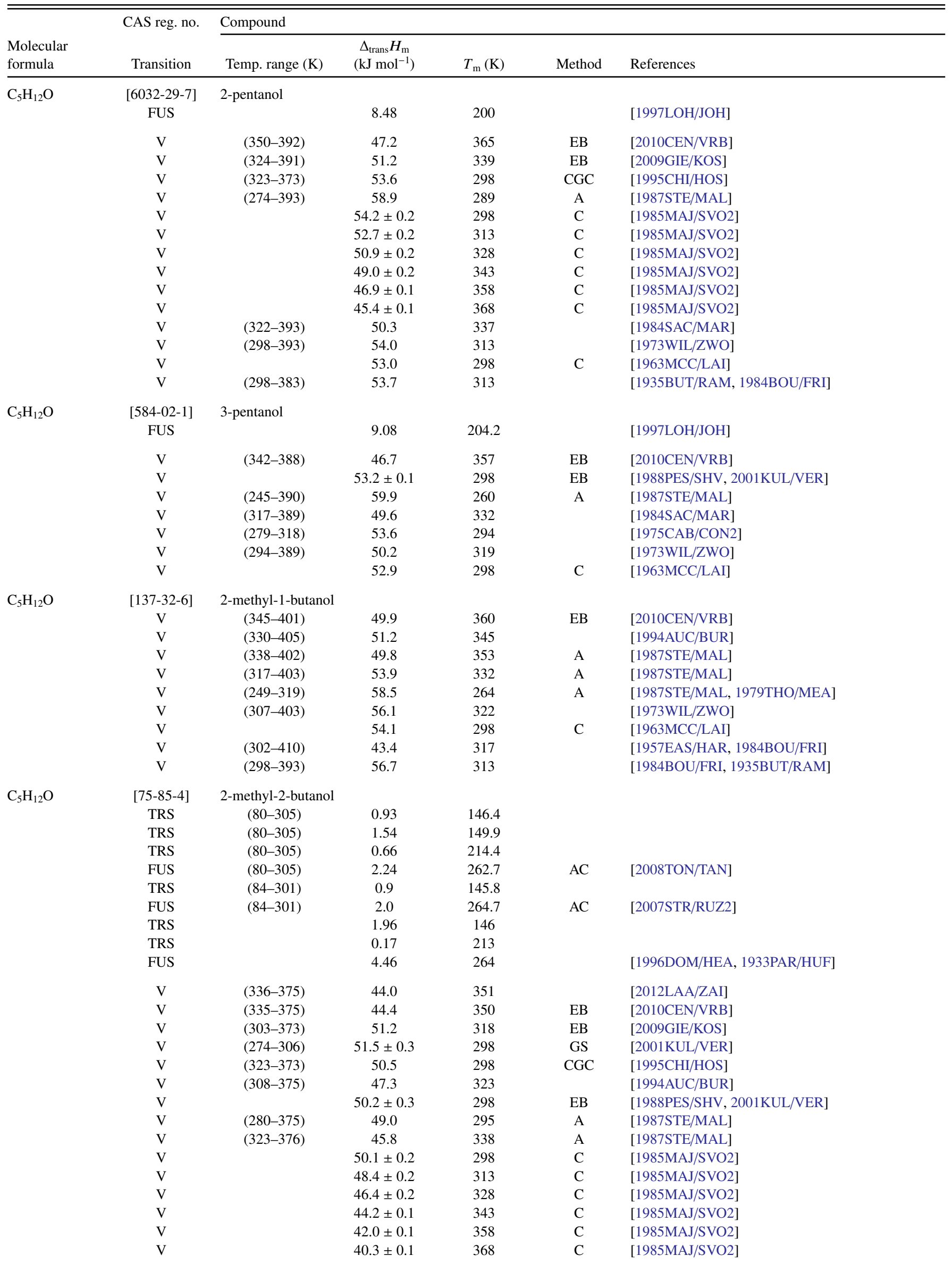


TABLE 8. Phase change enthalpies of $C_{5}$ organic compounds-Continued

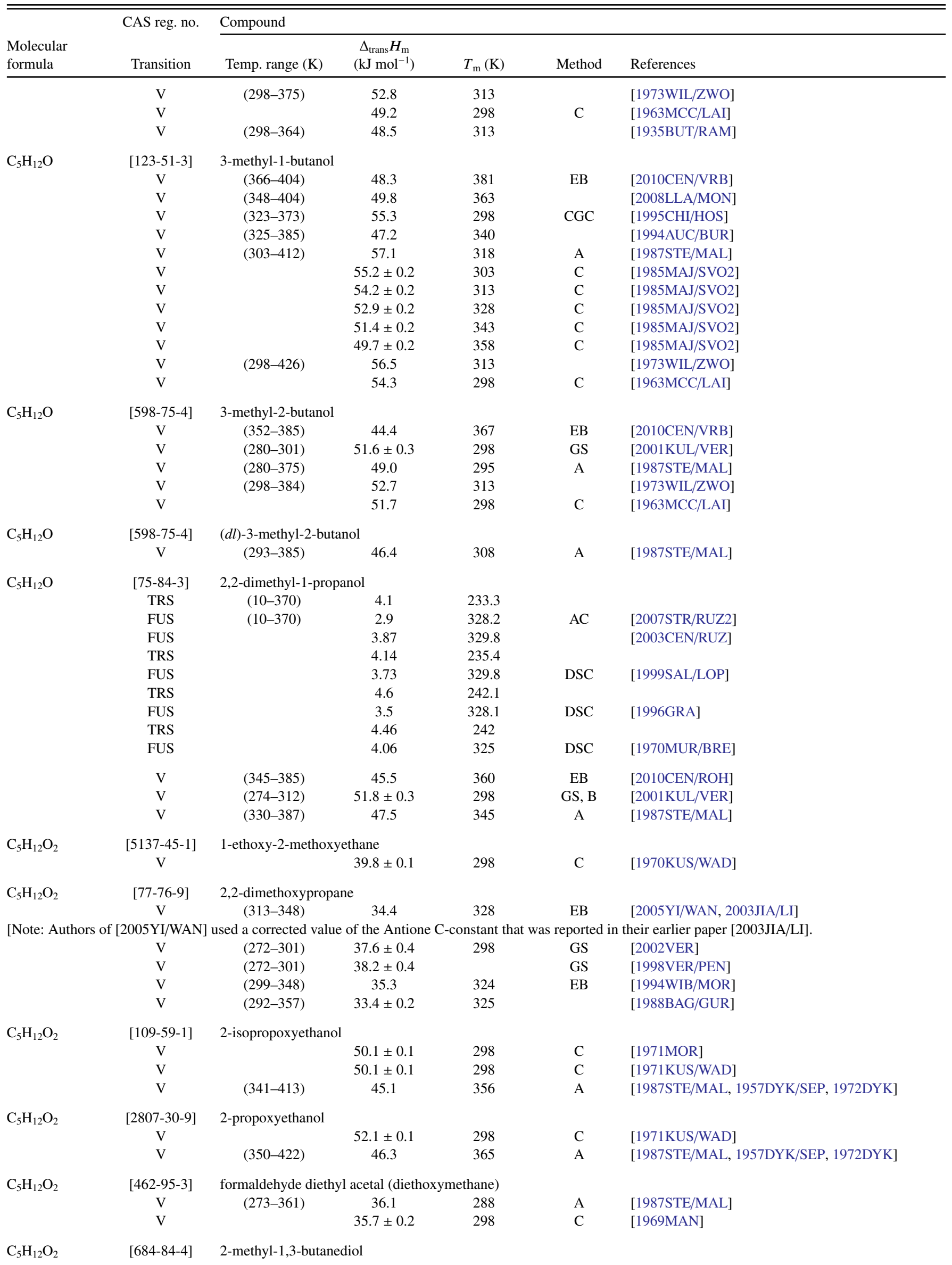


TABLE 8. Phase change enthalpies of $C_{5}$ organic compounds-Continued

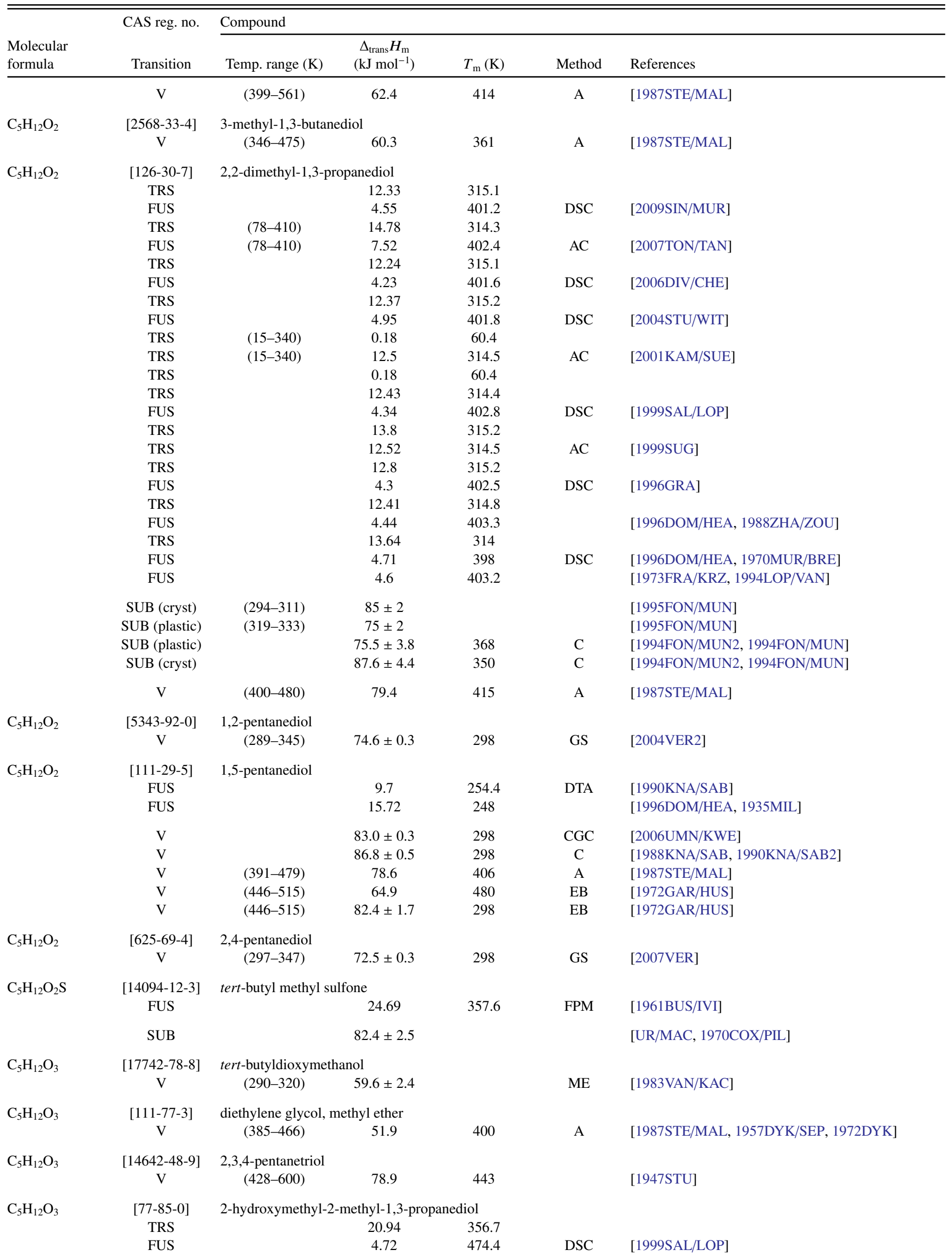


TABLE 8. Phase change enthalpies of $C_{5}$ organic compounds-Continued

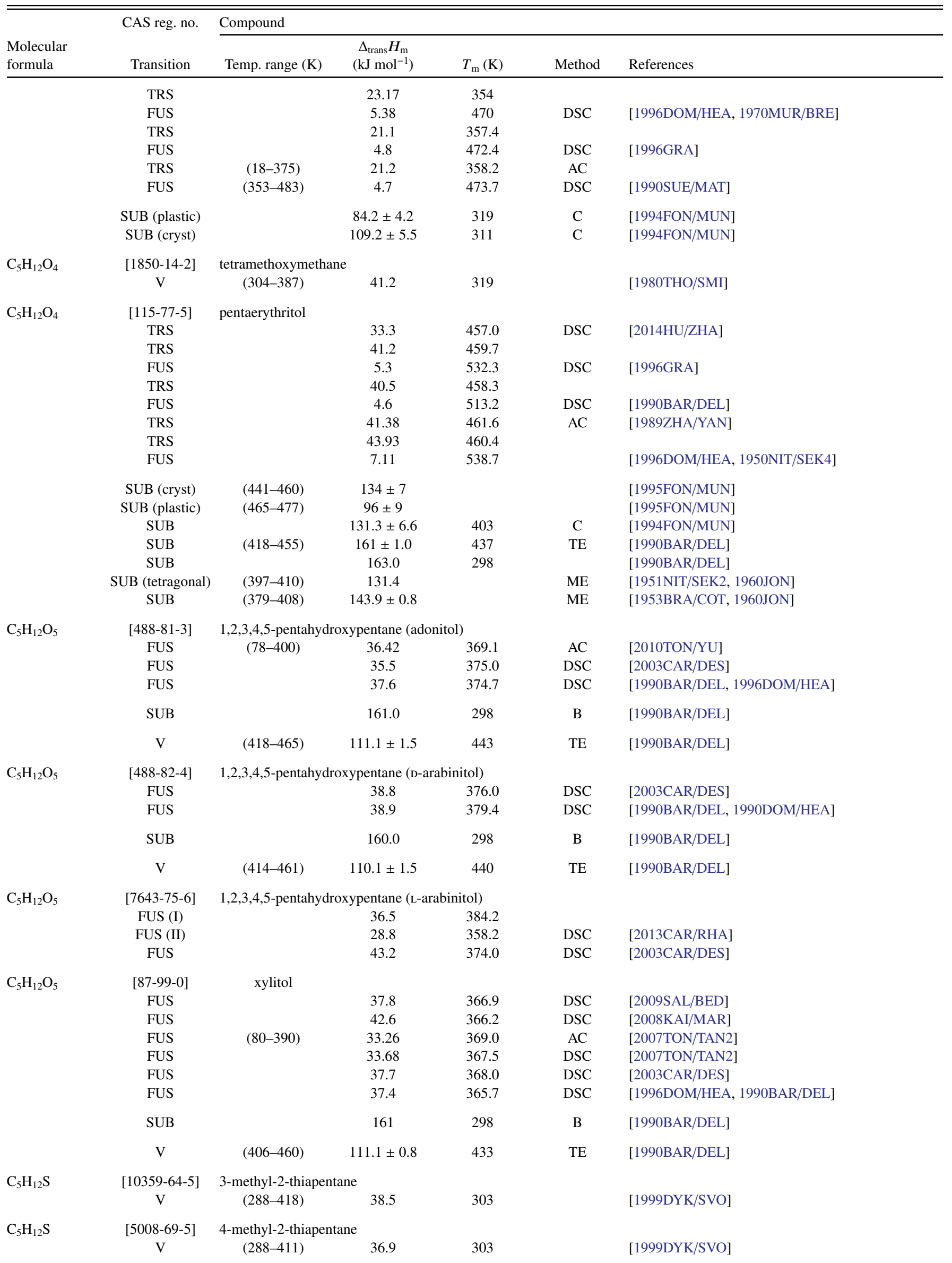


TABLE 8. Phase change enthalpies of $C_{5}$ organic compounds-Continued

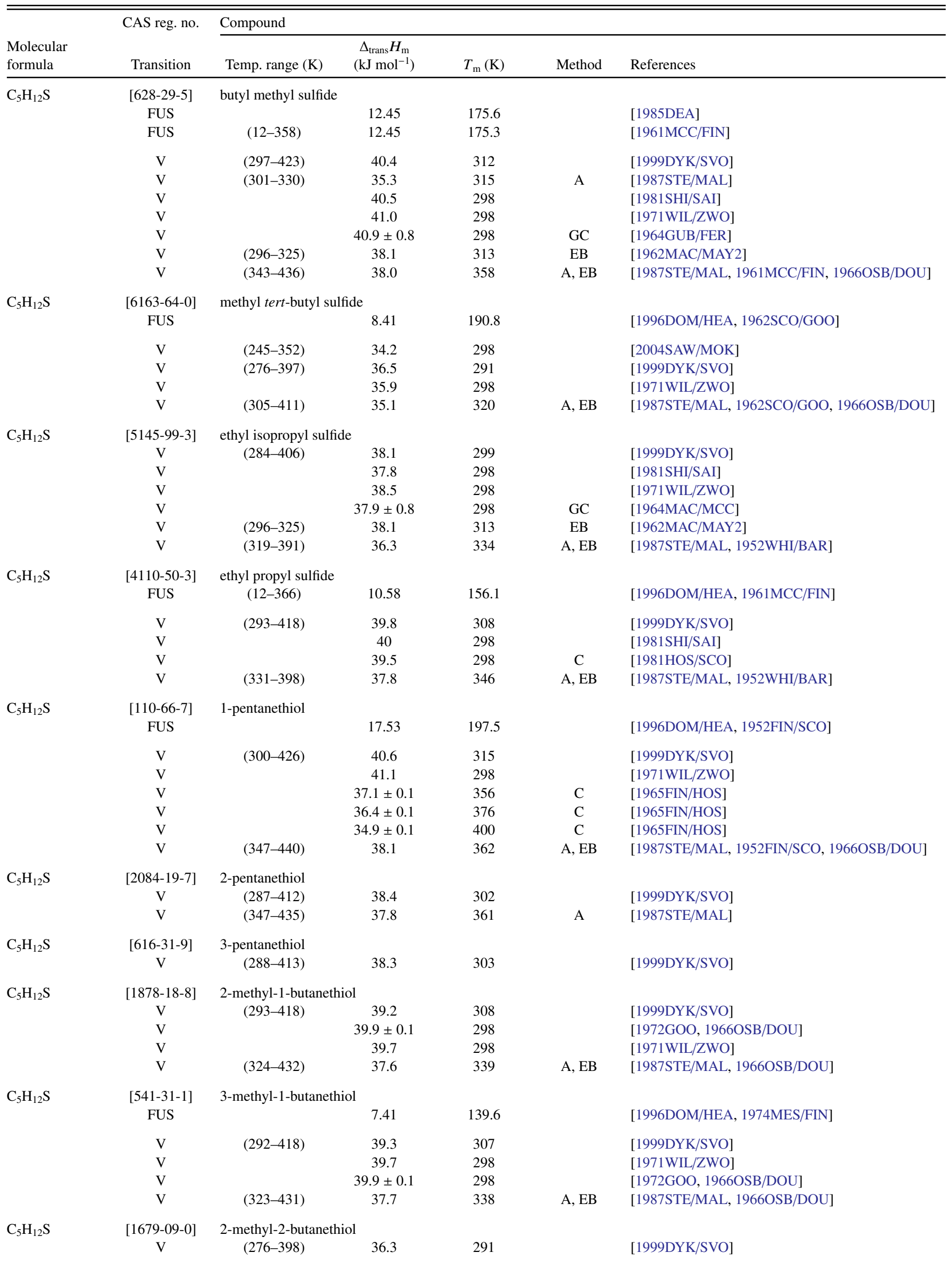


TABLE 8. Phase change enthalpies of $C_{5}$ organic compounds-Continued

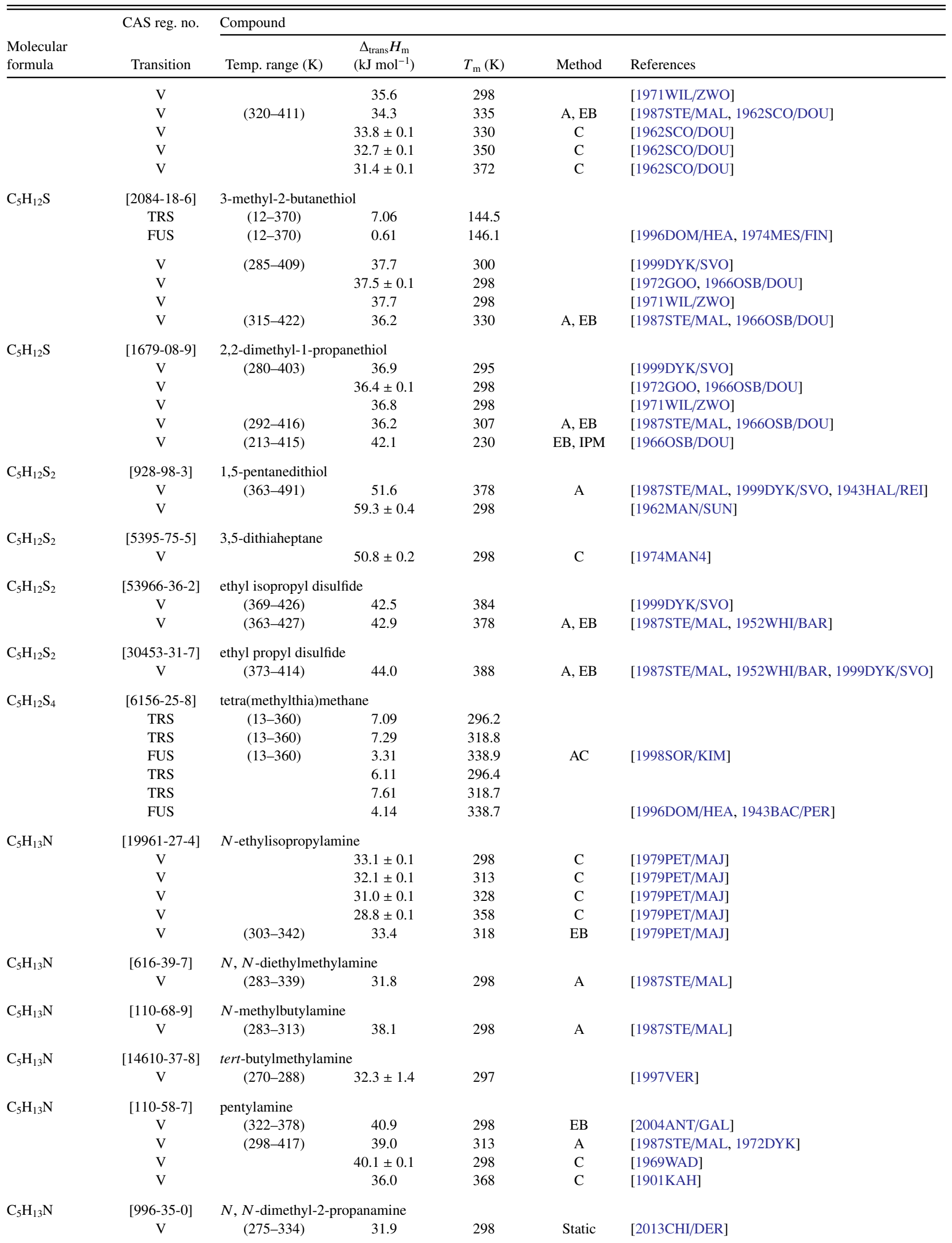


TABLE 8. Phase change enthalpies of $C_{5}$ organic compounds-Continued

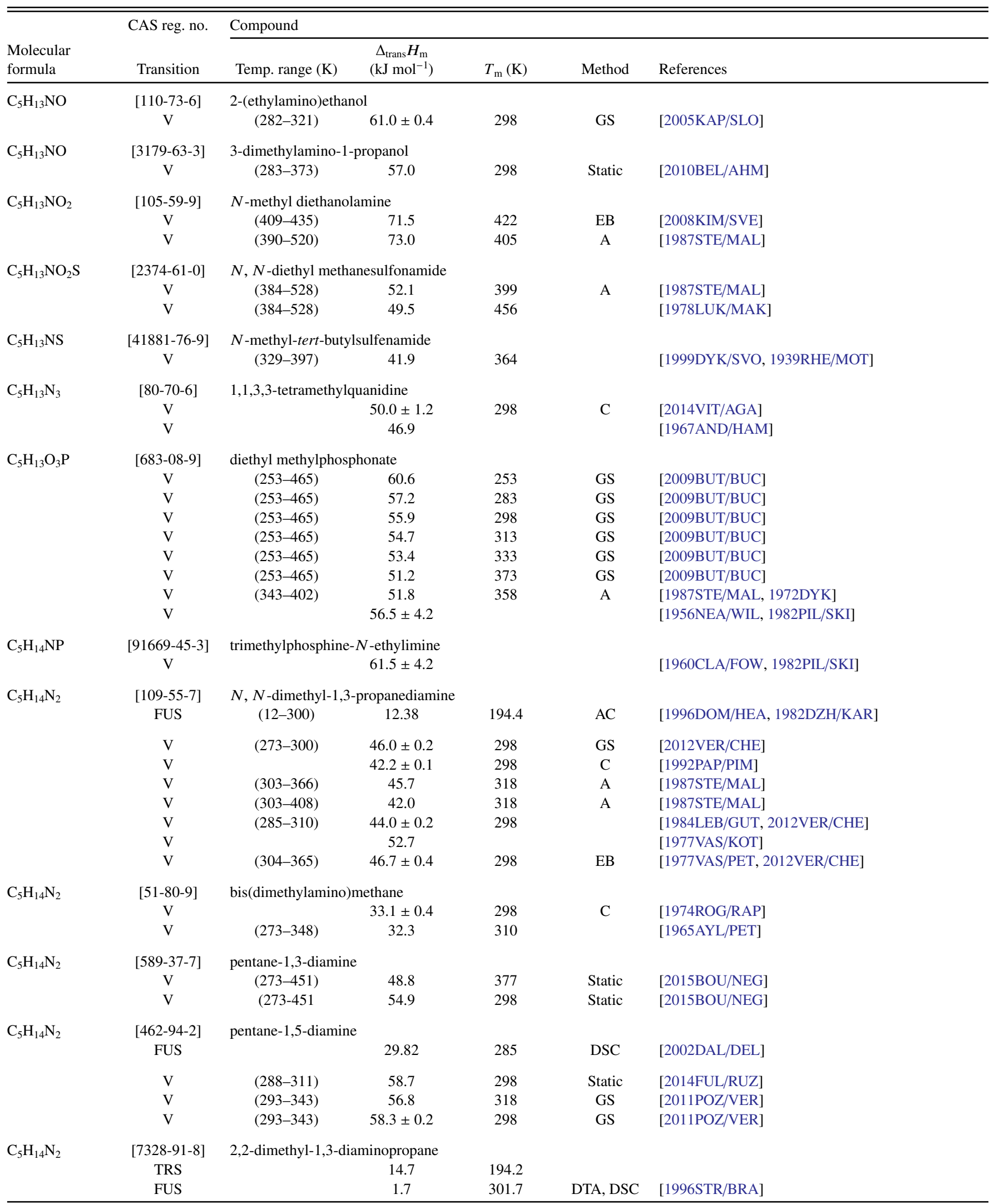


TABLE 9. Phase change enthalpies of $\mathrm{C}_{6}$ organic compounds

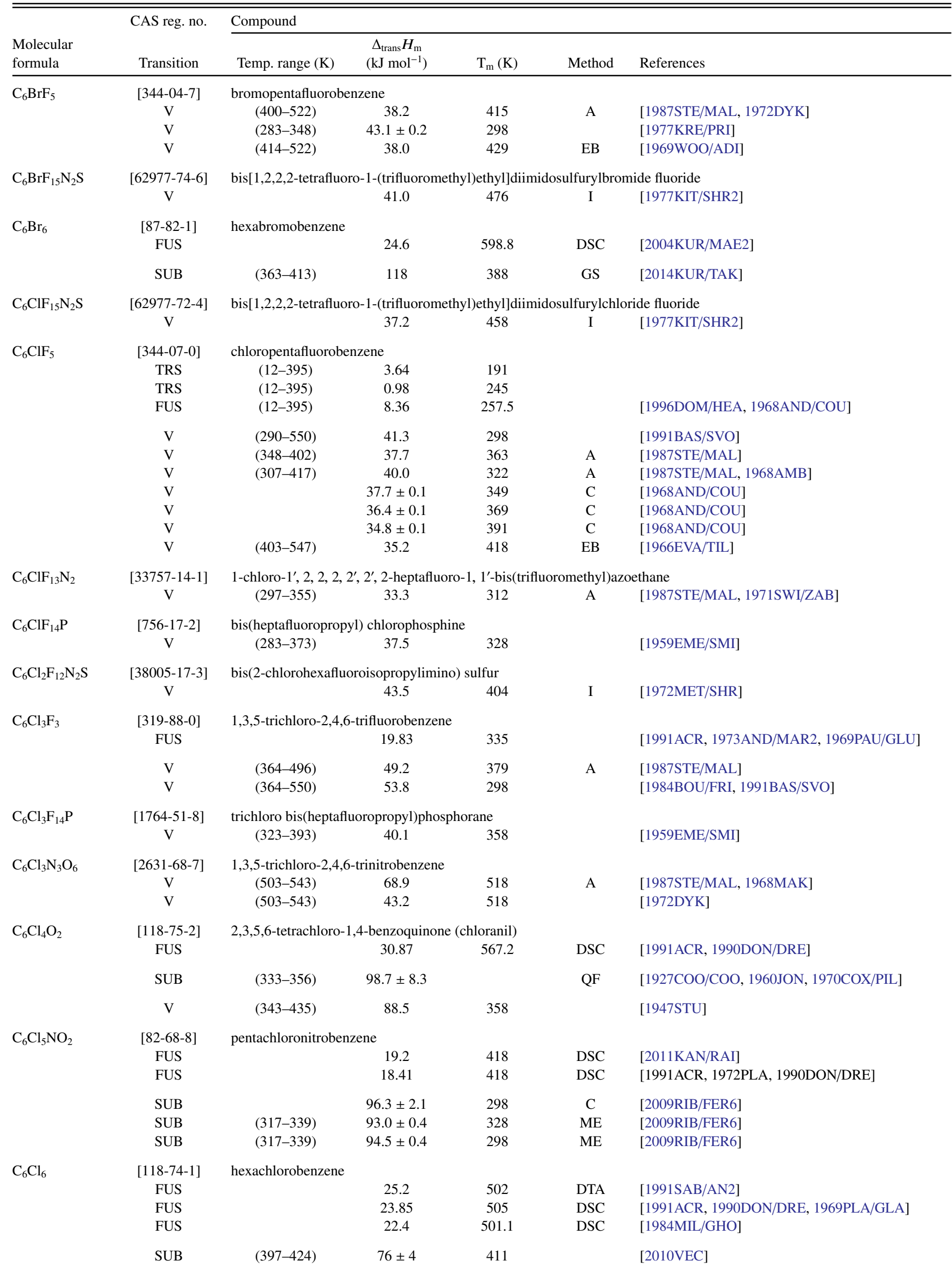


TABLE 9. Phase change enthalpies of $\mathrm{C}_{6}$ organic compounds-Continued

\begin{tabular}{|c|c|c|c|c|c|c|}
\hline \multirow[b]{2}{*}{$\begin{array}{l}\text { Molecular } \\
\text { formula }\end{array}$} & \multirow{2}{*}{$\begin{array}{l}\text { CAS reg. no. } \\
\text { Transition }\end{array}$} & \multicolumn{5}{|l|}{ Compound } \\
\hline & & Temp. range $(\mathrm{K})$ & $\begin{array}{c}\Delta_{\text {trans }} H_{\mathrm{m}} \\
\left(\mathrm{kJ} \mathrm{mol}^{-1}\right)\end{array}$ & $\mathrm{T}_{\mathrm{m}}(\mathrm{K})$ & Method & References \\
\hline & SUB & $(358-403)$ & $96.8 \pm 0.5$ & 298 & GS & [2007VER/EME] \\
\hline & SUB & $(258-313)$ & 105 & & & [1994LIU/DIC] \\
\hline & SUB & $(253-303)$ & $77.4 \pm 0.8$ & 278 & GS & [1994WAN/SHU] \\
\hline & SUB & & $89.6 \pm 0.2$ & 337 & $\mathrm{C}$ & [1991SAB/AN2] \\
\hline & SUB & & $90.5 \pm 0.2$ & 298 & $\mathrm{C}$ & [1991SAB/AN2] \\
\hline & SUB & $(461-506)$ & 85.5 & & & [1989LUB/JAN] \\
\hline & SUB & $(387-502)$ & 62.7 & 402 & A & [1987STE/MAL] \\
\hline & SUB & (314-373) & 94.7 & 344 & GS & [1986ROR/SAR, 1997DEL] \\
\hline & SUB & $(288-318)$ & 101.3 & 303 & GS & [1980FAR/YAN] \\
\hline & SUB & $(312-337)$ & $79.5 \pm 1.2$ & & & [1977STE2] \\
\hline \multicolumn{7}{|c|}{ Note: In [1977STE2] the sublimation surface was glass and hay } \\
\hline & SUB & $(369-397)$ & $92 \pm 8.2$ & & RG & [1949SEA/HOP2, 1970COX/PIL] \\
\hline & V & & $74.4 \pm 0.7$ & 298 & GS & [2001PUR/CHI] \\
\hline & $\mathrm{V}$ & $(413-453)$ & 76.8 & 298 & GC & [1994SPI/LUI] \\
\hline & $\mathrm{V}$ & $(258-313)$ & 81.3 & & GC & [1994LIU/DIC] \\
\hline & V & $(343-453)$ & 68.6 & 398 & GC & [1990HIN/BID2] \\
\hline & $\mathrm{V}$ & $(502-589)$ & 68.7 & 517 & A & [1987STE/MAL] \\
\hline & $\mathrm{V}$ & $(387-582)$ & 60.5 & 402 & & [1947STU] \\
\hline \multirow[t]{4}{*}{$\mathrm{C}_{6} \mathrm{D}_{10} \mathrm{O}$} & {$[51209-49-5]$} & cyclohexanone- $\mathrm{d}_{10}$ & & & & \\
\hline & TRS & & 7.1 & 216.8 & & \\
\hline & TRS & & 0.4 & 219.3 & & \\
\hline & FUS & & 1.19 & 241.5 & DTA & [1997BUS/HAM] \\
\hline \multirow[t]{2}{*}{$\mathrm{C}_{6} \mathrm{~F}_{5} \mathrm{NO}_{2}$} & [880-78-4] & pentafluoronitroben & & & & \\
\hline & FUS & & 11.81 & 250.5 & & [1996DOM/HEA, 1971PAU] \\
\hline \multirow[t]{22}{*}{$\mathrm{C}_{6} \mathrm{~F}_{6}$} & {$[392-56-3]$} & hexafluorobenzene & & & & \\
\hline & FUS & & 11.61 & 278.2 & DTA & [1994TAN/SAB3] \\
\hline & FUS & & 11.59 & 278.3 & & [1970MES/FIN] \\
\hline & FUS & & 11.59 & 278.3 & & [1996DOM/HEA, 1965COU/GRE] \\
\hline & SUB & $(215-278)$ & 49.2 & 263 & & [1987STE/MAL, 1965DOU/OSB] \\
\hline & SUB & $(238-268)$ & 49.8 & 253 & IPM, A & [1979SCO/OSB] \\
\hline & SUB & & 46.0 & 316 & $\mathrm{~B}$ & [1965COU/GRE] \\
\hline & $\mathrm{V}$ & $(288-333)$ & $36.1 \pm 0.1$ & 298 & & [2005DIA/GON] \\
\hline & V & $(318-376)$ & 34.4 & 333 & $\mathrm{~EB}$ & [1990AMB/EWI] \\
\hline & V & $(403-516)$ & 31.8 & 425 & & [1988DAV/EWI] \\
\hline & V & $(278-354)$ & 36.5 & 293 & A & [1987STE/MAL] \\
\hline & $\mathrm{V}$ & $(348-389)$ & 33.2 & 363 & A & [1987STE/MAL] \\
\hline & $\mathrm{V}$ & $(384-462)$ & 32.2 & 399 & A & [1987STE/MAL] \\
\hline & V & $(458-517)$ & 31.8 & 473 & A & [1987STE/MAL] \\
\hline & V & $(290-510)$ & 35.6 & 298 & & [1982INV, 1991BAS/SVO] \\
\hline & V & & 35.7 & 298 & $\mathrm{C}$ & [1981HOS/SCO] \\
\hline & V & $(293-323)$ & 35.7 & 308 & & [1980PAT/TOM] \\
\hline & $\mathrm{V}$ & $(281-335)$ & $36.4 \pm 0.1$ & 298 & & [1972KRE/PRI] \\
\hline & V & $(278-321)$ & 36.2 & 292 & MM & [1969FIN] \\
\hline & V & $(363-516)$ & 32.2 & 378 & EB & [1966EVA/TIL] \\
\hline & $\mathrm{V}$ & $(275-387)$ & 36.5 & 293 & & [1965DOU/OSB] \\
\hline & V & $(293-356)$ & 35.1 & 308 & & [1964PAT/PRO, 1984BOU/FRI] \\
\hline \multirow[t]{2}{*}{$\mathrm{C}_{6} \mathrm{~F}_{7} \mathrm{NOS}$} & {$[20094-84-2]$} & $N$-(pentafluoropher & 1)imidosulfu & ride & & \\
\hline & $\mathrm{V}$ & $(309-355)$ & 45.3 & 332 & & [1968GLE/VON] \\
\hline \multirow[t]{2}{*}{$\mathrm{C}_{6} \mathrm{~F}_{7} \mathrm{OP}$} & {$[59646-78-5]$} & pentafluorophenoxy & ifluorophosp & & & \\
\hline & $\mathrm{V}$ & $(310-363)$ & 42.4 & 325 & & [1976FAL/DES] \\
\hline \multirow[t]{2}{*}{$\mathrm{C}_{6} \mathrm{~F}_{7} \mathrm{O}_{2} \mathrm{P}$} & {$[59617-42-4]$} & pentafluorophenoxy & hosphoryl di & & & \\
\hline & $\mathrm{V}$ & $(323-367)$ & 46.4 & 338 & & [1976FAL/DES] \\
\hline \multirow[t]{2}{*}{$\mathrm{C}_{6} \mathrm{~F}_{8}$} & [5680-05-7] & perfluoro(2-methyl- & -methylenecy & ene) & & \\
\hline & V & $(243-306)$ & 31.0 & 258 & A, I & [1987STE/MAL, 1966BAN/BAR] \\
\hline $\mathrm{C}_{6} \mathrm{~F}_{10}$ & $\begin{array}{c}{[355-75-9]} \\
\mathrm{V}\end{array}$ & $\begin{array}{l}\text { perfluorocyclohexe } \\
\qquad(277-319)\end{array}$ & 31.0 & 298 & & [1979PRI/SAP] \\
\hline
\end{tabular}


TABLE 9. Phase change enthalpies of $\mathrm{C}_{6}$ organic compounds-Continued

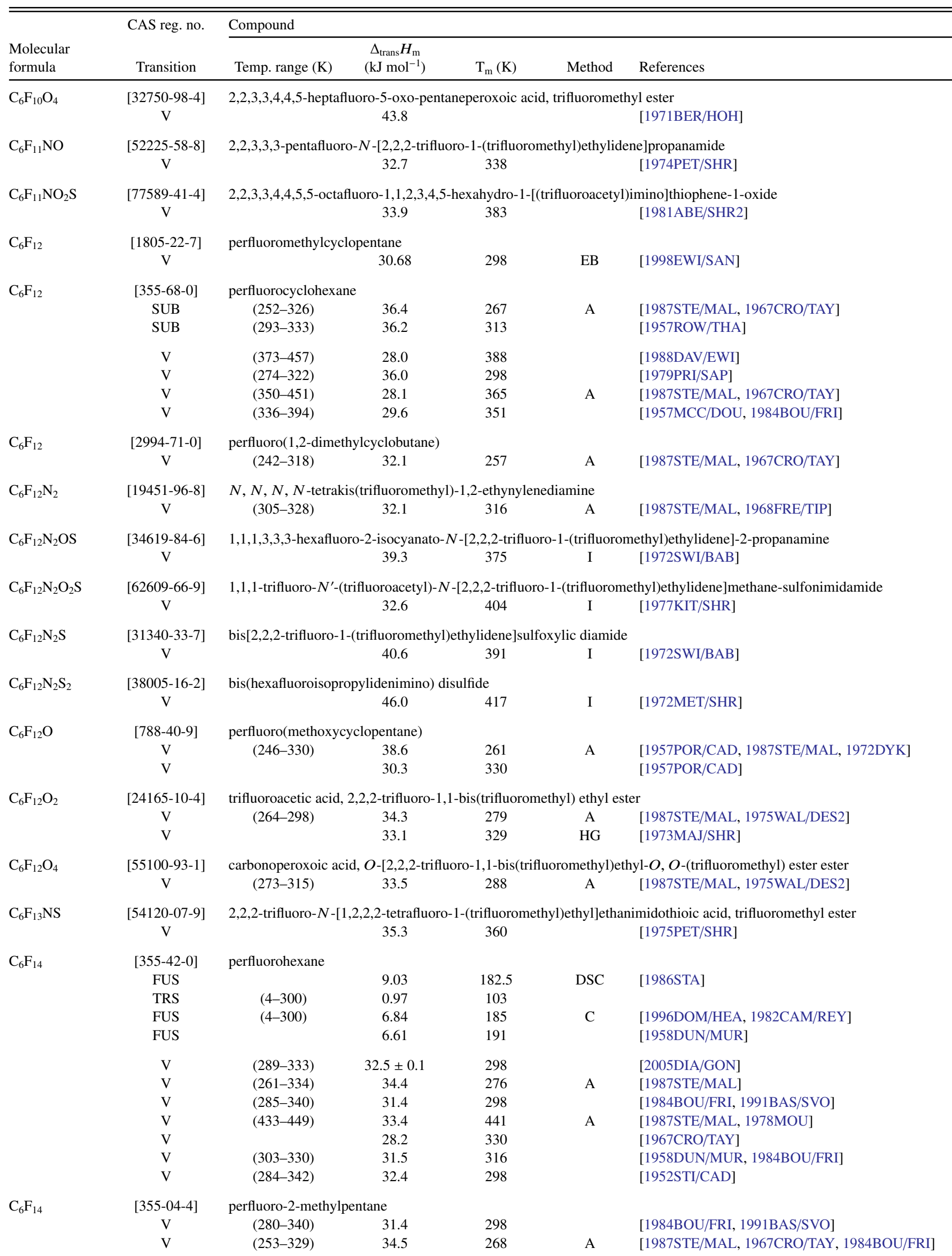


TABLE 9. Phase change enthalpies of $\mathrm{C}_{6}$ organic compounds-Continued

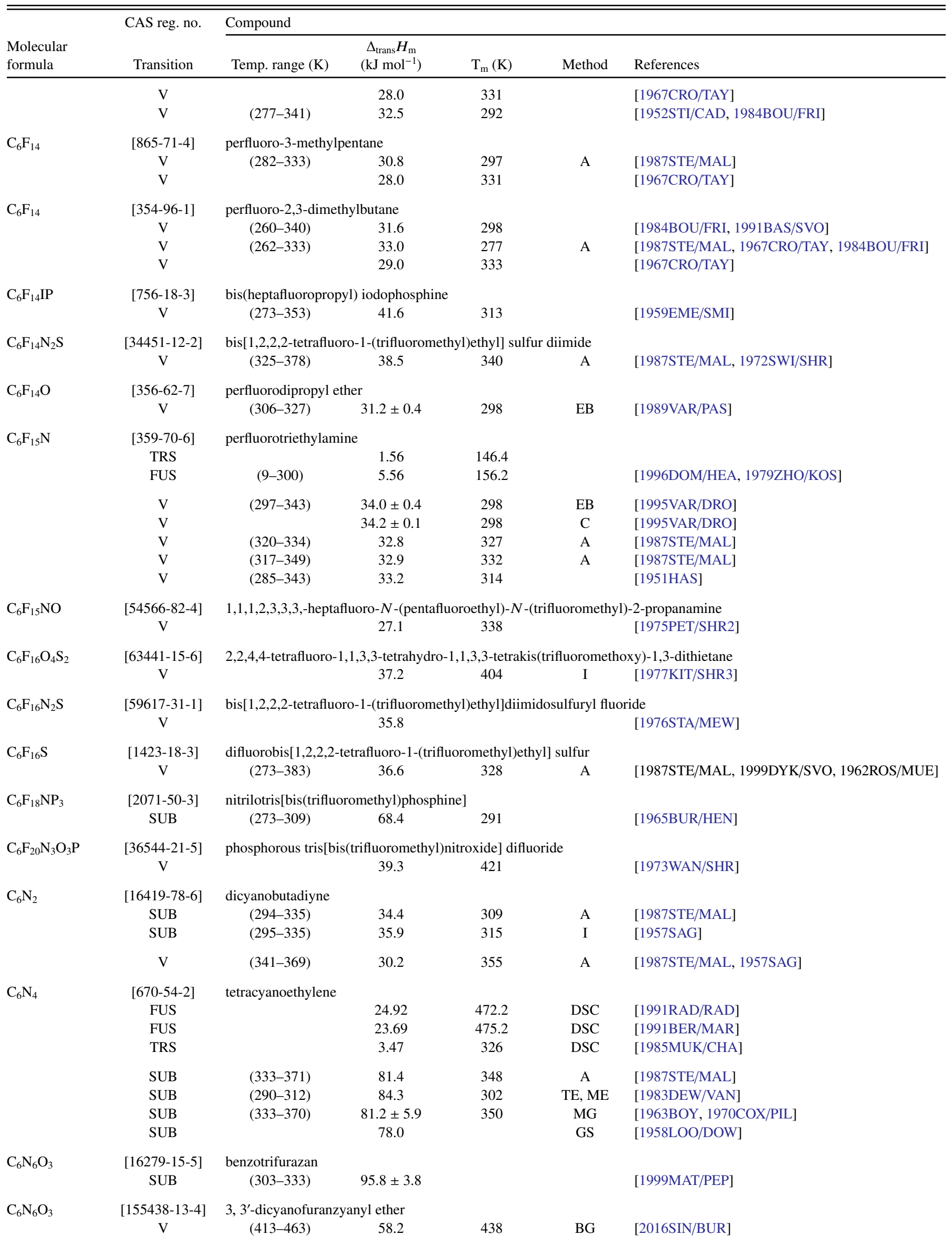


TABLE 9. Phase change enthalpies of $\mathrm{C}_{6}$ organic compounds-Continued

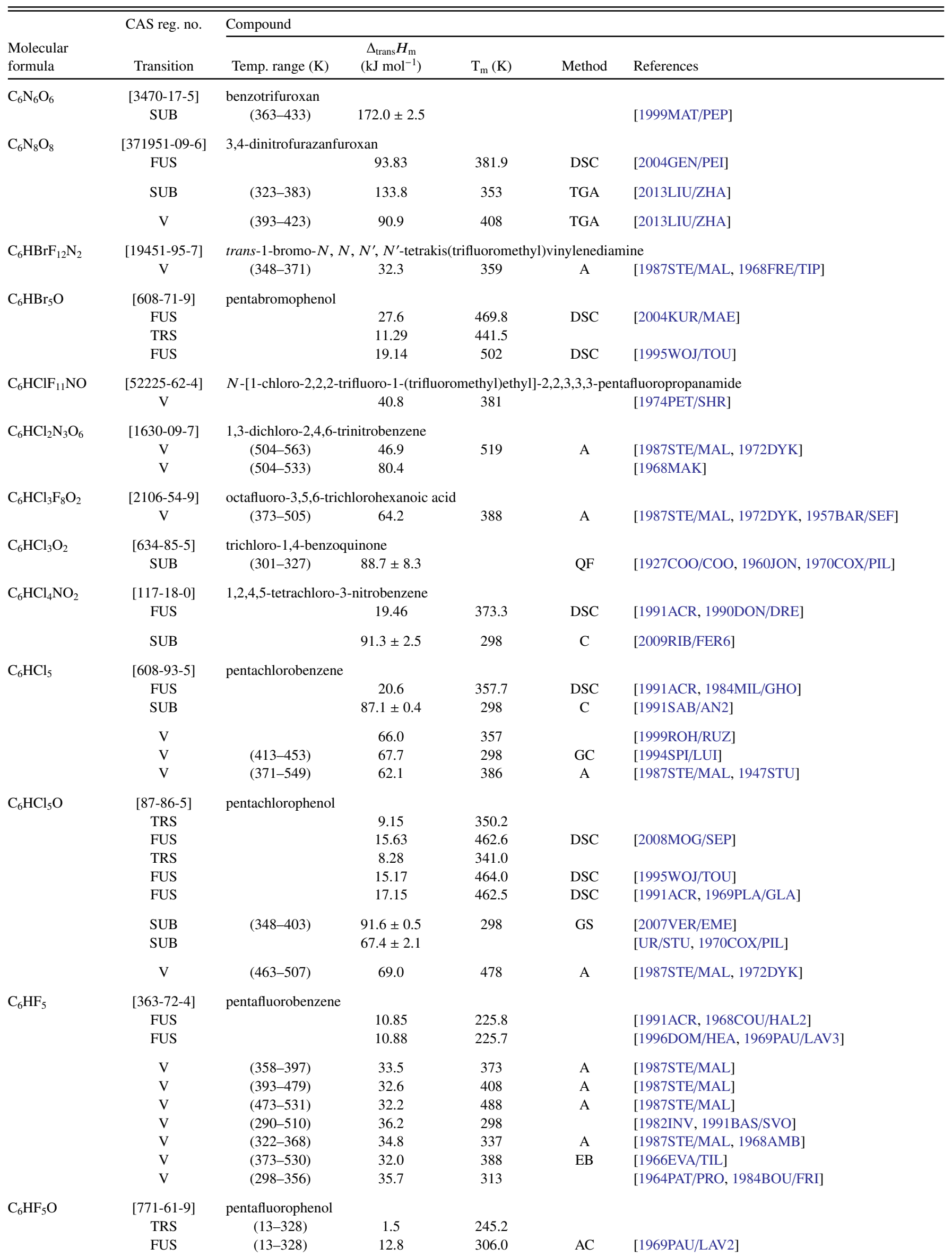


TABLE 9. Phase change enthalpies of $\mathrm{C}_{6}$ organic compounds-Continued

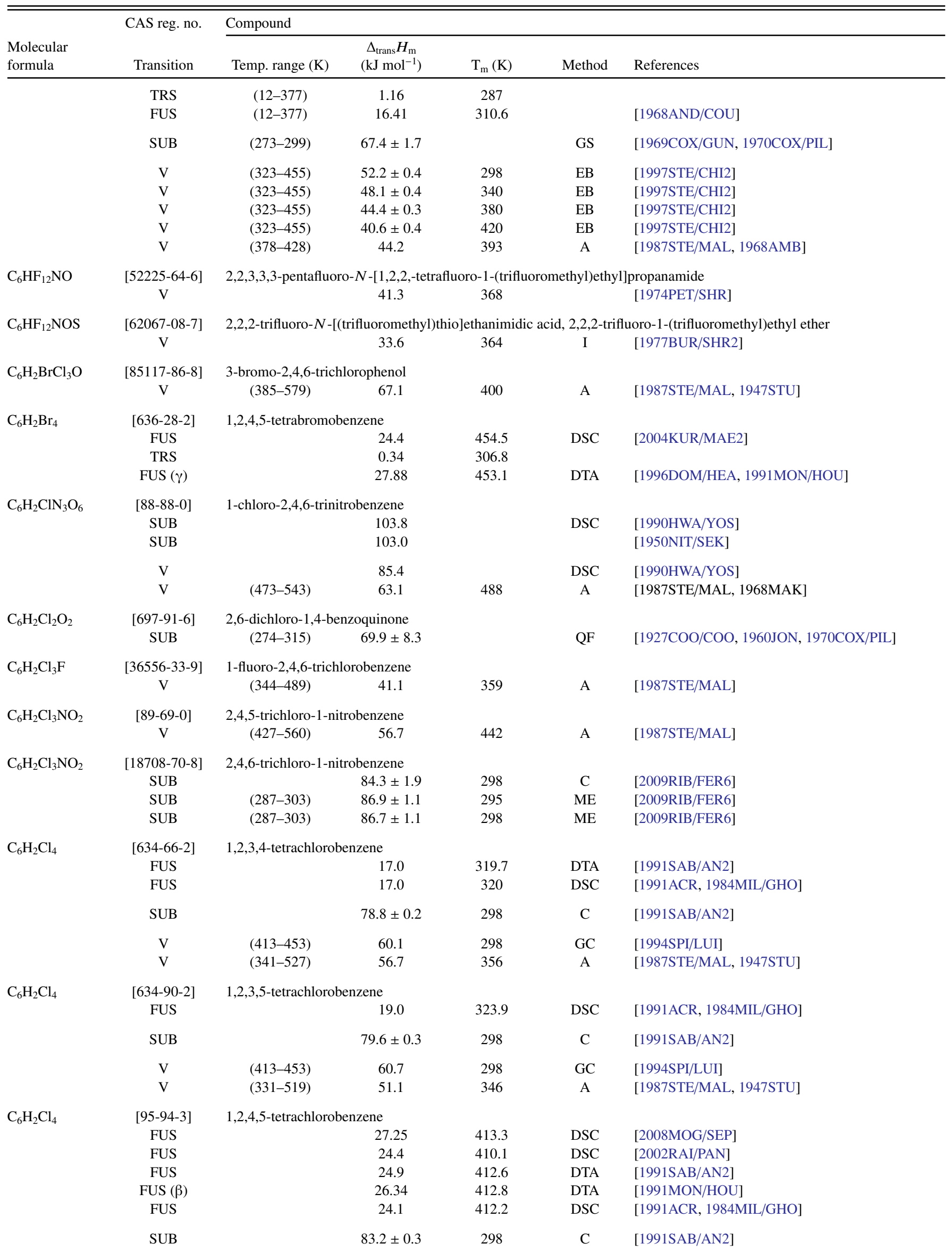


TABLE 9. Phase change enthalpies of $\mathrm{C}_{6}$ organic compounds-Continued

\begin{tabular}{|c|c|c|c|c|c|c|}
\hline $\begin{array}{l}\text { Molecular } \\
\text { formula }\end{array}$ & $\begin{array}{c}\text { CAS reg. no. } \\
\text { Transition }\end{array}$ & \multicolumn{5}{|l|}{ Compound } \\
\hline & $\mathrm{V}$ & $(419-518)$ & 52.0 & 434 & A & [1987STE/MAL, 1947STU] \\
\hline \multirow[t]{2}{*}{$\mathrm{C}_{6} \mathrm{H}_{2} \mathrm{Cl}_{4} \mathrm{O}$} & [58-90-2] & \multicolumn{2}{|c|}{ 2,3,4,6-tetrachlorophenol } & & & \\
\hline & $\mathrm{V}$ & $(373-548)$ & 64.8 & 388 & A & [1987STE/MAL, 1947STU] \\
\hline & $\mathrm{V}$ & $(293-323)$ & 77.9 & 308 & CGC & [1999LEI/WAN2] \\
\hline \multirow[t]{3}{*}{$\mathrm{C}_{6} \mathrm{H}_{2} \mathrm{Cl}_{4} \mathrm{O}_{2}$} & [87-87-6] & \multicolumn{2}{|c|}{ tetrachlorohydroquinone } & & & \\
\hline & SUB & $(298-359)$ & 89.0 & 313 & A & [1987STE/MAL] \\
\hline & SUB & $(333-356)$ & 88.7 & & QF & {$[1927 \mathrm{COO} / \mathrm{COO}, 1960 \mathrm{JON}]$} \\
\hline $\mathrm{C}_{6} \mathrm{H}_{2} \mathrm{Cl}_{5} \mathrm{~N}$ & {$[527-20-8]$} & \multicolumn{2}{|l|}{ pentachloroaniline } & & & \\
\hline \multirow{3}{*}{$\mathrm{C}_{6} \mathrm{H}_{2} \mathrm{~F}_{4}$} & $\mathrm{~V}$ & $(300-390)$ & 37.5 & 298 & & [1984BOU/FRI, 1991BAS/SVO] \\
\hline & $\mathrm{V}$ & $(300-392)$ & 36.8 & 315 & A & [1987STE/MAL, 1975AMB/ELL2, 1984BOU/FRI] \\
\hline & $\mathrm{V}$ & $(279-323)$ & 37.0 & 294 & MM & [1987STE/MAL, 1969FIN] \\
\hline \multirow[t]{6}{*}{$\mathrm{C}_{6} \mathrm{H}_{2} \mathrm{~F}_{4}$} & {$[2367-82-0]$} & \multicolumn{2}{|c|}{ 1,2,3,5-tetrafluorobenzene } & & & \\
\hline & FUS & $(11-353)$ & 10.67 & 226.9 & $\mathrm{AC}$ & [1973AND/MAR] \\
\hline & $\mathrm{V}$ & $(385-416)$ & 32.4 & 400 & A & [1987STE/MAL] \\
\hline & $\mathrm{V}$ & $(290-380)$ & 36.0 & 298 & & [1984BOU/FRI, 1991BAS/SVO] \\
\hline & $\mathrm{V}$ & $(287-382)$ & 36.0 & 302 & A & [1987STE/MAL, 1975AMB/ELL2, 1984BOU/FRI] \\
\hline & $\mathrm{V}$ & $(279-323)$ & 36.0 & 294 & MM & [1987STE/MAL, 1969FIN] \\
\hline $\mathrm{C}_{6} \mathrm{H}_{2} \mathrm{~F}_{4}$ & [327-54-8] & \multicolumn{2}{|c|}{ 1,2,4,5-tetrafluorobenzene } & & & \\
\hline \multirow{3}{*}{$\mathrm{C}_{6} \mathrm{H}_{2} \mathrm{~F}_{5} \mathrm{~N}$} & TRS & & 3.94 & 287.4 & & \\
\hline & FUS & & 14.27 & 306.8 & & [1996DOM/HEA, 1969PAU/LAV] \\
\hline & SUB & & $63.7 \pm 1.1$ & 298 & $\mathrm{C}$ & [2007RIB/FER] \\
\hline \multirow{2}{*}{$\mathrm{C}_{6} \mathrm{H}_{2} \mathrm{~F}_{12} \mathrm{O}$} & [176310-30-8] & $1,1,1,2,2,3,3$, -heptaf & uoro-3-(2,2,3,3 & entafluor & xy)propane & \\
\hline & $\mathrm{V}$ & $(288-344)$ & 34.8 & 303 & I & [2002MUR/YAM] \\
\hline $\mathrm{C}_{6} \mathrm{H}_{2} \mathrm{~F}_{12} \mathrm{O}_{3}$ & [205367-61-9] & 1,1'-oxybis[2-(diflu & romethoxy)-1, & -tetrafluo & & \\
\hline & $\mathrm{V}$ & $(268-283)$ & $38.5 \pm 0.8$ & & & [1999MAR/BAS] \\
\hline $\mathrm{C}_{6} \mathrm{H}_{2} \mathrm{~F}_{12} \mathrm{O}_{3} \mathrm{~S}$ & {$[53517-89-8]$} & $\operatorname{bis}(1,1,1,3,3,3$-hexa & uoro-2-propan & ulfite & & \\
\hline & $\mathrm{V}$ & & 42.4 & & & [1975DEM/KOV] \\
\hline $\mathrm{C}_{6} \mathrm{H}_{2} \mathrm{~F}_{12} \mathrm{O}_{4}$ & [249932-26-1] & $1,1,3,3,5,5,7,7,8,8,1$ & 10-dodecafluc & 2,4,6,9-te & adecane & \\
\hline & $\mathrm{V}$ & $(263-381)$ & $42.3 \pm 0.4$ & & & [1999MAR/BAS] \\
\hline $\mathrm{C}_{6} \mathrm{H}_{2} \mathrm{~F}_{14} \mathrm{NP}$ & [756-19-4] & amino bis(heptafluo & opropyl)phosp & & & \\
\hline & $\mathrm{V}$ & $(293-393)$ & 38.7 & 343 & & [1959EME/SMI] \\
\hline $\mathrm{C}_{6} \mathrm{H}_{2} \mathrm{~N}_{4}$ & [13481-25-9] & 2,3-dicyanopyrazine & & & & \\
\hline & FUS & & 19.8 & 405.1 & DSC & [2006MIR/MOR] \\
\hline & SUB & & $89.1 \pm 2.7$ & 298 & $\mathrm{C}$ & [2006MIR/MOR] \\
\hline $\mathrm{C}_{6} \mathrm{H}_{2} \mathrm{~N}_{4} \mathrm{O}_{6}$ & [5128-28-9] & 4,6-dinitrobenzofur & zan 1-oxide & & & \\
\hline & FUS & & 20.73 & 452.7 & & [1983RED/MUR] \\
\hline $\mathrm{C}_{6} \mathrm{H}_{3} \mathrm{BrCl}_{2} \mathrm{O}$ & [4524-77-0] & 2-bromo-4,6-dichlo & phenol & & & \\
\hline
\end{tabular}


TABLE 9. Phase change enthalpies of $\mathrm{C}_{6}$ organic compounds-Continued

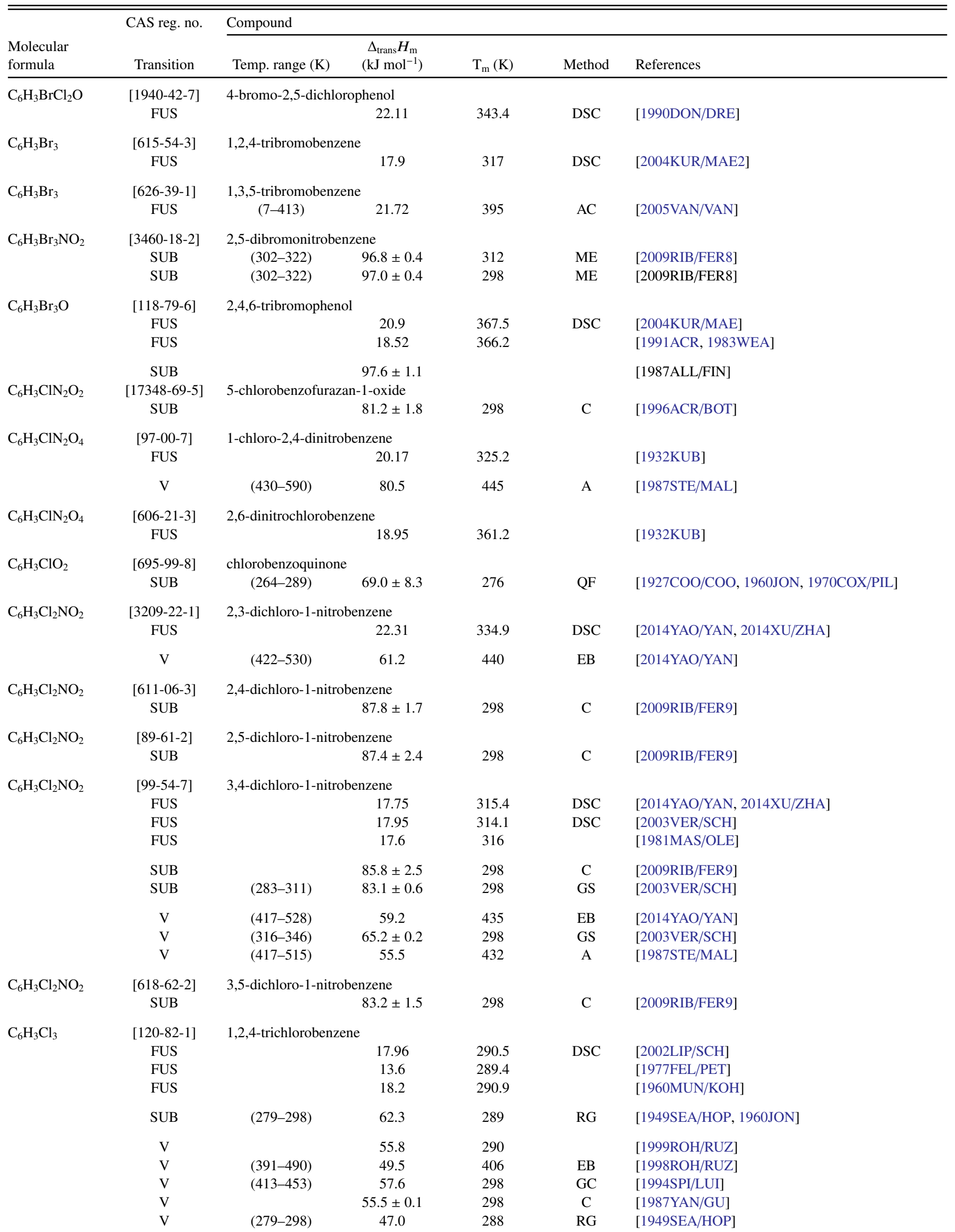


TABLE 9. Phase change enthalpies of $\mathrm{C}_{6}$ organic compounds-Continued

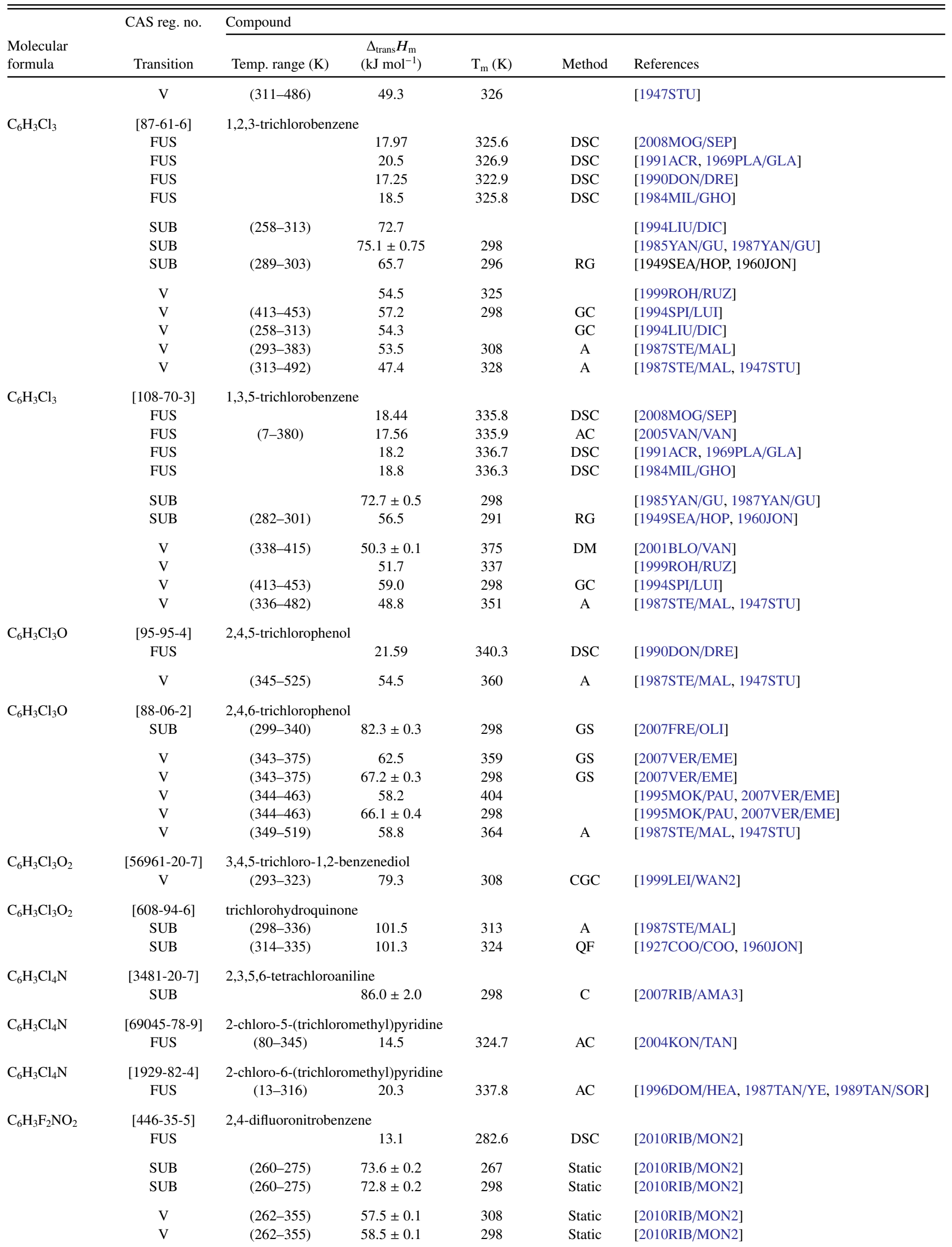


TABLE 9. Phase change enthalpies of $\mathrm{C}_{6}$ organic compounds-Continued

\begin{tabular}{|c|c|c|c|c|c|c|}
\hline \multirow[b]{2}{*}{$\begin{array}{l}\text { Molecular } \\
\text { formula }\end{array}$} & \multirow{2}{*}{$\begin{array}{l}\text { CAS reg. no. } \\
\text { Transition }\end{array}$} & \multicolumn{5}{|l|}{ Compound } \\
\hline & & Temp. range $(\mathrm{K})$ & $\begin{array}{c}\Delta_{\text {trans }} H_{\mathrm{m}} \\
\left(\mathrm{kJ} \mathrm{mol}^{-1}\right) \\
\end{array}$ & $\mathrm{T}_{\mathrm{m}}(\mathrm{K})$ & Method & References \\
\hline \multirow[t]{3}{*}{$\mathrm{C}_{6} \mathrm{H}_{3} \mathrm{~F}_{2} \mathrm{NO}_{2}$} & [364-74-9] & \multicolumn{5}{|c|}{ 2,5-difluoronitrobenzene } \\
\hline & $\mathrm{V}$ & $(262-353)$ & $57.8 \pm 0.1$ & 308 & Static & [2010RIB/MON2] \\
\hline & $\mathrm{V}$ & $(262-353)$ & $58.7 \pm 0.1$ & 298 & Static & [2010RIB/MON2] \\
\hline \multirow[t]{6}{*}{$\mathrm{C}_{6} \mathrm{H}_{3} \mathrm{~F}_{2} \mathrm{NO}_{2}$} & [369-34-6] & \multicolumn{5}{|c|}{ 3,4-difluoronitrobenzene } \\
\hline & FUS & & 16.4 & 268.0 & $\mathrm{DSC}$ & [2010RIB/MON2] \\
\hline & SUB & $(256-265)$ & $74.8 \pm 0.2$ & 260 & Static & [2010RIB/MON2] \\
\hline & SUB & $(256-265)$ & $73.8 \pm 0.4$ & 298 & Static & [2010RIB/MON2] \\
\hline & $\mathrm{V}$ & $(259-345)$ & $55.2 \pm 0.1$ & 302 & Static & [2010RIB/MON2] \\
\hline & $\mathrm{V}$ & $(259-345)$ & $55.5 \pm 0.1$ & 298 & Static & [2010RIB/MON2] \\
\hline \multirow[t]{3}{*}{$\mathrm{C}_{6} \mathrm{H}_{3} \mathrm{~F}_{3}$} & {$[372-38-3]$} & \multicolumn{5}{|c|}{ 1,3,5-trifluorobenzene } \\
\hline & $\mathrm{V}$ & $(280-320)$ & 33.9 & 298 & & [1984BOU/FRI, 1991BAS/SVO] \\
\hline & V & $(279-350)$ & 34.5 & 294 & $\mathrm{~A}, \mathrm{MM}$ & [1987STE/MAL, 1969FIN, 1972DYK] \\
\hline \multirow[t]{3}{*}{$\mathrm{C}_{6} \mathrm{H}_{3} \mathrm{~F}_{4} \mathrm{~N}$} & {$[5580-80-3]$} & \multicolumn{5}{|c|}{ 2,3,4,5-tetrafluoroaniline } \\
\hline & FUS & & 19.01 & 301.0 & DSC & [2007RIB/FER] \\
\hline & SUB & & $74.3 \pm 1.3$ & 298 & $\mathrm{C}$ & [2007RIB/FER] \\
\hline \multirow[t]{2}{*}{$\mathrm{C}_{6} \mathrm{H}_{3} \mathrm{~F}_{4} \mathrm{~N}$} & {$[363-73-5]$} & \multicolumn{5}{|c|}{ 2,3,4,6-tetrafluoroaniline } \\
\hline & $\mathrm{V}$ & & $50.4 \pm 0.6$ & 298 & $\mathrm{C}$ & [2007RIB/FER] \\
\hline \multirow[t]{3}{*}{$\mathrm{C}_{6} \mathrm{H}_{3} \mathrm{~F}_{4} \mathrm{~N}$} & {$[700-17-4]$} & \multicolumn{5}{|c|}{ 2,3,5,6-tetrafluoroaniline } \\
\hline & FUS & & 8.19 & 305.4 & $\mathrm{DSC}$ & [2007RIB/FER] \\
\hline & SUB & & $62.5 \pm 1.1$ & 298 & $\mathrm{C}$ & [2007RIB/FER] \\
\hline \multirow[t]{2}{*}{$\mathrm{C}_{6} \mathrm{H}_{3} \mathrm{~F}_{9} \mathrm{O}_{2}$} & {$[42031-16-3]$} & \multicolumn{5}{|c|}{ trifluoroacetic acid, 2,2,2-trifluoro-1-methyl-1-(trifluoromethyl)ethyl ester } \\
\hline & V & & 33.5 & 338 & HG & {$[1973 \mathrm{MAJ} / \mathrm{SHR}]$} \\
\hline \multirow[t]{2}{*}{$\mathrm{C}_{6} \mathrm{H}_{3} \mathrm{~F}_{9} \mathrm{O}_{2}$} & {$[24165-09-1]$} & \multicolumn{5}{|c|}{ acetic acid, 2,2,2-trifluoro-1,1-bis(trifluoromethyl)ethyl ester } \\
\hline & V & $(273-328)$ & 40.1 & 288 & A & [1987STE/MAL, 1975WAL/DES2] \\
\hline \multirow[t]{2}{*}{$\mathrm{C}_{6} \mathrm{H}_{3} \mathrm{~F}_{10} \mathrm{NS}$} & {$[54120-08-0]$} & \multicolumn{5}{|c|}{ 2,2,2-trifluoro- $N$-[1,2,2,2-tetrafluoro-1-(trifluoromethyl)ethyl]ethanimidothioic acid, methyl ester } \\
\hline & V & & 31.6 & 383 & & {$[1975 \mathrm{PET} / \mathrm{SHR}]$} \\
\hline $\mathrm{C}_{6} \mathrm{H}_{3} \mathrm{~F}_{11} \mathrm{O}$ & [176310-29-5] & 1,1,1,2,2,3,3-heptaf & oro-3- $(2,2,3, ?$ & fluoropro & propane & \\
\hline & V & $(288-357)$ & 37.2 & 303 & I & [2002MUR/YAM] \\
\hline $\mathrm{C}_{6} \mathrm{H}_{3} \mathrm{~F}_{11} \mathrm{O}$ & {$[181214-74-4]$} & $1,1,1,2,2,3,3,4,4,5,5$ & Indecafluoro- & hoxypent & & \\
\hline & V & $(288-358)$ & 36.6 & 303 & I & [2002MUR/YAM] \\
\hline $\mathrm{C}_{6} \mathrm{H}_{3} \mathrm{~F}_{11} \mathrm{O}$ & [203783-56-6] & $1,1,1,2,3,3,4,4$-octa & oro-4-metho & trifluoron & butane & \\
\hline & $\mathrm{V}$ & $(288-357)$ & 36 & & I & [2002MUR/YAM] \\
\hline $\mathrm{C}_{6} \mathrm{H}_{3} \mathrm{~F}_{11} \mathrm{O}$ & {$[290-28-8]$} & 1,1,1,2,3,3-hexafluo & $-3-(2,2,3,3,3$ & fluoropro & propane & \\
\hline & $\mathrm{V}$ & $(293-360)$ & 38.4 & 308 & I & [2002MUR/YAM] \\
\hline $\mathrm{C}_{6} \mathrm{H}_{3} \mathrm{~N}_{3} \mathrm{O}_{2} \mathrm{~S}$ & [6583-06-8] & 4-nitro-2,1,3-benzo & ladiazole & & & \\
\hline & FUS & & 22.6 & 381.4 & $\mathrm{DSC}$ & [2012RIB/FRE] \\
\hline & SUB & & $101.8 \pm 4.3$ & 298 & $\mathrm{C}$ & [2012RIB/FRE] \\
\hline $\mathrm{C}_{6} \mathrm{H}_{3} \mathrm{~N}_{3} \mathrm{O}_{4}$ & [18771-85-2] & 4-nitrobenzofurazan & -oxide & & & \\
\hline & SUB & & $97.3 \pm 1.6$ & 298 & $\mathrm{C}$ & [1996ACR/BOT] \\
\hline $\mathrm{C}_{6} \mathrm{H}_{3} \mathrm{~N}_{3} \mathrm{O}_{6}$ & {$[603-13-4]$} & 1,2,3-trinitrobenzen & & & & \\
\hline & $\mathrm{V}$ & $(523-573)$ & 60.3 & 538 & A & [1987STE/MAL, 1968MAK, 1972DYK] \\
\hline $\mathrm{C}_{6} \mathrm{H}_{3} \mathrm{~N}_{3} \mathrm{O}_{6}$ & {$[610-31-1]$} & 1,2,4-trinitrobenzen & & & & \\
\hline & V & $(523-573)$ & 82.6 & 538 & A & [1987STE/MAL, 1968MAK, 1972DYK] \\
\hline $\mathrm{C}_{6} \mathrm{H}_{3} \mathrm{~N}_{3} \mathrm{O}_{6}$ & {$[99-35-4]$} & 1,3,5-trinitrobenzen & & & & \\
\hline & TRS (I) & & 1.9 & 370 & & \\
\hline & FUS (I) & & 14.81 & 380.3 & & \\
\hline & FUS (II) & & 15.0 & 398.4 & DSC & [1996DOM/HEA, 1980RAD/RAD] \\
\hline & FUS & & 22.6 & 388.5 & DSC & [1975CAS/VEC] \\
\hline
\end{tabular}

Note: Authors of [1975CAS/VEC] give a melting point temperature of $12.1^{\circ} \mathrm{C}$, which is unrealistic. The value of 388.5 was calculated from the published enthalpy and entropy of fusion given in the paper. 
TABLE 9. Phase change enthalpies of $\mathrm{C}_{6}$ organic compounds - Continued

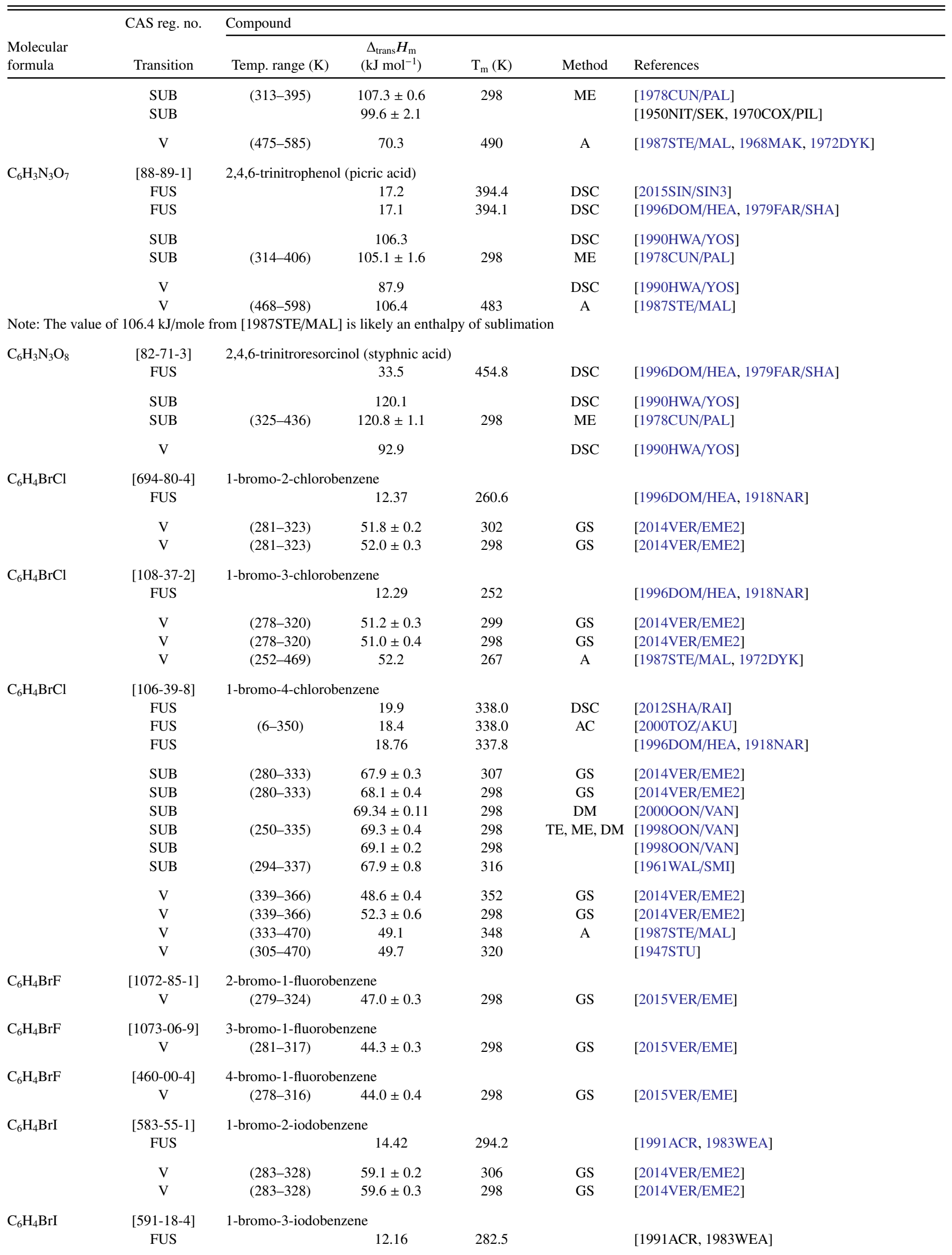


TABLE 9. Phase change enthalpies of $\mathrm{C}_{6}$ organic compounds-Continued

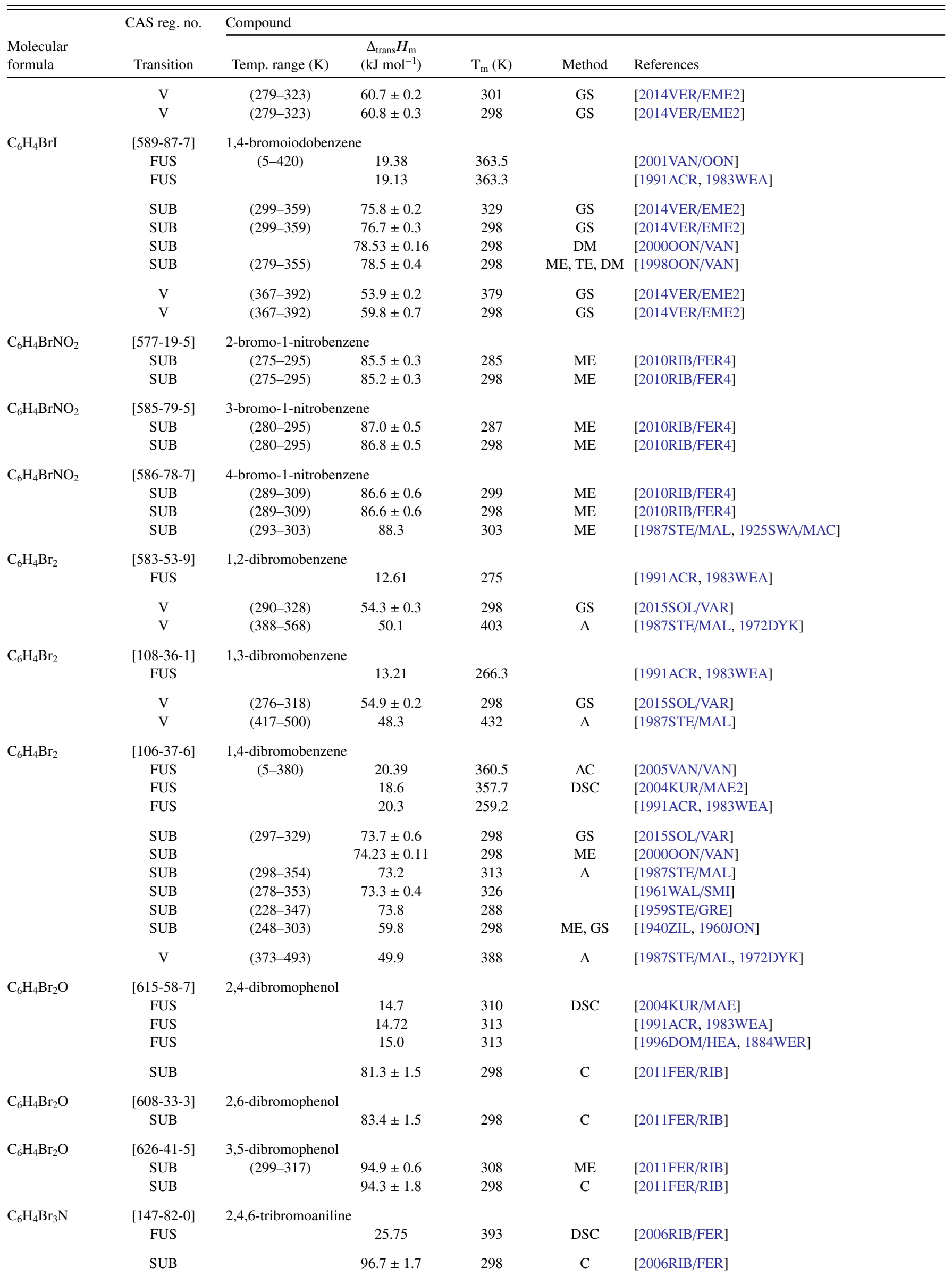


TABLE 9. Phase change enthalpies of $\mathrm{C}_{6}$ organic compounds - Continued

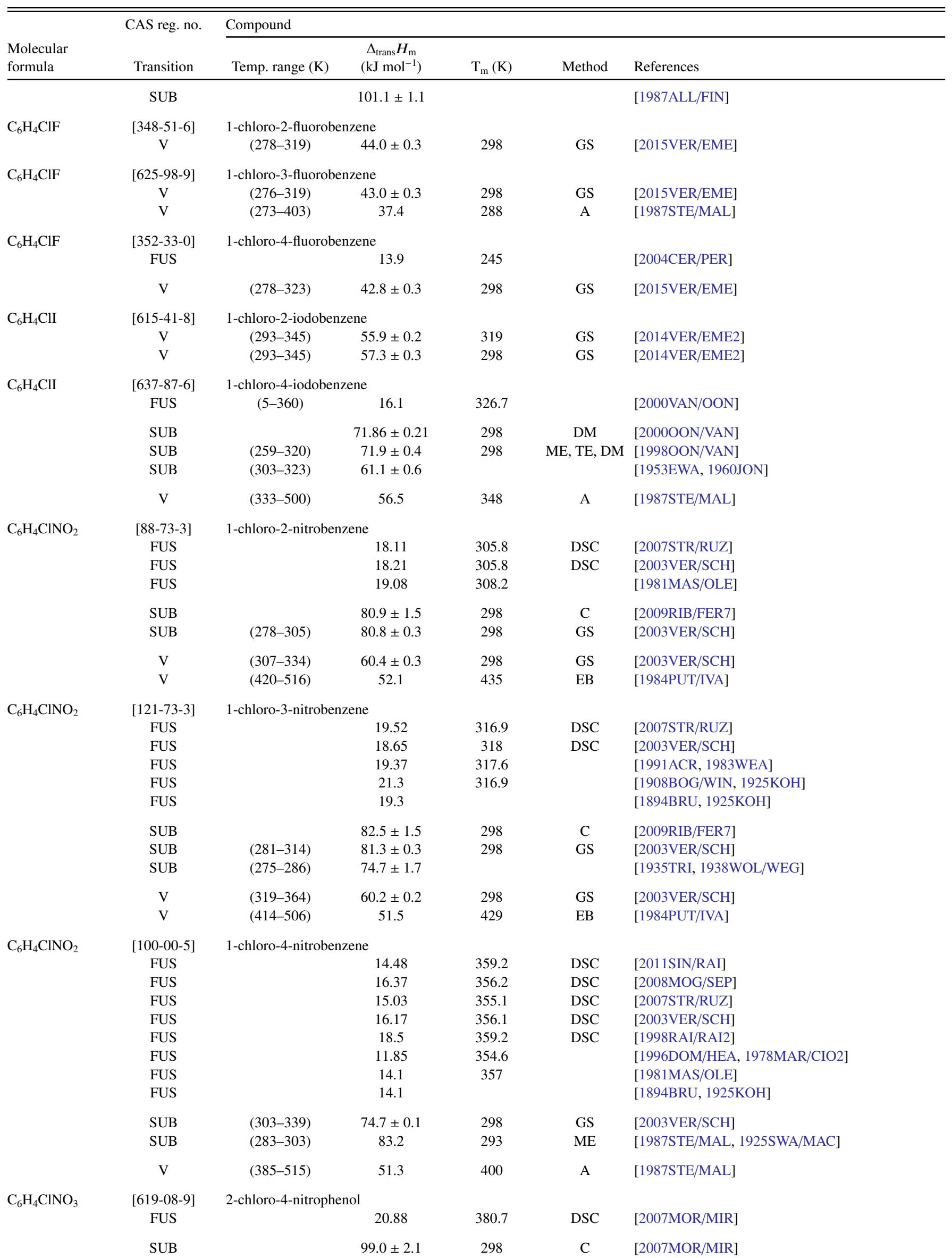


TABLE 9. Phase change enthalpies of $\mathrm{C}_{6}$ organic compounds-Continued

\begin{tabular}{|c|c|c|c|c|c|c|}
\hline \multirow[b]{2}{*}{$\begin{array}{l}\text { Molecular } \\
\text { formula }\end{array}$} & \multirow{2}{*}{$\begin{array}{c}\text { CAS reg. no. } \\
\text { Transition }\end{array}$} & \multicolumn{5}{|l|}{ Compound } \\
\hline & & Temp. range $(\mathrm{K})$ & $\begin{array}{c}\Delta_{\text {trans }} H_{\mathrm{m}} \\
\left(\mathrm{kJ} \mathrm{mol}^{-1}\right)\end{array}$ & $\mathrm{T}_{\mathrm{m}}(\mathrm{K})$ & Method & References \\
\hline \multirow[t]{3}{*}{$\mathrm{C}_{6} \mathrm{H}_{4} \mathrm{ClNO}_{3}$} & {$[89-64-5]$} & \multicolumn{5}{|c|}{ 4-chloro-2-nitrophenol } \\
\hline & FUS & & 22.69 & 360.3 & DSC & [2007MOR/MIR] \\
\hline & SUB & & $87.6 \pm 0.9$ & 298 & $\mathrm{C}$ & [2007MOR/MIR] \\
\hline \multirow[t]{3}{*}{$\mathrm{C}_{6} \mathrm{H}_{4} \mathrm{ClNO}_{3}$} & {$[610-78-6]$} & \multicolumn{5}{|c|}{ 4-chloro-3-nitrophenol } \\
\hline & FUS & & 25.97 & 399.4 & $\mathrm{DSC}$ & [2007MOR/MIR] \\
\hline & SUB & & $111.0 \pm 3.3$ & 298 & $\mathrm{C}$ & [2007MOR/MIR] \\
\hline \multirow[t]{3}{*}{$\mathrm{C}_{6} \mathrm{H}_{4} \mathrm{ClNO}_{3}$} & {$[54127-63-8]$} & 5-chloro-6-hydroxy & icotinic acid & & & \\
\hline & SUB & $(457-487)$ & $149.1 \pm 2.6$ & 472 & DSC & [2009SAN/FIG] \\
\hline & SUB & $(457-487)$ & $151.3 \pm 2.8$ & 298 & DSC & [2009SAN/FIG] \\
\hline \multirow[t]{3}{*}{$\mathrm{C}_{6} \mathrm{H}_{4} \mathrm{ClN}_{3} \mathrm{O}_{4}$} & [3531-19-9] & \multicolumn{5}{|c|}{ 2-chloro-4,6-dinitroaniline } \\
\hline & SUB & $(358-380)$ & $114.2 \pm 0.5$ & 369 & $\mathrm{ME}$ & [2010RIB/RIB] \\
\hline & SUB & $(358-380)$ & $115.0 \pm 0.9$ & 298 & $\mathrm{ME}$ & [2010RIB/RIB] \\
\hline \multirow[t]{3}{*}{$\mathrm{C}_{6} \mathrm{H}_{4} \mathrm{ClN}_{3} \mathrm{O}_{4}$} & {$[5388-62-5]$} & \multicolumn{5}{|c|}{ 4-chloro-2,6-dinitroaniline } \\
\hline & SUB & $(335-359)$ & $104.7 \pm 0.4$ & 347 & $\mathrm{ME}$ & [2010RIB/RIB] \\
\hline & SUB & $(335-359)$ & $105.2 \pm 0.7$ & 298 & ME & [2010RIB/RIB] \\
\hline \multirow[t]{3}{*}{$\mathrm{C}_{6} \mathrm{H}_{4} \mathrm{ClN}_{3} \mathrm{O}_{4}$} & {$[3531-19-9]$} & \multicolumn{5}{|c|}{ 2-chloro-4,6-dinitroaniline } \\
\hline & FUS (I) & & 21.1 & 433.2 & & \\
\hline & FUS (II) & & 24.2 & 435.2 & DSC & [2014KRI/RAJ] \\
\hline \multirow[t]{13}{*}{$\mathrm{C}_{6} \mathrm{H}_{4} \mathrm{Cl}_{2}$} & {$[95-50-1]$} & \multicolumn{5}{|l|}{ 1,2-dichlorobenzene } \\
\hline & FUS & & 13.09 & 255.9 & DSC & [2010WEI/WAN] \\
\hline & FUS & & 12.4 & 255.9 & DSC & [2009WEI/JIN, 2008WEI] \\
\hline & FUS & & 12.93 & 256.5 & & [1991ACR, 1983WEA, 1990DON/DRE] \\
\hline & $\mathrm{V}$ & & 51.2 & 256 & & [1999ROH/RUZ] \\
\hline & $\mathrm{V}$ & $(363-454)$ & 44.5 & 376 & $\mathrm{~EB}$ & [1998ROH/RUZ] \\
\hline & $\mathrm{V}$ & $(256-287)$ & 50.8 & 271 & & [1996POL/GUE] \\
\hline & $\mathrm{V}$ & $(413-453)$ & 50.9 & 298 & GC & [1994SPI/LUI] \\
\hline & $\mathrm{V}$ & $(258-313)$ & 51.2 & & GC & [1994LIU/DIC] \\
\hline & $\mathrm{V}$ & & $48.5 \pm 0.1$ & 298 & $\mathrm{C}$ & [1989AN/ZHE, 1989AN/ZHE2] \\
\hline & $\mathrm{V}$ & $(373-453)$ & 44.0 & 388 & A & [1987STE/MAL] \\
\hline & $\mathrm{V}$ & $(360-450)$ & 49.9 & 298 & & [1984BOU/FRI, 1991BAS/SVO] \\
\hline & $\mathrm{V}$ & $(301-343)$ & 50.0 & 322 & GS & {$[1982 \mathrm{GRA} / \mathrm{FOS}]$} \\
\hline \multirow[t]{10}{*}{$\mathrm{C}_{6} \mathrm{H}_{4} \mathrm{Cl}_{2}$} & {$[541-73-1]$} & 1,3-dichlorobenzen & & & & \\
\hline & FUS & & 12.51 & 248.3 & DSC & [2010WEI/WAN] \\
\hline & FUS & & 12.60 & 248.3 & DSC & [2009WEI/JIN, 2008WEI] \\
\hline & FUS & & 12.64 & 248.4 & & [1991ACR, 1983WEA] \\
\hline & $\mathrm{V}$ & & 50.4 & 248 & & [1999ROH/RUZ] \\
\hline & $\mathrm{V}$ & $(357-448)$ & 44.1 & 372 & $\mathrm{~EB}$ & [1998ROH/RUZ] \\
\hline & $\mathrm{V}$ & $(250-274)$ & 50.0 & 262 & & [1996POL/GUE] \\
\hline & $\mathrm{V}$ & $(413-453)$ & 53.9 & 298 & GC & [1994SPI/LUI] \\
\hline & $\mathrm{V}$ & $(360-450)$ & 47.0 & 298 & & [1984BOU/FRI, 1991BAS/SVO] \\
\hline & $\mathrm{V}$ & $(348-513)$ & 44.7 & 363 & A & [1987STE/MAL, 1972DYK] \\
\hline $\mathrm{C}_{6} \mathrm{H}_{4} \mathrm{Cl}_{2}$ & {$[106-46-7]$} & 1,4-dichlorobenzen & & & & \\
\hline & FUS & & 18.08 & 326.2 & DSC & [2010WEI/WAN] \\
\hline & FUS & & 18.19 & 326.2 & DSC & [2009WEI/JIN, 2008WEI] \\
\hline & TRS & $(5-380)$ & 1.24 & 275 & & \\
\hline & TRS & $(5-380)$ & 0.18 & 306 & & \\
\hline & FUS & $(5-380)$ & 17.91 & 326.2 & $\mathrm{AC}$ & [2005VAN/VAN] \\
\hline & TRS & $(20-330)$ & 1.26 & 271.8 & & \\
\hline & TRS & $(20-330)$ & 0.21 & 304.4 & & \\
\hline & FUS & $(20-330)$ & 18.19 & 326.2 & $\mathrm{AC}$ & [1976DWO/FIG] \\
\hline & FUS & & 18.2 & & DSC & [1972WAU/GET] \\
\hline & FUS & & 17.15 & 325.9 & DSC & [1991ACR, 1983WEA, 1969PLA/GLA] \\
\hline & FUS & & 19.0 & 326.7 & DSC & [1984MIL/GHO] \\
\hline & FUS & & 18.14 & 326.1 & & [1918NAR] \\
\hline & SUB & & $64.75 \pm 0.15$ & 298 & DM & [2000OON/VAN] \\
\hline & SUB & $(258-313)$ & 53.1 & & & [1994LIU/DIC] \\
\hline
\end{tabular}


TABLE 9. Phase change enthalpies of $\mathrm{C}_{6}$ organic compounds-Continued

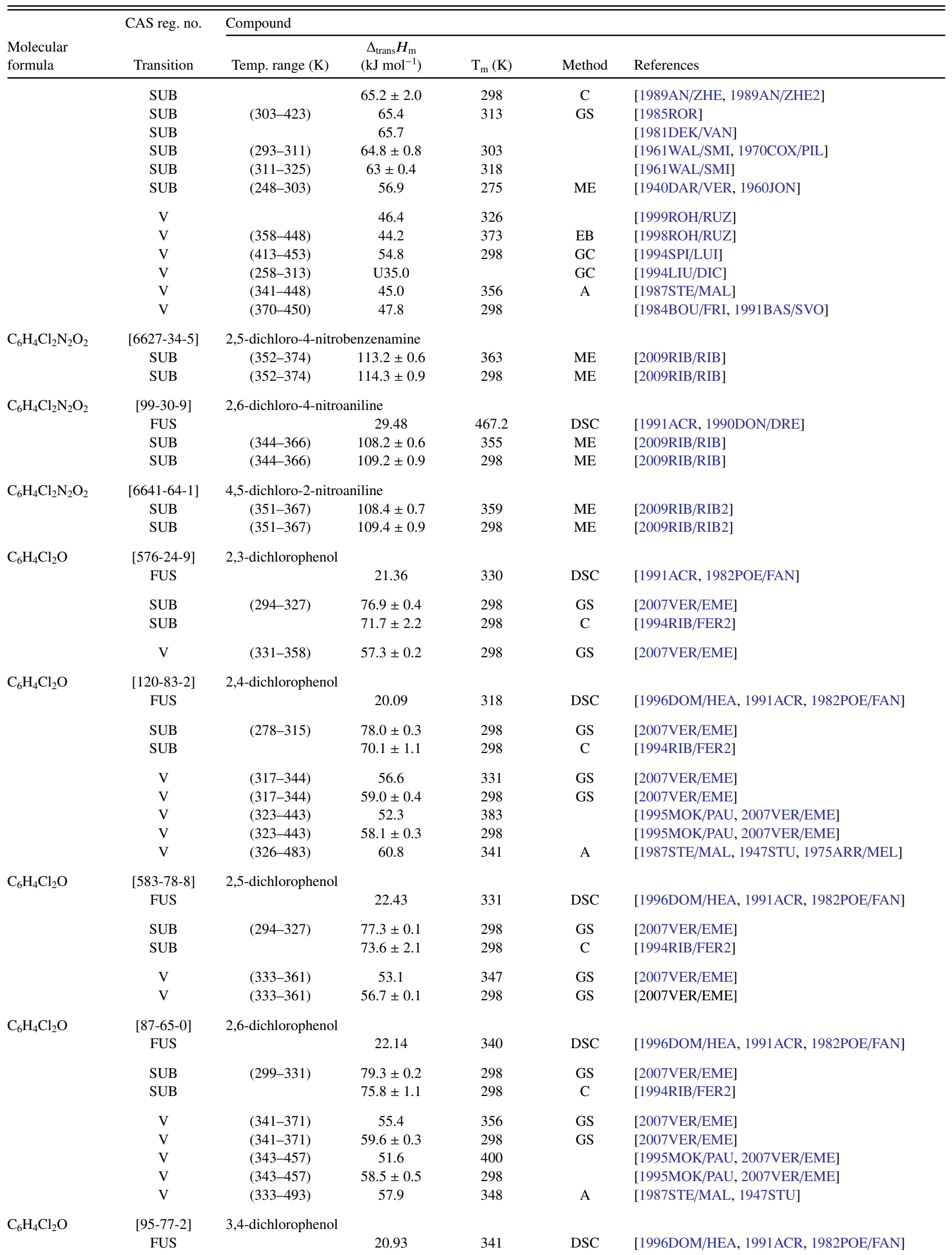


TABLE 9. Phase change enthalpies of $\mathrm{C}_{6}$ organic compounds-Continued

\begin{tabular}{|c|c|c|c|c|c|c|}
\hline \multirow[b]{2}{*}{$\begin{array}{l}\text { Molecular } \\
\text { formula }\end{array}$} & \multirow{2}{*}{$\begin{array}{l}\text { CAS reg. no. } \\
\text { Transition }\end{array}$} & \multicolumn{5}{|l|}{ Compound } \\
\hline & & Temp. range $(\mathrm{K})$ & $\begin{array}{c}\Delta_{\text {trans }} H_{\mathrm{m}} \\
\left(\mathrm{kJ} \mathrm{mol}^{-1}\right)\end{array}$ & $\mathrm{T}_{\mathrm{m}}(\mathrm{K})$ & Method & References \\
\hline & SUB & $(291-337)$ & $89.8 \pm 0.4$ & 298 & GS & [2007VER/EME] \\
\hline & SUB & & $81.3 \pm 2.3$ & 298 & $\mathrm{C}$ & [1994RIB/FER2] \\
\hline & $\mathrm{V}$ & $(341-368)$ & 66.7 & 355 & GS & [2007VER/EME] \\
\hline & $\mathrm{V}$ & $(341-368)$ & $70.8 \pm 0.2$ & 298 & GS & [2007VER/EME] \\
\hline \multirow[t]{4}{*}{$\mathrm{C}_{6} \mathrm{H}_{4} \mathrm{Cl}_{2} \mathrm{O}$} & {$[591-35-5]$} & 3,5-dichlorophenol & & & & \\
\hline & FUS & & 20.51 & 341 & DSC & [1996DOM/HEA, 1991ACR, 1982POE/FAN] \\
\hline & SUB & & $82.8 \pm 1.1$ & 298 & $\mathrm{C}$ & [1994RIB/FER2] \\
\hline & SUB & $(273-295)$ & 71.8 & 284 & A & [1987STE/MAL] \\
\hline \multirow[t]{2}{*}{$\mathrm{C}_{6} \mathrm{H}_{4} \mathrm{Cl}_{2} \mathrm{O}_{2}$} & {$[3428-24-8]$} & 4,5-dichloro-1,2-benze & enediol & & & \\
\hline & $\mathrm{V}$ & $(293-323)$ & 70.5 & 308 & CGC & [1999LEI/WAN2] \\
\hline \multirow[t]{2}{*}{$\mathrm{C}_{6} \mathrm{H}_{4} \mathrm{Cl}_{2} \mathrm{O}_{2}$} & {$[20103-10-0]$} & 2,6-dichlorohydroquin & lone & & & \\
\hline & SUB & $(324-345)$ & $92.0 \pm 8.3$ & & $\mathrm{QF}$ & [1927COO/COO, 1960JON, 1970COX/PIL] \\
\hline $\mathrm{C}_{6} \mathrm{H}_{4} \mathrm{Cl}_{2} \mathrm{O}_{3}$ & $\begin{array}{c}\text { [247097-56-9] } \\
\text { V }\end{array}$ & $\begin{array}{l}\text { vinyl mucochlorate } \\
\qquad(273-333)\end{array}$ & 63.9 & 288 & A & [1987STE/MAL, 1948RED/CHA5] \\
\hline \multirow[t]{2}{*}{$\mathrm{C}_{6} \mathrm{H}_{4} \mathrm{Cl}_{3} \mathrm{~N}$} & {$[634-67-3]$} & 2,3,4-trichloroaniline & & & & \\
\hline & SUB & & $92.4 \pm 1.7$ & 298 & $\mathrm{C}$ & [2002RIB/AMA] \\
\hline \multirow[t]{2}{*}{$\mathrm{C}_{6} \mathrm{H}_{4} \mathrm{Cl}_{3} \mathrm{~N}$} & {$[636-30-6]$} & 2,4,5-trichloroaniline & & & & \\
\hline & SUB & & $86.3 \pm 2.5$ & 298 & $\mathrm{C}$ & [2002RIB/AMA] \\
\hline \multirow[t]{3}{*}{$\mathrm{C}_{6} \mathrm{H}_{4} \mathrm{Cl}_{3} \mathrm{~N}$} & {$[634-93-5]$} & 2,4,6-trichloroaniline & & & & \\
\hline & SUB & & $85.3 \pm 1.9$ & 298 & $\mathrm{C}$ & [2002RIB/AMA] \\
\hline & V & $(407-535)$ & 92.9 & 422 & A & [1987STE/MAL, 1947STU] \\
\hline \multicolumn{7}{|c|}{ Note: Enthalpy of vaporization is likely in error } \\
\hline \multirow[t]{2}{*}{$\mathrm{C}_{6} \mathrm{H}_{4} \mathrm{Cl}_{3} \mathrm{~N}$} & {$[634-91-3]$} & 3,4,5-trichloroaniline & & & & \\
\hline & SUB & & $92.9 \pm 3.3$ & 298 & $\mathrm{C}$ & [2002RIB/AMA] \\
\hline \multirow[t]{3}{*}{$\mathrm{C}_{6} \mathrm{H}_{4} \mathrm{Cl}_{3} \mathrm{NO}$} & [35302-72-8] & 2-trichloroacetylpyrro & & & & \\
\hline & SUB & $(295-317)$ & $90.0 \pm 0.3$ & 306 & $\mathrm{ME}$ & [2010SAN/RIB3] \\
\hline & SUB & $(295-317)$ & $90.4 \pm 0.3$ & 298 & ME & [2010SAN/RIB3] \\
\hline \multirow[t]{2}{*}{$\mathrm{C}_{6} \mathrm{H}_{4} \mathrm{FI}$} & {$[348-52-7]$} & 2-iodo-1-fluorobenzen & & & & \\
\hline & V & $(283-328)$ & $51.5 \pm 0.3$ & 298 & GS & [2015VER/EME] \\
\hline \multirow[t]{2}{*}{$\mathrm{C}_{6} \mathrm{H}_{4} \mathrm{FI}$} & [1121-86-4] & 3-iodo-1-fluorobenzen & & & & \\
\hline & $\mathrm{V}$ & $(275-319)$ & $48.6 \pm 0.3$ & 298 & GS & [2015VER/EME] \\
\hline \multirow[t]{2}{*}{$\mathrm{C}_{6} \mathrm{H}_{4} \mathrm{FI}$} & [352-34-1] & 4-iodo-1-fluorobenzen & & & & \\
\hline & $\mathrm{V}$ & $(277-318)$ & $49.5 \pm 0.3$ & 298 & GS & [2015VER/EME] \\
\hline \multirow[t]{5}{*}{$\mathrm{C}_{6} \mathrm{H}_{4} \mathrm{FNO}_{2}$} & [1493-27-2] & 2-fluoronitrobenzene & & & & \\
\hline & SUB & $(257-261)$ & $79.3 \pm 0.8$ & 259 & Static & [2010RIB/MON] \\
\hline & SUB & $(257-261)$ & $78.4 \pm 0.8$ & 298 & Static & [2010RIB/MON] \\
\hline & $\mathrm{V}$ & $(267-359)$ & $57.9 \pm 0.1$ & 313 & Static & [2010RIB/MON] \\
\hline & $\mathrm{V}$ & $(267-359)$ & $59.5 \pm 0.1$ & 298 & Static & [2010RIB/MON] \\
\hline \multirow[t]{5}{*}{$\mathrm{C}_{6} \mathrm{H}_{4} \mathrm{FNO}_{2}$} & [402-67-5] & 3-fluoronitrobenzene & & & & \\
\hline & SUB & $(255-273)$ & $73.1 \pm 0.3$ & 264 & Static & [2010RIB/MON] \\
\hline & SUB & $(255-273)$ & $72.3 \pm 0.3$ & 298 & Static & [2010RIB/MON] \\
\hline & $\mathrm{V}$ & $(259-346)$ & $54.4 \pm 0.1$ & 302 & Static & [2010RIB/MON] \\
\hline & $\mathrm{V}$ & $(259-346)$ & $54.8 \pm 0.1$ & 298 & Static & [2010RIB/MON] \\
\hline \multirow[t]{5}{*}{$\mathrm{C}_{6} \mathrm{H}_{4} \mathrm{FNO}_{2}$} & [350-46-9] & 4-fluoronitrobenzene & & & & \\
\hline & SUB & $(259-291)$ & $71.5 \pm 0.3$ & 275 & Static & [2010RIB/MON] \\
\hline & SUB & $(259-291)$ & $70.9 \pm 0.3$ & 298 & Static & [2010RIB/MON] \\
\hline & $\mathrm{V}$ & $(272-349)$ & $55.1 \pm 0.1$ & 311 & Static & [2010RIB/MON] \\
\hline & $\mathrm{V}$ & $(272-349)$ & $56.0 \pm 0.1$ & 298 & Static & [2010RIB/MON] \\
\hline \multirow[t]{2}{*}{$\mathrm{C}_{6} \mathrm{H}_{4} \mathrm{~F}_{2}$} & [367-11-3] & 1,2-difluorobenzene & & & & \\
\hline & FUS & & 11.05 & 226.0 & & [1996DOM/HEA, 1963SCO/MES] \\
\hline
\end{tabular}


TABLE 9. Phase change enthalpies of $\mathrm{C}_{6}$ organic compounds-Continued

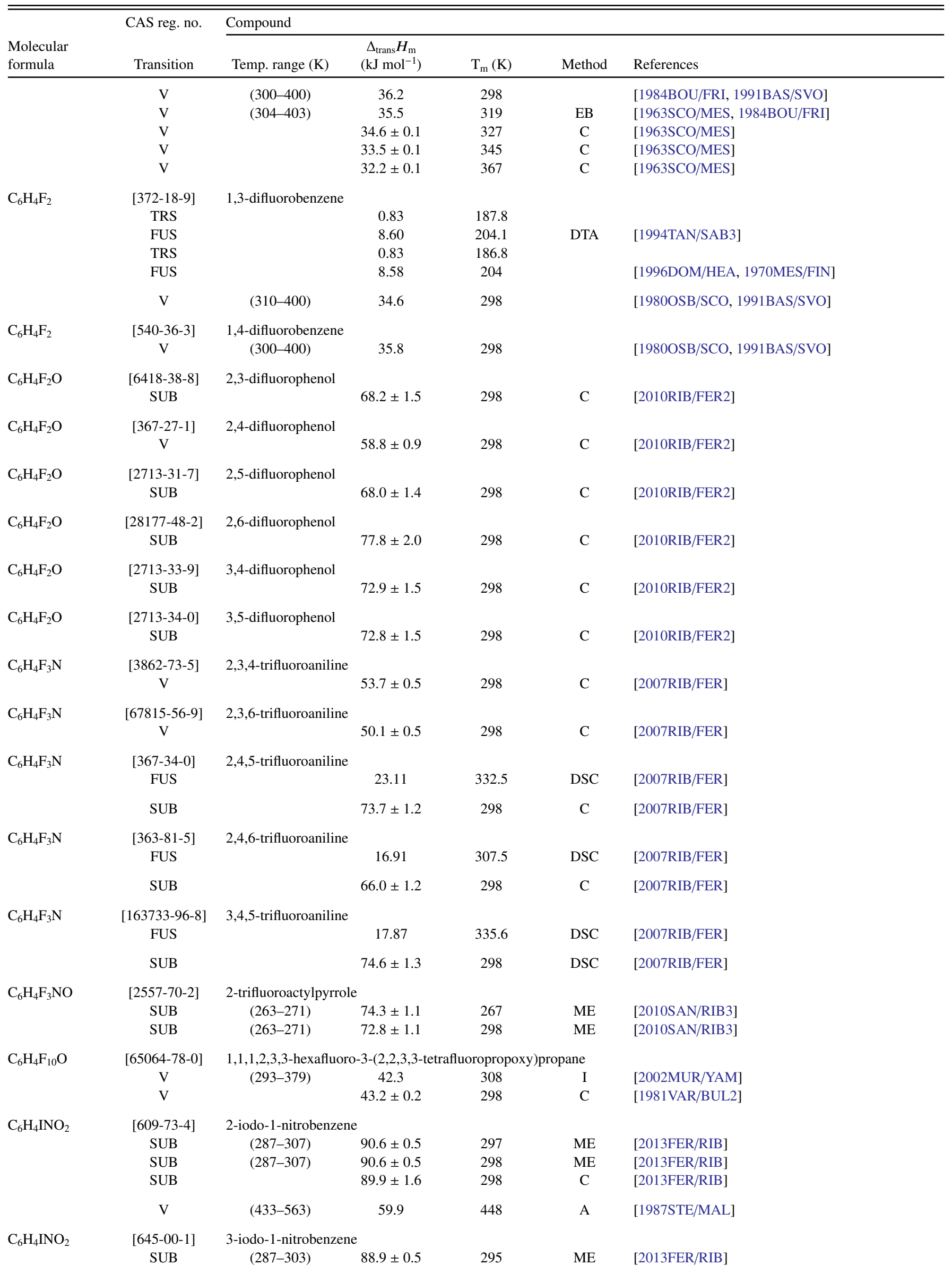


TABLE 9. Phase change enthalpies of $\mathrm{C}_{6}$ organic compounds-Continued

\begin{tabular}{|c|c|c|c|c|c|c|}
\hline \multirow[b]{2}{*}{$\begin{array}{l}\text { Molecular } \\
\text { formula }\end{array}$} & \multirow{2}{*}{$\begin{array}{l}\text { CAS reg. no. } \\
\text { Transition }\end{array}$} & \multicolumn{5}{|l|}{ Compound } \\
\hline & & Temp. range $(\mathrm{K})$ & $\begin{array}{c}\Delta_{\text {trans }} H_{\mathrm{m}} \\
\left(\mathrm{kJ} \mathrm{mol}^{-1}\right)\end{array}$ & $\mathrm{T}_{\mathrm{m}}(\mathrm{K})$ & Method & References \\
\hline & SUB & $(287-303)$ & $88.8 \pm 0.5$ & 298 & $\mathrm{ME}$ & [2013FER/RIB] \\
\hline & SUB & & $89.4 \pm 1.5$ & 298 & $\mathrm{C}$ & [2013FER/RIB] \\
\hline & SUB & $(295-306)$ & $83.2 \pm 1.2$ & 300 & & [1935TRI, 1938WOL/WEG, 1960JON] \\
\hline \multirow{4}{*}{$\mathrm{C}_{6} \mathrm{H}_{4} \mathrm{INO}_{2}$} & {$[636-98-6]$} & 4-iodo-1-nitrobenzene & & & & \\
\hline & SUB & $(311-333)$ & $93.9 \pm 0.5$ & 322 & ME & [2013FER/RIB] \\
\hline & SUB & $(311-333)$ & $94.6 \pm 0.6$ & 298 & $\mathrm{ME}$ & [2013FER/RIB] \\
\hline & SUB & & $98.2 \pm 1.7$ & 298 & $\mathrm{C}$ & [2013FER/RIB] \\
\hline \multirow[t]{4}{*}{$\mathrm{C}_{6} \mathrm{H}_{4} \mathrm{I}_{2}$} & {$[615-42-9]$} & 1,2-diiodobenzene & & & & \\
\hline & FUS & & 14.01 & 296.6 & & [1991ACR, 1983WEA] \\
\hline & $\mathrm{V}$ & $(299-348)$ & $63.8 \pm 0.4$ & 323 & GS & [2014VER/EME2] \\
\hline & $\mathrm{V}$ & $(299-348)$ & $65.6 \pm 0.5$ & 298 & GS & [2014VER/EME2] \\
\hline \multirow[t]{6}{*}{$\mathrm{C}_{6} \mathrm{H}_{4} \mathrm{I}_{2}$} & [626-00-6] & 1,3-diiodobenzene & & & & \\
\hline & FUS & & 15.93 & 307.4 & & [1991ACR, 1983WEA] \\
\hline & SUB & $(279-303)$ & $82.9 \pm 0.5$ & 291 & GS & [2014VER/EME2] \\
\hline & SUB & $(279-303)$ & $82.6 \pm 0.6$ & 298 & GS & [2014VER/EME2] \\
\hline & $\mathrm{V}$ & $(312-351)$ & $63.7 \pm 0.3$ & 331 & GS & [2014VER/EME2] \\
\hline & $\mathrm{V}$ & $(312-351)$ & $66.1 \pm 0.4$ & 298 & GS & [2014VER/EME2] \\
\hline \multirow[t]{9}{*}{$\mathrm{C}_{6} \mathrm{H}_{4} \mathrm{I}_{2}$} & [624-38-4] & 1,4-diiodobenzene & & & & \\
\hline & FUS & & 24.1 & 405.2 & DSC & [2010KAN/RED] \\
\hline & TRS & & 0.22 & 320 & & \\
\hline & FUS & $(5-420)$ & 22.3 & 402.4 & & [2001VAN/OON] \\
\hline & FUS & & 22.37 & 402 & & [1991ACR, 1983WEA] \\
\hline & SUB & $(298-347)$ & $84.4 \pm 0.3$ & 322 & GS & [2014VER/EME2] \\
\hline & SUB & $(298-347)$ & $85.4 \pm 0.4$ & 298 & GS & [2014VER/EME2] \\
\hline & SUB & $(372-401)$ & 63.4 & 386.5 & A & [1987STE/MAL] \\
\hline & $\mathrm{V}$ & $(402-560)$ & 52.6 & 417 & A & [1987STE/MAL] \\
\hline \multirow[t]{2}{*}{$\mathrm{C}_{6} \mathrm{H}_{4} \mathrm{~N}_{2}$} & {$[100-54-9]$} & nicotinic acid nitrile & & & & \\
\hline & $\mathrm{V}$ & $(453-479)$ & 45.0 & 466 & A & [1987STE/MAL, 1972DYK] \\
\hline \multirow[t]{2}{*}{$\mathrm{C}_{6} \mathrm{H}_{4} \mathrm{~N}_{2}$} & [100-70-9] & 2-cyanopyridine & & & & \\
\hline & SUB & $(233-323)$ & 76.6 & 298 & $\mathrm{ME}$ & [1991AUE/WEB] \\
\hline
\end{tabular}

Note: Authors of [1991AUE/WEB] refer to the value as an enthalpy of vaporization. The compound's melting point temperature fall within the range of measured VPs

$\begin{array}{ccccccc} & \text { SUB } & & 70.7 \pm 1.2 & 298 & \text { C } & \text { [1984BIC/PIL] } \\ \mathrm{C}_{6} \mathrm{H}_{4} \mathrm{~N}_{2} & {[100-54-9]} & \text { 3-cyanopyridine } & & & & \\ & \text { SUB } & & 72.1 \pm 1.8 & 298 & \mathrm{C} & {[1984 \mathrm{BIC} / \mathrm{PIL}]} \\ & \text { SUB } & (233-323) & 62.8 & 298 & \mathrm{ME} & {[1991 \mathrm{AUE} / \mathrm{WEB}]}\end{array}$

Note: Authors of [1991AUE/WEB] refer to the value as an enthalpy of vaporization. The compound's melting point temperature falls at the upper end of the range of measured VPS

SUB $79.0 \quad$ DSC $\quad[1989 \mathrm{SHI} / \mathrm{SHI}]$

\begin{tabular}{|c|c|c|c|c|c|c|}
\hline \multirow{3}{*}{$\mathrm{C}_{6} \mathrm{H}_{4} \mathrm{~N}_{2}$} & [100-48-1] & 4-cyanopyridine & & & & \\
\hline & SUB & & $73.2 \pm 0.6$ & 298 & $\mathrm{C}$ & [1984BIC/PIL] \\
\hline & SUB & $(233-323)$ & 54.0 & 298 & $\mathrm{ME}$ & [1991AUE/WEB] \\
\hline
\end{tabular}

Note: Authors of [1991AUE/WEB] refer to the value as an enthalpy of vaporization. The compound's melting point temperature falls above the range of measured VPs
SUB
75.6
DSC [1989SHI/SHI]

$\mathrm{C}_{6} \mathrm{H}_{4} \mathrm{~N}_{2} \mathrm{O}$

[14906-64-0] 3-cyanopyridine $N$-oxide

$\begin{array}{llllll}\text { SUB } & (345-392) & 101.9 \pm 2.0 & 298 & \text { ME } & \text { [1998RIB/MAT] }\end{array}$

$\mathrm{C}_{6} \mathrm{H}_{4} \mathrm{~N}_{2} \mathrm{O} \quad$ [14906-59-3] 4-cyanopyridine $N$-oxide

$\begin{array}{llllll}\text { SUB } & (345-392) & 104.4 \pm 4.3 & 298 & \text { ME } & \text { [1998RIB/MAT] }\end{array}$

$\mathrm{C}_{6} \mathrm{H}_{4} \mathrm{~N}_{2} \mathrm{O} \quad$ [273-09-6] benzofurazan

$\begin{array}{lllcl}\text { SUB } & 64.4 \pm 1.6 & 298 & \text { C } & \text { [1990LEI/PIL] } \\ \text { SUB } & 64.9 \pm 1.7 & 298 & \text { ME GS } & \text { [1980ARS] }\end{array}$

$\mathrm{C}_{6} \mathrm{H}_{4} \mathrm{~N}_{2} \mathrm{O}_{2} \quad$ [480-96-6] benzofurazan $N$-oxide

$\begin{array}{lllll}\text { SUB } & 79.6 \pm 1.7 & 298 & \mathrm{C} & \text { [1990LEI/PIL] }\end{array}$


TABLE 9. Phase change enthalpies of $\mathrm{C}_{6}$ organic compounds-Continued

\begin{tabular}{|c|c|c|c|c|c|c|}
\hline \multirow[b]{2}{*}{$\begin{array}{l}\text { Molecular } \\
\text { formula }\end{array}$} & \multirow{2}{*}{$\begin{array}{l}\text { CAS reg. no. } \\
\text { Transition }\end{array}$} & \multicolumn{5}{|l|}{ Compound } \\
\hline & & Temp. range $(\mathrm{K})$ & $\begin{array}{c}\Delta_{\text {trans }} H_{\mathrm{m}} \\
\left(\mathrm{kJ} \mathrm{mol}^{-1}\right)\end{array}$ & $\mathrm{T}_{\mathrm{m}}(\mathrm{K})$ & Method & References \\
\hline $\mathrm{C}_{6} \mathrm{H}_{4} \mathrm{~N}_{2} \mathrm{O}_{3}$ & SUB & $(323-343)$ & 95.5 & 333 & A & [1987STE/MAL, 1974PEP/LEB] \\
\hline \multirow[t]{8}{*}{$\mathrm{C}_{6} \mathrm{H}_{4} \mathrm{~N}_{2} \mathrm{O}_{4}$} & [528-29-0] & 1,2-dinitrobenzene & & & & \\
\hline & FUS & & 22.18 & 387.7 & DSC & [1993ACR, 1990HWA/TAM] \\
\hline & SUB & $(323-383)$ & 94.0 & & TGA & [2011FEL/RAM] \\
\hline & SUB & $(323-353)$ & $95.5 \pm 0.9$ & 298 & GS & [1997VER3] \\
\hline & SUB & $(323-353)$ & $93.1 \pm 0.9$ & 338 & GS & [1997VER3] \\
\hline & SUB & $(343-387)$ & 82.9 & 358 & A & [1987STE/MAL] \\
\hline & SUB & $(343-397)$ & $87.9 \pm 2.1$ & 298 & $\mathrm{TE}$ & [1976FER/PIA] \\
\hline & SUB & $(328-338)$ & $86.6 \pm 1.2$ & 309 & & [1935TRI, 1938WOL/WEG, 1960JON] \\
\hline \multirow{11}{*}{$\mathrm{C}_{6} \mathrm{H}_{4} \mathrm{~N}_{2} \mathrm{O}_{4}$} & FUS & & 16.0 & 364.0 & DSC & [2011RAI/MUD] \\
\hline & FUS & & 21.2 & 364.8 & DSC & [2010AGR/GUP] \\
\hline & FUS & & 24.2 & 363.0 & DSC & {$[2004 \mathrm{GUP} / \mathrm{SIN}]$} \\
\hline & FUS & & 13.8 & 363.0 & DSC & [2004SHA/TAN] \\
\hline & FUS & & 19.68 & 360.4 & DSC & [2002MUS/RAZ] \\
\hline & FUS & & 17.57 & 360.1 & DSC & [1993ACR, 1990HWA/TAM] \\
\hline & FUS & & 17.36 & 363.2 & & [1991ACR, 1983WEA] \\
\hline & SUB & $(335-356)$ & 76.1 & 345.5 & A & [1987STE/MAL] \\
\hline & SUB & $(332-383)$ & $87.0 \pm 0.8$ & 298 & TE & {$[1976$ FER/PIA $]$} \\
\hline & SUB & $(315-329)$ & $81.1 \pm 1.7$ & 323 & & [1935TRI, 1938WOL/WEG, 1960JON] \\
\hline & SUB & & $81.2 \pm 1.7$ & & & [1950NIT/SEK3, 1970COX/PIL] \\
\hline \multirow{4}{*}{$\mathrm{C}_{6} \mathrm{H}_{4} \mathrm{~N}_{2} \mathrm{O}_{4}$} & SUB & $(323-353)$ & $94.3 \pm 0.7$ & 298 & GS & [1997VER3] \\
\hline & SUB & $(339-398)$ & $96.2 \pm 2.5$ & 298 & TE & [1976FER/PIA] \\
\hline & SUB & $(345-368)$ & $89.1 \pm 1.7$ & 343 & & [1935TRI, 1938WOL/WEG, 1960JON] \\
\hline & $\mathrm{V}$ & $(445-572)$ & 60.3 & 460 & A & [1987STE/MAL, 1972DYK] \\
\hline \multirow[t]{4}{*}{$\mathrm{C}_{6} \mathrm{H}_{4} \mathrm{~N}_{2} \mathrm{O}_{5}$} & {$[66-56-8]$} & 2,3-dinitrophenol & & & & \\
\hline & FUS & & 22.67 & 419 & DSC & [2002MUS/RAZ] \\
\hline & FUS & & 26.24 & 417 & DSC & [1996DOM/HEA, 1982POE/FAN] \\
\hline & SUB & $(303-343)$ & 96.6 & 323 & & [1958HOY/PEP] \\
\hline \multirow[t]{2}{*}{$\mathrm{C}_{6} \mathrm{H}_{4} \mathrm{~N}_{2} \mathrm{O}_{5}$} & [51-28-5] & 2,4-dinitrophenol & & & & \\
\hline & FUS & & 25.3 & 388 & DSC & [2004SHA/TAN] \\
\hline
\end{tabular}

Note: Melting point temperature of $363.3 \mathrm{~K}$ was given in the experimental section of [2004SHA/TAN] for 2,4-dinitrophenol; however, a much higher was shown in the phase diagrams.

FUS

SUB

(293-333)

$\mathrm{C}_{6} \mathrm{H}_{4} \mathrm{~N}_{2} \mathrm{O}_{5}$

[329-71-5] 2,5-dinitrophenol FUS

$\mathrm{C}_{6} \mathrm{H}_{4} \mathrm{~N}_{2} \mathrm{O}_{5}$

$\begin{array}{cc}\text { SUB } & (278-333) \\ \text { [573-56-8] } & \text { 2,6-dinitrophenol } \\ \text { FUS } & \\ \text { FUS } & \\ \text { SUB } & (293-333)\end{array}$

$\begin{array}{cc}26.19 & 383.2 \\ 24.17 & 388 \\ 104.6 \pm 4.2 & 313\end{array}$

$23.73 \quad 381.0$

93.4

22.91

19.58

$112.1 \pm 4.2$

\author{
DSC [2002MUS/RAZ] \\ DSC [1996DOM/HEA, 1982POE/FAN] \\ [1958HOY/PEP, 1970COX/PIL]
}

DSC [1996DOM/HEA, 1982POE/FAN]

[1958HOY/PEP]

DSC [2002MUS/RAZ]

DSC [1996DOM/HEA, 1982POE/FAN]

[1958HOY/PEP, 1970COX/PIL] 
TABLE 9. Phase change enthalpies of $\mathrm{C}_{6}$ organic compounds-Continued

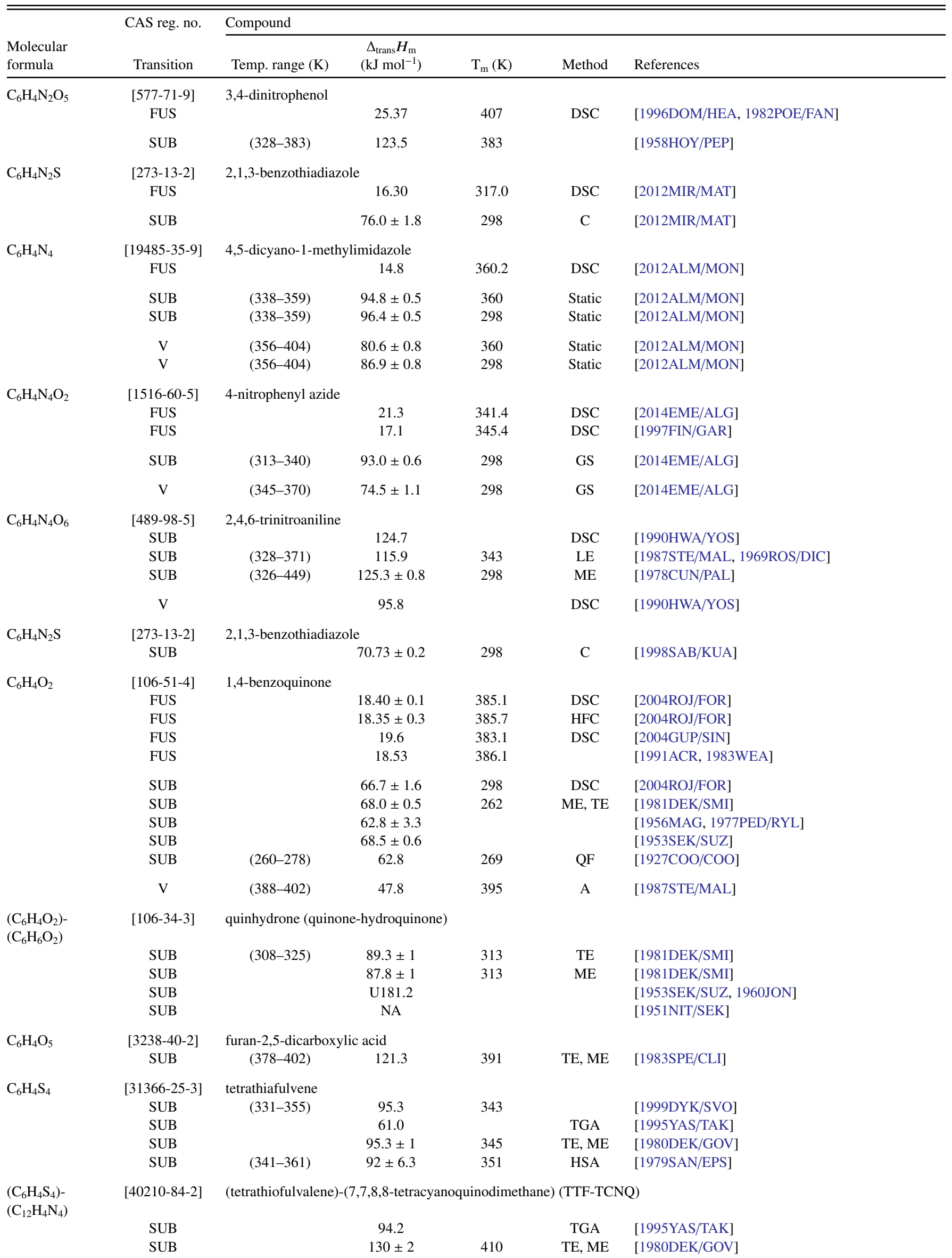


TABLE 9. Phase change enthalpies of $\mathrm{C}_{6}$ organic compounds-Continued

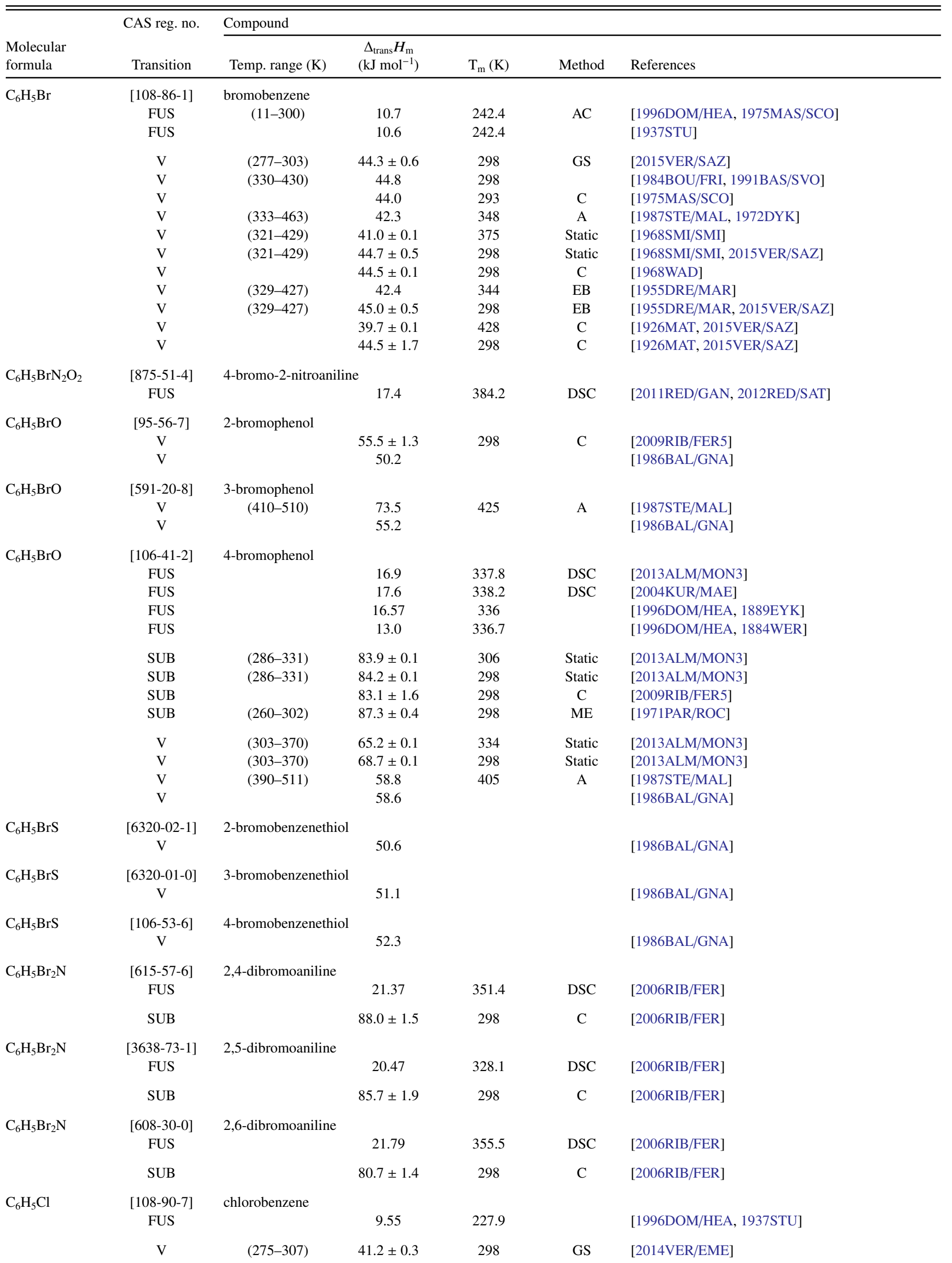


TABLE 9. Phase change enthalpies of $\mathrm{C}_{6}$ organic compounds-Continued

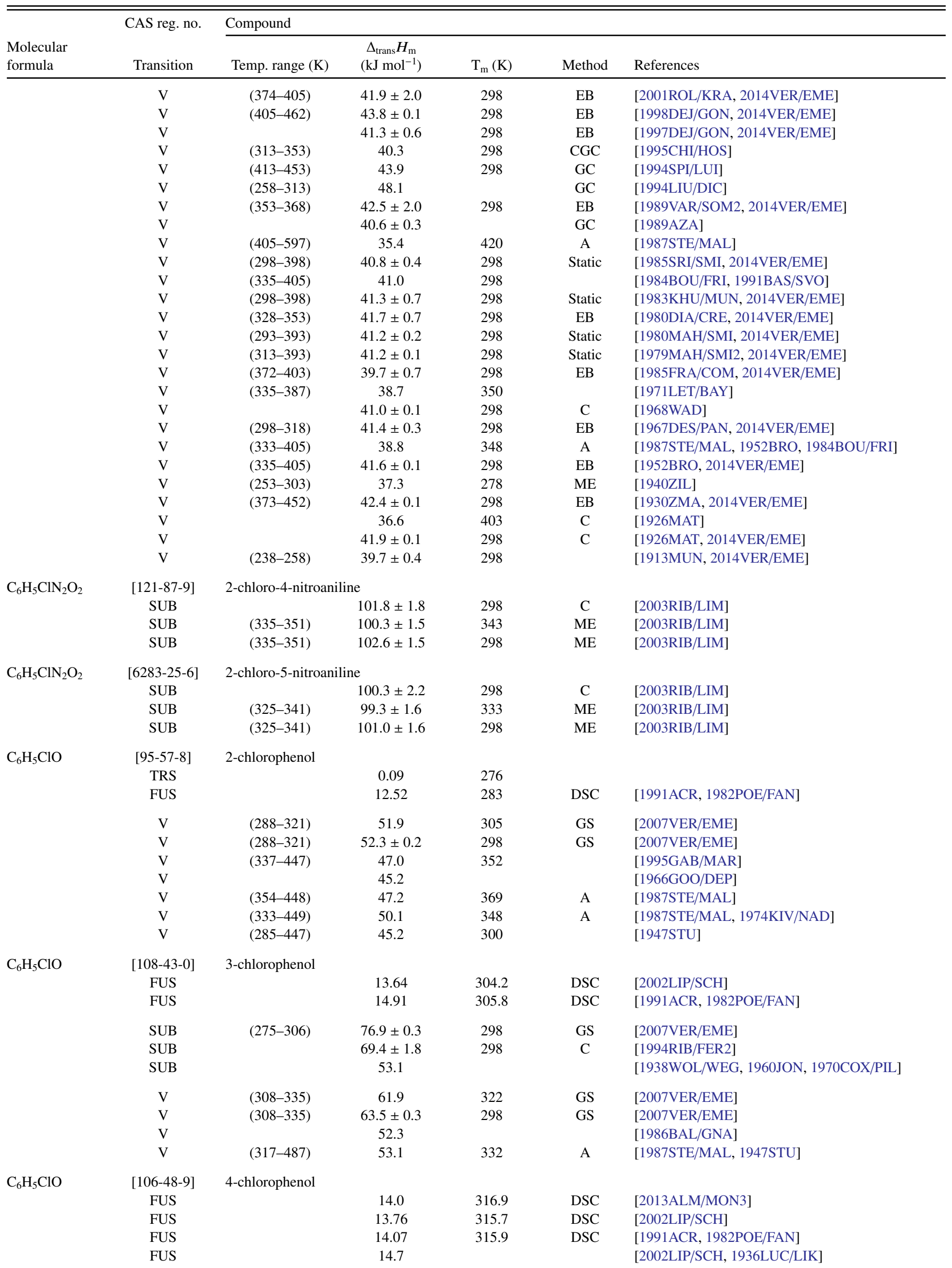


TABLE 9. Phase change enthalpies of $\mathrm{C}_{6}$ organic compounds-Continued

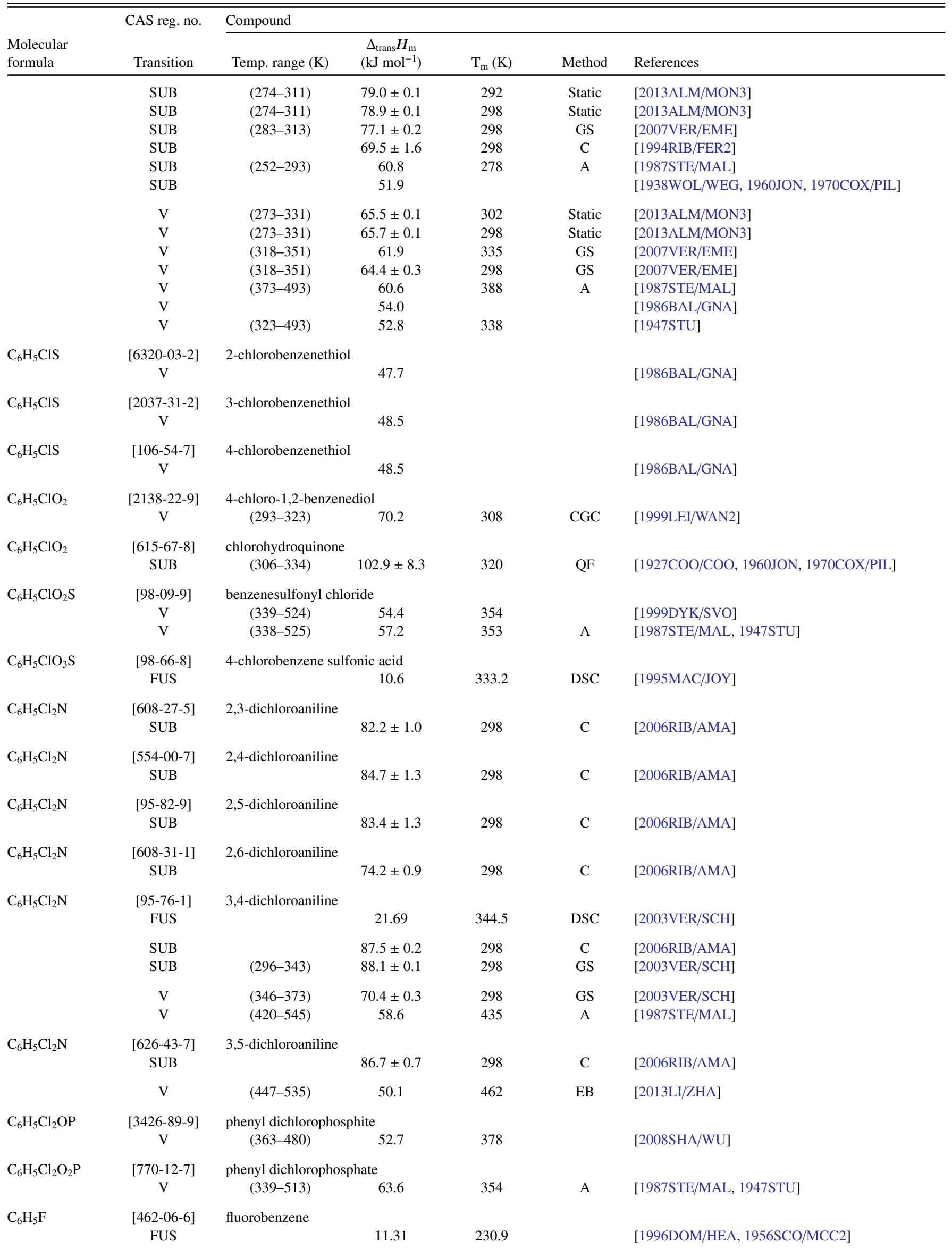


TABLE 9. Phase change enthalpies of $\mathrm{C}_{6}$ organic compounds-Continued

\begin{tabular}{|c|c|c|c|c|c|c|}
\hline \multirow[b]{2}{*}{$\begin{array}{l}\text { Molecular } \\
\text { formula }\end{array}$} & \multirow{2}{*}{$\begin{array}{c}\text { CAS reg. no. } \\
\text { Transition }\end{array}$} & \multicolumn{5}{|l|}{ Compound } \\
\hline & & Temp. range $(\mathrm{K})$ & $\begin{array}{c}\Delta_{\text {trans }} H_{\mathrm{m}} \\
\left(\mathrm{kJ} \mathrm{mol}^{-1}\right)\end{array}$ & $\mathrm{T}_{\mathrm{m}}(\mathrm{K})$ & Method & References \\
\hline & FUS & & 10.4 & 231.1 & & {$[1937 \mathrm{STU}]$} \\
\hline & $\mathrm{V}$ & $(274-303)$ & $34.5 \pm 0.2$ & 298 & GS & [2014VER/EME] \\
\hline & $\mathrm{V}$ & $(358-530)$ & 31.9 & 373 & A & [1987STE/MAL] \\
\hline & $\mathrm{V}$ & $(373-419)$ & 31.8 & 388 & A & [1987STE/MAL] \\
\hline & $\mathrm{V}$ & $(414-501)$ & 31.0 & 429 & A & [1987STE/MAL] \\
\hline & $\mathrm{V}$ & $(497-561)$ & 30.9 & 512 & A & [1987STE/MAL] \\
\hline & $\mathrm{V}$ & $(255-360)$ & 34.5 & 298 & & [1984BOU/FRI, 1991BAS/SVO] \\
\hline & $\mathrm{V}$ & $(313-353)$ & $35.2 \pm 0.1$ & 298 & $\mathrm{~EB}$ & [1980DIA/CRE, 2014VER/EME] \\
\hline & $\mathrm{V}$ & $(312-394)$ & 33.6 & 327 & $\mathrm{~EB}$ & [1987STE/MAL, 1956SCO/MCC2] \\
\hline & $\mathrm{V}$ & & $33.5 \pm 0.1$ & 318 & $\mathrm{C}$ & [1956SCO/MCC2] \\
\hline & $\mathrm{V}$ & & $32.4 \pm 0.1$ & 337 & $\mathrm{C}$ & [1956SCO/MCC2] \\
\hline & $\mathrm{V}$ & & $31.2 \pm 0.1$ & 358 & $\mathrm{C}$ & [1956SCO/MCC2] \\
\hline & $\mathrm{V}$ & & $29.7 \pm 0.1$ & 382 & $\mathrm{C}$ & [1956SCO/MCC2] \\
\hline & $\mathrm{V}$ & $(255-357)$ & $34.6 \pm 0.1$ & 298 & Static & [1889YOU, 2014VER/EME] \\
\hline \multirow[t]{2}{*}{$\mathrm{C}_{6} \mathrm{H}_{5} \mathrm{FO}$} & [367-12-4] & 2-fluorophenol & & & & \\
\hline & $\mathrm{V}$ & & $52.3 \pm 0.8$ & 298 & $\mathrm{C}$ & [2009RIB/FER] \\
\hline \multirow[t]{3}{*}{$\mathrm{C}_{6} \mathrm{H}_{5} \mathrm{FO}$} & {$[372-20-3]$} & 3-fluorophenol & & & & \\
\hline & V & & $60.1 \pm 0.9$ & 298 & $\mathrm{C}$ & [2009RIB/FER] \\
\hline & $\mathrm{V}$ & $(373-451)$ & 50.3 & 388 & A & [1987STE/MAL] \\
\hline \multirow[t]{8}{*}{$\mathrm{C}_{6} \mathrm{H}_{5} \mathrm{FO}$} & [371-41-5] & 4-fluorophenol & & & & \\
\hline & FUS & & 14.5 & 320.4 & DSC & [2013ALM/MON3] \\
\hline & SUB & $(265-317)$ & $74.3 \pm 0.1$ & 291 & Static & [2013ALM/MON3] \\
\hline & SUB & $(265-317)$ & $74.1 \pm 0.1$ & 298 & Static & [2013ALM/MON3] \\
\hline & SUB & & $73.9 \pm 1.4$ & 298 & $\mathrm{C}$ & [2009RIB/FER] \\
\hline & $\mathrm{V}$ & $(291-343)$ & $58.7 \pm 0.1$ & 317 & Static & [2013ALM/MON3] \\
\hline & $\mathrm{V}$ & $(291-343)$ & $60.0 \pm 0.2$ & 298 & Static & [2013ALM/MON3] \\
\hline & $\mathrm{V}$ & $(360-460)$ & 48.8 & 375 & A & [1987STE/MAL] \\
\hline \multirow[t]{2}{*}{$\mathrm{C}_{6} \mathrm{H}_{5} \mathrm{~F}_{2} \mathrm{~N}$} & [4519-40-8] & 2,3-difluoroaniline & & & & \\
\hline & $\mathrm{V}$ & & $49.3 \pm 0.5$ & 298 & $\mathrm{C}$ & [2007RIB/FER] \\
\hline \multirow[t]{2}{*}{$\mathrm{C}_{6} \mathrm{H}_{5} \mathrm{~F}_{2} \mathrm{~N}$} & {$[367-25-9]$} & 2,4-difluoroaniline & & & & \\
\hline & $\mathrm{V}$ & & $52.1 \pm 0.5$ & 298 & $\mathrm{C}$ & [2007RIB/FER] \\
\hline \multirow[t]{2}{*}{$\mathrm{C}_{6} \mathrm{H}_{5} \mathrm{~F}_{2} \mathrm{~N}$} & [367-30-6] & 2,5-difluoroaniline & & & & \\
\hline & $\mathrm{V}$ & & $52.5 \pm 0.5$ & 298 & $\mathrm{C}$ & [2007RIB/FER] \\
\hline \multirow[t]{2}{*}{$\mathrm{C}_{6} \mathrm{H}_{5} \mathrm{~F}_{2} \mathrm{~N}$} & [5509-65-9] & 2,6-difluoroaniline & & & & \\
\hline & $\mathrm{V}$ & & $47.5 \pm 0.5$ & 298 & $\mathrm{C}$ & [2007RIB/FER] \\
\hline \multirow[t]{2}{*}{$\mathrm{C}_{6} \mathrm{H}_{5} \mathrm{~F}_{2} \mathrm{~N}$} & [3863-11-4] & 3,4-difluoroaniline & & & & \\
\hline & $\mathrm{V}$ & & $53.3 \pm 0.5$ & 298 & $\mathrm{C}$ & [2007RIB/FER] \\
\hline \multirow[t]{3}{*}{$\mathrm{C}_{6} \mathrm{H}_{5} \mathrm{~F}_{2} \mathrm{~N}$} & {$[372-39-4]$} & 3,5-difluoroaniline & & & & \\
\hline & FUS & & 16.49 & 312.7 & $\mathrm{DSC}$ & [2007RIB/FER] \\
\hline & SUB & & $71.7 \pm 1.7$ & 298 & $\mathrm{C}$ & [2007RIB/FER] \\
\hline
\end{tabular}

Note: Authors indicate that this is a liquid to vapor phase transition; however, we believe it to be a solid to vapor transition given the size of the numerical value and that the calorimetric measurement was made below the melting point temperature.

\begin{tabular}{|c|c|c|c|c|c|c|}
\hline \multirow[t]{2}{*}{$\mathrm{C}_{6} \mathrm{H}_{5} \mathrm{~F}_{8} \mathrm{NOS}$} & [77984-30-6] & \multicolumn{5}{|c|}{ 1-(ethylimino)-2,2,3,3,4,4,5,5-octafluoro-1,1,2,3,4,5-hexahydrothiophene-1-oxide } \\
\hline & $\mathrm{V}$ & & 31.4 & 333 & & [1981ABE/SHR] \\
\hline \multirow[t]{2}{*}{$\mathrm{C}_{6} \mathrm{H}_{5} \mathrm{~F}_{9} \mathrm{O}$} & [163702-05-4] & \multicolumn{5}{|c|}{ 1-ethoxy-1,1,2,2,3,3,4,4,4-nonafluorobutane } \\
\hline & $\mathrm{V}$ & $(293-350)$ & 34.2 & 308 & I & [2002MUR/YAM] \\
\hline \multirow[t]{7}{*}{$\mathrm{C}_{6} \mathrm{H}_{5} \mathrm{I}$} & [591-50-4] & iodobenzene & & & & \\
\hline & FUS & & 9.75 & 241.8 & & [1996DOM/HEA, 1937STU] \\
\hline & $\mathrm{V}$ & $(284-314)$ & $48.5 \pm 0.4$ & 299 & GS & [2015VER/SAZ] \\
\hline & $\mathrm{V}$ & $(284-314)$ & $48.5 \pm 0.6$ & 298 & GS & [2015VER/SAZ] \\
\hline & $\mathrm{V}$ & $(313-353)$ & 47.4 & 298 & CGC & [1995CHI/HOS] \\
\hline & $\mathrm{V}$ & $(462-679)$ & 41.1 & 477 & $\mathrm{~A}$ & [1987STE/MAL] \\
\hline & $\mathrm{V}$ & $(320-460)$ & 48.9 & 298 & & [1984BOU/FRI, 1991BAS/SVO] \\
\hline
\end{tabular}


TABLE 9. Phase change enthalpies of $\mathrm{C}_{6}$ organic compounds - Continued

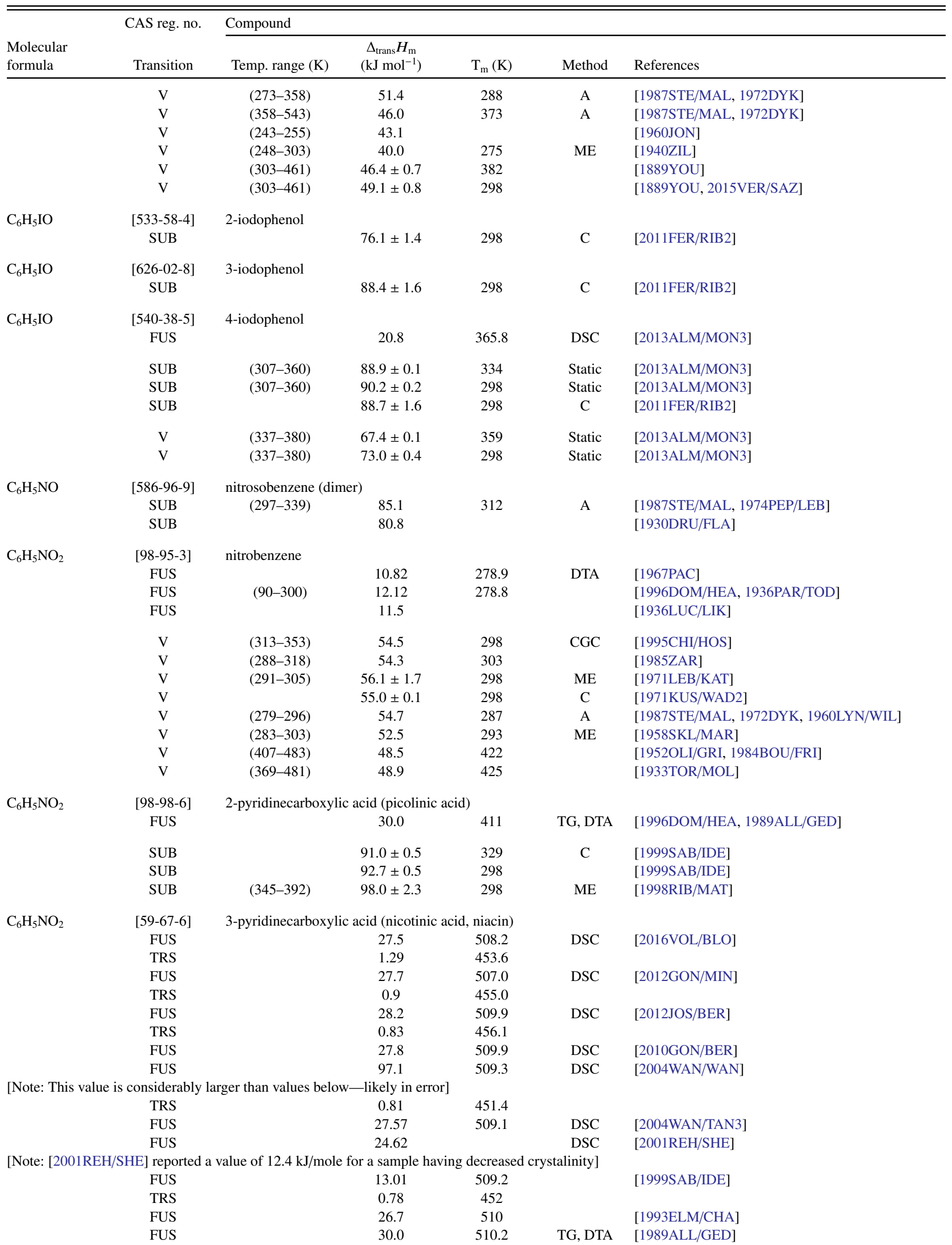


TABLE 9. Phase change enthalpies of $\mathrm{C}_{6}$ organic compounds-Continued

\begin{tabular}{|c|c|c|c|c|c|c|}
\hline \multirow[b]{2}{*}{$\begin{array}{l}\text { Molecular } \\
\text { formula }\end{array}$} & \multirow{2}{*}{$\begin{array}{c}\text { CAS reg. no. } \\
\text { Transition }\end{array}$} & \multicolumn{5}{|l|}{ Compound } \\
\hline & & Temp. range $(\mathrm{K})$ & $\begin{array}{c}\Delta_{\text {trans }} H_{\mathrm{m}} \\
\left(\mathrm{kJ} \mathrm{mol}^{-1}\right)\end{array}$ & $\mathrm{T}_{\mathrm{m}}(\mathrm{K})$ & Method & References \\
\hline & SUB & $(362-387)$ & $111.7 \pm 1.2$ & 298 & GS & [2016VOL/BLO] \\
\hline & SUB & & $112.1 \pm 0.5$ & 298 & $\mathrm{ME}$ & [2010GON/BER] \\
\hline & SUB & $(473-498)$ & 89.3 & & TG, DTA & [2002MEN/DOL] \\
\hline & SUB & $(352-360)$ & $123.9 \pm 3.7$ & 298 & $\mathrm{ME}$ & [2000RIB/GON] \\
\hline & SUB & & $101.1 \pm 0.6$ & 362 & $\mathrm{C}$ & [1999SAB/IDE] \\
\hline & SUB & & $105.2 \pm 0.6$ & 298 & & [1999SAB/IDE] \\
\hline & SUB & & $123.4 \pm 1.2$ & 298 & $\mathrm{C}$ & [1984BIC/PIL] \\
\hline \multirow{2}{*}{$\mathrm{C}_{6} \mathrm{H}_{5} \mathrm{NO}_{2}$} & {$[55-22-1]$} & \multicolumn{5}{|c|}{ 4-pyridinecarboxylic acid (isonicotinic acid) } \\
\hline & FUS & & 135 & 593 & TG, DTA & [1996DOM/HEA, 1989ALL/GED] \\
\hline \multicolumn{7}{|c|}{ Note: Enthalpy of fusion is much too large, compound likely decomposed } \\
\hline & SUB & & $107.7 \pm 0.7$ & 362 & $\mathrm{C}$ & [1999SAB/IDE] \\
\hline & SUB & & $111.3 \pm 0.6$ & 298 & & [1999SAB/IDE] \\
\hline & SUB & $(345-392)$ & $113.9 \pm 4.7$ & 298 & ME & {$[1998 \mathrm{RIB} / \mathrm{MAT}]$} \\
\hline \multirow[t]{14}{*}{$\mathrm{C}_{6} \mathrm{H}_{5} \mathrm{NO}_{3}$} & {$[88-75-5]$} & 2-nitrophenol & & & & \\
\hline & FUS & & U14.88 & 319.2 & DSC & [2014HAS/JIR] \\
\hline & FUS & & 21.77 & 318.7 & & [2012SHA/LAL] \\
\hline & FUS & & 17.05 & 316.3 & DSC & [2002MUS/RAZ] \\
\hline & FUS & & 17.45 & 318.2 & DTA & [1991ACR, 1994SAB/GOU] \\
\hline & FUS & & 17.88 & & DSC & [1992SHA/SHA] \\
\hline & FUS & & 17.91 & 318.6 & DSC & {$[1990 \mathrm{DON} / \mathrm{DRE}]$} \\
\hline & SUB & & 73.3 & 298 & $\mathrm{C}$ & [1994SAB/GOU] \\
\hline & SUB & $(273-292)$ & 54.8 & 282.5 & A & [1987STE/MAL] \\
\hline & SUB & $(298-310)$ & $73.2 \pm 1.3$ & & & [1935TRI, 1938WOL/WEG, 1960JON] \\
\hline & $\mathrm{V}$ & $(319-346)$ & $58.4 \pm 0.5$ & 298 & GS & [2007HEI/KAP] \\
\hline & $\mathrm{V}$ & $(366-490)$ & 55.9 & 381 & A & [1987STE/MAL] \\
\hline & $\mathrm{V}$ & $(324-347)$ & U43.3 & 298 & $\mathrm{ME}$ & [1958SKL/MAR, 2007HEI/KAP] \\
\hline & $\mathrm{V}$ & $(322-357)$ & 54.4 & 337 & A & {$[1947 \mathrm{STU}]$} \\
\hline \multirow[t]{13}{*}{$\mathrm{C}_{6} \mathrm{H}_{5} \mathrm{NO}_{3}$} & {$[554-84-7]$} & 3-nitrophenol & & & & \\
\hline & FUS & & 18.06 & 369 & DSC & [2002MUS/RAZ] \\
\hline & FUS & & 19.2 & 370 & DSC & [1982POE/FAN] \\
\hline & FUS & & 19.19 & 371.2 & DTA & [1991ACR, 1994SAB/GOU] \\
\hline & TRS & & 0.17 & 356 & & \\
\hline & FUS & & 19.96 & 370 & DSC & [1996DOM/HEA, 1989WOJ/MAR] \\
\hline & FUS & & 21.3 & 370.0 & & [1996DOM/HEA, 1972BOO/HAU] \\
\hline & SUB & & $91.2 \pm 0.5$ & 298 & $\mathrm{C}$ & {$[1994 \mathrm{SAB} / \mathrm{GOU}]$} \\
\hline & SUB & $(316-330)$ & $98.9 \pm 0.6$ & 321 & ME & [1992RIB/REI] \\
\hline & SUB & $(316-330)$ & $100.2 \pm 0.6$ & 298 & $\mathrm{ME}$ & [1992RIB/REI] \\
\hline & SUB & $(305-334)$ & 76.2 & 319.5 & & [1987STE/MAL] \\
\hline & SUB & $(303-328)$ & 91.8 & & ME & [1974LIO/HOP] \\
\hline & SUB & $(325-336)$ & $91.6 \pm 1.7$ & & & [1935TRI, 1938WOL/WEG, 1960JON] \\
\hline \multirow[t]{6}{*}{$\mathrm{C}_{6} \mathrm{H}_{5} \mathrm{NO}_{3}$} & {$[100-02-7]$} & 4-nitrophenol & & & & \\
\hline & FUS & & 16.69 & 385.2 & DSC & [2014HAS/JIR] \\
\hline & FUS & & 18.97 & 388.2 & DSC & [2013SIN/PAN] \\
\hline & FUS & & 19.22 & 387.2 & DSC & [2012KAN/RAI] \\
\hline & TRS & & 5.0 & & & \\
\hline & FUS & & 12.0 & & DSC & [2006WOJ/MOS] \\
\hline \multicolumn{7}{|c|}{$\begin{array}{l}\text { Note: Authors of [ } 2006 \mathrm{WOJ} / \mathrm{MOS}] \text { report that the } \beta \text {-phase to } \alpha \text {-phase was very sluggish, and difficult to quantify. The values given were referred to } \\
\text { crude estimates }\end{array}$} \\
\hline & FUS & & 11.0 & 386.4 & DSC & [2002MUS/RAZ] \\
\hline & FUS & & 18.3 & 388.2 & DSC & [1999RAI/RAI] \\
\hline & FUS & & 18.25 & 388.2 & DTA & [1996DOM/HEA, 1994SAB/GOU] \\
\hline & FUS & & 18.86 & 386.1 & DSC & [1990DON/DRE] \\
\hline & FUS & & U30.12 & 385.2 & DTA & [1986SIN/KUM] \\
\hline & FUS & & 19.3 & & & [1972BOO/HAU] \\
\hline & FUS & & 21.4 & 386.2 & DTA & [1958VAR] \\
\hline & SUB & & 92.4 & 298 & $\mathrm{C}$ & {$[1994 \mathrm{SAB} / \mathrm{GOU}]$} \\
\hline & SUB & $(303-328)$ & 87.9 & & $\mathrm{ME}$ & [1974LIO/HOP] \\
\hline
\end{tabular}


TABLE 9. Phase change enthalpies of $\mathrm{C}_{6}$ organic compounds - Continued

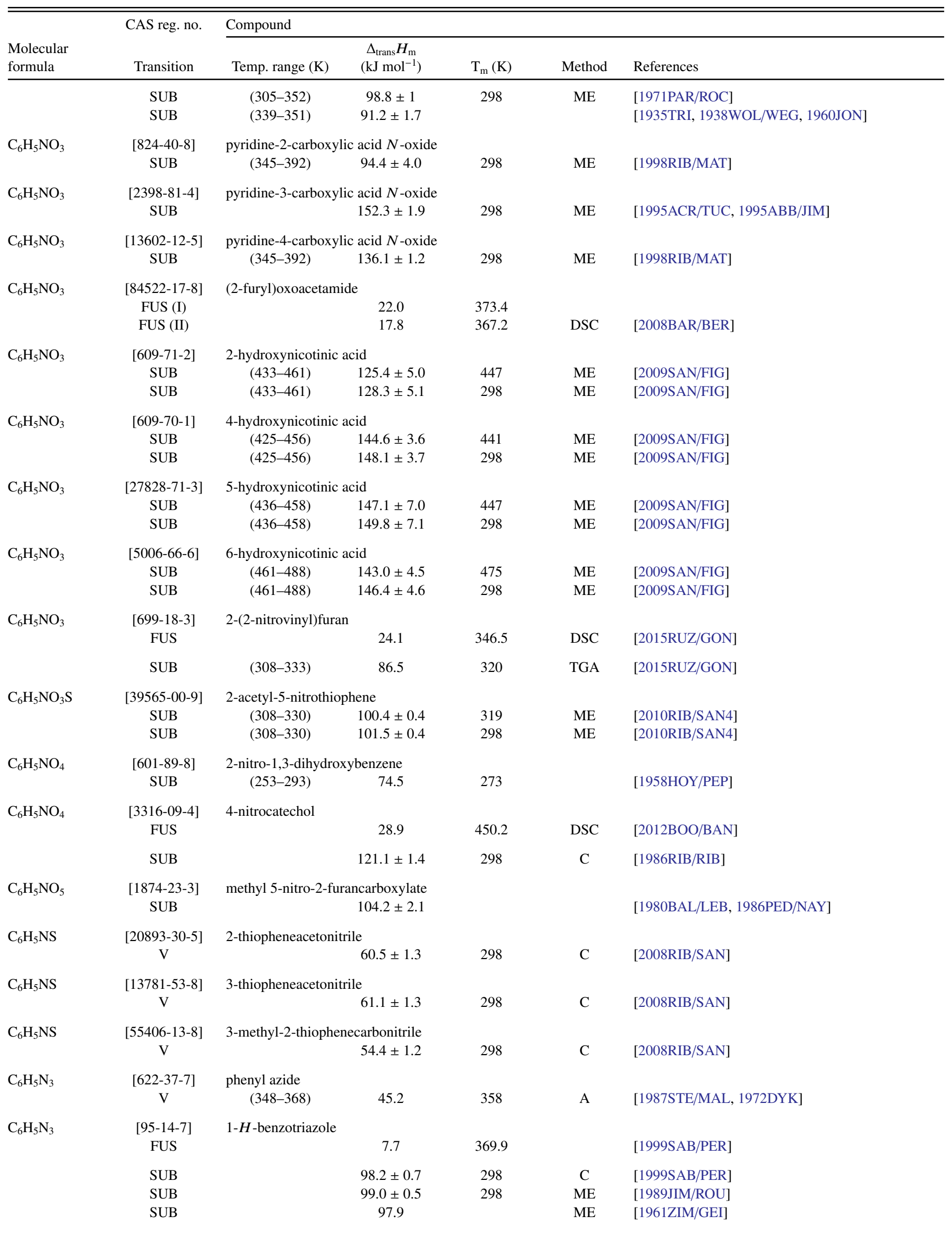

$\mathrm{C}_{6} \mathrm{H}_{5} \mathrm{~N}_{3}$

[274-79-3] imidazo[1,2-a]pyrazine 
TABLE 9. Phase change enthalpies of $\mathrm{C}_{6}$ organic compounds-Continued

\begin{tabular}{|c|c|c|c|c|c|c|}
\hline \multirow[b]{2}{*}{$\begin{array}{l}\text { Molecular } \\
\text { formula }\end{array}$} & \multirow{2}{*}{$\begin{array}{l}\text { CAS reg. no. } \\
\text { Transition }\end{array}$} & \multicolumn{5}{|l|}{ Compound } \\
\hline & & Temp. range $(\mathrm{K})$ & $\begin{array}{c}\Delta_{\text {trans }} H_{\mathrm{m}} \\
\left(\mathrm{kJ} \mathrm{mol}^{-1}\right)\end{array}$ & $\mathrm{T}_{\mathrm{m}}(\mathrm{K})$ & Method & References \\
\hline & FUS & & 15.3 & 359.2 & DSC & [2016MOR/RAT] \\
\hline & SUB & & $82.1 \pm 1.9$ & 298 & $\mathrm{~F}+\mathrm{V}$ & [2016MOR/RAT] \\
\hline & $\mathrm{V}$ & $(370-393)$ & $70.7 \pm 1.0$ & 298 & GS & [2016MOR/RAT] \\
\hline & $\mathrm{V}$ & & $67.9 \pm 1.4$ & 298 & CGC & [2016MOR/RAT] \\
\hline \multirow[t]{5}{*}{$\mathrm{C}_{6} \mathrm{H}_{5} \mathrm{~N}_{5} \mathrm{O}_{6}$} & [28930-29-2] & \multicolumn{5}{|c|}{ 1,3-diamino-2,4,6-trinitrobenzene } \\
\hline & SUB & & 146.9 & & DSC & [1990HWA/YOS] \\
\hline & SUB & $(335-382)$ & 140.0 & 350 & $\mathrm{LE}$ & [1987STE/MAL, 1969ROS/DIC] \\
\hline & SUB & & 143.5 & 298 & & [1978CUN/PAL] \\
\hline & $\mathrm{V}$ & & 110.9 & & DSC & [1990HWA/YOS] \\
\hline \multirow[t]{49}{*}{$\mathrm{C}_{6} \mathrm{H}_{6}$} & [71-43-2] & benzene & & & & \\
\hline & FUS & & 10.7 & 278 & DSC & [1999WAT/IIY] \\
\hline & FUS & & 9.94 & 278.6 & & [1948TSC] \\
\hline & FUS & & 9.87 & 278.7 & & [1948OLI/EAT] \\
\hline & FUS & & 9.92 & 278.7 & $\mathrm{C}$ & [1996DOM/HEA, 1942ZIE/AND] \\
\hline & FUS & $(92-300)$ & 9.80 & 278.6 & $\mathrm{C}$ & [1996DOM/HEA, 1930HUF/PAR2] \\
\hline & FUS & & 9.87 & 278.7 & $\mathrm{C}$ & [1996DOM/HEA, 1926AND/LYN] \\
\hline & FUS & & 10.00 & 278.6 & & [1925MAA/WAL] \\
\hline & FUS & & 9.78 & 279.0 & & [1924STR/PAR] \\
\hline & SUB & $(258-273)$ & 41.7 & & & [1994LIU/DIC] \\
\hline & SUB & $(223-279)$ & 45.2 & 264 & A & [1987STE/MAL, 1976HA/MOR] \\
\hline & SUB & & 45.1 & 278 & & [1984HES/WIS] \\
\hline & SUB & $(183-197)$ & 44.4 & 298 & $\mathrm{TE}, \mathrm{ME}$ & [1980DEK] \\
\hline & SUB & $(183-197)$ & $53.9 \pm 0.8$ & 193 & $\mathrm{TE}$ & [1977DEK/VAN] \\
\hline & SUB & $(183-197)$ & $49.4 \pm 0.4$ & 193 & $\mathrm{ME}$ & [1977DEK/VAN] \\
\hline & SUB & $(221-268)$ & 45.6 & 279 & MM & [1974JAC] \\
\hline & SUB & & 44.1 & 261 & & [1960JON] \\
\hline & SUB & & 43.1 & 229 & & [1960JON] \\
\hline & SUB & $(195-273)$ & 44.6 & 279 & MG & [1956MIL] \\
\hline & SUB & $(263-270)$ & 46.6 & 282 & A & [1947STU] \\
\hline & SUB & & 44.6 & 273 & & [1936DEB, 1974JAC] \\
\hline & SUB & $(184-200)$ & $\mathrm{U} 33.2$ & 192 & & [1933DEI] \\
\hline & SUB & $(214-238)$ & 43.3 & 226 & A & [1913MUN] \\
\hline & $\mathrm{V}$ & $(305-345)$ & 33.2 & 320 & & [2002LUB/BAN] \\
\hline & $\mathrm{V}$ & $(258-313)$ & 35.6 & & GC & [1994LIU/DIC] \\
\hline & $\mathrm{V}$ & $(296-377)$ & 33.5 & 311 & $\mathrm{~EB}$ & [1990AMB/EWI] \\
\hline & $\mathrm{V}$ & & $33.9 \pm 0.2$ & & GC & [1989AZA] \\
\hline & $\mathrm{V}$ & & 33.4 & 307 & $\mathrm{C}$ & [1988DON/LIN] \\
\hline & $\mathrm{V}$ & & 33.1 & 314 & $\mathrm{C}$ & [1988DON/LIN] \\
\hline & $\mathrm{V}$ & & 32.4 & 324 & $\mathrm{C}$ & [1988DON/LIN] \\
\hline & $\mathrm{V}$ & & 31.9 & 332 & $\mathrm{C}$ & [1988DON/LIN] \\
\hline & $\mathrm{V}$ & & 31.4 & 344 & $\mathrm{C}$ & [1988DON/LIN] \\
\hline & $\mathrm{V}$ & & 30.6 & 353 & $\mathrm{C}$ & [1988DON/LIN] \\
\hline & $\mathrm{V}$ & $(279-377)$ & 34.4 & 294 & A & [1987STE/MAL] \\
\hline & $\mathrm{V}$ & $(353-422)$ & 31.5 & 368 & A & [1987STE/MAL] \\
\hline & $\mathrm{V}$ & $(420-502)$ & 30.2 & 435 & A & [1987STE/MAL] \\
\hline & $\mathrm{V}$ & $(501-562)$ & 30.3 & 516 & A & [1987STE/MAL] \\
\hline & $\mathrm{V}$ & & 30.8 & 352 & & [1983NAT/VIS] \\
\hline & $\mathrm{V}$ & & 30.5 & 361 & & [1983NAT/VIS] \\
\hline & $\mathrm{V}$ & & 30.2 & 366 & & [1983NAT/VIS] \\
\hline & $\mathrm{V}$ & $(313-373)$ & 35.3 & 343 & & [1983TSO/WIL] \\
\hline & $\mathrm{V}$ & & 31.0 & 350 & & [1977RAO/VIU] \\
\hline & $\mathrm{V}$ & & $33.8 \pm 0.1$ & 298 & $\mathrm{C}$ & [1973SVO/VES] \\
\hline & $\mathrm{V}$ & & $33.0 \pm 0.1$ & 313 & $\mathrm{C}$ & [1973SVO/VES] \\
\hline & $\mathrm{V}$ & & $32.2 \pm 0.1$ & 328 & $\mathrm{C}$ & [1973SVO/VES] \\
\hline & $\mathrm{V}$ & & $31.8 \pm 0.1$ & 333 & $\mathrm{C}$ & [1973SVO/VES] \\
\hline & $\mathrm{V}$ & & $31.4 \pm 0.1$ & 343 & $\mathrm{C}$ & [1973SVO/VES] \\
\hline & $\mathrm{V}$ & & $30.9 \pm 0.1$ & 353 & $\mathrm{C}$ & [1973SVO/VES] \\
\hline & $\mathrm{V}$ & & $32.6 \pm 0.4$ & 313 & DSC & [1971MIT/IMA] \\
\hline
\end{tabular}


TABLE 9. Phase change enthalpies of $\mathrm{C}_{6}$ organic compounds-Continued

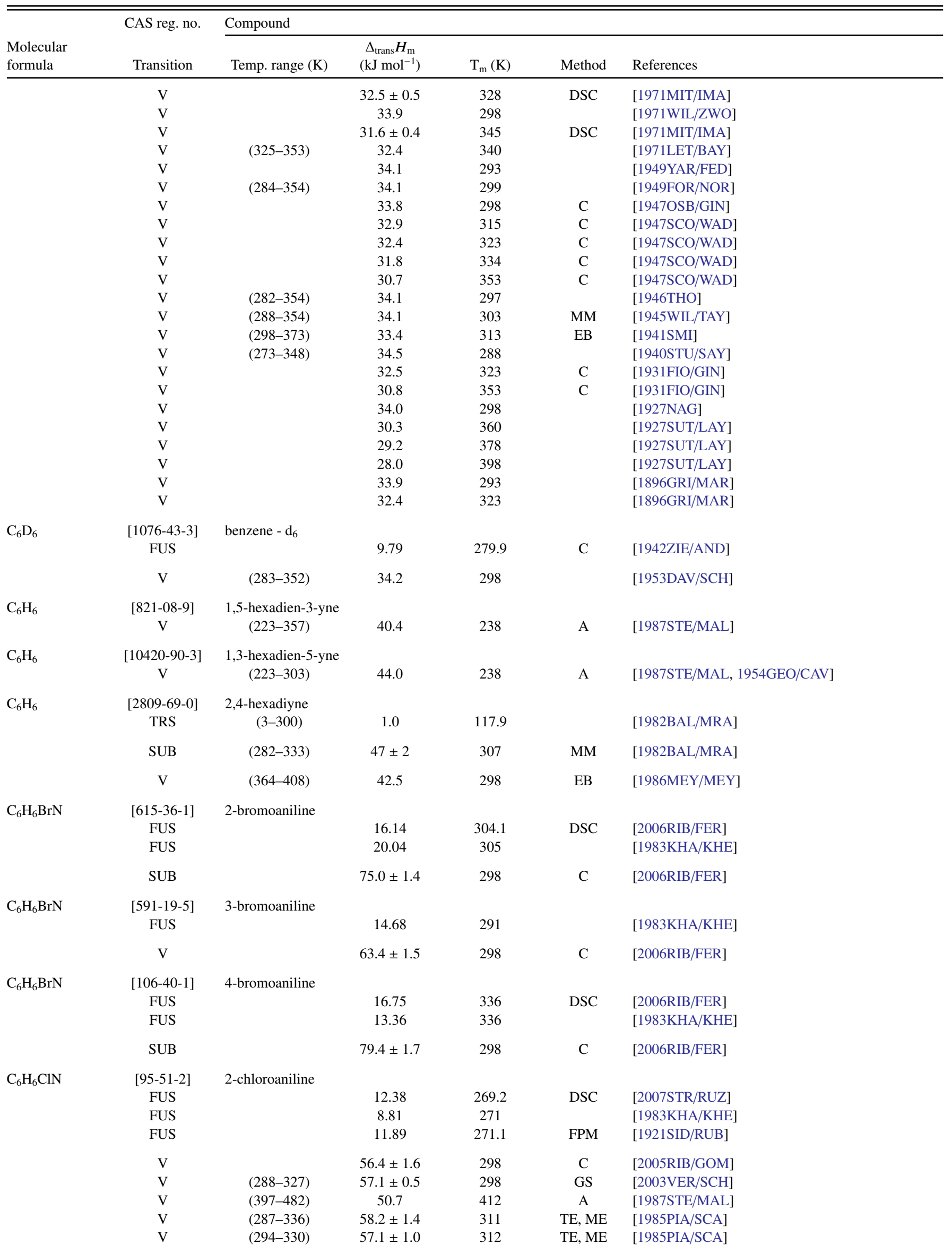


TABlE 9. Phase change enthalpies of $\mathrm{C}_{6}$ organic compounds-Continued

\begin{tabular}{|c|c|c|c|c|c|c|}
\hline \multirow[b]{2}{*}{$\begin{array}{l}\text { Molecular } \\
\text { formula }\end{array}$} & \multirow{2}{*}{$\begin{array}{l}\text { CAS reg. no. } \\
\text { Transition }\end{array}$} & \multicolumn{5}{|l|}{ Compound } \\
\hline & & Temp. range $(\mathrm{K})$ & $\begin{array}{c}\Delta_{\text {trans }} H_{\mathrm{m}} \\
\left(\mathrm{kJ} \mathrm{mol}^{-1}\right)\end{array}$ & $\mathrm{T}_{\mathrm{m}}(\mathrm{K})$ & Method & References \\
\hline \multirow[t]{8}{*}{$\mathrm{C}_{6} \mathrm{H}_{6} \mathrm{ClN}$} & [108-42-9] & 3-chloroaniline & & & & \\
\hline & FUS & & 12.0 & 263 & & [1983KHA/KHE] \\
\hline & FUS & & 11.64 & 262.8 & FPM & [1921SID/RUB] \\
\hline & $\mathrm{V}$ & & $61.1 \pm 2.8$ & 298 & $\mathrm{C}$ & [2005RIB/GOM] \\
\hline & $\mathrm{V}$ & $(291-340)$ & $60.2 \pm 0.1$ & 298 & GS & [2003VER/SCH] \\
\hline & $\mathrm{V}$ & $(398-573)$ & 53.6 & 413 & A & [1987STE/MAL, 1972DYK] \\
\hline & $\mathrm{V}$ & $(292-346)$ & $60.3 \pm 0.6$ & 319 & TE, ME & [1985PIA/SCA] \\
\hline & $\mathrm{V}$ & $(304-342)$ & $61.0 \pm 0.8$ & 323 & TE, ME & [1985PIA/SCA] \\
\hline \multirow[t]{9}{*}{$\mathrm{C}_{6} \mathrm{H}_{6} \mathrm{ClN}$} & [106-47-8] & 4-chloroaniline & & & & \\
\hline & FUS & & 20.47 & 342.8 & DSC & [2007STR/RUZ] \\
\hline & FUS & & 21.06 & 343.5 & DSC & [2003VER/SCH] \\
\hline & FUS & & 16.9 & 344 & & [1983KHA/KHE] \\
\hline & FUS & & 20.1 & 343.7 & FPM & [1921SID/RUB] \\
\hline & SUB & $(291-337)$ & $80.5 \pm 0.3$ & 298 & GS & [2003VER/SCH] \\
\hline & SUB & $(283-303)$ & 90.7 & 293 & $\mathrm{ME}$ & [1987STE/MAL, 1925SWA/MAC] \\
\hline & $\mathrm{V}$ & $(346-374)$ & $62.3 \pm 0.5$ & 298 & GS & [2003VER/SCH] \\
\hline & $\mathrm{V}$ & $(363-505)$ & 52.2 & 378 & A & [1987STE/MAL] \\
\hline \multirow[t]{2}{*}{$\mathrm{C}_{6} \mathrm{H}_{6} \mathrm{Cl}_{4}$} & {$[41992-55-6]$} & \multicolumn{5}{|c|}{$\alpha-3,4,5,6$-tetrachlorocyclohexene } \\
\hline & $\mathrm{V}$ & $(353-399)$ & 58.0 & 368 & A & [1987STE/MAL] \\
\hline \multirow[t]{4}{*}{$\mathrm{C}_{6} \mathrm{H}_{6} \mathrm{Cl}_{6}$} & [319-84-6] & \multicolumn{5}{|c|}{ ( $1 \alpha, 2 \alpha, 3 \beta, 4 \alpha, 5 \beta, 6 \beta)-1,2,3,4,5,6$-hexachlorocyclohexane ( $\alpha$-hexachlorocyclohexane) } \\
\hline & SUB & $(313-363)$ & 95.7 & 328 & A & [1987STE/MAL, 1960JON] \\
\hline & SUB & $(324-344)$ & 92.9 & 334 & TE & [1947BAL] \\
\hline & $\mathrm{V}$ & $(343-453)$ & 68.5 & 398 & GC & [1990HIN/BID2] \\
\hline \multirow[t]{4}{*}{$\mathrm{C}_{6} \mathrm{H}_{6} \mathrm{Cl}_{6}$} & [319-85-7] & \multicolumn{5}{|c|}{$\beta$-hexachlorocyclohexane $\left(\mathrm{mp} 314^{\circ} \mathrm{C}\right)$} \\
\hline & SUB & $(506-551)$ & 103.7 & & & [1989LUB/JAN] \\
\hline & SUB & $(313-363)$ & 107 & 328 & A & [1987STE/MAL, 1960JON] \\
\hline & SUB & $(368-390)$ & 102.9 & 379 & $\mathrm{TE}$ & [1947BAL] \\
\hline \multirow[t]{17}{*}{$\mathrm{C}_{6} \mathrm{H}_{6} \mathrm{Cl}_{6}$} & {$[58-89-9]$} & \multicolumn{5}{|c|}{$\gamma$-hexachlorocyclohexane (lindane) } \\
\hline & FUS & & 25.9 & 386.4 & DSC & [1991SAB/AN] \\
\hline & FUS & & 22.13 & 386.8 & DSC & [1990DON/DRE] \\
\hline & FUS & & 15.9 & 388.9 & DSC & [1969PLA/GLA] \\
\hline & SUB & $(347-384)$ & $89 \pm 3$ & 366 & & [2010VEC] \\
\hline & SUB & $(310-384)$ & $92.4 \pm 4.0$ & 298 & $\mathrm{ME}, \mathrm{TE}$ & [1998GIU/BRU] \\
\hline & SUB & $(292-326)$ & $97.7 \pm 0.6$ & 308 & ME & [1996BOE/MAR] \\
\hline & SUB & $(243-303)$ & $106.6 \pm 0.9$ & 273 & GS & [1994WAN/SHU] \\
\hline & SUB & & $90.1 \pm 0.7$ & 338 & $\mathrm{C}$ & [1991SAB/AN] \\
\hline & SUB & & $90.8 \pm 0.7$ & 298 & $\mathrm{C}$ & [1991SAB/AN] \\
\hline & SUB & $(313-363)$ & 99.2 & 328 & A & [1987STE/MAL, 1960JON] \\
\hline & SUB & $(293-313)$ & 88.9 & 303 & GS & [1983SPE/CLI, 1970SPE/CLI] \\
\hline & SUB & $(293-313)$ & 101.2 & 303 & & [1970SPE/CLI] \\
\hline & SUB & $(313-343)$ & 89.7 & 328 & & [1960SCH/LEG] \\
\hline & SUB & $(333-365)$ & 115.5 & & $\mathrm{TE}$ & {$[1947 \mathrm{BAL}]$} \\
\hline & $\mathrm{V}$ & $(387-428)$ & $72 \pm 2$ & 408 & & [2010VEC] \\
\hline & $\mathrm{V}$ & $(343-453)$ & 70.5 & 398 & GC & [1990HIN/BID2] \\
\hline \multirow[t]{3}{*}{$\mathrm{C}_{6} \mathrm{H}_{6} \mathrm{Cl}_{6}$} & {$[319-86-8]$} & \multicolumn{5}{|c|}{$\delta$-hexachlorocyclohexane $\left(\mathrm{mp} 142^{\circ} \mathrm{C}\right)$} \\
\hline & SUB & $(313-363)$ & 97.3 & 328 & A & [1987STE/MAL, 1960JON] \\
\hline & SUB & $(328-358)$ & 97.5 & & & {$[1947 \mathrm{BAL}]$} \\
\hline \multirow[t]{2}{*}{$\mathrm{C}_{6} \mathrm{H}_{6} \mathrm{FN}$} & {$[348-54-9]$} & 2-fluoroaniline & & & & \\
\hline & V & & $52.0 \pm 0.6$ & 298 & $\mathrm{C}$ & [2007RIB/FER] \\
\hline \multirow[t]{2}{*}{$\mathrm{C}_{6} \mathrm{H}_{6} \mathrm{FN}$} & [372-19-0] & 3-fluoroaniline & & & & \\
\hline & $\mathrm{V}$ & & $54.7 \pm 0.6$ & 298 & $\mathrm{C}$ & [2007RIB/FER] \\
\hline \multirow[t]{2}{*}{$\mathrm{C}_{6} \mathrm{H}_{6} \mathrm{FN}$} & [371-40-4] & 4-fluoroaniline & & & & \\
\hline & V & & $54.8 \pm 0.8$ & 298 & $\mathrm{C}$ & [2007RIB/FER] \\
\hline $\mathrm{C}_{6} \mathrm{H}_{6} \mathrm{~F}_{8} \mathrm{O}$ & {$[77527-96-9]$} & $1,1,2,2,3,3,4,4$-octa & doro-5-methox & tane & & \\
\hline
\end{tabular}


TABLE 9. Phase change enthalpies of $\mathrm{C}_{6}$ organic compounds - Continued

\begin{tabular}{|c|c|c|c|c|c|c|}
\hline \multirow[b]{2}{*}{$\begin{array}{l}\text { Molecular } \\
\text { formula }\end{array}$} & \multirow{2}{*}{$\begin{array}{l}\text { CAS reg. no. } \\
\text { Transition }\end{array}$} & \multicolumn{5}{|l|}{ Compound } \\
\hline & & Temp. range $(\mathrm{K})$ & $\begin{array}{c}\Delta_{\text {trans }} H_{\mathrm{m}} \\
\left(\mathrm{kJ} \mathrm{mol}^{-1}\right)\end{array}$ & $\mathrm{T}_{\mathrm{m}}(\mathrm{K})$ & Method & References \\
\hline $\mathrm{C}_{6} \mathrm{H}_{6} \mathrm{~F}_{8} \mathrm{O}_{2}$ & $\begin{array}{l}{[355-74-8]} \\
\text { SUB }\end{array}$ & \multicolumn{4}{|c|}{ 2,2,3,3,4,4,5,5-octafluoro-1,6-hexanediol } & [1974COX, 1977PED/RYL] \\
\hline $\mathrm{C}_{6} \mathrm{H}_{6} \mathrm{~F}_{9} \mathrm{~N}_{3} \mathrm{~S}$ & $\begin{array}{c}\text { [63265-76-9] } \\
\mathrm{V}\end{array}$ & \multicolumn{5}{|c|}{$N$-[N, $N^{\prime}$-dimethyl- $S$-(trifluoromethyl)sulfonodiimidoyl]-1,1,1,3,3,3-hexafluoro-2-propanimine } \\
\hline $\mathrm{C}_{6} \mathrm{H}_{6} \mathrm{IN}$ & $\begin{array}{l}\text { [615-43-0] } \\
\text { FUS } \\
\text { FUS }\end{array}$ & 2-iodoaniline & $\begin{array}{l}19.38 \\
13.95\end{array}$ & $\begin{array}{c}329.6 \\
333\end{array}$ & DSC & $\begin{array}{l}\text { [2006RIB/FER2] } \\
\text { [1983KHA/KHE] }\end{array}$ \\
\hline & $\mathrm{V}$ & & $67.5 \pm 1.4$ & 298 & $\mathrm{C}$ & [2006RIB/FER2] \\
\hline $\mathrm{C}_{6} \mathrm{H}_{6} \mathrm{IN}$ & $\begin{array}{l}\text { [540-37-4] } \\
\text { FUS } \\
\text { FUS }\end{array}$ & 4-iodoaniline & $\begin{array}{c}16.94 \\
15.1\end{array}$ & $\begin{array}{l}336 \\
334\end{array}$ & DSC & $\begin{array}{l}\text { [2006RIB/FER2] } \\
\text { [1983KHA/KHE] }\end{array}$ \\
\hline & SUB & & $84.8 \pm 1.4$ & 298 & $\mathrm{C}$ & [2006RIB/FER2] \\
\hline $\mathrm{C}_{6} \mathrm{H}_{6} \mathrm{~N}_{2}$ & $\begin{array}{c}{[1119-85-3]} \\
V\end{array}$ & $\begin{array}{l}\text { 3-hexenedinitrile } \\
\quad(353-448)\end{array}$ & 49.4 & 368 & A & [1987STE/MAL] \\
\hline $\mathrm{C}_{6} \mathrm{H}_{6} \mathrm{~N}_{2} \mathrm{O}$ & $\begin{array}{l}{[1452-77-3]} \\
\text { FUS } \\
\text { FUS }\end{array}$ & 2-pyridinecarboxam & $\begin{array}{l}19.1 \\
16.82\end{array}$ & $\begin{array}{c}379.3 \\
381\end{array}$ & DSC & $\begin{array}{l}\text { [2011CAS/RIB] } \\
\text { [1960NEG/MIK2] }\end{array}$ \\
\hline Note: Autho & $\begin{array}{l}\text { ort a large uncer } \\
\text { FUS } \\
\text { FUS } \\
\text { FUS }\end{array}$ & inty for form (I) corr & $\begin{array}{c}\text { ponding to } \pm \\
23.8 \\
25.5 \\
26.94\end{array}$ & $\begin{array}{c}\mathrm{J} / \mathrm{mole} \\
403.8 \\
401.6 \\
402\end{array}$ & $\begin{array}{l}\text { DSC } \\
\text { DSC }\end{array}$ & $\begin{array}{l}\text { [2009GOO/ROD] } \\
\text { [2008NIC/BEL] } \\
\text { [1960NEG/MIK2] }\end{array}$ \\
\hline & $\begin{array}{l}\text { SUB } \\
\text { SUB } \\
\text { SUB } \\
\text { SUB } \\
\text { SUB } \\
\text { SUB }\end{array}$ & $\begin{array}{l}(341-363) \\
(341-363) \\
(356-398) \\
(356-398) \\
(363-393)\end{array}$ & $\begin{array}{c}110.7 \pm 0.2 \\
112.0 \pm 0.2 \\
111.4 \pm 0.1 \\
113.2 \pm 0.1 \\
121.2 \pm 3.3 \\
111.8\end{array}$ & $\begin{array}{l}352 \\
298 \\
377 \\
298 \\
298 \\
378\end{array}$ & $\begin{array}{c}\text { ME } \\
\text { ME } \\
\text { DM } \\
\text { DM } \\
\text { C } \\
\text { ME }\end{array}$ & $\begin{array}{l}\text { [2015ALM/OLI] } \\
{[2015 \mathrm{ALM} / \mathrm{OLI}]} \\
{[2015 \mathrm{ALM} / \mathrm{OLI}]} \\
{[2015 \mathrm{ALM} / \mathrm{OLI}]} \\
{[2001 \mathrm{RIB} / \mathrm{GON}]} \\
{[1987 \mathrm{STE} / \mathrm{MAL}, 1960 \mathrm{NEG} / \mathrm{MIK}, 1959 \mathrm{HAR}]}\end{array}$ \\
\hline & $\begin{array}{l}\mathrm{V} \\
\mathrm{V}\end{array}$ & $\begin{array}{l}(396-433) \\
(396-433)\end{array}$ & $\begin{array}{l}84.5 \pm 0.1 \\
93.9 \pm 1.9\end{array}$ & $\begin{array}{l}415 \\
298\end{array}$ & $\begin{array}{l}\mathrm{DM} \\
\mathrm{DM}\end{array}$ & $\begin{array}{l}{[2015 \mathrm{ALM} / \mathrm{OLI}]} \\
{[2015 \mathrm{ALM} / \mathrm{OLI}]}\end{array}$ \\
\hline $\mathrm{C}_{6} \mathrm{H}_{6} \mathrm{~N}_{2} \mathrm{O}$ & $\begin{array}{c}\text { [1453-82-3] } \\
\text { FUS } \\
\text { FUS } \\
\text { FUS }\end{array}$ & 4-pyridinecarboxam & $\begin{array}{l}\text { de } \\
\begin{array}{c}23.6 \\
24.5 \\
26.81\end{array}\end{array}$ & $\begin{array}{c}429.8 \\
428.6 \\
431\end{array}$ & $\begin{array}{l}\text { DSC } \\
\text { DSC }\end{array}$ & $\begin{array}{l}\text { [2016VOL/BLO] } \\
\text { [2011CAS/RIB] } \\
\text { [1960NEG/MIK2] }\end{array}$ \\
\hline & $\begin{array}{l}\text { SUB } \\
\text { SUB } \\
\text { SUB }\end{array}$ & $\begin{array}{l}(351-377) \\
(383-412)\end{array}$ & $\begin{array}{c}117.0 \pm 1.0 \\
116.1 \pm 1.5 \\
99.9\end{array}$ & $\begin{array}{c}298 \\
298 \\
397.5\end{array}$ & $\begin{array}{c}\text { GS } \\
\text { C } \\
\text { ME }\end{array}$ & $\begin{array}{l}\text { [2016VOL/BLO] } \\
\text { [2001RIB/GON] } \\
\text { [1987STE/MAL, 1960NEG/MIK, 1959HAR] }\end{array}$ \\
\hline
\end{tabular}


TABLE 9. Phase change enthalpies of $\mathrm{C}_{6}$ organic compounds-Continued

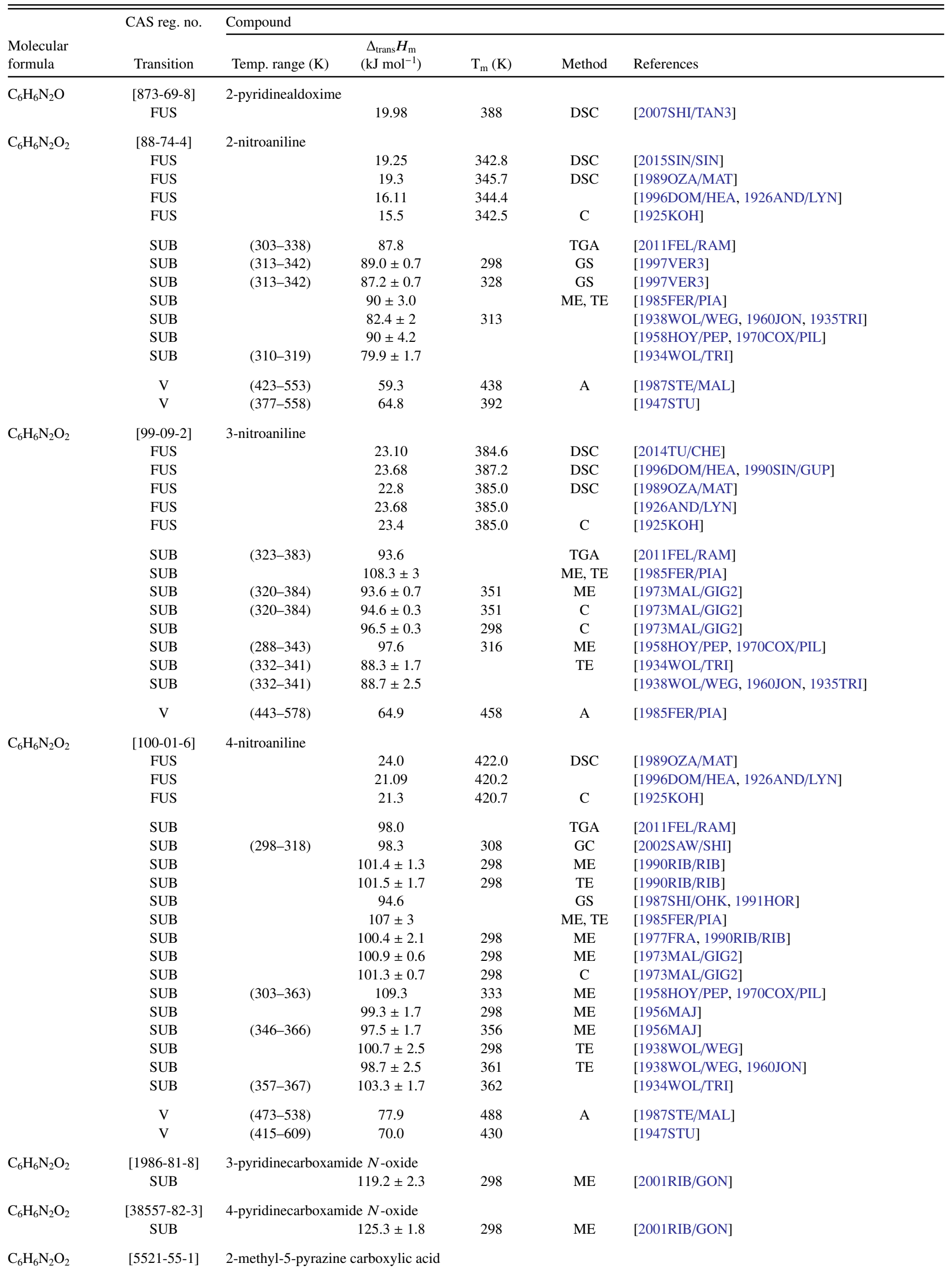


TABLE 9. Phase change enthalpies of $\mathrm{C}_{6}$ organic compounds-Continued

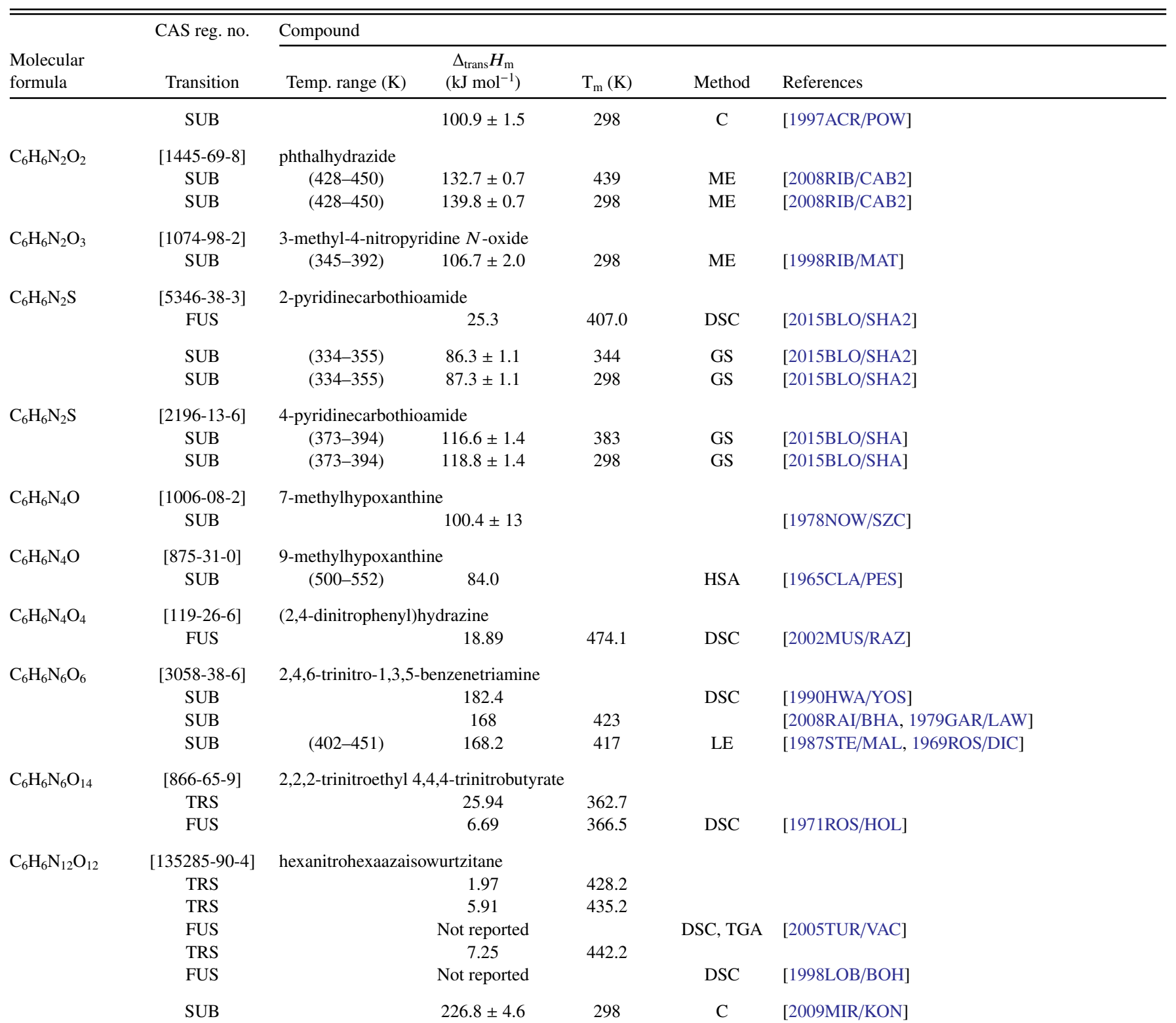

Note: Authors of [2009MIR/KON] tabulate the experimental value as an enthalpy of vaporization; however, they report that the compound is crystalline and use the value to calculate the standard molar enthalpy of formation. Given the crystaline state and how the value was used, we have tabulated the value as an enthalpy of sublimation.

\begin{tabular}{|c|c|c|c|c|c|c|}
\hline \multirow[t]{18}{*}{$\mathrm{C}_{6} \mathrm{H}_{6} \mathrm{O}$} & [108-95-2] & phenol & & & & \\
\hline & FUS & & 10.54 & 314.2 & DSC & [2014HAS/JIR] \\
\hline & FUS & & 11.49 & 314.1 & DSC & [1991WYR/PAL] \\
\hline & FUS & & 11.51 & 314 & & [1972INO/LIA, 1996DOM/HEA] \\
\hline & FUS & $(13-335)$ & 11.5 & 314.06 & $\mathrm{AC}$ & [1963AND/COU2] \\
\hline & FUS & & 11.3 & & $\mathrm{RC}$ & [1927STE/JOH] \\
\hline & FUS & & 11.4 & & $\mathrm{AC}$ & [1922STR/PAR, 1927STE/JOH] \\
\hline & FUS & & 11.8 & & & [1915BRI, 1927STE/JOH] \\
\hline & SUB & $(263-298)$ & $65.3 \pm 3.3$ & 280 & HSA & [1975CHI] \\
\hline & SUB & $(230-273)$ & $69.7 \pm 0.9$ & 298 & ME & [1971PAR/ROC] \\
\hline & SUB & $(282-313)$ & $68.7 \pm 0.5$ & & GS & [1960AND/BID, 1970COX/PIL] \\
\hline & SUB & $(283-303)$ & 68.2 & 293 & ME & [1958SKL/MAR] \\
\hline & SUB & $(273-310)$ & 64.8 & 292 & & [1958BID/MAR] \\
\hline & SUB & $(270-313)$ & 68.1 & 292 & & [1948NIT/SEK2] \\
\hline & SUB & $(278-305)$ & 67.8 & & $\mathrm{TE}$ & [1947BAL, 1960JON] \\
\hline & $\mathrm{V}$ & $(363-391)$ & 53.2 & 378 & $\mathrm{~EB}$ & [2001CHY/FRA] \\
\hline & $\mathrm{V}$ & $(393-433)$ & 58.8 & 298 & CGC & [1995CHI/HOS] \\
\hline & $\mathrm{V}$ & $(455-655)$ & 49.5 & 470 & A & [1987STE/MAL] \\
\hline
\end{tabular}


TABLE 9. Phase change enthalpies of $\mathrm{C}_{6}$ organic compounds-Continued

\begin{tabular}{|c|c|c|c|c|c|c|}
\hline \multirow[b]{2}{*}{$\begin{array}{l}\text { Molecular } \\
\text { formula }\end{array}$} & \multirow{2}{*}{$\begin{array}{l}\text { CAS reg. no. } \\
\text { Transition }\end{array}$} & \multicolumn{5}{|l|}{ Compound } \\
\hline & & Temp. range $(\mathrm{K})$ & $\begin{array}{c}\Delta_{\text {trans }} H_{\mathrm{m}} \\
\left(\mathrm{kJ} \mathrm{mol}^{-1}\right) \\
\end{array}$ & $\mathrm{T}_{\mathrm{m}}(\mathrm{K})$ & Method & References \\
\hline & $\mathrm{V}$ & $(314-395)$ & 57.4 & 329 & A & [1987STE/MAL] \\
\hline & $\mathrm{V}$ & $(387-456)$ & 50.9 & 402 & A & [1987STE/MAL] \\
\hline & $\mathrm{V}$ & $(449-526)$ & 46.8 & 464 & A & [1987STE/MAL] \\
\hline & $\mathrm{V}$ & $(520-625)$ & 43.8 & 535 & A & [1987STE/MAL] \\
\hline & $\mathrm{V}$ & & 51.1 & & & [1986BAL/GNA] \\
\hline & $\mathrm{V}$ & $(383-473)$ & 51.3 & 398 & EB, GS & $\begin{array}{l}\text { [1987STE/MAL, 1960AND/BID, 1972DYK, } \\
\text { 1958BID/MAR] }\end{array}$ \\
\hline & $\mathrm{V}$ & $(380-455)$ & 51.4 & 395 & & [1949DRE/SHR, 1949DRE/MAR, 1984BOU/FRI] \\
\hline & $\mathrm{V}$ & $(414-454)$ & 48.1 & 434 & & [1947GOL/MAR] \\
\hline & $\mathrm{V}$ & $(278-305)$ & 67.8 & 291 & & [1947BAL2] \\
\hline \multirow[t]{3}{*}{$\mathrm{C}_{6} \mathrm{H}_{6} \mathrm{O}$} & [1192-62-7] & 2-acetylfuran & & & & \\
\hline & FUS & & 15.5 & 301.6 & DSC & [2009FLO/CAM] \\
\hline & $\mathrm{V}$ & & $53.0 \pm 0.6$ & 298 & $\mathrm{C}$ & [2009RIB/AMA] \\
\hline \multirow[t]{3}{*}{$\mathrm{C}_{6} \mathrm{H}_{6} \mathrm{OS}$} & {$[88-15-3]$} & 2-acetylthiophene & & & & \\
\hline & V & & $58.8 \pm 1.2$ & 298 & $\mathrm{C}$ & [2007ROU/TEM] \\
\hline & $\mathrm{V}$ & & $55.4 \pm 5.4$ & 298 & CGC & [2007ROU/TEM] \\
\hline \multirow[t]{4}{*}{$\mathrm{C}_{6} \mathrm{H}_{6} \mathrm{OS}$} & [1468-83-3] & 3-acetylthiophene & & & & \\
\hline & FUS & & 18.9 & 333.6 & DSC & [2006TEM/ROU] \\
\hline & SUB & $(268-284)$ & $74.6 \pm 1.1$ & 298 & $\mathrm{ME}$ & [2007ROU/TEM] \\
\hline & $\mathrm{V}$ & & 55.2 & 298 & CGC & [2007ROU/TEM] \\
\hline \multirow[t]{2}{*}{$\mathrm{C}_{6} \mathrm{H}_{6} \mathrm{OS}$} & {$[13679-70-4]$} & 5-methyl-2-thiophe & carboxyaldehyde & & & \\
\hline & $\mathrm{V}$ & & $57.7 \pm 1.3$ & 298 & $\mathrm{C}$ & [2008RIB/SAN2] \\
\hline \multirow[t]{2}{*}{$\mathrm{C}_{6} \mathrm{H}_{6} \mathrm{OS}$} & [5834-16-2] & 3-methyl-2-thiophe & carboxyaldehyde & & & \\
\hline & $\mathrm{V}$ & & $56.2 \pm 1.2$ & 298 & $\mathrm{C}$ & [2008RIB/SAN2] \\
\hline \multirow[t]{3}{*}{$\mathrm{C}_{6} \mathrm{H}_{6} \mathrm{O}_{2}$} & {$[620-02-0]$} & 5-methyl-2-furaldel & & & & \\
\hline & V & $(303-443)$ & 49.7 & 373 & & [2014LOM/GIN2] \\
\hline & $\mathrm{V}$ & & $59.4 \pm 0.2$ & 298 & $\mathrm{C}$ & [2010RIB/AMA] \\
\hline \multirow[t]{17}{*}{$\mathrm{C}_{6} \mathrm{H}_{6} \mathrm{O}_{2}$} & [120-80-9] & 1,2-dihydroxybenze & (catechol) & & & \\
\hline & FUS & & 22.87 & 377.6 & $\mathrm{DSC}$ & [2008VER/KOZ] \\
\hline & FUS & & 18.55 & 377.6 & & [2000VER/SCH] \\
\hline & FUS & & 22.54 & 377.7 & DSC & [1997LEE/CHA] \\
\hline & FUS & & 22.74 & 377.7 & DTA & [1992MAN] \\
\hline & FUS & & 22.01 & 376.9 & $\mathrm{DSC}$ & [1989BRE/LIC] \\
\hline & FUS & & 22.8 & 375.2 & DTA & [1958VAR] \\
\hline & FUS & & 22.76 & 377.5 & $\mathrm{C}$ & [1926AND/LYN] \\
\hline & SUB & & $88.3 \pm 0.3$ & 298 & $\mathrm{C}$ & [2014GON/AGA] \\
\hline & SUB & & $87.5 \pm 0.3$ & 332 & $\mathrm{C}$ & [2014GON/AGA] \\
\hline & SUB & $(295-310)$ & $80.0 \pm 0.5$ & 302 & $\mathrm{ME}$ & [2006CHE/OJA] \\
\hline & SUB & & $87.5 \pm 0.3$ & 298 & $\mathrm{C}$ & [1991SAB/BUL] \\
\hline & SUB & & $86.6 \pm 1.6$ & 298 & $\mathrm{C}$ & [1984CAR] \\
\hline & SUB & & 80.8 & & & [1938WOL/WEG, 1960JON, 1935TRI] \\
\hline & $\mathrm{V}$ & $(378-389)$ & $71.9 \pm 0.8$ & 298 & GS & [2008VER/KOZ] \\
\hline & V & $(395-519)$ & 63.1 & 410 & A & [1987STE/MAL] \\
\hline & $\mathrm{V}$ & $(378-439)$ & 61.2 & 393 & GC & [1975KUN/LIL] \\
\hline \multirow[t]{11}{*}{$\mathrm{C}_{6} \mathrm{H}_{6} \mathrm{O}_{2}$} & {$[108-46-3]$} & 1,3-dihydroxybenz & (resorcinol) & & & \\
\hline & FUS & & 27.93 & 383.9 & DSC & [2015OSS/JUS] \\
\hline & FUS & & 19.28 & 382.3 & DSC & [2015SIN/SIN3] \\
\hline & FUS & & 21.68 & 383.2 & DTA & [1992MAN] \\
\hline & TRS & & 1.2 & 366.8 & & \\
\hline & FUS & & 18.9 & 382.6 & DSC & [1989BRE/LIC] \\
\hline & TRS & & 1.37 & 369.0 & & \\
\hline & FUS & & 20.89 & 382.7 & DSC & [1987EBI/ASK] \\
\hline & FUS & & 21.3 & 382.9 & $\mathrm{C}$ & [1926AND/LYN] \\
\hline & SUB & & $99.7 \pm 0.4$ & 298 & $\mathrm{C}$ & [2014GON/AGA] \\
\hline & SUB & & $99.3 \pm 0.4$ & 328 & $\mathrm{C}$ & [2014GON/AGA] \\
\hline
\end{tabular}


TABLE 9. Phase change enthalpies of $\mathrm{C}_{6}$ organic compounds-Continued

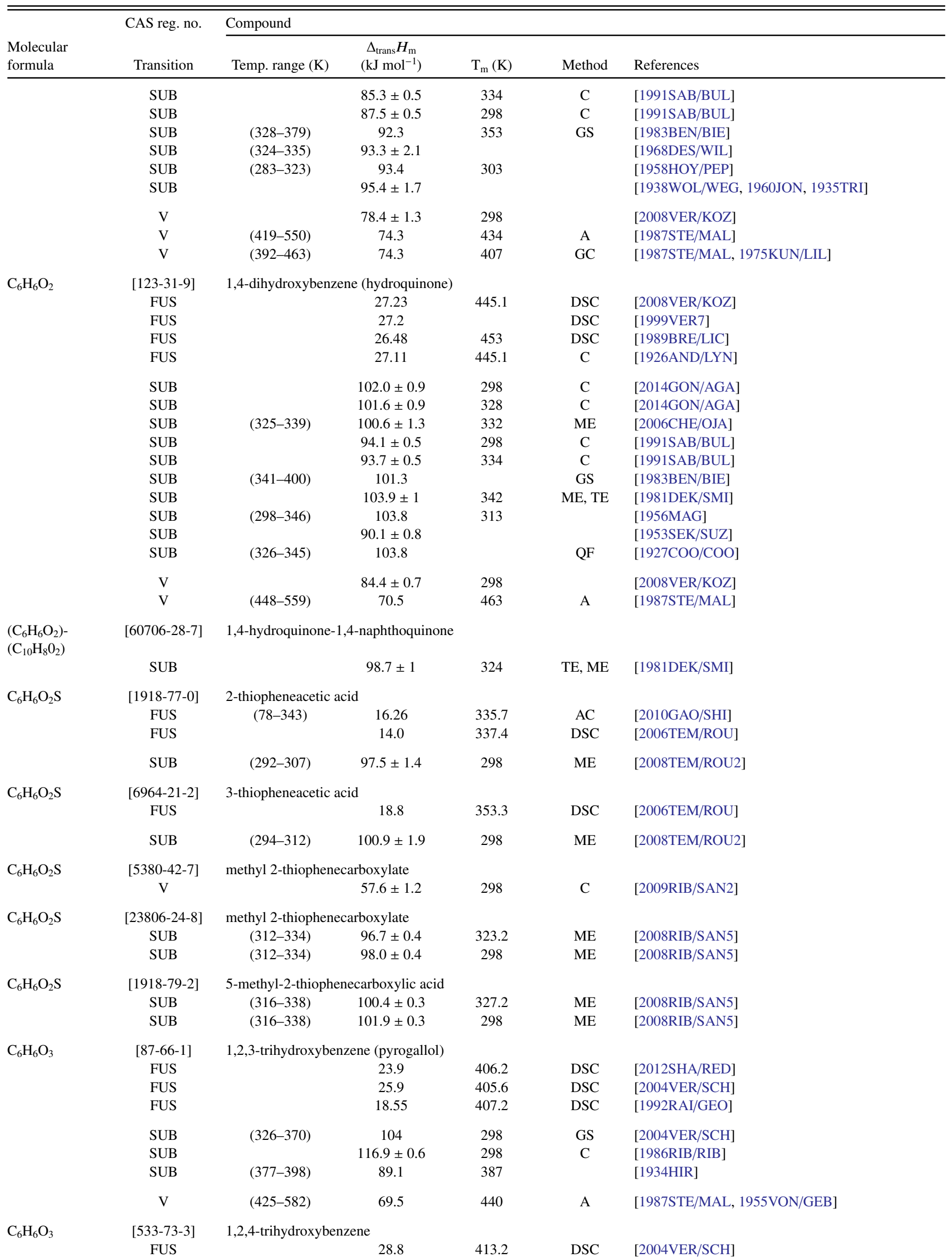


TABLE 9. Phase change enthalpies of $\mathrm{C}_{6}$ organic compounds-Continued

\begin{tabular}{|c|c|c|c|c|c|c|}
\hline \multirow[b]{2}{*}{$\begin{array}{l}\text { Molecular } \\
\text { formula }\end{array}$} & \multirow{2}{*}{$\begin{array}{l}\text { CAS reg. no. } \\
\text { Transition }\end{array}$} & \multicolumn{5}{|l|}{ Compound } \\
\hline & & Temp. range $(\mathrm{K})$ & $\begin{array}{c}\Delta_{\text {trans }} H_{\mathrm{m}} \\
\left(\mathrm{kJ} \mathrm{mol}^{-1}\right)\end{array}$ & $\mathrm{T}_{\mathrm{m}}(\mathrm{K})$ & Method & References \\
\hline & SUB & $(341-382)$ & $124.2 \pm 0.6$ & 298 & GS & [2004VER/SCH] \\
\hline & SUB & & $119.8 \pm 1.6$ & 298 & $\mathrm{C}$ & [1986RIB/RIB] \\
\hline \multirow[t]{5}{*}{$\mathrm{C}_{6} \mathrm{H}_{6} \mathrm{O}_{3}$} & [108-73-6] & 1,3,5-trihydroxybe & ene & & & \\
\hline & FUS & & 34.5 & 491.8 & DSC & [2004VER/SCH] \\
\hline & SUB & $(355-382)$ & $135.5 \pm 1.3$ & 298 & GS & [2004VER/SCH] \\
\hline & SUB & & $131.7 \pm 1.0$ & 298 & $\mathrm{C}$ & [1986RIB/RIB] \\
\hline & SUB & $(383-406)$ & 127.9 & & $\mathrm{TE}, \mathrm{ME}$ & [1983DEW/BOW] \\
\hline \multirow[t]{3}{*}{$\mathrm{C}_{6} \mathrm{H}_{6} \mathrm{O}_{3}$} & {$[67-47-0]$} & 5-hydroxymethylf & ural & & & \\
\hline & FUS & & 19.8 & 308.5 & DSC & [2009VER/EME] \\
\hline & $\mathrm{V}$ & $(314-368)$ & $83.4 \pm 0.2$ & 298 & GS & [2009VER/EME] \\
\hline \multirow[t]{2}{*}{$\mathrm{C}_{6} \mathrm{H}_{6} \mathrm{O}_{4}$} & {$[762-42-5]$} & butynedioic acid, & nethyl ester & & & \\
\hline & $\mathrm{V}$ & $(273-460)$ & 56.3 & 288 & A & [1987STE/MAL, 1972DYK, 1948RED/CHA5] \\
\hline \multirow[t]{11}{*}{$\mathrm{C}_{6} \mathrm{H}_{6} \mathrm{~S}$} & [108-98-5] & benzenethiol (thio & enol) & & & \\
\hline & FUS & & 11.48 & 258.2 & & [1996DOM/HEA, 1936PAR/TOD] \\
\hline & $\mathrm{V}$ & $(333-471)$ & 45.9 & 348 & & [1999DYK/SVO] \\
\hline & $\mathrm{V}$ & & 43.5 & & & [1986BAL/GNA] \\
\hline & $\mathrm{V}$ & $(385-486)$ & 43.1 & 400 & $\mathrm{~A}, \mathrm{~EB}$ & [1987STE/MAL, 1966OSB/DOU, 1956SCO/MCC] \\
\hline & V & & $43.8 \pm 0.1$ & 375 & $\mathrm{C}$ & {$[1956 \mathrm{SCO} / \mathrm{MCC}]$} \\
\hline & V & & $42.6 \pm 0.1$ & 395 & $\mathrm{C}$ & [1956SCO/MCC] \\
\hline & $\mathrm{V}$ & & $41.8 \pm 0.1$ & 407 & $\mathrm{C}$ & [1956SCO/MCC] \\
\hline & $\mathrm{V}$ & & $41.3 \pm 0.1$ & 417 & $\mathrm{C}$ & [1956SCO/MCC] \\
\hline & $\mathrm{V}$ & & $47.5 \pm 0.1$ & 298 & $\mathrm{C}$ & [1963MOR/SUN] \\
\hline & $\mathrm{V}$ & $(324-440)$ & 44.3 & 339 & & [1955VON/GEB, 1984BOU/FRI] \\
\hline \multirow[t]{2}{*}{$\mathrm{C}_{6} \mathrm{H}_{7} \mathrm{Cl}_{2} \mathrm{~N}$} & {$[137-04-2]$} & 2-chloroaniline hy & ochloride & & & \\
\hline & SUB & $(373-473)$ & 77.6 & 388 & A & [1987STE/MAL, 1975KON/SEL] \\
\hline \multirow[t]{5}{*}{$\mathrm{C}_{6} \mathrm{H}_{7} \mathrm{BrN}_{2} \mathrm{O}_{2}$} & {$[7033-39-8]$} & 1,3-dimethyl-5-brc & ouracil & & & \\
\hline & SUB & $(293-333)$ & $114.4 \pm 0.7$ & 314 & ME & [2015BRU/IRR] \\
\hline & SUB & $(293-333)$ & 114.9 & 298 & $\mathrm{ME}$ & [2015BRU/IRR] \\
\hline & SUB & $(328-360)$ & $110.3 \pm 0.5$ & 344 & $\mathrm{ME}$ & [2015BRU/IRR] \\
\hline & SUB & $(328-360)$ & 111.9 & 298 & ME & [2015BRU/IRR] \\
\hline \multirow[t]{2}{*}{$\mathrm{C}_{6} \mathrm{H}_{7} \mathrm{Cl}_{2} \mathrm{~N}$} & {$[141-85-5]$} & 3-chloroaniline hy & ochloride & & & \\
\hline & SUB & $(383-473)$ & 71.3 & 398 & A & [1987STE/MAL, 1975KON/SEL] \\
\hline \multirow[t]{2}{*}{$\mathrm{C}_{6} \mathrm{H}_{7} \mathrm{Cl}_{2} \mathrm{~N}$} & {$[20265-96-7]$} & 4-chloroaniline hy & ochloride & & & \\
\hline & SUB & $(373-483)$ & 77.8 & 388 & A & [1987STE/MAL, 1975KON/SEL] \\
\hline \multirow[t]{2}{*}{$\mathrm{C}_{6} \mathrm{H}_{7} \mathrm{Cl}_{3} \mathrm{OS}$} & {$[76619-93-7]$} & 2,3,3-trichloro-2-p & penethioic aci & ropyl es & & \\
\hline & V & $(383-433)$ & 69.4 & & GC & [1980PIT/KIS] \\
\hline \multirow[t]{8}{*}{$\mathrm{C}_{6} \mathrm{H}_{7} \mathrm{FN}_{2} \mathrm{O}_{2}$} & {$[3013-92-1]$} & 1,3-dimethyl-5-flu & ouracil & & & \\
\hline & SUB & $(288-326)$ & $98.4 \pm 0.7$ & 305 & ME & [2015BRU/IRR] \\
\hline & SUB & $(288-326)$ & 98.6 & 298 & $\mathrm{ME}$ & [2015BRU/IRR] \\
\hline & SUB & $(315-355)$ & $96.5 \pm 0.5$ & 335 & ME & [2015BRU/IRR] \\
\hline & SUB & $(315-355)$ & 97.6 & 298 & ME & [2015BRU/IRR] \\
\hline & SUB & $(317-339)$ & $95.9 \pm 0.6$ & 328 & $\mathrm{ME}$ & [2014AMA/SZT] \\
\hline & SUB & $(317-339)$ & $96.8 \pm 0.6$ & 298 & ME & [2014AMA/SZT] \\
\hline & SUB & $(338-373)$ & $119 \pm 4$ & & $\mathrm{TE}$ & [2002BRU/POR] \\
\hline \multirow[t]{2}{*}{$\mathrm{C}_{6} \mathrm{H}_{7} \mathrm{~F}_{3} \mathrm{~N}_{2} \mathrm{O}_{4}$} & {$[400-58-8]$} & $N-[N$-(trifluoroac & l)glycyl]glyc & & & \\
\hline & SUB & $(273-423)$ & 67.0 & 288 & A & [1987STE/MAL, 1960WEY/KLI] \\
\hline \multirow[t]{5}{*}{$\mathrm{C}_{6} \mathrm{H}_{7} \mathrm{IN}_{2} \mathrm{O}_{2}$} & {$[40738-83-8]$} & 1,3-dimethyl-5-ioc & aracil & & & \\
\hline & SUB & $(323-367)$ & $116.2 \pm 0.9$ & 345 & $\mathrm{ME}$ & [2015BRU/IRR] \\
\hline & SUB & $(323-367)$ & 117.8 & 298 & $\mathrm{ME}$ & [2015BRU/IRR] \\
\hline & SUB & $(354-392)$ & $116.7 \pm 0.7$ & 373 & ME & [2015BRU/IRR] \\
\hline & SUB & $(354-392)$ & 119.3 & 298 & $\mathrm{ME}$ & [2015BRU/IRR] \\
\hline \multirow[t]{2}{*}{$\mathrm{C}_{6} \mathrm{H}_{7} \mathrm{~N}$} & {$[15760-35-7]$} & \multicolumn{5}{|c|}{ 3-methylenecyclobutanecarbonitrile } \\
\hline & $\mathrm{V}$ & $(348-435)$ & 45.9 & 366 & BG & [1971HAL/BAL] \\
\hline
\end{tabular}


TABLE 9. Phase change enthalpies of $\mathrm{C}_{6}$ organic compounds-Continued

\begin{tabular}{|c|c|c|c|c|c|c|}
\hline \multirow[b]{2}{*}{$\begin{array}{l}\text { Molecular } \\
\text { formula }\end{array}$} & \multirow{2}{*}{$\begin{array}{l}\text { CAS reg. no. } \\
\text { Transition }\end{array}$} & \multicolumn{5}{|l|}{ Compound } \\
\hline & & Temp. range $(\mathrm{K})$ & $\begin{array}{c}\Delta_{\text {trans }} H_{\mathrm{m}} \\
\left(\mathrm{kJ} \mathrm{mol}^{-1}\right)\end{array}$ & $\mathrm{T}_{\mathrm{m}}(\mathrm{K})$ & Method & References \\
\hline \multirow[t]{2}{*}{$\mathrm{C}_{6} \mathrm{H}_{7} \mathrm{~N}$} & {$[31357-71-8]$} & \multicolumn{5}{|c|}{ bicyclo[2.1.0]pentane-1-carbonitrile } \\
\hline & $\mathrm{V}$ & $(332-390)$ & 41.8 & 343 & BG & [1971HAL/BAL] \\
\hline \multirow[t]{20}{*}{$\mathrm{C}_{6} \mathrm{H}_{7} \mathrm{~N}$} & {$[62-53-3]$} & aniline & & & & \\
\hline & FUS & & 9.16 & 265 & DSC & [1972AHM/EAD2] \\
\hline & FUS & $(14-313)$ & 10.54 & 267.1 & & [1962HAT/HIL, 1996DOM/HEA] \\
\hline & FUS & & 10.92 & 267.3 & $\mathrm{C}$ & [1942ZIE/AND] \\
\hline & FUS & & 10.56 & 266.8 & & [1933PAR/HUF] \\
\hline & $\mathrm{V}$ & $(350-499)$ & $51.0 \pm 0.2$ & 360 & $\mathrm{~EB}$ & [2002STE/CHI] \\
\hline & $\mathrm{V}$ & $(350-499)$ & $48.0 \pm 0.2$ & 400 & $\mathrm{~EB}$ & [2002STE/CHI] \\
\hline & $\mathrm{V}$ & $(350-499)$ & $45.2 \pm 0.2$ & 440 & $\mathrm{~EB}$ & [2002STE/CHI] \\
\hline & $\mathrm{V}$ & $(350-499)$ & $42.2 \pm 0.4$ & 480 & $\mathrm{~EB}$ & [2002STE/CHI] \\
\hline & $\mathrm{V}$ & $(421-591)$ & 45.8 & 444 & & [1992LEE/CHE] \\
\hline & V & $(273-338)$ & 52.2 & 288 & A & [1987STE/MAL] \\
\hline & $\mathrm{V}$ & $(304-485)$ & 53.6 & 319 & A & [1987STE/MAL] \\
\hline & $\mathrm{V}$ & $(373-458)$ & 48.6 & 388 & A & [1987STE/MAL] \\
\hline & $\mathrm{V}$ & $(455-523)$ & 46.3 & 470 & A & [1987STE/MAL] \\
\hline & $\mathrm{V}$ & $(313-386)$ & 51.4 & 350 & & [1979MAH/SMI] \\
\hline & $\mathrm{V}$ & & $55.8 \pm 0.1$ & 298 & $\mathrm{C}$ & [1971KUS/WAD2] \\
\hline & $\mathrm{V}$ & $(288-298)$ & 52.9 & 293 & & [1968RAV/DAN] \\
\hline & $\mathrm{V}$ & $(304-457)$ & 54.0 & 319 & & [1962HAT/HIL] \\
\hline & $\mathrm{V}$ & & 53.0 & 333 & $\mathrm{C}$ & [1962HAT/HIL] \\
\hline & $\mathrm{V}$ & & 40.4 & 456 & $\mathrm{C}$ & [1923AWB/GRI] \\
\hline \multirow[t]{23}{*}{$\mathrm{C}_{6} \mathrm{H}_{7} \mathrm{~N}$} & [109-06-8] & 2-methylpyridine & & & & \\
\hline & FUS & & 9.72 & 206.5 & & [1996DOM/HEA, 1963SCO/HUB] \\
\hline & $\mathrm{V}$ & $(308-441)$ & $41.2 \pm 0.1$ & 320 & $\mathrm{~EB}$ & [1999CHI/KNI] \\
\hline & $\mathrm{V}$ & $(308-441)$ & $38.8 \pm 0.1$ & 360 & $\mathrm{~EB}$ & [1999CHI/KNI] \\
\hline & $\mathrm{V}$ & $(308-441)$ & $36.4 \pm 0.1$ & 400 & $\mathrm{~EB}$ & [1999CHI/KNI] \\
\hline & $\mathrm{V}$ & $(308-441)$ & $33.7 \pm 0.3$ & 440 & $\mathrm{~EB}$ & [1999CHI/KNI] \\
\hline & $\mathrm{V}$ & $(323-373)$ & 43.6 & 298 & CGC & [1995CHI/HOS] \\
\hline & $\mathrm{V}$ & (292-403) & 42.0 & 307 & $\mathrm{~EB}$ & [1990LEN] \\
\hline & $\mathrm{V}$ & $(209-245)$ & 46.9 & 230 & A & [1987STE/MAL] \\
\hline & $\mathrm{V}$ & $(429-537)$ & 36.5 & 444 & A & [1987STE/MAL] \\
\hline & $\mathrm{V}$ & $(521-621)$ & 35.4 & 536 & A & [1987STE/MAL] \\
\hline & $\mathrm{V}$ & & $42.5 \pm 0.1$ & 298 & $\mathrm{C}$ & [1984MAJ/SVO2] \\
\hline & $\mathrm{V}$ & & $41.6 \pm 0.1$ & 313 & $\mathrm{C}$ & [1984MAJ/SVO2] \\
\hline & $\mathrm{V}$ & & $40.7 \pm 0.1$ & 328 & $\mathrm{C}$ & [1984MAJ/SVO2] \\
\hline & V & & $39.8 \pm 0.1$ & 343 & $\mathrm{C}$ & [1984MAJ/SVO2] \\
\hline & $\mathrm{V}$ & & $38.3 \pm 0.1$ & 368 & $\mathrm{C}$ & [1984MAJ/SVO2] \\
\hline & $\mathrm{V}$ & $(352-445)$ & 39.1 & 367 & $\mathrm{~EB}, \mathrm{IPM}$ & [1987STE/MAL, 1968OSB/DOU] \\
\hline & $\mathrm{V}$ & $(352-442)$ & 39.1 & 367 & $\mathrm{~EB}$ & [1987STE/MAL, 1963SCO/HUB] \\
\hline & $\mathrm{V}$ & & $38.8 \pm 0.1$ & 359 & $\mathrm{C}$ & [1963SCO/HUB] \\
\hline & $\mathrm{V}$ & & $37.7 \pm 0.1$ & 379 & $\mathrm{C}$ & [1963SCO/HUB] \\
\hline & V & & $36.2 \pm 0.1$ & 402 & $\mathrm{C}$ & [1963SCO/HUB] \\
\hline & $\mathrm{V}$ & $(337-403)$ & 39.8 & 352 & MG & [1953HER/MAR] \\
\hline & $\mathrm{V}$ & & 35.4 & 402 & $\mathrm{C}$ & {$[1901 \mathrm{KAH}]$} \\
\hline \multirow[t]{14}{*}{$\mathrm{C}_{6} \mathrm{H}_{7} \mathrm{~N}$} & [108-99-6] & 3-methylpyridine & & & & \\
\hline & FUS & & 14.18 & 255 & & [1996DOM/HEA, 1963SCO/GOO] \\
\hline & SUB & $(225-255)$ & 62.2 & 240 & A & [1987STE/MAL] \\
\hline & $\mathrm{V}$ & $(342-373)$ & $44.5 \pm 2.0$ & 298 & CGC & [2009LIP/CHI2] \\
\hline & $\mathrm{V}$ & $(314-457)$ & $43.2 \pm 0.1$ & 320 & $\mathrm{~EB}$ & [1999CHI/KNI] \\
\hline & $\mathrm{V}$ & $(314-457)$ & $40.9 \pm 0.1$ & 360 & $\mathrm{~EB}$ & [1999CHI/KNI] \\
\hline & $\mathrm{V}$ & $(314-457)$ & $38.6 \pm 0.1$ & 400 & EB & [1999CHI/KNI] \\
\hline & $\mathrm{V}$ & $(314-457)$ & $36.1 \pm 0.2$ & 440 & $\mathrm{~EB}$ & [1999CHI/KNI] \\
\hline & V & $(374-458)$ & 40.1 & 389 & A & [1987STE/MAL] \\
\hline & $\mathrm{V}$ & $(450-570)$ & 37.7 & 465 & A & [1987STE/MAL] \\
\hline & $\mathrm{V}$ & $(561-645)$ & 36.8 & 576 & A & [1987STE/MAL] \\
\hline & $\mathrm{V}$ & & $44.6 \pm 0.1$ & 298 & $\mathrm{C}$ & [1984MAJ/SVO2] \\
\hline & $\mathrm{V}$ & & $43.6 \pm 0.1$ & 313 & $\mathrm{C}$ & [1984MAJ/SVO2] \\
\hline & $\mathrm{V}$ & & $42.7 \pm 0.1$ & 328 & $\mathrm{C}$ & [1984MAJ/SVO2] \\
\hline
\end{tabular}


TABLE 9. Phase change enthalpies of $\mathrm{C}_{6}$ organic compounds-Continued

\begin{tabular}{|c|c|c|c|c|c|c|}
\hline \multirow[b]{2}{*}{$\begin{array}{l}\text { Molecular } \\
\text { formula }\end{array}$} & \multirow{2}{*}{$\begin{array}{l}\text { CAS reg. no. } \\
\text { Transition }\end{array}$} & \multicolumn{5}{|l|}{ Compound } \\
\hline & & Temp. range $(\mathrm{K})$ & $\begin{array}{c}\Delta_{\text {trans }} H_{\mathrm{m}} \\
\left(\mathrm{kJ} \mathrm{mol}^{-1}\right)\end{array}$ & $\mathrm{T}_{\mathrm{m}}(\mathrm{K})$ & Method & References \\
\hline & $\mathrm{V}$ & & $42.0 \pm 0.1$ & 343 & $\mathrm{C}$ & [1984MAJ/SVO2] \\
\hline & $\mathrm{V}$ & & $40.4 \pm 0.1$ & 368 & $\mathrm{C}$ & [1984MAJ/SVO2] \\
\hline & $\mathrm{V}$ & $(347-458)$ & 41.3 & 362 & $\mathrm{~EB}, \mathrm{IPM}$ & [1987STE/MAL, 1968OSB/DOU] \\
\hline & $\mathrm{V}$ & $(347-458)$ & 41.3 & 362 & EB & [1987STE/MAL, 1963SCO/GOO] \\
\hline & $\mathrm{V}$ & & $40.2 \pm 0.1$ & 372 & $\mathrm{C}$ & [1963SCO/GOO] \\
\hline & $\mathrm{V}$ & & $38.9 \pm 0.1$ & 393 & $\mathrm{C}$ & {$[1963 \mathrm{SCO} / \mathrm{GOO}]$} \\
\hline & $\mathrm{V}$ & & $37.4 \pm 0.1$ & 417 & $\mathrm{C}$ & {$[1963 \mathrm{SCO} / \mathrm{GOO}]$} \\
\hline & $\mathrm{V}$ & $(354-418)$ & 41.0 & 369 & MG & [1953HER/MAR] \\
\hline \multirow[t]{19}{*}{$\mathrm{C}_{6} \mathrm{H}_{7} \mathrm{~N}$} & $\begin{array}{c}{[108-89-4]} \\
\text { SUB }\end{array}$ & $\begin{array}{c}\text { 4-methylpyridine } \\
(213-239)\end{array}$ & 62.7 & 226 & A & [1987STE/MAL] \\
\hline & $\mathrm{V}$ & $(328-459)$ & $43.4 \pm 0.1$ & 320 & $\mathrm{~EB}$ & [1999CHI/KNI] \\
\hline & $\mathrm{V}$ & $(328-459)$ & $41.1 \pm 0.1$ & 360 & EB & [1999CHI/KNI] \\
\hline & $\mathrm{V}$ & $(328-459)$ & $38.8 \pm 0.1$ & 400 & EB & [1999CHI/KNI] \\
\hline & $\mathrm{V}$ & $(328-459)$ & $36.2 \pm 0.2$ & 440 & $\mathrm{~EB}$ & [1999CHI/KNI] \\
\hline & $\mathrm{V}$ & $(323-373)$ & 44.7 & 298 & CGC & [1995CHI/HOS] \\
\hline & $\mathrm{V}$ & $(348-460)$ & 41.4 & 363 & A & [1987STE/MAL] \\
\hline & V & $(348-367)$ & 42.1 & 347 & A & [1987STE/MAL] \\
\hline & $\mathrm{V}$ & $(381-460)$ & 40.0 & 396 & A & [1987STE/MAL] \\
\hline & $\mathrm{V}$ & $(452-573)$ & 37.9 & 467 & A & [1987STE/MAL] \\
\hline & $\mathrm{V}$ & $(564-646)$ & 37.2 & 579 & A & [1987STE/MAL] \\
\hline & $\mathrm{V}$ & & $44.9 \pm 0.1$ & 298 & $\mathrm{C}$ & [1984MAJ/SVO2] \\
\hline & $\mathrm{V}$ & & $43.9 \pm 0.1$ & 313 & $\mathrm{C}$ & [1984MAJ/SVO2] \\
\hline & $\mathrm{V}$ & & $42.9 \pm 0.1$ & 328 & $\mathrm{C}$ & [1984MAJ/SVO2] \\
\hline & $\mathrm{V}$ & & $42.1 \pm 0.1$ & 343 & $\mathrm{C}$ & [1984MAJ/SVO2] \\
\hline & $\mathrm{V}$ & & $44.8 \pm 0.1$ & 298 & $\mathrm{C}$ & [1981HOS/SCO] \\
\hline & $\mathrm{V}$ & $(283-298)$ & 45.5 & 298 & & [1979ARN/CHA] \\
\hline & $\mathrm{V}$ & $(348-459)$ & 41.4 & 363 & $\mathrm{~EB}, \mathrm{IPM}$ & [1987STE/MAL, 1968OSB/DOU] \\
\hline & $\mathrm{V}$ & $(350-418)$ & 41.3 & 365 & MG & [1953HER/MAR] \\
\hline \multirow[t]{2}{*}{$\mathrm{C}_{6} \mathrm{H}_{7} \mathrm{~N}$} & {$[26555-56-6]$} & 2-cyclopentene-1-c & bonitrile & & & \\
\hline & $\mathrm{V}$ & & $44.9 \pm 0.1$ & 298 & $\mathrm{C}$ & [1970PRO/KRE] \\
\hline \multirow[t]{2}{*}{$\left(\mathrm{C}_{6} \mathrm{H}_{7} \mathrm{~N}\right)-\left(\mathrm{SO}_{2}\right)$} & & aniline-sulfur diox & complex & & & \\
\hline & SUB & $(277-323)$ & 82.1 & 300 & & [1931HIL] \\
\hline \multirow[t]{10}{*}{$\mathrm{C}_{6} \mathrm{H}_{7} \mathrm{NO}$} & {$[95-55-6]$} & 2-aminophenol & & & & \\
\hline & FUS & & 26.74 & 448.7 & DSC & [2011MEL/PIN] \\
\hline & FUS & & 21.72 & 447.6 & & [2003HUA, 2005HUA/TAN] \\
\hline & FUS & & 31.4 & 443.2 & DSC & [2001ROT/GLA] \\
\hline & FUS & & 25.4 & 449.2 & $\mathrm{DSC}$ & [1996SAB/GOU] \\
\hline & FUS & & 34.0 & 447.4 & DSC & [1996DOM/HEA, 1989BRE/LIC] \\
\hline & SUB & & $93.5 \pm 0.8$ & 332 & $\mathrm{C}$ & {$[1996 \mathrm{SAB} / \mathrm{GOU}]$} \\
\hline & SUB & & $95.3 \pm 0.7$ & 337 & $\mathrm{C}$ & [1996SAB/GOU] \\
\hline & SUB & & $96.9 \pm 0.6$ & 298 & $\mathrm{C}$ & [1996SAB/GOU] \\
\hline & SUB & & $103.9 \pm 0.9$ & 298 & $\mathrm{C}$ & [1986NUN/BAR] \\
\hline \multirow[t]{10}{*}{$\mathrm{C}_{6} \mathrm{H}_{7} \mathrm{NO}$} & [591-27-5] & 3-aminophenol & & & & \\
\hline & FUS & & 27.6 & 395.0 & DSC & [2012SHA/RAI] \\
\hline & FUS & & 21.95 & 396.8 & & [2003HUA, 2005HUA/TAN] \\
\hline & FUS & & 23.9 & 390.7 & DSC & [2001ROT/GLA] \\
\hline & FUS & & 19.6 & 394.8 & DSC & [1996SAB/GOU] \\
\hline & FUS & & 22.98 & 399 & & [1991RAI/GEO] \\
\hline & FUS & & 24.7 & 394.2 & DSC & [1989BRE/LIC] \\
\hline & SUB & & $98.8 \pm 0.9$ & 335 & $\mathrm{C}$ & {$[1996 \mathrm{SAB} / \mathrm{GOU}]$} \\
\hline & SUB & & $101.6 \pm 0.9$ & 298 & $\mathrm{C}$ & [1996SAB/GOU] \\
\hline & SUB & & $104.7 \pm 1.2$ & 298 & $\mathrm{C}$ & [1986NUN/BAR] \\
\hline \multirow[t]{5}{*}{$\mathrm{C}_{6} \mathrm{H}_{7} \mathrm{NO}$} & {$[123-30-8]$} & 4-aminophenol & & & & \\
\hline & FUS & & 29.26 & 463.5 & DSC & [2002FAR/KAD] \\
\hline & FUS & & 23.8 & 455.2 & DSC & [2001ROT/GLA] \\
\hline & FUS & & 26.0 & 462.5 & & [1996SAB/GOU] \\
\hline & FUS & & 31.2 & 459.4 & DSC & [1989BRE/LIC, 1996DOM/HEA] \\
\hline
\end{tabular}


TABLE 9. Phase change enthalpies of $\mathrm{C}_{6}$ organic compounds-Continued

\begin{tabular}{|c|c|c|c|c|c|c|}
\hline \multirow[b]{2}{*}{$\begin{array}{l}\text { Molecular } \\
\text { formula }\end{array}$} & \multirow{2}{*}{$\begin{array}{l}\text { CAS reg. no. } \\
\text { Transition }\end{array}$} & \multicolumn{5}{|l|}{ Compound } \\
\hline & & Temp. range $(\mathrm{K})$ & $\begin{array}{c}\Delta_{\text {trans }} H_{\mathrm{m}} \\
\left(\mathrm{kJ} \mathrm{mol}^{-1}\right)\end{array}$ & $\mathrm{T}_{\mathrm{m}}(\mathrm{K})$ & Method & References \\
\hline & SUB & & $101.1 \pm 0.7$ & 335 & $\mathrm{C}$ & [1996SAB/GOU] \\
\hline & SUB & & $103.6 \pm 0.7$ & 298 & $\mathrm{C}$ & [1996SAB/GOU] \\
\hline & SUB & $(423-459)$ & 111.0 & 438 & A & [1987STE/MAL] \\
\hline & SUB & & $109.1 \pm 1.4$ & 298 & $\mathrm{C}$ & [1986NUN/BAR] \\
\hline & SUB & $(403-430)$ & 92.1 & 417 & I & [1954DUN, 1960JON] \\
\hline \multirow[t]{2}{*}{$\mathrm{C}_{6} \mathrm{H}_{7} \mathrm{NO}$} & {$[1121-25-1]$} & \multicolumn{5}{|c|}{ 2-methyl-3-hydroxypyridine } \\
\hline & SUB & & $89.3 \pm 1.3$ & 298 & $\mathrm{C}$ & [1982SUR/SAI, 1986PED/NAY] \\
\hline \multirow[t]{2}{*}{$\mathrm{C}_{6} \mathrm{H}_{7} \mathrm{NO}$} & {$[18615-86-6]$} & \multicolumn{5}{|c|}{ 2-methyl-4-hydroxypyridine } \\
\hline & SUB & & $113.0 \pm 1.3$ & 298 & & [1982SUR/SAI, 1986PED/NAY] \\
\hline \multirow[t]{2}{*}{$\mathrm{C}_{6} \mathrm{H}_{7} \mathrm{NO}$} & {$[1121-78-4]$} & \multicolumn{5}{|c|}{ 2-methyl-5-hydroxypyridine } \\
\hline & SUB & & $96.2 \pm 2.1$ & 298 & $\mathrm{C}$ & [1982SUR/SAI, 1986PED/NAY] \\
\hline \multirow[t]{2}{*}{$\mathrm{C}_{6} \mathrm{H}_{7} \mathrm{NO}$} & [3279-76-3] & \multicolumn{5}{|c|}{ 2-methyl-6-hydroxypyridine } \\
\hline & SUB & & $92.0 \pm 1.3$ & 298 & $\mathrm{C}$ & [1982SUR/SAI, 1986PED/NAY] \\
\hline \multirow[t]{4}{*}{$\mathrm{C}_{6} \mathrm{H}_{7} \mathrm{NO}$} & [931-19-1] & \multicolumn{5}{|c|}{ 2-methylpyridine $N$-oxide } \\
\hline & SUB & & $92.9 \pm 1.9$ & 298 & $\mathrm{C}$ & [2010CAB/MON] \\
\hline & SUB & & $78.2 \pm 2.2$ & 298 & $\mathrm{C}$ & {$[1995 \mathrm{ACR} / \mathrm{TUC}]$} \\
\hline & $\mathrm{V}$ & $(329-355)$ & $53.3 \pm 0.5$ & 298 & & [2010CAB/MON] \\
\hline \multirow[t]{2}{*}{$\mathrm{C}_{6} \mathrm{H}_{7} \mathrm{NO}$} & [1003-73-2] & \multicolumn{5}{|c|}{ 3-methylpyridine $N$-oxide } \\
\hline & SUB & & $82.2 \pm 2.4$ & 298 & $\mathrm{C}$ & [1995ACR/TUC] \\
\hline \multirow[t]{3}{*}{$\mathrm{C}_{6} \mathrm{H}_{7} \mathrm{NO}$} & {$[1003-67-4]$} & \multicolumn{5}{|c|}{ 4-methylpyridine $N$-oxide } \\
\hline & SUB & $(345-392)$ & $85.3 \pm 2.6$ & 298 & ME & [1998RIB/MAT] \\
\hline & SUB & $(316-341)$ & $79.1 \pm 1.3$ & & & [1995LEB/CHI] \\
\hline \multirow[t]{4}{*}{$\mathrm{C}_{6} \mathrm{H}_{7} \mathrm{NO}$} & {$[1628-89-3]$} & \multicolumn{5}{|l|}{ 2-methoxypyridine } \\
\hline & $\mathrm{V}$ & & $45.1 \pm 1.3$ & 298 & $\mathrm{C}$ & [2012AMA/RIB] \\
\hline & $\mathrm{V}$ & $(233-323)$ & 41.8 & 298 & $\mathrm{ME}$ & [1991AUE/WEB] \\
\hline & V & $(304-338)$ & 40.5 & 319 & A & [1987STE/MAL, 1974BEA/MUE] \\
\hline \multirow[t]{3}{*}{$\mathrm{C}_{6} \mathrm{H}_{7} \mathrm{NO}$} & [620-08-6] & \multicolumn{5}{|l|}{ 4-methoxypyridine } \\
\hline & V & & $57.4 \pm 1.3$ & 298 & $\mathrm{C}$ & [2012AMA/RIB] \\
\hline & $\mathrm{V}$ & $(233-323)$ & 52.7 & 298 & $\mathrm{ME}$ & [1991AUE/WEB] \\
\hline
\end{tabular}

Note: Authors of [1991AUE/WEB] refer to the value as an enthalpy of vaporization. The compound's melting point temperature falls within the temperature range of the measured VPs.

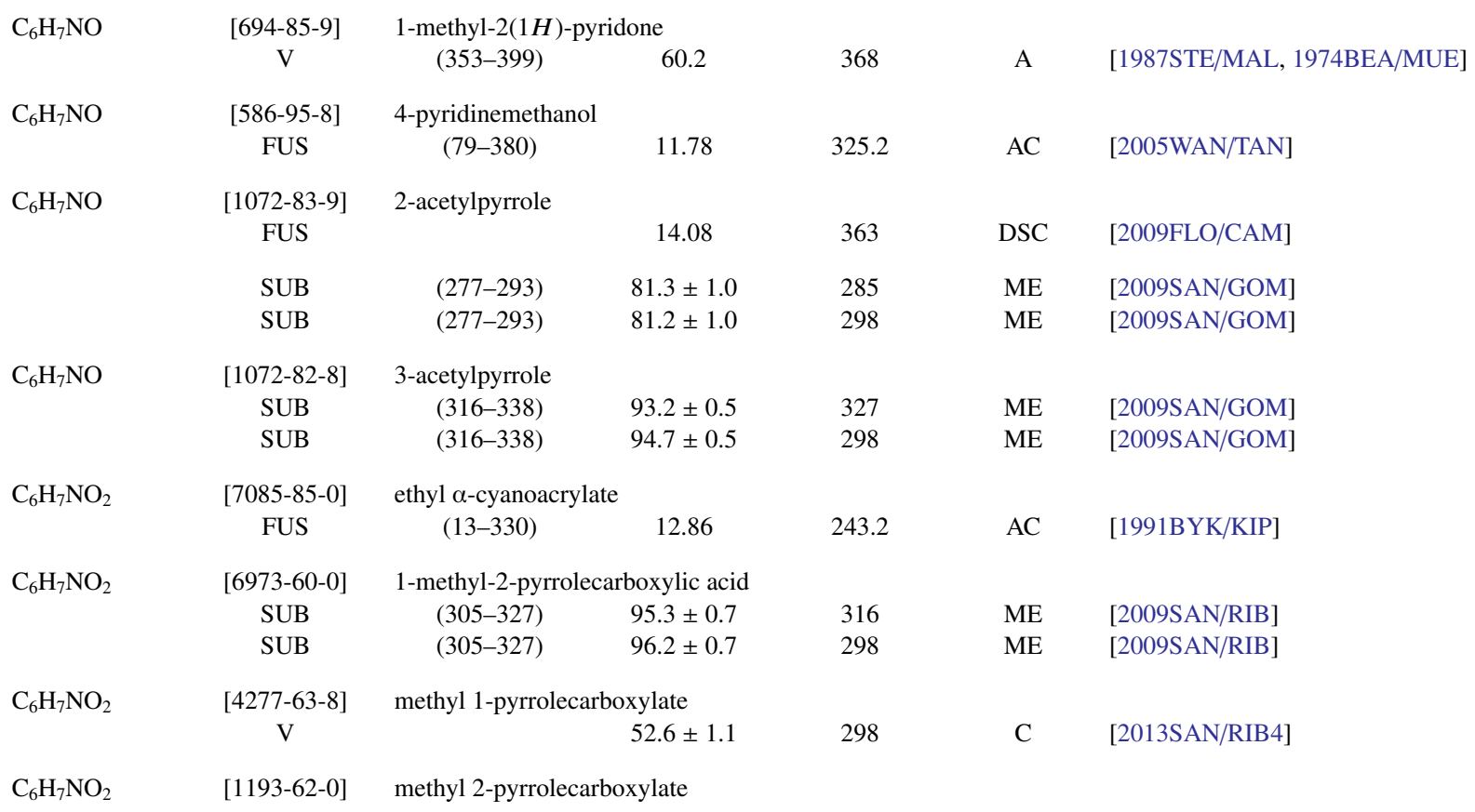


TABLE 9. Phase change enthalpies of $\mathrm{C}_{6}$ organic compounds-Continued

\begin{tabular}{|c|c|c|c|c|c|c|}
\hline \multirow[b]{2}{*}{$\begin{array}{l}\text { Molecular } \\
\text { formula }\end{array}$} & \multirow{2}{*}{$\begin{array}{l}\text { CAS reg. no. } \\
\text { Transition }\end{array}$} & \multicolumn{5}{|l|}{ Compound } \\
\hline & & Temp. range $(\mathrm{K})$ & $\begin{array}{c}\Delta_{\text {trans }} H_{\mathrm{m}} \\
\left(\mathrm{kJ} \mathrm{mol}^{-1}\right)\end{array}$ & $\mathrm{T}_{\mathrm{m}}(\mathrm{K})$ & Method & References \\
\hline & SUB & & $85.4 \pm 1.6$ & 298 & $\mathrm{C}$ & [2013SAN/RIB4] \\
\hline \multirow[t]{2}{*}{$\mathrm{C}_{6} \mathrm{H}_{7} \mathrm{NO}_{2} \mathrm{~S}$} & $\begin{array}{l}\text { [98-10-2] } \\
\text { FUS }\end{array}$ & \multicolumn{5}{|l|}{ benzenesulfonamide } \\
\hline & SUB & & $115.3 \pm 1.7$ & 298 & $\mathrm{C}$ & [2005MAT/MIR] \\
\hline $\mathrm{C}_{6} \mathrm{H}_{7} \mathrm{NO}_{3} \mathrm{~S}$ & $\begin{array}{c}{[121-57-3]} \\
\text { SUB }\end{array}$ & \multicolumn{5}{|c|}{ sulfanilic acid (4-aminobenzene sulfonic acid) } \\
\hline \multirow[t]{3}{*}{$\mathrm{C}_{6} \mathrm{H}_{7} \mathrm{NS}$} & $\begin{array}{c}{[22581-72-2]} \\
\text { FUS }\end{array}$ & 4-(methylthio)pyridin & 17.57 & & & {$[1974 \mathrm{BEA} / \mathrm{MUE}]$} \\
\hline & SUB & $(347-383)$ & $75.3 \pm 3.8$ & 365 & $\mathrm{~B}$ & [1974BEA/MUE] \\
\hline & $\mathrm{V}$ & $(346-383)$ & 55.8 & 361 & A & [1987STE/MAL, 1974BEA/MUE] \\
\hline \multirow[t]{2}{*}{$\mathrm{C}_{6} \mathrm{H}_{7} \mathrm{NS}$} & $\begin{array}{c}{[6887-59-8]} \\
\text { FUS }\end{array}$ & \multicolumn{4}{|c|}{ 1-methyl-4-thiopyridone } & [1974BEA/MUE] \\
\hline & SUB & $(440-465)$ & $188.3 \pm 9.2$ & 452 & B & [1974BEA/MUE] \\
\hline \multirow[t]{2}{*}{$\mathrm{C}_{6} \mathrm{H}_{7} \mathrm{~N}_{3} \mathrm{O}$} & $\begin{array}{c}\text { [54-85-3] } \\
\text { FUS } \\
\text { FUS } \\
\text { FUS }\end{array}$ & 4-pyridinecarbonylhy & $\begin{array}{c}\text { drazine } \\
31.1 \\
27.91 \\
28.39\end{array}$ & $\begin{array}{l}446.2 \\
445.8 \\
445.1\end{array}$ & $\begin{array}{l}\text { DSC } \\
\text { DSC } \\
\text { DSC }\end{array}$ & $\begin{array}{l}\text { [2015BLO/SHA] } \\
\text { [2012FOR/MEL, 2013MEL/BOG] } \\
\text { [2009FRE/ARA] }\end{array}$ \\
\hline & SUB & $(358-382)$ & $101.0 \pm 1.1$ & 298 & GS & [2015BLO/SHA] \\
\hline \multirow[t]{2}{*}{$\mathrm{C}_{6} \mathrm{H}_{7} \mathrm{~N}_{3} \mathrm{O}_{2}$} & $\begin{array}{c}\text { [3034-19-3] } \\
\text { SUB }\end{array}$ & \multicolumn{5}{|c|}{ 2-hydrazino-1-nitrobenzene } \\
\hline & $\mathrm{V}$ & & 99.6 & & DSC & [1990HWA/YOS] \\
\hline $\mathrm{C}_{6} \mathrm{H}_{7} \mathrm{~N}_{5}$ & $\begin{array}{l}{[5142-22-3]} \\
\text { SUB } \\
\text { SUB }\end{array}$ & $\begin{array}{l}\text { 1-methyladenine } \\
\quad(443-465) \\
(446-484)\end{array}$ & $\begin{array}{l}139.4 \pm 5.1 \\
136.9 \pm 2.8\end{array}$ & $\begin{array}{l}454 \\
465\end{array}$ & $\begin{array}{l}\mathrm{ME} \\
\mathrm{ME}\end{array}$ & $\begin{array}{l}\text { [2000ZIE] } \\
\text { [2000ZIE] }\end{array}$ \\
\hline $\mathrm{C}_{6} \mathrm{H}_{7} \mathrm{~N}_{5}$ & $\begin{array}{l}{[1445-08-5]} \\
\text { SUB } \\
\text { SUB }\end{array}$ & $\begin{array}{l}\text { 2-methyladenine } \\
\quad(412-437) \\
(421-437)\end{array}$ & $\begin{array}{l}120.6 \pm 1.3 \\
122.8 \pm 1.2\end{array}$ & $\begin{array}{l}424 \\
429\end{array}$ & $\begin{array}{l}\mathrm{ME} \\
\mathrm{ME}\end{array}$ & $\begin{array}{l}{[2000 \mathrm{ZIE}]} \\
{[2000 \mathrm{ZIE}]}\end{array}$ \\
\hline $\mathrm{C}_{6} \mathrm{H}_{7} \mathrm{~N}_{5}$ & $\begin{array}{c}\text { [5142-23-4] } \\
\text { SUB } \\
\text { SUB } \\
\text { SUB }\end{array}$ & $\begin{array}{c}\text { 3-methyladenine } \\
(392-412) \\
(395-411)\end{array}$ & $\begin{array}{c}117.3 \pm 1.1 \\
117.6 \pm 3.1 \\
83.7 \pm 9\end{array}$ & $\begin{array}{l}402 \\
403\end{array}$ & $\begin{array}{l}\text { ME } \\
\text { ME } \\
\text { HSA }\end{array}$ & $\begin{array}{l}{[2000 \mathrm{ZIE}]} \\
{[2000 \mathrm{ZIE}]} \\
{[1978 \mathrm{NOW} / \mathrm{SZC}]}\end{array}$ \\
\hline $\mathrm{C}_{6} \mathrm{H}_{7} \mathrm{~N}_{5}$ & $\begin{array}{l}\text { [443-72-1] } \\
\text { SUB }\end{array}$ & \multicolumn{3}{|l|}{$\begin{array}{c}N \text {-methyladenine } \\
(395-425)\end{array}$} & ME & [1984ZIE/ZIE] \\
\hline $\mathrm{C}_{6} \mathrm{H}_{7} \mathrm{~N}_{5}$ & $\begin{array}{c}{[22387-37-7]} \\
\text { SUB }\end{array}$ & $\begin{array}{l}\text { 8-methyladenine } \\
\qquad(424-455)\end{array}$ & $103.2 \pm 2.4$ & 439 & $\mathrm{ME}$ & [2000ZIE] \\
\hline $\mathrm{C}_{6} \mathrm{H}_{7} \mathrm{~N}_{5}$ & $\begin{array}{c}\text { [700-00-5] } \\
\text { SUB } \\
\text { SUB } \\
\text { SUB }\end{array}$ & $\begin{array}{l}\text { 9-methyladenine } \\
\quad(381-411) \\
(413-458)\end{array}$ & $\begin{array}{c}121.3 \pm 4.6 \\
121.7 \\
92 \pm 8\end{array}$ & 428 & $\begin{array}{l}\text { ME } \\
\text { HSA } \\
\text { HSA }\end{array}$ & $\begin{array}{l}{[1984 \mathrm{ZIE} / \mathrm{ZIE}]} \\
{[1987 \mathrm{STE} / \mathrm{MAL}, 1965 \mathrm{CLA} / \mathrm{PES}]} \\
{[1978 \mathrm{NOW} / \mathrm{SZC}]}\end{array}$ \\
\hline $\mathrm{C}_{6} \mathrm{H}_{7} \mathrm{NO}$ & $\begin{array}{c}{[1192-58-1]} \\
\mathrm{V}\end{array}$ & \multicolumn{4}{|c|}{ 1-methyl-2-pyrrolecarboxaldehyde } & [2011SAN/RIB3] \\
\hline $\mathrm{C}_{6} \mathrm{H}_{8}$ & $\begin{array}{l}\mathrm{V} \\
\mathrm{V}\end{array}$ & $\begin{array}{l}\text { cis, anti, cis-tricyclo[ } \\
\quad(273-329) \\
(273-329)\end{array}$ & $\begin{array}{l}\left.3.1 .0 .0^{2,4}\right] \text { hexa } \\
30.6 \\
29.7\end{array}$ & $\begin{array}{l}293 \\
313\end{array}$ & & $\begin{array}{l}\text { [1979LET/ORC] } \\
\text { [1979LET/ORC] }\end{array}$ \\
\hline \multirow[t]{2}{*}{$\mathrm{C}_{6} \mathrm{H}_{8}$} & $\begin{array}{c}{[592-57-4]} \\
\text { FUS }\end{array}$ & \multicolumn{4}{|l|}{ 1,3-cyclohexadiene } & [1996DOM/HEA, 1976GEI/WOL] \\
\hline & $\begin{array}{l}\mathrm{V} \\
\mathrm{V}\end{array}$ & $\begin{array}{l}(307-364) \\
(304-322)\end{array}$ & $\begin{array}{l}32.6 \\
32.4\end{array}$ & $\begin{array}{l}322 \\
308\end{array}$ & $\begin{array}{c}\text { A, EB } \\
\text { Static, MM }\end{array}$ & $\begin{array}{l}\text { [1987STE/MAL, 1973MEY/HOT] } \\
\text { [1974LET/MAR] }\end{array}$ \\
\hline $\mathrm{C}_{6} \mathrm{H}_{8}$ & {$[628-41-1]$} & 1,4-cyclohexadiene & & & & \\
\hline
\end{tabular}


TABLE 9. Phase change enthalpies of $\mathrm{C}_{6}$ organic compounds-Continued

\begin{tabular}{|c|c|c|c|c|c|c|}
\hline $\begin{array}{l}\text { Molecular } \\
\text { formula }\end{array}$ & $\begin{array}{l}\text { CAS reg. no. } \\
\text { Transition }\end{array}$ & \multicolumn{5}{|l|}{ Compound } \\
\hline & FUS & $(10-300)$ & 5.72 & 224 & & [1996DOM/HEA, 1976GEI/WOL] \\
\hline & $\mathrm{V}$ & $(304-360)$ & 34.0 & 319 & A & [1987STE/MAL] \\
\hline & $\mathrm{V}$ & $(304-322)$ & 33.9 & 308 & Static, MM & [1974LET/MAR] \\
\hline $\mathrm{C}_{6} \mathrm{H}_{8} \mathrm{ClN}$ & $\begin{array}{l}{[142-04-1]} \\
\text { SUB }\end{array}$ & $\begin{array}{l}\text { aniline hydrochloride } \\
\quad(383-471)\end{array}$ & 87.5 & 398 & A & [1987STE/MAL, 1975KON/SEL] \\
\hline & $\mathrm{V}$ & $(475-527)$ & 75 & 501 & & [2016HU/WU] \\
\hline $\mathrm{C}_{6} \mathrm{H}_{8} \mathrm{Cl}_{2} \mathrm{O}_{4}$ & $\begin{array}{c}{[6941-69-1]} \\
\mathrm{V}\end{array}$ & $\begin{array}{l}\text { ethylene glycol, bisch } \\
\quad(385-557)\end{array}$ & $\begin{array}{l}\text { loroacetate } \\
73.9\end{array}$ & 400 & A & [1987STE/MAL, 1947STU] \\
\hline $\mathrm{C}_{6} \mathrm{H}_{8} \mathrm{~F}_{8} \mathrm{~N}_{4}$ & $\begin{array}{c}\text { [18273-26-2] } \\
\text { FUS }\end{array}$ & 1,1,4,4-tetrakis(difluo & $\begin{array}{l}\text { roamino)cyc } \\
\quad 46.02\end{array}$ & $\begin{array}{l}\text { Ine } \\
382.2\end{array}$ & DSC & [2001OXL/SMI] \\
\hline $\mathrm{C}_{6} \mathrm{H}_{8} \mathrm{~N}_{2}$ & $\begin{array}{l}{[5910-89-4]} \\
\mathrm{V}\end{array}$ & 2,3-dimethylpyrazine & $52.6 \pm 1.7$ & 298 & $\mathrm{C}$ & [2003MOR/MIR] \\
\hline $\mathrm{C}_{6} \mathrm{H}_{8} \mathrm{~N}_{2}$ & $\begin{array}{c}{[123-32-0]} \\
\mathrm{V} \\
\mathrm{V} \\
\mathrm{V}\end{array}$ & $\begin{array}{l}\text { 2,5-dimethylpyrazine } \\
\qquad \begin{array}{l}(342-373) \\
(303-411)\end{array}\end{array}$ & $\begin{array}{c}47.6 \pm 2.7 \\
47.2 \pm 2.2 \\
\quad 44.5\end{array}$ & $\begin{array}{l}298 \\
298 \\
357\end{array}$ & $\begin{array}{l}\text { CGC } \\
\text { CGC }\end{array}$ & $\begin{array}{l}{[2010 \mathrm{LIP} / \mathrm{PLI}]} \\
{[2009 \mathrm{LIP} / \mathrm{CHI} 2]} \\
{[1995 \mathrm{SAK} / \mathrm{UEO}]}\end{array}$ \\
\hline $\mathrm{C}_{6} \mathrm{H}_{8} \mathrm{~N}_{2}$ & $\begin{array}{c}\text { [18364-47-1] } \\
\mathrm{V}\end{array}$ & $\begin{array}{l}\text { 3-methylaminopyridin } \\
(313-343)\end{array}$ & 57.2 & 326 & A & [1987STE/MAL] \\
\hline $\mathrm{C}_{6} \mathrm{H}_{8} \mathrm{~N}_{2}$ & $\begin{array}{c}{[1121-58-0]} \\
\mathrm{V}\end{array}$ & $\begin{array}{l}\text { 4-methylaminopyridin } \\
(313-343)\end{array}$ & ne 54.1 & 328 & A & [1987STE/MAL] \\
\hline $\mathrm{C}_{6} \mathrm{H}_{8} \mathrm{~N}_{2}$ & $\begin{array}{l}\text { [95-54-5] } \\
\text { FUS } \\
\text { FUS }\end{array}$ & 1,2-diaminobenzene & $\begin{array}{l}18.6 \\
23.1\end{array}$ & $\begin{array}{l}374.7 \\
373.9\end{array}$ & DSC & $\begin{array}{l}\text { [1997SAB/PER] } \\
\text { [1997LEE/CHA, 1989BRE/LIC] }\end{array}$ \\
\hline & $\begin{array}{l}\text { SUB } \\
\text { SUB } \\
\text { SUB }\end{array}$ & $\begin{array}{l}(292-314) \\
(292-314)\end{array}$ & $\begin{array}{l}89.7 \pm 0.4 \\
89.8 \pm 0.4 \\
85.5 \pm 0.3\end{array}$ & $\begin{array}{l}303 \\
298 \\
298\end{array}$ & $\begin{array}{l}\mathrm{ME} \\
\mathrm{ME} \\
\mathrm{C}\end{array}$ & $\begin{array}{l}\text { [2011SAN/RIB] } \\
{[2011 \mathrm{SAN} / \mathrm{RIB}]} \\
{[1997 \mathrm{SAB} / \mathrm{PER}]}\end{array}$ \\
\hline $\mathrm{C}_{6} \mathrm{H}_{8} \mathrm{~N}_{2}$ & $\begin{array}{l}\text { [108-45-2] } \\
\text { FUS } \\
\text { FUS }\end{array}$ & 1,3-diaminobenzene & $\begin{array}{c}12.03 \\
15.4\end{array}$ & $\begin{array}{l}336.3 \\
335.5\end{array}$ & DSC & $\begin{array}{l}\text { [1997SAB/PER] } \\
\text { [1996DOM/HEA, 1989BRE/LIC] }\end{array}$ \\
\hline & $\begin{array}{l}\text { SUB } \\
\text { SUB } \\
\text { SUB }\end{array}$ & $\begin{array}{l}(301-323) \\
(301-323)\end{array}$ & $\begin{array}{l}95.1 \pm 0.4 \\
95.5 \pm 0.4 \\
90.4 \pm 0.4\end{array}$ & $\begin{array}{l}312 \\
298 \\
298\end{array}$ & $\begin{array}{c}\mathrm{ME} \\
\mathrm{ME} \\
\mathrm{C}\end{array}$ & $\begin{array}{l}\text { [2011SAN/RIB] } \\
{[2011 \mathrm{SAN} / \mathrm{RIB}]} \\
{[1997 \mathrm{SAB} / \mathrm{PER}]}\end{array}$ \\
\hline & $\mathrm{V}$ & $(372-559)$ & 63.7 & 387 & A & [1987STE/MAL, 1947STU] \\
\hline $\mathrm{C}_{6} \mathrm{H}_{8} \mathrm{~N}_{2}$ & $\begin{array}{c}\text { [106-50-3] } \\
\text { FUS } \\
\text { FUS } \\
\text { FUS }\end{array}$ & 1,4-diaminobenzene & $\begin{array}{c}20.12 \\
24.86 \\
21.7\end{array}$ & $\begin{array}{c}414.3 \\
416 \\
412.3\end{array}$ & $\begin{array}{l}\text { DTA } \\
\text { DSC }\end{array}$ & $\begin{array}{l}\text { [1997SAB/PER] } \\
\text { [1992MAN] } \\
\text { [1996DOM/HEA, 1989BRE/LIC] }\end{array}$ \\
\hline
\end{tabular}


TABLE 9. Phase change enthalpies of $\mathrm{C}_{6}$ organic compounds-Continued

\begin{tabular}{|c|c|c|c|c|c|c|}
\hline \multirow[b]{2}{*}{$\begin{array}{l}\text { Molecular } \\
\text { formula }\end{array}$} & \multirow{2}{*}{$\begin{array}{l}\text { CAS reg. no. } \\
\text { Transition }\end{array}$} & \multicolumn{5}{|l|}{ Compound } \\
\hline & & Temp. range $(\mathrm{K})$ & $\begin{array}{c}\Delta_{\text {trans }} H_{\mathrm{m}} \\
\left(\mathrm{kJ} \mathrm{mol}^{-1}\right)\end{array}$ & $\mathrm{T}_{\mathrm{m}}(\mathrm{K})$ & Method & References \\
\hline & SUB & $(310-332)$ & $96.3 \pm 0.4$ & 321 & ME & [2011SAN/RIB] \\
\hline & SUB & $(310-332)$ & $96.8 \pm 0.4$ & 298 & $\mathrm{ME}$ & [2011SAN/RIB] \\
\hline & SUB & & $92.2 \pm 0.2$ & 298 & $\mathrm{C}$ & [1997SAB/PER] \\
\hline \multirow[t]{4}{*}{$\mathrm{C}_{6} \mathrm{H}_{8} \mathrm{~N}_{2}$} & $\begin{array}{c}\text { [100-63-0] } \\
\text { FUS }\end{array}$ & phenyl hydrazine & 16.43 & 292.8 & & [1996DOM/HEA, 1991ACR, 1911LOU/DUP] \\
\hline & $\mathrm{V}$ & $(413-518)$ & 57.3 & 428 & A & [1987STE/MAL, 1972DYK] \\
\hline & $\mathrm{V}$ & $(345-517)$ & 59.2 & 360 & & {$[1947 \mathrm{STU}]$} \\
\hline & $\mathrm{V}$ & $(378-465)$ & 57.2 & 393 & $\mathrm{~T}$ & [1942WIL/GIL] \\
\hline $\mathrm{C}_{6} \mathrm{H}_{8} \mathrm{~N}_{2}$ & $\begin{array}{c}{[13925-00-3]} \\
\mathrm{V}\end{array}$ & ethylpyrazine & $48.8 \pm 1.9$ & 298 & $\mathrm{C}$ & [2003MOR/MIR] \\
\hline \multirow[t]{11}{*}{$\mathrm{C}_{6} \mathrm{H}_{8} \mathrm{~N}_{2} \mathrm{O}_{2}$} & $\begin{array}{c}\text { [874-14-6] } \\
\text { FUS (I) } \\
\text { FUS (II) } \\
\text { FUS }\end{array}$ & 1,3-dimethyluracil & $\begin{array}{l}14.6 \\
18.4 \\
23.1\end{array}$ & $\begin{array}{c}399 \\
395.2 \\
392.5\end{array}$ & $\begin{array}{l}\text { DSC } \\
\text { DSC }\end{array}$ & $\begin{array}{l}\text { [1996DOM/HEA, 1989SAK/IMA] } \\
\text { [1984ZIE/ZIE2] }\end{array}$ \\
\hline & SUB & $(293-337)$ & $96.3 \pm 0.5$ & 317 & $\mathrm{ME}$ & [2015BRU/IRR] \\
\hline & SUB & $(293-337)$ & 96.8 & 298 & $\mathrm{ME}$ & [2015BRU/IRR] \\
\hline & SUB & $(293-337)$ & $95.9 \pm 0.9$ & 348 & ME & [2015BRU/IRR] \\
\hline & SUB & $(335-360)$ & 97.4 & 298 & $\mathrm{ME}$ & [2015BRU/IRR] \\
\hline & SUB & $(311-367)$ & $115.8 \pm 3.0$ & 338 & $\mathrm{TE}$ & [2000BRU/PIA] \\
\hline & SUB & $(340-368)$ & $96.9 \pm 1.2$ & 298 & $\mathrm{C}$ & [1989IMA/TAK] \\
\hline & SUB & & $96.4 \pm 1.4$ & 298 & $\mathrm{C}$ & [1985MUR/SAK] \\
\hline & SUB & $(313-363)$ & $101.7 \pm 2.1$ & 338 & QR & [1980TEP/YAN] \\
\hline & SUB & $(344-370)$ & 92 & & HSA & [1965CLA/PES] \\
\hline & $\mathrm{V}$ & $(400-454)$ & $46 \pm 4.2$ & 426 & HSA & [1978NOW/SZC] \\
\hline $\mathrm{C}_{6} \mathrm{H}_{8} \mathrm{~N}_{2} \mathrm{O}_{2}$ & $\begin{array}{l}{[26305-13-5]} \\
\text { SUB }\end{array}$ & $\begin{array}{l}\text { 5,6-dimethyluracil } \\
(416-457)\end{array}$ & $132.4 \pm 1.4$ & 298 & GS & [2013NOT/EME] \\
\hline $\mathrm{C}_{6} \mathrm{H}_{8} \mathrm{~N}_{2} \mathrm{O}_{2}$ & $\begin{array}{l}{[4160-72-9]} \\
\text { SUB }\end{array}$ & $\begin{array}{l}\text { 1-methylthymine } \\
\quad(378-428)\end{array}$ & $124.4 \pm 1.3$ & 398 & QR & [1980TEP/YAN] \\
\hline $\mathrm{C}_{6} \mathrm{H}_{8} \mathrm{~N}_{2} \mathrm{O}_{2}$ & $\begin{array}{l}{[4538-37-8]} \\
\text { FUS }\end{array}$ & $\begin{array}{l}\text { 1,4-diisocyanatobut } \\
(8-360)\end{array}$ & 20.76 & 231.2 & $\mathrm{AC}$ & [2006SMI/KAN] \\
\hline \multirow[t]{2}{*}{$\mathrm{C}_{6} \mathrm{H}_{8} \mathrm{~N}_{2} \mathrm{O}_{2}$} & $\begin{array}{l}{[4562-27-0]} \\
\text { FUS }\end{array}$ & 2,4-dimethoxypyrim & idine 16.64 & 279.6 & $\mathrm{DSC}$ & [2014GAL/RIB] \\
\hline & $\mathrm{V}$ & & $60.3 \pm 1.3$ & 298 & $\mathrm{C}$ & [2014GAL/RIB] \\
\hline \multirow[t]{5}{*}{$\mathrm{C}_{6} \mathrm{H}_{8} \mathrm{~N}_{2} \mathrm{O}_{2} \mathrm{~S}$} & {$[63-74-1]$} & 4-aminobenzene sul & honamide (sul & amide) & & \\
\hline & FUS & & 23.3 & 435.4 & DSC & [2002MAR/GOM, 2001MAR/GOM] \\
\hline & TRS & & 1.63 & 407 & & \\
\hline & FUS & & 24.02 & 439.3 & & [1996CIO/MEL] \\
\hline & FUS & & 23.0 & 438.7 & & [1985OHM/LIP] \\
\hline \multirow[t]{3}{*}{$\mathrm{C}_{6} \mathrm{H}_{8} \mathrm{~N}_{2} \mathrm{O}_{3}$} & {$[769-42-6]$} & 1,3-dimethylbarbitu & ic acid & & & \\
\hline & FUS & & 17.7 & 396.1 & DSC & [2011TEM/ROU] \\
\hline & SUB & $(340-390)$ & $92.3 \pm 0.6$ & 298 & GS & [2011ROU/NOT] \\
\hline \multirow[t]{4}{*}{$\mathrm{C}_{6} \mathrm{H}_{8} \mathrm{~N}_{2} \mathrm{O}_{3}$} & [24448-94-0] & 5,5-dimethylbarbitu & ic acid & & & \\
\hline & FUS & & 37.9 & 549.3 & DSC & [2011TEM/ROU] \\
\hline & FUS & & 34.8 & 548.7 & $\mathrm{DSC}$ & [2010ROU/NOT] \\
\hline & SUB & $(400-458)$ & $115.8 \pm 0.5$ & 298 & GS & [2010ROU/NOT] \\
\hline \multirow[t]{2}{*}{$\mathrm{C}_{6} \mathrm{H}_{8} \mathrm{~N}_{2} \mathrm{O}_{8}$} & [551-43-9] & 1,4:3,6-dianhydrom & nnitol dinitrate & mannide & & \\
\hline & FUS & & 20.5 & 337.2 & DSC & [1998HAT/SUZ] \\
\hline \multirow[t]{2}{*}{$\mathrm{C}_{6} \mathrm{H}_{8} \mathrm{~N}_{2} \mathrm{O}_{8}$} & {$[87-33-2]$} & 1,4:3,6-dianhydro-( & -glucitol dini & (isosorbic & trate) & \\
\hline & FUS & & 27.63 & 341.7 & $\mathrm{DSC}$ & [1998HAT/SUZ] \\
\hline \multirow[t]{2}{*}{$\mathrm{C}_{6} \mathrm{H}_{8} \mathrm{~N}_{2} \mathrm{O}_{8}$} & {$[38777-20-7]$} & 1,4:3,6-dianhydroid & ol dinitrate (is & e dinitrat & & \\
\hline & FUS & & 12.81 & 325.9 & DSC & [1998HAT/SUZ] \\
\hline
\end{tabular}


TABLE 9. Phase change enthalpies of $\mathrm{C}_{6}$ organic compounds-Continued

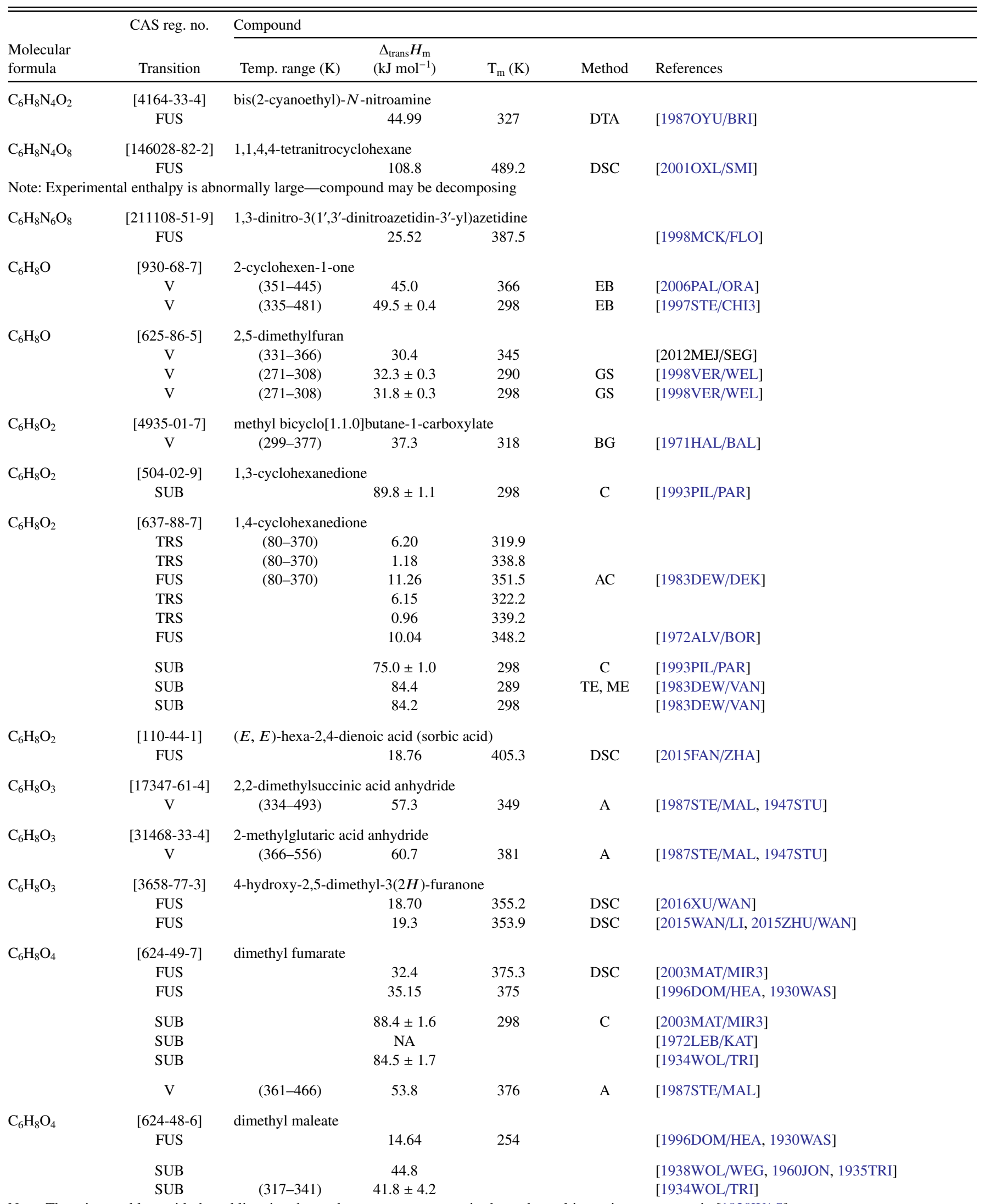

Note: There is a problem with the sublimation data — the temperature range is above the melting point temperture in [1930WAS]

$\begin{array}{lccccl}\mathrm{V} & & 64.4 \pm 1.6 & 298 & & \\ \mathrm{~V} & (385-421) & 52.0 & 400 & \text { A } & {[1987 \mathrm{MTT} / \mathrm{MAL} \text { ] }} \\ \mathrm{V} & (318-478) & 53.9 & 334 & & {[1947 \mathrm{STU}]}\end{array}$


TABLE 9. Phase change enthalpies of $\mathrm{C}_{6}$ organic compounds-Continued

\begin{tabular}{|c|c|c|c|c|c|c|}
\hline \multirow[b]{2}{*}{$\begin{array}{l}\text { Molecular } \\
\text { formula }\end{array}$} & \multirow{2}{*}{$\begin{array}{c}\text { CAS reg. no. } \\
\text { Transition }\end{array}$} & \multicolumn{5}{|l|}{ Compound } \\
\hline & & Temp. range $(\mathrm{K})$ & $\begin{array}{c}\Delta_{\text {trans }} H_{\mathrm{m}} \\
\left(\mathrm{kJ} \mathrm{mol}^{-1}\right) \\
\end{array}$ & $\mathrm{T}_{\mathrm{m}}(\mathrm{K})$ & Method & References \\
\hline \multirow[t]{2}{*}{$\mathrm{C}_{6} \mathrm{H}_{8} \mathrm{O}_{4}$} & $\begin{array}{c}\text { [5445-51-2] } \\
\text { FUS }\end{array}$ & \multicolumn{5}{|c|}{ cyclobutane-1,1-dicarboxylic acid } \\
\hline & $\begin{array}{l}\text { SUB } \\
\text { SUB }\end{array}$ & $(298-318)$ & $\begin{array}{c}84 \\
112.2 \pm 0.7\end{array}$ & 308 & $\begin{array}{c}\mathrm{ME} \\
\mathrm{C}\end{array}$ & $\begin{array}{l}\text { [2011BOO/MON] } \\
\text { [1983ALT/PIL] }\end{array}$ \\
\hline $\mathrm{C}_{6} \mathrm{H}_{8} \mathrm{O}_{4}$ & $\begin{array}{c}\text { [3396-14-3] } \\
\text { SUB }\end{array}$ & \multicolumn{5}{|c|}{ cyclobutane-1,2-dicarboxylic acid } \\
\hline \multirow[t]{2}{*}{$\mathrm{C}_{6} \mathrm{H}_{8} \mathrm{O}_{4}$} & $\begin{array}{l}{[95-96-5]} \\
\text { FUS }\end{array}$ & \multicolumn{5}{|c|}{$(d l)$-3,6-dimethyl-1,4-dioxane-2,5-dione ((DL)-lactide) } \\
\hline & SUB & $(329-384)$ & $91.6 \pm 0.4$ & 298 & GS & [2009EME/VER2] \\
\hline \multirow[t]{2}{*}{$\mathrm{C}_{6} \mathrm{H}_{8} \mathrm{O}_{4}$} & $\begin{array}{c}\text { [4511-42-6] } \\
\text { FUS } \\
\text { FUS }\end{array}$ & (l) 3,6-dimethyl-1 & $\begin{array}{c}\text { dioxane-2,5-d } \\
16.21 \\
16.94\end{array}$ & $\begin{array}{c}\text { (L)-lactid } \\
370.4 \\
366.6\end{array}$ & DSC & $\begin{array}{l}\text { [2013CHE/XIE] } \\
\text { [1999LEB/KUL] }\end{array}$ \\
\hline & SUB & $(324-362)$ & $86.2 \pm 0.4$ & 298 & GS & [2009EME/VER2] \\
\hline $\mathrm{C}_{6} \mathrm{H}_{8} \mathrm{O}_{4}$ & $\begin{array}{c}\text { [13076-17-0] } \\
\text { FUS }\end{array}$ & \multicolumn{5}{|c|}{ (d)-3,6-dimethyl-1,4-dioxane-2,5-dione ((D)-lactide) } \\
\hline $\mathrm{C}_{6} \mathrm{H}_{8} \mathrm{O}_{5}$ & $\begin{array}{c}{[3184-35-8]} \\
\text { SUB }\end{array}$ & $\begin{array}{c}\text { 2-oxohexanedioic } \\
(281-301)\end{array}$ & 127.0 & & TPTD & [2005CHA/ZIE] \\
\hline
\end{tabular}

Note: Values based on TPTD method are not consistent with values determined by other experimental methods

$\mathrm{C}_{6} \mathrm{H}_{8} \mathrm{O}_{5} \quad$ [689-31-6] 3-oxohexanedioic acid SUB (307-329) $151 \quad$ TPTD [2005CHA/ZIE]

Note: Values based on TPTD method are not consistent with values determined by other experimental methods

$\begin{array}{cccccc}\mathrm{C}_{6} \mathrm{H}_{8} \mathrm{O}_{6} & {[50-81-7]} & (l) \text {-ascorbic acid (vitamin C) } & & \\ & \text { FUS } & 45.2 & 467.4 & \text { DSC } & {[2014 \mathrm{SHA} / \mathrm{SRE}]} \\ & \text { FUS } & 37.0 & 464.0 & \text { DSC } & \text { [2012KLI/LEI] } \\ & \text { FUS } & 48.6 & 466.8 & \text { DSC } & \text { [2010PAR/LEE] } \\ & \text { FUS } & 45.6 & 466.7 & \text { DSC } & \text { [2003TAN] }\end{array}$

Note: Author of [2003TAN] noted that melting point temperature and enthalpy of fusion were dependent on scan rate, suggesting that the compound exhibited some decomposition. The value of $45.6 \mathrm{~kJ} / \mathrm{mole}$ was for the largest scan rate studied.

FUS $37.04 \quad 466.2 \quad$ DSC [1998MUR/BET]

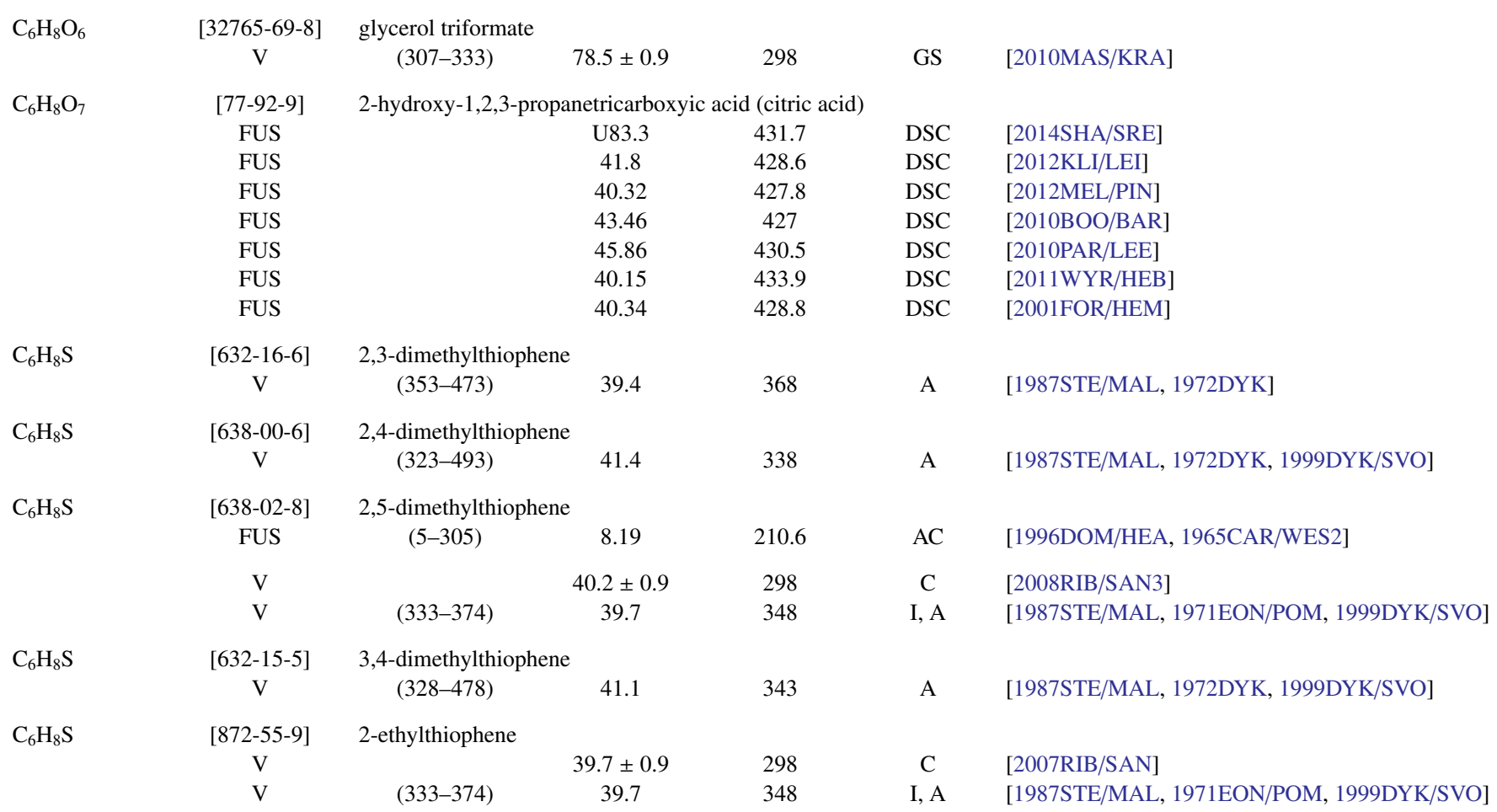


TABLE 9. Phase change enthalpies of $\mathrm{C}_{6}$ organic compounds-Continued

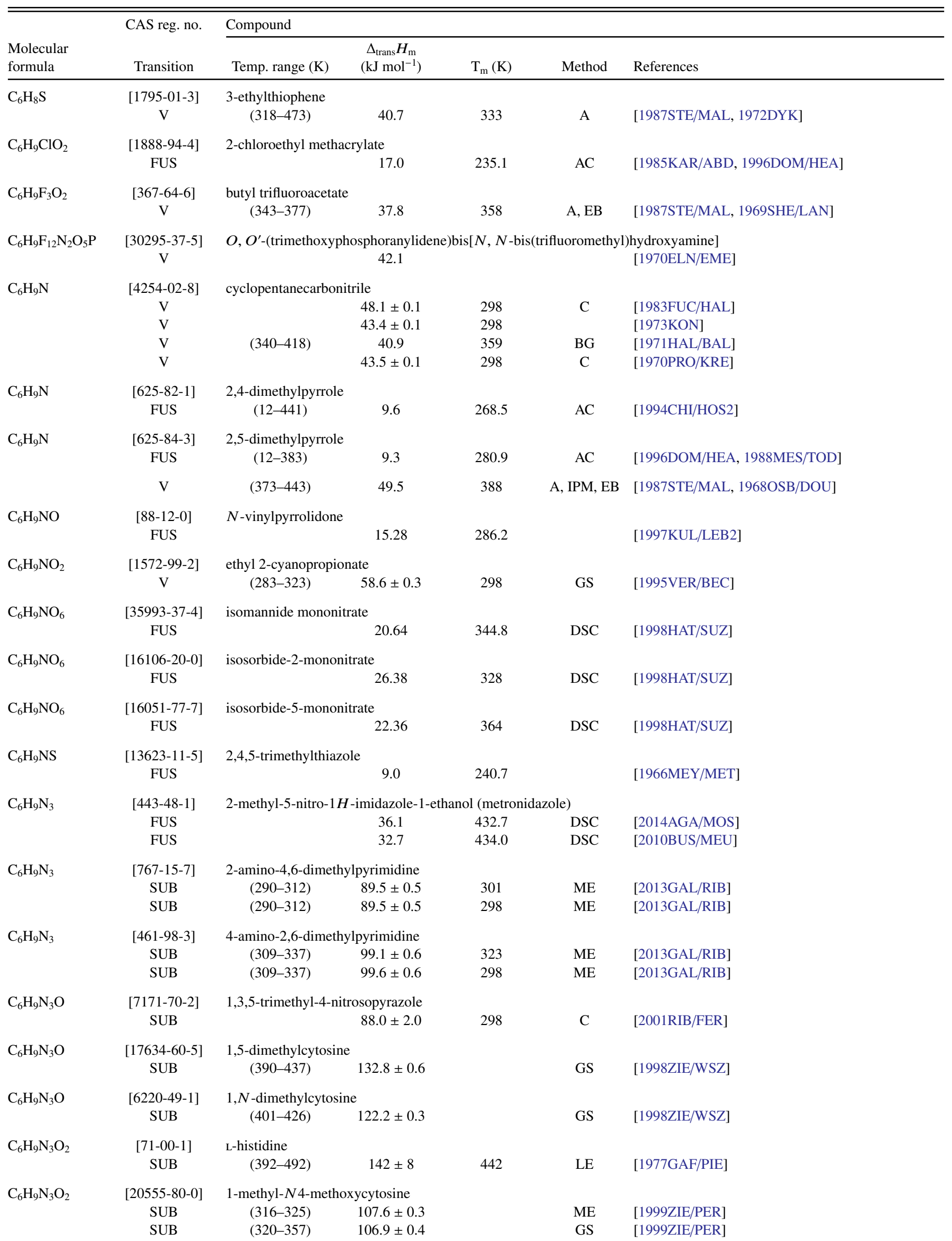


TABLE 9. Phase change enthalpies of $\mathrm{C}_{6}$ organic compounds-Continued

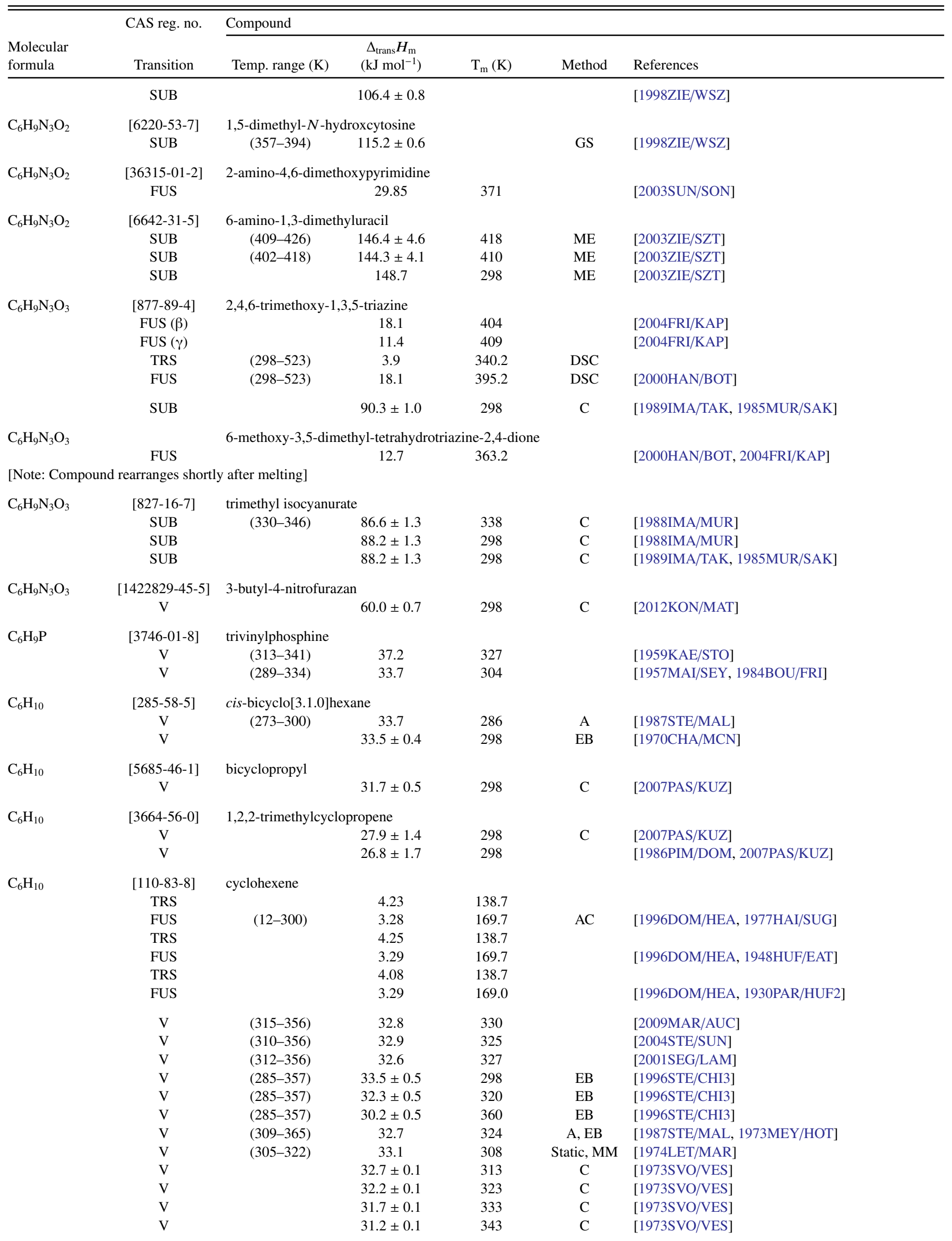


TABLE 9. Phase change enthalpies of $\mathrm{C}_{6}$ organic compounds - Continued

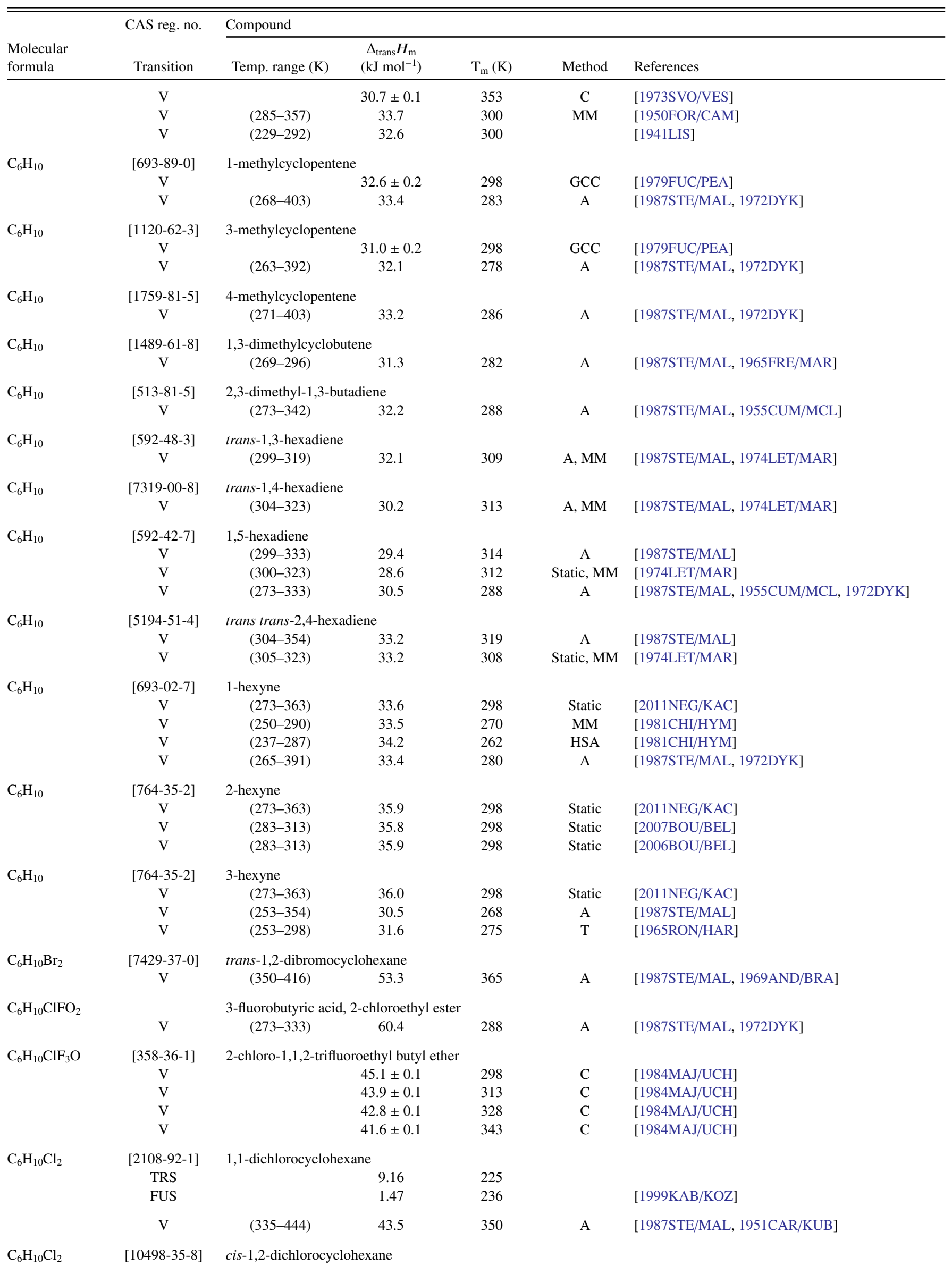


TABLE 9. Phase change enthalpies of $\mathrm{C}_{6}$ organic compounds-Continued

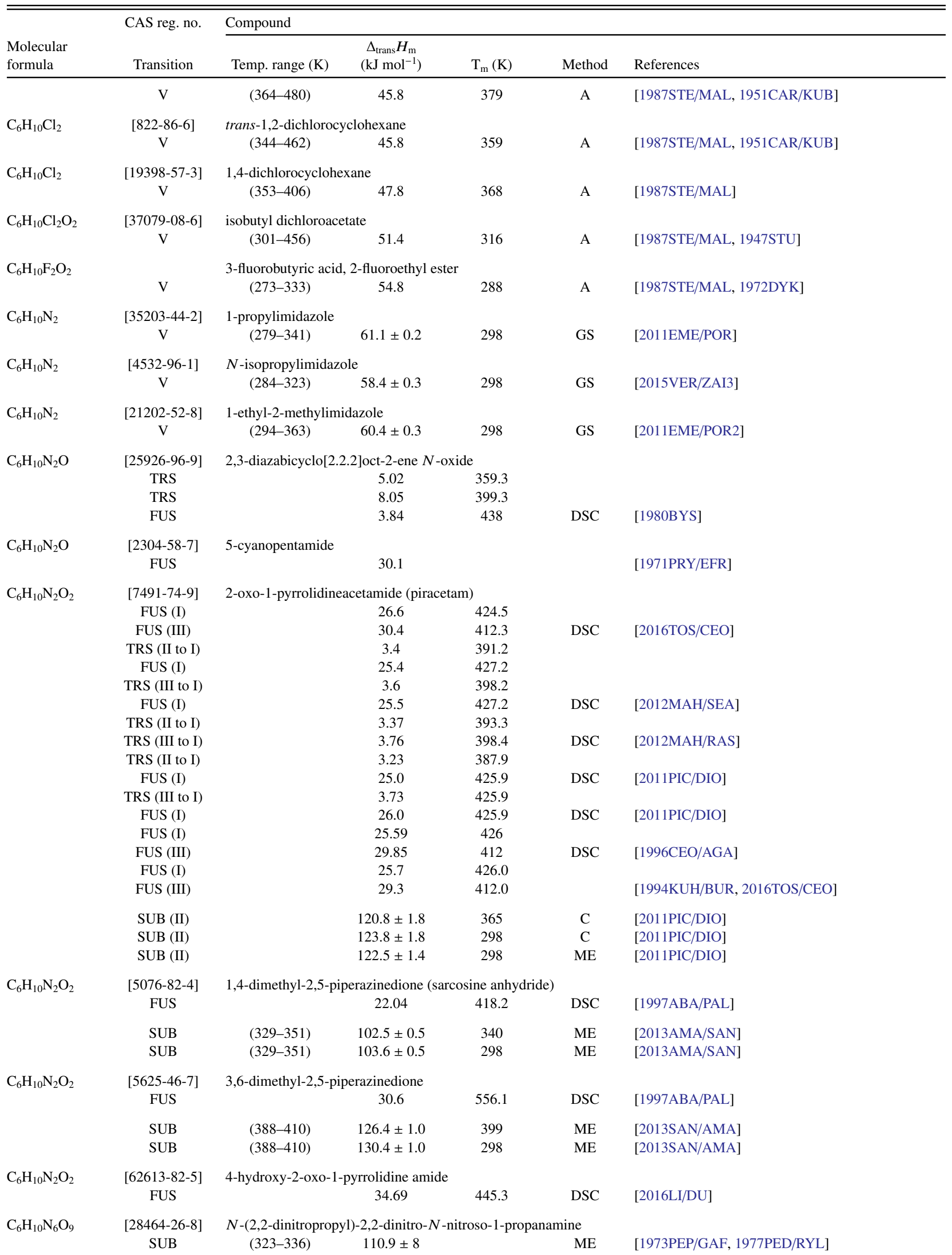


TABLE 9. Phase change enthalpies of $\mathrm{C}_{6}$ organic compounds-Continued

\begin{tabular}{|c|c|c|c|c|c|c|}
\hline \multirow[b]{2}{*}{$\begin{array}{l}\text { Molecular } \\
\text { formula }\end{array}$} & \multirow{2}{*}{$\begin{array}{l}\text { CAS reg. no. } \\
\text { Transition }\end{array}$} & \multicolumn{5}{|l|}{ Compound } \\
\hline & & Temp. range $(\mathrm{K})$ & $\begin{array}{c}\Delta_{\text {trans }} H_{\mathrm{m}} \\
\left(\mathrm{kJ} \mathrm{mol}^{-1}\right)\end{array}$ & $\mathrm{T}_{\mathrm{m}}(\mathrm{K})$ & Method & References \\
\hline $\mathrm{C}_{6} \mathrm{H}_{10} \mathrm{O}$ & $\begin{array}{c}\text { [279-49-2] } \\
\text { TRS } \\
\text { TRS } \\
\text { FUS }\end{array}$ & 7-oxabicyclo[2.2.1] & $\begin{array}{r}\text { eptane } \\
4.86 \\
0.98 \\
0.71\end{array}$ & $\begin{array}{c}180.5 \\
218.5 \\
244\end{array}$ & DSC & [1998PAR/GIL] \\
\hline $\mathrm{C}_{6} \mathrm{H}_{10} \mathrm{O}$ & $\begin{array}{c}\text { [1462-03-9] } \\
\text { FUS }\end{array}$ & \multicolumn{5}{|c|}{ 1-methylcyclopentanol } \\
\hline & $\begin{array}{l}\text { SUB (cryst III) } \\
\text { SUB (cryst III) } \\
\text { SUB (cryst III) }\end{array}$ & $(253-281)$ & $\begin{array}{l}73.7 \pm 0.4 \\
67.0 \pm 0.2 \\
67.4 \pm 0.2\end{array}$ & $\begin{array}{l}267 \\
298 \\
291\end{array}$ & $\begin{array}{l}\mathrm{ME} \\
\mathrm{C}\end{array}$ & $\begin{array}{l}{[1997 \mathrm{BLO} / \mathrm{KAB}]} \\
{[1997 \mathrm{BLO} / \mathrm{KAB}]} \\
{[1997 \mathrm{BLO} / \mathrm{KAB}]}\end{array}$ \\
\hline $\mathrm{C}_{6} \mathrm{H}_{10} \mathrm{O}$ & $\begin{array}{c}{[2270-61-3]} \\
\mathrm{V}\end{array}$ & \multicolumn{4}{|c|}{ 2,3-dihydro-4-methyl- $2 \boldsymbol{H}$-pyran } & [1987STE/MAL, 1968KAC/NEM, 1984BOU/FRI] \\
\hline $\mathrm{C}_{6} \mathrm{H}_{10} \mathrm{O}$ & $\begin{array}{c}{[35656-02-1]} \\
\mathrm{V}\end{array}$ & \multicolumn{5}{|c|}{ methylenetetrahydro- $2 \mathrm{H}$-pyran } \\
\hline $\mathrm{C}_{6} \mathrm{H}_{10} \mathrm{O}$ & $\begin{array}{c}\text { [108-94-1] } \\
\text { TRS } \\
\text { FUS } \\
\text { TRS } \\
\text { FUS } \\
\text { SUB } \\
\text { V } \\
\text { V } \\
\text { V } \\
\text { V } \\
\text { V } \\
\text { V } \\
\text { V } \\
\text { V } \\
\text { V } \\
\text { V } \\
\text { V } \\
\text { V } \\
\text { V } \\
\text { V } \\
\text { V } \\
\text { V } \\
\text { V } \\
\text { V } \\
\text { V }\end{array}$ & $\begin{array}{l}(345-458) \\
(395-426) \\
(296-368) \\
(381-446) \\
(362-439) \\
(293-353) \\
(273-298)\end{array}$ & $\begin{array}{c}8.66 \\
1.33 \\
8.74 \\
1.36 \\
49.3 \\
43.1 \\
46.6 \pm 0.4 \\
44.0 \\
44.4 \pm 0.1 \\
44.0 \pm 0.1 \\
43.4 \pm 0.1 \\
43.1 \pm 0.1 \\
42.2 \pm 0.1 \\
41.8 \pm 0.1 \\
41.4 \pm 0.1 \\
42.3 \pm 0.2 \\
42.2 \\
40.4 \\
43.8 \\
40.5 \\
41.5 \\
44.9 \pm 0.6 \\
45.1 \pm 0.1 \\
40.3\end{array}$ & $\begin{array}{l}220.8 \\
245.2 \\
220.6 \\
244.6 \\
254 \\
358 \\
298 \\
333 \\
308 \\
313 \\
323 \\
328 \\
338 \\
343 \\
348 \\
\\
360 \\
410 \\
330 \\
400 \\
377 \\
298 \\
298 \\
286\end{array}$ & $\begin{array}{c}\text { EB } \\
\text { CGC } \\
\text { C } \\
\text { C } \\
\text { C } \\
\text { C } \\
\text { C } \\
\text { C } \\
\text { C } \\
\text { GC } \\
\text { EB }\end{array}$ & 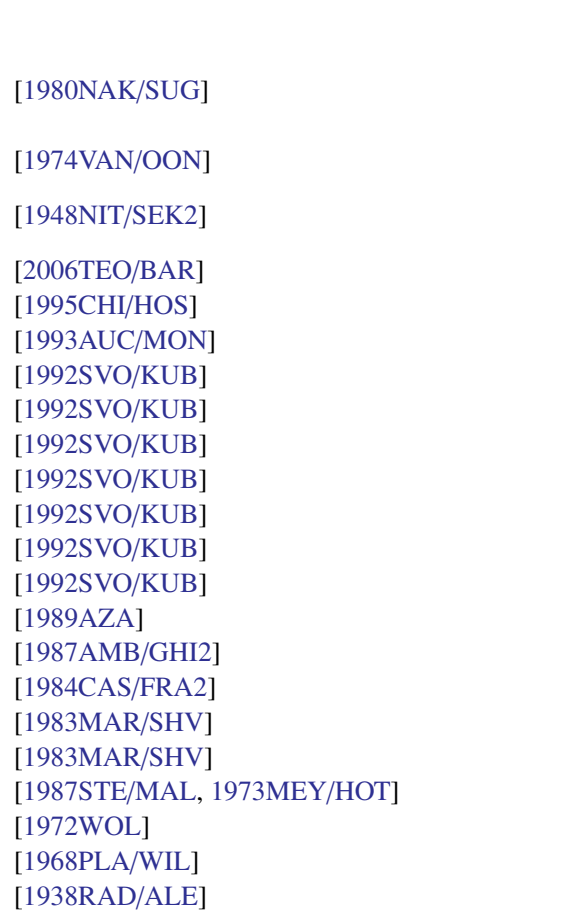 \\
\hline $\mathrm{C}_{6} \mathrm{H}_{10} \mathrm{O}$ & $\begin{array}{c}\text { [109-49-9] } \\
\text { V } \\
\text { V } \\
\text { V } \\
\text { V } \\
\text { V }\end{array}$ & $\begin{array}{r}5 \text {-hexen-2-one } \\
(317-440) \\
(317-440) \\
(317-440) \\
(317-440) \\
(449-561)\end{array}$ & $\begin{array}{c}42.1 \pm 0.1 \\
39.4 \pm 0.2 \\
36.6 \pm 0.3 \\
33.5 \pm 0.6 \\
34.6\end{array}$ & $\begin{array}{l}320 \\
360 \\
400 \\
440 \\
464\end{array}$ & $\begin{array}{c}\text { EB } \\
\text { EB } \\
\text { EB } \\
\text { EB } \\
\text { A }\end{array}$ & $\begin{array}{l}{[2002 \mathrm{STE} / \mathrm{CHI} 5]} \\
{[2002 \mathrm{STE} / \mathrm{CHI} 5]} \\
{[2002 \mathrm{STE} / \mathrm{CHI} 5]} \\
{[2002 \mathrm{STE} / \mathrm{CHI} 5]} \\
{[1987 \mathrm{STE} / \mathrm{MAL}]}\end{array}$ \\
\hline $\mathrm{C}_{6} \mathrm{H}_{10} \mathrm{O}$ & $\begin{array}{c}{[141-79-7]} \\
V\end{array}$ & mesityl oxide & 35.2 & 401 & & [1898LOU, 1997STE/CHI] \\
\hline $\mathrm{C}_{6} \mathrm{H}_{10} \mathrm{O}$ & $\begin{array}{c}{[3744-02-3]} \\
\mathrm{V}\end{array}$ & $\begin{array}{l}\text { 2-methyl-1-penten- } \\
(389-461)\end{array}$ & $\begin{array}{l}\text { one } \\
\quad 36.9\end{array}$ & 404 & A & [1987STE/MAL] \\
\hline
\end{tabular}


TABLE 9. Phase change enthalpies of $\mathrm{C}_{6}$ organic compounds-Continued

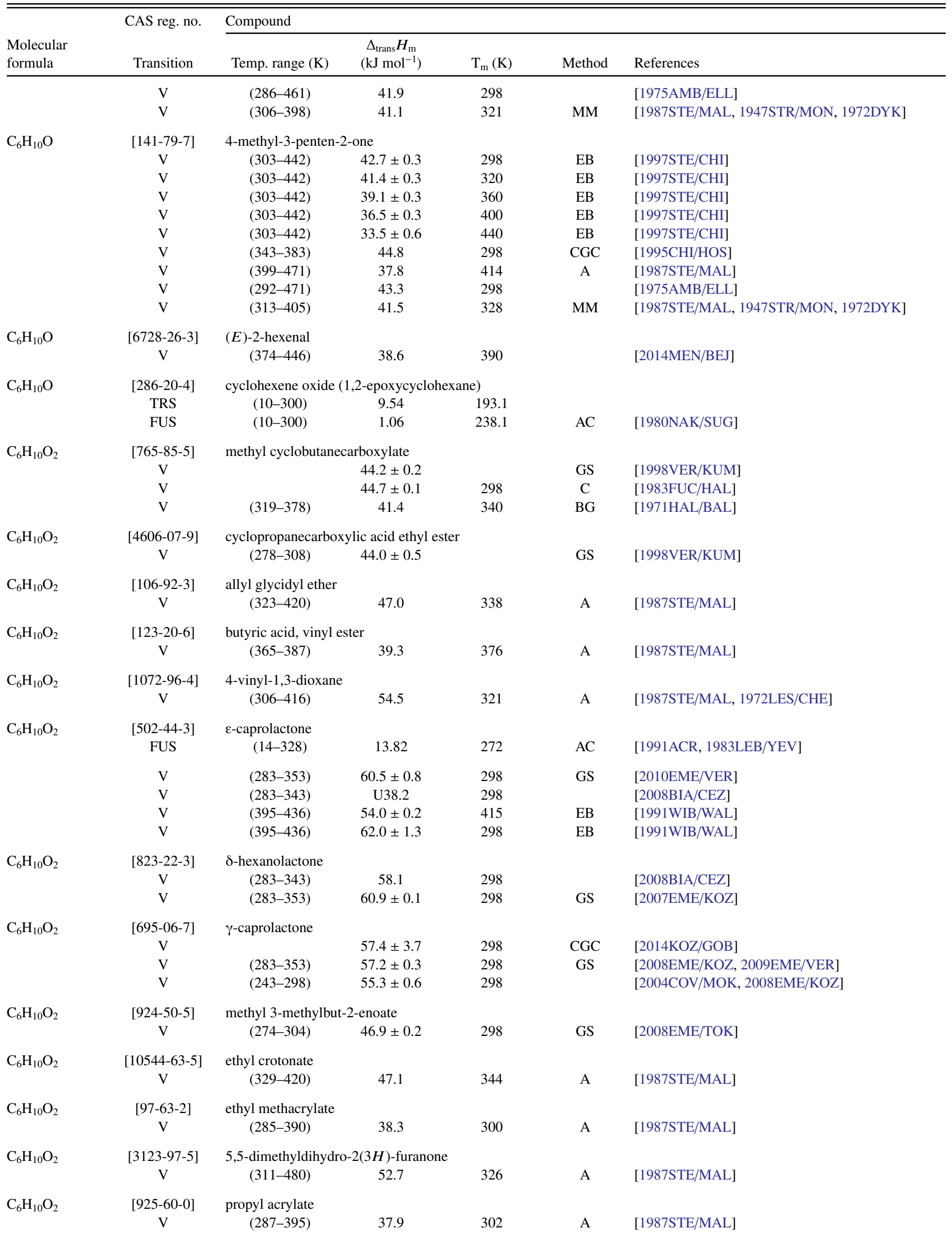


TABLE 9. Phase change enthalpies of $\mathrm{C}_{6}$ organic compounds-Continued

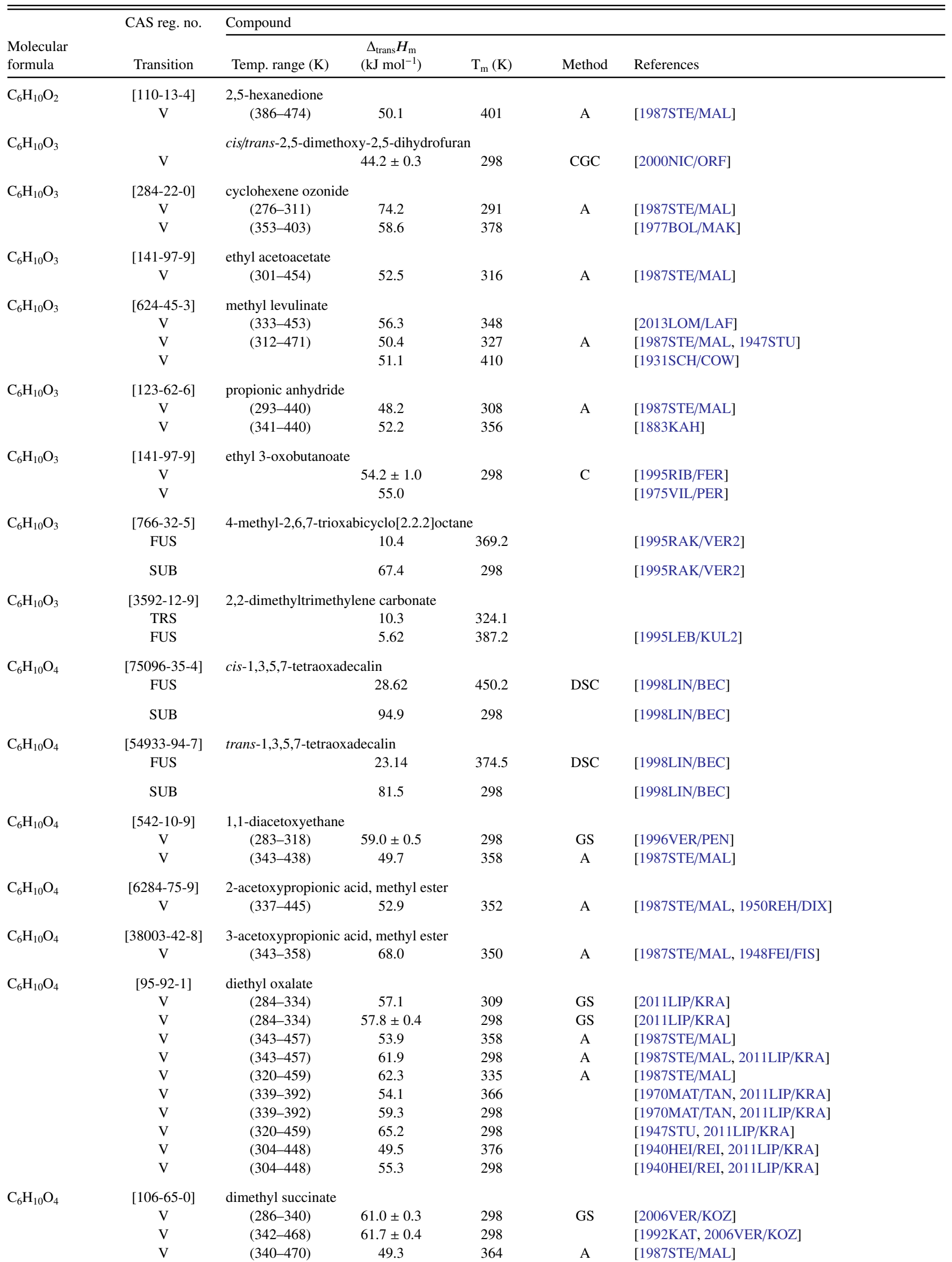


TABLE 9. Phase change enthalpies of $\mathrm{C}_{6}$ organic compounds-Continued

\begin{tabular}{|c|c|c|c|c|c|c|}
\hline \multirow[b]{2}{*}{$\begin{array}{l}\text { Molecular } \\
\text { formula }\end{array}$} & \multirow{2}{*}{$\begin{array}{l}\text { CAS reg. no. } \\
\text { Transition }\end{array}$} & \multicolumn{5}{|l|}{ Compound } \\
\hline & & Temp. range $(\mathrm{K})$ & $\begin{array}{c}\Delta_{\text {trans }} H_{\mathrm{m}} \\
\left(\mathrm{kJ} \mathrm{mol}^{-1}\right)\end{array}$ & $\mathrm{T}_{\mathrm{m}}(\mathrm{K})$ & Method & References \\
\hline & $\mathrm{V}$ & $(367-460)$ & $60.9 \pm 0.4$ & 298 & $\mathrm{~EB}$ & [1987DAU/JAL, 2006VER/KOZ] \\
\hline & $\mathrm{V}$ & $(398-468)$ & 62.4 & 298 & $\mathrm{~EB}$ & [1963VLA/GRA, 2006VER/KOZ] \\
\hline \multirow[t]{7}{*}{$\mathrm{C}_{6} \mathrm{H}_{10} \mathrm{O}_{4}$} & {$[111-55-7]$} & \multicolumn{5}{|c|}{ ethylene glycol diacetate } \\
\hline & $\mathrm{V}$ & $(295-325)$ & $60.2 \pm 1.1$ & & GS & [2011MAS/KRA] \\
\hline & $\mathrm{V}$ & $(291-334)$ & $61.4 \pm 0.2$ & 298 & GS & [2009VER/EME2] \\
\hline & $\mathrm{V}$ & $(311-464)$ & 55.2 & 326 & A & [1987STE/MAL] \\
\hline & $\mathrm{V}$ & & $61.4 \pm 0.2$ & 298 & $\mathrm{C}$ & [1986NIL/WAD] \\
\hline & $\mathrm{V}$ & & $61.0 \pm 0.1$ & 298 & $\mathrm{C}$ & [1970KUS/WAD] \\
\hline & $\mathrm{V}$ & $(373-463)$ & 57.6 & 388 & & [1926TAY/RIN, 1984BOU/FRI] \\
\hline \multirow{2}{*}{$\mathrm{C}_{6} \mathrm{H}_{10} \mathrm{O}_{4}$} & [609-02-9] & \multicolumn{5}{|c|}{ dimethyl methylmalonate } \\
\hline & $\mathrm{V}$ & $(278-308)$ & $57.8 \pm 0.8$ & 293 & GS & {$[1992 \mathrm{VER} / \mathrm{BEC}]$} \\
\hline \multirow[t]{3}{*}{$\mathrm{C}_{6} \mathrm{H}_{10} \mathrm{O}_{4}$} & {$[597-43-3]$} & \multicolumn{5}{|c|}{ 2,2-dimethylbutanedioic acid (2,2-dimethylsuccinic acid) } \\
\hline & FUS & & 38.1 & 411.0 & DSC & [2012WAN/LI] \\
\hline & SUB & $(350-365)$ & $122.7 \pm 2.7$ & 298 & ME & [2001RIB/MON2] \\
\hline \multirow[t]{3}{*}{$\mathrm{C}_{6} \mathrm{H}_{10} \mathrm{O}_{4}$} & {$[617-62-9]$} & \multicolumn{5}{|l|}{ 2-methylglutaric acid } \\
\hline & FUS & & 30.26 & 349 & DSC & {$[2010 \mathrm{BOO} / \mathrm{BAR}]$} \\
\hline & SUB & $(338-348)$ & $126.5 \pm 2.1$ & 298 & ME & [2001RIB/MON2] \\
\hline \multirow{3}{*}{$\mathrm{C}_{6} \mathrm{H}_{10} \mathrm{O}_{4}$} & {$[626-51-7]$} & \multicolumn{5}{|c|}{ 3-methylpentanedioic acid } \\
\hline & FUS & & 31.0 & 358.6 & DSC & [2012WAN/DEN, 2012WAN/LI] \\
\hline & FUS & & 27.4 & 356 & DSC & {$[2010 \mathrm{BOO} / \mathrm{BAR}]$} \\
\hline \multirow[t]{2}{*}{$\mathrm{C}_{6} \mathrm{H}_{10} \mathrm{O}_{4}$} & {$[13545-04-5]$} & \multicolumn{5}{|c|}{ 2,3-dimethylbutanedioic acid } \\
\hline & FUS & & 16.9 & 392.5 & DSC & [2012WAN/DEN, 2012WAN/LI] \\
\hline \multirow[t]{14}{*}{$\mathrm{C}_{6} \mathrm{H}_{10} \mathrm{O}_{4}$} & {$[124-04-9]$} & \multicolumn{5}{|l|}{ adipic acid } \\
\hline & FUS & & 32.0 & & DSC & [2014HAS/JIR] \\
\hline & FUS & & 35.89 & 423 & DSC & [2010BOO/BAR] \\
\hline & FUS & & 35.20 & 426.3 & DSC & [2010WAN/LAI, 2012WAN/LI] \\
\hline & FUS & & 33.7 & 419 & DSC & [2005ROU/TEM] \\
\hline & FUS & & 34.34 & 420.9 & DSC & [1984CHO/GO] \\
\hline & FUS & & 37.32 & 424.5 & DSC & [1982VAN/MUL] \\
\hline & FUS & & 34.85 & 426.4 & DSC & [1991ACR, 1974CIN/BER] \\
\hline & SUB & & $125 \pm 40$ & & ME, MS & [2009BOO/MAR] \\
\hline & SUB & $(353-373)$ & $124.7 \pm 20$ & & $\mathrm{ME}$ & [2009TAU/SIT] \\
\hline & SUB & $(328-368)$ & $145 \pm 4$ & & TPD & [2007CAP/LOV] \\
\hline & SUB & $(285-307)$ & 146.2 & & TPTD & [2005CHA/ZIE] \\
\hline & SUB & & NA & & & [2001ALB] \\
\hline & SUB & $(295-318)$ & 140 & & TPTD & [2001CHA/TOB] \\
\hline Note: Valu & on TPTD metl & $\mathrm{d}$ are not consistent & th values dete & d by othe & erimental $n$ & thods \\
\hline & SUB & & $133.6 \pm 1.3$ & 298 & $\mathrm{ME}$ & [1999RIB/MON, 1960DAV/THO] \\
\hline & SUB & (359-406) & $129.3 \pm 2.5$ & 383 & $\mathrm{ME}$ & [1960DAV/THO, 1960JON, 1970COX/PIL] \\
\hline & SUB & $(292-320)$ & $\mathrm{U} 37.2$ & 306 & A & [1947GRA] \\
\hline & $\mathrm{V}$ & $(424-503)$ & 105.2 & 298 & CGC & [2005ROU/TEM] \\
\hline & $\mathrm{V}$ & $(432-611)$ & 92.0 & 447 & A & [1987STE/MAL, 1947STU] \\
\hline $\mathrm{C}_{6} \mathrm{H}_{10} \mathrm{O}_{4}$ & {$[1070-34-4]$} & ethyl succinate & & & & \\
\hline & $\mathrm{V}$ & $(418-468)$ & 118.3 & 433 & $\mathrm{~EB}$ & [2011ORJ/YAN] \\
\hline $\mathrm{C}_{6} \mathrm{H}_{10} \mathrm{O}_{5}$ & {$[498-07-7]$} & 1,6 -anhydro- $\beta$-(D)- 8 & Icose (levoglu & & & \\
\hline & TRS & & 25.0 & 384 & & \\
\hline & FUS & & 2.9 & 454 & DSC & [2015KAB/PAU] \\
\hline & TRS & & 23.2 & 384.9 & & \\
\hline & FUS & & 3.3 & 455.4 & $\mathrm{DSC}$ & [2013ROC/GAL] \\
\hline & TRS & & $\mathrm{U} 13.2$ & 385.7 & & \\
\hline & FUS & & 3.2 & 455.3 & DSC & [2011BOO/MON] \\
\hline & TRS & & 24.95 & 385 & & \\
\hline & FUS & & 3.4 & 455 & DSC & [1970SHA/MCG] \\
\hline & SUB & $(353-383)$ & $125.7 \pm 1.6$ & 372 & $\mathrm{ME}$ & [2015KAB/PAU] \\
\hline & SUB & $(353-383)$ & $126.6 \pm 1.6$ & 298 & ME & [2015KAB/PAU] \\
\hline
\end{tabular}


TABLE 9. Phase change enthalpies of $\mathrm{C}_{6}$ organic compounds-Continued

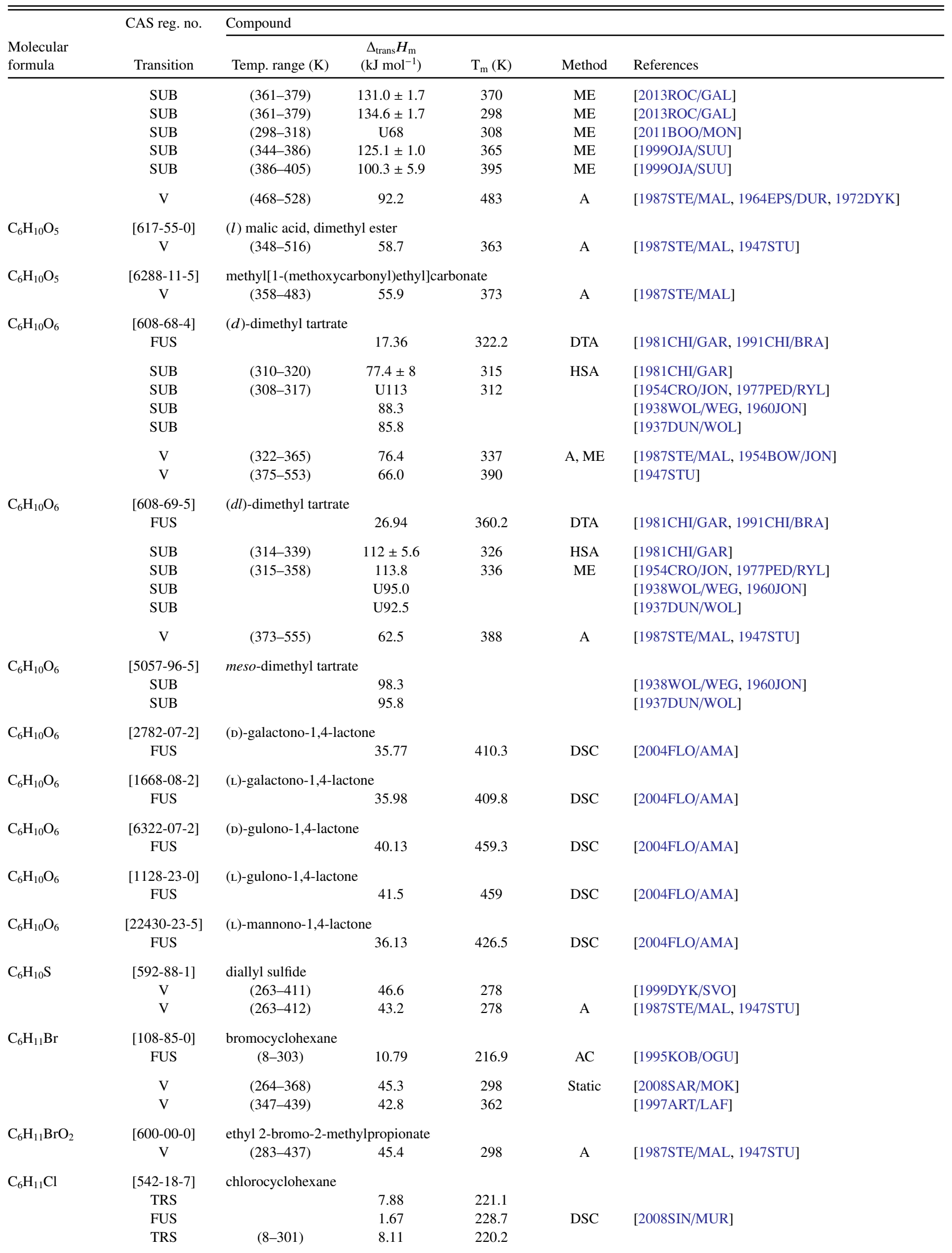


TABLE 9. Phase change enthalpies of $\mathrm{C}_{6}$ organic compounds-Continued

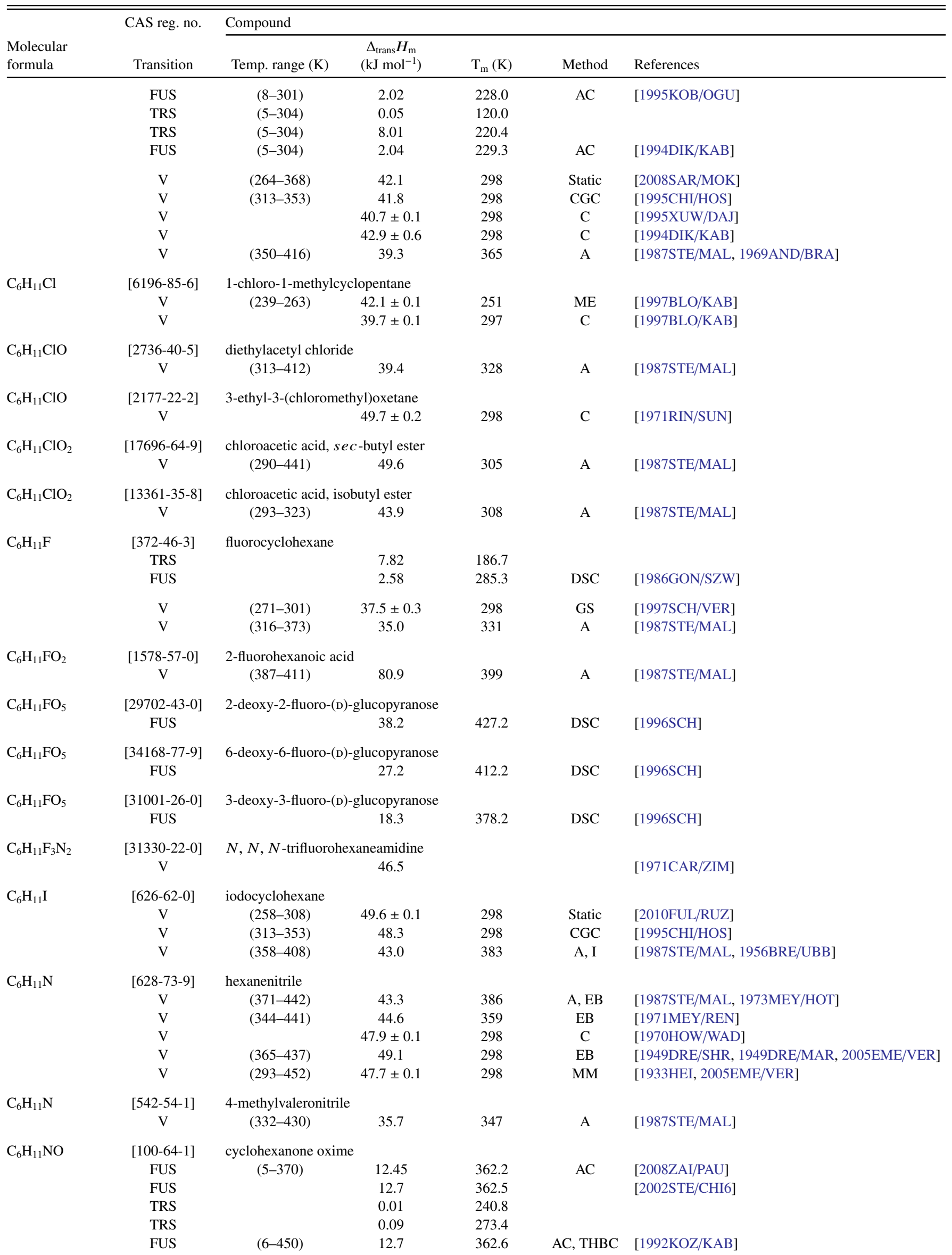


TABLE 9. Phase change enthalpies of $\mathrm{C}_{6}$ organic compounds-Continued

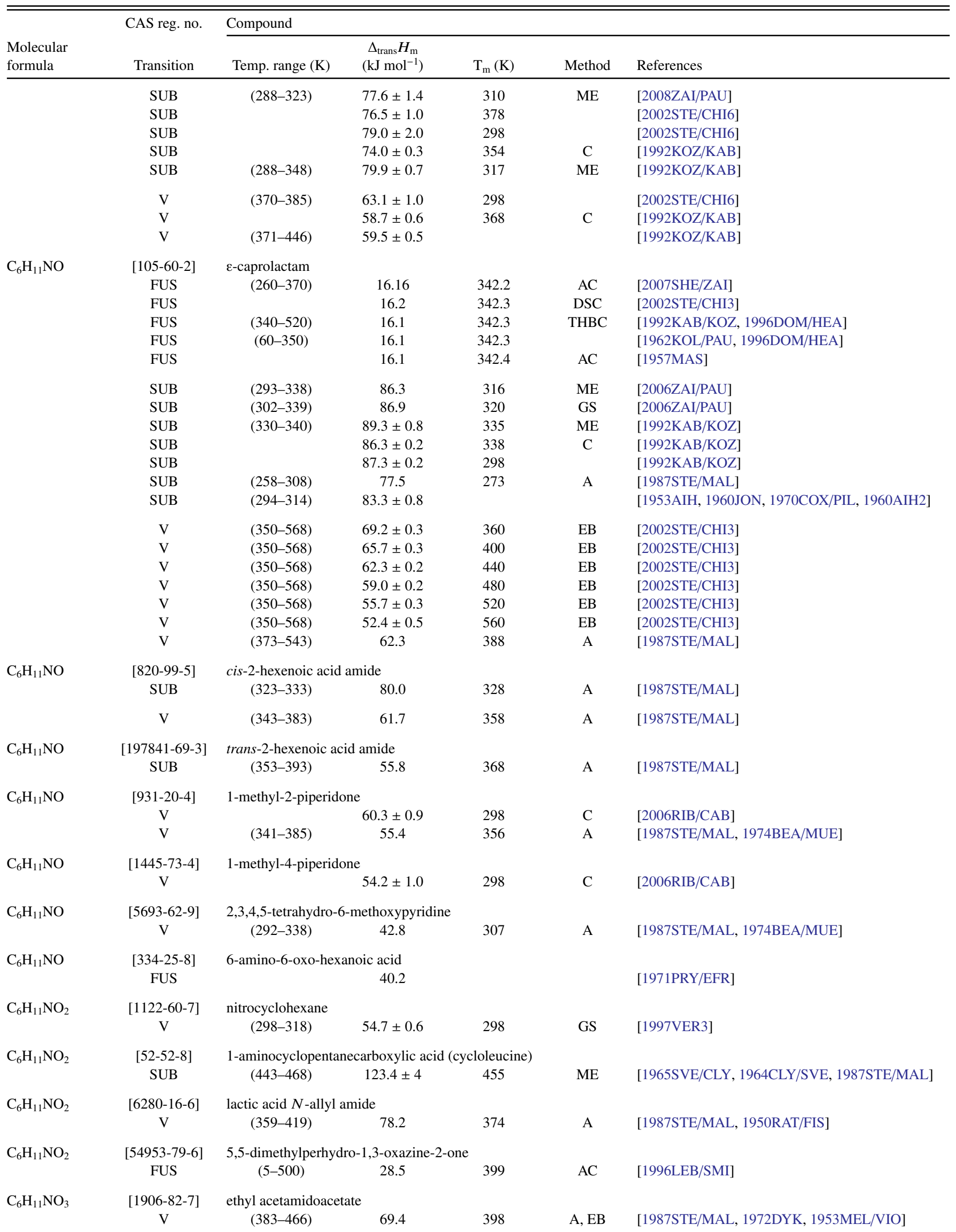


TABLE 9. Phase change enthalpies of $\mathrm{C}_{6}$ organic compounds-Continued

\begin{tabular}{|c|c|c|c|c|c|c|}
\hline $\begin{array}{l}\text { Molecular } \\
\text { formula } \\
\end{array}$ & $\begin{array}{l}\text { CAS reg. no. } \\
\text { Transition }\end{array}$ & \multicolumn{5}{|l|}{ Compound } \\
\hline \multirow[t]{2}{*}{$\mathrm{C}_{6} \mathrm{H}_{11} \mathrm{NS}$} & $\begin{array}{c}{[13070-07-0]} \\
\text { FUS }\end{array}$ & 2-piperidinethione & 16.32 & & & [1974BEA/MUE] \\
\hline & $\mathrm{V}$ & $(363-370)$ & 63.3 & 366 & A & [1987STE/MAL, 1974BEA/MUE] \\
\hline $\mathrm{C}_{6} \mathrm{H}_{11} \mathrm{NS}$ & $\begin{array}{c}\text { [19766-29-1] } \\
\mathrm{V}\end{array}$ & \multicolumn{4}{|c|}{ 2,3,4,5-tetrahydro-(methylthio)pyridine } & [1987STE/MAL, 1974BEA/MUE] \\
\hline $\mathrm{C}_{6} \mathrm{H}_{11} \mathrm{~N}_{2} \mathrm{O}_{3} \mathrm{PS}_{2}$ & $\begin{array}{l}\text { [950-37-8] } \\
\text { FUS }\end{array}$ & \multicolumn{5}{|c|}{$S$-2,3-dihydro-5-methoxy-2-oxo-1,3,4-thiadiazol-3-ylmethyl $O, O$-dimethyl phosphorodithioate } \\
\hline $\mathrm{C}_{6} \mathrm{H}_{11} \mathrm{~N}_{3} \mathrm{O}_{6}$ & $\begin{array}{c}\text { [62154-78-3] } \\
\text { SUB } \\
\text { SUB }\end{array}$ & 2,3,3-trinitro-2-metl & $\begin{array}{l}\text { lpentane } \\
92.0 \pm 0.8 \\
90.8\end{array}$ & $\begin{array}{l}298 \\
298\end{array}$ & $\mathrm{C}$ & $\begin{array}{l}\text { [2011MIR/KON] } \\
\text { [1999MIR/VOR] }\end{array}$ \\
\hline $\mathrm{C}_{6} \mathrm{H}_{11} \mathrm{~N}_{5} \mathrm{O}_{8}$ & $\begin{array}{c}\text { [1924-47-6] } \\
\text { SUB }\end{array}$ & $N$-(2,2-dinitropropy & $\begin{array}{l}\text {-2,2-dinitro-1 } \\
105.4 \pm 4.2\end{array}$ & anamine & & [1973DEK/OON, 1977PED/RYL] \\
\hline $\mathrm{C}_{6} \mathrm{H}_{12}$ & $\begin{array}{c}\text { [4806-61-5] } \\
\mathrm{V} \\
\mathrm{V}\end{array}$ & ethylcyclobutane & $\begin{array}{l}31.2 \pm 0.2 \\
32.6 \pm 0.8\end{array}$ & $\begin{array}{l}298 \\
298\end{array}$ & $\begin{array}{c}\mathrm{C} \\
\mathrm{EB}\end{array}$ & $\begin{array}{l}\text { [1983FUC/HAL] } \\
{[1974 \mathrm{GOO} / \mathrm{MOO}]}\end{array}$ \\
\hline $\mathrm{C}_{6} \mathrm{H}_{12}$ & $\begin{array}{l}\text { [110-82-7] } \\
\text { FUS } \\
\text { TRS } \\
\text { FUS } \\
\text { TRS } \\
\text { FUS } \\
\text { TRS } \\
\text { TRS } \\
\text { FUS } \\
\text { TRS } \\
\text { FUS } \\
\text { TRS } \\
\text { FUS } \\
\text { TRS } \\
\text { FUS } \\
\text { TRS } \\
\text { FUS }\end{array}$ & cyclohexane & $\begin{array}{l}2.60 \\
6.69 \\
2.63 \\
6.79 \\
2.68 \\
6.73 \\
6.99 \\
2.62 \\
6.69 \\
2.63 \\
6.74 \\
2.68 \\
6.82 \\
2.73 \\
6.23 \\
2.42\end{array}$ & $\begin{array}{l}279.8 \\
186.1 \\
279.7 \\
186.2 \\
279.8 \\
186.0 \\
186.1 \\
279.9 \\
186.1 \\
279.8 \\
186.1 \\
279.8 \\
186.4 \\
279.4 \\
185.9 \\
279.3\end{array}$ & $\begin{array}{l}\text { DSC } \\
\text { AC } \\
\text { DTA }\end{array}$ & $\begin{array}{l}\text { [2005HUA/SIM] } \\
\text { [2004NAN/TAN] } \\
\text { [1994TAN/SAB3] } \\
{[1984 \mathrm{DOM} / \mathrm{EVA}]} \\
{[1952 \mathrm{KAA} / \mathrm{COO}]} \\
{[1943 \mathrm{AST} / \mathrm{SZA}]} \\
{[1996 \mathrm{DOM} / \mathrm{HEA}, 1943 \mathrm{RUE} / \mathrm{HUF}]} \\
{[1942 \mathrm{ZIE} / \mathrm{AND}]} \\
{[1930 \mathrm{PAR} / \mathrm{HUF}]}\end{array}$ \\
\hline & SUB & $(223-280)$ & 27.6 & 265 & A & [1987STE/MAL] \\
\hline
\end{tabular}

Note: The Antoine coefficients given in [1987STE/MAL] for solid cyclohexane likely came from [1974JAC]—there is likely a typographical error in the C coefficient

$\begin{array}{lccccl}\text { SUB } & (223-278) & 36.4 \pm 0.7 & & \text { B } & {[1974 \mathrm{JAC}]} \\ \text { SUB } & & 46.6 & 186 & \mathrm{~B} & {[1963 \mathrm{BON}]} \\ \text { SUB } & (268-278) & 37.2 & 273 & & {[1960 \mathrm{JON}]} \\ \text { SUB } & (228-268) & 37.7 & 248 & \mathrm{~A} & {[1947 \mathrm{STU}]} \\ \text { SUB } & (269-279) & 36.5 & 274 & \mathrm{~A} & {[1934 \mathrm{ROT} / \mathrm{NAG}]} \\ \mathrm{V} & (296-353) & 33.1 & 315 & \mathrm{~EB} & {[2009 \mathrm{GIE} / \mathrm{KOS}]} \\ \mathrm{V} & (300-345) & 32.7 & 315 & & {[2002 \mathrm{LUB} / \mathrm{BAN}]} \\ \mathrm{V} & (360-470) & 32.2 & 375 & & {[1993 \mathrm{LEE} / \mathrm{HOL}]} \\ \mathrm{V} & (313-336) & 31.9 & 324 & \mathrm{~EB} & {[1995 \mathrm{DIO} / \mathrm{SAN}]} \\ \mathrm{V} & (313-336) & 33.1 & 298 & \mathrm{~EB} & {[1995 \mathrm{DIO} / \mathrm{SAN}]} \\ \mathrm{V} & & 32.3 & 314 & \mathrm{C} & {[1988 \mathrm{DON} / \mathrm{LIN}]} \\ \mathrm{V} & & 31.1 & 332 & \mathrm{C} & {[1988 \mathrm{DON} / \mathrm{LIN}]} \\ \mathrm{V} & & 30.3 & 345 & \mathrm{C} & {[1988 \mathrm{DON} / \mathrm{LIN}]} \\ \mathrm{V} & & 30.0 & 355 & \mathrm{C} & {[1988 \mathrm{DON} / \mathrm{LIN}]} \\ \mathrm{V} & (353-414) & 30.9 & 368 & \mathrm{~A} & {[1987 \mathrm{STE} / \mathrm{MAL}]}\end{array}$


TABLE 9. Phase change enthalpies of $\mathrm{C}_{6}$ organic compounds-Continued

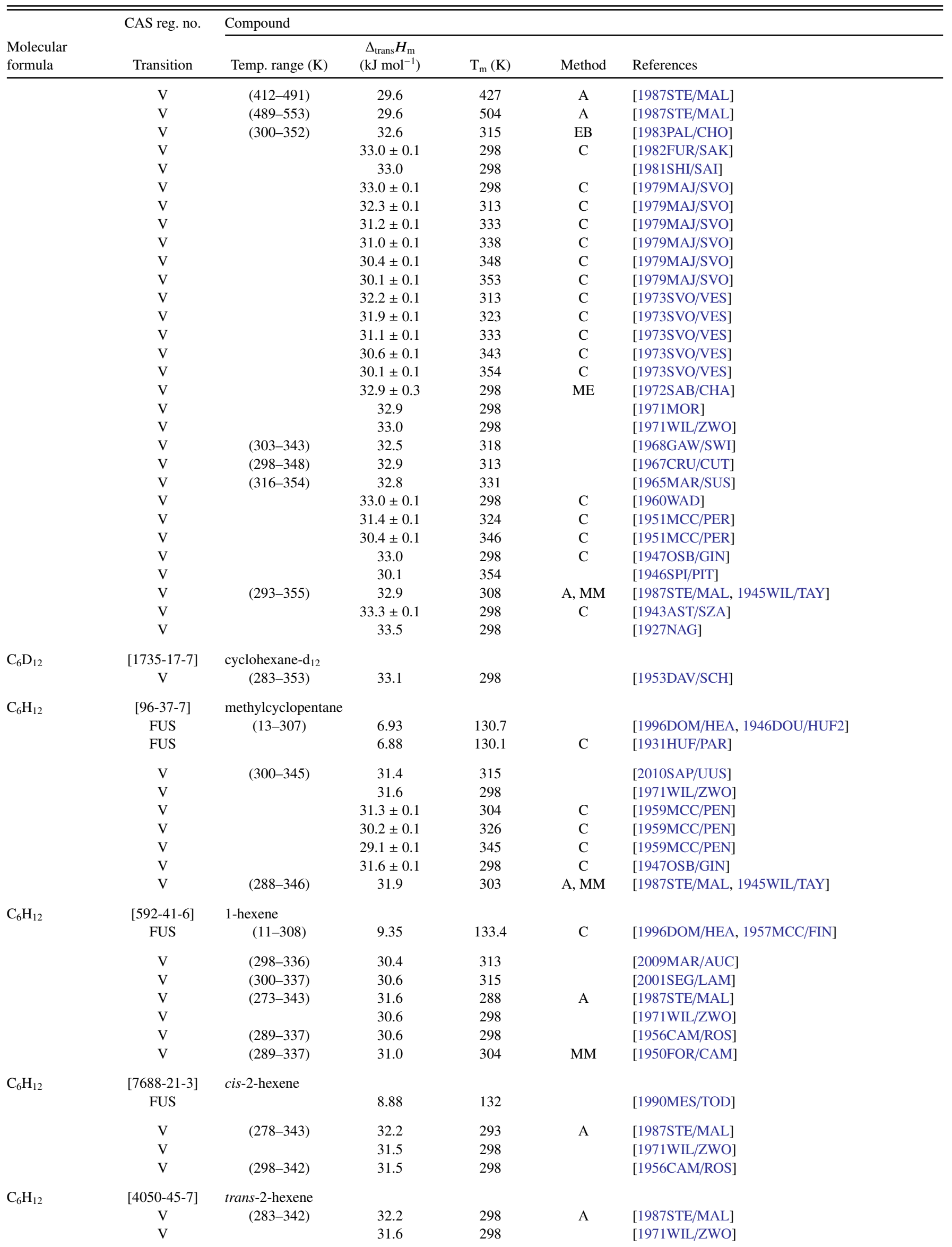


TABLE 9. Phase change enthalpies of $\mathrm{C}_{6}$ organic compounds-Continued

\begin{tabular}{|c|c|c|c|c|c|c|}
\hline \multirow[b]{2}{*}{$\begin{array}{l}\text { Molecular } \\
\text { formula }\end{array}$} & \multirow{2}{*}{$\begin{array}{l}\text { CAS reg. no. } \\
\text { Transition }\end{array}$} & \multicolumn{5}{|l|}{ Compound } \\
\hline & & Temp. range $(\mathrm{K})$ & $\begin{array}{c}\Delta_{\text {trans }} H_{\mathrm{m}} \\
\left(\mathrm{kJ} \mathrm{mol}^{-1}\right)\end{array}$ & $\mathrm{T}_{\mathrm{m}}(\mathrm{K})$ & Method & References \\
\hline & $\mathrm{V}$ & $(292-341)$ & 31.5 & 298 & & [1956CAM/ROS] \\
\hline $\mathrm{C}_{6} \mathrm{H}_{12}$ & $\begin{array}{c}{[7642-09-3]} \\
\text { V } \\
\text { V } \\
\text { V }\end{array}$ & $\begin{array}{l}\text { cis-3-hexene } \\
\quad(276-348) \\
(185-340)\end{array}$ & $\begin{array}{l}32.1 \\
31.3 \\
31.3\end{array}$ & $\begin{array}{l}291 \\
298 \\
298\end{array}$ & A & $\begin{array}{l}\text { [1987STE/MAL] } \\
{[1971 \mathrm{WIL} / \mathrm{ZWO}]} \\
{[1956 \mathrm{PEN} / \mathrm{SCO}]}\end{array}$ \\
\hline $\mathrm{C}_{6} \mathrm{H}_{12}$ & $\begin{array}{c}{[13269-52-8]} \\
\text { V } \\
\text { V } \\
\text { V }\end{array}$ & $\begin{array}{l}\text { trans-3-hexene } \\
\qquad \begin{array}{c}(278-341) \\
(291-341)\end{array}\end{array}$ & $\begin{array}{l}32.3 \\
31.6 \\
31.5\end{array}$ & $\begin{array}{l}293 \\
298 \\
298\end{array}$ & A & $\begin{array}{l}\text { [1987STE/MAL] } \\
{[1971 \mathrm{WIL} / \mathrm{ZWO}]} \\
{[1956 \mathrm{PEN} / \mathrm{SCO}]}\end{array}$ \\
\hline $\mathrm{C}_{6} \mathrm{H}_{12}$ & $\begin{array}{c}{[763-29-1]} \\
\mathrm{V} \\
\mathrm{V} \\
\mathrm{V}\end{array}$ & $\begin{array}{l}\text { 2-methyl-1-pentene } \\
\quad(272-341) \\
(300-335)\end{array}$ & $\begin{array}{l}31.6 \\
30.5 \\
30.5\end{array}$ & $\begin{array}{l}287 \\
298 \\
298\end{array}$ & A & $\begin{array}{l}\text { [1987STE/MAL] } \\
{[1971 \mathrm{WIL} / \mathrm{ZWO}]} \\
{[1956 \mathrm{CAM} / \mathrm{ROS}]}\end{array}$ \\
\hline $\mathrm{C}_{6} \mathrm{H}_{12}$ & $\begin{array}{c}{[760-20-3]} \\
\mathrm{V} \\
\mathrm{V} \\
\mathrm{V}\end{array}$ & $\begin{array}{l}\text { 3-methyl-1-pentene } \\
(265-333) \\
(287-328)\end{array}$ & $\begin{array}{l}30.0 \\
28.6 \\
28.6\end{array}$ & $\begin{array}{l}280 \\
298 \\
298\end{array}$ & A & $\begin{array}{l}\text { [1987STE/MAL] } \\
{[1971 \mathrm{WIL} / \mathrm{ZWO}]} \\
{[1956 \mathrm{CAM} / \mathrm{ROS}]}\end{array}$ \\
\hline $\mathrm{C}_{6} \mathrm{H}_{12}$ & $\begin{array}{c}\text { [691-37-2] } \\
\text { FUS }\end{array}$ & 4-methyl-1-pentene & 4.93 & 118.9 & & [1994LEB/SMI3] \\
\hline & $\begin{array}{l}\text { V } \\
\text { V } \\
\text { V } \\
\text { V } \\
\text { V } \\
\text { V } \\
\text { V }\end{array}$ & $\begin{array}{l}(310-360) \\
(310-360) \\
(310-360) \\
(310-360) \\
(265-333) \\
(287-328)\end{array}$ & $\begin{array}{c}28.6 \pm 0.2 \\
27.4 \pm 0.3 \\
26.2 \pm 0.4 \\
24.9 \pm 0.5 \\
30.1 \\
28.7 \\
28.7\end{array}$ & $\begin{array}{l}298 \\
320 \\
340 \\
360 \\
280 \\
298 \\
298\end{array}$ & $\begin{array}{c}\text { EB } \\
\text { EB } \\
\text { EB } \\
\text { EB } \\
\text { A }\end{array}$ & $\begin{array}{l}\text { [1997STE/CHI] } \\
{[1997 \mathrm{STE} / \mathrm{CHI}]} \\
{[1997 \mathrm{STE} / \mathrm{CHI}]} \\
{[1997 \mathrm{STE} / \mathrm{CHI}]} \\
{[1987 \mathrm{STE} / \mathrm{MAL}]} \\
{[1971 \mathrm{WIL} / \mathrm{ZWO}]} \\
{[1956 \mathrm{PEN} / \mathrm{SCO}]}\end{array}$ \\
\hline $\mathrm{C}_{6} \mathrm{H}_{12}$ & $\begin{array}{c}{[625-27-4]} \\
\mathrm{V} \\
\mathrm{V} \\
\mathrm{V}\end{array}$ & $\begin{array}{l}\text { 2-methyl-2-pentene } \\
(277-346) \\
(292-341)\end{array}$ & $\begin{array}{l}32.4 \\
31.6 \\
31.6\end{array}$ & $\begin{array}{l}292 \\
298 \\
298\end{array}$ & A & $\begin{array}{l}\text { [1987STE/MAL] } \\
{[1971 \mathrm{WIL} / \mathrm{ZWO}]} \\
{[1956 \mathrm{PEN} / \mathrm{SCO}]}\end{array}$ \\
\hline $\mathrm{C}_{6} \mathrm{H}_{12}$ & $\begin{array}{c}{[922-62-3]} \\
\mathrm{V} \\
\mathrm{V} \\
\mathrm{V}\end{array}$ & $\begin{array}{c}\text { cis-3-methyl-2-pente } \\
(277-347) \\
(300-344)\end{array}$ & $\begin{array}{l}32.2 \\
31.3 \\
32.1\end{array}$ & $\begin{array}{l}292 \\
298 \\
298\end{array}$ & A & $\begin{array}{l}\text { [1987STE/MAL] } \\
{[1971 \mathrm{WIL} / \mathrm{ZWO}]} \\
{[1956 \mathrm{CAM} / \mathrm{ROS}]}\end{array}$ \\
\hline $\mathrm{C}_{6} \mathrm{H}_{12}$ & $\begin{array}{c}{[616-12-6]} \\
\mathrm{V} \\
\mathrm{V} \\
\mathrm{V}\end{array}$ & $\begin{array}{l}\text { trans-3-methyl-2-per } \\
\qquad(280-349) \\
(292-341)\end{array}$ & $\begin{array}{r}32.8 \\
32.1 \\
31.3\end{array}$ & $\begin{array}{l}295 \\
298 \\
298\end{array}$ & A & $\begin{array}{l}\text { [1987STE/MAL] } \\
{[1971 \mathrm{WIL} / \mathrm{ZWO}]} \\
{[1956 \mathrm{CAM} / \mathrm{ROS}]}\end{array}$ \\
\hline $\mathrm{C}_{6} \mathrm{H}_{12}$ & $\begin{array}{c}{[691-38-3]} \\
\mathrm{V} \\
\mathrm{V} \\
\mathrm{V}\end{array}$ & $\begin{array}{c}\text { cis-4-methyl-2-pente } \\
\text { (267-330) } \\
(300-330)\end{array}$ & $\begin{array}{l}30.8 \\
29.5 \\
29.5\end{array}$ & $\begin{array}{l}282 \\
298 \\
298\end{array}$ & A & $\begin{array}{l}\text { [1987STE/MAL] } \\
{[1971 \mathrm{WIL} / \mathrm{ZWO}]} \\
{[1956 \mathrm{CAM} / \mathrm{ROS}]}\end{array}$ \\
\hline $\mathrm{C}_{6} \mathrm{H}_{12}$ & $\begin{array}{c}\text { [674-76-0] } \\
\mathrm{V} \\
\mathrm{V} \\
\mathrm{V}\end{array}$ & $\begin{array}{l}\text { trans-4-methyl-2-per } \\
\qquad(269-337) \\
(291-332)\end{array}$ & $\begin{array}{r}31.2 \\
30.0 \\
30.0\end{array}$ & $\begin{array}{l}284 \\
298 \\
298\end{array}$ & A & $\begin{array}{l}\text { [1987STE/MAL] } \\
{[1971 \mathrm{WIL} / \mathrm{ZWO}]} \\
{[1956 \mathrm{CAM} / \mathrm{ROS}]}\end{array}$ \\
\hline $\mathrm{C}_{6} \mathrm{H}_{12}$ & $\begin{array}{c}{[563-78-0]} \\
\mathrm{V} \\
\mathrm{V} \\
\mathrm{V}\end{array}$ & $\begin{array}{l}\text { 2,3-dimethyl-1-buten } \\
\text { (267-335) } \\
(289-329)\end{array}$ & $\begin{array}{l}30.5 \\
29.2 \\
29.2\end{array}$ & $\begin{array}{l}282 \\
298 \\
298\end{array}$ & A & $\begin{array}{l}\text { [1987STE/MAL] } \\
{[1971 \mathrm{WIL} / \mathrm{ZWO}]} \\
{[1956 \mathrm{CAM} / \mathrm{ROS}]}\end{array}$ \\
\hline $\mathrm{C}_{6} \mathrm{H}_{12}$ & $\begin{array}{c}\text { [558-37-2] } \\
\text { TRS } \\
\text { FUS }\end{array}$ & 3,3-dimethyl-1-buten & $\begin{array}{l}4.35 \\
1.09\end{array}$ & $\begin{array}{l}124.9 \\
158.4\end{array}$ & & [1996DOM/HEA, 1938KEN/SHO] \\
\hline & $\begin{array}{l}\mathrm{V} \\
\mathrm{V}\end{array}$ & $(254-316)$ & $\begin{array}{l}28.6 \\
26.6\end{array}$ & $\begin{array}{l}269 \\
298\end{array}$ & A & $\begin{array}{l}\text { [1987STE/MAL] } \\
\text { [1971WIL/ZWO] }\end{array}$ \\
\hline
\end{tabular}


TABLE 9. Phase change enthalpies of $\mathrm{C}_{6}$ organic compounds-Continued

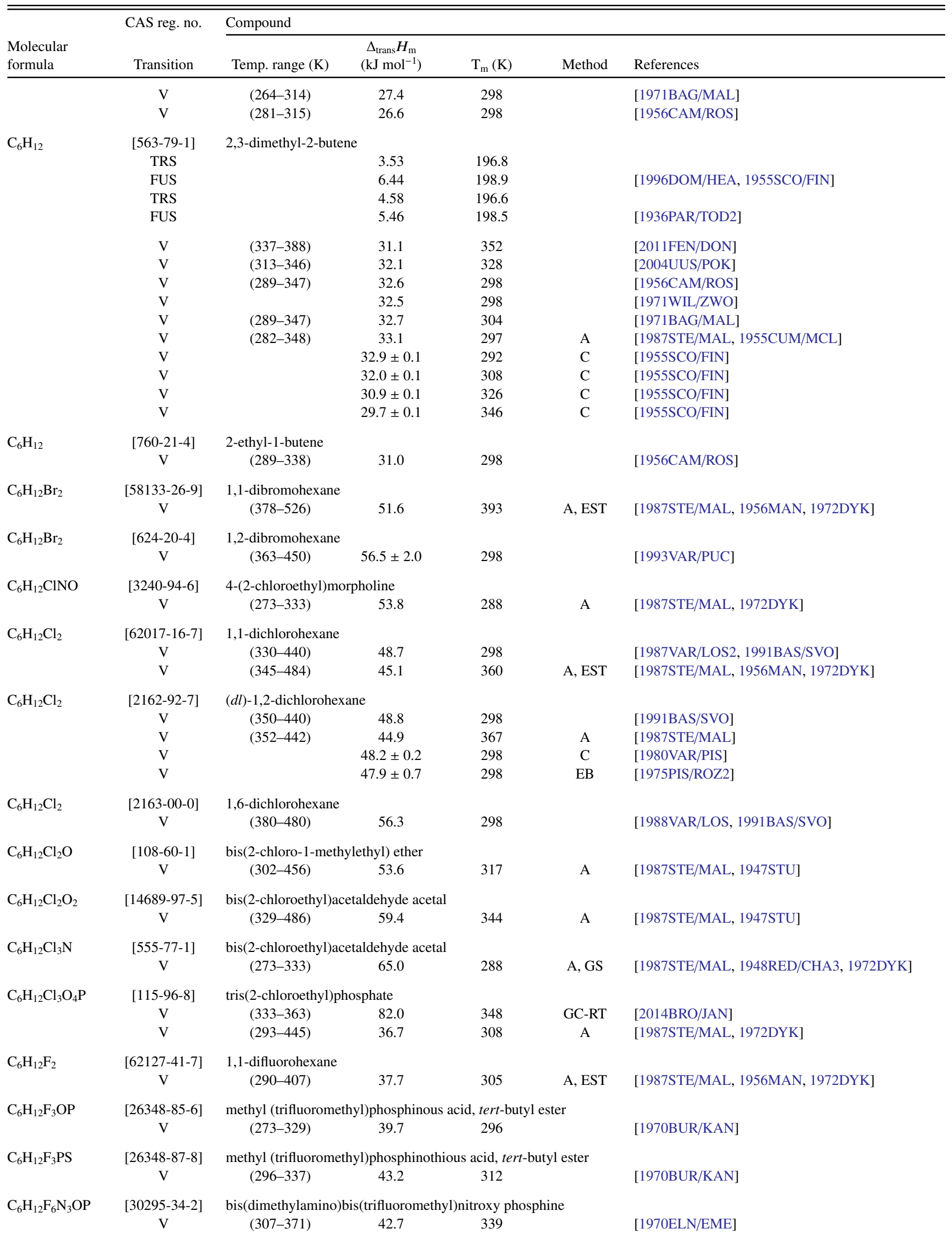


TABLE 9. Phase change enthalpies of $\mathrm{C}_{6}$ organic compounds-Continued

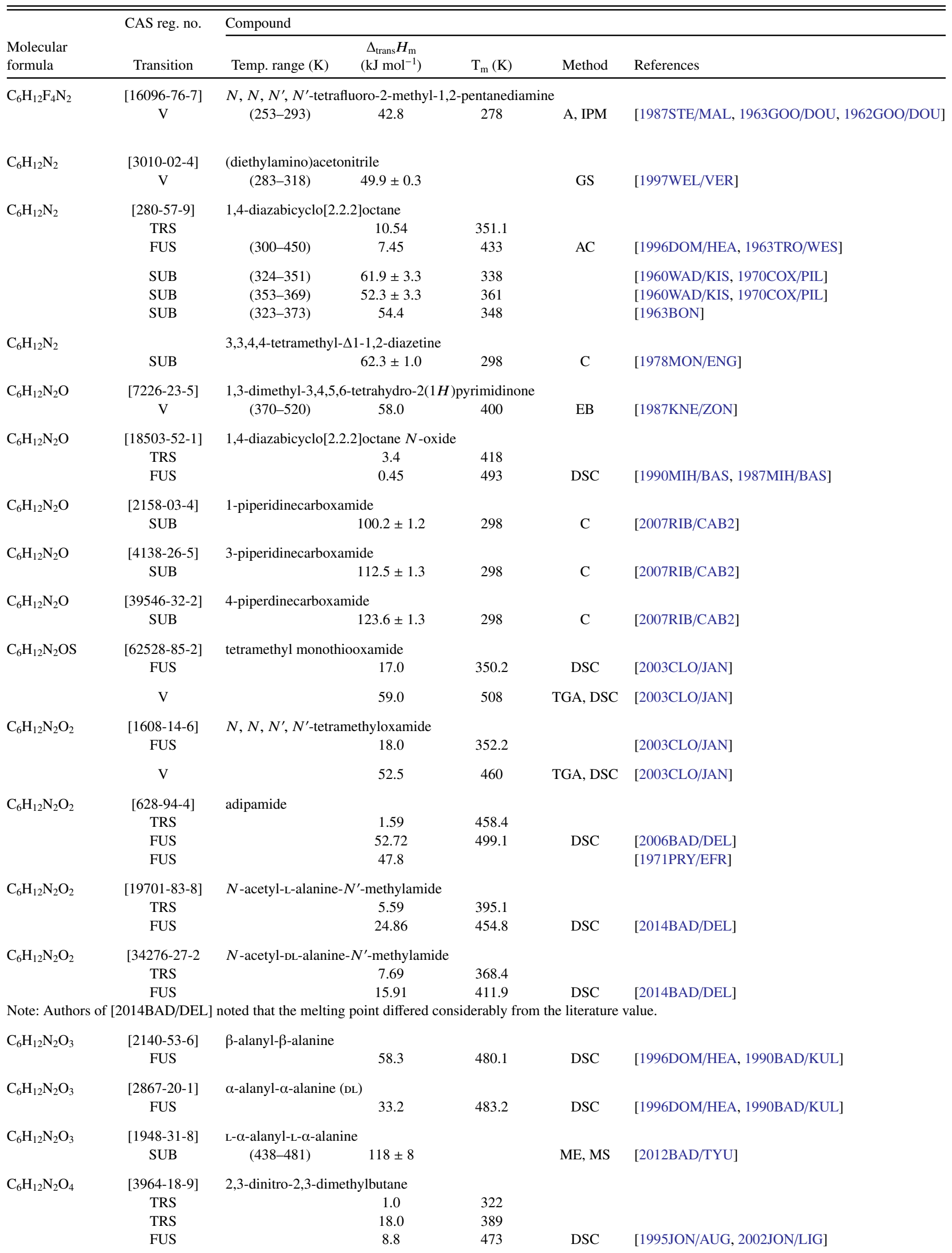


TABLE 9. Phase change enthalpies of $\mathrm{C}_{6}$ organic compounds - Continued

\begin{tabular}{|c|c|c|c|c|c|c|}
\hline \multirow[b]{2}{*}{$\begin{array}{l}\text { Molecular } \\
\text { formula }\end{array}$} & \multirow{2}{*}{$\begin{array}{l}\text { CAS reg. no. } \\
\text { Transition }\end{array}$} & \multicolumn{5}{|l|}{ Compound } \\
\hline & & Temp. range $(\mathrm{K})$ & $\begin{array}{c}\Delta_{\text {trans }} H_{\mathrm{m}} \\
\left(\mathrm{kJ} \mathrm{mol}^{-1}\right)\end{array}$ & $\mathrm{T}_{\mathrm{m}}(\mathrm{K})$ & Method & References \\
\hline & TRS & & 0.6 & 318 & & \\
\hline & TRS & & 23 & 388 & DTA & [1994SMI/MAT, 2002JON/LIG] \\
\hline & SUB & & $75.3 \pm 0.8$ & 298 & $\mathrm{C}$ & [2011MIR/KON] \\
\hline & SUB & & $74 \pm 5$ & & TGA & [2002JON/LIG] \\
\hline & SUB & & $79.5 \pm 0.8$ & 298 & & [1999MIR/VOR] \\
\hline & SUB & $(303-330)$ & $85 \pm 2$ & & ME & [1994SMI/MAT, 2002JON/LIG] \\
\hline & SUB & $(253-323)$ & 94 & & GC & [1991ELI, 2002JON/LIG] \\
\hline \multirow[t]{3}{*}{$\mathrm{C}_{6} \mathrm{H}_{12} \mathrm{~N}_{2} \mathrm{O}_{6}$} & [99115-63-6] & \multicolumn{2}{|c|}{ 2,5-hexanediol dinitrate } & & & \\
\hline & SUB & $(293-313)$ & 119 & 303 & A & [1987STE/MAL, 1957KEM/GOL, 1972DYK] \\
\hline & $\mathrm{V}$ & $(293-313)$ & 54.4 & 303 & $\mathrm{~B}, \mathrm{GS}$ & [1957KEM/GOL, 1972DYK] \\
\hline \multirow[t]{2}{*}{$\mathrm{C}_{6} \mathrm{H}_{12} \mathrm{~N}_{2} \mathrm{O}_{8}$} & [111-22-8] & \multicolumn{2}{|c|}{ triethylene glycol dinitrate } & & & \\
\hline & $\mathrm{V}$ & $(303-348)$ & 88.3 & 318 & A & [1987STE/MAL, 1972DYK, 1963WOO/ADI] \\
\hline \multirow[t]{3}{*}{$\mathrm{C}_{6} \mathrm{H}_{12} \mathrm{~N}_{2} \mathrm{~S}_{2}$} & [35840-78-9] & \multicolumn{2}{|c|}{ tetramethyl dithiooxamide } & & & \\
\hline & FUS & & 21.0 & 409.2 & DSC & {$[2003 \mathrm{CLO} / \mathrm{JAN}]$} \\
\hline & V & & 60.5 & 533 & TGA, DSC & {$[2003 \mathrm{CLO} / \mathrm{JAN}]$} \\
\hline \multirow[t]{9}{*}{$\mathrm{C}_{6} \mathrm{H}_{12} \mathrm{~N}_{4}$} & {$[100-97-0]$} & \multicolumn{3}{|c|}{ 1,3,5,7-tetraazatricyclo[3.3.1.1 $\left.1^{3,7}\right]$ decane } & & \\
\hline & SUB & $(339-378)$ & $77.7 \pm 0.4$ & 359 & GS & [2002VER2] \\
\hline & SUB & $(339-378)$ & $79.6 \pm 0.4$ & 298 & GS & [2002VER2] \\
\hline & SUB & $(298-453)$ & 76.8 & 313 & A & [1987STE/MAL] \\
\hline & SUB & $(302-328)$ & 78.8 & 316 & TE, ME & [1983DEW/VAN] \\
\hline & SUB & & $74.9 \pm 2.9$ & 298 & & [1960WAD/KIS, 1970MAN/RAP] \\
\hline & SUB & $(281-298)$ & $74.1 \pm 0.8$ & 289 & TE & [1960BUD] \\
\hline & SUB & $(293-553)$ & 75.4 & 423 & & [1958KLI/STR, 2002VER2, 1957STR/KLI] \\
\hline & SUB & $(293-553)$ & 79.4 & 298 & & [1958KLI/STR, 2002VER2] \\
\hline \multirow[t]{2}{*}{$\mathrm{C}_{6} \mathrm{H}_{12} \mathrm{O}$} & [1003-38-9] & \multicolumn{2}{|c|}{$(d l)$-2,5-dimethyltetrahydrofuran } & & & \\
\hline & $\mathrm{V}$ & $(278-370)$ & 35.4 & 293 & A & [1987STE/MAL] \\
\hline \multirow[t]{2}{*}{$\mathrm{C}_{6} \mathrm{H}_{12} \mathrm{O}$} & [1436-34-6] & \multicolumn{2}{|l|}{ 1,2-epoxyhexane } & & & \\
\hline & $\mathrm{V}$ & $(300-390)$ & 43.1 & 315 & A & [1987STE/MAL, 1969VOJ/CIH, 1984BOU/FRI] \\
\hline \multirow[t]{2}{*}{$\mathrm{C}_{6} \mathrm{H}_{12} \mathrm{O}$} & [1192-22-9] & \multicolumn{2}{|c|}{ 2-methyl-2,3-epoxypentane } & & & \\
\hline & $\mathrm{V}$ & $(306-369)$ & 40.6 & 321 & A & [1987STE/MAL, 1974LOG/FRO] \\
\hline \multirow{3}{*}{$\mathrm{C}_{6} \mathrm{H}_{12} \mathrm{O}$} & [6140-80-3] & \multicolumn{2}{|l|}{ allyl isopropyl ether } & & & \\
\hline & $\mathrm{V}$ & $(253-415)$ & 36.1 & 268 & A & [1987STE/MAL] \\
\hline & $\mathrm{V}$ & $(229-353)$ & 36.8 & 244 & A & [1987STE/MAL, 1947STU] \\
\hline \multirow[t]{3}{*}{$\mathrm{C}_{6} \mathrm{H}_{12} \mathrm{O}$} & [1471-03-0] & allyl propyl ether & & & & \\
\hline & $\mathrm{V}$ & $(261-428)$ & 37.5 & 276 & A & [1987STE/MAL] \\
\hline & $\mathrm{V}$ & $(234-364)$ & 36.4 & 249 & A & [1987STE/MAL, 1947STU] \\
\hline $\mathrm{C}_{6} \mathrm{H}_{12} \mathrm{O}$ & [111-34-2] & butyl vinyl ether & & & & \\
\hline & $\mathrm{V}$ & $(311-403)$ & $36.7 \pm 0.2$ & 298 & EB & [1996STE/CHI2] \\
\hline & $\mathrm{V}$ & $(311-403)$ & $35.2 \pm 0.2$ & 320 & EB & [1996STE/CHI2] \\
\hline & V & $(311-403)$ & $32.5 \pm 0.2$ & 360 & EB & [1996STE/CHI2] \\
\hline & $\mathrm{V}$ & $(311-403)$ & $29.6 \pm 0.2$ & 400 & EB & [1996STE/CHI2] \\
\hline & V & $(353-393)$ & 36.5 & 298 & CGC & [1995CHI/HOS] \\
\hline & V & $(269-368)$ & 36.1 & 284 & A & [1987STE/MAL] \\
\hline $\mathrm{C}_{6} \mathrm{H}_{12} \mathrm{O}$ & [109-53-5] & isobutyl vinyl ether & & & & \\
\hline & V & $(266-357)$ & 37.4 & 281 & A & [1987STE/MAL] \\
\hline $\mathrm{C}_{6} \mathrm{H}_{12} \mathrm{O}$ & [108-93-0] & cyclohexanol & & & & \\
\hline & TRS & & 8.7 & 245.2 & & \\
\hline & FUS & & 1.73 & 298.2 & DSC & [2009SIN/MUR] \\
\hline & TRS & $(170-320)$ & 8.66 & 264.9 & & \\
\hline & FUS & $(170-320)$ & 1.81 & 297.9 & $\mathrm{AC}$ & [1990MAY/RAC] \\
\hline
\end{tabular}

Note: Authors of [1990MAY/RAC] give the transition at $264.9 \mathrm{~K}$ as a II-> I transition. The paper also gives values of $8.62 \mathrm{~kJ} / \mathrm{mole}$ and $244.5 \mathrm{~K}$ for a III $\rightarrow$ I transition

$\begin{array}{lcc}\text { TRS } & 8.21 & 263.5 \\ \text { FUS } & 1.7 & 297\end{array}$


TABLE 9. Phase change enthalpies of $\mathrm{C}_{6}$ organic compounds-Continued

\begin{tabular}{|c|c|c|c|c|c|c|}
\hline \multirow[b]{2}{*}{$\begin{array}{l}\text { Molecular } \\
\text { formula }\end{array}$} & \multirow{2}{*}{$\begin{array}{l}\text { CAS reg. no. } \\
\text { Transition }\end{array}$} & \multicolumn{5}{|l|}{ Compound } \\
\hline & & Temp. range $(\mathrm{K})$ & $\begin{array}{c}\Delta_{\text {trans }} H_{\mathrm{m}} \\
\left(\mathrm{kJ} \mathrm{mol}^{-1}\right)\end{array}$ & $\mathrm{T}_{\mathrm{m}}(\mathrm{K})$ & Method & References \\
\hline & TRS & $(16-298)$ & 8.21 & 263.5 & & \\
\hline & FUS & $(16-298)$ & 1.7 & 297.0 & & [1929KEL5] \\
\hline & FUS & & 1.87 & & CR & {$[1912 \mathrm{DEF}]$} \\
\hline & SUB & $(272-298)$ & 60.7 & 285 & A & [1987STE/MAL, 1948NIT/SEK2] \\
\hline & $\mathrm{V}$ & $(322-433)$ & 60.1 & 337 & & [2004STE/SUN] \\
\hline & $\mathrm{V}$ & $(395-426)$ & 49.1 & 410 & $\mathrm{~EB}$ & [2002SII/KIR2] \\
\hline & $\mathrm{V}$ & $(390-430)$ & 49.8 & 405 & & [2002SWI/MAL] \\
\hline & $\mathrm{V}$ & & $62.0 \pm 0.3$ & 298 & $\mathrm{C}$ & [1999COS/EUS] \\
\hline & $\mathrm{V}$ & $(288-328)$ & $61.2 \pm 0.6$ & 308 & GS & [1998VER3] \\
\hline & $\mathrm{V}$ & $(288-328)$ & $61.8 \pm 0.6$ & 298 & GS & [1998VER3] \\
\hline & $\mathrm{V}$ & $(341-471)$ & $54.8 \pm 0.5$ & 360 & $\mathrm{~EB}$ & [1997STE/CHI3] \\
\hline & $\mathrm{V}$ & $(341-471)$ & $49.3 \pm 0.4$ & 400 & $\mathrm{~EB}$ & [1997STE/CHI3] \\
\hline & $\mathrm{V}$ & $(341-471)$ & $46.6 \pm 0.4$ & 420 & $\mathrm{~EB}$ & [1997STE/CHI3] \\
\hline & $\mathrm{V}$ & $(341-471)$ & $40.9 \pm 0.6$ & 460 & $\mathrm{~EB}$ & [1997STE/CHI3] \\
\hline & $\mathrm{V}$ & $(341-471)$ & $63.5 \pm 0.7$ & 298 & $\mathrm{~EB}$ & [1997STE/CHI3] \\
\hline & $\mathrm{V}$ & $(323-373)$ & 61.3 & 298 & CGC & [1995CHI/HOS] \\
\hline & $\mathrm{V}$ & $(350-456)$ & 55.0 & 365 & $\mathrm{~EB}$ & [1987AMB/GHI2] \\
\hline & $\mathrm{V}$ & $(318-434)$ & 59.9 & 333 & A & [1987STE/MAL] \\
\hline & $\mathrm{V}$ & $(300-434)$ & 62.7 & 315 & A & [1987STE/MAL] \\
\hline & $\mathrm{V}$ & $(404-432)$ & 49.3 & 418 & & [1984CAS/FRA2] \\
\hline & $\mathrm{V}$ & $(303-373)$ & 58.4 & 318 & & [1984SIP/WIE] \\
\hline & $\mathrm{V}$ & $(299-319)$ & 60.4 & 309 & & [1975CAB/CON2] \\
\hline & $\mathrm{V}$ & & $62.0 \pm 0.9$ & 298 & & [1975CAB/CON2] \\
\hline & $\mathrm{V}$ & & $62.0 \pm 0.2$ & 298 & $\mathrm{C}$ & [1968PLA/WIL] \\
\hline & $\mathrm{V}$ & & $62.0 \pm 0.3$ & 298 & & [1966WAD] \\
\hline & $\mathrm{V}$ & & $62.0 \pm 0.2$ & & $\mathrm{C}$ & [1962SEL/SUN] \\
\hline & $\mathrm{V}$ & & 45.4 & 434 & & [1962HEN/WEB, 1997STE/CHI3] \\
\hline & $\mathrm{V}$ & & 46.9 & 421 & & [1962HEN/WEB, 1997STE/CHI3] \\
\hline & $\mathrm{V}$ & & 53.9 & 362 & & [1962HEN/WEB, 1997STE/CHI3] \\
\hline & $\mathrm{V}$ & $(367-433)$ & 52.6 & 382 & & [1960NOV/MAT2, 1960NOV/MAT] \\
\hline & $\mathrm{V}$ & & 42.4 & 434 & & [1957GLA/RUL, 1997STE/CHI3] \\
\hline & $\mathrm{V}$ & $(307-422)$ & 54.8 & 322 & & [1946THO] \\
\hline & $\mathrm{V}$ & & $45.5 \pm 0.1$ & 432 & & [1931MAT/FEH, 1997STE/CHI3] \\
\hline & $\mathrm{V}$ & & 48.9 & 433 & & [1912DEF, 1997STE/CHI3] \\
\hline \multirow[t]{2}{*}{$\mathrm{C}_{6} \mathrm{H}_{12} \mathrm{O}$} & {$[4798-44-1]$} & 1-hexen-3-ol & & & & \\
\hline & V & $(238-308)$ & $55.2 \pm 0.1$ & 298 & Static & [2015STE/FUL] \\
\hline \multirow[t]{2}{*}{$\mathrm{C}_{6} \mathrm{H}_{12} \mathrm{O}$} & {$[928-96-1]$} & cis-3-hexen-1-ol & & & & \\
\hline & $\mathrm{V}$ & $(238-308)$ & $58.3 \pm 0.1$ & 298 & Static & [2015STE/FUL] \\
\hline \multirow[t]{2}{*}{$\mathrm{C}_{6} \mathrm{H}_{12} \mathrm{O}$} & {$[928-95-0]$} & trans-2-hexen-1-ol & & & & \\
\hline & $\mathrm{V}$ & $(238-308)$ & $59.6 \pm 0.1$ & 298 & Static & [2015STE/FUL] \\
\hline \multirow[t]{4}{*}{$\mathrm{C}_{6} \mathrm{H}_{12} \mathrm{O}$} & {$[821-41-0]$} & 5-hexen-1-ol & & & & \\
\hline & $\mathrm{V}$ & & $60.2 \pm 0.1$ & 298 & $\mathrm{C}$ & [1996ULB/KLU] \\
\hline & $\mathrm{V}$ & & $58.0 \pm 0.1$ & 343 & $\mathrm{C}$ & [1996ULB/KLU] \\
\hline & V & & $55.7 \pm 0.1$ & 358 & $\mathrm{C}$ & [1996ULB/KLU] \\
\hline \multirow[t]{5}{*}{$\mathrm{C}_{6} \mathrm{H}_{12} \mathrm{O}$} & {$[565-61-7]$} & $(d l)$-3-methyl-2-pen & none & & & \\
\hline & $\mathrm{V}$ & $(286-400)$ & 39.8 & 301 & A & [1987STE/MAL] \\
\hline & $\mathrm{V}$ & $(283-395)$ & 41.5 & 298 & A & [1987STE/MAL] \\
\hline & $\mathrm{V}$ & $(385-455)$ & 36.5 & 400 & A & [1987STE/MAL] \\
\hline & $\mathrm{V}$ & $(283-457)$ & 41.2 & 298 & & [1975AMB/ELL] \\
\hline \multirow[t]{9}{*}{$\mathrm{C}_{6} \mathrm{H}_{12} \mathrm{O}$} & [591-78-6] & 2-hexanone & & & & \\
\hline & FUS & $(12-382)$ & 14.9 & 217.7 & $\mathrm{AC}$ & [1996DOM/HEA, 1970AND/COU] \\
\hline & $\mathrm{V}$ & $(359-401)$ & 39.0 & 374 & $\mathrm{~EB}$ & [2002SII/KIR] \\
\hline & $\mathrm{V}$ & & $43.1 \pm 0.1$ & 298 & $\mathrm{C}$ & [1992SVO/KUB] \\
\hline & $\mathrm{V}$ & & $42.5 \pm 0.1$ & 308 & $\mathrm{C}$ & [1992SVO/KUB] \\
\hline & $\mathrm{V}$ & & $41.6 \pm 0.1$ & 323 & $\mathrm{C}$ & [1992SVO/KUB] \\
\hline & $\mathrm{V}$ & & $40.7 \pm 0.1$ & 338 & $\mathrm{C}$ & [1992SVO/KUB] \\
\hline & $\mathrm{V}$ & & $40.1 \pm 0.1$ & 348 & $\mathrm{C}$ & [1992SVO/KUB] \\
\hline & $\mathrm{V}$ & & $39.5 \pm 0.1$ & 358 & $\mathrm{C}$ & [1992SVO/KUB] \\
\hline
\end{tabular}


TABLE 9. Phase change enthalpies of $\mathrm{C}_{6}$ organic compounds-Continued

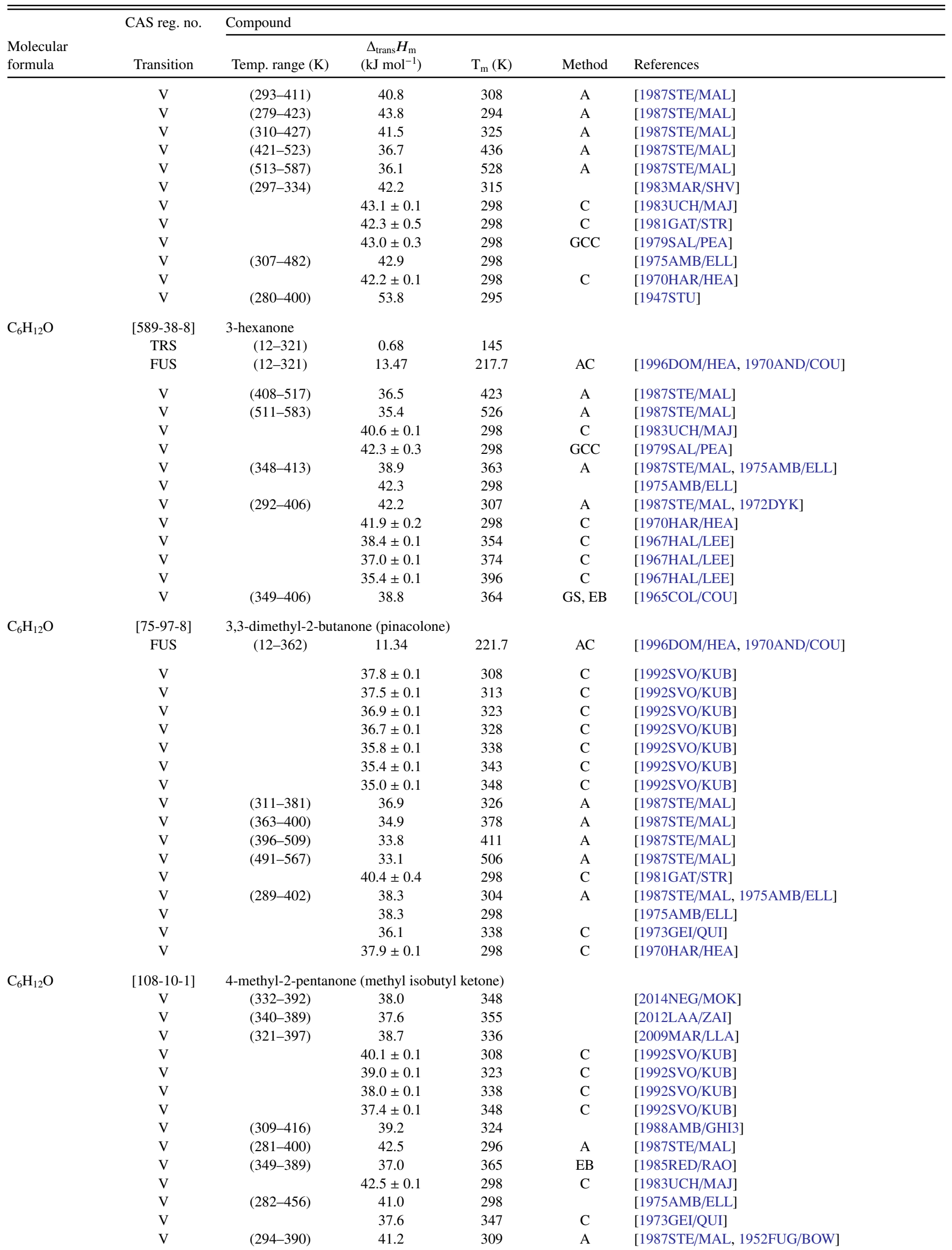


TABlE 9. Phase change enthalpies of $\mathrm{C}_{6}$ organic compounds-Continued

\begin{tabular}{|c|c|c|c|c|c|c|}
\hline \multirow[b]{2}{*}{$\begin{array}{l}\text { Molecular } \\
\text { formula }\end{array}$} & \multirow{2}{*}{$\begin{array}{c}\text { CAS reg. no. } \\
\text { Transition }\end{array}$} & \multicolumn{5}{|l|}{ Compound } \\
\hline & & Temp. range $(\mathrm{K})$ & $\begin{array}{c}\Delta_{\text {trans }} H_{\mathrm{m}} \\
\left(\mathrm{kJ} \mathrm{mol}^{-1}\right)\end{array}$ & $\mathrm{T}_{\mathrm{m}}(\mathrm{K})$ & Method & References \\
\hline \multirow[t]{6}{*}{$\mathrm{C}_{6} \mathrm{H}_{12} \mathrm{O}$} & {$[565-69-5]$} & \multicolumn{5}{|l|}{ 2-methyl-3-pentanone } \\
\hline & $\mathrm{V}$ & $(280-387)$ & 41.0 & 295 & A & [1987STE/MAL] \\
\hline & V & $(377-450)$ & 36.2 & 392 & A & [1987STE/MAL] \\
\hline & $\mathrm{V}$ & $(280-452)$ & 40.5 & 298 & & [1975AMB/ELL] \\
\hline & V & $(300-387)$ & 43.4 & 315 & A & [1987STE/MAL, 1974LOG/FRO] \\
\hline & $\mathrm{V}$ & & $39.8 \pm 0.2$ & 298 & $\mathrm{C}$ & [1970SEL2] \\
\hline \multirow[t]{8}{*}{$\mathrm{C}_{6} \mathrm{H}_{12} \mathrm{O}$} & {$[66-25-1]$} & \multicolumn{5}{|l|}{ hexanal } \\
\hline & FUS & & 13.3 & 214.9 & & \\
\hline & $\begin{array}{c}\text { TRS (liq } \\
\text { anomaly) }\end{array}$ & & 0.34 & 243.2 & $\mathrm{AC}$ & [1993LEB/VAS, 1991VAS/BYK] \\
\hline & $\mathrm{V}$ & $(358-425)$ & 38.6 & 375 & & [2014MEN/BEJ] \\
\hline & $\mathrm{V}$ & $(322-402)$ & 40.8 & 337 & EB & [2006PAL/ORA] \\
\hline & $\mathrm{V}$ & $(287-309)$ & $42.5 \pm 0.4$ & 298 & GS & [2003VER/KRA2] \\
\hline & $\mathrm{V}$ & & $42.3 \pm 0.1$ & 298 & & [1981DYA/KOR] \\
\hline & $\mathrm{V}$ & $(315-402)$ & 41.0 & 330 & & [1979MAR/SAC] \\
\hline \multirow[t]{2}{*}{$\mathrm{C}_{6} \mathrm{H}_{12} \mathrm{OS}$} & {$[999-90-6]$} & \multicolumn{5}{|l|}{$S$-butyl thiolacetate } \\
\hline & V & & $48.1 \pm 0.2$ & 298 & $\mathrm{C}$ & [1966WAD] \\
\hline \multirow[t]{2}{*}{$\mathrm{C}_{6} \mathrm{H}_{12} \mathrm{OS}$} & [999-90-6] & \multicolumn{5}{|c|}{$S$-tert-butyl thiolacetate } \\
\hline & V & & $42.9 \pm 0.2$ & 298 & $\mathrm{C}$ & [1966WAD] \\
\hline \multirow[t]{11}{*}{$\mathrm{C}_{6} \mathrm{H}_{12} \mathrm{O}_{2}$} & {$[1792-81-0]$} & \multicolumn{5}{|l|}{ cis-1,2-cyclohexanediol } \\
\hline & TRS & $(173-368)$ & 19.9 & 360.4 & & \\
\hline & FUS & $(173-368)$ & 3.3 & 371.6 & DSC & [2008MAR/EUS] \\
\hline & FUS & & 20.27 & 373.2 & DSC & [2002ZHO/PEN, 2003ZHO/ZHA] \\
\hline & TRS & & 19.89 & 360.4 & & \\
\hline & FUS & & 3.32 & 371.6 & DSC & [1995MAR/COS] \\
\hline & SUB & $(298-353)$ & $91.3 \pm 0.5$ & 298 & GS & [2015EME/VER3] \\
\hline & SUB & & $89.0 \pm 3.0$ & 298 & $\mathrm{C}$ & [1999COS/EUS] \\
\hline & SUB (cryst. I) & & $70.0 \pm 3.0$ & 366 & $\mathrm{C}$ & [1995MAR/COS] \\
\hline & SUB (cryst. III) & & $88.0 \pm 1.9$ & 343 & $\mathrm{C}$ & [1995MAR/COS] \\
\hline & SUB & $(289-320)$ & 43.7 & 304 & $\mathrm{ME}$ & [1987STE/MAL, 1940ZIL] \\
\hline \multirow[t]{7}{*}{$\mathrm{C}_{6} \mathrm{H}_{12} \mathrm{O}_{2}$} & {$[1460-57-7]$} & \multicolumn{5}{|c|}{ trans-1,2-cyclohexanediol } \\
\hline & FUS & $(173-373)$ & 21.0 & 382.6 & DSC & [2008MAR/EUS] \\
\hline & FUS & & 16.37 & 375.7 & DSC & [2002ZHO/PEN, 2003ZHO/ZHA] \\
\hline & FUS & & 18.51 & 372.3 & DSC & [1995MAR/COS] \\
\hline & SUB & $(296-350)$ & $90.4 \pm 0.4$ & 298 & GS & [2015EME/VER3] \\
\hline & SUB & & $85.9 \pm 1.4$ & 343 & $\mathrm{C}$ & [1995MAR/COS] \\
\hline & SUB & $(289-320)$ & 42.5 & 304 & $\mathrm{ME}$ & [1987STE/MAL, 1940ZIL] \\
\hline \multirow[t]{3}{*}{$\mathrm{C}_{6} \mathrm{H}_{12} \mathrm{O}_{2}$} & {$[931-71-5]$} & \multicolumn{5}{|l|}{ cis-1,4-cyclohexanediol } \\
\hline & TRS & & 14.1 & 374.3 & & \\
\hline & FUS & & 1.9 & 381.7 & DSC & [2014BEB/ROS] \\
\hline \multirow[t]{3}{*}{$\mathrm{C}_{6} \mathrm{H}_{12} \mathrm{O}_{2}$} & {$[126-39-6]$} & 2-ethyl-2-methyl-1,3- & dioxolane & & & \\
\hline & V & $(274-313)$ & $44.8 \pm 0.3$ & 298 & GS & [2002VER] \\
\hline & V & $(274-313)$ & $43.1 \pm 0.3$ & & GS & [1998VER/PEN] \\
\hline $\mathrm{C}_{6} \mathrm{H}_{12} \mathrm{O}_{2}$ & {$[3390-13-4]$} & 2-propyl-1,3-dioxolan & & & & \\
\hline & V & $(278-313)$ & $45.3 \pm 0.3$ & 298 & GS & [1998VER/PEN, 2002VER] \\
\hline $\mathrm{C}_{6} \mathrm{H}_{12} \mathrm{O}_{2}$ & {$[1121-61-5]$} & 4-ethyl-1,3-dioxane & & & & \\
\hline & $\mathrm{V}$ & $(362-412)$ & 39.3 & 377 & A & [1987STE/MAL] \\
\hline $\mathrm{C}_{6} \mathrm{H}_{12} \mathrm{O}_{2}$ & [766-20-1] & 2,4-dimethyl-1,3-diox & ane & & & \\
\hline & V & $(274-313)$ & $44.9 \pm 0.6$ & 298 & GS & [2002VER] \\
\hline $\mathrm{C}_{6} \mathrm{H}_{12} \mathrm{O}_{2}$ & {$[141-79-7]$} & 2,2-dimethyl-1,3-diox & ane & & & \\
\hline & FUS & & 12.1 & 229.6 & & [1975BOR] \\
\hline $\mathrm{C}_{6} \mathrm{H}_{12} \mathrm{O}_{2}$ & {$[766-15-4]$} & 4,4-dimethyl-1,3-diox & ane & & & \\
\hline & $\mathrm{V}$ & $(333-407)$ & 37.1 & 348 & A & [1987STE/MAL, 1968KAC/NEM] \\
\hline & $\mathrm{V}$ & $(363-406)$ & 38.8 & 378 & & [1969LES/MOR] \\
\hline
\end{tabular}


TABLE 9. Phase change enthalpies of $\mathrm{C}_{6}$ organic compounds-Continued

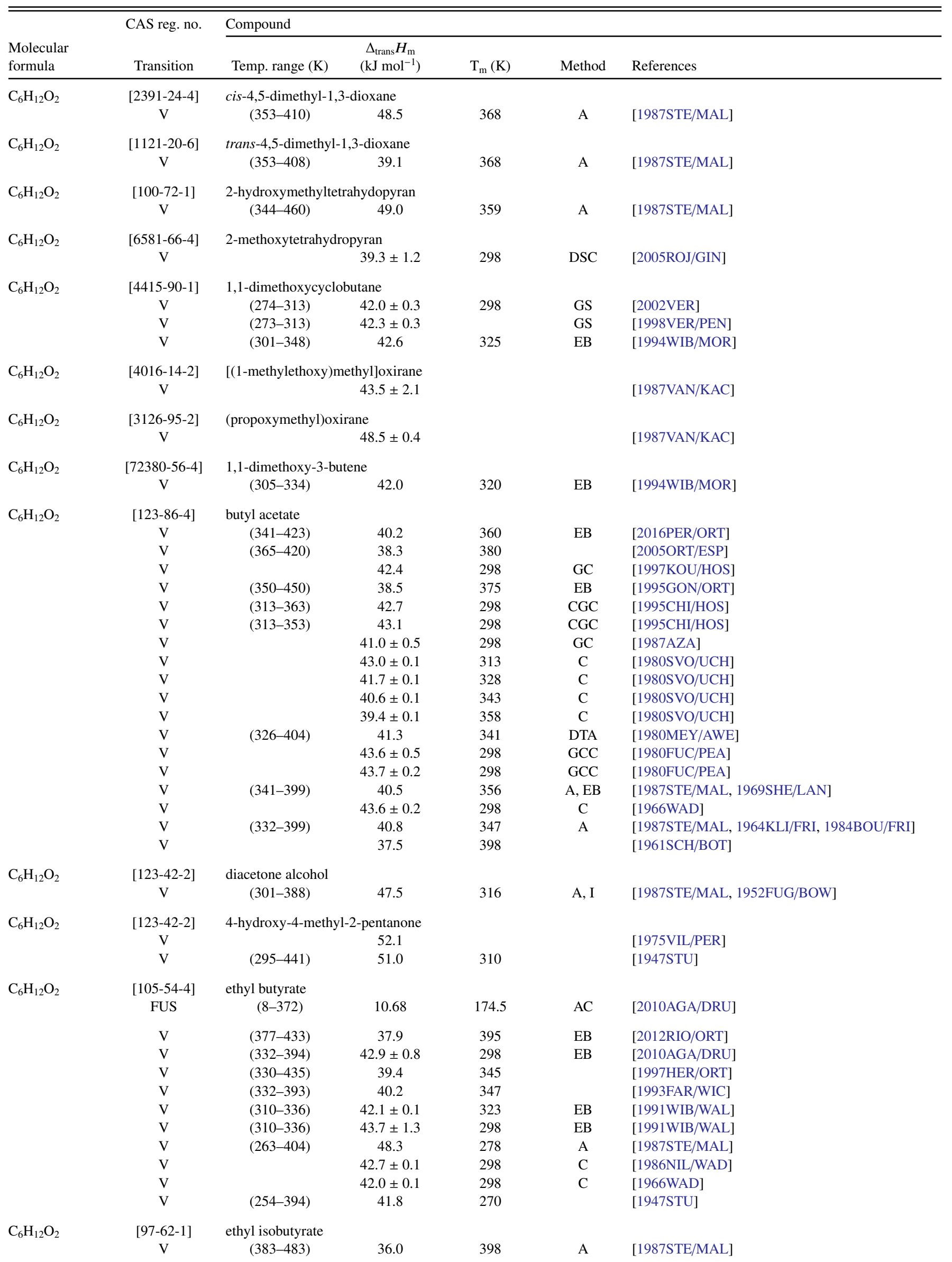


TABle 9. Phase change enthalpies of $\mathrm{C}_{6}$ organic compounds-Continued

\begin{tabular}{|c|c|c|c|c|c|c|}
\hline \multirow[b]{2}{*}{$\begin{array}{l}\text { Molecular } \\
\text { formula }\end{array}$} & \multirow{2}{*}{$\begin{array}{l}\text { CAS reg. no. } \\
\text { Transition }\end{array}$} & \multicolumn{5}{|l|}{ Compound } \\
\hline & & Temp. range $(\mathrm{K})$ & $\begin{array}{c}\Delta_{\text {trans }} H_{\mathrm{m}} \\
\left(\mathrm{kJ} \mathrm{mol}^{-1}\right) \\
\end{array}$ & $\mathrm{T}_{\mathrm{m}}(\mathrm{K})$ & Method & References \\
\hline & $\mathrm{V}$ & & $39.8 \pm 0.1$ & 298 & $\mathrm{C}$ & [1966WAD] \\
\hline & $\mathrm{V}$ & $(249-393)$ & 44.1 & 264 & A & [1987STE/MAL, 1947STU] \\
\hline $\mathrm{C}_{6} \mathrm{H}_{12} \mathrm{O}_{2}$ & $\begin{array}{c}{[105-46-4]} \\
\mathrm{V}\end{array}$ & 2-butyl acetate & 40.7 & 298 & & [2012ZHA/DU, 2014WIS/PER] \\
\hline $\mathrm{C}_{6} \mathrm{H}_{12} \mathrm{O}_{2}$ & $\begin{array}{c}{[110-19-0]} \\
\text { V } \\
\text { V } \\
\text { V } \\
\text { V }\end{array}$ & $\begin{array}{l}\text { isobutyl acetate } \\
\qquad \begin{array}{c}(300-516) \\
(308-391) \\
(325-393) \\
(252-391)\end{array}\end{array}$ & $\begin{array}{l}40.3 \\
39.9 \\
39.2 \\
39.8\end{array}$ & $\begin{array}{l}320 \\
323 \\
340 \\
267\end{array}$ & $\begin{array}{l}\mathrm{EB} \\
\mathrm{A}\end{array}$ & $\begin{array}{l}\text { [2012SUS/ROD] } \\
{[2005 \mathrm{MON} / \mathrm{MUN}]} \\
{[1996 \mathrm{BUR} / \mathrm{MON}]} \\
\text { [1987STE/MAL, 1947STU] }\end{array}$ \\
\hline $\mathrm{C}_{6} \mathrm{H}_{12} \mathrm{O}_{2}$ & $\begin{array}{c}{[540-88-5]} \\
\mathrm{V} \\
\mathrm{V}\end{array}$ & $\begin{array}{l}\text { tert-butyl acetate } \\
\qquad(308-372)\end{array}$ & $\begin{array}{c}36.7 \\
38.0 \pm 0.2\end{array}$ & $\begin{array}{l}323 \\
298\end{array}$ & $\mathrm{C}$ & $\begin{array}{l}\text { [2005MON/MUN] } \\
{[1966 \mathrm{WAD}, 1996 \mathrm{VER} / \mathrm{BEC}]}\end{array}$ \\
\hline $\mathrm{C}_{6} \mathrm{H}_{12} \mathrm{O}_{2}$ & $\begin{array}{c}{[598-98-1]} \\
\text { V } \\
\text { V } \\
\text { V } \\
\text { V } \\
\text { V }\end{array}$ & $\begin{array}{l}\text { methyl 2,2-dimethy } \\
\qquad(313-363) \\
(299-356)\end{array}$ & $\begin{array}{c}\text { ropanoate } \\
37.7 \\
39.0 \pm 0.5 \\
38.8 \\
39.7 \pm 0.3 \\
35.2\end{array}$ & $\begin{array}{l}298 \\
298 \\
298 \\
298 \\
319\end{array}$ & $\begin{array}{c}\text { CGC } \\
\text { GC } \\
\text { GCC } \\
\text { BG }\end{array}$ & $\begin{array}{l}{[1995 \mathrm{CHI} / \mathrm{HOS}]} \\
{[1987 \mathrm{AZA}]} \\
{[\mathrm{UR} / \mathrm{FUC}, 1985 \mathrm{MAJ} / \mathrm{SVO}]} \\
{[1980 \mathrm{FUC} / \mathrm{PEA}]} \\
{[1971 \mathrm{HAL} / \mathrm{BAL}]}\end{array}$ \\
\hline $\mathrm{C}_{6} \mathrm{H}_{12} \mathrm{O}_{2}$ & $\begin{array}{c}\text { [638-49-3] } \\
\mathrm{V} \\
\mathrm{V}\end{array}$ & $\begin{array}{r}\text { pentyl formate } \\
(274-313) \\
(274-313)\end{array}$ & $\begin{array}{c}45.6 \\
45.2 \pm 0.1\end{array}$ & $\begin{array}{l}294 \\
298\end{array}$ & $\begin{array}{l}\text { GS } \\
\text { GS }\end{array}$ & $\begin{array}{l}\text { [2012SAM/NAZ] } \\
{[2012 \text { SAM/NAZ] }}\end{array}$ \\
\hline $\mathrm{C}_{6} \mathrm{H}_{12} \mathrm{O}_{2}$ & $\begin{array}{c}{[110-45-2]} \\
\mathrm{V}\end{array}$ & $\begin{array}{l}\text { isopentyl formate } \\
\quad(255-397)\end{array}$ & 38.9 & 270 & A & [1987STE/MAL] \\
\hline $\mathrm{C}_{6} \mathrm{H}_{12} \mathrm{O}_{2}$ & $\begin{array}{c}{[556-24-1]} \\
\mathrm{V}\end{array}$ & $\begin{array}{l}\text { methyl isovalerate } \\
\quad(254-390)\end{array}$ & 41.2 & 269 & A & [1987STE/MAL, 1947STU] \\
\hline $\mathrm{C}_{6} \mathrm{H}_{12} \mathrm{O}_{2}$ & $\begin{array}{c}\text { [624-24-8] } \\
\text { V } \\
\text { V } \\
\text { V } \\
\text { V } \\
\text { V } \\
\text { V } \\
\text { V } \\
\text { V } \\
\text { V } \\
\text { V } \\
\text { V } \\
\text { V }\end{array}$ & $\begin{array}{l}\text { methyl valerate } \\
\qquad \begin{array}{c}(275-311) \\
(350-415) \\
(281-547) \\
(364-417)\end{array}\end{array}$ & $\begin{array}{c}43.7 \pm 0.3 \\
44.1 \pm 0.1 \\
44.4 \\
39.2 \\
41.3 \\
43.7 \pm 0.2 \\
42.5 \\
43.3 \pm 0.5 \\
44.3 \pm 0.5 \\
46.1 \pm 0.3 \\
44.1 \pm 0.1 \\
43.1 \pm 0.1\end{array}$ & $\begin{array}{l}298 \\
296 \\
379 \\
350 \\
298 \\
312 \\
298 \\
298 \\
298 \\
298 \\
298\end{array}$ & $\begin{array}{c}\text { GS } \\
\text { EB } \\
\\
\\
\\
\text { A } \\
\text { GC } \\
\text { C } \\
\text { GCC } \\
\text { GCC } \\
\text { C }\end{array}$ & $\begin{array}{l}\text { [2008VER/EME] } \\
\text { [2007CAM/MOL] } \\
\text { [2006CAM/MAR, 2006CAM] } \\
\text { [2003ORT/ESP2] } \\
\text { [2002VAN/VAN] } \\
\text { [2002VAN/VAN] } \\
\text { [1987STE/MAL] } \\
\text { [1987AZA] } \\
\text { [1981GAT/STR] } \\
\text { [1980FUC/PEA] } \\
\text { [1980FUC/PEA] } \\
\text { [1977MAN/SEL] }\end{array}$ \\
\hline $\mathrm{C}_{6} \mathrm{H}_{12} \mathrm{O}_{2}$ & $\begin{array}{c}{[106-36-5]} \\
\text { V } \\
\text { V } \\
\text { V } \\
\text { V } \\
\text { V } \\
\text { V } \\
\text { V } \\
\text { V } \\
\text { V } \\
\text { V } \\
\text { V }\end{array}$ & 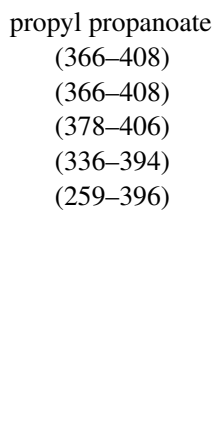 & $\begin{array}{c}38.3 \pm 0.1 \\
44.6 \pm 0.1 \\
37.6 \\
39.9 \\
43.1 \\
42.1 \pm 0.1 \\
43.2 \\
41.1 \pm 0.1 \\
40.0 \pm 0.1 \\
38.8 \pm 0.1 \\
42.2\end{array}$ & $\begin{array}{l}387 \\
298 \\
392 \\
351 \\
274 \\
313 \\
298 \\
328 \\
343 \\
358 \\
298\end{array}$ & $\begin{array}{l}\mathrm{A} \\
\mathrm{C} \\
\mathrm{C} \\
\mathrm{C} \\
\mathrm{C} \\
\mathrm{C}\end{array}$ & $\begin{array}{l}\text { [1999GON/ORT, 2012SAM/NAZ] } \\
\text { [1999GON/ORT, 2012SAM/NAZ] } \\
\text { [1994ORT/GAL] } \\
\text { [1993FAR/WIC] } \\
\text { [1987STE/MAL, 1947STU] } \\
\text { [1980SVO/UCH] } \\
\text { [1980SVO/UCH, 2012SAM/NAZ] } \\
\text { [1980SVO/UCH] } \\
\text { [1980SVO/UCH] } \\
\text { [1980SVO/UCH] } \\
\text { [1953WAL, 2012SAM/NAZ] }\end{array}$ \\
\hline $\mathrm{C}_{6} \mathrm{H}_{12} \mathrm{O}_{2}$ & $\begin{array}{c}{[142-62-1]} \\
\text { V } \\
\text { V } \\
\text { V } \\
\text { V } \\
\text { V } \\
\text { V }\end{array}$ & $\begin{array}{r}\text { hexanoic acid } \\
(297-328) \\
(297-328) \\
(353-393) \\
(270-280) \\
(335-487)\end{array}$ & $\begin{array}{c}68.4 \pm 0.9 \\
69.2 \pm 0.9 \\
71.3 \\
70.9 \\
73.2 \pm 2.0 \\
65.9\end{array}$ & $\begin{array}{l}313 \\
298 \\
298 \\
271 \\
298 \\
350\end{array}$ & $\begin{array}{c}\text { GS } \\
\text { GS } \\
\text { CGC } \\
\text { TE } \\
\text { A }\end{array}$ & $\begin{array}{l}\text { [2000VER2] } \\
\text { [2000VER2] } \\
\text { [1995CHI/HOS] } \\
\text { [1982DEK/SCH] } \\
{[1979 \mathrm{DEK} / \mathrm{OON}]} \\
{[1987 \mathrm{STE} / \mathrm{MAL}, 1972 \mathrm{DYK}]}\end{array}$ \\
\hline
\end{tabular}


TABlE 9. Phase change enthalpies of $\mathrm{C}_{6}$ organic compounds-Continued

\begin{tabular}{|c|c|c|c|c|c|c|}
\hline \multirow[b]{2}{*}{$\begin{array}{l}\text { Molecular } \\
\text { formula }\end{array}$} & \multirow{2}{*}{$\begin{array}{l}\text { CAS reg. no. } \\
\text { Transition }\end{array}$} & \multicolumn{5}{|l|}{ Compound } \\
\hline & & Temp. range $(\mathrm{K})$ & $\begin{array}{c}\Delta_{\text {trans }} H_{\mathrm{m}} \\
\left(\mathrm{kJ} \mathrm{mol}^{-1}\right)\end{array}$ & $\mathrm{T}_{\mathrm{m}}(\mathrm{K})$ & Method & References \\
\hline & $\mathrm{V}$ & $(371-452)$ & 66.6 & 386 & & [1957ROS/ACC, 1984BOU/FRI] \\
\hline & $\mathrm{V}$ & & 64.6 & 367 & $\mathrm{I}$ & [1943CRA] \\
\hline $\mathrm{C}_{6} \mathrm{H}_{12} \mathrm{O}_{2}$ & $\begin{array}{c}{[88-09-5]} \\
\mathrm{V}\end{array}$ & $\begin{array}{l}\text { 2-ethyl butyric acid } \\
\text { (373-466) }\end{array}$ & 58.2 & 388 & A & [1987STE/MAL] \\
\hline $\mathrm{C}_{6} \mathrm{H}_{12} \mathrm{O}_{2}$ & $\begin{array}{c}{[646-07-1]} \\
\mathrm{V}\end{array}$ & $\begin{array}{l}\text { 4-methylvaleric acid } \\
\text { (339-481) }\end{array}$ & 91.7 & 354 & A & [1987STE/MAL] \\
\hline $\mathrm{C}_{6} \mathrm{H}_{12} \mathrm{O}_{2}$ & $\begin{array}{c}{[595-37-9]} \\
\text { V } \\
\text { V } \\
\text { V } \\
\text { V }\end{array}$ & $\begin{array}{c}\text { 2,2-dimethylbutanoic } \\
(364-498) \\
(364-498) \\
(364-498) \\
(364-498)\end{array}$ & $\begin{array}{l}\text { acid } \\
59.4 \pm 0.3 \\
54.6 \pm 0.3 \\
50.0 \pm 0.4 \\
46.0 \pm 0.7\end{array}$ & $\begin{array}{l}370 \\
410 \\
450 \\
490\end{array}$ & $\begin{array}{l}\text { EB } \\
\text { EB } \\
\text { EB } \\
\text { EB }\end{array}$ & $\begin{array}{l}{[2002 \mathrm{STE} / \mathrm{CHI}]} \\
{[2002 \mathrm{STE} / \mathrm{CHI}]} \\
{[2002 \mathrm{STE} / \mathrm{CHI}]} \\
{[2002 \mathrm{STE} / \mathrm{CHI}]}\end{array}$ \\
\hline $\mathrm{C}_{6} \mathrm{H}_{12} \mathrm{O}_{2}$ & $\begin{array}{c}{[1070-83-3]} \\
\text { V } \\
\text { V }\end{array}$ & $\begin{array}{l}\text { 3,3-dimethylbutanoic } \\
\quad(283-325) \\
(283-325)\end{array}$ & $\begin{array}{l}\text { acid } \\
63.6 \pm 0.9 \\
64.0 \pm 0.9\end{array}$ & $\begin{array}{l}304 \\
298\end{array}$ & $\begin{array}{l}\text { GS } \\
\text { GS }\end{array}$ & $\begin{array}{l}\text { [2000VER2] } \\
{[2000 \mathrm{VER} 2]}\end{array}$ \\
\hline $\mathrm{C}_{6} \mathrm{H}_{12} \mathrm{O}_{3}$ & $\begin{array}{c}{[767-09-9]} \\
\mathrm{V}\end{array}$ & $\begin{array}{l}\text { 1-hexene ozonide } \\
\quad(353-373)\end{array}$ & 43.9 & 363 & $\mathrm{MM}$ & [1977BOL/MAK] \\
\hline $\mathrm{C}_{6} \mathrm{H}_{12} \mathrm{O}_{3}$ & $\begin{array}{c}{[37160-61-5]} \\
\mathrm{V}\end{array}$ & $\begin{array}{c}\text { sec-butyl glycolate } \\
(301-451)\end{array}$ & 52.3 & 316 & A & [1987STE/MAL, 1947STU] \\
\hline $\mathrm{C}_{6} \mathrm{H}_{12} \mathrm{O}_{3}$ & $\begin{array}{c}\text { [123-63-7] } \\
\text { TRS } \\
\text { TRS } \\
\text { FUS } \\
\text { FUS }\end{array}$ & $\begin{array}{l}2,4,6 \text {-trimethyl-1,3,5- } \\
\quad(80-310) \\
\quad(80-310) \\
(80-310)\end{array}$ & $\begin{array}{c}\text { trioxane (par } \\
0.26 \\
0.77 \\
13.52 \\
13.83\end{array}$ & $\begin{array}{l}\text { de) } \\
142.7 \\
147.5 \\
285.7 \\
285.8\end{array}$ & $\mathrm{AC}$ & $\begin{array}{l}\text { [1996DOM/HEA, 1969CLE/MEL] } \\
\text { [1911LOU/DUP] }\end{array}$ \\
\hline & $\begin{array}{l}\mathrm{V} \\
\mathrm{V}\end{array}$ & $(323-396)$ & $\begin{array}{c}41.5 \\
41.4 \pm 0.4\end{array}$ & 338 & A & $\begin{array}{l}\text { [1987STE/MAL] } \\
\text { [1959FLE/MOR] }\end{array}$ \\
\hline $\mathrm{C}_{6} \mathrm{H}_{12} \mathrm{O}_{3}$ & $\begin{array}{c}{[123-34-2]} \\
\mathrm{V}\end{array}$ & $\begin{array}{l}\text { glycerol 1-monoallyl } \\
\quad(323-383)\end{array}$ & $\begin{array}{l}\text { ether } \\
\qquad 74.7\end{array}$ & 338 & A & [1987STE/MAL, 1972DYK] \\
\hline $\mathrm{C}_{6} \mathrm{H}_{12} \mathrm{O}_{3}$ & $\begin{array}{c}{[817-95-8]} \\
\mathrm{V}\end{array}$ & $\begin{array}{l}\text { 2-ethoxyacetic acid, } \\
\quad(330-430)\end{array}$ & $\begin{array}{c}\text { thyl ester } \\
46.1\end{array}$ & 345 & A & [1987STE/MAL] \\
\hline $\mathrm{C}_{6} \mathrm{H}_{12} \mathrm{O}_{3}$ & $\begin{array}{c}{[14144-33-3]} \\
\mathrm{V}\end{array}$ & $\begin{array}{l}\text { 3-ethoxypropionic ac } \\
\quad(320-432)\end{array}$ & $\begin{array}{c}\text { id, methyl es } \\
44.3\end{array}$ & 335 & A & [1987STE/MAL, 1972DYK, 1948DIX/REH] \\
\hline $\mathrm{C}_{6} \mathrm{H}_{12} \mathrm{O}_{3}$ & $\begin{array}{c}{[111-15-9]} \\
\text { V } \\
\text { V } \\
\text { V } \\
\text { V }\end{array}$ & $\begin{array}{c}\text { 2-ethoxyethanol aceta } \\
(408-429) \\
(322-430) \\
(330-468)\end{array}$ & $\begin{array}{c}43.6 \\
50.9 \\
52.7 \pm 0.1 \\
52.6 \pm 0.4\end{array}$ & $\begin{array}{l}418 \\
337 \\
298 \\
298\end{array}$ & $\begin{array}{c}\mathrm{EB} \\
\mathrm{A} \\
\mathrm{C} \\
\mathrm{EB}\end{array}$ & $\begin{array}{l}\text { [2012RAN/BHA] } \\
\text { [1987STE/MAL] } \\
\text { [1970KUS/WAD] } \\
\text { [1966BOT/ADL] }\end{array}$ \\
\hline $\mathrm{C}_{6} \mathrm{H}_{12} \mathrm{O}_{3}$ & $\begin{array}{c}{[4897-95-4]} \\
\mathrm{V}\end{array}$ & $\begin{array}{l}\text { 3-hydroxypropionic a } \\
(350-375)\end{array}$ & $\begin{array}{c}\text { cid, propyl e } \\
60.9\end{array}$ & 362 & A & [1987STE/MAL] \\
\hline $\mathrm{C}_{6} \mathrm{H}_{12} \mathrm{O}_{3}$ & $\begin{array}{c}{[10606-42-5]} \\
\mathrm{V}\end{array}$ & $\begin{array}{l}\text { 3-methoxypropionic } \\
\quad(313-432)\end{array}$ & $\begin{array}{c}\text { acid, ethyl es } \\
44.6\end{array}$ & 328 & A & {$[1987 \mathrm{STE} / \mathrm{MAL}]$} \\
\hline $\mathrm{C}_{6} \mathrm{H}_{12} \mathrm{O}_{3}$ & $\begin{array}{c}{[616-09-1]} \\
\mathrm{V}\end{array}$ & $\begin{array}{l}\text { propyl lactate } \\
(334-442)\end{array}$ & 52.1 & 349 & A & [1987STE/MAL, 1950REH/DIX] \\
\hline $\mathrm{C}_{6} \mathrm{H}_{12} \mathrm{O}_{3}$ & $\begin{array}{c}{[617-51-6]} \\
\mathrm{V}\end{array}$ & $\begin{array}{c}\text { isopropyl lactate } \\
(356-430)\end{array}$ & 44.5 & 371 & & [2005PEN/MUR] \\
\hline $\mathrm{C}_{6} \mathrm{H}_{12} \mathrm{O}_{3}$ & $\begin{array}{c}{[54078-53-4]} \\
\mathrm{V}\end{array}$ & ethoxymethyl propior & $49.9 \pm 0.1$ & 298 & $\mathrm{C}$ & [1974MAN] \\
\hline $\mathrm{C}_{6} \mathrm{H}_{12} \mathrm{O}_{3}$ & $\begin{array}{c}{[5405-41-4]} \\
\mathrm{V}\end{array}$ & $\begin{array}{l}\text { ethyl 3-hydroxybutyr } \\
\qquad(363-393)\end{array}$ & $\begin{array}{l}\text { ate } \\
55.9 \pm 0.6\end{array}$ & 298 & CGC & [2005TEM/CHI] \\
\hline $\mathrm{C}_{6} \mathrm{H}_{12} \mathrm{O}_{4}$ & $\begin{array}{c}{[624-47-5]} \\
\mathrm{V}\end{array}$ & $\begin{array}{l}(d l) \text {-glycerol 1-propic } \\
\quad(388-456)\end{array}$ & 75.8 & 403 & A & [1987STE/MAL, 1972DYK] \\
\hline $\mathrm{C}_{6} \mathrm{H}_{12} \mathrm{O}_{4}$ & {$[1073-91-2]$} & diacetone diperoxide & & & & \\
\hline
\end{tabular}


TABLE 9. Phase change enthalpies of $\mathrm{C}_{6}$ organic compounds-Continued

\begin{tabular}{|c|c|c|c|c|c|c|}
\hline \multirow[b]{2}{*}{$\begin{array}{l}\text { Molecular } \\
\text { formula }\end{array}$} & \multirow{2}{*}{$\begin{array}{c}\text { CAS reg. no. } \\
\text { Transition }\end{array}$} & \multicolumn{5}{|l|}{ Compound } \\
\hline & & Temp. range $(\mathrm{K})$ & $\begin{array}{c}\Delta_{\text {trans }} H_{\mathrm{m}} \\
\left(\mathrm{kJ} \mathrm{mol}^{-1}\right)\end{array}$ & $\mathrm{T}_{\mathrm{m}}(\mathrm{K})$ & Method & References \\
\hline & SUB & $(288-328)$ & 81.9 & 308 & HSA & [2009OXL/SMI] \\
\hline \multirow[t]{2}{*}{$\mathrm{C}_{6} \mathrm{H}_{12} \mathrm{O}_{5}$} & & \multicolumn{5}{|c|}{ 1-deoxy-(D)-glucopyranose } \\
\hline & FUS & & 27.4 & 403.2 & $\mathrm{DSC}$ & [1996SCH] \\
\hline \multirow[t]{2}{*}{$\mathrm{C}_{6} \mathrm{H}_{12} \mathrm{O}_{5}$} & & \multicolumn{5}{|c|}{ 2-deoxy-(D)-glucopyranose } \\
\hline & FUS & & 34.5 & 398.7 & $\mathrm{DSC}$ & {$[1996 \mathrm{SCH}]$} \\
\hline \multirow[t]{2}{*}{$\mathrm{C}_{6} \mathrm{H}_{12} \mathrm{O}_{5}$} & & \multicolumn{5}{|c|}{ 3-deoxy-(D)-glucopyranose } \\
\hline & FUS & & 32.6 & 387.2 & DSC & [1996SCH] \\
\hline \multirow[t]{2}{*}{$\mathrm{C}_{6} \mathrm{H}_{12} \mathrm{O}_{5}$} & & \multicolumn{5}{|c|}{ 6-deoxy-(D)-glucopyranose } \\
\hline & FUS & & 22.7 & 409.2 & $\mathrm{DSC}$ & {$[1996 \mathrm{SCH}]$} \\
\hline \multirow[t]{10}{*}{$\mathrm{C}_{6} \mathrm{H}_{12} \mathrm{O}_{6}$} & {$[87-89-8]$} & myo-inositol & & & & \\
\hline & FUS & & 46.2 & 500.8 & DSC & [2006ZHO/LI] \\
\hline & FUS & & 47.9 & 496.9 & DSC & [1996DOM/HEA, 1990BAR/DEL] \\
\hline & SUB & $(438-458)$ & $174.0 \pm 2.6$ & 448 & $\mathrm{ME}$ & [2006CHE/OJA] \\
\hline & SUB & & $181.0 \pm 2.0$ & 298 & $\mathrm{C}$ & [1999COS/EUS] \\
\hline & SUB & & $154.7 \pm 1.4$ & 477 & $\mathrm{TE}$ & [1990BAR/DEL] \\
\hline & SUB & & 161 & 298 & & [1990BAR/DEL] \\
\hline & SUB & & 178 & 298 & $\mathrm{~B}$ & [1990BAR/DEL] \\
\hline & SUB & $(454-472)$ & 168 & & & [1983DEW/BOW] \\
\hline & $\mathrm{V}$ & $(497-524)$ & $119.0 \pm 1.4$ & 519 & $\mathrm{TE}$ & [1990BAR/DEL] \\
\hline \multirow[t]{8}{*}{$\mathrm{C}_{6} \mathrm{H}_{12} \mathrm{O}_{6}$} & {$[492-62-6]$} & $\alpha$-D-glucose & & & & \\
\hline & FUS $(0.05 \mathrm{~K} / \mathrm{min})$ & & 31.6 & 415.1 & DSC & \\
\hline & FUS $(0.5 \mathrm{~K} / \mathrm{min})$ & & 33.5 & 421.8 & DSC & \\
\hline & FUS (5 K/min) & & 36.1 & 431.3 & DSC & \\
\hline & FUS $(10 \mathrm{~K} / \mathrm{min})$ & & 36.6 & 435.2 & DSC & \\
\hline & FUS $(20 \mathrm{~K} / \mathrm{min})$ & & 36.9 & 438.7 & $\mathrm{DSC}$ & [2011MAG/PYD] \\
\hline & FUS & & 34.3 & 423.2 & & {$[1996 \mathrm{SCH}]$} \\
\hline & FUS & & 31.42 & 414 & & [1996DOM/HEA, 1934PAR/THO] \\
\hline $\mathrm{C}_{6} \mathrm{H}_{12} \mathrm{O}_{6}$ & FUS & D-(+)-glucose & 3335 & 4273 & $\mathrm{DSC}$ & [2011L FE/THO] \\
\hline
\end{tabular}

Note: The authors performed measuremetns as a function of heating rate, and the above values correspond to a heating rate of $2^{\circ} \mathrm{C} / \mathrm{min}$.

\begin{tabular}{|c|c|c|c|c|c|c|}
\hline $\mathrm{C}_{6} \mathrm{H}_{12} \mathrm{O}_{6}$ & $\begin{array}{c}\text { [50-99-7] } \\
\text { FUS }\end{array}$ & glucose & 32.11 & 433.5 & DSC & [2012BIT/MEN] \\
\hline $\mathrm{C}_{6} \mathrm{H}_{12} \mathrm{O}_{6}$ & $\begin{array}{c}\text { [57-48-7] } \\
\text { FUS }\end{array}$ & D-(-)-fructose & 31.15 & 390.1 & DSC & [2011LEE/THO] \\
\hline
\end{tabular}

Note: The authors performed measuremetns as a function of heating rate, and the above values correspond to a heating rate of $2^{\circ} \mathrm{C} / \mathrm{min}$.

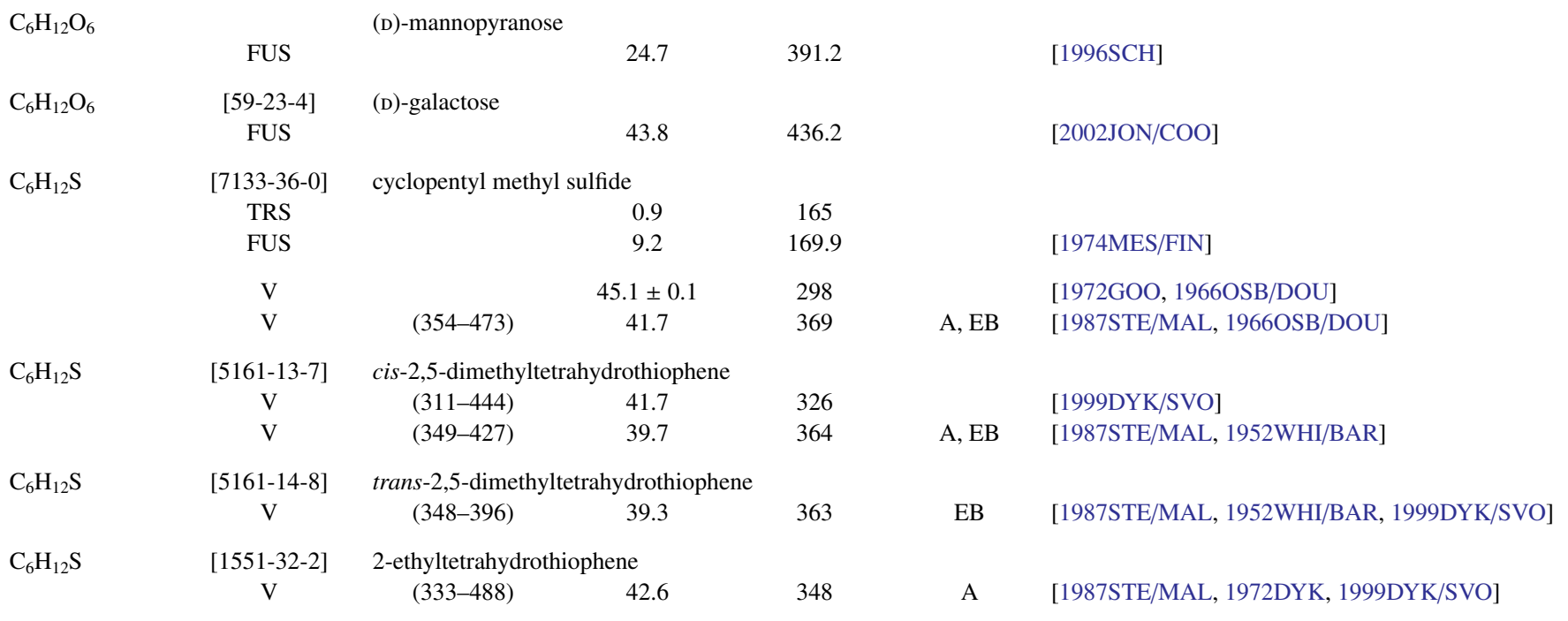

$\mathrm{C}_{6} \mathrm{H}_{12} \mathrm{~S} \quad$ [62184-67-2] 3-ethyltetrahydrothiophene 
TABLE 9. Phase change enthalpies of $\mathrm{C}_{6}$ organic compounds-Continued

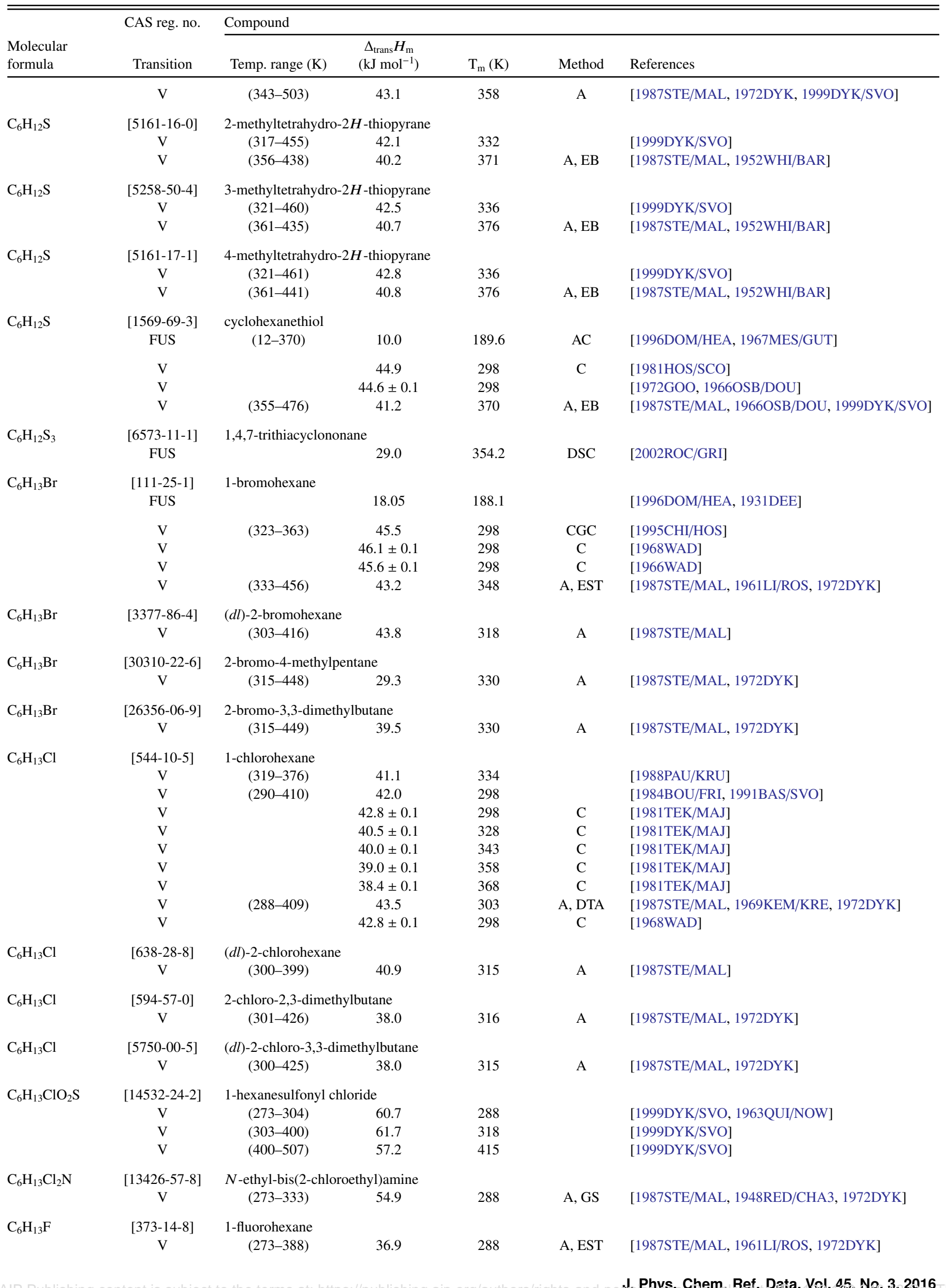


TABLE 9. Phase change enthalpies of $\mathrm{C}_{6}$ organic compounds-Continued

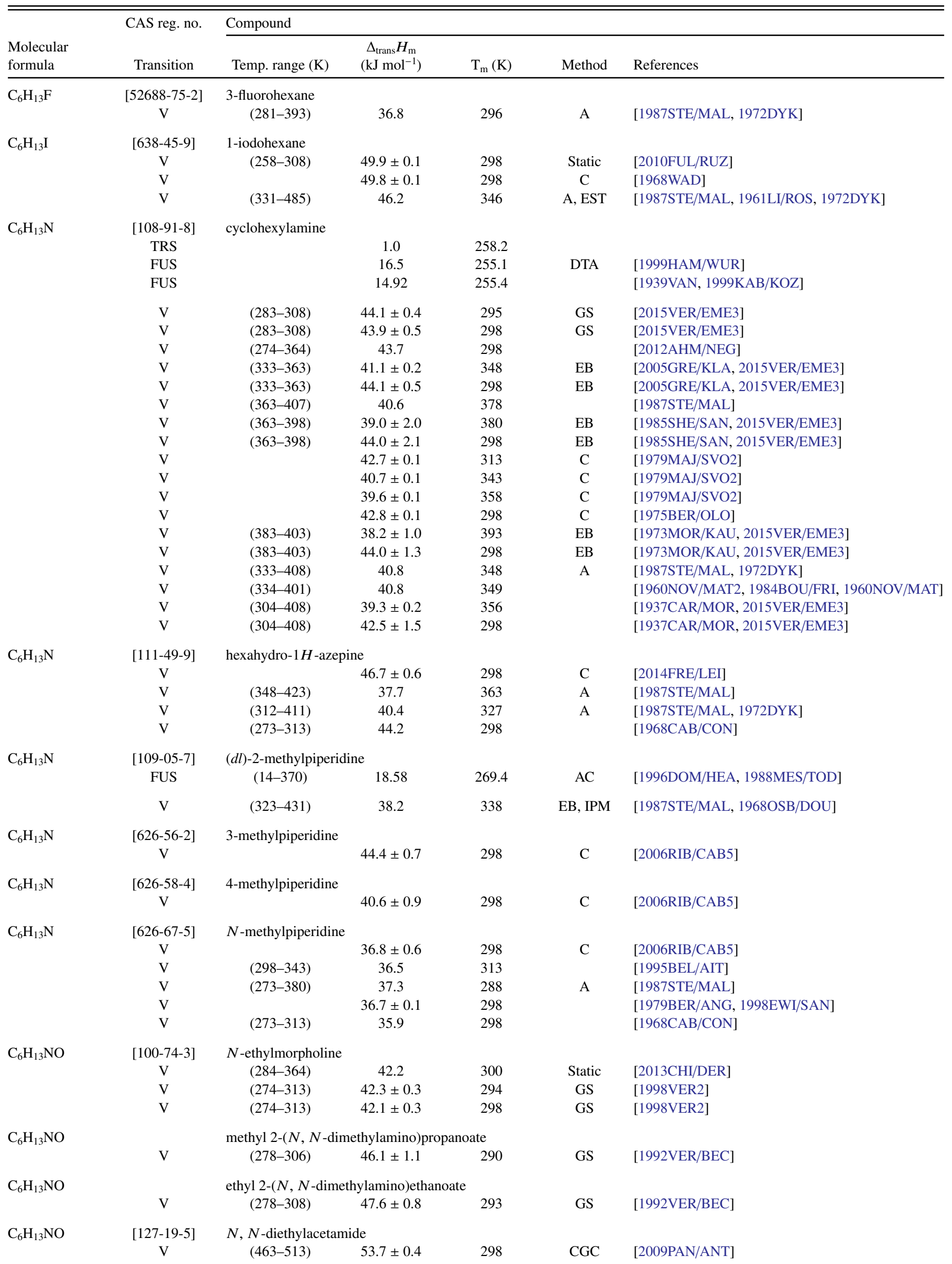


TABLE 9. Phase change enthalpies of $\mathrm{C}_{6}$ organic compounds-Continued

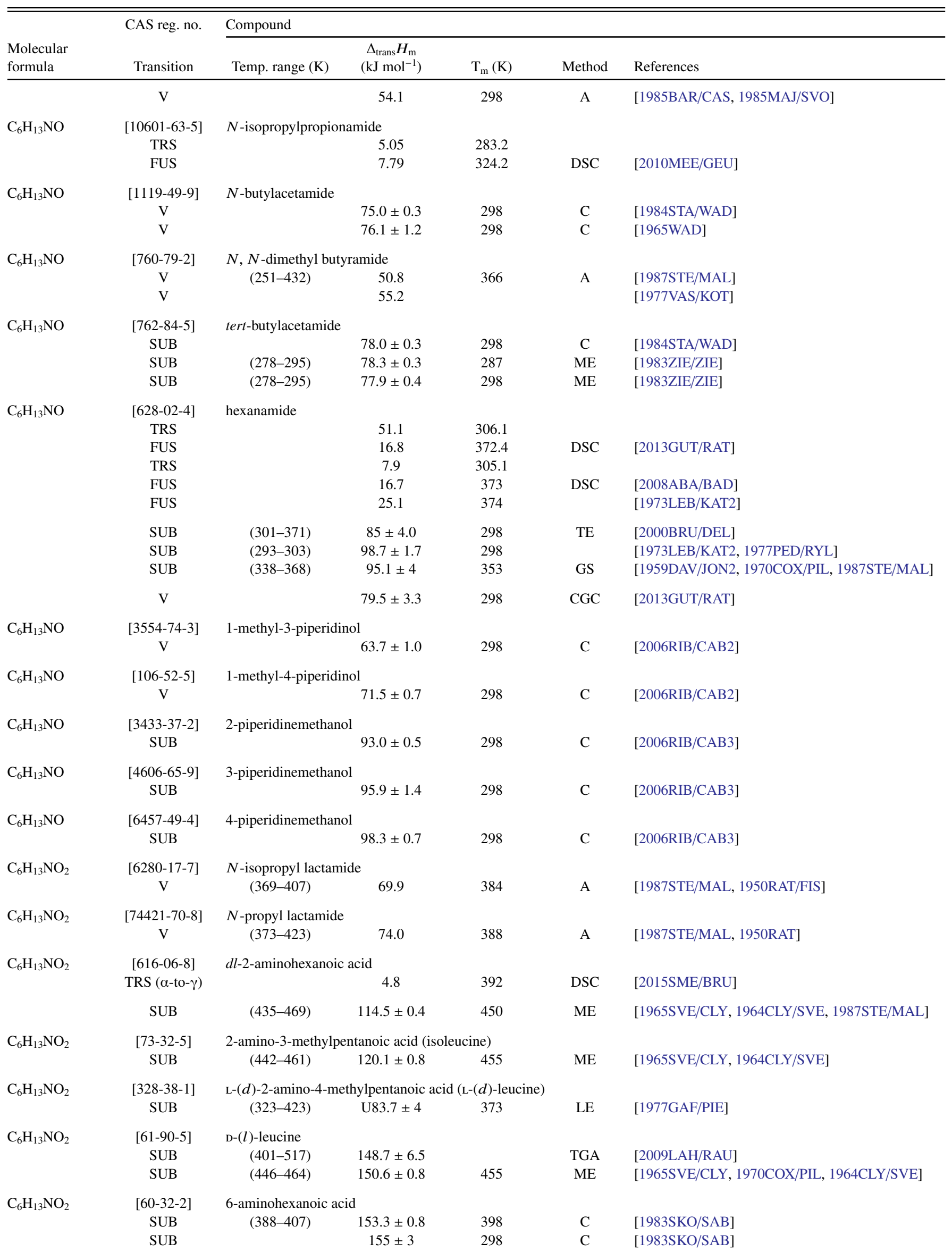


TABLE 9. Phase change enthalpies of $\mathrm{C}_{6}$ organic compounds-Continued

\begin{tabular}{|c|c|c|c|c|c|c|}
\hline \multirow[b]{2}{*}{$\begin{array}{l}\text { Molecular } \\
\text { formula }\end{array}$} & \multirow{2}{*}{$\begin{array}{l}\text { CAS reg. no. } \\
\text { Transition }\end{array}$} & \multicolumn{5}{|l|}{ Compound } \\
\hline & & Temp. range $(\mathrm{K})$ & $\begin{array}{c}\Delta_{\text {trans }} H_{\mathrm{m}} \\
\left(\mathrm{kJ} \mathrm{mol}^{-1}\right)\end{array}$ & $\mathrm{T}_{\mathrm{m}}(\mathrm{K})$ & Method & References \\
\hline \multirow[t]{40}{*}{$\mathrm{C}_{6} \mathrm{H}_{14}$} & [110-54-3] & hexane & & & & \\
\hline & FUS & & 13.03 & 177.8 & DTA & [1994TAN/SAB3] \\
\hline & FUS & & 13.08 & 177.8 & & [1996DOM/HEA, 1946DOU/HUF] \\
\hline & FUS & & 12.34 & 177.9 & & [1937STU] \\
\hline & FUS & & 13.03 & 177.9 & $\mathrm{C}$ & [1931HUF/PAR] \\
\hline & FUS & & 12.58 & 178.6 & & [1996DOM/HEA, 1930PAR/HUF] \\
\hline & SUB & & 50.8 & 178 & $\mathrm{~B}$ & {$[1963 \mathrm{BON}]$} \\
\hline & V & $(302-339)$ & $27.1 \pm 0.4$ & & $\mathrm{I}$ & [2010GER/PEL] \\
\hline & $\mathrm{V}$ & & $31.4 \pm 0.2$ & 298 & $\mathrm{C}$ & [2007PAS/KUZ] \\
\hline & $\mathrm{V}$ & & $31.5 \pm 0.1$ & 298 & $\mathrm{C}$ & [1996VAR/PAS] \\
\hline & V & & 31.5 & 298 & & [1994RUZ/MAJ] \\
\hline & $\mathrm{V}$ & $(283-323)$ & 32.1 & 298 & & [1992GRA/SAN] \\
\hline & $\mathrm{V}$ & & $31.3 \pm 0.3$ & & GC & [1989AZA] \\
\hline & V & $(238-298)$ & 34.9 & 253 & A & [1987STE/MAL] \\
\hline & $\mathrm{V}$ & $(189-259)$ & 35.7 & 244 & A & [1987STE/MAL] \\
\hline & V & $(298-343)$ & 31.5 & 313 & A & [1987STE/MAL] \\
\hline & V & $(341-377)$ & 30.1 & 356 & A & [1987STE/MAL] \\
\hline & $\mathrm{V}$ & $(374-451)$ & 29.3 & 389 & A & [1987STE/MAL] \\
\hline & V & $(445-508)$ & 29.4 & 460 & A & [1987STE/MAL] \\
\hline & V & & 26.6 & 373 & $\mathrm{C}$ & [1985WOR/YER] \\
\hline & $\mathrm{V}$ & & 22.5 & 423 & $\mathrm{C}$ & [1985WOR/YER] \\
\hline & V & & 15.7 & 473 & $\mathrm{C}$ & [1985WOR/YER] \\
\hline & $\mathrm{V}$ & & 8.9 & 498 & $\mathrm{C}$ & [1985WOR/YER] \\
\hline & $\mathrm{V}$ & & 31.6 & 298 & & [UR/FUC, 1985MAJ/SVO] \\
\hline & $\mathrm{V}$ & $(298-338)$ & 30.9 & 313 & & [1984MIC/JOS] \\
\hline & $\mathrm{V}$ & & $31.6 \pm 0.1$ & 298 & $\mathrm{C}$ & [1979MAJ/SVO] \\
\hline & $\mathrm{V}$ & & $30.7 \pm 0.1$ & 313 & $\mathrm{C}$ & [1979MAJ/SVO] \\
\hline & $\mathrm{V}$ & & $29.5 \pm 0.1$ & 333 & $\mathrm{C}$ & [1979MAJ/SVO] \\
\hline & $\mathrm{V}$ & & $28.2 \pm 0.1$ & 353 & $\mathrm{C}$ & [1979MAJ/SVO] \\
\hline & $\mathrm{V}$ & $(300-321)$ & 31.6 & 310 & Static, MM & [1974LET/MAR, 1984BOU/FRI] \\
\hline & $\mathrm{V}$ & $(178-265)$ & 32.5 & 250 & & [1973CAR/KOB] \\
\hline & $\mathrm{V}$ & & 31.55 & 298 & & [1971WIL/ZWO] \\
\hline & V & & $30.9 \pm 0.1$ & 309 & $\mathrm{C}$ & [1947WAD/DOU] \\
\hline & $\mathrm{V}$ & & $29.8 \pm 0.1$ & 328 & $\mathrm{C}$ & [1947WAD/DOU] \\
\hline & $\mathrm{V}$ & & $31.5 \pm 0.1$ & 298 & $\mathrm{C}$ & [1947OSB/GIN] \\
\hline & V & $(286-343)$ & 32.0 & 301 & A, MM & [1987STE/MAL, 1945WIL/TAY] \\
\hline & $\mathrm{V}$ & & $31.0 \pm 0.2$ & 298 & $\mathrm{C}$ & [1943LEM/FEL] \\
\hline & $\mathrm{V}$ & & $30.5 \pm 0.2$ & 313 & $\mathrm{C}$ & [1943LEM/FEL] \\
\hline & V & & $29.0 \pm 0.2$ & 333 & $\mathrm{C}$ & [1943LEM/FEL] \\
\hline & $\mathrm{V}$ & & $28.2 \pm 0.2$ & 353 & $\mathrm{C}$ & [1943LEM/FEL] \\
\hline \multirow[t]{16}{*}{$\mathrm{C}_{6} \mathrm{H}_{14}$} & {$[107-83-5]$} & 2-methylpentane & & & & \\
\hline & FUS & & 6.27 & 119.6 & & [1996DOM/HEA, 1946DOU/HUF] \\
\hline & V & $(290-333)$ & 30.2 & 305 & & [2010SAP/UUS] \\
\hline & $\mathrm{V}$ & $(301-333)$ & 30.0 & 316 & & [2002POK/UUS] \\
\hline & $\mathrm{V}$ & $(310-359)$ & 29.7 & 325 & & [1998AUC/LOR] \\
\hline & $\mathrm{V}$ & $(293-335)$ & 30.5 & 308 & A & [1987STE/MAL] \\
\hline & $\mathrm{V}$ & & 29.9 & 298 & & [1971WIL/ZWO] \\
\hline & $\mathrm{V}$ & & $29.9 \pm 0.1$ & 298 & $\mathrm{C}$ & [1949WAD/SMI] \\
\hline & $\mathrm{V}$ & & $28.7 \pm 0.1$ & 318 & $\mathrm{C}$ & [1949WAD/SMI] \\
\hline & $\mathrm{V}$ & & $27.8 \pm 0.1$ & 333 & $\mathrm{C}$ & [1949WAD/SMI] \\
\hline & $\mathrm{V}$ & & $29.9 \pm 0.1$ & 298 & $\mathrm{C}$ & [1947OSB/GIN] \\
\hline & $\mathrm{V}$ & $(286-334)$ & 30.4 & 301 & MM & [1945WIL/TAY] \\
\hline & V & & $29.8 \pm 0.2$ & 293 & $\mathrm{C}$ & [1943LEM/FEL] \\
\hline & $\mathrm{V}$ & & $29.0 \pm 0.2$ & 313 & $\mathrm{C}$ & [1943LEM/FEL] \\
\hline & $\mathrm{V}$ & & $27.6 \pm 0.2$ & 333 & $\mathrm{C}$ & [1943LEM/FEL] \\
\hline & $\mathrm{V}$ & & $26.9 \pm 0.2$ & 353 & $\mathrm{C}$ & [1943LEM/FEL] \\
\hline \multirow[t]{4}{*}{$\mathrm{C}_{6} \mathrm{H}_{14}$} & {$[96-14-0]$} & 3-methylpentane & & & & \\
\hline & FUS & & 5.31 & 110.3 & & [1996DOM/HEA, 1973FIN/MES] \\
\hline & V & $(316-361)$ & 29.9 & 331 & & [1999LOR/AUC] \\
\hline & $\mathrm{V}$ & $(293-338)$ & 30.5 & 308 & A & [1987STE/MAL] \\
\hline
\end{tabular}


TABLE 9. Phase change enthalpies of $\mathrm{C}_{6}$ organic compounds-Continued

\begin{tabular}{|c|c|c|c|c|c|c|}
\hline \multirow[b]{2}{*}{$\begin{array}{l}\text { Molecular } \\
\text { formula }\end{array}$} & \multirow{2}{*}{$\begin{array}{l}\text { CAS reg. no. } \\
\text { Transition }\end{array}$} & \multicolumn{5}{|l|}{ Compound } \\
\hline & & Temp. range $(\mathrm{K})$ & $\begin{array}{c}\Delta_{\text {trans }} H_{\mathrm{m}} \\
\left(\mathrm{kJ} \mathrm{mol}^{-1}\right)\end{array}$ & $\mathrm{T}_{\mathrm{m}}(\mathrm{K})$ & Method & References \\
\hline & $\mathrm{V}$ & & $29.5 \pm 0.1$ & 313 & $\mathrm{C}$ & [1979MAJ/SVO] \\
\hline & $\mathrm{V}$ & & $28.3 \pm 0.1$ & 333 & $\mathrm{C}$ & [1979MAJ/SVO] \\
\hline & V & & $27.0 \pm 0.1$ & 353 & $\mathrm{C}$ & [1979MAJ/SVO] \\
\hline & $\mathrm{V}$ & & $28.8 \pm 0.1$ & 324 & $\mathrm{C}$ & [1949WAD/SMI] \\
\hline & $\mathrm{V}$ & & $28.1 \pm 0.1$ & 336 & $\mathrm{C}$ & [1949WAD/SMI] \\
\hline & $\mathrm{V}$ & & $30.3 \pm 0.1$ & 298 & $\mathrm{C}$ & [1947OSB/GIN] \\
\hline & $\mathrm{V}$ & $(288-337)$ & 30.2 & 303 & MM & [1945WIL/TAY] \\
\hline \multirow[t]{12}{*}{$\mathrm{C}_{6} \mathrm{H}_{14}$} & [79-29-8] & 2,3-dimethylbutane & & & & \\
\hline & TRS & & 2.37 & 107 & & \\
\hline & V & & 29.1 & 298 & & [1971WIL/ZWO] \\
\hline & V & & $29.2 \pm 0.1$ & 296 & $\mathrm{C}$ & [1949WAD/SMI] \\
\hline & $\mathrm{V}$ & & $28.9 \pm 0.1$ & 303 & $\mathrm{C}$ & [1949WAD/SMI] \\
\hline & $\mathrm{V}$ & & $28.3 \pm 0.1$ & 313 & $\mathrm{C}$ & [1949WAD/SMI] \\
\hline & $\mathrm{V}$ & & $27.3 \pm 0.1$ & 331 & $\mathrm{C}$ & [1949WAD/SMI] \\
\hline & V & $(287-332)$ & 29.6 & 302 & MM & [1945WIL/TAY] \\
\hline & V & & $29.2 \pm 0.1$ & 293 & $\mathrm{C}$ & [1943LEM/FEL] \\
\hline & $\mathrm{V}$ & & $28.2 \pm 0.1$ & 313 & $\mathrm{C}$ & [1943LEM/FEL] \\
\hline & V & & $27.0 \pm 0.1$ & 333 & $\mathrm{C}$ & [1943LEM/FEL] \\
\hline & $\mathrm{V}$ & & $26.1 \pm 0.1$ & 353 & $\mathrm{C}$ & [1943LEM/FEL] \\
\hline \multirow[t]{7}{*}{$\mathrm{C}_{6} \mathrm{H}_{14}$} & {$[75-83-2]$} & 2,2-dimethylbutane & & & & \\
\hline & $\mathrm{V}$ & & 27.7 & 298 & & [1971WIL/ZWO] \\
\hline & $\mathrm{V}$ & $(273-318)$ & 28.7 & 288 & & [1949NIC/LAF, 1984BOU/FRI] \\
\hline & $\mathrm{V}$ & & $27.8 \pm 0.1$ & 296 & $\mathrm{C}$ & [1947WAD/DOU] \\
\hline & $\mathrm{V}$ & & $26.3 \pm 0.1$ & 323 & $\mathrm{C}$ & [1947WAD/DOU] \\
\hline & $\mathrm{V}$ & $(211-289)$ & 29.2 & 274 & & [1946KIL/PIT] \\
\hline & $\mathrm{V}$ & $(288-323)$ & 28.3 & 303 & MM & [1945WIL/TAY] \\
\hline \multirow[t]{2}{*}{$\mathrm{C}_{6} \mathrm{H}_{14} \mathrm{FO}_{3} \mathrm{P}$} & [55-91-4] & fluorophosphoric acic & , diisopropyl ester & & & \\
\hline & $\mathrm{V}$ & $(273-348)$ & 29.4 & 288 & A & [1987STE/MAL] \\
\hline \multirow[t]{4}{*}{$\mathrm{C}_{6} \mathrm{H}_{14} \mathrm{~N}_{2}$} & {$[821-67-0]$} & dipropyldiazene & & & & \\
\hline & $\mathrm{V}$ & & $39.9 \pm 0.4$ & 298 & $\mathrm{C}$ & [1976ENG/MEL] \\
\hline & $\mathrm{V}$ & $(295-305)$ & 39.5 & 300 & UV & [1974ENG/WOO] \\
\hline & $\mathrm{V}$ & & 41.1 & & & [1968BAC/NOV, 1974ENG/WOO] \\
\hline \multirow[t]{5}{*}{$\mathrm{C}_{6} \mathrm{H}_{14} \mathrm{~N}_{2}$} & [3880-49-7] & diisopropyldiazene & & & & \\
\hline & $\mathrm{V}$ & & $35.9 \pm 0.4$ & 298 & $\mathrm{C}$ & [1976ENG/MEL] \\
\hline & $\mathrm{V}$ & $(296-308)$ & 36.1 & 302 & UV & [1974ENG/WOO] \\
\hline & $\mathrm{V}$ & & 37.7 & & $\mathrm{I}$ & [1974ENG/WOO] \\
\hline & $\mathrm{V}$ & & 34.9 & & & [1968GEI/HOF, 1974ENG/WOO] \\
\hline \multirow[t]{2}{*}{$\mathrm{C}_{6} \mathrm{H}_{14} \mathrm{~N}_{2}$} & [3114-70-3] & 1,4-diaminocyclohex & ane & & & \\
\hline & $\mathrm{V}$ & $(383-473)$ & 48.2 & 398 & A & [1987STE/MAL] \\
\hline \multirow[t]{2}{*}{$\mathrm{C}_{6} \mathrm{H}_{14} \mathrm{~N}_{2}$} & [1436-59-5] & cis-1,2-cyclohexaned & lamine & & & \\
\hline & $\mathrm{V}$ & & $62.2 \pm 1.0$ & 298 & ME & [2007TOM/ROS] \\
\hline
\end{tabular}


TABLE 9. Phase change enthalpies of $\mathrm{C}_{6}$ organic compounds-Continued

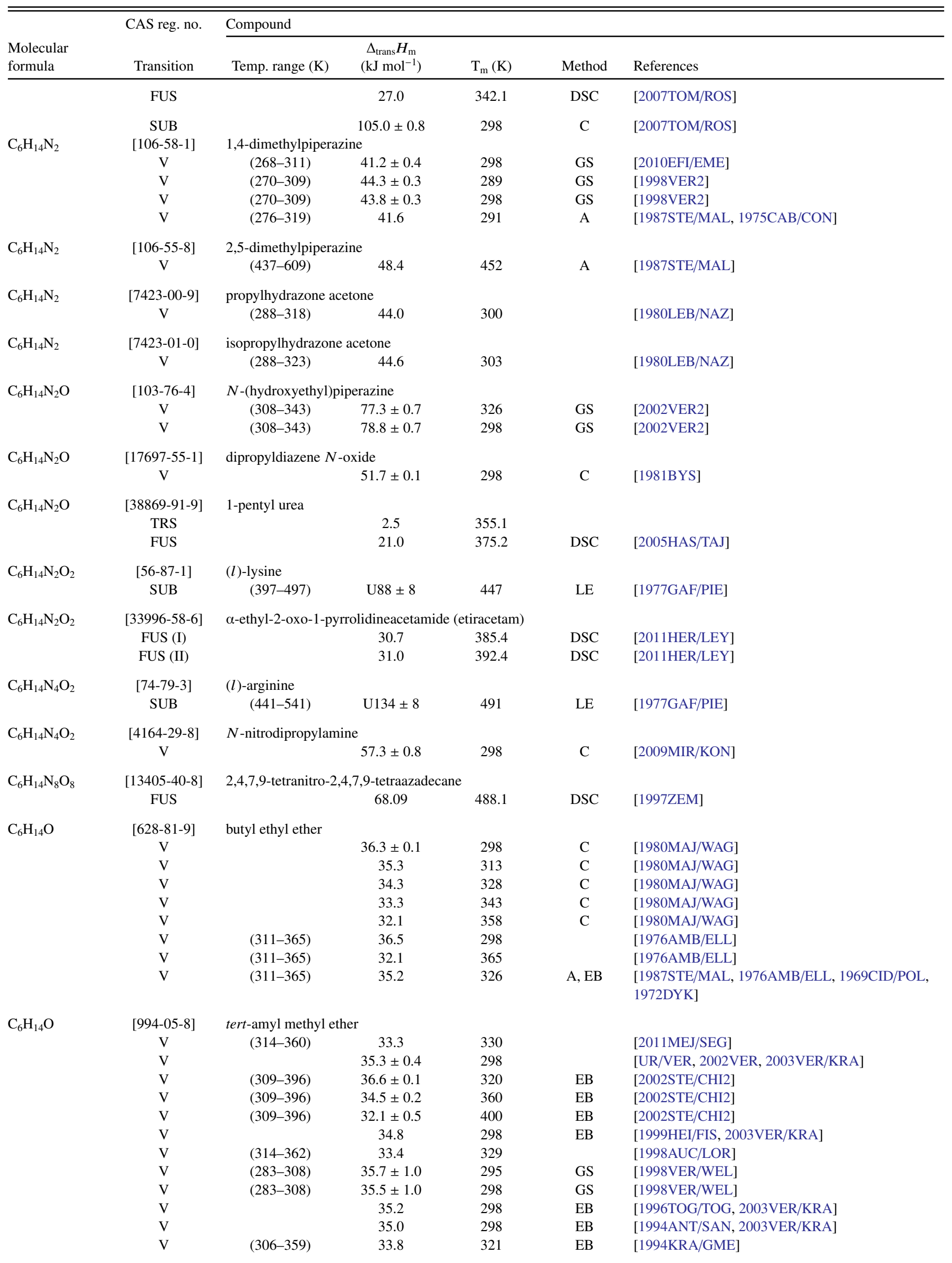


TABLE 9. Phase change enthalpies of $\mathrm{C}_{6}$ organic compounds-Continued

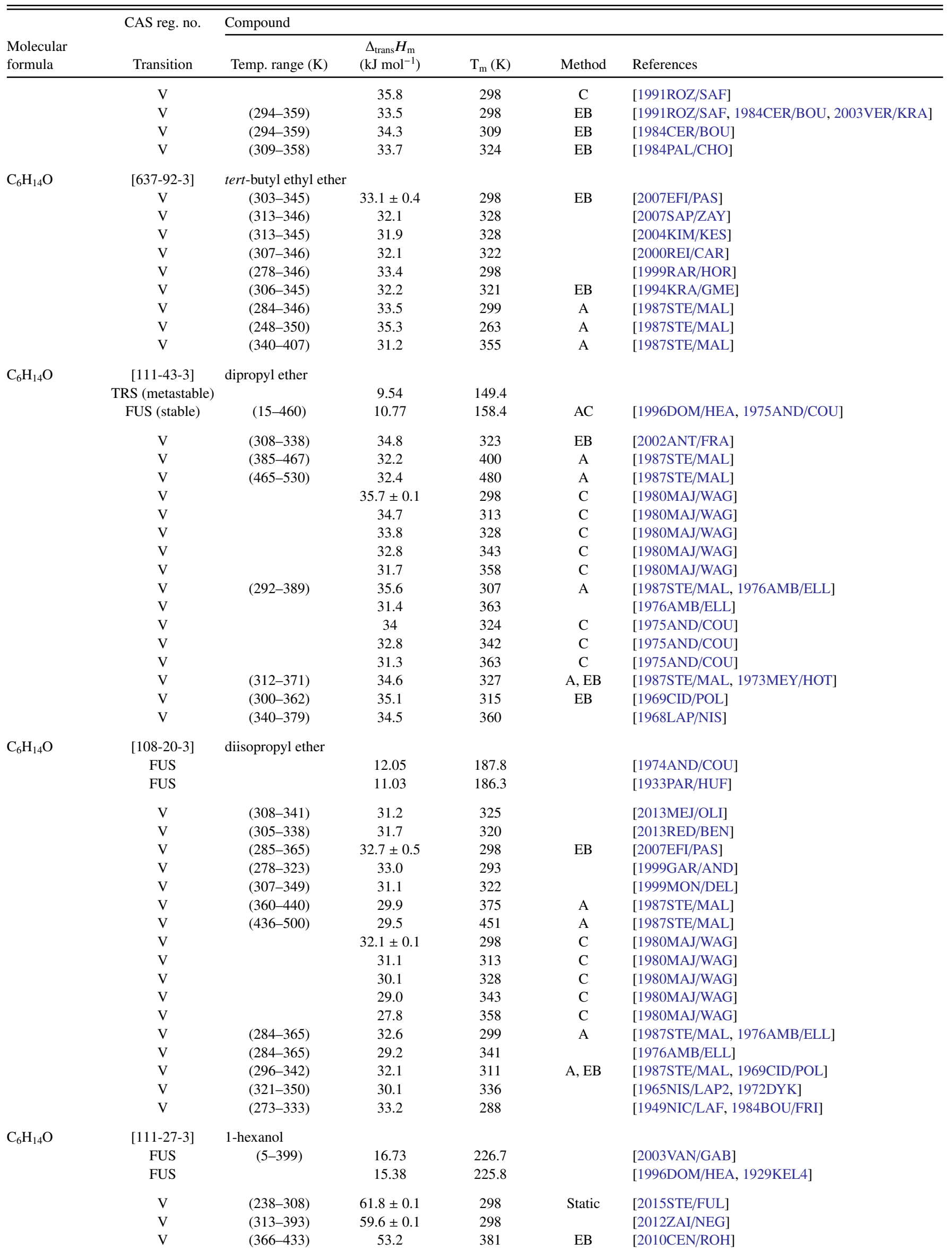


TABLE 9. Phase change enthalpies of $\mathrm{C}_{6}$ organic compounds-Continued

\begin{tabular}{|c|c|c|c|c|c|c|}
\hline \multirow[b]{2}{*}{$\begin{array}{l}\text { Molecular } \\
\text { formula }\end{array}$} & \multirow{2}{*}{$\begin{array}{l}\text { CAS reg. no. } \\
\text { Transition }\end{array}$} & \multicolumn{5}{|l|}{ Compound } \\
\hline & & Temp. range $(\mathrm{K})$ & $\begin{array}{c}\Delta_{\text {trans }} H_{\mathrm{m}} \\
\left(\mathrm{kJ} \mathrm{mol}^{-1}\right)\end{array}$ & $\mathrm{T}_{\mathrm{m}}(\mathrm{K})$ & Method & References \\
\hline & $\mathrm{V}$ & $(344-384)$ & 59.7 & 359 & $\mathrm{~EB}$ & [2009GIE/KOS] \\
\hline & $\mathrm{V}$ & $(328-423)$ & 59.9 & 298 & & [2006NAS/NEU] \\
\hline & $\mathrm{V}$ & $(265-363)$ & $61.7 \pm 0.3$ & 298 & GS & [2005ROG/PIS] \\
\hline & $\mathrm{V}$ & $(370-416)$ & 51.4 & 385 & $\mathrm{~EB}$ & [2004TAN/LI] \\
\hline & $\mathrm{V}$ & $(265-328)$ & 62.0 & 288 & GS & [2001KUL/VER2] \\
\hline & $\mathrm{V}$ & $(265-328)$ & 61.1 & 298 & GS & [2001KUL/VER2] \\
\hline & $\mathrm{V}$ & $(268-333)$ & $61.9 \pm 0.2$ & 301 & GS & [1998VER3] \\
\hline & $\mathrm{V}$ & $(268-333)$ & $62.1 \pm 0.2$ & 298 & GS & [1998VER3] \\
\hline & $\mathrm{V}$ & $(373-423)$ & 61.5 & 298 & CGC & [1995CHI/HOS] \\
\hline & $\mathrm{V}$ & $(323-373)$ & 61.6 & 298 & CGC & [1995CHI/HOS] \\
\hline & $\mathrm{V}$ & $(253-338)$ & 61.2 & 296 & & [1992NGU/KAS] \\
\hline & $\mathrm{V}$ & $(298-343)$ & 57.7 & 313 & A & [1987STE/MAL] \\
\hline & $\mathrm{V}$ & $(380-417)$ & 47.9 & 395 & $\mathrm{~EB}$ & [1985RED/RAO] \\
\hline & $\mathrm{V}$ & & $58.5 \pm 0.2$ & 328 & $\mathrm{C}$ & [1985MAJ/SVO3] \\
\hline & $\mathrm{V}$ & & $57.6 \pm 0.2$ & 343 & $\mathrm{C}$ & [1985MAJ/SVO3] \\
\hline & $\mathrm{V}$ & & $55.2 \pm 0.2$ & 358 & $\mathrm{C}$ & [1985MAJ/SVO3] \\
\hline & $\mathrm{V}$ & & $53.8 \pm 0.2$ & 368 & $\mathrm{C}$ & [1985MAJ/SVO3] \\
\hline & $\mathrm{V}$ & $(243-303)$ & 59.1 & 298 & & [1983SCH/STR] \\
\hline & $\mathrm{V}$ & & $60.8 \pm 0.2$ & 298 & $\mathrm{C}$ & [1977MAN/SEL] \\
\hline & $\mathrm{V}$ & $(308-430)$ & 57.9 & 323 & & [1973WIL/ZWO] \\
\hline & $\mathrm{V}$ & $(325-431)$ & 58.5 & 340 & DTA & [1987STE/MAL, 1969KEM/KRE] \\
\hline & $\mathrm{V}$ & & & & & [1972DYK] \\
\hline & $\mathrm{V}$ & & $61.6 \pm 0.2$ & 298 & $\mathrm{C}$ & [1966WAD] \\
\hline & $\mathrm{V}$ & $(334-381)$ & 56.0 & 349 & & [1961ROS/SUP] \\
\hline & $\mathrm{V}$ & $(308-428)$ & U55.8 & 323 & $\mathrm{I}$ & [1938HOV/LAN] \\
\hline & $\mathrm{V}$ & $(333-425)$ & 57.9 & 348 & & [1935BUT/RAM, 1984BOU/FRI] \\
\hline \multirow[t]{14}{*}{$\mathrm{C}_{6} \mathrm{H}_{14} \mathrm{O}$} & {$[626-93-7]$} & $(d l)$-2-hexanol & & & & \\
\hline & V & $(274-309)$ & $57.0 \pm 0.2$ & 298 & GS & [2005ROG/PIS] \\
\hline & $\mathrm{V}$ & $(274-309)$ & $58.3 \pm 0.3$ & 298 & GS & [2001KUL/VER] \\
\hline & $\mathrm{V}$ & $(224-323)$ & 61.8 & 239 & & [1999NGU/BER] \\
\hline & $\mathrm{V}$ & $(360-415)$ & 48.7 & 375 & A & [1987STE/MAL] \\
\hline & $\mathrm{V}$ & & $56.8 \pm 0.2$ & 313 & $\mathrm{C}$ & [1985MAJ/SVO2] \\
\hline & $\mathrm{V}$ & & $55.0 \pm 0.2$ & 328 & $\mathrm{C}$ & [1985MAJ/SVO2] \\
\hline & $\mathrm{V}$ & & $53.0 \pm 0.2$ & 343 & $\mathrm{C}$ & [1985MAJ/SVO2] \\
\hline & $\mathrm{V}$ & & $50.7 \pm 0.2$ & 358 & $\mathrm{C}$ & [1985MAJ/SVO2] \\
\hline & $\mathrm{V}$ & & $49.2 \pm 0.2$ & 368 & $\mathrm{C}$ & [1985MAJ/SVO2] \\
\hline & $\mathrm{V}$ & $(337-413)$ & 52.4 & 352 & & [1984SAC/MAR] \\
\hline & $\mathrm{V}$ & $(351-412)$ & 47.8 & 366 & A & [1987STE/MAL, 1975BRA/AND] \\
\hline & $\mathrm{V}$ & $(301-415)$ & 53.1 & 316 & & [1973WIL/ZWO] \\
\hline & $\mathrm{V}$ & $(298-413)$ & 49.7 & 356 & I & {$[1938 \mathrm{HOV} / \mathrm{LAN}]$} \\
\hline \multirow[t]{8}{*}{$\mathrm{C}_{6} \mathrm{H}_{14} \mathrm{O}$} & {$[623-37-0]$} & $(d l)$-3-hexanol & & & & \\
\hline & $\mathrm{V}$ & $(278-311)$ & $58.6 \pm 0.4$ & 298 & GS & [2001KUL/VER] \\
\hline & $\mathrm{V}$ & $(244-318)$ & U50.7 & 259 & & [1999NGU/BER] \\
\hline & $\mathrm{V}$ & $(354-410)$ & 46.1 & 369 & A & [1987STE/MAL] \\
\hline & $\mathrm{V}$ & $(280-320)$ & 57.5 & 295 & A & [1987STE/MAL] \\
\hline & $\mathrm{V}$ & $(333-409)$ & 51.5 & 348 & & [1984SAC/MAR] \\
\hline & $\mathrm{V}$ & $(280-316)$ & 57.4 & 295 & & [1975CAB/CON2] \\
\hline & V & $(298-408)$ & 46.4 & 353 & I & [1938HOV/LAN] \\
\hline \multirow[t]{10}{*}{$\mathrm{C}_{6} \mathrm{H}_{14} \mathrm{O}$} & {$[105-30-6]$} & \multicolumn{5}{|c|}{$(d l)$-2-methyl-1-pentanol } \\
\hline & $\mathrm{V}$ & $(275-313)$ & $59.4 \pm 0.3$ & 298 & GS & [2001KUL/VER] \\
\hline & $\mathrm{V}$ & $(367-423)$ & 49.3 & 382 & A & [1987STE/MAL] \\
\hline & $\mathrm{V}$ & $(261-294)$ & 64.9 & 279 & A & [1987STE/MAL, 1979THO/MEA] \\
\hline & $\mathrm{V}$ & & $57.4 \pm 0.2$ & 328 & $\mathrm{C}$ & [1985MAJ/SVO2] \\
\hline & $\mathrm{V}$ & & $55.7 \pm 0.2$ & 343 & $\mathrm{C}$ & [1985MAJ/SVO2] \\
\hline & $\mathrm{V}$ & & $53.9 \pm 0.2$ & 358 & $\mathrm{C}$ & [1985MAJ/SVO2] \\
\hline & $\mathrm{V}$ & & $52.7 \pm 0.2$ & 368 & $\mathrm{C}$ & [1985MAJ/SVO2] \\
\hline & $\mathrm{V}$ & $(298-423)$ & 54.2 & 313 & & [1973WIL/ZWO] \\
\hline & $\mathrm{V}$ & $(298-413)$ & 50.2 & 356 & I & {$[1938 \mathrm{HOV} / \mathrm{LAN}]$} \\
\hline \multirow[t]{3}{*}{$\mathrm{C}_{6} \mathrm{H}_{14} \mathrm{O}$} & {$[589-35-5]$} & \multicolumn{5}{|c|}{$(d l)$-3-methyl-1-pentanol } \\
\hline & $\mathrm{V}$ & $(280-316)$ & $61.7 \pm 0.3$ & 298 & GS & [2001KUL/VER] \\
\hline & $\mathrm{V}$ & $(328-427)$ & 54.8 & 343 & A & [1987STE/MAL] \\
\hline
\end{tabular}


TABLE 9. Phase change enthalpies of $\mathrm{C}_{6}$ organic compounds-Continued

\begin{tabular}{|c|c|c|c|c|c|c|}
\hline \multirow[b]{2}{*}{$\begin{array}{l}\text { Molecular } \\
\text { formula }\end{array}$} & \multirow{2}{*}{$\begin{array}{l}\text { CAS reg. no. } \\
\text { Transition }\end{array}$} & \multicolumn{5}{|l|}{ Compound } \\
\hline & & Temp. range $(\mathrm{K})$ & $\begin{array}{c}\Delta_{\text {trans }} H_{\mathrm{m}} \\
\left(\mathrm{kJ} \mathrm{mol}^{-1}\right)\end{array}$ & $\mathrm{T}_{\mathrm{m}}(\mathrm{K})$ & Method & References \\
\hline & $\mathrm{V}$ & $(298-423)$ & 47.2 & 360 & I & [1940HOV/LAN2] \\
\hline \multirow[t]{3}{*}{$\mathrm{C}_{6} \mathrm{H}_{14} \mathrm{O}$} & [626-89-1] & 4-methyl-1-pentanol & & & & \\
\hline & $\mathrm{V}$ & $(357-427)$ & 53.0 & 372 & A & [1987STE/MAL] \\
\hline & $\mathrm{V}$ & $(298-423)$ & 46.5 & 360 & I & [1940HOV/LAN2] \\
\hline \multirow[t]{8}{*}{$\mathrm{C}_{6} \mathrm{H}_{14} \mathrm{O}$} & [590-36-3] & 2-methyl-2-pentanol & & & & \\
\hline & $\mathrm{V}$ & $(341-396)$ & 44.2 & 356 & A & [1987STE/MAL] \\
\hline & $\mathrm{V}$ & $(330-397)$ & 48.9 & 345 & A & [1987STE/MAL] \\
\hline & $\mathrm{V}$ & & $54.7 \pm 0.2$ & 298 & $\mathrm{C}$ & [1985MAJ/SVO2] \\
\hline & $\mathrm{V}$ & & $44.4 \pm 0.2$ & 368 & $\mathrm{C}$ & [1985MAJ/SVO2] \\
\hline & $\mathrm{V}$ & $(288-396)$ & 58.3 & 303 & & [1973WIL/ZWO] \\
\hline & V & $(268-394)$ & 49.1 & 283 & & [1947STU] \\
\hline & $\mathrm{V}$ & $(288-396)$ & 51.3 & 303 & I & [1933HOR/LAN] \\
\hline \multirow[t]{5}{*}{$\mathrm{C}_{6} \mathrm{H}_{14} \mathrm{O}$} & [565-60-6] & $(d l)$-3-methyl-2-pent & nol & & & \\
\hline & $\mathrm{V}$ & $(275-310)$ & $58.2 \pm 0.3$ & 298 & GS & [2001KUL/VER] \\
\hline & $\mathrm{V}$ & $(314-409)$ & 54.4 & 329 & A & [1987STE/MAL] \\
\hline & V & $(255-295)$ & 60.4 & 280 & $\mathrm{~A}$ & [1987STE/MAL, 1979THO/MEA] \\
\hline & $\mathrm{V}$ & (296-408) & 54.8 & 311 & & [1973WIL/ZWO] \\
\hline \multirow[t]{2}{*}{$\mathrm{C}_{6} \mathrm{H}_{14} \mathrm{O}$} & [108-11-2] & $(d l)$-4-methyl-2-pent & nol & & & \\
\hline & $\mathrm{V}$ & $(274-301)$ & $57.3 \pm 0.3$ & 298 & GS & [2001KUL/VER] \\
\hline \multirow{2}{*}{$\mathrm{C}_{6} \mathrm{H}_{14} \mathrm{O}$} & $\mathrm{V}$ & $(298-401)$ & 52.0 & 313 & & {$[1973$ WIL/ZWO] } \\
\hline & $\mathrm{V}$ & $(298-399)$ & 44.4 & 349 & I & [1940HOV/LAN] \\
\hline \multirow[t]{6}{*}{$\mathrm{C}_{6} \mathrm{H}_{14} \mathrm{O}$} & {$[77-74-7]$} & 3-methyl-3-pentanol & & & & \\
\hline & $\mathrm{V}$ & $(275-301)$ & $55.7 \pm 0.3$ & 298 & GS & [2001KUL/VER] \\
\hline & $\mathrm{V}$ & & $56.7 \pm 0.8$ & 298 & $\mathrm{~EB}$ & [1991WIB/HAO] \\
\hline & $\mathrm{V}$ & $(322-397)$ & 40.1 & 337 & A & [1987STE/MAL] \\
\hline & $\mathrm{V}$ & $(338-396)$ & 46.4 & 353 & & [1973WIL/ZWO] \\
\hline & $\mathrm{V}$ & $(298-393)$ & 42.1 & 346 & I & [1940HOV/LAN] \\
\hline \multirow[t]{7}{*}{$\mathrm{C}_{6} \mathrm{H}_{14} \mathrm{O}$} & [1185-33-7] & 2,2-dimethyl-1-butan & & & & \\
\hline & TRS & & 5.77 & 194.5 & & \\
\hline & TRS & & 2.69 & 209.2 & & \\
\hline & FUS & & 4.42 & 233.6 & $\mathrm{AC}$ & [2013JUS/ZIE] \\
\hline & $\mathrm{V}$ & $(356-415)$ & 47.2 & 371 & A & [1987STE/MAL] \\
\hline & $\mathrm{V}$ & $(298-415)$ & 53.7 & 313 & & [1973WIL/ZWO] \\
\hline & $\mathrm{V}$ & $(298-408)$ & 52.1 & 313 & I & [1940HOV/LAN3] \\
\hline \multirow[t]{3}{*}{$\mathrm{C}_{6} \mathrm{H}_{14} \mathrm{O}$} & [19550-30-2] & $(d l)$-2,3-dimethyl-1-b & utanol & & & \\
\hline & $\mathrm{V}$ & $(324-431)$ & 51.4 & 339 & A & [1987STE/MAL] \\
\hline & $\mathrm{V}$ & $(373-422)$ & 49.6 & 388 & & [1973WIL/ZWO] \\
\hline \multirow[t]{3}{*}{$\mathrm{C}_{6} \mathrm{H}_{14} \mathrm{O}$} & [624-95-3] & 3,3-dimethyl-1-butan & & & & \\
\hline & FUS & $(13-302)$ & 9.54 & 235.7 & $\mathrm{AC}$ & [2004MAS/NAK] \\
\hline & $\mathrm{V}$ & $(276-312)$ & $58.0 \pm 0.2$ & 298 & GS & [2001KUL/VER] \\
\hline
\end{tabular}


TABLE 9. Phase change enthalpies of $\mathrm{C}_{6}$ organic compounds-Continued

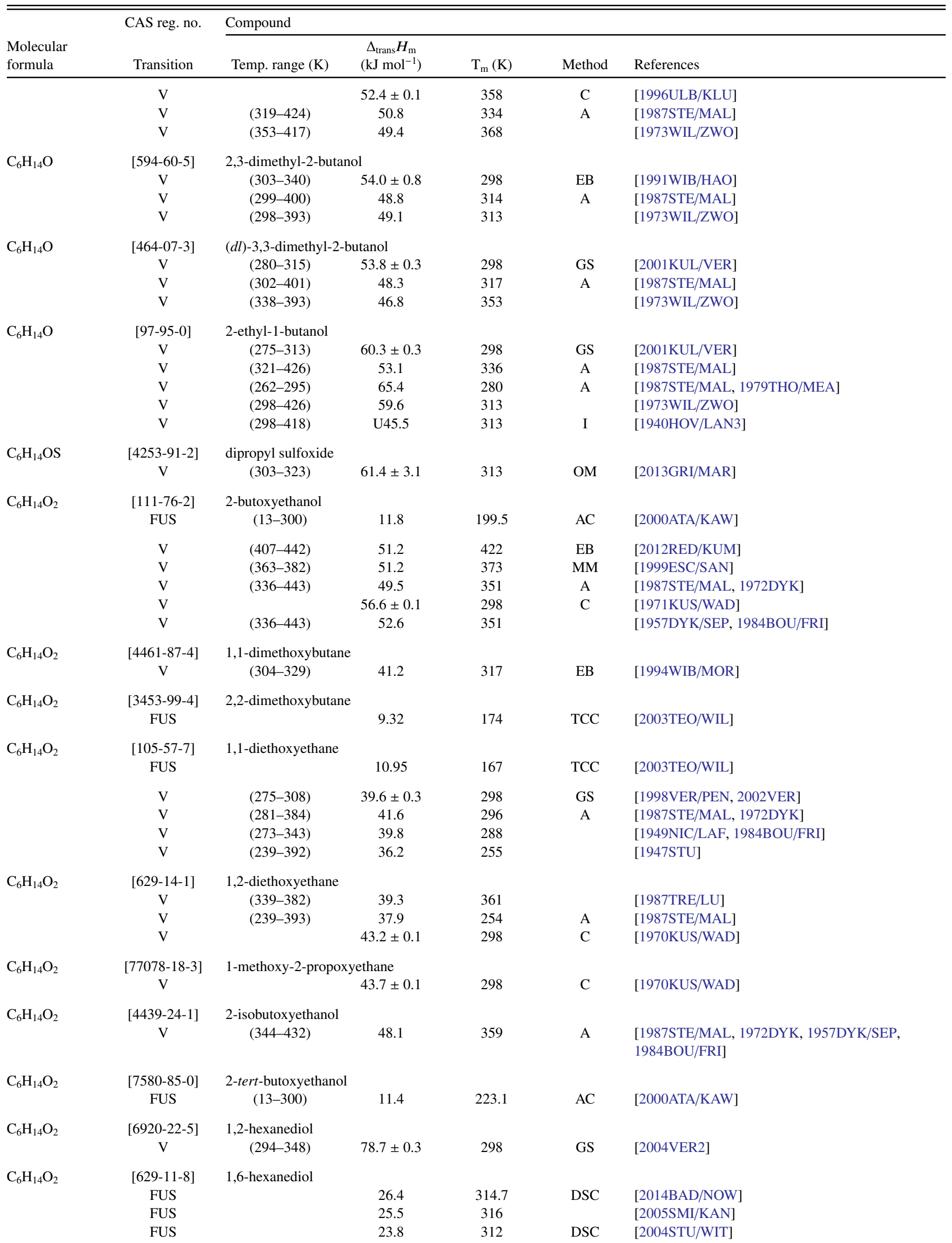


TABLE 9. Phase change enthalpies of $\mathrm{C}_{6}$ organic compounds-Continued

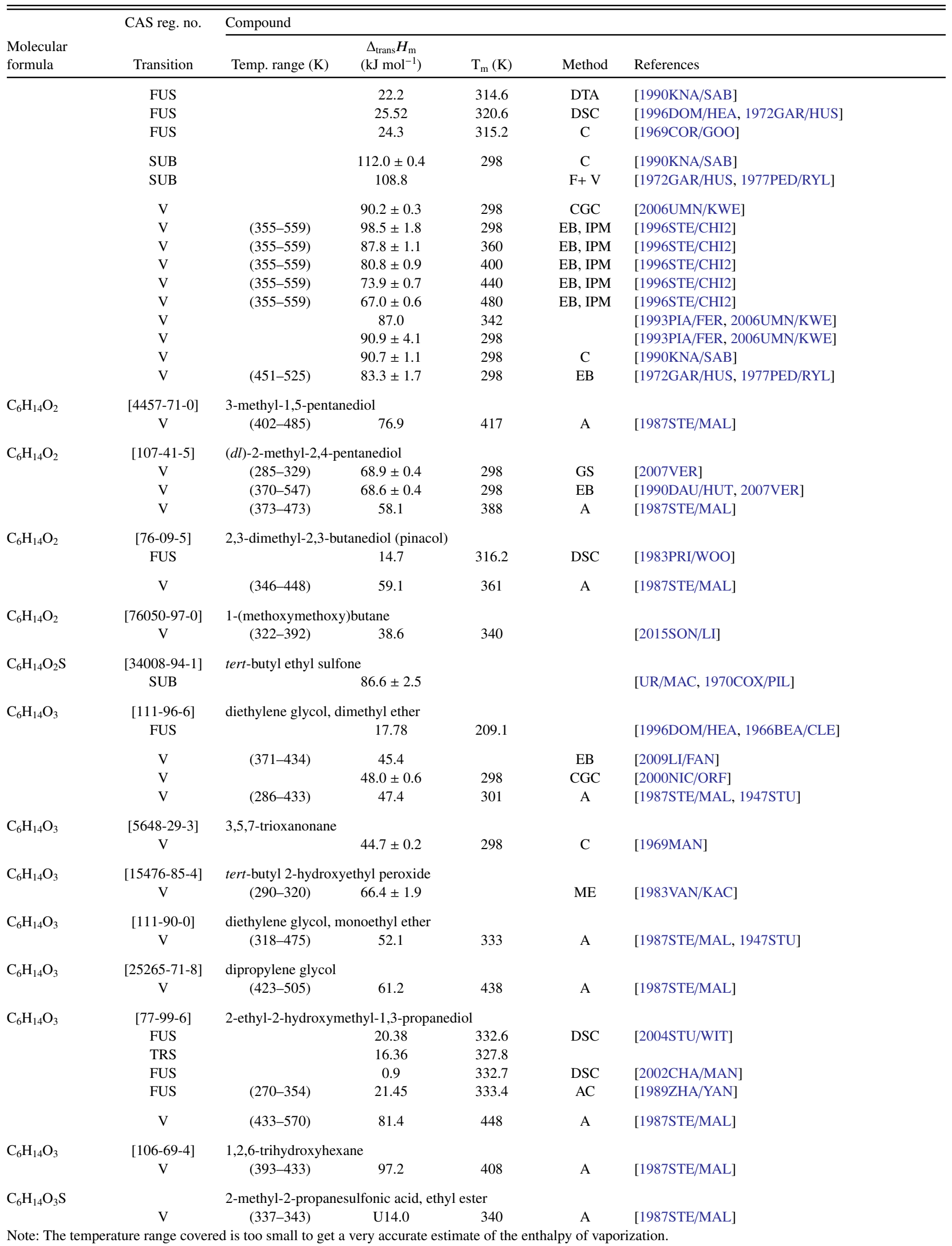

Note: The temperature range covered is too small to get a very accurate estimate of the enthalpy of vaporization. 
TABLE 9. Phase change enthalpies of $\mathrm{C}_{6}$ organic compounds-Continued

\begin{tabular}{|c|c|c|c|c|c|c|}
\hline \multirow[b]{2}{*}{$\begin{array}{l}\text { Molecular } \\
\text { formula }\end{array}$} & \multirow{2}{*}{$\begin{array}{l}\text { CAS reg. no. } \\
\text { Transition }\end{array}$} & \multicolumn{5}{|l|}{ Compound } \\
\hline & & Temp. range $(\mathrm{K})$ & $\begin{array}{c}\Delta_{\text {trans }} H_{\mathrm{m}} \\
\left(\mathrm{kJ} \mathrm{mol}^{-1}\right)\end{array}$ & $\mathrm{T}_{\mathrm{m}}(\mathrm{K})$ & Method & References \\
\hline \multirow[t]{2}{*}{$\mathrm{C}_{6} \mathrm{H}_{14} \mathrm{O}_{3} \mathrm{~S}$} & {$[623-98-3]$} & dipropyl sulfite & & & & \\
\hline & V & & $58.6 \pm 1.7$ & 298 & $\mathrm{BP}$ & [1969MAC/STE2] \\
\hline \multirow[t]{2}{*}{$\mathrm{C}_{6} \mathrm{H}_{14} \mathrm{O}_{4}$} & {$[2517-44-4]$} & \multicolumn{2}{|c|}{ 1,1,2,2-tetramethoxyethane } & & & \\
\hline & $\mathrm{V}$ & $(351-432)$ & 42.9 & 366 & A & [1987STE/MAL] \\
\hline \multirow[t]{9}{*}{$\mathrm{C}_{6} \mathrm{H}_{14} \mathrm{O}_{4}$} & [112-27-6] & triethylene glycol & & & & \\
\hline & V & $(442-562)$ & $72.2 \pm 0.3$ & 440 & $\mathrm{~EB}$ & [2002STE/CHI3] \\
\hline & $\mathrm{V}$ & $(442-562)$ & $68.5 \pm 0.3$ & 480 & $\mathrm{~EB}$ & [2002STE/CHI3] \\
\hline & $\mathrm{V}$ & $(442-562)$ & $64.6 \pm 0.3$ & 520 & $\mathrm{~EB}$ & [2002STE/CHI3] \\
\hline & $\mathrm{V}$ & $(442-562)$ & $60.8 \pm 0.5$ & 560 & $\mathrm{~EB}$ & [2002STE/CHI3] \\
\hline & $\mathrm{V}$ & $(278-323)$ & 77.0 & 300 & & [1972MCF/SOM] \\
\hline & $\mathrm{V}$ & $(288-303)$ & 67.7 & 295 & A & [1987STE/MAL, 1955ISH/MAT] \\
\hline & $\mathrm{V}$ & $(293-303)$ & 60.5 & 298 & & [1950WIS/PUC] \\
\hline & $\mathrm{V}$ & $(387-552)$ & 71.5 & 402 & A & [1987STE/MAL, 1947STU] \\
\hline \multirow[t]{2}{*}{$\mathrm{C}_{6} \mathrm{H}_{14} \mathrm{O}_{4} \mathrm{~S}$} & {$[598-05-0]$} & dipropyl sulfate & & & & \\
\hline & V & & $66.9 \pm 1.7$ & 298 & $\mathrm{BP}$ & [1969MAC/STE2] \\
\hline \multirow[t]{5}{*}{$\mathrm{C}_{6} \mathrm{H}_{14} \mathrm{O}_{6}$} & {$[608-66-2]$} & dulcitol & & & & \\
\hline & FUS & & 64.5 & 459.7 & DSC & [2015PAU/SHI] \\
\hline & FUS & & 65.1 & 460.3 & DSC & [1996DOM/HEA, 1990BAR/DEL] \\
\hline & SUB & & 205 & 298 & B & [1990BAR/DEL] \\
\hline & $\mathrm{V}$ & $(464-496)$ & $133.8 \pm 1.4$ & 482 & $\mathrm{TE}$ & [1990BAR/DEL] \\
\hline \multirow[t]{11}{*}{$\mathrm{C}_{6} \mathrm{H}_{14} \mathrm{O}_{6}$} & {$[69-65-8]$} & D-mannitol & & & & \\
\hline & FUS $(\beta)$ & & 44.8 & 440.2 & & \\
\hline & FUS ( $(\delta)$ & & 44.3 & 430.2 & DSC & [2013BAR/GIL] \\
\hline & FUS & & 59.5 & 441.0 & DSC & [2011KUM/VEI] \\
\hline & FUS & & 54.78 & 439.2 & DSC & [2011LEE/THO] \\
\hline & FUS & & 54.69 & 437.3 & DSC & [2010TON/LIU] \\
\hline & FUS & & 62.1 & 438.2 & DSC & [2008KAI/MAR] \\
\hline & FUS & & 61.57 & 438.2 & DSC & [2003GOM/SZA] \\
\hline & FUS & & 56.1 & 439.1 & DSC & [1996DOM/HEA, 1990BAR/DEL] \\
\hline & SUB & & 202 & 298 & B & [1990BAR/DEL] \\
\hline & $\mathrm{V}$ & $(458-501)$ & $135.6 \pm 1.1$ & 479 & $\mathrm{TE}$ & [1990BAR/DEL] \\
\hline \multirow[t]{11}{*}{$\mathrm{C}_{6} \mathrm{H}_{14} \mathrm{O}_{6}$} & {$[50-70-4]$} & (D)-sorbitol & & & & \\
\hline & FUS & & 35.9 & 372.0 & DSC & [2012JEG/PRA] \\
\hline & FUS & & 24.67 & 367.3 & DSC & [2012BIT/MEN] \\
\hline & FUS $(\alpha)$ & & 29.8 & 359.1 & DSC & [2009NEZ/AER] \\
\hline & FUS $(\beta)$ & & 31.7 & 371.2 & DSC & [2009NEZ/AER] \\
\hline & FUS & $(80-390)$ & 30.35 & 369.2 & $\mathrm{AC}$ & [2008TON/TAN2] \\
\hline & FUS & & $\mathrm{U} 20.0$ & 370.2 & DSC & [2008KAI/MAR] \\
\hline & FUS & & 39.53 & 370 & DSC & [2003GOM/SZA] \\
\hline & FUS & & 30.2 & 366.5 & DSC & [1996DOM/HEA, 1990BAR/DEL] \\
\hline & SUB & & 186 & 298 & $\mathrm{~B}$ & [1990BAR/DEL] \\
\hline & $\mathrm{V}$ & $(461-497)$ & $132.4 \pm 2.0$ & 477 & $\mathrm{TE}$ & [1990BAR/DEL] \\
\hline \multirow[t]{2}{*}{$\mathrm{C}_{6} \mathrm{H}_{14} \mathrm{O}_{6}$} & & (l)-iditol & & & & \\
\hline & FUS & & 30.9 & 352.8 & & [1993SIN/CAR] \\
\hline \multirow[t]{5}{*}{$\mathrm{C}_{6} \mathrm{H}_{14} \mathrm{~S}$} & [1741-83-9] & methyl pentyl sulfide & & & & \\
\hline & $\mathrm{V}$ & $(321-349)$ & 44.2 & 336 & & [1999DYK/SVO] \\
\hline & $\mathrm{V}$ & & 45.2 & 298 & & [1981SHI/SAI] \\
\hline & $\mathrm{V}$ & & $44.6 \pm 0.8$ & 298 & $\mathrm{GC}$ & [1964MAC/MCC] \\
\hline & $\mathrm{V}$ & $(321-350)$ & 42.6 & 308 & $\mathrm{~EB}$ & [1961NIC/KOB] \\
\hline \multirow[t]{4}{*}{$\mathrm{C}_{6} \mathrm{H}_{14} \mathrm{~S}$} & {$[638-46-0]$} & butyl ethyl sulfide & & & & \\
\hline & FUS & & 12.39 & 178.1 & & [1985DEA] \\
\hline & $\mathrm{V}$ & $(314-445)$ & 43.7 & 319 & & [1999DYK/SVO] \\
\hline & $\mathrm{V}$ & & 44.5 & 298 & & [1981SHI/SAI] \\
\hline
\end{tabular}


TABLE 9. Phase change enthalpies of $\mathrm{C}_{6}$ organic compounds-Continued

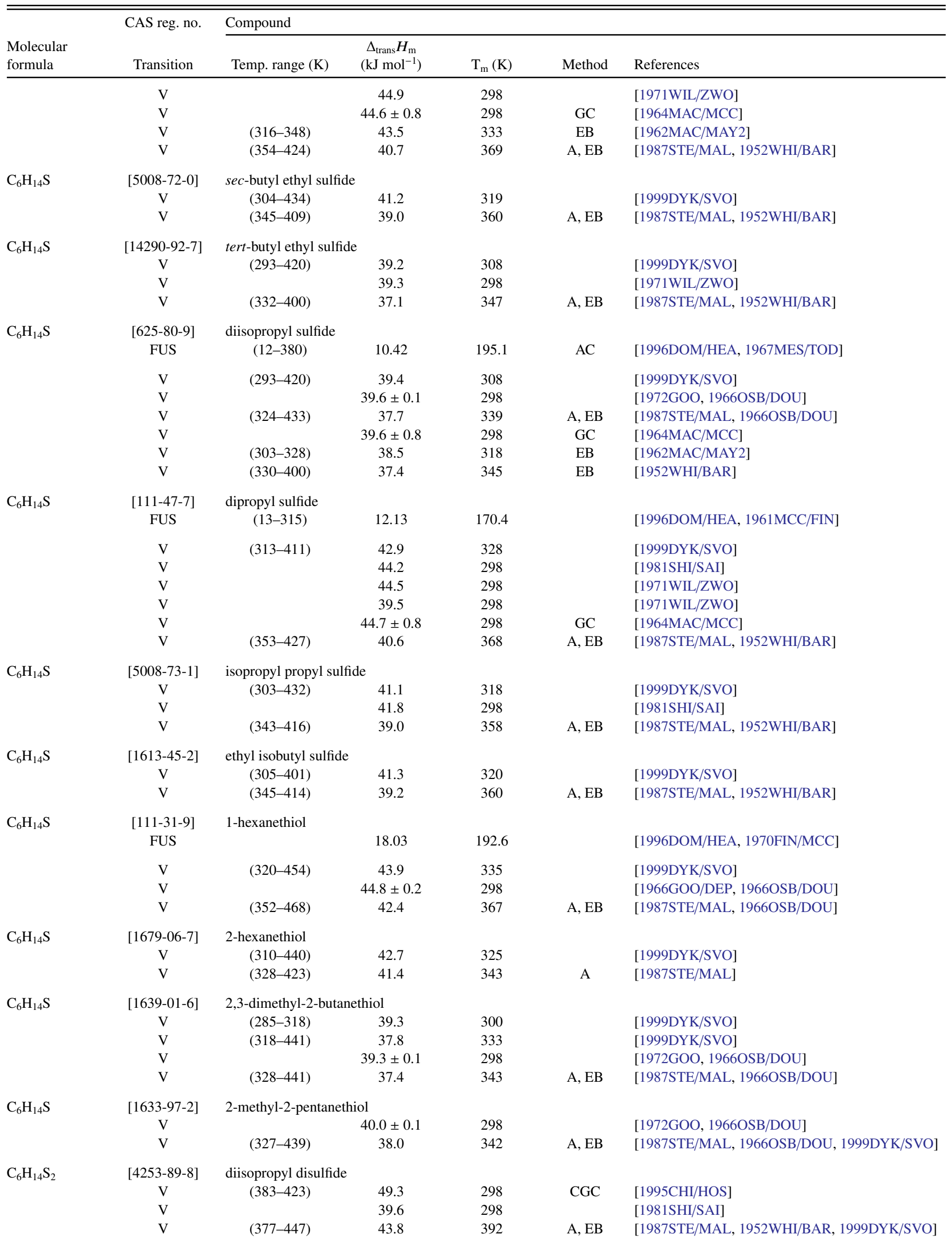


TABLE 9. Phase change enthalpies of $\mathrm{C}_{6}$ organic compounds-Continued

\begin{tabular}{|c|c|c|c|c|c|c|}
\hline \multirow[b]{2}{*}{$\begin{array}{l}\text { Molecular } \\
\text { formula }\end{array}$} & \multirow{2}{*}{$\begin{array}{l}\text { CAS reg. no. } \\
\text { Transition }\end{array}$} & \multicolumn{5}{|l|}{ Compound } \\
\hline & & Temp. range $(\mathrm{K})$ & $\begin{array}{c}\Delta_{\text {trans }} H_{\mathrm{m}} \\
\left(\mathrm{kJ} \mathrm{mol}^{-1}\right) \\
\end{array}$ & $\mathrm{T}_{\mathrm{m}}(\mathrm{K})$ & Method & References \\
\hline \multirow[t]{8}{*}{$\mathrm{C}_{6} \mathrm{H}_{14} \mathrm{~S}_{2}$} & {$[629-19-6]$} & dipropyl disulfide & & & & \\
\hline & FUS & & 13.81 & 187.7 & & [1996DOM/HEA, 1958HUB/DOU] \\
\hline & $\mathrm{V}$ & $(354-499)$ & 47.8 & 369 & & [1999DYK/SVO] \\
\hline & $\mathrm{V}$ & & $53.8 \pm 0.1$ & 298 & $\mathrm{C}$ & [1985KUS] \\
\hline & $\mathrm{V}$ & & 53.8 & 298 & & [1981SHI/SAI] \\
\hline & $\mathrm{V}$ & & $52.5 \pm 0.1$ & 298 & $\mathrm{C}$ & [1963MOR/SUN] \\
\hline & $\mathrm{V}$ & $(389-447)$ & 47.0 & 404 & $\mathrm{~A}, \mathrm{~EB}$ & [1987STE/MAL, 1958HUB/DOU, 1966OSB/DOU] \\
\hline & $\mathrm{V}$ & $(395-456)$ & 46.6 & 410 & $\mathrm{~EB}$ & {$[1952 \mathrm{WHI} / \mathrm{BAR}]$} \\
\hline \multirow{2}{*}{$\mathrm{C}_{6} \mathrm{H}_{14} \mathrm{~S}_{2}$} & {$[4151-69-3]$} & \multicolumn{5}{|c|}{ ethyl (1,1-dimethylethyl) disulfide } \\
\hline & V & $(373-461)$ & 43.4 & 388 & $\mathrm{~A}, \mathrm{~EB}$ & [1987STE/MAL, 1952WHI/BAR, 1999DYK/SVO] \\
\hline \multirow[t]{2}{*}{$\mathrm{C}_{6} \mathrm{H}_{14} \mathrm{~S}_{2}$} & {$[33672-51-4]$} & \multicolumn{5}{|c|}{ isopropyl propyl disulfide } \\
\hline & V & $(383-433)$ & 45.4 & 398 & A & [1987STE/MAL, 1999DYK/SVO] \\
\hline \multirow[t]{2}{*}{$\mathrm{C}_{6} \mathrm{H}_{14} \mathrm{~S}_{2}$} & {$[1191-43-1]$} & 1,6-hexanedithiol & & & & \\
\hline & $\mathrm{V}$ & $(379-511)$ & 55.7 & 394 & A & [1987STE/MAL, 1999DYK/SVO] \\
\hline \multirow[t]{2}{*}{$\mathrm{C}_{6} \mathrm{H}_{14} \mathrm{~S}_{2}$} & {$[5395-75-5]$} & 3,6-dithiaoctane & & & & \\
\hline & $\mathrm{V}$ & & $59.5 \pm 0.1$ & 298 & $\mathrm{C}$ & [1974MAN4] \\
\hline \multirow[t]{3}{*}{$\mathrm{C}_{6} \mathrm{H}_{14} \mathrm{~S}_{3}$} & {$[37460-04-1]$} & \multicolumn{5}{|c|}{ 2,5,8-trithianonane (trithiodiethylene glycol, dimethyl ether) } \\
\hline & $\mathrm{V}$ & $(391-533)$ & 116.4 & 406 & & [1999DYK/SVO] \\
\hline & $\mathrm{V}$ & $(391-418)$ & 103.7 & 404 & A & {$[1987 \mathrm{STE} / \mathrm{MAL}]$} \\
\hline \multirow[t]{5}{*}{$\mathrm{C}_{6} \mathrm{H}_{15} \mathrm{~N}$} & {$[111-26-2]$} & hexylamine & & & & \\
\hline & FUS & & 25.04 & 252.2 & DSC & [2005DOM/MAR] \\
\hline & $\mathrm{V}$ & $(323-373)$ & 45.0 & 298 & CGC & [1995CHI/HOS] \\
\hline & $\mathrm{V}$ & $(303-406)$ & 42.2 & 318 & A & [1987STE/MAL] \\
\hline & $\mathrm{V}$ & & $45.1 \pm 0.1$ & 298 & $\mathrm{C}$ & [1969WAD] \\
\hline \multirow[t]{2}{*}{$\mathrm{C}_{6} \mathrm{H}_{15} \mathrm{~N}$} & {$[21035-44-9]$} & \multicolumn{5}{|c|}{$(d l)$-sec-butyl ethyl amine } \\
\hline & V & $(283-372)$ & 37.9 & 298 & A & [1987STE/MAL, 1972DYK] \\
\hline \multirow[t]{12}{*}{$\mathrm{C}_{6} \mathrm{H}_{15} \mathrm{~N}$} & {$[108-18-9]$} & diisopropylamine & & & & \\
\hline & $\mathrm{V}$ & $(260-412)$ & 35.4 & 275 & A & [1987STE/MAL] \\
\hline & $\mathrm{V}$ & $(273-367)$ & 35.6 & 288 & A & [1987STE/MAL] \\
\hline & $\mathrm{V}$ & & $34.6 \pm 0.1$ & 298 & $\mathrm{C}$ & [1979PET/MAJ] \\
\hline & $\mathrm{V}$ & & $33.7 \pm 0.1$ & 313 & $\mathrm{C}$ & [1979PET/MAJ] \\
\hline & $\mathrm{V}$ & & $32.6 \pm 0.1$ & 328 & $\mathrm{C}$ & [1979PET/MAJ] \\
\hline & $\mathrm{V}$ & & $31.5 \pm 0.1$ & 343 & $\mathrm{C}$ & [1979PET/MAJ] \\
\hline & $\mathrm{V}$ & & $30.2 \pm 0.1$ & 358 & $\mathrm{C}$ & [1979PET/MAJ] \\
\hline & $\mathrm{V}$ & $(300-356)$ & 34.4 & 315 & EB & [1979PET/MAJ] \\
\hline & $\mathrm{V}$ & $(291-305)$ & 34.6 & 298 & & [1971LEB/KAT2] \\
\hline & $\mathrm{V}$ & & $34.5 \pm 0.1$ & 298 & $\mathrm{C}$ & [1969WAD] \\
\hline & $\mathrm{V}$ & $(273-333)$ & $33.8 \pm 0.2$ & 298 & I & [1969FRA/WAT] \\
\hline \multirow[t]{7}{*}{$\mathrm{C}_{6} \mathrm{H}_{15} \mathrm{~N}$} & {$[21968-17-2]$} & $N$-isopropyl propyl & nine & & & \\
\hline & $\mathrm{V}$ & & $37.3 \pm 0.1$ & 298 & $\mathrm{C}$ & [1979PET/MAJ] \\
\hline & $\mathrm{V}$ & & $36.2 \pm 0.1$ & 313 & $\mathrm{C}$ & [1979PET/MAJ] \\
\hline & $\mathrm{V}$ & & $35.2 \pm 0.1$ & 328 & $\mathrm{C}$ & [1979PET/MAJ] \\
\hline & $\mathrm{V}$ & & $34.1 \pm 0.1$ & 343 & $\mathrm{C}$ & [1979PET/MAJ] \\
\hline & $\mathrm{V}$ & & $33.0 \pm 0.1$ & 358 & $\mathrm{C}$ & [1979PET/MAJ] \\
\hline & $\mathrm{V}$ & $(312-369)$ & 36.2 & 327 & EB & [1979PET/MAJ] \\
\hline \multirow[t]{8}{*}{$\mathrm{C}_{6} \mathrm{H}_{15} \mathrm{~N}$} & {$[13360-63-9]$} & $N$-butylethylamine & & & & \\
\hline & $\mathrm{V}$ & & $40.2 \pm 0.1$ & 298 & $\mathrm{C}$ & [1979PET/MAJ] \\
\hline & $\mathrm{V}$ & & $39.1 \pm 0.1$ & 313 & $\mathrm{C}$ & [1979PET/MAJ] \\
\hline & V & & $38.0 \pm 0.1$ & 328 & $\mathrm{C}$ & [1979PET/MAJ] \\
\hline & $\mathrm{V}$ & & $36.9 \pm 0.1$ & 343 & $\mathrm{C}$ & [1979PET/MAJ] \\
\hline & $\mathrm{V}$ & & $35.8 \pm 0.1$ & 358 & $\mathrm{C}$ & [1979PET/MAJ] \\
\hline & $\mathrm{V}$ & $(313-375)$ & 39.9 & 328 & EB & [1979PET/MAJ] \\
\hline & $\mathrm{V}$ & $(283-382)$ & 41.4 & 298 & A & [1987STE/MAL, 1972DYK] \\
\hline $\mathrm{C}_{6} \mathrm{H}_{15} \mathrm{~N}$ & $\begin{array}{c}{[142-84-7]} \\
V\end{array}$ & $\begin{array}{r}\text { dipropylamine } \\
(321-382)\end{array}$ & 40.0 & 336 & & [2000RES/GON] \\
\hline
\end{tabular}


TABLE 9. Phase change enthalpies of $\mathrm{C}_{6}$ organic compounds-Continued

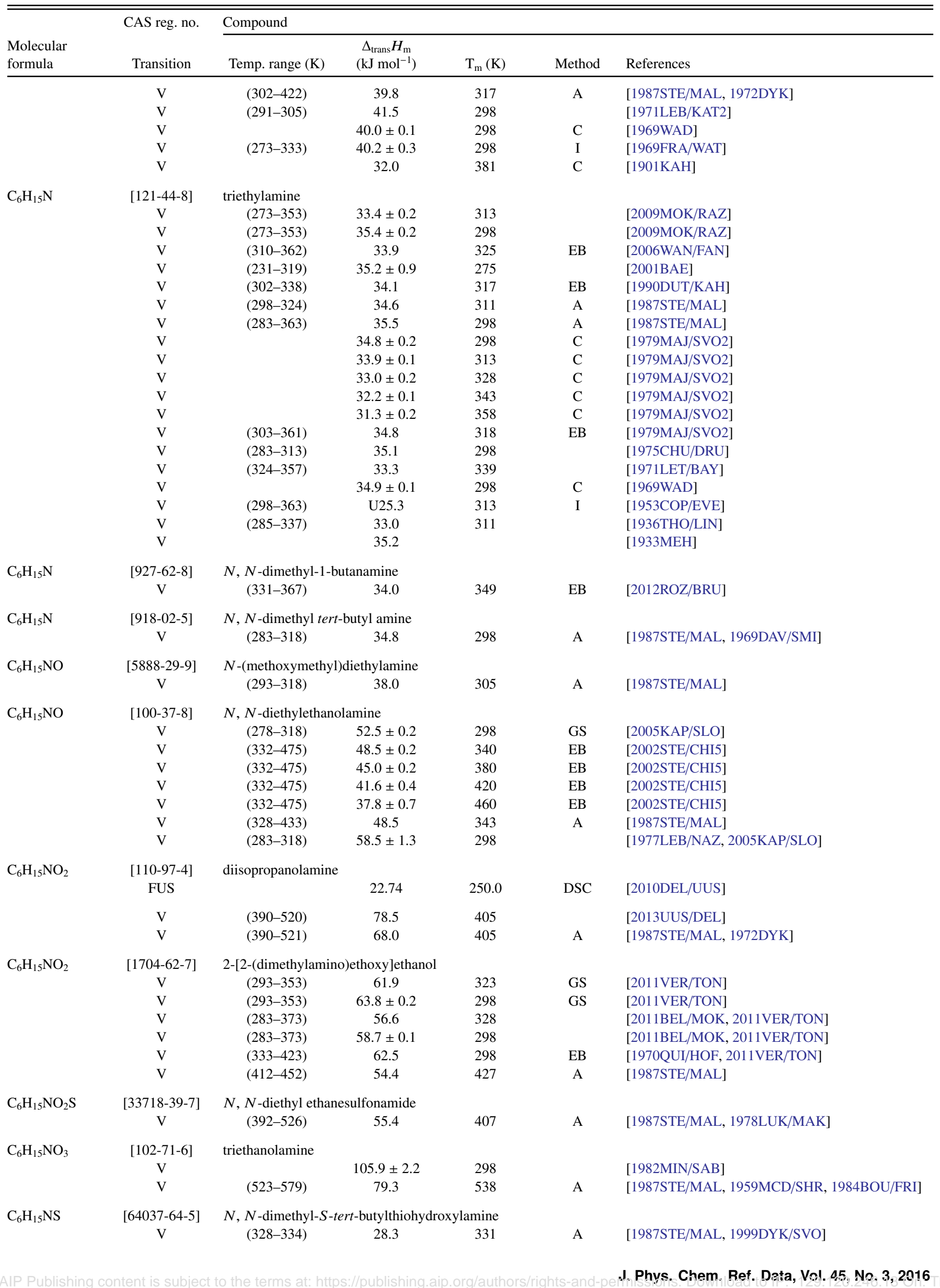


TABLE 9. Phase change enthalpies of $\mathrm{C}_{6}$ organic compounds-Continued

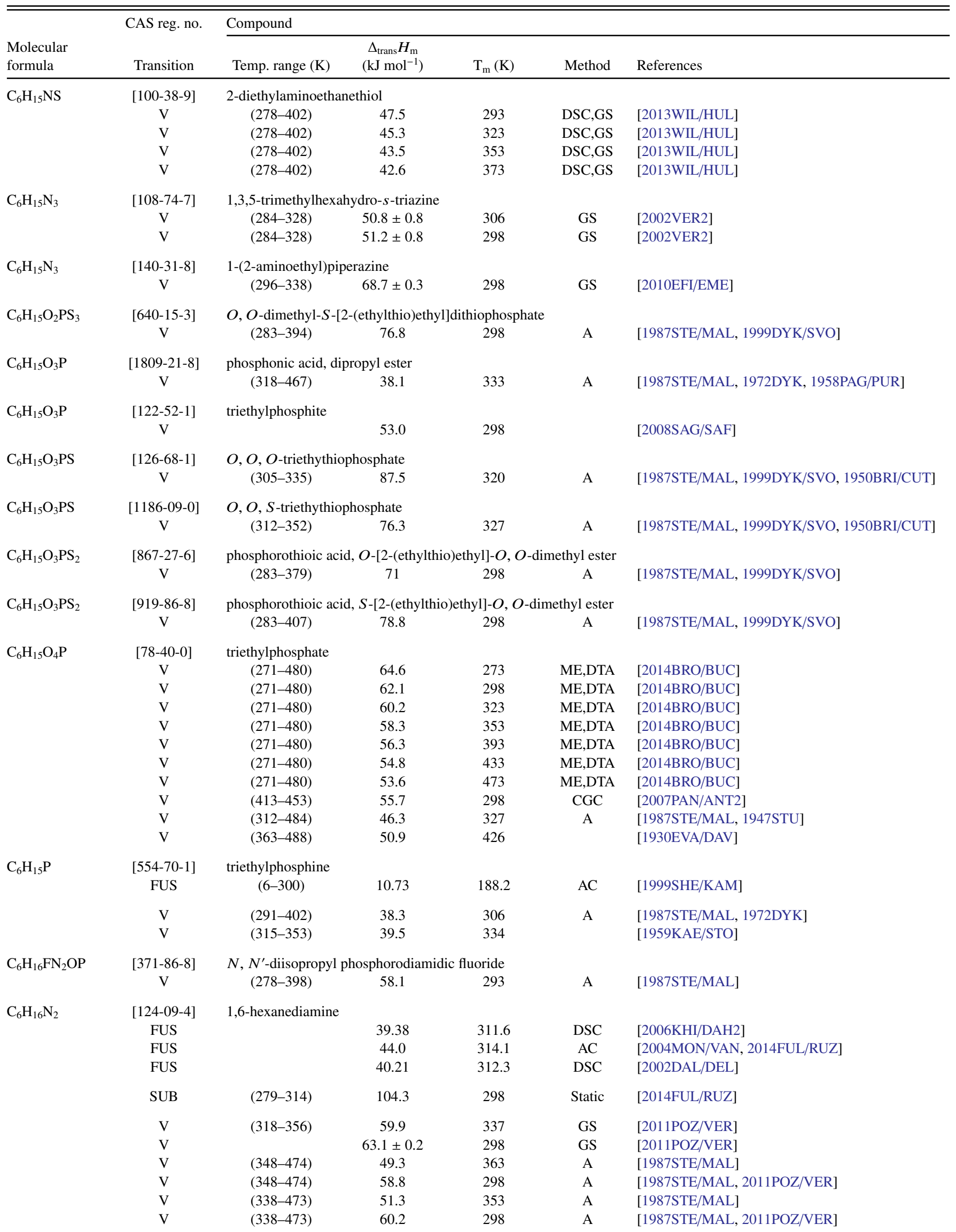

\footnotetext{
$\mathrm{C}_{6} \mathrm{H}_{16} \mathrm{~N}_{2}$ [15520-10-2] 1,5-diamino-2-methylpentane
} 
TABLE 9. Phase change enthalpies of $\mathrm{C}_{6}$ organic compounds-Continued

\begin{tabular}{|c|c|c|c|c|c|c|}
\hline \multirow[b]{2}{*}{$\begin{array}{l}\text { Molecular } \\
\text { formula }\end{array}$} & \multirow{2}{*}{$\begin{array}{l}\text { CAS reg. no. } \\
\text { Transition }\end{array}$} & \multicolumn{5}{|l|}{ Compound } \\
\hline & & Temp. range $(\mathrm{K})$ & $\begin{array}{c}\Delta_{\text {trans }} H_{\mathrm{m}} \\
\left(\mathrm{kJ} \mathrm{mol}^{-1}\right)\end{array}$ & $\mathrm{T}_{\mathrm{m}}(\mathrm{K})$ & Method & References \\
\hline & $\mathrm{V}$ & $(283-452)$ & 60.9 & 298 & Static & [2015BOU/NEG] \\
\hline \multirow[t]{2}{*}{$\mathrm{C}_{6} \mathrm{H}_{16} \mathrm{~N}_{2}$} & [110-18-9] & \multicolumn{5}{|c|}{ 1,2-bis(dimethylamino)ethane } \\
\hline & $\mathrm{V}$ & $(273-363)$ & 41.4 & 298 & Static & [2015BOU/NEG2] \\
\hline \multirow[t]{2}{*}{$\mathrm{C}_{6} \mathrm{H}_{16} \mathrm{~N}_{2}$} & [100-36-7] & \multicolumn{5}{|c|}{ 2-diethylaminoethylamine } \\
\hline & $\mathrm{V}$ & $(273-363)$ & 45.8 & 298 & & [2011KHI/DJE] \\
\hline \multirow[t]{4}{*}{$\mathrm{C}_{6} \mathrm{H}_{16} \mathrm{~N}_{2} \mathrm{O}_{2}$} & [4439-20-7] & \multicolumn{5}{|c|}{$N, N$-bis(2-hydroxyethyl)ethylenediamine } \\
\hline & FUS & & 49.7 & 373.2 & & [1997STE/CHI4] \\
\hline & $\mathrm{V}$ & $(399-500)$ & $87.7 \pm 0.2$ & 440 & EB,IPM & [1997STE/CHI2, 1997STE/CHI4] \\
\hline & $\mathrm{V}$ & $(399-500)$ & $84.8 \pm 0.2$ & 480 & EB,IPM & [1997STE/CHI2, 1997STE/CHI4] \\
\hline \multirow[t]{3}{*}{$\mathrm{C}_{6} \mathrm{H}_{16} \mathrm{~N}_{2} \mathrm{O}_{2}$} & [929-59-9] & \multicolumn{5}{|c|}{ 1,2-bis(2-aminoethoxy)ethane } \\
\hline & $\mathrm{V}$ & $(293-353)$ & 56.2 & 323 & Static & [2009RAZ/HAJ] \\
\hline & $\mathrm{V}$ & $(293-353)$ & 58.8 & 298 & Static & [2009RAZ/HAJ] \\
\hline \multirow[t]{2}{*}{$\mathrm{C}_{6} \mathrm{H}_{16} \mathrm{~N}_{2} \mathrm{O}_{2}$} & [3129-93-9] & \multicolumn{5}{|c|}{ diisopropyl ammonium nitrite } \\
\hline & SUB & $(288-299)$ & 39.0 & 293.5 & A & [1987STE/MAL, 1965MAR] \\
\hline \multirow[t]{2}{*}{$\mathrm{C}_{6} \mathrm{H}_{17} \mathrm{~N}_{3}$} & {$[56-18-8]$} & \multicolumn{5}{|l|}{ dipropylenetriamine } \\
\hline & $\mathrm{V}$ & $(293-442)$ & 70.2 & 298 & Static & [2016BOU/DER] \\
\hline $\mathrm{C}_{6} \mathrm{H}_{18} \mathrm{~N}_{3} \mathrm{P}$ & [1608-26-0] & \multicolumn{5}{|c|}{ tris(dimethylamino)phosphine } \\
\hline
\end{tabular}


TABLE 10. Phase change enthalpies of $\mathbf{C}_{7}$ organic compounds

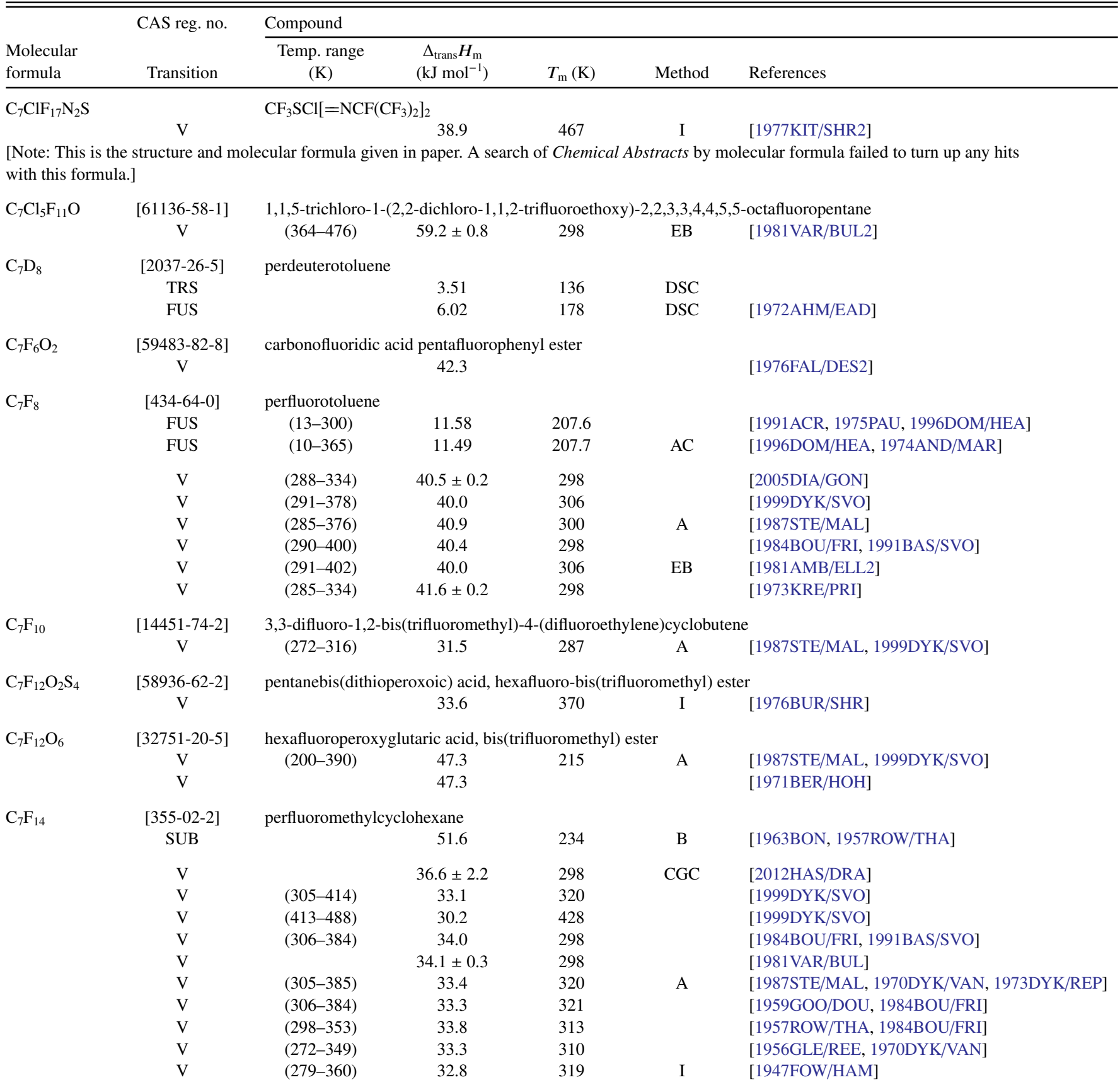

$\mathrm{C}_{7} \mathrm{~F}_{15} \mathrm{NS} \quad$ [77984-26-0] 2,2,3,3,4,4,5,5-octafluoro-1,1,2,3,4,5-hexahydro-1-[[1,2,2,2-tetrafluoro-1-(trifluoromethyl)ethyl]imino]thiophene $\begin{array}{llll}\mathrm{V} & 33.9 & 371 & {[1981 \mathrm{ABE} / \mathrm{SHR}]}\end{array}$

$\mathrm{C}_{7} \mathrm{~F}_{16} \quad$ [335-57-9] perfluoroheptane

$\begin{array}{ll}\mathrm{V} & (288-334) \\ \mathrm{V} & (291-378) \\ \mathrm{V} & (285-376) \\ \mathrm{V} & (290-400) \\ \mathrm{V} & (291-402) \\ \mathrm{V} & (285-334)\end{array}$

$6.95 \quad 221.9$

SUB 57.7

$\begin{array}{lccc}\text { V } & (363-474) & 32.6 & 378 \\ \text { V } & (304-390) & 34.4 \pm 0.3 & 320 \\ \text { V } & (304-390) & 32.5 \pm 0.4 & 340 \\ \text { V } & (304-390) & 30.4 \pm 0.6 & 360 \\ \text { V } & (304-390) & 28.1 \pm 0.8 & 380 \\ \text { V } & (304-390) & 36.3 \pm 0.3 & 298 \\ \text { V } & (290-355) & 35.9 & 298 \\ \text { V } & & 40.4 & 343 \\ \text { V } & & 33.3 & 373 \\ \text { V } & & 26.5 & 403 \\ \text { V } & & 19.7 & 433\end{array}$

DSC [1996DOM/HEA, 1986STA]

B [1963BON, 1951OLI/GRI]

[1999DYK/SVO]

EB [1997STE/CHI3]

EB [1997STE/CHI3]

EB [1997STE/CHI3]

EB [1997STE/CHI3]

EB [1997STE/CHI3]

[1984BOU/FRI, 1991BAS/SVO]

[1969ERM/SKR, 1997STE/CHI3]

[1969ERM/SKR, 1997STE/CHI3]

[1969ERM/SKR, 1997STE/CHI3]

[1969ERM/SKR, 1997STE/CHI3] 
TABLE 10. Phase change enthalpies of $\mathrm{C}_{7}$ organic compounds-Continued

\begin{tabular}{|c|c|c|c|c|c|c|}
\hline \multirow[b]{2}{*}{$\begin{array}{l}\text { Molecular } \\
\text { formula }\end{array}$} & \multirow{2}{*}{$\begin{array}{l}\text { CAS reg. no. } \\
\text { Transition }\end{array}$} & \multicolumn{5}{|l|}{ Compound } \\
\hline & & $\begin{array}{l}\text { Temp. range } \\
(\mathrm{K})\end{array}$ & $\begin{array}{c}\Delta_{\text {trans }} H_{\mathrm{m}} \\
\left(\mathrm{kJ} \mathrm{mol}^{-1}\right)\end{array}$ & $T_{\mathrm{m}}(\mathrm{K})$ & Method & References \\
\hline & $\mathrm{V}$ & & 12.8 & 463 & & [1969ERM/SKR, 1997STE/CHI3] \\
\hline & $\mathrm{V}$ & $(336-355)$ & 33.1 & 346 & & [1959YEN/REE] \\
\hline & $\mathrm{V}$ & $(293-355)$ & 34.9 & 324 & & [1956GLE/REE] \\
\hline & $\mathrm{V}$ & $(271-379)$ & 37.7 & 286 & A & [1987STE/MAL, 1951OLI/GRI, 1970DYK/VAN] \\
\hline & $\mathrm{V}$ & & 36.3 & 298 & & [1951OLI/GRI, 1997STE/CHI3] \\
\hline & $\mathrm{V}$ & & 31.6 & 356 & & [1951OLI/GRI, 1997STE/CHI3] \\
\hline & $\mathrm{V}$ & $(275-366)$ & 34.5 & 320 & $\mathrm{I}$ & [1947FOW/HAM] \\
\hline \multirow[t]{2}{*}{$\mathrm{C}_{7} \mathrm{~F}_{16} \mathrm{~N}_{2} \mathrm{OS}$} & {$[62609-64-7]$} & \multicolumn{5}{|c|}{$\begin{array}{l}\text { 1,1,1-trifluoro- } N^{\prime} \text {-[1,2,2,2-tetrafluoro-1-(trifluoromethyl)ethyl]- } N \text {-[2,2,2-trifluoro-1- } \\
\text { (trifluromethyl)ethylidene]methanesulfonimidamide }\end{array}$} \\
\hline & $\mathrm{V}$ & & 33.5 & 451 & & [1977KIT/SHR] \\
\hline \multirow[t]{2}{*}{$\mathrm{C}_{7} \mathrm{~F}_{17} \mathrm{~N}$} & {$[338-81-8]$} & perfluoro- $N, \Lambda$ & hylpropylam & & & \\
\hline & $\mathrm{V}$ & $(283-366)$ & 39.2 & 325 & & [1999DYK/SVO] \\
\hline \multirow[t]{2}{*}{$\mathrm{C}_{7} \mathrm{HF}_{5} \mathrm{O}_{2}$} & {$[602-94-8]$} & \multicolumn{5}{|c|}{ pentafluorobenzoic acid } \\
\hline & SUB & $(335-359)$ & $91.6 \pm 4.2$ & & GS & [1969COX/GUN, 1970COX/PIL] \\
\hline \multirow[t]{4}{*}{$\mathrm{C}_{7} \mathrm{HF}_{13} \mathrm{O}_{2}$} & {$[375-85-9]$} & \multicolumn{5}{|c|}{ tridecafluoroheptanoic acid } \\
\hline & $\mathrm{V}$ & $(359-485)$ & $61.4 \pm 0.3$ & 370 & $\mathrm{~EB}$ & [2002STE/CHI] \\
\hline & $\mathrm{V}$ & $(359-485)$ & $55.5 \pm 0.3$ & 410 & $\mathrm{~EB}$ & [2002STE/CHI] \\
\hline & $\mathrm{V}$ & $(359-485)$ & $48.7 \pm 0.7$ & 450 & $\mathrm{~EB}$ & [2002STE/CHI] \\
\hline \multirow[t]{4}{*}{$\mathrm{C}_{7} \mathrm{HF}_{15}$} & {$[375-83-7]$} & \multicolumn{5}{|c|}{$1,1,1,2,2,3,3,4,4,5,5,6,6,7,7$-pentafluoroheptane } \\
\hline & $\mathrm{V}$ & $(365-369)$ & 30.7 & 367 & & [1966CAR/STE] \\
\hline & $\mathrm{V}$ & $(292-322)$ & 37.7 & 298 & & [1956MCL/SCO] \\
\hline & $\mathrm{V}$ & $(292-370)$ & 37.1 & 307 & A & $\begin{array}{l}\text { [1987STE/MAL, 1953KAR/SAY, 1973DYK/REP, } \\
\text { 1999DYK/SVO] }\end{array}$ \\
\hline \multirow[t]{2}{*}{$\mathrm{C}_{7} \mathrm{H}_{2} \mathrm{~F}_{13} \mathrm{NO}$} & {$[54181-88-3]$} & \multicolumn{5}{|c|}{ (E)-1,1,1,2,3,3,3-heptafluoro- $N$-[2,2,2-trifluoro-1-(2,2,2-trifluoroethoxy)ethylidene]-2-propanamine } \\
\hline & V & & 35.7 & 369 & & [1975PET/SHR] \\
\hline \multirow[t]{2}{*}{$\mathrm{C}_{7} \mathrm{H}_{3} \mathrm{Br}_{2} \mathrm{NO}$} & {$[1689-84-5]$} & \multicolumn{5}{|c|}{ 3,5-dibromo-4-hydroxybenzonitrile } \\
\hline & FUS & & 32.03 & 464 & DSC & {$[1990 \mathrm{DON} / \mathrm{DRE}]$} \\
\hline \multirow[t]{2}{*}{$\mathrm{C}_{7} \mathrm{H}_{3} \mathrm{ClF}_{3} \mathrm{NO}_{2}$} & {$[777-37-7]$} & \multicolumn{5}{|c|}{ 1-(trifluoromethyl)-2-chloro-5-nitrobenzene } \\
\hline & $\mathrm{V}$ & $(364-508)$ & 58.1 & 379 & A & $\begin{array}{l}\text { [1987STE/MAL, 1953KAR/SAY, 1973DYK/REP, } \\
\text { 1999DYK/SVO] }\end{array}$ \\
\hline \multirow[t]{2}{*}{$\mathrm{C}_{7} \mathrm{H}_{3} \mathrm{ClF}_{3} \mathrm{NO}_{2}$} & {$[121-17-5]$} & \multicolumn{5}{|c|}{ 1-(trifluoromethyl)-4-chloro-3-nitrobenzene } \\
\hline & $\mathrm{V}$ & $(358-495)$ & 57.6 & 373 & A & [1987STE/MAL, 1973DYK/REP, 1999DYK/SVO] \\
\hline $\mathrm{C}_{7} \mathrm{H}_{3} \mathrm{Cl}_{2} \mathrm{~F}_{3}$ & {$[328-84-7]$} & 1-(trifluoromet & ,4-dichlorob & & & \\
\hline & $\mathrm{V}$ & $(353-453)$ & 44.1 & 368 & A & [1987STE/MAL] \\
\hline & $\mathrm{V}$ & $(284-446)$ & 41.8 & 299 & & [1947STU] \\
\hline & $\mathrm{V}$ & $(298-453)$ & 43.1 & 446 & & {$[1935 B O O / E L S]$} \\
\hline $\mathrm{C}_{7} \mathrm{H}_{3} \mathrm{Cl}_{2} \mathrm{~N}$ & {$[1194-65-6]$} & 2,6-dichlorober & trile & & & \\
\hline & FUS & & 24.56 & 421.2 & DSC & [2000ROD/VEC] \\
\hline & FUS & & 26.17 & 417.2 & DSC & [1991ACR, 1990DON/DRE] \\
\hline & FUS & & 25.94 & 416.7 & DSC & {$[1972 \mathrm{PLA}]$} \\
\hline $\mathrm{C}_{7} \mathrm{H}_{3} \mathrm{Cl}_{2} \mathrm{NO}$ & {$[102-36-3]$} & 3,4-dichloroph & ocyanate & & & \\
\hline & $\mathrm{V}$ & $(373-473)$ & 47.4 & 388 & A & [1987STE/MAL] \\
\hline & $\mathrm{V}$ & $(333-463)$ & 48.1 & 398 & & {$[1967 \mathrm{KON} / \mathrm{ZHU}]$} \\
\hline $\mathrm{C}_{7} \mathrm{H}_{3} \mathrm{Cl}_{3} \mathrm{O}_{2}$ & {$[50-31-7]$} & 2,3,6-trichlorob & ic acid & & & \\
\hline & FUS & & 23.85 & 402.7 & DSC & [1991ACR, 1972PLA] \\
\hline $\mathrm{C}_{7} \mathrm{H}_{3} \mathrm{Cl}_{5}$ & {$[13014-24-9]$} & 1-(trichloromet & 3,4-dichlorol & & & \\
\hline & $\mathrm{V}$ & $(438-663)$ & 59.3 & 453 & A & $\begin{array}{l}\text { [1987STE/MAL, 1970DYK/VAN, 1973DYK/REP, } \\
\text { 1999DYK/SVO] }\end{array}$ \\
\hline $\mathrm{C}_{7} \mathrm{H}_{3} \mathrm{~F}_{5}$ & {$[771-56-2]$} & 2,3,4,5,6-penta & toluene & & & \\
\hline & FUS & & 13.28 & 243.7 & & [1996DOM/HEA, 1971PAU2] \\
\hline & TRS & $(10-376)$ & 0.21 & 70.3 & & \\
\hline & FUS & $(10-376)$ & 12.99 & 243.3 & & [1996DOM/HEA, 1968COU/HAL] \\
\hline & $\mathrm{V}$ & $(403-523)$ & 36.1 & 418 & & {$[1999 \mathrm{DYK} / \mathrm{SVO}]$} \\
\hline
\end{tabular}


TABLE 10. Phase change enthalpies of $\mathrm{C}_{7}$ organic compounds-Continued

\begin{tabular}{|c|c|c|c|c|c|c|}
\hline \multirow[b]{2}{*}{$\begin{array}{l}\text { Molecular } \\
\text { formula }\end{array}$} & \multirow{2}{*}{$\begin{array}{l}\text { CAS reg. no. } \\
\text { Transition }\end{array}$} & \multicolumn{5}{|l|}{ Compound } \\
\hline & & $\begin{array}{l}\text { Temp. range } \\
(\mathrm{K})\end{array}$ & $\begin{array}{c}\Delta_{\text {trans }} H_{\mathrm{m}} \\
\left(\mathrm{kJ} \mathrm{mol}^{-1}\right)\end{array}$ & $T_{\mathrm{m}}(\mathrm{K})$ & Method & References \\
\hline & $\mathrm{V}$ & $(493-564)$ & 34.9 & 508 & & [1999DYK/SVO] \\
\hline & $\mathrm{V}$ & $(310-410)$ & 41.2 & 298 & & [1984BOU/FRI, 1991BAS/SVO] \\
\hline & $\mathrm{V}$ & $(312-416)$ & 39.9 & 327 & A & $\begin{array}{l}\text { [1987STE/MAL, 1968AMB, 1973DYK/REP, } \\
\text { 1999DYK/SVO] }\end{array}$ \\
\hline \multirow[t]{2}{*}{$\mathrm{C}_{7} \mathrm{H}_{3} \mathrm{I}_{2} \mathrm{NO}$} & [1689-83-4] & 4-hydroxy-3,5 & lobenzonitrile & & & \\
\hline & FUS & & 33.63 & 482.9 & DSC & [1990DON/DRE] \\
\hline \multirow[t]{2}{*}{$\mathrm{C}_{7} \mathrm{H}_{3} \mathrm{I}_{3} \mathrm{O}_{2}$} & {$[88-82-4]$} & 2,3,5-triiodobe & acid & & & \\
\hline & FUS & & 32.23 & 503.8 & DSC & [1991ACR, 1972PLA] \\
\hline \multirow[t]{3}{*}{$\mathrm{C}_{7} \mathrm{H}_{4} \mathrm{BrN}$} & {$[2042-37-7]$} & 2-bromobenzo & & & & \\
\hline & SUB & & $82.1 \pm 0.5$ & 281 & $\mathrm{ME}$ & [2013ROC/GAL] \\
\hline & SUB & & $81.8 \pm 0.5$ & 298 & $\mathrm{ME}$ & [2013ROC/GAL] \\
\hline \multirow{3}{*}{$\mathrm{C}_{7} \mathrm{H}_{4} \mathrm{BrN}$} & {$[6952-59-6]$} & 3-bromobenzo & & & & \\
\hline & SUB & & $78.3 \pm 0.5$ & 276 & ME & [2013ROC/GAL] \\
\hline & SUB & & $77.9 \pm 0.5$ & 298 & $\mathrm{ME}$ & [2013ROC/GAL] \\
\hline \multirow[t]{3}{*}{$\mathrm{C}_{7} \mathrm{H}_{4} \mathrm{BrN}$} & [623-00-7] & 4-bromobenzo & & & & \\
\hline & SUB & & $81.0 \pm 0.5$ & 288 & ME & [2013ROC/GAL] \\
\hline & SUB & & $80.8 \pm 0.5$ & 298 & $\mathrm{ME}$ & [2013ROC/GAL] \\
\hline \multirow[t]{5}{*}{$\mathrm{C}_{7} \mathrm{H}_{4} \mathrm{Br}_{2} \mathrm{O}_{2}$} & {$[610-71-9]$} & 2,5-dibromobe & acid & & & \\
\hline & FUS & & 35.8 & 428 & DSC & [2013VEC/BRU] \\
\hline & SUB & $(347-377)$ & $131.7 \pm 1.0$ & 362 & $\mathrm{TE}$ & [2013VEC/BRU] \\
\hline & SUB & $(347-377)$ & $133.6 \pm 1.0$ & 298 & $\mathrm{TE}$ & [2013VEC/BRU] \\
\hline & $\mathrm{V}$ & $(440-468)$ & $90.5 \pm 0.8$ & 455 & TGA & [2013VEC/BRU] \\
\hline \multirow[t]{5}{*}{$\mathrm{C}_{7} \mathrm{H}_{4} \mathrm{Br}_{2} \mathrm{O}_{2}$} & {$[618-58.6]$} & 3,5-dibromobe & acid & & & \\
\hline & FUS & & 30.1 & 491 & DSC & [2013VEC/BRU] \\
\hline & SUB & $(355-396)$ & $118.0 \pm 0.8$ & 376 & TE & [2013VEC/BRU] \\
\hline & SUB & $(355-396)$ & $120.3 \pm 0.8$ & 298 & $\mathrm{TE}$ & [2013VEC/BRU] \\
\hline & $\mathrm{V}$ & $(500-530)$ & $82.7 \pm 1.2$ & 514 & TGA & [2013VEC/BRU] \\
\hline \multirow[t]{4}{*}{$\mathrm{C}_{7} \mathrm{H}_{4} \mathrm{ClF}_{3}$} & {$[88-16-4]$} & 1-(trifluoromet & 2-chlorobenze & & & \\
\hline & FUS & & 11.6 & 264 & DSC & [1972AHM/EAD] \\
\hline & V & $(310-426)$ & 44.6 & 325 & A & $\begin{array}{l}\text { [1987STE/MAL, 1951POT/SAY, 1970DYK/VAN, } \\
\text { 1973DYK/REP] }\end{array}$ \\
\hline & $\mathrm{V}$ & $(293-431)$ & 39.5 & 426 & & {$[1935 \mathrm{BOO} / \mathrm{ELS}]$} \\
\hline \multirow[t]{3}{*}{$\mathrm{C}_{7} \mathrm{H}_{4} \mathrm{ClF}_{3}$} & {$[98-15-7]$} & 1-(trifluoromet & 3-chlorobenze & & & \\
\hline & $\mathrm{V}$ & $(302-411)$ & 43.0 & 317 & A & $\begin{array}{l}\text { [1987STE/MAL, 1951POT/SAY, 1970DYK/VAN, } \\
\text { 1973DYK/REP] }\end{array}$ \\
\hline & V & $(294-415)$ & 38.3 & 411 & & {$[1935 \mathrm{BOO} / \mathrm{ELS}]$} \\
\hline \multirow[t]{3}{*}{$\mathrm{C}_{7} \mathrm{H}_{4} \mathrm{ClF}_{3}$} & {$[98-56-6]$} & 1-(trifluoromet & 4-chlorobenze & & & \\
\hline & V & $(302-412)$ & 42.2 & 317 & A & $\begin{array}{l}\text { [1987STE/MAL, 1951POT/SAY, 1970DYK/VAN, } \\
\text { 1973DYK/REP] }\end{array}$ \\
\hline & V & $(293-416)$ & 37.6 & 412 & & {$[1935 \mathrm{BOO} / \mathrm{ELS}]$} \\
\hline \multirow[t]{2}{*}{$\mathrm{C}_{7} \mathrm{H}_{4} \mathrm{ClF}_{11} \mathrm{O}$} & {$[65064-84-8]$} & 5-(2-chloro-1, & ifluoroethoxy) & $, 2,3,3,4$ & luoropent & \\
\hline & $\mathrm{V}$ & & $54.1 \pm 0.2$ & 298 & $\mathrm{C}$ & [1981VAR/BUL2] \\
\hline \multirow[t]{5}{*}{$\mathrm{C}_{7} \mathrm{H}_{4} \mathrm{ClN}$} & [873-32-5] & 2-chlorobenzo & & & & \\
\hline & FUS & & 17.6 & 327.5 & DSC & [2014ROC/GAL] \\
\hline & SUB & & $72.9 \pm 0.8$ & 273 & $\mathrm{ME}$ & [2014ROC/GAL] \\
\hline & SUB & & $72.3 \pm 0.8$ & 298 & $\mathrm{ME}$ & [2014ROC/GAL] \\
\hline & $\mathrm{V}$ & $(378-486)$ & 53.5 & 393 & EB & [1994AIM2] \\
\hline \multirow[t]{4}{*}{$\mathrm{C}_{7} \mathrm{H}_{4} \mathrm{ClN}$} & [766-84-7] & 3-chlorobenzo & & & & \\
\hline & FUS & & 18.7 & 314.1 & DSC & [2014ROC/GAL] \\
\hline & SUB & & $77.4 \pm 0.5$ & 268 & $\mathrm{ME}$ & [2014ROC/GAL] \\
\hline & SUB & & $76.7 \pm 0.5$ & 298 & $\mathrm{ME}$ & [2014ROC/GAL] \\
\hline
\end{tabular}


TABLE 10. Phase change enthalpies of $\mathrm{C}_{7}$ organic compounds-Continued

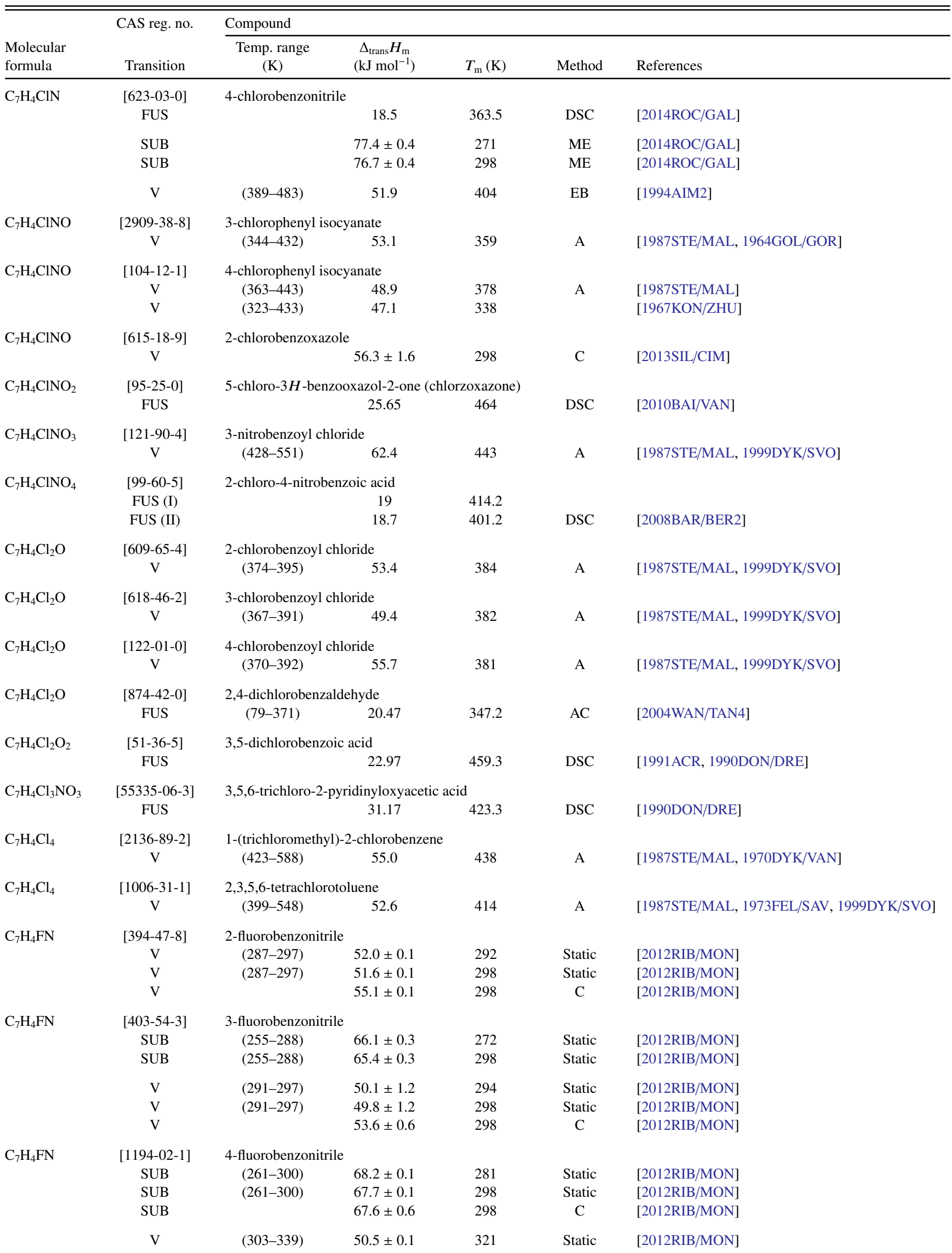


TABLE 10. Phase change enthalpies of $\mathrm{C}_{7}$ organic compounds-Continued

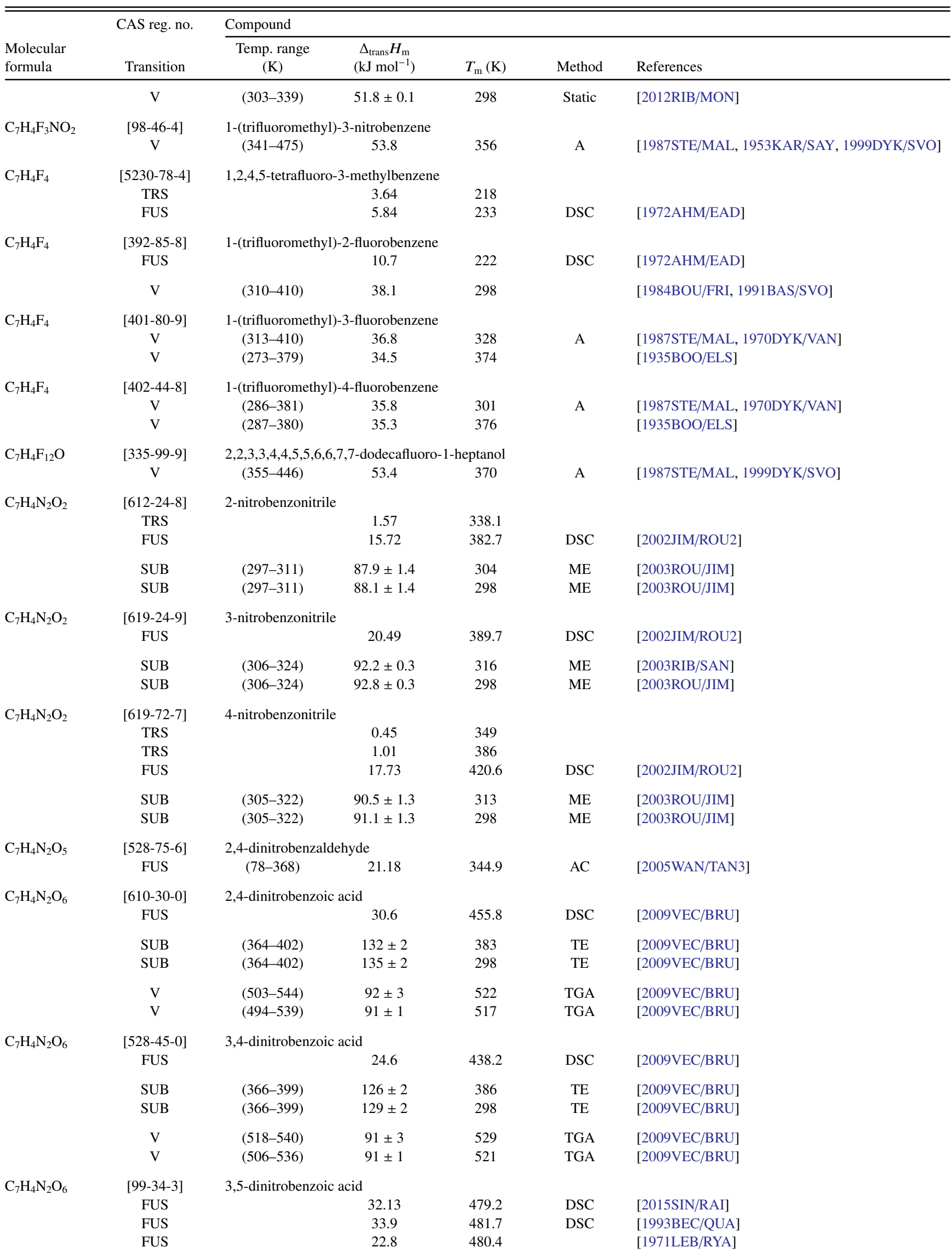


TABLE 10. Phase change enthalpies of $\mathrm{C}_{7}$ organic compounds-Continued

\begin{tabular}{|c|c|c|c|c|c|c|}
\hline \multirow[b]{2}{*}{$\begin{array}{l}\text { Molecular } \\
\text { formula }\end{array}$} & \multirow{2}{*}{$\begin{array}{l}\text { CAS reg. no. } \\
\text { Transition }\end{array}$} & \multicolumn{5}{|l|}{ Compound } \\
\hline & & $\begin{array}{c}\text { Temp. range } \\
(\mathrm{K})\end{array}$ & $\begin{array}{c}\Delta_{\text {trans }} H_{\mathrm{m}} \\
\left(\mathrm{kJ} \mathrm{mol}^{-1}\right)\end{array}$ & $T_{\mathrm{m}}(\mathrm{K})$ & Method & References \\
\hline & $\begin{array}{l}\text { SUB } \\
\text { SUB }\end{array}$ & $(350-361)$ & $\begin{array}{c}102.6 \pm 0.4 \\
107 \pm 0.4\end{array}$ & $\begin{array}{l}355 \\
298\end{array}$ & & $\begin{array}{l}\text { [1972GEI/RAU] } \\
{[1972 \mathrm{GEI} / \mathrm{RAU}]}\end{array}$ \\
\hline $\mathrm{C}_{7} \mathrm{H}_{4} \mathrm{~S}_{3}$ & $\begin{array}{c}\text { [934-36-1] } \\
\text { SUB }\end{array}$ & 4,5-benzo-1,3-c & $\begin{array}{l}\text { le-2-thione } \\
118.8 \pm 0.4\end{array}$ & 298 & & [1973RAU/GEI, 1977PED/RYL] \\
\hline $\mathrm{C}_{7} \mathrm{H}_{5} \mathrm{BrO}$ & $\begin{array}{c}{[618-32-6]} \\
V\end{array}$ & $\begin{array}{c}\text { benzoyl bromid } \\
\quad(320-492)\end{array}$ & 52.3 & 335 & A & [1987STE/MAL, 1947STU] \\
\hline $\mathrm{C}_{7} \mathrm{H}_{5} \mathrm{BrO}$ & $\begin{array}{c}{[1122-91-4]} \\
\text { FUS }\end{array}$ & 4-bromobenzal & 22.6 & 334.2 & $\mathrm{DSC}$ & [2008FAV/FRE] \\
\hline $\mathrm{C}_{7} \mathrm{H}_{5} \mathrm{BrO}_{2}$ & $\begin{array}{l}\text { [88-65-3] } \\
\text { FUS } \\
\text { FUS } \\
\text { SUB } \\
\text { SUB } \\
\text { SUB } \\
\text { SUB } \\
\text { SUB }\end{array}$ & $\begin{array}{l}(328-347) \\
(328-347)\end{array}$ & $\begin{array}{c}24.83 \\
23.0 \\
106.8 \pm 0.4 \\
108.5 \pm 0.6 \\
95.9 \pm 0.4 \\
110.9 \pm 1.1 \\
83.3 \pm 0.4\end{array}$ & $\begin{array}{l}338 \\
298 \\
298 \\
298\end{array}$ & $\begin{array}{l}\text { DSC } \\
\text { DSC } \\
\text { ME } \\
\mathrm{ME} \\
\mathrm{C} \\
\mathrm{C} \\
\mathrm{DSC}\end{array}$ & $\begin{array}{l}\text { [2005RIB/FON] } \\
{[1983 \mathrm{HOL}]} \\
{[2005 \mathrm{RIB} / \mathrm{FON}]} \\
{[2005 \mathrm{RIB} / \mathrm{FON}]} \\
{[1994 \mathrm{TAN} / \mathrm{SAB}]} \\
{[1987 \mathrm{FER} / \mathrm{PIL}]} \\
{[1983 \mathrm{HOL}]}\end{array}$ \\
\hline $\mathrm{C}_{7} \mathrm{H}_{5} \mathrm{BrO}_{2}$ & $\begin{array}{c}\text { [585-76-2] } \\
\text { FUS } \\
\text { SUB } \\
\text { SUB } \\
\text { SUB } \\
\text { SUB }\end{array}$ & $\begin{array}{c}\text { 3-bromobenzoi } \\
(328-347) \\
(328-347)\end{array}$ & $\begin{array}{c}24.91 \\
104.2 \pm 0.5 \\
105.9 \pm 0.7 \\
99.2 \pm 0.2 \\
105.0 \pm 1.1\end{array}$ & $\begin{array}{l}430.1 \\
338 \\
298 \\
298 \\
298\end{array}$ & $\begin{array}{l}\text { DSC } \\
\text { ME } \\
\text { ME } \\
\text { C } \\
\text { C }\end{array}$ & $\begin{array}{l}{[2005 \mathrm{RIB} / \mathrm{FON}]} \\
{[2005 \mathrm{RIB} / \mathrm{FON}]} \\
{[2005 \mathrm{RIB} / \mathrm{FON}]} \\
{[1994 \mathrm{TAN} / \mathrm{SAB}]} \\
{[1987 \mathrm{FER} / \mathrm{PIL}]}\end{array}$ \\
\hline $\mathrm{C}_{7} \mathrm{H}_{5} \mathrm{BrO}_{2}$ & $\begin{array}{c}\text { [586-76-5] } \\
\text { FUS } \\
\text { SUB } \\
\text { SUB } \\
\text { SUB } \\
\text { SUB }\end{array}$ & $\begin{array}{l}\text { 4-bromobenzoi } \\
\begin{array}{c}(349-366) \\
(349-366)\end{array}\end{array}$ & $\begin{array}{l}\text { d } 30.87 \\
107.4 \pm 0.5 \\
110.1 \pm 0.8 \\
103.1 \pm 0.6 \\
107.6 \pm 1.1\end{array}$ & $\begin{array}{l}526.3 \\
358 \\
298 \\
298 \\
298\end{array}$ & $\begin{array}{l}\text { DSC } \\
\text { ME } \\
\text { ME } \\
\text { C } \\
\text { C }\end{array}$ & $\begin{array}{l}{[2005 \mathrm{RIB} / \mathrm{FON}]} \\
{[2005 \mathrm{RIB} / \mathrm{FON}]} \\
{[2005 \mathrm{RIB} / \mathrm{FON}]} \\
{[1994 \mathrm{TAN} / \mathrm{SAB}]} \\
{[1987 \mathrm{FER} / \mathrm{PIL}]}\end{array}$ \\
\hline $\mathrm{C}_{7} \mathrm{H}_{5} \mathrm{ClO}$ & $\begin{array}{c}{[98-88-4]} \\
\mathrm{V}\end{array}$ & $\begin{array}{c}\text { benzoyl chlorid } \\
\quad(305-470)\end{array}$ & 49.6 & 320 & A & [1987STE/MAL, 1947STU, 1999DYK/SVO] \\
\hline $\mathrm{C}_{7} \mathrm{H}_{5} \mathrm{ClO}$ & $\begin{array}{c}{[89-98-5]} \\
\mathrm{V}\end{array}$ & $\begin{array}{c}\text { 2-chlorobenzal } \\
\text { (382-563) }\end{array}$ & de 49.8 & 397 & A & [1987STE/MAL, 1999DYK/SVO] \\
\hline $\mathrm{C}_{7} \mathrm{H}_{5} \mathrm{ClO}_{2}$ & $\begin{array}{c}\text { [118-91-2] } \\
\text { FUS } \\
\text { FUS } \\
\text { FUS } \\
\text { FUS } \\
\text { FUS } \\
\text { FUS } \\
\text { SUB } \\
\text { SUB } \\
\text { SUB } \\
\text { SUB } \\
\text { SUB } \\
\text { SUB } \\
\text { SUB }\end{array}$ & $\begin{array}{l}(338-402) \\
(338-402) \\
(320-339) \\
(320-339)\end{array}$ & $\begin{array}{c}24.67 \\
27.53 \\
25.25 \\
25.73 \\
26.3 \\
25.52 \\
99.9 \pm 0.4 \\
101.4 \pm 0.4 \\
105.0 \pm 0.4 \\
106.3 \pm 0.5 \\
100.9 \pm 0.5 \\
116.2 \pm 0.6 \\
79.5 \pm 3.3\end{array}$ & $\begin{array}{c}417.5 \\
414.2 \\
414 \\
413.4 \\
414\end{array}$ & $\begin{array}{c}\text { DSC } \\
\text { DSC } \\
\text { DSC } \\
\\
\text { DSC } \\
\text { GS } \\
\text { GS } \\
\text { ME } \\
\text { ME } \\
\text { C } \\
\text { DSC }\end{array}$ & $\begin{array}{l}\text { [2009MEL/PIN] } \\
\text { [2009DAS/SIN] } \\
\text { [2005RIB/FON] } \\
\text { [1991ACR, 1983WEA] } \\
\text { [1991SAB/HIR] } \\
{[1983 \mathrm{HOL}]} \\
{[2005 \mathrm{EME} / \mathrm{STR}]} \\
{[2005 \mathrm{EME} / \mathrm{STR}]} \\
{[2005 \mathrm{RIB} / \mathrm{FON}]} \\
{[2005 \mathrm{RIB} / \mathrm{FON}]} \\
{[1995 \mathrm{SAB} / \mathrm{AGU}]} \\
{[1983 \mathrm{HOL}]} \\
{[1938 \mathrm{WOL} / \mathrm{WEG}, 1960 J O N, 1970 \mathrm{COX} / \mathrm{PIL}]}\end{array}$ \\
\hline $\mathrm{C}_{7} \mathrm{H}_{5} \mathrm{ClO}_{2}$ & $\begin{array}{c}\text { [535-80-8] } \\
\text { FUS } \\
\text { FUS } \\
\text { FUS }\end{array}$ & 3-chlorobenzoi & $\begin{array}{c}23.67 \\
23.85 \\
22.0\end{array}$ & $\begin{array}{l}427.9 \\
427.4 \\
427.8\end{array}$ & DSC & $\begin{array}{l}\text { [2005RIB/FON] } \\
\text { [1991ACR, 1983WEA] } \\
\text { [1991SAB/HIR] }\end{array}$ \\
\hline & $\begin{array}{l}\text { SUB } \\
\text { SUB } \\
\text { SUB } \\
\text { SUB }\end{array}$ & $\begin{array}{l}(348-404) \\
(348-404) \\
(320-340) \\
(320-340)\end{array}$ & $\begin{array}{c}98.9 \pm 0.8 \\
100.6 \pm 0.8 \\
101.2 \pm 0.4 \\
102.5 \pm 0.5\end{array}$ & $\begin{array}{l}376 \\
298 \\
330 \\
298\end{array}$ & $\begin{array}{l}\text { GS } \\
\text { GS } \\
\mathrm{ME} \\
\mathrm{ME}\end{array}$ & $\begin{array}{l}\text { [2005EME/STR] } \\
{[2005 \mathrm{EME} / \mathrm{STR}]} \\
{[2005 \mathrm{RIB} / \mathrm{FON}]} \\
{[2004 \mathrm{RIB} / \mathrm{SAN} 3]}\end{array}$ \\
\hline
\end{tabular}


TABLE 10. Phase change enthalpies of $\mathrm{C}_{7}$ organic compounds-Continued

\begin{tabular}{|c|c|c|c|c|c|c|}
\hline \multirow[b]{2}{*}{$\begin{array}{l}\text { Molecular } \\
\text { formula }\end{array}$} & \multirow{2}{*}{$\begin{array}{c}\text { CAS reg. no. } \\
\text { Transition }\end{array}$} & \multicolumn{5}{|l|}{ Compound } \\
\hline & & $\begin{array}{c}\text { Temp. range } \\
(\mathrm{K})\end{array}$ & $\begin{array}{c}\Delta_{\text {trans }} H_{\mathrm{m}} \\
\left(\mathrm{kJ} \mathrm{mol}^{-1}\right) \\
\end{array}$ & $T_{\mathrm{m}}(\mathrm{K})$ & Method & References \\
\hline & SUB & & $101.4 \pm 0.4$ & 298 & $\mathrm{C}$ & [1995SAB/AGU] \\
\hline & SUB & & 99.6 & 413 & $\mathrm{C}$ & [1975ADE/BRO] \\
\hline & SUB & & 105.8 & 298 & $\mathrm{C}$ & [1975ADE/BRO] \\
\hline & SUB & & $80.8 \pm 3.3$ & & & [1938WOL/WEG, 1960JON, 1970COX/PIL] \\
\hline \multirow[t]{4}{*}{$\mathrm{C}_{7} \mathrm{H}_{5} \mathrm{ClO}_{2}$} & [74-11-3] & \multicolumn{5}{|c|}{ 4-chlorobenzoic acid } \\
\hline & FUS & & 32.0 & 513.4 & DSC & [2013ALM/MON] \\
\hline & FUS & & 30.91 & 512.5 & DSC & [2005RIB/FON] \\
\hline & FUS & $(80-580)$ & 13.5 & 512.3 & $\mathrm{AC}$ & [2002TAN/SUN] \\
\hline
\end{tabular}

[Note: This value is considerably lower than the other three reported enthalpies of fusion. This value is likely in error.]

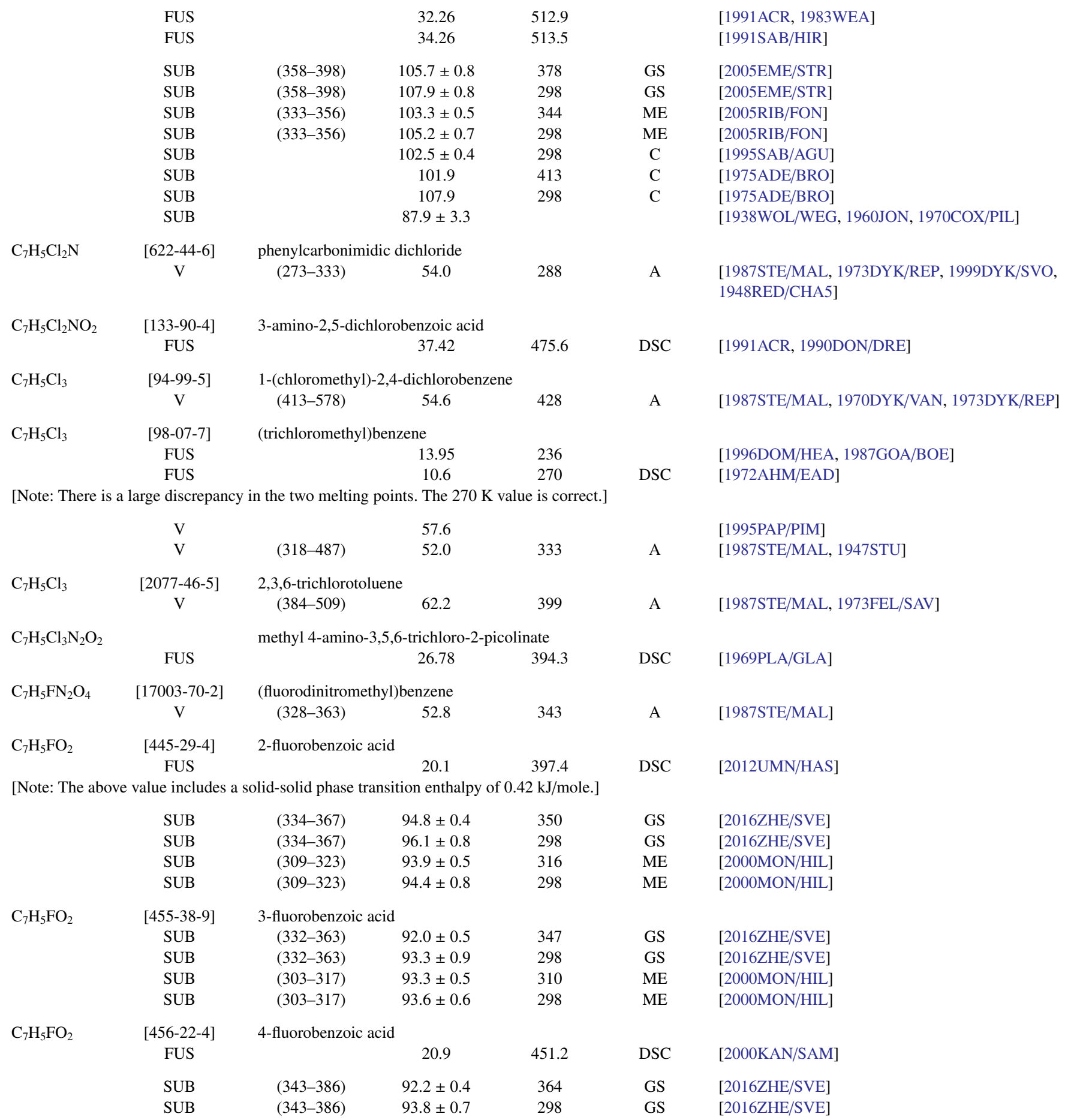


TABLE 10. Phase change enthalpies of $\mathrm{C}_{7}$ organic compounds-Continued

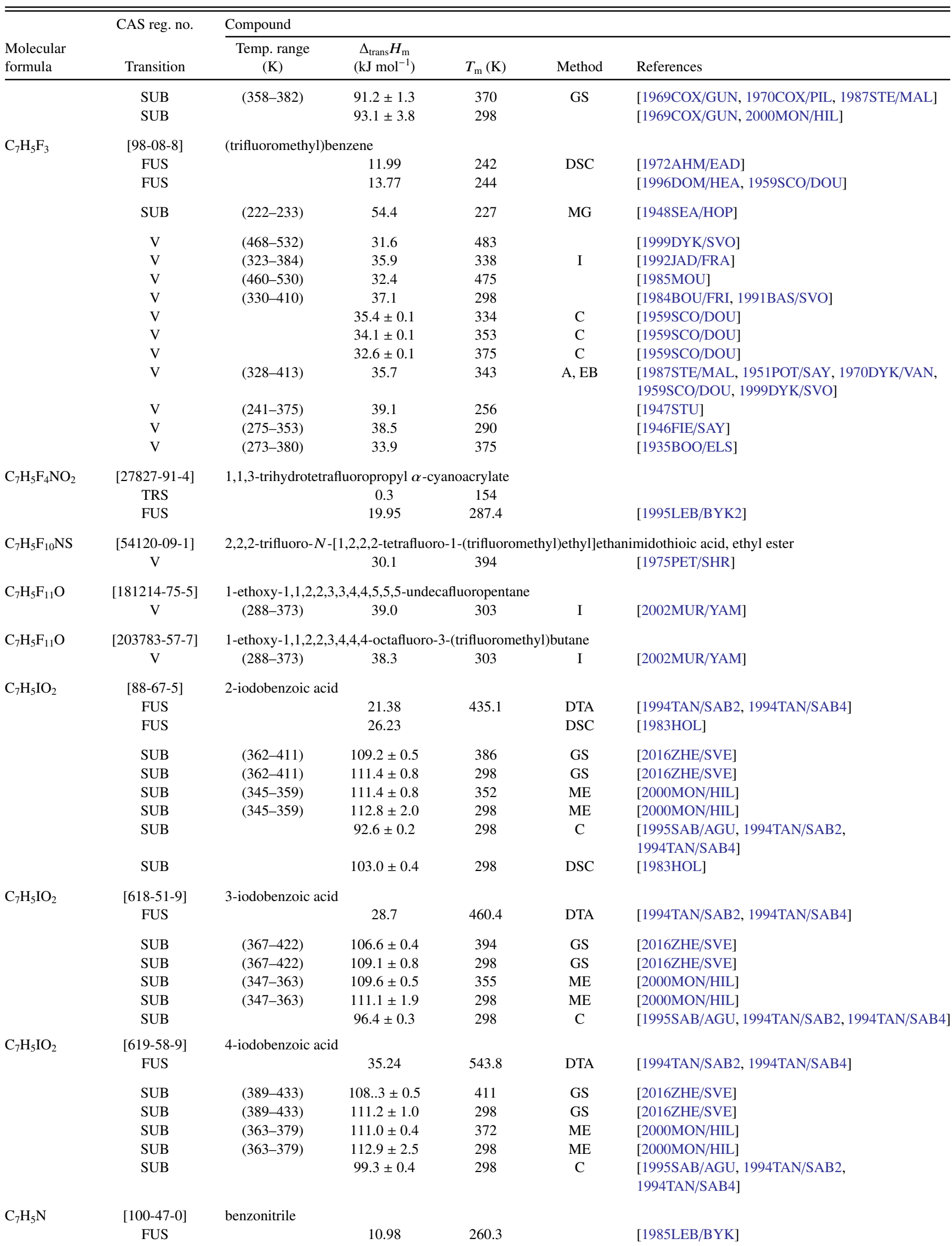


TABLE 10. Phase change enthalpies of $\mathrm{C}_{7}$ organic compounds-Continued

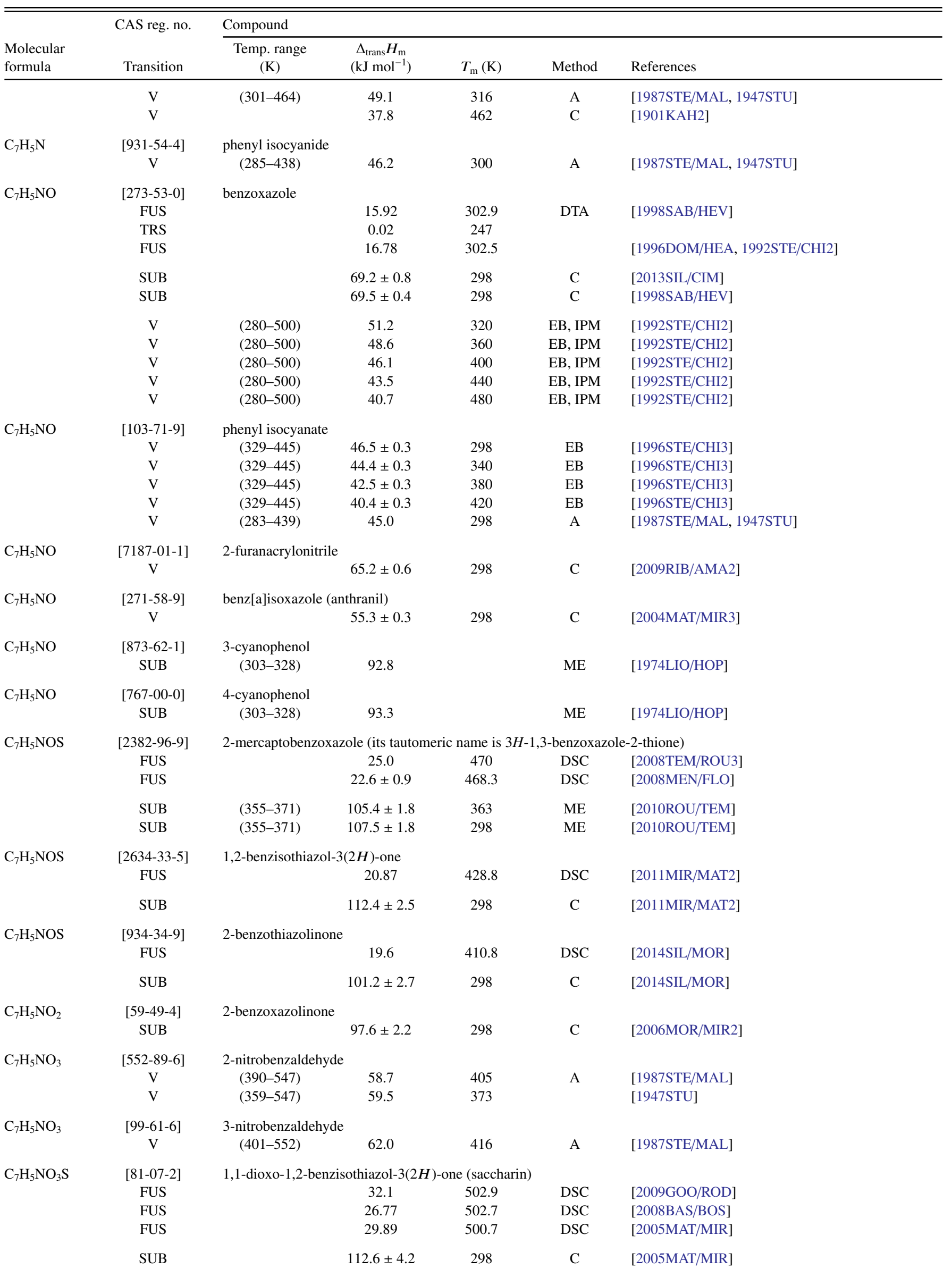


TABLE 10. Phase change enthalpies of $\mathrm{C}_{7}$ organic compounds-Continued

\begin{tabular}{|c|c|c|c|c|c|c|}
\hline \multirow[b]{2}{*}{$\begin{array}{l}\text { Molecular } \\
\text { formula }\end{array}$} & \multirow{2}{*}{$\begin{array}{l}\text { CAS reg. no. } \\
\text { Transition }\end{array}$} & \multicolumn{5}{|l|}{ Compound } \\
\hline & & $\begin{array}{c}\text { Temp. range } \\
\text { (K) }\end{array}$ & $\begin{array}{c}\Delta_{\text {trans }} H_{\mathrm{m}} \\
\left(\mathrm{kJ} \mathrm{mol}^{-1}\right)\end{array}$ & $T_{\mathrm{m}}(\mathrm{K})$ & Method & References \\
\hline \multirow[t]{4}{*}{$\mathrm{C}_{7} \mathrm{H}_{5} \mathrm{NO}_{4}$} & [552-16-9] & 2-nitrobenzoic & & & & \\
\hline & FUS & & 27.99 & 419 & & [1996DOM/HEA, 1926AND/LYN] \\
\hline & SUB & $(346-356)$ & $115.8 \pm 0.5$ & 356 & $\mathrm{ME}$ & [1999RIB/MAT] \\
\hline & SUB & $(346-356)$ & $118.7 \pm 0.5$ & 298 & $\mathrm{ME}$ & [1999RIB/MAT] \\
\hline \multirow[t]{5}{*}{$\mathrm{C}_{7} \mathrm{H}_{5} \mathrm{NO}_{4}$} & {$[121-92-6]$} & 3-nitrobenzoic & & & & \\
\hline & FUS & & 15.9 & 408.7 & DSC & [2004GUP/SIN] \\
\hline & FUS & & 19.33 & 414.3 & & [1996DOM/HEA, 1926AND/LYN] \\
\hline & SUB & $(347-361)$ & $107.2 \pm 0.4$ & 354 & $\mathrm{ME}$ & [1999RIB/MAT] \\
\hline & SUB & $(347-361)$ & $110.0 \pm 0.4$ & 298 & $\mathrm{ME}$ & [1999RIB/MAT] \\
\hline \multirow[t]{4}{*}{$\mathrm{C}_{7} \mathrm{H}_{5} \mathrm{NO}_{4}$} & {$[62-23-7]$} & 4-nitrobenzoic & & & & \\
\hline & FUS & & 36.9 & 512.4 & & [1996DOM/HEA, 1926AND/LYN] \\
\hline & SUB & $(367-381)$ & $115.4 \pm 0.6$ & 374 & $\mathrm{ME}$ & [1999RIB/MAT] \\
\hline & SUB & $(367-381)$ & $119.7 \pm 0.6$ & 298 & ME & [1999RIB/MAT] \\
\hline \multirow[t]{2}{*}{$\mathrm{C}_{7} \mathrm{H}_{5} \mathrm{NO}_{4}$} & {$[100-26-5]$} & pyridine-2,5-di & xylic acid & & & \\
\hline & SUB & & $163.6 \pm 2.7$ & 298 & $\mathrm{C}$ & [2005MAT/MOR] \\
\hline \multirow[t]{2}{*}{$\mathrm{C}_{7} \mathrm{H}_{5} \mathrm{NO}_{4}$} & [499-83-2] & pyridine-2,6-di & xylic acid & & & \\
\hline & SUB & & $137.1 \pm 5.7$ & 298 & $\mathrm{C}$ & [2005MAT/MOR] \\
\hline \multirow[t]{2}{*}{$\mathrm{C}_{7} \mathrm{H}_{5} \mathrm{NO}_{4}$} & {$[1874-22-2]$} & 3-(5-nitro-2-fur & -propenal & & & \\
\hline & SUB & & $97.9 \pm 2.1$ & & & [1980BAL/LEB, 1986PED/NAY] \\
\hline \multirow[t]{3}{*}{$\mathrm{C}_{7} \mathrm{H}_{5} \mathrm{NO}_{4}$} & {$[2620-44-2]$} & 5-nitro-1,3-ben & xole & & & \\
\hline & FUS & & 28.2 & 420.2 & DSC & [2007MAT/SOU] \\
\hline & SUB & & $97.4 \pm 2.2$ & 298 & $\mathrm{C}$ & [2007MAT/SOU] \\
\hline \multirow[t]{15}{*}{$\mathrm{C}_{7} \mathrm{H}_{5} \mathrm{NS}$} & {$[95-16-9]$} & benzothiazole & & & & \\
\hline & FUS & & 11.95 & 275.5 & DTA & [1998SAB/HEV] \\
\hline & TRS & & 0.04 & 245 & & \\
\hline & FUS & & 12.59 & 275.65 & & [1996DOM/HEA, 1992STE/CHI2] \\
\hline & FUS & $(6-320)$ & 12.8 & 275.6 & $\mathrm{AC}$ & [1996DOM/HEA, 1969GOU/WES] \\
\hline & FUS & & 13.3 & 276.0 & $\mathrm{CR}$ & [1963MEY, 1969GOU/WES] \\
\hline & SUB & & $72.9 \pm 0.6$ & 298 & B & {$[1998 \mathrm{SAB} / \mathrm{HEV}]$} \\
\hline & $\mathrm{V}$ & & $58.8 \pm 1.3$ & 298 & $\mathrm{C}$ & [2014SIL/CIM] \\
\hline & $\mathrm{V}$ & & $60.9 \pm 0.5$ & 298 & $\mathrm{C}$ & [1998SAB/HEV] \\
\hline & $\mathrm{V}$ & $(305-555)$ & 58.7 & 320 & $\mathrm{~EB}, \mathrm{IPM}$ & [1992STE/CHI2] \\
\hline & $\mathrm{V}$ & $(305-555)$ & 56.0 & 360 & $\mathrm{~EB}, \mathrm{IPM}$ & [1992STE/CHI2] \\
\hline & $\mathrm{V}$ & $(305-555)$ & 53.5 & 400 & $\mathrm{~EB}, \mathrm{IPM}$ & [1992STE/CHI2] \\
\hline & $\mathrm{V}$ & $(305-555)$ & 50.9 & 440 & $\mathrm{~EB}, \mathrm{IPM}$ & [1992STE/CHI2] \\
\hline & V & $(305-555)$ & 48.4 & 480 & $\mathrm{~EB}, \mathrm{IPM}$ & [1992STE/CHI2] \\
\hline & V & $(305-555)$ & 45.7 & 520 & EB, IPM & [1992STE/CHI2] \\
\hline \multirow[t]{2}{*}{$\mathrm{C}_{7} \mathrm{H}_{5} \mathrm{NS}$} & {$[103-72-0]$} & phenyl isothioc & & & & \\
\hline & V & $(320-492)$ & 52.6 & 335 & A & [1987STE/MAL, 1947STU] \\
\hline \multirow[t]{5}{*}{$\mathrm{C}_{7} \mathrm{H}_{5} \mathrm{NS}_{2}$} & [149-30-4] & 2-mercaptoben & azole (its taut & c name is & ,3-benzoth & ole-2-thione) \\
\hline & FUS & & $22.3 \pm 0.2$ & 455.9 & DSC & [2008TEM/ROU3] \\
\hline & FUS & & 20.56 & 453.5 & DSC & [2008MEN/FLO] \\
\hline & SUB & $(376-390)$ & $108.7 \pm 2.4$ & 383 & $\mathrm{ME}$ & [2010ROU/TEM] \\
\hline & SUB & $(376-390)$ & $111.0 \pm 2.4$ & 298 & $\mathrm{ME}$ & [2010ROU/TEM] \\
\hline \multirow[t]{2}{*}{$\mathrm{C}_{7} \mathrm{H}_{5} \mathrm{~N}_{3} \mathrm{O}$} & {$[90-16-4]$} & 1,2,3-benzotria & $(3 H)$-one & & & \\
\hline & SUB & & $111.3 \pm 3.4$ & 298 & $\mathrm{C}$ & [2011MIR/MAT3] \\
\hline \multirow[t]{3}{*}{$\mathrm{C}_{7} \mathrm{H}_{5} \mathrm{~N}_{3} \mathrm{O}_{2}$} & [5401-94-5] & 5-nitroindazole & & & & \\
\hline & SUB & (377-399) & $116.7 \pm 0.9$ & 388 & $\mathrm{ME}$ & [2010RIB/CAB] \\
\hline & SUB & (377-399) & $121.2 \pm 0.9$ & 298 & $\mathrm{ME}$ & [2010RIB/CAB] \\
\hline \multirow[t]{3}{*}{$\mathrm{C}_{7} \mathrm{H}_{5} \mathrm{~N}_{3} \mathrm{O}_{2}$} & [7597-18-4] & 6-nitroindazole & & & & \\
\hline & SUB & (359-381) & $113.4 \pm 0.6$ & 370 & $\mathrm{ME}$ & [2010RIB/CAB] \\
\hline & SUB & $(359-381)$ & $117.0 \pm 0.6$ & 298 & $\mathrm{ME}$ & [2010RIB/CAB] \\
\hline
\end{tabular}


TABLE 10. Phase change enthalpies of $\mathrm{C}_{7}$ organic compounds-Continued

\begin{tabular}{|c|c|c|c|c|c|c|}
\hline \multirow[b]{2}{*}{$\begin{array}{l}\text { Molecular } \\
\text { formula }\end{array}$} & \multirow{2}{*}{$\begin{array}{l}\text { CAS reg. no. } \\
\text { Transition }\end{array}$} & \multicolumn{5}{|l|}{ Compound } \\
\hline & & $\begin{array}{c}\text { Temp. range } \\
(\mathrm{K})\end{array}$ & $\begin{array}{c}\Delta_{\text {trans }} H_{\mathrm{m}} \\
\left(\mathrm{kJ} \mathrm{mol}^{-1}\right)\end{array}$ & $T_{\mathrm{m}}(\mathrm{K})$ & Method & References \\
\hline \multirow[t]{2}{*}{$\mathrm{C}_{7} \mathrm{H}_{5} \mathrm{~N}_{3} \mathrm{O}_{6}$} & {$[610-25-3]$} & 2,4,5-trinitrotoluene & & & & \\
\hline & FUS & & 24.7 & 376.2 & & [1996DOM/HEA, 1971CHI/THO] \\
\hline \multirow[t]{22}{*}{$\mathrm{C}_{7} \mathrm{H}_{5} \mathrm{~N}_{3} \mathrm{O}_{6}$} & {$[118-96-7]$} & 2,4,6-trinitrotoluene & & & & \\
\hline & FUS & & 22.0 & 354.0 & $\mathrm{DSC}$ & [2010RAM/FEL] \\
\hline & FUS & & 23.43 & 352.2 & DSC & [1993ACR, 1990HWA/TAM] \\
\hline & FUS & & 22.36 & 354.1 & & [1962CAD/ROD, 1969MAY/THO] \\
\hline & SUB & $(323-353)$ & 144.5 & 338 & TGA & [2013LIU/ZHA] \\
\hline & SUB & & $99.6 \pm 5$ & & UV/Vis & [2012HIK/WEE] \\
\hline & SUB & $(313-338)$ & 106.8 & & TGA & [2011FEL/RAM] \\
\hline & SUB & $(288-328)$ & 91 & 308 & HSA & [2009OXL/SMI] \\
\hline & SUB & & 104.2 & & DSC & [1990HWA/YOS] \\
\hline & SUB & $(293-353)$ & 112.4 & 308 & A & [1987STE/MAL] \\
\hline & SUB & & 81 & & & [1985DOB/CRA, 2009OXL/SMI] \\
\hline & SUB & $(301-349)$ & $113.2 \pm 1.5$ & 298 & ME & [1979KUD/KUD2] \\
\hline & SUB & $(297-330)$ & $99.2 \pm 2$ & & GS & [1976PEL, 1977PEL] \\
\hline & SUB & & $104.6 \pm 1.7$ & 298 & $\mathrm{ME}$ & [1971LEN/VEL] \\
\hline & SUB & $(327-349)$ & $103.3 \pm 2.5$ & 338 & & [1970LEN/VEL] \\
\hline & SUB & & U112-132 & & TGA & [1970MAY/VEN, 1978CUN/PAL] \\
\hline & SUB & $(323-353)$ & $118.4 \pm 4.2$ & & $\mathrm{ME}$ & [1950EDW, 1960JON, 1970COX/PIL] \\
\hline & SUB & & 102.2 & & & [1950NIT/SEK] \\
\hline & $\mathrm{V}$ & $(363-423)$ & 89.2 & 393 & TGA & [2013LIU/ZHA] \\
\hline & $\mathrm{V}$ & & 80.8 & & DSC & [1990HWA/YOS] \\
\hline & $\mathrm{V}$ & $(353-523)$ & 93.7 & 368 & A & [1987STE/MAL] \\
\hline & $\mathrm{V}$ & & $87.0 \pm 1.9$ & 298 & $\mathrm{ME}$ & [1978CUN/PAL] \\
\hline \multirow[t]{4}{*}{$\mathrm{C}_{7} \mathrm{H}_{5} \mathrm{~N}_{3} \mathrm{O}_{7}$} & {$[606-35-9]$} & 2,4,6-trinitroanisole & & & & \\
\hline & SUB & $(334-342)$ & 132.4 & 338 & A & [1987STE/MAL] \\
\hline & SUB & & $133.1 \pm 2.1$ & & & [1950NIT/SEK, 1970COX/PIL] \\
\hline & $\mathrm{V}$ & $(342-363)$ & 91.9 & 352 & A & {$[1987 \mathrm{STE} / \mathrm{MAL}]$} \\
\hline \multirow[t]{4}{*}{$\mathrm{C}_{7} \mathrm{H}_{5} \mathrm{~N}_{3} \mathrm{O}_{7}$} & {$[602-99-3]$} & 3-hydroxy-2,4,6-trin & iitrotoluene & & & \\
\hline & SUB & $(310-365)$ & $111.2 \pm 2.1$ & 298 & & [1978CUN/PAL] \\
\hline & SUB & $(325-350)$ & 103.3 & 337 & & [1970LEN/VEL] \\
\hline & SUB & & 104.6 & 298 & & [1970LEN/VEL] \\
\hline \multirow[t]{6}{*}{$\mathrm{C}_{7} \mathrm{H}_{5} \mathrm{~N}_{5} \mathrm{O}_{8}$} & {$[479-45-8]$} & $2,4,6-N$-tetranitro- $N$ & $V$-methylaniline & & & \\
\hline & FUS & & 22.93 & 402.6 & & [1973KRI/LIC] \\
\hline & FUS & & 25.86 & 402.6 & & [1996DOM/HEA, 1971HAL] \\
\hline & SUB & & 133.1 & & DSC & [1990HWA/YOS] \\
\hline & SUB & $(335-416)$ & $133.8 \pm 1.6$ & 298 & $\mathrm{ME}$ & [1978CUN/PAL] \\
\hline & $\mathrm{V}$ & & 108.4 & & DSC & [1990HWA/YOS] \\
\hline \multirow[t]{2}{*}{$\mathrm{C}_{7} \mathrm{H}_{6} \mathrm{ClF}$} & {$[443-83-4]$} & 1-chloro-3-fluoro-2-1 & methylbenzene & & & \\
\hline & FUS & & 12.6 & 246 & DSC & [1972AHM/EAD] \\
\hline \multirow[t]{2}{*}{$\mathrm{C}_{7} \mathrm{H}_{6} \mathrm{ClNO}_{2}$} & {$[612-23-7]$} & 1-(chloromethyl)-2-r & nitrobenzene & & & \\
\hline & SUB & & $96.2 \pm 3.5$ & & ME & [2006HOS/NAG] \\
\hline \multirow[t]{2}{*}{$\mathrm{C}_{7} \mathrm{H}_{6} \mathrm{Cl}_{2}$} & {$[98-87-3]$} & (dichloromethyl)ben & zene & & & \\
\hline & $\mathrm{V}$ & $(308-487)$ & 49.5 & 323 & A & [1987STE/MAL, 1947STU] \\
\hline \multirow[t]{2}{*}{$\mathrm{C}_{7} \mathrm{H}_{6} \mathrm{Cl}_{2}$} & {$[95-73-8]$} & 2,4-dichlorotoluene & & & & \\
\hline & V & $(346-475)$ & 50.6 & 361 & A & [1987STE/MAL, 1973FEL/SAV, 1999DYK/SVO] \\
\hline \multirow[t]{3}{*}{$\mathrm{C}_{7} \mathrm{H}_{6} \mathrm{Cl}_{2}$} & {$[118-69-4]$} & 2,6-dichlorotoluene & & & & \\
\hline & FUS & & 10.7 & 272 & DSC & [1972AHM/EAD] \\
\hline & $\mathrm{V}$ & $(275-305)$ & $51.6 \pm 0.3$ & 298 & GS & [2014VER/EME] \\
\hline \multirow[t]{2}{*}{$\mathrm{C}_{7} \mathrm{H}_{6} \mathrm{Cl}_{2}$} & {$[95-75-0]$} & 3,4-dichlorotoluene & & & & \\
\hline & V & $(378-543)$ & 49.4 & 393 & A & [1987STE/MAL, 1970DYK/VAN, 1999DYK/SVO] \\
\hline \multirow[t]{2}{*}{$\mathrm{C}_{7} \mathrm{H}_{6} \mathrm{Cl}_{2} \mathrm{O}$} & {$[1984-59-4]$} & 2,3-dichloroanisole & & & & \\
\hline & FUS & & 22.04 & 304.1 & DSC & [2008RIB/FER] \\
\hline
\end{tabular}


TABLE 10. Phase change enthalpies of $\mathrm{C}_{7}$ organic compounds-Continued

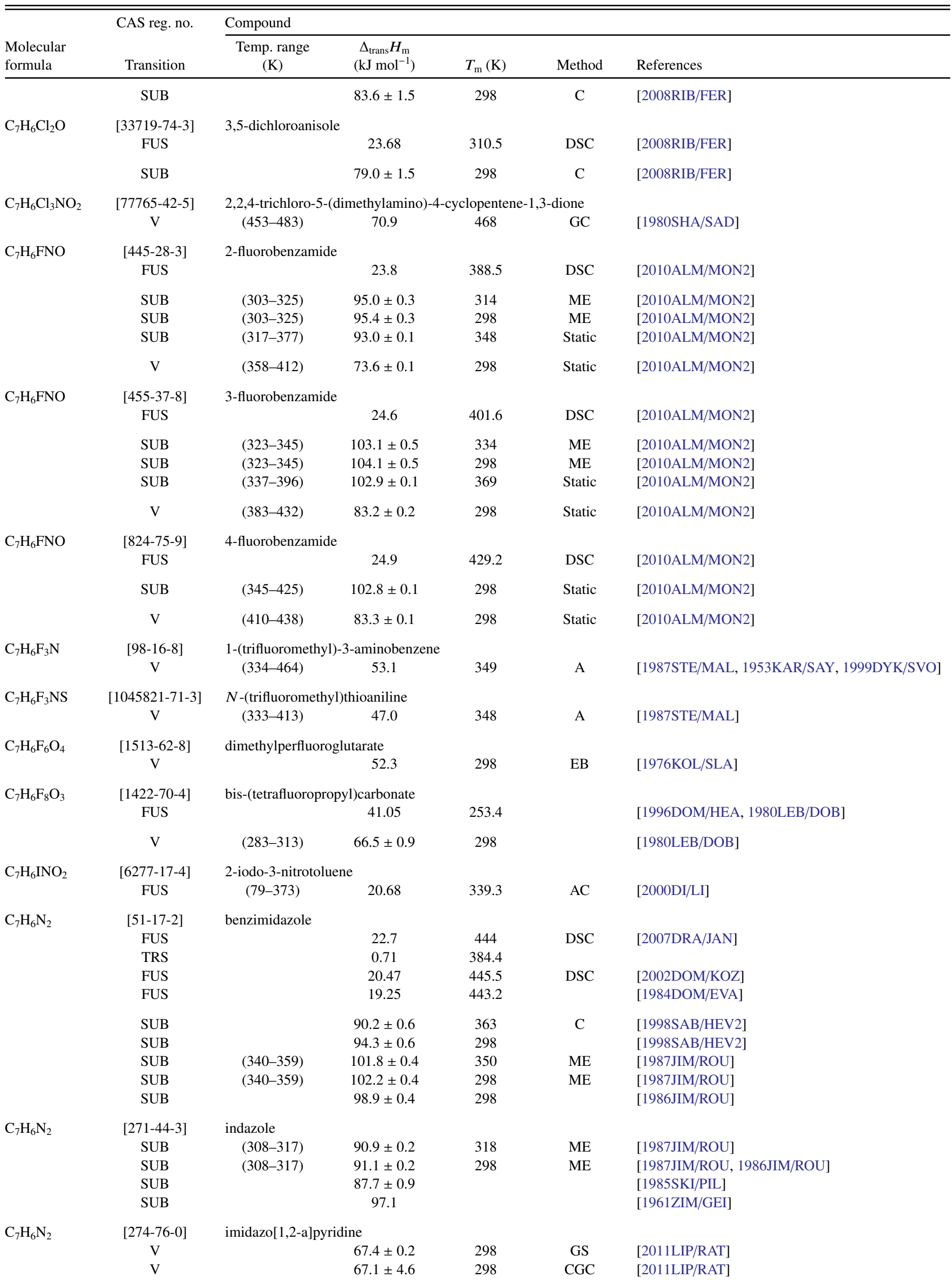


TABLE 10. Phase change enthalpies of $\mathrm{C}_{7}$ organic compounds-Continued

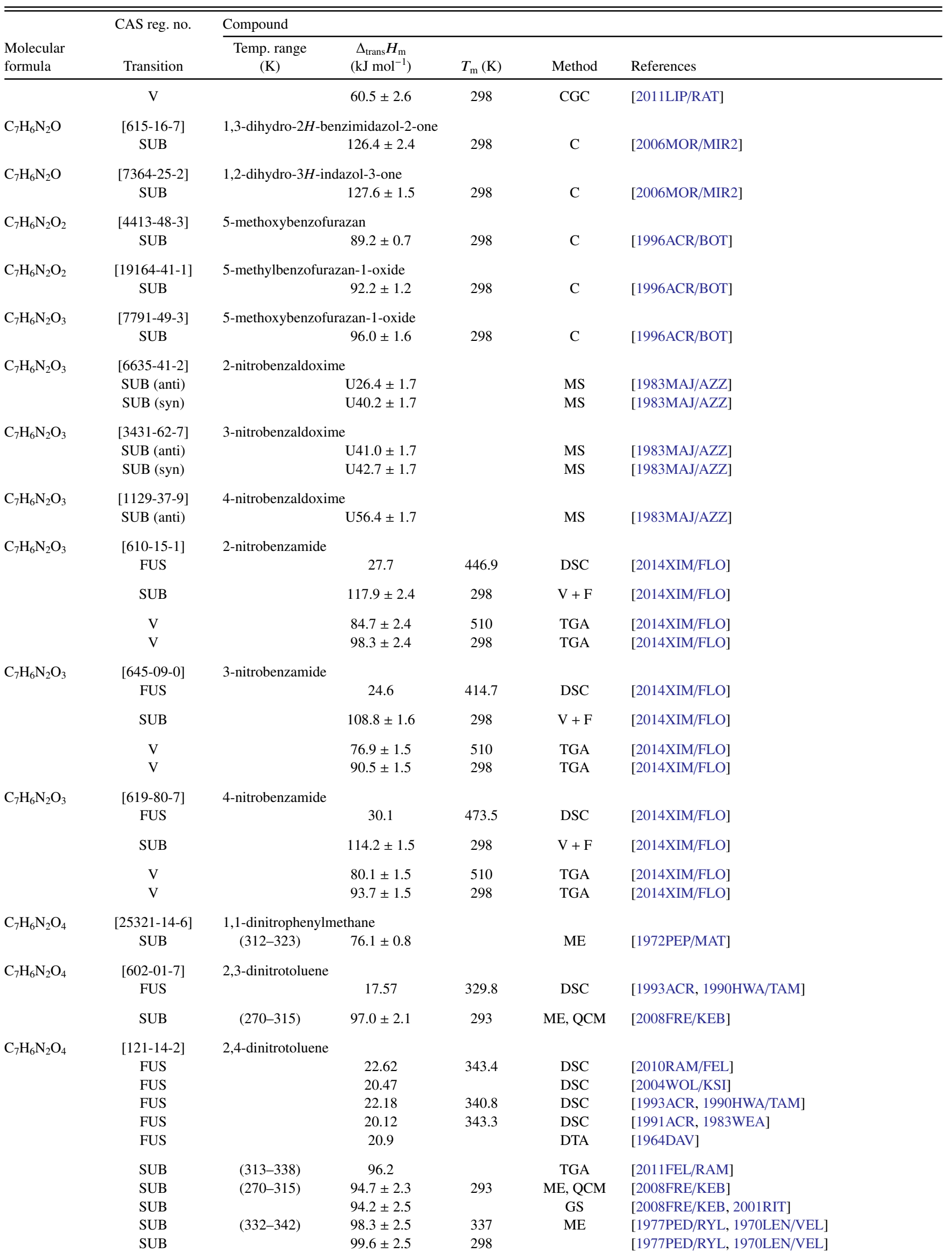


TABLE 10. Phase change enthalpies of $\mathrm{C}_{7}$ organic compounds-Continued

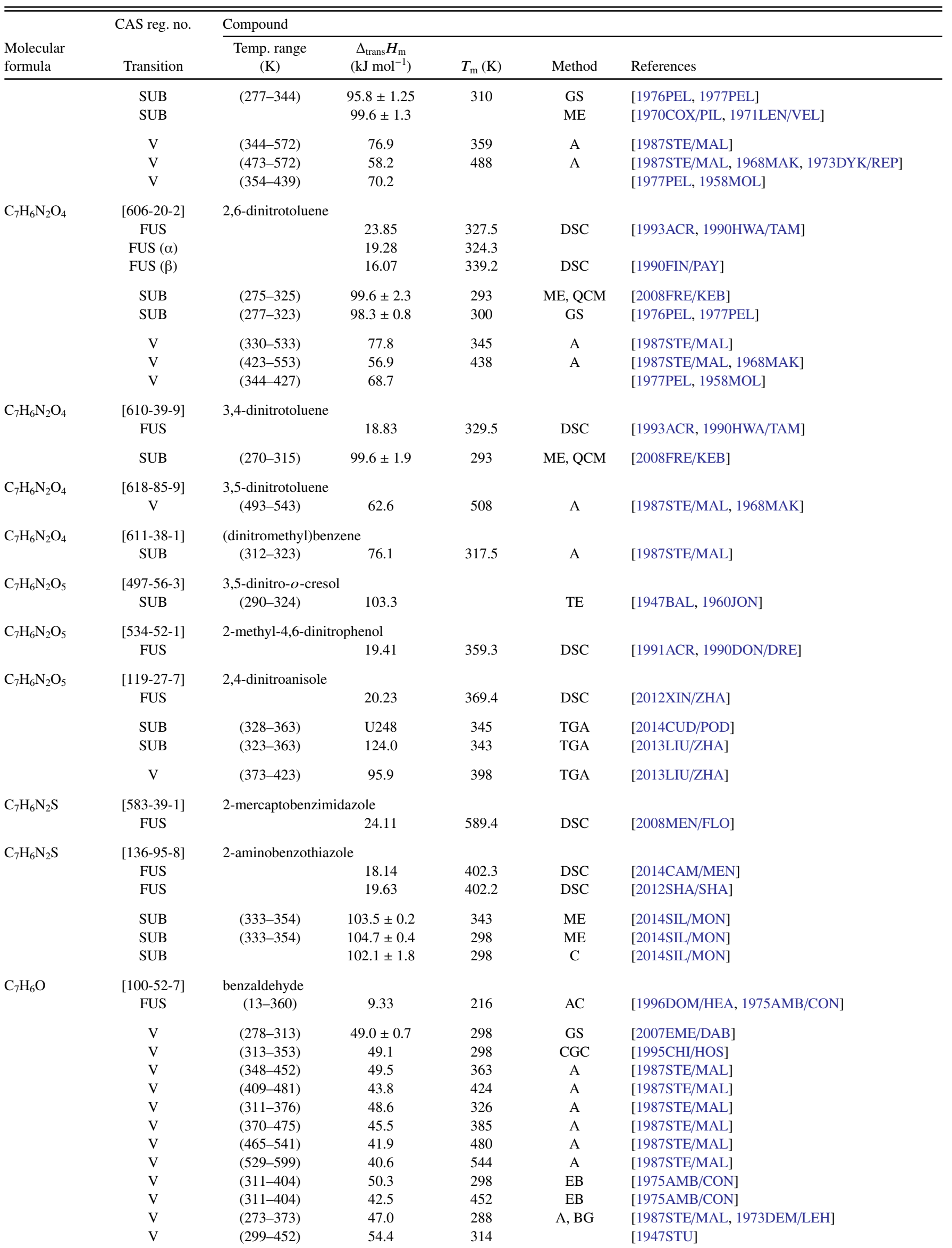


TABLE 10. Phase change enthalpies of $\mathrm{C}_{7}$ organic compounds-Continued

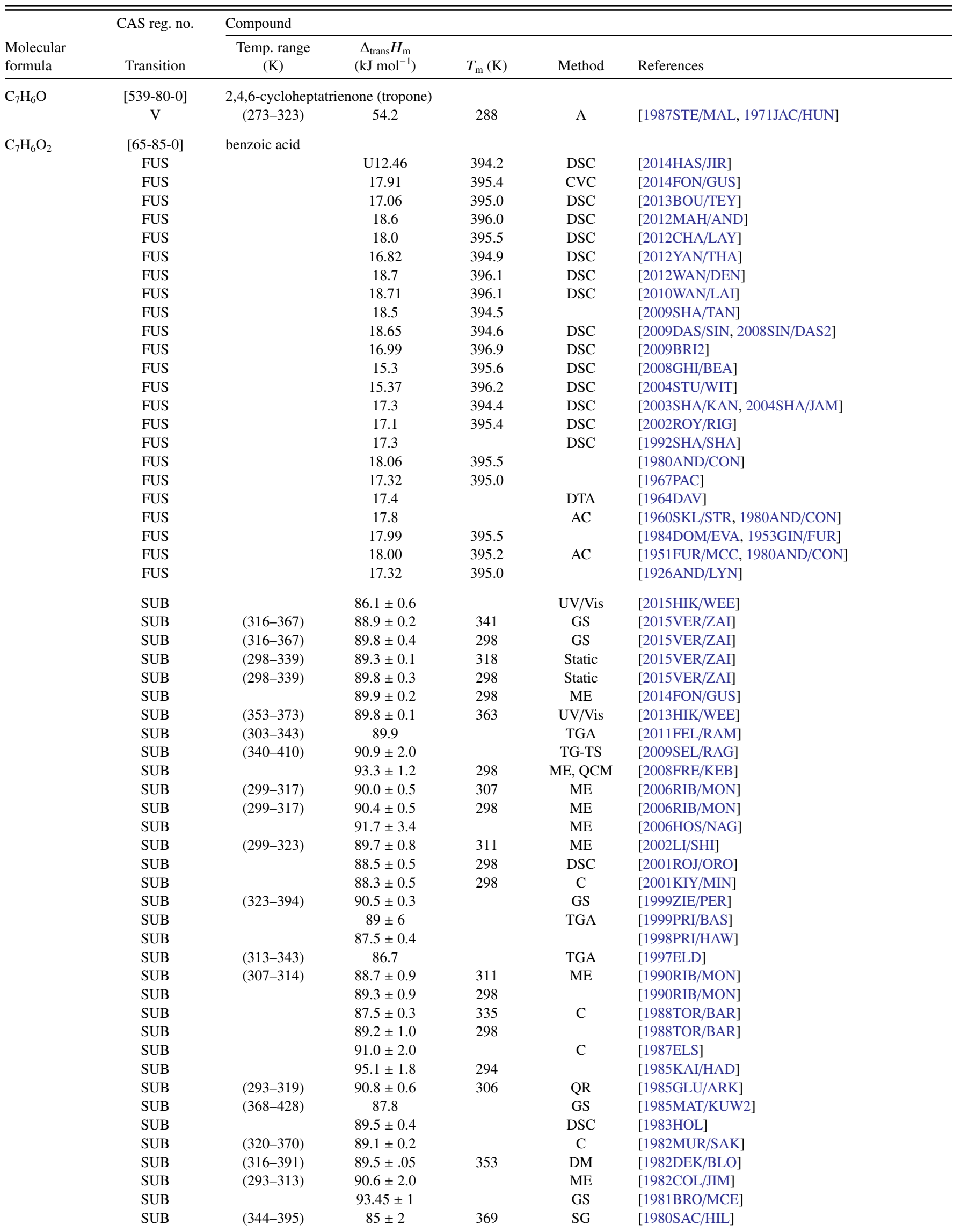


TABLE 10. Phase change enthalpies of $\mathrm{C}_{7}$ organic compounds-Continued

\begin{tabular}{|c|c|c|c|c|c|c|}
\hline \multirow[b]{2}{*}{$\begin{array}{l}\text { Molecular } \\
\text { formula }\end{array}$} & \multirow{2}{*}{$\begin{array}{c}\text { CAS reg. no. } \\
\text { Transition }\end{array}$} & \multicolumn{5}{|l|}{ Compound } \\
\hline & & $\begin{array}{c}\text { Temp. range } \\
(\mathrm{K})\end{array}$ & $\begin{array}{c}\Delta_{\text {trans }} H_{\mathrm{m}} \\
\left(\mathrm{kJ} \mathrm{mol}^{-1}\right)\end{array}$ & $T_{\mathrm{m}}(\mathrm{K})$ & Method & References \\
\hline & SUB & $(281-323)$ & $88.3 \pm 2.9$ & & LE & [1978NOW/SZC] \\
\hline & SUB & & $88.5 \pm 0.8$ & & $\mathrm{C}$ & [1976MIR/LEB] \\
\hline & SUB & $(294-331)$ & $92.5 \pm 4.0$ & & ME & [1975VAN/DEK] \\
\hline & SUB & $(293-318)$ & $88.5 \pm 1.6$ & & $\mathrm{TE}$ & [1975DEK/VAN] \\
\hline & SUB & $(273-318)$ & $92.9 \pm 0.2$ & 296 & ME & [1974ARS] \\
\hline & SUB & $(293-311)$ & $88.1 \pm 0.2$ & & TCM & [1973DEK/OON] \\
\hline & SUB & $(338-383)$ & $89.0 \pm 0.4$ & & ME & [1973MAL/GIG] \\
\hline & SUB & $(338-383)$ & $89.3 \pm 0.4$ & & $\mathrm{C}$ & [1973MAL/GIG] \\
\hline & SUB & $(290-315)$ & $86.6 \pm 1.3$ & & $\mathrm{ME}, \mathrm{C}$ & [1972WIE] \\
\hline & SUB & $(293-308)$ & $90.0 \pm 0.3$ & & ME & [1972COL/MON] \\
\hline & SUB & & $89.5 \pm 0.2$ & 298 & $\mathrm{C}$ & [1972MOR, 1971BEE/LIN] \\
\hline & SUB & $(299-329)$ & 89.1 & 314 & & [1971ASH] \\
\hline & SUB & $(290-315)$ & $86.6 \pm 1.7$ & 303 & ME & [1970WIE/WAU, 1999ZIE/PER] \\
\hline & SUB & $(324-392)$ & $90.4 \pm 0.8$ & 367 & HSA & [1970MEL/MER] \\
\hline & SUB & & $89.7 \pm 0.6$ & 298 & $\mathrm{C}$ & [1969CHA/STE] \\
\hline & SUB & $(348-378)$ & $88.9 \pm 0.5$ & 363 & GS & [1968MER] \\
\hline & SUB & $(291-307)$ & 90.9 & 299 & ME & [1965DAV/KYB] \\
\hline & SUB & $(243-387)$ & $91.5 \pm 0.5$ & 298 & GS & [1954DAV/JON, 1970COX/PIL, 1960JON] \\
\hline & SUB & & $84.2 \pm 0.8$ & 318 & $\mathrm{TE}$ & [1938WOL/WEG] \\
\hline & SUB & $(333-389)$ & 85.8 & 383 & $\mathrm{~T}$ & [1934HIR] \\
\hline & SUB & $(377-394)$ & $84.5 \pm 0.5$ & 364 & I & [1927KLO/WOO] \\
\hline & V & $(401-416)$ & $63.3 \pm 0.6$ & & & [2003PEN/RIB] \\
\hline & $\mathrm{V}$ & $(353-393)$ & 78.9 & 298 & CGC & [1995CHI/HOS] \\
\hline & V & $(368-428)$ & 67.8 & & GS & [1985MAT/KUW2] \\
\hline & $\mathrm{V}$ & $(405-523)$ & 66.3 & 420 & A & [1987STE/MAL] \\
\hline & V & & 65.4 & 428 & I & [1943CRA] \\
\hline & $\mathrm{V}$ & $(401-520)$ & 67.7 & 416 & $\mathrm{MM}, \mathrm{A}$ & [1927KLO/WOO] \\
\hline \multirow[t]{2}{*}{$\mathrm{C}_{7} \mathrm{H}_{6} \mathrm{O}_{2}$} & [90-02-8] & \multicolumn{5}{|c|}{ 2-hydroxybenzaldehyde } \\
\hline & FUS & & 13.3 & 278.7 & DSC & [2008BER/MIN] \\
\hline
\end{tabular}

[Note: The authors noted in the paper that their melting point temperature differed significantly from the published literature values.]

\begin{tabular}{|c|c|c|c|c|c|c|}
\hline & $\mathrm{V}$ & & $53.3 \pm 0.3$ & 298 & $\mathrm{C}$ & [2008BER/MIN] \\
\hline & $\mathrm{V}$ & & $50.4 \pm 1.3$ & 298 & $\mathrm{C}$ & [2007RIB/ARA] \\
\hline & V & $(383-470)$ & 30.6 & 398 & A & [1987STE/MAL] \\
\hline & V & & 47.7 & & & [1986BAL/GNA] \\
\hline & $\mathrm{V}$ & $(306-470)$ & 49.6 & 321 & & [1947STU] \\
\hline $\mathrm{C}_{7} \mathrm{H}_{6} \mathrm{O}_{2}$ & [100-83-4] & 3-hydroxybenz & yyde & & & \\
\hline & FUS & & 24.1 & 376.0 & DSC & [2012SHA/RAI] \\
\hline & SUB & $(312-330)$ & $99.7 \pm 0.6$ & 321 & ME & [2010RIB/GON] \\
\hline & SUB & $(312-330)$ & $100.1 \pm 0.6$ & 298 & ME & [2010RIB/GON] \\
\hline & SUB & $(303-328)$ & 93.6 & & ME & [1974LIO/HOP] \\
\hline $\mathrm{C}_{7} \mathrm{H}_{6} \mathrm{O}_{2}$ & [123-08-0] & 4-hydroxybenz & yde & & & \\
\hline & FUS (I) & $(298-400)$ & 22.2 & 391.1 & DSC & [2013SIM/BER2] \\
\hline & FUS & & 20.3 & 390.8 & DSC & [2008BER/MIN] \\
\hline & FUS & & 21.6 & 390.8 & DSC & [2008TEM/ROU] \\
\hline & SUB & $(324-341)$ & $101.8 \pm 0.5$ & 333 & ME & [2010RIB/GON] \\
\hline & SUB & $(324-341)$ & $102.5 \pm 0.5$ & 298 & ME & [2010RIB/GON] \\
\hline & SUB & & $99.7 \pm 0.4$ & 298 & $\mathrm{C}$ & [2008BER/MIN] \\
\hline & SUB & $(303-336)$ & $98.2 \pm 1.3$ & 298 & $\mathrm{ME}$ & [1987STE/MAL, 1971PAR/ROC] \\
\hline & SUB & $(303-328)$ & 94.4 & & ME & [1974LIO/HOP] \\
\hline & SUB & $(312-336)$ & 91.2 & 324 & & [1960AIH] \\
\hline & V & (394-583) & 72.3 & 409 & A & [1987STE/MAL, 1947STU] \\
\hline $\mathrm{C}_{7} \mathrm{H}_{6} \mathrm{O}_{2}$ & $\begin{array}{c}{[1864-94-4]} \\
V\end{array}$ & $\begin{array}{c}\text { phenylformate } \\
(287-305)\end{array}$ & $52.9+0.6$ & 298 & BG & [1976ANT/CAR, 1975ANT/CAR] \\
\hline $\mathrm{C}_{7} \mathrm{H}_{6} \mathrm{O}_{2}$ & [274-09-9] & 1,3-benzodiox & & & & \\
\hline & V & & 41.4 & & & [1958CAS/FLE2] \\
\hline $\mathrm{C}_{7} \mathrm{H}_{6} \mathrm{O}_{2}$ & {$[533-75-5]$} & 2-hydroxy-2,4, & oheptatrien- & (tropol & & \\
\hline
\end{tabular}


TABLE 10. Phase change enthalpies of $\mathrm{C}_{7}$ organic compounds-Continued

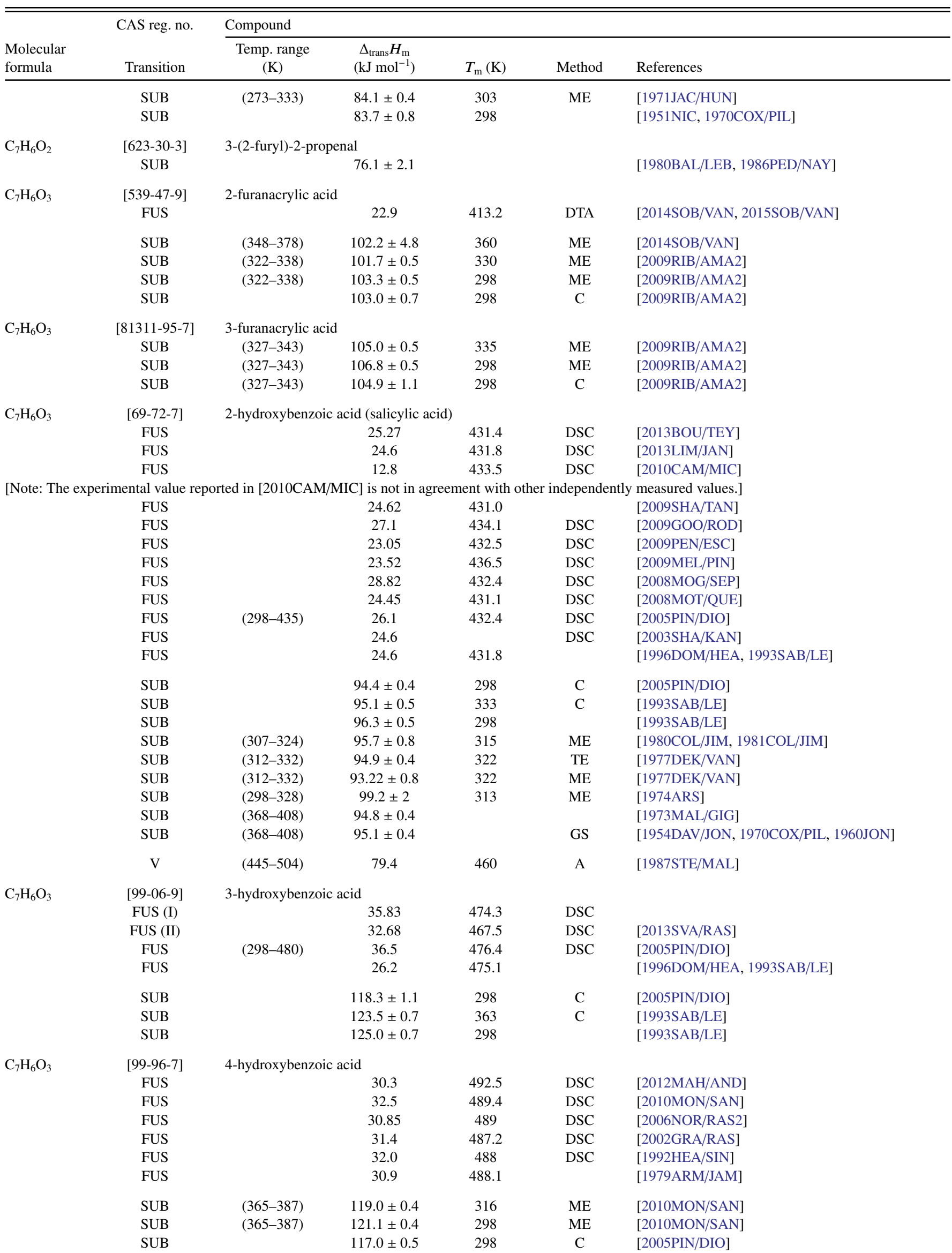


TABLE 10. Phase change enthalpies of $\mathrm{C}_{7}$ organic compounds-Continued

\begin{tabular}{|c|c|c|c|c|c|c|}
\hline \multirow[b]{2}{*}{$\begin{array}{l}\text { Molecular } \\
\text { formula }\end{array}$} & \multirow{2}{*}{$\begin{array}{l}\text { CAS reg. no. } \\
\text { Transition }\end{array}$} & \multicolumn{5}{|l|}{ Compound } \\
\hline & & $\begin{array}{c}\text { Temp. range } \\
(\mathrm{K})\end{array}$ & $\begin{array}{c}\Delta_{\text {trans }} H_{\mathrm{m}} \\
\left(\mathrm{kJ} \mathrm{mol}^{-1}\right) \\
\end{array}$ & $T_{\mathrm{m}}(\mathrm{K})$ & Method & References \\
\hline & SUB & & $112.4 \pm 0.7$ & 363 & $\mathrm{C}$ & [1993SAB/LE] \\
\hline & SUB & & $114.1 \pm 0.7$ & 298 & & [1993SAB/LE] \\
\hline & SUB & $(398-433)$ & 116.3 & & GS & [1954DAV/JON, 1960JON] \\
\hline \multirow[t]{4}{*}{$\mathrm{C}_{7} \mathrm{H}_{6} \mathrm{O}_{3}$} & [533-31-3] & \multicolumn{5}{|c|}{ 5-hydroxy-1,3-benzodioxole (sesamol) } \\
\hline & FUS & & 16.96 & 337.7 & DSC & [2004MAT/MON] \\
\hline & SUB & $(293-309)$ & $92.1 \pm 0.6$ & 301 & ME & [2004MAT/MON] \\
\hline & SUB & $(293-309)$ & $92.2 \pm 0.6$ & 298 & ME & [2004MAT/MON] \\
\hline \multirow[t]{3}{*}{$\mathrm{C}_{7} \mathrm{H}_{6} \mathrm{O}_{3} \mathrm{~S}$} & {$[4066-41-5]$} & \multicolumn{5}{|c|}{ 5-acetyl-2-thiophenecarboxylic acid } \\
\hline & SUB & $(364-387)$ & $119.6 \pm 0.6$ & 375.2 & ME & [2008RIB/SAN5] \\
\hline & SUB & $(364-387)$ & $123.5 \pm 0.6$ & 298 & $\mathrm{ME}$ & [2008RIB/SAN5] \\
\hline \multirow[t]{8}{*}{$\mathrm{C}_{7} \mathrm{H}_{6} \mathrm{O}_{4}$} & [303-38-8] & \multicolumn{5}{|c|}{ 2,3-dihydroxybenzoic acid } \\
\hline & FUS (triclinic) & & 29.69 & 481.3 & DSC & [2010SAR/SAN] \\
\hline & $\begin{array}{c}\text { FUS } \\
\text { (monoclinic) }\end{array}$ & & 21.63 & 481.7 & $\mathrm{DSC}$ & [2010SAR/SAN] \\
\hline & FUS & & 31.9 & 476.6 & DSC & [2010MON/GON] \\
\hline & SUB & $(345-363)$ & $109.1 \pm 0.8$ & 354 & ME & [2010MON/GON] \\
\hline & SUB & $(345-363)$ & $110.7 \pm 0.8$ & 298 & $\mathrm{ME}$ & [2010MON/GON] \\
\hline & SUB & & $116 \pm 4$ & & TGA & [1999PRI/BAS] \\
\hline & SUB & & $119.8 \pm 4.8$ & 298 & TGA & [1999PRI/BAS, 2015PRI] \\
\hline \multirow[t]{9}{*}{$\mathrm{C}_{7} \mathrm{H}_{6} \mathrm{O}_{4}$} & {$[89-86-1]$} & \multicolumn{5}{|c|}{ 2,4-dihydroxybenzoic acid } \\
\hline & FUS & & 31.0 & 501.7 & DSC & [2011VEC/BRU] \\
\hline & SUB & $(364-390)$ & $122 \pm 2$ & 298 & $\mathrm{TE}$ & [2011VEC/BRU] \\
\hline & SUB & & $117 \pm 7$ & 298 & TGA & [2011 VEC/BRU] \\
\hline & SUB & $(376-392)$ & $124.0 \pm 0.8$ & 384 & $\mathrm{ME}$ & [2010MON/GON] \\
\hline & SUB & $(376-392)$ & $126.4 \pm 0.8$ & 298 & $\mathrm{ME}$ & [2010MON/GON] \\
\hline & SUB & & $126 \pm 6$ & & TGA & [1999PRI/BAS] \\
\hline & SUB & & $127.9 \pm 6.2$ & 298 & TGA & [1999PRI/BAS, 2015PRI] \\
\hline & V & (511-534) & $79 \pm 4$ & 523 & TGA & [2011VEC/BRU] \\
\hline \multirow[t]{7}{*}{$\mathrm{C}_{7} \mathrm{H}_{6} \mathrm{O}_{4}$} & {$[490-79-9]$} & \multicolumn{5}{|c|}{ 2,5-dihydroxybenzoic acid } \\
\hline & FUS & & 20.8 & 476.2 & DSC & [2010MON/GON] \\
\hline & SUB & $(372-389)$ & $128.1 \pm 1.4$ & 380 & $\mathrm{ME}$ & [2010MON/GON] \\
\hline & SUB & $(372-389)$ & $130.4 \pm 1.3$ & 298 & ME & [2010MON/GON] \\
\hline & SUB & $(362-379)$ & $117.9 \pm 1.4$ & 370 & $\mathrm{ME}$ & [2006CHE/OJA] \\
\hline & SUB & & $109 \pm 3$ & 452 & TGA & [1999PRI/BAS] \\
\hline & SUB & & $114.4 \pm 2.5$ & 298 & TGA & [1999PRI/BAS, 2015PRI] \\
\hline \multirow[t]{9}{*}{$\mathrm{C}_{7} \mathrm{H}_{6} \mathrm{O}_{4}$} & {$[303-07-1]$} & \multicolumn{5}{|c|}{ 2,6-dihydroxybenzoic acid } \\
\hline & FUS & & 25.0 & 443.7 & DSC & [2011VEC/BRU] \\
\hline & SUB & $(346-369)$ & $111 \pm 2$ & 298 & $\mathrm{TE}$ & [2011VEC/BRU] \\
\hline & SUB & & $113 \pm 5$ & 298 & TGA & [2011VEC/BRU] \\
\hline & SUB & $(347-365)$ & $107.5 \pm 1.0$ & 356 & $\mathrm{ME}$ & [2010MON/GON] \\
\hline & SUB & $(347-365)$ & $109.1 \pm 1.0$ & 298 & ME & [2010MON/GON] \\
\hline & SUB & & $111 \pm 7$ & & TGA & [1999PRI/BAS] \\
\hline & SUB & & $109.6 \pm 2.6$ & 298 & TGA & [1999PRI/BAS, 2015PRI] \\
\hline & $\mathrm{V}$ & $(454-477)$ & $82 \pm 3$ & 465 & TGA & [2011VEC/BRU] \\
\hline \multirow[t]{9}{*}{$\mathrm{C}_{7} \mathrm{H}_{6} \mathrm{O}_{4}$} & {$[99-50-3]$} & \multicolumn{5}{|c|}{ 3,4-dihydroxybenzoic acid } \\
\hline & FUS & & 33.5 & 474.8 & DSC & [2013VEC] \\
\hline & FUS & & 34.0 & 474.9 & DSC & [2011VEC/BRU] \\
\hline & FUS (triclinic) & & 30.91 & 474.7 & DSC & [2010SAR/SAN] \\
\hline & FUS & & 31.2 & 472.3 & DSC & [2009QUE/MOT] \\
\hline & SUB & $(382-409)$ & $139 \pm 2$ & 298 & $\mathrm{TE}$ & [2011VEC/BRU] \\
\hline & SUB & & $146 \pm 7$ & 298 & TGA & [2011VEC/BRU] \\
\hline & SUB & $(387-403)$ & $132.3 \pm 1.2$ & 395 & ME & [2010MON/GON] \\
\hline & SUB & $(387-403)$ & $135.1 \pm 1.2$ & 298 & $\mathrm{ME}$ & [2010MON/GON] \\
\hline
\end{tabular}


TABLE 10. Phase change enthalpies of $\mathrm{C}_{7}$ organic compounds-Continued

\begin{tabular}{|c|c|c|c|c|c|c|}
\hline \multirow[b]{2}{*}{$\begin{array}{l}\text { Molecular } \\
\text { formula }\end{array}$} & \multirow{2}{*}{$\begin{array}{c}\text { CAS reg. no. } \\
\text { Transition }\end{array}$} & \multicolumn{5}{|l|}{ Compound } \\
\hline & & $\begin{array}{c}\text { Temp. range } \\
\text { (K) }\end{array}$ & $\begin{array}{c}\Delta_{\text {trans }} H_{\mathrm{m}} \\
\left(\mathrm{kJ} \mathrm{mol}^{-1}\right)\end{array}$ & $T_{\mathrm{m}}(\mathrm{K})$ & Method & References \\
\hline & SUB & & $153 \pm 9$ & 450 & TGA & [1999PRI/BAS] \\
\hline & SUB & & $157.8 \pm 10.3$ & 298 & TGA & [1999PRI/BAS] [2015PRI] \\
\hline & $\mathrm{V}$ & $(485-505)$ & $105 \pm 4$ & 495 & TGA & [2011VEC/BRU] \\
\hline \multirow[t]{7}{*}{$\mathrm{C}_{7} \mathrm{H}_{6} \mathrm{O}_{4}$} & {$[99-10-5]$} & \multicolumn{5}{|c|}{ 3,5-dihydroxybenzoic acid } \\
\hline & FUS & & 39.4 & 511.7 & DSC & [2010SAR/SAN] \\
\hline & FUS & & 38.3 & 508.3 & DSC & [2010MON/GON] \\
\hline & SUB & $(345-363)$ & $139.8 \pm 1.8$ & 416 & $\mathrm{ME}$ & [2010MON/GON] \\
\hline & SUB & $(345-363)$ & $143.2 \pm 1.8$ & 298 & ME & [2010MON/GON] \\
\hline & SUB & & $135 \pm 6$ & & TGA & [1999PRI/BAS] \\
\hline & SUB & & $144.4 \pm 8.4$ & 298 & TGA & [1999PRI/BAS, 2015PRI] \\
\hline \multirow[t]{4}{*}{$\mathrm{C}_{7} \mathrm{H}_{6} \mathrm{O}_{5}$} & [149-91-7] & \multicolumn{5}{|c|}{ 3,4,5-trihydroxybenzoic acid } \\
\hline & TRS & & 19.28 & 351.3 & & \\
\hline & FUS & & 62.38 & 524.2 & DSC & [2010BOG/GON] \\
\hline & SUB & $(391-421)$ & 75.1 & 406 & & [1934HIR] \\
\hline \multirow[t]{7}{*}{$\mathrm{C}_{7} \mathrm{H}_{7} \mathrm{Br}$} & [100-39-0] & benzylbromide & & & & \\
\hline & FUS & & 13.2 & 271.8 & & [1976ASH] \\
\hline & $\mathrm{V}$ & $(284-306)$ & $53.3 \pm 0.7$ & 298 & GS & [2002KRA/VAS] \\
\hline & V & & 53.7 & 298 & CGC & [2002KRA/VAS] \\
\hline & $\mathrm{V}$ & $(340-409)$ & 48.1 & 355 & $\mathrm{I}, \mathrm{A}$ & [1976ASH, 1987STE/MAL] \\
\hline & $\mathrm{V}$ & & $50.5 \pm 0.5$ & 298 & & [1976ASH] \\
\hline & V & $(305-472)$ & 46.9 & 320 & A & [1987STE/MAL, 1947STU, 1999DYK/SVO] \\
\hline \multirow[t]{7}{*}{$\mathrm{C}_{7} \mathrm{H}_{7} \mathrm{Br}$} & {$[95-46-5]$} & 2-bromotoluene & & & & \\
\hline & $\mathrm{V}$ & $(281-313)$ & $47.7 \pm 0.2$ & 297 & GS & [2015VER/SAZ] \\
\hline & $\mathrm{V}$ & $(281-313)$ & $47.7 \pm 0.5$ & 298 & GS & [2015VER/SAZ] \\
\hline & V & $(322-455)$ & 47.2 & 337 & & [1999DYK/SVO] \\
\hline & $\mathrm{V}$ & $(353-518)$ & 45.3 & 368 & A & [1987STE/MAL, 1970DYK/VAN, 1973DYK/REP] \\
\hline & $\mathrm{V}$ & $(297-455)$ & 52.6 & 312 & & [1947STU] \\
\hline & $\mathrm{V}$ & $(273-348)$ & 48.8 & 288 & & {$[1940 S T U / S A Y]$} \\
\hline \multirow[t]{6}{*}{$\mathrm{C}_{7} \mathrm{H}_{7} \mathrm{Br}$} & [591-17-3] & 3-bromotoluene & & & & \\
\hline & $\mathrm{V}$ & $(274-308)$ & $48.8 \pm 0.2$ & 291 & GS & [2015VER/SAZ] \\
\hline & $\mathrm{V}$ & $(274-308)$ & $48.4 \pm 0.5$ & 298 & GS & [2015VER/SAZ] \\
\hline & $\mathrm{V}$ & $(351-457)$ & 47.7 & 366 & & [1999DYK/SVO] \\
\hline & $\mathrm{V}$ & $(287-457)$ & 48.3 & 302 & A & [1987STE/MAL, 1947STU] \\
\hline & $\mathrm{V}$ & $(273-348)$ & 49.4 & 288 & & [1940STU/SAY] \\
\hline \multirow[t]{10}{*}{$\mathrm{C}_{7} \mathrm{H}_{7} \mathrm{Br}$} & [106-38-7] & 4-bromotoluene & & & & \\
\hline & FUS & $(150-320)$ & 15.13 & 301.2 & $\mathrm{AC}$ & [1996VAN/ALV] \\
\hline & FUS & & 15.3 & 299.7 & & [1911LOU/DUP] \\
\hline & SUB & $(275-301)$ & $62.6 \pm 0.3$ & 288 & GS & [2015VER/SAZ] \\
\hline & SUB & $(275-301)$ & $62.4 \pm 0.6$ & 298 & GS & [2015VER/SAZ] \\
\hline & $\mathrm{V}$ & $(302-335)$ & $46.2 \pm 0.3$ & 318 & GS & [2015VER/SAZ] \\
\hline & V & $(302-335)$ & $47.3 \pm 0.6$ & 298 & GS & [2015VER/SAZ] \\
\hline & V & $(320-458)$ & 47.1 & 335 & & [1999DYK/SVO] \\
\hline & $\mathrm{V}$ & $(273-472)$ & 55.3 & 288 & & [1999DYK/SVO] \\
\hline & $\mathrm{V}$ & $(358-523)$ & 45.8 & 373 & A & [1987STE/MAL, 1970DYK/VAN, 1973DYK/REP] \\
\hline \multirow[t]{3}{*}{$\mathrm{C}_{7} \mathrm{H}_{7} \mathrm{BrO}$} & [578-57-4] & 2-bromoanisole & & & & \\
\hline & $\mathrm{V}$ & & $61.8 \pm 1.3$ & 298 & $\mathrm{C}$ & [2009RIB/FER3] \\
\hline & $\mathrm{V}$ & & 52.3 & & & [1986BAL/GNA] \\
\hline \multirow[t]{3}{*}{$\mathrm{C}_{7} \mathrm{H}_{7} \mathrm{BrO}$} & [2398-37-0] & 3-bromoanisole & & & & \\
\hline & V & & $58.0 \pm 1.2$ & 298 & $\mathrm{C}$ & [2009RIB/FER3] \\
\hline & $\mathrm{V}$ & & 50.2 & & & [1986BAL/GNA] \\
\hline \multirow[t]{2}{*}{$\mathrm{C}_{7} \mathrm{H}_{7} \mathrm{BrO}$} & [104-92-7] & 4-bromoanisole & & & & \\
\hline & $\mathrm{V}$ & & $58.3 \pm 1.2$ & 298 & $\mathrm{C}$ & [2009RIB/FER3] \\
\hline
\end{tabular}


TABLE 10. Phase change enthalpies of $\mathrm{C}_{7}$ organic compounds-Continued

\begin{tabular}{|c|c|c|c|c|c|c|}
\hline \multirow[b]{2}{*}{$\begin{array}{l}\text { Molecular } \\
\text { formula }\end{array}$} & \multirow{2}{*}{$\begin{array}{l}\text { CAS reg. no. } \\
\text { Transition }\end{array}$} & \multicolumn{5}{|l|}{ Compound } \\
\hline & & $\begin{array}{c}\text { Temp. range } \\
(\mathrm{K})\end{array}$ & $\begin{array}{c}\Delta_{\text {trans }} H_{\mathrm{m}} \\
\left(\mathrm{kJ} \mathrm{mol}^{-1}\right)\end{array}$ & $T_{\mathrm{m}}(\mathrm{K})$ & Method & References \\
\hline & V & & 50.6 & & & [1986BAL/GNA] \\
\hline & V & $(318-496)$ & 48.9 & 333 & & {$[1947 \mathrm{STU}]$} \\
\hline \multirow[t]{2}{*}{$\mathrm{C}_{7} \mathrm{H}_{7} \mathrm{BrS}$} & {$[19614-16-5]$} & 2-bromothioani & & & & \\
\hline & $\mathrm{V}$ & & 56.5 & & & [1986BAL/GNA] \\
\hline \multirow[t]{2}{*}{$\mathrm{C}_{7} \mathrm{H}_{7} \mathrm{BrS}$} & {$[33733-73-2]$} & 3-bromothioani & & & & \\
\hline & $\mathrm{V}$ & & 54.4 & & & [1986BAL/GNA] \\
\hline \multirow[t]{2}{*}{$\mathrm{C}_{7} \mathrm{H}_{7} \mathrm{BrS}$} & [104-95-0] & 4-bromothioani & & & & \\
\hline & $\mathrm{V}$ & & 55.7 & & & [1986BAL/GNA] \\
\hline \multirow[t]{7}{*}{$\mathrm{C}_{7} \mathrm{H}_{7} \mathrm{Cl}$} & {$[100-44-7]$} & benzyl chloride & & & & \\
\hline & FUS & & 8.74 & 230 & $\mathrm{DSC}$ & [1972AHM/EAD] \\
\hline & $\mathrm{V}$ & $(276-309)$ & $50.1 \pm 0.3$ & 298 & GS & [2002KRA/VAS] \\
\hline & $\mathrm{V}$ & 392 & 49.9 & 298 & CGC & [2002KRA/VAS] \\
\hline & V & $(320-390)$ & 48.6 & 335 & A, I & [1987STE/MAL, 1976ASH] \\
\hline & $\mathrm{V}$ & & $50.1 \pm 0.5$ & 298 & & [1976ASH, 1999DYK/SVO] \\
\hline & $\mathrm{V}$ & $(295-453)$ & 48.6 & 310 & & [1987STE/MAL, 1947STU] \\
\hline \multirow[t]{11}{*}{$\mathrm{C}_{7} \mathrm{H}_{7} \mathrm{Cl}$} & {$[95-49-8]$} & 2-chlorotoluene & & & & \\
\hline & FUS & & 10.3 & 237 & $\mathrm{DSC}$ & [1972AHM/EAD] \\
\hline & $\mathrm{V}$ & $(274-306)$ & $46.4 \pm 0.4$ & 298 & GS & [2014VER/EME] \\
\hline & $\mathrm{V}$ & $(370-432)$ & 41.6 & 385 & & [1999DYK/SVO] \\
\hline & $\mathrm{V}$ & $(345-430)$ & 45.3 & 298 & & [1984BOU/FRI, 1991BAS/SVO] \\
\hline & $\mathrm{V}$ & $(345-430)$ & 42.5 & 361 & & [1984BOU/FRI] \\
\hline & $\mathrm{V}$ & $(338-493)$ & 42.8 & 353 & A & [1987STE/MAL, 1973DYK/REP, 1970DYK/VAN] \\
\hline & $\mathrm{V}$ & $(278-432)$ & 44.8 & 293 & & [1947STU] \\
\hline & $\mathrm{V}$ & $(273-348)$ & 45.8 & 288 & Static & [1940STU/SAY] \\
\hline & $\mathrm{V}$ & $(273-348)$ & $45.5 \pm 2.0$ & 298 & Static & [1940STU/SAY, 2014VER/EME] \\
\hline & V & & $46.1 \pm 0.1$ & 298 & $\mathrm{C}$ & [1926MAT, 2014VER/EME] \\
\hline \multirow[t]{6}{*}{$\mathrm{C}_{7} \mathrm{H}_{7} \mathrm{Cl}$} & [108-41-8] & 3-chlorotoluene & & & & \\
\hline & $\mathrm{V}$ & $(274-309)$ & $46.8 \pm 0.4$ & 298 & GS & [2014VER/EME] \\
\hline & $\mathrm{V}$ & $(373-435)$ & 41.9 & 388 & & [1999DYK/SVO] \\
\hline & $\mathrm{V}$ & $(277-436)$ & 43.7 & 292 & A & [1987STE/MAL, 1947STU] \\
\hline & $\mathrm{V}$ & $(273-348)$ & 46.2 & 288 & Static & [1940STU/SAY] \\
\hline & $\mathrm{V}$ & $(273-348)$ & $46.1 \pm 2.0$ & 298 & Static & [1940STU/SAY, 2014VER/EME] \\
\hline \multirow[t]{12}{*}{$\mathrm{C}_{7} \mathrm{H}_{7} \mathrm{Cl}$} & {$[106-43-4]$} & 4-chlorotoluene & & & & \\
\hline & FUS & & 13.6 & 280.6 & DSC & [1999MIK/MAR] \\
\hline & FUS & $(150-310)$ & 13.55 & 280.7 & $\mathrm{AC}$ & [1996VAN/ALV] \\
\hline & $\mathrm{V}$ & $(274-309)$ & $46.5 \pm 0.5$ & 298 & GS & [2014VER/EME] \\
\hline & $\mathrm{V}$ & $(362-435)$ & 41.8 & 375 & & [1999DYK/SVO] \\
\hline & V & $(304-436)$ & 41.7 & 319 & A & [1987STE/MAL] \\
\hline & $\mathrm{V}$ & $(340-430)$ & 46.0 & 298 & & [1984BOU/FRI, 1991BAS/SVO] \\
\hline & $\mathrm{V}$ & $(338-433)$ & 43.5 & 353 & & [1984BOU/FRI] \\
\hline & $\mathrm{V}$ & $(279-435)$ & 44.1 & 293 & & [1947STU] \\
\hline & $\mathrm{V}$ & $(277-348)$ & 45.3 & 313 & Static & [1940STU/SAY] \\
\hline & $\mathrm{V}$ & $(277-348)$ & $46.0 \pm 2.0$ & 298 & Static & [1940STU/SAY, 2014VER/EME] \\
\hline & $\mathrm{V}$ & & $46.4 \pm 0.1$ & 298 & $\mathrm{C}$ & [1926MAT, 2014VER/EME] \\
\hline \multirow[t]{2}{*}{$\mathrm{C}_{7} \mathrm{H}_{7} \mathrm{ClN}_{2} \mathrm{O}$} & {$[5814-05-1]$} & 2-chlorobenzoi & hydrazide & & & \\
\hline & FUS & & 25.6 & 392.2 & DSC & [2003CHI/ACR] \\
\hline \multirow[t]{2}{*}{$\mathrm{C}_{7} \mathrm{H}_{7} \mathrm{ClN}_{2} \mathrm{O}$} & {$[536-40-3]$} & 4-chlorobenzoi & d hydrazide & & & \\
\hline & FUS & & 32.9 & 437.2 & $\mathrm{DSC}$ & [2003CHI/ACR] \\
\hline \multirow[t]{2}{*}{$\mathrm{C}_{7} \mathrm{H}_{7} \mathrm{ClN}_{2} \mathrm{O}_{2}$} & {$[23042-32-2]$} & $N$-methyl- $N$ - ( & orophenyl)ni & & & \\
\hline & FUS & & 19.5 & 323.4 & DSC & [2002DAS/ZAL] \\
\hline \multirow[t]{2}{*}{$\mathrm{C}_{7} \mathrm{H}_{7} \mathrm{ClN}_{2} \mathrm{~S}$} & {$[5344-82-1]$} & 1-(o-chlorophe & hiourea & & & \\
\hline & FUS & & 22.29 & 413.5 & $\mathrm{DSC}$ & [1990DON/DRE] \\
\hline \multirow[t]{3}{*}{$\mathrm{C}_{7} \mathrm{H}_{7} \mathrm{ClO}$} & [766-51-8] & 2-chloroanisole & & & & \\
\hline & $\mathrm{V}$ & & $55.0 \pm 0.8$ & 298 & $\mathrm{C}$ & [2008RIB/FER2] \\
\hline & $\mathrm{V}$ & & 49.4 & & & [1986BAL/GNA] \\
\hline
\end{tabular}


TABLE 10. Phase change enthalpies of $\mathrm{C}_{7}$ organic compounds-Continued

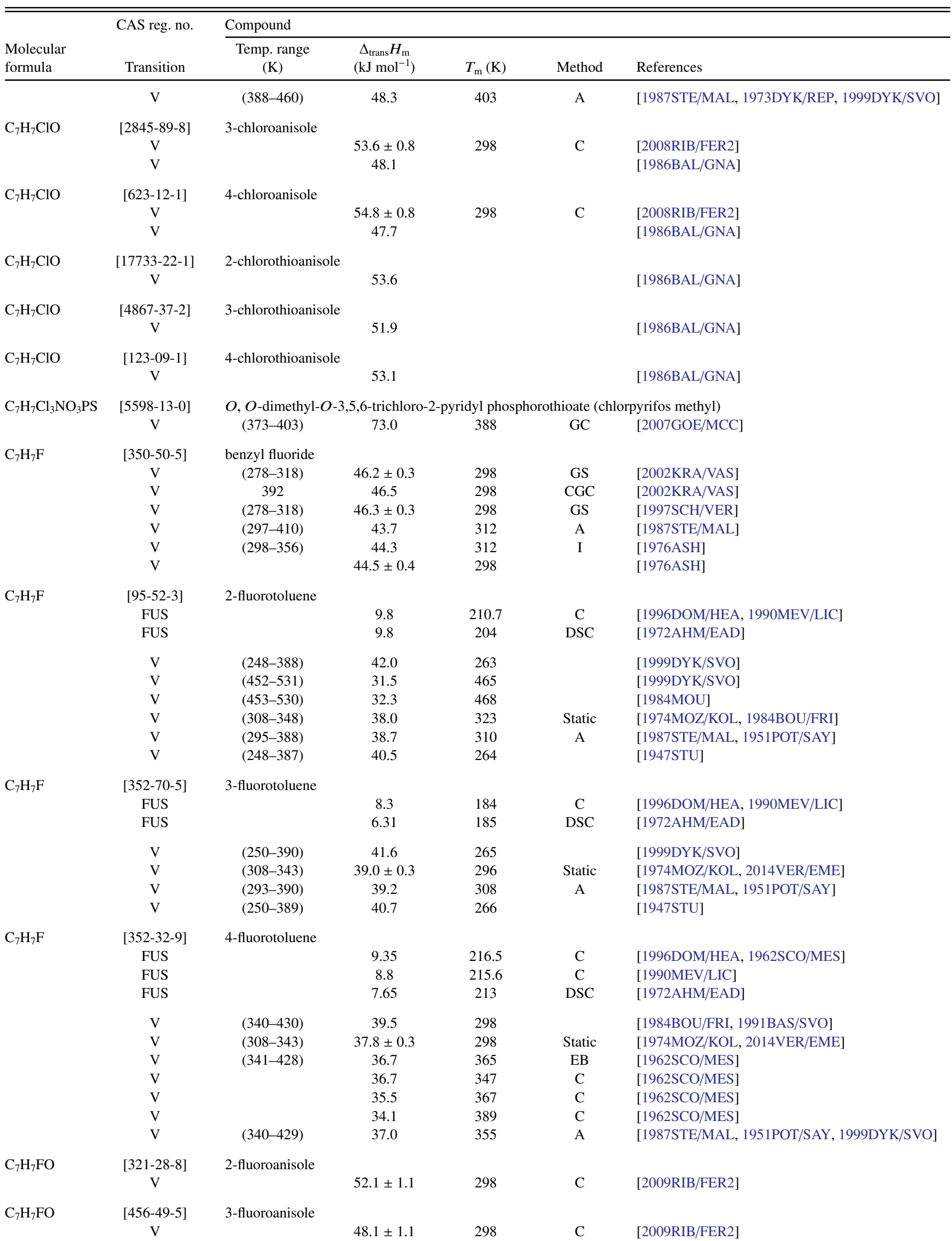


TABLE 10. Phase change enthalpies of $\mathrm{C}_{7}$ organic compounds-Continued

\begin{tabular}{|c|c|c|c|c|c|c|}
\hline \multirow[b]{2}{*}{$\begin{array}{l}\text { Molecular } \\
\text { formula }\end{array}$} & \multirow{2}{*}{$\begin{array}{l}\text { CAS reg. no. } \\
\text { Transition }\end{array}$} & \multicolumn{5}{|l|}{ Compound } \\
\hline & & $\begin{array}{c}\text { Temp. range } \\
\text { (K) }\end{array}$ & $\begin{array}{c}\Delta_{\text {trans }} H_{\mathrm{m}} \\
\left(\mathrm{kJ} \mathrm{mol}^{-1}\right)\end{array}$ & $T_{\mathrm{m}}(\mathrm{K})$ & Method & References \\
\hline \multirow[t]{2}{*}{$\mathrm{C}_{7} \mathrm{H}_{7} \mathrm{FO}$} & [459-60-9] & 4-fluoroanisole & & & & \\
\hline & $\mathrm{V}$ & & $48.7 \pm 1.2$ & 298 & $\mathrm{C}$ & [2009RIB/FER2] \\
\hline \multirow[t]{2}{*}{$\mathrm{C}_{7} \mathrm{H}_{7} \mathrm{~F}_{2} \mathrm{~N}$} & {$[23162-99-4]$} & \multicolumn{5}{|c|}{$N, N$-difluorobenzylamine } \\
\hline & $\mathrm{V}$ & $(313-333)$ & 77.8 & 323 & A & [1987STE/MAL] \\
\hline \multirow[t]{2}{*}{$\mathrm{C}_{7} \mathrm{H}_{7} \mathrm{~F}_{9} \mathrm{O}$} & {$[72372-80-6]$} & \multicolumn{5}{|c|}{ 1,1,1,2,2,3,3,4,4-nonafluoro-4-propoxybutane } \\
\hline & $\mathrm{V}$ & $(288-369)$ & 37.9 & 303 & I & [2002MUR/YAM] \\
\hline \multirow[t]{6}{*}{$\mathrm{C}_{7} \mathrm{H}_{7} \mathrm{~F}_{9} \mathrm{O}$} & {$[83310-97-8]$} & \multicolumn{5}{|c|}{ 3-(perfluorobutyl)-1-propanol } \\
\hline & $\mathrm{V}$ & $(254-309)$ & 75.8 & 256 & GS & [2013SCH/BUC] \\
\hline & $\mathrm{V}$ & $(254-309)$ & 69.6 & 273 & GS & [2013SCH/BUC] \\
\hline & $\mathrm{V}$ & $(254-309)$ & 66.9 & 282 & GS & [2013SCH/BUC] \\
\hline & $\mathrm{V}$ & $(254-309)$ & 64.1 & 293 & GS & [2013SCH/BUC] \\
\hline & $\mathrm{V}$ & $(254-309)$ & 60.7 & 309 & GS & {$[2013 \mathrm{SCH} / \mathrm{BUC}]$} \\
\hline \multirow[t]{6}{*}{$\mathrm{C}_{7} \mathrm{H}_{7} \mathrm{I}$} & {$[620-05-3]$} & benzyl iodide & & & & \\
\hline & FUS & & 13.2 & 299.5 & & [1976ASH] \\
\hline & V & $(301-337)$ & $57.4 \pm 0.3$ & 298 & GS & [2002KRA/VAS] \\
\hline & V & 392 & 57.7 & 298 & CGC & [2002KRA/VAS] \\
\hline & $\mathrm{V}$ & $(360-400)$ & 46.8 & 375 & $\mathrm{I}, \mathrm{A}$ & [1987STE/MAL, 1976ASH] \\
\hline & $\mathrm{V}$ & & $50.6 \pm 1.4$ & 298 & & {$[1976 \mathrm{ASH}]$} \\
\hline \multirow[t]{4}{*}{$\mathrm{C}_{7} \mathrm{H}_{7} \mathrm{I}$} & {$[615-37-2]$} & 2-iodotoluene & & & & \\
\hline & $\mathrm{V}$ & $(283-325)$ & $52.0 \pm 0.1$ & 304 & GS & [2015VER/SAZ] \\
\hline & $\mathrm{V}$ & $(283-325)$ & $52.3 \pm 0.4$ & 298 & GS & [2015VER/SAZ] \\
\hline & V & $(310-484)$ & 49.7 & 325 & A & [1987STE/MAL, 1947STU] \\
\hline \multirow[t]{3}{*}{$\mathrm{C}_{7} \mathrm{H}_{7} \mathrm{I}$} & {$[625-95-6]$} & 3-iodotoluene & & & & \\
\hline & $\mathrm{V}$ & $(279-318)$ & $53.0 \pm 0.4$ & 297 & GS & [2015VER/SAZ] \\
\hline & $\mathrm{V}$ & $(279-318)$ & $52.9 \pm 0.6$ & 298 & GS & [2015VER/SAZ] \\
\hline \multirow[t]{5}{*}{$\mathrm{C}_{7} \mathrm{H}_{7} \mathrm{I}$} & $\begin{array}{c}\text { [624-31-7] } \\
\text { FUS }\end{array}$ & $\begin{array}{c}\text { 4-iodotoluene } \\
\text { (150-320) }\end{array}$ & 14.96 & 306.7 & $\mathrm{AC}$ & [1996VAN/ALV] \\
\hline & SUB & $(280-303)$ & $68.2 \pm 0.7$ & 292 & GS & [2015VER/SAZ] \\
\hline & SUB & $(280-303)$ & $68.0 \pm 0.9$ & 298 & GS & [2015VER/SAZ] \\
\hline & V & $(308-333)$ & $51.2 \pm 0.7$ & 321 & GS & [2015VER/SAZ] \\
\hline & $\mathrm{V}$ & $(308-333)$ & $52.5 \pm 0.7$ & 298 & GS & [2015VER/SAZ] \\
\hline \multirow[t]{2}{*}{$\mathrm{C}_{7} \mathrm{H}_{7} \mathrm{IO}$} & {$[529-28-2]$} & 2-iodoanisole & & & & \\
\hline & $\mathrm{V}$ & & $65.3 \pm 1.4$ & 298 & $\mathrm{C}$ & [2012FER/RIB] \\
\hline \multirow[t]{2}{*}{$\mathrm{C}_{7} \mathrm{H}_{7} \mathrm{IO}$} & {$[766-85-8]$} & 3-iodoanisole & & & & \\
\hline & V & & $62.5 \pm 1.3$ & 298 & $\mathrm{C}$ & [2012FER/RIB] \\
\hline \multirow[t]{5}{*}{$\mathrm{C}_{7} \mathrm{H}_{7} \mathrm{IO}$} & {$[696-62-8]$} & 4-iodoanisole & & & & \\
\hline & SUB & & $82.4 \pm 0.8$ & 298 & $\mathrm{ME}$ & [2012FER/RIB] \\
\hline & SUB & & $82.6 \pm 1.5$ & 298 & $\mathrm{C}$ & [2012FER/RIB] \\
\hline & $\mathrm{V}$ & $(401-520)$ & 54.4 & 416 & A & [1987STE/MAL, 1999DYK/SVO] \\
\hline & $\mathrm{V}$ & $(401-479)$ & $53.1 \pm 0.4$ & 440 & I & {$[1956 \mathrm{BRE} / \mathrm{UBB}]$} \\
\hline \multirow[t]{2}{*}{$\mathrm{C}_{7} \mathrm{H}_{7} \mathrm{NO}$} & [6264-93-3] & 2-aminotropone & & & & \\
\hline & SUB & $(273-333)$ & $71.13 \pm 0.4$ & 303 & $\mathrm{ME}$ & [1971JAC/HUN] \\
\hline \multirow[t]{11}{*}{$\mathrm{C}_{7} \mathrm{H}_{7} \mathrm{NO}$} & {$[55-21-0]$} & benzamide & & & & \\
\hline & FUS & & 19.5 & 400.2 & DTA & [2013KAN] \\
\hline & FUS & & 22.80 & 400 & DSC & [2012BUT/MAR] \\
\hline & FUS & & 21.69 & 400 & DSC & [2010BAI/VAN] \\
\hline & FUS & & 23.18 & 401.3 & DSC & [2010ALM/MON] \\
\hline & FUS & & 19.15 & 402.1 & DSC & [2009BRI] \\
\hline & FUS & & 23.14 & & DSC & [2008SIN/DAS2] \\
\hline & FUS & & 18.49 & 402.3 & & [1991ACR, 1984SHA/BAS] \\
\hline & SUB & $(324-346)$ & $102.2 \pm 0.4$ & 335 & ME & [2010ALM/MON] \\
\hline & SUB & $(324-346)$ & $103.1 \pm 0.4$ & 298 & $\mathrm{ME}$ & [2010ALM/MON] \\
\hline & SUB & $(325-342)$ & 96.9 & 333.5 & & [1987STE/MAL, 1960AIH2] \\
\hline
\end{tabular}


TABLE 10. Phase change enthalpies of $\mathrm{C}_{7}$ organic compounds-Continued

\begin{tabular}{|c|c|c|c|c|c|c|}
\hline \multirow[b]{2}{*}{$\begin{array}{l}\text { Molecular } \\
\text { formula }\end{array}$} & \multirow{2}{*}{$\begin{array}{l}\text { CAS reg. no. } \\
\text { Transition }\end{array}$} & \multicolumn{5}{|l|}{ Compound } \\
\hline & & $\begin{array}{c}\text { Temp. range } \\
\text { (K) }\end{array}$ & $\begin{array}{c}\Delta_{\text {trans }} H_{\mathrm{m}} \\
\left(\mathrm{kJ} \mathrm{mol}^{-1}\right)\end{array}$ & $T_{\mathrm{m}}(\mathrm{K})$ & Method & References \\
\hline & SUB & $(323-349)$ & $101.7 \pm 1$ & 298 & $\mathrm{C}$ & {$[1982 \mathrm{TOR} / \mathrm{SAB} 2]$} \\
\hline & $\mathrm{V}$ & $(380-438)$ & $75.8 \pm 1.2$ & 409 & Static & [2010ALM/MON] \\
\hline & $\mathrm{V}$ & $(380-438)$ & $86.5 \pm 0.1$ & 298 & Static & [2010ALM/MON] \\
\hline \multicolumn{7}{|c|}{ [Note: VP measurements in [2010ALM/MON] include those for the subcooled liquid.] } \\
\hline \multirow[t]{2}{*}{$\mathrm{C}_{7} \mathrm{H}_{7} \mathrm{NO}$} & {$[103-70-8]$} & formanilide & & & & \\
\hline & SUB & $(298-318)$ & 77.8 & 308 & A & [1987STE/MAL, 1960AIH2] \\
\hline \multirow[t]{2}{*}{$\mathrm{C}_{7} \mathrm{H}_{7} \mathrm{NO}$} & [1122-62-9] & 2-acetylpyridin & & & & \\
\hline & $\mathrm{V}$ & & $60.5 \pm 0.3$ & 298 & $\mathrm{C}$ & [2007FRE/OLI] \\
\hline \multirow[t]{2}{*}{$\mathrm{C}_{7} \mathrm{H}_{7} \mathrm{NO}$} & {$[350-03-8]$} & 3-acetylpyridin & & & & \\
\hline & $\mathrm{V}$ & & $66.1 \pm 0.8$ & 298 & $\mathrm{C}$ & [2007FRE/OLI] \\
\hline \multirow[t]{2}{*}{$\mathrm{C}_{7} \mathrm{H}_{7} \mathrm{NO}$} & [1122-54-9] & 4-acetylpyridin & & & & \\
\hline & V & & $66.5 \pm 0.9$ & 298 & $\mathrm{C}$ & [2007FRE/OLI] \\
\hline \multirow[t]{2}{*}{$\mathrm{C}_{7} \mathrm{H}_{7} \mathrm{NO}_{2}$} & {$[622-42-4]$} & (nitromethyl)b & 538 & 378 & $A$ & [1087STE/MAI] \\
\hline & 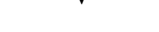 & (300 t10) & & 310 & A & [190/STL/NIRL] \\
\hline \multirow[t]{8}{*}{$\mathrm{C}_{7} \mathrm{H}_{7} \mathrm{NO}_{2}$} & {$[88-72-2]$} & 2-nitrotoluene & & & & \\
\hline & $\mathrm{V}$ & $(283-313)$ & $59.6 \pm 1.6$ & 298 & GS & [2010WID/BRU2] \\
\hline & $\mathrm{V}$ & $(274-323)$ & $59.0 \pm 0.3$ & 299 & GS & [2000VER/HEI] \\
\hline & $\mathrm{V}$ & $(274-323)$ & $59.1 \pm 0.3$ & 298 & GS & [2000VER/HEI] \\
\hline & $\mathrm{V}$ & $(388-448)$ & 52.0 & 403 & $\mathrm{~EB}$ & [1994AIM] \\
\hline & $\mathrm{V}$ & $(402-496)$ & 51.0 & 417 & A & [1987STE/MAL] \\
\hline & $\mathrm{V}$ & $(387-493)$ & 52.2 & 402 & & [1938LEV/SHT, 1994AIM] \\
\hline & $\mathrm{V}$ & $(323-490)$ & 48.1 & 406 & & [1926BER/MAY] \\
\hline \multirow[t]{5}{*}{$\mathrm{C}_{7} \mathrm{H}_{7} \mathrm{NO}_{2}$} & {$[99-08-1]$} & 3-nitrotoluene & & & & \\
\hline & $\mathrm{V}$ & $(293-313)$ & $56.6 \pm 2.5$ & 303 & GS & [2010WID/BRU2] \\
\hline & $\mathrm{V}$ & $(397-452)$ & 52.8 & 413 & $\mathrm{~EB}$ & [1994AIM] \\
\hline & $\mathrm{V}$ & $(353-505)$ & 49.8 & 368 & A & [1987STE/MAL] \\
\hline & $\mathrm{V}$ & (323-500) & 50.1 & 410 & & [1926BER/MAY] \\
\hline \multirow[t]{13}{*}{$\mathrm{C}_{7} \mathrm{H}_{7} \mathrm{NO}_{2}$} & [99-99-0] & 4-nitrotoluene & & & & \\
\hline & FUS & & 16.81 & 324.8 & $\mathrm{AC}$ & [1991ACR, 1980AMB/GUN, 1980AND/CON] \\
\hline & FUS & & 16.7 & 324.7 & DSC & [1979RIC/SAV] \\
\hline & FUS & & 13.31 & & $\mathrm{C}$ & [1963RAS/NIG] \\
\hline & SUB & $(303-321)$ & 81.3 & & TGA & [2011FEL/RAM] \\
\hline & SUB & $(283-313)$ & $74.8 \pm 1.0$ & 298 & GS & [2010WID/BRU2] \\
\hline & SUB & & $79.1 \pm 2.5$ & 298 & ME & [1971LEN/VEL] \\
\hline & SUB & $(298-310)$ & 79.1 & 298 & & [1970LEN/VEL] \\
\hline & $\mathrm{V}$ & $(407-457)$ & 52.8 & 422 & $\mathrm{~EB}$ & [1994AIM] \\
\hline & $\mathrm{V}$ & $(423-512)$ & 49.8 & 438 & A & [1987STE/MAL] \\
\hline & V & $(417-512)$ & 42.2 & 435 & $\mathrm{~EB}$ & [1980AMB/GUN] \\
\hline & $\mathrm{V}$ & $(387-493)$ & 54.2 & 402 & & [1938LEV/SHT, 1994AIM] \\
\hline & $\mathrm{V}$ & $(353-505)$ & 50.0 & 430 & & {$[1926 \mathrm{BER} / \mathrm{MAY}]$} \\
\hline \multirow[t]{12}{*}{$\mathrm{C}_{7} \mathrm{H}_{7} \mathrm{NO}_{2}$} & {$[118-92-3]$} & 2-aminobenzoi & d (anthranilic & & & \\
\hline & FUS (I) & & 20.4 & 422.2 & & \\
\hline & FUS (II) & & 20.5 & 421.8 & $\mathrm{DSC}$ & [2012BAG/RED] \\
\hline & FUS & & 22.86 & 420 & DSC & [2010BAI/VAN] \\
\hline & FUS (I) & & 21.6 & 419.3 & & \\
\hline & FUS (II) & & 18.3 & 419.4 & & \\
\hline & FUS (III) & & 20.1 & 419.3 & DSC & [2010JIA/JAN] \\
\hline & FUS & & 20.5 & 417.8 & & [1991ACR, 1974SAB/CHA, 1926AND/LYN] \\
\hline & SUB & & $100 \pm 1$ & 338 & TE, ME & [1979DEK/VOO] \\
\hline & SUB & & $99.6 \pm 0.5$ & 378 & $\mathrm{C}$ & [1974SAB/CHA] \\
\hline & SUB & & $104.9 \pm 1.0$ & 298 & $\mathrm{C}$ & [1974SAB/CHA] \\
\hline & SUB & & $111.6 \pm 1.7$ & 298 & & {$[1972 \mathrm{ARN} / \mathrm{JON}]$} \\
\hline \multirow[t]{3}{*}{$\mathrm{C}_{7} \mathrm{H}_{7} \mathrm{NO}_{2}$} & {$[99-05-8]$} & 3-aminobenzoi & & & & \\
\hline & FUS (I) & & 35.5 & 445.2 & & \\
\hline & FUS (II) & & 26.7 & 451.2 & DSC & [2010SVA/NOR] \\
\hline
\end{tabular}


TABLE 10. Phase change enthalpies of $\mathrm{C}_{7}$ organic compounds-Continued

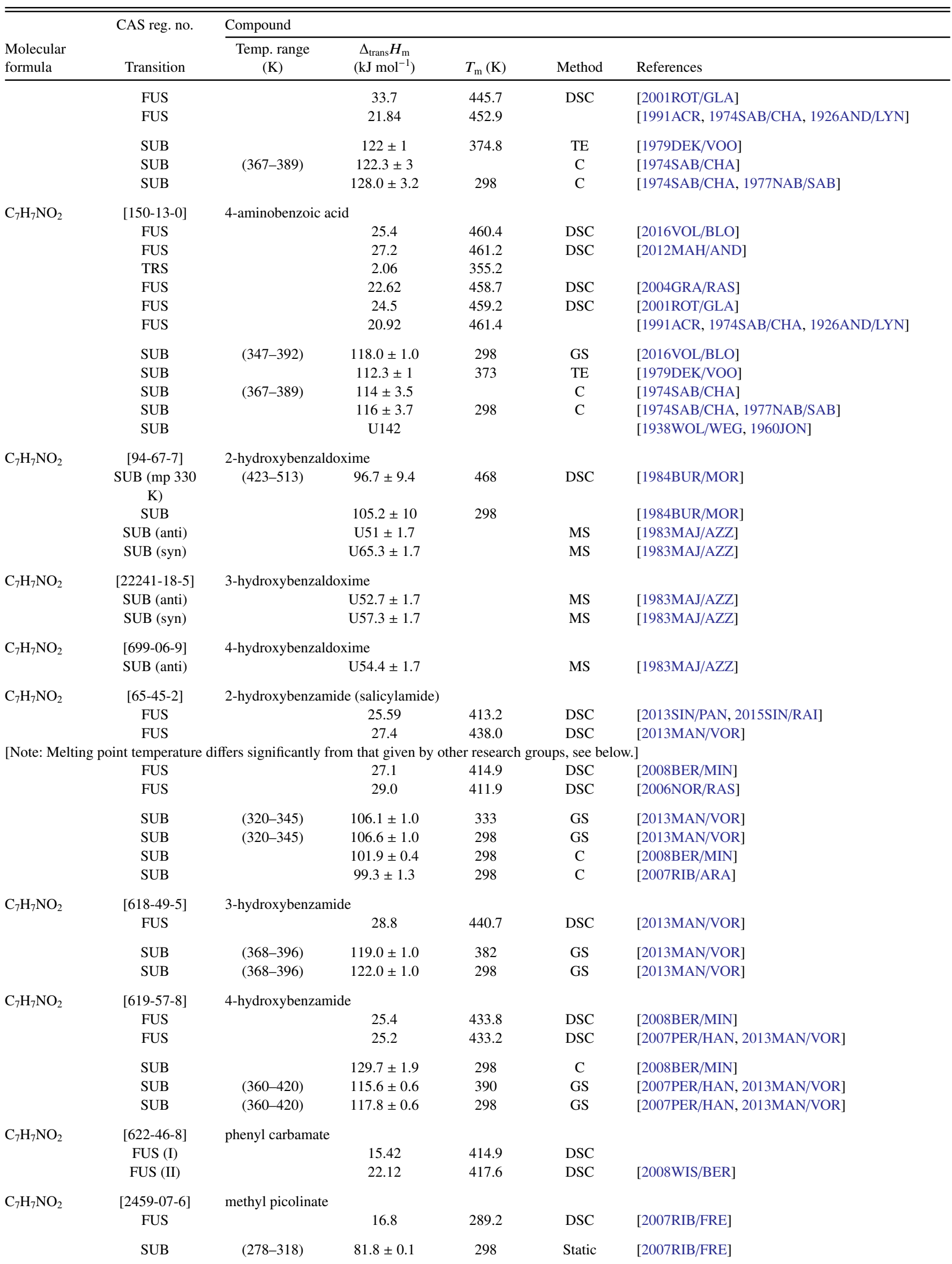


TABLE 10. Phase change enthalpies of $\mathrm{C}_{7}$ organic compounds-Continued

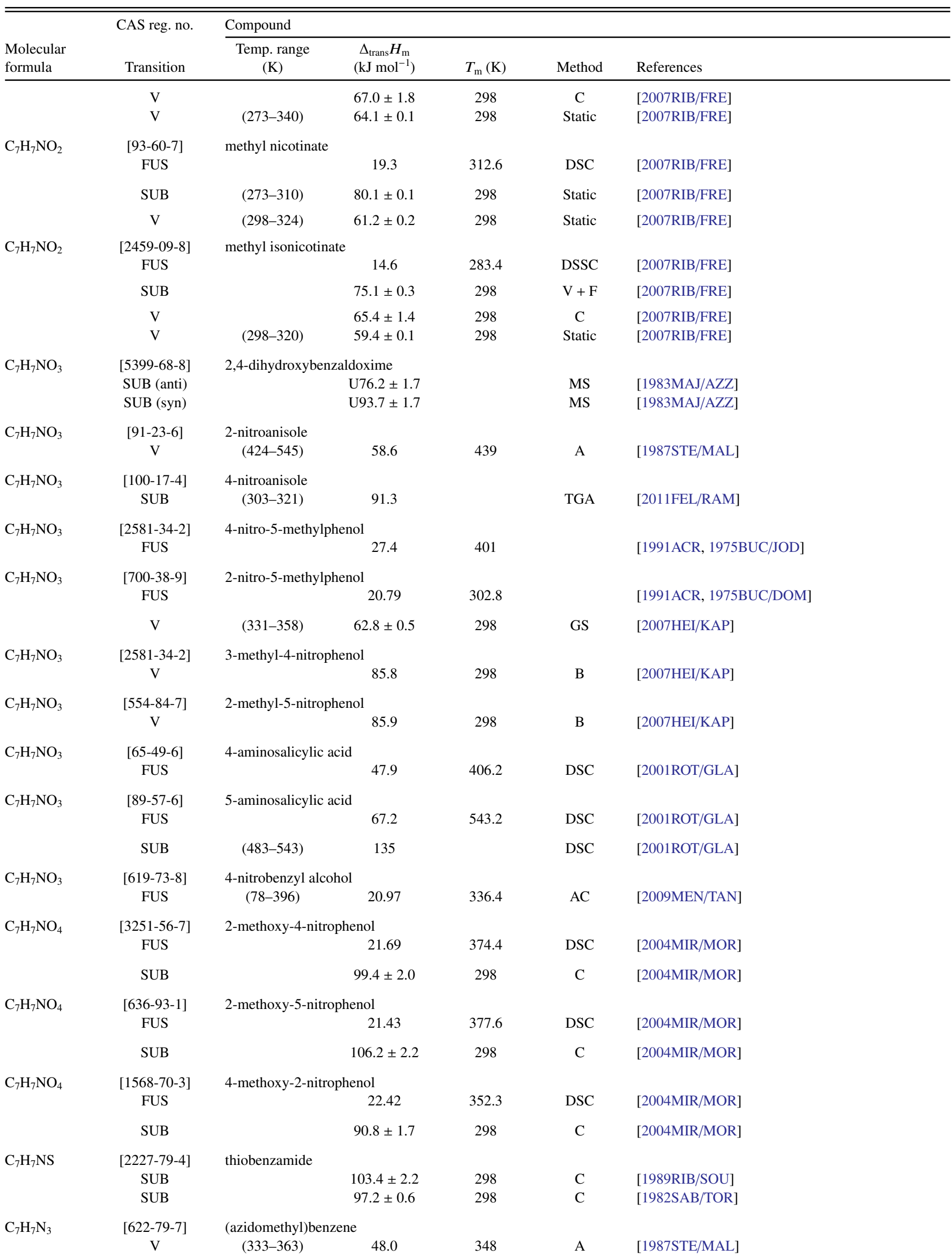


TABLE 10. Phase change enthalpies of $\mathrm{C}_{7}$ organic compounds-Continued

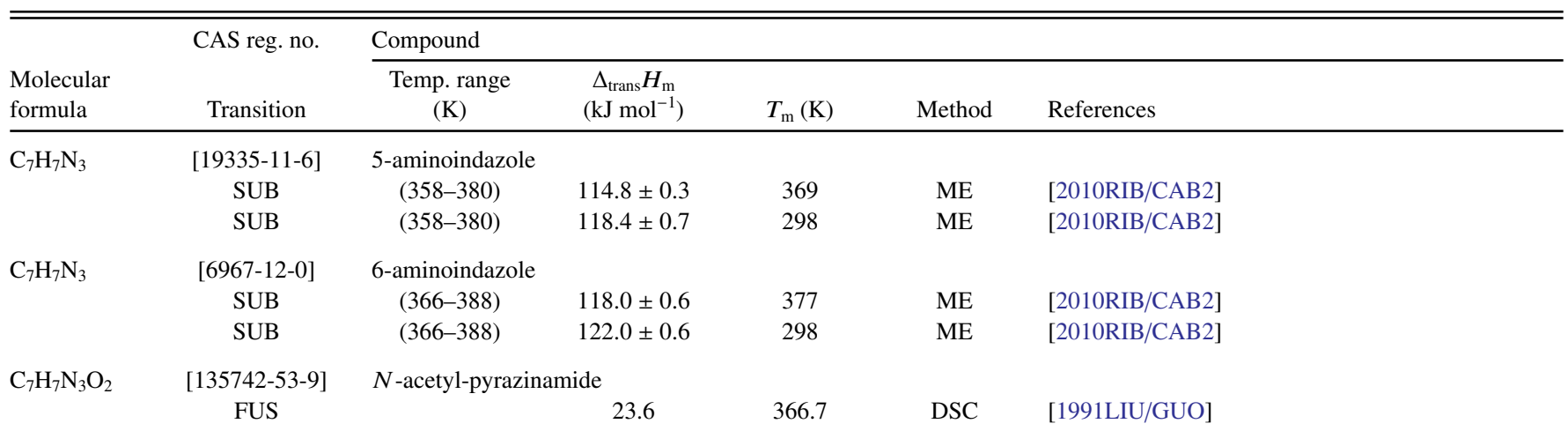

[Note: Authors of [1991LIU/GUO] give the name and molecular formula for $N$-acetyl-pyrazineamide; however, they draw the structure for $N$-(1-oxopropyl)pyrazinecarboxamide.]

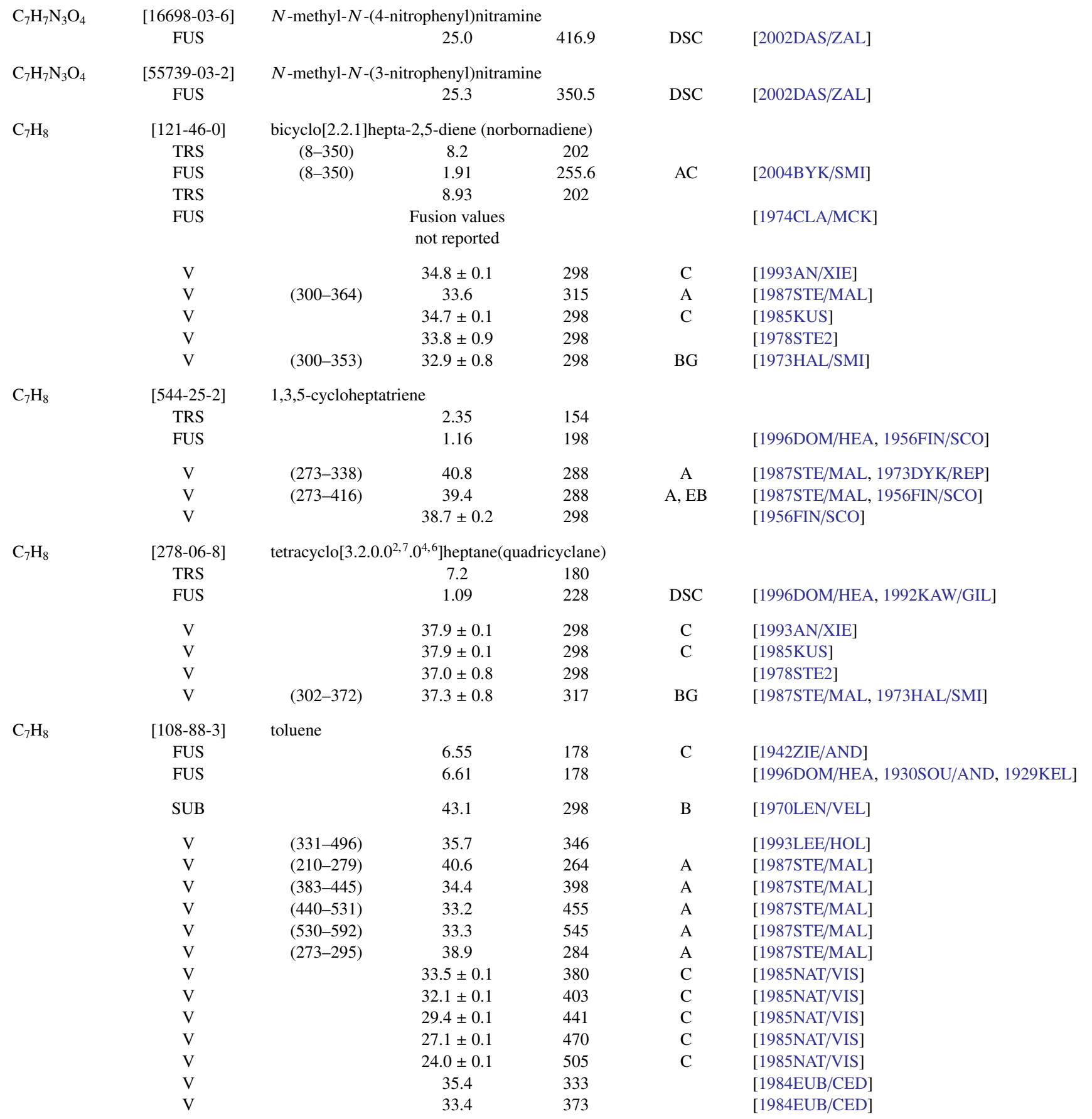


TABLE 10. Phase change enthalpies of $\mathrm{C}_{7}$ organic compounds-Continued

\begin{tabular}{|c|c|c|c|c|c|c|}
\hline \multirow[b]{2}{*}{$\begin{array}{l}\text { Molecular } \\
\text { formula }\end{array}$} & \multirow{2}{*}{$\begin{array}{c}\text { CAS reg. no. } \\
\text { Transition }\end{array}$} & \multicolumn{5}{|l|}{ Compound } \\
\hline & & $\begin{array}{c}\text { Temp. range } \\
(\mathrm{K})\end{array}$ & $\begin{array}{c}\Delta_{\text {trans }} H_{\mathrm{m}} \\
\left(\mathrm{kJ} \mathrm{mol}^{-1}\right)\end{array}$ & $T_{\mathrm{m}}(\mathrm{K})$ & Method & References \\
\hline & $\mathrm{V}$ & & 31.4 & 413 & & [1984EUB/CED] \\
\hline & $\mathrm{V}$ & & 28.4 & 453 & & [1984EUB/CED] \\
\hline & $\mathrm{V}$ & & 24.0 & 493 & & [1984EUB/CED] \\
\hline & $\mathrm{V}$ & $(343-383)$ & 35.4 & 360 & & [1975RIV] \\
\hline & $\mathrm{V}$ & & 38.0 & 298 & & [1971WIL/ZWO] \\
\hline & $\mathrm{V}$ & $(303-343)$ & 37.3 & 318 & & [1968GAW/SWI2] \\
\hline & V & $(288-348)$ & 36.9 & 303 & & [1967VAN/SOC] \\
\hline & $\mathrm{V}$ & $(210-293)$ & 37.8 & 278 & & [1956MIL] \\
\hline & $\mathrm{V}$ & $(308-386)$ & 37.0 & 323 & & [1987STE/MAL, 1949FOR/NOR] \\
\hline & $\mathrm{V}$ & & 38.0 & 298 & $\mathrm{C}$ & [1947OSB/GIN] \\
\hline & $\mathrm{V}$ & $(286-362)$ & 37.8 & 301 & & [1946THO] \\
\hline & $\mathrm{V}$ & $(308-384)$ & 37.0 & 323 & MM & [1945WIL/TAY] \\
\hline & $\mathrm{V}$ & $(273-323)$ & 38.8 & 288 & & [1943PIT/SCO] \\
\hline & $\mathrm{V}$ & $(273-323)$ & $38.1 \pm 0.2$ & 298 & & [1943PIT/SCO] \\
\hline
\end{tabular}

$\mathrm{C}_{7} \mathrm{H}_{8} \mathrm{ClN}_{3} \mathrm{O}_{4} \mathrm{~S}_{2}$ [58-93-5] 6-chloro-3,4-dihydro-2H-1,2,4-benzothiadiazine-7-sulfonamide-1,1-dioxide (hydrochlorthiazide)

$\begin{array}{lcccc}\text { FUS } & 37.6 & 539.4 & \text { DSC } & \text { [2010MUR/PIK2] } \\ \text { FUS } & 33.6 & 540.8 & \text { DSC } & \text { [2006WAS/HOL] } \\ \text { FUS } & 29.2 & 546 & \text { DSC } & \text { [2006ACE/AGA] } \\ \text { FUS } & 30.96 & 547.2 & & \end{array}$

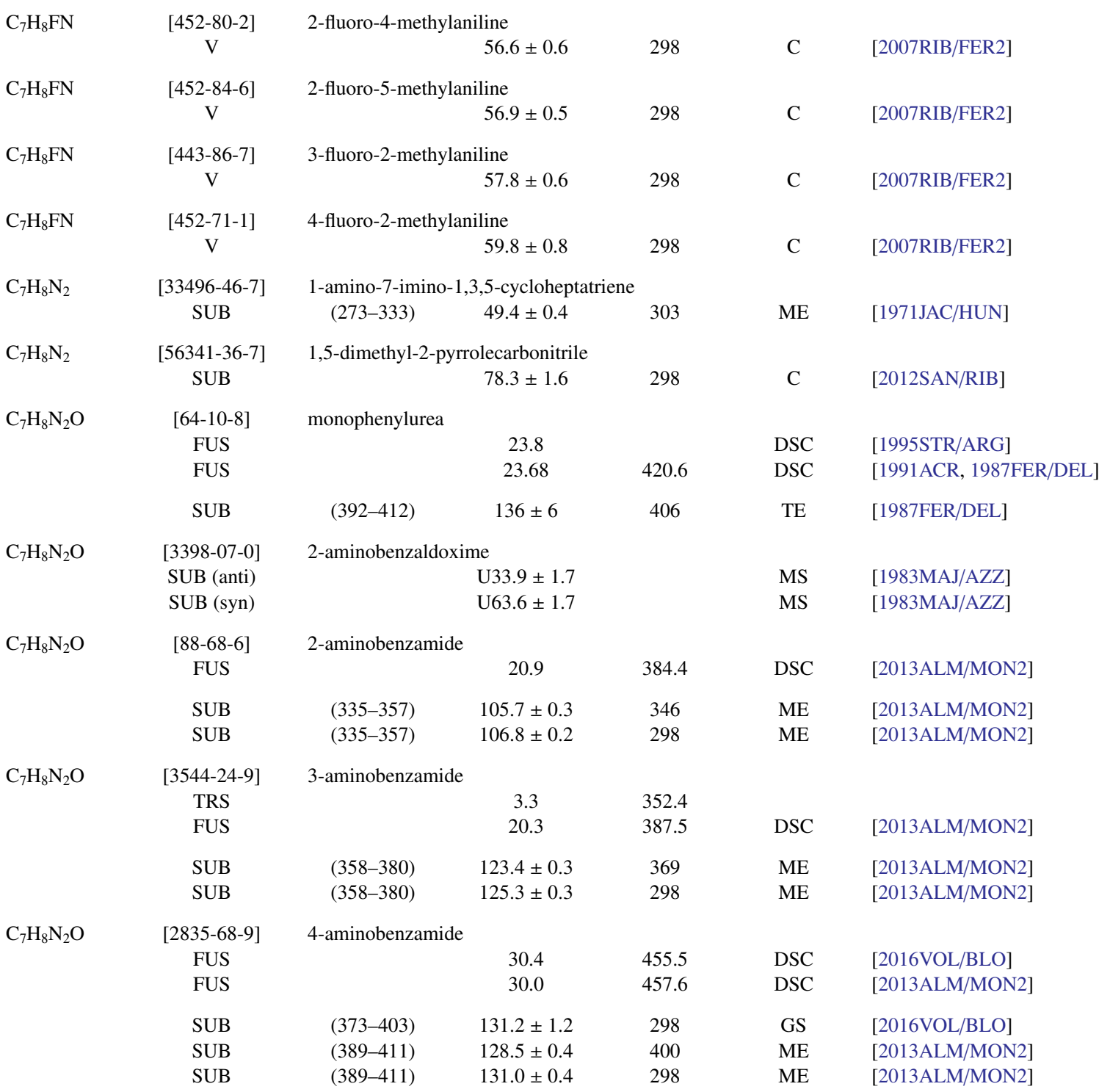

$\mathrm{C}_{7} \mathrm{H}_{8} \mathrm{~N}_{2} \mathrm{O} \quad$ [5231-96-9] (2-pyridyl)acetamide 
TABLE 10. Phase change enthalpies of $\mathrm{C}_{7}$ organic compounds-Continued

\begin{tabular}{|c|c|c|c|c|c|c|}
\hline \multirow[b]{2}{*}{$\begin{array}{l}\text { Molecular } \\
\text { formula }\end{array}$} & \multirow{2}{*}{$\begin{array}{l}\text { CAS reg. no. } \\
\text { Transition }\end{array}$} & \multicolumn{5}{|l|}{ Compound } \\
\hline & & $\begin{array}{c}\text { Temp. range } \\
(\mathrm{K})\end{array}$ & $\begin{array}{c}\Delta_{\text {trans }} H_{\mathrm{m}} \\
\left(\mathrm{kJ} \mathrm{mol}^{-1}\right)\end{array}$ & $T_{\mathrm{m}}(\mathrm{K})$ & Method & References \\
\hline & FUS & & 16.0 & 343 & $\mathrm{DSC}$ & [1979GON/CHA] \\
\hline & SUB & & 103.8 & 298 & $\mathrm{~B}, \mathrm{E}$ & [1979GON/CHA] \\
\hline & $\mathrm{V}$ & & 37.7 & 520 & DSC & [1979GON/CHA] \\
\hline \multirow[t]{2}{*}{$\mathrm{C}_{7} \mathrm{H}_{8} \mathrm{~N}_{2} \mathrm{O}$} & [613-94-5] & \multicolumn{5}{|c|}{ benzoic acid hydrazide } \\
\hline & FUS & & 25.7 & 388.2 & DSC & [2003CHI/ACR] \\
\hline \multirow[t]{8}{*}{$\mathrm{C}_{7} \mathrm{H}_{8} \mathrm{~N}_{2} \mathrm{O}$} & [114-33-0] & \multicolumn{5}{|c|}{$N$-methylnicotinamide } \\
\hline & FUS & & 22.0 & 379.1 & DSC & [2015ALM/OLI] \\
\hline & SUB & $(329-349)$ & $106.2 \pm 0.4$ & 339 & $\mathrm{ME}$ & [2015ALM/OLI] \\
\hline & SUB & $(329-349)$ & $107.4 \pm 0.4$ & 298 & $\mathrm{ME}$ & [2015ALM/OLI] \\
\hline & SUB & $(347-377)$ & $105.3 \pm 0.2$ & 362 & DM & [2015ALM/OLI] \\
\hline & SUB & $(347-377)$ & $107.1 \pm 0.2$ & 298 & DM & [2015ALM/OLI] \\
\hline & $\mathrm{V}$ & $(375-426)$ & $80.5 \pm 0.1$ & 401 & DM & [2015ALM/OLI] \\
\hline & V & $(375-426)$ & $88.2 \pm 0.7$ & 298 & DM & [2015ALM/OLI] \\
\hline \multirow[t]{2}{*}{$\mathrm{C}_{7} \mathrm{H}_{8} \mathrm{~N}_{2} \mathrm{O}_{2}$} & {$[3569-99-1]$} & \multicolumn{5}{|c|}{$N$-(hydroxymethyl)-3-pyridinecarboxamide } \\
\hline & FUS & & 32.9 & 426.2 & DSC & [2013AGA/MOS] \\
\hline \multirow{2}{*}{$\mathrm{C}_{7} \mathrm{H}_{8} \mathrm{~N}_{4}$} & {$[13753-57-6]$} & \multicolumn{5}{|c|}{ 4,4'-dipyrazolylmethane } \\
\hline & FUS & & 27.92 & 472 & DSC & [2009KEN/FLO] \\
\hline \multirow[t]{3}{*}{$\mathrm{C}_{7} \mathrm{H}_{8} \mathrm{~N}_{4} \mathrm{O}$} & {$[31010-51-2]$} & \multicolumn{5}{|c|}{ 9-ethylhypoxanthine } \\
\hline & SUB & & $108.8 \pm 13$ & & HSA & [1978NOW/SZC] \\
\hline & SUB & & U83.7 & & HSA & [1965CLA/PES] \\
\hline \multirow[t]{2}{*}{$\mathrm{C}_{7} \mathrm{H}_{8} \mathrm{~N}_{4} \mathrm{O}$} & {$[20535-82-4]$} & \multicolumn{5}{|c|}{ 1,9-dimethylhypoxanthine } \\
\hline & SUB & & $75.3 \pm 13$ & & HSA & {$[1978 N O W / S Z C]$} \\
\hline \multirow[t]{19}{*}{$\mathrm{C}_{7} \mathrm{H}_{8} \mathrm{~N}_{4} \mathrm{O}_{2}$} & {$[58-55-9]$} & \multicolumn{5}{|c|}{ 1,3-dimethylxanthine (theophylline) } \\
\hline & FUS & & 30.23 & 544.5 & DSC & [2014POB/DOM] \\
\hline & FUS & & 29.61 & 545 & DSC & [2010BAI/VAN] \\
\hline & FUS (form I) & & 29.6 & 547.2 & DSC & [2010SET/KHA] \\
\hline & FUS (form II, ort & orhombic) & 30.8 & 548.3 & DSC & [2010SET/KHA] \\
\hline & FUS (form IV) & & 31.9 & 547.3 & DSC & [2010SET/KHA] \\
\hline & FUS (form I) & & 28.02 & 547.9 & DSC & [2010SZT/LEG, 2009SZT] \\
\hline & FUS (form II) & & 30.1 & 543.9 & DSC & [2010SZT/LEG, 2009SZT] \\
\hline & FUS & & 19.0 & 546.8 & DSC & [2009GOO/ROD] \\
\hline & FUS & & 28.2 & 544 & & [1989GON/KRA] \\
\hline & FUS & & 28.2 & 542.3 & & [1989SUZ/SHI] \\
\hline & FUS & & 30.9 & 544.4 & DSC & [1984BRU/REI] \\
\hline & FUS & & 31.2 & 543.7 & DSC & [1983FOK/VAN] \\
\hline & SUB (form I) & $(413-453)$ & $132.0 \pm 0.3$ & 433 & $\mathrm{~T}$ & [1999GRI/SZE] \\
\hline & SUB (form I) & $(413-453)$ & 142 & 298 & $\mathrm{~T}$ & [1999GRI/SZE] \\
\hline & SUB (form II) & $(413-453)$ & $134.2 \pm 0.3$ & 433 & $\mathrm{~T}$ & [1999GRI/SZE] \\
\hline & SUB (form II) & $(413-453)$ & 144 & 298 & $\mathrm{~T}$ & [1999GRI/SZE] \\
\hline & SUB & & 126 & 421 & ME, TE & [1983FOK/VAN] \\
\hline & SUB & & 135 & 298 & & [1983FOK/VAN, 1999GRI/SZE] \\
\hline $\mathrm{C}_{7} \mathrm{H}_{8} \mathrm{O}$ & {$[100-66-3]$} & anisole & & & & \\
\hline & FUS & & 11.66 & 237 & & [1994LEE/LIE] \\
\hline & FUS & & 12.89 & 236 & & [1996DOM/HEA, 1987GOA/BOE] \\
\hline & FUS & & 17.03 & $\mathrm{U} 293.2$ & & [1996DOM/HEA, 1889EYK] \\
\hline & $\mathrm{V}$ & & $46.4 \pm 0.3$ & 298 & $\mathrm{C}$ & [2014SIM/AGA] \\
\hline & $\mathrm{V}$ & $(278-312)$ & $46.6 \pm 0.2$ & 298 & GS & [2005VAS/VER] \\
\hline & $\mathrm{V}$ & $(363-463)$ & 44.3 & 298 & $\mathrm{GC}$ & [2005HOS/GRY] \\
\hline & $\mathrm{V}$ & $(353-393)$ & 45.3 & 298 & CGC & [1995CHI/HOS] \\
\hline & $\mathrm{V}$ & $(382-429)$ & 41.8 & 397 & & [1993REI/SAN] \\
\hline & $\mathrm{V}$ & & 41.0 & & & [1986BAL/GNA] \\
\hline & $\mathrm{V}$ & $(382-437)$ & 41.9 & 397 & A & [1987STE/MAL, 1976AMB/ELL] \\
\hline & $\mathrm{V}$ & $(382-437)$ & 46.9 & 298 & & [1976AMB/ELL] \\
\hline & $\mathrm{V}$ & $(282-437)$ & 39.0 & 426 & & [1976AMB/ELL] \\
\hline & $\mathrm{V}$ & & $46.8 \pm 0.2$ & 298 & $\mathrm{C}$ & [1975FEN/HAR] \\
\hline
\end{tabular}


TABLE 10. Phase change enthalpies of $\mathrm{C}_{7}$ organic compounds-Continued

\begin{tabular}{|c|c|c|c|c|c|c|}
\hline \multirow[b]{2}{*}{$\begin{array}{l}\text { Molecular } \\
\text { formula }\end{array}$} & \multirow{2}{*}{$\begin{array}{c}\text { CAS reg. no. } \\
\text { Transition }\end{array}$} & \multicolumn{5}{|l|}{ Compound } \\
\hline & & $\begin{array}{l}\text { Temp. range } \\
(\mathrm{K})\end{array}$ & $\begin{array}{c}\Delta_{\text {trans }} H_{\mathrm{m}} \\
\left(\mathrm{kJ} \mathrm{mol}^{-1}\right) \\
\end{array}$ & $T_{\mathrm{m}}(\mathrm{K})$ & Method & References \\
\hline & $\mathrm{V}$ & $(363-383)$ & $38.2 \pm 0.4$ & 298 & & [1972LEB/KAT2, 2005VAS/VER] \\
\hline & $\mathrm{V}$ & & $42.9 \pm 0.1$ & 367 & $\mathrm{C}$ & [1967HAL/LEE] \\
\hline & $\mathrm{V}$ & & $42.0 \pm 0.1$ & 382 & $\mathrm{C}$ & [1967HAL/LEE] \\
\hline & $\mathrm{V}$ & & $40.5 \pm 0.1$ & 402 & $\mathrm{C}$ & [1967HAL/LEE] \\
\hline & $\mathrm{V}$ & & $38.9 \pm 0.1$ & 427 & $\mathrm{C}$ & [1967HAL/LEE] \\
\hline & $\mathrm{V}$ & & 39.4 & 298 & & [1957MCC/DOU, 2005VAS/VER] \\
\hline & V & $(382-437)$ & 41.9 & 397 & & [1955VON/GEB, 1965COL/COU] \\
\hline \multirow[t]{14}{*}{$\mathrm{C}_{7} \mathrm{H}_{8} \mathrm{O}$} & [100-51-6] & benzyl alcohol & & & & \\
\hline & FUS & & 8.79 & 257.6 & & [1991ACR, 1983WEA] \\
\hline & $\mathrm{V}$ & $(282-323)$ & $65.5 \pm 0.4$ & 298 & GS & [2005VAS/VER] \\
\hline & $\mathrm{V}$ & $(283-321)$ & $65.9 \pm 0.3$ & 298 & GS & [2004VER/VAS] \\
\hline & $\mathrm{V}$ & $(277-381)$ & $64.8 \pm 0.6$ & 298 & GS & [1999VER4] \\
\hline & $\mathrm{V}$ & $(323-373)$ & 69.5 & 298 & CGC & [1995CHI/HOS] \\
\hline & $\mathrm{V}$ & & 60.5 & & & [1995PAP/PIM] \\
\hline & $\mathrm{V}$ & $(404-507)$ & $62.5 \pm 0.3$ & 298 & EB & [1990AMB/GHI, 2005VAS/VER] \\
\hline & $\mathrm{V}$ & $(303-333)$ & 66.2 & 318 & GS & [1982GRA/FOS] \\
\hline & $\mathrm{V}$ & $(385-573)$ & 54.6 & 400 & A & [1987STE/MAL, 1973DYK/REP] \\
\hline & $\mathrm{V}$ & $(293-313)$ & 61.5 & 303 & A, ME & [1987STE/MAL, 1957SER/VOI, 1973DYK/REP] \\
\hline & $\mathrm{V}$ & $(396-478)$ & $62.1 \pm 0.3$ & 298 & EB & [1949DRE/SHR, 1949DRE/MAR, 2005VAS/VER] \\
\hline & $\mathrm{V}$ & $(312-348)$ & $63.0 \pm 2.2$ & 298 & $\mathrm{~EB}$ & [1937GAR/BRE, 2005VAS/VER] \\
\hline & V & & $60.3 \pm 0.4$ & 298 & $\mathrm{~EB}$ & [1926MAT, 2005VAS/VER] \\
\hline \multirow[t]{15}{*}{$\mathrm{C}_{7} \mathrm{H}_{8} \mathrm{O}$} & [95-48-7] & 2-hydroxytoluene & & & & \\
\hline & FUS & & 14.8 & 305.4 & DSC & [2007RIC/BER] \\
\hline & FUS & & 15.9 & 304.1 & DSC & [1998JAM/PAL] \\
\hline & FUS & & 15.82 & 304.2 & $\mathrm{AC}$ & [1996DOM/HEA, 1967AND/COU] \\
\hline & FUS & & 14.8 & 304.1 & $\mathrm{C}$ & {$[1990 \mathrm{MEV} / \mathrm{LIC}]$} \\
\hline & SUB & & $73.7 \pm 0.5$ & 298 & $\mathrm{C}$ & [2007RIC/BER] \\
\hline & SUB & $(273-303)$ & 74.8 & 288 & A & [1987STE/MAL] \\
\hline & SUB & $(273-303)$ & $76.0 \pm 0.8$ & 288 & & [1960AND/BID, 1970COX/PIL] \\
\hline & $\mathrm{V}$ & $(304-409)$ & 58.5 & 319 & A & [1987STE/MAL] \\
\hline & $\mathrm{V}$ & $(399-470)$ & 50.1 & 414 & A & [1987STE/MAL] \\
\hline & $\mathrm{V}$ & $(463-526)$ & 46.2 & 478 & A & [1987STE/MAL] \\
\hline & $\mathrm{V}$ & $(517-630)$ & 44.0 & 532 & A & [1987STE/MAL] \\
\hline & $\mathrm{V}$ & & 50.2 & & & [1986BAL/GNA] \\
\hline & $\mathrm{V}$ & $(383-473)$ & 51.3 & 398 & GS, EB & $\begin{array}{l}\text { [1987STE/MAL, 1960AND/BID, 1958BID/MAR, } \\
\text { 1973DYK/REP] }\end{array}$ \\
\hline & $\mathrm{V}$ & $(415-462)$ & 48.2 & 438 & & [1947GOL/MAR] \\
\hline \multirow[t]{19}{*}{$\mathrm{C}_{7} \mathrm{H}_{8} \mathrm{O}$} & [108-39-4] & 3-hydroxytoluene & & & & \\
\hline & FUS & & 8.9 & 282.3 & DSC & [2007RIC/BER] \\
\hline & FUS & & 10.67 & 285.3 & DSC & [1998JAM/PAL] \\
\hline & FUS & & 10.71 & 285.4 & $\mathrm{AC}$ & [1996DOM/HEA, 1967AND/COU] \\
\hline & FUS & & 9.1 & 280.8 & $\mathrm{C}$ & {$[1990 \mathrm{MEV} / \mathrm{LIC}]$} \\
\hline & SUB & $(273-285)$ & 56.1 & 279 & & [1987STE/MAL] \\
\hline & SUB & $(284-313)$ & $61.7 \pm 1.0$ & & GS & [1960AND/BID, 1958BID/MAR] \\
\hline & $\mathrm{V}$ & & $65.0 \pm 0.7$ & 298 & $\mathrm{C}$ & [2007RIC/BER] \\
\hline & $\mathrm{V}$ & $(393-433)$ & 62.5 & 298 & CGC & [1995CHI/HOS] \\
\hline & $\mathrm{V}$ & $(284-313)$ & 61.7 & 298 & A & [1987STE/MAL] \\
\hline & $\mathrm{V}$ & $(285-416)$ & 63.1 & 300 & A & [1987STE/MAL] \\
\hline & $\mathrm{V}$ & $(410-477)$ & 52.7 & 425 & A & [1987STE/MAL] \\
\hline & $\mathrm{V}$ & $(471-531)$ & 47.6 & 486 & A & [1987STE/MAL] \\
\hline & $\mathrm{V}$ & $(523-633)$ & 43.8 & 538 & A & [1987STE/MAL] \\
\hline & V & $(383-473)$ & 55.0 & 398 & GS, EB & [1987STE/MAL, 1960AND/BID, 1958BID/MAR] \\
\hline & $\mathrm{V}$ & $(388-429)$ & 60.6 & 409 & GS & [1980NAS/HWA, 1973DYK/REP] \\
\hline & $\mathrm{V}$ & & 61.7 & 298 & & [1958BID/MAR] \\
\hline & $\mathrm{V}$ & $(359-473)$ & 58.8 & 374 & & [1955VON/GEB, 1984BOU/FRI] \\
\hline & $\mathrm{V}$ & $(422-474)$ & 50.7 & 448 & & [1947GOL/MAR] \\
\hline
\end{tabular}


TABLE 10. Phase change enthalpies of $\mathrm{C}_{7}$ organic compounds-Continued

\begin{tabular}{|c|c|c|c|c|c|c|}
\hline \multirow[b]{2}{*}{$\begin{array}{l}\text { Molecular } \\
\text { formula }\end{array}$} & \multirow{2}{*}{$\begin{array}{l}\text { CAS reg. no. } \\
\text { Transition }\end{array}$} & \multicolumn{5}{|l|}{ Compound } \\
\hline & & $\begin{array}{l}\text { Temp. range } \\
(\mathrm{K})\end{array}$ & $\begin{array}{c}\Delta_{\text {trans }} H_{\mathrm{m}} \\
\left(\mathrm{kJ} \mathrm{mol}^{-1}\right)\end{array}$ & $T_{\mathrm{m}}(\mathrm{K})$ & Method & References \\
\hline & FUS & & 12.6 & 308.8 & DSC & [2007RIC/BER] \\
\hline & FUS & & 8.58 & 307.6 & DSC & [1998JAM/PAL] \\
\hline & FUS & & 12.72 & 307.9 & $\mathrm{AC}$ & [1996DOM/HEA, 1967AND/COU] \\
\hline & FUS & & 11.8 & 307.4 & $\mathrm{C}$ & [1990MEV/LIC] \\
\hline & FUS & & 12.25 & 309 & & [1996DOM/HEA, 1889EYK] \\
\hline & SUB & & $73.1 \pm 0.6$ & 298 & $\mathrm{C}$ & [2007RIC/BER] \\
\hline & SUB & $(273-307)$ & $73.9 \pm 1.5$ & 290 & & [1960AND/BID, 1970COX/PIL, 1958BID/MAR] \\
\hline & $\mathrm{V}$ & $(308-393)$ & 62.0 & 323 & A & {$[1987 \mathrm{STE} / \mathrm{MAL}]$} \\
\hline & $\mathrm{V}$ & $(385-477)$ & 55.4 & 400 & A & [1987STE/MAL] \\
\hline & $\mathrm{V}$ & $(463-533)$ & 49.2 & 478 & A & [1987STE/MAL] \\
\hline & $\mathrm{V}$ & $(523-635)$ & 46.0 & 538 & A & [1987STE/MAL] \\
\hline & $\mathrm{V}$ & & 54.0 & & & [1986BAL/GNA] \\
\hline & $\mathrm{V}$ & $(383-473)$ & 55.6 & 398 & A, GS, EB & $\begin{array}{l}\text { [1987STE/MAL, 1960AND/BID, 1973DYK/REP, } \\
\text { 1958BID/MAR] }\end{array}$ \\
\hline & $\mathrm{V}$ & $(419-474)$ & 51.3 & 446 & & {$[1947 \mathrm{GOL} / \mathrm{MAR}]$} \\
\hline \multirow[t]{2}{*}{$\mathrm{C}_{7} \mathrm{H}_{8} \mathrm{OS}$} & {$[106-53-6]$} & \multicolumn{5}{|c|}{ 4-methoxybenzenethiol } \\
\hline & $\mathrm{V}$ & & 52.3 & & & [1986BAL/GNA] \\
\hline \multirow[t]{2}{*}{$\mathrm{C}_{7} \mathrm{H}_{8} \mathrm{OS}$} & {$[13679-73-7]$} & \multicolumn{5}{|c|}{ 2-acetyl-4-methylthiophene } \\
\hline & V & & $63.0 \pm 2.6$ & 298 & $\mathrm{C}$ & [2008RIB/SAN4] \\
\hline \multirow[t]{2}{*}{$\mathrm{C}_{7} \mathrm{H}_{8} \mathrm{OS}$} & {$[13679-72-6]$} & \multicolumn{5}{|c|}{ 2-acetyl-3-methylthiophene } \\
\hline & $\mathrm{V}$ & & $57.1 \pm 2.4$ & 298 & $\mathrm{C}$ & [2008RIB/SAN4] \\
\hline \multirow[t]{2}{*}{$\mathrm{C}_{7} \mathrm{H}_{8} \mathrm{OS}$} & {$[13679-74-8]$} & \multicolumn{5}{|c|}{ 2-acetyl-5-methylthiophene } \\
\hline & V & & $62.0 \pm 2.6$ & 298 & $\mathrm{C}$ & [2008RIB/SAN4] \\
\hline \multirow[t]{2}{*}{$\mathrm{C}_{7} \mathrm{H}_{8} \mathrm{OS}$} & {$[36880-33-8]$} & \multicolumn{5}{|c|}{ 5-ethyl-2-thiophenecarboxaldehyde } \\
\hline & V & & $62.2 \pm 1.3$ & 298 & $\mathrm{C}$ & [2008RIB/SAN2] \\
\hline \multirow[t]{2}{*}{$\mathrm{C}_{7} \mathrm{H}_{8} \mathrm{O}_{2}$} & {$[488-17-5]$} & \multicolumn{5}{|c|}{ 3-methyl-1,2-dihydroxybenzene } \\
\hline & SUB & & $93.2 \pm 1.0$ & 298 & $\mathrm{C}$ & [1984CAR] \\
\hline \multirow[t]{4}{*}{$\mathrm{C}_{7} \mathrm{H}_{8} \mathrm{O}_{2}$} & [496-73-1] & \multicolumn{5}{|c|}{ 2,4-dihydroxytoluene } \\
\hline & SUB & (317-333) & $106.8 \pm 0.9$ & 325 & $\mathrm{ME}$ & [2009RIB/FER4] \\
\hline & SUB & $(317-333)$ & $107.3 \pm 3.0$ & 298 & $\mathrm{ME}$ & [2009RIB/FER4] \\
\hline & $\mathrm{V}$ & $(391-459)$ & 72.2 & 406 & $\mathrm{~A}, \mathrm{GC}$ & [1987STE/MAL, 1975KUN/LIL] \\
\hline \multirow[t]{4}{*}{$\mathrm{C}_{7} \mathrm{H}_{8} \mathrm{O}_{2}$} & {$[608-25-3]$} & \multicolumn{5}{|c|}{ 2,6-dihydroxytoluene } \\
\hline & SUB & $(309-329)$ & $98.8 \pm 0.5$ & 319 & $\mathrm{ME}$ & [2009RIB/FER4] \\
\hline & SUB & (309-329) & $99.2 \pm 2.3$ & 298 & $\mathrm{ME}$ & [2009RIB/FER4] \\
\hline & $\mathrm{V}$ & $(398-434)$ & 66.9 & 413 & $\mathrm{~A}, \mathrm{GC}$ & [1987STE/MAL, 1975KUN/LIL] \\
\hline \multirow[t]{3}{*}{$\mathrm{C}_{7} \mathrm{H}_{8} \mathrm{O}_{2}$} & {$[452-86-8]$} & 3,4-dihydroxyt & & & & \\
\hline & SUB & & $94.9 \pm 1.0$ & 298 & $\mathrm{C}$ & [1984CAR] \\
\hline & $\mathrm{V}$ & $(387-415)$ & 90.0 & 401 & A & [1987STE/MAL] \\
\hline $\mathrm{C}_{7} \mathrm{H}_{8} \mathrm{O}_{2}$ & {$[504-15-4]$} & 3,5-dihydroxyt & & & & \\
\hline & SUB & $(322-338)$ & $102.3 \pm 0.7$ & 330 & ME & [2009RIB/FER4] \\
\hline & SUB & $(322-338)$ & $102.9 \pm 3.5$ & 298 & $\mathrm{ME}$ & [2009RIB/FER4] \\
\hline & $\mathrm{V}$ & $(402-468)$ & 76.6 & 417 & $\mathrm{~A}, \mathrm{GC}$ & [1987STE/MAL, 1975KUN/LIL] \\
\hline $\mathrm{C}_{7} \mathrm{H}_{8} \mathrm{O}_{2}$ & {$[95-71-6]$} & 2-methyl-1,4-d & oxybenzene ( & hylhydro & one) & \\
\hline & FUS & & 27.6 & 404.2 & DSC & [1999VER7] \\
\hline & SUB & $(325-341)$ & $107.8 \pm 1.1$ & 333 & $\mathrm{ME}$ & [2009RIB/FER4] \\
\hline & SUB & $(325-341)$ & $108.4 \pm 3.9$ & 298 & $\mathrm{ME}$ & [2009RIB/FER4] \\
\hline & SUB & $(333-368)$ & $97.2 \pm 1.4$ & 351 & GS & [1999VER7] \\
\hline & SUB & $(333-368)$ & $100.4 \pm 1.4$ & 298 & GS & [1999VER7] \\
\hline $\mathrm{C}_{7} \mathrm{H}_{8} \mathrm{O}_{2}$ & [90-05-1] & 2-methoxypher & & & & \\
\hline & FUS & & 12.0 & 301.2 & DSC & [2003LEE/CHO] \\
\hline & $\mathrm{V}$ & $(286-341)$ & $61.4 \pm 0.3$ & 298 & GS & [2010VAR/ABA] \\
\hline
\end{tabular}


TABLE 10. Phase change enthalpies of $\mathrm{C}_{7}$ organic compounds-Continued

\begin{tabular}{|c|c|c|c|c|c|c|}
\hline \multirow[b]{2}{*}{$\begin{array}{l}\text { Molecular } \\
\text { formula }\end{array}$} & \multirow{2}{*}{$\begin{array}{l}\text { CAS reg. no. } \\
\text { Transition }\end{array}$} & \multicolumn{5}{|l|}{ Compound } \\
\hline & & $\begin{array}{c}\text { Temp. range } \\
(\mathrm{K})\end{array}$ & $\begin{array}{c}\Delta_{\text {trans }} H_{\mathrm{m}} \\
\left(\mathrm{kJ} \mathrm{mol}^{-1}\right)\end{array}$ & $T_{\mathrm{m}}(\mathrm{K})$ & Method & References \\
\hline & $\mathrm{V}$ & $(373-463)$ & 52.7 & 388 & EB & [2005LEE/SU] \\
\hline & $\mathrm{V}$ & $(373-463)$ & $59.2 \pm 0.4$ & 298 & EB & [2005LEE/SU, 2010VAR/ABA] \\
\hline & $\mathrm{V}$ & & $62.6 \pm 0.5$ & 298 & $\mathrm{C}$ & [2003MAT/MIR] \\
\hline & $\mathrm{V}$ & $(378-479)$ & 52.7 & 393 & A & [1987STE/MAL, 1973DYK/REP] \\
\hline & $\mathrm{V}$ & $(355-478)$ & 52.7 & 370 & EB & [1955VON/GEB] \\
\hline & $\mathrm{V}$ & $(355-478)$ & $58.9 \pm 0.3$ & 298 & EB & [1955VON/GEB, 2010VAR/ABA] \\
\hline \multirow[t]{5}{*}{$\mathrm{C}_{7} \mathrm{H}_{8} \mathrm{O}_{2}$} & {$[150-19-6]$} & 3-methoxyphenol & & & & \\
\hline & V & $(303-354)$ & $74.4 \pm 0.2$ & 298 & GS & [2010VAR/ABA] \\
\hline & $\mathrm{V}$ & & $75.9 \pm 1.2$ & 298 & $\mathrm{C}$ & [2003MAT/MIR] \\
\hline & $\mathrm{V}$ & $(413-518)$ & 64.8 & 428 & A & [1987STE/MAL, 1973DYK/REP] \\
\hline & $\mathrm{V}$ & $(413-518)$ & 73.2 & 298 & A & [1987STE/MAL, 1973DYK/REP, 2010VAR/ABA] \\
\hline \multirow[t]{11}{*}{$\mathrm{C}_{7} \mathrm{H}_{8} \mathrm{O}_{2}$} & {$[150-76-5]$} & 4-methoxyphenol & & & & \\
\hline & FUS & & 18.2 & 328.3 & DSC & [2010VAR/ABA] \\
\hline & FUS & & 18.3 & 328.4 & DSC & [1997LEE/CHA] \\
\hline & SUB & $(289-327)$ & $89.8 \pm 0.3$ & 298 & GS & [2010VAR/ABA] \\
\hline & SUB & & $94.4 \pm 1.2$ & 298 & $\mathrm{C}$ & [2003MAT/MIR] \\
\hline & SUB & $(278-300)$ & 88.7 & 289 & A & [1987STE/MAL, 1960AIH] \\
\hline & SUB & $(278-300)$ & 88.4 & 298 & A & [1987STE/MAL, 1960AIH, 2010VAR/ABA] \\
\hline & $\mathrm{V}$ & $(330-357)$ & $73.7 \pm 0.7$ & 298 & GS & [2010VAR/ABA] \\
\hline & $\mathrm{V}$ & & 58.6 & & & [1986BAL/GNA] \\
\hline & $\mathrm{V}$ & $(418-518)$ & 61.4 & 433 & A & [1987STE/MAL, 1973DYK/REP] \\
\hline & $\mathrm{V}$ & $(418-518)$ & 72.1 & 298 & A & [1987STE/MAL, 1973DYK/REP, 2010VAR/ABA] \\
\hline \multirow[t]{4}{*}{$\mathrm{C}_{7} \mathrm{H}_{8} \mathrm{O}_{2}$} & [90-01-7] & 2-hydroxybenzyl a & cohol & & & \\
\hline & FUS & & 21.5 & 358.3 & DSC & [2008PIN/DIO] \\
\hline & SUB & $(304-320)$ & $98.8 \pm 0.8$ & 312 & $\mathrm{ME}$ & [2010RIB/FER5] \\
\hline & SUB & $(304-320)$ & $99.5 \pm 1.5$ & 298 & $\mathrm{ME}$ & [2010RIB/FER5] \\
\hline \multirow[t]{3}{*}{$\mathrm{C}_{7} \mathrm{H}_{8} \mathrm{O}_{2}$} & {$[620-24-6]$} & 3-hydroxybenzyl a & cohol & & & \\
\hline & SUB & (324-340) & $114.3 \pm 0.6$ & 332 & $\mathrm{ME}$ & [2010RIB/FER5] \\
\hline & SUB & $(324-340)$ & $116.0 \pm 3.7$ & 298 & $\mathrm{ME}$ & [2010RIB/FER5] \\
\hline \multirow[t]{3}{*}{$\mathrm{C}_{7} \mathrm{H}_{8} \mathrm{O}_{2}$} & [623-05-2] & 4-hydroxybenzyl a & cohol & & & \\
\hline & SUB & $(333-349)$ & $127.1 \pm 1.2$ & 341 & $\mathrm{ME}$ & [2010RIB/FER5] \\
\hline & SUB & $(333-349)$ & $129.3 \pm 4.7$ & 298 & $\mathrm{ME}$ & [2010RIB/FER5] \\
\hline \multirow[t]{2}{*}{$\mathrm{C}_{7} \mathrm{H}_{8} \mathrm{O}_{2}$} & {$[63233-31-8]$} & 2,3-dimethyl-2H-p & ran-2-one & & & \\
\hline & V & $(352-518)$ & 64.9 & 367 & A & [1987STE/MAL, 1947STU] \\
\hline \multirow[t]{2}{*}{$\mathrm{C}_{7} \mathrm{H}_{8} \mathrm{O}_{2}$} & {$[1004-36-0]$} & 2,6-dimethyl-4-py & & & & \\
\hline & SUB & & $86.8 \pm 1.5$ & 298 & $\mathrm{C}$ & [2011SOU/MAT] \\
\hline \multirow[t]{2}{*}{$\mathrm{C}_{7} \mathrm{H}_{8} \mathrm{O}_{2}$} & [1193-79-9] & 5-methyl-2-acetylf & & & & \\
\hline & $\mathrm{V}$ & & $61.7 \pm 0.2$ & 298 & $\mathrm{C}$ & [2010RIB/AMA] \\
\hline \multirow[t]{2}{*}{$\mathrm{C}_{7} \mathrm{H}_{8} \mathrm{O}_{2}$} & [52480-43-0] & 4,5-dimethyl-2-fur & Idehyde & & & \\
\hline & $\mathrm{V}$ & & $57.7 \pm 0.6$ & 298 & $\mathrm{C}$ & [2011RIB/AMA] \\
\hline \multirow[t]{4}{*}{$\mathrm{C}_{7} \mathrm{H}_{8} \mathrm{O}_{2} \mathrm{~S}$} & [52911-98-5] & 6-methyl-4-metho & -2H-pyran-2 & & & \\
\hline & FUS & & 21.34 & & & [1974BEA/MUE] \\
\hline & SUB & $(402-415)$ & $130.5 \pm 5.9$ & 408 & $\mathrm{~B}$ & [1974BEA/MUE] \\
\hline & $\mathrm{V}$ & $(401-415)$ & 108.9 & 408 & A & [1987STE/MAL, 1999DYK/SVO, 1974BEA/MUE] \\
\hline \multirow[t]{4}{*}{$\mathrm{C}_{7} \mathrm{H}_{8} \mathrm{O}_{2} \mathrm{~S}$} & [52911-99-6] & 2-methyl-6-(methy & hio)- $4 H$-pyra & & & \\
\hline & FUS & & 25.10 & & & {$[1974 \mathrm{BEA} / \mathrm{MUE}]$} \\
\hline & SUB & $(388-433)$ & $87.4 \pm 3.8$ & 410 & $\mathrm{~B}$ & [1974BEA/MUE] \\
\hline & $\mathrm{V}$ & $(387-432)$ & 62.7 & 402 & A & [1987STE/MAL, 1999DYK/SVO, 1974BEA/MUE] \\
\hline \multirow[t]{2}{*}{$\mathrm{C}_{7} \mathrm{H}_{8} \mathrm{O}_{2} \mathrm{~S}$} & {$[3112-85-4]$} & methyl phenyl sulf & & & & \\
\hline & SUB & & $92 \pm 2.9$ & & & [UR/MAC, 1970COX/PIL] \\
\hline $\mathrm{C}_{7} \mathrm{H}_{8} \mathrm{O}_{2} \mathrm{~S}$ & {$[19432-68-9]$} & methyl 2-thiophen & acetate & & & \\
\hline
\end{tabular}


TABLE 10. Phase change enthalpies of $\mathrm{C}_{7}$ organic compounds-Continued

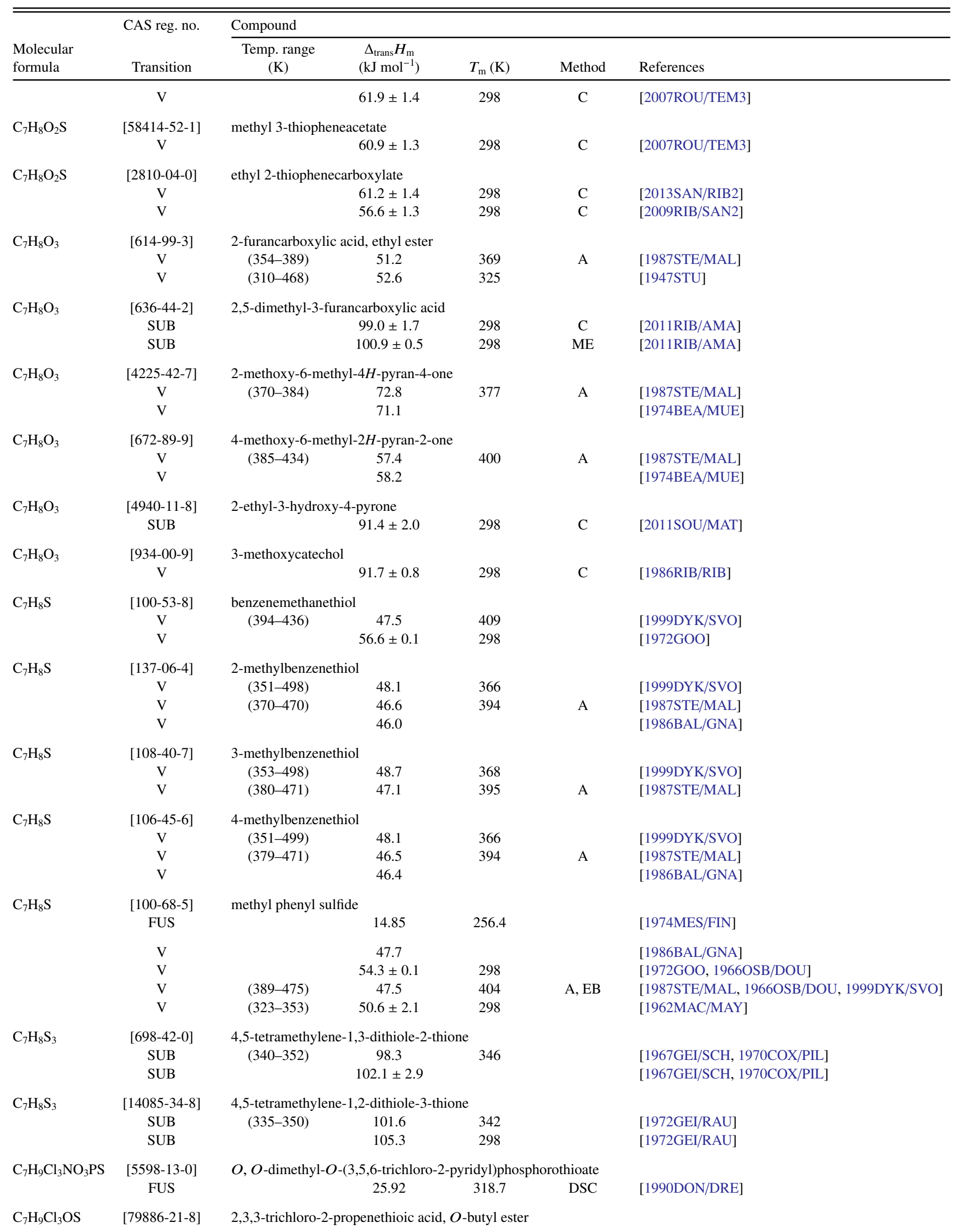


TABLE 10. Phase change enthalpies of $\mathrm{C}_{7}$ organic compounds-Continued

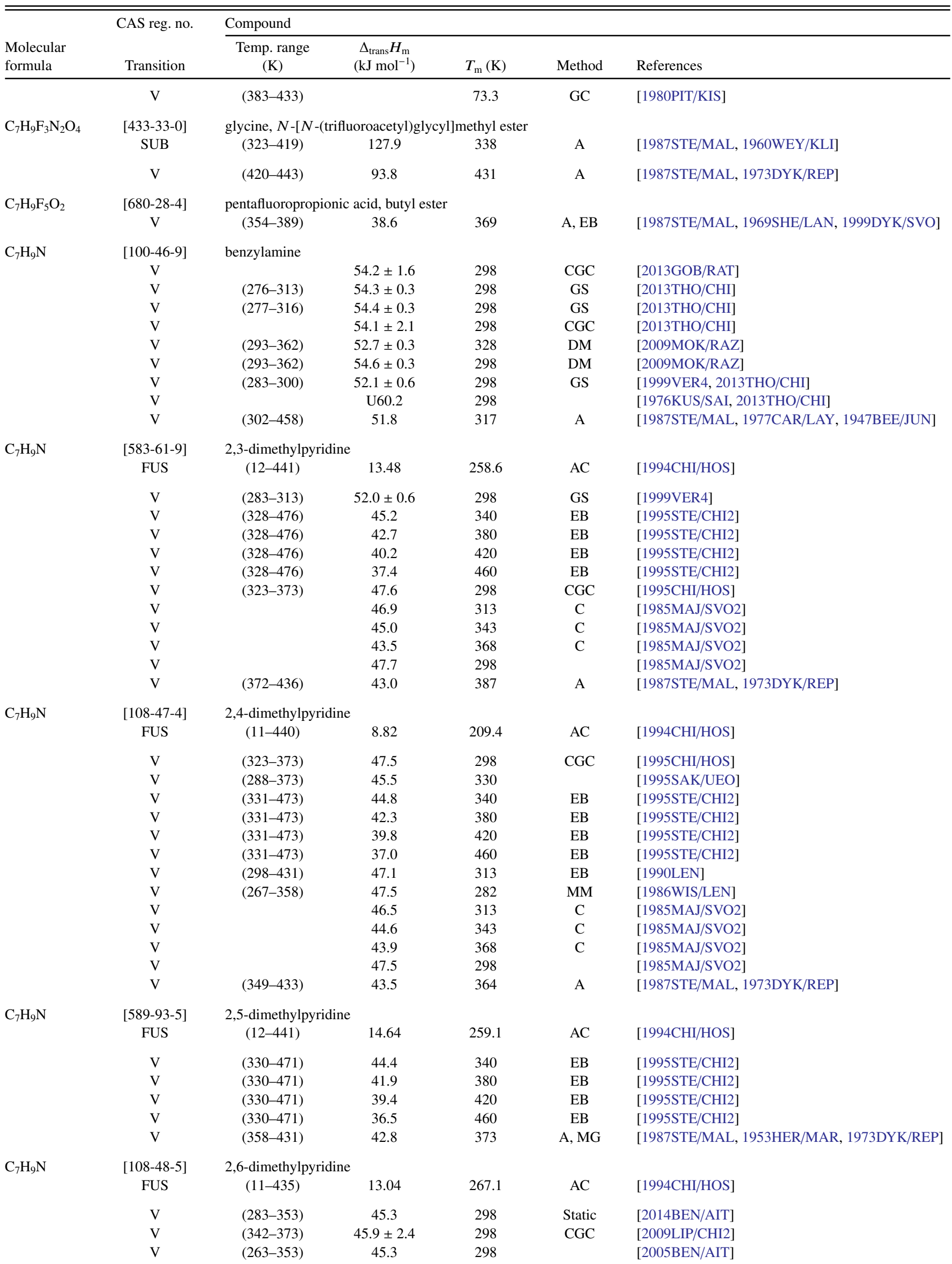


TABLE 10. Phase change enthalpies of $\mathrm{C}_{7}$ organic compounds-Continued

\begin{tabular}{|c|c|c|c|c|c|c|}
\hline \multirow[b]{2}{*}{$\begin{array}{l}\text { Molecular } \\
\text { formula }\end{array}$} & \multirow{2}{*}{$\begin{array}{c}\text { CAS reg. no. } \\
\text { Transition }\end{array}$} & \multicolumn{5}{|l|}{ Compound } \\
\hline & & $\begin{array}{c}\text { Temp. range } \\
(\mathrm{K})\end{array}$ & $\begin{array}{c}\Delta_{\text {trans }} H_{\mathrm{m}} \\
\left(\mathrm{kJ} \mathrm{mol}^{-1}\right)\end{array}$ & $T_{\mathrm{m}}(\mathrm{K})$ & Method & References \\
\hline & V & $(323-373)$ & 46.4 & 298 & CGC & [1995CHI/HOS] \\
\hline & $\mathrm{V}$ & $(288-373)$ & 43.7 & 330 & & [1995SAK/UEO] \\
\hline & $\mathrm{V}$ & $(315-457)$ & 43.9 & 320 & $\mathrm{~EB}$ & [1995STE/CHI2] \\
\hline & V & $(315-457)$ & 41.4 & 360 & $\mathrm{~EB}$ & [1995STE/CHI2] \\
\hline & $\mathrm{V}$ & $(315-457)$ & 38.8 & 400 & $\mathrm{~EB}$ & [1995STE/CHI2] \\
\hline & $\mathrm{V}$ & $(315-457)$ & 36.0 & 440 & $\mathrm{~EB}$ & [1995STE/CHI2] \\
\hline & V & $(295-417)$ & 45.0 & 310 & $\mathrm{~EB}$ & [1990LEN] \\
\hline & $\mathrm{V}$ & $(267-358)$ & 46.1 & 282 & MM & [1986WIS/LEN] \\
\hline & $\mathrm{V}$ & & 44.4 & 313 & $\mathrm{C}$ & [1985MAJ/SVO2] \\
\hline & V & & 42.5 & 343 & $\mathrm{C}$ & [1985MAJ/SVO2] \\
\hline & $\mathrm{V}$ & & 40.8 & 368 & $\mathrm{C}$ & [1985MAJ/SVO2] \\
\hline & $\mathrm{V}$ & & 45.4 & 298 & & [1985MAJ/SVO2] \\
\hline & $\mathrm{V}$ & $(352-418)$ & 41.6 & 367 & $\mathrm{~A}, \mathrm{MG}$ & [1987STE/MAL, 1953HER/MAR] \\
\hline \multirow[t]{12}{*}{$\mathrm{C}_{7} \mathrm{H}_{9} \mathrm{~N}$} & [583-58-4] & 3,4-dimethylpyridine & & & & \\
\hline & FUS & $(13-440)$ & 14.7 & 262.7 & $\mathrm{AC}$ & [1994CHI/HOS] \\
\hline & $\mathrm{V}$ & $(341-495)$ & 46.6 & 360 & EB & [1995STE/CHI2] \\
\hline & $\mathrm{V}$ & $(341-495)$ & 44.2 & 400 & $\mathrm{~EB}$ & [1995STE/CHI2] \\
\hline & $\mathrm{V}$ & $(341-495)$ & 41.7 & 440 & EB & [1995STE/CHI2] \\
\hline & $\mathrm{V}$ & $(341-495)$ & 39.0 & 480 & EB & [1995STE/CHI2] \\
\hline & $\mathrm{V}$ & $(288-422)$ & 47.6 & 355 & & [1995SAK/UEO] \\
\hline & $\mathrm{V}$ & & 48.8 & 328 & $\mathrm{C}$ & [1985MAJ/SVO2] \\
\hline & $\mathrm{V}$ & & 47.6 & 343 & $\mathrm{C}$ & [1985MAJ/SVO2] \\
\hline & $\mathrm{V}$ & & 45.9 & 368 & $\mathrm{C}$ & [1985MAJ/SVO2] \\
\hline & $\mathrm{V}$ & & 50.5 & 298 & & [1985MAJ/SVO] \\
\hline & $\mathrm{V}$ & $(385-454)$ & 44.8 & 400 & A & [1987STE/MAL, 1973DYK/REP] \\
\hline \multirow[t]{15}{*}{$\mathrm{C}_{7} \mathrm{H}_{9} \mathrm{~N}$} & {$[591-22-0]$} & 3,5-dimethylpyridine & & & & \\
\hline & FUS & $(13-444)$ & 13.11 & 266.9 & $\mathrm{AC}$ & [1994CHI/HOS] \\
\hline & $\mathrm{V}$ & $(273-353)$ & 48.5 & 298 & Static & [2005BEN/AIT, 2014BEN/AIT] \\
\hline & $\mathrm{V}$ & $(323-373)$ & 48.7 & 298 & CGC & [1995CHI/HOS] \\
\hline & $\mathrm{V}$ & $(288-392)$ & 47.0 & 340 & & [1995SAK/UEO] \\
\hline & $\mathrm{V}$ & $(335-487)$ & 46.7 & 340 & EB & [1995STE/CHI2] \\
\hline & $\mathrm{V}$ & $(335-487)$ & 44.3 & 380 & $\mathrm{~EB}$ & [1995STE/CHI2] \\
\hline & $\mathrm{V}$ & $(335-487)$ & 41.8 & 420 & $\mathrm{~EB}$ & [1995STE/CHI2] \\
\hline & $\mathrm{V}$ & $(335-487)$ & 39.2 & 460 & $\mathrm{~EB}$ & [1995STE/CHI2] \\
\hline & $\mathrm{V}$ & $(273-358)$ & 49.1 & 288 & MM & [1986WIS/LEN] \\
\hline & $\mathrm{V}$ & & 49.6 & 313 & $\mathrm{C}$ & [1985MAJ/SVO2] \\
\hline & $\mathrm{V}$ & & 46.5 & 343 & $\mathrm{C}$ & [1985MAJ/SVO2] \\
\hline & V & & 44.8 & 368 & $\mathrm{C}$ & [1985MAJ/SVO2] \\
\hline & $\mathrm{V}$ & & 49.5 & 298 & & [1985MAJ/SVO2] \\
\hline & $\mathrm{V}$ & $(373-446)$ & 44.3 & 388 & A & [1987STE/MAL, 1973DYK/REP] \\
\hline \multirow[t]{3}{*}{$\mathrm{C}_{7} \mathrm{H}_{9} \mathrm{~N}$} & {$[100-71-0]$} & 2-ethylpyridine & & & & \\
\hline & $\mathrm{V}$ & & $44.7 \pm 0.8$ & 298 & $\mathrm{C}$ & [2003MOR/MIR] \\
\hline & $\mathrm{V}$ & $(323-373)$ & 43.7 & 338 & A & [1987STE/MAL, 1973DYK/REP] \\
\hline \multirow[t]{2}{*}{$\mathrm{C}_{7} \mathrm{H}_{9} \mathrm{~N}$} & {$[536-78-7]$} & 3-ethylpyridine & & & & \\
\hline & $\mathrm{V}$ & $(334-373)$ & 44.6 & 349 & A & [1987STE/MAL, 1973DYK/REP] \\
\hline \multirow[t]{3}{*}{$\mathrm{C}_{7} \mathrm{H}_{9} \mathrm{~N}$} & {$[536-75-4]$} & 4-ethylpyridine & & & & \\
\hline & $\mathrm{V}$ & & $46.3 \pm 0.7$ & 298 & $\mathrm{C}$ & [2003MOR/MIR] \\
\hline & $\mathrm{V}$ & $(333-372)$ & 45.3 & 348 & A & [1987STE/MAL, 1973DYK/REP] \\
\hline \multirow[t]{9}{*}{$\mathrm{C}_{7} \mathrm{H}_{9} \mathrm{~N}$} & {$[100-61-8]$} & $N$-methylaniline & & & & \\
\hline & $\mathrm{V}$ & $(284-320)$ & $55.0 \pm 0.2$ & 298 & GS & [2015EME/PIM] \\
\hline & $\mathrm{V}$ & $(353-467)$ & 48.1 & 410 & Static & [1993XIE/CHE] \\
\hline & $\mathrm{V}$ & $(353-467)$ & $56.7 \pm 0.2$ & 298 & Static & [1993XIE/CHE, 2015EME/PIM] \\
\hline & $\mathrm{V}$ & $(309-469)$ & 53.6 & 324 & A & [1987STE/MAL] \\
\hline & $\mathrm{V}$ & & 45.4 & 467 & $\mathrm{C}$ & [1931MAT/FEH] \\
\hline & $\mathrm{V}$ & & $58.6 \pm 1.0$ & 298 & $\mathrm{C}$ & [1931MAT/FEH, 2015EME/PIM] \\
\hline & $\mathrm{V}$ & $(323-473)$ & 47.9 & 398 & Static & [1925NEL/WAL] \\
\hline & $\mathrm{V}$ & $(323-473)$ & $55.5 \pm 0.4$ & 298 & Static & [1925NEL/WAL, 2015EME/PIM] \\
\hline
\end{tabular}


TABLE 10. Phase change enthalpies of $\mathrm{C}_{7}$ organic compounds-Continued

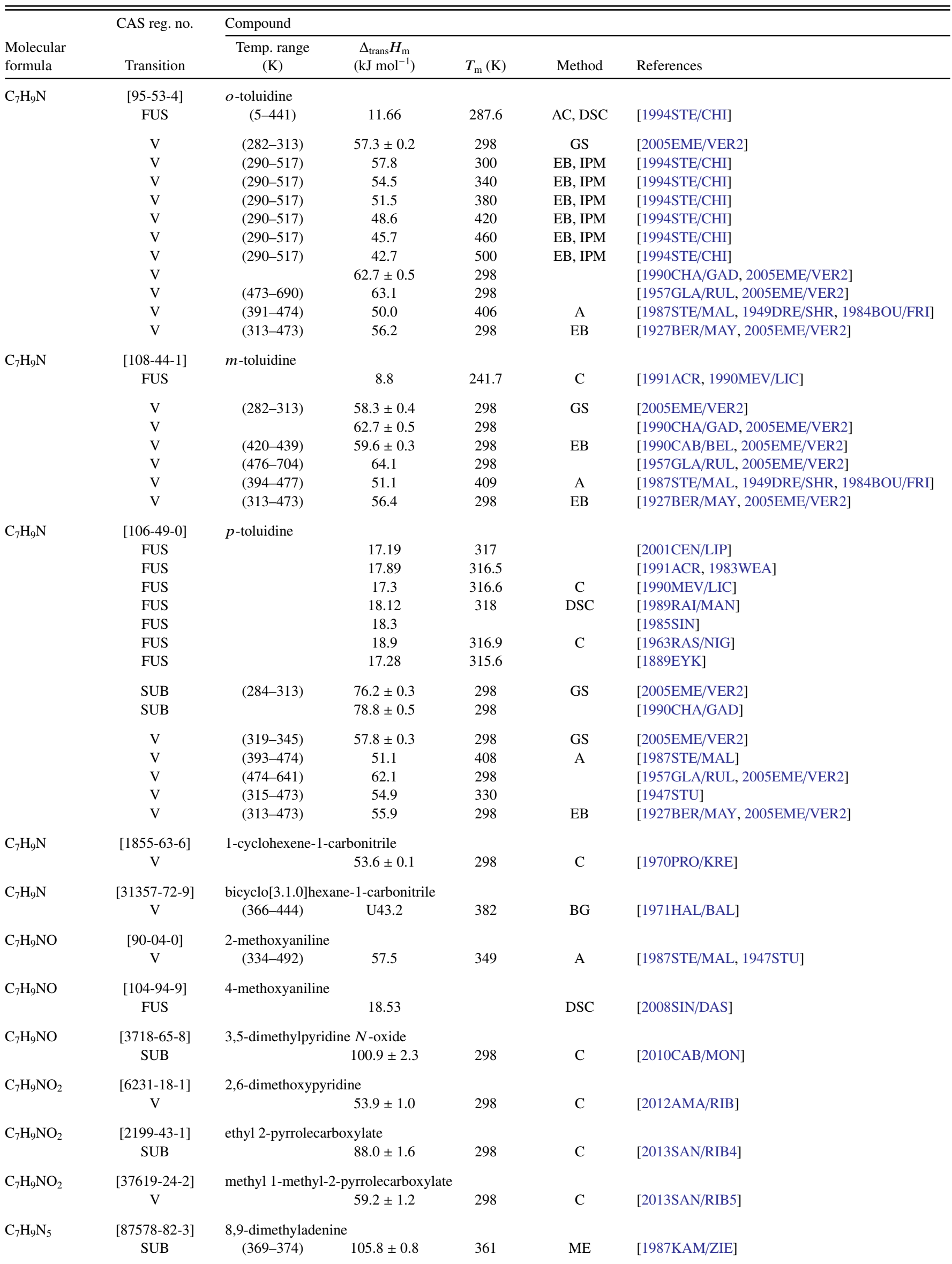


TABLE 10. Phase change enthalpies of $\mathrm{C}_{7}$ organic compounds-Continued

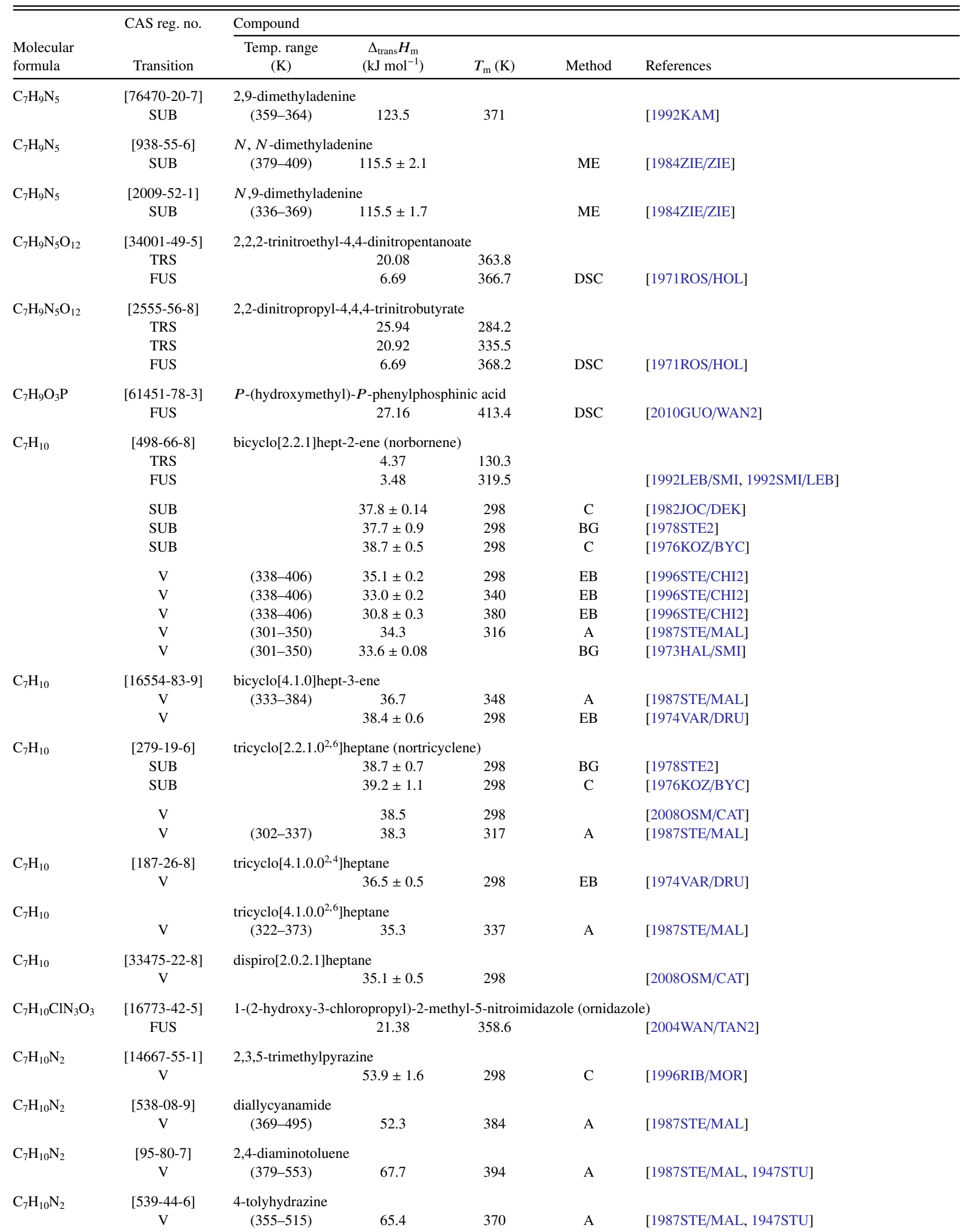


TABLE 10. Phase change enthalpies of $\mathrm{C}_{7}$ organic compounds-Continued

\begin{tabular}{|c|c|c|c|c|c|c|}
\hline \multirow[b]{2}{*}{$\begin{array}{l}\text { Molecular } \\
\text { formula }\end{array}$} & \multirow{2}{*}{$\begin{array}{c}\text { CAS reg. no. } \\
\text { Transition }\end{array}$} & \multicolumn{5}{|l|}{ Compound } \\
\hline & & $\begin{array}{c}\text { Temp. range } \\
(\mathrm{K})\end{array}$ & $\begin{array}{c}\Delta_{\text {trans }} H_{\mathrm{m}} \\
\left(\mathrm{kJ} \mathrm{mol}^{-1}\right)\end{array}$ & $T_{\mathrm{m}}(\mathrm{K})$ & Method & References \\
\hline \multirow[t]{2}{*}{$\mathrm{C}_{7} \mathrm{H}_{10} \mathrm{~N}_{2}$} & {$[4210-60-0]$} & \multicolumn{5}{|c|}{$\alpha$-tert-butylmalononitrile } \\
\hline & SUB & $(293-323)$ & $59.8 \pm 0.7$ & 298 & & {$[1990 \mathrm{BEC} / \mathrm{DOG}]$} \\
\hline \multirow[t]{3}{*}{$\mathrm{C}_{7} \mathrm{H}_{10} \mathrm{~N}_{2}$} & [646-20-8] & \multicolumn{5}{|l|}{ pimelonitrile } \\
\hline & FUS & & 15.0 & 241.7 & DSC & [2007BAD/BLA] \\
\hline & $\mathrm{V}$ & $(306-331)$ & 74.5 & 318 & A & [1987STE/MAL, 1960WOO/MUR] \\
\hline \multirow[t]{4}{*}{$\mathrm{C}_{7} \mathrm{H}_{10} \mathrm{~N}_{2}$} & {$[5683-33-0]$} & \multicolumn{5}{|c|}{ 2-N, N-dimethylaminopyridine } \\
\hline & $\mathrm{V}$ & $(288-348)$ & $55.2 \pm 0.1$ & 298 & GS & [2011LIP/RAT] \\
\hline & $\mathrm{V}$ & & $54.6 \pm 2.1$ & 298 & $\mathrm{CGC}$ & [2011LIP/RAT] \\
\hline & $\mathrm{V}$ & $(233-323)$ & 54.0 & 298 & $\mathrm{ME}$ & [1991AUE/WEB] \\
\hline \multirow[t]{2}{*}{$\mathrm{C}_{7} \mathrm{H}_{10} \mathrm{~N}_{2}$} & {$[18437-57-5]$} & \multicolumn{5}{|c|}{ 3-N, N-dimethylaminopyridine } \\
\hline & $\mathrm{V}$ & $(233-323)$ & 70.7 & 298 & $\mathrm{ME}$ & [1991AUE/WEB] \\
\hline \multirow[t]{4}{*}{$\mathrm{C}_{7} \mathrm{H}_{10} \mathrm{~N}_{2}$} & [1122-58-3] & \multicolumn{5}{|c|}{ 4-N, N-dimethylaminopyridine } \\
\hline & FUS & $(80-402)$ & 21.63 & 387.1 & $\mathrm{AC}$ & [2007SHI/TAN] \\
\hline & SUB & $(323-368)$ & $87.0 \pm 0.2$ & 298 & GS & [2011LIP/RAT] \\
\hline & SUB & $(233-323)$ & 81.6 & 298 & ME & [1991AUE/WEB] \\
\hline
\end{tabular}

[Note: Authors of [1991AUE/WEB] refer to the value as an enthalpy of vaporization. The compound's melting point temperature is above the temperature range of the measured VPs.]
V
$61.3 \pm 2.5$
298
CGC
[2011LIP/RAT]

$\mathrm{C}_{7} \mathrm{H}_{10} \mathrm{~N}_{2}$

[717908-74-2]

$N$-cyclopropylmethylimidazole

$$
\mathrm{V} \quad(303-343) \quad 59.8 \pm 0.3
$$

298

GS

[2015VER/ZAI3]

$\mathrm{C}_{7} \mathrm{H}_{10} \mathrm{~N}_{2} \mathrm{O}$

[25926-99-2]

FUS

6,7-diazatricyclo[3.2.2.0 $\left.0^{2,4}\right]$ non-6-ene- $N$-oxide

$15.8 \quad 372.6$

$2.6 \quad 411.4$

$\mathrm{C}_{7} \mathrm{H}_{10} \mathrm{~N}_{2} \mathrm{OS}$

[51-52-5]

SUB

6-propyl-2-thiouraci

$\begin{array}{lllll}(401-423) & 136.0 \pm 0.9 & 412 & \mathrm{ME} & {[2014 \mathrm{SZT} / \mathrm{GAL}]} \\ (401-423) & 139.7 \pm 0.9 & 298 & \mathrm{ME} & {[2014 \mathrm{SZT} / \mathrm{GAL}]}\end{array}$

$\mathrm{C}_{7} \mathrm{H}_{10} \mathrm{~N}_{2} \mathrm{O}$

[4401-71-2]

1,3-dimethylthymine

FUS

$23.6 \quad 426.5$

DSC

[1984ZIE/ZIE2]

SUB

(313-363)

$109.2 \pm 2.1$

338

QR

[1980TEP/YAN]

$\mathrm{C}_{7} \mathrm{H}_{10} \mathrm{~N}_{2} \mathrm{O}_{2}$

[4401-71-2]

1,3,5-trimethyluracil

FUS

16.1

428.7

DSC

[1996KAM/ZIE]

SUB

(348-391)

$94.9 \pm 0.6$

298

GS

[2013NOT/EME]

SUB

(321-331)

$103.5 \pm 1.5 \quad 326$

ME

[1996KAM/ZIE]

$\mathrm{C}_{7} \mathrm{H}_{10} \mathrm{~N}_{2} \mathrm{O}_{2} \quad$ [13509-52-9] 1,3,6-trimethyluracil

FUS

$21.2 \quad 384.5$

DSC

[1996DOM/HEA, 1984ZIE/ZIE2]

SUB

(300-340)

$106.7 \pm 2.5$

320

QR

[1980TEP/YAN]

$\mathrm{C}_{7} \mathrm{H}_{10} \mathrm{~N}_{2} \mathrm{O}$

[1709-52-0]

4-amino- $N$-methylbenzene sulfonamide

FUS

17.1

382.2

DSC

[2014PER/KAZ]

$\mathrm{C}_{7} \mathrm{H}_{10} \mathrm{~N}_{2} \mathrm{O}_{3}$

[7358-61-4]

1,3,5-trimethylbarbituric acid

FUS

13.7

362.6

DSC

[2011TEM/ROU]

SUB

$$
\text { (322-358) }
$$

$96.1 \pm 0.8$

298

GS

[2014NOT/ROU]

$\mathrm{C}_{7} \mathrm{H}_{10} \mathrm{~N}_{2} \mathrm{O}_{3}$

[702-47-6]

1,5,5-trimethylbarbituric acid

FUS

$$
30.2
$$

SUB

$(360-428)$

$106.2 \pm 0.4$

434.5

DSC

[2011TEM/ROU]

$\mathrm{C}_{7} \mathrm{H}_{10} \mathrm{~N}_{2} \mathrm{O}_{3}$

[7391-69-7]

SUB

SUB

5-isopropylbarbituric acid

SUB

$\begin{array}{ll} & 125.0 \pm 3.8 \\ (369-391) & 120.8 \pm 0.6 \\ (369-391) & 123.6 \pm 0.6\end{array}$

298

GS

[2014NOT/ROU]

$\mathrm{C}_{7} \mathrm{H}_{10} \mathrm{O}$

[17356-19-3]

1-ethynyl-1-cyclopentanol

$\mathrm{V}$ (323-373)

$\mathrm{V}$ (323-373)

62.1

298

380

298

C

ME

298

CGC

[1995CHI/HOS] 
TABLE 10. Phase change enthalpies of $\mathrm{C}_{7}$ organic compounds-Continued

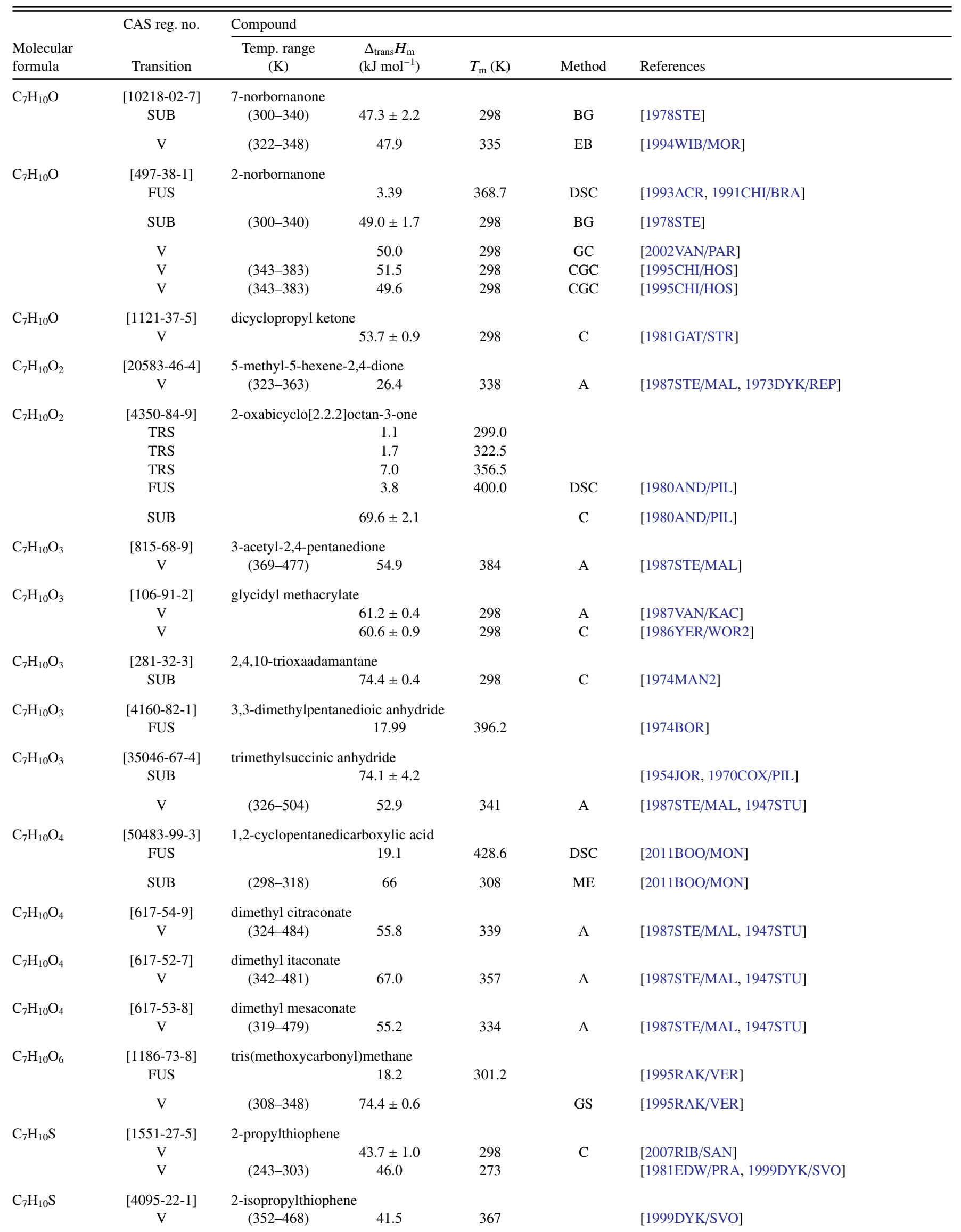


TABLE 10. Phase change enthalpies of $\mathrm{C}_{7}$ organic compounds-Continued

\begin{tabular}{|c|c|c|c|c|c|c|}
\hline \multirow[b]{2}{*}{$\begin{array}{l}\text { Molecular } \\
\text { formula }\end{array}$} & \multirow{2}{*}{$\begin{array}{l}\text { CAS reg. no. } \\
\text { Transition }\end{array}$} & \multicolumn{5}{|l|}{ Compound } \\
\hline & & $\begin{array}{c}\text { Temp. range } \\
(\mathrm{K})\end{array}$ & $\begin{array}{c}\Delta_{\text {trans }} H_{\mathrm{m}} \\
\left(\mathrm{kJ} \mathrm{mol}^{-1}\right)\end{array}$ & $T_{\mathrm{m}}(\mathrm{K})$ & Method & References \\
\hline \multirow[t]{3}{*}{$\mathrm{C}_{7} \mathrm{H}_{10} \mathrm{~S}_{3}$} & [2164-87-6] & \multicolumn{5}{|c|}{ 4,5-tetramethylene-1,3-dithiolan-2-thione } \\
\hline & SUB & $(353-369)$ & 99.0 & 360 & & [1967GEI/SCH, 1970COX/PIL] \\
\hline & SUB & & $103.9 \pm 2.9$ & 298 & & [1967GEI/SCH, 1970COX/PIL] \\
\hline \multirow[t]{2}{*}{$\mathrm{C}_{7} \mathrm{H}_{11} \mathrm{BrO}_{2}$} & {$[26918-14-9]$} & \multicolumn{5}{|c|}{ 4-bromo-3-methylcrotonic acid, ethyl ester } \\
\hline & $\mathrm{V}$ & $(346-381)$ & 43.1 & 361 & A & {$[1987 \mathrm{STE} / \mathrm{MAL}]$} \\
\hline \multirow[t]{2}{*}{$\mathrm{C}_{7} \mathrm{H}_{11} \mathrm{ClO}_{5}$} & & \multicolumn{5}{|c|}{ (2-chloroethyl)[(1-methoxycarbonyl)ethyl] carbonate } \\
\hline & $\mathrm{V}$ & $(365-525)$ & 66.8 & 380 & A & [1987STE/MAL, 1999DYK/SVO] \\
\hline \multirow[t]{2}{*}{$\mathrm{C}_{7} \mathrm{H}_{11} \mathrm{Cl}_{3} \mathrm{O}_{2}$} & {$[57392-56-0]$} & \multicolumn{5}{|c|}{ trichloracetic acid, neopentyl ester } \\
\hline & $\mathrm{V}$ & $(378-473)$ & 57.7 & 393 & A & [1987STE/MAL, 1999DYK/SVO, 1940QUA/NOR] \\
\hline \multirow[t]{5}{*}{$\mathrm{C}_{7} \mathrm{H}_{11} \mathrm{~N}$} & {$[766-05-2]$} & \multicolumn{5}{|c|}{ cyclohexanecarbonitrile } \\
\hline & TRS & & 7.43 & 215 & & \\
\hline & FUS & & 3.64 & 285.1 & & [1996DOM/HEA, 1991KIS/PIN] \\
\hline & $\mathrm{V}$ & $(333-427)$ & 39.4 & 351 & BG & {$[1971 \mathrm{HAL} / \mathrm{BAL}]$} \\
\hline & $\mathrm{V}$ & & $51.9 \pm 0.1$ & 298 & $\mathrm{C}$ & {$[1970 \mathrm{PRO} / \mathrm{KRE}]$} \\
\hline \multirow[t]{4}{*}{$\mathrm{C}_{7} \mathrm{H}_{11} \mathrm{~N}$} & {$[931-53-3]$} & \multicolumn{5}{|c|}{ isocyanocyclohexane } \\
\hline & FUS & & 3.98 & 277.7 & DSC & [2008SIN/MUR2] \\
\hline & TRS & & 6.18 & 192.6 & & \\
\hline & FUS & & 4.23 & 279.6 & & [1996DOM/HEA, 1991KIS/PIN] \\
\hline \multirow[t]{2}{*}{$\mathrm{C}_{7} \mathrm{H}_{11} \mathrm{~N}$} & {$[930-87-0]$} & \multicolumn{5}{|c|}{ 1,2,5-trimethylpyrrole } \\
\hline & $\mathrm{V}$ & & $50.7 \pm 1.3$ & 298 & $\mathrm{C}$ & [2014SAN/RIB] \\
\hline \multirow[t]{2}{*}{$\mathrm{C}_{7} \mathrm{H}_{11} \mathrm{NO}_{2}$} & & \multicolumn{5}{|c|}{ 2-methyl-2-acetoxybutyronitrile } \\
\hline & $\mathrm{V}$ & $(315-469)$ & 58.1 & 330 & A & [1987STE/MAL, 1947STU] \\
\hline $\mathrm{C}_{7} \mathrm{H}_{11} \mathrm{NO}_{2}$ & {$[7149-65-7]$} & 5-oxo-2-pyrroli & carboxylic aci & yl ester & & \\
\hline & $\mathrm{V}$ & $(418-511)$ & 73.7 & 433 & A, EB & [1987STE/MAL, 1953MEL/VIO] \\
\hline $\mathrm{C}_{7} \mathrm{H}_{11} \mathrm{~N}_{3} \mathrm{O}$ & {$[2228-27-5]$} & $1, N, N$-trimeth & tosine & & & \\
\hline & SUB & & $110.9 \pm 1.7$ & & & [1998ZIE/WSZ] \\
\hline $\mathrm{C}_{7} \mathrm{H}_{11} \mathrm{~N}_{3} \mathrm{O}$ & {$[25307-94-2]$} & 1,5,N-trimethy & sine & & & \\
\hline & SUB & $(396-431)$ & $108.0 \pm 2.0$ & & GS & [1998ZIE/WSZ] \\
\hline $\mathrm{C}_{7} \mathrm{H}_{11} \mathrm{~N}_{3} \mathrm{O}_{2}$ & [38691-19-9] & 1,5-dimethyl- $N$ & thoxycytosine & & & \\
\hline & SUB & $(327-365)$ & $95.6 \pm 0.7$ & & GS & [1998ZIE/WSZ] \\
\hline $\mathrm{C}_{7} \mathrm{H}_{11} \mathrm{~N}_{3} \mathrm{O}_{3}$ & [1077-93-6] & 3-(2-methyl-5-1 & imadazol-1-yl & an-1-ol (t & zole) & \\
\hline & FUS & & 25.65 & 333 & DSC & [2011MAH/PER] \\
\hline $\mathrm{C}_{7} \mathrm{H}_{11} \mathrm{~N}_{5} \mathrm{O}_{10}$ & [242800-94-8] & 1,1,1,4,4-pental & -2,2-dimethyl & & & \\
\hline & SUB & & $114.2 \pm 1.2$ & 298 & $\mathrm{C}$ & [2011MIR/KON] \\
\hline & SUB & & 103.8 & 298 & & [1999MIR/VOR] \\
\hline $\mathrm{C}_{7} \mathrm{H}_{12}$ & [279-23-2] & bicyclo[2.2.1]h & ne (norbornan & & & \\
\hline & FUS & & 4.45 & 360.8 & $\mathrm{DSC}$ & [2004VER/EME] \\
\hline & SUB & $(278-308)$ & $40.3 \pm 0.4$ & 293 & GS & [2004VER/EME] \\
\hline & SUB & $(278-308)$ & $40.1 \pm 0.4$ & 298 & GS & [2004VER/EME] \\
\hline & SUB & & $40.0 \pm 0.1$ & 298 & $\mathrm{C}$ & [1987AN/ZHU] \\
\hline & SUB & & $40.3 \pm 0.32$ & 298 & $\mathrm{C}$ & [1982JOC/DEK] \\
\hline & SUB & & $40.4 \pm 0.8$ & 298 & BG & [1978STE2] \\
\hline & SUB & $(284-326)$ & $40.0 \pm 0.8$ & 305 & TSGC & [1975CLA/KNO] \\
\hline & SUB & $(300-363)$ & $39.33 \pm 0.13$ & & BG & [1973HAL/SMI] \\
\hline & SUB & $(284-326)$ & $40.1 \pm 0.8$ & & $\mathrm{BG}$ & [1971BOY/SAN, 1977PED/RYL] \\
\hline $\mathrm{C}_{7} \mathrm{H}_{12}$ & {$[286-08-8]$} & cis-bicyclo[4.1. & ptane & & & \\
\hline & $\mathrm{V}$ & & $40.6 \pm 0.2$ & 298 & & [2008OSM/CAT] \\
\hline & V & $(298-385)$ & $38.0 \pm 0.8$ & 313 & A, EB & [1987STE/MAL, 1970CHA/MCN] \\
\hline $\mathrm{C}_{7} \mathrm{H}_{12}$ & {$[286-08-8]$} & $(d l)$-bicyclo[4.1 & eptane & & & \\
\hline & $\mathrm{V}$ & $(333-385)$ & 36.5 & 348 & A & [1987STE/MAL] \\
\hline $\mathrm{C}_{7} \mathrm{H}_{12}$ & {$[4625-24-5]$} & 1-methylbicycl & .0]hexane & & & \\
\hline & V & $(312-362)$ & 34.0 & 327 & A & [1987STE/MAL] \\
\hline
\end{tabular}


TABLE 10. Phase change enthalpies of $\mathrm{C}_{7}$ organic compounds-Continued

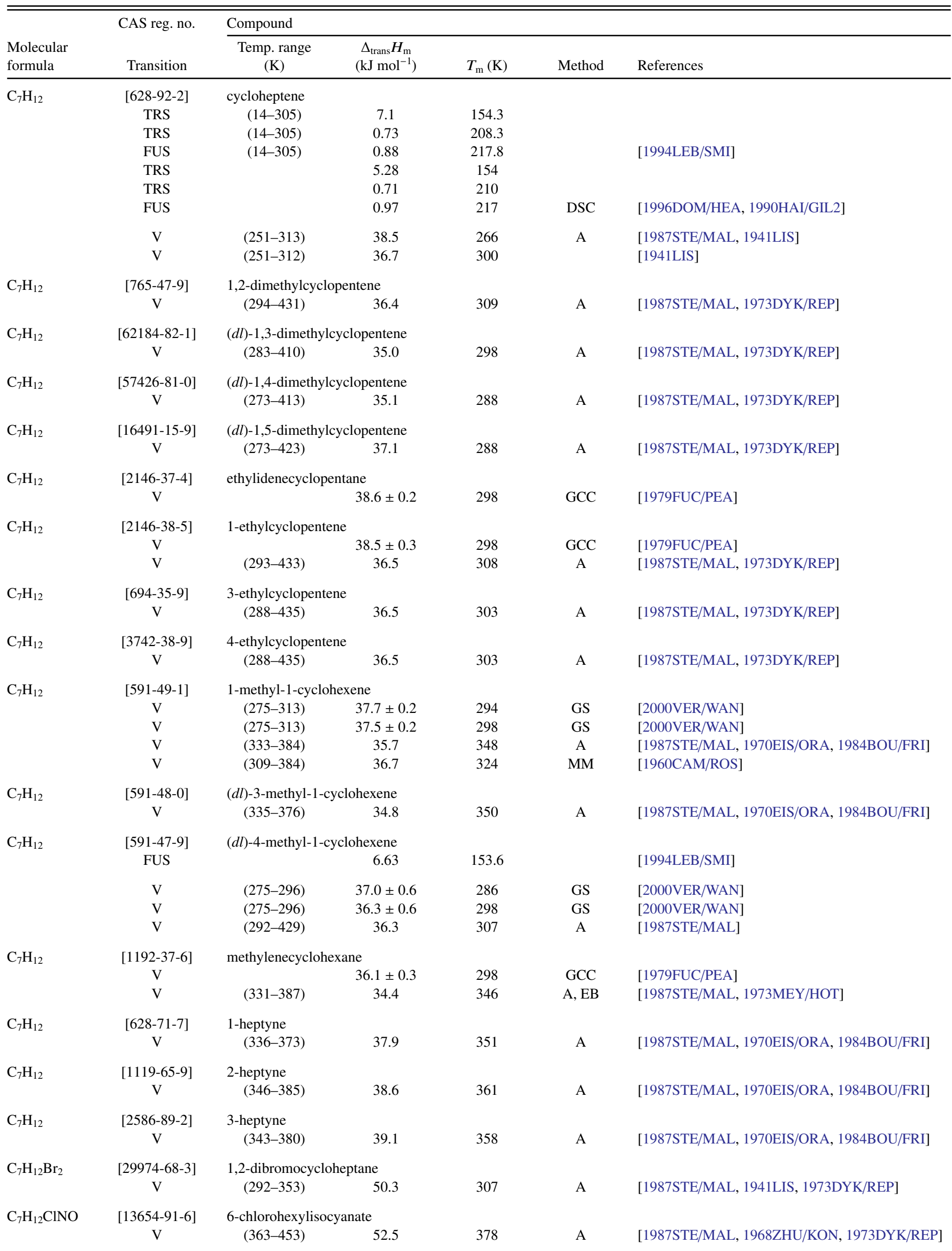


TABLE 10. Phase change enthalpies of $\mathrm{C}_{7}$ organic compounds-Continued

\begin{tabular}{|c|c|c|c|c|c|c|}
\hline \multirow[b]{2}{*}{$\begin{array}{l}\text { Molecular } \\
\text { formula }\end{array}$} & \multirow{2}{*}{$\begin{array}{l}\text { CAS reg. no. } \\
\text { Transition }\end{array}$} & \multicolumn{5}{|l|}{ Compound } \\
\hline & & $\begin{array}{c}\text { Temp. range } \\
(\mathrm{K})\end{array}$ & $\begin{array}{c}\Delta_{\text {trans }} H_{\mathrm{m}} \\
\left(\mathrm{kJ} \mathrm{mol}^{-1}\right)\end{array}$ & $T_{\mathrm{m}}(\mathrm{K})$ & Method & References \\
\hline \multirow[t]{4}{*}{$\mathrm{C}_{7} \mathrm{H}_{12} \mathrm{ClN}_{5}$} & [122-34-9] & \multicolumn{5}{|c|}{ 2-chloro-4,6-bis(ethylamino)- $s$-triazine (Simazin) } \\
\hline & FUS & & 47.35 & 502.5 & DSC & [1990DON/DRE] \\
\hline & FUS & & 48.2 & & DSC & [1971GET/WAR] \\
\hline & SUB & $(323-403)$ & 130.8 & 338 & GS-GC & [1987STE/MAL, 1964FRI/STA] \\
\hline \multirow[t]{2}{*}{$\mathrm{C}_{7} \mathrm{H}_{12} \mathrm{Cl}_{2} \mathrm{O}_{2}$} & {$[860540-85-8]$} & \multicolumn{5}{|c|}{ dichloroacetic acid, neopentyl ester } \\
\hline & V & $(368-463)$ & 57.4 & 383 & A & [1987STE/MAL, 1999DYK/SVO, 1940QUA/NOR] \\
\hline \multirow[t]{2}{*}{$\mathrm{C}_{7} \mathrm{H}_{12} \mathrm{Cl}_{2} \mathrm{~S}$} & {$[743438-46-2]$} & \multicolumn{5}{|c|}{ (2-chloroethyl)(2-chlorocyclopentyl) sulfide } \\
\hline & V & $(273-333)$ & 65.9 & 303 & $\mathrm{~A}, \mathrm{GS}$ & [1987STE/MAL, 1948RED/CHA, 1999DYK/SVO] \\
\hline \multirow[t]{3}{*}{$\mathrm{C}_{7} \mathrm{H}_{12} \mathrm{Cl}_{4}$} & {$[3922-36-9]$} & \multicolumn{5}{|c|}{ 1,1,1,7-tetrachloroheptane } \\
\hline & $\mathrm{V}$ & $(342-455)$ & 71.7 & 357 & & [1999DYK/SVO] \\
\hline & $\mathrm{V}$ & $(370-454)$ & 69.9 & 385 & A & {$[1987 \mathrm{STE} / \mathrm{MAL}]$} \\
\hline \multirow[t]{3}{*}{$\mathrm{C}_{7} \mathrm{H}_{12} \mathrm{~N}_{2}$} & {$[3010-03-5]$} & \multicolumn{5}{|c|}{ 1-piperidinoacetonitrile } \\
\hline & FUS & & 17.57 & 293.2 & & [1997WEL/VER] \\
\hline & $\mathrm{V}$ & $(303-338)$ & $56.0 \pm 0.5$ & & GS & [1997WEL/VER] \\
\hline \multirow[t]{5}{*}{$\mathrm{C}_{7} \mathrm{H}_{12} \mathrm{~N}_{2}$} & {$[3001-72-7]$} & \multicolumn{5}{|c|}{ 1,5-diazabicyclo[4.3.0]non-5-ene } \\
\hline & $\mathrm{V}$ & & 56.8 & 392 & DP-LPD & [2016OST/UUS] \\
\hline & $\mathrm{V}$ & & $62.6 \pm 1.3$ & 298 & DP-LPD & [2016OST/UUS] \\
\hline & $\mathrm{V}$ & & $61.9 \pm 0.2$ & 298 & GS & [2011LIP/RAT] \\
\hline & $\mathrm{V}$ & & $61.1 \pm 2.4$ & 298 & CGC & [2011LIP/RAT] \\
\hline \multirow[t]{2}{*}{$\mathrm{C}_{7} \mathrm{H}_{12} \mathrm{~N}_{2}$} & [33214-18-5] & \multicolumn{5}{|c|}{ 1-propyl-2-methylimidazole } \\
\hline & V & $(289-343)$ & $63.7 \pm 0.2$ & 298 & GS & [2011EME/POR2] \\
\hline \multirow[t]{2}{*}{$\mathrm{C}_{7} \mathrm{H}_{12} \mathrm{~N}_{2}$} & {$[4316-42-1]$} & \multicolumn{5}{|c|}{ 1-butylimidazole } \\
\hline & $\mathrm{V}$ & & $63.9 \pm 0.5$ & 298 & $\mathrm{C}$ & [2015VIT/AGA] \\
\hline $\mathrm{C}_{7} \mathrm{H}_{12} \mathrm{~N}_{2}$ & {$[16245-89-9]$} & $N$-isobutylimic & & & & \\
\hline & $\mathrm{V}$ & $(290-326)$ & $62.2 \pm 0.3$ & 298 & GS & [2015VER/ZAI3] \\
\hline $\mathrm{C}_{7} \mathrm{H}_{12} \mathrm{~N}_{2}$ & {$[20075-29-0]$} & $N$-sec-butylim & & & & \\
\hline & $\mathrm{V}$ & (296-330) & $64.6 \pm 0.3$ & 298 & GS & [2015VER/ZAI3] \\
\hline $\mathrm{C}_{7} \mathrm{H}_{12} \mathrm{~N}_{2}$ & {$[45676-04-8]$} & $N$-tert-butylim & & & & \\
\hline & $\mathrm{V}$ & $(297-343)$ & $61.9 \pm 0.4$ & 298 & GS & [2015VER/ZAI3] \\
\hline $\mathrm{C}_{7} \mathrm{H}_{12} \mathrm{~N}_{2} \mathrm{O}_{2}$ & [16395-58-7] & $N$-acetyl-L-pro & iide & & & \\
\hline & FUS & & 29.3 & 417.5 & DSC & [1997ABA/PAL] \\
\hline $\mathrm{C}_{7} \mathrm{H}_{12} \mathrm{~N}_{4} \mathrm{O}_{10}$ & [5917-61-3] & bis(2,4-dinitrop & 1)formal & & & \\
\hline & $\mathrm{V}$ & $(333-383)$ & $84.8 \pm 0.9$ & 358 & & [2007RAU/BEH] \\
\hline $\mathrm{C}_{7} \mathrm{H}_{12} \mathrm{O}$ & [497-37-0] & exo-norborneol & & & & \\
\hline & $\mathrm{V}$ & & 52.5 & 298 & GC & [2002VAN/PAR] \\
\hline $\mathrm{C}_{7} \mathrm{H}_{12} \mathrm{O}$ & [931-57-7] & 1-methoxycycl & & & & \\
\hline & $\mathrm{V}$ & $(274-313)$ & $44.0 \pm 0.2$ & 294 & GS & [1998VER/WEL] \\
\hline & $\mathrm{V}$ & $(274-313)$ & $43.7 \pm 0.2$ & 298 & GS & [1998VER/WEL] \\
\hline $\mathrm{C}_{7} \mathrm{H}_{12} \mathrm{O}$ & {$[502-42-1]$} & cycloheptanone & & & & \\
\hline & TRS & & 12.4 & 227 & & \\
\hline & TRS & & 0.43 & 232.6 & & \\
\hline & FUS & & 1.39 & 259.3 & DSC & [1998GON/SZW] \\
\hline & $\mathrm{V}$ & $(343-383)$ & 50.6 & 298 & CGC & [1995CHI/HOS] \\
\hline & $\mathrm{V}$ & $(343-383)$ & 51.9 & 298 & CGC & [1995CHI/HOS] \\
\hline & $\mathrm{V}$ & $(343-383)$ & 50.7 & 298 & CGC & [1995CHI/HOS] \\
\hline & $\mathrm{V}$ & $(313-453)$ & 48.5 & 328 & A & [1987STE/MAL] \\
\hline & $\mathrm{V}$ & $(373-465)$ & 44.8 & 388 & $\mathrm{~A}, \mathrm{~EB}$ & [1987STE/MAL, 1976MEY/HOT] \\
\hline & V & $(313-383)$ & $49.5 \pm 0.6$ & 298 & VP & {$[1972 \mathrm{WOL}]$} \\
\hline $\mathrm{C}_{7} \mathrm{H}_{12} \mathrm{O}$ & {$[583-60-8]$} & 2-methylcyclor & one & & & \\
\hline & $\mathrm{V}$ & $(339-437)$ & 44.0 & 298 & $\mathrm{~EB}$ & [2006PAL/ORA] \\
\hline & $\mathrm{V}$ & $(331-437)$ & 45.0 & 346 & & [1993BRU/MON] \\
\hline $\mathrm{C}_{7} \mathrm{H}_{12} \mathrm{O}$ & [591-24-2] & 3-methylcyclor & one & & & \\
\hline
\end{tabular}


TABLE 10. Phase change enthalpies of $\mathrm{C}_{7}$ organic compounds-Continued

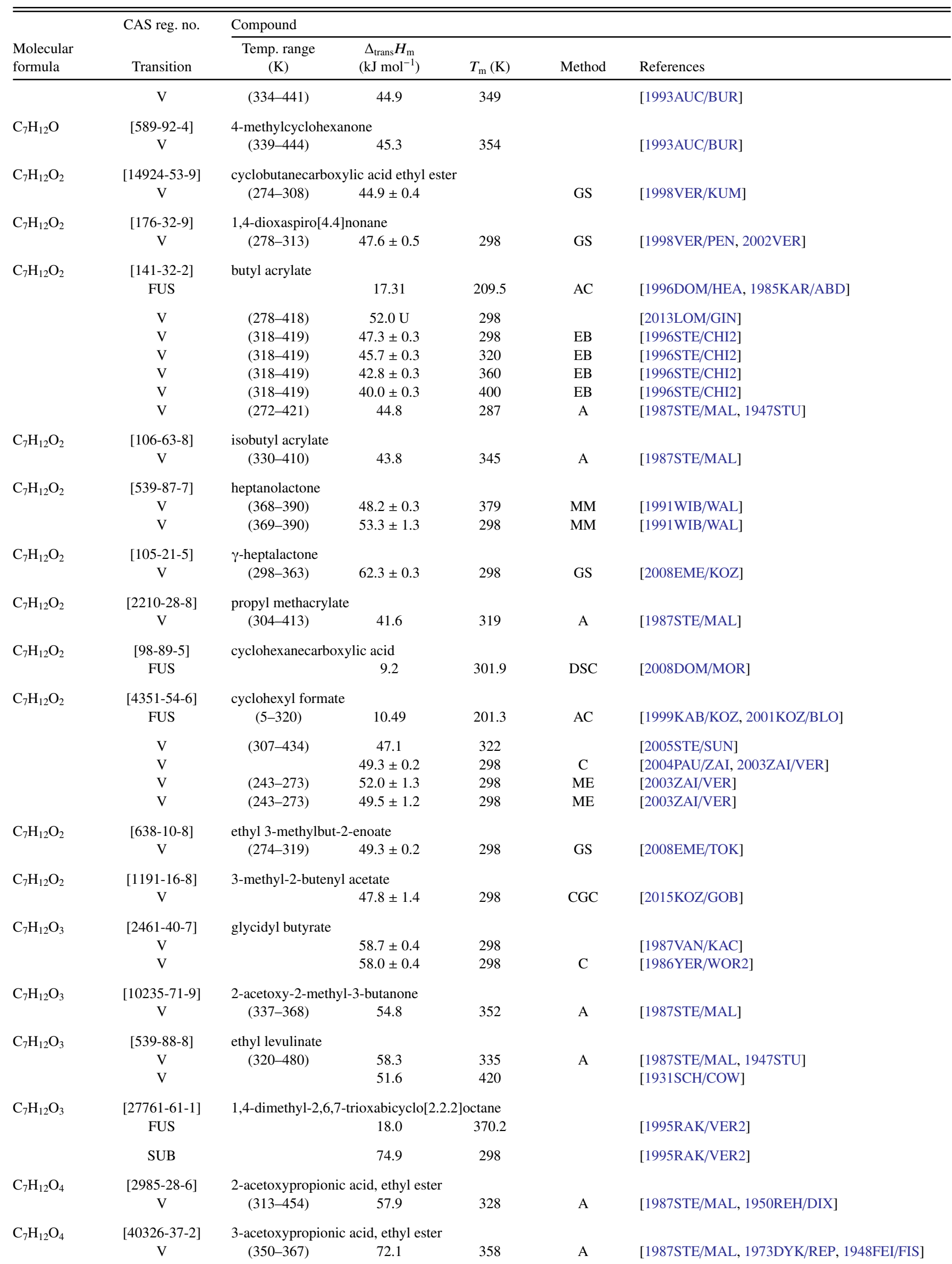


TABLE 10. Phase change enthalpies of $\mathrm{C}_{7}$ organic compounds-Continued

\begin{tabular}{|c|c|c|c|c|c|c|}
\hline \multirow[b]{2}{*}{$\begin{array}{l}\text { Molecular } \\
\text { formula }\end{array}$} & \multirow{2}{*}{$\begin{array}{c}\text { CAS reg. no. } \\
\text { Transition }\end{array}$} & \multicolumn{5}{|l|}{ Compound } \\
\hline & & $\begin{array}{c}\text { Temp. range } \\
(\mathrm{K})\end{array}$ & $\begin{array}{c}\Delta_{\text {trans }} H_{\mathrm{m}} \\
\left(\mathrm{kJ} \mathrm{mol}^{-1}\right)\end{array}$ & $T_{\mathrm{m}}(\mathrm{K})$ & Method & References \\
\hline \multirow[t]{2}{*}{$\mathrm{C}_{7} \mathrm{H}_{12} \mathrm{O}_{4}$} & [623-84-7] & \multicolumn{2}{|c|}{ 1,2-propylene glycol diacetate } & & & \\
\hline & $\mathrm{V}$ & $(318-367)$ & 54.9 & 323 & & [2001HOR/GAR] \\
\hline \multirow[t]{12}{*}{$\mathrm{C}_{7} \mathrm{H}_{12} \mathrm{O}_{4}$} & [105-53-3] & \multicolumn{2}{|l|}{ diethyl malonate } & & & \\
\hline & $\mathrm{V}$ & $(265-288)$ & 60.8 & 283 & ME & [2014BRO/BUC] \\
\hline & V & $(336-471)$ & 56.3 & 343 & DTA & [2014BRO/BUC] \\
\hline & V & $(336-471)$ & 54.7 & 373 & DTA & [2014BRO/BUC] \\
\hline & V & $(336-471)$ & 53 & 413 & DTA & [2014BRO/BUC] \\
\hline & $\mathrm{V}$ & $(336-471)$ & 51.7 & 453 & DTA & [2014BRO/BUC] \\
\hline & V & $(336-471)$ & 51.3 & 469 & DTA & [2014BRO/BUC] \\
\hline & V & $(284-316)$ & 61.1 & 300 & GS & [2011LIP/KRA] \\
\hline & V & $(284-316)$ & $61.7 \pm 0.3$ & 298 & GS & [2011LIP/KRA] \\
\hline & $\mathrm{V}$ & & $58.4 \pm 0.6$ & 298 & GC & [1992KAT, 2011LIP/KRA] \\
\hline & V & $(288-318)$ & $64.7 \pm 0.2$ & 293 & GS & [1992VER/BEC] \\
\hline & $\mathrm{V}$ & $(288-318)$ & $65.1 \pm 0.2$ & 298 & GS & [1992VER/BEC, 2011LIP/KRA] \\
\hline
\end{tabular}

[Note: Steele and coworkers in reference [2002STE/CHI6] refer to a personal communication with one of the authors of [1992VER/BEC]—stating that it was established that the compound studied was not diethyl malonate. In a subsequent paper [2011LIP/KRA] co-authored by one of the authors of [1992VER/BEC] the data are still taken to be diethyl malonate.]

$\begin{array}{cccc}\text { V } & (293-318) & 63.3 & 305 \\ \text { V } & (384-468) & 59.9 & 399 \\ \text { V } & (313-472) & 51.2 & 328 \\ \text { V } & (365-491) & 56.9 & 428 \\ \text { V } & (365-491) & 67.2 \pm 0.5 & 298 \\ \text { V } & (314-472) & 51.2 & 393 \\ \text { V } & (314-472) & 60.5 & 298 \\ \text { V } & (315-464) & 51.2 & 390 \\ \text { V } & (315-464) & 58.7 \pm 0.2 & 298\end{array}$

$\begin{array}{ll}\text { A } & {[1987 \text { STE/MAL }]} \\ \text { A } & {[1987 \text { STE/MAL }]} \\ \text { A } & {[1987 \text { STE/MAL }]} \\ \text { EB } & {[1978 \text { SMI/ZEL, 2011LIP/KRA }]} \\ \text { EB } & {[1978 \text { SMI/ZEL, 2011LIP/KRA }]} \\ & {[1947 \text { STU, 2011LIP/KRA }]} \\ & {[1947 \text { STU, 2011LIP/KRA }]} \\ & {[1940 H E I / R E I, 2011 \text { LIP/KRA }]} \\ & {[1940 \text { HEI/REI, 2011LIP/KRA }]}\end{array}$

\begin{tabular}{|c|c|c|c|c|c|c|}
\hline \multirow[t]{2}{*}{$\mathrm{C}_{7} \mathrm{H}_{12} \mathrm{O}_{4}$} & [6065-54-9] & \multicolumn{5}{|c|}{ dimethyl dimethylmalonate } \\
\hline & $\mathrm{V}$ & (278-307) & $55.6 \pm 0.8$ & 293 & GS & [1992VER/BEC] \\
\hline \multirow[t]{4}{*}{$\mathrm{C}_{7} \mathrm{H}_{12} \mathrm{O}_{4}$} & {$[1119-40-0]$} & \multicolumn{5}{|c|}{ glutaric acid, dimethyl ester } \\
\hline & $\mathrm{V}$ & $(283-348)$ & $65.7 \pm 0.4$ & 298 & GS & [2006VER/KOZ] \\
\hline & V & $(366-483)$ & 54.7 & 381 & A & [1987STE/MAL] \\
\hline & $\mathrm{V}$ & $(388-483)$ & 66.1 & 298 & EB & [1963VLA/GRA, 2006VER/KOZ] \\
\hline \multirow[t]{2}{*}{$\mathrm{C}_{7} \mathrm{H}_{12} \mathrm{O}_{4}$} & {$[627-91-8]$} & methyl adipate & & & & \\
\hline & V & $(453-503)$ & 82.9 & 468 & A & [1987STE/MAL] \\
\hline \multirow[t]{11}{*}{$\mathrm{C}_{7} \mathrm{H}_{12} \mathrm{O}_{4}$} & [111-16-0] & \multicolumn{5}{|c|}{ heptanedioic acid (pimelic acid) } \\
\hline & FUS & & 28.8 & 377.4 & DSC & [2012WAN/DEN, 2012WAN/LI] \\
\hline & FUS & & 31.78 & 378.0 & DSC & {$[2010 \mathrm{COO} / \mathrm{DAV}]$} \\
\hline & TRS & & 1.5 & 337.7 & & \\
\hline & FUS & & 30.3 & 377.5 & DSC & [2002STE/CHI6] \\
\hline & FUS & & 23.7 & 368.2 & DSC & [2005ROU/TEM] \\
\hline & FUS & & 27.62 & 377.5 & DSC & [1991ACR, 1974CIN/BER] \\
\hline & SUB & $(328-363)$ & $153 \pm 4$ & & TPD & [2007CAP/LOV] \\
\hline & SUB & $(283-300)$ & 124 & & TPTD & [2005CHA/ZIE] \\
\hline & SUB & $(318-336)$ & 80.8 & & TPTD & [2005CHA/ZIE] \\
\hline & SUB & $(288-308)$ & 178 & & TPTD & [2001CHA/TOB] \\
\hline
\end{tabular}

[Note: Values based on the TPTD method are not consistent with values determined by other experimental methods.]

$\begin{array}{cccccl}\text { SUB } & (358-371) & 136.6 \pm 1.0 & 365 & \text { ME } & \text { [1999RIB/MON] } \\ \text { SUB } & (358-371) & 139.9 \pm 1.0 & 298 & \text { ME } & \text { [1999RIB/MON] } \\ \text { V } & (424-503) & 112.0 \pm 0.8 & 298 & \text { CGC } & \text { [2005ROU/TEM] } \\ \text { V } & (436-615) & 88.6 & 451 & \text { A } & \text { [1987STE/MAL, 1947STU] }\end{array}$

\begin{tabular}{|c|c|c|c|c|c|c|}
\hline $\mathrm{C}_{7} \mathrm{H}_{12} \mathrm{O}_{4}$ & $\begin{array}{c}{[126-54-5]} \\
\mathrm{V}\end{array}$ & 2,4,8,10-tetraoxaspir & $\begin{array}{l}\text { o[5.5] undecane } \\
56.0\end{array}$ & & & [1959FLE/MOR] \\
\hline $\mathrm{C}_{7} \mathrm{H}_{12} \mathrm{O}_{4}$ & $\begin{array}{c}\text { [32786-47-3] } \\
\mathrm{V}\end{array}$ & $\begin{array}{l}\text { 2,2-diacetoxypropane } \\
\quad(283-318)\end{array}$ & $\begin{array}{l}\mathrm{e} \\
53.6 \pm 0.4\end{array}$ & 298 & GS & [1996VER/PEN] \\
\hline $\mathrm{C}_{7} \mathrm{H}_{12} \mathrm{O}_{4}$ & $\begin{array}{c}{[534-59-8]} \\
\text { SUB }\end{array}$ & $\begin{array}{l}\text { butylmalonic acid } \\
\quad(348-362)\end{array}$ & $124.6 \pm 2.3$ & 298 & ME & [2000RIB/MON] \\
\hline
\end{tabular}


TABLE 10. Phase change enthalpies of $\mathrm{C}_{7}$ organic compounds-Continued

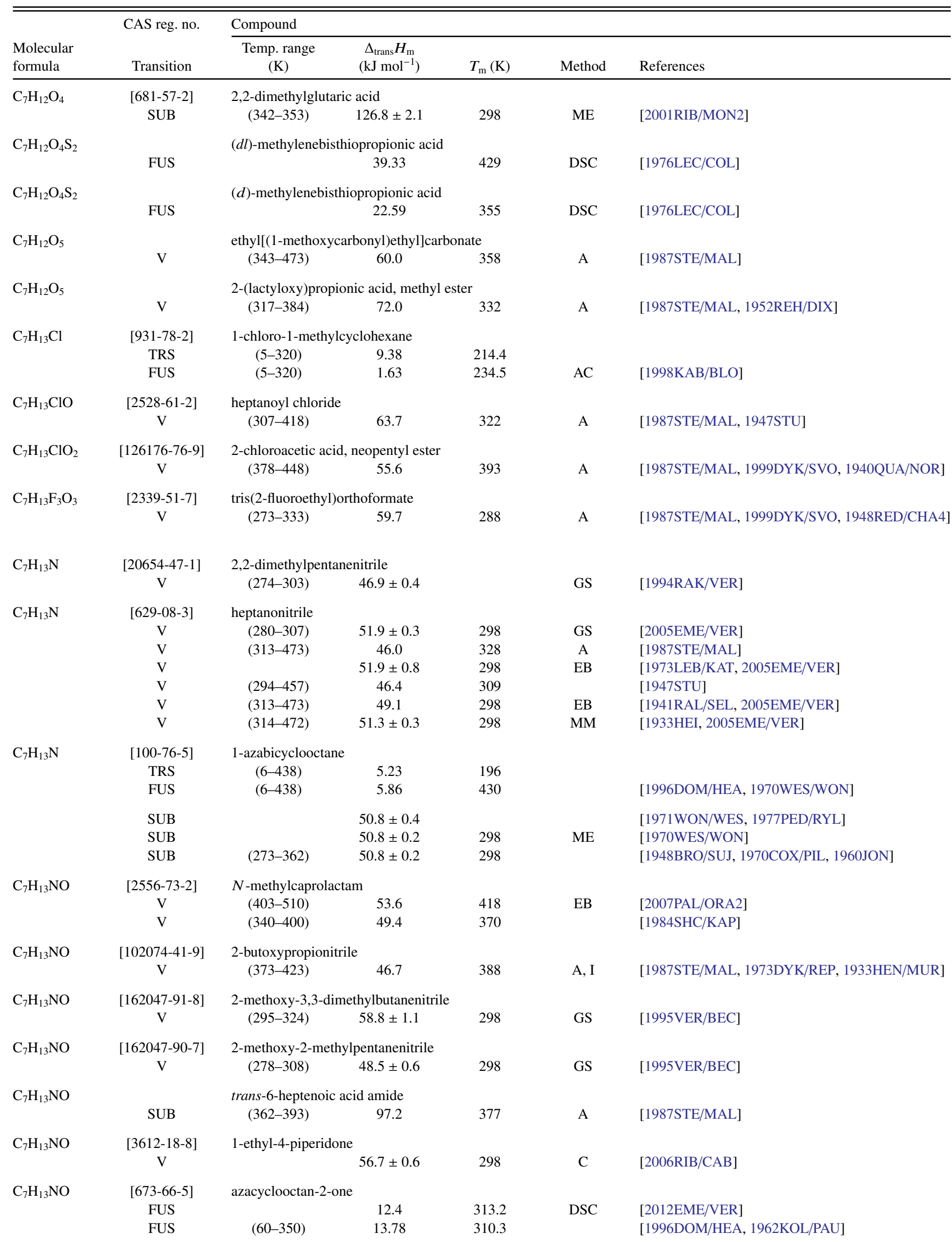


TABLE 10. Phase change enthalpies of $\mathrm{C}_{7}$ organic compounds-Continued

\begin{tabular}{|c|c|c|c|c|c|c|}
\hline \multirow[b]{2}{*}{$\begin{array}{l}\text { Molecular } \\
\text { formula }\end{array}$} & \multirow{2}{*}{$\begin{array}{l}\text { CAS reg. no. } \\
\text { Transition }\end{array}$} & \multicolumn{5}{|l|}{ Compound } \\
\hline & & $\begin{array}{c}\text { Temp. range } \\
(\mathrm{K})\end{array}$ & $\begin{array}{c}\Delta_{\text {trans }} H_{\mathrm{m}} \\
\left(\mathrm{kJ} \mathrm{mol}^{-1}\right)\end{array}$ & $T_{\mathrm{m}}(\mathrm{K})$ & Method & References \\
\hline & SUB & & $89.6 \pm 0.6$ & 298 & $\mathrm{~F}+\mathrm{V}$ & [2012EME/VER] \\
\hline & $\mathrm{V}$ & $(306-373)$ & $77.9 \pm 0.5$ & 298 & GS & [2012EME/VER] \\
\hline \multirow[t]{2}{*}{$\mathrm{C}_{7} \mathrm{H}_{13} \mathrm{NO}$} & [107-58-4] & \multicolumn{5}{|c|}{$N$-tert-butylacrylamide } \\
\hline & FUS & & 21.57 & 401.65 & DSC & [2015GAO/XUE] \\
\hline \multirow[t]{2}{*}{$\mathrm{C}_{7} \mathrm{H}_{13} \mathrm{NO}_{2}$} & {$[5468-39-3]$} & \multicolumn{5}{|c|}{ lactic acid $N$-(methallyl) amide } \\
\hline & $\mathrm{V}$ & $(360-428)$ & 81.8 & 375 & A & [1987STE/MAL, 1950RAT/FIS] \\
\hline $\mathrm{C}_{7} \mathrm{H}_{13} \mathrm{NO}_{2}$ & $\begin{array}{c}{[27097-66-1]} \\
\mathrm{V}\end{array}$ & \multicolumn{4}{|c|}{$N$-lactylmorpholine } & [1987STE/MAL, 1950RAT/FIS] \\
\hline \multirow[t]{2}{*}{$\mathrm{C}_{7} \mathrm{H}_{13} \mathrm{NO}_{3}$} & {$[5143-72-6]$} & \multicolumn{5}{|c|}{$(d l)$-N-acetylalanine ethyl ester } \\
\hline & V & $(372-460)$ & 65.2 & 387 & $\mathrm{~A}, \mathrm{~EB}$ & [1987STE/MAL, 1973DYK/REP, 1953MEL/VIO] \\
\hline \multirow[t]{2}{*}{$\mathrm{C}_{7} \mathrm{H}_{13} \mathrm{~N}_{3} \mathrm{O}_{3} \mathrm{~S}$} & {$[23135-22-0]$} & \multicolumn{5}{|c|}{$N, N$-dimethyl-2-methylcarbamoyloxyimino-2-(methylthio)acetamide } \\
\hline & FUS & & 30.17 & 372.2 & DSC & {$[1990 \mathrm{DON} / \mathrm{DRE}]$} \\
\hline \multirow[t]{2}{*}{$\mathrm{C}_{7} \mathrm{H}_{13} \mathrm{O}_{6} \mathrm{P}$} & {$[7786-34-7]$} & \multicolumn{5}{|c|}{ 2-methoxycarbonyl-1-methylvinyl dimethyl phosphate (mevinphos) } \\
\hline & $\mathrm{V}$ & $(293-383)$ & 68.1 & 308 & A & {$[1987 \mathrm{STE} / \mathrm{MAL}]$} \\
\hline \multirow[t]{13}{*}{$\mathrm{C}_{7} \mathrm{H}_{14}$} & {$[291-64-5]$} & cycloheptane & & & & \\
\hline & TRS & & 4.98 & 134.8 & & \\
\hline & TRS & & 0.29 & 198.2 & & \\
\hline & TRS & & 0.45 & 212.4 & & \\
\hline & FUS & & 1.88 & 265.1 & & [1996DOM/HEA, 1956FIN/SCO] \\
\hline & FUS & & 1.9 & 265.2 & & {$[1952 \mathrm{KAA} / \mathrm{COO}]$} \\
\hline & SUB & & 53.5 & 134 & & {$[1963 \mathrm{BON}]$} \\
\hline & V & $(282-333)$ & 38.6 & 297 & A & [1987STE/MAL] \\
\hline & V & $(476-604)$ & 31.7 & 491 & A & [1987STE/MAL] \\
\hline & V & $(333-398)$ & 36.4 & 348 & $\mathrm{~A}, \mathrm{~EB}$ & [1987STE/MAL, 1976MEY/HOT] \\
\hline & $\mathrm{V}$ & $(283-323)$ & 38.5 & 298 & & [1975ANA/GRO] \\
\hline & V & $(341-433)$ & 36.1 & 356 & $\mathrm{~A}, \mathrm{~EB}$ & [1987STE/MAL, 1956FIN/SCO] \\
\hline & $\mathrm{V}$ & & $38.5 \pm 0.2$ & 298 & & {$[1956 \mathrm{FIN} / \mathrm{SCO}]$} \\
\hline \multirow[t]{24}{*}{$\mathrm{C}_{7} \mathrm{H}_{14}$} & {$[108-87-2]$} & \multicolumn{5}{|c|}{ methylcyclohexane } \\
\hline & FUS & $(12-285)$ & 6.75 & 146.6 & & [1996DOM/HEA, 1946DOU/HUF] \\
\hline & FUS & & 6.67 & 146.2 & & [1930PAR/HUF2] \\
\hline & $\mathrm{V}$ & $(321-374)$ & 34.1 & 336 & EB & [2011XIN/YAN] \\
\hline & $\mathrm{V}$ & $(325-374)$ & 33.8 & 340 & & [2010SAP/UUS] \\
\hline & $\mathrm{V}$ & $(295-333)$ & 36.2 & 310 & & [1991WU/PIV] \\
\hline & $\mathrm{V}$ & & $35.1 \pm 0.4$ & 298 & GC & [1987AZA] \\
\hline & $\mathrm{V}$ & $(373-511)$ & 32.3 & 388 & A & [1987STE/MAL] \\
\hline & $\mathrm{V}$ & $(501-573)$ & 31.2 & 516 & A & [1987STE/MAL] \\
\hline & $\mathrm{V}$ & & 32.2 & 353 & & [1984EUB/CED] \\
\hline & V & & 29.9 & 393 & & [1984EUB/CED] \\
\hline & $\mathrm{V}$ & & 26.9 & 433 & & [1984EUB/CED] \\
\hline & V & & 23.4 & 473 & & [1984EUB/CED] \\
\hline & $\mathrm{V}$ & $(315-373)$ & 34.2 & 330 & $\mathrm{~EB}$ & [1983PAL/CHO] \\
\hline & $\mathrm{V}$ & & $35.4 \pm 0.1$ & 298 & $\mathrm{C}$ & [1979MAJ/SVO] \\
\hline & V & & $34.6 \pm 0.1$ & 313 & $\mathrm{C}$ & [1979MAJ/SVO] \\
\hline & $\mathrm{V}$ & & $33.5 \pm 0.1$ & 333 & $\mathrm{C}$ & [1979MAJ/SVO] \\
\hline & $\mathrm{V}$ & & $32.5 \pm 0.1$ & 353 & $\mathrm{C}$ & [1979MAJ/SVO] \\
\hline & V & & 35.4 & 298 & GCC & [1978FUC/PEA] \\
\hline & $\mathrm{V}$ & & 35.3 & 298 & & [1975KUS/SAI] \\
\hline & V & $(308-368)$ & 34.6 & 323 & A & [1987STE/MAL, 1970VAL/KIL, 1984BOU/FRI] \\
\hline & $\mathrm{V}$ & & 31.8 & 374 & & [1946SPI/PIT] \\
\hline & $\mathrm{V}$ & $(299-375)$ & 34.9 & 314 & MM & [1945WIL/TAY] \\
\hline & $\mathrm{V}$ & $(273-348)$ & 36.1 & 288 & & [1940STU/SAY] \\
\hline $\mathrm{C}_{7} \mathrm{H}_{14}$ & {$[1638-26-2]$} & 1,1-dimethylcy & ntane & & & \\
\hline & TRS & & 6.49 & 146.8 & & \\
\hline & FUS & & 1.09 & 203.7 & & [1996DOM/HEA, 1953GRO/OLI] \\
\hline & $\mathrm{V}$ & $(284-363)$ & 34.0 & 299 & A & [1987STE/MAL] \\
\hline
\end{tabular}


TABLE 10. Phase change enthalpies of $\mathrm{C}_{7}$ organic compounds-Continued

\begin{tabular}{|c|c|c|c|c|c|c|}
\hline \multirow[b]{2}{*}{$\begin{array}{l}\text { Molecular } \\
\text { formula }\end{array}$} & \multirow{2}{*}{$\begin{array}{l}\text { CAS reg. no. } \\
\text { Transition }\end{array}$} & \multicolumn{5}{|l|}{ Compound } \\
\hline & & $\begin{array}{c}\text { Temp. range } \\
\text { (K) }\end{array}$ & $\begin{array}{c}\Delta_{\text {trans }} H_{\mathrm{m}} \\
\left(\mathrm{kJ} \mathrm{mol}^{-1}\right)\end{array}$ & $T_{\mathrm{m}}(\mathrm{K})$ & Method & References \\
\hline & $\mathrm{V}$ & & 33.8 & 298 & & [1971WIL/ZWO] \\
\hline & $\mathrm{V}$ & $(289-362)$ & 33.8 & 304 & & [1949FOR/NOR] \\
\hline \multirow[t]{6}{*}{$\mathrm{C}_{7} \mathrm{H}_{14}$} & [1192-18-3] & \multicolumn{5}{|c|}{ cis-1,2-dimethylcyclopentane } \\
\hline & TRS & $(12-302)$ & 6.65 & 141.5 & & \\
\hline & FUS & $(12-302)$ & 1.66 & 219.4 & & [1996DOM/HEA, 1953GRO/OLI] \\
\hline & V & $(293-375)$ & 35.5 & 308 & A & [1987STE/MAL] \\
\hline & $\mathrm{V}$ & & 35.8 & 298 & & [1971WIL/ZWO] \\
\hline & $\mathrm{V}$ & $(298-373)$ & 35.2 & 313 & & [1949FOR/NOR] \\
\hline \multirow[t]{4}{*}{$\mathrm{C}_{7} \mathrm{H}_{14}$} & [822-50-4] & \multicolumn{5}{|c|}{ (dl)-trans-1,2-dimethylcyclopentane } \\
\hline & $\mathrm{V}$ & $(295-367)$ & 34.2 & 310 & A & [1987STE/MAL] \\
\hline & $\mathrm{V}$ & & 34.6 & 298 & & [1971WIL/ZWO] \\
\hline & $\mathrm{V}$ & $(299-366)$ & 34.0 & 314 & & {$[1949 F O R / N O R]$} \\
\hline \multirow[t]{7}{*}{$\mathrm{C}_{7} \mathrm{H}_{14}$} & {$[2532-58-3]$} & \multicolumn{5}{|c|}{ cis-1,3-dimethylcyclopentane } \\
\hline & V & $(295-366)$ & 34.2 & 310 & A & [1987STE/MAL] \\
\hline & $\mathrm{V}$ & & 34.3 & 298 & & [1971WIL/ZWO] \\
\hline & $\mathrm{V}$ & & $32.8 \pm 0.1$ & 323 & $\mathrm{C}$ & [1959MCC/PEN] \\
\hline & $\mathrm{V}$ & & $31.7 \pm 0.1$ & 342 & $\mathrm{C}$ & [1959MCC/PEN] \\
\hline & $\mathrm{V}$ & & $30.4 \pm 0.1$ & 364 & $\mathrm{C}$ & [1959MCC/PEN] \\
\hline & V & $(299-366)$ & 34.0 & 314 & & {$[1949 \mathrm{FOR} / \mathrm{NOR}]$} \\
\hline \multirow[t]{5}{*}{$\mathrm{C}_{7} \mathrm{H}_{14}$} & [1759-58-6] & \multicolumn{5}{|c|}{ (dl)-trans-1,3-dimethylcyclopentane } \\
\hline & FUS & $(13-304)$ & 7.41 & 139.5 & & [1996DOM/HEA, 1953GRO/OLI] \\
\hline & V & $(295-367)$ & 34.0 & 310 & $\mathrm{~A}$ & [1987STE/MAL] \\
\hline & V & & 34.5 & 298 & & [1971WIL/ZWO] \\
\hline & $\mathrm{V}$ & $(291-365)$ & 34.2 & 306 & & [1949FOR/NOR] \\
\hline \multirow[t]{12}{*}{$\mathrm{C}_{7} \mathrm{H}_{14}$} & {$[1640-89-7]$} & ethylcyclopent & & & & \\
\hline & FUS & $(12-302)$ & 6.86 & 134.7 & & [1996DOM/HEA, 1953GRO/OLI] \\
\hline & V & $(308-387)$ & 35.5 & 323 & A & [1987STE/MAL] \\
\hline & $\mathrm{V}$ & $(386-507)$ & 32.9 & 401 & A & [1987STE/MAL] \\
\hline & $\mathrm{V}$ & $(499-569)$ & 31.9 & 514 & A & [1987STE/MAL] \\
\hline & V & & $35.6 \pm 0.1$ & 313 & $\mathrm{C}$ & [1981SVO/CHA] \\
\hline & $\mathrm{V}$ & & $34.8 \pm 0.1$ & 328 & $\mathrm{C}$ & [1981SVO/CHA] \\
\hline & V & & $33.9 \pm 0.1$ & 343 & $\mathrm{C}$ & [1981SVO/CHA] \\
\hline & $\mathrm{V}$ & & $33.0 \pm 0.1$ & 358 & $\mathrm{C}$ & [1981SVO/CHA] \\
\hline & V & & $32.5 \pm 0.1$ & 368 & $\mathrm{C}$ & [1981SVO/CHA] \\
\hline & V & & 36.5 & 298 & & [1971WIL/ZWO] \\
\hline & $\mathrm{V}$ & $(302-377)$ & 35.7 & 317 & & [1949FOR/NOR] \\
\hline \multirow[t]{10}{*}{$\mathrm{C}_{7} \mathrm{H}_{14}$} & [592-76-7] & 1-heptene & & & & \\
\hline & FUS (I) & $(11-299)$ & 12.40 & 154.3 & & \\
\hline & FUS (II) & $(11-299)$ & 12.64 & 153.9 & $\mathrm{C}$ & [1957MCC/FIN] \\
\hline & FUS & & 12.64 & 153.4 & & [1996DOM/HEA, 1936PAR/TOD2] \\
\hline & V & $(311-368)$ & 34.6 & 326 & A & [1987STE/MAL] \\
\hline & $\mathrm{V}$ & $(327-367)$ & 33.9 & 342 & & [1970EIS/ORA, 1984BOU/FRI] \\
\hline & $\mathrm{V}$ & & 35.7 & 298 & & [1971WIL/ZWO] \\
\hline & $\mathrm{V}$ & $(295-318)$ & 35.3 & 310 & MM & [1950FOR/CAM] \\
\hline & $\mathrm{V}$ & $(255-312)$ & 35.9 & 300 & & [1941LIS] \\
\hline & $\mathrm{V}$ & $(273-362)$ & 34.5 & 288 & & {$[1936 \mathrm{BEN} / \mathrm{CUT}]$} \\
\hline \multirow[t]{6}{*}{$\mathrm{C}_{7} \mathrm{H}_{14}$} & [6443-92-1] & cis-2-heptene & & & & \\
\hline & $\mathrm{V}$ & $(276-304)$ & $39.0 \pm 0.3$ & 290 & GS & [2000VER/WAN] \\
\hline & $\mathrm{V}$ & $(276-304)$ & 38.6 & 298 & GS & [2000VER/WAN] \\
\hline & $\mathrm{V}$ & $(315-372)$ & 35.3 & 330 & A & [1987STE/MAL] \\
\hline & $\mathrm{V}$ & $(332-371)$ & 34.6 & 347 & & [1970EIS/ORA, 1984BOU/FRI] \\
\hline & $\mathrm{V}$ & & 36.0 & 298 & & [1971WIL/ZWO] \\
\hline \multirow[t]{4}{*}{$\mathrm{C}_{7} \mathrm{H}_{14}$} & [14686-13-6] & trans-2-hepten & & & & \\
\hline & $\mathrm{V}$ & $(314-373)$ & 35.3 & 329 & A & [1987STE/MAL] \\
\hline & $\mathrm{V}$ & & 36.0 & 298 & & [1971WIL/ZWO] \\
\hline & V & $(331-370)$ & 34.6 & 346 & & [1970EIS/ORA] \\
\hline
\end{tabular}


TABLE 10. Phase change enthalpies of $\mathrm{C}_{7}$ organic compounds-Continued

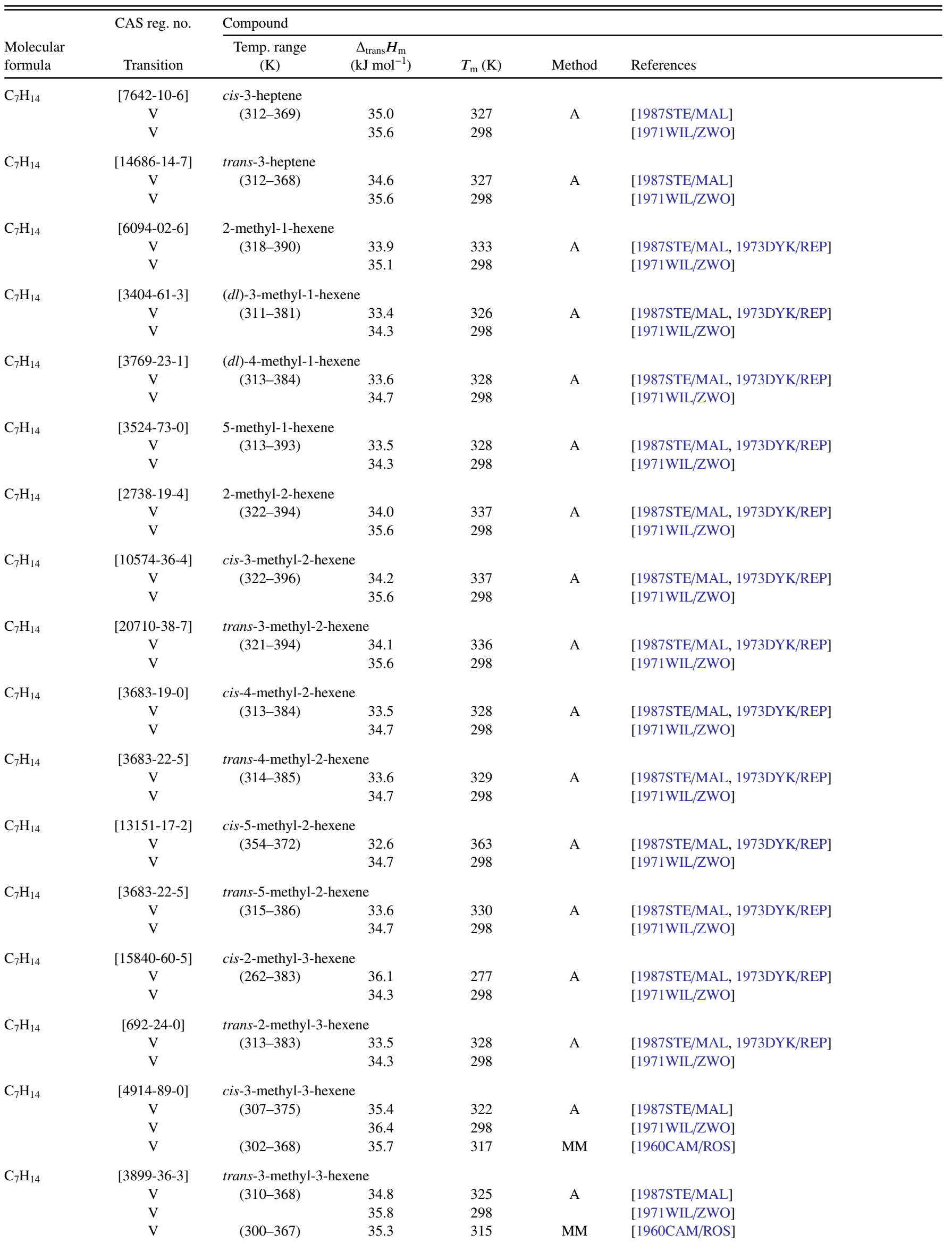


TABLE 10. Phase change enthalpies of $\mathrm{C}_{7}$ organic compounds-Continued

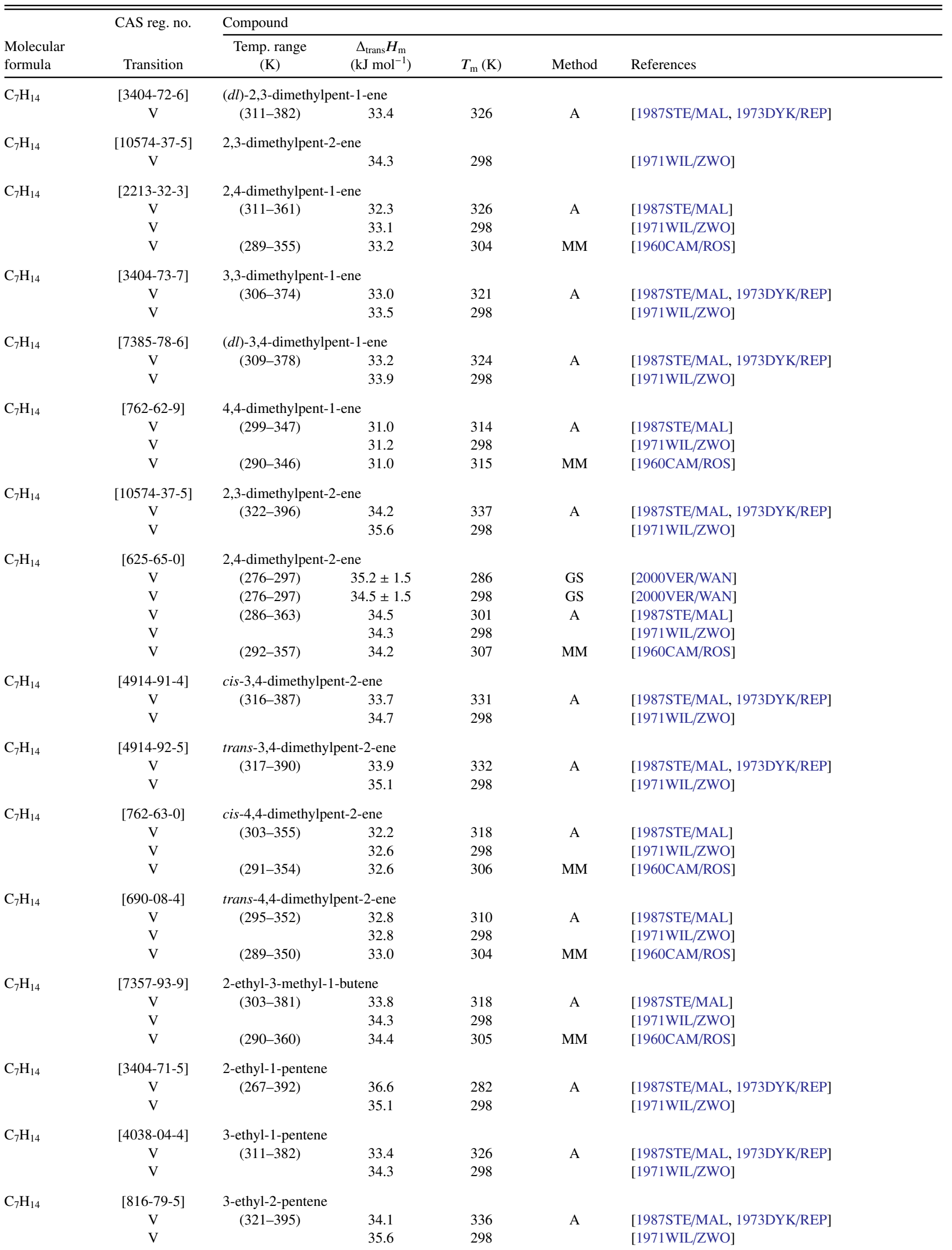


TABLE 10. Phase change enthalpies of $\mathrm{C}_{7}$ organic compounds-Continued

\begin{tabular}{|c|c|c|c|c|c|c|}
\hline \multirow[b]{2}{*}{$\begin{array}{l}\text { Molecular } \\
\text { formula }\end{array}$} & \multirow{2}{*}{$\begin{array}{l}\text { CAS reg. no. } \\
\text { Transition }\end{array}$} & \multicolumn{5}{|l|}{ Compound } \\
\hline & & $\begin{array}{c}\text { Temp. range } \\
(\mathrm{K})\end{array}$ & $\begin{array}{c}\Delta_{\text {trans }} H_{\mathrm{m}} \\
\left(\mathrm{kJ} \mathrm{mol}^{-1}\right)\end{array}$ & $T_{\mathrm{m}}(\mathrm{K})$ & Method & References \\
\hline \multirow[t]{4}{*}{$\mathrm{C}_{7} \mathrm{H}_{14}$} & {$[594-56-9]$} & \multicolumn{5}{|c|}{ 2,3,3-trimethyl-1-butene } \\
\hline & $\mathrm{V}$ & $(288-353)$ & 32.4 & 303 & A & [1987STE/MAL] \\
\hline & $\mathrm{V}$ & & 34.3 & 298 & & [1971WIL/ZWO] \\
\hline & $\mathrm{V}$ & $(288-351)$ & 32.1 & 303 & $\mathrm{MM}$ & [1960CAM/ROS] \\
\hline \multirow[t]{2}{*}{$\mathrm{C}_{7} \mathrm{H}_{14} \mathrm{Br}_{2}$} & {$[59104-79-9]$} & \multicolumn{5}{|l|}{ 1,1-dibromoheptane } \\
\hline & $\mathrm{V}$ & $(395-548)$ & 54.4 & 410 & $\mathrm{~A}, \mathrm{E}$ & [1987STE/MAL, 1956MAN, 1970DYK/VAN] \\
\hline \multirow[t]{3}{*}{$\mathrm{C}_{7} \mathrm{H}_{14} \mathrm{Br}_{2}$} & {$[42474-21-5]$} & \multicolumn{5}{|c|}{$(d l)$-1,2-dibromoheptane } \\
\hline & $\mathrm{V}$ & $(295-553)$ & 52.9 & 310 & A & [1987STE/MAL, 1973DYK/REP, 1999DYK/SVO] \\
\hline & $\mathrm{V}$ & $(295-355)$ & 54.4 & 300 & & [1941LIS] \\
\hline \multirow[t]{3}{*}{$\mathrm{C}_{7} \mathrm{H}_{14} \mathrm{Cl}_{2}$} & {$[821-25-0]$} & \multicolumn{5}{|l|}{ 1,1-dichloroheptane } \\
\hline & $\mathrm{V}$ & $(375-460)$ & 53.5 & 298 & & [1987VAR/LOS2, 1991BAS/SVO] \\
\hline & $\mathrm{V}$ & $(364-510)$ & 48.4 & 379 & $\mathrm{~A}, \mathrm{E}$ & [1987STE/MAL, 1956MAN, 1970DYK/VAN] \\
\hline \multirow[t]{3}{*}{$\mathrm{C}_{7} \mathrm{H}_{14} \mathrm{Cl}_{2}$} & {$[10575-87-8]$} & \multicolumn{5}{|l|}{ 1,2-dichloroheptane } \\
\hline & $\mathrm{V}$ & $(353-466)$ & 49.0 & 368 & & {$[1999 \mathrm{DYK} / \mathrm{SVO}]$} \\
\hline & $\mathrm{V}$ & $(350-470)$ & 53.2 & 298 & & [1982VAR/PUC, 1991BAS/SVO] \\
\hline \multirow[t]{3}{*}{$\mathrm{C}_{7} \mathrm{H}_{14} \mathrm{Cl}_{2}$} & {$[821-76-1]$} & \multicolumn{5}{|l|}{ 1,7-dichloroheptane } \\
\hline & $\mathrm{V}$ & $(406-491)$ & 52.3 & 421 & & [1999DYK/SVO] \\
\hline & $\mathrm{V}$ & $(410-490)$ & 61.2 & 298 & & [1988VAR/LOS, 1991BAS/SVO] \\
\hline \multirow[t]{2}{*}{$\mathrm{C}_{7} \mathrm{H}_{14} \mathrm{~F}_{2}$} & {$[407-96-5]$} & \multicolumn{5}{|l|}{ 1,1-difluoroheptane } \\
\hline & $\mathrm{V}$ & $(311-424)$ & 41.1 & 326 & $\mathrm{~A}, \mathrm{E}$ & [1987STE/MAL, 1956MAN, 1970DYK/VAN] \\
\hline \multirow[t]{2}{*}{$\mathrm{C}_{7} \mathrm{H}_{14} \mathrm{NO}_{5} \mathrm{P}$} & {$[6923-22-4]$} & \multicolumn{5}{|c|}{ dimethyl (E)-1-methyl-2-methylcarbamoylvinyl phosphate } \\
\hline & FUS & & 22.36 & 326.9 & DSC & {$[1990 \mathrm{DON} / \mathrm{DRE}]$} \\
\hline $\mathrm{C}_{7} \mathrm{H}_{14} \mathrm{~N}_{2}$ & {$[5351-04-2]$} & 3-(diethylamino)pro & pionitrile & & & \\
\hline & $\mathrm{V}$ & $(338-470)$ & 53.7 & 353 & A & [1987STE/MAL] \\
\hline $\mathrm{C}_{7} \mathrm{H}_{14} \mathrm{~N}_{2}$ & {$[82215-74-5]$} & 2-(diethylamino)pro & pionitrile & & & \\
\hline & $\mathrm{V}$ & $(278-315)$ & $50.8 \pm 0.3$ & & GS & [1997WEL/VER] \\
\hline $\mathrm{C}_{7} \mathrm{H}_{14} \mathrm{~N}_{2}$ & [2721-31-5] & 3,3,5,5-tetramethyl- & 1-pyrazoline & & & \\
\hline & SUB & & $61.6 \pm 0.2$ & 298 & & [1976ENG/MEL] \\
\hline $\mathrm{C}_{7} \mathrm{H}_{14} \mathrm{~N}_{2} \mathrm{O}_{2}$ & [37933-88-3] & $N$-acetyl L-valinami & & & & \\
\hline & FUS & & 39.1 & 509 & DSC & [1997ABA/PAL] \\
\hline & FUS & & 41.3 & 509.0 & DSC & [1990PUL/MAT] \\
\hline & SUB & & $129.8 \pm 1.9$ & 376 & $\mathrm{C}$ & [1999DEL/BAR] \\
\hline & SUB & & $133.1 \pm 2.2$ & 298 & & [1999DEL/BAR] \\
\hline & SUB & $(391-425)$ & $126 \pm 2.0$ & 418 & $\mathrm{TE}$ & [1990PUL/MAT] \\
\hline $\mathrm{C}_{7} \mathrm{H}_{14} \mathrm{~N}_{2} \mathrm{O}_{2}$ & & $N$-acetyl-DL- $\alpha$-amin & obutyric acid & ethylam & & \\
\hline & FUS & & 24.71 & 434.8 & $\mathrm{DSC}$ & [2014BAD/DEL] \\
\hline $\mathrm{C}_{7} \mathrm{H}_{14} \mathrm{~N}_{2} \mathrm{O}_{2}$ & {$[1740-56-3]$} & pimelamide & & & & \\
\hline & FUS & & 44.56 & 446.8 & DSC & [2006BAD/DEL] \\
\hline $\mathrm{C}_{7} \mathrm{H}_{14} \mathrm{~N}_{2} \mathrm{O}_{2} \mathrm{~S}$ & [116-06-3] & 2-methyl-2(methylth & iio)propanal, & nethylam & rbonyl]ox & \\
\hline & FUS & & 22.71 & 374 & DSC & [1990DON/DRE] \\
\hline & SUB & $(298-323)$ & 80.0 & 310 & $\mathrm{ME}$ & [1987STE/MAL] \\
\hline $\mathrm{C}_{7} \mathrm{H}_{14} \mathrm{O}$ & {$[5063-65-0]$} & 1,2-epoxyheptane & & & & \\
\hline & V & $(305-414)$ & 45.5 & 320 & A & [1987STE/MAL, 1970VOJ/CIH] \\
\hline $\mathrm{C}_{7} \mathrm{H}_{14} \mathrm{O}$ & {$[502-41-0]$} & cycloheptanol & & & & \\
\hline & TRS & & 0.45 & 227.9 & & \\
\hline & TRS & & 0.78 & 250.4 & & \\
\hline & FUS & & 1.51 & 278.3 & MDSC & [2003RUT/SAL] \\
\hline & TRS & & 2.93 & 172.2 & & \\
\hline & TRS & & 0.55 & 227.3 & & \\
\hline & TRS & & 0.88 & 258.4 & & \\
\hline & FUS & & 1.6 & 280.3 & & [1996DOM/HEA, 1972ADA/SUG] \\
\hline & $\mathrm{V}$ & $(284-323)$ & 64.7 & 299 & A & [1987STE/MAL] \\
\hline
\end{tabular}


TABLE 10. Phase change enthalpies of $\mathrm{C}_{7}$ organic compounds-Continued

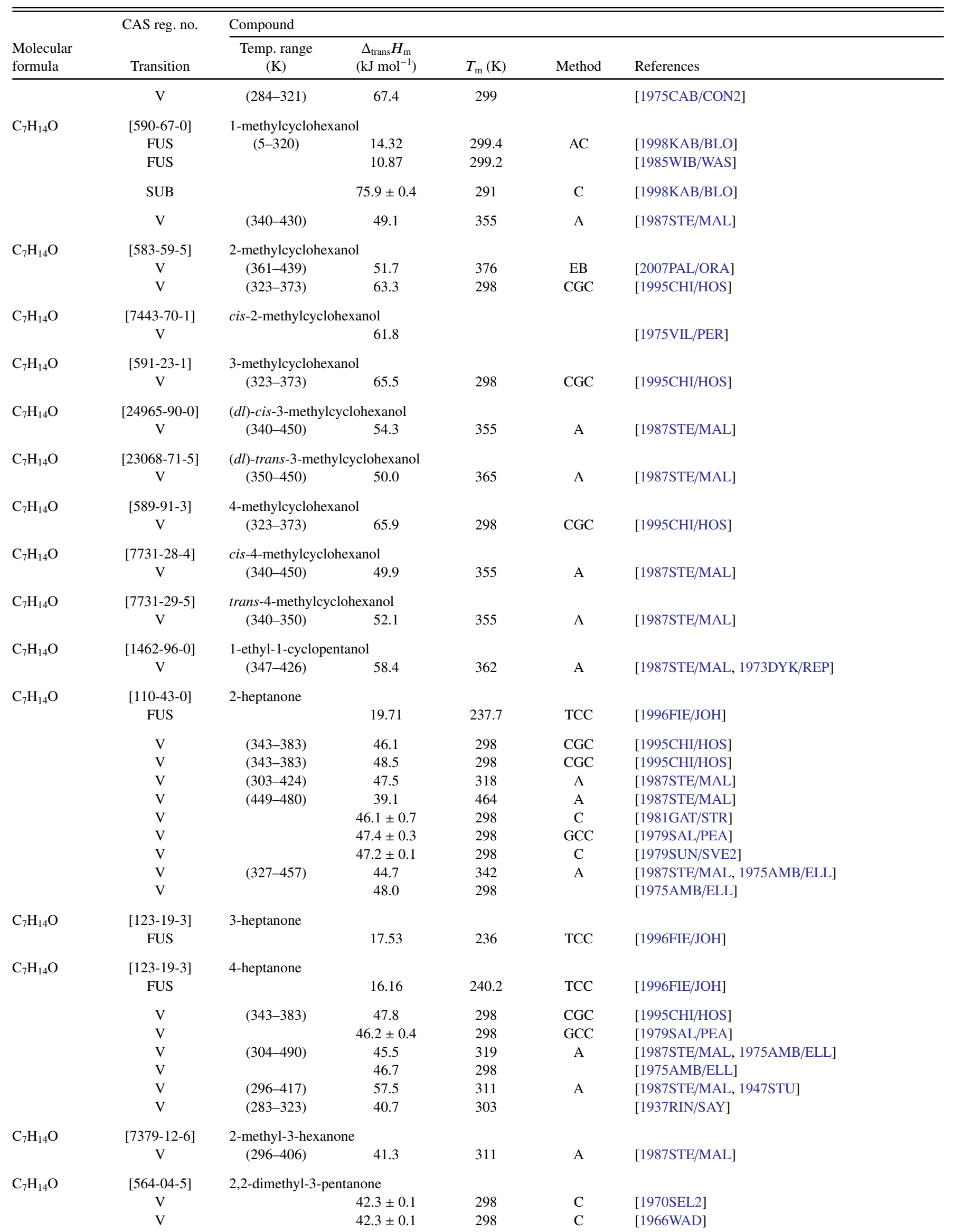


TABLE 10. Phase change enthalpies of $\mathrm{C}_{7}$ organic compounds-Continued

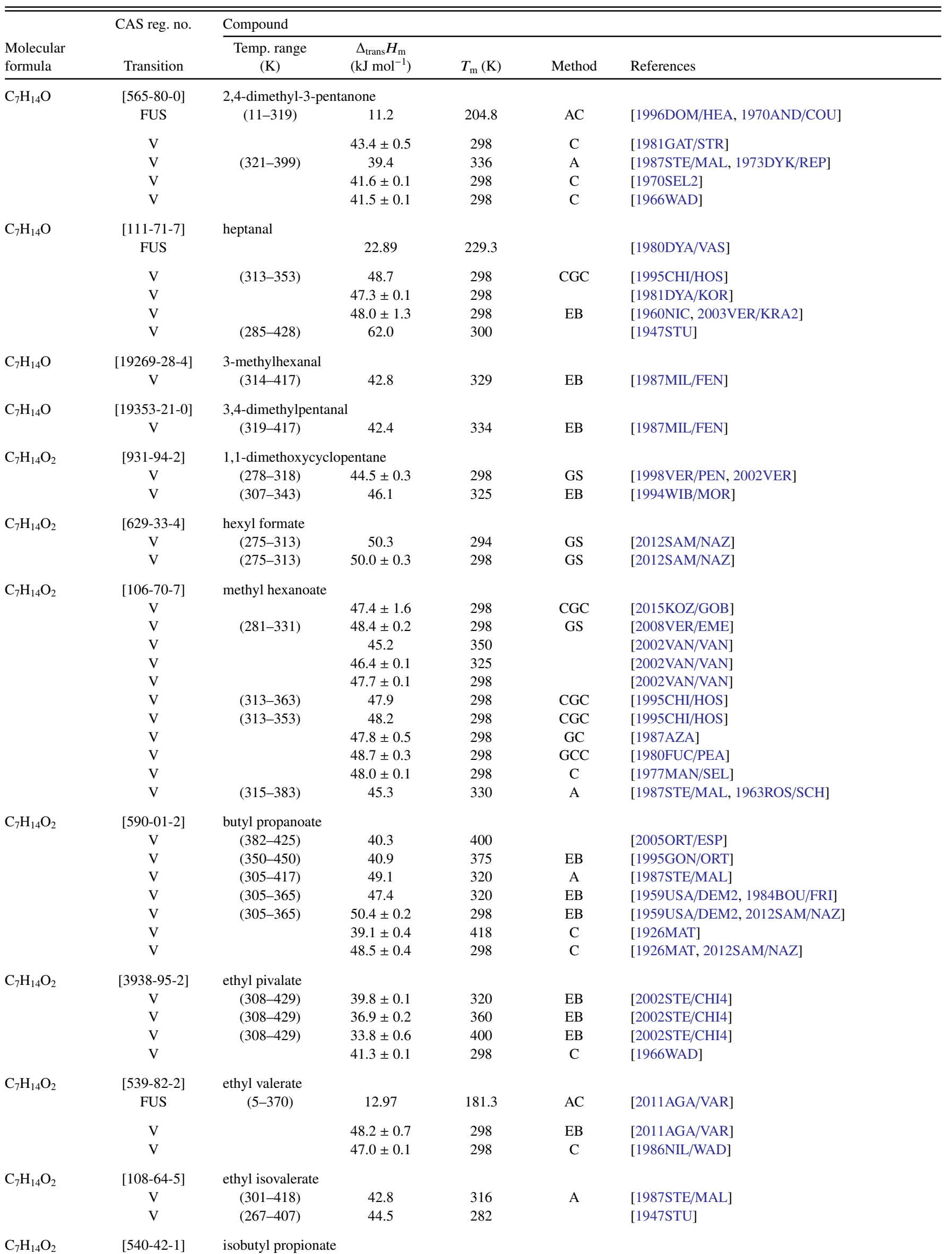


TABLE 10. Phase change enthalpies of $\mathrm{C}_{7}$ organic compounds-Continued

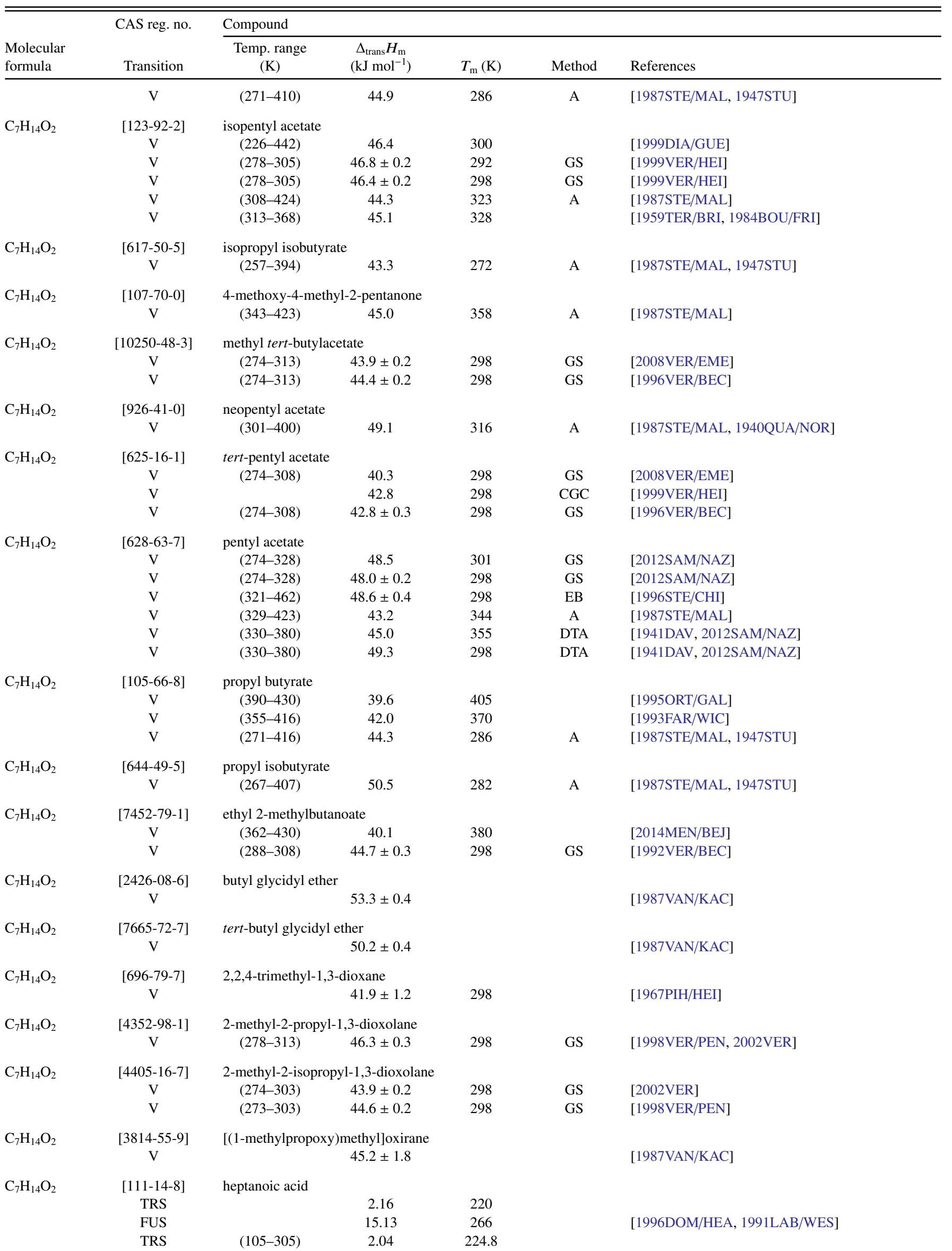


TABLE 10. Phase change enthalpies of $\mathrm{C}_{7}$ organic compounds-Continued

\begin{tabular}{|c|c|c|c|c|c|c|}
\hline \multirow[b]{2}{*}{$\begin{array}{l}\text { Molecular } \\
\text { formula }\end{array}$} & \multirow{2}{*}{$\begin{array}{l}\text { CAS reg. no. } \\
\text { Transition }\end{array}$} & \multicolumn{5}{|l|}{ Compound } \\
\hline & & $\begin{array}{c}\text { Temp. range } \\
(\mathrm{K})\end{array}$ & $\begin{array}{c}\Delta_{\text {trans }} H_{\mathrm{m}} \\
\left(\mathrm{kJ} \mathrm{mol}^{-1}\right)\end{array}$ & $T_{\mathrm{m}}(\mathrm{K})$ & Method & References \\
\hline & FUS & $(105-305)$ & 15.44 & 265.8 & $\mathrm{AC}$ & [1996DOM/HEA, 1982SCH/VAN2] \\
\hline & V & $(413-453)$ & 69.0 & 428 & & [2004CLI/RAM] \\
\hline & $\mathrm{V}$ & $(283-328)$ & $72.5 \pm 0.8$ & 306 & GS & [2000VER2] \\
\hline & V & $(283-328)$ & $72.9 \pm 0.8$ & 298 & GS & [2000VER2] \\
\hline & V & $(353-393)$ & 75.7 & 298 & CGC & [1995CHI/HOS] \\
\hline & $\mathrm{V}$ & & 76.0 & 266 & & [1982DEK/SCH] \\
\hline & $\mathrm{V}$ & $(271-291)$ & $72.0 \pm 1.5$ & 298 & $\mathrm{TE}$ & [1979DEK/OON] \\
\hline & $\mathrm{V}$ & $(351-495)$ & 68.3 & 366 & A & [1987STE/MAL, 1947STU] \\
\hline \multirow[t]{2}{*}{$\mathrm{C}_{7} \mathrm{H}_{14} \mathrm{O}_{3}$} & {$[33415-52-0]$} & \multicolumn{5}{|c|}{ tert-butylperoxymethyloxirane } \\
\hline & V & & $53.9 \pm 0.4$ & & & {$[1987 \mathrm{VAN} / \mathrm{KAC}]$} \\
\hline \multirow[t]{2}{*}{$\mathrm{C}_{7} \mathrm{H}_{14} \mathrm{O}_{3}$} & {$[20706-25-6]$} & \multicolumn{5}{|c|}{ 2-propoxyethylacetate } \\
\hline & V & & $55.6 \pm 0.1$ & 298 & $\mathrm{C}$ & [1970KUS/WAD] \\
\hline \multirow[t]{4}{*}{$\mathrm{C}_{7} \mathrm{H}_{14} \mathrm{O}_{3}$} & {$[138-22-7]$} & \multicolumn{5}{|l|}{$(d l)$-butyl lactate } \\
\hline & V & $(303-378)$ & 53.2 & 318 & & [2014LOM/GIN] \\
\hline & $\mathrm{V}$ & $(391-460)$ & 49.9 & 406 & & [2005PEN/MUR] \\
\hline & $\mathrm{V}$ & $(339-456)$ & 58.7 & 354 & A & [1987STE/MAL, 1950REH/DIX] \\
\hline \multirow[t]{2}{*}{$\mathrm{C}_{7} \mathrm{H}_{14} \mathrm{O}_{3}$} & {$[763-69-9]$} & \multicolumn{5}{|c|}{ 3-ethoxypropionic acid, ethyl ester } \\
\hline & $\mathrm{V}$ & $(312-446)$ & 45.5 & 327 & A & [1987STE/MAL, 1948DIX/REH] \\
\hline \multirow[t]{2}{*}{$\mathrm{C}_{7} \mathrm{H}_{14} \mathrm{O}_{3}$} & & \multicolumn{5}{|c|}{ 1-heptene ozonide } \\
\hline & $\mathrm{V}$ & $(353-373)$ & 44.4 & 363 & A & [1987STE/MAL, 1977BOL/MAK] \\
\hline \multirow[t]{2}{*}{$\mathrm{C}_{7} \mathrm{H}_{14} \mathrm{O}_{3}$} & {$[2018-45-3]$} & \multicolumn{5}{|c|}{ 4-(2-hydroxyethyl)-4-methyl-1,3-dioxane } \\
\hline & $\mathrm{V}$ & $(329-455)$ & 51.7 & 344 & A & [1987STE/MAL] \\
\hline \multirow[t]{2}{*}{$\mathrm{C}_{7} \mathrm{H}_{14} \mathrm{O}_{3}$} & {$[34451-19-9]$} & \multicolumn{5}{|c|}{ 3-hydroxypropionic acid, butyl ester } \\
\hline & V & $(361-382)$ & 60.3 & 371 & A & [1987STE/MAL, 1973DYK/REP] \\
\hline \multirow[t]{2}{*}{$\mathrm{C}_{7} \mathrm{H}_{14} \mathrm{O}_{3}$} & {$[5349-56-4]$} & \multicolumn{5}{|c|}{ 3-methoxypropionic acid, propyl ester } \\
\hline & $\mathrm{V}$ & $(323-433)$ & 47.0 & 338 & A & [1987STE/MAL] \\
\hline \multirow[t]{2}{*}{$\mathrm{C}_{7} \mathrm{H}_{14} \mathrm{O}_{3}$} & {$[14144-39-9]$} & 3-propoxypropi & acid, methyl & & & \\
\hline & V & $(323-453)$ & 46.6 & 338 & A & [1987STE/MAL] \\
\hline $\mathrm{C}_{7} \mathrm{H}_{14} \mathrm{O}_{3}$ & {$[557-25-5]$} & $(d l)$-butyric aci & -dihydroxyp & ester & & \\
\hline & $\mathrm{V}$ & $(392-449)$ & 80.4 & 407 & A & [1987STE/MAL] \\
\hline $\mathrm{C}_{7} \mathrm{H}_{14} \mathrm{O}_{3}$ & {$[14620-87-2]$} & 2-butoxypropio & & & & \\
\hline & V & $(373-473)$ & 52.8 & 388 & A & [1987STE/MAL, 1973DYK/REP] \\
\hline $\mathrm{C}_{7} \mathrm{H}_{14} \mathrm{O}_{3}$ & {$[623-96-1]$} & dipropyl carbor & & & & \\
\hline & V & $(274-318)$ & $53.2 \pm 0.3$ & 298 & GS & [2008KOZ/EME] \\
\hline $\mathrm{C}_{7} \mathrm{H}_{14} \mathrm{O}_{6}$ & {$[13224-94-7]$} & 3-methoxy- $\alpha$-( & ucopyranosic & & & \\
\hline & FUS & & 41.3 & 425.6 & DSC & {$[1996 \mathrm{SCH}]$} \\
\hline $\mathrm{C}_{7} \mathrm{H}_{14} \mathrm{O}_{6}$ & {$[617-04-9]$} & methyl $\alpha$-(D)-m & pyranoside & & & \\
\hline & FUS & & 44.7 & 455.2 & DSC & {$[1996 \mathrm{SCH}]$} \\
\hline $\mathrm{C}_{7} \mathrm{H}_{14} \mathrm{O}_{6}$ & {$[97-30-3]$} & methyl- $\alpha$-(D)-g & yranoside & & & \\
\hline & FUS & & 28.04 & 442.3 & $\mathrm{DSC}$ & [2016YAN/WAN] \\
\hline & FUS & & 35.11 & 436 & DSC & [2000AGU/GUA] \\
\hline & FUS & & 37.6 & 424.2 & DSC & [1996SCH] \\
\hline $\mathrm{C}_{7} \mathrm{H}_{14} \mathrm{O}_{6}$ & [709-50-2] & methyl- $\beta-(D)-g l$ & yranoside & & & \\
\hline & FUS & & 27.03 & 384.9 & DSC & [2000AGU/GUA] \\
\hline $\mathrm{C}_{7} \mathrm{H}_{14} \mathrm{O}_{6}$ & [3396-99-4] & methyl- $\alpha$-(D)-g & opyranoside & & & \\
\hline & FUS & & 27.95 & 397.6 & DSC & [2000AGU/GUA] \\
\hline $\mathrm{C}_{7} \mathrm{H}_{14} \mathrm{O}_{6}$ & [1824-94-8] & methyl- $\beta-(D)-g$ & pyranoside & & & \\
\hline & FUS & & 33.18 & 450.9 & DSC & [2000AGU/GUA] \\
\hline $\mathrm{C}_{7} \mathrm{H}_{14} \mathrm{~S}$ & $\begin{array}{c}{[37850-75-2]} \\
\mathrm{V}\end{array}$ & $\begin{array}{c}\text { allyl tert-butyl } \\
\text { (319-339) }\end{array}$ & 41.9 & 332 & & [1999DYK/SVO] \\
\hline
\end{tabular}


TABLE 10. Phase change enthalpies of $\mathrm{C}_{7}$ organic compounds-Continued

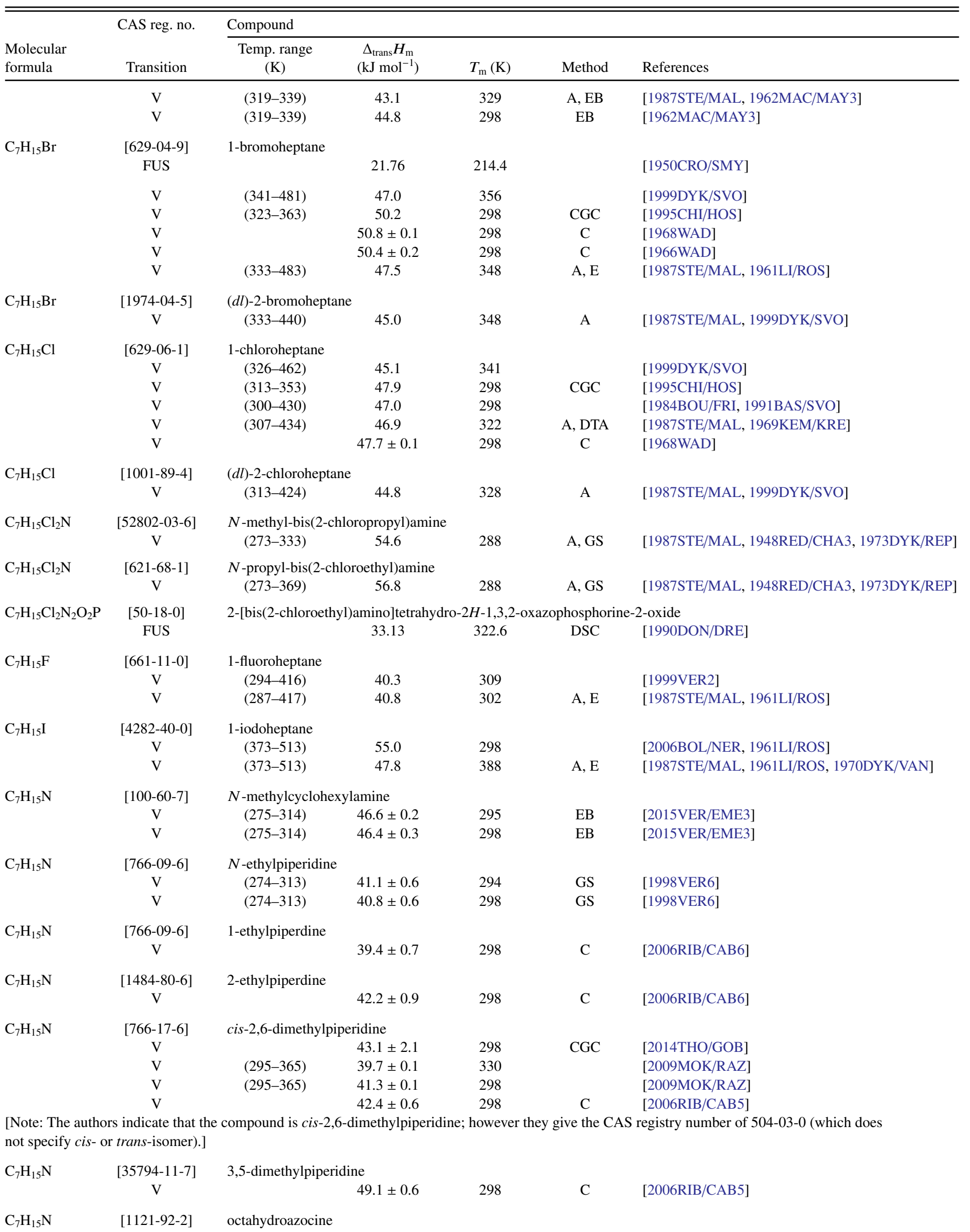


TABLE 10. Phase change enthalpies of $\mathrm{C}_{7}$ organic compounds-Continued

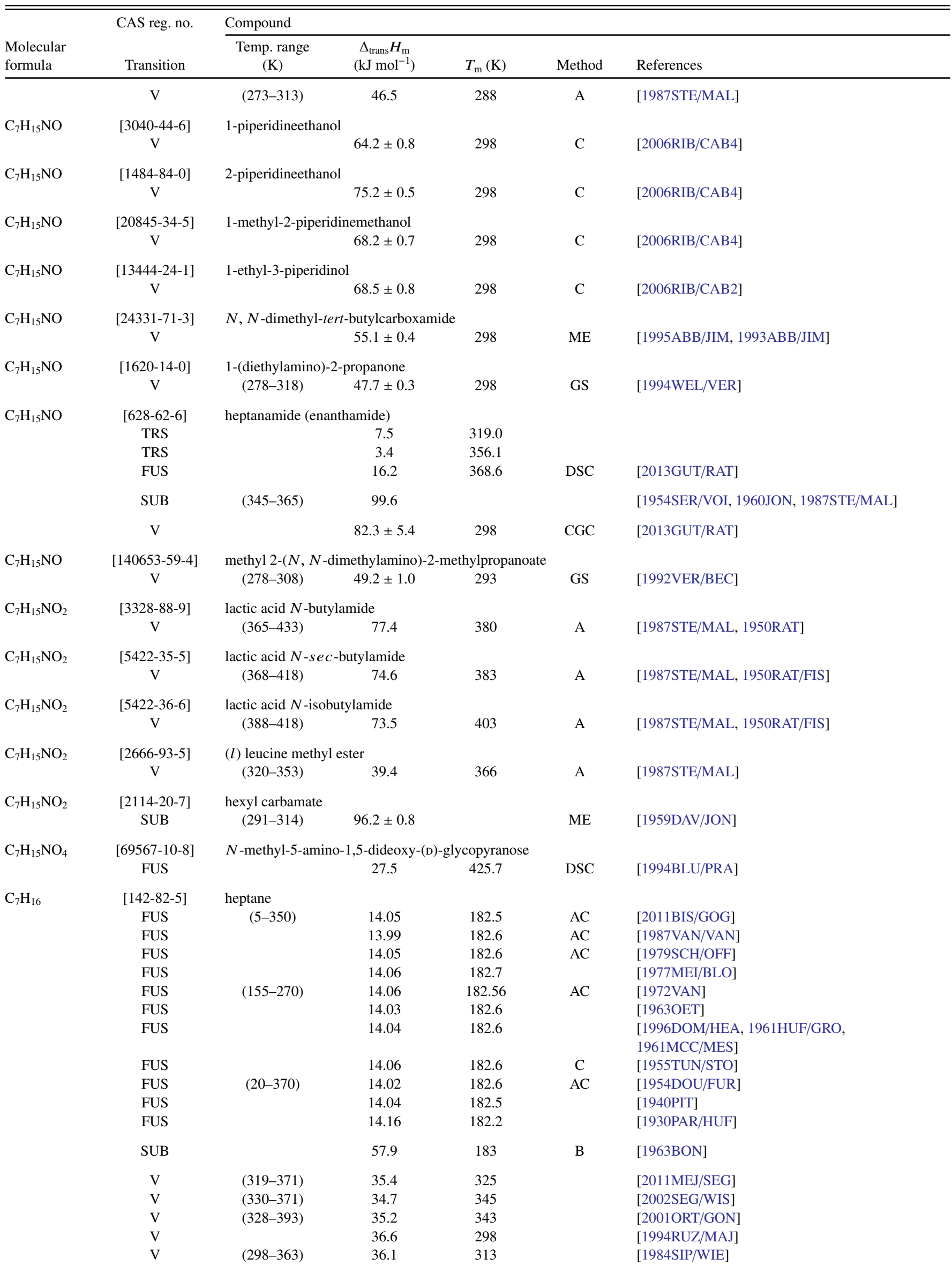


TABLE 10. Phase change enthalpies of $\mathrm{C}_{7}$ organic compounds-Continued

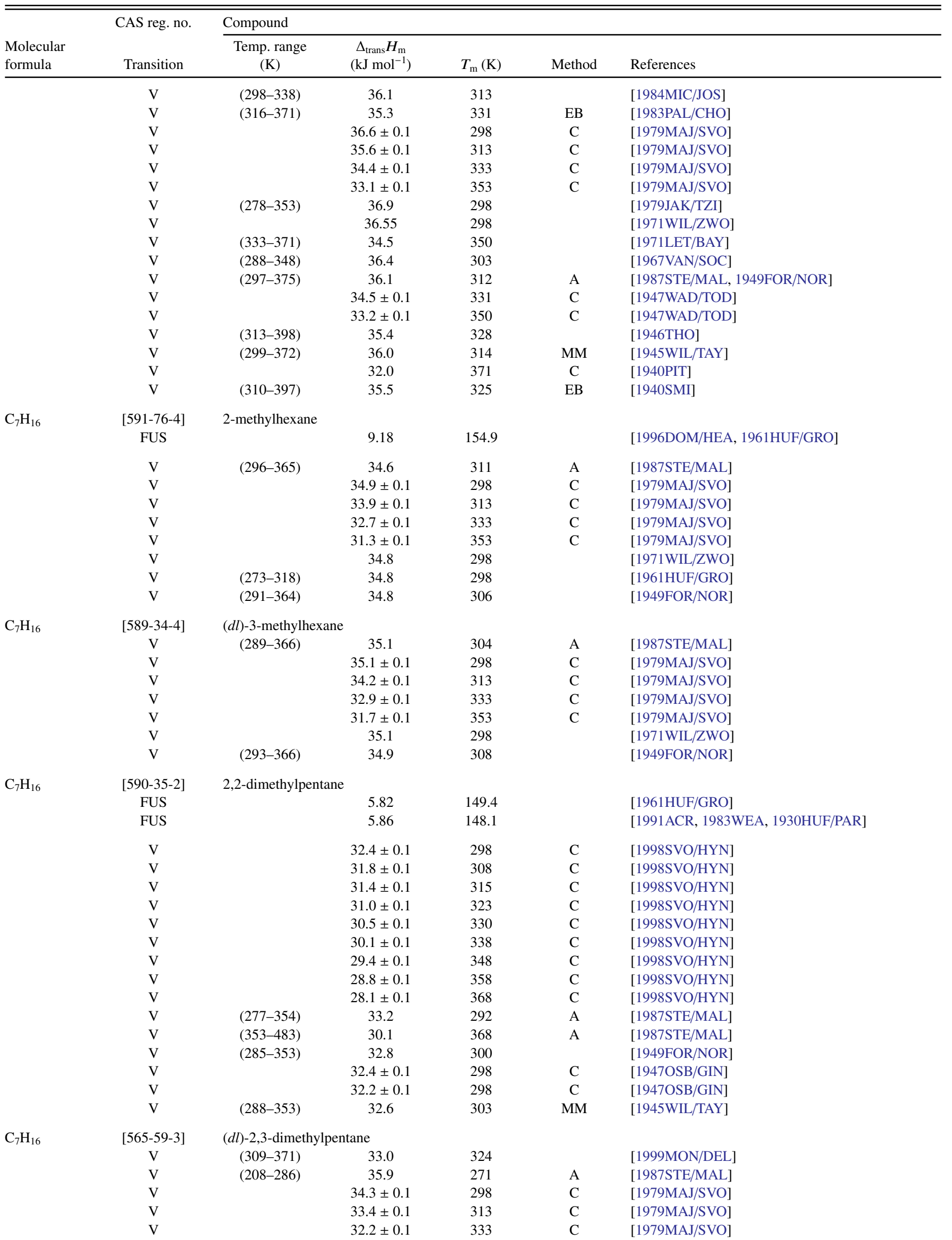


TABLE 10. Phase change enthalpies of $\mathrm{C}_{7}$ organic compounds-Continued

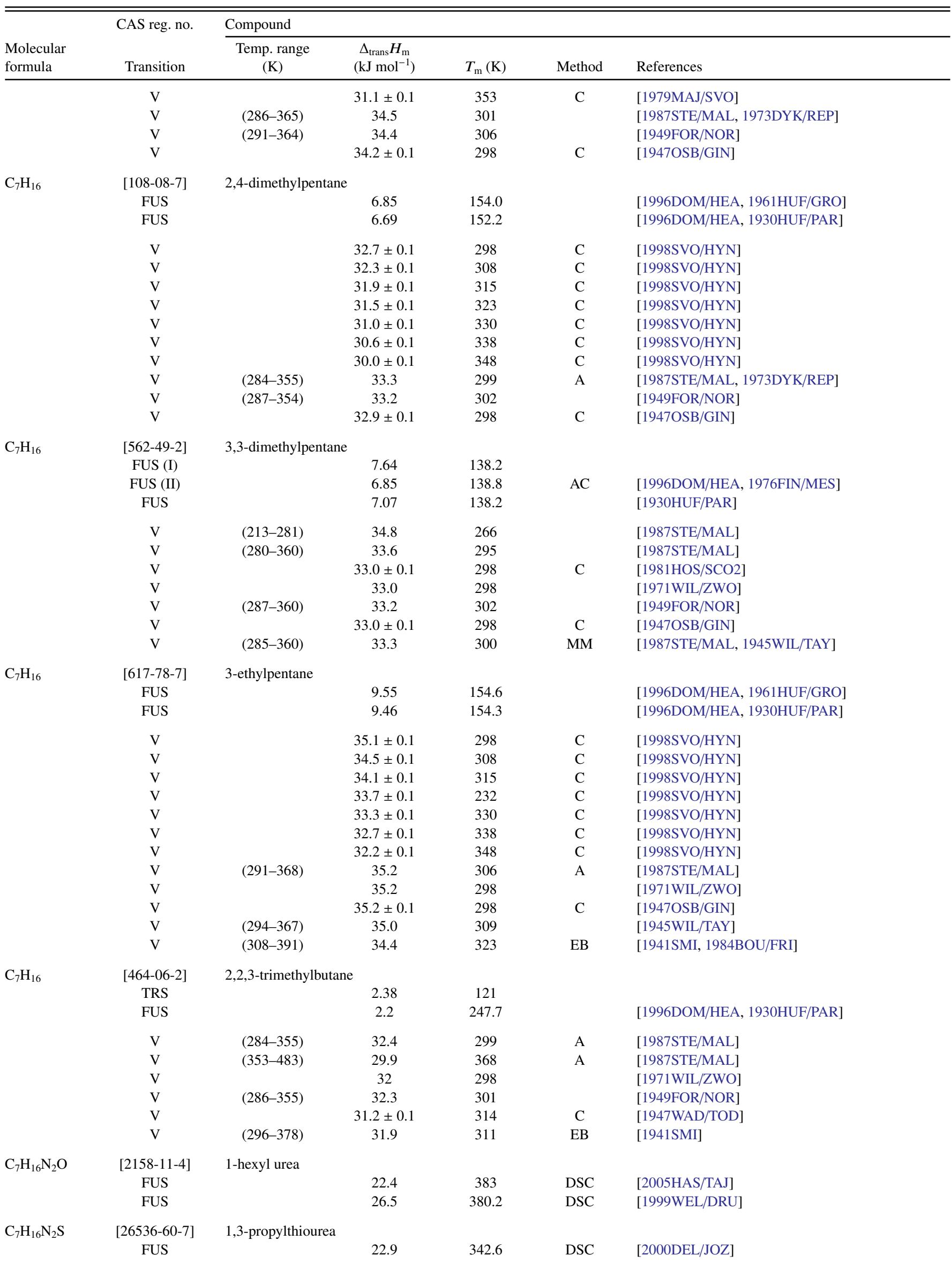


TABLE 10. Phase change enthalpies of $\mathrm{C}_{7}$ organic compounds-Continued

\begin{tabular}{|c|c|c|c|c|c|c|}
\hline \multirow[b]{2}{*}{$\begin{array}{l}\text { Molecular } \\
\text { formula }\end{array}$} & \multirow{2}{*}{$\begin{array}{l}\text { CAS reg. no. } \\
\text { Transition }\end{array}$} & \multicolumn{5}{|l|}{ Compound } \\
\hline & & $\begin{array}{l}\text { Temp. range } \\
(\mathrm{K})\end{array}$ & $\begin{array}{c}\Delta_{\text {trans }} H_{\mathrm{m}} \\
\left(\mathrm{kJ} \mathrm{mol}^{-1}\right)\end{array}$ & $T_{\mathrm{m}}(\mathrm{K})$ & Method & References \\
\hline & SUB & & $134.9 \pm 3.0$ & 298 & $\mathrm{~B}$ & [2000DEL/JOZ] \\
\hline & SUB & & $132.5 \pm 3.0$ & 298 & $\mathrm{C}$ & [1994TER/PIA] \\
\hline & V & $(346-394)$ & $107.0 \pm 3.0$ & 370 & $\mathrm{ME}, \mathrm{TE}$ & [1994TER/PIA] \\
\hline \multirow[t]{2}{*}{$\mathrm{C}_{7} \mathrm{H}_{16} \mathrm{~N}_{4} \mathrm{O}_{4}$} & {$[503152-73-6]$} & \multicolumn{5}{|c|}{$N, N^{\prime}$-bis(1-methylethyl)- $N, N^{\prime}$-dinitromethanediamine } \\
\hline & FUS & & 27.96 & 358.3 & DSC & [2003SPI/WAN] \\
\hline \multirow[t]{5}{*}{$\mathrm{C}_{7} \mathrm{H}_{16} \mathrm{O}$} & {$[919-94-8]$} & \multicolumn{5}{|l|}{ tert-amyl ethyl ether } \\
\hline & V & & $39.2 \pm 0.4$ & 298 & GS & [UR/VER, 2002VER, 2003VER/KRA] \\
\hline & $\mathrm{V}$ & & $38.2 \pm 0.2$ & 298 & $\mathrm{C}$ & [2002VAR/PAS] \\
\hline & $\mathrm{V}$ & $(318-374)$ & 35.7 & 333 & EB & [2002VAR/PAS] \\
\hline & $\mathrm{V}$ & $(320-374)$ & 35.6 & 335 & EB & [1994KRA/GME] \\
\hline \multirow[t]{5}{*}{$\mathrm{C}_{7} \mathrm{H}_{16} \mathrm{O}$} & {$[29072-93-3]$} & \multicolumn{5}{|l|}{ propyl tert-butyl ether } \\
\hline & FUS & & 9.87 & 179.6 & & [2001VAR/DRU, 2004DOR/YAN] \\
\hline & $\mathrm{V}$ & & 38.3 & 298 & CGC & [UR/VER, 2002VER, 2003VER/KRA] \\
\hline & $\mathrm{V}$ & $(315-370)$ & $37.2 \pm 0.6$ & 298 & $\mathrm{~EB}$ & [2002VAR/PAS2] \\
\hline & $\mathrm{V}$ & & $36.6 \pm 0.2$ & 298 & $\mathrm{C}$ & [2002VAR/PAS2, 2004DOR/YAN] \\
\hline \multirow[t]{6}{*}{$\mathrm{C}_{7} \mathrm{H}_{16} \mathrm{O}$} & {$[17348-59-3]$} & \multicolumn{5}{|c|}{ isopropyl tert-butyl ether } \\
\hline & FUS & & 8.46 & 184.8 & & [2001VAR/DRU, 2004DOR/YAN] \\
\hline & $\mathrm{V}$ & & 36.2 & 298 & CGC & [UR/VER, 2002VER, 2003VER/KRA] \\
\hline & $\mathrm{V}$ & $(305-360)$ & $34.4 \pm 0.6$ & 298 & EB & [2002VAR/PAS2] \\
\hline & $\mathrm{V}$ & & $34.5 \pm 0.2$ & 298 & $\mathrm{C}$ & [2002VAR/PAS2, 2004DOR/YAN] \\
\hline & $\mathrm{V}$ & $(307-360)$ & 34.0 & 322 & EB & [1994KRA/GME] \\
\hline \multirow[t]{14}{*}{$\mathrm{C}_{7} \mathrm{H}_{16} \mathrm{O}$} & {$[111-70-6]$} & 1-heptanol & & & & \\
\hline & FUS & $(5-370)$ & 18.35 & 239.9 & & [2003VAN/GAB] \\
\hline & FUS & $(78-300)$ & 18.16 & 240.4 & & [1996DOM/HEA, 1956PAR/KEN] \\
\hline & V & $(382-453)$ & 55.1 & 397 & EB & [2010CEN/ROH] \\
\hline & V & $(348-443)$ & 67.1 & 298 & & [2006NAS/NEU] \\
\hline & V & $(283-323)$ & $66.9 \pm 0.4$ & 298 & GS & [2005ROG/PIS] \\
\hline & V & $(323-373)$ & 66.5 & 298 & CGC & [1995CHI/HOS] \\
\hline & V & $(373-423)$ & 66.4 & 298 & $\mathrm{CGC}$ & [1995CHI/HOS] \\
\hline & V & $(258-363)$ & 65.2 & 310 & & [1992NGU/KAS] \\
\hline & V & $(335-450)$ & 62.5 & 350 & A & [1987STE/MAL] \\
\hline & V & & $66.8 \pm 0.2$ & 298 & $\mathrm{C}$ & [1977MAN/SEL] \\
\hline & V & $(333-449)$ & 65.2 & 348 & & [1973WIL/ZWO] \\
\hline & V & $(336-450)$ & 62.6 & 351 & DTA & [1987STE/MAL, 1969KEM/KRE] \\
\hline & V & $(333-425)$ & 62.9 & 348 & & [1935BUT/RAM, 1984BOU/FRI] \\
\hline \multirow[t]{6}{*}{$\mathrm{C}_{7} \mathrm{H}_{16} \mathrm{O}$} & {$[543-49-7]$} & $(d l)$-2-heptanol & & & & \\
\hline & $\mathrm{V}$ & $(275-312)$ & $62.1 \pm 0.4$ & 298 & GS & [2007VER/SCH] \\
\hline & $\mathrm{V}$ & $(244-338)$ & 66.1 & 259 & & [1999NGU/BER] \\
\hline & $\mathrm{V}$ & $(351-433)$ & 54.4 & 366 & & [1984SAC/MAR] \\
\hline & V & $(357-431)$ & 51.6 & 372 & A & [1987STE/MAL, 1975BRA/AND] \\
\hline & $\mathrm{V}$ & $(323-433)$ & 59.8 & 338 & & [1973WIL/ZWO] \\
\hline \multirow[t]{6}{*}{$\mathrm{C}_{7} \mathrm{H}_{16} \mathrm{O}$} & {$[589-82-2]$} & $(d l)$-3-heptanol & & & & \\
\hline & $\mathrm{V}$ & $(244-333)$ & 67.0 & 259 & & [1999NGU/BER] \\
\hline & $\mathrm{V}$ & $(325-430)$ & 60.3 & 340 & A & [1987STE/MAL] \\
\hline & $\mathrm{V}$ & $(263-295)$ & 64.7 & 280 & A & [1987STE/MAL, 1979THO/MEA] \\
\hline & V & $(349-430)$ & 53.1 & 364 & & [1984SAC/MAR] \\
\hline & $\mathrm{V}$ & $(328-429)$ & 59.2 & 343 & & [1973WIL/ZWO] \\
\hline \multirow[t]{6}{*}{$\mathrm{C}_{7} \mathrm{H}_{16} \mathrm{O}$} & {$[589-55-9]$} & 4-heptanol & & & & \\
\hline & $\mathrm{V}$ & & $62.4 \pm 0.3$ & 298 & & [2007VER/SCH] \\
\hline & $\mathrm{V}$ & $(320-428)$ & 58.2 & 335 & A & [1987STE/MAL] \\
\hline & $\mathrm{V}$ & $(349-428)$ & 53.1 & 364 & & [1984SAC/MAR] \\
\hline & V & $(282-320)$ & 63.1 & 297 & A & [1987STE/MAL, 1975CAB/CON2] \\
\hline & $\mathrm{V}$ & $(320-428)$ & 56.9 & 335 & & [1973WIL/ZWO] \\
\hline $\mathrm{C}_{7} \mathrm{H}_{16} \mathrm{O}$ & $\begin{array}{c}{[624-22-6]} \\
\mathrm{V}\end{array}$ & $\begin{array}{l}\text { 2-methyl-1-hexanol } \\
\quad(343-438)\end{array}$ & 53.5 & 390 & & [1973WIL/ZWO] \\
\hline
\end{tabular}


TABLE 10. Phase change enthalpies of $\mathrm{C}_{7}$ organic compounds-Continued

\begin{tabular}{|c|c|c|c|c|c|c|}
\hline \multirow[b]{2}{*}{$\begin{array}{l}\text { Molecular } \\
\text { formula }\end{array}$} & \multirow{2}{*}{$\begin{array}{l}\text { CAS reg. no. } \\
\text { Transition }\end{array}$} & \multicolumn{5}{|l|}{ Compound } \\
\hline & & $\begin{array}{l}\text { Temp. range } \\
\text { (K) }\end{array}$ & $\begin{array}{c}\Delta_{\text {trans }} H_{\mathrm{m}} \\
\left(\mathrm{kJ} \mathrm{mol}^{-1}\right)\end{array}$ & $T_{\mathrm{m}}(\mathrm{K})$ & Method & References \\
\hline $\mathrm{C}_{7} \mathrm{H}_{16} \mathrm{O}$ & $\begin{array}{c}{[818-49-5]} \\
\mathrm{V}\end{array}$ & $\begin{array}{l}\text { 4-methyl-1-hexanol } \\
\quad(348-448)\end{array}$ & 62.6 & 363 & & [1973WIL/ZWO] \\
\hline $\mathrm{C}_{7} \mathrm{H}_{16} \mathrm{O}$ & $\begin{array}{c}{[627-59-8]} \\
\mathrm{V}\end{array}$ & $\begin{array}{l}\text { 5-methyl-2-hexanol } \\
\quad(348-428)\end{array}$ & 49.4 & 388 & & [1973WIL/ZWO] \\
\hline $\mathrm{C}_{7} \mathrm{H}_{16} \mathrm{O}$ & $\begin{array}{c}{[617-29-8]} \\
\mathrm{V}\end{array}$ & $\begin{array}{l}\text { 2-methyl-3-hexanol } \\
\quad(323-420)\end{array}$ & 55.7 & 338 & & [1973WIL/ZWO] \\
\hline $\mathrm{C}_{7} \mathrm{H}_{16} \mathrm{O}$ & $\begin{array}{c}{[623-55-2]} \\
\mathrm{V}\end{array}$ & $\begin{array}{l}\text { 5-methyl-3-hexanol } \\
\quad(275-311)\end{array}$ & $59.8 \pm 0.3$ & 298 & GS & [2005ROG/PIS] \\
\hline $\mathrm{C}_{7} \mathrm{H}_{16} \mathrm{O}$ & $\begin{array}{c}{[6570-87-2]} \\
\mathrm{V}\end{array}$ & $\begin{array}{l}\text { 3,4-dimethyl-1-penta } \\
\quad(393-438)\end{array}$ & Inol 50.3 & 388 & & [1973WIL/ZWO] \\
\hline $\mathrm{C}_{7} \mathrm{H}_{16} \mathrm{O}$ & $\begin{array}{c}{[625-06-9]} \\
\mathrm{V}\end{array}$ & $\begin{array}{l}\text { 2,4-dimethyl-2-penta } \\
\quad(328-408)\end{array}$ & Inol 49.7 & 343 & & [1973WIL/ZWO] \\
\hline $\mathrm{C}_{7} \mathrm{H}_{16} \mathrm{O}$ & $\begin{array}{c}{[3970-62-5]} \\
\mathrm{V}\end{array}$ & $\begin{array}{l}\text { 2,2-dimethyl-3-penta } \\
\quad(318-411)\end{array}$ & Inol 51.4 & 333 & & [1973WIL/ZWO] \\
\hline $\mathrm{C}_{7} \mathrm{H}_{16} \mathrm{O}$ & $\begin{array}{c}{[595-41-5]} \\
\mathrm{V}\end{array}$ & $\begin{array}{l}\text { 2,3-dimethyl-3-penta } \\
\quad(318-413)\end{array}$ & Inol 53.2 & 333 & & [1973WIL/ZWO] \\
\hline $\mathrm{C}_{7} \mathrm{H}_{16} \mathrm{O}$ & $\begin{array}{c}{[18371-13-6]} \\
\mathrm{V}\end{array}$ & $\begin{array}{l}\text { 2-methyl-2-ethyl-1-b } \\
\quad(358-428)\end{array}$ & $\begin{array}{l}\text { utanol } \\
55.7\end{array}$ & 373 & & [1973WIL/ZWO] \\
\hline $\mathrm{C}_{7} \mathrm{H}_{16} \mathrm{O}$ & $\begin{array}{c}{[594-83-2]} \\
\mathrm{V}\end{array}$ & $\begin{array}{l}\text { 2,3,3-trimethyl-2-but } \\
\quad \text { (298-363) }\end{array}$ & $\begin{array}{l}\text { anol } \\
48.7\end{array}$ & 313 & MM & [1985WIE/SIP] \\
\hline $\mathrm{C}_{7} \mathrm{H}_{16} \mathrm{O}_{2}$ & $\begin{array}{c}{[13343-98-1]} \\
\mathrm{V}\end{array}$ & 1-butoxy-2-methoxy & $\begin{array}{l}\text { ethane } \\
47.8 \pm 0.1\end{array}$ & 298 & $\mathrm{C}$ & [1970KUS/WAD] \\
\hline $\mathrm{C}_{7} \mathrm{H}_{16} \mathrm{O}_{2}$ & $\begin{array}{c}{[18854-31-4]} \\
\mathrm{V}\end{array}$ & 1-propoxy-2-ethoxye & $\begin{array}{l}\text { thane } \\
46.8 \pm 0.1\end{array}$ & 298 & $\mathrm{C}$ & [1970KUS/WAD] \\
\hline $\mathrm{C}_{7} \mathrm{H}_{16} \mathrm{O}_{2}$ & $\begin{array}{c}{[3459-83-4]} \\
\mathrm{V}\end{array}$ & 1,3-diethoxypropane & $45.9 \pm 0.2$ & 298 & $\mathrm{C}$ & [1972MAN2] \\
\hline $\mathrm{C}_{7} \mathrm{H}_{16} \mathrm{O}_{2}$ & $\begin{array}{c}{[126-84-1]} \\
\text { V } \\
\text { V } \\
\text { V }\end{array}$ & $\begin{array}{l}\text { 2,2-diethoxypropane } \\
\quad(273-308) \\
\quad(273-308) \\
(286-304)\end{array}$ & $\begin{array}{c}43.2 \pm 0.4 \\
43.9 \\
\mathrm{U} 28.2\end{array}$ & $\begin{array}{l}298 \\
298 \\
295\end{array}$ & $\begin{array}{l}\text { GS } \\
\text { GS } \\
\text { A, I }\end{array}$ & $\begin{array}{l}\text { [2002VER] } \\
\text { [1998VER/PEN] } \\
\text { [1987STE/MAL, 1962STE/DOR] }\end{array}$ \\
\hline $\mathrm{C}_{7} \mathrm{H}_{16} \mathrm{O}_{2}$ & $\begin{array}{c}{[57018-52-7]} \\
\mathrm{V}\end{array}$ & $\begin{array}{l}\text { 1-tert-butoxy-2-prop } \\
\quad(346-420)\end{array}$ & $\begin{array}{l}\text { anol } \\
\quad 45.4\end{array}$ & 361 & $\mathrm{~EB}$ & [2001BER/WIC] \\
\hline $\mathrm{C}_{7} \mathrm{H}_{16} \mathrm{O}_{2}$ & $\begin{array}{c}{[141-73-1]} \\
\mathrm{V}\end{array}$ & $\begin{array}{l}\text { 4-methyl-4-methoxy } \\
\text { (343-423) }\end{array}$ & $\begin{array}{c}\text {-2-pentanol } \\
46.6\end{array}$ & 358 & A & [1987STE/MAL, 1973DYK/REP] \\
\hline
\end{tabular}


TABLE 10. Phase change enthalpies of $\mathrm{C}_{7}$ organic compounds-Continued

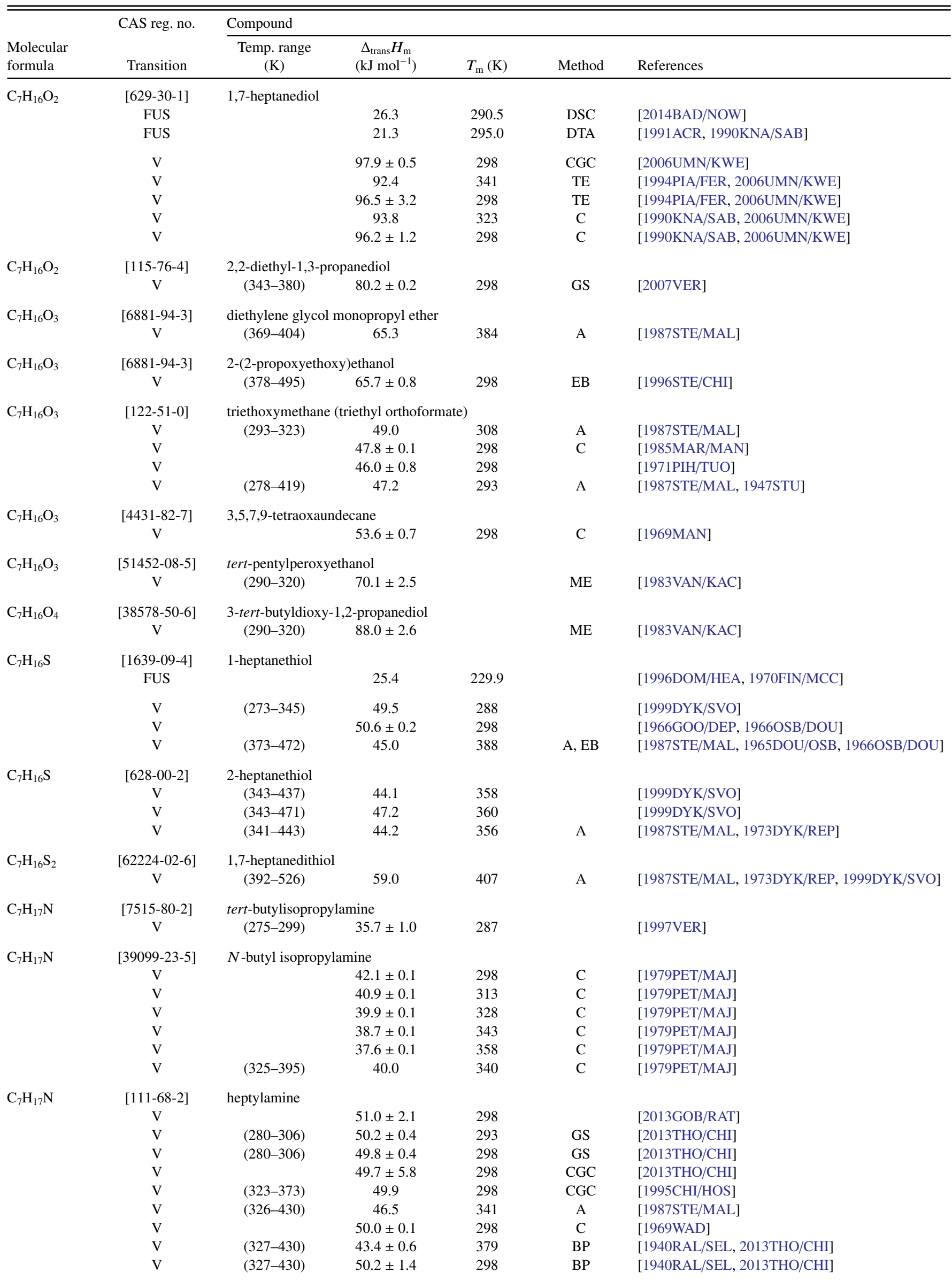


TABLE 10. Phase change enthalpies of $\mathrm{C}_{7}$ organic compounds-Continued

\begin{tabular}{|c|c|c|c|c|c|c|}
\hline \multirow[b]{2}{*}{$\begin{array}{l}\text { Molecular } \\
\text { formula }\end{array}$} & \multirow{2}{*}{$\begin{array}{l}\text { CAS reg. no. } \\
\text { Transition }\end{array}$} & \multicolumn{5}{|l|}{ Compound } \\
\hline & & $\begin{array}{c}\text { Temp. range } \\
\text { (K) }\end{array}$ & $\begin{array}{c}\Delta_{\text {trans }} H_{\mathrm{m}} \\
\left(\mathrm{kJ} \mathrm{mol}^{-1}\right)\end{array}$ & $T_{\mathrm{m}}(\mathrm{K})$ & Method & References \\
\hline \multirow[t]{2}{*}{$\mathrm{C}_{7} \mathrm{H}_{17} \mathrm{NO}$} & {$[7352-03-6]$} & \multicolumn{5}{|c|}{$N$-(ethoxymethyl)diethylamine } \\
\hline & $\mathrm{V}$ & $(285-400)$ & 39.6 & 300 & A & {$[1987 \mathrm{STE} / \mathrm{MAL}]$} \\
\hline \multirow[t]{2}{*}{$\mathrm{C}_{7} \mathrm{H}_{17} \mathrm{~N}_{3}$} & [934-98-5] & \multicolumn{5}{|c|}{ 1-(2-aminoethyl)-4-methylpiperazine } \\
\hline & $\mathrm{V}$ & $(298-332)$ & $64.0 \pm 0.2$ & 298 & GS & [2010EFI/EME] \\
\hline \multirow[t]{2}{*}{$\mathrm{C}_{7} \mathrm{H}_{17} \mathrm{O}_{2} \mathrm{PS}_{3}$} & [298-02-2] & \multicolumn{5}{|c|}{$O, O$-diethyl- $S$-[(ethylthio)methyl]dithiophosphate } \\
\hline & $\mathrm{V}$ & $(283-387)$ & 70.8 & 298 & A & [1987STE/MAL, 1973DYK/REP, 1999DYK/SVO] \\
\hline \multirow[t]{7}{*}{$\mathrm{C}_{7} \mathrm{H}_{17} \mathrm{O}_{3} \mathrm{P}$} & {$[1445-75-6]$} & \multicolumn{5}{|c|}{ diisopropyl methylphosphonate } \\
\hline & $\mathrm{V}$ & $(253-468)$ & 62.1 & 253 & GS & [2009BUT/BUC] \\
\hline & $\mathrm{V}$ & $(253-468)$ & 58.9 & 283 & GS & [2009BUT/BUC] \\
\hline & $\mathrm{V}$ & $(253-468)$ & 57.6 & 298 & GS & [2009BUT/BUC] \\
\hline & $\mathrm{V}$ & $(253-468)$ & 56.5 & 313 & GS & [2009BUT/BUC] \\
\hline & $\mathrm{V}$ & $(253-468)$ & 54.2 & 353 & GS & [2009BUT/BUC] \\
\hline & $\mathrm{V}$ & $(253-468)$ & 52.4 & 393 & GS & [2009BUT/BUC] \\
\hline \multirow[t]{7}{*}{$\mathrm{C}_{7} \mathrm{H}_{18} \mathrm{~N}_{2}$} & {$[646-19-5]$} & \multicolumn{5}{|c|}{ 1,7-heptanediamine } \\
\hline & FUS & & 43.5 & 301.5 & $\mathrm{AC}$ & [2004MON/VAN, 2014FUL/RUZ] \\
\hline & FUS & & 36.95 & 298.5 & DSC & [2002DAL/DEL] \\
\hline & SUB & $(274-300)$ & 105.3 & 298 & Static & [2014FUL/RUZ] \\
\hline & $\mathrm{V}$ & $(305-358)$ & 63.9 & 331 & GS & [2011POZ/VER] \\
\hline & $\mathrm{V}$ & $(305-358)$ & $67.1 \pm 0.2$ & 298 & GS & [2011POZ/VER] \\
\hline & $\mathrm{V}$ & $(273-313)$ & U46.5 & 288 & A & [1987STE/MAL] \\
\hline \multirow[t]{7}{*}{$\mathrm{C}_{7} \mathrm{H}_{18} \mathrm{~N}_{2}$} & [104-78-9] & \multicolumn{5}{|c|}{$N, N$-diethyl-1,3-propanediamine } \\
\hline & V & $(277-305)$ & $52.4 \pm 0.5$ & 298 & GS & [2012VER/CHE] \\
\hline & $\mathrm{V}$ & $(273-363)$ & 49.3 & 318 & & [2010KHI/DAH] \\
\hline & V & $(273-363)$ & $51.3 \pm 0.6$ & 298 & & [2010KHI/DAH, 2012VER/CHE] \\
\hline & $\mathrm{V}$ & $(303-343)$ & 49.0 & 323 & & [2008KHI/DAH] \\
\hline & $\mathrm{V}$ & $(303-343)$ & $51.0 \pm 0.3$ & 298 & & [2008KHI/DAH, 2012VER/CHE] \\
\hline & $\mathrm{V}$ & $(329-443)$ & 46.4 & 344 & A & [1987STE/MAL] \\
\hline \multirow[t]{3}{*}{$\mathrm{C}_{7} \mathrm{H}_{18} \mathrm{~N}_{2}$} & {$[110-95-2]$} & \multicolumn{5}{|c|}{$N, N, N^{\prime}, N^{\prime}$-tetramethyl-1,3-propanediamine } \\
\hline & $\mathrm{V}$ & $(273-363)$ & 44.1 & 298 & Static & [2015BOU/NEG2] \\
\hline & V & $(274-323)$ & $45.3 \pm 0.2$ & 298 & GS & [2012VER/CHE] \\
\hline \multirow[t]{2}{*}{$\mathrm{C}_{7} \mathrm{H}_{18} \mathrm{~N}_{2} \mathrm{O}$} & {$[5966-51-8]$} & \multicolumn{5}{|c|}{ 1,3-bis(dimethylamino)-2-propanol } \\
\hline & $\mathrm{V}$ & $(355-450)$ & 50.3 & 370 & A & [1987STE/MAL] \\
\hline \multirow[t]{3}{*}{$\mathrm{C}_{7} \mathrm{H}_{18} \mathrm{~N}_{2} \mathrm{O}$} & {$[2212-32-0]$} & \multicolumn{5}{|c|}{ 2-(2- $N, N$-dimethylaminoethyl- $N$-methylamino)ethanol } \\
\hline & $\mathrm{V}$ & $(293-353)$ & 63.2 & 323 & GS & [2011VER/TON] \\
\hline & $\mathrm{V}$ & $(293-353)$ & $65.2 \pm 0.2$ & 298 & GS & [2011VER/TON] \\
\hline \multirow[t]{2}{*}{$\mathrm{C}_{7} \mathrm{H}_{19} \mathrm{~N}_{3}$} & {$[67727-91-7]$} & \multicolumn{5}{|c|}{$N, N$-diethyl-2-(1-methylhydrazino)ethanamine } \\
\hline & $\mathrm{V}$ & $(283-313)$ & 61.8 & 298 & A & [1987STE/MAL] \\
\hline \multirow[t]{2}{*}{$\mathrm{C}_{7} \mathrm{H}_{20} \mathrm{~N}_{4}$} & {$[4741-99-5]$} & \multicolumn{5}{|c|}{ 1,4,8,11-tetraazaundecane } \\
\hline & $\mathrm{V}$ & $(332-348)$ & $98.3 \pm 1.3$ & 340 & $\mathrm{TE}$ & [1983CLA/COR] \\
\hline
\end{tabular}


TABLE 11. Phase change enthalpies of $\mathrm{C}_{8}$ organic compounds

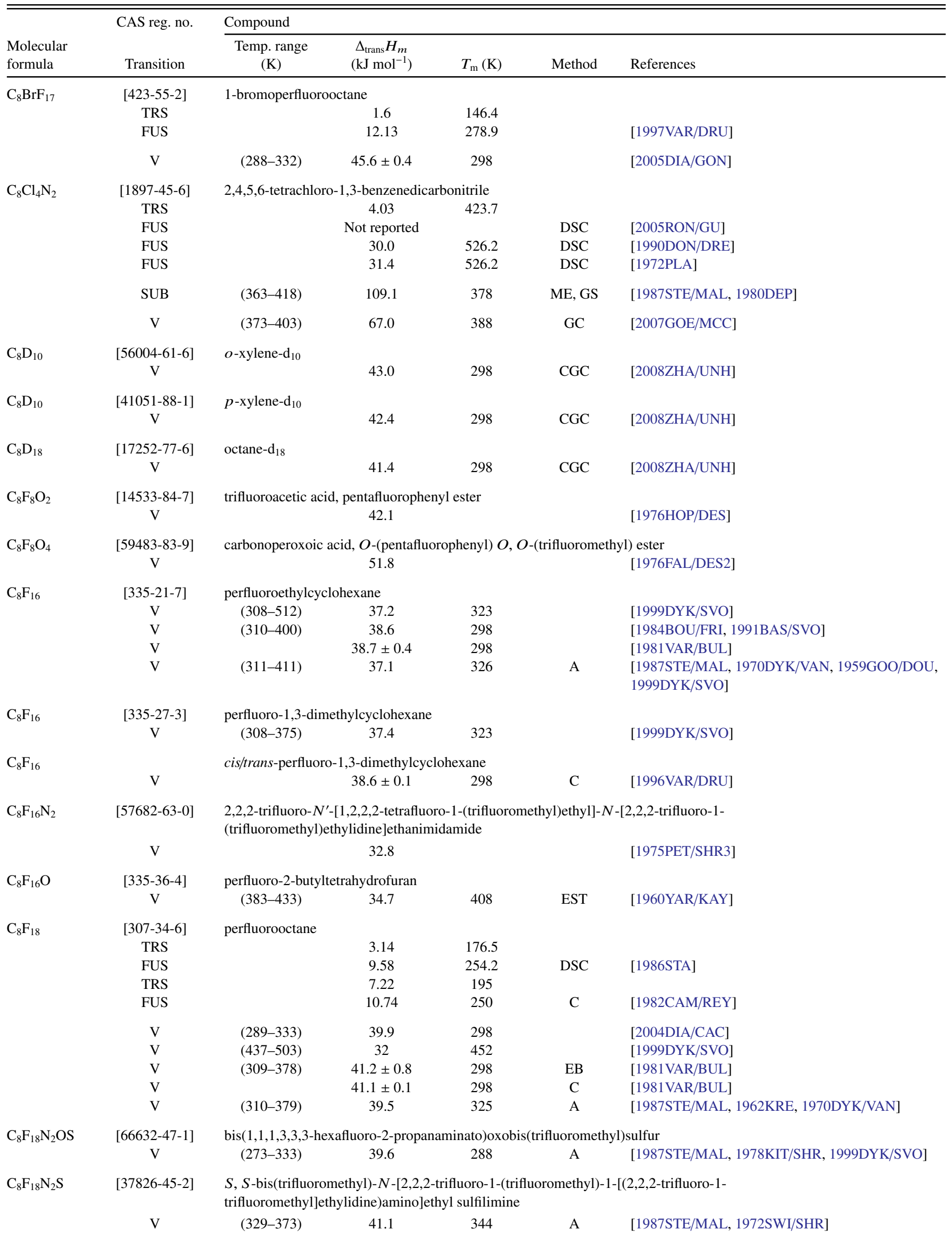


TABLE 11. Phase change enthalpies of $\mathrm{C}_{8}$ organic compounds-Continued

\begin{tabular}{|c|c|c|c|c|c|c|}
\hline \multirow[b]{2}{*}{$\begin{array}{l}\text { Molecular } \\
\text { formula }\end{array}$} & \multirow{2}{*}{$\begin{array}{c}\text { CAS reg. no. } \\
\text { Transition }\end{array}$} & \multicolumn{5}{|l|}{ Compound } \\
\hline & & $\begin{array}{l}\text { Temp. range } \\
(\mathrm{K})\end{array}$ & $\begin{array}{c}\Delta_{\text {trans }} H_{m} \\
\left(\mathrm{~kJ} \mathrm{~mol}^{-1}\right)\end{array}$ & $T_{\mathrm{m}}(\mathrm{K})$ & Method & References \\
\hline \multirow[t]{6}{*}{$\mathrm{C}_{8} \mathrm{~F}_{18} \mathrm{O}$} & [308-48-5] & \multicolumn{5}{|c|}{ bis(nonafluorobutyl) ether } \\
\hline & $\mathrm{V}$ & $(315-374)$ & $40.3 \pm 0.8$ & 298 & EB & [1989VAR/PAS] \\
\hline & $\mathrm{V}$ & & $40.7 \pm 0.1$ & 298 & $\mathrm{C}$ & [1989VAR/PAS] \\
\hline & $\mathrm{V}$ & $(343-375)$ & 36.6 & 358 & A & [1987STE/MAL] \\
\hline & $\mathrm{V}$ & $(288-313)$ & 42.2 & 300 & A & [1987STE/MAL] \\
\hline & $\mathrm{V}$ & $(374-413)$ & 56.3 & 389 & A & [1987STE/MAL, 1999DYK/SVO] \\
\hline \multirow[t]{2}{*}{$\mathrm{C}_{8} \mathrm{~F}_{18} \mathrm{O}_{2}$} & {$[647-40-5]$} & \multicolumn{5}{|c|}{ dodecafluoro-1,6-bis(trifluoromethoxy)hexane } \\
\hline & $\mathrm{V}$ & $(293-353)$ & 33.6 & 323 & & [1999DYK/SVO, 1964ROB] \\
\hline \multirow[t]{2}{*}{$\mathrm{C}_{8} \mathrm{~F}_{18} \mathrm{O}_{3} \mathrm{~S}$} & {$[53517-90-1]$} & \multicolumn{5}{|c|}{ bis(1,1,1,3,3,3-hexafluoro-2-(trifluoromethyl)-2-propanol) sulfite } \\
\hline & $\mathrm{V}$ & & 38.7 & & & [1975DEM/KOV] \\
\hline \multirow[t]{2}{*}{$\mathrm{C}_{8} \mathrm{~F}_{20} \mathrm{~N}_{2} \mathrm{~S}$} & {$[65844-11-3]$} & \multicolumn{5}{|c|}{$\begin{array}{l}\text { difluoro[1,1,1,3,3,3-hexafluoro- } N \text {-[2,2,2-trifluoro-1-(trifluoromethyl)-ethylidene-2,2-propanediaminato(2-)- } N] \text { - } \\
\text { bis(trifluoromethyl) sulfur }\end{array}$} \\
\hline & $\mathrm{V}$ & & 39.3 & 390 & I & [1978KIT/SHR] \\
\hline \multirow{2}{*}{$\begin{array}{l}\mathrm{C}_{8} \\
\mathrm{HCl}_{4} \mathrm{~F}_{11} \mathrm{O}_{2}\end{array}$} & {$[2923-68-4]$} & \multicolumn{5}{|c|}{ 3,5,7,8-tetrachloro-2,2,3,4,4,5,6,6,7,8,8-undecafluorooctanoic acid } \\
\hline & $\mathrm{V}$ & $(373-553)$ & 70.6 & 388 & A & [1987STE/MAL, 1957BAR/SEF, 1999DYK/SVO] \\
\hline $\mathrm{C}_{8} \mathrm{HF}_{15} \mathrm{O}_{2}$ & $\begin{array}{l}{[335-67-1]} \\
\text { SUB }\end{array}$ & $\begin{array}{l}\text { perfluorooctan } \\
\quad(298-318)\end{array}$ & \multicolumn{2}{|c|}{ perfluorooctanoic acid } & GS & [2008BAR/BOT] \\
\hline \multirow[t]{2}{*}{$\mathrm{C}_{8} \mathrm{HF}_{16} \mathrm{NO}$} & {$[54181-87-2]$} & \multicolumn{5}{|c|}{$\begin{array}{l}\text { 1,1,1,2,3,3,3-heptafluoro- } N \text {-[2,2,2-trifluoro-1-[2,2,2-trifluoro-1-(trifluoromethyl)ethoxy]ethylidene]-2- } \\
\text { propanamine }\end{array}$} \\
\hline & $\mathrm{V}$ & & 36.0 & 364 & & [1975PET/SHR] \\
\hline \multirow[t]{2}{*}{$\mathrm{C}_{8} \mathrm{HF}_{17}$} & {$[335-65-9]$} & \multicolumn{5}{|c|}{$1,1,1,2,2,3,3,4,4,5,5,6,6,7,7,8,8$-heptadecafluorooctane } \\
\hline & $\mathrm{V}$ & $(288-332)$ & $43.4 \pm 0.2$ & 298 & & {$[2005 \mathrm{DIA} / \mathrm{GON}]$} \\
\hline \multirow[t]{5}{*}{$\mathrm{C}_{8} \mathrm{H}_{2} \mathrm{Cl}_{4} \mathrm{~N}_{2}$} & [25983-14-6] & \multicolumn{5}{|c|}{ 2,3,6,7-tetrachloroquinoxaline } \\
\hline & FUS & & 29.6 & 446 & DSC & [2000MON/HIL2] \\
\hline & SUB & & $105.9 \pm 2.2$ & 298 & ME & [2004MOR/MIR] \\
\hline & SUB & $(347-361)$ & $106.2 \pm 0.3$ & 354 & ME & [2000MON/HIL2] \\
\hline & SUB & & $108.2 \pm 1.9$ & 298 & ME & [2000MON/HIL2] \\
\hline \multirow[t]{2}{*}{$\mathrm{C}_{8} \mathrm{H}_{2} \mathrm{~F}_{16}$} & [307-99-3] & \multicolumn{5}{|c|}{$1,1,2,2,3,3,4,4,5,5,6,6,7,7,8,8$-hexadecafluorooctane } \\
\hline & $\mathrm{V}$ & $(298-323)$ & 41.1 & 310 & A & [1987STE/MAL, 1999DYK/SVO, 1956MCL/SCO] \\
\hline $\mathrm{C}_{8} \mathrm{H}_{3} \mathrm{ClF}_{6}$ & {$[327-76-4]$} & 4-chloro-1,3-bi & luoromethyl) & & & \\
\hline & $\mathrm{V}$ & $(275-353)$ & 48.0 & 290 & A & $\begin{array}{l}\text { [1987STE/MAL, 1946FIE/SAY, 1970DYK/VAN, } \\
\text { 1999DYK/SVO] }\end{array}$ \\
\hline $\mathrm{C}_{8} \mathrm{H}_{3} \mathrm{ClF}_{6}$ & [328-72-3] & 5-chloro-1,3-bi & luoromethyl) & & & \\
\hline & $\mathrm{V}$ & $(275-353)$ & 46.2 & 290 & A & $\begin{array}{l}\text { [1987STE/MAL, 1946FIE/SAY, 1970DYK/VAN, } \\
\text { 1999DYK/SVO] }\end{array}$ \\
\hline $\mathrm{C}_{8} \mathrm{H}_{3} \mathrm{ClO}_{3}$ & [117-21-5] & 4-chloroisoben & an-1,3-dione & & & \\
\hline & FUS & & 18.65 & 398.8 & DSC & [2015WAN/WAN] \\
\hline & FUS & & 13.35 & 398.0 & DSC & [2010JI/MEN] \\
\hline $\mathrm{C}_{8} \mathrm{H}_{3} \mathrm{ClO}_{3}$ & [118-45-6] & 5-chloroisoben & an-1,3-dione & & & \\
\hline & FUS & & 21.45 & 367.3 & DSC & [2015WAN/WAN] \\
\hline & FUS & & 33.06 & 367.4 & DSC & [2010JI/MEN] \\
\hline & $\mathrm{V}$ & $(447-560)$ & 64.3 & 470 & EB & [2015LI/DU] \\
\hline $\mathrm{C}_{8} \mathrm{H}_{3} \mathrm{Cl}_{4} \mathrm{~F}_{3}$ & [328-82-5] & 1,1,1-trifluoro- & ichloro-2-(3, & lorophen & ane & \\
\hline & $\mathrm{V}$ & $(417-461)$ & 56.9 & 432 & A & [1987STE/MAL, 1999DYK/SVO, 1951PAR/BRO] \\
\hline $\mathrm{C}_{8} \mathrm{H}_{3} \mathrm{FO}_{3}$ & {$[319-03-9]$} & 4-fluorophthali & & & & \\
\hline & V & & 60.1 & 450 & EB & {$[2015 \mathrm{LI} / \mathrm{DU}]$} \\
\hline $\mathrm{C}_{8} \mathrm{H}_{3} \mathrm{~F}_{5} \mathrm{O}_{2}$ & {$[19220-93-0]$} & acidic acid, per & orophenyl es & & & \\
\hline & $\mathrm{V}$ & $(283-322)$ & 48.1 & 298 & A & [1987STE/MAL, 1999DYK/SVO] \\
\hline
\end{tabular}


TABLE 11. Phase change enthalpies of $\mathrm{C}_{8}$ organic compounds-Continued

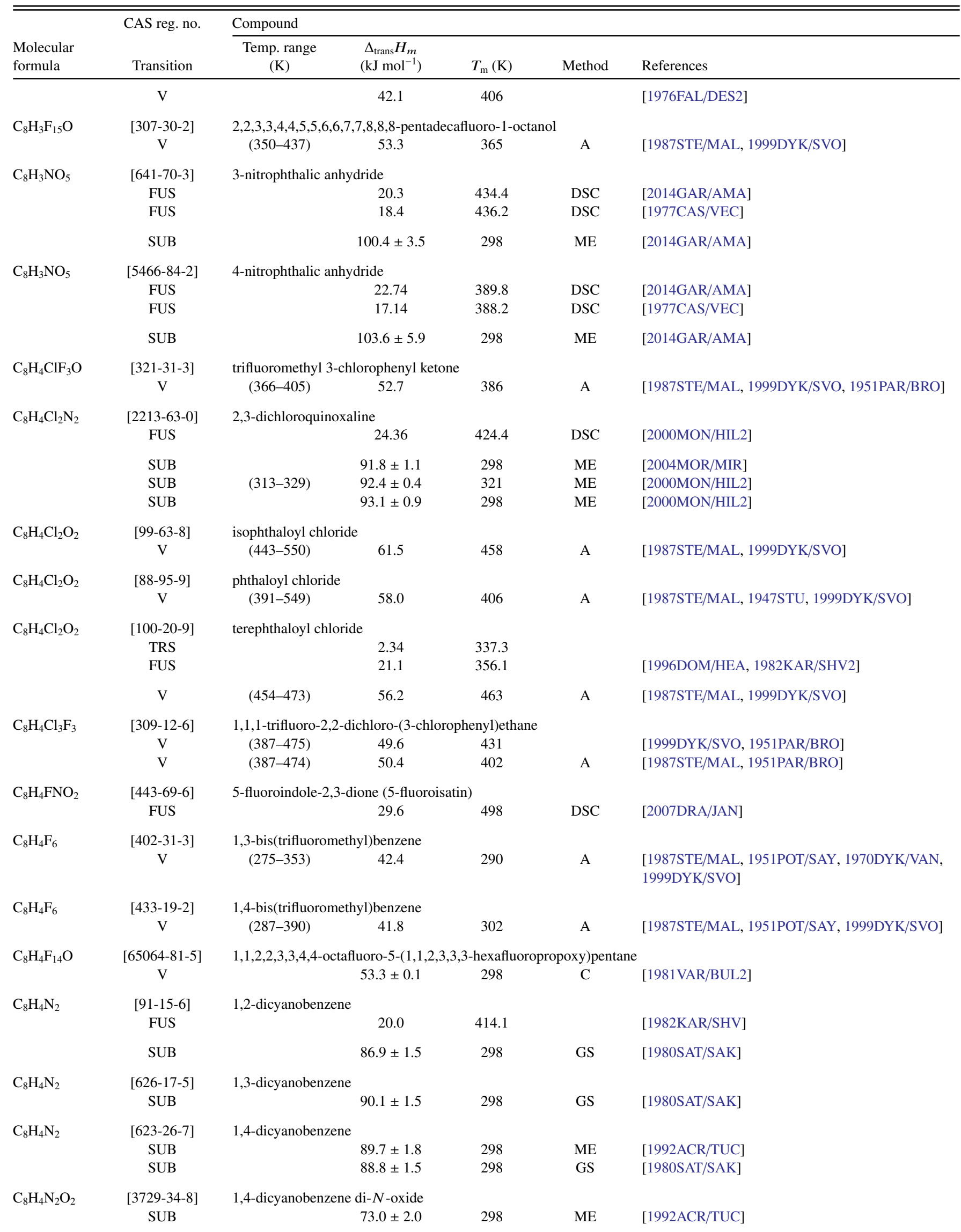


TABLE 11. Phase change enthalpies of $\mathrm{C}_{8}$ organic compounds-Continued

\begin{tabular}{|c|c|c|c|c|c|c|}
\hline \multirow[b]{2}{*}{$\begin{array}{l}\text { Molecular } \\
\text { formula }\end{array}$} & \multirow{2}{*}{$\begin{array}{l}\text { CAS reg. no. } \\
\text { Transition }\end{array}$} & \multicolumn{5}{|l|}{ Compound } \\
\hline & & $\begin{array}{l}\text { Temp. range } \\
(\mathrm{K})\end{array}$ & $\begin{array}{c}\Delta_{\text {trans }} H_{m} \\
\left(\mathrm{~kJ} \mathrm{~mol}^{-1}\right)\end{array}$ & $T_{\mathrm{m}}(\mathrm{K})$ & Method & References \\
\hline $\mathrm{C}_{8} \mathrm{H}_{4} \mathrm{~N}_{2} \mathrm{O}_{4}$ & $\begin{array}{l}\text { [603-62-3] } \\
\text { FUS }\end{array}$ & 3-nitrophthalin & 34.0 & 487 & DSC & [2007DRA/JAN] \\
\hline $\mathrm{C}_{8} \mathrm{H}_{4} \mathrm{O}_{2}$ & $\begin{array}{l}{[6383-11-5]} \\
\text { SUB }\end{array}$ & $\begin{array}{l}\text { benzocyclobut } \\
\text { (304-367) }\end{array}$ & U89.5 & 336 & & [1989ROR/RUT] \\
\hline $\mathrm{C}_{8} \mathrm{H}_{4} \mathrm{O}_{3}$ & $\begin{array}{l}\text { [85-44-9] } \\
\text { FUS } \\
\text { FUS }\end{array}$ & phthalic anhyd & $\begin{array}{c}23.09 \\
22.1\end{array}$ & $\begin{array}{l}403.3 \\
404.5\end{array}$ & $\begin{array}{l}\text { DSC } \\
\text { DSC }\end{array}$ & $\begin{array}{l}\text { [1991ACR, 1990DON/DRE] } \\
\text { [1979DAS/DHA] }\end{array}$ \\
\hline & $\begin{array}{l}\text { SUB } \\
\text { SUB } \\
\text { SUB } \\
\text { SUB } \\
\text { SUB }\end{array}$ & $\begin{array}{l}(313-383) \\
(333-403) \\
(303-333)\end{array}$ & $\begin{aligned} 87.9 & \pm 4.7 \\
87.9 & \\
84.4 & \pm 1.2 \\
81 & \pm 1 \\
88.4 & \pm 1.2\end{aligned}$ & $\begin{array}{l}298 \\
348 \\
388 \\
318\end{array}$ & $\begin{array}{c}\text { C } \\
\text { A } \\
\text { GS } \\
\text { C }\end{array}$ & $\begin{array}{l}\text { [2016FRE/SAN] } \\
\text { [1987STE/MAL, 1972AMI/VAK] } \\
\text { [1979DAS/DHA] } \\
\text { [1971BEE/LIN] } \\
\text { [1946CRO/FEE, 1970COX/PIL, 1960JON] }\end{array}$ \\
\hline & $\begin{array}{l}\text { V } \\
\text { V } \\
\text { V } \\
\text { V } \\
\text { V }\end{array}$ & $\begin{array}{l}(407-558) \\
(411-450) \\
(485-557)\end{array}$ & $\begin{array}{c}52.1 \\
63.9 \pm 2.5 \\
63.1 \\
65.3 \pm 0.8 \\
54.1\end{array}$ & $\begin{array}{l}422 \\
422 \\
521\end{array}$ & $\begin{array}{c}\text { A } \\
\text { GS }\end{array}$ & $\begin{array}{l}\text { [1987STE/MAL] } \\
{[1979 \mathrm{DAS} / \mathrm{DHA}]} \\
{[1952 \mathrm{GOT} / \mathrm{NIK}]} \\
{[1946 \mathrm{CRO} / \mathrm{FEE}, 1979 \mathrm{DAS} / \mathrm{DHA}]} \\
{[1920 \mathrm{MON}]}\end{array}$ \\
\hline $\mathrm{C}_{8} \mathrm{H}_{5} \mathrm{Br}_{3}$ & $\begin{array}{l}{[24162-65-0]} \\
\text { FUS }\end{array}$ & 2,4,5-tribromo & 25.1 & 340.3 & & [1993OIS/HOR] \\
\hline $\mathrm{C}_{8} \mathrm{H}_{5} \mathrm{Cl}_{2} \mathrm{~F}_{3}$ & $\begin{array}{c}{[309-10-4]} \\
\mathrm{V}\end{array}$ & $\begin{array}{c}\text { 1,1,1-trifluoro- } \\
\text { (365-446) }\end{array}$ & $\begin{array}{l}\text { ichloro-2-phe } \\
47.2\end{array}$ & $\begin{array}{l}\text { ane } \\
380\end{array}$ & A & [1987STE/MAL, 1999DYK/SVO, 1951PAR/BRO] \\
\hline $\mathrm{C}_{8} \mathrm{H}_{5} \mathrm{Cl}_{2} \mathrm{~N}$ & $\begin{array}{c}{[40626-45-7]} \\
\mathrm{V}\end{array}$ & $\begin{array}{c}\alpha, \alpha \text {-dichlorop } \\
\text { (329-497) }\end{array}$ & $\begin{array}{l}\text { acetonitrile } \\
57.2\end{array}$ & 344 & A & [1987STE/MAL, 1947STU] \\
\hline $\mathrm{C}_{8} \mathrm{H}_{5} \mathrm{Cl}_{3} \mathrm{O}_{2}$ & $\begin{array}{l}{[85-34-7]} \\
\text { FUS }\end{array}$ & 2,3,6-trichloro & $\begin{array}{c}\text { lacetic acid } \\
22.43\end{array}$ & 432.3 & DSC & [1991ACR, 1990DON/DRE] \\
\hline $\mathrm{C}_{8} \mathrm{H}_{5} \mathrm{Cl}_{3} \mathrm{O}_{3}$ & $\begin{array}{l}{[93-76-5]} \\
\text { FUS }\end{array}$ & (2,4,5-trichloro & $\begin{array}{c}\text { oxy)acetic ac } \\
38.0\end{array}$ & 428.7 & DSC & [1991ACR, 1990DON/DRE] \\
\hline $\mathrm{C}_{8} \mathrm{H}_{5} \mathrm{Cl}_{5}$ & $\begin{array}{c}{[606-07-5]} \\
\mathrm{V}\end{array}$ & $\begin{array}{c}\text { pentachloroeth } \\
\text { (369-572) }\end{array}$ & $\begin{array}{l}\text { zene } \\
\qquad 58.8\end{array}$ & 384 & A & [1987STE/MAL, 1947STU, 1999DYK/SVO] \\
\hline $\mathrm{C}_{8} \mathrm{H}_{5} \mathrm{~F}_{3} \mathrm{OS}_{2}$ & $\begin{array}{l}{[4552-64-1]} \\
\text { SUB }\end{array}$ & 1,1,1-trifluoro- & $\begin{array}{l}\text { hienyl)-4-me } \\
95.1 \pm 3.7\end{array}$ & $\begin{array}{l}\text {-3-buten } \\
298\end{array}$ & $\mathrm{C}$ & [1997RIB/SAN] \\
\hline $\mathrm{C}_{8} \mathrm{H}_{5} \mathrm{~F}_{3} \mathrm{O}_{2} \mathrm{~S}$ & $\begin{array}{l}{[15788-02-0]} \\
\text { SUB } \\
\text { SUB } \\
\text { SUB }\end{array}$ & $\begin{array}{l}\text { 1,1,1-trifluoro- } \\
(279-290) \\
(279-290)\end{array}$ & $\begin{array}{l}\text { hienyl)-4-hy } \\
86.2 \pm 0.6 \\
86.9 \pm 0.6 \\
86.2 \pm 0.6\end{array}$ & $\begin{array}{l}-3 \text {-buten- } \\
298 \\
285 \\
298\end{array}$ & $\begin{array}{c}\mathrm{C} \\
\mathrm{ME} \\
\mathrm{ME}\end{array}$ & $\begin{array}{l}{[1997 \mathrm{RIB} / \mathrm{SAN}]} \\
{[1992 \mathrm{RIB} / \mathrm{MON}]} \\
{[1992 \mathrm{RIB} / \mathrm{MON}]}\end{array}$ \\
\hline $\mathrm{C}_{8} \mathrm{H}_{5} \mathrm{~F}_{3} \mathrm{O}_{3}$ & $\begin{array}{c}\text { [326-90-9] } \\
\text { SUB }\end{array}$ & 4,4,4-trifluoro- & $\begin{array}{l}\text { uranyl)-buta } \\
70 \pm 10\end{array}$ & $\begin{array}{r}\text {-dione } \\
298\end{array}$ & & [1997RIB/GON] \\
\hline $\mathrm{C}_{8} \mathrm{H}_{5} \mathrm{~F}_{5} \mathrm{O}$ & $\begin{array}{c}{[434-45-7]} \\
\mathrm{V}\end{array}$ & $\begin{array}{c}\text { 2,2,2-trifluoroa } \\
\quad(342-425)\end{array}$ & $\begin{array}{r}\text { henone } \\
43.1\end{array}$ & 357 & A & [1987STE/MAL, 1999DYK/SVO] \\
\hline $\mathrm{C}_{8} \mathrm{H}_{5} \mathrm{~F}_{14} \mathrm{OP}$ & $\begin{array}{c}{[647-72-3]} \\
\mathrm{V}\end{array}$ & $\begin{array}{c}\text { ethyl bis(hepta } \\
\text { (303-393) }\end{array}$ & $\begin{array}{c}\text { ropyl)phosp } \\
41.9\end{array}$ & 348 & & [1959EME/SMI] \\
\hline $\mathrm{C}_{8} \mathrm{H}_{5} \mathrm{NO}$ & $\begin{array}{c}{[613-90-1]} \\
\text { SUB }\end{array}$ & $\begin{array}{c}\text { benzoylnitrile } \\
(292-304)\end{array}$ & $78.7 \pm 4.2$ & 298 & & [1969LEB/DNE, 1977PED/RYL, 1987STE/MAL] \\
\hline & $\mathrm{V}$ & $(318-481)$ & 52.0 & 333 & A & [1987STE/MAL, 1947STU] \\
\hline $\mathrm{C}_{8} \mathrm{H}_{5} \mathrm{NO}_{2}$ & $\begin{array}{l}{[85-41-6]} \\
\text { FUS }\end{array}$ & phthalimide & 28.6 & 507.2 & DSC & [1978CAN] \\
\hline & $\begin{array}{l}\text { SUB } \\
\text { SUB } \\
\text { SUB }\end{array}$ & $\begin{array}{l}(347-365) \\
(347-365) \\
(378-418)\end{array}$ & $\begin{array}{c}104.0 \pm 0.4 \\
106.3 \pm 1.3 \\
82.8\end{array}$ & $\begin{array}{l}356 \\
298 \\
393\end{array}$ & $\begin{array}{l}\text { ME } \\
\text { ME } \\
\text { RG }\end{array}$ & $\begin{array}{l}\text { [2006RIB/SAN] } \\
\text { [2006RIB/SAN] } \\
\text { [1987STE/MAL, 1956KLO] }\end{array}$ \\
\hline $\mathrm{C}_{8} \mathrm{H}_{5} \mathrm{NO}_{2}$ & $\begin{array}{l}{[91-56-5]} \\
\text { FUS }\end{array}$ & $1 H$-indole-2,3 & $\begin{array}{l}\text { (isatin) } \\
27.82\end{array}$ & 475.7 & DSC & [2003MAT/MIR2] \\
\hline
\end{tabular}


TABLE 11. Phase change enthalpies of $\mathrm{C}_{8}$ organic compounds-Continued

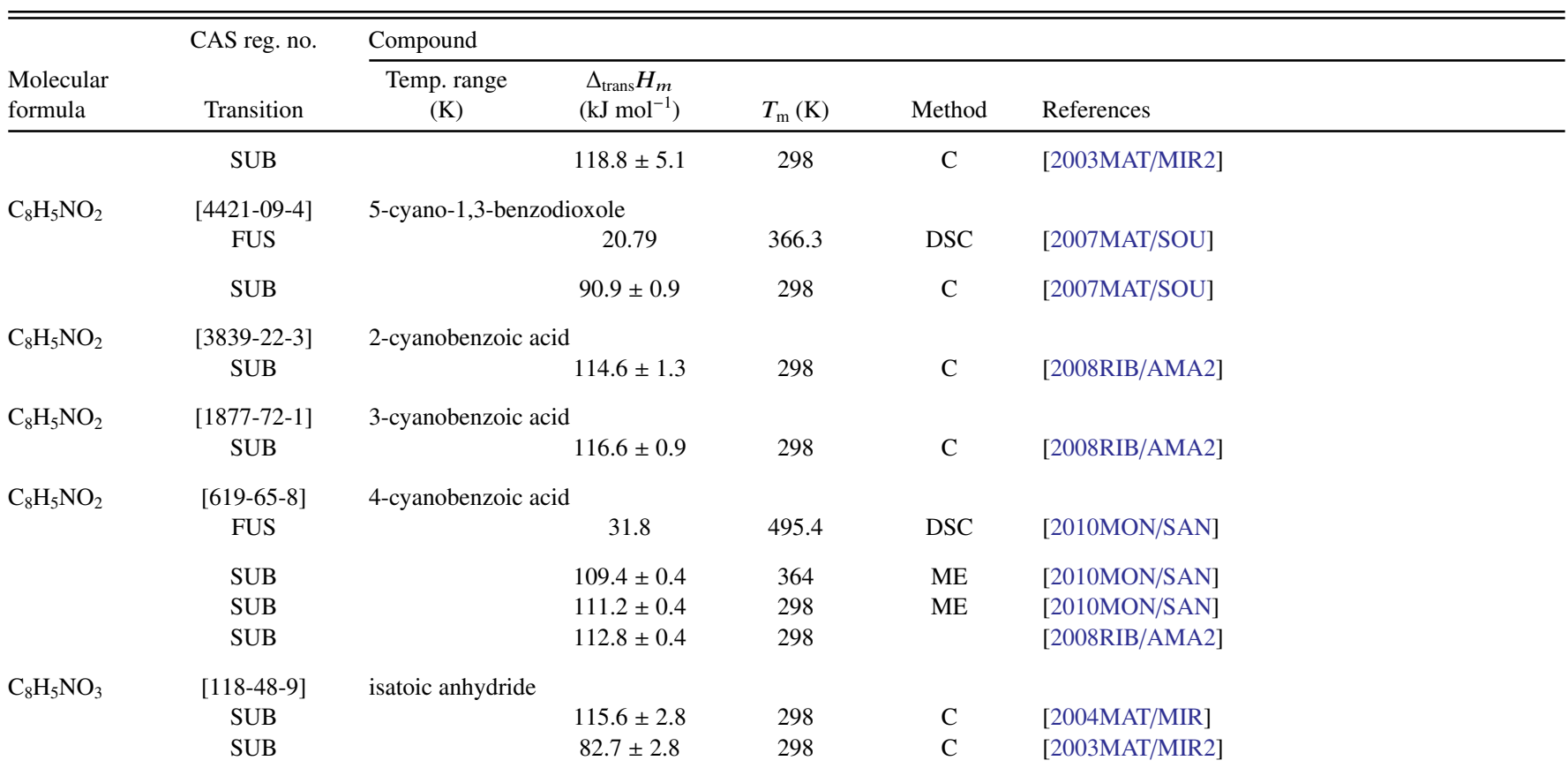

[Note: In [2004MAT/MIR] the authors state that an error was made in [2003MAT/MIR2] in converting the enthalpy of sublimation measured at a higher temperature back to $298 \mathrm{~K}$.]

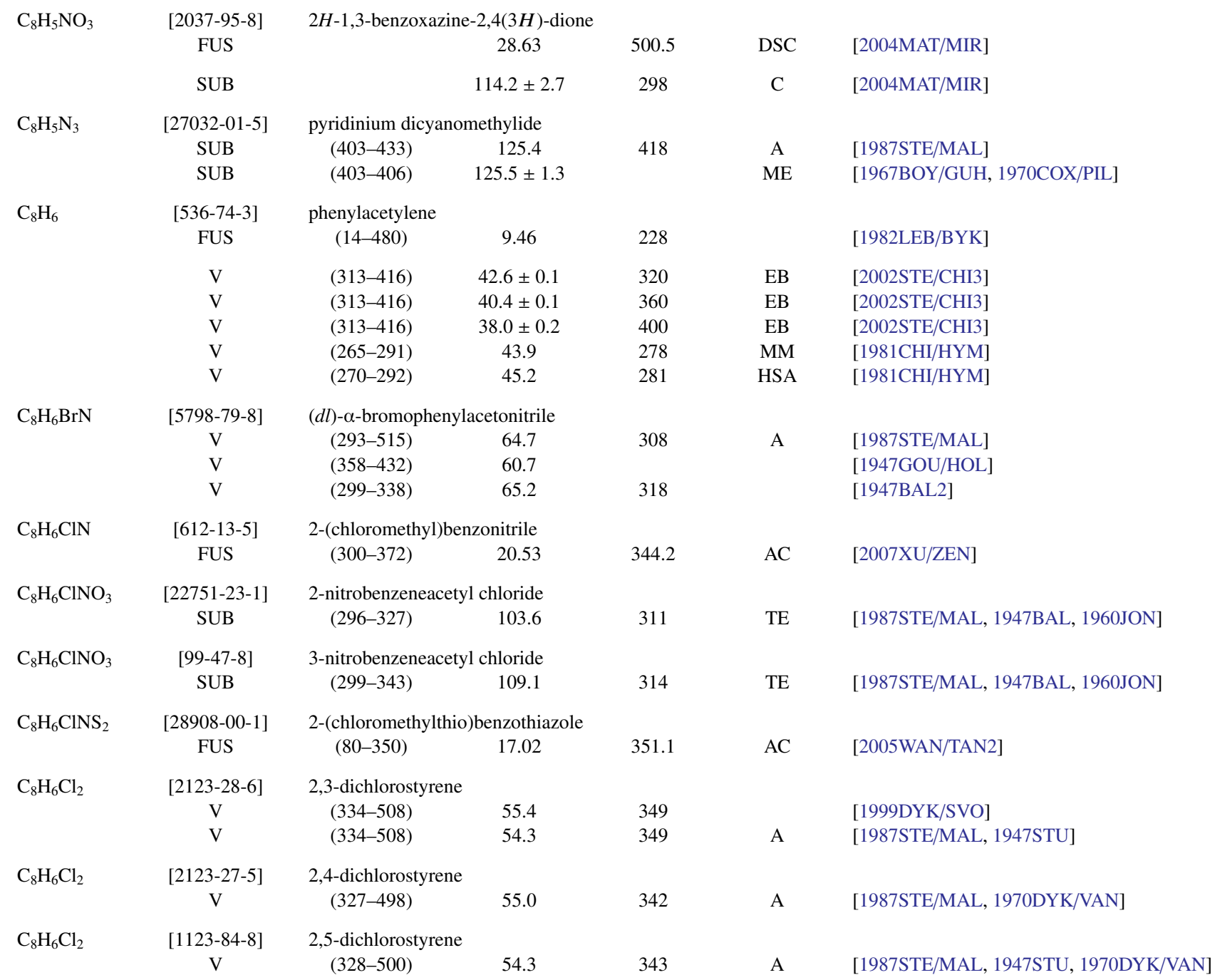


TABLE 11. Phase change enthalpies of $\mathrm{C}_{8}$ organic compounds-Continued

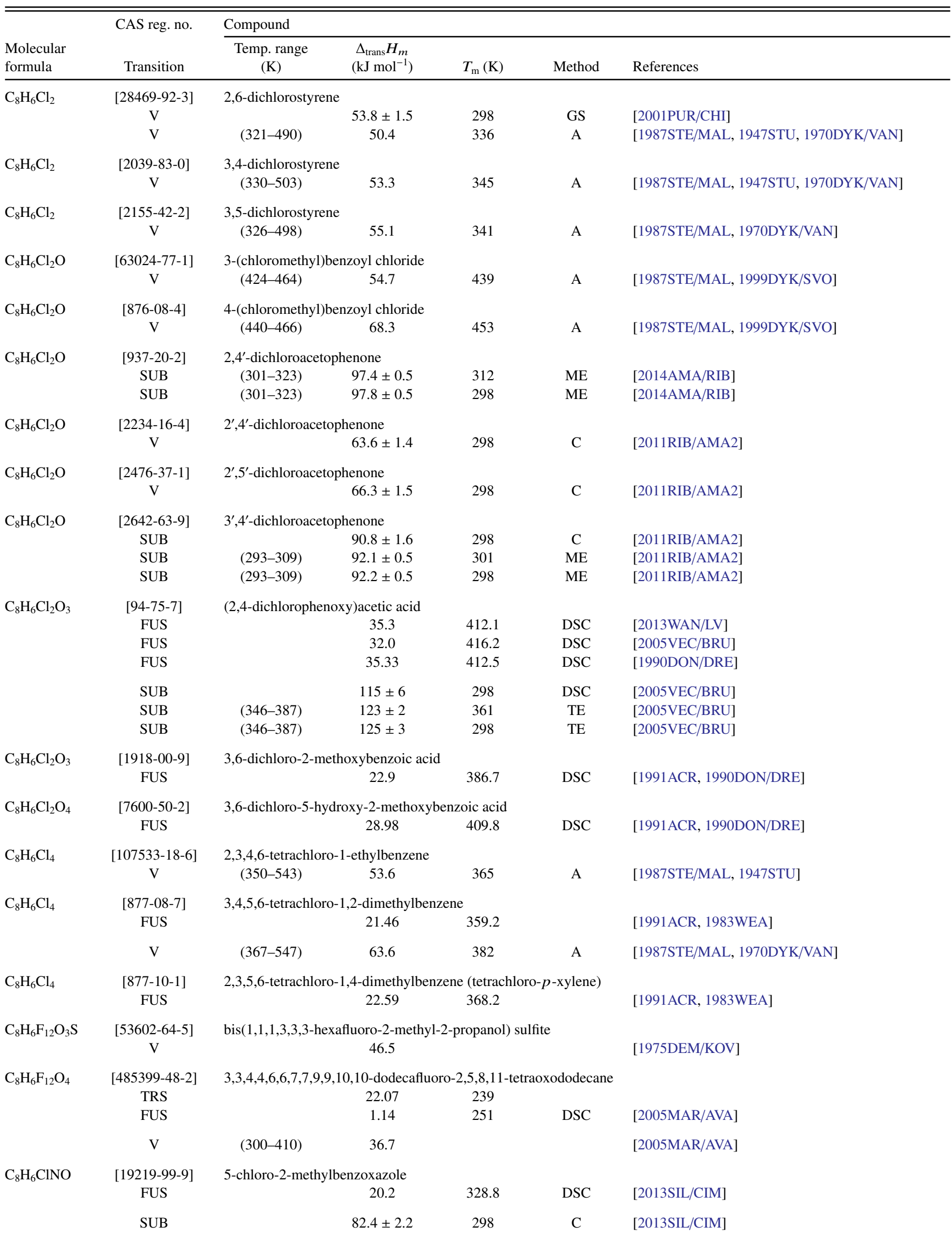

$\mathrm{C}_{8} \mathrm{H}_{6} \mathrm{CINO}$ [41014-43-1] 2-chloromethylbenzoxazole 
TABLE 11. Phase change enthalpies of $\mathrm{C}_{8}$ organic compounds-Continued

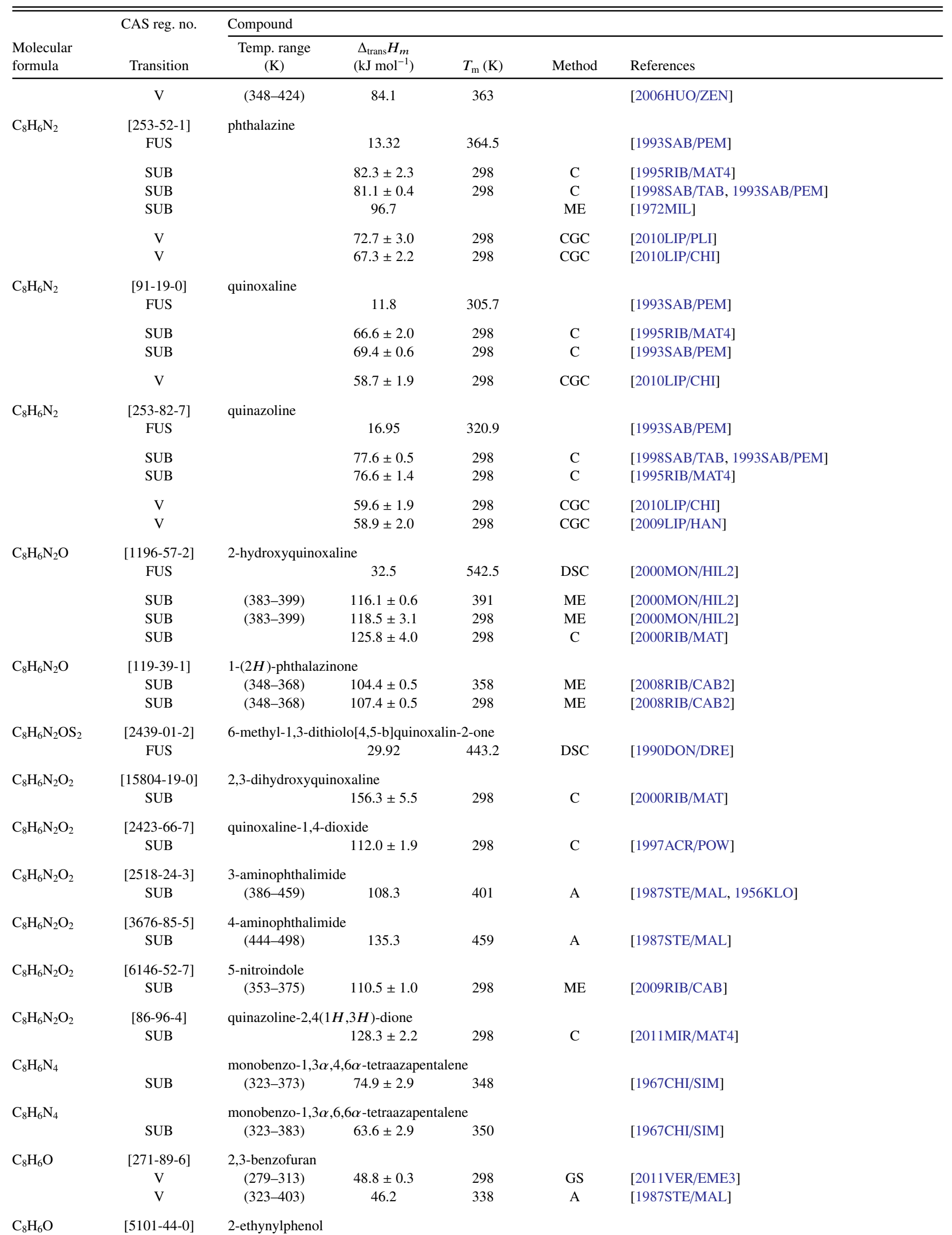


TABLE 11. Phase change enthalpies of $\mathrm{C}_{8}$ organic compounds-Continued

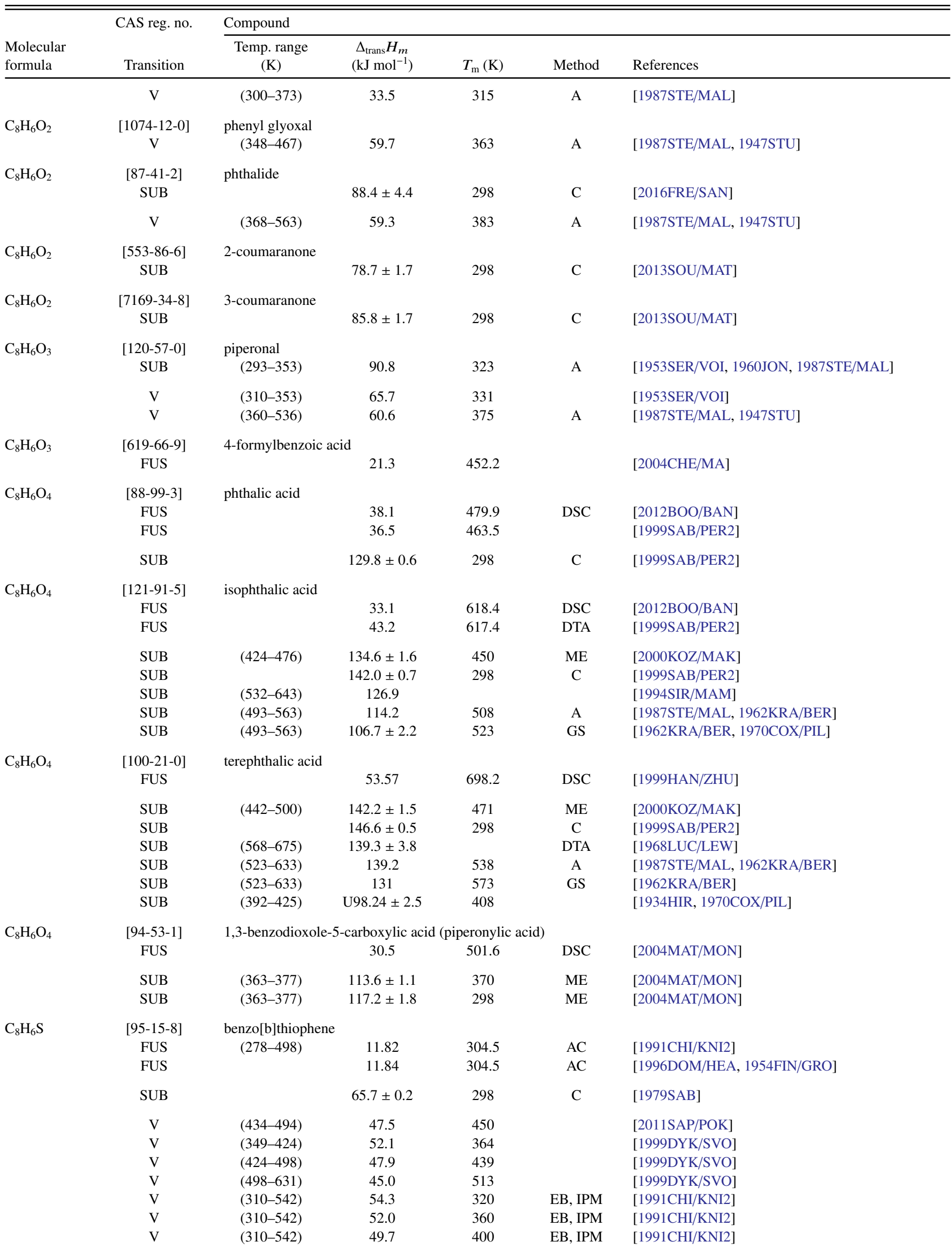


TABLE 11. Phase change enthalpies of $\mathrm{C}_{8}$ organic compounds-Continued

\begin{tabular}{|c|c|c|c|c|c|c|}
\hline \multirow[b]{2}{*}{$\begin{array}{l}\text { Molecular } \\
\text { formula }\end{array}$} & \multirow{2}{*}{$\begin{array}{c}\text { CAS reg. no. } \\
\text { Transition }\end{array}$} & \multicolumn{5}{|l|}{ Compound } \\
\hline & & $\begin{array}{c}\text { Temp. range } \\
(\mathrm{K})\end{array}$ & $\begin{array}{c}\Delta_{\text {trans }} H_{m} \\
\left(\mathrm{~kJ} \mathrm{~mol}^{-1}\right)\end{array}$ & $T_{\mathrm{m}}(\mathrm{K})$ & Method & References \\
\hline & $\mathrm{V}$ & $(310-542)$ & 46.2 & 460 & EB, IPM & [1991CHI/KNI2] \\
\hline & $\mathrm{V}$ & $(310-542)$ & 43.8 & 500 & EB, IPM & [1991CHI/KNI2] \\
\hline & $\mathrm{V}$ & $(310-542)$ & 41.2 & 540 & $\mathrm{~EB}, \mathrm{IPM}$ & [1991CHI/KNI2] \\
\hline & $\mathrm{V}$ & $(424-630)$ & 47.2 & 425 & & [1981WIE/KOB, 1980WIE/KOB] \\
\hline & $\mathrm{V}$ & $(424-630)$ & 42.8 & 505 & & [1981WIE/KOB, 1980WIE/KOB] \\
\hline & $\mathrm{V}$ & $(424-630)$ & 36.1 & 605 & & [1981WIE/KOB, 1980WIE/KOB] \\
\hline & $\mathrm{V}$ & $(306-346)$ & 53.8 & 326 & & [1981EDW/PRA, 1999DYK/SVO] \\
\hline \multirow[t]{4}{*}{$\mathrm{C}_{8} \mathrm{H}_{6} \mathrm{~S}_{2}$} & {$[492-97-7]$} & 2,2'-bithiophene & & & & \\
\hline & FUS & & 16.5 & 304.2 & DSC & [2006TEM/ROU] \\
\hline & SUB & $(275-291)$ & $86.0 \pm 0.4$ & 283 & $\mathrm{ME}$ & [2009RIB/SAN] \\
\hline & SUB & $(275-291)$ & $85.2 \pm 0.4$ & 298 & $\mathrm{ME}$ & [2009RIB/SAN] \\
\hline \multirow[t]{4}{*}{$\mathrm{C}_{8} \mathrm{H}_{6} \mathrm{~S}_{2}$} & {$[3172-56-3]$} & 3,3'-biothiophene & & & & \\
\hline & FUS & & 21.3 & 406.2 & $\mathrm{DSC}$ & [2011COS/LIM] \\
\hline & SUB & $(301-317)$ & $88.6 \pm 0.3$ & 309 & $\mathrm{ME}$ & [2009RIB/SAN] \\
\hline & SUB & $(301-317)$ & $89.2 \pm 0.3$ & 298 & $\mathrm{ME}$ & [2009RIB/SAN] \\
\hline \multirow[t]{2}{*}{$\mathrm{C}_{8} \mathrm{H}_{7} \mathrm{Br}$} & {$[2039-88-5]$} & 2-bromostyrene & & & & \\
\hline & $\mathrm{V}$ & $(378-543)$ & 48.7 & 393 & A & [1987STE/MAL, 1970DYK/VAN] \\
\hline \multirow[t]{3}{*}{$\mathrm{C}_{8} \mathrm{H}_{7} \mathrm{Br}$} & {$[2039-82-9]$} & 4-bromostyrene & & & & \\
\hline & $\mathrm{V}$ & $(393-420)$ & 48.5 & 406 & & [1999DYK/SVO] \\
\hline & $\mathrm{V}$ & $(383-543)$ & 49.9 & 398 & A & [1987STE/MAL, 1970DYK/VAN] \\
\hline \multirow[t]{2}{*}{$\mathrm{C}_{8} \mathrm{H}_{7} \mathrm{Br}$} & {$[70-11-1]$} & 2-bromoacetopher & & & & \\
\hline & SUB & & $90.9 \pm 1.1$ & 298 & $\mathrm{C}$ & [2014AMA/RIB2] \\
\hline \multirow[t]{2}{*}{$\mathrm{C}_{8} \mathrm{H}_{7} \mathrm{Br}$} & {$[2142-69-0]$} & 2'-bromoacetophe & & & & \\
\hline & $\mathrm{V}$ & & $64.9 \pm 1.3$ & 298 & $\mathrm{C}$ & [2014AMA/RIB2] \\
\hline \multirow[t]{2}{*}{$\mathrm{C}_{8} \mathrm{H}_{7} \mathrm{Br}$} & {$[2142-63-4]$} & 3'-bromoacetophe & & & & \\
\hline & $\mathrm{V}$ & & $66.6 \pm 1.0$ & 298 & $\mathrm{C}$ & [2014AMA/RIB2] \\
\hline \multirow[t]{7}{*}{$\mathrm{C}_{8} \mathrm{H}_{7} \mathrm{Br}$} & {$[99-90-1]$} & 4'-bromoacetophe & & & & \\
\hline & FUS & & & & DSC & [2016ALM/MON] \\
\hline & SUB & $(295-319)$ & $83.1 \pm 0.3$ & 307 & Static & [2016ALM/MON] \\
\hline & SUB & $(295-319)$ & $83.3 \pm 0.3$ & 298 & Static & [2016ALM/MON] \\
\hline & SUB & & $84.5 \pm 1.0$ & 298 & $\mathrm{C}$ & [2014AMA/RIB2] \\
\hline & $\mathrm{V}$ & $(305-378)$ & $63.3 \pm 0.1$ & 342 & Static & [2016ALM/MON] \\
\hline & $\mathrm{V}$ & $(305-378)$ & $66.4 \pm 0.1$ & 298 & Static & [2016ALM/MON] \\
\hline \multirow[t]{6}{*}{$\mathrm{C}_{8} \mathrm{H}_{7} \mathrm{BrO}_{2}$} & {$[619-42-1]$} & methyl 4-bromobe & oate & & & \\
\hline & FUS & & 25.8 & 351.2 & DSC & [2013ALM/MON] \\
\hline & SUB & $(293-349)$ & $88.7 \pm 0.1$ & 321 & Static & [2013ALM/MON] \\
\hline & SUB & $(293-349)$ & $89.3 \pm 0.2$ & 298 & Static & [2013ALM/MON] \\
\hline & $\mathrm{V}$ & $(355-378)$ & $60.6 \pm 0.2$ & 367 & Static & [2013ALM/MON] \\
\hline & $\mathrm{V}$ & $(355-378)$ & $66.1 \pm 0.4$ & 298 & Static & [2013ALM/MON] \\
\hline \multirow[t]{2}{*}{$\mathrm{C}_{8} \mathrm{H}_{7} \mathrm{Cl}$} & [2039-87-4] & 2-chlorostyrene & & & & \\
\hline & $\mathrm{V}$ & $(363-523)$ & 46.0 & 378 & A & [1987STE/MAL, 1970DYK/VAN] \\
\hline \multirow[t]{2}{*}{$\mathrm{C}_{8} \mathrm{H}_{7} \mathrm{Cl}$} & {$[2039-85-2]$} & 3-chlorostyrene & & & & \\
\hline & $\mathrm{V}$ & $(298-463)$ & 46.1 & 313 & A & [1987STE/MAL, 1947STU, 1970DYK/VAN] \\
\hline \multirow[t]{2}{*}{$\mathrm{C}_{8} \mathrm{H}_{7} \mathrm{Cl}$} & {$[1073-67-2]$} & 4-chlorostyrene & & & & \\
\hline & $\mathrm{V}$ & $(363-523)$ & 48.1 & 378 & A & [1987STE/MAL, 1947STU, 1970DYK/VAN] \\
\hline \multirow[t]{2}{*}{$\mathrm{C}_{8} \mathrm{H}_{7} \mathrm{ClN}_{2} \mathrm{O}_{2}$} & {$[14722-82-8]$} & $N$-(2-chloropheny & 2-(hydroxyil & acetamide & & \\
\hline & FUS & & 29.7 & 432.7 & DTA & {$[1982 \mathrm{CUE} / \mathrm{SOL}]$} \\
\hline \multirow[t]{2}{*}{$\mathrm{C}_{8} \mathrm{H}_{7} \mathrm{ClN}_{2} \mathrm{O}_{2} \mathrm{~S}$} & {$[364-98-7]$} & \multicolumn{5}{|c|}{ 7-chloro-3-methyl-2H-1,2,4-benzothiadiazine 1,1-dioxide (diazoxide) } \\
\hline & FUS & & 34.1 & 600.4 & DSC & [2006WAS/HOL] \\
\hline $\mathrm{C}_{8} \mathrm{H}_{7} \mathrm{ClO}$ & {$[532-27-4]$} & 2-chloroacetopher & & & & \\
\hline
\end{tabular}


TABLE 11. Phase change enthalpies of $\mathrm{C}_{8}$ organic compounds-Continued

\begin{tabular}{|c|c|c|c|c|c|c|}
\hline \multirow[b]{2}{*}{$\begin{array}{l}\text { Molecular } \\
\text { formula }\end{array}$} & \multirow{2}{*}{$\begin{array}{c}\text { CAS reg. no. } \\
\text { Transition }\end{array}$} & \multicolumn{5}{|l|}{ Compound } \\
\hline & & $\begin{array}{c}\text { Temp. range } \\
(\mathrm{K})\end{array}$ & $\begin{array}{c}\Delta_{\text {trans }} H_{m} \\
\left(\mathrm{~kJ} \mathrm{~mol}^{-1}\right)\end{array}$ & $T_{\mathrm{m}}(\mathrm{K})$ & Method & References \\
\hline & SUB & & $87.7 \pm 1.7$ & 298 & $\mathrm{C}$ & [2014AMA/RIB] \\
\hline & SUB & $(278-323)$ & 90.7 & 293 & $\mathrm{TE}$ & [1987STE/MAL, 1947BAL, 1960JON] \\
\hline \multirow[t]{2}{*}{$\mathrm{C}_{8} \mathrm{H}_{7} \mathrm{ClO}$} & {$[2142-68-9]$} & \multicolumn{5}{|c|}{ 2'-chloroacetophenone } \\
\hline & $\mathrm{V}$ & & $60.1 \pm 1.3$ & 298 & $\mathrm{C}$ & [2010RIB/AMA2] \\
\hline \multirow[t]{2}{*}{$\mathrm{C}_{8} \mathrm{H}_{7} \mathrm{ClO}$} & [99-02-5] & \multicolumn{5}{|c|}{ 3'-chloroacetophenone } \\
\hline & $\mathrm{V}$ & & $64.0 \pm 1.7$ & 298 & $\mathrm{C}$ & [2010RIB/AMA2] \\
\hline \multirow[t]{7}{*}{$\mathrm{C}_{8} \mathrm{H}_{7} \mathrm{ClO}$} & {$[99-91-2]$} & \multicolumn{5}{|c|}{ 4'-chloroacetophenone } \\
\hline & FUS & & 15.3 & 290.3 & DSC & [2016ALM/MON] \\
\hline & $\mathrm{V}$ & $(272-335)$ & $61.9 \pm 0.1$ & 303 & Static & [2016ALM/MON] \\
\hline & $\mathrm{V}$ & $(272-335)$ & $62.2 \pm 0.1$ & 298 & Static & [2016ALM/MON] \\
\hline & $\mathrm{V}$ & & $63.1 \pm 1.7$ & 298 & $\mathrm{C}$ & [2010RIB/AMA2] \\
\hline & $\mathrm{V}$ & $(404-623)$ & 54.0 & 419 & A & [1987STE/MAL, 1999DYK/SVO] \\
\hline & $\mathrm{V}$ & $(395-485)$ & 50.7 & 410 & & [1949DRE/SHR, 1949DRE/MAR, 1984BOU/FRI] \\
\hline $\mathrm{C}_{8} \mathrm{H}_{7} \mathrm{ClO}$ & $\begin{array}{c}{[103-80-0]} \\
\mathrm{V}\end{array}$ & $\begin{array}{c}\text { phenylacetyl ch } \\
\quad(321-483)\end{array}$ & 56.5 & 336 & A & [1987STE/MAL, 1947STU, 1999DYK/SVO] \\
\hline \multirow{2}{*}{$\mathrm{C}_{8} \mathrm{H}_{7} \mathrm{ClO}_{2}$} & {$[501-53-1]$} & \multicolumn{5}{|c|}{ benzyl chloroformate } \\
\hline & $\mathrm{V}$ & $(293-303)$ & $38.5 \pm 0.1$ & 298 & BG & [1990DAV/FIN] \\
\hline \multirow[t]{2}{*}{$\mathrm{C}_{8} \mathrm{H}_{7} \mathrm{ClO}_{2}$} & {$[2444-36-2]$} & \multicolumn{5}{|c|}{ (2-chlorophenyl)acetic acid } \\
\hline & FUS & & 24.33 & 367.4 & DSC & [2008RIB/FER3] \\
\hline \multirow[t]{2}{*}{$\mathrm{C}_{8} \mathrm{H}_{7} \mathrm{ClO}_{2}$} & {$[1878-65-5]$} & \multicolumn{5}{|c|}{ (3-chlorophenyl)acetic acid } \\
\hline & FUS & & 22.6 & 349.8 & DSC & [2008RIB/FER3] \\
\hline \multirow[t]{2}{*}{$\mathrm{C}_{8} \mathrm{H}_{7} \mathrm{ClO}_{2}$} & {$[1878-66-6]$} & \multicolumn{5}{|c|}{ (4-chlorophenyl)acetic acid } \\
\hline & FUS & & 23.57 & 377.9 & DSC & [2008RIB/FER3] \\
\hline \multirow[t]{6}{*}{$\mathrm{C}_{8} \mathrm{H}_{7} \mathrm{ClO}_{2}$} & [1126-46-1] & methyl 4-chlor & zoate & & & \\
\hline & FUS & & 21.3 & 315.1 & DSC & [2013ALM/MON] \\
\hline & SUB & $(277-312)$ & $83.5 \pm 0.1$ & 295 & Static & [2013ALM/MON] \\
\hline & SUB & $(277-312)$ & $83.4 \pm 0.1$ & 298 & Static & [2013ALM/MON] \\
\hline & $\mathrm{V}$ & $(285-334)$ & $61.6 \pm 0.1$ & 310 & Static & [2013ALM/MON] \\
\hline & $\mathrm{V}$ & $(285-334)$ & $62.4 \pm 0.1$ & 298 & Static & [2013ALM/MON] \\
\hline $\mathrm{C}_{8} \mathrm{H}_{7} \mathrm{ClO}_{3}$ & [10421-85-9] & 2-chloromande & & & & \\
\hline & FUS $(\alpha)$ & & 23.2 & 363.4 & DSC & [2011LOR/VON] \\
\hline & FUS $(\alpha)$ & & 23.1 & 363.1 & DSC & {$[2010 \mathrm{HE} / \mathrm{ROH}]$} \\
\hline & FUS & & 23.1 & 363.5 & DSC & [2009HE/ZHU] \\
\hline & FUS & & 20.08 & 358.5 & & [1991CHI/BRA] \\
\hline $\mathrm{C}_{8} \mathrm{H}_{7} \mathrm{ClO}_{3}$ & [52950-18-2] & $(R)$-2-chlorom & ic acid & & & \\
\hline & FUS & & 24.3 & 392.4 & DSC & [2011LOR/VON] \\
\hline & FUS & & 24.9 & 391.3 & DSC & [2009HE/ZHU, 2010HE/ROH] \\
\hline & FUS & & 24.69 & 392.5 & & [1991CHI/BRA] \\
\hline $\mathrm{C}_{8} \mathrm{H}_{7} \mathrm{ClO}_{3}$ & [61008-98-8] & $(R)$-3-chlorom & ic acid & & & \\
\hline & FUS & & 22.55 & 376.4 & DSC & [2010MIN/VON] \\
\hline & FUS & & 26.24 & 378.8 & DSC & [2009ZHA/RAY] \\
\hline $\mathrm{C}_{8} \mathrm{H}_{7} \mathrm{ClO}_{3}$ & [16273-37-3] & $(R, S)$-3-chlor & delic acid & & & \\
\hline & FUS & & 27.92 & 391.2 & DSC & [2010MIN/VON] \\
\hline & FUS & & 27.98 & 290.4 & DSC & [2009ZHA/RAY] \\
\hline $\mathrm{C}_{8} \mathrm{H}_{7} \mathrm{ClO}_{3}$ & [492-86-4] & $(d l)$-p-chlorom & lic acid & & & \\
\hline & FUS & & 27.2 & 394 & & [1991CHI/BRA] \\
\hline $\mathrm{C}_{8} \mathrm{H}_{7} \mathrm{ClO}_{3}$ & [32189-36-9] & $(d)$-p-chlorom & ic acid & & & \\
\hline & FUS & & 23.01 & 394 & & [1991CHI/BRA] \\
\hline $\mathrm{C}_{8} \mathrm{H}_{7} \mathrm{ClO}_{3}$ & [122-88-3] & 4-chlorophenox & tic acid & & & \\
\hline & FUS & & 36.27 & 429.6 & $\mathrm{DSC}$ & [1990DON/DRE] \\
\hline $\mathrm{C}_{8} \mathrm{H}_{7} \mathrm{ClO}_{3}$ & [19463-48-0] & 5-chloro-4-hyd & 3-methoxyb & ehyde & & \\
\hline
\end{tabular}


TABLE 11. Phase change enthalpies of $\mathrm{C}_{8}$ organic compounds-Continued

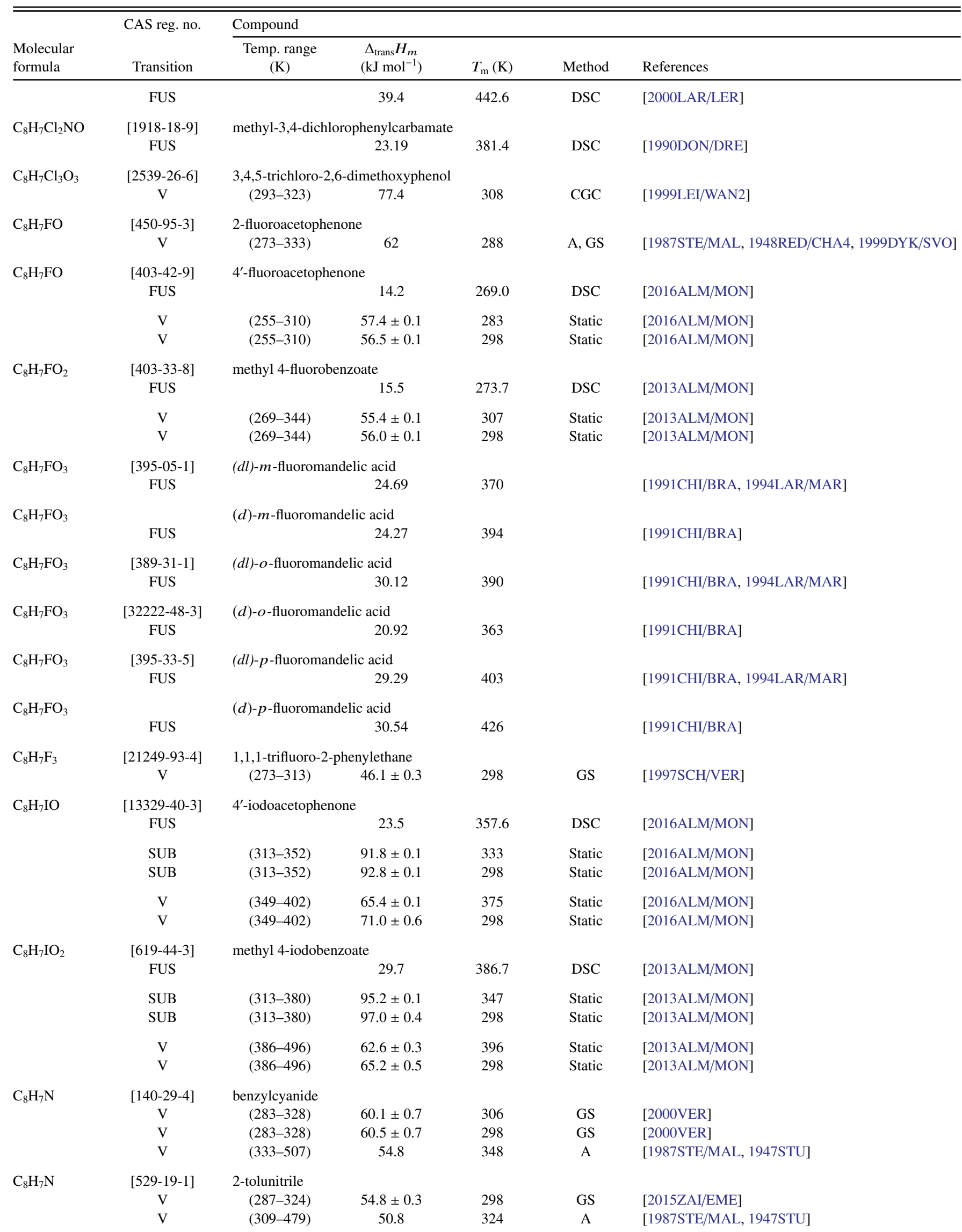


TABLE 11. Phase change enthalpies of $\mathrm{C}_{8}$ organic compounds-Continued

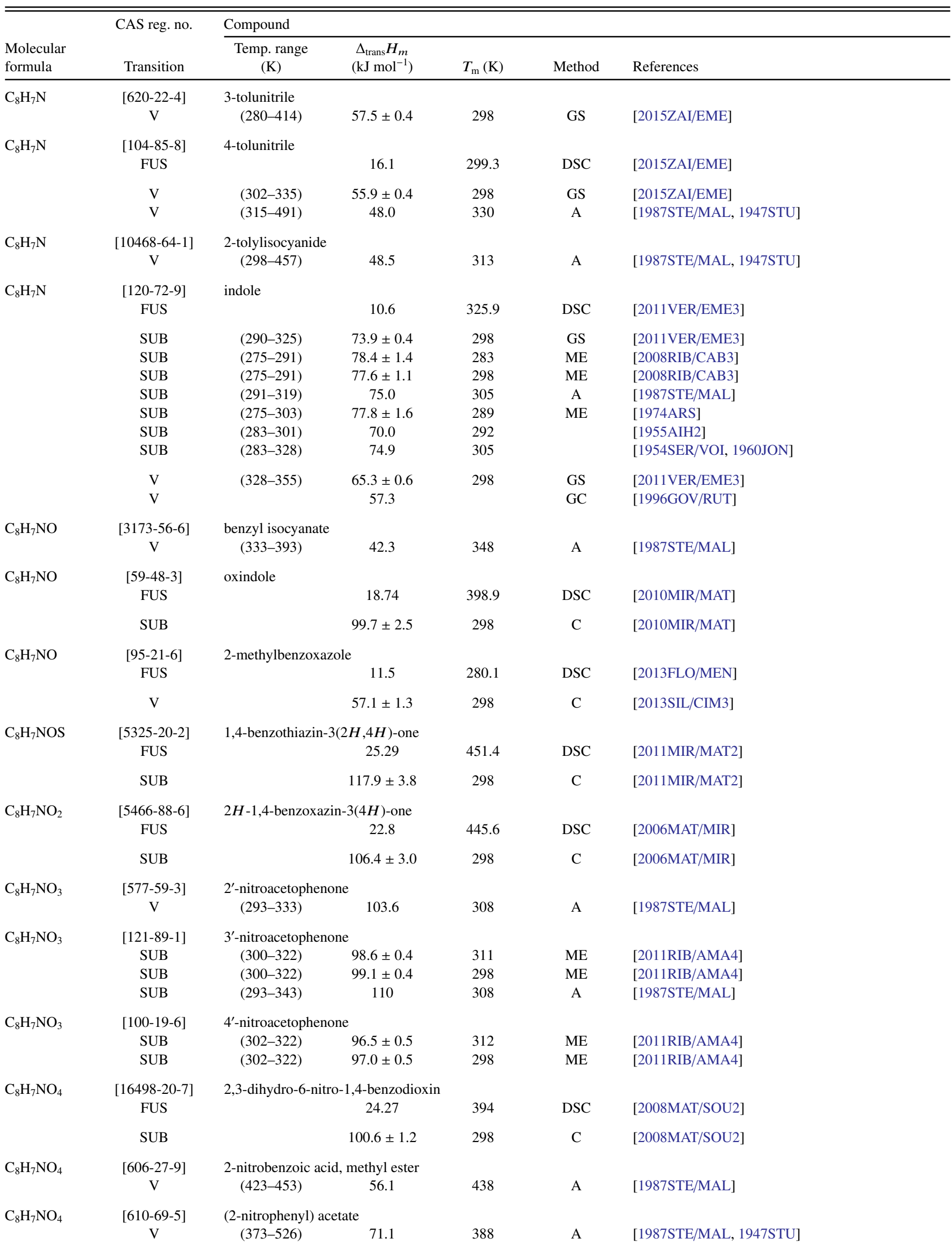


TABLE 11. Phase change enthalpies of $\mathrm{C}_{8}$ organic compounds-Continued

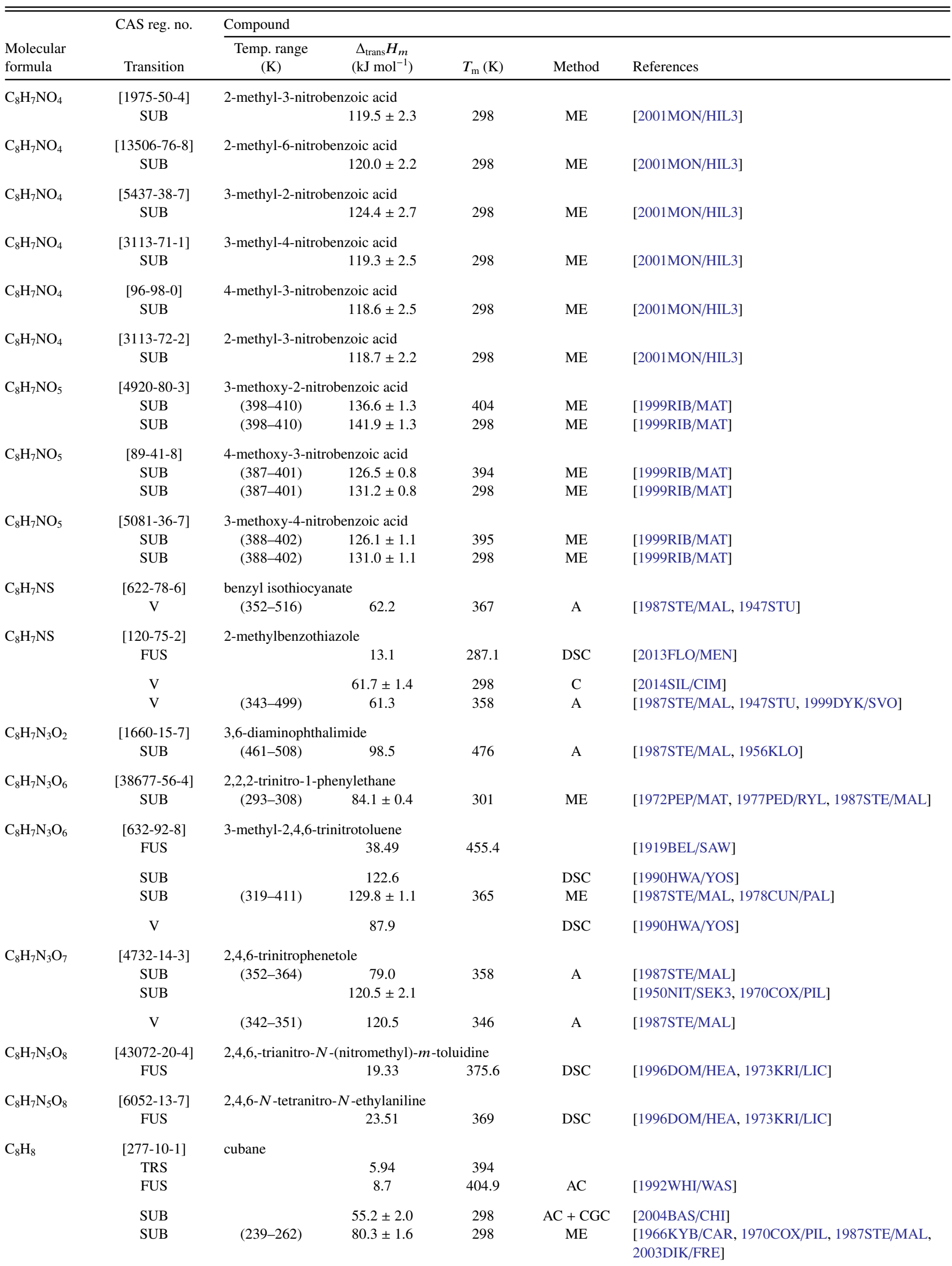


TABLE 11. Phase change enthalpies of $\mathrm{C}_{8}$ organic compounds-Continued

\begin{tabular}{lcccccc}
\hline \hline & CAS reg. no. & Compound & & \\
\cline { 3 - 6 } $\begin{array}{l}\text { Molecular } \\
\text { formula }\end{array}$ & Transition & Temp. range & $\Delta_{\text {trans }} H_{m}$ & & \\
$\left(\mathrm{~kJ} \mathrm{~mol}^{-1}\right)$ & $T_{\mathrm{m}}(\mathrm{K})$ & Method & References \\
\hline
\end{tabular}

[Note: In reference [2003DIK/FRE] the authors state that the value of $80.3 \mathrm{~kJ} / \mathrm{mole}$ from [1966KYB/CAR] pertains to the average temperature and not to $298 \mathrm{~K}$. The authors give a value of $79.1 \pm 1.7$ for the $298 \mathrm{~K}$ value.]

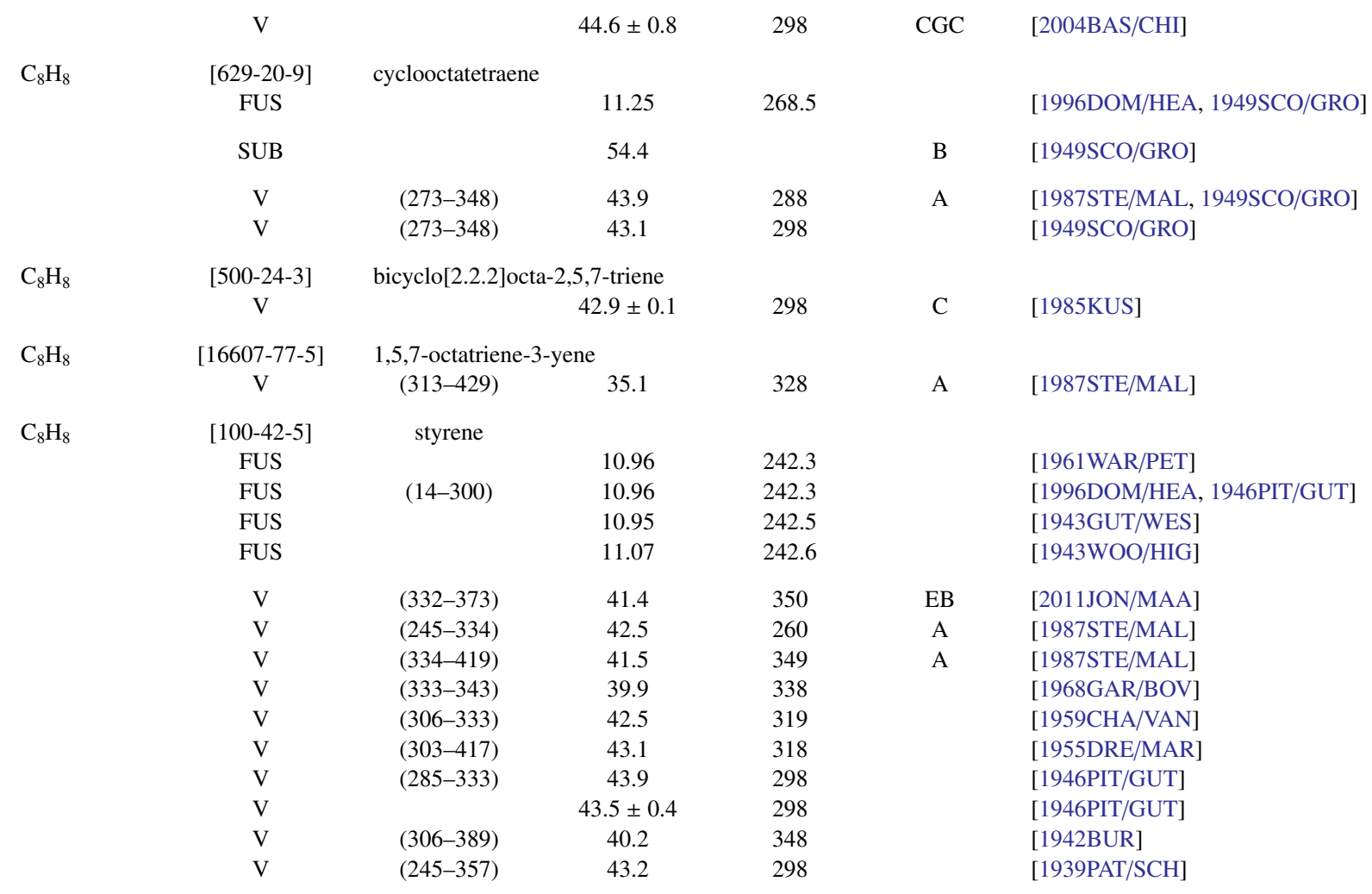

$\mathrm{C}_{8} \mathrm{H}_{8}$

[116316-76-8] 1-cyclopropyl-1,3-pentadiene

$\mathrm{V}$

$\mathrm{C}_{8} \mathrm{H}_{8} \mathrm{BrCl}_{2} \mathrm{O}_{3} \mathrm{PS} \quad$ [2104-96-3] FUS

$\mathrm{C}_{8} \mathrm{H}_{8} \mathrm{BrNO}$

[103-88-8]
FUS
FUS
SUB
V
V

$\mathrm{C}_{8} \mathrm{H}_{8} \mathrm{Br}_{2}$

[93-52-7]

$\mathrm{V}$

$\mathrm{C}_{8} \mathrm{H}_{8} \mathrm{Br}_{2}$

[91-13-4] FUS

$\mathrm{C}_{8} \mathrm{H}_{8} \mathrm{Br}_{2}$

[626-15-3] FUS

$\mathrm{C}_{8} \mathrm{H}_{8} \mathrm{ClNO}_{2} \quad$ [3942-54-9] FUS

$\mathrm{C}_{8} \mathrm{H}_{8} \mathrm{ClNO}_{4}$

[6940-53-0] FUS

$\mathrm{C}_{8} \mathrm{H}_{8} \mathrm{Cl}_{2}$

[1124-05-6]

$\mathrm{V}$

$\mathrm{C}_{8} \mathrm{H}_{8} \mathrm{Cl}_{2}$

[54484-61-6] $\mathrm{V}$
298

C

[2007PAS/KUZ]

$O$-(4-bromo-2,5-dichlorophenyl) $O, O$-dimethylphosphorothioate

$$
31.15 \quad 325.3 \quad \text { DSC } \quad \text { [1990DON/DRE] }
$$

4-bromoacetanilide

$26.0 \quad 440.3$

440.3

[2009BAR/ESP]

$25.8 \quad 441.2$

$110 \pm 4 \quad 298$

DSC [2004VEC/CAT]

$78 \pm 2$

480

$\mathrm{F}+\mathrm{V}$

[2009VEC/TOM]

$77 \pm 1$

460

TGA

[2009VEC/TOM]

[2009VEC/TOM]

(1,2-dibromoethyl)benzene

$$
\text { (359-527) } \quad 64.9
$$

374

A

[1987STE/MAL, 1947STU, 1970DYK/VAN]

$\alpha, \alpha^{\prime}$-dibromo-o-xylene

$$
26.78
$$

368.2

[1991ACR, 1983WEA]

$\alpha, \alpha^{\prime}$-dibromo- $m$-xylene

$$
23.69 \quad 350.2
$$

[1991ACR, 1983WEA]

$\mathrm{N}$-methyl-2-chlorophenylcarbamic acid ester

$$
\begin{array}{ll}
21.81 & 362.7
\end{array}
$$

DSC

[1990DON/DRE]

1-chloro-2,5-dimethoxy-4-nitrobenzene

$$
\begin{array}{ll}
28.02 & 413.7
\end{array}
$$

DSC

[2015ZHA/JIN]

2,5-dichloro-1,4-dimethylbenzene

$$
\text { (393-573) } \quad 52.7
$$

408

A

[1987STE/MAL, 1970DYK/VAN, 1999DYK/SVO]

2,5-dichloro-1,4-dimethylbenzene
(319-495)
48.9
334 
TABLE 11. Phase change enthalpies of $\mathrm{C}_{8}$ organic compounds-Continued

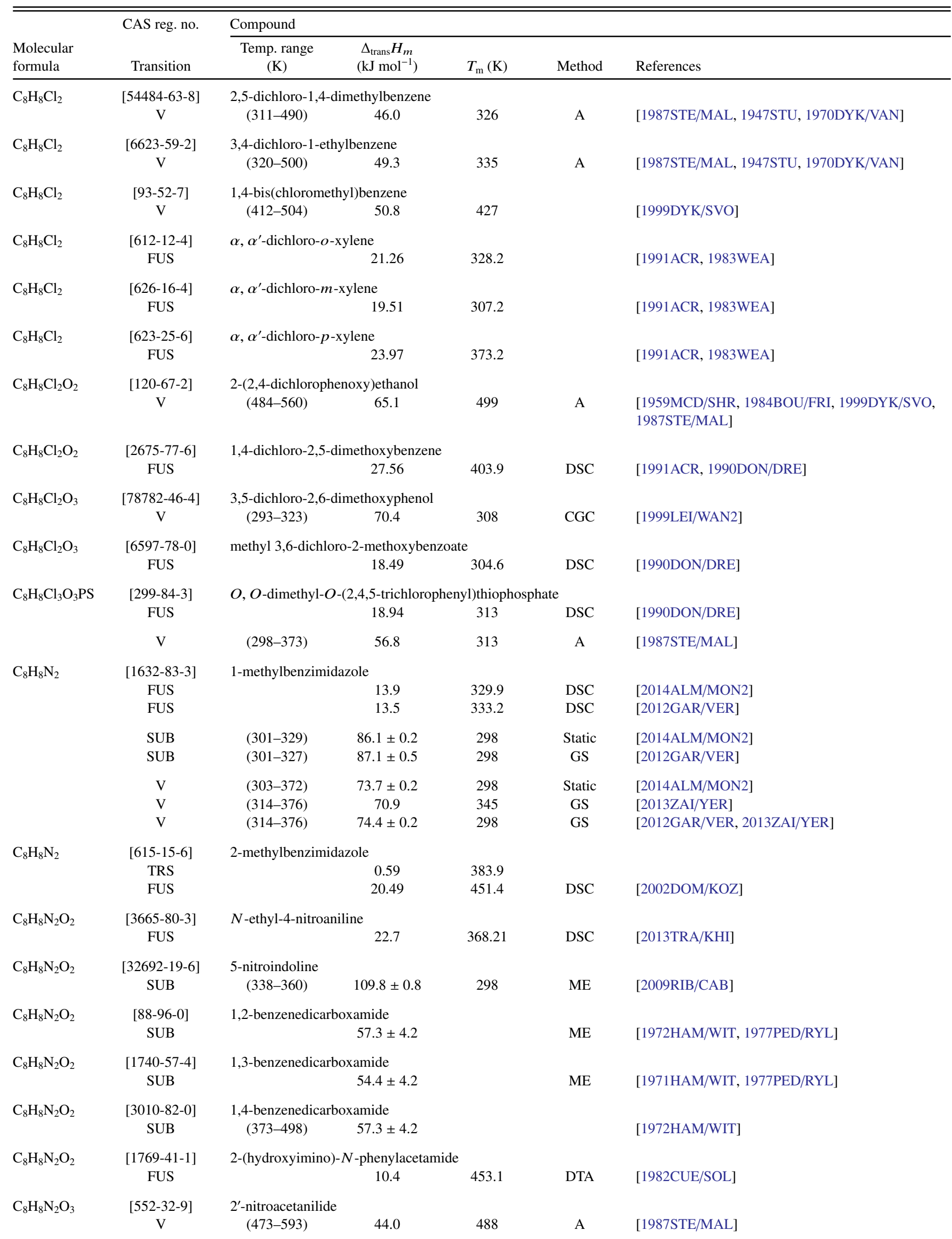


TABLE 11. Phase change enthalpies of $\mathrm{C}_{8}$ organic compounds-Continued

\begin{tabular}{|c|c|c|c|c|c|c|}
\hline \multirow[b]{2}{*}{$\begin{array}{l}\text { Molecular } \\
\text { formula }\end{array}$} & \multirow{2}{*}{$\begin{array}{l}\text { CAS reg. no. } \\
\text { Transition }\end{array}$} & \multicolumn{5}{|l|}{ Compound } \\
\hline & & $\begin{array}{c}\text { Temp. range } \\
(\mathrm{K})\end{array}$ & $\begin{array}{c}\Delta_{\text {trans }} H_{m} \\
\left(\mathrm{~kJ} \mathrm{~mol}^{-1}\right)\end{array}$ & $T_{\mathrm{m}}(\mathrm{K})$ & Method & References \\
\hline $\mathrm{C}_{8} \mathrm{H}_{8} \mathrm{~N}_{2} \mathrm{~S}$ & $\begin{array}{c}\text { [1477-42-5] } \\
\text { FUS }\end{array}$ & 2-amino-4-met & $\begin{array}{c}\text { nzothiazole } \\
15.91\end{array}$ & 410.7 & DSC & [2014CAM/MEN] \\
\hline $\mathrm{C}_{8} \mathrm{H}_{8} \mathrm{~N}_{2} \mathrm{~S}$ & $\begin{array}{c}\text { [2536-91-6] } \\
\text { FUS }\end{array}$ & 2-amino-6-met & $\begin{array}{l}\text { nzothiazole } \\
20.04\end{array}$ & 412.5 & DSC & [2014CAM/MEN] \\
\hline $\mathrm{C}_{8} \mathrm{H}_{8} \mathrm{~N}_{4} \mathrm{O}_{3}$ & $\begin{array}{c}{[1455-87-4]} \\
\text { SUB }\end{array}$ & 4-N, N-dimet & $\begin{array}{l}\text { hino-7-nitrobe } \\
134.2 \pm 3.4\end{array}$ & $\begin{array}{r}\text { razan } \\
298\end{array}$ & ME & [2014SAN/SIL] \\
\hline $\mathrm{C}_{8} \mathrm{H}_{8} \mathrm{O}$ & $\begin{array}{c}{[529-20-4]} \\
\mathrm{V}\end{array}$ & 2-methylbenza & $\begin{array}{l}\text { de } \\
52.5 \pm 0.2\end{array}$ & 298 & GS & [2013EME/VER] \\
\hline $\mathrm{C}_{8} \mathrm{H}_{8} \mathrm{O}$ & $\begin{array}{c}{[620-23-5]} \\
\mathrm{V}\end{array}$ & 3-methylbenza & $\begin{array}{l}\text { de } \\
53.7 \pm 0.3\end{array}$ & 298 & GS & [2013EME/VER] \\
\hline $\mathrm{C}_{8} \mathrm{H}_{8} \mathrm{O}$ & $\begin{array}{c}\text { [104-87-0] } \\
\mathrm{V}\end{array}$ & 4-methylbenza & $\begin{array}{l}\text { de } \\
53.5 \pm 0.4\end{array}$ & 298 & GS & [2013EME/VER] \\
\hline $\mathrm{C}_{8} \mathrm{H}_{8} \mathrm{O}$ & $\begin{array}{c}\text { [98-86-2] } \\
\text { TRS } \\
\text { FUS } \\
\text { V } \\
\text { V } \\
\text { V } \\
\text { V } \\
\text { V } \\
\text { V } \\
\text { V } \\
\text { V } \\
\text { V } \\
\text { V } \\
\text { V } \\
\text { V }\end{array}$ & $\begin{array}{l}(360-520) \\
(360-520) \\
(360-520) \\
(360-520) \\
(360-520) \\
(360-520) \\
(343-383) \\
(343-383) \\
(343-383) \\
(375-603) \\
(383-437) \\
(310-476)\end{array}$ & $\begin{array}{c}1.36 \\
16.65 \\
55.4 \pm 0.4 \\
52.6 \pm 0.4 \\
50.1 \pm 0.3 \\
47.5 \pm 0.3 \\
45.0 \pm 0.4 \\
42.2 \pm 0.4 \\
53.4 \\
52.7 \\
57.9 \\
49.7 \\
41.9 \\
51.2\end{array}$ & $\begin{array}{l}289.7 \\
292.7 \\
298 \\
340 \\
380 \\
420 \\
460 \\
500 \\
298 \\
298 \\
298 \\
390 \\
398 \\
325\end{array}$ & $\begin{array}{c}\text { EB } \\
\text { EB } \\
\text { EB } \\
\text { EB } \\
\text { EB } \\
\text { EB } \\
\text { CGC } \\
\text { CGC } \\
\text { CGC } \\
\text { A } \\
\text { GS, EB }\end{array}$ & $\begin{array}{l}\text { [2006SED/MAI] } \\
{[1911 \mathrm{LOU} / \mathrm{DUP}, 2006 \mathrm{SED} / \mathrm{MAI}]} \\
{[1996 \mathrm{STE} / \mathrm{CHI}]} \\
{[1996 \mathrm{STE} / \mathrm{CHI}]} \\
{[1996 \mathrm{STE} / \mathrm{CHI}]} \\
{[1996 \mathrm{STE} / \mathrm{CHI}]} \\
{[1996 \mathrm{STE} / \mathrm{CHI}]} \\
{[1996 \mathrm{STE} / \mathrm{CHI}]} \\
{[1995 \mathrm{CHI} / \mathrm{HOS}]} \\
{[1995 \mathrm{CHI} / \mathrm{HOS}]} \\
{[1995 \mathrm{CHI} / \mathrm{HOS}]} \\
{[1987 \mathrm{STE} / \mathrm{MAL}]} \\
{[1965 \mathrm{COL} / \mathrm{COU}]} \\
{[1947 \mathrm{STU}]}\end{array}$ \\
\hline $\mathrm{C}_{8} \mathrm{H}_{8} \mathrm{O}$ & $\begin{array}{c}\text { [496-14-0] } \\
\mathrm{V} \\
\mathrm{V} \\
\mathrm{V} \\
\mathrm{V} \\
\mathrm{V} \\
\mathrm{V}\end{array}$ & $\begin{array}{c}\text { 1,3-dihydroiso } \\
(285-510) \\
(285-510) \\
(285-510) \\
(285-510) \\
(285-510) \\
(285-510)\end{array}$ & $\begin{array}{c}\text { furan (phthal } \\
53.7 \pm 0.4 \\
52.2 \pm 0.4 \\
49.6 \pm 0.3 \\
47.0 \pm 0.3 \\
44.4 \pm 0.3 \\
41.6 \pm 0.5\end{array}$ & $\begin{array}{l}298 \\
320 \\
360 \\
400 \\
440 \\
480\end{array}$ & $\begin{array}{l}\text { EB } \\
\text { EB } \\
\text { EB } \\
\text { EB } \\
\text { EB } \\
\text { EB }\end{array}$ & $\begin{array}{l}\text { [1996STE/CHI3] } \\
\text { [1996STE/CHI3] } \\
{[1996 \mathrm{STE} / \mathrm{CHI} 3]} \\
{[1996 \mathrm{STE} / \mathrm{CHI} 3]} \\
{[1996 \mathrm{STE} / \mathrm{CHI} 3]} \\
{[1996 \mathrm{STE} / \mathrm{CHI} 3]}\end{array}$ \\
\hline $\mathrm{C}_{8} \mathrm{H}_{8} \mathrm{O}$ & $\begin{array}{c}\text { [496-16-2] } \\
\mathrm{V}\end{array}$ & $\begin{array}{l}\text { 2,3-dihydroben } \\
\text { (279-333) }\end{array}$ & $53.2 \pm 0.2$ & 298 & GS & [2011VER/EME] \\
\hline $\mathrm{C}_{8} \mathrm{H}_{8} \mathrm{O}$ & $\begin{array}{c}{[122-78-1]} \\
\mathrm{V} \\
\mathrm{V}\end{array}$ & $\begin{array}{l}\text { phenylacetalde } \\
\qquad \begin{array}{c}(293-343) \\
(283-333)\end{array}\end{array}$ & $\begin{array}{c}59.8 \pm 0.3 \\
54.5\end{array}$ & $\begin{array}{l}298 \\
298\end{array}$ & $\begin{array}{c}\text { GS } \\
\text { A }\end{array}$ & $\begin{array}{l}\text { [2007EME/DAB] } \\
{[1987 \mathrm{STE} / \mathrm{MAL}]}\end{array}$ \\
\hline $\mathrm{C}_{8} \mathrm{H}_{8} \mathrm{O}_{2}$ & $\begin{array}{c}\text { [493-09-4] } \\
\mathrm{V} \\
\mathrm{V}\end{array}$ & $\begin{array}{l}\text { 1,4-benzodiox } \\
(400-486)\end{array}$ & $\begin{array}{c}67.4 \pm 1.7 \\
50.4\end{array}$ & $\begin{array}{l}298 \\
415\end{array}$ & $\begin{array}{l}\mathrm{C} \\
\mathrm{A}\end{array}$ & $\begin{array}{l}\text { [2008MAT/SOU2] } \\
\text { [1987STE/MAL, 1958CAS/FLE2] }\end{array}$ \\
\hline $\mathrm{C}_{8} \mathrm{H}_{8} \mathrm{O}_{2}$ & $\begin{array}{c}{[104-57-4]} \\
\mathrm{V}\end{array}$ & $\begin{array}{l}\text { benzyl formate } \\
\quad(298-357)\end{array}$ & 51.6 & 313 & A & [1987STE/MAL] \\
\hline $\mathrm{C}_{8} \mathrm{H}_{8} \mathrm{O}_{2}$ & $\begin{array}{c}{[118-93-4]} \\
\text { FUS }\end{array}$ & 2'-hydroxyacet & $\begin{array}{l}\text { none } \\
\qquad 13.0\end{array}$ & 278.5 & DSC & [2008BER/MIN] \\
\hline & $\begin{array}{l}\mathrm{V} \\
\mathrm{V} \\
\mathrm{V}\end{array}$ & $(369-491)$ & $\begin{array}{c}58.3 \pm 0.3 \\
58.3 \\
50.2\end{array}$ & $\begin{array}{l}298 \\
384\end{array}$ & $\begin{array}{l}\mathrm{C} \\
\mathrm{A}\end{array}$ & $\begin{array}{l}{[2008 \mathrm{BER} / \mathrm{MIN}]} \\
{[1987 \mathrm{STE} / \mathrm{MAL}]} \\
{[1986 \mathrm{BAL} / \mathrm{GNA}]}\end{array}$ \\
\hline $\mathrm{C}_{8} \mathrm{H}_{8} \mathrm{O}_{2}$ & $\begin{array}{c}{[121-71-1]} \\
\text { FUS }\end{array}$ & 3'-hydroxyacet & none 23.4 & 366.7 & DSC & [2005CHE/TAN] \\
\hline $\mathrm{C}_{8} \mathrm{H}_{8} \mathrm{O}_{2}$ & $\begin{array}{l}\text { [99-93-4] } \\
\text { FUS } \\
\text { FUS }\end{array}$ & 4'-hydroxyacet & $\begin{array}{c}\text { none } \\
18.08 \\
17.0\end{array}$ & $\begin{array}{l}382.8 \\
381.3\end{array}$ & $\begin{array}{l}\text { DSC } \\
\text { DSC }\end{array}$ & $\begin{array}{l}\text { [2008BER/PIE] } \\
{[2005 \mathrm{CHE} / \mathrm{TAN}]}\end{array}$ \\
\hline
\end{tabular}


TABLE 11. Phase change enthalpies of $\mathrm{C}_{8}$ organic compounds-Continued

\begin{tabular}{|c|c|c|c|c|c|c|}
\hline \multirow[b]{2}{*}{$\begin{array}{l}\text { Molecular } \\
\text { formula }\end{array}$} & \multirow{2}{*}{$\begin{array}{l}\text { CAS reg. no. } \\
\text { Transition }\end{array}$} & \multicolumn{5}{|l|}{ Compound } \\
\hline & & $\begin{array}{c}\text { Temp. range } \\
(\mathrm{K})\end{array}$ & $\begin{array}{c}\Delta_{\text {trans }} H_{m} \\
\left(\mathrm{~kJ} \mathrm{~mol}^{-1}\right)\end{array}$ & $T_{\mathrm{m}}(\mathrm{K})$ & Method & References \\
\hline & $\begin{array}{c}\text { SUB } \\
\text { (monoclinic) }\end{array}$ & & $100.9 \pm 0.8$ & 348 & $\mathrm{C}$ & [2008BER/PIE] \\
\hline & $\begin{array}{c}\text { SUB } \\
\text { (monoclinic) }\end{array}$ & & $103.2 \pm 0.8$ & 298 & $\mathrm{C}$ & [2008BER/PIE] \\
\hline & SUB & & $104.3 \pm 0.4$ & 348 & $\mathrm{C}$ & [2008BER/PIE] \\
\hline & (orthorhombic) & & & & & \\
\hline & $\begin{array}{c}\text { SUB } \\
\text { (monoclinic) }\end{array}$ & & $101.8 \pm 2.7$ & 327 & $\mathrm{ME}$ & [2008BER/PIE] \\
\hline & $\begin{array}{c}\text { SUB } \\
\text { (monoclinic) }\end{array}$ & & $103.3 \pm 2.7$ & 298 & ME & [2008BER/PIE] \\
\hline & SUB & $(320-349)$ & 95.7 & 335 & A & [1987STE/MAL, 1960AIH] \\
\hline \multirow[t]{4}{*}{$\mathrm{C}_{8} \mathrm{H}_{8} \mathrm{O}_{2}$} & [123-11-5] & \multicolumn{5}{|c|}{ 4-methoxybenzaldehyde } \\
\hline & $\mathrm{V}$ & $(348-521)$ & 58.4 & 363 & $\mathrm{~A}, \mathrm{~EB}$ & [1985SCH/BRU] \\
\hline & $\mathrm{V}$ & $(283-323)$ & 60.4 & 298 & A & [1987STE/MAL, 1955SER/VOI] \\
\hline & $\mathrm{V}$ & $(346-521)$ & 57.1 & 361 & A & [1987STE/MAL, 1947STU] \\
\hline \multirow[t]{18}{*}{$\mathrm{C}_{8} \mathrm{H}_{8} \mathrm{O}_{2}$} & {$[93-58-3]$} & \multicolumn{5}{|c|}{ methyl benzoate } \\
\hline & FUS & $(5-320)$ & 14.8 & 260.8 & $\mathrm{AC}$ & [2002BLO/PAU] \\
\hline & FUS & & 14.83 & 260.8 & & [1998MAK/KAB] \\
\hline & FUS & & 13.9 & 261 & & [1978DOZ/FUJ] \\
\hline & $\mathrm{V}$ & $(358-517)$ & $51.1 \pm 0.2$ & 360 & EB & [2002STE/CHI2] \\
\hline & $\mathrm{V}$ & $(358-517)$ & $48.5 \pm 0.2$ & 400 & $\mathrm{~EB}$ & [2002STE/CHI2] \\
\hline & $\mathrm{V}$ & $(358-517)$ & $45.8 \pm 0.2$ & 440 & EB & [2002STE/CHI2] \\
\hline & V & $(358-517)$ & $43.0 \pm 0.4$ & 480 & EB & [2002STE/CHI2] \\
\hline & $\mathrm{V}$ & & $57.2 \pm 0.1$ & 303 & $\mathrm{C}$ & [1998MAK/KAB] \\
\hline & $\mathrm{V}$ & $(313-353)$ & 53.4 & 298 & $\mathrm{CGC}$ & [1995CHI/HOS] \\
\hline & $\mathrm{V}$ & (313-363) & 53.8 & 298 & CGC & [1995CHI/HOS] \\
\hline & $\mathrm{V}$ & $(433-473)$ & 54.7 & 298 & CGC & [1995CHI/HOS] \\
\hline & $\mathrm{V}$ & $(334-428)$ & 50.7 & 379 & BG & [1988KAT2] \\
\hline & V & $(334-428)$ & 48.3 & 410 & BG & [1988KAT2] \\
\hline & $\mathrm{V}$ & $(283-323)$ & 53.9 & 298 & A & [1987STE/MAL] \\
\hline & $\mathrm{V}$ & $(373-533)$ & 49.7 & 388 & A & [1987STE/MAL] \\
\hline & $\mathrm{V}$ & & $55.6 \pm 0.1$ & 298 & $\mathrm{C}$ & [1972COL/LAY, 1971KUS/WAD2] \\
\hline & V & $(341-433)$ & 52.8 & 363 & BG & [1971HAL/BAL] \\
\hline \multirow[t]{8}{*}{$\mathrm{C}_{8} \mathrm{H}_{8} \mathrm{O}_{2}$} & {$[118-90-1]$} & \multicolumn{5}{|c|}{ 2-methylbenzoic acid } \\
\hline & FUS & & 18.14 & 376.5 & DSC & [1985DOM/HOF, 1989DOM] \\
\hline & FUS & & 20.04 & & DSC & [1983HOL] \\
\hline & FUS & & 20.17 & 376.9 & & [1991ACR, 1926AND/LYN] \\
\hline & SUB & $(331-373)$ & $95.5 \pm 0.5$ & 352 & GS & [2015VER/ZAI] \\
\hline & SUB & $(331-373)$ & $96.9 \pm 0.6$ & 298 & GS & [2015VER/ZAI] \\
\hline & SUB & $(297-337)$ & $95.9 \pm 0.1$ & 298 & ME & [1986COL/JIM] \\
\hline & SUB & & $\mathrm{U} 137.7 \pm 0.5$ & & DSC & {$[1983 \mathrm{HOL}]$} \\
\hline \multirow[t]{4}{*}{$\mathrm{C}_{8} \mathrm{H}_{8} \mathrm{O}_{2}$} & [99-04-7] & \multicolumn{5}{|c|}{ 3-methylbenzoic acid } \\
\hline & FUS & & 15.73 & 381.9 & & [1991ACR, 1926AND/LYN] \\
\hline & SUB & $(303-323)$ & $97.0 \pm 0.3$ & 298 & $\mathrm{ME}$ & [1986COL/JIM] \\
\hline & $\mathrm{V}$ & $(473-533)$ & 62.8 & 503 & A & [1987STE/MAL, 1970MUL/GAL] \\
\hline \multirow[t]{10}{*}{$\mathrm{C}_{8} \mathrm{H}_{8} \mathrm{O}_{2}$} & {$[99-94-5]$} & \multicolumn{5}{|c|}{ 4-methylbenzoic acid } \\
\hline & FUS & & 22.3 & 453.3 & DSC & [2015VER/ZAI] \\
\hline & FUS & & 15.0 & 449.2 & DSC & [2000KAN/SAM] \\
\hline & FUS & & 22.72 & 452.8 & & [1991ACR, 1926AND/LYN] \\
\hline & SUB & $(347-408)$ & $94.1 \pm 1.1$ & 377 & TGA & [2015VER/ZAI] \\
\hline & SUB & $(347-408)$ & $96.2 \pm 1.3$ & 298 & TGA & [2015VER/ZAI] \\
\hline & SUB & $(340-399)$ & $95.8 \pm 0.2$ & 370 & GS & [2015VER/ZAI] \\
\hline & SUB & $(340-399)$ & $97.6 \pm 0.4$ & 298 & GS & [2015VER/ZAI] \\
\hline & SUB & $(320-337)$ & $98.6 \pm 0.6$ & 298 & $\mathrm{ME}$ & [2004MON/ALM] \\
\hline & SUB & $(318-337)$ & $98.8 \pm 0.3$ & 298 & $\mathrm{ME}$ & [1986COL/JIM] \\
\hline $\mathrm{C}_{8} \mathrm{H}_{8} \mathrm{O}_{2}$ & $\begin{array}{c}{[122-79-2]} \\
\mathrm{V}\end{array}$ & $\begin{array}{c}\text { phenyl acetate } \\
\text { (313-363) }\end{array}$ & 53.3 & 298 & CGC & [1995CHI/HOS] \\
\hline
\end{tabular}


TABLE 11. Phase change enthalpies of $\mathrm{C}_{8}$ organic compounds-Continued

\begin{tabular}{|c|c|c|c|c|c|c|}
\hline \multirow[b]{2}{*}{$\begin{array}{l}\text { Molecular } \\
\text { formula }\end{array}$} & \multirow{2}{*}{$\begin{array}{c}\text { CAS reg. no. } \\
\text { Transition }\end{array}$} & \multicolumn{5}{|l|}{ Compound } \\
\hline & & $\begin{array}{c}\text { Temp. range } \\
\text { (K) }\end{array}$ & $\begin{array}{c}\Delta_{\text {trans }} H_{m} \\
\left(\mathrm{~kJ} \mathrm{~mol}^{-1}\right)\end{array}$ & $T_{\mathrm{m}}(\mathrm{K})$ & Method & References \\
\hline \multirow{8}{*}{$\mathrm{C}_{8} \mathrm{H}_{8} \mathrm{O}_{2}$} & $\mathrm{~V}$ & $(433-473)$ & 53.6 & 298 & CGC & [1995CHI/HOS] \\
\hline & $\mathrm{V}$ & $(313-353)$ & 53.1 & 298 & CGC & [1995CHI/HOS] \\
\hline & $\mathrm{V}$ & $(311-469)$ & 51.7 & 326 & A & [1987STE/MAL, 1947STU] \\
\hline & [103-82-2] & phenylacetic acid & & & & \\
\hline & FUS & & 32.0 & & DSC & [2003SHA/KAN] \\
\hline & FUS & & 15.2 & 349.2 & DSC & [2002GRA/RAS] \\
\hline & FUS & & 16.5 & 350.8 & DSC & [2001MON/HIL] \\
\hline & FUS & & 14.49 & 349.9 & DSC & [1991ACR, 1983WEA] \\
\hline
\end{tabular}

[Note: There is a large discrepancy between the value of $32.0 \mathrm{~kJ} / \mathrm{mole}$ and the other reported literature values. Reference [2003SHA/KAN] quotes a literature value of $32.0 \mathrm{~kJ} / \mathrm{mole}$; however, the authors do not provide the source of the cited literature value.]

$\begin{array}{cccccc}\text { SUB } & (307-339) & 93.5 \pm 0.3 & 298 & \text { GS } & \text { [2004ROU/TEM] } \\ \text { SUB } & (305-321) & 98.6 \pm 0.4 & 313 & \text { ME } & \text { [2001MON/HIL] } \\ \text { SUB } & (305-321) & 99.0 \pm 0.6 & 298 & \text { ME } & \text { [2001MON/HIL] } \\ \text { V } & (353-392) & 79.1 \pm 0.3 & 298 & \text { GS } & \text { [2004ROU/TEM] } \\ \text { V } & (370-539) & 65.0 & 385 & \text { A } & \text { [1987STE/MAL] }\end{array}$

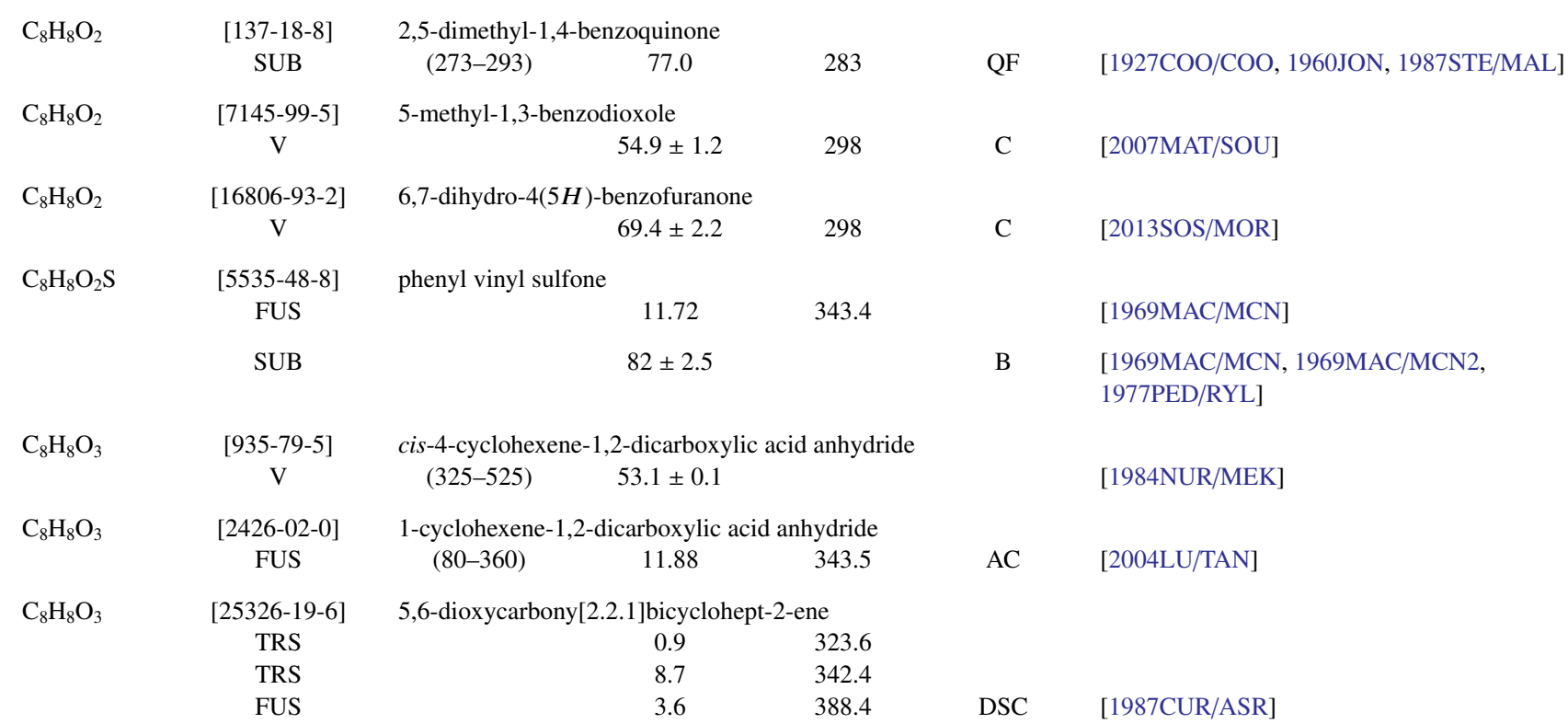

$\mathrm{C}_{8} \mathrm{H}_{8} \mathrm{O}_{3}$

[99-76-3] 4-hydroxybenzoic acid, methyl ester (methyl paraben)

\begin{tabular}{|c|c|c|c|c|c|}
\hline FUS & & 25.3 & 400.5 & DSC & [2014SUG/KAI] \\
\hline FUS & & 26.3 & 398.6 & DSC & [2011UMN/CHI] \\
\hline FUS & & 25.3 & 399.2 & DSC & [1999GIO/BET] \\
\hline FUS & & 24.31 & 398.5 & & [1990MAN/AHU] \\
\hline SUB & $(323-345)$ & $107.1 \pm 0.1$ & 334 & $\mathrm{ME}$ & [2014ALM/CUN] \\
\hline SUB & $(323-345)$ & $108.4 \pm 0.6$ & 298 & $\mathrm{ME}$ & [2014ALM/CUN] \\
\hline SUB & $(339-396)$ & $108.8 \pm 0.9$ & 367 & Static & [2014ALM/CUN] \\
\hline SUB & (339-396) & $106.4 \pm 0.1$ & 298 & Static & [2014ALM/CUN] \\
\hline SUB & (303-327) & $98.8 \pm 0.8$ & 298 & GS & [2005PER/ROD] \\
\hline $\mathrm{V}$ & $(383-423)$ & $87.1 \pm 1.6$ & 397 & Static & [2014ALM/CUN] \\
\hline V & $(383-423)$ & $79.0 \pm 0.1$ & 298 & Static & [2014ALM/CUN] \\
\hline V & & $79.5 \pm 0.5$ & 298 & CGC & [2011UMN/CHI] \\
\hline V & & 83.1 & 298 & CGC & [2005TEM/ROU, 2011UMN/CHI] \\
\hline $\mathrm{V}$ & $(446-517)$ & 81.5 & 461 & A & [1987STE/MAL] \\
\hline
\end{tabular}

$\mathrm{C}_{8} \mathrm{H}_{8} \mathrm{O}_{3}$

[119-36-8]

methyl salicylate

$\begin{array}{llll}\text { V } & (333-433) & \text { U56.2 } & 298 \\ \text { V } & & 52.3 & \\ \text { V } & (327-497) & 59.9 & 342 \\ \text { V } & (329-496) & 58.7 & 344 \\ \text { V } & (288-333) & 56.9 & 303\end{array}$

$\begin{array}{cl}\text { GC } & {[2005 \mathrm{HOS} / \mathrm{GRY}]} \\ \text { TG,DTA } & {[2001 \mathrm{CHE} / \mathrm{HUA}]} \\ \text { A } & {[1987 \mathrm{STE} / \mathrm{MAL}]} \\ \text { A } & {[1987 \mathrm{STE} / \mathrm{MAL}]} \\ & {[1987 \mathrm{STE} / \mathrm{MAL}]}\end{array}$


TABLE 11. Phase change enthalpies of $\mathrm{C}_{8}$ organic compounds-Continued

\begin{tabular}{|c|c|c|c|c|c|c|}
\hline \multirow[b]{2}{*}{$\begin{array}{l}\text { Molecular } \\
\text { formula }\end{array}$} & \multirow{2}{*}{$\begin{array}{c}\text { CAS reg. no. } \\
\text { Transition }\end{array}$} & \multicolumn{5}{|l|}{ Compound } \\
\hline & & $\begin{array}{c}\text { Temp. range } \\
(\mathrm{K})\end{array}$ & $\begin{array}{c}\Delta_{\text {trans }} H_{m} \\
\left(\mathrm{~kJ} \mathrm{~mol}^{-1}\right)\end{array}$ & $T_{\mathrm{m}}(\mathrm{K})$ & Method & References \\
\hline \multirow[t]{2}{*}{$\mathrm{C}_{8} \mathrm{H}_{8} \mathrm{O}_{3}$} & [148-53-8] & \multicolumn{5}{|c|}{ 2-hydroxy-3-methoxybenzaldehyde } \\
\hline & SUB & $(282-303)$ & 54.1 & 292.5 & A & [1987STE/MAL] \\
\hline \multirow[t]{11}{*}{$\mathrm{C}_{8} \mathrm{H}_{8} \mathrm{O}_{3}$} & {$[121-33-5]$} & \multicolumn{5}{|c|}{ 4-hydroxy-3-methoxybenzaldehyde (vanillin) } \\
\hline & FUS & & 24.8 & 354.8 & DSC & [2015SIN/SIN2] \\
\hline & FUS & & 19.52 & 357.2 & DTA & {$[2013 \mathrm{KAN}]$} \\
\hline & FUS (I) & & 22.4 & 355.2 & & \\
\hline & FUS (II) & & 20.7 & 353.2 & DSC & [2010BRA/GRE] \\
\hline & FUS & & 22.35 & 354.6 & DSC & [2008SIN/DAS, 2012GUP/AGR] \\
\hline & FUS & & 22.4 & 355.4 & DSC & [2008TEM/ROU] \\
\hline & FUS & & 16.13 & & DSC & [1992SHA/SHA] \\
\hline & SUB & $(293-353)$ & 88.7 & 323 & & [1953SER/VOI, 1960JON] \\
\hline & $\mathrm{V}$ & $(353-463)$ & 66.9 & 298 & GC & [2005HOS/GRY] \\
\hline & $\mathrm{V}$ & $(380-558)$ & 66.9 & 395 & A & [1987STE/MAL, 1947STU] \\
\hline \multirow[t]{16}{*}{$\mathrm{C}_{8} \mathrm{H}_{8} \mathrm{O}_{3}$} & {$[579-75-9]$} & \multicolumn{5}{|c|}{ 2-methoxybenzoic acid } \\
\hline & FUS & & 23.9 & 374.7 & DSC & [2015VER/ZAI] \\
\hline & FUS & & 24.0 & 374.6 & DSC & [2008PER/VOL] \\
\hline & SUB & $(342-371)$ & $106.3 \pm 0.9$ & 298 & GS & [2015JAK/SPO] \\
\hline & SUB & $(344-370)$ & $104.7 \pm 0.4$ & 357 & GS & [2015VER/ZAI] \\
\hline & SUB & $(344-370)$ & $106.7 \pm 0.6$ & 298 & GS & [2015VER/ZAI] \\
\hline & SUB & $(318-368)$ & $104.7 \pm 0.1$ & 343 & Static & [2015VER/ZAI] \\
\hline & SUB & $(318-368)$ & $106.2 \pm 0.4$ & 298 & Static & [2015VER/ZAI] \\
\hline & SUB & $(309-363)$ & $110.7 \pm 0.8$ & 298 & GS & [2008PER/VOL] \\
\hline & SUB & $(318-353)$ & 101.2 & 333 & A & [1987STE/MAL] \\
\hline & SUB & & $104.7 \pm 0.3$ & 298 & ME & [1978COL/JIM] \\
\hline & SUB & $(353-368)$ & $90.8 \pm 0.4$ & 360 & GS & [1973MAL/GIG, 1987STE/MAL] \\
\hline & SUB & $(353-368)$ & 90.9 & 360 & GS & [1954DAV/JON, 1960JON] \\
\hline & $\mathrm{V}$ & $(378-413)$ & $75.4 \pm 0.1$ & 394 & Static & [2015VER/ZAI] \\
\hline & $\mathrm{V}$ & $(378-413)$ & $86.0 \pm 0.8$ & 298 & Static & [2015VER/ZAI] \\
\hline & $\mathrm{V}$ & & 91.8 & 298 & S-F & [2008PER/VOL] \\
\hline \multirow[t]{8}{*}{$\mathrm{C}_{8} \mathrm{H}_{8} \mathrm{O}_{3}$} & [586-38-9] & \multicolumn{5}{|c|}{ 3-methoxybenzoic acid } \\
\hline & FUS & & 21.9 & 382.1 & DSC & [2015VER/ZAI] \\
\hline & FUS (I) & & 23.2 & 378.6 & & \\
\hline & FUS (II) & & 19.8 & 367.2 & DSC & [2015PER/CAS] \\
\hline & FUS & & 24.9 & 378.7 & DSC & [2008PER/VOL] \\
\hline & SUB & $(310-349)$ & $114.7 \pm 0.8$ & 298 & GS & [2008PER/VOL] \\
\hline & SUB & & $107.5 \pm 0.4$ & 298 & ME & [1987STE/MAL, 1978COL/JIM] \\
\hline & $\mathrm{V}$ & & 95 & 298 & S-F & [2008PER/VOL] \\
\hline \multirow[t]{14}{*}{$\mathrm{C}_{8} \mathrm{H}_{8} \mathrm{O}_{3}$} & [100-09-4] & \multicolumn{5}{|c|}{ 4-methoxybenzoic acid } \\
\hline & FUS & & 28.97 & 455.3 & DSC & {$[2010 \mathrm{FON} / \mathrm{SAN}]$} \\
\hline & FUS & & 29.9 & 455.6 & DSC & [2008PER/VOL] \\
\hline & FUS & & 24.2 & 449.2 & DSC & [2000KAN/SAM] \\
\hline & FUS & & 28.33 & 456.7 & & [1992SAB/ELW3, 2010FON/SAN] \\
\hline & FUS & & 29.72 & 456.5 & & [1986CAL/SUL, 2010FON/SAN] \\
\hline & FUS & & 28.4 & 457.8 & & [1991ACR, 1979ARM/JAM] \\
\hline & SUB & $(368-406)$ & $109.8 \pm 0.6$ & 387 & GS & [2015VER/ZAI] \\
\hline & SUB & $(368-406)$ & $112.8 \pm 0.8$ & 298 & GS & [2015VER/ZAI] \\
\hline & SUB & $(340-362)$ & $110.6 \pm 0.3$ & 351 & ME & [2010FON/SAN] \\
\hline & SUB & $(340-362)$ & $112.6 \pm 0.6$ & 298 & $\mathrm{ME}$ & [2010FON/SAN] \\
\hline & SUB & $(316-369)$ & $111.6 \pm 0.6$ & 298 & GS & [2008PER/VOL] \\
\hline & SUB & & $109.8 \pm 0.6$ & 298 & $\mathrm{ME}$ & [1987STE/MAL, 1978COL/JIM] \\
\hline & $\mathrm{V}$ & & 92 & 298 & S-F & [2008PER/VOL] \\
\hline \multirow[t]{5}{*}{$\mathrm{C}_{8} \mathrm{H}_{8} \mathrm{O}_{3}$} & [90-64-2] & \multicolumn{5}{|l|}{$(d l)$-mandelic acid } \\
\hline & FUS & & 26.46 & 392.6 & DSC & [2011RIE/BAR] \\
\hline & FUS & & 23.3 & 389.2 & DSC & [2004STU/WIT] \\
\hline & FUS & & 26.1 & 392.1 & MDSC & [2004PRO/RAS] \\
\hline & FUS & & 25.52 & 392 & & [1991CHI/BRA] \\
\hline
\end{tabular}


TABLE 11. Phase change enthalpies of $\mathrm{C}_{8}$ organic compounds-Continued

\begin{tabular}{|c|c|c|c|c|c|c|}
\hline \multirow[b]{2}{*}{$\begin{array}{l}\text { Molecular } \\
\text { formula }\end{array}$} & \multirow{2}{*}{$\begin{array}{l}\text { CAS reg. no. } \\
\text { Transition }\end{array}$} & \multicolumn{5}{|l|}{ Compound } \\
\hline & & $\begin{array}{c}\text { Temp. range } \\
(\mathrm{K})\end{array}$ & $\begin{array}{c}\Delta_{\text {trans }} H_{m} \\
\left(\mathrm{~kJ} \mathrm{~mol}^{-1}\right)\end{array}$ & $T_{\mathrm{m}}(\mathrm{K})$ & Method & References \\
\hline \multirow[t]{4}{*}{$\mathrm{C}_{8} \mathrm{H}_{8} \mathrm{O}_{3}$} & [611-71-2] & \multicolumn{5}{|c|}{$(d)$-mandelic acid $((R)$-mandelic acid $)$} \\
\hline & FUS & & 27.41 & 405.6 & DSC & [2011RIE/BAR] \\
\hline & FUS & & 26.2 & 404.1 & MDSC & [2004PRO/RAS] \\
\hline & FUS & & 26.36 & 406 & & [1991CHI/BRA] \\
\hline \multirow[t]{2}{*}{$\mathrm{C}_{8} \mathrm{H}_{8} \mathrm{O}_{3}$} & {$[17199-29-0]$} & \multicolumn{5}{|c|}{$(S)$-mandelic acid } \\
\hline & FUS & & 26.88 & 405.3 & DSC & [2011RIE/BAR] \\
\hline \multirow[t]{3}{*}{$\mathrm{C}_{8} \mathrm{H}_{8} \mathrm{O}_{3}$} & {$[156-38-7]$} & \multicolumn{5}{|c|}{ 4-hydroxyphenylacetic acid } \\
\hline & FUS & & 28.0 & 422.9 & DSC & [2002GRA/RAS] \\
\hline & FUS & & 28.4 & 423.6 & & [1991ACR, 1979ARM/JAM] \\
\hline \multirow[t]{4}{*}{$\mathrm{C}_{8} \mathrm{H}_{8} \mathrm{O}_{3}$} & {$[495-76-1]$} & \multicolumn{5}{|c|}{ 1,3-benzodioxole-5-methanol (piperonyl alcohol) } \\
\hline & FUS & & 18.05 & 327.1 & $\mathrm{DSC}$ & [2004MAT/MON] \\
\hline & SUB & $(305-319)$ & $103.0 \pm 0.6$ & 312 & ME & [2004MAT/MON] \\
\hline & SUB & $(305-319)$ & $103.7 \pm 0.7$ & 298 & ME & [2004MAT/MON] \\
\hline \multirow[t]{2}{*}{$\mathrm{C}_{8} \mathrm{H}_{8} \mathrm{O}_{4}$} & {$[520-45-6]$} & \multicolumn{5}{|c|}{ 2-acetyl-5-hydroxy-3-oxo-4-hexenoic acid- $d$-lactone (dehydroacetic acid) } \\
\hline & V & $(364-542)$ & 62.1 & 379 & A & [1987STE/MAL, 1947STU] \\
\hline \multirow[t]{4}{*}{$\mathrm{C}_{8} \mathrm{H}_{8} \mathrm{O}_{4}$} & {$[121-34-6]$} & \multicolumn{5}{|c|}{ 3-methoxy-4-hydroxybenzoic acid (vanillic acid) } \\
\hline & FUS & & 32.8 & 484.9 & DSC & [2016ZHA/GUO] \\
\hline & FUS & & 29.1 & 480.7 & DSC & [2012MAN/VIL] \\
\hline & FUS & & 25.6 & 484.7 & DSC & {$[2012 \mathrm{BOO} / \mathrm{BAN}]$} \\
\hline \multirow[t]{2}{*}{$\mathrm{C}_{8} \mathrm{H}_{8} \mathrm{O}_{4}$} & {$[28026-96-2]$} & \multicolumn{5}{|c|}{ 3,5-dihydroxy-4-methylbenzoic acid } \\
\hline & FUS & & 42.5 & 544.1 & DSC & {$[2012 \mathrm{BOO} / \mathrm{BAN}]$} \\
\hline \multirow[t]{8}{*}{$\mathrm{C}_{8} \mathrm{H}_{8} \mathrm{~S}$} & {$[4565-32-6]$} & \multicolumn{5}{|c|}{ 2,3-dihydrobenzo $[b]$ thiophene } \\
\hline & FUS & & 14.84 & 269.8 & & [2003STE/CHI] \\
\hline & $\mathrm{V}$ & $(345-557)$ & $59.1 \pm 0.2$ & 298 & IPM, EB & [2003STE/CHI] \\
\hline & $\mathrm{V}$ & $(345-557)$ & $56.3 \pm 0.2$ & 340 & IPM, EB & [2003STE/CHI] \\
\hline & $\mathrm{V}$ & $(345-557)$ & $53.8 \pm 0.2$ & 380 & IPM, EB & [2003STE/CHI] \\
\hline & $\mathrm{V}$ & $(345-557)$ & $51.4 \pm 0.2$ & 420 & IPM, EB & [2003STE/CHI] \\
\hline & $\mathrm{V}$ & $(345-557)$ & $49.0 \pm 0.2$ & 460 & IPM, EB & [2003STE/CHI] \\
\hline & $\mathrm{V}$ & $(345-557)$ & $46.4 \pm 0.3$ & 500 & IPM, EB & [2003STE/CHI] \\
\hline $\mathrm{C}_{8} \mathrm{H}_{9} \mathrm{Br}$ & {$[553-94-6]$} & 1-bromo-2,5-d & ylbenzene & & & \\
\hline & $\mathrm{V}$ & $(310-480)$ & 50.9 & 325 & & [1999DYK/SVO, 1947STU] \\
\hline & $\mathrm{V}$ & $(310-480)$ & 53.6 & 325 & A & [1987STE/MAL, 1970DYK/VAN] \\
\hline $\mathrm{C}_{8} \mathrm{H}_{9} \mathrm{Br}$ & {$[576-22-7]$} & 1-bromo-2,6-d & ylbenzene & & & \\
\hline & $\mathrm{V}$ & $(274-305)$ & $53.4 \pm 0.4$ & 290 & GS & [2015VER/SAZ] \\
\hline & $\mathrm{V}$ & $(274-305)$ & $52.7 \pm 0.6$ & 298 & GS & [2015VER/SAZ] \\
\hline $\mathrm{C}_{8} \mathrm{H}_{9} \mathrm{Br}$ & {$[585-71-7]$} & (1-bromoethyl) & ene & & & \\
\hline & $\mathrm{V}$ & $(298-333)$ & $56.4 \pm 0.3$ & 298 & GS & [2002KRA/VAS] \\
\hline & $\mathrm{V}$ & 392 & 52.4 & 298 & $\mathrm{CGC}$ & [2002KRA/VAS] \\
\hline $\mathrm{C}_{8} \mathrm{H}_{9} \mathrm{Br}$ & {$[103-63-9]$} & (2-bromoethyl) & & & & \\
\hline & $\mathrm{V}$ & $(348-401)$ & 51.5 & 363 & A & [1987STE/MAL, 1999DYK/SVO] \\
\hline $\mathrm{C}_{8} \mathrm{H}_{9} \mathrm{Br}$ & {$[1973-22-4]$} & 1-bromo-2-eth & zene & & & \\
\hline & $\mathrm{V}$ & $(368-523)$ & 48.1 & 383 & A & [1987STE/MAL, 1970DYK/VAN, 1999DYK/SVO] \\
\hline $\mathrm{C}_{8} \mathrm{H}_{9} \mathrm{Br}$ & {$[1585-07-5]$} & 1-bromo-4-eth & zene & & & \\
\hline & $\mathrm{V}$ & $(347-479)$ & 46.2 & 362 & & [1999DYK/SVO] \\
\hline & $\mathrm{V}$ & $(378-533)$ & 49.4 & 393 & A & [1987STE/MAL, 1970DYK/VAN] \\
\hline & $\mathrm{V}$ & $(303-479)$ & 52.0 & 318 & & [1947STU] \\
\hline $\mathrm{C}_{8} \mathrm{H}_{9} \mathrm{Cl}$ & {$[672-65-1]$} & $(d l)$-(1-chloroe & enzene & & & \\
\hline & $\mathrm{V}$ & $(281-319)$ & $52.8 \pm 0.2$ & 298 & GS & [2002KRA/VAS] \\
\hline & $\mathrm{V}$ & 392 & 52.4 & 298 & CGC & [2002KRA/VAS] \\
\hline & $\mathrm{V}$ & $(336-372)$ & 51.4 & 351 & & [1999DYK/SVO] \\
\hline & $\mathrm{V}$ & $(342-378)$ & 47.0 & 357 & A & [1987STE/MAL] \\
\hline $\mathrm{C}_{8} \mathrm{H}_{9} \mathrm{Cl}$ & $\begin{array}{c}{[622-24-2]} \\
\mathrm{V}\end{array}$ & $\begin{array}{c}\text { (2-chloroethyl) } \\
(356-480)\end{array}$ & 53.1 & 368 & & [1999DYK/SVO] \\
\hline
\end{tabular}


TABLE 11. Phase change enthalpies of $\mathrm{C}_{8}$ organic compounds-Continued

\begin{tabular}{|c|c|c|c|c|c|c|}
\hline \multirow[b]{2}{*}{$\begin{array}{l}\text { Molecular } \\
\text { formula }\end{array}$} & \multirow{2}{*}{$\begin{array}{l}\text { CAS reg. no. } \\
\text { Transition }\end{array}$} & \multicolumn{5}{|l|}{ Compound } \\
\hline & & $\begin{array}{l}\text { Temp. range } \\
(\mathrm{K})\end{array}$ & $\begin{array}{c}\Delta_{\text {trans }} H_{m} \\
\left(\mathrm{~kJ} \mathrm{~mol}^{-1}\right)\end{array}$ & $T_{\mathrm{m}}(\mathrm{K})$ & Method & References \\
\hline & V & $(356-380)$ & 51.7 & 368 & A & [1987STE/MAL] \\
\hline $\mathrm{C}_{8} \mathrm{H}_{9} \mathrm{Cl}$ & $\begin{array}{c}{[89-96-3]} \\
\mathrm{V} \\
\mathrm{V}\end{array}$ & $\begin{array}{l}\text { 1-chloro-2-ethy } \\
\quad(353-503) \\
(290-450)\end{array}$ & $\begin{array}{l}\text { zene } \\
46.1 \\
47.2\end{array}$ & $\begin{array}{l}368 \\
305\end{array}$ & A & $\begin{array}{l}\text { [1987STE/MAL, 1970DYK/VAN] } \\
\text { [1947STU] }\end{array}$ \\
\hline $\mathrm{C}_{8} \mathrm{H}_{9} \mathrm{Cl}$ & $\begin{array}{c}{[620-16-6]} \\
\mathrm{V} \\
\mathrm{V} \\
\mathrm{V}\end{array}$ & $\begin{array}{c}\text { 1-chloro-3-eth } \\
(348-457) \\
(358-508) \\
(291-454)\end{array}$ & $\begin{array}{l}\text { zene } \\
\begin{array}{r}46.4 \\
46.8 \\
46.4\end{array}\end{array}$ & $\begin{array}{l}363 \\
373 \\
307\end{array}$ & A & $\begin{array}{l}\text { [1999DYK/SVO] } \\
\text { [1987STE/MAL, 1970DYK/VAN] } \\
\text { [1947STU] }\end{array}$ \\
\hline $\mathrm{C}_{8} \mathrm{H}_{9} \mathrm{Cl}$ & $\begin{array}{c}{[622-98-0]} \\
\mathrm{V} \\
\mathrm{V} \\
\mathrm{V}\end{array}$ & $\begin{array}{c}\text { 1-chloro-4-eth } \\
(350-458) \\
(358-508) \\
(381-457)\end{array}$ & $\begin{array}{l}\text { zene } \\
\begin{array}{r}45.8 \\
46.8 \\
45.5\end{array}\end{array}$ & $\begin{array}{l}365 \\
373 \\
396\end{array}$ & A & $\begin{array}{l}\text { [1999DYK/SVO] } \\
\text { [1987STE/MAL, 1970DYK/VAN] } \\
\text { [1947STU] }\end{array}$ \\
\hline $\mathrm{C}_{8} \mathrm{H}_{9} \mathrm{Cl}$ & $\begin{array}{c}{[104-82-5]} \\
\mathrm{V}\end{array}$ & $\begin{array}{l}\text { 1-(chloromethy) } \\
\quad(376-457)\end{array}$ & $\begin{array}{c}\text { methylbenze } \\
44.9\end{array}$ & 391 & A & [1987STE/MAL, 1999DYK/SVO] \\
\hline $\mathrm{C}_{8} \mathrm{H}_{9} \mathrm{Cl}$ & $\begin{array}{c}{[6781-98-2]} \\
\mathrm{V}\end{array}$ & $\begin{array}{l}\text { 1-chloro-2,6-di } \\
\quad(275-305)\end{array}$ & $\begin{array}{l}\text { ylbenzene } \\
49.8 \pm 0.3\end{array}$ & 298 & GS & [2014VER/EME] \\
\hline $\mathrm{C}_{8} \mathrm{H}_{9} \mathrm{ClNO}_{5} \mathrm{PS}$ & $\begin{array}{c}{[500-28-7]} \\
\mathrm{V}\end{array}$ & $\begin{array}{l}O, O \text {-(dimethy } \\
\quad(283-409)\end{array}$ & $\begin{array}{c}\text { (3-chloro-4- } \\
92\end{array}$ & $\begin{array}{l}\text { henyl)thi } \\
346\end{array}$ & A & [1987STE/MAL, 1999DYK/SVO] \\
\hline $\mathrm{C}_{8} \mathrm{H}_{9} \mathrm{ClNO}_{5} \mathrm{PS}$ & $\begin{array}{l}{[2463-84-5]} \\
\text { FUS }\end{array}$ & $O$-(2-chloro-4- & $\begin{array}{c}\text { phenyl) } O, O \\
29.08\end{array}$ & $\begin{array}{l}\text { thyl pho } \\
323.9\end{array}$ & $\begin{array}{l}\text { thioate } \\
\text { DSC }\end{array}$ & [1990DON/DRE] \\
\hline $\mathrm{C}_{8} \mathrm{H}_{9} \mathrm{ClN}_{2} \mathrm{O}$ & $\begin{array}{l}{[52943-21-2]} \\
\text { FUS }\end{array}$ & $\begin{array}{l}\text { 2-chloro- } N, N \\
\quad(82-380)\end{array}$ & $\begin{array}{l}\text { ethylnicotina } \\
21.39\end{array}$ & 342.2 & $\mathrm{AC}$ & [2005SUN/LIU3] \\
\hline $\mathrm{C}_{8} \mathrm{H}_{9} \mathrm{ClO}$ & $\begin{array}{c}{[614-72-2]} \\
\mathrm{V}\end{array}$ & $\begin{array}{l}\text { 1-chloro-2-eth } \\
\quad(318-481)\end{array}$ & 52.4 & 333 & A & [1987STE/MAL, 1947STU] \\
\hline $\mathrm{C}_{8} \mathrm{H}_{9} \mathrm{ClO}$ & $\begin{array}{c}{[1875-88-3]} \\
\mathrm{V}\end{array}$ & $\begin{array}{l}\text { 4-chlorophenet } \\
\text { (426-673) }\end{array}$ & $\begin{array}{l}\text { lcohol } \\
59.3\end{array}$ & 411 & A & [1987STE/MAL, 1947STU] \\
\hline $\mathrm{C}_{8} \mathrm{H}_{9} \mathrm{ClO}$ & $\begin{array}{c}{[622-61-7]} \\
\mathrm{V}\end{array}$ & $\begin{array}{l}\text { 4-chloro-1-eth } \\
\quad(395-485)\end{array}$ & $\begin{array}{r}\text { nzene } \\
49.5\end{array}$ & 410 & A & [1987STE/MAL, 1947STU] \\
\hline $\mathrm{C}_{8} \mathrm{H}_{9} \mathrm{ClO}_{2}$ & $\begin{array}{c}{[7477-64-7]} \\
\mathrm{V}\end{array}$ & $\begin{array}{l}\text { ethylene glyco } \\
\quad(410-554)\end{array}$ & $\begin{array}{l}\text { alorophenyl } \\
68.5\end{array}$ & 425 & A & [1987STE/MAL, 1999DYK/SVO] \\
\hline $\mathrm{C}_{8} \mathrm{H}_{9} \mathrm{ClO}_{3}$ & $\begin{array}{c}{[18113-22-9]} \\
\mathrm{V}\end{array}$ & $\begin{array}{l}\text { 3-chloro-2,6-di } \\
\quad(293-323)\end{array}$ & $\begin{array}{c}\text { oxyphenol } \\
68.6\end{array}$ & 308 & CGC & [1999LEI/WAN2] \\
\hline $\mathrm{C}_{8} \mathrm{H}_{9} \mathrm{Cl}_{3} \mathrm{O}_{4}$ & $\begin{array}{c}{[98491-17-9]} \\
\mathrm{V}\end{array}$ & $\begin{array}{l}\text { 2-acetyl-4,4,4- } \\
\quad(374-409)\end{array}$ & $\begin{array}{l}\text { oro-3-oxobut } \\
53.1\end{array}$ & $\begin{array}{l}\text { id, ethyl } \\
389\end{array}$ & A & [1987STE/MAL, 1999DYK/SVO] \\
\hline $\mathrm{C}_{8} \mathrm{H}_{9} \mathrm{I}$ & $\begin{array}{c}{[10604-60-1]} \\
\mathrm{V}\end{array}$ & $\begin{array}{l}\text { (1-iodoethyl)b } \\
\quad(303-340)\end{array}$ & $59.9 \pm 0.4$ & 298 & GS & [2002KRA/VAS] \\
\hline $\mathrm{C}_{8} \mathrm{H}_{9} \mathrm{I}$ & $\begin{array}{c}{[4214-28-2]} \\
\mathrm{V} \\
\mathrm{V}\end{array}$ & $\begin{array}{l}\text { 1-iodo-2,4-dim } \\
\quad(294-328) \\
\quad(294-328)\end{array}$ & $\begin{array}{l}\text { benzene } \\
56.8 \pm 0.3 \\
57.7 \pm 0.6\end{array}$ & $\begin{array}{l}311 \\
298\end{array}$ & $\begin{array}{l}\text { GS } \\
\text { GS }\end{array}$ & $\begin{array}{l}{[2015 \mathrm{VER} / \mathrm{SAZ}]} \\
{[2015 \mathrm{VER} / \mathrm{SAZ}]}\end{array}$ \\
\hline $\mathrm{C}_{8} \mathrm{H}_{9} \mathrm{I}$ & $\begin{array}{c}{[608-28-6]} \\
\mathrm{V} \\
\mathrm{V}\end{array}$ & $\begin{array}{l}\text { 1-iodo-2,6-dim } \\
\quad(285-333) \\
\quad(285-333)\end{array}$ & $\begin{array}{l}\text { benzene } \\
56.9 \pm 0.1 \\
57.6 \pm 0.4\end{array}$ & $\begin{array}{l}309 \\
298\end{array}$ & $\begin{array}{l}\text { GS } \\
\text { GS }\end{array}$ & $\begin{array}{l}{[2015 \mathrm{VER} / \mathrm{SAZ}]} \\
{[2015 \mathrm{VER} / \mathrm{SAZ}]}\end{array}$ \\
\hline $\mathrm{C}_{8} \mathrm{H}_{9} \mathrm{~N}$ & $\begin{array}{c}{[140-76-1]} \\
\mathrm{V} \\
\mathrm{V}\end{array}$ & $\begin{array}{l}\text { 2-methyl-5-vin } \\
\quad(342-457) \\
(342-457)\end{array}$ & $\begin{array}{l}\text { idine } \\
55.2 \\
54.5\end{array}$ & $\begin{array}{l}357 \\
357\end{array}$ & A & $\begin{array}{l}\text { [1987STE/MAL] } \\
\text { [1961FRO/LOG, 1984BOU/FRI] }\end{array}$ \\
\hline $\mathrm{C}_{8} \mathrm{H}_{9} \mathrm{~N}$ & $\begin{array}{l}\mathrm{V} \\
\mathrm{V}\end{array}$ & $\begin{array}{l}N \text {-methylbenz } \\
\quad(283-318) \\
(283-318)\end{array}$ & $\begin{array}{l}\text { yde-imine } \\
51.1 \pm 0.2 \\
51.2 \pm 0.2\end{array}$ & $\begin{array}{l}301 \\
298\end{array}$ & $\begin{array}{l}\text { GS } \\
\text { GS }\end{array}$ & $\begin{array}{l}\text { [1997VER/MOR] } \\
\text { [1997VER/MOR] }\end{array}$ \\
\hline $\mathrm{C}_{8} \mathrm{H}_{9} \mathrm{~N}$ & $\begin{array}{c}{[496-15-1]} \\
\mathrm{V} \\
\mathrm{V}\end{array}$ & $\begin{array}{l}\text { indoline } \\
\qquad(281-338)\end{array}$ & $\begin{array}{l}60.9 \pm 0.2 \\
61.9 \pm 1.7\end{array}$ & $\begin{array}{l}298 \\
298\end{array}$ & $\begin{array}{c}\mathrm{GS} \\
\mathrm{C}\end{array}$ & $\begin{array}{l}\text { [2011VER/EME] } \\
\text { [2008RIB/CAB3] }\end{array}$ \\
\hline
\end{tabular}


TABLE 11. Phase change enthalpies of $\mathrm{C}_{8}$ organic compounds-Continued

\begin{tabular}{|c|c|c|c|c|c|c|}
\hline \multirow[b]{2}{*}{$\begin{array}{l}\text { Molecular } \\
\text { formula }\end{array}$} & \multirow{2}{*}{$\begin{array}{c}\text { CAS reg. no. } \\
\text { Transition }\end{array}$} & \multicolumn{5}{|l|}{ Compound } \\
\hline & & $\begin{array}{c}\text { Temp. range } \\
(\mathrm{K})\end{array}$ & $\begin{array}{c}\Delta_{\text {trans }} H_{m} \\
\left(\mathrm{~kJ} \mathrm{~mol}^{-1}\right)\end{array}$ & $T_{\mathrm{m}}(\mathrm{K})$ & Method & References \\
\hline \multirow[t]{14}{*}{$\mathrm{C}_{8} \mathrm{H}_{9} \mathrm{NO}$} & [103-84-4] & acetanilide $(N$ & ylacetamide) & & & \\
\hline & FUS & & 19.64 & 387.2 & DTA & {$[2013 \mathrm{KAN}]$} \\
\hline & FUS & & 21.83 & 387.5 & DSC & [2012CHA/LAY] \\
\hline & FUS & & 22.1 & 386.9 & DSC & [2012UMN/CHI] \\
\hline & FUS & & 21.4 & 386.9 & DSC & [2005CHE/TAN] \\
\hline & FUS & & 23.0 & 387.2 & DSC & [2004SHA/TAN] \\
\hline & FUS & & 18.3 & 387.2 & & [2004VEC/CAT] \\
\hline & FUS & & 20.3 & 389.0 & DSC & [1998BUS/ROM] \\
\hline & FUS & & 21.65 & 387.5 & & [1996DOM/HEA, 1980AND/CON] \\
\hline & SUB & $(303-324)$ & 80.6 & 313.5 & A & [1987STE/MAL, 1955AIH3] \\
\hline & SUB & $(317-336)$ & 87.2 & 326.5 & A & [1987STE/MAL, 1960AIH2] \\
\hline & V & & $82.1 \pm 3.0$ & 298 & $\mathrm{CGC}$ & [2012UMN/CHI] \\
\hline & $\mathrm{V}$ & $(473-577)$ & 64.8 & 488 & A & [1987STE/MAL] \\
\hline & $\mathrm{V}$ & $(387-577)$ & 66.3 & 402 & & [1947STU] \\
\hline \multirow[t]{2}{*}{$\mathrm{C}_{8} \mathrm{H}_{9} \mathrm{NO}$} & {$[41977-54-2]$} & anti 3-methylb & doxime & & & \\
\hline & SUB & & $\mathrm{U} 31 \pm 1.7$ & & MS & {$[1983 \mathrm{MAJ} / \mathrm{AZZ}]$} \\
\hline \multirow[t]{2}{*}{$\mathrm{C}_{8} \mathrm{H}_{9} \mathrm{NO}$} & [3235-02-7] & anti 4-methylb & doxime & & & \\
\hline & SUB & & $\mathrm{U} 36 \pm 1.7$ & & MS & {$[1983 \mathrm{MAJ} / \mathrm{AZZ}]$} \\
\hline \multirow[t]{2}{*}{$\mathrm{C}_{8} \mathrm{H}_{9} \mathrm{NO}$} & {$[103-81-1]$} & 2-phenylacetar & & & & \\
\hline & SUB & $(329-352)$ & 96.4 & 340.5 & A & [1987STE/MAL, 1960AIH2] \\
\hline \multirow[t]{2}{*}{$\mathrm{C}_{8} \mathrm{H}_{9} \mathrm{NO}$} & [99-03-6] & 3-aminoacetop & & & & \\
\hline & FUS & & 12.13 & 371.2 & & [1971LEB/GUT] \\
\hline \multirow[t]{5}{*}{$\mathrm{C}_{8} \mathrm{H}_{9} \mathrm{NO}$} & {$[99-92-3]$} & 4-aminoacetop & & & & \\
\hline & FUS & & 18.53 & 380.2 & DSC & [2013SIN/PAN] \\
\hline & FUS & & 19.6 & 378.2 & DSC & [2005CHE/TAN] \\
\hline & FUS & & 15.90 & 379.2 & & [1971LEB/GUT] \\
\hline & SUB & $(314-338)$ & 92.7 & 326 & A & [1987STE/MAL, 1960AIH2] \\
\hline \multirow[t]{8}{*}{$\mathrm{C}_{8} \mathrm{H}_{9} \mathrm{NO}$} & [613-93-4] & $N$-methylbenz & & & & \\
\hline & FUS & & 18.62 & 347.4 & DSC & [2010ALM/MON] \\
\hline & SUB & $(325-349)$ & $99.9 \pm 0.2$ & 337 & Static & [2010ALM/MON] \\
\hline & SUB & $(325-349)$ & $101.0 \pm 0.2$ & 298 & Static & [2010ALM/MON] \\
\hline & SUB & $(297-321)$ & 75.0 & 309 & A & [1987STE/MAL, 1955AIH] \\
\hline & SUB & $(307-329)$ & 85.7 & 318 & A & [1987STE/MAL, 1960AIH2] \\
\hline & $\mathrm{V}$ & $(323-388)$ & $79.2 \pm 0.1$ & 356 & Static & [2010ALM/MON] \\
\hline & $\mathrm{V}$ & $(323-388)$ & $84.9 \pm 0.5$ & 298 & Static & [2010ALM/MON] \\
\hline \multicolumn{7}{|c|}{ [Note: VP measurements in [2010ALM/MON] include those for the subcooled liquid.] } \\
\hline \multirow[t]{4}{*}{$\mathrm{C}_{8} \mathrm{H}_{9} \mathrm{NO}$} & {$[527-85-5]$} & $o$-methylbenz: & & & & \\
\hline & FUS & & 22.9 & 415.1 & DSC & [2012ALM/MAT] \\
\hline & SUB & $(325-347)$ & $105.0 \pm 0.3$ & 336 & $\mathrm{ME}$ & [2012ALM/MAT] \\
\hline & SUB & $(325-347)$ & $106.1 \pm 0.3$ & 298 & ME & [2012ALM/MAT] \\
\hline \multirow[t]{4}{*}{$\mathrm{C}_{8} \mathrm{H}_{9} \mathrm{NO}$} & {$[618-47-3]$} & $m$-methylbenz & & & & \\
\hline & FUS & & 21.6 & 366.7 & DSC & [2012ALM/MAT] \\
\hline & SUB & $(325-347)$ & $106.6 \pm 0.3$ & 336 & ME & [2012ALM/MAT] \\
\hline & SUB & $(325-347)$ & $107.8 \pm 0.3$ & 298 & $\mathrm{ME}$ & [2012ALM/MAT] \\
\hline \multirow[t]{4}{*}{$\mathrm{C}_{8} \mathrm{H}_{9} \mathrm{NO}$} & {$[619-55-6]$} & $p$-methylbenz & & & & \\
\hline & FUS & & 24.3 & 433.2 & $\mathrm{DSC}$ & [2012ALM/MAT] \\
\hline & SUB & $(339-361)$ & $108.3 \pm 0.3$ & 350 & $\mathrm{ME}$ & [2012ALM/MAT] \\
\hline & SUB & $(339-361)$ & $109.6 \pm 0.3$ & 298 & $\mathrm{ME}$ & [2012ALM/MAT] \\
\hline \multirow[t]{3}{*}{$\mathrm{C}_{8} \mathrm{H}_{9} \mathrm{NO}_{2}$} & [81-20-9] & 2-nitro-1,3-din & benzene & & & \\
\hline & V & $(284-323)$ & $57.2 \pm 0.8$ & 303 & GS & [2000VER/HEI] \\
\hline & $\mathrm{V}$ & $(284-323)$ & $57.5 \pm 0.8$ & 298 & GS & [2000VER/HEI] \\
\hline
\end{tabular}


TABLE 11. Phase change enthalpies of $\mathrm{C}_{8}$ organic compounds-Continued

\begin{tabular}{|c|c|c|c|c|c|c|}
\hline \multirow[b]{2}{*}{$\begin{array}{l}\text { Molecular } \\
\text { formula }\end{array}$} & \multirow{2}{*}{$\begin{array}{l}\text { CAS reg. no. } \\
\text { Transition }\end{array}$} & \multicolumn{5}{|l|}{ Compound } \\
\hline & & $\begin{array}{c}\text { Temp. range } \\
\text { (K) }\end{array}$ & $\begin{array}{c}\Delta_{\text {trans }} H_{m} \\
\left(\mathrm{~kJ} \mathrm{~mol}^{-1}\right)\end{array}$ & $T_{\mathrm{m}}(\mathrm{K})$ & Method & References \\
\hline & $\mathrm{V}$ & $(373-498)$ & 49.7 & 388 & A & [1987STE/MAL] \\
\hline \multirow{3}{*}{$\mathrm{C}_{8} \mathrm{H}_{9} \mathrm{NO}_{2}$} & {$[89-87-2]$} & \multicolumn{2}{|c|}{ 4-nitro-1,3-dimethylbenzene } & & & \\
\hline & V & $(368-518)$ & 56.7 & 383 & A & [1987STE/MAL] \\
\hline & $\mathrm{V}$ & $(338-517)$ & 57.3 & 353 & & [1947STU] \\
\hline \multirow[t]{2}{*}{$\mathrm{C}_{8} \mathrm{H}_{9} \mathrm{NO}_{2}$} & {$[83-41-0]$} & \multicolumn{2}{|c|}{ 1,2-dimethyl-3-nitrobenzene } & & & \\
\hline & $\mathrm{V}$ & $(383-518)$ & 59.4 & 398 & & [1984BOU/FRI] \\
\hline \multirow[t]{2}{*}{$\mathrm{C}_{8} \mathrm{H}_{9} \mathrm{NO}_{2}$} & {$[99-51-4]$} & \multicolumn{2}{|c|}{ 1,2-dimethyl-4-nitrobenzene } & & & \\
\hline & $\mathrm{V}$ & $(399-536)$ & 63.6 & 414 & & [1984BOU/FRI] \\
\hline \multirow[t]{4}{*}{$\mathrm{C}_{8} \mathrm{H}_{9} \mathrm{NO}_{2}$} & {$[612-22-6]$} & \multicolumn{2}{|c|}{ 2-nitro-1-ethylbenzene } & & & \\
\hline & V & $(284-323)$ & $62.7 \pm 0.4$ & 303 & GS & [2000VER/HEI] \\
\hline & $\mathrm{V}$ & $(284-323)$ & $63.0 \pm 0.4$ & 298 & GS & [2000VER/HEI] \\
\hline & $\mathrm{V}$ & $(353-422)$ & 56.3 & 368 & A & [1987STE/MAL] \\
\hline $\mathrm{C}_{8} \mathrm{H}_{9} \mathrm{NO}_{2}$ & $\begin{array}{c}{[100-12-9]} \\
\mathrm{V}\end{array}$ & \multicolumn{2}{|c|}{ 4-nitro-1-ethylbenzene } & 368 & A & [1987STE/MAL] \\
\hline \multirow[t]{3}{*}{$\mathrm{C}_{8} \mathrm{H}_{9} \mathrm{NO}_{2}$} & {$[103-01-5]$} & \multicolumn{2}{|c|}{$N$-phenylglycine } & & & \\
\hline & SUB & & $114.1 \pm 1.0$ & 365.4 & $\mathrm{C}$ & [1980SAB/SKO] \\
\hline & SUB & & $128.0 \pm 2.0$ & 298 & $\mathrm{C}$ & [1980SAB/SKO] \\
\hline \multirow[t]{3}{*}{$\mathrm{C}_{8} \mathrm{H}_{9} \mathrm{NO}_{2}$} & [875-74-1] & \multicolumn{3}{|c|}{$(d)$ - $\alpha$-phenylglycine } & & \\
\hline & SUB & & $148.9 \pm 2.2$ & 443 & $\mathrm{C}$ & [1980SAB/SKO] \\
\hline & SUB & & $165.0 \pm 6.0$ & 298 & $\mathrm{C}$ & [1980SAB/SKO] \\
\hline \multirow[t]{3}{*}{$\mathrm{C}_{8} \mathrm{H}_{9} \mathrm{NO}_{2}$} & {$[29577-53-5]$} & \multicolumn{3}{|c|}{ 2-methoxybenzaldoxime } & & \\
\hline & SUB (anti) & \multicolumn{3}{|c|}{$\mathrm{U} 20.1 \pm 1.7$} & MS & [1983MAJ/AZZ] \\
\hline & SUB (syn) & & $\mathrm{U} 32.6 \pm 1.7$ & & MS & [1983MAJ/AZZ] \\
\hline $\mathrm{C}_{8} \mathrm{H}_{9} \mathrm{NO}_{2}$ & [3235-04-9] & 4-methoxyben & xime & & & \\
\hline & SUB & & $\mathrm{U} 67.3 \pm 1.7$ & & MS & [1983MAJ/AZZ] \\
\hline $\mathrm{C}_{8} \mathrm{H}_{9} \mathrm{NO}_{2}$ & [134-20-3] & methyl 2-amin & zoate & & & \\
\hline & FUS & & 17.9 & 294.1 & DSC & [2012ALM/MON2] \\
\hline & SUB & $(287-298)$ & 78.4 & 292.5 & $\mathrm{ME}$ & [1987STE/MAL, 1954SER/VOI, 1960JON] \\
\hline & $\mathrm{V}$ & $(283-370)$ & $66.0 \pm 0.1$ & 326 & Static & [2012ALM/MON2] \\
\hline & $\mathrm{V}$ & $(283-370)$ & $67.1 \pm 0.1$ & 298 & Static & [2012ALM/MON2] \\
\hline & $\mathrm{V}$ & $(299-333)$ & 62.3 & 314 & $\mathrm{~A}, \mathrm{ME}$ & [1987STE/MAL, 1954SER/VOI] \\
\hline $\mathrm{C}_{8} \mathrm{H}_{9} \mathrm{NO}_{2}$ & {$[4518-10-9]$} & methyl 3-amin & zoate & & & \\
\hline & FUS & & 17.8 & 325.3 & DSC & [2012ALM/MON2] \\
\hline & SUB & $(309-324)$ & $94.1 \pm 0.1$ & 316 & Static & [2012ALM/MON2] \\
\hline & SUB & $(309-324)$ & $94.6 \pm 0.1$ & 298 & Static & [2012ALM/MON2] \\
\hline & $\mathrm{V}$ & $(312-376)$ & $75.4 \pm 0.1$ & 344 & Static & [2012ALM/MON2] \\
\hline & $\mathrm{V}$ & $(312-376)$ & $78.2 \pm 0.1$ & 298 & Static & [2012ALM/MON2] \\
\hline $\mathrm{C}_{8} \mathrm{H}_{9} \mathrm{NO}_{2}$ & {$[619-45-4]$} & methyl 4-amin & zoate & & & \\
\hline & FUS & & 24.9 & 284.2 & DSC & [2012ALM/MON2] \\
\hline & FUS & & 22.55 & 385.1 & & [1990MAN/AHU] \\
\hline & FUS & & 22.6 & 385.2 & DSC & [1990NEA/FLY] \\
\hline & SUB & $(319-341)$ & $104.2 \pm 0.3$ & 298 & ME & [2012ALM/MON2] \\
\hline & SUB & $(333-380)$ & $101.7 \pm 0.2$ & 356 & Static & [2012ALM/MON2] \\
\hline & SUB & $(333-380)$ & $103.4 \pm 0.2$ & 298 & Static & [2012ALM/MON2] \\
\hline & $\mathrm{V}$ & $(361-428)$ & $74.5 \pm 0.1$ & 394 & Static & [2012ALM/MON2] \\
\hline & $\mathrm{V}$ & $(361-428)$ & $84.0 \pm 0.1$ & 298 & Static & [2012ALM/MON2] \\
\hline $\mathrm{C}_{8} \mathrm{H}_{9} \mathrm{NO}_{2}$ & {$[614-80-2]$} & $o$-hydroxyacet & & & & \\
\hline & FUS & & 37.0 & 484.1 & DSC & [2008PER/VOL2] \\
\hline & FUS & & 21.25 & 364.5 & & [1996DOM/HEA, 1926AND/LYN] \\
\hline & SUB & $(330-378)$ & $120.9 \pm 0.9$ & 354 & GS & [2008PER/VOL2] \\
\hline
\end{tabular}


TABLE 11. Phase change enthalpies of $\mathrm{C}_{8}$ organic compounds-Continued

\begin{tabular}{|c|c|c|c|c|c|c|}
\hline \multirow[b]{2}{*}{$\begin{array}{l}\text { Molecular } \\
\text { formula }\end{array}$} & \multirow{2}{*}{$\begin{array}{c}\text { CAS reg. no. } \\
\text { Transition }\end{array}$} & \multicolumn{5}{|l|}{ Compound } \\
\hline & & $\begin{array}{c}\text { Temp. range } \\
(\mathrm{K})\end{array}$ & $\begin{array}{c}\Delta_{\text {trans }} H_{m} \\
\left(\mathrm{~kJ} \mathrm{~mol}^{-1}\right)\end{array}$ & $T_{\mathrm{m}}(\mathrm{K})$ & Method & References \\
\hline \multirow[t]{3}{*}{$\mathrm{C}_{8} \mathrm{H}_{9} \mathrm{NO}_{2}$} & [621-42-1] & \multicolumn{5}{|c|}{$m$-hydroxyacetanilide } \\
\hline & FUS & & 26.6 & 416.2 & DSC & [2008PER/VOL2] \\
\hline & SUB & $(345-397)$ & $109.6 \pm 1.1$ & 371 & GS & [2008PER/VOL2] \\
\hline \multirow[t]{40}{*}{$\mathrm{C}_{8} \mathrm{H}_{9} \mathrm{NO}_{2}$} & [103-90-2] & \multicolumn{5}{|c|}{ p-hydroxyacetanilide (acetaminophen, paracetamol) } \\
\hline & FUS & & 27.51 & 442.35 & DSC & [2015MAT/MOR] \\
\hline & FUS (I) & & 26.0 & 442.4 & & \\
\hline & FUS (II) & & 24.9 & 429.8 & DSC & [2014SAI/MUR] \\
\hline & FUS & & 27.35 & 441.9 & DSC & [2013BOU/TEY] \\
\hline & FUS (I) & & 27.8 & 441.9 & & \\
\hline & FUS (II) & & 26.7 & 429.4 & DSC & [2012KLI/LEI] \\
\hline & FUS & & 27.2 & 443.8 & DSC & [2010PIC/DIO] \\
\hline & \multicolumn{6}{|l|}{ (monoclinic) } \\
\hline & FUS & & 27.24 & 443 & DSC & [2010BAI/VAN] \\
\hline & FUS & & 28.1 & 441.1 & DSC & [2010AVU/ALE] \\
\hline & FUS & & 27.4 & 442.2 & DSC & [2010HAH/GRA] \\
\hline & FUS & & 27.6 & 443.2 & DSC & [2009MOT/CAR] \\
\hline & FUS & & 27.0 & 440.3 & DSC & [2009VEC/TOM] \\
\hline & FUS & & 27.6 & 443.4 & DSC & [2007MIS/MIS2] \\
\hline & \multicolumn{6}{|l|}{ (monoclinic) } \\
\hline & FUS & & 26.1 & 446.2 & DSC & [2007CAM/MAG] \\
\hline & TRS & & Not given & 149.9 & & \\
\hline & FUS & & 26.49 & 441.9 & $\mathrm{AC}, \mathrm{DSC}$ & {$[2006 X U / S U N]$} \\
\hline & FUS & & 22.8 & 447.6 & DSC & [2005TOM/CAT] \\
\hline & FUS & & 27.0 & 442.2 & DSC & [2004BOL/DRE] \\
\hline & \multicolumn{6}{|l|}{ (monoclinic) } \\
\hline & FUS & & 26.2 & 443 & DSC & [2004ROM/BUS] \\
\hline & FUS & & 26.0 & 442.2 & DSC & [2002FAR/KAD] \\
\hline & FUS & & 24.8 & 441.0 & DSC & [2002SCH/LEN] \\
\hline & FUS & & 28.2 & 442.5 & DSC & [1998BUS/ROM] \\
\hline & FUS & & 27.2 & 442.9 & DSC & [1993BEC/QUA] \\
\hline & FUS & & 26.02 & 441.2 & & [1990MAN/AHU] \\
\hline & SUB & & $127.0 \pm 1.9$ & 399 & $\mathrm{C}$ & [2010PIC/DIO] \\
\hline & (monoclinic) & & & & & \\
\hline & SUB & & $130.3 \pm 1.9$ & 298 & $\mathrm{C}$ & [2010PIC/DIO] \\
\hline & (monoclinic) & & & & & \\
\hline & SUB & $(394-427)$ & $125.7 \pm 3.0$ & 411 & $\mathrm{ME}$ & [2010PIC/DIO] \\
\hline & (monoclinic) & & & & & \\
\hline & $\begin{array}{c}\text { SUB } \\
\text { (monoclinic) }\end{array}$ & $(394-427)$ & $129.5 \pm 3.0$ & 298 & $\mathrm{ME}$ & [2010PIC/DIO] \\
\hline & SUB & & $138 \pm 3$ & 298 & $F+V$ & [2009VEC/TOM] \\
\hline & SUB & $(355-397)$ & $117.9 \pm 0.7$ & 298 & GS & [2007PER/VOL2] \\
\hline & (monoclinic) & & & & & \\
\hline & SUB & $(313-347)$ & 238.7 & & & [2003WIL/WIL, 2010PIC/DIO] \\
\hline & \multicolumn{6}{|c|}{ [Note: Authors of [2010PIC/DIO] state that the value of $238.8 \mathrm{~kJ} / \mathrm{mole}$ seemed unreasonably high.] } \\
\hline & $\mathrm{V}$ & & $103 \pm 3$ & 521 & TGA & [2009VEC/TOM] \\
\hline & V & & $99 \pm 1$ & 494 & TGA & [2009VEC/TOM] \\
\hline \multirow[t]{3}{*}{$\mathrm{C}_{8} \mathrm{H}_{9} \mathrm{NO}_{2}$} & {$[2603-10-3]$} & \multicolumn{5}{|c|}{ methyl $N$-phenylcarbamate } \\
\hline & FUS & & 14.56 & 325 & & [1971PRI, 1996DOM/HEA] \\
\hline & SUB & $(373-418)$ & 64.5 & 388 & EB & [2013XU/LI] \\
\hline \multirow[t]{4}{*}{$\mathrm{C}_{8} \mathrm{H}_{9} \mathrm{NO}_{2}$} & {$[4389-45-1]$} & \multicolumn{5}{|c|}{ 2-amino-3-methylbenzoic acid } \\
\hline & FUS & & 27.3 & 447.4 & DSC & [2001MON/HIL2] \\
\hline & SUB & $(343-357)$ & $107.3 \pm 1.8$ & 298 & ME & [2001MON/HIL2] \\
\hline & SUB & $(343-357)$ & $105.8 \pm 0.8$ & 350 & ME & [2001MON/HIL2] \\
\hline \multirow[t]{2}{*}{$\mathrm{C}_{8} \mathrm{H}_{9} \mathrm{NO}_{2}$} & [2941-78-8] & \multicolumn{5}{|c|}{ 2-amino-5-methylbenzoic acid } \\
\hline & FUS & & 27.63 & 450 & DSC & [2001MON/HIL2] \\
\hline
\end{tabular}


TABLE 11. Phase change enthalpies of $\mathrm{C}_{8}$ organic compounds-Continued

\begin{tabular}{|c|c|c|c|c|c|c|}
\hline \multirow[b]{2}{*}{$\begin{array}{l}\text { Molecular } \\
\text { formula }\end{array}$} & \multirow{2}{*}{$\begin{array}{c}\text { CAS reg. no. } \\
\text { Transition }\end{array}$} & \multicolumn{5}{|l|}{ Compound } \\
\hline & & $\begin{array}{c}\text { Temp. range } \\
(\mathrm{K})\end{array}$ & $\begin{array}{c}\Delta_{\text {trans }} H_{m} \\
\left(\mathrm{~kJ} \mathrm{~mol}^{-1}\right)\end{array}$ & $T_{\mathrm{m}}(\mathrm{K})$ & Method & References \\
\hline & SUB & $(345-361)$ & $110.6 \pm 1.9$ & 298 & $\mathrm{ME}$ & [2001MON/HIL2] \\
\hline & SUB & $(345-361)$ & $108.9 \pm 0.5$ & 353 & $\mathrm{ME}$ & [2001MON/HIL2] \\
\hline \multirow[t]{4}{*}{$\mathrm{C}_{8} \mathrm{H}_{9} \mathrm{NO}_{2}$} & {$[4389-50-8]$} & \multicolumn{5}{|c|}{ 2-amino-6-methylbenzoic acid } \\
\hline & FUS & & 27.49 & 398.7 & DSC & [2001MON/HIL2] \\
\hline & SUB & $(339-355)$ & $116.1 \pm 2.0$ & 298 & $\mathrm{ME}$ & [2001MON/HIL2] \\
\hline & SUB & $(339-355)$ & $114.7 \pm 1.2$ & 347 & ME & [2001MON/HIL2] \\
\hline \multirow[t]{4}{*}{$\mathrm{C}_{8} \mathrm{H}_{9} \mathrm{NO}_{2}$} & {$[52130-17-3]$} & \multicolumn{5}{|c|}{ 3-amino-2-methylbenzoic acid } \\
\hline & FUS & & 38.47 & 458.8 & DSC & [2001MON/HIL2] \\
\hline & SUB & $(367-381)$ & $127.8 \pm 2.6$ & 298 & $\mathrm{ME}$ & [2001MON/HIL2] \\
\hline & SUB & $(367-381)$ & $115.6 \pm 0.8$ & 374 & $\mathrm{ME}$ & [2001MON/HIL2] \\
\hline \multirow[t]{4}{*}{$\mathrm{C}_{8} \mathrm{H}_{9} \mathrm{NO}_{2}$} & {$[2458-12-0]$} & \multicolumn{5}{|c|}{ 3-amino-4-methylbenzoic acid } \\
\hline & FUS & & 26.89 & 438.8 & DSC & [2001MON/HIL2] \\
\hline & SUB & $(363-377)$ & $119.4 \pm 2.5$ & 298 & $\mathrm{ME}$ & [2001MON/HIL2] \\
\hline & SUB & $(363-377)$ & $117.3 \pm 0.9$ & 370 & $\mathrm{ME}$ & [2001MON/HIL2] \\
\hline \multirow[t]{4}{*}{$\mathrm{C}_{8} \mathrm{H}_{9} \mathrm{NO}_{2}$} & {$[2486-70-6]$} & \multicolumn{5}{|c|}{ 4-amino-3-methylbenzoic acid } \\
\hline & FUS & & 21.77 & 439.4 & DSC & [2001MON/HIL2] \\
\hline & SUB & $(367-383)$ & $122.0 \pm 2.6$ & 298 & $\mathrm{ME}$ & [2001MON/HIL2] \\
\hline & SUB & $(367-383)$ & $119.8 \pm 0.7$ & 375 & ME & [2001MON/HIL2] \\
\hline \multirow[t]{2}{*}{$\mathrm{C}_{8} \mathrm{H}_{9} \mathrm{NO}_{2}$} & {$[1197-55-3]$} & \multicolumn{5}{|c|}{ 4-aminophenylacetic acid } \\
\hline & FUS & & 42.7 & 468.2 & DSC & [2002GRA/RAS] \\
\hline \multirow[t]{4}{*}{$\mathrm{C}_{8} \mathrm{H}_{9} \mathrm{NO}_{2}$} & {$[10541-83-0]$} & \multicolumn{5}{|c|}{ 4-(methylamino)benzoic acid } \\
\hline & FUS & & 21.0 & 435.9 & DSC & [2010MON/SAN] \\
\hline & SUB & $(359-381)$ & $121.6 \pm 0.4$ & 328 & ME & [2010MON/SAN] \\
\hline & SUB & $(359-381)$ & $123.9 \pm 0.4$ & 298 & $\mathrm{ME}$ & [2010MON/SAN] \\
\hline \multirow[t]{4}{*}{$\mathrm{C}_{8} \mathrm{H}_{9} \mathrm{NO}_{2}$} & [2439-77-2] & \multicolumn{5}{|c|}{ 2-methoxybenzamide } \\
\hline & FUS & & 27.21 & 401.66 & DSC & [2014ALM/MON] \\
\hline & SUB & & $109.9 \pm 0.4$ & 346 & $\mathrm{ME}$ & [2014ALM/MON] \\
\hline & SUB & & $113.3 \pm 0.4$ & 298 & ME & [2014ALM/MON] \\
\hline \multirow[t]{4}{*}{$\mathrm{C}_{8} \mathrm{H}_{9} \mathrm{NO}_{2}$} & {$[5813-86-5]$} & \multicolumn{5}{|c|}{ 3-methoxybenzamide } \\
\hline & FUS & & 28.60 & 407.15 & DSC & [2014ALM/MON] \\
\hline & SUB & & $118.1 \pm 0.4$ & 358 & ME & [2014ALM/MON] \\
\hline & SUB & & $119.8 \pm 0.4$ & 298 & $\mathrm{ME}$ & [2014ALM/MON] \\
\hline \multirow[t]{4}{*}{$\mathrm{C}_{8} \mathrm{H}_{9} \mathrm{NO}_{2}$} & {$[3424-93-9]$} & 4-methoxybenz & & & & \\
\hline & FUS & & 29.04 & 440.64 & DSC & [2014ALM/MON] \\
\hline & SUB & & $117.3 \pm 0.4$ & 368 & $\mathrm{ME}$ & [2014ALM/MON] \\
\hline & SUB & & $119.3 \pm 0.4$ & 298 & ME & [2014ALM/MON] \\
\hline $\mathrm{C}_{8} \mathrm{H}_{9} \mathrm{NO}_{2} \mathrm{~S}_{2}$ & [949171-63-5] & $N$-theonylthioc & mic- $O$-ethyl & & & \\
\hline & FUS & & 21.9 & 345.9 & DSC & [2007RIB/MON] \\
\hline & SUB & & $143.2 \pm 3.1$ & 298 & $\mathrm{C}$ & [2007RIB/MON] \\
\hline $\mathrm{C}_{8} \mathrm{H}_{9} \mathrm{NO}_{3}$ & [3177-80-8] & 2-amino-3-met & benzoic acid & & & \\
\hline & FUS & & 24.21 & 443.0 & DSC & [2010MON/ALM] \\
\hline & SUB & $(349-369)$ & $113.3 \pm 0.7$ & 359.8 & $\mathrm{ME}$ & [2010MON/ALM] \\
\hline & SUB & $(349-369)$ & $115.5 \pm 0.7$ & 298 & ME & [2010MON/ALM] \\
\hline $\mathrm{C}_{8} \mathrm{H}_{9} \mathrm{NO}_{3}$ & [6705-03-9] & 2-amino-5-met & benzoic acid & & & \\
\hline & FUS & & 22.88 & 425 & DSC & [2010MON/ALM] \\
\hline & SUB & $(353-375)$ & $116.9 \pm 0.8$ & 364 & $\mathrm{ME}$ & [2010MON/ALM] \\
\hline & SUB & $(353-375)$ & $119.3 \pm 0.8$ & 298 & ME & [2010MON/ALM] \\
\hline $\mathrm{C}_{8} \mathrm{H}_{9} \mathrm{NO}_{3}$ & [2840-26-8] & 3-amino-4-met & benzoic acid & & & \\
\hline & FUS & & 25.34 & 477.9 & DSC & [2010MON/ALM] \\
\hline
\end{tabular}


TABLE 11. Phase change enthalpies of $\mathrm{C}_{8}$ organic compounds-Continued

\begin{tabular}{|c|c|c|c|c|c|c|}
\hline \multirow[b]{2}{*}{$\begin{array}{l}\text { Molecular } \\
\text { formula }\end{array}$} & \multirow{2}{*}{$\begin{array}{l}\text { CAS reg. no. } \\
\text { Transition }\end{array}$} & \multicolumn{5}{|l|}{ Compound } \\
\hline & & $\begin{array}{c}\text { Temp. range } \\
(\mathrm{K})\end{array}$ & $\begin{array}{c}\Delta_{\text {trans }} H_{m} \\
\left(\mathrm{~kJ} \mathrm{~mol}^{-1}\right)\end{array}$ & $T_{\mathrm{m}}(\mathrm{K})$ & Method & References \\
\hline & SUB & $(380-399)$ & $127.4 \pm 0.8$ & 389 & ME & [2010MON/ALM] \\
\hline & SUB & $(380-399)$ & $130.7 \pm 0.8$ & 298 & ME & [2010MON/ALM] \\
\hline \multirow{4}{*}{$\mathrm{C}_{8} \mathrm{H}_{9} \mathrm{NO}_{3}$} & {$[74165-74-5]$} & \multicolumn{5}{|c|}{ 3-amino-5-methoxybenzoic acid } \\
\hline & FUS & & 22.4 & 456.9 & DSC & [2010MON/ALM] \\
\hline & SUB & $(380-400)$ & $132.8 \pm 1.0$ & 390 & ME & [2010MON/ALM] \\
\hline & SUB & $(380-400)$ & $136.1 \pm 1.0$ & 298 & ME & {$[2010 \mathrm{MON} / \mathrm{ALM}]$} \\
\hline \multirow[t]{4}{*}{$\mathrm{C}_{8} \mathrm{H}_{9} \mathrm{NO}_{3}$} & {$[2486-69-3]$} & \multicolumn{5}{|c|}{ 4-amino-3-methoxybenzoic acid } \\
\hline & FUS & & 25.27 & 462.4 & DSC & {$[2010 \mathrm{MON} / \mathrm{ALM}]$} \\
\hline & SUB & $(373-395)$ & $128.9 \pm 1.2$ & 384 & $\mathrm{ME}$ & {$[2010 \mathrm{MON} / \mathrm{ALM}]$} \\
\hline & SUB & $(373-395)$ & $132.0 \pm 1.2$ & 298 & ME & {$[2010 \mathrm{MON} / \mathrm{ALM}]$} \\
\hline \multirow[t]{2}{*}{$\mathrm{C}_{8} \mathrm{H}_{9} \mathrm{NO}_{7}$} & {$[22401-53-2]$} & \multicolumn{5}{|c|}{ methyl 5-nitro-2-acetoxy-2,5-dihydro-2-furancarboxylate } \\
\hline & SUB & \multicolumn{4}{|c|}{$89.1 \pm 2.1$} & [1980BAL/LEB, 1986PED/NAY] \\
\hline \multirow[t]{2}{*}{$\mathrm{C}_{8} \mathrm{H}_{9} \mathrm{~N}_{3}$} & {$[62679-52-1]$} & \multicolumn{5}{|c|}{ 2,2-dicyanohexanenitrile } \\
\hline & $\mathrm{V}$ & $(288-323)$ & $61.0 \pm 0.2$ & & GS & [1994RAK/VER] \\
\hline \multirow[t]{2}{*}{$\mathrm{C}_{8} \mathrm{H}_{9} \mathrm{~N}_{3} \mathrm{O}_{4}$} & {$[3846-50-2]$} & \multicolumn{5}{|c|}{$N$-ethyl-2,4-dinitrobenzenamine } \\
\hline & FUS & & 19.67 & 387.2 & DSC & {$[2010 \mathrm{MEK} / \mathrm{KHI}]$} \\
\hline \multirow[t]{2}{*}{$\mathrm{C}_{8} \mathrm{H}_{9} \mathrm{O}_{3} \mathrm{PS}$} & {$[3811-49-2]$} & \multicolumn{5}{|c|}{ 2-methoxy-4H-1,3,2-benzodioxaphosphorin 2-sulfide } \\
\hline & FUS & & 16.92 & 327.9 & DSC & {$[1990 \mathrm{DON} / \mathrm{DRE}]$} \\
\hline \multirow[t]{17}{*}{$\mathrm{C}_{8} \mathrm{H}_{10}$} & {$[95-47-6]$} & 1,2-dimethylbe & & & & \\
\hline & FUS & & 13.68 & 248.0 & $\mathrm{C}$ & [1955TUN/STO] \\
\hline & FUS & & 13.6 & 247.8 & & [1996DOM/HEA, 1943PIT/SCO] \\
\hline & FUS & $(90-295)$ & 13.0 & 247.8 & $\mathrm{C}$ & [1930HUF/PAR2] \\
\hline & SUB & & 60.1 & 248 & $\mathrm{~B}$ & {$[1986 \mathrm{HES} / \mathrm{LIC}]$} \\
\hline & $\mathrm{V}$ & $(373-423)$ & 42.9 & 298 & CGC & {$[1995 \mathrm{CHI} / \mathrm{HOS}]$} \\
\hline & $\mathrm{V}$ & $(333-419)$ & 41.1 & 348 & A & [1987STE/MAL] \\
\hline & $\mathrm{V}$ & $(416-473)$ & 38.0 & 431 & A & [1987STE/MAL] \\
\hline & $\mathrm{V}$ & $(471-571)$ & 36.7 & 486 & A & [1987STE/MAL] \\
\hline & $\mathrm{V}$ & $(567-630)$ & 36.7 & 582 & A & [1987STE/MAL] \\
\hline & $\mathrm{V}$ & $(386-416)$ & 39.8 & 401 & & [1982CAS/FRA] \\
\hline & $\mathrm{V}$ & & 43.4 & 298 & & [1971WIL/ZWO] \\
\hline & $\mathrm{V}$ & $(333-342)$ & 41.5 & 338 & & [1968GAR/BOV] \\
\hline & $\mathrm{V}$ & & $43.4 \pm 0.1$ & 298 & $\mathrm{C}$ & [1947OSB/GIN] \\
\hline & $\mathrm{V}$ & $(337-419)$ & 40.8 & 352 & $\mathrm{MM}$ & [1945WIL/TAY, 1949FOR/NOR] \\
\hline & $\mathrm{V}$ & $(273-323)$ & 45.0 & 288 & & [1943PIT/SCO, 1984BOU/FRI] \\
\hline & $\mathrm{V}$ & $(273-323)$ & $43.8 \pm 0.2$ & 298 & & [1943PIT/SCO] \\
\hline $\mathrm{C}_{8} \mathrm{H}_{10}$ & {$[108-38-3]$} & 1,3-dimethylbe & & & & \\
\hline & FUS & & 11.67 & 225.3 & $\mathrm{C}$ & [1955TUN/STO] \\
\hline & FUS & & 11.59 & 225.3 & & [1996DOM/HEA, 1943PIT/SCO] \\
\hline & TRS & & 0.21 & 166 & & \\
\hline & FUS & $(96-275)$ & 11.44 & 219.6 & $\mathrm{C}$ & [1996DOM/HEA, 1930HUF/PAR2] \\
\hline & V & $(360-410)$ & 39.2 & 375 & & [2002SWI/MAL] \\
\hline & $\mathrm{V}$ & $(327-412)$ & 40.7 & 342 & & [1989PAR/GME] \\
\hline & $\mathrm{V}$ & $(267-301)$ & 44.7 & 282 & A & [1987STE/MAL] \\
\hline & $\mathrm{V}$ & $(412-462)$ & 37.5 & 427 & A & [1987STE/MAL] \\
\hline & $\mathrm{V}$ & $(461-554)$ & 36.4 & 476 & A & [1987STE/MAL] \\
\hline & $\mathrm{V}$ & $(550-617)$ & 36.2 & 565 & A & [1987STE/MAL] \\
\hline & $\mathrm{V}$ & $(380-411)$ & 38.7 & 395 & & [1983MAC] \\
\hline & $\mathrm{V}$ & & 42.7 & 298 & & [1971WIL/ZWO] \\
\hline & $\mathrm{V}$ & $(326-337)$ & 41 & 331 & & [1968GAR/BOV] \\
\hline & $\mathrm{V}$ & & $42.7 \pm 0.1$ & 298 & $\mathrm{C}$ & [1947OSB/GIN] \\
\hline & $\mathrm{V}$ & $(331-415)$ & 40.4 & 346 & MM & [1987STE/MAL, 1945WIL/TAY, 1949FOR/NOR] \\
\hline & $\mathrm{V}$ & $(273-333)$ & 43.2 & 288 & & [1943PIT/SCO, 1984BOU/FRI] \\
\hline & $\mathrm{V}$ & $(273-333)$ & $42.5 \pm 0.2$ & 298 & & [1943PIT/SCO] \\
\hline $\mathrm{C}_{8} \mathrm{H}_{10}$ & [106-42-3] & 1,4-dimethylbe & & & & \\
\hline & FUS & & 17.49 & 286.5 & $\mathrm{C}$ & [1955TUN/STO] \\
\hline
\end{tabular}


TABLE 11. Phase change enthalpies of $\mathrm{C}_{8}$ organic compounds-Continued

\begin{tabular}{|c|c|c|c|c|c|c|}
\hline \multirow[b]{2}{*}{$\begin{array}{l}\text { Molecular } \\
\text { formula }\end{array}$} & \multirow{2}{*}{$\begin{array}{l}\text { CAS reg. no. } \\
\text { Transition }\end{array}$} & \multicolumn{5}{|l|}{ Compound } \\
\hline & & $\begin{array}{c}\text { Temp. range } \\
(\mathrm{K})\end{array}$ & $\begin{array}{c}\Delta_{\text {trans }} H_{m} \\
\left(\mathrm{~kJ} \mathrm{~mol}^{-1}\right)\end{array}$ & $T_{\mathrm{m}}(\mathrm{K})$ & Method & References \\
\hline & FUS & & 17.11 & 286.3 & & [1996DOM/HEA, 1947COR/GIN, 1943PIT/SCO] \\
\hline & FUS & $(92-299)$ & 16.93 & 286.4 & $\mathrm{C}$ & [1930HUF/PAR2] \\
\hline & SUB & $(247-286)$ & 59.4 & 271 & A & [1987STE/MAL, 1974OSB/DOU] \\
\hline & SUB & & 60.8 & 286 & B & {$[1986 \mathrm{HES} / \mathrm{LIC}]$} \\
\hline & $\mathrm{V}$ & $(373-423)$ & 42.3 & 298 & $\mathrm{CGC}$ & [1995CHI/HOS] \\
\hline & $\mathrm{V}$ & $(293-323)$ & $43.0 \pm 0.1$ & 298 & & [1990SMI] \\
\hline & $\mathrm{V}$ & & $42.3 \pm 0.01$ & 298 & & [1988MES/FIN] \\
\hline & $\mathrm{V}$ & & 40.3 & 353 & & {$[1988 \mathrm{HOS} / \mathrm{ARC}]$} \\
\hline & $\mathrm{V}$ & $(411-463)$ & 37.3 & 426 & A & [1987STE/MAL] \\
\hline & $\mathrm{V}$ & $(460-553)$ & 36.1 & 475 & A & [1987STE/MAL] \\
\hline & $\mathrm{V}$ & $(551-616)$ & 36.2 & 566 & A & [1987STE/MAL] \\
\hline & $\mathrm{V}$ & & $36.0 \pm 0.1$ & 411 & $\mathrm{C}$ & [1985NAT/VIS] \\
\hline & $\mathrm{V}$ & & $34.5 \pm 0.1$ & 436 & $\mathrm{C}$ & [1985NAT/VIS] \\
\hline & $\mathrm{V}$ & & $30.5 \pm 0.1$ & 484 & $\mathrm{C}$ & [1985NAT/VIS] \\
\hline & $\mathrm{V}$ & & $24.7 \pm 0.1$ & 540 & $\mathrm{C}$ & [1985NAT/VIS] \\
\hline & $\mathrm{V}$ & $(380-410)$ & 37.3 & 395 & & [1982CAS/FRA] \\
\hline & $\mathrm{V}$ & $(348-439)$ & $39.6 \pm 0.1$ & 348 & $\mathrm{C}$ & [1981HOS/SCO3] \\
\hline & $\mathrm{V}$ & $(348-439)$ & $38.5 \pm 0.1$ & 366 & $\mathrm{C}$ & [1981HOS/SCO3] \\
\hline & V & $(348-439)$ & $37.2 \pm 0.1$ & 387 & $\mathrm{C}$ & [1981HOS/SCO3] \\
\hline & $\mathrm{V}$ & $(348-439)$ & $34.6 \pm 0.1$ & 427 & $\mathrm{C}$ & [1981HOS/SCO3] \\
\hline & $\mathrm{V}$ & $(348-439)$ & $33.8 \pm 0.1$ & 439 & $\mathrm{C}$ & [1981HOS/SCO3] \\
\hline & $\mathrm{V}$ & $(348-439)$ & $42.3 \pm 0.1$ & 298 & $\mathrm{C}$ & [1981HOS/SCO3] \\
\hline & V & & 42.6 & 298 & & [1974AMB/ELL] \\
\hline & $\mathrm{V}$ & $(286-453)$ & 42.4 & 301 & IPM, EB & [1987STE/MAL, 1974OSB/DOU] \\
\hline & V & & 42.4 & 298 & & [1971WIL/ZWO] \\
\hline & $\mathrm{V}$ & $(327-337)$ & 40.5 & 332 & & [1968GAR/BOV] \\
\hline & $\mathrm{V}$ & $(303-343)$ & 41.6 & 318 & & [1968GAW/SWI2] \\
\hline & $\mathrm{V}$ & & $42.4 \pm 0.1$ & 298 & $\mathrm{C}$ & [1947OSB/GIN] \\
\hline & $\mathrm{V}$ & $(332-413)$ & 40.1 & 347 & MM & [1945WIL/TAY, 1949FOR/NOR] \\
\hline & $\mathrm{V}$ & & $42.3 \pm 0.2$ & 298 & & {$[1943 \mathrm{PIT} / \mathrm{SCO}]$} \\
\hline \multirow[t]{22}{*}{$\mathrm{C}_{8} \mathrm{H}_{10}$} & {$[100-41-4]$} & ethylbenzene & & & & \\
\hline & FUS & & 9.16 & 178.2 & & [1996DOM/HEA, 1944GUT/SPI, 1946SCO/BRI] \\
\hline & FUS & & 9.16 & 178.0 & $\mathrm{C}$ & [1996DOM/HEA, 1930HUF/PAR2] \\
\hline & $\mathrm{V}$ & $(324-364)$ & 40.0 & 344 & $\mathrm{~EB}$ & [2011JON/MAA] \\
\hline & $\mathrm{V}$ & $(298-420)$ & 41.8 & 313 & A & [1987STE/MAL] \\
\hline & $\mathrm{V}$ & $(409-459)$ & 37.0 & 424 & A & [1987STE/MAL] \\
\hline & $\mathrm{V}$ & $(457-554)$ & 35.8 & 472 & A & [1987STE/MAL] \\
\hline & $\mathrm{V}$ & $(549-617)$ & 35.5 & 564 & A & [1987STE/MAL] \\
\hline & $\mathrm{V}$ & $(320-400)$ & 40.6 & 335 & & [1986PAU/KRU] \\
\hline & $\mathrm{V}$ & & $40.5 \pm 0.1$ & 328 & $\mathrm{C}$ & [1982SVO/CHA] \\
\hline & $\mathrm{V}$ & & $39.5 \pm 0.1$ & 343 & $\mathrm{C}$ & [1982SVO/CHA] \\
\hline & $\mathrm{V}$ & & $38.6 \pm 0.1$ & 358 & $\mathrm{C}$ & [1982SVO/CHA] \\
\hline & $\mathrm{V}$ & $(346-437)$ & $39.4 \pm 0.1$ & 346 & $\mathrm{C}$ & [1981HOS/SCO3] \\
\hline & $\mathrm{V}$ & $(346-437)$ & $38.4 \pm 0.1$ & 354 & $\mathrm{C}$ & [1981HOS/SCO3] \\
\hline & V & $(346-437)$ & $37.7 \pm 0.1$ & 376 & $\mathrm{C}$ & [1981HOS/SCO3] \\
\hline & $\mathrm{V}$ & $(346-437)$ & $36.3 \pm 0.1$ & 399 & $\mathrm{C}$ & [1981HOS/SCO3] \\
\hline & $\mathrm{V}$ & $(346-437)$ & $34.5 \pm 0.1$ & 425 & $\mathrm{C}$ & [1981HOS/SCO3] \\
\hline & $\mathrm{V}$ & $(346-437)$ & $42.4 \pm 0.1$ & 298 & $\mathrm{C}$ & [1981HOS/SCO3] \\
\hline & V & & 42.3 & 298 & & [1971WIL/ZWO] \\
\hline & $\mathrm{V}$ & & $42.2 \pm 0.1$ & 298 & $\mathrm{C}$ & [1947OSB/GIN] \\
\hline & $\mathrm{V}$ & $(330-410)$ & 40.0 & 345 & MM & [1945WIL/TAY, 1949FOR/NOR] \\
\hline & $\mathrm{V}$ & & 42.5 & 294 & & {$[1946 \mathrm{SCO} / \mathrm{BRI}]$} \\
\hline \multirow[t]{2}{*}{$\mathrm{C}_{8} \mathrm{H}_{10}$} & {$[27998-49-8]$} & 1,2-bicyclopro & etylene & & & \\
\hline & $\mathrm{V}$ & & $47.6 \pm 0.2$ & 298 & $\mathrm{C}$ & [2007PAS/KUZ] \\
\hline \multirow[t]{2}{*}{$\mathrm{C}_{8} \mathrm{H}_{10} \mathrm{ClNO}_{2}$} & {$[6358-64-1]$} & 4-chloro-2,5-di & oxyaniline & & & \\
\hline & FUS & & 26.68 & 391.9 & DSC & {$[2015 \mathrm{ZHA} / \mathrm{JIN}]$} \\
\hline \multirow[t]{2}{*}{$\mathrm{C}_{8} \mathrm{H}_{10} \mathrm{Cl}_{2} \mathrm{O}_{2}$} & {$[55701-05-8]$} & \multicolumn{5}{|c|}{ 3-(2,2-dichloroethenyl)-2,2-dimethylcyclopropanecarboxylic acid (ratio of cis/trans-is 35/65) } \\
\hline & FUS & $(78-389)$ & 16.32 & 331.5 & $\mathrm{AC}$ & [2007XUE/WAN] \\
\hline
\end{tabular}


TABLE 11. Phase change enthalpies of $\mathrm{C}_{8}$ organic compounds-Continued

\begin{tabular}{|c|c|c|c|c|c|c|}
\hline \multirow[b]{2}{*}{$\begin{array}{l}\text { Molecular } \\
\text { formula }\end{array}$} & \multirow{2}{*}{$\begin{array}{l}\text { CAS reg. no. } \\
\text { Transition }\end{array}$} & \multicolumn{5}{|l|}{ Compound } \\
\hline & & $\begin{array}{c}\text { Temp. range } \\
\text { (K) }\end{array}$ & $\begin{array}{c}\Delta_{\text {trans }} H_{m} \\
\left(\mathrm{~kJ} \mathrm{~mol}^{-1}\right)\end{array}$ & $T_{\mathrm{m}}(\mathrm{K})$ & Method & References \\
\hline \multirow[t]{2}{*}{$\mathrm{C}_{8} \mathrm{H}_{10} \mathrm{Cl}_{2} \mathrm{O}_{2}$} & {$[55701-03-6]$} & \multicolumn{5}{|c|}{ trans-( $R$ )-3-(2,2-dichloroethenyl)-2,2-dimethylcyclopropanecarboxylic acid } \\
\hline & FUS & $(78-389)$ & 13.75 & 344.8 & $\mathrm{AC}$ & [2008XUE/LI] \\
\hline \multirow[t]{2}{*}{$\mathrm{C}_{8} \mathrm{H}_{10} \mathrm{~F}_{3} \mathrm{NO}_{3}$} & {$[715-58-2]$} & \multicolumn{5}{|c|}{$N$-trifluoroacetyl- $l$-proline, methyl ester } \\
\hline & $\mathrm{V}$ & $(303-523)$ & 57.9 & 318 & A & [1987STE/MAL, 1999DYK/SVO, 1960WEY/KLI] \\
\hline \multirow{2}{*}{$\mathrm{C}_{8} \mathrm{H}_{10} \mathrm{~F}_{3} \mathrm{NO}_{5}$} & [81084-01-7] & \multicolumn{5}{|c|}{$N$-trifluoroacetyl- $l$-aspartic acid, dimethyl ester } \\
\hline & $\mathrm{V}$ & $(303-423)$ & 58.2 & 318 & A & [1987STE/MAL, 1999DYK/SVO, 1960WEY/KLI] \\
\hline \multirow{6}{*}{$\mathrm{C}_{8} \mathrm{H}_{10} \mathrm{NO}_{5} \mathrm{PS}$} & {$[298-00-0]$} & \multicolumn{5}{|c|}{$O, O$-dimethyl- $O$-(4-nitrophenyl) thiophosphate } \\
\hline & FUS & & 20.07 & 308.2 & DSC & [1991ACR, 1990DON/DRE] \\
\hline & SUB & $(298-308)$ & 125.1 & 303 & GS,A & [1984KIM/WOO] \\
\hline & SUB & $(278-288)$ & 108.7 & 283 & GS & [1983SPE/CLI, 1979SPE/SHO] \\
\hline & $\mathrm{V}$ & $(293-427)$ & 88.9 & 308 & A & [1987STE/MAL] \\
\hline & $\mathrm{V}$ & & 87.0 & & GS & [1979SPE/SHO] \\
\hline \multirow[t]{2}{*}{$\mathrm{C}_{8} \mathrm{H}_{10} \mathrm{~N}_{2} \mathrm{O}$} & [138-89-6] & \multicolumn{5}{|c|}{ 4-N, N-dimethylaminonitrosobenzene } \\
\hline & SUB & $(323-334)$ & $82.0 \pm 1.7$ & 298 & $\mathrm{ME}$ & [1994ACR/TUC] \\
\hline \multirow[t]{6}{*}{$\mathrm{C}_{8} \mathrm{H}_{10} \mathrm{~N}_{2} \mathrm{O}$} & {$[6972-69-6]$} & \multicolumn{5}{|c|}{$N, N$-dimethylnicotinamide } \\
\hline & FUS & & 19.4 & 317.3 & DSC & [2015ALM/OLI] \\
\hline & SUB & $(308-315)$ & $93.6 \pm 0.4$ & 312 & DM & [2015ALM/OLI] \\
\hline & SUB & $(308-315)$ & $94.0 \pm 0.8$ & 298 & DM & [2015ALM/OLI] \\
\hline & V & $(307-370)$ & $73.0 \pm 0.1$ & 338 & DM & [2015ALM/OLI] \\
\hline & $\mathrm{V}$ & $(307-370)$ & $76.1 \pm 0.2$ & 298 & DM & [2015ALM/OLI] \\
\hline \multirow[t]{4}{*}{$\mathrm{C}_{8} \mathrm{H}_{10} \mathrm{~N}_{2} \mathrm{O}_{2}$} & [619-31-8] & \multicolumn{5}{|c|}{ 3-nitro- $N, N$-dimethylaniline } \\
\hline & SUB & & $92.7 \pm 0.3$ & 298 & $\mathrm{C}$ & [1985MUR/SAK] \\
\hline & $\mathrm{V}$ & $(427-558)$ & 52.3 & 442 & A, GS, EB & [1987STE/MAL, 1960AND/BID] \\
\hline & V & $(357-492)$ & 48.2 & 372 & & [1955VON/GEB] \\
\hline \multirow[t]{4}{*}{$\mathrm{C}_{8} \mathrm{H}_{10} \mathrm{~N}_{2} \mathrm{O}_{2}$} & {$[100-23-2]$} & \multicolumn{5}{|c|}{$N, N$-dimethyl-4-nitroaniline } \\
\hline & SUB & & $102.7 \pm 1.1$ & 298 & $\mathrm{C}$ & [1985MUR/SAK] \\
\hline & SUB & $(344-366)$ & $98.7 \pm 1.7$ & 355 & $\mathrm{ME}$ & [1987STE/MAL, 1956MAJ] \\
\hline & SUB & $(372-393)$ & $101.3 \pm 2.0$ & 298 & ME & [1994ACR/TUC] \\
\hline $\mathrm{C}_{8} \mathrm{H}_{10} \mathrm{~N}_{2} \mathrm{O}_{3}$ & [22809-78-5] & $N$-methyl- $N$ - ( & thoxyphenyl) & nine & & \\
\hline & FUS & & 22.7 & 342.6 & DSC & [2002DAS/ZAL] \\
\hline $\mathrm{C}_{8} \mathrm{H}_{10} \mathrm{~N}_{2} \mathrm{O}_{3} \mathrm{~S}$ & [144-80-9] & $N$-[(4-aminoph & sulfonyl]acet & (sulface & & \\
\hline & FUS & & 29.8 & 455.2 & DSC & [2002MAR/GOM, 2001MAR/GOM] \\
\hline $\mathrm{C}_{8} \mathrm{H}_{10} \mathrm{~N}_{2} \mathrm{O}_{4} \mathrm{~S}$ & {$[156461-84-6]$} & $N$-methyl- $N-($ & thylsulfonylp & nitramin & & \\
\hline & FUS & & 26.1 & 377.8 & DSC & [2002DAS/ZAL] \\
\hline $\mathrm{C}_{8} \mathrm{H}_{10} \mathrm{~N}_{2} \mathrm{O}_{4} \mathrm{~S}$ & {$[23042-38-8]$} & $N$-methyl- $N$ - ( & thylsulfonylp & nitramin & & \\
\hline & FUS & & 19.2 & 438.1 & DSC & [2002DAS/ZAL] \\
\hline $\mathrm{C}_{8} \mathrm{H}_{10} \mathrm{~N}_{2} \mathrm{~S}$ & [536-33-4] & 2-ethyl-4-pyrid & rbothioamide & & & \\
\hline & SUB & $(364-381)$ & $164.2 \pm 1.8$ & 372 & GS & [2015BLO/SHA2] \\
\hline & SUB & $(364-381)$ & $166.5 \pm 1.8$ & 298 & GS & [2015BLO/SHA2] \\
\hline $\mathrm{C}_{8} \mathrm{H}_{10} \mathrm{~N}_{4} \mathrm{O}_{2}$ & [58-08-2] & caffeine $(1,3,7-$ & thylxanthine) & & & \\
\hline & TRS & & 3.29 & 421.5 & & \\
\hline & FUS & & 20.08 & 510.2 & DSC & [2015BOU/BOU] \\
\hline & FUS & & 19.52 & 510.3 & DSC & [2014DIC/LEG] \\
\hline & FUS & & 20.37 & 508.8 & DSC & [2012KLI/LEI] \\
\hline & FUS & & 19.6 & 508.7 & DSC & [2012AGA/MOS] \\
\hline & TRS & & 2.6 & 405.8 & & \\
\hline & FUS & & 17.9 & 505.4 & DSC & [2012MAN/VIL] \\
\hline & FUS & & 20.8 & 510.0 & DSC & [2010BAI/VAN] \\
\hline & FUS & & 24.8 & 507.7 & DSC & [2010GUO/SAD] \\
\hline & TRS & & 3.00 & 414.9 & & \\
\hline & FUS & & 23.46 & 509.6 & DSC & [2008MOG/SEP] \\
\hline
\end{tabular}


TABLE 11. Phase change enthalpies of $\mathrm{C}_{8}$ organic compounds-Continued

\begin{tabular}{|c|c|c|c|c|c|c|}
\hline \multirow[b]{2}{*}{$\begin{array}{l}\text { Molecular } \\
\text { formula }\end{array}$} & \multirow{2}{*}{$\begin{array}{c}\text { CAS reg. no. } \\
\text { Transition }\end{array}$} & \multicolumn{5}{|l|}{ Compound } \\
\hline & & $\begin{array}{c}\text { Temp. range } \\
(\mathrm{K})\end{array}$ & $\begin{array}{c}\Delta_{\text {trans }} H_{m} \\
\left(\mathrm{~kJ} \mathrm{~mol}^{-1}\right)\end{array}$ & $T_{\mathrm{m}}(\mathrm{K})$ & Method & References \\
\hline & TRS & & 3.43 & 420.9 & & \\
\hline & FUS & & 19.86 & 509.5 & DSC & [2007DON/LI] \\
\hline & TRS & & 4.02 & 428.2 & & \\
\hline & FUS & & 21.9 & 510.2 & DSC & [2006PIN/DIO] \\
\hline & TRS & & 3.9 & 428 & DSC & [2005DES/COR] \\
\hline & FUS & & 19.38 & 510.2 & DSC & [2005KLO/BRO] \\
\hline & TRS & & 3.94 & 426 & & \\
\hline & FUS & & 23.43 & 512 & DSC & [1990DOM/HEA, 1980CES/STA] \\
\hline & FUS & & 18.3 & 510 & & [1985OHM/LIP] \\
\hline & TRS & & 3.7 & 423.2 & & \\
\hline & FUS & & 21.5 & 509.2 & DSC & [1985SUZ/SHI] \\
\hline & TRS & & 1.71 & 420.8 & & \\
\hline & FUS & & 20.95 & 508.3 & $\mathrm{DSC}$ & [1984WEI/LEF] \\
\hline & SUB (I) & $(413-463)$ & $104.8 \pm 0.2$ & 438 & $\mathrm{~T}$ & [1999GRI/SZE] \\
\hline & SUB (I) & $(413-463)$ & 115 & 298 & $\mathrm{~T}$ & [1999GRI/SZE] \\
\hline & SUB (II) & $(413-463)$ & $113.6 \pm 0.2$ & 369 & $\mathrm{~T}$ & [1999GRI/SZE] \\
\hline & SUB (II) & $(413-463)$ & 119 & 298 & $\mathrm{~T}$ & [1999GRI/SZE] \\
\hline & SUB & $(315-364)$ & $112.6 \pm 2.4$ & & $\mathrm{ME}$ & [1998BOL/WIE] \\
\hline & SUB & & $105.1 \pm 0.7$ & & $\mathrm{ME}$ & [1985KAM/ZIE] \\
\hline & SUB & $(373-473)$ & 103.6 & 423 & UV & [1984EBE/FRA] \\
\hline & SUB (I) & $(446-509)$ & $100.0 \pm 0.6$ & 478 & MM & [1979BOT/CAM] \\
\hline & SUB (I) & & 110 & 298 & & [1979BOT/CAM, 1999GRI/SZE] \\
\hline & SUB (II) & $(446-509)$ & $110.7 \pm 0.7$ & 362 & MM & [1979BOT/CAM] \\
\hline & SUB (II) & & 114 & 298 & & [1979BOT/CAM, 1999GRI/SZE] \\
\hline & $\mathrm{V}$ & $(634-743)$ & $64.9 \pm 2.4$ & & DSC & [1998BOL/WIE] \\
\hline \multirow[t]{5}{*}{$\mathrm{C}_{8} \mathrm{H}_{10} \mathrm{O}$} & {$[526-75-0]$} & 2,3-dimethylphenol & & & & \\
\hline & FUS & & 20.29 & 345.8 & DSC & [1998JAM/PAL] \\
\hline & FUS & & 21.02 & 346 & DSC & [1991ACR, 1982POE/FAN] \\
\hline & SUB & $(283-323)$ & $84.0 \pm 1.0$ & & GS & [1960AND/BID, 1970COX/PIL, 1987STE/MAL] \\
\hline & $\mathrm{V}$ & $(433-492)$ & 52.1 & 448 & A, GS, EB & [1987STE/MAL, 1960AND/BID] \\
\hline \multirow[t]{6}{*}{$\mathrm{C}_{8} \mathrm{H}_{10} \mathrm{O}$} & {$[105-67-9]$} & 2,4-dimethylphenol & & & & \\
\hline & FUS & & 12.76 & 297.3 & DSC & [1998JAM/PAL] \\
\hline & V & $(393-433)$ & 64.6 & 298 & CGC & {$[1995 \mathrm{CHI} / \mathrm{HOS}]$} \\
\hline & $\mathrm{V}$ & $(282-318)$ & 65.9 & 297 & A & [1987STE/MAL] \\
\hline & $\mathrm{V}$ & $(282-318)$ & $65.9 \pm 0.2$ & & GS & [1960AND/BID, 1970COX/PIL] \\
\hline & $\mathrm{V}$ & $(429-486)$ & 51.8 & 444 & A, GS, EB & [1987STE/MAL, 1960AND/BID] \\
\hline \multirow[t]{5}{*}{$\mathrm{C}_{8} \mathrm{H}_{10} \mathrm{O}$} & {$[95-87-4]$} & 2,5-dimethylphenol & & & & \\
\hline & FUS & & 13.81 & 348.1 & DSC & [1998JAM/PAL] \\
\hline & FUS & & 23.38 & 348 & DSC & [1991ACR, 1982POE/FAN] \\
\hline & SUB & $(282-323)$ & $85.0 \pm 0.25$ & & GS & [1960AND/BID, 1970COX/PIL, 1987STE/MAL] \\
\hline & $\mathrm{V}$ & $(427-485)$ & 51.7 & 442 & A, GS, EB & [1987STE/MAL, 1960AND/BID] \\
\hline \multirow[t]{5}{*}{$\mathrm{C}_{8} \mathrm{H}_{10} \mathrm{O}$} & [576-26-1] & 2,6-dimethylphenol & & & & \\
\hline & FUS & & 18.83 & 318.6 & DSC & [1998JAM/PAL] \\
\hline & FUS & & 18.9 & 318.9 & DSC & [1991ACR, 1982POE/FAN] \\
\hline & SUB & & 75.6 & 298 & & [1971MOR] \\
\hline & SUB & & 75.1 & 298 & & [1968MOR] \\
\hline \multicolumn{7}{|c|}{ [Note: The author of [1968MOR] refers to the determined value as the enthalpy of vaporization even though the compound is a solid.] } \\
\hline & SUB & $(277-313)$ & $75.6 \pm 0.17$ & & GS & [1960AND/BID, 1970COX/PIL] \\
\hline & V & & 75.6 & 298 & & [1971MOR] \\
\hline & V & & 75.1 & 298 & & [1968MOR] \\
\hline & $\mathrm{V}$ & $(417-476)$ & 48.5 & 432 & A, GS, EB & [1987STE/MAL, 1960AND/BID] \\
\hline \multirow[t]{3}{*}{$\mathrm{C}_{8} \mathrm{H}_{10} \mathrm{O}$} & {$[95-65-8]$} & 3,4-dimethylphenol & & & & \\
\hline & FUS & & 19.04 & 338.5 & DSC & [1998JAM/PAL] \\
\hline & FUS & & 18.13 & 334 & DSC & [1991ACR, 1982POE/FAN] \\
\hline
\end{tabular}


TABLE 11. Phase change enthalpies of $\mathrm{C}_{8}$ organic compounds-Continued

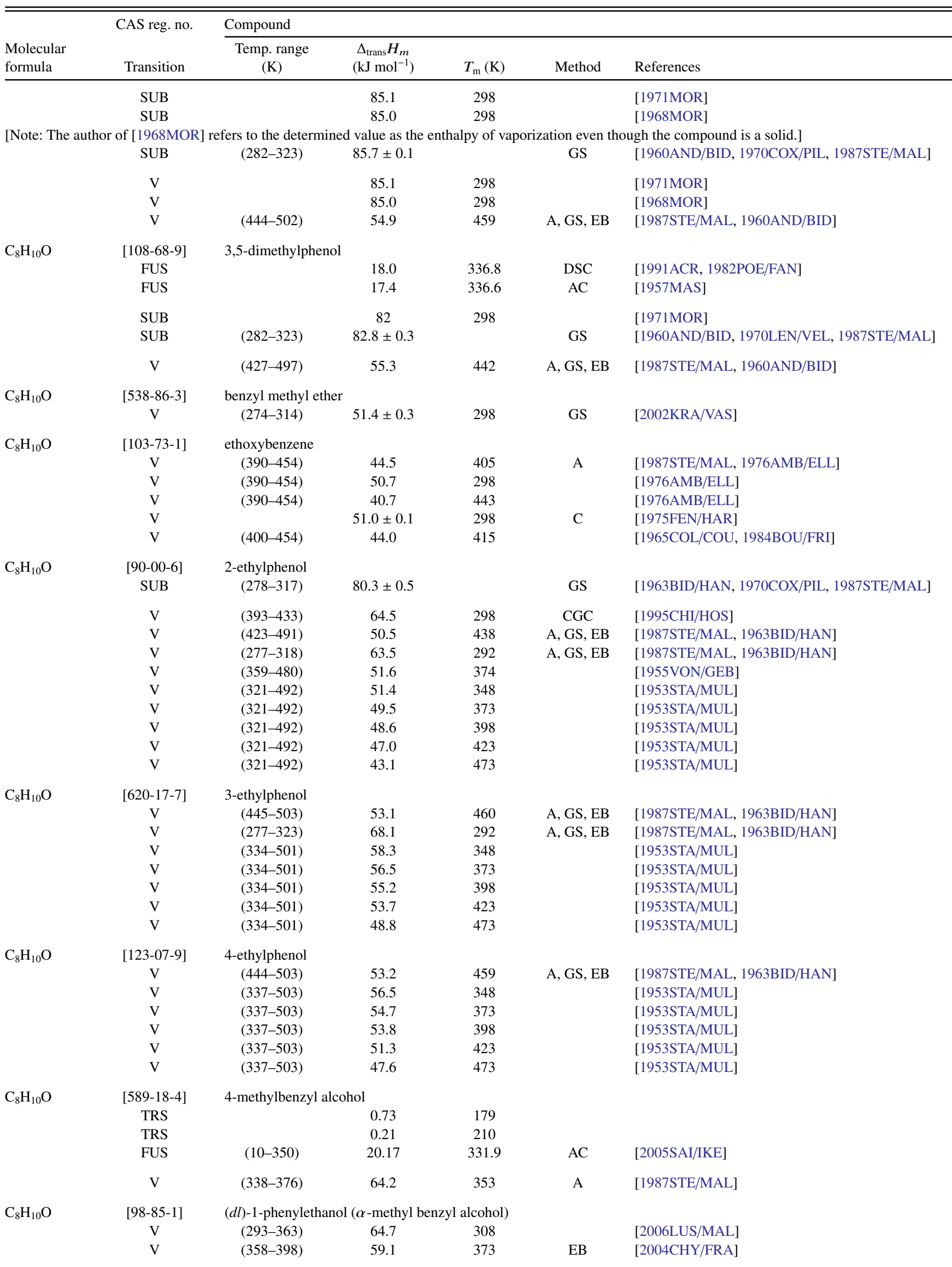


TABLE 11. Phase change enthalpies of $\mathrm{C}_{8}$ organic compounds-Continued

\begin{tabular}{|c|c|c|c|c|c|c|}
\hline \multirow[b]{2}{*}{$\begin{array}{l}\text { Molecular } \\
\text { formula }\end{array}$} & \multirow{2}{*}{$\begin{array}{c}\text { CAS reg. no. } \\
\text { Transition }\end{array}$} & \multicolumn{5}{|l|}{ Compound } \\
\hline & & $\begin{array}{c}\text { Temp. range } \\
(\mathrm{K})\end{array}$ & $\begin{array}{c}\Delta_{\text {trans }} H_{m} \\
\left(\mathrm{~kJ} \mathrm{~mol}^{-1}\right) \\
\end{array}$ & $T_{\mathrm{m}}(\mathrm{K})$ & Method & References \\
\hline & $\mathrm{V}$ & $(358-398)$ & 75.2 & 298 & EB & [2004CHY/FRA] \\
\hline & $\mathrm{V}$ & $(353-480)$ & 53.5 & 368 & A & [1987STE/MAL] \\
\hline \multirow[t]{9}{*}{$\mathrm{C}_{8} \mathrm{H}_{10} \mathrm{O}$} & {$[60-12-8]$} & 2-phenylethanol & & & & \\
\hline & V & $(288-363)$ & 66.7 & 298 & GS & [2007EME/DAB] \\
\hline & $\mathrm{V}$ & $(313-413)$ & U54.55 & 298 & GC & [2005HOS/GRY] \\
\hline & $\mathrm{V}$ & $(394-613)$ & 55.1 & 409 & A & [1987STE/MAL] \\
\hline & $\mathrm{V}$ & $(305-363)$ & $73.4 \pm 1.5$ & 298 & GC & [1981SHC/RUD, 2007EME/DAB] \\
\hline & $\mathrm{V}$ & $(284-287)$ & $69.7 \pm 1.6$ & 298 & $\mathrm{ME}$ & [1958SER/VOI, 2007EME/DAB] \\
\hline & $\mathrm{V}$ & $(283-318)$ & 68.4 & 298 & $\mathrm{~A}, \mathrm{ME}$ & [1987STE/MAL, 1954SER/VOI] \\
\hline & $\mathrm{V}$ & $(406-492)$ & $64.9 \pm 0.3$ & 298 & $\mathrm{~EB}$ & [1949DRE/SHR, 1949DRE/MAR, 2007EME/DAB] \\
\hline & $\mathrm{V}$ & $(331-492)$ & 68 & 298 & EB & [1947STU, 2007EME/DAB] \\
\hline \multirow[t]{3}{*}{$\mathrm{C}_{8} \mathrm{H}_{10} \mathrm{O}$} & {$[578-58-5]$} & 2-methylanisole & & & & \\
\hline & $\mathrm{V}$ & $(276-308)$ & $50.2 \pm 0.4$ & 298 & GS & [2015EME/ZAI] \\
\hline & $\mathrm{V}$ & & 45.2 & & & [1986BAL/GNA] \\
\hline \multirow[t]{2}{*}{$\mathrm{C}_{8} \mathrm{H}_{10} \mathrm{O}$} & {$[100-84-5]$} & 3-methylanisole & & & & \\
\hline & $\mathrm{V}$ & $(277-312)$ & $52.8 \pm 0.5$ & 298 & GS & [2015EME/ZAI] \\
\hline \multirow[t]{3}{*}{$\mathrm{C}_{8} \mathrm{H}_{10} \mathrm{O}$} & [104-93-8] & 4-methylanisole & & & & \\
\hline & $\mathrm{V}$ & $(275-308)$ & $53.3 \pm 0.4$ & 298 & GS & [2015EME/ZAI] \\
\hline & V & & 46.0 & & & [1986BAL/GNA] \\
\hline \multirow[t]{2}{*}{$\mathrm{C}_{8} \mathrm{H}_{10} \mathrm{OS}$} & [1879-16-9] & 4-methoxythioan & & & & \\
\hline & V & & 53.6 & & & [1986BAL/GNA] \\
\hline \multirow[t]{2}{*}{$\mathrm{C}_{8} \mathrm{H}_{10} \mathrm{OS}$} & {$[2530-10-1]$} & 3-acetyl-2,5-dim & lthiophene & & & \\
\hline & V & & $61.3 \pm 1.3$ & 298 & $\mathrm{C}$ & [2008RIB/SAN3] \\
\hline \multirow[t]{2}{*}{$\mathrm{C}_{8} \mathrm{H}_{10} \mathrm{O}_{2}$} & & ethyl trans- $b-(2-$ & 1)acrylate & & & \\
\hline & $\mathrm{V}$ & $(428-500)$ & 56.8 & 464 & & {$[1956 \mathrm{FRO} / \mathrm{LOE}]$} \\
\hline \multirow[t]{3}{*}{$\mathrm{C}_{8} \mathrm{H}_{10} \mathrm{O}_{2}$} & {$[105-13-5]$} & 4-methoxybenzy & cohol & & & \\
\hline & $\mathrm{V}$ & $(394-424)$ & 95.6 & 409 & A & [1987STE/MAL] \\
\hline & V & $(354-453)$ & 71.7 & 369 & $\mathrm{~EB}$ & [1985SCH/BRU] \\
\hline \multirow[t]{2}{*}{$\mathrm{C}_{8} \mathrm{H}_{10} \mathrm{O}_{2}$} & {$[488-87-9]$} & 1,3-dihydroxy-2, & imethylbenzene & & & \\
\hline & V & (393-459) & 74.7 & 408 & A, GC & [1987STE/MAL, 1975KUN/LIL] \\
\hline \multirow[t]{2}{*}{$\mathrm{C}_{8} \mathrm{H}_{10} \mathrm{O}_{2}$} & {$[527-55-9]$} & 1,3-dihydroxy-4, & imethylbenzene & & & \\
\hline & V & $(424-453)$ & 67.5 & 438 & $\mathrm{~A}, \mathrm{GC}$ & [1987STE/MAL, 1975KUN/LIL] \\
\hline \multirow[t]{2}{*}{$\mathrm{C}_{8} \mathrm{H}_{10} \mathrm{O}_{2}$} & {$[615-89-4]$} & 1,3-dihydroxy-4, & imethylbenzene & & & \\
\hline & $\mathrm{V}$ & $(388-466)$ & 74.7 & 403 & A, GC & [1975KUN/LIL] \\
\hline \multirow[t]{2}{*}{$\mathrm{C}_{8} \mathrm{H}_{10} \mathrm{O}_{2}$} & {$[615-90-7]$} & 1,4-dihydroxy-2, & imethylbenzene & & & \\
\hline & $\mathrm{V}$ & $(331-361)$ & 101.1 & 346 & A & [1987STE/MAL] \\
\hline \multirow[t]{2}{*}{$\mathrm{C}_{8} \mathrm{H}_{10} \mathrm{O}_{2}$} & {$[4299-72-3]$} & 1,3-dihydroxy-5- & ylbenzene & & & \\
\hline & $\mathrm{V}$ & $(408-479)$ & 81.3 & 423 & $\mathrm{~A}, \mathrm{GC}$ & [1987STE/MAL, 1975KUN/LIL] \\
\hline \multirow[t]{2}{*}{$\mathrm{C}_{8} \mathrm{H}_{10} \mathrm{O}_{2}$} & {$[615-90-7]$} & 2,5-dimethylhyd & dinone & & & \\
\hline & SUB & $(332-361)$ & 100.8 & & QF & [1927COO/COO, 1960JON] \\
\hline \multirow[t]{8}{*}{$\mathrm{C}_{8} \mathrm{H}_{10} \mathrm{O}_{2}$} & {$[91-16-7]$} & 1,2-dimethoxybe & & & & \\
\hline & FUS & & 12.6 & 295.7 & DSC & [2003LEE/CHO] \\
\hline & FUS & & 15.8 & 295.9 & & [1911LOU/DUP] \\
\hline & $\mathrm{V}$ & $(293-332)$ & $64.5 \pm 0.3$ & 298 & GS & [2010VAR/ABA] \\
\hline & $\mathrm{V}$ & $(373-468)$ & 52.7 & 388 & & [2002SU, 2004LEE/SU] \\
\hline & $\mathrm{V}$ & $(373-468)$ & $61.1 \pm 0.2$ & 298 & & [2002SU, 2004LEE/SU, 2010VAR/ABA] \\
\hline & $\mathrm{V}$ & & $68.1 \pm 1.4$ & 298 & $\mathrm{C}$ & [2000MAT/MIR] \\
\hline & $\mathrm{V}$ & & 66.9 & 298 & & [1958CAS/FLE2, 2010VAR/ABA] \\
\hline \multirow[t]{4}{*}{$\mathrm{C}_{8} \mathrm{H}_{10} \mathrm{O}_{2}$} & {$[151-10-0]$} & 1,3-dimethoxybe & & & & \\
\hline & V & $(296-332)$ & $59.7 \pm 0.2$ & 298 & GS & [2010VAR/ABA] \\
\hline & $\mathrm{V}$ & & $61.5 \pm 1.4$ & 298 & $\mathrm{C}$ & [2000MAT/MIR] \\
\hline & $\mathrm{V}$ & $(358-423)$ & 60.8 & 373 & A, GC & [1987STE/MAL, 1975KUN/LIL] \\
\hline
\end{tabular}

$\mathrm{C}_{8} \mathrm{H}_{10} \mathrm{O}_{2}$ 
TABLE 11. Phase change enthalpies of $\mathrm{C}_{8}$ organic compounds-Continued

\begin{tabular}{|c|c|c|c|c|c|c|}
\hline \multirow[b]{2}{*}{$\begin{array}{l}\text { Molecular } \\
\text { formula }\end{array}$} & \multirow{2}{*}{$\begin{array}{c}\text { CAS reg. no. } \\
\text { Transition }\end{array}$} & \multicolumn{5}{|l|}{ Compound } \\
\hline & & $\begin{array}{c}\text { Temp. range } \\
\text { (K) }\end{array}$ & $\begin{array}{c}\Delta_{\text {trans }} H_{m} \\
\left(\mathrm{~kJ} \mathrm{~mol}^{-1}\right)\end{array}$ & $T_{\mathrm{m}}(\mathrm{K})$ & Method & References \\
\hline & FUS & & 20.1 & 333.0 & DSC & [2010VAR/ABA] \\
\hline & SUB & $(283-327)$ & $80.3 \pm 0.2$ & 298 & GS & [2010VAR/ABA] \\
\hline & SUB & & $84.1 \pm 2.3$ & 298 & $\mathrm{C}$ & [2000MAT/MIR] \\
\hline & $\mathrm{V}$ & $(330-357)$ & $61.6 \pm 0.2$ & 298 & GS & [2010VAR/ABA] \\
\hline & $\mathrm{V}$ & $(298-357)$ & 62.1 & 313 & A & [1987STE/MAL] \\
\hline & $\mathrm{V}$ & & 51.5 & & & [1986BAL/GNA] \\
\hline \multirow[t]{2}{*}{$\mathrm{C}_{8} \mathrm{H}_{10} \mathrm{O}_{2}$} & [122-99-6] & 2-phenoxyethanol & & & & \\
\hline & $\mathrm{V}$ & $(351-519)$ & 66.0 & 366 & A & [1987STE/MAL, 1947STU] \\
\hline \multirow{2}{*}{$\mathrm{C}_{8} \mathrm{H}_{10} \mathrm{O}_{2}$} & {$[2896-67-5]$} & 3-methoxy-4-hydr & ytoluene & & & \\
\hline & $\mathrm{V}$ & $(356-495)$ & 53.2 & 371 & A & [1987STE/MAL] \\
\hline \multirow[t]{2}{*}{$\mathrm{C}_{8} \mathrm{H}_{10} \mathrm{O}_{2}$} & [135-02-4] & 2-methoxybenzald & yde & & & \\
\hline & $\mathrm{V}$ & & 55.2 & & & [1986BAL/GNA] \\
\hline \multirow[t]{2}{*}{$\mathrm{C}_{8} \mathrm{H}_{10} \mathrm{O}_{2}$} & [501-94-0] & 4-hydroxybenzene & hanol & & & \\
\hline & FUS & & 25.9 & 364 & DSC & [2009QUE/MOT] \\
\hline \multirow[t]{2}{*}{$\mathrm{C}_{8} \mathrm{H}_{10} \mathrm{O}_{2}$} & {$[10599-70-9]$} & 3-acetyl-2,5-dimet & lfuran & & & \\
\hline & $\mathrm{V}$ & & $57.5 \pm 1.5$ & 298 & $\mathrm{C}$ & [2011RIB/AMA] \\
\hline \multirow[t]{6}{*}{$\mathrm{C}_{8} \mathrm{H}_{10} \mathrm{O}_{2}$} & {$[94-71-3]$} & 2-ethoxyphenol & & & & \\
\hline & $\mathrm{V}$ & $(380-486)$ & 54.7 & 392 & EB & [2012PAL/ORA] \\
\hline & $\mathrm{V}$ & $(380-486)$ & 52.3 & 415 & $\mathrm{~EB}$ & [2012PAL/ORA] \\
\hline & V & $(380-486)$ & 50.9 & 428 & EB & [2012PAL/ORA] \\
\hline & $\mathrm{V}$ & $(380-486)$ & 49.3 & 445 & $\mathrm{~EB}$ & [2012PAL/ORA] \\
\hline & $\mathrm{V}$ & $(380-486)$ & 48.3 & 457 & EB & [2012PAL/ORA] \\
\hline \multirow{3}{*}{$\mathrm{C}_{8} \mathrm{H}_{10} \mathrm{O}_{2} \mathrm{~S}$} & {$[3112-90-1]$} & benzyl methyl sulf & & & & \\
\hline & FUS & & 25.52 & 400.5 & FPM & [1961BUS/IVI] \\
\hline & $\mathrm{V}$ & $(455-529)$ & 64.9 & 470 & $\mathrm{~A}, \mathrm{BG}$ & [1987STE/MAL, 1999DYK/SVO, 1961BUS/IVI] \\
\hline \multirow[t]{2}{*}{$\mathrm{C}_{8} \mathrm{H}_{10} \mathrm{O}_{2} \mathrm{~S}$} & [57382-97-5] & ethyl 2-thiophenea & etate & & & \\
\hline & $\mathrm{V}$ & & $61.8 \pm 1.3$ & 298 & $\mathrm{C}$ & [2009RIB/SAN2] \\
\hline \multirow[t]{2}{*}{$\mathrm{C}_{8} \mathrm{H}_{10} \mathrm{O}_{2} \mathrm{~S}$} & {$[37784-63-7]$} & ethyl 3-thiophenea & etate & & & \\
\hline & $\mathrm{V}$ & & $63.2 \pm 1.3$ & 298 & $\mathrm{C}$ & [2009RIB/SAN2] \\
\hline \multirow[t]{4}{*}{$\mathrm{C}_{8} \mathrm{H}_{10} \mathrm{O}_{3}$} & [13149-00-3] & cis-cyclohexane-1, & -dicarboxylic & anhydride & & \\
\hline & TRS & $(12-330)$ & 5.59 & 304 & & \\
\hline & FUS & $(12-330)$ & 0.85 & 310.5 & $\mathrm{AC}$ & [1983GEI/NUR] \\
\hline & $\mathrm{V}$ & $(325-525)$ & $48.8 \pm 0.1$ & & & [1984NUR/MEK] \\
\hline \multirow[t]{2}{*}{$\mathrm{C}_{8} \mathrm{H}_{10} \mathrm{O}_{3}$} & {$[85-42-7]$} & 1,2-cyclohexanedi & arboxylic anh & & & \\
\hline & FUS & $(80-390)$ & 14.71 & 303.8 & $\mathrm{AC}$ & [2008LU/GAO] \\
\hline \multirow[t]{2}{*}{$\mathrm{C}_{8} \mathrm{H}_{10} \mathrm{O}_{3}$} & {$[5150-42-5]$} & 2,3-dimethoxyphe & & & & \\
\hline & $\mathrm{V}$ & & $76.5 \pm 0.5$ & 298 & $\mathrm{C}$ & [2003MAT/MIR] \\
\hline \multirow[t]{2}{*}{$\mathrm{C}_{8} \mathrm{H}_{10} \mathrm{O}_{3}$} & {$[91-10-1]$} & 2,6-dimethoxyphe & & & & \\
\hline & SUB & & $98.4 \pm 1.1$ & 298 & $\mathrm{C}$ & [2003MAT/MIR] \\
\hline \multirow[t]{2}{*}{$\mathrm{C}_{8} \mathrm{H}_{10} \mathrm{O}_{3}$} & [500-99-2] & 3,5-dimethoxyphe & & & & \\
\hline & SUB & & $101.1 \pm 2.3$ & 298 & $\mathrm{C}$ & [2003MAT/MIR] \\
\hline \multirow[t]{3}{*}{$\mathrm{C}_{8} \mathrm{H}_{10} \mathrm{O}_{4}$} & & trans,trans-2,6-oct & diene-1,8-dio & & & \\
\hline & TRS & & 11.04 & 439 & & \\
\hline & FUS & & 27.77 & 541 & & [1969COR/FRA] \\
\hline \multirow[t]{2}{*}{$\mathrm{C}_{8} \mathrm{H}_{10} \mathrm{O}_{4}$} & & trans,cis-2,6-octad & ne-1,8-dioic & & & \\
\hline & FUS & & 22.78 & 380 & & [1969COR/FRA] \\
\hline \multirow[t]{2}{*}{$\mathrm{C}_{8} \mathrm{H}_{10} \mathrm{O}_{6}$} & [59743-08-7] & dioxobutanedioic a & id, diethyl es & & & \\
\hline & V & $(343-507)$ & 59.3 & 358 & A & [1987STE/MAL, 1947STU] \\
\hline $\mathrm{C}_{8} \mathrm{H}_{10} \mathrm{~S}$ & [766-92-7] & benzyl methyl sulf & & & & \\
\hline & $\mathrm{V}$ & (336-368) & 51.8 & 351 & & [1999DYK/SVO] \\
\hline
\end{tabular}


TABLE 11. Phase change enthalpies of $\mathrm{C}_{8}$ organic compounds-Continued

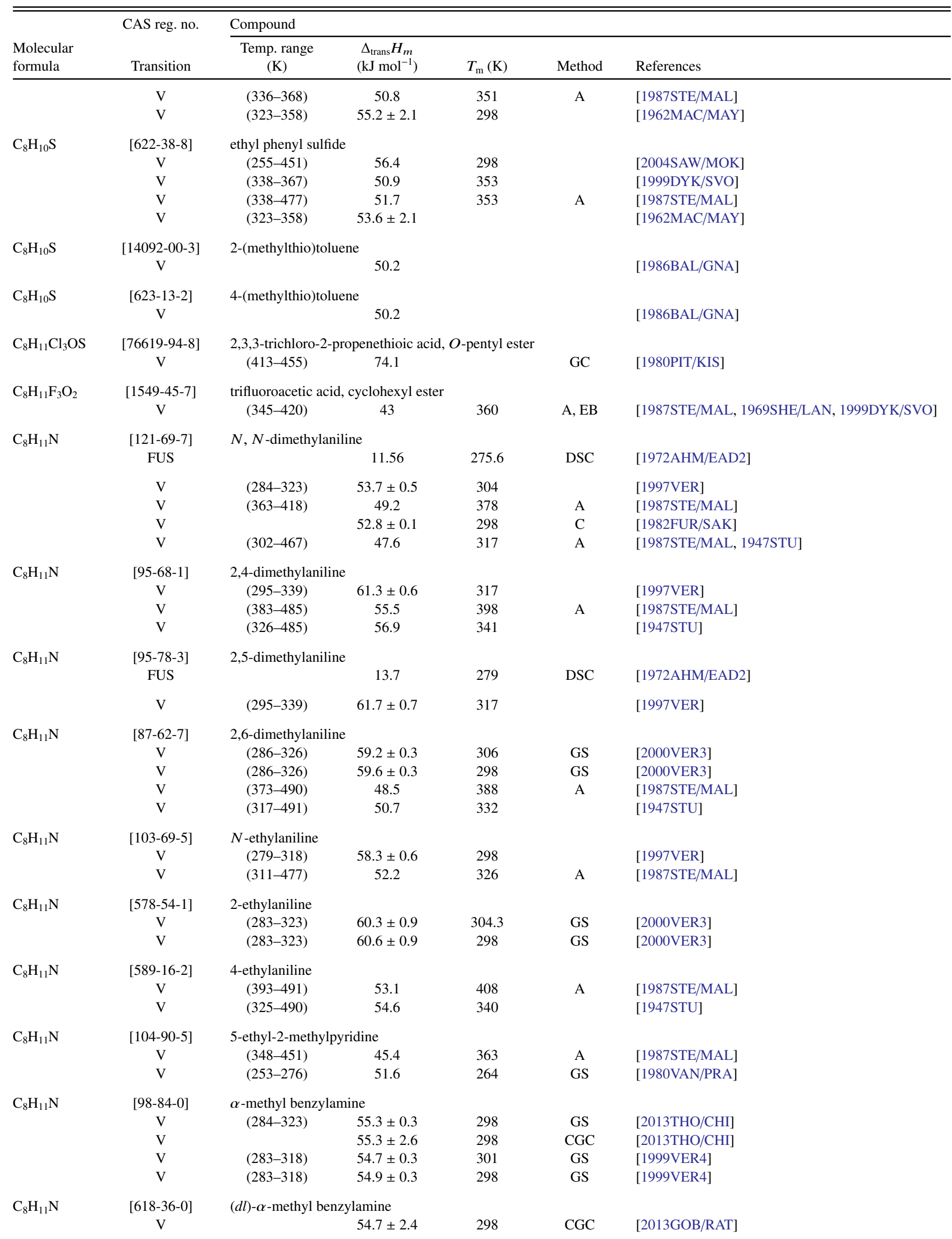


TABLE 11. Phase change enthalpies of $\mathrm{C}_{8}$ organic compounds-Continued

\begin{tabular}{|c|c|c|c|c|c|c|}
\hline \multirow[b]{2}{*}{$\begin{array}{l}\text { Molecular } \\
\text { formula }\end{array}$} & \multirow{2}{*}{$\begin{array}{l}\text { CAS reg. no. } \\
\text { Transition }\end{array}$} & \multicolumn{5}{|l|}{ Compound } \\
\hline & & $\begin{array}{c}\text { Temp. range } \\
\text { (K) }\end{array}$ & $\begin{array}{c}\Delta_{\text {trans }} H_{m} \\
\left(\mathrm{~kJ} \mathrm{~mol}^{-1}\right)\end{array}$ & $T_{\mathrm{m}}(\mathrm{K})$ & Method & References \\
\hline & $\mathrm{V}$ & $(292-318)$ & 36.7 & 305 & A & [1987STE/MAL] \\
\hline & $\mathrm{V}$ & & $54.5 \pm 0.1$ & 298 & $\mathrm{C}$ & [1987ATI/SAI] \\
\hline \multirow[t]{2}{*}{$\mathrm{C}_{8} \mathrm{H}_{11} \mathrm{~N}$} & [3886-69-9] & $(+)-\alpha$-methylbe & amine & & & \\
\hline & $\mathrm{V}$ & & $54.1 \pm 0.1$ & 298 & $\mathrm{C}$ & [1987ATI/SAI] \\
\hline \multirow[t]{2}{*}{$\mathrm{C}_{8} \mathrm{H}_{11} \mathrm{~N}$} & [2627-86-3] & (-)- $\alpha$-methylbe & amine & & & \\
\hline & $\mathrm{V}$ & & $54.6 \pm 0.1$ & 298 & $\mathrm{C}$ & [1987ATI/SAI] \\
\hline \multirow[t]{2}{*}{$\mathrm{C}_{8} \mathrm{H}_{11} \mathrm{~N}$} & {$[104-84-7]$} & 4-methylbenzyl & & & & \\
\hline & $\mathrm{V}$ & $(353-466)$ & 54.4 & 368 & A & [1987STE/MAL] \\
\hline \multirow[t]{6}{*}{$\mathrm{C}_{8} \mathrm{H}_{11} \mathrm{~N}$} & {$[64-04-0]$} & 2-phenylethylar & & & & \\
\hline & $\mathrm{V}$ & & $56.6 \pm 2.4$ & 298 & $\mathrm{CGC}$ & [2013GOB/RAT] \\
\hline & $\mathrm{V}$ & $(285-323)$ & $57.5 \pm 0.3$ & 298 & GS & [2013THO/CHI] \\
\hline & $\mathrm{V}$ & & $57.7 \pm 2.6$ & 298 & CGC & [2013THO/CHI] \\
\hline & $\mathrm{V}$ & $(277-351)$ & $55.7 \pm 0.2$ & 313 & DM & [2009MOK/RAZ] \\
\hline & $\mathrm{V}$ & $(277-351)$ & $56.8 \pm 0.2$ & 298 & DM & [2009MOK/RAZ] \\
\hline \multirow[t]{2}{*}{$\mathrm{C}_{8} \mathrm{H}_{11} \mathrm{~N}$} & {$[695-98-7]$} & 2,3,5-trimethyll & ine & & & \\
\hline & $\mathrm{V}$ & $(293-426)$ & 44.0 & 359 & & [1995SAK/UEO] \\
\hline \multirow[t]{5}{*}{$\mathrm{C}_{8} \mathrm{H}_{11} \mathrm{~N}$} & {$[1462-84-6]$} & 2,3,6-trimethyl & & & & \\
\hline & $\mathrm{V}$ & & 48.5 & 328 & $\mathrm{C}$ & [1985MAJ/SVO2] \\
\hline & $\mathrm{V}$ & & 47.5 & 343 & $\mathrm{C}$ & [1985MAJ/SVO2] \\
\hline & $\mathrm{V}$ & & 45.7 & 368 & $\mathrm{C}$ & [1985MAJ/SVO2] \\
\hline & $\mathrm{V}$ & & 50.6 & 298 & & [1985MAJ/SVO2] \\
\hline \multirow[t]{11}{*}{$\mathrm{C}_{8} \mathrm{H}_{11} \mathrm{~N}$} & {$[108-75-8]$} & 2,4,6-trimethyl & & & & \\
\hline & FUS & & 9.54 & 229 & $\mathrm{AC}$ & [1996DOM/HEA, 1957MAS] \\
\hline & $\mathrm{V}$ & & $51.4 \pm 2.8$ & 298 & CGC & [2010LIP/PLI] \\
\hline & $\mathrm{V}$ & $(342-373)$ & $50.4 \pm 2.9$ & 298 & CGC & [2009LIP/CHI2] \\
\hline & $\mathrm{V}$ & $(323-373)$ & 50.2 & 298 & CGC & [1995CHI/HOS] \\
\hline & $\mathrm{V}$ & $(303-424)$ & 46.5 & 363 & & [1995SAK/UEO] \\
\hline & $\mathrm{V}$ & $(298-444)$ & 51.2 & 313 & $\mathrm{~EB}$ & [1990LEN] \\
\hline & $\mathrm{V}$ & & $50.3 \pm 0.2$ & 298 & & [1985MAJ/SVO2] \\
\hline & $\mathrm{V}$ & & 48.3 & 328 & $\mathrm{C}$ & [1985MAJ/SVO2] \\
\hline & $\mathrm{V}$ & & 47.2 & 343 & $\mathrm{C}$ & [1985MAJ/SVO2] \\
\hline & $\mathrm{V}$ & & 45.5 & 368 & $\mathrm{C}$ & [1985MAJ/SVO2] \\
\hline \multirow[t]{2}{*}{$\mathrm{C}_{8} \mathrm{H}_{11} \mathrm{~N}$} & [622-39-9] & 2-propylpyridin & & & & \\
\hline & $\mathrm{V}$ & $(338-445)$ & 46.6 & 353 & A & [1987STE/MAL] \\
\hline \multirow[t]{2}{*}{$\mathrm{C}_{8} \mathrm{H}_{11} \mathrm{~N}$} & [4673-31-8] & 3-propylpyridin & & & & \\
\hline & $\mathrm{V}$ & $(350-450)$ & 49.9 & 365 & A & [1987STE/MAL] \\
\hline \multirow[t]{2}{*}{$\mathrm{C}_{8} \mathrm{H}_{11} \mathrm{~N}$} & [1122-81-2] & 4-propypyridin & & & & \\
\hline & $\mathrm{V}$ & $(354-465)$ & 47.8 & 369 & A & [1987STE/MAL] \\
\hline \multirow[t]{2}{*}{$\mathrm{C}_{8} \mathrm{H}_{11} \mathrm{~N}$} & [103434-09-9] & 1-norbornylisoc & & & & \\
\hline & SUB & & $60.6 \pm 0.5$ & 298 & & [1987MEI/DOG] \\
\hline \multirow[t]{3}{*}{$\mathrm{C}_{8} \mathrm{H}_{11} \mathrm{~N}$} & [3211-90-3] & exo-2-cyanobic & 2.2.1] heptane & & & \\
\hline & TRS & & 7.95 & 237.7 & & \\
\hline & FUS & & 2.93 & 298.8 & $\mathrm{AC}$ & [1996DOM/HEA, 1995KOL] \\
\hline \multirow[t]{3}{*}{$\mathrm{C}_{8} \mathrm{H}_{11} \mathrm{~N}$} & {$[3211-87-8]$} & endo-2-cyanobi & [2.2.1]heptane & & & \\
\hline & TRS & & 2.25 & 177.3 & & \\
\hline & FUS & & 2.96 & 331.2 & $\mathrm{AC}$ & [1996DOM/HEA, 1995KOL] \\
\hline \multirow[t]{2}{*}{$\mathrm{C}_{8} \mathrm{H}_{11} \mathrm{NO}$} & [122-98-5] & 2-anilinoethano & & & & \\
\hline & $\mathrm{V}$ & $(377-553)$ & 69.9 & 392 & A & [1987STE/MAL, 1947STU] \\
\hline \multirow[t]{2}{*}{$\mathrm{C}_{8} \mathrm{H}_{11} \mathrm{NO}$} & {$[94-70-2]$} & 2-ethoxyaniline & & & & \\
\hline & $\mathrm{V}$ & $(373-458)$ & 57.3 & 388 & A & [1987STE/MAL] \\
\hline \multirow[t]{2}{*}{$\mathrm{C}_{8} \mathrm{H}_{11} \mathrm{NO}$} & [156-43-4] & 4-ethoxyaniline & & & & \\
\hline & $\mathrm{V}$ & $(421-523)$ & 61.2 & 436 & A & [1987STE/MAL] \\
\hline
\end{tabular}


TABLE 11. Phase change enthalpies of $\mathrm{C}_{8}$ organic compounds-Continued

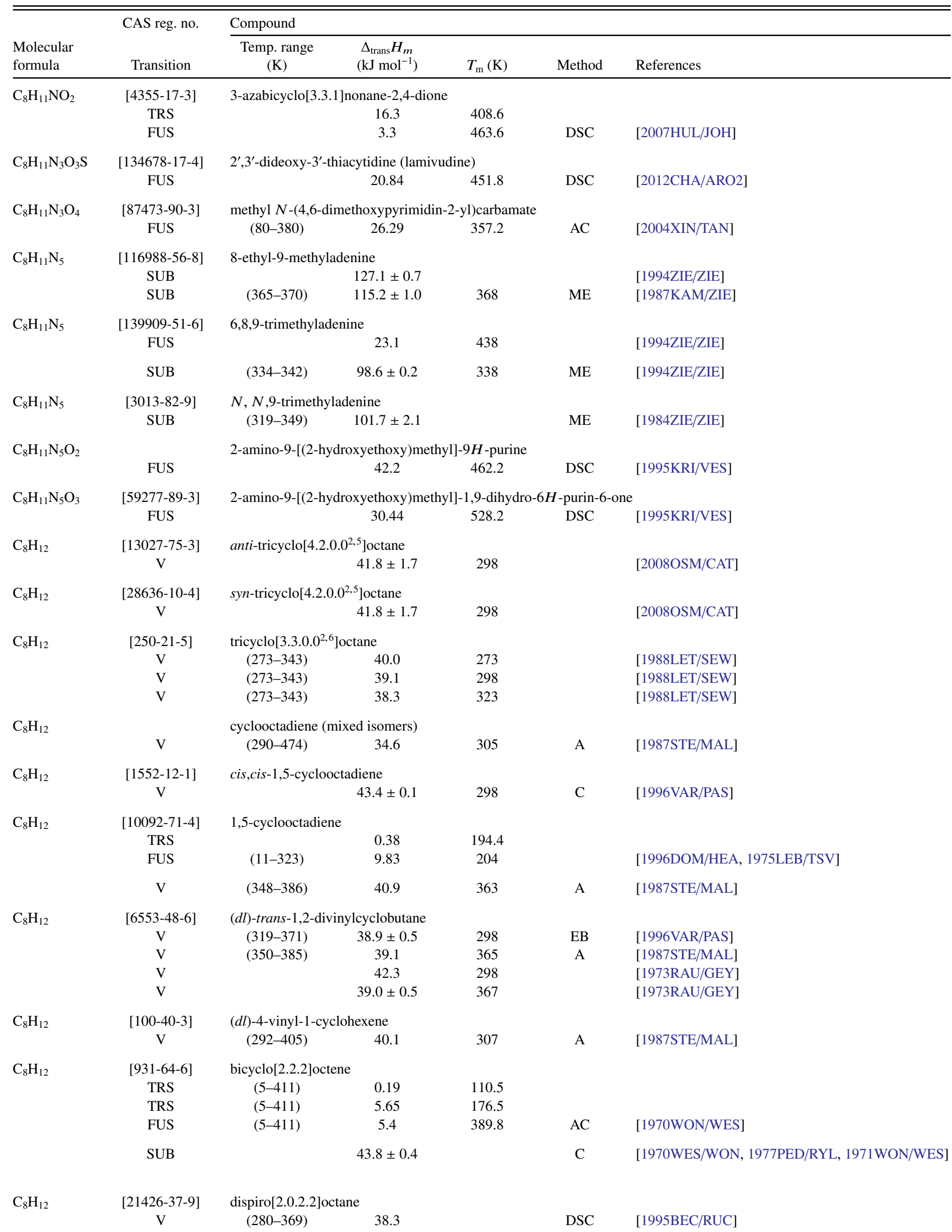


TABLE 11. Phase change enthalpies of $\mathrm{C}_{8}$ organic compounds-Continued

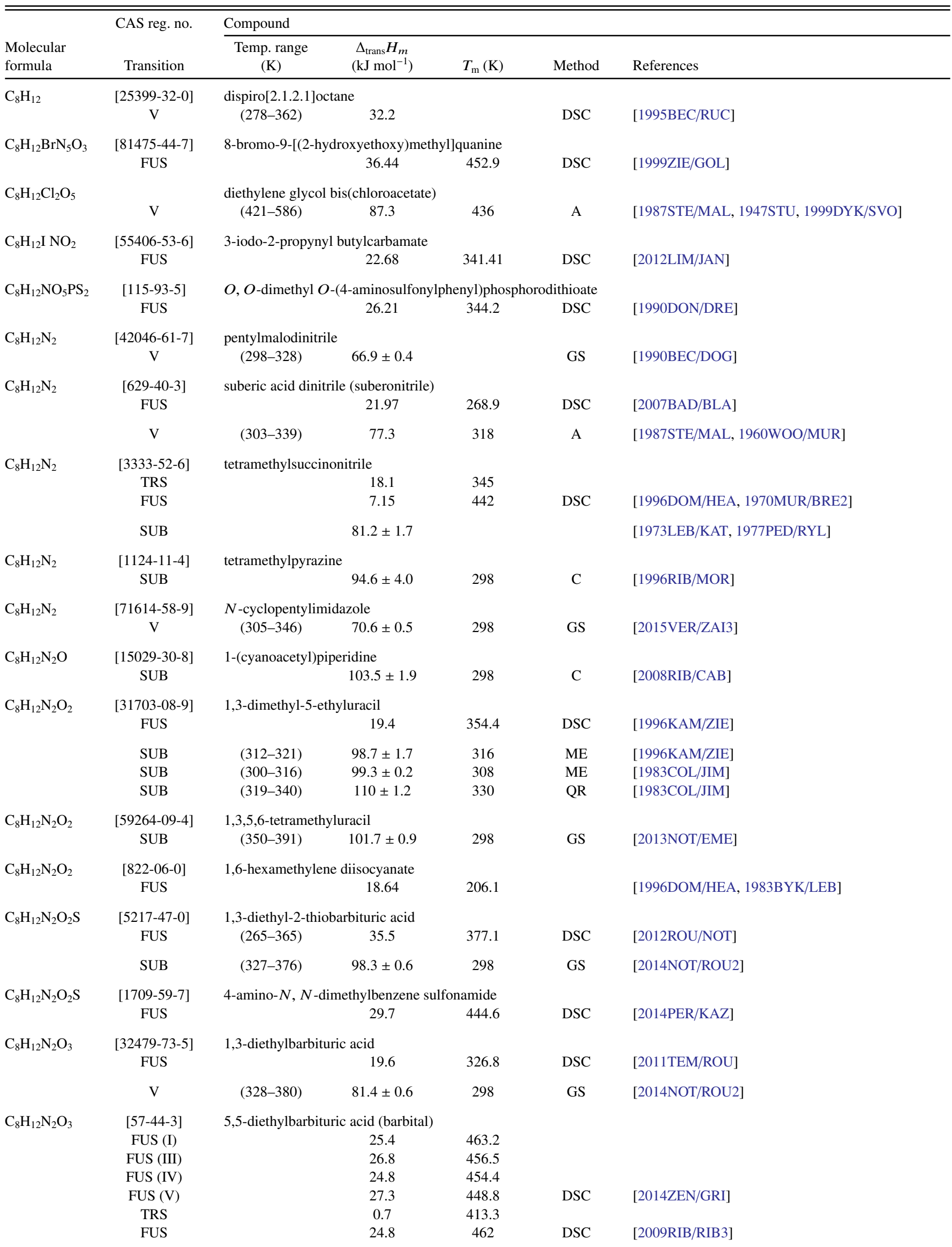


TABLE 11. Phase change enthalpies of $\mathrm{C}_{8}$ organic compounds-Continued

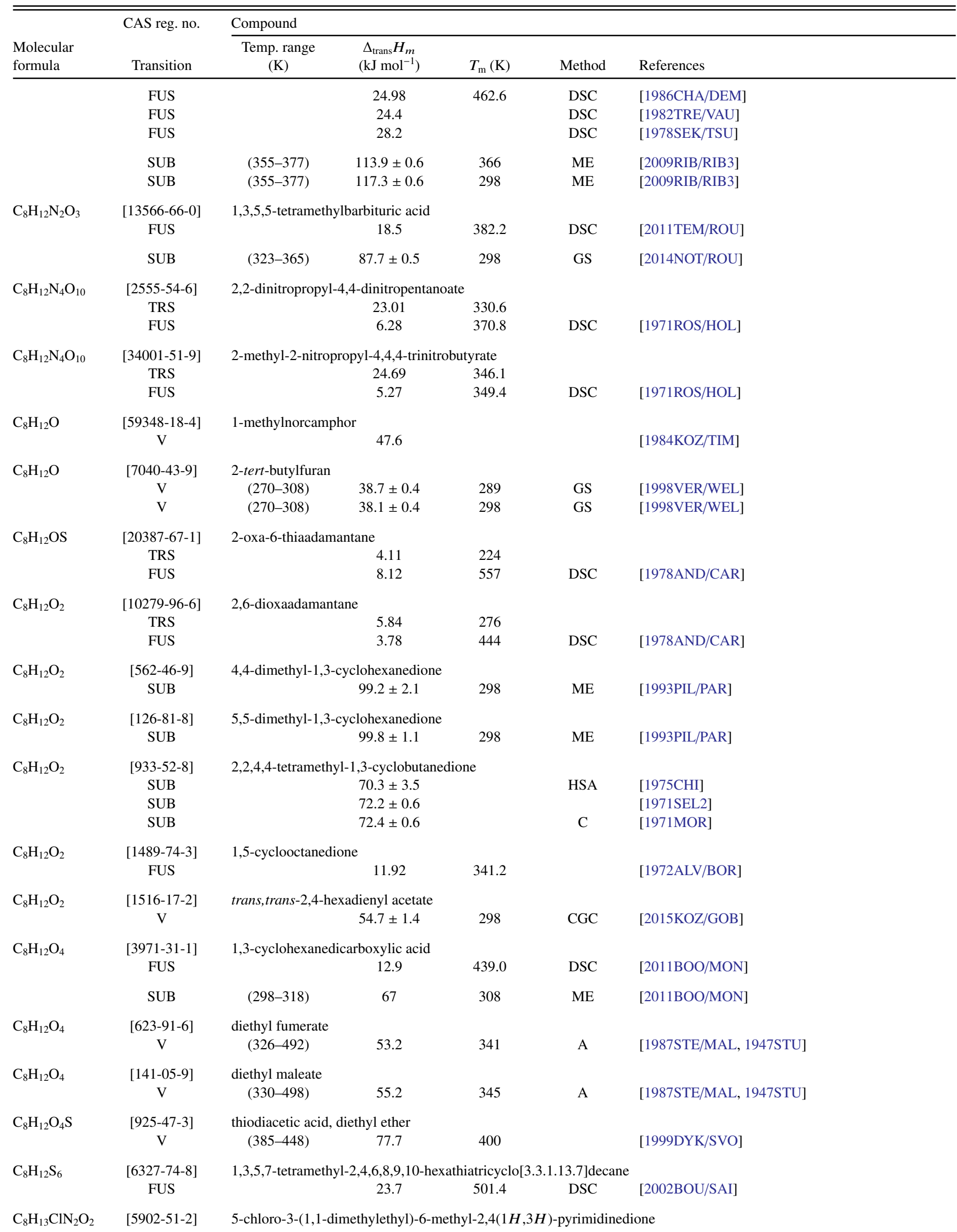


TABLE 11. Phase change enthalpies of $\mathrm{C}_{8}$ organic compounds-Continued

\begin{tabular}{|c|c|c|c|c|c|c|}
\hline \multirow[b]{2}{*}{$\begin{array}{l}\text { Molecular } \\
\text { formula }\end{array}$} & \multirow{2}{*}{$\begin{array}{c}\text { CAS reg. no. } \\
\text { Transition }\end{array}$} & \multicolumn{5}{|l|}{ Compound } \\
\hline & & $\begin{array}{c}\text { Temp. range } \\
\text { (K) }\end{array}$ & $\begin{array}{c}\Delta_{\text {trans }} H_{m} \\
\left(\mathrm{~kJ} \mathrm{~mol}^{-1}\right)\end{array}$ & $T_{\mathrm{m}}(\mathrm{K})$ & Method & References \\
\hline & FUS & & 12.51 & 448 & DSC & [1990DON/DRE] \\
\hline \multirow[t]{7}{*}{$\mathrm{C}_{8} \mathrm{H}_{14}$} & [280-33-1] & \multicolumn{5}{|c|}{ bicyclo[2.2.2] octane } \\
\hline & TRS & & 4.6 & 164 & & [1984DOM/EVA] \\
\hline & TRS & $(5-465)$ & 4.6 & 164.3 & & \\
\hline & FUS & $(5-465)$ & 8.37 & 447.5 & $\mathrm{AC}$ & [1991ACR, 1970WON/WES] \\
\hline & SUB & $(323-363)$ & $46.3 \pm 0.8$ & 343 & BG & [1971BOY/SAN, 1977PED/RYL, 1987STE/MAL] \\
\hline & SUB & & $47.7 \pm 0.8$ & 298 & & [1971BOY/SAN, 1977PED/RYL] \\
\hline & SUB & & $48.0 \pm 2$ & & $\mathrm{C}$ & [1970WES/WON, 1971BOY/SAN, 1977PED/RYL] \\
\hline
\end{tabular}

$\mathrm{C}_{8} \mathrm{H}_{14} \quad$ [1755-05-1] cis-bicyclo[3.3.0]octane
$\mathrm{V}$
(298-318) 42.0
308
A
[1987STE/MAL]

[Note: The coefficients in [1987STE/MAL] came from [1970CHA/MCN]. The coefficients pertain to $\log \mathrm{P}=\mathrm{A}-\mathrm{B} / \mathrm{T}+\mathrm{C} \log \mathrm{T}$, and not the Antoine equation.]

$\begin{array}{lllll}\mathrm{V} & 41.5 \pm 0.4 & 318 & \text { EB } & \text { [1970CHA/MCN] } \\ \mathrm{V} & 43.1 \pm 0.8 & 298 & \text { EB } & \text { [1970CHA/MCN] }\end{array}$

$\mathrm{C}_{8} \mathrm{H}_{14}$

[5597-89-7] trans-bicyclo[3.3.0]octane
$\mathrm{V}$
(298-320)
41.4
$309 \quad$ A
[1987STE/MAL]

[Note: The coefficients in [1987STE/MAL] came from [1970CHA/MCN]. The coefficients pertain to $\log \mathrm{P}=\mathrm{A}-\mathrm{B} / \mathrm{T}+\mathrm{C} \log \mathrm{T}$, and not the Antoine equation.]

$$
\begin{array}{lllll}
\mathrm{V} & 41.3 \pm 0.4 & 320 & \mathrm{~EB} & {[1970 \mathrm{CHA} / \mathrm{MCN}]} \\
\mathrm{V} & 42.7 \pm 0.8 & 298 & \mathrm{~EB} & {[1970 \mathrm{CHA} / \mathrm{MCN}]}
\end{array}
$$

$\mathrm{C}_{8} \mathrm{H}_{14} \quad$ [28282-35-1] cis-bicyclo[4.2.0]octane
$\mathrm{V}$
(298-347)
40.7
313
A [1987STE/MAL]

[Note: The coefficients in [1987STE/MAL] came from [1970CHA/MCN]. The coefficients pertain to log P = A -B/T + C log T, and not the Antoine equation.]

$\begin{array}{lllll}\text { V } & 39.5 \pm 0.4 & 347 & \text { EB } & \text { [1970CHA/MCN] } \\ \text { V } & 42.7 \pm 1.2 & 298 & \text { EB } & \text { [1970CHA/MCN] }\end{array}$

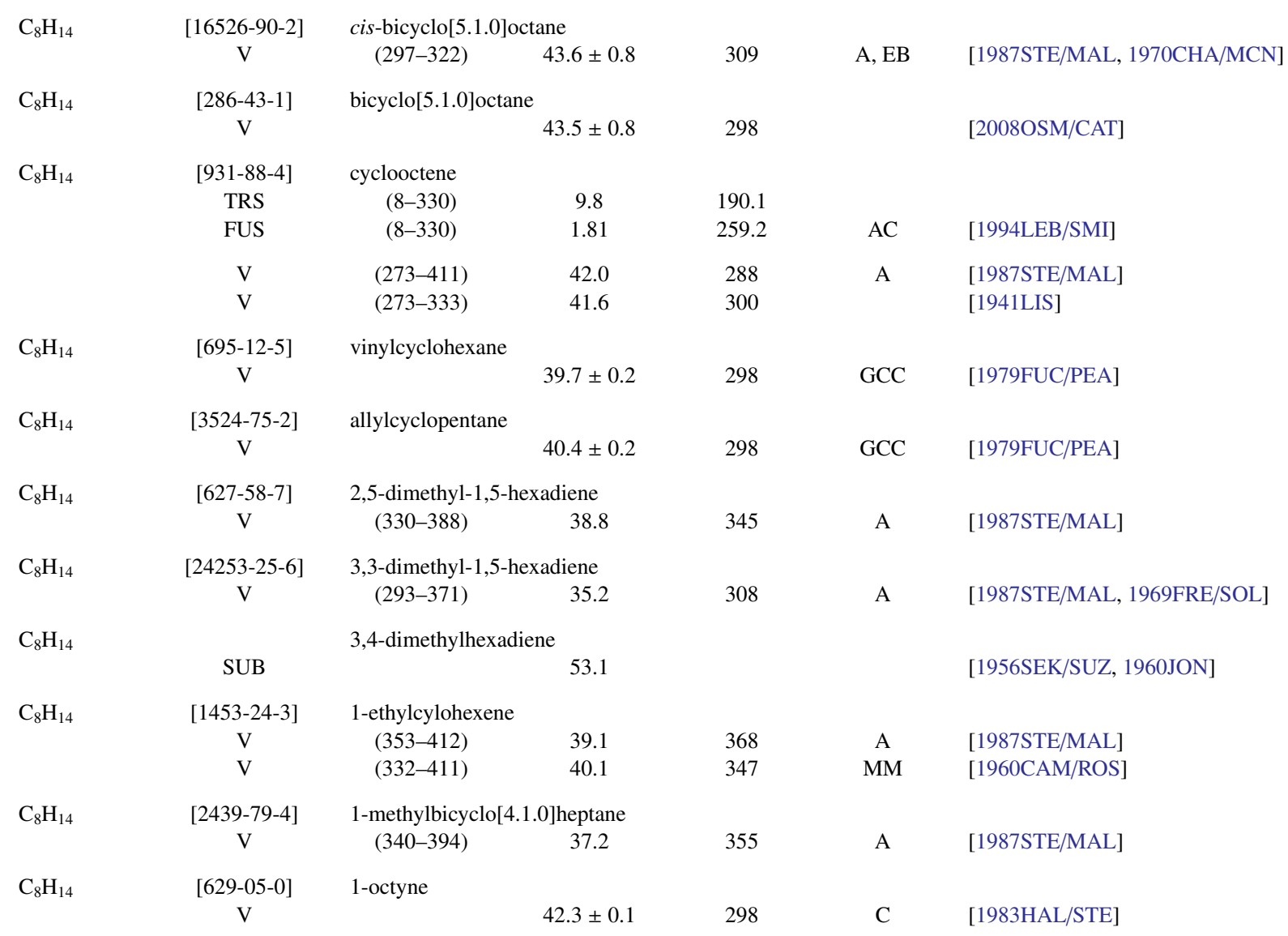


TABLE 11. Phase change enthalpies of $\mathrm{C}_{8}$ organic compounds-Continued

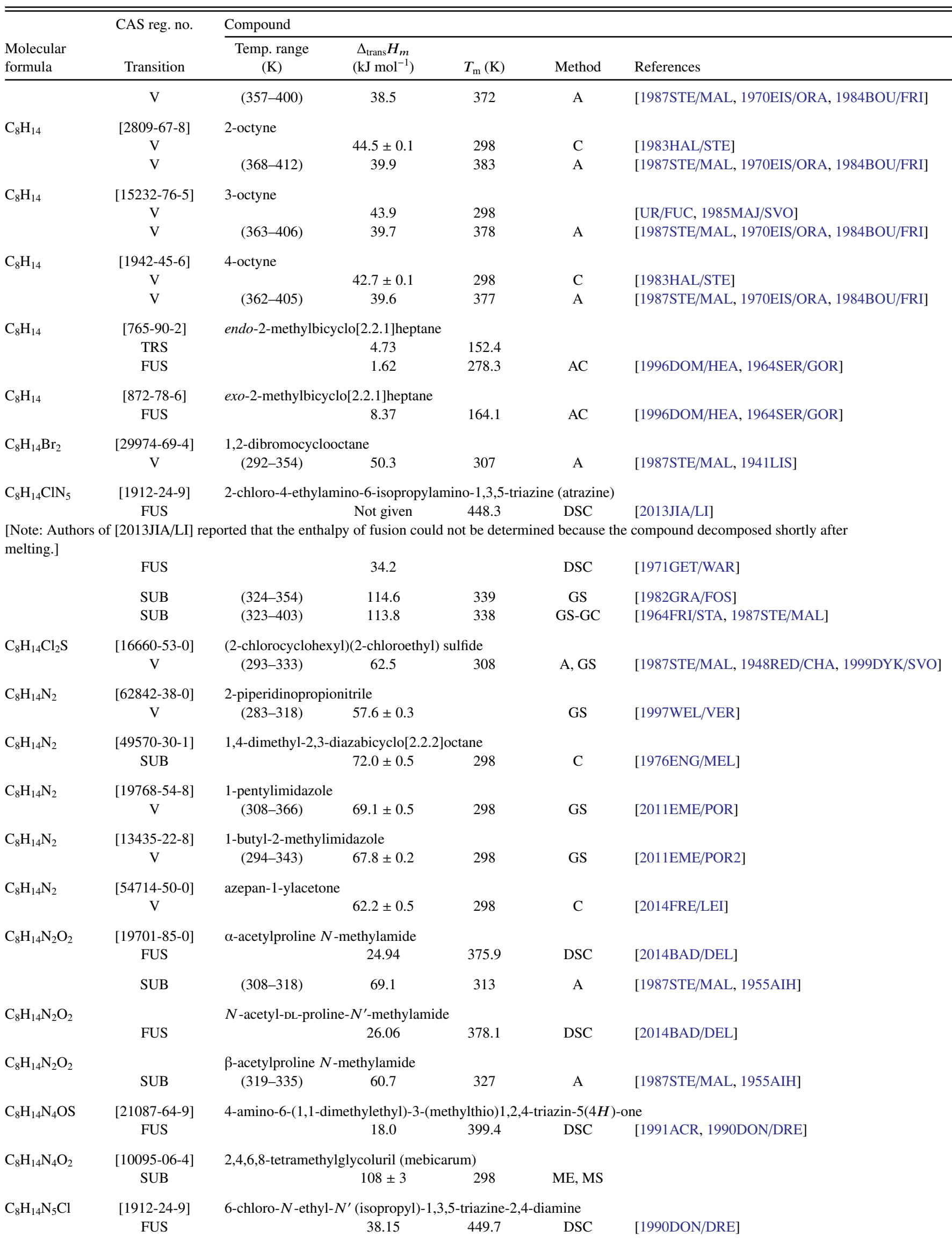


TABLE 11. Phase change enthalpies of $\mathrm{C}_{8}$ organic compounds-Continued

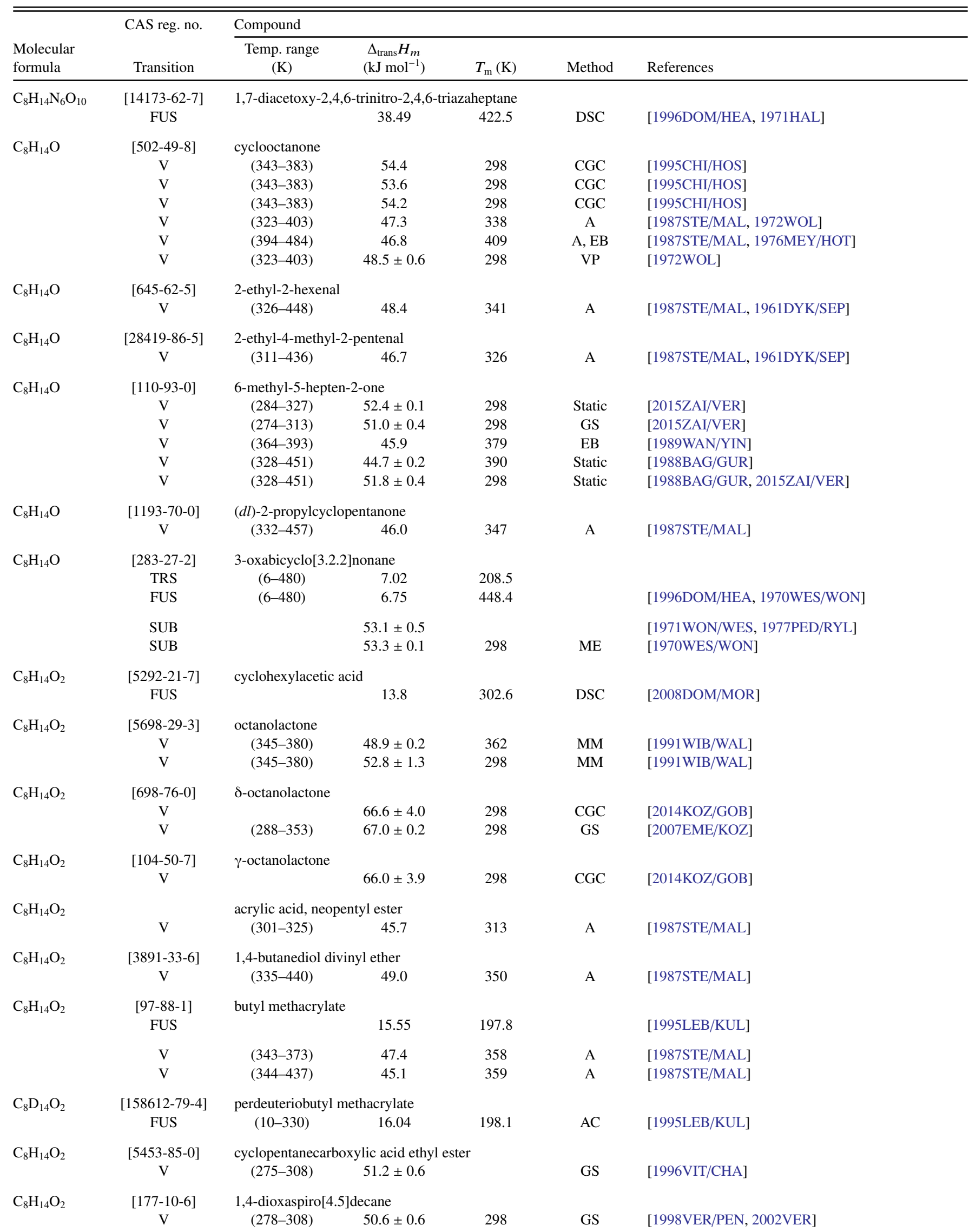


TABLE 11. Phase change enthalpies of $\mathrm{C}_{8}$ organic compounds-Continued

\begin{tabular}{|c|c|c|c|c|c|c|}
\hline \multirow[b]{2}{*}{$\begin{array}{l}\text { Molecular } \\
\text { formula }\end{array}$} & \multirow{2}{*}{$\begin{array}{l}\text { CAS reg. no. } \\
\text { Transition }\end{array}$} & \multicolumn{5}{|l|}{ Compound } \\
\hline & & $\begin{array}{c}\text { Temp. range } \\
(\mathrm{K})\end{array}$ & $\begin{array}{c}\Delta_{\text {trans }} H_{m} \\
\left(\mathrm{~kJ} \mathrm{~mol}^{-1}\right)\end{array}$ & $T_{\mathrm{m}}(\mathrm{K})$ & Method & References \\
\hline $\mathrm{C}_{8} \mathrm{H}_{14} \mathrm{O}_{2}$ & $\begin{array}{c}{[622-45-7]} \\
\text { FUS }\end{array}$ & cyclohexyl acetate & 3.38 & 221.45 & DSC & [2008DOM/MOR] \\
\hline \multicolumn{7}{|c|}{ [Note: The value reported in [2008DOM/MOR] seems abnormally small.] } \\
\hline & TRS & & 5.23 & 221.8 & & \\
\hline & FUS & $(5-320)$ & 8.0 & 224.6 & $\mathrm{AC}$ & [1999KAB/KOZ, 2001KOZ/BLO] \\
\hline & $\mathrm{V}$ & & $52.3 \pm 0.2$ & 298 & $\mathrm{C}$ & [2004PAU/ZAI, 2003ZAI/VER] \\
\hline & $\mathrm{V}$ & $(253-283)$ & $56.5 \pm 0.5$ & 298 & ME & [2003ZAI/VER] \\
\hline & $\mathrm{V}$ & $(253-283)$ & $52.6 \pm 0.5$ & 298 & ME & [2003ZAI/VER] \\
\hline & $\mathrm{V}$ & $(274-318)$ & $52.3 \pm 0.8$ & 298 & GS & [2003ZAI/VER] \\
\hline & $\mathrm{V}$ & $(333-378)$ & 51.7 & 298 & CGC & [1999VER/HEI] \\
\hline & $\mathrm{V}$ & $(278-318)$ & $51.7 \pm 0.2$ & 298 & GS & [1996VER/BEC] \\
\hline & $\mathrm{V}$ & $(368-446)$ & 46.7 & 383 & A, EB & [1987STE/MAL, 1969SHE/LAN] \\
\hline \multirow[t]{2}{*}{$\mathrm{C}_{8} \mathrm{H}_{14} \mathrm{O}_{2}$} & {$[585-07-9]$} & methylacrylic acid, & ert-butyl ester & & & \\
\hline & $\mathrm{V}$ & $(313-410)$ & 42.9 & 328 & A & [1987STE/MAL] \\
\hline \multirow[t]{2}{*}{$\mathrm{C}_{8} \mathrm{H}_{14} \mathrm{O}_{2}$} & [2998-23-4] & pentyl acrylate & & & & \\
\hline & V & $(325-440)$ & 44.9 & 340 & A & [1987STE/MAL] \\
\hline \multirow[t]{2}{*}{$\mathrm{C}_{8} \mathrm{H}_{14} \mathrm{O}_{2}$} & [142-30-3] & 2,5-dimethyl-3-hexy & ne-2,5-diol & & & \\
\hline & V & & $82.8 \pm 1.0$ & 298 & CGC & [2006UMN/KWE] \\
\hline \multirow[t]{2}{*}{$\mathrm{C}_{8} \mathrm{H}_{14} \mathrm{O}_{2}$} & {$[56922-71-5]$} & propyl 3-methylbut- & 2-enoate & & & \\
\hline & V & $(278-311)$ & $53.0 \pm 0.2$ & 298 & GS & [2008EME/TOK] \\
\hline \multirow[t]{2}{*}{$\mathrm{C}_{8} \mathrm{H}_{14} \mathrm{O}_{2}$} & {$[25859-51-2]$} & isopropyl 3-methylb & ut-2-enoate & & & \\
\hline & V & $(279-313)$ & $50.0 \pm 0.2$ & 298 & GS & [2008EME/TOK] \\
\hline \multirow[t]{2}{*}{$\mathrm{C}_{8} \mathrm{H}_{14} \mathrm{O}_{3}$} & [106-31-0] & butyric anhydride & & & & \\
\hline & V & $(349-470)$ & 49.1 & 364 & A & [1987STE/MAL] \\
\hline \multirow[t]{2}{*}{$\mathrm{C}_{8} \mathrm{H}_{14} \mathrm{O}_{3}$} & [764-99-8] & diethylene glycol di & vinyl ether & & & \\
\hline & V & $(336-470)$ & 50.0 & 351 & A & [1987STE/MAL] \\
\hline \multirow[t]{2}{*}{$\mathrm{C}_{8} \mathrm{H}_{14} \mathrm{O}_{3}$} & [607-97-6] & 2-ethylacetoacetic a & cid, ethyl ester & & & \\
\hline & V & $(313-471)$ & 53.3 & 328 & A & [1987STE/MAL, 1947STU] \\
\hline \multirow[t]{3}{*}{$\mathrm{C}_{8} \mathrm{H}_{14} \mathrm{O}_{3}$} & {$[21884-26-4]$} & isopropyl levulinate & & & & \\
\hline & $\mathrm{V}$ & $(321-481)$ & 56.6 & 336 & A & [1987STE/MAL, 1947STU] \\
\hline & V & & 52.0 & 422 & & [1931SCH/COW] \\
\hline \multirow[t]{2}{*}{$\mathrm{C}_{8} \mathrm{H}_{14} \mathrm{O}_{3}$} & $\begin{array}{c}\text { [645-67-0] } \\
\mathrm{V}\end{array}$ & propyl levulinate & & & & \\
\hline & $\begin{array}{l}\mathrm{V} \\
\mathrm{V}\end{array}$ & $(332-495)$ & $\begin{array}{l}56.3 \\
540\end{array}$ & $\begin{array}{l}347 \\
436\end{array}$ & A & $\begin{array}{l}\text { [1987STE/MAL] } \\
\text { [1931SCH/COW] }\end{array}$ \\
\hline \multirow{2}{*}{$\mathrm{C}_{8} \mathrm{H}_{14} \mathrm{O}_{4}$} & ז007727281 & ? potoyunronioni & id nronulotor & & & \\
\hline & [20473-73-8] & 2-acetoxypropionic & acid, propyl ester & 333 & A & [1987STE/MAL 1950REH/DIX] \\
\hline \multirow[t]{2}{*}{$\mathrm{C}_{8} \mathrm{H}_{14} \mathrm{O}_{4}$} & {$[200867-13-6]$} & 3-acetoxypropionic & acid, propyl ester & & & \\
\hline & V & $(361-373)$ & 74.7 & 367 & A & [1987STE/MAL, 1948FEI/FIS] \\
\hline \multirow[t]{10}{*}{$\mathrm{C}_{8} \mathrm{H}_{14} \mathrm{O}_{4}$} & {$[123-25-1]$} & diethyl succinate & & & & \\
\hline & V & $(393-468)$ & 51.2 & 408 & & [2011MAT/KIM] \\
\hline & $\mathrm{V}$ & $(290-346)$ & 63.5 & & GS & [2011LIP/KRA] \\
\hline & V & $(290-346)$ & $65.1 \pm 0.3$ & 298 & GS & [2011LIP/KRA] \\
\hline & $\mathrm{V}$ & $(327-490)$ & 56.5 & 342 & A & [1987STE/MAL] \\
\hline & $\mathrm{V}$ & $(327-490)$ & 64.5 & 298 & A & [1987STE/MAL, 2011LIP/KRA] \\
\hline & $\mathrm{V}$ & $(328-490)$ & 54.7 & 409 & & [1947STU, 2011LIP/KRA] \\
\hline & $\mathrm{V}$ & $(328-490)$ & 64.5 & 298 & & [1947STU, 2011LIP/KRA] \\
\hline & $\mathrm{V}$ & $(335-455)$ & 54.7 & 395 & & [1940HEI/REI, 2011LIP/KRA] \\
\hline & $\mathrm{V}$ & $(335-455)$ & $63.8 \pm 1.1$ & 298 & & [1940HEI/REI, 2011LIP/KRA] \\
\hline \multirow[t]{5}{*}{$\mathrm{C}_{8} \mathrm{H}_{14} \mathrm{O}_{4}$} & {$[615-81-6]$} & diisopropyl oxalate & & & & \\
\hline & $\mathrm{V}$ & $(288-330)$ & $60.2 \pm 0.4$ & 298 & GS & [2011POR/KRA] \\
\hline & $\mathrm{V}$ & $(418-501)$ & 57.8 & 433 & A & [1987STE/MAL] \\
\hline & $\mathrm{V}$ & $(316-467)$ & 62.3 & 298 & A & [1987STE/MAL, 2011POR/KRA] \\
\hline & $\mathrm{V}$ & $(316-467)$ & 57.6 & 331 & A & [1987STE/MAL, 1947STU] \\
\hline
\end{tabular}


TABLE 11. Phase change enthalpies of $\mathrm{C}_{8}$ organic compounds-Continued

\begin{tabular}{|c|c|c|c|c|c|c|}
\hline \multirow[b]{2}{*}{$\begin{array}{l}\text { Molecular } \\
\text { formula }\end{array}$} & \multirow{2}{*}{$\begin{array}{l}\text { CAS reg. no. } \\
\text { Transition }\end{array}$} & \multicolumn{5}{|l|}{ Compound } \\
\hline & & $\begin{array}{c}\text { Temp. range } \\
\text { (K) }\end{array}$ & $\begin{array}{c}\Delta_{\text {trans }} H_{m} \\
\left(\mathrm{~kJ} \mathrm{~mol}^{-1}\right)\end{array}$ & $T_{\mathrm{m}}(\mathrm{K})$ & Method & References \\
\hline \multirow[t]{7}{*}{$\mathrm{C}_{8} \mathrm{H}_{14} \mathrm{O}_{4}$} & [627-93-0] & \multicolumn{5}{|l|}{ dimethyl adipate } \\
\hline & $\mathrm{V}$ & $(353-443)$ & 74.6 & 368 & & [2007LEE/LAI] \\
\hline & $\mathrm{V}$ & $(294-373)$ & $69.0 \pm 0.2$ & 298 & GS & [2006VER/KOZ] \\
\hline & $\mathrm{V}$ & $(293-344)$ & $67.1 \pm 0.3$ & 298 & GS & [2006VAS/VER, 2006VER/KOZ] \\
\hline & $\mathrm{V}$ & $(293-323)$ & $\mathrm{U} 55.9 \pm 2.0$ & 298 & $\mathrm{TE}$ & [1997CHE/LIA, 2006VER/KOZ] \\
\hline & $\mathrm{V}$ & $(382-500)$ & 58.8 & 397 & A & [1987STE/MAL] \\
\hline & $\mathrm{V}$ & $(428-498)$ & 73.4 & 298 & & [1963VLA/GRA, 2006VER/KOZ] \\
\hline \multirow[t]{2}{*}{$\mathrm{C}_{8} \mathrm{H}_{14} \mathrm{O}_{4}$} & {$[615-98-5]$} & \multicolumn{5}{|l|}{ dipropyl oxalate } \\
\hline & $\mathrm{V}$ & $(326-487)$ & 57.8 & 341 & A & [1987STE/MAL, 1947STU] \\
\hline \multirow[t]{3}{*}{$\mathrm{C}_{8} \mathrm{H}_{14} \mathrm{O}_{4}$} & {$[123-80-8]$} & \multicolumn{5}{|c|}{ ethylene dipropionate } \\
\hline & $\mathrm{V}$ & $(295-323)$ & $63.1 \pm 1.0$ & & GS & [2011MAS/KRA] \\
\hline & $\mathrm{V}$ & & $67.6 \pm 0.5$ & 298 & $\mathrm{C}$ & [1986NIL/WAD] \\
\hline \multirow[t]{3}{*}{$\mathrm{C}_{8} \mathrm{H}_{14} \mathrm{O}_{4}$} & {$[609-08-5]$} & \multicolumn{5}{|c|}{ 2-methylmalonic acid, diethyl ester } \\
\hline & $\mathrm{V}$ & $(373-468)$ & 55.5 & 388 & & [2005LEE/SU] \\
\hline & $\mathrm{V}$ & $(312-475)$ & 52.5 & 327 & A & [1987STE/MAL, 1947STU] \\
\hline \multirow[t]{11}{*}{$\mathrm{C}_{8} \mathrm{H}_{14} \mathrm{O}_{4}$} & {$[505-48-6]$} & \multicolumn{5}{|c|}{ octanedioic acid (suberic acid) } \\
\hline & TRS & & 2.0 & 335.9 & & \\
\hline & TRS & & 9.1 & 403.6 & & \\
\hline & FUS & & 30.7 & 413.2 & DSC & [2005ROU/TEM] \\
\hline & FUS & & 28.82 & 415.3 & DSC & [1991ACR, 1974CIN/BER] \\
\hline & SUB & $(348-378)$ & $168 \pm 7$ & & TPD & [2007CAP/LOV] \\
\hline & SUB & $(310-320)$ & 148 & & TPTD & [2001CHA/TOB] \\
\hline & SUB & & $147.8 \pm 3.8$ & 298 & & [1999RIB/MON, 1960DAV/THO] \\
\hline & SUB & $(379-407)$ & $143.1 \pm 3.8$ & 393 & M & [1960DAV/THO, 1970COX/PIL, 1987STE/MAL] \\
\hline & $\mathrm{V}$ & $(424-503)$ & $116.7 \pm 0.8$ & 298 & CGC & [2005ROU/TEM] \\
\hline & $\mathrm{V}$ & $(445-619)$ & 91.4 & 460 & A & [1987STE/MAL, 1947STU] \\
\hline \multirow[t]{3}{*}{$\mathrm{C}_{8} \mathrm{H}_{14} \mathrm{O}_{4}$} & {$[630-51-3]$} & \multicolumn{5}{|c|}{ tetramethysuccinic acid } \\
\hline & TRS & & 13.43 & 383 & & \\
\hline & FUS & & 6.47 & 464 & DSC & [1996DOM/HEA, 1970MUR/BRE2] \\
\hline \multirow{2}{*}{$\mathrm{C}_{8} \mathrm{H}_{14} \mathrm{O}_{4} \mathrm{~S}$} & {$[925-47-3]$} & \multicolumn{5}{|c|}{ thiodiacetic acid, diethyl ester } \\
\hline & $\mathrm{V}$ & $(384-448)$ & 77.3 & 399 & A & [1987STE/MAL] \\
\hline \multirow[t]{2}{*}{$\mathrm{C}_{8} \mathrm{H}_{14} \mathrm{O}_{5}$} & & \multicolumn{5}{|c|}{ isopropyl[1-(methoxycarbonyl)ethyl] carbonate } \\
\hline & $\mathrm{V}$ & $(330-493)$ & 55.5 & 345 & A & [1987STE/MAL] \\
\hline $\mathrm{C}_{8} \mathrm{H}_{14} \mathrm{O}_{5}$ & & 2-(lactyloxy)propi & nic acid, ethyl e & & & \\
\hline & $\mathrm{V}$ & $(321-389)$ & 72.8 & 336 & A & [1987STE/MAL, 1952REH/DIX] \\
\hline $\mathrm{C}_{8} \mathrm{H}_{14} \mathrm{O}_{5}$ & {$[7554-12-3]$} & malic acid, diethyl & ester & & & \\
\hline & $\mathrm{V}$ & $(353-527)$ & 59.6 & 368 & A & [1987STE/MAL, 1947STU] \\
\hline $\mathrm{C}_{8} \mathrm{H}_{14} \mathrm{O}_{5}$ & {$[902263-88-1]$} & propyl[1-(methoxy & arbonyl)ethyl] & onate & & \\
\hline & V & $(373-495)$ & 58.0 & 388 & A & [1987STE/MAL] \\
\hline $\mathrm{C}_{8} \mathrm{H}_{14} \mathrm{O}_{6}$ & {$[13811-71-7]$} & $(d)$-diethyl tartrate & & & & \\
\hline & $\mathrm{V}$ & $(375-553)$ & 65.9 & 390 & & [1947STU] \\
\hline $\mathrm{C}_{8} \mathrm{H}_{14} \mathrm{O}_{6}$ & {$[87-91-2]$} & $(d l)$-diethyl tartrate & & & & \\
\hline & $\mathrm{V}$ & $(375-553)$ & 67.3 & 390 & A & [1987STE/MAL, 1947STU] \\
\hline $\mathrm{C}_{8} \mathrm{H}_{14} \mathrm{O}_{6}$ & & $(d)$-dimethoxysucc & nic acid dimeth & ster & & \\
\hline & SUB & & 53.1 & & & [1937DUN/WOL] \\
\hline $\mathrm{C}_{8} \mathrm{H}_{14} \mathrm{O}_{6}$ & & $(d l)$-dimethoxysuc & inic acid dimetl & ester & & \\
\hline & SUB & & 57.7 & & & [1937DUN/WOL] \\
\hline $\mathrm{C}_{8} \mathrm{H}_{14} \mathrm{O}_{6}$ & & meso-dimethoxysu & cinic acid dime & ester & & \\
\hline & SUB & & 74.1 & & & [1937DUN/WOL] \\
\hline $\mathrm{C}_{8} \mathrm{H}_{14} \mathrm{O}_{6} \mathrm{~S}$ & [29771-87-7] & sulfonyldiacetic ac & d, diethyl ester & & & \\
\hline & $\mathrm{V}$ & $(421-494)$ & 88.2 & 426 & & [1999DYK/SVO] \\
\hline & $\mathrm{V}$ & $(421-494)$ & 87.6 & 436 & A & [1987STE/MAL] \\
\hline
\end{tabular}


TABLE 11. Phase change enthalpies of $\mathrm{C}_{8}$ organic compounds-Continued

\begin{tabular}{|c|c|c|c|c|c|c|}
\hline \multirow[b]{2}{*}{$\begin{array}{l}\text { Molecular } \\
\text { formula }\end{array}$} & \multirow{2}{*}{$\begin{array}{l}\text { CAS reg. no. } \\
\text { Transition }\end{array}$} & \multicolumn{5}{|l|}{ Compound } \\
\hline & & $\begin{array}{c}\text { Temp. range } \\
(\mathrm{K})\end{array}$ & $\begin{array}{c}\Delta_{\text {trans }} H_{m} \\
\left(\mathrm{~kJ} \mathrm{~mol}^{-1}\right)\end{array}$ & $T_{\mathrm{m}}(\mathrm{K})$ & Method & References \\
\hline \multirow[t]{2}{*}{$\mathrm{C}_{8} \mathrm{H}_{14} \mathrm{O}_{6} \mathrm{~S}$} & {$[5450-67-9]$} & \multicolumn{5}{|c|}{ dimethyl 3,3'-sulfonyldipropionate } \\
\hline & FUS & & 41.1 & 390.3 & DSC & [1994WAN/KUO] \\
\hline \multirow[t]{2}{*}{$\mathrm{C}_{8} \mathrm{H}_{15} \mathrm{Br}$} & {$[1647-26-3]$} & \multicolumn{5}{|c|}{ (2-bromoethyl)cyclohexane } \\
\hline & V & $(311-486)$ & 54.2 & 326 & A & [1987STE/MAL, 1947STU, 1970DYK/VAN] \\
\hline \multirow[t]{2}{*}{$\mathrm{C}_{8} \mathrm{H}_{15} \mathrm{ClO}$} & {$[111-64-8]$} & \multicolumn{5}{|c|}{ octanoyl chloride } \\
\hline & $\mathrm{V}$ & $(343-373)$ & 74.5 & 358 & A & [1987STE/MAL, 1999DYK/SVO] \\
\hline \multirow[t]{2}{*}{$\mathrm{C}_{8} \mathrm{H}_{15} \mathrm{ClO}$} & {$[99222-85-2]$} & \multicolumn{5}{|c|}{ 5-methylheptanoyl chloride } \\
\hline & $\mathrm{V}$ & $(338-373)$ & 66.3 & 353 & A & [1987STE/MAL, 1999DYK/SVO] \\
\hline \multirow{2}{*}{$\mathrm{C}_{8} \mathrm{H}_{15} \mathrm{Cl}_{3} \mathrm{O}_{4}$} & & \multicolumn{5}{|c|}{ trichlorohydrine pentaerythritol } \\
\hline & $\mathrm{V}$ & $(404-449)$ & 80.4 & 419 & & {$[1965 \mathrm{LUT} / \mathrm{KOL}]$} \\
\hline \multirow[t]{5}{*}{$\mathrm{C}_{8} \mathrm{H}_{15} \mathrm{~N}$} & {$[283-24-9]$} & \multicolumn{5}{|c|}{ 3-azabicyclo[3.2.2]nonane } \\
\hline & TRS & $(5-350)$ & 14.48 & 297.8 & $\mathrm{AC}$ & [1963BAR/WES] \\
\hline & FUS & $(280-490)$ & 6.92 & 466.6 & $\mathrm{AC}$ & [1996DOM/HEA, 1964WUL/WES] \\
\hline & SUB & & $57.8 \pm 1.3$ & 298 & $\mathrm{C}$ & [1970WES/WON] \\
\hline & SUB & $(303-443)$ & 52.2 & 318 & A & [1987STE/MAL, 1964WUL/WES] \\
\hline \multirow[t]{9}{*}{$\mathrm{C}_{8} \mathrm{H}_{15} \mathrm{~N}$} & {$[124-12-9]$} & \multicolumn{5}{|l|}{ octanenitrile } \\
\hline & V & (283-310) & $55.7 \pm 0.2$ & 298 & GS & [2005EME/VER] \\
\hline & $\mathrm{V}$ & $(373-480)$ & 50.0 & 388 & A & [1987STE/MAL] \\
\hline & V & & $56.8 \pm 0.3$ & 298 & $\mathrm{C}$ & [1977STRI/SUN] \\
\hline & $\mathrm{V}$ & $(374-420)$ & 49.8 & 389 & $\mathrm{~EB}$ & [1971MEY/REN] \\
\hline & $\mathrm{V}$ & $(420-479)$ & 48.0 & 435 & $\mathrm{~EB}$ & [1971MEY/REN] \\
\hline & $\mathrm{V}$ & $(316-477)$ & 56.7 & 331 & & {$[1947 \mathrm{STU}]$} \\
\hline & $\mathrm{V}$ & $(322-460)$ & 53.5 & 298 & $\mathrm{~EB}$ & [1941RAL/SEL, 2005EME/VER] \\
\hline & $\mathrm{V}$ & $(294-477)$ & $57.2 \pm 0.3$ & 298 & MM & [1933HEI, 2005EME/VER] \\
\hline \multirow[t]{2}{*}{$\mathrm{C}_{8} \mathrm{H}_{15} \mathrm{NO}$} & {$[4747-81-3]$} & \multicolumn{5}{|c|}{ heptyl isocyanate } \\
\hline & V & $(326-461)$ & 47.5 & 341 & A & [1987STE/MAL] \\
\hline \multirow[t]{2}{*}{$\mathrm{C}_{8} \mathrm{H}_{15} \mathrm{NO}$} & {$[6554-73-0]$} & \multicolumn{5}{|c|}{ methacrylic acid $N$-tert-butylamide } \\
\hline & $\mathrm{V}$ & $(340-467)$ & 49.6 & 355 & A & [1987STE/MAL] \\
\hline $\mathrm{C}_{8} \mathrm{H}_{15} \mathrm{NO}$ & & trans-2-octenoi & d amide & & & \\
\hline & SUB & $(373-393)$ & 73.5 & 383 & A & [1987STE/MAL] \\
\hline $\mathrm{C}_{8} \mathrm{H}_{15} \mathrm{NO}$ & [935-30-8] & azacyclononan- & & & & \\
\hline & FUS & & 18.9 & 348.5 & DSC & [2012EME/VER] \\
\hline & SUB & $(309-344)$ & $102.3 \pm 0.5$ & 298 & GS & [2012EME/VER] \\
\hline & $\mathrm{V}$ & $(353-373)$ & $85.3 \pm 0.5$ & 298 & GS & [2012EME/VER] \\
\hline $\mathrm{C}_{8} \mathrm{H}_{15} \mathrm{NO}_{2}$ & [2867-47-2] & methacrylic aci & (dimethylami & hyl ester & & \\
\hline & FUS & & 16.85 & 237.7 & $\mathrm{AC}$ & [1996DOM/HEA, 1985KAR/ABD] \\
\hline & $\mathrm{V}$ & $(372-460)$ & 48.8 & 387 & A & [1987STE/MAL] \\
\hline $\mathrm{C}_{8} \mathrm{H}_{15} \mathrm{NO}_{2}$ & & 1-lactopiperidir & & & & \\
\hline & $\mathrm{V}$ & $(346-408)$ & 62.1 & 361 & A & [1987STE/MAL] \\
\hline $\mathrm{C}_{8} \mathrm{H}_{15} \mathrm{NO}_{2}$ & [1563-86-6] & $N$-acetyl- $N$-bu & etamide & & & \\
\hline & $\mathrm{V}$ & & $64.4 \pm 0.4$ & 298 & $\mathrm{C}$ & [1965WAD] \\
\hline $\mathrm{C}_{8} \mathrm{H}_{15} \mathrm{NO}_{3}$ & [5411-58-5] & $N, N$-diethylo & c acid, ethyl & & & \\
\hline & $\mathrm{V}$ & $(349-525)$ & 60.5 & 364 & A & [1987STE/MAL, 1947STU] \\
\hline $\mathrm{C}_{8} \mathrm{H}_{15} \mathrm{~N}_{5} \mathrm{O}$ & [673-04-1] & 2-methoxy-4,6 & thylamino)-1 & riazine $(\mathrm{s}$ & & \\
\hline & FUS & & 24.7 & & DSC & [1971GET/WAR] \\
\hline & SUB & $(323-403)$ & 98.2 & 338 & GS-GC & [1987STE/MAL, 1964FRI/STA] \\
\hline $\mathrm{C}_{8} \mathrm{H}_{15} \mathrm{~N}_{5} \mathrm{~S}$ & {$[1014-70-6]$} & 2-methylthio-4 & (ethylamino) & -triazine & ryn) & \\
\hline & FUS & & 24.0 & 353.2 & & [2007VEC/BRU] \\
\hline
\end{tabular}


TABLE 11. Phase change enthalpies of $\mathrm{C}_{8}$ organic compounds-Continued

\begin{tabular}{|c|c|c|c|c|c|c|}
\hline \multirow[b]{2}{*}{$\begin{array}{l}\text { Molecular } \\
\text { formula }\end{array}$} & \multirow{2}{*}{$\begin{array}{c}\text { CAS reg. no. } \\
\text { Transition }\end{array}$} & \multicolumn{5}{|l|}{ Compound } \\
\hline & & $\begin{array}{c}\text { Temp. range } \\
(\mathrm{K})\end{array}$ & $\begin{array}{c}\Delta_{\text {trans }} H_{m} \\
\left(\mathrm{~kJ} \mathrm{~mol}^{-1}\right)\end{array}$ & $T_{\mathrm{m}}(\mathrm{K})$ & Method & References \\
\hline & SUB & & $120 \pm 6$ & 298 & DSC & [2007VEC/BRU] \\
\hline & SUB & & $115 \pm 4$ & 298 & DSC & [2007VEC/BRU] \\
\hline & SUB & $(323-355)$ & 101.3 & 338 & GS-GC & [1987STE/MAL, 1964FRI/STA] \\
\hline & $\mathrm{V}$ & & $88 \pm 4$ & 461 & $\mathrm{DSC}$ & [2007VEC/BRU] \\
\hline & $\mathrm{V}$ & & $83.7 \pm 1.3$ & 453 & TGA & [2007VEC/BRU] \\
\hline \multirow[t]{2}{*}{$\mathrm{C}_{8} \mathrm{H}_{15} \mathrm{~N}_{5} \mathrm{~S}$} & [1014-69-3] & \multicolumn{5}{|c|}{ 2-methylthio-4-methylamino-6-isopropyl-1,3,5-triazine } \\
\hline & SUB & $(323-357)$ & 101.5 & 338 & GS-GC & [1987STE/MAL, 1964FRI/STA] \\
\hline \multirow[t]{8}{*}{$\mathrm{C}_{8} \mathrm{H}_{15} \mathrm{~N}_{7} \mathrm{O}_{2} \mathrm{~S}_{3}$} & {$[76824-35-6]$} & \multicolumn{5}{|c|}{$3-[[[2-[($ aminoiminomethyl)amino]-4-thiazolyl $]$ methyl $]$ thio $]-N$-(aminosulfonyl)propanimidamide (famotidine) } \\
\hline & FUS (I) & & 48.17 & 445.65 & & \\
\hline & FUS (II) & & 50.28 & 439.35 & DSC & [2015MAT/MOR] \\
\hline & FUS (I) & & 49.7 & 447.0 & & \\
\hline & FUS (II) & & 48.6 & 438.6 & DSC & [2007LU/WAN] \\
\hline & FUS (I) & & 45.81 & 444.4 & & \\
\hline & FUS (II) & & 43.92 & 436.6 & DSC & [2002ROU/DAV] \\
\hline & SUB & & 207 & & TGA & [1997ELD] \\
\hline \multirow[t]{7}{*}{$\mathrm{C}_{8} \mathrm{H}_{16}$} & {$[16747-50-5]$} & 1-ethyl-1-meth & lopentane & & & \\
\hline & $\mathrm{V}$ & $(332-422)$ & $36.9 \pm 0.1$ & 332 & $\mathrm{C}$ & [1981HOS/SCO3] \\
\hline & $\mathrm{V}$ & $(332-422)$ & $35.9 \pm 0.1$ & 350 & $\mathrm{C}$ & [1981HOS/SCO3] \\
\hline & $\mathrm{V}$ & $(332-422)$ & $34.7 \pm 0.1$ & 371 & $\mathrm{C}$ & [1981HOS/SCO3] \\
\hline & $\mathrm{V}$ & $(332-422)$ & $33.9 \pm 0.1$ & 384 & $\mathrm{C}$ & [1981HOS/SCO3] \\
\hline & $\mathrm{V}$ & $(332-422)$ & $32.2 \pm 0.1$ & 410 & $\mathrm{C}$ & [1981HOS/SCO3] \\
\hline & $\mathrm{V}$ & $(332-422)$ & 38.8 & 298 & $\mathrm{C}$ & [1981HOS/SCO3] \\
\hline \multirow[t]{14}{*}{$\mathrm{C}_{8} \mathrm{H}_{16}$} & [292-64-8] & cyclooctane & & & & \\
\hline & TRS & & 6.32 & 166.5 & & \\
\hline & TRS & & 0.48 & 183.8 & & \\
\hline & FUS & & 2.41 & 288 & & [1991ACR, 1956FIN/SCO] \\
\hline & TRS & & 5.72 & 167.2 & & \\
\hline & FUS & & 2.51 & 288 & & [1952KAA/COO] \\
\hline & SUB & & 58.7 & 166 & B & {$[1963 \mathrm{BON}]$} \\
\hline & $\mathrm{V}$ & $(358-413)$ & 40.3 & 373 & & [1991WU/LOC] \\
\hline & V & & $43.1 \pm 0.2$ & & GC & [1989AZA] \\
\hline & $\mathrm{V}$ & $(289-369)$ & 43.3 & 304 & A & [1987STE/MAL] \\
\hline & $\mathrm{V}$ & $(373-434)$ & 39.3 & 388 & EB & [1976MEY/HOT] \\
\hline & $\mathrm{V}$ & $(291-323)$ & 43.1 & 306 & & [1975ANA/GRO] \\
\hline & $\mathrm{V}$ & & $43.3 \pm 0.2$ & 298 & & [1956FIN/SCO] \\
\hline & $\mathrm{V}$ & $(369-467)$ & 39.4 & 384 & A, EB & [1987STE/MAL, 1956FIN/SCO] \\
\hline \multirow[t]{8}{*}{$\mathrm{C}_{8} \mathrm{H}_{16}$} & {$[590-66-9]$} & 1,1-dimethylcy & xane & & & \\
\hline & TRS & & 5.98 & 153.2 & & \\
\hline & FUS & $(12-303)$ & 2.01 & 239.8 & $\mathrm{AC}$ & [1996DOM/HEA, 1949HUF/TOD] \\
\hline & $\mathrm{V}$ & $(271-303)$ & $39.6 \pm 0.1$ & 287 & GS & [1995CHI/HES] \\
\hline & $\mathrm{V}$ & & $38.8 \pm 0.1$ & 298 & & [1995CHI/HES] \\
\hline & $\mathrm{V}$ & & 37.9 & 298 & & [1975KUS/SAI] \\
\hline & $\mathrm{V}$ & & 37.8 & 298 & & [1971WIL/ZWO] \\
\hline & $\mathrm{V}$ & $(313-395)$ & 36.6 & 328 & A & [1987STE/MAL, 1949FOR/NOR] \\
\hline \multirow[t]{9}{*}{$\mathrm{C}_{8} \mathrm{H}_{16}$} & {$[2207-01-4]$} & cis-1,2-dimethy & ohexane & & & \\
\hline & TRS & & 8.26 & 172.5 & & \\
\hline & FUS & $(12-298)$ & 1.64 & 223.3 & $\mathrm{AC}$ & [1996DOM/HEA, 1949HUF/TOD] \\
\hline & $\mathrm{V}$ & & 39.4 & 298 & & [1975KUS/SAI] \\
\hline & $\mathrm{V}$ & & 39.7 & 298 & & [1971WIL/ZWO] \\
\hline & $\mathrm{V}$ & & $35.5 \pm 0.1$ & 370 & $\mathrm{C}$ & [1951MCC/PER] \\
\hline & $\mathrm{V}$ & & $34.5 \pm 0.1$ & 387 & $\mathrm{C}$ & [1951MCC/PER] \\
\hline & $\mathrm{V}$ & & $39.7 \pm 0.1$ & 298 & $\mathrm{C}$ & [1947OSB/GIN] \\
\hline & $\mathrm{V}$ & $(322-405)$ & 38.0 & 337 & A, MM & [1987STE/MAL, 1945WIL/TAY] \\
\hline
\end{tabular}


TABLE 11. Phase change enthalpies of $\mathrm{C}_{8}$ organic compounds-Continued

\begin{tabular}{|c|c|c|c|c|c|c|}
\hline \multirow[b]{2}{*}{$\begin{array}{l}\text { Molecular } \\
\text { formula }\end{array}$} & \multirow{2}{*}{$\begin{array}{c}\text { CAS reg. no. } \\
\text { Transition }\end{array}$} & \multicolumn{5}{|l|}{ Compound } \\
\hline & & $\begin{array}{c}\text { Temp. range } \\
\text { (K) }\end{array}$ & $\begin{array}{c}\Delta_{\text {trans }} H_{m} \\
\left(\mathrm{~kJ} \mathrm{~mol}^{-1}\right)\end{array}$ & $T_{\mathrm{m}}(\mathrm{K})$ & Method & References \\
\hline \multirow[t]{8}{*}{$\mathrm{C}_{8} \mathrm{H}_{16}$} & [6876-23-9] & \multicolumn{5}{|c|}{$(d l)$-trans-1,2-dimethylcyclohexane } \\
\hline & FUS & $(12-301)$ & 10.5 & 185 & $\mathrm{AC}$ & [1996DOM/HEA, 1949HUF/TOD] \\
\hline & $\mathrm{V}$ & & 38.3 & 298 & & [1975KUS/SAI] \\
\hline & $\mathrm{V}$ & & 38.4 & 298 & & [1971WIL/ZWO] \\
\hline & $\mathrm{V}$ & & $34.4 \pm 0.1$ & 373 & $\mathrm{C}$ & [1951MCC/PER] \\
\hline & $\mathrm{V}$ & & $33.5 \pm 0.1$ & 387 & $\mathrm{C}$ & [1951MCC/PER] \\
\hline & $\mathrm{V}$ & & $38.4 \pm 0.1$ & 298 & $\mathrm{C}$ & [1947OSB/GIN] \\
\hline & $\mathrm{V}$ & $(316-399)$ & 37.0 & 331 & $\mathrm{~A}, \mathrm{MM}$ & [1987STE/MAL, 1945WIL/TAY] \\
\hline \multirow[t]{9}{*}{$\mathrm{C}_{8} \mathrm{H}_{16}$} & [638-04-0] & \multicolumn{5}{|c|}{ cis-1,3-dimethylcyclohexane } \\
\hline & FUS & $(12-299)$ & 10.82 & 197.6 & $\mathrm{AC}$ & [1996DOM/HEA, 1949HUF/TOD] \\
\hline & $\mathrm{V}$ & $(318-396)$ & 36.8 & 333 & A & [1987STE/MAL] \\
\hline & $\mathrm{V}$ & & 38.1 & 298 & & [1975KUS/SAI] \\
\hline & $\mathrm{V}$ & & 38.2 & 298 & & [1971WIL/ZWO] \\
\hline & $\mathrm{V}$ & & $34.9 \pm 0.1$ & 363 & $\mathrm{C}$ & [1951MCC/PER] \\
\hline & $\mathrm{V}$ & & $33.3 \pm 0.1$ & 385 & $\mathrm{C}$ & [1951MCC/PER] \\
\hline & $\mathrm{V}$ & & $38.2 \pm 0.1$ & 298 & $\mathrm{C}$ & [1947OSB/GIN] \\
\hline & $\mathrm{V}$ & $(316-398)$ & 37.7 & 331 & MM & [1945WIL/TAY] \\
\hline \multirow[t]{7}{*}{$\mathrm{C}_{8} \mathrm{H}_{16}$} & [2207-03-6] & \multicolumn{5}{|c|}{$(d l)$-trans-1,3-dimethylcyclohexane } \\
\hline & FUS & $(13-299)$ & 9.87 & 183.1 & $\mathrm{AC}$ & [1996DOM/HEA, 1949HUF/TOD] \\
\hline & V & $(314-400)$ & 37.9 & 329 & A & [1987STE/MAL] \\
\hline & $\mathrm{V}$ & & 39.1 & 298 & & [1975KUS/SAI] \\
\hline & $\mathrm{V}$ & & 39.2 & 298 & & [1971WIL/ZWO] \\
\hline & $\mathrm{V}$ & & $39.2 \pm 0.1$ & 298 & $\mathrm{C}$ & [1947OSB/GIN] \\
\hline & $\mathrm{V}$ & $(314-394)$ & 37.4 & 329 & MM & [1945WIL/TAY] \\
\hline \multirow[t]{6}{*}{$\mathrm{C}_{8} \mathrm{H}_{16}$} & [624-29-3] & \multicolumn{5}{|c|}{ cis-1,4-dimethylcyclohexane } \\
\hline & FUS & $(12-303)$ & 9.29 & 185.7 & $\mathrm{AC}$ & [1996DOM/HEA, 1949HUF/TOD] \\
\hline & $\mathrm{V}$ & & 39.0 & 298 & & [1975KUS/SAI] \\
\hline & $\mathrm{V}$ & & 39.0 & 298 & & [1971WIL/ZWO] \\
\hline & $\mathrm{V}$ & & $39.0 \pm 0.1$ & 298 & $\mathrm{C}$ & [1947OSB/GIN] \\
\hline & V & $(317-400)$ & 37.6 & 332 & $\mathrm{~A}, \mathrm{MM}$ & [1987STE/MAL, 1945WIL/TAY] \\
\hline \multirow[t]{9}{*}{$\mathrm{C}_{8} \mathrm{H}_{16}$} & {$[2207-04-7]$} & \multicolumn{5}{|c|}{$(d l)$-trans-1,4-dimethylcyclohexane } \\
\hline & FUS & $(12-299)$ & 12.34 & 236.2 & $\mathrm{AC}$ & [1996DOM/HEA, 1949HUF/TOD] \\
\hline & $\mathrm{V}$ & & 37.6 & 298 & & [1975KUS/SAI] \\
\hline & $\mathrm{V}$ & & 37.9 & 298 & & [1971WIL/ZWO] \\
\hline & $\mathrm{V}$ & & $35.6 \pm 0.1$ & 341 & $\mathrm{C}$ & [1951MCC/PER] \\
\hline & $\mathrm{V}$ & & $34.6 \pm 0.1$ & 357 & $\mathrm{C}$ & [1951MCC/PER] \\
\hline & $\mathrm{V}$ & & $33.5 \pm 0.1$ & 377 & $\mathrm{C}$ & [1951MCC/PER] \\
\hline & $\mathrm{V}$ & & $39.9 \pm 0.1$ & 298 & $\mathrm{C}$ & [1947OSB/GIN] \\
\hline & $\mathrm{V}$ & $(313-395)$ & 36.7 & 328 & $\mathrm{~A}, \mathrm{MM}$ & [1987STE/MAL, 1945WIL/TAY] \\
\hline \multirow[t]{15}{*}{$\mathrm{C}_{8} \mathrm{H}_{16}$} & {$[1678-91-7]$} & \multicolumn{5}{|c|}{ ethylcyclohexane } \\
\hline & FUS & & 8.45 & 161.9 & $\mathrm{AC}$ & [2015RAM/CHI] \\
\hline & FUS & & 8.5 & 161.5 & Quasi-AC & [2006MAN/CUT] \\
\hline & FUS & $(12-299)$ & 8.33 & 161.4 & & [1996DOM/HEA, 1949HUF/TOD] \\
\hline & $\mathrm{V}$ & & $39.2 \pm 0.4$ & 298 & GC & [1987AZA] \\
\hline & $\mathrm{V}$ & & $39.8 \pm 0.1$ & 313 & $\mathrm{C}$ & [1981SVO/CHA] \\
\hline & $\mathrm{V}$ & & $38.9 \pm 0.1$ & 328 & $\mathrm{C}$ & [1981SVO/CHA] \\
\hline & V & & $37.9 \pm 0.1$ & 343 & $\mathrm{C}$ & [1981SVO/CHA] \\
\hline & $\mathrm{V}$ & & $37.0 \pm 0.1$ & 358 & $\mathrm{C}$ & [1981SVO/CHA] \\
\hline & $\mathrm{V}$ & & $36.3 \pm 0.1$ & 368 & $\mathrm{C}$ & [1981SVO/CHA] \\
\hline & $\mathrm{V}$ & & $40.0 \pm 0.4$ & 298 & GCC & [1978FUC/PEA] \\
\hline & $\mathrm{V}$ & & 40.4 & 298 & & [1975KUS/SAI] \\
\hline & $\mathrm{V}$ & & 40.5 & 298 & & [1971WIL/ZWO] \\
\hline & $\mathrm{V}$ & & $40.5 \pm 0.1$ & 298 & $\mathrm{C}$ & [1947OSB/GIN] \\
\hline & $\mathrm{V}$ & $(323-407)$ & 38.6 & 338 & A, MM & [1987STE/MAL, 1945WIL/TAY] \\
\hline
\end{tabular}


TABLE 11. Phase change enthalpies of $\mathrm{C}_{8}$ organic compounds-Continued

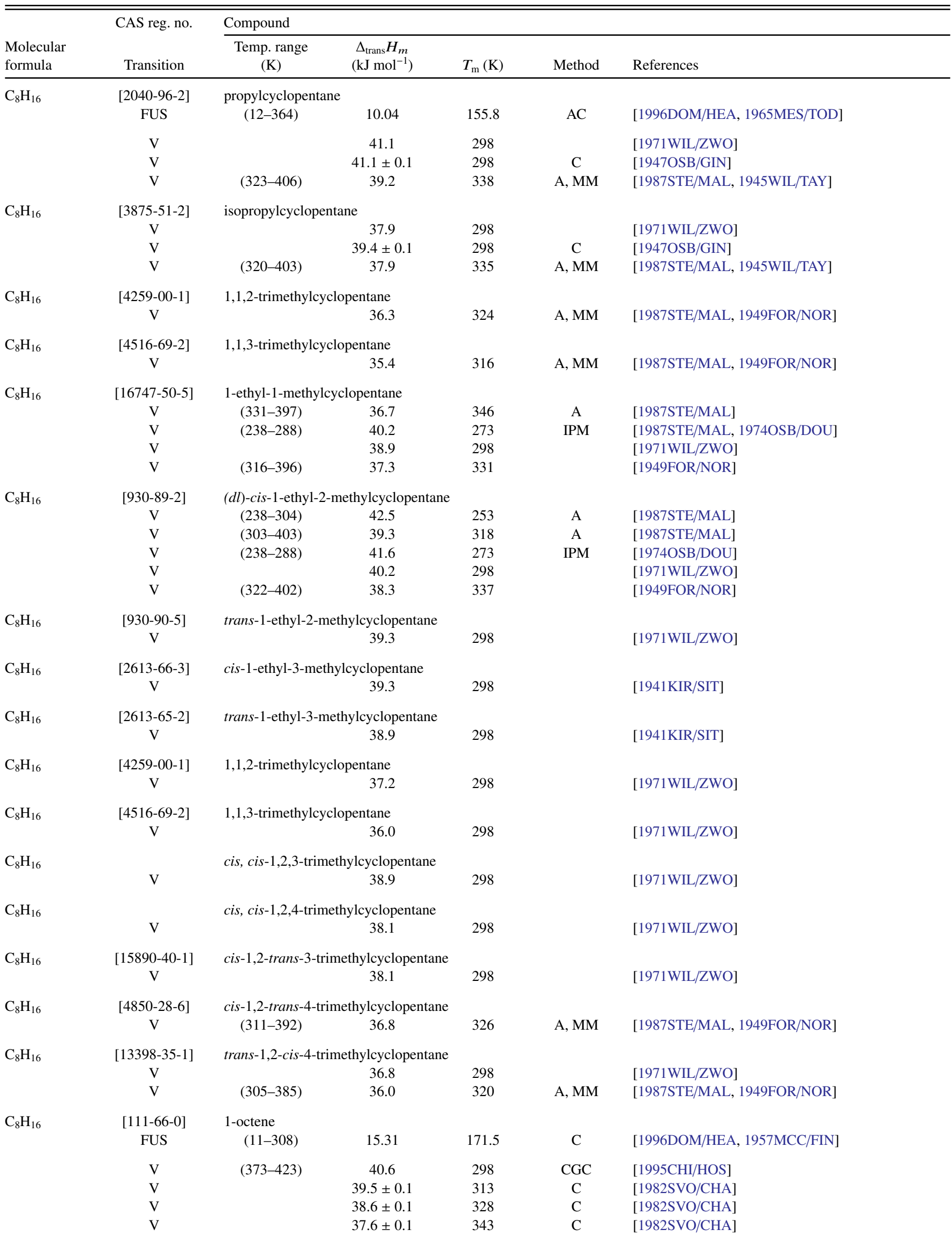


TABLE 11. Phase change enthalpies of $\mathrm{C}_{8}$ organic compounds-Continued

\begin{tabular}{|c|c|c|c|c|c|c|}
\hline \multirow[b]{2}{*}{$\begin{array}{l}\text { Molecular } \\
\text { formula }\end{array}$} & \multirow{2}{*}{$\begin{array}{l}\text { CAS reg. no. } \\
\text { Transition }\end{array}$} & \multicolumn{5}{|l|}{ Compound } \\
\hline & & $\begin{array}{l}\text { Temp. range } \\
\text { (K) }\end{array}$ & $\begin{array}{c}\Delta_{\text {trans }} H_{m} \\
\left(\mathrm{~kJ} \mathrm{~mol}^{-1}\right)\end{array}$ & $T_{\mathrm{m}}(\mathrm{K})$ & Method & References \\
\hline & $\mathrm{V}$ & & $36.6 \pm 0.1$ & 358 & $\mathrm{C}$ & [1982SVO/CHA] \\
\hline & $\mathrm{V}$ & & $35.8 \pm 0.1$ & 368 & $\mathrm{C}$ & {$[1982 \mathrm{SVO} / \mathrm{CHA}]$} \\
\hline & $\mathrm{V}$ & $(263-291)$ & 40.2 & 277 & MM & [1981CHI/HYM] \\
\hline & $\mathrm{V}$ & $(260-291)$ & 41.2 & 275 & HSA & [1981CHI/HYM] \\
\hline & $\mathrm{V}$ & & $40.3 \pm 0.2$ & 298 & $\mathrm{C}$ & [1977MAN/SEL] \\
\hline & $\mathrm{V}$ & & 38.0 & 298 & & [1971WIL/ZWO] \\
\hline & $\mathrm{V}$ & $(317-395)$ & 38.8 & 332 & $\mathrm{~A}, \mathrm{MM}$ & [1987STE/MAL, 1950FOR/CAM] \\
\hline \multirow[t]{3}{*}{$\mathrm{C}_{8} \mathrm{H}_{16}$} & {$[7642-04-8]$} & cis-2-octene & & & & \\
\hline & $\mathrm{V}$ & & 40.2 & 298 & & [1971WIL/ZWO] \\
\hline & $\mathrm{V}$ & $(356-400)$ & 37.8 & 371 & A & [1987STE/MAL, 1983ELV/KUU] \\
\hline \multirow[t]{3}{*}{$\mathrm{C}_{8} \mathrm{H}_{16}$} & [13389-42-9] & trans-2-octene & & & & \\
\hline & $\mathrm{V}$ & (356-399) & 37.9 & 371 & A & [1987STE/MAL, 1983ELV/KUU] \\
\hline & $\mathrm{V}$ & & 40.2 & 298 & & [1971WIL/ZWO] \\
\hline \multirow[t]{3}{*}{$\mathrm{C}_{8} \mathrm{H}_{16}$} & {$[14850-22-7]$} & cis-3-octene & & & & \\
\hline & $\mathrm{V}$ & & 37.3 & 298 & & [1983ELV/KUU] \\
\hline & $\mathrm{V}$ & & 39.7 & 298 & & [1971WIL/ZWO] \\
\hline \multirow{3}{*}{$\mathrm{C}_{8} \mathrm{H}_{16}$} & [14919-01-8] & trans-3-octene & & & & \\
\hline & $\mathrm{V}$ & $(354-396)$ & 37.6 & 369 & A & [1987STE/MAL, 1983ELV/KUU] \\
\hline & $\mathrm{V}$ & & 40.2 & 298 & & [1971WIL/ZWO] \\
\hline \multirow[t]{3}{*}{$\mathrm{C}_{8} \mathrm{H}_{16}$} & {$[7642-15-1]$} & cis-4-octene & & & & \\
\hline & $\mathrm{V}$ & (353-395) & 37.2 & 368 & A & [1987STE/MAL, 1983ELV/KUU] \\
\hline & $\mathrm{V}$ & & 39.7 & 298 & & [1971WIL/ZWO] \\
\hline \multirow[t]{5}{*}{$\mathrm{C}_{8} \mathrm{H}_{16}$} & {$[14850-23-8]$} & trans-4-octene & & & & \\
\hline & $\mathrm{V}$ & $(276-308)$ & $43.2 \pm 0.3$ & 292 & GS & [2000VER/WAN] \\
\hline & $\mathrm{V}$ & $(276-308)$ & $42.9 \pm 0.3$ & 298 & GS & [2000VER/WAN] \\
\hline & $\mathrm{V}$ & $(353-396)$ & 37.4 & 368 & A & [1987STE/MAL, 1983ELV/KUU] \\
\hline & $\mathrm{V}$ & & 39.7 & 298 & & [1971WIL/ZWO] \\
\hline \multirow[t]{2}{*}{$\mathrm{C}_{8} \mathrm{H}_{16}$} & [15870-10-7] & 2-methyl-1-hep & & & & \\
\hline & $\mathrm{V}$ & & 39.3 & 298 & & [1971WIL/ZWO] \\
\hline \multirow[t]{2}{*}{$\mathrm{C}_{8} \mathrm{H}_{16}$} & [4810-09-7] & 3-methyl-1-hep & & & & \\
\hline & $\mathrm{V}$ & & 38.5 & 298 & & [1971WIL/ZWO] \\
\hline \multirow[t]{2}{*}{$\mathrm{C}_{8} \mathrm{H}_{16}$} & [13151-05-8] & 4-methyl-1-hep & & & & \\
\hline & $\mathrm{V}$ & & 38.9 & 298 & & [1971WIL/ZWO] \\
\hline \multirow[t]{2}{*}{$\mathrm{C}_{8} \mathrm{H}_{16}$} & [13151-04-7] & 5-methyl-1-hep & & & & \\
\hline & $\mathrm{V}$ & & 38.9 & 298 & & [1971WIL/ZWO] \\
\hline \multirow[t]{2}{*}{$\mathrm{C}_{8} \mathrm{H}_{16}$} & {$[5026-76-6]$} & 6-methyl-1-hep & & & & \\
\hline & $\mathrm{V}$ & & 38.9 & 298 & & [1971WIL/ZWO] \\
\hline \multirow[t]{3}{*}{$\mathrm{C}_{8} \mathrm{H}_{16}$} & {$[627-97-4]$} & 2-methyl-2-hep & & & & \\
\hline & $\mathrm{V}$ & & 39.7 & 298 & & [1971WIL/ZWO] \\
\hline & $\mathrm{V}$ & $(257-396)$ & 41.2 & 272 & A & [1987STE/MAL, 1947STU] \\
\hline \multirow[t]{2}{*}{$\mathrm{C}_{8} \mathrm{H}_{16}$} & [22768-19-0] & 3-methyl-cis-2- & & & & \\
\hline & $\mathrm{V}$ & & 39.7 & 298 & & [1971WIL/ZWO] \\
\hline \multirow[t]{2}{*}{$\mathrm{C}_{8} \mathrm{H}_{16}$} & [22768-20-3] & 3-methyl-trans- & ptene & & & \\
\hline & $\mathrm{V}$ & & 39.7 & 298 & & [1971WIL/ZWO] \\
\hline \multirow[t]{2}{*}{$\mathrm{C}_{8} \mathrm{H}_{16}$} & [66225-16-9] & 4-methyl-cis-2- & ene & & & \\
\hline & $\mathrm{V}$ & & 38.9 & 298 & & [1971WIL/ZWO] \\
\hline \multirow[t]{2}{*}{$\mathrm{C}_{8} \mathrm{H}_{16}$} & {$[66225-17-0]$} & 4-methyl-trans- & ptene & & & \\
\hline & $\mathrm{V}$ & & 38.9 & 298 & & [1971WIL/ZWO] \\
\hline $\mathrm{C}_{8} \mathrm{H}_{16}$ & $\begin{array}{c}{[24608-84-2]} \\
V\end{array}$ & 5-methyl-cis-2- & ene 39.3 & 298 & & [1971WIL/ZWO] \\
\hline
\end{tabular}


TABLE 11. Phase change enthalpies of $\mathrm{C}_{8}$ organic compounds-Continued

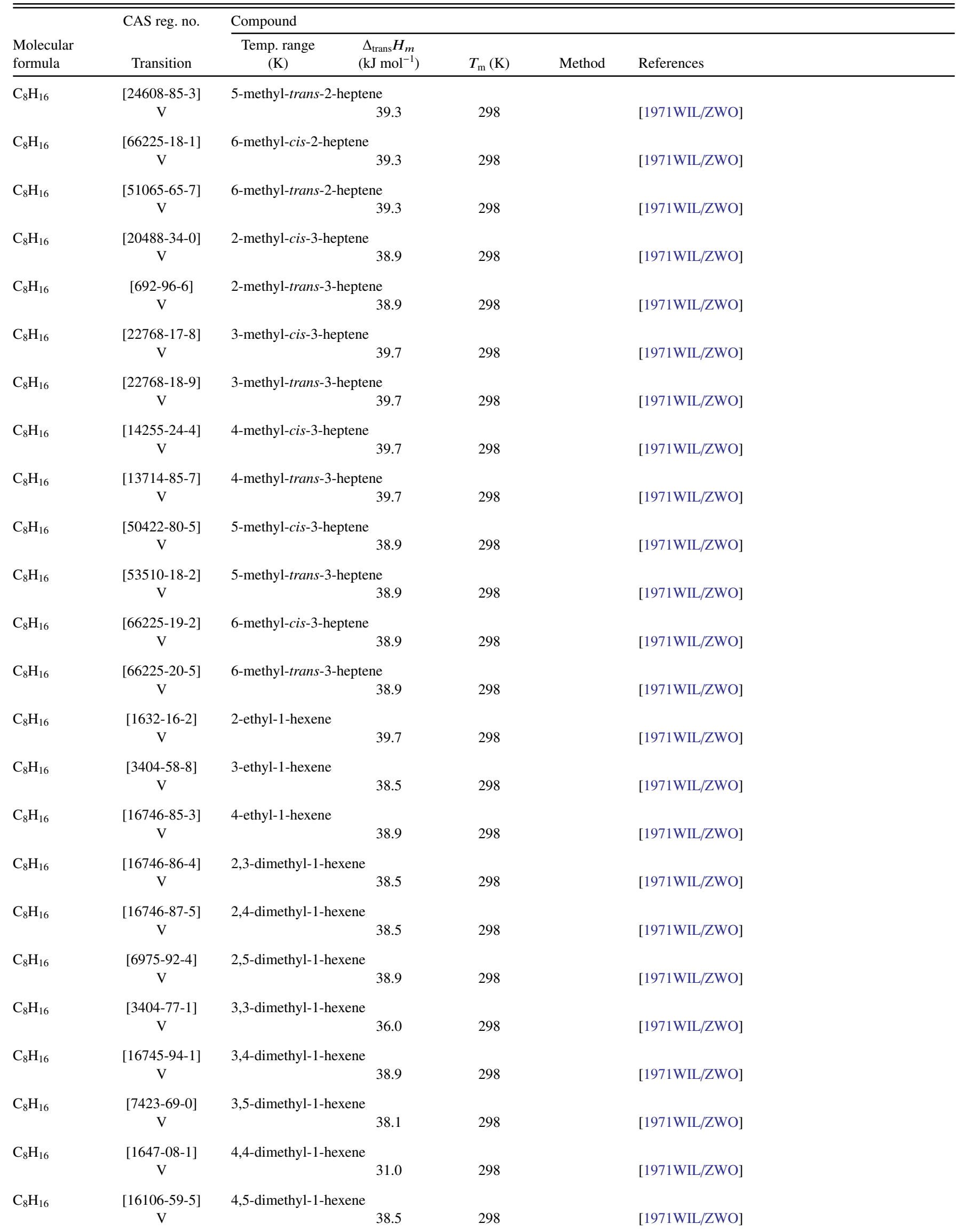


TABLE 11. Phase change enthalpies of $\mathrm{C}_{8}$ organic compounds-Continued

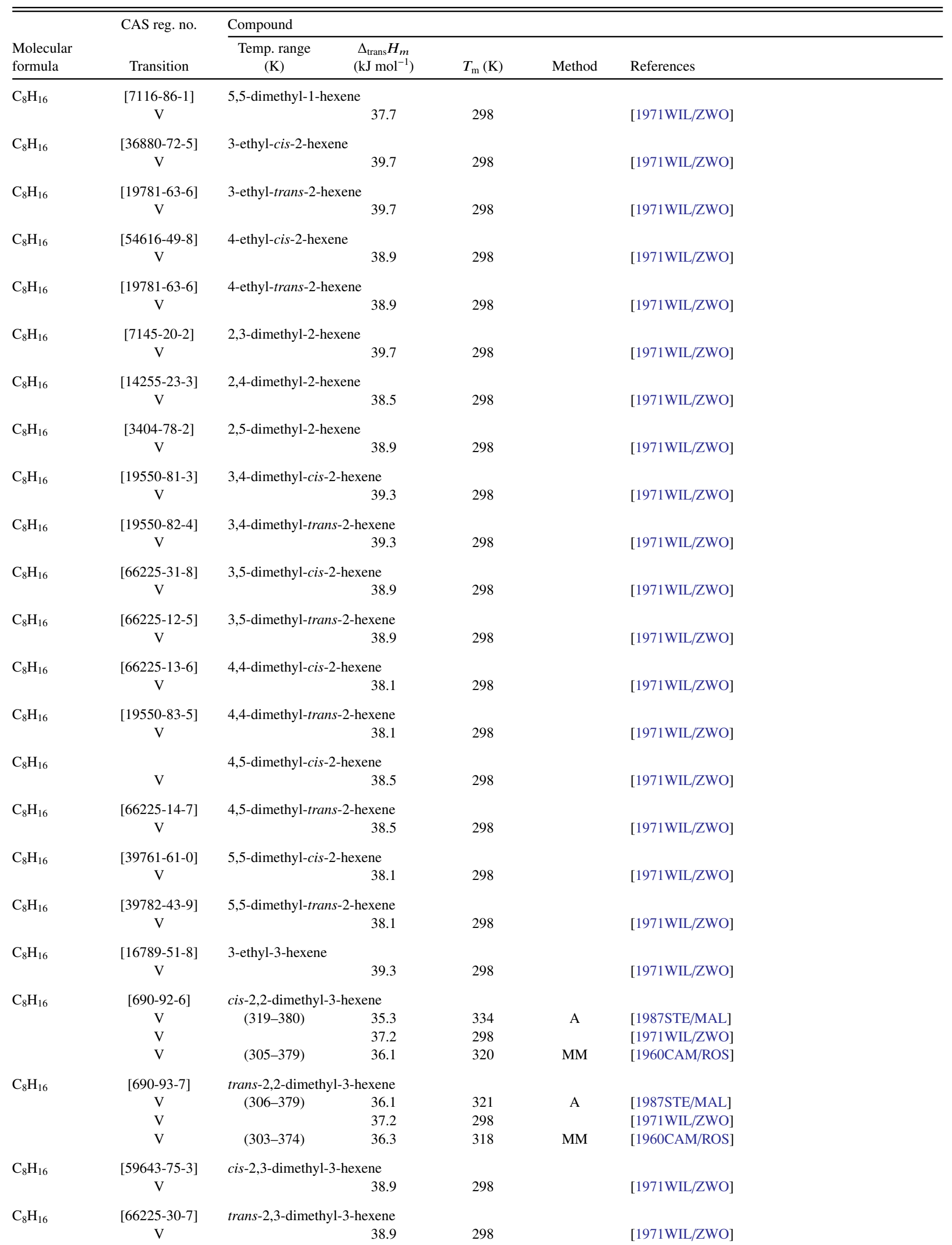


TABLE 11. Phase change enthalpies of $\mathrm{C}_{8}$ organic compounds-Continued

\begin{tabular}{|c|c|c|c|c|c|c|}
\hline \multirow[b]{2}{*}{$\begin{array}{l}\text { Molecular } \\
\text { formula }\end{array}$} & \multirow{2}{*}{$\begin{array}{l}\text { CAS reg. no. } \\
\text { Transition }\end{array}$} & \multicolumn{5}{|l|}{ Compound } \\
\hline & & $\begin{array}{c}\text { Temp. range } \\
\text { (K) }\end{array}$ & $\begin{array}{c}\Delta_{\text {trans }} H_{m} \\
\left(\mathrm{~kJ} \mathrm{~mol}^{-1}\right)\end{array}$ & $T_{\mathrm{m}}(\mathrm{K})$ & Method & References \\
\hline \multirow[t]{2}{*}{$\mathrm{C}_{8} \mathrm{H}_{16}$} & [37549-89-6] & cis-2,4-dimethy & hexene & & & \\
\hline & $\mathrm{V}$ & & 38.5 & 298 & & [1971WIL/ZWO] \\
\hline \multirow[t]{2}{*}{$\mathrm{C}_{8} \mathrm{H}_{16}$} & [61847-78-7] & trans-2,4-dime & -3-hexene & & & \\
\hline & $\mathrm{V}$ & & 38.5 & 298 & & [1971WIL/ZWO] \\
\hline \multirow[t]{2}{*}{$\mathrm{C}_{8} \mathrm{H}_{16}$} & {$[10557-44-5]$} & cis-2,5-dimethy & hexene & & & \\
\hline & $\mathrm{V}$ & & 37.2 & 298 & & [1971WIL/ZWO] \\
\hline \multirow[t]{2}{*}{$\mathrm{C}_{8} \mathrm{H}_{16}$} & [692-70-6] & trans-2,5-dime & -3-hexene & & & \\
\hline & V & & 37.5 & 298 & & [1971WIL/ZWO] \\
\hline \multirow[t]{2}{*}{$\mathrm{C}_{8} \mathrm{H}_{16}$} & [19550-87-9] & cis-3,4-dimethy & hexene & & & \\
\hline & $\mathrm{V}$ & & 39.7 & 298 & & [1971WIL/ZWO] \\
\hline \multirow[t]{2}{*}{$\mathrm{C}_{8} \mathrm{H}_{16}$} & [19550-88-0] & trans-3,4-dime & -3-hexene & & & \\
\hline & $\mathrm{V}$ & & 39.7 & 298 & & [1971WIL/ZWO] \\
\hline \multirow[t]{2}{*}{$\mathrm{C}_{8} \mathrm{H}_{16}$} & [15918-08-8] & 2-propyl-1-pen & & & & \\
\hline & V & & 39.3 & 298 & & [1971WIL/ZWO] \\
\hline \multirow[t]{2}{*}{$\mathrm{C}_{8} \mathrm{H}_{16}$} & [61847-79-8] & 2-isopropyl-1-1 & ene & & & \\
\hline & $\mathrm{V}$ & & 38.8 & 298 & & [1971WIL/ZWO] \\
\hline \multirow[t]{4}{*}{$\mathrm{C}_{8} \mathrm{H}_{16}$} & [3404-67-9] & 2-ethyl-3-meth & -pentene & & & \\
\hline & $\mathrm{V}$ & $(307-389)$ & 36.4 & 322 & A & [1987STE/MAL] \\
\hline & $\mathrm{V}$ & & 38.9 & 298 & & [1971WIL/ZWO] \\
\hline & $\mathrm{V}$ & $(308-383)$ & 36.4 & 323 & $\mathrm{MM}$ & [1960CAM/ROS] \\
\hline \multirow[t]{2}{*}{$\mathrm{C}_{8} \mathrm{H}_{16}$} & [3404-80-6] & 2-ethyl-4-meth & -pentene & & & \\
\hline & $\mathrm{V}$ & & 38.5 & 298 & & [1971WIL/ZWO] \\
\hline \multirow[t]{2}{*}{$\mathrm{C}_{8} \mathrm{H}_{16}$} & [19780-66-6] & 3-ethyl-2-meth & -pentene & & & \\
\hline & $\mathrm{V}$ & & 37.7 & 298 & & [1971WIL/ZWO] \\
\hline \multirow[t]{2}{*}{$\mathrm{C}_{8} \mathrm{H}_{16}$} & [6196-60-7] & 3-ethyl-3-meth & -pentene & & & \\
\hline & $\mathrm{V}$ & & 38.9 & 298 & & [1971WIL/ZWO] \\
\hline \multirow[t]{2}{*}{$\mathrm{C}_{8} \mathrm{H}_{16}$} & [61847-80-1] & 3-ethyl-4-meth & -pentene & & & \\
\hline & $\mathrm{V}$ & & 38.5 & 298 & & [1971WIL/ZWO] \\
\hline \multirow[t]{2}{*}{$\mathrm{C}_{8} \mathrm{H}_{16}$} & [560-23-6] & 2,3,3-trimethyl & entene & & & \\
\hline & $\mathrm{V}$ & & 38.5 & 298 & & [1971WIL/ZWO] \\
\hline \multirow[t]{2}{*}{$\mathrm{C}_{8} \mathrm{H}_{16}$} & [565-76-4] & 2,3,4-trimethyl & entene & & & \\
\hline & $\mathrm{V}$ & & 38.5 & 298 & & [1971WIL/ZWO] \\
\hline \multirow[t]{2}{*}{$\mathrm{C}_{8} \mathrm{H}_{16}$} & [107-39-1] & 2,4,4-trimethyl & entene & & & \\
\hline & FUS & & 8.79 & 178.9 & & [1996DOM/HEA, 1936PAR/TOD2] \\
\hline \multirow{2}{*}{\multicolumn{7}{|c|}{$\begin{array}{l}\text { [Note: The authors give enthalpy of fusion data for two isomers of diisobutylene. It is speculated in the manuscript that the low boiling point is } \\
\text { is 2,2,4-trimethyl-1-pentene; whereas the high boiling point isomer is believed to be 2,2,4-trimethyl-2-pentene. The value above is for the low } \\
\text { boiling isomer.] }\end{array}$}} \\
\hline & & & & & & \\
\hline & $\mathrm{V}$ & $(306-356)$ & 35.7 & 298 & EB & [2007MAL] \\
\hline & $\mathrm{V}$ & $(343-381)$ & 33.5 & 358 & A & [1987STE/MAL] \\
\hline & $\mathrm{V}$ & & 35.7 & 298 & & [1971WIL/ZWO] \\
\hline & $\mathrm{V}$ & $(301-375)$ & 35.1 & 316 & $\mathrm{MM}$ & [1960CAM/ROS] \\
\hline \multirow[t]{2}{*}{$\mathrm{C}_{8} \mathrm{H}_{16}$} & [564-03-4] & 3,3,4-trimethyl & entene & & & \\
\hline & $\mathrm{V}$ & & 38.1 & 298 & & [1971WIL/ZWO] \\
\hline $\mathrm{C}_{8} \mathrm{H}_{16}$ & [19780-67-7] & 2-methyl-3-eth & -pentene & & & \\
\hline & $\mathrm{V}$ & & 39.3 & 298 & & [1971WIL/ZWO] \\
\hline $\mathrm{C}_{8} \mathrm{H}_{16}$ & [42067-48-1] & 4-methyl-3-eth & is-2-pentene & & & \\
\hline & $\mathrm{V}$ & & 39.3 & 298 & & [1971WIL/ZWO] \\
\hline $\mathrm{C}_{8} \mathrm{H}_{16}$ & [42067-49-2] & 4-methyl-3-eth & ans-2-pentene & & & \\
\hline & $\mathrm{V}$ & & 38.9 & 298 & & [1971WIL/ZWO] \\
\hline
\end{tabular}


TABLE 11. Phase change enthalpies of $\mathrm{C}_{8}$ organic compounds-Continued

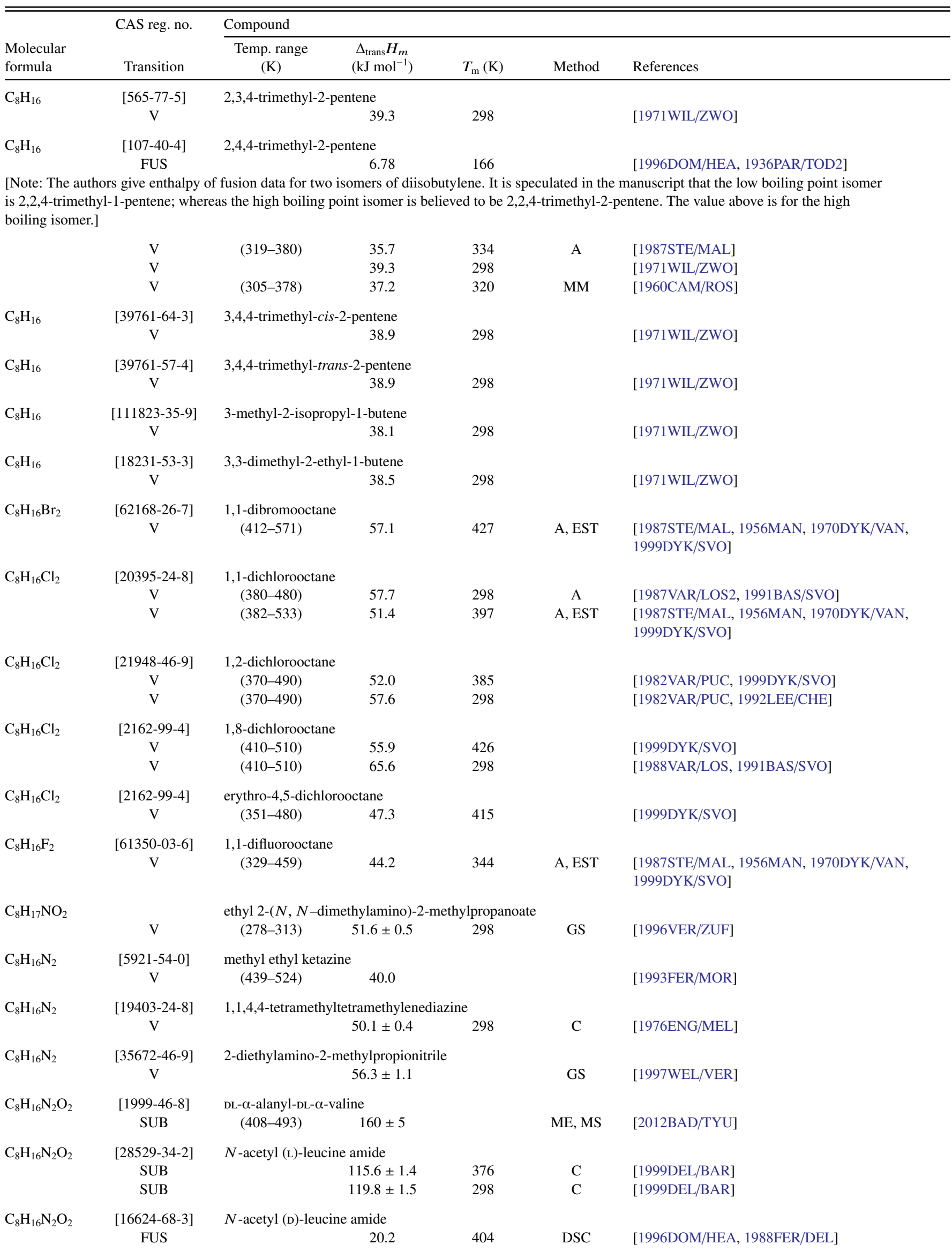


TABLE 11. Phase change enthalpies of $\mathrm{C}_{8}$ organic compounds-Continued

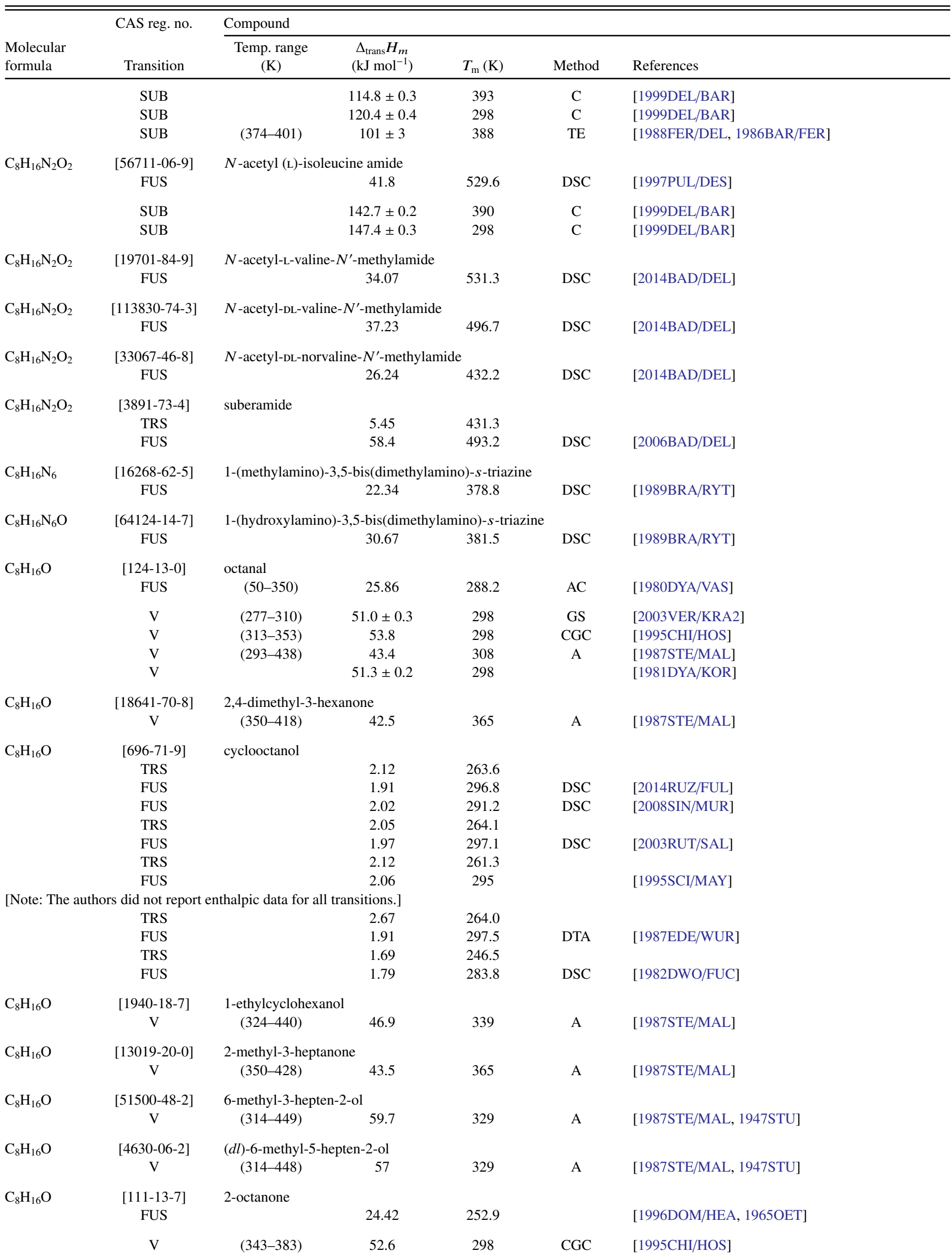


TABLE 11. Phase change enthalpies of $\mathrm{C}_{8}$ organic compounds-Continued

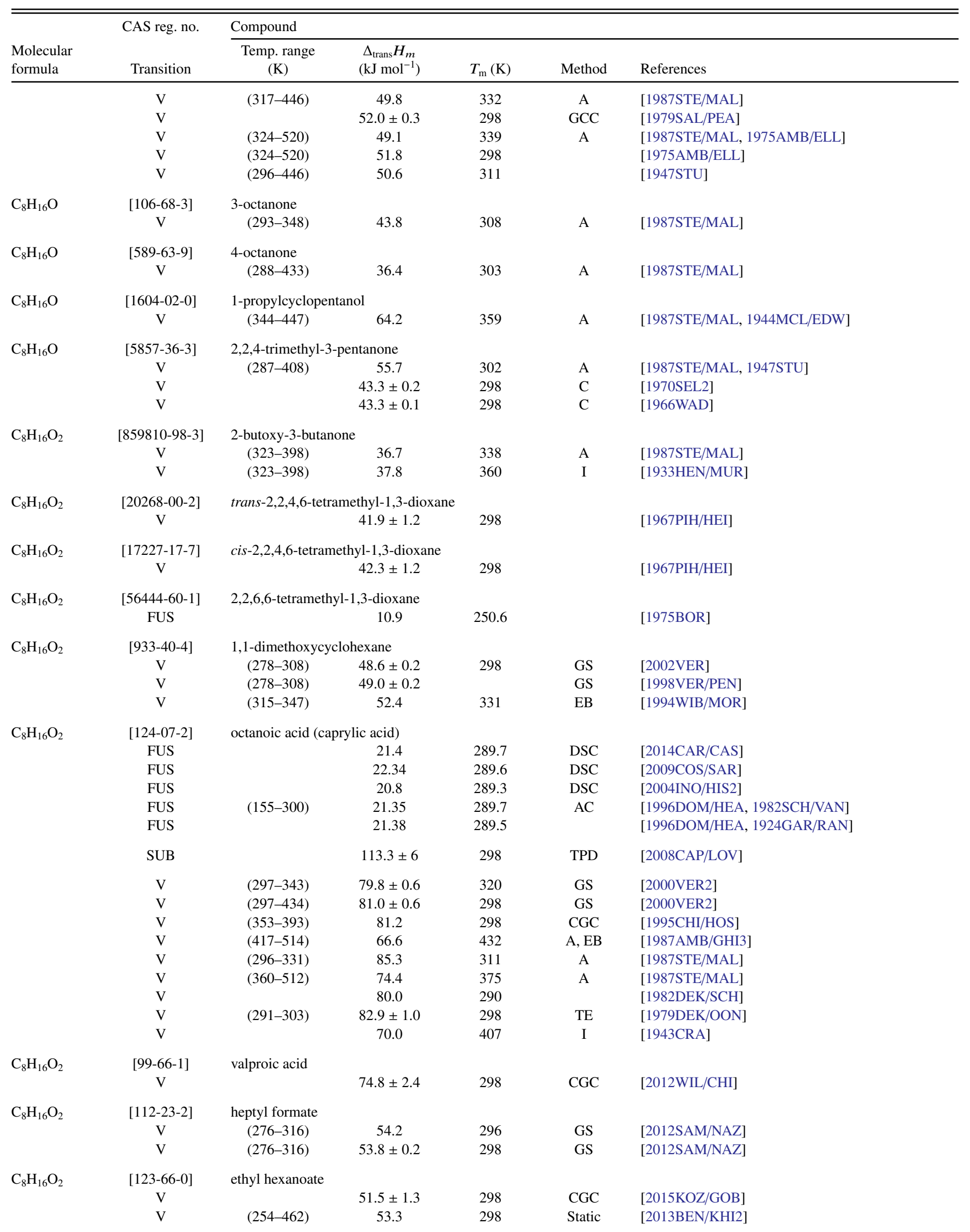


TABLE 11. Phase change enthalpies of $\mathrm{C}_{8}$ organic compounds-Continued

\begin{tabular}{|c|c|c|c|c|c|c|}
\hline \multirow[b]{2}{*}{$\begin{array}{l}\text { Molecular } \\
\text { formula }\end{array}$} & \multirow{2}{*}{$\begin{array}{l}\text { CAS reg. no. } \\
\text { Transition }\end{array}$} & \multicolumn{5}{|l|}{ Compound } \\
\hline & & $\begin{array}{c}\text { Temp. range } \\
(\mathrm{K})\end{array}$ & $\begin{array}{c}\Delta_{\text {trans }} H_{m} \\
\left(\mathrm{~kJ} \mathrm{~mol}^{-1}\right)\end{array}$ & $T_{\mathrm{m}}(\mathrm{K})$ & Method & References \\
\hline & $\mathrm{V}$ & $(408-440)$ & 43.1 & 423 & EB & [2011MAT/YAM] \\
\hline & $\mathrm{V}$ & $(253-462)$ & $49.5 \pm 0.5$ & 358 & Static & [2011BEN/KHI] \\
\hline & $\mathrm{V}$ & $(253-462)$ & $54.1 \pm 0.5$ & 298 & Static & [2011BEN/KHI] \\
\hline & $\mathrm{V}$ & $(279-309)$ & $50.8 \pm 0.4$ & 294 & GS & [1999VER/HEI] \\
\hline & $\mathrm{V}$ & $(279-309)$ & $50.6 \pm 0.4$ & 298 & GS & [1999VER/HEI] \\
\hline & $\mathrm{V}$ & $(345-374)$ & $47.4 \pm 0.3$ & 359 & $\mathrm{~EB}$ & [1991WIB/WAL] \\
\hline & V & $(345-379)$ & $51.5 \pm 1.3$ & 298 & $\mathrm{~EB}$ & [1991WIB/WAL] \\
\hline & V & $(396-449)$ & 51.8 & 311 & A & [1987STE/MAL] \\
\hline & $\mathrm{V}$ & $(300-376)$ & 48.6 & 315 & A & [1987STE/MAL] \\
\hline & $\mathrm{V}$ & & $51.7 \pm 0.1$ & 298 & $\mathrm{C}$ & [1986NIL/WAD] \\
\hline \multirow{8}{*}{$\mathrm{C}_{8} \mathrm{H}_{16} \mathrm{O}_{2}$} & {$[149-57-5]$} & \multicolumn{5}{|c|}{$(d l)$-2-ethylhexanoic acid } \\
\hline & $\mathrm{V}$ & $(397-514)$ & $67.0 \pm 0.6$ & 400 & $\mathrm{~EB}$ & [1997STE/CHI3] \\
\hline & $\mathrm{V}$ & $(397-514)$ & $64.8 \pm 0.6$ & 420 & $\mathrm{~EB}$ & [1997STE/CHI3] \\
\hline & $\mathrm{V}$ & $(397-514)$ & $59.9 \pm 0.5$ & 460 & $\mathrm{~EB}$ & [1997STE/CHI3] \\
\hline & $\mathrm{V}$ & $(397-514)$ & $54.2 \pm 0.7$ & 500 & $\mathrm{~EB}$ & [1997STE/CHI3] \\
\hline & $\mathrm{V}$ & $(397-514)$ & $76.3 \pm 0.9$ & 298 & $\mathrm{~EB}$ & [1997STE/CHI3] \\
\hline & $\mathrm{V}$ & $(403-500)$ & 61.8 & 418 & A & [1987STE/MAL] \\
\hline & $\mathrm{V}$ & & $75.6 \pm 0.5$ & 298 & $\mathrm{C}$ & [1976STR] \\
\hline \multirow[t]{7}{*}{$\mathrm{C}_{8} \mathrm{H}_{16} \mathrm{O}_{2}$} & [142-92-7] & hexyl acetate & & & & \\
\hline & FUS & & 19.83 & 212.1 & & [1996DOM/HEA, 1984VAS/PET] \\
\hline & $\mathrm{V}$ & $(274-309)$ & $51.9 \pm 0.3$ & 298 & GS & [2006KRA/VER] \\
\hline & $\mathrm{V}$ & & 52.1 & 298 & GC & [1997KOU/HOS] \\
\hline & $\mathrm{V}$ & $(303-444)$ & 50.9 & 318 & & [1995ARC/BLA] \\
\hline & $\mathrm{V}$ & $(304-381)$ & 48.9 & 319 & A & [1987STE/MAL] \\
\hline & $\mathrm{V}$ & $(378-459)$ & 46.2 & 387 & DTA & [1980MEY/AWE] \\
\hline \multirow[t]{4}{*}{$\mathrm{C}_{8} \mathrm{H}_{16} \mathrm{O}_{2}$} & [109-21-7] & butyl butanoate & & & & \\
\hline & FUS & & 14.93 & 181.7 & & [1996DOM/HEA, 1984VAS/PET] \\
\hline & $\mathrm{V}$ & $(400-460)$ & 41.8 & 420 & & [2005ORT/ESP] \\
\hline & $\mathrm{V}$ & $(400-450)$ & 42.2 & 425 & EB & {$[1995 \mathrm{GON} / \mathrm{ORT}]$} \\
\hline \multirow[t]{2}{*}{$\mathrm{C}_{8} \mathrm{H}_{16} \mathrm{O}_{2}$} & {$[539-90-2]$} & isobutyl butyrate & & & & \\
\hline & $\mathrm{V}$ & $(277-430)$ & 41.7 & 292 & A & [1987STE/MAL, 1947STU] \\
\hline \multirow[t]{4}{*}{$\mathrm{C}_{8} \mathrm{H}_{16} \mathrm{O}_{2}$} & {$[97-85-8]$} & isobutyl isobutyrate & & & & \\
\hline & $\mathrm{V}$ & $(274-319)$ & 48.5 & 298 & GS & [2008VER/EME] \\
\hline & $\mathrm{V}$ & $(278-313)$ & $44.5 \pm 0.1$ & 298 & GS & [1996VER/BEC] \\
\hline & $\mathrm{V}$ & $(277-421)$ & 46.9 & 292 & A & [1987STE/MAL, 1947STU] \\
\hline \multirow[t]{2}{*}{$\mathrm{C}_{8} \mathrm{H}_{16} \mathrm{O}_{2}$} & {$[105-68-0]$} & isopentyl propionate & & & & \\
\hline & $\mathrm{V}$ & $(281-434)$ & 44.1 & 296 & A & [1987STE/MAL] \\
\hline \multirow[t]{3}{*}{$\mathrm{C}_{8} \mathrm{H}_{16} \mathrm{O}_{2}$} & {$[624-54-4]$} & pentyl propanoate & & & & \\
\hline & $\mathrm{V}$ & $(274-328)$ & 52.1 & 301 & GS & [2012SAM/NAZ] \\
\hline & $\mathrm{V}$ & $(274-328)$ & $52.2 \pm 0.1$ & 298 & GS & [2012SAM/NAZ] \\
\hline \multirow[t]{14}{*}{$\mathrm{C}_{8} \mathrm{H}_{16} \mathrm{O}_{2}$} & {$[106-73-0]$} & methyl heptanoate & & & & \\
\hline & $\mathrm{V}$ & $(421-444)$ & 46.3 & 433 & & [2009POS/MAR] \\
\hline & $\mathrm{V}$ & $(278-310)$ & $53.2 \pm 0.2$ & 298 & GS & [2008VER/EME] \\
\hline & $\mathrm{V}$ & & 49.1 & 350 & & [2002VAN/VAN] \\
\hline & $\mathrm{V}$ & & $50.2 \pm 0.1$ & 326 & & [2002VAN/VAN] \\
\hline & $\mathrm{V}$ & & $51.8 \pm 0.1$ & 298 & & [2002VAN/VAN] \\
\hline & $\mathrm{V}$ & $(313-363)$ & 53.4 & 298 & $\mathrm{CGC}$ & [1995CHI/HOS] \\
\hline & $\mathrm{V}$ & $(433-473)$ & 53.7 & 298 & CGC & [1995CHI/HOS] \\
\hline & $\mathrm{V}$ & $(313-353)$ & 53.5 & 298 & CGC & [1995CHI/HOS] \\
\hline & $\mathrm{V}$ & & $49.7 \pm 0.5$ & 298 & GC & [1987AZA] \\
\hline & $\mathrm{V}$ & & $53.1 \pm 0.4$ & 298 & GCC & [1980FUC/PEA] \\
\hline & $\mathrm{V}$ & & $53.1 \pm 0.1$ & 298 & $\mathrm{C}$ & [1980FUC/PEA] \\
\hline & $\mathrm{V}$ & & $51.6 \pm 0.5$ & 298 & $\mathrm{C}$ & [1977MAN/SEL] \\
\hline & $\mathrm{V}$ & $(332-402)$ & 49.0 & 347 & A, EST & [1987STE/MAL, 1963ROS/SCH] \\
\hline \multirow[t]{2}{*}{$\mathrm{C}_{8} \mathrm{H}_{16} \mathrm{O}_{2}$} & {$[25415-67-2]$} & \multicolumn{5}{|c|}{ 4-methylvaleric acid, ethyl ester } \\
\hline & $\mathrm{V}$ & $(284-434)$ & 45.4 & 299 & A & [1987STE/MAL, 1947STU] \\
\hline
\end{tabular}


TABLE 11. Phase change enthalpies of $\mathrm{C}_{8}$ organic compounds-Continued

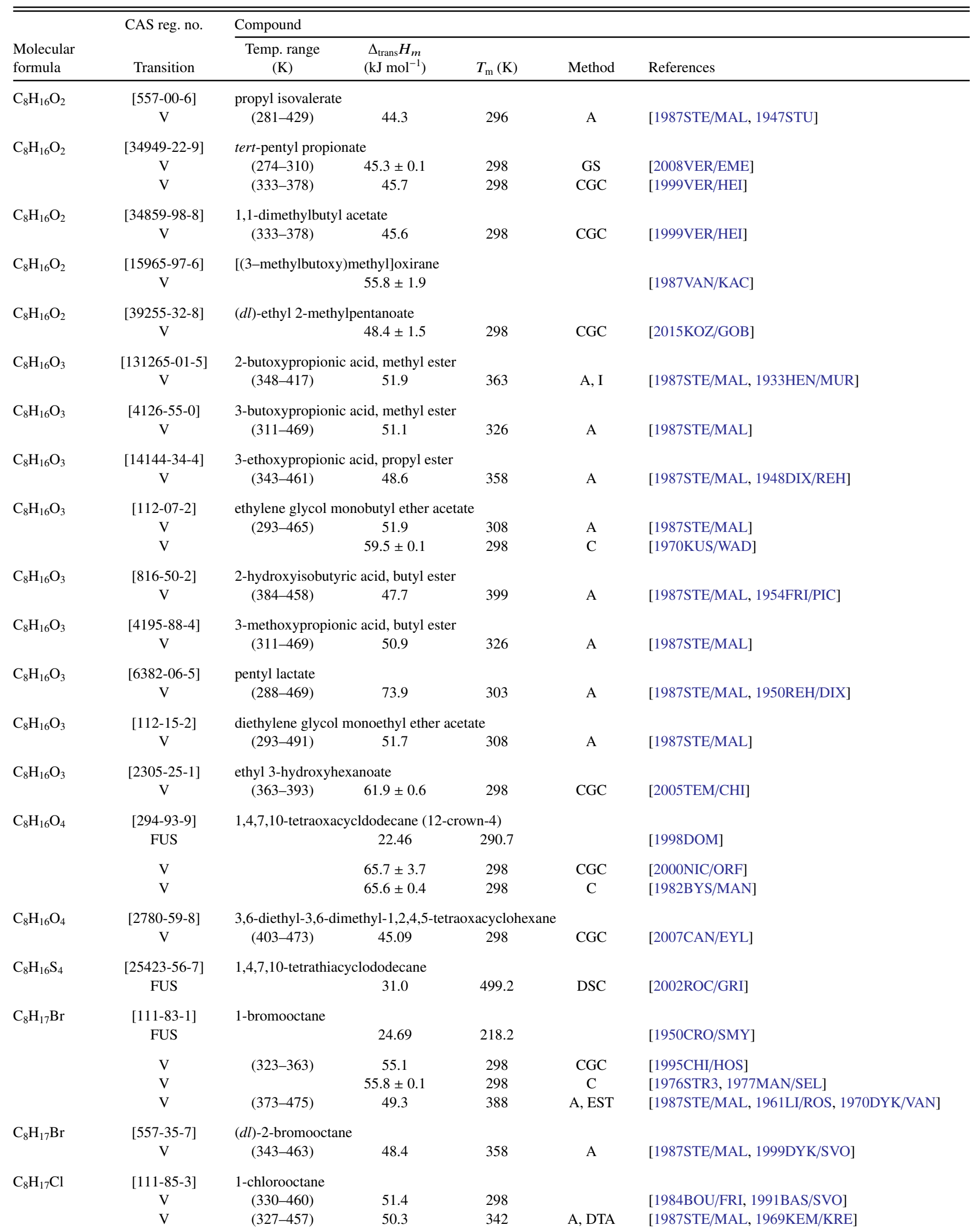


TABLE 11. Phase change enthalpies of $\mathrm{C}_{8}$ organic compounds-Continued

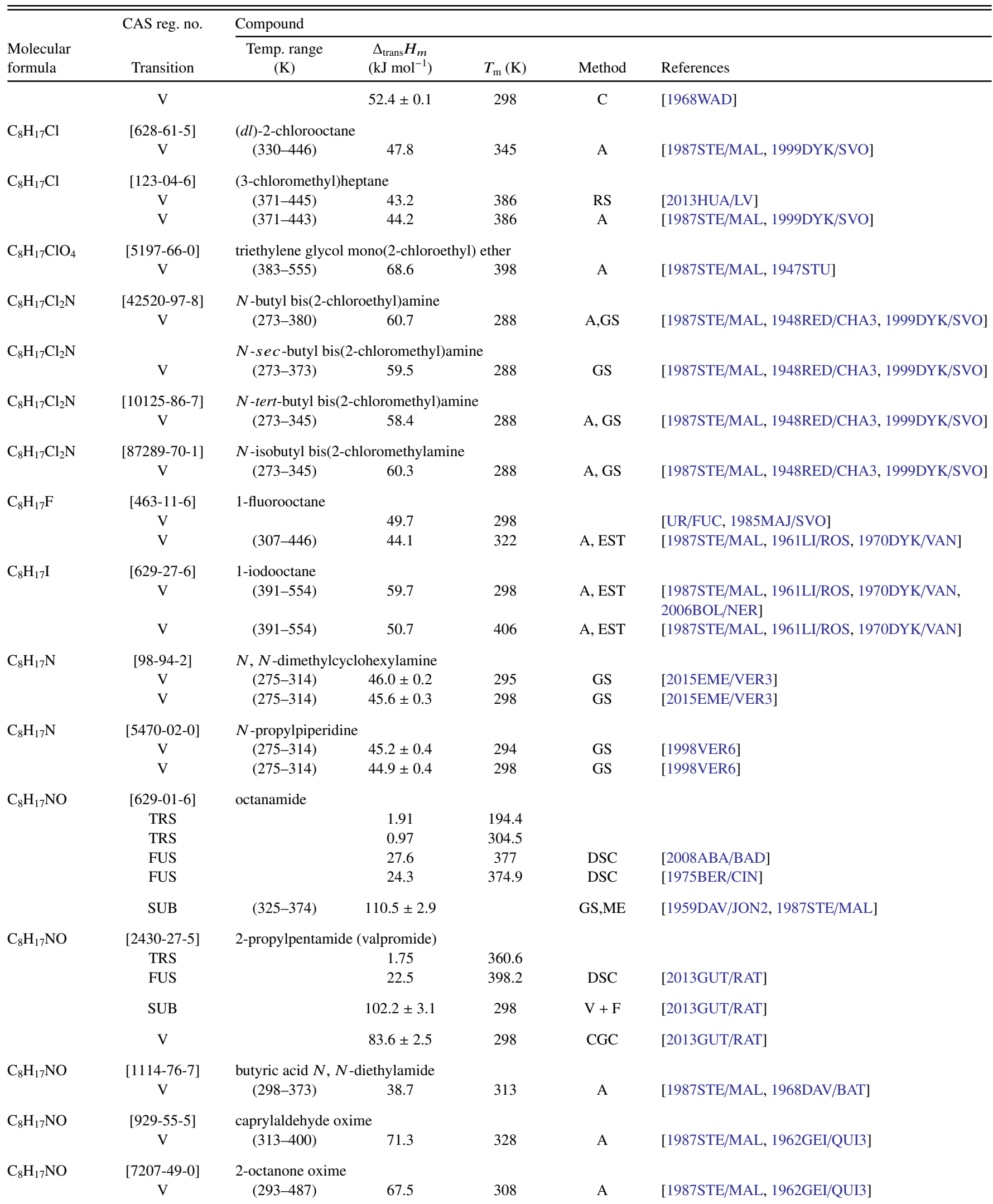


TABLE 11. Phase change enthalpies of $\mathrm{C}_{8}$ organic compounds-Continued

\begin{tabular}{|c|c|c|c|c|c|c|}
\hline \multirow[b]{2}{*}{$\begin{array}{l}\text { Molecular } \\
\text { formula }\end{array}$} & \multirow{2}{*}{$\begin{array}{l}\text { CAS reg. no. } \\
\text { Transition }\end{array}$} & \multicolumn{5}{|l|}{ Compound } \\
\hline & & $\begin{array}{c}\text { Temp. range } \\
\text { (K) }\end{array}$ & $\begin{array}{c}\Delta_{\text {trans }} H_{m} \\
\left(\mathrm{~kJ} \mathrm{~mol}^{-1}\right)\end{array}$ & $T_{\mathrm{m}}(\mathrm{K})$ & Method & References \\
\hline $\mathrm{C}_{8} \mathrm{H}_{17} \mathrm{NO}$ & $\begin{array}{c}{[7207-50-3]} \\
\mathrm{V}\end{array}$ & $\begin{array}{l}\text { 3-octanone oxime } \\
\quad(293-400)\end{array}$ & 67.2 & 308 & A & [1987STE/MAL, 1962GEI/QUI3] \\
\hline $\mathrm{C}_{8} \mathrm{H}_{17} \mathrm{NO}$ & $\begin{array}{c}{[7207-51-4]} \\
\mathrm{V}\end{array}$ & $\begin{array}{l}\text { 4-octanone oxime } \\
\quad(293-400)\end{array}$ & 68.8 & 308 & A & [1987STE/MAL, 1962GEI/QUI3] \\
\hline $\mathrm{C}_{8} \mathrm{H}_{17} \mathrm{NO}_{2}$ & $\begin{array}{c}{[5342-78-9]} \\
\text { V } \\
\text { V }\end{array}$ & $\begin{array}{l}\text { 2,4,4-trimethyl-2-nit } \\
\quad(288-324) \\
(288-324)\end{array}$ & $\begin{array}{l}\text { ropentane } \\
54.2 \pm 0.8 \\
54.7 \pm 0.8\end{array}$ & $\begin{array}{l}306 \\
298\end{array}$ & $\begin{array}{l}\text { GS } \\
\text { GS }\end{array}$ & $\begin{array}{l}\text { [1997VER3] } \\
\text { [1997VER3] }\end{array}$ \\
\hline $\mathrm{C}_{8} \mathrm{H}_{17} \mathrm{NO}_{2}$ & $\begin{array}{c}{[5395-35-7]} \\
\mathrm{V}\end{array}$ & $\begin{array}{l}\text { lactic acid } N \text {-isopen } \\
\quad(386-433)\end{array}$ & $\begin{array}{c}\text { tylamide } \\
77.9\end{array}$ & 401 & A & [1987STE/MAL, 1950RAT/FIS] \\
\hline $\mathrm{C}_{8} \mathrm{H}_{17} \mathrm{NO}_{2}$ & $\begin{array}{c}{[5323-54-6]} \\
\mathrm{V}\end{array}$ & $\begin{array}{l}\text { lactic acid } N \text {-pentyl } \\
\quad(373-448)\end{array}$ & $\begin{array}{l}\text { amide } \\
81.8\end{array}$ & 388 & A & [1987STE/MAL, 1950RAT] \\
\hline $\mathrm{C}_{8} \mathrm{H}_{17} \mathrm{NO}_{2}$ & $\begin{array}{c}{[2743-60-4]} \\
\text { V }\end{array}$ & $\begin{array}{l}\text { (l) leucine ethyl este } \\
\text { (333-449) }\end{array}$ & 43.5 & 348 & A & [1987STE/MAL] \\
\hline $\mathrm{C}_{8} \mathrm{H}_{17} \mathrm{NO}_{2}$ & $\mathrm{~V}$ & ethyl 2-(N,N-dime & $\begin{array}{l}\text { thylamino) }-2 \\
55.6 \pm 0.4\end{array}$ & $\begin{array}{l}\text { ylpropion } \\
283\end{array}$ & DSC & [1993SCH/BEC] \\
\hline $\mathrm{C}_{8} \mathrm{H}_{17} \mathrm{NO}_{2}$ & $\begin{array}{c}{[7214-62-2]} \\
\mathrm{V}\end{array}$ & $\begin{array}{l}\text { (1-methylheptyl)nitr } \\
\text { (303-338) }\end{array}$ & ite 44.9 & 318 & A & [1987STE/MAL] \\
\hline $\mathrm{C}_{8} \mathrm{H}_{17} \mathrm{NO}_{2}$ & $\begin{array}{l}{[1002-57-9]} \\
\text { SUB } \\
\text { SUB }\end{array}$ & $\begin{array}{l}\text { 8-aminooctanoic aci } \\
\text { (391-402) }\end{array}$ & $\begin{array}{l}\mathrm{d} \\
166.2 \pm 0.9 \\
170 \pm 4\end{array}$ & $\begin{array}{l}397 \\
298\end{array}$ & $\begin{array}{l}\mathrm{C} \\
\mathrm{C}\end{array}$ & $\begin{array}{l}{[1983 \mathrm{SKO} / \mathrm{SAB}]} \\
{[1983 \mathrm{SKO} / \mathrm{SAB}]}\end{array}$ \\
\hline $\mathrm{C}_{8} \mathrm{H}_{17} \mathrm{NO}_{4}$ & $\begin{array}{c}{[72458-42-5]} \\
\text { FUS }\end{array}$ & $N$-ethyl-5-amino-1, & $\begin{array}{l}\text { 5-dideoxy-(D) } \\
26.3\end{array}$ & $\begin{array}{l}\text { opyranose } \\
429.1\end{array}$ & DSC & [1994BLU/PRA] \\
\hline $\mathrm{C}_{8} \mathrm{H}_{17} \mathrm{~N}_{3}$ & $\begin{array}{c}{[7438-05-3]} \\
\mathrm{V}\end{array}$ & $\begin{array}{l}\text { 1-octylazide } \\
(276-326)\end{array}$ & $58.9 \pm 0.3$ & 298 & GS & [2014EME/ALG] \\
\hline $\mathrm{C}_{8} \mathrm{H}_{18}$ & $\begin{array}{c}{[590-73-8]} \\
\mathrm{V} \\
\mathrm{V} \\
\mathrm{V} \\
\mathrm{V}\end{array}$ & $\begin{array}{l}\text { 2,2-dimethylhexane } \\
\text { (243-380) } \\
(302-381)\end{array}$ & $\begin{array}{c}37.3 \\
39.7 \\
37.3 \pm 0.1 \\
36.6\end{array}$ & $\begin{array}{l}298 \\
258 \\
298 \\
317\end{array}$ & $\begin{array}{c}\mathrm{C} \\
\mathrm{A}, \mathrm{MM}\end{array}$ & $\begin{array}{l}{[1971 \mathrm{WIL} / \mathrm{ZWO}]} \\
{[1947 \mathrm{STU}]} \\
{[1947 \mathrm{OSB} / \mathrm{GIN}]} \\
\text { [1987STE/MAL, 1945WIL/TAY] }\end{array}$ \\
\hline $\mathrm{C}_{8} \mathrm{H}_{18}$ & $\begin{array}{c}\text { [584-94-1] } \\
\mathrm{V} \\
\mathrm{V} \\
\mathrm{V} \\
\mathrm{V}\end{array}$ & $\begin{array}{l}\text { 2,3-dimethylhexane } \\
\text { (250-388) } \\
(310-390)\end{array}$ & $\begin{array}{c}38.8 \\
41.4 \\
38.8 \pm 0.1 \\
37.6\end{array}$ & $\begin{array}{l}298 \\
265 \\
298 \\
325\end{array}$ & $\begin{array}{c}\mathrm{C} \\
\mathrm{A}, \mathrm{MM}\end{array}$ & $\begin{array}{l}{[1971 \mathrm{WIL} / \mathrm{ZWO}]} \\
{[1947 \mathrm{STU}]} \\
{[1947 \mathrm{OSB} / \mathrm{GIN}]} \\
{[1987 \mathrm{STE} / \mathrm{MAL}, 1945 \mathrm{WIL} / \mathrm{TAY}]}\end{array}$ \\
\hline $\mathrm{C}_{8} \mathrm{H}_{18}$ & $\begin{array}{c}{[589-43-5]} \\
\mathrm{V} \\
\mathrm{V} \\
\mathrm{V} \\
\mathrm{V}\end{array}$ & $\begin{array}{l}\text { 2,4-dimethylhexane } \\
\text { (246-382) } \\
(305-385)\end{array}$ & $\begin{array}{c}37.8 \\
41 \\
37.8 \pm 0.1 \\
36.9\end{array}$ & $\begin{array}{l}298 \\
261 \\
298 \\
320\end{array}$ & $\begin{array}{c}\mathrm{C} \\
\mathrm{A}, \mathrm{MM}\end{array}$ & $\begin{array}{l}\text { [1971WIL/ZWO] } \\
{[1947 \mathrm{STU}]} \\
{[1947 \mathrm{OSB} / \mathrm{GIN}]} \\
{[1987 \mathrm{STE} / \mathrm{MAL}, 1945 \mathrm{WIL} / \mathrm{TAY}]}\end{array}$ \\
\hline $\mathrm{C}_{8} \mathrm{H}_{18}$ & $\begin{array}{c}{[592-13-2]} \\
\mathrm{V} \\
\mathrm{V} \\
\mathrm{V} \\
\mathrm{V}\end{array}$ & $\begin{array}{l}\text { 2,5-dimethylhexane } \\
(246-382) \\
(307-383)\end{array}$ & $\begin{array}{c}37.9 \\
41.1 \\
37.9 \pm 0.1 \\
36.9\end{array}$ & $\begin{array}{l}298 \\
261 \\
298 \\
322\end{array}$ & $\begin{array}{c}\mathrm{C} \\
\mathrm{A}, \mathrm{MM}\end{array}$ & $\begin{array}{l}\text { [1971WIL/ZWO] } \\
{[1947 \mathrm{STU}]} \\
{[1947 \mathrm{OSB} / \mathrm{GIN}]} \\
\text { [1987STE/MAL, 1945WIL/TAY] }\end{array}$ \\
\hline $\mathrm{C}_{8} \mathrm{H}_{18}$ & $\begin{array}{c}{[563-16-6]} \\
\mathrm{V} \\
\mathrm{V} \\
\mathrm{V}\end{array}$ & $\begin{array}{l}\text { 3,3-dimethylhexane } \\
\quad(247-385) \\
(308-386)\end{array}$ & $\begin{array}{c}41.2 \\
37.5 \pm 0.1 \\
36.6\end{array}$ & $\begin{array}{l}262 \\
298 \\
323\end{array}$ & $\begin{array}{c}\mathrm{C} \\
\mathrm{A}, \mathrm{MM}\end{array}$ & $\begin{array}{l}\text { [1947STU] } \\
\text { [1947OSB/GIN] } \\
\text { [1987STE/MAL, 1945WIL/TAY] }\end{array}$ \\
\hline $\mathrm{C}_{8} \mathrm{H}_{18}$ & $\begin{array}{c}{[583-48-2]} \\
\mathrm{V} \\
\mathrm{V} \\
\mathrm{V}\end{array}$ & $\begin{array}{l}\text { 3,4-dimethylhexane } \\
\quad(251-390) \\
(313-392)\end{array}$ & $\begin{array}{c}41.3 \\
39.0 \pm 0.1 \\
37.7\end{array}$ & $\begin{array}{l}266 \\
298 \\
328\end{array}$ & $\begin{array}{c}\mathrm{C} \\
\mathrm{A}, \mathrm{MM}\end{array}$ & $\begin{array}{l}\text { [1947STU] } \\
\text { [1947OSB/GIN] } \\
\text { [1987STE/MAL, 1945WIL/TAY] }\end{array}$ \\
\hline
\end{tabular}


TABLE 11. Phase change enthalpies of $\mathrm{C}_{8}$ organic compounds-Continued

\begin{tabular}{|c|c|c|c|c|c|c|}
\hline \multirow[b]{2}{*}{$\begin{array}{l}\text { Molecular } \\
\text { formula }\end{array}$} & \multirow{2}{*}{$\begin{array}{l}\text { CAS reg. no. } \\
\text { Transition }\end{array}$} & \multicolumn{5}{|l|}{ Compound } \\
\hline & & $\begin{array}{c}\text { Temp. range } \\
(\mathrm{K})\end{array}$ & $\begin{array}{c}\Delta_{\text {trans }} H_{m} \\
\left(\mathrm{~kJ} \mathrm{~mol}^{-1}\right)\end{array}$ & $T_{\mathrm{m}}(\mathrm{K})$ & Method & References \\
\hline \multirow[t]{5}{*}{$\mathrm{C}_{8} \mathrm{H}_{18}$} & [619-99-8] & 3-ethylhexane & & & & \\
\hline & $\mathrm{V}$ & & 39.7 & 298 & & [1971WIL/ZWO] \\
\hline & $\mathrm{V}$ & $(251-391)$ & 42.4 & 268 & & [1947STU] \\
\hline & V & & $39.6 \pm 0.1$ & 298 & $\mathrm{C}$ & [1947OSB/GIN] \\
\hline & $\mathrm{V}$ & $(314-393)$ & 38.2 & 329 & $\mathrm{~A}, \mathrm{MM}$ & [1987STE/MAL, 1945WIL/TAY] \\
\hline \multirow[t]{4}{*}{$\mathrm{C}_{8} \mathrm{H}_{18}$} & [609-26-7] & 3-ethyl-2-meth & tane & & & \\
\hline & $\mathrm{V}$ & & 38.5 & 298 & & [1971WIL/ZWO] \\
\hline & $\mathrm{V}$ & & $38.5 \pm 0.1$ & 298 & $\mathrm{C}$ & [1947OSB/GIN] \\
\hline & $\mathrm{V}$ & $(311-390)$ & 37.4 & 326 & $\mathrm{~A}, \mathrm{MM}$ & [1987STE/MAL, 1945WIL/TAY] \\
\hline \multirow[t]{5}{*}{$\mathrm{C}_{8} \mathrm{H}_{18}$} & [1067-08-9] & 3-ethyl-3-meth & tane & & & \\
\hline & $\mathrm{V}$ & & 38.0 & 298 & & [1971WIL/ZWO] \\
\hline & $\mathrm{V}$ & $(249-391)$ & 40.2 & 264 & & [1947STU] \\
\hline & $\mathrm{V}$ & & $38.0 \pm 0.1$ & 298 & $\mathrm{C}$ & [1947OSB/GIN] \\
\hline & $\mathrm{V}$ & $(312-393)$ & 36.9 & 327 & $\mathrm{~A}, \mathrm{MM}$ & [1987STE/MAL, 1945WIL/TAY] \\
\hline \multirow[t]{11}{*}{$\mathrm{C}_{8} \mathrm{H}_{18}$} & {$[592-27-8]$} & 2-methylheptai & & & & \\
\hline & FUS & & 11.92 & 164.2 & & [1996DOM/HEA, 1971MES/FIN] \\
\hline & V & $(285-392)$ & 39.8 & 300 & A & [1987STE/MAL] \\
\hline & V & & $39.7 \pm 0.1$ & 298 & $\mathrm{C}$ & [1979MAJ/SVO] \\
\hline & $\mathrm{V}$ & & $38.7 \pm 0.1$ & 313 & $\mathrm{C}$ & [1979MAJ/SVO] \\
\hline & V & & $37.3 \pm 0.1$ & 333 & $\mathrm{C}$ & [1979MAJ/SVO] \\
\hline & $\mathrm{V}$ & & $36.0 \pm 0.1$ & 353 & $\mathrm{C}$ & [1979MAJ/SVO] \\
\hline & V & $(233-283)$ & 41.6 & 268 & IPM & [1987STE/MAL, 1974OSB/DOU] \\
\hline & $\mathrm{V}$ & & 39.7 & 298 & & [1971WIL/ZWO] \\
\hline & $\mathrm{V}$ & & $39.8 \pm 0.1$ & 298 & $\mathrm{C}$ & [1947OSB/GIN] \\
\hline & $\mathrm{V}$ & $(315-391)$ & 38.1 & 330 & MM & [1945WIL/TAY] \\
\hline \multirow[t]{8}{*}{$\mathrm{C}_{8} \mathrm{H}_{18}$} & {$[589-81-1]$} & 3-methylheptas & & & & \\
\hline & FUS & & 11.67 & 152.6 & & [1996DOM/HEA, 1973FIN/MES] \\
\hline & $\mathrm{V}$ & & $39.8 \pm 0.2$ & 298 & $\mathrm{C}$ & [1987AN/HU] \\
\hline & $\mathrm{V}$ & $(286-393)$ & 40.1 & 301 & A & [1987STE/MAL] \\
\hline & $\mathrm{V}$ & $(238-286)$ & 41.6 & 271 & IPM & [1987STE/MAL, 1974OSB/DOU] \\
\hline & $\mathrm{V}$ & & 39.8 & 298 & & [1971WIL/ZWO] \\
\hline & V & & $39.8 \pm 0.1$ & 298 & $\mathrm{C}$ & [1947OSB/GIN] \\
\hline & $\mathrm{V}$ & $(316-393)$ & 38.3 & 331 & $\mathrm{MM}$ & [1945WIL/TAY] \\
\hline \multirow[t]{10}{*}{$\mathrm{C}_{8} \mathrm{H}_{18}$} & {$[589-53-7]$} & 4-methylheptai & & & & \\
\hline & FUS & & 10.84 & 152.2 & & [1991ACR, 1983WEA] \\
\hline & $\mathrm{V}$ & & $39.7 \pm 0.1$ & 298 & $\mathrm{C}$ & [1979MAJ/SVO] \\
\hline & $\mathrm{V}$ & & $38.7 \pm 0.1$ & 313 & $\mathrm{C}$ & [1979MAJ/SVO] \\
\hline & $\mathrm{V}$ & & $37.4 \pm 0.1$ & 333 & $\mathrm{C}$ & {$[1979 \mathrm{MAJ} / \mathrm{SVO}]$} \\
\hline & $\mathrm{V}$ & & $36.1 \pm 0.1$ & 353 & $\mathrm{C}$ & [1979MAJ/SVO] \\
\hline & $\mathrm{V}$ & & 39.7 & 298 & & [1971WIL/ZWO] \\
\hline & $\mathrm{V}$ & $(253-391)$ & 42.3 & 268 & & [1947STU] \\
\hline & $\mathrm{V}$ & & $39.7 \pm 0.1$ & 298 & $\mathrm{C}$ & [1947OSB/GIN] \\
\hline & $\mathrm{V}$ & $(312-392)$ & 38.2 & 327 & $\mathrm{~A}, \mathrm{MM}$ & [1987STE/MAL, 1945WIL/TAY] \\
\hline \multirow[t]{12}{*}{$\mathrm{C}_{8} \mathrm{H}_{18}$} & [111-65-9] & octane & & & & \\
\hline & FUS & & 21.8 & 216.6 & DSC & [2004MON/RAJ] \\
\hline & FUS & & 20.32 & 215.8 & DSC & [2005HUA/SIM] \\
\hline & FUS & & 21.0 & 216.4 & $\mathrm{C}$ & [1955TUN/STO] \\
\hline & FUS & & 20.74 & 216.4 & & [1996DOM/HEA, 1954FIN/GRO2] \\
\hline & FUS & & 20.62 & 215.8 & $\mathrm{C}$ & [1996DOM/HEA, 1931HUF/PAR] \\
\hline & FUS & & 20.09 & 215.6 & & [1996DOM/HEA, 1930PAR/HUF] \\
\hline & SUB & & 68.1 & 216 & $\mathrm{~B}$ & {$[1963 \mathrm{BON}]$} \\
\hline & $\mathrm{V}$ & $(323-563)$ & 39.4 & 338 & $\mathrm{~EB}$ & [2003EWI/OCH] \\
\hline & $\mathrm{V}$ & & 41.6 & 298 & & [1994RUZ/MAJ] \\
\hline & $\mathrm{V}$ & $(297-400)$ & 41.0 & 312 & A & [1987STE/MAL] \\
\hline & $\mathrm{V}$ & $(216-278)$ & 44.4 & 263 & A & [1987STE/MAL] \\
\hline
\end{tabular}


TABLE 11. Phase change enthalpies of $\mathrm{C}_{8}$ organic compounds-Continued

\begin{tabular}{|c|c|c|c|c|c|c|}
\hline \multirow[b]{2}{*}{$\begin{array}{l}\text { Molecular } \\
\text { formula }\end{array}$} & \multirow{2}{*}{$\begin{array}{l}\text { CAS reg. no. } \\
\text { Transition }\end{array}$} & \multicolumn{5}{|l|}{ Compound } \\
\hline & & $\begin{array}{c}\text { Temp. range } \\
(\mathrm{K})\end{array}$ & $\begin{array}{c}\Delta_{\text {trans }} H_{m} \\
\left(\mathrm{~kJ} \mathrm{~mol}^{-1}\right)\end{array}$ & $T_{\mathrm{m}}(\mathrm{K})$ & Method & References \\
\hline & $\mathrm{V}$ & $(396-432)$ & 36.3 & 411 & A & [1987STE/MAL] \\
\hline & V & $(428-510)$ & 35.5 & 443 & A & [1987STE/MAL] \\
\hline & V & $(506-569)$ & 34.9 & 521 & A & [1987STE/MAL] \\
\hline & V & $(295-402)$ & 41.2 & 310 & & [1986PAU/KRU] \\
\hline & V & $(298-333)$ & 41.9 & 313 & & [1984MIC/JOS] \\
\hline & V & & $41.5 \pm 0.1$ & 298 & $\mathrm{C}$ & [1981HOS/SCO2] \\
\hline & $\mathrm{V}$ & & $41.5 \pm 0.1$ & 298 & $\mathrm{C}$ & [1979MAJ/SVO] \\
\hline & V & & $40.5 \pm 0.1$ & 313 & $\mathrm{C}$ & [1979MAJ/SVO] \\
\hline & V & & $39.1 \pm 0.1$ & 333 & $\mathrm{C}$ & [1979MAJ/SVO] \\
\hline & V & & $37.8 \pm 0.1$ & 353 & $\mathrm{C}$ & [1979MAJ/SVO] \\
\hline & $\mathrm{V}$ & $(217-297)$ & 43.0 & 282 & & [1973CAR/KOB] \\
\hline & $\mathrm{V}$ & & 41.5 & 298 & & [1971WIL/ZWO] \\
\hline & V & & $41.0 \pm 0.5$ & 298 & $\mathrm{C}$ & [1963MOR/SUN] \\
\hline & V & & $38.0 \pm 0.1$ & 311 & $\mathrm{C}$ & [1960MCK/SAG] \\
\hline & $\mathrm{V}$ & & $36.7 \pm 0.1$ & 328 & $\mathrm{C}$ & [1960MCK/SAG] \\
\hline & V & & $35.4 \pm 0.1$ & 344 & $\mathrm{C}$ & [1960MCK/SAG] \\
\hline & V & & $41.5 \pm 0.1$ & 298 & $\mathrm{C}$ & [1947OSB/GIN] \\
\hline & $\mathrm{V}$ & $(326-400)$ & 39.2 & 341 & $\mathrm{MM}$ & [1945WIL/TAY] \\
\hline \multirow[t]{13}{*}{$\mathrm{C}_{8} \mathrm{H}_{18}$} & {$[560-21-4]$} & $(d l)$-2,2,3-trime & entane & & & \\
\hline & $\mathrm{V}$ & & $37.7 \pm 0.1$ & 298 & $\mathrm{C}$ & [1998SVO/HYN] \\
\hline & $\mathrm{V}$ & & $37.1 \pm 0.1$ & 308 & $\mathrm{C}$ & [1998SVO/HYN] \\
\hline & V & & $36.6 \pm 0.1$ & 315 & $\mathrm{C}$ & [1998SVO/HYN] \\
\hline & V & & $36.0 \pm 0.1$ & 323 & $\mathrm{C}$ & [1998SVO/HYN] \\
\hline & V & & $35.5 \pm 0.1$ & 330 & $\mathrm{C}$ & [1998SVO/HYN] \\
\hline & $\mathrm{V}$ & & $35.1 \pm 0.1$ & 338 & $\mathrm{C}$ & [1998SVO/HYN] \\
\hline & $\mathrm{V}$ & & $34.8 \pm 0.1$ & 348 & $\mathrm{C}$ & [1998SVO/HYN] \\
\hline & V & & $34.1 \pm 0.1$ & 358 & $\mathrm{C}$ & [1998SVO/HYN] \\
\hline & $\mathrm{V}$ & & $33.5 \pm 0.1$ & 368 & $\mathrm{C}$ & [1998SVO/HYN] \\
\hline & V & & 36.9 & 298 & & [1971WIL/ZWO] \\
\hline & $\mathrm{V}$ & & $36.9 \pm 0.1$ & 298 & $\mathrm{C}$ & [1947OSB/GIN] \\
\hline & $\mathrm{V}$ & $(306-384)$ & 36.1 & 321 & $\mathrm{~A}, \mathrm{MM}$ & [1987STE/MAL, 1945WIL/TAY] \\
\hline \multirow[t]{26}{*}{$\mathrm{C}_{8} \mathrm{H}_{18}$} & {$[540-84-1]$} & 2,2,4-trimethyl & & & & \\
\hline & FUS & & 9.08 & 165.8 & DTA & [1994TAN/SAB3] \\
\hline & FUS & & 9.21 & 165.8 & & [1940PIT] \\
\hline & FUS & & 9.04 & 165.3 & & [1996DOM/HEA, 1930PAR/HUF] \\
\hline & $\mathrm{V}$ & $(373-423)$ & 34.9 & 298 & CGC & [1995CHI/HOS] \\
\hline & V & $(289-333)$ & 36.1 & 304 & & [1991WU/PIV] \\
\hline & $\mathrm{V}$ & $(423-523)$ & 31.6 & 438 & A & [1987STE/MAL] \\
\hline & $\mathrm{V}$ & $(372-416)$ & 32.2 & 387 & A & [1987STE/MAL] \\
\hline & $\mathrm{V}$ & $(413-494)$ & 31.5 & 428 & A & [1987STE/MAL] \\
\hline & $\mathrm{V}$ & $(490-544)$ & 31.4 & 505 & A & [1987STE/MAL] \\
\hline & $\mathrm{V}$ & & $35.2 \pm 0.1$ & 298 & $\mathrm{C}$ & [1982SVO/CHA] \\
\hline & $\mathrm{V}$ & & $34.4 \pm 0.1$ & 313 & $\mathrm{C}$ & [1982SVO/CHA] \\
\hline & $\mathrm{V}$ & & $33.4 \pm 0.1$ & 328 & $\mathrm{C}$ & [1982SVO/CHA] \\
\hline & V & & $32.6 \pm 0.1$ & 343 & $\mathrm{C}$ & [1982SVO/CHA] \\
\hline & V & & $31.7 \pm 0.1$ & 358 & $\mathrm{C}$ & [1982SVO/CHA] \\
\hline & $\mathrm{V}$ & & $31.0 \pm 0.1$ & 368 & $\mathrm{C}$ & [1982SVO/CHA] \\
\hline & $\mathrm{V}$ & & $35.1 \pm 0.1$ & 298 & $\mathrm{C}$ & [1979MAJ/SVO] \\
\hline & $\mathrm{V}$ & & $34.3 \pm 0.1$ & 313 & $\mathrm{C}$ & [1979MAJ/SVO] \\
\hline & V & & $33.2 \pm 0.1$ & 333 & $\mathrm{C}$ & [1979MAJ/SVO] \\
\hline & $\mathrm{V}$ & & $32.0 \pm 0.1$ & 353 & $\mathrm{C}$ & [1979MAJ/SVO] \\
\hline & V & & 35.1 & 298 & & [1971WIL/ZWO] \\
\hline & $\mathrm{V}$ & (194-299) & 40.7 & 209 & A & [1987STE/MAL, 1956MIL] \\
\hline & V & & $35.1 \pm 0.1$ & 298 & $\mathrm{C}$ & [1947OSB/GIN] \\
\hline & V & $(297-374)$ & 34.8 & 312 & $\mathrm{~A}, \mathrm{MM}$ & [1987STE/MAL, 1945WIL/TAY] \\
\hline & V & & 31.0 & 371 & $\mathrm{C}$ & [1940PIT] \\
\hline & $\mathrm{V}$ & $(318-399)$ & 33.9 & 333 & EB & [1940SMI] \\
\hline \multirow[t]{3}{*}{$\mathrm{C}_{8} \mathrm{H}_{18}$} & [560-21-4] & \multicolumn{5}{|c|}{ 2,3,3-trimethylpentane } \\
\hline & V & & $37.6 \pm 0.1$ & 298 & $\mathrm{C}$ & [1998SVO/HYN] \\
\hline & $\mathrm{V}$ & & $36.9 \pm 0.1$ & 308 & $\mathrm{C}$ & [1998SVO/HYN] \\
\hline
\end{tabular}


TABLE 11. Phase change enthalpies of $\mathrm{C}_{8}$ organic compounds-Continued

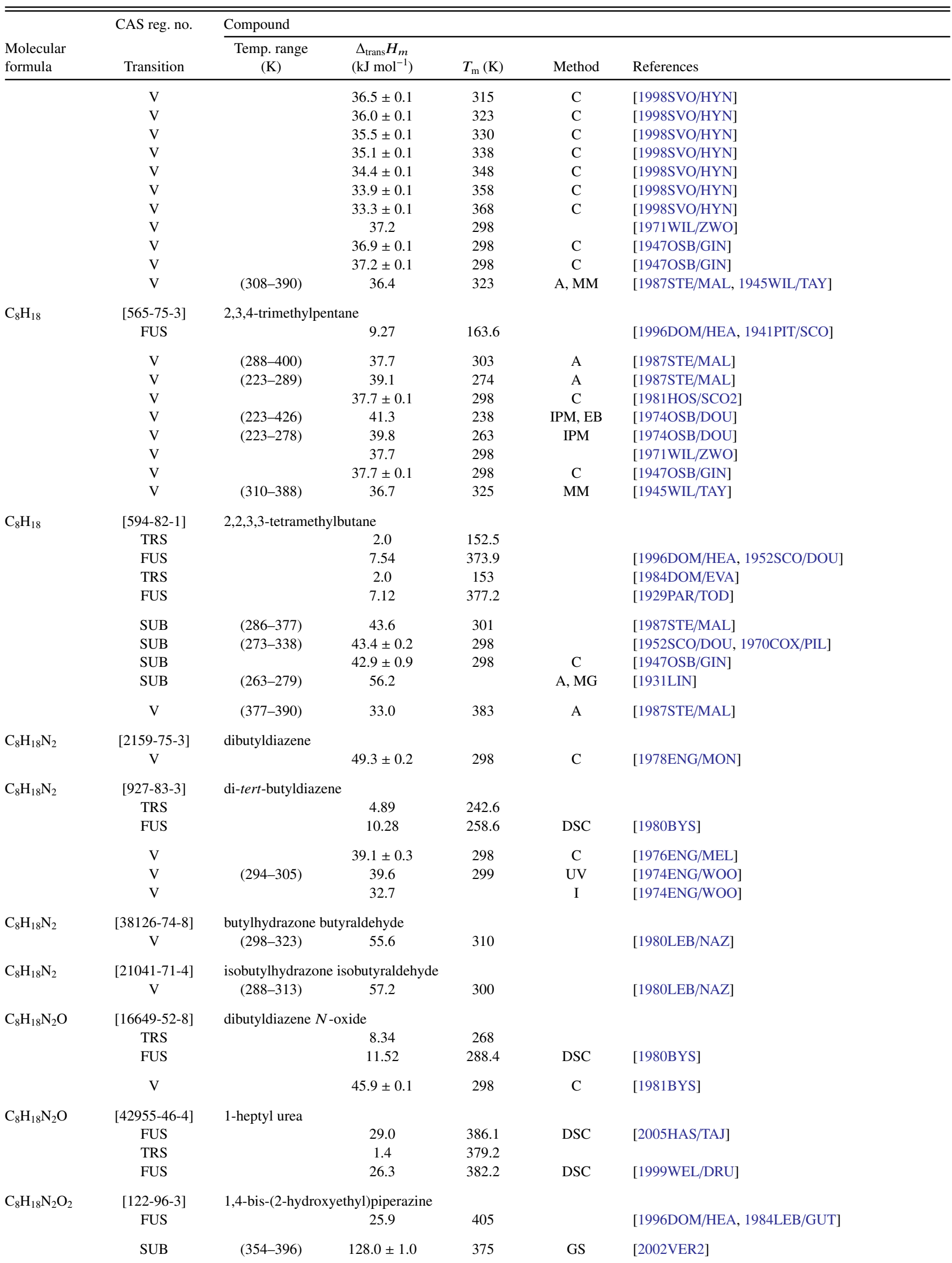


TABLE 11. Phase change enthalpies of $\mathrm{C}_{8}$ organic compounds-Continued

\begin{tabular}{|c|c|c|c|c|c|c|}
\hline \multirow[b]{2}{*}{$\begin{array}{l}\text { Molecular } \\
\text { formula }\end{array}$} & \multirow{2}{*}{$\begin{array}{c}\text { CAS reg. no. } \\
\text { Transition }\end{array}$} & \multicolumn{5}{|l|}{ Compound } \\
\hline & & $\begin{array}{c}\text { Temp. range } \\
(\mathrm{K})\end{array}$ & $\begin{array}{c}\Delta_{\text {trans }} H_{m} \\
\left(\mathrm{~kJ} \mathrm{~mol}^{-1}\right)\end{array}$ & $T_{\mathrm{m}}(\mathrm{K})$ & Method & References \\
\hline \multirow{6}{*}{$\mathrm{C}_{8} \mathrm{H}_{18} \mathrm{~N}_{4} \mathrm{O}_{4}$} & SUB & $(354-396)$ & $130.5 \pm 1.0$ & 298 & GS & [2002VER2] \\
\hline & SUB & $(334-356)$ & 104.1 & & & [1984LEB/GUT] \\
\hline & V & $(413-507)$ & $67.8 \pm 5.3$ & & & [1998ABD/MEI] \\
\hline & [35823-10-0] & \multicolumn{5}{|c|}{$N, N^{\prime}$-dimethyl- $N, N^{\prime}$ dinitro-1,6-hexanediamine } \\
\hline & FUS & & 61.68 & 331 & DTA & {$[19870 Y U / B R I]$} \\
\hline & SUB & & $125.5 \pm 1.7$ & 298 & $\mathrm{C}$ & [2009MIR/KON] \\
\hline
\end{tabular}

[Note: The authors of [2009MIR/KON] tabulate the experimental value as an enthalpy of vaporization; however, they report that the compound is crystalline and use the value to calculate the standard molar enthalpy of formation. Given the crystaline state and how the value was used, we have tabulated the value as an enthalpy of sublimation.]

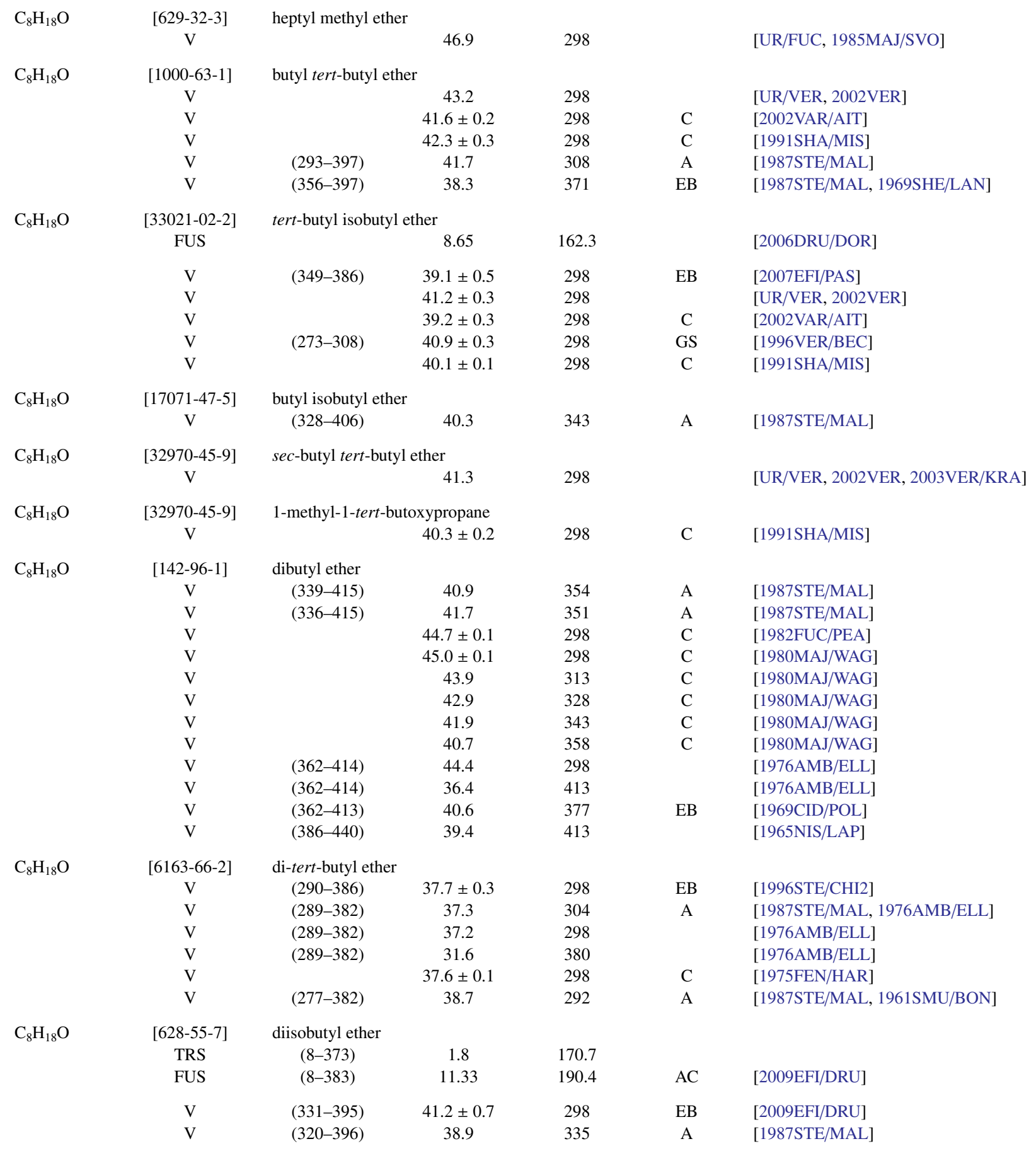


TABLE 11. Phase change enthalpies of $\mathrm{C}_{8}$ organic compounds-Continued

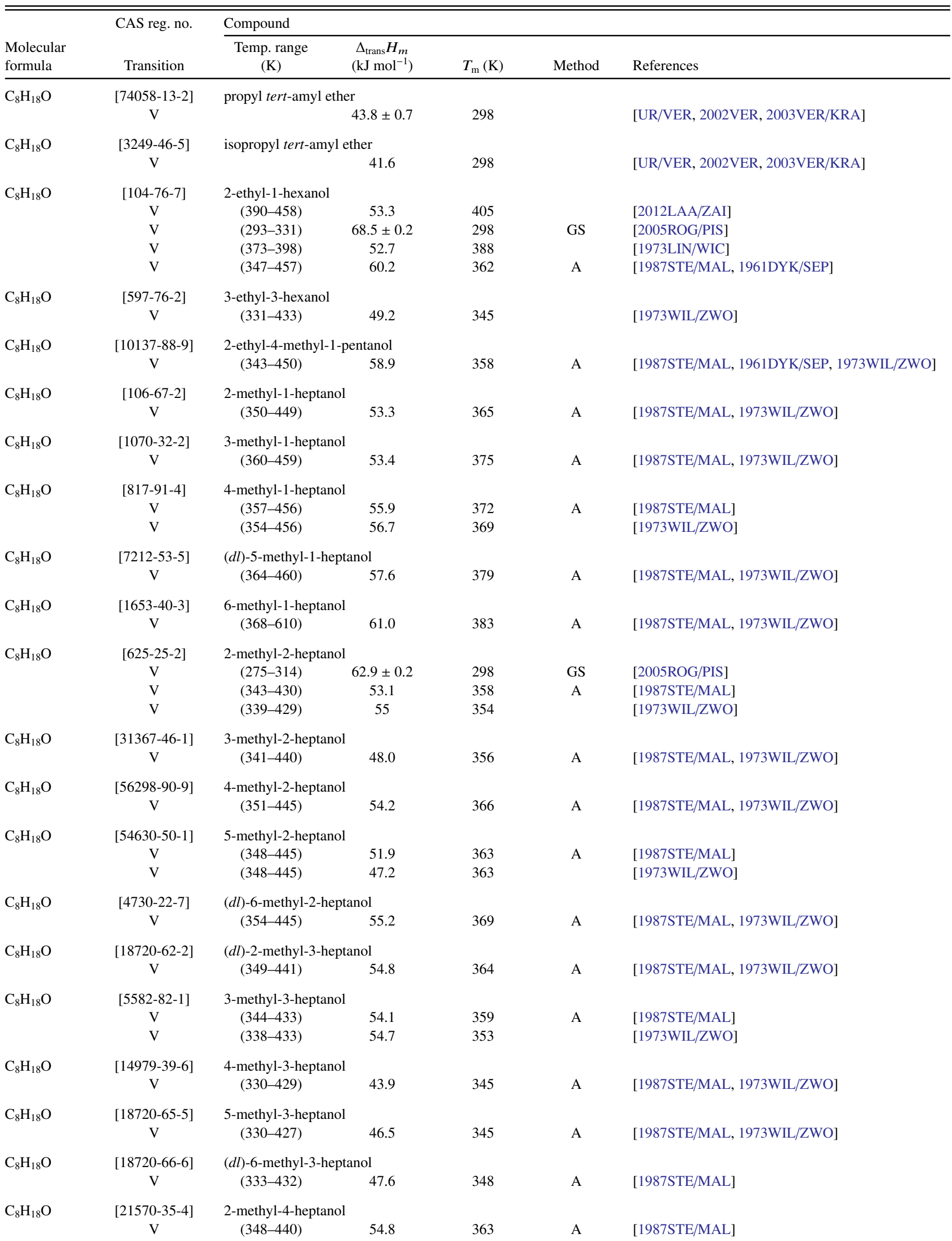


TABLE 11. Phase change enthalpies of $\mathrm{C}_{8}$ organic compounds-Continued

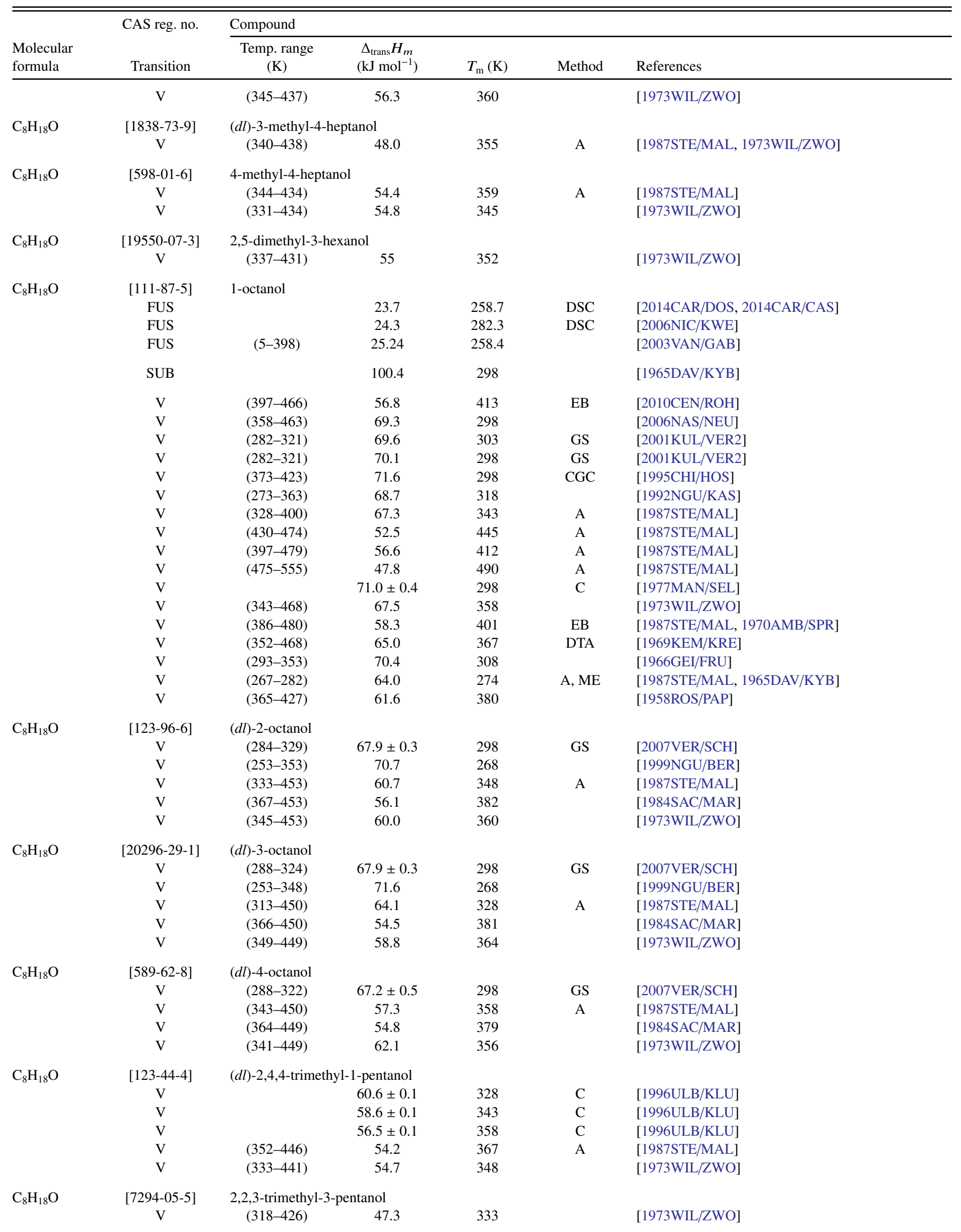


TABLE 11. Phase change enthalpies of $\mathrm{C}_{8}$ organic compounds-Continued

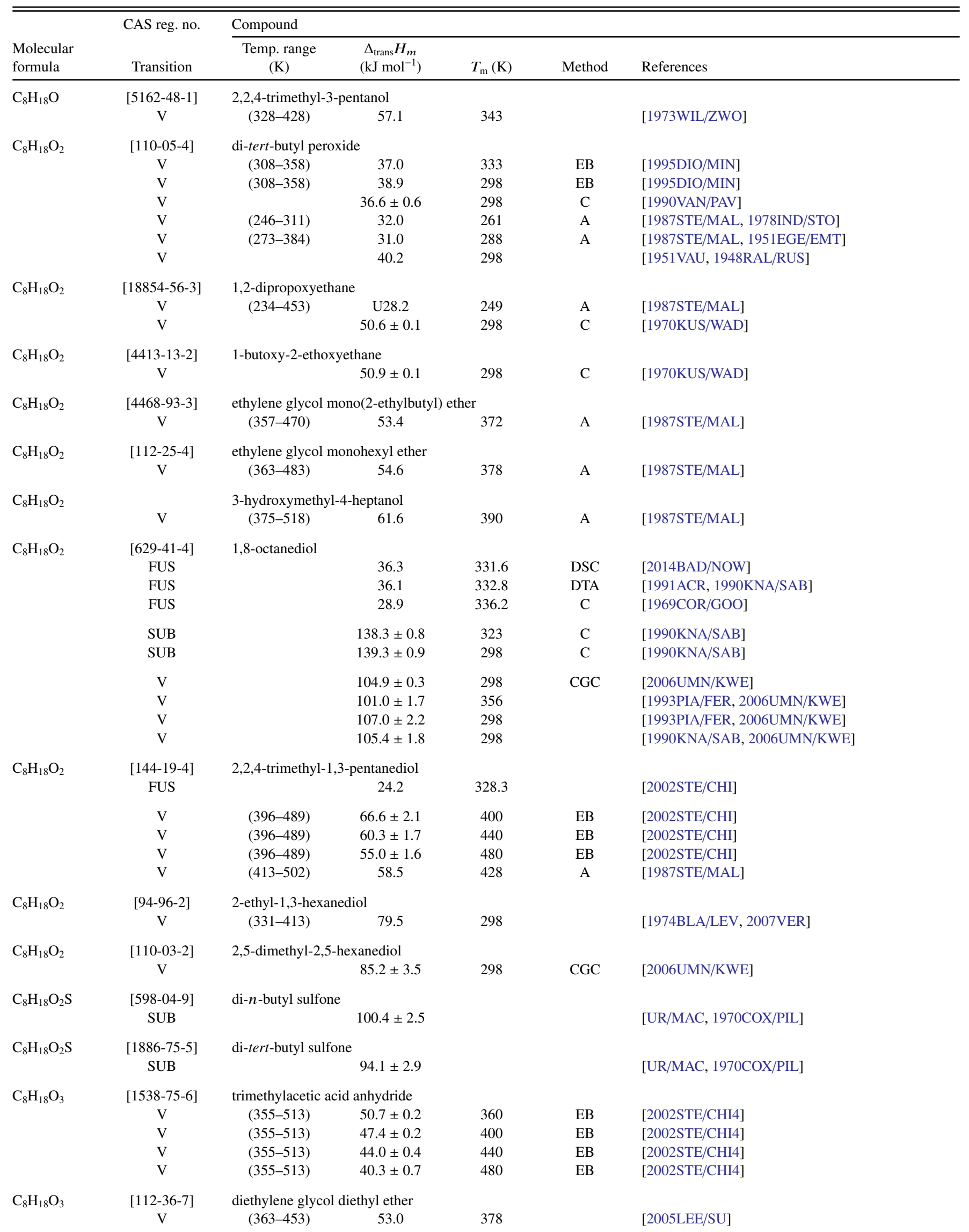


TABLE 11. Phase change enthalpies of $\mathrm{C}_{8}$ organic compounds-Continued

\begin{tabular}{|c|c|c|c|c|c|c|}
\hline \multirow[b]{2}{*}{$\begin{array}{l}\text { Molecular } \\
\text { formula }\end{array}$} & \multirow{2}{*}{$\begin{array}{l}\text { CAS reg. no. } \\
\text { Transition }\end{array}$} & \multicolumn{5}{|l|}{ Compound } \\
\hline & & $\begin{array}{c}\text { Temp. range } \\
\text { (K) }\end{array}$ & $\begin{array}{c}\Delta_{\text {trans }} H_{m} \\
\left(\mathrm{~kJ} \mathrm{~mol}^{-1}\right)\end{array}$ & $T_{\mathrm{m}}(\mathrm{K})$ & Method & References \\
\hline & $\mathrm{V}$ & & $56.4 \pm 1.4$ & 298 & CGC & [2000NIC/ORF] \\
\hline & $\mathrm{V}$ & $(330-461)$ & 48.3 & 345 & A & [1987STE/MAL] \\
\hline \multirow[t]{2}{*}{$\mathrm{C}_{8} \mathrm{H}_{18} \mathrm{O}_{3}$} & {$[112-34-5]$} & \multicolumn{5}{|c|}{ diethylene glycol monobutyl ether } \\
\hline & $\mathrm{V}$ & $(415-505)$ & 55.7 & 430 & A & [1987STE/MAL] \\
\hline \multirow{2}{*}{$\mathrm{C}_{8} \mathrm{H}_{18} \mathrm{O}_{3} \mathrm{~S}$} & {$[626-85-7]$} & dibutyl sulfite & & & & \\
\hline & $\mathrm{V}$ & & $67.8 \pm 1.7$ & 298 & $\mathrm{BP}$ & [1969MAC/STE2] \\
\hline \multirow[t]{2}{*}{$\mathrm{C}_{8} \mathrm{H}_{18} \mathrm{O}_{4}$} & {$[112-49-2]$} & \multicolumn{5}{|c|}{ 1,2-bis(2-methoxyethoxy)ethane (triglyme) } \\
\hline & $\mathrm{V}$ & & $63.7 \pm 3.3$ & 298 & CGC & {$[2000 \mathrm{NIC} / \mathrm{ORF}]$} \\
\hline \multirow[t]{2}{*}{$\mathrm{C}_{8} \mathrm{H}_{18} \mathrm{O}_{4}$} & {$[112-49-2]$} & \multicolumn{5}{|c|}{ 2,5,8,11-tetraoxadodecane } \\
\hline & FUS & & 23.71 & 229.3 & & [1996DOM/HEA, 1966BEA/CLE] \\
\hline \multirow{2}{*}{$\mathrm{C}_{8} \mathrm{H}_{18} \mathrm{O}_{4}$} & {$[52894-25-4]$} & \multicolumn{5}{|c|}{ 1,2,7,8-octanetetraol (1,2,7,8-tetrahydroxyoctane) } \\
\hline & FUS & & 36.7 & 352.2 & & [1991HEN/TSC, 1994HEN/DIE] \\
\hline \multirow[t]{2}{*}{$\mathrm{C}_{8} \mathrm{H}_{18} \mathrm{O}_{4} \mathrm{~S}$} & {$[625-22-9]$} & \multicolumn{5}{|l|}{ dibutyl sulfate } \\
\hline & $\mathrm{V}$ & & $75.5 \pm 1.7$ & 298 & $\mathrm{BP}$ & [1969MAC/STE2] \\
\hline \multirow[t]{3}{*}{$\mathrm{C}_{8} \mathrm{H}_{18} \mathrm{O}_{4} \mathrm{~S}_{2}$} & {$[76-20-0]$} & \multicolumn{5}{|c|}{ 2,2-butanediol bis(ethylsulfonate) (trional) } \\
\hline & V & $(443-493)$ & 75.7 & 458 & A & [1987STE/MAL, 1999DYK/SVO] \\
\hline & V & & 78.2 & 448 & I & [1943CRA] \\
\hline \multirow[t]{2}{*}{$\mathrm{C}_{8} \mathrm{H}_{18} \mathrm{O}_{5}$} & {$[112-60-7]$} & tetraethylene glycol & & & & \\
\hline & V & $(426-581)$ & 92.2 & 441 & A & [1987STE/MAL, 1947STU] \\
\hline \multirow[t]{7}{*}{$\mathrm{C}_{8} \mathrm{H}_{18} \mathrm{~S}$} & {$[544-40-1]$} & dibutyl sulfide & & & & \\
\hline & FUS & $(12-355)$ & 19.41 & 198.1 & & [1996DOM/HEA, 1961MCC/FIN] \\
\hline & $\mathrm{V}$ & $(255-422)$ & 44.8 & 339 & & [2004SAW/MOK] \\
\hline & $\mathrm{V}$ & $(283-390)$ & 40.3 & 298 & & [1999DYK/SVO] \\
\hline & $\mathrm{V}$ & & 53 & 298 & & [1981SHI/SAI] \\
\hline & $\mathrm{V}$ & & $54.2 \pm 0.8$ & 298 & GC & [1964MAC/MCC] \\
\hline & $\mathrm{V}$ & $(390-470)$ & 46.5 & 405 & $\mathrm{~A}, \mathrm{~EB}$ & [1987STE/MAL, 1952WHI/BAR] \\
\hline \multirow[t]{10}{*}{$\mathrm{C}_{8} \mathrm{H}_{18} \mathrm{~S}$} & {$[626-26-6]$} & di-tert-butyl sulfide & & & & \\
\hline & $\mathrm{V}$ & $(264-329)$ & 44.9 & 279 & & [1999DYK/SVO] \\
\hline & $\mathrm{V}$ & $(329-470)$ & 41.4 & 344 & & [1999DYK/SVO] \\
\hline & $\mathrm{V}$ & $(390-470)$ & 46.4 & 405 & & [1999DYK/SVO] \\
\hline & $\mathrm{V}$ & $(278-308)$ & 44.8 & 293 & & [1998STO/NG] \\
\hline & $\mathrm{V}$ & $(324-420)$ & 42.4 & 339 & A & [1987STE/MAL] \\
\hline & $\mathrm{V}$ & & 43.8 & 298 & & [1981SHI/SAI] \\
\hline & $\mathrm{V}$ & & $43.8 \pm 0.1$ & 298 & & [1972GOO] \\
\hline & $\mathrm{V}$ & & $49.3 \pm 0.8$ & 298 & GC & [1964MAC/MCC] \\
\hline & $\mathrm{V}$ & $(325-350)$ & 42.3 & 333 & $\mathrm{~EB}$ & [1962MAC/MAY2] \\
\hline $\mathrm{C}_{8} \mathrm{H}_{18} \mathrm{~S}$ & [592-65-4] & diisobutyl sulfide & & & & \\
\hline & $\mathrm{V}$ & $(325-346)$ & 46.4 & 335 & A & [1987STE/MAL, 1999DYK/SVO] \\
\hline & $\mathrm{V}$ & & 48.7 & 298 & & [1981SHI/SAI] \\
\hline & $\mathrm{V}$ & & $48.5 \pm 0.8$ & 298 & GC & [1964MAC/MCC] \\
\hline & $\mathrm{V}$ & $(326-346)$ & 43.1 & 336 & EB & [1962MAC/MAY2] \\
\hline $\mathrm{C}_{8} \mathrm{H}_{18} \mathrm{~S}$ & [626-26-6] & di-sec-butyl sulfide & & & & \\
\hline & V & $(255-422)$ & 47.0 & 298 & & [2004SAW/MOK] \\
\hline $\mathrm{C}_{8} \mathrm{H}_{18} \mathrm{~S}$ & [111-88-6] & 1-octanethiol & & & & \\
\hline & FUS & & 24.27 & 224 & & [1996DOM/HEA, 1985DEA] \\
\hline & $\mathrm{V}$ & $(372-473)$ & 49.6 & 387 & A & [1987STE/MAL, 1999DYK/SVO, 1932ELL/REI] \\
\hline $\mathrm{C}_{8} \mathrm{H}_{18} \mathrm{~S}$ & {$[3001-66-9]$} & 2-octanethiol & & & & \\
\hline & $\mathrm{V}$ & $(347-489)$ & 49.0 & 362 & & {$[1999 \mathrm{DYK} / \mathrm{SVO}]$} \\
\hline $\mathrm{C}_{8} \mathrm{H}_{18} \mathrm{~S}$ & {$[10435-81-1]$} & $(d l)$-2-octanethiol & & & & \\
\hline & $\mathrm{V}$ & $(361-460)$ & 48.0 & 376 & A & [1987STE/MAL, 1999DYK/SVO, 1932ELL/REI] \\
\hline $\mathrm{C}_{8} \mathrm{H}_{18} \mathrm{~S}_{2}$ & {$[629-45-8]$} & dibutyl disulfide & & & & \\
\hline & V & $(383-423)$ & 64.1 & 298 & CGC & [1995CHI/HOS] \\
\hline
\end{tabular}


TABLE 11. Phase change enthalpies of $\mathrm{C}_{8}$ organic compounds-Continued

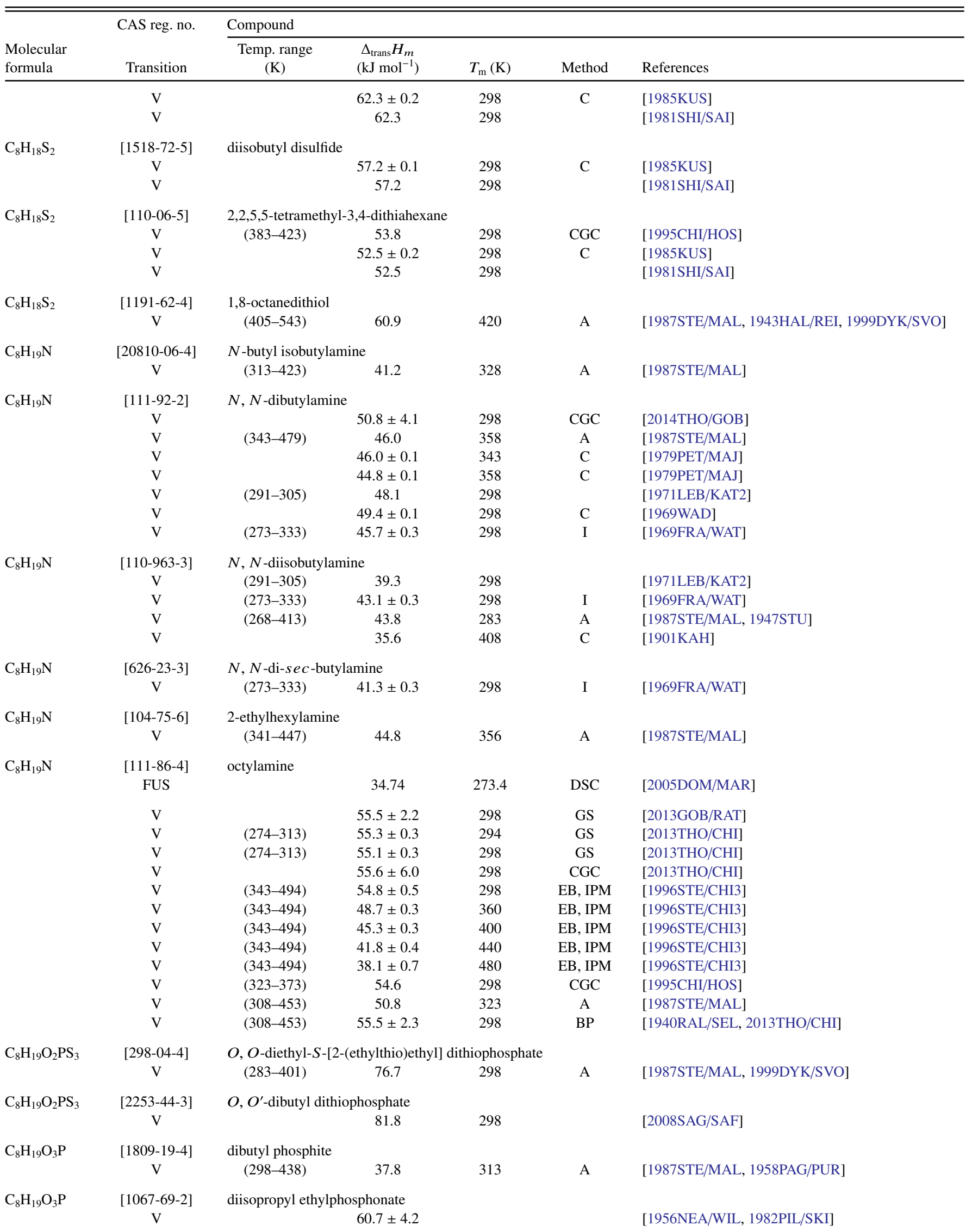


TABLE 11. Phase change enthalpies of $\mathrm{C}_{8}$ organic compounds-Continued

\begin{tabular}{|c|c|c|c|c|c|c|}
\hline \multirow[b]{2}{*}{$\begin{array}{l}\text { Molecular } \\
\text { formula }\end{array}$} & \multirow{2}{*}{$\begin{array}{l}\text { CAS reg. no. } \\
\text { Transition }\end{array}$} & \multicolumn{5}{|l|}{ Compound } \\
\hline & & $\begin{array}{c}\text { Temp. range } \\
\text { (K) }\end{array}$ & $\begin{array}{c}\Delta_{\text {trans }} H_{m} \\
\left(\mathrm{~kJ} \mathrm{~mol}^{-1}\right)\end{array}$ & $T_{\mathrm{m}}(\mathrm{K})$ & Method & References \\
\hline \multirow[t]{2}{*}{$\mathrm{C}_{8} \mathrm{H}_{19} \mathrm{O}_{3} \mathrm{PS}_{2}$} & [298-03-3] & \multicolumn{5}{|c|}{$O, O$-diethyl- $O$-[2-(ethylthio)ethyl] thiophosphate } \\
\hline & $\mathrm{V}$ & $(283-411)$ & 78.7 & 298 & A & [1987STE/MAL, 1999DYK/SVO] \\
\hline \multirow[t]{2}{*}{$\mathrm{C}_{8} \mathrm{H}_{19} \mathrm{O}_{3} \mathrm{PS}_{2}$} & {$[126-75-0]$} & \multicolumn{5}{|c|}{$O, O$-diethyl- $S$-[2-(ethylthio)ethyl] thiophosphate } \\
\hline & $\mathrm{V}$ & $(283-401)$ & 76.4 & 298 & A & [1987STE/MAL, 1999DYK/SVO] \\
\hline \multirow[t]{2}{*}{$\mathrm{C}_{8} \mathrm{H}_{20} \mathrm{ClN}$} & {$[6287-40-7]$} & \multicolumn{5}{|c|}{ dibutylammonium chloride } \\
\hline & $\mathrm{V}$ & $(553-563)$ & 116.7 & 558 & A & [1987STE/MAL, 1999DYK/SVO, 1967KIS] \\
\hline \multirow[t]{6}{*}{$\mathrm{C}_{8} \mathrm{H}_{20} \mathrm{~N}_{2}$} & [373-44-4] & \multicolumn{5}{|c|}{ octane-1,8-diamine } \\
\hline & FUS & & 50.51 & 324.9 & DSC & [2006KHI/DAH2] \\
\hline & FUS & & 52.4 & 325.6 & $\mathrm{AC}$ & [2004MON/VAN, 2014FUL/RUZ] \\
\hline & FUS & & 50.98 & 324.8 & DSC & [2002DAL/DEL] \\
\hline & SUB & $(289-313)$ & 116.7 & 298 & Static & [2014FUL/RUZ] \\
\hline & $\mathrm{V}$ & $(328-363)$ & $71.7 \pm 0.6$ & 298 & GS & [2011POZ/VER] \\
\hline \multirow[t]{2}{*}{$\mathrm{C}_{8} \mathrm{H}_{20} \mathrm{~N}_{2}$} & {$[4267-00-9]$} & \multicolumn{5}{|c|}{ tetraethylhydrazine } \\
\hline & $\mathrm{V}$ & $(308-368)$ & 33.4 & 323 & A & [1987STE/MAL, 1943WES/EUC] \\
\hline \multirow[t]{4}{*}{$\mathrm{C}_{8} \mathrm{H}_{20} \mathrm{~N}_{2}$} & {$[97-84-7]$} & \multicolumn{5}{|c|}{$N, N, N^{\prime}, N^{\prime}$-tetramethyl-1,3-butanediamine } \\
\hline & $\mathrm{V}$ & $(273-363)$ & 49.2 & 288 & & [2002DAH/MOK] \\
\hline & $\mathrm{V}$ & $(273-363)$ & $47.9 \pm 0.4$ & 298 & & [2002DAH/MOK, 2012VER/CHE] \\
\hline & $\mathrm{V}$ & $(335-439)$ & 42.7 & 350 & A & [1987STE/MAL] \\
\hline \multirow[t]{6}{*}{$\mathrm{C}_{8} \mathrm{H}_{20} \mathrm{~N}_{2} \mathrm{O}$} & {$[5336-24-3]$} & \multicolumn{5}{|c|}{ 1,3-di-tert-butyl urea } \\
\hline & TRS & $(5-320)$ & 1.9 & 301.7 & $\mathrm{AC}$ & [1995KAB/KOZ2] \\
\hline & SUB & $(323-372)$ & $91.9 \pm 0.9$ & 348 & $\mathrm{ME}$ & [2003ZAI/KAB] \\
\hline & SUB & $(323-372)$ & $91.9 \pm 0.9$ & 350 & $\mathrm{ME}$ & [2003ZAI/KAB] \\
\hline & SUB & & $90.0 \pm 1.0$ & 350 & $\mathrm{C}$ & [2003ZAI/KAB] \\
\hline & SUB & & $91.7 \pm 0.7$ & 298 & & [2003ZAI/KAB] \\
\hline \multirow[t]{2}{*}{$\mathrm{C}_{8} \mathrm{H}_{20} \mathrm{~N}_{2} \mathrm{O}_{2} \mathrm{~S}$} & [2832-49-7] & \multicolumn{5}{|c|}{$N, N, N^{\prime}, N^{\prime}$-tetraethylsulfamide } \\
\hline & $\mathrm{V}$ & $(407-528)$ & 59.1 & 422 & A & [1987STE/MAL, 1978LUK/MAK] \\
\hline \multirow[t]{2}{*}{$\mathrm{C}_{8} \mathrm{H}_{20} \mathrm{~N}_{2} \mathrm{O}_{3}$} & {$[6487-26-5]$} & \multicolumn{5}{|c|}{$N, N, N^{\prime}$-tris(2-hydroxyethyl)ethylenediamine } \\
\hline & $\mathrm{V}$ & $(373-472)$ & 90.0 & 423 & GS & [1998ABD/MEI] \\
\hline \multirow[t]{2}{*}{$\mathrm{C}_{8} \mathrm{H}_{20} \mathrm{O}_{5} \mathrm{P}_{2} \mathrm{~S}_{2}$} & [3689-24-5] & \multicolumn{5}{|c|}{ dithiopyrophosphoric acid, tetraethyl ester } \\
\hline & $\mathrm{V}$ & $(293-409)$ & 80.6 & 308 & A & [1987STE/MAL, 1999DYK/SVO] \\
\hline \multirow[t]{2}{*}{$\mathrm{C}_{8} \mathrm{H}_{20} \mathrm{O}_{7} \mathrm{P}_{2}$} & {$[107-49-3]$} & \multicolumn{5}{|c|}{ pyrophosphoric acid, tetraethyl ester } \\
\hline & $\mathrm{V}$ & $(283-411)$ & 82.2 & 298 & A & [1987STE/MAL] \\
\hline \multirow[t]{2}{*}{$\mathrm{C}_{8} \mathrm{H}_{23} \mathrm{~N}_{5}$} & [112-57-2] & \multicolumn{5}{|c|}{ tetraethylene pentamine } \\
\hline & $\mathrm{V}$ & $(464-615)$ & 71.3 & 478 & A & [1987STE/MAL] \\
\hline $\mathrm{C}_{8} \mathrm{H}_{24} \mathrm{~N}_{4} \mathrm{O}_{3} \mathrm{P}_{2}$ & [152-16-9] & pyrophosphoric & tetrakis (dim & mide) & & \\
\hline & $\mathrm{V}$ & $(273-415)$ & 65.5 & 288 & A & [1987STE/MAL] \\
\hline
\end{tabular}


TABle 12. Phase change enthalpies of $\mathrm{C}_{9}$ organic compounds

\begin{tabular}{|c|c|c|c|c|c|c|}
\hline \multirow[b]{2}{*}{$\begin{array}{l}\text { Molecular } \\
\text { formula }\end{array}$} & \multirow{2}{*}{$\begin{array}{l}\text { CAS reg. no. } \\
\text { Transition }\end{array}$} & \multicolumn{5}{|l|}{ Compound } \\
\hline & & Temp. range $(\mathrm{K})$ & $\begin{array}{c}\Delta_{\text {trans }} H_{\mathrm{m}} \\
\left(\mathrm{kJ} \mathrm{mol}^{-1}\right)\end{array}$ & $T_{\mathrm{m}}(\mathrm{K})$ & Method & References \\
\hline $\mathrm{C}_{9} \mathrm{~F}_{16}$ & $\begin{array}{c}{[75240-06-1]} \\
\mathrm{V}\end{array}$ & trans-perfluorohyd & $\begin{array}{l}\text { dane } \\
45.2 \pm 0.1\end{array}$ & 298 & $\mathrm{C}$ & [1996VAR/DRU] \\
\hline $\mathrm{C}_{9} \mathrm{~F}_{16}$ & $\begin{array}{c}\text { [75262-87-2] } \\
\text { TRS } \\
\text { TRS } \\
\text { FUS }\end{array}$ & $\begin{array}{c}\text { cis-perfluorobicycl } \\
(5-350) \\
(5-350) \\
(5-350)\end{array}$ & $\begin{array}{c}\text { 4.3.0]nonane } \\
8.76 \\
1.27 \\
2.72\end{array}$ & $\begin{array}{l}200.6 \\
245.6 \\
291.3\end{array}$ & $\mathrm{AC}$ & [1998VAR/DRU] \\
\hline $\mathrm{C}_{9} \mathrm{~F}_{16}$ & $\begin{array}{l}\text { [75240-06-1] } \\
\text { TRS } \\
\text { FUS }\end{array}$ & $\begin{array}{l}\text { trans-perfluorobicy } \\
\qquad \begin{array}{c}(5-350) \\
(5-350)\end{array}\end{array}$ & $\begin{array}{c}\text { [4.3.0]nona } \\
8.91 \\
2.63\end{array}$ & $\begin{array}{l}236.6 \\
248.1\end{array}$ & $\mathrm{AC}$ & [1998VAR/DRU] \\
\hline $\mathrm{C}_{9} \mathrm{~F}_{17} \mathrm{NO}_{3} \mathrm{~S}$ & $\begin{array}{c}{[34834-20-3]} \\
\mathrm{V}\end{array}$ & $\begin{array}{l}\text { perfluoro-1-octanes } \\
\qquad(324-470)\end{array}$ & $\begin{array}{l}\text { fonylisocyan } \\
67.7\end{array}$ & 339 & A & [1987STE/MAL, 1999DYK/SVO, 1974BEH/HAA] \\
\hline $\mathrm{C}_{9} \mathrm{~F}_{18}$ & $\begin{array}{c}{[374-59-4]} \\
\text { V } \\
\text { V } \\
\text { V } \\
\text { V }\end{array}$ & $\begin{array}{l}\text { perfluoro(propylcy } \\
\qquad(321-396)\end{array}$ & $\begin{array}{l}\text { hexane) } \\
40.4 \\
43.1 \pm 0.1 \\
43.1 \pm 0.5 \\
43.1 \pm 0.1\end{array}$ & $\begin{array}{l}336 \\
298 \\
298 \\
298\end{array}$ & $\begin{array}{c}\mathrm{C} \\
\mathrm{EB} \\
\mathrm{C}\end{array}$ & $\begin{array}{l}\text { [1999DYK/SVO] } \\
\text { [1996VAR/DRU] } \\
\text { [1981VAR/BUL] } \\
\text { [1981VAR/BUL] }\end{array}$ \\
\hline $\mathrm{C}_{9} \mathrm{~F}_{18}$ & $\begin{array}{c}{[423-02-9]} \\
\mathrm{V}\end{array}$ & perfluoro(isopropyl & $\begin{array}{l}\text { clohexane) } \\
46.7 \pm 0.1\end{array}$ & 298 & $\mathrm{C}$ & [1996VAR/DRU] \\
\hline $\mathrm{C}_{9} \mathrm{~F}_{18} \mathrm{~N}_{2}$ & $\begin{array}{c}{[34451-14-4]} \\
\mathrm{V}\end{array}$ & $\begin{array}{l}\text { 1,1,1,3,3,3-hexaflu } \\
\quad(314-381)\end{array}$ & $\begin{array}{l}-N, N^{\prime}-\text { bis }[2 \\
35.5\end{array}$ & $\begin{array}{l}\text { fluoro-1- }( \\
329\end{array}$ & $\begin{array}{c}\text { omethy) } \\
\text { A }\end{array}$ & $\begin{array}{l}\text { hylidene]-2,2-propanediamine } \\
\text { [1987STE/MAL, 1972SWI/SHR] }\end{array}$ \\
\hline $\mathrm{C}_{9} \mathrm{~F}_{18} \mathrm{O}_{3}$ & $\begin{array}{c}{[40719-69-5]} \\
\mathrm{V}\end{array}$ & $\begin{array}{l}\text { carbonic acid, bis }[1 \\
\quad(316-358)\end{array}$ & $\begin{array}{c}\text { 1,3,3,3-hexa } \\
39.7\end{array}$ & -2-(trifluc & $\begin{array}{c}\text { thyl)-2-pr } \\
\text { A }\end{array}$ & $\begin{array}{l}\text { yl ester } \\
\text { [1987STE/MAL, 1975WAL/DES2] }\end{array}$ \\
\hline $\mathrm{C}_{9} \mathrm{~F}_{19} \mathrm{NO}$ & $\begin{array}{l}{[54120-06-8]} \\
\mathrm{V}\end{array}$ & $\begin{array}{l}\text { 2,2,2-trifluoro- } N \text { - }[ \\
\text { 1,2,2,2-tetrafluoro- }\end{array}$ & $\begin{array}{l}\text {,2,2-tetrafluc } \\
\text { trifluorometl } \\
\quad 37.6\end{array}$ & $\begin{array}{l}\text { (trifluoror } \\
\text { hyl ester } \\
385\end{array}$ & ethyl]eth & $\begin{array}{l}\text { imidic acid, } \\
\text { [1975PET/SHR] }\end{array}$ \\
\hline $\mathrm{C}_{9} \mathrm{~F}_{20}$ & $\begin{array}{c}{[375-96-2]} \\
\mathrm{V} \\
\mathrm{V}\end{array}$ & $\begin{array}{l}\text { perfluorononane } \\
\qquad \begin{array}{l}(288-333) \\
(387-524)\end{array}\end{array}$ & $\begin{array}{l}45.3 \pm 0.2 \\
32.8\end{array}$ & $\begin{array}{l}298 \\
402\end{array}$ & A & $\begin{array}{l}\text { [2005DIA/GON] } \\
\text { [1987STE/MAL, 1967BER/WES, 1999DYK/SVO] }\end{array}$ \\
\hline $\mathrm{C}_{9} \mathrm{~F}_{21} \mathrm{~N}$ & $\begin{array}{c}{[514-03-4]} \\
\mathrm{V} \\
\mathrm{V}\end{array}$ & $\begin{array}{l}\text { perfluoro- } N \text {-methy } \\
\quad(339-407)\end{array}$ & $\begin{array}{c}V, N \text {-dibutyla } \\
48.8 \pm 0.8 \\
48.2 \pm 0.1\end{array}$ & $\begin{array}{l}298 \\
298\end{array}$ & $\begin{array}{c}\mathrm{EB} \\
\mathrm{C}\end{array}$ & $\begin{array}{l}{[1995 \mathrm{VAR} / \mathrm{DRO}]} \\
{[1995 \mathrm{VAR} / \mathrm{DRO}]}\end{array}$ \\
\hline $\mathrm{C}_{9} \mathrm{~F}_{21} \mathrm{~N}$ & $\begin{array}{c}{[338-83-0]} \\
\mathrm{V} \\
\mathrm{V} \\
\mathrm{V}\end{array}$ & $\begin{array}{l}\text { tris(heptafluoropro } \\
\qquad \begin{array}{l}(329-403) \\
(333-403)\end{array}\end{array}$ & $\begin{array}{l}\text { amine } \\
46.6 \pm 0.3 \\
46.9 \pm 0.7 \\
40.6\end{array}$ & $\begin{array}{l}298 \\
298 \\
348\end{array}$ & $\begin{array}{l}\mathrm{C} \\
\mathrm{EB} \\
\mathrm{A}\end{array}$ & $\begin{array}{l}\text { [1995VAR/DRO] } \\
\text { [1995VAR/DRO] } \\
\text { [1987STE/MAL, 1951HAS] }\end{array}$ \\
\hline $\mathrm{C}_{9} \mathrm{H}_{2} \mathrm{Cl}_{6} \mathrm{O}_{3}$ & $\begin{array}{c}{[7365-74-4]} \\
\text { TRS } \\
\text { FUS }\end{array}$ & $4,5,6,7,8,8$-hexachl & $\begin{array}{c}\text { o-3a, } 4,7,7 \mathrm{a}-\mathrm{t} \\
10.64 \\
2.67\end{array}$ & $\begin{array}{c}\text { dro- } 4,7-\mathrm{m} \\
385.4 \\
506\end{array}$ & $\begin{array}{l}\text { oisobenze } \\
\text { DSC }\end{array}$ & $\begin{array}{l}\text { ran-1,3-dione } \\
\text { [1984WEI/LEF] }\end{array}$ \\
\hline $\mathrm{C}_{9} \mathrm{H}_{4} \mathrm{ClF}_{15} \mathrm{O}$ & $\begin{array}{c}{[65064-85-9]} \\
\text { V } \\
\text { V }\end{array}$ & $\begin{array}{l}7 \text {-(2-chloro-1,1,2-t } \\
\quad(358-460)\end{array}$ & $\begin{array}{l}\text { uoroethoxy) } \\
64.6 \pm 1.1 \\
63.8 \pm 0.4\end{array}$ & $\begin{array}{c}2,3,3,4,4, \\
298 \\
298\end{array}$ & $\begin{array}{c}\text { 5-dodecaf } \\
\text { EB } \\
\text { C }\end{array}$ & $\begin{array}{l}\text { roheptane } \\
\text { [1981 VAR/BUL2] } \\
\text { [1981VAR/BUL2] }\end{array}$ \\
\hline $\mathrm{C}_{9} \mathrm{H}_{4} \mathrm{Cl}_{3} \mathrm{NO}_{2} \mathrm{~S}$ & $\begin{array}{l}{[133-07-3]} \\
\text { FUS }\end{array}$ & $2-[$ (trichloromethyl & $\begin{array}{l}\text { io]- } 1 H \text {-isoil } \\
35.49\end{array}$ & $\begin{array}{l}1,3(2 H)- \\
454.2\end{array}$ & DSC & [1991ACR, 1990DON/DRE] \\
\hline $\mathrm{C}_{9} \mathrm{H}_{4} \mathrm{Cl}_{4} \mathrm{O}_{4}$ & $\begin{array}{l}{[887-54-7]} \\
\text { FUS }\end{array}$ & methyl tetrachlorot & $\begin{array}{l}\text { ephthalic acic } \\
16.89\end{array}$ & 444.3 & DSC & [1990DON/DRE] \\
\hline $\mathrm{C}_{9} \mathrm{H}_{4} \mathrm{Cl}_{8} \mathrm{O}$ & $\begin{array}{l}\text { [76341-69-0] } \\
\text { FUS }\end{array}$ & $1,3,4,5,6,7,8,8$-octa & $\begin{array}{l}\text { loro- } 2,3,3 \mathrm{a}, 4 \\
25.94\end{array}$ & $\begin{array}{c}\text { hexahydr } \\
395.4\end{array}$ & $\begin{array}{l}\text { methanoi } \\
\text { DSC }\end{array}$ & $\begin{array}{l}\text { enzofuran } \\
\text { [1969PLA/GLA] }\end{array}$ \\
\hline $\mathrm{C}_{9} \mathrm{H}_{4} \mathrm{O}_{5}$ & $\begin{array}{c}{[552-30-7]} \\
\text { FUS } \\
\text { V }\end{array}$ & $\begin{array}{l}\text { trimellitic acid anh } \\
\qquad(558-596)\end{array}$ & $\begin{array}{l}\text { ride } \\
\qquad \begin{array}{c}10.46 \\
65.6\end{array}\end{array}$ & $\begin{array}{l}385 \\
573\end{array}$ & A & $\begin{array}{l}\text { [1996DOM/HEA, 1978MAR/CIO] } \\
\text { [1987STE/MAL] }\end{array}$ \\
\hline $\mathrm{C}_{9} \mathrm{H}_{5} \mathrm{BrClNO}$ & $\begin{array}{l}{[7640-33-7]} \\
\text { SUB }\end{array}$ & $\begin{array}{l}\text { 7-bromo-5-chloro- } \\
\quad(353-368)\end{array}$ & $\begin{array}{l}\text { ydroxyquinc } \\
110.1 \pm 0.8\end{array}$ & 361 & $\mathrm{ME}$ & [1992RIB/MON2] \\
\hline
\end{tabular}


TABLE 12. Phase change enthalpies of $\mathrm{C}_{9}$ organic compounds-Continued

\begin{tabular}{|c|c|c|c|c|c|c|}
\hline \multirow[b]{2}{*}{$\begin{array}{l}\text { Molecular } \\
\text { formula }\end{array}$} & \multirow{2}{*}{$\begin{array}{l}\text { CAS reg. no. } \\
\text { Transition }\end{array}$} & \multicolumn{5}{|l|}{ Compound } \\
\hline & & Temp. range $(\mathrm{K})$ & $\begin{array}{c}\Delta_{\text {trans }} H_{\mathrm{m}} \\
\left(\mathrm{kJ} \mathrm{mol}^{-1}\right)\end{array}$ & $T_{\mathrm{m}}(\mathrm{K})$ & Method & References \\
\hline & SUB & $(353-368)$ & $113.2 \pm 0.8$ & 298 & $\mathrm{ME}$ & [1992RIB/MON2] \\
\hline $\mathrm{C}_{9} \mathrm{H}_{5} \mathrm{Br}_{2} \mathrm{NO}$ & $\begin{array}{c}\text { [521-74-4] } \\
\text { SUB } \\
\text { SUB } \\
\text { SUB }\end{array}$ & $\begin{array}{c}\text { 5,7-dibromo-8-hydr } \\
(365-380) \\
(365-380) \\
(363-393)\end{array}$ & $\begin{array}{c}\text { xyquinoline } \\
113.6 \pm 1.3 \\
117.3 \pm 1.3 \\
94.1\end{array}$ & $\begin{array}{l}372 \\
298\end{array}$ & $\begin{array}{l}\mathrm{ME} \\
\mathrm{ME}\end{array}$ & $\begin{array}{l}\text { [1992RIB/MON2] } \\
\text { [1992RIB/MON2] } \\
\text { [1963HOR/WEN] }\end{array}$ \\
\hline $\mathrm{C}_{9} \mathrm{H}_{5} \mathrm{ClINO}$ & $\begin{array}{c}\text { [130-26-7] } \\
\text { SUB } \\
\text { SUB }\end{array}$ & $\begin{array}{c}\text { 5-chloro-7-iodo-8-h } \\
(359-378) \\
(359-378)\end{array}$ & $\begin{array}{l}\text { droxyquinolin } \\
111.3 \pm 0.4 \\
114.8 \pm 0.4\end{array}$ & $\begin{array}{l}368 \\
298\end{array}$ & $\begin{array}{l}\mathrm{ME} \\
\mathrm{ME}\end{array}$ & $\begin{array}{l}\text { [1992RIB/MON2] } \\
\text { [1992RIB/MON2] }\end{array}$ \\
\hline $\mathrm{C}_{9} \mathrm{H}_{5} \mathrm{ClINO}$ & $\begin{array}{c}\text { [35048-13-6] } \\
\text { SUB }\end{array}$ & $\begin{array}{l}\text { 5-iodo-7-chloro-8-h } \\
\quad(383-414)\end{array}$ & $\begin{array}{l}\text { droxyquinolin } \\
131\end{array}$ & & & [1963HOR/WEN] \\
\hline $\mathrm{C}_{9} \mathrm{H}_{5} \mathrm{ClN}_{2} \mathrm{O}_{2}$ & $\begin{array}{c}{[15166-26-4]} \\
\text { V } \\
\text { V }\end{array}$ & $\begin{array}{c}\text { 5-chloro-2,4-diisocy } \\
(373-433) \\
(373-433)\end{array}$ & $\begin{array}{l}\text { nato-1-methy } \\
66.7 \\
60.2 \pm 0.2\end{array}$ & $\begin{array}{l}\text { zene } \\
388 \\
403\end{array}$ & A & $\begin{array}{l}\text { [1987STE/MAL] } \\
{[1972 S T R / N O V]}\end{array}$ \\
\hline $\mathrm{C}_{9} \mathrm{H}_{5} \mathrm{Cl}_{2} \mathrm{~N}$ & $\begin{array}{l}{[86-98-6]} \\
\text { SUB }\end{array}$ & 4,7-dichloroquinoli & $89.5 \pm 2.3$ & 298 & $\mathrm{C}$ & [2006RIB/MAT] \\
\hline $\mathrm{C}_{9} \mathrm{H}_{5} \mathrm{Cl}_{2} \mathrm{NO}$ & $\begin{array}{c}\text { [773-76-2] } \\
\text { SUB } \\
\text { SUB } \\
\text { SUB }\end{array}$ & $\begin{array}{c}\text { 5,7-dichloro-8-hydr } \\
(351-366) \\
(351-366) \\
(363-393)\end{array}$ & $\begin{array}{l}\text { xyquinoline } \\
106.3 \pm 0.7 \\
109.3 \pm 0.7 \\
92.9\end{array}$ & $\begin{array}{l}358 \\
298\end{array}$ & $\begin{array}{l}\mathrm{ME} \\
\mathrm{ME}\end{array}$ & $\begin{array}{l}\text { [1992RIB/MON2] } \\
\text { [1992RIB/MON2] } \\
\text { [1963HOR/WEN] }\end{array}$ \\
\hline $\mathrm{C}_{9} \mathrm{H}_{5} \mathrm{Cl}_{3} \mathrm{~N}_{4}$ & $\begin{array}{l}\text { [101-05-3] } \\
\text { FUS }\end{array}$ & 4,6-dichloro- $N$-(2 & $\begin{array}{l}\text { lorophenyl)-1 } \\
31.48\end{array}$ & $\begin{array}{c}\text { triazin-2 } \\
431\end{array}$ & DSC & [1990DON/DRE] \\
\hline $\mathrm{C}_{9} \mathrm{H}_{5} \mathrm{I}_{2} \mathrm{NO}$ & $\begin{array}{l}{[83-73-8]} \\
\text { SUB } \\
\text { SUB } \\
\text { SUB }\end{array}$ & $\begin{array}{c}\text { 5,7-diiodo-8-hydrox } \\
(389-404) \\
(389-404) \\
(403-423)\end{array}$ & $\begin{array}{l}\text { quinoline } \\
121.9 \pm 0.8 \\
126.8 \pm 0.8 \\
110.9\end{array}$ & $\begin{array}{l}396 \\
298\end{array}$ & $\begin{array}{l}\text { ME } \\
\text { ME }\end{array}$ & $\begin{array}{l}\text { [1992RIB/MON2] } \\
\text { [1992RIB/MON2] } \\
\text { [1963HOR/WEN] }\end{array}$ \\
\hline $\mathrm{C}_{9} \mathrm{H}_{6} \mathrm{BrN}$ & $\begin{array}{c}{[5332-24-1]} \\
\mathrm{V}\end{array}$ & 3-bromoquinoline & $70.7 \pm 2.3$ & 298 & $\mathrm{C}$ & [2008RIB/AMA] \\
\hline $\mathrm{C}_{9} \mathrm{H}_{6} \mathrm{ClN}$ & $\begin{array}{l}{[612-62-4]} \\
\text { SUB }\end{array}$ & 2-chloroquinoline & $84.3 \pm 2.6$ & 298 & $\mathrm{C}$ & [2006RIB/MAT] \\
\hline $\mathrm{C}_{9} \mathrm{H}_{6} \mathrm{ClN}$ & $\begin{array}{l}{[611-35-8]} \\
\text { SUB }\end{array}$ & 4-chloroquinoline & $78.6 \pm 1.7$ & 298 & $\mathrm{C}$ & [2006RIB/MAT] \\
\hline $\mathrm{C}_{9} \mathrm{H}_{6} \mathrm{ClN}$ & $\begin{array}{c}{[612-57-7]} \\
\text { SUB }\end{array}$ & 6-chloroquinoline & $80.8 \pm 1.9$ & 298 & $\mathrm{C}$ & [2006RIB/MAT] \\
\hline $\mathrm{C}_{9} \mathrm{H}_{6} \mathrm{ClNO}$ & $\begin{array}{c}{[130-16-5]} \\
\text { SUB } \\
\text { SUB }\end{array}$ & $\begin{array}{c}\text { 5-chloro-8-hydroxy } \\
(317-327) \\
(317-327)\end{array}$ & $\begin{array}{l}\text { uinoline } \\
\qquad 97.5 \pm 0.9 \\
98.7 \pm 0.9\end{array}$ & $\begin{array}{l}322 \\
298\end{array}$ & $\begin{array}{l}\mathrm{ME} \\
\mathrm{ME}\end{array}$ & $\begin{array}{l}\text { [1992RIB/MON2] } \\
\text { [1992RIB/MON2] }\end{array}$ \\
\hline $\mathrm{C}_{9} \mathrm{H}_{6} \mathrm{ClNO}_{2}$ & $\begin{array}{c}\text { [17564-64-6] } \\
\text { SUB } \\
\text { SUB }\end{array}$ & $\begin{array}{c}N \text {-chloromethylpht } \\
(323-343)\end{array}$ & $\begin{array}{l}\text { alimide } \\
103.5 \pm 1.1 \\
103.6 \pm 0.9\end{array}$ & $\begin{array}{l}298 \\
298\end{array}$ & $\begin{array}{c}\mathrm{C} \\
\mathrm{ME}\end{array}$ & $\begin{array}{l}\text { [2007RIB/SAN3] } \\
\text { [2007RIB/SAN3] }\end{array}$ \\
\hline $\mathrm{C}_{9} \mathrm{H}_{6} \mathrm{Cl}_{2} \mathrm{~N}_{2} \mathrm{O}_{3}$ & $\begin{array}{c}\text { [20354-26-1] } \\
\text { FUS }\end{array}$ & 2-(3,4-dichlorophe & $\begin{array}{l}\text {-4-methyl-1 } \\
29.5\end{array}$ & $\begin{array}{c}\text { oxadiazo } \\
396.3\end{array}$ & $\begin{array}{l}\text {-3,5-dione } \\
\text { DSC }\end{array}$ & [1990DON/DRE] \\
\hline $\mathrm{C}_{9} \mathrm{H}_{6} \mathrm{Cl}_{2} \mathrm{O}_{3}$ & $\begin{array}{c}\text { [17812-11-2] } \\
\text { TRS } \\
\text { FUS }\end{array}$ & 2,3-dichloro-5-norb & $\begin{array}{c}\text { nene-2,3-dic } \\
17.94 \\
5.36\end{array}$ & $\begin{array}{l}\text { ylic anh } \\
339.1 \\
457.6\end{array}$ & DSC & [1984WEI/LEF] \\
\hline $\mathrm{C}_{9} \mathrm{H}_{6} \mathrm{Cl}_{6} \mathrm{O}_{3} \mathrm{~S}$ & $\begin{array}{c}\text { [959-98-8] } \\
\text { FUS }\end{array}$ & endosulfan I & 10.0 & 380 & DSC & [1990DON/DRE] \\
\hline $\mathrm{C}_{9} \mathrm{H}_{6} \mathrm{Cl}_{6} \mathrm{O}_{3} \mathrm{~S}$ & $\begin{array}{c}\mathrm{V} \\
{[33213-65-9]} \\
\mathrm{V}\end{array}$ & $\begin{array}{c}(343-453) \\
\text { endosulfan II } \\
(343-453)\end{array}$ & $\begin{array}{l}80.4 \\
82.4\end{array}$ & $\begin{array}{l}398 \\
398\end{array}$ & GC & $\begin{array}{l}\text { [1990HIN/BID2] } \\
\text { [1990HIN/BID2] }\end{array}$ \\
\hline $\mathrm{C}_{9} \mathrm{H}_{6} \mathrm{Cl}_{6} \mathrm{O}_{3} \mathrm{~S}$ & $\begin{array}{c}{[115-29-7]} \\
\text { FUS }\end{array}$ & $6,7,8,9,10,10$-hex & $\begin{array}{l}\text { ro- } 1,5,5 \mathrm{a}, 6, \\
16.52\end{array}$ & $\begin{array}{c}\text { hexahyd } \\
368\end{array}$ & $\begin{array}{l}\text {-methano- } \\
\text { DSC }\end{array}$ & $\begin{array}{l}\text { 4,3-benzodioxathiepin-3-oxide (endosulfan) } \\
\text { [2000ROD/VEC] }\end{array}$ \\
\hline
\end{tabular}


TABLE 12. Phase change enthalpies of $\mathrm{C}_{9}$ organic compounds-Continued

\begin{tabular}{|c|c|c|c|c|c|c|}
\hline \multirow[b]{2}{*}{$\begin{array}{l}\text { Molecular } \\
\text { formula }\end{array}$} & \multirow{2}{*}{$\begin{array}{l}\text { CAS reg. no. } \\
\text { Transition }\end{array}$} & \multicolumn{5}{|l|}{ Compound } \\
\hline & & Temp. range $(\mathrm{K})$ & $\begin{array}{c}\Delta_{\text {trans }} H_{\mathrm{m}} \\
\left(\mathrm{kJ} \mathrm{mol}^{-1}\right)\end{array}$ & $T_{\mathrm{m}}(\mathrm{K})$ & Method & References \\
\hline $\mathrm{C}_{9} \mathrm{H}_{6} \mathrm{Cl}_{6} \mathrm{O}_{4} \mathrm{~S}$ & $\begin{array}{c}{[1031-07-8]} \\
\mathrm{V}\end{array}$ & $\begin{array}{l}\text { endosulfan sulfate } \\
\qquad(343-453)\end{array}$ & 85.6 & 398 & GC & [1990HIN/BID2] \\
\hline $\mathrm{C}_{9} \mathrm{H}_{6} \mathrm{Cl}_{6} \mathrm{O}_{4} \mathrm{~S}$ & $\begin{array}{c}\text { [1031-07-8] } \\
\text { FUS }\end{array}$ & \multicolumn{5}{|c|}{ 6,7,8,9,10,10-hexachloro-6,9-methano-2,4,3-benzodioxathiapin-3,3-dioxide } \\
\hline $\mathrm{C}_{9} \mathrm{H}_{6} \mathrm{INO}$ & $\begin{array}{c}{[13207-63-1]} \\
\text { SUB }\end{array}$ & \multicolumn{4}{|c|}{ 5-iodo-8-hydroxyquinoline } & [1963HOR/WEN] \\
\hline $\mathrm{C}_{9} \mathrm{H}_{6} \mathrm{~N}_{2} \mathrm{O}_{2}$ & $\begin{array}{c}\text { [584-84-9] } \\
\text { V } \\
\text { V } \\
\text { V } \\
\text { V }\end{array}$ & $\begin{array}{l}\text { 2,4-toluene diisocy } \\
\quad(373-530) \\
(393-530) \\
(373-530) \\
(373-433)\end{array}$ & $\begin{array}{l}\text { ate } \\
\qquad \begin{array}{r}59.7 \\
59.5 \\
61.3 \\
57.7 \pm 0.2\end{array}\end{array}$ & $\begin{array}{l}388 \\
408 \\
388 \\
403\end{array}$ & $\begin{array}{l}\text { A } \\
\text { A } \\
\text { I }\end{array}$ & $\begin{array}{l}\text { [1987STE/MAL] } \\
{[1987 \mathrm{STE} / \mathrm{MAL}]} \\
{[1975 \mathrm{FRE} / \mathrm{ADA}]} \\
{[1972 \mathrm{STR} / \mathrm{NOV}]}\end{array}$ \\
\hline $\mathrm{C}_{9} \mathrm{H}_{6} \mathrm{~N}_{2} \mathrm{O}_{2}$ & $\begin{array}{c}{[91-08-7]} \\
\mathrm{V}\end{array}$ & $\begin{array}{l}\text { 2,6-toluene diisocy } \\
\quad(373-463)\end{array}$ & ate 60.4 & 388 & A & [1987STE/MAL] \\
\hline $\mathrm{C}_{9} \mathrm{H}_{6} \mathrm{~N}_{2} \mathrm{O}_{2}$ & $\begin{array}{l}{[607-34-1]} \\
\text { SUB } \\
\text { SUB }\end{array}$ & $\begin{array}{l}\text { 5-nitroquinoline } \\
\quad(310-324) \\
(310-324)\end{array}$ & $\begin{array}{l}93.2 \pm 0.7 \\
94.2 \pm 0.7\end{array}$ & $\begin{array}{l}317 \\
298\end{array}$ & $\begin{array}{l}\mathrm{ME} \\
\mathrm{ME}\end{array}$ & $\begin{array}{l}\text { [1997RIB/MAT5] } \\
\text { [1997RIB/MAT5] }\end{array}$ \\
\hline $\mathrm{C}_{9} \mathrm{H}_{6} \mathrm{~N}_{2} \mathrm{O}_{2}$ & $\begin{array}{l}{[613-50-3]} \\
\text { SUB } \\
\text { SUB }\end{array}$ & $\begin{array}{l}\text { 6-nitroquinoline } \\
(336-350) \\
(336-350)\end{array}$ & $\begin{array}{l}101.5 \pm 1.0 \\
103.8 \pm 1.0\end{array}$ & $\begin{array}{l}343 \\
298\end{array}$ & $\begin{array}{l}\mathrm{ME} \\
\mathrm{ME}\end{array}$ & $\begin{array}{l}\text { [1997RIB/MAT5] } \\
\text { [1997RIB/MAT5] }\end{array}$ \\
\hline $\mathrm{C}_{9} \mathrm{H}_{6} \mathrm{~N}_{2} \mathrm{O}_{2}$ & $\begin{array}{l}{[607-35-2]} \\
\text { SUB } \\
\text { SUB }\end{array}$ & $\begin{array}{l}\text { 8-nitroquinoline } \\
\quad(338-352) \\
(338-352)\end{array}$ & $\begin{array}{l}104.3 \pm 0.9 \\
106.7 \pm 0.9\end{array}$ & $\begin{array}{l}345 \\
298\end{array}$ & $\begin{array}{l}\mathrm{ME} \\
\mathrm{ME}\end{array}$ & $\begin{array}{l}\text { [1997RIB/MAT5] } \\
\text { [1997RIB/MAT5] }\end{array}$ \\
\hline $\mathrm{C}_{9} \mathrm{H}_{6} \mathrm{~N}_{2} \mathrm{O}_{3}$ & $\begin{array}{c}\text { [4008-48-4] } \\
\text { FUS } \\
\text { FUS } \\
\text { SUB } \\
\text { SUB }\end{array}$ & $\begin{array}{l}(413-453) \\
(413-453)\end{array}$ & $\begin{array}{l}\text { noline } \\
\qquad \begin{array}{c}19.61 \\
24.7 \\
81.66 \\
86.14\end{array}\end{array}$ & $\begin{array}{c}455.2 \\
453.2 \\
298\end{array}$ & $\begin{array}{l}\text { DSC } \\
\text { DSC } \\
\text { TGA } \\
\text { TGA }\end{array}$ & $\begin{array}{l}{[2010 \mathrm{GAO} / \mathrm{LIN}]} \\
{[2001 \mathrm{ZOR} / \mathrm{COS}]} \\
{[2010 \mathrm{GAO} / \mathrm{LIN}]} \\
{[2010 \mathrm{GAO} / \mathrm{LIN}]}\end{array}$ \\
\hline Note: The aut & $\begin{array}{l}\text { f }[2010 \mathrm{GAO} / \mathrm{LI}] \\
\text { SUB } \\
\text { SUB }\end{array}$ & $\begin{array}{l}\text { did note that their ex } \\
\qquad(352-362)\end{array}$ & $\begin{array}{l}114.1 \pm 2.2 \\
111.2 \pm 3.0\end{array}$ & $\begin{array}{l}\text { fered sign } \\
298 \\
298\end{array}$ & $\begin{array}{l}\text { ntly from } \\
\text { ME } \\
\text { C }\end{array}$ & $\begin{array}{l}\text { e earlier published literature values. } \\
\text { [1989RIB/MON] } \\
\text { [1989RIB/MON] }\end{array}$ \\
\hline $\mathrm{C}_{9} \mathrm{H}_{6} \mathrm{~N}_{4} \mathrm{O}_{2}$ & $\begin{array}{c}\text { [23190-84-3] } \\
\text { SUB }\end{array}$ & \multicolumn{4}{|c|}{ 3-amino-2-quinoxalinecarbonitrile 1,4-dioxide } & [2004RIB/GOM] \\
\hline \multirow[t]{3}{*}{$\mathrm{C}_{9} \mathrm{H}_{6} \mathrm{O}_{2}$} & $\begin{array}{l}\text { [91-64-5] } \\
\text { FUS } \\
\text { FUS } \\
\text { FUS } \\
\text { FUS } \\
\text { FUS }\end{array}$ & coumarin & $\begin{array}{c}16.04 \\
19.54 \\
18.63 \\
19.6 \\
19.14\end{array}$ & $\begin{array}{l}344.4 \\
341.7 \\
342.3 \\
342.0 \\
342.1\end{array}$ & $\begin{array}{l}\text { DSC } \\
\text { DSC } \\
\text { DSC } \\
\text { DSC }\end{array}$ & $\begin{array}{l}\text { [2015HUA/WAN] } \\
\text { [2013DOS/MOR] } \\
\text { [2009MAT/SOU4] } \\
\text { [2004STU/WIT] } \\
\text { [1996DOM/HEA, 1991ELW/SAB, 1992SAB/WAT] }\end{array}$ \\
\hline & $\begin{array}{l}\text { SUB } \\
\text { SUB } \\
\text { SUB }\end{array}$ & $(293-353)$ & $\begin{array}{l}95.4 \pm 2.6 \\
83.1 \\
86.2\end{array}$ & $\begin{array}{l}298 \\
298 \\
323\end{array}$ & $\begin{array}{c}\mathrm{C} \\
\mathrm{C} \\
\mathrm{ME}\end{array}$ & $\begin{array}{l}\text { [2009MAT/SOU4] } \\
\text { [1991ELW/SAB, 1992SAB/WAT] } \\
\text { [1953SER/VOI, 1960JON, 1987STE/MAL] }\end{array}$ \\
\hline & $\mathrm{V}$ & $(379-463)$ & 63.2 & 394 & A & [1987STE/MAL, 1947STU] \\
\hline \multirow[t]{2}{*}{$\mathrm{C}_{9} \mathrm{H}_{6} \mathrm{O}_{2}$} & $\begin{array}{l}\text { [491-38-3] } \\
\text { FUS } \\
\text { FUS }\end{array}$ & chromone & $\begin{array}{l}15.44 \\
17.31\end{array}$ & $\begin{array}{l}329.9 \\
330.3\end{array}$ & $\mathrm{DSC}$ & $\begin{array}{l}\text { [2009MAT/SOU4] } \\
\text { [1991ACR, 1996DOM/HEA, 1988SAB/ELW] }\end{array}$ \\
\hline & $\begin{array}{l}\text { SUB } \\
\text { SUB }\end{array}$ & & $\begin{array}{l}86.5 \pm 1.1 \\
81.3 \pm 0.2\end{array}$ & $\begin{array}{l}298 \\
298\end{array}$ & $\begin{array}{l}\mathrm{C} \\
\mathrm{C}\end{array}$ & $\begin{array}{l}\text { [2009MAT/SOU4] } \\
{[1988 \mathrm{SAB} / \mathrm{ELW}]}\end{array}$ \\
\hline \multirow[t]{2}{*}{$\mathrm{C}_{9} \mathrm{H}_{6} \mathrm{O}_{2}$} & $\begin{array}{l}{[606-23-5]} \\
\text { FUS }\end{array}$ & 1,3-indandione & 21.8 & 401.5 & DSC & [2007MAT/MIR] \\
\hline & SUB & & $97.3 \pm 1.8$ & 298 & $\mathrm{C}$ & [2007MAT/MIR] \\
\hline $\mathrm{C}_{9} \mathrm{H}_{6} \mathrm{O}_{2}$ & $\begin{array}{l}{[637-44-5]} \\
\text { FUS }\end{array}$ & 3-phenylpropiolic a & 25.95 & 410.54 & DSC & [2001MON/HIL5] \\
\hline
\end{tabular}


TABLE 12. Phase change enthalpies of $\mathrm{C}_{9}$ organic compounds-Continued

\begin{tabular}{|c|c|c|c|c|c|c|}
\hline \multirow[b]{2}{*}{$\begin{array}{l}\text { Molecular } \\
\text { formula }\end{array}$} & \multirow{2}{*}{$\begin{array}{l}\text { CAS reg. no. } \\
\text { Transition }\end{array}$} & \multicolumn{5}{|l|}{ Compound } \\
\hline & & Temp. range $(\mathrm{K})$ & $\begin{array}{c}\Delta_{\text {trans }} H_{\mathrm{m}} \\
\left(\mathrm{kJ} \mathrm{mol}^{-1}\right)\end{array}$ & $T_{\mathrm{m}}(\mathrm{K})$ & Method & References \\
\hline & SUB & $(329-343)$ & $103.9 \pm 0.6$ & 336 & ME & [2001MON/HIL5] \\
\hline & SUB & $(329-343)$ & $105.0 \pm 1.4$ & 298 & $\mathrm{ME}$ & [2001MON/HIL5] \\
\hline \multirow[t]{3}{*}{$\mathrm{C}_{9} \mathrm{H}_{6} \mathrm{O}_{3}$} & {$[93-35-6]$} & 1-hydroxycoumarin & & & & \\
\hline & FUS & & 31.25 & 503.7 & DSC & [2011SOU/MAT2] \\
\hline & SUB & & $133.6 \pm 1.5$ & 298 & $\mathrm{C}$ & [2011SOU/MAT2] \\
\hline \multirow[t]{3}{*}{$\mathrm{C}_{9} \mathrm{H}_{6} \mathrm{O}_{3}$} & [939-19-5] & 3-hydroxycoumarin & & & & \\
\hline & FUS & & 27.10 & 425.6 & $\mathrm{DSC}$ & [2010SOU/MOR] \\
\hline & SUB & & $91.9 \pm 0.6$ & 298 & $\mathrm{C}$ & [2010SOU/MOR] \\
\hline \multirow[t]{4}{*}{$\mathrm{C}_{9} \mathrm{H}_{6} \mathrm{O}_{3}$} & [1076-38-6] & 4-hydroxycoumarin & & & & \\
\hline & FUS & & 24.49 & 488.3 & DSC & [2013DOS/MOR] \\
\hline & FUS & & 23.17 & 483.6 & DSC & [2010SOU/MOR] \\
\hline & SUB & & $128.5 \pm 1.8$ & 298 & $\mathrm{C}$ & [2010SOU/MOR] \\
\hline \multirow[t]{2}{*}{$\mathrm{C}_{9} \mathrm{H}_{6} \mathrm{O}_{6}$} & [528-44-9] & 1,2,4-benzenetricarb & oxylic acid & & & \\
\hline & FUS & & 30.8 & 499.6 & DSC & [2012BOO/BAN] \\
\hline \multirow[t]{2}{*}{$\mathrm{C}_{9} \mathrm{H}_{6} \mathrm{O}_{6}$} & {$[554-95-0]$} & 1,3,5-benzenetricarb & oxylic acid & & & \\
\hline & SUB & $(553-593)$ & 159.4 & 573 & GS & [1987STE/MAL, 1962KRA/BER] \\
\hline \multirow[t]{3}{*}{$\mathrm{C}_{9} \mathrm{H}_{6} \mathrm{~S}_{3}$} & {$[3445-76-9]$} & 5-phenyl-1,2-dithiole & -3-thione & & & \\
\hline & SUB & $(363-373)$ & $117.4 \pm 0.4$ & & & [1972GEI/RAU] \\
\hline & SUB & & $123.3 \pm 0.4$ & 298 & & [1972GEI/RAU] \\
\hline \multirow[t]{2}{*}{$\mathrm{C}_{9} \mathrm{H}_{6} \mathrm{~S}_{3}$} & {$[3445-76-9]$} & 5-phenyldithiolethio & & & & \\
\hline & FUS & & 26.27 & 398 & DSC & [1999DOL/LEC] \\
\hline \multirow[t]{2}{*}{$\mathrm{C}_{9} \mathrm{H}_{7} \mathrm{BrO}_{2}$} & {$[14473-91-7]$} & 3-bromo-trans-cinna & mic acid & & & \\
\hline & FUS & & 31.06 & 443.2 & DSC & [2001AHN/HAR] \\
\hline \multirow[t]{2}{*}{$\mathrm{C}_{9} \mathrm{H}_{7} \mathrm{Cl}_{3} \mathrm{O}_{3}$} & {$[93-72-1]$} & 2-(2,4,5-trichlorophe & noxy)propano & & & \\
\hline & FUS & & 39.58 & 450.6 & DSC & [1990DON/DRE] \\
\hline \multirow[t]{3}{*}{$\mathrm{C}_{9} \mathrm{H}_{7} \mathrm{Cl}_{3} \mathrm{O}_{3}$} & {$[1928-37-6]$} & 2,4,5-trichloropheno & xyacetic acid, & hyl ester & & \\
\hline & FUS & & 30.46 & 361.9 & DSC & {$[1990 D O N / D R E]$} \\
\hline & $\mathrm{V}$ & $(444-573)$ & 76.9 & 459 & A & [1987STE/MAL, 1999DYK/SVO] \\
\hline \multirow[t]{2}{*}{$\mathrm{C}_{9} \mathrm{H}_{7} \mathrm{~F}_{3} \mathrm{O}_{2}$} & [1736-09-0] & trifluoroacetic acid, & -tolyl ester & & & \\
\hline & V & $(363-439)$ & 47.4 & 378 & $\mathrm{~A}, \mathrm{~EB}$ & [1987STE/MAL, 1969SHE/LAN, 1999DYK/SVO] \\
\hline \multirow[t]{2}{*}{$\mathrm{C}_{9} \mathrm{H}_{7} \mathrm{~F}_{3} \mathrm{O}_{2}$} & {$[1813-29-2]$} & trifluoroacetic acid, & -tolyl ester & & & \\
\hline & $\mathrm{V}$ & $(365-442)$ & 47.8 & 380 & $\mathrm{~A}, \mathrm{~EB}$ & [1987STE/MAL, 1969SHE/LAN, 1999DYK/SVO] \\
\hline \multirow[t]{10}{*}{$\mathrm{C}_{9} \mathrm{H}_{7} \mathrm{~N}$} & {$[119-65-3]$} & isoquinoline & & & & \\
\hline & FUS & & 13.54 & 299.6 & & [1996DOM/HEA, 1988STE/ARC] \\
\hline & V & & 53.3 & & GC & [1996GOV/RUT] \\
\hline & $\mathrm{V}$ & $(313-566)$ & $58.9 \pm 0.1$ & 320 & IPM, EB & [1988STE/ARC] \\
\hline & V & $(313-566)$ & $56.4 \pm 0.1$ & 360 & IPM, EB & [1988STE/ARC] \\
\hline & $\mathrm{V}$ & $(313-566)$ & $54.1 \pm 0.1$ & 400 & IPM, EB & [1988STE/ARC] \\
\hline & $\mathrm{V}$ & $(313-566)$ & $51.7 \pm 0.1$ & 440 & IPM, EB & [1988STE/ARC] \\
\hline & $\mathrm{V}$ & $(313-566)$ & $49.4 \pm 0.2$ & 480 & IPM, EB & [1988STE/ARC] \\
\hline & V & $(313-566)$ & $47.0 \pm 0.3$ & 520 & IPM, EB & [1988STE/ARC] \\
\hline & $\mathrm{V}$ & $(439-517)$ & 51.0 & 454 & $\mathrm{~A}, \mathrm{~EB}$ & [1987STE/MAL, 1961MAL] \\
\hline \multirow[t]{10}{*}{$\mathrm{C}_{9} \mathrm{H}_{7} \mathrm{~N}$} & [91-22-5] & quinoline & & & & \\
\hline & TRS & & 0.07 & 220 & & \\
\hline & FUS & & 10.66 & 258.4 & & [1996DOM/HEA, 1988STE/ARC] \\
\hline & FUS & & 10.72 & 257.9 & $\mathrm{AC}$ & [1957MAS] \\
\hline & FUS & & 10.80 & 258.4 & & {$[1936 \mathrm{PAR} / \mathrm{TOD}]$} \\
\hline & $\mathrm{V}$ & & 53.3 & & GC & [1996GOV/RUT] \\
\hline & $\mathrm{V}$ & $(573-668)$ & 46.9 & 588 & DSC & [1996BAC/GRZ] \\
\hline & $\mathrm{V}$ & $(504-616)$ & 46.5 & 519 & & [1992LEE/CHE] \\
\hline & $\mathrm{V}$ & $(298-559)$ & $57.9 \pm 0.1$ & 320 & IPM, EB & [1988STE/ARC] \\
\hline & $\mathrm{V}$ & $(298-559)$ & $55.5 \pm 0.1$ & 360 & IPM, EB & [1988STE/ARC] \\
\hline
\end{tabular}


TABLE 12. Phase change enthalpies of $\mathrm{C}_{9}$ organic compounds-Continued

\begin{tabular}{|c|c|c|c|c|c|c|}
\hline \multirow[b]{2}{*}{$\begin{array}{l}\text { Molecular } \\
\text { formula }\end{array}$} & \multirow{2}{*}{$\begin{array}{c}\text { CAS reg. no. } \\
\text { Transition }\end{array}$} & \multicolumn{5}{|l|}{ Compound } \\
\hline & & Temp. range $(\mathrm{K})$ & $\begin{array}{c}\Delta_{\text {trans }} H_{\mathrm{m}} \\
\left(\mathrm{kJ} \mathrm{mol}^{-1}\right)\end{array}$ & $T_{\mathrm{m}}(\mathrm{K})$ & Method & References \\
\hline & $\mathrm{V}$ & $(298-559)$ & $53.1 \pm 0.1$ & 400 & IPM, EB & [1988STE/ARC] \\
\hline & $\mathrm{V}$ & $(298-559)$ & $50.7 \pm 0.1$ & 440 & IPM, EB & [1988STE/ARC] \\
\hline & $\mathrm{V}$ & $(298-559)$ & $48.4 \pm 0.2$ & 480 & IPM, EB & [1988STE/ARC] \\
\hline & $\mathrm{V}$ & $(298-559)$ & $46.0 \pm 0.3$ & 520 & IPM, EB & [1988STE/ARC] \\
\hline & $\mathrm{V}$ & $(473-548)$ & U65.4 & 488 & & [1987KLA/MOH] \\
\hline & $\mathrm{V}$ & $(463-794)$ & 46.1 & 478 & A & [1987STE/MAL] \\
\hline & $\mathrm{V}$ & $(286-309)$ & 58.1 & 298 & GS & [1980VAN/PRA] \\
\hline & $\mathrm{V}$ & $(433-511)$ & 49.2 & 448 & $\mathrm{~EB}$ & [1987STE/MAL, 1961MAL] \\
\hline \multirow[t]{3}{*}{$\mathrm{C}_{9} \mathrm{H}_{7} \mathrm{NO}$} & {$[59-31-4]$} & 2-hydroxyquinoline & & & & \\
\hline & SUB & $(375-390)$ & $115.2 \pm 0.6$ & 383 & $\mathrm{ME}$ & [1990RIB/MAT] \\
\hline & SUB & $(375-390)$ & $119.4 \pm 0.6$ & 298 & ME & [1990RIB/MAT] \\
\hline \multirow[t]{3}{*}{$\mathrm{C}_{9} \mathrm{H}_{7} \mathrm{NO}$} & [611-36-9] & 4-hydroxyquinoline & & & & \\
\hline & SUB & $(415-433)$ & $128.8 \pm 1.1$ & 424 & ME & [1990RIB/RIB] \\
\hline & SUB & $(415-433)$ & $135.1 \pm 1.1$ & 298 & $\mathrm{ME}$ & [1990RIB/RIB] \\
\hline \multirow[t]{6}{*}{$\mathrm{C}_{9} \mathrm{H}_{7} \mathrm{NO}$} & [148-24-3] & 8-hydroxyquinoline & & & & \\
\hline & FUS & $(70-370)$ & 40.3 & 345.7 & $\mathrm{AC}$ & [2008WAN/TAN] \\
\hline & FUS & & 22.1 & 346.8 & DSC & [2001ZOR/COS] \\
\hline & SUB & $(293-303)$ & $89.5 \pm 0.9$ & 298 & ME & [1989RIB/MON] \\
\hline & SUB & & $89.0 \pm 1.4$ & 298 & $\mathrm{C}$ & [1989RIB/MON] \\
\hline & SUB & $(308-328)$ & $108.8 \pm 1.7$ & & $\mathrm{ME}$ & [1963HOR/WEN, 1970COX/PIL, 1987STE/MAL] \\
\hline \multirow[t]{2}{*}{$\mathrm{C}_{9} \mathrm{H}_{7} \mathrm{NO}$} & {$[491-30-5]$} & 1-hydroxyisoquinolir & & & & \\
\hline & SUB & & $113.6 \pm 2.2$ & 298 & $\mathrm{C}$ & [2005RIB/MAT] \\
\hline \multirow[t]{2}{*}{$\mathrm{C}_{9} \mathrm{H}_{7} \mathrm{NO}$} & {$[2439-04-5]$} & 5-hydroxyisoquinolir & & & & \\
\hline & SUB & & $109.6 \pm 2.1$ & 298 & $\mathrm{C}$ & [2005RIB/MAT] \\
\hline \multirow[t]{4}{*}{$\mathrm{C}_{9} \mathrm{H}_{7} \mathrm{NO}$} & {$[614-16-4]$} & $\Omega-$ cyanoacetopheno & & & & \\
\hline & SUB & & $98.5 \pm 2.1$ & 298 & $\mathrm{C}$ & [2015AMA/SZT] \\
\hline & SUB & $(318-333)$ & 99.8 & 325.5 & A & [1987STE/MAL] \\
\hline & SUB & & $92.5 \pm 4.2$ & & ME & [1969LEB/DNE, 1977PED/RYL] \\
\hline \multirow[t]{2}{*}{$\mathrm{C}_{9} \mathrm{H}_{7} \mathrm{NO}$} & [6136-68-1] & 3-acetylbenzonitrile & & & & \\
\hline & SUB & & $94.7 \pm 1.5$ & 298 & $\mathrm{C}$ & [2015AMA/SZT] \\
\hline \multirow[t]{3}{*}{$\mathrm{C}_{9} \mathrm{H}_{7} \mathrm{NO}$} & [487-89-8] & indole-3-carboxyalde & ehyde & & & \\
\hline & SUB & $(372-394)$ & $119.1 \pm 1.0$ & 383 & ME & [2014AMA/DEC] \\
\hline & SUB & $(372-394)$ & $120.3 \pm 1.0$ & 298 & ME & [2014AMA/DEC] \\
\hline \multirow[t]{3}{*}{$\mathrm{C}_{9} \mathrm{H}_{7} \mathrm{NO}_{2}$} & [696-04-8] & indole-2-carboxylic a & acid & & & \\
\hline & SUB & $(360-382)$ & $118.2 \pm 1.0$ & 371 & ME & [2014AMA/DEC] \\
\hline & SUB & $(360-382)$ & $121.9 \pm 1.0$ & 298 & $\mathrm{ME}$ & [2014AMA/DEC] \\
\hline \multirow[t]{2}{*}{$\mathrm{C}_{9} \mathrm{H}_{7} \mathrm{NO}_{2}$} & [5154-02-9] & 1,5-dihydroxyisoquir & noline & & & \\
\hline & SUB & & $123.6 \pm 2.2$ & 298 & $\mathrm{C}$ & [2005RIB/MAT] \\
\hline \multirow[t]{3}{*}{$\mathrm{C}_{9} \mathrm{H}_{7} \mathrm{NO}_{2}$} & {$[550-44-7]$} & $N$-methylphthalimid & & & & \\
\hline & SUB & $(298-316)$ & $91.1 \pm 0.5$ & 307 & ME & [1997ROU/JIM] \\
\hline & SUB & $(298-316)$ & $91.1 \pm 0.5$ & 298 & ME & [1997ROU/JIM] \\
\hline \multirow[t]{3}{*}{$\mathrm{C}_{9} \mathrm{H}_{7} \mathrm{NO}_{2}$} & [2058-74-4] & 1-methyl-1H-indole- & -2,3-dione $(N$ & hylisatin) & & \\
\hline & FUS & & 19.5 & 403.3 & DSC & [2003MAT/MIR2] \\
\hline & SUB & & $105.6 \pm 3.3$ & 298 & $\mathrm{C}$ & [2003MAT/MIR2] \\
\hline \multirow[t]{3}{*}{$\mathrm{C}_{9} \mathrm{H}_{7} \mathrm{~N}_{3} \mathrm{O}_{2}$} & {$[35975-00-9]$} & 5-amino-6-nitroquin & oline & & & \\
\hline & SUB & $(400-424)$ & $130.7 \pm 0.8$ & 412 & $\mathrm{ME}$ & [1998RIB/CAR] \\
\hline & SUB & $(400-424)$ & $136.4 \pm 0.8$ & 298 & ME & [1998RIB/CAR] \\
\hline \multirow[t]{3}{*}{$\mathrm{C}_{9} \mathrm{H}_{7} \mathrm{~N}_{3} \mathrm{O}_{2} \mathrm{~S}$} & {$[473-42-7]$} & 4-nitro- $N$-2-thiazoly & lbenzene sulf & ide & & \\
\hline & FUS & & 31.8 & 529.8 & $\mathrm{DSC}$ & {$[2014 \mathrm{PER} / \mathrm{KAZ}]$} \\
\hline & SUB & $(452-509)$ & $144.0 \pm 2.0$ & 298 & GS & {$[2016 \mathrm{VOL} / \mathrm{BLO}]$} \\
\hline \multirow[t]{2}{*}{$\mathrm{C}_{9} \mathrm{H}_{7} \mathrm{~N}_{3} \mathrm{~S}$} & {$[41814-78-2]$} & \multicolumn{5}{|c|}{ 5-methyl-1,2,4-triazolo[3,4-b]benzothiazole } \\
\hline & FUS & & 24.07 & 460.2 & DSC & [1990DON/DRE] \\
\hline
\end{tabular}


TABLE 12. Phase change enthalpies of $\mathrm{C}_{9}$ organic compounds-Continued

\begin{tabular}{|c|c|c|c|c|c|c|}
\hline \multirow[b]{2}{*}{$\begin{array}{l}\text { Molecular } \\
\text { formula }\end{array}$} & \multirow{2}{*}{$\begin{array}{l}\text { CAS reg. no. } \\
\text { Transition }\end{array}$} & \multicolumn{5}{|l|}{ Compound } \\
\hline & & Temp. range $(\mathrm{K})$ & $\begin{array}{c}\Delta_{\text {trans }} H_{\mathrm{m}} \\
\left(\mathrm{kJ} \mathrm{mol}^{-1}\right)\end{array}$ & $T_{\mathrm{m}}(\mathrm{K})$ & Method & References \\
\hline \multirow[t]{6}{*}{$\mathrm{C}_{9} \mathrm{H}_{8}$} & {$[95-13-6]$} & indene & & & & \\
\hline & FUS & $(15-320)$ & 10.20 & 271.7 & & [1996DOM/HEA, 1961STU/SIN] \\
\hline & $\mathrm{V}$ & $(275-339)$ & $50.3 \pm 0.1$ & 298 & GS & [2011VER/EME3] \\
\hline & $\mathrm{V}$ & $(369-457)$ & 45.3 & 384 & A & [1987STE/MAL] \\
\hline & $\mathrm{V}$ & $(289-455)$ & 43.6 & 304 & A & [1987STE/MAL, 1947STU] \\
\hline & $\mathrm{V}$ & $(329-454)$ & 43.9 & 392 & & [1942BUR] \\
\hline \multirow[t]{2}{*}{$\mathrm{C}_{9} \mathrm{H}_{8} \mathrm{Cl}_{2} \mathrm{O}_{3}$} & {$[6597-78-0]$} & \multicolumn{5}{|c|}{ methyl 3,6-dichloro-2-methoxybenzoate } \\
\hline & FUS & & 18.49 & 304.6 & DSC & [1991ACR, 1990DON/DRE] \\
\hline \multirow[t]{6}{*}{$\mathrm{C}_{9} \mathrm{H}_{8} \mathrm{Cl}_{2} \mathrm{O}_{3}$} & {$[120-36-5]$} & \multicolumn{5}{|c|}{ 2-(2,4-dichlorophenoxy)propanoic acid } \\
\hline & FUS & & 32.0 & 391.3 & DSC & [2005VEC/BRU] \\
\hline & FUS & & 30.43 & 389.2 & DSC & [1991ACR, 1990DON/DRE] \\
\hline & SUB & & $116 \pm 6$ & 298 & DSC & [2005VEC/BRU] \\
\hline & SUB & $(343-375)$ & $128 \pm 2$ & 359 & $\mathrm{TE}$ & [2005VEC/BRU] \\
\hline & SUB & $(343-375)$ & $130 \pm 3$ & 298 & $\mathrm{TE}$ & [2005VEC/BRU] \\
\hline \multirow[t]{4}{*}{$\mathrm{C}_{9} \mathrm{H}_{8} \mathrm{Cl}_{2} \mathrm{O}_{3}$} & [1928-38-7] & \multicolumn{5}{|c|}{ 2,4-dichlorophenoxyacetic acid, methyl ester } \\
\hline & FUS & & 20.0 & 313.4 & DSC & [2005VEC/BRU] \\
\hline & FUS & & 25.1 & 315.4 & $\mathrm{DSC}$ & [1969PLA/GLA] \\
\hline & $\mathrm{V}$ & $(403-548)$ & 68.0 & 418 & A & [1987STE/MAL, 1999DYK/SVO] \\
\hline \multirow[t]{2}{*}{$\mathrm{C}_{9} \mathrm{H}_{8} \mathrm{Cl}_{2} \mathrm{O}_{4}$} & {$[76330-06-8]$} & \multicolumn{5}{|c|}{ 2,6-dichlorosyringaldehyde } \\
\hline & V & $(293-323)$ & 82.2 & 308 & CGC & [1999LEI/WAN2] \\
\hline \multirow[t]{2}{*}{$\mathrm{C}_{9} \mathrm{H}_{8} \mathrm{Cl}_{3} \mathrm{NO}_{3}$} & {$[75907-45-8]$} & \multicolumn{5}{|c|}{ 2,2,4-trichloro-5-(4-morpholinyl)-4-cyclopentene-1,3-dione } \\
\hline & $\mathrm{V}$ & $(453-483)$ & 79.6 & 468 & GC & [1980SHA/SAD] \\
\hline \multirow[t]{3}{*}{$\mathrm{C}_{9} \mathrm{H}_{8} \mathrm{~N}_{2}$} & {$[1126-00-7]$} & \multicolumn{5}{|l|}{ 1-phenylpyrazole } \\
\hline & $\mathrm{V}$ & & $68.6 \pm 2.9$ & 298 & CGC & [2010LIP/PLI] \\
\hline & $\mathrm{V}$ & & $70.2 \pm 3.4$ & 298 & $\mathrm{C}$ & [2000RIB/RIB2] \\
\hline \multirow[t]{3}{*}{$\mathrm{C}_{9} \mathrm{H}_{8} \mathrm{~N}_{2}$} & {$[7164-98-9]$} & \multicolumn{5}{|l|}{ 1-phenylimidazole } \\
\hline & $\mathrm{V}$ & & $74.3 \pm 2.6$ & 298 & CGC & [2010LIP/PLI] \\
\hline & $\mathrm{V}$ & & $84.6 \pm 3.7$ & 298 & $\mathrm{C}$ & [2000RIB/RIB2] \\
\hline \multirow[t]{2}{*}{$\mathrm{C}_{9} \mathrm{H}_{8} \mathrm{~N}_{2}$} & [670-96-2] & 2-phenylimidazole & & & & \\
\hline & FUS & & 17.81 & 420 & DSC & [2007SIF/AIT] \\
\hline $\mathrm{C}_{9} \mathrm{H}_{8} \mathrm{~N}_{2}$ & [580-17-6] & 3-aminoquinoline & & & & \\
\hline & SUB & $(329-345)$ & $101.1 \pm 0.9$ & 337 & ME & [1993RIB/MAT] \\
\hline & SUB & $(329-345)$ & $103.1 \pm 0.9$ & 298 & $\mathrm{ME}$ & [1993RIB/MAT] \\
\hline & SUB & & $104.8 \pm 4.8$ & 298 & $\mathrm{C}$ & [1993RIB/MAT] \\
\hline $\mathrm{C}_{9} \mathrm{H}_{8} \mathrm{~N}_{2}$ & [611-34-7] & 5-aminoquinoline & & & & \\
\hline & SUB & $(329-349)$ & $102.9 \pm 0.7$ & 339 & ME & [1993RIB/MAT] \\
\hline & SUB & $(329-349)$ & $105.0 \pm 0.7$ & 298 & ME & [1993RIB/MAT] \\
\hline & SUB & & $103.3 \pm 3.4$ & 298 & $\mathrm{C}$ & [1993RIB/MAT] \\
\hline $\mathrm{C}_{9} \mathrm{H}_{8} \mathrm{~N}_{2}$ & {$[580-15-4]$} & 6-aminoquinoline & & & & \\
\hline & SUB & $(333-349)$ & $103.6 \pm 1.0$ & 341 & ME & [1993RIB/MAT] \\
\hline & SUB & $(333-349)$ & $105.7 \pm 1.0$ & 298 & $\mathrm{ME}$ & [1993RIB/MAT] \\
\hline $\mathrm{C}_{9} \mathrm{H}_{8} \mathrm{~N}_{2}$ & [578-66-5] & 8-aminoquinoline & & & & \\
\hline & SUB & $(296-314)$ & $93.0 \pm 0.5$ & 305 & ME & [1993RIB/MAT] \\
\hline & SUB & (296-314) & $93.33 \pm 0.5$ & 298 & $\mathrm{ME}$ & [1993RIB/MAT] \\
\hline $\mathrm{C}_{9} \mathrm{H}_{8} \mathrm{~N}_{2} \mathrm{O}$ & [14003-34-0] & 2-methyl-3-hydroxy & uinoxaline & & & \\
\hline & FUS & & 33.4 & 522.9 & $\mathrm{DSC}$ & [2000MON/HIL2] \\
\hline & SUB & $(375-391)$ & $117.2 \pm 0.4$ & 383 & $\mathrm{ME}$ & [2000MON/HIL2] \\
\hline & SUB & $(375-391)$ & $119.7 \pm 2.8$ & 298 & $\mathrm{ME}$ & [2000MON/HIL2] \\
\hline & SUB & & $123.0 \pm 4.4$ & 298 & $\mathrm{C}$ & [2000RIB/MAT] \\
\hline $\mathrm{C}_{9} \mathrm{H}_{8} \mathrm{~N}_{2} \mathrm{O}_{2}$ & [6639-86-7] & 2-methylquinoxalin & 1,4-dioxide & & & \\
\hline & SUB & & $107.0 \pm 6.2$ & 298 & $\mathrm{C}$ & [1997ACR/POW] \\
\hline $\mathrm{C}_{9} \mathrm{H}_{8} \mathrm{~N}_{2} \mathrm{O}_{2}$ & [5972-09-8] & 3-methylaminophth & imide & & & \\
\hline
\end{tabular}


TABLE 12. Phase change enthalpies of $\mathrm{C}_{9}$ organic compounds-Continued

\begin{tabular}{|c|c|c|c|c|c|c|}
\hline \multirow[b]{2}{*}{$\begin{array}{l}\text { Molecular } \\
\text { formula }\end{array}$} & \multirow{2}{*}{$\begin{array}{l}\text { CAS reg. no. } \\
\text { Transition }\end{array}$} & \multicolumn{5}{|l|}{ Compound } \\
\hline & & Temp. range $(\mathrm{K})$ & $\begin{array}{c}\Delta_{\text {trans }} H_{\mathrm{m}} \\
\left(\mathrm{kJ} \mathrm{mol}^{-1}\right)\end{array}$ & $T_{\mathrm{m}}(\mathrm{K})$ & Method & References \\
\hline & SUB & $(402-450)$ & 104.9 & 417 & RG & [1987STE/MAL, 1956KLO] \\
\hline \multirow[t]{6}{*}{$\mathrm{C}_{9} \mathrm{H}_{8} \mathrm{O}$} & {$[83-33-0]$} & 1-indanone & & & & \\
\hline & FUS & & 17.6 & 314.1 & $\mathrm{DSC}$ & [2007MAT/MIR] \\
\hline & FUS & & 17.78 & 312.9 & DSC & [1998VER4] \\
\hline & SUB & & $78.7 \pm 2.8$ & 298 & $\mathrm{C}$ & [2007MAT/MIR] \\
\hline & SUB & & $83.5 \pm 0.7$ & 298 & GS & [1998VER4] \\
\hline & $\mathrm{V}$ & $(318-348)$ & $60.3 \pm 0.4$ & & GS & [1998GUD/TOR] \\
\hline \multirow[t]{3}{*}{$\mathrm{C}_{9} \mathrm{H}_{8} \mathrm{O}$} & {$[615-13-4]$} & 2-indanone & & & & \\
\hline & FUS & & 16.89 & 330 & DSC & [2007MAT/MIR] \\
\hline & SUB & & $78.3 \pm 1.1$ & 298 & $\mathrm{C}$ & [2007MAT/MIR] \\
\hline \multirow[t]{5}{*}{$\mathrm{C}_{9} \mathrm{H}_{8} \mathrm{O}$} & [104-55-2] & 3-phenyl-2-propen & cinnamaldeh & & & \\
\hline & $\mathrm{V}$ & & 62.4 & 298 & $\mathrm{GC}$ & [2002VAN/PAR] \\
\hline & $\mathrm{V}$ & $(408-482)$ & 51.7 & 444 & TGA & [2002HAZ/DOL] \\
\hline & $\mathrm{V}$ & $(349-519)$ & 58.2 & 364 & A & [1987STE/MAL, 1947STU] \\
\hline & $\mathrm{V}$ & $(353-373)$ & 72.7 & 363 & A & [1987STE/MAL] \\
\hline \multirow[t]{3}{*}{$\mathrm{C}_{9} \mathrm{H}_{8} \mathrm{O}_{2}$} & [621-82-9] & cinnamic acid & & & & \\
\hline & FUS & & 11.97 & 408.7 & DSC & [2009MUR/BER] \\
\hline & FUS & & 22.63 & 406.2 & DTA & [1986SIN/KUM] \\
\hline
\end{tabular}

Note: In both instances, the authors did not specify cis-or trans, and Chemical Abstracts gave a CARN of [621-82-9] when indexing the compound.

\begin{tabular}{|c|c|c|c|c|c|c|}
\hline \multirow[t]{5}{*}{$\mathrm{C}_{9} \mathrm{H}_{8} \mathrm{O}_{2}$} & [140-10-3] & \multicolumn{5}{|l|}{ trans-cinnamic acid } \\
\hline & FUS & & 22.21 & 406.1 & DSC & [2008MOT/QUE] \\
\hline & FUS & & 25.7 & 405.5 & DSC & [2004STU/WIT] \\
\hline & FUS & & 22.6 & 404.8 & DSC & [2004SHA/JAM] \\
\hline & FUS & & 22.63 & 406.2 & & [1991ACR, 1983WEA] \\
\hline & SUB & $(333-347)$ & $107.1 \pm 0.8$ & 298 & ME & [1999MON/HIL] \\
\hline & $\mathrm{V}$ & $(430-573)$ & 73.9 & 445 & A & [1987STE/MAL] \\
\hline \multirow[t]{4}{*}{$\mathrm{C}_{9} \mathrm{H}_{8} \mathrm{O}_{2}$} & {$[102-94-3]$} & \multicolumn{5}{|c|}{ allocinnamic acid (cis-cinnamic acid) } \\
\hline & FUS & $(255-360)$ & 16.8 & 340.1 & DSC & [2016DAV/LIM] \\
\hline & FUS & & 16.95 & 341.2 & & [1991ACR, 1983WEA] \\
\hline & SUB & $(306-326)$ & $101.0 \pm 1.2$ & 298 & $\mathrm{ME}$ & [2016DAV/LIM] \\
\hline \multirow[t]{2}{*}{$\mathrm{C}_{9} \mathrm{H}_{8} \mathrm{O}_{2}$} & {$[39869-70-0]$} & \multicolumn{5}{|c|}{ 7,7-dimethoxynorborane } \\
\hline & $\mathrm{V}$ & $(321-357)$ & 49.0 & 339 & $\mathrm{~EB}$ & [1994WIB/MOR] \\
\hline \multirow[t]{2}{*}{$\mathrm{C}_{9} \mathrm{H}_{8} \mathrm{O}_{2}$} & [119-84-6] & \multicolumn{5}{|l|}{ 3,4-dihydrocoumarin } \\
\hline & $\mathrm{V}$ & & $69.9 \pm 0.5$ & 298 & $\mathrm{C}$ & [2009MAT/SOU2] \\
\hline \multirow[t]{3}{*}{$\mathrm{C}_{9} \mathrm{H}_{8} \mathrm{O}_{2}$} & [491-37-2] & chromanone & & & & \\
\hline & FUS & & 16.7 & 312.3 & DSC & [2009MAT/SOU] \\
\hline & SUB & & $84.6 \pm 1.3$ & 298 & $\mathrm{C}$ & [2009MAT/SOU] \\
\hline \multirow[t]{3}{*}{$\mathrm{C}_{9} \mathrm{H}_{8} \mathrm{O}_{2}$} & {$[4385-35-7]$} & 3-isochromanone & & & & \\
\hline & FUS & & 18.3 & 355.9 & DSC & [2009MAT/SOU] \\
\hline & SUB & & $97.3 \pm 1.4$ & 298 & $\mathrm{C}$ & [2009MAT/SOU] \\
\hline \multirow[t]{3}{*}{$\mathrm{C}_{9} \mathrm{H}_{8} \mathrm{O}_{2}$} & {$[621-82-9]$} & 3-phenylacrylic acid & & & & \\
\hline & SUB & $(380-404)$ & $110.7 \pm 6.3$ & 392 & ME & [2014DIB/RAE] \\
\hline & SUB & $(380-404)$ & $113.8 \pm 6.3$ & 298 & ME & [2014DIB/RAE] \\
\hline \multirow[t]{2}{*}{$\mathrm{C}_{9} \mathrm{H}_{8} \mathrm{O}_{2} \mathrm{~S}$} & {$[2525-42-0]$} & \multicolumn{5}{|c|}{ phenyl propadienyl sulfone } \\
\hline & SUB & & $105.4 \pm 2.5$ & & & [1969MAC/STE, 1970COX/PIL] \\
\hline \multirow[t]{2}{*}{$\mathrm{C}_{9} \mathrm{H}_{8} \mathrm{O}_{2} \mathrm{~S}$} & [2525-41-9] & \multicolumn{5}{|c|}{ phenyl prop-1-ynyl sulfone } \\
\hline & SUB & & $95.4 \pm 2.5$ & & $\mathrm{~B}$ & [1969MAC/STE, 1970COX/PIL] \\
\hline \multirow[t]{2}{*}{$\mathrm{C}_{9} \mathrm{H}_{8} \mathrm{O}_{2} \mathrm{~S}$} & {$[2525-40-8]$} & \multicolumn{5}{|c|}{ phenyl prop-2-ynyl sulfone } \\
\hline & SUB & & $105 . \pm 2.5$ & & B & [1969MAC/STE, 1970COX/PIL] \\
\hline $\mathrm{C}_{9} \mathrm{H}_{8} \mathrm{O}_{3}$ & [129-64-6] & \multicolumn{5}{|c|}{ endo-5-norbornene-2,3-dicarboxylic anhydride } \\
\hline
\end{tabular}


TABLE 12. Phase change enthalpies of $\mathrm{C}_{9}$ organic compounds-Continued

\begin{tabular}{|c|c|c|c|c|c|c|}
\hline \multirow[b]{2}{*}{$\begin{array}{l}\text { Molecular } \\
\text { formula }\end{array}$} & \multirow{2}{*}{$\begin{array}{l}\text { CAS reg. no. } \\
\text { Transition }\end{array}$} & \multicolumn{5}{|l|}{ Compound } \\
\hline & & Temp. range $(\mathrm{K})$ & $\begin{array}{c}\Delta_{\text {trans }} H_{\mathrm{m}} \\
\left(\mathrm{kJ} \mathrm{mol}^{-1}\right)\end{array}$ & $T_{\mathrm{m}}(\mathrm{K})$ & Method & References \\
\hline & FUS & & 3.71 & 437.2 & DSC & [1967PIN/WIL] \\
\hline & SUB & & $97 \pm 4.2$ & 298 & MG & [1973ROG/QUA, 1977PED/RYL] \\
\hline \multirow{3}{*}{$\mathrm{C}_{9} \mathrm{H}_{8} \mathrm{O}_{3}$} & [3162-29-6] & 5-oxoethyl-1,3-ben & dioxole & & & \\
\hline & FUS & & 26.23 & 358.9 & DSC & [2007MAT/SOU] \\
\hline & SUB & & $104.4 \pm 2.2$ & 298 & $\mathrm{C}$ & [2007MAT/SOU] \\
\hline $\mathrm{C}_{9} \mathrm{H}_{8} \mathrm{O}_{3}$ & [29668-44-8] & 2,3-dihydro-1,4-be & odioxin-6-carb & xaldehyde & & \\
\hline \multirow{3}{*}{$\mathrm{C}_{9} \mathrm{H}_{8} \mathrm{O}_{3}$} & FUS (I) & & 30.5 & 395.3 & & \\
\hline & FUS (II) & & 30.5 & 368.0 & & \\
\hline & FUS (racemic) & & 26.5 & 366.6 & DSC & [2011NAV/MAC] \\
\hline \multirow[t]{2}{*}{$\mathrm{C}_{9} \mathrm{H}_{8} \mathrm{O}_{3}$} & [7400-08-0] & 3-(4-hydroxypheny & propenoic acid & p-coumaric ac & & \\
\hline & SUB & & $130.9 \pm 3.3$ & 298 & ME & [2012DAV/HER] \\
\hline \multirow[t]{3}{*}{$\mathrm{C}_{9} \mathrm{H}_{8} \mathrm{O}_{4}$} & [331-39-5] & 3,4-dihydroxycinna & ic acid (caffei & acid) & & \\
\hline & FUS & & 27.53 & 496.5 & DSC & [2010PAR/LEE] \\
\hline & SUB & $(409-424)$ & $170.2 \pm 4.6$ & 411 & ME & [2006CHE/OJA] \\
\hline \multirow[t]{2}{*}{$\mathrm{C}_{9} \mathrm{H}_{8} \mathrm{O}_{4}$} & [1679-64-7] & monomethyl tereph & Ialate & & & \\
\hline & FUS & & 39.73 & 493.7 & DSC & [2013ZHA/XIA] \\
\hline \multirow[t]{4}{*}{$\mathrm{C}_{9} \mathrm{H}_{8} \mathrm{O}_{4}$} & [4376-18-5] & monomethyl phthal & & & & \\
\hline & FUS & & 21.63 & 357.5 & DSC & [2005MON/SOU] \\
\hline & SUB & $(335-355)$ & $115.9 \pm 0.6$ & 345.3 & ME & [2005MON/SOU] \\
\hline & SUB & $(335-355)$ & $117.9 \pm 0.8$ & 298 & ME & [2005MON/SOU] \\
\hline \multirow[t]{4}{*}{$\mathrm{C}_{9} \mathrm{H}_{8} \mathrm{O}_{4}$} & [1877-71-0] & monomethyl isopht & alate & & & \\
\hline & FUS & & 36.5 & 466.7 & DSC & [2005MON/SOU] \\
\hline & SUB & $(359-379)$ & $122.6 \pm 0.7$ & 369.2 & ME & [2005MON/SOU] \\
\hline & SUB & $(359-379)$ & $125.6 \pm 1.0$ & 298 & ME & [2005MON/SOU] \\
\hline \multirow[t]{7}{*}{$\mathrm{C}_{9} \mathrm{H}_{8} \mathrm{O}_{4}$} & [50-78-2] & 2-acetoxybenzoic a & id (aspirin) & & & \\
\hline & FUS & & 33.51 & 408.65 & DSC & [2015MAT/MOR] \\
\hline & FUS & & 33.85 & 407.7 & DSC & [2015ALM/SOU] \\
\hline & FUS (I) & & 30.9 & 410 & & \\
\hline & FUS (II) & & 29.9 & 406 & DSC & [2012BAG/RED] \\
\hline & FUS & & 25.9 & 415.1 & DSC & [2011GOR/WOJ] \\
\hline & FUS & & U19.1 & 408.8 & DSC & [2010CAM/MIC] \\
\hline \multicolumn{7}{|c|}{ Note: The value reported in [2010CAM/MIC] is not in agreement with other independently measured values } \\
\hline & FUS & & 32.56 & 409.0 & DSC & [2010HAH/GRA] \\
\hline & FUS & & 29.17 & 409.2 & DSC & {$[2004 \mathrm{XU} / \mathrm{SUN} 2]$} \\
\hline & FUS & & 31.01 & 412.7 & DSC & [2001PER/BAU, 2003PER/BAU] \\
\hline & FUS & & 27.6 & 414 (Graph) & DSC & {$[2000 \mathrm{JOH} / \mathrm{PYK}]$} \\
\hline & FUS & & 29.8 & 414 & DSC & [2000KIR] \\
\hline & SUB & $(341-361)$ & $116.6 \pm 1.0$ & 351 & ME & [2015ALM/SOU] \\
\hline
\end{tabular}


TABLE 12. Phase change enthalpies of $\mathrm{C}_{9}$ organic compounds-Continued

\begin{tabular}{|c|c|c|c|c|c|c|}
\hline \multirow[b]{2}{*}{$\begin{array}{l}\text { Molecular } \\
\text { formula }\end{array}$} & \multirow{2}{*}{$\begin{array}{l}\text { CAS reg. no. } \\
\text { Transition }\end{array}$} & \multicolumn{5}{|l|}{ Compound } \\
\hline & & Temp. range $(\mathrm{K})$ & $\begin{array}{c}\Delta_{\text {trans }} H_{\mathrm{m}} \\
\left(\mathrm{kJ} \mathrm{mol}^{-1}\right)\end{array}$ & $T_{\mathrm{m}}(\mathrm{K})$ & Method & References \\
\hline \multirow[t]{2}{*}{$\mathrm{C}_{9} \mathrm{H}_{8} \mathrm{O}_{4}$} & $\begin{array}{c}\text { [6304-89-8] } \\
\text { FUS }\end{array}$ & \multicolumn{4}{|c|}{ 3-acetoxybenzoic acid } & {$[2015 \mathrm{ALM} / \mathrm{SOU}]$} \\
\hline & $\begin{array}{l}\text { SUB } \\
\text { SUB }\end{array}$ & $\begin{array}{l}(344-362) \\
(344-362)\end{array}$ & $\begin{array}{l}119.3 \pm 0.7 \\
121.1 \pm 0.7\end{array}$ & $\begin{array}{l}353 \\
298\end{array}$ & $\begin{array}{l}\text { ME } \\
\text { ME }\end{array}$ & $\begin{array}{l}{[2015 \mathrm{ALM} / \mathrm{SOU}]} \\
{[2015 \mathrm{ALM} / \mathrm{SOU}]}\end{array}$ \\
\hline \multirow[t]{2}{*}{$\mathrm{C}_{9} \mathrm{H}_{8} \mathrm{O}_{4}$} & $\begin{array}{c}\text { [2345-34-8] } \\
\text { FUS } \\
\text { FUS }\end{array}$ & 4-acetoxybenzoic a & $\begin{array}{c}30.5 \\
26.35\end{array}$ & $\begin{array}{l}464.8 \\
467.2\end{array}$ & $\begin{array}{l}\text { DSC } \\
\text { DSC }\end{array}$ & $\begin{array}{l}\text { [2010MON/SAN] } \\
\text { [1997YUA/ZHA] }\end{array}$ \\
\hline & $\begin{array}{c}\text { SUB } \\
\text { SUB(II) }\end{array}$ & $\begin{array}{l}(351-373) \\
(351-373)\end{array}$ & $\begin{array}{l}113.9 \pm 0.3 \\
116.1 \pm 0.3\end{array}$ & $\begin{array}{c}314.4 \\
298\end{array}$ & $\begin{array}{l}\mathrm{ME} \\
\mathrm{ME}\end{array}$ & $\begin{array}{l}{[2010 \mathrm{MON} / \mathrm{SAN}]} \\
{[2010 \mathrm{MON} / \mathrm{SAN}]}\end{array}$ \\
\hline \multirow[t]{2}{*}{$\mathrm{C}_{9} \mathrm{H}_{8} \mathrm{O}_{4}$} & $\begin{array}{c}{[2861-28-1]} \\
\text { FUS }\end{array}$ & \multicolumn{5}{|c|}{ 1,3-benzodioxole-5-acetic acid (homopiperonylic acid) } \\
\hline & $\begin{array}{l}\text { SUB } \\
\text { SUB }\end{array}$ & $\begin{array}{l}(346-364) \\
(346-364)\end{array}$ & $\begin{array}{l}120.1 \pm 0.8 \\
122.9 \pm 1.4\end{array}$ & $\begin{array}{l}355 \\
298\end{array}$ & $\begin{array}{l}\mathrm{ME} \\
\mathrm{ME}\end{array}$ & $\begin{array}{l}\text { [2004MAT/MON] } \\
{[2004 \mathrm{MAT} / \mathrm{MON}]}\end{array}$ \\
\hline $\mathrm{C}_{9} \mathrm{H}_{8} \mathrm{O}_{4}$ & $\begin{array}{c}{[3663-80-7]} \\
\text { SUB }\end{array}$ & \multicolumn{5}{|c|}{$(d l)$-1,4-benzodioxan-2-carboxylic acid } \\
\hline $\mathrm{C}_{9} \mathrm{H}_{8} \mathrm{O}_{4}$ & $\begin{array}{c}\text { [4316-23-8] } \\
\text { FUS }\end{array}$ & \multicolumn{5}{|c|}{ 4-methylphthalic acid } \\
\hline $\mathrm{C}_{9} \mathrm{H}_{9} \mathrm{BrO}_{3}$ & $\begin{array}{l}\text { [32019-08-2] } \\
\text { FUS }\end{array}$ & \multicolumn{5}{|c|}{$(d l)-2-(p$-bromophenoxy)propanoic acid } \\
\hline $\mathrm{C}_{9} \mathrm{H}_{9} \mathrm{BrO}_{3}$ & FUS & \multicolumn{5}{|c|}{$(d)-2-(p$-bromophenoxy)propanoic acid } \\
\hline $\mathrm{C}_{9} \mathrm{H}_{9} \mathrm{BrO}_{3}$ & $\begin{array}{c}{[40620-67-5]} \\
\text { FUS }\end{array}$ & \multicolumn{5}{|c|}{$(d l)-3-(m$-bromophenyl)-3-hydroxypropanoic acid } \\
\hline $\mathrm{C}_{9} \mathrm{H}_{9} \mathrm{BrO}_{3}$ & $\begin{array}{c}{[40620-57-3]} \\
\text { FUS }\end{array}$ & $(d)-3$-( $m$-bromoph & $\begin{array}{l}\text { hyl)-3-hydrox } \\
23.85\end{array}$ & $\begin{array}{c}\text { panoic ac } \\
350\end{array}$ & & {$[1991 \mathrm{CHI} / \mathrm{BRA}]$} \\
\hline $\mathrm{C}_{9} \mathrm{H}_{9} \mathrm{BrO}_{3}$ & $\begin{array}{l}\text { [40620-68-6] } \\
\text { FUS }\end{array}$ & \multicolumn{4}{|c|}{$(d l)$-3-( $p$-bromophenyl)-3-hydroxypropanoic acid } & {$[1991 \mathrm{CHI} / \mathrm{BRA}]$} \\
\hline $\mathrm{C}_{9} \mathrm{H}_{9} \mathrm{BrO}_{3}$ & $\begin{array}{c}{[40620-58-4]} \\
\text { FUS }\end{array}$ & \multicolumn{5}{|c|}{ (d)-3-(p-bromophenyl)-3-hydroxypropanoic acid } \\
\hline $\mathrm{C}_{9} \mathrm{H}_{9} \mathrm{ClO}_{3}$ & $\begin{array}{c}\text { [25140-86-7] } \\
\text { FUS }\end{array}$ & \multicolumn{5}{|c|}{$(d l)$-2-(o-chlorophenoxy)propanoic acid } \\
\hline $\mathrm{C}_{9} \mathrm{H}_{9} \mathrm{ClO}_{3}$ & FUS & \multicolumn{5}{|c|}{$(d)-2-(o$-chlorophenoxy)propanoic acid } \\
\hline $\mathrm{C}_{9} \mathrm{H}_{9} \mathrm{ClO}_{3}$ & $\begin{array}{l}\text { [101-10-0] } \\
\text { FUS }\end{array}$ & $(d l)-2-(m$-chloroph & \multicolumn{3}{|c|}{$(d l)-2-(m$-chlorophenoxy)propanoic acid } & {$[1991 \mathrm{CHI} / \mathrm{BRA}]$} \\
\hline $\mathrm{C}_{9} \mathrm{H}_{9} \mathrm{ClO}_{3}$ & FUS & \multicolumn{4}{|c|}{$(d)-2-(m$-chlorophenoxy)propanoic acid } & {$[1991 \mathrm{CHI} / \mathrm{BRA}]$} \\
\hline $\mathrm{C}_{9} \mathrm{H}_{9} \mathrm{ClO}_{3}$ & FUS & \multicolumn{5}{|c|}{$(d l)-3-(p$-chlorophenyl)-3-hydroxypropanoic acid } \\
\hline $\mathrm{C}_{9} \mathrm{H}_{9} \mathrm{ClO}_{3}$ & $\begin{array}{l}{[40620-55-1]} \\
\text { FUS }\end{array}$ & \multicolumn{5}{|c|}{ (d)-3-( $p$-chlorophenyl)-3-hydroxypropanoic acid } \\
\hline $\mathrm{C}_{9} \mathrm{H}_{9} \mathrm{ClO}_{3}$ & $\begin{array}{c}\text { [40620-64-2] } \\
\text { FUS }\end{array}$ & \multicolumn{5}{|c|}{$(d l)-3-(m$-chlorophenyl)-3-hydroxypropanoic acid } \\
\hline $\mathrm{C}_{9} \mathrm{H}_{9} \mathrm{ClO}_{3}$ & $\begin{array}{c}\text { [40620-54-0] } \\
\text { FUS }\end{array}$ & \multicolumn{5}{|c|}{ (d)-3-(m-chlorophenyl)-3-hydroxypropanoic acid } \\
\hline $\mathrm{C}_{9} \mathrm{H}_{9} \mathrm{ClO}_{3}$ & $\begin{array}{l}\text { [94-74-6] } \\
\text { FUS }\end{array}$ & (4-chloro- $o$-tolylox & $\begin{array}{c}\text { acetic acid } \\
29.98\end{array}$ & 392.9 & $\mathrm{DSC}$ & [1991ACR, 1990DON/DRE] \\
\hline $\mathrm{C}_{9} \mathrm{H}_{9} \mathrm{ClO}_{4}$ & $\begin{array}{c}{[76341-69-0]} \\
\mathrm{V}\end{array}$ & $\begin{array}{l}\text { 2-chlorosyringalde } \\
(293-323)\end{array}$ & 77.7 & 308 & CGC & [1999LEI/WAN2] \\
\hline
\end{tabular}


TABLE 12. Phase change enthalpies of $\mathrm{C}_{9}$ organic compounds-Continued

\begin{tabular}{|c|c|c|c|c|c|c|}
\hline \multirow[b]{2}{*}{$\begin{array}{l}\text { Molecular } \\
\text { formula }\end{array}$} & \multirow{2}{*}{$\begin{array}{l}\text { CAS reg. no. } \\
\text { Transition }\end{array}$} & \multicolumn{5}{|l|}{ Compound } \\
\hline & & Temp. range $(\mathrm{K})$ & $\begin{array}{c}\Delta_{\text {trans }} H_{\mathrm{m}} \\
\left(\mathrm{kJ} \mathrm{mol}^{-1}\right)\end{array}$ & $T_{\mathrm{m}}(\mathrm{K})$ & Method & References \\
\hline $\mathrm{C}_{9} \mathrm{H}_{9} \mathrm{Cl}_{2} \mathrm{NO}$ & $\begin{array}{l}\text { [709-98-8] } \\
\text { FUS }\end{array}$ & 3', 4'-dichloropropi & $\begin{array}{l}\text { anilide } \\
18.26\end{array}$ & 363.7 & DSC & [1991ACR, 1990DON/DRE] \\
\hline $\mathrm{C}_{9} \mathrm{H}_{9} \mathrm{FO}_{3}$ & $\begin{array}{c}\text { [40620-61-9] } \\
\text { FUS }\end{array}$ & $(d l)-3-(m$-fluoroph & $\begin{array}{l}\text { yl)-3-hydroxy } \\
20.5\end{array}$ & $\begin{array}{l}\text { anoic acid } \\
290\end{array}$ & & [1991CHI/BRA] \\
\hline $\mathrm{C}_{9} \mathrm{H}_{9} \mathrm{FO}_{3}$ & $\begin{array}{l}\text { [40620-51-7] } \\
\text { FUS }\end{array}$ & (d)-3-(m-fluoroph & $\begin{array}{c}\text { yl)-3-hydroxy } \\
24.27\end{array}$ & $\begin{array}{l}\text { anoic acid } \\
311\end{array}$ & & [1991CHI/BRA] \\
\hline $\mathrm{C}_{9} \mathrm{H}_{9} \mathrm{FO}_{3}$ & FUS & $(d l)$-3-(o-fluorophe & $\begin{array}{l}\text { 1)-3-hydroxy } \\
27.2\end{array}$ & $\begin{array}{l}\text { anoic acid } \\
\quad 342\end{array}$ & & {$[1991 \mathrm{CHI} / \mathrm{BRA}]$} \\
\hline $\mathrm{C}_{9} \mathrm{H}_{9} \mathrm{FO}_{3}$ & $\begin{array}{l}{[40620-50-6]} \\
\text { FUS }\end{array}$ & (d)-3-(o-fluorophe & $\begin{array}{l}\text { 1)-3-hydroxy1 } \\
22.59\end{array}$ & $\begin{array}{l}\text { anoic acid } \\
348\end{array}$ & & [1991CHI/BRA] \\
\hline $\mathrm{C}_{9} \mathrm{H}_{9} \mathrm{FO}_{3}$ & $\begin{array}{l}\text { [40620-62-0] } \\
\text { FUS }\end{array}$ & $(d l)-3$-(p-fluoroph & $\begin{array}{c}\text { y)-3-hydroxy } \\
27.61\end{array}$ & $\begin{array}{l}\text { anoic acid } \\
\quad 362\end{array}$ & & [1991CHI/BRA] \\
\hline $\mathrm{C}_{9} \mathrm{H}_{9} \mathrm{FO}_{3}$ & FUS & $(d)$-3-(p-fluorophe & $\begin{array}{l}\text { 1)-3-hydroxy } \\
30.96\end{array}$ & $\begin{array}{l}\text { anoic acid } \\
381\end{array}$ & & {$[1991 \mathrm{CHI} / \mathrm{BRA}]$} \\
\hline $\mathrm{C}_{9} \mathrm{H}_{9} \mathrm{~F}_{6} \mathrm{NO}_{5}$ & $\begin{array}{c}{[1548-45-4]} \\
V\end{array}$ & $\begin{array}{c}\text { (l) } \mathrm{N}, \mathrm{O} \text {-bis(trifluor } \\
(323-413)\end{array}$ & $\begin{array}{l}\text { cetal)-threoni } \\
72.5\end{array}$ & $\begin{array}{l}\text { ethyl ester } \\
338\end{array}$ & A & [1987STE/MAL, 1999DYK/SVO, 1960WEY/KLI] \\
\hline $\mathrm{C}_{9} \mathrm{H}_{9} \mathrm{~N}$ & $\begin{array}{c}{[603-76-9]} \\
\text { FUS }\end{array}$ & 1-methylindole & 10.9 & 276.5 & DSC & [2014ALM/MON2] \\
\hline & SUB & $(267-275)$ & $73.9 \pm 0.3$ & 298 & Static & [2014ALM/MON2] \\
\hline & $\begin{array}{l}\text { V } \\
\text { V } \\
\text { V } \\
\text { V }\end{array}$ & $\begin{array}{l}(269-342) \\
(296-338)\end{array}$ & $\begin{array}{l}61.8 \pm 0.1 \\
64.3 \pm 0.4 \\
61.1 \pm 3.1 \\
62.2 \pm 1.6\end{array}$ & $\begin{array}{l}298 \\
298 \\
298 \\
298\end{array}$ & $\begin{array}{l}\text { Static } \\
\text { GS } \\
\text { CGC } \\
\text { C }\end{array}$ & $\begin{array}{l}\text { [2014ALM/MON2] } \\
\text { [2011 VER/EME3] } \\
\text { [2010LIP/PLI] } \\
\text { [2009RIB/CAB2] }\end{array}$ \\
\hline $\mathrm{C}_{9} \mathrm{H}_{9} \mathrm{~N}$ & $\begin{array}{l}{[95-20-5]} \\
\text { FUS }\end{array}$ & 2-methylindole & 15.72 & 329.4 & & [1997PEY/LET] \\
\hline & SUB & & $88.7 \pm 2.4$ & 298 & $\mathrm{C}$ & [2009RIB/CAB2] \\
\hline $\mathrm{C}_{9} \mathrm{H}_{9} \mathrm{~N}$ & $\begin{array}{l}{[83-34-1]} \\
\text { SUB } \\
\text { SUB }\end{array}$ & $\begin{array}{c}\text { 3-methylindole (sk } \\
\text { (288-333) }\end{array}$ & $\begin{array}{l}90.4 \pm 1.9 \\
83.3\end{array}$ & $\begin{array}{l}298 \\
303\end{array}$ & $\begin{array}{l}\mathrm{C} \\
\mathrm{A}\end{array}$ & $\begin{array}{l}\text { [2009RIB/CAB2] } \\
\text { [1987STE/MAL] }\end{array}$ \\
\hline & $\mathrm{V}$ & $(368-540)$ & 64.5 & 383 & A & [1987STE/MAL, 1947STU] \\
\hline $\mathrm{C}_{9} \mathrm{H}_{9} \mathrm{~N}$ & $\begin{array}{c}{[1823-91-2]} \\
\mathrm{V} \\
\mathrm{V}\end{array}$ & $\begin{array}{c}\alpha \text {-methylbenzylcy } \\
(284-318) \\
(284-318)\end{array}$ & $\begin{array}{l}\text { ide } \\
60.8 \pm 0.7 \\
60.9 \pm 0.7\end{array}$ & $\begin{array}{l}301 \\
298\end{array}$ & $\begin{array}{l}\text { GS } \\
\text { GS }\end{array}$ & $\begin{array}{l}\text { [2000VER] } \\
{[2000 \mathrm{VER}]}\end{array}$ \\
\hline $\mathrm{C}_{9} \mathrm{H}_{9} \mathrm{~N}$ & $\begin{array}{c}\text { [21789-36-6] } \\
\text { SUB }\end{array}$ & 2,6-dimethylbenzo & $\begin{array}{l}\text { rile } \\
83.9 \pm 2.8\end{array}$ & 298 & $\mathrm{C}$ & [1991ACR/TUC] \\
\hline $\mathrm{C}_{9} \mathrm{H}_{9} \mathrm{NO}$ & $\begin{array}{c}{[5676-58-4]} \\
\mathrm{V}\end{array}$ & 2,5-dimethylbenzo & $\begin{array}{l}\text { zole } \\
60.1 \pm 1.3\end{array}$ & 298 & $\mathrm{C}$ & [2013SIL/CIM3] \\
\hline $\mathrm{C}_{9} \mathrm{H}_{9} \mathrm{NO}_{2}$ & $\begin{array}{c}{[122-85-0]} \\
\text { SUB }\end{array}$ & $\begin{array}{c}\text { 4-acetomidobenzal } \\
(328-346)\end{array}$ & $\begin{array}{l}\text { hyde } \\
999.0\end{array}$ & 337 & A & [1987STE/MAL, 1960AIH2] \\
\hline $\mathrm{C}_{9} \mathrm{H}_{9} \mathrm{NO}_{3}$ & $\begin{array}{l}{[89-52-1]} \\
\text { FUS } \\
\text { FUS }\end{array}$ & 2-(acetylamino)ber & $\begin{array}{c}\text { oic acid } \\
29.32 \\
49.4\end{array}$ & $\begin{array}{l}455.1 \\
458.4\end{array}$ & $\begin{array}{l}\text { DSC } \\
\text { DSC }\end{array}$ & $\begin{array}{l}\text { [2015ALM/SOU] } \\
\text { [2014MAN/VOR] }\end{array}$ \\
\hline & $\begin{array}{l}\text { SUB } \\
\text { SUB } \\
\text { SUB }\end{array}$ & $\begin{array}{l}(367-389) \\
(367-389) \\
(345-393)\end{array}$ & $\begin{array}{c}124.8 \pm 1.3 \\
127.6 \pm 1.3 \\
116 \pm 1\end{array}$ & $\begin{array}{l}378 \\
298 \\
298\end{array}$ & $\begin{array}{l}\mathrm{ME} \\
\mathrm{ME} \\
\mathrm{GS}\end{array}$ & $\begin{array}{l}\text { [2015ALM/SOU] } \\
\text { [2015ALM/SOU] } \\
\text { [2014MAN/VOR] }\end{array}$ \\
\hline $\mathrm{C}_{9} \mathrm{H}_{9} \mathrm{NO}_{3}$ & $\begin{array}{c}{[587-48-4]} \\
\text { FUS } \\
\text { FUS }\end{array}$ & 3-(acetylamino)ber & $\begin{array}{c}\text { oic acid } \\
42.28 \\
39.4\end{array}$ & $\begin{array}{l}522.2 \\
518.2\end{array}$ & $\begin{array}{l}\mathrm{DSC} \\
\mathrm{DSC}\end{array}$ & $\begin{array}{l}\text { [2015ALM/SOU] } \\
\text { [2014MAN/VOR] }\end{array}$ \\
\hline & $\begin{array}{l}\text { SUB } \\
\text { SUB }\end{array}$ & $\begin{array}{l}(423-441) \\
(423-441)\end{array}$ & $\begin{array}{l}152.6 \pm 1.3 \\
157.3 \pm 1.3\end{array}$ & $\begin{array}{l}432 \\
298\end{array}$ & $\begin{array}{l}\mathrm{ME} \\
\mathrm{ME}\end{array}$ & $\begin{array}{l}\text { [2015ALM/SOU] } \\
{[2015 \mathrm{ALM} / \mathrm{SOU}]}\end{array}$ \\
\hline
\end{tabular}


TABLE 12. Phase change enthalpies of $\mathrm{C}_{9}$ organic compounds-Continued

\begin{tabular}{|c|c|c|c|c|c|c|}
\hline \multirow[b]{2}{*}{$\begin{array}{l}\text { Molecular } \\
\text { formula }\end{array}$} & \multirow{2}{*}{$\begin{array}{l}\text { CAS reg. no. } \\
\text { Transition }\end{array}$} & \multicolumn{5}{|l|}{ Compound } \\
\hline & & Temp. range $(\mathrm{K})$ & $\begin{array}{c}\Delta_{\text {trans }} H_{\mathrm{m}} \\
\left(\mathrm{kJ} \mathrm{mol}^{-1}\right)\end{array}$ & $T_{\mathrm{m}}(\mathrm{K})$ & Method & References \\
\hline & SUB & $(407-443)$ & $137 \pm 1$ & 298 & GS & [2014MAN/VOR] \\
\hline $\mathrm{C}_{9} \mathrm{H}_{9} \mathrm{NO}_{3}$ & $\begin{array}{l}\text { [556-08-1] } \\
\text { FUS } \\
\text { FUS }\end{array}$ & 4-(acetylamino)ber & $\begin{array}{r}\text { oic acid } \\
34.2 \\
42.4\end{array}$ & $\begin{array}{l}535.3 \\
531.7\end{array}$ & $\begin{array}{l}\text { DSC } \\
\text { DSC }\end{array}$ & $\begin{array}{l}\text { [2014MAN/VOR] } \\
\text { [2010MON/SAN] }\end{array}$ \\
\hline & $\begin{array}{l}\text { SUB } \\
\text { SUB } \\
\text { SUB }\end{array}$ & $\begin{array}{l}(395-440) \\
(423-443) \\
(423-443)\end{array}$ & $\begin{array}{c}137 \pm 1 \\
144.2 \pm 1.1 \\
148.9 \pm 1.1\end{array}$ & $\begin{array}{l}298 \\
325 \\
298\end{array}$ & $\begin{array}{l}\text { GS } \\
\text { ME } \\
\text { ME }\end{array}$ & $\begin{array}{l}\text { [2014MAN/VOR, 2014MAN/VOR2] } \\
\text { [2010MON/SAN] } \\
{[2010 \mathrm{MON} / \mathrm{SAN}]}\end{array}$ \\
\hline $\mathrm{C}_{9} \mathrm{H}_{9} \mathrm{NO}_{4}$ & $\begin{array}{c}{[618-98-4]} \\
\mathrm{V}\end{array}$ & $\begin{array}{l}\text { 3-nitrobenzoic acid } \\
(381-571)\end{array}$ & $\begin{array}{c}\text { ethyl ester } \\
65.1\end{array}$ & 396 & A & [1987STE/MAL, 1947STU] \\
\hline $\mathrm{C}_{9} \mathrm{H}_{9} \mathrm{NO}_{4}$ & $\begin{array}{c}{[5251-93-4]} \\
\text { FUS }\end{array}$ & [(benzoylamino)ox & $\begin{array}{c}\text { acetic acid } \\
31.46\end{array}$ & 416.9 & DSC & [1991ACR, 1990DON/DRE] \\
\hline $\mathrm{C}_{9} \mathrm{H}_{9} \mathrm{NO}_{4}$ & $\begin{array}{c}{[5453-67-8]} \\
\text { SUB }\end{array}$ & dimethyl pyridine- & $\begin{array}{l}\text { 5-dicarboxylate } \\
113.5 \pm 3.8\end{array}$ & 298 & $\mathrm{C}$ & [2005MAT/MOR] \\
\hline $\mathrm{C}_{9} \mathrm{H}_{9} \mathrm{NO}_{5}$ & FUS & $(d l)-2-(p$-nitropher & $\begin{array}{l}\text { xy)propanoic a } \\
32.22\end{array}$ & 411.4 & & [1991CHI/BRA] \\
\hline $\mathrm{C}_{9} \mathrm{H}_{9} \mathrm{NO}_{5}$ & FUS & $(d)-2-(p$-nitrophen & $\begin{array}{l}\text { xy)propanoic ac } \\
20.92\end{array}$ & 362 & & [1991CHI/BRA] \\
\hline $\mathrm{C}_{9} \mathrm{H}_{9} \mathrm{NS}$ & $\begin{array}{l}{[95-26-1]} \\
\text { FUS }\end{array}$ & 2,5-dimethylbenzo & $\begin{array}{l}\text { iazole } \\
18.3\end{array}$ & 313.0 & $\mathrm{DSC}$ & [2014SIL/CIM] \\
\hline & SUB & & $84.9 \pm 1.5$ & 298 & $\mathrm{C}$ & [2014SIL/CIM] \\
\hline $\mathrm{C}_{9} \mathrm{H}_{9} \mathrm{~N}_{3} \mathrm{O}_{2}$ & $\begin{array}{c}\text { [107023-66-5] } \\
\text { FUS }\end{array}$ & 2-[methyl(4-nitrop] & $\begin{array}{l}\text { nyl)amino]acet } \\
22.96\end{array}$ & $\begin{array}{l}\text { trile } \\
388.8\end{array}$ & DSC & {$[2006 \mathrm{GAO} / \mathrm{CHE}]$} \\
\hline $\mathrm{C}_{9} \mathrm{H}_{9} \mathrm{~N}_{3} \mathrm{O}_{2} \mathrm{~S}_{2}$ & $\begin{array}{l}\text { [72-14-0] } \\
\text { FUS } \\
\text { FUS } \\
\text { FUS }\end{array}$ & 4-amino- $N$-2-thiaz & $\begin{array}{l}\text { ylbenzenesulfo } \\
27.4 \\
30.3 \\
\\
24.1\end{array}$ & $\begin{array}{c}\text { nide (sulf } \\
474.2 \\
473 \\
\\
473.4\end{array}$ & $\begin{array}{l}\text { zole) } \\
\text { DSC } \\
\text { DSC }\end{array}$ & $\begin{array}{l}\text { [2015GAU/VAN] } \\
\text { [2003MAR/AVI, 2002MAR/GOM, } \\
\text { 2001MAR/GOM] } \\
\text { [1983KHA] }\end{array}$ \\
\hline $\mathrm{C}_{9} \mathrm{H}_{9} \mathrm{~N}_{3} \mathrm{O}_{6}$ & $\begin{array}{c}{[602-96-0]} \\
\text { SUB }\end{array}$ & $\begin{array}{c}\text { 2,4,6-trinitromesity } \\
(319-397)\end{array}$ & $103.6 \pm 1.2$ & & $\mathrm{ME}$ & [1987STE/MAL, 1978CUN/PAL] \\
\hline $\mathrm{C}_{9} \mathrm{H}_{10}$ & $\begin{array}{c}\text { [496-11-7] } \\
\text { TRS } \\
\text { FUS } \\
\text { FUS } \\
\text { V } \\
\text { V } \\
\text { V } \\
\text { V } \\
\text { V } \\
\text { V } \\
\text { V } \\
\text { V } \\
\text { V } \\
\text { V } \\
\text { V } \\
\text { V } \\
\text { V } \\
\text { V } \\
\text { V }\end{array}$ & $\begin{array}{l}\text { indane } \\
\qquad \begin{array}{c}(5-445) \\
(5-445) \\
(15-320) \\
(338-495) \\
(338-495) \\
(338-495) \\
(338-495) \\
(338-495) \\
(338-495) \\
(284-338) \\
(374-466) \\
(382-451) \\
(382-451) \\
(382-451) \\
(382-451) \\
(382-451) \\
(382-451) \\
(355-482)\end{array}\end{array}$ & $\begin{array}{l}0.15 \\
8.55 \\
8.6 \\
48.9 \pm 0.2 \\
46.3 \pm 0.2 \\
44.0 \pm 0.2 \\
41.7 \pm 0.2 \\
39.1 \pm 0.4 \\
37.8 \pm 0.5 \\
49.0 \pm 0.2 \\
44.0 \\
43.9 \pm 0.1 \\
41.9 \pm 0.1 \\
41.3 \pm 0.1 \\
40.4 \pm 0.1 \\
39.6 \pm 0.1 \\
49.0 \\
45.0\end{array}$ & $\begin{array}{l}73.83 \\
221.8 \\
221.8 \\
298 \\
340 \\
380 \\
420 \\
460 \\
480 \\
298 \\
389 \\
382 \\
415 \\
424 \\
440 \\
451 \\
298 \\
370\end{array}$ & $\begin{array}{l}\text { EB } \\
\text { EB } \\
\text { EB } \\
\text { EB } \\
\text { EB } \\
\text { EB } \\
\text { GS } \\
\text { A } \\
C \\
C \\
C \\
C \\
C \\
C\end{array}$ & $\begin{array}{l}\text { [2016CHI/STE] } \\
{[1996 \mathrm{DOM} / \mathrm{HEA}, 1961 \mathrm{STU} / \mathrm{SIN}]} \\
{[2016 \mathrm{CHI} / \mathrm{STE}]} \\
{[2016 \mathrm{CHI} / \mathrm{STE}]} \\
{[2016 \mathrm{CHI} / \mathrm{STE}]} \\
{[2016 \mathrm{CHI} / \mathrm{STE}]} \\
{[2016 \mathrm{CHI} / \mathrm{STE}]} \\
{[2016 \mathrm{CHI} / \mathrm{STE}]} \\
{[2011 \mathrm{VER} / \mathrm{EME}]} \\
{[1987 \mathrm{STE} / \mathrm{MAL}]} \\
{[1981 \mathrm{HOS} / \mathrm{SCO} 3]} \\
{[1981 \mathrm{HOS} / \mathrm{SCO} 3]} \\
{[1981 \mathrm{HOS} / \mathrm{SCO} 3]} \\
{[1981 \mathrm{HOS} / \mathrm{SCO} 3]} \\
{[1981 \mathrm{HOS} / \mathrm{SCO} 3]} \\
{[1981 \mathrm{HOS} / \mathrm{SCO} 3]} \\
{[1976 \mathrm{AMB} / \mathrm{SPR}]}\end{array}$ \\
\hline $\mathrm{C}_{9} \mathrm{H}_{10}$ & $\begin{array}{c}{[611-15-4]} \\
V\end{array}$ & $\begin{array}{l}\text { 2-methylstyrene } \\
(305-385)\end{array}$ & 47.9 & 320 & A & [1987STE/MAL, 1953CLE/WIS] \\
\hline $\mathrm{C}_{9} \mathrm{H}_{10}$ & $\begin{array}{c}{[100-80-1]} \\
\mathrm{V}\end{array}$ & $\begin{array}{l}\text { 3-methylstyrene } \\
\qquad(314-385)\end{array}$ & 47.5 & 329 & A & [1987STE/MAL, 1953CLE/WIS] \\
\hline $\mathrm{C}_{9} \mathrm{H}_{10}$ & $\begin{array}{c}{[622-97-9]} \\
\mathrm{V}\end{array}$ & $\begin{array}{l}\text { 4-methylstyrene } \\
\qquad(304-390)\end{array}$ & 47.6 & 319 & A & [1987STE/MAL, 1953CLE/WIS] \\
\hline
\end{tabular}


TABLE 12. Phase change enthalpies of $\mathrm{C}_{9}$ organic compounds-Continued

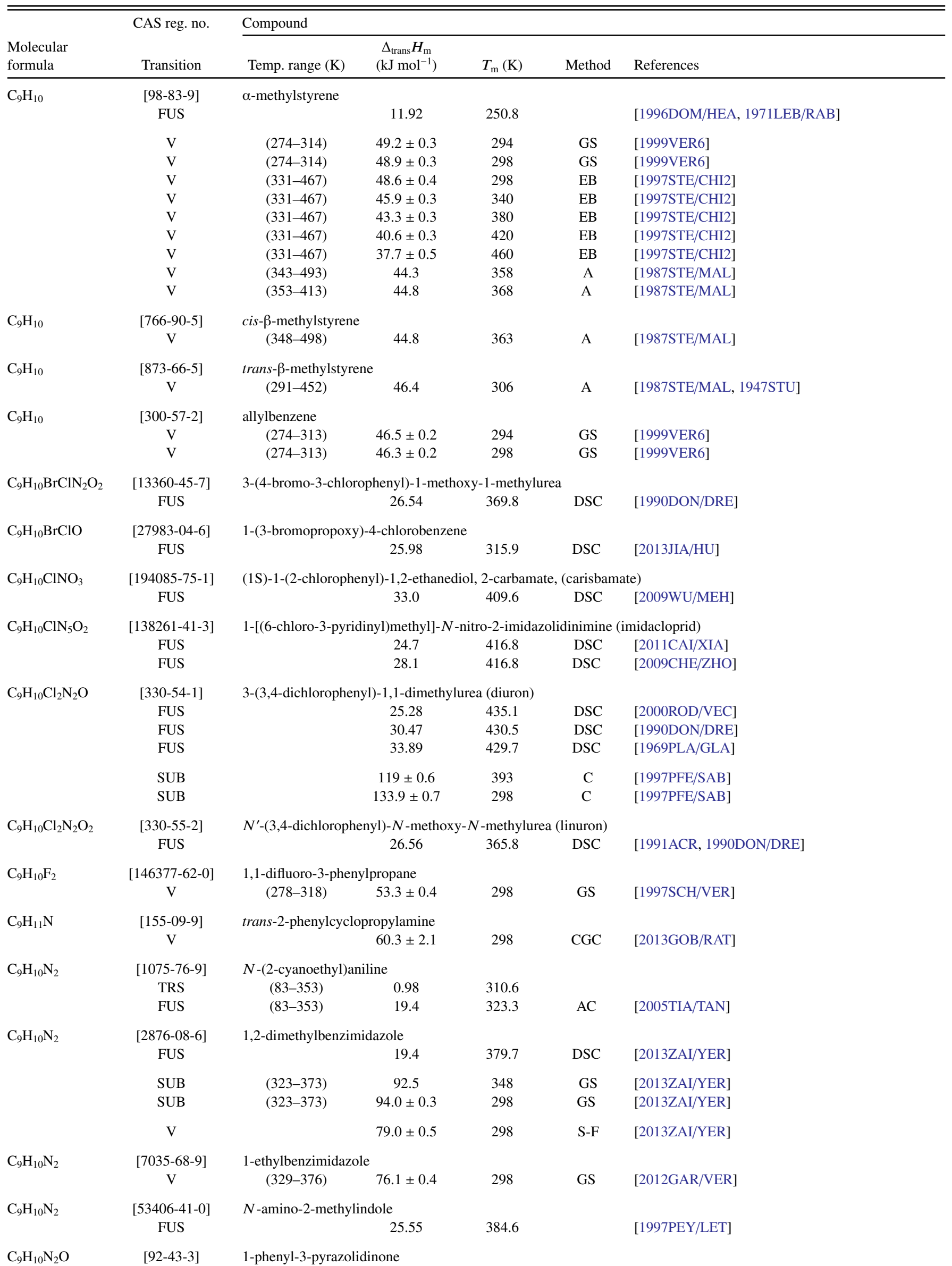


TABLE 12. Phase change enthalpies of $\mathrm{C}_{9}$ organic compounds-Continued

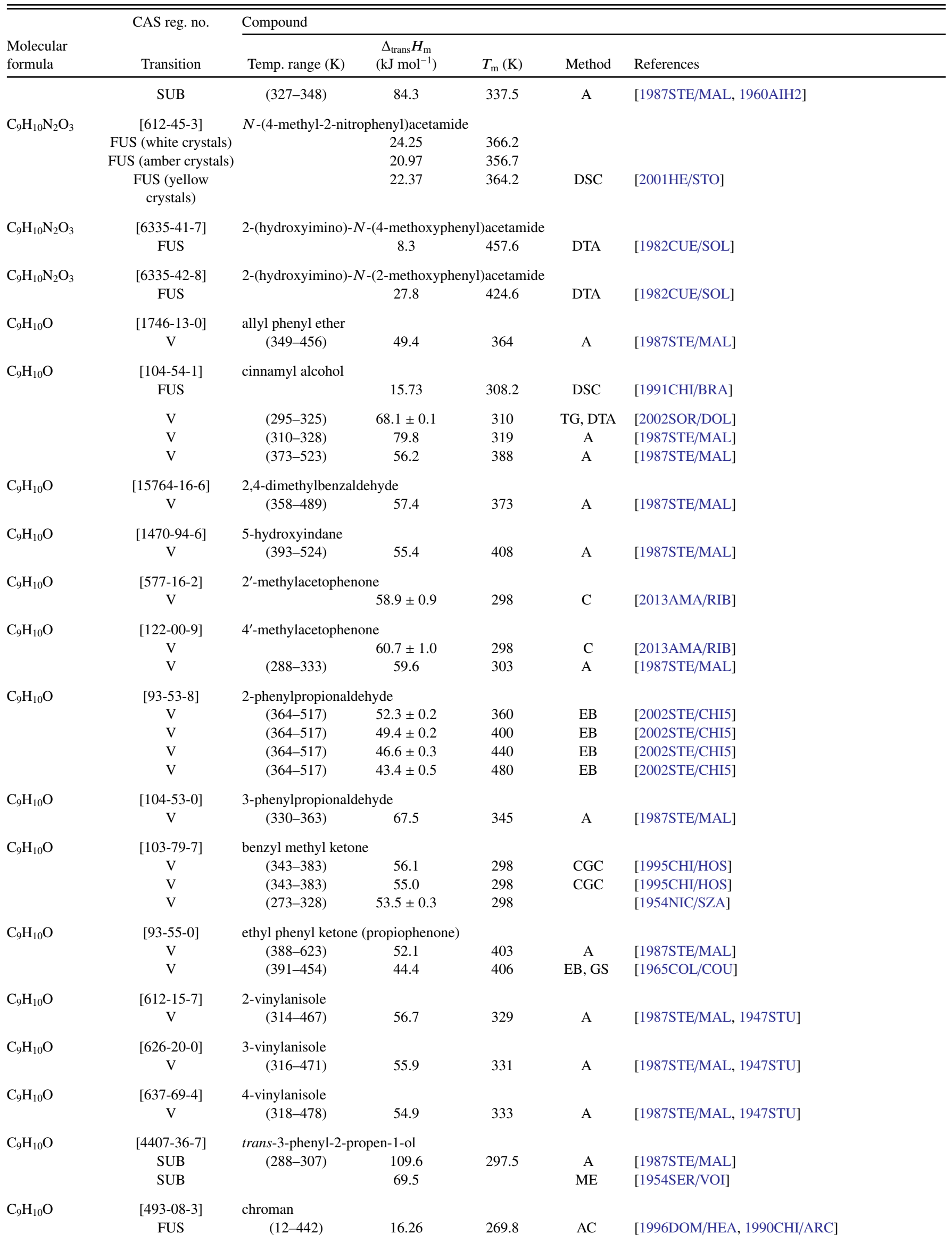


TABLE 12. Phase change enthalpies of $\mathrm{C}_{9}$ organic compounds-Continued

\begin{tabular}{|c|c|c|c|c|c|c|}
\hline \multirow[b]{2}{*}{$\begin{array}{l}\text { Molecular } \\
\text { formula }\end{array}$} & \multirow{2}{*}{$\begin{array}{l}\text { CAS reg. no. } \\
\text { Transition }\end{array}$} & \multicolumn{5}{|l|}{ Compound } \\
\hline & & Temp. range $(\mathrm{K})$ & $\begin{array}{c}\Delta_{\text {trans }} H_{\mathrm{m}} \\
\left(\mathrm{kJ} \mathrm{mol}^{-1}\right)\end{array}$ & $T_{\mathrm{m}}(\mathrm{K})$ & Method & References \\
\hline & $\mathrm{V}$ & $(293-535)$ & $55.2 \pm 0.1$ & 320 & IPM, EB & [1990CHI/ARC] \\
\hline & $\mathrm{V}$ & $(293-535)$ & $52.7 \pm 0.1$ & 360 & IPM, EB & [1990CHI/ARC] \\
\hline & $\mathrm{V}$ & $(293-535)$ & $50.2 \pm 0.1$ & 400 & IPM, EB & [1990CHI/ARC] \\
\hline & $\mathrm{V}$ & $(293-535)$ & $42.5 \pm 0.5$ & 520 & IPM, EB & [1990CHI/ARC] \\
\hline \multirow[t]{5}{*}{$\mathrm{C}_{9} \mathrm{H}_{10} \mathrm{O}$} & {$[493-05-0]$} & isochroman & & & & \\
\hline & FUS & $(12-440)$ & 16.75 & 277.5 & $\mathrm{AC}$ & [1996DOM/HEA, 1990CHI/ARC] \\
\hline & $\mathrm{V}$ & $(295-536)$ & $57.1 \pm 0.1$ & 298 & IPM, EB & [1990CHI/ARC] \\
\hline & $\mathrm{V}$ & $(295-536)$ & $55.6 \pm 0.1$ & 320 & IPM, EB & [1990CHI/ARC] \\
\hline & $\mathrm{V}$ & $(295-536)$ & $42.6 \pm 0.5$ & 520 & IPM, EB & [1990CHI/ARC] \\
\hline \multirow[t]{4}{*}{$\mathrm{C}_{9} \mathrm{H}_{10} \mathrm{O}_{2}$} & [89-71-4] & methyl $o$-toluate & & & & \\
\hline & FUS & $(5-320)$ & 12.47 & 228.8 & $\mathrm{AC}$ & [2002BLO/PAU] \\
\hline & FUS & & 12.5 & 228.8 & $\mathrm{AC}$ & [1998MAK/KAB] \\
\hline & $\mathrm{V}$ & & $57.3 \pm 0.2$ & 293 & $\mathrm{C}$ & [1998MAK/KAB] \\
\hline \multirow[t]{5}{*}{$\mathrm{C}_{9} \mathrm{H}_{10} \mathrm{O}_{2}$} & [99-36-5] & methyl $m$-toluate & & & & \\
\hline & FUS & $(5-320)$ & 17.14 & 270.6 & $\mathrm{AC}$ & [2002BLO/PAU] \\
\hline & FUS & & 21.15 & 269.9 & $\mathrm{AC}$ & [1998MAK/KAB] \\
\hline & $\mathrm{V}$ & & $60.3 \pm 0.2$ & 296 & $\mathrm{C}$ & [1998MAK/KAB] \\
\hline & $\mathrm{V}$ & $(359-500)$ & 54.8 & 374 & A & [1987STE/MAL] \\
\hline \multirow{4}{*}{$\mathrm{C}_{9} \mathrm{H}_{10} \mathrm{O}_{2}$} & SUB & & $83.3 \pm 0.3$ & 298 & $\mathrm{C}$ & [1998MAK/KAB] \\
\hline & SUB & $(403-493)$ & 51.6 & 418 & & [1998SEM/WIL] \\
\hline & $\mathrm{V}$ & $(293-357)$ & $57.8 \pm 0.1$ & 325 & Static & [2011ALM/MON] \\
\hline & $\mathrm{V}$ & $(293-357)$ & $59.8 \pm 0.2$ & 298 & Static & [2011ALM/MON] \\
\hline $\mathrm{C}_{9} \mathrm{H}_{10} \mathrm{O}_{2}$ & $\begin{array}{l}\text { [122-46-3] } \\
\text { SUB }\end{array}$ & $\begin{array}{c}\text { acetic acid, 3-tolyl } \\
(274-317)\end{array}$ & 60.7 & 295 & $\mathrm{TE}$ & [1947BAL, 1960JON] \\
\hline
\end{tabular}

[Note: The above value is likely an enthalpy of vaporization as the melting point temperature is listed as $285 \mathrm{~K}$ ]

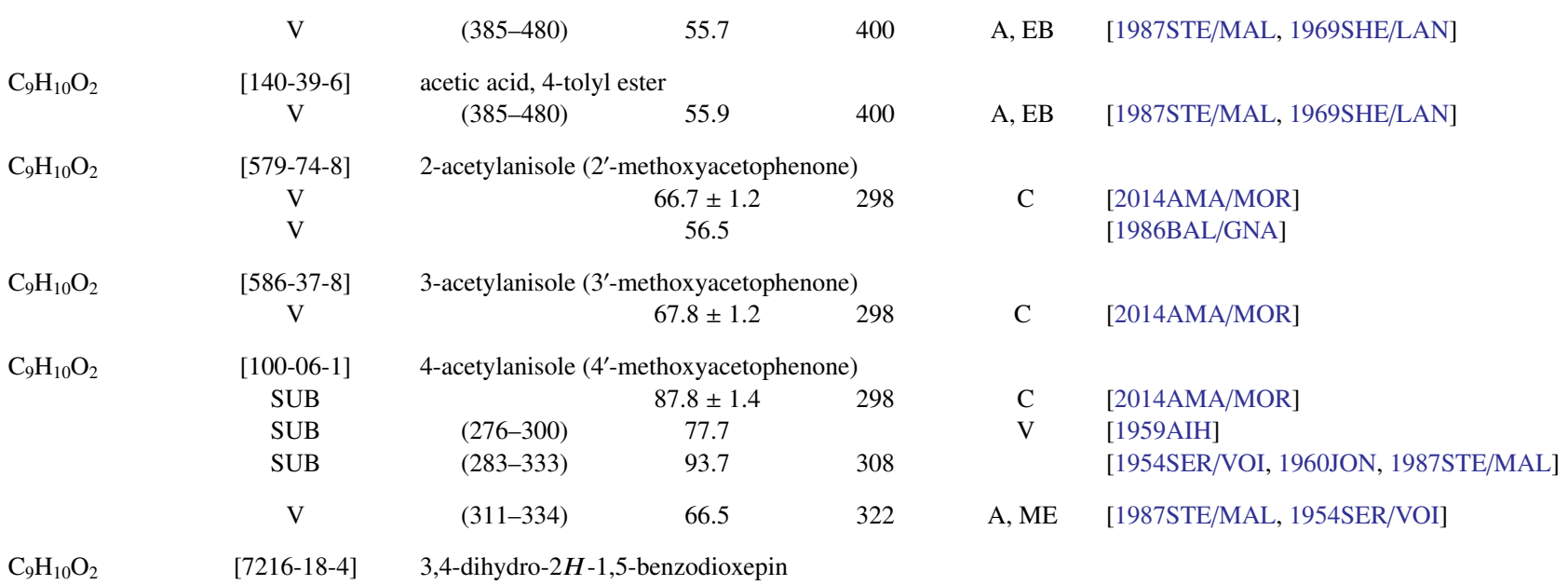


TABLE 12. Phase change enthalpies of $\mathrm{C}_{9}$ organic compounds-Continued

\begin{tabular}{|c|c|c|c|c|c|c|}
\hline \multirow[b]{2}{*}{$\begin{array}{l}\text { Molecular } \\
\text { formula }\end{array}$} & \multirow{2}{*}{$\begin{array}{l}\text { CAS reg. no. } \\
\text { Transition }\end{array}$} & \multicolumn{5}{|l|}{ Compound } \\
\hline & & Temp. range $(\mathrm{K})$ & $\begin{array}{c}\Delta_{\text {trans }} H_{\mathrm{m}} \\
\left(\mathrm{kJ} \mathrm{mol}^{-1}\right)\end{array}$ & $T_{\mathrm{m}}(\mathrm{K})$ & Method & References \\
\hline & $\mathrm{V}$ & & 55.6 & & & [1958CAS/FLE2] \\
\hline \multirow[t]{3}{*}{$\mathrm{C}_{9} \mathrm{H}_{10} \mathrm{O}_{2}$} & {$[140-11-4]$} & benzyl acetate & & & & \\
\hline & $\mathrm{V}$ & $(283-490)$ & 55.5 & 298 & A & [1987STE/MAL] \\
\hline & $\mathrm{V}$ & $(283-328)$ & 60.4 & 305 & ME & [1954SER/VOI] \\
\hline \multirow[t]{11}{*}{$\mathrm{C}_{9} \mathrm{H}_{10} \mathrm{O}_{2}$} & [93-89-0] & ethylbenzoate & & & & \\
\hline & V & $(283-332)$ & $61.1 \pm 0.3$ & 298 & GS & [2006VAS/VER] \\
\hline & $\mathrm{V}$ & $(369-531)$ & $52.5 \pm 0.2$ & 380 & EB & [2002STE/CHI2] \\
\hline & $\mathrm{V}$ & $(369-531)$ & $49.6 \pm 0.2$ & 420 & EB & [2002STE/CHI2] \\
\hline & $\mathrm{V}$ & $(369-531)$ & $46.7 \pm 0.3$ & 460 & $\mathrm{~EB}$ & [2002STE/CHI2] \\
\hline & $\mathrm{V}$ & $(369-531)$ & $43.6 \pm 0.5$ & 500 & EB & [2002STE/CHI2] \\
\hline & $\mathrm{V}$ & $(344-440)$ & 57.0 & 356 & $\mathrm{BG}$ & [1988KAT2] \\
\hline & $\mathrm{V}$ & $(344-440)$ & 50.5 & 419 & BG & [1988KAT2] \\
\hline & $\mathrm{V}$ & $(288-333)$ & 55.9 & 303 & A & [1987STE/MAL] \\
\hline & $\mathrm{V}$ & $(358-487)$ & 50.4 & 373 & A & [1987STE/MAL] \\
\hline & $\mathrm{V}$ & $(317-486)$ & 51.9 & 332 & & [1947STU] \\
\hline \multirow[t]{6}{*}{$\mathrm{C}_{9} \mathrm{H}_{10} \mathrm{O}_{2}$} & {$[501-52-0]$} & \multicolumn{5}{|c|}{ 3-phenylpropionic acid (hydrocinnamic acid) } \\
\hline & FUS & & 15.61 & 321.2 & DSC & [2001MON/HIL4] \\
\hline & FUS & & 17.68 & 321.2 & & [1991ACR, 1983WEA] \\
\hline & SUB & $(305-315)$ & $102.0 \pm 0.7$ & 310 & $\mathrm{ME}$ & [2001MON/HIL4] \\
\hline & SUB & $(305-315)$ & $102.4 \pm 0.8$ & 298 & ME & [2001MON/HIL4] \\
\hline & $\mathrm{V}$ & $(375-553)$ & 67.0 & 390 & A & [1987STE/MAL, 1947STU] \\
\hline \multirow[t]{8}{*}{$\mathrm{C}_{9} \mathrm{H}_{10} \mathrm{O}_{2}$} & {$[122-60-1]$} & \multicolumn{5}{|c|}{ (phenoxymethyl)oxirane } \\
\hline & $\mathrm{V}$ & $(400-532)$ & $69.9 \pm 0.7$ & 298 & $\mathrm{~EB}$ & [1997STE/CHI] \\
\hline & $\mathrm{V}$ & $(400-532)$ & $60.3 \pm 0.5$ & 400 & EB & [1997STE/CHI] \\
\hline & $\mathrm{V}$ & $(400-532)$ & $56.7 \pm 0.4$ & 440 & $\mathrm{~EB}$ & [1997STE/CHI] \\
\hline & $\mathrm{V}$ & $(400-532)$ & $53.1 \pm 0.4$ & 480 & EB & [1997STE/CHI] \\
\hline & $\mathrm{V}$ & $(400-532)$ & $51.3 \pm 0.5$ & 500 & $\mathrm{~EB}$ & [1997STE/CHI] \\
\hline & $\mathrm{V}$ & $(400-532)$ & $49.4 \pm 0.6$ & 520 & $\mathrm{~EB}$ & [1997STE/CHI] \\
\hline & $\mathrm{V}$ & $(343-373)$ & $65.6 \pm 0.1$ & & & [1976KUZ/MIR] \\
\hline \multirow[t]{2}{*}{$\mathrm{C}_{9} \mathrm{H}_{10} \mathrm{O}_{2}$} & {$[101-41-7]$} & \multicolumn{5}{|l|}{ methyl phenylacetate } \\
\hline & $\mathrm{V}$ & $(333-433)$ & 57.4 & 298 & GC & {$[2005 \mathrm{HOS} / \mathrm{GRY}]$} \\
\hline \multirow[t]{3}{*}{$\mathrm{C}_{9} \mathrm{H}_{10} \mathrm{O}_{2}$} & {$[936-51-6]$} & \multicolumn{5}{|c|}{ 2-phenyl-1,3-dioxolane } \\
\hline & $\mathrm{V}$ & $(285-333)$ & $62.6 \pm 0.7$ & 298 & GS & [2002VER] \\
\hline & $\mathrm{V}$ & $(298-333)$ & $62.1 \pm 0.3$ & 316 & GS & [1995VER/DOG] \\
\hline \multirow[t]{4}{*}{$\mathrm{C}_{9} \mathrm{H}_{10} \mathrm{O}_{2}$} & {$[612-19-1]$} & \multicolumn{5}{|l|}{ 2-ethylbenzoic acid } \\
\hline & SUB & $(298-313)$ & 100.5 & 305.5 & $\mathrm{ME}$ & [1987STE/MAL, 1976COL/JIM] \\
\hline & SUB & & $101.1 \pm 0.4$ & 298 & $\mathrm{ME}$ & [1984COL/JIM] \\
\hline & SUB & $(298-313)$ & $100.7 \pm 2.5$ & 298 & $\mathrm{ME}$ & [1976COL/JIM] \\
\hline \multirow[t]{3}{*}{$\mathrm{C}_{9} \mathrm{H}_{10} \mathrm{O}_{2}$} & $\begin{array}{c}\text { [619-20-5] } \\
\text { SUB }\end{array}$ & \multicolumn{5}{|l|}{ 3-ethylbenzoic acid } \\
\hline & $\begin{array}{l}\text { SUB } \\
\text { SUB }\end{array}$ & $(500-510)$ & $99.7 \pm 0.4$ & $\begin{array}{l}309 \\
298\end{array}$ & ME & [1984COL/JIM] \\
\hline & SUB & $(300-318)$ & $99.1 \pm 2.5$ & 298 & $\mathrm{ME}$ & [1976COL/JIM] \\
\hline \multirow[t]{8}{*}{$\mathrm{C}_{9} \mathrm{H}_{10} \mathrm{O}_{2}$} & [619-64-7] & 4-ethylbenzoic acid & & & & \\
\hline & FUS & & 13.2 & 380.2 & $\mathrm{DSC}$ & [2000KAN/SAM] \\
\hline & FUS & & 14.06 & 386.2 & DSC & [1991CHI/BRA] \\
\hline & SUB & $(321-335)$ & $101.2 \pm 0.8$ & 298 & $\mathrm{ME}$ & [2004MON/ALM] \\
\hline & SUB & $(310-329)$ & 98.2 & 319.5 & $\mathrm{ME}$ & [1987STE/MAL, 1976COL/JIM] \\
\hline & SUB & & $98.9 \pm 0.2$ & 298 & $\mathrm{ME}$ & [1984COL/JIM] \\
\hline & SUB & $(311-330)$ & $97.6 \pm 0.2$ & 321 & $\mathrm{ME}$ & [1984COL/JIM] \\
\hline & SUB & $(320-329)$ & $97.5 \pm 2.5$ & 298 & $\mathrm{ME}$ & [1976COL/JIM] \\
\hline $\mathrm{C}_{9} \mathrm{H}_{10} \mathrm{O}_{2}$ & {$[603-79-2]$} & 2,3-dimethylbenzoic & acid & & & \\
\hline & FUS & & 18.3 & 417.6 & $\mathrm{CVC}$ & [1996BEL/UFN] \\
\hline & SUB & (316-337) & $102.3 \pm 0.4$ & 326 & $\mathrm{ME}$ & [1984COL/JIM2] \\
\hline & SUB & (316-337) & $104.6 \pm 0.4$ & 298 & $\mathrm{ME}$ & [1984COL/JIM2] \\
\hline
\end{tabular}

$\mathrm{C}_{9} \mathrm{H}_{10} \mathrm{O}_{2}$

[611-01-8] 2,4-dimethylbenzoic acid 
TABLE 12. Phase change enthalpies of $\mathrm{C}_{9}$ organic compounds-Continued

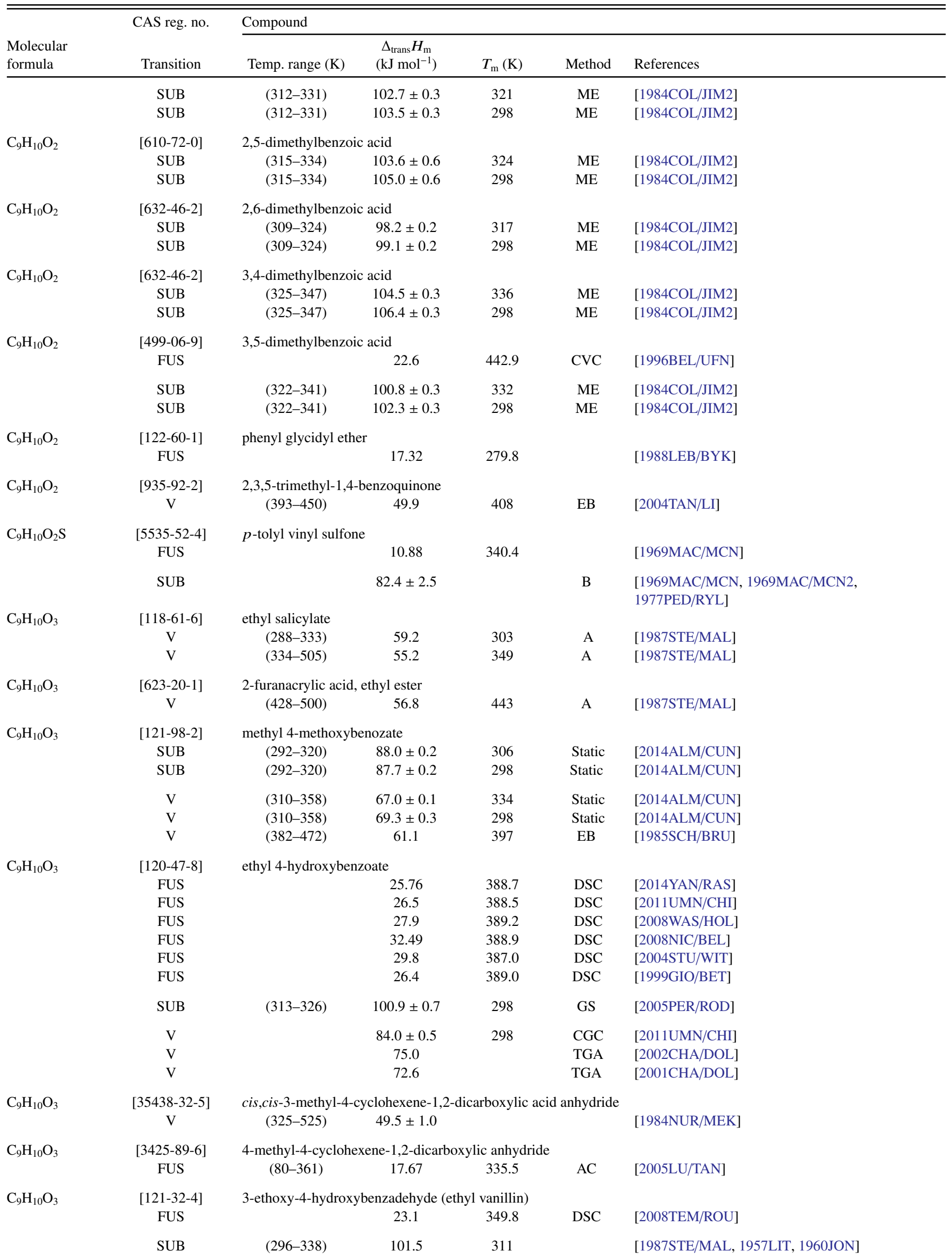


TABLE 12. Phase change enthalpies of $\mathrm{C}_{9}$ organic compounds-Continued

\begin{tabular}{|c|c|c|c|c|c|c|}
\hline \multirow[b]{2}{*}{$\begin{array}{l}\text { Molecular } \\
\text { formula }\end{array}$} & \multirow{2}{*}{$\begin{array}{l}\text { CAS reg. no. } \\
\text { Transition }\end{array}$} & \multicolumn{5}{|l|}{ Compound } \\
\hline & & Temp. range $(\mathrm{K})$ & $\begin{array}{c}\Delta_{\text {trans }} H_{\mathrm{m}} \\
\left(\mathrm{kJ} \mathrm{mol}^{-1}\right) \\
\end{array}$ & $T_{\mathrm{m}}(\mathrm{K})$ & Method & References \\
\hline $\mathrm{C}_{9} \mathrm{H}_{10} \mathrm{O}_{3}$ & $\begin{array}{c}\text { [120-14-9] } \\
\text { FUS }\end{array}$ & \multicolumn{5}{|c|}{ 3,4-dimethoxybenzaldehyde } \\
\hline $\mathrm{C}_{9} \mathrm{H}_{10} \mathrm{O}_{3}$ & FUS & & \multicolumn{3}{|c|}{$(d l)$-3-phenyl-3-hydroxypropanoic acid } & [1991CHI/BRA] \\
\hline $\mathrm{C}_{9} \mathrm{H}_{10} \mathrm{O}_{3}$ & $\begin{array}{c}{[2768-42-5]} \\
\text { FUS }\end{array}$ & \multicolumn{5}{|c|}{ (d)-3-phenyl-3-hydroxypropanoic acid } \\
\hline $\mathrm{C}_{9} \mathrm{H}_{10} \mathrm{O}_{3}$ & $\begin{array}{l}\text { [940-31-8] } \\
\text { FUS }\end{array}$ & \multicolumn{5}{|c|}{$(d l)$-2-phenoxypropionic acid } \\
\hline $\mathrm{C}_{9} \mathrm{H}_{10} \mathrm{O}_{3}$ & FUS & \multicolumn{4}{|c|}{$(d)$-2-phenoxypropionic acid } & [1991CHI/BRA] \\
\hline $\mathrm{C}_{9} \mathrm{H}_{10} \mathrm{O}_{3}$ & $\begin{array}{l}\text { [104-01-8] } \\
\text { FUS }\end{array}$ & 4-methoxyphenylac & $\begin{array}{l}\text { tic acid } \\
21.8\end{array}$ & 358.1 & & [1991ACR, 1979ARM/JAM] \\
\hline $\mathrm{C}_{9} \mathrm{H}_{10} \mathrm{O}_{3}$ & $\begin{array}{l}\text { [501-97-3] } \\
\text { FUS }\end{array}$ & 4-hydroxyphenylpr & \multicolumn{4}{|c|}{ 4-hydroxyphenylpropionic acid } \\
\hline $\mathrm{C}_{9} \mathrm{H}_{10} \mathrm{O}_{3}$ & $\begin{array}{c}\text { [619-86-3] } \\
\text { FUS } \\
\text { FUS } \\
\text { FUS } \\
\text { SUB } \\
\text { SUB } \\
\text { SUB }\end{array}$ & $\begin{array}{l}(349-373) \\
(349-373)\end{array}$ & $\begin{array}{c}35.07 \\
28.9 \\
29.4 \\
119.4 \pm 0.5 \\
121.9 \pm 1.0 \\
123.3 \pm 0.9\end{array}$ & $\begin{array}{l}471.8 \\
465.2 \\
472.8 \\
361 \\
298 \\
298\end{array}$ & $\begin{array}{l}\text { DSC } \\
\text { DSC }\end{array}$ & $\begin{array}{l}\text { [2010FON/SAN] } \\
\text { [2000KAN/SAM] } \\
{[1991 \mathrm{ACR}, 1979 A R M / J A M]} \\
{[2010 \mathrm{FON} / \mathrm{SAN}]} \\
{[2010 \mathrm{FON} / \mathrm{SAN}]} \\
{[2010 \mathrm{RIB} / \mathrm{FER} 3]}\end{array}$ \\
\hline \multirow[t]{2}{*}{$\mathrm{C}_{9} \mathrm{H}_{10} \mathrm{O}_{3}$} & $\begin{array}{c}\text { [3663-82-9] } \\
\text { FUS }\end{array}$ & 1,4-benzodioxan-2 & \multicolumn{3}{|c|}{ 1,4-benzodioxan-2-hydroxymethyl } & [2008MAT/SOU] \\
\hline & SUB & & $106.9 \pm 0.8$ & 298 & $\mathrm{C}$ & [2008MAT/MIR2] \\
\hline $\mathrm{C}_{9} \mathrm{H}_{10} \mathrm{O}_{3}$ & $\begin{array}{c}{[53282-12-5]} \\
\mathrm{V}\end{array}$ & \multicolumn{5}{|c|}{ ethyl trans-b-(2-furyl)acrylate } \\
\hline $\mathrm{C}_{9} \mathrm{H}_{10} \mathrm{O}_{4}$ & $\begin{array}{c}{[1521-38-6]} \\
\text { SUB } \\
\text { SUB }\end{array}$ & $\begin{array}{c}\text { 2,3-dimethoxybenz } \\
(336-356) \\
(336-356)\end{array}$ & $\begin{array}{l}\text { ic acid } \\
115.1 \pm 0.3 \\
116.6 \pm 0.3\end{array}$ & $\begin{array}{l}346 \\
298\end{array}$ & $\begin{array}{l}\mathrm{ME} \\
\mathrm{ME}\end{array}$ & $\begin{array}{l}\text { [1985COL/JIM] } \\
{[1985 \mathrm{COL} / \mathrm{JIM}]}\end{array}$ \\
\hline $\mathrm{C}_{9} \mathrm{H}_{10} \mathrm{O}_{4}$ & $\begin{array}{l}{[91-52-1]} \\
\text { SUB } \\
\text { SUB }\end{array}$ & $\begin{array}{c}\text { 2,4-dimethoxybenz } \\
(346-367) \\
(346-367)\end{array}$ & $\begin{array}{l}\text { ic acid } \\
120.5 \pm 0.4 \\
123.4 \pm 0.4\end{array}$ & $\begin{array}{l}357 \\
298\end{array}$ & $\begin{array}{l}\mathrm{ME} \\
\mathrm{ME}\end{array}$ & $\begin{array}{l}\text { [1985COL/JIM] } \\
{[1985 \mathrm{COL} / \mathrm{JIM}]}\end{array}$ \\
\hline $\mathrm{C}_{9} \mathrm{H}_{10} \mathrm{O}_{4}$ & $\begin{array}{c}\text { [1466-76-8] } \\
\text { SUB } \\
\text { SUB }\end{array}$ & $\begin{array}{c}\text { 2,6-dimethoxybenz } \\
(335-378) \\
(335-378)\end{array}$ & $\begin{array}{l}\text { ic acid } \\
118.4 \pm 0.4 \\
121.7 \pm 0.4\end{array}$ & $\begin{array}{l}367 \\
298\end{array}$ & $\begin{array}{l}\mathrm{ME} \\
\mathrm{ME}\end{array}$ & $\begin{array}{l}{[1985 \mathrm{COL} / \mathrm{JIM}]} \\
{[1985 \mathrm{COL} / \mathrm{JIM}]}\end{array}$ \\
\hline \multirow[t]{2}{*}{$\mathrm{C}_{9} \mathrm{H}_{10} \mathrm{O}_{4}$} & $\begin{array}{l}\text { [93-07-2] } \\
\text { FUS }\end{array}$ & \multicolumn{5}{|c|}{ 3,4-dimethoxybenzoic acid } \\
\hline & $\begin{array}{l}\text { SUB } \\
\text { SUB }\end{array}$ & $\begin{array}{l}(359-378) \\
(359-378)\end{array}$ & $\begin{array}{l}126.1 \pm 0.6 \\
129.8 \pm 0.6\end{array}$ & $\begin{array}{l}369 \\
298\end{array}$ & $\begin{array}{l}\mathrm{ME} \\
\mathrm{ME}\end{array}$ & $\begin{array}{l}\text { [1985COL/JIM] } \\
{[1985 \mathrm{COL} / \mathrm{JIM}]}\end{array}$ \\
\hline $\mathrm{C}_{9} \mathrm{H}_{10} \mathrm{O}_{4}$ & $\begin{array}{c}{[2785-98-0]} \\
\text { SUB } \\
\text { SUB }\end{array}$ & $\begin{array}{c}\text { 2,5-dimethoxybenz } \\
(324-342) \\
(324-342)\end{array}$ & $\begin{array}{l}113.3 \pm 0.7 \\
116.1 \pm 0.7\end{array}$ & $\begin{array}{l}333 \\
298\end{array}$ & $\begin{array}{l}\text { ME } \\
\mathrm{ME}\end{array}$ & $\begin{array}{l}\text { [1996JIM/ROU] } \\
\text { [1996JIM/ROU] }\end{array}$ \\
\hline $\mathrm{C}_{9} \mathrm{H}_{10} \mathrm{O}_{4}$ & $\begin{array}{c}{[1132-21-4]} \\
\text { SUB } \\
\text { SUB }\end{array}$ & $\begin{array}{c}\text { 3,5-dimethoxybenz } \\
(356-376) \\
(356-376)\end{array}$ & $\begin{array}{l}\text { ic acid } \\
124.5 \pm 0.6 \\
127.1 \pm 0.6\end{array}$ & $\begin{array}{l}369 \\
298\end{array}$ & $\begin{array}{l}\text { ME } \\
\text { ME }\end{array}$ & $\begin{array}{l}{[1985 \mathrm{COL} / \mathrm{JIM}]} \\
{[1985 \mathrm{COL} / \mathrm{JIM}]}\end{array}$ \\
\hline $\mathrm{C}_{9} \mathrm{H}_{10} \mathrm{O}_{4}$ & FUS & $( \pm)$-bicyclo[2.2.1]h & $\begin{array}{l}\text { t-5-ene-trans } \\
29.8\end{array}$ & $\begin{array}{c}\text { dicarboxy } \\
458.2\end{array}$ & $\begin{array}{l}\text { cid } \\
\text { DSC }\end{array}$ & [1971PIN/TON] \\
\hline $\mathrm{C}_{9} \mathrm{H}_{10} \mathrm{O}_{4}$ & $\begin{array}{c}\text { [32216-02-7] } \\
\text { FUS }\end{array}$ & (+)-bicyclo[2.2.1]h & $\begin{array}{l}\text { t-5-ene-trans } \\
22.5\end{array}$ & $\begin{array}{c}\text { dicarboxy } \\
449.2\end{array}$ & $\begin{array}{l}\text { cid } \\
\text { DSC }\end{array}$ & [1971PIN/TON] \\
\hline $\mathrm{C}_{9} \mathrm{H}_{10} \mathrm{O}_{4}$ & FUS & $(d l)$-erythro phenyl & $\begin{array}{c}\text { yceric acid } \\
31.38\end{array}$ & 395 & & [1991CHI/BRA] \\
\hline
\end{tabular}


TABLE 12. Phase change enthalpies of $\mathrm{C}_{9}$ organic compounds-Continued

\begin{tabular}{|c|c|c|c|c|c|c|}
\hline \multirow[b]{2}{*}{$\begin{array}{l}\text { Molecular } \\
\text { formula }\end{array}$} & \multirow{2}{*}{$\begin{array}{l}\text { CAS reg. no. } \\
\text { Transition }\end{array}$} & \multicolumn{5}{|l|}{ Compound } \\
\hline & & Temp. range $(\mathrm{K})$ & $\begin{array}{c}\Delta_{\text {trans }} H_{\mathrm{m}} \\
\left(\mathrm{kJ} \mathrm{mol}^{-1}\right)\end{array}$ & $T_{\mathrm{m}}(\mathrm{K})$ & Method & References \\
\hline \multirow[t]{2}{*}{$\mathrm{C}_{9} \mathrm{H}_{10} \mathrm{O}_{4}$} & & \multicolumn{5}{|c|}{ (d)-erythro phenylglyceric acid } \\
\hline & FUS & & 23.43 & 371.5 & & [1991CHI/BRA] \\
\hline \multirow[t]{2}{*}{$\mathrm{C}_{9} \mathrm{H}_{10} \mathrm{O}_{5}$} & {$[613-75-2]$} & \multicolumn{5}{|c|}{ 2-(diacetoxymethyl)furan } \\
\hline & SUB & \multicolumn{4}{|c|}{$109.6 \pm 2.5$} & [1980BAL/LEB, 1986PED/NAY] \\
\hline \multirow[t]{3}{*}{$\mathrm{C}_{9} \mathrm{H}_{10} \mathrm{O}_{5}$} & {$[530-57-4]$} & \multicolumn{5}{|c|}{ 3,5-dimethoxy-4-hydroxybenzoic acid (syringic acid) } \\
\hline & FUS & & 28.1 & 482.5 & DSC & [2012BOO/BAN] \\
\hline & FUS & & 33.7 & 480.3 & DSC & [2009QUE/MOT] \\
\hline \multirow[t]{3}{*}{$\mathrm{C}_{9} \mathrm{H}_{11} \mathrm{Br}$} & [7073-94-1] & \multicolumn{5}{|c|}{ 1-bromo-2-isopropylbenzene } \\
\hline & $\mathrm{V}$ & $(404-484)$ & 48.4 & 419 & & [1999DYK/SVO] \\
\hline & $\mathrm{V}$ & $(378-528)$ & 49.8 & 393 & A & [1987STE/MAL, 1970DYK/VAN] \\
\hline \multirow[t]{3}{*}{$\mathrm{C}_{9} \mathrm{H}_{11} \mathrm{Br}$} & {$[586-61-8]$} & \multicolumn{5}{|c|}{ 1-bromo-4-isopropylbenzene } \\
\hline & $\mathrm{V}$ & $(362-493)$ & 51.1 & 377 & & [1999DYK/SVO] \\
\hline & $\mathrm{V}$ & $(388-528)$ & 50.4 & 403 & A & [1987STE/MAL, 1970DYK/VAN] \\
\hline \multirow[t]{2}{*}{$\mathrm{C}_{9} \mathrm{H}_{11} \mathrm{Br}$} & {$[3575-19-7]$} & \multicolumn{5}{|l|}{ cumyl bromide } \\
\hline & V & 392 & 58.0 & 298 & CGC & [2002KRA/VAS] \\
\hline \multirow{2}{*}{$\mathrm{C}_{9} \mathrm{H}_{11} \mathrm{BrN}_{2} \mathrm{O}$} & {$[3060-89-7]$} & \multicolumn{5}{|c|}{$N^{\prime}$-(4-bromophenyl)- $N$-methoxy- $N$-methylurea } \\
\hline & FUS & & 24.44 & 368.3 & DSC & [1991ACR, 1990DON/DRE] \\
\hline \multirow[t]{2}{*}{$\mathrm{C}_{9} \mathrm{H}_{11} \mathrm{BrO}_{2}$} & {$[109417-60-9]$} & \multicolumn{5}{|c|}{ 1-bromo-2-(2-methoxyethoxy)benzene } \\
\hline & V & $(302-368)$ & $64.5 \pm 0.3$ & 298 & GS & [2006DAB/SPO] \\
\hline \multirow[t]{2}{*}{$\mathrm{C}_{9} \mathrm{H}_{11} \mathrm{BrO}_{3}$} & {$[63834-58-2]$} & (racemic) 3-(2-bro & phenoxy)pro & 1,2-diol & & \\
\hline & FUS & & 33.4 & 353.5 & DSC & [2006ZAK/LAZ] \\
\hline $\mathrm{C}_{9} \mathrm{H}_{11} \mathrm{BrO}_{3}$ & {$[386702-67-6]$} & $(R)$-3-(2-bromoop & loxy)propane & diol & & \\
\hline & FUS & & 38.4 & 374.3 & DSC & [2006ZAK/LAZ] \\
\hline $\mathrm{C}_{9} \mathrm{H}_{11} \mathrm{Cl}$ & {$[2077-13-6]$} & 1-chloro-2-isoprop & enzene & & & \\
\hline & V & $(341-465)$ & 48.1 & 356 & & [1999DYK/SVO] \\
\hline & $\mathrm{V}$ & $(363-508)$ & 47.7 & 378 & A & [1987STE/MAL, 1970DYK/VAN] \\
\hline $\mathrm{C}_{9} \mathrm{H}_{11} \mathrm{Cl}$ & [2621-46-7] & 1-chloro-4-isoprop & enzene & & & \\
\hline & $\mathrm{V}$ & $(307-472)$ & 51.4 & 322 & & [1999DYK/SVO] \\
\hline & $\mathrm{V}$ & $(368-513)$ & 48.5 & 383 & A & [1987STE/MAL, 1970DYK/VAN] \\
\hline $\mathrm{C}_{9} \mathrm{H}_{11} \mathrm{Cl}$ & {$[934-53-2]$} & cumyl chloride & & & & \\
\hline & $\mathrm{V}$ & 392 & 54.7 & 298 & CGC & [2002KRA/VAS] \\
\hline $\mathrm{C}_{9} \mathrm{H}_{11} \mathrm{ClN}_{2} \mathrm{O}$ & {$[150-68-5]$} & 3-(4-chlorophenyl) & 1-dimethylu & ionuron) & & \\
\hline & FUS & & 29.3 & 447.6 & & [2004KON/TAN2] \\
\hline & FUS & & 29.46 & 447.6 & DSC & [1991ACR, 1990DON/DRE] \\
\hline & SUB & $(303-379)$ & $114.6 \pm 4.9$ & 341 & $\mathrm{ME}, \mathrm{C}$ & [1987STE/MAL, 1972WIE] \\
\hline $\mathrm{C}_{9} \mathrm{H}_{11} \mathrm{ClN}_{2} \mathrm{O}_{2}$ & {$[1746-81-2]$} & $N^{\prime}$-(4-chloropheny & $N$-methoxy- & thylurea & & \\
\hline & FUS & & 22.54 & 353.4 & DSC & [1991ACR, 1990DON/DRE] \\
\hline $\mathrm{C}_{9} \mathrm{H}_{11} \mathrm{ClO}_{2}$ & {$[67146-43-4]$} & propylene glycol n & o(4-chloropl & ether & & \\
\hline & $\mathrm{V}$ & $(417-542)$ & 64.9 & 432 & A & [1987STE/MAL, 1999DYK/SVO] \\
\hline $\mathrm{C}_{9} \mathrm{H}_{11} \mathrm{ClO}_{3}$ & {$[93-65-2]$} & 2-(4-chloro-2-metl & phenoxy)pro & acid & & \\
\hline & FUS & & 26.43 & 366.2 & DSC & [1990DON/DRE] \\
\hline $\mathrm{C}_{9} \mathrm{H}_{11} \mathrm{ClO}_{3}$ & [5112-21-0] & (racemic) 3-(2-chlc & phenoxy)pro & 1,2-diol & & \\
\hline & FUS & & 29.0 & 344.9 & DSC & [2006ZAK/LAZ] \\
\hline $\mathrm{C}_{9} \mathrm{H}_{11} \mathrm{ClO}_{3}$ & [153547-60-5] & $(R)-3$-(2-chloroph & xy)propane- & & & \\
\hline & FUS & & 38.2 & 363.3 & DSC & [2006ZAK/LAZ] \\
\hline $\mathrm{C}_{9} \mathrm{H}_{11} \mathrm{ClS}$ & [4332-51-8] & benzyl (2-chloroet) & sulfide & & & \\
\hline & V & $(293-333)$ & 52.3 & 308 & A, GS & [1987STE/MAL, 1948RED/CHA, 1999DYK/SVO] \\
\hline $\mathrm{C}_{9} \mathrm{H}_{11} \mathrm{Cl}_{3} \mathrm{NO}_{3} \mathrm{PS}$ & $\begin{array}{c}{[330-55-2]} \\
\text { FUS }\end{array}$ & $O, O$-diethyl- $O-(3$ & $\begin{array}{c}\text {-trichloro-2- } \\
24.53\end{array}$ & $\begin{array}{l}\text { 1)phosph } \\
315\end{array}$ & $\begin{array}{l}\text { ioate } \\
\text { DSC }\end{array}$ & [1991ACR, 1990DON/DRE] \\
\hline
\end{tabular}


TABLE 12. Phase change enthalpies of $\mathrm{C}_{9}$ organic compounds-Continued

\begin{tabular}{|c|c|c|c|c|c|c|}
\hline \multirow[b]{2}{*}{$\begin{array}{l}\text { Molecular } \\
\text { formula }\end{array}$} & \multirow{2}{*}{$\begin{array}{c}\text { CAS reg. no. } \\
\text { Transition }\end{array}$} & \multicolumn{5}{|l|}{ Compound } \\
\hline & & Temp. range $(\mathrm{K})$ & $\begin{array}{c}\Delta_{\text {trans }} H_{\mathrm{m}} \\
\left(\mathrm{kJ} \mathrm{mol}^{-1}\right) \\
\end{array}$ & $T_{\mathrm{m}}(\mathrm{K})$ & Method & References \\
\hline \multirow[t]{2}{*}{$\mathrm{C}_{9} \mathrm{H}_{11} \mathrm{Cl}_{3} \mathrm{NO}_{4} \mathrm{P}$} & {$[5598-15-2]$} & \multicolumn{5}{|c|}{ 3,5,6-trichloro-2-pyridyl diethylphosphate (chlorpyrifos oxon) } \\
\hline & SUB & $(373-403)$ & 79.0 & 388 & GC & {$[2007 \mathrm{GOE} / \mathrm{MCC}]$} \\
\hline \multirow[t]{2}{*}{$\mathrm{C}_{9} \mathrm{H}_{11} \mathrm{FO}_{3}$} & {$[399-28-0]$} & \multicolumn{5}{|c|}{ (racemic) 3-(2-fluorophenoxy)propane-1,2-diol } \\
\hline & FUS & & 20.5 & 318.2 & DSC & [2006ZAK/LAZ] \\
\hline \multirow[t]{2}{*}{$\mathrm{C}_{9} \mathrm{H}_{11} \mathrm{FO}_{3}$} & [912556-93-5] & \multicolumn{5}{|c|}{$(R)$-3-(2-fluorophenoxy)propane-1,2-diol } \\
\hline & FUS & & 26.0 & 333.7 & DSC & {$[2006 \mathrm{ZAK} / \mathrm{LAZ}]$} \\
\hline \multirow[t]{2}{*}{$\mathrm{C}_{9} \mathrm{H}_{11} \mathrm{~F}_{5} \mathrm{O}_{2}$} & {$[24262-73-5]$} & \multicolumn{5}{|c|}{ pentafluoropropionic acid, cyclohexyl ester } \\
\hline & $\mathrm{V}$ & $(335-428)$ & 46.4 & 350 & $\mathrm{~A}, \mathrm{~EB}$ & [1987STE/MAL, 1969SHE/LAN] \\
\hline \multirow[t]{2}{*}{$\mathrm{C}_{9} \mathrm{H}_{11} \mathrm{~F}_{9}$} & {$[1190430-21-7]$} & \multicolumn{5}{|c|}{ 1,1,1,2,2,3,3,4,4-nonafluorononane } \\
\hline & $\mathrm{V}$ & $(278-328)$ & $45.7 \pm 0.1$ & 298 & Static & {$[2015 \mathrm{MOR} / \mathrm{DAS}]$} \\
\hline \multirow[t]{2}{*}{$\mathrm{C}_{9} \mathrm{H}_{11} \mathrm{I}$} & {$[54290-22-1]$} & \multicolumn{5}{|l|}{ cumyl iodide } \\
\hline & V & 392 & 63.3 & 298 & CGC & [2002KRA/VAS] \\
\hline \multirow[t]{2}{*}{$\mathrm{C}_{9} \mathrm{H}_{11} \mathrm{IO}_{3}$} & {$[55169-06-7]$} & \multicolumn{5}{|c|}{ (racemic) 3-(2-iodophenoxy)propane-1,2-diol } \\
\hline & FUS & & 34.2 & 362.5 & DSC & {$[2006 \mathrm{ZAK} / \mathrm{LAZ}]$} \\
\hline \multirow[t]{2}{*}{$\mathrm{C}_{9} \mathrm{H}_{11} \mathrm{IO}_{3}$} & {$[912556-94-6]$} & \multicolumn{5}{|c|}{$(R)$-3-(2-iodophenoxy)propane-1,2-diol } \\
\hline & FUS & & 37.4 & 383.5 & DSC & {$[2006 \mathrm{ZAK} / \mathrm{LAZ}]$} \\
\hline \multirow[t]{9}{*}{$\mathrm{C}_{9} \mathrm{H}_{11} \mathrm{~N}$} & {$[635-46-1]$} & 1,2,3,4-tetrahydroq & noline & & & \\
\hline & FUS & $(12-441)$ & 11.81 & 290 & $\mathrm{AC}$ & [1991ACR, 1989STE/CHI3] \\
\hline & $\mathrm{V}$ & $(323-572)$ & $65.3 \pm 0.2$ & 298 & IPM, EB & [1989STE/CHI3] \\
\hline & $\mathrm{V}$ & $(323-572)$ & $62.1 \pm 0.1$ & 340 & IPM, EB & [1989STE/CHI3] \\
\hline & $\mathrm{V}$ & $(323-572)$ & $59.2 \pm 0.1$ & 380 & IPM, EB & [1989STE/CHI3] \\
\hline & V & $(323-572)$ & $56.3 \pm 0.1$ & 420 & IPM, EB & [1989STE/CHI3] \\
\hline & $\mathrm{V}$ & $(323-572)$ & $53.5 \pm 0.2$ & 460 & IPM, EB & [1989STE/CHI3] \\
\hline & $\mathrm{V}$ & $(323-572)$ & $50.8 \pm 0.3$ & 500 & IPM, EB & [1989STE/CHI3] \\
\hline & $\mathrm{V}$ & $(323-572)$ & $47.9 \pm 0.4$ & 540 & IPM, EB & [1989STE/CHI3] \\
\hline $\mathrm{C}_{9} \mathrm{H}_{11} \mathrm{~N}$ & {$[10500-57-9]$} & 5,6,7,8-tetrahydroq & noline & & & \\
\hline & FUS & $(5-442)$ & 9.08 & 222.7 & $\mathrm{AC}$ & [1991ACR, 1989STE/CHI3] \\
\hline & $\mathrm{V}$ & $(303-544)$ & $57.6 \pm 0.2$ & 298 & IPM, EB & [1989STE/CHI3] \\
\hline & $\mathrm{V}$ & $(303-544)$ & $56.1 \pm 0.1$ & 320 & IPM, EB & [1989STE/CHI3] \\
\hline & V & $(303-544)$ & $53.6 \pm 0.1$ & 360 & IPM, EB & [1989STE/CHI3] \\
\hline & V & $(303-544)$ & $51.1 \pm 0.1$ & 400 & IPM, EB & [1989STE/CHI3] \\
\hline & $\mathrm{V}$ & $(303-544)$ & $48.7 \pm 0.3$ & 440 & IPM, EB & [1989STE/CHI3] \\
\hline & $\mathrm{V}$ & $(303-544)$ & $46.2 \pm 0.4$ & 480 & IPM, EB & [1989STE/CHI3] \\
\hline & $\mathrm{V}$ & $(303-544)$ & $43.5 \pm 0.5$ & 520 & IPM, EB & [1989STE/CHI3] \\
\hline $\mathrm{C}_{9} \mathrm{H}_{11} \mathrm{~N}$ & {$[824-21-5]$} & $N$-methylindoline & & & & \\
\hline & V & $(296-338)$ & $64.2 \pm 0.4$ & 298 & GS & [2011VER/EME] \\
\hline $\mathrm{C}_{9} \mathrm{H}_{11} \mathrm{NO}$ & {$[579-10-2]$} & $N$-methylacetanilic & & & & \\
\hline & $\mathrm{V}$ & $(383-519)$ & 60.1 & 398 & A & [1987STE/MAL] \\
\hline & $\mathrm{V}$ & $(377-526)$ & 56.7 & 392 & & {$[1947 \mathrm{STU}]$} \\
\hline $\mathrm{C}_{9} \mathrm{H}_{11} \mathrm{NO}$ & {$[120-66-1]$} & $N$-(2-methylpheny & cetamide & & & \\
\hline & SUB & $(315-340)$ & 96.8 & 327.5 & & [1987STE/MAL, 1960AIH2] \\
\hline $\mathrm{C}_{9} \mathrm{H}_{11} \mathrm{NO}$ & [103-89-9] & $N$-(4-methylpheny & cetamide & & & \\
\hline & SUB & $(331-350)$ & 99.0 & 341 & & [1960AIH2] \\
\hline $\mathrm{C}_{9} \mathrm{H}_{11} \mathrm{NO}$ & [611-74-5] & $N, N$-dimethylbenza & ide & & & \\
\hline & FUS & & 21.22 & 316.9 & DSC & [2010ALM/MON] \\
\hline & SUB & $(297-315)$ & $88.6 \pm 0.3$ & 306 & Static & [2010ALM/MON] \\
\hline & SUB & $(297-315)$ & $88.8 \pm 0.3$ & 298 & Static & [2010ALM/MON] \\
\hline & SUB & $(289-305)$ & $89.7 \pm 0.3$ & 298 & & [1995ABB/JIM] \\
\hline & $\mathrm{V}$ & $(302-362)$ & $68.7 \pm 0.1$ & 332 & Static & [2010ALM/MON] \\
\hline & $\mathrm{V}$ & $(302-362)$ & $72.5 \pm 0.1$ & 298 & Static & [2010ALM/MON] \\
\hline
\end{tabular}

Note: VP measurements reported in [2010ALM/MON] include those for the subcooled liquid

[1978BEA/LEE] 
TABLE 12. Phase change enthalpies of $\mathrm{C}_{9}$ organic compounds-Continued

\begin{tabular}{|c|c|c|c|c|c|c|}
\hline \multirow[b]{2}{*}{$\begin{array}{l}\text { Molecular } \\
\text { formula }\end{array}$} & \multirow{2}{*}{$\begin{array}{l}\text { CAS reg. no. } \\
\text { Transition }\end{array}$} & \multicolumn{5}{|l|}{ Compound } \\
\hline & & Temp. range $(\mathrm{K})$ & $\begin{array}{c}\Delta_{\text {trans }} H_{\mathrm{m}} \\
\left(\mathrm{kJ} \mathrm{mol}^{-1}\right) \\
\end{array}$ & $T_{\mathrm{m}}(\mathrm{K})$ & Method & References \\
\hline \multirow[t]{2}{*}{$\mathrm{C}_{9} \mathrm{H}_{11} \mathrm{NO}$} & {$[120-66-1]$} & \multicolumn{5}{|c|}{ 2-(acetylamino)toluene } \\
\hline & FUS & & 21.7 & 382.7 & $\mathrm{DSC}$ & [2003HUA, 2005HUA/TAN] \\
\hline \multirow[t]{4}{*}{$\mathrm{C}_{9} \mathrm{H}_{11} \mathrm{NO}$} & {$[103-89-9]$} & \multicolumn{5}{|c|}{ 4-(acetylamino)toluene } \\
\hline & FUS & & 29.4 & 421.3 & DSC & [2012UMN/CHI] \\
\hline & FUS & & 28.93 & 424 & DSC & [2003HUA, 2005HUA/TAN] \\
\hline & $\mathrm{V}$ & & $86.2 \pm 3.2$ & 298 & CGC & [2012UMN/CHI] \\
\hline \multirow[t]{2}{*}{$\mathrm{C}_{9} \mathrm{H}_{11} \mathrm{NO}$} & {$[100-10-7]$} & \multicolumn{5}{|c|}{ 4-(N,N-dimethylamino)benzaldehyde } \\
\hline & FUS & $(80-360)$ & 19.07 & 346.2 & $\mathrm{AC}$ & [1999MEN/LIA] \\
\hline \multirow[t]{3}{*}{$\mathrm{C}_{9} \mathrm{H}_{11} \mathrm{NO}_{2}$} & {$[6526-72-3]$} & \multicolumn{5}{|c|}{ 1-nitro-2-isopropylbenzene } \\
\hline & $\mathrm{V}$ & $(278-323)$ & $65.5 \pm 0.7$ & 301 & GS & [2000VER/HEI] \\
\hline & $\mathrm{V}$ & $(278-323)$ & $65.6 \pm 0.7$ & 298 & GS & [2000VER/HEI] \\
\hline \multirow{2}{*}{$\mathrm{C}_{9} \mathrm{H}_{11} \mathrm{NO}_{2}$} & {$[87-25-2]$} & \multicolumn{5}{|c|}{ ethyl 2-aminobenzoate (ethyl anthranilate) } \\
\hline & $\mathrm{V}$ & $(433-593)$ & 59.6 & 448 & A & [1987STE/MAL] \\
\hline \multirow[t]{11}{*}{$\mathrm{C}_{9} \mathrm{H}_{11} \mathrm{NO}_{2}$} & {$[94-09-7]$} & \multicolumn{5}{|c|}{ ethyl 4-aminobenzoate (benzocaine) } \\
\hline & FUS (II) & & 23.3 & 362.4 & DSC & [2013GAN/BAR] \\
\hline & FUS & & 17.81 & 362.7 & DTA & [2013FUL/VLA] \\
\hline & FUS & & 22.55 & 362 & DSC & [2010BAI/VAN] \\
\hline & FUS & & 25.65 & 362.6 & DSC & [2010MIY/KHA] \\
\hline & FUS & & 24.6 & 362.6 & DSC & [2008WAS/HOL] \\
\hline & FUS & & 21.4 & 362.5 & DSC & [2004STU/WIT] \\
\hline & FUS & & 21.16 & & DSC & [1995YAM/KIT] \\
\hline & FUS & & 23.56 & 362.8 & & $\begin{array}{l}\text { [1991ACR, 1990MAN/AHU, 1989NEA/FLY, } \\
\text { 1990NEA/FLY] }\end{array}$ \\
\hline & SUB & & $112.9 \pm 4.3$ & 298 & $\mathrm{~V}+\mathrm{F}$ & [2013GOB/GUT] \\
\hline & $\mathrm{V}$ & & $91.8 \pm 4.2$ & 298 & CGC & [2013GOB/GUT] \\
\hline \multirow[t]{2}{*}{$\mathrm{C}_{9} \mathrm{H}_{11} \mathrm{NO}_{2}$} & {$[101-99-5]$} & \multicolumn{5}{|l|}{ ethyl carbanilate } \\
\hline & $\mathrm{V}$ & $(380-510)$ & 84.2 & 395 & A & [1987STE/MAL, 1947STU] \\
\hline \multirow[t]{2}{*}{$\mathrm{C}_{9} \mathrm{H}_{11} \mathrm{NO}_{2}$} & {$[603-71-4]$} & \multicolumn{5}{|c|}{ 2,4,6-trimethylnitrobenzene } \\
\hline & SUB & & $78.6 \pm 1.0$ & 298 & $\mathrm{C}$ & [1993ACR/TUC2] \\
\hline \multirow[t]{6}{*}{$\mathrm{C}_{9} \mathrm{H}_{11} \mathrm{NO}_{2}$} & {$[63-91-2]$} & L- $(l)$-phenylalanine & & & & \\
\hline & SUB & & $158.3 \pm 2.0$ & 418 & ME,MS & [2014TYU/KRA] \\
\hline & SUB & & $160.6 \pm 3.5$ & 298 & ME,MS & [2014TYU/KRA] \\
\hline & SUB & & $145 \pm 6$ & 439 & ME,MS & [2013TYU/BAD] \\
\hline & SUB & $(342-442)$ & $\mathrm{U} 90 \pm 6.3$ & 392 & $\mathrm{LE}$ & [1977GAF/PIE] \\
\hline & SUB & $(451-469)$ & $154.0 \pm 0.8$ & 455 & $\mathrm{ME}$ & $\begin{array}{l}\text { [1965SVE/CLY, 1970COX/PIL, 1987STE/MAL, } \\
\text { 1964CLY/SVE] }\end{array}$ \\
\hline $\mathrm{C}_{9} \mathrm{H}_{11} \mathrm{NO}_{2}$ & [101-99-5] & ethyl phenyl carbar & & & & \\
\hline & FUS & & 16.27 & 326 & & [1971PRI] \\
\hline $\mathrm{C}_{9} \mathrm{H}_{11} \mathrm{NO}_{2}$ & {$[51-66-1]$} & $p$-methoxyacetanil & & & & \\
\hline & FUS & & 25.4 & 398.2 & DSC & [2012UMN/CHI] \\
\hline & FUS & & 27.82 & 400.3 & & [1990MAN/AHU] \\
\hline & $\mathrm{V}$ & & $92.0 \pm 3.4$ & 298 & CGC & [2012UMN/CHI] \\
\hline & $\mathrm{V}$ & & 85.7 & 466 & I & [1943CRA] \\
\hline $\mathrm{C}_{9} \mathrm{H}_{11} \mathrm{NO}_{2}$ & [610-16-2] & 2-(dimethylamino) & nzoic acid & & & \\
\hline & FUS & & 14.1 & 341.9 & DSC & [2012BOO/BAN] \\
\hline $\mathrm{C}_{9} \mathrm{H}_{11} \mathrm{NO}_{2}$ & [99-64-9] & 3-(dimethylamino) & nzoic acid & & & \\
\hline & FUS & & 21.8 & 423.3 & DSC & [2012BOO/BAN] \\
\hline $\mathrm{C}_{9} \mathrm{H}_{11} \mathrm{NO}_{2}$ & [619-84-1] & 4-(dimethylamino) & nzoic acid & & & \\
\hline & FUS & & 34.1 & 512.5 & $\mathrm{DSC}$ & [2010MON/SAN] \\
\hline & SUB & $(369-385)$ & $117.4 \pm 0.5$ & 309 & $\mathrm{ME}$ & [2010MON/SAN] \\
\hline & SUB & $(369-385)$ & $120.3 \pm 0.5$ & 298 & $\mathrm{ME}$ & [2010MON/SAN] \\
\hline $\mathrm{C}_{9} \mathrm{H}_{11} \mathrm{NO}_{2} \mathrm{~S}_{2}$ & $\begin{array}{l}{[949171-64-6]} \\
\text { FUS }\end{array}$ & $N$-theonylthiocarb & $\begin{array}{l}\text { nic- } O \text {-propyl } \\
26.1\end{array}$ & 370.1 & DSC & [2007RIB/MON] \\
\hline
\end{tabular}


TABLE 12. Phase change enthalpies of $\mathrm{C}_{9}$ organic compounds-Continued

\begin{tabular}{|c|c|c|c|c|c|c|}
\hline \multirow[b]{2}{*}{$\begin{array}{l}\text { Molecular } \\
\text { formula }\end{array}$} & \multirow{2}{*}{$\begin{array}{l}\text { CAS reg. no. } \\
\text { Transition }\end{array}$} & \multicolumn{5}{|l|}{ Compound } \\
\hline & & Temp. range $(\mathrm{K})$ & $\begin{array}{c}\Delta_{\text {trans }} H_{\mathrm{m}} \\
\left(\mathrm{kJ} \mathrm{mol}^{-1}\right) \\
\end{array}$ & $T_{\mathrm{m}}(\mathrm{K})$ & Method & References \\
\hline & SUB & & $136.5 \pm 1.8$ & 298 & $\mathrm{C}$ & [2007RIB/MON] \\
\hline $\mathrm{C}_{9} \mathrm{H}_{11} \mathrm{NO}_{3}$ & $\begin{array}{c}\text { [60-18-4] } \\
\text { SUB } \\
\text { SUB } \\
\text { SUB }\end{array}$ & $\begin{array}{l}(l) \text {-tyrosine } \\
\quad(412-512)\end{array}$ & $\begin{aligned} 200.0 & \pm 1.8 \\
202.4 & \pm 3.6 \\
101 & \pm 8\end{aligned}$ & $\begin{array}{l}471 \\
298 \\
462\end{array}$ & $\begin{array}{l}\text { ME,MS } \\
\text { ME,MS } \\
\text { LE }\end{array}$ & $\begin{array}{l}\text { [2014TYU/KRA] } \\
{[2014 \mathrm{TYU} / \mathrm{KRA}]} \\
{[1977 \mathrm{GAF} / \mathrm{PIE}]}\end{array}$ \\
\hline $\mathrm{C}_{9} \mathrm{H}_{11} \mathrm{NS}$ & $\begin{array}{c}\text { [15482-60-7] } \\
\text { FUS }\end{array}$ & $N, N$-dimethylbenze & $\begin{array}{c}\text { ecarbothioam } \\
25.1\end{array}$ & & & [1978BEA/LEE] \\
\hline & SUB & & $94.8 \pm 2.0$ & 298 & $\mathrm{C}$ & [1989RIB/SOU] \\
\hline & $\mathrm{V}$ & & $73.6 \pm 4.0$ & & & [1978BEA/LEE] \\
\hline $\mathrm{C}_{9} \mathrm{H}_{11} \mathrm{NS}$ & $\begin{array}{c}{[40780-82-3]} \\
\mathrm{V}\end{array}$ & $N$-methylthiobenzi & $\begin{array}{l}\text { idate } \\
666.1 \pm 3.3\end{array}$ & & & [1978BEA/LEE] \\
\hline $\mathrm{C}_{9} \mathrm{H}_{11} \mathrm{O}_{4} \mathrm{P}$ & $\begin{array}{c}\text { [14657-64-8] } \\
\text { FUS }\end{array}$ & 2-carboxyethyl(phe & $\begin{array}{l}\text { yl)phosphinic } \\
33.58\end{array}$ & 432.66 & DSC & [2010GUO/WAN2] \\
\hline $\mathrm{C}_{9} \mathrm{H}_{12}$ & $\begin{array}{c}{[38451-18-2]} \\
\text { V } \\
\text { V }\end{array}$ & $\begin{array}{l}\text { cis-bicyclo[4.3.0]n } \\
\quad(356-429)\end{array}$ & $\begin{array}{c}\text { a-3,7-diene } \\
41.8 \\
44.9\end{array}$ & $\begin{array}{l}371 \\
298\end{array}$ & A & $\begin{array}{l}\text { [1987STE/MAL] } \\
{[1972 \mathrm{KOZ} / \mathrm{TIM}]}\end{array}$ \\
\hline $\mathrm{C}_{9} \mathrm{H}_{12}$ & $\begin{array}{c}\text { [28304-66-7] } \\
\text { V } \\
\text { V } \\
\text { V } \\
\text { V } \\
\text { V }\end{array}$ & $\begin{array}{c}(Z)-5 \text {-ethylidene-2 } \\
(315-462) \\
(315-462) \\
(315-462) \\
(315-462) \\
(315-462)\end{array}$ & $\begin{array}{l}\text { orbornene } \\
44.3 \pm 0.3 \\
43.0 \pm 0.3 \\
40.5 \pm 0.3 \\
38.0 \pm 0.3 \\
35.2 \pm 0.5\end{array}$ & $\begin{array}{l}298 \\
320 \\
360 \\
400 \\
440\end{array}$ & $\begin{array}{l}\text { EB } \\
\text { EB } \\
\text { EB } \\
\text { EB } \\
\text { EB }\end{array}$ & $\begin{array}{l}{[1997 \mathrm{STE} / \mathrm{CHI}]} \\
{[1997 \mathrm{STE} / \mathrm{CHI}]} \\
{[1997 \mathrm{STE} / \mathrm{CHI}]} \\
{[1997 \mathrm{STE} / \mathrm{CHI}]} \\
{[1997 \mathrm{STE} / \mathrm{CHI}]}\end{array}$ \\
\hline $\mathrm{C}_{9} \mathrm{H}_{12}$ & $\begin{array}{c}{[3048-64-4]} \\
\text { V }\end{array}$ & $\begin{array}{l}\text { 5-ethylidene-2-nork } \\
\quad(314-420)\end{array}$ & $\begin{array}{l}\text { rnene } \\
422.3 \pm 0.3\end{array}$ & 298 & $\mathrm{~EB}$ & [1996STE/CHI2] \\
\hline $\mathrm{C}_{9} \mathrm{H}_{12}$ & $\begin{array}{c}{[28304-67-8]} \\
\mathrm{V}\end{array}$ & $\begin{array}{l}\text { trans-5-ethylidene- } \\
\quad(346-416)\end{array}$ & $\begin{array}{c}\text { norbornene } \\
41.2\end{array}$ & 361 & A & [1987STE/MAL] \\
\hline $\mathrm{C}_{9} \mathrm{H}_{12}$ & $\begin{array}{c}\text { [611-14-3] } \\
\mathrm{V} \\
\mathrm{V} \\
\mathrm{V}\end{array}$ & $\begin{array}{l}\text { 2-ethyltoluene } \\
\text { (353-443) }\end{array}$ & $\begin{array}{l}46.9 \\
47.7 \\
43.6\end{array}$ & $\begin{array}{l}298 \\
298 \\
368\end{array}$ & A & $\begin{array}{l}\text { [1994RUZ/ZAB] } \\
\text { [1971WIL/ZWO] } \\
\text { [1987STE/MAL, 1949FOR/NOR] }\end{array}$ \\
\hline $\mathrm{C}_{9} \mathrm{H}_{12}$ & $\begin{array}{c}{[620-14-4]} \\
\text { V } \\
\text { V } \\
\text { V }\end{array}$ & $\begin{array}{l}\text { 3-ethyltoluene } \\
\text { (348-438) }\end{array}$ & $\begin{array}{l}46.6 \\
46.9 \\
43.4\end{array}$ & $\begin{array}{l}298 \\
298 \\
363\end{array}$ & A & $\begin{array}{l}\text { [1994RUZ/ZAB] } \\
\text { [1971WIL/ZWO] } \\
\text { [1987STE/MAL, 1949FOR/NOR] }\end{array}$ \\
\hline $\mathrm{C}_{9} \mathrm{H}_{12}$ & $\begin{array}{c}{[622-96-8]} \\
\mathrm{V} \\
\mathrm{V} \\
\mathrm{V}\end{array}$ & $\begin{array}{l}\text { 4-ethyltoluene } \\
\text { (349-442) }\end{array}$ & $\begin{array}{l}46.5 \\
46.6 \\
43.2\end{array}$ & $\begin{array}{l}298 \\
298 \\
364\end{array}$ & A & $\begin{array}{l}\text { [1994RUZ/ZAB] } \\
\text { [1971WIL/ZWO] } \\
\text { [1987STE/MAL, 1949FOR/NOR] }\end{array}$ \\
\hline $\mathrm{C}_{9} \mathrm{H}_{12}$ & $\begin{array}{l}{[98-82-8]} \\
\text { FUS }\end{array}$ & isopropylbenzene & 7.32 & 177.1 & $\mathrm{AC}$ & [1973KIS/SUG] \\
\hline & $\begin{array}{l}\text { V } \\
\text { V } \\
\text { V } \\
\text { V } \\
\text { V } \\
\text { V } \\
\text { V } \\
\text { V }\end{array}$ & $\begin{array}{l}(349-426) \\
(339-433)\end{array}$ & $\begin{array}{c}45.1 \\
41.2 \\
42.1 \\
45.1 \pm 0.1 \\
44.0 \\
45.1 \\
45.1 \\
41.9\end{array}$ & $\begin{array}{l}298 \\
364 \\
354 \\
298 \\
298 \\
298 \\
298 \\
358\end{array}$ & $\begin{array}{c}\mathrm{C} \\
\mathrm{MM}\end{array}$ & $\begin{array}{l}\text { [1994RUZ/ZAB] } \\
{[1989 \mathrm{CEP} / \mathrm{GON}]} \\
{[1987 \mathrm{STE} / \mathrm{MAL}]} \\
{[1982 \mathrm{FUC} / \mathrm{HAL}]} \\
{[1975 \mathrm{KUS} / \mathrm{SAI}]} \\
{[1971 \mathrm{WIL} / \mathrm{ZWO}]} \\
{[1947 \mathrm{OSB} / \mathrm{GIN}]} \\
{[1949 \mathrm{FOR} / \mathrm{NOR}, 1945 \mathrm{WIL} / \mathrm{TAY}]}\end{array}$ \\
\hline $\mathrm{C}_{9} \mathrm{H}_{12}$ & $\begin{array}{c}\text { [103-65-1] } \\
\text { FUS }\end{array}$ & propylbenzene & 9.27 & 173.6 & & [1991ACR, 1965MES/TOD2] \\
\hline & $\begin{array}{l}\text { V } \\
\text { V } \\
\text { V }\end{array}$ & $(340-391)$ & $\begin{array}{l}46.2 \\
43.8 \\
45.0\end{array}$ & $\begin{array}{l}298 \\
355 \\
298\end{array}$ & & $\begin{array}{l}\text { [1994RUZ/ZAB] } \\
{[1986 \mathrm{PAU} / \mathrm{KRU}]} \\
{[1975 \mathrm{KUS} / \mathrm{SAI}]}\end{array}$ \\
\hline
\end{tabular}


TABLE 12. Phase change enthalpies of $\mathrm{C}_{9}$ organic compounds-Continued

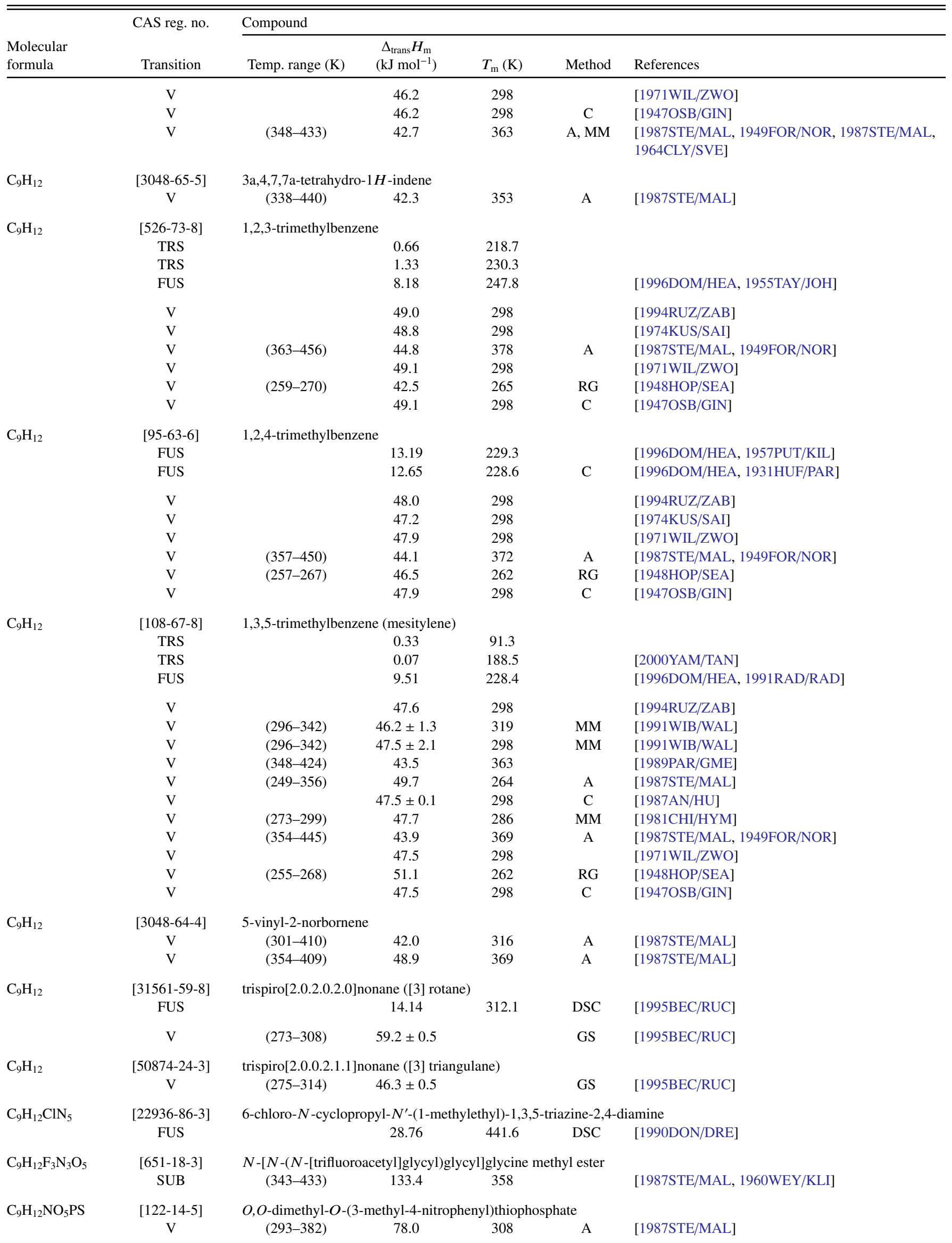


TABLE 12. Phase change enthalpies of $\mathrm{C}_{9}$ organic compounds-Continued

\begin{tabular}{|c|c|c|c|c|c|c|}
\hline \multirow[b]{2}{*}{$\begin{array}{l}\text { Molecular } \\
\text { formula }\end{array}$} & \multirow{2}{*}{$\begin{array}{l}\text { CAS reg. no. } \\
\text { Transition }\end{array}$} & \multicolumn{5}{|l|}{ Compound } \\
\hline & & Temp. range $(\mathrm{K})$ & $\begin{array}{c}\Delta_{\text {trans }} H_{\mathrm{m}} \\
\left(\mathrm{kJ} \mathrm{mol}^{-1}\right)\end{array}$ & $T_{\mathrm{m}}(\mathrm{K})$ & Method & References \\
\hline \multirow[t]{2}{*}{$\mathrm{C}_{9} \mathrm{H}_{12} \mathrm{~N}_{2}$} & [103-02-6] & \multicolumn{5}{|c|}{ phenylhydrazone acetone } \\
\hline & $\mathrm{V}$ & $(413-436)$ & 74.6 & 424 & A & [1987STE/MAL, 1913BLA] \\
\hline \multirow[t]{2}{*}{$\mathrm{C}_{9} \mathrm{H}_{12} \mathrm{~N}_{2}$} & [1502-10-9] & \multicolumn{5}{|c|}{$N$-methyl-7-(methylimino)-1,3,5-cycloheptatrienylamine } \\
\hline & SUB & & $49.4 \pm 4$ & & & [1971JAC/HUN, 1977PED/RYL] \\
\hline \multirow[t]{2}{*}{$\mathrm{C}_{9} \mathrm{H}_{12} \mathrm{~N}_{2}$} & {$[31529-46-1]$} & \multicolumn{5}{|c|}{$N$-amino-2-methylindoline } \\
\hline & FUS & & 24.45 & 318.2 & & [1997PEY/LET] \\
\hline \multirow[t]{2}{*}{$\mathrm{C}_{9} \mathrm{H}_{12} \mathrm{~N}_{2} \mathrm{O}$} & {$[101-42-8]$} & \multicolumn{5}{|c|}{ 1,1-dimethyl-3-phenylurea } \\
\hline & FUS & & 22.81 & 404.8 & $\mathrm{DSC}$ & [1991ACR, 1990DON/DRE] \\
\hline \multirow[t]{2}{*}{$\mathrm{C}_{9} \mathrm{H}_{12} \mathrm{~N}_{2} \mathrm{O}_{2}$} & {$[13142-86-4]$} & \multicolumn{5}{|l|}{ 3-ethoxyphenylurea } \\
\hline & SUB & \multicolumn{4}{|c|}{$75.3 \pm 8.3$} & [1954TSU/KAT, 1970COX/PIL] \\
\hline \multirow[t]{2}{*}{$\mathrm{C}_{9} \mathrm{H}_{12} \mathrm{~N}_{2} \mathrm{O}_{2}$} & {$[150-69-6]$} & \multicolumn{4}{|c|}{ 4-ethoxyphenylurea (dulcin) } & \\
\hline & SUB & \multicolumn{3}{|c|}{$83.7 \pm 8.3$} & & [1954TSU/KAT, 1970COX/PIL] \\
\hline \multirow[t]{2}{*}{$\mathrm{C}_{9} \mathrm{H}_{12} \mathrm{~N}_{2} \mathrm{O}_{2} \mathrm{~S}_{2}$} & {$[2651-16-3]$} & \multicolumn{4}{|c|}{$S$-methyl- $N^{\prime}$-tosylisothiourea } & \\
\hline & FUS & & 31.2 & 401.2 & DSC & [1992REI/HAN] \\
\hline $\mathrm{C}_{9} \mathrm{H}_{12} \mathrm{~N}_{2} \mathrm{~S}$ & [14222-60-7] & 2-propyl-4-pyridin & arbothioamid & & & \\
\hline & FUS & & 23.21 & 414.1 & & [2007WAN/ZHA] \\
\hline $\mathrm{C}_{9} \mathrm{H}_{12} \mathrm{~N}_{4} \mathrm{O}_{2}$ & [5770-28-5] & 8-ethyltheophyllin & & & & \\
\hline & FUS & & 37.2 & 545.3 & DSC & [1989GON/KRA] \\
\hline $\mathrm{C}_{9} \mathrm{H}_{12} \mathrm{~N}_{4} \mathrm{O}_{3}$ & [519-37-9] & 1,3-dimethyl-7-(2- & droxyethyl)x & & & \\
\hline & FUS & & 32.62 & 435.8 & $\mathrm{DSC}$ & [2014POB/DOM] \\
\hline $\mathrm{C}_{9} \mathrm{H}_{12} \mathrm{O}$ & [4013-34-7] & (1-methoxyethyl)b & zene & & & \\
\hline & $\mathrm{V}$ & $(298-313)$ & $49.2 \pm 0.4$ & 296 & GS & [2001 VER/HEI] \\
\hline & $\mathrm{V}$ & $(298-313)$ & $49.1 \pm 0.4$ & 298 & GS & [2001 VER/HEI] \\
\hline $\mathrm{C}_{9} \mathrm{H}_{12} \mathrm{O}$ & [539-30-0] & benzyl ethyl ether & & & & \\
\hline & $\mathrm{V}$ & $(278-314)$ & $53.5 \pm 0.4$ & 298 & GS & [2002KRA/VAS] \\
\hline & $\mathrm{V}$ & $(299-460)$ & 48.0 & 314 & A & [1987STE/MAL, 1947STU] \\
\hline $\mathrm{C}_{9} \mathrm{H}_{12} \mathrm{O}$ & {$[14804-32-1]$} & 2-ethylanisole & & & & \\
\hline & $\mathrm{V}$ & $(302-460)$ & 49.8 & 317 & A & [1987STE/MAL, 1947STU] \\
\hline $\mathrm{C}_{9} \mathrm{H}_{12} \mathrm{O}$ & {$[10568-38-4]$} & 3-ethylanisole & & & & \\
\hline & V & $(306-470)$ & 49.3 & 321 & A & [1987STE/MAL, 1947STU] \\
\hline $\mathrm{C}_{9} \mathrm{H}_{12} \mathrm{O}$ & {$[1515-95-3]$} & 4-ethylanisole & & & & \\
\hline & $\mathrm{V}$ & $(306-470)$ & 51.9 & 321 & A & [1987STE/MAL, 1947STU] \\
\hline $\mathrm{C}_{9} \mathrm{H}_{12} \mathrm{O}$ & {$[698-71-5]$} & 5-ethyl-3-methyph & & & & \\
\hline & V & $(468-521)$ & 55.0 & 483 & A, GS, EB & [1987STE/MAL, 1964HAN/HAR] \\
\hline & $\mathrm{V}$ & $(385-506)$ & 58.5 & 58.5 & & [1955VON/GEB] \\
\hline $\mathrm{C}_{9} \mathrm{H}_{12} \mathrm{O}$ & [88-69-7] & 2-isopropylphenol & & & & \\
\hline & $\mathrm{V}$ & $(375-493)$ & 63.5 & 390 & $\mathrm{~EB}$ & [1990NES/NAZ] \\
\hline & $\mathrm{V}$ & $(370-489)$ & 55.1 & 385 & A & [1987STE/MAL] \\
\hline & $\mathrm{V}$ & $(375-493)$ & 56.1 & 390 & & [1986TSV/NAZ] \\
\hline & V & $(335-501)$ & 57.3 & 350 & & [1947STU] \\
\hline $\mathrm{C}_{9} \mathrm{H}_{12} \mathrm{O}$ & [618-45-1] & 3-isopropylphenol & & & & \\
\hline & $\mathrm{V}$ & $(377-497)$ & 64.3 & 392 & A & [1987STE/MAL] \\
\hline $\mathrm{C}_{9} \mathrm{H}_{12} \mathrm{O}$ & [99-89-8] & 4-isopropylphenol & & & & \\
\hline & $\mathrm{V}$ & $(391-507)$ & 63.7 & 406 & $\mathrm{~EB}$ & [1990NES/NAZ] \\
\hline & $\mathrm{V}$ & $(380-496)$ & 63.1 & 395 & $\mathrm{~A}$ & [1987STE/MAL] \\
\hline $\mathrm{C}_{9} \mathrm{H}_{12} \mathrm{O}$ & [2741-16-4] & isopropyl phenyl e & & & & \\
\hline & $\mathrm{V}$ & $(345-448)$ & 49.5 & 360 & A & [1987STE/MAL, 1965HEI/SUR, 1984BOU/FRI] \\
\hline $\mathrm{C}_{9} \mathrm{H}_{12} \mathrm{O}$ & {$[122-97-4]$} & 3-phenyl-1-propan & & & & \\
\hline & $\mathrm{V}$ & $(284-328)$ & 62.8 & 299 & A & [1987STE/MAL] \\
\hline & $\mathrm{V}$ & $(347-508)$ & 62.6 & 362 & & {$[1947 \mathrm{STU}]$} \\
\hline $\mathrm{C}_{9} \mathrm{H}_{12} \mathrm{O}$ & {$[617-94-7]$} & 2-phenyl-2-propan & & & & \\
\hline
\end{tabular}


TABLE 12. Phase change enthalpies of $\mathrm{C}_{9}$ organic compounds-Continued

\begin{tabular}{|c|c|c|c|c|c|c|}
\hline \multirow[b]{2}{*}{$\begin{array}{l}\text { Molecular } \\
\text { formula }\end{array}$} & \multirow{2}{*}{$\begin{array}{l}\text { CAS reg. no. } \\
\text { Transition }\end{array}$} & \multicolumn{5}{|l|}{ Compound } \\
\hline & & Temp. range $(\mathrm{K})$ & $\begin{array}{c}\Delta_{\text {trans }} H_{\mathrm{m}} \\
\left(\mathrm{kJ} \mathrm{mol}^{-1}\right)\end{array}$ & $T_{\mathrm{m}}(\mathrm{K})$ & Method & References \\
\hline & $\mathrm{V}$ & $(391-423)$ & 52.9 & 406 & A & [1987STE/MAL] \\
\hline $\mathrm{C}_{9} \mathrm{H}_{12} \mathrm{O}$ & $\begin{array}{c}{[622-85-5]} \\
\mathrm{V}\end{array}$ & $\begin{array}{l}\text { phenyl propyl ether } \\
\text { (374-463) }\end{array}$ & 46.5 & 389 & A & [1987STE/MAL] \\
\hline $\mathrm{C}_{9} \mathrm{H}_{12} \mathrm{O}$ & $\begin{array}{c}\text { [644-35-9] } \\
\mathrm{V} \\
\mathrm{V} \\
\mathrm{V} \\
\mathrm{V}\end{array}$ & $\begin{array}{c}\text { 2-propylphenol } \\
(377-495) \\
(381-504) \\
(381-504) \\
(381-504)\end{array}$ & $\begin{array}{l}56.9 \\
59.9 \\
57.2 \\
53.0\end{array}$ & $\begin{array}{l}392 \\
398 \\
423 \\
473\end{array}$ & A & $\begin{array}{l}\text { [1987STE/MAL] } \\
\text { [1953STA/MUL] } \\
\text { [1953STA/MUL] } \\
\text { [1953STA/MUL] }\end{array}$ \\
\hline $\mathrm{C}_{9} \mathrm{H}_{12} \mathrm{O}$ & $\begin{array}{c}{[621-27-2]} \\
\mathrm{V} \\
\mathrm{V} \\
\mathrm{V} \\
\mathrm{V}\end{array}$ & $\begin{array}{c}\text { 3-propylphenol } \\
(408-538) \\
(386-512) \\
(386-512) \\
(386-512)\end{array}$ & $\begin{array}{l}60.2 \\
59.9 \\
57.2 \\
53.0\end{array}$ & $\begin{array}{l}423 \\
398 \\
423 \\
473\end{array}$ & A & $\begin{array}{l}\text { [1987STE/MAL] } \\
{[1953 \mathrm{STA} / \mathrm{MUL}]} \\
\text { [1953STA/MUL] } \\
\text { [1953STA/MUL] }\end{array}$ \\
\hline $\mathrm{C}_{9} \mathrm{H}_{12} \mathrm{O}$ & $\begin{array}{c}{[645-56-7]} \\
\text { V } \\
\text { V } \\
\text { V } \\
\text { V } \\
\text { V } \\
\text { V }\end{array}$ & $\begin{array}{c}\text { 4-propylphenol } \\
(383-508) \\
(347-517) \\
(347-517) \\
(347-517) \\
(347-517) \\
(347-517)\end{array}$ & $\begin{array}{l}56.7 \\
61.3 \\
59.5 \\
58.4 \\
56.2 \\
51.5\end{array}$ & $\begin{array}{l}398 \\
348 \\
373 \\
398 \\
423 \\
473\end{array}$ & A & $\begin{array}{l}\text { [1987STE/MAL] } \\
\text { [1953STA/MUL] } \\
\text { [1953STA/MUL] } \\
\text { [1953STA/MUL] } \\
\text { [1953STA/MUL] } \\
\text { [1953STA/MUL] }\end{array}$ \\
\hline $\mathrm{C}_{9} \mathrm{H}_{12} \mathrm{O}$ & $\begin{array}{c}{[697-82-5]} \\
\mathrm{V} \\
\mathrm{V}\end{array}$ & $\begin{array}{l}\text { 2,3,5-trimethylphenol } \\
\quad(459-521) \\
(379-506)\end{array}$ & $\begin{array}{l}53.9 \\
55.1\end{array}$ & $\begin{array}{l}474 \\
394\end{array}$ & $\mathrm{~A}, \mathrm{GS}, \mathrm{EB}$ & $\begin{array}{l}\text { [1987STE/MAL, 1964HAN/HAR] } \\
\text { [1955VON/GEB] }\end{array}$ \\
\hline $\mathrm{C}_{9} \mathrm{H}_{12} \mathrm{O}$ & $\begin{array}{l}{[2416-94-6]} \\
\text { FUS }\end{array}$ & 2,3,6-trimethylphenol & 22.05 & 331.2 & & [1999VER] \\
\hline & SUB & & $86.7 \pm 0.6$ & 298 & GS & [1999VER] \\
\hline $\mathrm{C}_{9} \mathrm{H}_{12} \mathrm{O}$ & $\begin{array}{c}{[496-78-6]} \\
V\end{array}$ & $\begin{array}{l}\text { 2,4,5-trimethylphenol } \\
\quad(379-505)\end{array}$ & 56.5 & 394 & A & [1987STE/MAL, 1955VON/GEB] \\
\hline $\mathrm{C}_{9} \mathrm{H}_{12} \mathrm{O}$ & $\begin{array}{c}\text { [527-60-6] } \\
\text { SUB } \\
\text { SUB }\end{array}$ & 2,4,6-trimethylphenol & $\begin{array}{c}82.8 \pm 0.3 \\
95.0\end{array}$ & $\begin{array}{l}298 \\
298\end{array}$ & $\begin{array}{l}\text { GS } \\
\mathrm{C}\end{array}$ & $\begin{array}{l}\text { [1999VER] } \\
\text { [1971BER/GIR, 1999VER] }\end{array}$ \\
\hline & $\mathrm{V}$ & $(367-494)$ & 53.2 & 382 & A & [1987STE/MAL, 1955VON/GEB] \\
\hline $\mathrm{C}_{9} \mathrm{H}_{12} \mathrm{O}$ & $\begin{array}{c}{[2416-94-6]} \\
V\end{array}$ & $\begin{array}{l}\text { 2,5,6-trimethylphenol } \\
\quad(359-503)\end{array}$ & $51.1 \pm 0.2$ & 431 & Static & [1988BAG/GUR] \\
\hline $\mathrm{C}_{9} \mathrm{H}_{12} \mathrm{O}$ & $\begin{array}{c}{[527-54-8]} \\
\mathrm{V}\end{array}$ & $\begin{array}{l}\text { 3,4,5-trimethylphenol } \\
\quad(396-521)\end{array}$ & 61.1 & 411 & A & [1987STE/MAL] \\
\hline $\mathrm{C}_{9} \mathrm{H}_{12} \mathrm{O}$ & $\begin{array}{c}\text { [617-94-7] } \\
\text { SUB } \\
\text { SUB }\end{array}$ & $\begin{array}{c}\alpha, \alpha \text {-dimethylbenzyl a } \\
(276-302) \\
(276-302)\end{array}$ & $\begin{array}{l}\text { lcohol } \\
82.8 \pm 0.7 \\
82.3 \pm 0.7\end{array}$ & $\begin{array}{l}289 \\
298\end{array}$ & $\begin{array}{l}\text { GS } \\
\text { GS }\end{array}$ & $\begin{array}{l}{[1999 \mathrm{VER} 4]} \\
{[1999 \mathrm{VER} 4]}\end{array}$ \\
\hline & $\begin{array}{l}\mathrm{V} \\
\mathrm{V}\end{array}$ & $\begin{array}{l}(311-338) \\
(311-338)\end{array}$ & $\begin{array}{l}63.4 \pm 0.5 \\
65.0 \pm 0.5\end{array}$ & $\begin{array}{l}325 \\
298\end{array}$ & $\begin{array}{l}\text { GS } \\
\text { GS }\end{array}$ & $\begin{array}{l}{[1999 V E R 4]} \\
{[1999 V E R 4]}\end{array}$ \\
\hline $\mathrm{C}_{9} \mathrm{H}_{12} \mathrm{O}_{2}$ & $\begin{array}{c}{[700-13-0]} \\
\mathrm{V}\end{array}$ & $\begin{array}{l}\text { trimethylhydroquinon } \\
\qquad(450-501)\end{array}$ & $45.5 \pm 0.3$ & 475 & Static & [1988BAG/GUR] \\
\hline $\mathrm{C}_{9} \mathrm{H}_{12} \mathrm{O}_{2}$ & $\begin{array}{c}{[80-15-9]} \\
\mathrm{V} \\
\mathrm{V}\end{array}$ & $\begin{array}{l}\text { cumene hydroperoxide } \\
\qquad(283-333) \\
(347-390)\end{array}$ & $\begin{array}{l}69.9 \\
74.0\end{array}$ & $\begin{array}{l}298 \\
362\end{array}$ & $\begin{array}{l}\text { A } \\
\text { A }\end{array}$ & $\begin{array}{l}\text { [1987STE/MAL] } \\
\text { [1987STE/MAL] }\end{array}$ \\
\hline $\mathrm{C}_{9} \mathrm{H}_{12} \mathrm{O}_{2}$ & $\mathrm{~V}$ & $\begin{array}{l}\text { 1,3-dihydroxy-5-meth } \\
\text { (388-453) }\end{array}$ & $\begin{array}{l}\text { yl-2-ethylbe } \\
77.1\end{array}$ & 403 & $\mathrm{~A}, \mathrm{GC}$ & [1987STE/MAL, 1975KUN/LIL] \\
\hline $\mathrm{C}_{9} \mathrm{H}_{12} \mathrm{O}_{2}$ & $\begin{array}{c}{[4179-19-5]} \\
\mathrm{V}\end{array}$ & $\begin{array}{l}\text { 3,5-dimethoxytoluene } \\
(374-520)\end{array}$ & 59.5 & 389 & A & [1987STE/MAL] \\
\hline $\mathrm{C}_{9} \mathrm{H}_{12} \mathrm{O}_{2}$ & {$[622-08-2]$} & ethylene glycol monob & benzyl ether & & & \\
\hline
\end{tabular}


TABLE 12. Phase change enthalpies of $\mathrm{C}_{9}$ organic compounds-Continued

\begin{tabular}{|c|c|c|c|c|c|c|}
\hline \multirow[b]{2}{*}{$\begin{array}{l}\text { Molecular } \\
\text { formula }\end{array}$} & \multirow{2}{*}{$\begin{array}{l}\text { CAS reg. no. } \\
\text { Transition } \\
\end{array}$} & \multicolumn{5}{|l|}{ Compound } \\
\hline & & Temp. range $(\mathrm{K})$ & $\begin{array}{c}\Delta_{\text {trans }} H_{\mathrm{m}} \\
\left(\mathrm{kJ} \mathrm{mol}^{-1}\right) \\
\end{array}$ & $T_{\mathrm{m}}(\mathrm{K})$ & Method & References \\
\hline & $\mathrm{V}$ & $(453-530)$ & 58.6 & 468 & A & [1987STE/MAL] \\
\hline $\mathrm{C}_{9} \mathrm{H}_{12} \mathrm{O}_{2}$ & $\begin{array}{c}{[770-35-4]} \\
\mathrm{V}\end{array}$ & $\begin{array}{l}\text { propylene glycol 1- } \\
\qquad(389-509)\end{array}$ & $\begin{array}{l}\text { nenyl ether } \\
59.5\end{array}$ & 404 & A & [1987STE/MAL] \\
\hline $\mathrm{C}_{9} \mathrm{H}_{12} \mathrm{O}_{2}$ & V & $\begin{array}{l}\text { isopropyl catechol ( } \\
\quad(393-453)\end{array}$ & $\begin{array}{c}\text { omer not spe } \\
65.3\end{array}$ & 423 & & [1965GAK/BAB] \\
\hline $\mathrm{C}_{9} \mathrm{H}_{12} \mathrm{O}_{2}$ & $\begin{array}{c}{[1125-88-8]} \\
\mathrm{V} \\
\mathrm{V}\end{array}$ & $\begin{array}{l}\text { benzaldehyde dimet } \\
(278-318) \\
(283-318)\end{array}$ & $\begin{array}{l}y l \text { acetal } \\
60.9 \pm 0.5 \\
56.5 \pm 0.7\end{array}$ & $\begin{array}{l}298 \\
300\end{array}$ & $\begin{array}{l}\text { GS } \\
\text { GS }\end{array}$ & $\begin{array}{l}\text { [2002VER] } \\
{[1995 \mathrm{VER} / \mathrm{DOG}]}\end{array}$ \\
\hline $\mathrm{C}_{9} \mathrm{H}_{12} \mathrm{O}_{2}$ & $\begin{array}{c}{[2138-48-9]} \\
\text { SUB }\end{array}$ & 3-isopropyl-1,2-dih & $\begin{array}{l}\text { lroxybenzen } \\
97.8 \pm 1.7\end{array}$ & 298 & $\mathrm{C}$ & [1984RIB/RIB2] \\
\hline $\mathrm{C}_{9} \mathrm{H}_{12} \mathrm{O}_{2}$ & $\begin{array}{l}{[1125-21-9]} \\
\text { FUS }\end{array}$ & 2,6,6-trimethyl-2-cy & $\begin{array}{c}\text { lohexene- } 1,4 \\
7.2\end{array}$ & 297.3 & DSC & [1992BAB/HWA] \\
\hline $\mathrm{C}_{9} \mathrm{H}_{12} \mathrm{O}_{3}$ & $\begin{array}{c}\text { [634-36-6] } \\
\text { SUB }\end{array}$ & 1,2,3-trimethoxyber & $\begin{array}{l}\text { ene } \\
98.0 \pm 0.3\end{array}$ & 298 & $\mathrm{C}$ & [2000MAT/MIR] \\
\hline $\mathrm{C}_{9} \mathrm{H}_{12} \mathrm{O}_{3}$ & $\begin{array}{c}{[621-23-8]} \\
\text { SUB }\end{array}$ & 1,3,5-trimethoxyber & $\begin{array}{l}\text { ene } \\
100.6 \pm 1.9\end{array}$ & 298 & $\mathrm{C}$ & [2000MAT/MIR] \\
\hline & V & & $68.2 \pm 2.0$ & 298 & CGC & {$[2000 \mathrm{NIC} / \mathrm{ORF}]$} \\
\hline $\mathrm{C}_{9} \mathrm{H}_{12} \mathrm{O}_{3}$ & $\begin{array}{l}\text { [538-43-2] } \\
\text { FUS }\end{array}$ & (racemic) 3-phenox & $\begin{array}{l}\text { ropane-1,2-c } \\
28.0\end{array}$ & 331.7 & $\mathrm{DSC}$ & [2008BRE/BRE, 2006ZAK/LAZ] \\
\hline $\mathrm{C}_{9} \mathrm{H}_{12} \mathrm{O}_{3}$ & $\begin{array}{c}{[82430-38-4]} \\
\text { FUS }\end{array}$ & $(R)$-3-phenoxyprop & $\begin{array}{l}\text { e-1,2-diol } \\
31.8\end{array}$ & 341.5 & DSC & [2008BRE/BRE, 2006ZAK/LAZ] \\
\hline $\mathrm{C}_{9} \mathrm{H}_{12} \mathrm{O}_{3}$ & $\begin{array}{c}{[5662-95-3]} \\
\text { FUS }\end{array}$ & 3,3-tetramethyleneg & $\begin{array}{c}\text { taric acid an } \\
15.3\end{array}$ & $\begin{array}{l}\text { de } \\
338.4\end{array}$ & DSC & [2008MAT/MIR] \\
\hline & SUB & & $96.4 \pm 1.1$ & 298 & $\mathrm{C}$ & [2008MAT/MIR] \\
\hline $\mathrm{C}_{9} \mathrm{H}_{12} \mathrm{O}_{4}$ & $\begin{array}{l}\text { [642-71-7] } \\
\text { FUS }\end{array}$ & 3,4,5-trimethoxyphe & 31.94 & 420.2 & DSC & [2008MAT/MIR2] \\
\hline & SUB & & $125.3 \pm 3.1$ & 298 & $\mathrm{C}$ & [2008MAT/MIR2] \\
\hline $\mathrm{C}_{9} \mathrm{H}_{12} \mathrm{~S}$ & $\begin{array}{c}{[6263-62-3]} \\
\mathrm{V} \\
\mathrm{V} \\
\mathrm{V}\end{array}$ & $\begin{array}{c}\text { benzyl ethyl sulfide } \\
(346-370) \\
(345-500)\end{array}$ & $\begin{array}{c}56.0 \\
54.8 \\
56.9 \pm 2.1\end{array}$ & $\begin{array}{l}358 \\
360 \\
298\end{array}$ & A & $\begin{array}{l}\text { [1999DYK/SVO] } \\
\text { [1987STE/MAL] } \\
\text { [1962MAC/MAY] }\end{array}$ \\
\hline $\mathrm{C}_{9} \mathrm{H}_{12} \mathrm{~S}$ & $\begin{array}{c}{[20760-06-9]} \\
V\end{array}$ & $\begin{array}{c}\text { 2-ethylthioanisole } \\
(481-511)\end{array}$ & 44.3 & 496 & & [1999DYK/SVO] \\
\hline $\mathrm{C}_{9} \mathrm{H}_{12} \mathrm{~S}$ & $\begin{array}{c}{[34786-24-8]} \\
\mathrm{V}\end{array}$ & $\begin{array}{l}\text { ethyl } m \text {-tolyl sulfid } \\
\quad(472-502)\end{array}$ & 43.5 & 487 & & [1999DYK/SVO] \\
\hline $\mathrm{C}_{9} \mathrm{H}_{12} \mathrm{~S}$ & $\begin{array}{c}{[622.63-9]} \\
\mathrm{V}\end{array}$ & $\begin{array}{l}\text { ethyl } p \text {-tolyl sulfide } \\
\quad(473-503)\end{array}$ & 43.6 & 488 & & [1999DYK/SVO] \\
\hline $\mathrm{C}_{9} \mathrm{H}_{12} \mathrm{~S}$ & $\begin{array}{c}{[3019-20-3]} \\
\mathrm{V}\end{array}$ & $\begin{array}{c}\text { (isopropylthio)benz } \\
(461-491)\end{array}$ & $\mathrm{U} 23.6$ & 476 & & [1999DYK/SVO] \\
\hline $\mathrm{C}_{9} \mathrm{H}_{12} \mathrm{~S}$ & $\begin{array}{c}{[874-79-3]} \\
\mathrm{V}\end{array}$ & $\begin{array}{l}\text { (propylthio)benzene } \\
\quad(473-503)\end{array}$ & 44.3 & 488 & & [1999DYK/SVO] \\
\hline $\mathrm{C}_{9} \mathrm{H}_{13} \mathrm{BrN}_{2} \mathrm{O}_{2}$ & $\begin{array}{l}\text { [314-40-9] } \\
\text { FUS }\end{array}$ & 5-bromo-6-methyl-? & $\begin{array}{l}\text { (1-methylpro } \\
22.02\end{array}$ & $\begin{array}{c}2,4(1 H, ? \\
428.3\end{array}$ & $\begin{array}{l}\text { yrimidine } \\
\text { DSC }\end{array}$ & $\begin{array}{l}\text { ione } \\
\text { [1991ACR, 1990DON/DRE] }\end{array}$ \\
\hline $\mathrm{C}_{9} \mathrm{H}_{13} \mathrm{ClN}_{6}$ & $\begin{array}{l}{[21725-46-2]} \\
\text { FUS }\end{array}$ & 2-[[4-chloro-6-(ethy & $\begin{array}{l}\operatorname{mino})-1,3,5 \\
41.96\end{array}$ & $\begin{array}{c}\text { n-2-yl]at } \\
437.9\end{array}$ & $\begin{array}{l}\text {-2-methyl } \\
\text { DSC }\end{array}$ & $\begin{array}{l}\text { ropanenitrile } \\
\text { [1991ACR, 1990DON/DRE] }\end{array}$ \\
\hline $\mathrm{C}_{9} \mathrm{H}_{13} \mathrm{ClN}_{6}$ & $\begin{array}{l}{[21725-46-2]} \\
\text { SUB }\end{array}$ & $\begin{array}{c}\text { 2-[(4-chloro-6-ethy }] \\
(339-365)\end{array}$ & $\begin{array}{l}\text { mino- } s \text {-triazi } \\
90.7\end{array}$ & $\begin{array}{l}\text { 1) } \operatorname{amino} \\
352\end{array}$ & $\begin{array}{l}\text { ethylpropi } \\
\text { GS }\end{array}$ & $\begin{array}{l}\text { hitrile (cyanazine) } \\
\text { [1982GRA/FOS] }\end{array}$ \\
\hline $\mathrm{C}_{9} \mathrm{H}_{13} \mathrm{Cl}_{3} \mathrm{NO}_{4} \mathrm{P}$ & $\begin{array}{l}{[5598-15-2]} \\
\text { FUS }\end{array}$ & $O, O$-diethyl- $O$ - $(3$, & $\begin{array}{l}\text {-trichloro-2- } \\
15.61\end{array}$ & $\begin{array}{l}\text { l) phosp } \\
312.5\end{array}$ & DSC & [1990DON/DRE] \\
\hline
\end{tabular}


TABLE 12. Phase change enthalpies of $\mathrm{C}_{9}$ organic compounds-Continued

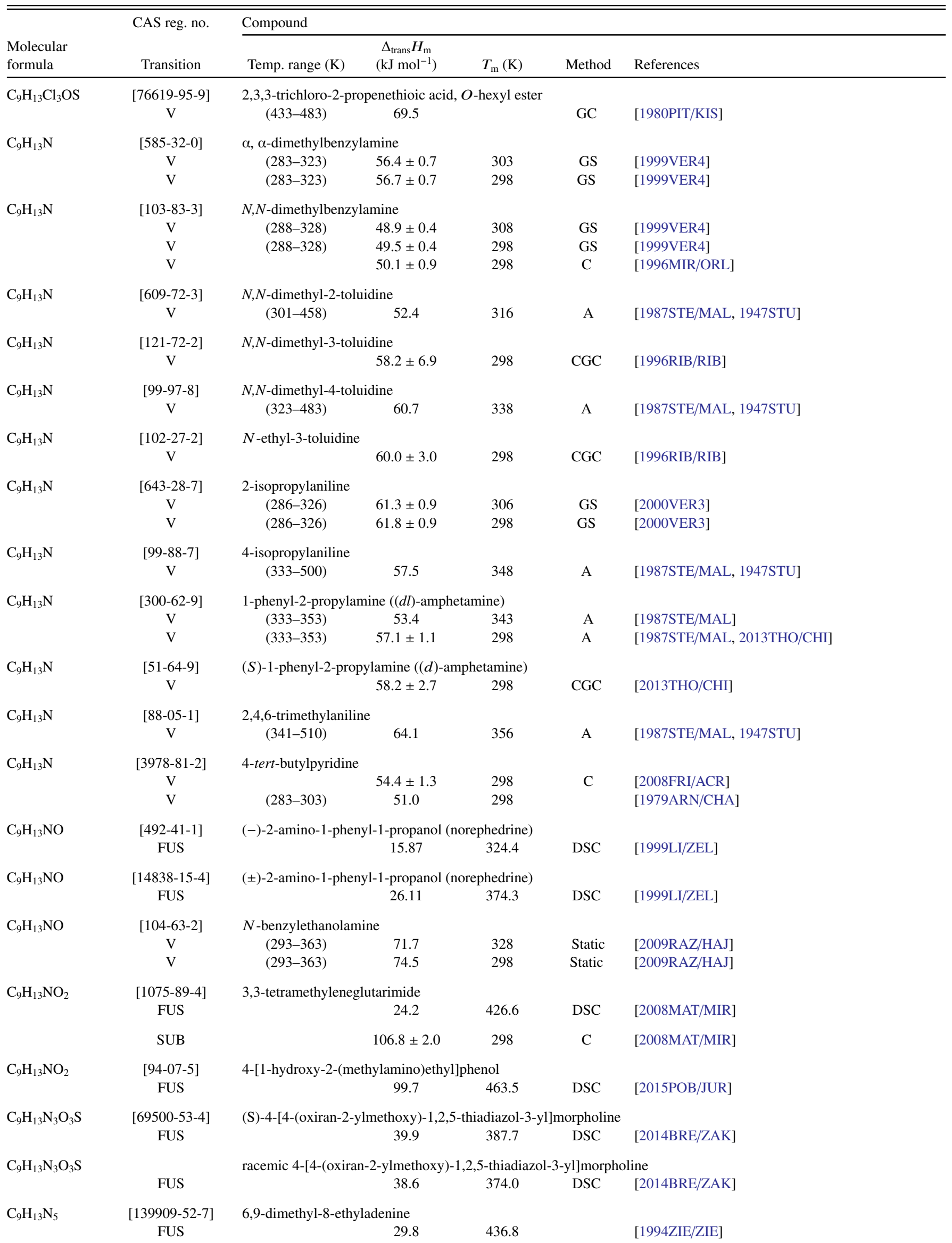


TABLE 12. Phase change enthalpies of $\mathrm{C}_{9}$ organic compounds-Continued

\begin{tabular}{|c|c|c|c|c|c|c|}
\hline \multirow[b]{2}{*}{$\begin{array}{l}\text { Molecular } \\
\text { formula }\end{array}$} & \multirow{2}{*}{$\begin{array}{l}\text { CAS reg. no. } \\
\text { Transition }\end{array}$} & \multicolumn{5}{|l|}{ Compound } \\
\hline & & Temp. range $(\mathrm{K})$ & $\begin{array}{c}\Delta_{\text {trans }} H_{\mathrm{m}} \\
\left(\mathrm{kJ} \mathrm{mol}^{-1}\right) \\
\end{array}$ & $T_{\mathrm{m}}(\mathrm{K})$ & Method & References \\
\hline & SUB & $(345-351)$ & $94.1 \pm 0.1$ & 348 & ME & [1994ZIE/ZIE] \\
\hline $\mathrm{C}_{9} \mathrm{H}_{13} \mathrm{~N}_{5}$ & $\begin{array}{c}{[117954-97-9]} \\
\text { SUB }\end{array}$ & $\begin{array}{l}\text { 8-propyl-9-methyla } \\
(364-370)\end{array}$ & $\begin{array}{l}\text { enine } \\
124.2 \pm 0.8\end{array}$ & 367 & ME & [1987KAM/ZIE] \\
\hline $\mathrm{C}_{9} \mathrm{H}_{13} \mathrm{~N}_{5} \mathrm{O}_{4}$ & $\begin{array}{l}{[82410-32-0]} \\
\text { FUS }\end{array}$ & 9-[(1,3-dihydroxy- & $\begin{array}{l}\text { propoxy)metl } \\
37.88\end{array}$ & $\begin{array}{l}\text { lanine } \\
509.2\end{array}$ & DSC & [1999ZIE/GOL] \\
\hline $\mathrm{C}_{9} \mathrm{H}_{14}$ & V & 1-ethylnortricyclo[ & $\begin{array}{l}\left.2,1,0^{2,6}\right] \text { hepta } \\
42.0 \pm 0.1\end{array}$ & 298 & $\mathrm{C}$ & [1996VAR/PAS] \\
\hline $\mathrm{C}_{9} \mathrm{H}_{14}$ & $\begin{array}{c}{[2972-20-5]} \\
\mathrm{V}\end{array}$ & 2-methylenebicyclc & $\begin{array}{c}2.2 .2 \text { ]octane } \\
45.2\end{array}$ & & & {$[1974 \mathrm{KOZ} / \mathrm{BYC}]$} \\
\hline $\mathrm{C}_{9} \mathrm{H}_{14}$ & $\begin{array}{c}{[4893-13-4]} \\
\text { V } \\
\text { V }\end{array}$ & $\begin{array}{l}\text { 2-methylbicyclo }[2 . \\
\quad(363-402)\end{array}$ & $\begin{array}{l}\text { 2]oct-2-ene } \\
40.2 \\
43.5 \pm 0.4\end{array}$ & $\begin{array}{l}378 \\
298\end{array}$ & $\begin{array}{r}\mathrm{A} \\
\mathrm{EB}\end{array}$ & $\begin{array}{l}\text { [1987STE/MAL] } \\
\text { [1974VAR/DRU, 1974KOZ/BYC] }\end{array}$ \\
\hline $\mathrm{C}_{9} \mathrm{H}_{14}$ & $\begin{array}{c}{[2146-39-6]} \\
\mathrm{V}\end{array}$ & $\begin{array}{l}\text { 2-vinylbicyclo }[2.2 . \\
(350-385)\end{array}$ & $\begin{array}{r}\text { heptane } \\
38.6\end{array}$ & 365 & A & [1987STE/MAL] \\
\hline $\mathrm{C}_{9} \mathrm{H}_{14}$ & $\begin{array}{c}{[7124-86-9]} \\
\text { SUB }\end{array}$ & bicyclo[3.2.2]non- & $\begin{array}{l}\text { ene } \\
\qquad 48 \pm 1.0\end{array}$ & 298 & $\mathrm{C}$ & [1982JOC/DEK2] \\
\hline $\mathrm{C}_{9} \mathrm{H}_{14}$ & $\begin{array}{c}\text { [6671-66-5] } \\
\text { SUB }\end{array}$ & bicyclo[3.3.1]non-2 & $\begin{array}{l}\text { ene } \\
\qquad 48.2 \pm 0.4\end{array}$ & 298 & $\mathrm{C}$ & [1982JOC/DEK2] \\
\hline $\mathrm{C}_{9} \mathrm{H}_{14}$ & $\begin{array}{l}{[16456-33-0]} \\
\text { SUB }\end{array}$ & bicyclo[4.2.1]non-? & $\begin{array}{l}\text { ene } \\
\qquad 49.7 \pm 0.8\end{array}$ & 298 & $\mathrm{C}$ & [1982JOC/DEK2] \\
\hline $\mathrm{C}_{9} \mathrm{H}_{14} \mathrm{ClN}_{5}$ & $\begin{array}{c}\text { [139-40-2] } \\
\text { FUS } \\
\text { FUS }\end{array}$ & 2-chloro-4,6-bis(isc & $\begin{array}{c}\text { ropylamino)- } \\
41.87 \\
42.1\end{array}$ & $\begin{array}{c}\text {-triazine } \\
490.3\end{array}$ & $\begin{array}{l}\text { azine) } \\
\text { DSC } \\
\text { DSC }\end{array}$ & $\begin{array}{l}\text { [1991ACR, 1990DON/DRE] } \\
\text { [1971GET/WAR] }\end{array}$ \\
\hline $\mathrm{C}_{9} \mathrm{H}_{14} \mathrm{~F}_{3} \mathrm{NO}_{3}$ & $\begin{array}{c}{[1115-39-5]} \\
\mathrm{V}\end{array}$ & $\begin{array}{c}N \text {-trifluoroacetyl- } l \\
\quad(273-463)\end{array}$ & $\begin{array}{c}\text { eucine, methy } \\
55.9\end{array}$ & 288 & A & [1987STE/MAL, 1999DYK/SVO, 1960WEY/KLI] \\
\hline $\mathrm{C}_{9} \mathrm{H}_{14} \mathrm{~N}_{2}$ & $\begin{array}{c}{[1675-69-0]} \\
\text { FUS }\end{array}$ & azelaic acid dinitril & 18.68 & 251.1 & DSC & [2007BAD/BLA] \\
\hline & $\mathrm{V}$ & $(308-341)$ & 80.4 & 323 & A & [1987STE/MAL, 1960WOO/MUR] \\
\hline $\mathrm{C}_{9} \mathrm{H}_{14} \mathrm{~N}_{2} \mathrm{O}_{2}$ & $\begin{array}{c}{[82413-39-6]} \\
\text { FUS }\end{array}$ & 1,3-dimethyl-5-pro & $\begin{array}{l}\text { yluracil } \\
26.3\end{array}$ & 355 & DSC & [1996KAM/ZIE] \\
\hline & SUB & $(317-327)$ & $111.0 \pm 1.6$ & 322 & $\mathrm{ME}$ & [1996KAM/ZIE] \\
\hline $\mathrm{C}_{9} \mathrm{H}_{14} \mathrm{~N}_{2} \mathrm{O}_{2}$ & $\begin{array}{c}\text { [175412-48-3] } \\
\text { FUS }\end{array}$ & 1,3-dimethyl-5-iso & $\begin{array}{c}\text { opyluracil } \\
22.4\end{array}$ & 354.7 & DSC & [1996KAM/ZIE] \\
\hline & SUB & $(316-328)$ & $102.9 \pm 1.6$ & 322 & ME & [1996KAM/ZIE] \\
\hline $\mathrm{C}_{9} \mathrm{H}_{14} \mathrm{~N}_{2} \mathrm{O}_{2}$ & $\begin{array}{c}\text { [21472-93-5] } \\
\text { FUS }\end{array}$ & 1,3-diethylthymine & 21.6 & 327 & DSC & [1984ZIE/ZIE2] \\
\hline & $\begin{array}{l}\text { SUB } \\
\text { SUB }\end{array}$ & $(307-325)$ & $\begin{array}{l}89.8 \pm 0.4 \\
95.0 \pm 2.1\end{array}$ & $\begin{array}{l}298 \\
317\end{array}$ & $\begin{array}{c}\mathrm{C} \\
\mathrm{QR}\end{array}$ & $\begin{array}{l}\text { [1980SAB/KOM] } \\
{[1980 \mathrm{TEP} / \mathrm{YAN}]}\end{array}$ \\
\hline $\mathrm{C}_{9} \mathrm{H}_{14} \mathrm{~N}_{2} \mathrm{O}_{3}$ & $\begin{array}{l}{[50-11-3]} \\
\text { FUS }\end{array}$ & metharbital & 30.1 & & DSC & [1978SEK/TSU] \\
\hline $\mathrm{C}_{9} \mathrm{H}_{14} \mathrm{O}$ & [281-24-3] & 2-oxaadamantane & & & & \\
\hline & FUS & & 8.12 & 567 & DSC & [1978AND/CAR] \\
\hline $\mathrm{C}_{9} \mathrm{H}_{14} \mathrm{O}$ & $\begin{array}{c}\text { [17931-55-4] } \\
\text { TRS } \\
\text { TRS }\end{array}$ & bicyclo[3.3.1]nona & $\begin{array}{r}\text { 9-one } \\
13.99 \\
14.11\end{array}$ & $\begin{array}{c}299 \\
300.5\end{array}$ & $\begin{array}{l}\mathrm{DSC} \\
\mathrm{AC}\end{array}$ & $\begin{array}{l}\text { [1998PAR/GIL2] } \\
\text { [1991WHI/PER] }\end{array}$ \\
\hline $\mathrm{C}_{9} \mathrm{H}_{14} \mathrm{O}$ & $\begin{array}{c}{[5689-04-3]} \\
\mathrm{V}\end{array}$ & cis-2-hexahydroind & none 57.5 & 298 & & [1971SEL3] \\
\hline $\mathrm{C}_{9} \mathrm{H}_{14} \mathrm{O}$ & $\begin{array}{c}{[16484-17-6]} \\
\mathrm{V}\end{array}$ & trans-2-hexahydroi & $\begin{array}{l}\text { danone } \\
56.1\end{array}$ & 298 & & [1971SEL3] \\
\hline
\end{tabular}


TABLE 12. Phase change enthalpies of $\mathrm{C}_{9}$ organic compounds-Continued

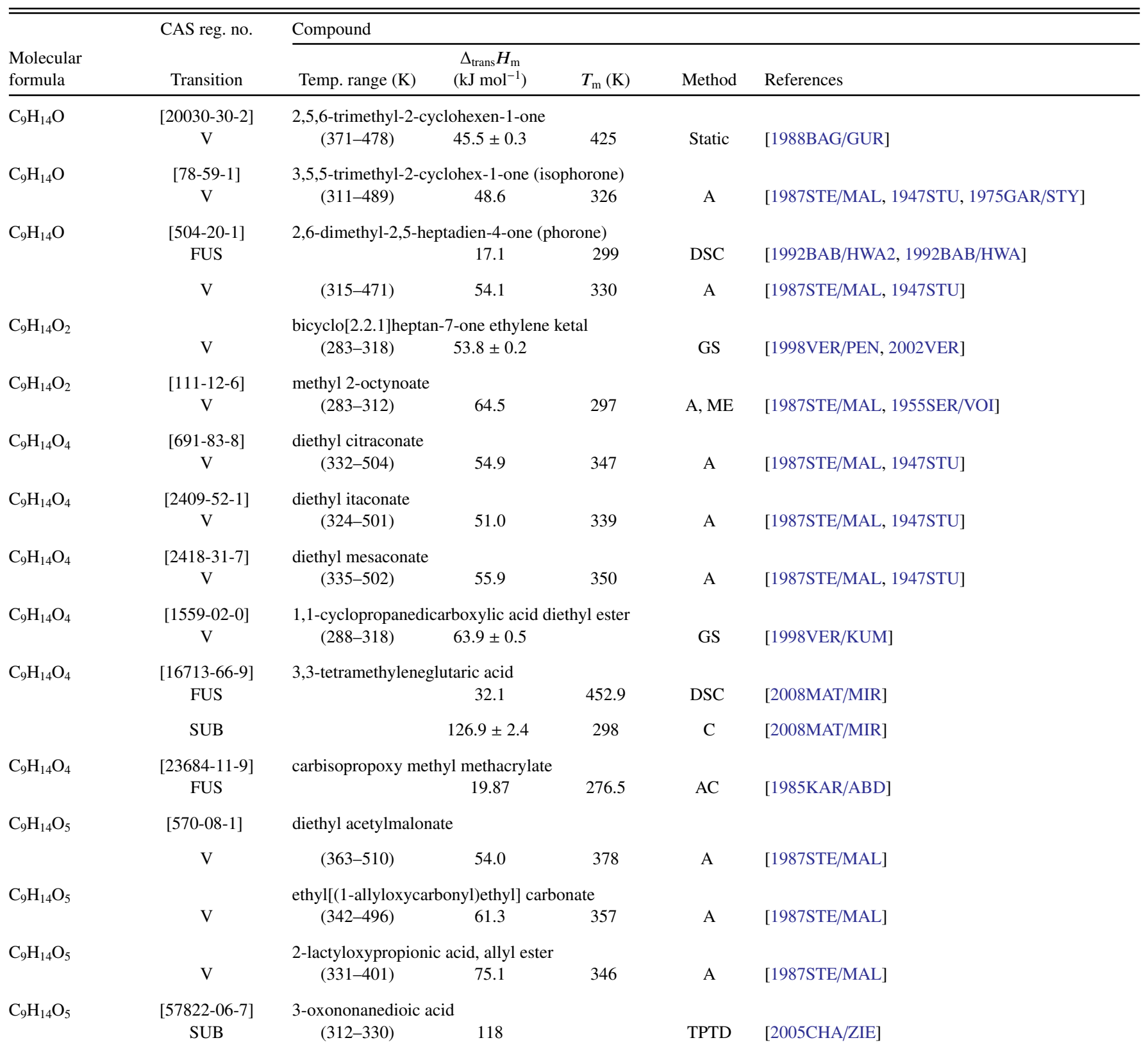

Note: Values based on TPTD method are not consistent with values determined by other experimental methods

\begin{tabular}{|c|c|c|c|c|c|c|}
\hline \multirow{8}{*}{$\mathrm{C}_{9} \mathrm{H}_{14} \mathrm{O}_{6}$} & {$[102-76-1]$} & \multicolumn{5}{|c|}{ glycerol triacetate (triacetin) } \\
\hline & FUS & & 25.8 & 275.3 & & [1996DOM/HEA, 1983RAB/KHL] \\
\hline & $\mathrm{V}$ & $(300-328)$ & $83.9 \pm 0.8$ & 298 & GS & [2010MAS/KRA] \\
\hline & $\mathrm{V}$ & $(320-361)$ & $81.9 \pm 0.3$ & 298 & GS & [2009VER/EME2] \\
\hline & $\mathrm{V}$ & $(440-590)$ & $83.8 \pm 0.9$ & 298 & $\mathrm{~EB}$ & [1990DAU/HUT, 2009VER/EME2] \\
\hline & $\mathrm{V}$ & $(284-319)$ & 82.0 & 299 & A & [1987STE/MAL, 1963WOO/ADI] \\
\hline & $\mathrm{V}$ & & $85.7 \pm 0.3$ & 298 & $\mathrm{C}$ & [1986NIL/WAD] \\
\hline & $\mathrm{V}$ & & $83.4 \pm 1.0$ & 298 & GCC & [1980FUC/PEA] \\
\hline \multirow[t]{2}{*}{$\mathrm{C}_{9} \mathrm{H}_{14} \mathrm{O}_{7}$} & {$[1587-20-8]$} & trimethyl citrate & & & & \\
\hline & V & $(379-560)$ & 617.4 & 394 & A & [1987STE/MAL, 1947STU] \\
\hline \multirow[t]{3}{*}{$\mathrm{C}_{9} \mathrm{H}_{14} \mathrm{~S}$} & {$[281-25-4]$} & 2-thiaadamantane & & & & \\
\hline & TRS & & 1.95 & 211 & & \\
\hline & FUS & & 8.1 & 597 & DSC & [1978AND/CAR] \\
\hline \multirow[t]{2}{*}{$\mathrm{C}_{9} \mathrm{H}_{14} \mathrm{~S}$} & [4861-58-9] & 2-pentylthiophene & & & & \\
\hline & $\mathrm{V}$ & & $52.0 \pm 1.2$ & 298 & $\mathrm{C}$ & [2007RIB/SAN] \\
\hline \multirow[t]{2}{*}{$\mathrm{C}_{9} \mathrm{H}_{15} \mathrm{Cl}_{3} \mathrm{O}_{2}$} & \multicolumn{6}{|c|}{ 3-chloro-2,2-bis(chloromethyl)propyl butyrate } \\
\hline & V & $(426-482)$ & 73.6 & 441 & A & [1987STE/MAL, 1999DYK/SVO] \\
\hline
\end{tabular}


TABLE 12. Phase change enthalpies of $\mathrm{C}_{9}$ organic compounds-Continued

\begin{tabular}{|c|c|c|c|c|c|c|}
\hline \multirow[b]{2}{*}{$\begin{array}{l}\text { Molecular } \\
\text { formula }\end{array}$} & \multirow{2}{*}{$\begin{array}{l}\text { CAS reg. no. } \\
\text { Transition }\end{array}$} & \multicolumn{5}{|l|}{ Compound } \\
\hline & & Temp. range $(\mathrm{K})$ & $\begin{array}{c}\Delta_{\text {trans }} H_{\mathrm{m}} \\
\left(\mathrm{kJ} \mathrm{mol}^{-1}\right)\end{array}$ & $T_{\mathrm{m}}(\mathrm{K})$ & Method & References \\
\hline \multirow[t]{2}{*}{$\mathrm{C}_{9} \mathrm{H}_{15} \mathrm{Cl}_{6} \mathrm{O}_{4} \mathrm{P}$} & {$[13674-87-8]$} & \multicolumn{5}{|c|}{ tris(1,3-dichloroisopropyl)phosphate } \\
\hline & $\mathrm{V}$ & $(383-413)$ & 91.3 & 398 & GC-RT & {$[2014 \mathrm{BRO} / \mathrm{JAN}]$} \\
\hline \multirow[t]{2}{*}{$\mathrm{C}_{9} \mathrm{H}_{15} \mathrm{NOS}$} & {$[59300-33-3]$} & \multicolumn{5}{|c|}{ carbamothioic acid, (1-methylethyl)-2-propynyl- $S$-ethyl ester } \\
\hline & $\mathrm{V}$ & $(298-313)$ & 72.8 & 305 & A & [1987STE/MAL, 1999DYK/SVO, 1976DEP] \\
\hline \multirow[t]{2}{*}{$\mathrm{C}_{9} \mathrm{H}_{15} \mathrm{NOS}$} & {$[59300-32-2]$} & \multicolumn{5}{|c|}{ carbamothioic acid, propyl-2-propynyl- $S$-ethyl ester } \\
\hline & $\mathrm{V}$ & $(298-313)$ & 64.6 & 305 & A & [1987STE/MAL, 1976DEP] \\
\hline \multirow[t]{3}{*}{$\mathrm{C}_{9} \mathrm{H}_{15} \mathrm{NO}_{3} \mathrm{~S}$} & {$[62571-86-2]$} & \multicolumn{5}{|c|}{ 1-[(2S)-3-mercapto-2-methyl-1-oxopropyl]- $(l)$-proline } \\
\hline & FUS & & 26.68 & 378.7 & DSC & [2015MAR/PIN] \\
\hline & FUS & & 20.38 & 379.5 & DSC & [2008STU/ROR] \\
\hline \multirow{2}{*}{$\mathrm{C}_{9} \mathrm{H}_{15} \mathrm{NO}_{5}$} & {$[1068-90-2]$} & \multicolumn{5}{|c|}{ diethyl acetamidomalonate } \\
\hline & FUS & & 34.3 & 368.7 & DSC & {$[2013 \mathrm{LI} / \mathrm{TAN}]$} \\
\hline \multirow[t]{3}{*}{$\mathrm{C}_{9} \mathrm{H}_{15} \mathrm{~N}_{3} \mathrm{O}_{3}$} & {$[82859-98-1]$} & \multicolumn{5}{|c|}{$N$-acetylglycyl- $(l)$-prolinamide } \\
\hline & TRS & & 5.6 & 450.6 & & \\
\hline & FUS & & 27.0 & 457.8 & DSC & [1996PUL/BAR] \\
\hline \multirow[t]{2}{*}{$\mathrm{C}_{9} \mathrm{H}_{15} \mathrm{~N}_{3} \mathrm{O}_{3}$} & {$[52186-41-1]$} & \multicolumn{5}{|c|}{$N$-acetyl- $(l)$-prolyl-glycinamide } \\
\hline & FUS & & 32.2 & 434.1 & $\mathrm{DSC}$ & [1992BAR/GIA] \\
\hline \multirow{2}{*}{$\mathrm{C}_{9} \mathrm{H}_{15} \mathrm{~N}_{3} \mathrm{O}_{8}$} & {$[34001-52-0]$} & \multicolumn{5}{|c|}{ neopentyl-4,4,4-trinitrobutyrate } \\
\hline & FUS & & 22.59 & 333.5 & DSC & [1971ROS/HOL] \\
\hline $\mathrm{C}_{9} \mathrm{H}_{16}$ & [3452-09-3] & 1-nonyne & & & & \\
\hline & V & $(320-464)$ & $45.6 \pm 0.2$ & 320 & EB & [2002STE/CHI4] \\
\hline & $\mathrm{V}$ & $(320-464)$ & $42.7 \pm 0.2$ & 360 & EB & [2002STE/CHI4] \\
\hline & $\mathrm{V}$ & $(320-464)$ & $39.7 \pm 0.3$ & 400 & $\mathrm{~EB}$ & [2002STE/CHI4] \\
\hline & $\mathrm{V}$ & $(320-464)$ & $36.4 \pm 0.5$ & 440 & EB & [2002STE/CHI4] \\
\hline $\mathrm{C}_{9} \mathrm{H}_{16}$ & [39124-79-3] & trans-bicyclo $[6.1 .0$ & onane & & & \\
\hline & V & & $42.7 \pm 0.6$ & & EB & [1978COR/PER] \\
\hline $\mathrm{C}_{9} \mathrm{H}_{16}$ & {$[13757-43-2]$} & cis-bicyclo[6.1.0]n & ane & & & \\
\hline & $\mathrm{V}$ & & $49.8 \pm 0.8$ & & EB & [1978COR/PER] \\
\hline & V & $(297-360)$ & $50.4 \pm 0.8$ & 312 & $\mathrm{~A}$ & [1987STE/MAL, 1970CHA/MCN] \\
\hline $\mathrm{C}_{9} \mathrm{H}_{16}$ & {$[20454-81-3]$} & 1,4-dimethylbicycl & 2.2.1]heptane & & & \\
\hline & $\mathrm{V}$ & $(328-393)$ & 36.8 & 343 & A & [1987STE/MAL, 1970VAR/BEL, 1984BOU/FRI] \\
\hline $\mathrm{C}_{9} \mathrm{H}_{16}$ & {$[20558-16-1]$} & trans-2,3-dimethyll & zyclo[2.2.1]h & & & \\
\hline & $\mathrm{V}$ & $(345-411)$ & 39.3 & 360 & A & [1987STE/MAL, 1970VAR/BEL, 1984BOU/FRI] \\
\hline $\mathrm{C}_{9} \mathrm{H}_{16}$ & {$[2146-41-0]$} & 2-ethylbicyclo[2.2. & heptane & & & \\
\hline & $\mathrm{V}$ & $(349-396)$ & 44.4 & 364 & A & [1987STE/MAL] \\
\hline $\mathrm{C}_{9} \mathrm{H}_{16}$ & {$[4551-51-3]$} & cis-hexahydroindan & & & & \\
\hline & TRS & & 8.26 & 182.3 & & \\
\hline & TRS & & 0.39 & 184.5 & & \\
\hline & FUS & & 1.4 & 236.5 & & [1972FIN/MCC] \\
\hline & $\mathrm{V}$ & $(263-293)$ & 47.1 & 278 & A & [1987STE/MAL] \\
\hline & $\mathrm{V}$ & $(290-366)$ & 45.9 & 305 & A & [1987STE/MAL] \\
\hline & $\mathrm{V}$ & $(363-463)$ & 41.9 & 378 & A & [1987STE/MAL] \\
\hline & $\mathrm{V}$ & $(350-442)$ & 42.6 & 365 & GS & [1955CAM/ROS] \\
\hline $\mathrm{C}_{9} \mathrm{H}_{16}$ & [3296-50-2] & trans-hexahydroind & & & & \\
\hline & FUS & & 10.9 & 213.9 & & [1972FIN/MCC] \\
\hline & $\mathrm{V}$ & $(281-362)$ & 45.1 & 296 & A & [1987STE/MAL] \\
\hline & $\mathrm{V}$ & $(356-457)$ & 41.1 & 371 & A & [1987STE/MAL] \\
\hline & $\mathrm{V}$ & $(262-283)$ & 45.9 & 272 & A & [1987STE/MAL] \\
\hline & V & $(358-479)$ & 41.0 & 373 & A & [1987STE/MAL] \\
\hline & $\mathrm{V}$ & $(345-435)$ & 41.6 & 360 & GS & [1955CAM/ROS] \\
\hline $\mathrm{C}_{9} \mathrm{H}_{16}$ & [2114-42-3] & allylcyclohexane & & & & \\
\hline & $\mathrm{V}$ & & $44.0 \pm 0.2$ & 298 & GCC & [1979FUC/PEA] \\
\hline $\mathrm{C}_{9} \mathrm{H}_{16}$ & [1003-64-1] & ethylidenecyclohex & & & & \\
\hline
\end{tabular}


TABLE 12. Phase change enthalpies of $\mathrm{C}_{9}$ organic compounds-Continued

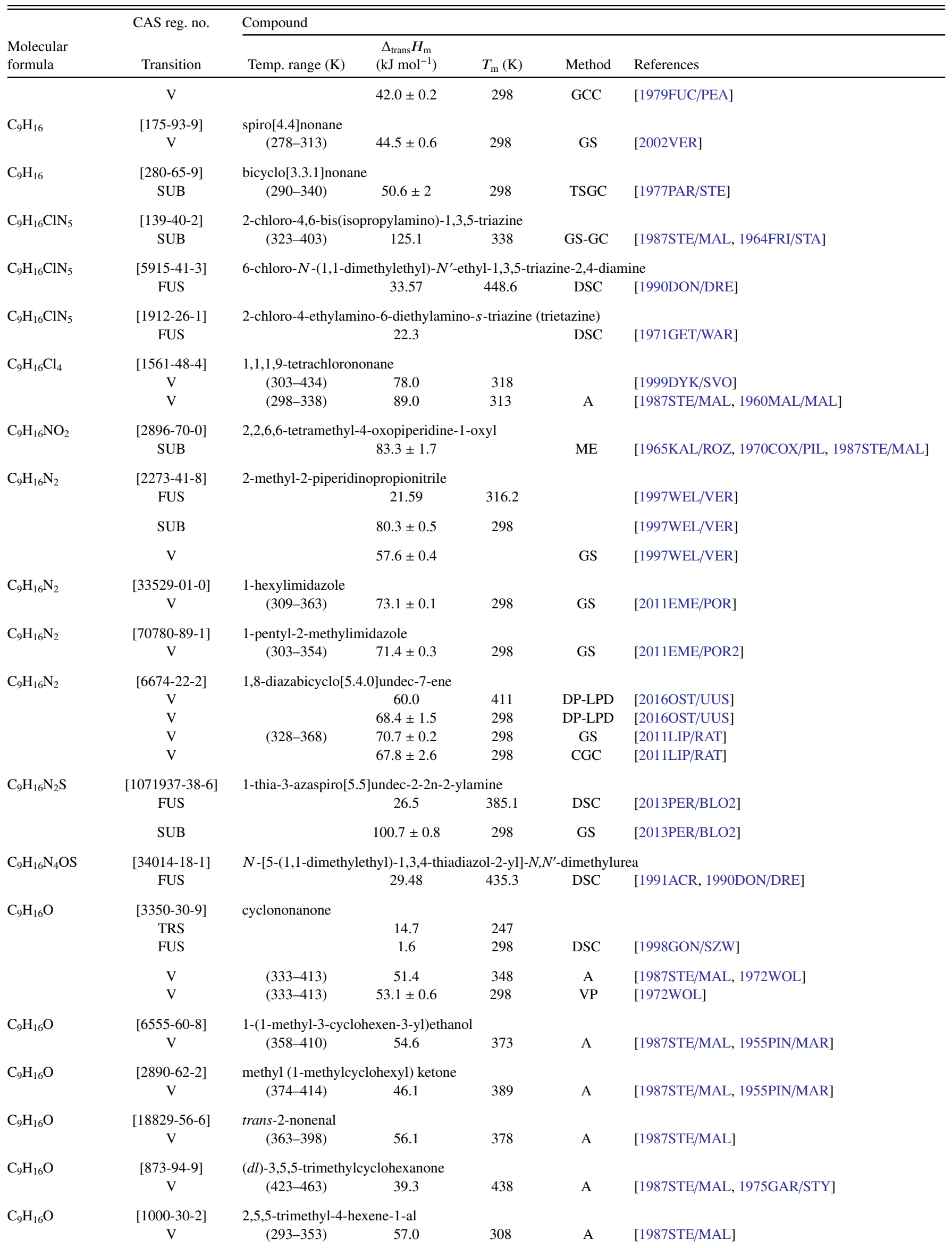


TABLE 12. Phase change enthalpies of $\mathrm{C}_{9}$ organic compounds-Continued

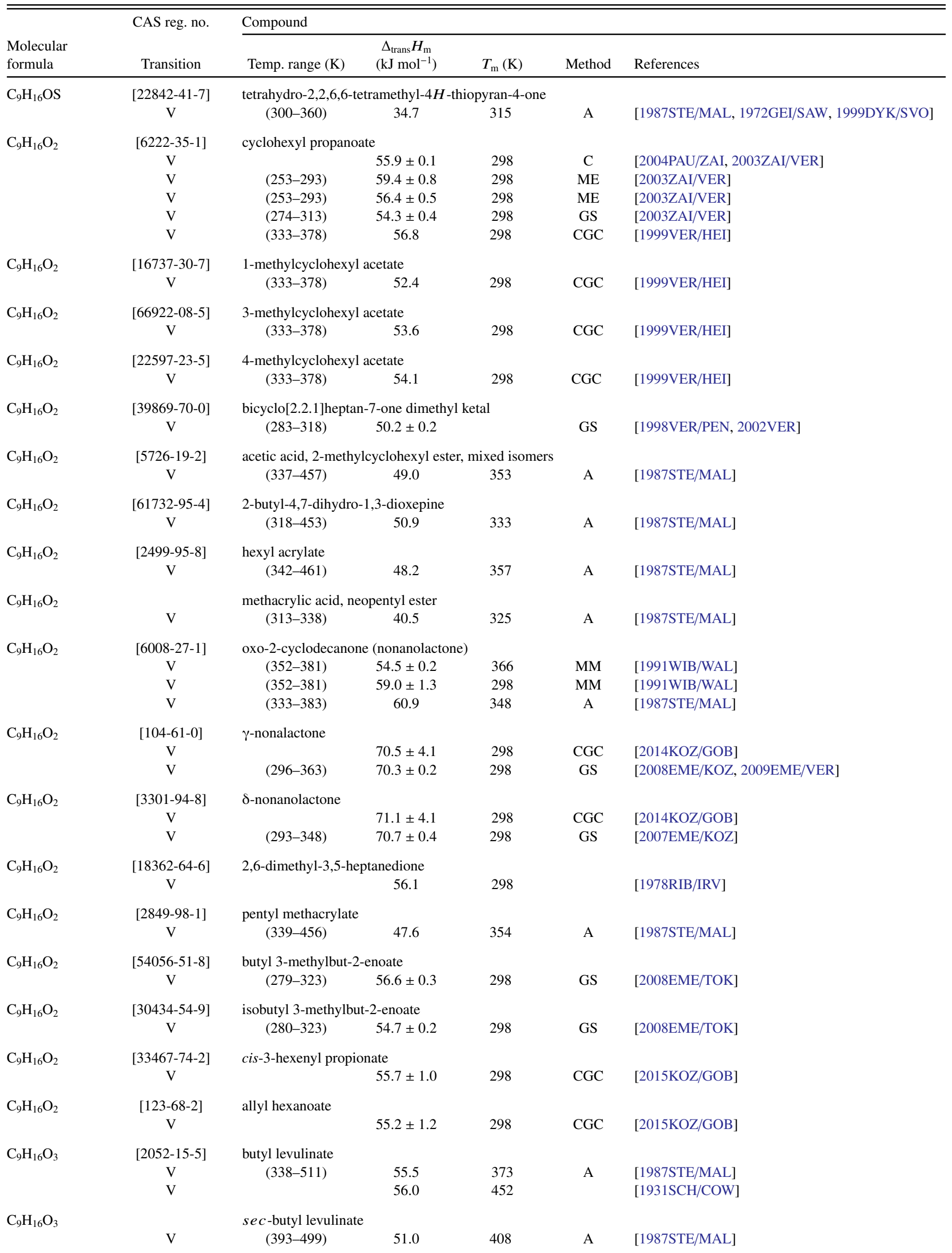


TABLE 12. Phase change enthalpies of $\mathrm{C}_{9}$ organic compounds-Continued

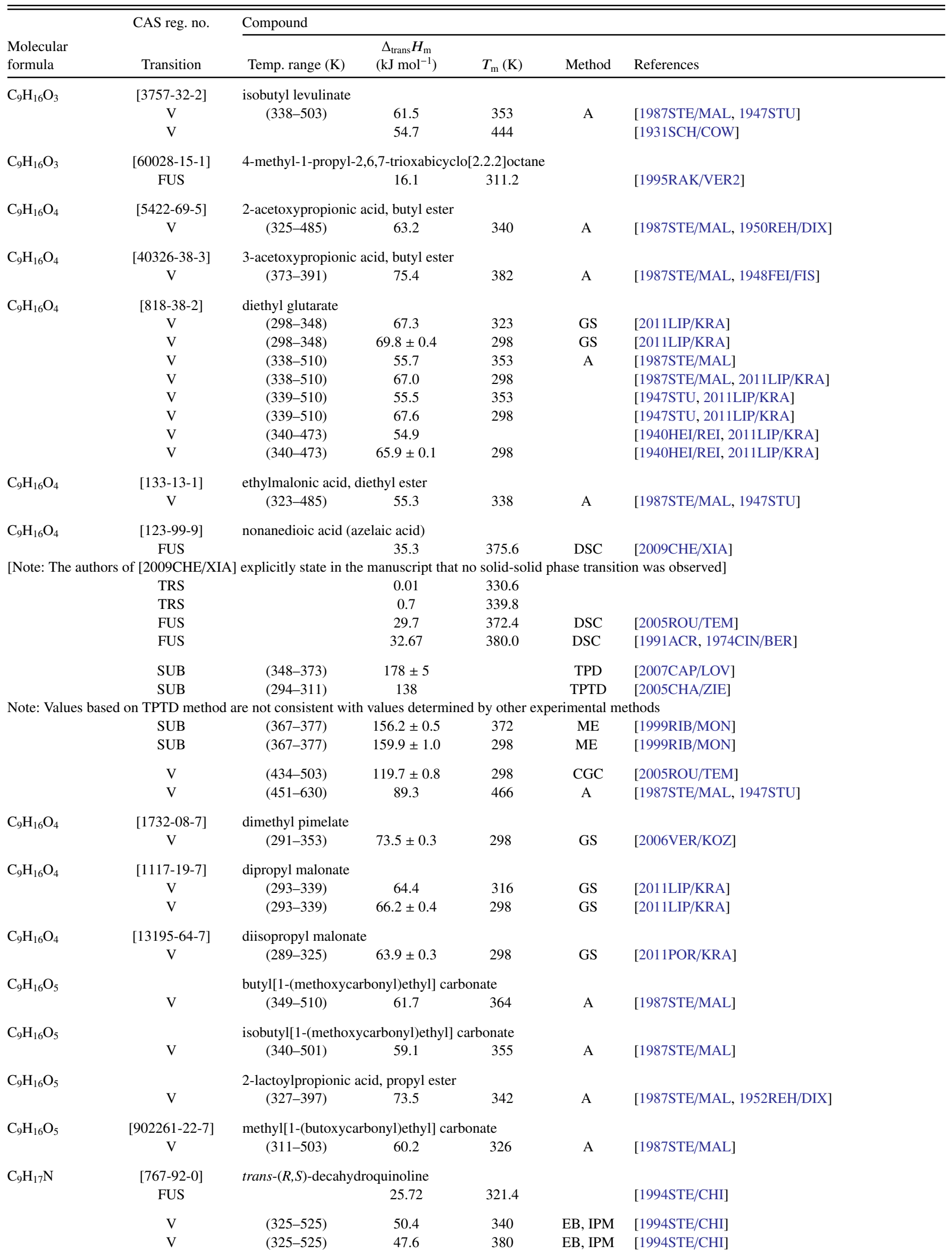


TABLE 12. Phase change enthalpies of $\mathrm{C}_{9}$ organic compounds-Continued

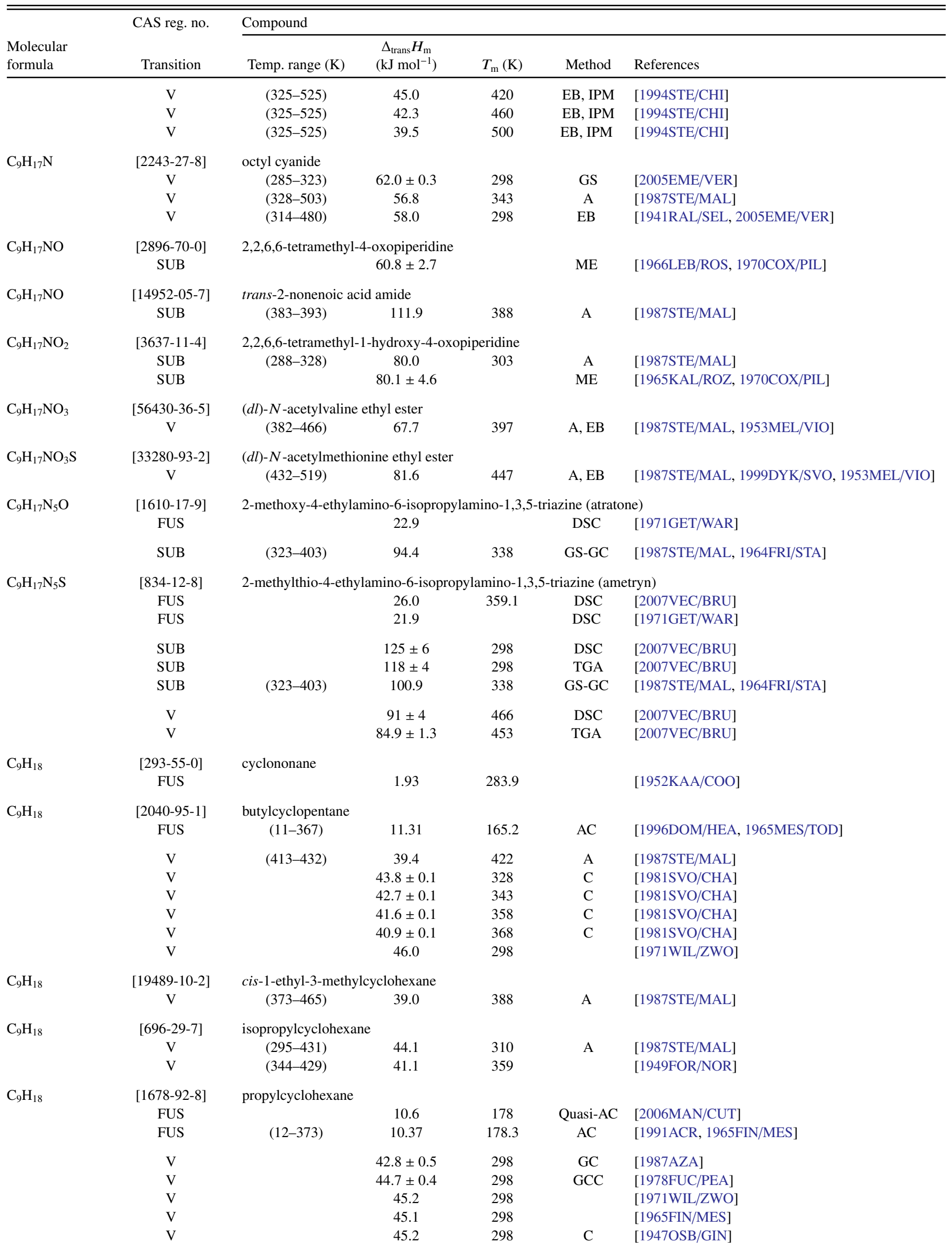


TABLE 12. Phase change enthalpies of $\mathrm{C}_{9}$ organic compounds-Continued

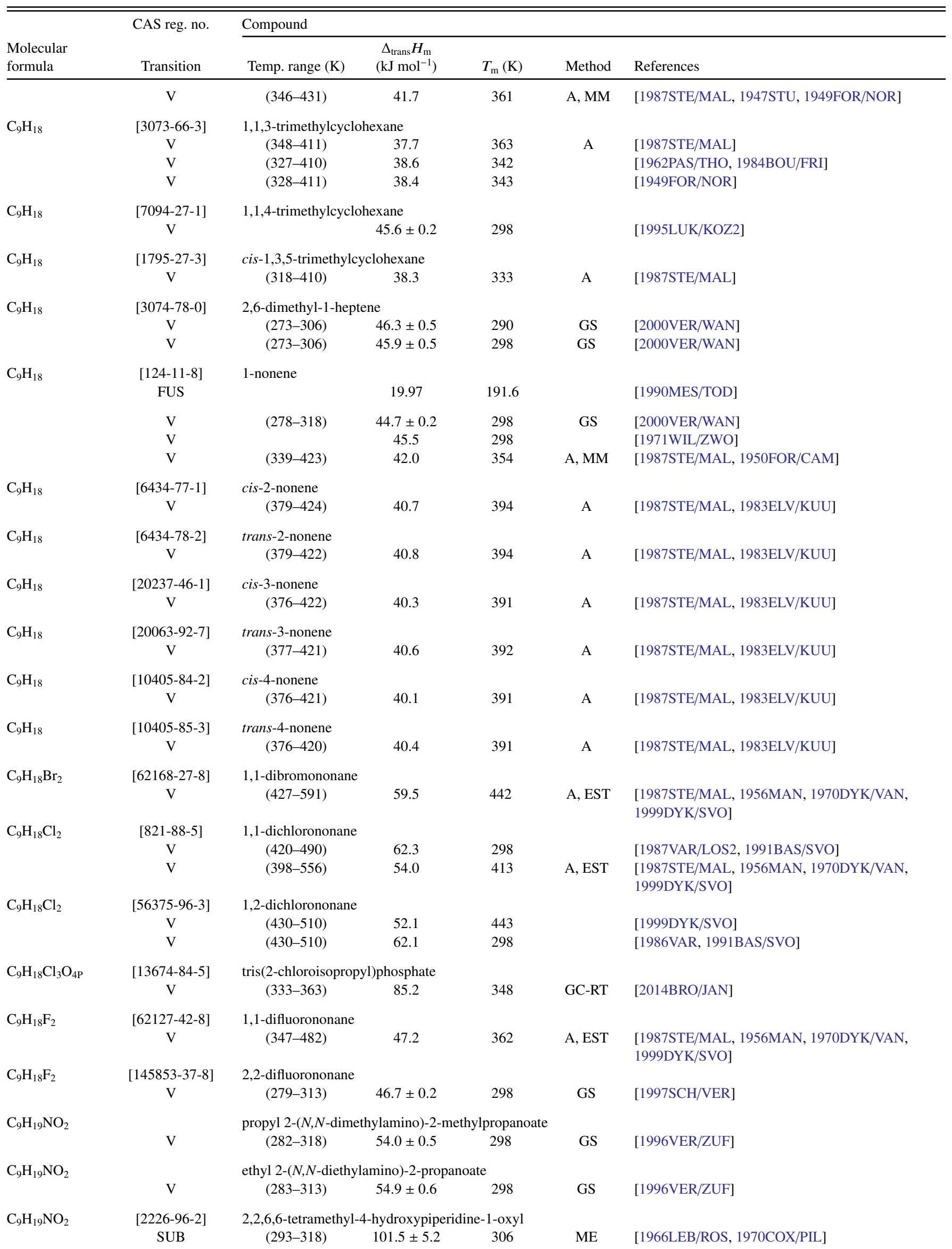


TABLE 12. Phase change enthalpies of $\mathrm{C}_{9}$ organic compounds-Continued

\begin{tabular}{|c|c|c|c|c|c|c|}
\hline \multirow[b]{2}{*}{$\begin{array}{l}\text { Molecular } \\
\text { formula }\end{array}$} & \multirow{2}{*}{$\begin{array}{l}\text { CAS reg. no. } \\
\text { Transition }\end{array}$} & \multicolumn{5}{|l|}{ Compound } \\
\hline & & Temp. range $(\mathrm{K})$ & $\begin{array}{c}\Delta_{\text {trans }} H_{\mathrm{m}} \\
\left(\mathrm{kJ} \mathrm{mol}^{-1}\right)\end{array}$ & $T_{\mathrm{m}}(\mathrm{K})$ & Method & References \\
\hline \multirow[t]{3}{*}{$\mathrm{C}_{9} \mathrm{H}_{18} \mathrm{~N}_{2}$} & [19340-91-1] & 2-(diethylamino)pe & anenitrile & & & \\
\hline & $\mathrm{V}$ & $(283-318)$ & $57.4 \pm 0.4$ & & GS & [1997WEL/VER] \\
\hline & V & $(283-326)$ & 58.8 & 298 & A & [1987STE/MAL] \\
\hline \multirow[t]{2}{*}{$\mathrm{C}_{9} \mathrm{H}_{18} \mathrm{~N}_{2} \mathrm{OS}$} & {$[95600-05-8]$} & $N, N$-diethyl- $N^{\prime}$-iso & Itanoylthiour & & & \\
\hline & SUB & 363 & $120.8 \pm 2.5$ & 298 & $\mathrm{C}$ & [2001RIB/RIB] \\
\hline \multirow{2}{*}{$\mathrm{C}_{9} \mathrm{H}_{18} \mathrm{~N}_{2} \mathrm{O}_{2}$} & {$[1842-72-4]$} & azelamide & & & & \\
\hline & FUS & & 55.0 & 450.4 & DSC & [2006BAD/DEL] \\
\hline \multirow[t]{2}{*}{$\mathrm{C}_{9} \mathrm{H}_{18} \mathrm{~N}_{2} \mathrm{O}_{2}$} & [32483-15-1] & $N$-acetyl-L-leucine & $V^{\prime}$-methylami & & & \\
\hline & FUS & & 23.19 & 438.2 & DSC & [2014BAD/DEL] \\
\hline \multirow[t]{2}{*}{$\mathrm{C}_{9} \mathrm{H}_{18} \mathrm{~N}_{2} \mathrm{O}_{2}$} & {$[26090-94-8]$} & $N$-acetyl-DL-leucin & $N^{\prime}$-methylam & & & \\
\hline & FUS & & 27.16 & 432.1 & DSC & [2014BAD/DEL] \\
\hline \multirow[t]{2}{*}{$\mathrm{C}_{9} \mathrm{H}_{18} \mathrm{~N}_{2} \mathrm{O}_{2}$} & {$[27777-86-2]$} & $N$-acetyl-DL-norleu & ne- $N^{\prime}$-methy & & & \\
\hline & FUS & & 34.34 & 444.0 & DSC & [2014BAD/DEL] \\
\hline \multirow[t]{2}{*}{$\mathrm{C}_{9} \mathrm{H}_{18} \mathrm{~N}_{2} \mathrm{O}_{2}$} & [32483-16-2] & $N$-acetyl-L-isoleuci & e- $N^{\prime}$-methyla & & & \\
\hline & FUS & & 38.13 & 525.7 & DSC & [2014BAD/DEL] \\
\hline \multirow[t]{2}{*}{$\mathrm{C}_{9} \mathrm{H}_{18} \mathrm{~N}_{2} \mathrm{O}_{2}$} & {$[120328-70-3]$} & $N$-acetyl-DL-isoleu & ne- $N^{\prime}$-methyl & & & \\
\hline & FUS & & 27.13 & 482.0 & DSC & [2014BAD/DEL] \\
\hline \multirow[t]{3}{*}{$\mathrm{C}_{9} \mathrm{H}_{18} \mathrm{~N}_{2} \mathrm{O}_{2} \mathrm{~S}$} & {$[39196-18-4]$} & 3,3-dimethyl-1-(me & ylthio)-2-but & $O-((\mathrm{me}$ & mino)carb & xyl)oxime \\
\hline & FUS & & 19.83 & 330.2 & DSC & [1991ACR, 1990DON/DRE] \\
\hline & SUB & $(298-328)$ & $93.5 \pm 6$ & 308 & ME & [1987STE/MAL, 1976DEP] \\
\hline \multirow{3}{*}{$\mathrm{C}_{9} \mathrm{H}_{18} \mathrm{~N}_{2} \mathrm{O}_{4}$} & {$[57-53-4]$} & 2-methyl-2-propyl- & 3-propanedio & dicarban & & \\
\hline & FUS (I) & & 35.64 & 377.8 & & \\
\hline & FUS (II) & & 32.97 & 372.7 & & [1991LEF/GUI] \\
\hline \multirow[t]{2}{*}{$\mathrm{C}_{9} \mathrm{H}_{18} \mathrm{~N}_{6}$} & {$[645-05-6]$} & 1,3,5-tris(dimethyla & aino)-s-triazi & & & \\
\hline & FUS & & 23.01 & 444.4 & DSC & [1991ACR, 1989BRA/RYT] \\
\hline \multirow[t]{2}{*}{$\mathrm{C}_{9} \mathrm{H}_{18} \mathrm{~N}_{6}$} & {$[16268-64-7]$} & 1-(ethylamino)-3,5 & is(dimethylar & $-s$-triazin & & \\
\hline & FUS & & 16.74 & 333 & DSC & [1991ACR, 1989BRA/RYT] \\
\hline \multirow[t]{2}{*}{$\mathrm{C}_{9} \mathrm{H}_{18} \mathrm{O}$} & {$[1462-97-1]$} & 1-butylcyclopentan & & & & \\
\hline & V & $(359-466)$ & 63.5 & 374 & A & [1987STE/MAL] \\
\hline \multirow[t]{4}{*}{$\mathrm{C}_{9} \mathrm{H}_{18} \mathrm{O}$} & {$[815-24-7]$} & 2,2,4,4-tetramethyl & -pentanone & & & \\
\hline & $\mathrm{V}$ & & $45.5 \pm 0.4$ & 298 & $\mathrm{C}$ & [1977PEA/FUC] \\
\hline & $\mathrm{V}$ & & $45.4 \pm 0.1$ & 298 & $\mathrm{C}$ & [1970SEL2] \\
\hline & $\mathrm{V}$ & & $45.4 \pm 0.1$ & 298 & $\mathrm{C}$ & [1966WAD] \\
\hline \multirow[t]{12}{*}{$\mathrm{C}_{9} \mathrm{H}_{18} \mathrm{O}$} & {$[108-83-8]$} & 2,6-dimethyl-4-hep & none & & & \\
\hline & $\mathrm{V}$ & & $49.8 \pm 0.1$ & 308 & $\mathrm{C}$ & [1992SVO/KUB] \\
\hline & $\mathrm{V}$ & & $49.3 \pm 0.1$ & 313 & $\mathrm{C}$ & [1992SVO/KUB] \\
\hline & $\mathrm{V}$ & & $48.4 \pm 0.1$ & 323 & $\mathrm{C}$ & [1992SVO/KUB] \\
\hline & $\mathrm{V}$ & & $47.9 \pm 0.1$ & 328 & $\mathrm{C}$ & [1992SVO/KUB] \\
\hline & $\mathrm{V}$ & & $47.1 \pm 0.1$ & 338 & $\mathrm{C}$ & [1992SVO/KUB] \\
\hline & $\mathrm{V}$ & & $46.6 \pm 0.1$ & 343 & $\mathrm{C}$ & [1992SVO/KUB] \\
\hline & $\mathrm{V}$ & & $46.1 \pm 0.1$ & 348 & $\mathrm{C}$ & [1992SVO/KUB] \\
\hline & $\mathrm{V}$ & & $45.2 \pm 0.1$ & 358 & $\mathrm{C}$ & [1992SVO/KUB] \\
\hline & $\mathrm{V}$ & $(322-471)$ & 51.0 & 298 & & [1975AMB/ELL] \\
\hline & $\mathrm{V}$ & & $50.9 \pm 0.1$ & 298 & $\mathrm{C}$ & [1970SEL2] \\
\hline & $\mathrm{V}$ & $(336-451)$ & 46.8 & 351 & $\mathrm{~A}, \mathrm{MM}$ & [1987STE/MAL, 1947STR/GAB] \\
\hline \multirow[t]{2}{*}{$\mathrm{C}_{9} \mathrm{H}_{18} \mathrm{O}$} & {$[6555-61-9]$} & 1-(1-methylcyclohe & yl)ethanol & & & \\
\hline & $\mathrm{V}$ & $(358-408)$ & 55.5 & 373 & A & [1987STE/MAL, 1955PIN/MAR] \\
\hline $\mathrm{C}_{9} \mathrm{H}_{18} \mathrm{O}$ & [124-19-6] & nonanal & & & & \\
\hline & FUS & & 29.6 & 253.9 & & [1980DYA/VAS] \\
\hline & $\mathrm{V}$ & $(276-309)$ & $55.3 \pm 0.3$ & 298 & GS & [2003VER/KRA2] \\
\hline & $\mathrm{V}$ & $(323-343)$ & 55.2 & 298 & CGC & [1996KOU/HOS, 2000OVA/KOU] \\
\hline & $\mathrm{V}$ & $(313-353)$ & 58.9 & 298 & CGC & [1995CHI/HOS] \\
\hline
\end{tabular}


TABLE 12. Phase change enthalpies of $\mathrm{C}_{9}$ organic compounds-Continued

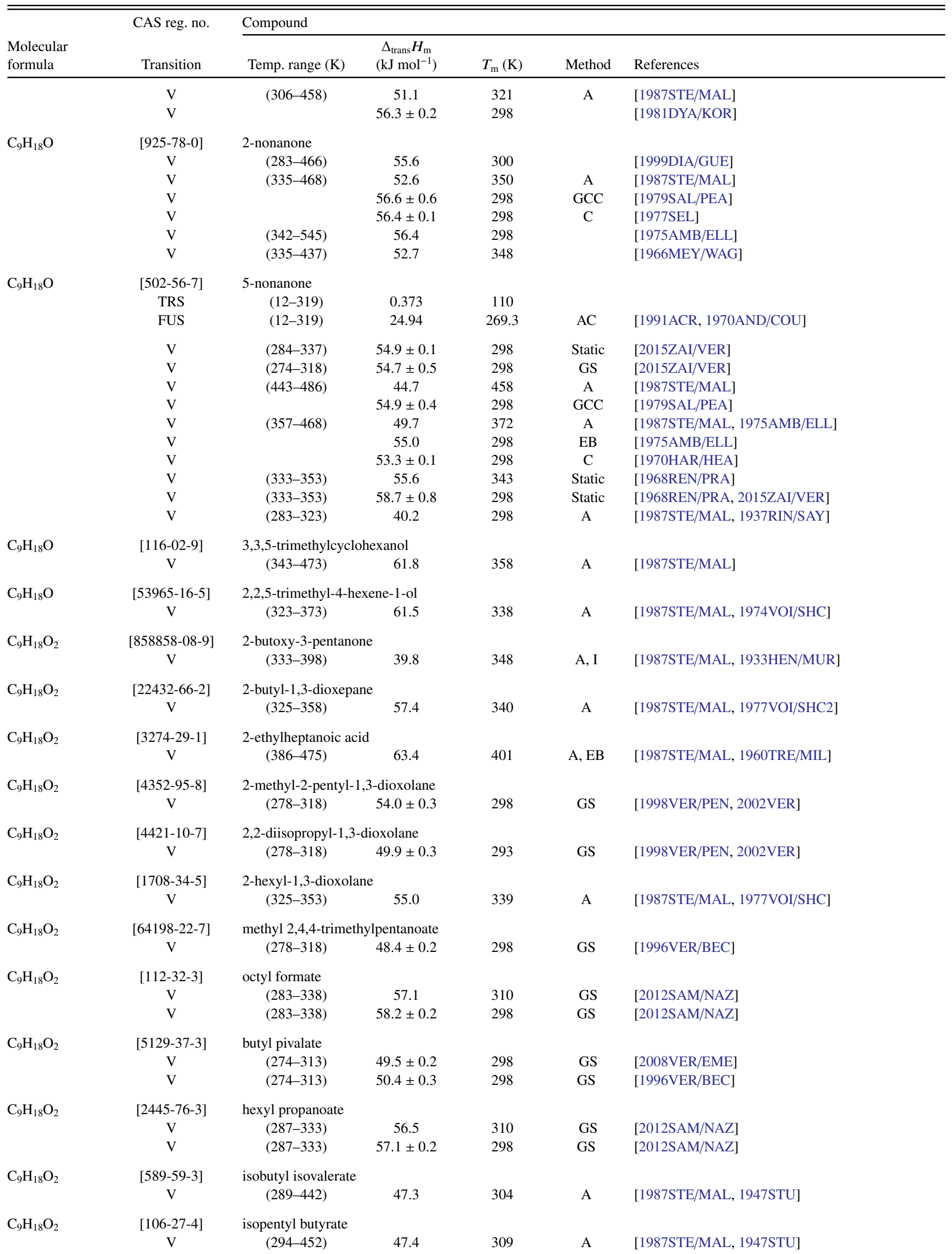


TABLE 12. Phase change enthalpies of $\mathrm{C}_{9}$ organic compounds-Continued

\begin{tabular}{|c|c|c|c|c|c|c|}
\hline \multirow[b]{2}{*}{$\begin{array}{l}\text { Molecular } \\
\text { formula }\end{array}$} & \multirow{2}{*}{$\begin{array}{l}\text { CAS reg. no. } \\
\text { Transition }\end{array}$} & \multicolumn{5}{|l|}{ Compound } \\
\hline & & Temp. range $(\mathrm{K})$ & $\begin{array}{c}\Delta_{\text {trans }} H_{\mathrm{m}} \\
\left(\mathrm{kJ} \mathrm{mol}^{-1}\right)\end{array}$ & $T_{\mathrm{m}}(\mathrm{K})$ & Method & References \\
\hline \multirow[t]{3}{*}{$\mathrm{C}_{9} \mathrm{H}_{18} \mathrm{O}_{2}$} & {$[2050-01-3]$} & \multicolumn{5}{|l|}{ isopentyl isobutyrate } \\
\hline & $\mathrm{V}$ & & $51.7 \pm 1.6$ & 298 & CGC & [2015KOZ/GOB] \\
\hline & $\mathrm{V}$ & $(287-442)$ & 47.4 & 302 & A & [1987STE/MAL, 1947STU] \\
\hline \multirow[t]{2}{*}{$\mathrm{C}_{9} \mathrm{H}_{18} \mathrm{O}_{2}$} & {$[2311-46-8]$} & \multicolumn{5}{|l|}{ isopropyl caproate } \\
\hline & $\mathrm{V}$ & $(307-383)$ & 51.6 & 322 & A & [1987STE/MAL] \\
\hline \multirow[t]{11}{*}{$\mathrm{C}_{9} \mathrm{H}_{18} \mathrm{O}_{2}$} & [111-11-5] & \multicolumn{5}{|c|}{ methyl octanoate (methyl caprylate) } \\
\hline & $\mathrm{V}$ & & 53.3 & 350 & & [2002VAN/VAN] \\
\hline & $\mathrm{V}$ & & $52.6 \pm 0.1$ & 363 & & [2002VAN/VAN] \\
\hline & $\mathrm{V}$ & & $56.9 \pm 0.1$ & 298 & & [2002VAN/VAN] \\
\hline & $\mathrm{V}$ & & $54.7 \pm 0.6$ & 298 & GC & [1987AZA] \\
\hline & $\mathrm{V}$ & & $57.3 \pm 0.4$ & 298 & GCC & [1980FUC/PEA] \\
\hline & $\mathrm{V}$ & & $57.9 \pm 0.4$ & 298 & $\mathrm{C}$ & [1977PEA/FUC] \\
\hline & $\mathrm{V}$ & & $56.4 \pm 0.5$ & 298 & $\mathrm{C}$ & [1977MAN/SEL] \\
\hline & $\mathrm{V}$ & $(347-470)$ & 52.4 & 362 & A, EST & [1987STE/MAL, 1963ROS/SCH] \\
\hline & $\mathrm{V}$ & $(373-419)$ & 50.8 & 388 & & [1961ROS/SUP, 1984BOU/FRI] \\
\hline & $\mathrm{V}$ & $(307-350)$ & 55.2 & 322 & $\mathrm{MG}, \mathrm{OM}$ & {$[1952 \mathrm{SCO} / \mathrm{MAC}]$} \\
\hline \multirow[t]{9}{*}{$\mathrm{C}_{9} \mathrm{H}_{18} \mathrm{O}_{2}$} & {$[112-05-0]$} & \multicolumn{5}{|l|}{ nonanoic acid } \\
\hline & TRS & & 8.15 & 263.0 & & \\
\hline & FUS & $(90-305)$ & 19.82 & 285.5 & $\mathrm{AC}$ & [1982SCH/VAN2] \\
\hline & TRS & & 5.61 & 268 & & \\
\hline & FUS & & 20.31 & 285.5 & & [1991ACR, 1924GAR/RAN] \\
\hline & $\mathrm{V}$ & $(381-528)$ & 76.9 & 396 & A & [1987STE/MAL] \\
\hline & $\mathrm{V}$ & $(292-313)$ & $85.3 \pm 2.0$ & 304 & ME, TE & [1982DEK/SCH] \\
\hline & $\mathrm{V}$ & $(293-303)$ & $82.4 \pm 0.4$ & 298 & & [1968BAC/NOV] \\
\hline & $\mathrm{V}$ & $(387-483)$ & 64.2 & & EB & [1960TRE/MIL] \\
\hline \multirow[t]{3}{*}{$\mathrm{C}_{9} \mathrm{H}_{18} \mathrm{O}_{2}$} & {$[626-77-7]$} & \multicolumn{5}{|l|}{ propyl caproate } \\
\hline & $\mathrm{V}$ & $(315-394)$ & 52.8 & 330 & A & [1987STE/MAL] \\
\hline & $\mathrm{V}$ & $(315-394)$ & 52.1 & 330 & & [1961ROS/SUP, 1984BOU/FRI] \\
\hline \multirow[t]{4}{*}{$\mathrm{C}_{9} \mathrm{H}_{18} \mathrm{O}_{2}$} & {$[112-06-1]$} & \multicolumn{5}{|l|}{ heptyl acetate } \\
\hline & V & $(274-306)$ & $57.1 \pm 0.2$ & 298 & GS & [2006KRA/VER] \\
\hline & $\mathrm{V}$ & & 56.9 & 298 & $\mathrm{GC}$ & [1997KOU/HOS] \\
\hline & $\mathrm{V}$ & $(387-478)$ & 49.2 & 402 & DTA & [1980MEY/AWE] \\
\hline \multirow[t]{4}{*}{$\mathrm{C}_{9} \mathrm{H}_{18} \mathrm{O}_{2}$} & {$[2050-00-2]$} & \multicolumn{5}{|l|}{ tert-amyl butyrate } \\
\hline & $\mathrm{V}$ & $(274.5-309)$ & $50.3 \pm 0.2$ & 298 & GS & [2008VER/EME] \\
\hline & $\mathrm{V}$ & $(333-378)$ & 48.9 & 298 & CGC & [1999VER/HEI] \\
\hline & V & $(278-308)$ & $50.8 \pm 0.6$ & 298 & GS & [1996VER/BEC] \\
\hline \multirow[t]{2}{*}{$\mathrm{C}_{9} \mathrm{H}_{18} \mathrm{O}_{2}$} & [194784-93-5] & tert-amyl isobutyrate & & & & \\
\hline & $\mathrm{V}$ & $(333-378)$ & 47.8 & 298 & CGC & [1999VER/HEI] \\
\hline $\mathrm{C}_{9} \mathrm{H}_{18} \mathrm{O}_{2}$ & [245658-26-8] & 2-methyl-2-pentanol $\mathrm{p}$ & propanoate & & & \\
\hline & $\mathrm{V}$ & $(333-378)$ & 49.8 & 298 & CGC & [1999VER/HEI] \\
\hline $\mathrm{C}_{9} \mathrm{H}_{18} \mathrm{O}_{2}$ & [245658-31-5] & 2,3-dimethyl-2-butanc & ol 2-propanoate & & & \\
\hline & $\mathrm{V}$ & $(333-378)$ & 49.9 & 298 & CGC & [1999VER/HEI] \\
\hline $\mathrm{C}_{9} \mathrm{H}_{18} \mathrm{O}_{2}$ & [15706-73-7] & butyl 2-methylbutano & & & & \\
\hline & $\mathrm{V}$ & $(274-319)$ & $54.4 \pm 0.3$ & 298 & GS & [2008VER/EME] \\
\hline & $\mathrm{V}$ & $(278-313)$ & $50.6 \pm 0.5$ & 298 & GS & [1996VER/BEC] \\
\hline $\mathrm{C}_{9} \mathrm{H}_{18} \mathrm{O}_{2}$ & [540-18-1] & pentyl butyrate & & & & \\
\hline & V & & $53.6 \pm 0.6$ & 298 & CGC & [2015KOZ/GOB] \\
\hline $\mathrm{C}_{9} \mathrm{H}_{18} \mathrm{O}_{3}$ & [109857-47-8] & 2-butoxypropionic aci & id, ethyl ester & & & \\
\hline & $\mathrm{V}$ & $(348-438)$ & 80.3 & 363 & $\mathrm{~A}, \mathrm{I}$ & [1987STE/MAL, 1933HEN/MUR] \\
\hline $\mathrm{C}_{9} \mathrm{H}_{18} \mathrm{O}_{3}$ & [14144-35-5] & 3-ethoxypropionic aci & id, butyl ester & & & \\
\hline & $\mathrm{V}$ & $(346-479)$ & 51.8 & 361 & A & [1987STE/MAL, 1948DIX/REH] \\
\hline $\mathrm{C}_{9} \mathrm{H}_{18} \mathrm{O}_{3}$ & {$[93282-65-6]$} & 3-hydroxypropionic a & cid, hexyl ester & & & \\
\hline & $\mathrm{V}$ & $(408-432)$ & 69.6 & 420 & A & [1987STE/MAL] \\
\hline
\end{tabular}


TABLE 12. Phase change enthalpies of $\mathrm{C}_{9}$ organic compounds-Continued

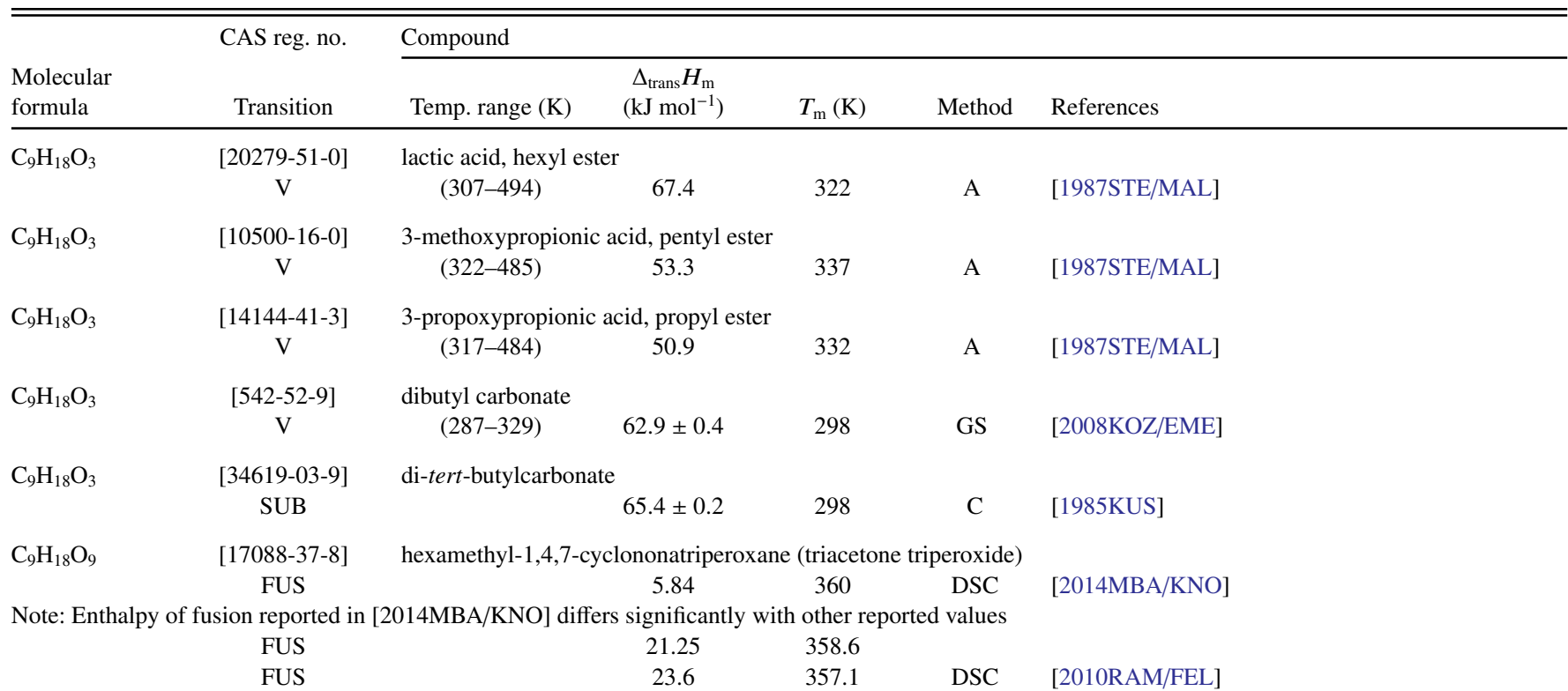

Note: The values in the text for the measured enthalpy of fusion and melting point temperture were different than the value in Table 1 of [2010RAM/FEL]

\begin{tabular}{|c|c|c|c|c|c|c|}
\hline & SUB & & $71 \pm 4.8$ & & HSA & [2015MBA/KNO] \\
\hline & SUB & & $65 \pm 6.1$ & & HSA & [2015MBA/KNO] \\
\hline & SUB & (298-327) & 71.7 & 313 & TGA & [2014MBA/KNO] \\
\hline & SUB & $(303-338)$ & 72.1 & & TGA & [2011FEL/RAM] \\
\hline & SUB & $(314-332)$ & 91.7 & 323 & TGA & [2010OXL/SMI] \\
\hline & SUB & $(288-323)$ & 73 & 304 & HSA & [2009OXL/SMI] \\
\hline & SUB & $(288-333)$ & 109.3 & 309 & HSA & [2005OXL/SMI] \\
\hline $\mathrm{C}_{9} \mathrm{H}_{18} \mathrm{~S}_{4}$ & [25423-58-9] & 1,5,9-trithiacyclodod & ecane & & & \\
\hline & TRS & & 12 & 349.2 & & \\
\hline & FUS & & 19.4 & 373.2 & DSC & [2002ROC/GRI] \\
\hline $\mathrm{C}_{9} \mathrm{H}_{19} \mathrm{Br}$ & [693-58-3] & 1-bromononane & & & & \\
\hline & FUS & & 30.12 & 243.2 & & [1950CRO/SMY] \\
\hline & $\mathrm{V}$ & $(376-525)$ & 53.1 & 391 & & [1999DYK/SVO] \\
\hline & $\mathrm{V}$ & $(391-549)$ & 52.2 & 406 & A, EST & [1987STE/MAL, 1961LI/ROS, 1970DYK/VAN] \\
\hline $\mathrm{C}_{9} \mathrm{H}_{19} \mathrm{Cl}$ & {$[2473-01-0]$} & 1-chlorononane & & & & \\
\hline & $\mathrm{V}$ & $(363-509)$ & 51.5 & 378 & & [1999DYK/SVO] \\
\hline & $\mathrm{V}$ & $(340-480)$ & 55.9 & 298 & & [1984BOU/FRI, 1991BAS/SVO] \\
\hline & $\mathrm{V}$ & $(342-478)$ & 53.4 & 357 & A, DTA & [1987STE/MAL, 1969KEM/KRE] \\
\hline $\mathrm{C}_{9} \mathrm{H}_{19} \mathrm{~F}$ & [463-18-3] & 1-fluorononane & & & & \\
\hline & $\mathrm{V}$ & $(278-313)$ & $50.8 \pm 0.9$ & 298 & GS & [1994STE/CHI] \\
\hline & $\mathrm{V}$ & $(333-473)$ & 46.8 & 348 & A, EST & $\begin{array}{l}\text { [1987STE/MAL, 1961LI/ROS, 1970DYK/VAN, } \\
\text { 1999DYK/SVO] }\end{array}$ \\
\hline $\mathrm{C}_{9} \mathrm{H}_{19} \mathrm{I}$ & [4282-42-2] & 1-iodononane & & & & \\
\hline & $\mathrm{V}$ & & 64.5 & 298 & & [2006BOL/NER, 1961LI/ROS] \\
\hline & $\mathrm{V}$ & $(391-551)$ & 54.6 & 406 & & [1999DYK/SVO] \\
\hline & $\mathrm{V}$ & $(408-577)$ & 53.5 & 423 & A & [1987STE/MAL, 1970DYK/VAN] \\
\hline & $\mathrm{V}$ & $(343-493)$ & 64.3 & 358 & & [1947STU] \\
\hline $\mathrm{C}_{9} \mathrm{H}_{19} \mathrm{~N}$ & [768-66-1] & 2,2,6,6-tetramethylp & peridine & & & \\
\hline & $\mathrm{V}$ & $(288-313)$ & $44.5 \pm 0.5$ & 300 & & [1997VER] \\
\hline $\mathrm{C}_{9} \mathrm{H}_{19} \mathrm{~N}$ & [4945-48-6] & $N$-butylpiperidine & & & & \\
\hline & $\mathrm{V}$ & $(275-313)$ & $49.2 \pm 0.2$ & 294 & GS & [1998VER6] \\
\hline & $\mathrm{V}$ & $(275-313)$ & $48.9 \pm 0.2$ & 298 & GS & [1998VER6] \\
\hline $\mathrm{C}_{9} \mathrm{H}_{19} \mathrm{~N}$ & [13173-21-2] & $N, N$-diethyl-4-penter & ylamine & & & \\
\hline & $\mathrm{V}$ & $(338-430)$ & 41.5 & 353 & A & [1987STE/MAL] \\
\hline $\mathrm{C}_{9} \mathrm{H}_{19} \mathrm{NO}$ & [103-00-4] & 1-(cyclohexylamino) & -2-propanol & & & \\
\hline & $\mathrm{V}$ & $(423-512)$ & 56.6 & 438 & A & [1987STE/MAL, 1984BOU/FRI, 1959MCD/SHR] \\
\hline $\mathrm{C}_{9} \mathrm{H}_{19} \mathrm{NO}$ & [1120-07-6] & nonanamide & & & & \\
\hline & SUB & $(353-370)$ & $114.6 \pm 3.3$ & 361 & ME, A & [1959DAV/JON2, 1987STE/MAL] \\
\hline
\end{tabular}


TABLE 12. Phase change enthalpies of $\mathrm{C}_{9}$ organic compounds-Continued

\begin{tabular}{|c|c|c|c|c|c|c|}
\hline \multirow[b]{2}{*}{$\begin{array}{l}\text { Molecular } \\
\text { formula }\end{array}$} & \multirow{2}{*}{$\begin{array}{c}\text { CAS reg. no. } \\
\text { Transition }\end{array}$} & \multicolumn{5}{|l|}{ Compound } \\
\hline & & Temp. range $(\mathrm{K})$ & $\begin{array}{c}\Delta_{\text {trans }} H_{\mathrm{m}} \\
\left(\mathrm{kJ} \mathrm{mol}^{-1}\right)\end{array}$ & $T_{\mathrm{m}}(\mathrm{K})$ & Method & References \\
\hline \multirow[t]{2}{*}{$\mathrm{C}_{9} \mathrm{H}_{19} \mathrm{NO}_{2}$} & [35601-84-4] & \multicolumn{5}{|c|}{ heptylcarbamic acid, methyl ester } \\
\hline & $\mathrm{V}$ & $(368-408)$ & 109.8 & 383 & A & [1987STE/MAL] \\
\hline \multirow{2}{*}{$\mathrm{C}_{9} \mathrm{H}_{19} \mathrm{NO}_{2}$} & [3637-10-3] & \multicolumn{5}{|c|}{ 2,2,6,6-tetramethyl-1,4-dihydroxypiperidine } \\
\hline & SUB & $(318-348)$ & $100.4 \pm 0.6$ & 328 & $\mathrm{ME}$ & [1966LEB/ROS, 1970COX/PIL] \\
\hline \multirow[t]{5}{*}{$\mathrm{C}_{9} \mathrm{H}_{19} \mathrm{NS}$} & {$[5842-07-9]$} & \multicolumn{5}{|c|}{ diisopropylaminoethanethiol } \\
\hline & $\mathrm{V}$ & $(275-423)$ & 55.6 & 293 & GS,DSC & [2013WIL/HUL] \\
\hline & $\mathrm{V}$ & $(275-423)$ & 52.7 & 323 & GS,DSC & [2013WIL/HUL] \\
\hline & $\mathrm{V}$ & $(275-423)$ & 50.6 & 353 & GS,DSC & [2013WIL/HUL] \\
\hline & V & $(275-423)$ & 48.4 & 393 & GS,DSC & [2013WIL/HUL] \\
\hline \multirow[t]{21}{*}{$\mathrm{C}_{9} \mathrm{H}_{20}$} & {$[111-84-2]$} & nonane & & & & \\
\hline & TRS & & 8.39 & 217.2 & & \\
\hline & FUS & & 13.24 & 219.2 & DSC & [2005HUA/SIM] \\
\hline & TRS & & 6.2 & 218.2 & & \\
\hline & FUS & & 15.0 & 219.5 & $\mathrm{DSC}$ & [2004MON/RAJ] \\
\hline & TRS & & 6.28 & 217.2 & & \\
\hline & FUS & & 15.48 & 219.7 & & [1991ACR, 1996DOM/HEA, 1954FIN/GRO2] \\
\hline & SUB & & 74.6 & 219 & B & {$[1963 \mathrm{BON}]$} \\
\hline & $\mathrm{V}$ & & $46.5 \pm 0.2$ & 298 & $\mathrm{C}$ & [2007PAS/KUZ] \\
\hline & $\mathrm{V}$ & & 46.7 & 299 & $\mathrm{C}$ & [1996VIT/CHA] \\
\hline & $\mathrm{V}$ & & 46 & 314 & $\mathrm{C}$ & [1996VIT/CHA] \\
\hline & V & & $46.6 \pm 0.2$ & 298 & $\mathrm{C}$ & [1996VAR/PAS] \\
\hline & $\mathrm{V}$ & & 46.6 & 298 & & [1994RUZ/MAJ] \\
\hline & $\mathrm{V}$ & $(322-413)$ & 43.9 & 337 & & [1986PAU/KRU] \\
\hline & $\mathrm{V}$ & & 44.3 & 328 & $\mathrm{C}$ & [1984MAJ/SVO3] \\
\hline & V & & 43.2 & 343 & $\mathrm{C}$ & [1984MAJ/SVO3] \\
\hline & $\mathrm{V}$ & & 42.1 & 358 & $\mathrm{C}$ & [1984MAJ/SVO3] \\
\hline & $\mathrm{V}$ & & 46.4 & 298 & & [1971WIL/ZWO] \\
\hline & $\mathrm{V}$ & $(219-308)$ & 48.3 & 234 & A & [1987STE/MAL, 1973CAR/KOB] \\
\hline & $\mathrm{V}$ & & 46.4 & 298 & $\mathrm{C}$ & [1947OSB/GIN] \\
\hline & $\mathrm{V}$ & $(344-426)$ & 42.7 & 359 & $\mathrm{~A}, \mathrm{MM}$ & [1987STE/MAL, 1945WIL/TAY, 1949FOR/NOR] \\
\hline \multirow[t]{4}{*}{$\mathrm{C}_{9} \mathrm{H}_{20}$} & {$[3221-61-2]$} & 2-methyloctane & & & & \\
\hline & FUS & & 17.99 & 192.8 & & {$[1998 \mathrm{HEL} / \mathrm{OWE}]$} \\
\hline & $\mathrm{V}$ & $(305-417)$ & 43.2 & 320 & A & [1987STE/MAL] \\
\hline & $\mathrm{V}$ & & 44.9 & 298 & & [1971WIL/ZWO, 1961LAB/GRE] \\
\hline \multirow[t]{3}{*}{$\mathrm{C}_{9} \mathrm{H}_{20}$} & {$[2216-33-3]$} & 3-methyloctane & & & & \\
\hline & FUS & & 16.99 & 165.6 & & [1998HEL/OWE] \\
\hline & $\mathrm{V}$ & & 44.9 & 298 & & [1971WIL/ZWO, 1961LAB/GRE] \\
\hline \multirow[t]{2}{*}{$\mathrm{C}_{9} \mathrm{H}_{20}$} & {$[2216-34-4]$} & 4-methyloctane & & & & \\
\hline & $\mathrm{V}$ & & 44.5 & 298 & & [1971WIL/ZWO, 1961LAB/GRE] \\
\hline \multirow[t]{2}{*}{$\mathrm{C}_{9} \mathrm{H}_{20}$} & {$[15869-80-4]$} & 3-ethylheptane & & & & \\
\hline & $\mathrm{V}$ & & 44.5 & 298 & & [1971WIL/ZWO, 1961LAB/GRE] \\
\hline \multirow[t]{2}{*}{$\mathrm{C}_{9} \mathrm{H}_{20}$} & {$[2216-32-2]$} & 4-ethylheptane & & & & \\
\hline & $\mathrm{V}$ & & 44.1 & 298 & & {$[1961 \mathrm{LAB} / \mathrm{GRE}]$} \\
\hline \multirow[t]{2}{*}{$\mathrm{C}_{9} \mathrm{H}_{20}$} & {$[1071-26-7]$} & 2,2-dimethylheptane & & & & \\
\hline & $\mathrm{V}$ & & 42.3 & 298 & & [1971WIL/ZWO, 1961LAB/GRE] \\
\hline \multirow[t]{2}{*}{$\mathrm{C}_{9} \mathrm{H}_{20}$} & {$[3074-71-3]$} & 2,3-dimethylheptane & & & & \\
\hline & $\mathrm{V}$ & & 43.6 & 298 & & [1971WIL/ZWO, 1961LAB/GRE] \\
\hline \multirow[t]{2}{*}{$\mathrm{C}_{9} \mathrm{H}_{20}$} & {$[2213-23-2]$} & 2,4-dimethylheptane & & & & \\
\hline & V & & 42.9 & 298 & & [1971WIL/ZWO, 1961LAB/GRE] \\
\hline $\mathrm{C}_{9} \mathrm{H}_{20}$ & {$[2216-30-0]$} & 2,5-dimethylheptane & & & & \\
\hline & V & & 43.3 & 298 & & [1971WIL/ZWO, 1961LAB/GRE] \\
\hline $\mathrm{C}_{9} \mathrm{H}_{20}$ & {$[1072-05-5]$} & 2,6-dimethylheptane & & & & \\
\hline & $\mathrm{V}$ & & 43.3 & 298 & & [1971WIL/ZWO, 1961LAB/GRE] \\
\hline
\end{tabular}


TABLE 12. Phase change enthalpies of $\mathrm{C}_{9}$ organic compounds-Continued

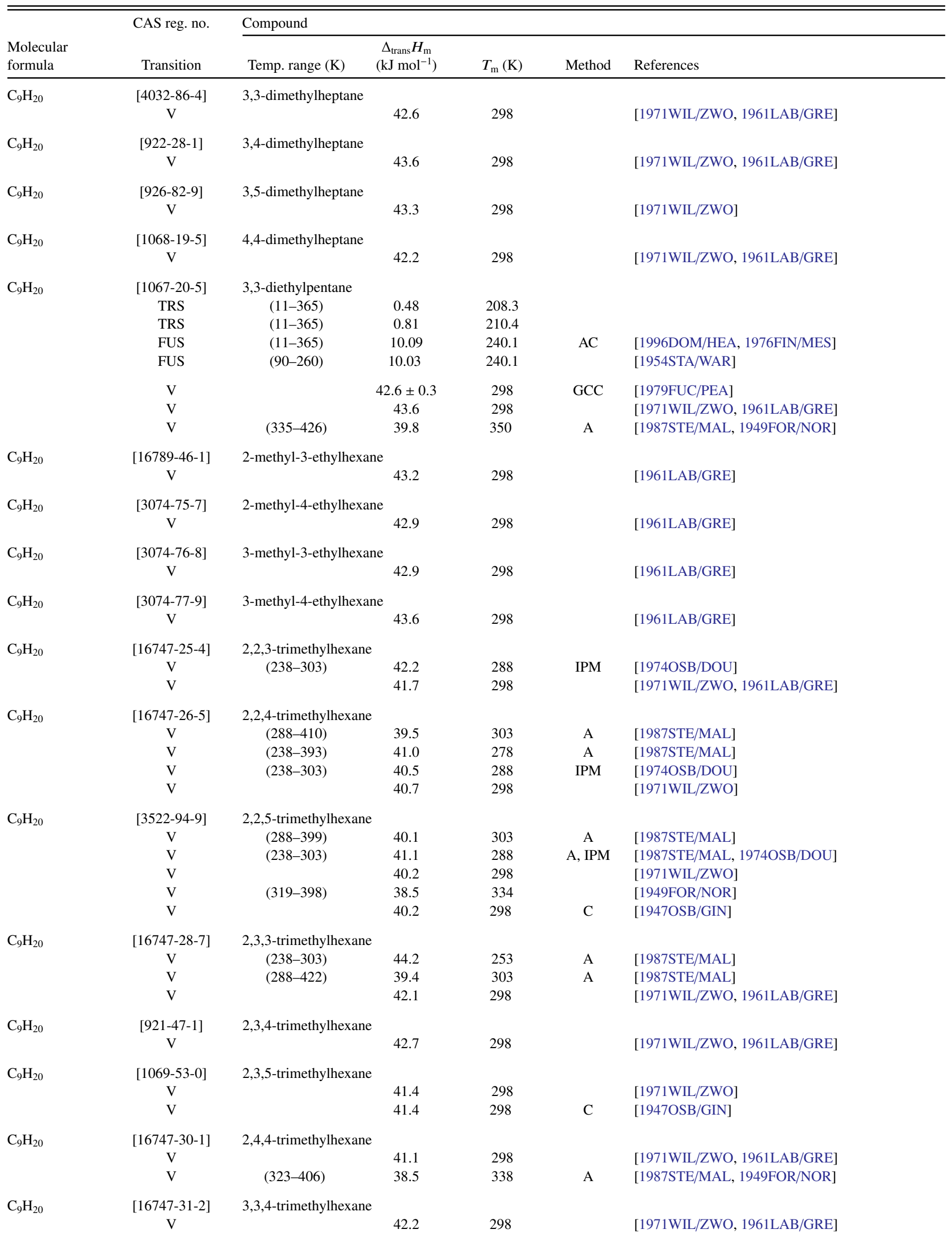


TABLE 12. Phase change enthalpies of $\mathrm{C}_{9}$ organic compounds-Continued

\begin{tabular}{|c|c|c|c|c|c|c|}
\hline \multirow[b]{2}{*}{$\begin{array}{l}\text { Molecular } \\
\text { formula }\end{array}$} & \multirow{2}{*}{$\begin{array}{l}\text { CAS reg. no. } \\
\text { Transition }\end{array}$} & \multicolumn{5}{|l|}{ Compound } \\
\hline & & Temp. range $(\mathrm{K})$ & $\begin{array}{c}\Delta_{\text {trans }} H_{\mathrm{m}} \\
\left(\mathrm{kJ} \mathrm{mol}^{-1}\right)\end{array}$ & $T_{\mathrm{m}}(\mathrm{K})$ & Method & References \\
\hline \multirow[t]{2}{*}{$\mathrm{C}_{9} \mathrm{H}_{20}$} & {$[16747-32-3]$} & \multicolumn{4}{|c|}{ 2,2-dimethyl-3-ethylpentane } & \\
\hline & $\mathrm{V}$ & & 41.7 & 298 & & [1971WIL/ZWO, 1961LAB/GRE] \\
\hline \multirow[t]{2}{*}{$\mathrm{C}_{9} \mathrm{H}_{20}$} & {$[16747-33-4]$} & \multicolumn{4}{|c|}{ 2,3-dimethyl-3-ethylpentane } & \\
\hline & $\mathrm{V}$ & & 42.7 & 298 & & [1971WIL/ZWO, 1961LAB/GRE] \\
\hline \multirow[t]{2}{*}{$\mathrm{C}_{9} \mathrm{H}_{20}$} & {$[1068-87-7]$} & \multicolumn{4}{|c|}{ 2,4-dimethyl-3-ethylpentane } & \\
\hline & $\mathrm{V}$ & & 42.3 & 298 & & [1971WIL/ZWO, 1961LAB/GRE] \\
\hline \multirow[t]{5}{*}{$\mathrm{C}_{9} \mathrm{H}_{20}$} & {$[7154-79-2]$} & \multicolumn{4}{|c|}{ 2,2,3,3-tetramethylpentane } & \\
\hline & TRS & $(12-400)$ & 7.33 & 174.5 & & \\
\hline & FUS & $(12-400)$ & 2.33 & 263.4 & $\mathrm{AC}$ & [1996DOM/HEA, 1976FIN/MES] \\
\hline & $\mathrm{V}$ & & 41.2 & 298 & & [1971WIL/ZWO, 1961LAB/GRE] \\
\hline & $\mathrm{V}$ & $(328-415)$ & 39.2 & 343 & A & [1987STE/MAL, 1949FOR/NOR] \\
\hline \multirow[t]{3}{*}{$\mathrm{C}_{9} \mathrm{H}_{20}$} & {$[1186-53-4]$} & \multicolumn{4}{|c|}{ 2,2,3,4-tetramethylpentane } & \\
\hline & $\mathrm{V}$ & & 40.8 & 298 & & [1971WIL/ZWO, 1961LAB/GRE] \\
\hline & V & $(325-413)$ & 38.4 & 340 & A & [1987STE/MAL, 1949FOR/NOR] \\
\hline \multirow[t]{8}{*}{$\mathrm{C}_{9} \mathrm{H}_{20}$} & {$[1070-87-7]$} & \multicolumn{2}{|c|}{ 2,2,4,4-tetramethylpentane } & & & \\
\hline & FUS & $(12-379)$ & 9.75 & 206.7 & $\mathrm{AC}$ & [1996DOM/HEA, 1976FIN/MES] \\
\hline & $\mathrm{V}$ & & $38.5 \pm 0.1$ & 298 & $\mathrm{C}$ & [1982FUC/PEA] \\
\hline & $\mathrm{V}$ & & $38.5 \pm 0.3$ & 298 & GCC & [1979FUC/PEA] \\
\hline & $\mathrm{V}$ & & 38.2 & 298 & & [1971WIL/ZWO, 1961LAB/GRE] \\
\hline & $\mathrm{V}$ & $(313-397)$ & 37.2 & 328 & A & [1987STE/MAL, 1949FOR/NOR] \\
\hline & $\mathrm{V}$ & $(331-375)$ & 36.5 & 346 & $\mathrm{~EB}$ & [1941SMI, 1984BOU/FRI] \\
\hline & $\mathrm{V}$ & $(375-422)$ & 34.8 & 390 & & [1941SMI] \\
\hline \multirow{3}{*}{$\mathrm{C}_{9} \mathrm{H}_{20}$} & {$[16747-38-9]$} & \multicolumn{4}{|c|}{ 2,3,3,4-tetramethylpentane } & \\
\hline & $\mathrm{V}$ & & 41.8 & 298 & & [1971WIL/ZWO, 1961LAB/GRE] \\
\hline & $\mathrm{V}$ & $(331-416)$ & 39.3 & 346 & A & [1987STE/MAL, 1949FOR/NOR] \\
\hline \multirow[t]{2}{*}{$\mathrm{C}_{9} \mathrm{H}_{20} \mathrm{ClF}_{3} \mathrm{~N}_{2} \mathrm{~S}$} & {$[63265-72-5]$} & \multicolumn{5}{|c|}{ chlorobis( $N$-ethylethanaminato)(trifluoromethyl) sulfur } \\
\hline & $\mathrm{V}$ & & 39.8 & 479 & I & [1977KIT/SHR2] \\
\hline $\mathrm{C}_{9} \mathrm{H}_{20} \mathrm{ClF}_{3} \mathrm{~N}_{2} \mathrm{OS}$ & {$[63265-74-7]$} & chlorobis $(N$-ethyl & lanaminato) $\mathrm{o}$ & fluorome & sulfur & \\
\hline & $\mathrm{V}$ & & 44.4 & 486 & I & [1977KIT/SHR2] \\
\hline $\mathrm{C}_{9} \mathrm{H}_{20} \mathrm{~N}_{2} \mathrm{O}$ & {$[1792-17-2]$} & 1,3-dibutylurea & & & & \\
\hline & TRS & & 11.1 & 311.5 & & \\
\hline & FUS & & 14.87 & 346.9 & & [1996DOM/HEA, 1987DEL/FER] \\
\hline & SUB & $(323-372)$ & $91.9 \pm 0.9$ & 348 & $\mathrm{ME}$ & [2003ZAI/KAB] \\
\hline & SUB & $(323-372)$ & $91.9 \pm 0.9$ & 350 & $\mathrm{ME}$ & [2003ZAI/KAB] \\
\hline & SUB & & $90.0 \pm 1.0$ & 350 & $\mathrm{C}$ & [2003ZAI/KAB] \\
\hline & $\mathrm{V}$ & $(379-413)$ & $101.1 \pm 1.6$ & 396 & & [1990PIA/FER] \\
\hline $\mathrm{C}_{9} \mathrm{H}_{20} \mathrm{~N}_{2} \mathrm{O}$ & {$[2158-10-3]$} & 1-octyl urea & & & & \\
\hline & TRS & & 11.8 & 353.2 & & \\
\hline & FUS & & 24.4 & 374.6 & DSC & [2005HAS/TAJ] \\
\hline & TRS & & 11.5 & 350.2 & & \\
\hline & FUS & & 24.6 & 372.2 & $\mathrm{DSC}$ & [1999WEL/DRU] \\
\hline $\mathrm{C}_{9} \mathrm{H}_{20} \mathrm{~N}_{2} \mathrm{O}$ & [1187-03-7] & 1,1,3,3-tetraethylu & & & & \\
\hline & FUS & & 20.55 & 253 & & $\begin{array}{l}\text { [1990KOZ/SIM2, 1996DOM/HEA, } \\
\text { 1995KAB/KOZ2] }\end{array}$ \\
\hline $\mathrm{C}_{9} \mathrm{H}_{20} \mathrm{~N}_{2} \mathrm{~S}$ & {$[109-46-6]$} & 1,3-butylthiourea & & & & \\
\hline & FUS & & 28.34 & 338 & DSC & [2000DEL/JOZ] \\
\hline & SUB & & $141.0 \pm 2$ & 298 & B & [2000DEL/JOZ] \\
\hline & SUB & & $137 \pm 3.0$ & 298 & $\mathrm{C}$ & [1994TER/PIA] \\
\hline & $\mathrm{V}$ & $(368-403)$ & $105 \pm 2.0$ & 386 & ME, TE & [1994TER/PIA] \\
\hline $\mathrm{C}_{9} \mathrm{H}_{20} \mathrm{~N}_{2} \mathrm{~S}_{2}$ & [1518-58-7] & diethylammonium & ethyldithioca & & & \\
\hline & SUB & & $209.9 \pm 3.0$ & & $\mathrm{C}$ & [1988RIB/REI] \\
\hline & SUB & & $111.8 \pm 3.0$ & & & [1979CAV/HIL] \\
\hline
\end{tabular}

Note: The authors of [1988RIB/REI] state compound decomposes on sublimation 
TABLE 12. Phase change enthalpies of $\mathrm{C}_{9}$ organic compounds-Continued

\begin{tabular}{|c|c|c|c|c|c|c|}
\hline \multirow[b]{2}{*}{$\begin{array}{l}\text { Molecular } \\
\text { formula }\end{array}$} & \multirow{2}{*}{$\begin{array}{l}\text { CAS reg. no. } \\
\text { Transition }\end{array}$} & \multicolumn{5}{|l|}{ Compound } \\
\hline & & Temp. range $(\mathrm{K})$ & $\begin{array}{c}\Delta_{\text {trans }} H_{\mathrm{m}} \\
\left(\mathrm{kJ} \mathrm{mol}^{-1}\right)\end{array}$ & $T_{\mathrm{m}}(\mathrm{K})$ & Method & References \\
\hline $\mathrm{C}_{9} \mathrm{H}_{20} \mathrm{~N}_{4} \mathrm{O}_{4}$ & FUS & & 31.0 & 345.2 & DSC & [2003SPI/WAN] \\
\hline \multirow[t]{8}{*}{$\mathrm{C}_{9} \mathrm{H}_{20} \mathrm{O}$} & [143-08-8] & 1-nonanol & & & & \\
\hline & FUS & & 24.54 & 268.1 & DSC & [2005DOM/MAR] \\
\hline & V & & 72.2 & 298 & $\mathrm{CGC}$ & [2000OVA/KOU] \\
\hline & V & $(373-423)$ & 76.7 & 298 & $\mathrm{CGC}$ & [1995CHI/HOS] \\
\hline & V & $(273-323)$ & 77.4 & 298 & & [1992NGU/KAS] \\
\hline & $\mathrm{V}$ & $(368-500)$ & 65.0 & 383 & $\mathrm{~A}$ & [1987STE/MAL] \\
\hline & V & (381-495) & 62.9 & 396 & A & [1987STE/MAL] \\
\hline & V & & $76.9 \pm 0.8$ & 298 & $\mathrm{C}$ & [1977MAN/SEL] \\
\hline \multirow{3}{*}{$\mathrm{C}_{9} \mathrm{H}_{20} \mathrm{O}$} & $\mathrm{V}$ & $(286-324)$ & $72.9 \pm 0.6$ & 298 & GS & [2007VER/SCH] \\
\hline & $\mathrm{V}$ & $(253-353)$ & 79.6 & 268 & & [1999NGU/BER] \\
\hline & $\mathrm{V}$ & $(364-471)$ & 55.5 & 379 & & [1973WIL/ZWO] \\
\hline \multirow[t]{4}{*}{$\mathrm{C}_{9} \mathrm{H}_{20} \mathrm{O}$} & [624-51-1] & 3-nonanol & & & & \\
\hline & $\mathrm{V}$ & $(263-363)$ & $70.9 \pm 0.3$ & 298 & GS & [2007VER/SCH] \\
\hline & $\mathrm{V}$ & $(263-363)$ & 75.5 & 278 & & [1999NGU/BER] \\
\hline & $\mathrm{V}$ & $(366-468)$ & 57.1 & 381 & & [1973WIL/ZWO] \\
\hline \multirow[t]{2}{*}{$\mathrm{C}_{9} \mathrm{H}_{20} \mathrm{O}$} & [5932-79-6] & 4-nonanol & & & & \\
\hline & $\mathrm{V}$ & (285-324) & $71.5 \pm 0.3$ & 298 & GS & [2007VER/SCH] \\
\hline $\mathrm{C}_{9} \mathrm{H}_{20} \mathrm{O}$ & [623-93-8] & 5-nonanol & & & & \\
\hline $\mathrm{C}_{9} \mathrm{H}_{20} \mathrm{O}$ & V & $(353-388)$ & 53.2 & 368 & & [1973WIL/ZWO] \\
\hline \multirow[t]{2}{*}{$\mathrm{C}_{9} \mathrm{H}_{20} \mathrm{O}$} & & 2,2-dimethyl-4-hept & & & & \\
\hline & $\mathrm{V}$ & $(320-445)$ & 50.2 & 335 & & [1973WIL/ZWO] \\
\hline \multirow[t]{4}{*}{$\mathrm{C}_{9} \mathrm{H}_{20} \mathrm{O}$} & [108-82-7] & 2,6-dimethyl-4-hept & & & & \\
\hline & $\mathrm{V}$ & $(278-321)$ & $65.2 \pm 0.3$ & 298 & GS & [2005ROG/PIS] \\
\hline & $\mathrm{V}$ & $(363-453)$ & 54.5 & 378 & & [1973WIL/ZWO] \\
\hline & $\mathrm{V}$ & $(374-452)$ & 52.8 & 389 & $\mathrm{~A}, \mathrm{MM}$ & [1987STE/MAL, 1947STR/GAB] \\
\hline \multirow[t]{2}{*}{$\mathrm{C}_{9} \mathrm{H}_{20} \mathrm{O}$} & [5340-41-0] & 2,2,3-trimethyl-3-he & anol & & & \\
\hline & $\mathrm{V}$ & $(343-441)$ & 55.1 & 358 & & [1973WIL/ZWO] \\
\hline \multirow[t]{2}{*}{$\mathrm{C}_{9} \mathrm{H}_{20} \mathrm{O}$} & [3452-97-9] & 3,5,5-trimethyl-1-he & anol & & & \\
\hline & $\mathrm{V}$ & $(288-324)$ & $67.9 \pm 0.4$ & 298 & GS & [2005ROG/PIS] \\
\hline \multirow[t]{2}{*}{$\mathrm{C}_{9} \mathrm{H}_{20} \mathrm{O}$} & [3970-59-0] & 2,4-dimethyl-3-ethy & 3-pentanol & & & \\
\hline & $\mathrm{V}$ & $(369-451)$ & 50.0 & 384 & & [1973WIL/ZWO] \\
\hline \multirow[t]{2}{*}{$\mathrm{C}_{9} \mathrm{H}_{20} \mathrm{O}$} & [29772-39-2] & 2,2,3,4-tetramethyl- & -pentanol & & & \\
\hline & $\mathrm{V}$ & $(329-448)$ & 60.8 & 344 & & [1973WIL/ZWO] \\
\hline $\mathrm{C}_{9} \mathrm{H}_{20} \mathrm{O}$ & [14609-79-1] & di-tert-butylmethan & & & & \\
\hline & SUB & $(274-322)$ & $62.7 \pm 0.9$ & 298 & GS & [1998VER3] \\
\hline $\mathrm{C}_{9} \mathrm{H}_{20} \mathrm{O}$ & [14609-79-1] & 2,2,4,4-tetramethylp & ntan-3-ol & & & \\
\hline & TRS & & 1.9 & 263 & & \\
\hline & FUS & & 7.3 & 322 & & [1996DOM/HEA, 1990BAT/JAK] \\
\hline
\end{tabular}


TABLE 12. Phase change enthalpies of $\mathrm{C}_{9}$ organic compounds-Continued

\begin{tabular}{|c|c|c|c|c|c|c|}
\hline \multirow[b]{2}{*}{$\begin{array}{l}\text { Molecular } \\
\text { formula }\end{array}$} & \multirow{2}{*}{$\begin{array}{l}\text { CAS reg. no. } \\
\text { Transition }\end{array}$} & \multicolumn{5}{|l|}{ Compound } \\
\hline & & Temp. range $(\mathrm{K})$ & $\begin{array}{c}\Delta_{\text {trans }} H_{\mathrm{m}} \\
\left(\mathrm{kJ} \mathrm{mol}^{-1}\right)\end{array}$ & $T_{\mathrm{m}}(\mathrm{K})$ & Method & References \\
\hline \multirow[t]{2}{*}{$\mathrm{C}_{9} \mathrm{H}_{20} \mathrm{O}$} & [3249-47-6] & \multicolumn{5}{|c|}{ butyl 1,1-dimethylpropyl ether } \\
\hline & $\mathrm{V}$ & $(278-308)$ & $46.1 \pm 0.3$ & 298 & GS & [1996VER/BEC] \\
\hline \multirow[t]{2}{*}{$\mathrm{C}_{9} \mathrm{H}_{20} \mathrm{O}$} & {$[62108-41-2]$} & \multicolumn{5}{|c|}{ 2-methoxy-2,4,4-trimethylpentane } \\
\hline & $\mathrm{V}$ & $(381-418)$ & 38.5 & 396 & & [2001UUS/POK] \\
\hline \multirow[t]{3}{*}{$\mathrm{C}_{9} \mathrm{H}_{20} \mathrm{O}$} & {$[62108-41-2]$} & \multicolumn{5}{|c|}{ methyl tert-octyl ether } \\
\hline & V & & 45.3 & 298 & & [UR/VER, 2002VER, 2003VER/KRA] \\
\hline & $\mathrm{V}$ & & 45.33 & 298 & EB & [2001UUS/POK, 2003VER/KRA] \\
\hline \multirow[t]{4}{*}{$\mathrm{C}_{9} \mathrm{H}_{20} \mathrm{O}$} & {$[10100-95-5]$} & \multicolumn{5}{|c|}{ pentyl tert-butyl ether } \\
\hline & V & & 48.3 & 298 & & [UR/VER, 2002VER, 2003VER/KRA] \\
\hline & $\mathrm{V}$ & $(319-365)$ & 43.7 & 334 & EB & [1990ROZ/BAR] \\
\hline & $\mathrm{V}$ & $(319-365)$ & $46.9 \pm 1.0$ & 298 & $\mathrm{~EB}$ & [1990ROZ/BAR] \\
\hline \multirow[t]{2}{*}{$\mathrm{C}_{9} \mathrm{H}_{20} \mathrm{O}$} & {$[10100-95-5]$} & \multicolumn{5}{|c|}{ isobutyl tert-amyl ether } \\
\hline & $\mathrm{V}$ & & 46.3 & 298 & & [UR/VER, 2002VER, 2003VER/KRA] \\
\hline \multirow[t]{2}{*}{$\mathrm{C}_{9} \mathrm{H}_{20} \mathrm{O}$} & & \multicolumn{5}{|c|}{ sec-butyl tert-amyl ether } \\
\hline & $\mathrm{V}$ & & 46.8 & 298 & & [UR/VER, 2002VER, 2003VER/KRA] \\
\hline \multirow[t]{2}{*}{$\mathrm{C}_{9} \mathrm{H}_{20} \mathrm{O}$} & {$[3249-47-6]$} & \multicolumn{5}{|l|}{ butyl tert-amyl ether } \\
\hline & V & & 48.3 & 298 & & [UR/VER, 2002VER, 2003VER/KRA] \\
\hline \multirow[t]{2}{*}{$\mathrm{C}_{9} \mathrm{H}_{20} \mathrm{O} \mathrm{S}$} & {$[3079-27-4]$} & \multicolumn{5}{|c|}{ methyl octyl sulfoxide } \\
\hline & FUS & & 36.8 & & $\mathrm{C}$ & {$[1969 \mathrm{COR} / \mathrm{GOO}]$} \\
\hline $\mathrm{C}_{9} \mathrm{H}_{20} \mathrm{O}_{2}$ & {$[22419-28-9]$} & 2,6,6-trimethyl-5-o & -2-heptanol & & & \\
\hline & $\mathrm{V}$ & $(329-454)$ & 53.3 & 344 & & [1968KAC/NEM, 1984BOU/FRI] \\
\hline $\mathrm{C}_{9} \mathrm{H}_{20} \mathrm{O}_{2}$ & {$[2568-90-3]$} & dibutyoxymethane & & & & \\
\hline & $\mathrm{V}$ & $(366-452)$ & 47.9 & 381 & $\mathrm{~EB}$ & [2000PAL/SZA] \\
\hline & $\mathrm{V}$ & $(363-452)$ & 48.1 & 298 & $\mathrm{~EB}$ & [2000PAL/SZA] \\
\hline $\mathrm{C}_{9} \mathrm{H}_{20} \mathrm{O}_{2}$ & [18854-58-5] & 1-butyoxy-2-propo & ethane & & & \\
\hline & $\mathrm{V}$ & & $54.7 \pm 0.1$ & 298 & $\mathrm{C}$ & [1970KUS/WAD] \\
\hline $\mathrm{C}_{9} \mathrm{H}_{20} \mathrm{O}_{2}$ & [115-84-4] & 2-butyl-2-ethyl-1,3 & opanediol & & & \\
\hline & FUS & & 20.8 & 317.3 & & [2002STE/CHI] \\
\hline & $\mathrm{V}$ & $(424-523)$ & $74.3 \pm 0.3$ & 420 & $\mathrm{~EB}$ & [2002STE/CHI] \\
\hline & $\mathrm{V}$ & $(424-523)$ & $67.2 \pm 0.3$ & 460 & $\mathrm{~EB}$ & [2002STE/CHI] \\
\hline & $\mathrm{V}$ & $(424-523)$ & $61.4 \pm 0.6$ & 500 & $\mathrm{~EB}$ & [2002STE/CHI] \\
\hline $\mathrm{C}_{9} \mathrm{H}_{20} \mathrm{O}_{2}$ & [22419-28-9] & 4-tert-butoxy-2-me & yl-2-butanol & & & \\
\hline & V & $(367-483)$ & 61.5 & 382 & A & [1987STE/MAL] \\
\hline $\mathrm{C}_{9} \mathrm{H}_{20} \mathrm{O}_{2}$ & [3937-56-2] & 1,9-nonanediol & & & & \\
\hline & FUS & & 36.7 & 318.7 & $\mathrm{DSC}$ & [2014BAD/NOW] \\
\hline & FUS & & 36.4 & 319.6 & DTA & [1991ACR, 1990KNA/SAB] \\
\hline & FUS & & 33.5 & 318.6 & $\mathrm{C}$ & [1969COR/GOO] \\
\hline & SUB & & 148.7 & & & [1990KNA/SAB] \\
\hline & $\mathrm{V}$ & & $112.5 \pm 0.2$ & 298 & CGC & [2006UMN/KWE] \\
\hline & $\mathrm{V}$ & & 104.4 & 360 & $\mathrm{TE}$ & [1994PIA/FER, 2006UMN/KWE] \\
\hline & $\mathrm{V}$ & & $111.4 \pm 7.0$ & 298 & TE & [1994PIA/FER, 2006UMN/KWE] \\
\hline & $\mathrm{V}$ & & $110.0 \pm 1.0$ & 323 & $\mathrm{C}$ & [1990KNA/SAB, 2006UMN/KWE] \\
\hline & $\mathrm{V}$ & & $112.8 \pm 2.1$ & 298 & $\mathrm{C}$ & [1990KNA/SAB, 2006UMN/KWE] \\
\hline $\mathrm{C}_{9} \mathrm{H}_{20} \mathrm{O}_{2}$ & [3089-24-5] & 2,2,4-trimethyl-1,6 & exanediol & & & \\
\hline & $\mathrm{V}$ & $(419-541)$ & 68.0 & 434 & A & [1987STE/MAL] \\
\hline $\mathrm{C}_{9} \mathrm{H}_{20} \mathrm{O}_{2} \mathrm{~S}$ & {$[54581-77-0]$} & 3-(hexylthio)-1,2-p & panediol & & & \\
\hline & FUS & & 48.5 & 290.8 & $\mathrm{DSC}$ & [1993ACR, 1990VAN/VAN] \\
\hline $\mathrm{C}_{9} \mathrm{H}_{20} \mathrm{O}_{2} \mathrm{~S}_{2}$ & & meso-1,3-bis(propy & afinyl)propan & & & \\
\hline & FUS & & 40.58 & 411.3 & DSC & [2001CAL/MEL] \\
\hline $\mathrm{C}_{9} \mathrm{H}_{20} \mathrm{O}_{2} \mathrm{~S}_{2}$ & & racemic 1,3 -bis(pro & lsufinyl)prop & & & \\
\hline & FUS & & 35.15 & 387.8 & $\mathrm{DSC}$ & [2001CAL/MEL] \\
\hline
\end{tabular}


TABLE 12. Phase change enthalpies of $\mathrm{C}_{9}$ organic compounds-Continued

\begin{tabular}{|c|c|c|c|c|c|c|}
\hline \multirow[b]{2}{*}{$\begin{array}{l}\text { Molecular } \\
\text { formula }\end{array}$} & \multirow{2}{*}{$\begin{array}{l}\text { CAS reg. no. } \\
\text { Transition }\end{array}$} & \multicolumn{5}{|l|}{ Compound } \\
\hline & & Temp. range $(\mathrm{K})$ & $\begin{array}{c}\Delta_{\text {trans }} H_{\mathrm{m}} \\
\left(\mathrm{kJ} \mathrm{mol}^{-1}\right)\end{array}$ & $T_{\mathrm{m}}(\mathrm{K})$ & Method & References \\
\hline & $\mathrm{V}$ & $(319-479)$ & 55.0 & 334 & A & [1987STE/MAL, 1947STU] \\
\hline \multirow[t]{2}{*}{$\mathrm{C}_{9} \mathrm{H}_{20} \mathrm{O}_{3}$} & {$[10305-38-1]$} & \multicolumn{2}{|c|}{ 3-(hexyloxy)-1,2-propanediol } & & & \\
\hline & FUS & & 10.2 & 272.9 & DSC & [1990VAN/VAN] \\
\hline & $\mathrm{V}$ & $(369-541)$ & 63.3 & 384 & A & [1987STE/MAL] \\
\hline \multirow{2}{*}{$\mathrm{C}_{9} \mathrm{H}_{20} \mathrm{O}_{4}$} & [4161-32-4] & \multicolumn{3}{|c|}{ 3,3'-[1,3-propanediylbis(oxy)]bis-1-propanol } & & \\
\hline & FUS & & 21.15 & 263.1 & DSC & [1991BED/BOO] \\
\hline \multirow[t]{2}{*}{$\mathrm{C}_{9} \mathrm{H}_{20} \mathrm{O}_{4}$} & [78-09-1] & tetraethoxymethane & & & & \\
\hline & $\mathrm{V}$ & & $52.9 \pm 0.2$ & 298 & $\mathrm{C}$ & [1985MAR/MAN] \\
\hline \multirow[t]{2}{*}{$\mathrm{C}_{9} \mathrm{H}_{20} \mathrm{~S}$} & [13281-11-3] & 2-nonanethiol & & & & \\
\hline & V & (379-482) & 50.3 & 394 & & [1999DYK/SVO, 1932ELL/REI] \\
\hline \multirow[t]{2}{*}{$\mathrm{C}_{9} \mathrm{H}_{20} \mathrm{~S}_{2}$} & [3489-28-9] & 1,9-nonanedithiol & & & & \\
\hline & $\mathrm{V}$ & $(418-557)$ & 63.6 & 433 & A & [1987STE/MAL, 1999DYK/SVO, 1943HAL/REI] \\
\hline \multirow[t]{2}{*}{$\mathrm{C}_{9} \mathrm{H}_{21} \mathrm{~N}$} & [2439-54-5] & $N$-methyl octylamin & & & & \\
\hline & $\mathrm{V}$ & $(365-508)$ & 49.2 & 380 & A & [1987STE/MAL] \\
\hline \multirow[t]{2}{*}{$\mathrm{C}_{9} \mathrm{H}_{21} \mathrm{~N}$} & {$[112-20-9]$} & nonylamine & & & & \\
\hline & $\mathrm{V}$ & $(377-478)$ & 50.7 & 392 & A & [1987STE/MAL] \\
\hline $\mathrm{C}_{9} \mathrm{H}_{21} \mathrm{~N}$ & {$[102-69-2]$} & tripropylamine & & & & \\
\hline \multirow{4}{*}{$\mathrm{C}_{9} \mathrm{H}_{21} \mathrm{O}_{4} \mathrm{P}$} & $\mathrm{V}$ & $(401-527)$ & 60.4 & 453 & DSC & [2014BRO/BUC] \\
\hline & $\mathrm{V}$ & $(401-527)$ & 58.4 & 493 & DSC & [2014BRO/BUC] \\
\hline & $\mathrm{V}$ & $(401-527)$ & 57.2 & 525 & DSC & [2014BRO/BUC] \\
\hline & $\mathrm{V}$ & $(394-525)$ & 56.7 & 409 & A & [1987STE/MAL, 1930EVA/DAV] \\
\hline \multirow[t]{2}{*}{$\mathrm{C}_{9} \mathrm{H}_{21} \mathrm{P}$} & [2234-97-1] & tripropylphosphine & & & & \\
\hline & $\mathrm{V}$ & $(324-368)$ & $39.4 \pm 0.2$ & 346 & & [2001BAE] \\
\hline \multirow[t]{2}{*}{$\mathrm{C}_{9} \mathrm{H}_{22} \mathrm{ClN}_{2} \mathrm{PS}$} & [58023-20-4] & \multicolumn{5}{|c|}{$P$-(chloromethyl)- $N, N^{\prime}$-bis(1-methylpropyl)phosphorothioic diamide } \\
\hline & $\mathrm{V}$ & $(333-368)$ & 66.8 & 348 & A & [1987STE/MAL, 1999DYK/SVO, 1975KOR/VIA] \\
\hline \multirow[t]{6}{*}{$\mathrm{C}_{9} \mathrm{H}_{22} \mathrm{~N}_{2}$} & [646-24-2] & nonane-1,9-diamine & & & & \\
\hline & FUS & & 49.2 & 310.4 & $\mathrm{AC}$ & [2004MON/VAN, 2014FUL/RUZ] \\
\hline & TRS & & 7.77 & 301.7 & & \\
\hline & FUS & & 36.24 & 308.1 & DSC & [2002DAL/DEL] \\
\hline & $\mathrm{V}$ & $(318-365)$ & 70.6 & 341 & GS & [2011POZ/VER] \\
\hline & $\mathrm{V}$ & $(318-365)$ & $75.5 \pm 0.3$ & 298 & GS & [2011POZ/VER] \\
\hline
\end{tabular}


Table 13. Phase change enthalpies of $\mathrm{C}_{10}$ organic compounds

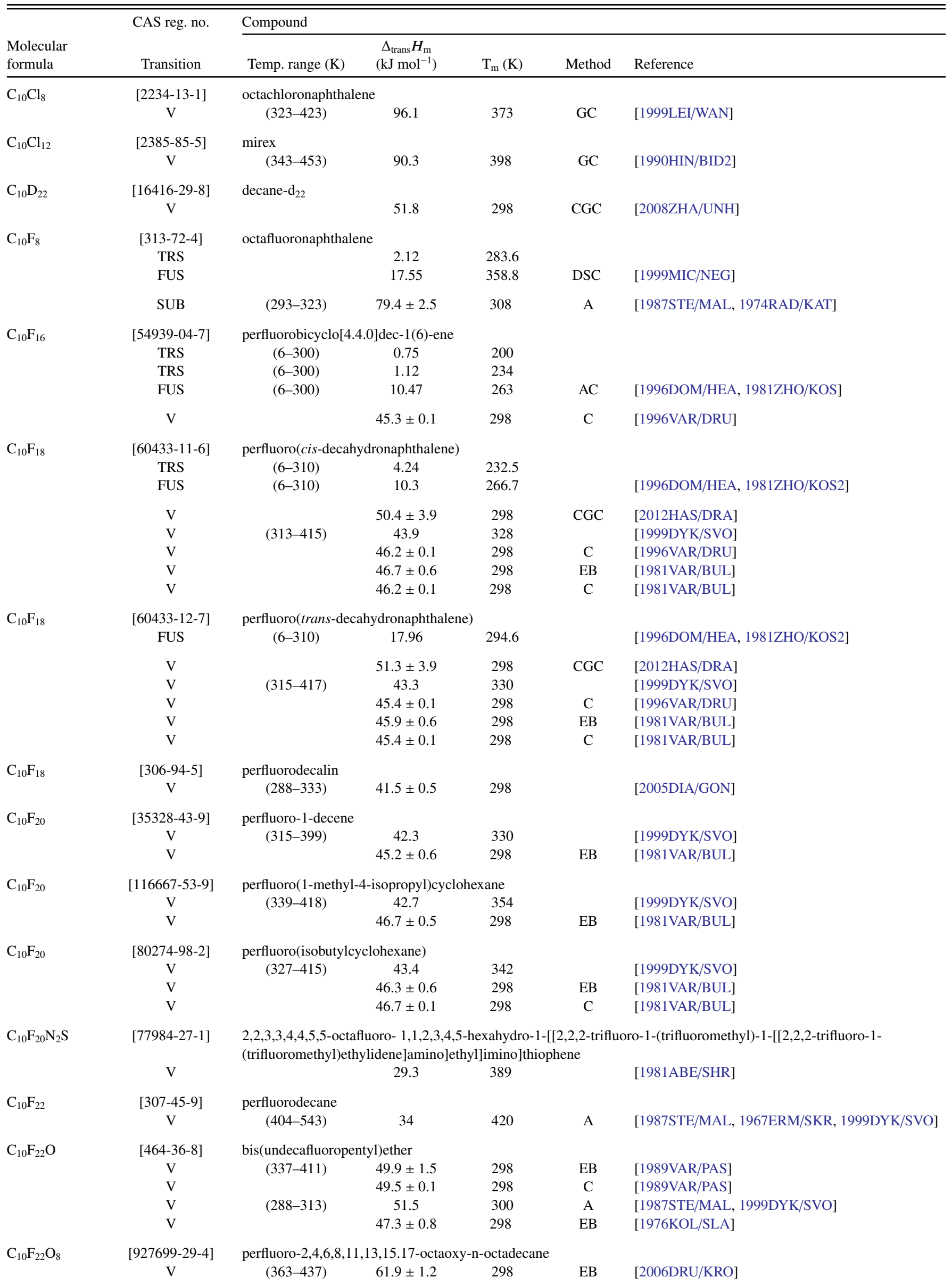


TABLE 13. Phase change enthalpies of $\mathrm{C}_{10}$ organic compounds-Continued

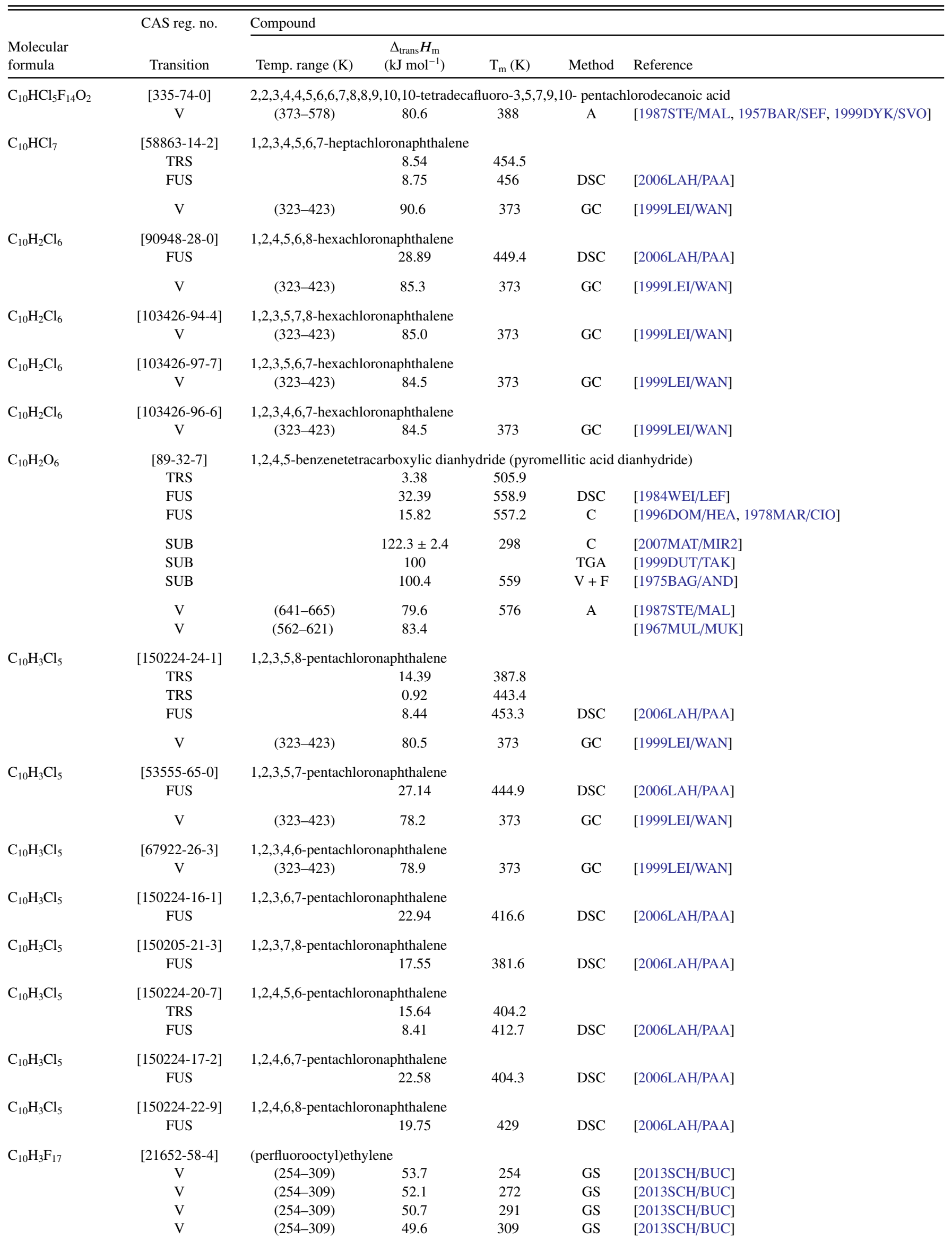


TABLE 13. Phase change enthalpies of $\mathrm{C}_{10}$ organic compounds-Continued

\begin{tabular}{|c|c|c|c|c|c|c|}
\hline \multirow[b]{2}{*}{$\begin{array}{l}\text { Molecular } \\
\text { formula }\end{array}$} & \multirow{2}{*}{$\begin{array}{l}\text { CAS reg. no. } \\
\text { Transition }\end{array}$} & \multicolumn{5}{|l|}{ Compound } \\
\hline & & Temp. range $(\mathrm{K})$ & $\begin{array}{c}\Delta_{\text {trans }} H_{\mathrm{m}} \\
\left(\mathrm{kJ} \mathrm{mol}^{-1}\right)\end{array}$ & $\mathrm{T}_{\mathrm{m}}(\mathrm{K})$ & Method & Reference \\
\hline $\mathrm{C}_{10} \mathrm{H}_{4} \mathrm{Cl}_{2} \mathrm{O}_{2}$ & $\begin{array}{l}{[117-80-6]} \\
\text { FUS }\end{array}$ & \multicolumn{4}{|c|}{ 2,3-dichloro-1,4-naphthalenedione } & [1991ACR, 1990DON/DRE] \\
\hline $\mathrm{C}_{10} \mathrm{H}_{4} \mathrm{Cl}_{4}$ & $\begin{array}{c}{[67922-21-8]} \\
\mathrm{V}\end{array}$ & \multicolumn{4}{|c|}{ 1,2,4,7-tetrachloronaphthalene } & [1999LEI/WAN] \\
\hline \multirow[t]{2}{*}{$\mathrm{C}_{10} \mathrm{H}_{4} \mathrm{Cl}_{4}$} & $\begin{array}{l}{[53555-63-8]} \\
\text { FUS }\end{array}$ & 1,2,3,5-tetrachloro & $\begin{array}{r}\text { phthalene } \\
27.14\end{array}$ & 453 & DSC & [2006LAH/PAA] \\
\hline & $\mathrm{V}$ & $(323-423)$ & 73.4 & 373 & GC & [1999LEI/WAN] \\
\hline \multirow[t]{2}{*}{$\mathrm{C}_{10} \mathrm{H}_{4} \mathrm{Cl}_{4}$} & $\begin{array}{c}\text { [20020-02-4] } \\
\text { TRS } \\
\text { TRS } \\
\text { FUS }\end{array}$ & 1,2,3,4-tetrachloro & $\begin{array}{r}\text { phthalene } \\
10.53 \\
1.33 \\
11.54\end{array}$ & $\begin{array}{l}440.5 \\
454.3 \\
470.8\end{array}$ & DSC & [2006LAH/PAA] \\
\hline & $\mathrm{V}$ & $(323-423)$ & 73.2 & 373 & GC & [1999LEI/WAN] \\
\hline $\mathrm{C}_{10} \mathrm{H}_{4} \mathrm{Cl}_{4}$ & $\begin{array}{c}\text { [149864-82-4] } \\
\text { FUS }\end{array}$ & \multicolumn{5}{|c|}{ 1,2,7,8-tetrachloronaphthalene } \\
\hline $\mathrm{C}_{10} \mathrm{H}_{4} \mathrm{Cl}_{8} \mathrm{O}$ & $\begin{array}{c}{[27304-13-8]} \\
V\end{array}$ & \multicolumn{5}{|c|}{$2,3,4,5,6,6 \mathrm{a}, 7,7$-octachloro-1a,1b,5,5a,6,6a -hexahydro-2,5-methano- $2 \boldsymbol{H}$-indeno-[1,2-b]oxirene (oxychlordane) } \\
\hline \multirow[t]{2}{*}{$\mathrm{C}_{10} \mathrm{H}_{5} \mathrm{Cl}_{3}$} & $\begin{array}{c}{[50402-52-3]} \\
\text { FUS }\end{array}$ & \multicolumn{4}{|c|}{ 1,2,3-trichloronaphthalene } & [2006LAH/PAA] \\
\hline & $\mathrm{V}$ & $(323-423)$ & 68 & 373 & GC & [1999LEI/WAN] \\
\hline \multirow[t]{2}{*}{$\mathrm{C}_{10} \mathrm{H}_{5} \mathrm{Cl}_{7}$} & $\begin{array}{c}\text { [76-44-8] } \\
\text { FUS } \\
\text { TRS } \\
\text { FUS }\end{array}$ & $1,4,5,6,7,8,8$-hepta & $\begin{array}{c}\text { loro- } 3 \mathrm{a}, 4,7,7 \mathrm{a} \\
20.72 \\
23.4 \\
2.09\end{array}$ & $\begin{array}{c}\text { ahydro-4 } \\
362.2 \\
358.2 \\
371\end{array}$ & $\begin{array}{l}\text { endometha } \\
\text { TGA, DSC } \\
\text { DSC }\end{array}$ & $\begin{array}{l}\text { oindene (heptachlor) } \\
\text { [2000ROD/VEC] } \\
\text { [1995KSI/NAG] }\end{array}$ \\
\hline & $\mathrm{V}$ & $(343-453)$ & 76.5 & 398 & GC & [1990HIN/BID2] \\
\hline \multirow[t]{2}{*}{$\mathrm{C}_{10} \mathrm{H}_{5} \mathrm{Cl}_{7} \mathrm{O}$} & $\begin{array}{c}\text { [1024-57-3] } \\
\text { TRS } \\
\text { FUS }\end{array}$ & 1,4,5,6,7,8,8-hepta & $\begin{array}{c}\text { loro-2,3-epoxy } \\
18.9 \\
2.85\end{array}$ & $\begin{array}{c}4,7,7 \mathrm{a}-\mathrm{t} \\
385.2 \\
434.9\end{array}$ & $\begin{array}{c}\text { hydro-4,7-c } \\
\text { DSC }\end{array}$ & $\begin{array}{l}\text { ado-methanoindan } \\
\text { [1995KSI/NAG] }\end{array}$ \\
\hline & $\mathrm{V}$ & $(373-423)$ & 75 & 398 & GC & [2007GOE/MCC] \\
\hline $\mathrm{C}_{10} \mathrm{H}_{5} \mathrm{Cl}_{9}$ & $\begin{array}{c}{[5103-73-1]} \\
\mathrm{V}\end{array}$ & \multicolumn{4}{|l|}{ cis-nonachlor } & [1990HIN/BID2] \\
\hline $\mathrm{C}_{10} \mathrm{H}_{5} \mathrm{Cl}_{9}$ & $\begin{array}{c}{[39765-80-5]} \\
\mathrm{V}\end{array}$ & \multicolumn{4}{|l|}{ trans-nonachlor } & [1990HIN/BID2] \\
\hline $\mathrm{C}_{10} \mathrm{H}_{5} \mathrm{~F}_{15} \mathrm{O}_{2}$ & $\begin{array}{c}\text { [3108-24-5] } \\
\text { V } \\
\text { V } \\
\text { V } \\
\text { V }\end{array}$ & $\begin{array}{l}\text { ethyl perfluoroocta } \\
(254-309) \\
(254-309) \\
(254-309) \\
(254-309)\end{array}$ & $\begin{array}{l}64.1 \\
60.4 \\
57.4 \\
54.9\end{array}$ & $\begin{array}{l}263 \\
278 \\
293 \\
308\end{array}$ & $\begin{array}{l}\text { GS } \\
\text { GS } \\
\text { GS } \\
\text { GS }\end{array}$ & $\begin{array}{l}{[2013 \mathrm{SCH} / \mathrm{BUC}]} \\
{[2013 \mathrm{SCH} / \mathrm{BUC}]} \\
{[2013 \mathrm{SCH} / \mathrm{BUC}]} \\
{[2013 \mathrm{SCH} / \mathrm{BUC}]}\end{array}$ \\
\hline $\mathrm{C}_{10} \mathrm{H}_{6} \mathrm{BrNO}_{2}$ & $\begin{array}{l}{[13380-67-1]} \\
\text { SUB }\end{array}$ & $\begin{array}{l}\text { 1-(4-bromophenyl) } \\
\quad(350-370)\end{array}$ & $\begin{array}{l}H \text {-pyrrole- } 2,5 \\
105.9 \pm 0.7\end{array}$ & & $\mathrm{C}$ & [1998KIS/KAS] \\
\hline \multirow[t]{2}{*}{$\mathrm{C}_{10} \mathrm{H}_{6} \mathrm{Br}_{2}$} & $\begin{array}{c}{[83-53-4]} \\
\text { FUS }\end{array}$ & \multicolumn{5}{|c|}{ 1,4-dibromonaphthalene } \\
\hline & SUB & $(297-322)$ & $90.8 \pm 1.7$ & & $\mathrm{ME}$ & [2008GOL/SUU2] \\
\hline $\mathrm{C}_{10} \mathrm{H}_{6} \mathrm{Cl}_{2}$ & $\begin{array}{c}{[2050-69-3]} \\
\mathrm{V}\end{array}$ & \multicolumn{5}{|c|}{ 1,2-dichloronaphthalene } \\
\hline $\mathrm{C}_{10} \mathrm{H}_{6} \mathrm{Cl}_{2}$ & $\begin{array}{c}{[1825-31-6]} \\
\mathrm{V}\end{array}$ & $\begin{array}{l}\text { 1,4-dichloronaphth } \\
\text { (323-423) }\end{array}$ & $\begin{array}{l}\text { ene } \\
58.7\end{array}$ & 373 & GC & [1999LEI/WAN] \\
\hline $\mathrm{C}_{10} \mathrm{H}_{6} \mathrm{Cl}_{4} \mathrm{O}_{4}$ & $\begin{array}{l}{[1861-32-1]} \\
\text { FUS }\end{array}$ & dimethyl-2,3,5,6-te & $\begin{array}{l}\text { achloro-1,4-be } \\
30.23\end{array}$ & $\begin{array}{l}\text { edicarbo } \\
431.7\end{array}$ & $\begin{array}{l}\text { ate (chlorth } \\
\text { DSC }\end{array}$ & [1991ACR, 1990DON/DRE] \\
\hline & SUB & $(348-433)$ & $104.9 \pm 1.4$ & 390 & ME, GS & [1981DEP] \\
\hline
\end{tabular}


TABLE 13. Phase change enthalpies of $\mathrm{C}_{10}$ organic compounds-Continued

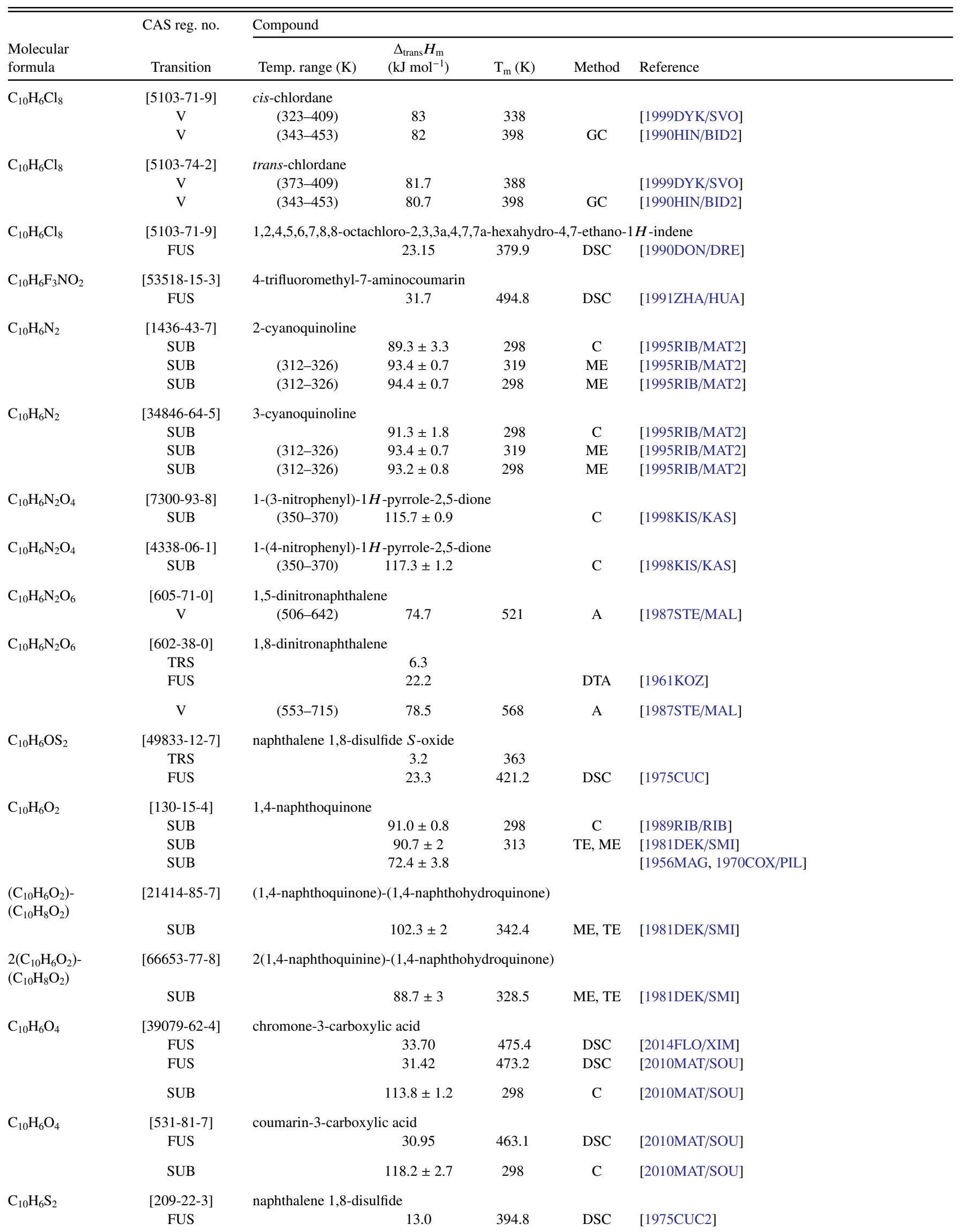


TABLE 13. Phase change enthalpies of $\mathrm{C}_{10}$ organic compounds-Continued

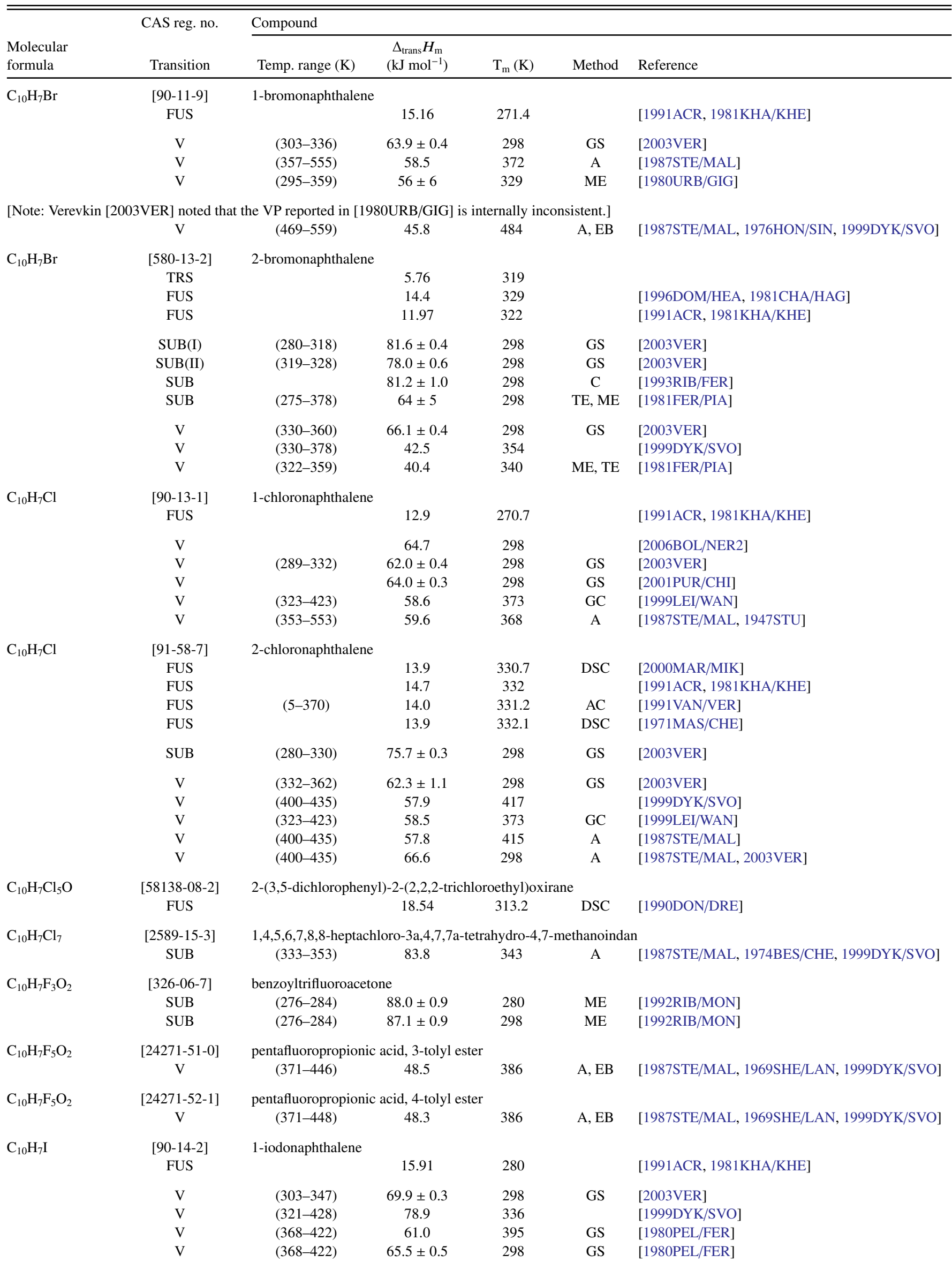


TABLE 13. Phase change enthalpies of $\mathrm{C}_{10}$ organic compounds-Continued

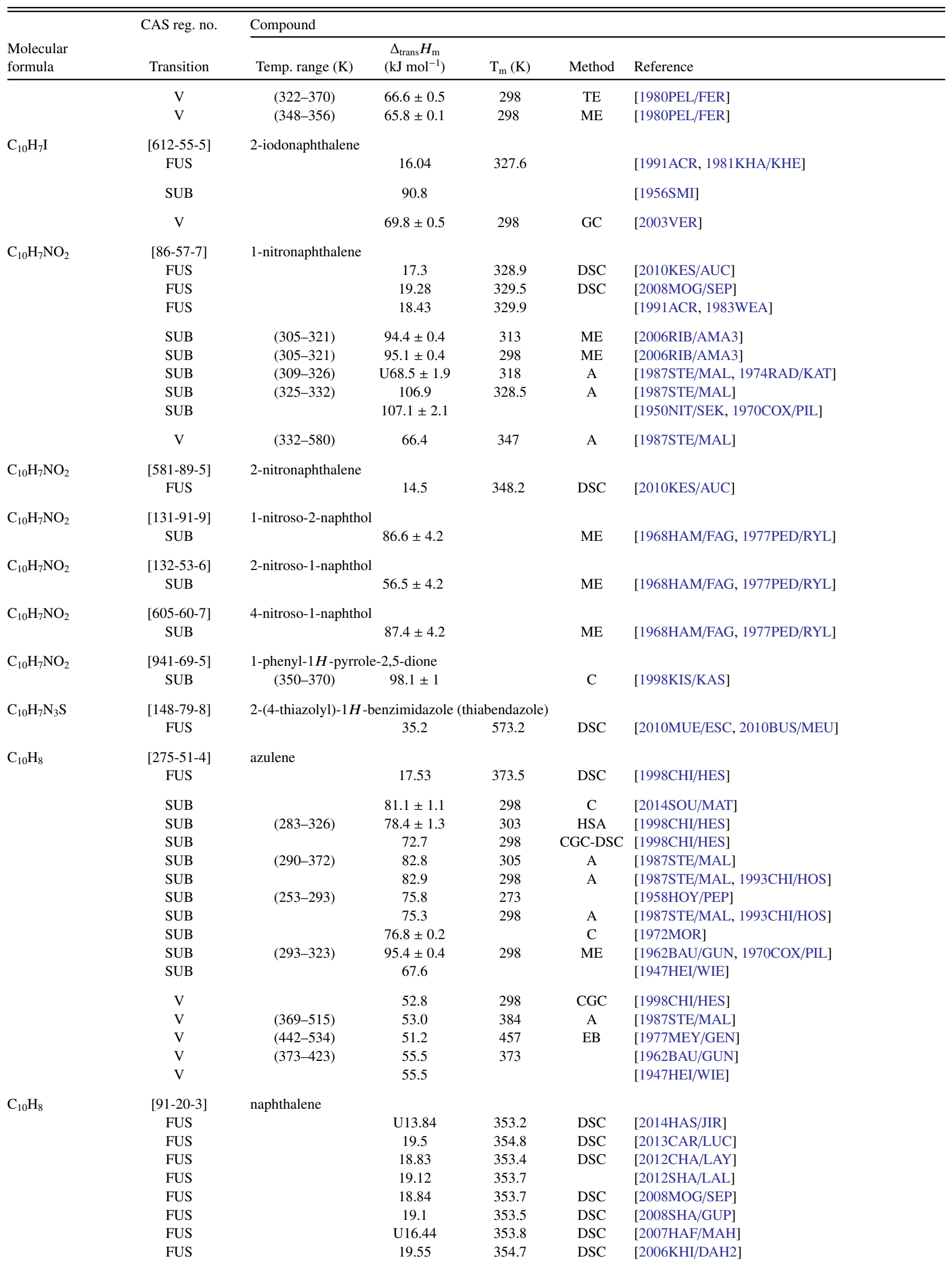


TABLE 13. Phase change enthalpies of $\mathrm{C}_{10}$ organic compounds-Continued

\begin{tabular}{|c|c|c|c|c|c|c|}
\hline \multirow[b]{2}{*}{$\begin{array}{l}\text { Molecular } \\
\text { formula }\end{array}$} & \multirow{2}{*}{$\begin{array}{l}\text { CAS reg. no. } \\
\text { Transition }\end{array}$} & \multicolumn{5}{|l|}{ Compound } \\
\hline & & Temp. range $(\mathrm{K})$ & $\begin{array}{c}\Delta_{\text {trans }} H_{\mathrm{m}} \\
\left(\mathrm{kJ} \mathrm{mol}^{-1}\right)\end{array}$ & $\mathrm{T}_{\mathrm{m}}(\mathrm{K})$ & Method & Reference \\
\hline & FUS & & 19.55 & 354.7 & DSC & [2006KHI/DAH] \\
\hline & FUS & & 18.71 & 253.2 & DSC & [2004STU/WIT] \\
\hline & FUS & & 19.1 & & DSC & [2003SHA/KAN] \\
\hline & FUS & & 19.03 & 353.4 & $\mathrm{AC}$ & [2002VAN/VAN3] \\
\hline & FUS & $(5-440)$ & 19.0 & 353.4 & $\mathrm{AC}$ & {$[2002 \mathrm{CHI} / \mathrm{KNI}]$} \\
\hline & FUS & & 18.2 & 356.2 & DSC & [1998RAI/RAI2] \\
\hline & FUS & & 19.11 & & DSC & [1992SHA/SHA] \\
\hline & FUS & & 19.1 & 353.4 & & {$[1980 \mathrm{AND} / \mathrm{CON}]$} \\
\hline & FUS & & 19.0 & 353.3 & DSC & [1980RAD/RAD] \\
\hline & FUS & & 18.96 & 353.4 & DSC & [1980KRA/PIG] \\
\hline & FUS & & 19.1 & 353.4 & DSC & [1991ACR, 1973CAS/VEC] \\
\hline & FUS & & 19.1 & 353.5 & & [1964RAS/BAS] \\
\hline & FUS & & 19.4 & 351.2 & DTA & [1958VAR] \\
\hline & FUS & & 18.81 & 353.4 & $\mathrm{AC}$ & [1957MAS] \\
\hline & FUS & & 19.37 & 353.5 & $\mathrm{C}$ & [1955TUN/STO] \\
\hline & FUS & & 18.79 & 353.0 & & [1950UEB/ORT] \\
\hline & FUS & & 18.79 & 353.4 & & [1944EIB] \\
\hline & FUS & & 19.04 & 353.4 & & [1941SCH] \\
\hline & FUS & & 19.20 & 353.0 & $\mathrm{RC}$ & [1932SPA/THO] \\
\hline & FUS & & 19.00 & 353.2 & & {$[1926 \mathrm{AND} / \mathrm{LYN}]$} \\
\hline & SUB & & $69.1 \pm 0.3$ & & $\mathrm{UV} / \mathrm{Vis}$ & [2015HIK/WEE] \\
\hline & SUB & $(303-316)$ & $68.9 \pm 0.5$ & 309 & UV/Vis & [2013HIK/WEE] \\
\hline & SUB & $(303-348)$ & 69.9 & & TGA & [2011FEL/RAM] \\
\hline & SUB & $(303-313)$ & $71.8 \pm 1.0$ & 308 & TGA & [2007SID/ATA] \\
\hline & SUB & & $71.7 \pm 1.3$ & 298 & DSC & [2001ROJ/ORO] \\
\hline & SUB & $(267-303)$ & $88.0 \pm 2.5$ & & $\mathrm{ME}$ & [1998BOL/WIE] \\
\hline & SUB & & 70.4 & 298 & CGC-DSC & [1998CHI/HES] \\
\hline & SUB & $(313-353)$ & 71.7 & 333 & GS & [1995NAS/LEN] \\
\hline & SUB & $(243-273)$ & $73.7 \pm 1.0$ & 258 & GS & [1994WAN/SHU] \\
\hline & SUB & $(337-352)$ & $78.2 \pm 1$ & & $\mathrm{GC}$ & [1988KHU] \\
\hline & SUB & & $70.9 \pm 0.4$ & 323 & DSC & [1988TOR/BAR] \\
\hline & SUB & & $72.3 \pm 0.4$ & 298 & DSC & [1988TOR/BAR] \\
\hline & SUB & $(299-331)$ & 73.4 & 315 & GS & [1986SAT/INO] \\
\hline & SUB & (333-393) & 69.9 & & GS & [1985MAT/KUW2] \\
\hline & SUB & & $73.7 \pm 0.6$ & & & [1985KIS/VEI] \\
\hline & SUB & $(293-331)$ & $72.3 \pm 0.8$ & & QR & [1985GLU/ARK] \\
\hline & SUB & $(283-323)$ & $75.8 \pm 1.1$ & 303 & GS & [1983SON/ZOL] \\
\hline & SUB & & $72.6 \pm 0.4$ & & DSC & [1983HOL] \\
\hline & SUB & & $72.6 \pm 0.1$ & 298 & $\begin{array}{l}\text { TE, ME, } \\
\text { DM }\end{array}$ & [1983VAN/JAC, 1981DEK/KUI] \\
\hline & SUB & $(302-352)$ & 72.8 & 327 & GS & [1982GRA/FOS] \\
\hline & SUB & $(271-285)$ & $72.8 \pm 0.3$ & & $\mathrm{ME}$ & [1982COL/JIM] \\
\hline & SUB & & $72.4 \pm 0.7$ & 298 & $\mathrm{C}$ & [1982MUR/SAK] \\
\hline & SUB & $(274-353)$ & $72.5 \pm 0.1$ & & DM & [1981DEK/KUI] \\
\hline & SUB & $(328-398)$ & $76.0 \pm 2.0$ & & DSC & [1980MUR/CAV] \\
\hline & SUB & $(253-273)$ & $72.6 \pm 0.6$ & 298 & $\mathrm{TE}$ & [1980DEK] \\
\hline & SUB & $(280-305)$ & 71.3 & 293 & GS & [1979MAC/PRA] \\
\hline & SUB & $(253-273)$ & $74.77 \pm 0.4$ & & $\mathrm{TE}$ & [1977DEK/VAN] \\
\hline & SUB & $(253-273)$ & $73.9 \pm 0.2$ & & $\mathrm{ME}$ & [1977DEK/VAN] \\
\hline & SUB & $(303-329)$ & $74.35 \pm 1.7$ & & TSGC & [1975MCE/SAN] \\
\hline & SUB & & $72.3 \pm 0.4$ & & $\mathrm{C}$ & [1976FER/PIA] \\
\hline & SUB & $(263-343)$ & $72.5 \pm 0.3$ & & DM & [1975AMB/LAW] \\
\hline & SUB & & $67.8 \pm 3.5$ & 280 & HSA & [1975CHI] \\
\hline & SUB & & 72.5 & 298 & GS & [1974SIN] \\
\hline & SUB & & $72.7 \pm 1.7$ & & & [1974RAD/KAT] \\
\hline & SUB & $(281-290)$ & $64 \pm .5$ & & LE & [1973MCE/SAN] \\
\hline & SUB & & $72.1 \pm 0.25$ & 298 & $\mathrm{C}$ & [1972MOR] \\
\hline & SUB & & $73.0 \pm 0.3$ & 298 & $\mathrm{C}$ & [1972IRV] \\
\hline & SUB & $(283-323)$ & 72.7 & & ME & [1971RAD] \\
\hline & SUB & & 66.5 & & & [1968KAR/RAB] \\
\hline & SUB & $(230-260)$ & $72.7 \pm 0.3$ & & KG & [1963MIL, 1970COX/PIL] \\
\hline & SUB & $(276-283)$ & 66.3 & & $\mathrm{~V}$ & [1959AIH] \\
\hline & SUB & $(283-303)$ & 65.8 & 293 & $\mathrm{ME}$ & [1958SKL/MAR] \\
\hline
\end{tabular}


TABLE 13. Phase change enthalpies of $\mathrm{C}_{10}$ organic compounds-Continued

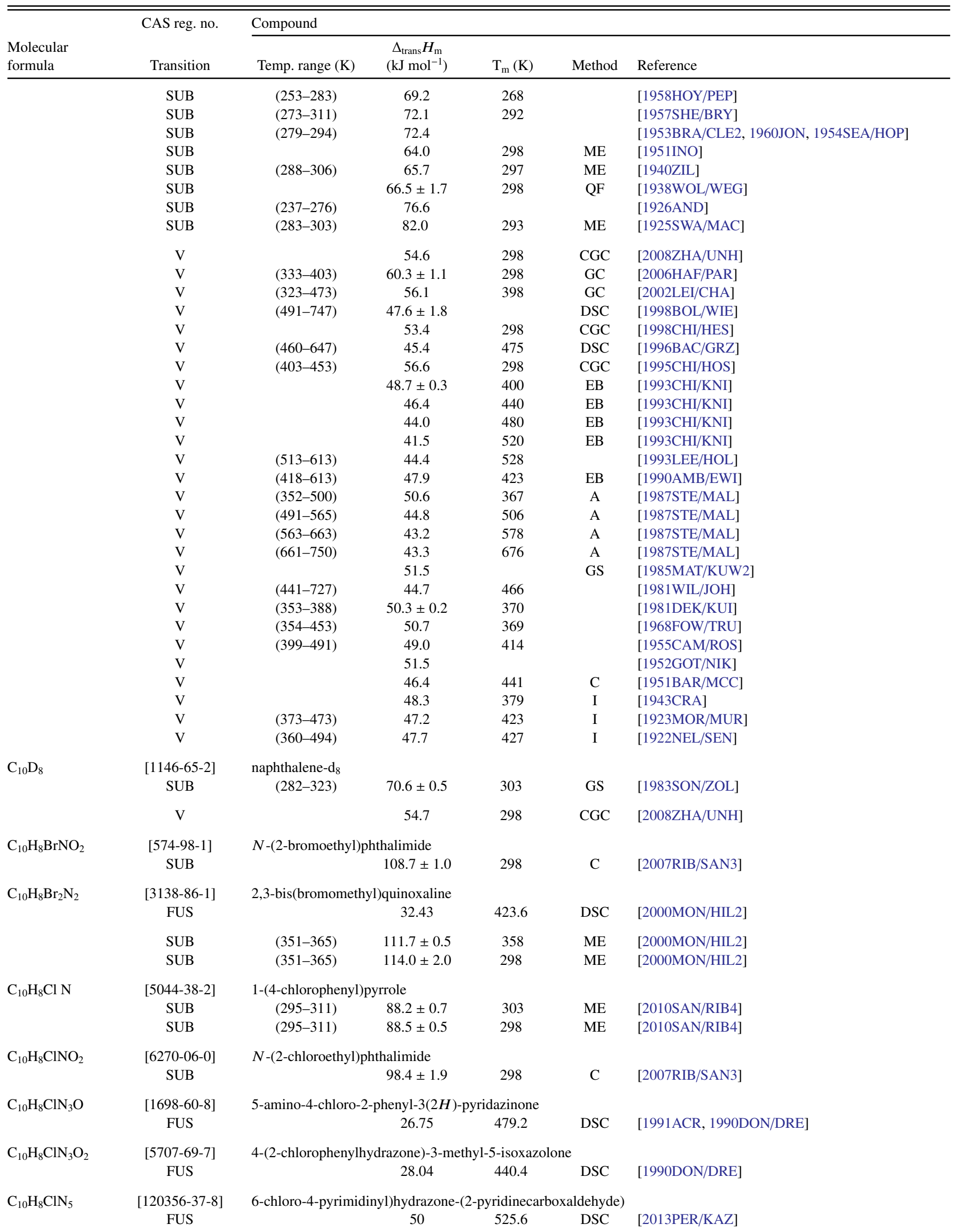


TABLE 13. Phase change enthalpies of $\mathrm{C}_{10}$ organic compounds-Continued

\begin{tabular}{|c|c|c|c|c|c|c|}
\hline \multirow[b]{2}{*}{$\begin{array}{l}\text { Molecular } \\
\text { formula }\end{array}$} & \multirow{2}{*}{$\begin{array}{l}\text { CAS reg. no. } \\
\text { Transition }\end{array}$} & \multicolumn{5}{|l|}{ Compound } \\
\hline & & Temp. range $(\mathrm{K})$ & $\begin{array}{c}\Delta_{\text {trans }} H_{\mathrm{m}} \\
\left(\mathrm{kJ} \mathrm{mol}^{-1}\right)\end{array}$ & $\mathrm{T}_{\mathrm{m}}(\mathrm{K})$ & Method & Reference \\
\hline $\mathrm{C}_{10} \mathrm{H}_{8} \mathrm{Cl}_{2} \mathrm{O}_{6}$ & $\begin{array}{c}\text { [24648-18-8] } \\
\text { TRS } \\
\text { FUS (white) } \\
\text { FUS (white) } \\
\text { TRS } \\
\text { (yellow-white) }\end{array}$ & 3,6-dichloro-2,5-dih & $\begin{array}{c}\text { droxyterepht } \\
1.7 \\
41.0 \\
44.6 \\
2.6\end{array}$ & $\begin{array}{l}380 \\
455 \\
461 \\
403\end{array}$ & $\begin{array}{l}\text { DSC } \\
\text { DSC } \\
\text { DSC }\end{array}$ & $\begin{array}{l}\text { [1990RIC/YAN] } \\
{[1972 B Y R / C U R]} \\
{[1972 B Y R / C U R]}\end{array}$ \\
\hline $\mathrm{C}_{10} \mathrm{H}_{8} \mathrm{Cl} \mathrm{N}$ & $\begin{array}{c}\text { [81329-31-9] } \\
\text { SUB } \\
\text { SUB }\end{array}$ & $\begin{array}{c}\text { 1-(4-chlorophenyl)p } \\
(271-287) \\
(271-287)\end{array}$ & $\begin{array}{l}\text { rrole } \\
79.8 \pm 1.0 \\
78.9 \pm 1.0\end{array}$ & $\begin{array}{l}279 \\
298\end{array}$ & $\begin{array}{l}\text { ME } \\
\mathrm{ME}\end{array}$ & $\begin{array}{l}\text { [2010SAN/RIB4] } \\
\text { [2010SAN/RIB4] }\end{array}$ \\
\hline $\mathrm{C}_{10} \mathrm{H}_{8} \mathrm{I} \mathrm{N}$ & $\begin{array}{c}\text { [92636-36-7] } \\
\text { SUB } \\
\text { SUB }\end{array}$ & $\begin{array}{c}\text { 1-(4-iodophenyl)pyr } \\
(319-341) \\
(319-341)\end{array}$ & $\begin{array}{l}\text { ole } \\
91.5 \pm 0.5 \\
100.7 \pm 0.5\end{array}$ & $\begin{array}{l}330 \\
298\end{array}$ & $\begin{array}{l}\text { ME } \\
\text { ME }\end{array}$ & $\begin{array}{l}\text { [2010SAN/RIB4] } \\
\text { [2010SAN/RIB4] }\end{array}$ \\
\hline $\mathrm{C}_{10} \mathrm{H}_{8} \mathrm{NO}_{2}$ & $\begin{array}{l}{[87-51-4]} \\
\text { SUB }\end{array}$ & $\begin{array}{c}\text { indole-3-acetic acid } \\
\quad(313-423)\end{array}$ & $64.0 \pm 1.5$ & 368 & $\mathrm{ME}$ & [1988TOR/BAR] \\
\hline $\mathrm{C}_{10} \mathrm{H}_{8} \mathrm{~N}_{2}$ & $\begin{array}{c}\text { [366-18-7] } \\
\text { FUS } \\
\text { FUS }\end{array}$ & 2,2'-bipyridine & $\begin{array}{l}20.4 \\
19.9\end{array}$ & $\begin{array}{c}345 \\
342\end{array}$ & $\begin{array}{l}\text { DSC } \\
\text { DSC }\end{array}$ & $\begin{array}{l}\text { [2009LIP/HAN] } \\
\text { [1986AIR/SIL] }\end{array}$ \\
\hline & $\begin{array}{l}\text { SUB } \\
\text { SUB } \\
\text { SUB }\end{array}$ & & $\begin{array}{l}81.8 \pm 2.3 \\
75.0 \pm 5.0 \\
81.9 \pm 0.3\end{array}$ & $\begin{array}{l}298 \\
298\end{array}$ & $\begin{array}{l}\mathrm{C} \\
\mathrm{B}\end{array}$ & $\begin{array}{l}\text { [1995RIB/MOR] } \\
\text { [1996CHA/EMM] } \\
\text { [1985SKI/PIL] }\end{array}$ \\
\hline & $\mathrm{V}$ & & $67.0 \pm 2.3$ & 298 & CGC & [2009LIP/CHI, 2009LIP/HAN] \\
\hline $\mathrm{C}_{10} \mathrm{H}_{8} \mathrm{~N}_{2}$ & $\begin{array}{c}{[581-47-5]} \\
\text { FUS }\end{array}$ & 2,4'-bipyridine & 17.4 & 332.8 & DSC & [2009LIP/HAN] \\
\hline & SUB & & $87.9 \pm 1.7$ & 298 & $\mathrm{C}$ & [1995RIB/MOR] \\
\hline & $\mathrm{V}$ & & $70.9 \pm 1.6$ & 298 & CGC & [2009LIP/CHI, 2009LIP/HAN] \\
\hline $\mathrm{C}_{10} \mathrm{H}_{8} \mathrm{~N}_{2}$ & $\begin{array}{c}{[553-26-4]} \\
\text { FUS } \\
\text { FUS }\end{array}$ & 4,4'-bipyridine & $\begin{array}{l}24.7 \\
16.1\end{array}$ & $\begin{array}{l}385.0 \\
377.5\end{array}$ & $\begin{array}{l}\text { DSC } \\
\text { DSC }\end{array}$ & $\begin{array}{l}\text { [2015SUR/SIM] } \\
\text { [2009LIP/HAN] }\end{array}$ \\
\hline & SUB & & $106.3 \pm 2.8$ & 298 & $\mathrm{C}$ & [1995RIB/MOR] \\
\hline & $\mathrm{V}$ & & $71.1 \pm 2.6$ & 298 & CGC & [2009LIP/CHI, 2009LIP/HAN] \\
\hline $\mathrm{C}_{10} \mathrm{H}_{8} \mathrm{~N}_{2}$ & $\begin{array}{c}\text { [3438-48-0] } \\
\text { FUS }\end{array}$ & 4-phenylpyrimidine & 18.8 & 334.1 & DSC & [2009LIP/HAN] \\
\hline & $\mathrm{V}$ & & $68.8 \pm 2.5$ & 298 & CGC & [2009LIP/HAN] \\
\hline $\mathrm{C}_{10} \mathrm{H}_{8} \mathrm{~N}_{2} \mathrm{O}$ & $\begin{array}{c}{[33421-43-1]} \\
\text { SUB } \\
\text { SUB }\end{array}$ & $\begin{array}{c}2,2^{\prime} \text {-bipyridine- } N \text {-or } \\
(319-331) \\
(319-331)\end{array}$ & $\begin{array}{l}\text { ide } \\
103.7 \pm 0.8 \\
105.1 \pm 0.8\end{array}$ & $\begin{array}{l}325 \\
298\end{array}$ & $\begin{array}{l}\mathrm{ME} \\
\mathrm{ME}\end{array}$ & $\begin{array}{l}\text { [2011SAN/MON] } \\
\text { [2011SAN/MON] }\end{array}$ \\
\hline $\mathrm{C}_{10} \mathrm{H}_{8} \mathrm{~N}_{2} \mathrm{O}_{2}$ & $\begin{array}{c}\text { [7275-43-6] } \\
\text { SUB }\end{array}$ & $\begin{array}{c}2,2^{\prime} \text {-bipyridine- } N, N \\
(410-450)\end{array}$ & $\begin{array}{l}\text { dioxide } \\
125.0 \pm 2.0\end{array}$ & 298 & $\mathrm{ME}$ & [2011SAN/MON] \\
\hline $\mathrm{C}_{10} \mathrm{H}_{8} \mathrm{~N}_{2} \mathrm{O}_{2}$ & $\begin{array}{c}{[3634-83-1]} \\
\mathrm{V}\end{array}$ & $\begin{array}{c}\text { 1,3-bis(isocyanatom } \\
(403-473)\end{array}$ & $\begin{array}{c}\text { thyl)benzene } \\
46.7\end{array}$ & 418 & A & [1987STE/MAL] \\
\hline $\mathrm{C}_{10} \mathrm{H}_{8} \mathrm{~N}_{2} \mathrm{O}_{2}$ & $\begin{array}{c}{[1014-98-8]} \\
\mathrm{V}\end{array}$ & $\begin{array}{c}\text { 1,4-bis(isocyanatom } \\
(403-473)\end{array}$ & $\begin{array}{l}\text { thyl)benzene } \\
56.9\end{array}$ & 418 & A & [1987STE/MAL] \\
\hline $\mathrm{C}_{10} \mathrm{H}_{8} \mathrm{~N}_{2} \mathrm{O}_{2}$ & $\begin{array}{c}{[64711-83-7]} \\
\mathrm{V}\end{array}$ & $\begin{array}{c}\text { benzene, ethyldiisoc } \\
\quad(363-473)\end{array}$ & $\begin{array}{l}\text { anato (mixed } \\
60.7\end{array}$ & $\begin{array}{l}\text { lers) } \\
378\end{array}$ & A & [1987STE/MAL, 1977ZHU/MEL] \\
\hline $\mathrm{C}_{10} \mathrm{H}_{8} \mathrm{~N}_{2} \mathrm{O}_{2}$ & $\begin{array}{c}{[881-07-2]} \\
\text { SUB } \\
\text { SUB }\end{array}$ & $\begin{array}{c}\text { 8-nitroquinaldine } \\
(346-360) \\
(346-360)\end{array}$ & $\begin{array}{l}108.3 \pm 0.8 \\
111.0 \pm 0.8\end{array}$ & $\begin{array}{l}353 \\
298\end{array}$ & $\begin{array}{l}\mathrm{ME} \\
\mathrm{ME}\end{array}$ & $\begin{array}{l}\text { [1997RIB/MAT5] } \\
\text { [1997RIB/MAT5] }\end{array}$ \\
\hline $\mathrm{C}_{10} \mathrm{H}_{8} \mathrm{~N}_{2} \mathrm{O}_{2}$ & $\begin{array}{c}{[33265-60-0]} \\
\text { SUB } \\
\text { SUB }\end{array}$ & $\begin{array}{c}\text { 1-(2-nitrophenyl)pyr } \\
(306-326) \\
(306-326)\end{array}$ & $\begin{array}{l}\text { ole } \\
100.4 \pm 0.4 \\
101.3 \pm 0.4\end{array}$ & $\begin{array}{l}316 \\
298\end{array}$ & $\begin{array}{l}\mathrm{ME} \\
\mathrm{ME}\end{array}$ & $\begin{array}{l}\text { [2010SAN/RIB2] } \\
\text { [2010SAN/RIB2] }\end{array}$ \\
\hline
\end{tabular}


TABLE 13. Phase change enthalpies of $\mathrm{C}_{10}$ organic compounds-Continued

\begin{tabular}{|c|c|c|c|c|c|c|}
\hline \multirow[b]{2}{*}{$\begin{array}{l}\text { Molecular } \\
\text { formula }\end{array}$} & \multirow{2}{*}{$\begin{array}{c}\text { CAS reg. no. } \\
\text { Transition }\end{array}$} & \multicolumn{5}{|l|}{ Compound } \\
\hline & & Temp. range $(\mathrm{K})$ & $\begin{array}{c}\Delta_{\text {trans }} H_{\mathrm{m}} \\
\left(\mathrm{kJ} \mathrm{mol}^{-1}\right)\end{array}$ & $\mathrm{T}_{\mathrm{m}}(\mathrm{K})$ & Method & Reference \\
\hline \multirow[t]{3}{*}{$\mathrm{C}_{10} \mathrm{H}_{8} \mathrm{~N}_{2} \mathrm{O}_{2}$} & {$[4533-42-0]$} & \multicolumn{5}{|c|}{ 1-(4-nitrophenyl)pyrrole } \\
\hline & SUB & $(344-366)$ & $109.6 \pm 0.4$ & 355 & ME & [2010SAN/RIB2] \\
\hline & SUB & $(344-366)$ & $112.4 \pm 0.4$ & 298 & $\mathrm{ME}$ & [2010SAN/RIB2] \\
\hline \multirow[t]{2}{*}{$\mathrm{C}_{10} \mathrm{H}_{8} \mathrm{~N}_{2} \mathrm{O}_{3}$} & {$[6118-65-6]$} & \multicolumn{5}{|c|}{ 3-acetamidophthalimide } \\
\hline & SUB & $(428-468)$ & 108.5 & 443 & A & [1987STE/MAL, 1956KLO] \\
\hline \multirow[t]{19}{*}{$\mathrm{C}_{10} \mathrm{H}_{8} \mathrm{O}$} & {$[90-15-3]$} & \multicolumn{5}{|l|}{ 1-naphthol } \\
\hline & FUS & $(5-445)$ & 24.26 & 368.5 & $\mathrm{AC}$ & [2015CHI/STE] \\
\hline & FUS & & 21.77 & 368.3 & DSC & [2015SIN/SIN] \\
\hline & FUS & & 20.76 & 367.2 & DSC & [2014HAS/JIR] \\
\hline & FUS & & 21.62 & 368.2 & DSC & [2012GUP/AGR] \\
\hline & FUS & & 23.54 & 368.2 & & [2012SHA/LAL] \\
\hline & FUS & & 23.3 & 368.7 & DSC & [2002RAI/PAN] \\
\hline & FUS & & 24.4 & 369.7 & DSC & [1998RAI/RAI] \\
\hline & FUS & & 23.93 & & DSC & [1992SHA/SHA] \\
\hline & FUS & & 23.47 & 368.7 & DTA & [1992MAN] \\
\hline & FUS & & 22.2 & 367.2 & DSC & [1990DON/DRE] \\
\hline & FUS & & 23.49 & 368 & & [1983WEA] \\
\hline & FUS & & 23.33 & 367.2 & $\mathrm{C}$ & [1979KHE/LAL, 1991ACR] \\
\hline & FUS & & 23.22 & 369 & DSC & [1967PAC] \\
\hline & FUS & & 23.47 & 368.2 & $\mathrm{C}$ & [1926AND/LYN] \\
\hline & SUB & $(296-313)$ & $91.2 \pm 0.4$ & & ME & [1974COL/ROU2] \\
\hline & SUB & $(279-328)$ & $89.1 \pm 1.7$ & 304 & $\mathrm{ME}$ & [1974ARS] \\
\hline & SUB & $(298-312)$ & 93.3 & 305 & A & [1987STE/MAL, 1960AIH] \\
\hline & SUB & $(314-324)$ & 84.3 & 319 & A & [1987STE/MAL, 1960AIH] \\
\hline \multicolumn{7}{|c|}{ [Note: In reference [1960AIH] the author mentions that there may be a small phase transition at $39.4^{\circ} \mathrm{C}$ as evidenced in the log $\mathrm{P}$ versus $1 / \mathrm{T}$ graph.] } \\
\hline & SUB & & $91.5 \pm 3.8$ & 298 & B & [1926AND/LYN, 1970COX/PIL, 1927MAY/BER] \\
\hline & $\mathrm{V}$ & $(370-569)$ & $67.3 \pm 0.3$ & 380 & IPM & [2015CHI/STE] \\
\hline & $\mathrm{V}$ & $(370-569)$ & $64.0 \pm 0.2$ & 420 & IPM & [2015CHI/STE] \\
\hline & $\mathrm{V}$ & $(370-569)$ & $60.9 \pm 0.2$ & 460 & IPM & [2015CHI/STE] \\
\hline & $\mathrm{V}$ & $(370-569)$ & $57.8 \pm 0.3$ & 500 & IPM & [2015CHI/STE] \\
\hline & $\mathrm{V}$ & $(370-569)$ & $56.3 \pm 0.3$ & 520 & IPM & [2015CHI/STE] \\
\hline & V & $(399-556)$ & 58.5 & 414 & $\mathrm{~A}$ & [1987STE/MAL] \\
\hline & $\mathrm{V}$ & $(423-563)$ & 60.8 & 473 & & [1927MAY/BER] \\
\hline \multirow[t]{15}{*}{$\mathrm{C}_{10} \mathrm{H}_{8} \mathrm{O}$} & {$[135-19-3]$} & \multicolumn{5}{|l|}{ 2-naphthol } \\
\hline & FUS & & 21.9 & 394.2 & DSC & [2014HAS/JIR] \\
\hline & FUS & & 17.51 & 392.2 & DTA & [2013KAN] \\
\hline & FUS & & 21.08 & 394.2 & DSC & [2010AGR/GUP, 2012GUP/AGR] \\
\hline & FUS & & 24.1 & 393.9 & DSC & [2008MOG/SEP] \\
\hline & FUS & $(363-413)$ & 20.9 & 392.5 & DSC & [2003ROJ/ORO] \\
\hline & FUS & & 21.2 & 395.2 & DSC & [1998RAI/RAI2] \\
\hline & FUS & & 17.51 & 396.2 & $\mathrm{C}$ & [1979KHE/LAL] \\
\hline & FUS & & 18.79 & 393.6 & $\mathrm{C}$ & [1991ACR, 1926AND/LYN] \\
\hline & SUB & & $85.5 \pm 1.2$ & 298 & DSC & [2003ROJ/ORO] \\
\hline & SUB & $(305-323)$ & $94.2 \pm 0.5$ & & $\mathrm{ME}$ & [1974COL/ROU2] \\
\hline & SUB & $(277-324)$ & $87.4 \pm 2.5$ & 300 & $\mathrm{ME}$ & [1974ARS] \\
\hline & SUB & $(283-323)$ & $78.7 \pm 0.8$ & 298 & & [1968KAR/RAB, 1977PED/RYL, 1987STE/MAL] \\
\hline & SUB & $(298-312)$ & 97.8 & 305 & A & [1987STE/MAL, 1960AIH] \\
\hline & SUB & $(314-332)$ & 87.8 & 323 & A & [1987STE/MAL, 1960AIH] \\
\hline
\end{tabular}

[Note: In reference [1960AIH] the author mentions that there may be a small phase transition at $39.1^{\circ} \mathrm{C}$ as evidenced in the log $\mathrm{P}$ versus $1 / \mathrm{T}$ graph.]

$\begin{array}{cccccc}\text { SUB } & & 83.0 \pm 3.8 & 298 & \text { B } & \text { [1926AND/LYN, } \\ \text { V } & (393-433) & 76.2 & 298 & \text { CGC } & \text { [1995CHI/HOS] } \\ \text { V } & (401-561) & 59.7 & 416 & \text { A } & \text { [1987STE/MAL] } \\ \text { V } & (426-473) & 64.2 & 449 & \text { EB } & \text { [1974MAN/LOG] } \\ \text { V } & (417-561) & 59.7 & 432 & & \text { [1955VON/GEB] } \\ \text { V } & (423-563) & 61.8 & 473 & & \end{array}$

$\mathrm{C}_{10} \mathrm{H}_{8} \mathrm{O} \quad$ [4759-11-9] 1,6-oxido[10]annulene

SUB $80.4 \pm 8.4$

B [1969BRE/HAG, 1977PED/RYL] 
TABLE 13. Phase change enthalpies of $\mathrm{C}_{10}$ organic compounds-Continued

\begin{tabular}{|c|c|c|c|c|c|c|}
\hline \multirow[b]{2}{*}{$\begin{array}{l}\text { Molecular } \\
\text { formula }\end{array}$} & \multirow{2}{*}{$\begin{array}{l}\text { CAS reg. no. } \\
\text { Transition }\end{array}$} & \multicolumn{5}{|l|}{ Compound } \\
\hline & & Temp. range $(\mathrm{K})$ & $\begin{array}{c}\Delta_{\text {trans }} H_{\mathrm{m}} \\
\left(\mathrm{kJ} \mathrm{mol}^{-1}\right)\end{array}$ & $\mathrm{T}_{\mathrm{m}}(\mathrm{K})$ & Method & Reference \\
\hline \multirow{2}{*}{$\mathrm{C}_{10} \mathrm{H}_{8} \mathrm{OS}_{3}$} & {$[532-11-6]$} & \multicolumn{5}{|c|}{ 5-(4-methoxyphenyl)-3H-1,2-dithiole-3-thione } \\
\hline & FUS & & 24.39 & 382.2 & DSC & [1999DOL/LEC] \\
\hline \multirow[t]{2}{*}{$\mathrm{C}_{10} \mathrm{H}_{8} \mathrm{O}_{2}$} & {$[571-60-8]$} & \multicolumn{5}{|c|}{ 1,4-naphthohydroquinone } \\
\hline & SUB & & $119 \pm 1$ & 381 & ME, TE & [1981DEK/SMI] \\
\hline \multirow{2}{*}{$\mathrm{C}_{10} \mathrm{H}_{8} \mathrm{O}_{2}$} & {$[574-00-5]$} & \multicolumn{5}{|c|}{ 1,2-dihydroxynaphthalene } \\
\hline & SUB & & $109.3 \pm 0.9$ & 298 & $\mathrm{C}$ & [1988RIB/RIB] \\
\hline \multirow[t]{2}{*}{$\mathrm{C}_{10} \mathrm{H}_{8} \mathrm{O}_{2}$} & {$[132-86-5]$} & \multicolumn{5}{|c|}{ 1,3-dihydroxynaphthalene } \\
\hline & SUB & & $116.0 \pm 1.1$ & 298 & $\mathrm{C}$ & [1988RIB/RIB] \\
\hline \multirow[t]{3}{*}{$\mathrm{C}_{10} \mathrm{H}_{8} \mathrm{O}_{2}$} & {$[92-44-4]$} & \multicolumn{5}{|c|}{ 2,3-dihydroxynaphthalene } \\
\hline & SUB & & $109.6 \pm 1.0$ & 298 & $\mathrm{C}$ & [1988RIB/RIB] \\
\hline & SUB & $(341-359)$ & $109.4 \pm 0.5$ & 350 & ME & [1979COL/JIM2] \\
\hline \multirow{3}{*}{$\mathrm{C}_{10} \mathrm{H}_{8} \mathrm{O}_{3}$} & {$[90-33-5]$} & \multicolumn{5}{|c|}{ 4-methyl-7-hydroxycoumarin } \\
\hline & FUS & & 31.41 & 462.0 & DSC & [2011AMA/PIN] \\
\hline & FUS & & 29.14 & 460.7 & DSC & [1996DOM/HEA, 1989ZHA/HUA] \\
\hline \multirow[t]{3}{*}{$\mathrm{C}_{10} \mathrm{H}_{8} \mathrm{O}_{3}$} & {$[531-59-9]$} & \multicolumn{5}{|l|}{ 7-methoxycoumarin } \\
\hline & FUS & & 23.16 & 391.0 & DSC & [2010MOR/SOU] \\
\hline & SUB & & $107.3 \pm 1.8$ & 298 & $\mathrm{C}$ & [2010MOR/SOU] \\
\hline \multirow[t]{3}{*}{$\mathrm{C}_{10} \mathrm{H}_{8} \mathrm{O}_{4}$} & {$[32846-66-5]$} & \multicolumn{5}{|c|}{ pentacyclo[4.2.0.0 $\left.0^{2,5} \cdot 0^{3,8} \cdot 0^{4,7}\right]$ octane-1,4-dicarboxylic acid (1,4-cubanedicarboxylic acid) } \\
\hline & FUS & & 17.4 & 495.0 & DSC & [2011ROU/MAR] \\
\hline & $\mathrm{V}$ & & $122.5 \pm 8.0$ & 298 & CGC & [2011ROU/MAR] \\
\hline $\mathrm{C}_{10} \mathrm{H}_{9} \mathrm{Br}$ & [207844-51-7] & bromobullvalene & & & & \\
\hline & FUS & & 14.2 & 317.2 & DSC & [1998LUS/OLI] \\
\hline $\mathrm{C}_{10} \mathrm{H}_{9} \mathrm{Cl}$ & {$[27576-94-9]$} & chlorobullvalene & & & & \\
\hline & FUS & & 13.5 & 287.2 & DSC & [1998LUS/OLI] \\
\hline $\mathrm{C}_{10} \mathrm{H}_{9} \mathrm{Cl}_{2} \mathrm{NO}$ & [2164-09-2] & $N$-(3,4-dichloroph & yl)-2-methyl- & spenamid & & \\
\hline & FUS & & 32.04 & 395.5 & $\mathrm{DSC}$ & [1991ACR, 1990DON/DRE] \\
\hline $\mathrm{C}_{10} \mathrm{H}_{9} \mathrm{Cl}_{3} \mathrm{O}_{3}$ & {$[1928-39-8]$} & (2,4,5-trichlorophe & xy)acetic acic & yl ester & & \\
\hline & $\mathrm{V}$ & $(444-573)$ & 76.4 & 459 & A & [1987STE/MAL, 1999DYK/SVO] \\
\hline & $\mathrm{V}$ & $(444-573)$ & 68.7 & 508 & GC & [1966JEN/SCH] \\
\hline $\mathrm{C}_{10} \mathrm{H}_{9} \mathrm{Cl}_{3} \mathrm{O}_{3}$ & {$[4841-20-7]$} & methyl 2-(2,4,5-tric & orophenoxy) & onate & & \\
\hline & FUS & & 31.95 & 360.6 & DSC & [1991ACR, 1990DON/DRE] \\
\hline $\mathrm{C}_{10} \mathrm{H}_{9} \mathrm{Cl}_{3} \mathrm{O}_{3}$ & {$[93-80-1]$} & 4-(2,4,5-trichloropl & oxy)butanoic & & & \\
\hline & FUS & & 30.28 & 386.7 & DSC & [1991ACR, 1990DON/DRE] \\
\hline $\mathrm{C}_{10} \mathrm{H}_{9} \mathrm{Cl}_{4} \mathrm{NO}_{2} \mathrm{~S}$ & {$[2425-06-1]$} & $N$-[(1,1,2,2-tetrach & roethyl)thio]- & clohexene & 2-dicarboxi & mide \\
\hline & FUS & & 40.22 & 432.7 & DSC & [1990DON/DRE] \\
\hline & FUS & & 43.1 & 432 & DSC & [1969PLA/GLA] \\
\hline $\mathrm{C}_{10} \mathrm{H}_{9} \mathrm{I}$ & {$[207844-52-8]$} & iodobullvalene & & & & \\
\hline & FUS & & 15.5 & 376.2 & $\mathrm{DSC}$ & [1998LUS/OLI] \\
\hline $\mathrm{C}_{10} \mathrm{H}_{9} \mathrm{IO}_{2}$ & {$[122200-58-2]$} & methyl-4-iodocuba & carboxylate & & & \\
\hline & FUS & & 22.3 & 395 & DSC & [2010GRI/TSA] \\
\hline $\mathrm{C}_{10} \mathrm{H}_{9} \mathrm{~N}$ & [91-63-4] & 2-methylquinoline & dinaldine) & & & \\
\hline & FUS & & 12.52 & 270.5 & $\mathrm{AC}, \mathrm{DSC}$ & [2005CHI/STE] \\
\hline & V & $(319-553)$ & $62.6 \pm 0.1$ & 298 & IPM, EB & [2005CHI/STE] \\
\hline & $\mathrm{V}$ & $(319-553)$ & $61.0 \pm 0.1$ & 320 & IPM, EB & [2005CHI/STE] \\
\hline & $\mathrm{V}$ & $(319-553)$ & $58.2 \pm 0.1$ & 360 & IPM, EB & [2005CHI/STE] \\
\hline & V & $(319-553)$ & $55.6 \pm 0.1$ & 400 & IPM, EB & [2005CHI/STE] \\
\hline & $\mathrm{V}$ & $(319-553)$ & $53.0 \pm 0.1$ & 440 & IPM, EB & [2005CHI/STE] \\
\hline & $\mathrm{V}$ & $(319-553)$ & $50.4 \pm 0.1$ & 480 & IPM, EB & [2005CHI/STE] \\
\hline & $\mathrm{V}$ & $(319-553)$ & $47.7 \pm 0.2$ & 520 & IPM, EB & [2005CHI/STE] \\
\hline & $\mathrm{V}$ & & $66.1 \pm 1.9$ & 298 & $\mathrm{C}$ & [1995RIB/MAT] \\
\hline & $\mathrm{V}$ & $(281-313)$ & 61.2 & 297 & GS & [1980VAN/PRA] \\
\hline
\end{tabular}


TABLE 13. Phase change enthalpies of $\mathrm{C}_{10}$ organic compounds-Continued

\begin{tabular}{|c|c|c|c|c|c|c|}
\hline $\begin{array}{l}\text { Molecular } \\
\text { formula }\end{array}$ & $\begin{array}{l}\text { CAS reg. no. } \\
\text { Transition } \\
\end{array}$ & \multicolumn{5}{|l|}{ Compound } \\
\hline $\mathrm{C}_{10} \mathrm{H}_{9} \mathrm{~N}$ & $\begin{array}{c}{[612-58-8]} \\
\mathrm{V} \\
\mathrm{V}\end{array}$ & $\begin{array}{l}\text { 3-methylquinoline } \\
(443-528) \\
(449-528)\end{array}$ & $\begin{array}{l}55.8 \\
55.1\end{array}$ & $\begin{array}{l}458 \\
466\end{array}$ & A & $\begin{array}{l}\text { [1987STE/MAL, 1961MAL2] } \\
\text { [1964MAL/WEC] }\end{array}$ \\
\hline $\mathrm{C}_{10} \mathrm{H}_{9} \mathrm{~N}$ & $\begin{array}{c}{[91-62-3]} \\
\mathrm{V} \\
\mathrm{V} \\
\mathrm{V}\end{array}$ & $\begin{array}{l}\text { 6-methylquinoline } \\
\begin{array}{c}(453-540) \\
(459-540)\end{array}\end{array}$ & $\begin{array}{l}67.7 \pm 1.8 \\
56.1 \\
55.7\end{array}$ & $\begin{array}{l}298 \\
468 \\
474\end{array}$ & $\begin{array}{l}\mathrm{C} \\
\mathrm{A}\end{array}$ & $\begin{array}{l}\text { [1995RIB/MAT] } \\
{[1987 \mathrm{STE} / \mathrm{MAL}]} \\
{[1964 \mathrm{MAL} / \mathrm{WEC}]}\end{array}$ \\
\hline $\mathrm{C}_{10} \mathrm{H}_{9} \mathrm{~N}$ & $\begin{array}{l}{[611-32-5]} \\
\text { FUS }\end{array}$ & 8-methylquinoline & 10.73 & 246.9 & AC, DSC & [2005CHI/STE] \\
\hline & $\begin{array}{l}\text { V } \\
\text { V } \\
\text { V } \\
\text { V } \\
\text { V } \\
\text { V } \\
\text { V } \\
\text { V } \\
\text { V }\end{array}$ & $\begin{array}{l}(324-553) \\
(324-553) \\
(324-553) \\
(324-553) \\
(324-553) \\
(324-553) \\
(324-553) \\
(493-523)\end{array}$ & $\begin{array}{l}62.1 \pm 0.1 \\
59.2 \pm 0.1 \\
56.6 \pm 0.1 \\
54.0 \pm 0.1 \\
51.3 \pm 0.1 \\
49.0 \pm 0.2 \\
46.2 \pm 0.3 \\
65.7 \pm 1.9 \\
52.2\end{array}$ & $\begin{array}{l}298 \\
340 \\
380 \\
420 \\
460 \\
500 \\
540 \\
298 \\
508\end{array}$ & $\begin{array}{l}\text { IPM, EB } \\
\text { IPM, EB } \\
\text { IPM, EB } \\
\text { IPM, EB } \\
\text { IPM, EB } \\
\text { IPM, EB } \\
\text { IPM, EB } \\
\text { C } \\
\text { A, EB }\end{array}$ & $\begin{array}{l}{[2005 \mathrm{CHI} / \mathrm{STE}]} \\
{[2005 \mathrm{CHI} / \mathrm{STE}]} \\
{[2005 \mathrm{CHI} / \mathrm{STE}]} \\
{[2005 \mathrm{CHI} / \mathrm{STE}]} \\
{[2005 \mathrm{CHI} / \mathrm{STE}]} \\
{[2005 \mathrm{CHI} / \mathrm{STE}]} \\
{[2005 \mathrm{CHI} / \mathrm{STE}]} \\
{[1995 \mathrm{RIB} / \mathrm{MAT}]} \\
{[1987 \mathrm{STE} / \mathrm{MAL}, 1961 \mathrm{MAL}]}\end{array}$ \\
\hline $\mathrm{C}_{10} \mathrm{H}_{9} \mathrm{~N}$ & $\begin{array}{c}\text { [134-32-7] } \\
\text { FUS } \\
\text { FUS } \\
\text { FUS } \\
\text { FUS } \\
\text { FUS }\end{array}$ & 1-naphthylamine & $\begin{array}{c}13.3 \\
16.18 \\
13.30 \\
14.49 \\
14.0\end{array}$ & $\begin{array}{c}321.2 \\
323 \\
323.2 \\
322.2\end{array}$ & $\begin{array}{l}\text { DSC } \\
\text { DSC } \\
\text { DSC } \\
\text { C } \\
\text { DTA }\end{array}$ & $\begin{array}{l}\text { [2014HAS/JIR] } \\
\text { [1993TIE, 2007VER/GEO] } \\
\text { [1992SHA/SHA] } \\
\text { [1979KHE/LAL, 1991ACR] } \\
\text { [1958VAR] }\end{array}$ \\
\hline & $\begin{array}{l}\mathrm{V} \\
\mathrm{V}\end{array}$ & $\begin{array}{l}(323-353) \\
(377-574)\end{array}$ & $\begin{array}{l}73.3 \pm 0.4 \\
63.6\end{array}$ & $\begin{array}{l}298 \\
392\end{array}$ & $\begin{array}{c}\text { GS } \\
\text { A }\end{array}$ & $\begin{array}{l}\text { [2007VER/GEO] } \\
\text { [1987STE/MAL, 1947STU] }\end{array}$ \\
\hline $\mathrm{C}_{10} \mathrm{H}_{9} \mathrm{~N}$ & $\begin{array}{l}{[91-59-8]} \\
\text { FUS } \\
\text { FUS } \\
\text { FUS }\end{array}$ & 2-naphthylamine & $\begin{array}{c}25.8 \\
23.61 \\
23.33\end{array}$ & $\begin{array}{l}386.2 \\
386.2\end{array}$ & $\begin{array}{c}\text { DTA } \\
\text { C }\end{array}$ & $\begin{array}{l}\text { [1999RAI/SHE] } \\
\text { [1979KHE/LAL] } \\
\text { [1991ACR, 1970CHA/BOU] }\end{array}$ \\
\hline & $\begin{array}{l}\text { SUB } \\
\text { SUB } \\
\text { SUB }\end{array}$ & $(283-323)$ & $\begin{array}{c}73.9 \\
74.1 \pm 1.7 \\
88.3 \pm 4.2\end{array}$ & 298 & $\begin{array}{c}\text { A } \\
\mathrm{ME}\end{array}$ & $\begin{array}{l}\text { [1987STE/MAL] } \\
\text { [1968KAR/RAB, 1977PED/RYL] } \\
\text { [1947STU, 1970COX/PIL] }\end{array}$ \\
\hline & $\mathrm{V}$ & $(388-579)$ & 63.5 & 403 & A & [1987STE/MAL, 1947STU] \\
\hline $\mathrm{C}_{10} \mathrm{H}_{9} \mathrm{~N}$ & $\begin{array}{l}{[635-90-5]} \\
\text { SUB } \\
\text { SUB }\end{array}$ & $\begin{array}{l}\text { 1-phenylpyrrole } \\
\quad(271-287) \\
(271-287)\end{array}$ & $\begin{array}{l}81.8 \pm 0.6 \\
80.8 \pm 0.6\end{array}$ & $\begin{array}{l}279 \\
298\end{array}$ & $\begin{array}{l}\text { ME } \\
\text { ME }\end{array}$ & $\begin{array}{l}{[2010 \mathrm{SAN} / \mathrm{RIB}]} \\
{[2010 \mathrm{SAN} / \mathrm{RIB}]}\end{array}$ \\
\hline $\mathrm{C}_{10} \mathrm{H}_{9} \mathrm{NO}$ & $\begin{array}{c}{[5263-87-6]} \\
\mathrm{V}\end{array}$ & 6-methoxyquinoline & $78.1 \pm 2.3$ & 298 & $\mathrm{C}$ & [2003RIB/SAN] \\
\hline $\mathrm{C}_{10} \mathrm{H}_{9} \mathrm{NO}$ & $\begin{array}{c}{[5343-98-6]} \\
\text { SUB } \\
\text { SUB }\end{array}$ & $\begin{array}{l}\beta-\text { cyanopropiophen } \\
\quad(318-333) \\
(318-333)\end{array}$ & $\begin{array}{l}\text { one } \\
101.7 \pm 4.2 \\
\quad 108.5\end{array}$ & 325.5 & $\begin{array}{c}\mathrm{ME} \\
\mathrm{A}\end{array}$ & $\begin{array}{l}\text { [1969LEB/DNE, 1977PED/RYL] } \\
\text { [1987STE/MAL] }\end{array}$ \\
\hline $\mathrm{C}_{10} \mathrm{H}_{9} \mathrm{NO}$ & $\begin{array}{c}{[18615-86-6]} \\
\text { SUB } \\
\text { SUB }\end{array}$ & $\begin{array}{c}\text { 2-methyl-4-hydroxyc } \\
(424-442) \\
(424-442)\end{array}$ & $\begin{array}{l}\text { uinoline } \\
132.2 \pm 1.0 \\
139.0 \pm 1.0\end{array}$ & $\begin{array}{l}433 \\
298\end{array}$ & $\begin{array}{l}\text { ME } \\
\text { ME }\end{array}$ & $\begin{array}{l}\text { [1990RIB/MAT] } \\
\text { [1990RIB/MAT] }\end{array}$ \\
\hline
\end{tabular}


TABLE 13. Phase change enthalpies of $\mathrm{C}_{10}$ organic compounds-Continued

\begin{tabular}{|c|c|c|c|c|c|c|}
\hline \multirow[b]{2}{*}{$\begin{array}{l}\text { Molecular } \\
\text { formula }\end{array}$} & \multirow{2}{*}{$\begin{array}{l}\text { CAS reg. no. } \\
\text { Transition }\end{array}$} & \multicolumn{5}{|l|}{ Compound } \\
\hline & & Temp. range $(\mathrm{K})$ & $\begin{array}{c}\Delta_{\text {trans }} H_{\mathrm{m}} \\
\left(\mathrm{kJ} \mathrm{mol}^{-1}\right) \\
\end{array}$ & $\mathrm{T}_{\mathrm{m}}(\mathrm{K})$ & Method & Reference \\
\hline \multirow[t]{4}{*}{$\mathrm{C}_{10} \mathrm{H}_{9} \mathrm{NO}$} & [826-81-3] & \multicolumn{5}{|c|}{ 2-methyl-8-hydroxyquinoline } \\
\hline & SUB & $(296-307)$ & $90.4 \pm 0.7$ & 298 & ME & [1989RIB/MON] \\
\hline & SUB & & $87.2 \pm 1.9$ & 298 & $\mathrm{C}$ & [1989RIB/MON] \\
\hline & SUB & $(308-333)$ & 87.9 & & $\mathrm{ME}$ & [1987STE/MAL, 1963HOR/WEN] \\
\hline \multirow[t]{3}{*}{$\mathrm{C}_{10} \mathrm{H}_{9} \mathrm{NO}$} & {$[607-66-9]$} & \multicolumn{5}{|c|}{ 4-methyl-2-hydroxyquinoline } \\
\hline & SUB & $(391-405)$ & $123.1 \pm 1.6$ & 398 & $\mathrm{ME}$ & [1990RIB/MAT] \\
\hline & SUB & $(391-405)$ & $128.1 \pm 1.6$ & 298 & ME & [1990RIB/MAT] \\
\hline \multirow[t]{2}{*}{$\mathrm{C}_{10} \mathrm{H}_{9} \mathrm{NO}_{2}$} & {$[87-51-4]$} & \multicolumn{5}{|l|}{ indole-3-acetic acid } \\
\hline & SUB & $(313-423)$ & $64.0 \pm 1.4 \mathrm{U}$ & 368 & $\mathrm{ME}$ & {$[1988 \mathrm{GAL} / \mathrm{GON}]$} \\
\hline \multirow[t]{2}{*}{$\mathrm{C}_{10} \mathrm{H}_{9} \mathrm{NO}_{2}$} & {$[26093-31-2]$} & \multicolumn{5}{|c|}{ 4-methyl-7-aminocoumarin } \\
\hline & FUS & & 32.09 & 499.9 & DSC & [1996DOM/HEA, 1989ZHA/HUA] \\
\hline \multirow[t]{2}{*}{$\mathrm{C}_{10} \mathrm{H}_{9} \mathrm{NO}_{2}$} & {$[5022-29-7]$} & \multicolumn{5}{|c|}{$N$-ethylphthalimide } \\
\hline & SUB & & $90.9 \pm 1.2$ & 298 & $\mathrm{C}$ & [2006RIB/SAN] \\
\hline \multirow[t]{2}{*}{$\mathrm{C}_{10} \mathrm{H}_{9} \mathrm{NO}_{2}$} & {$[6563-13-9]$} & \multicolumn{5}{|c|}{ 6-methoxyquinoline $N$-oxide } \\
\hline & SUB & & $117.9 \pm 1.0$ & 298 & $\mathrm{C}$ & [2003RIB/SAN] \\
\hline \multirow[t]{3}{*}{$\mathrm{C}_{10} \mathrm{H}_{9} \mathrm{NO}_{2}$} & [942-24-5] & \multicolumn{5}{|c|}{ methyl $1 H$-indole-3-carboxylate } \\
\hline & SUB & $(353-375)$ & $114.6 \pm 0.9$ & 364 & $\mathrm{ME}$ & [2016CAR/AMA] \\
\hline & SUB & $(353-375)$ & $116.8 \pm 0.9$ & 298 & ME & [2016CAR/AMA] \\
\hline \multirow[t]{2}{*}{$\mathrm{C}_{10} \mathrm{H}_{9} \mathrm{~N}_{3} \mathrm{O}_{2}$} & {$[929692-87-5]$} & \multicolumn{5}{|c|}{ 2-carbamoyl-3-methylquinoxaline $N$-oxide } \\
\hline & SUB & & $138.0 \pm 0.6$ & 298 & $\mathrm{C}$ & [2012VIV/FRE] \\
\hline \multirow[t]{2}{*}{$\mathrm{C}_{10} \mathrm{H}_{9} \mathrm{~N}_{3} \mathrm{O}_{3}$} & {$[23433-66-1]$} & \multicolumn{5}{|c|}{ 3-methylquinoxaline-2-carboxamide-1,4-dioxide } \\
\hline & SUB & & $166.6 \pm 3.7$ & 298 & $\mathrm{ME}$ & [2007GOM/SOU2] \\
\hline \multirow[t]{4}{*}{$\mathrm{C}_{10} \mathrm{H}_{10}$} & {$[5603-34-9]$} & \multicolumn{5}{|c|}{ pentacyclo $\left[4.4 .0 .0^{2,5} 0^{3,8} 0^{4,7}\right] \mathrm{dec}-9$-ene (basketene) } \\
\hline & FUS & & 2.72 & 331.8 & DSC & [2002VER/KUM] \\
\hline & SUB & $(274-313)$ & $55.4 \pm 0.5$ & 294 & GS & [2002VER/KUM] \\
\hline & SUB & $(274-313)$ & $55.3 \pm 0.5$ & 298 & GS & [2002VER/KUM] \\
\hline \multirow[t]{4}{*}{$\mathrm{C}_{10} \mathrm{H}_{10}$} & {$[21604-76-2]$} & \multicolumn{5}{|c|}{ tricyclo[4.2.2.0 $\left.0^{2,5}\right] \mathrm{dec}-3,7,9$-triene } \\
\hline & FUS & & 1.46 & 293.7 & DSC & [2002VER/KUM] \\
\hline & $\mathrm{V}$ & $(296-326)$ & $47.2 \pm 0.4$ & 311 & GS & [2002VER/KUM] \\
\hline & $\mathrm{V}$ & $(296-326)$ & $47.9 \pm 0.4$ & 298 & GS & [2002VER/KUM] \\
\hline $\mathrm{C}_{10} \mathrm{H}_{10}$ & [26934-61-2] & $2,2 \mathrm{a}, 2 \mathrm{~b}, 3,5 \mathrm{a}, 5 \mathrm{~b}-\mathrm{hex}$ & hydro-1,2,3-m & no- $1 H$-cy & $\operatorname{prop}[\mathrm{cd}]$ in & lene (snoutene) \\
\hline & FUS & & 7.87 & 334 & DSC & [2002VER/KUM] \\
\hline & SUB & $(274-313)$ & $58.9 \pm 0.4$ & 294 & GS & [2002VER/KUM] \\
\hline & SUB & $(274-313)$ & $58.7 \pm 0.4$ & 298 & GS & [2002VER/KUM] \\
\hline $\mathrm{C}_{10} \mathrm{H}_{10}$ & [108-57-6] & 1,3-divinylbenzene & & & & \\
\hline & V & $(305-453)$ & 48.3 & 320 & A & [1987STE/MAL, 1947STU] \\
\hline $\mathrm{C}_{10} \mathrm{H}_{10}$ & {$[77-73-6]$} & dicyclopentadiene & & & & \\
\hline & V & $(307-440)$ & 42.4 & 322 & & [1947STU] \\
\hline $\mathrm{C}_{10} \mathrm{H}_{10}$ & $\begin{array}{c}{[447-53-0]} \\
\text { FUS }\end{array}$ & $\begin{array}{l}\text { 1,2-dihydronaphthal } \\
(5-444)\end{array}$ & ene 10.53 & 264.4 & $\mathrm{AC}$ & [2008CHI/STE] \\
\hline
\end{tabular}

[Note: The authors report two solid/solid phase transitions having negligible enthalpy of transition.]

\begin{tabular}{|c|c|c|c|c|c|c|}
\hline & V & & $54.8 \pm 0.1$ & 298 & IPM, EB & [2008CHI/STE] \\
\hline & V & & $53.4 \pm 0.1$ & 320 & IPM, EB & [2008CHI/STE] \\
\hline & V & & $50.8 \pm 0.1$ & 360 & IPM, EB & [2008CHI/STE] \\
\hline & $\mathrm{V}$ & & $48.4 \pm 0.1$ & 400 & IPM, EB & [2008CHI/STE] \\
\hline & V & & $45.9 \pm 0.1$ & 440 & IPM, EB & [2008CHI/STE] \\
\hline & V & $(274-319)$ & $51.9 \pm 0.4$ & 296 & GS & [1999VER6] \\
\hline & V & $(274-319)$ & $51.9 \pm 0.4$ & 298 & GS & [1999VER6] \\
\hline $\mathrm{C}_{10} \mathrm{H}_{10}$ & [612-17-9] & 1,4-dihydronap & & & & \\
\hline & FUS & & 9.35 & 298.1 & DSC & [1999VER6] \\
\hline & SUB & & $63.6 \pm 1.6$ & 298 & & [1999VER6] \\
\hline
\end{tabular}


TABLE 13. Phase change enthalpies of $\mathrm{C}_{10}$ organic compounds-Continued

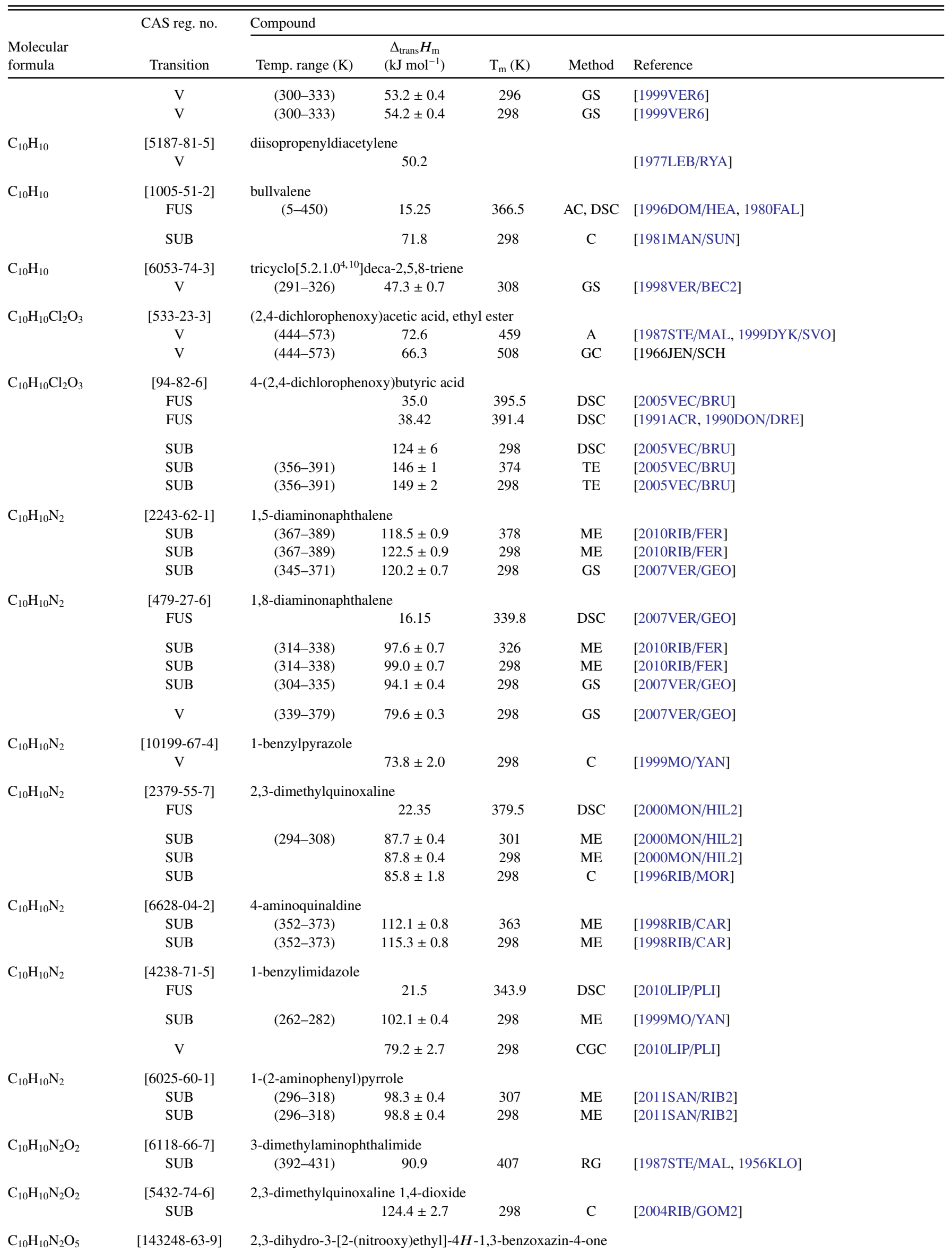


TABLE 13. Phase change enthalpies of $\mathrm{C}_{10}$ organic compounds-Continued

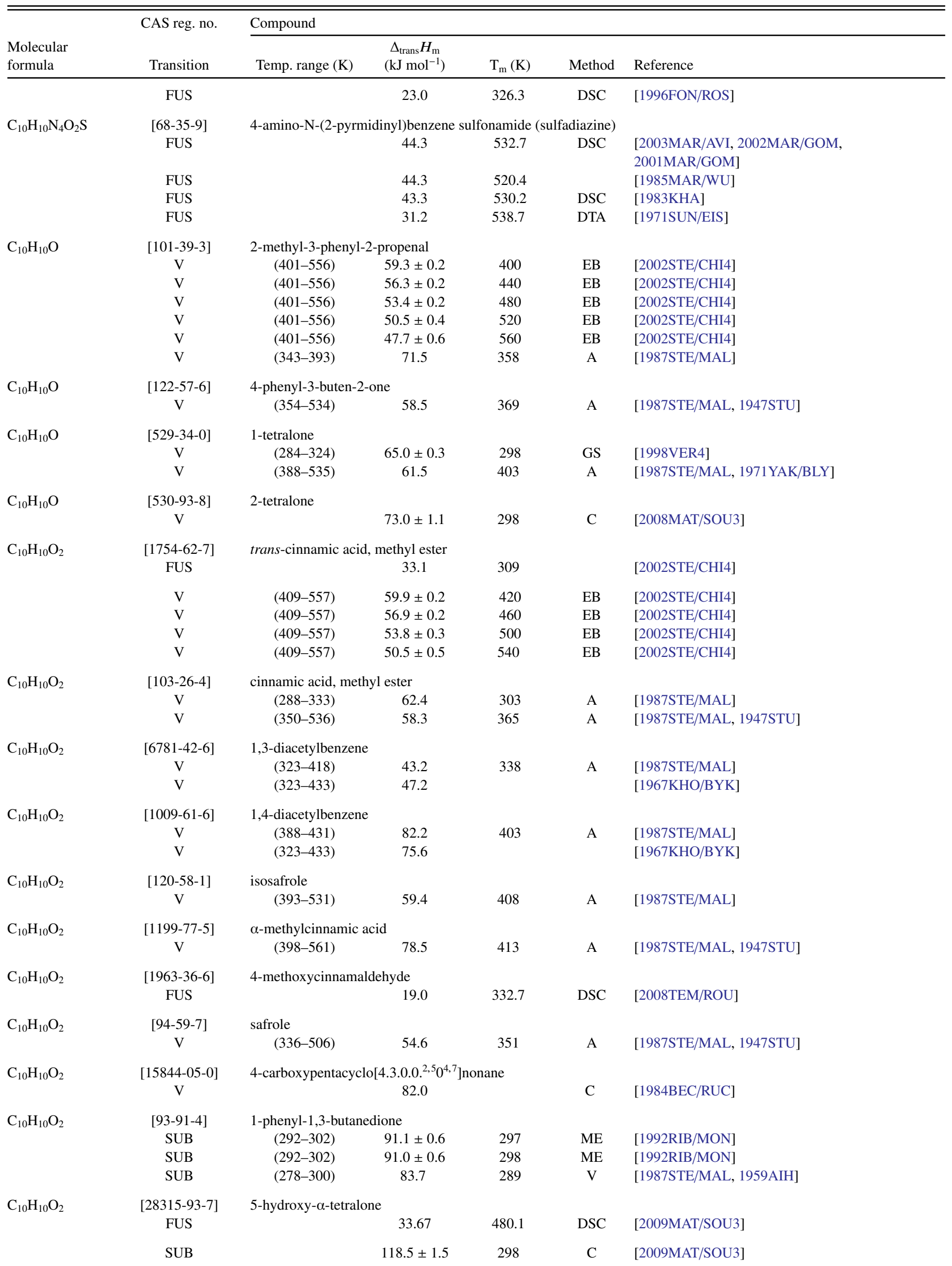


TABLE 13. Phase change enthalpies of $\mathrm{C}_{10}$ organic compounds-Continued

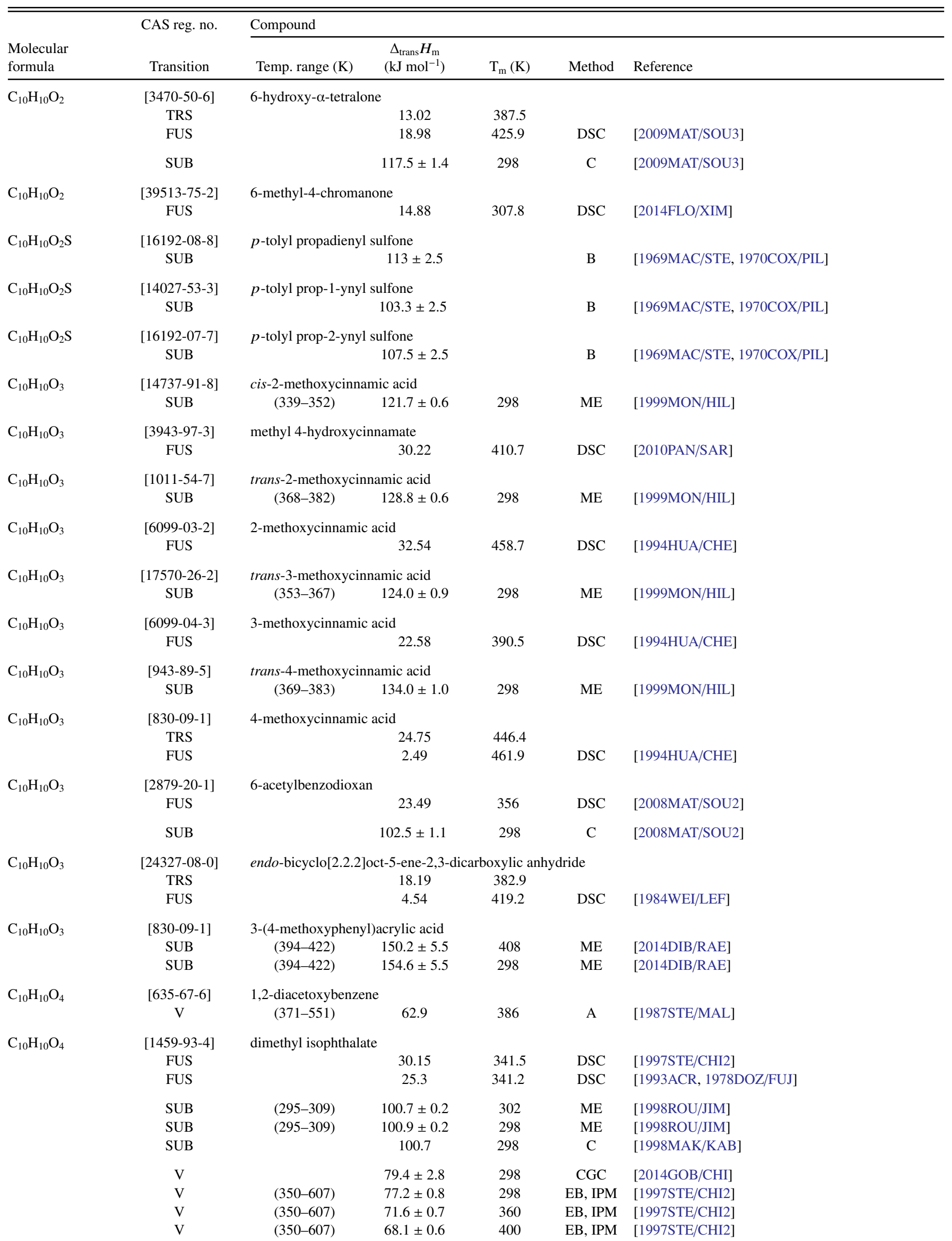


TABLE 13. Phase change enthalpies of $\mathrm{C}_{10}$ organic compounds-Continued

\begin{tabular}{|c|c|c|c|c|c|c|}
\hline \multirow[b]{2}{*}{$\begin{array}{l}\text { Molecular } \\
\text { formula }\end{array}$} & \multirow{2}{*}{$\begin{array}{l}\text { CAS reg. no. } \\
\text { Transition }\end{array}$} & \multicolumn{5}{|l|}{ Compound } \\
\hline & & Temp. range $(\mathrm{K})$ & $\begin{array}{c}\Delta_{\text {trans }} H_{\mathrm{m}} \\
\left(\mathrm{kJ} \mathrm{mol}^{-1}\right)\end{array}$ & $\mathrm{T}_{\mathrm{m}}(\mathrm{K})$ & Method & Reference \\
\hline & V & $(350-607)$ & $64.7 \pm 0.5$ & 440 & $\mathrm{~EB}, \mathrm{IPM}$ & [1997STE/CHI2] \\
\hline & $\mathrm{V}$ & $(350-607)$ & $61.3 \pm 0.5$ & 480 & $\mathrm{~EB}, \mathrm{IPM}$ & [1997STE/CHI2] \\
\hline & $\mathrm{V}$ & $(350-607)$ & $57.7 \pm 0.5$ & 520 & $\mathrm{~EB}, \mathrm{IPM}$ & [1997STE/CHI2] \\
\hline & $\mathrm{V}$ & $(350-607)$ & $53.9 \pm 0.7$ & 560 & $\mathrm{~EB}, \mathrm{IPM}$ & [1997STE/CHI2] \\
\hline & $\mathrm{V}$ & $(393-550)$ & 60.5 & 408 & A, GS & [1987STE/MAL, 1963VOI] \\
\hline \multirow[t]{15}{*}{$\mathrm{C}_{10} \mathrm{H}_{10} \mathrm{O}_{4}$} & {$[131-11-3]$} & dimethyl phthalate & & & & \\
\hline & FUS & & 16.95 & 274.2 & & [1998MAK/KAB, 1986RAB/NOV] \\
\hline & FUS & & 15.7 & 273.2 & DSC & [1993ACR, 1978DOZ/FUJ] \\
\hline & $\mathrm{V}$ & & $84.2 \pm 3.4$ & 298 & CRT & [2015GOB/CHI] \\
\hline & $\mathrm{V}$ & & $66.4 \pm 12$ & 298 & $\mathrm{CGC}$ & [2015GOB/CHI] \\
\hline & $\mathrm{V}$ & & $76.7 \pm 1.7$ & 298 & CGC & [2014GOB/CHI] \\
\hline & $\mathrm{V}$ & $(466-518)$ & 61.5 & 481 & EB & [1999ROH/MUS] \\
\hline & $\mathrm{V}$ & & $69.4 \pm 0.1$ & 365 & $\mathrm{C}$ & [1998MAK/KAB] \\
\hline & $\mathrm{V}$ & & $72.5 \pm 0.6$ & 344 & $\mathrm{C}$ & [1998MAK/KAB] \\
\hline & $\mathrm{V}$ & & $74.5 \pm 0.3$ & 326 & $\mathrm{C}$ & [1998MAK/KAB] \\
\hline & $\mathrm{V}$ & $(304-371)$ & 78.7 & 319 & A & [1987STE/MAL] \\
\hline & V & $(371-547)$ & 63.7 & 386 & A & [1987STE/MAL] \\
\hline & $\mathrm{V}$ & $(377-440)$ & 68.6 & 409 & & [1969DAV/MAK] \\
\hline & $\mathrm{V}$ & & 64.8 & & Static & [1968DAV/BAT] \\
\hline & V & & 78.7 & & & [1948SMA/SMA] \\
\hline \multirow[t]{11}{*}{$\mathrm{C}_{10} \mathrm{H}_{10} \mathrm{O}_{4}$} & [120-61-6] & dimethyl terephtha & & & & \\
\hline & FUS & & 34.87 & 414.3 & DSC & [2013ZHA/XIA] \\
\hline & FUS & & 32.9 & 413.8 & DSC & [1993ACR, 1978DOZ/FUJ] \\
\hline & FUS & & 31.63 & 413.7 & $\mathrm{AC}$ & [1968ELL/CHR] \\
\hline & SUB & $(311-330)$ & $103.8 \pm 0.3$ & 321 & $\mathrm{ME}$ & [1998ROU/JIM] \\
\hline & SUB & $(311-330)$ & $104.6 \pm 0.3$ & 298 & $\mathrm{ME}$ & [1998ROU/JIM] \\
\hline & SUB & $(373-413)$ & 94.4 & 388 & A & [1987STE/MAL] \\
\hline & SUB & $(373-413)$ & 88.3 & 393 & GS & [1962KRA/BER] \\
\hline & SUB & & 105.3 & & $\mathrm{C}$ & [1998MAK/KAB] \\
\hline & $\mathrm{V}$ & & $78.6 \pm 1.1$ & 298 & CGC & [2014GOB/CHI] \\
\hline & $\mathrm{V}$ & $(413-523)$ & 62.0 & 428 & A & [1987STE/MAL] \\
\hline \multirow[t]{8}{*}{$\mathrm{C}_{10} \mathrm{H}_{10} \mathrm{O}_{4}$} & [1135-24-6] & 3-(4-hydroxy-3-me & oxyphenyl)-2 & enoic ac & erulic acid & \\
\hline & FUS & & 34.7 & 445.9 & DSC & [2016EME/YER] \\
\hline & FUS & & 31.9 & 444.9 & $\mathrm{DSC}$ & [2008MOT/QUE, 2012MAN/VIL] \\
\hline & FUS & & 17.89 & 435.3 & DSC & [1994HUA/CHE] \\
\hline & SUB & $(385-442)$ & $133.2 \pm 1.8$ & 413 & TGA & [2016EME/YER] \\
\hline & SUB & $(385-442)$ & $137.4 \pm 1.9$ & 298 & TGA & [2016EME/YER] \\
\hline & SUB & & $131.8 \pm 4.0$ & 298 & ME & [2012DAV/HER] \\
\hline & SUB & $(369-390)$ & $132.4 \pm 1.3$ & 379 & ME & [2006CHE/OJA] \\
\hline \multirow[t]{2}{*}{$\mathrm{C}_{10} \mathrm{H}_{10} \mathrm{O}_{4}$} & {$[635-51-8]$} & $(R S)$-phenylsuccir & acid & & & \\
\hline & FUS & & 37.37 & 440.1 & DSC & [2006PRO/RAS] \\
\hline \multirow[t]{2}{*}{$\mathrm{C}_{10} \mathrm{H}_{10} \mathrm{O}_{4}$} & {$[4036-30-0]$} & $(S)$-phenylsuccinic & cid & & & \\
\hline & FUS & & 41.84 & 446.9 & DSC & [2006PRO/RAS] \\
\hline \multirow[t]{3}{*}{$\mathrm{C}_{10} \mathrm{H}_{11} \mathrm{ClN}_{2} \mathrm{O}_{4}$} & [310412-18-1] & ethyl (2-chloromet & 1-2,3-dihydro- & oxazolo[ & a]-pyrimid & n-5-one)-6-carboxylate \\
\hline & TRS & & 5.38 & 379.7 & & \\
\hline & FUS & & 10.77 & 413.4 & DSC & [2000CHA/SOS] \\
\hline \multirow[t]{2}{*}{$\mathrm{C}_{10} \mathrm{H}_{11} \mathrm{ClO}_{3}$} & & $(d l)$-2-(2-chloro-3- & ethylphenoxy) & ionic aci & & \\
\hline & FUS & & 30.54 & 391.5 & DSC & [1976LEC/COL] \\
\hline \multirow[t]{2}{*}{$\mathrm{C}_{10} \mathrm{H}_{11} \mathrm{ClO}_{3}$} & & $(d)-2$-(2-chloro-3- & ethylphenoxy) & lonic aci & & \\
\hline & FUS & & 22.18 & 359.5 & DSC & [1976LEC/COL] \\
\hline \multirow[t]{2}{*}{$\mathrm{C}_{10} \mathrm{H}_{11} \mathrm{~F}_{3} \mathrm{~N}_{2} \mathrm{O}$} & {$[2164-17-2]$} & $N, N$-dimethyl- $N^{\prime}-$ & -(trifluorometh & henyl]ur & & \\
\hline & FUS & & 29.82 & 434.1 & $\mathrm{DSC}$ & [1991ACR, 1990DON/DRE] \\
\hline \multirow{2}{*}{$\mathrm{C}_{10} \mathrm{H}_{11} \mathrm{~F}_{3} \mathrm{~N}_{2} \mathrm{O}_{3} \mathrm{~S}$} & {$[47000-92-0]$} & \multicolumn{5}{|c|}{$N$-[4-methyl-3-[[(trifluoromethyl)sulfonyl]amino]phenyl]acetamide } \\
\hline & FUS & & 40.47 & 455.7 & DSC & {$[1990 \mathrm{DON} / \mathrm{DRE}]$} \\
\hline
\end{tabular}


TABLE 13. Phase change enthalpies of $\mathrm{C}_{10}$ organic compounds-Continued

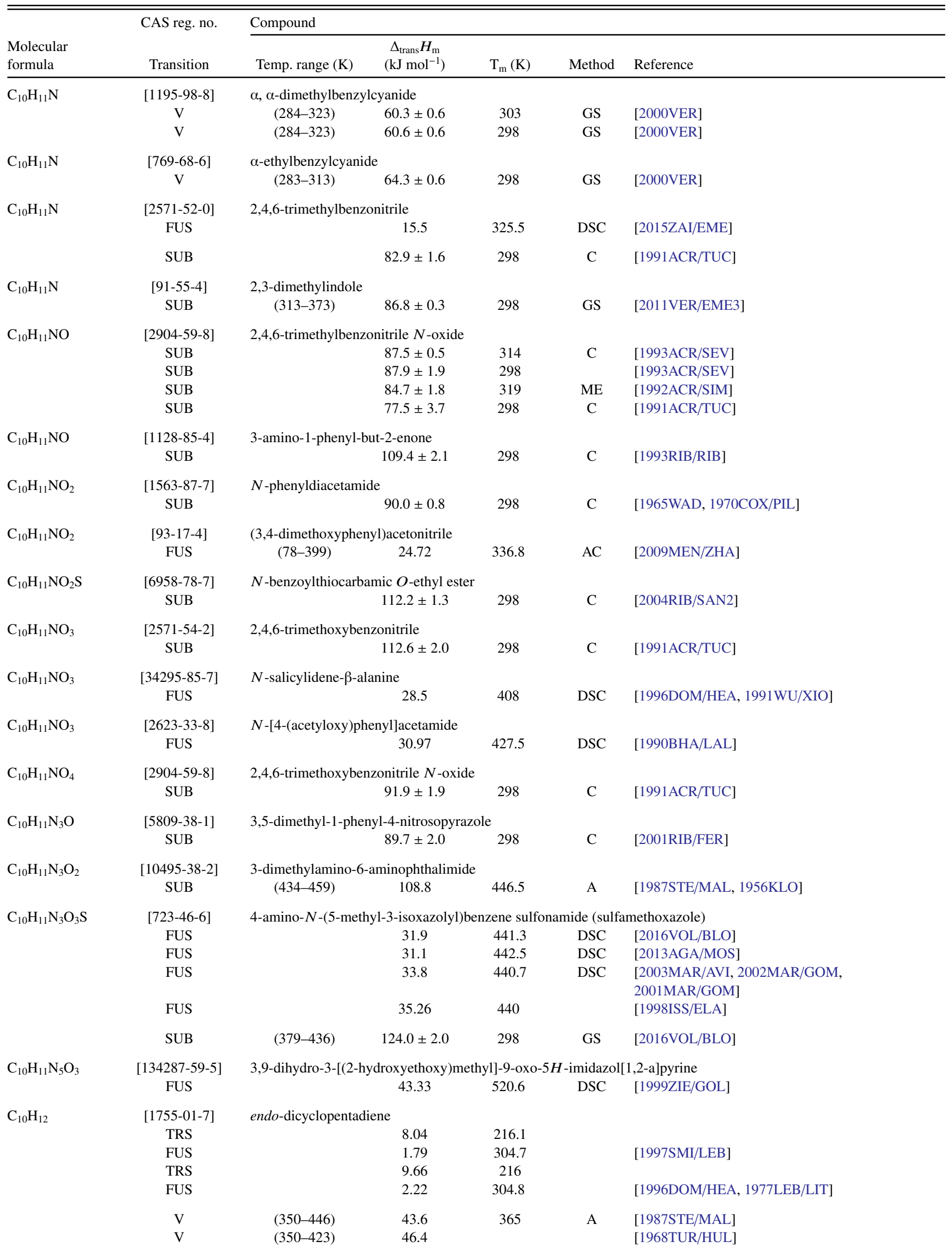


TABLE 13. Phase change enthalpies of $\mathrm{C}_{10}$ organic compounds-Continued

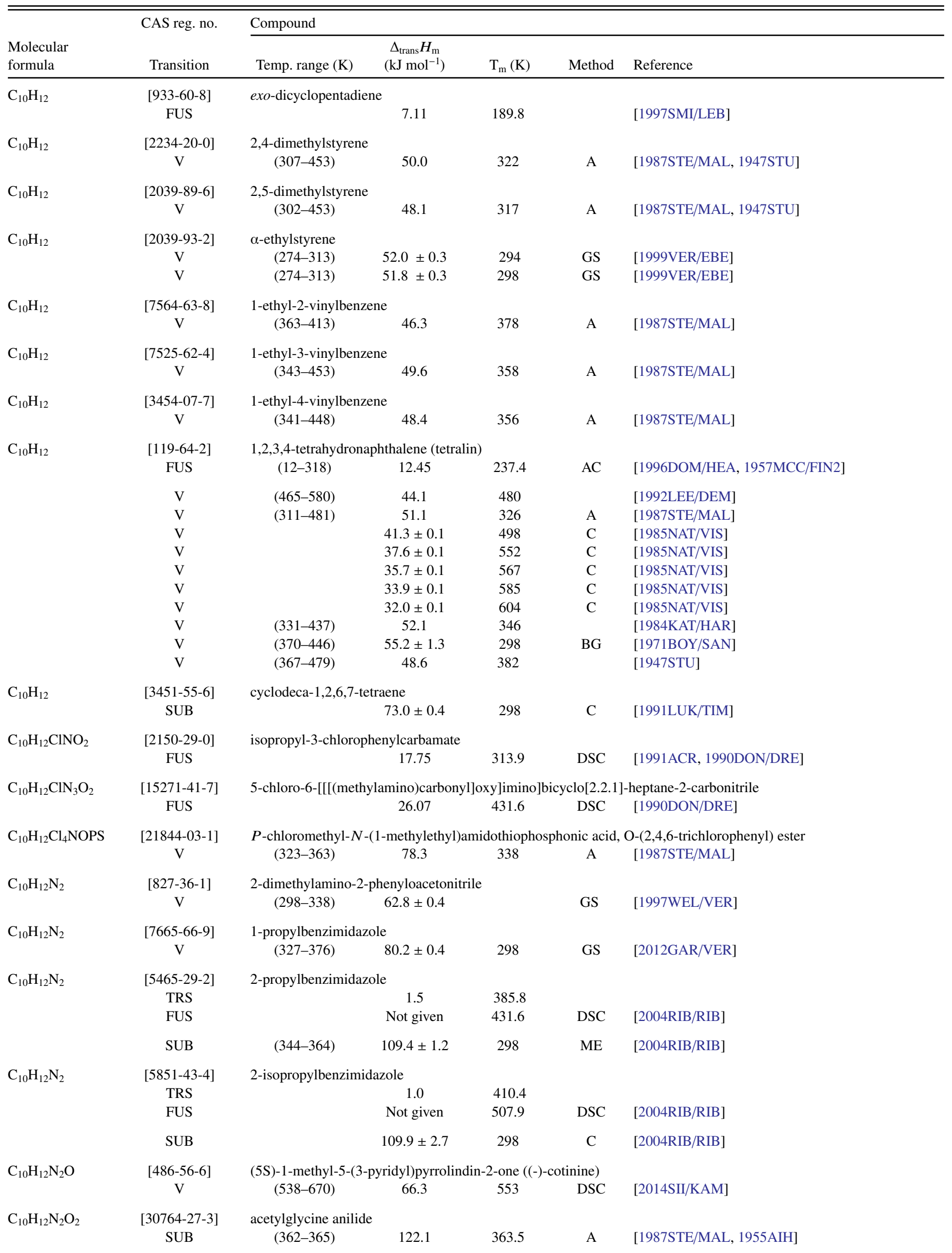


TABLE 13. Phase change enthalpies of $\mathrm{C}_{10}$ organic compounds-Continued

\begin{tabular}{|c|c|c|c|c|c|c|}
\hline \multirow[b]{2}{*}{$\begin{array}{l}\text { Molecular } \\
\text { formula }\end{array}$} & \multirow{2}{*}{$\begin{array}{l}\text { CAS reg. no. } \\
\text { Transition }\end{array}$} & \multicolumn{5}{|l|}{ Compound } \\
\hline & & Temp. range $(\mathrm{K})$ & $\begin{array}{c}\Delta_{\text {trans }} H_{\mathrm{m}} \\
\left(\mathrm{kJ} \mathrm{mol}^{-1}\right)\end{array}$ & $\mathrm{T}_{\mathrm{m}}(\mathrm{K})$ & Method & Reference \\
\hline $\mathrm{C}_{10} \mathrm{H}_{12} \mathrm{~N}_{2} \mathrm{O}_{3}$ & $\begin{array}{l}\text { [52-43-7] } \\
\text { FUS } \\
\text { FUS } \\
\text { FUS }\end{array}$ & allobarbital & $\begin{array}{c}32.31 \\
24.9 \\
31.7\end{array}$ & 442.6 & $\begin{array}{l}\text { DSC } \\
\text { DSC } \\
\text { DSC }\end{array}$ & $\begin{array}{l}\text { [1986CHA/DEM] } \\
\text { [1982TRE/VAU] } \\
\text { [1978SEK/TSU] }\end{array}$ \\
\hline $\mathrm{C}_{10} \mathrm{H}_{12} \mathrm{~N}_{2} \mathrm{O}_{3}$ & $\begin{array}{c}\text { [38423-62-0] } \\
\text { FUS }\end{array}$ & 2-ethoxyisonitroso & $\begin{array}{r}\text { etanilide } \\
23.0\end{array}$ & 407.3 & DTA & [1996DOM/HEA, 1982CUE/SOL] \\
\hline $\mathrm{C}_{10} \mathrm{H}_{12} \mathrm{~N}_{2} \mathrm{O}_{3}$ & $\begin{array}{c}\text { [17122-74-6] } \\
\text { FUS }\end{array}$ & 4-ethoxyisonitroso & $\begin{array}{r}\text { etanilide } \\
7.6\end{array}$ & 491.2 & DTA & [1982CUE/SOL, 1996DOM/HEA] \\
\hline $\mathrm{C}_{10} \mathrm{H}_{12} \mathrm{~N}_{2} \mathrm{O}_{4}$ & $\begin{array}{c}\text { [3056-17-5] } \\
\text { FUS }\end{array}$ & $2,3^{\prime}$-didehydro-3'-c & $\begin{array}{l}\text { xythymidine } \\
26.91\end{array}$ & $\begin{array}{l}\text { udine) } \\
443.2\end{array}$ & DSC & [2000GAN/BOG] \\
\hline $\mathrm{C}_{10} \mathrm{H}_{12} \mathrm{~N}_{2} \mathrm{O}_{4} \mathrm{~S}$ & $\begin{array}{c}{[138517-12-1]} \\
\text { FUS }\end{array}$ & (4-nitrophenyl)-2-( & $\begin{array}{l}\text { ethylthio)ethy } \\
31.27\end{array}$ & $\begin{array}{r}\text { bamate } \\
349.4\end{array}$ & DSC & [1993TIE/FRA] \\
\hline $\mathrm{C}_{10} \mathrm{H}_{12} \mathrm{~N}_{2} \mathrm{O}_{5}$ & $\begin{array}{l}{[88-85-7]} \\
\text { FUS }\end{array}$ & 2-sec-butyl-4,6-din & $\begin{array}{l}\text { ophenol } \\
21.81\end{array}$ & 313.7 & DSC & [1990DON/DRE] \\
\hline $\mathrm{C}_{10} \mathrm{H}_{12} \mathrm{~N}_{4} \mathrm{O}_{3}$ & $\begin{array}{l}\text { [74392-01-1] } \\
\text { SUB }\end{array}$ & 4- $N, N$-diethylamin & $\begin{array}{l}\text { 7-nitrobenzofi } \\
139.2 \pm 1.6\end{array}$ & 298 & ME & [2014SAN/SIL] \\
\hline $\mathrm{C}_{10} \mathrm{H}_{12} \mathrm{~N}_{2} \mathrm{~S}$ & $\begin{array}{c}\text { [7341-63-1] } \\
\text { FUS }\end{array}$ & $N$-allyl- $N$-phenyl & $\begin{array}{l}\text { ourea } \\
\quad 27.61\end{array}$ & 375 & & [1996DOM/HEA, 1928SHI] \\
\hline $\mathrm{C}_{10} \mathrm{H}_{12} \mathrm{O}$ & $\begin{array}{c}{[25679-28-1]} \\
\mathrm{V}\end{array}$ & $\begin{array}{l}\text { cis-anethole } \\
\quad \text { (333-363) }\end{array}$ & 68.7 & 348 & A & [1987STE/MAL] \\
\hline $\mathrm{C}_{10} \mathrm{H}_{12} \mathrm{O}$ & $\begin{array}{c}{[4180-23-8]} \\
\mathrm{V}\end{array}$ & $\begin{array}{r}\text { trans-anethole } \\
(333-363)\end{array}$ & 78.3 & 348 & A & [1987STE/MAL] \\
\hline $\mathrm{C}_{10} \mathrm{H}_{12} \mathrm{O}$ & $\begin{array}{c}{[140-67-0]} \\
\mathrm{V}\end{array}$ & $\begin{array}{l}\text { estragole } \\
\qquad(325-488)\end{array}$ & 56.3 & 340 & A & [1987STE/MAL] \\
\hline $\mathrm{C}_{10} \mathrm{H}_{12} \mathrm{O}$ & $\begin{array}{c}{[2142-64-5]} \\
\mathrm{V} \\
\mathrm{V}\end{array}$ & $\begin{array}{c}\text { 2'-ethylacetopheno } \\
(363-397) \\
(293-423)\end{array}$ & $\begin{array}{c}52.8 \\
\mathrm{U} 23.7\end{array}$ & $\begin{array}{l}378 \\
368\end{array}$ & A & $\begin{array}{l}\text { [1987STE/MAL] } \\
\text { [1968KHO/BYK] }\end{array}$ \\
\hline $\mathrm{C}_{10} \mathrm{H}_{12} \mathrm{O}$ & $\begin{array}{c}{[937-30-4]} \\
\mathrm{V} \\
\mathrm{V}\end{array}$ & $\begin{array}{l}\text { 4'-ethylacetopheno } \\
\begin{array}{c}(294-368) \\
(293-423)\end{array}\end{array}$ & $\begin{array}{l}42.2 \\
39.8\end{array}$ & $\begin{array}{l}309 \\
368\end{array}$ & A & $\begin{array}{l}\text { [1987STE/MAL] } \\
\text { [1968KHO/BYK] }\end{array}$ \\
\hline $\mathrm{C}_{10} \mathrm{H}_{12} \mathrm{O}$ & $\begin{array}{c}{[122-03-2]} \\
\mathrm{V}\end{array}$ & $\begin{array}{l}\text { 4-isopropylbenzalc } \\
\quad(331-505)\end{array}$ & $\begin{array}{l}\text { hyde (cuminal) } \\
55.3\end{array}$ & 346 & A & [1987STE/MAL, 1947STU] \\
\hline $\mathrm{C}_{10} \mathrm{H}_{12} \mathrm{O}$ & $\begin{array}{c}{[5445-77-2]} \\
\mathrm{V}\end{array}$ & $\begin{array}{c}\text { 2-methyl-3-phenyl } \\
(333-373)\end{array}$ & $\begin{array}{l}\text { opanal } \\
59.1\end{array}$ & 348 & A & [1987STE/MAL] \\
\hline $\mathrm{C}_{10} \mathrm{H}_{12} \mathrm{O}$ & $\begin{array}{c}\text { [5337-93-9] } \\
\mathrm{V}\end{array}$ & $\begin{array}{c}\text { 4'-methypropiophe } \\
\quad(332-512)\end{array}$ & 52.6 & 347 & A & [1987STE/MAL, 1947STU] \\
\hline
\end{tabular}


TABLE 13. Phase change enthalpies of $\mathrm{C}_{10}$ organic compounds-Continued

\begin{tabular}{|c|c|c|c|c|c|c|}
\hline \multirow[b]{2}{*}{$\begin{array}{l}\text { Molecular } \\
\text { formula }\end{array}$} & \multirow{2}{*}{$\begin{array}{l}\text { CAS reg. no. } \\
\text { Transition }\end{array}$} & \multicolumn{5}{|l|}{ Compound } \\
\hline & & Temp. range $(\mathrm{K})$ & $\begin{array}{c}\Delta_{\text {trans }} H_{\mathrm{m}} \\
\left(\mathrm{kJ} \mathrm{mol}^{-1}\right) \\
\end{array}$ & $\mathrm{T}_{\mathrm{m}}(\mathrm{K})$ & Method & Reference \\
\hline $\mathrm{C}_{10} \mathrm{H}_{12} \mathrm{O}$ & $\begin{array}{c}{[5459-40-5]} \\
\mathrm{V}\end{array}$ & $\begin{array}{c}\text { 4-vinylphenetole } \\
(337-498)\end{array}$ & 59.2 & 352 & A & [1987STE/MAL, 1947STU] \\
\hline $\mathrm{C}_{10} \mathrm{H}_{12} \mathrm{O}$ & $\begin{array}{c}\text { [1712-69-2] } \\
\text { FUS }\end{array}$ & 4-methoxy- $\alpha$-meth & $\begin{array}{l}\text { tyrene } \\
19.07\end{array}$ & 309.2 & & [1999VER6] \\
\hline & SUB & & $81.2 \pm 0.4$ & 298 & & [1999VER6] \\
\hline & $\begin{array}{l}\text { V } \\
\text { V }\end{array}$ & $\begin{array}{l}(308-343) \\
(308-343)\end{array}$ & $\begin{array}{l}60.6 \pm 0.3 \\
62.1 \pm 0.3\end{array}$ & $\begin{array}{l}326 \\
298\end{array}$ & $\begin{array}{l}\text { GS } \\
\text { GS }\end{array}$ & $\begin{array}{l}\text { [1999VER6] } \\
\text { [1999VER6] }\end{array}$ \\
\hline $\mathrm{C}_{10} \mathrm{H}_{12} \mathrm{O}_{2}$ & $\begin{array}{c}\text { [3674-77-9] } \\
\text { SUB }\end{array}$ & $\begin{array}{l}\text { 2-phenyl-2-methyl } \\
\quad(293-324)\end{array}$ & $\begin{array}{l}\text { 3-dioxolane } \\
81.9 \pm 0.5\end{array}$ & 308 & $\mathrm{~T}$ & [1995VER/DOG] \\
\hline $\mathrm{C}_{10} \mathrm{H}_{12} \mathrm{O}_{2}$ & $\begin{array}{c}{[103-45-7]} \\
\text { V } \\
\text { V } \\
\text { V }\end{array}$ & $\begin{array}{l}\text { acetic acid, phenet } \\
\qquad \begin{array}{c}(283-318) \\
(422-506)\end{array}\end{array}$ & $\begin{array}{l}\text { ester } \\
61.3 \pm 1.3 \\
67.4 \\
52.2\end{array}$ & $\begin{array}{l}298 \\
298 \\
437\end{array}$ & $\begin{array}{c}\text { CGC } \\
\text { A } \\
\text { A }\end{array}$ & $\begin{array}{l}{[2015 \mathrm{KOZ} / \mathrm{GOB}]} \\
{[1987 \mathrm{STE} / \mathrm{MAL}]} \\
{[1987 \mathrm{STE} / \mathrm{MAL}]}\end{array}$ \\
\hline $\mathrm{C}_{10} \mathrm{H}_{12} \mathrm{O}_{2}$ & $\begin{array}{c}\text { [31508-44-8] } \\
\text { V } \\
\text { V }\end{array}$ & $\begin{array}{c}\text { methyl 2-phenylpr } \\
(284-318) \\
(284-318)\end{array}$ & $\begin{array}{l}\text { onate } \\
61.8 \pm 0.7 \\
62.0 \pm 0.7\end{array}$ & $\begin{array}{c}301 \\
298\end{array}$ & $\begin{array}{l}\text { GS } \\
\text { GS }\end{array}$ & $\begin{array}{l}\text { [1999VER8] } \\
\text { [1999VER8] }\end{array}$ \\
\hline $\mathrm{C}_{10} \mathrm{H}_{12} \mathrm{O}_{2}$ & $\begin{array}{c}{[97-53-0]} \\
\mathrm{V} \\
\mathrm{V} \\
\mathrm{V} \\
\mathrm{V}\end{array}$ & $\begin{array}{l}\text { 4-allyl-2-methoxy! } \\
\qquad \begin{array}{l}(395-527) \\
(285-333) \\
(351-526)\end{array}\end{array}$ & $\begin{array}{l}\text { nol (eugenol) } \\
\qquad 66.3 \\
57.7 \\
66.1 \\
60.3\end{array}$ & $\begin{array}{l}298 \\
410 \\
300 \\
366\end{array}$ & $\begin{array}{c}\mathrm{GC} \\
\mathrm{A} \\
\mathrm{ME}\end{array}$ & $\begin{array}{l}\text { [2002VAN/PAR] } \\
{[1987 \mathrm{STE} / \mathrm{MAL}]} \\
{[1987 \mathrm{STE} / \mathrm{MAL}, 1959 \mathrm{SCO} / \mathrm{DOU}]} \\
{[1947 \mathrm{STU}]}\end{array}$ \\
\hline $\mathrm{C}_{10} \mathrm{H}_{12} \mathrm{O}_{2}$ & $\begin{array}{c}\text { [122-63-4] } \\
\mathrm{V}\end{array}$ & $\begin{array}{c}\text { benzyl propionate } \\
(298-378)\end{array}$ & 59.0 & 313 & A & [1987STE/MAL] \\
\hline $\mathrm{C}_{10} \mathrm{H}_{12} \mathrm{O}_{2}$ & $\begin{array}{c}\text { [501-19-9] } \\
\mathrm{V}\end{array}$ & $\begin{array}{c}\text { 5-allyl-2-methoxy } \\
(345-527)\end{array}$ & $\begin{array}{l}\text { nol } \\
61.4\end{array}$ & 371 & A & [1987STE/MAL] \\
\hline $\mathrm{C}_{10} \mathrm{H}_{12} \mathrm{O}_{2}$ & $\begin{array}{c}{[97-54-1]} \\
\mathrm{V}\end{array}$ & $\begin{array}{c}\text { 2-methoxy-4-(1-pr } \\
\quad(359-540)\end{array}$ & $\begin{array}{l}\text { enyl)phenol (i } \\
60.7\end{array}$ & $\begin{array}{c}\text { igenol) } \\
374\end{array}$ & & [1957DYK/SEP] \\
\hline $\mathrm{C}_{10} \mathrm{H}_{12} \mathrm{O}_{2}$ & $\begin{array}{c}{[5912-86-7]} \\
\mathrm{V}\end{array}$ & $\begin{array}{r}c i s-\text { isoeugenol } \\
(373-403)\end{array}$ & 69.7 & 388 & A & [1987STE/MAL] \\
\hline $\mathrm{C}_{10} \mathrm{H}_{12} \mathrm{O}_{2}$ & $\begin{array}{c}{[5932-68-3]} \\
\mathrm{V}\end{array}$ & $\begin{array}{c}\text { trans-isoeugenol } \\
\quad(363-420)\end{array}$ & 69.1 & 378 & A & [1987STE/MAL] \\
\hline $\mathrm{C}_{10} \mathrm{H}_{12} \mathrm{O}_{2}$ & $\begin{array}{c}{[3674-77-9} \\
\text { FUS }\end{array}$ & acetophenone ethy & $\begin{array}{c}\text { e glycol ketal } \\
25.2\end{array}$ & 333.6 & & [1995VER/DOG] \\
\hline $\mathrm{C}_{10} \mathrm{H}_{12} \mathrm{O}_{2}$ & $\begin{array}{c}{[16108-50-2]} \\
\text { FUS }\end{array}$ & 2-acetyl-3,5-dimet & $\begin{array}{r}\text { phenol } \\
1.36\end{array}$ & 333.2 & DTA & [1989SAL/ABA] \\
\hline
\end{tabular}

[Note: Reported enthalpy of fusion is too small, and the published enthalpy and entropy of fusion data are internally inconsistent.]

\begin{tabular}{|c|c|c|c|c|c|c|}
\hline \multirow[t]{5}{*}{$\mathrm{C}_{10} \mathrm{H}_{12} \mathrm{O}_{2}$} & [101-97-3] & \multicolumn{5}{|c|}{ phenylacetic acid, ethyl ester } \\
\hline & $\mathrm{V}$ & $(333-433)$ & 60.7 & 298 & GC & [2005HOS/GRY] \\
\hline & $\mathrm{V}$ & $(288-328)$ & $63.9 \pm 0.4$ & 308 & GS & [1999VER8] \\
\hline & $\mathrm{V}$ & $(288-328)$ & $64.5 \pm 0.4$ & 298 & GS & [1999VER8] \\
\hline & $\mathrm{V}$ & $(393-500)$ & 54 & 408 & $\mathrm{~A}$ & [1987STE/MAL] \\
\hline \multirow[t]{4}{*}{$\mathrm{C}_{10} \mathrm{H}_{12} \mathrm{O}_{2}$} & {$[2315-68-6]$} & propylbenzoate & & & & \\
\hline & $\mathrm{V}$ & $(359-458)$ & 60.2 & 379 & $\mathrm{BG}$ & [1988KAT2] \\
\hline & $\mathrm{V}$ & $(359-458)$ & 52.7 & 440 & BG & [1988KAT2] \\
\hline & $\mathrm{V}$ & $(327-504)$ & 53.8 & 342 & A & [1987STE/MAL, 1947STU] \\
\hline \multirow[t]{2}{*}{$\mathrm{C}_{10} \mathrm{H}_{12} \mathrm{O}_{2}$} & [2930-05-4] & \multicolumn{5}{|c|}{ [(phenylmethoxy)methyl]oxirane } \\
\hline & $\mathrm{V}$ & & $71.0 \pm 0.4$ & & & [1987VAN/KAC] \\
\hline \multirow[t]{3}{*}{$\mathrm{C}_{10} \mathrm{H}_{12} \mathrm{O}_{2}$} & {$[2529-36-4]$} & \multicolumn{5}{|c|}{ 2,3,6-trimethylbenzoic acid } \\
\hline & SUB & $(314-336)$ & $104.4 \pm 0.2$ & 298 & $\mathrm{ME}$ & [1987COL/JIM2] \\
\hline & SUB & $(314-336)$ & $103.6 \pm 0.2$ & 325 & $\mathrm{ME}$ & [1987COL/JIM2] \\
\hline \multirow[t]{2}{*}{$\mathrm{C}_{10} \mathrm{H}_{12} \mathrm{O}_{2}$} & {$[480-63-7]$} & \multicolumn{5}{|c|}{ 2,4,6-trimethylbenzoic acid } \\
\hline & SUB & $(316-340)$ & $103.6 \pm 0.3$ & 298 & $\mathrm{ME}$ & [1987COL/JIM2] \\
\hline
\end{tabular}


TABLE 13. Phase change enthalpies of $\mathrm{C}_{10}$ organic compounds-Continued

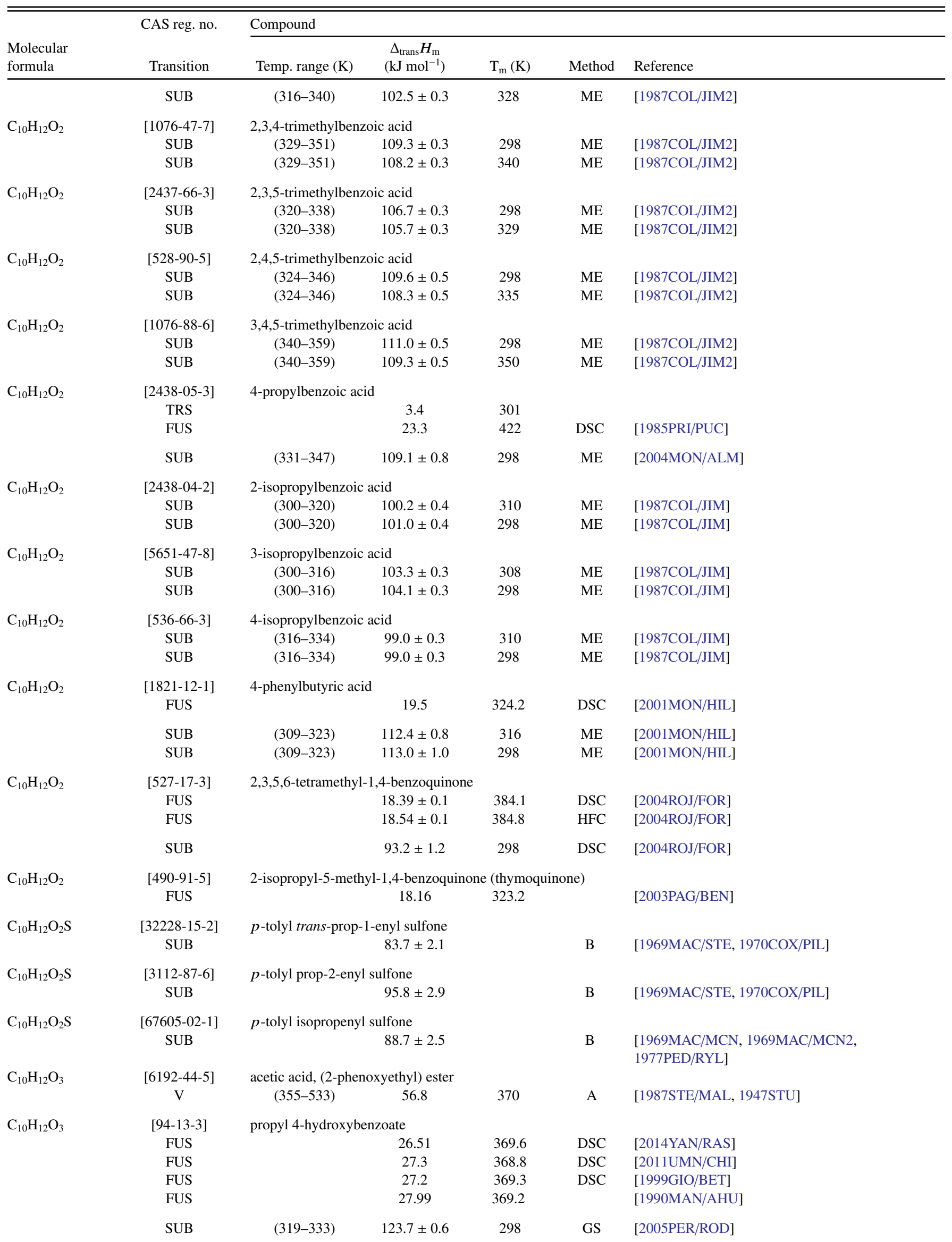


TABLE 13. Phase change enthalpies of $\mathrm{C}_{10}$ organic compounds-Continued

\begin{tabular}{|c|c|c|c|c|c|c|}
\hline \multirow[b]{2}{*}{$\begin{array}{l}\text { Molecular } \\
\text { formula }\end{array}$} & \multirow{2}{*}{$\begin{array}{l}\text { CAS reg. no. } \\
\text { Transition }\end{array}$} & \multicolumn{5}{|l|}{ Compound } \\
\hline & & Temp. range $(\mathrm{K})$ & $\begin{array}{c}\Delta_{\text {trans }} H_{\mathrm{m}} \\
\left(\mathrm{kJ} \mathrm{mol}^{-1}\right)\end{array}$ & $\mathrm{T}_{\mathrm{m}}(\mathrm{K})$ & Method & Reference \\
\hline & $\mathrm{V}$ & & $89.5 \pm 0.6$ & 298 & $\mathrm{CGC}$ & [2011UMN/CHI] \\
\hline & V & & 79.1 & & TGA & [2002CHA/DOL] \\
\hline & $\mathrm{V}$ & & 76.5 & & TGA & [2001CHA/DOL] \\
\hline $\mathrm{C}_{10} \mathrm{H}_{12} \mathrm{O}_{3}$ & $\begin{array}{l}{[3759-31-7]} \\
\text { FUS }\end{array}$ & $(d l)$-3-hydroxy-3-p & $\begin{array}{c}\text { enylbutyric ac } \\
19.66\end{array}$ & 330 & & [1991CHI/BRA] \\
\hline $\mathrm{C}_{10} \mathrm{H}_{12} \mathrm{O}_{3}$ & FUS & (d)-3-hydroxy-3-p & $\begin{array}{c}\text { nylbutyric aci } \\
22.59\end{array}$ & 357 & & [1991CHI/BRA] \\
\hline $\mathrm{C}_{10} \mathrm{H}_{12} \mathrm{O}_{3}$ & $\begin{array}{l}\text { [4919-33-9] } \\
\text { FUS }\end{array}$ & 4-ethoxyphenylace & 23.0 & 360.2 & & [1991ACR, 1979ARM/JAM] \\
\hline $\mathrm{C}_{10} \mathrm{H}_{12} \mathrm{O}_{3}$ & $\begin{array}{l}{[6342-77-4]} \\
\text { FUS }\end{array}$ & 3-(2-methoxyphen & $\begin{array}{l}\text { propionic aci } \\
25.33\end{array}$ & 360.5 & DSC & [2001MON/HIL4] \\
\hline & $\begin{array}{l}\text { SUB } \\
\text { SUB }\end{array}$ & $\begin{array}{l}(331-347) \\
(331-347)\end{array}$ & $\begin{array}{l}116.0 \pm 0.4 \\
117.8 \pm 1.4\end{array}$ & $\begin{array}{c}339 \\
298\end{array}$ & $\begin{array}{l}\text { ME } \\
\text { ME }\end{array}$ & $\begin{array}{l}\text { [2001MON/HIL4] } \\
\text { [2001MON/HIL4] }\end{array}$ \\
\hline $\mathrm{C}_{10} \mathrm{H}_{12} \mathrm{O}_{3}$ & $\begin{array}{c}\text { [1929-29-9] } \\
\text { FUS } \\
\text { FUS }\end{array}$ & 3-(4-methoxyphen & $\begin{array}{c}\text { propionic aci } \\
29.57 \\
28.5\end{array}$ & $\begin{array}{l}376.1 \\
376.9\end{array}$ & DSC & $\begin{array}{l}\text { [2001MON/HIL4] } \\
\text { [1991ACR, 1979ARM/JAM] }\end{array}$ \\
\hline & $\begin{array}{l}\text { SUB } \\
\text { SUB }\end{array}$ & $\begin{array}{l}(341-357) \\
(341-357)\end{array}$ & $\begin{array}{l}122.3 \pm 0.3 \\
124.5 \pm 1.7\end{array}$ & $\begin{array}{c}349 \\
298\end{array}$ & $\begin{array}{l}\text { ME } \\
\mathrm{ME}\end{array}$ & $\begin{array}{l}\text { [2001MON/HIL4] } \\
\text { [2001MON/HIL4] }\end{array}$ \\
\hline $\mathrm{C}_{10} \mathrm{H}_{12} \mathrm{O}_{3}$ & $\begin{array}{c}\text { [5438-19-7] } \\
\text { TRS (liq cryst) } \\
\text { TRS (liq cryst) } \\
\text { TRS } \\
\text { (liq cryst-to-liq) }\end{array}$ & 4-propoxybenzoic & $\begin{array}{ll}\text { id } & \\
& 7.95 \\
16.74 \\
\\
2.51\end{array}$ & $\begin{array}{l}394.2 \\
419.9 \\
426.7\end{array}$ & DSC & [1967HER] \\
\hline & $\begin{array}{c}\text { SUB } \\
\text { SUB(II) }\end{array}$ & $\begin{array}{l}(345-365) \\
(345-365)\end{array}$ & $\begin{array}{l}123.8 \pm 0.5 \\
126.0 \pm 0.5\end{array}$ & $\begin{array}{c}355 \\
298\end{array}$ & $\begin{array}{l}\mathrm{ME} \\
\mathrm{ME}\end{array}$ & $\begin{array}{l}\text { [2010FON/SAN] } \\
\text { [2010FON/SAN] }\end{array}$ \\
\hline $\mathrm{C}_{10} \mathrm{H}_{12} \mathrm{O}_{4}$ & $\begin{array}{c}\text { [999-21-3] } \\
\mathrm{V}\end{array}$ & $\begin{array}{l}\text { maleic acid, dially } \\
\quad(392-426)\end{array}$ & $\begin{array}{l}\text { ster } \\
77.7\end{array}$ & 407 & A & [1987STE/MAL, 1958MOR/YOS] \\
\hline $\mathrm{C}_{10} \mathrm{H}_{12} \mathrm{O}_{4}$ & $\begin{array}{c}\text { [20765-04-2] } \\
\text { FUS }\end{array}$ & 2,5-diethoxy-1,4-b & $\begin{array}{c}\text { zoquinone } \\
28.7\end{array}$ & 459.3 & $\mathrm{DSC}$ & [1996KEE/VAN] \\
\hline $\mathrm{C}_{10} \mathrm{H}_{12} \mathrm{O}_{5}$ & $\begin{array}{c}\text { [490-64-2] } \\
\text { FUS }\end{array}$ & 2,4,5-trimethoxybe & $\begin{array}{l}\text { zoic acid } \\
31.15\end{array}$ & 417.9 & $\mathrm{DSC}$ & [2003HUA, 2005HUA/TAN] \\
\hline $\mathrm{C}_{10} \mathrm{H}_{12} \mathrm{O}_{5}$ & $\begin{array}{c}\text { [118-41-2] } \\
\text { FUS }\end{array}$ & 3,4,5-trimethoxybe & $\begin{array}{r}\text { zoic acid } \\
29.9\end{array}$ & 444.5 & DSC & [2003HUA, 2005HUA/TAN] \\
\hline & $\begin{array}{l}\text { SUB } \\
\text { SUB }\end{array}$ & $(354-372)$ & $\begin{array}{l}127.9 \pm 0.8 \\
131.2 \pm 0.8\end{array}$ & $\begin{array}{l}363 \\
298\end{array}$ & $\begin{array}{l}\mathrm{ME} \\
\mathrm{ME}\end{array}$ & $\begin{array}{l}\text { [2001ROU/JIM2] } \\
\text { [2001ROU/JIM2] }\end{array}$ \\
\hline $\mathrm{C}_{10} \mathrm{H}_{12} \mathrm{O}_{6}$ & $\begin{array}{c}\text { [6289-46-9] } \\
\text { FUS } \\
\text { FUS }\end{array}$ & dimethyl 2,5-dioxo & $\begin{array}{c}\text {,4-cyclohexar } \\
27.69 \\
26.52\end{array}$ & $\begin{array}{c}\text { arboxylate } \\
429.9 \\
427.7\end{array}$ & $\begin{array}{l}\text { DSC } \\
\text { DSC }\end{array}$ & $\begin{array}{l}\text { [2014LIU/ZOU] } \\
\text { [2011CHE/YAN] }\end{array}$ \\
\hline $\mathrm{C}_{10} \mathrm{H}_{13} \mathrm{Br}$ & $\begin{array}{c}{[2437-76-5]} \\
\mathrm{V}\end{array}$ & $\begin{array}{c}\text { 2-bromo-4-isoprop } \\
(400-510)\end{array}$ & $\begin{array}{l}\text { toluene } \\
50.2\end{array}$ & 415 & $\mathrm{~A}$ & [1987STE/MAL, 1970DYK/VAN, 1999DYK/SVO] \\
\hline $\mathrm{C}_{10} \mathrm{H}_{13} \mathrm{Br}$ & $\begin{array}{c}{[4478-10-8]} \\
\mathrm{V}\end{array}$ & $\begin{array}{c}\text { 3-bromo-4-isoprop } \\
\quad(400-510)\end{array}$ & $\begin{array}{r}\text { toluene } \\
48.3\end{array}$ & 415 & A & [1987STE/MAL, 1970DYK/VAN, 1999DYK/SVO] \\
\hline $\mathrm{C}_{10} \mathrm{H}_{13} \mathrm{BrO}$ & $\begin{array}{c}{[54514-31-7]} \\
\mathrm{V}\end{array}$ & $\begin{array}{l}\text { 2-bromophenyl iso } \\
\quad(293-323)\end{array}$ & $\begin{array}{l}\text { Ityl ether } \\
\quad 64.0 \pm 0.2\end{array}$ & 298 & GS & [2005STR/SPO] \\
\hline $\mathrm{C}_{10} \mathrm{H}_{13} \mathrm{BrO}$ & $\begin{array}{c}{[223564-75-8]} \\
\mathrm{V}\end{array}$ & $\begin{array}{l}\text { 3-bromophenyl iso } \\
\quad(290-323)\end{array}$ & $\begin{array}{l}\text { tyl ether } \\
65.3 \pm 0.2\end{array}$ & 298 & GS & [2005STR/SPO] \\
\hline $\mathrm{C}_{10} \mathrm{H}_{13} \mathrm{BrO}$ & $\begin{array}{c}{[30752-23-9]} \\
\mathrm{V}\end{array}$ & $\begin{array}{l}\text { 4-bromophenyl iso } \\
\quad(293-333)\end{array}$ & $\begin{array}{l}\text { Ityl ether } \\
66.8 \pm 0.6\end{array}$ & 298 & GS & [2005STR/SPO] \\
\hline $\mathrm{C}_{10} \mathrm{H}_{13} \mathrm{Cl}$ & $\begin{array}{c}{[4395-79-3]} \\
\mathrm{V}\end{array}$ & $\begin{array}{c}\text { 2-chloro-4-isoprop } \\
(400-490)\end{array}$ & $\begin{array}{l}\text { toluene } \\
49.3\end{array}$ & 415 & A & [1987STE/MAL, 1970DYK/VAN, 1999DYK/SVO] \\
\hline
\end{tabular}


TABLE 13. Phase change enthalpies of $\mathrm{C}_{10}$ organic compounds-Continued

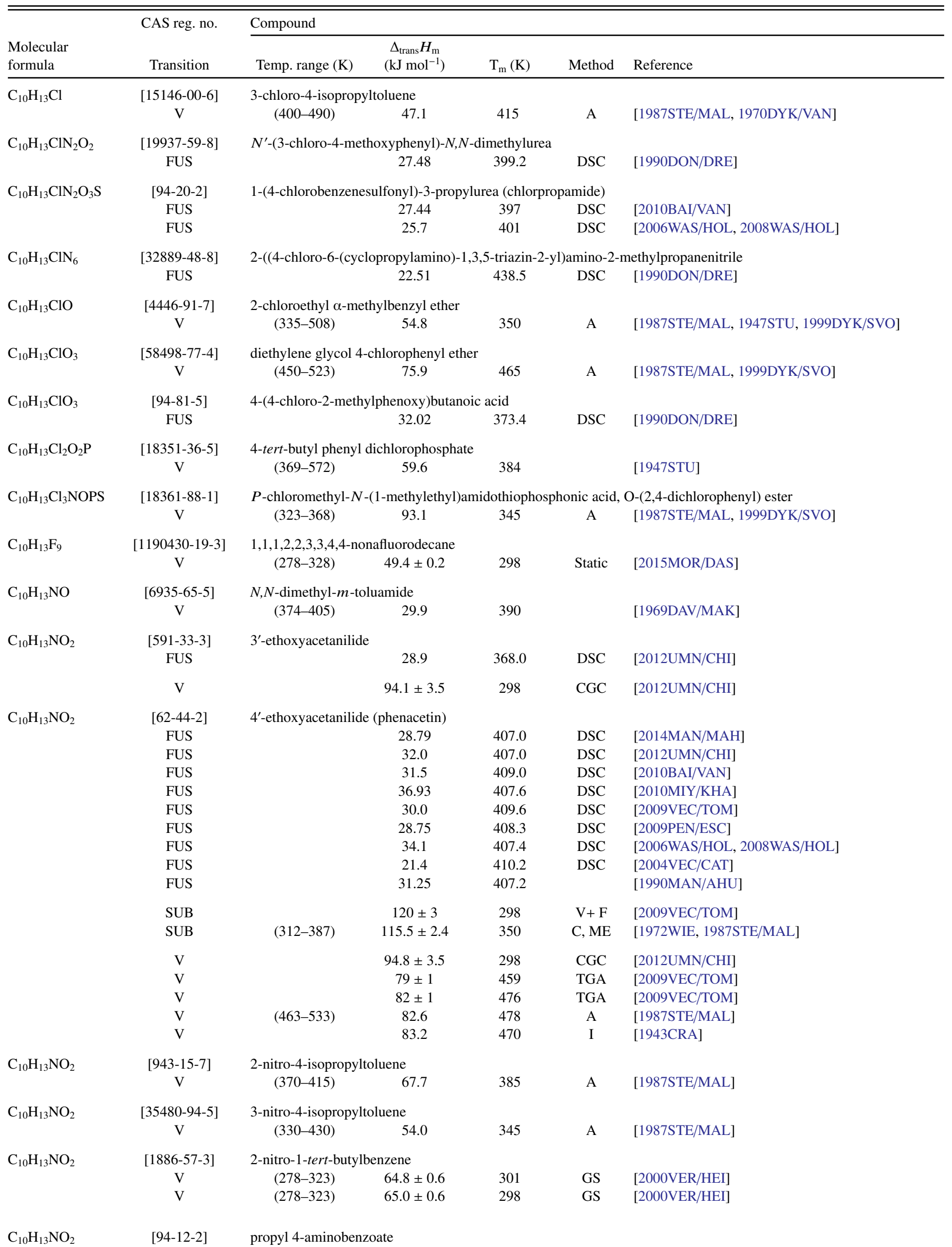


TABLE 13. Phase change enthalpies of $\mathrm{C}_{10}$ organic compounds-Continued

\begin{tabular}{|c|c|c|c|c|c|c|}
\hline \multirow[b]{2}{*}{$\begin{array}{l}\text { Molecular } \\
\text { formula }\end{array}$} & \multirow{2}{*}{$\begin{array}{c}\text { CAS reg. no. } \\
\text { Transition }\end{array}$} & \multicolumn{5}{|l|}{ Compound } \\
\hline & & Temp. range $(\mathrm{K})$ & $\begin{array}{c}\Delta_{\text {trans }} H_{\mathrm{m}} \\
\left(\mathrm{kJ} \mathrm{mol}^{-1}\right)\end{array}$ & $\mathrm{T}_{\mathrm{m}}(\mathrm{K})$ & Method & Reference \\
\hline & FUS & & 20.54 & 347.1 & $\mathrm{DSC}$ & $\begin{array}{l}\text { [1991ACR, 1990MAN/AHU, 1989NEA/FLY, } \\
\text { 1990NEA/FLY] }\end{array}$ \\
\hline \multirow{8}{*}{$\mathrm{C}_{10} \mathrm{H}_{13} \mathrm{NO}_{2}$} & {$[1202-25-1]$} & \multicolumn{5}{|c|}{ methyl $p$ - $N, N$-dimethylaminobenzoate } \\
\hline & FUS & & 23.4 & 371.3 & DSC & [2011ALM/MON] \\
\hline & FUS & & 26.07 & 371.8 & & [1991ACR, 1990MAN/AHU] \\
\hline & SUB & $(311-333)$ & $100.1 \pm 0.3$ & 298 & ME & [2011ALM/MON] \\
\hline & SUB & $(325-368)$ & $100.9 \pm 0.2$ & 298 & Static & [2011ALM/MON] \\
\hline & SUB & $(325-368)$ & $99.0 \pm 0.2$ & 347 & Static & [2011ALM/MON] \\
\hline & $\mathrm{V}$ & $(353-390)$ & $81.0 \pm 0.1$ & 298 & Static & [2011ALM/MON] \\
\hline & $\mathrm{V}$ & $(353-390)$ & $74.0 \pm 0.1$ & 371 & Static & [2011ALM/MON] \\
\hline \multirow[t]{2}{*}{$\mathrm{C}_{10} \mathrm{H}_{13} \mathrm{NO}_{2}$} & {$[5532-90-1]$} & \multicolumn{5}{|c|}{ propyl $N$-phenylcarbamate } \\
\hline & FUS & & 21.08 & 331 & & [1971PRI] \\
\hline \multirow[t]{2}{*}{$\mathrm{C}_{10} \mathrm{H}_{13} \mathrm{NO}_{2}$} & {$[122-42-9]$} & \multicolumn{5}{|c|}{ isopropyl phenylcarbamate } \\
\hline & FUS & & 19.37 & 359.5 & DSC & [1991ACR, 1990DON/DRE] \\
\hline \multirow[t]{2}{*}{$\mathrm{C}_{10} \mathrm{H}_{13} \mathrm{NO}_{2}$} & [2425-10-7] & \multicolumn{5}{|c|}{ 3,4-dimethylphenyl methylcarbamate } \\
\hline & FUS & & 24.97 & 350.8 & DSC & [1991ACR, 1990DON/DRE] \\
\hline \multirow[t]{3}{*}{$\mathrm{C}_{10} \mathrm{H}_{13} \mathrm{NO}_{2}$} & {$[5426-62-0]$} & \multicolumn{5}{|l|}{$N$-benzyl- $\beta$-alanine } \\
\hline & FUS & & 42.7 & 457.0 & DSC & [2011ROU/NOT2] \\
\hline & SUB & & $171.6 \pm 3.7$ & 298 & $\mathrm{C}$ & [2011NOT/ROU] \\
\hline \multirow[t]{3}{*}{$\mathrm{C}_{10} \mathrm{H}_{13} \mathrm{NO}_{2} \mathrm{~S}_{2}$} & [949171-65-7] & \multicolumn{5}{|c|}{$N$-theonylthiocarbamic- $O$-butyl ester } \\
\hline & FUS & & 23.89 & 364.3 & DSC & {$[2007 \mathrm{RIB} / \mathrm{MON}]$} \\
\hline & SUB & & $147.5 \pm 1.9$ & 298 & $\mathrm{C}$ & {$[2007 \mathrm{RIB} / \mathrm{MON}]$} \\
\hline \multirow[t]{2}{*}{$\mathrm{C}_{10} \mathrm{H}_{13} \mathrm{NO}_{4}$} & {$[6988-21-2]$} & \multicolumn{5}{|c|}{ 2-(1,3-dioxolan-2-yl)phenyl methylcarbamate } \\
\hline & FUS & & 23.82 & 387.2 & DSC & [1990DON/DRE] \\
\hline \multirow[t]{2}{*}{$\mathrm{C}_{10} \mathrm{H}_{13} \mathrm{~N}_{5} \mathrm{O}_{3}$} & & \multicolumn{5}{|c|}{ 2-acetylamino-9-[(2-hydroxyethoxy)methyl]-9H-purine } \\
\hline & FUS & & 54.92 & 454.2 & DSC & [1995KRI/VES] \\
\hline $\mathrm{C}_{10} \mathrm{H}_{13} \mathrm{~N}_{5} \mathrm{O}_{3}$ & & $9-[(2$-acetoxyethox & methyl]-2-an & $9 H$-purin & & \\
\hline & FUS & & 42.69 & 408.2 & DSC & [1995KRI/VES] \\
\hline $\mathrm{C}_{10} \mathrm{H}_{13} \mathrm{~N}_{5} \mathrm{O}_{4}$ & [110104-37-5] & 2-acetylamino-9-[( & hydroxyethox & thyl] $-1,9$ & $y d r o-6 H$ & urin-6-one \\
\hline & FUS & & 53.83 & 490.2 & DSC & {$[1995 \mathrm{KRI} / \mathrm{VES}]$} \\
\hline $\mathrm{C}_{10} \mathrm{H}_{13} \mathrm{~N}_{5} \mathrm{O}_{4}$ & {$[102728-64-3]$} & 2-amino-9-[(2-acet & yethoxy)met & 1,9-dihyd & $H$-purin- & one \\
\hline & FUS & & 49.9 & 515.2 & DSC & [1995KRI/VES] \\
\hline $\mathrm{C}_{10} \mathrm{H}_{13} \mathrm{~N}_{5} \mathrm{O}_{4}$ & {$[30516-87-1]$} & 3'-azido-2,3'-dideo & thymidine $(\mathrm{z}$ & dine) & & \\
\hline & FUS & & 31.12 & 296.8 & DSC & [2010ARA/DOS] \\
\hline & FUS & & 33.03 & 395.8 & DSC & [2003ARA/STO] \\
\hline $\mathrm{C}_{10} \mathrm{H}_{14}$ & {$[104-51-8]$} & butylbenzene & & & & \\
\hline & FUS & & 11.22 & 185.3 & & [1996DOM/HEA, 1965MES/TOD2] \\
\hline & FUS & & 10.98 & 184.6 & $\mathrm{C}$ & [1996DOM/HEA, 1931HUF/PAR] \\
\hline & $\mathrm{V}$ & $(343-501)$ & $47.4 \pm 0.2$ & 350 & EB & [2002STE/CHI, 2006VER] \\
\hline & $\mathrm{V}$ & $(343-501)$ & $43.5 \pm 0.2$ & 410 & EB & [2002STE/CHI] \\
\hline & $\mathrm{V}$ & $(343-501)$ & $40.6 \pm 0.4$ & 450 & EB & [2002STE/CHI] \\
\hline & $\mathrm{V}$ & $(343-501)$ & $37.5 \pm 0.7$ & 490 & EB & [2002STE/CHI] \\
\hline & $\mathrm{V}$ & & 50.8 & 298 & & [1994RUZ/ZAB] \\
\hline & $\mathrm{V}$ & $(243-403)$ & 53.5 & 258 & & [1993KAS/MOK] \\
\hline & $\mathrm{V}$ & & $48.0 \pm 0.1$ & 343 & $\mathrm{C}$ & [1982SVO/CHA] \\
\hline & $\mathrm{V}$ & & $46.8 \pm 0.1$ & 358 & $\mathrm{C}$ & [1982SVO/CHA] \\
\hline & $\mathrm{V}$ & & $46.0 \pm 0.1$ & 368 & $\mathrm{C}$ & [1982SVO/CHA] \\
\hline & $\mathrm{V}$ & & 50.1 & 298 & & [1971WIL/ZWO] \\
\hline & $\mathrm{V}$ & $(374-454)$ & 45.2 & 389 & & [1965LIN/FRI, 1984BOU/FRI] \\
\hline & $\mathrm{V}$ & $(369-463)$ & 45.7 & 384 & A & [1987STE/MAL, 1949FOR/NOR] \\
\hline $\mathrm{C}_{10} \mathrm{H}_{14}$ & {$[135-98-8]$} & $(d l)$-sec-butylbenze & & & & \\
\hline & $\mathrm{V}$ & $(335-491)$ & $45.7 \pm 0.2$ & 340 & EB & [2002STE/CHI] \\
\hline
\end{tabular}


TABLE 13. Phase change enthalpies of $\mathrm{C}_{10}$ organic compounds-Continued

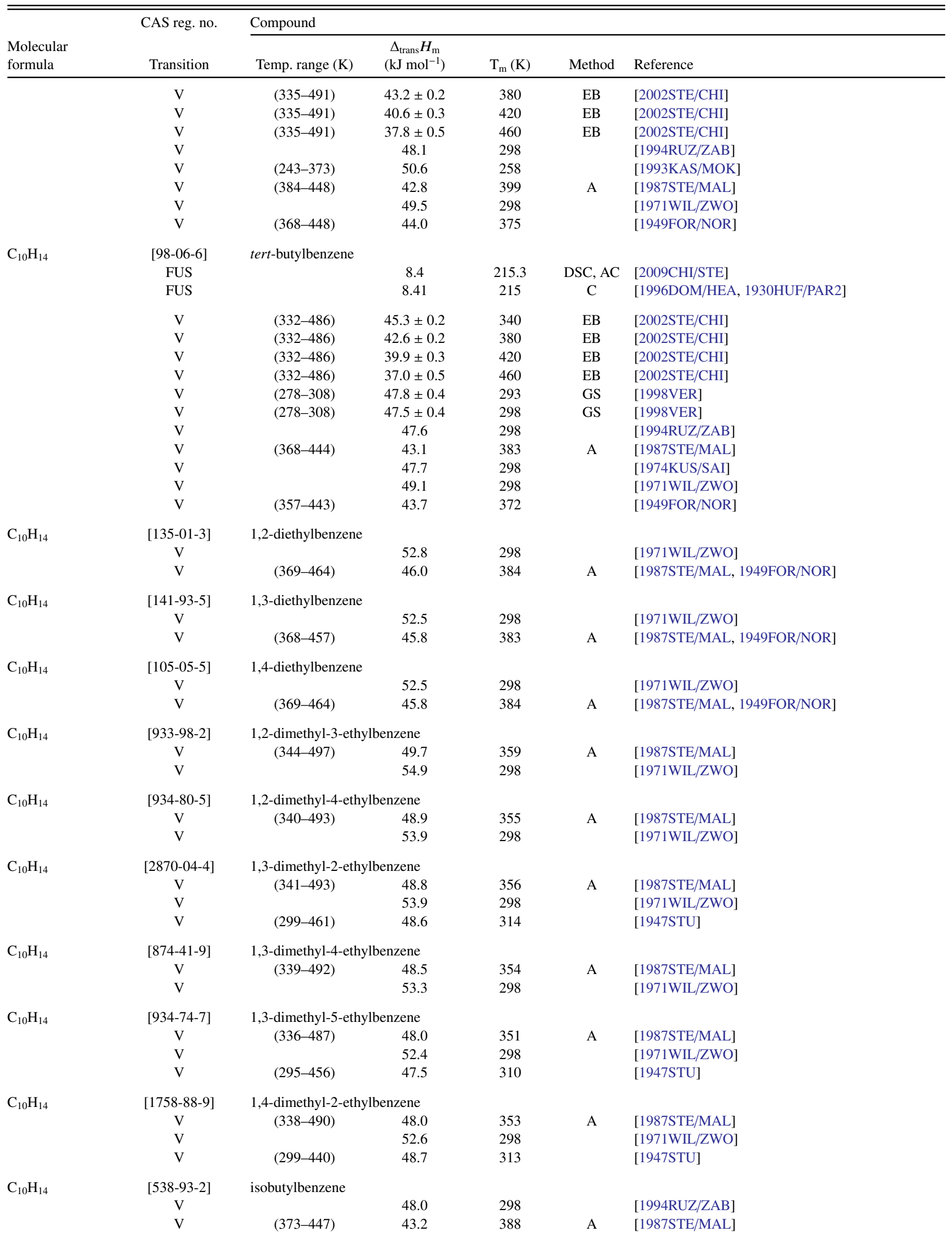


TABLE 13. Phase change enthalpies of $\mathrm{C}_{10}$ organic compounds-Continued

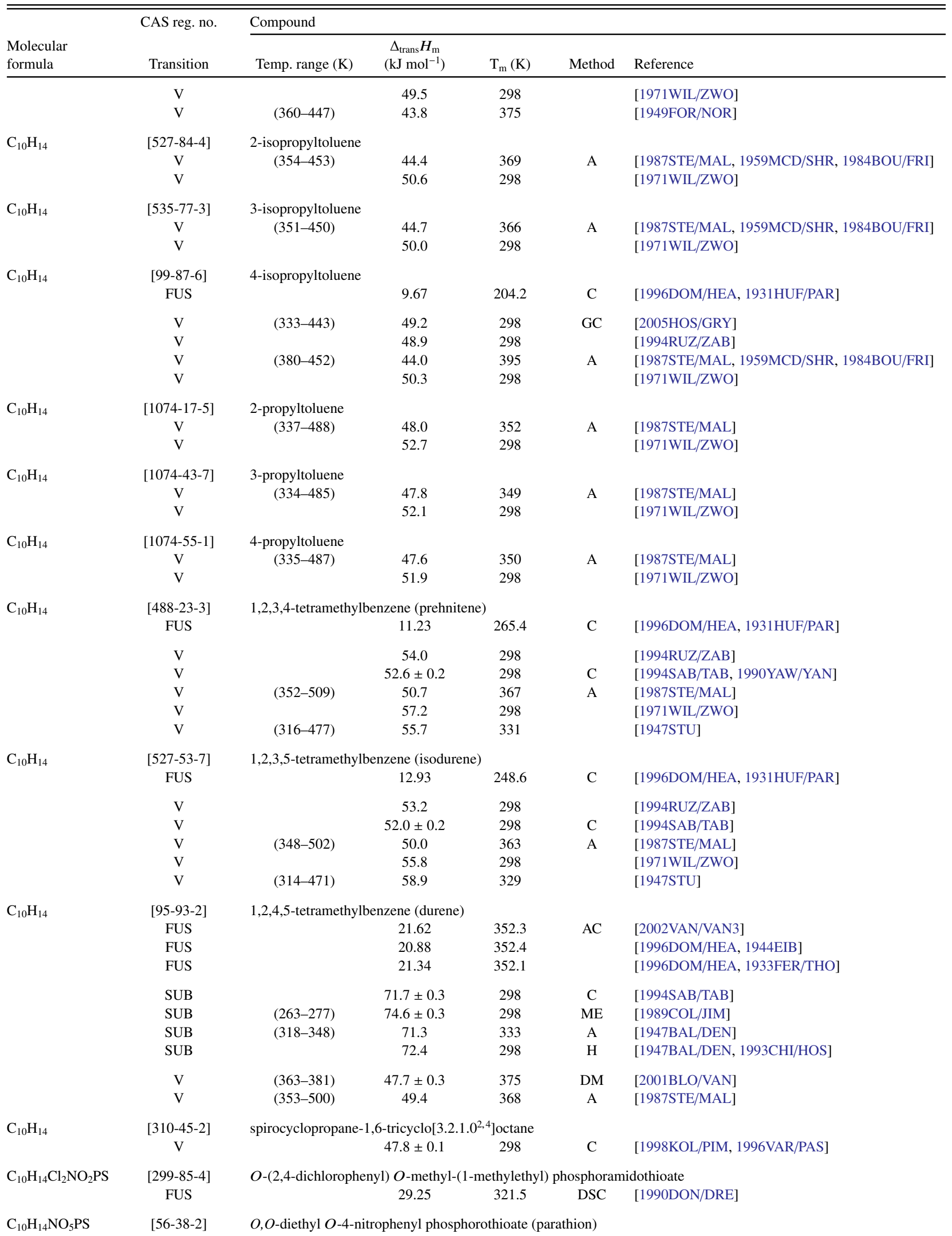


TABLE 13. Phase change enthalpies of $\mathrm{C}_{10}$ organic compounds-Continued

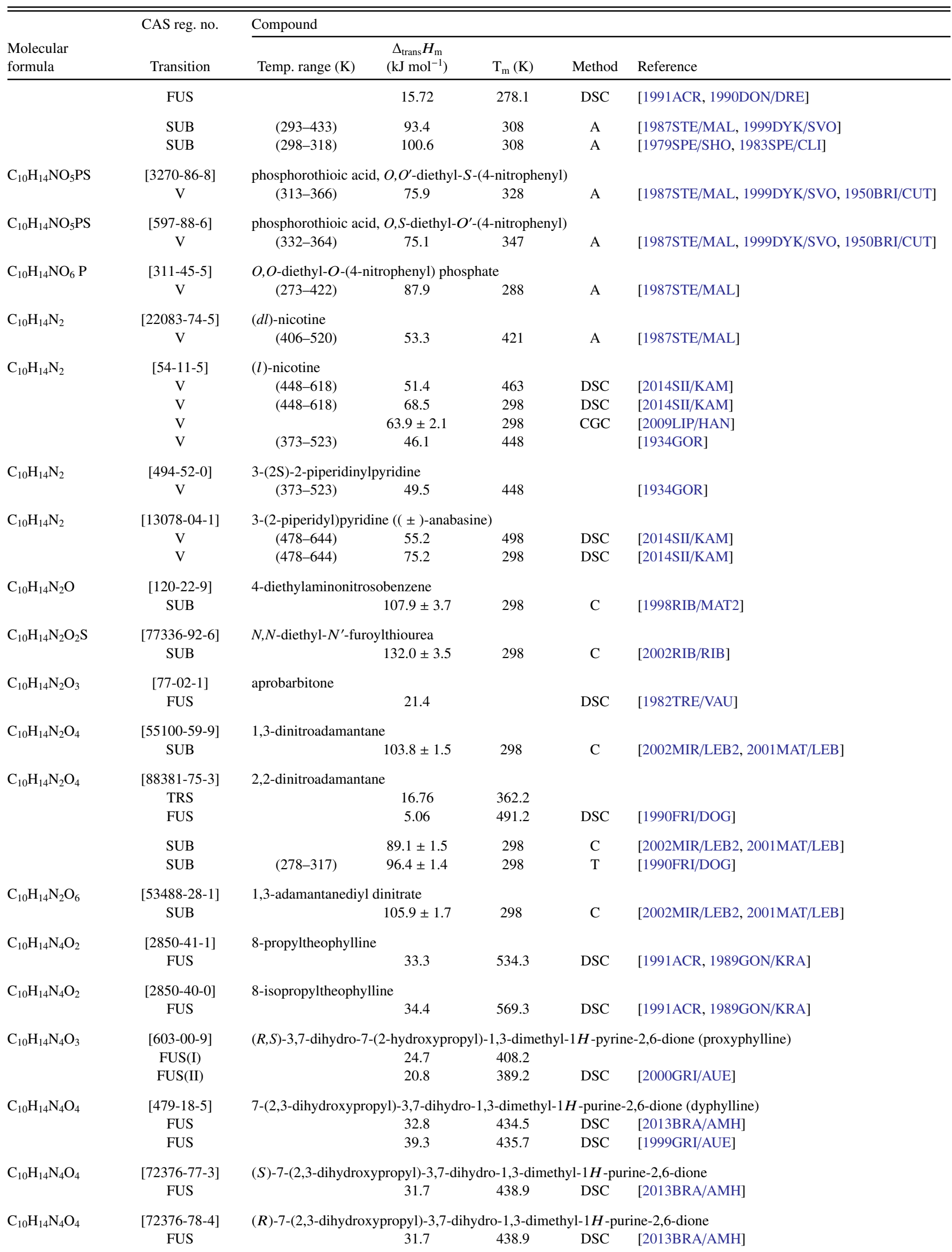


TABLE 13. Phase change enthalpies of $\mathrm{C}_{10}$ organic compounds-Continued

\begin{tabular}{|c|c|c|c|c|c|c|}
\hline \multirow[b]{2}{*}{$\begin{array}{l}\text { Molecular } \\
\text { formula }\end{array}$} & \multirow{2}{*}{$\begin{array}{l}\text { CAS reg. no. } \\
\text { Transition }\end{array}$} & \multicolumn{5}{|l|}{ Compound } \\
\hline & & Temp. range $(\mathrm{K})$ & $\begin{array}{c}\Delta_{\text {trans }} H_{\mathrm{m}} \\
\left(\mathrm{kJ} \mathrm{mol}^{-1}\right)\end{array}$ & $\mathrm{T}_{\mathrm{m}}(\mathrm{K})$ & Method & Reference \\
\hline \multirow[t]{5}{*}{$\mathrm{C}_{10} \mathrm{H}_{14} \mathrm{O}$} & [3180-09-4] & 2-butylphenol & & & & \\
\hline & $\mathrm{V}$ & $(403-533)$ & 55.1 & 418 & A & [1987STE/MAL, 1975ARR/MEL] \\
\hline & $\mathrm{V}$ & $(382-520)$ & 52.9 & 398 & & [1953STA/MUL] \\
\hline & $\mathrm{V}$ & $(382-520)$ & 51.0 & 423 & & [1953STA/MUL] \\
\hline & $\mathrm{V}$ & $(382-520)$ & 47.0 & 473 & & [1953STA/MUL] \\
\hline \multirow[t]{2}{*}{$\mathrm{C}_{10} \mathrm{H}_{14} \mathrm{O}$} & {$[89-72-5]$} & 2-sec-butylphenol & & & & \\
\hline & $\mathrm{V}$ & $(451-513)$ & 52.1 & 466 & A, GS, EB & [1987STE/MAL, 1964HAN/HAR] \\
\hline \multirow[t]{10}{*}{$\mathrm{C}_{10} \mathrm{H}_{14} \mathrm{O}$} & {$[88-18-6]$} & 2-tert-butylphenol & & & & \\
\hline & $\mathrm{V}$ & $(289-329)$ & $62.6 \pm 0.2$ & 309 & GS & [1999VER2] \\
\hline & $\mathrm{V}$ & $(289-329)$ & $63.2 \pm 0.2$ & 298 & GS & [1999VER2] \\
\hline & $\mathrm{V}$ & $(409-467)$ & 74.1 & 424 & EB & [1990NES/NAZ] \\
\hline & $\mathrm{V}$ & $(409-465)$ & 52.9 & 424 & & [1986TSV/NAZ] \\
\hline & $\mathrm{V}$ & $(353-498)$ & 54.9 & 368 & $\mathrm{~A}$ & [1987STE/MAL] \\
\hline & $\mathrm{V}$ & $(330-507)$ & 55.6 & 348 & & [1953STA/MUL] \\
\hline & $\mathrm{V}$ & $(330-507)$ & 53.9 & 373 & & [1953STA/MUL] \\
\hline & $\mathrm{V}$ & $(330-507)$ & 51.0 & 423 & & [1953STA/MUL] \\
\hline & V & $(330-507)$ & 47.0 & 473 & & [1953STA/MUL] \\
\hline \multirow[t]{5}{*}{$\mathrm{C}_{10} \mathrm{H}_{14} \mathrm{O}$} & {$[4074-43-5]$} & 3-butylphenol & & & & \\
\hline & $\mathrm{V}$ & $(396-533)$ & 62.5 & 411 & A & [1987STE/MAL] \\
\hline & $\mathrm{V}$ & $(396-533)$ & 56.6 & 398 & & [1953STA/MUL] \\
\hline & $\mathrm{V}$ & $(396-533)$ & 54.4 & 423 & & [1953STA/MUL] \\
\hline & $\mathrm{V}$ & $(396-533)$ & 49.9 & 473 & & [1953STA/MUL] \\
\hline \multirow[t]{10}{*}{$\mathrm{C}_{10} \mathrm{H}_{14} \mathrm{O}$} & {$[585-34-2]$} & 3-tert-butylphenol & & & & \\
\hline & SUB & & $88.9 \pm 0.5$ & 298 & $\mathrm{C}$ & [1999RIB/MAT2] \\
\hline & SUB & $(278-319)$ & $86.0 \pm 0.5$ & 298 & GS & [1999VER2] \\
\hline & SUB & $(266-299)$ & 70.7 & 281 & & [1987STE/MAL] \\
\hline & $\mathrm{V}$ & $(320-348)$ & $69.1 \pm 0.8$ & 334 & GS & [1999VER2] \\
\hline & $\mathrm{V}$ & $(320-348)$ & $71.3 \pm 0.8$ & 298 & GS & [1999VER2] \\
\hline & $\mathrm{V}$ & $(391-524)$ & 62.4 & 406 & A & [1987STE/MAL] \\
\hline & $\mathrm{V}$ & $(391-524)$ & 56.6 & 398 & & [1953STA/MUL] \\
\hline & $\mathrm{V}$ & $(391-524)$ & 54.4 & 423 & & [1953STA/MUL] \\
\hline & $\mathrm{V}$ & $(391-524)$ & 49.9 & 473 & & [1953STA/MUL] \\
\hline \multirow[t]{6}{*}{$\mathrm{C}_{10} \mathrm{H}_{14} \mathrm{O}$} & [1638-22-8] & 4-butylphenol & & & & \\
\hline & $\mathrm{V}$ & (395-653) & 61.7 & 410 & A & [1987STE/MAL] \\
\hline & $\mathrm{V}$ & $(357-529)$ & 57.6 & 373 & & [1953STA/MUL] \\
\hline & $\mathrm{V}$ & $(357-529)$ & 56.6 & 398 & & [1953STA/MUL] \\
\hline & $\mathrm{V}$ & $(357-529)$ & 54.4 & 423 & & [1953STA/MUL] \\
\hline & $\mathrm{V}$ & $(357-529)$ & 49.9 & 473 & & [1953STA/MUL] \\
\hline \multirow[t]{2}{*}{$\mathrm{C}_{10} \mathrm{H}_{14} \mathrm{O}$} & {$[99-71-8]$} & 4-sec-butylphenol & & & & \\
\hline & V & $(344-516)$ & 59.0 & 359 & A & [1987STE/MAL, 1947STU] \\
\hline \multirow[t]{13}{*}{$\mathrm{C}_{10} \mathrm{H}_{14} \mathrm{O}$} & [98-54-4] & 4-tert-butylphenol & & & & \\
\hline & FUS & & 14.52 & 373.2 & DTA & [1972INO/LIA] \\
\hline & SUB & & $89.4 \pm 2.5$ & 298 & $\mathrm{C}$ & [1999RIB/MAT2] \\
\hline & SUB & $(293-334)$ & $85.0 \pm 0.5$ & 313 & GS & [1999VER2] \\
\hline & SUB & $(293-334)$ & $85.9 \pm 0.5$ & 298 & GS & [1999VER2] \\
\hline & SUB & $(280-304)$ & 84.3 & 292 & & [1987STE/MAL, 1960AIH] \\
\hline & $\mathrm{V}$ & & $67.9 \pm 1.0$ & 298 & $\mathrm{C}$ & [1999RIB/MAT2] \\
\hline & $\mathrm{V}$ & $(471-525)$ & 54.3 & 486 & $\mathrm{~A}, \mathrm{GS}, \mathrm{EB}$ & [1987STE/MAL, 1947STU, 1964HAN/HAR] \\
\hline & $\mathrm{V}$ & $(346-523)$ & 59.6 & 348 & & [1953STA/MUL] \\
\hline & $\mathrm{V}$ & $(346-523)$ & 57.6 & 373 & & [1953STA/MUL] \\
\hline & $\mathrm{V}$ & $(346-523)$ & 56.6 & 398 & & [1953STA/MUL] \\
\hline & $\mathrm{V}$ & $(346-523)$ & 54.4 & 423 & & [1953STA/MUL] \\
\hline & $\mathrm{V}$ & $(346-523)$ & 49.9 & 473 & & [1953STA/MUL] \\
\hline $\mathrm{C}_{10} \mathrm{H}_{14} \mathrm{O}$ & $\begin{array}{c}{[1126-79-0]} \\
\mathrm{V}\end{array}$ & $\begin{array}{l}\text { butyl phenyl ether } \\
\text { (391-483) }\end{array}$ & 48.9 & 406 & A & [1987STE/MAL, 1949DRE/SHR, 1984BOU/FRI] \\
\hline
\end{tabular}


TABLE 13. Phase change enthalpies of $\mathrm{C}_{10}$ organic compounds-Continued

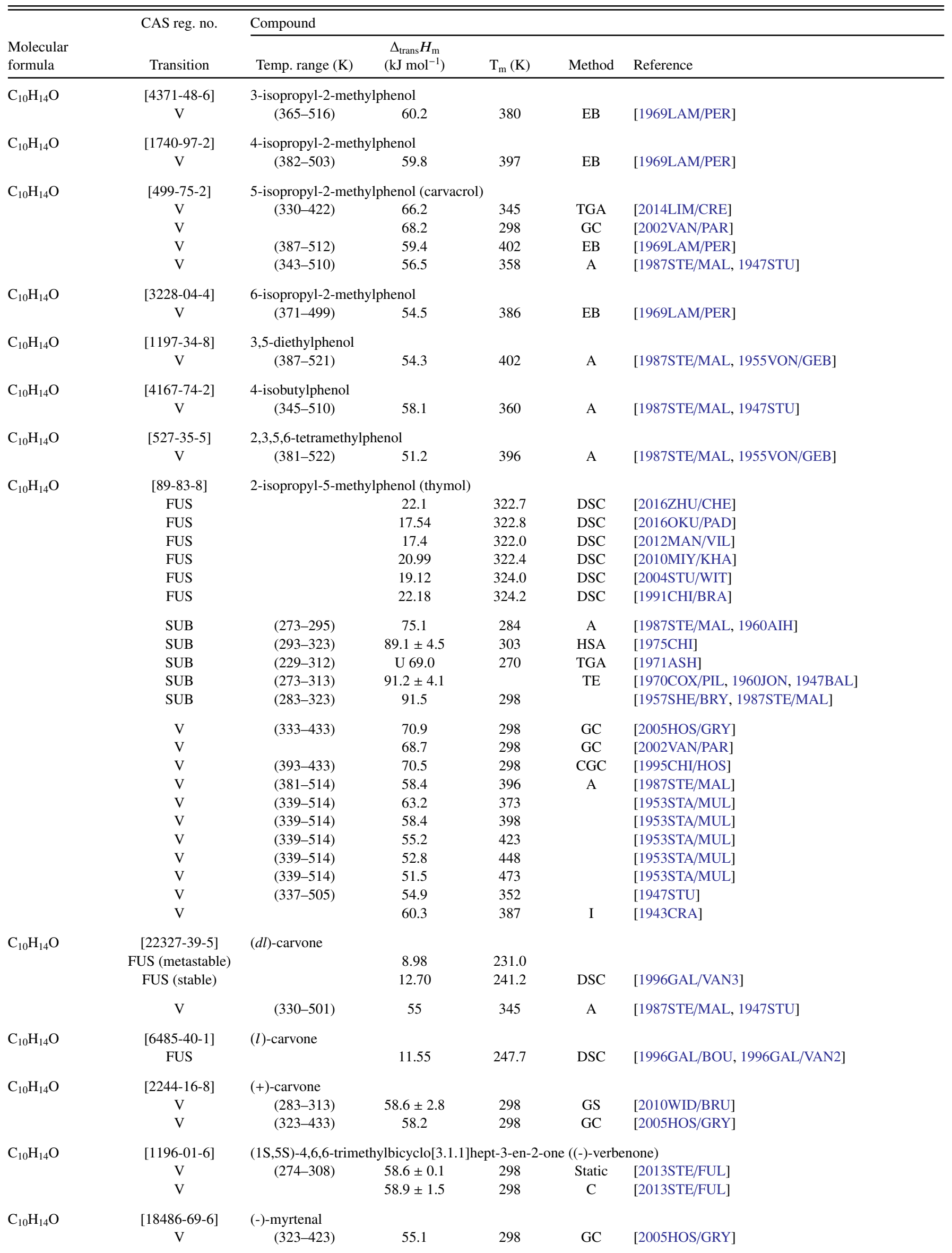


TABLE 13. Phase change enthalpies of $\mathrm{C}_{10}$ organic compounds-Continued

\begin{tabular}{|c|c|c|c|c|c|c|}
\hline \multirow[b]{2}{*}{$\begin{array}{l}\text { Molecular } \\
\text { formula }\end{array}$} & \multirow{2}{*}{$\begin{array}{l}\text { CAS reg. no. } \\
\text { Transition }\end{array}$} & \multicolumn{5}{|l|}{ Compound } \\
\hline & & Temp. range $(\mathrm{K})$ & $\begin{array}{c}\Delta_{\text {trans }} H_{\mathrm{m}} \\
\left(\mathrm{kJ} \mathrm{mol}^{-1}\right)\end{array}$ & $\mathrm{T}_{\mathrm{m}}(\mathrm{K})$ & Method & Reference \\
\hline $\mathrm{C}_{10} \mathrm{H}_{14} \mathrm{O}$ & $\begin{array}{c}{[1585-06-4]} \\
\mathrm{V}\end{array}$ & $\begin{array}{l}\text { 4-ethylphenetole } \\
\quad(321-481)\end{array}$ & 54.3 & 336 & A & [1987STE/MAL, 1947STU] \\
\hline $\mathrm{C}_{10} \mathrm{H}_{14} \mathrm{O}$ & $\begin{array}{c}{[22545-12-6]} \\
\mathrm{V}\end{array}$ & $\begin{array}{l}\text { 2-(2-ethylphenyl)e } \\
\quad(420-653)\end{array}$ & $\begin{array}{l}\text { nol } \\
59.5\end{array}$ & 435 & A & [1987STE/MAL, 1949DRE/MAR, 1949DRE/SHR] \\
\hline $\mathrm{C}_{10} \mathrm{H}_{14} \mathrm{O}$ & $\begin{array}{c}{[22545-13-7]} \\
\mathrm{V}\end{array}$ & $\begin{array}{l}\text { 2-(4-ethylphenyl)e } \\
\quad(420-653)\end{array}$ & $\begin{array}{l}\text { nol } \\
59.1\end{array}$ & 435 & A & [1987STE/MAL, 1949DRE/MAR, 1949DRE/SHR] \\
\hline $\mathrm{C}_{10} \mathrm{H}_{14} \mathrm{O}$ & $\begin{array}{c}{[536-60-7]} \\
\mathrm{V}\end{array}$ & $\begin{array}{c}\text { 4-isopropylbenzyl } \\
(347-520)\end{array}$ & $\begin{array}{l}\text { ohol } \\
59.7\end{array}$ & 362 & A & [1987STE/MAL, 1947STU] \\
\hline $\mathrm{C}_{10} \mathrm{H}_{14} \mathrm{O}$ & $\begin{array}{c}\text { [7384-80-7] } \\
\mathrm{V}\end{array}$ & $\begin{array}{c}\text { 2-methyl-3-pheny } \\
(343-393)\end{array}$ & $\begin{array}{c}\text { propanol } \\
71.9\end{array}$ & 358 & A & [1987STE/MAL] \\
\hline $\mathrm{C}_{10} \mathrm{H}_{14} \mathrm{O}$ & $\begin{array}{c}{[3299-05-6]} \\
\text { V } \\
V\end{array}$ & $\begin{array}{c}\text { (1-ethoxyethyl)be } \\
(286-318) \\
(286-318)\end{array}$ & $\begin{array}{l}52.4 \pm 0.2 \\
52.6 \pm 0.2\end{array}$ & $\begin{array}{l}302 \\
298\end{array}$ & $\begin{array}{l}\text { GS } \\
\text { GS }\end{array}$ & $\begin{array}{l}\text { [2001 VER/HEI] } \\
\text { [2001 VER/HEI] }\end{array}$ \\
\hline $\mathrm{C}_{10} \mathrm{H}_{14} \mathrm{O}$ & $\begin{array}{c}\text { [700-58-3] } \\
\text { TRS } \\
\text { FUS }\end{array}$ & $\begin{array}{c}\text { 2-adamantanone } \\
\qquad(5-310)\end{array}$ & $\begin{array}{c}7.63 \\
11.77\end{array}$ & $\begin{array}{l}216.4 \\
557.5\end{array}$ & $\begin{array}{c}\mathrm{AC} \\
\mathrm{DSC}\end{array}$ & $\begin{array}{l}\text { [2006BAZ/BLO] } \\
{[2006 \mathrm{BAZ} / \mathrm{BLO}]}\end{array}$ \\
\hline & $\begin{array}{l}\text { SUB } \\
\text { SUB } \\
\text { SUB }\end{array}$ & $(280-333)$ & $\begin{array}{l}66.4 \pm 0.3 \\
66.3 \pm 0.8 \\
76.1 \pm 1.5\end{array}$ & $\begin{array}{l}298 \\
298 \\
298\end{array}$ & $\begin{array}{c}\mathrm{C} \\
\mathrm{ME} \\
\mathrm{C}\end{array}$ & $\begin{array}{l}\text { [2006BAZ/BLO] } \\
\text { [2006BAZ/BLO] } \\
\text { [2002MIR/LEB, 2006BAZ/BLO, 2002MIR/LEB2, } \\
\text { 2001MAT/LEB] }\end{array}$ \\
\hline & SUB & $(320-370)$ & $80.3 \pm 2.5$ & 298 & BG & {$[1978 \mathrm{ARO} / \mathrm{STE}]$} \\
\hline & V & & $60.7 \pm 0.2$ & 298 & GC & [2002VAN/PAR] \\
\hline $\mathrm{C}_{10} \mathrm{H}_{14} \mathrm{O}$ & $\begin{array}{c}{[935-67-1]} \\
\mathrm{V} \\
\mathrm{V}\end{array}$ & $\begin{array}{c}\text { (1-methoxy-1-met } \\
(278-313) \\
(278-313)\end{array}$ & $\begin{array}{l}\text { ethyl)-benzene } \\
53.0 \pm 0.5 \\
52.9 \pm 0.5\end{array}$ & $\begin{array}{l}296 \\
298\end{array}$ & $\begin{array}{l}\text { GS } \\
\text { GS }\end{array}$ & $\begin{array}{l}\text { [2001HEI/VER] } \\
\text { [2001HEI/VER] }\end{array}$ \\
\hline $\mathrm{C}_{10} \mathrm{H}_{14} \mathrm{O}_{2}$ & $\begin{array}{c}{[4026-05-5]} \\
\mathrm{V} \\
\mathrm{V}\end{array}$ & $\begin{array}{c}\text { 1,2-dihydroxy-3-te } \\
(334-384) \\
(334-384)\end{array}$ & $\begin{array}{l}\text { butylbenzene } \\
70.1 \pm 0.8 \\
73.5 \pm 0.8\end{array}$ & $\begin{array}{l}359 \\
298\end{array}$ & $\begin{array}{l}\text { GS } \\
\text { GS }\end{array}$ & $\begin{array}{l}{[2000 \mathrm{VER} / \mathrm{SCH}]} \\
{[2000 \mathrm{VER} / \mathrm{SCH}]}\end{array}$ \\
\hline $\mathrm{C}_{10} \mathrm{H}_{14} \mathrm{O}_{2}$ & $\begin{array}{l}\text { [98-29-3] } \\
\text { FUS } \\
\text { FUS }\end{array}$ & 1,2-dihydroxy-4-te & $\begin{array}{c}\text { butylbenzene } \\
15.1 \\
4.5\end{array}$ & $\begin{array}{c}330.4 \\
327\end{array}$ & DSC & $\begin{array}{l}\text { [2000VER/SCH] } \\
{[1997 \mathrm{STE} / \mathrm{CHI} 2]}\end{array}$ \\
\hline
\end{tabular}

[Note: Reference [2000VER/SCH] notes the large discrepancy between their measured enthalpy of fusion and that reported in [1997STE/CHI2]. The authors of [2000VER/SCH] attribute the difference to the solid sample in [1997STE/CHI2] being only partly crystalline.]

$\begin{array}{cccccl}\text { SUB } & (303-323) & 98.7 \pm 0.9 & 313 & \text { GS } & {[2000 \mathrm{VER} / \mathrm{SCH}]} \\ \text { SUB } & (303-323) & 99.2 \pm 0.9 & 298 & \text { GS } & {[2000 \mathrm{VER} / \mathrm{SCH}]} \\ \text { SUB } & & 99.3 \pm 1.4 & 298 & \text { C } & {[1984 \mathrm{CAR}]} \\ \text { V } & (439-516) & 96.5 \pm 2.8 & 298 & \text { EB } & {[1997 \mathrm{STE} / \mathrm{CHI} 2]} \\ \text { V } & (439-516) & 69.6 \pm 0.6 & 440 & \text { EB } & {[1997 \mathrm{STE} / \mathrm{CHI} 2]} \\ \mathrm{V} & (439-516) & 65.2 \pm 0.5 & 480 & \text { EB } & {[1997 \mathrm{STE} / \mathrm{CHI} 2]}\end{array}$

\begin{tabular}{|c|c|c|c|c|c|}
\hline \multirow[t]{3}{*}{$\mathrm{C}_{10} \mathrm{H}_{14} \mathrm{O}_{2}$} & $\begin{array}{c}\text { [1948-33-0] } \\
\text { FUS }\end{array}$ & \multicolumn{4}{|l|}{ 2-tert-butyl-1,4-dihydroxybenzene } \\
\hline & SUB & $101.2 \pm 1.3$ & 351 & GS & [1999VER7] \\
\hline & SUB & $104.4 \pm 1.3$ & 298 & GS & [1999VER7] \\
\hline \multirow[t]{2}{*}{$\mathrm{C}_{10} \mathrm{H}_{14} \mathrm{O}_{2}$} & [13331-20-9] & \multicolumn{4}{|l|}{ 1,3-dihydroxy-2-butylbenzene } \\
\hline & $\mathrm{V}$ & $(413-469)$ & 428 & $\mathrm{~A}, \mathrm{GC}$ & [1987STE/MAL, 1975KUN/LIL] \\
\hline \multirow[t]{2}{*}{$\mathrm{C}_{10} \mathrm{H}_{14} \mathrm{O}_{2}$} & [2785-87-7] & \multicolumn{4}{|l|}{ 2-methoxy-4-propylphenol } \\
\hline & $\mathrm{V}$ & $(373-413)$ & 388 & A & [1987STE/MAL] \\
\hline \multirow[t]{2}{*}{$\mathrm{C}_{10} \mathrm{H}_{14} \mathrm{O}_{2}$} & & \multicolumn{4}{|c|}{ tert-butylcatechol (isomer not specified) } \\
\hline & $\mathrm{V}$ & $(421-466)$ & 443 & & [1965GAK/BAB] \\
\hline \multirow[t]{2}{*}{$\mathrm{C}_{10} \mathrm{H}_{14} \mathrm{O}_{2}$} & [490-06-2] & \multicolumn{4}{|c|}{ 6-methyl-3-isopropyl-1,2-dihydroxybenzene } \\
\hline & SUB & $96.6 \pm 0.9$ & 298 & $\mathrm{C}$ & [1984CAR] \\
\hline
\end{tabular}


TABLE 13. Phase change enthalpies of $\mathrm{C}_{10}$ organic compounds-Continued

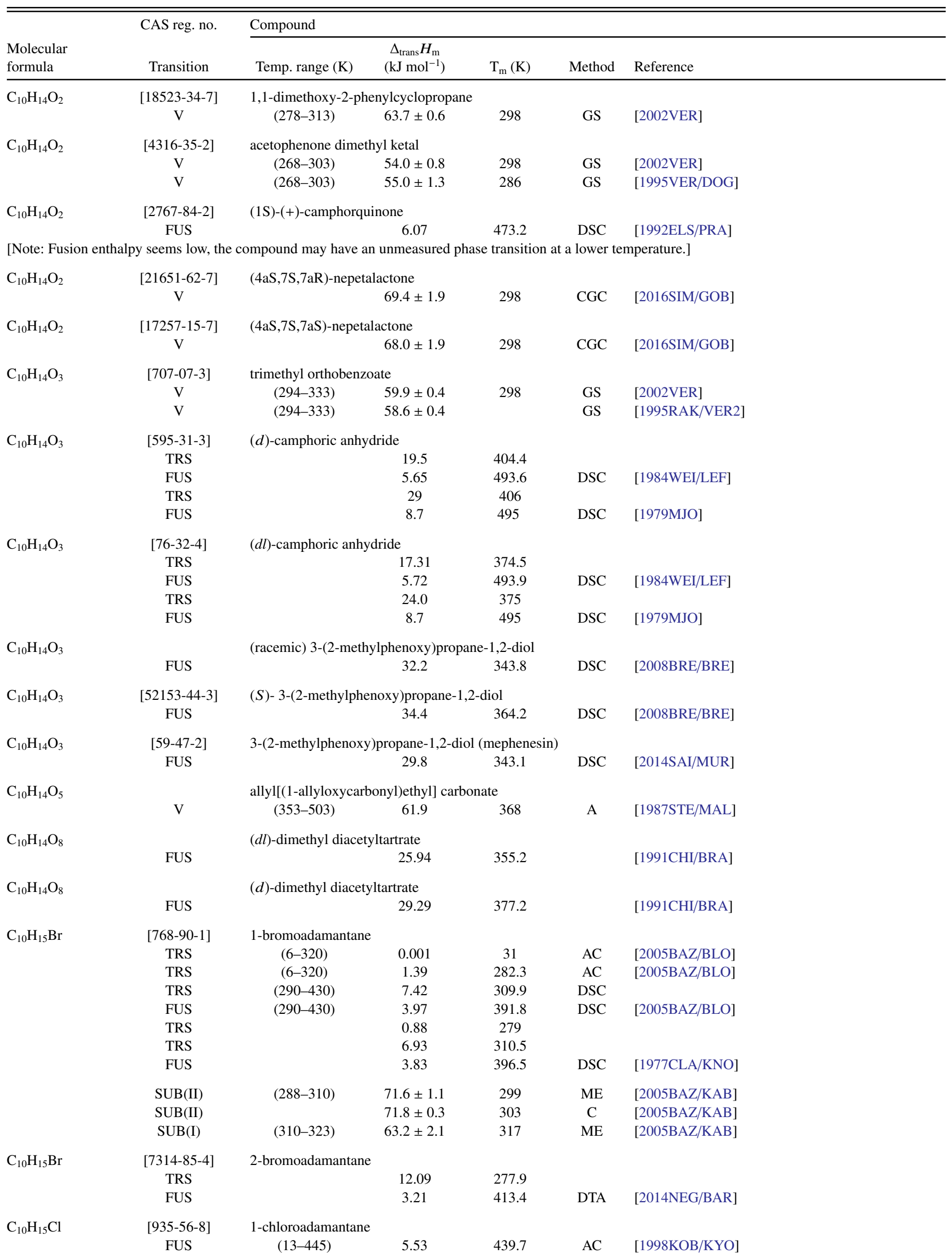


TABLE 13. Phase change enthalpies of $\mathrm{C}_{10}$ organic compounds-Continued

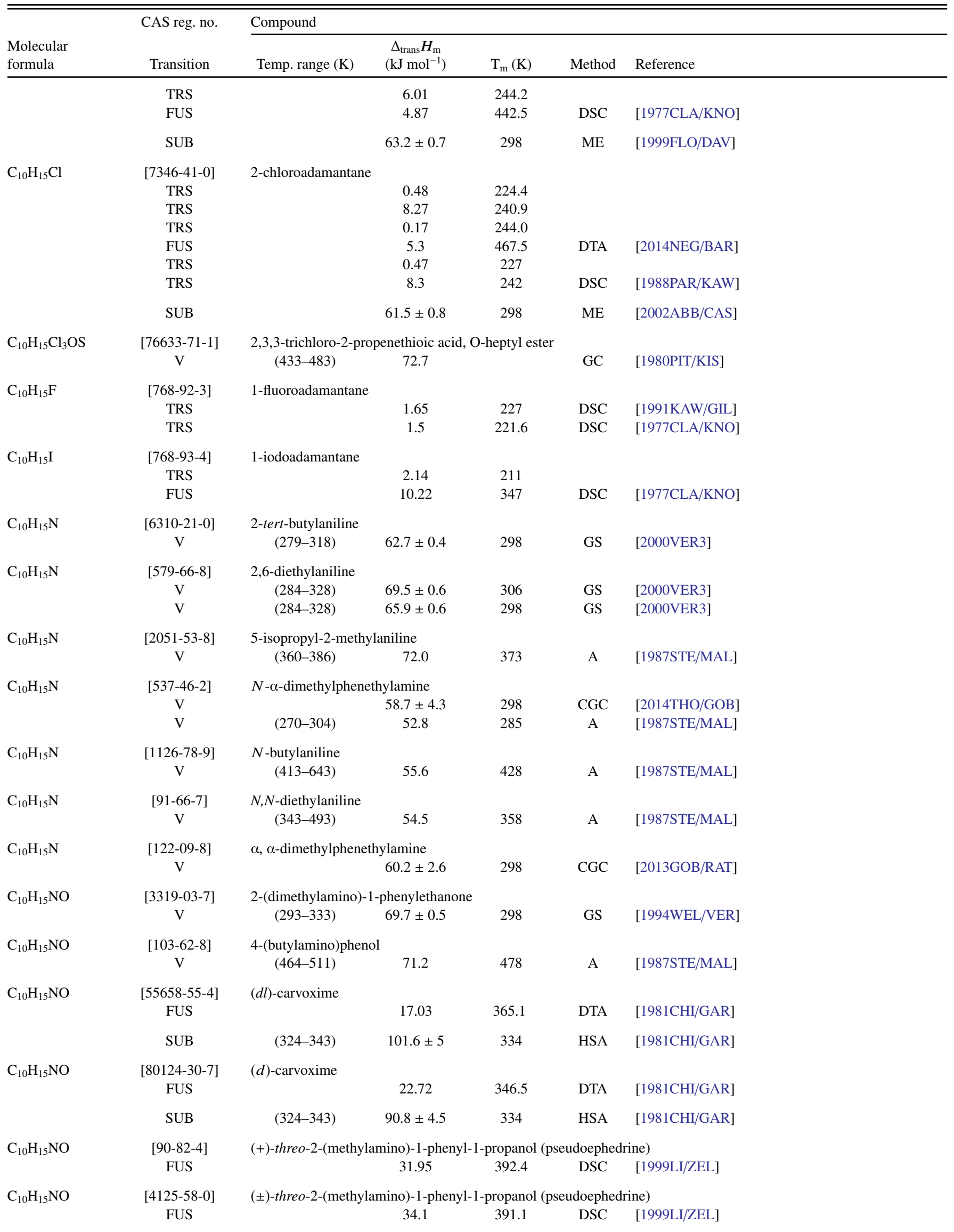


TABLE 13. Phase change enthalpies of $\mathrm{C}_{10}$ organic compounds-Continued

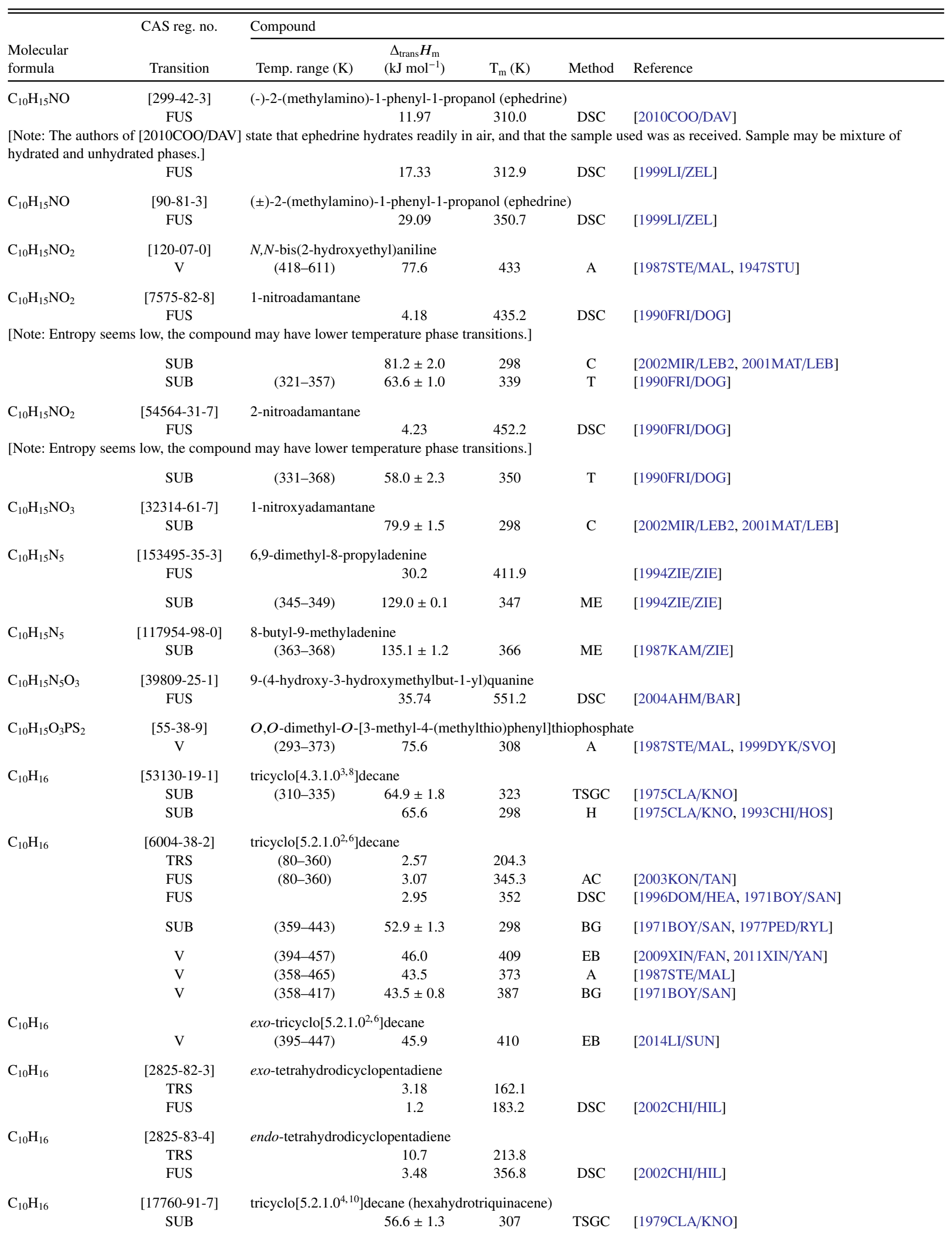


TABLE 13. Phase change enthalpies of $\mathrm{C}_{10}$ organic compounds-Continued

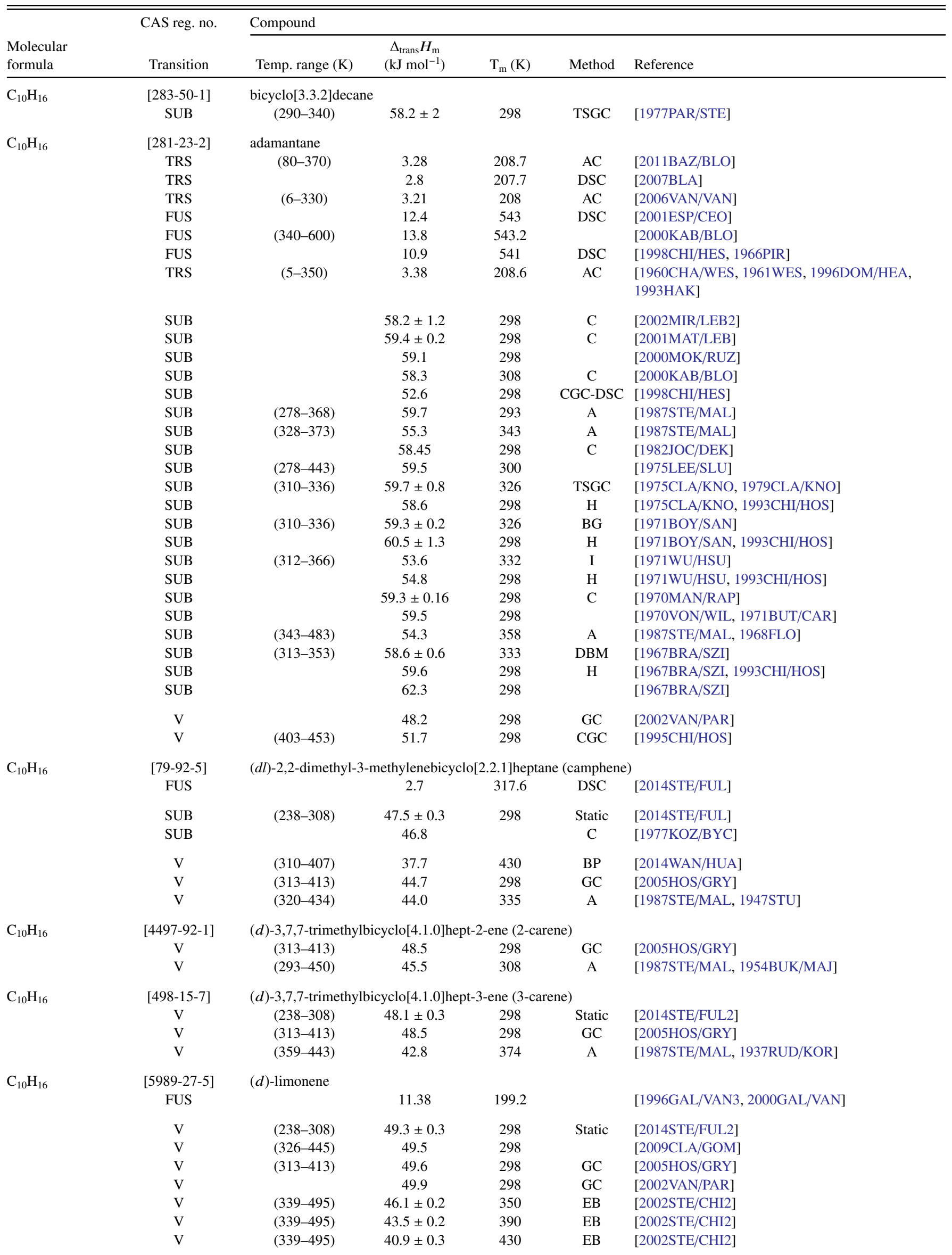


TABLE 13. Phase change enthalpies of $\mathrm{C}_{10}$ organic compounds-Continued

\begin{tabular}{|c|c|c|c|c|c|c|}
\hline \multirow[b]{2}{*}{$\begin{array}{l}\text { Molecular } \\
\text { formula }\end{array}$} & \multirow{2}{*}{$\begin{array}{l}\text { CAS reg. no. } \\
\text { Transition }\end{array}$} & \multicolumn{5}{|l|}{ Compound } \\
\hline & & Temp. range $(\mathrm{K})$ & $\begin{array}{c}\Delta_{\text {trans }} H_{\mathrm{m}} \\
\left(\mathrm{kJ} \mathrm{mol}^{-1}\right)\end{array}$ & $\mathrm{T}_{\mathrm{m}}(\mathrm{K})$ & Method & Reference \\
\hline & $\mathrm{V}$ & $(339-495)$ & $37.9 \pm 0.6$ & 470 & $\mathrm{~EB}$ & [2002STE/CHI2] \\
\hline & V & $(243-461)$ & 49.2 & 300 & & [1999DIA/GUE] \\
\hline & V & $(373-423)$ & 49.6 & 298 & CGC & [1995CHI/HOS] \\
\hline & V & & $48.9 \pm 0.1$ & 298 & $\mathrm{C}$ & [1987ATI/SAI] \\
\hline & V & $(287-448)$ & 44.5 & 302 & A & [1987STE/MAL] \\
\hline & $\mathrm{V}$ & $(288-323)$ & 47.7 & 303 & A & [1987STE/MAL] \\
\hline \multirow[t]{6}{*}{$\mathrm{C}_{10} \mathrm{H}_{16}$} & [5989-54-8] & (l)-limonene & & & & \\
\hline & V & $(238-308)$ & $49.3 \pm 0.3$ & 298 & Static & [2014STE/FUL2] \\
\hline & V & $(320-451)$ & 47.4 & 335 & & [1996ROD/BER] \\
\hline & V & $(325-450)$ & 47.0 & 340 & & [1993NAD/BER] \\
\hline & V & & $49.0 \pm 0.1$ & 298 & $\mathrm{C}$ & [1987ATI/SAI] \\
\hline & V & $(303-363)$ & 45.5 & 318 & A & [1987STE/MAL, 1954BUK/MAJ] \\
\hline \multirow[t]{3}{*}{$\mathrm{C}_{10} \mathrm{H}_{16}$} & [138-86-3] & $(d l)$-limonene & & & & \\
\hline & $\mathrm{V}$ & $(347-420)$ & 45.3 & 370 & $\mathrm{BP}$ & [2014WAN/HUA] \\
\hline & $\mathrm{V}$ & $(287-448)$ & 45.9 & 302 & A & [1987STE/MAL] \\
\hline \multirow{2}{*}{$\mathrm{C}_{10} \mathrm{H}_{16}$} & & limonene & & & & \\
\hline & $\mathrm{V}$ & $(353-405)$ & 39.4 & 379 & TGA & [2002HAZ/DOL] \\
\hline \multirow{2}{*}{$\mathrm{C}_{10} \mathrm{H}_{16}$} & & $\beta$-myrcene & & & & \\
\hline & $\mathrm{V}$ & $(303-363)$ & 47.0 & 318 & & [1954BUK/MAJ] \\
\hline \multirow[t]{3}{*}{$\mathrm{C}_{10} \mathrm{H}_{16}$} & {$[123-35-3]$} & \multicolumn{5}{|c|}{ 7-methyl-3-methylene-1,6-octadiene (myrcene) } \\
\hline & $\mathrm{V}$ & & 50.6 & 298 & GC & [2002VAN/PAR] \\
\hline & $\mathrm{V}$ & $(287-445)$ & 45.7 & 302 & A & [1987STE/MAL, 1947STU] \\
\hline \multirow[t]{2}{*}{$\mathrm{C}_{10} \mathrm{H}_{16}$} & [99-83-2] & \multicolumn{5}{|c|}{ 5-isopropyl-2-methyl-1,3-cyclohexadiene } \\
\hline & $\mathrm{V}$ & $(293-448)$ & 47.7 & 308 & A & [1987STE/MAL] \\
\hline \multirow[t]{2}{*}{$\mathrm{C}_{10} \mathrm{H}_{16}$} & {$[555-10-2]$} & \multicolumn{5}{|c|}{ 3-isopropyl-6-methylenecyclohexene } \\
\hline & $\mathrm{V}$ & $(303-363)$ & 47.7 & 318 & A & [1987STE/MAL, 1954BUK/MAJ] \\
\hline \multirow[t]{2}{*}{$\mathrm{C}_{10} \mathrm{H}_{16}$} & [7785-26-4] & (-)- $\alpha$-pinene & & & & \\
\hline & $\mathrm{V}$ & $(238-308)$ & $44.6 \pm 0.1$ & 298 & Static & [2013STE/FUL] \\
\hline \multirow[t]{7}{*}{$\mathrm{C}_{10} \mathrm{H}_{16}$} & & $\alpha$-pinene & & & & \\
\hline & $\mathrm{V}$ & $(320-429)$ & 42.5 & 335 & & [1996ROD/BER] \\
\hline & $\mathrm{V}$ & $(365-430)$ & 40.2 & 380 & & [1993REI/SAN] \\
\hline & $\mathrm{V}$ & & $44.6 \pm 0.1$ & 298 & $\mathrm{C}$ & {$[1987 \mathrm{AN} / \mathrm{HU}]$} \\
\hline & $\mathrm{V}$ & $(327-363)$ & 43.6 & & & [1954TUC/HAW] \\
\hline & V & $(363-395)$ & 40.8 & & & [1954TUC/HAW] \\
\hline & $\mathrm{V}$ & $(395-413)$ & 39.6 & & & [1954TUC/HAW] \\
\hline \multirow[t]{4}{*}{$\mathrm{C}_{10} \mathrm{H}_{16}$} & {$[80-56-8]$} & $(d)$ - $\alpha$-pinene & & & & \\
\hline & $\mathrm{V}$ & $(308-427)$ & 45.4 & 298 & & [2009CLA/GOM] \\
\hline & $\mathrm{V}$ & $(292-433)$ & 45.0 & 307 & A & [1987STE/MAL] \\
\hline & $\mathrm{V}$ & $(293-363)$ & 43.4 & 308 & & [1954BUK/MAJ] \\
\hline \multirow[t]{6}{*}{$\mathrm{C}_{10} \mathrm{H}_{16}$} & [127-91-3] & $\beta$-pinene & & & & \\
\hline & $\mathrm{V}$ & $(290-439)$ & 46.0 & 305 & & [1996ROD/BER] \\
\hline & $\mathrm{V}$ & $(364-439)$ & 41.6 & 379 & & [1993REI/SAN] \\
\hline & $\mathrm{V}$ & & $45.8 \pm 0.1$ & 298 & $\mathrm{C}$ & [1987AN/HU] \\
\hline & V & $(353-371)$ & 44.0 & & & [1954TUC/HAW] \\
\hline & $\mathrm{V}$ & $(372-404)$ & 41.5 & & & [1954TUC/HAW] \\
\hline \multirow[t]{3}{*}{$\mathrm{C}_{10} \mathrm{H}_{16}$} & & $(l)$ - $\beta$-pinene & & & & \\
\hline & V & $(291-441)$ & 46.1 & 306 & $\mathrm{~A}$ & [1987STE/MAL] \\
\hline & V & $(293-363)$ & 44.9 & 308 & & [1954BUK/MAJ] \\
\hline $\mathrm{C}_{10} \mathrm{H}_{16}$ & [18172-67-3] & $(-)-\beta$-pinene & & & & \\
\hline & $\mathrm{V}$ & (248-308) & $45.9 \pm 0.1$ & 298 & Static & [2013STE/FUL] \\
\hline $\mathrm{C}_{10} \mathrm{H}_{16}$ & [586-62-9] & terpinolene & & & & \\
\hline & $\mathrm{V}$ & $(313-363)$ & 50.8 & 328 & & [1954BUK/MAJ] \\
\hline & $\mathrm{V}$ & $(305-458)$ & 50.5 & 320 & A & [1987STE/MAL, 1947STU] \\
\hline
\end{tabular}


TABLE 13. Phase change enthalpies of $\mathrm{C}_{10}$ organic compounds-Continued

\begin{tabular}{|c|c|c|c|c|c|c|}
\hline \multirow[b]{2}{*}{$\begin{array}{l}\text { Molecular } \\
\text { formula }\end{array}$} & \multirow{2}{*}{$\begin{array}{l}\text { CAS reg. no. } \\
\text { Transition }\end{array}$} & \multicolumn{5}{|l|}{ Compound } \\
\hline & & Temp. range $(\mathrm{K})$ & $\begin{array}{c}\Delta_{\text {trans }} H_{\mathrm{m}} \\
\left(\mathrm{kJ} \mathrm{mol}^{-1}\right)\end{array}$ & $\mathrm{T}_{\mathrm{m}}(\mathrm{K})$ & Method & Reference \\
\hline & V & $(313-413)$ & 51.4 & 298 & $\mathrm{GC}$ & [2005HOS/GRY] \\
\hline $\mathrm{C}_{10} \mathrm{H}_{16}$ & $\begin{array}{c}{[4221-98-1]} \\
\mathrm{V}\end{array}$ & $\begin{array}{l}\text { sabinene } \\
\qquad(313-413)\end{array}$ & 46.9 & 298 & $\mathrm{GC}$ & [2005HOS/GRY] \\
\hline $\mathrm{C}_{10} \mathrm{H}_{16}$ & $\begin{array}{c}{[4221-98-1]} \\
\mathrm{V}\end{array}$ & $\begin{array}{c}(-)-\alpha \text {-phellandrene } \\
(313-423)\end{array}$ & 48.3 & 298 & $\mathrm{GC}$ & [2005HOS/GRY] \\
\hline $\mathrm{C}_{10} \mathrm{H}_{16}$ & $\begin{array}{c}{[2867-05-2]} \\
\mathrm{V}\end{array}$ & $\begin{array}{l}\alpha \text {-thujene } \\
\quad(313-413)\end{array}$ & 44.8 & 298 & GC & [2005HOS/GRY] \\
\hline $\mathrm{C}_{10} \mathrm{H}_{16} \mathrm{Cl}_{3} \mathrm{NOS}$ & $\begin{array}{c}{[2303-17-5]} \\
\text { FUS }\end{array}$ & carbamothioic acid, & $\begin{array}{l}\text { is(isopropyl), } \\
27.11\end{array}$ & 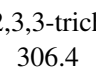 & $\begin{array}{l}\text { allyl) este } \\
\text { DSC }\end{array}$ & [1991ACR, 1990DON/DRE] \\
\hline & $\begin{array}{l}\mathrm{V} \\
\mathrm{V}\end{array}$ & $\begin{array}{l}(293-318) \\
(293-318)\end{array}$ & $\begin{array}{l}84.3 \\
84.0\end{array}$ & 305 & A & $\begin{array}{l}\text { [1987STE/MAL] } \\
\text { [1983SPE/CLI, 1978GRO/SPE] }\end{array}$ \\
\hline $\mathrm{C}_{10} \mathrm{H}_{16} \mathrm{NO}_{4} \mathrm{PS}$ & $\begin{array}{l}{[52-85-7]} \\
\text { FUS }\end{array}$ & $O$-[4-(dimethylami & $\begin{array}{l}\text { )sulfonyl]phe } \\
26.5\end{array}$ & $\begin{array}{l}O, O-\operatorname{dim} \\
326.8\end{array}$ & $\begin{array}{l}\text { lphosphor } \\
\text { DSC }\end{array}$ & $\begin{array}{l}\text { thionate } \\
\text { [1990DON/DRE] }\end{array}$ \\
\hline $\mathrm{C}_{10} \mathrm{H}_{16} \mathrm{~N}_{2}$ & $\begin{array}{l}{[1871-96-1]} \\
\text { FUS }\end{array}$ & sebaconitrile & 28.2 & 281.2 & DSC & [2007BAD/BLA] \\
\hline & V & $(303-343)$ & 83.7 & 318 & A & [1987STE/MAL] \\
\hline $\mathrm{C}_{10} \mathrm{H}_{16} \mathrm{~N}_{2}$ & $\begin{array}{c}{[33089-74-6]} \\
\mathrm{V}\end{array}$ & $N^{\prime}$-(2,4-dimethylph & $\begin{array}{l}\text { nyl)- } N \text {-methy } \\
89.2\end{array}$ & $\begin{array}{l}\text { namidine } \\
303\end{array}$ & & [1998ZHA/MO] \\
\hline $\mathrm{C}_{10} \mathrm{H}_{16} \mathrm{~N}_{2}$ & $\begin{array}{c}{[85688-96-6]} \\
\text { SUB }\end{array}$ & methyl(1,1,1-trimet & $\begin{array}{l}\text { lpropyl)prop } \\
62.0 \pm 0.7\end{array}$ & $\begin{array}{r}\text { initrile } \\
298\end{array}$ & & [1990BEC/DOG] \\
\hline $\mathrm{C}_{10} \mathrm{H}_{16} \mathrm{~N}_{2}$ & $\begin{array}{c}{[85688-95-5]} \\
\text { FUS }\end{array}$ & (1,1-dimethylpropy & $\begin{array}{c}\text { ethylpropaned } \\
19.25\end{array}$ & 307.5 & & [1990BEC/DOG] \\
\hline & SUB & & $76.2 \pm 0.8$ & 298 & & [1990BEC/DOG] \\
\hline $\mathrm{C}_{10} \mathrm{H}_{16} \mathrm{~N}_{2}$ & $\begin{array}{c}\text { [85688-81-9] } \\
\text { FUS }\end{array}$ & meso-2,3-diethyl-2, & $\begin{array}{c}\text { dimethylsucc } \\
26.78\end{array}$ & $\begin{array}{l}\text { itrile } \\
370.2\end{array}$ & & [1983BAR/BEC] \\
\hline $\mathrm{C}_{10} \mathrm{H}_{16} \mathrm{~N}_{2}$ & $\begin{array}{c}{[71621-00-6]} \\
\mathrm{V}\end{array}$ & $\begin{array}{c}N \text {-cyclohexylmethy } \\
\quad(314-353)\end{array}$ & $\begin{array}{l}\text { imidazole } \\
77.5 \pm 0.4\end{array}$ & 298 & GS & [2015VER/ZAI3] \\
\hline $\mathrm{C}_{10} \mathrm{H}_{16} \mathrm{~N}_{2} \mathrm{O}_{2}$ & $\begin{array}{c}\text { [82413-40-9] } \\
\text { FUS }\end{array}$ & 1,3-dimethyl-5-buty & $\begin{array}{l}\text { aracil } \\
22.0\end{array}$ & 312.1 & DSC & [1996KAM/ZIE] \\
\hline & SUB & $(306-311)$ & $106.3 \pm 1.3$ & 309 & $\mathrm{ME}$ & [1996KAM/ZIE] \\
\hline $\mathrm{C}_{10} \mathrm{H}_{16} \mathrm{~N}_{2} \mathrm{O}_{2}$ & $\begin{array}{c}\text { [125-40-6] } \\
\text { FUS }\end{array}$ & 5-ethyl-5-(1-methyl & $\begin{array}{c}\text { ropyl })-2,4,6(1 \\
23.1\end{array}$ & $H, 5 H)$ & $\begin{array}{l}\text { midinetric } \\
\text { DSC }\end{array}$ & $\begin{array}{l}\text { e (butabarbitone) } \\
\text { [1982TRE/VAU] }\end{array}$ \\
\hline $\mathrm{C}_{10} \mathrm{H}_{16} \mathrm{~N}_{2} \mathrm{O}_{2} \mathrm{~S}$ & $\begin{array}{c}\text { [1709-39-3] } \\
\text { FUS }\end{array}$ & 4-amino- $N, N$-diethy & $\begin{array}{c}\text { benzene sulfo } \\
19.4\end{array}$ & $\begin{array}{l}\text { de } \\
378.8\end{array}$ & $\mathrm{DSC}$ & [2014PER/KAZ] \\
\hline $\mathrm{C}_{10} \mathrm{H}_{16} \mathrm{~N}_{2} \mathrm{O}_{3}$ & $\begin{array}{c}\text { [77-28-1] } \\
\text { FUS(I) } \\
\text { FUS(II) } \\
\text { FUS(III) } \\
\text { FUS }\end{array}$ & 5-butyl-5-ethylbarb & $\begin{array}{l}\text { aric acid (buto } \\
\qquad \begin{array}{c}13.6 \\
14.7 \\
17.3 \\
16.8\end{array}\end{array}$ & $\begin{array}{l}\text { ital) } \\
394.2 \\
392.7 \\
396.2\end{array}$ & $\begin{array}{l}\text { DSC } \\
\text { DSC }\end{array}$ & $\begin{array}{l}\text { [1989CHA/DEM] } \\
\text { [1982TRE/VAU] }\end{array}$ \\
\hline $\mathrm{C}_{10} \mathrm{H}_{16} \mathrm{~N}_{4} \mathrm{O}_{2} \mathrm{~S}$ & $\begin{array}{c}\text { [55511-98-3] } \\
\text { FUS }\end{array}$ & 3-(5-(1,1-dimethyle & $\begin{array}{l}\text { yl)-1,3,4-thia } \\
25.46\end{array}$ & $\begin{array}{l}1-2-y 1)-4 \\
408.9\end{array}$ & $\begin{array}{l}\text { lroxy-1-m } \\
\text { DSC }\end{array}$ & $\begin{array}{l}\text { thyl-2-imidazolidinone } \\
\text { [1990DON/DRE] }\end{array}$ \\
\hline $\mathrm{C}_{10} \mathrm{H}_{16} \mathrm{~N}_{6} \mathrm{~S}$ & $\begin{array}{c}\text { [51481-61-9] } \\
\text { FUS } \\
\text { FUS } \\
\text { FUS } \\
\text { FUS } \\
\text { FUS(I) } \\
\text { FUS(II) } \\
\text { FUS(A) } \\
\text { FUS(B) }\end{array}$ & $N$-cyano- $N^{\prime}$-methy & $\begin{array}{c}N^{\prime \prime}-[2-[(5-\mathrm{m} \\
35.49 \\
\mathrm{U} 79.7 \\
41.36 \\
41.97 \\
41.0 \\
39.7 \\
34.8 \\
35.4\end{array}$ & $\begin{array}{c}1 H \text {-imic } \\
414.15 \\
415.1 \\
414.9 \\
416.6 \\
413.8 \\
413.5 \\
413.7 \\
413.6\end{array}$ & $\begin{array}{l}\text { 1-4-yl)-thi } \\
\text { DSC } \\
\text { DSC } \\
\text { DSC } \\
\text { DSC }\end{array}$ & $\begin{array}{l}\text { ethyl]quanidine (cimetidine) } \\
\text { [2015MAT/MOR] } \\
\text { [2011DOM/POB] } \\
\text { [2002SOU/DAV] } \\
\text { [1999BAU/MAR] }\end{array}$ \\
\hline
\end{tabular}


TABLE 13. Phase change enthalpies of $\mathrm{C}_{10}$ organic compounds-Continued

\begin{tabular}{|c|c|c|c|c|c|c|}
\hline \multirow[b]{2}{*}{$\begin{array}{l}\text { Molecular } \\
\text { formula }\end{array}$} & \multirow{2}{*}{$\begin{array}{l}\text { CAS reg. no. } \\
\text { Transition }\end{array}$} & \multicolumn{5}{|l|}{ Compound } \\
\hline & & Temp. range $(\mathrm{K})$ & $\begin{array}{c}\Delta_{\text {trans }} H_{\mathrm{m}} \\
\left(\mathrm{kJ} \mathrm{mol}^{-1}\right)\end{array}$ & $\mathrm{T}_{\mathrm{m}}(\mathrm{K})$ & Method & Reference \\
\hline & FUS(C) & & 36.6 & 417.7 & & \\
\hline & FUS(D) & & 38.2 & 412.2 & DSC & [1996BAU] \\
\hline
\end{tabular}

[Note: Cimetidine is reported to have seven different crystalline forms [1990SUD/SAT], with only two being used in the pharmaceutical industry.]

\begin{tabular}{|c|c|c|c|c|c|c|}
\hline \multirow[t]{4}{*}{$\mathrm{C}_{10} \mathrm{H}_{16} \mathrm{O}$} & [29171-20-8] & \multicolumn{3}{|c|}{ 3,7-dimethyl-6-octen-1-yn-3-ol (dehydrolinalool) } & \multirow[b]{2}{*}{ EB } & \multirow[b]{2}{*}{ [2001ZHU/LI] } \\
\hline & $\mathrm{V}$ & $(406-471)$ & 52.1 & 421 & & \\
\hline & V & $(359-381)$ & Unreliable & & & [1999ZAR/CHA] \\
\hline & $\mathrm{V}$ & $(369-445)$ & $50.4 \pm 0.1$ & 407 & Static & [1988BAG/GUR] \\
\hline \multirow[t]{4}{*}{$\mathrm{C}_{10} \mathrm{H}_{16} \mathrm{O}$} & & camphor & & & & \\
\hline & V & $(343-383)$ & 54.4 & 298 & CGC & [1995CHI/HOS] \\
\hline & V & $(343-383)$ & 54.5 & 298 & CGC & [1995CHI/HOS] \\
\hline & $\mathrm{V}$ & $(343-383)$ & 55.2 & 298 & CGC & {$[1995 \mathrm{CHI} / \mathrm{HOS}]$} \\
\hline \multirow[t]{5}{*}{$\mathrm{C}_{10} \mathrm{H}_{16} \mathrm{O}$} & {$[464-48-2]$} & (-)-camphor & & & & \\
\hline & TRS & & 11.0 & 243.9 & & \\
\hline & TRS & & 0.2 & 373.4 & & \\
\hline & FUS & & 4.9 & 448.5 & DSC & [2014STE/FUL] \\
\hline & SUB & $(273-308)$ & $56.9 \pm 0.3$ & 298 & Static & [2014STE/FUL] \\
\hline \multirow[t]{13}{*}{$\mathrm{C}_{10} \mathrm{H}_{16} \mathrm{O}$} & {$[464-49-3]$} & (d)-camphor & & & & \\
\hline & TRS & & 11.2 & 242.7 & & \\
\hline & TRS & & 0.16 & 370.0 & & \\
\hline & FUS & & 6.3 & 451.8 & DSC & [2010RIE/BAR] \\
\hline & TRS & & 16.0 & 242.0 & & \\
\hline & TRS & & 0.23 & 374.0 & & \\
\hline & FUS & & 6.0 & 450.8 & DSC & [2003ABR/SMI, 2010RIE/BAR] \\
\hline & TRS & & 11.3 & 244.0 & & \\
\hline & FUS & & 5.4 & 450.0 & DSC & [1983SEK/TSU, 2010RIE/BAR] \\
\hline & TRS & & 10.65 & 243.0 & & [1970ROB/ROS, 2010RIE/BAR] \\
\hline & FUS & & 5.3 & 452.0 & DSC & {$[1979 \mathrm{MJO}]$} \\
\hline & TRS & & 11.5 & 245.0 & & [1956SCH/WAG, 2010RIE/BAR] \\
\hline & FUS & & 6.0 & 450.8 & & [1899HUL, 2010RIE/BAR] \\
\hline \multirow[t]{15}{*}{$\mathrm{C}_{10} \mathrm{H}_{16} \mathrm{O}$} & {$[76-22-2]$} & $(d l)$-camphor & & & & \\
\hline & TRS & & 1.3 & 206.1 & & \\
\hline & TRS & & 0.17 & 364 & & \\
\hline & FUS & & 5.9 & 448.0 & DSC & [2010RIE/BAR] \\
\hline & FUS & & 5.6 & 443.0 & DSC & [2003ABR/SMI, 2010RIE/BAR] \\
\hline & TRS & & 0.72 & 203.8 & & \\
\hline & TRS & & 0.23 & 350.0 & & [1958SCH/WAG, 2010RIE/BAR] \\
\hline & TRS & & 0.84 & 210.0 & & \\
\hline & FUS & & 6.86 & 452.0 & & [1952SCH/FRE, 2010RIE/BAR] \\
\hline & SUB & & $51.8 \pm 0.8$ & & & [1977STE] \\
\hline & SUB & $(273-293)$ & $51.5 \pm 2.6$ & 283 & HSA & [1975CHI] \\
\hline & SUB & $(273-298)$ & $\mathrm{U} 65.8$ & & & [1960JON, 1940ZIL] \\
\hline & SUB & & 50.7 & & & [1960JON, 1937DEW] \\
\hline & SUB & $(273-453)$ & 53.6 & 363 & & [1960JON] \\
\hline & SUB & $(285-318)$ & 54.7 & 301 & & [1957SHE/BRY] \\
\hline \multirow[t]{2}{*}{$\mathrm{C}_{10} \mathrm{H}_{16} \mathrm{O}$} & {$[464-49-3]$} & $(+)$-camphor & & & & \\
\hline & $\mathrm{V}$ & & 55.3 & 298 & GC & [2002VAN/PAR] \\
\hline \multirow[t]{2}{*}{$\mathrm{C}_{10} \mathrm{H}_{16} \mathrm{O}$} & {$[547-61-5]$} & (-)-trans-pinocarveol & & & & \\
\hline & V & $(283-323)$ & $62.5 \pm 1.0$ & 298 & GS & [2010WID/BRU] \\
\hline \multirow[t]{2}{*}{$\mathrm{C}_{10} \mathrm{H}_{16} \mathrm{O}$} & [1686-14-2] & $\alpha$-pinene oxide & & & & \\
\hline & V & & 53.6 & 298 & $\mathrm{GC}$ & [2002VAN/PAR] \\
\hline \multirow[t]{5}{*}{$\mathrm{C}_{10} \mathrm{H}_{16} \mathrm{O}$} & {$[13854-85-8]$} & (d)-3-bornanone & & & & \\
\hline & SUB & $(273-408)$ & 54.2 & 288 & A & [1987STE/MAL] \\
\hline & SUB & $(323-339)$ & 55.0 & 331 & A & [1987STE/MAL] \\
\hline & SUB & $(408-451)$ & 49.8 & 423 & A & [1987STE/MAL] \\
\hline & $\mathrm{V}$ & $(452-488)$ & 44.6 & 467 & $\mathrm{~A}$ & [1987STE/MAL] \\
\hline
\end{tabular}


TABLE 13. Phase change enthalpies of $\mathrm{C}_{10}$ organic compounds-Continued

\begin{tabular}{|c|c|c|c|c|c|c|}
\hline \multirow[b]{2}{*}{$\begin{array}{l}\text { Molecular } \\
\text { formula }\end{array}$} & \multirow{2}{*}{$\begin{array}{c}\text { CAS reg. no. } \\
\text { Transition }\end{array}$} & \multicolumn{5}{|l|}{ Compound } \\
\hline & & Temp. range $(\mathrm{K})$ & $\begin{array}{c}\Delta_{\text {trans }} H_{\mathrm{m}} \\
\left(\mathrm{kJ} \mathrm{mol}^{-1}\right)\end{array}$ & $\mathrm{T}_{\mathrm{m}}(\mathrm{K})$ & Method & Reference \\
\hline \multirow[t]{4}{*}{$\mathrm{C}_{10} \mathrm{H}_{16} \mathrm{O}$} & {$[4695-62-9]$} & \multicolumn{5}{|c|}{ (d)-1,3,3-trimethylbicyclo[2.2.1]heptan-2one (fenchone) } \\
\hline & FUS & & 17.4 & 278.6 & DSC & [2014STE/FUL] \\
\hline & SUB & $(238-278)$ & $67.3 \pm 0.4$ & 298 & Static & [2014STE/FUL] \\
\hline & $\mathrm{V}$ & $(243-308)$ & $51.8 \pm 0.3$ & 298 & Static & [2014STE/FUL] \\
\hline \multicolumn{7}{|c|}{ [Note: VP measurements in [2014STE/FUL] include values for the subcooled liquid.] } \\
\hline & V & $(365-384)$ & 47.0 & 374 & & {$[2002 \mathrm{BAT}]$} \\
\hline & $\mathrm{V}$ & & $51.7 \pm 0.1$ & 298 & $\mathrm{C}$ & [1987ATI/SAI] \\
\hline & $\mathrm{V}$ & & $51.4 \pm 0.1$ & 298 & $\mathrm{C}$ & {$[1987 \mathrm{ATI} / \mathrm{SAI}]$} \\
\hline & $\mathrm{V}$ & & $51.1 \pm 0.1$ & 298 & $\mathrm{C}$ & {$[1985 \mathrm{KUS}]$} \\
\hline & $\mathrm{V}$ & $(301-464)$ & 48.9 & 316 & A & [1987STE/MAL, 1947STU] \\
\hline \multirow[t]{4}{*}{$\mathrm{C}_{10} \mathrm{H}_{16} \mathrm{O}$} & {$[7787-20-4]$} & \multicolumn{5}{|c|}{ (l)-1,3,3-trimethylbicyclo[2.2.1]heptan-2one (fenchone) } \\
\hline & $\mathrm{V}$ & & $51.1 \pm 0.1$ & 298 & $\mathrm{C}$ & {$[1987 \mathrm{ATI} / \mathrm{SAI}]$} \\
\hline & $\mathrm{V}$ & & $51.3 \pm 0.1$ & 298 & $\mathrm{C}$ & [1987ATI/SAI] \\
\hline & $\mathrm{V}$ & & $51.4 \pm 0.1$ & 298 & $\mathrm{C}$ & {$[1985 \mathrm{KUS}]$} \\
\hline \multirow[t]{4}{*}{$\mathrm{C}_{10} \mathrm{H}_{16} \mathrm{O}$} & {$[89-82-7]$} & pulegone & & & & \\
\hline & $\mathrm{V}$ & $(353-453)$ & 58.0 & 298 & GC & {$[2005 \mathrm{HOS} / \mathrm{GRY}]$} \\
\hline & V & & 62.0 & 298 & GC & [2002VAN/PAR] \\
\hline & $\mathrm{V}$ & $(331-494)$ & U99.8 & 346 & A & [1987STE/MAL, 1947STU] \\
\hline \multirow[t]{2}{*}{$\mathrm{C}_{10} \mathrm{H}_{16} \mathrm{O}$} & {$[471-15-8]$} & \multicolumn{5}{|c|}{ (d)-1-isopropyl-4-methylbicyclo[3.1.0]hexan-3-one (thujone) } \\
\hline & $\mathrm{V}$ & $(311-474)$ & 51.8 & 326 & A & [1987STE/MAL, 1947STU] \\
\hline \multirow[t]{2}{*}{$\mathrm{C}_{10} \mathrm{H}_{16} \mathrm{O}$} & {$[499-74-1]$} & \multicolumn{5}{|c|}{$(d l)$-3-isopropyl-6-methyl-2-cyclohexene-1-one } \\
\hline & V & $(364-507)$ & 56.9 & 379 & A & [1987STE/MAL] \\
\hline \multirow[t]{2}{*}{$\mathrm{C}_{10} \mathrm{H}_{16} \mathrm{O}$} & {$[43205-82-9]$} & \multicolumn{5}{|c|}{ 5-isopropyl-2-methyl-2-cyclohexen-1-one } \\
\hline & V & $(361-503)$ & 56.8 & 376 & A & {$[1987 \mathrm{STE} / \mathrm{MAL}]$} \\
\hline \multirow[t]{2}{*}{$\mathrm{C}_{10} \mathrm{H}_{16} \mathrm{O}$} & {$[21218-11-1]$} & \multicolumn{5}{|c|}{ 3,4-epoxycarane ( $\alpha$-isomer) } \\
\hline & $\mathrm{V}$ & & 49.4 & & & [1977ALE/KOZ] \\
\hline \multirow[t]{2}{*}{$\mathrm{C}_{10} \mathrm{H}_{16} \mathrm{O}$} & {$[35671-18-2]$} & \multicolumn{5}{|c|}{ 3,4-epoxycarane ( $\beta$-isomer) } \\
\hline & $\mathrm{V}$ & & 50.2 & & & {$[1977 \mathrm{ALE} / \mathrm{KOZ}]$} \\
\hline $\mathrm{C}_{10} \mathrm{H}_{16} \mathrm{O}$ & {$[4584-09-2]$} & $(d l)$-dihydrocarvon & & & & \\
\hline & $\mathrm{V}$ & $(319-496)$ & 51.2 & 334 & A & [1987STE/MAL, 1947STU] \\
\hline $\mathrm{C}_{10} \mathrm{H}_{16} \mathrm{O}$ & {$[5392-40-5]$} & citral & & & & \\
\hline & V & $(372-473)$ & 55.9 & 298 & & [2009CLA/GOM] \\
\hline $\mathrm{C}_{10} \mathrm{H}_{16} \mathrm{O}$ & {$[141-27-5]$} & geranial (trans-citra & & & & \\
\hline & $\mathrm{V}$ & $(343-453)$ & 62.5 & 298 & GC & [2005HOS/GRY] \\
\hline & V & $(283-333)$ & 61 & 298 & A & [1987STE/MAL] \\
\hline & $\mathrm{V}$ & $(373-501)$ & 54.9 & 388 & A & [1987STE/MAL] \\
\hline $\mathrm{C}_{10} \mathrm{H}_{16} \mathrm{O}$ & [13040-03-4] & $(+)$-cis-verbenol & & & & \\
\hline & $\mathrm{V}$ & $(323-433)$ & 54.9 & 298 & GC & [2005HOS/GRY] \\
\hline $\mathrm{C}_{10} \mathrm{H}_{16} \mathrm{O}$ & [18881-04-4] & (-)-cis-verbenol & & & & \\
\hline & SUB & $(278-313)$ & $81.2 \pm 0.1$ & 298 & Static & [2013STE/FUL] \\
\hline & SUB & & $80.0 \pm 1.2$ & 298 & $\mathrm{C}$ & [2013STE/FUL] \\
\hline $\mathrm{C}_{10} \mathrm{H}_{16} \mathrm{O}$ & [22339-08-8] & $(+)$-trans-verbenol & & & & \\
\hline & $\mathrm{V}$ & $(323-433)$ & 55.0 & 298 & GC & [2005HOS/GRY] \\
\hline $\mathrm{C}_{10} \mathrm{H}_{16} \mathrm{O}$ & [106-26-3] & neral & & & & \\
\hline & $\mathrm{V}$ & $(343-453)$ & 60.2 & 298 & GC & [2005HOS/GRY] \\
\hline $\mathrm{C}_{10} \mathrm{H}_{16} \mathrm{O}$ & [768-95-6] & 1-adamantanol & & & & \\
\hline & TRS & $(5-600)$ & 11.29 & 357.1 & & \\
\hline & FUS & $(5-600)$ & 12.36 & 552.9 & & [2003CHA/BLO] \\
\hline & TRS & & 13.81 & 365 & DSC & [1991CHI/BRA] \\
\hline & TRS & & 2.5 & 369.2 & DTA & [1989SAL/ABA2, 1987SAL/SAI] \\
\hline & SUB & & $86.8 \pm 0.2$ & 298 & $\mathrm{C}$ & [2003CHA/BLO2] \\
\hline
\end{tabular}


TABLE 13. Phase change enthalpies of $\mathrm{C}_{10}$ organic compounds-Continued

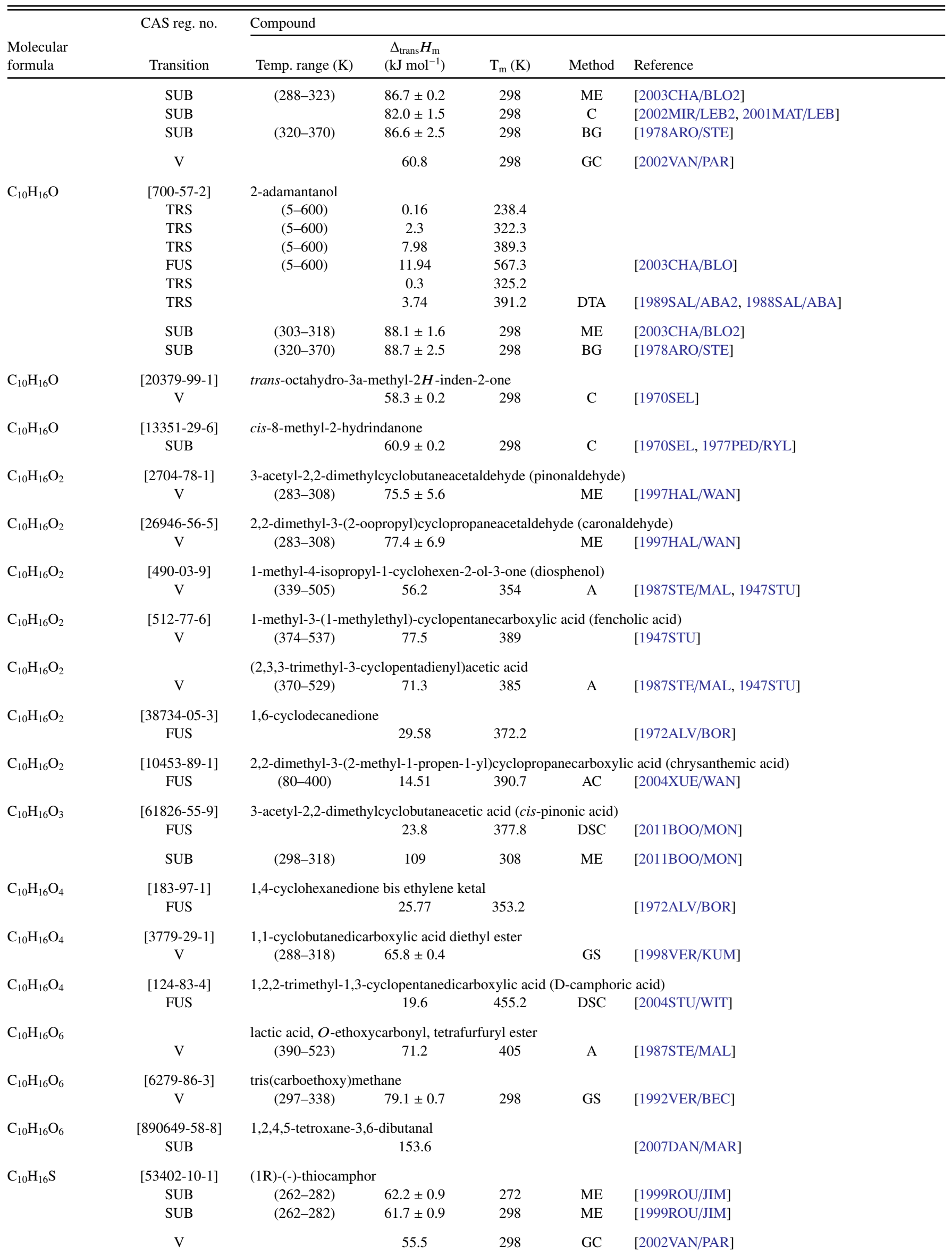


TABLE 13. Phase change enthalpies of $\mathrm{C}_{10}$ organic compounds-Continued

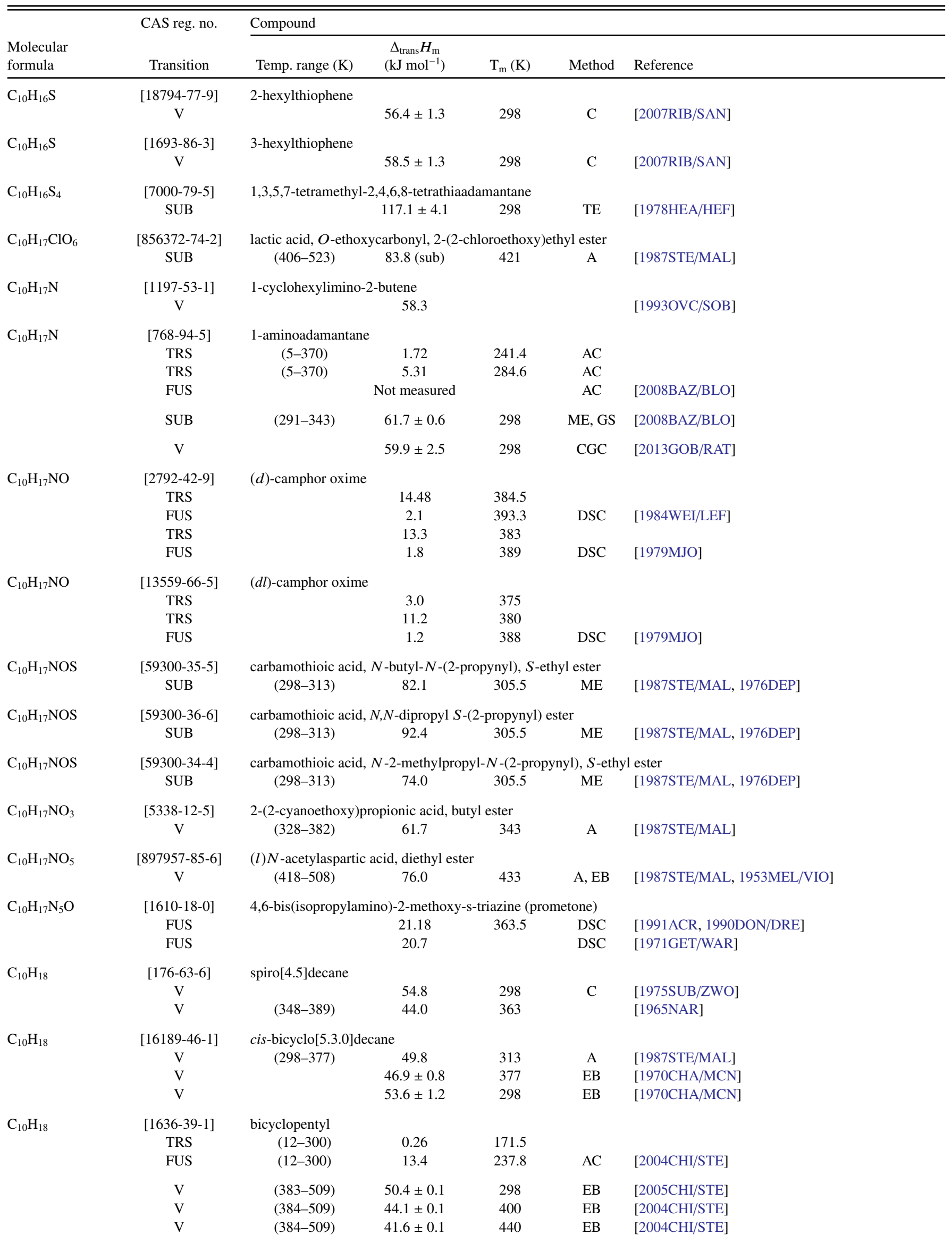


TABLE 13. Phase change enthalpies of $\mathrm{C}_{10}$ organic compounds-Continued

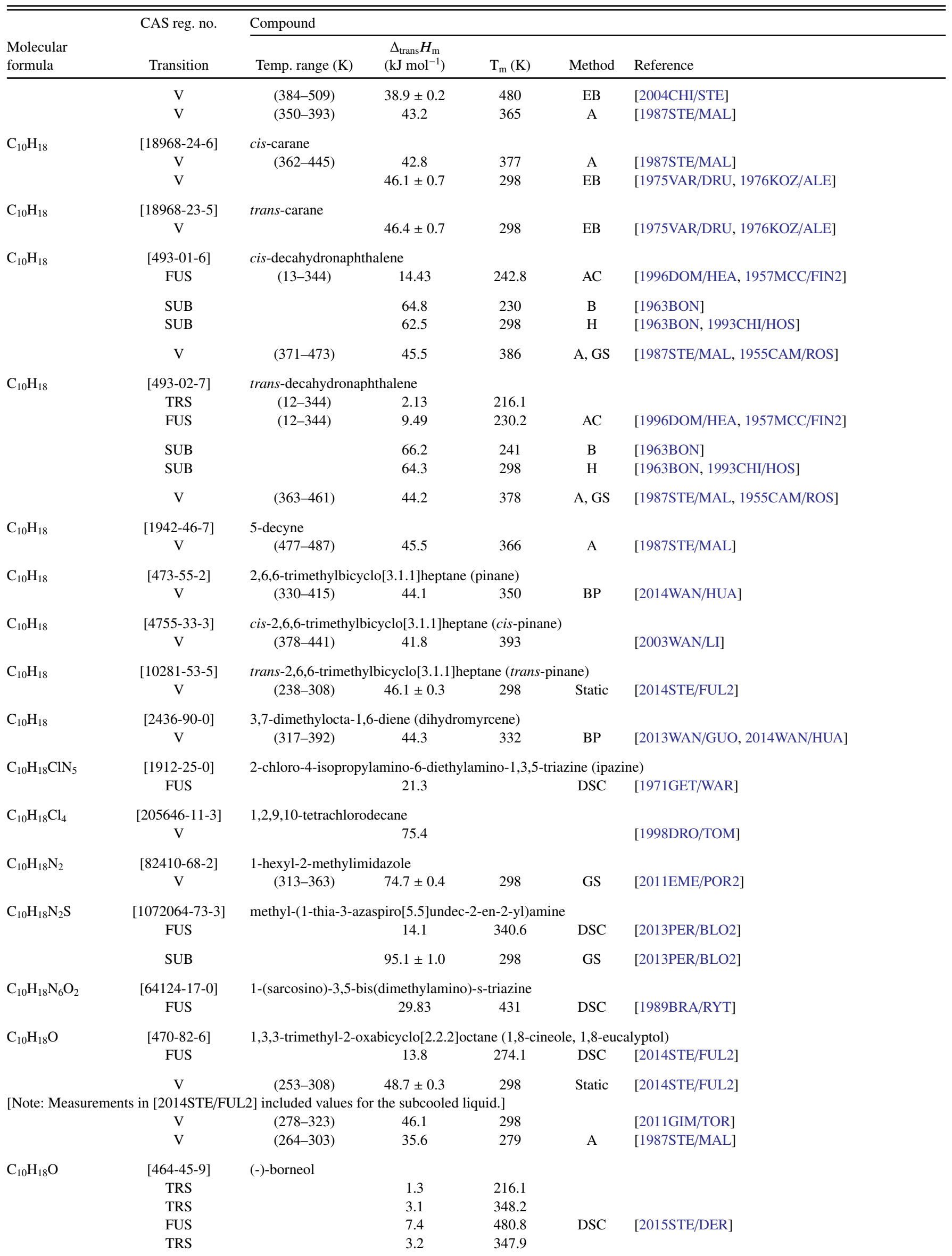


TABLE 13. Phase change enthalpies of $\mathrm{C}_{10}$ organic compounds-Continued

\begin{tabular}{|c|c|c|c|c|c|c|}
\hline \multirow[b]{2}{*}{$\begin{array}{l}\text { Molecular } \\
\text { formula }\end{array}$} & \multirow{2}{*}{$\begin{array}{l}\text { CAS reg. no. } \\
\text { Transition }\end{array}$} & \multicolumn{5}{|l|}{ Compound } \\
\hline & & Temp. range $(\mathrm{K})$ & $\begin{array}{c}\Delta_{\text {trans }} H_{\mathrm{m}} \\
\left(\mathrm{kJ} \mathrm{mol}^{-1}\right)\end{array}$ & $\mathrm{T}_{\mathrm{m}}(\mathrm{K})$ & Method & Reference \\
\hline & FUS & & 7.3 & 480.2 & $\mathrm{DSC}$ & [2014STE/FUL] \\
\hline & SUB(I) & $(348-363)$ & $76.1 \pm 0.6$ & 298 & Static & \\
\hline & SUB(II) & $(293-347)$ & $75.6 \pm 0.2$ & 298 & Static & \\
\hline & SUB & $(273-308)$ & $74.7 \pm 0.4$ & 298 & Static & [2014STE/FUL] \\
\hline \multirow[t]{3}{*}{$\mathrm{C}_{10} \mathrm{H}_{18} \mathrm{O}$} & {$[6627-72-1]$} & $(d l)$-borneol & & & & \\
\hline & SUB & $(350-475)$ & 69.3 & 365 & A & [1987STE/MAL] \\
\hline & $\mathrm{V}$ & $(477-487)$ & 50.9 & 482 & A & [1987STE/MAL] \\
\hline \multirow[t]{2}{*}{$\mathrm{C}_{10} \mathrm{H}_{18} \mathrm{O}$} & {$[470-67-7]$} & 1,4-cineole & & & & \\
\hline & V & $(288-449)$ & 46.1 & 303 & A & [1987STE/MAL] \\
\hline \multirow[t]{5}{*}{$\mathrm{C}_{10} \mathrm{H}_{18} \mathrm{O}$} & {$[470-82-6]$} & 1,8-cineole & & & & \\
\hline & $\mathrm{V}$ & $(327-420)$ & 46.3 & 342 & & [2011TOR/GAR] \\
\hline & $\mathrm{V}$ & $(313-413)$ & 49.0 & 298 & GC & [2005HOS/GRY] \\
\hline & $\mathrm{V}$ & & 53.2 & 298 & GC & [2002VAN/PAR] \\
\hline & $\mathrm{V}$ & $(353-403)$ & 41.1 & 378 & TGA & [2002HAZ/DOL] \\
\hline \multirow[t]{2}{*}{$\mathrm{C}_{10} \mathrm{H}_{18} \mathrm{O}$} & [619-01-2] & (d)-dihydrocarveol & & & & \\
\hline & V & $(336-498)$ & 58.2 & 351 & A & [1987STE/MAL] \\
\hline \multirow[t]{2}{*}{$\mathrm{C}_{10} \mathrm{H}_{18} \mathrm{O}$} & [2217-01-8] & $(d l)$-fenchyl alcohol & & & & \\
\hline & $\mathrm{V}$ & $(318-474)$ & 89.1 & 333 & A & [1987STE/MAL] \\
\hline \multirow[t]{4}{*}{$\mathrm{C}_{10} \mathrm{H}_{18} \mathrm{O}$} & [106-24-1] & geraniol & & & & \\
\hline & $\mathrm{V}$ & $(273-368)$ & $76.9 \pm 0.2$ & 298 & Static & [2015STE/DER] \\
\hline & $\mathrm{V}$ & $(288-333)$ & 62.9 & 303 & A & [1987STE/MAL] \\
\hline & $\mathrm{V}$ & $(342-503)$ & 59.1 & 357 & A & [1987STE/MAL] \\
\hline \multirow[t]{2}{*}{$\mathrm{C}_{10} \mathrm{H}_{18} \mathrm{O}$} & [7786-67-6] & (d)-isopulegol & & & & \\
\hline & V & $(335-485)$ & 49.8 & 350 & A & [1987STE/MAL] \\
\hline \multirow[t]{3}{*}{$\mathrm{C}_{10} \mathrm{H}_{18} \mathrm{O}$} & [126-90-9] & (d)-linalool & & & & \\
\hline & $\mathrm{V}$ & $(273-323)$ & 65.4 & 298 & & [1999DIA/GUE] \\
\hline & $\mathrm{V}$ & $(313-471)$ & 52.4 & 328 & A & [1987STE/MAL] \\
\hline \multirow[t]{7}{*}{$\mathrm{C}_{10} \mathrm{H}_{18} \mathrm{O}$} & {$[78-70-6]$} & linalool & & & & \\
\hline & $\mathrm{V}$ & $(285-353)$ & $65.6 \pm 0.1$ & 298 & Static & [2015ZAI/VER] \\
\hline & $\mathrm{V}$ & $(285-328)$ & $66.2 \pm 0.6$ & 298 & GS & [2015ZAI/VER] \\
\hline & $\mathrm{V}$ & $(352-468)$ & 65.0 & 298 & & [2009CLA/GOM] \\
\hline & $\mathrm{V}$ & $(333-433)$ & 55.3 & 298 & $\mathrm{GC}$ & [2005HOS/GRY] \\
\hline & $\mathrm{V}$ & $(368-428)$ & 51.4 & 399 & TGA & [2002HAZ/DOL] \\
\hline & $\mathrm{V}$ & $(409-465)$ & 50.3 & 424 & EB & [2002DEN/LI] \\
\hline \multirow[t]{2}{*}{$\mathrm{C}_{10} \mathrm{H}_{18} \mathrm{O}$} & [106-25-2] & cis-3,7-dimethyl-2,6 & octadien-1-ol & & & \\
\hline & $\mathrm{V}$ & $(334-499)$ & 55.4 & 349 & A & [1987STE/MAL] \\
\hline \multirow[t]{3}{*}{$\mathrm{C}_{10} \mathrm{H}_{18} \mathrm{O}$} & {$[98-55-5]$} & $(d l)$ - $\alpha$-terpineol & & & & \\
\hline & SUB & $(287-308)$ & 80.1 & 297.5 & A & [1987STE/MAL] \\
\hline & $\mathrm{V}$ & $(325-491)$ & 54.0 & 340 & A & [1987STE/MAL] \\
\hline \multirow[t]{2}{*}{$\mathrm{C}_{10} \mathrm{H}_{18} \mathrm{O}$} & {$[7785-53-7]$} & $(+)$ - $\alpha$-terpineol & & & & \\
\hline & $\mathrm{V}$ & & 60.7 & 298 & GC & [2002VAN/PAR] \\
\hline \multirow[t]{3}{*}{$\mathrm{C}_{10} \mathrm{H}_{18} \mathrm{O}$} & {$[10482-56-1]$} & $\alpha$-terpineol & & & & \\
\hline & FUS & $(83-344)$ & 15.78 & 308.5 & $\mathrm{AC}$ & [2016MAR/SMI] \\
\hline & SUB & $(283-328)$ & 80.3 & 305 & $\mathrm{ME}$ & [1954SER/VOI, 1960JON] \\
\hline \multirow[t]{2}{*}{$\mathrm{C}_{10} \mathrm{H}_{18} \mathrm{O}$} & {$[562-74-3]$} & terpinen-4-ol & & & & \\
\hline & $\mathrm{V}$ & $(323-433)$ & 55.5 & 298 & GC & [2005HOS/GRY] \\
\hline \multirow[t]{2}{*}{$\mathrm{C}_{10} \mathrm{H}_{18} \mathrm{O}$} & {$[124-76-5]$} & $(d l)$-isoborneol & & & & \\
\hline & SUB & $(373-457)$ & 41.1 & 388 & A & [1987STE/MAL, 1936GRE] \\
\hline \multirow[t]{2}{*}{$\mathrm{C}_{10} \mathrm{H}_{18} \mathrm{O}$} & {$[1196-00-5]$} & \multicolumn{5}{|c|}{$(1 \mathrm{R}, 2 \mathrm{R}, 3 \mathrm{R}, 5 \mathrm{~S})-(-)$-isopinocamheol } \\
\hline & SUB & $(283-313)$ & $80.5 \pm 1.1$ & 298 & GS & [2010WID/BRU] \\
\hline
\end{tabular}


TABLE 13. Phase change enthalpies of $\mathrm{C}_{10}$ organic compounds-Continued

\begin{tabular}{|c|c|c|c|c|c|c|}
\hline \multirow[b]{2}{*}{$\begin{array}{l}\text { Molecular } \\
\text { formula }\end{array}$} & \multirow{2}{*}{$\begin{array}{l}\text { CAS reg. no. } \\
\text { Transition }\end{array}$} & \multicolumn{5}{|l|}{ Compound } \\
\hline & & Temp. range $(\mathrm{K})$ & $\begin{array}{c}\Delta_{\text {trans }} H_{\mathrm{m}} \\
\left(\mathrm{kJ} \mathrm{mol}^{-1}\right)\end{array}$ & $\mathrm{T}_{\mathrm{m}}(\mathrm{K})$ & Method & Reference \\
\hline $\mathrm{C}_{10} \mathrm{H}_{18} \mathrm{O}$ & $\begin{array}{c}{[132203-71-5]} \\
\mathrm{V}\end{array}$ & $\begin{array}{c}(+) \text {-trans-myrtanol } \\
(283-313)\end{array}$ & $73.0 \pm 2.4$ & 298 & GS & [2010WID/BRU] \\
\hline $\mathrm{C}_{10} \mathrm{H}_{18} \mathrm{O}$ & $\begin{array}{c}{[854885-23-7]} \\
\mathrm{V}\end{array}$ & $\begin{array}{l}\text { 1-(1-methylcyclohex } \\
\quad(397-422)\end{array}$ & $\begin{array}{l}\text { 3-enyl)-1-pr } \\
53.6\end{array}$ & 409 & A & [1987STE/MAL, 1955PIN/MAR] \\
\hline $\mathrm{C}_{10} \mathrm{H}_{18} \mathrm{O}$ & $\begin{array}{c}\text { [1502-06-3] } \\
\text { FUS } \\
\text { V } \\
\text { V }\end{array}$ & $\begin{array}{l}(353-423) \\
(353-423)\end{array}$ & $\begin{array}{c}24.3 \\
55.2 \\
58.4 \pm 0.6\end{array}$ & $\begin{array}{l}294.9 \\
368 \\
298\end{array}$ & $\begin{array}{l}\text { DSC } \\
\text { A } \\
\text { VP }\end{array}$ & $\begin{array}{l}\text { [1998GON/SZW] } \\
\text { [1987STE/MAL, 1972WOL] } \\
\text { [1972WOL] }\end{array}$ \\
\hline $\mathrm{C}_{10} \mathrm{H}_{18} \mathrm{O}$ & $\begin{array}{c}{[2890-65-5]} \\
\mathrm{V}\end{array}$ & $\begin{array}{l}\text { ethyl (1-methylcyclc } \\
\quad(388-431)\end{array}$ & $\begin{array}{l}\text { hexyl) ketone } \\
45.2\end{array}$ & 403 & A & [1987STE/MAL, 1955PIN/MAR] \\
\hline $\mathrm{C}_{10} \mathrm{H}_{18} \mathrm{O}$ & $\begin{array}{c}{[10458-14-7]} \\
\text { V } \\
\text { V }\end{array}$ & $\begin{array}{c}\text { 2-isopropyl-5-methy } \\
(372-397) \\
(350-483)\end{array}$ & $\begin{array}{c}\text { cyclohexanor } \\
50.1 \\
51.2\end{array}$ & $\begin{array}{l}\text { enthone) } \\
385 \\
365\end{array}$ & A & $\begin{array}{l}{[2002 \mathrm{BAT}]} \\
{[1987 \mathrm{STE} / \mathrm{MAL}]}\end{array}$ \\
\hline $\mathrm{C}_{10} \mathrm{H}_{18} \mathrm{O}$ & $\begin{array}{c}{[2385-77-5]} \\
\mathrm{V} \\
\mathrm{V}\end{array}$ & $\begin{array}{r}(d) \text {-citronellal } \\
(288-333) \\
(317-480)\end{array}$ & $\begin{array}{l}54.9 \\
53.2\end{array}$ & $\begin{array}{l}303 \\
332\end{array}$ & $\begin{array}{l}\text { A } \\
\text { A }\end{array}$ & $\begin{array}{l}\text { [1987STE/MAL] } \\
\text { [1987STE/MAL, 1947STU] }\end{array}$ \\
\hline $\mathrm{C}_{10} \mathrm{H}_{18} \mathrm{O}$ & $\begin{array}{c}{[69891-94-7]} \\
\mathrm{V}\end{array}$ & $\begin{array}{r}(Z)-3 \text {-decenal } \\
(323-343)\end{array}$ & 59.2 & 298 & CGC & [1996KOU/HOS, 2000OVA/KOU] \\
\hline $\mathrm{C}_{10} \mathrm{H}_{18} \mathrm{O}$ & $\begin{array}{c}{[68676-85-7]} \\
\mathrm{V}\end{array}$ & $\begin{array}{r}(E)-3 \text {-decenal } \\
(323-343)\end{array}$ & 59.8 & 298 & CGC & [1996KOU/HOS, 2000OVA/KOU] \\
\hline $\mathrm{C}_{10} \mathrm{H}_{18} \mathrm{O}$ & $\begin{array}{c}{[21662-09-9]} \\
\mathrm{V}\end{array}$ & $\begin{array}{r}(Z)-4 \text {-decenal } \\
(323-343)\end{array}$ & 59.3 & 298 & CGC & [1996KOU/HOS, 2000OVA/KOU] \\
\hline $\mathrm{C}_{10} \mathrm{H}_{18} \mathrm{O}$ & $\begin{array}{c}{[65405-70-1]} \\
\mathrm{V}\end{array}$ & $\begin{array}{r}(E)-4 \text {-decenal } \\
(323-343)\end{array}$ & 60 & 298 & CGC & [1996KOU/HOS, 2000OVA/KOU] \\
\hline $\mathrm{C}_{10} \mathrm{H}_{18} \mathrm{O}$ & $\begin{array}{c}{[21662-08-8]} \\
\mathrm{V}\end{array}$ & $\begin{array}{r}(Z)-5 \text {-decenal } \\
(323-343)\end{array}$ & 58.5 & 298 & CGC & [1996KOU/HOS, 2000OVA/KOU] \\
\hline $\mathrm{C}_{10} \mathrm{H}_{18} \mathrm{O}$ & $\begin{array}{c}{[21662-11-3]} \\
V\end{array}$ & $\begin{array}{r}(E)-5 \text {-decenal } \\
(323-343)\end{array}$ & 59.2 & 298 & CGC & [1996KOU/HOS, 2000OVA/KOU] \\
\hline $\mathrm{C}_{10} \mathrm{H}_{18} \mathrm{O}$ & $\begin{array}{c}{[147159-48-6]} \\
\text { V }\end{array}$ & $\begin{array}{r}(Z)-6-\text { decenal } \\
(323-343)\end{array}$ & 59.3 & 298 & CGC & [1996KOU/HOS, 2000OVA/KOU] \\
\hline $\mathrm{C}_{10} \mathrm{H}_{18} \mathrm{O}$ & $\begin{array}{c}\text { [147159-48-6] } \\
\mathrm{V}\end{array}$ & $\begin{array}{r}(E)-6 \text {-decenal } \\
(323-343)\end{array}$ & 59.5 & 298 & CGC & [1996KOU/HOS, 2000OVA/KOU] \\
\hline $\mathrm{C}_{10} \mathrm{H}_{18} \mathrm{O}$ & $\begin{array}{c}{[21661-97-2]} \\
\mathrm{V}\end{array}$ & $\begin{array}{r}(Z)-7-\text { decenal } \\
(323-343)\end{array}$ & 59.9 & 298 & CGC & [1996KOU/HOS, 2000OVA/KOU] \\
\hline $\mathrm{C}_{10} \mathrm{H}_{18} \mathrm{O}$ & $\begin{array}{c}{[21662-10-2]} \\
\mathrm{V}\end{array}$ & $\begin{array}{r}(E)-7 \text {-decenal } \\
(323-343)\end{array}$ & 59.8 & 298 & CGC & [1996KOU/HOS, 2000OVA/KOU] \\
\hline $\mathrm{C}_{10} \mathrm{H}_{18} \mathrm{O}$ & $\begin{array}{c}{[174155-46-5]} \\
\mathrm{V}\end{array}$ & $\begin{array}{r}(Z)-8 \text {-decenal } \\
(323-343)\end{array}$ & 60.5 & 298 & CGC & [1996KOU/HOS, 2000OVA/KOU] \\
\hline $\mathrm{C}_{10} \mathrm{H}_{18} \mathrm{O}$ & $\begin{array}{c}{[174155-47-6]} \\
\mathrm{V}\end{array}$ & $\begin{array}{r}(E)-8 \text {-decenal } \\
(323-343)\end{array}$ & 60.2 & 298 & CGC & [1996KOU/HOS, 2000OVA/KOU] \\
\hline $\mathrm{C}_{10} \mathrm{H}_{18} \mathrm{O}_{2}$ & $\begin{array}{c}{[502-47-6]} \\
\mathrm{V}\end{array}$ & $\begin{array}{l}\text { 3,7-dimethyl-6-octe } \\
\text { (372-530) }\end{array}$ & $\begin{array}{r}\text { oic acid } \\
68.7\end{array}$ & 387 & A & [1987STE/MAL, 1947STU] \\
\hline $\mathrm{C}_{10} \mathrm{H}_{18} \mathrm{O}_{2}$ & $\begin{array}{c}{[702-75-0]} \\
\mathrm{V}\end{array}$ & $\begin{array}{l}\text { 8,8-dimethyl-6,10-d } \\
\text { (283-313) }\end{array}$ & $\begin{array}{l}\text { xaspiro }[4.5] \\
53.7 \pm 0.5\end{array}$ & 298 & GS & [1998VER/PEN, 2002VER] \\
\hline $\mathrm{C}_{10} \mathrm{H}_{18} \mathrm{O}_{2}$ & $\begin{array}{c}{[7333-23-5]} \\
\mathrm{V}\end{array}$ & 2,2,6-dimethyl-3,5-1 & $\begin{array}{l}\text { ptanedione } \\
57.7\end{array}$ & 298 & & [1976BUR/SHR] \\
\hline $\mathrm{C}_{10} \mathrm{H}_{18} \mathrm{O}_{2}$ & $\begin{array}{l}\mathrm{V} \\
\mathrm{V}\end{array}$ & $\begin{array}{l}\text { L-decanolactone } \\
\begin{array}{c}(365-387) \\
(365-387)\end{array}\end{array}$ & $\begin{array}{l}57.7 \pm 0.8 \\
63.0 \pm 1.5\end{array}$ & $\begin{array}{l}376 \\
298\end{array}$ & $\begin{array}{l}\mathrm{MM} \\
\mathrm{MM}\end{array}$ & $\begin{array}{l}\text { [1991WIB/WAL] } \\
\text { [1991WIB/WAL] }\end{array}$ \\
\hline
\end{tabular}


TABLE 13. Phase change enthalpies of $\mathrm{C}_{10}$ organic compounds-Continued

\begin{tabular}{|c|c|c|c|c|c|c|}
\hline \multirow[b]{2}{*}{$\begin{array}{l}\text { Molecular } \\
\text { formula }\end{array}$} & \multirow{2}{*}{$\begin{array}{l}\text { CAS reg. no. } \\
\text { Transition }\end{array}$} & \multicolumn{5}{|l|}{ Compound } \\
\hline & & Temp. range $(\mathrm{K})$ & $\begin{array}{c}\Delta_{\text {trans }} H_{\mathrm{m}} \\
\left(\mathrm{kJ} \mathrm{mol}^{-1}\right)\end{array}$ & $\mathrm{T}_{\mathrm{m}}(\mathrm{K})$ & Method & Reference \\
\hline $\mathrm{C}_{10} \mathrm{H}_{18} \mathrm{O}_{2}$ & $\begin{array}{c}\text { [706-14-9] } \\
\mathrm{V} \\
\mathrm{V}\end{array}$ & $\begin{array}{c}\gamma \text {-decanolactone } \\
(298-365)\end{array}$ & $\begin{array}{l}75.0 \pm 4.3 \\
75.6 \pm 0.3\end{array}$ & $\begin{array}{l}298 \\
298\end{array}$ & $\begin{array}{c}\text { CGC } \\
\text { GS }\end{array}$ & $\begin{array}{l}\text { [2014KOZ/GOB] } \\
\text { [2008EME/KOZ] }\end{array}$ \\
\hline $\mathrm{C}_{10} \mathrm{H}_{18} \mathrm{O}_{2}$ & $\begin{array}{c}{[705-86-2]} \\
\mathrm{V} \\
\mathrm{V}\end{array}$ & 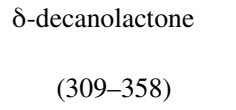 & $\begin{array}{l}75.5 \pm 4.3 \\
74.2 \pm 0.3\end{array}$ & $\begin{array}{l}298 \\
298\end{array}$ & $\begin{array}{l}\text { CGC } \\
\text { GS }\end{array}$ & $\begin{array}{l}\text { [2014KOZ/GOB] } \\
\text { [2007EME/KOZ] }\end{array}$ \\
\hline $\mathrm{C}_{10} \mathrm{H}_{18} \mathrm{O}_{2}$ & $\begin{array}{c}{[2499-58-3]} \\
\mathrm{V}\end{array}$ & $\begin{array}{c}\text { heptyl acrylate } \\
(359-481)\end{array}$ & 51.1 & 374 & A & [1987STE/MAL] \\
\hline $\mathrm{C}_{10} \mathrm{H}_{18} \mathrm{O}_{2}$ & $\begin{array}{c}{[142-09-6]} \\
\mathrm{V}\end{array}$ & $\begin{array}{l}\text { hexyl methacrylate } \\
\quad(354-475)\end{array}$ & 50.5 & 369 & A & [1987STE/MAL] \\
\hline $\mathrm{C}_{10} \mathrm{H}_{18} \mathrm{O}_{2}$ & $\begin{array}{c}{[512-77-6]} \\
\mathrm{V}\end{array}$ & $\begin{array}{c}\text { 1-methyl-3-isopropy } \\
\text { (374-538) }\end{array}$ & $\begin{array}{c}\text { cyclopentane } \\
91.6\end{array}$ & $\begin{array}{c}\text { oxylic ac } \\
389\end{array}$ & A & [1987STE/MAL] \\
\hline $\mathrm{C}_{10} \mathrm{H}_{18} \mathrm{O}_{2}$ & $\begin{array}{c}\text { [1551-44-6] } \\
\text { FUS } \\
\text { V } \\
\text { V } \\
\text { V } \\
\text { V } \\
\text { V } \\
\text { V } \\
\text { V }\end{array}$ & $\begin{array}{l}(273-310) \\
(273-310) \\
(278-313) \\
(333-378) \\
(283-313)\end{array}$ & $\begin{array}{c}16.57 \\
60.0 \pm 0.2 \\
60.1 \pm 0.2 \\
64.1 \pm 0.6 \\
59.8 \pm 0.6 \\
58.4 \pm 0.7 \\
60.0 \\
60.0 \pm 0.6\end{array}$ & $\begin{array}{l}219.6 \\
298 \\
298 \\
298 \\
298 \\
298 \\
298 \\
298\end{array}$ & $\begin{array}{c}\text { AC } \\
\text { C } \\
\text { C } \\
\text { ME } \\
\text { ME } \\
\text { GS } \\
\text { CGC } \\
\text { GS }\end{array}$ & $\begin{array}{l}\text { [2001KOZ/BLO] } \\
{[2004 \mathrm{PAU} / \mathrm{ZAI}]} \\
{[2003 \mathrm{ZAI} / \mathrm{VER}]} \\
{[2003 \mathrm{ZAI} / \mathrm{VER}]} \\
{[2003 \mathrm{ZAI} / \mathrm{VER}]} \\
{[2003 \mathrm{ZAI} / \mathrm{VER}]} \\
{[1999 \mathrm{VER} / \mathrm{HEI}]} \\
{[1996 \mathrm{VER} / \mathrm{BEC}]}\end{array}$ \\
\hline $\mathrm{C}_{10} \mathrm{H}_{18} \mathrm{O}_{2}$ & $\begin{array}{c}{[1129-47-1]} \\
\mathrm{V}\end{array}$ & $\begin{array}{c}\text { cyclohexyl isobutyra } \\
(333-378)\end{array}$ & 57.7 & 298 & CGC & [1999VER/HEI] \\
\hline $\mathrm{C}_{10} \mathrm{H}_{18} \mathrm{O}_{2}$ & $\mathrm{~V}$ & $\begin{array}{c}\text { 1-methylcyclohexyl } \\
(333-378)\end{array}$ & $\begin{array}{c}\text { ropanoate } \\
55.8\end{array}$ & 298 & CGC & [1999VER/HEI] \\
\hline $\mathrm{C}_{10} \mathrm{H}_{18} \mathrm{O}_{2}$ & $\mathrm{~V}$ & $\begin{array}{c}\text { 3-methylcyclohexyl } \\
(333-378)\end{array}$ & $\begin{array}{c}\text { ropanoate } \\
58.3\end{array}$ & 298 & $\mathrm{CGC}$ & [1999VER/HEI] \\
\hline $\mathrm{C}_{10} \mathrm{H}_{18} \mathrm{O}_{2}$ & $\mathrm{~V}$ & $\begin{array}{c}\text { 4-methylcyclohexyl } \\
(333-378)\end{array}$ & $\begin{array}{c}\text { ropanoate } \\
58.9\end{array}$ & 298 & CGC & [1999VER/HEI] \\
\hline $\mathrm{C}_{10} \mathrm{H}_{18} \mathrm{O}_{2}$ & $\begin{array}{c}{[56922-72-6]} \\
\mathrm{V}\end{array}$ & pentyl 3-methylbut- & $\begin{array}{l}\text { enoate } \\
61.8 \pm 0.4\end{array}$ & 298 & GS & [2008EME/TOK] \\
\hline $\mathrm{C}_{10} \mathrm{H}_{18} \mathrm{O}_{2}$ & $\begin{array}{c}\text { [71697-84-2] } \\
\text { FUS }\end{array}$ & (-)-5-hydroxy- $a, a$, & $\begin{array}{c}\text { trimethyl-3-c } \\
34.69\end{array}$ & $\begin{array}{c}\text { hexene-1- } \\
423.6\end{array}$ & $\begin{array}{l}\text { hanol (trat } \\
\text { DSC }\end{array}$ & $\begin{array}{l}s \text {-sobrerol) } \\
\text { [1999LI/ZEL, 1990BET/GIO] }\end{array}$ \\
\hline $\mathrm{C}_{10} \mathrm{H}_{18} \mathrm{O}_{2}$ & $\begin{array}{l}{[42370-41-2]} \\
\text { FUS }\end{array}$ & $( \pm)$-5-hydroxy- $a, a$ & $\begin{array}{l}\text {-trimethyl-3- } \\
34.39\end{array}$ & $\begin{array}{l}\text { hexene-1 } \\
404.9\end{array}$ & $\begin{array}{l}\text { thanol }(t r c \\
\text { DSC }\end{array}$ & $\begin{array}{l}\text { us-sobrerol) } \\
\text { [1999LI/ZEL, 1990BET/GIO] }\end{array}$ \\
\hline $\mathrm{C}_{10} \mathrm{H}_{18} \mathrm{O}_{2}$ & $\begin{array}{l}\text { [772-36-1] } \\
\text { FUS }\end{array}$ & (-)-5-hydroxy- $a, a$, & $\begin{array}{l}\text { trimethyl-3-c } \\
23.18\end{array}$ & $\begin{array}{l}\text { hexene-1- } \\
382.9\end{array}$ & $\begin{array}{l}\text { hanol (cis } \\
\text { DSC }\end{array}$ & $\begin{array}{l}\text { obrerol) } \\
\text { [1999LI/ZEL, 1990BET/GIO] }\end{array}$ \\
\hline $\mathrm{C}_{10} \mathrm{H}_{18} \mathrm{O}_{2}$ & $\begin{array}{l}{[54164-89-5]} \\
\text { FUS }\end{array}$ & $( \pm)$-5-hydroxy- $a, a$ & $\begin{array}{l}\text {-trimethyl-3- } \\
25.86\end{array}$ & $\begin{array}{l}\text { hexene-1 } \\
378.9\end{array}$ & $\begin{array}{l}\text { thanol }(c i s \\
\text { DSC }\end{array}$ & $\begin{array}{l}\text { sobrerol) } \\
\text { [1999LI/ZEL, 1990BET/GIO] }\end{array}$ \\
\hline $\mathrm{C}_{10} \mathrm{H}_{18} \mathrm{O}_{2}$ & $\begin{array}{c}{[16491-36-4]} \\
\mathrm{V}\end{array}$ & cis-3-hexenyl butyra & $59.9 \pm 1.7$ & 298 & $\mathrm{CGC}$ & [2015KOZ/GOB] \\
\hline $\mathrm{C}_{10} \mathrm{H}_{18} \mathrm{O}_{3}$ & $\begin{array}{c}{[103985-60-0]} \\
\text { V }\end{array}$ & $\begin{array}{l}\text { 3-hydroxy-2,3-dime } \\
(362-387)\end{array}$ & $\begin{array}{l}\text { yyl-4-hexeno } \\
57.4\end{array}$ & $\begin{array}{c}\text { d, ethyl e } \\
374\end{array}$ & A & [1987STE/MAL] \\
\hline $\mathrm{C}_{10} \mathrm{H}_{18} \mathrm{O}_{3}$ & $\begin{array}{c}\text { [71172-75-3] } \\
\text { V } \\
\text { V }\end{array}$ & $\begin{array}{l}\text { isopentyl levulinate } \\
(403-521)\end{array}$ & $\begin{array}{l}59.4 \\
56.3\end{array}$ & $\begin{array}{l}418 \\
461\end{array}$ & A & $\begin{array}{l}\text { [1987STE/MAL] } \\
{[1931 \mathrm{SCH} / \mathrm{COW}]}\end{array}$ \\
\hline $\mathrm{C}_{10} \mathrm{H}_{18} \mathrm{O}_{3}$ & $\mathrm{~V}$ & $\begin{array}{l}\text { 1-ethylpropyl levuliı } \\
\text { (397-513) }\end{array}$ & 58.6 & 412 & A & [1987STE/MAL] \\
\hline $\mathrm{C}_{10} \mathrm{H}_{18} \mathrm{O}_{3}$ & V & $\begin{array}{l}\text { 1-methylbutyl levuli } \\
\text { (397-513) }\end{array}$ & ate 57.2 & 412 & A & [1987STE/MAL] \\
\hline
\end{tabular}


TABLE 13. Phase change enthalpies of $\mathrm{C}_{10}$ organic compounds-Continued

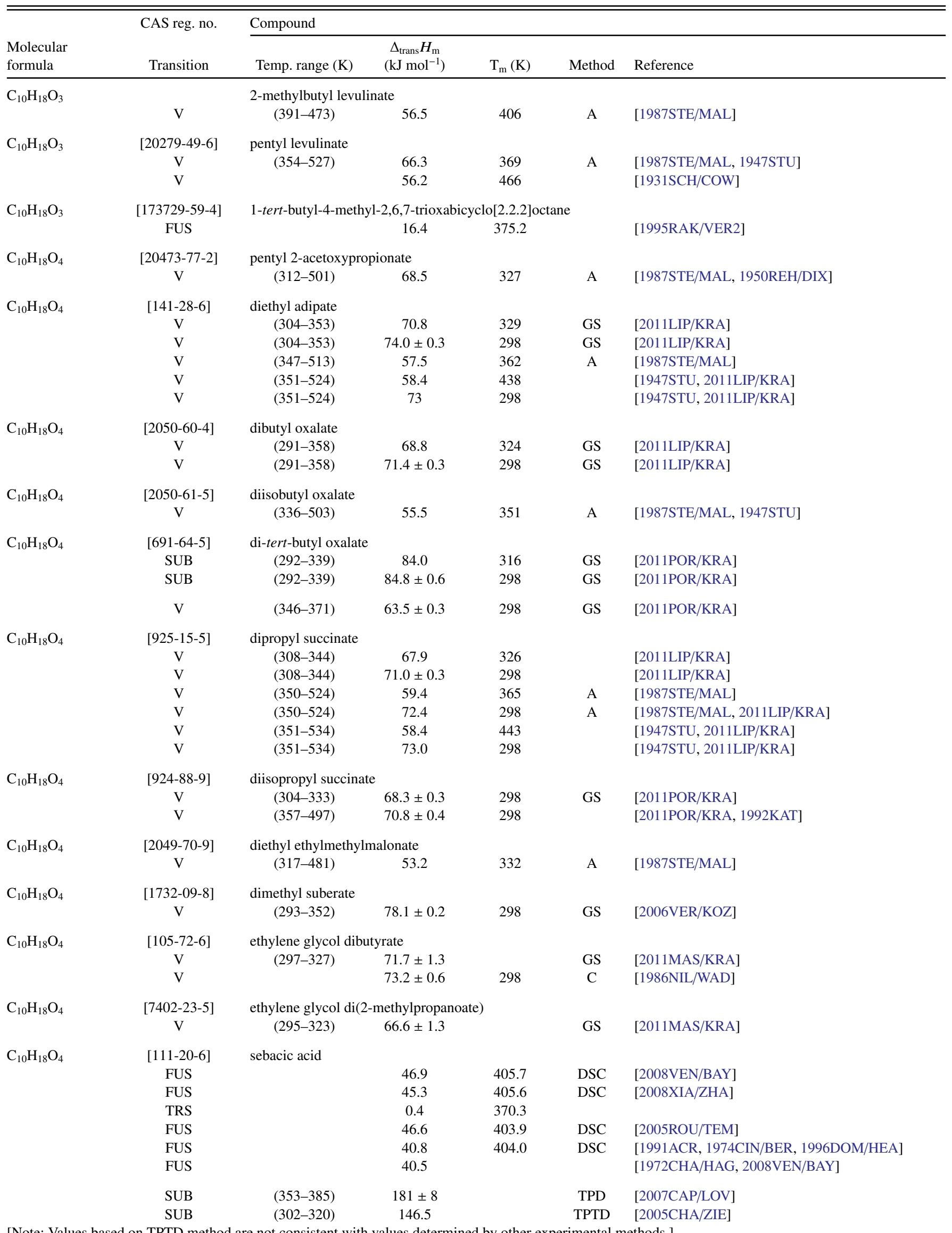

[Note: Values based on TPTD method are not consistent with values determined by other experimental methods.] 
TABLE 13. Phase change enthalpies of $\mathrm{C}_{10}$ organic compounds-Continued

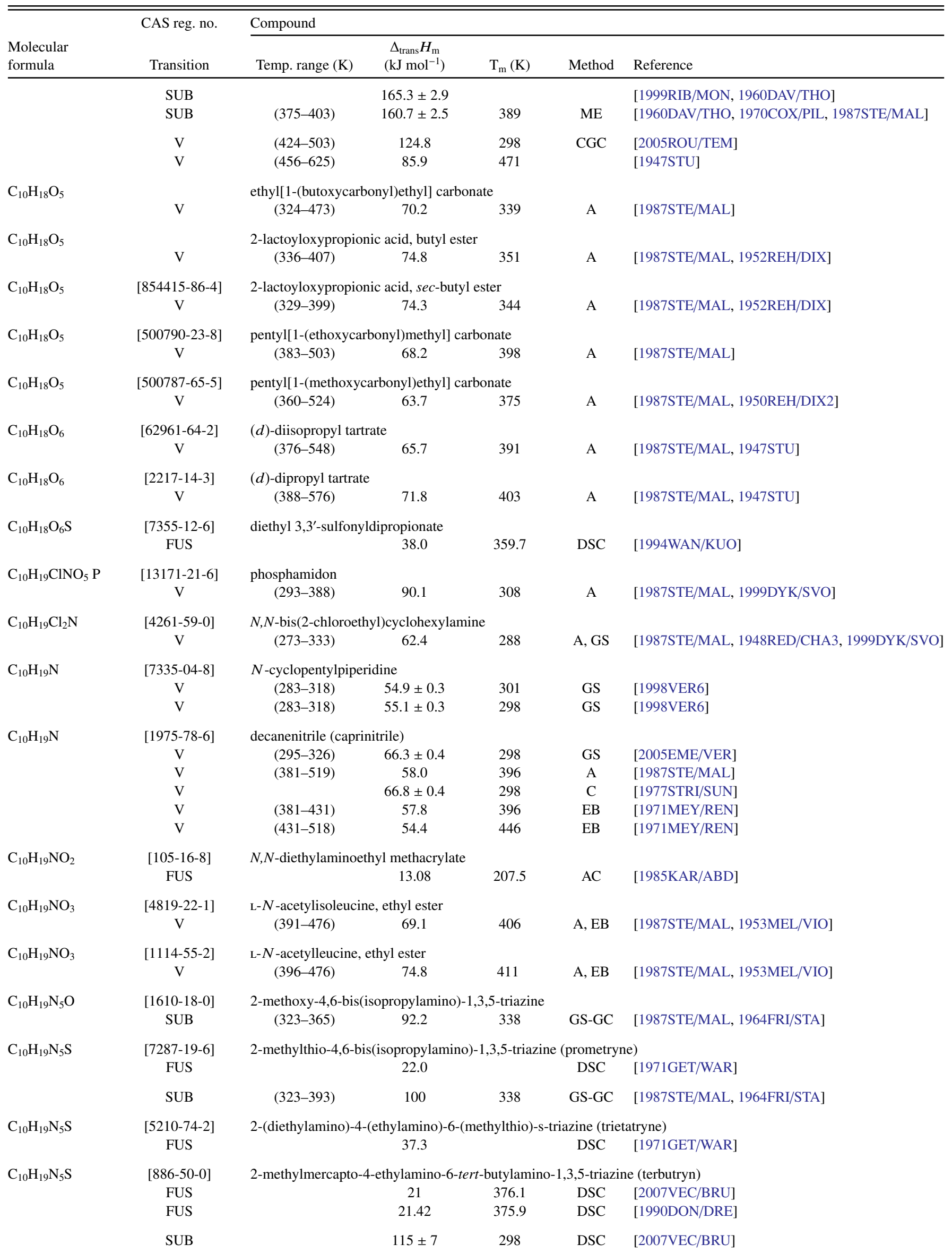


TABLE 13. Phase change enthalpies of $\mathrm{C}_{10}$ organic compounds-Continued

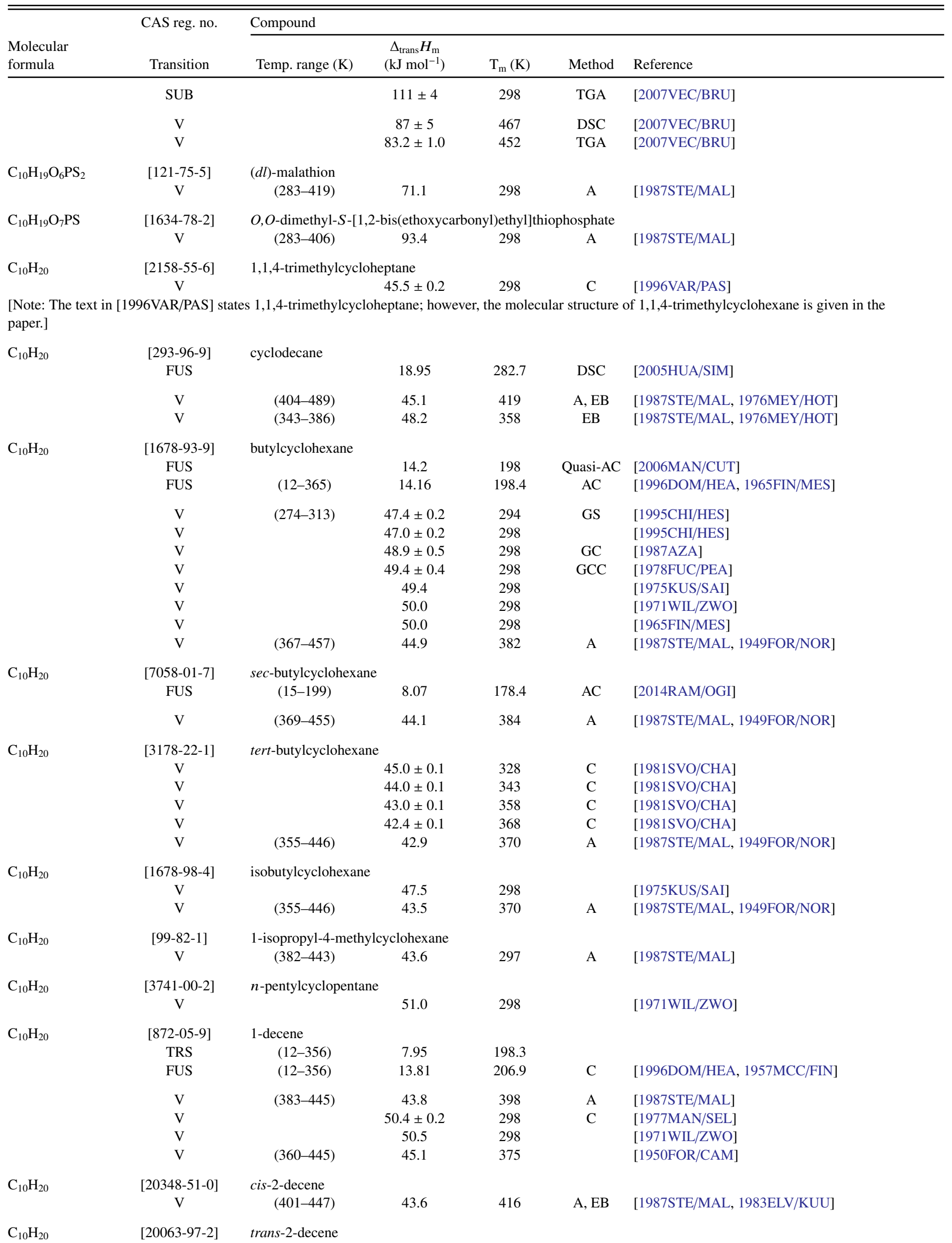


TABLE 13. Phase change enthalpies of $\mathrm{C}_{10}$ organic compounds-Continued

\begin{tabular}{|c|c|c|c|c|c|c|}
\hline \multirow[b]{2}{*}{$\begin{array}{l}\text { Molecular } \\
\text { formula }\end{array}$} & \multirow{2}{*}{$\begin{array}{l}\text { CAS reg. no. } \\
\text { Transition }\end{array}$} & \multicolumn{5}{|l|}{ Compound } \\
\hline & & Temp. range $(\mathrm{K})$ & $\begin{array}{c}\Delta_{\text {trans }} H_{\mathrm{m}} \\
\left(\mathrm{kJ} \mathrm{mol}^{-1}\right)\end{array}$ & $\mathrm{T}_{\mathrm{m}}(\mathrm{K})$ & Method & Reference \\
\hline & $\mathrm{V}$ & $(401-447)$ & 43.7 & 416 & A, EB & [1987STE/MAL, 1983ELV/KUU] \\
\hline $\mathrm{C}_{10} \mathrm{H}_{20}$ & $\begin{array}{c}{[19398-86-8]} \\
\text { V }\end{array}$ & $\begin{array}{l}\text { cis-3-decene } \\
\text { (398-444) }\end{array}$ & 43.1 & 413 & $\mathrm{~A}, \mathrm{~EB}$ & [1987STE/MAL, 1983ELV/KUU] \\
\hline $\mathrm{C}_{10} \mathrm{H}_{20}$ & $\begin{array}{c}{[19150-21-1]} \\
\mathrm{V}\end{array}$ & $\begin{array}{c}\text { trans-3-decene } \\
\quad(398-445)\end{array}$ & 43.4 & 413 & $\mathrm{~A}, \mathrm{~EB}$ & [1987STE/MAL, 1983ELV/KUU] \\
\hline $\mathrm{C}_{10} \mathrm{H}_{20}$ & $\begin{array}{c}{[19398-88-0]} \\
V\end{array}$ & $\begin{array}{l}\text { cis-4-decene } \\
(397-444)\end{array}$ & 43.0 & 412 & $\mathrm{~A}, \mathrm{~EB}$ & [1987STE/MAL, 1983ELV/KUU] \\
\hline $\mathrm{C}_{10} \mathrm{H}_{20}$ & $\begin{array}{c}{[19398-89-1]} \\
\text { V }\end{array}$ & $\begin{array}{c}\text { trans-4-decene } \\
(398-444)\end{array}$ & 43.2 & 413 & $\mathrm{~A}, \mathrm{~EB}$ & [1987STE/MAL, 1983ELV/KUU] \\
\hline $\mathrm{C}_{10} \mathrm{H}_{20}$ & $\begin{array}{c}{[7433-78-5]} \\
\mathrm{V}\end{array}$ & $\begin{array}{l}\text { cis-5-decene } \\
(397-443)\end{array}$ & 42.9 & 412 & $\mathrm{~A}, \mathrm{~EB}$ & [1987STE/MAL, 1983ELV/KUU] \\
\hline $\mathrm{C}_{10} \mathrm{H}_{20}$ & $\begin{array}{c}{[7433-56-9]} \\
\mathrm{V}\end{array}$ & $\begin{array}{c}\text { trans-5-decene } \\
(398-444)\end{array}$ & 43.2 & 413 & $\mathrm{~A}, \mathrm{~EB}$ & [1987STE/MAL, 1983ELV/KUU] \\
\hline $\mathrm{C}_{10} \mathrm{H}_{20}$ & $\begin{array}{c}{[4485-13-6]} \\
\mathrm{V}\end{array}$ & $\begin{array}{l}\text { 4-propyl-3-heptene } \\
(333-371)\end{array}$ & 43.7 & 348 & A, MG & [1987STE/MAL, 1955SCH/WHI] \\
\hline $\mathrm{C}_{10} \mathrm{H}_{20}$ & $\begin{array}{c}{[692-48-8]} \\
\mathrm{V}\end{array}$ & trans-2,2,5,5-tetrame & $\begin{array}{l}\text { thyl-3-hexene } \\
42.0 \pm 0.2\end{array}$ & 298 & GCC & [1979FUC/PEA] \\
\hline $\mathrm{C}_{10} \mathrm{H}_{20}$ & $\begin{array}{c}\text { [22808-06-6] } \\
\text { TRS } \\
\text { TRS } \\
\text { FUS }\end{array}$ & 2,2,5,5-tetramethylhe & $\begin{array}{r}\text { x-3-ene } \\
1.21 \\
4.33 \\
10.25\end{array}$ & $\begin{array}{l}235.8 \\
243.5 \\
268.9\end{array}$ & DSC & {$[1980 B Y S]$} \\
\hline $\mathrm{C}_{10} \mathrm{H}_{20} \mathrm{Br}_{2}$ & $\begin{array}{c}{[59104-80-2]} \\
\mathrm{V}\end{array}$ & $\begin{array}{l}\text { 1,1-dibromodecane } \\
(442-610)\end{array}$ & 62.2 & 457 & $\mathrm{~A}, \mathrm{EST}$ & $\begin{array}{l}\text { [1987STE/MAL, 1956MAN, 1970DYK/VAN, } \\
\text { 1999DYK/SVO] }\end{array}$ \\
\hline $\mathrm{C}_{10} \mathrm{H}_{20} \mathrm{Br}_{2}$ & $\begin{array}{l}{[28467-71-2]} \\
V\end{array}$ & $\begin{array}{l}\text { 1,2-dibromodecane } \\
\text { (368-524) }\end{array}$ & 67.0 & 383 & A & [1987STE/MAL, 1947STU, 1970DYK/VAN] \\
\hline $\mathrm{C}_{10} \mathrm{H}_{20} \mathrm{Cl}_{2}$ & $\begin{array}{c}{[3162-62-7]} \\
\mathrm{V}\end{array}$ & $\begin{array}{l}\text { 1,1-dichlorodecane } \\
(415-577)\end{array}$ & 56.9 & 430 & A, EST & [1987STE/MAL, 1956MAN, 1970DYK/VAN] \\
\hline $\mathrm{C}_{10} \mathrm{H}_{20} \mathrm{Cl}_{2}$ & $\begin{array}{c}\text { [2162-98-3] } \\
\text { V } \\
\text { V } \\
\text { V }\end{array}$ & $\begin{array}{c}\text { 1,10-dichlorodecane } \\
(441-520) \\
(440-540)\end{array}$ & $\begin{array}{l}61.1 \\
67.3 \\
73.1\end{array}$ & $\begin{array}{l}456 \\
298\end{array}$ & & $\begin{array}{l}\text { [1999DYK/SVO] } \\
\text { [1998DRO/TOM] } \\
\text { [1991BAS/SVO] }\end{array}$ \\
\hline $\mathrm{C}_{10} \mathrm{H}_{20} \mathrm{Cl}_{2} \mathrm{~N}_{3} \mathrm{O}_{4} \mathrm{P}_{3}$ & $\begin{array}{c}\text { [131471-44-8] } \\
\text { FUS }(\alpha) \\
\text { FUS }(\beta)\end{array}$ & 2,2,4,4-bis $\left(2^{\prime}, 2^{\prime}\right.$-dim & $\begin{array}{l}\text { thylpropane-1' } \\
32.7 \\
29.4\end{array}$ & $\begin{array}{c}\text { dioxy) }-6, \\
484.1 \\
484.6\end{array}$ & $\begin{array}{l}\text { lichlorocyclo } \\
\text { DSC } \\
\text { DSC }\end{array}$ & $\begin{array}{l}\text { triphosphazene } \\
\text { [2011HAC/MON] } \\
\text { [2011HAC/MON] }\end{array}$ \\
\hline $\mathrm{C}_{10} \mathrm{H}_{20} \mathrm{~F}_{2}$ & $\begin{array}{l}{[62127-43-9]} \\
\mathrm{V}\end{array}$ & $\begin{array}{l}\text { 1,1-difluorodecane } \\
\text { (364-504) }\end{array}$ & 50.2 & 379 & A, EST & $\begin{array}{l}\text { [1987STE/MAL, 1956MAN, 1970DYK/VAN, } \\
\text { 1999DYK/SVO] }\end{array}$ \\
\hline $\mathrm{C}_{10} \mathrm{H}_{20} \mathrm{~N}_{2}$ & $\begin{array}{l}{[53657-09-3]} \\
\mathrm{V}\end{array}$ & $\begin{array}{l}\text { 1-heptylimidazole } \\
\text { (299-366) }\end{array}$ & $76.8 \pm 0.3$ & 298 & GS & [2011EME/POR] \\
\hline $\mathrm{C}_{10} \mathrm{H}_{20} \mathrm{~N}_{2} \mathrm{OS}$ & $\begin{array}{l}{[398995-30-7]} \\
\text { SUB }\end{array}$ & $\begin{array}{l}N, N \text {-diethyl- } N^{\prime} \text {-isov } \\
363\end{array}$ & $\begin{array}{l}\text { leroylthiourea } \\
121.5 \pm 3.2\end{array}$ & 298 & $\mathrm{C}$ & [2001RIB/RIB] \\
\hline $\mathrm{C}_{10} \mathrm{H}_{20} \mathrm{~N}_{2} \mathrm{OS}$ & $\begin{array}{l}{[398995-31-8]} \\
\text { SUB }\end{array}$ & $\begin{array}{l}N, N \text {-diethyl- } N^{\prime} \text {-piva } \\
366\end{array}$ & $\begin{array}{l}\text { oylthiourea } \\
114.9 \pm 2.7\end{array}$ & 298 & $\mathrm{C}$ & [2001RIB/RIB] \\
\hline $\mathrm{C}_{10} \mathrm{H}_{20} \mathrm{~N}_{2} \mathrm{O}_{2}$ & $\begin{array}{l}{[14288-05-2]} \\
\text { FUS }\end{array}$ & tetraethyloxamide & 17.0 & 310.2 & DSC & [2003CLO/JAN] \\
\hline & $\mathrm{V}$ & & 63 & 464 & TGA, DSC & [2003CLO/JAN] \\
\hline $\mathrm{C}_{10} \mathrm{H}_{20} \mathrm{~N}_{2} \mathrm{O}_{2}$ & $\begin{array}{l}{[1740-54-1]} \\
\text { FUS }\end{array}$ & sebacamide & 68.7 & 484.3 & DSC & [2006BAD/DEL] \\
\hline $\mathrm{C}_{10} \mathrm{H}_{20} \mathrm{~N}_{6}$ & $\begin{array}{c}\text { [16268-73-8] } \\
\text { FUS }\end{array}$ & 1-(ethylmethylamino & $\begin{array}{l}\text {-3,5-bis(dime } \\
21.3\end{array}$ & $\begin{array}{l}\text { amino)-s } \\
384\end{array}$ & $\begin{array}{l}\text { azine } \\
\text { DSC }\end{array}$ & [1991ACR, 1989BRA/RYT] \\
\hline
\end{tabular}


TABLE 13. Phase change enthalpies of $\mathrm{C}_{10}$ organic compounds-Continued

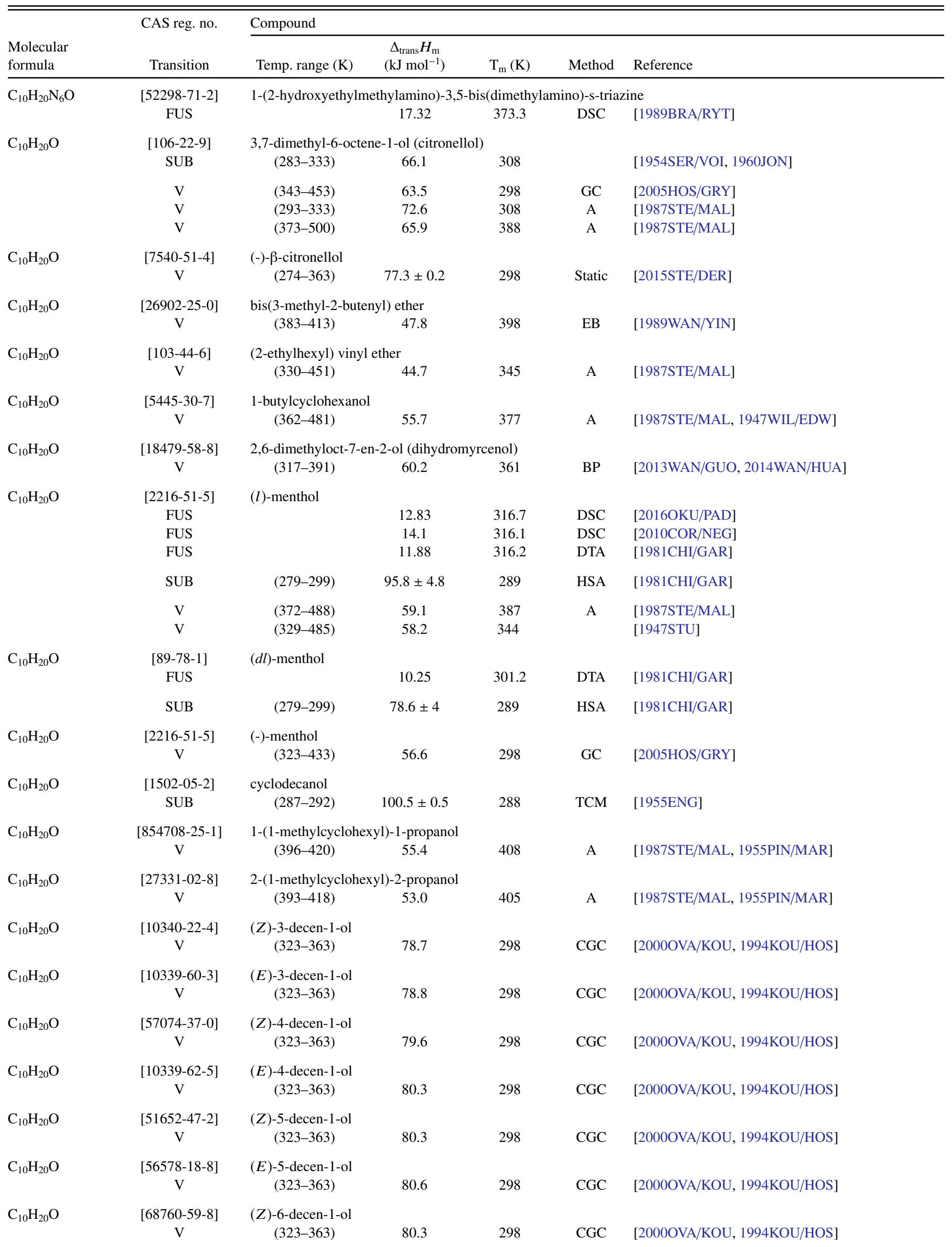


TABLE 13. Phase change enthalpies of $\mathrm{C}_{10}$ organic compounds-Continued

\begin{tabular}{|c|c|c|c|c|c|c|}
\hline \multirow[b]{2}{*}{$\begin{array}{l}\text { Molecular } \\
\text { formula }\end{array}$} & \multirow{2}{*}{$\begin{array}{c}\text { CAS reg. no. } \\
\text { Transition }\end{array}$} & \multicolumn{5}{|l|}{ Compound } \\
\hline & & Temp. range $(\mathrm{K})$ & $\begin{array}{c}\Delta_{\text {trans }} H_{\mathrm{m}} \\
\left(\mathrm{kJ} \mathrm{mol}^{-1}\right)\end{array}$ & $\mathrm{T}_{\mathrm{m}}(\mathrm{K})$ & Method & Reference \\
\hline $\mathrm{C}_{10} \mathrm{H}_{20} \mathrm{O}$ & $\begin{array}{c}{[38421-92-0]} \\
\mathrm{V}\end{array}$ & $\begin{array}{c}(E)-6 \text {-decen-1-ol } \\
\quad(323-363)\end{array}$ & 80.6 & 298 & CGC & [2000OVA/KOU, 1994KOU/HOS] \\
\hline $\mathrm{C}_{10} \mathrm{H}_{20} \mathrm{O}$ & $\begin{array}{c}{[16504-66-8]} \\
\mathrm{V}\end{array}$ & $\begin{array}{c}(Z)-7 \text {-decen-1-ol } \\
\quad(323-363)\end{array}$ & 80.8 & 298 & CGC & [2000OVA/KOU, 1994KOU/HOS] \\
\hline $\mathrm{C}_{10} \mathrm{H}_{20} \mathrm{O}$ & $\begin{array}{c}{[52957-12-7]} \\
\mathrm{V}\end{array}$ & $\begin{array}{c}(E)-7 \text {-decen-1-ol } \\
(323-363)\end{array}$ & 81.1 & 298 & CGC & [2000OVA/KOU, 1994KOU/HOS] \\
\hline $\mathrm{C}_{10} \mathrm{H}_{20} \mathrm{O}$ & $\begin{array}{c}{[83799-67-1]} \\
\mathrm{V}\end{array}$ & $\begin{array}{c}(Z)-8 \text {-decen-1-ol } \\
\quad(323-363)\end{array}$ & 81.6 & 298 & CGC & [2000OVA/KOU, 1994KOU/HOS] \\
\hline $\mathrm{C}_{10} \mathrm{H}_{20} \mathrm{O}$ & $\begin{array}{c}{[83799-68-2]} \\
\mathrm{V}\end{array}$ & $\begin{array}{c}(E)-8 \text {-decen-1-ol } \\
(323-363)\end{array}$ & 81.5 & 298 & CGC & [2000OVA/KOU, 1994KOU/HOS] \\
\hline $\mathrm{C}_{10} \mathrm{H}_{20} \mathrm{O}$ & $\begin{array}{c}\text { [693-54-9] } \\
\text { V } \\
\text { V } \\
\text { V } \\
\text { V }\end{array}$ & $\begin{array}{l}\text { 2-decanone } \\
\quad(317-484) \\
(357-560) \\
(358-568)\end{array}$ & $\begin{array}{c}51.7 \\
55.1 \\
60.9 \pm 0.5 \\
44.6\end{array}$ & $\begin{array}{l}332 \\
372 \\
298 \\
487\end{array}$ & $\begin{array}{c}\text { A } \\
\text { A } \\
\text { GCC }\end{array}$ & $\begin{array}{l}\text { [1987STE/MAL, 1947STU] } \\
\text { [1987STE/MAL] } \\
\text { [1979SAL/PEA] } \\
\text { [1975AMB/ELL] }\end{array}$ \\
\hline $\mathrm{C}_{10} \mathrm{H}_{20} \mathrm{O}$ & $\begin{array}{c}\text { [868-91-7] } \\
\mathrm{V}\end{array}$ & 2,2,5,5-tetramethyl & $\begin{array}{l}\text {-hexanone } \\
48.8 \pm 0.2\end{array}$ & 298 & $\mathrm{C}$ & {$[1970$ SEL2] } \\
\hline $\mathrm{C}_{10} \mathrm{H}_{20} \mathrm{O}$ & $\begin{array}{c}\text { [112-31-2] } \\
\text { FUS } \\
\text { V } \\
\text { V } \\
\text { V } \\
\text { V } \\
\text { V } \\
\text { V }\end{array}$ & $\begin{array}{l}\text { decanal } \\
\quad(50-350) \\
(280-316) \\
(308-353) \\
(288-333) \\
(293-358) \\
(324-482)\end{array}$ & $\begin{array}{c}30.6 \\
59.5 \pm 0.4 \\
60.5 \\
57.3 \\
57.3 \\
60.4 \pm 0.3 \\
56.3\end{array}$ & $\begin{array}{l}268.2 \\
298 \\
298 \\
303 \\
308 \\
298 \\
339\end{array}$ & $\begin{array}{c}\mathrm{AC} \\
\mathrm{GS} \\
\mathrm{CGC} \\
\mathrm{A} \\
\mathrm{A}\end{array}$ & $\begin{array}{l}\text { [1980DYA/VAS] } \\
\text { [2003VER/KRA2] } \\
\text { [1996KOU/HOS, 2000OVA/KOU] } \\
\text { [1987STE/MAL] } \\
\text { [1987STE/MAL] } \\
\text { [1981DYA/KOR] } \\
\text { [1987STE/MAL, 1947STU] }\end{array}$ \\
\hline $\mathrm{C}_{10} \mathrm{H}_{20} \mathrm{O}_{2}$ & $\begin{array}{c}{[4359-57-3]} \\
\mathrm{V}\end{array}$ & $\begin{array}{c}\text { 2-heptyl-1,3-dioxo } \\
(318-453)\end{array}$ & 62.0 & 333 & A & [1987STE/MAL, 1977VOI/SHC] \\
\hline $\mathrm{C}_{10} \mathrm{H}_{20} \mathrm{O}_{2}$ & $\begin{array}{c}{[4359-47-1]} \\
\mathrm{V}\end{array}$ & $\begin{array}{c}\text { 2-(1-ethylpentyl)-1 } \\
(333-453)\end{array}$ & $\begin{array}{c}\text { dioxolane } \\
55.3\end{array}$ & 348 & A & [1987STE/MAL, 1977VOI/SHC] \\
\hline $\mathrm{C}_{10} \mathrm{H}_{20} \mathrm{O}_{2}$ & $\begin{array}{c}{[2244-85-1]} \\
\mathrm{V}\end{array}$ & $\begin{array}{c}\text { 4-hexyl-1,3-dioxan } \\
(318-453)\end{array}$ & 56.9 & 333 & A & [1987STE/MAL, 1977VOI/SHC] \\
\hline $\mathrm{C}_{10} \mathrm{H}_{20} \mathrm{O}_{2}$ & $\begin{array}{c}{[61827-60-9]} \\
\mathrm{V}\end{array}$ & $\begin{array}{c}\text { 3-pentyl-4-hydroxy } \\
(383-453)\end{array}$ & $\begin{array}{c}\text { trahydropyran } \\
72.6\end{array}$ & 398 & A & [1987STE/MAL, 1977VOI/SHC] \\
\hline $\mathrm{C}_{10} \mathrm{H}_{20} \mathrm{O}_{2}$ & $\begin{array}{c}{[859773-58-3]} \\
\text { V }\end{array}$ & $\begin{array}{c}\text { 2-butoxy-3-hexano } \\
\quad(333-418)\end{array}$ & 39.5 & 348 & A, I & [1987STE/MAL, 1933HEN/MUR] \\
\hline $\mathrm{C}_{10} \mathrm{H}_{20} \mathrm{O}_{2}$ & $\begin{array}{c}{[107-75-5]} \\
\mathrm{V}\end{array}$ & $\begin{array}{l}\text { hydroxycitronellal } \\
\quad(283-333)\end{array}$ & 75.3 & 298 & $\mathrm{~A}, \mathrm{ME}$ & [1987STE/MAL, 1955SER/VOI] \\
\hline $\mathrm{C}_{10} \mathrm{H}_{20} \mathrm{O}_{2}$ & $\begin{array}{c}\text { [112-14-1] } \\
\mathrm{V} \\
\mathrm{V} \\
\mathrm{V} \\
\mathrm{V}\end{array}$ & $\begin{array}{l}\text { octyl acetate } \\
\qquad \begin{array}{r}(274-309) \\
(334-417) \\
(345-472)\end{array}\end{array}$ & $\begin{array}{c}60.7 \pm 0.4 \\
61.7 \\
54.9 \\
47.8\end{array}$ & $\begin{array}{l}298 \\
298 \\
349 \\
360\end{array}$ & $\begin{array}{c}\text { GS } \\
\text { GC } \\
\text { A } \\
\text { A }\end{array}$ & $\begin{array}{l}\text { [2006KRA/VER] } \\
{[1997 \mathrm{DEF} / \mathrm{CAR}]} \\
{[1987 \mathrm{STE} / \mathrm{MAL}]} \\
{[1987 \mathrm{STE} / \mathrm{MAL}]}\end{array}$ \\
\hline $\mathrm{C}_{10} \mathrm{H}_{20} \mathrm{O}_{2}$ & $\begin{array}{c}\text { [106-32-1] } \\
\text { V } \\
\text { V } \\
\text { V } \\
\text { V } \\
\text { V }\end{array}$ & $\begin{array}{c}\text { ethyl octanoate } \\
(284-462) \\
(284-462) \\
(382-412) \\
(382-412) \\
(330-480)\end{array}$ & $\begin{array}{c}55.0 \pm 0.6 \\
62.0 \pm 0.6 \\
52.5 \pm 0.2 \\
59.5 \pm 1.3 \\
53.2\end{array}$ & $\begin{array}{l}373 \\
298 \\
397 \\
298 \\
345\end{array}$ & $\begin{array}{l}\text { Static } \\
\text { Static } \\
\text { EB } \\
\text { EB } \\
\text { A }\end{array}$ & $\begin{array}{l}\text { [2011BEN/KHI] } \\
\text { [2011BEN/KHI] } \\
{[1991 \mathrm{WIB} / \mathrm{WAL}]} \\
\text { [1991WIB/WAL] } \\
\text { [1987STE/MAL] }\end{array}$ \\
\hline $\mathrm{C}_{10} \mathrm{H}_{20} \mathrm{O}_{2}$ & $\begin{array}{c}\text { [103-09-3] } \\
\mathrm{V}\end{array}$ & $\begin{array}{c}\text { 2-ethylhexyl acetat } \\
(333-472)\end{array}$ & 50.1 & 348 & A & [1987STE/MAL] \\
\hline $\mathrm{C}_{10} \mathrm{H}_{20} \mathrm{O}_{2}$ & $\begin{array}{c}{[659-70-1]} \\
\mathrm{V}\end{array}$ & $\begin{array}{c}\text { isopentyl isovalera } \\
(341-479)\end{array}$ & 46.4 & 356 & A & [1987STE/MAL] \\
\hline
\end{tabular}


TABLE 13. Phase change enthalpies of $\mathrm{C}_{10}$ organic compounds-Continued

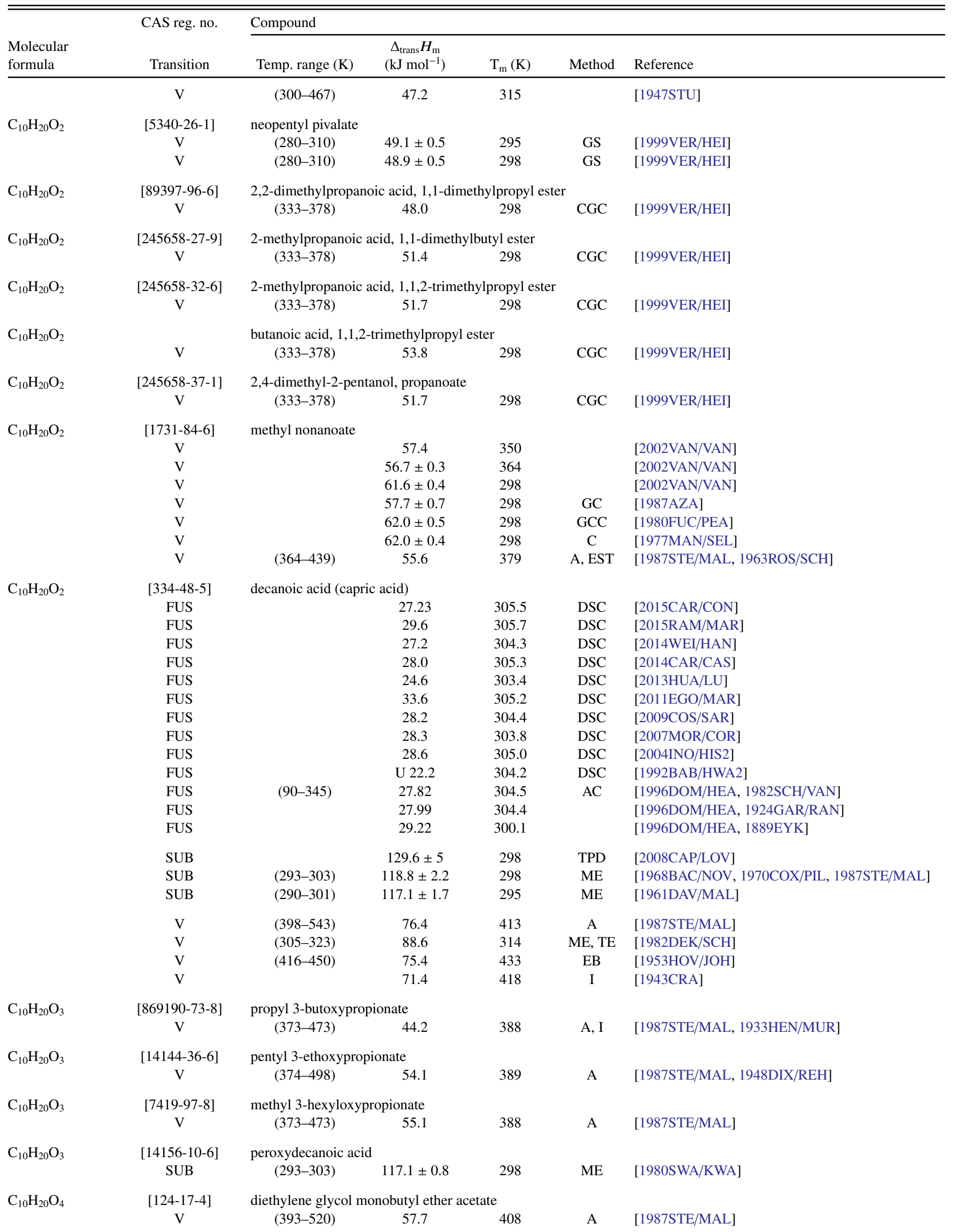


TABLE 13. Phase change enthalpies of $\mathrm{C}_{10}$ organic compounds-Continued

\begin{tabular}{|c|c|c|c|c|c|c|}
\hline \multirow[b]{2}{*}{$\begin{array}{l}\text { Molecular } \\
\text { formula }\end{array}$} & \multirow{2}{*}{$\begin{array}{c}\text { CAS reg. no. } \\
\text { Transition }\end{array}$} & \multicolumn{5}{|l|}{ Compound } \\
\hline & & Temp. range $(\mathrm{K})$ & $\begin{array}{c}\Delta_{\text {trans }} H_{\mathrm{m}} \\
\left(\mathrm{kJ} \mathrm{mol}^{-1}\right)\end{array}$ & $\mathrm{T}_{\mathrm{m}}(\mathrm{K})$ & Method & Reference \\
\hline \multirow[t]{2}{*}{$\mathrm{C}_{10} \mathrm{H}_{20} \mathrm{O}_{4}$} & [33785-99-8] & \multicolumn{5}{|c|}{ 3,3,6,6-tetraethyl-1,2,4,5-tetraoxacyclohexane } \\
\hline & $\mathrm{V}$ & $(403-473)$ & 50.1 & 298 & CGC & {$[2007 \mathrm{CAN} / \mathrm{EYL}]$} \\
\hline \multirow[t]{3}{*}{$\mathrm{C}_{10} \mathrm{H}_{20} \mathrm{O}_{5}$} & {$[33100-27-5]$} & \multicolumn{5}{|c|}{ 1,4,7,10,13-pentaoxacyclopentadecane (15-crown-5) } \\
\hline & $\mathrm{V}$ & & $75.7 \pm 1.7$ & 298 & CGC & [2000NIC/ORF] \\
\hline & $\mathrm{V}$ & & $79.6 \pm 0.3$ & 298 & $\mathrm{C}$ & {$[1982 \mathrm{BYS} / \mathrm{MAN}]$} \\
\hline \multirow[t]{3}{*}{$\mathrm{C}_{10} \mathrm{H}_{20} \mathrm{~S}_{4}$} & {$[24194-61-4]$} & \multicolumn{5}{|c|}{ 1,4,8,11-tetrathiacyclotetradecane } \\
\hline & TRS & & 2.7 & 345.2 & & \\
\hline & FUS & & 33.0 & 393.2 & DSC & [2002ROC/GRI] \\
\hline \multirow[t]{4}{*}{$\mathrm{C}_{10} \mathrm{H}_{20} \mathrm{~S}_{5}$} & {$[36338-04-2]$} & \multicolumn{5}{|c|}{ 1,4,7,10,13-pentathiacyclopentadecane } \\
\hline & TRS & & 11.0 & 318.2 & & \\
\hline & TRS & & 4.2 & 340.2 & & \\
\hline & FUS & & 17.0 & 391.2 & DSC & [2002ROC/GRI] \\
\hline \multirow[t]{3}{*}{$\mathrm{C}_{10} \mathrm{H}_{21} \mathrm{Br}$} & {$[112-29-8]$} & 1-bromodecane & & & & \\
\hline & V & $(391-545)$ & 56.1 & 406 & & [1999DYK/SVO] \\
\hline & $\mathrm{V}$ & $(383-570)$ & 56.6 & 398 & A, EST & [1987STE/MAL, 1961LI/ROS] \\
\hline \multirow[t]{4}{*}{$\mathrm{C}_{10} \mathrm{H}_{21} \mathrm{Cl}$} & {$[1002-69-3]$} & 1-chlorodecane & & & & \\
\hline & $\mathrm{V}$ & & $64.0 \pm 0.2$ & 298 & GS & [2001PUR/CHI] \\
\hline & V & $(379-530)$ & 54.4 & 394 & & [1999DYK/SVO] \\
\hline & $\mathrm{V}$ & $(359-499)$ & 56.2 & 374 & A, DTA & [1987STE/MAL, 1969KEM/KRE] \\
\hline \multirow[t]{2}{*}{$\mathrm{C}_{10} \mathrm{H}_{21} \mathrm{~F}$} & {$[334-56-5]$} & 1-fluorodecane & & & & \\
\hline & V & $(342-503)$ & 50.4 & 357 & A & [1987STE/MAL, 1961LI/ROS] \\
\hline \multirow[t]{4}{*}{$\mathrm{C}_{10} \mathrm{H}_{21} \mathrm{I}$} & {$[2050-77-3]$} & 1-iododecane & & & & \\
\hline & V & $(397-598)$ & 69.8 & 298 & $\mathrm{~A}, \mathrm{EST}$ & [1987STE/MAL, 1961LI/ROS, 2006BOL/NER] \\
\hline & $\mathrm{V}$ & $(407-571)$ & 57.4 & 422 & & [1999DYK/SVO] \\
\hline & $\mathrm{V}$ & $(397-598)$ & 58.1 & 412 & $\mathrm{~A}, \mathrm{EST}$ & [1987STE/MAL, 1961LI/ROS] \\
\hline \multirow[t]{2}{*}{$\mathrm{C}_{10} \mathrm{H}_{21} \mathrm{~N}$} & {$[101-40-6]$} & \multicolumn{5}{|c|}{$N, \alpha$-dimethylcyclohexanethylamine } \\
\hline & $\mathrm{V}$ & $(270-300)$ & 50.2 & 285 & A & {$[1987 \mathrm{STE} / \mathrm{MAL}]$} \\
\hline \multirow[t]{2}{*}{$\mathrm{C}_{10} \mathrm{H}_{21} \mathrm{NO}$} & [6282-97-9] & $N, N$-diethylhexana & ide & & & \\
\hline & $\mathrm{V}$ & $(373-443)$ & 47.7 & 388 & A & [1987STE/MAL] \\
\hline $\mathrm{C}_{10} \mathrm{H}_{21} \mathrm{NO}$ & {$[2319-29-1]$} & decanamide & & & & \\
\hline & TRS & & 1.05 & 218.7 & & \\
\hline & TRS & & 18.8 & 366.6 & & \\
\hline & FUS & & 15.1 & 370.6 & DSC & [2008ABA/BAD] \\
\hline & SUB & $(353-370)$ & $125.9 \pm 1.3$ & 361.5 & ME & [1959DAV/JON2] \\
\hline $\mathrm{C}_{10} \mathrm{H}_{21} \mathrm{NO}_{2}$ & & ethyl 2-(N,N-diethy & mino)butano & & & \\
\hline & $\mathrm{V}$ & $(283-313)$ & $57.3 \pm 0.2$ & 298 & GS & {$[1996 \mathrm{VER} / \mathrm{ZUF}]$} \\
\hline $\mathrm{C}_{10} \mathrm{H}_{21} \mathrm{~N}_{3}$ & {$[62103-13-3]$} & 1-decylazide & & & & \\
\hline & $\mathrm{V}$ & $(299-344)$ & $67.8 \pm 0.4$ & 298 & GS & [2014EME/ALG] \\
\hline $\mathrm{C}_{10} \mathrm{H}_{22}$ & {$[124-18-5]$} & decane & & & & \\
\hline & FUS & & 26.48 & 243.5 & DSC & [2005HUA/SIM] \\
\hline & FUS & & 27.6 & 243.0 & DSC & [2004MAR/KAI] \\
\hline & FUS & & 28.7 & 243.5 & & [1996DOM/HEA, 1954FIN/GRO2] \\
\hline & FUS & & 28.78 & 243.1 & $\mathrm{C}$ & [1996DOM/HEA, 1931HUF/PAR] \\
\hline & SUB & & 80.3 & 298 & B & [1980SWA/KWA] \\
\hline & SUB & & 84.8 & 243 & $\mathrm{~B}$ & {$[1963 \mathrm{BON}]$} \\
\hline & SUB & & 82.4 & 298 & $\mathrm{H}$ & [1963BON, 1993CHI/HOS] \\
\hline & $\mathrm{V}$ & $(324-402)$ & 48.3 & 339 & GC & [2007MOK/RAZ] \\
\hline & $\mathrm{V}$ & $(337-376)$ & 46.6 & 352 & & [2002BAT] \\
\hline & $\mathrm{V}$ & & $51.1 \pm 3.9$ & 298 & CGC & {$[2000 \mathrm{NIC} / \mathrm{ORF}]$} \\
\hline & $\mathrm{V}$ & & 51.5 & 299 & $\mathrm{C}$ & [1996VIT/CHA] \\
\hline & V & & 50.5 & 314 & $\mathrm{C}$ & [1996VIT/CHA] \\
\hline & $\mathrm{V}$ & & 50.1 & 324 & $\mathrm{C}$ & [1996VIT/CHA] \\
\hline & $\mathrm{V}$ & & 49.2 & 334 & $\mathrm{C}$ & [1996VIT/CHA] \\
\hline
\end{tabular}


TABLE 13. Phase change enthalpies of $\mathrm{C}_{10}$ organic compounds-Continued

\begin{tabular}{|c|c|c|c|c|c|c|}
\hline \multirow[b]{2}{*}{$\begin{array}{l}\text { Molecular } \\
\text { formula }\end{array}$} & \multirow{2}{*}{$\begin{array}{l}\text { CAS reg. no. } \\
\text { Transition }\end{array}$} & \multicolumn{5}{|l|}{ Compound } \\
\hline & & Temp. range $(\mathrm{K})$ & $\begin{array}{c}\Delta_{\text {trans }} H_{\mathrm{m}} \\
\left(\mathrm{kJ} \mathrm{mol}^{-1}\right)\end{array}$ & $\mathrm{T}_{\mathrm{m}}(\mathrm{K})$ & Method & Reference \\
\hline & $\mathrm{V}$ & $(423-473)$ & 51.5 & 298 & CGC & [1995CHI/HOS] \\
\hline & $\mathrm{V}$ & & 51.4 & 298 & & [1994RUZ/MAJ] \\
\hline & $\mathrm{V}$ & $(409-584)$ & 42.5 & 424 & & [1992LEE/DEM] \\
\hline & $\mathrm{V}$ & $(252-383)$ & 53.8 & 267 & A & [1987STE/MAL] \\
\hline & $\mathrm{V}$ & $(447-526)$ & 41.7 & 462 & A & [1987STE/MAL] \\
\hline & $\mathrm{V}$ & $(524-617)$ & 38.6 & 539 & A & [1987STE/MAL] \\
\hline & $\mathrm{V}$ & $(298-347)$ & 50.3 & 313 & GS & [1986ALL/JOS] \\
\hline & $\mathrm{V}$ & $(308-351)$ & $49.8 \pm 1.7$ & & & [1984BEC/RUC] \\
\hline & V & & $51.4 \pm 0.1$ & 298 & $\mathrm{C}$ & [1982FUR/SAK] \\
\hline & $\mathrm{V}$ & & 51.4 & 298 & $\mathrm{C}$ & [1947OSB/GIN] \\
\hline & $\mathrm{V}$ & $(368-440)$ & 45.5 & 383 & MM & [1945WIL/TAY] \\
\hline \multirow[t]{7}{*}{$\mathrm{C}_{10} \mathrm{H}_{22}$} & [871-83-0] & 2-methylnonane & & & & \\
\hline & FUS & & 17.49 & 198.8 & & [1996DOM/HEA, 1941PAR/WES] \\
\hline & $\mathrm{V}$ & $(324-441)$ & $46.4 \pm 0.2$ & 339 & A & [1987STE/MAL] \\
\hline & $\mathrm{V}$ & & $47.3 \pm 0.2$ & 328 & $\mathrm{C}$ & [1984MAJ/SVO3] \\
\hline & $\mathrm{V}$ & & $46.2 \pm 0.2$ & 343 & $\mathrm{C}$ & [1984MAJ/SVO3] \\
\hline & $\mathrm{V}$ & & $45.0 \pm 0.2$ & 358 & $\mathrm{C}$ & [1984MAJ/SVO3] \\
\hline & $\mathrm{V}$ & & 51.0 & 298 & & [1971WIL/ZWO] \\
\hline \multirow[t]{2}{*}{$\mathrm{C}_{10} \mathrm{H}_{22}$} & [5911-04-6] & 3-methylnonane & & & & \\
\hline & FUS & & 18.7 & 188.5 & & [1996DOM/HEA, 1941PAR/WES] \\
\hline $\mathrm{C}_{10} \mathrm{H}_{22}$ & $\mathrm{~V}$ & & 49.5 & 298 & & [1961LAB/GRE] \\
\hline \multirow[t]{6}{*}{$\mathrm{C}_{10} \mathrm{H}_{22}$} & {$[15869-85-9]$} & 5-methylnonane & & & & \\
\hline & FUS & & 16.65 & 186.7 & & [1996DOM/HEA, 1941PAR/WES] \\
\hline & $\mathrm{V}$ & & $47.0 \pm 0.2$ & 328 & $\mathrm{C}$ & [1984MAJ/SVO3] \\
\hline & $\mathrm{V}$ & & $45.9 \pm 0.2$ & 343 & $\mathrm{C}$ & [1984MAJ/SVO3] \\
\hline & $\mathrm{V}$ & & $44.6 \pm 0.2$ & 358 & $\mathrm{C}$ & [1984MAJ/SVO3] \\
\hline & $\mathrm{V}$ & & 49.8 & 298 & & [1971WIL/ZWO] \\
\hline \multirow[t]{2}{*}{$\mathrm{C}_{10} \mathrm{H}_{22}$} & [5881-17-4] & 3-ethyloctane & & & & \\
\hline & $\mathrm{V}$ & & 49.0 & 298 & & [1971WIL/ZWO] \\
\hline \multirow[t]{2}{*}{$\mathrm{C}_{10} \mathrm{H}_{22}$} & {$[15869-86-0]$} & 4-ethyloctane & & & & \\
\hline & $\mathrm{V}$ & & 48.1 & 298 & & [1971WIL/ZWO] \\
\hline \multirow[t]{3}{*}{$\mathrm{C}_{10} \mathrm{H}_{22}$} & {$[3178-29-8]$} & 4-propylheptane & & & & \\
\hline & $\mathrm{V}$ & & 48.5 & 298 & & [1971WIL/ZWO] \\
\hline & $\mathrm{V}$ & $(331-430)$ & 44.1 & 346 & $\mathrm{~A}, \mathrm{MG}$ & [1987STE/MAL, 1955SCH/WHI] \\
\hline \multirow[t]{2}{*}{$\mathrm{C}_{10} \mathrm{H}_{22}$} & [52896-87-4] & 4-isopropylheptane & & & & \\
\hline & V & & 47.3 & 298 & & [1971WIL/ZWO] \\
\hline \multirow[t]{2}{*}{$\mathrm{C}_{10} \mathrm{H}_{22}$} & {$[15869-87-1]$} & 2,2-dimethyloctane & & & & \\
\hline & V & & 49.0 & 298 & & [1971WIL/ZWO] \\
\hline $\mathrm{C}_{10} \mathrm{H}_{22}$ & $\begin{array}{c}\text { [7146-60-3] } \\
\mathrm{V}\end{array}$ & 2,3-dimethyloctane & 48.1 & 298 & & [1971WIL/ZWO] \\
\hline
\end{tabular}


TABLE 13. Phase change enthalpies of $\mathrm{C}_{10}$ organic compounds-Continued

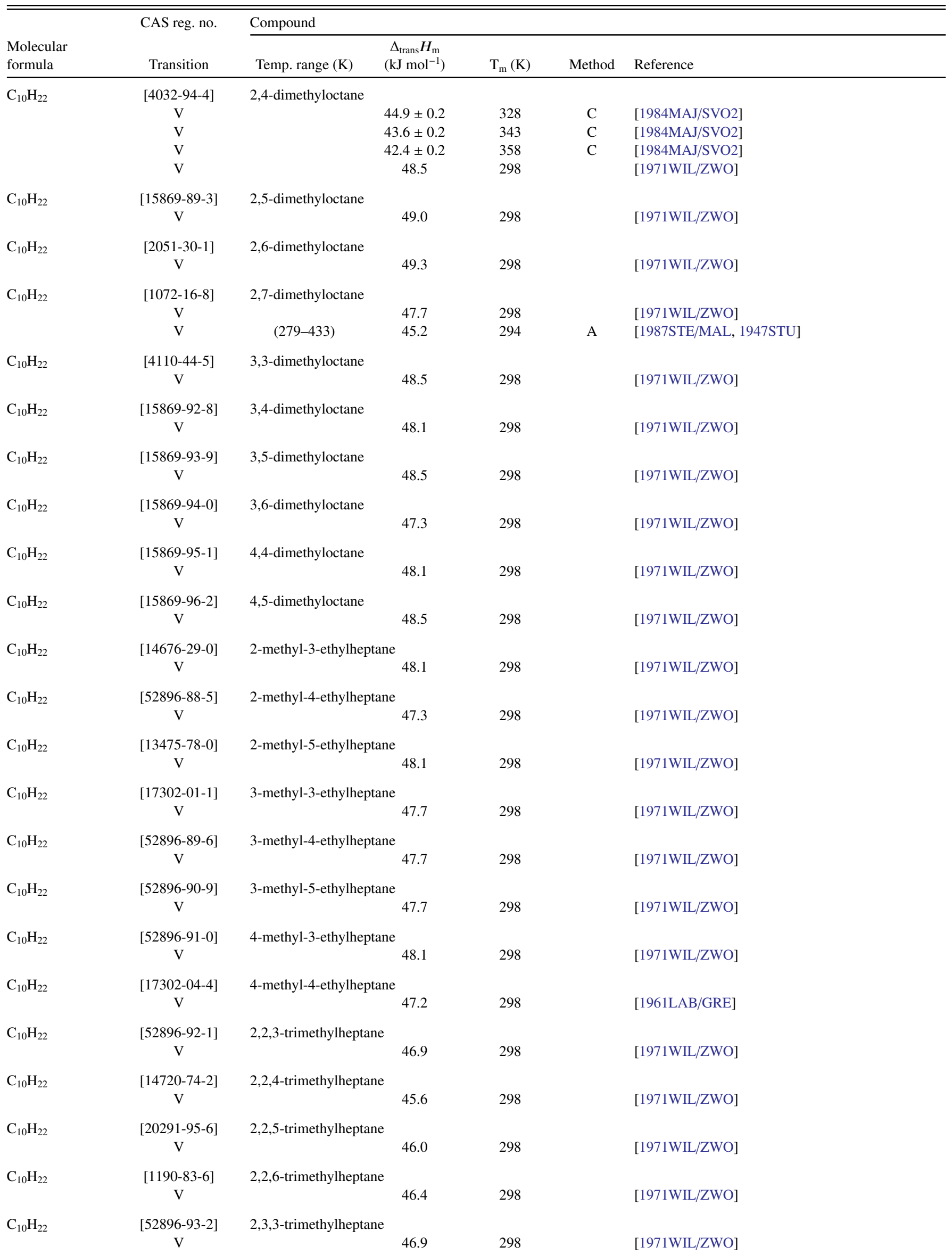


TABLE 13. Phase change enthalpies of $\mathrm{C}_{10}$ organic compounds-Continued

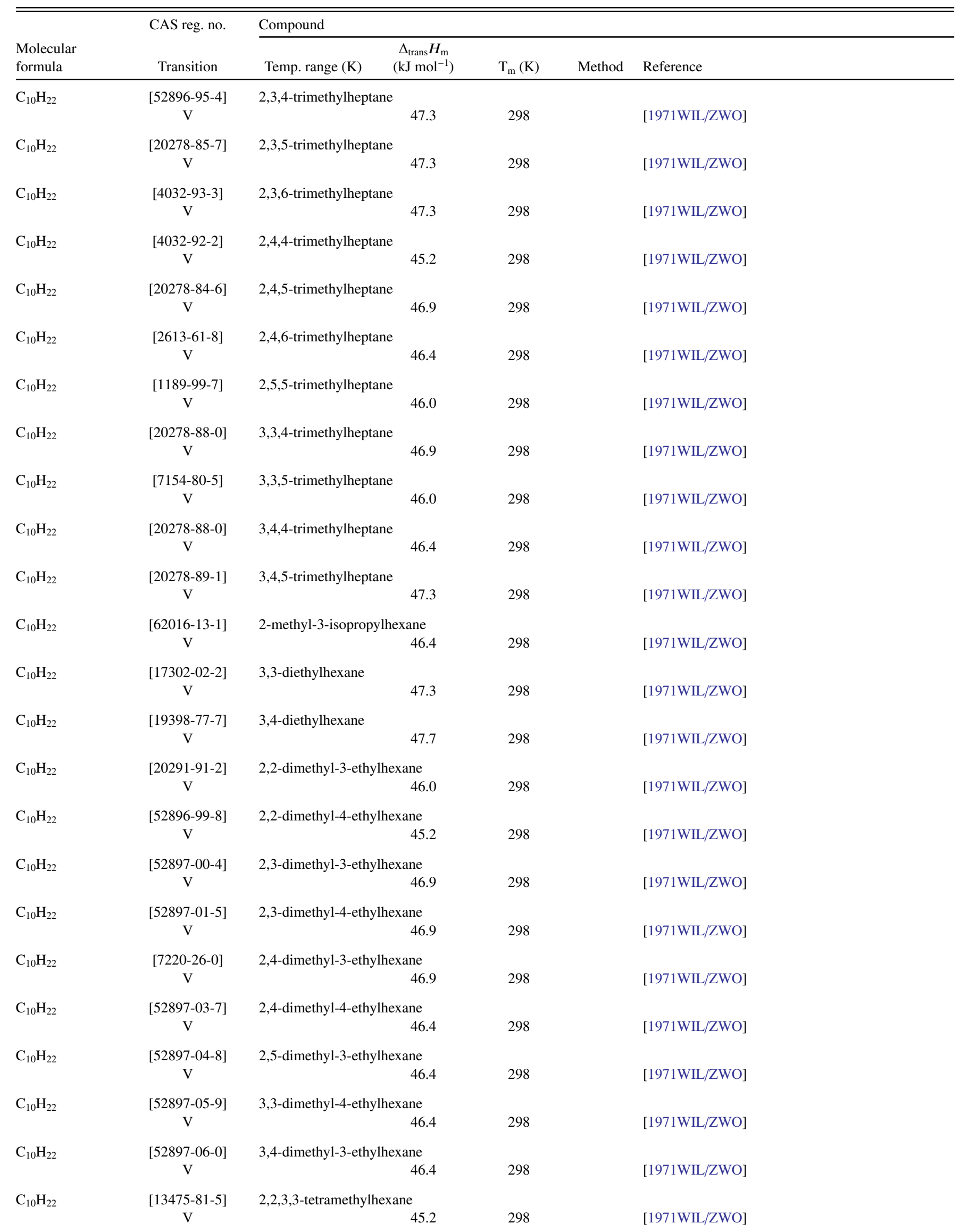


TABLE 13. Phase change enthalpies of $\mathrm{C}_{10}$ organic compounds-Continued

\begin{tabular}{|c|c|c|c|c|c|c|}
\hline \multirow[b]{2}{*}{$\begin{array}{l}\text { Molecular } \\
\text { formula }\end{array}$} & \multirow{2}{*}{$\begin{array}{l}\text { CAS reg. no. } \\
\text { Transition }\end{array}$} & \multicolumn{5}{|l|}{ Compound } \\
\hline & & Temp. range $(\mathrm{K})$ & $\begin{array}{c}\Delta_{\text {trans }} H_{\mathrm{m}} \\
\left(\mathrm{kJ} \mathrm{mol}^{-1}\right)\end{array}$ & $\mathrm{T}_{\mathrm{m}}(\mathrm{K})$ & Method & Reference \\
\hline \multirow[t]{2}{*}{$\mathrm{C}_{10} \mathrm{H}_{22}$} & [52897-08-2] & 2,2,3,4-tetramethyll & xane & & & \\
\hline & $\mathrm{V}$ & & 45.6 & 298 & & [1971WIL/ZWO] \\
\hline \multirow[t]{2}{*}{$\mathrm{C}_{10} \mathrm{H}_{22}$} & [52897-09-3] & 2,2,3,5-tetramethyll & xane & & & \\
\hline & V & & 45.2 & 298 & & [1971WIL/ZWO] \\
\hline \multirow[t]{2}{*}{$\mathrm{C}_{10} \mathrm{H}_{22}$} & {$[51750-65-3]$} & 2,2,4,4-tetramethylh & xane & & & \\
\hline & $\mathrm{V}$ & & 43.5 & 298 & & [1971WIL/ZWO] \\
\hline \multirow[t]{2}{*}{$\mathrm{C}_{10} \mathrm{H}_{22}$} & {$[16747-42-5]$} & 2,2,4,5-tetramethylh & xane & & & \\
\hline & $\mathrm{V}$ & & 44.4 & 298 & & [1971WIL/ZWO] \\
\hline \multirow[t]{2}{*}{$\mathrm{C}_{10} \mathrm{H}_{22}$} & [1071-81-4] & 2,2,5,5-tetramethyll & xane & & & \\
\hline & $\mathrm{V}$ & & 43.5 & 298 & & [1971WIL/ZWO] \\
\hline \multirow[t]{2}{*}{$\mathrm{C}_{10} \mathrm{H}_{22}$} & [52897-10-6] & 2,3,3,4-tetramethyll & xane & & & \\
\hline & $\mathrm{V}$ & & 46.4 & 298 & & [1971WIL/ZWO] \\
\hline \multirow[t]{2}{*}{$\mathrm{C}_{10} \mathrm{H}_{22}$} & [52897-11-7] & 2,3,3,5-tetramethyll & xane & & & \\
\hline & $\mathrm{V}$ & & 45.2 & 298 & & [1971WIL/ZWO] \\
\hline \multirow[t]{2}{*}{$\mathrm{C}_{10} \mathrm{H}_{22}$} & {$[52897-12-8]$} & 2,3,4,4-tetramethyll & xane & & & \\
\hline & $\mathrm{V}$ & & 46.0 & 298 & & [1971WIL/ZWO] \\
\hline \multirow[t]{2}{*}{$\mathrm{C}_{10} \mathrm{H}_{22}$} & {$[52897-15-1]$} & 2,3,4,5-tetramethyll & xane & & & \\
\hline & V & & 46.0 & 298 & & [1971WIL/ZWO] \\
\hline \multirow[t]{2}{*}{$\mathrm{C}_{10} \mathrm{H}_{22}$} & [5171-84-6] & 3,3,4,4-tetramethyll & xane & & & \\
\hline & V & & 42.3 & 298 & & [1971WIL/ZWO] \\
\hline \multirow[t]{2}{*}{$\mathrm{C}_{10} \mathrm{H}_{22}$} & {$[13475-79-1]$} & 2,4-dimethyl-3-isop & opylpentane & & & \\
\hline & V & & 45.6 & 298 & & [1971WIL/ZWO] \\
\hline \multirow[t]{2}{*}{$\mathrm{C}_{10} \mathrm{H}_{22}$} & [52897-16-2] & 2-methyl-3,3-diethy & pentane & & & \\
\hline & $\mathrm{V}$ & & 47.3 & 298 & & [1971WIL/ZWO] \\
\hline \multirow[t]{2}{*}{$\mathrm{C}_{10} \mathrm{H}_{22}$} & {$[52897-17-3]$} & 2,2,3-trimethyl-3-et & ylpentane & & & \\
\hline & $\mathrm{V}$ & & 46.0 & 298 & & [1971WIL/ZWO] \\
\hline \multirow[t]{2}{*}{$\mathrm{C}_{10} \mathrm{H}_{22}$} & [52897-18-4] & 2,2,4-trimethyl-3-et & ylpentane & & & \\
\hline & $\mathrm{V}$ & & 44.8 & 298 & & [1971WIL/ZWO] \\
\hline \multirow[t]{2}{*}{$\mathrm{C}_{10} \mathrm{H}_{22}$} & [52897-19-5] & 2,3,4-trimethyl-3-et & ylpentane & & & \\
\hline & $\mathrm{V}$ & & 46.4 & 298 & & [1971WIL/ZWO] \\
\hline \multirow[t]{2}{*}{$\mathrm{C}_{10} \mathrm{H}_{22}$} & [16747-44-7] & 2,2,3,3,4-pentameth & lpentane & & & \\
\hline & $\mathrm{V}$ & & 45.2 & 298 & & [1971WIL/ZWO] \\
\hline \multirow[t]{2}{*}{$\mathrm{C}_{10} \mathrm{H}_{22}$} & {$[16747-45-8]$} & 2,2,3,4,4-pentameth & lpentane & & & \\
\hline & $\mathrm{V}$ & & 43.5 & 298 & & [1971WIL/ZWO] \\
\hline \multirow[t]{2}{*}{$\mathrm{C}_{10} \mathrm{H}_{22} \mathrm{~N}_{2} \mathrm{O}$} & [28141-55-1] & 1-nonyl urea & & & & \\
\hline & FUS & & 38.9 & 380.3 & DSC & [2005HAS/TAJ] \\
\hline $\mathrm{C}_{10} \mathrm{H}_{22} \mathrm{O}$ & {$[69775-79-7]$} & hexyl tert-butyl eth & & & & \\
\hline & $\mathrm{V}$ & & 53.2 & 298 & $\mathrm{CGC}$ & [UR/VER, 2002VER, 2003VER/KRA] \\
\hline $\mathrm{C}_{10} \mathrm{H}_{22} \mathrm{O}$ & & pentyl tert-amyl eth & & & & \\
\hline & $\mathrm{V}$ & & 53.5 & 298 & CGC & [UR/VER, 2002VER, 2003VER/KRA] \\
\hline $\mathrm{C}_{10} \mathrm{H}_{22} \mathrm{O}$ & {$[187103-12-4]$} & ethyl tert-octyl ethe & & & & \\
\hline & V & & $45.3 \pm 0.3$ & 298 & GS & [UR/VER, 2002VER, 2003VER/KRA] \\
\hline $\mathrm{C}_{10} \mathrm{H}_{22} \mathrm{O}$ & {$[693-65-2]$} & dipentyl ether & & & & \\
\hline & $\mathrm{V}$ & $(373-460)$ & 46.2 & 388 & A & [1987STE/MAL] \\
\hline & $\mathrm{V}$ & $(423-480)$ & 45.6 & 451 & & [1968LAP/NIS] \\
\hline $\mathrm{C}_{10} \mathrm{H}_{22} \mathrm{O}$ & [54459-71-1] & butyl hexyl ether & & & & \\
\hline & V & & $53.2 \pm 0.1$ & 298 & $\mathrm{C}$ & [1985KUS] \\
\hline $\mathrm{C}_{10} \mathrm{H}_{22} \mathrm{O}$ & [544-01-4] & diisopentyl ether & & & & \\
\hline
\end{tabular}


TABLE 13. Phase change enthalpies of $\mathrm{C}_{10}$ organic compounds-Continued

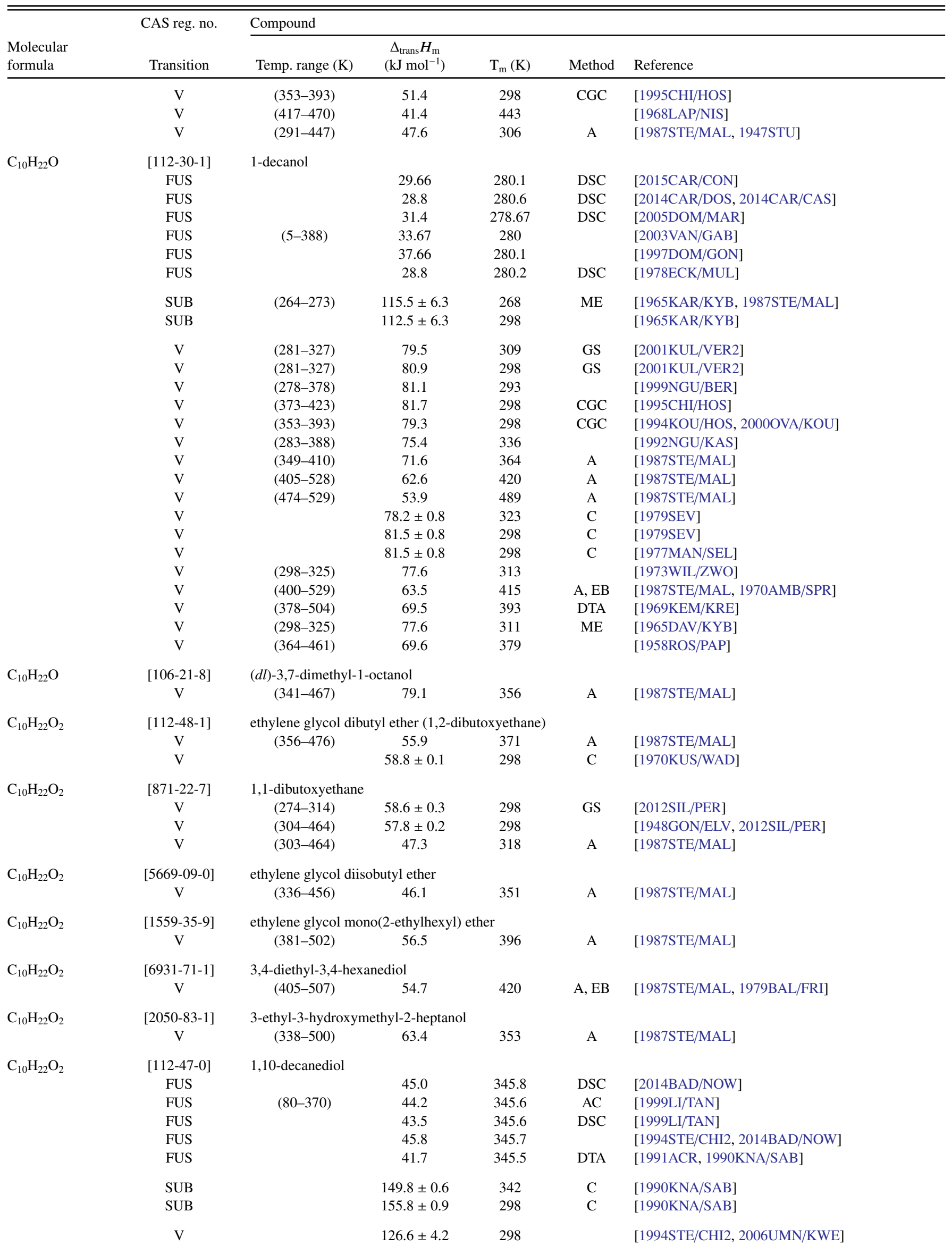


TABLE 13. Phase change enthalpies of $\mathrm{C}_{10}$ organic compounds-Continued

\begin{tabular}{|c|c|c|c|c|c|c|}
\hline \multirow[b]{2}{*}{$\begin{array}{l}\text { Molecular } \\
\text { formula }\end{array}$} & \multirow{2}{*}{$\begin{array}{l}\text { CAS reg. no. } \\
\text { Transition }\end{array}$} & \multicolumn{5}{|l|}{ Compound } \\
\hline & & Temp. range $(\mathrm{K})$ & $\begin{array}{c}\Delta_{\text {trans }} H_{\mathrm{m}} \\
\left(\mathrm{kJ} \mathrm{mol}^{-1}\right)\end{array}$ & $\mathrm{T}_{\mathrm{m}}(\mathrm{K})$ & Method & Reference \\
\hline & $\mathrm{V}$ & & 112.4 & 364 & & [1993PIA/FER, 2006UMN/KWE] \\
\hline & $\mathrm{V}$ & & $120.4 \pm 4.9$ & 298 & & [1993PIA/FER, 2006UMN/KWE] \\
\hline & $\mathrm{V}$ & & $113.7 \pm 2.1$ & 298 & & [1990KNA/SAB, 2006UMN/KWE] \\
\hline \multirow[t]{3}{*}{$\mathrm{C}_{10} \mathrm{H}_{22} \mathrm{O}_{2} \mathrm{~S}$} & {$[126835-71-0]$} & \multicolumn{5}{|c|}{ 3-(heptylthio)-1,2-propanediol } \\
\hline & TRS & & 27.3 & 289.5 & & \\
\hline & FUS & & 1.7 & 292.5 & $\mathrm{DSC}$ & [1993ACR, 1990VAN/VAN] \\
\hline \multirow[t]{4}{*}{$\mathrm{C}_{10} \mathrm{H}_{22} \mathrm{O}_{3}$} & {$[112-59-4]$} & \multicolumn{5}{|c|}{ diethylene glycol monohexyl ether } \\
\hline & $\mathrm{V}$ & $(413-473)$ & 62.8 & 428 & & [2005LEE/SU] \\
\hline & $\mathrm{V}$ & $(403-423)$ & 86.5 & 298 & EB & [2004CHY/FRA2] \\
\hline & $\mathrm{V}$ & $(406-531)$ & 62.7 & 421 & A & [1987STE/MAL] \\
\hline \multirow[t]{2}{*}{$\mathrm{C}_{10} \mathrm{H}_{22} \mathrm{O}_{3}$} & {$[24083-03-2]$} & \multicolumn{5}{|c|}{ dipropylene glycol monobutyl ether } \\
\hline & $\mathrm{V}$ & $(337-500)$ & 63.2 & 352 & A & [1987STE/MAL, 1947STU] \\
\hline \multirow[t]{2}{*}{$\mathrm{C}_{10} \mathrm{H}_{22} \mathrm{O}_{3}$} & {$[127748-41-8]$} & \multicolumn{5}{|c|}{ 3-(heptyloxy)-1,2-propanediol } \\
\hline & FUS & & 28.8 & 288 & DSC & [1993ACR, 1990VAN/VAN] \\
\hline \multirow[t]{2}{*}{$\mathrm{C}_{10} \mathrm{H}_{22} \mathrm{O}_{4}$} & {$[20324-33-8]$} & \multicolumn{5}{|c|}{ tripropylene glycol monomethyl ether } \\
\hline & $\mathrm{V}$ & $(308-515)$ & 58.7 & 323 & A & [1987STE/MAL] \\
\hline \multirow[t]{3}{*}{$\mathrm{C}_{10} \mathrm{H}_{22} \mathrm{O}_{5}$} & {$[143-24-8]$} & \multicolumn{5}{|c|}{ tetraethylene glycol dimethyl ether (tetraglyme) } \\
\hline & $\mathrm{V}$ & & $76.9 \pm 2.6$ & 298 & CGC & [2000NIC/ORF] \\
\hline & $\mathrm{V}$ & $(419-553)$ & 58 & 434 & A & [1987STE/MAL] \\
\hline \multirow[t]{2}{*}{$\mathrm{C}_{10} \mathrm{H}_{22} \mathrm{~S}$} & {$[3698-94-0]$} & \multicolumn{5}{|l|}{ 1-ethylthiooctane } \\
\hline & V & $(384-545)$ & $63.9 \pm 0.6$ & 298 & EB & [1996STE/CHI] \\
\hline \multirow[t]{6}{*}{$\mathrm{C}_{10} \mathrm{H}_{22} \mathrm{~S}$} & {$[143-10-2]$} & 1-decanethiol & & & & \\
\hline & FUS & & 33.3 & 247.9 & & [1996DOM/HEA, 1970FIN/MCC] \\
\hline & $\mathrm{V}$ & $(390-544)$ & 56.4 & 405 & & [1999DYK/SVO] \\
\hline & $\mathrm{V}$ & $(283-293)$ & 58.6 & 288 & A & [1987STE/MAL] \\
\hline & $\mathrm{V}$ & $(413-534)$ & 54.6 & 428 & A & [1987STE/MAL] \\
\hline & $\mathrm{V}$ & & $65.5 \pm 0.5$ & 298 & $\mathrm{C}$ & [1977MAN/SEL] \\
\hline $\mathrm{C}_{10} \mathrm{H}_{22} \mathrm{~S}$ & {$[13402-60-3]$} & 2-decanethiol & & & & \\
\hline & $\mathrm{V}$ & $(380-534)$ & 54.6 & 395 & & [1999DYK/SVO] \\
\hline $\mathrm{C}_{10} \mathrm{H}_{22} \mathrm{~S}$ & {$[544-02-5]$} & diisopentyl sulfide & & & & \\
\hline & $\mathrm{V}$ & $(339-366)$ & 57.9 & 352 & A & [1987STE/MAL, 1999DYK/SVO] \\
\hline & V & $(340-365)$ & 56.9 & 352 & $\mathrm{C}$ & [1962MAC/MAY2] \\
\hline $\mathrm{C}_{10} \mathrm{H}_{22} \mathrm{~S}$ & {$[872-10-6]$} & dipentyl sulfide & & & & \\
\hline & $\mathrm{V}$ & $(346-365)$ & U66.3 & 356 & & [1999DYK/SVO] \\
\hline & $\mathrm{V}$ & $(346-366)$ & 58.7 & 356 & A & [1987STE/MAL] \\
\hline & $\mathrm{V}$ & $(346-366)$ & 57.5 & 358 & EB & [1962MAC/MAY2] \\
\hline $\mathrm{C}_{10} \mathrm{H}_{22} \mathrm{~S}_{2}$ & {$[112-51-6]$} & dipentyl disulfide & & & & \\
\hline & V & $(410-571)$ & 59.8 & 425 & & [1999DYK/SVO] \\
\hline & $\mathrm{V}$ & & $71.1 \pm 0.2$ & 298 & $\mathrm{C}$ & [1985KUS] \\
\hline $\mathrm{C}_{10} \mathrm{H}_{22} \mathrm{~S}_{2}$ & [1191-67-9] & 1,10-decanedithiol & & & & \\
\hline & V & $(434-571)$ & 72.3 & 449 & A & [1987STE/MAL, 1999DYK/SVO, 1943HAL/REI] \\
\hline $\mathrm{C}_{10} \mathrm{H}_{23} \mathrm{~N}$ & {$[2016-57-1]$} & decylamine & & & & \\
\hline & FUS & & 42.70 & 289.2 & $\mathrm{DSC}$ & [2005DOM/MAR] \\
\hline & $\mathrm{V}$ & & $65.3 \pm 2.3$ & 298 & CGC & [2013GOB/RAT] \\
\hline & $\mathrm{V}$ & $(299-343)$ & $64.9 \pm 0.3$ & 298 & GS & {$[2013 \mathrm{THO} / \mathrm{CHI}]$} \\
\hline & $\mathrm{V}$ & & $64.8 \pm 4.6$ & 298 & CGC & [2013THO/CHI] \\
\hline & $\mathrm{V}$ & $(410-506)$ & 52.4 & 425 & A, EST & [1987STE/MAL, 1956MAN2] \\
\hline & $\mathrm{V}$ & $(329-431)$ & $56.1 \pm 0.6$ & 380 & $\mathrm{BP}$ & [1940RAL/SEL, 2013THO/CHI] \\
\hline & $\mathrm{V}$ & $(329-431)$ & $65.1 \pm 0.6$ & 380 & $\mathrm{BP}$ & [1940RAL/SEL, 2013THO/CHI] \\
\hline $\mathrm{C}_{10} \mathrm{H}_{23} \mathrm{~N}$ & [7378-99-6] & $N, N$-methyloctylam & & & & \\
\hline & $\mathrm{V}$ & $(420-458)$ & 46.9 & 439 & EB & [2012ROZ/BRU] \\
\hline & $\mathrm{V}$ & $(284-323)$ & $54.0 \pm 0.5$ & 303 & & [1997VER] \\
\hline & $\mathrm{V}$ & $(371-517)$ & 50.2 & 386 & A & [1987STE/MAL] \\
\hline
\end{tabular}


TABLE 13. Phase change enthalpies of $\mathrm{C}_{10}$ organic compounds-Continued

\begin{tabular}{|c|c|c|c|c|c|c|}
\hline $\begin{array}{l}\text { Molecular } \\
\text { formula }\end{array}$ & $\begin{array}{l}\text { CAS reg. no. } \\
\text { Transition }\end{array}$ & \multicolumn{5}{|l|}{ Compound } \\
\hline \multirow{3}{*}{$\mathrm{C}_{10} \mathrm{H}_{23} \mathrm{~N}$} & $\mathrm{~V}$ & & $61.2 \pm 2.6$ & 298 & CGC & [2014THO/GOB] \\
\hline & $\mathrm{V}$ & $(379-527)$ & 51.2 & 394 & A & [1987STE/MAL] \\
\hline & $\mathrm{V}$ & $(379-527)$ & $61.6 \pm 1.5$ & 298 & A & [1987STE/MAL, 2014THO/GOB] \\
\hline $\mathrm{C}_{10} \mathrm{H}_{23} \mathrm{NO}_{2}$ & FUS & & 28.8 & 324.9 & DSC & [1993ACR, 1990VAN/VAN] \\
\hline \multirow[t]{2}{*}{$\mathrm{C}_{10} \mathrm{H}_{23} \mathrm{~N}_{3}$} & {$[67752-90-3]$} & \multicolumn{5}{|c|}{ [2-(dimethylamino)ethyl]methylhydrazone-2-propanone } \\
\hline & $\mathrm{V}$ & $(288-315)$ & 62.3 & 301 & A & [1987STE/MAL, 1980LEB/NAZ] \\
\hline \multirow[t]{2}{*}{$\mathrm{C}_{10} \mathrm{H}_{24} \mathrm{NO}_{3} \mathrm{PS}$} & {$[78-53-5]$} & \multicolumn{5}{|c|}{$O, O$-diethyl-S-[2-(diethylamino)ethyl]thiophosphate } \\
\hline & $\mathrm{V}$ & $(358-407)$ & 94.5 & 373 & A & [1987STE/MAL, 1999DYK/SVO] \\
\hline $\mathrm{C}_{10} \mathrm{H}_{24} \mathrm{~N}_{2}$ & $\mathrm{~V}$ & $(338-353)$ & $80.2 \pm 0.3$ & 298 & GS & [2011POZ/VER] \\
\hline \multirow[t]{2}{*}{$\mathrm{C}_{10} \mathrm{H}_{24} \mathrm{~N}_{4}$} & [996-70-3] & \multicolumn{5}{|c|}{ tetrakis(dimethylamino)ethylene } \\
\hline & $\mathrm{V}$ & $(358-485)$ & $53.9 \pm 0.5$ & 298 & EB & [1997STE/CHI4] \\
\hline \multirow[t]{2}{*}{$\mathrm{C}_{10} \mathrm{H}_{24} \mathrm{~N}_{4}$} & {$[295-37-4]$} & \multicolumn{5}{|c|}{ 1,4,8,11-tetraazacyclotetradecane } \\
\hline & SUB & $(352-372)$ & $133.9 \pm 2.5$ & 362 & $\mathrm{TE}$ & [1983CLA/COR] \\
\hline \multirow[t]{4}{*}{$\mathrm{C}_{10} \mathrm{H}_{27} \mathrm{~N}_{5} \mathrm{O}_{6}$} & [114606-56-3] & \multicolumn{5}{|c|}{$\begin{array}{l}\text { 8-[[(1R)-1-(3,4-dimethoxyphenyl)-2-hydroxyethyl]amino]-3,7-dihydro-7-(2-methoxyethyl)- } \\
\text { 1,3-dimethyl-1 } \boldsymbol{H} \text {-pyrine-2,6-dione }\end{array}$} \\
\hline & FUS(I) & & 40.31 & 384.2 & & \\
\hline & FUS(II) & & 39.88 & 401.2 & & \\
\hline & FUS(III) & & 38.58 & 391.2 & DSC & [1999GIR/PIE] \\
\hline
\end{tabular}

\section{Acknowledgment}

The authors would like to thank Dr. Don Burgess for all the help he provided in preparation of this manuscript for publication.

\section{References}

1871REG 1873BER

1879PET

$1883 \mathrm{KAH}$

1884WER

1886RIC

1889COL

1889EYK

1889YOU

1894BRU

$1894 \mathrm{KAH}$

1895PIC

1896GRI/MAR

$1898 \mathrm{KAH}$

1898LOU

1899HUL

1901DEF

1901DEF2

$1901 \mathrm{KAH}$

$1901 \mathrm{KAH} 2$

1906REX
M. Regnault, Ann. Chim. Phys. 23, 73 (1871).

M. Berthelot, Compt. Rend. 77, 26 (1873).

Petterson, Ges. Wiss. Upsala 36 (1879).

G. W. A. Kahlbaum, Ber. Dtsch. Chem. Ges. 16, 2476-2484 (1883).

E. Werner, Ann. Chim. Phys. 2, 567 (1884).

A. Richardson, J. Chem. Soc., Trans. 49, 761-776(1886).

N. Collie, J. Chem. Soc., Trans. 55, 110 (1889).

J. F. Eykman, Z. Phys. Chem. 4, 497-519 (1889).

S. Young, J. Chem. Soc. 55, 486 (1889).

L. Bruner, Chem. Ber. 27, 2102 (1894).

G. W. A. Kahlbaum, Z. Phys. Chem. 13, 14-55 (1894).

S. U. Pickering, J. Chem. Soc. 67, 664 (1895).

E. H. Griffiths and D. Marshall, Philos. Mag. J. Sci. 41, 1 (1896).

G. W. A. Kahlbaum, Z. Phys. Chem. 26, 577 (1898).

W. Louguinine, Ann. Chim. 7, 334-337 (1898).

G. A. Hulett, Z. Phys. Chem. 28, 629 (1899).

M. de Forcrand, Comp. Rend. 132, 688-690 (1901).

M. de Forcrand, Comp. Rend. 132, 569-571 (1901).

L. Kahlenberg, J. Phys. Chem. 5, 284 (1901).

L. Kahlenberg, J. Phys. Chem. 5, 215 (1901).

A. Rex, Z. Phys. Chem. 55, 355-370 (1906).
$1907 \mathrm{MCI}$

1908BOG/WIN

1909MAS/FAU

1910BIN

1910MEY

1911LOU/DUP

1912DEF

1913BLA

1913FLE/TYR

1913HER/RAT

1913MUN

1914MUL

1915BRI

1916TER

1918NAR

1919BEL/SAW

1919MOL/BAT

1920ATK/HEY

1920BAX/BEZ

1920GIB/PAR

1920MON

1921MAA/WRI

1921SID/RUB
D. McIntosh, J. Phys. Chem. 11, 306 (1907).

A. Bogojawlenski and N. Windogradow, Z. Phys. Chem. 64, 251 (1908).

G. Massol and A. Faucon, Compt. Rend. 149, 345 (1909). E. C. Bingham, Am. Chem. J. 43, 287-309 (1910). J. Meyer, Z. Phys. Chem. 72, 225-254 (1910). W. Louguinine and G. Dupont, Bull. Soc. Chim. Fr. 9, 219-224 (1911); Chem. Abstr. 5, 10166 (1911).

M. de Forcrand, C. R. Hebd. Seances Acad. Sci. 154, 1766 (1912).

J. J. Blanksma, Chem. Weekbl. 9, 924 (1913).

J. F. Fletcher and D. Tyrer, J. Chem. Soc. 103, 513 (1913).

W. Herz and W. Rathmann, Chem.-Ztg. 36, 1417 (1913).

C. F. Mündel, Z. Phys. Chem. 85, 435-465 (1913).

A. H. R. Muller, Z. Phys. Chem. 86, 177 (1914).

P. W. Bridgman, Phys. Rev. 6, 94 (1915).

J. W. Terwen, Z. Phys. Chem. 91, 469-499 (1916).

J. Narbutt, Z. Elektrochem. Angew. Phys. Chem. 24, 339 (1918).

J. M. Bell and J. P. Sawyer, J. Ind. Eng. Chem. 11, 1025-1028 (1919).

E. Moles and T. J. Batuecas, Chim. Phys. Phys.-Chim. Biol. 17, 537-588 (1919).

R. H. Atkinson, C. T. Heycock, and W. J. Pope, J. Chem. Soc., Trans. 117, 1410 (1920).

G. P. Baxter, F. K. Bezzenberger, and C. H. Wilson, J. Am. Chem. Soc. 42, 1386-1392 (1920).

G. E. Gibson, G. S. Parks, and W. M. Latimer, J. Am. Chem. Soc. 42, 1542 (1920).

K. P. Monroe, J. Ind. Eng. Chem. 12, 969-971 (1920).

O. Maass and C. H. Wright, J. Am. Chem. Soc. 43, 1098-1111 (1921).

N. V. Sidgwick and H. E. Rubie, J. Chem. Soc., Faraday Trans. 119, 1013 (1921). 
1921STO/HEN A. Stock, F. Henning, and E. Kuss, Ber. Dtsch. Chem. Ges. 54, 1119-1129 (1921).

1922LAT

1922NEL/SEN

W. M. Latimer, J. Am. Chem. Soc. 44, 90 (1922).

1922STR/PAR $\quad$ K. Stratton and J. R. Partington, Philos. Mag. 43, 436 (1922).

1922TAY/SMI R. S. Taylor and L. B. Smith, J. Am. Chem. Soc. 44, 2450-2463 (1922).

1923AWB/GRI J. H. Awbery and E. Griffiths, Proc. Phys. Soc., London 36, 303 (1923).

1923GIB/GIA G. E. Gibson and W. F. Giauque, J. Am. Chem. Soc. 45, 93 (1923).

1923JEN/SHO C. F. Jenkin and D. N. Shorthose, Special Report 14, Department of Scientific and Industrial Research, Food Investigation Board, Great Britain, 1923.

1923MOR/MUR F. S. Mortimer and R. V. Murphy, Ind. Eng. Chem. 15, 1140-1142 (1923).

1923WEI/DOW R. M. Weis and C. R. Downs, J. Am. Chem. Soc. 45, 1003 (1923).

1924EUC/KAR A. Eucken and E. Karwat, Z. Phys. Chem. 112, 467 (1924).

1924GAR/RAN W. E. Garner and F. C. Randall, J. Chem. Soc., Trans. 125, 881 (1924).

1924SHO

D. N. Shorthose, Special Report 19, Department of Scientific and Industrial Research, Food Investigation Board, Great Britain, 1924.

1924STR/PAR K. Stratton and J. R. Partington, Philos. Mag. 48, 1085 (1924).

1925KOH G. T. Kohman, J. Phys. Chem. 29, 1048 (1925).

1925MAA/WAL O. Maas and L. J. Walbauer, J. Am. Chem. Soc. 47, 1 (1925).

1925NEL/WAL O. A. Nelson and H. Wales, J. Am. Chem. Soc. 47, 867 (1925).

1925PAR G. S. Parks, J. Am. Chem. Soc. 47, 338 (1925).

1925PAR/KEL G. S. Parks and K. K. Kelley, J. Am. Chem. Soc. 47, 2089-2097 (1925).

1925PER/BAR J. H. Perry and D. C. Bardwell, J. Am. Chem. Soc. 47, 2629-2632 (1925).

1925SWA/MAC T. H. Swan and H. Mack, Jr., J. Am. Chem. Soc. 47, 2112-2116 (1925)

1925WIL

1926AND

J. W. A. Williams, J. Am. Chem. Soc. 47, 2644 (1925).

1926AND/LYN $\quad$ D. H. Andrews, G. Lynn, and J. Johnston, J. Am. Chem. Soc. 48, 1274-1287 (1926).

1926BER/MAY J. F. T. Berliner and O. E. May, J. Am. Chem. Soc. 48, 2630 (1926).

1926DAN/JEN L. I. Dana, A. C. Jenkins, J. N. Burdick, and R. C. Timm, Refrig. Eng. 12, 387 (1926).

1926EVA/AYL W. V. Evans and M. B Aylesworth, Ind. Eng. Chem. 18, 24-27 (1926).

1926FEL/DUR W. A. Felsing and S. A. Durban, J. Am. Chem. Soc. 48, 2885-2893 (1926).

1926LOO/WAL A. G. Loomis and J. E. Walters, J. Am. Chem. Soc. 48, 2051-2055 (1926).

1926MAT J. H. Mathews, J. Am. Chem. Soc. 48, 562-576 (1926).

1926NOY/WEB W. A. Noyles, Jr. and D. E. Webbe, J. Am. Chem. Soc. 48, 1882-1887 (1926).

1926PAR/AND G. S. Parks and C. T. Anderson, J. Am. Chem. Soc. 48, 1506 (1926).

1926PER/POR J. H. Perry and F. Porter, J. Am. Chem. Soc. 48, 299-302 (1926).

1926SIN/HAR H. Sinozaki, R. Hara, and S. Mitsukuri, Bull. Chem. Soc. Jpn. 1, 59-61 (1926).

1926STR

1926TAY/RIN

1926YAT

1927BER/MAY

F. Straus, Ber. Dtsch. Chem. Ges. 59, 1664-1681 (1926).

C. A. Taylor and W. H. Rinkenbach, J. Chem. Soc. 48, 1305-1309 (1926).

G. W. C. Yates, Philos. Mag. 2, 817-826 (1926).

1927COO/COO A. S. Coolidge and M. S. Coolidge, J. Am. Chem. Soc. 49, 100-104 (1927).
1927DAN/BAR L. I. Dana, J. N. Bardick, and A. C. Jenkins, J. Am. Chem. Soc. 49, 2801 (1927).

1927EDW/WIL M. J. Edwards and J. M. Williams, J. Chem. Soc. 1927, 855.

1927KLO/WOO S. Klosky, L. P. L. Woo, and R. J. Flanigan, J. Am. Chem. Soc. 49, 1280-1284 (1927).

1927MAY/BER O. E. May, J. F. T. Berliner, and D. F. J. Lynch, J. Am. Chem. Soc. 49, 1012-1016 (1927).

1927NAG N. N. Nagornov, Anal. Instrum. Anal. Phys. Chem. 3, 562-583 (1927); Chem. Zentr. II 2668 (1927); Chem. Abstr. 22, 4298 (1928).

1927PER J. H. Perry, J. Phys. Chem. 31, 1737-1741 (1927).

1927RIN W. H. Rinkenbach, Ind. Eng. Chem. 19, 474-476 (1927).

1927STE/JOH L. E. Steiner and J. J. Johnston, J. Phys. Chem. 32, 912-939 (1927).

1927SUT/LAY J. A. Sutcliffe, F. C. Lay, and W. L. Prichard, Proc. R. Soc. A 115, 88 (1927).

1928EUC/HAU A. Eucken and F. Hauck, Z. Phys. Chem. 134, 161 (1928).

1928 NEL

1928NEL2

1928PAR/BAR

O. A. Nelson, Ind. Eng. Chem. 20, 1382-1384 (1928).

O. A. Nelson, Ind. Eng. Chem. 20, 1380-1382 (1928).

G. S. Parks and B. J. Barton, J. Am. Chem. Soc. 50, 24-27 (1928).

1928PAR/KEL G. S. Parks and K. K. Kelley, J. Phys. Chem. 32, 734 (1928).

1928ROL M. Roland, Bull. Soc. Chim. Belg. 37, 117-140 (1928)

1928SHI V. P. Shishokina, Izv. Inst. Fiz.-Khim. Anal., Akad. Nauk SSSR 4, 183 (1928).

1928SKA/SAX E. L. Skau and B. Saxton, J. Am. Chem. Soc. 50, 2693 (1928).

1928STE

1929BAB/JAC

D. F. Stedman, Trans. Faraday Soc. 24, 289 (1928).

1929CLU

V. S. Babasinian and J. G. Jackson, J. Am. Chem. Soc. 51 2147 (1929).

1929HAG/WEI C. F. Haggerty and J. F. Weiler, J. Am. Chem. Soc. 51, 1623-1626 (1929).

1929KEL

1929KEL2

1929KEL3

1929KEL4

1929KEL5

1929MOO/LUD

K. K. Kelley, J. Am. Chem. Soc. 51, 2738 (1929).

K. K. Kelley, J. Am. Chem. Soc. 51, 180 (1929).

K. K. Kelley, J. Am. Chem. Soc. 51, 1145 (1929).

K. K. Kelley, J. Am. Chem. Soc. 51, 779 (1929).

K. K. Kelley, J. Am. Chem. Soc. 51, 1400 (1929).

1929PAR/TOD

R. B. Mooney and E. B. Ludlam, Proc. R. Soc. Edinburgh 49, 160 (1929).

1929PEA/PET

G. S. Parks and S. S. Todd, J. Ind. Eng. Chem. 21, 1235 (1929).

1929SMY/ENG

J. N. Pearce and P. E. Peters, J. Phys. Chem. 33, 873-878 (1929).

$1930 \mathrm{COO}$

1930DRU/FLA

C. P. Smyth and E. W. Engel, J. Am. Chem. Soc. 51, 2646-2660 (1929).

1930EVA/DAV

A. S. Coolidge, J. Am. Chem. Soc. 52, 1874-1887 (1930). 1930, 1310.

1930HUF/PAR H. M. Huffman, G. S. Parks, and S. B. Thomas, J. Am. Chem. Soc. 52, 3241 (1930).

1930HUf/PAR2 H. M. Huffman, G. S. Parks, and A. C. Daniels, J. Am. Chem. Soc. 52, 1547 (1930).

1930LIV/HEI R. Livingston and J. B. Heisig, J. Am. Chem. Soc. 52, 2409-2410 (1930).

1930NEL

1930PAR/HUF

O. A. Nelson, Ind. Eng. Chem. 22, 971-972 (1930).

1930PAR/HUF2 G. S. Parks and H. M. Huffman, J. Am. Chem. Soc. 52, 4381 (1930).

1930PHI/WAT J. C. Philip and S. C. Waterton, J. Chem. Soc. 1930, 2783.

1930 SCH/THO H. A. Schuette and R. W. Thomas, J. Am. Chem. Soc. 52, 2028-2030 (1930).

1930SOU/AND J. C. Southland and D. H. Andrews, J. Franklin Inst. 209, 349-360 (1930).

1930WAS A. Wasserman, Z. Phys. Chem. A146, 418 (1930).

1930WIE/HUB R. Wiebe, K. H. Hubbard, and M. J. Brevoort, J. Am. Chem. Soc. 52, 611 (1930). 
1930ZMA

1931BUF/FLE R. M. Buffington and J. Fleischer, J. Ind. Eng. Chem. 23,

M. A. Zmaczynski, J. Chim. Phys. 27, 503-517 (1930). 1290-1292 (1931).

1931COS V. E. Cosslett, Z. Anorg. Allg. Chem. 201, 75-80 (1931); Chem. Abstr. 26, 1535 (1932).

1931CRO/BIJ C. A. Crommelin, W. J. Bijleveld, and E. G. Brown, Proc. Acad. Sci. Amsterdam 34, 1314-1317 (1931); Chem. Abstr. 26, 2904 (1931).

1931DEE R. F. Deese, J. Am. Chem. Soc. 53, 3673 (1931).

1931FIO/GIN E. F. Fiock, D. C. Ginnings, and W. B. Holton, J. Res. Natl. Bur. Stand. 6, 881 (1931).

1931HIL A. E. Hill, J. Am. Chem. Soc. 53, 2598-2608 (1931).

1931HUF/PAR H. M. Huffman, G. S. Parks, and M. Barmore, J. Am. Chem. Soc. 53, 3876 (1931).

1931LIN E. G. Lindner, J. Phys. Chem. 35, 531-535 (1931).

1931MAT/FEH J. E. Mathews and P. R. Fehlandt, J. Am. Chem. Soc. 53, 3212 (1931).

1931PAR/HUF G. S. Parks and H. M. Huffman, Ind. Eng. Chem. 23, 1138 (1931).

1931SCH/COW H. A. Schuette and M. A. Cowley, J. Am. Chem. Soc. 53, 3485-3489 (1931).

1931VAN/MAN P. A. van der Meulen and R. F. Mann, J. Am. Chem. Soc. 53, 451-453 (1931).

1932CLA/GIA J. O. Clayton and W. F. Giauque, J. Am. Chem. Soc. 54, 2610-2626 (1932).

1932ELL/REI L. M. Ellis, Jr. and E. E. Reid, J. Am. Chem. Soc. 54, 1674-1687 (1932).

1932GRI/AWB E. Griffiths and J. H. Awbery, Proc. Phys. Soc. 44, 121 (1932).

1932 KUB

1932SPA/THO

T. Kubota, J. Chem. Soc. Jpn. 53, 404-417 (1932).

1932VAU

Chem. 36, 882-888 (1932).

W. E. Vaughan, J. Am. Chem. Soc. 54, 3863-3876 (1932).

1933BAR/BUR $\quad$ E. G. V. Barrett and J. L. Burrage, J. Phys. Chem. 37, 1029-1035 (1933).

1933BOO/BUR H. S. Booth, P. E. Burchfield, E. M. Bixly, and J. B. MacKelvey, J. Am. Chem. Soc. 53, 2231-2235 (1933).

1933BRO/FRA L. F. Broadway and R. G. J. Fraser, J. Chem. Soc. 429-430 (1933).

1933DEI

1933FER/THO

V. R. Deitz, J. Am. Chem. Soc. 55, 472-475 (1933).

1933HEI

1933HEI/MUR

1933HEI2

1933HEN/MUR

1933HOR/GEI

1933HOR/LAN

1933JOH

1933LEN

1933MAR/CUT

1933MEH

1933MEN/MOH

1933MIL/MEN

1933NEL/YOU

1933PAR/HUF

1933ROT/MEY

1933RUF/BRE

1933TAN

1933TAY/LAY

J. Marsden and A. C. Cuthbertson, Can. J. Res. 9, 419 (1933).

W. Mehl, Beih., Z. Ges. Kalte-Ind. Ser. 1, 5 (1933). 257-263 (1933)

F. T. Miles and A. W. C. Menzies, J. Phys. Chem. 37, 425-430 (1933).

O. A. Nelson and H. D. Young, J. Am. Chem. Soc. 55, 2429-2431 (1933).

G. S. Parks, H. M. Huffman, and M. Barmore, J. Am. Chem. Soc. 55, 2733-2740 (1933).

W. A. Roth and I. Meyer, Z. Electrochem. 39, 35 (1933). O. Ruff and O. Bretschneider, Z. Anorg. Allg. Chem. 210, 173 (1933).

H. Tanneberger, Ber. Dtsch. Chem. Ges. 66, 484-486 (1933); Chem. Abstr. 27, 3374 (1933).

A. Taylor and E. T. Layng, J. Chem. Phys. 1, 798-808
1933THO/BUR

N. V. Thornton, A. B. Burg, and H. I. Schlesinger, J. Am. Chem. Soc. 55, 3177 (1933).

1933TOR/MOL M. T. Toral and E. Moles, An. R. Soc. Esp. Fis. Quim. 31, 735-745 (1933); Chem. Abstr. 28, 954 (1934).

1933WHI/FLE $\quad$ F. C. Whitmore and G. H. Fleming, J. Am. Chem. Soc. 55, 3803-3806 (1933).

1933 YOS/STO D. M. Yost and W. E. Stone, J. Am. Chem. Soc. 55, 1889-1895 (1933).

1934CAM/CAM A. N. Campbell and A. J. R. Campbell, Trans. Faraday Soc. 30, 1109-1114 (1934).

1934GOO

J. W. Goodeve, Trans. Faraday Soc. 30, 501-504 (1934), Note: Vapor pressure data is presented graphically in paper as $\log \mathrm{P}$ versus $1 / \mathrm{T}$.

1934GOR S. V. Gorbachev, Zh. Prikl. Khim. 7, 388-391 (1934); Chem. Abstr. 29, 13996 (1934).

1934HIE/WOE W. Hieber and A. Woerner, Z. Elektrochem. Angew. Phys. Chem. 40, 252-256 (1934).

1934HIR

1934JAC/PAR

H. Hirsbrunner, Helv. Chim. Acta 17, 477-504 (1934).

\section{(1934)}

1934JOH/LON H. L. Johnston and E. A. Long, J. Am. Chem. Soc. 56, 31 (1934).

1934LEW/SCH G. N. Lewis and P. W. Schutz, J. Am. Chem. Soc. 56, 1002 (1934).

1934MEH

1934MIL/DIT

W. Mehl, Z. Phys. Chem. A164, 312-313 (1934)

$19340 \mathrm{GU} / \mathrm{ANJ}$ F. W. Miller, Jr. and H. R. Dittmar, J. Am. Chem. Soc. 56, 848 (1934).

1934PAR/THO

S. Oguri, S. Anjo, and Y. Kuwabara, Waseda Appl. Chem. Soc. Bull. 22, 1-4 (1934); Chem. Abstr. 28, 4298 (1934). (1934).

1934POU/WIL J. R. Pound and A. M. Wilson, J. Phys. Chem. 39, 1135 (1934).

1934RIG/FEL N. E. Rigler, W. A. Felsing, and H. R. Henze, J. Am. Chem. Soc. 56, 1499 (1934).

1934ROT/NAG L. Rotinjanz and N. Nagornow, Z. Phys. Chem. A 169, 20-30 (1934).

1934RUF/MIL O. Ruff and G. Miltschitzky, Z. Anorg. Allg. Chem. 221, 154 (1934).

1934WOL/TRI K. L. Wolf and H. G. Trieschmann, Z. Phys. Chem. B 27 376-380 (1934).

1935BAU/BUR H. Bauer and K. Burschkies, Ber. Dtsch. Chem. Ges. 68 , 1238-1243 (1935).

1935BOO/ELS H. S. Booth, H. M. Elsey, and P. E. Burchfield, J. Am. Chem. Soc. 57, 2066 (1935).

1935BUT/RAM J. A. V. Butler, C. N. Ramchandani, and D. W. Thompson, J. Chem. Soc. 1935, 280-285.

1935CAR/DIC J. L. Carrico and R. G. Dickinson, J. Am. Chem. Soc. 57, 1343-1348 (1935).

1935HUF/ELL H. M. Huffman and E. L. Ellis, J. Am. Chem. Soc. 57, 46 (1935).

1935 KIR/POP V. A. Kireev and A. A. Popov, J. Gen. Chem. (USSR) 5, 1399-1401 (1935); Chem. Abstr. 30, 2441 (1936).

1935LEE P. Z. Lee, Z. Anorg. Allg. Chem. 223, 213-217 (1935); Chem. Abstr. 29, 6118 (1935).

1935MIL

$1935 \mathrm{SCH} / \mathrm{STA}$

P. Miller, Iowa State Coll. J. Sci. 10, 91 (1935). (1933).
1935SPE/WIL

1935THO/LIN

1935TIM

1935TRI

1935WIB/SUT

1935WIL 1936AST/MES

1936BEN/CUT
H. Schierholtz and M. L. Staples, J. Am. Chem. Soc. 57, 2709-2711 (1935).

R. Spence and W. Wild, J. Chem. Soc. 138, 506-509 (1935).

H. W. Thompson and J. W. Linnett, Trans. Faraday Soc. 31, 1743-1747 (1935).

J. Timmermans, Bull. Soc. Chem. Belg. 44, 17 (1935).

H. G. Trieschmann, Ph.D. dissertation (Inst. Fur Phys. Chem. and Electrochem. der Universitat Kiel, Germany, 1935).

E. Wiberg and W. Sutterlin, Z. Elektrochem. Angew. Phys. Chem. 41, 151 (1935).

A. L. Wilson, Ind. Eng. Chem. 27, 867-871 (1935).

J. G. Aston and G. H. Messerly, J. Am. Chem. Soc. 58, 2354-2361 (1936).

H. E. Bent, G. R. Cuthbertson, M. Dorfman, and R. E. Leary, J. Am. Chem. Soc. 58, 165-170 (1936). 
1936DEB

1936EWE

1936GRE

$1936 \mathrm{HOV} / \mathrm{SCH}$

1936LUC/LIK

1936PAR/TOD

J. H. De Boer, Trans. Faraday Soc. 32, 10 (1936).

M. Ewert, Bull. Soc. Chim. Belg. 45, 493-515 (1936).

M. A. Grekhnev, Lesokhim. Prom-st. 5, 11-12 (1936); Chem. Abstr. 32, 5271 (1938).

F. Hovorka, R. A. Schaefer, and D. Dreisback, J. Am. Chem. Soc. 58, 2264 (1936).

G. P. Luchinskii and A. I. Likhacheva, Zh. Fiz. Khim. 7, 723 (1936).

G. S. Parks, S. S. Todd, and W. A. Moore, J. Am. Chem. Soc. 58, 398 (1936).

1936PAR/TOD2 G. S. Parks, S. S. Todd, and C. H. Shomate, J. Am. Chem. Soc. 58, 2505 (1936)

1936PEA/BAK J. N. Pearce and H. M. Bakke, Proc. Iowa Acad. Sci. 43, 171 (1936)

1936RUF/BRE O. Ruff, O. Bretschneider, W. Luchsinger, and G. Mitschitzky, Ber. Dtsch. Chem. Ges. B 69, 299 (1936).

1936RUF/GIE O. Ruff and M. Giese, Ber. Dtsch. Chem. Ges. B 69, 684 (1936); Chem. Abstr. 30, 31302 (1936).

1936THO/LIN H. W. Thompson and J. W. Linnett, Trans. Faraday Soc. 32, 681-685 (1936).

1936TOD/PAR S. S. Todd and G. S. Parks, J. Am. Chem. Soc. 58, 134 (1956).

1937AST/SIL J. G. Aston, C. W. Siller, and G. H. Messerly, J. Am. Chem. Soc. 59, 1743-1751 (1937).

1937BEK/WOO N. Bekkedahl and L. A. Wood, J. Res. Natl. Bur. Stand 19, 551 (1937).

1937BRO/MAN O. L. I. Brown and G. G. Manov, J. Am. Chem. Soc. 59, 500 (1937).

1937CAR/MOR T. S. Carswell and H. L. Morrill, Ind. Eng. Chem. 29, 1247 (1937).

1937DEW J. H. de Wilde, Z. Anorg. Allg. Chem. 233, 411-414 (1937).

1937DUN/WOL H. Dunken and K. L. Wolf, Z. Phys. Chem. B 38, 441-450 (1937).

1937EGA/KEM C. J. Egan and J. D. Kemp, J. Am. Chem. Soc. 59, 1264-1268 (1937)

1937GAL/HIB A. F. Gallaugher and H. Hibbert, J. Am. Chem. Soc. 59, 2521-2525 (1937)

1937GAR/BRE G. S. Gardner and J. E. Brewer, Ind. Eng. Chem. 29, 179-181 (1937).

1937GIA/EGA W. F. Giauque and C. J. Egan, J. Chem. Phys. 5, 45-54 (1937).

1937GUY/SCH A. Guyer, W. Schutze, and M. Weidenmann, Helv. Chim. Acta 20, 936-949 (1937).

1937HEN A. L. Henne, J. Am. Chem. Soc. 59, 1200 (1937).

1937HRY/SMO K. Hrynakowski and A. Smoczkiewiczowa, Rocz. Chem. 17, 165 (1937)

1937ISH/TAN F. Ishikawa and C. Tanobe, Rikagaku Kenkyusho Iho 16, 1318 (1937); Chem. Abstr. 32, 41687 (1938).

1937KEM/GIA J. D. Kemp and W. F. Giauque, J. Am. Chem. Soc. 59, 79-84 (1937).

1937KLE/WAG A. Klemenc and G. Wagner, Ber. Dtsch. Chem. Ges. 70, 1880-1882 (1937); Chem. Abstr. 31, 8412 (1937).

1937 MOO/KAN V. G. Moor, E. K. Kanep, and I. E. Dobkin, "Materials on cracking and chemical treatment of cracking products," Trans. Exptl. Research Lab. Khemgas USSR 3, 320-328 (1937); Chem. Abstr. 31, 6072 (1937).

1937PAR/SHO G. S. Parks, C. H. Shomate, W. D. Kennedy, and B. L. Crawford, Jr., J. Chem. Phys. 5, 359 (1937).

1937PER A. Perlick, Bull. Int. Inst. Refrig. 18, 1 (1937).

1937RIN/SAY J. C. Rintelen, Jr., J. H. Saylor, and P. M. Gross, J. Am. Chem. Soc. 59, 1129-1130 (1937).

1937RUD/KOR G. A. Rudakov and S. Ya. Korotov, Zh. Prikl. Khim. 10, 312 (1937); Chem. Abstr. 31, 32399 (1937)

1937STU

1937THO/DAI H. W. Thompson and F. S. Dainton, Trans. Faraday Soc. 33, 1546-1555 (1937).

1937WIT/KEM R. K. Witt and J. D. Kemp, J. Am. Chem. Soc. 59, 273-276 (1937).

1938BAS/EME L. A. Bashford, H. J. Emeleus, and H. V. A. Briscoe, J. Chem. Soc. 1938, 1358.

1938BRA
1938EFT

1938EGA/KEM

1938EUC/SCH

1938HOV/LAN

1938KEM/EGA

1938KEN/SHO

1938KUC

1938LEV/SHT

1938LIN

1938RAD/ALE

1938REI

1938SCA/RAY

1938WOL/WEG

1939AST/EID

1939FRA/CLU

1939FRE/HIL

1939GIA/RUE

1939PAT/SCH

1939POW/GIA

1939RAI

1939REI

1939RHE/MOT

1939RUE/GIA

1939VAN

1940AST/KEN

1940AST/MES

1940BEL

1940BEN/MCH

1940BIR/BUC

1940DAR/VER

1940HEI/REI

1940HOD

$1940 \mathrm{HOV} / \mathrm{LAN}$

1940HOV/LAN2

1940HOV/LAN3

1940LAM/ROP

1940MES/AST

1940MES/KEN

1940PIT

1940PIT2
E. Eftring, Ph.D. dissertation, University of Lund, 1938, as quoted in [1956KIR].

C. G. Egan and J. D. Kemp, J. Am. Chem. Soc. 60, 2097-2101 (1938).

A. Eucken and E. Schroder, Z. Phys. Chem. B41, 307 (1938).

F. Hovorka, H. P. Lankelma, and S. C. Stanford, J. Am. Chem. Soc. 60, 820-827 (1938).

J. D. Kemp and C. J. Egan, J. Am. Chem. Soc. 60, 1521-1525 (1938).

W. D. Kennedy, C. H. Shomate, and G. P. Parks, J. Am. Chem. Soc. 60, 1507 (1938).

K. Kuchinskaya, Sbornik Trudov. Opytnogo Zavoda im. Akad. S. V. Lebedeva 27-30 (1938); Chem. Abstr. 34 3147 (1940).

E. Levin and I. Shtern, Zh. Prikl. Khim. (Moscow) 11, 426-441 (1938).

M. Linhard, Z. Anorg. Allg. Chem. 236, 200-208 (1938).

D. Radalescu and M. Alexa, Bull. Chem. Soc. Rom. 20A, 89-113 (1938).

L. Reidel, Z. Ges. Kalte-Ind. 45, 221 (1938).

G. Scatchard and C. L. Raymond, J. Am. Chem. Soc. 60 1278-1287 (1938); Erratum, 60, 3099 (1938).

K. L. Wolf and H. Z. Weghofer, Z. Phys. Chem. B 39, 194-208 (1938).

J. G. Aston, M. L. Eidinoff, and W. S. Forster, J. Am. Chem. Soc. 61, 1539-1543 (1939).

A. Frank and K. Clusius, Z. Phys. Chem. 42B, 395-421 (1939).

K. J. Frederick and J. H. Hildebrand, J. Am. Chem. Soc. 61, 1555 (1939).

W. F. Giauque and R. A. Ruehrwein, J. Am. Chem. Soc. 61, 2626 (1939).

W. Patnode and W. J. Scheiber, J. Am. Chem. Soc. 61, 3449-3451 (1939).

T. M. Powell and W. F. Giauque, J. Am. Chem. Soc. 61, 2366-2370 (1939).

W. E. Railing, J. Am. Chem. Soc. 61, 3349 (1939).

L. Reidel, Bull. Int. Inst. Refrig. 20, 1-10 (1939).

H. Rheinboldt and E. Motzkus, Ber. Dtsch. Chem. Ges. B 72, 657 (1939).

R. A. Ruehrwein and W. F. Giauque, J. Am. Chem. Soc. 61, 2940-2944 (1939).

A. Van de Vloed, Bull. Soc. Chem. Belg. 48, 229-268 (1939).

J. G. Aston, R. M. Kennedy, and S. C. Schumann, J. Am. Chem. Soc. 62, 2059-2063 (1940).

J. G. Aston and G. H. Messerly, J. Am. Chem. Soc. 62, 1917-1923 (1940).

A. F. Belyaev, Zh. Fiz. Khim. 14, 1009 (1940).

A. F. Benning and R. C. McHarness, Ind. Eng. Chem. 32, 497-499 (1940).

L. Birckenbach and E. Buchner, Ber. Dtsch. Chem. Ges. B 73, 1153 (1940).

F. R. Darkis, H. E. Vermillion, and P. M. Gross, Ind. Eng. Chem. 32, 946-949 (1940).

W. Heiber and E. Reindl, Ztschr. Electrochim. 10, 559-570 (1940)

E. B. Hodge, Ind. Eng. Chem. 32, 748 (1940).

F. Hovorka, H. P. Lankelma, and A. E. Axelrod, J. Am. Chem. Soc. 62, 187-189 (1940).

F. Horvorka, H. P. Lankelma, and I. Schneider, J. Am. Chem. Soc. 62, 1096-1098 (1940).

F. Horvorka, H. P. Lankelma, and W. R. Smith, J. Am. Chem. Soc. 62, 2372-2374 (1940).

A. B. Lamb and E. E. Roper, J. Am. Chem. Soc. 62, 806-814 (1940).

G. H. Messerly and J. G. Aston, J. Am. Chem. Soc. 62, 886-890 (1940).

G. H. Messerly and R. M. Kennedy, J. Am. Chem. Soc. 62, 2988-2991 (1940).

K. S. Pitzer, J. Am. Chem. Soc. 62, 1224-1227 (1940).

K. S. Pitzer, J. Am. Chem. Soc. 62, 331 (1940). 
1940QUA/NOR O. R. Quayle and H. M. Norton, J. Am. Chem. Soc. 62, 1170 (1940).

1940RAL/SEL A. W. Ralston, W. M. Selby, W. O. Pool, and R. H. Potts, Ind. Eng. Chem. 32, 1093 (1940).

1940ROS/SAN E. J. Rosenbaum and C. R. Sandberg, J. Am. Chem. Soc. 62, $1622(1940)$.

1940SMI

1940STU/SAY J. M. Stuckey and J. H. Saylor, J. Am. Chem. Soc. 62,

E. R. Smith, J. Res. Natl. Bur Stand. 24, 229-234 (1940). 2922-2925 (1940).

1940ZIL A. A. Zil'berman-Granovskaya, J. Phys. Chem. USSR 14, 759-767 (1940); 14, 768-773 (1940); 14, 1004-1006 (1940).; Chem. Abstr. 35, 3867 (1941).

1941BEL A. F. Belyaev, Acta Physicochem. U.S.S.R. 14, 523 (1941).

1941DAV

1941HEI

1941KEN/SAG

D. S. Davis, Ind. Eng. Chem. 33, 401 (1941).

C. B. Heisig, J. Am. Chem. Soc. 63, 1698-1699 (1941).

R. M. Kennedy, M. Sagenkahn, and J. G. Aston, J. Am. Chem. Soc. 63, 2267-2272 (1941).

1941 KIR/SIT V. A. Kireev and I. P. Sitnikov, J. Appl. Chem. USSR 14, 483-485 (1941); Chem. Abstr. 36, 2189 (1942).

1941LIS

1941MIL

1941NIT/SEK

M. W. Lister, J. Am. Chem. Soc. 63, 143-149 (1941)

G. Milazzo, Boll. Sci. Fac. Chim. Ind. Bologna 94-98 (1941).

I. Nitta and S. Seki, J. Chem. Soc. Jpn. 62, 581-586 (1941); Chem. Abstr. 36, 15 (1942).

1941 OSB/GAR D. W. Osborne, C. S. Garner, R. N. Doecher, and D. M. Yost, J. Am. Chem. Soc. 63, 3496-3499 (1941).

1941PAR/WES G. S. Parks, T. J. West, and G. E. Moore, J. Am. Chem. Soc. 63, 1133 (1941).

1941PIT/GWI K. S. Pitzer and W. D. Gwinn, J. Am. Chem. Soc. 63, 3313 (1941).

1941PIT/SCO K. S. Pitzer and D. W. Scott, J. Am. Chem. Soc. 63, 2419 (1941).

1941RAL/SEL A. W. Ralston, W. M. Selby, and W. O. Pool, J. Ind. Eng. Chem. 33, 682-683 (1941).

1941RIE

$1941 \mathrm{SCH}$

1941SEK/NIT

L. Riedel, Z. Ges. Kalte-Ind. 48, 9-13 (1941).

W. R. Schmidt, M.S. thesis, Washington University, St. Louis, 1941.

1941SMI

1941STO/FIS

S. Seki and I. Nitta, J. Chem. Soc. Jpn. 62, 907-912 (1941).

E. R. Smith, J. Res. Natl. Bur. Stand. 26, 129-134 (1941).

J. W. Stout and L. H. Fisher, J. Chem. Phys. 9, 163-168 (1941).

1941YOS/OSB $\quad$ D. M. Yost, D. W. Osborne, and C. S. Garner, J. Am. Chem. Soc. 63, 3492 (1941).

1942BEA/ING J. A. Beattie, H. G. Ingersoll, and W. H. Stockmayer, J. Am. Chem. Soc. 64, 546-548 (1942).

1942BEN S. W. Benson, Ind. Eng. Chem., Anal. Ed. 13, 189-191 (1942).

1942BEN/KIS S. W. Benson and G. B. Kistiakowsky, J. Am. Chem. Soc. 64, 80-86 (1942).

1942BUR

1942DAV/BRO

P. E. Burchfield, J. Am. Chem. Soc. 64, 2501 (1942).

1942OSB/DOE D. W. Osborne, R. W. Doescher, and D. M. Yost, J. Am. D. W. Osborne, R. W. Doescher,
Chem. Soc. 64, 169-172 (1942).

1942RUS/OSB H. D. Russell, Jr., D. W. Osborne, and D. M. Yost, J. Am. Chem. Soc. 64, 165-169 (1942).

1942 SCH/AST S. C. Schumann, J. G. Aston, and M. Sagenkahn, J. Am. Chem. Soc. 64, 1039-1043 (1942); Erratum, 64, 30690 (1942).

1942WIL/GIL G. E. Williams and E. C. Gilbert, J. Am. Chem. Soc. 64, 2776-2777 (1942).

1942ZIE/AND W. T. Ziegler and D. H. Andrews, J. Am. Chem. Soc. 64, 2482-2485 (1942).

1943AST/FIN J. G. Aston, H. L. Finke, and S. C. Schumann, J. Am. Chem. Soc. 65, 341-346 (1943).

1943AST/SZA J. G. Aston, G. J. Szasz, and H. L. Finke, J. Am. Chem. Soc. 65, 1135-1139 (1943).

1943BAC/PER H. J. Backer and W. G. Perdok, Recl. Trav. Chim. PaysBas Belg. 62, 533 (1943).

1943BUR P. Z. Burbo, J. Phys. USSR 7, 286-289 (1943); J. Phys. Chem. USSR 18, 253-257 (1944); Chem. Abstr. 39, 1581 (1945).
1943BUR2

1943CRA

1943GUT/WES

1943HAL/REI

1943HOL/MEL

1943KET/KRU

1943LEM/FEL

1943NIT/SEK

1943PIT/SCO

1943RUE/HUF

1943WES/EUC

1943WOO/HIG

1944AST/SAG

1944EIB

1944GUT/SPI

1944HIC/HOO

1944IOF/YAM

1944MCD

1944MCL/EDW

1944MIL

1944MIL2

1944ODA/MID

1944RUB/LEV

1944RUS/GOL

1944SCO/FER

1944YOS

1945DAV/WIE

1945FAW/RAS

1945GUT/PIT

1945SCO/MEY

1945SWI/HOC

1945WAC/LIN

1945WIL/TAY

1946AST/FIN

1946CRO/FEE

1946DOU/HUF

1946DOU/HUF2
A. B. Burg, J. Am. Chem. Soc. 65, 1629-1635 (1943).

K. S. N. Cramer, Recl. Trav. Chim. Pays-Bas 62, 606-610 (1943); Chem. Zentr. II 2234 (1943); Chem. Abstr. 38, 6148 (1944).

L. Guttman, E. F. Westrum, and K. S. Pitzer, J. Am. Chem. Soc. 65, 1246 (1943).

W. P. Hall and E. E. Reid, J. Am. Chem. Soc. 65, 1466-1468 (1943).

G. Holst and L. Melander, Svensk. Kem. Tid. 55, 131-135 (1943); Chem. Abstr. 40, 2706 (1946).

J. A. A. Ketelaar and S. Kruyer, Rec. Trav. Chim. 62, 550-552 (1943); Chem. Abstr. 38, 1926 (1944).

J. F. Lemons and W. A. Felsing, J. Am. Chem. Soc. 65, 46-48 (1943).

I. Nitta and S. Seki, J. Chem. Soc. Jpn. 64, 475-482 (1943); Chem. Abstr. 41, 3359a (1947).

K. S. Pitzer and D. W. Scott, J. Am. Chem. Soc. 65, 803-811 (1943).

R. A. Ruehrwein and H. M. Huffman, J. Am. Chem. Soc. 65, 1620 (1943).

O. Westphal and M. Eucken, Ber. Dtsch. Chem. Ges. B 76, 1137 (1943)

L. A. Wood and C. F. Higgins, India Rubber World $\mathbf{1 0 7}$ 475 (1943); Chem. Abstr. 37, 13815 (1943).

J. G. Aston, M. L. Sagenkahn, G. J. Szasz, G. W. Moessen, and H. F. Zuhr, J. Am. Chem. Soc. 66, 1171-1177 (1944).

J. Eibert, M.S. thesis, Washington University, St. Louis, 1944.

G. B. Guthrie, Jr., R. W. Spitzer, and H. M. Huffman, J. Am. Chem. Soc. 66, 2120 (1944).

J. F. G. Hicks, J. G. Hooley, and C. C. Stephenson, J. Am. Chem. Soc. 66, 1054 (1944).

I. I. Ioffe and E. S. Yamp'olskaya, Zh. Prikl. Khim. 17, 527-528 (1944); Chem. Abstr. 39, 3986 (1945).

H. J. McDonald, J. Phys. Chem. 48, 47-50 (1944).

C. R. McLellan and W. R. Edwards, Jr., J. Am. Chem. Soc. 66, 409 (1944).

G. Milazzo, Gazz. Chim. Ital. 74, 58-62 (1944).

G. Milazzo, Gazz. Chim. Ital. 74, 49-57 (1944).

M. Odan, S.-I. Midzushima, and Y. Morino, Sci. Pap. Inst. Phys. Chem. Res. (Jpn.) 42, 27 (1944); Chem. Abstr. 41, 30419 (1947).

T. R. Rubin, B. D. Levedahl, and D. M Yost, J. Am. Chem. Soc. 66, 279-282 (1944).

H. Russell, Jr., D. R. V. Golding, and D. M. Yost, J. Am. Chem. Soc. 66, 16-20 (1944).

R. B. Scott, W. J. Ferguson, and F. G. Brickwedde, J. Res. Natl. Bur. Stand. 33, 1-20 (1944).

U. Yoshida, Mem. Coll. Sci. Eng., Kyoto Imp. Univ. 24A, 135 (1944).

H. S. Davis and O. F. Wiedeman, Ind. Eng. Chem. 37, 482-485 (1945).

F. S. Fawcett and H. E. Rasmussen, J. Am. Chem. Soc. 67, 1705-1709 (1945).

L. Guttman and K. S. Pitzer, J. Am. Chem. Soc. 67, 324-327 (1945).

R. B. Scott, C. H. Meyers, R. D. Rands, Jr., F. G. Brickwedde, and N. Bekkedahl, J. Res. Natl. Bur. Stand. 35, 39 (1945).

E. S. Swift, Jr. and H. P. Hochanadel, J. Am. Chem. Soc. 67, 880-881 (1945).

R. C. Wackher, C. B. Linn, and A. V. Grosse, Ind. Eng. Chem. 37, 464-468 (1945).

C. B. Willingham, W. J. Taylor, J. M. Pignocco, and F. D. Rossini, J. Res. Natl. Bur. Stand. 35, 219-224 (1945).

J. G. Aston, H. L. Finke, A. B. Bestul, E. L. Pace, and G. J. Szasz, J. Am. Chem. Soc. 68, 52-57 (1946).

D. A. Crooks and F. M. Feetham, J. Chem. Soc. 1946, 899-901.

D. R. Douslin and H. M. Huffman, J. Am. Chem. Soc. 68, 1704 (1946).

173 (1946). 
1946DZU L. S. Dzung, Brown Boveri Rev. 33, 158-163 (1946).

1946FIE/SAY F. H. Field and J. H. Saylor, J. Am. Chem. Soc. 68, 2649-2650 (1946).

1946KIL/PIT J. E. Kilpatrick and K. S. Pitzer, J. Am. Chem. Soc. 68, 1066-1072 (1946).

1946KNO/SCH J. W. Knowlton, N. C. Schieltz, and D. MacMillan, J. Am. Chem. Soc. 68, 208-210 (1946).

1946PIT/GUT K. S. Pitzer, L. Guttman, and E. F. Westrum, Jr., J. Am. Chem. Soc. 68, 2209-2212 (1946).

1946RUE/POW R. A. Ruehrkein and T. M. Powell, J. Am. Chem. Soc. 68 , 1063-1066 (1946).

1946SCO/BRI R. B. Scott and R. G. Brickwedde, J. Res. Natl. Bur. Stand. 35, 501-512 (1946).

1946SPI/PIT R. Spitzer and K. S. Pitzer, J. Am. Chem. Soc. 68, 2537-2538 (1946).

1946 THO G. W. Thompson, Chem. Rev. 38, 1-39 (1946).

1947AST/FIN J. G. Aston, H. L. Fink, J. W. Tooke, and M. R. Cines, Anal. Chem. 19, 218 (1947).

1947AST/SZA J. G. Aston and G. J. Szasz, J. Am. Chem. Soc. 69, 3108-3114 (1947).

1947BAL $\quad$ E. W. Balson, Trans. Faraday Soc. 43, 54-60 (1947).

1947BAL/DEN E. W. Balson, K. G. Denbigh, and N. K. Adam, Trans. Faraday Soc. 43, 42-48 (1947).

1947BAL2 E. W. Balson, Trans. Faraday Soc. 43, 48 (1947).

1947BEE/JUN J. Beersmans and J. C. Jungers, Bull. Soc. Chim. Belg. 56, 238-250 (1947).

1947BOL/COL J. M. Bolling, A. R. Collett, and C. L. Lazzell, Proc. W. Va. Acad. Sci. 19, 61 (1947).

1947COR/GIN R. J. Corruccini and D. C. Ginnings, J. Am. Chem. Soc. 69, 2271 (1947).

1947DOU/WIN D. E. Douglass and C. A. Winkier, Can. J. Res. 25B, 381 (1947).

1947 EFR/BLO A. Efron and R. E. Blom, J. Phys. Chem. 51, 480 (1947).

1947FOW/HAM R. D. Fowler, J. M. Hamilton, Jr., J. S. Kasper, C. E. Weber, W. B. Burford III, and H. C. Anderson, Ind. Eng. Chem. 39, 375-378 (1947).

1947GOL/MAR K. B. Goldblum, R. W. Martin, and R. B. Young, Ind. Eng. Chem. 39, 1474-1476 (1947).

1947GOU/HOL C. Gould, Jr., G. Holzman, and C. Niemann, Anal. Chem. 19, 204-206 (1947).

1947GRA

1947HEI/WIE

A. Granovskaya, J. Phys. Chem. USSR 21, 967-968 (1947); Chem. Abstr. 42, 2486d (1948).

E. Heilbronner and K. Wieland, Helv. Chim. Acta 30, 947-956 (1947).

1947IVI/DAI K. J. Ivin and F. S. Dainton, Trans. Faraday Soc. 43, 32-35 (1947).

1947JON/GIA W. M. Jones and W. F. Giauque, J. Am. Chem. Soc. 69, 983-987 (1947).

1947KET/VAN J. A. A. Ketalaar, P. F. Van Velden, and J. S. Zalm, Recl. Trav. Chim. Pays-Bas 66, 721-732 (1947).

1947LEI/SHO A. G. Leibush and E. D. Shorina, Zh. Prikl. Khim. 20, 69-76 (1947).

1947OSB/GIN N. S. Osborne and D. C. Ginnings, J. Res. Natl. Bur. Stand. 39, 453-477 (1947); Chem. Abstr. 42, $1795 i$ (1948).

1947 SCO/WAD D. W. Scott, G. Waddington, J. C. Smith, and H. M. Huffman, J. Chem. Phys. 15, 565 (1947).

1947STR/GAB F. H. Stross, C. M. Gable, and G. C. Rounds, J. Am. Chem. Soc. 69, 1629-1630 (1947).

1947STR/MON F. H. Stross, J. M. Monger, and H. de V. Finch, J. Am. Chem. Soc. 69, 1627-1628 (1947).

1947STU $\quad$ D. R. Stull, Ind. Eng. Chem. 39, 517-540 (1947).

1947SZA/MOR G. J. Szasz, J. A. Morrison, E. L. Pace, and J. G. Aston, J. Chem. Phys. 15, 562 (1947).

1947TOD/OLI S. S. Todd, G. D. Oliver, and H. M. Huffman, J. Am. Chem. Soc. 69, 1519 (1947).

1947WAD/DOU G. Waddington and D. R. Douslin, J. Am. Chem. Soc. 69, 2275-2279 (1947).

1947WAD/TOD G. Waddington, S. S. Todd, and H. M. Huffman, J. Am. Chem. Soc. 69, 22-30 (1947).

1947WIL/EDW H. B. Williams and W. R. Edwards, J. Am. Chem. Soc. 69, 336 (1947)

1948BAN/EME A. A. Banks, H. J. Emeleus, R. N. Haszeldine, and V. Kerrigan, J. Chem. Soc. 1948, 2188-2190.
1948BEN/FRA

H. E. Bent and R. J. Francel, J. Am. Chem. Soc. 70, 634-637 (1948).

1948BOO/MAR H. S. Booth, D. R. Martin, and F. E. Kendall, J. Am. Chem. Soc. 70, 2523 (1948).

1948BRO/SUJ H. C. Brown and S. Sujishi, J. Am. Chem. Soc. 1948, 2878-2881.

1948CAT/ELL J. B. Catch, D. F. Elliot, D. H. Hey, and E. R. H. Jones, J. Chem. Soc. 272 (1948).

1948DAY/NIC H. O. Day, D. E. Nicholson, and W. A. Felsing, J. Am. Chem. Soc. 70, 1784-1785 (1948).

1948DIX/REH M. B. Dixon, C. E. Rehberg, and C. E. Fisher, J. Am. Chem. Soc. 70, 3733 (1948).

1948DOU T. B. Douglas, J. Am. Chem. Soc. 70, 2001-2002 (1948).

1948EME/WOO H. J. Emeleus and J. F. Wood, J. Chem. Soc. 1948, 2183-2188.

1948FEI/FIS M. L. Fein and C. M. Fisher, J. Org. Chem. 13, 749 (1948).

1948GAN/JUN J. F. Ganeff and J. C. Jungers, Bull. Soc. Chim. Belg. 57, 82-87 (1948).

1948GIA/JON W. F. Giauque and W. M. Jones, J. Am. Chem. Soc. 70, 120-124 (1948).

1948GLE/HAU O. Glemser and V. Haüser, Z. Naturforsch., B 3, 159-163 (1948).

1948GON/ELV A. Z. Gonner, P. J. Elving, and S. Steingiser, Ind. Eng. Chem. 40, 497 (1948).

1948GOR/GIA J. Gordon and W. F. Giauque, J. Am. Chem. Soc. 70, 1506-1510 (1948).

1948HOP/SEA E. R. Hopke and G. W. Sears, J. Am. Chem. Soc. 70, 3801-3803 (1948).

1948HUF/EAT H. M. Huffman, M. Eaton, and G. D. Oliver, J. Am. Chem. Soc. 70, 2911 (1948).

1948KEL/CAD K. B. Kellogg and G. H. Cady, J. Am. Chem. Soc. 70, 3986-3990 (1948).

1948KLA/MOH F. Klages and K. Mohler, Chem. Ber. 81, 411-417 (1948).

1948MIC/WAS A. Michels and T. Wassenaar, Physica (Amsterdam) 14, 104-110 (1948).

1948NIT/SEK I. Nitta and S. Seki, J. Chem. Soc. Jpn. 69, 85-87 (1948); Chem. Abstr. 44, 9204e (1950).

1948NIT/SEK2 I. Nitta and S. Seki, J. Chem. Soc. Jpn. Pure Chem. Sect. 69, 141-143 (1948); 69, 143-145 (1948).; Chem. Abstr. 46, 1320i (1952).

1948OLI/EAT G. D. Oliver, M. Eaton, and H. M. Huffman, J. Am. Chem. Soc. 70, 1502 (1948).

1948PAC/AST $\quad$ E. L. Pace and J. G. Aston, J. Am. Chem. Soc. 70, 566-570 (1948).

1948RAL/RUS J. H. Raley, F. F. Rust, and W. E. Vaughan, J. Am. Chem. Soc. 70, 88-94 (1948).

1948RED/CHA C. E. Redemann, S. W. Chaikin, and R. B. Fearing, J. Am. Chem. Soc. 70, 631-634 (1948).

1948RED/CHA3 C. E. Redemann, S. W. Chaikin, and R. B. Fearing, J. Am. Chem. Soc. 70, 1648-1650 (1948).

1948RED/CHA4 C. E. Redemann, S. W. Chaikin, R. B. Fearing, J. W. Rotariu, J. Savit, and D. Van Hoesen, J. Am. Chem. Soc. 70, 3604-3606 (1948).

1948RED/CHA5 C. E. Redemann, S. W. Chaikin, and R. B. Fearing, J. Am. Chem. Soc. 70, 2582 (1948).

1948SEA/HOP G. W. Sears and E. R. Hopke, J. Phys. Chem. 52, 1137-1142 (1948).

1948SMA/SMA P. A. Small, K. W. Small, and P. Cowley, Trans. Faraday Soc. 44, 810-816 (1948).

1948TSC H. Tschamler, Monatsh. Chem. 79, 162 (1948)

1949COL/DEV C. F. Coleman and T. De Vries, J. Am. Chem. Soc. 71, 2839 (1949).

1949DRE/MAR R. R. Dreisbach and R. A. Martin, Ind. Eng. Chem. 41, 2875-2878 (1949).

1949DRE/SHR R. R. Dreisbach and S. A. Shrader, Ind. Eng. Chem. 41, 2879-2880 (1949).

1949EME/HAS H. J. Emeleus and R. N. Haszeldine, J. Chem. Soc. 1949, 2948.

1949FOR/NOR A. F. Forziati, W. R. Norris, and F. D. Rossini, J. Res. Natl. Bur. Stand. 43, 555-563 (1949).

1949GIA/GOR W. F. Giauque and J. Gordon, J. Am. Chem. Soc. 71, 2176-2181 (1949). 
1949HIG/END T. Higuchi, N. Endow, and J. E. Willard, J. Am. Chem. Soc. 71, 365-366 (1949).

1949HOL/DOR D. E. Holcomb and C. L. Dorsey, Jr., Ind. Eng. Chem. 41, 2788-2792 (1949); Erratum, 42, 570 (1950).

1949HUF/TOD H. M. Huffman, S. S. Todd, and G. D. Oliver, J. Am. Chem. Soc. 71, 584 (1949).

1949KRE/WIE C. B. Kretschmer and R. Wiebe, J. Am. Chem. Soc. 71, 1793-1797 (1949).

1949NIC

1949NIC/LAF

J. C. Nicholson, J. Chem. Soc. 1949, 1553-1555.

$\begin{array}{ll}\text { 1949SCO/GRO } & \text { (1949); 229, 935-936 (1949). } \\ \text { D. W. Scott, M. E. Gross, G. D. Oliver, and H. M. Huff- }\end{array}$ man, J. Am. Chem. Soc. 71, 1634-1636 (1949).

1949SCO/WAD D. W. Scott, G. Waddington, J. C. Smith, and H. M. Huffman, J. Am. Chem. Soc. 71, 2767-2773 (1949).

1949SEA/HOP G. W. Sears and E. R. Hopke, J. Am. Chem. Soc. 71, 2575-2576 (1949).

1949SEA/HOP2 G. W. Sears and E. R. Hopke, J. Am. Chem. Soc. 71, 1632-1634 (1949)

1949STA/GUP L. A. K. Staveley and A. K. Gupta, Trans. Faraday Soc. 45, 50 (1949).

1949THO/EME J. Thompson and H. J. Emeleus, J. Chem. Soc. 1949, 3080 .

1949WAD/KNO G. Waddington, J. W. Knowlton, D. W. Scott, S. D. Oliver, S. S. Todd, W. N. Hubbard, J. C. Smith, and H. M. Huffman, J. Am. Chem. Soc. 71, 797-808 (1949).

1949WAD/SMI G. Waddington, J. C. Smith, D. W. Scott, and H. M. Huffman, J. Am. Chem. Soc. 71, 3902-3906 (1949).

1949 WIN/KUL L. O. Winstrom and L. Kulp, Ind. Eng. Chem. 41, 2584-2586 (1949).

1949YAR/FED N. L. Yarym-Agaev, N. N. Fedos'ev, and K. G. Skorikov, Zh. Fiz. Khim. 11, 1257-1265 (1949).

1950AST/MAS J. G. Aston, S. V. R. Mastrangelo, and G. W. Moessen, J. Am. Chem. Soc. 72, 5287-5291 (1950).

1950BRI/CUT N. F. H. Bright, J. C. Cuthill, and N. H. Woodbury, J. Sci. Food Agric. 1, 344 (1950).

1950COL/POP K. F. Coles and F. Popper, Ind. Eng. Chem. 42, 1434-1438 (1950).

$1950 \mathrm{CON} / \mathrm{ELV} \quad$ A. Z. Conner, P. J. Elving, J. Benischeck, P. E. Tobias, and S. Steingiser, Ind. Eng. Chem. 42, 106-110 (1950).

1950CRO/SMY R. W. Crowe and C. P. Smyth, J. Am. Chem. Soc. 72, 1098-1106 (1950).

1950CRO/SMY2 R. W. Crowe and C. P. Smyth, J. Am. Chem. Soc. 72, 4009 (1950).

1950DAY/FEL H. O. Day and W. A. Felsing, J. Am. Chem. Soc. 72, 1698-1699 (1950).

1950EDW G. T. Edwards, Trans. Faraday Soc. 46, 423-427 (1950).

1950FOR/CAM A. F. Forziati, D. L. Camin, and F. D. Rossini, J. Res. Natl. Bur. Stand. 45, 406-410 (1950).

1950IWA B. L. Iwanclow, Ph.D. dissertation, University of Wisconsin, 1950.

1950 KUS/CRO L. M. Kushner, R. W. Crowe, and C. P. Smyth, J. Am. Chem. Soc. 72, 1091 (1950).

1950MAT/SUM J. B. Matthews, J. F. Sumner, and E. A. Moellyn-Hughes, Trans. Faraday Soc. 46, 797-803 (1950).

$1950 \mathrm{MCK} / \mathrm{COP} \quad$ C. McKinley and J. P. Copes, J. Am. Chem. Soc. 72, 5331 (1950).

1950MIC/WAS A. Michels and T. Wassennar, Physica (Amsterdam) 16, 221-224 (1950).

1950MIC/WAS2 A. Michels, T. Wassenaar, Th. Zwietering, and P. Smits, Physica 16, 501 (1950).

1950NIT/SEK I. Nitta, S. Seki, M. Momotani, and K. Sato, J. Chem. Soc. Jpn. 71, 378-382 (1950); Chem. Abstr. 45, 6448g (1951).

1950 NIT/SEK2 I. Nitta, S. Seki, M. Momotani, K. Suzuki, and S. Nakagawa, Proc. Jpn. Acad. 26, 11-18 (1950); Chem. Abstr. 45, 4545b (1950).

1950NIT/SEK3 I. Nitta, S. Seki, and M. Momotani, J. Chem. Soc. Jpn., Pure Chem. Sect. 71, 430-433 (1950); Chem. Abstr. 1951, 45; 1951, 6448i.

1950NIT/SEK4 I. Nitta, S. Seki, and M. Momotani, Proc. Jpn. Acad. 26, 25-26 (1950).

1950NOY/NOY R. M. Noyes, W. A. Noyes, and H. Steinmetz, J. Am. Chem. Soc. 72, 33-34 (1950).
1950RAT

1950RAT/FIS

1950REH/DIX

1950REH/DIX2

1950SCO/FIN

1950SCO/FIN2

W. R. Ratchford, J. Org. Chem. 15, 326 (1950)

W. P. Ratchford and C. H. Fisher, J. Org. Chem. 15, 317 (1950).

C. E. Rehberg and M. B. Dixon, J. Am. Chem. Soc. 72, 1918-1922 (1950).

(1950).

D. W. Scott, H. L. Finke, M. E. Gross, and H. M. Huffman, J. Am. Chem. Soc. 72, 2424-2430 (1950).

D. W. Scott, H. L. Finke, W. N. Hubbard, J. P. McCullough, M. E. Gross, K. D. Williamson, G. Waddington, and H. M. Huffman, J. Am. Chem. Soc. 72, 4664-4666 (1950).

1950SCO/WAD D. W. Scott and G. Waddington, J. Am. Chem. Soc. 72 4310-4311 (1950).

1950SEK/MOM S. Seki and M. Momotani, Bull. Chem. Soc. Jpn. 23, 30 (1950).

1950SIL/CAD G. A. Silvey and G. H. Cady, J. Am. Chem. Soc. 72, 3624 (1950).

1950UEB/ORT K. Ueberreiter and H.-J. Orthmann, Z. Natursforsch. 5A 101 (1950).

1950WIS/PUC H. Wise, T. T. Puck, and C. F. Farley, J. Phys. Chem. 54, 734-741 (1950).

1951AST/FIN

J. G. Aston, H. L. Finke, G. J. Janz, and K. E. Russell, J. Am. Chem. Soc. 73, 1939-1943 (1951).

1951AST/JAN J. G. Aston, G. J. Janz, and K. E. Russell, J. Am. Chem. Soc. 73, 1943-1945 (1951).

1951BAR/MCC G. M. Barrow and A. L. McClellan, J. Am. Chem. Soc. 73, 573-575 (1951).

1951BRY/HOW D. Bryce-Smith and K. E. Howlett, J. Chem. Soc. 1141-1142 (1951).

1951BUR/CAD L. L. Burger and G. H. Cady, J. Am. Chem. Soc. 73, 4243-4246 (1951).

1951CAR/KUB B. Carroll, D. G. Kubler, H. W. Davis, and A. M. Whaley, J. Am. Chem. Soc. 73, 5382 (1951).

1951COA/HAR G. E. Coates, J. Harris, and T. Sutcliffe, J. Chem. Soc. 2762-2763 (1951).

1951EGE/EMT A. C. Egerton, W. Emte, and G. J. Minkoff, Discuss. Faraday Soc. 10, 278-282 (1951).

1951FLO/ALP $\quad$ D. G. Flom, N. Alpert, and P. J. Elving, Ind. Eng. Chem. 43, 1178-1181 (1951).

1951FUR/MCC G. T. Furukawa, R. E. McCoskey, and G. T. King, J. Res. Natl. Bur. Stand. 47, 256 (1951).

1951HAS R. N. Haszeldine, J. Chem. Soc. 1951, 102.

1951HAS2

1951INO

1951MCC/PER

R. N. Haszeldine, J. Chem. Soc. 1951, 588.

H. Inokuchi, Nippon Kagaku Zassi 72, 552-555 (1951).

1951MCE/RIG

J. P. McCullough, W. B. Person, and R. Spitzer, J. Am. Chem. Soc. 73, 4069-4071 (1951).

1951 NIC

W. S. McErvan and M. W. Rigg, J. Am. Chem. Soc. 73, 4725-4727 (1951).

G. R. Nicholson, as quoted by, J. W. Cook, A. R. Gibb, R. A. Raphael, and A. R. Somervile, J. Chem. Soc. 503-511 (1951).

1951NIT/SEK I. Nitta, S. Seki, H. Chihara, and K. Suzuki, Science Papers Osaka University No. 29, 1951; Chem. Abstr. 46 3820 (1952).

1951NIT/SEK2 I. Nitta, S. Seki, and K. Suzuki, Bull. Chem. Soc. Jpn. 24, 63-69 (1951).

1951OLI/GRI G. D. Oliver and J. W. Grisard, J. Am. Chem. Soc. 73, 1688-1690 (1951).

1951OLI/GRI2 G. D. Oliver, J. W. Grisard, and C. W. Cunningham, J. Am. Chem. Soc. 73, 5719 (1951).

1951PAR/BRO J. D. Park, H. A. Brown, and J. R. Lacher, J. Am. Chem. Soc. 73, 709 (1951).

1951POT/SAY J. C. Potter and J. H. Saylor, J. Am. Chem. Soc. 73, 90-91 (1951).

1951SCO/FIN $\quad$ D. W. Scott, H. L. Finke, J. P. McCullough, M. E. Gross, K. D. Williamson, G. Waddington, and H. M. Huffman, J. Am. Chem. Soc. 73, 261-265 (1951).

1951SMI/BON T. F. Smith and R. F. Bonner, Ind. Eng. Chem. 43, 1169-1173 (1951).

1951TIC/LOS A. W. Tickner and F. P. Lossing, J. Phys. Chem. 55, 733-741 (1951). 
1951VAU W. E. Vaughan, Discuss. Faraday Soc. 10, 330 (1951).

$1952 \mathrm{AIH}$

1952BAC/SIM K. C. Bachman and E. L. Simons, Ind. Eng. Chem. 44, 202 (1952)

1952BAN J. Banus, Nature 171, 173 (1952).

1952BRA/COT R. S. Bradley, S. Cotson, and E. G. Cox, J. Chem. Soc. 740-741 (1952).

1952BRA/EME G. A. R. Brandt, H. J. Emeleus, and R. N. Haszeldine, J. Chem. Soc. 1952, 2198-2205.

1952BRO

1952BYW

1952EDW

1952 FIN/SCO

I. Brown, Aust. J. Sci. Res. 5, 530-540 (1952).

S. Bywater, J. Polym. Sci. 9, 417-422 (1952).

G. Edwards, Trans. Faraday Soc. 48, 513-515 (1952).

H. L. Finke, D. W. Scott, M. E. Gross, G. Waddington, and H. M. Huffman, J. Am. Chem. Soc. 74, 2804-2806 (1952).

1952FUG/BOW E. T. G. Fuge, S. T. Bowden, and W. J. Jones, J. Phys. Chem. 56, 1013-1016 (1952).

1952GOT/NIK R. Goto snd M. Nikki, Bull. Inst. Chem. Res., Kyoto Univ. 28, 68-69 (1952); Chem. Abstr. 47, 34027 (1953).

1952 GUT/SCO $\quad$ D. W. Scott, W. N. Hubbard, C. Katz, J. P. McCullough, M. E. Gross, K. D. Williamson, and G. Waddington, J. Am. Chem. Soc. 74, 4662-4669 (1952).

1952 GUT/SCO2 G. B. Guthrie, D. W. Scott, and G. Waddington, J. Am. Chem. Soc. 74, 2795-2800 (1952).

1952HAS/LEE R. N. Haszeldine and K. Leedham, J. Chem. Soc. 1952, 3483 .

1952HUB/FIN W. N. Hubbard, H. L. Finke, D. W. Scott, J. P. McCullough, C. Katz, M. E. Gross, J. F. Messerly, R. E. Pennington, and G. Waddington, J. Am. Chem. Soc. 74, 6025-6030 (1952).

1952JON/TAM W. S. Jones and W. S. Tamplin, "Physical properties of propylene glycol," in Glycols, edited by G. O. Curme, Jr., American Chemical Society Monograph (Reinhold, New York, 1952), Vol. 114, Chap. 9, pp. 210-240.

1952KAA/COO S. Kaarsemaker and J. Coops, Recl. Trav. Chim. Pays-Bas 71, 261 (1952).

1952LEI/MOR L. C. Leitch and A. T. Morse, Can. J. Chem. 30, 924-932 (1952).

1952MCC/SCO J. P. McCullough, D. W. Scott, H. L. Finke, M. E. Gross, K. D. Williamson, R. E. Pennington, G. Waddington, and H. M. Huffman, J. Am. Chem. Soc. 74, 2801-2804 (1952).

1952MCK/MOE J. S. McKinley-McKee and E. A. Moelwyn-Hughes, Trans. Faraday Soc. 48, 247 (1952).

1952MIC/WAS A. Michels, T. Wassenaar, and Th. N. Zwietering, Physica 18, 160 (1952)

1952OLI/GRI G. D. Oliver and J. W. Grisard, J. Am. Chem. Soc. 74, 2705-2707 (1952).

1952REH/DIX C. E. Rehberg and M. B. Dixon, J. Am. Chem. Soc. 74, 1609 (1952).

1952SCH/FRE K. Schafer and O. Frey, Z. Elektrochem. 56, 882 (1952).

1952SCO/DOU D. W. Scott, D. R. Douslin, M. E. Gross, G. D. Oliver, and H. M. Huffman, J. Am. Chem. Soc. 74, 883-887 (1952).

1952SCO/FIN D. W. Scott, H. L. Finke, W. N. Hubbard, J. P. MuCullough, G. D. Oliver, M. E. Gross, C. Katz, K. D. Williamson, G. Waddington, and H. M. Huffman, J. Am. Chem. Soc. 74, 4656-4662 (1952).

1952SCO/FIN2 D. W. Scott, H. L. Finke, J. P. McCullough, M. E. Gross, R. E. Pennington, and G. Waddington, J. Am. Chem. Soc. 74, 2478-2848 (1952)

1952SCO/MAC T. A. Scott, Jr., D. MacMillan, and E. H. Melvin, Ind. Eng. Chem. 44, 172-175 (1952).

1952SIM/MAU J. H. Simons and J. W. Mausteller, J. Chem. Phys. 20, 1516-1519 (1952).

1952SPE/TAM H. T. Spengler and W. S. Tamplin, Anal. Chem. 24, 941 (1952).

1952STI/CAD V. E. Stiles and G. H. Cady, J. Am. Chem. Soc. 74, 3771-3773 (1952)

1952WHI G. H. Whipple, Ind. Eng. Chem. 44, 1664-1667 (1952).

1952 WHI/BAR P. T. White, D. G. Barnard-Smith, and F. A. Fidler, Ind. Eng. Chem. 44, 1430-1438 (1952).

1953AIH A. Aihara, J. Chem. Soc. Jpn., Pure Chem. Sect. 74, 437-441 (1953); 74, 631-634 (1953); 74, 634-635 (1953); Chem. Abstr. 48, 1088h (1954).
1953AST/WOO J. G. Aston, J. L. Wood, and T. P. Zolki, J. Am. Chem. Soc. 75, 6202-6204 (1953).

1953ATK/TRE $\quad$ B. Atkinson and A. B. Trenwith, J. Chem. Soc. 1953, 2082.

1953BAN J. Banus, J. Chem. Soc. 1953, 3755.

1953BAR/BRO J. A. Barker, I. Brown, and F. Smith, Discuss. Faraday Soc. 15, 142-150 (1953).

1953BEN/EME F. W. Bennet, H. J. Emeleus, and R. N. Haszeldine, J. Chem. Soc. 1953, 1565.

1953BRA/CAR R. S. Bradley and A. D. Care, J. Chem. Soc. 1953, 1688-1690.

1953BRA/CLE R. S. Bradley and T. G. Cleasby, J. Chem. Soc. 1953 , 1690-1692.

1953BRA/CLE2 R. S. Bradley and T. G. Cleasby, J. Chem. Soc. 1953 , $1681-1684$.

1953BRA/COT R. S. Bradley and S. Cotson, J. Chem. Soc. 1953 1684-1688.

1953CLE/WIS H. E. Clements, K. V. Wise, and S. E. Johnson, J. Am. Chem. Soc. 75, 1593-5395 (1953).

1953COP/EVE J. L. Copp and D. H. Everett, Discuss. Faraday Soc. 15, 174-188 (1953).

1953DAV/SCH R. T. Davis, Jr. and R. W. Schiessler, J. Phys. Chem. 57, 966-968 (1953).

1953EDW

1953EWA

1953FRA/DUV

G. Edwards, Trans. Faraday Soc. 49, 152-154 (1953). 1066 (1953)

1953FUR/MCC G. T. Furukawa, R. E. McCoskey, and M. L. Reilly, J. Res. Natl. Bur. Stand. 51, 69-72 (1953).

1953 GIN/FUR D. C. Ginnings and G. T. Furukawa, J. Am. Chem. Soc. 75, 522 (1953).

1953GRA/SMI P. Gray and P. L. Smith, J. Chem. Soc. 1953, 2380.

1953GRO/OLI M. E. Gross, G. D. Oliver, and H. M. Huffman, J. Am. Chem. Soc. 75, 2801 (1953).

1953HAS/KID R. N. Haszeldine and J. M. Kidd, J. Chem. Soc. 1953, 3219.

1953HER/MAR E. F. G. Herington and J. F. Martin, Trans. Faraday Soc. 49, 154-162 (1953).

1953HOV/JOH $\quad$ S. R. Hoover, H. John, and E. F. Mellon, Anal. Chem. 25 1940 (1953)

1953JAN/HAS J. Jander and R. N. Haszeldine, Naturwissenschaften 40, 579 (1953); Chem. Abstr. 49, 23487 (1955).

1953JEN/STY A. D. Jenkins and D. W. G. Style, J. Chem. Soc. 1953, 2337-2340.

1953KAR/SAY S. Kardon and J. H. Saylor, J. Am. Chem. Soc. 75, 1997 (1953).

1953MCC/SCO J. P. McCullough, D. W. Scott, H. L. Finke, W. N. Hubbard, M. E. Gross, C. Katz, R. E. Pennington, J. F. Messerly, and G. Waddington, J. Am. Chem. Soc. 75, 1818-1824 (1953).

1953MEL/VIO E. F. Mellon, S. J. Viola, and S. R. Hooper, J. Phys. Chem. 57, 607-608 (1953).

1953MIC/WAS A. Michels, T. Wassenaar, P. Louwerse, R. J. Lunbeck, and G. W. Wolkers, Physica 19, 287-289 (1953).

1953MIL/PAO G. Milazzo and L. Paoloni, Gazz. Chim. Ital. 83, 867-874 (1953).

1953RAT/GWI G. W. Rathjens and W. D. Gwinn, J. Am. Chem. Soc. 75, 5629-5633 (1953).

1953 SCO/FIN $\quad$ D. W. Scott, H. L. Finke, W. N. Hubbard, J. P. McCullough, C. Katz, M. E. Gross, J. P. Messerly, R. E. Pennington, and G. Waddington, J. Am. Chem. Soc. 75, 2795-2800 (1953).

1953SEK/SUZ S. Seki and K. Suzuki, Bull. Chem. Soc. Jpn. 26, 209-213 (1953); 26, 372-380 (1953).

1953SER/VOI V. V. Serpinskii, S. A. Voitkevich, and N. Y. Lyuboshits, Zh. Fiz. Khim. 27, 1032-1038 (1953); Chem. Abstr. 49, 3594e (1955).

1953STA/MUL H. Stage, E. Müller, and P. Faldix, Erdöle Kohle 6, 375-380 (1953), Note: Enthalpies of vaporization were calculated by fitting published vapor pressure data, taken from various sources to the Antoine equation. Some of the references are to unpublished data measured by chemical companies. 
1953WAG/BUR R. I. Wagner and A. B. Burg, J. Am. Chem. Soc. 75, 3869-3871 (1953).

1953WAL

K. A. Walsh, U.S. Atomic Energy Commission Report LA-1649, U.S. Atomic Energy Commission (1953); Chem. Abstr. 55, 19319c-19319d (1961).

1954ABR/DAV A. Abrams and T. W. Davis, J. Am. Chem. Soc. 76, 5993-5995 (1954); R. B. Mooney and E. B. Ludlam, Proc. R. Soc. Edinburgh 49, 160-169 (1929).

1954BOW/JON T. I. Bowell and G. L. Jones, Jr., J. Phys. Chem. 58, 666-667 (1954).

1954BUK/MAJ M. Bukala, J. Majewski, and W. Rodzinski, Przem. Chem. 10, 6-11 (1954).

1954BUR/WOO A. B. Burg and H. W. Woodrow, J. Am. Chem. Soc. 76, 219 (1954)

1954CRO/JON T. I. Crowell and G. L. Jones, J. Phys. Chem. 58, 666-667 (1954).

1954DAV/JEN M. Davies and D. G. Jenkin, J. Chem. Soc. 1954, 2374-2377.

1954DAV/JON M. Davies and J. I. Jones, Trans. Faraday Soc. 50, 1042-1047 (1954).

1954DOU/FUR T. B. Douglas, G. T. Furukawa, R. E. McCoskey, and A. F. Ball, J. Res. Natl. Bur. Stand. 53, 139 (1954).

1954DUN

1954FIN/GRO

1954FIN/GRO2

S. A. Dunn, J. Am. Chem. Soc. 76, 6191-6192 (1954).

H. L. Finke, M. E. Gross, J. F. Messerly, and G. Waddington, J. Am. Chem. Soc. 76, 854 (1954).

1954FRI/PIC V. Fried, J. Pick, E. Hala, and O. Vilim, Chem. Listy 48, Huffman, J. Am. Chem. Soc. 76, 333 (1954) 774-775 (1954).

1954FUR/MCC G. T. Furukawa, R. E. McCoskey, and M. L. Reilly, J. Res. Natl. Bur. Stand. 52, 11-16 (1954).

1954GEO/CAV K. K. Georgleff, W. T. Cave, and K. G. Blaikie, J. Am. Chem. Soc. 76, 5494 (1954).

1954GRA/SMI P. Gray and P. L. Smith, J. Chem. Soc. 1954, 769.

1954HUB/WAD W. N. Hubbard and G. Waddington, Recl. Trav. Chim. Pays-Bas 73, 910-913 (1954).

1954JAN/HAS J. Jander and R. N. Haszeldine, J. Chem. Soc. 1954, 696-698.

1954JAN/HAS2 J. Jander and R. N. Haszeldine, J. Chem. Soc. 1954, 919.

1954JOR

1954LOR/WOO G. Lord and A. A. Woolf, J. Chem. Soc. 1954, 2546-2551.

1954MCC/FIN J. P. McCullough, H. L. Finke, W. N. Hubbard, W. D. Good, R. E. Pennington, J. F. Messerly, and G. Waddington, J. Am. Chem. Soc. 76, 2661-2664 (1954).

1954MCC/FIN2 J. P. McCullough, H. L. Tinke, D. W. Scott, M. E. Gross, J. F. Messerly, R. E. Pennington, and G. Waddington, J. Am. Chem. Soc. 76, 4796 (1954).

1954MCC/SCO J. P. McCullough, D. W. Scott, R. E. Pennington, I. A. Hossenlopp, and G. Waddington, J. Am. Chem. Soc. 76, 4791-4796 (1954).

1954MEN/CAD A. Menefee and G. H. Cady, J. Am. Chem. Soc. 76, 2020 (1954).

1954NIC/SZA G. R. Nicholson, M. Szarc, and J. W. Taylor, J. Chem. Soc. 1954, 2767-2769.

1954POT/RIT A. E. Potter, Jr. and H. L. Ritter, J. Phys. Chem. 58, 1040-1042 (1954).

1954SEA/HOP G. W. Sears and E. R. Hopke, J. Am. Chem. Soc. 76, 2026 (1954).

1954SER/VOI V. V. Serpinskii, S. A. Voitkevich, and N. Y. Lyuboshits, Zh. Fiz. Khim. 28, 810-813 (1954); 28, 1969-1974 (1954); Chem. Abstr. 49, 6677C (1955); 50, 4573h-4573i (1956).

1954STA/WAR L. A. K. Staveley, J. B. Warren, H. P. Paget, and D. J. Dowrick, J. Chem. Soc. 1992 (1954).

1954TSU/KAT Y. Tsuzuki, S. Kata, and H. Okazaki, Kagaku 24, 523-524 (1954); Chem. Abstr. 48, 13740h (1954).

1954TUC/HAW W. C. Tucker, Jr. and J. E. Hawkins, Ind. Eng. Chem. 46, 2387 (1954)

1954WIS K. V. Wise, J. Am. Chem. Soc. 76, 3094-3095 (1954).

1954WIT

C. R. Witschonke, Anal. Chem. 26, 562 (1954).
$1955 \mathrm{AIH}$

1955AIH2

1955AIH3

1955ARM/BRI

1955AST/WIL

1955AST/ZOL

1955CAM/ROS

1955CUM/MCL

1955DRE/MAR

1955ENG

1955FOZ/MOR

1955HAR/SWI

1955ISH/MAT

1955JAS/MIL

$1955 \mathrm{KOS}$

1955LON/SAC2

1955MCC/FIN

1955MEA/STA

1955MOR/AYS

1955NEA/WIL

1955NEA/WIL

1955PIN/MAR

$1955 \mathrm{SCH} / \mathrm{WHI}$

1955SCO/FIN

1955SER/VOI

1955TAY/JOH

1955TUN/STO

1955 VON/GEB

1955WEL

1956AMB

1956BAR/CAD

1956BAR/HAS

1956BAR/HAS2

1956BRE/UBB

1956CAM/ROS

1956CAP/JAC
A. Aihara, J. Chem. Soc. Jpn., Pure Chem. Sect. 76, 495-497 (1955).

A. Aihara, J. Chem. Soc. Jpn., Pure Chem. Sect. 76, 497-499 (1955).

A. Aihara, Nippon Kagaku Zassi 76, 492-494 (1955); Chem. Abstr. 49, 15323c (1955).

G. T. Armstrong, F. G. Brickwedde, and R. B. Scott, J. Res. Natl. Bur. Stand. 55, 39-52 (1955).

J. G. Aston, P. E. Wills, and T. P Zolki, J. Am. Chem. Soc. 77, 3939-3941 (1955).

J. G. Aston, T. P. Zolki, and J. L. Wood, J. Am. Chem. Soc. 77, 281-284 (1955).

D. L. Camin and F. D. Rossini, J. Phys. Chem. 59, 1173-1179 (1955).

G. A. N. Cummings and E. McLaughlin, J. Chem. Soc. 1391-1392 (1955).

R. Dreyer, W. Martin, and U. von Weber, J. Prakt. Chem. 273, 324-328 (1955).

J. J. Engelsman, Ph. D.
sterdam, Holland, 1955.

O. R. Foz Gazulla, J. Morcilio, A. Perez-Masia, and A. Mendes, An. R. Soc. Esp. Fis. Quim. 50B, 23-34 (1955).

N. H. Hartshorne and P. McL. Swift, J. Chem. Soc. 1955, 3705-3720.

T. Ishiguro and K. Matsumoto, Yakugaku Zasshi 75, 1414-1417 (1955); Chem. Abstr. 50, 53875 (1956).

J. J. Jasper and G. B. Miller, J. Phys. Chem. 59, 441-442 (1955).

G. H. Kosolapoff, J. Chem. Soc. 2964-2965 (1955).

L. H. Long and J. F. Sackman, Res. Corresp. Suppl. Research (London) 8, 523 (1955); Chem. Abstr. 50, 47201 (1956).

J. P. McCullough, H. L. Finke, J. F. Messerly, R. E. Pennington, I. A. Hossenlopp, and G. Waddington, J. Am. Chem. Soc. 77, 6119-6125 (1955).

W. H. Mears, R. F. Stahl, S. R. Orfeo, R. C. Shair, L. F. Kells, W. Thompson, and H. McCann, Ind. Eng. Chem. 47, 1449-1454 (1955).

A. T. Morse, P. B. Ayscough, and L. C. Leitch, Can. J. Chem. 33, 453-457 (1955).

E. Neale and L. T. D. Williams, J. Chem. Soc. 1955, 2485-2490.

H. Pines and J. Marechal, J. Am. Chem. Soc. 77, 2819 (1955).

R. W. Schiessler and F. C. Whitmore, Ind. Eng. Chem. 47, 1660-1665 (1955), data is deposited as supplementary material, Document No. 4597.

D. W. Scott, H. L. Finke, J. P. McCullough, M. E. Gross, J. F. Messerly, R. E. Pennington, and G. Waddington, J. Am. Chem. Soc. 77, 4993-4998 (1955).

V. V. Serpinskii, S. A. Voitkevich, and N. Yu. Lyuboshits, Zh. Fiz. Khim. 29, 653-657 (1955); Chem. Abstr. 50, 16231e (1956).

R. D. Taylor, B. H. Johnson, and J. E. Kilpatrick, J. Chem. Phys. 23, 1225 (1955).

D. D. Tunnicliff and H. Stone, Anal. Chem. 27, 73 (1955).

E. Von Terres, F. Gebert, H. Hulsemann, H. Petereit, H. Toepsch, and W. Ruppert, Brennst.-Chem. 36, 272-274 (1955).

W. Weltner, J. Am. Chem. Soc. 77, 3941 (1955).

D. Ambrose, Trans. Faraday Soc. 52, 772-781 (1956).

E. J. Barber and C. H. Cady, J. Phys. Chem. 60, 504-506 (1956).

D. A. Barr and R. N. Haszeldine, J. Chem. Soc. 1956, 3416-3428.

D. A. Barr and R. N. Haszeldine, J. Chem. Soc. 1956, 3428.

D. Brennan and A. R. Ubbelohde, J. Chem. Soc. 1956, 3011-3016.

D. L. Camin and F. D. Rossini, J. Phys. Chem. 60, 1446-1351 (1956).
R. H. Capps and W. M. Jackson, J. Phys. Chem. 60, 811-812 (1956). 
1956FIN/SCO H. L. Finke, D. W. Scott, M. E. Gross, J. F. Messerly, and G. Waddington, J. Am. Chem. Soc. 78, 5469-5476 (1956).

1956FRO/LOE F. Fromm and M. C. Loeffler, J. Phys. Chem. 60, 252-253 (1956).

1956GAR/SCH D. Garvin and C. Schubert, J. Phys. Chem. 60, 807-808 (1956).

1956GLE/REE D. N. Glew and L. W. Reeves, J. Phys. Chem. 60, 615 (1956).

1956GRA/HAS T. Gramstad and R. N. Haszeldine, J. Chem. Soc. 1956, 173.

1956GRA/PRA P. Gray, M. W. Pratt, and M. J. Larkin, J. Chem. Soc. 1956, 210-212.

1956KIR F. W. Kirkbride, J. Appl. Chem. 6, 11-21 (1956).

1956KLO V. P. Klochkov, Zh. Fiz. Khim. 30, 2823-2824 (1956); Chem. Abstr. 51, 13495g (1957).

1956LI/PIT J. C. M. Li and K. S. Pitzer, J. Am. Chem. Soc. 78, 1077-1080 (1956).

1956MAG A. Magnus, Z. Phys. Chem. 9, 141-161 (1956).

1956MAJ T. G. Majury, Chem. Ind. (London) 349-350 (1956), data also published in J. Soc. Dyers Col. 72, 41-49 (1956).

1956MAN Manufacturing Chemists Association Research Project, Tables 23-9-2(2.0111)-k and 23-9-2(2.0112)-k; Tables 23-10-2(2.0111)-k and 23-10-2(2.0112)-k; Tables 2311-2(2.0111)-k and 23-11-2(2.0112)-k, (Manufacturing Chemists Association, College Station, TX, 1956); Note: The authors suspect that the tabulated Antoine Constants for the various compounds were generated in the manner discussed in [1961LI/ROS].

1956MAN2 Manufacturing Chemists Association Research Project, Tables 23-18-2(1.01111)-k and 23-18-2(1.01112)-k (Manufacturing Chemists Association, College Station, TX, 1956); Note: The authors suspect that the tabulated Antoine Constants for the various compounds were generated in the manner discussed in [1961LI/ROS].

1956MAR/STA J. G. Marshall, L. A. K. Staveley, and K. R. Hart, Trans. Faraday Soc. 52, 19 (1956).

$1956 \mathrm{MCL} / \mathrm{SCO} \quad$ E. P. McLaughlin and R. L. Scott, J. Phys. Chem. 60, 674 (1956).

1956MIL G. Milazzo, Ann. Chim. (Roma) 46, 1105-1111 (1956); Chem. Abstr. 51, 7791b (1957).

1956 NEA/WIL E. Neale, L. T. D. Williams, and V. T. Moores, J. Chem. Soc. 1956, 422-427.

1956 PAR/KEN $\quad$ G. S. Parks, W. D. Kennedy, R. R. Gates, J. R. Mosely, G. E. Moore, and M. L. Renquist, J. Am. Chem. Soc. 78, 56 (1956).

1956PEN/FIN R. E. Pennington, H. L. Finke, W. N. Hubbard, J. F. Messerly, F. R. Frow, I. A. Hossenlopp, and G. Waddington, J. Am. Chem. Soc. 78, 2055-2060 (1956).

1956 PEN/SCO R. E. Pennington, D. W. Scott, H. L. Finke, J. P. McCullough, J. F. Messerly, I. A. Hossenlopp, and G. Waddington, J. Am. Chem. Soc. 78, 3266-3272 (1956).

1956PIC/FRI J. Pick, V. Fried, E. Hala, and O. Vilim, Collect. Czech. Chem. Commun. 21, 260-261 (1956).

1956PLA R. Plank, Kaltetechnik 8, 127 (1956).

1956SCH/WAG K. Schafer, U. Wagner, and H. Engelbach, Chem. Ber. 89, 327 (1956).

1956SCO/MCC D. W. Scott, J. P. McCullough, W. N. Hubbard, J. F. Messerly, I. A. Hossenlopp, F. R. Frow, and G. Waddington, J. Am. Chem. Soc. 78, 5463-5468 (1956).

1956SCO/MCC2 D. W. Scott, J. P. McCullough, W. D. Good, J. F. Messerly, R. E. Pennington, T. C. Kincheloe, I. A. Hossenlopp, D. R. Douslin, and G. Waddington, J. Am. Chem. Soc. 78, 5457-5463 (1956).

1956SEK/SUZ S. Seki, K. Suzuki, and T. Koide, J. Chem. Soc. Jpn., Pure Chem. Sect. 77, 346-349 (1956); Chem. Abstr. 50, 5343i (1956).

1956SMI L. Smith, Acta Chem. Scand. 10, 884-886 (1956).

1956SUZ/ONI K. Suzuki, S. Onishi, T. Koide, and S. Seki, Bull. Chem. Soc. Jpn. 29, 127-131 (1956).

1956 TOO $\quad$ E. M. Toops, Jr., J. Phys. Chem. 60, 304-306 (1956).

1956 VON/JEN A. Von Brockhaus and E. Jenckel, Macromol. Chem. 18, 262-293 (1956).
1957AYL

1957BAR/SEF

1957BES/KOC S. D. Beskov, L. I. Kochetkova, and R. M. Golubeva, Uch.

W. S. Barnhart, R. J. Seffl, R. F. Wade, F. W. West, and J. L. Zollinger, J. Chem. Eng. Data 2, 80-83 (1957). Zap. - Mosk. Gos. Pedagog. Inst. Im. V. I. Lenina 99, 147-149 (1957); Chem. Abstr. 53, 20993d (1959).

1957BIS/PAR T. C. Bissot, R. W. Parry, and D. H. Campbell, J. Am. Chem. Soc. 79, 796-800 (1957).

1957BRO/SMI I. Brown and F. Smith, Aust. J. Chem. 10, 423-428 (1957).

1957CAL $\quad$ F. Call, J. Sci. Food Agric. 8, 81-85 (1957).

1957CAR/DAV E. L. Carpenter and H. S. Davis, J. Appl. Chem. 7, 671-676 (1957).

1957DYK/SEP J. Dykyj, M. Seprakova, and J. Paulech, Chem. Zvesti 11 461-466 (1957).

1957EAS/HAR $\quad$ B. C. Easton, M. K. Hargreaves, and R. K. S. Mitchell, J. Appl. Chem. (London) 7, 198-204 (1957).

1957FRA/JOH D. M. Frankel, C. E. Johnson, and H. M. Pitt, J. Org. Chem. 22, 1119 (1957).

1957FRA/SAN J. W. Frazer and R. H. Sanborn, U.S. Atomic Energy Commission Report UCRL-4978, U.S. Atomic Energy Commission (1957); Chem. Abstr. 52, 8936a (1958).

1957GLA/RUL F. Glaser and H. Ruland, Chem. Ing. Tech. 29, 772-775 (1957).

1957GRA/PRA P. Gray and M. W. T. Pratt, J. Chem. Soc. 1957, 2163-2168.

1957KEM/GOL M. D. Kemp, S. Goldhagen, and F. A. Zihlman, J. Phys. Chem. 61, 240-242 (1957).

1957LIT R. Littlewood, J. Chem. Soc. 2419-2420 (1957).

1957LON/SAC L. H. Long and J. F. Sackman, Trans. Faraday Soc. 53, 1606-1611 (1957).

1957MAI/SEY L. Maier, O. Seyforth, F. C. A. Stone, and E. Rochov, J. Am. Chem. Soc. 79, 5884-5889 (1957).

1957MAS S. V. R. Mastrangelo, Anal. Chem. 29, 841 (1957).

1957MCC/DOU J. P. McCullough, D. R. Douslin, J. F. Messerly, I. A. Hossenlopp, T. C. Kincheloe, and G. Waddington, J. Am. Chem. Soc. 79, 4289-4295 (1957).

1957 MCC/FIN J. P. McCullough, H. L. Finke, M. E. Gross, J. F. Messerly, and G. Waddington, J. Phys. Chem. 61, 289 (1957).

1957MCC/FIN2 J. P. McCullough, H. L. Finke, J. F. Messerly, S. S. Todd, T. C. Kincheloe, and G. Waddington, J. Phys. Chem. 61, 1105 (1957).

1957MCC/HUB J. P. McCullough, W. N. Hubbard, F. R. Frow, I. A. Hossenlopp, and G. Waddington, J. Am. Chem. Soc. 79, 561-566 (1957).

1957NEI/WHI E. F. Neilson and D. White, J. Am. Chem. Soc. 79, 5618-5621 (1957).

1957PEN/KOB R. E. Pennington and K. A. Kobe, J. Am. Chem. Soc. 79, 300 (1957).

1957POR/CAD R. S. Porter and G. H. Cady, J. Am. Chem. Soc. 79, 5625-5627 (1957).

1957PUT/KIL W. E. Putnam and J. E. Kilpatrick, J. Chem. Phys. 27, 1075 (1957).

1957ROS/ACC A. Rose, J. A. Acciari, R. C. Johnson, and W. W. Sanders, Ind. Eng. Chem. 49, 104-109 (1957).

1957ROW/THA J. S. Rowlinson and R. Thacker, Trans. Faraday Soc. 53, $1-8$ (1957).

1957SAG

1957SCO/FIN

A. J. Saggiomo, J. Org. Chem. 22, 1171-1175 (1957). D. W. Scott, H. L. Finke, J. P. McCullough, J. F. Messerly, R. E. Pennington, I. A. Hossenlopp, and G. Waddington, J. Am. Chem. Soc. 79, 1062-1068 (1957).

1957SER/VOI V. V. Serpinskii, S. A. Voitkevich, and N. Yu. Lyuboshits, Zh. Fiz. Khim. 31, 1278-1284 (1957); Chem. Abstr. 52, 3270f (1958).

1957SHE/BRY T. K. Sherwood and H. S. Bryant, Jr., Can. J. Chem. Eng. 35, 51-57 (1957); Chem. Abstr. 52, 92a (1958).

1957STR/KLI I. N. Stranski, G. Klipping, A. F. Bogenschuetz, H. J. Heinrick, and H. Maennig, Adv. Catal. 9, 406 (1957); Chem. Abstr. 53, 1793 (1959).

1957WIL/HAR K. D. Williamson and R. H. Harrison, J. Chem. Phys. 26, 1409-1411 (1957).

1958BID/MAR D. P. Biddiscombe and J. F. Martin, Trans. Faraday Soc. 54, 1316-1322 (1958). 
1958BIL/NOL J. J. Billings and A. W. Nolle, J. Chem. Phys. 29, 214 (1958).

1958BOO/MOR H. S. Booth and W. C. Morris, J. Phys. Chem. 62, 875 (1958).

1958BRO/MEA J. A. Brown and W. H. Mears, J. Phys. Chem. 62, 960-962 (1958).

1958BUR/SLO A. B. Burg and P. J. Slota, Jr., J. Am. Chem. Soc. 80, 1107-1109 (1958).

1958CAS/FLE R. C. Cass, S. E. Fletcher, C. T. Mortimer, P. G. Quincey, and H. D. Springall, J. Chem. Soc. 1958, 958-963.

1958CAS/FLE2 R. C. Cass, S. E. Fletcher, C. T. Mortimer, P. G. Quincey, and H. D. Springall, J. Chem. Soc. 1958, 2595-2599.

1958CAS/FLE3 R. C. Cass, S. E. Fletcher, C. T. Mortimer, H. D. Springall, and T. R. White, J. Chem. Soc. 1958, 1406-1410.

1958DIC/HIL F. Dickinson, R. Hill, and J. Murray, J. Chem. Soc. 1958, 1441.

1958DUN/MUR R. D. Dunlap, C. J. Murphy, Jr., and R. C. Bedford, J. Am. Chem. Soc. 80, 83-85 (1958).

1958GRA/PRA P. Gray and M. W. T. Pratt, J. Chem. Soc. 1958, 3403-3412.

1958HOY/PEP H. Hoyer and W. Peperle, Z. Electrochem. 62, 61-66 (1958).

1958HUB/DOU W. N. Hubbard, D. R. Douslin, J. P. McCullough, D. W. Scott, S. S. Todd, J. F. Messerly, I. A. Hossenlopp, A. George, and G. Waddington, J. Am. Chem. Soc. 80, 3547-3554 (1958).

1958 KLI/STR V. G. Klipping and I. N. Stranski, Z. Anorg. Allg. Chem. 297, 23-31 (1958)

1958KOS/SAM V. N. Kostryukov, O. P. Samorukov, and P. G. Strelkov, Zh. Fiz. Khim. 32, 1354 (1958).

1958LOO/DOW C. E. Looney and J. R. Downey, J. Am. Chem. Soc. 80, 2840-2844 (1958).

1958MAH/BUR W. Mahler and A. B. Burg, J. Am. Chem. Soc. 80, 6161-6167 (1958).

1958MCC/FIN J. P. McCullough, H. L. Finke, D. W. Scott, R. E. Pennington, M. E. Gross, J. F. Messerly, and G. Waddington, J. Am. Chem. Soc. 80, 4786-4793 (1958).

1958MOL

L. Molard, Mem. Poudres 40, 13 (1958), as quoted in [1977PEL].

1958MOR/YOS T. Morikawa and K. Yoshida, Kagaku to Kogyo 32, 228 (1958).

1958PAG/PUR F. M. Page and J. H. Purnell, J. Chem. Soc. 1958, 621.

1958PEP

W. J. Peppel, Ind. Eng. Chem. 50, 767-770 (1958).

1958ROS/PAP A. Rose, B. T. Paphronis, and E. T. Williams, J. Chem. Eng. Data 3, 216-219 (1958).

1958SCH/WAG K. Schafer and U. Wagner, Z. Elektrochem. 62, 328 (1958).

1958SCO/MCC D. W. Scott, J. P. McCullough, J. F. Messerly, R. E. Pennington, I. A. Hossenlopp, H. L. Finke, and G. Waddington, J. Am. Chem. Soc. 80, 55-59 (1958).

1958SER/VOI V. V. Serpinskii, S. A. Voitkevich, and N. Yu. Lyuboshits, Trudy Vsesoyuz. Nauch.- Issledovatel. Inst. Sintet. I. Natural. Dushistykh Veshchestv 4, 125-130 (1958).

1958SKL/MAR S. I. Sklyarenko, B. I. Markin, and L. B. Belyaeva, Zh. Fiz. Khim. 32, 1916-1921 (1958); Chem. Abstr. 53, 4848f (1959).

1958UNG/MCB H. Ungnade and E. T. McBee, Chem. Rev. 58, 249-320 (1958).

1958VAR M. C. P. Varma, J. Appl. Chem. 8, 117 (1958).

1959AIH

1959ARO/KAS

A. Aihara, Bull. Chem. Soc. Jpn. 32, 1242-1248 (1959).

Kh.A. Aronovich, L. P. Kastorskii, and K. F. Fedorova, Zh. Fiz. Khim. 41, 20 (1959).

1959BIS/FIN E. R. Bissell and M. Finger, J. Org. Chem. 24, 1259-1261 (1959).

1959BRA/DRU R. S. Bradley and T. Drury, Trans. Faraday Soc. 55, 1844-1847 (1959).

1959BRO/SMI I. Brown and F. Smith, Aust. J. Chem. 12, 407-412 (1959).

1959CHA/VAN P. Chaiyavech and M. Van Winkle, J. Chem. Eng. Data 4, 53-57 (1959).

1959DAV/JON M. Davies and A. H. Jones, Trans. Faraday Soc. 55, 1329-1332 (1959).
1959DAV/JON2

1959DEA/EVA

M. Davies, A. H. Jones, and
Soc. 55, 1100-1108 (1959).

G. H. Deal, H. D. Evans, E. D. Oliver, and M. N. Papdopoulous, Pet. Ref. 38, 185 (1959).

1959EME/SMI H. J. Emeleus and J. D. Smith, J. Chem. Soc. 1959, 375-381.

1959EVA/SKI F. W. Evans and H. A. Skinner, Trans. Faraday Soc. 55, 255-259 (1959).

1959FLE/MOR S. E. Fletcher, C. T. Mortimer, and H. D. Springall, J. Chem. Soc. 1959, 580-584.

1959GOO/DOU W. D. Good, D. R. Douslin, D. W. Scott, A. George, J. L. Lacina, J. P. Dawson, and G. Waddington, J. Phys. Chem. 63, 1133-1138 (1959).

1959HAR N. Harutada, Takamine Kenkyusho Nempo 11, 66-75 (1959); Chem. Abstr. 55, 10026e (1961).

1959 HIL/MCD $\quad$ D. L. Hildenbrand and R. A. McDonald, J. Phys. Chem. 68, 1521-1523 (1959).

1959HIL/SIN $\quad$ D. L. Hildenbrand, G. C. Sinke, R. A. McDonald, and W. R. Kramer, J. Chem. Phys. 31, 650-654 (1959).

1959KAE/STO H. D. Kaesz and F. G. A. Stone, J. Org. Chem. 24, 635 (1959).

1959KOL/PAU V. P. Kolesov, I. E. Paukov, S. M. Skuratov, and E. A Seregin, Dokl. Akad. Nauk SSSR 128, 130 (1959).

1959MCC/DOU J. P. McCullough, D. R. Douslin, W. N. Hubbard, S. S. Todd, J. F. Messerly, I. A. Hossenlopp, F. R. Frow, J. P. Dawson, and G. Waddington, J. Am. Chem. Soc. 81, 5884-5890 (1959).

1959MCC/PEN J. P. McCullough, R. E. Pennington, J. C. Smith, I. A. Hossenlopp, and G. Waddington, J. Am. Chem. Soc. 81, 5880-5883 (1959).

1959MCD/SHR R. A. McDonald, S. A. Shrader, and D. R. Stull, J. Chem. Eng. Data 4, 311-313 (1959).

1959SCO/DOU ～D. W. Scott, D. R. Douslin, J. F. Messerly, S. S. Todd, I. A. Hossenlopp, T. C. Kincheloe, and J. P. McCullough, J. Am. Chem. Soc. 81, 1015-1020 (1959).

1959SEE/BAL F. Seel and K. Ballreich, Chem. Ber. 92, 344 (1959).

1959SEP/PAU M. Seprakova, J. Paulech, and J. Dykyj, Chem. Zvesti 313-316 (1959).

1959STE/GRE J. H. Stern and N. W. Gregory, J. Phys. Chem. 63, 556-559 (1959).

1959TAK/CHI S. Takagi, H. Chihara, and S. Seki, Bull. Chem. Soc. Jpn. 32, 84-88 (1959).

1959TAK/SHI S. Takagi, R. Shintani, H. Chihara, and S. Seki, Bull. Chem. Soc. Jpn. 32, 137-142 (1959); Chem. Abstr. 54, 1958g (1960).

1959TER/BRI E. Terres, L. Brinkmann, D. Fischer, D. Hüllstrung, W. Loz, and G. Weisbrod, Brennst.-Chem. 40, 279-280 (1959).

1959URB L. Urbancova, Chem. Zvesti 13, 224-227 (1959).

1959USA/DEM M. Usanovich and A. I. Dembitskii, Zh. Obshch. Khim. 29, 1781-1785 (1959).

1959USA/DEM2 M. Usanovich and A. I. Dembitskii, Zh. Obshch. Khim. 29, 1771-1781 (1959).

1959VAC/STA J. Vacek and J. Stanek, Chem. Prum. 9, 286 (1959).

1959YEN/REE L. C. Yen and T. M. Reed III, J. Chem. Eng. Data 4, 102-107 (1959).

1960AIH A. Aihara, Bull. Chem. Soc. Jpn. 33, 194-200 (1960).

1960AIH2

1960AND/BID

A. Aihara, Bull. Chem. Soc. Jpn. 33, 1188-1194 (1960).

R. J. L. Andon, D. P. Biddiscombe, J. D. Cox, R. Handley, D. Harrop, E. F. G. Herington, and J. F. Martin, J. Chem. Soc. 1960, 5246-5255.

1960BUD S. Budurov, Izv. Khim. Inst., Bulg. Akad. Nauk. 7, 281-301 (1960).

1960BUR/SLO A. B. Burg and P. J. Slota, Jr., J. Am. Chem. Soc. 82, 2148 (1960).

1960CAM/ROS D. L. Camin and F. D. Rossini, J. Chem. Eng. Data 5, 368-372 (1960).

1960CHA/WES S. S. Chang and E. F. Westrum, J. Phys. Chem. 94, 1547-1551 (1960).

1960CLA/FOW A. P. Claydon, P. A. Fowell, and C. T. Mortimer, J. Chem. Soc. 1960, 3284-3287.

1960DAV/THO M. Davies and G. H. Thomas, Trans. Faraday Soc. 56, 185-192 (1960). 
1960DIN/PAC R. E. Dininny and E. L. Pace, J. Chem. Phys. 32, 805 (1960).

1960DYK/PAU J. Dykyj, J. Paulech, and M. Seprakova, Chem. Zvesti 14, 327-333 (1960).

1960EME/NAB H. J. Emeleus and S. N. Nabi, J. Chem. Soc. 1960, $1103-1108$

1960EME/PUG H. J. Emeleus and H. Pugh, J. Chem. Soc. 1960, $1108-1112$.

1960FRA

1960GIA/OTT

J. W. Frazer, J. Inorg. Nucl. Chem. 16, 63-66 (1960).

1960GLE

W. F. Giauque and J. B. Ott, J. Am. Chem. Soc. 82, 2689-2695 (1960).

D. N. Glew, Can. J. Chem. 38, 208-211 (1960)

1960GRE J. H. S. Green, Chem. Ind. (London) 1215-1216 (1960).

1960GRI/HAZ C. E. Griffin and R. N. Hazeldine, J. Chem. Soc. 1960, $1398-1406$.

1960HIR/STE R. C. Hirt, J. E. Steger, and G. L. Simard, J. Polym. Sci. 43, 319-323 (1960); Chem. Abstr. 54, 16968b (1960).

1960JON

1960KLY/MIS

A. H. Jones, J. Chem. Eng. Data 5, 196-200 (1960).

M. L. Klyueva, K. P. Mischenko, and M. K. Fedorov, Zh. Prikl. Khim. 3, 473-475 (1960); Chem. Abstr. 54, 11616g (1960).

1960LYN/WIL E. J. Lynch and C. R. Wilke, J. Chem. Eng. Data 5, 300 (1960).

1960MAC/MAY H. Mackle, R. G. Mayrick, and J. J. Rooney, Trans. Faraday Soc. 56, 115-117 (1960).

1960MAL/MAL N. A. Malafeev, V. A. Malyusov, N. N. Umnik, I. V. Podgornaya, and V. M. Zhavoronkov, Dokl. Akad. Nauk SSSR 135, 659 (1960); Chem. Abstr. 56, 5115 (1962).

$1960 \mathrm{MCK} / \mathrm{SAG} \quad$ R. A. Mckay and B. H. Sage, J. Chem. Eng. Data 5, 21-24 (1960).

1960MUE/IGN C. R. Mueller and J. Ignatowski, J. Chem. Phys. 32, 1430-1434 (1960).

1960MUN/KOH R. J. Munn and F. Kohler, Monatsh. Chem. 91, 381 (1960).

1960NEG/MIK H. Negoro, T. Miki, and S. Ueda, Yakugaku Zasshi 80, 665-669 (1960); Chem. Abstr. 54, 20424f (1960).

1960NEG/MIK2 H. Negoro, T. Miki, S. Ueda, T. Sanada, and R. Okada, Yakugaku Zasshi 80, 670-673 (1960).

1960NIC G. R. Nicholson, J. Chem. Soc. 2377-2378 (1960).

1960NOV/MAT J. Novak, J. Matous, and J. Pick, Collect. Czech. Chem. Commun. 25, 583-584 (1960).

1960NOV/MAT2 J. Novak, J. Matous, and J. Pick, Collect. Czech. Chem. Commun. 25, 2405-2413 (1960).

1960SCH/LEG K. Schwabe and C. Legler, Z. Elektrochem. 64, 902-905 (1960).

1960SKL/STR A. A. Sklyankin and P. G. Strelkov, Zh. Prikl. Mekh. Tekh. Fiz. 2, 100 (1960).

1960THO L. H. Thomas, J. Chem. Soc. 1960, 4906-4914 as quoted in [2000BRU/DEL].

1960TRE/MIL F. M. Trent, F. D. Miller, and G. H. Brown, J. Chem. Eng. Data 5, 110-111 (1960).

1960VAN/CAD W. P. Van Meter and G. H. Cady, J. Am. Chem. Soc. 82, 6005-6008 (1960).

1960WAD I. Wadso, Acta Chem. Scand. 14, 566-572 (1960).

1960WAD/KIS T. Wada, E. Kishida, Y. Tomiie, H. Suga, S. Seki, and I. Nitta, Bull. Chem. Soc. Jpn. 33, 1317-1318 (1960).

1960WEY/KLI F. Weygand, G. Klipping, and D. Palm, Chem. Ber. 93, 2619-2625 (1960).

1960WOO/MUR A. L. Woodman, W. L. Murbach, and M. H. Kaufman, J. Phys. Chem. 64, 658-660 (1960).

1960 YAR/KAY R. M. Yarrington and W. B. Kay, J. Chem. Eng. Data 5, 24-29 (1960).

1961BAN/GIN R. E. Banks, A. E. Ginsberg, and R. N. Haszeldine, J. Chem. Soc. 1961, 1740-1743.

1961BAR/HAS D. A. Barr, R. N. Haszeldine, and C. J. Willis, J. Chem. Soc. 1961, 1351-1362.

1961BER/SCO W. T. Berg, D. W. Scott, W. N. Hubbard, S. S. Todd, J. F. Messerly, I. A. Hossenlopp, A. Osborn, D. R. Douslin, and J. P. McCullough, J. Phys. Chem. 65, 1425-1430 (1961).

1961BUR/GRI A. B. Burg and J. E. Griffiths, J. Am. Chem. Soc. 83, 4333-4335 (1961).

1961BUS/IVI W. K. Busfield, K. J. Ivin, H. Mackle, and P. A. G. O’Hare, Trans. Faraday Soc. 1058-1063 (1961).
1961CAS/RAY

J. R. Case, N. H. Ray, and H. L. Roberts, J. Chem. Soc. 1961, 2070-2075.

1961CAS/RAY2 J. R. Case, N. H. Ray, and H. L. Roberts, J. Chem. Soc. 1961, 2066-2070.

1961DAV/MAL M. Davies and V. E. Malpass, J. Chem. Soc. 1961, 1048-1055.

1961DYK/SEP

J. Dykvy, M. Seprakova, and J. Paulech, Chem. Zvesti 15, 465-468 (1961); Chem. Abstr. 56, 10930b (1962).

1961FRO/LOG A. F. Frolov, M. A. Loginova, and M. M. Kiseleva, Zh. Phys. Khim. 35, 1784-1788 (1961).

1961 GLA/TIM A. R. Glasgow and J. Timmerman, Bull. Soc. Chem. Belg. 70, 599 (1961).

1961GOO/LAC W. D. Good, J. L. Lacina, and J. P. McCullough, J. Phys. Chem. 65, 2229-2231 (1961).

1961HEI/ILA J. Heinrich, J. Ilavsky, and J. Surovy, Chem. Zvesti 15, 414-418 (1961).

1961HES/WHI P. Hestermans and D. White, J. Phys. Chem. 65, 362-365 (1961).

1961HUF/GRO H. M. Huffman, M. E. Gross, D. W. Scott, and J. P. McCullough, J. Phys. Chem. 65, 495-503 (1961).

$1961 \mathrm{KOZ}$ V. M. Kozhin, Zh. Strukt. Khim. 2, 46 (1961).

1961LAB/GRE A. Labbauf, J. B. Greenshields, and F. D. Rossini, J. Chem. Eng. Data 6, 261-263 (1961).

1961Li/ROS J. C. M. Li and F. D. Rossini, J. Chem. Eng. Data 6 , 268-270 (1961).

1961MAL S. Malanowski, Bull. Acad. Pol. Sci., Ser. Sci. Chim. 9, 71-76 (1961).

1961MAL2 S. Malanowski, Bull. Acad. Pol. Sci., Ser. Sci. Chim. 9 83 (1961).

1961MAT/MCK J. F. Mathews and J. J. McKetta, J. Phys. Chem. 65, 758-762 (1961).

1961MCC/FIN J. P. McCullough, H. L. Finke, W. N. Hubbard, S. S. Todd, J. F. Messerly, D. R. Douslin, and G. Waddington, J. Phys. Chem. 65, 784-791 (1961).

1961MCC/MES J. P. McCullough and J. F. Messerly, Bull. Bur. Mines 596 (1961).

1961NIC/KOB J. K. Nickerson, K. A. Kobe, and J. J. McKetta, J. Phys. Chem. 65, 1037-11043 (1961).

1961PAC/BOB E. L. Pace and R. J. Bobka, J. Chem. Phys. 35, 454-457 (1961).

1961ROS/SUP A. Rose and W. R. Supina, J. Chem. Eng. Data 6, 173-179 (1961).

1961SCH/BOT I. M. Schiopu, O. Bot, and V. Onu, Bull. Inst. Politeh. Iasi. 7, 115-118 (1961); Chem. Abstr. 58, 10793c (1963).

1961SHR/CAD J. M. Shreeve and G. H. Cady, J. Am. Chem. Soc. 83, 4521 (1961).

1961SMU/BON E. J. Smutny and A. Bondi, J. Phys. Chem. 65, 546-550 (1961).

1961SPE

1961STU/SIN

1961VAN

1961WAL/SMI

1961WAR/PET

A. Sperandio, Ph.D. thesis, University of Zurich, 1961.

D. R. Stull, G. C. Sinke, R. A. McDonald, W. E. Hatton, and D. L. Hildenbrand, Pure Appl. Chem. 2, 315 (1961). G. Van dyke Tiers, J. Org. Chem. 26, 2538-2539 (1961). P. N. Walsh and N. O. Smith, J. Chem. Eng. Data 6, 33-35 (1961).

1961WES

1961ZIM/GEI

R. W. Warfield and M. C. Petree, J. Polym. Sci. 55, 497 (1961).

$\begin{array}{ll}\text { 1962BAN/CHE } & \text { 368-371 (1961). } \\ \text { R. E. Banks, W. M. Cheng, and R. N. Haszeldine, J. Chem. }\end{array}$ Soc. 1962, 3407.

1962BAU/GUN A. Bauder and H. H. Gunthard, Helv. Chim. Acta 45 , 1698-1702 (1962).

1962BER/MCK N. S. Berman and J. J. McKetta, J. Phys. Chem. 66 1444-1448 (1962).

1962BIR/BLO J. M. Birchall, A. J. Bloom, P. N. Haszeldine, and C. J. Willis, J. Chem. Soc. 1962, 3021.

1962BIT/KAU H.-J. Bittrich and E. Kauer, Z. Phys. Chem. 219, 224-238 (1962).

1962BIT/KAU2 H.-J. Bittrich, E. kauer, M. Kraft, G. Schoeppe, W. Soell, and A. Ullrich, J. Prakt. Chem. 17, 250-262 (1962).

1962CAD/ROD H. H. Cady and W. H. Rodgers, U.S. Atomic Energy Commission Report LA-2696, U.S. Atomic Energy Commission, 1962, p. 16. 
1962DAI/EVA F. S. Dainton, D. M. Evans, F. E. Hoare, and T. P. Melia, Polymer 3, 271 (1962).

1962DAV/SUN J. V. Davies and S. Sunner, Acta Chem. Scand. 16, 1870 (1962).

1962DOW A. J. Downs, J. Chem. Soc. 1962, 4361-4366.

1962EME/PAC H. J. Emeleus, K. H. Packer, and N. Welcman, J. Chem. Soc. 2529-2531 (1962).

1962GEI/QUI3 G. Geiseler, K. Quitzsch, R. Gesemann, and H. J. Gesemann, Z. Phys. Chem. 35, 10 (1962).

1962GOO/DOU W. D. Good, D. R. Douslin, and J. P. McCullough, J. Phys. Chem. 66, 958-959 (1962).

1962GRI/BUR J. E. Griffiths and A. B. Burg, J. Am. Chem. Soc. 84, 3442-3450 (1962).

1962HAT/HIL W. E. Hatton, D. L. Hildenbrand, G. C. Sinke, and D. R. Stull, J. Chem. Eng. Data 7, 229-231 (1962).

1962HEN/WEB V. P. Hennings and U. V. Weber, J. Prakt. Chem. 18, 91 (1962).

1962JON H. E. Jones, J. Chem. Eng. Data 7, 13 (1962).

1962 KOL/PAU V. P. Kolesov, J. Ye. Paukov, and S. M. Skuratov, Zh. Fiz. Khim. 36, 770 (1962).

1962KRA/BER M. Kraus, L. Beranek, K. Kochloeff, and V. Bazant, Chem. Prum. 12, 649-652 (1962); Chem. Abstr. 58, 10746c (1963).

1962KRE A. Kreglewski, Bull. Acad. Pol. Sci., Ser. Sci. Chim. 10, 629-633 (1962).

1962LEB/MIR Y. A. Lebedev, E. A. Miroshnichenko, and A. M. Chaikin, Dokl. Akad. Nauk SSSR 145, 1288-1289 (1962); Chem. Abstr. 58, 2906g (1963).

1962MAC/MAY H. Mackle and R. G. Mayrick, Trans. Faraday Soc. 58, 33-39 (1962).

1962MAC/MAY2 H. Mackle and R. G. Mayrick, Trans. Faraday Soc. 58, 230-237 (1962).

1962MAC/MAY3 H. Mackle and R. G. Mayrick, Trans. Faraday Soc. 58, 238-243 (1962).

1962MAN/SUN M. Mansson and S. Sunner, Acta Chem. Scand. 16, 1863-1869 (1962).

1962MAR J. J. Martin, J. Chem. Eng. Data 7, 68-72 (1962).

1962PAS/THO G. J. Pasek and G. L. Thodes, J. Chem. Eng. Data 7, 21-26 (1962).

1962ROS/HEI G. R. Ross and W. G. Heideger, J. Chem. Eng. Data 7, 505-507 (1962).

1962ROS/MUE R. M. Rosenberg and E. L. Muetterties, Inorg. Chem. 1, 756 (1962).

1962SCO/DOU D. W. Scott, D. R. Douslin, H. L. Finke, W. N. Hubbard, J. F. Messerly, I. A. Hossenlopp, and J. P. McCullough, J. Phys. Chem. 66, 1334-1341 (1962).

1962SCO/GOO D. W. Scott, W. D. Good, S. S. Todd, J. F. Messerly, W. T. Berg, I. A. Hossenlop, J. L. Lucina, A. G. Osborn, and J. P. McCullough, J. Chem. Phys. 36, 406-412 (1962).

1962SCO/MES D. W. Scott, J. F. Messerly, S. S. Todd, I. A. Hossenlopp, D. R. Douslin, and J. P. McCullough, J. Chem. Phys. 37, 867 (1962).

1962SEL/SUN P. Sellers and S. Sunner, Acta Chem. Scand. 16, 46-52 (1962).

1962SIN/HIL G. C. Sinke and D. L. Hildenbrand, J. Chem. Eng. Data 7, 74 (1962).

1962STE/DOR J. H. Stern and F. H. Dorer, J. Phys. Chem. 66, 97-99 (1962).

1962SUN/MEI W. Sundermeyer and W. Meise, Z. Anorg. Allg. Chem. 317, 334 (1962).

1962TJE

1962TJE2

1962VAL/BRO R. H. Valentini, G. E. Brodale, and W. R. Giauque, J. Phys.

J. Tebbes, Acta Chem. Scand. 16, 916-921 (1962).

J. Tebbes, Acta Chem. Scand. 16, 953-957 (1962). Chem. 66, 392-395 (1962).

1962 VOH/KAN S. P. Vohra, T. L. Kang, K. A. Kobe, and J. J. McKetta, J. Chem. Eng. Data 7, 150-155 (1962).

1962VON/AYM A. J. Von Arvia, P. J. Aymonino, and H. J. Schumacher, Z. Anorg. Allg. Chem. 316, 327-334 (1962).

1962WAD/SMI G. Waddington, J. C. Smith, K. D. Williamson, and D. W. Scott, J. Phys. Chem. 66, 1074-1077 (1962).

1962 WEB/KIL L. A. Weber and J. E. Kilpatrick, J. Chem. Phys. 36, 829 (1962).
1962WIL/CAD S. M. Williamson and G. H. Cady, Inorg. Chem. 1, 673-677 (1962).

1963AMB/TOW D. Ambrose and R. J. Townsend, J. Chem. Soc. 1963, 3614-3625.

1963AND/COU R. J. L. Andon, J. F. Counsell, and J. F. Martin, Trans. Faraday Soc. 59, 1555 (1963).

1963AND/COU2 R. J. L. Andon, J. F. Counsell, E. F. G. Herington, and J. F. Martin, Trans. Faraday Soc. 59, 830 (1963).

1963BAR/WES C. M. Barber and E. F. Westrum, Jr., J. Phys. Chem. 67, 2373 (1963).

1963BEN/MCK E. T. Benyon, Jr. and J. J. McKetta, J. Phys. Chem. 67, 2761-2765 (1963).

1963BID/COL D. P. Biddiscombe, R. R. Collerson, R. Handley, E. F. G. Herington, J. F. Martin, and C. H. S. Sprake, J. Chem. Soc. 1963, 1954-1957.

1963BID/HAN D. P. Biddiscombe, R. Handley, D. Harrop, A. J. Head, G. B. Lewis, J. F. Martin, and C. H. S. Sprake, J. Chem. Soc. 1963, 5764-5768.

1963BON

1963BOT/SEI

A. Bondi, J. Chem. Eng. Data 8, 371-381 (1963).

1963BOY G. A. Bottomley and G. H. F. Seiflow, J. Appl. Chem. 13, 399-409 (1963).

1963BRO R. H. Boyd, J. Chem. Phys. 38, 2529-2535 (1963).

1963BUL/SER J. A. Brown, J. Chem. Eng. Data 8, 106-108 (1963).

1963CAP/FRI Prom. (Moscow) 7, 507-509 (1963).

$\begin{array}{ll}\text { 1963DAN/FLU } & \text { Wun. 28, 2235-2239 (1963). } \\ \text { W. Dannhauser and A. F. Fluechkinger, J. Chem. Phys. } 38,\end{array}$ W. Dannhause
$69-72(1963)$.

1963EME/HAA H. J. Emeleus and A. Haas, J. Chem. Soc. 1963, $1272-1275$.

1963GIL/CAD W. P. Gilbreath and G. H. Cady, Inorg. Chem. 2, 496-499 (1963).

1963GOO/DOU W. D. Good, D. R. Douslin, and J. P. McCullough, J. Phys. Chem. 67, 1312-1314 (1963).

1963GOO/TOD W. D. Good, S. S. Todd, J. F. Messerly, J. L. Lacina, J. P. Dawson, D. W. Scott, and J. P. McCullough, J. Phys. Chem. 67, 1306-1311 (1963).

1963HAL/COX J. L. Hales, J. D. Cox, and E. B. Lees, Trans. Faraday Soc. 59, 1544-1554 (1963).

1963HIR/HIL H. Hiraoka and J. H. Hildebrand, J. Phys. Chem. 67, 916-918 (1963).

1963HOL/WAG R. R. Holmes and R. P. Wagner, Inorg. Chem. 2, 384-388 (1963).

1963HOR/WEN G. R. Horton and W. W. Wendlandt, J. Inorg. Nucl. Chem. 25, 241-245 (1963).

1963KOR/GEL N. G. Koral'nik and P. E. Geller, Zh. Prikl. Khim. 36, 1627 (1963); Chem. Abstr. 59, 81936 (1963).

1963MCC/LAI K. G. McCurdy and K. J. Laidler, Can. J. Chem. 41, 1867-1871 (1963).

1963MEY

1963MIL

1963MOR/SUN P. Meyer, D.E.S. dissertation, University of AixMarseilles, France, 1963.

G. A. Miller, J. Chem. Eng. Data 8, 69-72 (1963).

1963NOB/REE P. Noble, Jr., W. I. Reed, C. J. Hoffman, J. A. Gallaghan, P. Noble, Jr., W. I. Reed, C. J. Hoffman, J. A. Gallaghan,
and F. G. Borgardt, AIAA J. 1, 395-397 (1963); Chem. Abstr. 58, 13701b (1963).

1963OET F. L. Oetting, J. Phys. Chem. 67, 2757 (1963).

1963QUI/NOW K. Quitzsch, C. Nowak, P. Winkler, and G. Geiseler, J. Prakt. Chem. 20, 92-98 (1963).

1963RAS/NIG R. P. Rastogi, R. P. Nigam, R. N. Sharma, and H. L. Girdhar, J. Chem. Phys. 39, 3042-3045 (1963).

1963ROS/SCH A. Rose and V. N. Schrodt, J. Chem. Eng. Data 8, 9-13 (1963).

$19635 C O / G O O \quad$ D. W. Scott, W. D. Good, G. B. Gutherie, S. S. Todd, I. A Hossenlopp, A. G. Osborn, and J. P. McCullough, J. Phys. Chem. 67, 685-689 (1963).

1963SCO/HUB D. W. Scott, W. N. Hubbard, J. F. Messerly, S. S. Todd, I. A. Hossenlopp, W. D. Good, D. R. Douslin, and J. P. McCullough, J. Phys. Chem. 67, 680-685 (1963).

1963SCO/MES D. W. Scott, J. F. Messerly, S. S. Todd, I. A. Hossenlopp, A. Osborn, and J. P. McCullough, J. Chem. Phys. 38, 532-539 (1963). 
1963SOK/ZHI V. V. Sokolov, L. P. Zhilina, and K. P. Mischenko, Zh. Prikl Khim. 36, 750-754 (1963).

1963TRO/WES J. C. Trowbridge and E. F. Westrum, Jr., J. Phys. Chem. 67, 2381 (1963).

1963VIN/MAR C. G. Vinson, Jr. and J. J. Martin, J. Chem. Eng. Data 8, 74-75 (1963).

1963VLA/GRA O. N. Vlasov, V. A. Granzhan, and L. M. Savenko, Zh. Prikl. Khim. 36, 2311-2313 (1963).

1963VOI S. A. Voitkevich, Tr. Vses. Nauchn.-Issled. Inst. Sintetich. i Natural'n Dushistykh Veshchestv. 91-92 (1963); Chem. Abstr. 61, 13121e (1964).

1963WOJ J. G. Wojtasinski, J. Chem. Eng. Data 8, 381-385 (1963).

1963 WOO/ADI A. L. Woodman and A. Adicoff, J. Chem. Eng. Data 8, 241-242 (1963).

1963 WUL/WES C. A. Wulff and E. F. Westrum, Jr., J. Phys. Chem. 67, 2376-2381 (1963).

1964AMB/TOW D. Ambrose and R. Townsend, Trans. Faraday Soc. 60, 1025-1029 (1964).

1964BAN/CHE R. E. Banks, W. M. Cheng, and R. N. Haszeldine, J. Chem. Soc. 2485-2486 (1964).

1964BER/LAR N. S. Berman, C. W. Larkam, and J. J. McKetta, J. Chem. Eng. Data 9, 218-219 (1964).

$1964 B O N / J O R \quad$ O. D. Bonner, C. F. Jordan, and K. W. Bunzl, J. Phys. Chem. 68, 2450 (1964).

1964CAV R. G. Cavell, J. Chem. Soc. 1964, 1992-1995.

1964CAV/EME R. G. Cavell and H. J. Emeleus, J. Chem. Soc. 1964, $5825-5832$.

1964CAV/EME2 R. G. Cavell and H. J. Emeleus, J. Chem. Soc. Suppl. 5896-5897 (1964).

1964CLY/SVE D. D. Clyde and H. Svec, U.S. Atomic Energy Commission Report IS-790, U.S. Atomic Energy Commission (1964); Chem. Abstr. 61, 1293b (1964).

1964DAV D. J. David, Anal. Chem. 36, 2162 (1964).

1964DUN/CAD L. C. Duncan and G. H. Cady, Inorg. Chem. 3, 850-852 (1964).

1964DUN/CAD2 L. C. Duncan and G. H. Cady, Inorg. Chem. 3, 1045-1047 (1964).

1964EME/TAT H. J. Emeleus and B. W. Tattershall, Z. Anorg. Allg. Chem. 327, 147 (1964).

1964EPS/DUR Yu. V. Epsgtein, L. I. Durynina, and A. S. Pashinkin, Zh. Prikl. Khim. 37, 2543-2545 (1964).

1964FAW/LIP $\quad$ F. S. Fawcett and R. D. Lipscomb, J. Am. Chem. Soc. 86, 2576-2579 (1964)

1964FIS/BUC G. Fisher and A. S. Buchanan, Aust. J. Chem. 17, 481 (1964).

1964FRI/STA K. Friedrich and K. Stammbach, J. Chromatogr. 16, 22-28 (1964).

1964FUR/REI G. T. Furukawa, M. L. Reilly, H. H. Piccirelli, and M. J. Tenenbaum, J. Res. Natl. Bur. Stand., Sect. A 68, 367-379 (1964).

1964GEI/KON G. Geiseler and W. Konig, Z. Phys. Chem. (Leipzig) 227, 81-90 (1964).

1964 GOL/GOR N. A. Goldberg, V. A. Gorbushchenkov, and Z. G. Teplova, Zh. Prikl. Khim. 37, 745-747 (1964).

1964GUB/FER A. N. Gubkov, N. A. Fermor, and N. I. Smirnov, Zh. Prikl. Khim. 37, 2204-2210 (1964).

1964HAN/HAR R. Handley, D. Harrop, J. F. Martin, and C. H. S. Sprake, J. Chem. Soc. 1964, 4404-4406.

1964KLE A. V. Kletskii, Inzh. Fiz. Zh. Akad. Nauk. Beldrussk. SSR 7, 40-43 (1964).

1964KLI/FRI V. Kliment, V. Fried, and J. Pick, Collect. Czech. Chem. Commun. 29, 2008-2015 (1964).

1964MAC/MCC H. Mackle and R. T. B. McClean, Trans. Faraday Soc. 60, 817-821 (1964).

1964MAL/WEC B. Malanowska and J. Wecsile, Bull. de L'Acad. Polon. des Sci. 12, 239 (1964).

1964MCE/KIL D. M. McEachern, Jr. and J. E. Kilpatrick, J. Chem. Phys. 41, 3127 (1964).

1964MUR K. P. Murphy, J. Chem. Eng. Data 9, 259-260 (1964).

1964NIX

1964OET

1964PAT/PRO

J. F. Nixon, J. Chem. Soc. 2469 (1964).

F. L. Oetting, J. Chem. Phys. 41, 149 (1964).

C. R. Patrick and G. S. Prosser, Trans. Faraday Soc. 60,
1964SER/TIM 700-704 (1964).
1964PET/BUR

1964RAS/BAS

1964RAT/SHR

1964ROB

1964SER/GOR

1964SEV/SOK

1964TRO/WES

1964WUL/WES

1965AYL/PET

1965BAN/BAR

1965BAN/BUR

1965BAN/HAS

1965BUR/GOS

1965BUR/HEN

1965CAR/WES

1965CAR/WES2

1965CLA/PES

1965CLE/WON

1965CLE/WUL

1965CLI/KOB

1965COL/COU

1965COU/GRE

1965COU/HAL

1965DAV/KYB

1965DIN/HAS

1965DIN/HAS2

1965DIN/HAS3

1965DOU/OSB

1965DRE/MER

1965FIN/HOS

1965FIN/MES

1965FRA/SHR

1965FRE/MAR

1965GAK/BAB

L. K. Peterson and A. B. Burg, J. Am. Chem. Soc. 86, 2587-2591 (1964).

R. P. Rastogi and P. S. Bassi, J. Phys. Chem. 68, 2398 (1964).

C. T. Ratcliffe and J. M. Shreeve, Inorg. Chem. 3, 631-633 (1964).

H. L. Roberts, J. Chem. Soc. 1964, 4538.

E. A. Seregin, N. N. Goroshko, V. P. Kolesov, N. A. Belikova, S. M. Skuratov, and A. F. Plate, Dokl. Akad. Nauk SSSR 159, 1381 (1964).

L. A. Serafimov, V. S. Timofeev, M. P. Strukova, and S. V. L'vov, Russ. J. Phys. Chem. 38, 1018-1019 (1964).

N. N. Sevrugova, V. A. Sokorskii, and Zhavoronkov, Zh. Prikl. Khim. (Leningrad) 37, 1989-1993 (1964).

J. C. Trowbridge and E. F. Westrum, Jr., J. Phys. Chem. 68, 255 (1964).

C. A. Wulff and E. F. Westrum, Jr., J. Phys. Chem. 68, 430 (1964).

B. J. Aylett and L. K. Peterson, J. Chem. Soc. 1965, 4043-4048.

R. E. Banks, M. G. Barlow, and R. N. Haszeldine, J. Chem. Soc. 1965, 6149-6163.

R. E. Banks and E. D. Burling, J. Chem. Soc. 1965, 6077.

R. E. Banks, R. N. Haszeldine, and D. R. Taylor, J. Chem. Soc. 1965, 5602.

A. B. Burg and K. Gosling, J. Am. Chem. Soc. 87, 2113 (1965).

A. B. Burg and J. Heners, J. Am. Chem. Soc. 87, 3092-3096 (1965).

H. G. Carlson and E. F. Westrum, Jr., J. Chem. Eng. Data 10, 134-135 (1965)

H. G. Carlson and E. F. Westsrum, Jr., J. Phys. Chem. 68, 1524 (1965).

L. B. Clark, G. G. Peschel, and I. Tinoco, J. Phys. Chem. 69, 3615-3618 (1965).

H. L. Clever, W.-K. Wong, and E. F. Westrum, Jr., J. Phys. Chem. 69, 1209 (1965).

H. L. Clever, C. A. Wulff, and E. F. Westrum, Jr., J. Phys. Chem. 69, 1983 (1965).

A. F. Clifford and C. S. Kobayashi, Inorg. Chem. 4, 571 (1965).

R. R. Collerson, R. Handley, J. F. Martin, and C. H. S. Sprake, J. Chem. Soc. 1965, 3697-3700.

J. F. Counsell, J. H. S. Green, J. L. Hales, and J. F. Martin, Trans. Faraday Soc. 61, 212-218 (1965).

J. F. Counsell, J. L. Hales, and J. F. Martin, Trans. Faraday Soc. 61, 1869-1875 (1965).

M. Davies and B. Kybett, Trans. Faraday Soc. 61, 1608-1617 (1965); 61, 1893-1896 (1965).

A. H. Dinwoodie and R. N. Haszeldine, J. Chem. Soc. 2266 (1965).

A. H. Dinwoodie and R. N. Haszeldine, J. Chem. Soc. $\mathbf{1 9 6 5}, 1675$.

A. H. Dinwoodie and R. N. Haszeldine, J. Chem. Soc. 1965, 1681.

D. R. Douslin and A. Osborn, Rev. Sci. Instrum. 42, 369-373 (1965).

R. D. Dresdner, J. Merritt, and J. P. Royal, Inorg. Chem. 4, 1228 (1965).

H. L. Finke, I. A. Hossenlopp, and W. T. Berg, J. Phys. Chem. 69, 3030-3031 (1965).

H. L. Finke, J. F. Messerly, and S. S. Todd, J. Phys. Chem. 60, 2094-2100 (1965).

G. W. Fraser and J. M. Shreeve, Inorg. Chem. 4, 1497-1498 (1965).

H. M. Frey, D. C. Marshall, and R. F. Skinner, Trans. Faraday Soc. 61, 861 (1965).

1965HAS/TIP R. N. Haszeldine and A. E. Tipping, J. Chem. Soc. 1965, 6141-6149.

1965HEI/SUR J. Heinrich, J. Surovy, and J. Dojcansky, Chem. Zvesti 19, 462-464 (1965). 
1965HUL/REI H. S. Hull, A. F. Reid, and A. G. Turnbull, Aust. J. Chem. 18, 249-252 (1965).

$1965 \mathrm{KAL} /$ ROZ L. A. Kalashnikova, E. G. Rozantsev, and A. M. Chaikin, Izv. Akad. Nauk SSSR 800-805 (1965); Chem. Abstr. 63, 4972c (1965).

$1965 \mathrm{KAR} / \mathrm{KYB} \quad$ H. A. Karnes, B. Kybett, M. H. Wilson, J. L. Margrave, and M. S. Newman, J. Am. Chem. Soc. 87, 5554-5558 (1965).

1965KIL/BIT H. Kilian and H. J. Bittrich, Z. Phys. Chem. 230, 383 (1965).

1965KUD/SAV I. V. Kudryashov and R. I. Savechenko, Izv. Vysh. Ucheb. Zav. Khim. Khim. Tekhnol. 8, 602 (1965).

1965LIN/FRI J. Linek, V. Fried, and J. Pick, Collect. Czech. Chem. Commun. 30, 1358-1365 (1965).

1965LUS/RUF M. Lustig and J. K. Ruff, Inorg. Chem. 4, 1441 (1965).

1965LUT/KOL N. V. Lutugina, V. N. Kolbina, and L. I. Reshetova, Zh. Prikl. Khim. 38, 1541-1546 (1965).

1965MAR P. Martin, J. Chem. Eng. Data 10, 292-294 (1965).

1965 MAR/SUS A. N. Marinichev and M. P. Susarev, Zh. Prikl. Khim. 38, 378-383 (1965).

$1965 \mathrm{MCD} / \mathrm{KIL} \quad$ L. A. Mcdougall and J. E. Kilpatrick, J. Chem. Phys. 42, 2311-2321 (1965).

1965 MCD/KIL2 L. A. Mcdougall and J. E. Kilpatrick, J. Chem. Phys. 42, 2307 (1965).

1965MER/POL I. Mertl and J. Polak, Collect. Czech. Chem. Commun. 30, 3526-3528 (1965).

1965 MES/TOD J. F. Messerly, S. S. Todd, and H. L. Finke, J. Phys. Chem. 69, 353 (1965).

1965MES/TOD2 J. F. Messerly, S. S. Todd, and H. L. Finke, J. Phys. Chem. 69, 4304 (1965).

1965NAR

1965NIS/LAP

1965NIS/LAP2 L. A. Nisel'son and I. I. Lapivus, Russ. J. Phys. Chem. 39, 931-932 (1965).

1965NIX

$1965 \mathrm{NOF} / \mathrm{CAD}$

J. F. Nixon, J. Inorg. Nucl. Chem. 27, 1281-1287 (1965).

1965OET

1965PES/NAS

R. Noftle and G. H. Cady, Inorg. Chem. 4, 1010-1012 (1965). 38, 1623 (1965).

1965PUT/MCE W. E. Putnam, D. M. McEachern, and J. E. Kiplatrick, J. Chem. Phys. 42, 749-755 (1965).

1965RON/HAR R. E. Rondeau and L. A. Harrah, J. Chem. Eng. Data 10, 84-85 (1965).

1965SER/BYK I. I. Serebryannaya and S. Sh. Byk, Khim. Prom. 23, 828-829 (1965).

1965SHR/DUN J. M. Shreeve, L. C. Duncan, and G. H. Cady, Inorg. Chem. 4, 1516-1517 (1965).

$1965 \mathrm{SOM} / \mathrm{COO}$ G. Somsen and J. Coops, Recl. Trav. Chim. 84, 985 (1965).

1965SVE/CLY H. Svec and D. D. Clyde, J. Chem. Eng. Data 10, 151-152 (1965).

1965SWA/VAN P. A. Swamy and M. J. Van Winkle, J. Chem. Eng. Data 10, 214-215 (1965).

1965WAD

1965WAR/PET

I. Wadso, Acta Chem. Scand. 9, 1079-1087 (1965).

R. W. Warfield and M. C. Petree, Die Makromol. Chem. 84, 1 (1965).

1966BAN/BAR R. W. Banks, M. G. Barlow, W. R. Deem, R. N. Haszeldine, and D. R. Taylor, J. Chem. Soc. C 1966, 981-984.

1966BAN/HAS R. E. Banks, G. M. Haslam, R. N. Haszeldine, and A. Peppin, J. Chem. Soc. C 1966, 1171.

1966BAN/MOO R. E. Banks and G. J. Moore, J. Chem. Soc. C 1966, 2304.

1966BEA/CLE R. H. Beaumont, B. Clegg, G. Gee, J. B. M. Herbert, D. J. Marks, R. C. Roberts, and D. Sims, Polymer 7, 401 (1966).

1966BOR/NAK B. Börjesson, Y. Nakase, and S. Sunner, Acta Chem. Scand. 20, 803-810 (1966).

1966BOT/ADL T. R. Bott and H. N. Adler, J. Chem. Eng. Data 11, 25-30 (1966).

1966BRO/FRA D. H. Brown, G. W. Fraser, and G. W. A. Sharp, J. Chem. Soc. A 1966, 171-174.

1966BUR
1966BUR/JOS

1966CAR/STE

1966COH/MAC

1966DEL/SHR

1966DES/CAD

1966DOB

1966DOB/EME

1966DOB/EME2

1966DWO/GUI

1966EAR/HIL

1966EME/ONA

1966EME/TAT

1966EVA/TIL

1966FOX/FRA

1966GAN/SEM

1966GEI/FRU

1966GEI/QUI

A. B. Burg, K. K. Joshi, and J. F. Nixon, J. Am. Chem. Soc. 88, 31 (1966).

J. L. Carson, R. C. Stewart, and A. G. Williamson, J. Chem. Eng. Data 11, 231-233 (1966).

B. Cohen and A. G. MacDiarmid, J. Chem. Soc. 1966, 1780.

J. J. Delfino and J. M. Shreeve, Inorg. Chem. 5, 308-309 (1966).

D. D. DesMarteau and G. H. Cady, Inorg. Chem. 5, 169-171 (1966).

R. C. Dobbie, J. Chem. Soc. A 1966, 1555-1556.

R. C. Dobbie and H. J. Emeleus, J. Chem. Soc. A 1966 933-936.

R. C. Dobbie and H. J. Emeleus, J. Chem. Soc. A 1966 367.

A. Dworkin and M. Guillamin, J. Chim. Phys. Phys.Chim. Biol. 63, 53 (1966).

B. L. Earl, B. K. Hill, and J. M. Shreeve, Inorg. Chem. 5 , 2184-2186 (1966).

H. J. Emeleus and T. Onak, J. Chem. Soc. A 1966, 1291-1292.

H. J. Emeleus and B. W. Tattershall, J. Inorg. Nucl. Chem. 28, 1823-1827 (1966).

F. D. Evans and P. F. Tiley, J. Chem. Soc. B 1966 , 134-136.

W. B. Fox and G. Franz, Inorg. Chem. 5, 946 (1966).

V. A. Ganzhan, S. V. Semenko, and O. G. Kirillova, Zh Prikl. Khim. 39, 1399 (1966); Chem. Abstr. 65, 9820f (1966).

G. Geiseler, J. Fruwert, and R. Huetting, Chem. Ber. 99, 1594-1601 (1966).

G. Geiseler, K. Quitzsch, H.-J. Rauh, H. Schaffernicht, and H. Walther, Ber. Bunsenges Phys. Chem. 70, 551-556 (1966).

1966GOO/DEP W. D. Good and B. L. DePrater, J. Phys. Chem. 70, 3606-3609 (1966).

1966HAS/TIP R. N. Haszeldine and A. E. Tipping, J. Chem. Soc. C 1966 , 1236-1241.

1966JEN/SCH D. J. Jensen and E. D. Schall, J. Agric. Food Chem. 14, 123-126 (1966).

1966KRE

1966KYB/CAR $\quad$ B. D. Kybett, S. Carroll, P. Natalis, D. W. Bonnell, J. L. Margrave, and J. L. Franklin, J. Am. Chem. Soc. 88, 626 (1966).

1966LEB/ROS Y. A. Lebedev, E. G. Rosantsev, L. A. Kalashnikova, V. P. Lebedev, M. B. Nieman, and Y. A. Apin, Dokl. Acad. Nauk SSSR 168, 104 (1966) [Engl. Ed. 460].

1966LIU/ZIE K. F. Liu and W. T. Ziegler, J. Chem. Eng. Data 11, 187 (1966).

1966LUS

1966MAN/SUN

1966MAT/RAS

M. Lustig, Inorg. Chem. 5, 1317 (1966).

1966MEA/ROS

1966MEY/FRA M. Mansson and
$845-848$ (1966).

A. Matejick and J. Raska, Chem. Prum. 16, 82 (1966).

W. H. Mears, E. Rosenthal, and J. V. Sinka, J. Chem. Eng. Data 11, 338-342 (1966).

1966MEY/MET

M. D. Meyers and S. Frank, Inorg. Chem. 5, 1455-1457 (1966).

1966MEY/WAG E. F. Meyer and R. E. Wagner, J. Phys. Chem. 70, 3162-3168 (1966).

1966MOR/TAM H. L. Morris, M. Tamres, and S. Searles, Inorg. Chem. 5, 2156-2160 (1966).

1966 OSB/DOU A. N. Osborn and D. R. Douslin, J. Chem. Eng. Data 11, 502-509 (1966).

1966PIR J. Pirsch, Monatsh. Chem. 97, 260-270 (1966).

1966REI/SHE R. C. Reid and T. K. Sherwood, The Properties of Gases and Liquids, Their Estimation and Correlation (McGraw Hill, New York, 1966).

1966RIN/ONE M. A. Ring, H. E. O'Neal, A. H. Kadhim, and F. J. Jappe, Organomet. Chem. 5, 124-129 (1966).

1966THO/MEA L. M. Thomas and R. Meatyard, J. Chem. Soc. A 1966, 92-96. 
1966WAD

1966WAD2

I. Wadso, Acta Chem. Scand. 20, 544-552 (1966)

1966WAG/WIL

I. Wadso, Acta Chem. Scand. 20, 536 (1966).

1966WIE/RUF

R. I. Wagner and C. O. Wilson, Jr., Inorg. Chem. 5, 1009 (1966).

1966ZIM/ROB M. F. Zimmer, R. A. Robb, and G. A. Carpenter, J. Chem. Eng. Data 11, 577-579 (1966)

1967AND/COU R. J. L. Andon, J. F. Counsell, E. B. Lees, J. F. Martin, and C. J. Mash, Trans. Faraday Soc. 63, 1115 (1967).

1967AND/FOX L. R. Anderson and W. B. Fox, J. Am. Chem. Soc. 89, 4313 (1967).

1967AND/HAM M. L. Anderson and R. N. Hammer, J. Chem. Eng. Data 12, 442-447 (1967).

1967BAB/SHR D. P. Babb and J. M. Shreeve, Inorg. Chem. 6, 351 (1967).

1967BAN/HAS R. E. Banks, R. N. Haszeldine, and R. Hatton, J. Chem. Soc. C 1967, 427-430.

1967BER/WES H. A. Berman and E. D. West, J. Chem. Eng. Data 12, 197-199 (1967).

1967BOY/GUH R. H. Boyd, K. R. Guha, and R. Wuthrich, J. Phys. Chem. 71, 2187-2191 (1967).

1967BRA/SZI W. K. Bratton, I. Szilard, and C. A. Cupas, J. Org. Chem. 32, 2019-2022 (1967).

1967BUC/COX E. Buckley and J. D. Cox, Trans. Faraday Soc. 63, 895-901 (1967).

1967CAV R. G. Cavell, Can. J. Chem. 45, 1309 (1967).

1967CHI/SIM Y. T. Chia and H. E. Simmons, J. Am. Chem. Soc. 89, 2638-2643 (1967).

1967CRO/TAY G. A. Crowder, Z. L. Taylor, T. M. Reed III, and J. A. Young, J. Chem. Eng. Data 12, 481-484 (1967).

1967 CRU/CUT A. J. B. Cruickshank and A. J. B. Cutler, J. Chem. Eng. Data 12, 326-329 (1967).

1967DAN/GOL S. M. Danov and Y. D. Golubev, Tr. Khim. Khim. Tekhnol. 52-55 (1967); Chem. Abstr. 68, 95253a (1968).

1967DEM/KEN G. C. Demitras, R. A. Kent, and R. G. MacDiarmid, Inorg. Chem. 6, 1903 (1967).

1967DES/PAN D. D. Deshpande and M. V. Pandya, Trans. Faraday Soc. 63, 2149 (1967).

1967ERM/SKR N. V. Ermakov and V. P. Skripov, Zh. Fiz. Khim. 41, 77-81 (1967).

1967FRA/SHR G. W. Fraser and J. M. Shreeve, Inorg. Chem. 6, 1711 (1967).

1967FRI/GAL V. Fried, P. Gallant, and G. B. Schneier, J. Chem. Eng. Data 12, 504-508 (1967).

1967GEI/SCH G. Geiseler, H. Schaffernicht, and H. Walther, Ber. Bunsenges Phys. Chem. 71, 196-199 (1967).

1967GLE/BIE O. Glemser, U. Biermann, and M. Fild, Chem. Ber. 100, 1082-1086 (1967)

1967GLE/BIE2 O. Glemser and U. Biermann, Inorg. Nucl. Chem. Lett. 3, 223 (1967)

1967GLE/BIE3 O. Glemser, U. Biermann, and A. Hoff, Z. Naturforsch. Anorg. Chem. B 22, 893 (1967).

$1967 \mathrm{GOE} / \mathrm{SCH} \quad$ K. H. Goebel, J. Schaffenger, and G. Opel, Chem. Technol. 19, 307-308 (1967).

1967HAC/MAT M. Haccuria and M. P. Mathieu, Ind. Chim. Belge 32, 165-167 (1967).

1967HAL/LEE J. L. Hales, E. B. Lees, and D. J. Ruxton, Trans. Faraday Soc. 63, 1876-1879 (1967).

1967HAS/TIP R. N. Haszeldine and A. E. Tipping, J. Chem. Soc. C 1967, 1241

1967HER A. J. Herbert, Trans. Faraday Soc. 63, 555-560 (1967).

1967 HIR/SUD M. Hirata and S. Suda, Kagaku Kogaku 31, 339-342 (1967).

1967HYN/BIS J. B. Hynes, B. C. Bishop, and L. A. Bigelow, Inorg. Chem. 6, 417 (1967).

1967KHO/BYK A. S. Khorevskaya and S. Sh. Byk, Zh. Prikl. Khim. 40, 464 (1967).

1967KIS A. Kisza, Rocz. Chim. 41, 351 (1967).

1967KLE/PET A. B. Kletshii and L. E. Petric, Zh. Fiz. Khim. 41, 1183-1184 (1967).

1967KON/ZHU I. I. Konstantinov, E. Z. Zhuravlev, and Z. G. Teplova, Zh. Prikl. Khim. 40, 1084-1087 (1967).
1967LEB/RAB B. V. Lebedev, I. B. Rabinovich, and V. A. Budarina, Polym. Sci. U.S.S.R. 9, 545 (1967).

1967LES/OGO T. M. Lesteva, S. K. Ogorodnikov, and A. I. Morozova, Zh. Prikl. Khim. (Leningrad) 40, 891-894 (1967).

1967LUS

1967MEE/GOL

M. Lustig, Inorg. Chem. 6, 1064-1065 (1967).

1967MES/GUT 196 (1967). $\quad$ J. Messerly, G. B. Guthrie, S. S. Todd, and H. L. Finke, J. Chem. Eng. Data 12, 338 (1967).

1967MES/TOD J. F. Messerly, S. S. Todd, and G. B. Guthrie, J. Chem. Eng. Data 12, 426 (1967).

1967MIR/LEB $\quad$ E. A. Miroshnichenko, Y. A. Lebendev, S. A. Shevelev, V. I. Gulevskaya, A. A. Fainzil'berg, and A. Y. Apin, Zh. Fiz. Khim. 41, 1477-1479 (1967); Russ. J. Phys. Chem. 41, 783-785 (1967).

1967MUL/MUK F. I. Mullayanov, R. K. Mukhutdinov, M. F. Mazitov, M. F. Buz'ko, V. P. Borschenko, and V. N. Krashennikov, Khim. Prom. (Moscow) 43, 505-506 (1967); Chem. Abstr. 68, 113943 (1968).

1967MUR/KIV J. Murto and A. Kivinen, Suom. Kemistil. B 40, 258-264 (1967).

1967NIK/RAB $\quad$ P. N. Nikolaev and I. B. Rabinovich, Zh. Fiz. Khim. 49, 2191-2194 (1967).

1967NIK/RAB2 P. N. Nikolaev, I. B. Rabinovich, and B. V. Lebedev, Zh. Fiz. Khim. 41, 1294 (1967).

1967PAC

1967PAC/PLA

P. Pacor, Anal. Chim. Acta 37, 200 (1967).

1967PIH/HEI (1967).

1967PIN/WIL P. E. Pincock, K. R. Wilson, and T. E. Kiovsky, J. Am.

K. Pihlaja and J. Heikkila, Acta Chem. Scand. 21, 2430-2434 (1967). Chem. Soc. 89, 6890-6897 (1967).

1967PLA/PAC A. C. Plaush and E. L. Pace, J. Chem. Phys. 47, 44-48 (1967).

1967QUI

1967QUI/HOF

K. Quitzsch, Z. Phys. Chem. (Leipzig) 236, 241 (1967).

1967RIB/WES

K. Quitzsch, H. P. Hofmann, R. Pfestorf, and G. Geiseler, Z. Phys. Chem. (Leipzig) 235, 99 (1967).

A. Ribner and E. F. Westrum, Jr., J. Phys. Chem. 71, 1208-1215 (1967).

1967SCO/BER D. W. Scott, W. T. Berg, I. A. Hossenlopp, W. N. Hubbard, J. F. Messerly, S. S. Todd, D. R. Douslin, J. P. McCullough, and G. Waddington, J. Phys. Chem. 71, 2263-2270 (1967).

1967SHA

1967SIM/KNO

R. L. Shak, J. Chem. Eng. Data 12, 474-480 (1967).

1967SIV/MAT

M. Simon, C. M. Knobler, and A. G. Duncan, Cryogenics 7, 138-140 (1967).

1967TSE/GOD M. A. Tseneva and Yu. K. Godovskii, Zh. Fiz. Khim. 41, 1827 (1967).

1967VAN

1967VAN/SOC

W. A. Van Hook, J. Chem. Phys. 46, 1907-1918 (1967).

1967WES/RIB

H. C. Van Ness, C. A. Soczek, G. L. Peloquin, and R. L. Machado, J. Chem. Eng. Data 12, 217-223 (1967).

1968ADA/SUG E. F. Westrum an

1968AMB K. Adachi, H. Suga, and S. Seki, Bull. Chem. Soc. Jpn. 41, 1073-1087 (1968).

1968AND/COU R. J. L. Andon, J. F. Counsell, J. L. Hales, E. B. Lees, and J. F. Martin, J. Chem. Soc. A 1968, 2357-2361.

1968AND/COU2 R. J. L. Andon, J. F. Counsell, and J. F. Martin, J. Chem. Soc. A 1968, 1894.

1968BAC/NOV D. P. Baccanari, J. A. Novinski, Y.-C. Pan, M. M. Yevitz, and H. A. Swain, Trans. Faraday Soc. 64, 1201-1205 (1968).

1968BAN/BRA R. E. Banks, A. Braithwaite, R. N. Haszeldine, and D. R. Taylor, J. Chem. Soc. C 1968, 2593.

1968BAR/CAR E. E. Baroody, G. A. Carpenter, R. A. Robb, and M. F. Zimmer, J. Chem. Eng. Data 13, 215-217 (1968).

1968BRI/PRI A. R. Briden, D. Price, and H. Sutcliffe, J. Chem. Soc. B 1968, 387.

1968CAB/CON S. Cabani, G. Conti, and L. Lepori, Ric. Sci. 38, 1039 (1968). 
1968CHA/CAV T. L. Charlton and R. G. Cavell, Inorg. Chem. 7, 2195 (1968).

1968CHE/TUR E. L. Cherkasskaya, A. M. Tur, Z. F. Petrenkova, and V. I. Lyubomilov, Zh. Prikl. Khim. 41, 2553-2554 (1968).

1968CIH/VOJ M. Cihova, J. Vojtko, and M. Hrusovsky, Zb. Pr. Chemickotechnol. Fak. SVST (Slov. Vys. SK. Tech.) 135-138 (1968); Chem. Abstr. 72, 136676u (1970).

1968CIH/VOJ2 M. Cihova, J. Vojtko, and M. Hrusovsky, Chem. Zvesti 22, 599 (1968).

1968COU/HAL J. F. Counsell, J. L. Hales, E. B. Lees, and J. F. Martin, J. Chem. Soc. A 1968, 2994-2996.

1968COU/HAL2 J. F. Counsell, J. L. Hales, and J. F. Martin, J. Chem. Soc. A 1968, 2042.

1968COU/LEE J. F. Counsell, E. B. Lees, and J. F. Martin, J. Chem. Soc. A 1968, 1819-1823.

1968DAV/BAT Zh. V. Davydova, P. S. Bataev, I. A. Makolkin, and M. E. Yanitskaya, Tr., Mosk. Inst. Nar. Khoz. 46, 32 (1968).

1968DES/WIL P. D. Desai, R. C. Wilhoit, and B. J. Zwolinski, J. Chem. Eng. Data 13, 334-335 (1968).

1968ELL/CHR J. H. Elliott and M. D. Chris, J. Chem. Eng. Data 13, 475 (1968).

1968FLO W. Z. Florian, Phys. Chem. (Frankfurt am Main) 61, 319-321 (1968); Chem. Abstr. 70, 81136u (1969).

1968FOW/TRU L. Fowler, W. N. Trump, and C. E. Vogler, J. Chem. Eng. Data 13, 209-210 (1968).

1968FRE/TIP J. Freear and A. E. Tipping, J. Chem. Soc. C 1968, 1096-1103.

1968GAR/BOV Yu. N. Garber and R. A. Bovkun, Zh. Prikl. Khim. 41, 318 (1968).

1968GAW/SWI W. J. Gaw and F. L. Swinton, Trans. Faraday Soc. 64, 637-647 (1968).

1968GAW/SWI2 W. J. Gaw and F. L. Swinton, Trans. Faraday Soc. 64, 2023-2034 (1968).

1968GEI/HOF G. Geiseler and J. Hoffmann, Z. Phys. Chem. (Frankfurt am Main) 57, 318-330 (1968).

1968GIR/WES H. L. Girdhar, E. F. Westrum, Jr., and C. A. Wulff, J. Chem. Eng. Data 13, 239 (1968).

1968GLE/VON O. Glemser and S. P. von Halasz, Z. Naturforsch. B 23, 743-745 (1968).

1968GLE/VON2 O. Glemser, S. P. von Halasz, and U. Biermann, Z. Naturforsch. B 23, 1381-1382 (1968).

1968GOP/RIZ F. Gopal and S. A. Rizvi, J. Indian Chem. Soc. 45, 13-16 (1968).

1968GOS/BUR K. Gosling and A. B. Burg, J. Am. Chem. Soc. 90, 2011 (1968).

1968GOU/WES P. Goursot and E. F. Westrum, Jr., C. R. Acad. Sci. Paris Ser. C 266, 667-668 (1968).

1968GOU/WES2 P. Goursot and E. F. Westrum, Jr., C. R. Acad. Sci. Paris Ser. C 266, 590-592 (1968).

1968HAM/FAG J. V. Hamilton and T. F. Fagley, J. Chem. Eng. Data 13, 523-527 (1968).

1968HAS/TIP R. N. Haszeldine and A. E. Tipping, J. Chem. Soc. C 1968, 398-405.

$1968 \mathrm{HOH} / \mathrm{SHR} \quad$ F. A. Hohorst and J. M. Shreeve, Inorg. Chem. 7, 624 (1968).

1968KAC/NEM R. V. Kachalova and M. S. Nemtsov, Zh. Prikl. Khim. (Leningrad) 41, 2315-2319 (1968).

1968KAR/RAB N. V. Karyakin, I. B. Rabinovich, and L. G. Pakhomov, Russ. J. Phys. Chem. 42, 954-955 (1968).

1968 KHO/BYK A. S. Khorevskaya and S. S. Byk, Zh. Prikl. Khim. (Leningrad) 41, 2566-2568 (1968); Chem. Abstr. 70, 61317a (1969).

1968KUS/MIS V. V. Kushchenko and K. P. Mishchenko, Zh. Prikl. Khim. 41, 646-648 (1968); Chem. Abstr. 68, 117314a (1968).

1968LAP/NIS I. I. Lapidus and L. A. Nisel'son, Russ. J. Phys. Chem. 42, 733-734 (1968).

1968LUC/LEW C. A. Lucchesi and W. T. Lewis, J. Chem. Eng. Data 13, 389-391 (1968).

1968MAK Y. Y. Maksimov, Russ. J. Phys. Chem. 42, 1550-1552 (1968).

1968MAL/MEU P. F. Malbrunot, P. A. Meunier, G. M. Scatena, W. H. Mears, K. P. Murphy, and J. V. Sinka, J. Chem. Eng. Data 13, 16-21 (1968).
1968MAN/NAK M. Mansson, Y. Nakase, and S. Sunner, Acta Chem. Scand. 22, 171-174 (1968).

1968MAS/RAE R. Mason and A. I. M. Rae, Proc. R. Soc. A 304, 501-512 (1968).

1968MER I. Mertl, Chem. Listy 62, 584-587 (1968).

1968MOR

E. Morawetz, Acta Chem. Scand. 22, 1509-1531 (1968).

1968NAS/BAB L. L. Nash, D. P. Babb, J. J. Couville, and J. M. Shreeve, J. Inorg. Nucl. Chem. 30, 3373-3375 (1968).

$1968 \mathrm{NOF}$

$1968 \mathrm{NOF} / \mathrm{SHR}$

R. E. Noftle, Inorg. Chem. 7, 2167 (1968).

19680SB/DOU A. G. Osborn and D. R. Douslin, J. Chem. Eng. Data 13, 534-537 (1968).

1968PAC/REN E. L. Pace and M. A. Reno, J. Chem. Phys. 48, 1231-1235 (1968).

1968PEP/MIR V. I. Pepekin, E. A. Miroshnichenko, Y. A. Levedev, and A. Y. Apin, Russ. J. Phys. Chem. 42, 1583-1584 (1968).

1968PLA/WIL R. D. Place and S. M. Williamson, J. Am. Chem. Soc. 90, 2550-3556 (1968).

1968RAT/SHR C. Ratcliffe and J. M. Shreeve, J. Am. Chem. Soc. 90, 5403-5408 (1968).

1968RAV/DAN A. A. Ravdel and V. V. Danilov, Izv. Vyssbikh. Uchebnykh. Zaved., Khim. Khimichesk. Tekhnol. 11, 642-645 (1968); Chem. Abstr. 69, 90232 (1968).

1968REN/PRA H. Renon and J. M. Prasunitz, Ind. Eng. Chem. Process Des. Dev. 7, 220 (1968).

1968ROE

1968SMI/SMI

H. W. Roesky, Angew. Chem. 7, 218 (1968).

$\begin{array}{ll}\text { 1968TUR/HUL } & \text { A. G. Turnbull and H. S. Hull, Aust. J. Chem. 21, } 1789\end{array}$ (1968).

1968WAD I. Wadso, Acta Chem. Scand. 22, 2438-2444 (1968).

1968ZHU/KON E. Z. Zhuravlev and I. I. Konstantinov, Zh. Prikl. Khim. 41, 1170-1172 (1968).

1969AND/BRA～D. N. Andreeskii, M. M. Brazhnikov, and V. N. Il'nchik, Vest. Beloruss Univ. 3-5 (1969); Chem. Abstr. 73, 113607 (1970).

1969BAN/BAR R. E. Banks, M. G. Barlow, W. D. Davies, R. N. Haszeldine, and D. R. Taylor, J. Chem. Soc. C 1969, 1104.

1969BAN/HAS R. E. Banks, R. N. Haszeldine, and W. D. Morton, J. Chem. Soc. C 1969, 1947.

1969BRE/HAG W. Bremser, R. Hagen, E. Heilbronner, and E. Vogel, Helv. Chim. Acta 52, 418-431 (1969).

1969BRO/FOC I. Brown, W. Fock, and F. Smith, J. Chem. Thermodyn. 1, 273-291 (1969).

1969BUR/MIS A. B. Burg and I. B. Mishra, Inorg. Chem. 8, 1199 (1969).

1969CAL/VAL G. C. Calero, M. M. Valle, and C. G. Losa, Rev. Acad. Cienc. Exactas, Fis., Quim. Nat. Zaragoza 24, 137-158 (1969).

1969CHA/STE R. Chastel, F. Steckel, and H. Tachoire, Proceedings of the First International Conference on Calorimetry Thermodynamics Paper 5/2 (Polish Scientific Publishers, Warsaw, 1969); as cited in [1982COL/JIM].

1969CID/POL J. Cidlinsky and J. Polak, Collect. Czech. Chem. Commun. 34, 1317-1321 (1969).

1969CIH/VOJ M. Cihova, J. Vojtko, and M. Hrusovsky, Chem. Zvesti 4, 270 (1969).

1969CLE/MEL G. A. Clegg and T. P. Melia, Makromol. Chem. 123, 194 (1969).

1969CLE/MEL2 $\quad$ G. A. Clegg and T. P. Melia, Polymer 10, 912 (1969).

1969CLE/MEL3 G. A. Clegg and T. P. Melia, Makromol. Chem. 130, 258 (1969).

1969COR/FRA P. Corradini, A. Frasci, and E. Martuscelli, J. Chem. Soc. D 1969, 778-780.

1969COR/GOO J. M. Corkill, J. F. Goodman, and J. R. Tate, Trans. Faraday Soc. 65, 1742 (1969).

1969COX/GUN J. D. Cox, H. A. Gundry, D. Harrop, and A. J. Head, J. Chem. Thermodyn. 1, 77-87 (1969).

1969CRI F. T. Crimmons, U.S. Atomic Energy Commission Report No. UCRL-50704, 1969; Chem. Abstr. 72, 70876a (1970).

1969DAN/MAT S. M. Danov, V. B. Matin, P. V. Efremov, and N. K. Slashtshina, Zh. Fiz. Khim. 43, 733-736 (1969); Chem. Abstr. 71, 6729g (1969). 
1969DAV/MAK Z. V. Davydova, I. A. Makolkin, and P. S. Bataev, Zh. Obshch. Khim. 39, 1668-1669 (1969).

1969DAV/SMI R. R. Davidson and W. H. Smith, Chem. Eng. Sci. 24, 1589 (1969).

1969DEV/ONE J. A. Devore and H. E. O’Neal, J. Phys. Chem. 73, 2644-2648 (1969).

1969ENO/SHI H. Enokido, T. Shinoda, and Y. Mashiko, Bull. Chem. Soc. Jpn. 42, 3415 (1969)

1969ERM/SKR G. V. Ermakov and V. P. Skripov, Russ. J. Phys. Chem. 43, 726 (1969).

1969FIN

1969FRA/WAT

T. J. V. Findlay, J. Chem. Eng. Data 14, 229-231 (1969).

F. Franks and B. Watson, Trans. Faraday Soc. 65, 2339-2349 (1969)

1969FRE/SOL H. M. Frey and R. K. Solly, Trans. Faraday Soc. 65, 1372 (1969).

1969FRE/TIP J. Freear and A. E. Tipping, J. Chem. Soc. C 1969, 411-416.

1969FRE/TIP2 J. Freear and A. E. Tipping, J. Chem. Soc. C 1969, 1955.

1969GLE/MEW2 O. Glemser, R. Mews, and S. P. Von Halasz, Inorg. Nucl. Chem. Lett. 5, 321 (1969).

1969GLE/VON O. Glemser and S. P. Von Halasz, Inorg. Nucl. Chem. Lett. 5, 393-398 (1969).

1969GOU/AND D. E. Gould, L. R. Anderson, D. E. Young, and W. B. Fox, J. Am. Chem. Soc. 91, 1310 (1969).

1969GOU/WES P. Goursot and E. F. Westrum, J. Chem. Eng. Data 14, 1 (1969).

1969KEM/KRE R. H. Kemme and S. I. Kreps, J. Chem. Eng. Data 14, 98-102 (1969).

1969KON/PRO J. Konicek, M. Prochazka, V. Krestanova, and M. Smisek, Collect. Czech. Chem. Commun. 34, 2249-2257 (1969).

1969KRE/WOO W. Kreis and R. H. Wood, J. Chem. Thermodyn. 1, 523-525 (1969).

1969KUD/REE A. P. Kudchadker, C. O. Reed, Jr., L. J. Clague, and B. J. Zwolinski, Tex. J. Sci. 20, 367 (1969).

1969LAM/PER R. Lamantine and R. Perrin, Bull. Soc. Chim. Fr. 443-445 (1969).

1969LEB/DNE N. D. Lebedeva, A. S. Dneprovskii, and Y. A. Katin, Russ. J. Phys. Chem. 43, 770-773 (1969).

1969LES/MOR T. M. Lesteva, A. I. Morozova, V. I. Morozova, S. K. Ogorodnikov, and T. M. Tyvina, Zh. Prikl. Khim. 42, 533-538 (1969).

1969MAC/MCN H. Mackle, D. V. McNally, and W. V. Steele, Trans. Faraday Soc. 65, 2060-2068 (1969).

1969MAC/MCN2 H. Mackle and D. V. McNally, Trans. Faraday Soc. 65, 1738-1741 (1969).

1969MAC/STE H. Mackle and W. V. Steele, Trans. Faraday Soc. 65, 2069-2077 (1969).

1969MAC/STE2 H. Mackle and W. V. Steele, Trans. Faraday Soc. 65, 2053 (1969).

1969MAN M. Mansson, J. Chem. Thermodyn. 1, 141-151 (1969).

1969MAN/MOR M. Mansson, E. Morawetz, Y. Nakase, and S. Sunner, Acta Chem. Scand. 23, 56-60 (1969).

1969MAS J. Mason, J. Chem. Soc. A 1969, 1587-1592.

1969MAY/THO F. G. J. May, B. W. Thorpe, and W. Connick, J. Cryst. Growth 5, 312 (1969).

1969MEL/MER T. P. Melia and R. Merrifield, J. Appl. Chem. (London) 19, 79-82 (1969).

1969MES S. Meszaros, Period. Polytech., Chem. Eng. (Budapest) 13, 79-88 (1969); Chem. Abstr. 72, 66308z (1970).

$1969 \mathrm{MIR} / \mathrm{LEB} \quad$ E. A. Miroshnichenko and Y. A. Lebedev, Khim. Geterotsikl. Soedin. 5, 963-964 (1969).

1969OSB/DOU A. G. Osborn and D. R. Douslin, J. Chem. Eng. Data 14, 208-209 (1969).

1969OTO/BES P. P. Otopkov, N. A. Bessarab, Ya. I. Rotshtein, and R. V. Dzhagatspanyan, Zh. Priklad. Khim. 42, 2367 (1969).

1969PAU/GLU I. E. Paukov and L. K. Glukhikh, Russ. J. Phys. Chem. 43, 120 (1969).

1969PAU/LAV I. E. Paukov, M. N. Lavrent'eva, and M. P. Anisimov, Zh. Fiz. Khim. 43, 2941 (1969).

1969PAU/LAV2 L. E. Paukov, M. N. Lavrent'eva, and M. P. Anisimov, Zh. Fiz. Khim. 43, 785 (1969).

1969PAU/LAV3 L. E. Paukov and M. N. Lavrent'eva, Zh. Fiz. Khim. 43, 2938 (1969).
1969PIH/HEI

K. Pihlaja and J. Heikkila, Acta Chem. Scand. 23, 1053 (1969); Suom. Kemistil. 148 (1972) (as quoted in [2003CHO/DHA])

1969PLA/GLA C. Plato and A. R. Glasgow, Jr., Anal. Chem. 41, 330-336 (1969).

1969ROS

1969ROS/DIC

J. M. Rosen, Microscope (London) 117, 141-144 (1969).

1969ROS/HOL

J. M. Rosen and C. Dickinson, J. Chem. Eng. Data 14, 120-124 (1969).

1969RUE

1969SCH/MAY

J. M. Rosen, J. R. Holden, and H. T. Simmons, Mol. Cryst. Liq. Cryst. 6, 205-210 (1969).

B. G. Rueben, J. Chem. Eng. Data 14, 235-236 (1969).

1969SHE/LAN

$$
\text { (1969). }
$$

1969SMI/PAC J. H. Smith and E. L. Pace, J. Phys. Chem. 73, 4232-4236 248-250 (1969). (1969).

1969SOU/GOU M. A. Soulie, P. Goursot, A. Peneloux, and J. Metzger, J. Chim. Phys. Physicochim. Biol. 66, 607-610 (1969).

1969SOU/GOU2 M. A. Soulie, P. Goursot, A. Peneloux, and J. Metzger, J. Chim. Phys. Physicochim. Biol. 66, 603-606 (1969).

1969STU/WES D. R. Stull, E. F. Westrum, Jr., and G. C. Sinke, The Chemical Thermodyanmics of Organic Compounds (John Wiley, New York, 1969).

1969VOJ/CIH J. Vojtko, M. Cihova, and M. Hrusovsky, Zb. Pr. Chem.Tech. Fak. Svst. 179 (1969); Chem. Abstr. 76, 76492b (1972).

1969WAD

1969WOO/ADI

I. Wadso, Acta Chem. Scand. 23, 2061-2064 (1969).

1970AMB/COU 479-480 (1969)

1970AMB/SPR

D. Ambrose, J. F. Counsell, and A. J. Davenport, J. Chem. Thermodyn. 2, 283-294 (1970).

1970AND/BRA 631-645 (1970).

D. N. Andreevskii and M. M. Brazhnikov, Vestn. Beloruss. Univ. 2, 14-18 (1970); Chem. Abstr. 76, 158651s (1972).

1970AND/COU R. J. L. Andon, J. F. Counsell, E. B. Lees, and J. F. Martin, J. Chem. Soc. A 1970, 833.

1970BAN/BRI R. E. Banks, M. Bridge, R. N. Haszeldine, D. W. Roberts, and N. I. Tucker, J. Chem. Soc. C 1970, 2531.

1970BON/CAT D. Bonderman, E. D. Cater, and W. Bennett, J. Chem. Eng. Data 15, 396-400 (1970).

1970BUR/KAN A. B. Burg and D.-K. Kang, J. Am. Chem. Soc. 92, 1901-1908 (1970).

1970BUR/PAR A. B. Burg and D. M. Parker, J. Am. Chem. Soc. 92, 1898-1901 (1970).

1970CAR/ZIM G. A. Carpenter, M. F. Zimmer, E. E. Baroody, and R. A. Robb, J. Chem. Eng. Data 15, 553-556 (1970).

1970CHA/BOU N. B. Chanh, Y. Bouillaud, and P. Lencrerat, J. Chim. Phys. Phys.-Chim. Biol. 67, 1198 (1970).

1970CHA/CAV T. L. Charlton and R. G. Cavell, Inorg. Chem. 9, 379 (1970).

1970CHA/MCN S.-J. Chang, D. McNally, S. Sharry-Tehrany, M. J. Hickey, and R. H. Boyd, J. Am. Chem. Soc. 92, 3109-3118 (1970).

1970CHA/WES E. T. Chang and E. F. Westrum, J. Phys. Chem. 74, 2528 (1970).

1970CLE/WES H. L. Clever and E. F. Westrum, Jr., J. Phys. Chem. 74 1309 (1970).

1970COU/FEN J. F. Counsell, J. O. Fenwick, and E. B. Lees, J. Chem. Thermodyn. 2, 367-372 (1970).

1970COX/PIL J. D. Cox and G. Pilcher, Thermochemistry of Organic and Organometallic Compounds (Academic Press, London, 1970).

1970DES

1970DUN

1970DYK

D. D. Des Marteau, Inorg. Chem. 9, 2179 (1970).

L. C. Duncan, Inorg. Chem. 9, 987 (1970).

J. Dykyj, J. Petrochemia 10, 51-59 (1970); J. Petrochemica 10, 123-146 (1970); J. Petrochemica 10, 187-206 (1970).

1970DYK/VAN J. Dykyj and A. Vanko, Petrochemica 10, 3-22 (1970).

1970EIS/ORA
O. Eisen and A. Orav, Eesti Tead. Akad. Toim., Keem. Geol. 19, 202-205 (1970). 
1970ELN/EME Y. O. El Nigumi and H. J. Emeleus, J. Inorg. Nucl. Chem. 32, 3213 (1970).

1970EME/SPA H. J. Emeleus, P. M. Spaziante, and S. M. Williamson, J. Inorg. Nucl. Chem. 32, 3219 (1970).

1970FET/EFR Yu. M. Fetisov, A. A. Efremov, Yu. I. Levin, and Ya. D. Zel'venskii, Tr. Inst. - Mosk. Khim.-Tekhnol. Inst. im. D. I. Mendeleeva 67, 34-37 (1970); Chem. Abstr. 75, 80864 (1971).

1970FIN/MCC H. L. Finke, J. P. McCullough, J. F. Messerly, G. B. Guthrie, and D. R. Douslin, J. Chem. Thermodyn. 2, 27 (1970).

1970GEN/DUV B. Genot and X. Duval, J. Chim. Phys. Physicochim. Biol. 67, 1332-1335 (1970).

1970GOO/MOO W. D. Good and R. T. Moore, J. Chem. Eng. Data 15, 150-153 (1970).

1970HAR/HEA D. Harrop, A. J. Head, and G. B. Lewis, J. Chem. Thermodyn. 2, 203-210 (1970).

1970HAS/KEE R. N. Haszeldine, D. W. Keen, and A. E. Tipping, J. Chem. Soc. C 1970, 414.

1970HER R. Herchl, Can. Anaesth. Soc. J. 17, 541 (1970).

1970HOW/WAD P. B. Howard and I. Wadso, Acta Chem. Scand. 24, 145-152 (1970).

1970IRV/WAD R. J. Irving and I. Wadso, Acta Chem. Scand. 24, 589-593 (1970).

1970KOI/OUC E. Koizumi and S. Ouchi, Nippon Kagaku Zasshi 91, 501-503 (1970); Chem. Abstr. 74, 46115x (1971).

1970KON/WAD J. Konicek and I. Wadso, Acta Chem. Scand. 24, 2612-2616 (1970).

1970KRI/LIC G. Krien, H. H. Licht, and F. Trimborn, Explosivstoffe 18, 203 (1970).

1970KUS/WAD K. Kusano and I. Wadso, Acta Chem. Scand. 24, 2037-2042 (1970).

1970LAW

1970LEN/VEL

E. W. Lawless, Inorg. Chem. 9, 2796 (1970).

1970LIN/SIL D. C.-K. Lin, I. H. Silberberg, and J. J. McKetta, J. Chem. Eng. Data 15, 483-492 (1970).

1970LUS M. Lustig, Inorg. Chem. 9, 104 (1970).

1970MAN/RAP M. Mansson, N. Rapport, and E. F. Westrum, Jr., J. Am. Chem. Soc. 92, 7296-7299 (1970).

1970MAT/TAN A. Matsuda, Y. Tanaka, and T. Munakata, Kyushu Univ. Fukuoka Jpn. Kogaku Shuho-Kyushu Daigaku 43, 834-841 (1970).

1970MAY/VEN J. N. Maycock and V. R. P. Venecker, Thermochim. Acta 1, 191-198 (1970).

1970MEL/MER T. P. Melia and R. Merrifield, J. Inorg. Nucl. Chem. 32, 1489-1493 (1970).

1970MES/FIN J. F. Messerly and H. L. Finke, J. Chem. Thermodyn. 2, 867 (1970).

1970MES/TOD J. F. Messerly, S. S. Todd, and G. B. Guthrie, J. Chem. Eng. Data 15, 227 (1970).

1970MOI/ANT V. D. Moisev and N. D. Antonova, Zh. Fiz. Khim. 44, 2912-2913 (1970); Chem. Abstr. 74, 80487e (1971).

1970 MUL/GAL F. I. Mullayanov, G. V. Galagan, M. F. Buz'ko, Y. V. Churkin, and A. A. Shiryaeva, Tr. Nauch-Issled. Inst. Neftekhim. Proizvod. 2, 95-97 (1970); Chem. Abstr. 74, 91930v (1971).

1970MUR/BRE E. Murrill and L. Breed, Thermochim. Acta 1, 239-246 (1970).

1970MUR/BRE2 E. Murrill and L. W. Breed, Thermochim. Acta 1, 409 (1970).

1970 NOF

1970POL/MUR J. Polak, S. Murakami, V. T. Lam, and G. C. Benson, J. Chem. Eng. Data 15, 323-328 (1970).

1970PRO/KRE M. Prochazka, V. Krestanova, J. Konicek, and M. Smisek, Collect. Czech. Chem. Commun. 35, 727-732 (1970).

1970QUI/HOF K. Quitzsch, H. P. Hofmann, D. Hering, R. Salzer, and G. Geiseler, Z. Phys. Chem. (Leipzig) 243, 321-339 (1970).

1970ROB/ROS P. M. Robinson, H. J. Rossell, and H. G. Scott, Mol. Cryst. Liq. Cryst. 10, 61 (1970).

1970SCO 1970SEL 1970SEL2

D. W. Scott, J. Chem. Thermodyn. 2, 833-837 (1970)

P. Sellers, Acta Chem. Scand. 24, 2453-2458 (1970).

P. Sellers, J. Chem. Thermodyn. 2, 211-219 (1970).
1970SHA/MCG
1970SIL/RUD

1970SMI/THO

1970SPE/CLI

1970VAL/KIL

1970VAR/BEL

1970VOJ/CIH

1970VON/GLE

1970VON/GLE2

1970VON/WIL

1970WES/WON

1970WIE/WAU

1970WON/WES

1970ZAB/SHR

1971ADA/SUG

1971AND/CON

1971ASH

1971BAG/MAL

1971BEE/LIN

1971BER/GIR

1971BER/HOH

1971BOY/SAN

1971BUT/CAR

1971CAR/WES

1971CAR/ZIM

1971CAV/CHA

1971CHI/THO

1971CHO/JON

1971COU/LEE

1971DEM/SHR

1971DIN/STA

1971DYK

1971EON/POM

1971GEL/SIM

F. Shafizadeh, G. D. McGinnis, R. A. Susott, and C. W. Philpot, Carbohydr. Res. 15, 165-178 (1970).

L. Silver and R. Rudman, J. Phys. Chem. 74, 3134 (1970).

E. D. Smith and W. L. Thornsberry, J. Chem. Eng. Data 15, 296-297 (1970).

W. F. Spencer and M. M. Cliath, J. Agric. Food Chem. 18, 529-530 (1970).

A. J. Valerja and J. E. Kilpatrick, J. Chem. Phys. 52, 4545-4549 (1970).

R. M. Varushchenko, N. A. Belikova, S. M. Skuratov, and A. F. Plate, Zh. Fiz. Khim. 44, 3022-3025 (1970).

J. Vojtko, M. Cihova, and M. Hrusovsky, Chem. Zvesti 24, 173-176 (1970).

S. P. Von Halasz and O. Glemser, Chem. Ber. 103, 553-560 (1970).

S. P. Von Halasz and O. Glemser, Chem. Ber. 103, 594-602 (1970).

P. R. von Schleyer, J. E. Williams, and K. R. Blanchard, J. Am. Chem. Soc. 92, 2377-2386 (1970).

E. F. Westrum, Jr., W. E. Wong, and E. Morawetz, J. Phys. Chem. 74, 2542-2547 (1970).

A. G. Wiedemann and H. P. Waughna, 3rd Proc. Toronto Symp. Therm. Anal. 233 (1970); as quoted in [1999ZIE/PER].

W.-K. Wong and E. F. Westrum, Jr., J. Phys. Chem. 74, 1303 (1970).

L. M. Zaborowski and J. M. Shreeve, J. Am. Chem. Soc. 92, 3665-3668 (1970).

K. Adachi, H. Suga, and S. Seki, Bull. Chem. Soc. Jpn. 44, 78 (1971).

R. J. L. Andon, J. E. Connett, J. F. Counsell, E. B. Lees, and J. F. Martin, J. Chem. Soc. A 1971, 661-664.

S. J. Ashcroft, Thermochim. Acta 2, 512-514 (1971).

A. Baghdoyan, J. Malik, and V. Fried, J. Chem. Eng. Data 16, 96-97 (1971).

G. Beech and R. M. Lintonbon, Thermochim. Acta 2, 86-88 (1971).

G. Bertholon, M. Giray, R. Perrin, and M. F. VincentFalquet-Berny, Bull. Soc. Chem. Fr. 3180-3187 (1971).

P. A. Bernstein, F. A. Hohorst, and D. D. DesMarteau, J. Am. Chem. Soc. 93, 3882 (1971).

R. H. Boyd, S. N. Sanwal, S. Shary-Tehrany, and D. McNally, J. Phys. Chem. 75, 1264-1271 (1971).

R. S. Butler, A. S. Carson, P. G. Laye, and W. V. Steele, J. Chem. Thermodyn. 3, 277-280 (1971).

H. G. Carlson and E. F. Westrum, Jr., J. Phys. Chem. 54, 1464 (1971).

G. A. Carpenter, M. F. Zimmer, E. E. Baroody, and R. A. Robb, J. Chem. Eng. Data 16, 46 (1971).

R. G. Cavell, T. L. Charlton, and W. Sim, J. Am. Chem. Soc. 93, 1130 (1971).

M. C. Chick and B. W. Thorpe, Aust. J. Chem. 24, 181 (1971).

J. K. Choi and M. J. Joncich, J. Chem. Eng. Data 16, 87-90 (1971).

J. F. Counsell, D. A. Lee, and J. F. Martin, J. Chem. Soc. A 1971, 313-316.

R. A. De Marco and J. M. Shreeve, Inorg. Chem. 10, 911-913 (1971).

R. H. Dinegar and M. Stammier, Explosivstoffe 1, 14 (1971).

J. Dykyj, Petrochemia 11, 27-45 (1971).

C. Eon, C. Pommier, and G. Guiochon, J. Chem. Eng. Data 16, 408-410 (1971).

Z. I. Geller, V. D. Simonov, M. V. Lyubarskii, E. A. Uyssh. Ucheb. Zaved., Nept. Gaz 7, 71-74 (1971).

1971 GET/WAR F. W. Getzen and T. M. Ward, Ind. Eng. Chem. Proc. Res. Dev. 10, 122 (1971).

1971GOO/MOO W. D. Good and R. T. Moore, J. Chem. Thermodyn. 3, 701-705 (1971).

1971HAL P. G. Hall, Trans. Faraday Soc. 67, 556 (1971). 
1971HAL/BAL H. K. Hall, Jr. and J. H. Baldt, J. Am. Chem. Soc. 93, 140-145 (1971).

1971HAM/WIT W. S. Hamilton and L. C. Witt, J. Chem. Eng. Data 16, 234-235 (1971).

1971HOD P. E. Hodge, Ph.D. dissertation, Case Western Reserve, 1971; Diss. Abstr. 32, 1482 (1971).

1971JAC/HUN W. Jackson, T. S. Hung, and H. P. Hopkins, Jr., J. Chem. Thermodyn. 3, 347-353 (1971).

1971 KOL/VOL V. P. Kolesov and V. N. Vorob'ev, Zh. Fiz. Khim. 45, 1293 (1971).

1971KUS/WAD K. Kusano and I. Wadso, Acta Chem. Scand. 25, 219-224 (1971).

1971KUS/WAD2 K. Kusano and I. Wadso, Bull. Chem. Soc. Jpn. 44, 1705-1707 (1971).

1971LEB/GUT N. D. Lebedeva, N. M. Gutner, and V. L. Ryadnenko, Russ. J. Phys. Chem. 45, 561 (1971).

1971LEB/KAT N. D. Lebedeva, Y. A. Katin, and G. Y. Akhmedova, Russ. J. Phys. Chem. 45, 1192-1193 (1971).

1971LEB/KAT2 N. D. Lebedeva, Y. A. Katin, and G. Y. Akhmedova, Russ. J. Phys. Chem. 45, 771-772 (1971).

1971LEB/RAB B. V. Lebedev and I. B. Rabinovich, Tr. Khim. Khim. Tekhnol. 1, 12 (1971).

1971LEB/RAB2 B. V. Lebedev and I. B. Rabinovich, Tr. Khim. Khim. Tekhnol. 1, 8 (1971).

1971LEB/RYA N. D. Lebedeva, V. L. Ryadnenko, and I. N. Kuznetsova, Russ. J. Phys. Chem. 45, 549 (1971)

1971LEN/VEL C. Lenchitz, R. W. Velicky, G. Silvestro, and L. P. Schlosberg, J. Chem. Thermodyn. 3, 689-692 (1971).

1971LES/KHR T. M. Lesteva, E. I. Khrapkov, V. A. Gil'mutdinova, and E. M. Sire, Zh. Priklad. Khim. 44, 2594 (1971).

1971LET/BAY T. M. Letcher and J. W. Bayles, J. Chem. Eng. Data 16, 266 (1971)

1971MAN/RIN M. Mansson, B. Ringner, and S. Sunner, J. Chem. Thermodyn. 3, 547-551 (1971).

1971MAS/CHE W. L. Masterton, C. H. Chen, C. E. Waring, R. Oksala, L. Beatty, and A. Krause, J. Chem. Thermodyn. 3, 243 (1971).

1971MAT/PEP Y. N. Matyushin, V. I. Pepekin, S. P. Golova, T. I. Godovikova, and L. I. Khmelnitskii, Bull. Acad. Sci. USSR, Div. Chem. Sci. 20, 162-164 (1971).

1971MES/FIN J. F. Messerly and H. L. Finke, J. Chem. Thermodyn. 3, 675 (1971).

1971MEY/REN E. F. Meyer, T. A. Renner, and K. S. Stec, J. Phys. Chem. 76, 642-648 (1971).

1971MIT/IMA I. Mita, I. Imai, and I. Kambe, Thermochim. Acta 2, 337-344 (1971).

1971MOR E. Morawetz, Chem. Scr. 1, 103-111 (1971).

1971MOR2 E. Morawetz, Dissertation, University of Lund, 1971; see [1983DEW/VAN].

1971PAR/ROC G. H. Parsons, C. H. Rochester, and C. E. C. Wood, J. Chem. Soc. B 1971, 533-536.

1971PAU

1971PAU2

1971PIH/TUO

I. E. Paukov, Zh. Fiz. Khim. 45, 1293 (1971).

I. E. Paukov, Zh. Fiz. Khim. 45, 1297 (1971).

K. Pihlaja and M. -L. Tuomi, Acta Chem. Scand. 25, 465-469 (1971).

1971PIN/TON R. E. Pincock, M. -M. Tong, and K. R. Wilson, J. Am. Chem. Soc. 93, 1669-1672 (1971).

1971POL/BEN J. Polak and G. C. Benson, J. Chem. Thermodyn. 3, 235-242 (1971).

1971PRI V. P. Privalko, Russ. J. Phys. Chem. 45, 900 (1971).

1971PRY/EFR R. O. Pryanikova and G. D. Efremova, Tr. Nauch-Issled. Proekt. Inst. Azotn. Prom. Prod. Org. Sln. 12, 112 (1971).

1971RAD

L. G. Radchenko, Zh. Fiz. Khim. 45, 1310-1311 (1971); Chem. Abstr. 75, 122104f (1971).

1971RAP/WES N. J. Rapport, E. F. Westrum, Jr., and J. T. S. Andrews, J. Am. Chem. Soc. 93, 4363-4374 (1971).

1971RAT/HAR C. T. Ratcliffe, C. V. Hardin, L. R. Anderson, and W. B. Fox, J. Am. Chem. Soc. 93, 3886 (1971).

1971RIN/SUN B. Ringner, S. Sunner, and H. Watanabe, Acta Chem. Scand. 25, 141-146 (1971).

1971ROS/HOL J. M. Rosen, J. R. Holden, and H. T. Simmons, Sr., Microscope 19, 151-156 (1971).
1971SAU/SHR

D. T. Sauer and J. M. Shreeve, J. Fluorine Chem. 1, 1-11 (1971).

1971SAU/SHR2 D. T. Sauer and J. M. Shreeve, Inorg. Chem. 10, 358-362 (1971).

1971SAU/SHR3 $\quad$ D. T. Sauer and J. M. Shreeve, Z. Anorg. Allgem. Chem. 385, 113 (1971).

1971SEL2 P. Sellers, Acta Chem. Scand. 25, 2194-2198 (1971); Acta Chem. Scand. 25, 2291-2294 (1971).

1971SEL3

1971SUN/EIS

P. Sellers, Acta Chem. Scand. 25, 2189-2193 (1971).

1971SWI/ZAB

C. Sunwoo and H. Eisen, J. Pharm. Sci. 60, 238-244 (1971).

1971WEE/GAR

F. Swindell, L. M. Zaborowski, and J. M. Shreeve, Inorg. Chem. 10, 1635-1638 (1971).

1971WIL/ZWO

P. Weeks and G. L. Gard, J. Fluorine Chem. 1, 295 (1971). R. C. Wilhoit and B. J. Zwolinski, "Handbook of vapor pressures and heats of vaporization of hydrocarbons and related compounds," in API 44-TRC Publications in Science and Engineering (API, College Station, TX, 1971).

1971 WON/WES W. -K. Wong and E. F. Westrum, Jr., J. Chem. Thermodyn 3, 105-124 (1971).

1971WU/HSU P. -J. Wu, L. Hsu, and D. A. Dows, J. Chem. Phys. 54, 2714-2721 (1971).

1971YAK/BLY A. M. Yakubson, L. I. Blyakhman, M. Ya Mel'nichenko, Z. V. Efimova, and V. I. Trofimov, Zh. Fiz. Khim. 44, 1198 (1971).

1971ZAB/SHR L. M. Zaborowski and J. M. Shreeve, Inorg. Chem. 10, 407-409 (1971).

1972ACK/HAL M. N. Ackermann, M. R. Hallmark, S. K. Hammond, and N. Roe, Inorg. Chem. 11, 3076 (1972).

1972ADA/SUG K. Adachi, H. Suga, and S. Seki, Bull. Chem. Soc. Jpn. 45, 1960 (1972).

1972AHM/EAD A. M. I. Ahmed and R. G. Eades, J. Chem. Soc., Faraday Trans. 2 68, 1623-1633 (1972).

1972AHM/EAD2 A. M. I. Ahmed and R. G. Eades, J. Chem. Soc., Faraday Trans. 2 68, 2017-2022 (1972).

1972ALV/BOR T. Alvik, G. Borgen, and J. Dale, Acta Chem. Scand. 126, 1805-1816 (1972).

1972AMB/SPR D. Ambrose, C. H. S. Sprake, and R. Townsend, J. Chem. Thermodyn. 4, 247-254 (1972).

1972AMI/VAK A. V. Amitin, E. A. Vakurova, V. K. Katunin, and N. S. Afanas'eva, Zh. Fiz. Khim. 46, 1054 (1972); Chem. Abstr. 77, 66425p (1972).

1972ARE/VAN J. G. Arentsen and J. C. van Miltenburg, J. Chem. Thermodyn. 4, 789 (1972).

1972ARN/JON P. R. Arnold and F. Jones, Mol. Cryst. Liq. Cryst. 19, 133-140 (1972).

1972BOO/HAU H. J. Booss and K. R. Hauschildt, Fresenius' Z. Anal. Chem. 261, 32 (1972).

1972BOU/AIM T. Boublik and K. Aim, Collect. Czech. Chem. Commun. 37, 3513-3521 (1972).

1972B YR/CUR S. R. Byrn, D. Y. Curtin, and I. C. Paul, J. Am. Chem. Soc. 94, 890-898 (1972).

1972CHA/HAG N. B. Chanh, Y. Haget, and J. Bedouin, J. Bull. Soc. Fr. Mineral. Cristallogr. 95, 281 (1972).

1972COL/LAY M. Colomina, J. L. Laynez, R. Perez-Ossorio, and C. Turrion, J. Chem. Thermodyn. 4, 499-506 (1972).

1972COL/MON M. Colomina, C. Monzon, C. Turrion, and J. Laynez, Paper presented at the the Fifth Experimental Thermodynamics Conference (Lancaster, 1972); as cited in [1982COL/JIM].

1972COU/LEE J. F. Counsell and D. A. Lee, J. Chem. Thermodyn. 4, 915-917 (1972).

1972DEM/SHR R. A. De Marco and J. M. Shreve, J. Fluorine Chem. 1, 269-276 (1972).

1972DES

1972DUB/DEV C. Dubois and C. Devin, C. R. Seances l'Acad. Sci. Ser. C 274, 1289 (1972).

1972DYK J. Dykyj, J. Petrochemia 12, 13-31 (1972); Petrochemia 12, 34-63 (1972).

1972FIN/MCC H. L. Finke, J. P. McCullough, J. F. Messerly, A. Osborn, and D. R. Douslin, J. Chem. Thermodyn. 4, 477-494 (1972). 
1972FIN/MES H. L. Finke, J. F. Messerly, and S. S. Todd, J. Chem. Thermodyn. 4, 359-374 (1972).

1972GAR/HUS P. J. Gardner and K. S. Hussain, J. Chem. Thermodyn. 4, 819-827 (1972).

1972GEI/RAU G. Geiseler and H. J. Rauh, Z. Phys. Chem. (Leipzig) 249, 376-382 (1972).

1972GEI/SAW G. Geiseler and J. Sawistowsky, Z. Phys. Chem (Leipzig) 250, 43-48 (1972).

1972 GOO W. D. Good, J. Chem. Eng. Data 17, 158-162 (1972).

1972GRA/SAL V. A. Granzhan and E. A. Salganikov, Tr. Nauch.Issled. Proekt. Inst. Azotn. Prom. Prod. Org. Sin. 8587 (1972); Chem. Abstr. 79, 118908m (1973).

1972HAM/WIT W. S. Hamilton and L. C. Witt, J. Chem. Eng. Data 17, 138-139 (1972).

1972 HU/SIN $\quad$ A. T. Hu, G. C. Sinke, and M. J. Mintz, J. Chem. Thermodyn. 4, 239 (1972).

1972INO/LIA P. P. Inozemtsev, A. G. Liakumovich, and Z. D. Gracheva, Russ. J. Phys. Chem. 46, 914 (1972).

1972IRV

1972JAK/VAN

R. J. Irving, J. Chem. Thermodyn. 4, 793-794 (1972).

G. Jakli and W. A van Hook, J. Chem. Thermodyn. 4, 857-864 (1972).

1972 KOL/VOR V.P. Kolesov and V. N. Vorob'ev, Dokl. Akad. Nauk SSSR 203, 116 (1972).

1972KOZ/TIM M. P. Kozina, L. P. Timofeeva, S. M. Pimenova, V. A. Aleshina, N. A. Belikova, A. A. Bobyleva, and A. F. Plate, Zh. Fiz. Khim. 46, 2959 (1972); Chem. Abstr. 78, 63105 (1973).

1972KRE/PRI M. Krech, S. J. W. Price, and W. F. Yared, Can. J. Chem. 50, 2935-2938 (1972).

1972LAY/WAD J. Laynez and I. Wadso, Acta Chem. Scand. 26, 3148-3152 (1972).

1972LEB/KAT N. D. Lebedeva and Y. A. Katin, Zh. Fiz. Khim. 46, 1060-1061 (1972) [Engl. Ed. 623].

1972LEB/KAT2 N. D. Lebedeva and Y. Katin, Zh. Fiz. Khim. 46, 1088-1089 (1972).

1972LES/CHE T. M. Lesteva and V. I. Chernaya, Zh. Priklad. Khim. 45, 459 (1972).

1972MAN M. Mansson, J. Chem. Thermodyn. 4, 865-871 (1972).

1972MAN2 M. Mansson, Acta Chem. Scand. 26, 1707-1708 (1972).

1972 MCF/SOM F. R. McFeely and G. A. Somorjee, J. Phys. Chem. 76, 914-918 (1972).

1972MET/SHR S. G. Metcalf and J. M. Shreeve, Inorg. Chem. 11, 1631-1634 (1972).

1972MIL G. D. Mills, Diss. Abstr. Int. B 33, 1485 (1972); Chem. Abstr. 78, 42653f (1973).

1972MOR E. Morawetz, J. Chem. Thermodyn. 4, 455-460 (1972).

1972NAK/TOY K. Nakanishi and O. Toyama, Bull. Chem. Soc. Jpn. 45, 3210-3211 (1972).

1972NEE/HAL W. C. Neely and T. D. Hall, J. Chem. Eng. Data 17, 294-295 (1972).

1972NIS/HAK M. Nishimura, M. Hakayama, and T. Yano, J. Chem. Eng. Jpn. 5, 223-226 (1972); Chem. Abstr. 78, 48659j (1973).

1972PAC/HOD E. L. Pace and P. E. Hodge, J. Chem. Thermodyn. 4, 441-447 (1972).

1972PEP/MAT V. I. Pepekin, Y. N. Matyushin, G. G. Rozantsev, S. A. Shevelev, and A. Y. Apin, Izv. Akad. Nauk SSSR Ser. Khim. 21, 2703-2705 (1972) [Engl. Ed. 2634-2636].

1972PIN/CAV A. A. Pinkerton and R. G. Cavell, J. Am. Chem. Soc. 94, 1870 (1972).

1972PLA

1972PRY/GOO R. Prydz and R. D. Goodwin, J. Chem. Thermodyn. 4, R. Prydz and R.

1972REG J. Regnier, J. Chim. Phys. Physicochim. Biol. 69, 942-944 (1972).

1972ROG/ORT R. N. Rogers and L. W. Ortiz, Thermochim. Acta 3, 379 (1972).

1972ROM/SUK B. C. Romanov, B. I. Sukkhorukov, E. A. Miroshnichenko, and Y. A. Lebedev, Fourth Int. Biophys. Congr. Abstr. E7, a4/7 (1972); as quoted in [2000BRU/PIA].

1972SAB/CHA R. Sabbah, R. Chastel, and M. Laffitte, Thermochim. Acta 5, 117-127 (1972).

1972 SAU/SHR $\quad$ D. T. Sauer and J. M. Shreeve, Inorg. Chem. 11, 238-242 (1972).
1972SOK/TSY

1972STR/NOV

N. M. Sokolov, L. N. Tsygankova, M. I. Shtrom, and N. M. Zhavoronkov, Sov. Chem. Ind. 48, 427 (1972).

Y. A. Strepikheev, O. P. Novikova, and A. L. Chimishkyan, Khim. Prom. (Moscow) 48, 230-231 (1972); Chem. Abstr. 69, 145717t (1972).

1972SWI/BAB R. F. Swindell, D. B. Babb, T. J. Ouellette, and J. M. Shreeve, Inorg. Chem. 11, 242-245 (1972).

1972SWI/SHR R. F. Swindell and J. M. Shreeve, J. Am. Chem. Soc. 94 5713-5718 (1972).

1972URB/JAN S. Urban, J. A. Janik, J. Lenik, J. Mayer, T. Baluga, and S. Wrobel, Phys. Status Solidi A 10, 271-280 (1972).

1972VAN

1972VAR/DRU J. C. van Miltenburg, J. Chem. Thermodyn. 4, 773 (1972).

1972VOR/KOL 46, 1313 (1972).

1972WAU/GET

Y. N. Vorob'ev, V. P. Kolesov, V. M. Kuznets, and T. A. Babushkina, Zh. Obshch. Khim. 42, 391 (1972).

1972WIE 38 (1972).

1972WIE H. G. Wiedemann, Thermochim. Acta 3, 355-366

1972WOL

1972ZOR/HUR (1972).

G. Wolf, Helv. Chim. Acta 55, 1446-1458 (1972).

1973ABE/SHR

T. A. Zordan, D. G. Hurkot, M. Peterson, and L. G. Hepler, Thermochim. Acta 5, 21 (1972).

1973ABE/SHR2

T. Abe and J. M. Shreeve, J. Fluorine Chem. 3, 17-26 (1973).

1973AMB/SPR

T. Abe and J. M. Shreeve, J. Fluorine Chem. 3, 187-196 (1973).

1973AND/COU

D. Ambrose, C. H. S. Sprake, and R. J. Townsend, J. Chem. Soc., Faraday Trans. 1 69, 839-841 (1973).

1973AND/MAR

R. J. L. Andon, J. F. Counsell, D. A. Lee, and J. F. Martin, J. Chem. Soc., Faraday Trans. 1 69, 1721-1726 (1973).

Trans. 1 69, 761-770 (1973).

1973AND/MAR2 J. L. Andon and J. F. Martin, J. Chem. Soc., Faraday Trans. 1 69, 871 (1973).

1973BAR/MOR2 D. S. Barnes, C. T. Mortimer, and E. Mayer, J. Chem. Thermodyn. 5, 481-483 (1973).

1973BEH/GAT

W. Behrendt and G. Gattow, Z. Anorg. Allegm. Chem. 398, 198-206 (1973).

1973BER/DES P. A. Bernstein and D. D. DesMarteau, J. Fluorine Chem. 2, 315-321 (1973).

1973CAR/KOB G. F. Carruth and R. Kobayashi, J. Chem. Eng. Data 18, 115-126 (1973).

1973CAS/VEC F. Casellato, C. Vecchi, A. Girell, and B. Casu, Thermochim. Acta 6, 361-368 (1973).

1973COU/LEE J. F. Counsell and D. A. Lee, J. Chem. Thermodyn. 5, 583-589 (1973).

1973DAW/SIL P. D. Dawson, Jr., I. M. Silberberg, and J. J. McKetta, J. Chem. Eng. Data 18, 7-15 (1973).

1973DEK/OON C. G. DeKruif and H. A. Oonk, J. Chem. Eng. Technol. 45, 455-461 (1973); Chem. Abstr. 78, 152198u (1973).

1973DEM/LEH G. R. De Mare, T. Lehman, and M. Termonia, J. Chem. Thermodyn. 5, 829-832 (1973).

1973DEM/SHR R. A. De Marco and J. M. Shreeve, Inorg. Chem. 12, 1896-1899 (1973).

1973DIE/MAR E. A. Dietz and D. R. Martin, J. Inorg. Nucl. Chem. 35, 3681-3684 (1973).

1973DYK/REP J. Dykyj and M. Repas, Petrochemia 13, 179-198 (1973).

1973FEL/SAV I. N. Fel'dman, V. V. Savko, U. I. Mamai, and M. F. Finkel'shtein, Russ. J. Phys. Chem. 47, 1531 (1973).

1973FIN/MES H. L. Finke and J. F. Messerley, J. Chem. Thermodyn. 5, 247 (1973).

1973FRA/KRZ H. P. Frank, K. Krzemicki, and H. Voellenkle, Chem.-Ztg. 97, 206 (1973).

1973GEI/QUI G. Geiseler, K. Quitzsch, H.-P. Hofmann, and R. Z. Pfestorf, Z. Phys. Chem. (Leipzig) 252, 170-176 (1973).

1973GOT/MEN F. A. Gothard, D. C. Mentianu, D. G. Breban, and C. I. Cristea, J. Chem. Eng. Data 18, 381-384 (1973).

1973HAL/SMI H. K. Hall, C. D. Smith, and J. H. Baldt, J. Am. Chem. Soc. 95, 3197-3201 (1973).

1973HAM/AYE J. V. Hamilton and D. A. Ayers, J. Chem. Eng. Data 18, 366-367 (1973). 
1973HAM/MIT J. V. Hamilton and G. M. Mitchell, J. Chem. Eng. Data 18, 38-39 (1973).

1973HOH/DES F. A. Hohorst, D. D. DesMarteau, L. R. Anderson, D. E. Gould, and W. B. Fox, J. Am. Chem. Soc. 95, 3866 (1973).

1973KIS/SUG K. Kishimoto, H. Suga, and S. Seki, Bull. Chem. Soc. Jpn. 46, 3020 (1973).

1973KON J. Konicek, Acta Chem. Scand. 27, 1496-1502 (1973).

1973KRE/PRI M. J. Krech, S. J. W. Price, and W. F. Yared, Can. J. Chem. 51, 3662-3664 (1973).

1973KRI/LIC G. Krien, H. H. Licht, and J. Zierath, Thermochim. Acta 6, 465-472 (1973).

1973LEB/KAT N. D. Lebedeva and Y. A. Katin, Zh. Fiz. Khim. 47, 1620 (1973); Russ. J. Phys. Chem. (Engl. Trans.) 47, 922-923 (1973).

1973LEB/KAT2 N. D. Lebedeva and Y. A. Katin, J. Appl. Chem. USSR 46, 2131 (1973).

1973LIN/CEN E. L. Lines and L. F. Centofanti, Inorg. Chem. 12, 598 (1973).

1973LIN/WIC J. Linek and I. Wichterle, Collect. Czech. Chem. Commun. 38, 1846-1852 (1973).

1973MAJ/SHR A. Majid and J. M. Shreeve, J. Org. Chem. 38, 4028-4031 (1973).

1973MAL/GIG L. Malaspina, R. Gigli, and G. Bardi, J. Chem. Phys. 59, 387-394 (1973).

1973MAL/GIG2 L. Malaspina, R. Gigli, G. Bardi, and G. DeMaria, J. Chem. Thermodyn. 5, 699-706 (1973).

1973MCE/SAN D. M. McEachern and O. Sandoval, J. Phys. E: Sci. Instrum. 6, 155-161 (1973).

1973MEY/HOT E. F. Meyer and R. D. Hotz, J. Chem. Eng. Data 18, 359-362 (1973).

1973MOR/KAU K. Morke, E. Kauer, J. Kornig, and H.-J. Bittrich, Z. Phys. Chem. Leipzig 252, 323 (1973).

1973PEP/GAF V. I. Pepekin, R. G. Gafurov, Y. A. Lebedev, L. T. Eremenko, E. M. Sogomonyan, and A. Y. Apin, Izv. Akad. Nauk SSSR Ser. Khim. 22, 318-322 (1973) [Engl. Ed. 304].

1973PEP/LEB V. I. Pepekin, Y. A. Lebedev, and A. Y. Apin, Dokl. Phys. Chem. 208, 35-37 (1973).

1973PHI/JAM R. Philippe, C. Jambon, and P. Clechet, J. Chem. Thermodyn. 5, 431 (1973).

1973RAU/GEI H.-J. Rauh and G. Geiseler, Z. Phys. Chem. (Leipzig) 252, 395-397 (1973).

1973RAU/GEY H.-J. Rauh, W. Geyer, H. Schmidt, and G. Geiseler, Z. Phys. Chem. (Leipzig) 253, 43-48 (1973).

1973ROC/SYM D. H. Rochester and J. R. Symonds, J. Chem. Soc., Faraday Trans. 1 1973, 1267-1273.

1973ROG/QUA F. E. Rogers and S. W. Quan, J. Phys. Chem. 77, 828-831 (1973).

1973SCH/PIL C. J. Schack, D. Pilipovich, and J. F. Hon, Inorg. Chem. 12, 897-900 (1973).

1973SPR/WRI G. H. Sprenger, K. J. Wright, and J. M. Shreeve, Inorg. Chem. 12, 2890-2893 (1973).

1973SVO/VES V. Svoboda, F. Vesely, R. Holub, and J. Pick, Collect. Czech. Chem. Commun. 38, 3539-3543 (1973).

1973VAS/KOR I. A. Vasil'ev and A. D. Korkhov, Zh. Fiz. Khim. 47, 2710 (1973).

1973WAN/SHR C. S.-C. Wang and J. M. Shreeve, Inorg. Chem. 12, 81-83 (1973).

1973WAR/SKU W. Waradzin and P. Skubla, Chem. Prum. 23, 556-557 (1973); Chem. Abstr. 80, 112780n (1974).

1973WIL/ZWO R. C. Wilhoit and B. J. Zwolinski, J. Phys. Chem. Ref. Data 2 Suppl. 1, 1-420 (1973); Note: The authors report Antoine constants for numerous alcohols that were calculated from vapor pressure data taken from the published literature. For several of the alcohols, the authors had only a very limited number of experimental data points that were pooled from several sources.

1973WRI/SHR K. J. Wright and J. M. Shreeve, Inorg. Chem. 12, 77-80 (1973).

1974AMB/ELL D. Ambrose and J. H. Ellender, J. Chem. Thermodyn. 6, 909-914 (1974).
1974AMB/SPR

1974AMB/SPR2

D. Ambrose and C. H. S. Sprake, J. Chem. Thermodyn. 6 453-456 (1974).

1974AND/COU

D. Ambrose, C. H. S. Sprake, and R. J. Townsend, J. Chem. Thermodyn. 6, 693-700 (1974).

1974AND/MAR

R. J. L. Andon, J. F. Counsell, D. A. Lee, and J. F.
J. Chem. Soc., Faraday Trans. 1 70, 1914 (1974).

1974ARS Trans. 1 70, 605 (1974).

1974BEA/MUE M. R. Arshadi, J. Chem. Soc., Faraday Trans. 1 70, 1569-1571 (1974).

1974BEH/HAA

1974BES/CHE

P. Beak, D. S. Mueller, and J. Lee, J. Am. Chem. Soc. 96, 3867-3874 (1974).

1974BESCHE

1974BLA/LEV

E. Behrend and A. Haas, J. Fluorine Chem. 4, 83 (1974). N. A. Bessarab, F. S. Chernoglazova, and Y. M. Martynov, Zh. Fiz. Khim. 48, 235-236 (1974); Chem. Abstr. 80, $112833 \mathrm{~g}$ (1974).

1974BLA/OGO (1974).

1974BOR

1974BRY/CAZ

Y. M. Blazhin, S. K. Ogorodnikov, A. I. Morzova, S. V. Kazakova, and L. N. Volkova, Zh. Prikl. Khim. 47, 184 (1974).

$\begin{array}{ll} & \text { Chem. Eng. Data 19, 107-110 (1974). } \\ \text { 1974CAF/SIC } & \text { L. F. R. Cafferata and J. E. Sicre, Inorg. Chem. 13, } 242\end{array}$

G. Borgen, Acta Chem. Scand. 28B, 13-17 (1974). (1974).

1974CIN/BER A. Cingolani and G. Berchiesi, J. Therm. Anal. 6, 87 (1974).

1974CLA/MCK T. Clark, M. A. McKervey, H. Mackle, and J. Rooney, J. Chem. Soc., Faraday Trans. 1 70, 1279-1291 (1974).

1974COL/ROU2 M. Colomina, M. V. Roux, and C. Turrion, J. Chem. Thermodyn. 6, 571-576 (1974).

1974COX C. D. Cox, Pure Appl. Chem. 40, 399-450 (1974).

1974DOJ/HEI J. Dojcansky and J. Heinrich, Chem. Zvesti 28, 157-159 (1974); Chem. Abstr. 82, 64723p (1975).

1974ENG/WOO P. S. Engel, J. L. Wood, J. A. Sweet, and J. L. Margrave, J. Am. Chem. Soc. 96, 2381-2387 (1974).

1974GIB/CRE H. F. Gibbard and J. L. Creek, J. Chem. Eng. Data 19, 308-310 (1974).

1974GOL/PEP O. F. Golovanova, V. I. Pepekin, B. L. Korsunskii, R. G. Gafurov, L. T. Eremenko, and F. I. Dubovitskii, Izv. Akad. Nauk SSSR Ser. Khim. 1495 (1974).

1974GOO/MOO W. D. Good, R. T. Moore, A. G. Osborn, and D. R. Douslin, J. Chem. Thermodyn. 6, 303-310 (1974).

1974GUS/REN J. L. Gustin and H. Renon, Bull. Soc. Chim. Fr. 2719-2722 (1974).

1974HIN/KLU J. Hine and A. W. Klueppet, J. Am. Chem. Soc. 96, 2924 (1974).

1974HOH/DES F. A. Hohorst and D. D. DesMarteau, Inorg. Chem. 13, 715 (1974).

1974JAC A. W. Jackowski, J. Chem. Thermodyn. 6, 49-52 (1974).

1974KIV/NAD N. N. Kiva, S. I. Nadutkina, L. V. Ivanova, and V. S. Bogdanov, Russ. J. Phys. Chem. 148, 297 (1974).

1974KOL/VOR V. P. Kolesov, V. N. Vorob'ev, E. A. Szrzhina, Yu. A. Pentin, and Yu. D. Timoshenkova, J. Chem. Thermodyn. 6, 613 (1974).

1974KOZ/BYC M. P. Kozina, L. V. Bychikhina, G. L. Gal'chenko, A. N Kalinichenko, A. A. Bobyleva, N. A. Belikova, and A. F. Plate, Zh. Fiz. Khim. 48, 2075-2077 (1974); Chem. Abstr. 81, 159978u (1974).

1974KUS/SAI K. Kusano and Y. Saito, Preprints, 10th Conf. Chem. Thermodyn. Therm. Anal. Jpn. 155 (1974); as quoted in [1985MAJ/SVO].

1974LET/MAR T. M. Letcher and F. Marsicano, J. Chem. Thermodyn. 6, 509-514 (1974).

1974LIN/CEN E. L. Lines and L. F. Centofanti, Inorg. Chem. 13, 1517 (1974).

1974LIO/HOP C. L. Liotta, H. P. Hopkins, Jr., and T. P. Kasudia, J. Am. Chem. Soc. 96, 7153 (1974).

1974LOG/FRO M. A. Loginova, A. F. Frolov, L. N. Bol'shakova, N. A. Simanov, and L. V. Ob'edkova, Zh. Priklad. Khim. 47, 99-102 (1974). 
1974MAJ/SHR A. Majid and J. M. Shreeve, Inorg. Chem. 13, 2710-2714 (1974).

1974MAN M. Mansson, Acta Chem. Scand. Ser. B 28, 905-908 (1974).

1974MAN/LOG G. D. Mantyukov, M. A. Loginova, B. N. Bychkov, and S. S. Rozhkov, Zh. Priklad. Khim. 47, 2585 (1974).

1974MAN2 M. Mansson, Acta Chem. Scand. Ser. B 28, 895-899 (1974).

1974MAN3 M. Mansson, Acta Chem. Scand. Ser. B 28, 677-680 (1974).

1974MAN4 M. Mansson, J. Chem. Thermodyn. 6, 1153-1159(1974).

1974MES/FIN J. F. Messerly, H. L. Finke, and S. S. Todd, J. Chem. Thermodyn. 6, 635-657 (1974).

1974MOZ/KOL L. V. Mozhginskay and L. E. Kolysko, Zh. Fiz. Khim. 48, 1506-1507 (1974).

1974MUR/TUD R. A. Murogova, G. L. Tudorovskaya, V. V. Laufer, V. D. Koxlova, and L. A. Seraphimov, Zh. Prikl. Khim. 47, 2024-2027 (1974).

1974MYA/SCH L. F. Myasnikova, V. A. Schmelev, I. L. Vaisman, V. I. Bushinskii, and D. A. Novokhatka, Zh. Prikl. Khim. (Leningrad) 47, 2604-2606 (1974).

1974OSB/DOU A. G. Osborn and D. R. Douslin, J. Chem. Eng. Data 19, 114-117 (1974).

1974PEP/LEB V. I. Pepekin, V. P. Lebedev, A. A. Balepin, and Y. A. Lebedev, Dokl. Akad. Nauk SSSR 221, 1118-1121 (1974).

1974PEP/MAT V. I. Pepekin, Y. N. Matyushin, and Y. A. Lebedev, Izv. Akad. Nauk SSSR Ser. Khim. 23, 1786-1790 (1974) [Engl. Ed. 1707].

1974PEP/NAT V. I. Pepekin, F. Ya. Natsibullin, L. T. Eremenko, and Yu. A. Lebedev, Izv. Akad. Nauk SSSR Ser. Khim. 925 (1974).

1974PET/SHR K. E. Peterman and J. M. Shreeve, Inorg. Chem. 13, 2705-2709 (1974).

1974RAD/KAT L. G. Radchenko and A. I. Kataigorodskii, Zh. Fiz. Khim. 48, 2702-2074 (1972) [Engl. Ed. 1595-1597 (1974)].

1974ROG/RAP $\quad$ F. E. Rogers and R. J. Rapiejko, J. Phys. Chem. 78, 599 (1974).

1974SAB/CHA R. Sabbah, R. Chastel, and M. Laffite, Can. J. Chem. 52, 2201-2205 (1974).

1974SAS/KON Y. Sassa, R. Konishi, and T. Katayama, J. Chem. Eng. Data 19, 44-48 (1974).

1974SIN G. C. Sinke, J. Chem. Thermodyn. 6, 311-316 (1974).

1974SPR/SHR G. H. Sprenger and J. M. Shreeve, J. Fluorine Chem. 4, 201-205 (1974).

1974SUN/WUL S. Sunner and C. A. Wulff, J. Chem. Thermodyn. 6, 287-292 (1974).

1974 TEP/SUK $\quad$ A. B. Teplitskii, L. F. Sukhodub, and I. K. Yanson, Fiz. Kondens. Sostoyanuja 32, 68-73 (1974); Chem. Abstr. 84, 170535e (1976).

1974VAN/HOU A. J. G. Van Diemen, C. J. M. Houten, and H. N. Stein, J. Chem. Thermodyn. 6, 805 (1974).

1974VAN/OON J. C. van Miltenburg and H. A. J. Oonk, Mol. Cryst. Liq. Cryst. 28, 167 (1974).

1974VAR/BUL R. M. Varushchenko and L. L. Bulgakova, Tr. Khim. Khim. Tekhnol. 1, 69-74 (1974).

1974VAR/DRU R. M. Varushchenko, A. I. Druzhimina, O. Y. Kovner, E. M. Mil'vitskaya, A. A. Bobyleva, N. A. Belikova, and G. L. Gal'chenko, Russ. J. Phys. Chem. 48, 1121 (1974).

1974 VAS/KOR I. A. Vasil'ev and A. D. Korkhov, Tr. Khim. Khim. Tekhnol. 103 (1974); Chem. Abstr. 82, 65233r (1975).

1974VOI/SHC S. A. Voitkevich, M. M. Shchedrina, K. K. Prilepskaya, and T. A. Rudol'fi, Masiozhirovanya Prom. 29 (1974).

1974 VOR/KOL V. N. Vorob'ev, V. P. Kolesov, E. A. Sarzhina, G. M. Kuramshina, and Yu. A. Pentin, Zh. Fiz. Khim. 48, 239 (1974).

1974YAN/VER I. K. Yanson, B. I. Verkin, O. I. Shlyarevskii, and A. B. Teplitskii, Stud. Biophys. 46, 29-44 (1974); Chem. Abstr. 83, 8884a (1975).

1974ZHU/MON E. Z. Zhuravlev, N. M. Moncharzh, and V. G. Vodop'yanov, Tr. Khim. Khim. Tecknol. 102-105 (1974).

1975ADE/BRO F. A. Adedeji, D. L. S. Brown, J. A. Connor, M. L. Leung, I. M. Paz-Andrade, and H. A. Skinner, J. Organomet. Chem. 97, 221-228 (1975).
1975AMB/CON

D. Ambrose, J. E. Connett, J. H. S. Green, J. L. Hales, A. J. Head, and J. F. Martin, J. Chem. Thermodyn. 7, 1143-1157 (1975).

1975AMB/ELL D. Ambrose, J. H. Ellender, E. B. Lees, C. H. S. Sprake, and R. J. Townsend, J. Chem. Thermodyn. 7, 453-472 (1975).

1975AMB/ELL2 D. Ambrose, J. W. Ellender, C. H. S. Sprake, and R. I. Townsend, J. Chem. Soc., Faraday Trans. 71, 35-41 (1975).

1975AMB/LAW D. Ambrose, I. J. Lawrenson, and C. H. S. Sprake, J. Chem. Thermodyn. 7, 1173-1176 (1975).

1975ANA/GRO S. C. Anand, J.-P. E. Grolier, O. Kiyohara, C. J. Halpin, and G. C. Benson, J. Chem. Eng. Data 20, 184-189 (1975).

1975AND/COU R. J. L. Andon, J. F. Counsell, D. A. Lee, and J. F. Martin, J. Chem. Thermodyn. 7, 587 (1975).

1975AND/MAR R. J. L. Andon and J. F. Martin, J. Chem. Thermodyn. 7, 593 (1975).

1975ANT/CAR M. E. Anthoney, A. S. Carson, and P. G. Laye, 4th Conf. Int. Thermodyn. Chim. [C.R.] 1, 99-103 (1975).

1975ARR/MEL J. Arro, L. Melder, and H. Tamvelius, Tr. Tallin. Inst. Toimetict. 37-43 (1975).

1975BAG/AND G. V. Bagrov, S. N. Andreev, M. F. Smirnova, and L. A. Vol'f, Deposited Doc. VINITI 1196 (1975); Chem. Abstr. 87, 59208y (1977).

1975BAR/PIL $\quad$ D. S. Barnes and G. Pilcher, J. Chem. Thermodyn. 7, 377-382 (1975).

1975BER/CIN G. Berchiesi, A. Cingolani, and D. Leonesi, J. Therm. Anal. 8, 427 (1975)

1975BER/OLO S. Bergstrom and G. Olofsson, J. Solution Chem. 4, 535-555 (1975).

1975BOG/BER K. A. Bogdanova, A. A. Berlin, V. Z. Kompanets, G. V. Rakova, E. A. Miroshnichenko, Y. A. Lebedev, and N. S. Enikolopyan, Vysokomol. Soedin. Ser. A 17, 658-664 (1975); , Chem. Abstr. 83, 43841f (1975).

1975BOR

1975BRA/AND

G. Borgen, Acta Chem. Scand. 29B, 265-272 (1975).

M. M. Brazhnikov, D. N. Andreevskii, A. I. Sachek, and A. D. Peshchenko, Zh. Prikl. Khim. (Leningrad) 48, 2181-2185 (1975).

1975BUC/DOM H. Buchowski, U. Domanska, A. Ksiazczak, and A. Maczynski, Rocz. Chem. 49, 1889 (1975).

1975BUC/JOD H. Buchowski, W. Jodzewicz, R. Milek, W. Ufnalski, and A. Maczynski, Rocz. Chem. 49, 1879 (1975).

1975CAB/CON S. Cabani, G. Conti, D. Giannessi, and L. Lepori, J. Chem. Soc. Faraday Trans. I 71, 1154-1160 (1975).

1975CAB/CON2 S. Cabani, G. Conti, V. Mollica, and L. Lepori, J. Chem. Soc., Faraday Trans. 1 71, 1943-1945 (1975).

1975CAS/VEC F. Casellato, C. Vecchi, A. Girelli, and P. G. Farrell, Thermochim. Acta 13, 37 (1975).

$1975 \mathrm{CHI}$

1975CHU/DRU J. S. Chickos, J. Chem. Educ. 52, 134-136 (1975).

$\begin{array}{ll}1975 C L A / K N O & \text { Davison, J. Chem. Eng. Data 20, 58-61 (1975). } \\ \text { T. Clark, T. Knox, H. Mackle, M. A. McKervey, and J. J. }\end{array}$ Rooney, J. Chem. Soc., Faraday Trans. 1 71, 2107-2110 (1975).

1975CUC

1975CUC2

1975DEK/VAN

A. Cuccuru, Thermochim. Acta 13, 96-99 (1975).

A. Cuccuru, Thermochim. Acta 11, 247-251 (1975).

C. G. DeKruif, C. H. D. van Ginkel, and J. Voogd, 4th Conf. Int. Thermodyn. Chim. [C.R.] 8, 11-18 (1975), as cited in [1982COL/JIM]..

1975DEM/KOV R. A. De Marco, T. A. Kovacina, and W. B. Fox, J. Fluorine Chem. 5, 221-230 (1975).

1975DEM/KOV2 R. A. De Marco, T. A. Kovacina, and W. B. Fox, J. Fluorine Chem. 6, 93-104 (1975).

1975FEN/HAR J. O. Fenwick, D. Harrop, and A. J. Head, J. Chem. Thermodyn. 7, 943-954 (1975).

1975FRE/ADA H. K. Frensdorff and R. K. Adams, J. Chem. Eng. Data 20, 13-15 (1975).

1975GAR/STY V. I. Garanin, G. F. Sytchinskii, and O. D. Sinyakina, Neftepererab. Neftekhim. 39 (1975); Chem. Abstr. 84, 50413.

1975GOM/SEE W. Gombler and F. Seel, Z. Naturforsch. 30B, 169 (1975). 
1975GOO/MES W. D. Good, J. F. Messerly, A. G. Osborn, and D. R. Douslin, J. Chem. Thermodyn. 7, 285-291 (1975).

1975GRO

$1975 \mathrm{HOE} / \mathrm{HOE} \quad$ C. Hoerner, A. Hoepfner, and B. Schmeiser, Ber. Bunsenges Phys. Chem. 79, 222-225 (1975).

1975HOE/PAR A. Hoepfner, N. Parekh, C. Hoerner, and A. Abdel-Hamid, Ber. Bunsenges Phys. Chem. 79, 216-222 (1975).

1975IRV/RIB R. J. Irving and M. A. V. Ribeiro da Silva, J. Chem. Soc., Dalton Trans. 1975, 798-800.

1975IWA/DAT H. Iwasaki and K. Date, Koatsu Gasu 12, 374-380 (1975).

1975KIR/LAS R. L. Kirchmeier, U. I. Lasouris, and J. M. Shreeve, Inorg. Chem. 14, 592-596 (1975).

$1975 \mathrm{KON} / \mathrm{SEL} \quad$ I. I. Konstantinov, V. D. Selivanov, and T. I. Melent'eva, Zh. Fiz. Khim. 49, 1058-1060 (1975).

1975KOR/VIA A. I. Korovin and O. N. Viasov, Zh. Fiz. Khim. 49, 2149 (1975).

1975KUN/LIL H. Kundel, U. Lille, and N. Kaidas, Tr. Tallin. Politekh. Inst. 390, 107-115 (1975).

1975KUS/SAI K. Kusano and Y. Saito, Preprints, 33rd Annu. Meeting Chem. Soc., Jpn. 1, 123 (1975), as quoted in [1985MAJ/SVO].

1975LEB/TSV $\quad$ B. V. Lebedev, L. Y. Tsvetkova, E. G. Kiparisova, and N. K. Lebedev, Russ. J. Phys. Chem. 49, 1265 (1975).

1975LEE/SLU W. Y. Lee and L. J. Slutsky, J. Phys. Chem. 79, 2602-2604 (1975).

1975MAN/KOR J. Manczinger and G. Kortum, Z. Phys. Chem. Neue Folge 95, 177 (1975).

1975MAS/SCO J. F. Masi and R. B. Scott, J. Res. Natl. Bur. Stand. Sect. A 79, 619-628 (1975).

$1975 \mathrm{MCE} / \mathrm{SAN} \quad$ D. M. McEachern, O. Sandoval, and J. C. Iniguez, J. Chem. Thermodyn. 7, 299-306 (1975).

1975MES/BAE U. Messow, J. Baer, K. Quitzsch, and G. Geiseler, J. Prakt. Chem. 317, 114-122 (1975).

1975MES/FIN J. F. Messerly, H. L. Finke, A. G. Osborn, and D. R. Douslin, J. Chem. Thermodyn. 7, 1029-1046 (1975).

1975PAU

1975PEP/MAT

1975PET/SAN

L. E. Paukov, Russ. J. Phys. Chem. 49, 265 (1975).

V. I. Pepekin, Y. N. Matyushin, A. D. Nikolaeva, A. P. Kirsanov, L. V. Platonova, and Y. A. Lebedev, Izv. Akad. Nauk SSSR Ser. Khim. 1870-1871 (1975).

V. M. Petrov and L. Sandler, Zh. Fiz. Khim. 49, 2797-2800 (1975).

1975PET/SHR K. E. Peterman and J. M. Shreeve, Inorg. Chem. 14, 1106-1110 (1975).

1975PET/SHR2 K. E. Peterman and J. M. Shreeve, Inorg. Chem. 14, 1223-1228 (1975).

1975PET/SHR3 K. E. Peterman and J. M. Shreeve, J. Fluorine Chem. 6, 83-92 (1975).

1975PIS/ROZ V. V. Pisarev, A. M. Rozhnov, R. M. Varushchenko, and A. G. Sarkisov, Russ. J. Phys. Chem. 49, 1450-1451 (1975).

1975PIS/ROZ2 V. V. Pisarev, A. M. Rozhnov, R. M. Varushchenko, and A. G. Sarkisov, Russ. J. Phys. Chem. 49, 1605 (1975).

1975RAK/GUT S. S. Rakhmenkulov, S. A. Gutov, and I. E. Paulov, Zh. Fiz. Khim. 49, 2722 (1975).

1975RIV F. Rivenq, Bull. Soc. Chim. Fr. 2433-2434 (1975).

1975SOU/BAR M. A. Soulie, D. A. Bares, and J. Metzer, C. R. Seances Acad. Sci., Ser. C 281, 341 (1975).

1975SUB/ZWO D. J. Subach and B. J. Zwolinski, J. Chem. Eng. Data 20, 232-234 (1975).

1975TEP/YAN A. B. Teplitskii and I. K. Yanson, Biofizik 20, 189-193 (1975); Chem. Abstr. 83, 16712h (1975).

1975URB/TOM S. Urban, Z. Tomkowicz, J. Mayer, and T. Waluga, Acta Phys. Pol., A 48, 61 (1975).

1975VAN/DEK C. H. D. van Ginkel, G. C. DeKruif, and F. E. B. DeWaal, J. Phys. E: Sci. Instrum. 8, 490-492 (1975).

1975VAR/DRU R. M. Varushchenko and A. I. Druzhinina, Zh. Fiz. Khim. 49, 2446 (1975).

1975VIL/PER R. Vilcu, S. Perisanu, and I. Ciocazanu, 4th Conf. Int. Thermodyn. Chim. [C.R.] 1, 105-112 (1975).

1975WAL/DES N. S. Walker and D. D. DesMarteau, J. Fluorine Chem. 5, 127-133 (1975).

1975WAL/DES2 N. S. Walker and D. D. DesMarteau, J. Fluorine Chem. 5, 135-139 (1975).
1975YAN/TEP

I. K. Yanson and A. B. Teplitskii, Zh. Fiz. Khim. 49, 736-738 (1975) [Engl. Ed. 428-429 (1975)].

1976AMB/ELL

D. Ambrose, J. H. Ellender, C. H. S. Sprake, and R. J. Townsend, J. Chem. Thermodyn. 8, 165-178 (1976).

1976AMB/SPR D. Ambrose and C. H. S. Sprake, J. Chem. Thermodyn. 8, 601-602 (1976).

1976AMM/BUL M. M. Ammar, L. L. Bulgakova, and R. M. Varushchenko, Russ. J. Phys. Chem. 50, 1466-1467 (1976).

1976ANT/CAR M. E. Anthoney, A. S. Carson, and P. G. Laye, J. Chem. Soc., Perkin Trans. 2 1976, 1032-1036.

1976ANT/CAR2 M. E. Anthoney, A. S. Carson, P. G. Laye, and M. Yurekli, J. Chem. Thermodyn. 8, 1009-1110 (1976).

1976ASH S. J. Ashcroft, J. Chem. Eng. Data 21, 397-398 (1976).

1976ATA/CHI T. Atake and H. Chihara, Chem. Lett. 1976, 683-688.

1976BAR/BOU M. A. Barnard, Y. Boukari, and F. Busnot, Thermochim. Acta 16, 267-275 (1976).

1976BER/BOU M. A. Bernard, Y. Boukari, and F. Busnot, Thermochim. Acta 16, 267 (1976).

1976BOR/CHU G. K. Borisov and S. G. Chugunova, Russ. J. Phys. Chem. 50, 1791 (1976).

1976BRA/PES

M. M. Brazhnikov, A. D. Peshchenko, and O. V. Ral'ko, Zh. Prikl. Khim. (Leningrad) 49, 1041-1044 (1976).

1976BUR/SHR C. A. Burton and J. M. Shreeve, J. Am. Chem. Soc. 98, 6545-6547 (1976).

1976CIH/HYN J. Cihlar, V. Hynek, V. Svoboda, and R. Holub, Collect. Czech. Chem. Commun. 41, 1-6 (1976).

1976COL/JIM M. Colomina, P. Jimenez, R. Perez-Ossorio, and C. Turrion, J. Chem. Thermodyn. 8, 439-444 (1976).

1976CON/COU J. E. Connett, J. F. Counsell, and D. A. Lee, J. Chem. Thermodyn. 8, 1199-1203 (1976).

1976DAV/FIN R. H. Davies, A. Finch, P. J. Gardner, A. Hameed, and M. Stephens, J. Chem. Soc., Dalton Trans. 1976, 556-558.

1976DEP

1976DWO/FIG R. S. DePablo, J. Chem. Eng. Data 21, 141-143 (1976).

1976ENG/MEL A. Dworkin, P. Figuiere, M. Ghelfenstein, and H. Szwarc, J. Chem. Thermodyn. 8, 835 (1976).

P. S. Engel, R. A. Melaugh, M. Mansson, J. W. Timberlake, A. Garner, and F. D. Rossini, J. Chem. Thermodyn. 8, 607-621 (1976).

1976FAL/DES E. R. Falardeau and D. D. DesMarteau, J. Fluorine Chem. 7, 185-195 (1976).

1976FAL/DES2 $\quad$ E. R. Falardeau and D. D. DesMarteau, J. Fluorine Chem. 7, 409-414 (1976).

1976FAL/DES3 $\quad$ E. R. Falardeau and D. D. DesMarteau, J. Am. Chem. Soc. 98, 3529 (1976).

1976FER/PIA D. Ferro, V. Piacente, R. Gigli, and G. D’Ascenzo, J. Chem. Thermodyn. 8, 1137-1143 (1976).

1976FIN/MES H. L. Finke, J. F. Messerly, and D. R. Douslin, J. Chem. Thermodyn. 8, 965 (1976).

1976GEI/WOL G. Geipel and G. Wolf, Z. Phys. Chem. 257, 587 (1976).

1976HA/MOR H. Ha, J. A. Morrison, and E. L. Richards, J. Chem. Soc., Faraday Trans. 1 72, 1051-1057 (1976).

1976HAM/THO W. S. Hamilton, P. Thomspon, and S. Pustejovsky, J. Chem. Eng. Data 21, 428-429 (1976).

1976 HON/SIN $\quad$ H. C. Hon, R. P. Singh, and A. P. Kudchadker, J. Chem. Eng. Data 21, 430-431 (1976).

1976HOP/DES M. J. Hopkinson and D. D. DesMarteau, J. Fluorine Chem. 7, 501-510 (1976).

1976KOL/SLA V. P. Kolesov, G. M. Slavutskaya, and L. N. Dityat'eva, J. Chem. Thermodyn. 8, 907-914 (1976).

1976KOZ/ALE M. P. Kozina, V. A. Aleshina, G. L. Gal'chenko, E. F. Buinova, and I. I. Bardyshev, Vestsi Akad. Navuk BSSR Ser. Khim. Vavuk 14 (1976); Chem. Abstr. 85, 33197 (1976).

1976KOZ/BYC M. P. Kozina, L. V. Bychikhina, G. V. Gal'chenko, E. M. Milwitskaya, M. Ordubade, and A. F. Plate, Dokl. Akad. Nauk SSSR 226, 1105-1108 (1976) [Engl. Ed. 143 (1976)].

1976KUS/SAI Joint meeting Kyushu, Chungoku and Skikoku Branches of Chem. Soc. Jpn. 60, (1976).

1976KUZ/MIR V. P. Kuznetsova, E. A. Miroshinichenko, A. Z. Zelenetskii, G. V. Rokova, Y. A. Lebedev, and N. S. Enikolopyan, Dokl. Phys. Chem. 226, 147-150 (1976). 
1976LEC/COL M. Leclercq, A. Collet, and J. Jacques, Tetrahedron 32, 821-828 (1976).

1976MEY/HOT E. F. Meyer and C. A. Hotz, J. Chem. Eng. Data 21, 274-279 (1976).

1976MIR/LEB E. A. Miroshnichenko and Y. A. Lebedev, Vzaimodeistvie Konform. Mol. Tezisy Dokl. Vses. Simp. 3rd 4041 (1976), in Mezhnol.; Chem. Abstr. 91, 56104 h (1979).

1976MIS/RIE S. Miskiewicz, K. Rieser, and T. Dorfmuller, Ber. Bunsenges. Phys. Chem. 80, 395-405 (1976).

1976MOR/RIC J. A. Morrison and E. J. Richards, J. Chem. Thermodyn. 8, 1033 (1976).

1976PEL

1976RAO/CHI

P. A. Pella, Anal. Chem. 48, 1632-1637 (1976).

$\begin{array}{ll}\text { 1976STA/MEW } & \text { 393-394 (1976). } \\ \text { I. Stahl, R. Mews, and O. Glemser, J. Fluorine Chem. 7, }\end{array}$ 55-64 (1976).

1976STE/POL K. Steele, B. E. Poling, and D. B. Manley, J. Chem. Eng. Data 21, 399-403 (1976).

1976STR G. Stridh, J. Chem. Thermodyn. 8, 193-194 (1976).

1976STR3 G. Stridh, J. Chem. Thermodyn. 8, 901-906 (1976).

1976TAY/CRO J. W. Taylor and R. J. Crookes, J. Chem. Soc., Faraday Trans. 1 72, 723-729 (1976).

1976VAR/BUL R. M. Varushchenko and L. L. Bulgakova, Tr. Khim. Khim. Tekhnol. 3, 29-33 (1976).

1976VOG/PIT G. J. Vogt and K. S. Pitzer, J. Chem. Thermodyn. 8, 1011 (1976).

1977ALE/KOZ V. A. Aleshina, M. P. Kozina, and G. L. Gal'chenko, Vses. Konf. Kalorim. [Rasshir. Tezisy Dokl.] 7th 1, 85-91 (1977); Chem. Abstr. 92, 94582r (1980).

1977BIG/FUK J. Bigeleisen, S. Fuks, S. W. Ribnikar, and Y. Yato, J. Chem. Phys. 66, 1689 (1977).

1977BOL/MAK N. I. Boldenkov and Y. Y. Maksimov, Zh. Fiz. Khim. 51, 1502-1504 (1977).

1977BUR

1977BUR/SHR C. A. Burton and J. M. Shreeve, Inorg. Chem. 16, 1039-1042 (1977).

1977BUR/SHR2 C. A. Burton and J. M. Shreeve, Inorg. Chem. 16, 1408-1411 (1977).

1977CAM/SCH H. K. Cammenga, F. W. Schulze, and W. Theuerl, J. Chem. Eng. Data 22, 131-134 (1977).

1977CAR/LAY A. S. Carson, P. G. Laye, and M. Yurkeli, J. Chem. Thermodyn. 9, 827-829 (1977).

1977CAS/VEC F. Casellato, C. Vecchi, and A. Girelli, Thermochim. Acta 21, 195-199 (1977).

1977CLA/KNO T. Clark, T. M. O. Knox, H. Mackle, and M. A. McKervey, J. Chem. Soc. Faraday Trans. I 73, 1224-1231 (1977).

1977DAS/REE T. R. Das, C. O. Reed, Jr., and P. T. Eubank, J. Chem. Eng. Data 22, 3-9 (1977).

1977DAS/REE2 T. R. Das, C. O. Reed, Jr., and P. T. Eubank, J. Chem. Eng. Data 22, 9-15 (1977).

1977DAS/REE3 T. R. Das, C. O. Reed, Jr., and P. T. Eubank, J. Chem. Eng. Data 22, 16-21 (1977).

1977DEK/VAN C. G. DeKruif and C. H. D. van Ginkel, J. Chem. Thermodyn. 9, 725-730 (1977).

1977DIT/KOL L. N. Dityat'eva and V. P. Kolesov, Vses. Konf. Kalorim. [Rasshir. Tezisy Dokl.] 7th 1, 95-97 (1977); Chem. Abstr. 92, 58009a (1980).

1977FEL/PET I. N. Fel'dman, D. S. Petrenko, V. O. Avilov, G. S. Stuchkov, M. Kh Aizenshtadt, and V. S. Freger, Zh. Fiz. Khim. 51, 1002 (1977).

1977FRA J. Franklin, Ph.D. thesis, University of Leeds, 1977.

1977GAF/PIE J. S. Gaffney, R. C. Pierce, and L. Friedman, J. Am. Chem. Soc. 99, 4293-4298 (1977).

$1977 \mathrm{GOM}$ W. Gombler, Angew. Chem., Int. Ed. Engl. 16, 723 (1977).

1977HAI/SUG O. Haida, H. Suga, and S. Seki, Bull. Chem. Soc. Jpn. 50, 802 (1977).

1977KIM/KIM $\quad$ B. C. Kim and D. H. Kim, Hwahak Kwa Hwahak Kongop 20, 232-234 (1977); Chem. Abstr. 88, 95565s (1978).

1977KIS

1977KIT/SHR
1977KIT/SHR2

T. Kitazume and J. M. Shreeve, J. Am. Chem. Soc. 99, 3690-3695 (1977).

1977KIT/SHR3 T. Kitazume and J. M. Shreeve, J. Am. Chem. Soc. 99 , 4194-4196 (1977).

1977KOR/VAS A. D. Korkhov and I. A. Vasil'ev, Termodin. Org. Soedin. 34 (1977).

1977KOZ/BYC M. P. Kozina, V. L. Bychikhina, and G. L. Gal'chenko, Zh. Fiz. Khim. 51, 2142 (1977) [Engl. Ed. 1258-1259].

1977KRE/PRI M. J. Krech, S. W. Price, and H. Sapiano, Can. J. Chem. 55, 4222-4226 (1977).

1977LEB/LIT $\quad$ B. V. Lebedev and V. Ya Lityagov, Vysokomol. Soedin. B19, 558 (1977).

1977LEB/NAZ N. D. Lebedeva, L. F. Nazarova, and Y. Katin, Termodin. Org. Soedin. 6, 72-73 (1977).

1977LEB/RYA N. D. Lebedeva, V. L. Ryadnenko, N. N. Kiseleva, and L. F. Nazarova, Vses. Konf. Kalorim. [Rasshir. Tezisy Dokl.] 7th 1, 91-95 (1977); Chem. Abstr. 92, 75617q (1980).

1977LYU/SMO M. V. Lyubarskii and R. I. Smolyanets, Vses. Konf. Kalorim. [Rasshir. Tezisy Dokl.] 7th 1, 119-121 (1977); Chem. Abstr. 92, 75619s (1980).

1977MAN/SEL M. Mansson, P. Sellers, G. Stridh, and S. Sunner, J. Chem. Thermodyn. 9, 91-97 (1977).

1977MEI/BLO E. L. Meijer, J. G. Blok, J. Kroon, and H. A. J. Oonk, Thermochim. Acta 20, 325 (1977).

1977MEY/GEN E. F. Meyer and T. H. Gens, J. Chem. Eng. Data 22, 30-31 (1977).

1977NAB/SAB M. Nabavian, R. Sabbah, R. Chastel, and M. Laffitte, J. Chim. Phys. Phys.-Chim. 74, 115-126 (1977).

1977 NGA/SAB S. N. Ngauv, R. Sabbah, and M. Laffite, Thermochim. Acta 20, 371-380 (1977).

1977NOF/FOX R. E. Noftle and W. B. Fox, J. Fluorine Chem. 9, 219 (1977).

1977NOV/NOV N. Novakova and J. Novak, J. Chromatogr. 135, 13-24 (1977).

1977PAR/STE W. Parker, W. V. Steele, and I. Watt, J. Chem. Thermodyn. 9, 307-310 (1977).

1977PEA/FUC L. A. Peacock and R. Fuchs, J. Am. Chem. Soc. 99, 5524-5525 (1977).

1977PED/RYL J. B. Pedley and J. Rylance, N. P. L. Computer Analysed Thermochemical Data: Organic and Organometallic Compounds (School of Molecular Sciences, University Of Sussex, Brighton, UK, 1977).

1977PEL

1977PIS/ROZ P. A. Pella, J. Chem. Thermodyn. 9, 301-305 (1977).

1977RAO/VIU J. J. Rao and D. S. Viuswanath, J. Chem. Eng. Data 22 , J. Phys. Chem. 51, 323-324 (1977) 36-38 (1977).

1977SAB/NAB R. Sabbah, M. Nabavian, and M. Laffitte, C. R. Hebd. Seances Acad. Ser. C 284, 953-954 (1977); Chem. Abstr. 87, 151519y (1977)

1977SEL P. Sellers, J. Chem. Thermodyn. 9, 139-142 (1977).

1977STE

1977STE2

1977STRI/SUN W. V. Steele, J. Chem. Thermodyn. 9, 311-314 (1977).

H. Steinwandter, Chemosphere 6, 59-65 (1977); Chem. Abstr. 87, 17285r (1977).

dyn. 9, 1005-1010 (1977).

1977SVO/MAJ V. Svoboda, V. Majer, F. Vesely, and J. Pick, Collect. Czech. Chem. Commun. 42, 1755-1760 (1977).

1977SVO/VES V. Svoboda, F. Vesely, R. Holub, and J. Pick, Collect. Czech. Chem. Commun. 42, 943-951 (1977).

1977VAR/AMM R. M. Varushchenko, M. M. Ammar, and L. L. Bulgakova, Zh. Fiz. Khim. 51, 278-279 (1977).

1977VAR/AMM2 R. M. Varushchenko, M. M. Ammar, and L. L. Bulgakova, Russ. J. Phys. Chem. 51, 167-168 (1977).

1977VAS/KOT T. F. Vasil'eva and V. I. Kotov, Vses. Konf. Kalorim. [Rasshir. Tezisy Dokl.] 7th 1, 102-106 (1977); Chem. Abstr. 92, 58010u (1980).

1977VAS/PET T. F. Vasil'eva and V. M. Petrov, Termodin. Org. Soedin. 6, 74 (1977).

1977VOI/SHC S. A. Voitkevich, M. M. Shchedrina, K. K. Prilepskaya, N P. Solov'eva, T. M. Tsirkel, and T. A. Rudol'fi, Maslozhir. Prom-st. 23 (1977). 
1977VOI/SHC2 S. A. Voitkevich, M. M. Shchedrina, K. K. Prilepskaya, N. P. Solov'eva, T. M. Tsirkel, and T. A. Rudol'fi, Maslozhir. Prom-st. 35 (1977).

1977ZHU/MEL $\quad$ E. Z. Zhuravlev, T. L. Melent'eva, P. V. Mulyanov, A. I. Kormushechkina, T. M. Bogdanova, and I. I. Konstantinov, Zh. Prikl Khim. (Leningrad) 50, 2032-2035 (1977).

1978AND/CAR J. T. S. Andrews, R. E. Carpenter, T. M. Martinko, R. C. Fort, R. C. Jr., T. A. Flood, and M. G. Adlington, Mol. Cryst. Liq. Cryst. 41, 257-261 (1978).

1978ARO/STE M. Arora and W. V. Steele, J. Chem. Thermodyn. 10, 403-407 (1978).

1978BEA/LEE P. Beak, J.-K. Lee, and J. M. Zeigler, J. Org. Chem. 43, 1536 (1978).

1978BER/SPI C. V. Berney and D. Spickerman, J. Chem. Thermodyn. 10, 637-640 (1978).

1978BOU/LEC D. Bougeard, N. A. Le Calve, A. Novak, and B. C. Nguyen, Mol. Cryst. Liq. Cryst. 44, 113-123 (1978).

1978CAB/MOL S. Cabani, V. Mollica, and L. Lepori, J. Chem. Soc., Faraday Trans. 1 74, 2667 (1978).

1978CAL/CAL C. D. H. Calis-Van Ginkel, G. H. M. Calis, C. W. M. Timmermans, C. G. DeKruif, and H. A. J. Oonk, J. Chem. Thermodyn. 10, 1083-1088 (1978).

1978CAN

$1978 \mathrm{CAV} / \mathrm{CHA} \quad$ S. D. Cave, A. Chainese, and A. Prantera, J. Chem. Eng. Data 23, 279-281 (1978).

1978CHI/SHE J. S. Chickos and D. E. Sherwood, J. Org. Chem. 43, 1146-1150 (1978).

1978COL/JIM M. Colomina, P. Jimenez, M. V. Roux, and C. Turrion, J. Chem. Thermodyn. 10, 661-665 (1978).

1978COR/PER R. P. Corbally, M. J. Perkins, A. S. Carson, P. G. Laye, and W. V. Steele, J. Chem. Soc., Chem. Commun. 1978, 778-779.

1978CUN/PAL R. B. Cundall, T. F. Palmer, and C. E. C. Wood, J. Chem. Soc., Faraday Trans. 1 74, 1339-1345 (1978).

1978DEM/FOX R. A. De Marco and W. B. Fox, J. Fluorine Chem. 12, 137-151 (1978).

1978DEP R. S. DePablo, J. Phys. D: Appl. Phys. 11, 193-199 (1978).

1978DOZ/FUJ Y. Dozen, H. Fujishima, and H. Shingu, Thermochim. Acta 25, 209-216 (1978).

1978ECK/MUL T. Eckert and J. Muller, Arch. Pharm. 311, 31 (1978).

$1978 \mathrm{ENG} / \mathrm{MON} \quad$ P. S. Engel, R. L. Mongomery, M. Mansson, R. A. Leckonby, H. L. Foyt, and F. D. Rossini, J. Chem. Thermodyn. 10, 205-211 (1978).

1978FUC/PEA R. Fuchs and L. A. Peacock, Can. J. Chem. 56, 2493-2498 (1978).

1978GOD/RAC M. Godleweka and M. Rachwalska, Rap.-Inst. Fiz. Jad (Krakow) 997/PS, 1-14 (1978).

1978GOD/RAC2 M. Godleweka and M. Rachwalska, Phys. Status Solidi A 47, 661 (1978).

1978GRO/SPE R. Grover, W. F. Spencer, W. J. Farmer, and T. D. Shoup, Weed Sci. 26, 505-508 (1978).

1978GUN/HEA H. A. Gundry and A. J. Head, J. Chem. Thermodyn. 10, 195 (1978).

1978HAM/BEN W. S. Hamilton, S. Benton, J. French, D. McCormick, S. Pustejovsky, and P. J. Thomspon, J. Chem. Eng. Data 23, 201-203 (1978).

1978HEA/HEF G. A. Heath, G. T. Hefter, and W. V. Steele, J. Chem. Thermodyn. 10, 395-401 (1978).

1978 IND/STO D. Indritz, J. Stone, and F. Williams, J. Chem. Eng. Data 23, 6-7 (1978).

1978KAC/SOL A. J. Kacmarek, I. J. Solomon, and M. Lustig, J. Inorg. Nucl. Chem. 40, 574 (1978).

1978KAR/RAB N. V. Karyakin, I. B. Rabinovich, and N. G. Pal'tseva, Vysok. Soedin. Ser. A 20, 2025 (1978).

1978KIS/SUG K. Kishimoto, H. Suga, and S. Seki, Bull. Chem. Soc. Jpn. 51, 1691-1696 (1978).

1978KIT/SHR T. Kitazume and J. M. Shreeve, Inorg. Chem. 17, 2173-2176 (1978).

1978KOL/DIT V. P. Kolesov and L. N. Dityat'eva, Termodin. Org. Soedin. 7, 44-47 (1978); Chem. Abstr. 92, 75657c (1980).
1978KOS/KOL E. A. Kosarukina, V. P. Kolesov, V. N. Vorob'ev, and L. Fogel, Zh. Fiz. Khim. 52, 509 (1978).

1978LEB/RAB2 B. V. Lebedev, I. B. Rabinovich, V. I. Milov, and V. Ya Lityagov, J. Chem. Thermodyn. 10, 321 (1978).

1978LEB/TSV $\quad$ B. V. Lebedev, L. Y. Tsvetkova, and I. B. Rabinovich, J. Chem. Thermodyn. 10, 809-815 (1978).

1978LEB/TSV2 B. V. Lebedev, L. Y. Tsvetkova, I. B. Rabinovich, E. S. Finkel'shtein, and B. S. Strel'chick, Termodin. Org. Soedin. 7, 3-7 (1978); Chem. Abstr. 92, 83507u (1980).

1978LUK/MAK V. M. Luk'yanets, R. G. Makitra, and A. Yas'kovyak, Ukr. Khim. Zhur. 44, 392 (1978).

1978MAR/CIO $\quad$ D. I. Marchidan and M. Ciopec, Rev. Roum. Chim. 23, 19 (1978).

1978MAR/CIO2 D. I. Marchidan and M. Ciopec, J. Therm. Anal. 14, 131 (1978).

1978MAT/TRA N. B. Matin, V. I. Tranchenko, E. Grigoryan, S. M Danov, and E. N. Zil'berman, Zh. Priklad. Khim. 51, 1900 (1978).

1978MCC/HAM D. G. McCormick and W. S. Hamilton, J. Chem. Thermodyn. 10, 275-278 (1978).

1978MON/ENG R. L. Montgomery, P. S. Engel, R. A. Leckonby, F. D Rossini, M. Mansson, S. Szilagyi, and J. W. Timberlake, J. Chem. Eng. Data 23, 129-131 (1978).

1978MOS/RAB E. M. Moseeva, I. B. Rabinovich, G. I. Busygina, V. A. Safonov, and E. Yu Ovchinnikov, Termodin. Org. Soedin. 7, 8 (1978).

1978MOU A. H. N. Mousa, J. Chem. Eng. Data 23, 133-134 (1978).

1978NOW/SZC M. J. Nowak, K. Szczepaniak, A. Barski, and D. Z. Shugar, Natureforsch. C: Biosci. 33C, 876-883 (1978).

1978RIB/IRV M. A. V. Ribeiro da Silva and R. G. Irving, Rev. Port. Quim. 20, 36-46 (1978); Chem. Abstr. 90, 211054s (1979).

1978ROD/HIL R. C. Rodgers and G. E. Hill, Br. J. Anaesth. 50, 415 (1978).

1978SAB/LAF R. Sabbah and M. Laffite, Bull. Soc. Chem. France, Ptie 1, 50-52 (1978).

1978SEK/TSU K. Sekiguchi, Y. Tsuda, M. Kanke, E. Suzuki, and M. Iwatsuru, Chem. Pharm. Bull. 26, 1279 (1978).

1978SMI/RAE J. H. Smith and A. I. M. Rae, J. Phys. C: Solid State Phys. 11, 1771 (1978).

1978SMI/ZEL G. E. Smirnova, Ya. D. Zel'venski, V. A. Shalygin, G. A. Sukhorukova, and T. X. Dzhekseneva, Mosk. Khim.Teknol. Inst. Mockow USSR VINITI 3298-78, 9 (1978).

1978STA/MEW I. Stahl, R. Mews, and O. J. Glemser, Fluorine Chem. 11, 455-465 (1978).

1978STE W. V. Steele, J. Chem. Thermodyn. 10, 585-590 (1978).

1978STE2

1978STR/ROG W. V. Steele, J. Chem. Thermodyn. 10, 919-927 (1978). R. Stryjek, M. Rogalski, T. Treszczanowicz, and M. Luszczyk, Bull. Acad. Pol. Sci., Ser. Sci. Chim. 26, 327 (1978).

1978SUN/VIS S. Sundaram and D. S. Viswanath, J. Chem. Eng. Data 23, 63-64 (1978)

1978TRI/VOO L. E. Trimble and R. J. H. Voorhoeve, Analyst 103, 759-765 (1978).

1978VIL R. Vilcu, Bull. Inst. Politek "Gheorghe Gheorghiu-Dej" Bucuresti Ser. Chim.-Metal 40, 2 (1978).

1978VIL/PER R. Vilcu, S. Perisanu, and I. Ciocazanu, Bull. Inst. Politek "Gheorghe Gheorghiu-Dej" Bucuresti Ser. Chim.-Metal 40, 9-12 (1978); Chem. Abstr. 91, 28258t (1979).

1979ARM/JAM N. A. Armstrong, K. C. James, and C. K. Wong, J. Pharm. Pharmacol. 31, 627-631 (1979).

1979ARN/CHA E. M. Arnett and B. Chawla, J. Am. Chem. Soc. 101, 7141 (1979).

1979BAL/FRI M. Balish and V. J. Fried, Chem. Eng. Data 24, 91-92 (1979).

1979BER/ANG G. Berthon, V. Angot, V. Beden, and O. Enea, J. Chem. Thermodyn. 11, 539-546 (1979).

1979BLU/BAE V. I. Bludilina, A. K. Baev, V. K. Matveev, I. L. Gaidym, and E. I. Shcherbina, Zh. Fiz. Khim. 53, 1052-1053 (1979); Chem. Abstr. 91, 9585f (1979).

1979BOT/CAM H. Bothe and H. K. Cammenga, J. Therm. Anal. 16, 267-275 (1979). 
1979BRI/VAN W. J. Briels and J. C. van Miltenburg, J. Chem. Phys. 70, 1064 (1979).

1979CAV/HIL K. J. Cavell, J. O. Hill, and R. J. Magee, Thermochim. Acta 33, 155-160 (1979).

1979CAV/PIN R. G. Cavell, A. A. Pinkerton, W. Sim, and A. Tomlinson, Inorg. Chem. 18, 2901 (1979).

1979CLA/KNO T. Clark, T. M. Knox, M. A. McKervey, H. Mackle, and J. Rooney, J. Am. Chem. Soc. 101, 2404-2410 (1979).

1979COL/JIM2 M. Colomina, P. Jimenez, M. V. Roux, and C. Turrion, An. Quim. 75, 620-624 (1979); Chem. Abstr. 92, 75685k (1980).

1979DAA/VAN H. Daamen, H. Van der Poel, D. J. Stufkens, and A. Oskam, Thermochim. Acta 34, 69-83 (1979).

1979DAS/DHA D. Das, S. R. Dharwadkar, and M. S. Chandrasekharaiah, Thermochim. Acta 30, 371-376 (1979).

1979DEK/OON C. G. De Kruif and H. A. Oonk, J. Chem. Thermodyn. 11, 287-290 (1979).

1979DEK/VOO C. G. DeKruif, J. Voogd, and J. C. A. Offringa, J. Chem. Thermodyn. 11, 651-656 (1979).

1979DUM J. P. Dumas, J. Phys. C: Solid State Phys. 12, 2225 (1979).

1979FAR/SHA R. G. Farrell, F. Shahidi, F. Casellato, C. Vecchi, and A. Girelli, Thermochim. Acta 33, 275 (1979).

1979FUC/PEA R. Fuchs and L. A. Peacock, Can. J. Chem. 57, 2302-2304 (1979).

1979GAR/LAW R. G. Garza, Lawrence Livermore National Laboratory UCRL, 1979; study referenced in [2008RAI/BHA].

1979 GON/CHA A. S. Goncalves, A. P. Chagas, and C. Airoldi, J. Chem. Soc., Dalton Trans. 1979, 159-161.

1979JAK/TZI G. Jakli, P. Tzias, and W. A. Van Hook, Acta Chim. Acad. Sci. Hung. 99, 121-125 (1979).

1979KHE/LAL $\quad$ S. C. Khetarpal, K. Lal, and H. L. Bhainagar, Aust. J. Chem. 32, 49 (1979).

1979KUD/KUD A. P. Kudchadker, S. A. Kudchadker, R. P. Shukla, and P. R. J. Patnaik, J. Phys. Chem. Ref. Data 8, 499-513 (1979).

1979KUD/KUD2 S. A. Kudchadker, A. P. Kudchadker, and B. J. Zwolinski, J. Chem. Thermodyn. 11, 1051-1059 (1979).

1979LET/ORC T. M. Letcher, S. W. Orchard, and M. J. Albers, J. Chem. Thermodyn. 11, 173-175 (1979).

1979LIN J. Linek, Collect. Czech. Chem. Commun. 44, 3501 (1979).

1979MAC/PRA A. B. Macknick and J. M. Prausnitz, J. Chem. Eng. Data 24, 175-178 (1979).

1979MAH/SMI P. J. Maher and B. D. Smith, J. Chem. Eng. Data 24, 16-22 (1979).

1979MAH/SMI2 P. J. Maher and B. D. Smith, J. Chem. Eng. Data 24, 363 (1979).

1979MAJ/SVO V. Majer, V. Svoboda, S. Hala, and J. Pick, J. Collect. Czech. Chem. Commun. 44, 637-651 (1979).

1979MAJ/SVO2 V. Majer, V. Svoboda, J. Koubek, and J. Pick, J. Collect. Czech. Chem. Commun. 44, 3521-3528 (1979).

1979MAN/LAU C. Mandec, J. Lauransan, C. Garrigou-Lagrange, J. Housty, and N. B. Chanh, C. R. Acad. Sci. Paris C 289, 413 (1979).

1979MAR/SAC V. S. Markovnik, A. I. Sachek, A. D. Peshchenko, O. V. Shvaro, D. N. Andreevskii, and N. M. Olizarevich, Termodin. Org. Soedin. 107-110 (1979).

1979MJO C. C. Mjojo, J. Chem. Soc., Faraday Trans. 2 75, 692-703 (1979).

1979NIS/BAB V. P. Nistratov, A. G. Babinkov, K. G. Shvetsova, and S. A. Lapteva, Termodin. Org. Soedin. 33 (1979).

1979OLO G. Olofsson, in Combustion Calorimetry, edited by S. Sunner and M. Mansson (Pergamon, New York, 1979), Chap. 6.

1979PET/MAJ L. Petros, V. Majer, J. Koubek, V. Svoboda, and J. Pick, Collect. Czech. Chem. Commun. 44, 3533-3540 (1979).

1979PRI/SAP $\quad$ S. J. W. Price and H. J. Sapiano, Can. J. Chem. 57, 685-688 (1979).

1979RIC/SAV M. J. Richardson and N. G. Savill, Thermochim. Acta 30, 327 (1979).

1979SAB $\quad$ R. Sabbah, Bull. Soc. Chem. Fr. 434-437 (1979).

1979SAL/PEA P. P. S. Saluja, L. A. Peacock, and R. Fuchs, J. Am. Chem. Soc. 101, 1958-1962 (1979).
1979SAN/EPS

D. J. Sandman, A. J. Epstein, J. S. Chickos, J. Ketchum, J. S. Fu, and H. A. Scheraga, J. Chem. Phys. 70, 305-313 (1979).

1979SCH/OFF R. C. F. Schaake, J. C. A. Offringa, G. J. K. van der Berg, and J. C. van Miltenburg, J. R. Neth. Chem. Soc. 98, 408 (1979).

1979SCO/OSB D. W. Scott and A. G. Osborn, J. Phys. Chem. 83, 2714-2723 (1979).

1979SEK/DES A. Sekiya and D. D. DesMarteau, J. Fluorine Chem. 14, 289-297 (1979).

1979SEV

1979SPE/SHO

C. Sevensson, J. Chem. Thermodyn. 11, 593-596 (1979).

W. F. Spencer, T. D. Shoup, M. M. Cliath, W. J. Farmer, and R. J. Hague, J. Agric. Food Chem. 27, 273-278 (1979).

1979SUK/VLA S. I. Sukhova, O. N. Vlasov, and L. V. Li, Nov. Khim. Sredstva Azsch Rast. 51-52 (1979); Chem. Abstr. 92, 83359x (1980).

1979SUN/SVE2 S. Sunner, C. Svensson, and A. S. Zelepuga, J. Chem. Thermodyn. 11, 491-495 (1979).

1979SUN/VIS S. Sundaram and D. S. Viswanath, Can. J. Chem. Eng. 57, 233 (1979).

1979THO/MEA L. H. Thomas, R. Meatyard, H. Smith, and G. H. Davies, J. Chem. Eng. Data 24, 159-161 (1979).

1979YAN/TEP I. K. Yanson, A. B. Teplitsky, and L. F. Sukhodub, Biopolymers 18, 1149-1170 (1979).

1979ZHO/KOS D. Yu. Zhogin, E. A. Kosarukina, V. P. Kolesov, I. I. Baburina, Y. A. Pentin, and I. V. Izmest'ev, Zh. Obshch. Khim. 49, 629 (1979).

1980ABE/SHR T. Abe and J. M. Shreeve, Inorg. Chem. 19, 3063-3067 (1980).

1980AMB/GUN D. Ambrose and H. A. Gundry, J. Chem. Thermodyn. 12, 559 (1980).

1980AND/CON R. J. L. Andon and J. E. Connett, Thermochim. Acta 42 , 241 (1980).

1980AND/PIL F. Andruzzi, G. Pilcher, J. M. Hacking, and S. Cavell, Makromol. Chem. 181, 923-929 (1980); Chem. Abstr. 93, 8632y (1980).

1980ARS M. R. Arshadi, J. Chem. Thermodyn. 12, 903-906 (1980).

1980BAL/LEB A. A. Balepin, V. P. Lebedev, A. A. Kuznetsova, K. K. Venter, M. A. Trusule, D. O. Lolya, and Y. A. Lebedev, Isvest. Akaud. Nauk. S.S.S.R. Ser. Khim. 4, 848 (1980).

1980BAR/BEN G. Bardi, L. Bencivenni, D. Ferro, B. Martini, C. S. Nunziante, and R. Teghil, Thermochim. Acta 40, 275-282 (1980).

1980BYS K. Bystrom, J. Chem. Soc., Faraday Trans. 1 76, 1986-1990 (1980).

1980CES/STA

1980COL/JIM

A. Cesaro and G. Starec, J. Phys. Chem. 84, 1345 (1980).

M. Colomina, P. Jimenez, M. V. Roux, and C. Turrion, J. Calorim. Anal. Therm. 11, 1-6 (1980); Chem. Abstr. 95, 121878 n (1981), see [1981COL/JIM].

1980DAV/FIN R. H. Davies, A. Finch, and P. G. Gardner, J. Chem. Thermodyn. 12, 291-296 (1980).

1980DEK C. G. DeKruif, J. Chem. Thermodyn. 12, 243-248 (1980).

1980DEK/GOV C. G. DeKruif and H. A. J. Govers, J. Chem. Phys. 73, 553-555 (1980).

1980DEP

1980DIA/CRE

R. S. DePablo, J. Phys. D: Appl. Phys. 13, 313-319 (1980).

1980DYA/VAS

M. Diaz-Pena, A. Crespo-Colin, A. Compositzo, and I. Escuder, J. Chem. Eng. Data 25, 17 (1980).

G. N. D'yakova and I. A. Vasil'ev, Neftepererab. Neftekhim. (Moscow) 51 (1980); Chem. Abstr. 92, 170269m (1980).

1980FAL B. Falk, J. Chem. Thermodyn. 12, 967 (1980).

1980FAR/YAN W. J. Farmer, M. S. Yang, J. Letey, and W. F. Spencer, Soil Sci. Soc. Am. J. 44, 676-680 (1980).

1980FER/BEN $\quad$ D. Ferro, L. Bencivenni, R. Teghil, and R. Mastromarino, Thermochim. Acta 42, 75-83 (1980).

1980FER/BEN2 D. Ferro, L. Bencivenni, R. Teghil, and M. Pelino, J. Indian Chem. Soc. 57, 629-632 (1980).

1980FRA/CAS R. Francesconi, C. Castellari, A. Arcelli, and F. Comelli, Can. J. Chem. Eng. 58, 113-115 (1980). 
1980FUC/PEA R. Fuchs and L. A. Peacock, Can. J. Chem. 58, 2796-2799 (1980).

1980JAR/AFA N. L. Jarim-Agaev, L. D Afanasienko, V. P. Kalinichenko, and G. B. Tolmacheva, Ukr. Khim. Zhur. 46, 1331-1333 (1980).

1980KRA/PIG A. Krajewska and K. Pigon, Thermochim. Acta 41, 187-197 (1980).

1980LEB/DOB N. D. Lebedeva, S. L. Dobychin, V. B. Stepanov, Yu. A. Katin, and N. M. Kozlova, Zh. Prikl. Khim. 53, 803 (1980).

1980LEB/KAL N. D. Lebedeva, Y. A. Kalin, V. L. Raydnenko, V. B. Stapanov, and N. M. Kozlova, Zh. Prikl. Khim. (Leningrad) 53, 2588-2590 (1980); Chem. Abstr. 94, 156270q (1981).

1980LEB/NAZ N. D. Lebedeva, L. F. Nazarova, and Y. A. Katin, Zh. Prikl. Khim. (Leningrad) 53, 1394-1395 (1980).

1980MAH/SMI P. J. Maher and B. D. Smith, J. Chem. Eng. Data 25, 61 (1980).

1980MAJ/SVA V. Majer, L. Svab, and V. Svoboda, J. Chem. Thermodyn. 12, 843-847 (1980).

$1980 \mathrm{MAJ} / \mathrm{SVO}$ V. Majer, V. Svoboda, A. Posta, and J. Pick, Collect. Czech. Chem. Commun. 45, 3063-3069 (1980).

$1980 \mathrm{MAJ} /$ WAG V. Majer, Z. Wagner, V. Svoboda, and V. Cadek, J. Chem. Thermodyn. 12, 387-391 (1980).

1980MEY/AWE E. F. Meyer, M. J. Awe, and R. E. Wagner, J. Chem. Eng. Data 25, 371-374 (1980).

1980MUR/CAV J. P. Murray, K. J. Cavell, and J. O. Hill, Thermochim. Acta 36, 97-101 (1980).

1980NAK/SUG N. Nakamura, H. Suga, and H. S. Seki, Bull. Chem. Soc. Jpn. 53, 2755-2761 (1980).

1980NAS/HWA P. Nasir, S. C. Hwang, and R. Kobayashi, J. Chem. Eng. Data 25, 298-301 (1980).

$1980 \mathrm{OSB} / \mathrm{SCO} \quad$ A. G. Osborn and D. W. Scott, J. Chem. Thermodyn. 12, 429-438 (1980).

1980PAT/TOM C. R. Patrick and F. J. Tomes, Fluorine Chem. 15, 267-278 (1980).

1980PEL/FER M. Pelino, D. Ferro, and V. Piacente, Thermochim. Acta 41, 297 (1980).

1980PIT/KIS V. S. Pityugin, T. L. Kislitsyna, R. F. Shakirov, L. N. Sharif'yanova, and E. N. Maslennikov, Khim. Prom-st. Ser. Khlornaya Prom-st. 11-14 (1980).

1980RAD/RAD M. Radomska and R. Radomski, Thermochim. Acta 40, 405 (1980).

1980SAB R. Sabbah, Thermochim. Acta 35, 73-77 (1980).

1980SAB/KOM R. Sabbah and S. Komorowski, Thermochim. Acta 41, 379-381 (1980).

1980SAB/SKO R. Sabbah and S. Skoulika, Thermochim. Acta 36, 179-187 (1980).

1980SAB2 R. Sabbah, Thermochim. Acta 41, 33-40 (1980).

1980SAC/HIL J. Sachinides and J. O. Hill, Thermochim. Acta 35, 59-66 (1980).

1980SAT/SAK T. Sato-Toshima, M. Sakiyama, and S. Saki, Bull. Chem. Soc. Jpn. 53, 2762-2767 (1980).

1980SHA/SAD L. N. Sharif'yanova, F. M. Sadykova, and S. G. Akhmerova, Khim. Prom-st. Ser. Khlornaya Prom-st. 16-18 (1980).

1980SVO/UCH V. Svoboda, V. Uchtyilova, V. Majer, and J. Pick, Collect. Czech. Chem. Commun. 45, 3233-3240 (1980).

1980SWA/KWA H. A. Swain, Jr., Y. C.-Y. Kwan, and H.-N. Sung, J. Phys. Chem. 84, 1347-1349 (1980).

1980TEP/YAN A. B. Teplitskii, I. K. Yanson, O. T. Glukhova, A. Zielenkiewicz, W. Zielenkiewicz, and K. L. Wierzchowski, Biophys. Chem. 11, 17-21 (1980).

1980THO/SMI L. H. Thomas, H. Smith, and G. H. Davis, J. Chem. Technol. Biotechnol. 30, 476-480 (1980).

1980URB/GIG M. Urbani, R. Gigli, and V. Picente, J. Chem. Eng. Data 25, 97-100 (1980).

1980VAN/PRA C. Van de Rostyne and J. M. Prausnitz, J. Chem. Eng. Data 25, 1-3 (1980).

1980VAR/PIS R. M. Varushchenko, V. V. Pisarev, and T. A. Kotel'nikova, Zh. Fiz. Khim. 54, 2666 (1980).

1980VIL/PER R. Vilcu and S. Perisanu, Rev. Roum. Chim. 25, 619-624 (1980).
1980VOG/SCH

1980WIE/KOB

1980YEV/LEB

1980ZHO/KOS

1981ABE/SHR

1981ABE/SHR2

1981AMB/ELL

1981AMB/ELL2

1981AMB/HAL

1981BRO/MCE

1981BYS

1981CHA/HAG

1981CHI/GAR

1981CHI/HYM

1981COL/JIM

1981DEK/KUI

1981DEK/SMI

1981DEK/VAN

1981DEP

1981DIT/SKO

1981DYA/KOR

1981EDW/PRA

1981FER/PIA

1981FIN/MES

1981GAT/STR

1981HAL/COG

1981HOS/SCO

1981HOS/SCO2

1981HOS/SCO3

1981INO/ARA

1981JOO/ARL

1981KHA/KHE

1981KOL/KOS

1981MAJ/SVO
L. Vogel and H. Schuberth, Chem. Technol. (Leipzig) 32, 143 (1980).

S. A. Wieczorek and R. Kobayashi, J. Chem. Eng. Data 25, 302 (1980).

A. A. Yevstropov, B. V. Lebedev, Y. G. Kiparisova, V. A. Alekseyev, and G. A. Stashina, Polym. Sci. USSR 22, 2685-2692 (1980).

D. Yu. Zhogin, E. A. Kosarukina, and V. P. Kolesov, Zh. Obshch. Khim. 50, 249 (1980).

T. Abe and J. M. Shreeve, Inorg. Chem. 20, 2894-2899 (1981).

T. Abe and J. M. Shreeve, Inorg. Chem. 20, 2432-2434 (1981).

D. Ambrose, J. H. Ellender, H. A. Gundry, D. A. Lee, and R. J. Townsend, J. Chem. Thermodyn. 13, 795-802 (1981).

D. Ambrose and J. H. Ellender, J. Chem. Thermodyn. 13, 901 (1981).

D. Ambrose and D. J. Hall, J. Chem. Thermodyn. 13, 61-66 (1981).

W. Brostow, D. M. McEachern, and J. A. Valdez, Mater Chem. 6, 187-195 (1981)

K. Bystrom, J. Chem. Thermodyn. 13, 139-145 (1981).

N. B. Chanh, Y. Haget, A. Meresse, D. Louer, and R. Shirley, J. Phys. Chem. Solids 42, 217 (1981).

J. S. Chickos, D. L. Garin, M. Hitt, and G. Schilling, Tetrahedron 37, 2255-2259 (1981).

J. S. Chickos, A. S. Hyman, L. H. Ladon, and J. F. Liebman, J. Org. Chem. 46, 4294-4296 (1981).

M. Colomina, P. Jimenez, M. V. Roux, and C. Turrion, An. Quim. Ser. A 77, 114 (1981).

C. G. De Kruif, T. Kuipers, J. C. van Miltenburg, R. C. F. Schaake, and G. Stevens, J. Chem. Thermodyn. 13, 1081-1086 (1981).

C. G. DeKruif, E. J. Smit, and H. A. Gover, J. Chem. Phys. 74, 5838-5841 (1981).

C. G. De Kruif, A. C. G. Van Genderen, J. C. W. G. Bink, and H. A. J. Oonk, J. Chem. Thermodyn. 13, 457-463 (1981).

R. S. DePablo, J. Chem. Eng. Data 26, 237-239 (1981). V. E. Ditsent, I. I. Skorokhodov, M. N. Zolotareva, V. I. Savuskina, and B. N. Tabenko, Zh. Prikl. Khim. (Leningrad) 54, 1617-1619 (1981).

G. N. D'yakova, G. L. Korichev, A. D. Korkhova, T. F. Vasil'eva, and I. A. Vasil'ev, Zh. Prikl. Khim. (Leningrad) 54, 1644-1646 (1981).

D. R. Edwards and J. M. Prausnitz, J. Chem. Eng. Data 26, 121-124 (1981).

D. Ferro, V. Piacente, and M. Pelino, Rev. Roum. Chim. 26, 9-15 (1981).

H. L. Finke, J. F. Meserly, and S. H. Lee-Bechtold, J. Chem. Thermodyn. 13, 345 (1981).

G. D. Gatta, L. Stradella, and P. Venturello, J. Solution Chem. 10, 209-220 (1981).

J. L. Hales, R. C. Cogman, and W. J. Frith, J. Chem. Thermodyn. 13, 591-601 (1981).

I. A. Hossenlopp and D. W. Scott, J. Chem. Thermodyn. 13, 405-414 (1981).

13, 415-421 (1981)

I. A. Hossenlopp and D. W. Scott, J. Chem. Thermodyn. 13, 423-428 (1981)

M. Inoue, Y. Arai, S. Saito, and J. Suzuki, J. Chem. Eng. Data 26, 287-293 (1981).

H.-J. Joo and W. Arlt, J. Chem. Eng. Data 26, 138-140 (1981).

M. S. Khanna, S. C. Khetarpal, K. Lal, and H. L. Bhatnagar, Ind. J. Chem. A 20, 544 (1981).

V. P. Kolesov, E. A. Kosarukina, D. Yu. Zhogin, M. E. Poloznikova, and Yu. A. Pentin, J. Chem. Thermodyn. 13, 115 (1981).

V. Majer, V. Svoboda, A. Posta, and J. Pick, Collect. Czech. Chem. Commun. 46, 817-822 (1981). 
1981MAN/SUN M. Mansson and S. Sunner, J. Chem. Thermodyn. 13, 671-675 (1981).

1981MAR/SAC V. S. Markovnik, A. I. Sachek, A. D. Peshchenko, O. V. Shvaro, and D. N. Andreevskii, Termodin. Org. Soedin. Gor'kii 10, 54-57 (1981).

1981MAS/OLE T. N. Masalitinova, T. P. Oleinikova, V. L. Ryadnenko, N. N. Kiseleva, and N. D. Lebedeva, Zh. Prikl. Khim. (Leningrad) 54, 1799-1802 (1981).

1981NAV/HAU P. Navard and J. M. Haudin, J. Therm. Anal. 22, 107-118 (1981).

1981PAP/ERA T. S. Papina, P. A. Erastov, and V. P. Kolevsov, J. Chem. Thermodyn. 13, 683 (1981).

1981PAV/BES F. Pavese and L. M. Besley, J. Chem. Thermodyn. 13, 1095 (1981).

1981SAB/MIN R. Sabbah and C. Minadakis, Thermochim. Acta 43, 269-277 (1981).

1981SHC/RUD M. M. Shchedrina, T. A. Rudol'fi, L. O. Mindlin, and K. K. Prilepskaya, Maslozhirovays Prom. 10, 33-40 (1981).

1981SHI/SAI M. Shimizu, Y. Saito, and K. Kusano, Preprints, 17th Conference on Chemical Thermodynamics and Thermal Analysis, Japan (1981), p. 50 as quoted in [1985MAJ/SVO].

1981SVO/CHA V. Svoboda, V. Charvatova, V. Majer, and J. Pick, Collect. Czech. Chem. Commun. 46, 2983-2988 (1981).

$1981 T E K / M A J \quad$ V. Tekac, V. Majer, V. Svoboda, and V. Hynek, J. Chem. Thermodyn. 13, 659-662 (1981).

1981 VAR/BUL R. M. Varushchenko, L. L. Bulgakova, P. S. Minzabekyants, and K. N. Makarov, Russ. J. Phys. Chem. 55, 1480-1482 (1981).

1981VAR/BUL2 R. M. Varushchenko, L. L. Bulgakova, and M. M. Ammar, Vestnik Moskov. Univer. Ser. 2 Khim. 22, 54 (1981).

1981 WIE/KOB $\quad$ S. A. Wieczorek and P. Kobayashi, J. Chem. Eng. Data 26, 8-11 (1981); 26, 11-13 (1981).

1981 WIL/JOH G. M. Wilson, R. H. Johnston, S.-C. Hwang, and C. Tsonopoulos, Ind. Eng. Chem., Process Des. Dev. 20, 94-104 (1981).

1981YAR/KAL N. L. Yarym-Agaev and V. P. Kalinichenko, Zh. Prikl. Khim. 54, 1172 (1982).

$1981 Z$ HO/KOS D. Yu. Zhogin, E. A. Kosarukina, V. P. Kolesov, I. P. Prokudin, and B. A. Mel'nichenko, Zh. Fiz. Khim. 55, 98 (1981).

1981ZHO/KOS2 D. Yu. Zhogin, E. A. Kosarukina, and V. P. Kolesov, Zh. Fiz. Khim. 55, 1955 (1981).

1982 AIR/CHA C. Airoldi, A. P. Chagas, and F. P. Assuncao, Can. J. Chem. 60, 2132-2136 (1982).

1982APP/VAN G. T. Appleton and W. A. Van Hook, J. Chem. Eng. Data 27, 363 (1982).

1982BAL/MRA M. Balty, S. Mraw, L. A. K. Staveley, A. H. Overs, M. C. Owen, R. K. Thomas, and J. W. White, Mol. Phys. 45, 1015-1034 (1982).

1982BYS K. Bystroem, J. Chem. Thermodyn. 14, 865-870 (1982).

1982BYS/MAN K. Bystrom and J. Mansson, J. Chem. Soc., Faraday Trans. 2 1982, 565-569.

1982CAM/REY M. Campos-Vallette, M. Rey-Lafon, and R. Lagnier, Chem. Phys. Lett. 89, 189 (1982).

1982CAS/FRA C. Castellari, R. Francesconi, and F. Comelli, J. Chem. Eng. Data 27, 156-158 (1982).

$1982 \mathrm{COL} / \mathrm{JIM} \quad$ M. Colomina, P. Jimenez, and C. Turrion, J. Chem. Thermodyn. 14, 779-784 (1982).

1982CUE/SOL M. A. Cuevas, X. Solans, and L. Artus, Afinidad 39, 406-408 (1982).

1982DEK/BLO C. G. Dekruif and J. G. Blok, J. Chem. Thermodyn. 14, 201-206 (1982).

1982DEK/SCH G. C. Dekruif, R. C. F. Schaake, J. C. Van Meltenburg, K. Van der Klauw, and J. G. Blok, J. Chem. Thermodyn. 14, 791-798 (1982).

1982DEM/FOX R. A. De Marco and W. B. Fox, J. Org. Chem. 47, 3772-3773 (1982).

1982DWO/FUC A. Dworkin, A. H. Fuchs, M. Ghelfenstein, and H. Szwarc, J. Phys., Lett. 43, L21-L27 (1982).

1982DZH/KAR O. I. Dzhafarov, K. A. Karasharli, and A. M. Kuliev, Azerb. Khim. Zh. 111 (1982).
1982FUC/HAL

1982FUC/PEA

1982FUR/SAK

1982GRA/FOS

1982GUT/KNA

1982HON/WAK

1982INA/MUR

1982INI/LOP

1982INV

1982JOC/DEK

1982JOC/DEK2

1982JOH/KAT

1982KAR/SHV

R. Fuchs, J. H. Hallman, and M. O. Perlman, Can. J. Chem. 60, 1832-1835 (1982).

R. Fuchs, L. A. Peacock, and W. K. Stephenson, Can. J. Chem. 60, 1953-1958 (1982).

J. Furukawa, M. Sakiyama, S. Seki, Y. Saito, and K. Kusano, Bull. Chem. Soc. Jpn. 55, 3329-3330 (1982).

B. T. Grayson and L. A. Fosbraey, Pestic. Sci. 13, 269-278 (1982).

B. Gutsche and H. Knapp, Fluid Phase Equilib. 8, 285-300 (1982).

G. S. Hong, R. Wakslak, H. Finston,
Eng. Data 27, 146-148 (1982).

S. Inagaki, S. Murata, and M. Sakiyama, Bull. Chem. Soc. Jpn. 55, 2808-2813 (1982).

J. C. Iniguez, M. E. Lopez, and D. M. McEachern, Rev. Soc. Quim. Mex. 26, 122-124 (1982); Chem. Abstr. 97, 144252 j (1982).

C. Invernizzi, Termotecnica 4, 78-85 (1982).

R. Jochems, H. Dekker, C. Mosselman, and G. Somsen, J. Chem. Thermodyn. 14, 395-398 (1982).

R. Jochems, H. Dekker, C. Mosselman, and G. Somsen, J. Chem. Thermodyn. 14, 799-800 (1982).

K. K. Hohri, Y. Katsuhara, and D. D. Desmarteau, J. Fluorine Chem. 19, 227 (1982). $\begin{array}{ll} & \text { N. V. Karyakin, K. G. Shvetsova, and E. S. Dzhari- } \\ \text { mova, Termodin. Org. Soedin. } 33 \text { (1982); Chem. Abstr. }\end{array}$ 99, 129172f (1983).

1982KAR/SHV2 N. V. Karyakin and K. G. Shvetsova, Deposited Doc. SPSTL 861, 1982, p. 14..

1982KOS/KOL E. A. Kosarukina, V. P. Kolesov, G. M. Kuramshina, and Y. A. Pentin, Termodin. Org. Soedin. 11 (1982).

1982KOS/ZHO E. A. Kosarukina, D. Yu. Zhogin, V. P. Kolesov, G. M. Kuramshina, Yu. A. Pentin, I. V. Izmest'ev, and A. V. Danilov, Zh. Fiz. Khim. 56, 1892 (1982).

1982KUL/LEB T. G. Kulagina, B. V. Lebedev, E. G. Kiparisova, E. B. Lyudvig, and I. G. Barskaya, Vysokom. Soedin. Ser. A 24, 1496 (1982).

1982LEB/BYK $\quad$ B. V. Lebedev, T. A. Bykova, N. N. Smirnova, and T. G. Kulagina, Zh. Obshch. Khim. 52, 2630-2636 (1982).

1982LEB/KUL $\quad$ B. V. Lebedev, T. G. Kulagina, Ye. B. Lyudvig, and T. N. Ovchinnikova, Vysokomol. Soedin. 24A, 1490 (1982).

1982MAR/AND J. F. Martin and R. J. L. Andon, J. Chem. Thermodyn. 14, 679-688 (1982).

1982MEN J. Menaucourt, J. Chim. Phys. Phys.-Chim. Biol. 79, 531-535 (1982).

1982MIN/SAB C. Minadakis and R. Sabbah, Thermochim. Acta 55, 147-159 (1982).

1982MUR/SAK S. Murata, M. Sakiyama, and S. Seki, J. Chem. Thermodyn. 14, 707-721 (1982); 14, 723-731 (1982).

1982PIL/SKI G. Pilcher and H. A. Skinner, in The Chemistry of the Metal-Carbon Bond, edited by F. R. Hartley and S. Patai (John Wiley Sons, New York, 1982).

1982POE/FAN G. Poeti, E. Faneli, and M. Braghetti, J. Therm. Anal. 24, 273-279 (1982)

1982ROO F. L. Rook, J. Chem. Eng. Data 27, 72-73 (1982).

1982SAB/TOR R. Sabbah and L. A. Torres Gomez, Thermochim. Acta 52, 285-295 (1982).

1982 SAC/PES A. I. Sachek, A. D. Peshenenko, V. S. Markovnik, O. V. Ral'ko, D. N. Andreevskii, and A. A. Leont'eva, Termodin. Org. Soedin. 94-98 (1982).

1982SCH/VAN R. C. F. Schaake, J. C. van Miltenberg, and C. G. De Kruif, J. Chem. Thermodyn. 14, 771 (1982).

1982SCH/VAN2 R. C. F. Schaake, J. C. van Miltenberg, and C. G. De Kruif, J. Chem. Thermodyn. 14, 763 (1982).

1982SUR/SAI S. Suradi, N. El Saiad, G. Pilcher, and H. A. Skinner, J. Chem. Thermodyn. 14, 45-50 (1982).

1982SVO/CHA V. Svoboda, V. V. Charvatova, V. Majer, and V. Hynek, Collect. Czech. Chem. Commun. 47, 543-549 (1982).

1982TOR/SAB L. A. Torres Gomez and R. Sabbah, Thermochim. Acta. 57, 67-81 (1982).

1982TOR/SAB2 L. A. Torres Gomez and R. Sabbah, Thermochim. Acta 58, 311-315 (1982). 
1982TOU/OKA H. Touhara, S. Okazaki, F. Okino, H. Tanaka, K. Ikari, and K. Nakanishi, J. Chem. Thermodyn. 14, 145-156 (1982).

1982TRE/VAU C. Treiner, C. Vaution, and G. N. Cave, J. Pharm. Pharmacol. 34, 539 (1982).

1982VAN/MUL A. A. Van Dooren and B. W. Muller, Thermochim. Acta 54, 115 (1982).

1982VAR/PUC R. M. Varushchenko, S. S. Puchkov, and A. I. Druzinina, Zh. Fiz. Khim. 56, 2934-2937 (1982).

1982WRE/VIK D. J. Wren and A. C. Vikis, J. Chem. Thermodyn. 14, 435-437 (1982).

1983ALT/PIL G. Al-Takhin, G. Pilcher, J. Bickerton, and A. A. Zaki, J. Chem. Soc., Dalton Trans. 1983, 2657.

1983AN/MAN X.-W. An and M. Mansson, J. Chem. Thermodyn. 15, 287 (1983).

1983BAR/BEC W. Barbe, H.-D. Beckhaus, H. J. Lindner, and C. Rüchardt, Chem. Ber. 116, 1017 (1983).

1983BEN/BIE R. Bender, V. Bieling, and G. Mauer, J. Chem. Thermodyn. 15, 585 (1983).

1983BRO/GAM J. P. Bros, M. Gambino, M. Gaune-Escard, and N. T. Huu, Calorim. Anal. Therm. 14, 92 (1983).

1983BYK/LEB T. A. Bykova and B. V. Lebedev, Fiz.-Khim. Osnovy Sinteza i Pererab. Polimerov 39 (1983).

1983CHA/HAL J. Chao, K. R. Hall, and J.-M. Yao, Thermochim. Acta 64, 1354 (1983).

1983CLA/COR R. M. Clay, S. Corr, G. Keenan, and W. V. Steele, J. Am. Chem. Soc. 105, 2070 (1983).

1983COL/JIM M. Colomina, P. Jimenez, C. Turrion, M. Kaminski, and W. Zielenkiewicz, Thermochim. Acta 68, 371 (1983).

1983DEW/BOW H. G. M. Dewit, J. G. Bowstra, and C. G. Dekruif, J. Chem. Phys. 78, 1470 (1983).

1983DEW/DEK H. G. M. Dewit, C. G. De Kruif, and J. C. Van Miltenburg, J. Chem. Thermodyn. 15, 891 (1983).

1983DEW/OFF H. G. M. Dewit, J. C. A. Offringa, C. G. Dekruif, and J. C. Miltenburg, Thermochim. Acta 65, 43 (1983).

1983DEW/VAN H. G. M. Dewit, J. C. Van Miltenburg, and C. G. Dekruif, J. Chem. Thermodyn. 15, 651 (1983).

1983 ELV/KUU A. Elvelt, M. Kuus, and L. S. Kudryavtseva, Eesti NSV Tead. Akad. Toim. Keem. 32, 172 (1983).

1983FOK/VAN J. G. Fokkens, J. G. M. Van Amelsfoort, C. J. De Blaey, C. G. De Kruif, and J. Wilting, Int. J. Pharm. 14, 79 (1983).

1983FUC/HAL R. Fuchs and J. H. Hallman, Can. J. Chem. 61, 503 (1983).

1983GEI/NUR Kh. I. Geidarov, G. G. Nurullaev, M. S. Salakhov, Z. Guseinov, and K. A. Karasharli, Azerb. Khim. Zh. 120 (1983).

1983HAL/STE J. H. Hallman, W. K. Stepenson, and R. Fuchs, Can. J. Chem. 61, 2044 (1983).

1983HOL M. R. Holdiness, Thermochim. Acta 68, 375 (1983).

1983KHA F. I. Khattab, Thermochim. Acta 61, 253 (1983).

1983KHA/KHE M. S. Khanna, S. C. Khetarpal, K. Lal, and H. L. Bhatnagar, Indian J. Pure Appl. Phys. 20, 503 (1983).

1983KHU/MUN J. R. Khurma, S. Munjal, and B. D. Smith, J. Chem. Eng. Data 28, 100 (1983).

1983LEB/YEV $\quad$ B. V. Lebedev and A. A. Yevstropov, J. Chem. Thermodyn. 15, 115 (1983).

1983MAC V. Machat, Ph.D. thesis, Utzcht Institut of Chemical Technology, Prague, 1983.

1983MAJ/AZZ J. R. Majer and A. S. P. Azzouz, J. Chem. Soc., Faraday Trans. 1 79, 675 (1983).

1983MAR/SHV V. S. Markovnik, O. V. Shvaro, A. I. Sachek, and A. D. Peshchenko, Izvest. Vyssh. Ucheb. Zaved. Khim. Khim. Technol. 26, 698 (1983).

1983NAT/VIS G. Natarajan and D. S. Viswanath, Rev. Sci. Instrum. 54, 1175 (1983).

1983OI/SHU T. Oi, J. Shulman, A. Popowicz, and T. Ishida, J. Phys. Chem. 87, 3153 (1983).

1983PAL/CHO M. Palczewska-Tulinska, J. Cholinski, A. M. Szafranski, and D. Wyrzkowska-Stankiewicz, Fluid Phase Equilib. 11, 233 (1983).

1983PRI/WOO J. G. Priest, E. M. Wooley, J. B. Ott, and J. R. Goates, J. Chem. Thermodyn. 15, 357 (1983).

1983RAB/KHL I. B. Rabinovich, T. B. Khlyustova, A. N. Mochalov, and N. Y. Kokurina, Zh. Fiz. Khim. 57, 2867 (1983).
1983RED/MUR

G. Om. Reddy, B. K. M. Murali, and A. K. Chatterjee, Propellants, Explos., Pyrotech. 8, 29 (1983).

1983SCH/STR T. Schmeling and R. Strey, Ber. Bunsen-Ges. Phys. Chem. 87, 871 (1983).

1983SEK/TSU K. Sekiguchi, Y. Tsuda, K. Chihara, and E. Suzuki, Yakugaku Zasshi 103, 213 (1983).

1983SKO/SAB S. Skoulika and R. Sabbah, Thermochim. Acta 61, 203 (1983).

1983SON/ZOL W. J. Sonnefeld, W. H. Zoller, and W. E. May, Anal. Chem. $\mathbf{5 5}, 275$ (1983).

1983SPE/CLI $\quad$ W. F. Spencer and M. M. Cliath, Residue Rev. 85, 57 (1983).

1983TAM/DRA A. Tamir, C. Dragoescu, A. Apelblat, and J. Wisniak, Fluid Phase Equilib. 10, 9 (1983).

1983TSO/WIL C. Tsonopoulos and G. M. Wilson, AIChE J. 29, 990 (1983).

1983UCH/MAJ V. Uchytilova, V. Majer, V. Svoboda, and V. Hynek, J. Chem. Thermodyn. 15, 853 (1983).

1983VAN/JAC P. J. Van Ekeren, M. H. G. Jacobs, J. C. A. Offringa, and C. G. J. Dekruif, J. Chem. Thermodyn. 15, 409 (1983).

1983 VAN/KAC Yu.Ya. Van-Chin-Syan, N. S. Kachurina, G. A. Petrovskaya, and S. K. Chuchmarev, Russ. J. Phys. Chem. 57, 1751 (1983).

1983VAS T. F. Vasel'eva, in Teplofiz, edited by G. I. Cherednichenko (Svoistva Uglevodororov Nefteprod, USSR, 1983), pp. 85-94.

1983WEA CRC Handbook of Chemistry and Physics, 64th ed., edited by R. C. Weast (CRC Press, Boca Raton, FL, 1983).

1983ZIE/ZIE A. Zielenkiewicz, W. Zielenkiewicz, M. Colomina, P. Jimenez, E. Cesari, A. Sanahiya, and V. Torra, An. Quim. Ser. A 79/3(Suppl. 2), 677 (1983); Chem. Abstr. 101, 22920f (1984).

1984BEC/RUC H.-D. Beckhaus, C. Rüchardt, and M. Smisek, Thermochim. Acta 79, 149 (1984).

1984BIC/MIN J. Bickerton, M. E. Minas da Piedade, and G. Pilcher, J. Chem. Thermodyn. 16, 661 (1984).

1984BIC/PIL J. Bickerton, G. Pilcher, and G. Al-Takhin, J. Chem. Thermodyn. 16, 373 (1984).

1984BOS/TUR S. R. Bosco and A. G. Turner, J. Chem. Eng. Data 29, 242 (1984).

1984BOU/FRI T. Boublik, V. Fried, and E. Hala, The Vapour Pressures of Pure Substances: Selected Values of the Temperature Dependence of the Vapour Pressures of Some Pure Substances in the Normal and Low Pressure Region, Second Revised Edition (Elsevier, Amsterdan, 1984), Note: The vaporization enthalpies were calculated from the vapor pressures obtained from the Antoine constants reported in this compendium. In cases where the Antoine constant $\mathrm{C}=0$ the Antoine Equation $(\log 10 \mathrm{P}=\mathrm{A} \mathrm{B} /(\mathrm{C}+\mathrm{T}))$ reduces to the integrated form of the Clausius Clapeyron equation directly. This was the case for many vaporization enthalpies. In those cases where $\mathrm{C}^{1} 0$, the vaporization enthalpy was calculated as DvapHm $(\mathrm{T})=2303 \mathrm{R} \mathrm{B}[\mathrm{T} / \mathrm{T}$ $+\mathrm{C})] 2$.

1984BRU/REI S. Bruns, J. Reichelt, and H. K. Cammenga, Thermochim. Acta 72, 31 (1984).

1984BUR/MOR P. M. Burkinshaw and C. T. Mortimer, J. Chem. Soc., Dalton Trans. 1984, 75.

1984CAR

1984CAS/FRA

A. S. Carson, J. Chem. Thermodyn. 16, 427 (1984).

1984CAS/FRA2 $\quad$ C. Castellari, R. Francesconi, and F. Comelli, J. Chem. Eng. Data 29, 90 (1984).

1984CAS/FRA3 C. Castellari, R. Francesconi, F. Comelli, and S. Ottani, J. Chem. Eng. Data 29, 283 (1984).

1984CER/BOU I. Cervenkova and T. Boublik, J. Chem. Eng. Data 29, 425 (1984).

1984CHO/GO K. Y. Chow, J. Go, M. Mehdizadeh, and D. J. W. Grant, Int. J. Pharm. 20, 3 (1984).

1984COL/JIM M. Colomina, P. Jimenez, M. V. Roux, and C. Turrion, J. Chem. Thermodyn. 16, 379 (1984).

1984COL/JIM2 M. Colomina, P. Jimenez, M. V. Roux, and C. Turrion, J. Chem. Thermodyn. 16, 1121 (1984). 
1984DOM/EVA E. S. Domalski, W. H. Evans, and E. D. Hearing, J. Phys. Chem. Ref. Data 13(Suppl. 1), 1-286 (1984).

1984EBE/FRA H. Ebeling and E. U. Franck, Ber. Bunsen-Ges. Phys. Chem. 88, 862 (1984).

1984ENG/SAN R. Eng and S. I. Sandler, J. Chem. Eng. Data 29, 156 (1984).

1984EUB/CED P. T. Eubank, L. E. Cediel, J. C. Holste, and K. R. Hall, J. Chem. Eng. Data 29, 389 (1984).

1984FER/DEL E. Fernandez-Fassnacht and F. Del Rio, J. Chem. Thermodyn. 16, 469 (1984).

1984GOL/KOL Y. G. Golovanova and V. P. Kolesov, Vestn. Mosk. Univ. Ser. 2 Khim. 24, 244 (1984).

1984HES/WIS W. Hessler, Wiss. Z. Wilhelm-Pieck-Univ. Rostich 33, 9 (1984).

1984HUL/LU D. M. Hull and B.C.-Y. Lu, J. Chem. Eng. Data 29, 417 (1984).

1984KAT/HAR H. Katayama and Y. Harada, J. Chem. Eng. Data 29, 373 (1984).

1984KAU/RUT $\quad$ W. F. Kaukler and J. Rutter, Mater. Sci. Eng. 65, L1 (1984).

1984KIM/WOO Y. H. Kim, J. E. Woodrow, and J. N. Seiber, J. Chromatogr. 314, 37 (1984).

1984KOZ/TIM M. P. Kozina, L. P. Timofeeva, G. L. Gal'chenko, E. S. Balenkova, and M. D. Ordubadi, Vestn. Mosk. Univ. Ser. 2 Khim. 25, 364 (1984); Chem. Abstr. 101, 190955q (1984).

1984LEB/GUT N. D. Lebedeva, N. M. Gutner, N. M. Katin, Yu. A. Yu, N. M. Kozlova, N. N. Kiseleva, E. F. Machinya, and S. L. Dobychin, Zh. Prikl. Khim. (Lenningrad) 57, 2297 (1984).

1984LEB/GUT2 N. V. Lebedeva, N. M. Gutner, Yu. A. Katin, N. M. Kozlova, N. N. Kiseleva, E. F. Makhina, and S. L. Dobychin, J. Appl. Chem. (USSR) 57, 2118 (1984).

$1984 \mathrm{MAJ} / \mathrm{SVO} \quad$ V. Majer, V. Svoboda, and V. Hynek, J. Chem. Thermodyn. 16, 1059 (1984).

1984MAJ/SVO2 V. Majer, V. Svoboda, and M. Lencka, J. Chem. Thermodyn. 16, 1019 (1984).

1984MAJ/SVO3 V. Majer, V. Svoboda, J. Pechacek, and S. Hala, J. Chem. Thermodyn. 16, 567 (1984).

1984MAJ/UCH V. Majer, V. Uchytilova, V. Svoboda, and A. Posta, J. Chem. Thermodyn. 16, 761 (1984).

1984MIC/JOS M.-A. Michou-Saucet, J. Jose, C. Michou-Saucet, and J. C. Merlin, Thermochim. Acta 75, 85 (1984).

1984MIL/GHO M. M. Miller, S. Ghodbane, S. P. Wasik, Y. B. Tewari, and D. E. Martire, J. Chem. Eng. Data 29, 184 (1984).

1984MOU A. H. N. Mousa, J. Fluorine Chem. 25, 165 (1984).

1984NUR/MEK G. G. Nurullaev, S. A. Mekhtiev, K. A. Karasharli, N. F. Musaeva, and M. S. Salakhov, Azerb. Khim. Zh. 132 (1984).

1984PAL/CHO M. Palczewska-Tulinska, J. Cholinski, A. M. Szafranski, and D. Wyrzkowska-Stankiewicz, Fluid Phase Equilib. 15, 295 (1984).

1984PIN/POS N. Pingel, U. Poser, and A. Wurflinger, J. Chem. Soc., Faraday Trans. 1 80, 3221 (1984).

1984PUT/IVA S. Putcha, R. V. Ivanturi, and R. Machiraju, J. Chem. Eng. Data 29, 135 (1984).

1984RIB/RIB2 M. D. M. C. Ribeiro Da Silva, M. A. V. Ribeiro Da Silva, and G. Pilcher, J. Chem. Thermodyn. 16, 1149 (1984).

1984SAC/MAR A. I. Sachek, V. S. Markovnik, A. D. Peshchenko, A. V. Shvaro, and D. N. Andreevskii, Khim. Prom.-St. (Moscow) 337 (1984).

1984SHA/BAS $\quad$ B. L. Sharma and P. S. Bassi, Ind. J. Chem. A 23, 303 (1984).

1984 SHC/KAP A. E. Shcherbina, L. M. Kaporovskii, and E. A. Shcherbina, Zh. Prikl. Khim. (Leningrad) 57, 1910 (1984); Chem. Abstr. 101, 177895a (1984).

1984SIP/WIE J. T. Sipowska and S. A. Wieczorek, J. Chem. Thermodyn. 16, 693 (1984).

1984STA/WAD P. Starzewski, I. Wadso, and W. Zielenkiewicz, J. Chem. Thermodyn. 16, 331 (1984).

1984TSE/YAO W. S. Tse, Y. D. Yao, and W. W. Lin, Annu. Rep. Inst. Phys., Acad. Sin. 14, 65 (1984).
1984UCH/MAJ V. Uchytilova, V. Majer, V. Svoboda, and I. Hemer, J. Chem. Thermodyn. 16, 475 (1984).

1984VAS/PET I. A. Vasil'ev and V. M. Petrov, Thermodynamic Properties of Oxygen-Containing Organic Compounds (Leningrad Khimya Linengradskoe, Soedinenii, 1984).

1984WEI/LEF $\quad$ D. I. Weinstein, A. J. Leffler, and J. A. Currie, Mol. Cryst. Liq. Cryst. 104, 95 (1984).

1984WIS/TAM J. Wisniak and A. Tamir, J. Chem. Eng. Data 29, 19 (1984).

1984ZIE/ZIE $\quad$ A. Zielenkiewicz, W. Zielenkiewicz, L. F. Sukhodub, O. T. Glukhova, A. B. Teplitsky, and K. L. Wierzchowski, J. Solution Chem. 13, 757 (1984).

1984ZIE/ZIE2 A. Zielenkiewicz, W. Zielenkiewicz, and S. Malanowski, Thermochim. Acta 74, 95 (1984).

1985APP/MEN E. H. Appelman, M. H. Mendelsohn, and H. Kim, J. Am. Chem. Soc. 107, 6515 (1985).

1985BAR/CAS G. Barone, G. Castronuovo, G. Della Gatta, V. Elia, and A. Iannone, Fluid Phase Equilib. 21, 157 (1985).

1985BRO/INI W. Brostow, J. C. Iniguez, M. A. Shmorhun, and J. A. Valdez, Mater. Chem. Phys. 12, 557 (1985).

1985CAO/BAC J.-R. Cao and R. A. Back, Can. J. Chem. 63, 2945 (1985).

1985COL/JIM M. Colomina, P. Jimenez, M. V. Roux, and C. Turrion, J. Chem. Thermodyn. 17, 1091 (1985).

1985DEA J. A. Dean, Lange's Handbook of Chemistry, 13th ed. (McGraw-Hill, New York, 1985).

1985DOB/CRA $\quad$ B. M. Dobratz and P. L. Crawford, Explosives Handbook: Principles of Chemical Explosives and Explosive Simulants (Lawrence Livermore National Laboratory, Livermore, CA, 1985).

1985DOM/HOF U. Domańska and T. Hofman, J. Solution Chem. 14, 531 (1985).

1985FER/PIA D. Ferro and V. Piacente, Thermochim. Acta 90, 387 (1985).

1985FIG/SZW P. Figuiere, H. Szwarc, M. Oguni, and H. Suga, J. Chem. Thermodyn. 17, 949 (1985).

1985FRA/COM R. Francesconi and P. Comelli, J. Chem. Eng. Data 30, 352 (1985).

1985GLU/ARK O. T. Glukhova, N. M. Arkhangelova, A. B. Teplitsky, L. F. Sukhodub, I. K. Yanson, and M. Kaminski, Thermochim. Acta 95, 133 (1985).

1985HAN Y. P. Handa, Can. J. Chem. 63, 68 (1985).

1985KAI/HAD E. Kaiserberger, W. Hadrich, and W.-D. Emmerich, Thermochim. Acta 95, 331 (1985)

1985KAM/ZIE M. Kaminski and W. Zielenkiewicz, Calorim. Anal. Therm. 16, 281 (1985); Chem. Abstr. 104, 96844t (1986).

1985KAR/ABD M. K. Karabaev, T. P. Abduzhaminov, M. M. Kenisarin, and A. A. Saidov, Izv. Akad. Nauk Uzb. SSR Ser. Fiz-Mat. Nauk 74 (1985).

1985KIS/VEI V. D. Kiselev, E. A. Veisman, B. N. Solomonov, and A. I. Konovalov, Zh. Obsh. Khim. 55, 1965 (1985).

1985KUS K. Kusano, Thermochim. Acta 88, 109 (1985).

1985LEB/BYK $\quad$ B. V. Lebedev, T. A. Bykova, E. G. Kiparisova, Y. A. Chernomordik, A. S. Kurapov, and V. A. Sergeev, Bull. Acad. Sci. USSR, Div. Chem. Sci. 34, 274 (1985).

$1985 \mathrm{MAJ} / \mathrm{SVO} \quad$ V. Majer and V. Svoboda, Enthalpies of Vaporization of Organic Compounds: A Critical Review and Data Compilation (Blackwell Scientific Publishers, Oxford, 1985).

1985MAJ/SVO2 V. Majer, V. Svoboda, and M. Lencka, J. Chem. Thermodyn. 17, 365 (1985).

1985 MAJ/SVO3 V. Majer, V. Svoboda, V. Uchytilova, and M. Finke, Fluid Phase Equilib. 20, 111 (1985).

1985MAR/MAN K. N. Marsh and M. Mansson, J. Chem. Thermodyn. 17, 995 (1985).

1985MAR/WU A. Martin, P. L. Wu, and T. Velasquez, J. Pharm. Sci. 74, 277 (1985).

1985MAT/KUW2 N. Matsubara and T. Kuwamoto, Thermochim. Acta 83 193 (1985).

1985MOU A. H. N. Mousa, J. Fluorine Chem. 30, 29 (1985).

1985MUK/CHA R. Mukhopadhyay, S. L. Chaplot, and K. R. Rao, Phys. Status Solidi A 92, 467 (1985).

1985MUR/SAK S. Murata, M. Sakiyama, and S. Seki, Thermochim. Acta 88, 121 (1985). 
1985NAT/VIS G. Natarajan and D. S. Viswanath, J. Chem. Eng. Data 30, 137 (1985).

1985OHM/LIP A. Ohm and B. C. Lippold, Int. J. Pharm. Tech. Prod. Mfg. 6, 1 (1985).

1985OZA

1985PAS/VAR

T. Ozawa, Thermochim. Acta 92, 27 (1985).

L. L. Pashchenko and R. M. Varushchenko, Zh. Obsh. Khim. 55, 721 (1985).

1985PIA/SCA V. Piacente, P. Scardala, D. Ferro, and R. Gigli, J. Chem. Eng. Data 30, 372 (1985).

1985PRI/PUC V. P. Privalko, G. A. Puchkovskaya, E. N. Shermatov, and A. A. Yakubov, Mol. Cryst. Liq. Cryst. 126, 289 (1985).

1985RAV/RAO A. Raviprasad and K. V. Rao, J. Chem. Thermodyn. 17, 117 (1985).

1985RED/RAO K. D. Reddy, M. V. P. Rao, and M. Ramakrishna, J. Chem. Eng. Data 30, 394 (1985).

1985ROR B. F. Rordorf, Thermochim. Acta 85, 435 (1985).

1985SCH/BRU A. G. R. Scholz and E. Brunner, J. Chem. Eng. Data 30, 72 (1985).

1985SHE/SAN G. S. Shealy and S. I. Sandler, J. Chem. Eng. Data 30, 455 (1985).

1985SIN

1985SKI/PIL

1985SRI/SMI

$1985 \mathrm{SUZ} / \mathrm{SHI}$

1985WIB/WAS

N. Singh, Bull. Soc. Chim. Fr. 626 (1985). comment to, M. Faour, and T. S. Akasheh, J. Chem. Soc. Perkin Trans. II 811 (1985).

R. Srivastava and B. D. Smith, J. Chem. Eng. Data 30, 313 (1985).

E. Suzuki, K.-I. Shirotani, Y. Tsuda, and K. Sekiguchi, Chem. Pharm. Bull. 33, 5028 (1985).

$\begin{array}{ll}\text { 1985WIE/SIP } & \text { Murcko, J. Am. Chem. Soc. 107, } 6019 \text { (1985). } \\ \text { S. Aieczorek and J. T. Sipowska, J. Chem. Thermodyn. }\end{array}$ 17, 255 (1985).

1985WOR/YER C. J. Wormald and T. K. Yerlett, J. Chem. Thermodyn. 17, $1171(1985)$

1985YAN/GU H. K. Yan, J. K. Gu, and R. H. Fu, Wuli Huaxue Xuebao 1, 543 (1985).

1985ZAR A. P. Zaraiskii, Zh. Fiz. Khim. 59, 2087 (1985).

1986AIR/SIL C. Airoldi, M. L. C. P. Silva, and A. P. Chagas, J. Chem. Soc., Dalton Trans. 1986, 1913.

1986ALL/JOS N. Allemand, J. Jose, and J. C. Merlin, Thermochim. Acta 105, 79 (1986).

1986BAL/GNA V. Baliah and K. Gnanasekaran, Indian J. Chem. 25A, 673 (1986).

1986BAR/FER G. Barone, D. Ferro, P. Fiorani, C. A. Mattia, and R. Puliti, Journ. Calorim., Anal. Therm. Thermodyn. Chim. 17, 471 (1986).

1986CAL/SUL J. E. Callanan, S. A. Sullivan, and D. F. Vecchia, J. Res. Natl. Bur. Stand. 91, 123 (1986)

1986CHA/DEM A. Chuvet, G. De Maury, A. Terol, and J. Masse, Thermochim. Acta 97, 143 (1986).

1986COL/JIM M. Colomina, P. Jimenez, M. V. Roux, and C. Turrion, An. Quim. 82, 126 (1986).

1986DMI/KAC Yu. G. Dmitriev, N. S. Kachurina, C. H. Wang, and V. V. Kouchukei, Vestn. L'vov. Politekh. Inst. 201, 29 (1986).

1986EMO/NAU H. H. Emons, R. Naumann, K. Jahn, and H.-J. Flammersheim, Thermochim. Acta 104, 127 (1986).

1986GON/SZW A. Gonthier-Vassal and H. Szwarc, Chem. Phys. Lett. 129, 5 (1986).

1986HES/LIC W. Hessler and W. Lichtenstein, Wiss. Z. Wilhelm-PieckUniv. Rostich 35, 27 (1986).

1986JIM/ROU P. Jimenez, M. V. Roux, C. Turrion, and F. Gomis, Journ. Calorim., Anal. Therm. Thermodyn. Chim. 17, 469 (1986).

1986KOZ/DAL A. A. Kozyro, S. V. Dalidovich, and A. P. Krausulin, Zh. Prikl. Khim. 59, 1456 (1986).

1986KRA/KOZ A. P. Krasulin, A. A. Kozyro, and S. V. Dalidovich, Zh. Fiz. Khim. 60, 2580 (1986).

1986KRA/KOZ2 A. P. Krasulin and A. A. Kozyro, Proceedings of the 11th All-Union Conference on Calorimetry and Chemical Thermodynamics (Institute of Neorganicheskoj Khimii, Novosibirsk, 1986), Vol. 17, p. 129 , as quoted in [1990PIA/FER].
1986KRE/PRA

D. H. Krevor and J. M. Prausnitz, J. Chem. Eng. Data 31, 349 (1986).

1986MEY/MEY E. F. Meyer and J. J. Meyer, J. Chem. Eng. Data 31, 273 (1986).

1986MIC/JOS M.-A. Michou-Saucet, J. Jose, and C. Michou-Saucet, Thermochim. Acta 102, 271 (1986).

1986NIL/WAD S.-O. Nilsson and I. Wadso, J. Chem. Thermodyn. 18, 673 (1986).

1986NOV/RAB N. V. Novoselova, I. B. Rabinovich, L. Ya. Tsvetkova, E. M. Moseeva, and A. G. Babinkov, Zh. Fiz. Khim. 60, 1627 (1986).

1986NUN/BAR L. Nunez, L. Barral, L. S. Gavelanes, and G. Pilcher, J. Chem. Thermodyn. 18, 575 (1986).

1986OBR/LAM R. A. O’Brien, W. Y. Lam, and D. D. Desmarteau, J. Org. Chem. 51, 4466 (1986).

1986PAU/KRU H.-I. Paul, J. Krug, and H. Knapp, Thermochim. Acta 108, 9 (1986).

1986PAV/PES A. L. Pavlov, A. D. Peschenko, and V. I. Zubkov, Termodin. Org. Soedin. Gorky 85, 77 (1986).

1986PED/NAY J. P. Pedley, R. D. Naylor, and S. P. Kirby, Thermochemical Data of Organic Compounds, 2nd ed. (Chapman and Hall, New York, 1986).

1986PIM/DOM S. M. Pimenova, I. N. Domnin, A. M. Lakshin, and V. V. Takhistov, Abstract of Papers XI All-Union Conference on Calorimetry and Thermodynamics (Novosibirsk, 1986), Vol. 2, p. 41.

1986RAB/NOV I. B. Rabinovich, I. V. Novoselova, E. M. Moiseeva, A T. Babinkov, and A. T. Tsvetkova, Zh. Fiz. Khim. 60, 545 (1986).

1986RIB/RIB M. D. M. C. Ribeiro Da Silva, M. A. V. Ribeiro Da Silva, and G. Pilcher, J. Chem. Thermodyn. 18, 295 (1986).

1986ROR/SAR $\quad$ B. F. Rordorf, L. P. Sarna, and G. R. B. Webster, Chemosphere 15, 2073 (1986).

1986SAT/INO N. Sato, H. Inomata, K. Arai, and S. Saito, J. Chem. Eng. Jpn. 19, 145 (1986).

1986SIN/KUM N. B. Singh and P. Kumar, J. Chem. Eng. Data 31, 406 (1986).

1986STA H. W. Starkweather, Jr., Macromolecules 19, 1131 (1986).

1986TSV/NAZ V. S. Tsvetkov, A. G. Nazmutdinov, K. S. Sharonov, and A. M. Rozhnov, Termodin. Org. Soedin. 71 (1986).

1986VAR R. M. Varushchenko, Zh. Fiz. Khim. 60, 291 (1986).

1986WEN/SCH U. Wenzel and G. M. Schneider, Thermochim. Acta 109 , 111 (1986).

1986WIS/LEN B. Wisniewska, M. Lencka, and M. Rogalski, J. Chem. Thermodyn. 18, 703 (1986).

1986YER/WOR Y. K. Yerlett and C. J. Wormald, J. Chem. Thermodyn. 18, 719 (1986)

1986YER/WOR2 T. K. Yerlett and C. J. Wormald, J. Chem. Thermodyn. 18, 371 (1986)

1987ALL/FIN $\quad$ P. H. Allot, A. Finch, G. Pilcher, L. Numez, and L. Barral, J. Chem. Thermodyn. 19, 771 (1987).

1987ALN/ALS H. Al-Najjar and D. Al-Sammerrai, J. Chem. Tech. Biotechnol. 37, 145 (1987).

1987AMB/GHI D. Ambrose and N. B. Ghiassee, J. Chem. Thermodyn. 19, 911 (1987).

1987AMB/GHI2 D. Ambrose and N. B. Ghiassee, J. Chem. Thermodyn. 19, 903 (1987).

1987AMB/GHI3 D. Ambrose and N. B. Ghiassee, J. Chem. Thermodyn. 19, 505 (1987).

1987AN/HU O. An, R. Hu, H. Wang, M. Wu, and Y. Zou, Acta Phys.Chim. Sin. 3, 668 (1987).

1987AN/ZHU X. An, I. Zhu, and R. Hu, Thermochim. Acta 121, 473 (1987).

1987ATI/SAI Z. Atik, Y. Saito, and K. Kusano, J. Chem. Thermodyn. 19, 99 (1987).

1987AZA E. C. Azandegbe, Analusis 15, 370 (1987).

1987CHI J. S. Chickos, in Molecular Structure and Energetics, Volume 2, Physical Measurements, edited by J. F. Liebman and A. Greenberg (VCH Publishers, New York, 1987).

1987COL/JIM M. Colomina, P. Jimenez, R. Perez-Ossorlo, M. V. Roux, and C. Turrion, J. Chem. Thermodyn. 19, 155 (1987). 
1987COL/JIM2 M. Colomina, P. Jimenez, M. V. Roux, and C. Turrion, J. Chem. Thermodyn. 19,1139 (1987).

1987CUR/ASR S. A. Curran and J. Asrar, Mol. Cryst. Liq. Cryst. 148, 255 (1987).

1987DAU/JAL T. E. Daubert, J. W. Jalowka, and V. Goren, AIChE Symp. Ser. 83, 128 (1987).

1987DEL/FER G. Della Gatta and D. Ferro, Thermochim. Acta 122, 143 (1987).

1987EBI/ASK Y. Ebisuzaki, L. H. Askari, A. M. Bryan, and M. F. Nicol, J. Chem. Phys. 87, 6659 (1987).

1987EDE/WUR R. Edelmann and A. Wurflinger, Mol. Cryst. Liq. Cryst. 148, 249 (1987).

1987ELS N. I. El-Sayed, Indian J. Technol. 25, 28 (1987).

1987FER/DEL D. Ferro and G. Della Gatta, Thermochim. Acta 122, 189 (1987).

1987FER/DEL2 D. Ferro, G. Della Gatta, and V. Piacente, J. Chem. Thermodyn. 19, 915 (1987).

1987FER/PIL M. L. C. C. H. Ferrao and G. Pilcher, J. Chem. Thermodyn. 19, 543 (1987).

1987FIO/FER P. Fiorani and D. Ferro, Thermochim. Acta 112, 387 (1987).

1987FUC/CHA R. Fuchs, E. J. Chambers, and W. K. Stepenson, Can. J. Chem. 65, 2624 (1987).

1987GAM/GAU M. Gambino, P. Gaune, M. Habavian, M. Gaune-Escard, and J. P. Bros, Thermochim. Acta 111, 37 (1987).

1987GAR/TRE F. Garcia-Sanchez and A. Trejo, J. Chem. Thermodyn. 19, 359 (1987).

1987GOA/BOE J. R. Goates, J. Boerio-Goates, S. R. Goates, and J. B. Ott, J. Chem. Thermodyn. 19, 103 (1987).

1987HAU/WU T. Hauschild, H. S. Wu, and S. I. Sandler, J. Chem. Eng. Data 32, 226 (1987).

1987JIM/ROU P. Jimenez, M. V. Roux, C. Turrion, and F. Gomis, J. Chem. Thermodyn. 19, 985 (1987).

1987KAF/DOR V. V. Kafarov, I. N. Dorokhov, V. N. Vetokhin, and L. P. Volkov, Dokl. Phys. Chem. 298, 77 (1987).

1987KAM/ZIE M. Kaminski and W. Zielenkiewicz, Bull. Pol. Acad. Sci., Chem. 35, 583 (1987).

1987KAN/OI A. Kanungo, T. Oi, A. Popowicz, and T. Ishida, J. Phys. Chem. 91, 4198 (1987).

1987KHO/BUG M. G. Khodzhaeva, Yu.V. Bugakov, and T. S. Ismailov, Khim.-Farm. Zh. 21, 760 (1987).

1987KLA/MOH S. M. Klara, R. S. Mohamed, D. M. Dempsey, and G. D. Holder, J. Chem. Eng. Data 32, 143 (1987).

1987KNE/ZON P. Kneisi and J. W. Zondio, J. Chem. Eng. Data 21, 11 (1987).

1987KRA/KOZ A. P. Krasulin, A. A. Kozyro, and G. Ya. Kabo, Zh. Prikl. Khim. 60, 104 (1987).

1987MEI/DOG M. Meier, B. Dogan, H. D. Beckhaus, and C. Rüchardt, New J. Chem. 11, 1 (1987).

1987MIH/BAS P. Mihailovic, P. Bassoul, and J. Simon, Chem. Phys. Lett. 141, 462 (1987).

1987MIL/FEN P. L. Mills and R. L. Fenton, J. Chem. Eng. Data 32, 266 (1987).

1987OTT/WOO J. B. Ott, B. F. Woodfield, C. Guanquan, J. Boerio-Goates, and J. R. Goates, J. Chem. Thermodyn. 19, 177 (1987).

1987OYU/BRI Y. Oyumi and T. B. Brill, Thermochim. Acta 116, 125 (1987).

1987SAL/SAI $\quad$ S. R. Salman, E. Z. Said, and K. F. Abas, Thermochim. Acta 111, 21 (1987).

1987SHI/OHK T. Shimizu, S. Ohkubo, M. Kimura, I. Tabata, and T. Hori, J. Soc. Dyers Colour. 103, 132 (1987).

1987SMI/TER G. G. Smirnova, G. F. Tereshchenko, and M. A. Blagushina, Zh. Prikl. Khim. 62, 182 (1987).

1987STE/MAL R. M. Stephenson and S. Malanowski, Handbook of the Thermodynamics of Organic Compounds (Elsevier, New York, 1987), Note: The data in this listing was obtained directly from this compendium which does not contain literature references. The sublimation enthalpies were calculated from the vapor pressures obtained from the Antoine constants reported in this compendium. In cases where the Antoine constant $\mathrm{C}=0$ the Antoine Equation $(\log 10 \mathrm{P}=\mathrm{A}-\mathrm{B} /(\mathrm{C}+\mathrm{T}))$ reduces to the integrated form of the Clausius Clapeyron equation directly. This was the case for most sublimation enthalpies. In those cases where this condition was not met, vapor pressures were calculated over a $30 \mathrm{~K}$ temperature range closest to $\mathrm{T}=$ $298 \mathrm{~K}$ and the temperature dependence was reformulated in terms of the integrated form of the Clausius Clapeyron equation.

1987TAN/YE Z. Tan, J. Ye, A. Yin, S. Chen, and W. Wang, Kexue Tongbao 32, 240 (1987).

1987TRE/LU T. Treszczanowicz and B. C.-Y. Lu., J. Chem. Thermodyn. 19, 391 (1987).

1987VAN/KAC Yu.Ya. Van-Chin-Syan and N. S. Kachurina, Russ. J. Phys. Chem. 61, 622 (1987).

1987VAN/VAN J. C. van Miltenburg, G. J. K. van den Berg, and M. J. van Bommel, J. Chem. Thermodyn. 19, 1129 (1987).

1987VAR/LOS R. M. Varushchenko, O. L. Loseva, and A. I. Druzhinina, Zh. Fiz. Khim. 61, 31 (1987).

1987VAR/LOS2 R. M. Varushchenko, O. L. Loseva, and A. I. Druzhinina, Zh. Fiz. Khim. 61, 638 (1987).

1987YAN/GU H. Yan, J. Gu, X. An, and R.-H. Hu, Huaxue Xuebao 45 1184 (1987).

1988AFZ/BUT M. Afzal, P. K. Butt, and M. J. Iqbal, Pak. J. Sci. Ind. Res. 31, 404-407 (1988).

1988 AMB/GHI2 $\quad$ D. Ambrose and N. B. Ghiassee, J. Chem. Thermodyn. 20, 765-766 (1988).

1988AMB/GHI3 D. Ambrose, N. B. Ghiassee, and R. Tuckerman, J. Chem. Thermodyn. 20, 767-768 (1988).

1988ASH S. J. Ashcroft, J. Chem. Eng. Data 33, 73 (1988).

1988ASK/DAU $\quad$ C. F. Askonas and T. E. Daubert, J. Chem. Eng. Data 33, 225-229 (1988).

1988BAG/GUR A. K. Baglay, L. L. Gurarly, and G. G. Kuleshov, J. Chem. Eng. Data 33, 512-518 (1988).

1988BAS/NIL M. Bastos, S. O. Nilsson, M. D. M. C. Ribeiro Da Silva, M. A. V. Ribeiro Da Silva, and J. Wadso, J. Chem. Thermodyn. 20, 1353-1359 (1988).

1988DAV/EWI D. R. M. Davies, B. Ewing, J. A. Hugill, and M. L. McGlashan, Can. J. Chem. 66, 760-762 (1988).

1988DON/LIN J.-Q. Dong, R.-S. Lin, and W.-H. Yen, Can. J. Chem. 66, 783-790 (1988).

1988FER/DEL D. Ferro, G. Della Gatta, and G. Barone, Therm. Anal. 34, 835-841 (1988).

1988GAL/GON F. Galan-Estella, J. Gonzalez-Julian, and P. Aquado-Rodriquez, Phytochemistry 27, 3069-3073 (1988); Chem. Abstr. 110, 102153h (1989).

1988GAM/BRO M. Gambino and J. P. Bros, Thermochim. Acta 127, 223 (1988).

1988GOL/SIT O. F. Golovanova, G. V. Sitonina, V. I. Pepekin, and F. I. Korsunskii, Izv. Akad. Nauk. SSSR Ser. Khim. 1012-1016 (1988); Chem. Abstr. 110, 38466j (1989).

1988HOS/ARC I. A. Hossenlopp and D. G. Archer, J. Chem. Thermodyn. 20, 1061-1068 (1988).

1988IMA/MUR A. Imamura, S. Murata, and M. Sakiyama, J. Chem. Thermodyn. 20, 389-396 (1988).

1988ISM/GAB T. S. Ismailov, N. R. Gabzalilova, and Kh. M. Makhkamov, Uzbekskii Khim. Zh. 4, 48-50 (1988).

1988KAT2 H. Katayama, J. Chem. Eng. Data 33, 75-77 (1988).

1988KHU V. L. Khudyakov, Russ. J. Phys. Chem. 62, 1743-1744 (1988).

1988KNA/SAB P. Knauth and R. Sabbah, Bull. Soc. Chem. Fr. 834-836 (1988).

1988LEB/BYK $\quad$ B. V. Lebedev, T. A. Bykova, E. G. Kiparisova, M. Frenkel, A. M. Fainleib, and V. A. Pankratov, Bull. Acad. Sci. USSR, Div. Chem. Sci. 37, 1082 (1988).

1988LEB/KUL $\quad$ B. V. Lebedev, T. G. Kulagina, and N. N. Smirnova, J. Chem. Thermodyn. 20, 1383-1396 (1988).

1988LEB/VAS B. V. Lebedev and V. G. Vasil'ev, Zh. Fiz. Khim. 62, 3099 (1988).

1988LET/SEW T. M. Letcher, J. Sewry, and S. W. Orchard, J. Chem. Thermodyn. 20, 1231-1232 (1988).

1988LIC/RIT H. H. Licht and H. Ritter, Propellants, Explos., Pyrotech. 13, 25-29 (1988).

1988LUS/RUB V. N. Lushnikov, Y. I. Rubstov, L. T. Dremenko, and A. M. Korolev, Zh. Fiz. Khim. 62, 1209 (1988). 
1988MES/FIN

J. F. Messerly, H. L. Finke, W. D. Good, and B. Gammon, J. Chem. Thermodyn. 20, 485-501 (1988).

1988MES/TOD J. F. Messerly, S. S. Todd, H. L. Finke, W. D. Good, and B. E. Gammon, J. Chem. Thermodyn. 20, 209 (1988).

1988 MIR/KOR E. A. Miroshnichenko, L. I. Korchatova, V. P. Shelaputina, S. A. Zyuz'kevich, and Yu. A. Lebedev, Bull. Acad. Sci. USSR, Div. Chem. Sci. 37, 1778 (1988).

1988NUN/BAR L. Nunez, L. Barral, and G. Pilcher, J. Chem. Thermodyn. 20, 1211-1216 (1988).

1988PAR/KAW R. M. Paroli, N. T. Kawai, I. S. Butler, and D. F. R. Gilson, Can. J. Chem. 66, 1973-1978 (1988).

1988PAU/KRU H. I. Paul, J. Krug, and H. Knapp, J. Chem. Eng. Data 33, 435-460 (1988).

1988PES/SHV A. D. Peschenko, O. V. Shvaro, and V. I. Zubkov, Termodin. Org. Soedin. (Gorky) 39-41 (1988).

1988PET/TSY N. N. Petropavlov, I. G. Tsygankova, and L. A. Teslenko, Sov. Phys. Crystallogr. 33, 853 (1988).

1988RIB/REI M. A. V. Ribeiro Da Silva, A. M. Reis, and G. Pilcher, Thermochim. Acta 124, 319-328 (1988).

1988RIB/RIB M. A. V. Ribeiro Da Silva, M. D. M. C. Ribeiro Da Silva, and G. Pilcher, J. Chem. Thermodyn. 20, 969-974 (1988).

1988SAB/ELW R. Sabbah and L. El Watik, Bull. Soc. Chim. Fr. 626-630 (1988).

1988SAL/ABA $\quad$ S. R. Salman and K. F. Abas, Thermochim. Acta 136, 81 (1988).

1988SHA/DIE R. H. Shay, B. N. Diel, D. M. Schubert, and A. D. Norman, Inorg. Chem. 27, 2378-2382 (1988).

1988SHA/PIL L. Shaofeng and G. Pilcher, J. Chem. Thermodyn. 20, 463-465 (1988).

1988STE/ARC W. V. Steele, D. G. Archer, R. D. Chirico, W. B. Collier, I. A. Hossenlopp, A. Nguyen, K. N. Smith, and B. E. Gammon, J. Chem. Thermodyn. 20, 1233-1264 (1988).

1988TOR/BAR L. A. Torres-Gomez, G. Barreiro-Rodriguez, and A. Galarza-Mondragon, Thermochim. Acta 124, 229-233 (1988).

1988VAN/VAN M. J. Van Bommel, J. C. Van Miltenburg, and A. Schuijj, J. Chem. Thermodyn. 20, 397 (1988).

1988VAR/LOS R. M. Varushchenko, O. L. Loseva, A. I. Druzhinina, and E. F. Zorina, Zh. Fiz. Khim. 62, 1776-1780 (1988).

1988WHI/PER M. A. White and A. Perrott, Can. J. Chem. 66, 729-733 (1988).

1988ZHA/ZOU A. Zhang, H. Zou, and M. Yang, Gaodeng Xuexia Huaxue Suebao 9, 1085 (1988)

1989ABB/JIM J.-L Abboud, P. Jimenez, M. V. Roux, C. Turrion, and C. Lopez-Mardomingo, J. Chem. Thermodyn. 21, 859-865 (1989).

1989ALL/GED J. R. Allan, W. C. Geddes, C. S. Hindle, and A. E. Orr, Thermochim. Acta 153, 249 (1989).

1989AN/HU X. An and H. Hu, Wuli Huaxue Xuebao 5, 565-571 (1989); Chem. Abstr. 112, 118206 (1990).

1989AN/ZHE X. An and X. Zheng, Wuli Hauxue Xuebao 5, 487-491 (1989).

1989AN/ZHE2 X.-W. An and X.-L. Zheng, Acta Phys.-Chim. Sin. 5, 487-491 (1989).

1989AZA

1989BRA/RYT $\quad$ B. K. Braxton and J. H. Rytting, Thermochim. Acta 154, 27-47 (1989).

1989BRE/LIC P. Bret-Dibat and A. Lichanot, Thermochim. Acta 147, 261-271 (1989).

1989BRO/CON J. M. Brown, A. D. Conn, G. Pilcher, M. L. P. Leitao, and M. Y. J. Yang, J. Chem. Soc., Chem. Commun. 1817-1819 (1989).

1989CAT/CAB J. Catalan, P. Cabildo, J. Elguero, J. Gomez, and J. Laynez, J. Phys. Org. Chem. 2, 646 (1989).

1989 CEP/GON E. Cepeda, C. Gonzalez, and J. M. Resa, J. Chem. Eng. Data 34, 270-273 (1989).

1989CHA/DEM A. Chauvet, A. De Maury, and J. Masse, Thermochim. Acta 147, 17-32 (1989).

1989CHI/GRO M. P. Chiarelli and M. L. Gross, Anal. Chem. 61, 1895-1900 (1989).

$1989 \mathrm{CHI} / \mathrm{NGU} \quad$ R. D. Chirico, A. Nguyen, W. V. Steele, M. M. Strube, and C. Tsonopoulos, J. Chem. Eng. Data 34, 149-156 (1989).
1989COL/JIM M. Colomina, P. Jimenez, M. V. Roux, and C. Turrion, J. Chem. Thermodyn. 21, 275-281 (1989).

1989COX/WAG J. D. Cox, D. D. Wagman, and V. A. Medvedev, CODATA Key Values for Thermodynamics (Hemisphere, New York, 1989).

1989DOM U. Domanska, J. Solution Chem. 18, 1153 (1989).

1989GON/KRA T. Gondova, P. Kralik, and J. Gonda, Thermochim. Acta 156, 147-155 (1989).

1989HAN M. I. Hansford, Ph.D. thesis, Durham University, 1989, as quoted in [2000BRO/DU].

1989 HIL/MOU F. R. Hilgeman, F. Y. N. Mouroux, D. Mok, and M. K. Holan, J. Chem. Eng. Data 34, 220 (1989).

1989IMA/TAK A. Imamura, K. Takahashi, S. Murata, and M. Sakiyama, J. Chem. Thermodyn. 21, 237-246 (1989).

1989JIM/ROU P. Jimenez, M. V. Roux, and C. Turrion, J. Chem. Thermodyn. 21, 759-764 (1989).

1989LUB/JAN J. Lubkowski, T. Janiak, J. Czerminski, and J. Blazejowski, Thermochim. Acta 155, 7-28 (1989).

1989NEA/FLY S. H. Neau, G. L. Flynn, and S. H. Yalkowsky, Int. J. Pharm. 49, 223 (1989).

1989NIK/TRI R. Nikolic, J. Tripkovic, and D. H. Kerridge, Thermochim. Acta 146, 353 (1989).

1989OZA/MAT R. Ozawa and M. Matsuoka, J. Cryst. Growth 98, 411 (1989).

1989PAR/GME S. J. Park and J. Gmehling, J. Chem. Eng. Data 34, 399-401 (1989).

1989RAI/MAN U. S. Rai, K. D. Mandal, and N. P. Singh, J. Therm. Anal. 35, 1687 (1989).

1989RIB/MON M. A. V. Ribeiro Da Silva, M. J. S. Monte, and M. A. R. Matos, J. Chem. Thermodyn. 21, 159-166 (1989).

1989RIB/RIB M. A. V. Ribeiro Da Silva, M. D. M. C. Ribeiro Da Silva, J. A. S. Taixeira, J. M. Bruce, P. M. Guyan, and G. Pilcher, J. Chem. Thermodyn. 21, 265-274 (1989).

1989RIB/SOU M. D. M. C. Ribeiro Da Silva, P. Souza, and G. Pilcher, J. Chem. Thermodyn. 21, 173-178 (1989).

1989ROR/RUT $\quad$ B. F. Rordorf, S. Rutschmann, and P. Schiess, Int. J. Mass Spectrom. Ion Processes 95, 211-222 (1989).

1989SAK/IMA M. Sakiyama and A. Imamura, Thermochim. Acta 142, 365 (1989).

1989SAL/ABA S. R. Salman and K. F. Abas, Thermochim. Acta 152, 381-386 (1989).

1989SAL/ABA2 S. R. Salman and K. F. Abas, Thermochim. Acta 142 245-249 (1989).

1989SHI/SHI Y. Shibutani and K. Shinra, Bull. Chem. Soc. Jpn. 62, 1477-1481 (1989).

1989STE/CHI2 W. V. Steele, R. D. Chirico, A. Nguyen, I. A. Hossenlopp, and N. K. Smith, AIChE Symp. Ser. 85, 140-162 (1989).

1989STE/CHI3 W. V. Steele, R. D. Chirico, I. A. Hossenlopp, A. Nguyen, N. K. Smith, and B. E. Gammon, J. Chem. Thermodyn. 21, 1121-1149 (1989).

1989SUZ/SHI E. Suzuki, K. Shimomura, and K. Sekiguchi, Chem. Pharm. Bull. 37, 493-497 (1989).

1989TAN/SOR Z.-C. Tan, M. Sorai, and H. Suga, Sci. China, Ser. B 32, 1194 (1989)

1989UTS/GAE H. Utschick, R. Gaedeke, and H. Sohr, Z. Chem. 29, 257 (1989).

1989VAR/PAS R. M. Varushchenko and L. L. Pashchenko, Russ. J. Phys. Chem. 63, 964-967 (1989).

1989VAR/SOM $\quad$ B. Varughese and J. T. Sommerfeld, J. Chem. Eng. Data 34, 25-26 (1989).

1989VAR/SOM2 B. Varughese and J. T. Sommerfeld, J. Chem. Eng. Data 34, 21-25 (1989).

1989VAS/LEB V. G. Vasil'ev and B. V. Lebedev, Zh. Obshch. Khim. 59, 2415-2420 (1989).

1989VIN/WOR M. D. Vine and C. J. Wormald, J. Chem. Thermodyn. 21, 1151-1157 (1989).

1989VOR/KLY M. G. Voronkov, V. A. Klyuchnikov, S. N. Kolabin, G. N. Shvets, P. I. Varushin, E. N. Deryagina, N. A. Korchevin, and S. I. Tsvetnitskaya, Dokl. Phys. Chem. 307, 650-653 (1989).

1989WAN/YIN K. Wang, X.-G. Ying, M.-S. Xia, J.-Z. Xia, and Y. Hu, J. Chem. Eng. Data 34, 126-130 (1989). 
1989WOJ/MAR G. Wojcik and Y. Marqueton, Mol. Cryst. Liq. Cryst. 168, 247 (1989).

1989WU/SAN H. S. Wu and S. I. Sandler, J. Chem. Eng. Data 34, 209-213 (1989).

1989ZHA/HUA X. Zhang, W. Huang, F. Tan, G. Xu, and Z. Wu, Chin. Sci. Bull. 34, 1844 (1989).

1989ZHA/YAN Z. Y. Zhang and M. L. Yang, Thermochim. Acta 156, 157-161 (1989).

1990AMB/EWI D. Ambrose, M. B. Ewing, N. B. Ghiassee, and J. C. S. Ochoa, J. Chem. Thermodyn. 22, 589 (1990).

1990AMB/GHI D. Ambrose and N. B. Ghiasse, J. Chem. Thermodyn. 22, 307 (1990)

1990AN/HE X. An, J. He, and R.-H. Hu, Thermochim. Acta 169, 331 (1990).

1990BAD/KUL V. G. Badelin, O. V. Kulikov, V. S. Vatagin, E. Udzig, A. Zielenkiewicz, W. Zielenkiewicz, and G. A. krestov, Thermochim. Acta 169, 81 (1990).

1990BAR/DEL G. G. Barone, Della Gatta, D. Ferro, and V. Piacente, J. Chem. Soc., Faraday Trans. 86, 75 (1990).

1990BAT/JAK G. Bator, R. Jakubas, Z. Malarski, Z. Galewski, J. Matuszewski, and A. Miniewicz, J. Mol. Struct. 240, 39 (1990).

1990BEC/DOG H. D. Beckhaus, B. Dogan, J. Pakusch, S. P. Verevkin, and C. Rüchardt, Chem. Ber. 123, 2153 (1990).

1990BET/GIO G. Bettinetti, F. Giordano, G. Fronza, A. Italia, R. Pellegata, M. Villa, and P. Ventura, J. Pharm. Sci. 79, 470 (1990).

1990BHA/LAL D. V. Bhalla and J. K. Lalla, Drug Dev. Ind. Pharm. 16, 115 (1990).

1990CAB/BEL J. L. Cabezas and S. Beltran, J. Chem. Eng. Data 35, 389 (1990).

1990CEO/SZW R. Ceolin, H. Szwarc, and F. Lepage, Thermochim. Acta 158, 347 (1990).

1990CHA/GAD J. Chao, N. A. M. Gadalla, B. E. Gammon, K. N. Marsh, A. S. Rodgers, G. R. Somayajulu, and R. C. Wilhoit, J. Phys. Chem. Ref. Data 19, 1547 (1990).

1990CHI/ARC R. D. Chirico, D. G. Archer, I. A. Hossenlopp, A. Nguyen, W. V. Steele, and B. E. Gammon, J. Chem. Thermodyn. 22, 665 (1990).

1990CHI/HOS J. S. Chickos, S. Hosseini, D. G. Hesse, and J. F. Liebman, Struct. Chem. 4, 271-278 (1993).

1990DAU/HUT T. E. Daubert and G. Hutchison, AIChE Symp. Ser. 279, 93 (1990).

1990DAV/FIN R. H. Davies, A. Finch, and K. Salem, Thermochim. Acta 170, 213 (1990).

1990DOM/HEA E. S. Domalski and E. D. Hearing, J. Phys. Chem. Ref. Data 19, 881 (1990).

1990DON/DRE J. R. Donnelly, L. A. Drewes, R. L. Johnson, W. D. Munslow, K. K. Knapp, and G. W. Sovocool, Thermochim. Acta 167, 155 (1990).

1990DUT/KAH N. V. Dutt, A. P. Kahol, P. J. Reddy, and K. S. Raghunandanan, J. Chem. Eng. Data 35, 114 (1990).

1990FIN/PAY A. Finch and J. Payne, Thermochim. Acta 164, 55 (1990).

1990FRI/DOG K. Fritzsch, B. Dogan, H.-D. Beckhaus, and C. Rücuardt, Thermochim. Acta 160, 147 (1990).

1990HAI/GIL J. Haines and D. F. R. Gilson, J. Chem. Soc., Faraday Trans. 86, 2617 (1990).

1990HAI/GIL2 J. Haines and D. F. R. Gilson, Can. J. Chem. 68, 604 (1990).

1990HIN/BID2 H. D. Hinckley, T. F. Bidleman, W. T. Foreman, and J. R. Tuschall, J. Chem. Eng. Data 35, 232 (1990).

1990HWA/TAM D.-R. Hwang, M. Tamura, T. Yoshida, N. Tanaka, and F. Hosoya, J. Energ. Mater. 8, 85-98 (1990).

1990HWA/YOS D. R. Hwang, F. Yoshizawa, and M. Tamura, Anzen Kogaku 29, 168 (1990); Chem. Abstr. 115, 186382x (1991).

1990KAB/MIR G. Y. Kabo, E. A. Miroshnichenko, M. L. Frenkel, A. A. Kozyro, V. V. Simirsky, A. P. Krasulin, V. P. Vorob'eva, and Y. A. Lebedev, Izv. Akad. Nauk SSSR Ser. Khim. 39, 750 (1990)

1990KAB/MIR2 G. Y. Kabo, E. A. Miroshnichenko, M. L. Frenkel, A. A. Kozyro, V. V. Simirskii, A. P. Krasulin, V. P. Vorob'eva, and Y. A. Lebedev, Bull. Acad. Sci. USSR, Div. Chem. Sci. 39, 662 (1990).
1990KNA/SAB 1990KNA/SAB2 1990KNA/SAB3

1990KOZ/SIM

P. Knauth and R. Sabbah, Can. J. Chem. 68, 731 (1990). P. Knauth and R. Sabbah, Struct. Chem. 1, 43 (1990).

P. Knauth and R. Sabbah, Thermochim. Acta 164, 145 (1990).

A. A. Kozyro, V. V. Simirskil, A. P. Krasulin, V. M Sevruk, G. Y. Kabo, M. L. Frenkel, P. N. Gaponik, and Y. V. Grigor'ev, Russ. J. Phys. Chem. 64, 348 (1990).

1990KOZ/SIM2 A. A. Kozyro, V. V. Simirskii, G. Y. Kabo, M. L. Frenkel, A. P. Krasulin, V. M. Sevruk, and N. A. Sokolov, Zh. Fiz. Khim. 64, 2360 (1990).

1990 KOZ/SIM3 A. A. Kozyro, V. V. Simirskii, A. P. Krasulin, V. M. Sevruk, G. Y. Kabo, M. L. Frenkel, P. N. Gaponik, and Y. V. Grigor'ev, Zh. Fiz. Khim. 64, 656 (1990).

1990LEI/PIL M. L. P. Leitao, G. Pilcher, W. E. Acree, Jr., A. I. Zvaigzne, S. A. Tucker, and M. D. M. C. Ribeiro Da Silva, J. Chem. Thermodyn. 22, 923 (1990).

1990LEI/PIL2 M. L. P. Leitao, G. Pilcher, Y. Meng-Yan, J. M. Brown, and A. D. Conn, J. Chem. Thermodyn. 22, 885 (1990).

1990LEN

1990LYM/REE M. Lenka, J. Chem. Thermodyn. 22, 473 (1990).

J. D. Birkett, in Handbook of Chemical Property Estimation Methods, edited by W. J. Lyman, W. F. Reehl, and D. H. Rosaenblatt (American Chemical Society, 1990), p. 23-1.

1990MAN/AHU R. H. Manzo and A. A. Ahumanda, J. Pharm. Sci. 79, 1109 (1990).

1990MAY/RAC J. Mayer, M. Rachwalska, E. Scresinska, and J. Sciesinski, J. Phys. 51, 857 (1990).

1990MEN/PIL Y. Meng-Yan and G. Pilcher, J. Chem. Thermodyn. 22, 893 (1990).

1990MES/TOD J. F. Messerly, S. S. Todd, H. L. Finke, S. H. Lee-Bechtold, G. B. Guthrie, W. V. Steele, and R. D. R. D. Chirico, J. Chem. Thermodyn. 22, 1107 (1990).

1990MEV/LIC I. M. Meva'a and A. Lichanot, Thermochim. Acta 158 335 (1990).

1990MID/KAT Y. Mido, H. Katano, T. Isono, M. Hashimoto, and H. Matsuura, J. Phys. Chem. 94, 8070 (1990).

1990MIH/BAS P. Mihailovic, P. Bassoul, and J. Simon, J. Phys. Chem. 94, 2815 (1990).

1990NEA/FLY 1990NES/NAZ S. H. Neau and G. L. Flynn, Pharm. Res. 7, 1157 (1990). T. N. Nesterova, A. G. Nazmutdinov, V. S. Tsvetkov, A. M. Rozhnov, and I. Yu. Roshchupkina, J. Chem. Thermodyn. 22, 365 (1990).

1990ORT/SUS J. Ortego, P. Susial, and C. de Alfonso, J. Chem. Eng. Data 35, 216 (1990).

1990PIA/FER V. Piacente, D. Ferro, and G. Della Gatta, Thermochim. Acta 158, 79 (1990).

1990PUL/MAT R. Puliti, C. A. Mattia, G. Barone, G. Della Gatta, and D. Ferro, Thermochim. Acta 162, 229 (1990).

1990RAM/KUD D. H. S. Ramkumar and A. P. Kudchadker, Fluid Phase Equilib. 55, 207 (1990).

1990RIB/MAT M. A. V. Ribeiro Da Silva, M. A. R. Matos, and M. J. S. Monte, J. Chem. Thermodyn. 22, 609 (1990).

1990RIB/MON M. A. V. Ribeiro Da Silva and M. J. S. Monte, Thermochim. Acta 171, 169 (1990).

1990RIB/RIB M. A. V. Ribeiro Da Silva, M. D. M. C. Ribeiro Da Silva, M. C. S. S. Rangel, G. Pilcher, M. J. Akello, A. S. Carson, and E. H. Jamea, Thermochim. Acta 160, 267 (1990).

1990RIC/YAN M. F. Richardson, O.-C. Yang, E. Novotny-Bregger, and J. D. Dunitz, Acta Crystallogr., Sect. B 46, 653 (1990).

1990ROZ/BAR A. M. Rozhnov, V. I. Barkov, K. G. Sharonov, and V. S. Tsvetkov, J. Chem. Thermodyn. 22, 327 (1990).

1990SIN/GLI N. B. Singh and M. E. Glicksman, Thermochim. Acta 159, 93 (1990).

1990 SIN/GUP R. N. Singh, J. P. Gupta, N. Singh, N. P. Singh, O. P. Singh, N. B. Singh, R. H. Hopkins, and R. Mazelsky, Thermochim. Acta 165, 297 (1990).

1990SMI N. O. Smith, J. Chem. Eng. Data 35, 387 (1990).

1990SOL/KAB T. V. Soldatova, G. Y. Kabo, A. A. Kozyro, and M. L. Frenkel, Russ. J. Phys. Chem. 64, 177 (1990).

1990SUE/MAT K. Suenaga, T. Matsuo, and H. Suga, Thermochim. Acta 163, 263 (1990).

1990SUH/MAN M. Suhara and K. Mano, Z. Naturforsch., A 45, 339 (1990). 
1990VAN/PAV Y. Y. Van-Chin-Syan, Y. P. Pavlovskii, N. S. Kachurina, M. A. Dikii, Y. V. Panchenko, and G. A. Petrovskaya, Russ. J. Phys. Chem. 64, 295 (1990).

1990VAN/VAN H. A. Van Doren, R. van der Geest, R. M. Kellog, and H. Wynberg, Recl. Trav. Chim. Pays-Bas 109, 197 (1990).

1990VOR/ZVE V. M. Vorotyntsev, Y. B. Zverev, and D. A. Shamrakov, Vysokochist Veshchestra 123 (1990).

1990YAW/YAN C. L. Yaws, H. C. Yang, and W. A. Cowley, Hydrocarbon Process., Int. Ed. 69, 87 (1990).

1990YIN/LIN Z. Z. Ying and Y. M. Lin, J. Chem. Thermodyn. 22, 617 (1990).

1990ZHA/YAN Z. Zhang and M. L. Yang, Thermochim. Acta 169, 263 (1990).

1991 ACR $\quad$ W. E. Acree, Jr., Thermochim. Acta 189, 37 (1991), and references therein.

1991ACR/TUC $\quad$ W. E. Acree, Jr., S. A. Tucker, A. I. Zvaigzne, Y. MengYan, G. Pilcher, and M. D. M. C. Ribeiro Da Silva, J. Chem. Thermodyn. 23, 31 (1991).

1991AUE/WEB $\quad$ D. H. Aue, H. M. Webb, W. R. Davidson, P. Toure, H. P. Hopekins, Jr., S. P. Moulik, and D. V. Jahagirdar, J. Am. Chem. Soc. 113, 1770-1780 (1991).

1991BAS/SVO P. Basarova and V. Svoboda, Fluid Phase Equilib. 68, 13 (1991).

1991BED/BOO A. D. Bedells, C. Booth, and K. Viras, Makromol. Chem. 192, 2099 (1991).

1991BER/MAR V. Berbenni, A. Marini, V. Massarotti, D. Capsoni, R. Riccardi, C. Margheritis, and M. Conte, Thermochim. Acta 181, 315 (1991).

1991BYK/KIP T. A. Bykova, Y. G. Kiparisova, B. V. Lebedev, K. A. Mager, and Y. G. Gololobov, Polym. Sci. U.S.S.R. 33, 537 (1991).

1991CHI/BRA J. S. Chickos, C. M. Braton, D. G. Hesse, and J. F. Liebman, J. Org. Chem. 56, 927 (1991), and references and supplementary material therein.

1991CHI/KNI2 R. D. Chirico, S. E. Knipmeyer, A. Nguyen, and W. V. Steele, J. Chem. Thermodyn. 23, 759 (1991).

1991DIK/KAB V. V. Dikii and G. Ya. Kabo, Dokl. Akad. Nauk BSSR 35, 158 (1991); Chem. Abstr. 114, 206233j (1991).

1991ELG/YRA J. Elguero, G. I. Yranzo, J. Laynez, P. Jimenez, M. Menendez, J. Catalan, J. L. G. de Paz, F. Anvia, and R. W. Taft, J. Org. Chem. 56, 3942 (1981).

1991ELI L. Elias, Conference on International Civil Aviation Organization paper AH-DE/5-WP/17, Montreal, 23-27 September 1991, as quoted in [2002JON/LIG].

1991ELW/SAB $\quad$ L. El Watik and R. Sabbah, Bull. Soc. Chim. Fr. 128, 344 (1991).

1991EWI/GOO M. B. Ewing and A. R. H. Goodwin, J. Chem. Thermodyn. 23, 1163 (1991).

1991FUK/MAT M. Fukai, T. Matsuo, and H. Suga, Thermochim. Acta 183, 215 (1991).

1991HEN/TSC F. Hentrich, C. Tschierske, and H. Zaschke, Angew. Chem., Int. Ed. Engl. 30, 440 (1991).

1991HOR T. Hori, Shikizai Kyokaishi 64, 83 (1991).

1991KAW/GIL N. T. Kawai, D. F. R. Gilson, and I. S. Butler, Can. J. Chem. 69, 1758 (1991).

1991KIS/PIN I. Kishimoto, J. J. Pinvidic, T. Matsuo, and H. Suga, Proc. Jpn. Acad., Ser. B 67, 66 (1991).

$1991 \mathrm{KOL} / \mathrm{ROZ}$ M. Kol, S. Rozen, and E. Appelman, J. Am. Chem. Soc. 113, 2648 (1991).

1991LAB/WES A. K. Labban, E. F. Westrum, Jr., and J. A. R. Cheda, Can. J. Chem. 69, 1796 (1991).

1991LEF/GUI C. Lefebvre, F. Guillaume, R. Bouche, R. Bouaziz, and J. C. Guyot, Pharm. Acta Helv. 66, 90 (1991).

1991LIU/GUO Z. Z. Liu, X. D. Guo, L. E. Straub, G. Erdos, R. J. Prankerd, R. J. Gonzalez-Rothi, and H. Schreier, Drug Des. Discovery 8, 57 (1991).

1991LUK/TIM V. A. Luk'yanova, L. P. Timofeeva, M. P. Kozina, V. N. Kirin, and A. V. Tarakanova, Russ. J. Phys. Chem. 65, 439 (1991).

1991 MON/HOU D. Mondieig, J. R. Housty, Y. Haget, M. A. Cuevas-Diarte, and H. A. J. Oonk, Thermochim. Acta 177, 169 (1977).

1991PIC/RYL M. Pickering, J. Rylance, R. W. H. Small, and D. Stubley, Acta Crystallogr., Sect. B 47, 782 (1991).
1991RAD/RAD M. Radomska and R. Radomski, J. Therm. Anal. 37, 693 (1991).

1991RAI/GEO U. S. Rai and S. George, Cryst. Res. Technol. 26, 511 (1991).

1991ROZ/SAF A. M. Rozhnov, V. V. Safronov, S. P. Verevkin, K. G. Sharonov, and V. I. Alenin, J. Chem. Thermodyn. 23, 629 (1991).

1991SAB/AN R. Sabbah and X. W. An, Thermochim. Acta 178, 339 (1991).

1991SAB/AN2 R. Sabbah and X. W. An, Thermochim. Acta 179, 81 (1991).

1991SAB/BUL R. Sabbah and E. N. L. E. Buluku, Can. J. Chem. 69, 481 (1991).

1991SAB/HIR R. Sabbah and H. Hirtz, Bull. Soc. Chim. Fr. 26 (1991).

1991SHA/MIS K. G. Sharonov, Y. B. Mishentseva, A. M. Rozhnov, E. A. Miroshnichenko, and L. I. Korchatova, J. Chem. Thermodyn. 23, 636 (1991).

1991STE/CHI2 W. V. Steele, R. D. Chirico, A. Nguyen, I. A. Hossenlopp, and N. K. Smith, U.S. DOE Report NIPER-514 (1991), pp. $1-80$.

1991VAN/VER J. C. van Miltenburg and M. L. Verdonk, J. Chem. Thermodyn. 23, 273 (1991).

1991VAS/BYK V. G. Vasil'ev, T. A. Bykova, and B. V. Lebedev, Zh. Fiz. Khim. 65, 51 (1991).

1991WHI/PER M. A. White and A. Perrott, J. Solid State Chem. 90, 87 (1991).

1991WIB/HAO K. B. Wiberg and S. Hao, J. Org. Chem. 56, 5108 (1991).

1991 WIB/WAL K. B. Wiberg and R. F. Waldron, J. Am. Chem. Soc. 113, 7697 (1991).

1991WIL/WIL W. V. Wilding, L. C. Wilson, and G. M. Wilson, AIChE Data Ser. 1, 6 (1991).

1991 WU/LOC H. S. Wu, W. E. Locke III, and S. I. Sandler, J. Chem. Eng. Data 36, 127 (1991).

1991WU/PIV H. S. Wu, K. A. Pividal, and S. I. Sandler, J. Chem. Eng. Data 36, 418 (1991).

1991 WU/XIO $\quad$ S. Wu and W. Xiong, Xibei Daxue Xuebao, Ziran Kexueban 21, 39 (1991).

1991WYR/PAL D. Wyrzykowska-Stankiewicz and M. PalczewskaTulinska, Thermochim. Acta 190, 209 (1991).

1991ZHA/HUA X. Zhang, W. Huang, F. Tan, G. Xu, and Z. Wu, Acta Phys.-Chim. Sin. 1, 106 (1991).

1992 ACR/SIM $\quad$ W. E. Acree, Jr., V. V. Simirsky, A. A. Kozyro, A. P. Krasulin, G. J. Kabo, and M. L. Frenkel, J. Chem. Eng. Data 37, 131 (1992).

1992ACR/TUC W. E. Acree, Jr., S. A. Tucker, and G. Pilcher, J. Chem. Thermodyn. 24, 213 (1992).

1992BAB/HWA M. W. Babich, S. W. Hwang, and R. D. Mounts, Thermochim. Acta 210, 83 (1992).

1992BAB/HWA2 M. W. Babich, S. W. Hwang, and R. D. Mounts, Thermochim. Acta 210, 77 (1992).

1992BAR/GIA G. Barone, C. Giancola, T. H. Lilley, C. A. Mattia, and R. Puliti, J. Therm. Anal. 38, 2771 (1992).

1992ELS/PRA M. Elsabee and R. Prankerd, Int. J. Pharm. 86, 211 (1992), Note: Experimental fusion enthalpy (in J g-1) was taken from graph.

1992GRA/SAN M. Gracia, F. Sanchez, P. Perez, J. Valero, and C. Gutierrez Losa, J. Chem. Thermodyn. 24, 463 (1992).

1992HE/AN J. He, X.-W. An, and R.-H. Hu, Acta Chim. Sin. 50, 943 (1992).

1992HEA/SIN E. A. Heath, P. Singh, and Y. Ebisuzaki, Acta Crystallogr., Sect. C 48, 1960 (1992).

1992JAD/FRA R. Jadot and M. Fraiha, J. Chem. Eng. Data 37, 509 (1992).

1992JIM/ROU P. Jimenez, M. V. Roux, and C. Turrion, J. Chem. Thermodyn. 24, 1145 (1992).

1992KAB/KOZ G. J. Kabo, A. A. Kozyro, V. S. Krouk, V. M. Sevruk, I. A. Yursha, V. V. Simirsky, and V. I. Gogolinsky, J. Chem. Thermodyn. 24, 1 (1992).

1992KAM M. Kaminski, Bull. Pol. Acad. Sci., Chem. 40, 149 (1992).

1992KAT H. Katayama, J. Chem. Eng. Jpn. 25, 366 (1992).

1992KAW/GIL N. T. Kawai, D. F. R. Gilson, and I. S. Butler, Mol. Cryst. Liq. Cryst. 211, 59 (1992). 
1992KIM/SZY W. Kimizuka and J. Szydlowski, Fluid Phase Equilib. 77, 261 (1992).

1992KOZ/KAB A. A. Kozyro, G. J. Kabo, V. S. Krouk, M. S. Sheiman, I. A. Yursha, V. V. Simirsky, A. P. Krasulin, V. M. Sevruk, and V. I. Gogolinsky, J. Chem. Thermodyn. 24, 883 (1992).

1992LEB/KUL B. V. Lebedev and T. G. Kulagina, J. Chem. Thermodyn. 24, 751 (1992).

1992LEB/SMI $\quad$ B. Lebedev, N. Smirnova, Y. Kiparisova, and K. Makovetsky, Makromol. Chem. 193, 1399 (1992).

1992LEE/CHE C.-H. Lee, Q. Chen, R. S. Mohamed, and G. D. Holder, J. Chem. Eng. Data 37, 179 (1992).

1992LEE/DEM C.-H. Lee, D. M. Dempsey, R. S. Mohamed, and G. D. Holder, J. Chem. Eng. Data 37, 183 (1992).

1992MAK

1992MAN

Yu. Ya. Maksimov, Zh. Fiz. Khim. 66, 540 (1992).

K. D. Mandal, Pol. J. Chem. 66, 1673 (1992)

1992 NGU/KAS J. N'Guimbi, H. Kasehgari, I. Mokbel, and J. Jose, Thermochim. Acta 196, 367 (1992).

19920GU/YAM K. Oguchi, M. Yamagishi, and A. Murano, Fluid Phase Equilib. 80, 131 (1992).

19920KA/OGU N. Okamoto, M. Oguni, and H. Suga, Thermochim. Acta 202, 215 (1992).

1992PAP/PIM T. S. Papina, S. M. Pimenova, V. Yu. Zakharov, and V. P. Kolesov, Zh. Khim. Termodin. Termodin. 1, 207 (1992).

1992RAA/BRO J. D. Raal and C. J. Broucharet, Fluid Phase Equilib. 74, 253 (1992).

1992RAH/GME U. Rahm and E. Gmelin, J. Therm. Anal. 38, 335 (1992). 1992RAI/GEO U. S. Rai and S. George, Can. J. Chem. 70, 2869 (1992).

1992REI/HAN H. Reinke, M. Hans, and H. Dehne, Mikrochim. Acta 108, 143 (1992)

1992RIB/MAT M. A. V. Ribeiro Da Silva, A. R. Matos, Y. Meng-Yan, and G. Pilcher, J. Chem. Thermodyn. 24, 107 (1992).

1992RIB/MON M. A. V. Ribeiro Da Silva and M. J. S. Monte, J. Chem. Thermodyn. 24, 1219 (1992).

1992RIB/MON2 M. A. V. Ribeiro Da Silva and M. J. S. Monte, J. Chem. Thermodyn. 24, 715 (1992).

1992RIB/REI M. A. V. Ribeiro Da Silva, A. M. V. Reis, M. J. S. Monte, M. S. S. F. Bartolo, and J. A. Rodriques, J. Chem. Thermodyn. 24, 653 (1992).

1992SAB/ELW3 R. Sabbah and L. El Watik, J. Therm. Anal. 38, 855 (1992).

1992SAB/WAT R. Sabbah and L. E. Watik, J. Therm. Anal. 38, 803 (1992).

1992SAL/WAN M. Salvi-Narkhede, B.-H. Wang, J. L. Adcock, and W. H. Van Hook, J. Chem. Thermodyn. 24, 1065 (1992).

1992SHA/SHA $\quad$ B. L. Sharma, N. K. Sharma, and M. Rambal, Thermochim. Acta 206, 71 (1992).

1992SMI/LEB N. N. Smirnova, B. V. Lebedev, E. G. Kiparisova, K. L. Makovetskii, and L. I. Gorbacheva, Polym. Sci. U.S.S.R. 34, 72 (1992).

1992STE/CHI2 W. V. Steele, R. D. Chirico, S. E. Knipmeyer, and A. Nguyen, J. Chem. Thermodyn. 24, 499 (1992).

$1992 \mathrm{SVO} / \mathrm{KUB} \quad$ V.Svoboda, V. Kubes, and P. Basarova, J. Chem. Thermodyn. 24, 333 (1992).

1992 SVO/KUB2 V. Svoboda, V. Kubes, and P. Basarova, J. Chem. Thermodyn. 24, 555 (1992).

1992VER/BEC S. P. Verevkin, H.-D. Beckhaus, and C. Rüchardt, Thermochim. Acta 197, 27 (1992).

1992WEB L. A. Weber, Fluid Phase Equilib. 80, 141 (1992).

1992WHI/WAS M. A. White, R. E. Wasylishen, P. E. Eaton, Y. Xiong, K. Pramod, and N. Nodari, J. Phys. Chem. 96, 421 (1992).

1992ZHU/WU M.-S. Zhu, J. Wu, and Y.-D. Fu, Fluid Phase Equilib. 80, 99 (1992).

1993ABB/JIM J.-L.M. Abboud, P. Jimenez, M. V. Roux, C. Turrion, and C. Lopez-Mardomingo, Calorim. Anal. Therm. 24, 181 (1993).

1993ACR W. E. Acree, Jr., Thermochim. Acta 219, 97 (1993), and references therein.

1993ACR/SEV W. E. Acree, Jr., V. M. Sevruk, A. A. Kozyro, A. P. Krasulin, G. J. Kabo, and M. L. Frenkel, J. Chem. Eng. Data 38, 101 (1993).

1993ACR/TUC2 $\quad$ W. E. Acree, Jr., S. A. Tucker, G. Pilcher, A. Chowdhary, M. D. C. Ribeiro Da Silva, and M. J. S. Monte, J. Chem. Thermodyn. 25, 1253 (1993).
1993AN/XIE

X.-W. An and Y.-D. Xie, Thermochim. Acta 220, 17 (1993).

1993AUC/BUR A. Aucejo, M. C. Burguet, R. Munoz, and M. I. Vazquez, J. Chem. Eng. Data 38, 379 (1993).

1993AUC/MON A. Aucejo, J. B. Monton, R. Munoz, and M. Sanchotello, J. Chem. Eng. Data 38, 160 (1993).

1993BEC/QUA

G. Becket, S. B. Quah, and J. O. Hill, J. Therm. Anal. 40, 537 (1993).

1993BRU/MON M. C. Burguet, J. B. Monton, M. Sanchotello, and M. I. Vazquez, J. Chem. Eng. Data 38, 328 (1993).

1993CHI/HOS J. S. Chickos, S. Hosseini, D. G. Hesse, and J. F. Liebman, Struct. Chem. 4, 261 (1993), Note: Heat capacity corrections to $298 \mathrm{~K}$ were estimated by the procedure outlined in the article.

1993CHI/HOS2 J. S. Chickos, S. Hosseini, D. G. Hesse, and J. F. Liebman, Struct. Chem. 4, 261-269 (1993).

1993CHI/KNI R. D. Chirico, S. E. Knipmeyer, A. Nguyen, and W. V. Steele, J. Chem. Thermodyn. 25, 1461 (1993).

1993DEM/BUC D. De Marco, R. Bucci, and C. Gallo, J. Therm. Anal. 39, 239 (1993).

1993DIK/KAB V. V. Diky, G. J. Kabo, A. A. Kozyro, A. P. Krasulin, and V. M. Sevruk, J. Chem. Thermodyn. 25, 1169 (1993).

1993ELM/CHA A. El Moussaoui, A. Chauvet, and J. Masse, J. Therm. Anal. 39, 373 (1993).

1993FAR/WIC J. Farkova and I. Wichterle, Fluid Phase Equilib. 90, 143 (1993).

1993FER/MOR N. C. Ferreira, C. A. Moreira dos Santos, and I. C. C. Calegao, Ecl. Quim. Sao Paulo 18, 49 (1993).

1993FUJ/OGU H. Fujimori and M. Oguni, J. Phys. Chem. Solids 54, 607 (1993).

1993FUJ/OGU2 H. Fujimori and M. Oguni, J. Phys. Chem. Solids 54, 271 (1993).

1993HAK G. Hakvoort, Calorim. Anal. Therm. 24, 177 (1993).

1993HE/AN J. He, X. An, and R. Hu, Huaxue Xuebao 51, 1059 (1993); Chem. Abstr. 120, 87930w (1994).

1993IGA/LOP J. M. Igartua, A. Lopez-Echarri, T. Breczewski, and I. Ruiz-Larrea, Phase Transitions 46, 47 (1993).

1993KAB/KOZ G. J. Kabo, A. A. Kozyro, A. P. Krasulin, V. M. Sevruk, and L. S. Ivashkevich, J. Chem. Thermodyn. 25, 485 (1993).

1993KAS/MOK H. Kasehgari, I. Mokbel, C. Viton, and J. Jose, Fluid Phase Equilib. 87, 133 (1993).

1993KOZ/KAB A. A. Kozyro, G. J. Kabo, A. P. Krasulin, V. M. Sevruk, V. V. Simirsky, M. S. Sheiman, and M. L. Frenkel, J. Chem. Thermodyn. 25, 1409 (1993).

1993LEB/VAS B. Lebedev, V. Vasil'yev, and N. Novosyolova, Makromol. Chem. 194, 739 (1993).

1993LEE/HOL C.-H. Lee and G. D. Holder, J. Chem. Eng. Data 38, 320 (1993).

1993NAD/BER M. H. Nadais and M. G. Bernardo-Gil, Fluid Phase Equilib. 91, 321 (1993).

1993OIS/HOR T. Oishi, H. Horie, and H. Shuyama, Polym. J. 25, 781 (1993).

1993 OVC/SOB V. V. Ovchinnikov, A. A. Sobanov, and A. N. Pudovik, Dokl. Phys. Chem. 333, 483 (1993).

1993PIA/FER V. Piacente, D. Ferro, and G. Della Gatta, Thermochim. Acta 223, 65 (1993).

1993PIL/PAR G. Pilcher, O. G. Parchment, I. H. Hillier, F. Heatley, D. Fletcher, M. A. V. Ribeiro Da Silva, M. L. C. C. H. Ferrao, M. J. S. Monte, and F. Jiye, J. Phys. Chem. 97, 243 (1993).

1993RAN/LAK H. S. Randhawa and R. Lakhani, J. Therm. Anal. 39, 437-445 (1993).

1993REI/SAN R. Reich and V. Sanhueza, J. Chem. Eng. Data 38, 341 (1993).

1993RIB/FER M. A. V. Ribeiro Da Silva, M. L. C. C. H. Ferrao, and A. J. M. Lopes, J. Chem. Thermodyn. 25, 229 (1993).

1993RIB/MAT M. A. V. Ribeiro Da Silva, M. A. R. Matos, M. J. S. Monte, M. C. B. Alves, and J. M. A. P. Vieira, J. Chem. Thermodyn. 25, 579 (1993).

1993RIB/RIB M. A. V. Ribeiro Da Silva, M. D. M. C. Ribeiro Da Silva, J. P. A. Paiva, I. M. C. S. Nogueira, A. M. Damas, J. V. Barley, M. M. Harding, M. J. Akello, and G. Pilcher, J. Chem. Soc., Perkin Trans. 2 1993, 1765. 
1993SAB/LE R. Sabbah and T. H. D. Le, Can. J. Chem. 71, 1378 (1993).

1993SAB/PEM R. Sabbah and O. Pemenzi, C. R. Acad. Sci. Paris Ser. II 317, 575 (1993).

1993SCH/BEC R. Schulze, H.-D. Beckhaus, and C. Rüchardt, Chem. Ber. 126, 1031 (1993).

1993SIL/WEB A. M. Silva and L. A. Weber, J. Chem. Eng. Data 38, 644 (1993).

1993SIN/CAR M. Siniti, J. Carre, J. M. Letoffe, J. P. Bastide, and P. Claudy, Thermochim. Acta 224, 97 (1993).

1993STR/ARG L. Stradella and M. Argeniero, Thermochim. Acta 219 , 315 (1993).

1993SUS/ORT P. Susial and J. Ortego, J. Chem. Eng. Data 38, 434 (1993).

1993SUS/ORT2 P. Susial and J. Ortego, J. Chem. Eng. Data 38, 647 (1993).

1993SZA/CZAＭ. Szafanski, P. Czarnecki, W. Dollhopf, G. W. H. Hone, G. Brackenhofer, and W. Nawrocik, J. Phys.: Condens. Matter 5, 7425 (1993).

1993TIE G. V. D. Tiers, Thermochim. Acta 226, 317 (1993).

1993TIE/FRA G. V. D. Tiers and C. V. Francis, Thermochim. Acta 226, 311 (1993).

1993VAR/PUC R. M. Varushchenko, G. A. Puchkova, and A. I. Druzhinina, Zh. Fiz. Khim. 67, 897 (1993).

1993WEB/GOO L. A. Weber and A. R. H. Goodwin, J. Chem. Eng. Data 38, 254 (1993).

1993WOL/KIM H. Wolff, W. Kimizuka, and J. Szydlowski, Fluid Phase Equilib. 90, 163 (1993).

1993XIE/CHE R. Xie and C. J. Chen, J. Chem. Eng. Chin. Univ. 7, 267 (1993).

1994ACR/TUC W. E. Acree, Jr., S. A. Tucker, G. Pilcher, and G. Toole, J. Chem. Thermodyn. 26, 85 (1994).

1994AIM K. Aim, J. Chem. Eng. Data 39, 591 (1994).

1994AIM2 K. Aim, J. Chem. Thermodyn. 26, 977 (1994).

1994ANT/SAN M. Antosik and S. I. Sandler, J. Chem. Eng. Data 39, 584 (1994).

1994AUC/BUR A. Aucejo, M. C. Burguet, J. B. Monton, R. Munoz, M. Sanchotello, and M. I. Vazquez, J. Chem. Eng. Data 39, 578 (1994).

1994BLA/BEL B. Blanco, S. Beltran, J. L. Cabezas, and J. Coca, J. Chem. Eng. Data 39, 23 (1994).

1994BLU/PRAＤ. Blunk, K. Praefcke, and G. Legler, Liq. Cryst. 17, 841 (1993).

1994CAR/LAY A. S. Carson, P. G. Laye, J. B. Pedley, A. M. Welsby, J. S. Chickos, and S. Hosseini, J. Chem. Thermodyn. 26, 1103 (1994).

1994CHI/HOS R. D. Chirico, I. A. Hossenlopp, B. E. Gammon, S. E. Knipmeyer, and W. V. Steele, J. Chem. Thermodyn. 26, 1187 (1994).

1994CHI/HOS2 R. D. Chirico, I. A. Hossenlopp, B. E. Gammon, S. E. Knipmeyer, and W. V. Steele, J. Chem. Thermodyn. 26, 1219 (1994).

1994CUN/JON Experimental Results for DIPPR 1990-1991 Projects on Phase Equilibria and Pure Component Properties, edited by J. R. Cunningham and D. K. Jones, DIPPR Data Series (AIChE, New York, NY, 1994), Vol. 2, p. 154.

1994DIK/KAB V. V. Diky, G. J. Kabo, A. A. Kozyro, A. P. Krasulin, and V. M. Sevruk, J. Chem. Thermodyn. 26, 1001 (1994).

1994DOU/FUE S. Q. Dou, H. Fuess, W. Schmahl, R. Strauss, and A. Weiss, Z. Naturforsch. 49A, 594 (1994).

1994FER/MAR D. Ferro, R. Martino, and G. Della Gatta, J. Chem. Thermodyn. 26, 183 (1994).

1994FON/MUN J. Font and J. Muntasell, Mater. Res. Bull. 29, 1091 (1994).

1994FON/MUN2 J. Font and J. Muntasell, Thermochim. Acta 246, 57 (1994).

1994FUJ/OGU H. Fujimori and M. Oguni, J. Chem. Thermodyn. 26, 367 (1994).

1994GRA/PER M. Gracia, P. Perez, and J. Valero, Fluid Phase Equilib. 97, 147 (1994)

1994HEN/DIE F. Hentrich, S. Diele, and C. Tschierske, Liq. Cryst. 17, 827 (1994).

1994HUA/CHE W. Huang, S. Chen, F. Tan, and Z. Yang, Wuli Huaxue Xuebao 10, 151 (1994).
1994KIM/LEE K.-J. Kim, C.-H. Lee, and S.-K. Ryu, J. Chem. Eng. Data 39, 228 (1994).

1994KOU/HOS $\quad$ B. Koutek, M. Hoskovec, P. Vrkova, K. Konecny, and L. Feltl, J. Chromatogr. A 679, 307 (1994).

1994KRA/GME M. A. Krähenbühl and J. Gmehling, J. Chem. Eng. Data 39, 759 (1994).

1994KUH/BUR M. Kuhnert-Brandstatter, A. Burger, and R. Vollenklee, Sci. Pharm. 62, 307 (1994).

1994LAR/MAR S. Larsen and K. Marthi, Acta Crystallogr., Sect. B 50, 373 (1994).

1994LEB/SMI B. Lebedev and N. Smirnova, Macromol. Chem. Phys. 195, 35 (1994).

1994LEB/SMI3 B. V. Lebedev, N. N. Smirnova, V. G. Yasil'ev, E. G. Kiparisova, and V. I. Kleiner, Polym. Sci. USSR Ser. A 36, 1171 (1994).

1994LEE/LIE M.-J. Lee, P.-J. Lien, and W.-K. Huang, Ind. Eng. Chem. Res. 33, 2853 (1994).

1994LIU/DIC K. Liu and R. M. Dickhut, Chemosphere 29, 581 (1994).

1994LOP/VAN D. O. Lopez, J. Van Braak, J. L. L. Tamarit, and H. A. J. Oonk, Calphad 18, 387 (1994).

1994ORT/GAL J. Ortega and S. Galvan, J. Chem. Eng. Data 39, 907 (1994).

1994PIA/FER V. Piacente, D. Ferro, and G. Della Gatta, Thermochim. Acta 232, 317 (1994); 235, 292 (1994).

1994RAK/VER K. Rakus, S. P. Verevkin, H.-D. Beckhaus, and C. Rüchardt, Chem. Ber. 127, 2225 (1994).

1994RIB/FER2 M. A. V. Riberio Da Silva, M. L. C. C. H. Ferrao, J. H. Fang, and F. Jiye, J. Chem. Thermodyn. 26, 839 (1994).

1994RUZ/MAJ K. Ruzicka and V. Majer, J. Phys. Chem. Ref. Data 23, 1 (1994).

1994RUZ/ZAB V. Ruzicka, Jr., M. Zabransky, K. Ruzicka, and V. Majer, Thermochim. Acta 245, 121 (1994).

1994SAB/GOU R. Sabbah and M. Gouali, Aust. J. Chem. 47, 1651 (1994).

1994SAB/TAB R. Sabbah, D. Tabet, and S. T. Belaadi, Thermochim. Acta 247, 193 (1994).

1994SIR/MAM G. Siracusa, A. Mamo, and M. Bellomo, Thermochim. Acta 244, 105 (1994).

1994SMI/MAT S. P. Smirnov, Y. N. Matiushin, and I. Z. Akmetov, Conference on International Civil Aviation Organization paper AH-DE/8-WP/12, Montreal, 14-18 February 1994, as quoted in [2002JON/LIG].

1994SPI/LUI W. Spieksma, R. Luijk, and H. A. J. Govers, J. Chromatogr. A 672, 141 (1994).

1994STE/CHI W. V. Steele, R. D. Chirico, A. Nguyen, and S. E. Knipmeyer, J. Chem. Thermodyn. 26, 515 (1994).

1994STE/CHI2 W. V. Steele, R. D. Chirico, I. A. Hossenlopp, S. E. Knipmeyer, A. Nguyen, and N. K. Smith, "Determination of ideal-gas phase enthalpies of formation for key compounds," in Experimental Results for DIPPR 1990-1991 Projects on Phase Equilibria Pure Component Properties, edited by J. R. Cunningham and D. K. Jones (AIChE, New York, 1994), pp. 188-215.

1994TAN/SAB $\quad$ Z. C. Tan and R. Sabbah, Sci. China, Ser. B: Chem., Life Sci., Earth Sci. 37, 641 (1994).

1994TAN/SAB2 Z. C. Tan and R. Sabbah, Thermochim. Acta 231, 109 (1994).

1994TAN/SAB3 $\quad$ Z. C. Tan and R. Sabbah, J. Therm. Anal. 41, 1577 (1994). 1994TAN/SAB4 Z. C. Tan and R. Sabbah, Chin. Sci. Bull. 39, 1003 (1994). 1994TER/PIA L. Terenzi and V. Piacenti, Thermochim. Acta 235, 61 (1994).

1994TIP/JIM A. E. Tipping, P. Jimenez, E. Ballesteros, J.-L. M. Abboud, M. Yanez, M. Esseffar, and J. Elguero, J. Org. Chem. 59, 1039 (1994).

1994TOR/HER L. A. Torres, I. Hernadez-Contreras, J. A. Guardado, and M. G. Gonzalez, Meas. Sci. Technol. 5, 51 (1994).

1994WAN/KUO C.-C. Wang, J.-F. Kuo, and C.-Y. Chen, Macromol. Chem. Phys. 195, 1493 (1994).

1994WAN/SHU F. Wania, W.-Y. Shui, and D. Mackay, J. Chem. Eng. Data 39, 572 (1994).

1994WEL/VER F. Welle, S. P. Verevkin, M. Keller, H.-D. Beckhaus, and C. Rüchardt, Chem. Ber. 127, 697 (1994). 
1994WIB/MOR K. B. Wiberg, K. M. Morgan, and H. Maltz, J. Am. Chem. Soc. 116, 11067 (1994).

1994ZIE/ZIE $\quad$ W. Zielenkiewicz, A. Zielenkiewicz, and K. L. Wierzchowski, J. Solution Chem. 23, 1125 (1994).

1995ABB/JIM J.-L. M. Abboud, P. Jimenez, M. V. Roux, C. Turrion, C. Lopez-Mardomingo, A. Podosenin, D. W. Rogers, and J. F. Liebman, J. Phys. Org. Chem. 8, 15 (1995).

1995 ACR/TUC W. E. Acree, Jr., S. A. Tucker, M. D. M. C. Ribeiro Da Silva, M. A. R. Matos, J. M. Goncalves, M. A. V. Ribeiro Da Silva, and G. Pilcher, J. Chem. Thermodyn. 27, 391 (1995).

1995ALM/FIN H. M. A. Al-Maydama, A. Finch, P. J. Gardner, and A. J. Head, J. Chem. Thermodyn. 27, 575 (1995).

1995ALM/FIN2 H. M. A. Al-Maydama, A. Finch, P. J. Gardner, and A. J. Head, J. Chem. Thermodyn. 27, 273 (1995).

1995ARC/BLA A. Arce, A. Blanco, J. Martinez-Ageitos, and A. Soto, J. Chem. Eng. Data 40, 515 (1995).

1995AUC/GON A. Aucejo, V. Gonzalez-Alfaro, J. B. Monton, and M. I. Vazquez, J. Chem. Eng. Data 40, 332 (1995).

1995BAH/DUP A. Bah and N. Dupont-Pavlovsky, J. Chem. Eng. Data 40, 869 (1995).

1995BAR/LOP M. Barrio, D. O. Lopez, J. Ll. Tamarit, and Y. Haget, Mater. Res. Bull. 30, 659 (1995).

1995BEC/RUC H.-D. Beckhaus, C. Rüchardt, S. I. Kozhushkov, V. N. Belov, S. P. Verevkin, and A. de Meijere, J. Am. Chem. Soc. 117, 11854 (1995).

1995BEL/AIT $\quad$ F. B. Belaribi, A. Ait-Kaci, and J. Jose, J. Therm. Anal. 44, 1177 (1995).

1995CHE/WAN G.-H. Chen, Q. Wang, Z.-M. Ma, X.-H. Yan, and S.-J. Han, J. Chem. Eng. Data 40, 361 (1995).

1995CHI/HES J. S. Chickos, D. G. Hesse, S. Hosseini, J. F. Liebman, G. D. Mendenhall, S. P. Verevkin, K. Rakus, H.-D. Beckhaus, and C. Rüchardt, J. Chem. Thermodyn. 27, 693 (1995).

$1995 \mathrm{CHI} / \mathrm{HOS} \quad$ J. S. Chickos, S. Hosseini, and D. G. Hesse, Thermochim. Acta 249, 41 (1995).

1995DEJ/BUR A. Dejoz, M. C. Burguet, R. Munoz, and M. Sanchotello, J. Chem. Eng. Data 40, 290 (1995).

1995DEL/SAB P. Del Vecchio, R. Sabbah, L. Abate, G. Garone, and G. Della Gatta, Calorim. Anal. Therm. 26, 158 (1995).

1995DIO/MIN H. P. Diogo, M. E. Minas da Piedade, J. A. Martinho Simoes, and Y. Nagana, J. Chem. Thermodyn. 27, 597 (1995).

1995DIO/SAN H. P. Diogo, R. C. Santos, P. M. Nunes, and M. E. Minas da Piedade, Thermochim. Acta 249, 113 (1995).

1995FER/DEL P. Ferloni and G. Della Gatta, Thermochim. Acta 266, 203 (1995).

1995FON/MUN J. Font and J. Muntasell, J. Mater. Chem. 5, 1137 (1995).

1995GAB/MAR C. Gabaldon, P. Marzal, and J. B. Monton, J. Chem. Eng. Data 40, 190 (1995).

1995GON/ORT E. Gonzales and J. Ortega, J. Chem. Eng. Data 40, 1178 (1995).

1995GUT/LIU J. P. Guthrie and Z. Liu, Can. J. Chem. 73, 1395 (1995).

1995JAB/LET $\quad$ S. Jabrane, J. M. Letoffe, and P. Claudy, Thermochim. Acta 258, 33 (1995).

1995JON/AUG $\quad$ D. E. G. Johnes, R. A. Augsten, K. P. Murnaghan, Y. P. Handa, and C. I. Ratcliffe, J. Therm. Anal. 44, 547 (1995).

1995 KAB/KOZ2 G. J. Kabo, A. A. Kozyro, V. V. Diky, and V. V. Simirsky, J. Chem. Eng. Data 40, 371 (1995).

1995KOB/OGU K. Kobashi and M. Oguni, J. Chem. Thermodyn. 27, 979 (1995).

1995KOL K. P. Kolesov, Thermochim. Acta 226, 129 (1995).

1995KRI/VES A. Kristl and G. Vesnaver, J. Chem. Soc., Faraday Trans. 91, 995 (1995).

1995KSI/NAG A. Ksiazczak and I. Nagata, Thermochim. Acta 254, 31 (1995).

1995LEB/BYK $\quad$ B. V. Lebedev, T. A. Bykova, E. A. Kiparisova, B. G. Velen'kaya, and V. N. Filatova, Polym. Sci. USSR Ser. A 37, 126 (1995).

1995LEB/BYK2 B. V. Lebedev, T. A. Bykova, N. V. Novoselova, N. G. Senchenya, and K. A. Mager, Polym. Sci. USSR Ser. A 37, 886 (1995).

1995LEB/CHI L. P. Lebedev, V. V. Chironov, A. N. Kizin, I. F. Falyakhov, I. Sh. Saifullin, O. R. Klyuchnikov, Y. D. Orlov, and A.
Y. Lebedev, Izv. Akad. Nauk SSSR Ser. Khim. 4, 660 (1995).

1995LEB/KUL $\quad$ B. V. Lebedev, T. G. Kulagina, N. N. Smirnova, E. G. Kiparisova, and N. V. Novoselova, Russ. J. Phys. Chem. 69, 525 (1995).

1995LEB/KUL2 B. V. Lebedev, T. G. Kulagina, V. I. Telnoy, and V. G. Vasil'ev, Macromol. Chem. Phys. 196, 3487 (1995).

1995 LUK/KOZ2 V. A. Luk'yanova and M. P. Kozina, Zh. Fiz. Khim. 69 2094 (1995); Chem. Abstr. 124, 68127f (1996).

1995MAC/JOY J. I. Macnab and J. A. Joy, Thermochim. Acta 259, 31 (1995).

1995MAR/COS T. M. R. Maria, F. S. Costa, M. L. P. Leitao, and J. S. Redinha, Thermochim. Acta 269-270, 405 (1995).

1995MAR/GAB P. Marzal, C. Gabaldon, A. Seco, and J. B. Monton, J. Chem. Eng. Data 40, 589 (1995).

1995MOK/PAU I. Mokbel, V. Pauchon, and J. Jose, ELDATA: Int. Electron. J. Phys.-Chem. Data 1, 53 (1995).

1995NAS/LEN K. Nass, D. Lenoir, and A. Kettrup, Angew. Chem., Int. Ed. Engl. 34, 1735 (1995).

1995OHT/YAM T. Ohta, O. Yamamuro, and T. Matsuo, J. Phys. Chem. 99, 2403 (1995).

1995ORT/GAL J. Ortego and S. Galvan, J. Chem. Eng. Data 40, 699 (1995).

1995PAP/PIM T. S. Papina, S. M. Pimenova, V. A. Luk'yanova, and V. P. Kolesov, Zh. Fiz. Khim. 69, 2148 (1995); Chem. Abstr. 124, 68148p (1996).

1995RAK/VER K. Rakus, S. P. Verevkin, M. Keller, H.-D. Beckhaus, and C. Rüchardt, Liebigs Ann. 1995, 1483.

1995RAK/VER2 K. Rakus, S. P. Verevkin, W.-H. Peng, H.-D. Beckhaus, and C. Rüchardt, Liebigs Ann. 1995, 2059.

1995RIB/FER M. A. V. Ribeiro Da Silva, M. L. C. C. H. Ferrao, and F. Jiye, J. Chem. Eng. Data 40, 426 (1995).

1995RIB/MAT M. A. V. Ribeiro Da Silva, M. A. R. Matos, and L. M. P. F. Amaral, J. Chem. Thermodyn. 27, 565 (1995).

1995RIB/MAT2 M. A. V. Ribeiro Da Silva, M. A. R. Matos, and L. M. P. F. Amaral, J. Chem. Thermodyn. 27, 1187 (1995).

1995RIB/MAT4 M. A. V. Ribeiro Da Silva, M. A. R. Matos, and V. M. F. Morais, J. Chem. Soc., Faraday Trans. 91, 1907 (1995).

1995RIB/MOR M. A. V. Ribeiro Da Silva, V. M. F. Morais, M. A. R. Matos, and C. M. A. Rio, J. Org. Chem. 60, 5291 (1995).

1995SAB/AGU R. Sabbah and A. R. Aguilar, Can. J. Chem. 73, 1538 (1995).

1995SAK/UEO A. Sakoguchi, R. Ueoka, Y. Kato, and Y. Arai, Kagaku Kogaku Ronbunshu 21, 219 (1995).

1995SCH/PUS J. Schmelzer and J. Pusch, Fluid Phase Equilib. 110, 183 (1995).

1995SCI/MAY J. Sciesinski, J. Mayer, T. Wasiutynski, E. Sciesinska, and J. Wojtowicz, Phase Transitions 54, 15 (1995).

1995SKA/GOL S. A. Skackelford and J. F. Goldman, Propellants, Explos., Pyrotech. 20, 1 (1995).

1995STE/CHI2 W. V. Steele, R. D. Chirico, A. Nguyen, and S. E. Knipmeyer, J. Chem. Thermodyn. 27, 311 (1995).

1995STR/ARG L. Stradella and M. Argentero, Thermochim. Acta 268, 1 (1995).

1995TAK/YAM S. Takahara, O. Yamamuro, and T. Matsuo, J. Phys. Chem. 99, 9589 (1995).

1995VAR/DRO R. M. Varushchenko and A. I. Droujinina, J. Chem. Thermodyn. 27, 355 (1995).

1995VER/BEC S. P. Verevkin, H.-D. Beckhaus, and C. Rüchardt, Thermochim. Acta 249, 1 (1995).

1995VER/DOG S. P. Verevkin, B. Dogan, J. Hadrich, H.-D. Beckhaus, and C. Rüchardt, J. Prakt. Chem. 337, 93 (1995).

1995VIE/DEQ E. F. S. Vieira, J. C. de Queiroz, and F. S. Dias, Thermochim. Acta 256, 249 (1995).

1995WOJ/TOU G. Wojcik, L. Toupet, C. Gors, and M. Foulon, Phys. Status Solidi A 147, 99 (1995).

1995WOL/LAN H. Wolff, H. Landeck, H.-P. Frerichs, and E. Wolff, Fluid Phase Equilib. 109, 245 (1995).

1995XUW/DAJ A. Xu-Wu and G. Da-Jun, Thermochim. Acta 253, 235 (1995).

1995YAM/KIT K. Yamamoto, H. Kitamura, M. Momota, and K. Narita, Thermochim. Acta 267, 313 (1995). 
1995YAS/TAK K. Yase, Y. Takahashi, N. Ara-kato, and A. Kawazu, Jpn. J. Appl. Phys., Part 1 34, 636 (1995).

1995YIN/LIU C.-M. Yin, Z.-R. Liu, Y.-H. Kong, C.-L. Jia, and X.-M. Guo, Thermochim. Acta 262, 185 (1995).

1996ACR/BOT W. E. Acree, Jr., S. G. Bott, S. A. Tucker, M. D. M. C. Ribeiro Da Silva, M. A. R. Matos, and G. Pilcher, J. Chem. Thermodyn. 28, 673 (1996).

1996BAC/GRZ D. D. Back, L. R. Grzyll, and M. Corrigan, Thermochim. Acta 272, 53 (1996).

1996BAU A. Bauer-Brandl, Int. J. Pharm. 140, 195 (1996).

1996BEL/UFN B. Belhachemi, W. Ufnalsky, and Z. Derriche, J. Chim. Phys. 93, 1117 (1996)

1996BOE/MAR A. Boehncke, K. Martin, M. G. Muller, and H. G. Cammenga, J. Chem. Eng. Data 41, 543 (1996).

1996BUR/MON M. C. Burguet, J. B. Monton, R. Munoz, J. Wisniak, and H. Segura, J. Chem. Eng. Data 41, 1191 (1996).

1996CEO/AGA R. Ceolin, V. Agafonov, D. Louer, V. A. Dzyabchenko, S. Toscani, and J. M. Cense, J. Solid State Chem. 122, 186 (1996).

1996CHA/EMM P. Chassot and F. Emmenegger, Inorg. Chem. 35, 5931 (1996).

1996CHI/SAB J. S. Chickos, R. Sabbah, S. Hosseini, and J. F. Liebman, Struct. Chem. 7, 391 (1996).

1996CHI/STE R. D. Chirico, W. V. Steele, A. Nguyen, T. D. Klots, and S. E. Knipmeyer, J. Chem. Thermodyn. 28, 797 (1996).

1996CIO/MEL I. Ciocazanu and V. Meltzer, J. Therm. Anal. 47, 1755 (1996).

1996DAH/WIC O. Dahmani, I. Wichterle, and A. Ait-Kaci, Fluid Phase Equilib. 124, 135 (1996)

1996DOM/HEA E. S. Domalski and E. D. Hearing, J. Phys. Chem. Ref. Data 25, 1 (1996), and references therein.

1996DOM/MOO U. Domanska, W. C. Moollan, and T. M. Letcher, J. Chem. Eng. Data 41, 261 (1996).

1996DOU/FUE S.-Q. Dou, H. Fuess, R. Straub, and A. Weiss, Z. Naturforsch. 51A, 777 (1996).

1996FIE/JOH C. Fiege, R. Joh, M. Petri, and J. Gmehling, J. Chem. Eng. Data 41, 1431 (1996).

1996FON/ROS L. Fontana, L. Rosati, A. Sala, and L. Dobetti, Pharm. Acta Helv. 71, 341 (1996)

1996GAL/BOU H. E. Gallis, F. Bougrioua, H. A. J. Oonk, P. J. van Ekeren, and J. C. van Miltenburg, Thermochim. Acta 274, 231 (1996).

1996GAL/VAN2 H. E. Gallis, J. C. van Miltenburg, H. A. J. Oonk, and J. P. van der Eerden, Thermochim. Acta 286, 307 (1996).

1996GAL/VAN3 H. E. Gallis and J. C. van Miltenburg, Thermochim. Acta 274, 223 (1996).

1996GIL/WIL N. F. Giles, H. L. Wilson, and W. V. Wilding, J. Chem. Eng. Data 41, 1223 (1996).

1996GOV/RUT H. A. J. Govers, E. Ruts, F. W. M. van der Wielen, and A. G. van Haelst, Polycyclic Aromat. Compd. 9, 75 (1996).

1996GRA

1996JIM/ROU

B. Granzow, J. Mol. Struct. 381, 127 (1996).

$\begin{array}{ll}\text { 1996KAM/ZIE } & \text { Chem. 7, } 375 \text { (1996). } \\ \text { M. Kaminski and W. Zielenkiewicz, J. Chem. Thermodyn. }\end{array}$

28, 153 (1996).

1996KEE/VAN E. M. D. Keegstra, V. van der Mieden, J. W. Zwikker, L. W. Jenneskens, A. Schouten, H. Kooijman, N. Veldman, and A. L. Spek, Chem. Mater. 8, 1092 (1996).

1996KOU/HOS B. Koutek, M. Hoskovec, P. Vrkocova, K. Konecny, L. Feltl, and J. Vrkoc, J. Chromatogr. A 719, 391 (1996).

1996LEB/SMI B. Lebedev, N. Smirnova, and V. Telnoy, Macromol. Chem. Phys. 197, 3807 (1996).

1996LIN/WIC J. Linek, I. Wichterle, and K. Marsh, J. Chem. Eng. Data 41, 1212 (1996).

1996LUE/MAG T. O. Lueddecke and J. W. Magee, Int. J. Thermophys. 17, 823 (1996).

1996MIR/ORL E. A. Miroshinchenko, Yu. D. Orlov, L. I. Korchatova, V. P. Vorob'eva, and Yu. A. Lebedev, Zh. Fiz. Khim. 70, 1583 (1996).

1996OLS J. D. Olson, Fluid Phase Equilib. 116, 414 (1996).

1996POL/GUE M. Polednicek, T. Guetachew, J. Jose, V. Ruzicka, V. Rohac, and M. Zabransky, ELDATA: Int. Electron. J. Phys.Chem. Data 2, 41 (1996).
1996PUL/BAR

1996RIB/MOR Struct. 382, 197 (1996).

M. A. V. Ribeiro Da Silva, V. M. F. Morais, M. A. R. Matos, C. M. A. Rio, and C. M. G. S. Piedade, Struct. Chem. 7, 329 (1996).

1996RIB/RIB M. A. V. Ribeiro Da Silva, M. D. M. C. Ribeiro Da Silva, M. F. B. M. Monteiro, M. L. A. C. N. Gomes, J. S. Chickos, A. P. Smith, and J. F. Liebman, Struct. Chem. 7, 367 (1996).

1996ROD/BER M. F. Rodrigues and M. G. Bernardo-Gil, J. Chem. Eng. Data 41, 581 (1996).

1996ROU/JIM2 M. V. Roux, P. Jimenez, J. Z. Davalos, O. Castano, M. T. Molina R. Notario, M. Herreros, and J. L. M. Abboud, J. Am. Chem. Soc. 118, 12735 (1996).

1996SAB/GOU R. Sabbah and M. Gouali, Can. J. Chem. 74, 500 (1996). $1996 \mathrm{SCH}$ 1996STE/CHI F. P. Schwarz, J. Solution Chem. 25, 471 (1996).

1996STE/CHI2 W. V. Steele, R. D. Chirico, S. E. Knipmeyer, and A. Nguyen, J. Chem. Eng. Data 41, 1255 (1996).

1996STE/CHI3 W. V. Steele, R. D. Chirico, S. E. Knipmeyer, A. Nguyen, W. V. Steele, R. D. Chirico, S. E. Knipmeyer, A. Nguyen, and N. K. Smith, J. Chem. Eng. Data 41, 1285 (1996). N. K. Smith, and I. R. Tasker, J. Chem. Eng. Data 41, 1269 (1996).

1996STE/REV S. G. Stepanian, I. D. Reva, E. D. Radchenko, and G. C. Sheina, Vib. Spectrosc. 11, 123-133 (1996).

1996STR/BRA R. Strauss, S. Braun, S.-Q. Dou, H. Fuess, and A. Weiss, Z. Naturforsch. 51A, 871 (1996).

1996SUS/SMI S. R. Susay, M. A. Smith, and G. G. Lockwood, Anesth. Analg. 83, 864 (1996).

1996TOG/TOG R. K. Toghiani, H. Toghiani, and G. Verkateswarlu, Fluid Phase Equilib. 122, 157 (1996).

1996ULB/KLU P. Ulbig, M. Klüppel, and S. Schulz, Thermochim. Acta 271, 9 (1996).

1996USH/SED V. S. Ushakov, S. M. Sedov, B. A. Knyazev, and B. I. Kuchkaev, Zh. Fiz. Khim. 70, 1573 (1996).

1996VAN/ALV J. C. van Miltenburg, A. Alvarez-Larena, M. Labrador, L. Palacios, J. Rodriquez-Romero, E. Tauler, and E. Estop, Thermochim. Acta 273, 31 (1996).

1996VAN/YU Yu.Ya. Van-Chin-Syan, V. V. Kochubei, V. V. Sergeev, Yu. A. Raevskii, S. I. Gerasimchuk, and Kh. Z. Kotovich, J. Sov. Phys. Chem. (Engl. Transl.) 70, 1789 (1996).

1996VAR/DRU R. M. Varushchenko, A. I. Druzhinina, and I. L. Pashchenko, Fluid Phase Equilib. 126, 93 (1996).

1996VAR/PAS R. M. Varushchenko, L. L. Pashchenko, and A. I. Druzhinina, Russ. J. Phys. Chem. 70, 208 (1996).

1996VER/BEC S. P. Verevkin, H.-D. Beckhaus, R. S. Belen'kaya, K Rakus, and C. Rüchardt, Thermochim. Acta 279, 47 (1996).

1996VER/PEN S. P. Verevkin, W.-H. Peng, H.-D. Beckhaus, and C. Rüchardt, Struct. Chem. 7, 397 (1996).

1996VER/ZUF S. P. Verevkin, S. Züffle, H.-D. Beckhaus, and C. Rüchardt, Thermochim. Acta 285, 1 (1996).

1996VIT/CHA C. Viton, M. Chavret, and J. Jose, ELDATA: Int. Electron. J. Phys.-Chem. Data 2, 103 (1996).

1996WEB/DEF L. A. Weber and D. R. Defibaugh, J. Chem. Eng. Data 41, 1477 (1996).

1996WEB/DEF2 L. A. Weber and D. R. Defibaugh, J. Chem. Eng. Data 41, 382 (1996).

1996ZAB/RUZ M. Zabransky, V. Ruzicka, Jr., V. Majer, and E. S. Domalski, J. Phys. Chem. Ref. Data, Monogr. 6, 1-1596 (1996).

1996ZHA/HU J. Zhang, R. Hu, C. Zhu, F. Geng, and Q. Long, Theory Pract. Energ. Mater. [Proceedings of the International Autumn Seminar on Propellants, Explosives and Pyrotechnics], Beijing, Oct. 7-10, 1996 (1996), 133-138; Chem. Abstr. 126, 133206p (1997).

1997ABA/PAL L. Abate, B. Palecz, C. Giancola, and G. D. Gatta, J. Chem. Thermodyn. 29, 359 (1997).

1997ACR/POW W. E. Acree, Jr., J. R. Powell, S. A. Tucker, M. D. M. C. Ribeiro Da Silva, M. A. R. Matos, J. M. Goncalves, L. M. N. B. F. Santos, V. M. F. Morais, and G. Pilcher, J. Org. Chem. 62, 3722 (1997).

1997ART/LAF H. Artigas, C. Lafuente, P. Cea, F. M. Royo, and J. S. Urieta, J. Chem. Eng. Data 42, 132 (1997). 
1997BAE A. E. Baev, Russ. J. Inorg. Chem. 42, 587 (1997).

1997BLA/BEL B. Blanco, S. Beltran, J. L. Cabezas, and J. Coca, J. Chem. Eng. Data 42, 938 (1997).

1997BLO/KAB A. V. Blokhin, G. K. Kabo, A. A. Kozyro, L. S. Ivashkevich, A. P. Krasulin, V. V. Diky, V. Yu, and V. Vaksimuk, Thermochim. Acta 292, 19 (1997).

1997BUS/HAM D. Busing, F. Hamann, and A. Wurflinger, Thermochim. Acta 299, 33 (1997).

1997CAL/FIL J. C. G. Calado, E. J. M. Filipe, and J. N. C. Lopes, J. Chem. Thermodyn. 29, 1435 (1997).

1997CHE/LIA J.Chen, Y. Liang, and P. Ma, Huagong Xuebao (Chin. Ed.) 48, 622 (1997).

1997DEF/CAR D. R. Defibaugh, E. Carrillo-Nava, J. J. Hurly, M. R. Moldover, J. W. Schmidt, and L. A. Weber, J. Chem. Eng. Data 42, 488 (1997).

1997DEJ/GON A. Dejoz, V. Gonzalez-Alfaro, F. J. Llopis, P. J. Migue, and M. I. Vazquez, Fluid Phase Equilib. 134, 151 (1997).

1997DEL

1997DOM/GON U. Domanska and J. A. Gonzalez, Fluid Phase Equilib. 129, 139. (1997), Secondary reference.

1997DUA/HWA H. A. Duarte-Garza, C.-A. Hwang, S. A. Kellerman, R. C. Miller, K. R. Hall, J. C. Holste, K. N. Marsh, and G. E. Gammon, J. Chem. Eng. Data 42, 497 (1997).

1997ELD J. P. Elder, J. Therm. Anal. 49, 897 (1997).

1997FIN/GAR A. Finch, P. J. Gardner, A. J. Head, and W. Xiaoping, Thermochim. Acta 298, 191 (1997).

1997HAL/WAN M. Hallquist, I. Wangberg, and E. Ljungstrom, Environ. Sci. Technol. 31, 3166 (1997).

1997HER/ORT P. Hernández and J. Ortega, J. Chem. Eng. Data 42, 1090 (1997).

1997JEN/SAN M. Jenau, M. Sandmann, A. Wurflinger, and J. L. Tamarit, Z. Naturforsch. 52A, 493 (1997).

1997KLE A. Klein, "Thermodynamics of liquid mixtures with lactones," Fortschr.-Beric VDI Reihe 3: Verfahrenstechnik 479, 1-206 (1997).

1997KOP/BEU R. Kopitzky, M. Beuleke, G. Balzer, and H. Willner, Inorg. Chem. 36, 1994 (1997).

1997KOR/API B. L. Korsounskii and T. A. Apina, Int. Annu. Conf. ICT 28(1), 46 (1997); Chem. Abstr. 127, 163997k (1997).

1997 KOU/HOS $\quad$ B. Koutek, M. Hoskovec, P. Vrkocova, and L. Feltl, J. Chromatogr. A 759, 93 (1997).

1997KUL/LEB T. T. Kulagina and B. V. Lebedev, Russ. J. Phys. Chem. 71, 520 (1997).

1997KUL/LEB2 T. G. Kulagina and B. V. Lebedev, Russ. J. Phys. Chem. 71, 709 (1997).

1997LEE/CHA M.-J. Lee, Y.-K. Chang, H.-M. Lin, and C.-H. Chen, J. Chem. Eng. Data 42, 349 (1997).

1997LIM/TUN $\quad$ L.-T. Lim and M. A. Tung, J. Food Sci. 62, 1061 (1997).

1997LOH/JOH J. Lohmann, R. Joh, and J. Gmehling, J. Chem. Eng. Data 42, 1170 (1997).

1997MIN/BEH L. M. G. Minier and R. Behrens, Jr., Propellants, Explos., Pyrotech. 22, 23 (1997).

1997MON/BUR J. B. Monton, M. C. Burguet, R. Munoz, J. Wisniak, and H. Segura, J. Chem. Eng. Data 42, 1195 (1997).

1997PEY/LET L. Peyrot, J. M. Letoffe, M. Elkhatib, J. P. Scharff, and H. Delalu, Calorim. Anal. Therm. 28, 298 (1997).

1997PFE/SAB G. Pfefer, R. Sabbah, and R. Boistelle, J. Appl. Crystallogr. 30, 527 (1997).

1997PUL/DES R. Puliti, C. De Sena, and C. Giancola, J. Therm. Anal. 48, 1249 (1997).

1997REU/BUS J. Reuter, D. Büsing, J. Ll. Tamarit, and A. Würflinger, J. Mater. Chem. 7, 41 (1997).

1997RIB/GON M. A. V. Ribeiro Da Silva, J. M. Goncalves, and G. Pilcher, J. Chem. Thermodyn. 29, 253 (1997).

1997RIB/MAT M. A. V. Ribeiro Da Silva, M. A. R. Matos, and L. M. P. F. Amaral, J. Chem. Thermodyn. 29, 1545 (1997).

1997RIB/MAT2 M. A. V. Ribeiro Da Silva, M. A. R. Matos, and L. M. P. F. Amaral, J. Chem. Thermodyn. 29, 1535 (1997).

1997RIB/MAT5 M. A. V. Ribeiro Da Silva, M. A. R. Matos, and L. M. P. F. Amaral, J. Chem. Thermodyn. 29, 295 (1997).

1997RIB/SAN M. A. V. Ribeiro Da Silva, L. M. N. B. F. Santos, and G. Pilcher, J. Chem. Thermodyn. 29, 757 (1997).
1997ROU/JIM

M. V. Roux, P. Jimenez, M. A. Martin-Luengo, J. Z. Davalos, Z. Sun, R. S. Hosmane, and J. F. Liebman, J. Org. Chem. 62, 2732 (1997).

1997SAB/PER R. Sabbah and L. Perez, Can. J. Chem. 75, 357 (1997).

1997SAK/HOR T. Sako, S. Horiguchi, H. Ichimaru, and S. Nakagawa, J. Chem. Eng. Data 42, 169 (1997).

1997SCH/VER F. Schaffer, S. P. Verevkin, H.-J. Rieger, H.-D. Beckhaus, and C. Rüchardt, Liebigs Ann. 1997, 1333.

1997SMI/LEB N. N. Smirnova, B. V. Lebedev, E. G. Kiparisova, K. L. Makovetskii, and I. Y. Ostrovskay, Polym. Sci. USSR Ser. A 39, 893 (1997).

1997STE/CHI W. V. Steele, R. D. Chirico, A. B. Cowell, S. E. Knipmeyer, and A. Nguyen, J. Chem. Eng. Data 42, 1053 (1997).

1997STE/CHI2 W. V. Steele, R. D. Chirico, S. E. Knipmeyer, and A. Nguyen, J. Chem. Eng. Data 42, 1008 (1997).

1997STE/CHI3 W. V. Steele, R. D. Chirico, S. E. Knipmeyer, and A. Nguyen, J. Chem. Eng. Data 42, 1021 (1997).

1997STE/CHI4 W. V. Steele, R. D. Chirico, S. E. Knipmeyer, A. Nguyen, and N. K. Smith, J. Chem. Eng. Data 42, 1037 (1997).

1997UKR/SOL E. A. Ukraintseva, D. V. Soldatov, and Yu. A. Dyadin, Zh. Neorgan. Khim. 42, 283 (1997).

1997URY/MOC V. F. Ur'yash, A. N. Mochalov, V. F. Kupriyanov, A. G. Smirnov, T. M. Kuleshova, and V. I. Samashkin, Russ. J. Gen. Chem. 67, 550 (1997).

1997VAR/DRU R. M. Varushchenko, A. I. Druzhinina, and E. L. Sorkin, J. Chem. Thermodyn. 29, 623 (1997).

1997VAR/PAS R. M. Varushchenko, L. L. Pashchenko, V. M. Yuldasheva, and A. P. Orlov, Russ. J. Phys. Chem. 71, 539 (1997).

1997VER

1997VER/MOR S. P. Verevkin, J. Chem. Thermodyn. 29, 891 (1997).

1997VER3

1997WEL/VER F. M. Welle, S. P. Verevkin, H.-D. Beckhaus, and C. Thermodyn. 29, 1175 (1997). Rüchardt, Liebigs Ann. 1997, 155.

1997YUA/ZHA J. Yuan, G.-L. Zhang, D.-Y. Huang, and H. Z. Zhang, Liq. Cryst. 22, 693 (1997).

1997ZEM S. Zeman, Thermochim. Acta 302, 11 (1997)

1997ZHA/HU J. Zhang, R. Hu, C. Zhu, G. Feng, and Q. Long, Thermochim. Acta 298, 31 (1997).

1997ZHA/TAN J.-B. Zhang, Z.-C. Tan, S.-H. Meng, S.-H. Li, and L.-M. Zhang, Thermochim. Acta 307, 11 (1997).

1998ABD/MEI M. A. Abdi and A. Meisen, J. Chem. Eng. Data 43, 133 (1998).

1998AUC/LOR A. Aucejo, S. Loras, R. Munoz, P. Reich, and H. Segura, J. Chem. Eng. Data 43, 973 (1998).

1998BLA/KLI W. Blanke, G. Klingenberg, and F. Weber, Int. J. Thermophys. 19, 653 (1998).

1998BOL/WIE A. Boller and H. G. Wiedemann, J. Therm. Anal. 53, 431 (1998).

1998BUS/ROM P. Bustamante, S. Romero, A. Pena, B. Escalera, and A. Reillo, J. Pharm. Sci. 87, 1590 (1998).

1998CHI/HES J. S. Chickos, D. Hesse, S. Hosseini, G. Nichols, and P. Webb, Thermochim. Acta 313, 101 (1998).

1998DEJ/GON A. Dejoz, V. Gonzalez-Alfaro, F. J. Llopis, and M. I. Vazquez, Fluid Phase Equilib. 145, 287 (1998).

1998DOM U. Domanska, Pol. J. Chem. 72, 925 (1998).

1998DRO/TOM K. G. Drouillard, G. T. Tomy, D. C. G. Muir, and K. Friesen, J. Environ. Toxicol. Chem. 17, 1252 (1998).

1998EWI/SAN M. B. Ewing and J. C. Sanchez Ochoa, J. Chem. Thermodyn. 30, 189 (1998).

1998 GEO/YOU M. A. George, K. M. Young, E. A. Robertson III, S. E. Beck, and G. Voloshin, J. Chem. Eng. Data 43, 60 (1998).

1998 GIU/BRU A. Giustini, B. Brunetti, and V. Piacenti, J. Chem. Eng. Data 43, 447 (1998).

1998 GON/SZW A. Gonthier-Vassal and H. Szwarc, Thermochim. Acta 320, 141 (1998).

1998GUD/TOR R. Gudino, L. A. Torres, R. L. Santillan, and N. Farfan, J. Chem. Thermodyn. 30, 671 (1998).

1998HAT/SUZ T. Hatanaka, R. Suzuki, K. Katayama, and T. Koizumi, Int. J. Pharm. 168, 199 (1998), Note: Experimental enthalpies of fusion and melting point temperatures were obtained through correspondence with the authors. 
1998HEL/OWE H. C. Helgeson, C. E. Owens, A. M. Knox, and L. Richard, Geochim. Cosmochim. Acta 62, 985 (1998)

1998ISS/ELA Y. M. Issa, A. L. El-Ansary, and W. Selim, Anal. Lett. 31, 131 (1998).

1998JAB/LET S. Jabrane, J. M. Letoffe, and P. Claudy, Thermochim. Acta 311, 121 (1998).

1998JAM/PAL M. E. Jamroz, M. Palczewska-Tulinska, D. Wyrzykowska-Stankiewicz, A. M. Szafranski, J. Polaczek, J. C. Dobrowolski, M. H. Jamroz, and A. P. Mazurek, Fluid Phase Equilib. 152, 307 (1998).

1998KAB/BLO G. J. Kabo, A. V. Blokhin, A. A. Kozyro, V. V. Diky, L. S. Ivashkevich, A. P. Krasulin, V. M. Sevruk, and M. Frenkel, Thermochim. Acta 313, 111 (1998).

1998KIS/KAS V. D. Kiselev, E. A. Kashaeva, and A. I. Konovalov, Russ. J. Gen. Chem. 68, 1246 (1998).

1998KOB/KYO K. Kobashi, T. Kyomen, and M. Oguni, J. Phys. Chem. Solids 59, 667 (1998).

1998KOL/PIM V. P. Kolesov, S. M. Pimenova, V. A. Lukyanova, T. S. Kuznetsova, and M. P. Kozina, J. Chem. Thermodyn. 30, 1455 (1998).

1998LEB/CHI V. P. Lebedev, V. V. Chirnonov, V. P. Vorob'eva, and Yu. N. Matyushin, Khim. Fiz. 17, 54 (1998).

1998LEB/KUL B. V. Lebedev and T. G. Kulagina, J. Therm. Anal. Calorim. 54, 731 (1998).

1998LIN/BEC A. Linden, H.-D. Beckhaus, S. P. Verevkin, C. Rüchardt, B. Ganguly, and B. Fuchs, J. Org. Chem. 63, 8205 (1998).

1998LOB/BOH S. Löbbecke, M. A. Bohn, A. Pfeil, and H. Krause, Proceedings of the 29th International Annual Conference of ICT, Karlsruhe, 1998.

1998LUS/OLI Z. Lus, L. Olivier, R. Poupko, K. Muller, C. Krieger, and H. Zimmerman, J. Am. Chem. Soc. 120, 5526 (1998).

1998MAG J. W. Magee, Int. J. Thermophys. 19, 1397 (1998).

1998MAK/KAB Y. V. Maksimuk, G. J. Kabo, V. V. Simirsky, A. A. Kozyro, and V. M. Sevruck, J. Chem. Eng. Data 43, 293 (1998).

1998MCK/FLO R. L. McKenney, Jr., T. G. Floyd, W. E. Stevens, T. G. Archibald, A. P. Marchand, G. V. M. Sharma, and S. G. Bott, J. Energ. Mater. 16, 1 (1998).

1998 MOR/KOP K. M. Morgan and D. A. Kopp, J. Chem. Soc., Perkin Trans. 2 1998, 2759.

1998MUR/BET P. Mura, G. P. Bettinetti, M. T. Faucci, and P. L. Parrini, Thermochim. Acta 321, 59 (1998).

1998NOL/VAL O. Noll, A. Valtz, D. Richon, T. Getachew-Sawaya, I. Mokbel, and J. Jose, ELDATA: Int. Electron. J. Phys.Chem. Data 4, 105 (1998).

1998OLS J. D. Olson, Fluid Phase Equilib. 150-151, 713 (1998)

199800 N/VAN H. A. J. Oonk, P. R. van der Linde, J. Huinink, and J. G. Blok, J. Chem. Thermodyn. 30, 897 (1998).

1998PAR/GIL R. M. Paroli, D. F. R. Gilson, and I. S. Butler, Can. J. Chem. 76, 1365 (1998), Note: Authors report only an entropy of transition and a temperature range for the first two transitions. The temperature that is listed is the midpoint of the reported temperature range, and the transition enthalpy is computed as the product of the reported transition entropy times transition temperature as defined above.

1998PAR/GIL2 R. M. Paroli, D. F. R. Gilson, and I. S. Butler, J. Solid State Chem. 136, 16 (1998).

1998PRI/HAW D. M. Price and M. Hawkins, Thermochim. Acta 315, 19 (1998).

1998RAI/RAI U. S. Rai and R. N. Rai, J. Therm. Anal. 53, 883 (1998). 1998RAI/RAI2 U. S. Rai and R. N. Rai, Asian J. Chem. 10, 421 (1998).

1998RIB/CAR M. A. V. Ribeiro Da Silva, A. P. S. M. C. Carvalho, M. J. S. Monte, and E. Giera, J. Chem. Thermodyn. 30, 815 (1998).

1998RIB/MAT M. D. M. C. Ribeiro Da Silva, M. A. R. Matos, M. C. Vas, L. M. N. B. F. Santos, G. Pilcher, W. E. Acree, Jr., and J. R. Powell, J. Chem. Thermodyn. 30, 869 (1998).

1998RIB/MAT2 M. D. M. C. Ribeiro Da Silva, M. A. R. Matos, G. Pilcher, and W. E. Acree, Jr., J. Chem. Thermodyn. 30, 271 (1998).

1998ROH/RUZ V. Rohac, V. Ruzicka, K. Ruzicka, and K. Aim, J. Chem. Eng. Data 43, 770 (1998).
1998ROU/JIM

M. V. Roux, P. Jimenez, J. Z. Davalos, C. Turrion, H. Y. Afeefy, and J. F. Liebman, J. Chem. Soc., Faraday Trans. 94, 887 (1998).

1998SAB/DAS R. Sabbah and M. E. da silva Eusebio, Can. J. Chem. 76, 18 (1998).

1998SAB/HEV R. Sabbah and R. Hevia, Thermochim. Acta 313, 131 (1998).

1998SAB/HEV2 R. R. Sabbah, Hevia, and D. Tabet, Thermochim. Acta 316, 1 (1998).

1998SAB/KUA R. Sabbah, D. N. Kuakuvi, and L. Perez, Thermochim. Acta 316, 137 (1998).

1998SAB/TAB R. Sabbah, D. Tabet, and M. E. S. Eusebio, Thermochim. Acta 315, 93 (1998).

1998SEM/WIL B. Semeniuk and H. Wilczura-Wachnik, Fluid Phase Equilib. 152, 337 (1998).

1998SOR/KIM M. Sorai, K. Kimura, A. Weiss, and R. Strauss, J. Chem. Thermodyn. 30, 1441 (1998).

1998STO/NG L. D. Stockton, T. L. Ng, N. Maung, I. B. Poole, J. O. Williams, A. C. Wright, D. R. Foster, and D. J. ColeHamilton, J. Cryst. Growth 183, 95 (1998).

1998 SVO/HYN V. V. Svoboda, Hynek, and B. Koutek, J. Chem. Thermodyn. 30, 1411 (1998).

1998VAR/DRU R. M. Varushchenko and A. I. Druzhinina, J. Chem. Thermodyn. 30, 697 (1998).

1998VAS/LEB V. G. Vasil'ev and B. V. Lebedev, Polym. Sci. Ser. A 40, 464 (1998).

1998VER

1998VER/BEC2

S. P. Verevkin, J. Chem. Thermodyn. 30, 1029 (1998).

S. P. Verevkin, H.-D. Beckhaus, C. Rüchardt, R. Haag, S. I. Kozhushkov, T. Zywietz, A. De Meijere, H. Jiao, and P. v. R. Schleyer, J. Am. Chem. Soc. 120, 11130 (1998).

1998VER/KUM S. P. Verevkin, M. Kümmerlin, H.-D. Beckhaus, C. Galli, and C. Rüchardt, Eur. J. Org. Chem. 1998, 579.

1998VER/PEN S. P. Verevkin, W.-H. Peng, H. D. Beckhaus, and C. Rücuardt, Eur. J. Org. Chem. 1998, 2323.

1998VER/WEL S. P. Verevkin and F. M. Welle, Struct. Chem. 9, 215 (1998).

1998VER2 S. P. Verevkin, J. Chem. Thermodyn. 30, 1069 (1998).

1998VER3 S. P. Verevkin, Struct. Chem. 9, 375 (1998).

1998VER4

1998VER6

1998WIL/LIU

S. P. Verevkin, Thermochim. Acta 310, 229 (1998).

S. P. Verevkin, Struct. Chem. 9, 113 (1998).

G. O. Williams III and J. Liu, Int. J. Pharm. 166, 99 (1998).

1998ZHA/MO X. Zhang, H. Mo, K. Yang, and F. An, Huanjing Huaxue 17, 50 (1998); Chem. Abstr. 128, 318311d (1998), Value is referred to as a vaporization enthalpy.

1998ZIE/WSZ A. Zielenkiewicz, M. Wszelaka-Rylik, J. Poznanski, and W. Zielenkiewicz, J. Solution Chem. 27, 235 (1998).

1999AHL/LOH J. Ahlers, J. Lohmann, and J. Gmehling, J. Chem. Eng. Data 44, 727 (1999).

1999ANT/FRA M. Antosik, Z. Fras, and S. K. Malanowski, J. Chem. Eng. Data 44, 368 (1999).

1999AUC/LOR A. Aucejo, S. Loras, R. Munoz, and L. M. Ordonez, Fluid Phase Equilib. 156, 173 (1999).

1999BAU/MAR A. Bauer-Brandl, E. Marti, A. Geoffroy, A. Poso, J. Suurkuusk, E. Wappler, and K. H. Bauer, J. Therm. Anal. Calorim. 57, 7 (1999).

1999BRU/PIA B. Brunetti and V. Piacenti, J. Chem. Eng. Data 44, 809 (1999).

1999CHI/KNI R. D. Chirico, S. E. Knipmeyer, A. Nguyen, and W. V. Steele, J. Chem. Thermodyn. 31, 339 (1999).

1999COS/EUS F. S. Costa, M. E. Eusebio, J. S. Redinha, and M. L. P. Leitao, J. Chem. Thermodyn. 31, 895 (1999).

1999DAV/FLO J. Z. Davalos, H. Flores, P. Jimenez, R. Notano, M. V. Roux, E. Juaristi, R. S. Hosmane, and J. F. Liebman, J. Org. Chem. 64, 9328 (1999).

1999DEF R. F. de Farias, Quim. Nova 22, 509 (1999).

1999DEF/DEO R. F. de Farias, O. A. de Oliveira, J. V. Medeiros, and C. Airoldi, Thermochim. Acta 328, 241 (1999).

1999DEL/BAR P. Del Vecchio, B. Barone, R. Sabbah, G. Della Gatta, and L. Abate, J. Chem. Thermodyn. 31, 1001 (1999).

1999DIA/GUE M. A. E. Diaz, T. Guetachew, P. Landy, J. Jose, and A. Voilley, Fluid Phase Equilib. 157, 257 (1999). 
1999DOL/LEC G. Dollo, P. Le Corre, M. Chollet, F. Chevanne, M. Bertault, J.-L. Burgot, and R. Le Verge, J. Pharm. Sci. 88, 889 (1999).

1999DRU/VAR A. I. Druzhinina and R. M. Varushchenko, Russ. J. Phys. Chem. 73, 1367 (1999).

1999DUT/TAK R. Dutt, Y. Takahashi, and M. Iijima, Jpn. J. Appl. Phys., Part 2 38, L687-L690 (1999).

1999DYK/SVO J. Dykyj, J. Svoboda, R. C. Wilhoit, M. L. Frenkel, and K. R. Hall, Vapor Pressure of Chemicals: Part A. Vapor Pressure and Antoine Constants for Hydrocarbons and Sulfur, Selenium, Tellurium and Hydrogen Containing Organic Compounds (Springer, Berlin, 1999), Note: The vaporization enthalpies were calculated from the vapor pressures obtained from the Antoine constants reported in this compendium. In cases where the Antoine constant $\mathrm{C}=0$ the Antoine Equation $(\log 10 \mathrm{P}=\mathrm{A} \mathrm{B} /(\mathrm{C}+\mathrm{T}))$ reduces to the integrated form of the Clausius Clapeyron equation directly. This was the case for many vaporization enthalpies. In those cases where this condition was not met the vaporization enthalpy was calculated as $\operatorname{DvapHm}(\mathrm{T})$ $=2303 \mathrm{R} \mathrm{B}[\mathrm{T} /(\mathrm{T}+\mathrm{C})] 2$.

1999ESC/SAN G. N. Escobedo-Alvarado and S. I. Sandler, J. Chem. Eng. Data 44, 319 (1999).

1999FAT D. Fatu, J. Therm. Anal. Calorim. 56, 739 (1999).

1999FLO/DAV H. Flores, J. Z. Davalos, J. L. M. Abboud, O. Castano, R. Gomperts, P. Jimenez, R. Notario, and M. V. Roux, J. Phys. Chem. A 103, 7555 (1999).

1999GAR/AND R. Garriga, A. C. Andres, P. Perez, and M. Gracia, J. Chem. Eng. Data 44, 296 (1999).

1999GIO/BET F. Giordano, R. Bettini, C. Donini, A. Gazzaniga, M. R. Caira, G. G. Z. Zhang, and D. J. W. Grant, J. Pharm. Sci. 88, 1210 (1999).

1999GIR/PIE D. Giron, P. Piechon, C. Goldbronn, and S. Pfeffer, J. Therm. Anal. Calorim. 57, 61 (1999).

1999 GON/ORT C. G. Gonzalez, J. P. Ortega, P. Hernandez, and S. Galvan, J. Chem. Eng. Data 44, 772 (1999).

1999GOT/BUH A. Gotze, C. Buhrmester, I. Svoboda, and H. Fuess, Croatica Chim. Acta 72, 443 (1999).

1999GRI/AUE U. J. Griesser, M. E. Auer, and A. Burger, Sci. Pharm. 67, 319 (1999)

1999GRI/SZE U. J. Griesser, M. Szelagiewicz, U. Ch. Hofmeier, C. Pitt, and S. Ciaferani, J. Therm. Anal. 57, 45 (1999).

1999HAM/WUR F. Hamann and A. Wurflinger, Z. Phys. Chem. 211, 85 (1999).

1999HAN/ZHU N. Han, L. Zhu, and R. Fu, Sep. Purif. Technol. 16, 175 (1999).

1999HEI/FIS A. Heine, K. Fischer, and J. Gmehling, J. Chem. Eng. Data 44, 373 (1999)

1999JON/FEN D. E. G. Jones, H. T. Feng, R. A. Austen, and R. C. Fouchard, J. Therm. Anal. Calorim. 55, 9 (1999); Chem. Abstr. 132, 182729u (2000).

1999KAB/KOZ G. J. Kabo, A. A. Kozyro, M. Frenkel, and A. V. Blokhin, Mol. Cryst. Liq. Cryst. 326, 333 (1999).

1999KOB/OGU K. Kobashi and M. Oguni, J. Phys. Chem. B 103, 7687 (1999).

1999LEB/KUL ～B. V. Lebedev, T. G. Kulagina, and E. G. Kiparisova, Russ. J. Phys. Chem. 73, 521 (1999).

1999LEI/WAN Y. D. Lei, F. Wania, and W. Y. Shiu, J. Chem. Eng. Data 44, 577 (1999).

1999LEI/WAN2 Y. D. Lei, F. Wania, W. Y. Shiu, and D. G. B. Boocock, J. Chem. Eng. Data 44, 200 (1999).

1999LI/TAN L. Li, Z. C. Tan, S. H. Meng, and Y. J. Song, Thermochim. Acta 342, 53 (1999).

1999LI/ZEL Z. J. Li, M. T. Zell, E. J. Munson, and D. J. W. Grant, J. Pharm. Sci. 88, 337 (1999).

1999LIM/PAR J. S. Lim, Y.-Y. Park, B.-G. Lee, Y.-W. Lee, and J.-D. Kim, J. Chem. Eng. Data 44, 1226 (1999).

1999LOR/AUC S. Loras, A. Aucejo, and R. Munoz, Fluid Phase Equilib. 156, 185 (1999).

1999MAR/BAS G. Marchionni, M. Bassi, G. Fontana, P. Maccone, and G. Ajroldi, J. Fluorine Chem. 98, 41 (1999).

1999MAT/PEP Y. N. Matyushin, V. I. Pepekin, V. P. Lebedev, V. V. Chironov, L. M. Kostikova, Y. O. Inozemtcev, T. S. Pivina, and A. B. Sheremetev, Int. Annu. Conf. ICT 30, 77/1 (1999).
1999MCK/STE

R. L. McKenney, Jr., W. E. Stevens, and T. G. Floyd, J. Energ. Mater. 17, 113-140 (1999).

1999MEN/LIA S. H. Meng, P. Liang, Z. C. Tan, Y. J. Song, L. Li, and L. Wang, Thermochim. Acta 342, 47 (1999).

1999MIC/NEG F. Michaud, Ph. Negrier, D. Mikailitchenko, A. Marbeuf, Y. Haget, M. Cuevas-Diarte, and H. A. J. Oonk, Mol. Cryst. Liq. Cryst. 326, 409 (1999).

1999MIK/MAR D. Mikailitchenko, A. Marbeug, and H. A. J. Oonk, Chem. Mater. 11, 2866 (1999).

1999MIR/VOR E. A. Miroshnichenko and V. P. Vorob'eva, Russ. J. Phys. Chem. 73, 349 (1999).

1999MIR/VOR2 E. A. Miroshnichenko and V. P. Vorob'eva, Zh. Fiz. Khim. 73, 419 (1999).

1999MO/YAN O. Mo, M. Yanez, M. V. Roux, P. Jimenez, J. Z. Davalos, M. A. V. Ribeiro da Silva, M. D. M. C. Ribeiro da Silva, M. A. R. Matos, L. M. P. F. Amaral, A. Sanchez-Migallon, P. Cabildo, R. Claramunt, J. Elguero, and J. F. Liebman, J. Phys. Chem. A 103, 9336 (1999); Erratum, J. Phys. Chem. A 117, 10569 (2013).

1999MOK/PRC L. Mokbel, S. Prcedda, T. Guetachew, B. Marongiu, and J. Jose, ELDATA: Int. Electron. J. Phys.-Chem. Data 5, 79 (1999).

1999MON/DEL J. B. Monton, J. de la Torre, M. C. B urguet, R. Munoz, and S. J. Loras, J. Chem. Eng. Data 44, 1158 (1999).

1999MON/HIL M. J. S. Monte and D. M. Hillesheim, J. Chem. Thermodyn. 31, 1433 (1999).

1999MUN/LEE S. Y. Mun and H. Lee, J. Chem. Eng. Data 44, 1231 (1999).

1999NGU/BER J. N'Guimbi, C. Berro, I. Mokbel, E. Rauzy, and J. Jose, Fluid Phase Equilib. 162, 143 (1999).

1999OJA/SUU V. Oja and E. M. Suuberg, J. Chem. Eng. Data 44, 26 (1999).

1999ORT/HER J. Ortega and P. Hernandez, J. Chem. Eng. Data 44, 757 (1999).

1999PAR/BAR L. C. Pardo, M. Barrio, J. Ll. Tamarit, D. O. Lopez, J. Salud, P. Negrier, and D. Mondieig, Chem. Phys. Lett. 308, 204 (1999).

1999PRI/BAS $\quad$ D. M. Price, S. Bashir, and P. R. Derrick, Thermochim. Acta 327, 167 (1999).

1999RAI/RAI 1999RAI/SHE U. S. Rai and R. N. Rai, J. Mater. Res. 14, 1299 (1999). U. S. Rai and H. Shekhar, Asian J. Chem. 11, 453 (1999).

1999RAR/HOR J. Rarey, S. Horstmann, and J. Gmehling, J. Chem. Eng. Data 44, 532 (1999).

1999RIB/MAT M. A. V. Ribeiro da Silva, M. A. R. Matos, M. J. S. Monte, D. M. Hillesheim, M. C. P. O. Marques, and N. F. T. G. Vieira, J. Chem. Thermodyn. 31, 1429 (1999).

1999RIB/MAT2 M. A. V. Ribeiro da Silva, M. A. R. Matos, V. M. F. Morais, and M. S. Miranda, J. Org. Chem. 64, 8816 (1999).

1999RIB/MON M. A. V. Ribeiro da Silva, M. J. S. Monte, and J. R. Ribeiro, J. Chem. Thermodyn. 31, 1093 (1999).

1999RIB/RIB M. A. V. Ribeiro da Silva, M. D. M. C. Ribeiro da Silva, M. A. R. Matos, P. Jimenez, M. V. Roux, J. Elguero, R. Claramunt, P. Cabildo, and A. Sanchez-Migallon, J. Chem. Thermodyn. 31, 129 (1999).

1999ROH/MUS V. Rohac, J. E. Musgrove, K. Ruzicka, V. Ruzicka, M. Zabransky, and K. Aim, J. Chem. Thermodyn. 31, 971 (1999).

1999ROH/RUZ V. Rohac, V. Ruzicka, K. Ruzicka, M. Polednicek, K. Aim, J. Jose, and M. Zabransky, Fluid Phase Equilib. 157, 121 (1999).

1999ROU/DAV M. V. Roux, J. Z. Davalos, P. Jimenez, H. Flores, J. L. Saiz, J. L. M. Abboud, and E. Juaristi, J. Chem. Thermodyn. 31, 635 (1999).

1999ROU/JIM M. V. Roux, P. Jimenez, J. Z. Davalos, R. Notaro, and J.-L. M. Abboud, J. Chem. Thermodyn. 31, 1457 (1999).

1999SAB/IDE 1999SAB/PER 1999SAB/PER2 1999SAL/LOP R. Sabbah and S. Ider, Can. J. Chem. 77, 249 (1999). R. Sabbah and L. Perez, Aust. J. Chem. 52, 235 (1999). R. Sabbah and L. Perez, Can. J. Chem. 77, 1508 (1999). J. Salud, D. O. Lopez, M. Barrio, and J. Lltamarit, J. Mater. Chem. 9, 909 (1999).

1999SHA/BUD S. D. Sharma, D. Buddhi, and R. L. Sawhney, Sol. Energy 66, 483 (1999). 
1999SHE/KAM M. S. Sheiman, G. P. Kamelova, I. A. Zelyaev, and V. M. Sheiman, Russ. J. Phys. Chem. 73, 512 (1999).

1999SUG S. Suga, Thermochim. Acta 328, 9 (1999).

1999TOC/AKI K. Tochigi, K. Akimoto, K. Ochi, F. Liu, and Y. Kawase, J. Chem. Eng. Data 44, 588 (1999).

1999VER S. P. Verevkin, J. Chem. Thermodyn. 31, 1397 (1999).

1999VER/EBE S. P. Verevkin and J. Ebenhoch, Struct. Chem. 10, 401 (1999).

1999VER/HEI S. P. Verevkin and A. Heintz, J. Chem. Eng. Data 44, 1240 (1999).

1999VER2

1999VER4

1999VER6

1999VER7

1999VER8

1999WAT/IIY

1999WEB

1999WEL/DRU

S. P. Verevkin, J. Chem. Thermodyn. 31, 559 (1999).

S. P. Verevkin, J. Chem. Eng. Data 44, 1245 (1999).

S. P. Verevkin, Thermochim. Acta 326, 17 (1999).

S. P. Verevkin, Phys. Chem. Chem. Phys. 1, 127 (1999).

S. P. Verevkin, Thermochim. Acta 332, 27 (1999).

A. Watanabe, T. Iiyama, and K. Kaneko, Chem. Phys. Lett. 305, 71 (1999).

F. Weber, PTB-Mitteilungen 109, 469 (1999).

1999ZAR/CHA M. I. Zaretskii, E. M. Chartov, L. A. Pushkina, and V. V. Elkin, Russ. J. Appl. Chem. 72, 1702 (1999).

1999ZAR/CHA2 M. I. Zaretskii, E. M. Chartov, L. A. Pushkina, V. V. Elkin, and V. V. Rusak, Zh. Prikl. Khim. 72, 1436 (1999).

$1999 Z H E / K A T \quad$ V.P.Zhelezny, Y. A. Katchurka, and M. V. Pybnikov, High Temp.-High Pressures 31, 169 (1999).

1999ZIE/GOL W. Zielenkiewicz, B. Golankiewicz, G. L. Pelovich, and M. Kozbial, J. Sol. Chem. 28, 731 (1999).

1999ZIE/PER W. Zielenkiewicz, G. L. Perlovich, and M. WszelakaRylik, J. Therm. Anal. Calorim. 57, 225 (1999).

2000AGU/GUA A. R. Aguilar and E. O. Guareno, J. Chem. Thermodyn. 32, 767 (2000).

2000ATA/KAW T. Atake, H. Kawaji, T. Tojo, K. Kawasaki, Y. Ootsuka, M. Katou, and Y. Koga, Bull. Chem. Soc. Jpn. 73, 1987 (2000).

2000BEL/BEL B. F. Belaribi, G. Belaribi-Boukais, A. A. Kaci, and J. Jose, J. Therm. Anal. Calorim. 61, 787 (2000).

2000BOB/CAM S. Bobbo, R. Camporese, and R. Stryjek, J. Chem. Thermodyn. 32, 1647 (2000).

2000BOU/YE E. Bourret-Courchesne, Q. Ye, D. W. Peters, J. Arnold, M. Ahmed, S. J. C. Irvine, R. Kanjolia, L. M. Smith, and S. A. Rushworth, J. Cryst. Growth 217, 47 (2000).

2000BRO/DU S. Brownridge, H. Du, S. A. Fairhurst, R. C. Haddon, H. Oberhammer, S. Parson, J. Passmore, M. J. Schriver, L. H. Sutcliffe, and N. P. C. Westwood, J. Chem. Soc., Dalton Trans. 2000, 3365.

2000BRU/DEL B. Brunetti, G. Della Gatta, and V. Piacente, J. Chem. Eng. Data 45, 237 (2000).

2000BRU/PIA B. Brunetti, V. Piacente, and G. Portalone, J. Chem. Eng. Data 45, 242 (2000).

$2000 \mathrm{CHA} / \mathrm{SOS} \quad$ C. Chaimbault, J. J. Sosc, J. M. Leger, P. Negrier, F. Capelle, and C. Jarry, J. Pharm. Sci. 89, 1496 (2000).

2000DEL/JOZ G. Della Gatta, M. Jozwiak, B. Brunetti, and L. Abate, J. Chem. Thermodyn. 32, 979 (2000).

2000DI/LI Y.-Y. Di, S. Li, S.-H. Meng, Z.-C. Tan, and S.-S. Qu, Huaxue Xuebao 58, 1380 (2000); Chem. Abstr. 134, 10187r (2001).

2000DI/TAN Y. Y. Di, Z.-C. Tan, X.-M. Xu, S.-H. Meng, and S.-S. Qu, Thermochim. Acta 356, 143 (2000).

2000GAL/VAN H. E. Gallis, J. C. van Miltenburg, and H. A. J. Oonk, Phys. Chem. Chem. Phys. 2, 5619 (2000).

2000GAN/BOG R. B. Gandhi, J. B. Bogardus, D. E. Bugay, R. K. Perrone, and M. A. Kaplan, Int. J. Pharm. 201, 221 (2000).

2000GRI/AUE U. J. Griesser, M. E. Auer, and A. Burger, Microchem. J. 65, 283 (2000).

2000HAN/BOT E. Handelsman-Benory, M. Botoshansky, M. Greenberg, V. Shteiman, and M. Kaftory, Tetrahedron 56, 6887 (2000).

2000HAN/PAR B. C. Hancock and M. Parks, Pharm. Res. 17, 397 (2000).

2000JAR/MAR S. Jarmelo, T. M. R. Maria, M. L. P. Leitao, and R. Fausto, Phys. Chem. Chem. Phys. 2, 1155 (2000).

2000JOH/PYK G. P. Johari and D. Pyke, Phys. Chem. Chem. Phys. 2, 5479 (2000)
2000KAB/BLO

G. J. Kabo, A. V. Blokhin, M. B. Charapennikau, A. G. Kabo, and V. M. Sevruk, Thermochim. Acta 345, 125 (2000).

2000KAN/SAM S. K. Kang and E. T. Samulski, Liq. Cryst. 27, 371 (2000).

2000KIR

2000KOZ/MAK

D. Kirklin, J. Chem. Thermodyn. 32, 701 (2000).

2000LAR/LER L. Larachi, M. Leroux, S. Hamoudi, A. Bernis, and A Sayari, J. Chem. Eng. Data 45, 404 (2000).

2000LIS/JAM Z. Lisicki and M. E. Jamroz, J. Chem. Thermodyn. 32 , 1335 (2000).

2000MAR/MIK A. Marbeuf, D. Mikailitchenko, P. Negrier, M.-A. CuevasDiarte, and T. Calvet-Pallas, Chem. Mater. 12, 3280 (2000).

2000MAT/MIR M. A. R. Matos, M. S. Miranda, and V. M. F. Morais, J. Phys. Chem. A 104, 9260 (2000).

2000MOK/RUZ I. Mokbel, K. Ruzicka, V. V. Majer, Ruzicka, M. Ribeiro, J. Jose, and M. Zabransky, Fluid Phase Equilib. 169, 191 (2000).

2000MON/HIL M. J. S. Monte and D. M. Hillesheim, J. Chem. Thermodyn. 32, 1727 (2000).

2000MON/HIL2 M. J. S. Monte and D. M. Hillesheim, J. Chem. Eng. Data 45, 1088 (2000)

2000NIC/ORF G. Nichols, J. Orf, S. M. Reiter, J. Chickos, and G. W. Gokel, Thermochim. Acta 346, 15 (2000).

200000 N/VAN H. A. J. Oonk, A. C. G. van Genderen, J. G. Blok, and P. R. van der Linde, Phys. Chem. Chem. Liq. 2, 5614 (2000).

$2000 \mathrm{OVA} / \mathrm{KOU} \quad$ P. V. Ova, B. Koultek, and M. Hoskovec, in Practice Oriented Results on Use and Production of Neem-Ingredients and Pheromones VI, edited by H. Kleeberg and C. P. W. Zebitz (Druck and Graphic, Giessen, 2000), pp. 211-218.

2000PAL/SZA M. Palczewska-Tulinska and A. M. Szafranski, J. Chem. Eng. Data 45, 988 (2000).

2000REI/CAR R. Reich, M. Cartes, H. Segura, and J. Wisniak, Phys. Chem. Liq. 38, 217 (2000).

2000RES/GON J. M. Resa, C. Gonzalez, S. Ortiz de Landaluce, and J. Lanz, J. Chem. Eng. Data 45, 867 (2000).

2000RIB/GON M. D. M. C. Ribeiro da Silva, J. M. Goncalves, and W. E. Acree, Jr., J. Chem. Thermodyn. 32, 1071 (2000).

2000RIB/MAT M. A. V. Ribeiro da Silva, M. A. R. Matos, C. M. A. Rio, M. S. Miranda, and V. M. F. Morais, J. Phys. Chem. A 104, 6644 (2000).

2000RIB/MON M. A. V. Ribeiro da Silva, M. J. S. Monte, and J. R. Ribeiro, J. Chem. Eng. Data 45, 756 (2000).

2000RIB/RIB2 M. A. V. Ribeiro da Silva, M. D. M. C. Ribeiro da Silva, M. A. R. Matos, P. Jimenez, M. V. Roux, M. A. MartinLuengo, J. Elguero, R. Claramut, and P. Cabildo, J. Chem. Thermodyn. 32, 237 (2000).

2000ROD/ART S. Rodriguez, H. Artigas, C. Lafuente, A. M. Mainar, and F. M. Royo, Thermochim. Acta 362, 153 (2000).

2000ROD/VEC F. Rodante, S. Vecchio, G. Catalani, and M. Guidotti, J. Therm. Anal. Calorim. 60, 605 (2000).

2000TAM/LOP J. Ll. Tamarit, D. O. Lopez, X. Alcobe, M. Barrio, J. Salud, and L. C. Pardo, Chem. Mater. 12, 555 (2000).

2000TOZ/AKU Y. Tozuka, H. Akutsu, Y. Yamamura, K. Saito, and M. Sorai, Bull. Chem. Soc. Jpn. 73, 2279 (2000).

2000URY/KUP V. F. Ur'yash, V. F. Kupriyanov, N. Y. Kokurina, A. G. Smirnov, and T. M. Kuleshova, Russ. J. Gen. Chem. 70, 719 (2000).

2000VAN/OON J. C. van Miltenburg, H. A. J. Oonk, and G. J. K. van den Berg, J. Chem. Eng. Data 45, 704 (2000).

2000VER S. P. Verevkin, J. Chem. Thermodyn. 32, 207 (2000).

2000VER/HEI S. P. Verevkin and A. Heintz, J. Chem. Thermodyn. 32 , 1169 (2000).

2000VER/SCH S. P. Verevkin and C. Schick, J. Chem. Eng. Data 45, 946 (2000).

2000VER/WAN S. P. Verevkin, D. Wandschneder, and A. Heintz, J. Chem. Eng. Data 45, 618 (2000).

2000VER2 S. P. Verevkin, J. Chem. Eng. Data 45, 953 (2000).

2000VER3

2000WOR/FEN
S. P. Verevkin, J. Chem. Thermodyn. 32, 247 (2000). C. J. Wormald and D. P. Fennell, Int. J. Thermophys. 21, 767 (2000). 
2000WOR/JAM C. J. Wormald and G. F. James, J. Chem. Eng. Data 45, 348 (2000).

2000WOR/VIN C. J. Wormald and M. D. Vine, J. Chem. Thermodyn. 32, $329(2000)$.

2000WOR/VIN2 C. J. Wormald and M. D. Vine, J. Chem. Thermodyn. 32, 659-669 (2000).

2000YAM/TAN M. Yamazaki, M. Tanaka, T. Inoue, Y. Suzuki, Y. Nibu, H. Shimada, and R. Shimadu, Bull. Chem. Soc. Jpn. 73, 837 (2000).

2000ZIE W. Zielenkiewicz, J. Chem. Eng. Data 45, 626 (2000).

2001AHN/HAR S. Ahn, K. D. M. Harris, B. M. Kariuki, and D. M. S. Zin, J. Solid State Chem. 156, 10 (2001).

2001ALB K. C. Albyn, J. Chem. Eng. Data 46, 1415 (2001).

2001ALB/HAH M. Albert, I. Hahnenstein, H. Hasse, and G. Maurer, J. Chem. Eng. Data 46, 897 (2001).

2001BAE A. K. Baev, Izv. Vyssh. Uchebn. Zaved., Khim. Khim. Tekhnol. 44, 3 (2001).

2001BER/WIC S. Bernatova and I. Wichterle, Fluid Phase Equilib. 189, 111 (2001).

2001BLO/VAN J. G. Blok, A. C. G. van Genderen, P. R. van der Linde, and H. A. J. Oonk, J. Chem. Thermodyn. 33, 1097 (2001).

2001CAL/MEL M. Calligaris, A. Melchior, and S. Geremia, Inorg. Chim. Acta 323, 89 (2001).

2001CEN/LIP ～M. Censky, M. Lipovska, H.-G. Schmidt, V. Ruzicka, and G. Wolf, J. Therm. Anal. Calorim. 63, 879 (2001).

2001CHA/DOL K. Chatterjee, D. Dollimore, and K. Alexander, Instrum. Sci. Technol. 29, 133 (2001); Chem. Abstr. 135, 16629t (2001), reported values are referred to as enthalpies of vaporization.

2001CHA/TOB S. Chattopadhyay, H. J. Tobias, and P. J. Ziemann, Anal. Chem. 73, 3797 (2001).

2001CHE/HUA Y. Cheng, Y. Huang, K. Alexander, and D. Dollimore, Thermochim. Acta 367/368, 23 (2001).

2001CHY/FRA K. Chylinski, Z. Fras, and S. K. Malanowski, J. Chem. Eng. Data 46, 29 (2001).

2001DI/SUN2 Y.-Y. Di, X.-H. Sun, Z.-C. Tan, Y.-F. Liu, S.-H. Meng, S.L. Gao, and S.-S. Qu, Thermochim. Acta 369, 25 (2001).

2001ESP/CEO P. Espeau and R. Ceolin, J. Phys. IV 11, 207 (2001).

2001FOR/HEM A. Forster, J. Hempenstall, I. Tucker, and T. Rades, Int. J. Pharm. 226, 147 (2001).

2001HE/STO X. He, J. G. Stowell, K. R. Morris, R. R. Pfeiffer, H. Li, P. Stahly, and S. R. Byrn, Cryst. Growth Des. 1, 305 (2001).

2001HEI/VER A. Heintz and S. P. Verevkin, Fluid Phase Equilib. 179, 85 (2001).

2001HOR/GAR S. Horstmann, H. Gardeler, K. Fischer, F. Köster, and J. Gmehling, J. Chem. Eng. Data 46, 337 (2001).

2001JAM/DOB M. E. Jamroz, J. C. Dobrowolski, J. Placzek, A. M. Szafranski, J. K. Kazimirski, and Z. Lisicki, J. Chem. Thermodyn. 33, 565 (2001).

2001KAM/SUE R. Kamae, K. Suenaga, T. Matsuo, and H. Suga, J. Chem. Thermodyn. 33, 471 (2001).

2001KIY/MIN T. Kiyobayashi and M. E. Minas da Piedade, J. Chem. Thermodyn. 33, 11 (2001).

$2001 \mathrm{KOZ} /$ BLO A. A. Kozyro, A. V. Blokhin, G. J. Kabo, and Y. U. Paulechka, J. Chem. Thermodyn. 33, 305 (2001).

2001 KUL/DES I. Kul, D. D. DesMarteau, and A. L. Beyerlein, Fluid Phase Equilib. 185, 241 (2001).

2001 KUL/VER D. Kulikov, S. P. Verevkin, and A. Heintz, J. Chem. Eng. Data 46, 1593 (2001).

2001KUL/VER2 D. Kulikov, S. P. Verevkin, and A. Heintz, Fluid Phase Equilib. 192, 187 (2001).

2001LAG/DIO A. L. C. Lagoa, H. P. Diogo, M. P. Dias, M. E. Minas de Piedade, L. M. P. E. Amaral, M. A. V. Ribeiro da Silva, J. A. M. Simoes, R. C. Guedes, B. J. Costa Cabral, K. Schwarz, and M. Eppli, Chem. Eur. J. 7, 483 (2001).

2001LOR/AUC S. Loras, A. Aucejo, J. B. Monton, J. Wisniak, and H. Segura, J. Chem. Eng. Data 46, 1351 (2001).

2001MAR/GOM F. Martinez and A. Gomez, J. Sol. Chem. 30, 909 (2001). 2001MAT/LEB Y. N. Matyushin, V. P. Lebedev, E. A. Miroshnichenko, L. M. Kostikova, and Y. O. Inosemzev, Int. Annu. Conf. ICT 32, 102/1 (2001).

2001MON/HIL M. J. S. Monte and D. M. Hillesheim, J. Chem. Eng. Data 46, 1601 (2001).
2001MON/HIL2 M. J. S. Monte and D. M. Hillesheim, J. Chem. Thermodyn. 33, 745 (2001).

2001MON/HIL3 M. J. S. Monte and D. M. Hillesheim, J. Chem. Thermodyn. 33, 103 (2001)

2001MON/HIL4 M. J. S. Monte and D. M. Hillesheim, J. Chem. Thermodyn. 33, 837 (2001).

2001MON/HIL5 M. J. S. Monte and D. M. Hillesheim, J. Chem. Thermodyn. 33, 849 (2001).

2001 MUN/KRA L. A. L. Munoz and M. A. Krahenbuhl, J. Chem. Eng. Data 46, 120 (2001).

2001ORT/GON J. Ortega, C. Gonzalez, and S. Galvan, J. Chem. Eng. Data 46, 904 (2001).

2001OXL/SMI J. C. Oxley, J. L. Smith, J. Zhang, and C. Bedford, J. Phys. Chem. A 105, 579 (2001). Note: Authors report that compounds exhibit melting endotherm well below their decomposition exotherm.

2001PER/BAU G. L. Perlovich and A. Bauer-Brandl, J. Therm. Anal. Calorim. 63, 653 (2001).

2001PER/HAN G. L. Perlovich, L. K. Hansen, and A. Bauer-Brandl, J. Therm. Anal. Calorim. 66, 699 (2001).

2001PUR/CHI S. Puri, J. S. Chickos, and W. Welsh, J. Anal. Chem. 73 1480 (2001)

2001REH/SHE M. Rehman, B. Y. Skekunov, P. York, and P. Colthorpe, J. Pharm. Sci. 90, 1570 (2001).

2001RIB/FER M. D. M. C. Ribeiro da Silva, S. C. C. Ferreira, I. A. P. Rodrigues, L. C. M. da Silva, W. E. Acree, Jr., S. Pandey, and L. E. Roy, J. Chem. Thermodyn. 33, 1227 (2001).

2001RIB/GON M. D. M. C. Ribeiro da Silva, J. M. Goncalves, S. C. C. Ferreira, L. C. M. da Silva, M. J. Sottomayor, G. Pilcher, W. E. Acree, Jr., and L. E. Roy, J. Chem. Thermodyn. 33, 1263 (2001).

2001RIB/MON2 M. A. V. Riberio da Silva, M. J. S. Monte, and J. R. Ribeiro, J. Chem. Thermodyn. 33, 23 (2001).

2001RIB/RIB M. A. V. Ribeiro da Silva, M. D. M. C. Ribeiro da Silva, L. C. M. da Silva, F. Dietze, E. Hoyer, L. Beyer, B. Schröder, A. M. Damas, and J. F. Liebman, J. Chem. Soc., Perkin Trans. 2 2001, 2174.

2001RIT L. Rittfeldt, Anal. Chem. 73, 2405 (2001).

2001ROJ/ORO A. Rojas-Aguilar, E. Orozco-Guareno, and M. Martinezherra, J. Chem. Thermodyn. 33, 1405 (2001).

2001ROL/KRA M. P. Rolemberg and M. A. Krahnbuhl, J. Chem. Eng. Data 46, 256 (2001).

2001ROT/GLA M. K. Rotich, B. D. Glass, and M. E. Brown, J. Therm. Anal. Calorim. 64, 681 (2001).

2001ROU/JIM M. V. Roux, P. Jimenez, J. Z. Davalos, R. Notario, and E. Juaristi, J. Org. Chem. 66, 5343 (2001).

2001ROU/JIM2 M. V. Roux, P. P. Jimenez, A. Mayorga, J. Z. Davalos, S. Böhm, and O. Exner, J. Phys. Chem. A 105, 7926 (2001).

2001SEG/LAM H. Segura, E. Lam, R. Reich, and J. Wisniak, Phys. Chem. Liq. 39, 43 (2001).

2001UUS/POK P. Uusi-Kyyny, J.-P. Pokki, J. Aittaman, and S. Liukkonen, J. Chem. Eng. Data 46, 1244 (2001).

2001VAN/OON J. C. van Miltenburg, H. A. J. Oonk, and B. J. K. Van den Berg, J. Chem. Eng. Data 46, 84 (2001).

2001VAR/DRU R. M. Varushchenko, A. I. Druzhinina, A. Yu. Churkina, and Z.-C. Tan, Russ. J. Phys. Chem. 75, 1223 (2001).

2001VER/HEI S. P. Verevkin and A. Heintz, J. Chem. Eng. Data 46, 984 (2001).

2001 VER/VAZ E. Vercher, M. I. Vazquez, and A. Martinez-Andreu, J. Chem. Eng. Data 46, 1584 (2001).

2001XIE/CHE R. Xie and Y. Cheng, Huagong Xuebao (Chin. Ed.) 52, 650 (2001).

2001ZAB/RUZ M. Zábranský, V. Růžička, Jr., and E. S. Domalski, J. Phys. Chem. Ref. Data 30, 1199-1689 (2001).

2001ZHU/LI L. Zhu, H. Li, C. Wang, and S. Han, J. Chem. Eng. Data 46, 1231 (2001).

2001ZOR/COS H. E. Zorel, Jr., A. G. C. Costalonga, M. S. Crespi, and C. A. Ribiero, Quim. Nova 24, 599 (2001).

2002ABB/CAS J.-L. M. Abboud, O. Castao, J. Z. Davalos, P. Jimenez, R. Gomperts, P. Muller, and M. V. Roux, J. Org. Chem. 67, 1057 (2002).

2002ANT/FRA M. Antosik, Z. Fras, and S. K. Malanowski, J. Chem. Eng. Data 47, 757 (2002). 
2002BAT I. Batiu, Fluid Phase Equilib. 198, 111 (2002).

2002BLO/PAU A. V. Blokhin, Y. U. Paulechka, G. J. Kabo, and A. A. Kozyro, J. Chem. Thermodyn. 34, 29 (2002).

2002BOB/ART S. Bobbo, G. Artico, L. Fedele, M. Scattolini, and R. Camporese, J. Chem. Eng. Data 47, 839 (2002).

2002BOB/FED S. Bobbo, L. Fedele, M. Scattolini, and R. Camporese, J. Chem. Eng. Data 47, 179 (2002).

2002BOU/SAI M. Bouvet and H. Said, Phys. Chem. News 8, 79 (2002); Chem. Abstr. 138, 344563 (2002).

2002BRU/POR B. Brunetti, G. Portalone, and V. Piacente, J. Chem. Eng. Data 47, 17 (2002).

2002CHA/DOL K. Chatterjee, D. Dollimore, and K. S. Alexander, Thermochim. Acta 392-393, 107 (2002).

2002CHA/MAN D. Chandra, H. Mandalia, W.-M. Chien, D. W. Lindle, and R. Rudman, Z. Phys. Chem. (Muenchen) 216, 1389 (2002).

2002CHI/HIL J. S. Chickos, D. M. Hillesheim, G. Nichols, and M. J. Zehe, J. Chem. Thermodyn. 34, 1647 (2002).

$2002 \mathrm{CHI} / \mathrm{KNI} \quad$ R. D. Chirico, S. E. Knipmeyer, and W. V. Steele, J. Chem. Thermodyn. 34, 1873 (2002).

2002CON/WIC D. Constantinesku and I. Wichterle, Fluid Phase Equilib. 203, 71 (2002).

2002DAH/MOK A. Dahmani, I. Mokbel, and J. Jose, Fluid Phase Equilib. 202, 193 (2002).

2002DAL/DEL L. Dall'acqua, G. Della Gatta, B. Nowicka, and P. Ferloni, J. Chem. Thermodyn. 34, 1 (2002).

2002DAS/ZAL Z. Daszkiewicz, J. Zaleski, E. M. Nowakowska, and J. B. Kyziol, Pol. J. Chem. 76, 1113 (2002).

2002DEN/LI D. Deng, H. Li, and S. Han, J. Chem. Thermodyn. 34, 1431 (2002).

2002DIN/PAS G. Di Nicola and G. Passerini, J. Chem. Eng. Data 47, 882 (2002).

2002DOM/KOZ U. Domanska, M. K. Kozlowska, and M. Rogalski, J. Chem. Eng. Data 47, 456 (2002).

2002DOM/KOZ2 U. Domanska, M. K. Kozlowska, and M. Rogalski, J. Chem. Eng. Data 47, 8 (2002).

2002FAR/KAD D. Faroongsarng, N. Kadejinda, and A. Sunthornpit, AAPS PharmSciTech 1, 23 (2002).

2002GRA/RAS S. Gracin and A. C. Rasmuson, J. Chem. Eng. Data 47, 1379 (2002).

2002HAZ/DOL A. Hazra, D. Dollimore, and K. Alexander, Thermochim. Acta 392/393, 221 (2002).

2002JIM/ROU2 P. Jimenez, M. V. Roux, J. Z. Davalos, and M. Temprado, Thermochim. Acta 394, 25 (2002).

2002JON/COO S. O. Jonsdottir, S. A. Cooke, and E. A. Macedo, Carbohydrate Res. 337, 1563 (2002).

2002JON/LIG $\quad$ D. E. G. Jones, P. D. Lightfoot, R. C. Fouchard, and Q. S. M Kwok, Thermochim. Acta 388, 159 (2002).

2002KOS/MIR L. M. Kostikova, E. A. Miroshnichenko, Y. N. Matyushin, A. B. Vorob'ev, and J. O. Inozemtsev, Int. Annu. Conf. ICT 33, 86/1 (2002).

2002KRA/VAS E. L. Krasnykh, T. V. Vasiltsova, S. P. Verevkin, and A. Heintz, J. Chem. Eng. Data 47, 1372 (2002).

2002LAG/DIO A. L. C. Lagoa, H. P. Diogo, M. E. Minas da Piedade, L. M. P. F. Amaral, R. C. Guedes, B. J. C. Cabral, D. V. Kulikov, S. P. Verevkin, M. Siedler, and M. Epple, J. Phys. Chem. 106, 9855 (2002).

2002LEI/CHA Y. D. Lei, R. Chankalal, A. Chan, and F. Wania, J. Chem. Eng. Data 47, 801 (2002).

2002LI/SHI X.-W. Li, E. Shibata, E. Kasai, and T. Nakamura, Mater. Trans. 43, 2903 (2002).

2002LIP/SCH M. Lipovska, H.-G. Schmidt, V. Rohac, V. Ruicka, G. Wolf, and M. Zabranska, J. Therm. Anal. Calorim. 68, 753 (2002).

2002LOR/AUC S. Loras, A. Aucejo, J. B. Monton, J. Wisniak, and H. Segura, J. Chem. Eng. Data 47, 1256 (2002).

2002LUB/BAN M. Lubomska, A. Banas, and S. K. Malanowski, J. Chem. Eng. Data 47, 1466 (2002).

2002MAN J. A. Manion, J. Phys. Chem. Ref. Data 31, 123 (2002).

2002MAR/GOM F. Martinez and A. Gomez, Phys. Chem. Liq. 40, 411 (2002).

2002MAR/OLI I. M. Marrucho, N. S. Oliveira, and R. Dohrn, J. Chem. Eng. Data 47, 554 (2002).
2002MAT/LEB

2002MEN/DOL

2002MIR/LEB

2002MIR/LEB2

2002MUR/YAM

2002MUS/RAZ

2002PFO/RIE

2002POK/UUS

2002RAI/PAN

2002RIB/AMA

2002RIB/RIB

2002ROC/GRI

2002ROD/CAN

2002ROD/CAN2

2002ROU/DAV

2002ROY/RIG

2002SAW/SHI

2002SCH/LEN

2002SEG/GAL

2002SEG/WIS

2002SII/KIR

2002SII/KIR2

2002SOR/DOL

2002SOU/DAV

2002STE/CHI

2002STE/CHI2

2002STE/CHI3

2002STE/CHI4

2002STE/CHI5

2002STE/CHI6

2002SU

2002SWI/MAL

2002SZT/KAM

2002TAN/SUN
Y. N. Matyushin, V. P. Lebedev, E. A. Miroshnichenko, V. P. Vorob'eva, V. V. Chironov, L. M. Kostikova, and J. O. Inozemtcev, Int. Annu. Conf. ICT 33, 84/1 (2002).

D. Menon, D. Dollimore, and K. S. Alexander, Thermochim. Acta 392/393, 237 (2002).

E. A. Miroshnichenko, V. P. Lebedev, and Y. N. Matyushin, Dokl. Akad. Nauk 382, 497 (2002).

E. A. Miroshnichenko, V. P. Lebedev, and Yu. N. Matyushin, Dokl. Russ. Chem. 382, 40 (2002).

J. Murata, S. Yamashita, M. Akiyama, S. Katayama, T. Hiaki, and A. Sekiya, J. Chem. Eng. Data 47, 911 (2002).

A. M. Musuc, D. Razus, and D. Oancea, An. Univ. Bucuresti, Chim. 11, 147 (2002).

O. Pfohl, C. Riebesell, and R. Dohrn, Fluid Phase Equilib. 202, 289 (2002).

J.-P. Pokki, P. Uusi-Kyyny, J. Aittamaa, and S. Liukkonen, J. Chem. Eng. Data 47, 371 (2002).

U. S. Rai, P. Pandey, and R. N. Rai, Mater. Lett. 53, 83 (2002).

M. A. V. Ribeiro da Silva, L. M. P. F. Amaral, and I. M. C. L. Ferreira, J. Chem. Thermodyn. 34, 119 (2002).

M. A. V. Ribeiro da Silva, M. D. M. C. Ribeiro da Silva, L. C. M. da Silva, and F. Dietze, J. Chem. Thermodyn. 34, 155 (2002).

G. D. Rockwell, T. B. Grindley, K. C. Smith, and M. A. White, J. Phys. Chem. B 106, 12311 (2002).

A. Rodriquez, J. Canosa, A. Dominquez, and J. Tojo, Fluid Phase Equilib. 198, 95 (2002).

A. Rodriquez, J. Canosa, A. Dominquez, and A. Tojo, J. Chem. Eng. Data 47, 1098 (2002).

M. V. Roux, J. Z. Davalos, and P. Jimenez, Thermochim. Acta 394, 19 (2002).

S. Roy, A. T. Riga, and K. S. Alexander, Thermochim. Acta 392/393, 399 (2002).

Y. Sawanoi, Y. Shimbo, I. Tabata, K. Hisada, and T. Hori, Dyes Pigm. 52, 29 (2002).

E. Schnitzler, K. Lencone, and M. Kobelnik, Exact Soil Sci., Agrarian S. Eng. 8, 91 (2002).

H. Segura, G. Galindo, R. Reich, J. Wisniak, and S. Loras, Phys. Chem. Liq. 40, 277 (2002).

H. Segura, J. Wisniak, G. Galindo, and R. Reich, Phys. Chem. Liq. 40, 67 (2002).

E. Siimer, H. Kirss, M. Kuus, and L. Kudryavtseva, Proc. Est. Acad. Sci., Chem. 51, 19 (2002).

E. Siimer, H. Kirrs, M. Kuus, and L. Kudryavtseva, J. Chem. Eng. Data 47, 52 (2001).

T. V. Sorokina, D. Dollimore, and K. S. Alexander, Thermochim. Acta 392/393, 315 (2002).

P. O. Souillac, P. Dave, and J. H. Rytting, Int. J. Pharm. 231, 185 (2002).

W. V. Steele, R. D. Chirico, S. E. Knipmeyer, and A. Nguyen, J. Chem. Eng. Data 47, 648 (2002).

W. V. Steele, R. D. Chirico, A. B. Cowell, S. E. Knipmeyer, and A. Nguyen, J. Chem. Eng. Data 47, 667 (2002).

W. V. Steele, R. D. Chirico, S. E. Knipmeyer, and A. Nguyen, J. Chem. Eng. Data 47, 689 (2002).

W. V. Steele, R. D. Chirico, A. B. Cowell, S. E. Knipmeyer, and A. Nguyen, J. Chem. Eng. Data 47, 700 (2002).

W. V. Steele, R. D. Chirico, S. E. Knipmeyer, and A. Nguyen, J. Chem. Eng. Data 47, 715 (2002).

W. V. Steele, R. D. Chirico, A. B. Cowell, S. E. Knipmeyer, and A. Nguyen, J. Chem. Eng. Data 47, 725 (2002).

C. C. Su, M.S. thesis, National Taiwan University of Science and Technology, Taipei, 2002.

B. E. Swiatek and S. K. Malanowski, J. Chem. Eng. Data 47, 478 (2002).

P. Szterner, M. Kaminski, and A. Zielenkiewicz, J. Chem. Thermodyn. 34, 1005 (2002).

Z.-C. Tan, L.-X. Sun, S.-H. Meng, L. Li, F. Xu, P. Yu, B.P. Liu, and J.-B. Zhang, J. Chem. Thermodyn. 34, 1417 (2002). 
2002TAT/DOL A. S. Tatavarti, D. Dollimore, and K. S. Alexander, Pharm. Sci. 4, 1 (2002).

2002TEM/ROU M. Temprado, M. V. Roux, P. Jimenez, J. Z. Davalos, and R. Notario, J. Phys. Chem. A 106, 11173 (2002).

2002VAL/COQ A. Valtz, C. Coquelet, A. Baba-Ahmed, and D. Richon, Fluid Phase Equilib. 202, 29 (2002).

2002VAN/PAR A. van Roon, J. R. Parsons, and H. A. J. Govers, J. Chromatogr. A 955, 105 (2002).

2002VAN/VAN A. C. G. van Genderen, J. C. van Miltenburg, J. G. Blok, M. J. van Bommel, P. J. van Ekeren, G. J. K. van den Berg, and H. A. J. Oonk, Fluid Phase Equilib. 202, 109 (2002).

2002VAN/VAN2 J. C. van Miltenburg and P. J. van Ekeren, Thermochim. Acta 385, 11 (2002).

2002VAN/VAN3 J. C. van Miltenburg, G. J. K. van den Berg, and A. C. G. Genderen, Thermochim. Acta 383, 13 (2002).

2002VAR/AIT R. M. Varuschenko, C. A. Aitkeeva, A. I. Druzhinina, Y. A. Myshenseva, and L. L. Pashchenko, Russ. J. Phys. Chem. 76, 724-728 (2002).

2002VAR/DRU R. M. Varushchenko and A. I. Druzhinina, Fluid Phase Equilib. 199, 109 (2002).

2002VAR/PAS R. M. Varushchenko, L. L. Pashchenko, A. Y. Churkina, and A. V. Shabanova, Russ. J. Phys. Chem. 76, 915 (2002).

2002VAR/PAS2 R. M. Varuschenko, L. L. Pashchenko, A. I. Druzhinina, A. Yu. Churkina, I. I. Vorob'ev, and S. N. Kravhun, Russ. J. Phys. Chem. 76, 546-551 (2002).

2002VER S. P. Verevkin, J. Chem. Eng. Data 47, 1071 (2002).

2002VER/KUM S. P. Verevkin, M. Kummerlin, E. Hickl, H.-D. Beckhaus, C. Rüchardt, S. I. Kozhushkov, R. Haag, R. Boese, J. Benet-Bucholz, K. Nordhoff, and A. de Meijere, Eur. J. Org. Chem. 2280 (2002).

2002VER2 S. P. Verevkin, J. Chem. Thermodyn. 34, 263 (2002).

2002 WIL/VON G. M. Wilson, D. M. Von Niederhausern, and N. F. Giles, J. Chem. Eng. Data 47, 761 (2002).

2002ZHO/PEN C.-R. Zhou, G.-S. Peng, Y.-D. Zhang, D. G. Jiang, F. Wang, J.-J. Li, and X.-Q. Shen, Gaoxiao Huaxue Gongeheng Xuebao 16, 237 (2002); Chem. Abstr. 137, 316567d (2002).

2003ABR/SMI V. K. Abrosimov, V. I. Smirnov, V. A. Kuznetsov, T. M. Okhrimenko, and E. P. Efremova, Russ. J. Phys. Chem. 77, 1191 (2003).

2003ARA/STO A. A. S. Araujo, S. Storpirtis, L. P. Mercuri, F. M. S. Carvalho, M. dos Santos Filho, and J. R. Matos, Int. J. Pharm. 260, 303 (2003).

2003CAC/BAU C. Cacela, A. Baudot, M. L. Duarte, A. M. Matos-Beja, M. R. Silva, J. A. Paixo, and R. Fausto, J. Mol. Struct. 649, 143 (2003).

2003CAR/DES L. Carpentier, S. Desprex, and M. Descamps, J. Therm. Anal. Calorim. 73, 577 (2003).

2003CEN/RUZ M. Censky, K. Ruzicka, Z. Ruzicka, and M. Zabransky, Thermochim. Acta 408, 45 (2003).

2003CHA/BLO M. B. Charapennikau, A. V. Blokhin, A. G. Kabo, and G. J. Kabo, J. Chem. Thermodyn. 35, 145 (2003).

2003CHA/BLO2 M. B. Charapennikau, A. V. Blokhin, G. J. Kabo, V. M. Sevruk, and A. P. Krasulin, Thermochim. Acta 405, 85 (2003).

2003CHI/ACR J. S. Chickos and W. E. Acree, Jr., Thermochim. Acta 395, 59 (2003).

2003CHI/KNI R. D. Chirico, S. E. Knipmeyer, and W. V. Steele, J. Chem. Thermodyn. 35, 1059 (2003).

2003CHO/DHA S. P. Chopade, A. D. Dhale, A. M. Clark, C. W. Kiesling, L. K. Myrant, J. E. Jackson, and D. J. Miller, J. Chem. Eng. Data 48, 44 (2003).

2003CLO/JAN K. Clou, J. F. Janssens, N. Blaton, A. T. H. Lenstra, and H. O. Desseyn, Thermochim. Acta 298, 47 (2003).

2003DER/MIC S. O. Derawi, M. L. Michelsen, G. M. Kontogeorgis, and E. H. Stenby, Fluid Phase Equilib. 209, 163 (2003).

2003DIK/FRE V. V. Diky, M. Frenkel, and L. S. Karpushenkava, Thermochim. Acta 408, 115 (2003).

2003EUS/LOP $\quad$ E. M. Eusebio, A. J. Lopes Jesus, M. S. C. Cruz, M. L. P. Leitao, and R. J. Simoes, J. Chem. Thermodyn. 35, 123 (2003).
2003EWI/OCH

M. B. Ewing and J. C. S. Ochoa, Fluid Phase Equilib. 210, 277 (2003).

2003GOB/RAT C. Gobble, N. Rath, and J. Chickos, J. Chem. Eng. Data 58, 2600-2009 (2013).

2003GOM/SZA A. Gombas, P. Szabo-Revesz, G. Regdon, Jr., and I. Eros, J. Therm. Anal. Calorim. 73, 615 (2003).

2003HUA

2003JIA/LI

C.-Y. Huang, M.S. thesis, Chin Culture University, Taipei, 2003.

2003KON/TAN L.-G. Kong, Z.-C. Tan, J. Xu, S.-H. Meng, and X.-H. Bao,

H. Jiang, H. Li, C. Wang, T. Tan, and S. Han, J. Chem. Thermodyn. 35, 1567 (2003). J. Chem. Thermodyn. 35, 1897 (2003).

2003LEE/CHO M.-J. Lee, Y.-H. Chou, and H.-M. Lin, Ind. Eng. Chem. Res. 42, 3434 (2003).

2003LEI/LI Y.Lei, H. Li, L. Zhu, and S. Han, Fluid Phase Equilib. 206, 87 (2003).

2003MAR/AVI F. Martinez, C. M. Avila, and A. Gomez, J. Braz. Chem. Soc. 4, 803 (2003).

2003MAT/MIR M. A. R. Matos, M. S. Miranda, and V. M. F. Morais, J. Chem. Eng. Data 48, 669 (2003).

2003MAT/MIR2 M. A. R. Matos, M. S. Miranda, V. M. F. Morais, and J. F. Liebman, Org. Biomol. Chem. 1, 2566 (2003).

2003MAT/MIR3 M. A. R. Matos, M. S. Miranda, V. M. F. Morais, and J. F. Liebman, Org. Biomol. Chem. 1, 2930 (2003).

2003MCK/KRA R. L. McKenney, Jr. and T. R. Krawietz, Energy Mater. 21, 141 (2003).

2003MIR/LEB E. A. Miroshnichenko, V. P. Lebedev, L. M. Kostikova, V. P. Vorob'eva, A. B. Vorob'ev, and J. O. Inozemtcev, Int. Annu. Conf. ICT 34, 122/1 (2003).

2003MOR/MIR V. M. F. Morais, M. S. Miranda, and M. A. R. Matos, Org. Biomol. Chem. 1, 4329 (2003).

2003ORT/ESP J. Ortega, F. Espiau, and M. Postigo, J. Chem. Eng. Data 48, 916 (2003).

2003ORT/ESP2 J. Ortega, F. Espiau, J. Togo, J. Canosa, and A. Rodriguez, J. Chem. Eng. Data 48, 1183 (2003).

2003PAG/BEN S. Pagola, A. Benavente, A. Raschi, E. Romano, M. A. A. Molina, and P. W. Stephens, AAPS PharmSciTech 5, 1 (2003).

2003PEN/RIB R. Rena, J. P. Ribet, J. L. Maural, L. Valat, F. Lacouloncha, and A. Chauvet, Thermochim. Acta 408, 85 (2003).

2003PER/BAU G. L. Perlovich and A. Bauer-Brandl, Pharm. Res. 20, 471 (2003). Note: Authors calculated the ideal mole fraction solubility of $\mathrm{x}=0.0311$ at $298.2 \mathrm{~K}$ using their previously published enthalpy of fusion data, which was presumably reported previously. [2001PER/BAU] The referenced paper does not contain the experimental enthalpy of fusion data. The enthalpy of fusion value for acetylsalicylic acid was calculated using the thermodynamic relationship: $\ln \mathrm{x}=-\mathrm{DfusHm}(\mathrm{T})($ Tfus -298.2$) /\left(8.314^{*} 2982^{*}\right.$ Tfus $)$.

2003RIB/LIM M. A. V. Ribeiro da Silva, L. M. S. S. Lima, L. M. P. F. Amaral, A. I. M. C. L. Ferreira, and J. R. B. Gomes, J. Chem. Thermodyn. 35, 1343 (2003).

2003RIB/SAN M. D. M. C. Ribeiro da Silva, L. M. N. B. F. Santos, A. L. R. Silva, O. Fernandez, and W. E. Acree, Jr., J. Chem. Thermodyn. 35, 1093 (2003).

2003ROJ/ORO A. Rojas and E. Orozco, Thermochim. Acta 405, 93 (2003).

2003ROM/LEI J. M. Romero, L. C. Leiva, N. L. Jorge, M. E. Gomez Vara, and E. A. Castro, Acta Chim. Slov. 50, 579 (2003).

2003ROU/JIM M. V. Roux, P. Jimenez, J. Z. Davalos, M. Temperado, and J. F. Liebman, J. Chem. Thermodyn. 35, 803 (2003).

2003ROU/TEM M. V. Roux, M. Temprado, P. Jimenez, J. Z. Davalos, C. Foces-Foces, M. V. Garcia, and M. I. Redondo, Thermochim. Acta 404, 235 (2003).

2003ROU/TEM2 M. V. Roux, M. Temprado, P. Jimenez, R. Guzman, E. Juaristi, and J. S. Chickos, Thermochim. Acta 406, 9 (2003).

2003ROU/TEM3 M. V. Roux, M. Temprado, P. Jimenez, J. Perez-Parajon, and R. Notario, J. Phys. Chem. A 107, 11460 (2003).

2003RUT/SAL M. A. Rute, J. Salud, P. Negrier, D. O. Lopez, J. L1. Tamarit, R. Puertas, M. Barrio, and D. Mondieig, J. Phys. Chem. B 107, 5914 (2003). 
2003SHA/KAN $\quad$ B. L. Sharma, R. Kant, R. Sharma, and S. Tandon, Mater. Chem. Phys. 82, 216 (2003).

2003SPI/WAN D. Spitzer, B. Wanders, M. R. Schafer, and R. Welter, J. Mol. Struct. 644, 37 (2003).

2003STE/CHI W. V. Steele, R. D. Chirico, A. B. Cowell, A. Nguyen, and S. E. Knipmeyer, J. Chem. Thermodyn. 35, 1253 (2003).

2003SUC/RAJ M. Suceska, M. Rajic, S. Matecic-Musanic, S.Zeman, and Z. Jalovy, J. Therm. Anal. Calorim. 74, 853 (2003).

2003SUN/SON X.-H. Sun, J.-R. Song, Z.-C. Tan, Y.-Y. Di, H.-X. Ma, M.-H. Wang, and L.-X. Sun, Huaxue Xuebao 61, 1897 (2003).

2003TAN Y. Tan, Wuxi Qinggong Daxue Xuebao 22, 102 (2003).

2003TEO/WIL M. Teodorescu, M. Wilken, R. Wittig, and J. Gmehling, Fluid Phase Equilib. 204, 267 (2003).

2003VAN/GAB J. C. van Miltenburg, H. Gabrielova, and K. Ruzicka, J. Chem. Eng. Data 48, 1323 (2003).

2003VER S. P. Verevkin, J. Chem. Thermodyn. 35, 1237 (2003).

2003VER/KRA S. P. Verevkin, E. L. Krasnykh, T. V. Vasiltsova, and A. Heintz, J. Chem. Eng. Data 48, 591 (2003).

2003VER/KRA2 S. P. Verevkin, E. L. Krasnykh, T. V. Vasiltsova, B. Koutek, J. Doubsky, and A. Heintz, Fluid Phase Equilib. 206, 331 (2003).

2003VER/SCH S. P. Verevkin and C. Schick, Fluid Phase Equilib. 211, 161 (2003)

2003WAN/LI C. Wang, H. Li, L. Ma, and S. Han, J. Chem. Thermodyn. 35, 131 (2003).

2003WIL/WIL D. Williams, D. Wilson, R. Storey, and P. Basford, "Vapour pressure determination of pharmaceutical powders," in 7th International Conference/Workshop on Pharmacy and Applied Physical Chemistry (European Society for Applied Physical Chemistry, Innsbruck, 2003).

2003ZAI/KAB Dz. Zaitsau, G. J. Kabo, A. A. Kozyro, and V. M. Sevruk, Thermochim. Acta 406, 17 (2003).

2003ZAI/VER D. H. Zaitsau, S. P. Verevkin, Y. U. Paulechka, G. J. Kabo, and V. M. Sevuk, J. Chem. Eng. Data 48, 1393 (2003).

2003ZHO/ZHA C. Zhou, Y. Zhang, and D. Jiang, Chin. J. Chem. Eng. 11, 598 (2003).

2003ZIE/SZT W. Zielenkiewicz, P. Szterner, and M. Kaminski, J. Chem. Eng. Data 48, 1132 (2003).

2004AHM/BAR A. Ahmed, B. W. Barry, A. C. Williams, and A. F. Davis, J. Pharm. Biomed. Anal. 34, 945 (2004).

2004AHM/GIE S. Ahmad, R. Giesen, and K. Lucas, J. Chem. Eng. Data 49, 826 (2004).

2004ANT/GAL M. Antosik, M. Galka, and S. K. Malanowski, J. Chem. Eng. Data 49, 11 (2004).

2004BAR/PAR M. Barrio, L. C. Pardo, J. Ll. Tamarit, P. Negrier, D. O. Lopez, J. Salad, and D. Mondieig, J. Phys. Chem. B 108, 11089-11096 (2004).

2004BAS/CHI A. Bashir-Hashemi, J. S. Chickos, W. Hanshaw, H. Zhao, B. S. Farivar, and J. F. Liebman, Thermochim. Acta 424, 91 (2004).

2004BOL/DRE E. V. Boldyreva, V. A. Drebushchak, I. E. Paukov, Y. A. Kovalevskaya, and T. N. Drebushchak, J. Therm. Anal. Calorim. 77, 607 (2004).

2004BYK/SMI T. A. Bykova, N. N. Smirnova, L. V. Nikishchenkova, G. P. Belova, and E. V. Novikova, Russ. J. Phys. Chem. 78, 1712 (2004).

2004CER/PER L. Cerioni, S. Perez, and A. Wolfenson, J. Phys. Chem. Solids 65, 1133 (2004).

2004CHE/CLE Y. Chernyak and J. H. Clements, J. Chem. Eng. Data 49, 1180 (2004).

2004CHE/MA M. Chen and P. Ma, J. Chem. Eng. Data 49, 756 (2004); Secondary reference.

2004CHI/STE R. D. Chirico and W. V. Steele, J. Chem. Thermodyn. 36, 633 (2004)

2004CHY/FRA K. Chylinski, Z. Fras, and S. K. Malanowski, J. Chem. Eng. Data 49, 2 (2004).

2004CHY/FRA2 C. Chylinski, Z. Fras, and S. K. Malanowski, J. Chem. Eng. Data 49, 18 (2004).

2004CLI/RAM C. L. Clifford, D. Ramjugernath, and J. D. Raal, J. Chem. Eng. Data 49, 1189 (2004).

2004COV/MOK M. Covarrubias-Cervantes, I. Mokbel, A. Champion, J. Jose, and A. Voilley, Food Chem. 85, 221 (2004).
2004DIA/CAC

A. M. A. Dias, A. I. Caco, J. A. P. Coutinho, L. M. N. B. F. Santos, M. M. Pineiro, L. F. Vega, M. F. Costa Gomes, and I. M. Manucho, Fluid Phase Equilib. 225, 39 (2004).

2004DIN

2004DOR/YAN M. S. Ding, J. Chem. Eng. Data 49, 276 (2004).

2004EWI/SAN D. V. Dorofeeva, V. S. Yangman, R. M. Varushehenko, and A. I. Druzhinina, Int. J. Thermophys. 25, 1097 (2004).

2004FLO/AMA M. B. Ewing and J. C. Sanchez Ochoa, J. Chem. Eng. Data 49, 486 (2004).

2004FRI/KAP

H. Flores and P. Amador, J. Chem. Thermodyn. 36, 1019 (2004).

2004FUJ/TOD

N. Fridman, M. Kapon, Y. Sheynin, and M. Kaftory, Acta Crystallogr., Sect. B 60, 97 (2004).

2004GEN/PEI Conf. Proc. 708, 685 (2004).

Z. Geng-qu, C. Pei, H. Rong-zu, L. Yang, Z. Zhi-zhong, Z. Yan-shui, Y. Xu-wu, G. Yin, G. Sheng-li, and S. Qu-zhen, J. Hazard. Mater. 113, 67 (2004).

2004GRA/RAS S. Gracin and A. C. Rasmuson, Cryst. Growth Des. 4, 1013 (2004).

2004GUP/SIN R. K. Gupta and R. A. Singh, J. Cryst. Growth 267, 340 (2004).

2004HAN/BEY A. R. Hansen and K. D. Beyer, J. Phys. Chem. A 108, 3457 (2004).

2004HOR/FIS S. Horstman, K. Fisher, and J. Gmehling, J. Chem. Eng. Data 49, 1494 (2004).

2004INO/HIS2 T. Inoue, Y. Hisatsugu, M. Suzuki, Z. Wang, and L. Zeng, Chem. Phys. Lipids 132, 225 (2004).

2004KAO/SIE C.-P. C. Kao, A. C. Sievert, M. Schuller, and J. F. Sturgis, J. Chem. Eng. Data 49, 532 (2004).

2004KIM/KES Y. Kim, K. I. Keskinen, and J. Aittamaa, J. Chem. Eng. Data 49, 1273 (2004).

2004KON/TAN L. G. Kong, Z. C. Tan, W. Zhang, F. Xu, M. H. Wang, X. H Bao, T. Zhang, and L. X. Sun, Chem. Pap. 58, 295 (2004).

2004KON/TAN2 L.-G. Kong, Z.-C. Tan, J.-T. Mei, L.-X. Sun, and X.-H. Bao, Thermochim. Acta 414, 131 (2004).

2004KUR/MAE H. Kuramochi, K. Maeda, and K. Kawamoto, Environ. Toxicol. Chem. 23, 1386 (2004).

2004KUR/MAE2 H. Kuramochi, K. Maeda, and K. Kawamoto, J. Chem. Eng. Data 49, 720 (2004).

2004LAU/HIL K. H. Lau, D. L. Hildenbrand, S. Crouch-Baker, and A. Sanjurjo, J. Chem. Eng. Data 49, 544 (2004).

2004LEE/SU M.-J. Lee, C.-C. Su, and H.-M. Lin, J. Chem. Eng. Data 49, 588 (2004).

2004LU/TAN X.-C. Lu, Z.-C. Tan, Y.-Y. Di, Q. Shi, L.-X. Shi, L.-X. Sun, and T. Zhang, J. Chem. Thermodyn. 36, 787 (2004).

2004LUB/MAL M. Lubomska and S. K. Malanowski, J. Chem. Eng. Data 49, 1488 (2004).

2004MA/LIU X.-B. Ma, X.-G. Liu, Z.-H. Li, and G.-H. Xu, Fluid Phase Equilib. 221, 51 (2004).

2004MAR/KAI E. Marti, E. Kaisersberger, and W.-D. Emmerich, J. Therm. Anal. Calorim. 77, 905 (2004).

2004MAS/NAK M. Massalska-Arodz, T. Nakamoto, T. Wasiutynski, J. Mayer, J. Krawczyk, and M. Sorai, J. Chem. Thermodyn. 36, 877 (2004).

2004MAT/MIR M. A. R. Matos, M. S. Miranda, V. M. F. Morais, and J. F. Liebman, Org. Biomol. Chem. 2, 1647 (2004).

2004MAT/MIR3 M. A. R. Matos, M. S. Miranda, V. M. F. Morais, and J. F. Liebman, Eur. J. Org. Chem. 2004, 3340.

2004MAT/MON M. A. R. Matos, M. J. S. Monte, C. C. S. Sousa, A. R. R. P. Almeida, and V. M. F. Morais, Org. Biomol. Chem. 2, 908 (2004).

2004MIR/MOR M. S. Miranda, V. M. F. Morais, and M. A. R. Matos, J. Chem. Thermodyn. 36, 431 (2004).

2004MON/ALM M. J. S. Monte, A. R. R. P. Almeida, and M. A. V. Ribeiro da Silva, J. Chem. Thermodyn. 36, 385 (2004).

2004MON/RAJ D. Mondieig, F. Rajabalee, V. Metivaud, H. A. J. Oonk, and M. A. Cuevas-Diarte, Chem. Mater. 16, 786 (2004).

2004MON/VAN L. Montinaro and J. C. van Miltenburg, The Chemical Thermodynamics Group (University of Utrecht, Ultrecht, 2004), as quoted in [2014FUL/RUZ].

2004MOR/MAT V. M. F. Morais, M. A. R. Matos, M. S. Miranda, and J. F. Liebman, Mol. Phys. 102, 525 (2004). 
2004MOR/MIR V. M. F. Morais, M. S. Miranda, and M. A. R. Matos, J. Chem. Thermodyn. 36, 377 (2004).

2004NAN/TAN Z. D. Nan and Z. C. Tan, J. Therm. Anal. Calorim. 76, 955 (2004).

2004NAS/ZIM K. Nasirzadeh, D. Zimin, R. Neueder, and W. Kunz, J. Chem. Eng. Data 49, 607 (2004).

2004PAU/ZAI Y. U. Paulechka, Dz. H. Zaitsau, and G. J. Kabo, J. Mol. Liq. 115, 105 (2004).

2004PRO/RAS V. M. Profir and A. C. Rasmuson, Cryst. Growth Des. 4, 315 (2004)

2004RAI R. N. Rai, J. Mater. Res. 19, 1348 (2004).

2004RIB/GOM M. D. M. C. Ribeiro da Silva, J. R. B. Gomes, J. M. Gancalves, E. A. Sousa, S. Pandey, and W. E. Acree, Jr., Org. Biomol. Chem. 2, 2507 (2004).

2004RIB/GOM2 M. D. M. C. Ribeiro da Silva, J. R. B. Gomes, J. M. Goncalves, E. A. Sousa, S. Pandey, and W. E. Acree, Jr., J. Org. Chem. 69, 2785 (2004).

2004RIB/MAT M. D. M. C. Ribeiro da Silva, M. A. R. Matos, M. S. Miranda, V. M. F. Morais, and W. E. Acree, Jr., J. Chem. Thermodyn. 36, 107 (2004).

2004RIB/RIB M. A. V. Ribeiro da Silva, M. D. M. C. Ribeiro da Silva, L. M. P. F. Amaral, P. Jimenez, M. V. Roux, J. Z. Davalos, M. Temprado, P. Cabildo, R. M. Claramunt, J. Elguero, O. Mó, and M. Yánéz, J. Chem. Thermodyn. 36, 533 (2004).

2004RIB/SAN2 M. A. V. Ribeiro da Silva, L. M. N. B. F. Santos, B. Schroder, F. Dietze, and L. Beyer, J. Chem. Thermodyn. 36, 491 (2004).

2004RIB/SAN3 M. A. V. Ribiero da Silva and L. M. N. B. F. Santos, J. Chem. Thermodyn. 36, 447 (2004).

2004ROJ/FOR A. Rojas-Aguilar, H. Fores-Lara, M. Martinez-Herrera, and F. Ginez-Carbajal, J. Chem. Thermodyn. 36, 453 (2004).

2004ROM/BUS S. Romero, P. Bustamante, B. Escalera, M. Cirri, and P. Mura, J. Therm. Anal. Calorim. 77, 541 (2004).

2004ROU/TEM M. V. Roux, M. Temprado, R. Notario, S. P. Verevkin, V. N. Emel'yanenko, D. Demasters, and J. F. Liebman, Mol. Phys. 102, 1909 (2004).

2004ROU/TEM2 M. V. Roux, M. Temprado, P. Jimenez, C. Foces-Foces, M. V. Garcia, and M. I. Redondo, Thermochim. Acta 420, 59 (2004).

2004ROU/TEM3 M. V. Roux, M. Temprado, P. Jimenez, R. Notario, R. Guzman-Mejia, and E. Juaristi, J. Org. Chem. 69, 1670 (2004).

2004ROU/TEM4 M. V. Roux, M. Temprado, P. Jimenez, J. Z. Davalos, R. Notario, G. Martin-Valcarcet, L. Garrido, R. GuzmanHejia, and E. Juaristi, J. Org. Chem. 69, 5454 (2004).

2004SAW/MOK T. Sawaya, I. Mokbel, E. Rauzy, J. Saab, C. Berro, and J. Jose, Fluid Phase Equilib. 226, 283 (2004).

2004SHA/JAM $\quad$ B. L. Sharma, R. Jamwal, and R. Kant, Cryst. Res. Technol. 39, 454 (2004).

2004SHA/TAN $\quad$ B. L. Sharma, S. Tandon, R. Kant, and R. Sharma, Thermochim. Acta 421, 161 (2004).

2004SMI/MAR N. N. Smirnova, A. V. Markin, K. V. Kandeev, H. Hocker, and H. Keul, Thermochim. Acta 409, 55 (2004).

2004STE/SUN F. Steyer and K. Sundmacher, J. Chem. Eng. Data 49, 1675 (2004).

2004STU/WIT L. Sturz, V. T. Witusiewicz, U. Hecht, and S. Rex, J. Cryst. Growth 270, 273 (2004).

2004TAN/LI T. Tan, H. Li, C. Wang, H. Jiang, and S. Han, Fluid Phase Equilib. 224, 279 (2004).

2004UUS/POK P. Uusi-Kyyny, J.-P. Pokki, Y. Khim, and J. Aittamaa, J. Chem. Eng. Data 49, 251 (2004).

2004VAN/VAN J. C. Van Miltenburg and G. J. K. Van den Berg, J. Chem. Eng. Data 49, 735 (2004).

2004VEC/CAT S. Vecchio, A. Catalani, V. Rossi, and M. Tomassetti, Thermochim. Acta 420, 99 (2004).

2004VER/EME S. P. Verevkin and V. E. Emel'yanenko, J. Phys. Chem. A 108, 6575 (2004).

2004VER/SCH S. P. Verevkin and C. Schick, Thermochim. Acta 415, 35 (2004).

2004VER/VAS S. P. Verevkin and T. V. Vasiltsova, J. Chem. Eng. Data 49, 1717 (2004).

2004VER2
2004WAN/TAN2 M.-H. Wang, Z.-C. Tan, X.-H. Sun, F. Xu, Y.-F. Liu, L.-X. Sun, and T. Zhang, Thermochim. Acta 414, 25 (2004).

2004WAN/TAN3 S. X. Wang, Z. C. Tan, Y. Y. Di, F. Xu, M. H. Wang, L. X. Sun, and T. Zhang, J. Therm. Anal. Calorim. 76, 335 (2004).

2004WAN/TAN4 S.-X. Wang, Z.-C. Tan, Y.-Y. Di, F. Xu, H.-T. Zhang, L.-X Sun, and T. Zhang, J. Chem. Thermodyn. 36, 393 (2004).

2004WAN/WAN L.-C. Wang and F. A. Wang, Fluid Phase Equilib. 226, 289 (2004).

2004WAN/WIE X. Wang, H. Wiehler, and C. B. Ching, Chirality 16, 220 (2004).

2004WOL/KSI T. Wolszakiewicz, A. Ksiazezak, and T. Ksiqzczak, J. Therm. Anal. Calorim. 77, 353 (2004).

2004XIN/TAN J. Xing, Z.-C. Tan, Y.-Y. Di, X.-H. Sun, L.-X. Sun, and Z. Tao, Huaxue Xuebao 62, 2415 (2004).

2004XU/SUN2 F. Xu, L.-X. Sun, Z.-C. Tan, J.-G. Liang, D.-H. Zhou, Y.-Y. Di, X. Z. Lan, and T. Zhang, Wuli Huaxue Xuebao 20, 50 (2004); Chem. Abstr. 141, 42690 (2004).

2004XUE/WAN B. Xue, Y.-Y. Wang, Z.-C. Tan, S.-W. Lu, and S.-H. Meng, J. Therm. Anal. Calorim. 76, 965 (2004).

2004ZAB/RUZ M. Zábranský and V. Růžička, Jr., J. Phys. Chem. Ref. Data 33, 1071-1081 (2004).

2004ZIE/SZT ～W. Zielenkiewicz and P. Szterner, J. Chem. Eng. Data 49 1197 (2004).

2005BAZ/BLO A. B. Bazyleva, A. V. Blokhin, G. J. Kabo, A. G. Kabo, and Y. U. Paulechka, J. Chem. Thermodyn. 37, 643 (2005).

2005BAZ/KAB A. B. Bazyleva, G. J. Kabo, Y. U. Paulechka, D. H. Zaitsau, A. V. Blokhin, and V. M. Sevruk, Thermochim. Acta 436, 56 (2005).

2005BEN/AIT H. Ben-Makhlouf-Hakem, A. Ait-Kaci, and J. Jose, Fluid Phase Equilib. 232, 189 (2005).

2005BOE/HOP J. A. Boerio-Goates, S. D. Hopkins, R. A. R. Monteiro, M. D. M. C. Ribeiro da Silva, M. A. V. Ribeiro da Silva, and R. N. Goldberg, J. Chem. Thermodyn. 37, 1239-1249 (2005).

2005CHA/ZIE S. Chattopadhyay and P. Ziemann, J. Aerosol Sci. Technol. 39, 1085 (2005).

2005CHE/TAN Y.-P. Chen, M. Tang, and J.-C. Kuo, Fluid Phase Equilib. 232, 182 (2005)

2005CHI/STE R. D. Chirico and W. V. Steele, J. Chem. Eng. Data 50, 697 (2005).

2005CON/CHI J. Contineanu, L. Chivu, and S. Perisanu, Rev. Chim. 56, 719 (2005).

2005DES/COR M. Descamps, N. T. Correia, P. Derollez, F. Danede, and F. Capet, J. Phys. Chem. B 109, 16092 (2005).

2005DIA/GON A. M. A. Dias, C. M. B. Goncalves, A. I. Caco, L. M. N B. F. Santos, M. M. Pineiro, L. F. Vega, J. A. P. Coutinho, and I. M. Marrucho, J. Chem. Eng. Data 50, 1328 (2005).

2005DOM/LAC U. Domanska and J. Lachwa, J. Chem. Thermodyn. 37, 692 (2005).

2005DOM/MAR U. Domanska and M. Marciniak, Fluid Phase Equilib. 235, 30 (2005).

2005EME/STR V. N. Emel'yanenko, A. Strutynska, and S. P. Verevkin, J. Phys. Chem. A 109, 4375 (2005).

2005EME/VER V. N. Emel'yanenko, S. P. Verevkin, B. Koutek, and J. Doubsky, J. Chem. Thermodyn. 37, 73 (2005).

2005EME/VER2 V. N. Emel'yanenko and S. P. Verevkin, J. Phys. Chem. A 109, 3960 (2005).

2005ERB/DEL M. F. Erben, C. O. Della Vedova, H. Willner, F. Trautner, H. Oberhammer, and R. Boese, Inorg. Chem. 44, 7070 (2005).

2005GOM/AMA J. R. B. Gomes, L. M. P. F. Amaral, and M. A. V. Ribeiro da Silva, Chem. Phys. Lett. 406, 154 (2005).

2005GRE/KLA A. Grenner, M. Klauck, and J. Schmelzer, Fluid Phase Equilib. 233, 170 (2005).

2005HAS/TAJ M. Hashimoto, T. Tajima, K. Eda, K. Yamamura, and T. Okazaki, J. Mol. Struct. 734, 23 (2005).

2005HOS/GRY M. Hoskovec, D. Grygarova, J. Cvacka, L. Strinz, J. Zima, S. P. Verevkin, and B. Koutek, J. Chromatogr. A 1083, 161 (2005).

2005HUA/SIM D. Huang, S. L. Simon, and G. B. Mckenna, J. Chem. Phys. 122, 084907 (2005). 
2005HUA/TAN C.-Y. Huang, M. Tang, and Y.-P. Chen, J. Chem. Eng. Data 50, 40 (2005). Note: Numerical values of the enthalpy of fusion obtained through correspondence with Y.-P. Chen.

2005KAP/SLO S. Kapteina, K. Slowik, S. P. Verevkin, and A. Heintz, J. Chem. Eng. Data 50, 398 (2005).

2005KLO/BRO M. G. Klous, G. M. Bronner, B. Nuijen, J. M. van Ree, and J. H. Beijnen, J. Pharm. Biomed. Anal. 39, 944 (2005).

2005LEE/SU M.-J. Lee, C.-C. Su, and H.-M. Lin, J. Chem. Eng. Data 50, 1535 (2005).

2005LOP/TOM A. J. Lopes Jesus, L. I. N. Tomé, M. E. Eusébio, and J. S. Redinha, J. Phys. Chem. B 109, 18055 (2005).

2005LU/TAN X.-C. Lu, Z.-C. Tan, Q. Shi, H.-T. Zhang, L.-X. Sun, and T. Zhang, J. Chem. Eng. Data 50, 932 (2005).

2005LUC/FER S. Lucas, D. Ferry, B. Demirdjian, and J. Suzanne, J. Phys. Chem. B 109, 18103 (2005).

2005MAR/AVA G. Marchionni, M. Avataneo, U. De Patto, P. Maccone, and G. Pezzin, J. Fluorine Chem. 126, 465 (2005).

2005MAR/ZAT S. A. Markarian, A. L. Zatikyan, V. V. Grigoryan, and G. S. Grigoryan, J. Chem. Eng. Data 50, 23 (2005).

2005MAT/MIR M. A. R. Matos, M. S. Miranda, V. M. F. Morais, and J. F. Liebman, Mol. Phys. 103, 221 (2005).

2005MAT/MOR M. A. R. Matos, V. M. F. Morais, M. D. M. C. Ribeiro da Silva, M. C. F. Margues, E. A. Sousa, J. P. Castieiras, C. P. Santos, and W. E. Acree, Jr., J. Chem. Eng. Data 50, 1184 (2005).

2005MON/MUN J. B. Monton, R. Munoz, M. C. Burguet, and J. de la Torre, Fluid Phase Equilib. 227, 19 (2005).

$2005 \mathrm{MON} / \mathrm{SOU}$ M. J. S. Monte and C. A. D. Sousa, J. Chem. Eng. Data 50, 2101 (2005).

2005MUN/MON R. Munoz, J. B. Monton, M. C. Burguet, and J. de la Torre, Fluid Phase Equilib. 232, 62 (2005).

2005NAS/NEU K. Nasirzadeh, R. Neueder, and W. Kunz, J. Chem. Eng. Data 50, 26 (2005).

2005ORT/ESP J. Ortega, F. Espiau, and M. Postigo, J. Chem. Eng. Data 50, 444 (2005).

2005OXL/SMI J. C. Oxley, J. L. Smith, K. Schinde, and J. Moran, Propellants, Explos., Pyrotech. 30, 127 (2005).

2005PEN/MUR S. Pena-Tejedor, R. Murga, M. T. Sanz, and S. Beltran, Fluid Phase Equilib. 230, 197 (2005).

2005PER/ROD G. L. Perlovich, S. V. Rodionov, and A. Bauer-Brandl, Eur. J. Pharm. Sci. 24, 25 (2005).

2005PIN/DIO S. S. Pinto, H. P. Diogo, R. C. Guedes, B. J. C. Cabral, M. E. Minas de Piedade, and J. A. M. Simoes, J. Phys. Chem. A 109, 9700 (2005).

2005RES/GON J. M. Resa, C. Gonzalez, S. Ortiz de Landaluce, and J. M. Goenaga, J. Chem. Eng. Data 50, 319 (2005).

2005RIB/FON M. A. V. Ribeiro da Silva, J. M. S. Fonseca, R. P. B. M. Carvalho, and M. J. S. Monte, J. Chem. Thermodyn. 37, 271 (2005).

2005RIB/GOM M. A. V. Ribeiro da Silva, J. R. B. Gomes, and A. I. M. C. L. Ferreira, J. Phys. Chem. B 109, 13356 (2005).

2005RIB/MAT M. A. V. Ribeiro da Silva, M. A. R. Matos, and L. M. P. F. Amaral, J. Chem. Thermodyn. 37, 1312 (2005).

2005RIB/MIR M. D. M. C. Ribeiro da Silva, M. S. Miranda, C. M. V. Vaz, M. A. R. Matos, and W. E. Acree, Jr., J. Chem. Thermodyn. 37, 49 (2005).

2005ROG/PIS G. N. Roganov, P. N. Pisarev, V. N. Emel'yanenko, and S. P. Verevkin, J. Chem. Eng. Data 50, 1114 (2005).

2005ROJ/GIN A. Rojas-Aguilar, F. Ginez-Carbajal, E. Orozco-Guareno, and H. Flores-Sejura, J. Therm. Anal. Calorim. 79, 95 (2005).

2005RON/GU H.-R. Rong and H. Gu, Thermochim. Acta 428, 19 (2005).

2005ROU/TEM M. V. Roux, M. Temprado, and J. S. Chickos, J. Chem. Thermodyn. 37, 941 (2005).

2005SAI/IKE H. Saitoh, S. Ikeuchi, and K. Saito, J. Therm. Anal. Calorim. 81, 511 (2005).

2005SMI/KAN N. N. Smirnova, K. V. Kandeev, and T. A. Bykova, Russ. J. Phys. Chem. 79, 857 (2005).

2005STE/SUN F. Steyer and K. Sundmacher, J. Chem. Eng. Data 50, 1277 (2005).

2005STR/SPO A. Strutynska, A. Sporzynski, J. Serwatowski, and S. P. Verevkin, Fluid Phase Equilib. 227, 283 (2005).
2005SUN/LIU3 X.-H. Sun, F.-Y. Liu, Z.-C. Tan, P.-J. Ji, and M.-H. Wang, Chin. J. Chem. 23, 1490 (2005).

2005TEM/CHI M. Temprado and J. S. Chickos, Thermochim. Acta $\mathbf{4 3 5}$, 49 (2005).

2005TEM/ROU M. Temprado, M. V. Roux, P. Umnahanant, H. Zhao, and J. S. Chickos, J. Phys. Chem. B 109, 12590 (2005).

2005TIA/TAN Q.-F. Tian, Z.-C. Tan, Q. Shi, F. Xu, L.-X. Sun, and T. Zhang, Thermochim. Acta 430, 53 (2005).

2005TOM/CAT M. Tomassetti, A. Catalani, V. Rossi, and S. Vecchio, J. Pharm. Biomed. Anal. 37, 949 (2005).

2005TUR/VAC R. Turcotte, M. Vachon, Q. S. M. Kwok, R. Wang, and D. E. G. Jones, Thermochim. Acta 433, 105 (2005).

2005VAL/QUI E. Valez, J. Quijano, J. Vaviria, M. V. Roux, P. Jimenez, M. Temprado, G. Martin-Valcarcel, J. Perez-Parajon, and R. Notario, J. Phys. Chem. A 109, 7832 (2005).

2005VAN/VAN P. R. van der Linde, J. C. van Miltenburg, G. J. K. van den Berg, and H. A. J. Oonk, J. Chem. Eng. Data 50, 164 (2005).

2005VAS/VER T. V. Vasiltsova, S. P. Verevkin, E. Bich, A. Heintz, R. Bogel-Lukasik, and U. Domanska, J. Chem. Eng. Data 50, 142 (2005).

2005VEC/BRU S. Vecchio and B. Brunetti, J. Chem. Eng. Data 50, 666 (2005). Complete chemical numbers for several of the compounds were obtained through personal corresponence with $\mathrm{S}$. Vecchio.

2005VON/GAR S. Von Ahsen, P. Garcia, H. Willner, and G. A. Argueello, Inorg. Chem. 44, 5713 (2005).

2005WAN/TAN M.-H. Wang, Z.-C. Tan, X.-H. Sun, L.-X. Sun, Y.-F. Liu, and T. Zhang, Wuli Huaxue Xuebao 21, 573 (2005).

2005WAN/TAN2 M.-H. Wang, Z.-C. Tan, X.-H. Sun, H.-T. Zhang, B.-P. Liu, L.-X. Sun, and T. Zhang, J. Chem. Eng. Data 50, 270 (2005).

2005WAN/TAN3 S.-X. Wang, Z.-C. Tan, Q. Shi, Y.-Y. Di, H.-T. Zhang, F. Xu, L.-X. Sun, and T. Zhang, J. Chem. Thermodyn. 37, 349 (2005).

2005YI/WAN W. Yi, C. Wang, H. Li, and S. Han, J. Chem. Eng. Data 50, 1837 (2005)

2005ZIE/SZT W. Zielenkiewicz and P. Szterner, J. Chem. Eng. Data 50, 1139 (2005).

2006ACE/AGA J. M. Aceves-Hernandez, E. Agacino-Valdes, M. Paz, and J. Hinojosa-Torres, J. Mol. Struct. 786, 1 (2006).

2006ACR/CHI W. E. Acree, Jr. and J. S. Chickos, J. Phys. Chem. Ref. Data 35, 1051 (2006).

2006BAD/DEL E. Badea, G. Della Gata, D. D. Angelo, B. Brunetti, and Z. Reckova, J. Chem. Thermodyn. 38, 1546 (2006).

2006BAZ/BLO A. B. Bazyleva, A. V. Blokhin, G. J. Kabo, A. G. Kabo, and V. M. Sevruk, Thermochim. Acta 451, 65 (2006).

2006BOL/NER M. F. Bolotnikov and Yu. A. Neruchev, Fluid Phase Equilib. 243, 121 (2006).

2006BOL/NER2 M. F. Bolotnikov and Yu. A. Neruchev, Russ. J. Phys. Chem. 80, 1191 (2006).

2006BOU/BEL G. Boukais-Belaribi, B. F. Belaribi, A. Ait-Kaci, and J. Jose, Fluid Phase Equilib. 248, 181 (2006).

2006BUC/BUE J. H. Buchaman, L. C. Buettner, and D. E. Trevault, J. Chem. Eng. Data 51, 1331 (2006).

2006BUH/MIE C. Buhrmester, G. Miehe, H. Ehrenberg, and H. Fuess, Cryst. Res. Technol. 41, 48 (2006).

2006CAM A. G. Camacho, Doctoral thesis, Universidad Nacional de Tucuman, Tucuman, Argentina, 2006.

2006CAM/MAR A. G. Camacho, A. Mariano, L. Mussari, and M. A. Postigo, J. Chem. Eng. Data 51, 1536 (2006).

2006CHE/OJA X. Chen, V. Oja, W. G. Chan, and M. R. Hajaligol, J. Chem. Eng. Data 51, 386 (2006).

2006DAB/SPO A. Dabrowska, A. Sporzynski, and S. P. Verevkin, Fluid Phase Equilib. 249, 115 (2006).

2006DEB/MED A. L. F. de Barros, A. Medina, F. Zappa, J. M. Pereira, E. Bessa, M. H. P. Martins, L. F. S. Coelho, W. Wolff, and N. V. de Castro Faria, Nucl. Instrum. Methods Phys. Res., Sect. A 560, 219 (2006).

2006DIV/CHE S. Divi, R. Chellappa, and D. Chandra, J. Chem. Thermodyn. 38, 1312 (2006).

2006DOM/MOR U. Domansak, P. Morawski, and R. Wierzbicki, Fluid Phase Equilib. 242, 154 (2006). 
2006DRE/KOV V. A. Drebushchak, Yu. A. Kovalevskaya, I. E. Paukov, and E. V. Boldyreva, J. Therm. Anal. Calorim. 85, 485 (2006).

2006DRU/DOR A. I. Druzhinina, O. V. Dorofeeva, R. M. Varushchenk, and E. L. Krasnykh, J. Chem. Thermodyn. 38, 10 (2006).

2006DRU/KRO A. I. Druzhinina, O. V. Krol, A. A. Efimova, R. M. Varushchenko, and L. L. Gervits, Russ. J. Phys. Chem. 80, 1742 (2006).

2006EME/KAB V. N. Emel'yanenko, G. J. Kabo, and S. P. Verevkin, J. Chem. Eng. Data 51, 79 (2006).

2006FUJ/MAT H. Fujimori, K. Matsuda, A. Todoroki, T. Asaji, and M. Oguni, J. Non-Cryst. Solids 352, 4790 (2006).

2006GAO/CHE S. Gao, W. Chen, G. Wang, and J. Chen, J. Cryst. Growth 297, 361 (2006).

2006GAO/ZHA H.-X. Gao, F.-Q. Zhao, R.-Z. Hu, Q. Pan, B.-Z. Wang, X.W. Yang, Y. Gao, S.-L. Gao, and Q.-Z. Shi, Chin. J. Chem. 24, 177 (2006).

2006HAF/PAR J. J. H. Haftka, J. R. Parsons, and H. A. J. Govers, J. Chromatogr. A 1135, 91 (2006).

2006HOS/NAG S. M. A. Hosseini, H. Naghibi, M. H. Peikar, and E. Jamalizadeh, Asian J. Chem. 18, 351 (2006).

2006HUO/ZEN W.-L. Huo, Z.-X. Zeng, and W.-L. Xue, J. Chem. Eng. Data 51, 2110 (2006).

2006KHI/DAH K. Khimeche and A. Dahmani, J. Therm. Anal. Calorim. 84, 47 (2006).

2006KHI/DAH2 K. Khimeche and A. Dahmani, J. Chem. Eng. Data 51, 382 (2006).

2006KRA/VER E. L. Krasnykh, S. P. Verevkin, B. Koutek, and J. Doubsky, J. Chem. Thermodyn. 38, 717 (2006).

2006LAH/PAA A. Lahtinen, J. Paasivirta, and V. A. Nikiforov, Thermochim. Acta 447, 5 (2006).

2006LUS/MAL M. Luszczyk and S. K. Malanowski, J. Chem. Eng. Data 51, 1735 (2006).

2006MAN/CUT A. Mandanici, M. Cutroni, A. Triolo, V. Rodriguez-Mora, and M. A. Ramos, J. Chem. Phys. 125, 054514 (2006).

2006MAT/MIR M. A. R. Matos, M. S. Miranda, V. M. F. Morais, and J. F. Liebman, Mol. Phys. 104, 1833 (2006).

2006MIR/MOR M. S. Miranda, V. M. F. Morais, and M. A. R. Matos, J. Chem. Thermodyn. 38, 559 (2006).

2006 MOR/MIR V. M. F. Morais, M. S. Miranda, and M. A. R. Matos, J. Chem. Thermodyn. 38, 450 (2006).

2006MOR/MIR2 V. M. F. Morais, M. S. Miranda, M. A. R. Matos, and J. F. Liebman, Mol. Phys. 104, 325 (2006).

2006NAS/NEU K. Nasurzadeh, R. Neueder, and W. Kunz, J. Chem. Eng. Data 51, 7 (2006).

2006NIC/KWE G. Nichols, S. Kweskin, M. Frericks, S. Reiter, G. Wang, J. Orf, B. Carvallo, D. Hillesheim, and J. S. Chickos, J. Chem. Eng. Data 51, 475 (2006).

2006NOR/RAS F. L. Nordstrom and A. C. Rasmuson, J. Chem. Eng. Data 51, 1775 (2006).

2006NOR/RAS2 F. L. Nordstrom and A. C. Rasmuson, J. Pharm. Sci. 95, 748 (2006)

2006PAL/ORA M. Palczewska-Tuliuska and P. Oracz, J. Chem. Eng. Data 51, 639 (2006).

2006PIN/DIO S. P. Pinto and H. P. Diogo, J. Chem. Thermodyn. 38, 1515 (2006).

2006PRO/RAS V. M. Profir and A. C. Rasmuson, Cryst. Growth Des. 6, 1143 (2006).

2006RIB/AMA M. A. V. Ribeiro da Silva, L. M. P. F. Amaral, and J. R. B. Gomes, J. Phys. Chem. B 110, 9301 (2006).

2006RIB/AMA3 M. A. V. Ribeiro da Silva, L. M. P. F. Amaral, A. F. L. O. M. Santos, and J. R. B. Gomes, J. Chem. Thermodyn. 38, 748 (2006).

2006RIB/CAB M. A. V. Ribeiro da Silva and J. I. T. A. Cabral, J. Chem. Eng. Data 51, 1556 (2006).

2006RIB/CAB2 M. A. V. Ribeiro da Silva and J. I. T. A. Cabral, J. Chem. Eng. Data 51, 767 (2006).

2006RIB/CAB3 M. A. V. Ribeiro da Silva and J. I. T. A. Cabral, J. Chem. Thermodyn. 38, 1008 (2006).

2006RIB/CAB4 M. A. V. Ribeiro da Silva and J. L. T. A. Cabral, J. Chem. Thermodyn. 38, 1461 (2006).

2006RIB/CAB5 M. A. V. Ribeiro da Silva, J. I. T. A. Cabral, P. Gomes, and J. R. B. Gomes, J. Org. Chem. 71, 3677 (2006).
2006RIB/CAB6

M. A. V. Ribeiro da Silva, J. I. T. A. Cabral, and J. R. B. Gomes, J. Chem. Thermodyn. 38, 1072 (2006).

2006RIB/FER

M. A. V. Ribeiro da Silva, A. I. M. C. L. Ferreira, and J. R. B. Gomes, Bull. Chem. Soc. Jpn. 79, 1852 (2006).

2006RIB/FER2

M. A. V. Ribeiro da Silva, A. I. M. C. L. Ferreira, and J. R. B. Gomes, Chem. Phys. Lett. 422, 565 (2006).

2006RIB/MAT M. A. V. Ribeiro da Silva, M. A. R. Matos, and L. M. P. F. Amaral, J. Chem. Thermodyn. 38, 49 (2006)

2006RIB/MON M. A. V. Ribeiro da Silva, M. J. S. Monte, and L. M. N. B. F. Santos, J. Chem. Thermodyn. 38, 778 (2006).

2006RIB/SAN M. A. V. Ribeiro da Silva, C. P. F. Santos, M. J. S. Monte, and C. A. D. Sousa, J. Therm. Anal. Calorim. 83, 533 (2006).

2006ROD/GIN S. Rodriquez, B. Giner, M. Haro, S. Martin, and H. Artigas, Phys. Chem. Liq. 44, 275 (2006).

2006ROU/TEM M. V. Roux, M. Temprado, P. Jimenez, R. Notario, R. Guzman-Mejia, and E. Juaristi, J. Org. Chem. 71, 2581 (2006).

2006SED/MAI Z. Sedlakova, I. Maijevska, K. Rehak, and P. Vrbka, Collect. Czech. Chem. Commun. 71, 1350 (2006).

2006SMI/KAN N. N. Smirnova, K. V. Kandeev, T. A. Bykova, and T. G. Kulagaina, J. Chem. Thermodyn. 38, 376 (2006).

2006TEM/ROU M. Temprado, M. V. Roux, P. Jimenéz, R. Guzmán-Mejía, and E. Juaristi, Thermochim. Acta 441, 20 (2006).

2006TEO/BAR M. Teodorescu, A. Barhala, and D. Dragoescu, J. Chem. Thermodyn. 38, 1432 (2006).

2006UMN/KWE P. Umnahanant, S. Kweskin, G. Nichols, M. J. Dunn, H. Smart-Ebinne, and J. S. Chickos, J. Chem. Eng. Data 51, 2246 (2006).

2006VAN/VAN P. J. van Ekeren, A. C. G. van Genderen, and G. J. K. van den Berg, Thermochim. Acta 446, 33 (2006).

2006VAS/VER T. V. Vasiltsova, S. P. Verevkin, E. Bich, A. Heintz, R. Bogel-Lukasik, and U. Domanska, J. Chem. Eng. Data 51, 213 (2006).

2006VER S. P. Verevkin, J. Chem. Thermodyn. 38, 1111 (2006).

2006VER/KOZ S. P. Verevkin, S. A. Kozlova, V. N. Emel'yanenko, E. D. Nikitin, A. P. Popov, and E. L. Krasnykh, J. Chem. Eng. Data 51, 1896 (2006).

2006WAN/FAN Z. Wang, W. Fang, R. Lin, Y. Guo, and X. Zhou, Fuel 85, 1794 (2006).

2006WAN/NAN X.-R. Wang, Z.-D. Nan, and Z.-C. Tan, Chin. J. Chem. 24, 1301 (2006).

2006WAS/HOL C. M. Wassvik, A. G. Holmen, C. A. S. Bergstrom, I. Zamora, and P. Artursson, Eur. J. Pharm. Sci. 29, 294 (2006).

2006WOJ/MOS G. Wojcik and I. Mossakowska, Acta Crystallogr., Sect. B 62, 143 (2006).

2006XU/SUN $\quad$ F. Xu, L. X. Sun, Z. C. Tan, J. G. Liang, and T. Zhang, J. Therm. Anal. Calorim. 83, 187 (2006).

2006ZAI/PAU D. H. Zaitsau, Y. U. Paulechka, G. J. Kabo, A. N. Kolpikau, V. N. Emel'yanenko, A. Heintz, and S. P. Verevkin, J. Chem. Eng. Data 51, 130 (2006).

$2006 Z A K / L A Z \quad$ D. V. Zakharychev, S. N. Lazarev, Z. A. Bredikhina, and A. A. Bredikhin, Russ. Chem. Bull. 55, 230 (2006).

2006ZHO/LI C.-R. Zhou, J. Li, X.-H. Shi, and H.-F. Wang, Zhengzhou Daxue Xuebao, Gongxueban 27, 29 (2006).

2007BAD/BLA E. Badea, I. Blanco, and G. Della Gatta, J. Chem. Thermodyn. 39, 1392 (2007).

2007BAR/COZ F. Barontini and V. Cozzani, J. Therm. Anal. Calorim. 89, 309 (2007).

2007BER/MIN C. E. S. Bernardes, M. E. Minas da Piedade, L. M. P. F. Amaral, A. I. M. C. L. Ferreira, M. A. V. Ribeiro da Silva, H. P. Diogo, and B. J. C. Cabral, J. Phys. Chem. A 111, 1713 (2007).

2007BLA

2007BOU/BEL

R. L. Blaine, Proc. NATAS Annu. Conf. Therm. Anal Appl. 35, 1-7 (2007); Chem. Abstr. 148, 223167 (2007).

G. Boukais-Belaribi, B. F. Belaribi, J. Lohmann, and J. Jose, Fluid Phase Equilib. 262, 180 (2007).

2007CAM/MAG L. Campanella, A. L. Magri, M. Tomassetti, V. Rossi, and S. Vecchio, Drug Develop. Ind. Pharm. 33, 830 (2007).

2007CAM/MOL A. G. Camacho, J. M. Moll, S. Canzonieri, and M. A. Postigo, J. Chem. Eng. Data 52, 871 (2007). 
2007CAN/EYL A. I. Canizo, G. N. Eyler, and G. P. Barreto, Chromatographia 65, 21 (2007).

2007CAP/LOV C. D. Cappa, E. R. Lovejoy, and A. R. Ravishankara, J. Phys. Chem. A 111, 3099 (2007).

2007DAN/MAR A. A. Daniela, P. I. Mariela, R. M. Jorge, J. L. Nelly, G. Vara, E. Manuel, C. A. Eduardo, and J. H. Alicia, Global J. Mol. Sci. 2, 8 (2007).

2007DON/LI J.-X. Dong, Q. Li, Z.-C. Tan, Z.-H. Zhang, and Y. Liu, J. Chem. Thermodyn. 39, 108 (2007).

2007DRA/JAN L. C. Draucker, M. Janakat, M. J. Lazzaroni, D. Bush, C. A. Eckert, T. C. Frank, and J. D. Olson, Ind. Eng. Chem. Res. 46, 2198 (2007).

2007ECK/HUA B. Eckl, Y.-L. Huang, J. Vrabec, and H. Hasse, Fluid Phase Equilib. 260, 177 (2007)

2007EFI/PAS A. A. Efimova, L. L. Pashchenko, R. M. Varushchenko, E. Krasnyh, and S. V. Levanova, J. Chem. Thermodyn. 39, 142-147 (2007).

2007EME/DAB V. N. Emel'yanenko, A. Dabrowska, S. P. Verevkin, M. O. Hertel, H. Scheuren, and K. Sommer, J. Chem. Eng. Data 52, 468 (2007).

2007EME/KOZ V. N. Emel'yanenko, S. A. Kozlova, S. P. Verevkin, and G. N. Roganov, J. Chem. Thermodyn. 39, 10 (2007).

2007FER/BAD D. Ferro, E. Badea, M. Jozwiak, and J. V. Rau, Thermochim. Acta 460, 50 (2007).

2007FRE/OLI V. L. S. Freitas, L. I. P. Oliveira, and M. D. M. C. Ribeiro da Silva, J. Chem. Thermodyn. 39, 39 (2007).

2007GOE/MCC A. Goel, L. L. McConnell, and A. Torrents, J. Environ. Sci. Health, Part B 42, 343 (2007).

$2007 \mathrm{GOM} / \mathrm{SOU} 2$ J. R. B. Gomes, E. A. Sousa, P. Gomes, N. Vale, J. M. Goncalves, S. Pandey, W. E. Acree, Jr., and M. D. M. C. Ribeiro da Silva, J. Phys. Chem. B 111, 2075 (2007).

2007HAF/MAH S. L. Hafsaoui and R. Mahmoud, J. Therm. Anal. Calorim. 88, 565 (2007).

2007HEI/KAP A. Heintz, S. Kapteina, and S. P. Verevkin, J. Phys. Chem. A 111, 6552 (2007).

2007HUL/JOH A. T. Hulme, A. Johnston, A. J. Florence, P. Fernandes, K. Shankland, C. T. Bedford, G. W. A. Welch, G. Sadiq, D. A. Haynes, W. D. S. Motherwell, D. A. Toucher, and S. L. Price, J. Am. Chem. Soc. 129, 3649 (2007).

2007HWA/HAN H.-C. Hwang, K.-J. Han, and S.-J. Park, J. Chem. Eng. Data 52, 1118 (2007).

2007LEE/LAI M.-J. Lee, C.-H. Lai, T.-B. Wang, and H.-M. Lin, J. Chem. Eng. Data 52, 1291 (2007).

2007LIU/LIU L.-S. Liu, W.-S. Liu, N. Zhou, X.-N. Fan, H. Song, C. Fu, and Y.-Y. Yang, Gaoxiao Huaxue Gongcheng Xuebao 21, 875 (2007); Chem. Abstr. 148, 222587 (2007).

2007LU/WAN J. Lu, X.-J. Wang, X. Yang, and C.-B. Ching, Cryst. Growth Des. 7, 1590 (2007).

2007MAL

2007MAT/MIR

S. K. Malanowski, J. Chem. Eng. Data 52, 239 (2007).

M. A. R. Matos, M. S. Miranda, M. J. S. Monte, L. M. N. B. F. Santos, V. M. F. Morais, J. S. Chickos, P. Umnahanant, and J. F. Liebman, J. Phys. Chem. A 111, 11153 (2007).

2007MAT/MIR2 M. A. R. Matos, M. S. Miranda, S. M. M. Pereira, V. M. F. Morais, and J. F. Liebman, J. Phys. Chem. 111, 7181 (2007).

2007MAT/SOU M. A. R. Matos, C. C. S. Sousa, and V. M. F. Morais, J. Chem. Eng. Data 52, 1089 (2007).

2007MIS/MIS2 A. K. Misra, M. Misra, G. M. Panpalia, and A. K. Dorle, Pharm. Dev. Technol. 12, 423 (2007).

2007MOK/RAZ M. Mokbel, A. Razzouk, A. Hajjaji, N. Misakni, and J. Jose, J. Chem. Eng. Data 52, 1720 (2007).

2007MOR/COR E. Moreno, R. Cordobilla, T. Calvet, M. A. Cuevas-Diarte, G. Gbabode, P. Negrier, D. Mondieig, and H. A. J. Oonk, New J. Chem. 31, 947 (2007).

2007 MOR/MIR V. M. F. Morais, M. S. Miranda, and M. A. R. Matos, J. Chem. Eng. Data 52, 627 (2007).

2007PAL/ORA M. Palczewska-Tulinska and P. Oracz, J. Chem. Eng. Data 52, 645 (2007).

2007PAL/ORA2 M. Palczewska-Tuliuska and P. Oracz, J. Chem. Eng. Data 52, 2468 (2007).

2007PAN/ANT2 K. Panneerselvam, M. P. Antony, T. G. Srinivasan, and P. R. Vasudeva Rao, Thermochim. Acta 466, 49 (2007).
2007PAS/KUZ

2007PER/HAN

2007PER/VOL2

2007RAU/BEH

2007RIB/AMA

2007RIB/AMA3

2007RIB/ARA

2007RIB/CAB2

2007RIB/FER

2007RIB/FER2

2007RIB/FRE

2007RIB/MON

2007RIB/SAN

2007RIB/SAN3

2007RIC/BER

2007ROU/NOT

2007ROU/TEM

2007ROU/TEM

2007ROU/TEM2

2007ROU/TEM3

2007SAP/ZAY

2007SCH/DOE

2007SHE/ZAI

2007SHI/TAN

2007SHI/TAN2

2007SHI/TAN3

2007SID/ATA

2007SIF/AIT

2007SMI/TSV

2007STR/RUZ

2007STR/RUZ2

2007TOM/ROS
L. L. Pashchenko and T. S. Kuznetsova, Russ. J. Phys. Chem. A 81, 1238 (2007).

G. L. Perlovich, L. Kr. Hansen, T. V. Volkova, S. Mirza, A. N. Manin, and A. Bauer-Brandl, Cryst. Growth Des. 7, 2643 (2007).

G. L. Perlovich, T. V. Volkova, and A. Bauer-Brandl, J. Therm. Anal. Calorim. 89, 767 (2007).

R. B. Rauch and R. Behrens, Propellants, Explos., Pyrotech. 32, 97 (2007).

M. A. V. Ribeiro da Silva, L. M. P. F. Amaral, and J. R. B. Gomes, J. Phys. Chem. B 111, 792 (2007).

M. A. V. Ribeiro da Silva, L. M. P. F. Amaral, and J. R. B. Gomes, Pol. J. Chem. 81, 865 (2007).

M. D. M. C. Ribeiro da Silva and N. R. M. Araujo, J. Chem. Thermodyn. 39, 1372 (2007).

M. A. V. Ribeiro da Silva and J. I. T. A. Cabral, Thermochim. Acta 453, 147 (2007).

M. A. V. Ribeiro da Silva, A. I. M. C. L. Ferreira, and J. R. B. Gomes, J. Phys. Chem. B 111, 2052 (2007).

M. A. V. Ribeiro da Silva, A. I. M. C. L. Ferreira, and J. R. B. Gomes, J. Phys. Chem. B 111, 6444 (2007).

M. D. M. C. Ribeiro da Silva, V. L. S. Freitas, L. M. N. B. F. Santos, M. Fulem, M. J. Sottomayor, M. J. S. Monte, and W. E. Acree, Jr., J. Chem. Eng. Data 52, 580 (2007). M. A. V. Ribeiro da Silva, I. M. M. Monteiro, L. M. N. B. F. Santos, and B. Schroder, J. Chem. Thermodyn. 39, 767 (2007).

M. A. V. Ribeiro da Silva and A. F. L. O. M. Santos, J. Therm. Anal. Calorim. 88, 7 (2007).

M. A. V. Ribeiro da Silva, C. P. F. Santos, M. J. S. Monte, and C. A. D. Sousa, J. Chem. Thermodyn. 39, 1363 (2007).

L. S. Richard, C. E. S. Bernardes, H. P. Diogo, J. P. Leal, and M. E. Minas da Piedade, J. Phys. Chem. A 111, 8741 (2007).

M. V. Roux, R. Notario, E. Velez, M. Temprado, A. Guerrero, S. P. Verevkin, J. Quijano, and J. Gaviria, J. Chem. Thermodyn. 39, 1377 (2007).

M. V. Roux, M. Temprado, P. Jimenez, R. Notario, J. S. Chickos, A. F. L. O. M. Santos, and M. A. V. Ribeiro da Silva, J. Phys. Chem. A 111, 11084 (2007).

M. V. Roux, M. Temprado, P. Jimenez, R. Notario, R. Guzman-Mejia, and E. Juaristi, J. Org. Chem. 72, 1143 (2007).

M. V. Roux, M. Temprado, R. Notario, J. S. Chickos, A. F. L. O. M. Santos, and M. A. V. Ribeiro da Silva, J. Phys. Chem. A 111, 5280 (2007).

E. Sapei, A. Zaytseva, P. Uusi-Kyyny, K. I. Keshinen, and J. Aittamaa, Fluid Phase Equilib. 252, 130 (2007).

B. Schmid, M. Doeker, and J. Gmehling, Fluid Phase Equilib. 258, 115 (2007).

M. P. Shevelyova, D. H. Zaitsau, Y. U. Paulechka, A. V. Blokhin, G. J. Kabo, S. P. Verevkin, and A. Heintz, J. Chem. Eng. Data 52, 1360 (2007).

Q. Shi, Z.-C. Tan, Y.-Y. Di, B. Tong, Y.-S. Li, and S.-X. Wang, J. Chem. Eng. Data 52, 941 (2007).

Q. Shi, Z.-C. Tan, Y.-Y. Di, B. Tong, S.-X. Wang, and Y.-Z. Li, Thermochim. Acta 463, 6 (2007).

Q. Shi, Z.-C. Tan, B. Tong, Y.-Y. Di, Z.-H. Zhang, J.-L. Zeng, L.-X. Sun, and Y.-S. Li, J. Chem. Thermodyn. 39, 817 (2007).

M. A. Siddiqi and B. Atakan, Thermochim. Acta 452, 128 (2007).

H. Sifaoui, A. Ait-Kaci, A. Mondarressi, and M. Rogalski, Thermochim. Acta 456, 114 (2007).

N. N. Smirnova, L. Ya. Tsvtkova, T. A. Bykova, and Y. Marcus, J. Chem. Thermodyn. 39, 1508 (2007).

M. Straka, K. Ruzicka, and V. Ruzicka, J. Chem. Eng. Data 52, 1375 (2007).

M. Straka, A. van Genderen, K. Ruzicka, and V. Ruzicka, J. Chem. Eng. Data 52, 794 (2007).

L. I. N. Tome, M. T. S. Rosado, S. C. C. Nunes, T. M. R. Maria, J. Canotilho, and M. E. S. Eusebio, J. Chem. Thermodyn. 39, 1354 (2007). 
2007TON/TAN B. Tong, Z.-C. Tan, X. C. Lv, L. X. Sun, F. Xu, Q. Shi, and Y. S. Li, J. Therm. Anal. Calorim. 90, 217 (2007).

2007TON/TAN2 B. Tong, Z.-C. Tan, Q. Shi, Y.-S. Li, D.-T. Yue, and S.-X. Wang, Thermochim. Acta 457, 20 (2007).

2007VAR/DRU R. M. Varushchenko, A. I. Druzhinina, G. M. Kuramshina, and O. V. Dorofeeva, Fluid Phase Equilib. 256, 112 (2007).

2007VEC/BRU S. Vecchio and B. Brunetti, J. Chem. Eng. Data 52, 1585 (2007).

2007VER S. P. Verevkin, J. Chem. Eng. Data 52, 301 (2007).

2007VER/EME S. P. Verevkin, V. N. Emel'yanenko, and A. Klamt, J. Chem. Eng. Data 52, 499 (2007).

2007VER/GEO S. P. Verevkin, M. Georgieva, and S. V. Melkhanova, J. Chem. Eng. Data 52, 286 (2007).

2007VER/SCH S. P. Verevkin and C. Schick, J. Chem. Thermodyn. 39, 758 (2007)

2007WAN/ZHA S.-Z. Wang, X.-S. Zhao, Z.-C. Tan, Y.-S. Lu, B. Tong, Q. Shi, and Y. Li, Wuli Huaxue Xuebao 23, 1459 (2007).

2007XU/ZEN X.-G. Xu, Z.-X. Zeng, W.-I. Xue, and H. Y. Zhang, J. Chem. Eng. Data 52, 1189 (2007).

2007XUE/WAN B. Xue, J. Y. Wang, Z.-C. Tan, and T.-H. Wu, Chem. Res. Chin. Univ. 23, 460 (2007).

2008ABA/BAD L. Abate, E. Badea, I. Blando, and G. Della Gatta, J. Chem. Eng. Data 53, 959 (2008).

2008BAE $\quad$ A. K. Baev, Russ. J. Phys. Chem. A 82, 1266 (2008).

2008BAR/BER I. Barsky and J. Bernstein, Cryst. Eng. Commun. 10, 669 (2008).

2008BAR/BER2 I. Barsky, J. Bernstein, P. W. Stephens, and K. H. Stone, New J. Chem. 32, 1747 (2008).

2008BAR/BOT C. A. Barton, M. A. Botelho, and M. A. Kaiser, J. Chem. Eng. Data 53, 939 (2008).

2008BAS/BOS S. Basavoju, D. Bostrom, and S. Velaga, Pharm. Res. 25, 530 (2008)

2008BAZ/BLO A. B. Bazleva, A. V. Blokhin, A. G. Kabo, G. J. Kabo, V. N. Emel'yanenko, and S. P. Verevkin, J. Chem. Thermodyn. 40, 509 (2008).

2008BER/MIN C. E. S. Bernardes and M. E. Minas da Piedade, J. Phys. Chem. A 112, 10029 (2008).

2008BER/PIE C. E. S. Bernardes, M. F. M. Piedade, and M. E. Minas da Piedade, Cryst. Gowth Des. 8, 2419 (2008).

2008BIA/CEZ T. F. Biazus, A. M. Cezaro, G. R. Borges, J. P. Bender, E. Franceschi, M. L. Corazza, and V. Oliveira, J. Chem. Thermodyn. 40, 437 (2008).

2008BRE/BRE A. A. Bredikhin, Z. A. Bredikhina, V. G. Novikova, A. V. Pashagin, D. V. Zakharychev, and A. T. Gubaidullin, Chirality 20, 1092 (2008).

2008CAP/LOV C. D. Cappa, E. R. Lovejoy, and A. R. Ravishankara, J. Phys. Chem. A 112, 3959 (2008).

2008CHI/STE R. D. Chirico and W. V. Steele, J. Chem. Thermodyn. 40, 806 (2008).

2008DIC/SGH $\quad$ E. Dichi, M. Sghaier, B. Fraisse, F. Bonhomme, and G. Keller, J. Alloys Compd. 458, 595 (2008).

2008DOM/MOR U. Domanska, P. Morawski, and M. Piekarska, J. Chem. Thermodyn. 40, 710 (2008).

2008EME/KOZ V. N. Emel'yanenko, S. A. Kozlova, S. P. Verevkin, and G. N. Roganov, J. Chem. Thermodyn. 40, 911 (2008).

2008EME/TOK V. N. Emel'yanenko, A. V. Toktonov, S. A. Kozlova, S. P. Verevkin, V. Andrushko, N. Andrushko, and A. Borner, J. Phys. Chem. A 112, 4036 (2008).

2008EME/VER V. N. Emel'yanenko, S. P. Verevkin, E. N. Burakova, G. N. Roganov, and M. K. Georgieva, Russ. J. Phys. Chem. A 82, 1521 (2008).

2008FAV/FRE R. Favareto, P. H. Fregadolli, V. F. Cabral, O. A. C. Antunes, and L. Cardozo-Filho, J. Chem. Eng. Data 53, 1080 (2008).

2008FRE/KEB A. Freedman, P. L. Kebabian, Z. Li, W. A. Robinson, and J. C. Wormhoudt, Meas. Sci. Technol. 19, 125102 (2008).

2008FRI/ACR V. L. S. Frietas, W. E. Acree, Jr., and M. D. M. C. Ribeiro da Silva, J. Chem. Eng. Data 53, 1820 (2008).

2008GHI/BEA O. R. Ghita, M. A. Beard, J. McCabe, R. Bottom, J. Richmond, and P. E. Evans, J. Mater. Sci. 43, 4988 (2008).

2008 GOL/SUU2 J. L. Goldfarb and E. M. Suuberg, J. Chem. Thermodyn. 40, 460 (2008).
2008KAI/MAR

A. Kaizawa, N. Maruoka, A. Kawai, H. Kamano, T. Jozuka, T. Senda, and T. Akiyama, Heat Mass Transfer 44, 763 (2008).

2008KHI/DAH K. Khimeche, A. Dahmani, and J. Jose, J. Chem. Thermodyn. 40, 777 (2008).

2008KIM/SVE I. Kim, H. F. Svendsen, and E. Borresen, J. Chem. Eng. Data 53, 2521 (2008).

2008KOZ/EME S. A. Kozlova, V. N. Emel'yanenko, M. Georgieva, S. P. Verevkin, Y. Chernyak, B. Schaffner, and A. Borner, J. Chem. Thermodyn. 40, 1136 (2008).

2008LEV/BAR R. Levit, M. Barrio, N. Beglio, J. Ll. Tamarit, P. Negrier, L. C. Pardo, J. Sanchez-Marcos, and D. Mondieig, J. Phys. Chem. B 112, 13916 (2008).

2008LLA/MON E. Lladosa, J. B. Monton, and M. C. Burguet, J. Chem. Eng. Data 53, 1897 (2008).

2008LU/GAO X.-C. Lu, X.-H. Gao, Z.-C. Tan, Y.-S. Li, and L.-X. Sun, J. Therm. Anal. Calorim. 92, 523 (2008).

2008MAC/SHI A. D. MacKerell, Jr., J. H. Shim, and V. M. Anisimov, J. Chem. Theory Comput. 4, 1307 (2008).

2008MAR/EUS T. M. R. Maria and M. E. S. Eusebio, J. Chem. Eng. Data 53, 1316 (2008).

2008MAT/IVA V. E. Matulis, O. A. Ivashkevich, P. N. Gaponik, P. D. Elkind, G. T. Sukhanov, A. B. Bazyleva, and D. H. Zaitsau, J. Mol. Struct.: THEOCHEM 854, 18 (2008).

2008MAT/MIR M. A. R. Matos, M. S. Miranda, D. A. P. Fonseca, V. M. F. Morais, and J. F. Liebman, J. Phys. Chem. A 112, 10053 (2008).

2008MAT/MIR2 M. A. R. Matos, M. S. Miranda, and V. M. F. Morais, J. Chem. Thermodyn. 40, 625 (2008).

2008MAT/SOU M. A. R. Matos, C. C. S. Sousa, and V. M. F. Morais, J. Chem. Thermodyn. 40, 1485 (2008).

2008MAT/SOU2 M. A. R. Matos, C. C. S. Sousa, and V. M. F. Morais, J. Phys. Chem. 112, 7961 (2008).

2008MAT/SOU3 M. A. R. Matos, C. C. S. Sousa, and V. M. F. Morais, J. Chem. Thermodyn. 40, 1552 (2008).

2008MEN/FLO J. Mentado, H. Flores, and P. Amador, J. Chem. Thermodyn. 40, 1106 (2008).

2008MOG/SEP E. Mogalian, K. Sepassi, and P. B. Myrdal, Drug Dev. Ind. Pharm. 34, 930 (2008).

2008MOT/QUE F. L. Mota, A. J. Queimada, S. P. Pinho, and E. A. Macedo, Ind. Eng. Chem. Res. 47, 5182 (2008).

2008NIC/BEL S. Nicoli, S. Belzi, P. Santi, M. R. Caira, J. Li, and R. Bettini, J. Pharm. Sci. 97, 4830 (2008).

2008OSM/CAT A. Osmont, L. Catoire, and I. Gokalp, Energy Fuels 22, 2241 (2008).

2008PER/VOL G. L. Perlovich, T. V. Volkova, A. N. Manin, and A. BauerBrandl, J. Pharm. Sci. 97, 3883 (2008).

2008PER/VOL2 G. L. Perlovich, T. V. Volkova, A. N. Manin, and A. BauerBrandl, AAPS PharmSciTech 9, 205 (2008).

2008PIN/DIO S. S. Pinto and H. P. Diogo, J. Pharm. Sci. 97, 5354 (2008).

2008RAI/BHA N. Rai, D. Bhatt, J. I. Siepmann, and L. E. Fried, J. Chem. Phys. 129, 194510 (2008).

2008RIB/AMA M. A. V. Ribeiro da Silva and L. M. P. F. Amaral, J. Therm. Anal. Calorim. 92, 53 (2008).

2008RIB/AMA2 M. A. V. Ribeiro da Silva, L. M. P. F. Amaral, C. R. P. Boaventura, and J. R. B. Gomes, J. Chem. Thermodyn. 40, 1226 (2008)

2008RIB/AMA3 M. A. V. Ribeiro da Silva, L. M. P. F. Amaral, and A. F. L. O. M. Santos, J. Chem. Thermodyn. 40, 1588 (2008).

2008RIB/CAB M. A. V. Ribeiro da Silva and J. I. T. A. Cabral, J. Therm. Anal. Calorim. 92, 59 (2008).

2008RIB/CAB2 M. A. V. Ribeiro da Silva and J. I. T. A. Cabral, J. Chem. Thermodyn. 40, 829 (2008).

2008RIB/CAB3 M. A. V. Ribeiro da Silva, J. I. T. A. Cabral, and J. R. B. Gomes, J. Phys. Chem. 112, 12263 (2008).

2008RIB/FER M. A. V. Ribeiro da Silva and A. I. M. C. L. Ferreira, J. Chem. Thermodyn. 40, 924 (2008).

2008RIB/FER2 M. A. V. Ribeiro da Silva and A. I. M. C. L. Ferreira, J. Chem. Thermodyn. 40, 362 (2008).

2008RIB/FER3 M. A. V. Ribeiro da Silva, A. I. M. C. L. Ferreira, L. M. S. S. Lima, and S. M. M. Sousa, J. Chem. Thermodyn. 40, 137 (2008). 
2008RIB/FIG M. A. V. Ribeiro da Silva, D. F. Figueriedo, and J. I. T. A. Cabral, J. Chem. Thermodyn. 40, 369 (2008).

2008RIB/RIB M. D. M. C. M. A. V. Ribeiro da Silva, Ribeiro da Silva, V. L. S. Freitas, M. V. Roux, P. Jimenez, M. Temprado, J. Z. Davalos, P. Cabildo, R. M. Claramunt, and J. Elguero, J. Chem. Thermodyn. 40, 386 (2008).

2008RIB/RIB2 M. D. M. C. M. A. V. Ribeiro da Silva, Ribeiro da Silva, V. L. S. Freitas, M. V. Roux, P. Jimenez, J. Z. Davalas, P. Cabildo, R. M. Claramunt, and J. Elguero, J. Chem. Thermodyn. 40, 1378 (2008).

2008RIB/SAN M. A. V. Ribeiro da Silva and A. F. L. O. M. Santos, J. Chem. Thermodyn. 40, 225 (2008).

2008RIB/SAN2 M. A. V. Ribeiro da Silva and A. F. L. O. M. Santos, J. Chem. Thermodyn. 40, 917 (2008).

2008RIB/SAN3 M. A. V. Ribeiro da Silva and A. F. L. O. M. Santos, J. Chem. Thermodyn. 40, 1217 (2008).

2008RIB/SAN4 M. A. V. Ribeiro da Silva and A. F. L. O. M. Santos, J. Chem. Thermodyn. 40, 1309 (2008).

2008RIB/SAN5 M. A. V. Ribeiro da Silva and A. F. L. O. M. Santos, J. Chem. Thermodyn. 40, 1451 (2008).

2008ROU/TEM2 M. V. Roux, M. Temprado, R. Notario, C. Foces-Foces, V. N. Emel'yanenko, and S. P. Verevkin, J. Phys. Chem. A 112, 7455 (2008).

2008SAG/SAF $\quad$ E. V. Sagadeev and Yu. G. Safina, Russ. J. Gen. Chem. 78, 65-68 (2008).

2008SAR/MOK S. Sarraute, I. Mokbel, M. F. Costa Gomes, V. Majer, and J. Jose, Atmos. Environ. 42, 4724 (2008).

2008SHA/GUP $\quad$ B. L. Sharma, S. Gupta, S. Tandon, and R. Kant, Mater. Chem. Phys. 111, 423 (2008).

2008SHA/WU X.-Z. Shao, J.-S. Wu, G.-Q. Zhang, and L.-S. Wang, J. Chem. Eng. Data 53, 1012 (2008).

2008SIN/DAS N. B. Singh, S. S. Das, P. Gupta, and M. K. Dwivedi, J. Cryst. Growth 311, 118 (2008).

2008SIN/DAS2 N. B. Singh, S. S. Das, N. P. Singh, and T. J. Agrawal, Cryst. Growth 310, 2878 (2008).

2008SIN/MUR L. P. Singh and S. S. N. Murthy, J. Phys. Chem. B 112, 2606 (2008).

2008SIN/MUR2 L. P. Singh and S. S. N. Murthy, J. Chem. Phys. 129, 094501 (2008).

2008SON/RAM M. Soni, D. Ramjugernath, and J. D. Raal, J. Chem. Eng. Data 53, 745 (2008).

2008STU/ROR H. K. Stulzer, P. O. Roriguez, T. M. Cardoso, J. S. R. Matos, and M. A. S. Silva, J. Therm. Anal. Calorim. 91, 323 (2008).

2008SZT P. Szterner, J. Chem. Eng. Data 53, 1892-1896 (2008).

2008TEM/ROU M. Temprado, M. V. Roux, and J. S. Chickos, J. Therm. Anal. Calorim. 94, 257 (2008).

2008TEM/ROU2 M. Temprado, M. V. Roux, P. Jimenez, C. Foces-Foces, and R. Notario, J. Phys. Chem. A 112, 10378 (2008).

2008TEM/ROU3 M. Temprado, M. V. Roux, A. R. Parameswar, A. V. Demchenko, J. S. Chickos, and J. F. Liebman, J. Therm. Anal. Calorim. 91, 471 (2008).

2008TON/TAN B. Tong, Z.-C. Tan, and S.-X. Wang, Chin. J. Chem. 26, 1561 (2008).

2008TON/TAN2 B. Tong, Z. C. Tan, Q. Shi, Y. S. Li, and S. X. Wang, J. Therm. Anal. Calorim. 91, 463 (2008).

2008VEN/BAY L. Ventola, L. Bayes, R. Benzges, F. J. Novegil-Anleo, M. A. Cuevas-Diarte, T. Calvet, and D. Mondieig, Helv. Chim. Acta 91, 1286 (2008).

2008VER/EME S. P. Verevkin and V. N. Emel'yanenko, Fluid Phase Equilib. 266, 64 (2008).

2008VER/KOZ S. P. Verevkin and S. A. Kozlova, Thermochim. Acta 471, 33 (2008).

2008VER/TOK S. P. Verevkin, A. V. Toktonov, Y. Chernyak, B. Schaffner, and A. Borner, Fluid Phase Equilib. 268, 1 (2008).

2008WAN/TAN S.-X. Wang, Z.-C. Tan, Y.-S. Li, B. Tong, Y. Li, Q. Shi, and J.-N. Zhang, Chin. J. Chem. 26, 2016 (2008).

2008WAS/HOL C. M. Wassvik, A. G. Holmen, R. Draheim, P. Artursson, and C. A. S. Bergstrom, J. Med. Chem. 51, 3035 (2008).

2008WEI

2008WIS/BER S. Wishkerman and J. Bernstein, Chem. Eur. J. 14, 197 (2008).
2008XIA/ZHA

2008XUE/LI

Q. Xia, F.-B. Zhang, G.-L. Zhang, J.-C. Ma, and L. Zhao, J. Chem. Eng. Data 53, 838 (2008).

2008YAN/SUP

B. Xue, X.-F. Li, J.-Y. Wang, S.-J. Yu, Z.-C. Tan, and L.-X. Sun, J. Therm. Anal. Calorim. 94, 529 (2008).

2008ZAI/PAU

W. Yan and G. J. Suppes, J. Chem. Eng. Data 53, 2033 (2008).

V. N. Emel'yanenko, S. P. Verevkin, and A. J. Heintz,
C. Chem. Eng. Data 53, 694 (2008).

2008ZHA/UNH H. Zhao, P. Unhannanant, W. Hanshaw, and J. S. Chickos, J. Chem. Eng. Data 53, 1545 (2008).

2009AST/DYU A. M. Astakhov, K. P. Dyugaev, A. A. Kuzubov, V. A. Nasluzov, A. D. Vasiliev, and E. S. Buka, J. Struct. Chem. 50, 201 (2009).

2009BAR/ESP M. Barrio, P. Espeau, J. L. Tamarit, M.-A. Perrin, N. Veglio, and R. Ceolin, J. Pharm. Sci. 98, 1657 (2009).

2009BAR/GAM C. Baraldi, M. C. Gamberini, A. Tinti, F. Palazzoli, and V. Ferioli, J. Mol. Struct. 918, 88 (2009).

2009BEL/RAZ A. Belabbaci, A. Razzouk, I. Mokbel, J. Jose, and L. Negadi, J. Chem. Eng. Data 54, 2312 (2009).

2009BOO/MAR A. M. Booth, T. Markus, G. McFiggans, C. J. Percival, M. R. McGillen, and D. O. Topping, Atmos. Meas. Sci. 2, 355 (2009).

2009BRI

2009BRI2 H. G. Brittain, Cryst. Growth Des. 9, 2492 (2009).

2009BUT/BUC H. G. Brittain, Cryst. Growth Des. 9, 3497 (2009).

2009CHE/PET E. Y. Cheung and M. L. Peterson, Thermochim. Acta 482 , Eng. Data 54, 1876 (2009). 57 (2009).

2009CHE/XIA

S. Chen, Q. Xia, D. Li, W.-G. Yan, F.-B. Zhang, and G.-L. Zhang, J. Chem. Eng. Data 54, 1395 (2009).

2009CHE/ZHO L.-L. Chen, C.-R. Zhou, and X.-H. Shi, Gaoxiao Huaxue Gangcheng Xuebao 23, 730 (2009).

2009CHI/STE R. D. Chirico and W. V. Steele, J. Chem. Thermodyn. 41, 392 (2009).

2009CLA/GOM R. A. Clara, A. C. Gomez Marigliano, and H. N. Solimo, J. Chem. Eng. Data 54, 1087 (2009).

2009COS/SAR M. C. Costa, M. Sardo, M. P. Rolemberg, J. A. P. Coutinho, A. J. R. Meirelles, P. Ribeiro-Claro, and M. A. Krahenbuhl, Chem. Phys. Lipids 160, 85 (2009).

2009DAS/SIN $\quad$ S. S. Das, N. P. Sing, T. Agrawal, P. Gupta, S. N. Tiwari, and N. B. Singh, Mol. Cryst. Liq. Cryst. 501, 107 (2009).

2009EFI/DRU A. A. Efimova, A. I. Druzhinina, R. M. Varushchenko, O. V. Dorofeeva, and E. L. Krasnyh, J. Chem. Eng. Data 54, 2457 (2009)

2009EME/VER V. N. Emel'yanenko, S. P. Verevkin, E. N. Burakova, G. N. Roganov, and M. K. Georgieva, Russ. J. Phys. Chem. A 83, 598 (2009).

2009EME/VER2 V. N. Emel'yanenko, S. P. Verevkin, and A. A. Pimerzin, Russ. J. Phys. Chem. 83, 2013 (2009).

2009FLO/CAM H. Flores, E. A. Camarillo, and J. Mentado, Thermochim. Acta 493, 76 (2009).

2009FRE/ARA F. D. Freire, C. F. S. Aragao, T. F. A. D. Moura, and F. N Raffin, J. Therm. Anal. Calorim. 97, 333-336 (2009).

2009FRE/GOM2 V. L. S. Freitas, J. R. B. Gomes, and M. D. M. C. Ribeiro da Silva, J. Therm. Anal. Calorim. 97, 827 (2009).

2009GIE/KOS P. Gierycz, A. Kosowski, and R. Swietlik, J. Chem. Eng. Data 54, 2996 (2009).

2009GOO/ROD D. J. Good and N. Rodriguez-Hornedo, Cryst. Growth Des. 9, 2252 (2009).

2009HA/HAN J.-M. Ha, B. D. Hamilton, M. A. Hillmyer, and M. D. Ward, Cryst. Growth Des. 9, 4766 (2009).

2009HE/ZHU Q. He, J. Zhu, H. Gomaa, M. Jennings, and S. Rohani, J. Pharm. Sci. 98, 1835 (2009).

2009KEN/FLO A. R. Kennedy, A. J. Florence, F. J. McInnes, and N. J. Wheate, Dalton Trans. 2009, 7695.

2009LAH/RAU A. Lahde, J. Raula, J. Malm, E. I. Kauppinen, and M. Karppinen, Thermochim. Acta 482, 17 (2009).

2009LEG/BAC B. Legendre, P. Bac, M. German, and Y. Feutelais, J. Therm. Anal. Calorim. 98, 91 (2009).

2009LI/FAN $\quad$ D. Li, W. Fang, W. Xie, Y. Wing, Y. Guo, and R. Lin, Energy Fuels 23, 794 (2009). 
2009LIP/CHI D. Lipkind and J. S. Chickos, "38TH Great Lakes Regional Meeting," 87, 58 (2009).

2009LIP/CHI2 D. Lipkind and J. S. Chickos, Struct. Chem. 20, 49 (2009).

2009LIP/HAN D. Lipkind, W. Hanshaw, and J. S. Chickos, J. Chem. Eng. Data 54, 2930 (2009).

2009MAR/AUC B. Marrufo, A. Aucejo, M. Sanchotello, and S. Loras, Fluid Phase Equilib. 279, 11 (2009).

2009MAR/LLA N. F. Martinez, E. Lladosa, M. C. Burguet, J. B. Monton, and M. Yazimon, Fluid Phase Equilib. 277, 49 (2009).

2009MAT/SOU M. A. R. Matos, C. C. S. Sousa, and V. M. F. Morais, J. Chem. Thermodyn. 41, 308 (2009).

2009MAT/SOU2 M. A. R. Matos, C. C. S. Sousa, and V. M. F. Morais, J. Chem. Thermodyn. 41, 69 (2009).

2009MAT/SOU3 M. A. R. Matos, C. C. S. Sousa, and V. M. F. Morais, J. Chem. Eng. Data 54, 2189 (2009).

2009MAT/SOU4 M. A. R. Matos, C. C. S. Sousa, M. S. Miranda, V. M. F. Morais, and J. F. Liebman, J. Phys. Chem. B 113, 11216 (2009).

2009MEL/PIN V. Meltzer and E. Pincu, Rev. Roum. Chim. 54, 333 (2009).

2009MEN/TAN Q. Meng, Z. Tan, X. Wang, Y. Dong, W. Li, and Q. Shi, Chin. J. Chem. 27, 1225 (2009).

2009MEN/ZHA Q.-F. Meng, J.-N. Zhang, Z.-C. Tan, Y.-P. Dong, W. Li, and Q. Shi, J. Chem. Eng. Data 54, 232 (2009).

2009MIR/KON E. A. Miroshnichenko, T. S. Kon'kova, Yu. N. Matyushin, and Ya. O. Inozemtsev, Russ. Chem. Bull. (Int. Ed.) 58, 2015 (2009).

2009MOK/RAZ I. Mokhel, A. Razzouk, T. Sawaya, and J. Jose, J. Chem. Eng. Data 54, 819-822 (2009).

2009MOT/CAR F. L. Mota, A. P. Carneiro, A. J. Queimada, S. P. Pinho, and E. A. Macedo, Eur. J. Pharm. Sci. 37, 499 (2009).

2009MUR/BER F. S. Murakami, L. S. Bernardi, R. N. Pereira, B. R. Valente, E. C. Vasconcelos, M. A. S. Carvalho Filho, and M. A. S. Silva, Pharm. Chem. J. 43, 716 (2009).

2009NEZ/AER A. Nezzal, L. Aert, M. Verspaille, G. Hendrickx, and A. Redl, J. Cryst. Growth 311, 3863 (2009).

2009OXL/SMI J. C. Oxley, J. L. Smith, W. Luo, and J. Brady, Propellants, Explos., Pyrotech. 34, 539 (2009).

2009PAN/ANT K. Panneerselvam, M. P. Antony, T. G. Srinivasan, and P. R. V. Rao, Thermochim. Acta 495, 1 (2009).

2009PEN/ESC M. A. Pena, A. Escalera, A. Reillo, A. B. Sanchez, and P. Bustamante, J. Pharm. Sci. 98, 1129 (2009).

2009PER/MAG R. A. Perkins and J. W. Magee, J. Chem. Eng. Data 54, 2646 (2009).

2009PER/OCH R. A. Perkins, J. C. S. Ochoa, and J. W. Magee, J. Chem. Eng. Data 54, 3192 (2009).

2009POS/MAR M. A. Postigo, A. B. Mariano, A. F. Jara, and N. Zurakowski, J. Chem. Eng. Data 54, 1575 (2009).

2009QUE/MOT A. J. Queimada, F. L. Mota, S. P. Pinho, and E. A. Macedo, J. Phys. Chem. B 113, 3469 (2009).

2009RAI/RED R. N. Rai and R. S. B. Reddi, Thermochim. Acta 496, 13 (2009).

2009RAZ/HAJ A. Razzouk, A. Hajjaji, I. Mokbel, P. Mougin, and J. Jose, Fluid Phase Equilib. 282, 11 (2009).

2009RIB/AMA M. A. V. Ribeiro da Silva and L. M. P. F. Amaral, J. Chem. Thermodyn. 41, 26 (2009).

2009RIB/AMA2 M. A. V. Ribeiro da Silva and L. M. P. F. Amaral, J. Chem. Thermodyn. 41, 349 (2009).

2009RIB/CAB M. A. V. Ribeiro da Silva and J. I. T. A. Cabral, J. Chem. Thermodyn. 41, 355 (2009).

2009RIB/CAB2 M. A. V. Ribeiro da Silva, J. I. T. A. Cabral, and J. R. B. Gomes, J. Chem. Thermodyn. 41, 1193 (2009).

2009RIB/FER M. A. V. Ribeiro da Silva and A. I. M. C. L. Ferreira, J. Chem. Eng. Data 54, 2517 (2009).

2009RIB/FER2 M. A. V. Ribeiro da Silva and A. I. M. C. L. Ferreira, J. Chem. Thermodyn. 41, 361 (2009).

2009RIB/FER3 M. A. V. Ribeiro da Silva and A. I. M. C. L. Ferreira, J. Chem. Thermodyn. 41, 499 (2009).

2009RIB/FER4 M. A. V. Ribeiro da Silva and A. I. M. C. L. Ferreira, J. Chem. Thermoydn. 41, 1096 (2009); Corrigendum, J. Chem. Thermoydn. 43, 1564 (2011).
2009RIB/FER5

2009RIB/FER6

M. A. V. Ribeiro da Silva and A. I. M. C. L. Ferreira, J. Chem. Thermodyn. 41, 1104 (2009).

M. A. V. Ribeiro da Silva, A. I. M. C. L. Ferreira, J. I. T. A. Cabral, A. F. L. O. M. Santos, A. R. G. Moreno, T. L. P. Galvao, I. M. Rocha, P. M. V. Fernandes, S. Q. Salgueiro, V. A. F. de Moura, I. M. S. C. Oliveira, P. C. Cotelo, and M. R. A. Ribeiro, J. Chem. Thermodyn. 41, 984 (2009).

2009RIB/FER7 M. A. V. Ribeiro da Silva, A. I. M. C. L. Ferreira, and A. R. G. Moreno, J. Chem. Thermodyn. 41, 109 (2009).

2009RIB/FER8 M. A. V. Ribeiro da Silva, A. I. M. F. Ferreira, A. F. L. O. M. Santos, and I. M. Rocha, J. Chem. Thermodyn. 41, 1239 (2009).

2009RIB/FER9 M. A. V. Ribeiro da Silva, A. I. M. C. L. Ferreira, and A. R. G. Moreno, J. Chem. Thermodyn. 41, 904 (2009).

2009RIB/RIB M. A. V. Ribeiro da Silva, M. D. M. C. Ribeiro da Silva, A. I. M. C. L. Ferreira, A. F. L. O. M. Santos, and T. L. P. Galvao, J. Chem. Thermodyn. 41, 1074 (2009).

2009RIB/RIB2 M. A. V. Ribeiro da Silva, M. D. M. C. Ribeiro da Silva, A. I. M. C. L. Ferreira, A. F. L. O. M. Santos, and T. L. P. Galvao, J. Chem. Thermodyn. 41, 1247 (2009).

2009RIB/RIB3 M. D. M. C. Ribeiro da Silva, M. A. V. Ribeiro da Silva, V. L. S. Freitas, M. V. Roux, P. Jimenez, M. Temprado, J. Z. Davalas, P. Cabildo, R. M. Calramunt, and J. Elguero, J. Chem. Thermodyn. 41, 1400 (2009).

2009RIB/SAN M. A. V. Ribeiro da Silva, A. F. L. O. M. Santos, J. R. B. Gomes, M. V. Roux, M. Temprado, P. Jimenez, and R. Notario, J. Phys. Chem. A 113, 11042 (2009).

2009RIB/SAN2 M. A. V. Ribeiro da Silva and A. F. L. O. M. Santos, J. Chem. Thermodyn. 41, 926 (2009).

2009ROU/TEM M. V. Roux, M. Temprado, P. Jimenez, C. Foces-Foces, R. Notario, A. R. Parameswar, A. V. Demchenko, J. S. Chickos, C. A. Deakyne, A. K. Ludden, and J. F. Liebman, J. Phys. Chem. A 113, 10772 (2009).

2009SAL/BED F. Salaun, G. Bedek, E. Devaux, D. Dupont, and D. Deranton, Int. J. Thermophys. 30, 1242 (2009).

2009SAN/FIG R. C. Santos, R. M. B. B. M. Figueira, M. F. M. Piedade, H. P. Diogo, and M. E. Minas da Piedade, J. Phys. Chem. B 113, 14291 (2009).

2009SAN/GOM A. F. L. O. M. Santos, J. R. B. Gomes, and M. A. V. Ribeiro da Silva, J. Phys. Chem. A 113, 3630 (2009).

2009SAN/RIB A. F. L. O. M. Santos and M. A. V. Ribeiro da Silva, J. Phys. Chem. A 113, 9741 (2009).

2009SAP/UUS E. Sapei, P. Uusi-Kyyny, K. I. Keskinen, and J. Aittamaa, Fluid Phase Equilib. 279, 81 (2009).

2009SEL/RAG J. Selvakumar, V. S. Raghunathan, and K. S. Nagaraja, J. Phys. Chem. C 113, 19011 (2009).

2009SHA/TAN B. L. Sharma, S. Tandon, and S. Gupta, Cryst. Res. Technol. 44, 258 (2009).

2009SIN/MUR ～L. P. Singh and S. S. N. Murthy, Phys. Chem. Chem. Phys. 11, 5110 (2009)

2009SZT P. Szterner, J. Therm. Anal. Calorim. 98, 337 (2009).

2009TAU/SIT P. Taulelle, G. Sitja, G. Pepe, E. Garcia, C. Hoff, and S. Veesler, Cryst. Growth Des. 9, 4706 (2009).

2009VEC/BRU S. Vecchio and B. Brunetti, J. Chem. Thermodyn. 41, 880 (2009).

2009VEC/TOM S. Vecchio and M. Tomassetti, Fluid Phase Equilib. 279, 64 (2009).

2009VER/EME S. P. Verevkin, V. N. Emel'yanenko, E. N. Stepurko, R. V. Rulys, D. H. Zaitsau, and A. Stark, Ind. Eng. Chem. Res. 48, 10087 (2009).

2009VER/EME2 S. P. Verevkin, V. N. Emel'yanenko, A. V. Toktonov, A. S. Leolko, J. Duwensee, U. Kragl, and S. M. Sarge, Ind. Eng. Chem. Res. 48, 7388 (2009).

2009VER/EME4 S. P. Verevkin, V. N. Emel'yanenko, A. V. Toktonov, P. Goodrich, and C. Hardacre, Ind. Eng. Chem. Res. 48, 9809 (2009).

2009VER/KOZ S. P. Verevkin, S. A. Kozlova, V. N. Emel'yanenko, and G. Nell, J. Chem. Thermodyn. 41, 1125 (2009).

2009WEI/JIN D. Wei and K. Jin, J. Chem. Thermodyn. 41, 145 (2009).

2009WU/MEH W. Wu, S. J. Mehrman, Y. Zhou, S. X. Pu, L. Huang, A. Fermier, and S. Karki, Cryst. Growth 311, 3435-3444 (2009). 
2009XIN/FAN Y. Xing, W. Fang, D. Li, Y. Guo, and R. Lin, J. Chem. Eng. Data 54, 1865 (2009).

2009ZEN/GST N. Zencirci, E. Gstrein, C. Langes, and U. J. Griesser, Thermochim. Acta 485, 33 (2009).

2009ZHA/RAY Y. Zhang, A. Ray, and S. Rohani, Chem. Eng. Sci. 64, 192 (2009).

2010ACR/CHI W. E. Acree, Jr. and J. S. Chickos, J. Phys. Chem. Ref. Data 39, 1-940 (2010).

2010AGA/DRU L. E. Agafonova, A. I. Druzhinina, R. M. Varushchenko, and O. V. Polyakova, Vestn. Mosk. Univ., Ser. 2: Khim. 51, 347 (2010).

2010AGR/GUP T. Agrawal, P. Gupta, S. S. Das, A. Gupta, and N. B. Singh, J. Chem. Eng. Data 55, 4206 (2010).

2010ALM/MON A. R. R. P. Almeida and M. J. S. Monte, J. Chem. Eng. Data 55, 3507 (2010).

2010ALM/MON2 A. R. R. P. Almeida and M. J. S. Monte, J. Chem. Eng. Data 55, 5230 (2010).

2010ARA/DOS A. A. S. Araujo, M. dos Santos Bezerra, S. Storpirtis, and J. do Rosario Matos, Braz. J. Pharm. Sci. 46, 37 (2010).

2010AVU/ALE S. G. Avula, K. Alexander, and A. Riga, J. Therm. Anal. Calorim. 99, 655 (2010).

2010BAB/KUZ T. S. Bakkina and A. V. Kuznetsov, J. Therm. Anal. Calorim. 101, 33 (2010).

2010BAI/VAN J. A. Baird, B. van Eerdenbrugh, and L. S. Taylor, J. Pharm. Sci. 99, 3787 (2010).

2010BEL/AHM A. Belabbaci, N. C.-B. Ahmed, I. Mokbel, and L. Negadi, J. Chem. Thermodyn. 42, 1158 (2010).

2010BOG/GON R. Bogel-Lukasik, L. M. N. Goncalves, and E. BogelLukasik, Green Chem. 12, 1947 (2010).

2010BOO/BAR A. M. Booth, M. H. Barley, D. O. Topping, G. McFiggans, A. Garforth, and C. J. Percival, Atmos. Chem. Phys. 10, 4879 (2010).

2010BRA/GRE D. Braga, F. Grepioni, L. Maini, P. P. Mazzeo, and K. Rubini, Thermochim. Acta 507/508, 1 (2010).

2010BUS/MEU P. Bustamante, S. Meula, B. Escalera, and A. Pena, Chem. Pharm. Bull. 58, 644 (2010).

2010CAB/MON J. I. T. A. Cabral, R. A. R. Monteiro, M. A. A. Rocha, L. M. N. B. F. Santos, W. E. Acree, Jr., and M. D. M. C. Ribeiro da Silva, J. Therm. Anal. Calorim. 100, 431 (2010).

2010CAM/MIC L. Campanella, V. Micieli, M. Tomassetti, and S. Vechio, J. Therm. Anal. Calorim. 99, 887 (2010).

2010CAS/MAR R. A. E. Castro, T. M. R. Maria, A. O. L. Evora, J. C. Feiteira, M. R. Silva, A. M. Beja, J. Canotilho, and M. E. S. Eusebio, Cryst. Growth Des. 10, 274 (2010).

2010CEN/ROH M. Censky, V. Rohac, K. Ruzicka, M. Fulem, and K. Aim, Fluid Phase Equilib. 298, 192 (2010).

2010CEN/VRB M. Censky, P. Vrbka, K. Ruzicka, and M. Fulem, Fluid Phase Equilib. 298, 199 (2010).

2010CHA/PAP P. Chatzigeorgiou, N. Papakonstantopoulos, N. Togaroulia, E. Pollatos, P. Xynogalas, and K. Viras, J. Phys. Chem. B 114, 1294 (2010).

2010CLA/MAR R. A. Clara, A. C. G. Marigliano, D. Morales, and H. N. Solimo, J. Chem. Eng. Data 55, 5862 (2010).

2010CON/NEA I. Contineanu, A. Neascu, and S. T. Perisanu, Thermochim. Acta 497, 96 (2010).

2010COO/DAV C. L. Cooke, D. J. Davey, S. Black, C. Muryn, and R. G. Prichard, Cryst. Growth Des. 10, 5270 (2010).

2010COR/NEG Y. Corvis, P. Negrier, M. Lazerges, S. Massip, J.-M. Leger, and P. Espeau, J. Phys. Chem. B 114, 5420 (2010).

2010DEL/UUS C. Dell'Era, P. Uusi-Kyyny, E.-L. Rautama, M. Pakkanen, and V. Alopaeus, Fluid Phase Equilib. 299, 51 (2010).

2010EFI/EME A. A. Efimova, V. N. Emel'yanenko, S. P. Verevkin, and Y. Chernyak, J. Chem. Thermodyn. 42, 330 (2010).

2010EME/VER V. N. Emel'yankenko, S. P. Verevkin, E. N. Stepurko, G. N. Roganov, and M. K. Georgieva, Russ. J. Phys. Chem. A 84, 356 (2010).

2010EME/VER2 V. N. Emel'yankenko, S. P. Verevkin, E. N. Stepurko, G. N. Roganov, and M. K. Georgieva, Russ. J. Phys. Chem. A 84, 1301 (2010).

2010EME/VER3 V. N. Emel'yankenko, S. P. Verevkin, S. Schick, E. N. Stepurko, G. N. Roganov, and M. K. Georgieva, Russ. J. Phys. Chem. A 84, 1491 (2010).
2010FAN/WAN

C. L. Fan and L. S. Wang, J. Chem. Eng. Data 55, 479 (2010).

2010FAR/RAJ B. V. Farahani, F. H. Rajabi, B. Hosseindoust, and H. Zenooz, J. Phase Equilib. Diff. 31, 536 (2010).

2010FON/SAN J. M. S. Fonseca, L. M. N. B. F. Santos, and M. J. S. Monte, J. Chem. Eng. Data 55, 2238 (2010).

2010FRE/GOM3 V. L. S. Freitas, J. R. B. Gomes, L. Gales, A. M. Damas, and M. D. M. C. Ribeiro da Silva, J. Chem. Eng. Data 55, 5009 (2010).

2010FUL/RUZ M. Fulem, K. Ruzicka, P. Moravek, J. Pangrac, E. Hulicius, B. Kozyrkin, and V. Shatunov, J. Chem. Eng. Data 55, $4780(2010)$

2010GAO/LIN G.-Y. Gao and S.-Y. Lin, J. Pharm. Sci. 99, 255 (2010).

2010GAO/SHI

Z. Gao, Q. Shi, Z.-C. Tan, and X. Lan, Huaxue Xuebao 68, 227 (2010).

2010GER/PEL $\quad$ A. P. Gerola, L. S. Peloi, N. Hioka, O. S. Filho, and L. A. Kato, Quim. Nova 33, 482 (2010).

2010GON/BER E. M. Goncalves, C. E. S. Bernardes, H. P. Diogo, and M. E. Minas da Piedade, J. Phys. Chem. B 114, 5475 (2010).

2010GRI/TSA J. R. Griffiths, J. Tsanaktsidis, G. P. Savage, and R. Priefer, Thermochim. Acta 499, 15 (2010).

2010GUO/SAD ～K. Guo, G. Sadiq, C. Seaton, R. Davey, and Q. Yin, Cryst. Growth Des. 10, 268 (2010).

2010GUO/WAN2 X.-Z. Guo and L.-S. Wang, J. Chem. Eng. Data 55, 4709 (2011).

2010HAH/GRA I. Hahnenkamp, G. Graubner, and J. Gmehling, Int. J. Pharm. 388, 73 (2010).

2010HE/ROH Q. He, S. Rohani, J. Zhu, and H. Gomaa, Cryst. Growth Des. 10, 5136 (2010).

2010HEN/CAM A. Hengstermann and L. Cameretti, J. Chem. Eng. Data $\mathbf{5 5}, 4597(2010)$

2010HU/CHE Y. Hu, X. Chen, W. Yang, Z. Lei, and C. Zhao, J. Phys. Chem. Solids 71, 170 (2010).

2010JI/MEN H.-Z. Ji, X.-C. Meng, and H.-K. Zhao, J. Chem. Eng. Data 55, 2590 (2010).

2010JIA/JAN S. Jiang, P. J. Jansen, and J. H. ter Horst, Cryst. Growth Des. 10, 2123 (2010).

2010KAN/RED S. Kant, R. S. B. Reddi, and R. N. Rai, Fluid Phase Equilib. 291, 71 (2010).

2010KES/AUC V. Kestens, G. Auclair, K. Drozdzewska, A. Held, G. Roebben, and T. Linsinger, J. Therm. Anal. Calorim. 99, 245 (2010).

2010KHI/DAH K. Khimeche, A. Dahmani, and I. Mokbel, J. Chem. Thermodyn. 42, 829 (2010).

2010LEO/PIN ～L. Leon, M. Pink, J. A. Kaduk, J. M. Delgado, and G. Diaz de Delgado, J. Chem. Crystallogr. 40, 104 (2010).

2010LIP/CHI D. Lipkind, J. S. Chickos, and J. F. Liebman, J. Chem. Eng. Data 55, 1628 (2010).

2010LIP/PLI D. Lipkind, G. Plienrasri, and J. S. Chickos, J. Phys. Chem. B 114, 16959 (2010).

2010MAR/JES T. M. R. Maria, A. J. L. Jesus, and M. E. S. Eusebio, J. Therm. Anal. Calorim. 100, 385 (2010).

2010MAR/LOR B. Marrufo, S. Loras, and M. Sanchotello, J. Chem. Eng. Data 55, 62 (2010).

2010MAS/KRA A. S. Maslakova, E. L. Krasnykh, and S. V. Levanova, Russ. J. Phys. Chem. 84, 163 (2010).

2010MAT/SOU M. A. R. Matos, C. C. S. Sousa, and V. M. F. Morais, J. Therm. Anal. Calorim. 100, 519 (2010).

2010MEE/GEU F. Meersman, B. Geukens, M. Wubbenhorst, J. Leys, S. Napolitano, Y. Filinchuk, G. van Assche, B. van Mele, and E. Nies, J. Phys. Chem. B 114, 13944 (2010).

2010MEJ/SEG A. Mejia, H. Segura, and M. Cartes, J. Chem. Eng. Data 55, 428 (2010).

2010MEK/KHI A. Mekki, K. Khimeche, and A. Dahmani, J. Chem. Thermodyn. 42, 1050 (2010).

2010MIN/VON T. L. Minh, J. Von Langermann, H. Lorenz, and A. SeidelMorgenstern, J. Pharm. Sci. 99, 4084 (2010).

2010MIR/MAT M. S. Miranda, M. A. R. Matos, V. M. F. Morais, and J. F. Liebman, J. Chem. Thermodyn. 42, 1101 (2010).

2010MIY/KHA Y. Miyako, N. Khalef, K. Matsuzaki, and R. Pinal, Int. J. Pharm. 393, 48 (2010).

2010MON/ALM M. J. S. Monte, A. R. R. P. Almeida, and M. A. R. Matos, J. Chem. Eng. Data 55, 419 (2010). 
2010MON/GON M. J. S. Monte, M. V. Goncalves, and M. D. M. C. Ribeiro da Silva, J. Chem. Eng. Data 55, 2246 (2009).

2010MON/SAN M. J. S. Monte, L. M. N. B. F. Santos, J. M. S. Fonesca, and C. A. D. Sousa, J. Therm. Anal. Calorim. 100, 465 (2010).

$2010 \mathrm{MOR} / \mathrm{SOU} \quad$ V. M. F. Morais, C. C. S. Sousa, and M. A. R. Matos, J. Mol. Struct.: THEOCHEM 946, 13 (2010).

2010MUE/ESC S. Muela, B. Escalera, M. A. Pena, and P. Bustamante, Int. J. Pharm. 394, 93 (2010).

2010MUR/PIK2 $\quad$ S. B. Murdande, M. J. Pikal, R. M. Shanker, and R. H. Bogner, Pharm. Sci. 99, 2704 (2010).

2010OXL/SMI J. Oxley, J. L. Smith, J. Brady, and S. Naik, Propellants, Explos., Pyrotech. 35, 278 (2010).

2010PAN/SAR E. Panteli, P. Saratsioti, H. Stamatis, and E. Voutsas, J. Chem. Eng. Data 55, 745 (2010).

2010PAR/LEE K. A. Park, H. J. Lee, and I. K. Hong, J. Ind. Eng. Chem. 16, 490 (2010).

2010PAU/KOV I. E. Paukov, Y. A. Kovalevskaya, and E. V. Boldyreva, J. Therm. Anal. Calorim. 100, 295-301 (2010).

2010PEE/HUD Z. Peeters, R. L. Hudson, M. H. Moore, and A. Lewis, Icarus 210, 480 (2010).

2010PET/REY M. Petitjean, E. Reyes-Perez, D. Perez, Ph. Mariabel, and C. Le Calve, J. Chem. Eng. Data 55, 852 (2010).

2010PIC/DIO R. Picciochi, H. P. Diogo, and M. E. Minas da Piedade, J. Therm. Anal. Calorim. 100, 391 (2010).

2010RAM/FEL M. L. Ramierz, H. Felix-Rivera, R. A. Sanchez-Cuprill, and S. P. Hernandez-Rivera, J. Therm. Anal. Calorim. 202, 549 (2010).

2010RIB/AMA M. A. V. Ribeiro da Silva and L. M. P. F. Amaral, J. Therm. Anal. Calorim. 100, 375 (2010).

2010RIB/AMA2 M. A. V. Ribeiro da Silva and L. M. P. F. Amaral, J. Chem. Thermodyn. 42, 1473 (2010).

2010RIB/CAB M. A. V. Ribeiro da Silva and J. I. T. A. Cabral, J. Therm. Anal. Calorim. 100, 457 (2010).

2010RIB/CAB2 M. A. V. Ribeiro da Silva, J. I. T. Cabral, and A. Cimas, J. Chem. Thermodyn. 42, 1240 (2010).

2010RIB/FER M. A. V. Ribeiro da Silva, A. I. M. C. L. Ferreira, A. F. L. O. M. Santos, C. M. A. Ferreira, D. C. B. Barros, J. A. C. Reis, J. C. S. Costa, M. M. G. Calvinho, S. I. A. Rocha, S. P. Pinto, S. S. L. Freire, S. M. Almeida, V. S. Guimaraes, and V. N. M. Almeida, J. Chem. Thermodyn. 42, 371 (2010).

2010RIB/FER2 M. A. V. Ribeiro da Silva and A. I. M. C. L. Ferreira, J. Chem. Thermodyn. 42, 182 (2010).

2010RIB/FER3 M. A. V. Ribeiro da Silva, A. I. M. C. L. Ferreira, and F. M. Maciel, J. Chem. Thermodyn. 42, 220 (2010).

2010RIB/FER4 M. A. V. Ribeiro da Silva, A. I. M. L. Ferreira, A. F. L. O. M. Santos, and I. M. Rocha, J. Chem. Thermodyn. 42, 169 (2010).

2010RIB/FER5 M. A. V. Ribeiro da Silva and A. I. M. C. L. Ferreira, J. Therm. Anal. Calorim. 100, 447 (2010).

2010RIB/GON M. D. M. C. Ribeiro da Silva, M. V. Goncalves, and M. J. S. Monte, J. Chem. Thermodyn. 42, 472 (2010).

2010RIB/MON M. A. V. Ribeiro da Silva, M. J. S. Monte, A. I. M. C. L. Ferreira, J. A. S. A. Oliveira, and A. Cimas, J. Phys. Chem. B 114, 7909 (2010).

2010RIB/MON2 M. A. V. Ribeiro da Silva, M. J. S. Monte, A. I. M. C. L. Ferreira, J. A. S. A. Oliveira, and A. Cimas, J. Phys. Chem. B 114, 12914 (2010)

2010RIB/RIB M. A. V. Ribeiro da Silva, M. D. M. C. Ribeiro da Silva, A. F. L. O. M. Santos, A. I. M. C. L. Ferreira, and T. L. P. Galvao, J. Chem. Thermodyn. 42, 496 (2010).

2010RIB/RIB2 M. A. V. Ribeiro da Silva, M. D. M. C. Ribeiro da Silva, A. F. L. O. M. Santos, M. V. Roux, C. Foces-Foces, R. Notario, R. Guzman-Mejia, and E. Juaristi, J. Phys. Chem. B 114, 16471 (2010).

2010RIB/SAN3 M. A. V. Ribeiro da Silva, A. F. L. O. M. Santos, and L. M. P. F. Amaral, J. Chem. Thermodyn. 42, 564 (2010).

2010RIB/SAN4 M. A. V. Ribeiro da Silva and A. F. L. O. M. Santos, J. Therm. Anal. Calorim. 100, 403 (2010).

2010RIE/BAR I. B. Rietveld, M. Barrio, N. Veglio, P. Espeau, J. Ll. Tamarit, and R. Ceolin, Thermochim. Acta 511, 43 (2010).
2010ROU/FOC

M. V. Roux, C. Foces-Foces, R. Notario, M. A. V. Ribeiro da Silva, M. D. M. C. Ribeiro da Silva, A. F. L. O. M. Santos, and E. Juaristi, J. Phys. Chem. B 114, 10530 (2010).

2010ROU/NOT M. V. Roux, R. Notario, C. Foces-Foces, M. Temprado, F. Ros, V. N. Emel'yanenko, and S. P. Verevkin, J. Phys. Chem. A 114, 3583 (2010).

2010ROU/TEM M. V. Roux, M. Temprado, P. Jimenez, C. Foces-Foces, R. Notario, A. R. Parameswar, A. V. Demchenko, J. S. Chickos, C. A. Deakyne, and J. F. Liebman, J. Phys. Chem. A 114, 6336 (2010).

2010SAN/RIB $\quad$ A. F. L. O. M. Santos and M. A. V. Ribeiro da Silva, J. Chem. Thermodyn. 42, 734 (2010).

2010 SAN/RIB2 A. F. L. O. M. Santos and M. A. V. Ribeiro da Silva, J. Chem. Thermodyn. 42, 1016 (2010).

2010SAN/RIB3

A. F. L. O. M. Santos and M. A. V. Ribeiro da Silva, J. Chem. Thermodyn. 42, 1079 (2010).

2010SAN/RIB4

A. F. L. O. M. Santos and M. A. V. Ribeiro da Silva, J. Chem. Thermodyn. 42, 1441 (2010).

2010SAN/ROC

L. M. N. B. F. Santos, M. A. A. Rocha, L. R. Gomes, B. Schroeder, and J. A. P. Coutinho, J. Chem. Eng. Data 55, 2799-2808 (2010).

2010SAP/UUS E. Sapei, P. Uusi-Kyyny, K. I. Keskinen, and V. Alopaeus, Fluid Phase Equilib. 288, 155 (2010).

2010SAR/SAN $\quad$ B. Sarma, P. Sanphui, and A. Nangia, Cryst. Growth Des. 10, 2388 (2010).

2010SET/KHA L. Seton, D. Khamar, I. J. Bradshaw, and G. A. Hutcheon, Cryst. Growth Des. 10, 3879 (2010).

2010SHA/REN

Y.-H. Shao, X.-N. Ren, and Z.-R. Liu, J. Therm. Anal. Calorim. 101, 1135 (2010).

2010SOU/MOR

C. C. S. Sousa, V. F. M. Morais, and M. M. Matos, J. Chem. Thermodyn. 42, 1372 (2010).

2010SOU/SAT

J. Soujanya, B. Satyavathi, and T. E. Vittal Prasad, J. Chem. Thermodyn. 42, 621 (2010).

2010SUC/MUS

M. Suceska, S. M. Musanic, and I. F. Houra, Thermochim. Acta 510, 9 (2010).

2010SVA/NOR

M. Svard, F. L. Nordstrom, T. Jasnobulka, and A. C. Rasmuson, Cryst. Growth Des. 10, 195 (2010).

2010SZT/LEG P. Szterner, B. Legendre, and M. Sghaier, J. Therm. Anal. Calorim. 99, 325 (2010).

2010TON/LIU B. Tong, R.-B. Liu, C.-G. Meng, F.-Y. Yu, S.-H. Ji, and Z.-C. Tan, J. Chem. Eng. Data 55, 119 (2010).

2010TON/TAN B. Tong, Z.-C. Tan, R.-B. Liu, C.-G. Meng, and J.-N. Zhang, Energy Convers. Manage. 51, 1905 (2010).

2010TON/YU B. Tong, Y. Yu, Z.-C. Tan, C.-G. Meng, L.-J. Cui, G. Xia, and R.-B. Liu, Thermochim. Acta 499, 117 (2010).

2010VAR/ABA M. A. Varfolomeev, D. I. Abaidullina, B. N. Solomonov, S. P. Verevkin, and V. N. Emel'yanenko, J. Phys. Chem. B 114, 16503 (2010).

2010VEC

2010WAN/LAI S. Vecchio, Thermochim. Acta 499, 27 (2010).

2010WAN/ZEN T.-C. Wang, T.-Y. Lai, and Y.-P. Chen, J. Chem. Eng. Data 55, 5797 (2010).

2010WEI/WAN D. Wei, L. Wang, F. Yan, and C. Zhang, Fluid Phase Q.-Y. Wang, H. Zeng, H. Song, Q.-S. Liu, and S. Yao, J. Chem. Eng. Data 55, 5271 (2010). Equilib. 291, 66 (2010).

2010WID/BRU J. A. Widegren and T. J. Bruno, Environ. Sci. Technol. 44, 388 (2010).

2010WID/BRU2 J. A. Widegren and T. J. Bruno, J. Chem. Eng. Data 55, 159 (2010).

2010WU/REE H. Wu, N. Reeves-McLaren, J. Pokorny, J. Yarwood, and A. R. West, Cryst. Growth Des. 10, 3141 (2010).

2010YAN/LEI W. Yang, Z. Lei, Y. Hu, X. Chen, and S. Fu, Ind. Eng. Chem. Res. 49, 11170 (2010).

2010ZAB/KOL M. M. Zabransky, Z. Kolska, V. Ruzicka, Jr., and E. S. Domalski, J. Phys. Chem. Ref. Data 39, 013103 (2010).

2010ZEN/LI Z.-X. Zeng, X.-N. Li, W.-L. Xue, C.-S. Zhang, and S.-C. Bian, Ind. Eng. Chem. Res. 49, 5543 (2010).

2011AGA/VAR L. E. Agafonova, R. M. Varushchenko, A. I. Druzhinina, O. V. Polyakova, and Yu. S. Kolesov, Russ. J. Phys. Chem. 85, 1516 (2011).

2011AHM/NEG N. C.-B. Ahmed, L. Negadi, I. Mokbel, and J. Jose, J. Chem. Thermodyn. 43, 719 (2011). 
2011ALM/MON A. R. R. P. Almeida and M. J. S. Monte, J. Chem. Eng. Data 58, 4862 (2011).

2011AMA/PIN P. Amador, B. Pineda, A. Lopez, and H. Flores, J. Chem. Thermodyn. 43, 1414 (2011).

2011BAZ/BLO A. B. Bazyleva, A. V. Blokhin, G. J. Kabo, M. B. Chiarapennikau, V. N. Emel'yanenko, S. P. Verevkin, and V. Diky, J. Phys. Chem. B 115, 10064 (2011).

2011BEL/MOK A. Belabbaci, I. Mokbel, A. A. Kaci, J. Jose, and L. Negadi, J. Chem. Thermodyn. 43, 17 (2011).

2011BEN/KHI M. Benziane, K. Khimeche, I. Mokbel, T. Sawaya, A. Dahmani, and J. Jose, J. Chem. Eng. Data 56, 4736 (2011).

2011BIS/GOG M. R. Bissengaliyeva, D. B. Gogol, S. T. Taymasova, and N. S. Bekturganov, J. Chem. Eng. Data 56, 195 (2011).

2011BOG/DAO E. Bogdani, R. Daoussi, S. Vessot, J. Jose, and J. Andrieu, Chem. Eng. Res. Des. 89, 2606 (2011).

2011BOO/MON A. M. Booth, W. J. Montague, M. H. Barley, D. O. Topping, G. McFiggans, A. Garforth, and C. J. Percival, Atmos. Chem. Phys. 11, 655 (2011).

2011CAI/XIA Z. Cairong, S. Xiaohua, C. Lingling, and W. Haifeng, Fluid Phase Equilib. 302, 123 (2011).

2011CAS/RIB R. A. E. Castro, J. D. B. Ribeiro, T. M. R. Maria, M. R. Silva, C. Yueste-Vivas, J. Canotilho, and M. E. S. Eusebio, Cryst. Growth Des. 11, 5396 (2011).

2011CHE/YAN Z.-G. Chen, W.-G. Yang, Y.-H. Hu, Z.-Y. Lei, Y.-H. Wang, and W.-Q. Wan, J. Chem. Eng. Data 56, 2726 (2011).

2011 COS/LIM J. C. S. Costa, C. F. R. A. C. Lima, M. A. A. Rocha, L. R. Gomes, and L. M. N. B. F. Santos, J. Chem. Thermodyn. 43, 133 (2011).

2011DOM/POB U. Domanska, A. Pobudkowska, P. Pelczarska, and L. Zukowski, Int. J. Pharm. 403, 115 (2011).

2011EGO/MAR V. M. Egorov, V. A. Marikhin, and L. P. Myasnikova, Polym. Sci. Ser. A 53, 906 (2011).

2011EME/POR V. N. Emel'yanenko, S. V. Portnova, S. P. Verevkin, A. Skrzypczak, and T. Schubert, J. Chem. Thermodyn. 43, 1500 (2011).

2011EME/POR2 V. N. Emel'yanenko, S. V. Portnova, S. P. Verevkin, and A. Skrzypczak, J. Chem. Eng. Data 56, 3532 (2011).

2011EME/STEＶ. N. Emel'yanenko, E. N. Stepurko, S. P. Verevkin, and G. N. Roganov, Russ. J. Phys. Chem. 85, 179 (2011).

2011EME/VER2 V. N. Emel'yanenko, S. P. Verevkin, M. A. Varfolomeev, V. V. Turovtsev, and Y. D. Orlov, J. Chem. Eng. Data 56, 4183 (2011).

2011FAN/LI D. Fan, J. Li, J. Shi, C. Peng, H. Liu, Y. Hu, and P. Paricaud, J. Chem. Eng. Data 56, 1323 (2011).

2011FEL/RAM H. Felix-Rivera, M. L. Ramirez-Cedeno, R. A. SanchezCuprill, and S. P. Hernandez-Rivera, Thermochim. Acta 514, 37 (2011).

2011FEN/DON C. Feng, J. Dong, and Y. Li, Fluid Phase Equilib. 309, 201 (2011).

2011FER/RIB A. I. M. C. L. Ferreira and M. A. V. Ribeiro da Silva, J. Chem. Thermodyn. 43, 227 (2011).

2011FER/RIB2 A. I. M. C. L. Ferreira and M. A. V. Ribeiro da Silva, J. Chem. Eng. Data 56, 4881 (2011).

2011FUL/RUZ M. Fulem, K. Ruzicka, and M. Ruzicka, Fluid Phase Equilib. 303, 205 (2011).

$2011 \mathrm{GIM} / \mathrm{TOR} \quad$ B. Gimeno, M. Torcal, A. M. Mainar, and P. Perez, Fluid Phase Equilib. 309, 83 (2011).

$2011 \mathrm{GOR} /$ WOJ A. Gorniak, A. Wojakowska, B. Karolewicz, and J. Pluta, J. Therm. Anal. Calorim. 104, 1195 (2011).

2011HAC/MON F. Hacivelioglu, R. Montis, D. B. Davies, A. Kilic, M. B. Hursthouse, and S. J. Coles, Cryst. Eng. Commun. 13, 4102 (2011).

2011HER/LEY C. Herman, T. Leyssens, V. Vermylen, V. Halloin, and B. Haut, J. Chem. Thermodyn. 43, 677 (2011).

2011JON/MAA M. T. G. Jongmans, J. I. W. Maassen, A. J. Luijks, B. Schuur, and A. B. de Haan, J. Chem. Eng. Data 56, 3510 (2011).

2011KAN/RAI S. Kant and R. N. Rai, Thermochim. Acta 512, 49 (2011).

2011KHI/DJE K. Khimeche, F. Djellouli, A. Dahmani, and I. Mokbel, J. Chem. Eng. Data 56, 4972 (2011).

2011KUM/VEI G. Kumaresan, R. Veiraj, and S. Iniyan, J. Appl. Sci. 11, 3044-3048 (2011).
2011LEE/THO

2011LI/BOU2

2011LIP/KRA

2011LIP/RAT

2011LOR/VON

2011MAG/PYD

2011MAH/PER

2011MAS/KRA

2011MAT/KIM

2011MAT/YAM

2011MEJ/SEG

2011MEL/PIN

2011MIR/KON

2011MIR/MAT2

2011MIR/MAT3

2011MIR/MAT4

2011NAV/MAC

2011NEG/KAC

$2011 \mathrm{NOT} / \mathrm{ROU}$

2011ORJ/YAN

2011PAU/KAB

2011PIC/DIO

2011POR/KRA

2011POZ/VER

2011RAI/MUD

2011RED/GAN

2011RIB/AMA

2011RIB/AMA2

2011RIB/AMA4

2011RIE/BAR

2011ROU/MAR

2011ROU/NOT
J. W. Lee, L. C. Thomas, and S. J. Schmidt, J. Agric. Food Chem. 59, 684 (2011).

J. Li, S. A. Bourne, and M. R. Caira, Chem. Commun. 47, 1530 (2011).

S. V. Lipp, E. L. Krasnykh, and S. P. Verevkin, J. Chem. Eng. Data 56, 800 (2011).

D. Lipkind, N. Rath, J. S. Chickos, V. A. Pozdeev, and S. P. Verevkin, J. Phys. Chem. B 115, 8785 (2011).

H. Lorenz, J. von Langermann, G. Sadiq, C. C. Seaton, R. J. Davey, and A. Seidel-Morgenstern, Cryst. Growth Des. 11, 1549 (2011).

A. Magon and M. Pyda, Carbohydr. Res. 346, 2558 (2011).

N. Mahe, M.-A. Perrin, M. Barrio, B. Nicolai, I. B. Rietveld, J.-L. Tamarit, and R. Ceolin, J. Pharm. Sci. 100, 2258 (2011).

A. S. Maslakova, E. L. Krasnykh, and S. V. Levanova, Russ. J. Phys. Chem. 85, 1695-1700 (2011).

T. Mathuni, J.-I. Kim, and S.-J. Park, J. Chem. Eng. Data 56, 89 (2011)

H. Matsuda, H. Yamada, R. Takahashi, A. Koda, K. Kurihara, K. Tochigi, and K. Ochi, J. Chem. Eng. Data 56, 5045 (2011).

A. Mejia, H. Segura, and M. Cartes, J. Chem. Eng. Data 56, $3142(2011)$

V. Meltzer and E. Pincu, J. Chem. Thermodyn. 23, 1011 (2011).

E. A. Miroshnichenko, T. S. Kon'kova, Ya. O. Inozemtsev, and Yu. N. Matyushin, Russ. Chem. Bull. 60, 36 (2011).

M. S. Miranda, M. A. R. Matos, V. M. F. Morais, and J. F. Liebman, J. Chem. Thermodyn. 43, 635 (2011).

M. S. Miranda, M. A. R. Matos, V. M. F. Morais, and J. F. Liebman, J. Phys. Chem. B 115, 6616 (2011).

M. S. Miranda, M. A. R. Matos, V. M. F. Morais, and J. F. Liebman, J. Chem. Eng. Data 56, 4516 (2011).

P. S. Navare and J. C. MacDonald, Cryst. Growth Des. 11, 2422 (2011).

L. Negadi, A. A. Kaci, A. Negadi, and J. Jose, Fluid Phase Equilib. 300, 135 (2011).

R. Notario, M. V. Roux, C. Foces-Foces, M. A. V. Ribeiro da Silva, M. D. M. C. Ribeiro da Silva, A. F. L. O. M. Santos, R. Guzman-Mejia, and E. Juaristi, J. Phys. Chem. B 115, 9401 (2011).

A. Orjuela, A. J. Yanez, J. Evans, A. M. Hassan, D. J. Miller, and C. T. Lira, Fluid Phase Equilib. 309, 121 (2011).

Y. U. Paulechka, G. J. Kabo, A. V. Blokhin, and D. S. Firaha, J. Chem. Eng. Data 56, 4891 (2011).

R. Picciochi, H. P. Diogo, and M. E. Minas da Piedade, J. Pharm. Sci. 100, 594 (2011).

S. V. Portnova, E. L. Krasnykh, and S. P. Verevkin, Fluid Phase Equilib. 309, 114 (2011).

V. A. Pozdeev and S. P. Verevkin, J. Chem. Thermodyn. 43, 1791 (2011).

R. N. Rai, S. R. Mudunuri, R. S. B. Reddi, V. S. A. Kumar Satuluri, S. Ganeshmoorthy, and P. K. Gupta, J. Cryst. Growth 321, 72 (2011).

R. S. Reddi, S. Ganesamoorthy, P. K. Gupta, and R. N. Rai, Fluid Phase Equilib. 313, 121 (2011).

M. A. V. Ribeiro da Silva and L. M. P. F. Amaral, J. Chem. Thermodyn. 43, 1 (2011).

M. A. V. Ribeiro da Silva and L. M. P. F. Amaral, J. Chem. Thermodyn. 43, 255 (2011).

M. A. V. Ribeiro da Silva and L. M. P. F. Amaral, J. Chem. Thermodyn. 43, 876 (2011).

I. B. Rietveld, M. Barrio, J.-L. Tamarit, B. Do, and R. Ceolin, J. Phys. Chem. B 115, 14698 (2011).

M. V. Roux, G. Martin-Valcarcel, R. Notario, S. Kini, J. S. Chickos, and J. F. Liebman, J. Chem. Eng. Data 56, 1220 (2011).

M. V. Roux, R. Notario, C. Foces-Foces, M. Temprado, F. Rox, V. N. Emel'yanenko, and S. P. Verevkin, J. Phys. Chem. A 115, 3167 (2011). 
2011ROU/NOT2 M. V. Roux, R. Notario, M. Segura, R. Guzman-Mejia, E. Juaristi, and J. S. Chickos, J. Chem. Eng. Data 56, 3807 (2011).

2011ROU/TEM M. V. Roux, M. Temprado, P. Jimenez, R. Notario, A. R. Parameswar, A. V. Demchenko, J. S. Chickos, C. A. Deakyne, and J. F. Liebman, J. Chem. Eng. Data 56, 4725 (2011).

2011SAN/MON L. M. B. F. Santos, A. R. Monteiro, J. M. Goncalves, W. E. Acree, Jr., and M. D. M. C. Ribeiro da Silva, J. Chem. Thermodyn. 43, 1044 (2011).

2011SAN/RIB $\quad$ A. F. L. O. M. Santos and M. A. V. Ribeiro da Silva, J. Phys. Chem. B 115, 4939 (2011).

2011 SAN/RIB2 A. F. L. O. M. Santos and M. A. V. Ribeiro da Silva, J. Chem. Thermodyn. 43, 1480 (2011).

2011SAN/RIB3 A. F. L. O. M. Santos and M. A. V. Ribeiro da Silva, J. Phys. Chem. B 115, 12549 (2011).

2011SAP/POK E. Sapei, J.-P. Pokki, P. Uusi-Kyyny, K. I. Keskinen, and V. Alopaeus, Fluid Phase Equilib. 307, 180 (2011).

2011SAP/UUS E. Sapei, P. Uusi-Kyyny, K. I. Keskinen, J.-P. Pokki, and V. Alopaeus, Fluid Phase Equilib. 301, 200 (2011).

2011SIN/RAI M. Singh, R. N. Rai, and U. S. Rai, Am. J. Anal. Chem. 2, 953 (2011).

2011 SOU/MAT $\quad$ C. C. S. Sousa, M. A. R. Matos, and V. M. F. Morais, J. Chem. Thermodyn. 43, 1159 (2011).

2011 SOU/MAT2 $\quad$ C. C. S. Sousa, M. A. R. Matos, and V. M. F. Morais, J. Chem. Thermodyn. 43, 1435 (2011).

2011SOU/NIS K. Sou, K. Nishikawa, Y. Koga, and K.-I. Tozaki, Chem. Phys. Lett. 506, 217 (2011).

2011TAN/FUJ N. Tanimoto and H. Fujimori, J. Non-Cryst. Solids 357, 731 (2011).

2011TEM/ROU M. Temprado, M. V. Roux, F. Ros, R. Notario, M. Segura, and J. S. Chickos, J. Chem. Eng. Data 56, 263 (2011).

2011TOR/GAR M. Torcal, S. Garcia-Abarrio, J. F. Pardo, A. M. Mainar, and J. S. Urieta, J. Chem. Eng. Data 56, 3208 (2011).

2011UMN/CHI P. Umnahanant and J. S. Chickos, J. Pharm. Sci. 100, 1847 (2011).

2011VEC/BRU S. Vecchio and B. Brunetti, Thermochim. Acta 515, 84 (2011).

2011VER/EME S. P. Verevkin, V. N. Emel'yanenko, A. A. Pimerzin, and E. E. Vishnevskay, J. Phys. Chem. A 115, 1992 (2011).

2011VER/EME2 S. P. Verevkin, V. N. Emelyanenko, M. Algarra, J. M. Lopez-Romero, F. Aguiar, J. E. Rodriguez-Borges, and J. C. G. Esteves da Silva, J. Chem. Thermodyn. 43, 1652 (2011).

2011VER/EME3 S. P. Verevkin, V. N. Emel'yanenko, A. A. Pimerzin, and E. E. Vishnevskay, J. Phys. Chem. A 115, 12271 (2011).

2011 VER/TON S. P. Verevkin, B. Tong, U. Welz-Biermann, and Y. Chernyak, J. Chem. Eng. Data 56, 4400 (2011).

2011 VER/ZAI S. P. Verevkin, D. H. Zaitsau, V. N. Emel'yanenko, Y. U. Paulechka, A. V. Blokhin, A. B. Bazyleva, and G. J. Kabo, J. Phys. Chem. B 115, 4404 (2011).

2011WYR/HEB D. Wyrzykowski, E. Hebanowska, G. Nowak-Wiczy, M. Makowski, and L. Chmyurzynski, J. Therm. Anal. Calorim. 104, 731 (2011).

2011 XIN/YAN Y. Xing, X. Yang, W. Fang, Y. Guo, and R. Lin, Fluid Phase Equilib. 305, 192 (2011).

2012AGA/MOS E. V. Agafonova, V. Yu. Moshchenskii, and M. L. Tkachenko, Russ. J. Phys. Chem. 86, 1035 (2012).

2012AGA/VAR L. E. Agafonova, R. M. Varushchenko, A. I. Druzhinina, and O. V. Polyakova, J. Chem. Thermodyn. 47, 120 (2012).

2012AHM/NEG N. C.-B. Ahmed, L. Negadi, I. Mokbel, A. A. Kaci, and J. Jose, J. Chem. Thermodyn. 44, 116 (2012).

2012ALM/MAT A. R. R. P. Almeida, M. A. R. Matos, M. J. S. Monte, and V. M. F. Morais, J. Chem. Thermodyn. 47, 81 (2012).

2012ALM/MON A. R. R. P. Almeida and M. J. S. Monte, J. Chem. Thermodyn. 44, 163 (2012).

2012ALM/MON2 A. R. R. P. Almeida and M. J. S. Monte, J. Chem. Thermodyn. 53, 100 (2012).

2012AMA/RIB $\quad$ L. M. P. F. Amaral and M. A. V. Ribeiro da Silva, J. Chem. Thermodyn. 48, 65 (2012).

2012BAD/TYU V. G. Badelin, E. Yu. Tyunina, A. V. Krasnov, V. V. Tyunina, N. I. Giricheva, and A. V. Girichev, Russ. J. Phys. Chem. A 86, 457 (2012).
2012BAG/RED

P. P. Bag and C. M. Reddy, Cryst. Growth Des. 12, 2740 (2012).

2012BIT/MEN K. Bitchikh, A.-H. Menial, and W. Louaer, Energy Procedia 18, 1152 (2012).

2012BOO/BAN A. M. Booth, T. Bannan, M. R. McGillen, M. H. Barley, D. O. Topping, G. McFiggans, and C. J. Percival, RSC Adv. 2, 4430 (2012).

2012BRU/MAI G. Bruni, M. Maielta, L. Maggi, M. Bini, D. Capsoni, S. Ferrari, M. Boiocchi, V. Berbenni, C. Milanesi, and A. Marini, Cryst. Eng. Commun. 14, 6035 (2012).

2012BUT/MAR C. Butterhof, T. Martin, P. Ectors, D. Zahn, P. Niemietz, J. Senker, C. Nather, and J. Breu, Cryst. Growth Des. 12, 5365 (2012).

2012CHA/ARO2 R. Chadha, P. Arora, and S. Bhandari, ISRN Thermodyn. 2012, 671027 (2012).

2012CHA/LAY

E. L. Charsley, P. G. Laye, and H. M. Markham, Thermochim. Acta 539, 115 (2012).

2012DAV/HER J. Z. Davalos, R. Herrero, A. Chana, A. Guerrero, P. Jimenez, and J. M. Santiuste, J. Phys. Chem. A 116, 2261 (2012).

2012EME/VER V. N. Emel'yanenko, S. P. Verevkin, R. V. Ralys, V. V. Turovtsev, and V. Yu. Orlov, Russ. J. Phys. Chem. A 86, 1493 (2012).

2012 FER/RIB $\quad$ A. I. M. C. L. Ferreira and M. A. V. Ribeiro da Silva, J. Chem. Thermodyn. 48, 84 (2012).

2012FOR/MEL A. Forte, C. I. Melo, R. Bogel-Lukasik, and E. BogelLukasik, Fluid Phase Equilib. 318, 89 (2012).

2012GAR/VER I. V. Garist, S. P. Verevkin, J. E. Bara, M. S. Hindman, an S. P. O. Danielson, J. Chem. Eng. Data 57, 1803 (2012).

2012GIM/MAR B. Gimeno, S. Martinez, J. S. Urieta, and P. Perez, J. Chem. Eng. Data 57, 3026 (2012).

2012GON/MIN E. M. Goncalves and M. E. Minas de Piedade, J. Chem. Thermodyn. 47, 362 (2012).

2012GOR/ALE C. H. Gorbitz, F. Alebachew, and V. Petricek, J. Phys. Chem. B 116, 10715 (2012).

2012GUP/AGR P. Gupta, T. Agrawal, S. S. Das, and N. B. Singh, J. Chem. Thermodyn. 48, 291 (2012).

2012HAN/SOL R. Hani, R. Solimando, L. Negadi, J. Jose, and A. AitKaci, J. Chem. Thermodyn. 54, 83 (2012).

2012HAN/ZHO X.-W. Han, C.-R. Zhou, and X.-H. Shi, J. Therm. Anal. Calorim. 109, 441 (2012).

2012HAS/DRA D. Hasty, J. Drapekin, T. Subramanian, T. C. Winter, J. S. Chickos, A. A. Samarov, A. V. Yermalayeu, and S. P. Verevkin, J. Chem. Eng. Data 57, 2350 (2012).

2012HIK/WEE W. M. Hikal and B. L. Weeks, J. Therm. Anal. Calorim. 110, 955 (2012).

2012HUL/BAS R. J. Hulse, R. S. Basu, R. R. Singh, and R. H. P. Thomas, J. Chem. Eng. Data 57, 3581 (2012); Erratum, 59, 531 (2014).

2012JEG/PRA B. Jeganathan and V. Prakya, Iranian J. Pharm. Sci. 8, 83 (2012).

2012JOS/BER A. Joseph, C. E. S. Bernardes, and M. E. Minas da Piedade, J. Chem. Thermodyn. 55, 23 (2012).

2012KAN/RAI S. Kant, U. S. Rai, and R. N. Rai, J. Therm. Anal. Calorim. 110, 551 (2012).

2012KLI/LEI K. Klimova and J. Leitner, Thermochim. Acta 550, 59 (2012).

2012KON/MAT T. S. Kon'kova, Yu. N. Matyushin, E. A. Miroshnichenko, S. V. Prokhorova, J. O. Inozemtsev, A. V. Inozemtsev, A. B. Sheremetev, A. M. Kozeev, and L. L. Pasheneno, Int. Annu. Conf. ICT 43, 76/1 (2012).

2012LAA/ZAI H. Laavi, A. Zaitseva, J.-P. Pokki, P. Uusi-Kyyny, Y. Kim, and V. Alopaeus, J. Chem. Eng. Data 57, 3092 (2012).

2012LIM/JAN J. Lim, S. Jang, M. S. Shin, and H. Kim, Fluid Phase Equilib. 332, 144 (2012).

2012LOU/MEL C. Lourenco, C. I. Melo, R. Bogel-Lukasik, and E. BogelLukasik, J. Chem. Eng. Data 57, 1525 (2012).

2012MAH/AND C. Maheshwari, V. Andre, S. Reddy, L. Roy, T. Duarte, and N. Rodriguez-Hornedo, CrystEngComm 14, 4801 (2012).

2012MAH/RAS A. Maher, A. C. Rasmuson, D. M. Croker, and B. K. Hodnett, J. Chem. Eng. Data 57, 3525 (2012). 
2012MAH/SEA A. Maher, C. C. Seaton, S. Hudson, D. M. Croker, A. C. Rasmuson, and B. K. Hodnett, Cryst. Growth Des. 12, 6223 (2012).

2012MAN/VIL M. S. Manic, D. Villanueva, T. Fornari, A. J. Queimada, E. A. Macedo, and V. Najdanovic-Visak, J. Chem. Thermodyn. 48, 93 (2012).

2012MEL/PIN V. Meltzer and E. Pincu, Central Eur. J. Chem. 10, 1584 (2012).

2012MIR/MAT M. S. Miranda, M. A. R. Matos, V. M. F. Morais, and J. F. Liebman, J. Chem. Thermodyn. 50, 30 (2012).

2012MOK/SAW I. Mokbel, T. Sawaya, M.-L. Zanota, R. A. Naccoul, J. Jose, and C. de Bellefon, J. Chem. Eng. Data 57, 284 (2012).

2012PAL/ORA M. Palczewska-Tulinska and P. Oracz, J. Chem. Eng. Data 57, 3176 (2012).

2012RAM/ULI I. A. Ramos, S. E. Ulic, R. M. Romano, Y. V. Vishnevskiy, R. J. F. Berger, N. W. Mitzel, H. Beckers, H. Willner, S. Tong, M. Ge, and C. O. Della Vedova, J. Phys. Chem. A 116, 11586 (2012).

2012RAN/BHA B. K. Rana, N. V. Bhate, S. M. Mahajani, and S. P. Dabke, J. Chem. Eng. Data 57, 3483 (2012).

2012RED/KUM K. R. Reddy, D. B. K. Kumar, G. S. Rao, P. S. S. Sri, and C. Rambabu, Fluid Phase Equilib. 336, 52 (2012).

2012RED/SAT R. S. B. Reddi, V. S. A. K. Satuluri, U. S. Rai, and R. N. Rai, J. Therm. Anal. Calorim. 107, 377-385 (2012).

2012RIB/CAB M. A. V. Ribeiro da Silva and J. I. T. A. Cabral, J. Chem. Thermodyn. 47, 138 (2012).

2012RIB/FRE M. D. M. C. Ribeiro da Silva, V. L. S. Freitas, M. A. A. Vieira, M. J. Sottomayor, and W. E. Acree, Jr., J. Chem. Thermodyn. 49, 146-153 (2012).

2012RIB/GAL M. A. V. Ribeiro da Silva, T. L. P. Galvao, I. M. Rocha, and A. F. L. O. M. Santos, J. Chem. Thermodyn. 54, 330 (2012).

2012RIB/MON M. A. V. Ribeiro da Silva, M. J. S. Monte, I. M. Rocha, and A. Cimas, J. Org. Chem. 77, 4312 (2012).

2012RIO/ORT R. Rios, J. Ortega, and L. Fernandez, J. Chem. Eng. Data $\mathbf{5 7 ,} 3210$ (2012).

2012ROD/SOL M. N. Rodnikova, I. A. Solonina, M. R. Kiselev, and S. V. Makaev, Russ. J. Phys. Chem. A 86, 1745 (2012).

2012ROU/NOT M. V. Roux, R. Notario, M. Segura, and J. S. Chickos, J. Chem. Eng. Data 57, 249 (2012).

2012ROU/NOT2 M. V. Roux, R. Notario, D. H. Zaitsau, V. E. Emel'yanenko, and S. P. Verevkin, J. Phys. Chem. A 116, 4639 (2012).

2012ROU/NOT3 M. V. Roux, R. Notario, M. Segura, J. S. Chickos, and J. F. Liebman, J. Phys. Org. Chem. 25, 916 (2012).

2012ROZ/BRU J. Rozmus, I. Brunella, P. Mougin, and J. C. de Hemptinne, J. Chem. Eng. Data 57, 2915 (2012).

2012SAM/NAZ A. A. Samarov, A. G. Nazmutdinov, and S. P. Verevkin, Fluid Phase Equilib. 334, 70 (2012).

2012SAN/RIB $\quad$ A. F. L. O. M. Santos and M. A. V. Ribeiro da Silva, J. Chem. Thermodyn. 48, 194 (2012).

2012SHA/LAL $\quad$ B. L. Sharma, P. Lal, M. Sharma, and A. K. Sharma, J. Therm. Anal. Calorim. 109, 447 (2012).

2012SHA/RAI K. P. Sharma and R. N. Rai, Thermochim. Acta 535, 66 (2012).

2012SHA/RED K. P. Sharma, R. S. B. Reddi, and R. N. Rai, J. Therm. Anal. Calorim. 110, 545 (2012).

2012SHA/SHA K. P. Sharma, P. R. Shakya, and R. Rai, Sci. World 10, 91 (2012).

2012SIL/PER V. M. T. M. Silva, C. S. M. Pereira, A. E. Rodrigues, S. P. Verevkin, V. N. Emel'yanenko, I. V. Garist, and J. Gmehling, Ind. Eng. Chem. Res. 39, 12723 (2012).

2012SUS/ROD P. Susial, J. J. Rodriguez-Henriquez, J. C. Apolinario, V. D. Castillo, and H. J. Estupinan, J. Serb. Chem. Soc. 77, 1243 (2012).

2012UMN/CHI P. Umnahanant and J. Chickos, J. Chem. Eng. Data 57, 1331 (2012)

2012UMN/HAS P. Umnahanant, D. Hasty, and J. S. Chickos, J. Pharm. Sci. 101, 2045 (2012).

2012VER/CHE S. P. Verevkin and Y. Chernyak, J. Chem. Thermodyn. 47, 328 (2012).
2012VER/EME S. P. Verevkin and V. N. Emel'yanenko, J. Chem. Thermodyn. 46, 94 (2012).

2012VER/EME4 S. P. Verevkin, V. N. Emel'yanenko, R. Notario, M. V. Roux, J. S. Chickos, and J. F. Liebman, J. Phys. Chem. Lett. 3, 3454 (2012).

2012VIV/FRE M. L. F. Viveiros, V. L. S. Freitas, N. Vale, J. R. B. Gomes, P. Gomes, and M. D. M. C. Ribeiro da Silva, J. Phys. Org. Chem. 25, 420 (2012).

2012WAN/DEN T.-C. Wang and K.-C. Deng, J. Chem. Eng. Data 57, 78 (2012).

2012WAN/HU K. Wang, Y. Hu, W. Yang, Y. Shi, and Y. Li, Thermochim. Acta 538, 79 (2012).

2012WAN/LI T.-C. Wang, Y.-J. Li, and Y.-P. Chen, J. Chem. Eng. Data $\mathbf{5 7}, 3519$ (2012)

2012WIL/CHI J. E. Wilson and J. Chickos, J. Chem. Eng. Data 57, 2281 (2012).

2012XIN/ZHA X. Xing, F. Zhao, S. Ma, K. Xu, L. Xiao, H. Gao, T. An, and R. Hu, Propellants, Explos., Pyrotech. 37, 179 (2012).

2012YAN/THA H. Yang, J. Thati, and A. C. Rasmuson, J. Chem. Thermodyn. 48, 150 (2012).

2012ZAI/NEG L. K. Zaitri, L. Negadi, I. Mokbel, N. Msakni, and J. Jose, Fuel 95, 438 (2012).

2012ZEN/CHE Z.-X. Zeng, J. Chen, W.-1. Xue, P. Zhang, and Y. Xie, Can. J. Chem. Eng. 90, 570 (2012).

2012ZHA/DU W. Zhang, W. Du, N. Meng, R. Sun, Y. Shao, and C. Li, Energy Procedia 16, 1078 (2012).

2013AGA/MOS E. V. Agafonova, Yu. V. Moshchenskii, and M. L. Tkachenko, Russ. J. Phys. Chem. A 87, 1291 (2013).

2013ALM/MON A. R. R. P. Almeida and M. J. S. Monte, J. Chem. Thermodyn. 57, 160 (2013).

2013ALM/MON2 A. R. R. P. Almeida, M. J. S. Monte, M. A. R. Matos, and V. M. F. Morais, J. Chem. Thermodyn. 59, 222 (2013).

2013ALM/MON3 A. R. R. P. Almeida and M. J. S. Monte, J. Chem. Thermodyn. 65, 150 (2013).

2013AMA/RIB L. M. P. F. Amaral and M. A. V. Ribeiro da Silva, J. Chem. Thermodyn. 57, 301 (2013).

2013AMA/SAN L. M. P. F. Amaral, A. F. L. O. M. Santos, M. D. M. C. Ribeiro da Silva, and R. Notario, J. Chem. Thermodyn. 58, 315 (2013).

2013AMA/SZT L. M. P. F. Amaral, P. Szterner, and M. A. V. Ribeiro da Silva, J. Chem. Thermodyn. 64, 187 (2013).

2013BAR/GIL C. Barreneche, A. Gil, F. Sheth, A. I. Fernandez, and L. F. Cabeza, Solar Energy 94, 344 (2013).

2013BEN/KHI2 M. Benziane, K. Khimeche, I. Mokbel, A. Dahmani, and J. Jose, J. Chem. Eng. Data 58, 492 (2013).

2013BLO/KOH A. V. Blokhin, S. V. Kohut, G. J. Kabo, E. N. Stepurko, Y. U. Paulechka, and O. V. Voitkevich, Thermochim. Acta 565, 221 (2013).

2013BOU/TEY B. Bouillot, S. Teychene, and B. Biscans, Ind. Eng. Chem. Res. 52, 9276 (2013).

2013BRA/AMH Ｃ. Brandel, Y. Amharar, J. M. Rollinger, U. J. Griesser, Y. Cartigny, S. Petit, and G. Coquerel, Mol. Pharm. 10, 3850 (2013).

2013BUR/STR A. U. Burman and K. H. U. Strom, J. Chem. Eng. Data 58, 257 (2013).

2013CAR/LUC S.P. Carvalho, E. F. Lucas, G. Gonzalez, and L. S. Spinelli, J. Braz. Chem. Soc. 24, 1998 (2013).

2013CAR/RHA L. Carpentier, K. F. Rharrassi, P. Derollez, and Y. Guinet, Thermochim. Acta 556, 63 (2013).

2013CHE/XIE Z. Chen, C. Xie, Z. Xu, Y. Wang, H. Zhao, and H. Hao, J. Chem. Eng. Data 58, 143 (2013).

2013CHI/DER N. Chiali-Baba-Ahmed, F. Dergal, L. Negadi, and I. Mokbel, J. Chem. Thermodyn. 63, 44 (2013).

2013DAV/KIL P. S. Davis and J. E. Kilpatrick, J. Chem. Thermodyn. 58, 134 (2013).

2013DOS/MOR A. D. dos Santos, A. R. C. Morais, C. Melo, R. BogelLukasik, and E. Bogel-Lukasik, Fluid Phase Equilib. 356, 18 (2013).

2013EME/VER V. N. Emel'yanenko and S. P. Verevkin, Vest. St. Peter. Ser. Fiz. Khim. 113 (2013).

2013FER/RIB A. I. M. C. L. Ferreira and M. A. V. Ribeiro da Silva, J. Chem. Thermodyn. 59, 94 (2013). 
2013FLO/MEN H. Flores, J. Mentado, E. A. Camarillo, A. Ximello, and M. A. Amador, Struct. Chem. 24, 2083 (2013).

2013FUL/VLA A. Fulias, G. Vlase, C. Grigorie, I. Ledeti, P. Albu, M. Bilanin, and T. Vlase, J. Therm. Anal. Calorim. 113, 265 (2013).

2013GAL/RIB T. L. P. Galvao, M. D. M. C. Ribeiro da Silva, and M. A. V. Ribeiro da Silva, J. Chem. Thermodyn. 62, 186 (2013).

2013GAL/ROC T. L. P. Galvao, I. M. Rocha, M. D. M. C. Ribeiro da Silva, and M. A. V. Ribeiro da Silva, J. Phys. Chem. C 117, 5826 (2013).

2013GAN/BAR I. Gana, M. Barrio, B. Do, J.-L. Tamarit, R. Ceolin, and I. B. Rietveld, Int. J. Pharm. 456, 480 (2013).

2013GOB/GUT C. Gobble, A. Gutterman, and J. S. Chickos, Struct. Chem. 24, 1903 (2013).

2013GOB/RAT C. Gobble, N. Rath, and J. Chickos, J. Chem. Eng. Data 58, 2600 (2013).

2013GRI/MAR G. S. Grigoryan and Sh.L.A. Markaryan, Russ. J. Phys. Chem. 87, 191 (2013).

2013GUT/RAT A. Gutterman, N. Rath, and J. Chickos, J. Chem. Eng. Data 58, 749 (2013).

2013HIK/WEE W. M. Hikal and B. L. Weeks, Chem. Phys. 415, 228 (2013).

2013HUA/LU J. Huang, S. Lu, X. Kong, S. Liu, and Y. Li, Materials 6, 4758 (2013).

2013HUA/LV G. Huang and C. Lv, J. Chem. Eng. Data 58, 279 (2013).

2013HUN/SUT S. Hunter, T. Sutinen, S. F. Parker, C. A. Morrison, D. M. Williamson, S. Thompson, P. J. Gould, and C. R. Pulham, J. Phys. Chem. C 117, 8062 (2013).

2013JIA/HU X.-M. Jiang, Y.-H. Hu, W.-G. Yang, Z.-Y. Lei, R.-R. Tang, and F. Shen, J. Sol. Chem. 42, 272 (2013).

2013JIA/LI D. Jia, Y. Li, Y. Li, and C. Li, J. Chem. Eng. Data 58, 3183 (2013).

2013JUS/ZIE $\quad$ E. Juszynska-Galazka, P. M. Zielinski, M. MassalskaArodz, and J. Krawczyk, Acta Phys. Pol., A 124, 917 (2013).

2013KAN V. Kant, Bull. Pharm. Med. Sci. 1, 117 (2013).

2013KUM/NAN S. S. Kumar and A. Nangia, Cryst. Eng. Commun. 15, 6498 (2013).

2013LI/LU Q. Li, F. Lu, Y. Tian, S. Feng, Y. Shen, and B. Wang, J. Chem. Eng. Data 58, 1020 (2013).

2013LI/TAN R.-C. Li and Z.-C. Tan, J. Chem. Eng. Data 58, 2137 (2013).

2013LI/ZHA Y. Li, H. Zhao, M. Mao, and Z. Yang, J. Chem. Eng. Data 58, 1629 (2013).

2013LIM/JAN J. Lim, S. Jang, H. K. Cho, M. S. Shin, and H. Kim, J. Chem. Thermodyn. 57, 295 (2013).

2013LIU/ZHA R. Liu, T. Zhang, Y. Liu, L. Yang, and Z. Zhou, J. Therm. Anal. Calorim. 112, 1523 (2013).

2013LOM/GIN L. Lomba, B. Giner, C. Lafuente, S. Martin, and H. Artigas, J. Chem. Eng. Data 58, 1193 (2013).

2013LOM/LAF L. Lomba, C. Lafuente, M. Garcia-Mardones, I. Gascon, and B. Giner, J. Chem. Thermodyn. 65, 34 (2013).

2013MAN/VOR A. N. Manin, A. P. Voronin, and G. L. Perlovich, Thermochim. Acta 551, 17 (2013).

2013MEJ/OLI A. Mejia, M. B. Oliveira, H. Segura, M. Cartes, and J. A. P. Coutinho, Fluid Phase Equilib. 345, 60 (2013).

2013MEL/BOG C. I. Melo, R. Bogel-Lukasik, M. N. Ponte, and E. BogelLukasik, Fluid Phase Equilib. 338, 209 (2013).

2013MIR/MAT M. S. Miranda, M. A. R. Matos, and V. M. F. Morais, J. Chem. Thermodyn. 62, 170 (2013).

2013MOR/ELL K. M. Morgan, J. A. Ellis, J. Lee, A. Fulton, S. L. Wilson, P. S. Dupart, and R. Dastorri, J. Org. Chem. 78, 4303 (2013).

2013NAT/JES C. Nather, I. Jess, P. G. Jones, C. Taouss, and N. Teschmit, Cryst. Growth Des. 13, 1676 (2013).

2013NEG/BAR Ph. Negrier, M. Barrio, J. Ll. Tamarit, D. Mondieig, M. J. Zuriaga, and S. C. Perez, Cryst. Growth Des. 13, 2143 (2013).

2013NOT/EME R. Notario, V. N. Emel'yanenko, M. V. Roux, F. Ros, S. P. Verevkin, J. S. Chickos, and J. F. Liebman, J. Phys. Chem. A 117, 244 (2013).

2013PAL/MCC K. J. Paluch, T. McCabe, H. Muller-Bunz, O. I. Corrigan, A. M. Healy, and L. Tajber, Mol. Pharm. 10, 3640 (2013).
2013PER/BLO2

G. L. Perlovich, S. V. Blokhina, M. V. Ol'khovich, A. V. Sharapova, and A. N. Proshin, J. Sol. Chem. 42, 2057 (2013).

2013PER/KAZ G. L. Perlovich, V. P. Kazachenko, N. N. Strakhova, K.-J. Schaper, and O. A. Raevsky, J. Chem. Eng. Data 58, 2659 (2013).

2013RED/BEN P. Reddy, T. P. Benecke, and D. Ramjugernath, J. Chem. Thermodyn. 58, 330 (2013).

2013RIB/AMA M. A. V. Ribeiro da Silva, L. M. P. F. Amaral, and P. Szterner, J. Chem. Thermodyn. 57, 380 (2013).

2013ROC/GAL I. M. Rocha, T. L. P. Galvao, M. D. M. C. Ribeiro da Silva, and M. A. V. Ribeiro da Silva, Struct. Chem. 24, 1935 (2013).

2013SAN/AMA A. F. L. O. M. Santos, L. M. P. F. Amaral, M. D. M. C. Ribeiro da Silva, M. V. Roux, and R. Notario, J. Chem. Thermodyn. 58, 29 (2013).

2013 SAN/RIB $\quad$ A. F. L. M. Santos and M. A. V. Ribeiro da Silva, J. Chem. Thermodyn. 57, 454 (2013).

2013SAN/RIB2 A. F. L. M. Santos and M. A. V. Ribeiro da Silva, J. Chem. Thermodyn. 58, 476 (2013).

2013SAN/RIB4 A. F. L. O. M. Santos and M. A. V. Ribeiro da Silva, J. Phys. Chem. A 117, 5195 (2013).

2013 SAN/RIB5 A. F. L. O. M. Santos and M. A. V. Ribeiro da Silva, J. Chem. Thermodyn. 67, 190 (2013).

2013 SCH/BUC $\quad$ B. J. Schindler, J. H. Buchanan, J. H. Mahle, G. W. Peterson, and T. G. Glover, J. Chem. Eng. Data 58, 1806 (2013).

2013SIL/CIM A. L. R. Silva, A. Cimas, and M. D. M. C. Ribeiro da Silva, J. Chem. Thermodyn. 57, 212 (2013).

2013 SIL/CIM2 A. L. R. Silva, A. Cimas, N. Vale, P. Gomes, M. J. S. Monte, and M. D. M. C. Ribeiro da Silva, J. Chem. Thermodyn. 58, 158 (2013).

2013SIL/CIM3 A. L. R. Silva, A. Cimas, and M. D. M. C. Ribeiro da Silva, Struct. Chem. 24, 1863 (2013).

2013SIM/BER R. G. Simoes, C. E. S. Bernardes, H. P. Diogo, F. Agapito, and M. E. Minas da Piedade, Mol. Pharm. 10, 2713-2722 (2013).

2013SIM/BER2 R. G. Simoes, C. E. S. Bernardes, and M. E. Minas da Piedade, Cryst. Growth Des. 13, 2803 (2013).

2013SIN/PAN M. Singh, P. Pandey, R. N. Rai, and U. S. Rai, J. Therm. Anal. Calorim. 113, 977 (2013).

2013 SOS/MOR $\quad$ C. C. S. Sosa, V. M. F. Morais, and M. A. R. Matos, J. Chem. Thermodyn. 56, 83 (2013).

2013SOU/MAT C. C. S. Sousa, M. A. R. Matos, L. M. N. B. F. Santos, and V. M. F. Morais, J. Chem. Thermodyn. 67, 210 (2013).

2013STE/FUL V. Stejfa, M. Fulem, K. Ruzicka, C. Cervinka, M. A. A. Rocha, L. M. N. B. F. Santos, and B. Schroder, J. Chem. Thermodyn. 60, 117 (2013).

2013SVA/RAS M. Svard and A. C. Rasmuson, Cryst. Growth Des. 13, 1140 (2013).

2013THO/CHI M. Thornton, J. Chickos, I. V. Garist, M. A. Varfolomeev, A. A. Svetlov, and S. P. Verevkin, J. Chem. Eng. Data 58, 2018 (2013).

2013TRA/KHI D. Trache, K. Khimeche, R. Benelmir, and A. Dahmani, Thermochim. Acta 565, 8 (2013).

2013TYU/BAD E. Yu. Tyunina and V. G. Badelin, Russ. J. Gen. Chem. 83, 708 (2013).

2013UUS/DEL P. Uusi-Kyyny, C. Dell'era, A. Penttila, M. Pakkanen, and V. Alopaeus, Fluid Phase Equilib. 338, 164 (2013).

2013VEC

2013VEC/BRU S. Vecchio and B. Brunetti, Fluid Phase Equilib. 338, 148 (2013).

2013VEN/BEN J. G. Veneral, T. Benazzi, M. A. Mazutti, F. A. P. Voll, L. Cardozo-Filho, M. I. Corazza, R. Guirardello, and J. V. Oliveira, J. Chem. Thermodyn. 58, 398 (2013).

2013WAN L. Wang, J. Mol. Liq. 188, 55 (2013).

2013WAN/GUO Y. Wang, C. Guo, X. Liu, S. Huang, B. Wang, and H. Zheng, Fluid Phase Equilib. 342, 47 (2013).

2013WAN/LI H. Wang, L. Li, and C. Ye, Fluid Phase Equilib. 342, 42 (2013).

2013WAN/LV

2013WIL/HUL
L. Wang and T.-T. Lv, J. Mol. Liq. 181, 29 (2013).

B. R. Williams, M. S. Hulet, A. Brozena, R. W. Miles, Jr., and D. E. Tavault, J. Chem. Eng. Data 58, 1679 (2013). 
2013XU/LI D. Xu, H. Li, and Z. Li, J. Chem. Eng. Data 58, 3110 (2013).

2013 YE/DON C. S. Ye, J. Dong, and L. Li, J. Chem. Eng. Chin. Univ. 27, 21 (2013).

2013ZAI/YER ～D. H. Zaitsau, A. V. Yermalayeu, V. N. Emel'yanenko, C. Schick, S. P. Verevkin, A. A. Samarov, S. Schlenk, and P. Wasserscheid, Z. Phys. Chem. 227, 205 (2013).

2013ZHA/LV Z. Zhang, M. Lv, D. Huang, P. Jia, D. Sun, and W. Li, J. Chem. Eng. Data 58, 3054 (2013).

2013ZHA/XIA H. Zhang, Q. Xia, Y. Yang, F.-B. Zhang, and G. L. Zhang, Ind. Eng. Chem. Res. 52, 5230 (2013).

2013ZHA/YAN W. Zhang, Z. Q. Yang, J. Lu, and J. Lu, J. Chem. Eng. Data 58, 2307 (2013).

2014ACH/EME F. Achrainer, V. N. Emel'yanenko, W. Tantawy, S. P. Verevkin, and H. Zipse, J. Phys. Chem. B 118, 10426 (2014).

2014AGA/MOS E. V. Agafonova, Y. V. Moshchenskiy, and M. L. Tkachenko, Thermochim. Acta 580, 1 (2014).

2014ALM/CUN A. R. R. P. Almeida, A. F. G. Cunha, M. A. R. Matos, V. M. F. Morais, and M. J. S. Monte, J. Chem. Thermodyn. 78, 43 (2014).

2014ALM/MON A. R. R. P. Almeida, M. J. S. Monte, M. A. R. Matos, and V. M. F. Morais, J. Chem. Thermodyn. 73, 12 (2014).

2014ALM/MON2 A. R. R. P. Almeida and M. J. S. Monte, J. Chem. Thermodyn. 77, 46 (2014).

2014AMA/DEC L. M. P. F. Amaral, T. M. T. de Carvalho, J. I. T. A. Cabral, M. D. M. C. Ribeiro da Silva, and M. A. V. Ribeiro da Silva, J. Therm. Anal. Calorim. 151, 803 (2014).

2014AMA/MOR L. M. P. F. Amaral, V. M. F. Morais, and M. A. V. Ribeiro da Silva, J. Chem. Thermodyn. 74, 22 (2014).

2014AMA/RIB $\quad$ L. M. P. F. Amaral and M. A. V. Ribeiro da Silva, J. Chem. Thermodyn. 73, 44 (2014).

2014AMA/RIB2 L. M. P. F. Amaral and M. A. V. Ribeiro da Silva, J. Chem. Thermodyn. 78, 254 (2014).

2014AMA/SZT L. M. P. F. Amaral, P. Szterner, M. S. Miranda, and M. A. V. Ribeiro da Silva, J. Chem. Thermodyn. 75, 106 (2014).

2014BAD/DEL E. Badea, G. Della Gatta, and B. Palecz, J. Chem. Thermodyn. 73, 178 (2014).

2014BAD/NOW E. Badea, B. Nowicka, and G. Della Gatta, J. Chem. Thermodyn. 68, 90 (2014).

2014BEB/ROS S. V. S. Bebiano, M. T. S. Rosado, R. A. E. Castro, M. Ramos Cilva, J. Cantilho, T. M. R. Maria, and M. E. S. Eusebio, J. Mol. Struct. 1078, 10 (2014).

2014BEN/AIT H. Ben-Makhlouf-Hakem, A. Ait-Kaci, and J. Jose, Fluid Phase Equilib. 373, 34 (2014).

2014BEN/NEG L. Bendiaf, A. Negadi, I. Mokbel, and L. Negadi, Fuel 122, 247 (2014).

2014BER/SIM C. E. S. Bernardes, R. G. H. P. Simoes diogo, and M. E. Minas da Piedade, J. Chem. Thermodyn. 73, 140-147 (2014).

2014BRE/ZAK A. A. Bredikhin, D. V. Zakharychev, A. T. Gubaidullin, R. R. Fayzullin, A. V. Pashagin, and Z. A. Bredikhina, Cryst. Growth Des. 14, 1676 (2014).

2014BRO/BUC A. Brozena, J. H. Buchanan, R. W. Miles, Jr., B. R. Williams, and M. S. Hulet, J. Chem. Eng. Data 59, 2649 (2014).

2014BRO/JAN $\quad$ S. Brommer, L. M. Jantunen, T. F. Bidleman, S. Harrad, and M. L. Diamond, J. Chem. Eng. Data 59, 1441 (2014).

2014BRO/TEV A. Brozena, D. E. Tevault, and K. Irwin, J. Chem. Eng. Data 59, 307 (2014).

2014CAM/MEN E. A. Camarillo, J. Mentado, H. Flores, and J. M. Hernandez-Perez, J. Chem. Thermodyn. 73, 269 (2014).

2014CAR/CAS N. D. D. Carareto, T. Castagnaro, M. C. Costa, and A. J. A. Meirelles, J. Chem. Thermodyn. 78, 99 (2014).

2014CAR/DOS N. D. D. Carareto, A. O. dos Santos, M. P. Rolemberg, L. P. Cardoso, M. C. Costa, and A. J. A. Meirelles, Thermochim. Acta 589, 137 (2014).

2014CHE/LI ～L. Chen, H.-R. Li, R.-J. Xu, Y. Xiong, T. Xu, B.-Y. Huang, and Y.-J. Shu, Propellants, Explos., Pyrotech. 39, 217 (2014).

2014COR/GUI Y. Corvis, N. Guiblin, and P. Espeau, J. Phys. Chem. B 118, 1925 (2014).
2014CUD/POD

2014DIB/RAE

2014DIC/LEG

2014EME/ALG

2014FLO/XIM

2014FON/GUS

2014FRE/GOM2

2014FRE/LEI

2014FUL/RUZ

2014GAL/RIB

2014GAL/ROC

2014GAR/AMA

2014GOB/CHI

2014GON/AGA

2014GUO/YIN

2014HAS/JIR

2014HU/ZHA

2014KHE/MUT

2014KOZ/GOB

2014KRI/RAJ

2014KUR/TAK

2014LI/SUN

2014LIM/CRE

2014LIU/ZOU

2014LOM/GIN

2014LOM/GIN2

2014MAN/MAH

2014MAN/VOR

2014MAN/VOR2

2014MBA/KNO

2014MEA/SVA

2014MEN/BEJ

2014NEG/BAR

2014NEG/MOK
M. F. Cuddy, A. R. Poda, and M. A. Chappell, Propellants, Explos., Pyrotech. 39, 236 (2014).

V. N. Dibrivnyl, Yu. A. Raevskii, and V. V. Sergeev, Russ. J. Gen. Chem. 84, 1069 (2014).

E. Dichi, B. Legendre, and M. Sghaier, J. Therm. Anal. Calorim. 115, 1551 (2014).

V. N. Emel'yaneko, M. Algarra, J. C. G. Esteves da Silva, J. Hierrezuelo, J. M. Lopez-Romero, and S. P. Verevkin, Thermochim. Acta 597, 78 (2014).

H. Flores, A. Ximello, E. A. Camarillo, and P. Amador, J. Therm. Anal. Calorim. 117, 433 (2014).

J. M. S. Fonseca, N. Gushterov, and R. Dohrn, J. Chem. Thermodyn. 73, 148 (2014).

V. L. S. Freitas, J. R. B. Gomes, and M. D. M. C. Ribeiro da Silva, J. Chem. Eng. Data 59, 312 (2014).

V. L. S. Freitas, S. Leirosa, R. Notario, and M. D. M. C. Ribeiro da Silva, J. Org. Chem. 79, 11583 (2014).

M. Fulem, K. Ruzicka, C. Cervinka, A. Bazyleva, and G. Della Gatta, Fluid Phase Equilib. 371, 93 (2014).

T. L. P. Galvao, M. D. M. C. Ribeiro da Silva, and M. A. V. Ribeiro da Silva, J. Phys. Chem. A 118, 4816 (2014).

T. L. P. Galvao, I. M. Rocha, M. D. M. C. Ribeiro da Silva, and M. A. V. Ribeiro da Silva, J. Phys. Chem. A 118, 3360 (2014).

M. A. Garcia-Castro, P. Amador, J. M. Hernandez-Perez, A. E. Medina-Vavela, and H. Flores, J. Phys. Chem. A 118, 3820 (2014).

C. Gobble, J. Chickos, and S. P. Verevkin, J. Chem. Eng. Data 59, 1353 (2014)

E. M. Goncalves, F. Agapito, T. S. Almeida, and J. A. M. Simoes, J. Chem. Thermodyn. 73, 90 (2014).

Y. Guo, Q. Yin, H. Hao, M. Zhang, Y. Bao, B. Hou, W. Chen, H. Zhang, and W. Cong, J. Chem. Eng. Data 59, 1326 (2014).

T. Hasl and I. Jiricek, Energy Procedia 46, 301 (2014).

P. Hu, P.-P. Zhao, Y. Jin, and Z.-S. Chen, Solar Energy 102, 91 (2014).

A. Khelassi-Sefaoui, F. Mutelet, L. Negadi, and I. Mokbel, J. Chem. Thermodyn. 72, 134 (2014).

M. Kozlovskiy, C. Gobble,
Thermodyn. 73, 262 (2014).

V. Krishnakumar and M. Ragaboopathi, J. Therm. Anal. Calorim. 115, 723 (2014).

H. Kuramochi, H. Takigami, M. Scheringer, and S.-I. Sakai, J. Chem. Eng. Data 59, 8 (2014).

D. Li, X. Sun, L. Li, S. Wang, L. Wang, and X. Liu, J. Taiwan Inst. Chem. Eng. 45, 823 (2014).

C. E. Lima de Oliveira and M. A. Cremasco, Thermochim. Acta 577, 1 (2014).

T. Liu, W. Zou, W. Zhao, F.-B. Zhang, G.-L. Zhang, and Q. Xia, Fluid Phase Equilib. 374, 102 (2014).

L. Lomba, B. Giner, E. Zuriaga, I. Gascon, and C. Lafuente, Thermochim. Acta 575, 305 (2014).

L. Lomba, B. Giner, M. C. Lopez, L. Aldea, and C. Lafuente, J. Chem. Eng. Data 59, 329 (2014).

D. R. Mantheni, M. P. K. Maheswaram, R. Munigeti, I. Perera, A. Riga, and K. S. Alexander, J. Therm. Anal. Calorim. 115, 2253 (2014).

A. N. Manin, A. P. Voronin, and G. L. Perlovich, Thermochim. Acta 583, 72 (2014).

A. N. Manin, A. P. Voronin, N. G. Manin, M. V. Vener, A V. Shishkina, A. S. Lermontov, and German L. Perlovich, J. Phys. Chem. B 118, 6803 (2014).

J. Mbah, D. Knot, and S. Steward, Talanta 129, 586 (2014).

D. Mealeya, M. Svärd, and Ake C. Rasmuson, Fluid Phase Equilib. 375, 73-79 (2014).

D. A. Meneses, A. Bejarano, and J. C. de la Fuente, J. Chem. Thermodyn. 74, 16 (2014).

P. Negrier, M. Barrio, J. Ll. Tamarit, and D. Mondieig, J. Phys. Chem. B 118, 9595 (2014).

L. Negadi, I. Mokbel, N. Chiali-Baba-Ahmed, and L. Kara-Zaitri, J. Chem. Thermodyn. 70, 233 (2014). 
2014NOG/ILD B. A. Nogueira, G. O. Ildiz, J. Canotilho, E. M. S. Eusebio, and R. Fausto, J. Phys. Chem. A 118, 5994 (2014).

2014NOT/ROU R. Notario, M. V. Roux, F. Ros, V. N. Emel'yanenko, and S. P. Verevkin, J. Chem. Thermodyn. 74, 144 (2014).

2014NOT/ROU2 R. Notario, M. V. Roux, F. Ros, V. N. Emel'yanenko, D. H. Zaitsau, and S. P. Verevkin, J. Chem. Thermodyn. 77, 151 (2014).

2014PEN/UUS A. Penttila, P. Uusi-Kyyny, A. Salminen, J. Seppala, and V. Alopaeus, Int. J. Greenhouse Gas Control 22, 313 (2014).

2014PER/KAZ G. L. Perlovich, V. P. Kazachenko, N. N. Strakhova, and O. A. Raevsky, J. Chem. Eng. Data 59, 4217 (2014).

2014POB/DOM A. Pobudkowski, U. Domanska, and J. A. Kryska, J. Chem. Thermodyn. 79, 41 (2014).

2014RAM/OGI S. L. L. M. Ramos, M. Ogino, and M. Oguni, J. Therm. Anal. Calorim. 115, 1353 (2014).

2014RIO/ORT R. Rios, J. Ortega, L. Fernandez, I. de Nuez, and J. Wisniak, J. Chem. Eng. Data 59, 125 (2014).

2014ROC/GAL I. M. Rocha, T. L. P. Galvao, M. D. M. C. Ribeiro da Silva, and M. A. V. Ribeiro da Silva, J. Phys. Chem. A 118, 1502 (2014).

2014RUZ/FUL K. Ruzicka, M. Fulem, P. B. P. Serra, O. Vlk, and I. Krakovsky, Thermochim. Acta 596, 98 (2014).

2014SAI/MUR M. K. Saini and S. S. N. Murthy, Thermochim. Acta 575, 195 (2014).

2014SAN/RIB $\quad$ A. F. L. O. M. Santos and M. A. V. Ribeiro da Silva, J. Chem. Thermodyn. 75, 1 (2014).

2014SAN/SIL $\quad$ A. F. L. O. M. Santos, A. L. R. Silva, O. D. F. Santiago, J. M. Goncalves, S. Pandey, W. E. Acree, Jr., and M. D. M. C. Ribeiro da Silva, J. Chem. Thermodyn. 73, 62 (2014).

2014SHA/SRE S. Shantikumar, G. Sreekanth, K. V. S. Nath, S. J. Valli, and N. Satheeshkumar, J. Therm. Anal. Calorim. 115, 2423 (2014).

2014SII/KAM C. Siitsman, I. Kamenev, and V. Oja, Thermochim. Acta $\mathbf{5 9 5}, 35$ (2014).

2014SIL/CIM A. L. R. Silva, A. Cimas, and M. D. M. C. Ribeiro da Silva, J. Chem. Thermodyn. 73, 3 (2014).

2014SIL/MON A. L. R. Silva, M. J. S. Monte, V. M. F. Morais, and M. D. M. C. Ribeiro da Silva, J. Chem. Thermodyn. 74, 67 (2014).

2014SIL/MOR A. L. R. Silva, V. M. F. Morais, and M. D. M. C. Ribeiro da Silva, J. Mol. Struct. 1078, 197 (2014).

2014SIM/AGA R. G. Simoes, F. Agapito, H. P. Diogo, and M. E. Minas da Piedade, J. Phys. Chem. A 118, 11026 (2014).

2014SOB/VAN I. B. Sobechko, Yu. Ya. Van-Chin-Syan, V. V. Kochubei, R. T. Prokop, N. I. Velychkivska, Yu.I. Gorak, V. N. Dibrivnyi, and M. D. Obushak, Russ. J. Phys. Chem. 88, 2046 (2014).

2014SOU/MAT ～C. C. S. Sousa, M. A. R. Matos, and V. M. F. Morais, J. Chem. Thermodyn. 73, 101 (2014).

2014STE/FUL V. Ch. Stejfa, M. Fulem, K. Ruzicka, and C. Cervinka, J. Chem. Thermodyn. 79, 272 (2014).

2014STE/FUL2 V. Ch. Stejfa, M. Fulem, K. Ruzicka, and C. Cervinka, J. Chem. Thermodyn. 79, 280 (2014).

2014SUG/KAI K. Sugandha, S. Kaity, S. Mukherjee, J. Isaac, and A. Ghosh, Cryst. Growth Des. 14, 4475 (2014).

2014SZT/GAL P. Szterner, T. L. P. Galvao, L. M. P. F. Amaral, M. D. M. C. Ribeiro da Silva, and M. A. V. Ribeiro da Silva, Thermochim. Acta 588, 68 (2014).

2014TEM/NOT M. Temprado, R. Notario, M. V. Roux, and S. P. Verevkin, J. Chem. Thermodyn. 73, 97 (2014).

2014THO/GOB M. Thornton, C. Gobble, and J. Chickos, J. Chem. Thermodyn. 73, 51 (2014).

2014TU/CHE W. Tu, Z. Chen, Y. Gao, Z. Li, Y. Zhang, R. Liu, Y. Tian, and L.-M. Wang, Phys. Chem. Chem. Phys. 16, 3586 (2014).

2014TYU/KRA V. V. Tyunina, A. V. Krasnov, E. Y. Tyunina, V. G. Baldelin, and G. V. Girichev, J. Chem. Thermodyn. 74, 221 (2014).

2014VER/EME S. P. Verevkin, V. N. Emel'yanenko, M. A. Varfolomeev, B. N. Solomonov, and K. V. Zherikova, Fluid Phase Equilib. 380, 67 (2014).
2014VER/EME2 S. P. Verevkin, V. N. Emel'yanenko, M. A. Varfolomeev, B. N. Solomonov, K. V. Zherikova, and S. V. Melkhanova, J. Phys. Chem. A 118, 14479 (2014).

2014VIT/AGA J. Vitorino, F. Agapito, M. F. M. Piedade, C. E. S. Bernardes, H. P. Diogo, J. P. Leal, and M. E. Minas da Piedade, J. Chem. Thermodyn. 77, 179 (2014).

2014WAN/HUA Y.-S. Wang, N.-R. Huan, B.-H. Xu, B.-Y. Wang, and Z. S. Bai, J. Chem. Eng. Data 59, 494 (2014).

2014WEI/HAN D. Wei, S. Han, and B. Wang, Fluid Phase Equilib. 373, 84 (2014).

2014WIS/PER J. Wisniak, J. R. Perez-Correa, A. Mejia, and H. Segura, J. Chem. Eng. Data 59, 942 (2014).

2014WU/DAN H. Wu, L. Dang, and H. Wei, Ind. Eng. Chem. Res. 53, 1707 (2014).

2014XIM/FLO A. Ximello, H. Flores, A. Rojas, E. A. Carmarillo, and M. P. Amador, J. Chem. Thermodyn. 79, 33 (2014).

2014XU/ZHA H. Xu, B. Zhang, Z. Yang, G. Yao, and H. Zhao, J. Chem. Eng. Data 59, 1281 (2014).

2014YAN/KOU Z.-Q. Yang, L.-G. Kou, W. Mao, J. Lu, W. Zhang, and J. Lu, J. Chem. Eng. Data 59, 157 (2014).

2014YAN/RAS H. Yang and A. C. Rasmuson, Fluid Phase Equilib. 376, 69 (2014).

2014YAN/WAN ～W. Yang, C. Wang, Y. Hu, Y. Li, and K. Wang, Fluid Phase Equilib. 363, 276 (2014).

2014YAO/YAN G. Yao, Z. Yang, B. Zhang, H. Xu, and H. Zhao, Fluid Phase Equilib. 367, 103 (2014).

2014ZEN/GRI N. Zencirci, U. J. Griesser, T. Gelbrich, D. C. Apperley, and R. K. Harris, Mol. Pharm. 11, 338 (2014).

2014ZEN/YAN Z. Zeng, Z. Yang, W. Xue, and X. Li, Chin. J. Chem. Eng. 22, 1145 (2014).

2014ZHA/QIA X. Zhang, G. Qian, X. Yang, C. Hu, and X. Zhou, Fluid Phase Equilib. 363, 228 (2014).

2014ZHA/WEI W. Zhang, Z.-Q. Wei, J. Lu, and J. Lu, Gaoxiao Xauxue Gongcheng Xuebao 28, 449 (2014).

2015ALM/OLI A. R. R. P. Almeida, J. A. S. A. Oliveira, and M. J. S. Monte, J. Chem. Thermodyn. 82, 108 (2015).

2015ALM/SOU A. R. R. P. Almeida, C. A. D. Sousa, L. M. N. B. F. Santos, and M. J. S. Monte, J. Chem. Thermodyn. 86, 6 (2015).

2015AMA/SZT L. M. P. F. Amaral, P. Szterner, V. M. F. Morais, and M. A. V. Ribeiro da Silva, J. Chem. Thermodyn. 91, 452 (2015).

2015BLO/SHA S. Blokhina, A. Sharapova, M. Ol'khovich, T. Volkova, and G. Perlovich, J. Therm. Anal. Calorim. 120, 1053 (2015).

2015BLO/SHA2 S. Blokhina, A. Sharapova, M. Ol'khovich, T. Volkova, and G. Perlovich, Thermochim. Acta 622, 97 (2015).

2015BOU/BOU Y. Boumrah, S. Bouanani, K. Khimeche, and A. Dahmani, J. Therm. Anal. Calorim. 120, 583 (2015).

2015BOU/NEG Z. Bouzina, A. Negadi, I. Mokbel, J. Jose, and L. Negadi, J. Chem. Thermodyn. 84, 81 (2015).

2015BOU/NEG2 Z. Bouzina, A. Negadi, F. Dergal, I. Mokbel, J. Jose, and L. Negadi, J. Mol. Liq. 201, 83 (2015).

2015BRU/IRR B. Brunetti, S. Irrera, and G. Portalone, J. Chem. Eng. Data 60, 74 (2015).

2015CAR/CON F. Cardoso de Matos, M. Conceicao da Costa, A. J. A Meirelles, and E. A. C. Batista, Fluid Phase Equilib. 464, 1 (2015).

2015CHI/STE R. D. Chirico, W. V. Steele, and A. F. Kazakov, J. Chem. Thermodyn. 86, 106 (2015).

2015EME/PIM V. N. Emel'yanenko, A. A. Pimerzin, V. V. Turovtsev, and S. P. Verevkin, J. Phys. Chem. A 119, 2142 (2015).

2015EME/TUR V. N. Emel'yanenko, V. V. Turovtsev, and Yu. D. Orlov, Russ. J. Phys. Chem. A 89, 1146 (2015).

2015EME/VER V. N. Emel'yanenko and S. P. Verevkin, J. Chem. Thermodyn. 85, 111 (2015).

2015EME/VER2 V. N. Emel'yanenko, S. P. Verevkin, and R. Notario, J. Chem. Thermodyn. 87, 129 (2015).

2015EME/VER3 V. N. Emel'yanenko and S. P. Verevkin, Russ. J. Phys. Chem. A 89, 1740 (2015).

2015EME/ZAI V. N. Emel'yanenko, K. V. Zaitseva, F. Agapito, J. A. Martinho, and S. P. Verevkin, J. Chem. Thermodyn. 85, 155 (2015).

2015EME/ZAI2 V. N. Emel'yanenko, D. H. Zaitsau, E. Shoifet, F. Meurer, S. P. Verevkin, C. Schick, and C. Held, J. Phys. Chem. A 119, 9680 (2015). 
2015FAN/ZHA J. Fang, M. Zhang, P. Zhu, J. Ouyang, J. Gong, W. Chen, and F. Xu, J. Chem. Thermodyn. 85, 202 (2015).

2015GAO/XUE X. Gao, W.-L. Xue, Z.-X. Zeng, and X.-R. Fan, J. Chem. Eng. Data 60, 2273 (2015).

2015GAU/VAN N. Gautschi, P. Van Hoogevest, and M. Kuentz, Int. J. Pharm. 491, 218 (2015).

2015GOB/CHI C. Gobble and J. S. Chickos, J. Chem. Eng. Data 60, 2739 (2015).

2015HIK/WEE W. M. Kikal and B. L. Weeks, J. Therm. Anal. Calorim. 122, 1055 (2015).

2015HUA/JIA Z. Huang, H. Jiang, L. Li, H. Wang, and T. Qui, J. Chem. Thermodyn. 86, 75 (2015).

2015HUA/WAN X. Huang, J. Wang, H. Hao, J. Ouyang, Y. Gao, Y. Bao, Y. Wang, and Q. Yin, Fluid Phase Equilib. 394, 148 (2015).

2015JAK/SPO M. Jakubczk, A. Sporznski, V. N. Emel'yanenko, M. A. Varfolomeev, and S. P. Verevkin, Thermochim. Acta 615, 88 (2015).

2015KAB/PAU G. J. Kabo, Y. U. Paulechka, O. V. Voitkevich, A. V. Blokhin, E. N. Stepurko, S. V. Kohut, and Y. V. Voznyi, J. Chem. Thermodyn. 85, 101 (2015).

2015KOZ/GOB M. Kozlovskiy, C. Gobble, and J. S. Chickos, J. Chem. Thermodyn. 86, 65 (2015).

2015LI/DU R.-R. Li, C.-B. Du, S. Han, J. Wang, G.-B. Yao, and H.-K. Zhao, Fluid Phase Equilib. 396, 35 (2015).

2015LI/YAN H.-Y. Li, B. Yan, K.-Q. Bai, H. Liu, H.-X. Ma, J.-R. Song, and L. Yan, J. Chem. Thermodyn. 91, 240 (2015).

2015MAR/PIN D.-C. Marinescu, E. Pincu, P. Oancea, G. Bruni, A. Marini, and V. Meltzer, J. Therm. Anal. Calorim. 120, 829 (2015).

2015MAT/MOR H. Matsuda, K. Mori, M. Tomioka, K. Kariyasu, T. Fukami, K. Kurihara, K. Tochigi, and K. Tomono, Fluid Phase Equilib. 406, 116 (2015).

2015MBA/KNO J. Mbah, D. Kno, S. Steward, and D. Cornett, Int. J. Energ. Mater. Chem. Propul. 14, 321 (2015).

2015MOR/DAS P. Morgado, G. Das, C. McCabe, and E. J. M. Filipe, J. Phys. Chem. B 119, 1623 (2015).

2015OSS/JUS M. D. Ossowska-Chrusciel, E. Juszynska-Galazka, W. Zajac, A. Rudzki, and J. Chrusciel, J. Mol. Struct. 1082, 103 (2015).

2015PAU/SHI A. Paul, L. Shi, and C. W. Bielawski, Energy Convers. Manage. 103, 139 (2015).

2015PER/CAS P. S. Pereira Silva, R. A. E. Castro, E. Melro, M. R. Silva, T. M. R. Maria, J. Canotilho, and M. E. S. Eusebio, J. Therm. Anal. Calorim. 120, 667 (2015).

2015POB/JUR A. Pobudkowska, B. A. Jurkowska, and M. A. Wiatrowski, Fluid Phase Equilib. 406, 209 (2015).

2015PRI D. M. Price, Thermochim. Acta 622, 44 (2015).

2015RAM/CHI S. L. L. M. Ramos, A. K. Chigira, and M. Oguni, J. Phys. Chem. B 119, 4076 (2015).

2015RAM/MAR M. Ramos-Riesco, F. J. Martinez-Casado, J. A. R. Cheda, M. I. R. Yelamos, A. Fernandez-Mertinez, and S. LopezAndres, Cryst. Growth Des. 15, 497 (2015).

2015ROT/VRB J. Rotrekl, P. Vrbka, Z. Sedlakova, Z. Wagner, J. Jacquemin, and M. Bendova, Pure Appl. Chem. 87, 453 (2015).

2015RUZ/GON V. Ruz, M. M. Gonzalez, D. Winant, Z. Rodriguez, and G. Van den Mooter, Molecules 20, 15175 (2015).

2015SHA/GE X. Shao, H. Ge, Z. Li, C. Ren, and J. Wang, Fluid Phase Equilib. 390, 7 (2015).

2015SIN/RAI M. Singh, R. N. Rai, and U. S. Rai, J. Cryst. Growth 419, 114 (2015).

2015SIN/SIN J. Singh and N. B. Singh, Fluid Phase Equilib. 386, 168 (2015).

2015 SIN/SIN2 J. Singh and N. B. Singh, J. Chem. Thermodyn. 89, 197 (2015).

2015SIN/SIN3 J. Singh and N. B. Singh, J. Cryst. Growth 424, 14 (2015).

2015SME/BRU M. M. H. Smets, S. J. T. Brugman, E. R. H. van Eck, A. van den Ende, H. Meekes, and H. M. Cuppen, Cryst. Growth Des. 15, 5157 (2015).

2015SOB/VAN I. B. Sobechko, Yu. Ya. Van-Chin-Syan, Yu. I. Gorak, V. V. Kochubei, R. T. Prokop, N. I. Velychkivska, Yu. I. Gorak, V. N. Dibrivnyi, and M. D. Obushak, Russ. J. Phys. Chem. 89, 919 (2015).
2015SOL/ROD

2015SOL/VAR V. Khoroshilov, Russ. J. Phys. Chem. 89, 910 (2015).

B. N. Solomonov, M. A. Varfolomeev, R. N. Nagrimanov, V. B. Norikov, M. A. Ziganshin, A. V. Gerasimov, and S. P. Verevkin, J. Chem. Eng. Data 60, 748 (2015).

2015SON/LI Y. Song, J. Q. Li, X. Sun, Z. Feng, Y. Lin, J. Wang, J. Ding, H. Zhang, and W. Li, Fluid Phase Equilib. 388, 16 (2015).

2015STE/DER V. Stejfa, F. Dergal, I. Mokbel, M. Fulem, J. Jose, and K. Ruzicka, Fluid Phase Equilib. 406, 124 (2015).

2015STE/FUL V. Stejfa, M. Fulem, K. Ruzicka, and P. Matejka, Fluid Phase Equilib. 402, 18 (2015).

2015SUN/YIN X. Sun, Q. Yin, S. Ding, Z. Shen, Y. Bao, J. Gong, B. Hou, H. Hao, Y. Wang, J. Wang, and C. Xie, J. Chem. Thermodyn. 85, 171 (2015).

2015SUR/SIM A. O. Surov, A. A. Simagina, N. G. Manin, L. G. Kuzmina, A. V. Churakov, and G. L. Perlovich, Cryst. Growth Des. 15, 228 (2015).

2015VER/EME S. P. Verevkin, V. N. Emel'yanenko, M. A. Varfolomeev, B. N. Solomonov, K. V. Zherikova, and S. V. Melkhanova, Fluid Phase Equilib. 387, 160 (2015).

2015VER/EME2 S. P. Verevkin, V. N. Emel'yanenko, and I. V. Garist, J. Chem. Thermodyn. 87, 34 (2015).

2015VER/EME3 S. P. Verevkin and V. N. Emel'yanenko, Thermochim. Acta 608, 40 (2015).

2015VER/SAZ S. P. Verevkin, A. Yu. Sazonova, V. E. Emel'yanenko, D. H. Zaitsau, M. A. Varfolomeev, B. N. Solomonov, and K. V. Zerkikova, J. Chem. Eng. Data 60, 89 (2015).

2015VER/ZAI S. P. Verevkin, D. H. Zaitsau, V. E. Emel'uanenko, E. N. Stepurko, and K. S. Zherikova, Thermochim. Acta 622, 18 (2015).

2015VER/ZAI2 S. P. Verevkin, D. H. Zaitsau, V. N. Emel'yanenko, and A. A. Zhabina, Fluid Phase Equilib. 397, 87 (2015).

2015VER/ZAI3 S. P. Verevkin, K. V. Zaitseva, A. D. Stanton, M. S. Hindman, A. C. Irvin, and J. E. Bara, Ind. Eng. Chem. Res. 54, 9850 (2015).

2015VIT/AGA J. Vitorino, F. Agapita, C. E. S. Bernardes, and M. E. Minas da Piedade, J. Chem. Thermodyn. 80, 59 (2015).

2015WAN/LI L. Y. Wang, X.-C. Li, L. Zhu, Z.-L. Sha, Y.-F. Wang, and L.-B. Yang, J. Mol. Liq. 208, 211 (2015).

2015WAN/WAN C. Wang, Y. Wang, Q. Yin, Z. Xu, Y. Bao, B. Hou, W. Liu, and H. Hao, J. Chem. Eng. Data 60, 3053 (2015).

2015ZAI/EME K. V. Zaitseva, V. N. Emel'yanenko, F. Agapito, A. A Pimerzin, M. A. Varfolomeev, and S. P. Verevkin, J. Chem. Thermodyn. 91, 186 (2015).

2015ZAI/PAU D. Zaitsau, E. Paulechka, D. S. Firaha, A. V. Blokhin, F. J. Kabo, A. Bazyleva, A. G. Kabo, M. A. Varfolomeev, and V. M. Sevruk, J. Chem. Thermodyn. 91, 459 (2015).

$2015 Z A I / V E R \quad$ D. H. Zaitsau, S. P. Verevkin, and A. Yu. Sazonova, Fluid Phase Equilib. 386, 140 (2015).

2015ZHA/JIN L. Zhang, H. Jing, W. Zhu, F.-B. Zhang, G.-L. Zhang, and Q. Xia, J. Chem. Thermodyn. 80, 142 (2015).

2015ZHA/QIA X. Zhang, G. Qian, R. Wang, X. Yang, L. Hao, H. Wei, and X. Zhou, Fluid Phase Equilib. 388, 59 (2015).

2015ZHU/WAN L. Zhu, L.-Y. Wang, X.-C. Li, Z. L. Sha, Y.-F. Wang, and L.-B. Yang, J. Chem. Thermodyn. 91, 369 (2015).

2016ALM/MON A. R. R. P. Almeida and M. J. S. Monte, J. Chem. Thermodyn. 92, 118 (2016).

2016AMA/SZT L. M. P. F. Amaral, P. Szterner, V. M. F. Morais, M. D. M. C. Ribeiro da Silva, and M. A. V. Ribeiro da Silva, J. Chem. Thermodyn. 96, 93 (2016).

2016BOU/DER Z. Bouzina, F. Dergal, I. Mokbel, A. Negadi, J. Saab, J. Jose, and L. Negadi, Fluid Phase Equilib. 414, 164 (2016).

2016CAR/AMA T. M. T. Carvalho, L. M. P. F. Amaral, V. M. F. Morais, and M. C. Ribeiro da Silva, J. Chem. Thermodyn. 97, 70 (2016).

2016CHI/STE R. D. Chirico, W. V. Steele, and A. F. Kazakov, J. Chem. Thermodyn. 96, 41 (2016).

2016DAV/LIM J. Z. Davalos, C. F. R. A. C. Lima, A. M. S. Silva, L. M. N. B. F. Santos, R. Erra-Balsells, and M. L. Salum, J. Chem. Thermodyn. 95, 195 (2016).

2016EME/YER V. N. Emel'yanenko, A. V. Yermalayeu, M. Voges, C. Held, G. Sadowski, and S. P. Verevkin, Fluid Phase Equilib. 422, 99 (2016). 
2016FRE/SAN V. L. S. Freitas, C. P. F. Santos, M. D. M. C. Ribeiro da Silva, and M. A. V. Ribeiro da Silva, J. Chem. Thermodyn. 96, 74 (2016).

2016HU/WU H.-S. Hu, Y.-L. Wu, and M.-D. Yang, Fluid Phase Equilib. 414, 133 (2016).

2016LI/DU K. Li, S. Du, S. Wu, D. Cai, J. Wang, D. Zhang, K. Zhao, P. Yang, B. Yu, B. Gao, D. Li, and J. Gong, J. Chem. Thermodyn. 96, 12 (2016).

2016MAR/SMI A. V. Markin, N. V. Smirnova, I. S. Il'ichev, T. I. Dolinsky, and A. B. Radbil, J. Therm. Anal. Calorim. 123, 1451 (2016).

2016MAT/INA H. Matsuda, K. Inaba, H. Sumida, K. Kurihara, K. Tochigi, and K. Ochi, Fluid Phase Equilib. 420, 50 (2016).

2016MOR/RAT M. Mori, N. Rath, C. Gobble, J. Chickos, A. A. Samarov, and S. P. Verevkin, J. Chem. Eng. Data 61, 370 (2016).

2016OKU/PAD M. Okuniewski, K. Paduszynski, and U. Domanska, Fluid Phase Equilib. 422, 66 (2016).

2016OST/UUS A. Ostonen, P. Uusi-Kyyny, M. Pakkanen, and V. Olopaeus, Fluid Phase Equilib. 408, 79 (2016).

2016PER/ORT E. Perez, J. Ortega, L. Fernandez, J. Wisniak, and J. Canosa, Fluid Phase Equilib. 412, 79 (2016)

2016SIL/MOR A. L. R. Silva, V. M. F. Morais, M. D. M. C. Ribeiro da Silva, R. G. Simoes, C. E. S. Bernardes, M. F. M. Piedade, and M. E. Minas da Piedade, J. Chem. Thermodyn. 95, 35 (2016).

2016SIM/GOB $\quad$ D. Simmons, C. Gobble, and J. Chickos, J. Chem. Thermodyn. 92, 126 (2016).

2016SIN/BUR V. P. Sinditskii, A. V. Burzhava, A. N. Chernyi, D. S. Shmelev, V. N. Apalkova, N. V. Palysaeva, and A. B. Sheremetev, J. Therm. Anal. Calorim. 123, 1431 (2016).

2016SZT/GAL P. Szterner, T. L. P. Galvao, L. M. P. F. Amaral, M. D. M. C. Ribeiro da Silva, and M. A. V. Ribeiro da Silva, Thermochim. Acta 625, 36 (2016).
2016TOS/CEO

S. Toscani, R. Ceolin, L. Ter Minassian, M. Barrio, N. Veglio, J.-L. Tamarit, D. Louer, and I. B. Rietveld, Int. J. Pharm. 497, 96 (2016).

2016VOL/BLO T. V. Volkova, S. V. Blokhina, A. M. Ryzhakov, A. V. Sharapova, M. V. Ol'khovich, and G. L. Perlovich, J. Therm. Anal. Calorim. 123, 841 (2016).

2016VRB/DOH P. Vrbka and V. Dohnal, Fluid Phase Equilib. 411, 59 (2016).

2016WAN/YAO D.-C. Wang, S. Yao, Y. Cao, T. Yao, and H. Song, J. Chem. Thermodyn. 92, 55 (2016).

2016WAN/YIN Y. Wang, Q. Yin, X. Sun, Y. Bao, J. Gong, B. Hou, Y. Wang, M. Zhang, C. Xie, and H. Hao, J. Chem. Thermodyn. 94, 110 (2016).

2016XU/WAN J. Xu, Y. Wang, G. Wang, C. Huang, H. Hao, and Q. Yin, J. Chem. Thermodyn. 92, 12 (2016).

2016YAN/WAN X. Yang, J. Wang, H. Song, and W. Zou, Fluid Phase Equilib. 409, 417 (2016).

2016ZHA/GUO T. Zhang, F. Guo, Q. Cui, M. Lu, X. Song, H. Tang, and Q. Li, J. Chem. Eng. Data 61, 420 (2016).

2016ZHE/SVE K. V. Zherikova, A. A. Svetlov, M. A. Varfolomeev, S. P. Verevkin, and C. Held, Fluid Phase Equilib. 409, 399 (2016).

2016ZHU/CHE P. Zhu, Y. Chen, J. Fang, Z. Wang, C. Xie, B. Hou, W. Chen, and F. Xu, J. Chem. Thermodyn. 92, 198 (2016).

2016 ZHU/CHE2 P. Zhu, Y. Chen, M. Zhang, Y. Bao, C. Xie, B. Hou, J. Gong, and W. Chen, J. Chem. Thermodyn. 97, 9 (2016).

$\mathrm{UR} / \mathrm{CHI}$

$\mathrm{UR} / \mathrm{FUC}$

UR/MAC

UR/STU

UR/VER J. S. Chickos, (unpublished). R. H. Fuchs (unpublished). H. Mackle, W. V. Steele, and D. V. McNally (unpublished).

D. Stull (unpublished).

S. P. Verevkin, E. Krasnykh, T. Vasiltsova, and A. Heintz (unpublished); as quoted in [2002VER]. 